NIST-SP 1119 Volume II

\title{
Technical Study of the Sofa Super Store Fire - South Carolina, June 18, 2007 - Appendices
}

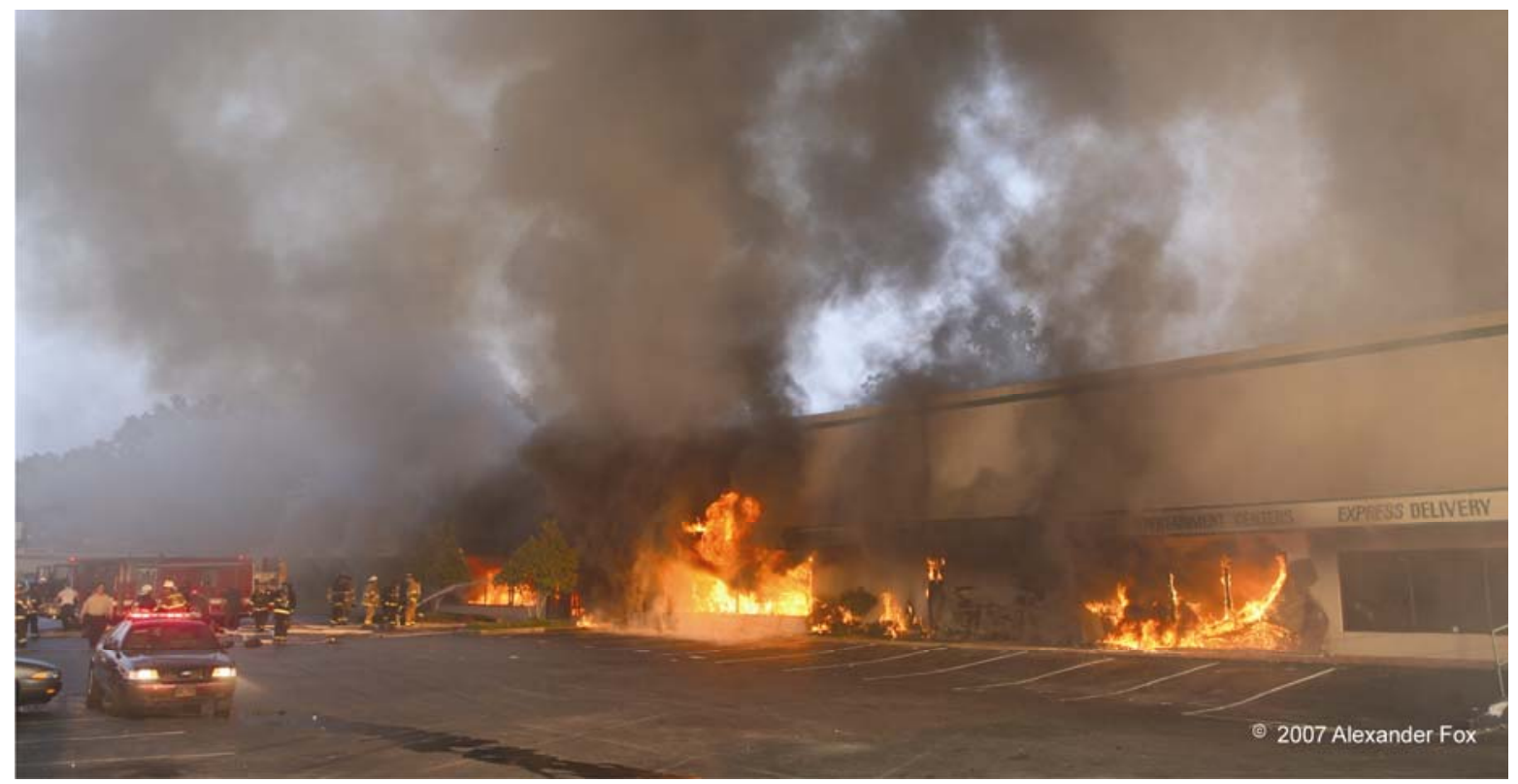

Nelson P. Bryner

Stephen P. Fuss

Bryan W. Klein

Anthony D. Putorti 

NIST-SP 1119 Volume II

\section{Technical Study of the Sofa Super Store Fire - South Carolina, June 18, 2007}

Nelson P. Bryner

Stephen P. Fuss

Bryan W. Klein

Anthony D. Putorti

Fire Research Division

Engineering Laboratory

National Institute of Standards and Technology

March 2011

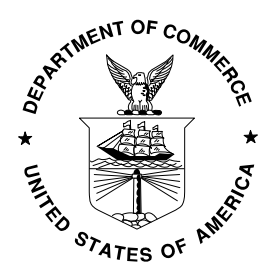

U.S. Department of Commerce

Gary Locke, Secretary 


\section{$\underline{\text { Disclaimer }}$}

Certain commercial entities, equipment, products, or materials are identified in this document in order to describe a procedure or concept adequately or to trace the history of the procedures and practices used. Such identification is not intended to imply recommendation, endorsement, or implication that the entities, products, materials, or equipment are necessarily the best available for the purpose.

\section{$\underline{\text { Disclaimer }}$}

The policy of NIST is to use the International System of Units (metric units) in all publications. In this document, however, units are presented in metric units or the inch-pound system, whichever is prevalent to the discipline. Conversion tables are provided in this report.

National Institute of Standards and Technology NIST-SP

Natl. Inst. of Std., 738 pages (March 2011)

CODEN: XXXXXX NSPUE2

\section{U.S. GOVERNMENT PRINTING OFFICE} WASHINGTON: 2008

For sale by the Superintendent of Documents, U.S. Government Printing Office Internet: bookstore.gpo.gov — Phone: (202) 512-1800 — Fax: (202) 512-2250 Mail: Stop SSOP, Washington, DC 20402-0001 


\section{ABSTRACT}

A fire occurred on the evening of June 18, 2007, in the Sofa Super Store in Charleston, SC. NIST analyzed the fire ground, consulted with other experts, and performed computer simulations of fire growth alternatives. Based on these analyses, the following sequence of events is likely to have occurred. A fire began in packing material and discarded furniture outside an enclosed loading dock area. The fire spread to the loading dock, then into both the retail showroom and warehouse spaces. During the early stages of the fire in the two latter locations, the fire spread was slowed by the limited supply of fresh air. This under-ventilation led to generation of a large mass of pyrolyzed and only partially oxidized effluent. The smoke and combustible gases flowed into the interstitial space below the roof and above the suspended ceiling of the main retail showroom. As this space filled with unburned fuel, the hot smoke also seeped through the suspended ceiling into the main showroom and formed a hot smoke layer below the suspended ceiling. Up to this time, the extent of fire spread into the interstitial space was not visible to fire fighters in the store. If the fire spread had been visible to the fire fighters in the store, it would have provided a direct indication of a fire hazard in the showroom. Meanwhile, the fire at the back of the main showroom and the gas mixture below the suspended ceiling were both still fuel rich. When the front windows were broken out or vented, the inflow of additional air allowed the heat release rate of the fire to intensify rapidly and added air to the layer of unburned fuel below the suspended ceiling enabling the ignition of the unburned fuel/air mixture. The fire swept from the rear to the front of the main showroom extremely quickly, and then into the west and east showrooms. Nine fire fighters were killed in the Sofa Super Store fire. Based on NIST's simulation of events, this report includes eleven recommendations to help mitigate such future losses.

Keywords: fire investigation, fire modeling, fire spread, fire sprinklers, furniture flammability, retail store fire, sprinklers 


\section{TABLE OF CONTENTS (VOL. I)}

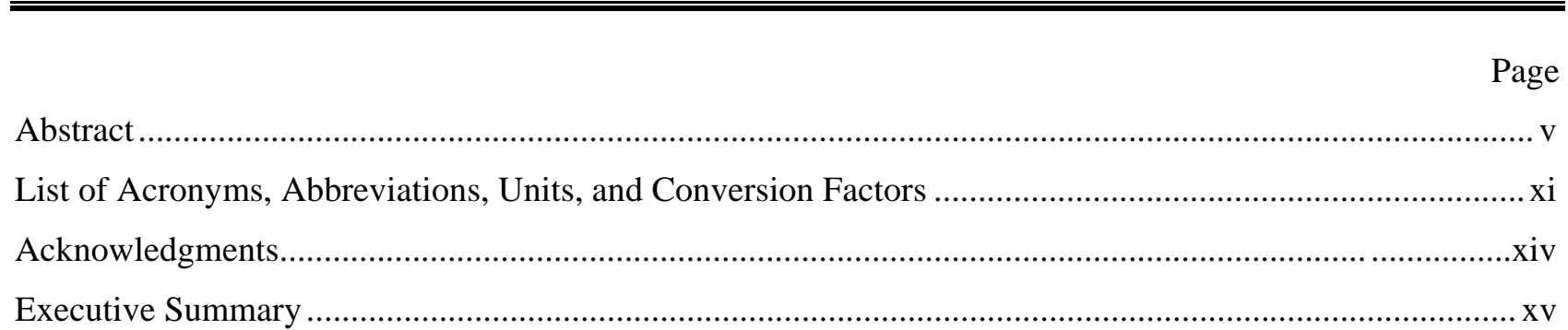

\section{Chapter 1 Background}

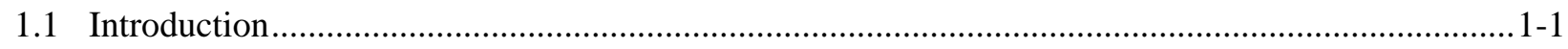

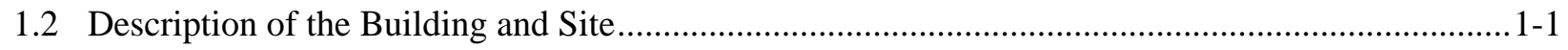

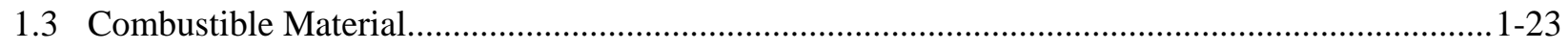

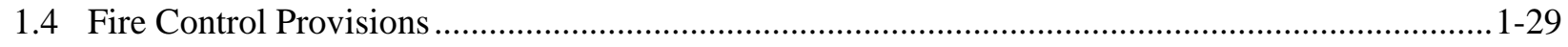

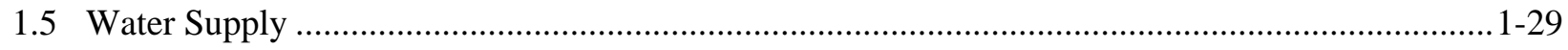

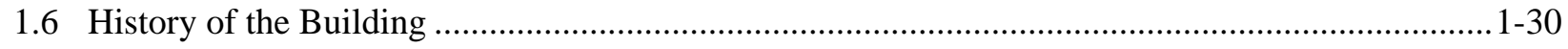

1.7 Aerial Photography of the Sofa Super Store ............................................................................... 1-35

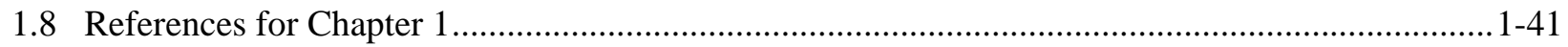

\section{Chapter 2 Description and Time Line of the Incident}

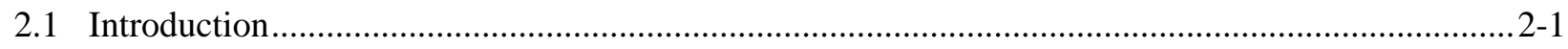

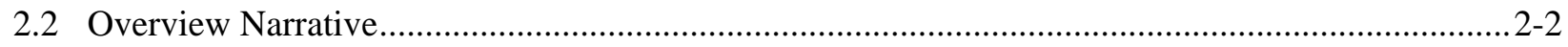

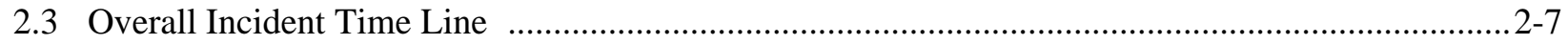

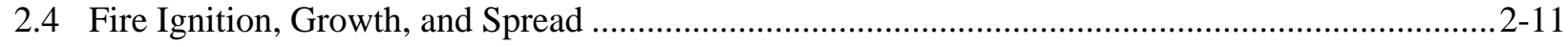

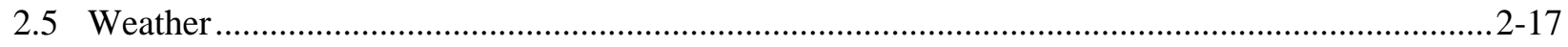

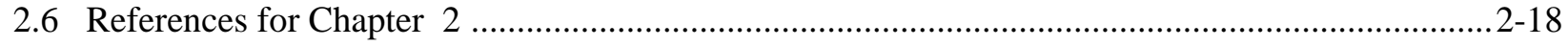

\section{Chapter 3 The Emergency Incident Response}

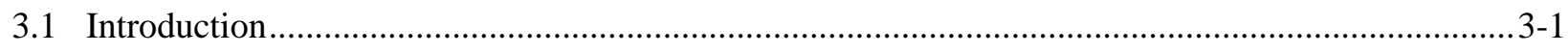

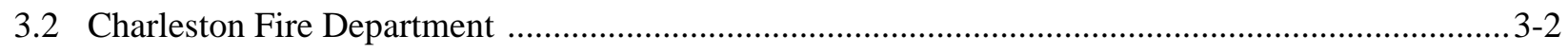

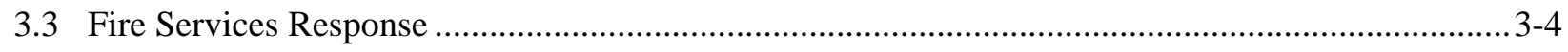

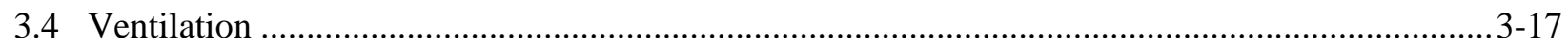

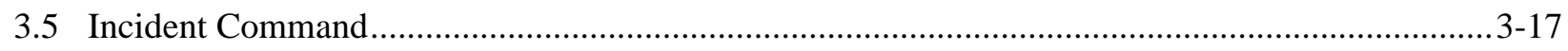

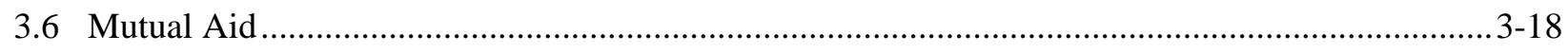

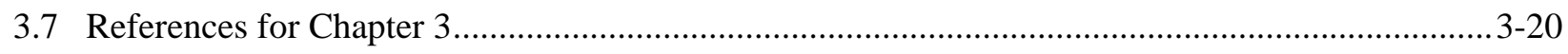




\section{Chapter 4 Computer Simulation Of Fire And Smoke Spread}

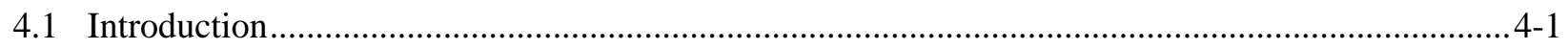

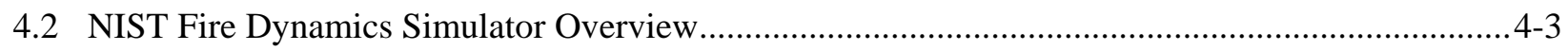

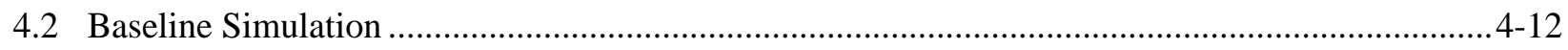

4.4 Baseline Simulation With Sprinklers Added Inside the Loading Dock...................................... 4-29

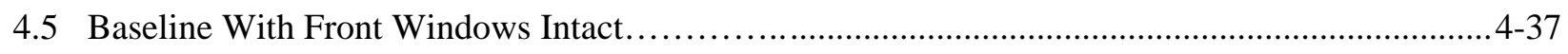

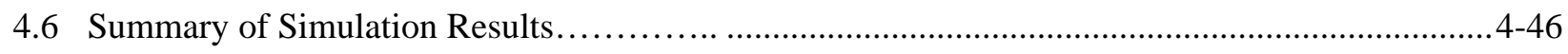

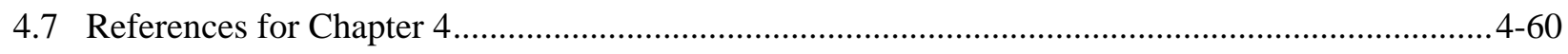

Chapter 5 Model Codes, Standards, And Practices

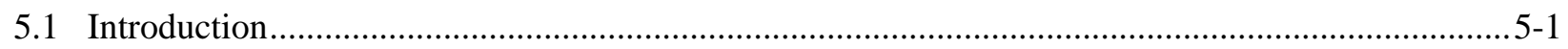

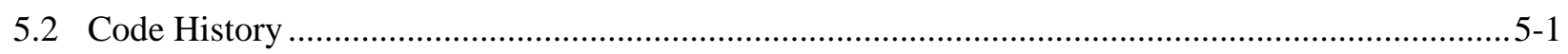

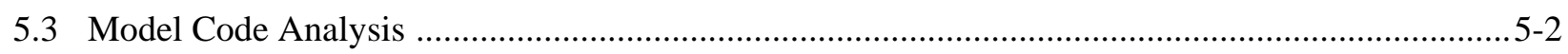

5.4 Limiting Fire Spread in Large Furniture Display Areas ...........................................................5-10

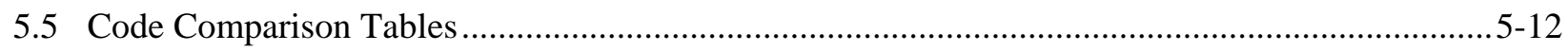

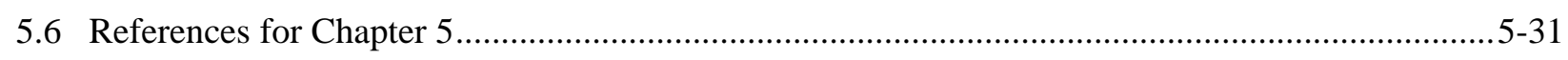

\section{Chapter 6 Summary, Findings and Recommendations}

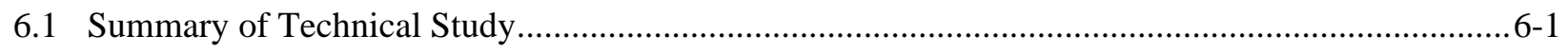

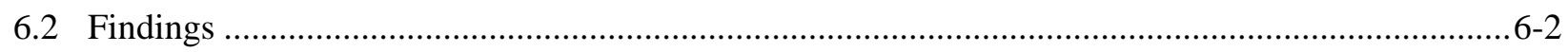

6.3 Recommendations for Improving Model Standards, Codes. and Practices ..................................6-11

6.4 Research Recommendations and Other Appropriate Actions.......................................................6-14

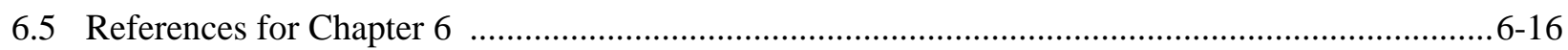




\section{TABLE OF CONTENTS (VoL. II)}

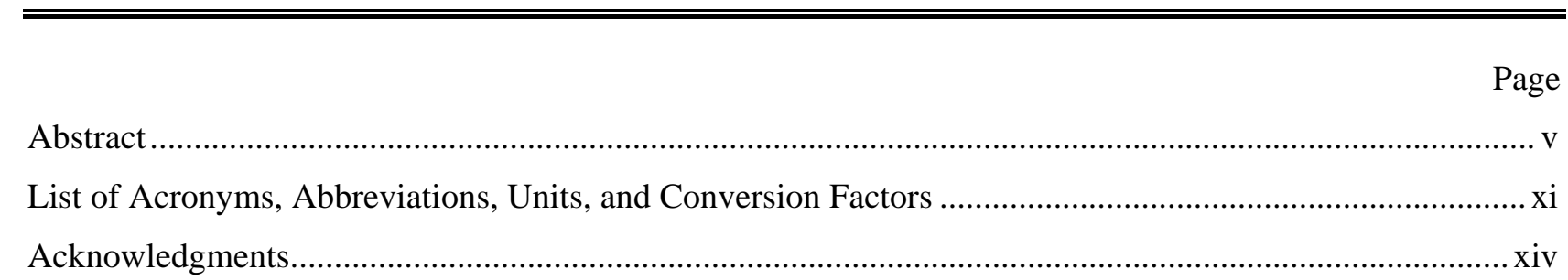

\section{Appendix A Weather Data}

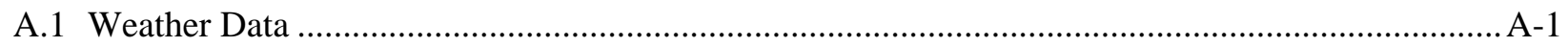

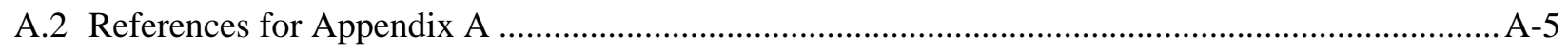

\section{Appendix B Overall Scene Photos}

B.1 Overall Scene Photos

\section{Appendix C Loading Dock Area}

C.1 Loading Dock Area Photos- Before Removal of Roof ................................................................. C-1

C.2 Loading Dock Area Photos- After Removal of Roof ................................................................... C-6

\section{Appendix D Main Showroom}

D.1 Main Showroom Exterior Photos - Before Removal of Wall and Roof......................................... D-1

D.2 Main Showroom Exterior Photos - After Removal of Wall and Roof .......................................D-11

\section{Appendix E West Showroom}

E.1 West Showroom Exterior Photos ................................................................................................... E-1

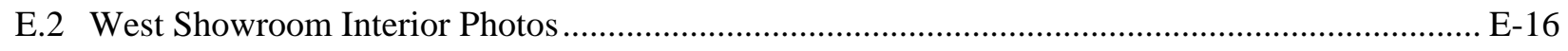

\section{Appendix F East Showroom}

F.1 East Showroom Exterior Photos ….................................................................................................

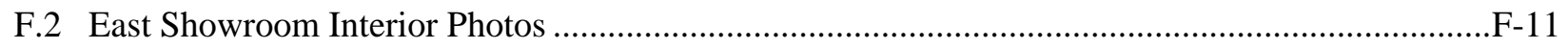

\section{Appendix G Warehouse Photographs}

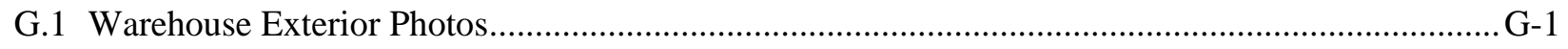

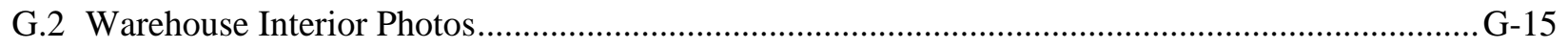




\section{Appendix H Repair Shops}

H.1 Repair Shop Photos H-1

\section{Appendix I Rear Wall Of Main Showroom}

I.1 Rear Wall of Main Showroom Photos .......................................................................................... I

I.2 Rear of Main Showroom After Removal of Warehouse........................................................... I-12

\section{Appendix J Retail Merchandise Estimates}

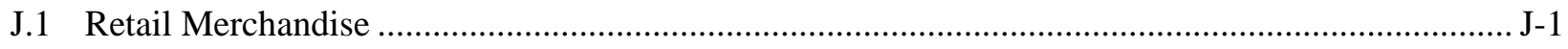

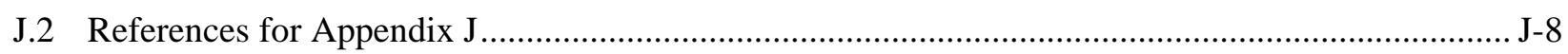

\section{Appendix K Computer Simulation of Fire and Smoke Spread}

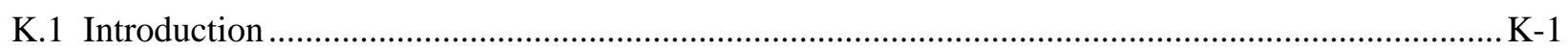

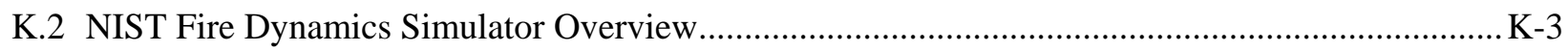

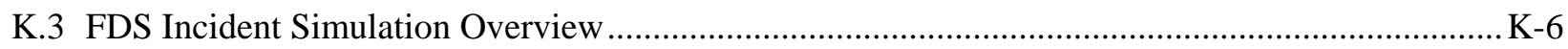

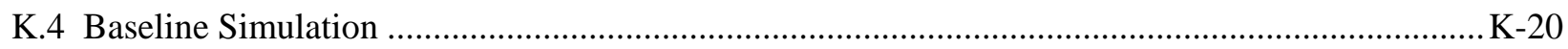

K.5 Baseline with Sprinklers Added on Loading Dock...................................................................42

K.6 Baseline with Front Windows Intact........................................................................................ K-52

K.7 Vertical Ventilation - Small Roof Vent..................................................................................... K-62

K.8 Vertical Ventilation - Large Roof Vent................................................................................

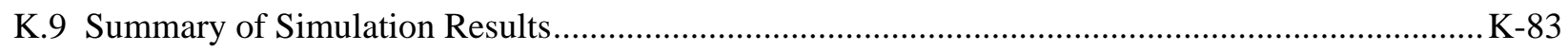

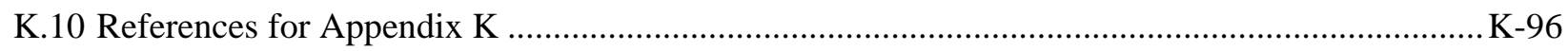

\section{Appendix L Computer Simulation Input Files}

L.1 Fire Dynamic Simulator Version 5.1.0 …................................................................................. L-1

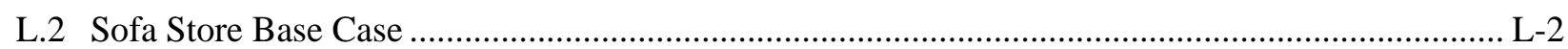

L.3 Base Case with Sprinklers …........................................................................................ L-120

L.4 Base Case with Windows Intact ........................................................................................ L-122

L.5 Base Case with Windows Intact with Small Vent ..................................................................... L-123

L.6 Base Case with Windows Intact with Large Vent ............................................................... L-124

\section{Appendix M Fire Inspection and Building Pre-Plan}

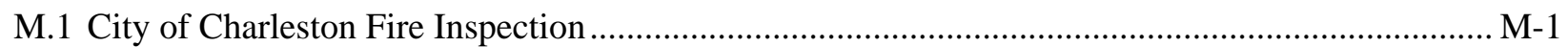

M.2 City of Charleston Fire Department Pre-Planning .................................................................... M-3

M.3 City of Charleston Fire Department Pre-Planning Sketch.......................................................... M-4 


\section{Appendix N Additional Aerial Images}

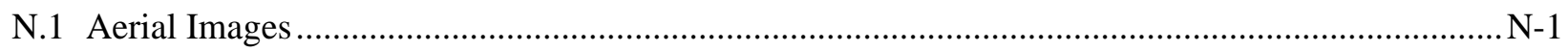

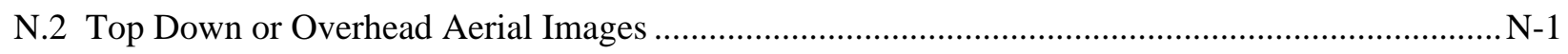

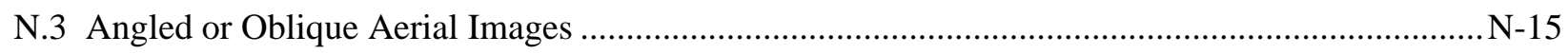

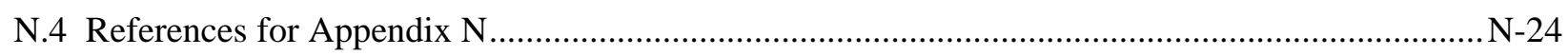

\section{Appendix O Additional Aerial Images}

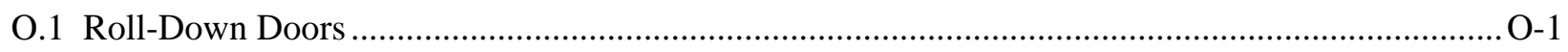

O.2 Warehouse Roll-Down Doors .................................................................................................

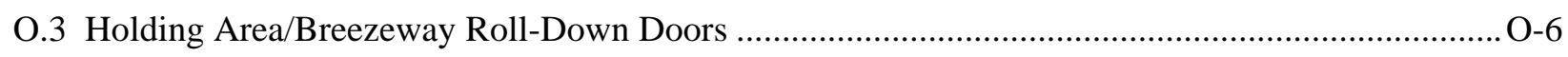

O.4 Holding Area/Loading Dock Roll-Down Door......................................................................... O-12

O.5 Main Showroom/West Showroom Roll-Down Door ................................................................. O-17

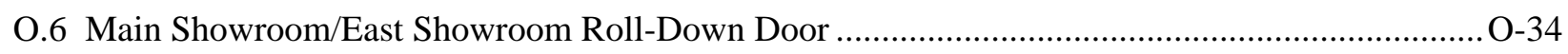

\section{Appendix P Loading Dock Fuels}

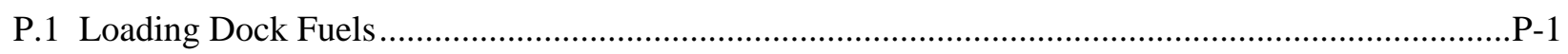

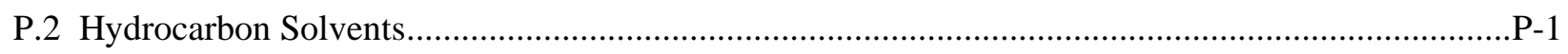

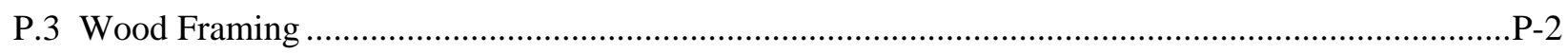

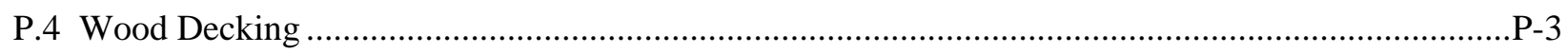

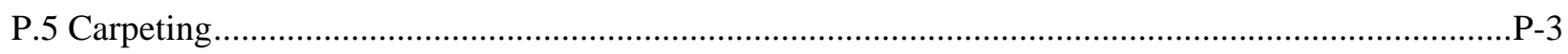

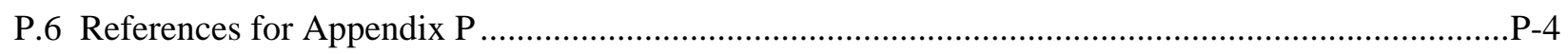

\section{Appendix Q Technical Reports, Media Articles, Web Pages, and Other}

\section{Resources}

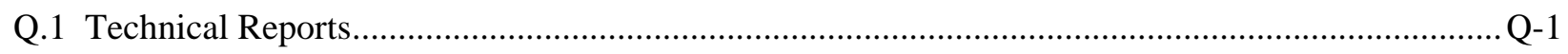

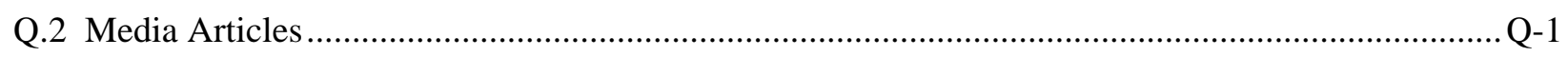

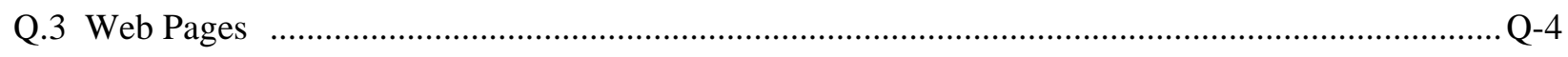

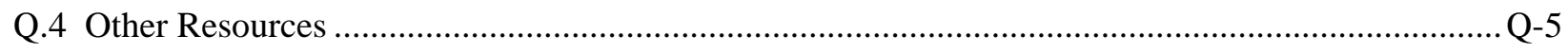




\section{LIST OF ACRONYMS, ABBREVIATIONS, UNITS, AND CONVERSION FACTORS}

\begin{tabular}{ll} 
AHJ & Authority Having Jurisdiction \\
ASTM & American Society for Testing and Materials \\
ATF & Bureau of Alcohol, Tobacco and Firearms \\
BBC & Basic Building Code \\
CCSO & Charleston County Sheriffs Office \\
CSMO & City of Charleston Safety Management Division \\
CFD & computational fluid dynamics, and Charleston Fire Department \\
CPD & Charleston Police Department \\
DHS & Department of Homeland Security \\
EST & Eastern Standard Time \\
FDS & Fire Dynamics Simulator \\
FEMA & Federal Emergency Management Agency \\
HRR & heat release rate \\
IBC & International Building Code \\
IFC & International Fire Code \\
IC & Incident Command \\
ICC & International Code Council \\
IFSTA & International Fire Service Training Association \\
LLR & South Carolina Department of Labor, Licensing and Regulation \\
NFPA & National Fire Protection Association \\
NIOSH & National Institute for Occupational Safety and Health \\
NIST & National Institute of Standards and Technology \\
RTI & response time index \\
SBC & Standard Building Code \\
SFC & Standard Fire Code \\
\hline
\end{tabular}




$\begin{array}{ll}\mathrm{t} & \text { time } \\ \mathrm{TC} & \text { thermocouple } \\ \mathrm{UL} & \text { Underwriters Laboratories } \\ \text { USC } & \text { United States Code } \\ \text { USFA } & \text { United States Fire Administration }\end{array}$

\section{Units}

$\begin{array}{ll}{ }^{\circ} \mathrm{C} & \text { degrees Centigrade } \\ { }^{\circ} \mathrm{F} & \text { degrees Fahrenheit } \\ \mathrm{ft} & \text { feet } \\ \mathrm{GJ} & \text { Gigajoule } \\ \mathrm{gpm} & \text { gallons/minute } \\ \text { in } & \text { inch } \\ \mathrm{kg} & \text { kilogram } \\ \mathrm{kPa} & \text { kilopascal } \\ \mathrm{kW} & \text { kilowatt } \\ \mathrm{L} & \text { liter } \\ \mathrm{m} & \text { meter } \\ \mathrm{mm} & \text { millimeter } \\ \mathrm{min} & \text { minute } \\ \mathrm{MW} & \text { megawatt } \\ \mathrm{psi} & \text { pounds/in }{ }^{2} \\ \mathrm{~s} & \text { second } \\ \mathrm{W} & \text { Watt } \\ \mu \mathrm{m} & \text { micrometer }\end{array}$




\section{Conversion Factors}

$$
\begin{aligned}
& { }^{\circ} \mathrm{F}=1.8 \mathrm{x}{ }^{\circ} \mathrm{C}+32 \\
& 1 \mathrm{foot}=0.3048 \mathrm{~m} \\
& 1 \mathrm{inch}=2.54 \mathrm{~cm} \\
& 1 \mathrm{~m}=3.281 \mathrm{ft} \\
& 1 \mathrm{~mm}=0.03937 \mathrm{in} \\
& 1 \mathrm{~L}=0.2642 \mathrm{gal} \\
& 1 \mathrm{~kg}=2.204 \mathrm{lb} \text { (mass) } \\
& 1 \mathrm{~kg} / \mathrm{m}^{3}=0.06243 \mathrm{lb} / \mathrm{ft}^{3} \\
& 1 \mathrm{kPa}=0.1450 \mathrm{psi} \\
& 1 \mathrm{~kJ}=0.9479 \mathrm{Btu} \\
& 1 \mathrm{~kJ}=1,000 \mathrm{~J} \\
& 1 \mathrm{MJ}=1,000,000 \mathrm{~J} \\
& 1 \mathrm{GJ}=1,000,000,000 \mathrm{~J} \\
& 1 \mathrm{~kJ} / \mathrm{kg}=0.4301 \mathrm{Btu} / \mathrm{lb} \\
& 1 \mathrm{~kJ} / \mathrm{kg}-{ }^{\circ} \mathrm{C}=0.2389 \mathrm{Btu} / \mathrm{lb}-{ }^{\circ} \mathrm{F} \\
& 1 \mathrm{~kW}=3413 \mathrm{Btu} / \mathrm{hr} \\
& 1 \mathrm{~kW} / \mathrm{m}^{2}=317.1 \mathrm{Btu} / \mathrm{hr} / \mathrm{ft}{ }^{2} \\
& 1 \mathrm{~kW} / \mathrm{m}^{2}-{ }^{\circ} \mathrm{C}=176.1 \mathrm{Btu} / \mathrm{hr}-\mathrm{ft}{ }^{2}-{ }^{\circ} \mathrm{F} \\
& 1 \mathrm{~W} / \mathrm{m}-{ }^{\circ} \mathrm{C}=0.5778 \mathrm{Btu} / \mathrm{hr}-\mathrm{ft}-{ }^{\circ} \mathrm{F} \\
& 1 \mathrm{~kg}=1000 \mathrm{~g} \\
& 1 \mathrm{~m}=1000 \mathrm{~mm} \\
& 1 \mu \mathrm{m}=0.001 \mathrm{~mm} \\
& 1 \mathrm{KW}=1,000 \mathrm{~W} \\
& 1 \mathrm{MW}=1,000,000 \mathrm{~W} \\
& \hline{ }^{2}
\end{aligned}
$$




\section{ACKNOWLEDGMENTS}

The collaboration with the National Institute for Occupational Safety and Health, including Tim Merinar, Matt Bowyer, Jay Tarley, Dawn Castillo, and Robert Koedam, provided on-scene assistance, access to interviews, and support for computer fire model simulations. The cooperation of the Bureau of Alcohol Tobacco and Firearms, including John Golder, Chris Porreca, and David Sheppard provided on-scene assistance and discussions on computer simulations and fire origin and cause. The NIST team also held valuable discussions with Ken Farmer, Robert Neale, and Kenneth Kuntz of the Department of Homeland Security, United States Fire Administration, concerning previous large fire incidents. Lee Baughman of the South Carolina Department of Labor, Licensing, and Regulation provided access to the fire scene. The entire Charleston Fire Department is acknowledged for providing access to staff, fire stations, and fire apparatus to make possible this technical study. The Charleston Police Department provided access to scene photographs and videos. The on-scene assistance and discussion with City of Charleston Safety Management Division, Ted Austin, is also acknowledged. The City of Charleston Zoning Division, Edye Graves, provided background information on building codes for the City of Charleston.

A number of people from NIST assisted the official team members. Of particular note were David Stroup, Glenn Forney, and Anthony Hamins, who devoted considerable time on the fire scene documenting dimensions, materials of construction, and burn patterns. William Grosshandler, Anthony Hamins, and Richard Gann devoted significant time to reviewing and improving the report. The assistance of Adam Barowy and Jonathan Kent in improving the figures, slice files, and digital images was critical and much appreciated. The support that Kelly Opert provided for documenting the reports and media citation significantly improved the readability of the appendices. The energetic assistance of Sue Haga in formatting, assembling, and proofreading these two volumes is also gratefully acknowledged. 


\section{ApPendix A. Weather Data}

\section{A.1 WEATHER DATA}

Local climatological data was obtained from the National Climatic Data Center [1] for the nearest weather monitoring station which was located at Charleston Air Force Base/International Airport. The Charleston International Airport at 5500 International Boulevard, Charleston, was approximately $13.6 \mathrm{~km}$ (8.5 miles) north of the Sofa Super Store.

The weather data was collected once an hour throughout the day. The data included sky conditions, visibility, temperatures, dew points, relative humidity, wind speed and wind velocity, barometric pressures and precipitation. 
CHARLESTON AFB INTERNATIONAL AIRPORT $(\mathbf{1 3 8 8 0})$ CHARLESTON, SC

Elevation: $39 \mathrm{ft}$. above sea level $(06 / 2007)$

atitude: 32.899

Longitude: -80.041

Version. VER2

\begin{tabular}{|c|c|c|c|c|c|c|c|c|c|c|c|c|c|c|c|c|c|c|c|c|c|c|}
\hline \multirow[t]{2}{*}{ Date } & \multirow[t]{2}{*}{$\begin{array}{l}\text { Time } \\
\text { (LST) }\end{array}$} & \multirow[t]{2}{*}{$\begin{array}{l}\text { Station } \\
\text { Type }\end{array}$} & \multirow[t]{2}{*}{$\begin{array}{c}\text { Sky } \\
\text { Conditions }\end{array}$} & \multirow[t]{2}{*}{$\begin{array}{l}\text { Msibility } \\
\text { (SM) }\end{array}$} & \multirow[t]{2}{*}{$\begin{array}{l}\text { Weather } \\
\text { Type }\end{array}$} & \multicolumn{2}{|r|}{$\begin{array}{l}\text { Dry } \\
\text { Bulb } \\
\text { Temp }\end{array}$} & \multicolumn{2}{|c|}{$\begin{array}{l}\text { Wiet } \\
\text { Bulb } \\
\text { Temp }\end{array}$} & \multicolumn{2}{|c|}{$\begin{array}{l}\text { Dew } \\
\text { Point } \\
\text { Temp }\end{array}$} & \multirow[t]{2}{*}{$\begin{array}{l}\text { Rel } \\
\text { Humd } \\
x\end{array}$} & \multirow[t]{2}{*}{$\begin{array}{l}\text { Minnd } \\
\text { Speed } \\
\text { (MPH) }\end{array}$} & \multirow[t]{2}{*}{$\begin{array}{l}\text { Whind } \\
\text { Dir }\end{array}$} & \multirow[t]{2}{*}{$\begin{array}{l}\text { Whind } \\
\text { Gusts } \\
\text { (MPH) }\end{array}$} & \multirow[t]{2}{*}{$\begin{array}{l}\text { Station } \\
\text { Pressure } \\
\text { (in. hig) }\end{array}$} & \multirow[t]{2}{*}{$\begin{array}{l}\text { Press } \\
\text { Tend }\end{array}$} & \multirow[t]{2}{*}{$\begin{array}{l}\text { Net } \\
\text { 3-hr } \\
\text { Chg } \\
(\mathrm{mb})\end{array}$} & \multirow{2}{*}{$\begin{array}{c}\text { Sea } \\
\text { Level } \\
\text { Pressure } \\
\text { (in. hg) }\end{array}$} & \multirow[t]{2}{*}{$\begin{array}{l}\text { Report } \\
\text { Type }\end{array}$} & \multirow{2}{*}{$\begin{array}{l}\text { Precip. } \\
\text { Total } \\
\text { (in) }\end{array}$} & \multirow{2}{*}{$\begin{array}{l}\text { Ati- } \\
\text { meter } \\
\text { (in. hg. }\end{array}$} \\
\hline & & & & & & (F & $\Rightarrow \quad$ (C) & (F) & (C) & (F) & (C) & & & & & & & & & & & \\
\hline 1 & 2 & 3 & 4 & 5 & 6 & 7 & 8 & 9 & 10 & 11 & 12 & 13 & 14 & 15 & 16 & 17 & 18 & 19 & 20 & 21 & 22 & 23 \\
\hline 18 & 0056 & 11 & CLR & 10.00 & & 74 & 23.3 & 71 & 21.8 & 70 & 21.1 & 87 & 6 & 200 & & 30.04 & 0 & 000 & 30.08 & AA & & 30.09 \\
\hline 18 & 0156 & 11 & CLR & 10.00 & & 74 & 23.3 & 71 & 21.8 & 70 & 21.1 & 87 & 3 & 140 & & 30.03 & & & 30.08 & AA & & 30.08 \\
\hline $\begin{array}{l}18 \\
18\end{array}$ & 0256 & 11 & CLR & 10.00 & & 73 & 22.8 & 71 & 21.6 & 70 & 21.1 & 90 & 3 & 190 & & 30.03 & & & 30.07 & AA & & 30.08 \\
\hline $\begin{array}{l}18 \\
18 \\
18\end{array}$ & 0356 & 11 & CLR & 7.00 & & 72 & 22.2 & 70 & 21.1 & 69 & ${ }^{20.6}$ & 90 & 0 & 000 & & 30.02 & 7 & 006 & 30.06 & AA & & 30.07 \\
\hline 18 & $\begin{array}{l}0.556 \\
0556\end{array}$ & 11 & CLR & $\begin{array}{l}9.00 \\
10.00\end{array}$ & & ${ }_{73}^{73}$ & $\begin{array}{l}22.8 \\
22.8\end{array}$ & 71 & $\begin{array}{l}21.6 \\
21.3\end{array}$ & 80 & $\begin{array}{l}21.1 \\
20.8\end{array}$ & ${ }_{87}^{90}-x-3$ & $\begin{array}{l}5 \\
5\end{array}$ & ${ }_{230}^{230}$ & & $\begin{array}{l}30.03 \\
3004\end{array}$ & & & 30.08 & AA & & 30.08 \\
\hline 18 & 0656 & 11 & CLR & 10.00 & & 75 & 23.9 & 72 & 22.0 & 70 & $\begin{array}{l}20.0 \\
21.1\end{array}$ & 85 & 5 & ${ }_{220}^{200}$ & & 30.05 & 1 & 009 & 30.09 & $A A$ & & 30.10 \\
\hline 18 & 0756 & 11 & CLR & 10.00 & & 80 & 26.7 & 72 & 22.2 & 68 & 20.0 & 67 & 9 & 230 & & 30.05 & & & 30.10 & AA & & 30.10 \\
\hline 18 & 0856 & 11 & FEN1035 & 10.00 & & 83 & 28.3 & 73 & 22.7 & 68 & 20.0 & 61 & 9 & 240 & & 30.05 & & & 30.10 & $A A$ & & 30.10 \\
\hline 18 & 0956 & 11 & FEN0 35 & 10.00 & & 86 & 30.0 & 74 & 23.2 & 68 & 20.0 & 55 & 7 & 250 & & 30.06 & 3 & 005 & 30.11 & $A A$ & & 30.11 \\
\hline 18 & 1056 & 11 & FEM045 SCT075 & 10.00 & & 86 & 30.0 & 74 & 23.5 & 69 & 20.6 & 57 & 5 & vR & & 30.06 & & & 30.10 & $A A$ & & 30.11 \\
\hline 18 & 1156 & 11. & SСТ0 48 SСT095 & 10.00 & & 88 & 31.1 & 75 & 23.8 & 69 & 20.6 & 53 & 6 & VR & & 30.05 & & & 30.10 & $A A$ & & 30,10 \\
\hline 18 & 1256 & 11 & SCT045 & 10.00 & & 90 & 32.2 & 76 & 24.5 & 70 & 21.1 & 52 & 6 & VR & & 30.04 & 8 & 007 & 30.09 & $A A$ & & 3009 \\
\hline 18 & 1356 & 11. & GKN046 & 10.00 & & 91. & 32.8 & 76 & 24.6 & 70 & 21.1 & 50 & 3 & VR & & 30.02 & & & 30.07 & AA & & 30.07 \\
\hline 18 & 1456 & 11 & EKN046TCU & 10.00 & & 91 & 32.8 & 78 & 25.6 & 73 & 22.8 & 56 & 11 & 190 & & 30.00 & & & 30.05 & $A A$ & & 30.05 \\
\hline 18 & 1556 & 11 & FENU045TCU & 10.00 & & 90 & 32.2 & 79 & 25.8 & 74 & 23.3 & 59 & 14 & 190 & 17 & 29.98 & 8 & 019 & 30.03 & $A A$ & & 30.03 \\
\hline 18 & 1656 & 11 & FEMO045 & 10.00 & & 89 & 31.7 & 78 & 25.3 & 73 & 22.8 & 59 & 11 & 180 & & 29.98 & & & 30.03 & AA & & 30.03 \\
\hline 18 & 1756 & 11 & FEND050 & 10.00 & & 86 & 30.0 & 76 & 24.5 & 72 & 22.2 & 63 & 11 & 190 & & 29.98 & & & 30.03 & $A A$ & & 30.03 \\
\hline 18 & 1856 & 11 & FEMN070 & 10.00 & & 82 & 27.8 & 74 & 23.6 & 71 & 21.7 & 69 & 9 & 190 & & 30.00 & 3 & 005 & 30.05 & $A A$ & & 30.05 \\
\hline 18 & 1956 & 11 & CLR & 10.00 & & 79 & 26.1 & 75 & 23.8 & 73 & 22.8 & 82 & 10 & 190 & & 30.02 & & & 30.07 & $A A$ & & 30.07 \\
\hline 18 & 2056 & 11 & CLR & 10.00 & & 78 & 25.6 & 75 & 23.6 & 73 & 22.8 & 85 & 10 & 200 & & 30.05 & 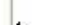 & $6 a^{2}$ & 30.09 & $A \mathrm{~A}$ & & 30.10 \\
\hline 18 & 2156 & 11 & FENO050 & 10.00 & & 78 & 25.6 & 73 & 22.9 & 71 & 21.7 & 79 & 8 & 190 & & 30.05 & 1 & 017 & 30.10 & AA & & 30.10 \\
\hline $\begin{array}{l}18 \\
18 \\
-18\end{array}$ & $\begin{array}{l}2356 \\
2356\end{array}$ & $\begin{array}{l}11 \\
11\end{array}$ & 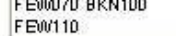 & $\begin{array}{l}10.00 \\
10.00\end{array}$ & & $\begin{array}{l}78 \\
78\end{array}$ & $\begin{array}{l}26.1 \\
25.6\end{array}$ & ${ }_{73}^{72}$ & $\begin{array}{l}22.4 \\
22.9\end{array}$ & $\begin{array}{l}69 \\
71\end{array}$ & $\begin{array}{l}20.6 \\
21.7\end{array}$ & $\begin{array}{l}72 \\
79\end{array}$ & 8 & $\begin{array}{l}230 \\
210\end{array}$ & & $\begin{array}{l}30.040 \\
30.03\end{array}$ & & & $\begin{array}{l}30.09 \\
30.08\end{array}$ & $A A$ & & $\begin{array}{l}30.09 \\
30.08\end{array}$ \\
\hline
\end{tabular}

Dynamically generated Mon Jul 16 10:57:41 EDT 2007 via htfp://cdo.ncdc.noaa.gov/gclcd/QCLCD

Figure A-1. Climatological data for Charleston International Airport on June 18, 2007. 


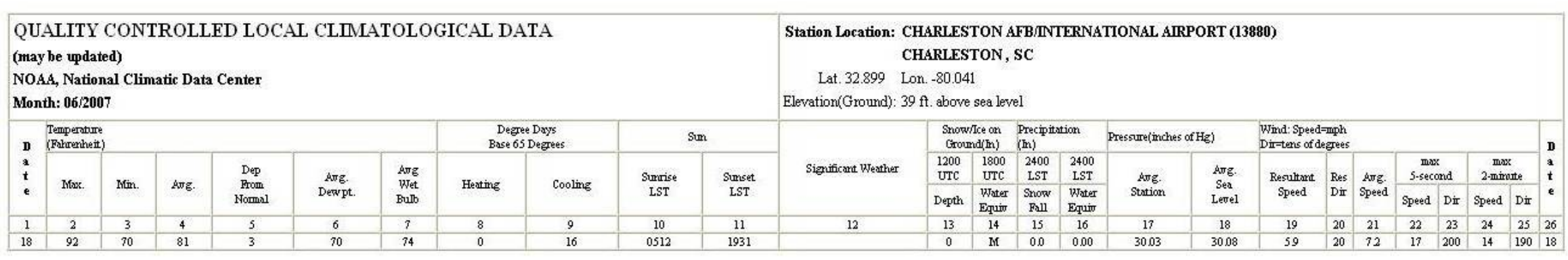

Figure A-2. Minimum, maximum, and average climatological data for Charleston International Airport on June $18,2007$. 


$$
\begin{aligned}
& (06 / 2007) \\
&
\end{aligned}
$$

Elevation: $39 \mathrm{ft}$. above sea level

Longitude: -80041

Data Version: VER2

\begin{tabular}{|l|l|l|l|}
\hline Date & Time & Remarks \\
\hline 18 &
\end{tabular}

$18 \quad 0056$ A02 SLP187 T02330211 102612023350000 (AiniS)

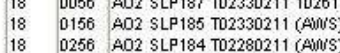

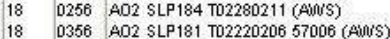

180456 A02 SLP185 TO2280211 (ANuils)

180556 A02 SLP188 T02280206 (ANuIS

0656 A02 SLP190 T02390211 102392021151009 (ANuIS)

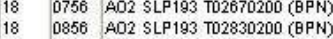

180956 A02 SLP 196 T03000200 53005 (BPN)

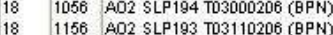

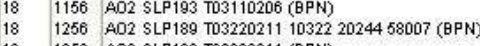

$\begin{array}{lll}18 & 1356 & \text { A02 SLP182 T03280211 (BPN) } \\ 18 & 1456 & \text { A02 SLP176 TCU E TO3280228 (BPN) }\end{array}$

$\begin{array}{lll}18 & 1456 & \text { A02 SLP176 TCU E TO3280228 (BPN) } \\ 18 & 1556 & \text { A02 SLP170 TCU NE TO3220233 58019 (NOD) }\end{array}$

18 1656 A02 SLP168 CB DSNT N AND NE TO3170228 (NOD)

\begin{tabular}{l|l|l}
18 & 1756 & A02 SLP168 CB DSNT N TO3000222 (WVD) \\
18 & 1856 & A02 SLP175 T02780217 103332027853005 (MiD)
\end{tabular}

$18 \quad 2056$ A02 SLP 190 T202502028 (NiU)

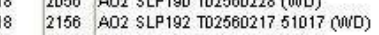

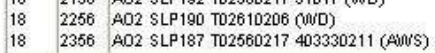

Dynamically generated Mon Jul 16 10:59:19 EDT 2007 via hitp://cdo.ncde.noaa. gov/gclcd/QCLCD

Figure A-3. Hourly remarks for climatological data for Charleston International Airport on June $18,2007$. 


\section{A.2 REFERENCES FOR APPENDIX A}

[1] http://cdo.ncdc.noaa.gov/gcldc/QCLCD 
A-6 


\section{Appendix B. Overall Scene Photos}

\section{B.1 OVERALL SCENE PHOTOS}

A limited series of photographs were combined to provide panoramic images. These images allow an overall perspective, but the process of combining separate images can introduce some distortion into a panoramic image. For example, the image below shows the east side of the warehouse and was a combination of three separate photographs. Although this provides an overall perspective of the collapsed warehouse, the chain link fences, one of the left and another on the right, appear to be almost parallel in this panoramic image. In reality, the two fences intersected at 90 degrees; this is the type of distortion which can occur as multiple images are combined.

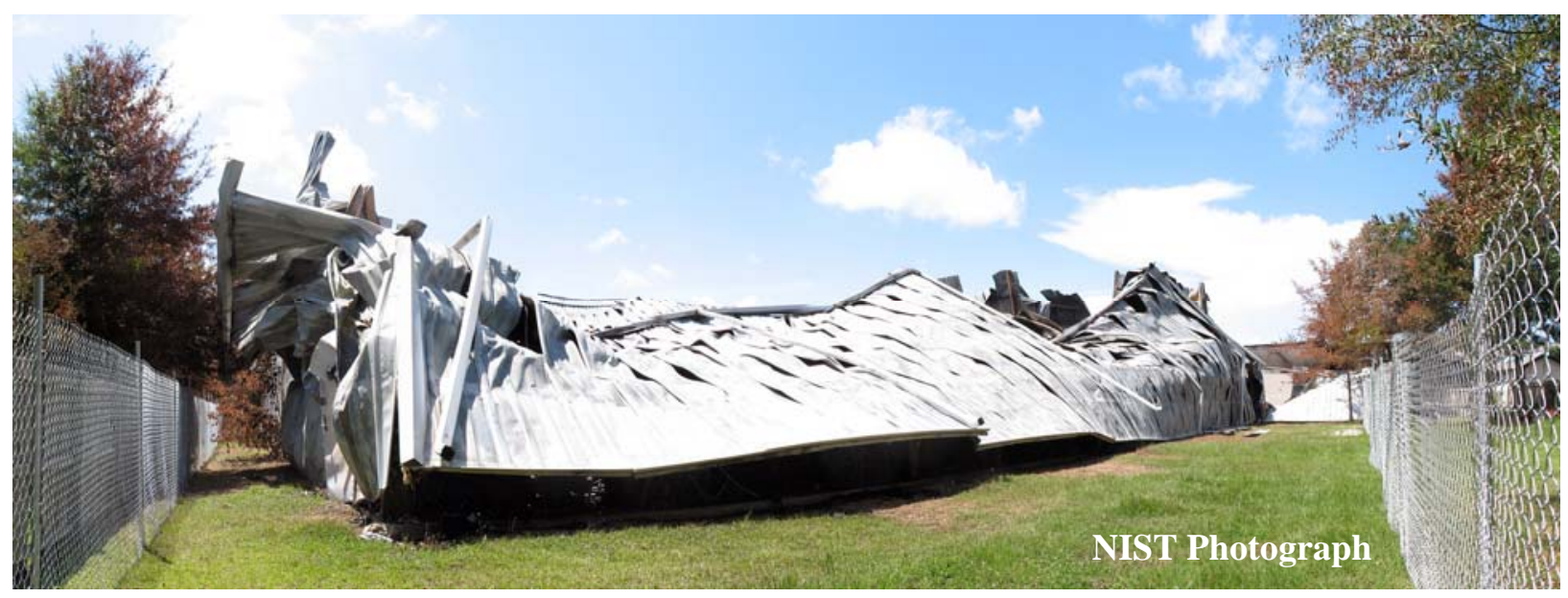

Figure B-1. Combining multiple images into a single panoramic image can introduce distortions. 


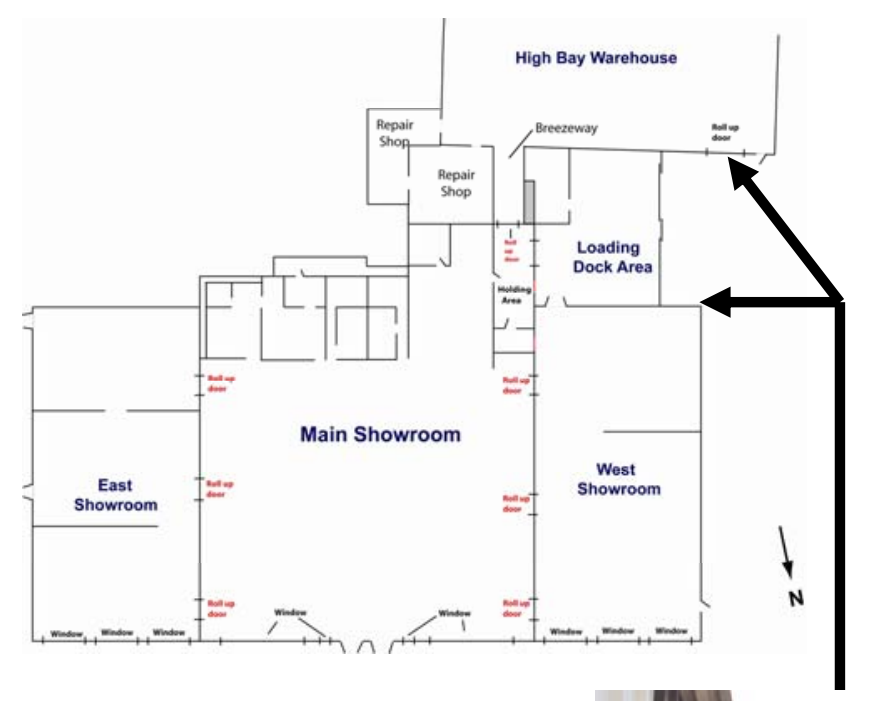

Figure B-2. Loading dock area.

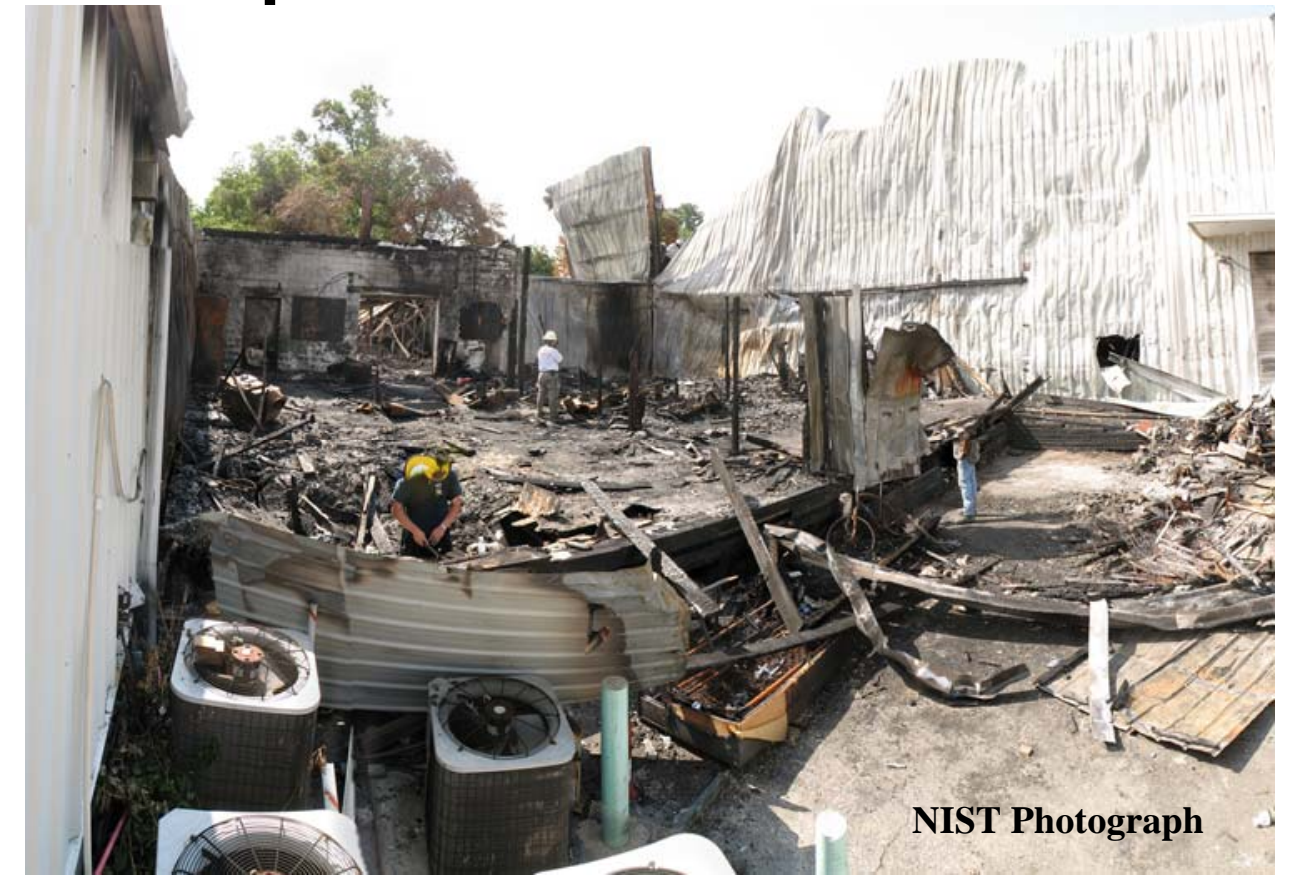




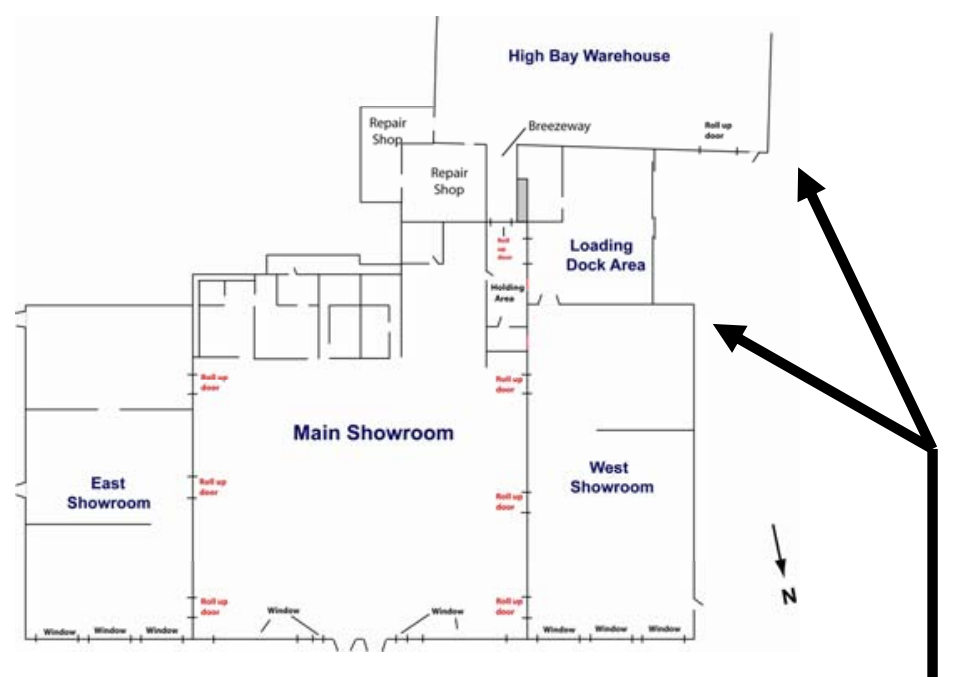

Figure B-3. Loading dock area looking toward warehouse.

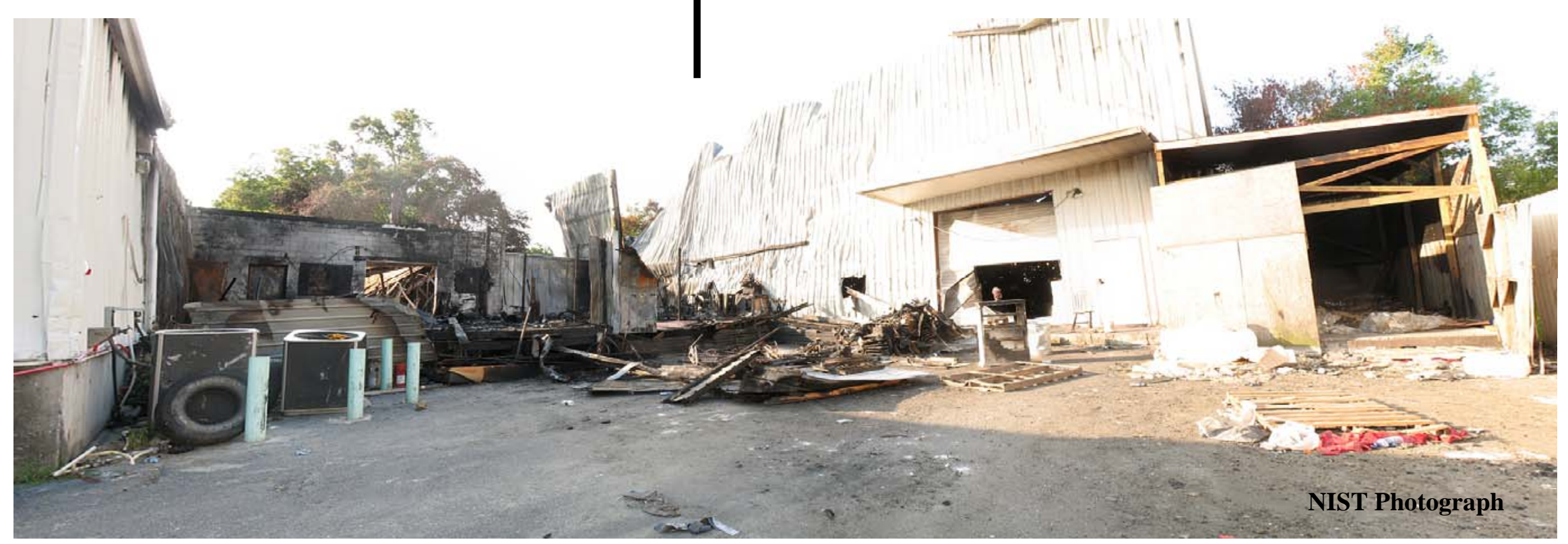




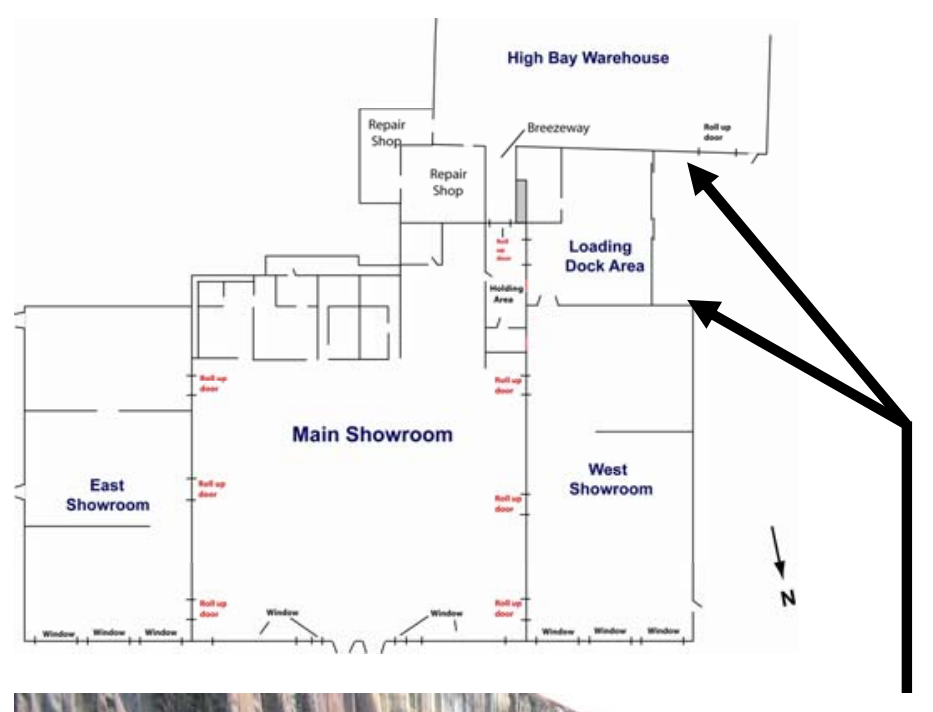

Figure B-4. Loading dock area looking toward open roll-down door into holding area.

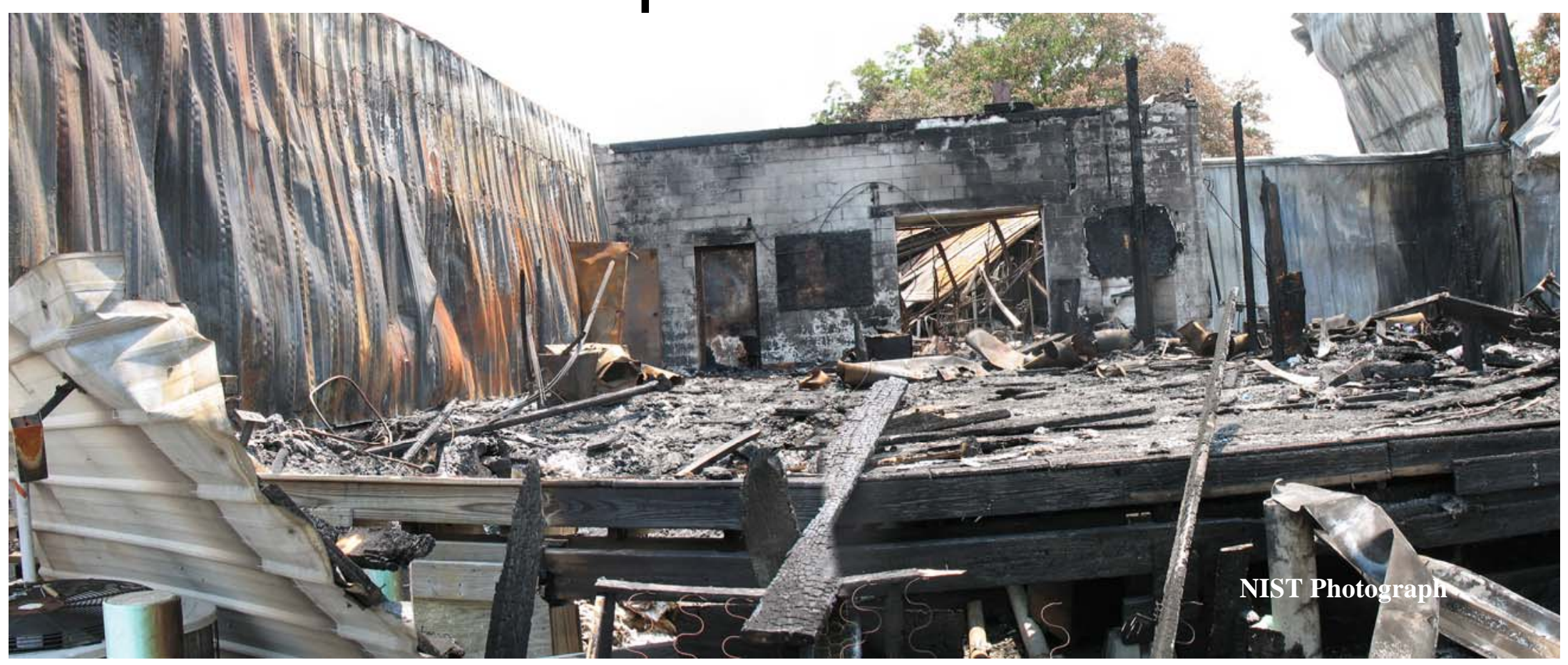



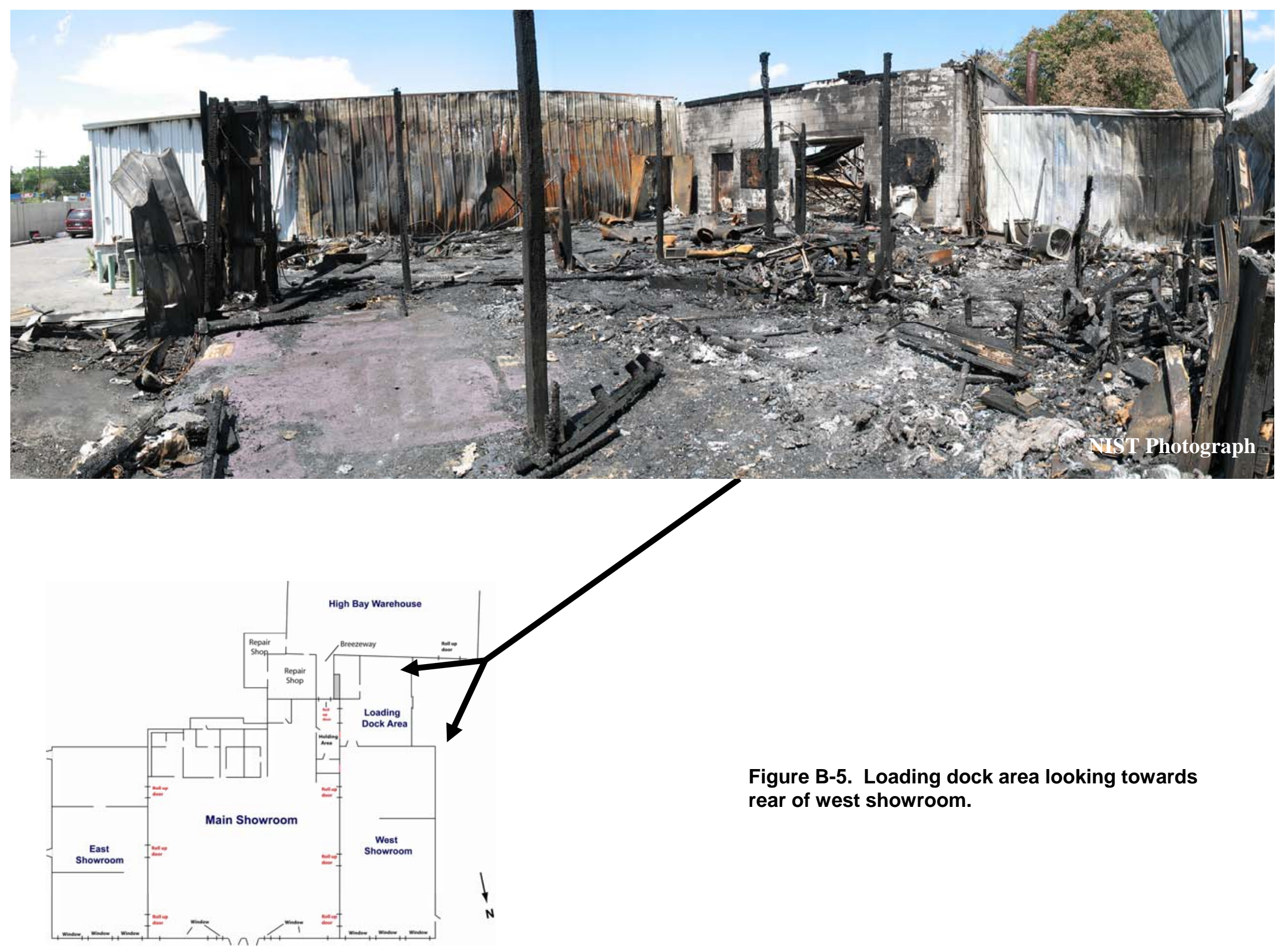

Figure B-5. Loading dock area looking towards rear of west showroom. 

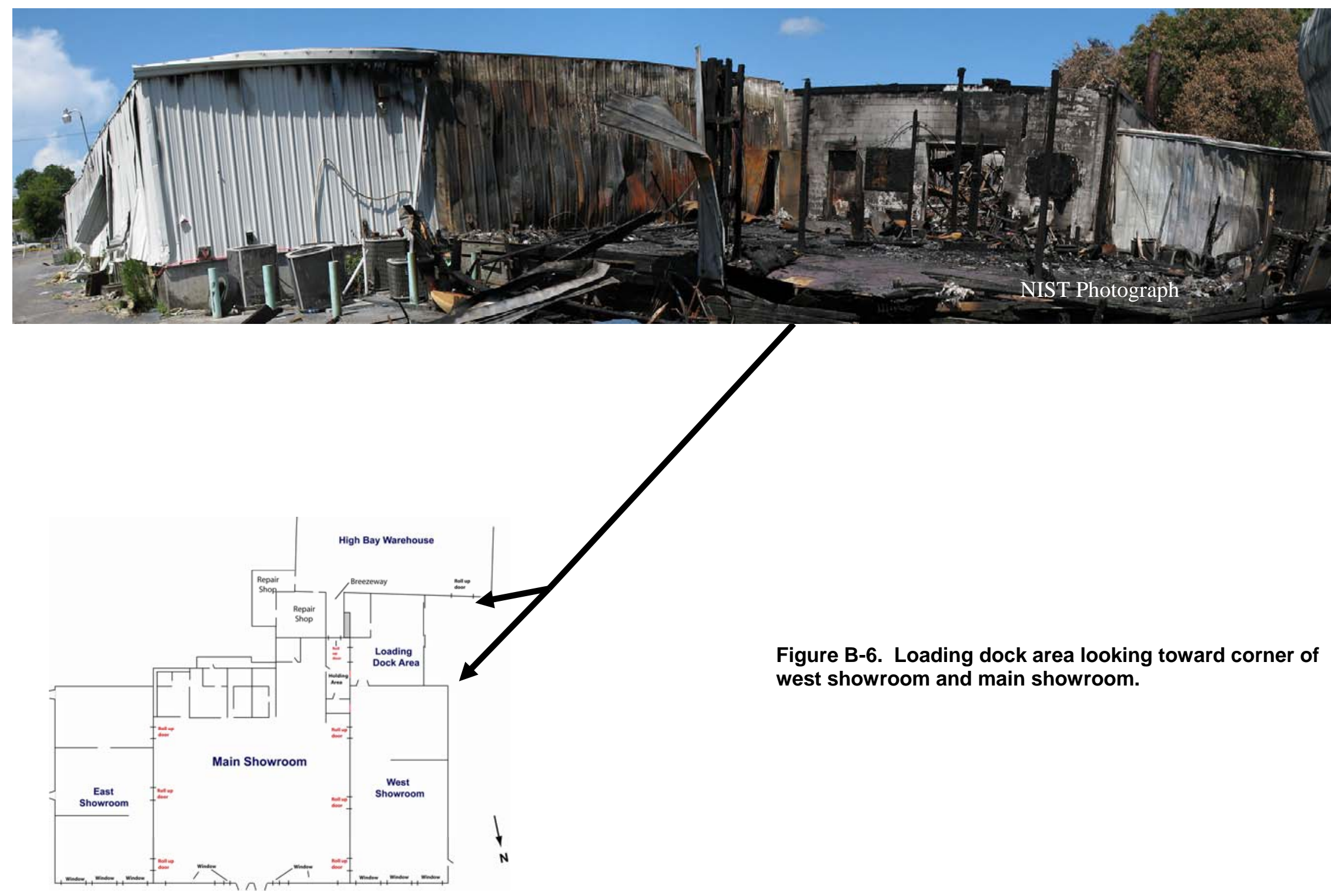

Figure B-6. Loading dock area looking toward corner of west showroom and main showroom. 


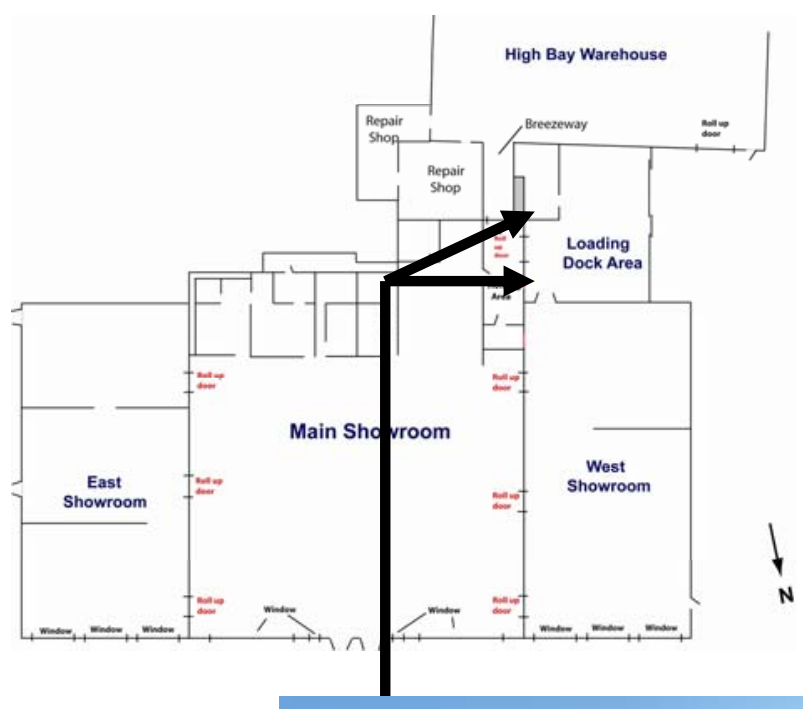

Figure B-7. Loading dock area looking along rear wall of west showroom.

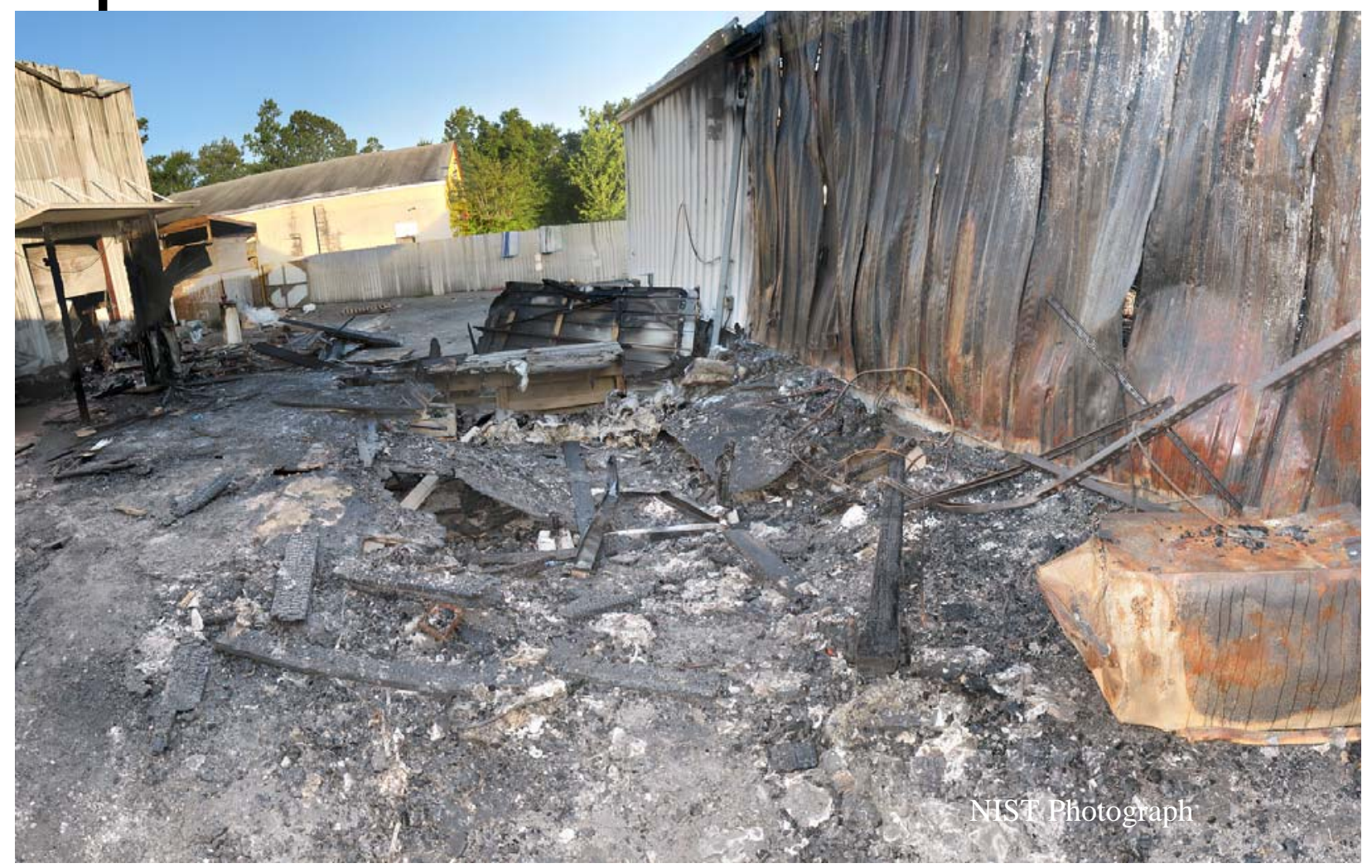




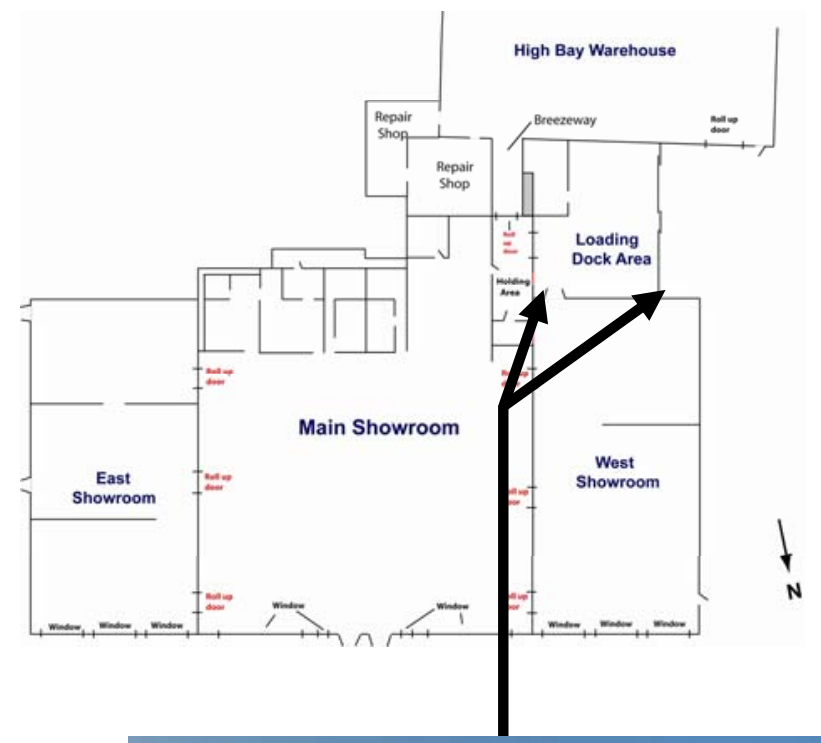

Figure B-8. Loading dock area looking toward warehouse.

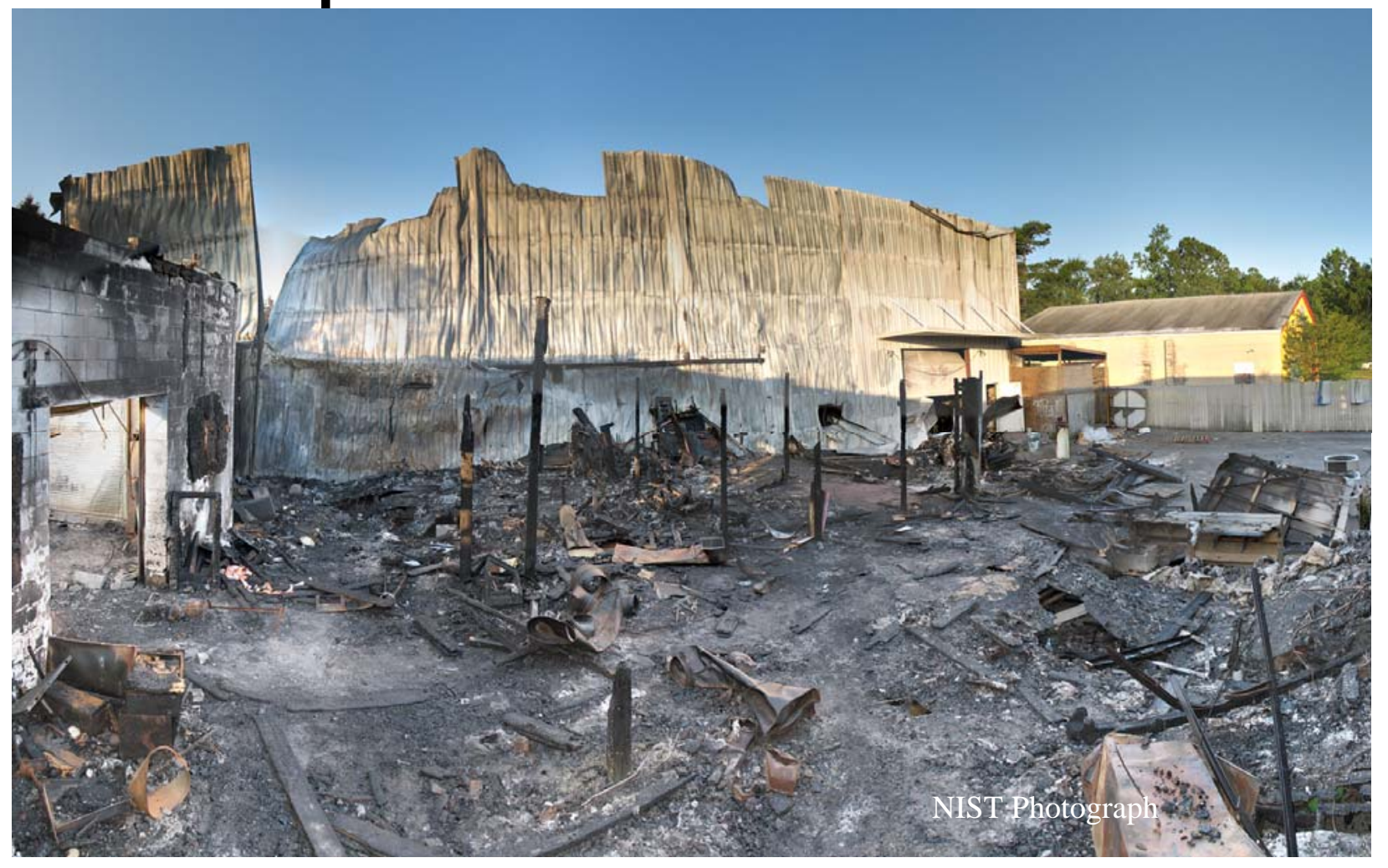




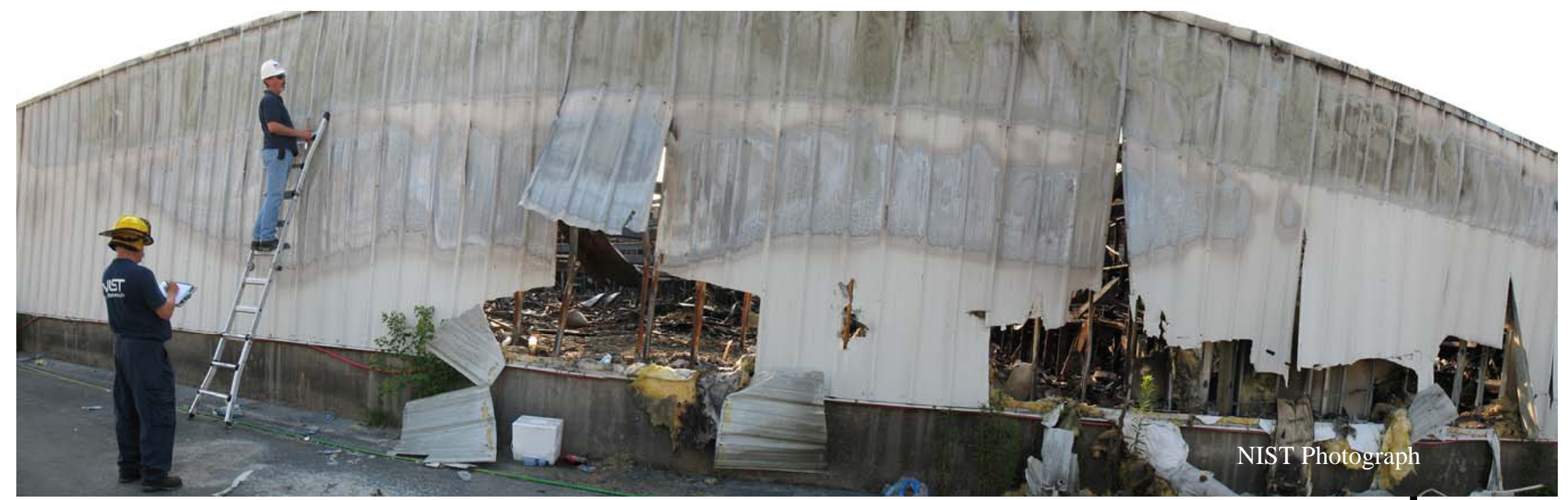

Figure B-9. West wall of west showroom.

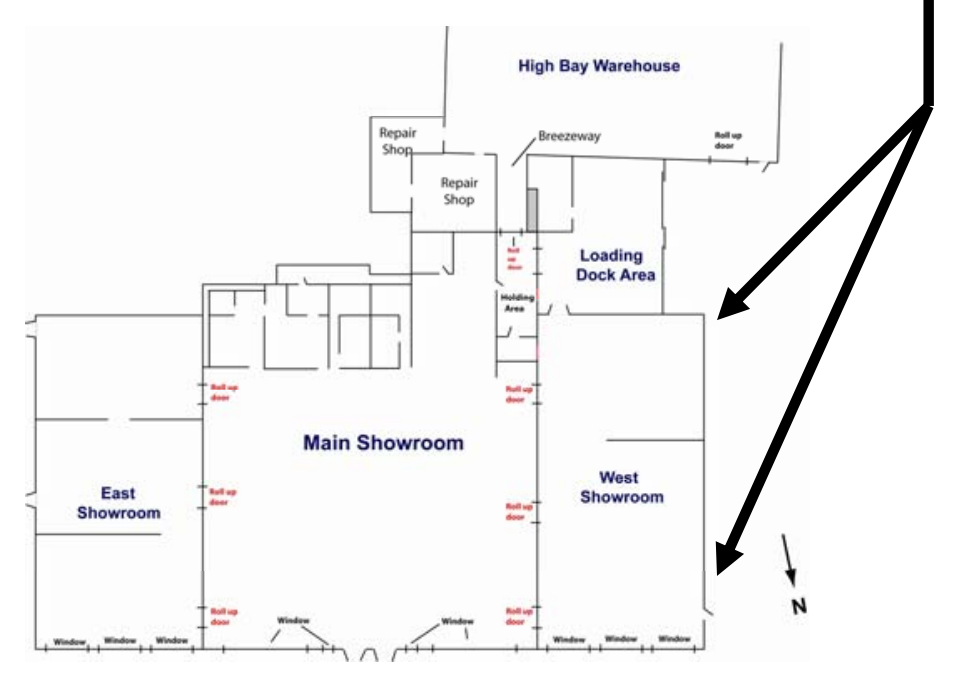


Figure B-10. Rear wall of west showroom. Double steel doors visible in left of photograph.
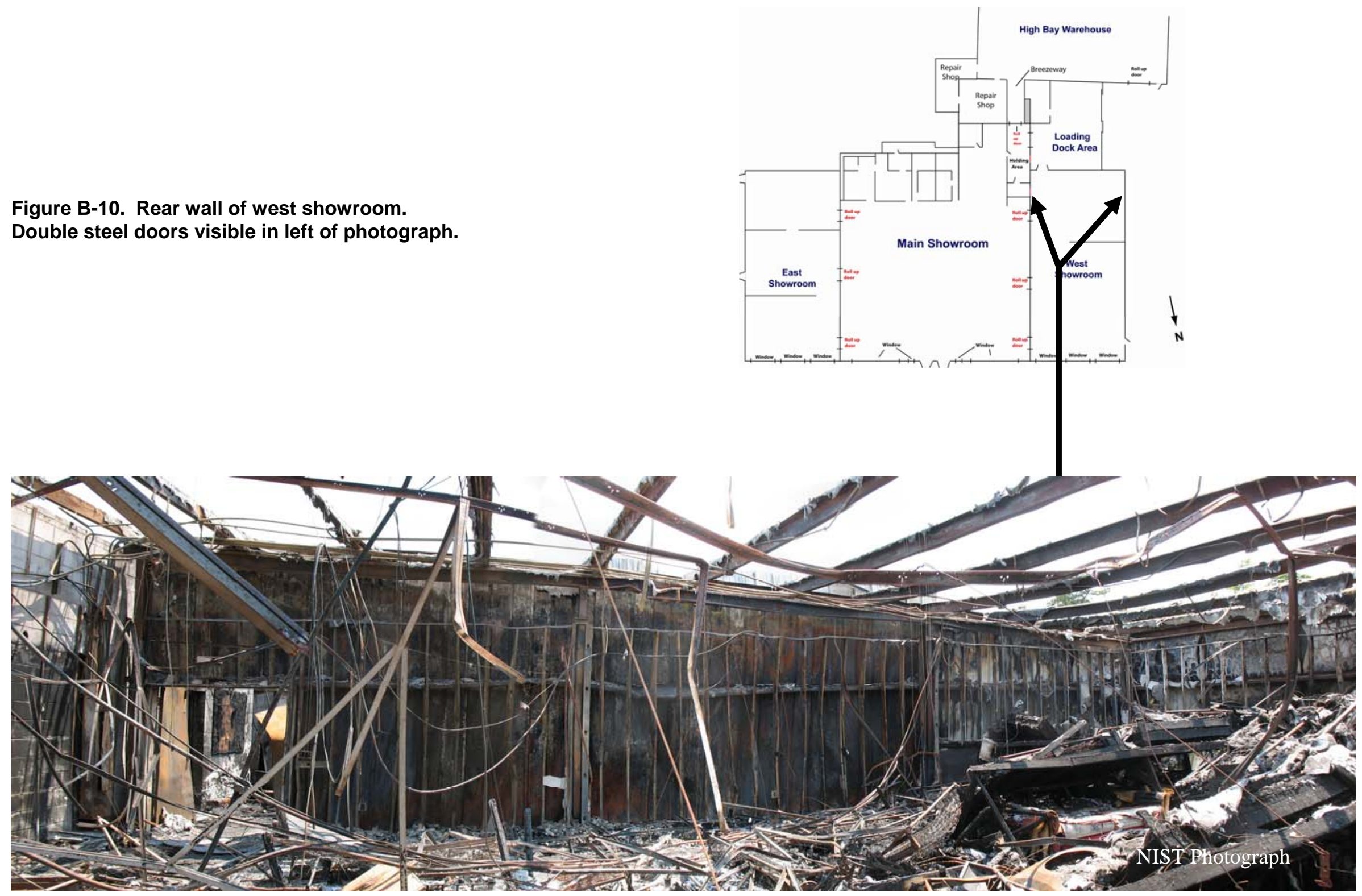


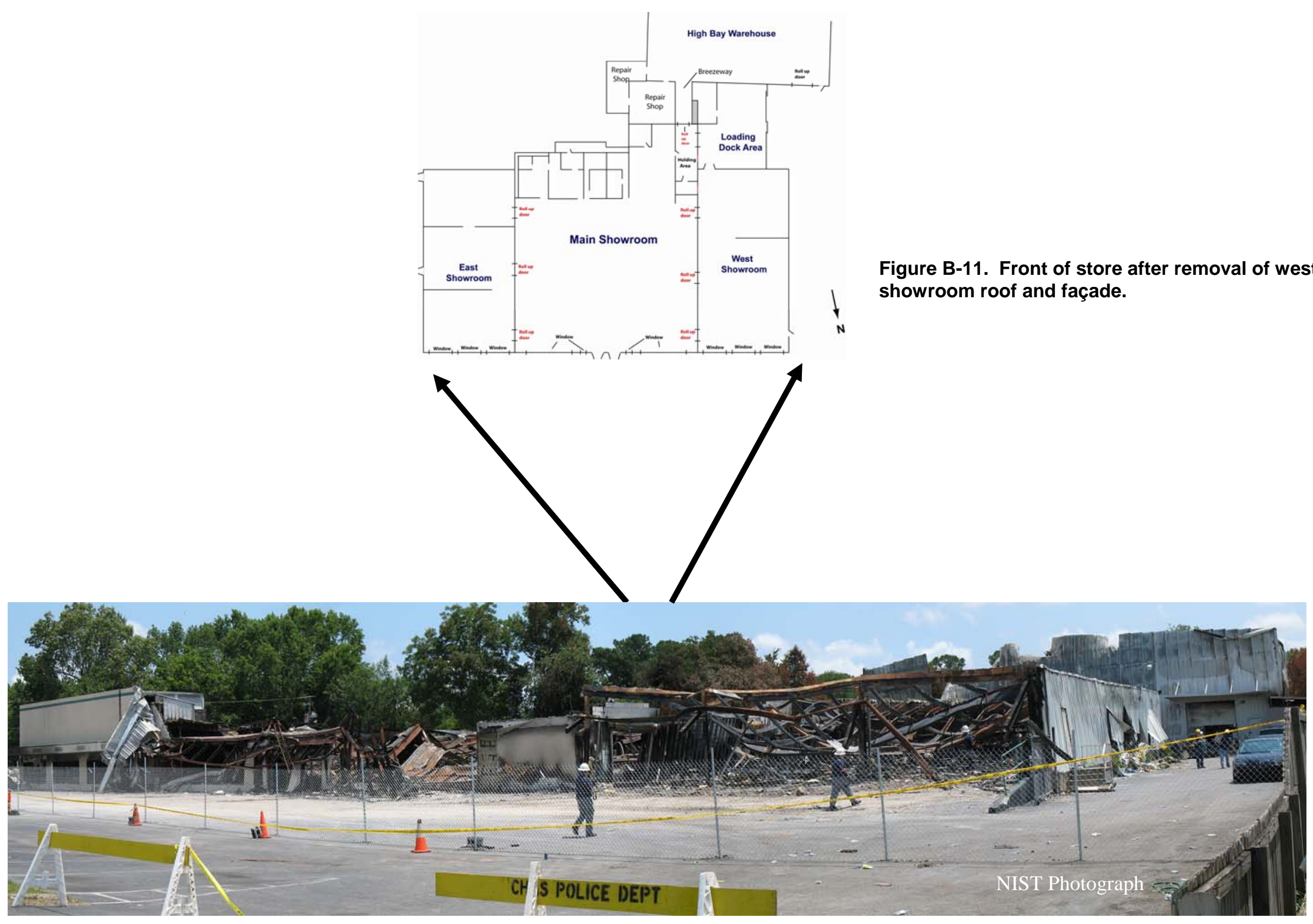




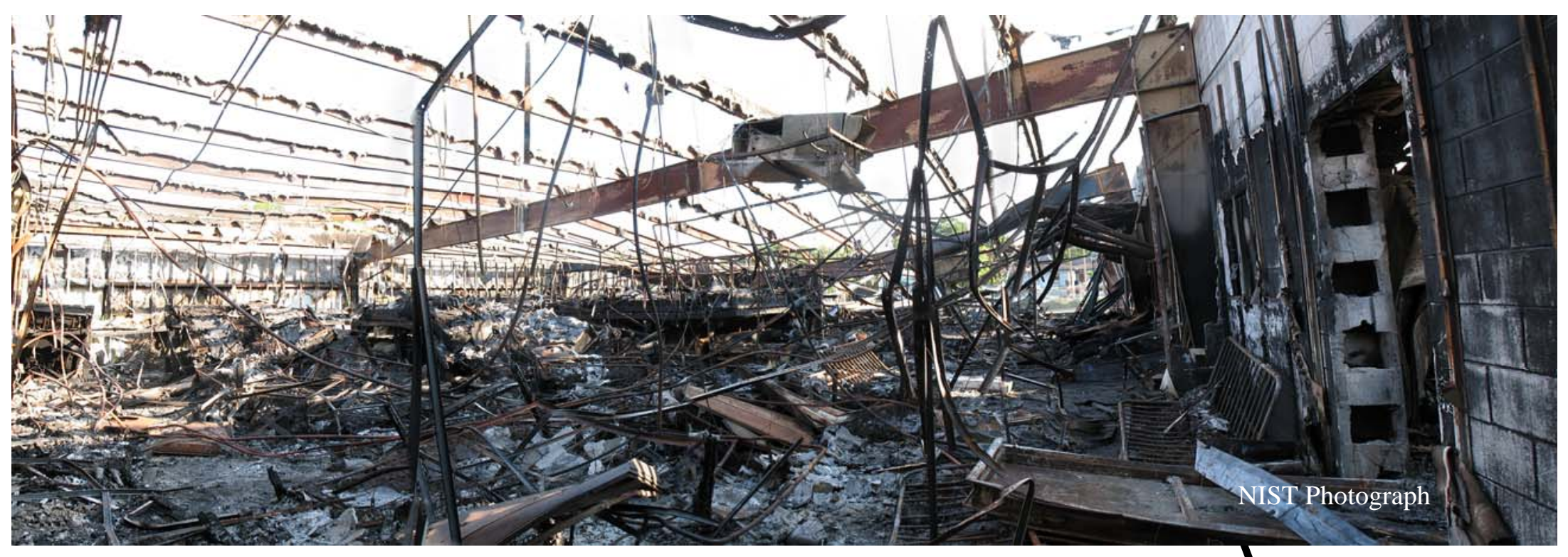

Figure B-12. Interior of west showroom looking toward front of store.

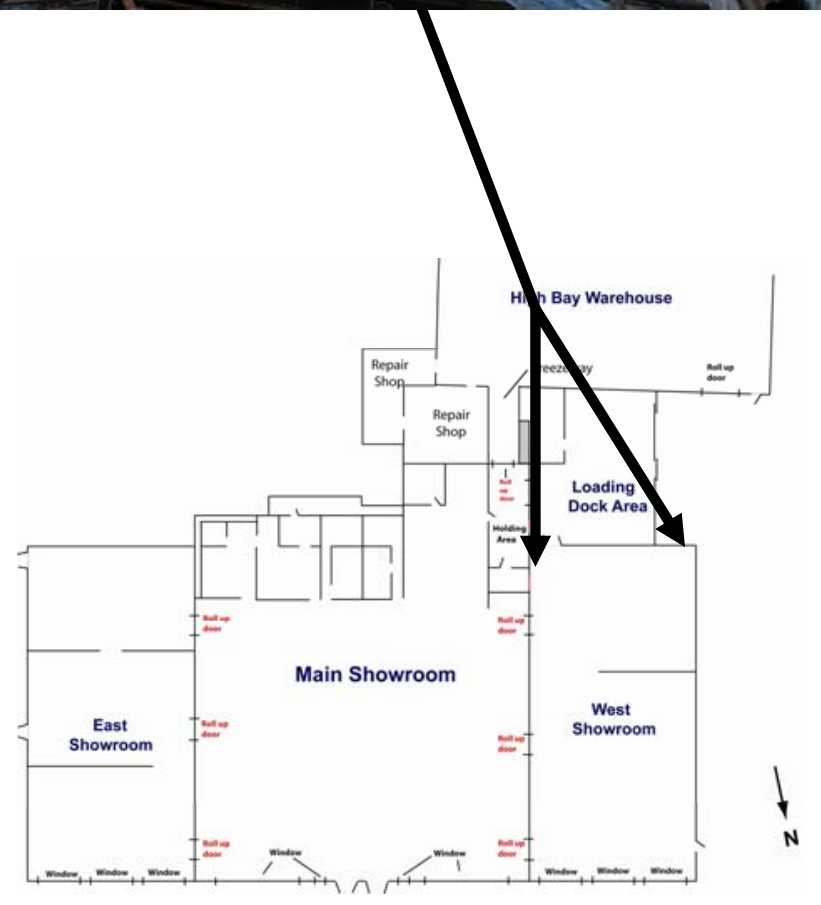




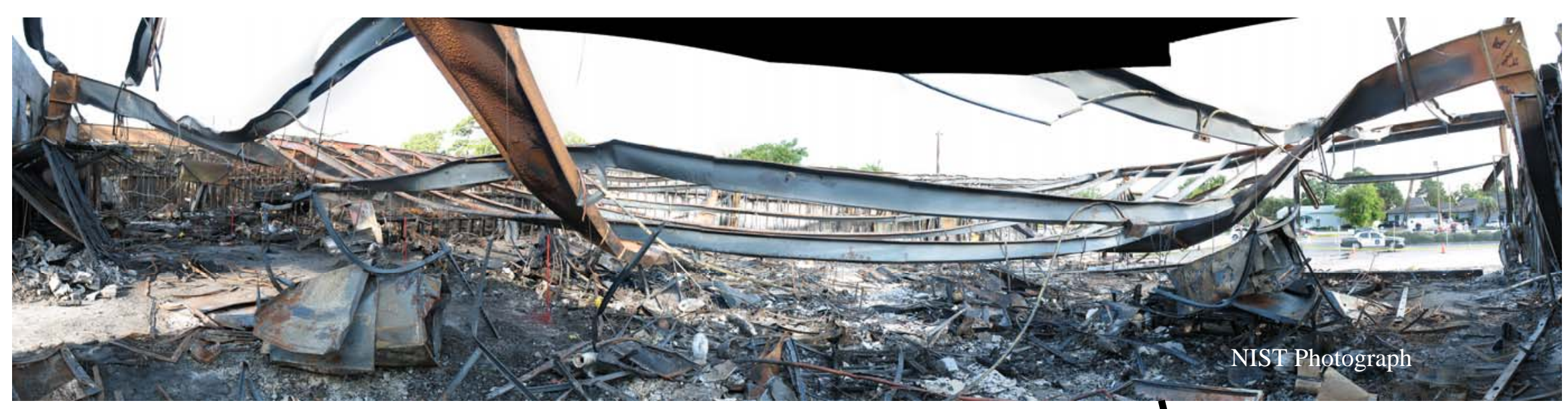

Figure B-13. Interior of west showroom looking toward front of store.

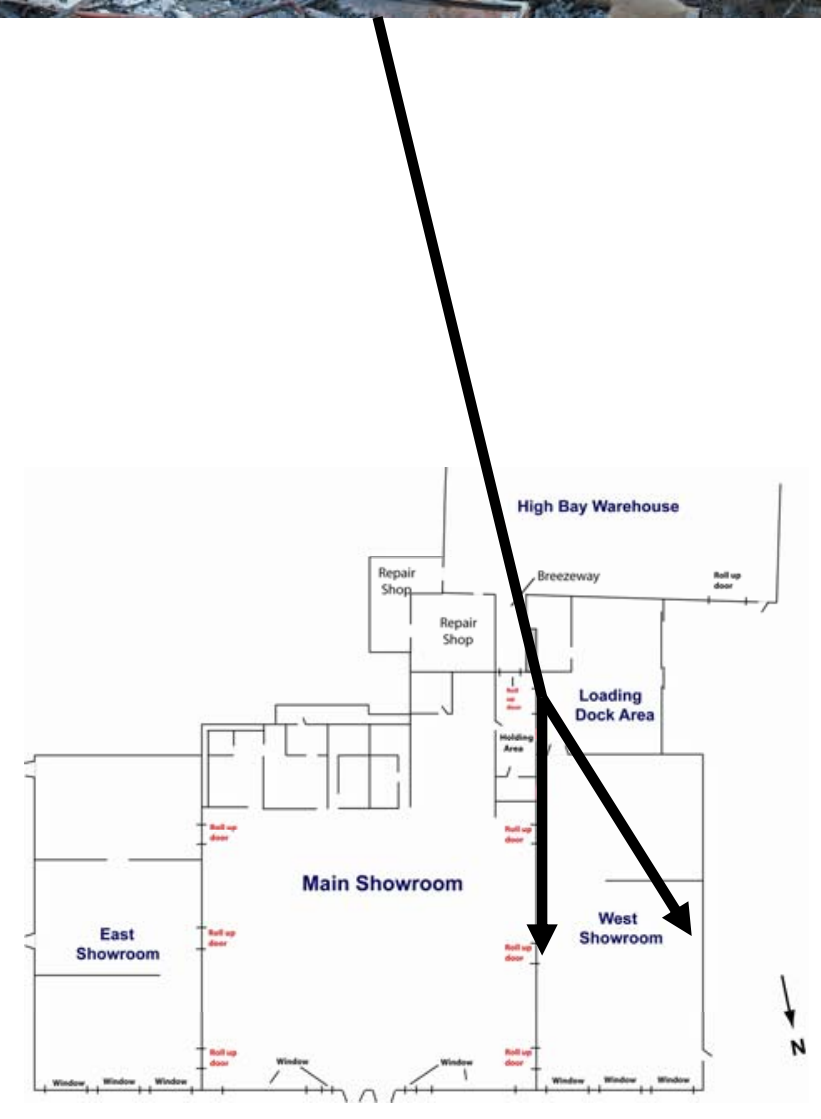


Figure B-14. Front of west showroom.
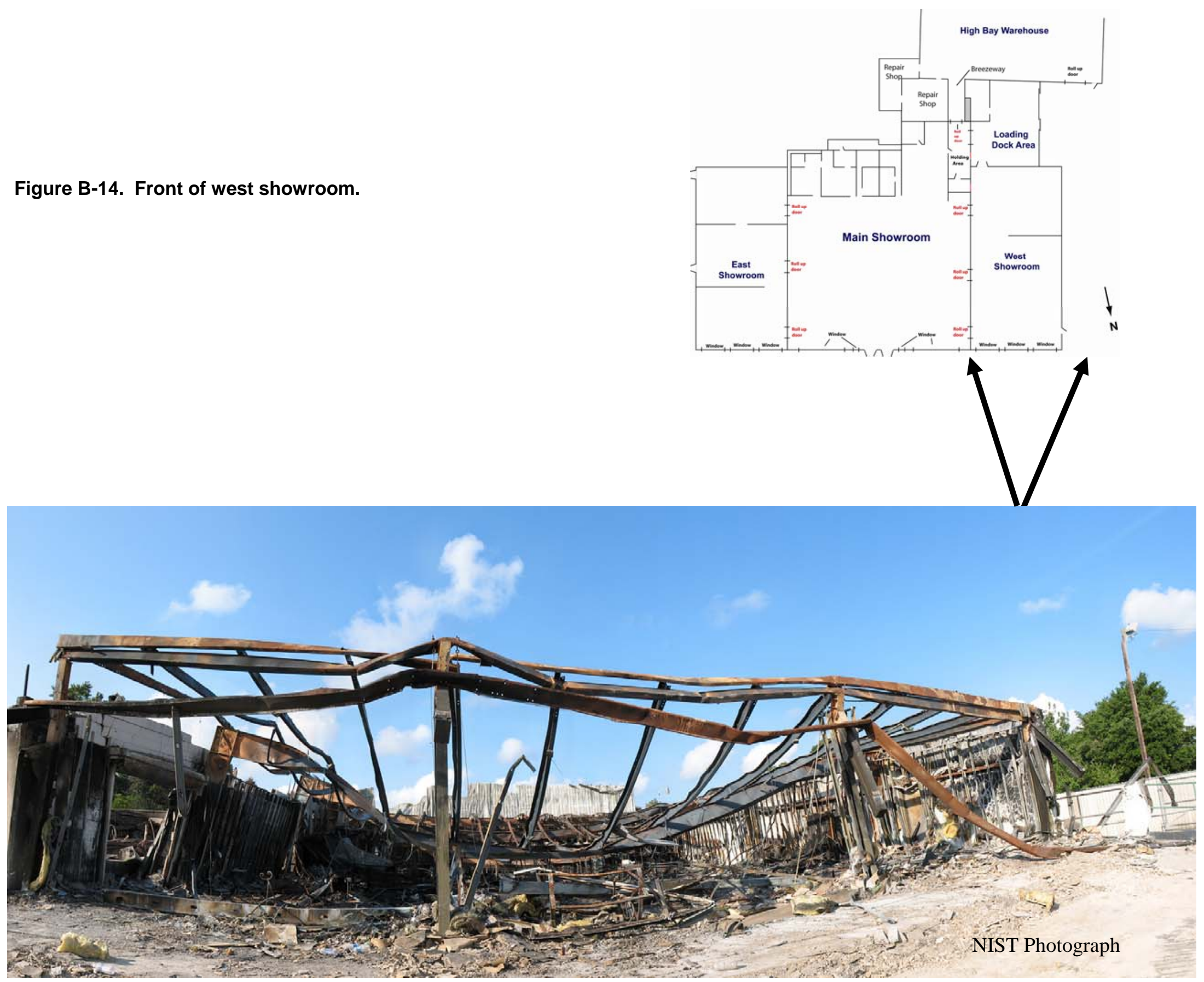
Figure B-15. Interior of main showroom looking toward east, rear, and west of showroom.
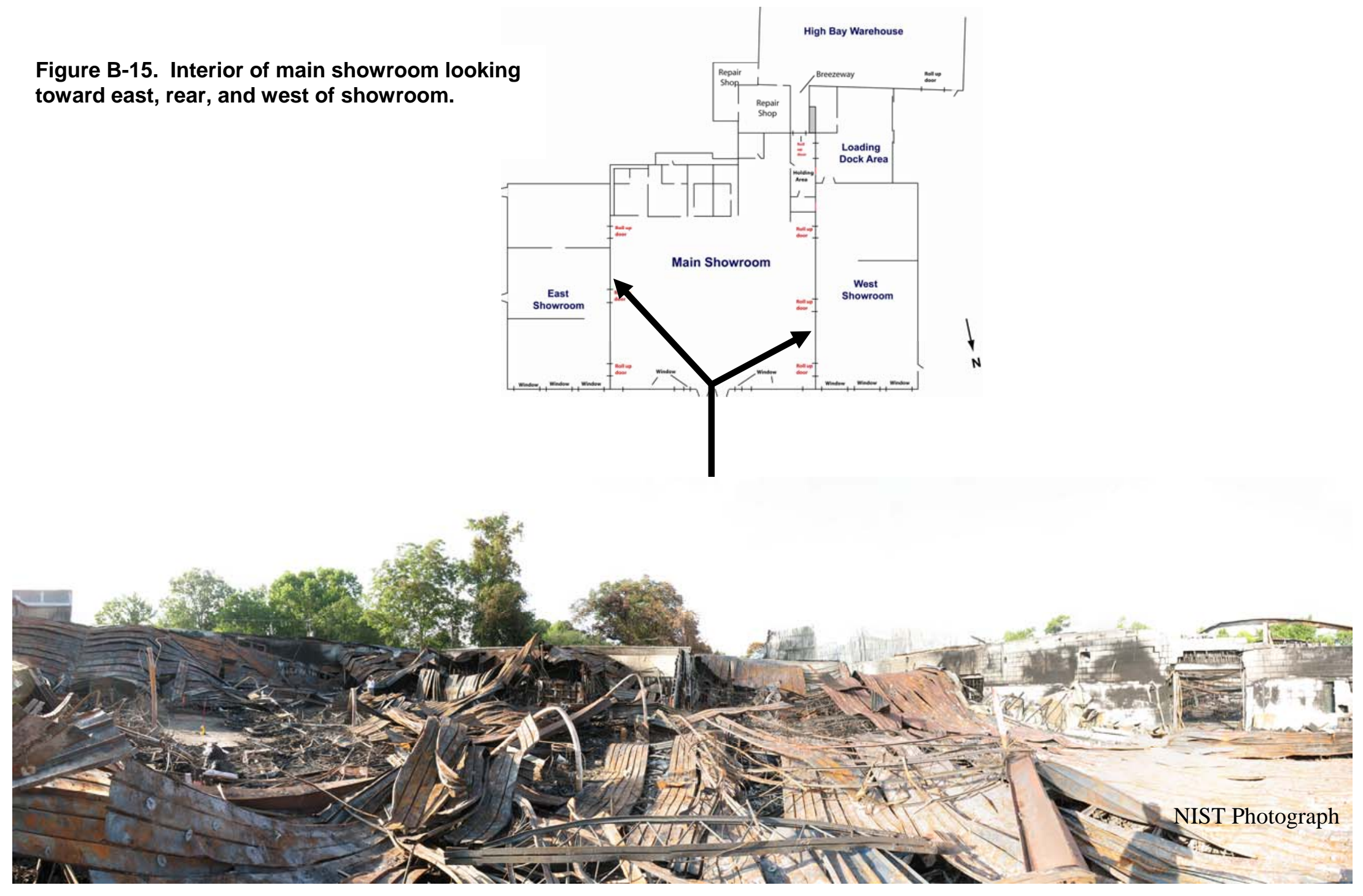
Figure B-16. Interior of main showroom looking toward front, east, and rear of the showroom.
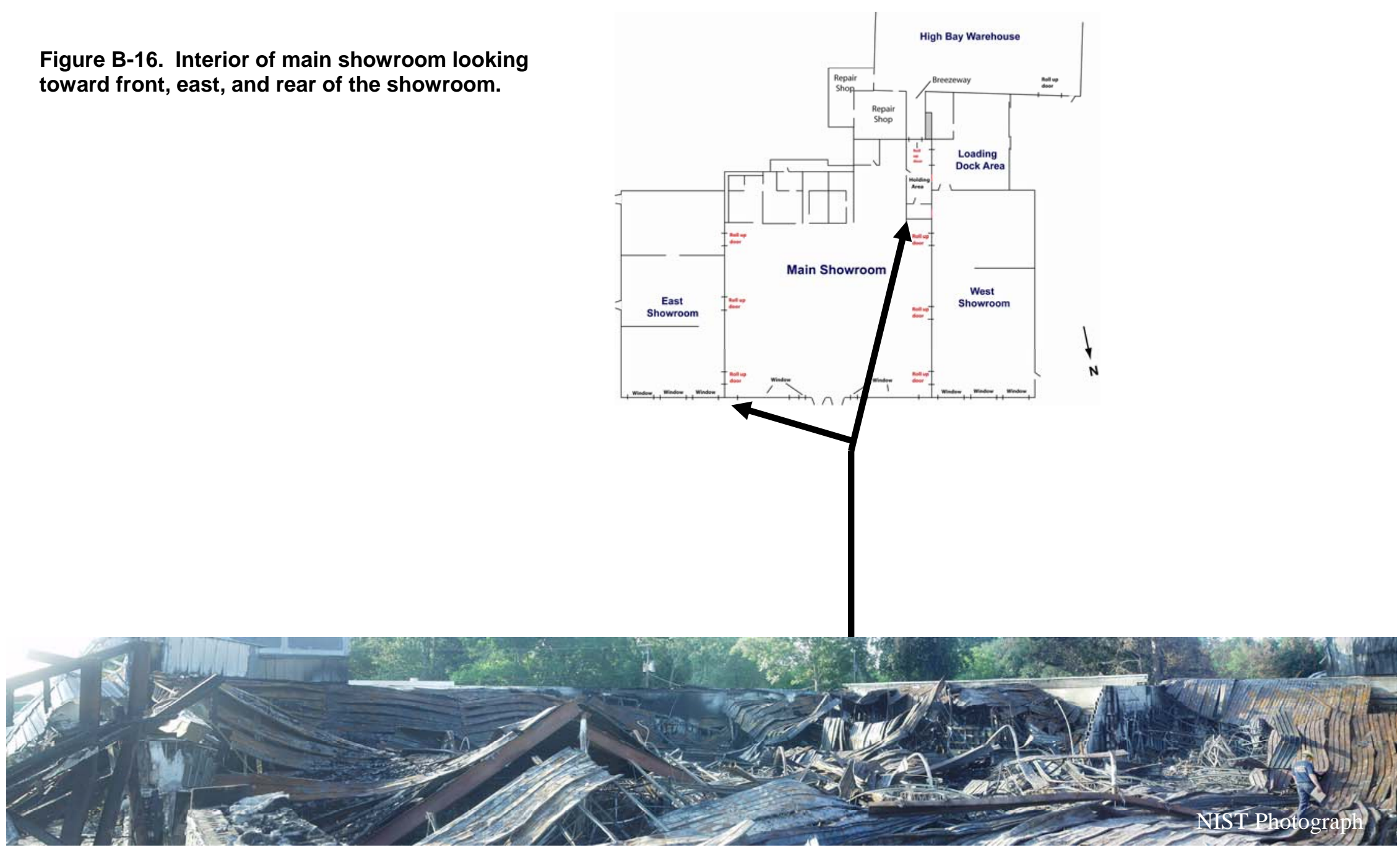


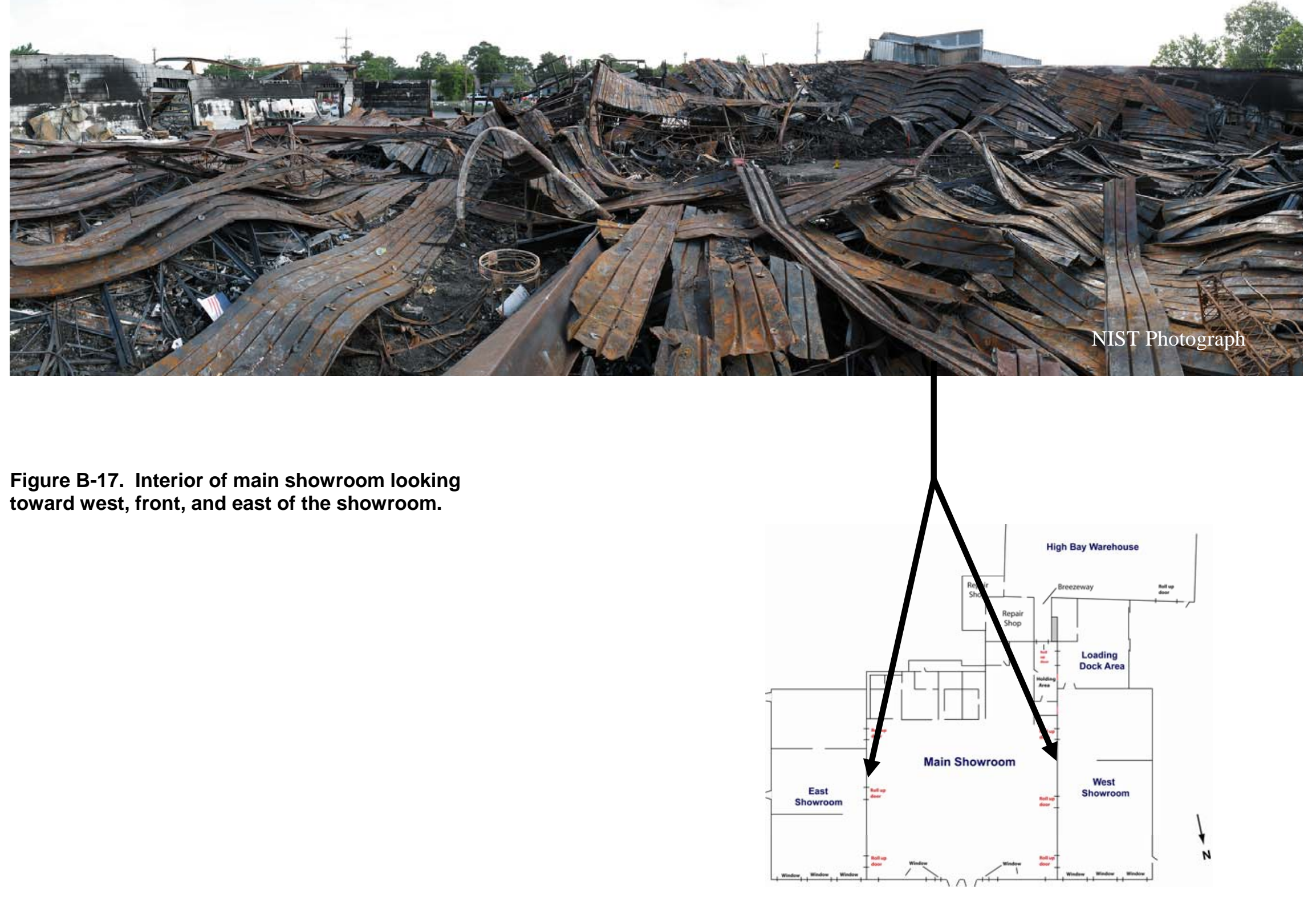




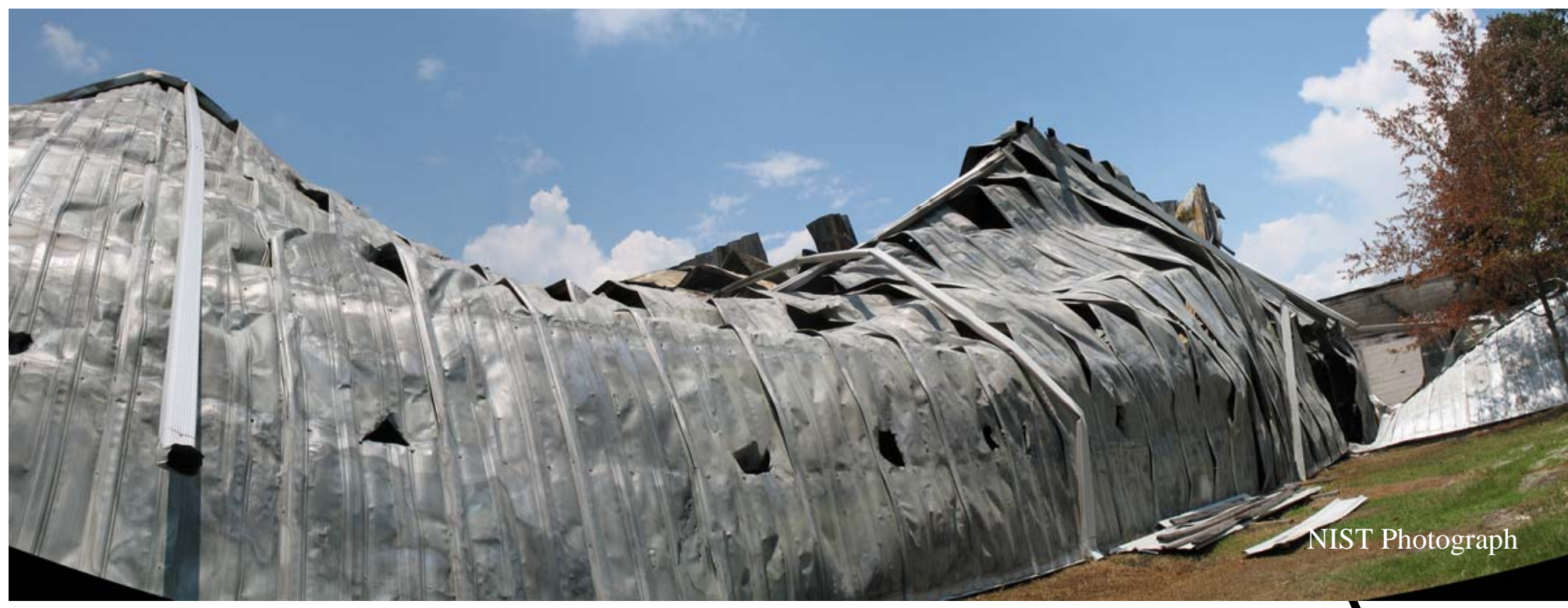

Figure B-18. East wall of warehouse looking toward loading dock.

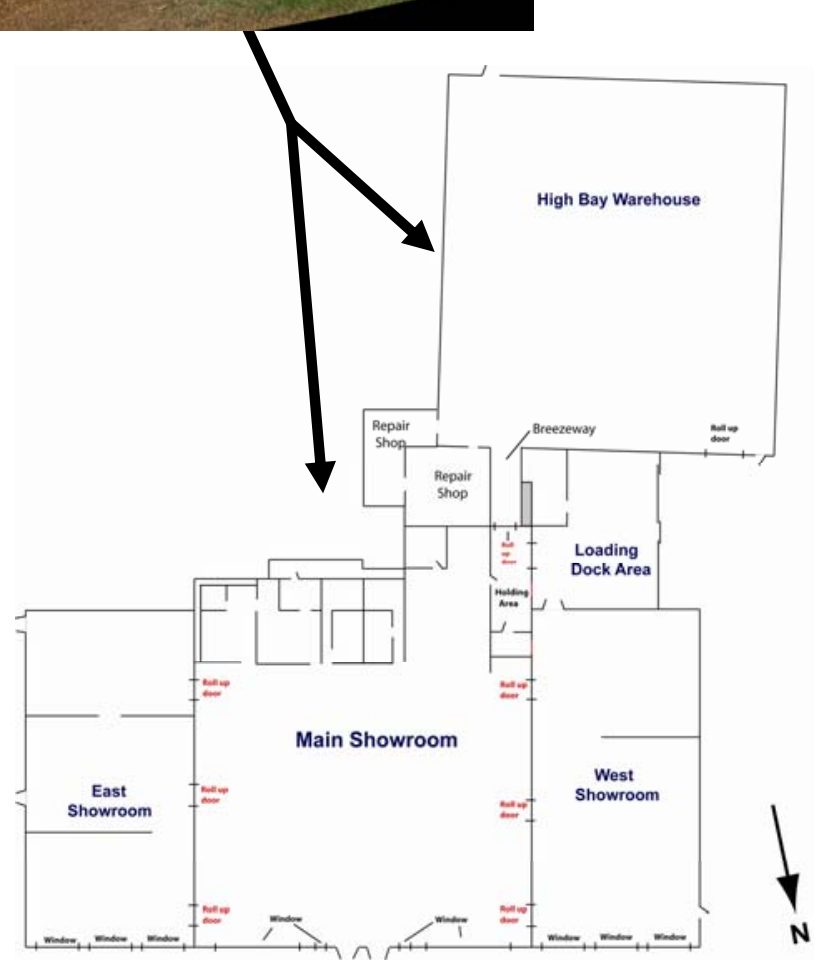




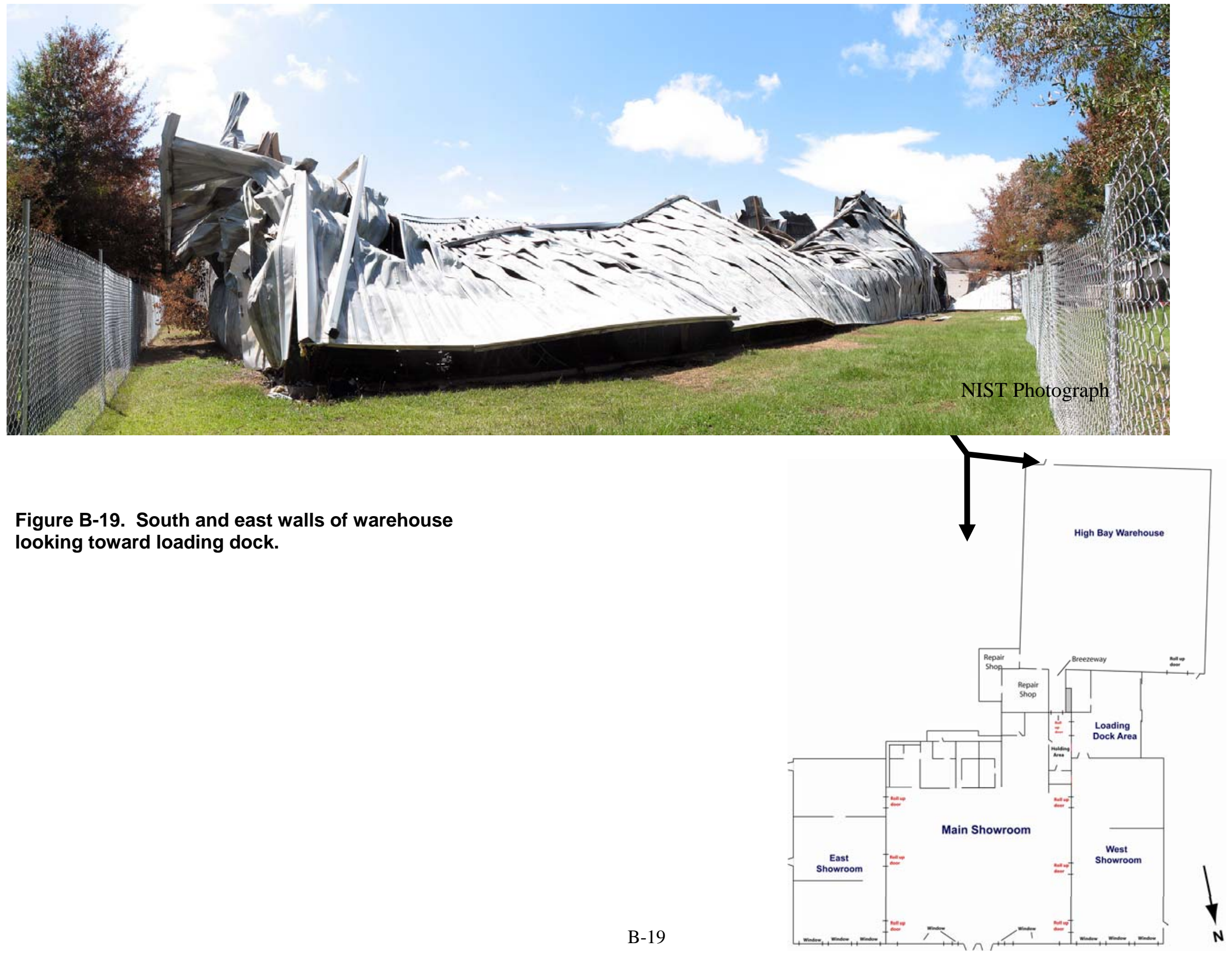




\section{ApPendix C. LoAding Dock AREA}

\section{C.1 LOADING DOCK AREA PHOTOS - BEFORE REMOVAL OF ROOF}

A limited series of photographs were obtained before the roof was removed from the enclosed loading dock area. The roof was lifted off the dock area for safety reasons. Another series of photographs were taken after removal of the roof.

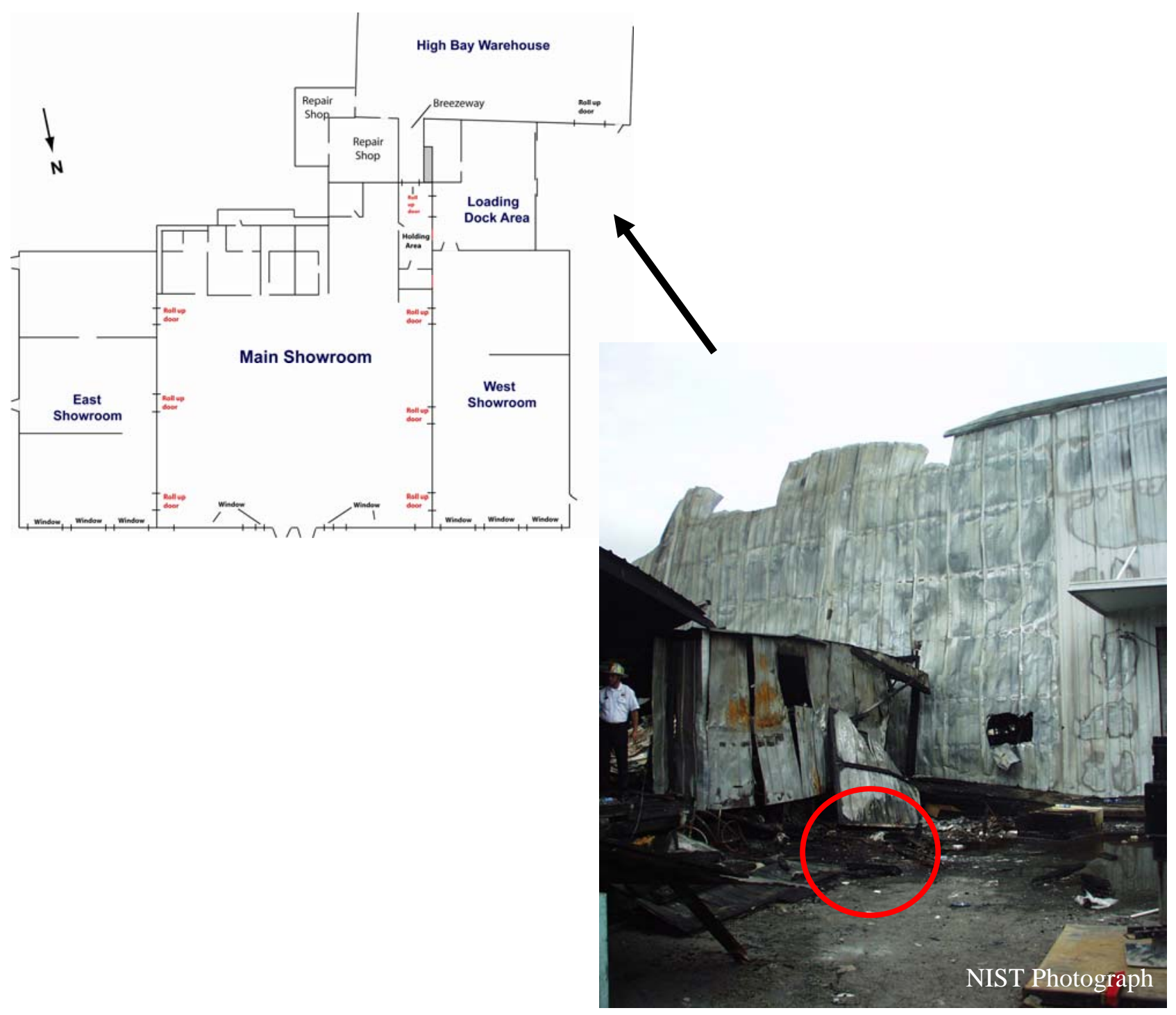

Figure C-1. West wall of loading dock area intersecting north wall of distribution warehouse. Fire ignited in pile of trash outside loading dock (within red circle). 

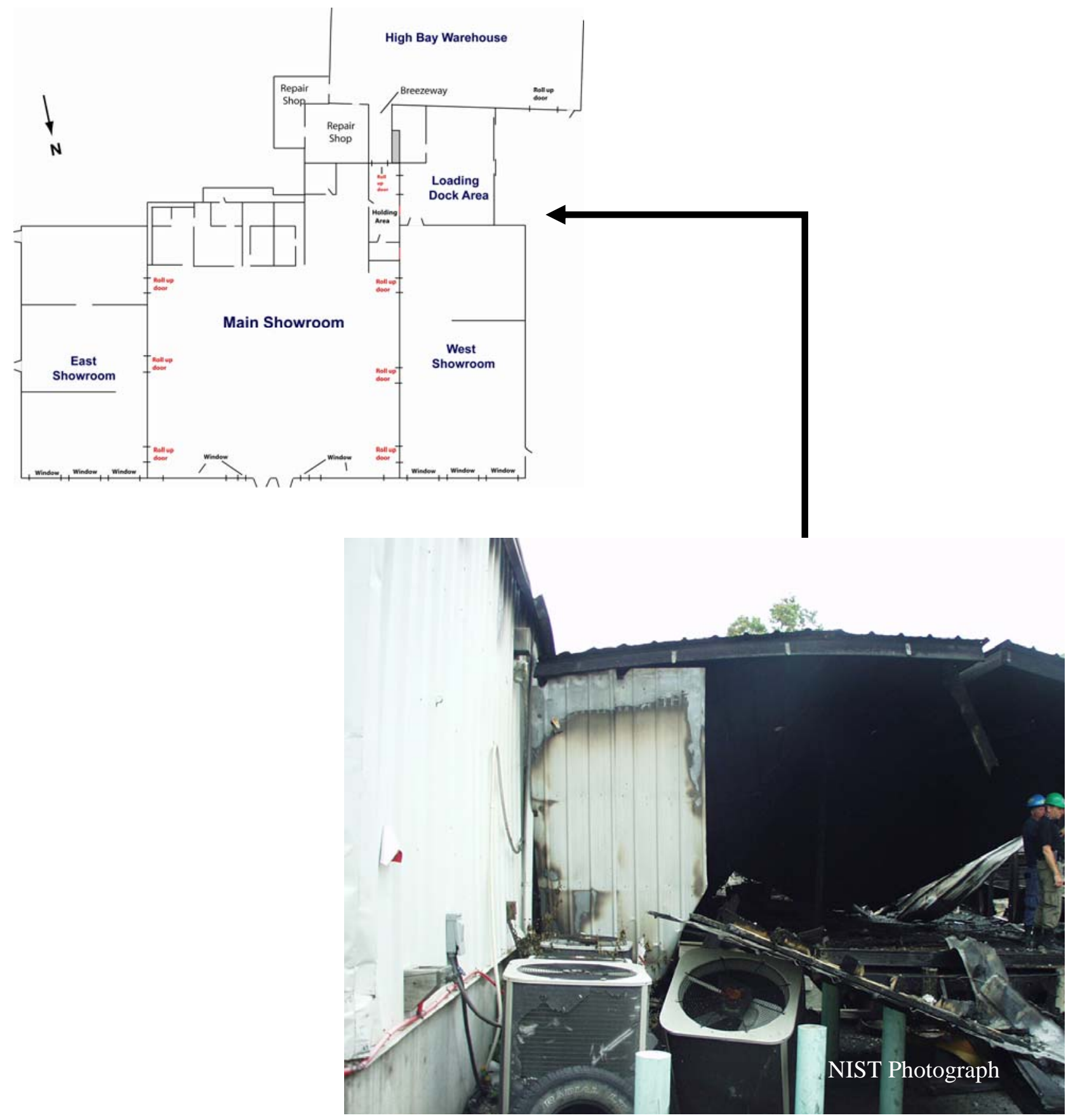

Figure C-2. West wall of loading dock intersecting with rear wall of west showroom. 


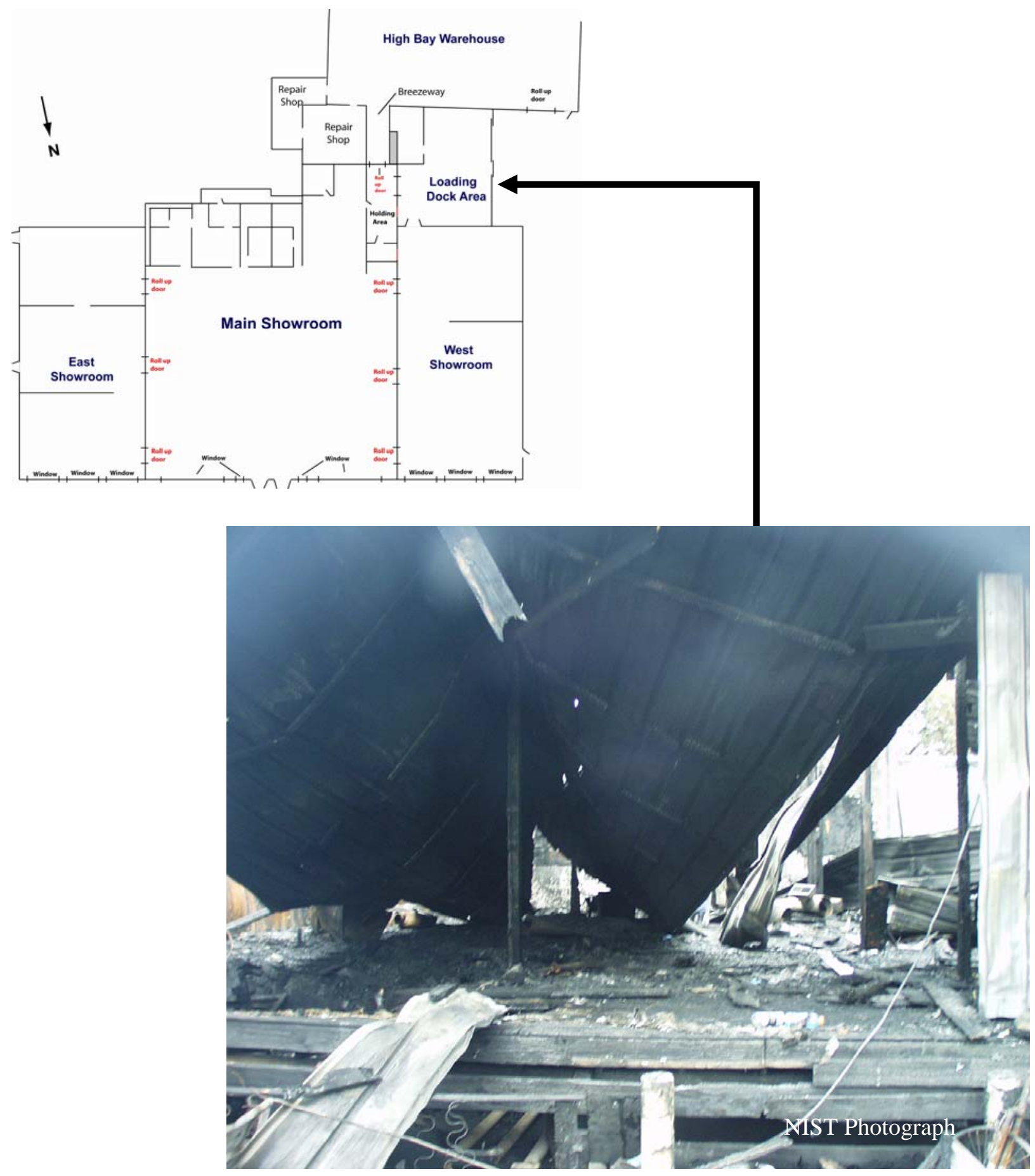

Figure C-3. Remains of center portion of west wall of loading dock. Line of studs on right supported $2.7 \mathrm{~m} \mathrm{(9} \mathrm{ft)} \mathrm{high} \mathrm{section} \mathrm{while} \mathrm{line} \mathrm{of} \mathrm{studs} \mathrm{in}$ left center of image supported $3.7 \mathrm{~m}(12 \mathrm{ft})$ high section. 


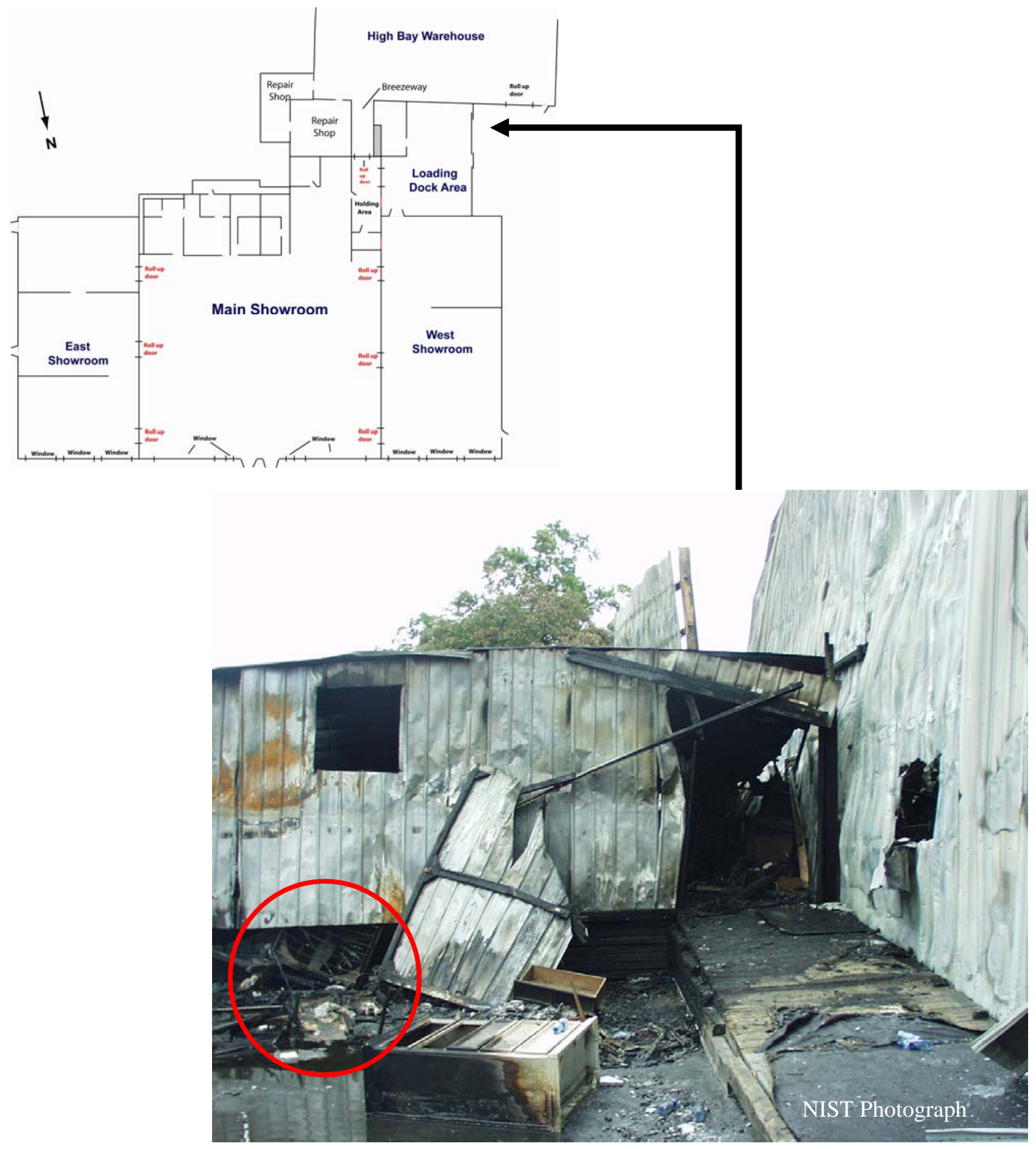

Figure C-4. Right side of west wall of loading dock. Inclined ramp led from warehouse loading dock. Fire ignited in pile of trash outside loading dock (within red circle). 


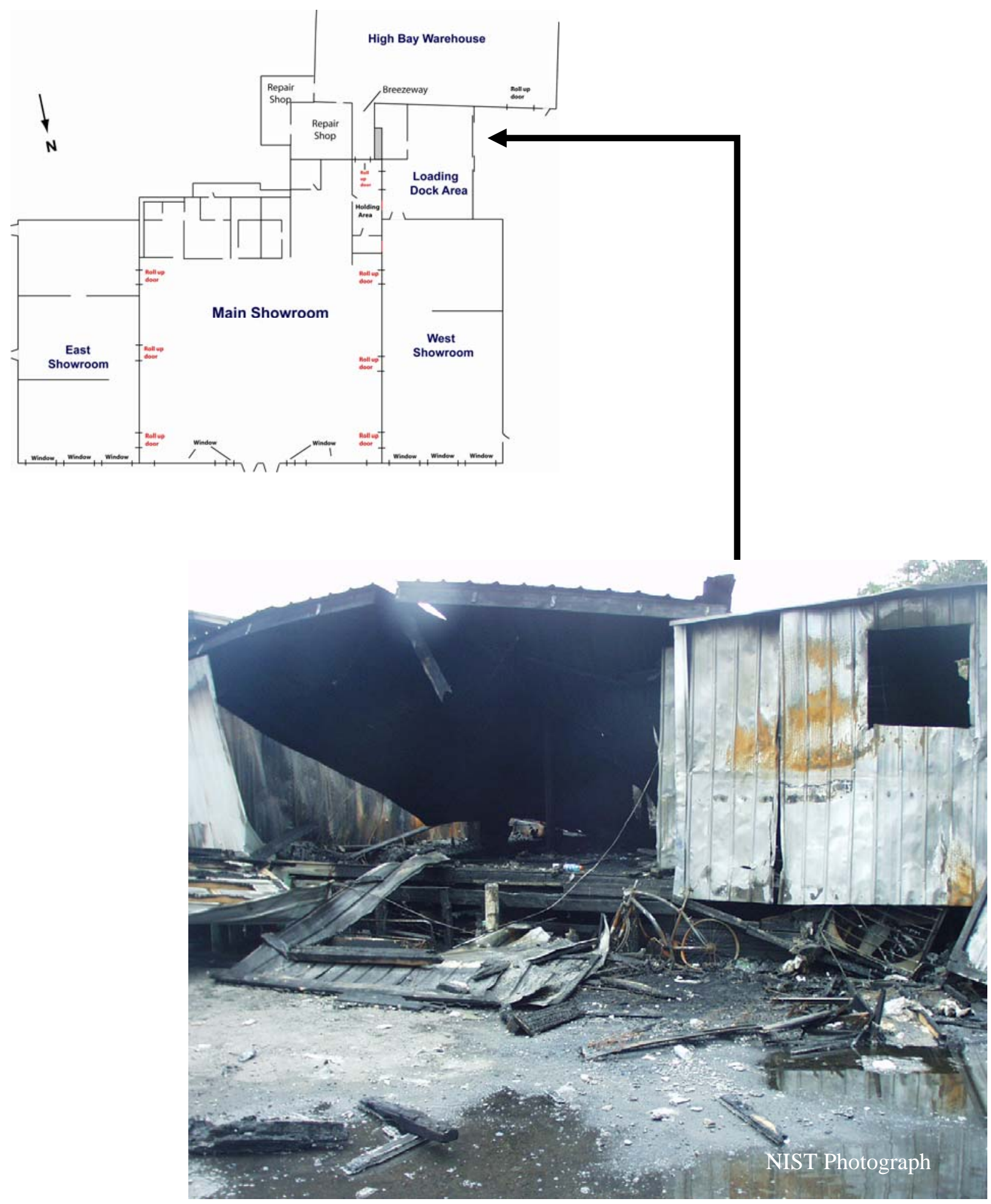

Figure C-5. Left center portion of west wall of loading dock. 


\section{C.2 LOADING DOCK AREA PHOTOS- AFTER REMOVAL OF ROOF}

After the roof was removed from the enclosed loading dock area, west showroom, and the rear of the main showroom, additional photographs were obtained.

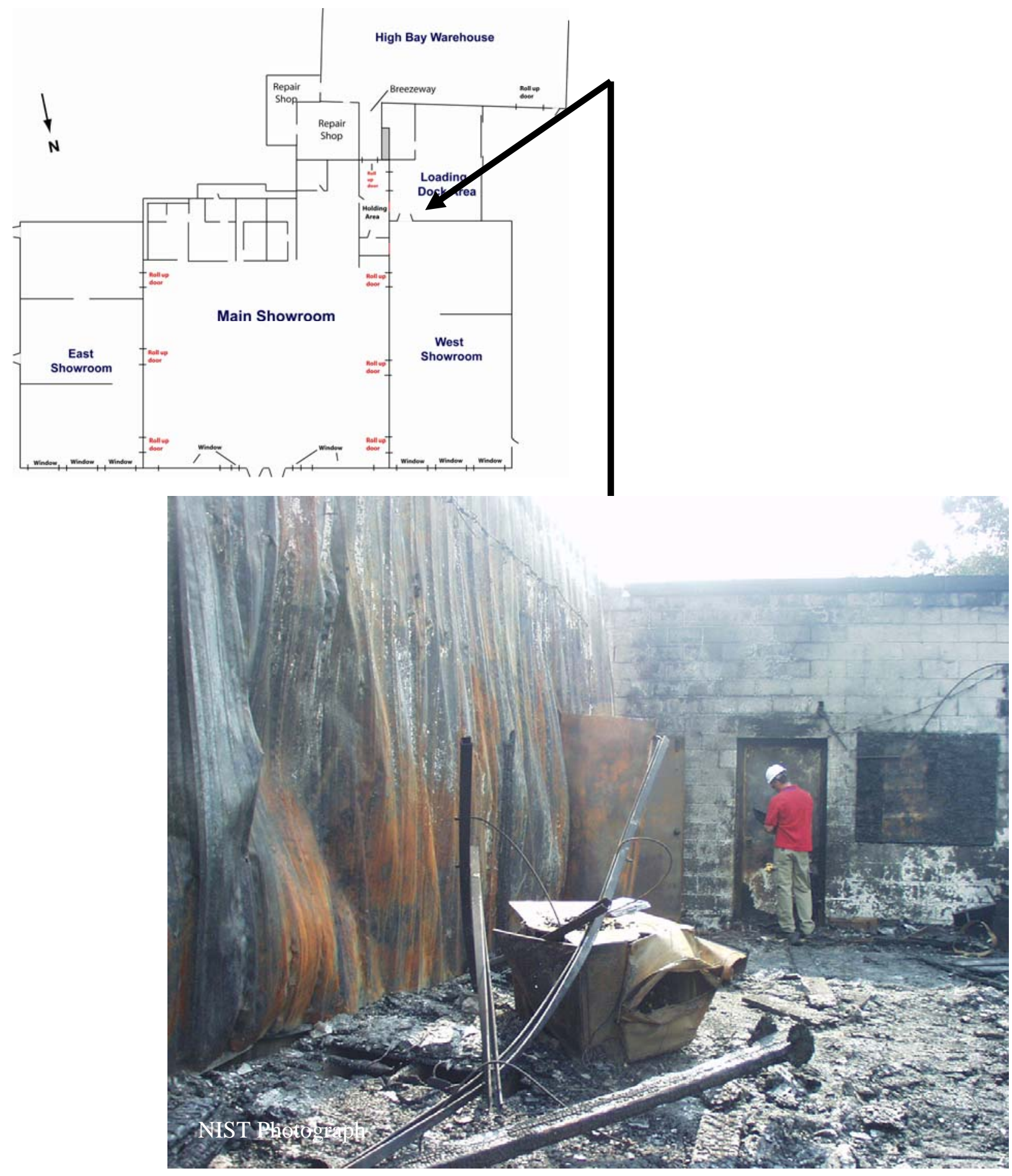

Figure C-6. Inside loading dock area, rear wall of west showroom intersects east wall of loading dock area. 


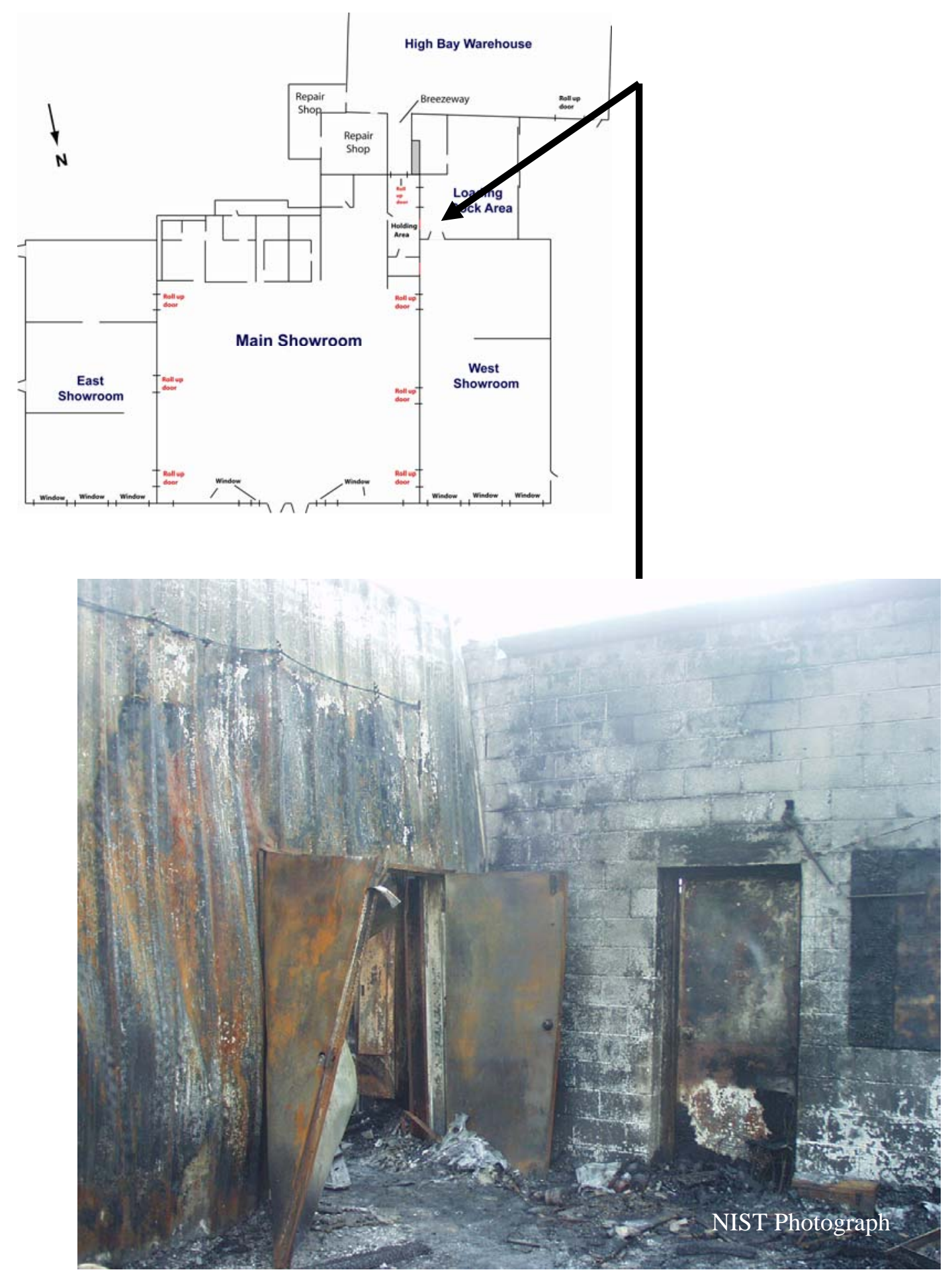

Figure C-7. Rear wall of west showroom intersects rear wall (east wall) of loading dock area. 


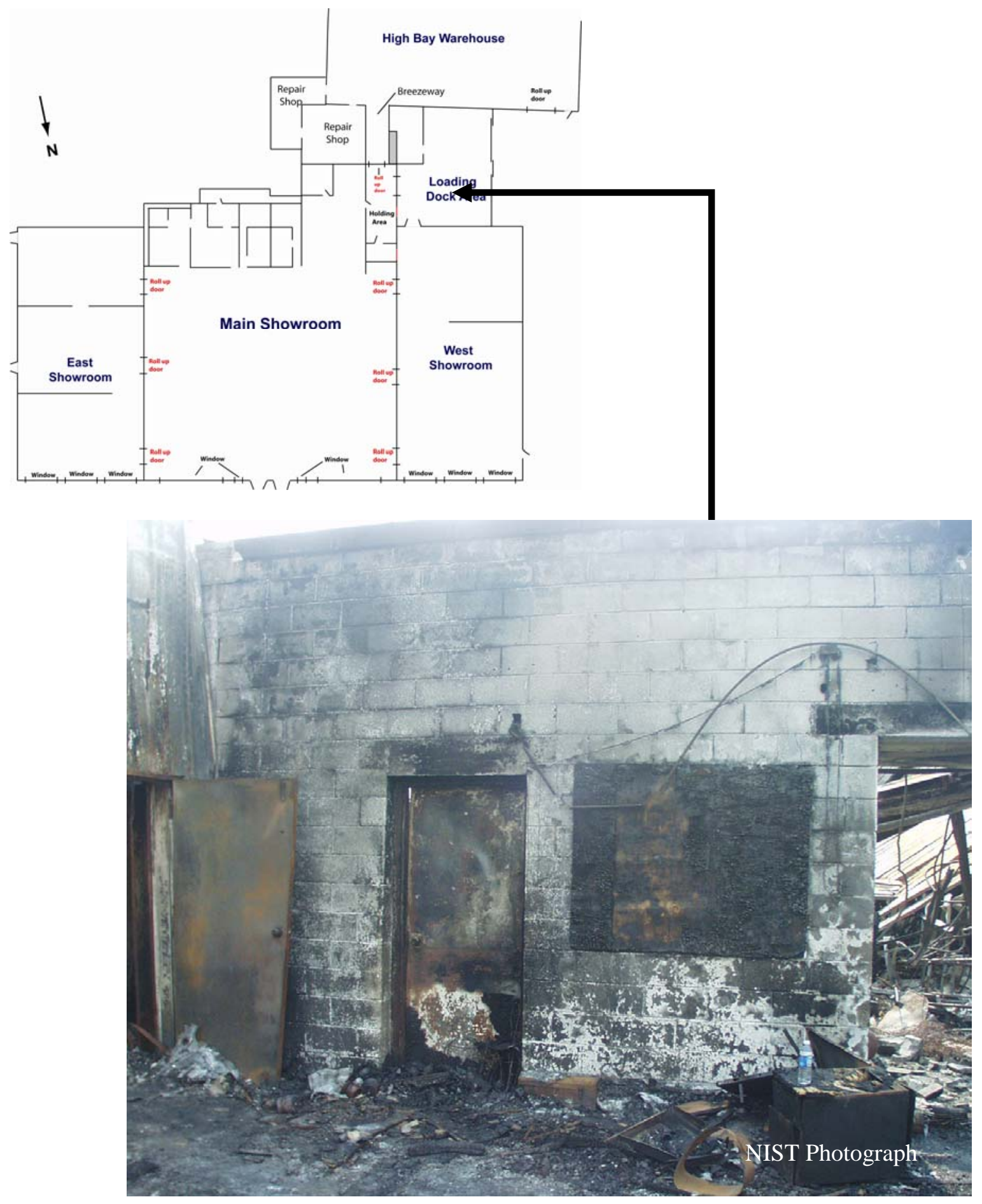

Figure C-8. Rear (east) wall of loading dock area shared with west wall of main showroom. 


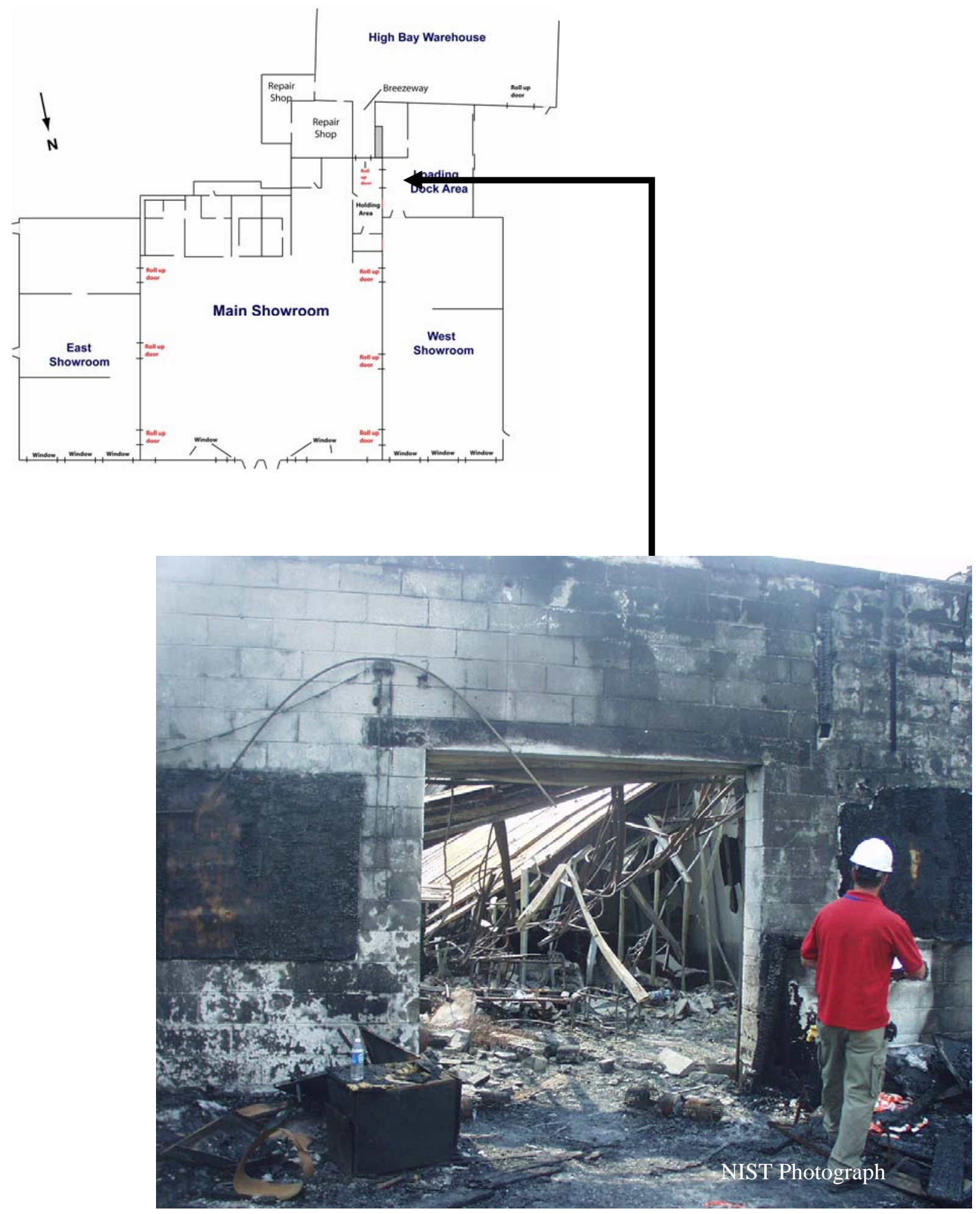

Figure C-9. Non-fire roll-down door between loading dock and holding area as viewed from loading dock. 


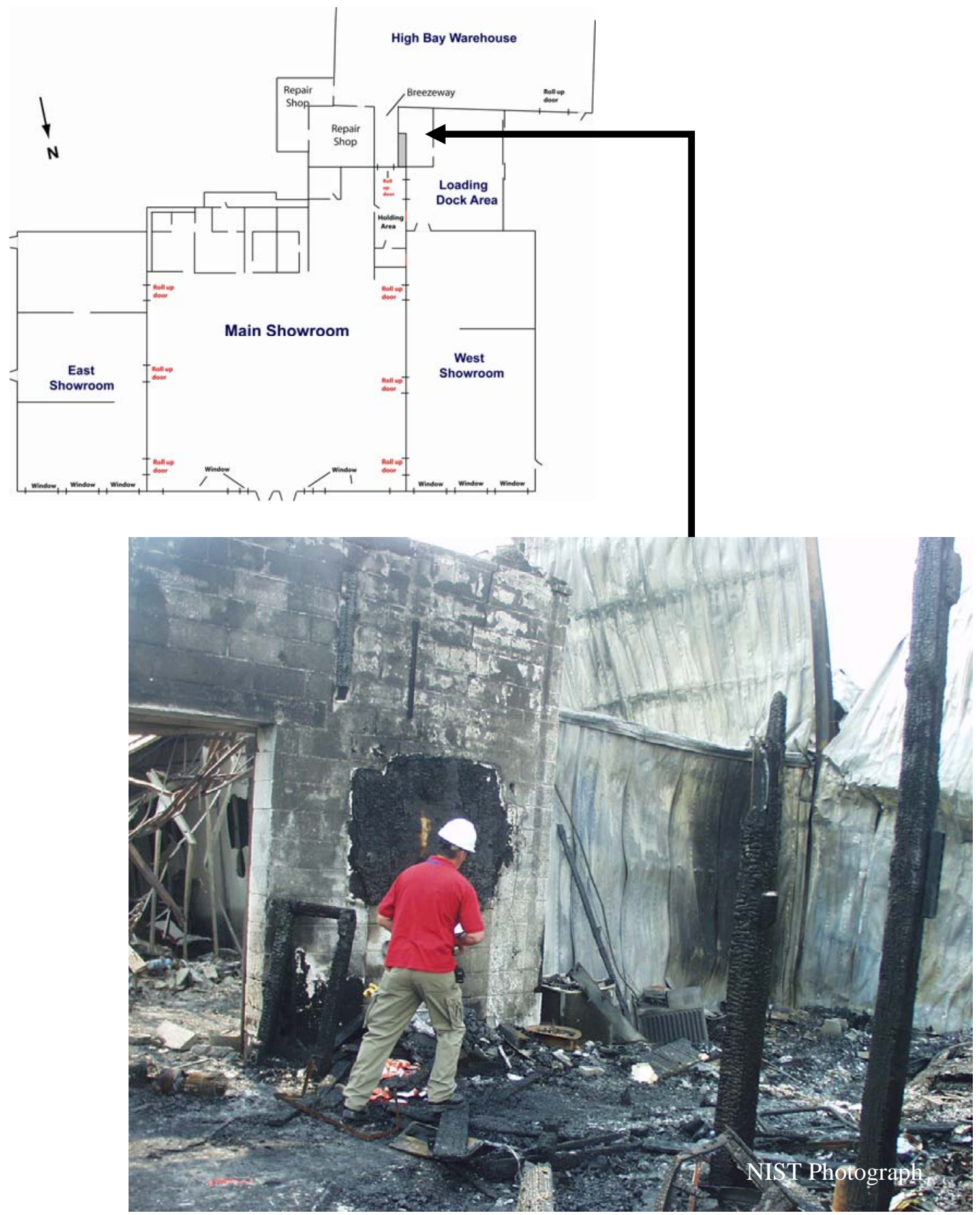

Figure C-10. Rear wall of loading dock area adjacent to holding area. Metal clad breezeway led from rear of holding area to distribution warehouse. 

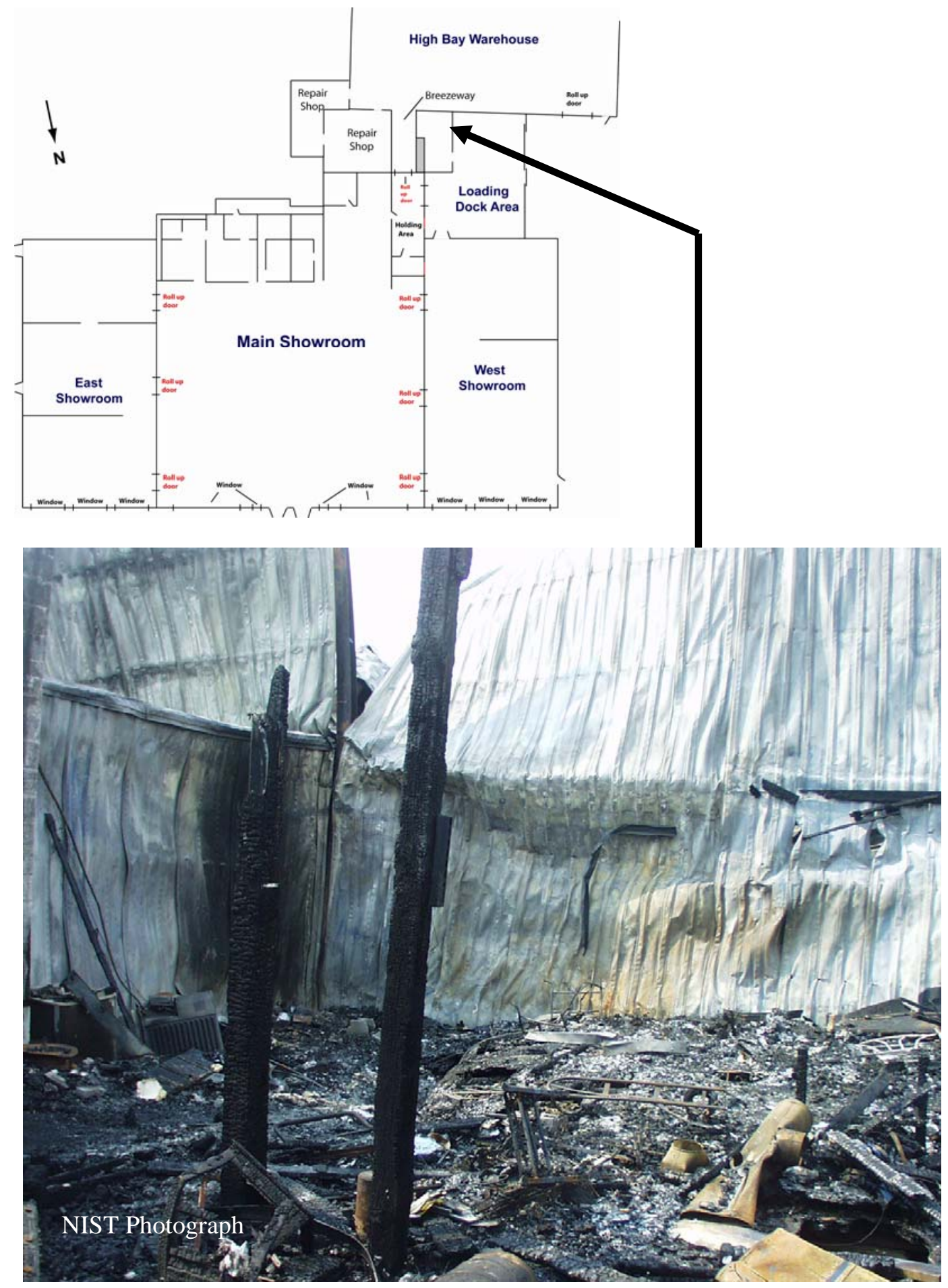

Figure C-11. Rear (east) wall of loading dock area/metal breezeway wall intersect with north wall of warehouse. 


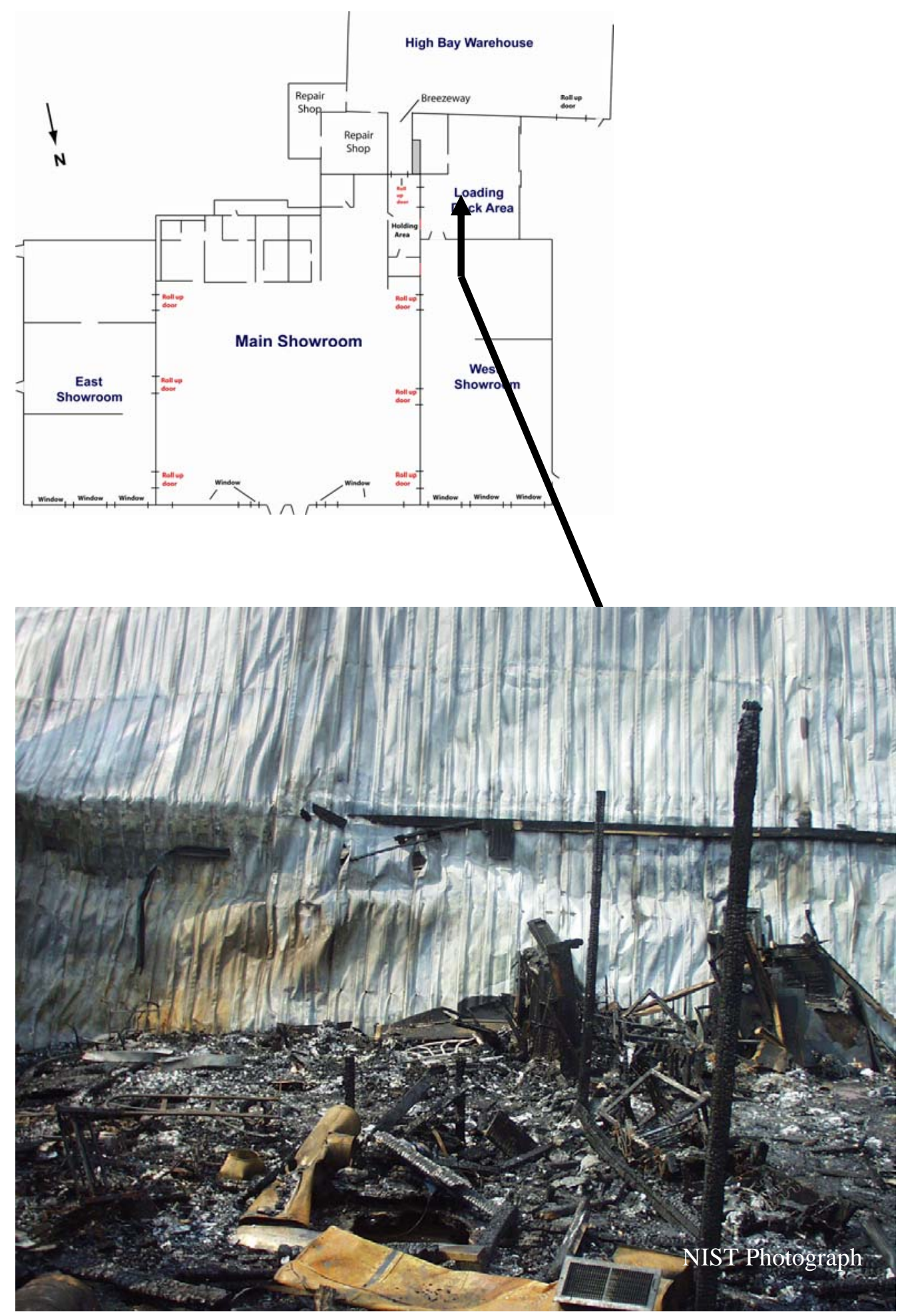

Figure C-12. North wall of warehouse was also south wall of loading dock area. 


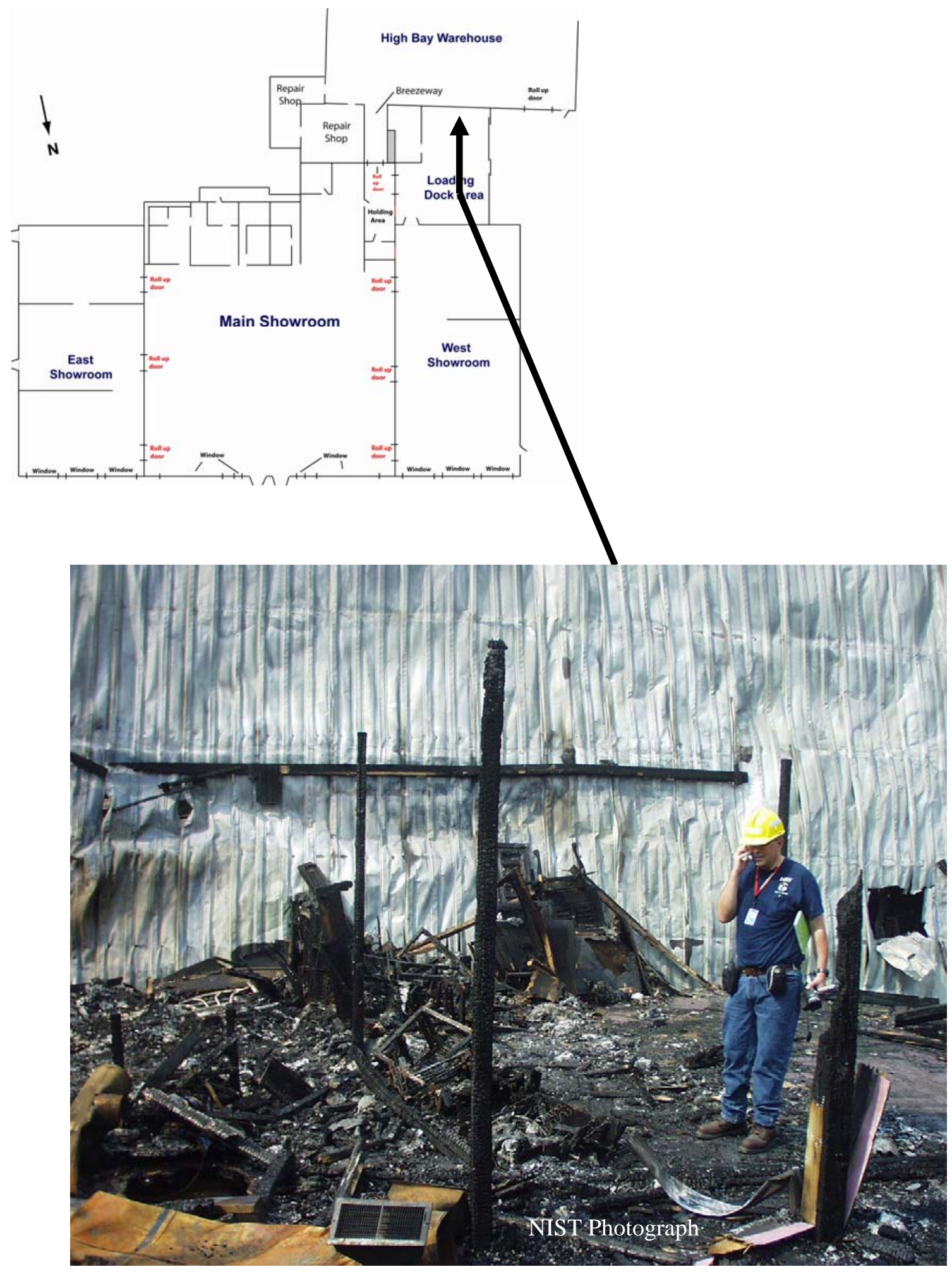

Figure C-13. North wall of warehouse was also south wall of loading dock area. 


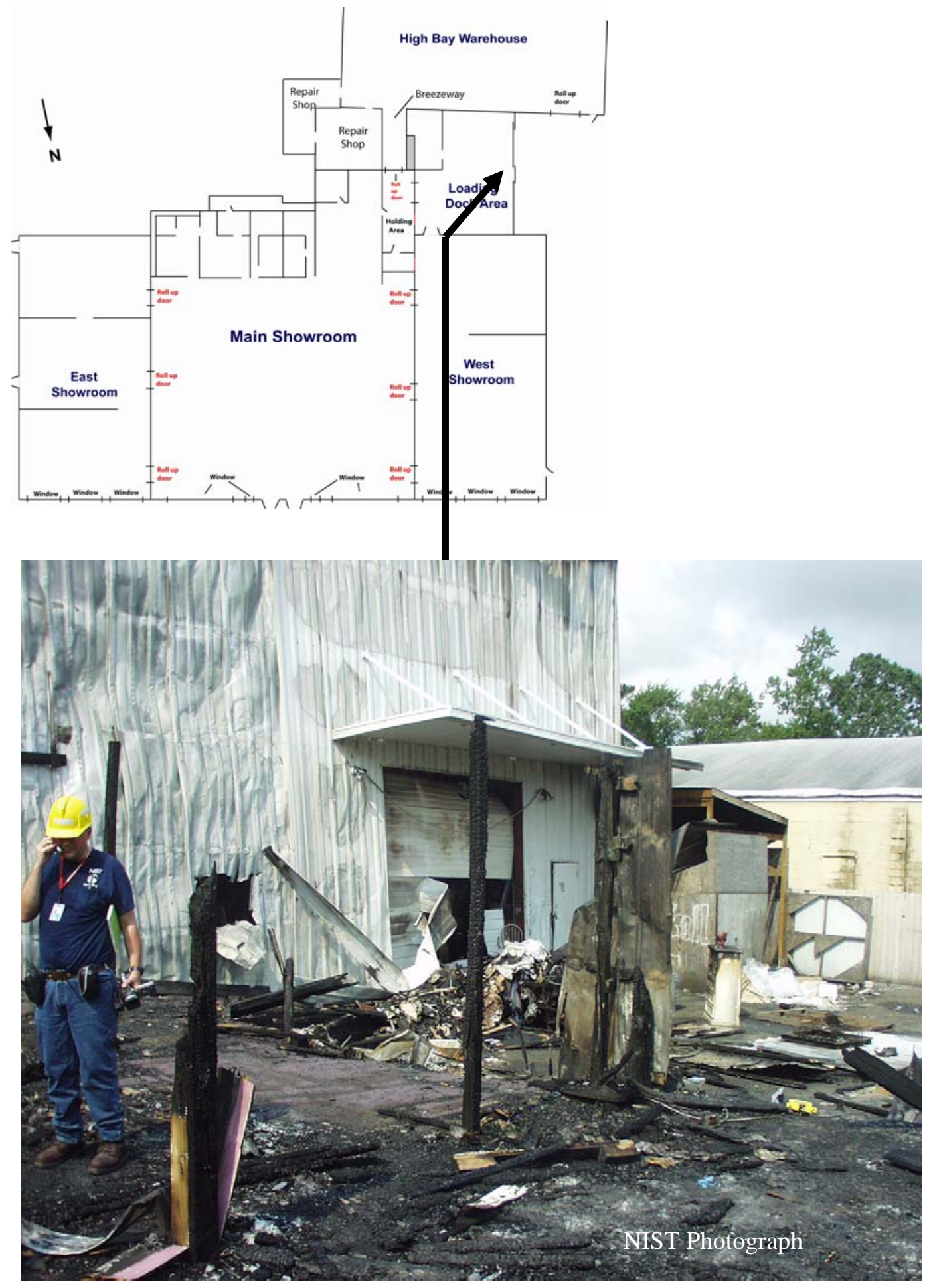

Figure C-14. Loading dock area in foreground and warehouse loading area in background. 


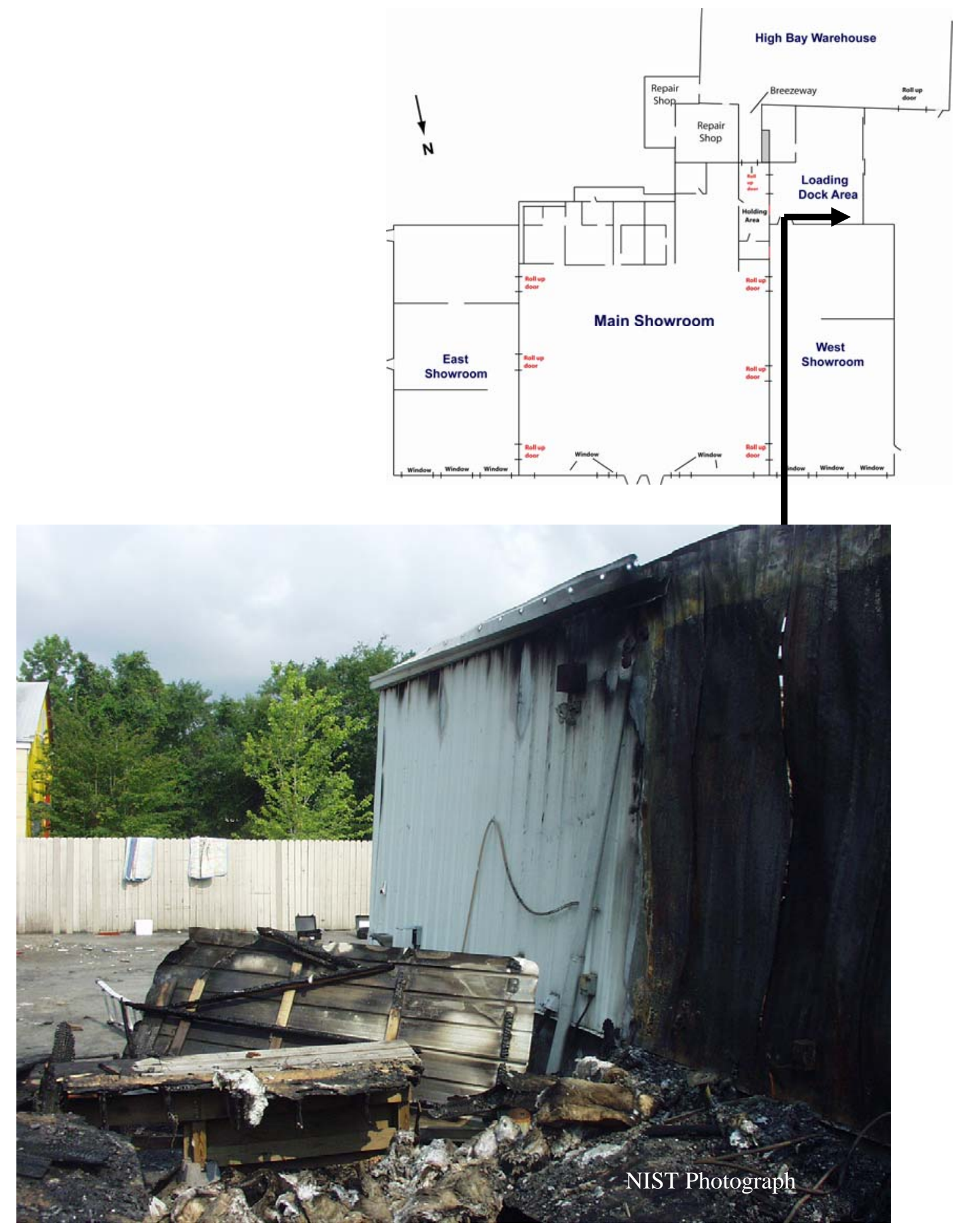

Figure C-15. Loading dock floor in foreground. Rear wall of west showroom on right of photograph. Darker section of rear wall was inside the enclosed loading dock area. Lighter portion of wall was outside enclosed loading dock. 


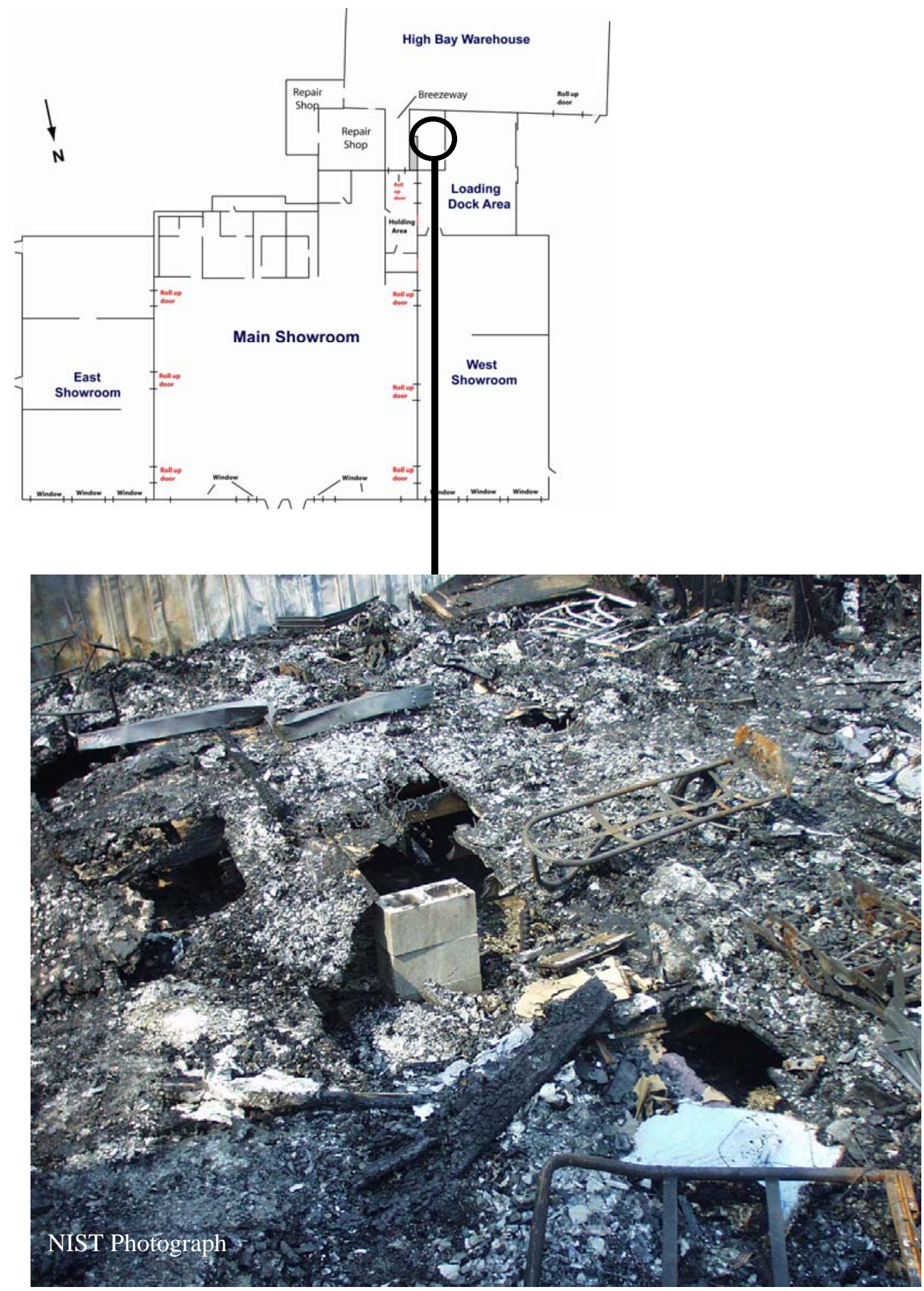

Figure C-16. Portion of enclosed loading dock floor. 


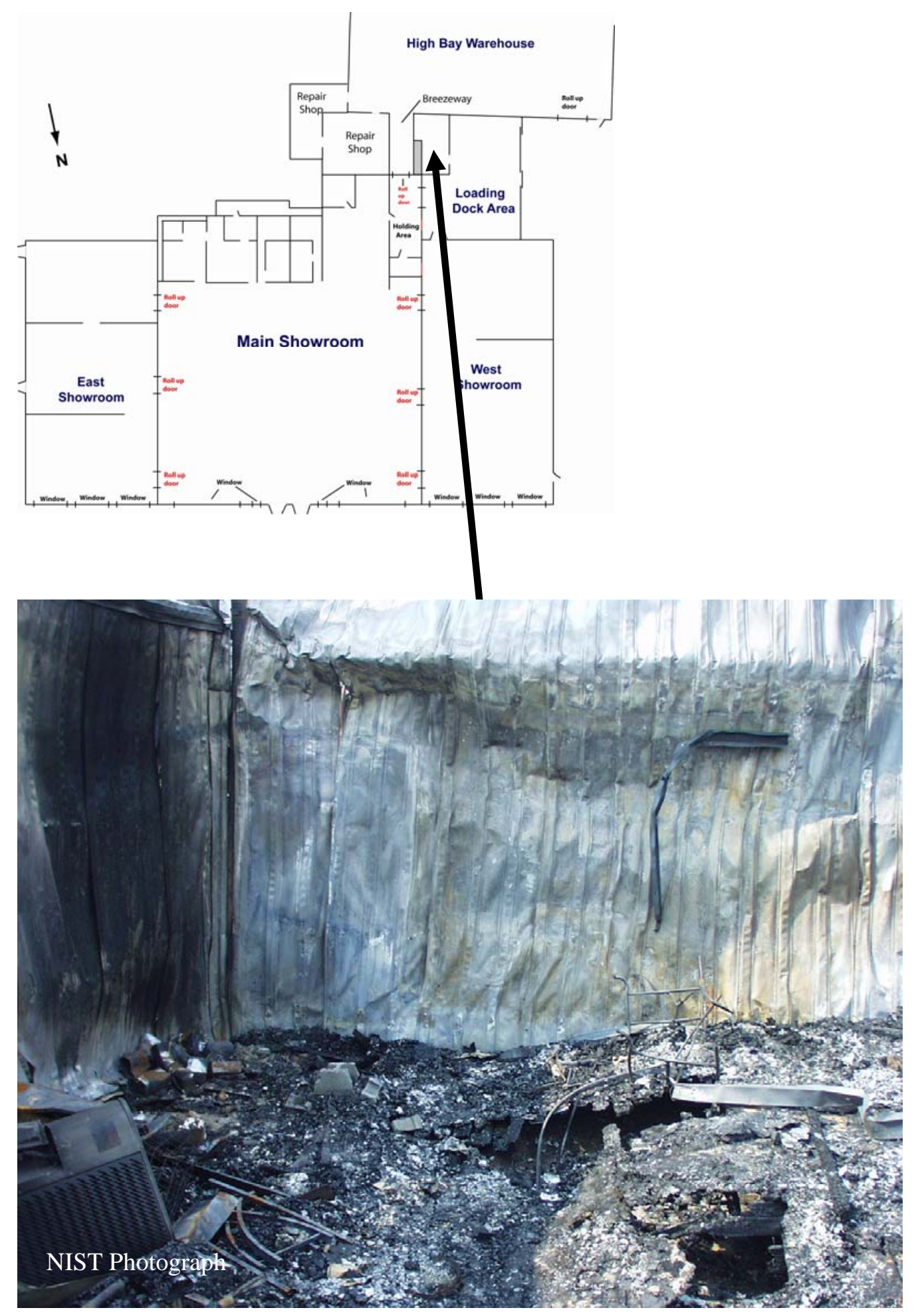

Figure C-17. Intersection of metal clad breezeway wall and north wall of warehouse. 


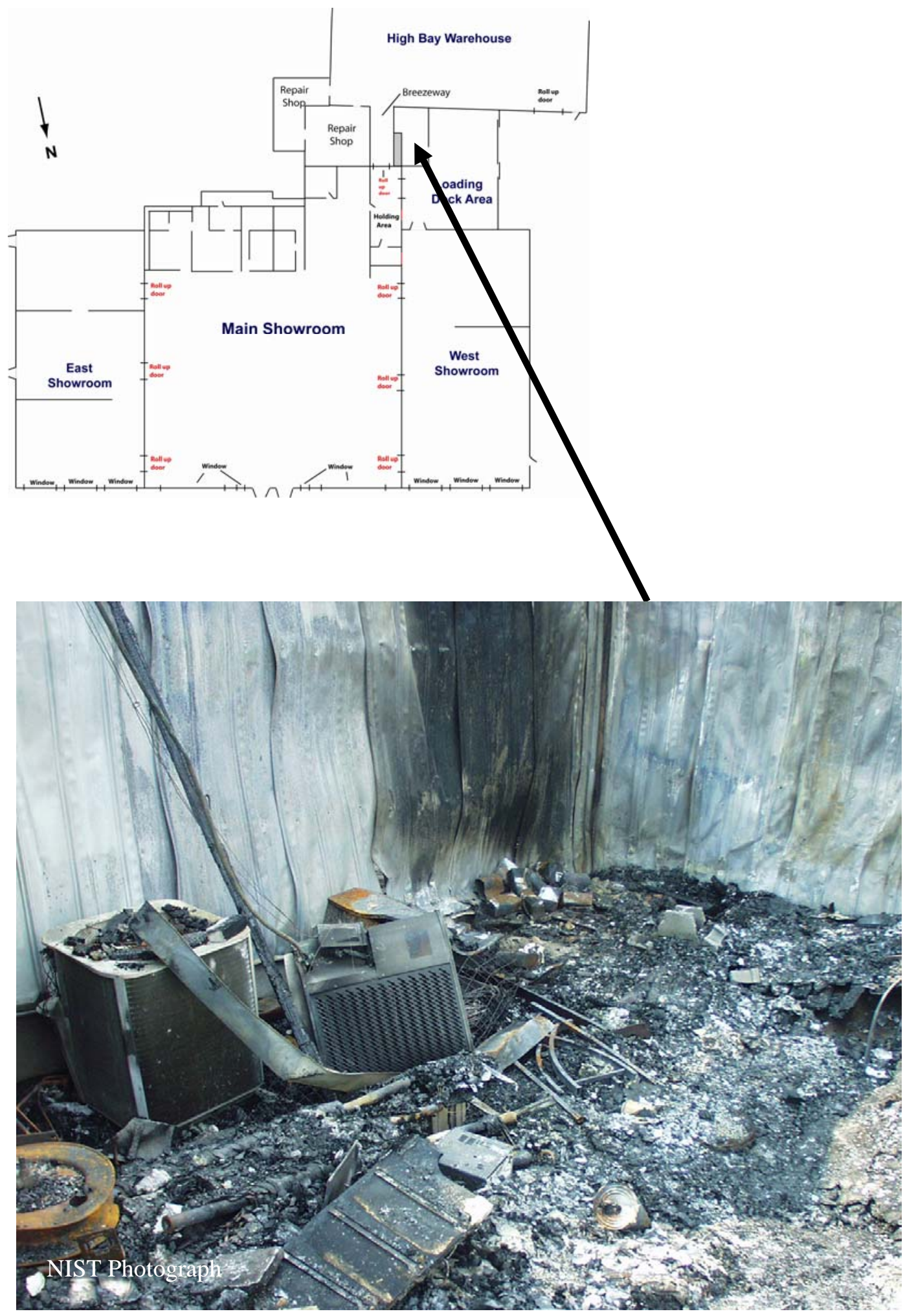

Figure C-18. Intersection of breezeway wall and north wall of warehouse. Heat pumps were located in small courtyard area between loading dock and breezeway wall. Gallon containers of hydrocarbon solvent can be seen at base of breezeway wall. 

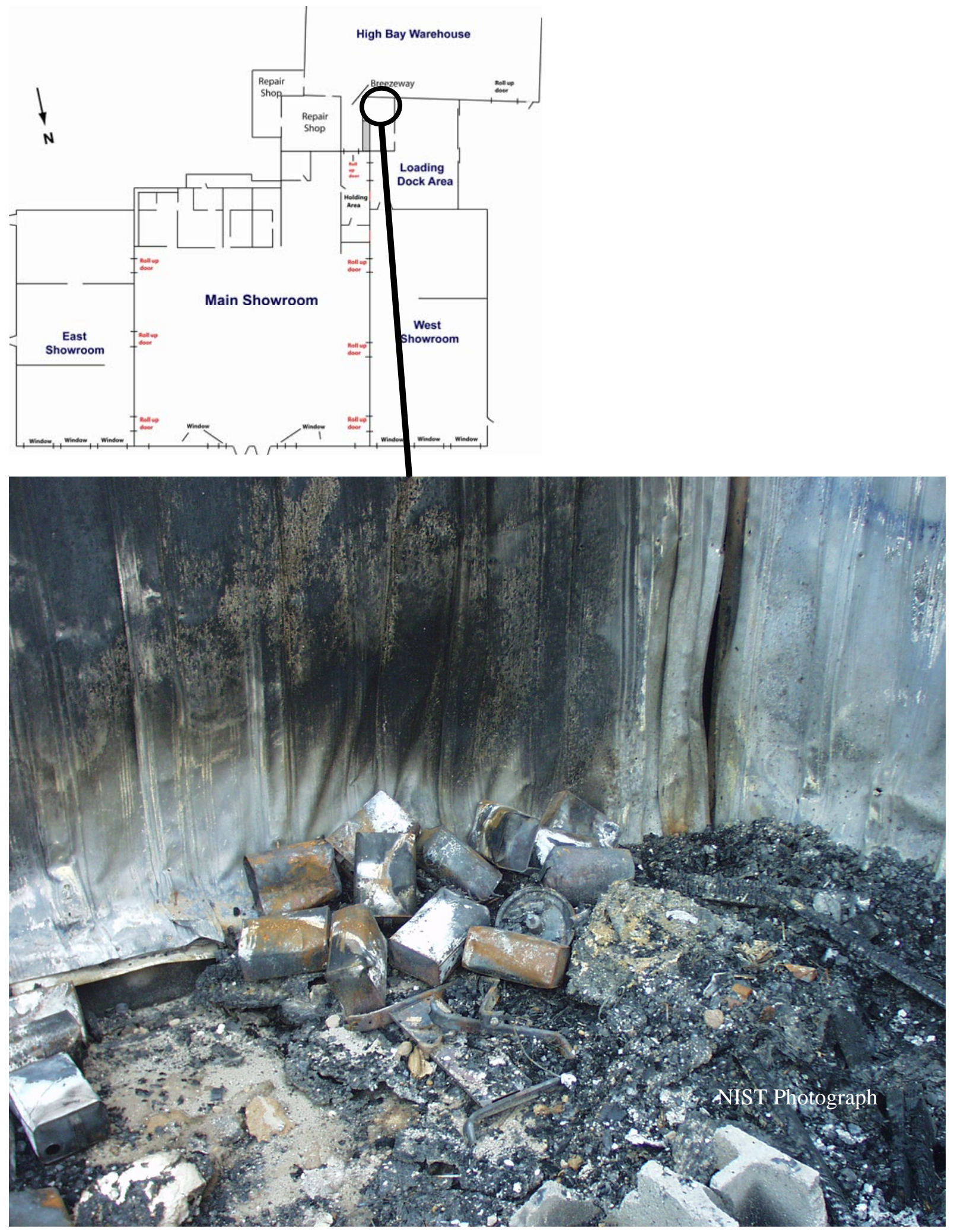

Figure C-19. Close up photograph of some of the solvent containers in rear of loading dock. 


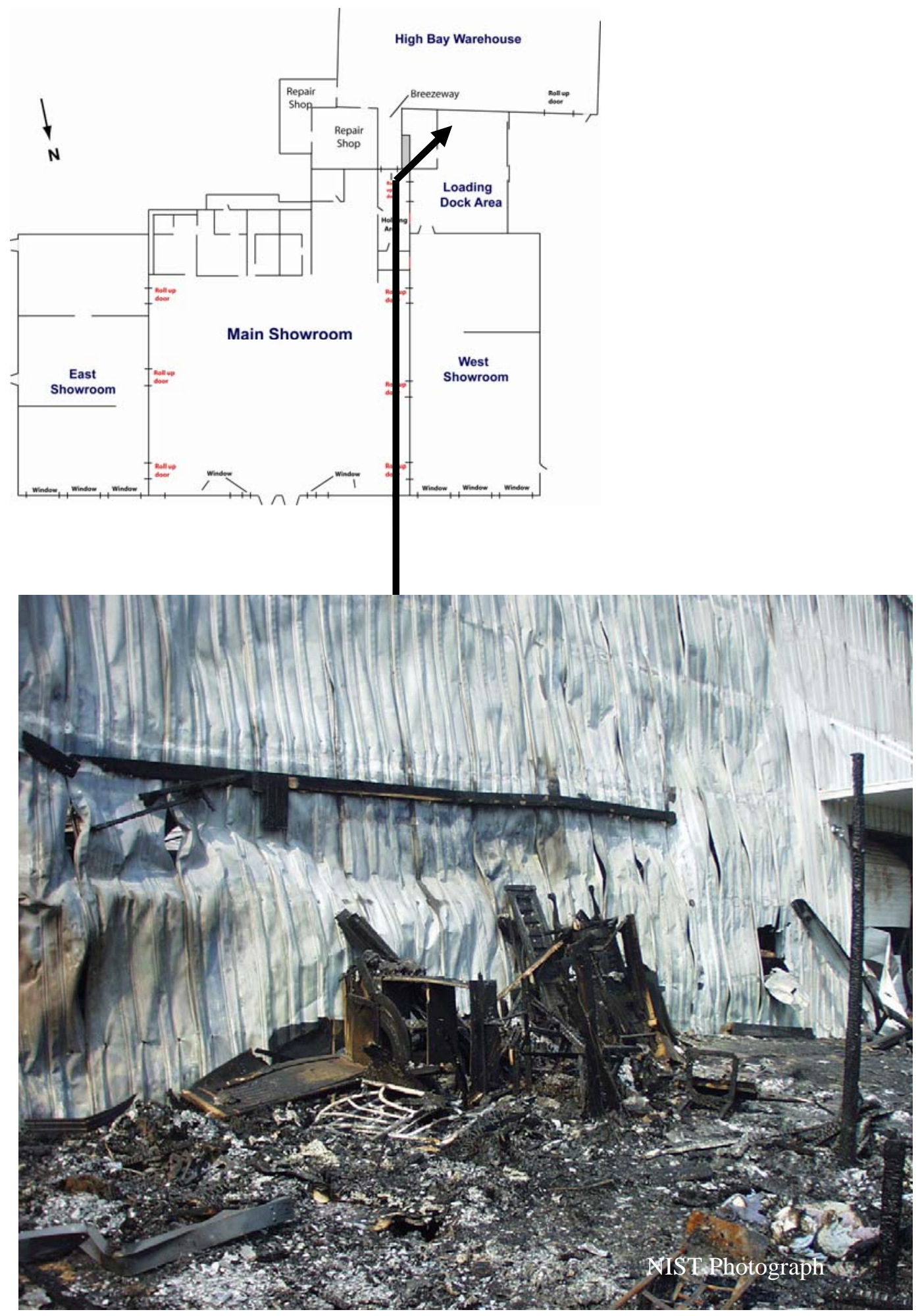

Figure C-20. North wall of warehouse adjacent to loading dock area. 


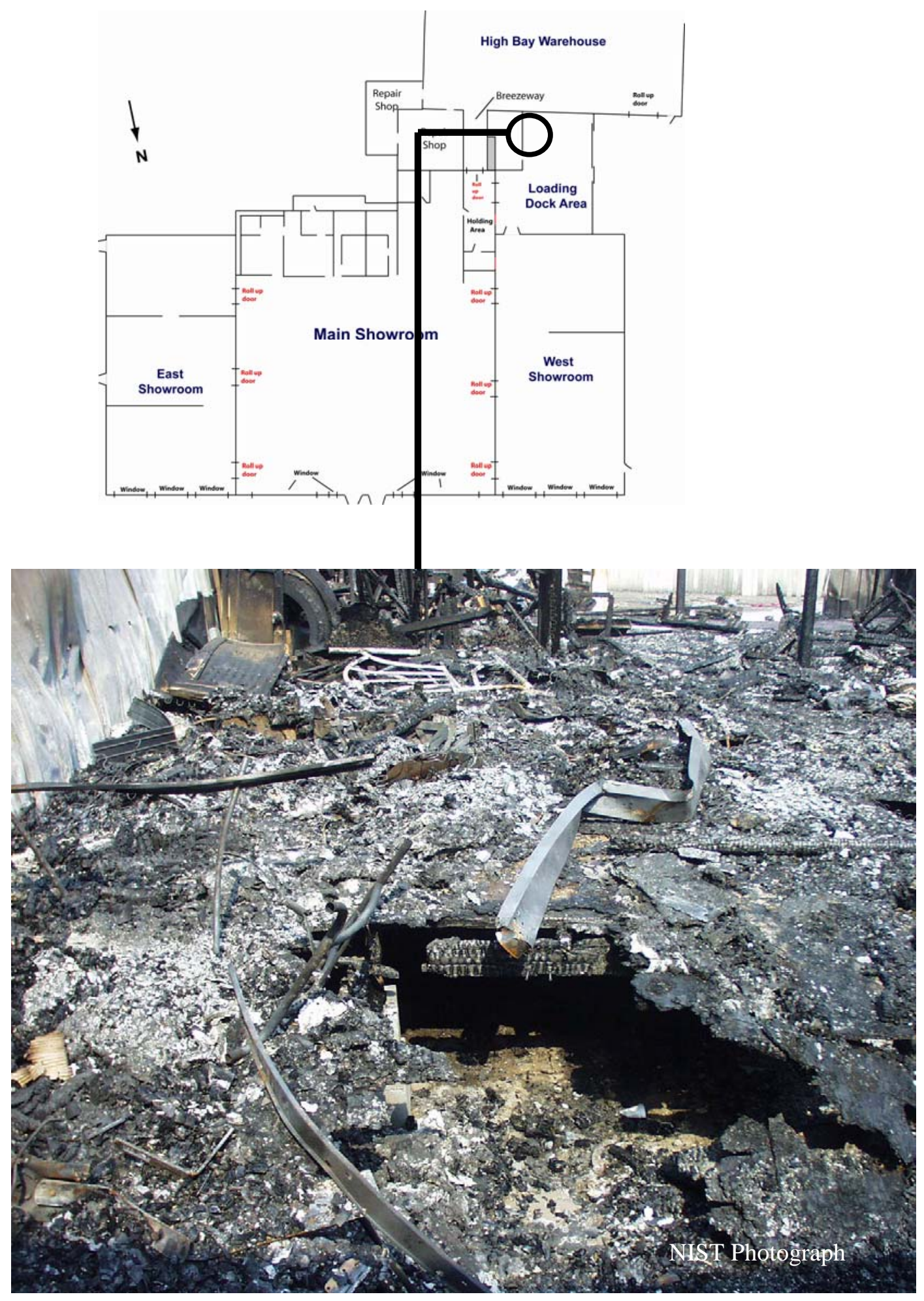

Figure C-21. Floor of enclosed loading dock near warehouse wall. 


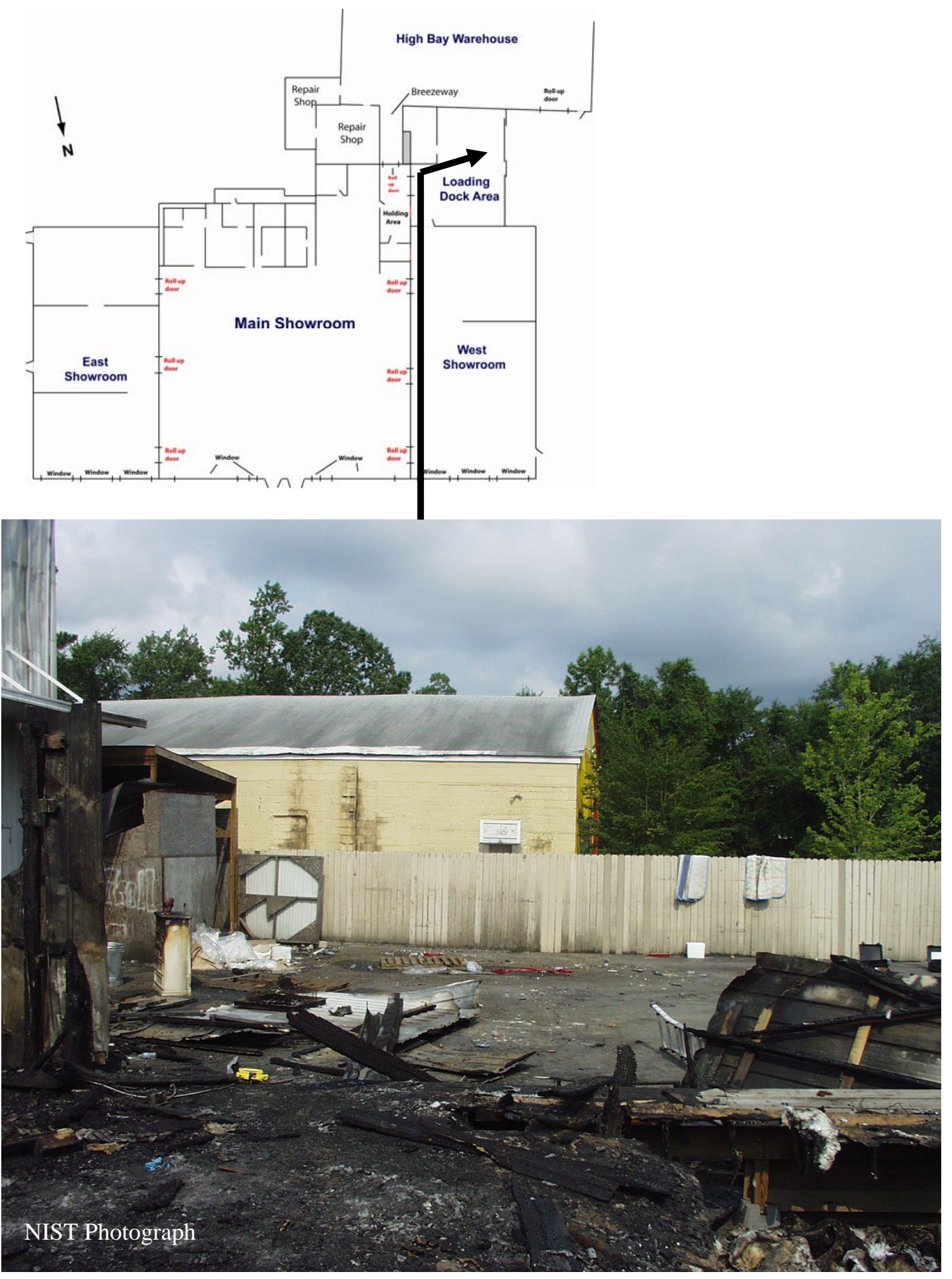

Figure C-22. View of loading area from loading dock. Warehouse loading door is in left of photograph. 


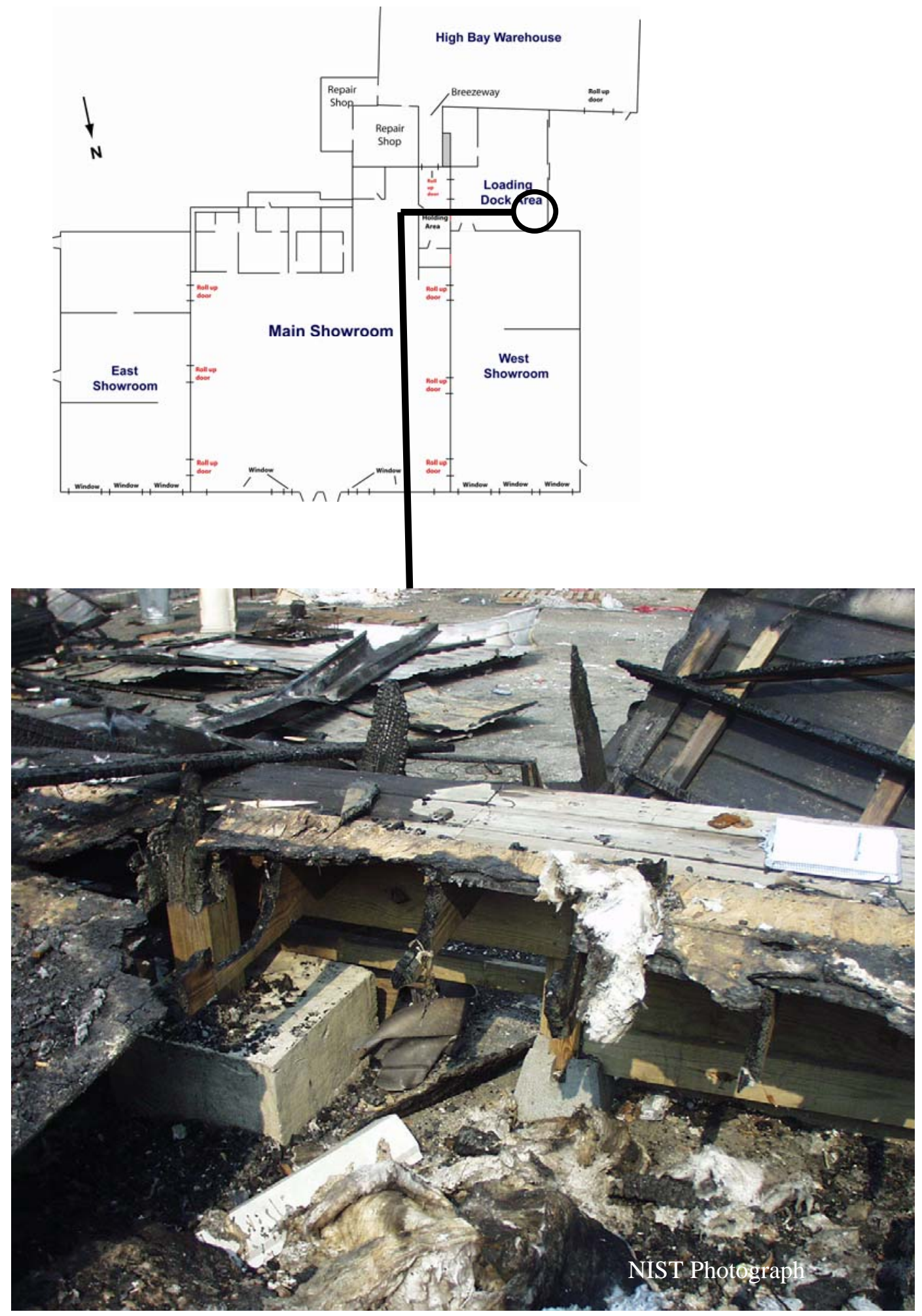

Figure C-23. Floor of enclosed loading dock near rear of west showroom. 


\section{APPENDIX D. MAIN SHOWROOM}

\section{D.1 MAIN SHOWROOM EXTERIOR PHOTOS - BEFORE REMOVAL OF WALL AND ROOF}

A limited series of photographs were obtained of the front (north) and rear (south) walls of the main showroom before the collapsed façade was removed from the front of the store.

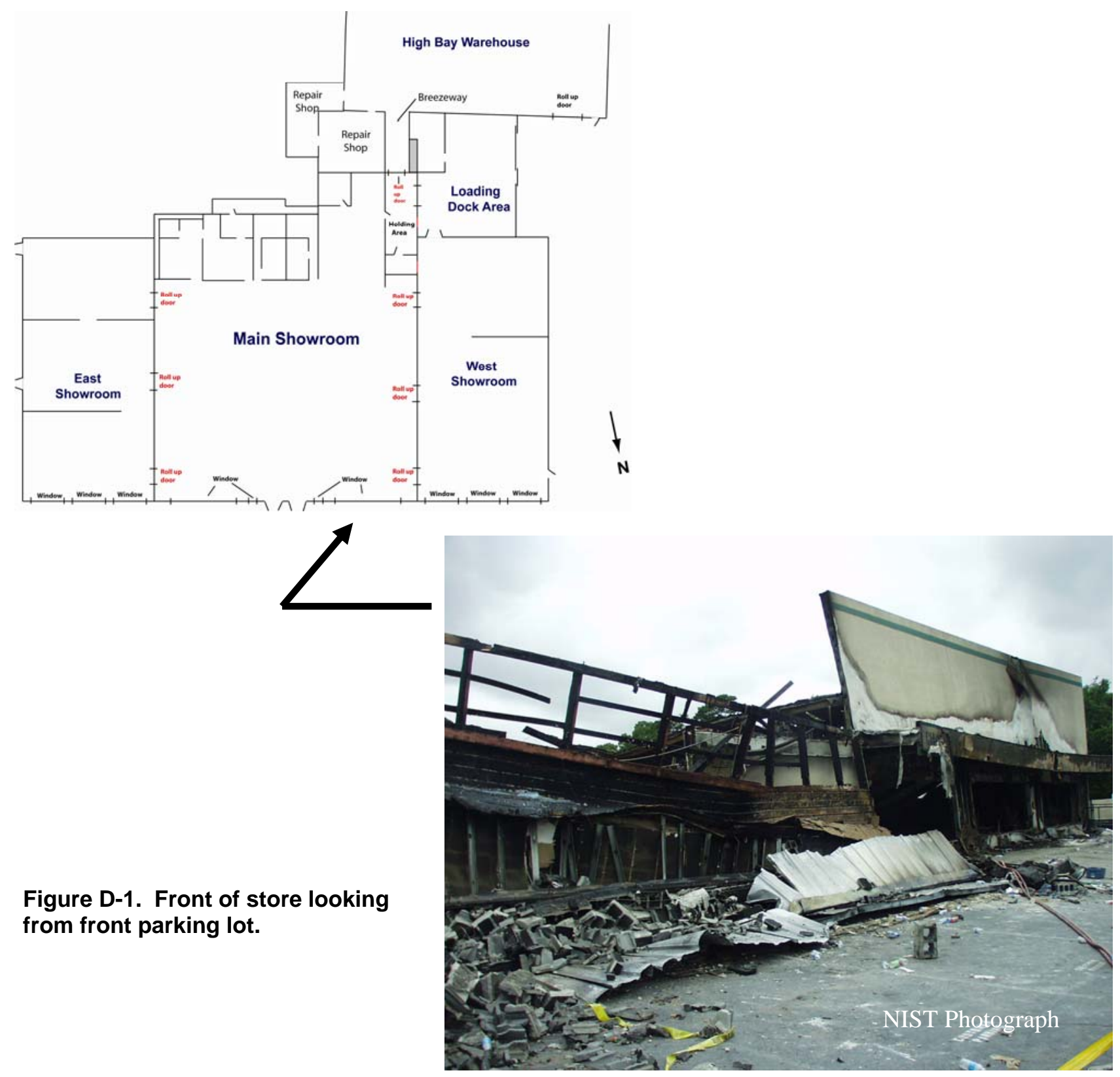



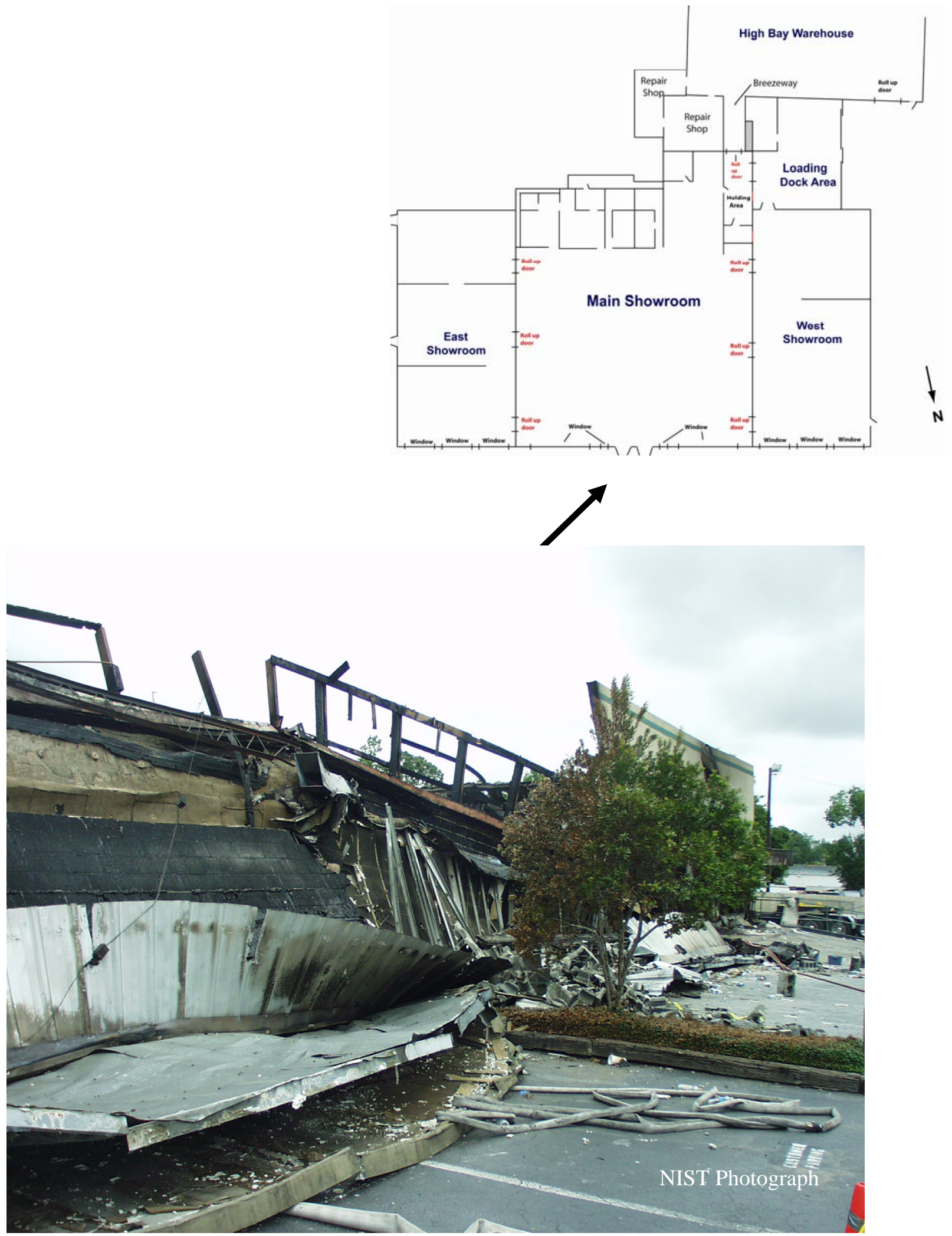

Figure D-2. Front of main showroom. Façade and parapet wall of main showroom collapsed into parking spaces. 


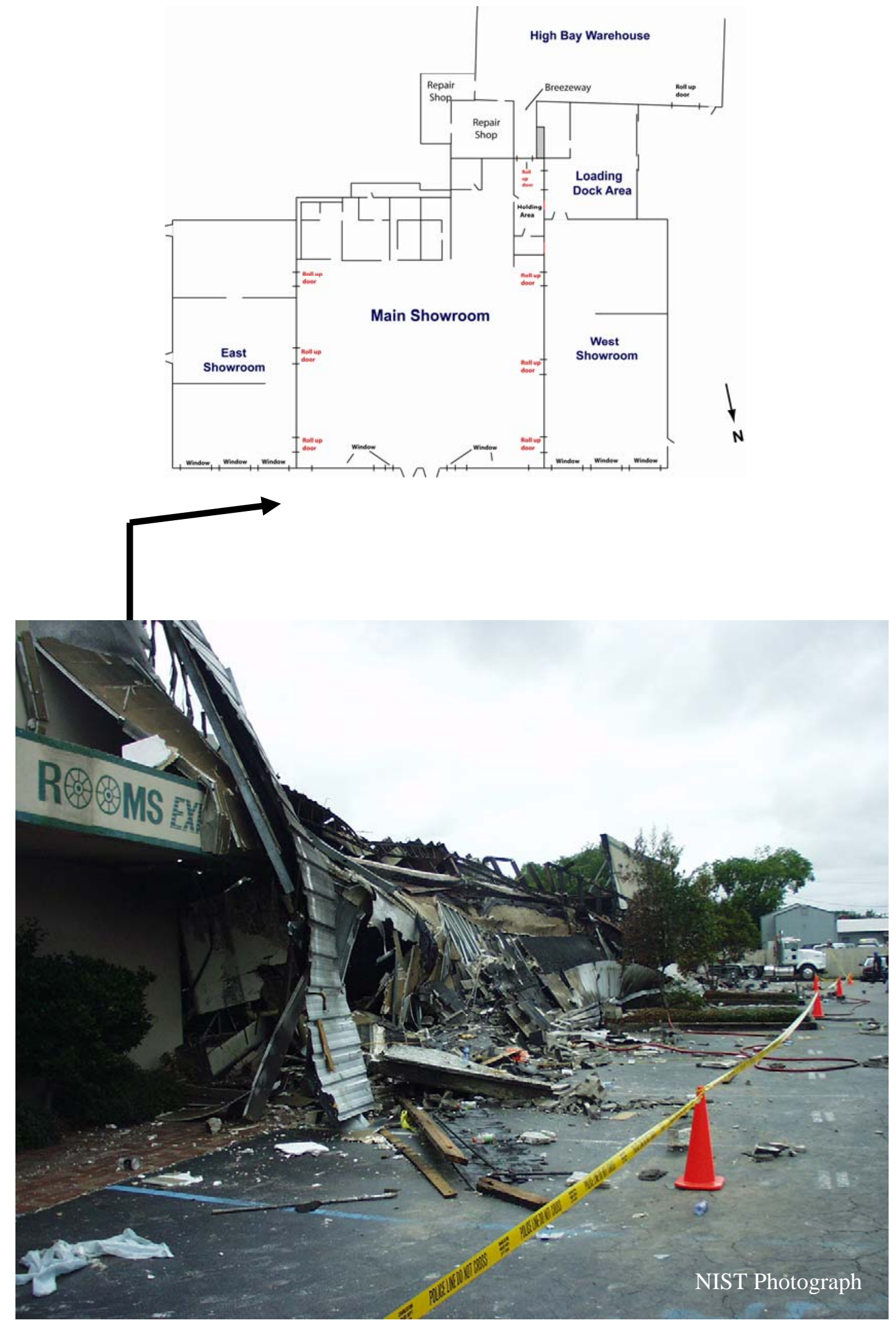

Figure D-3. Front of main showroom. Facade and parapet wall of main showroom collapsed into parking spaces. 

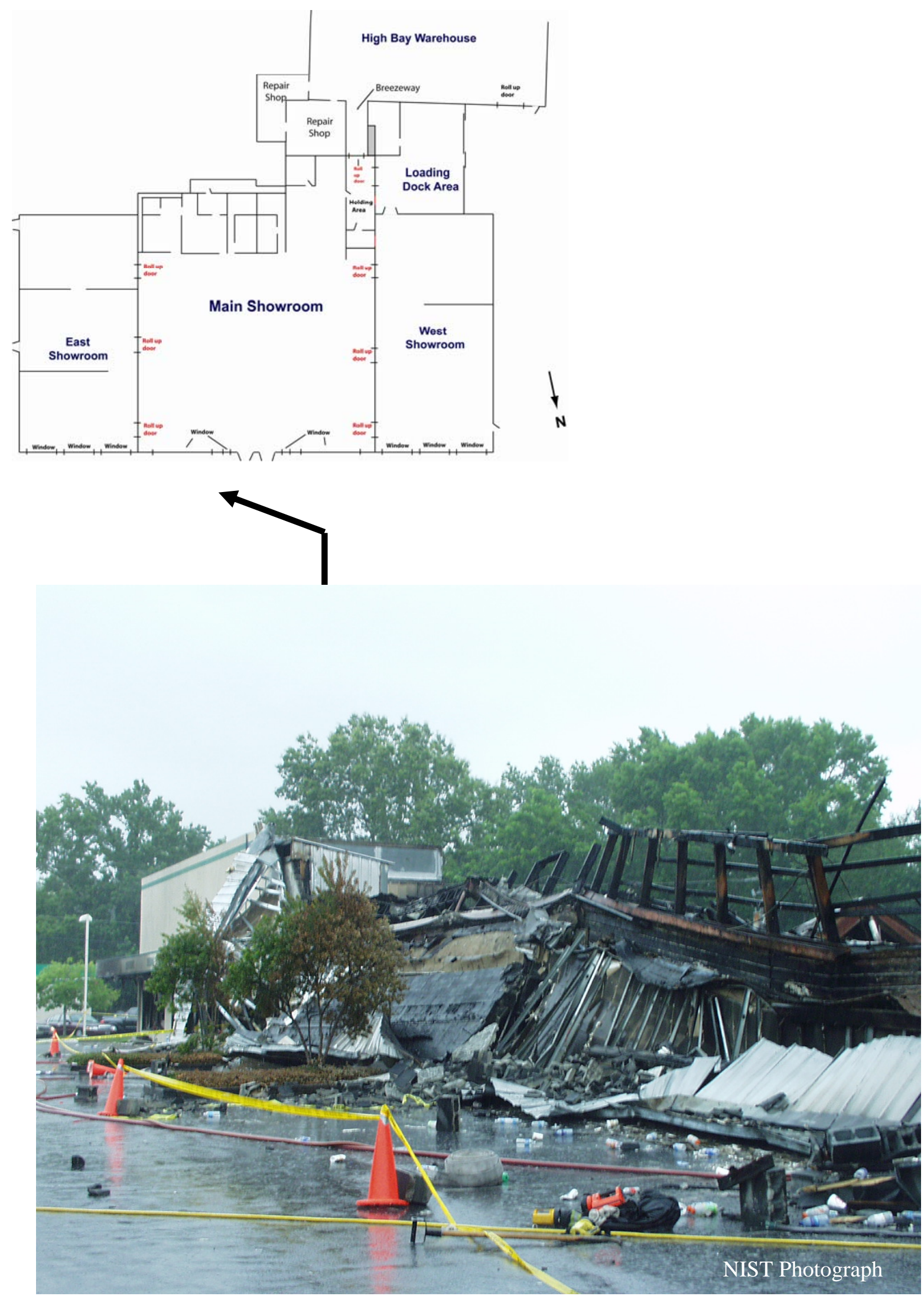

Figure D-4. Front of main showroom looking towards east showroom. 


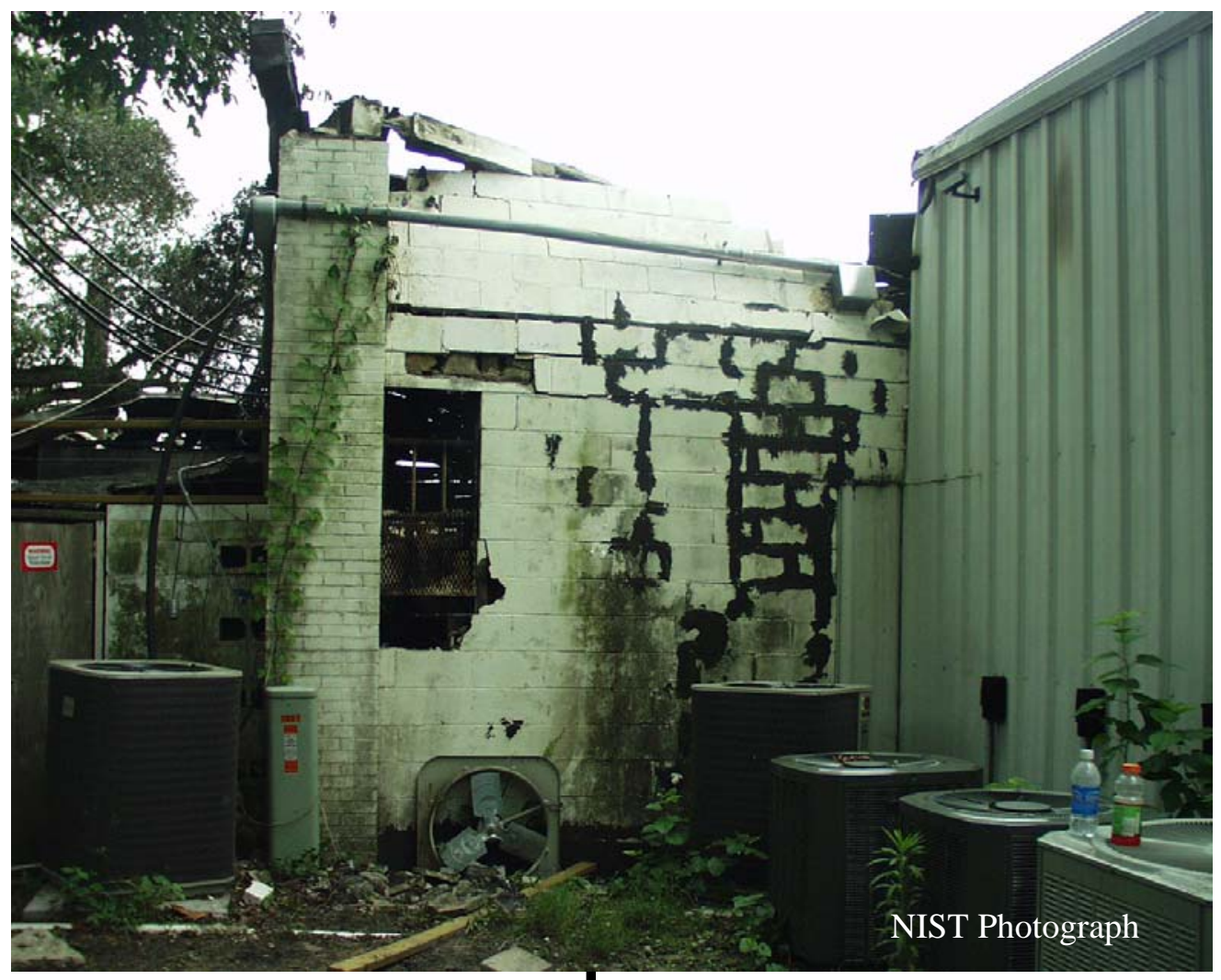

Figure D-5. Rear of main showroom.

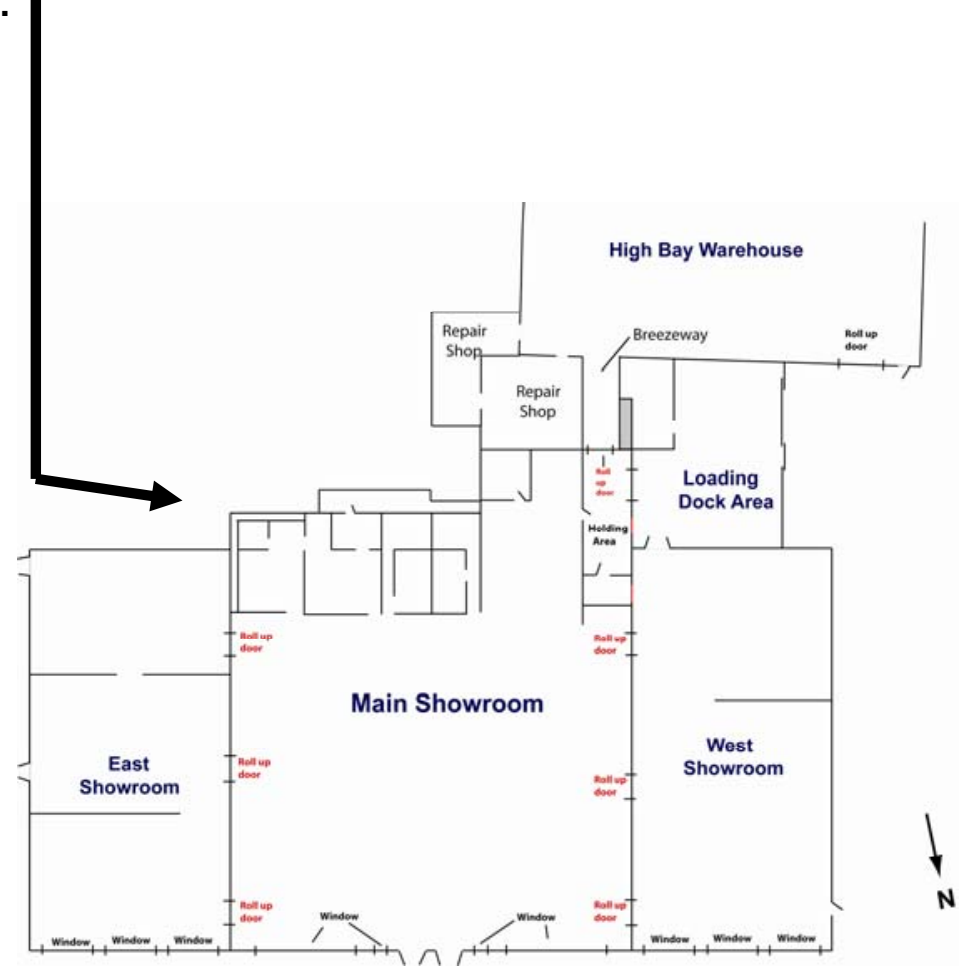




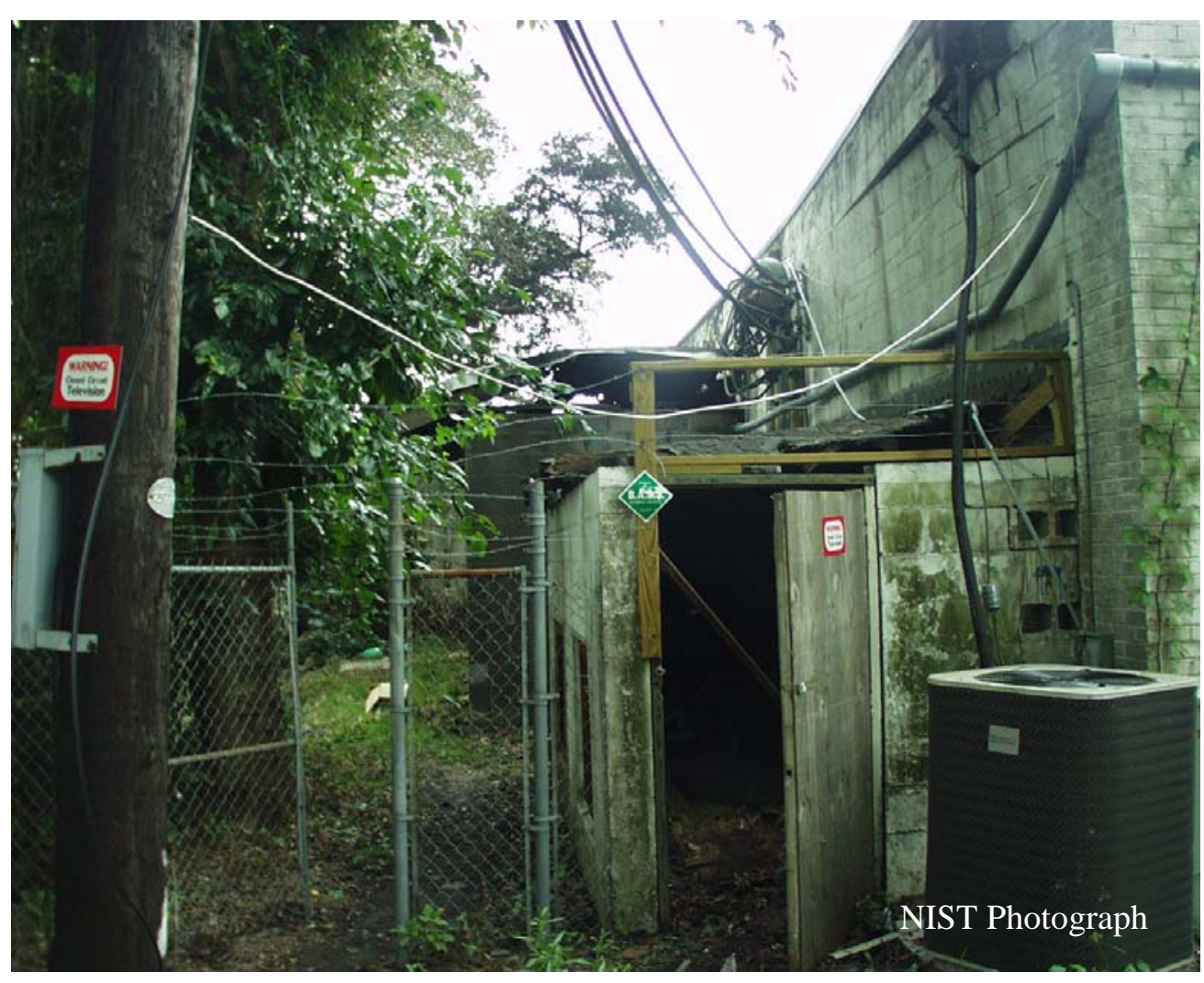

Figure D-6. Rear of main showroom.

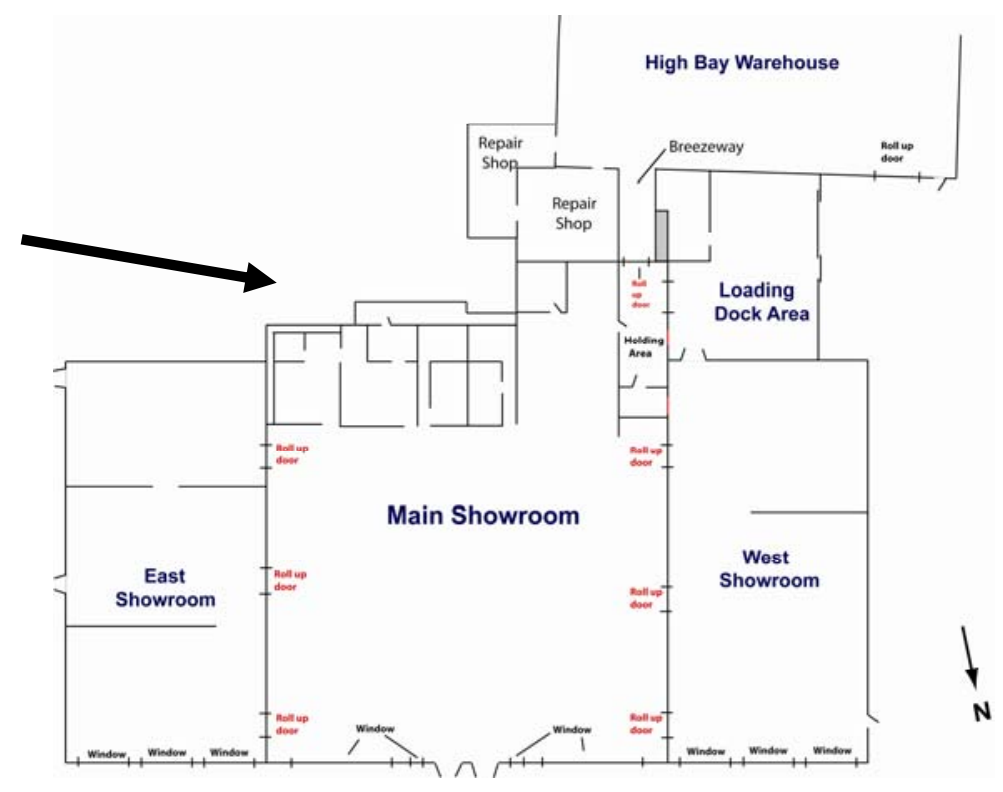




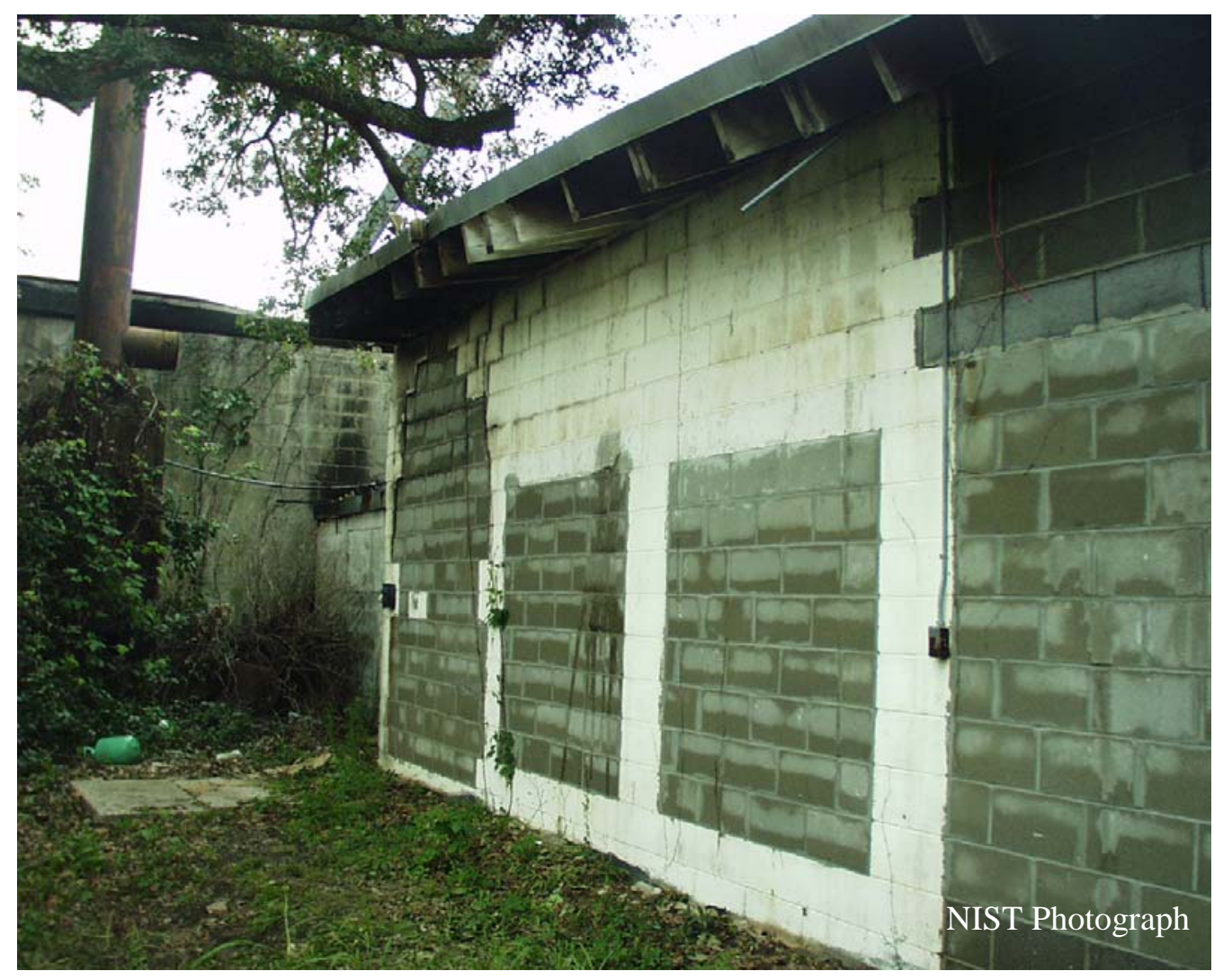

Figure D-7. Rear of main showroom. Openings in original wall have been filled in with concrete masonry blocks.

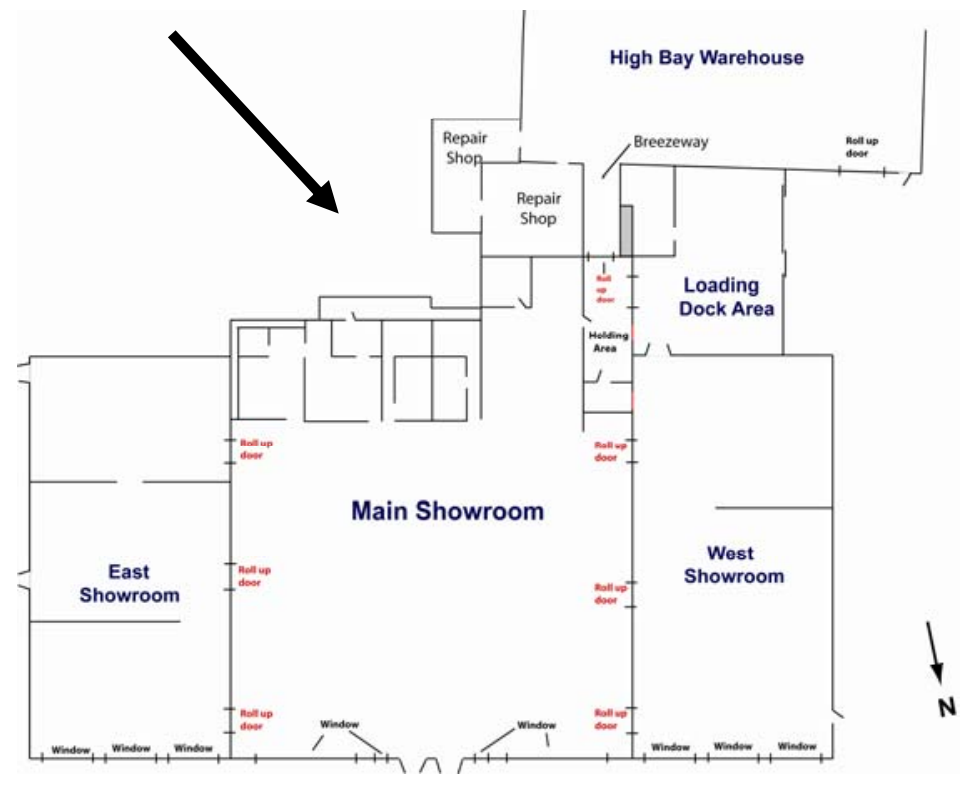




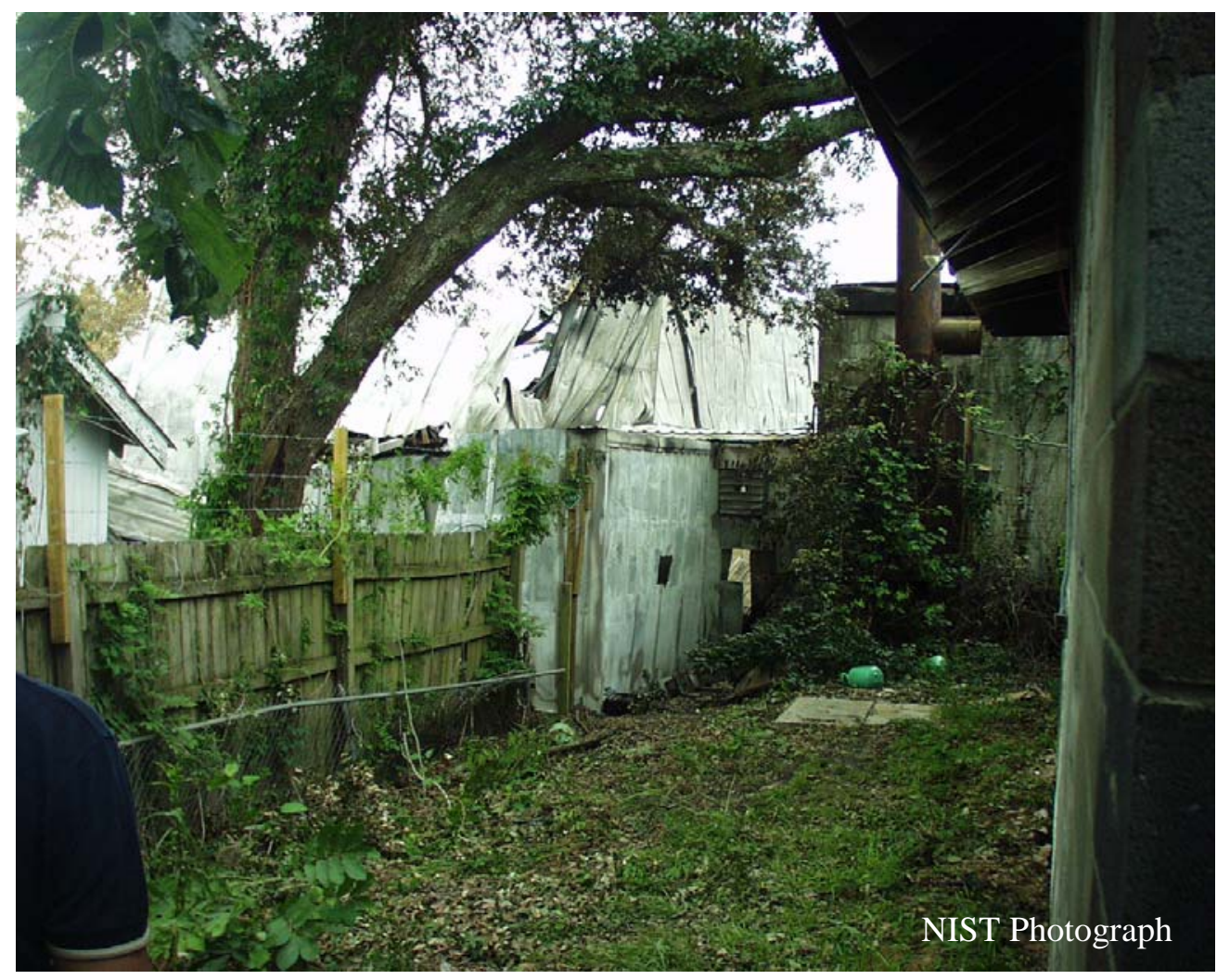

Figure D-8. Area behind main showroom. Rear wall of main showroom is visible on right side of photograph. North wall of repair shop is visible in center of photograph. Fence separates store from private residences located behind store.

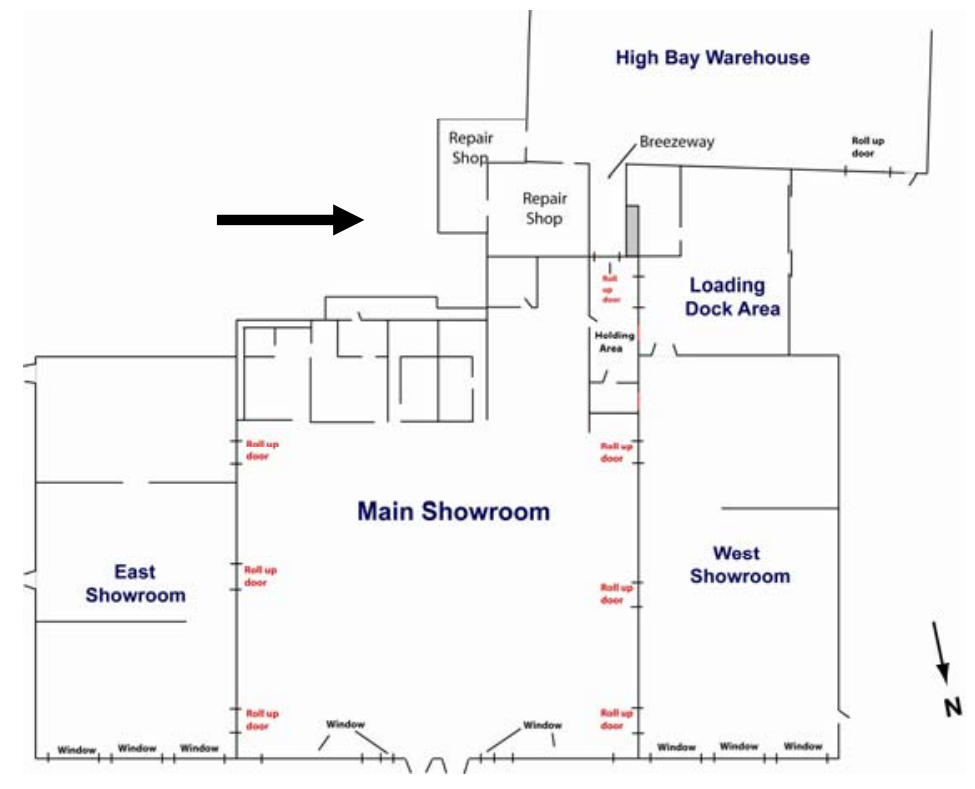




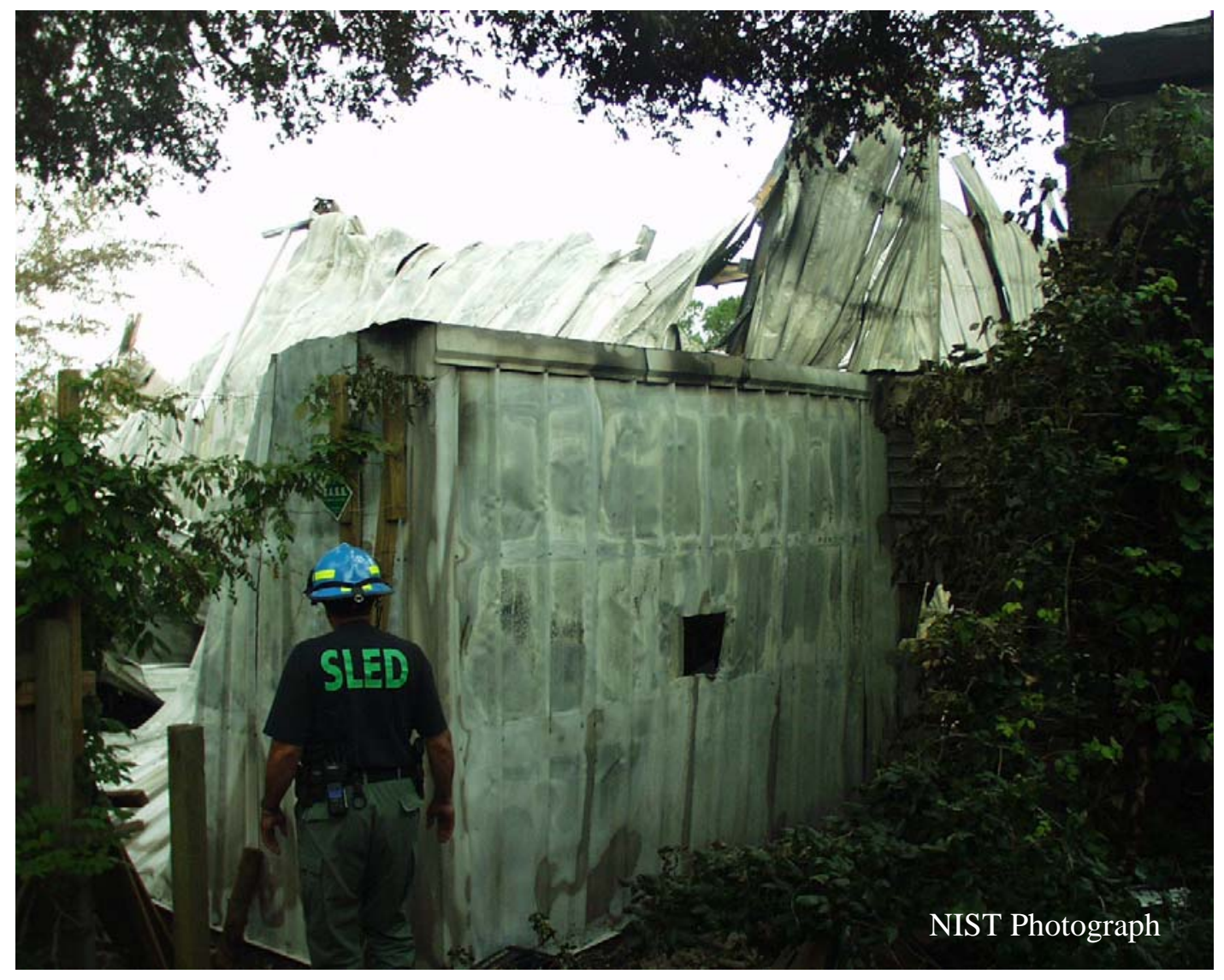

Figure D-9. North east corner of repair shop. Collapsed warehouse is visible in background.

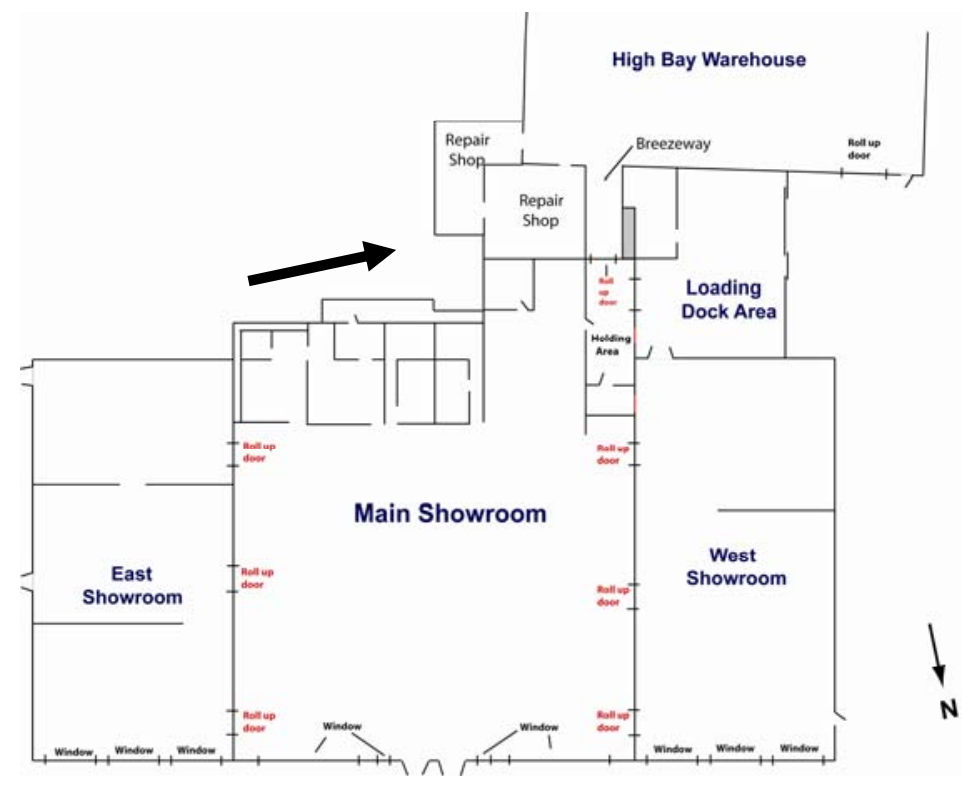




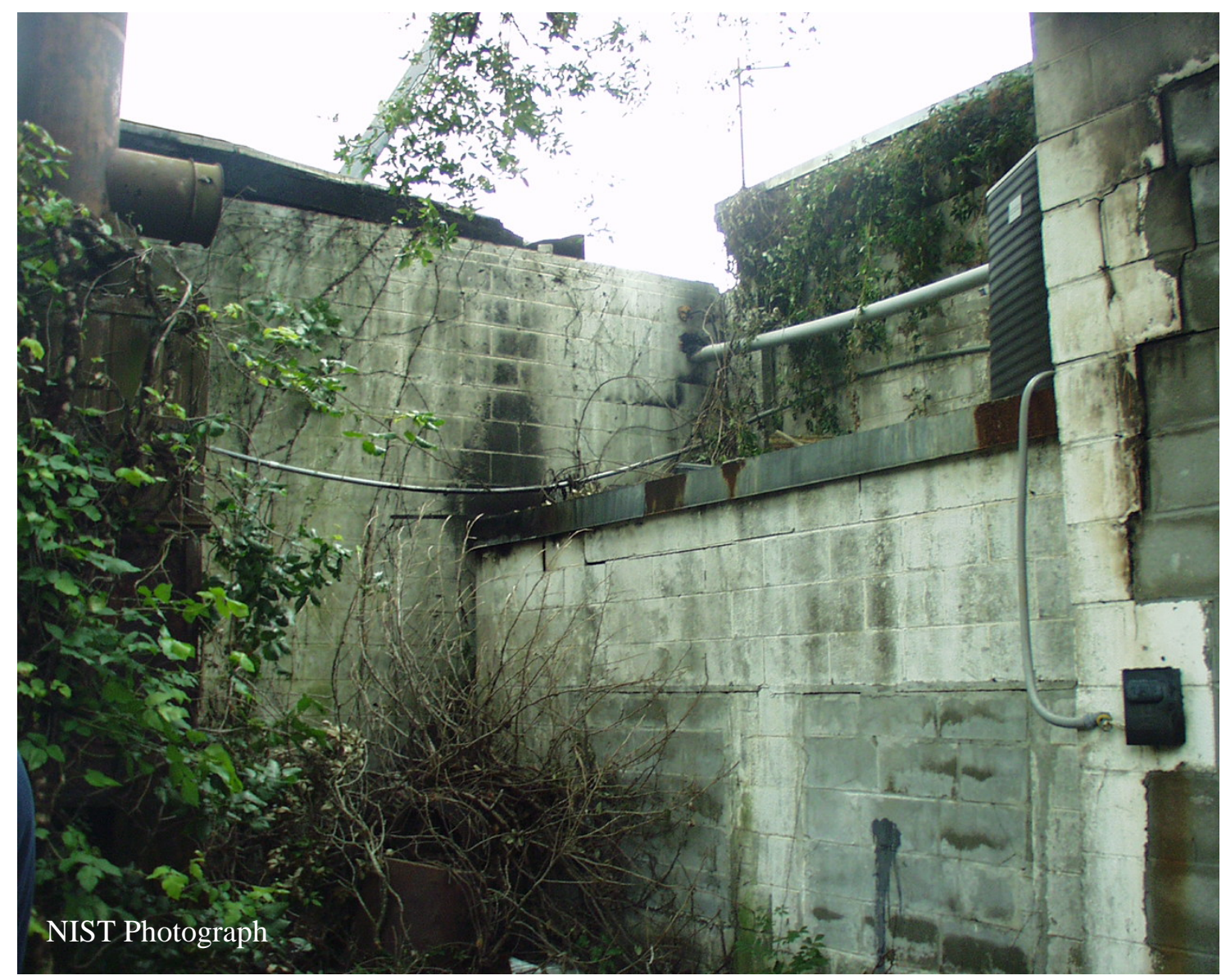

Figure D-10. Rear of main showroom. Incinerator exhaust stack visible in upper left corner of photograph.

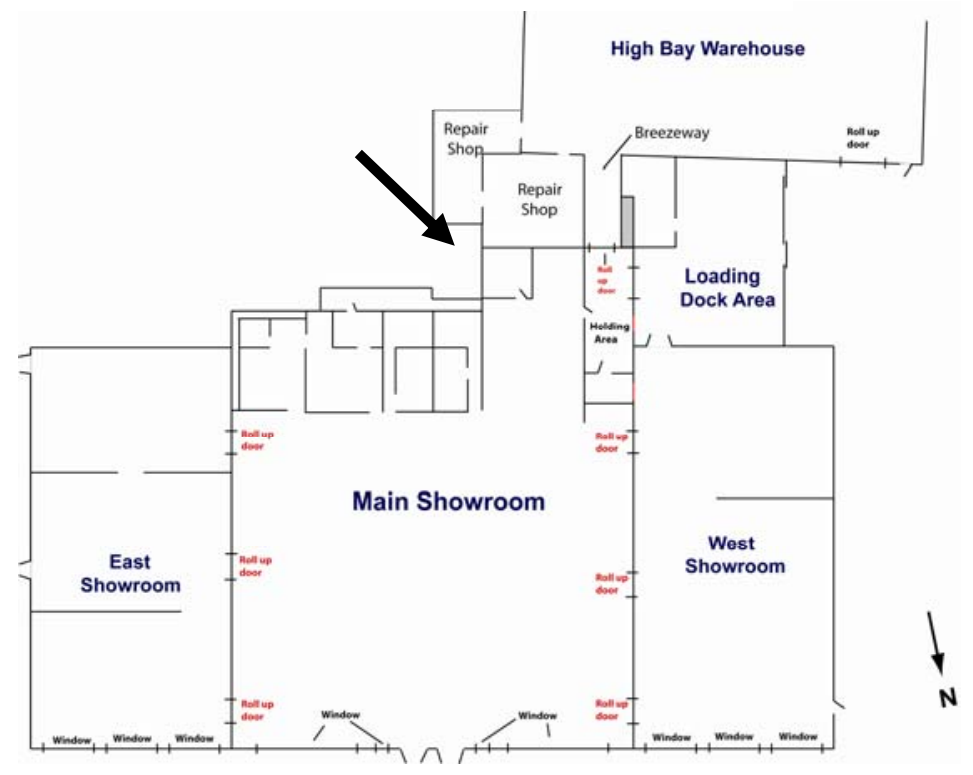




\section{D.2 MAIN SHOWROOM EXTERIOR PHOTOS - AFTER REMOVAL OF WALL AND ROOF}

The collapsed façade and parapet wall were removed from the front of the store. Portions of the roof were also removed from the main showroom. The collapsed façade and roof sections were removed for safety reasons. Additional photos were taken after removal of façade rubble and roof section. The east and west walls of the original structure became interior walls after the addition of the east and west showroom.

Figure D-11. North-west corner of main showroom looking from front parking lot.

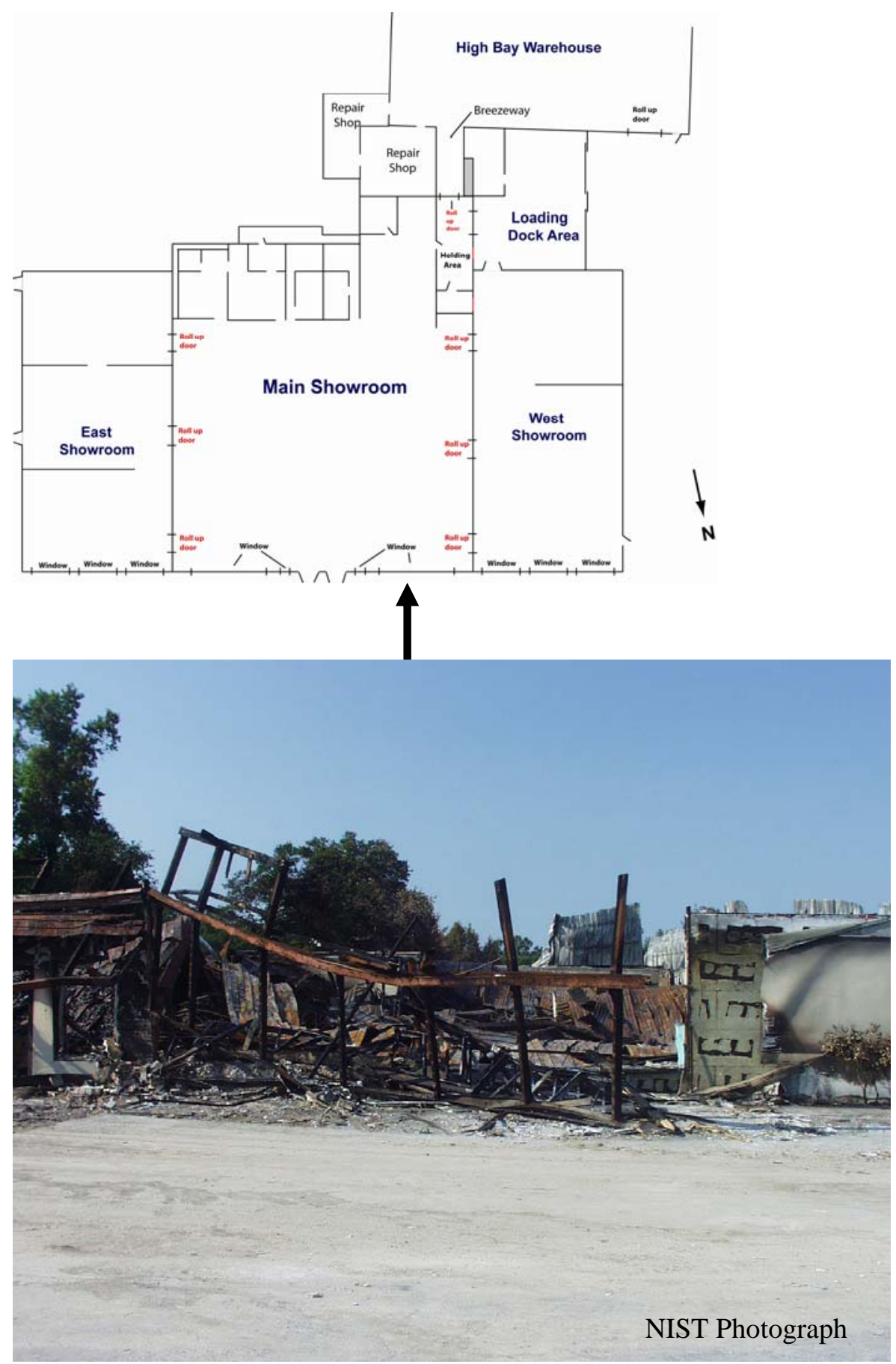



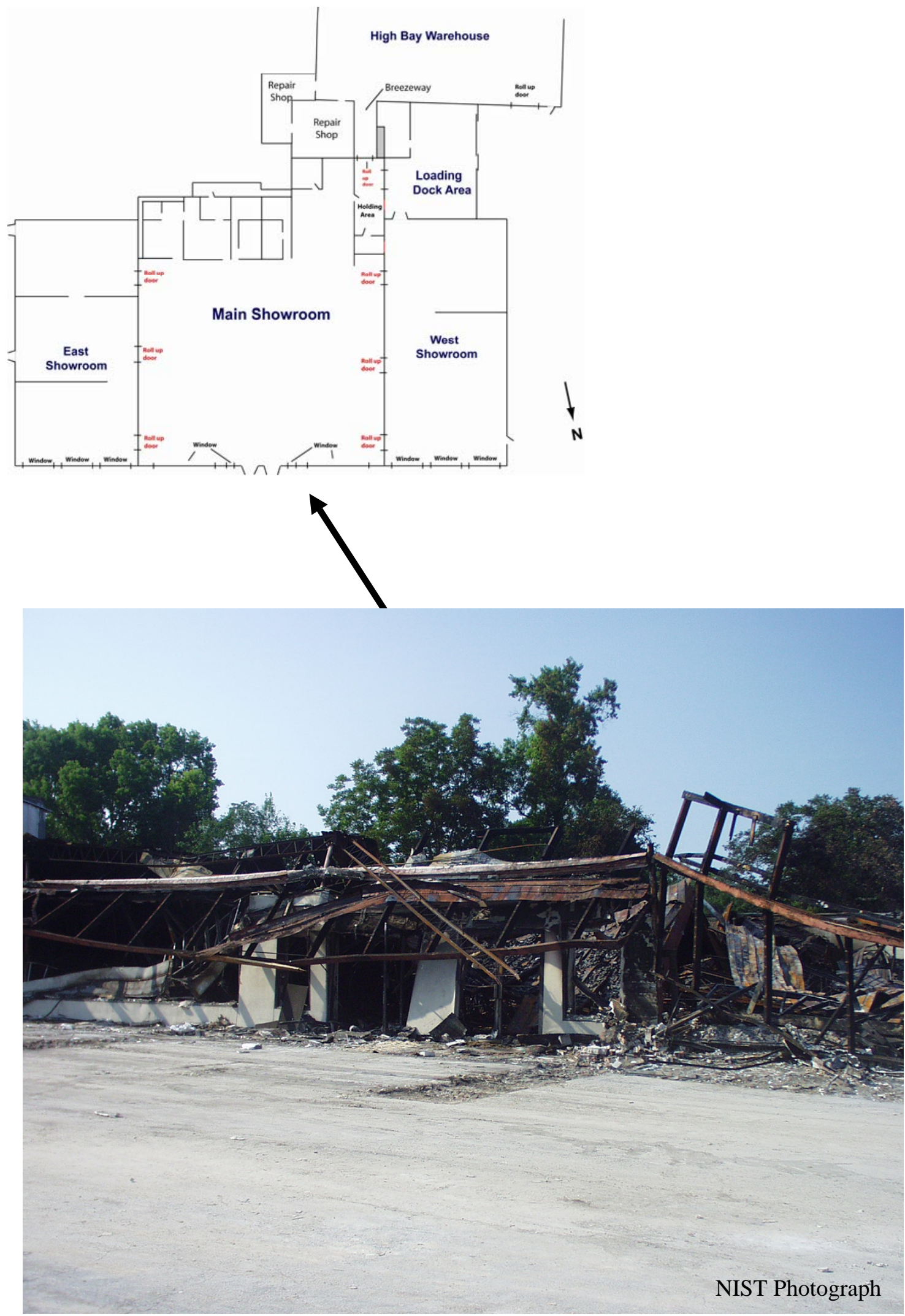

Figure D-12. Front of main showroom after removal of façade and parapet wall rubble. 

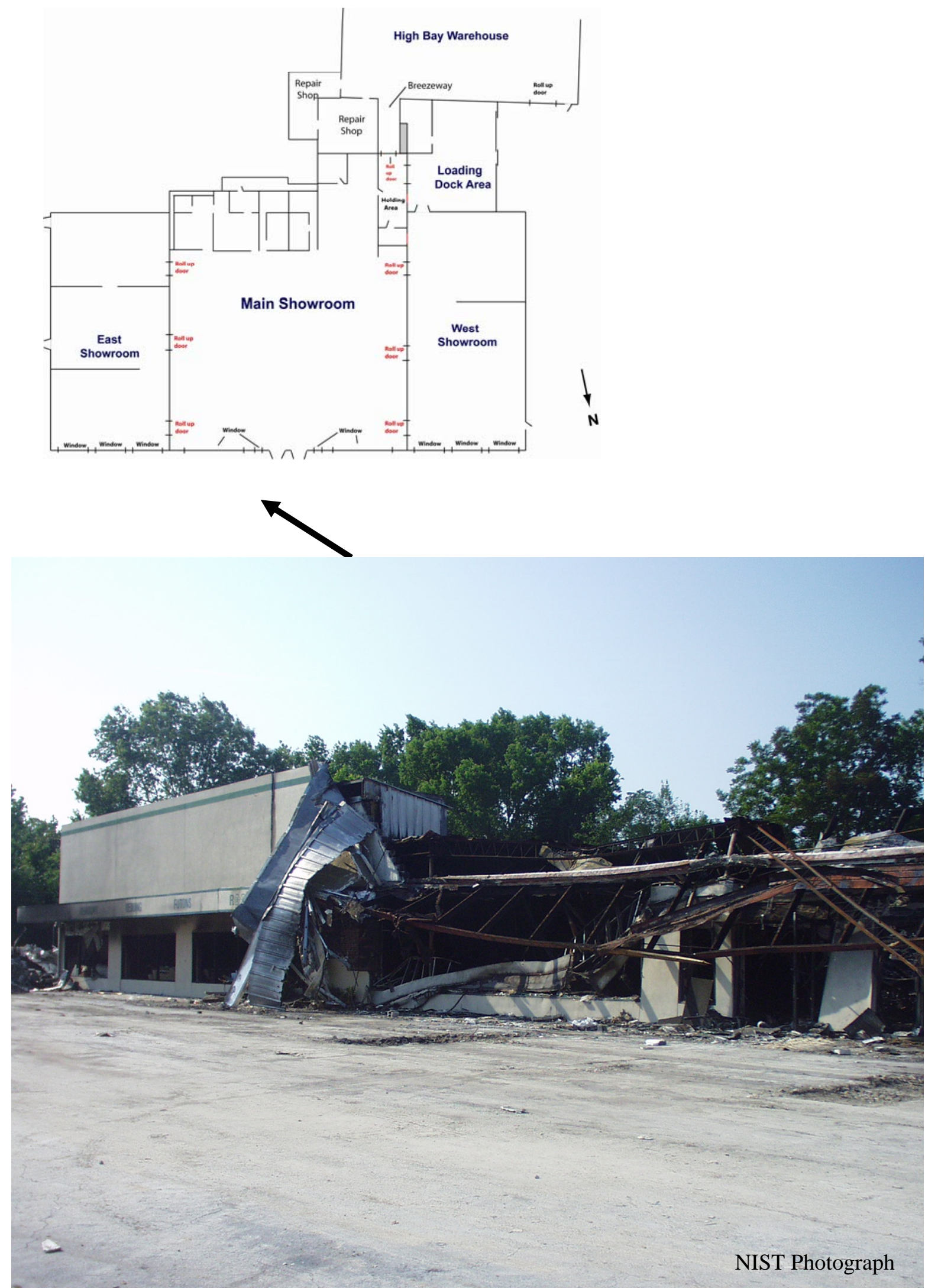

Figure D-13. Front of main showroom after removal of collapsed façade. Façade and parapet wall of east showroom still in place. 

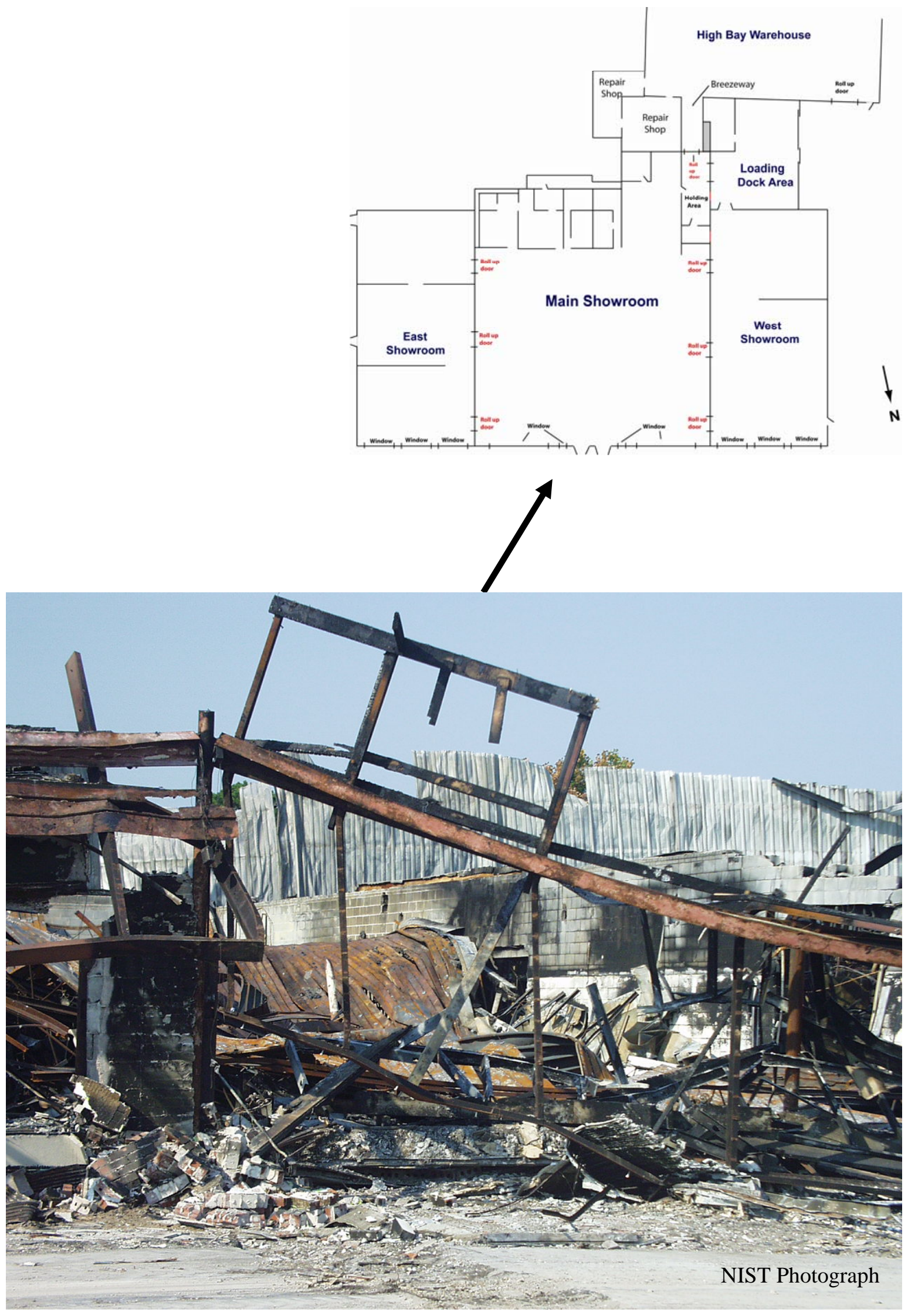

Figure D-14. Front of main showroom. View is looking toward warehouse. 


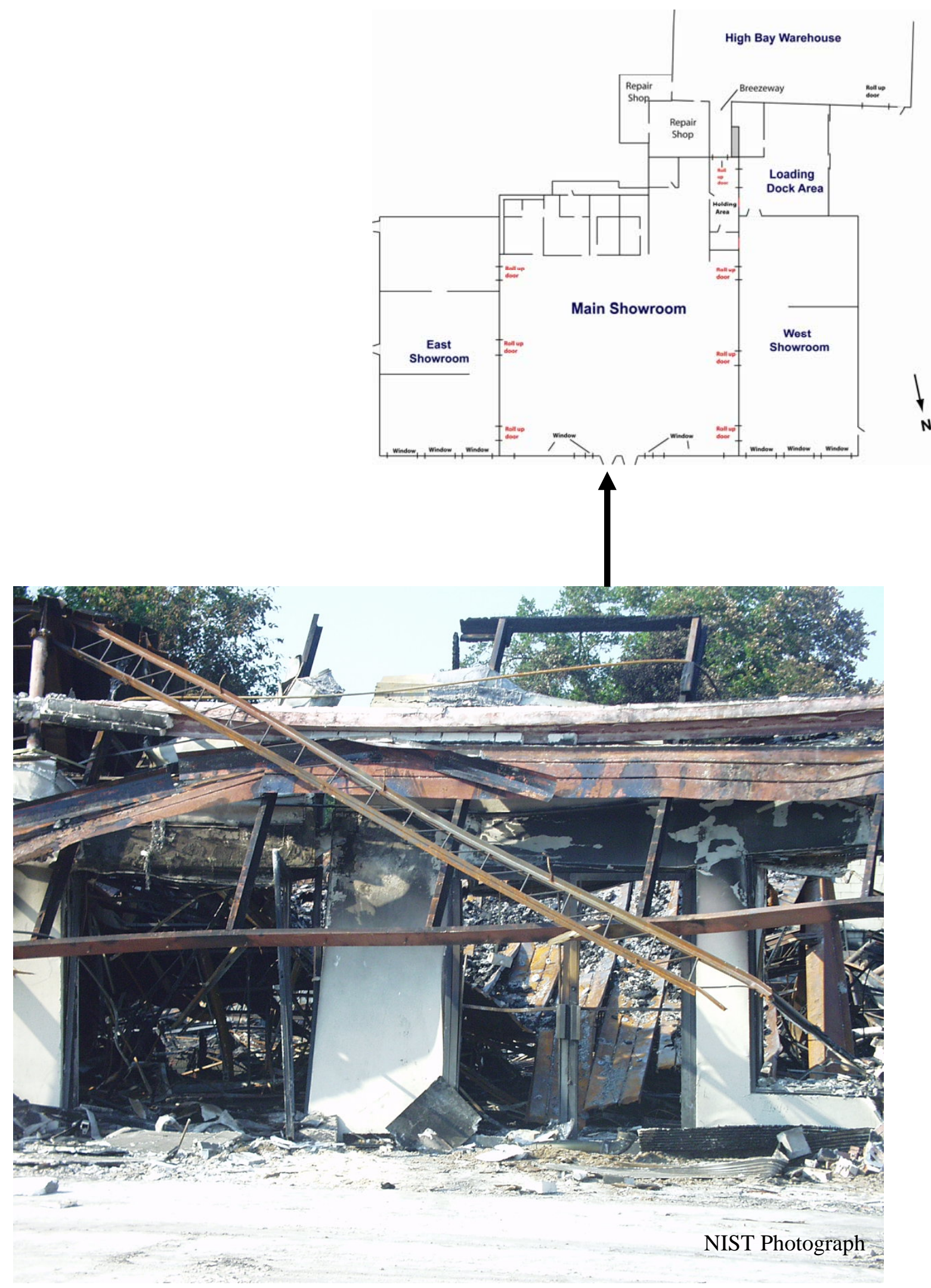

Figure D-15. Front of main showroom. View is looking through front doors into interior. 

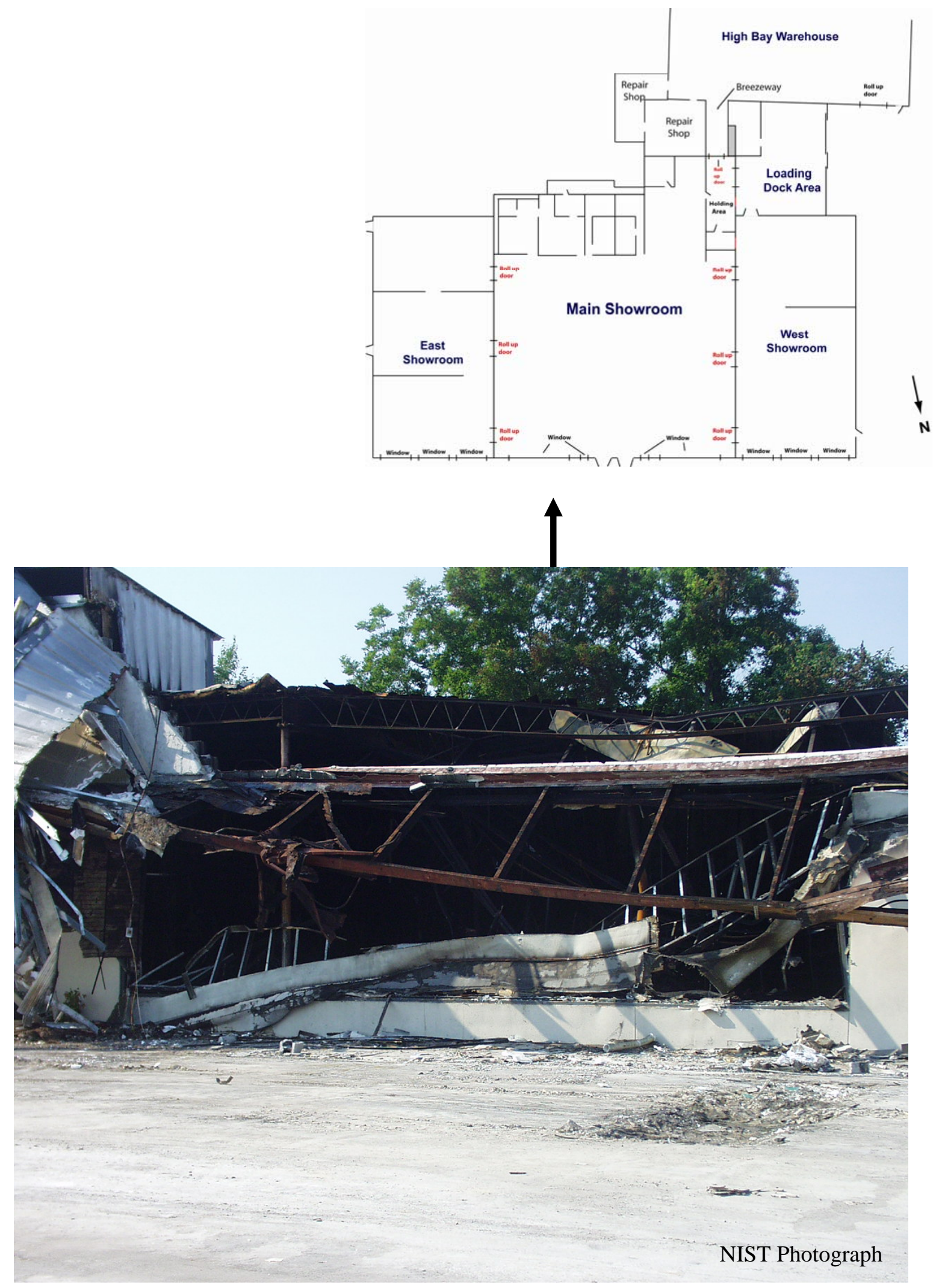

Figure D-16. Front of main showroom. View is looking through display windows east of front doors. 


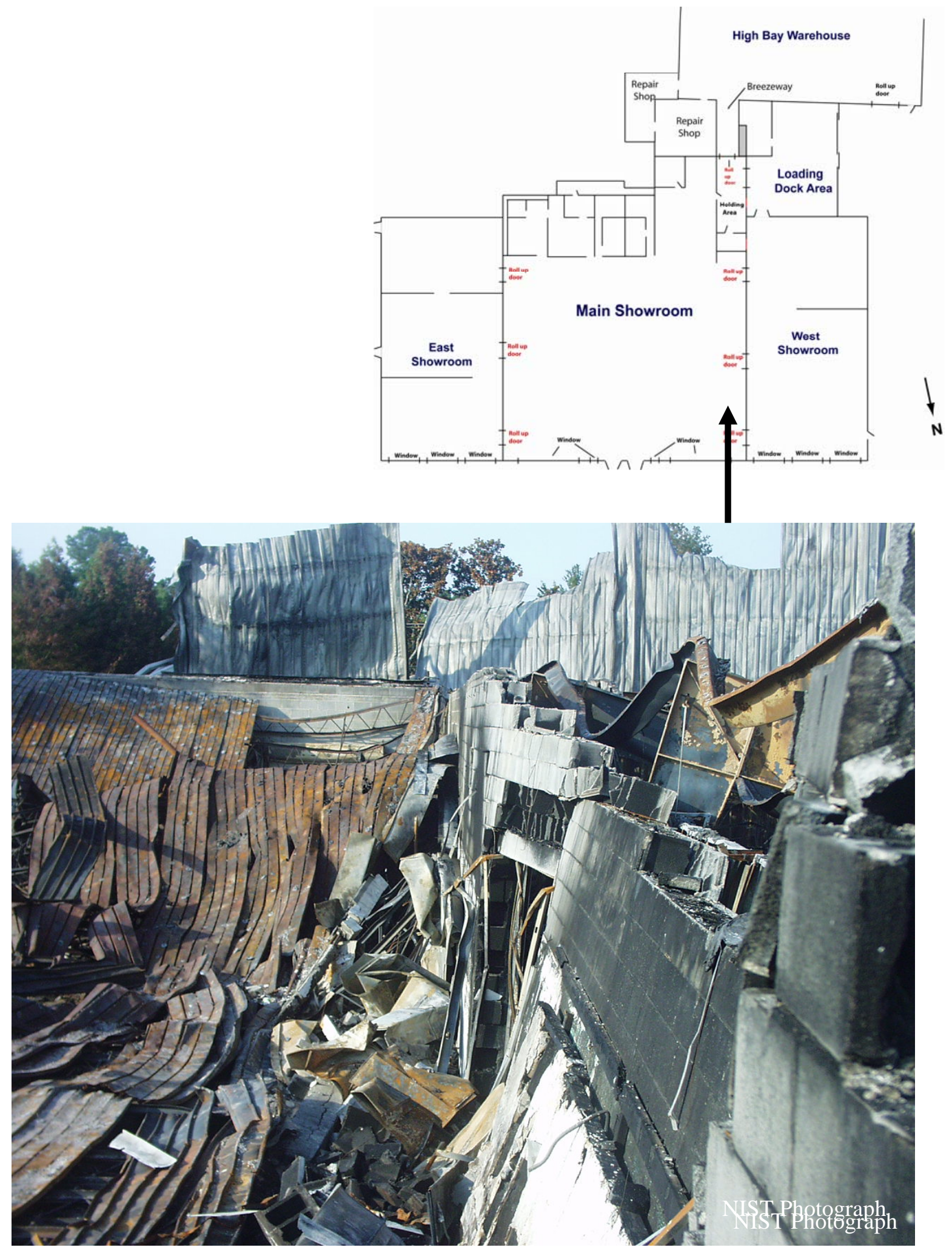

Figure D-17. Interior of main showroom looking along west wall toward warehouse. 


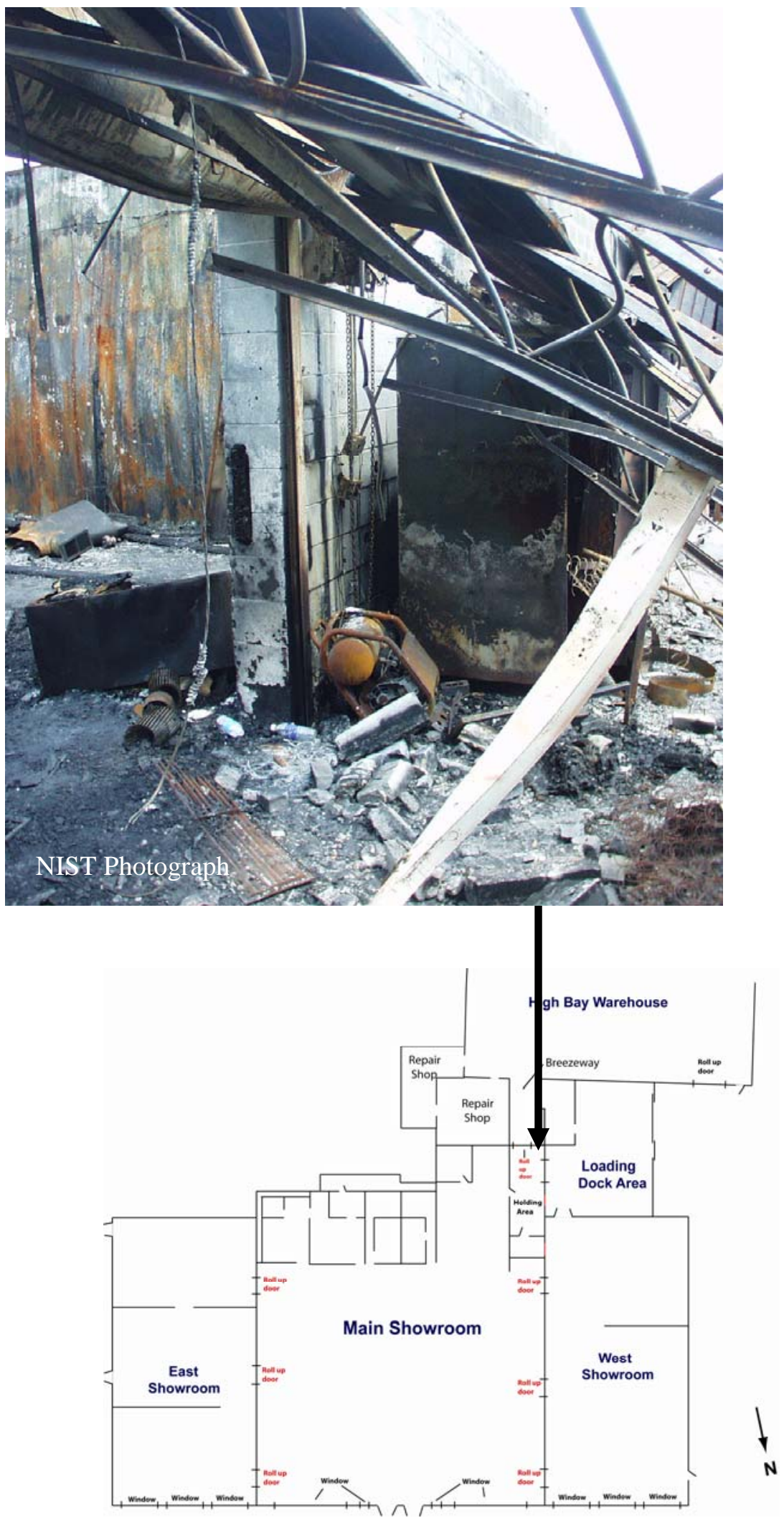

Figure D-18. Interior of holding area along west wall. Open non-fire roll-down door and vending machines visible along west wall. 


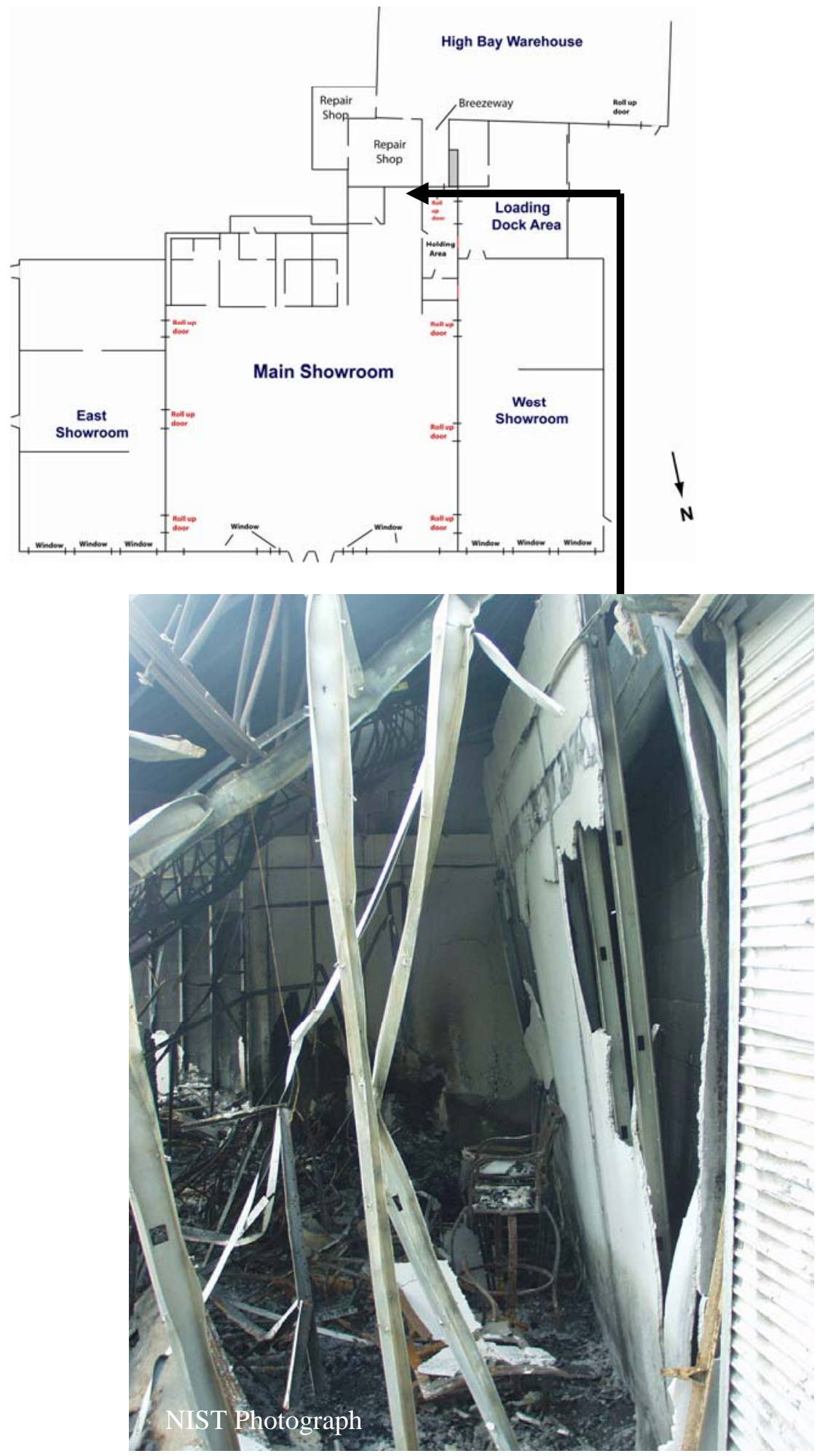

Figure D-19. Interior of main showroom looking rear wall of showroom. 

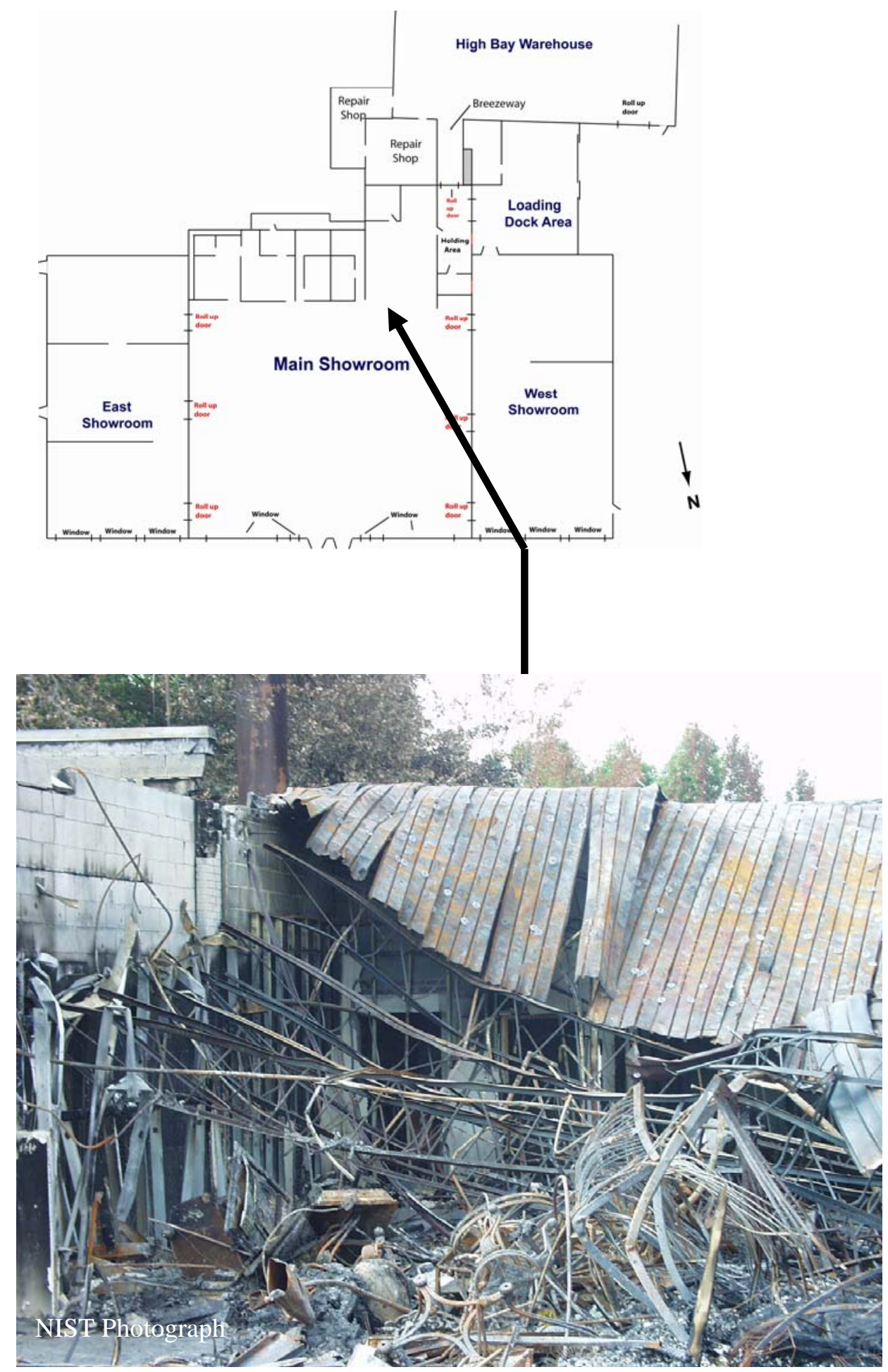

Figure D-20. Interior of main showroom toward rear of showroom. 


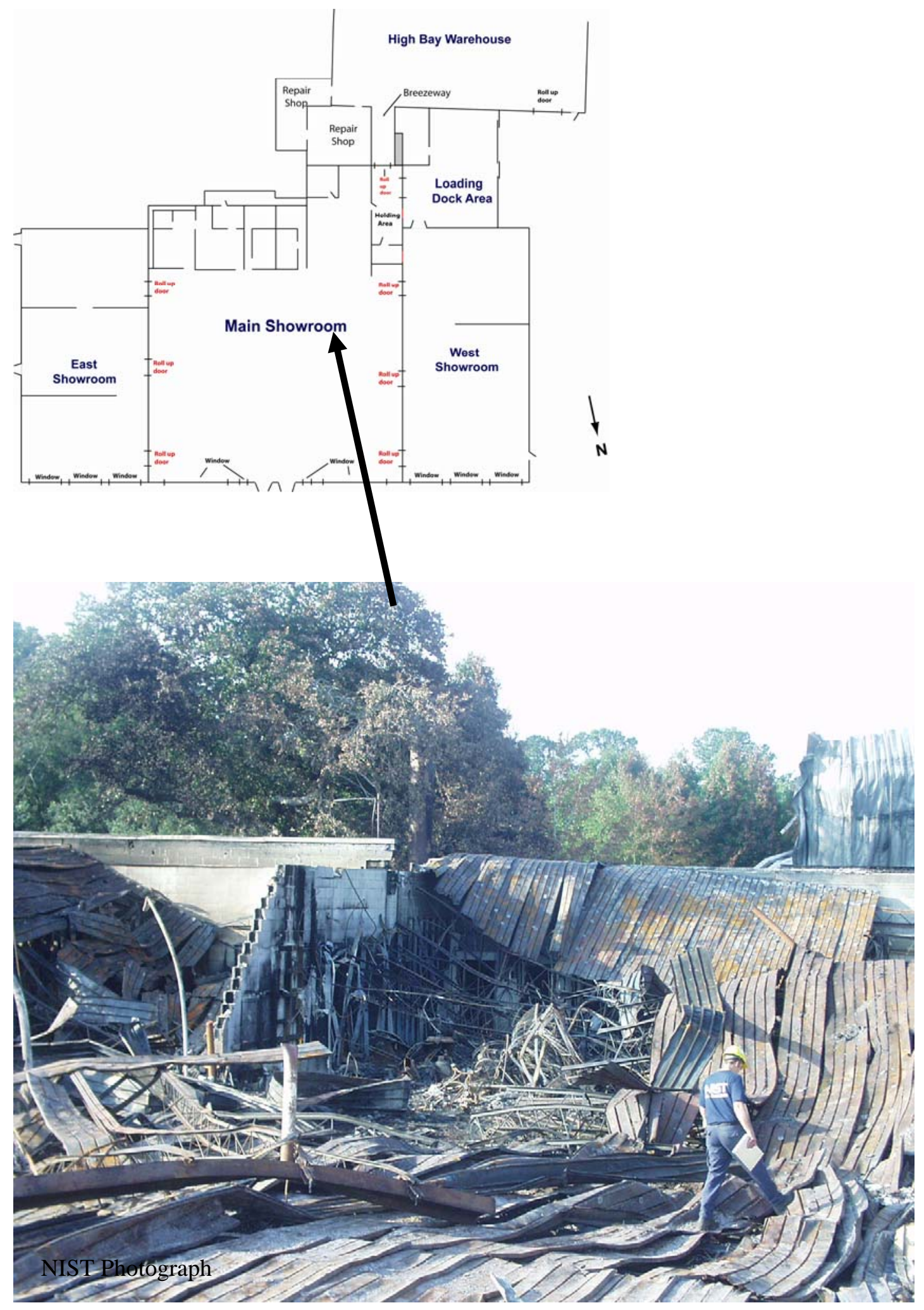

Figure D-21. Interior of main showroom looking toward office area at rear of showroom. 


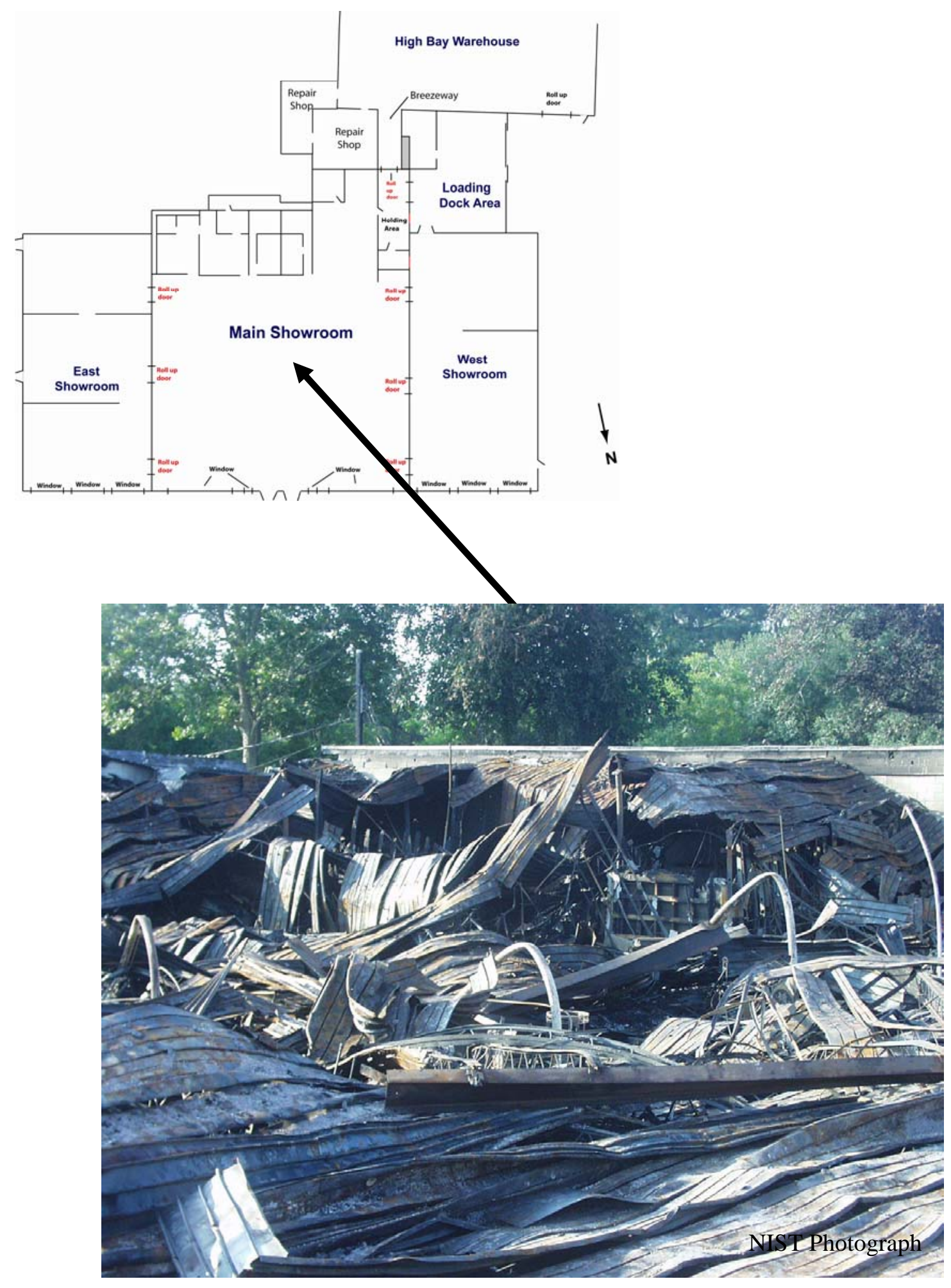

Figure D-22. Interior of main showroom looking toward office area at rear of showroom. 

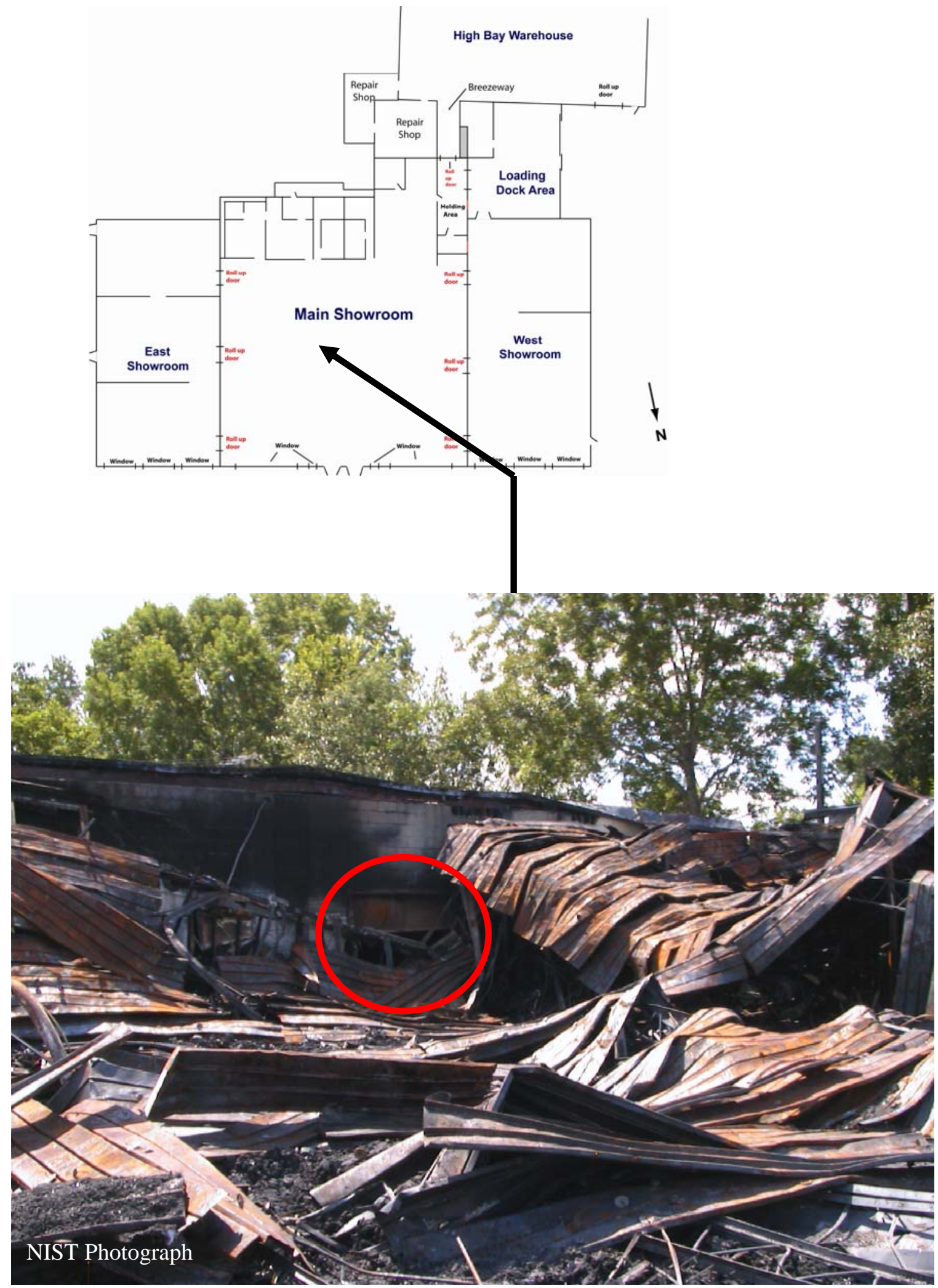

Figure D-23. Interior of main showroom looking toward east wall. Partially closed rolldown fire door circled. 

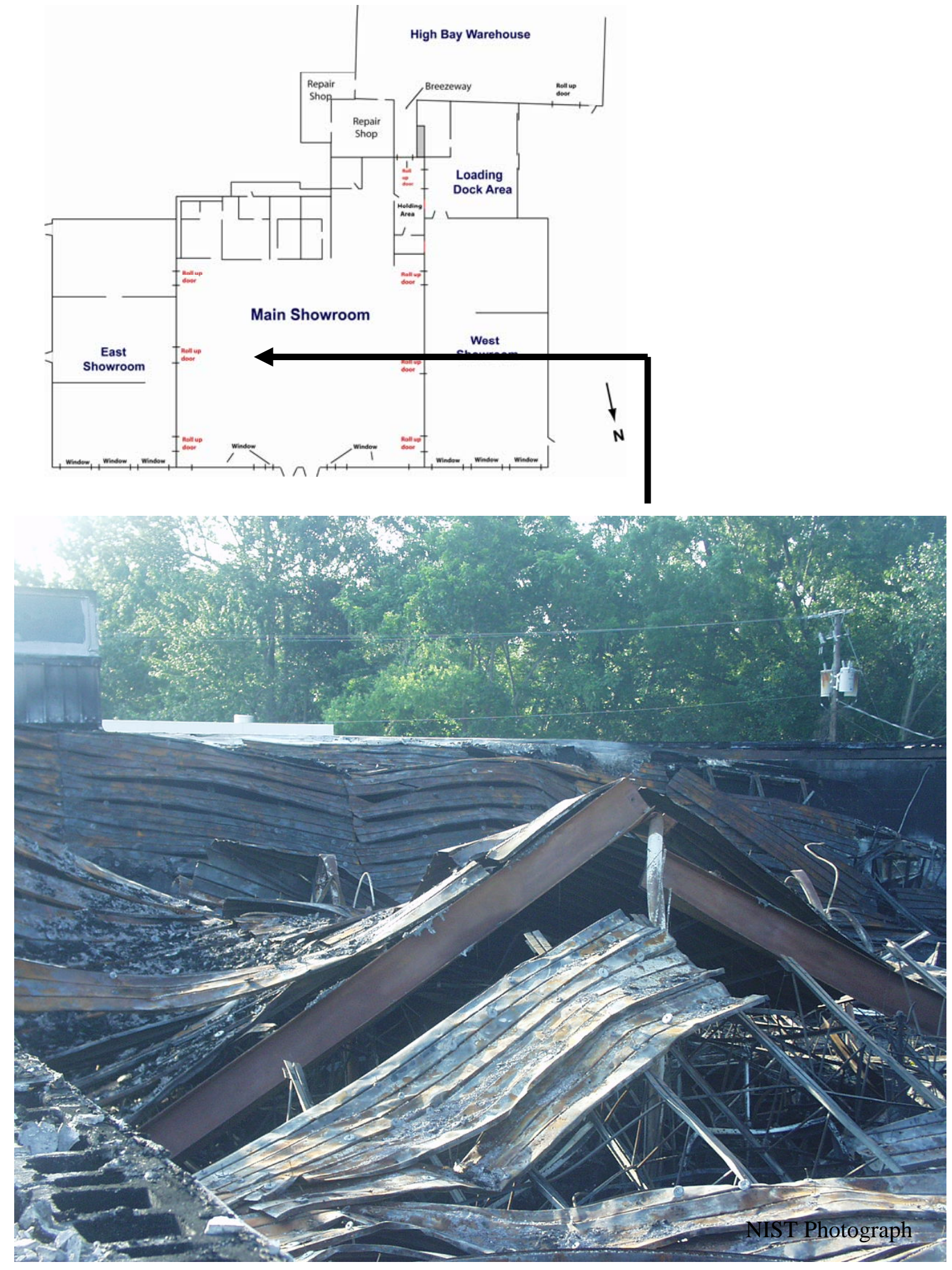

Figure D-24. Interior of main showroom looking toward east wall. 

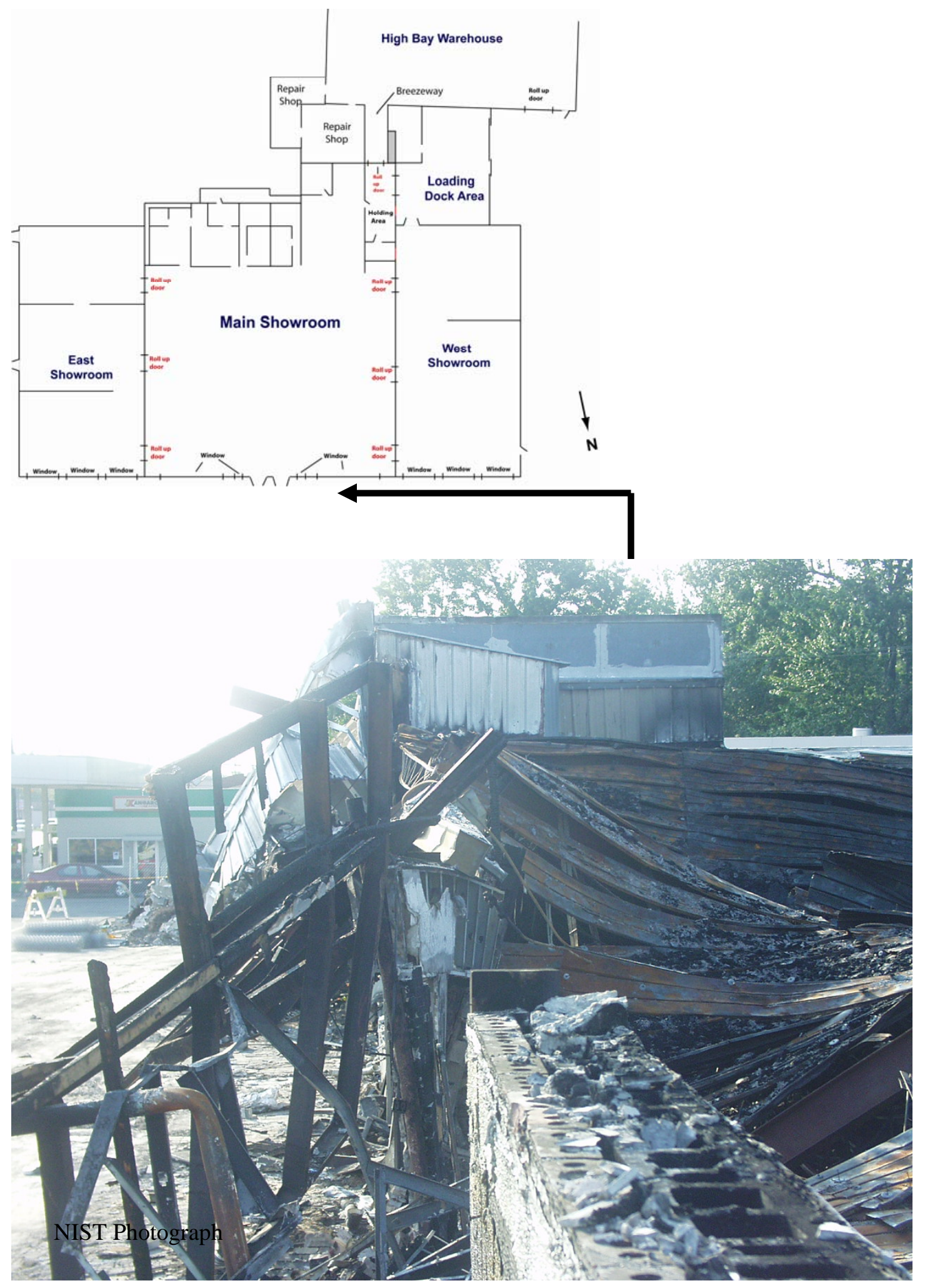

Figure D-25. Interior of main showroom looking along front wall toward east showroom. 

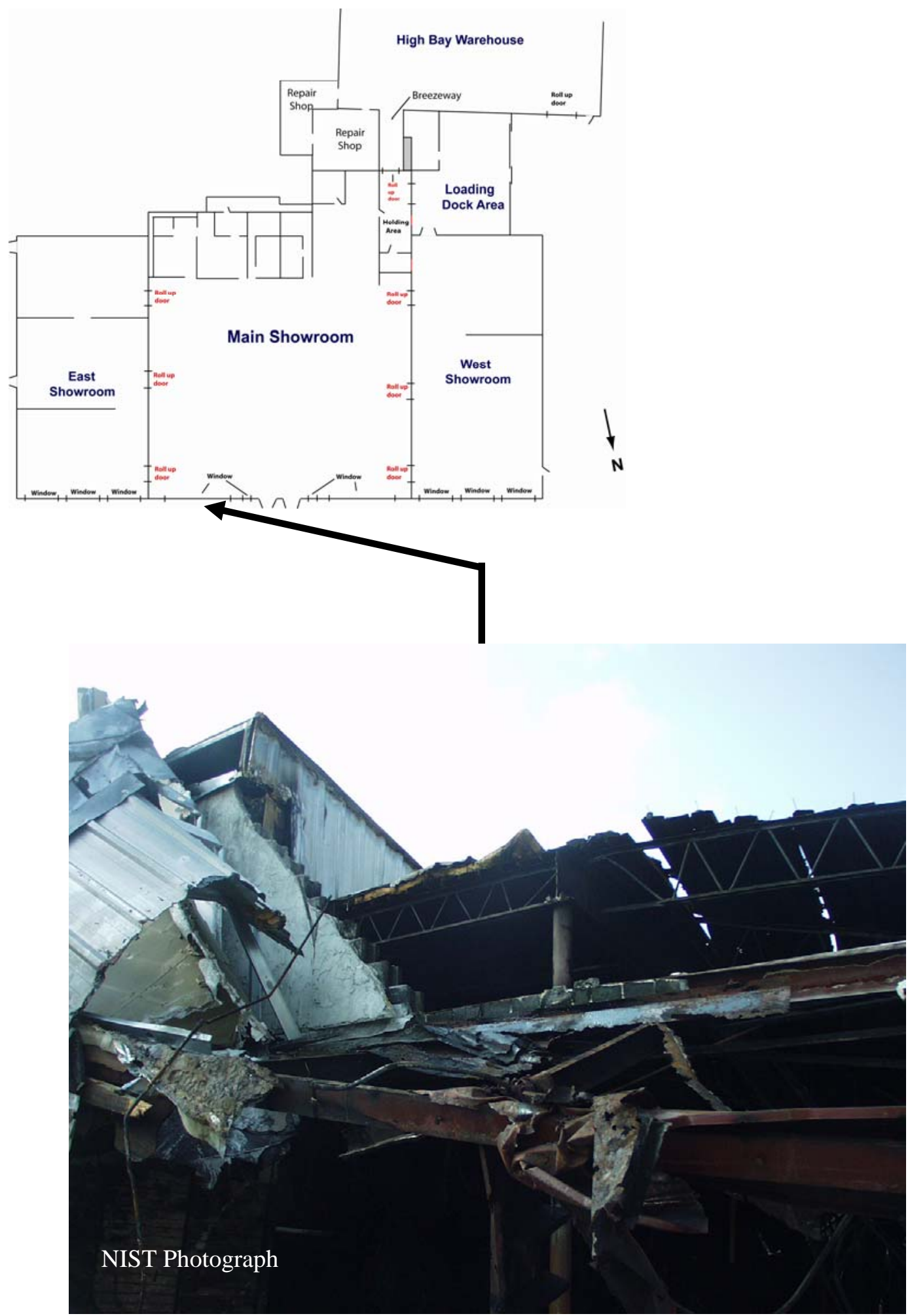

Figure D-26. North-east corner of main showroom looking toward east showroom. 


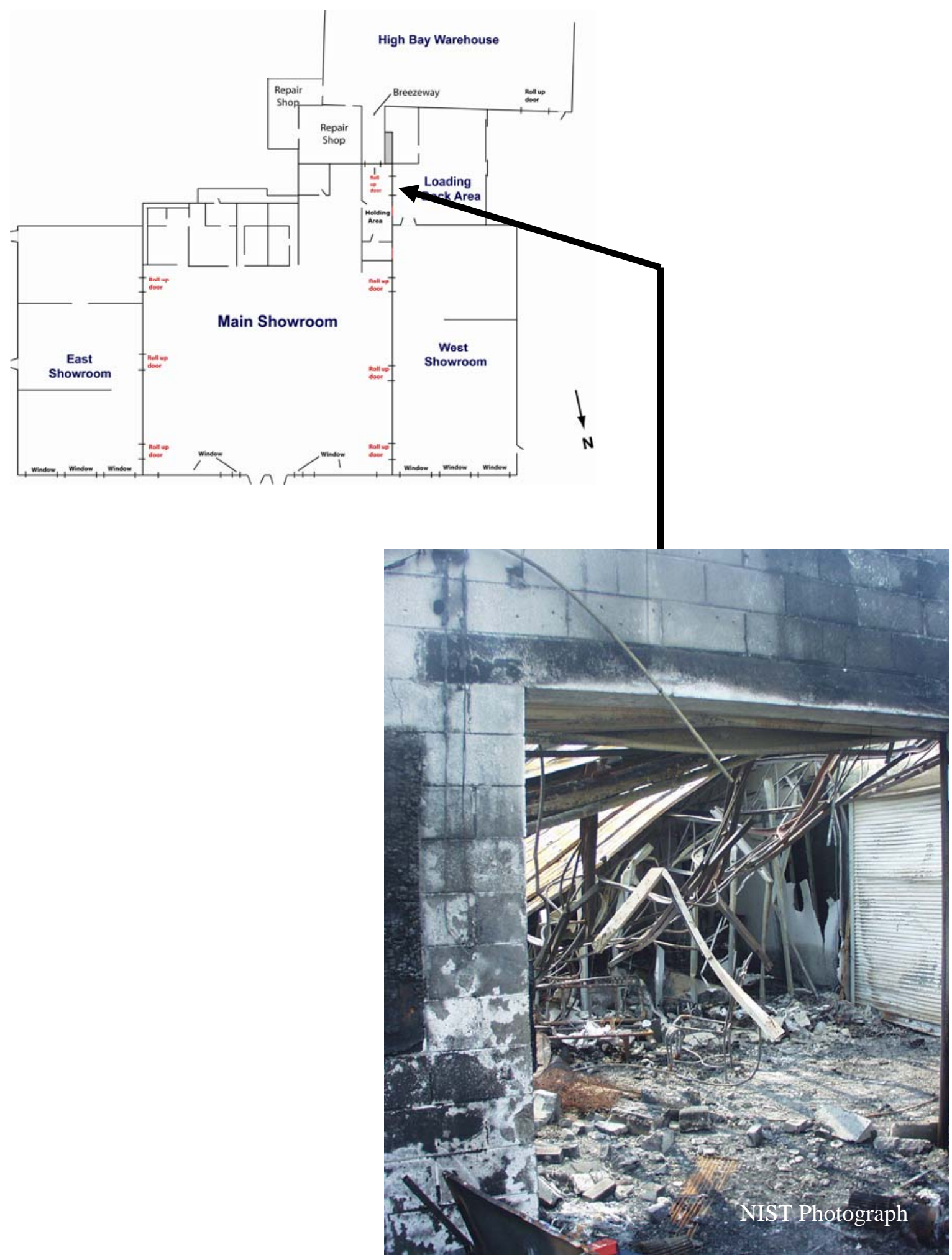

Figure D-27. Holding area viewed through non-fire roll-down door. 


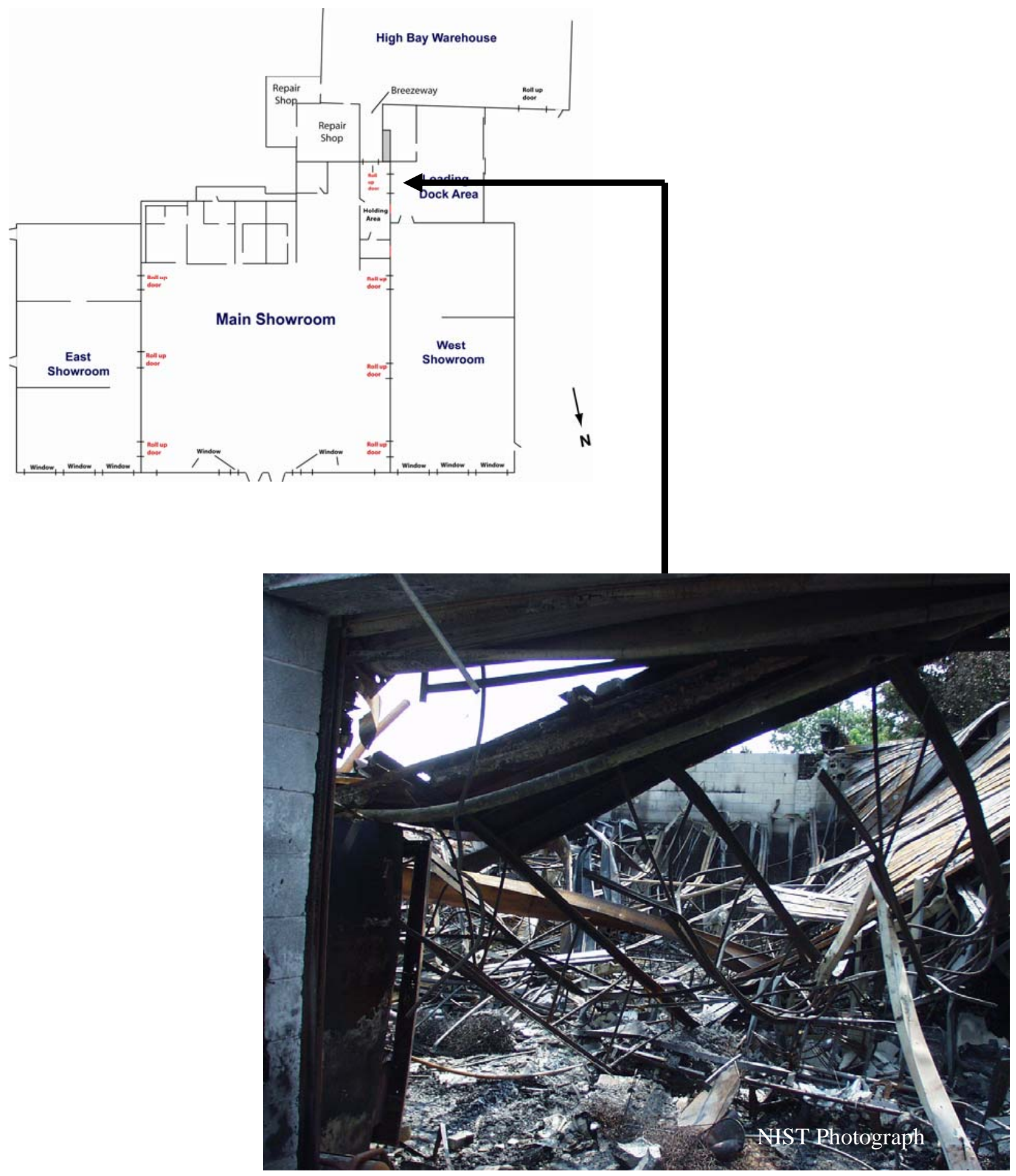

Figure D-28. Holding area viewed through non-fire roll-down door. 

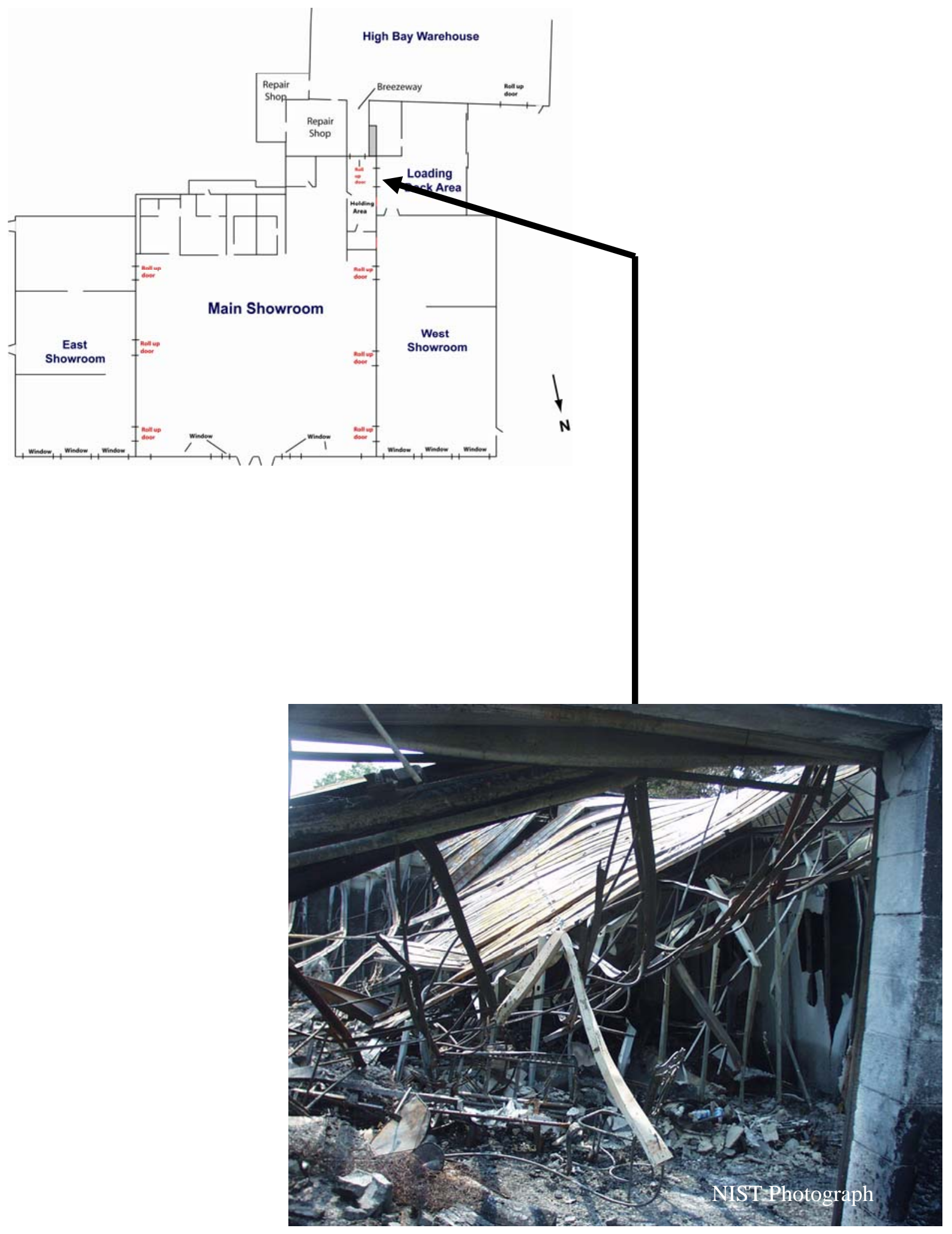

Figure D-29. Holding area and rear of main showroom. 
D-30 


\section{APPENDIX E. WeSt SHOWROOM}

\section{E.1 WEST SHOWROOM EXTERIOR PHOTOS}

A series of photos were taken of the front, rear, and west side of the west showroom. Early photos were taken before the roof and front façade were removed.

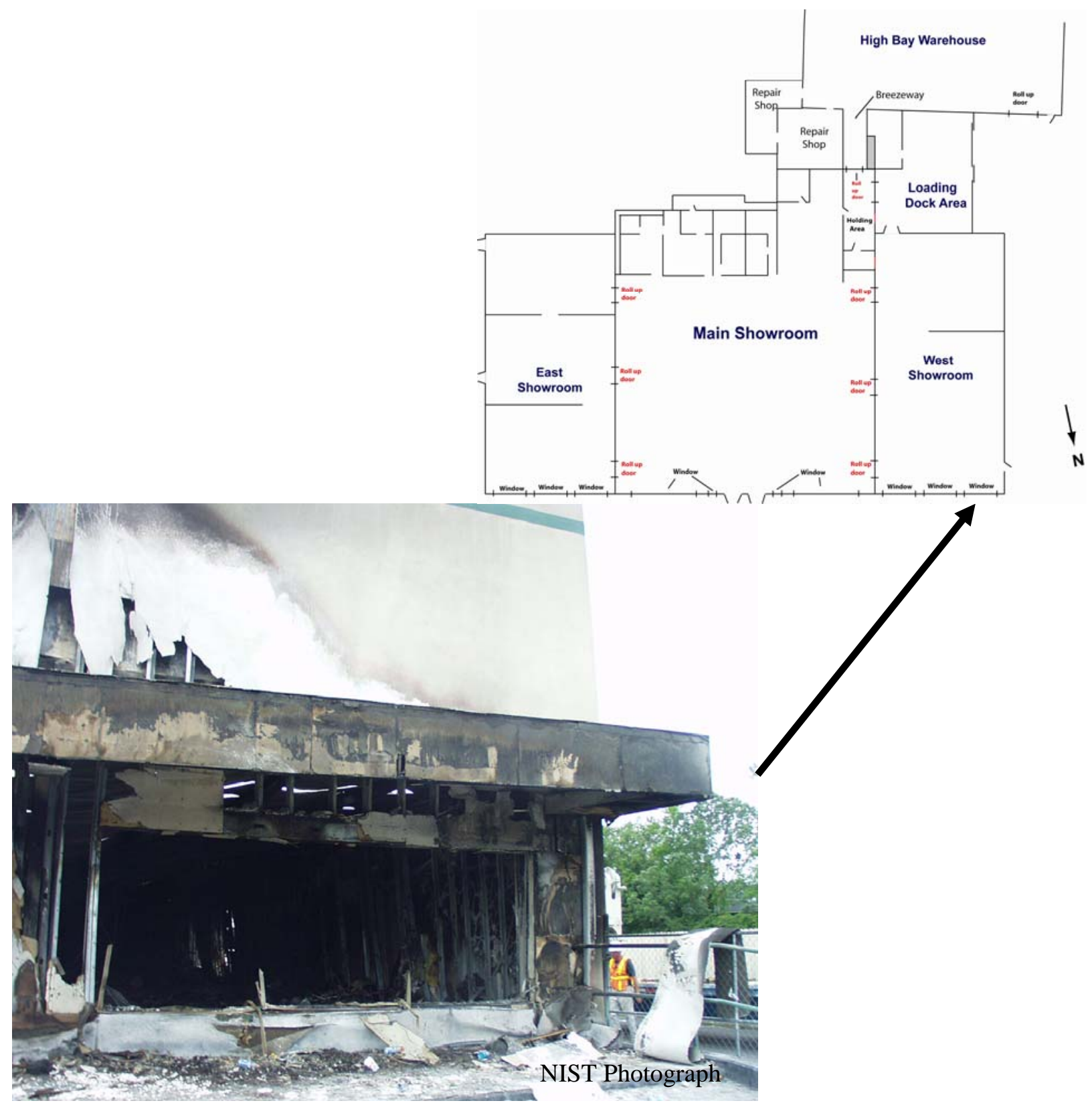

Figure E-1. Exterior of west showroom. Remains of west-front window section visible. 

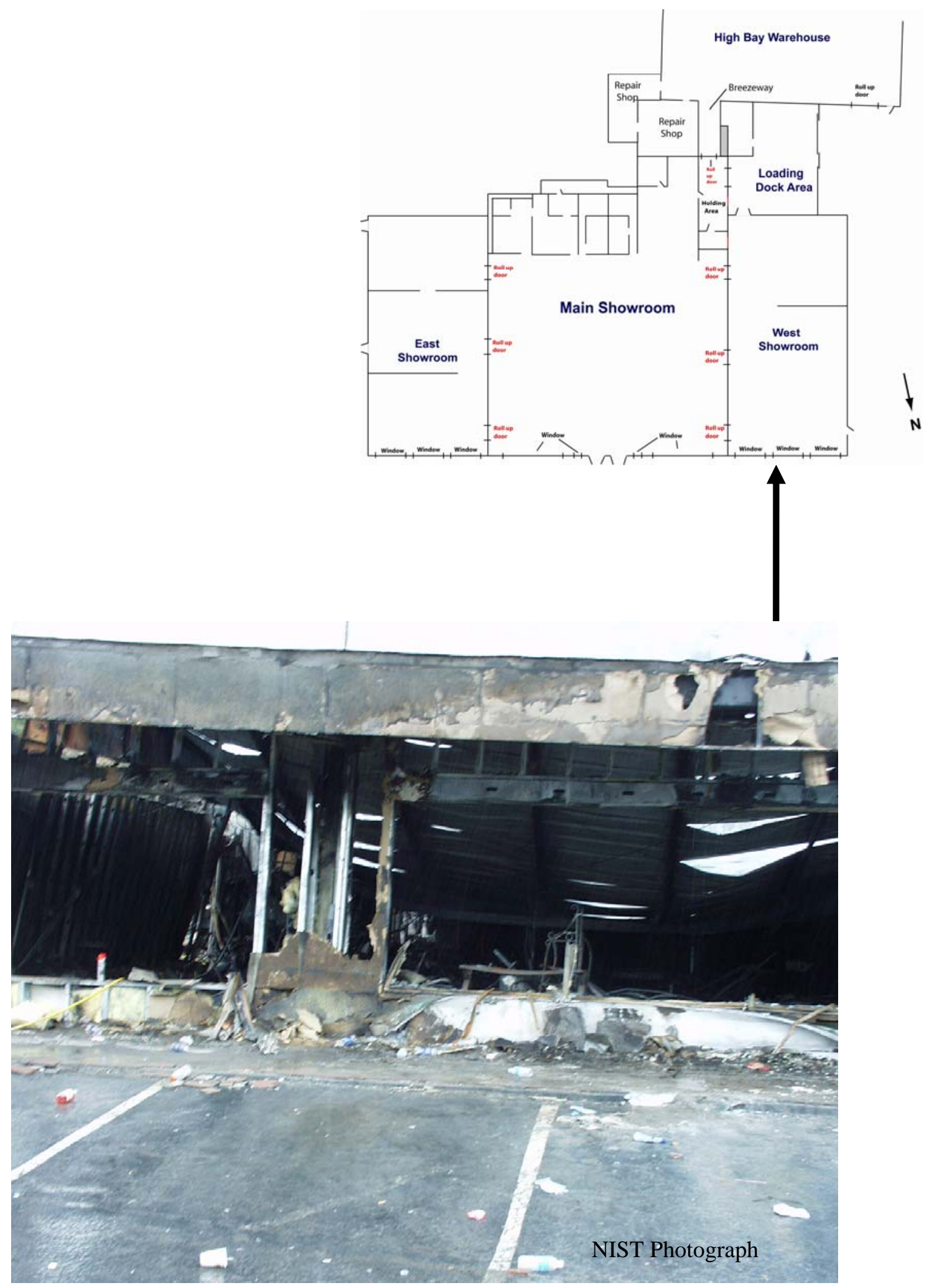

Figure E-2. Exterior of west showroom. Remains of east- and center-front window sections visible. 


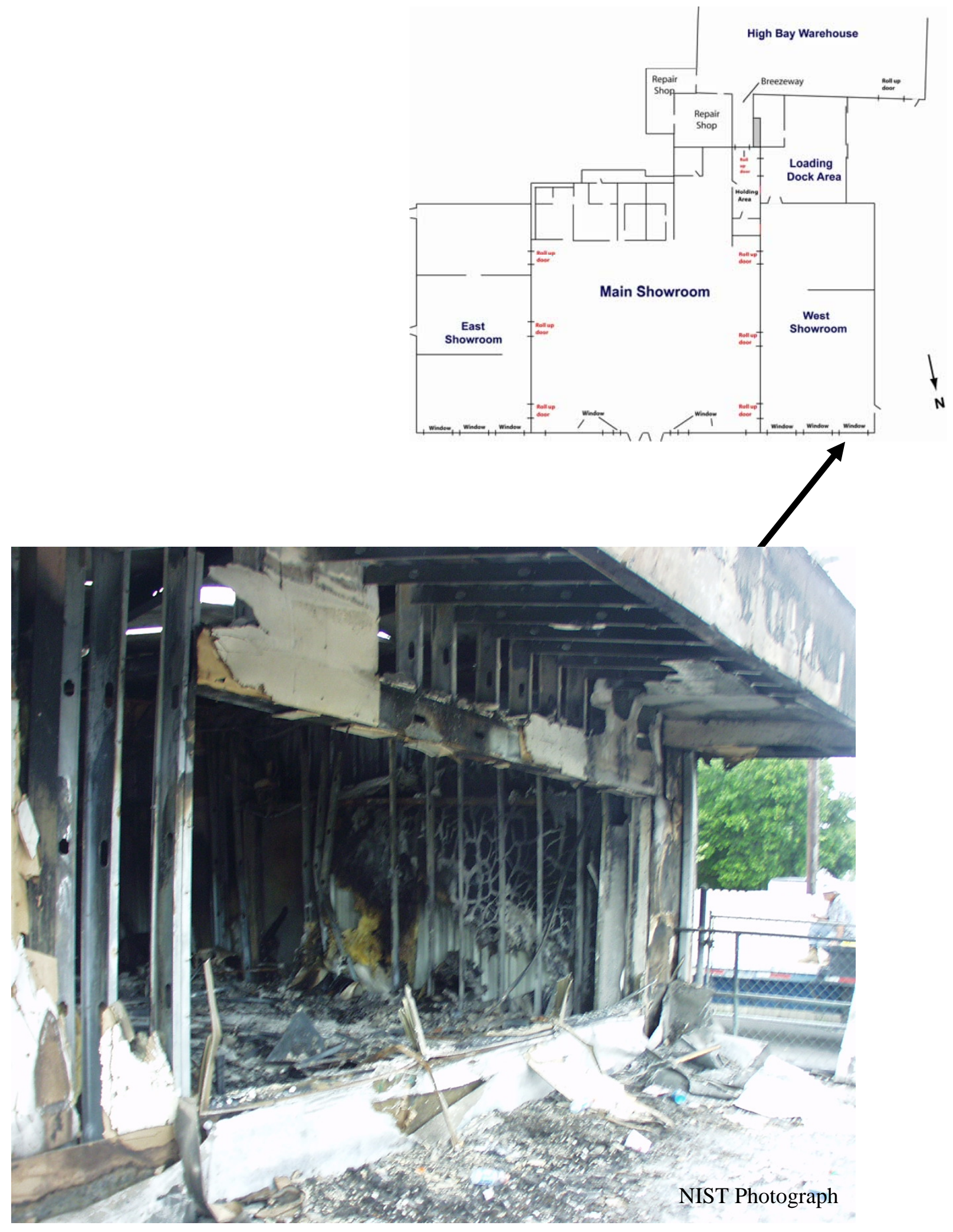

Figure E-3. Exterior view of front of west showroom. Metal stud walls visible. 


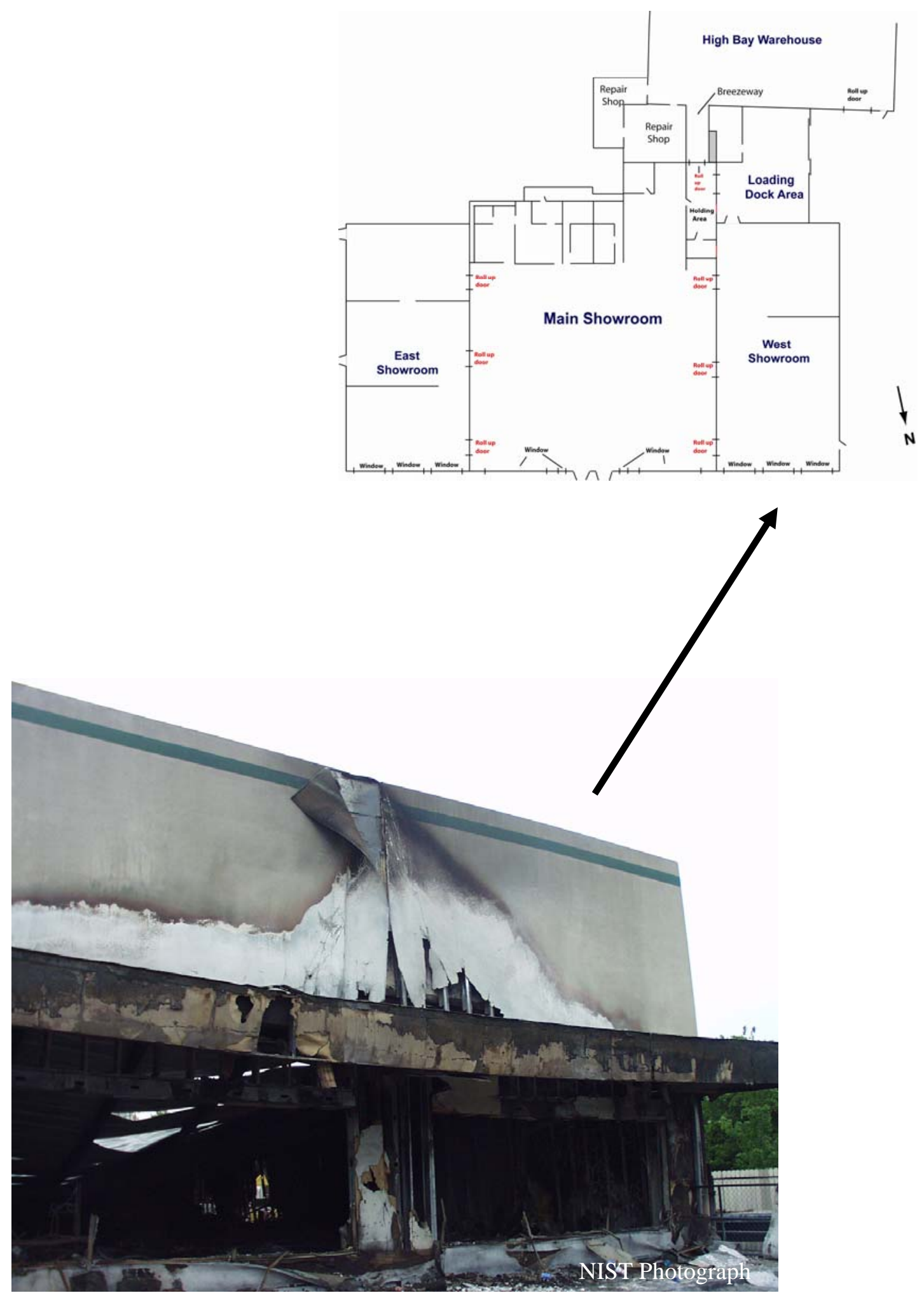

Figure E-4. Exterior front wall above windows of west showroom. 


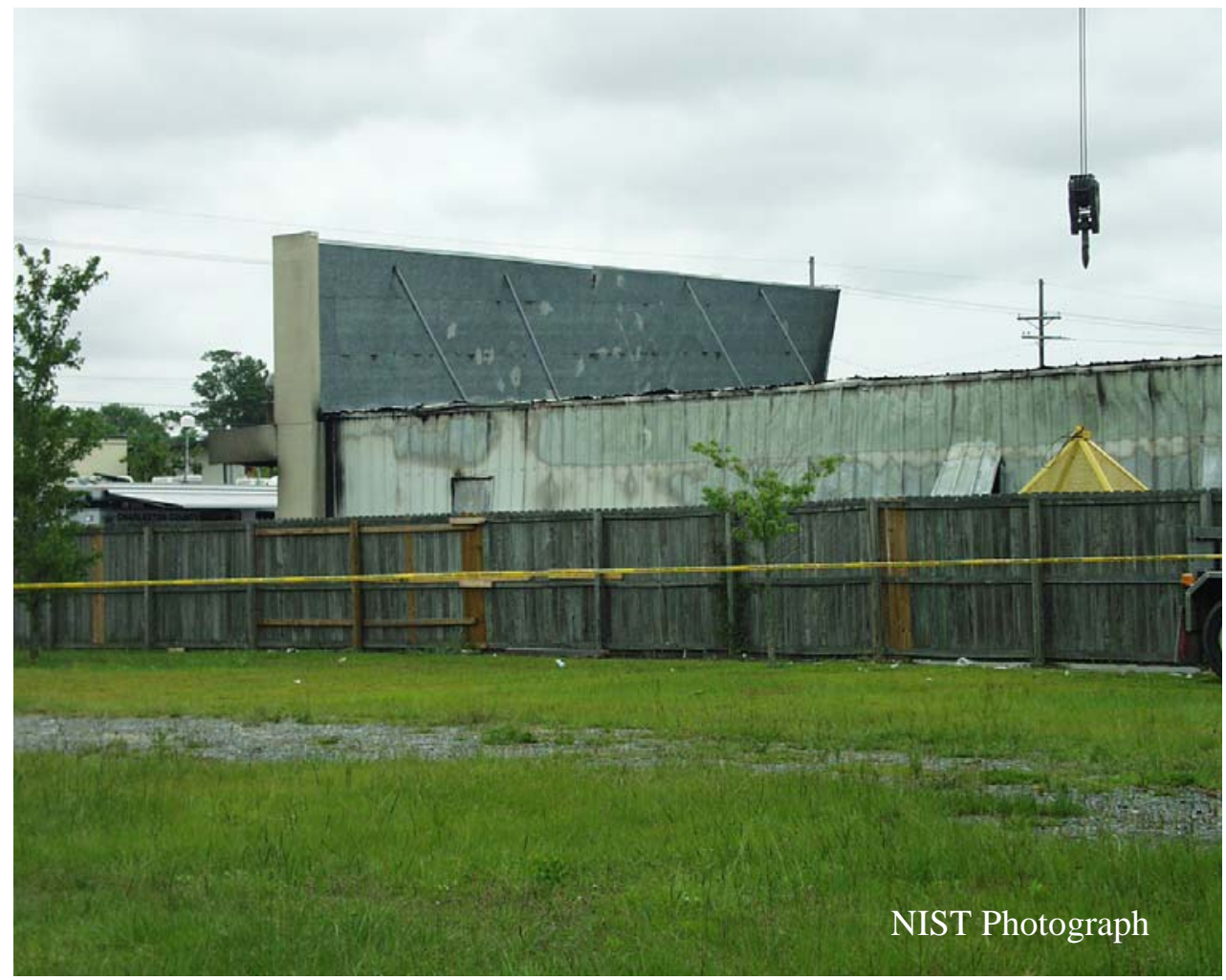

Figure E-5. Rear of front wall of west showroom. Metal sided west wall of showroom also visible in center of photo. Wooden fence separates furniture store from an adjacent property.

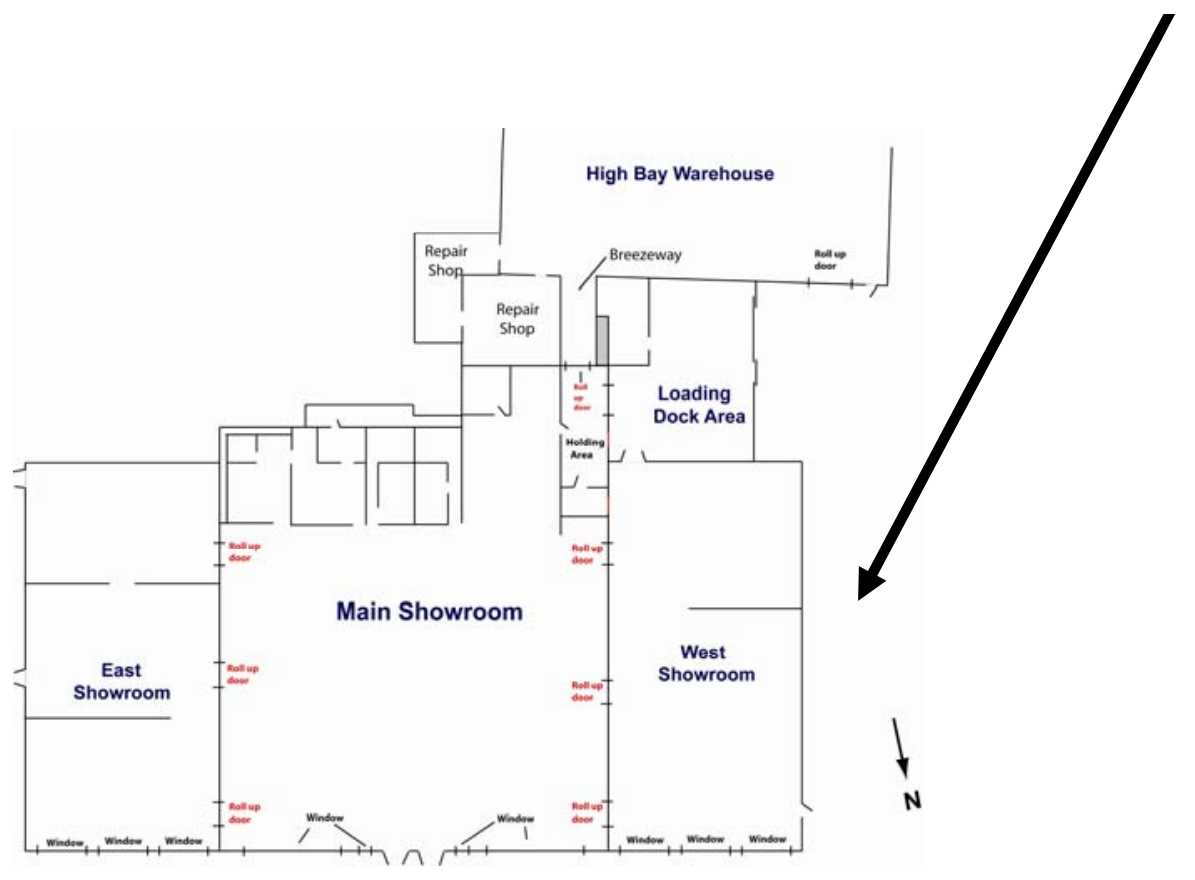




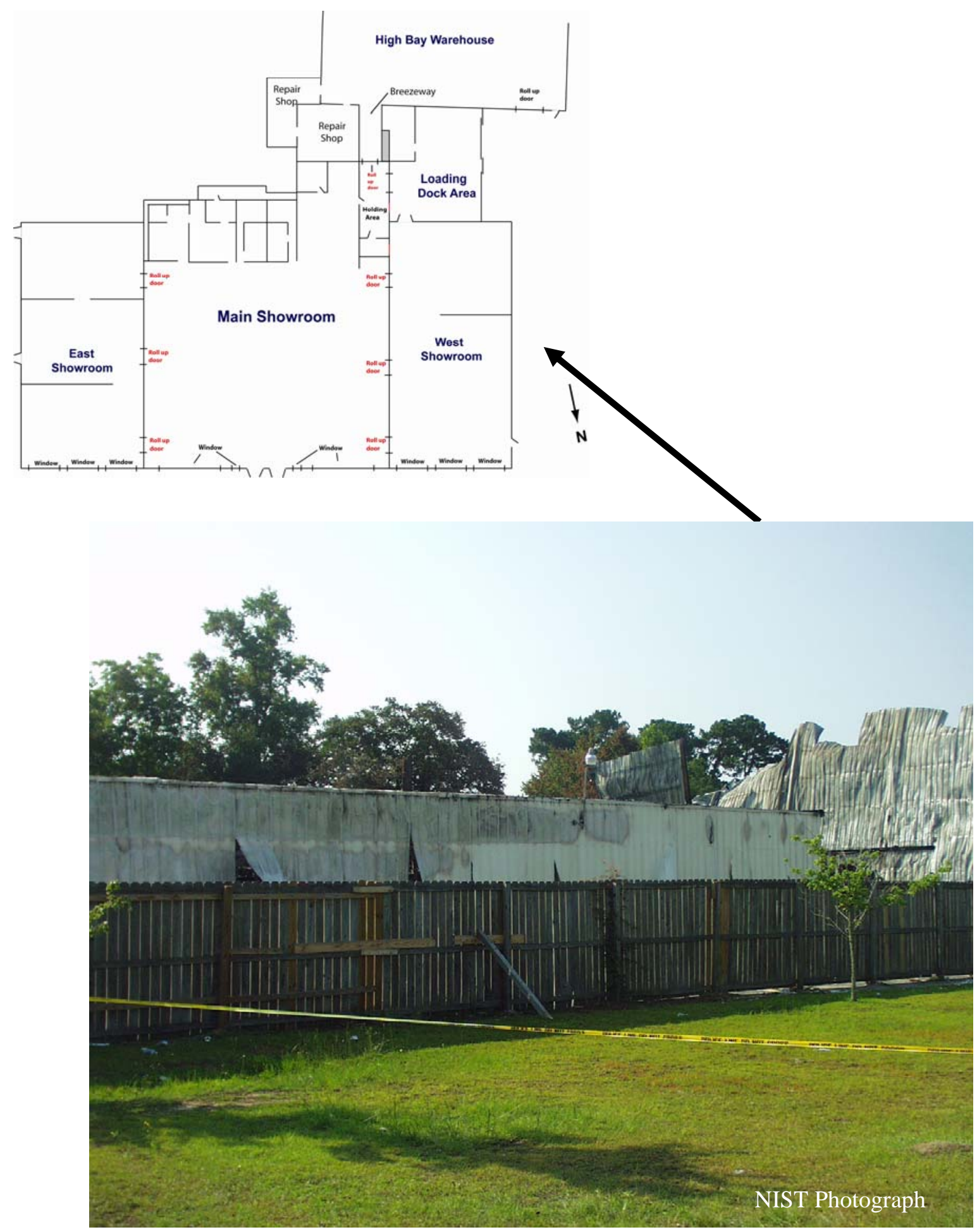

Figure E-6. West wall of west showroom. Discoloration patterns due to exposure to high temperatures visible on metal siding. Front section of west showroom displayed more discoloration than rear section of west showroom. Wooden fence separates furniture store from adjacent property. 

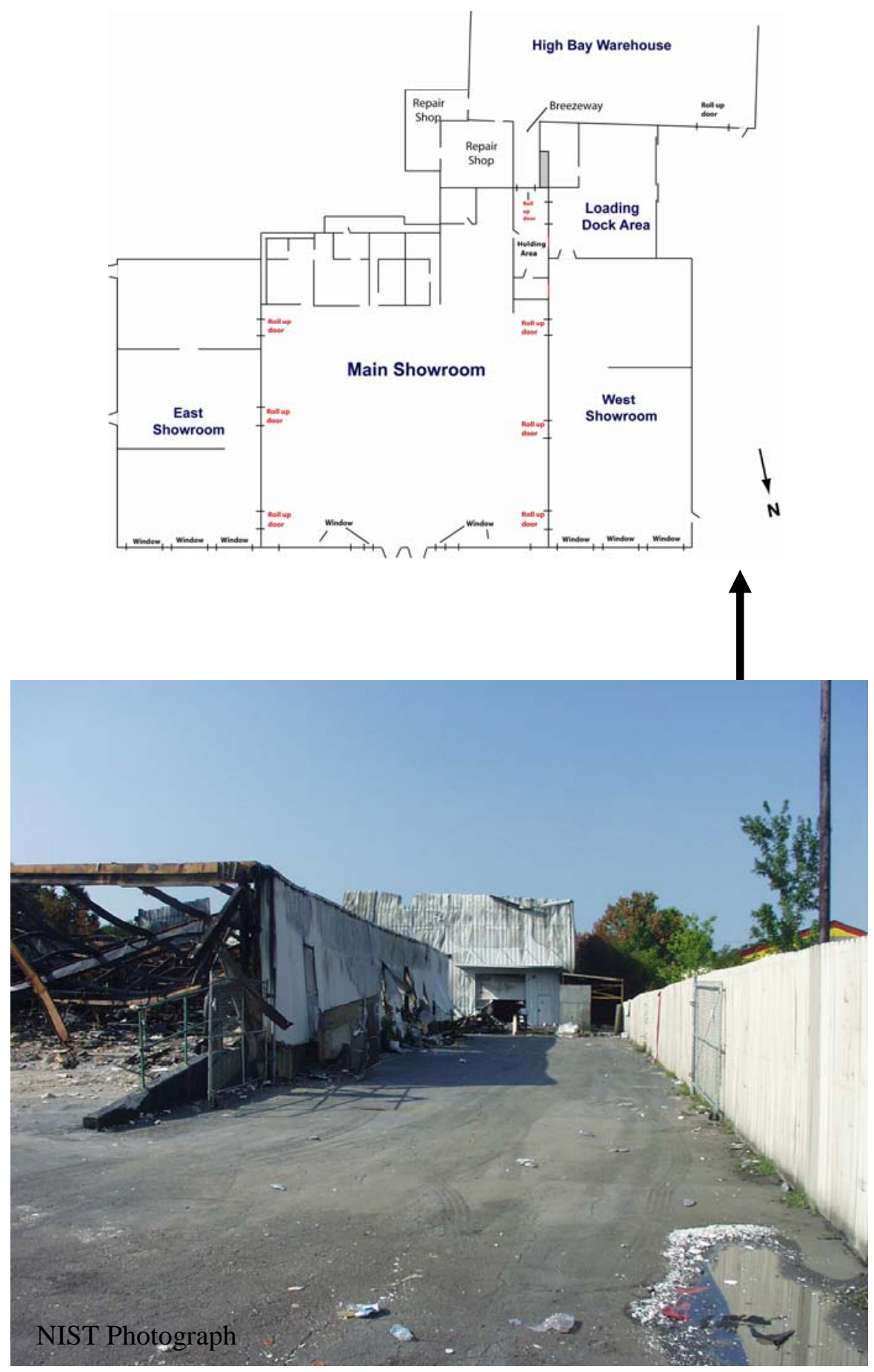

Figure E-7. Exterior view of west wall of west showroom. Driveway along side of west wall provides access to enclosed loading dock (not visible in photo) and warehouse loading area (visible in background of photo). Wood fence on right of the driveway separates furniture store from an adjacent property. 

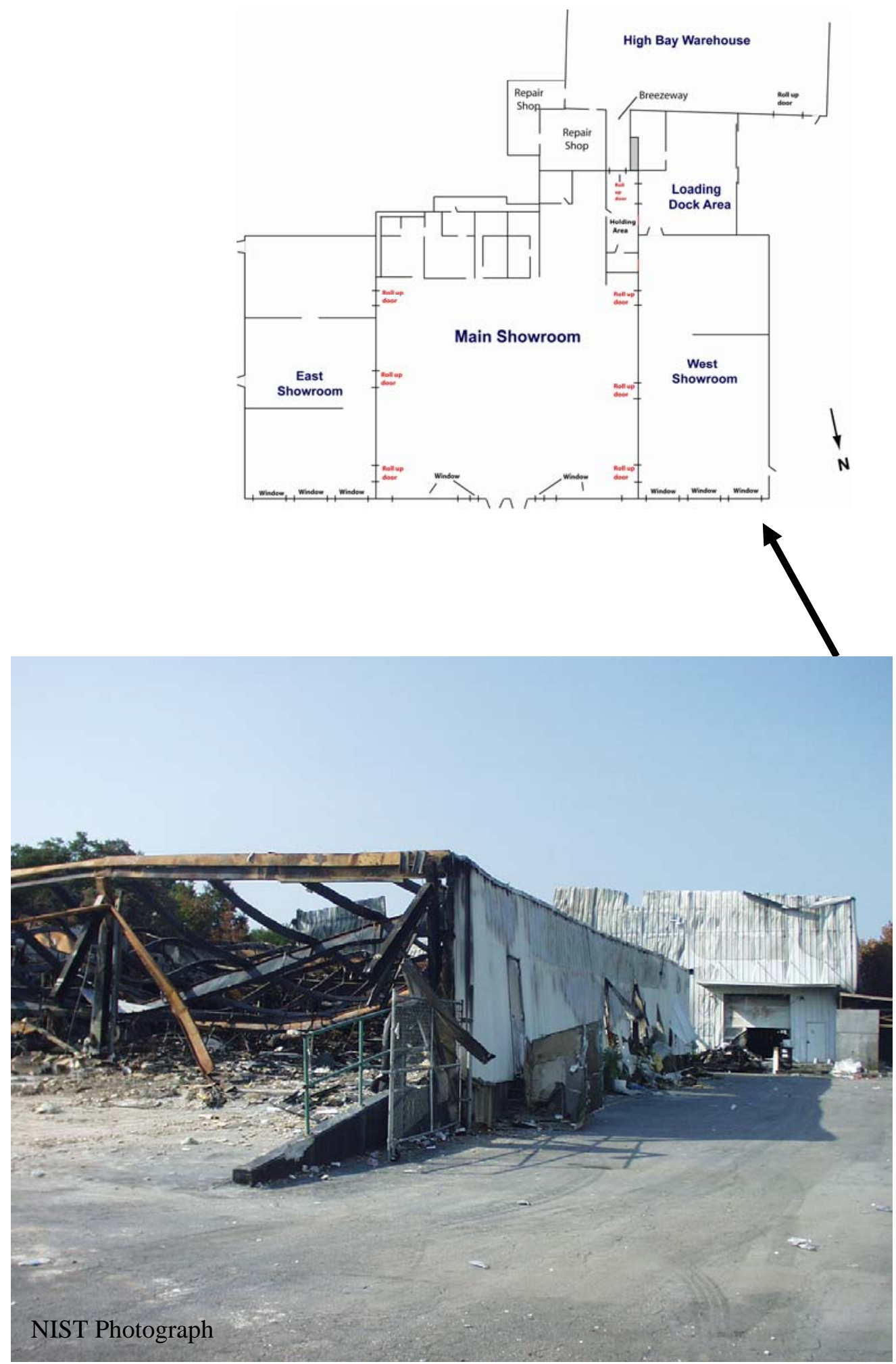

Figure E-8. Northwest corner of west showroom. Exit door on west wall visible in center of photo. 

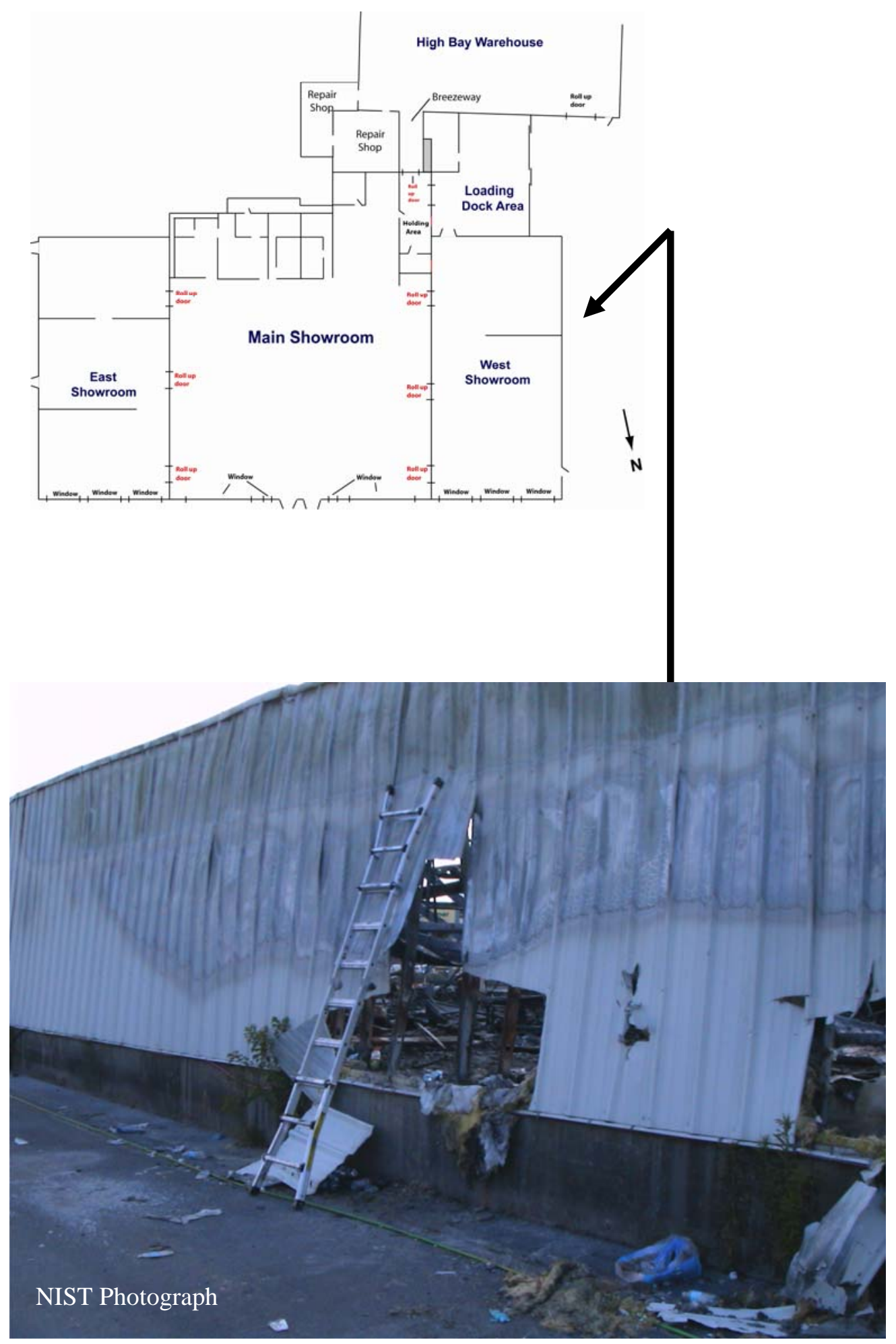

Figure E-9. Exterior of west wall of west showroom. Discoloration of metal siding visible at top of wall. Fire department cut access holes through metal siding during the fire. 


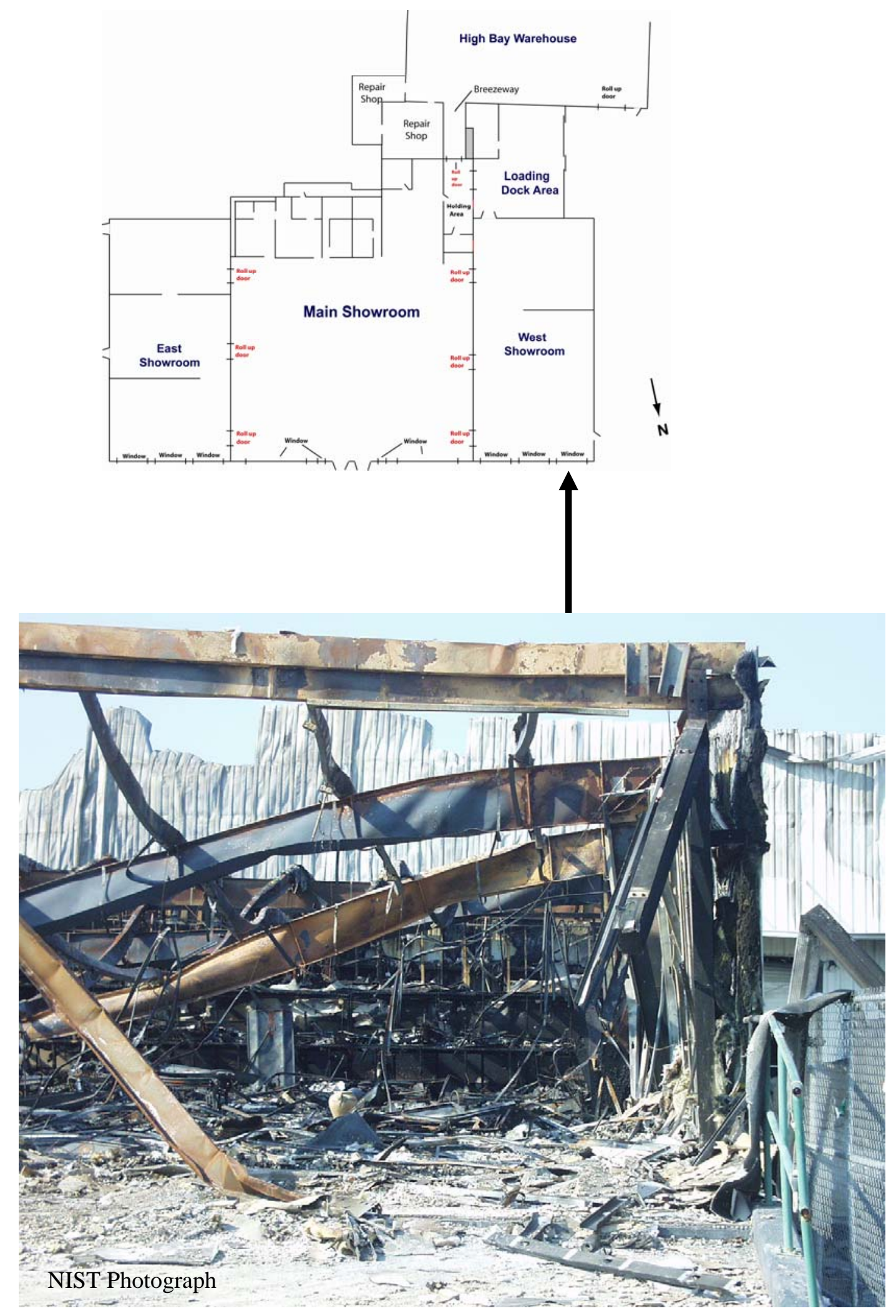

Figure E-10. Northwest corner of west showroom after removal of façade and roof. 


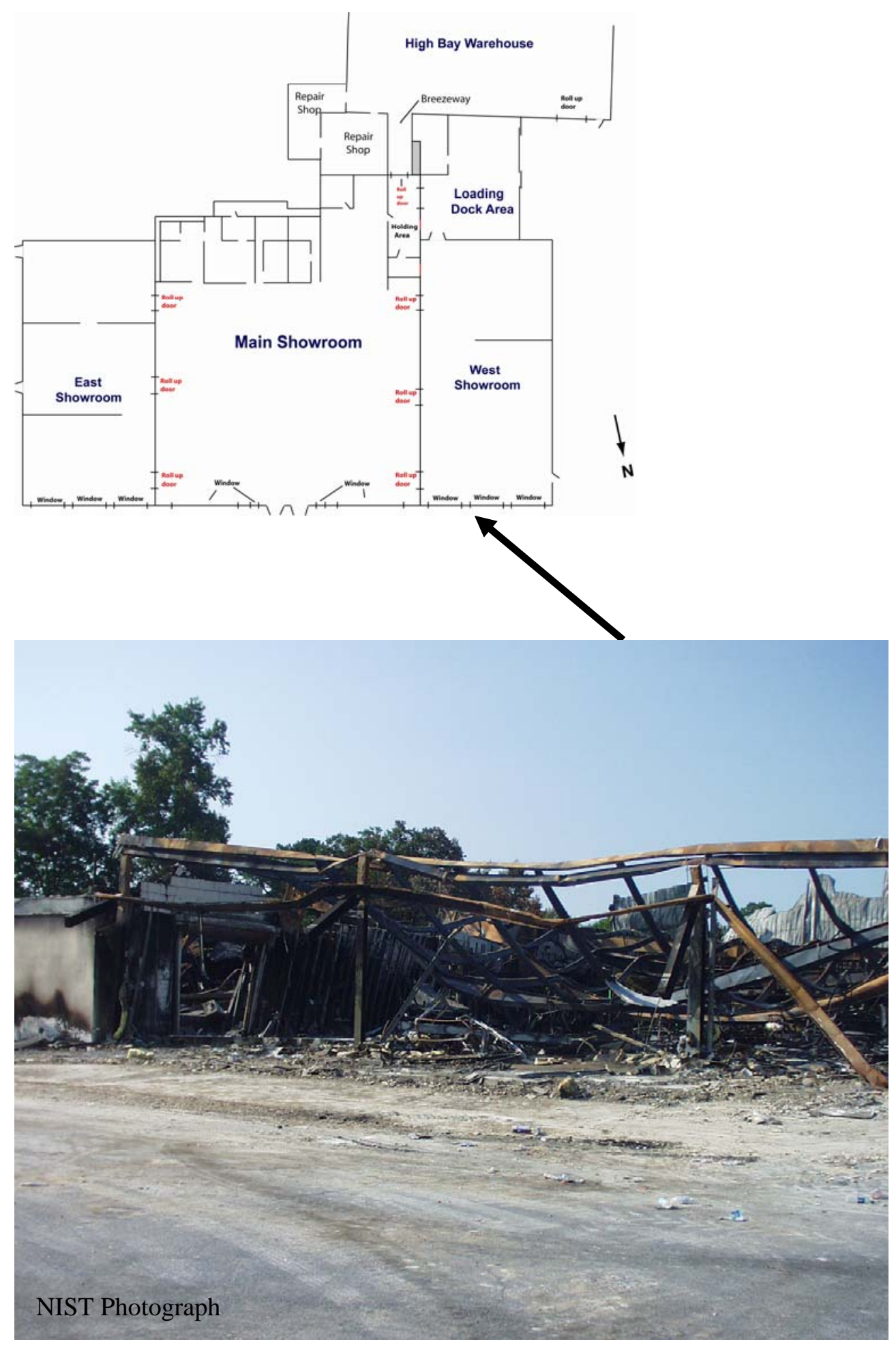

Figure E-11. Exterior view of west showroom looking toward main showroom. Open roll-down fire door visible in left center of photo. 

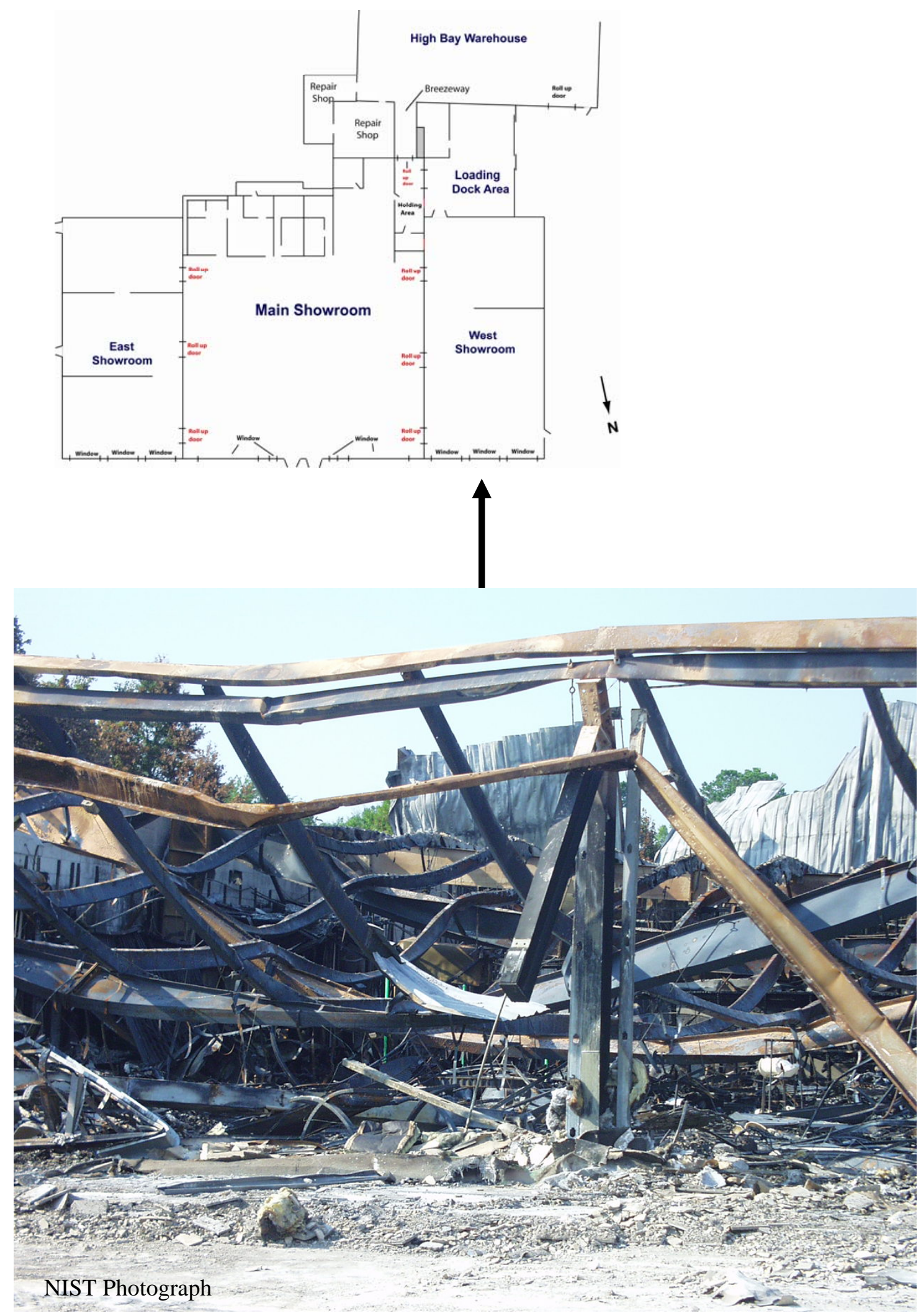

Figure E-12. Exterior view of west showroom looking into enter-front portion of showroom. Roof Z-channel and I-beam supports sagged to within $1 \mathrm{~m}(3.3 \mathrm{ft})$ of showroom floor. 


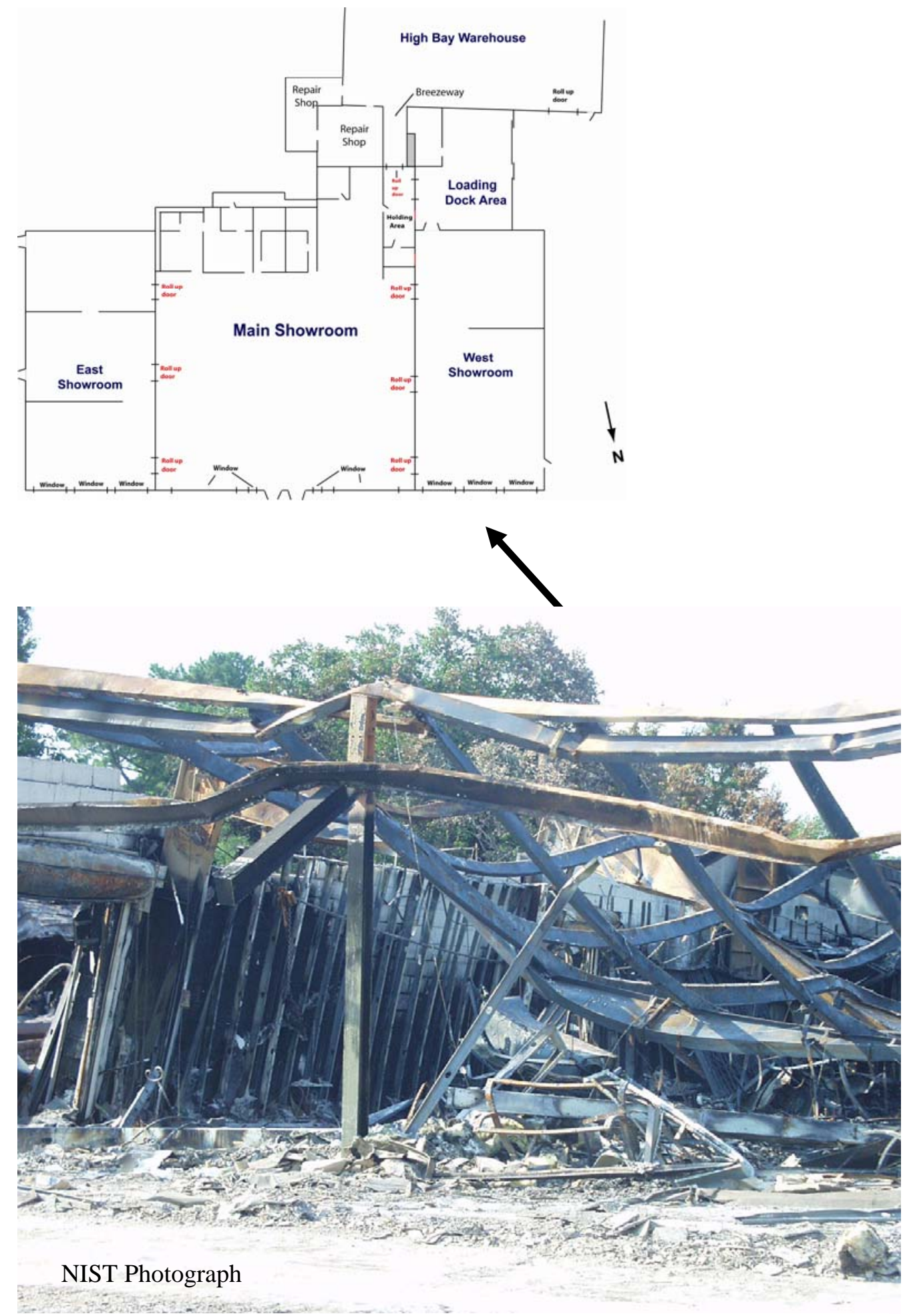

Figure E-13. Exterior view of west showroom looking into east-front portion of showroom. Metal stud wall attached to west wall of main showroom visible in center of photo. Open roll-down fire door partially visible at left center of photo. 

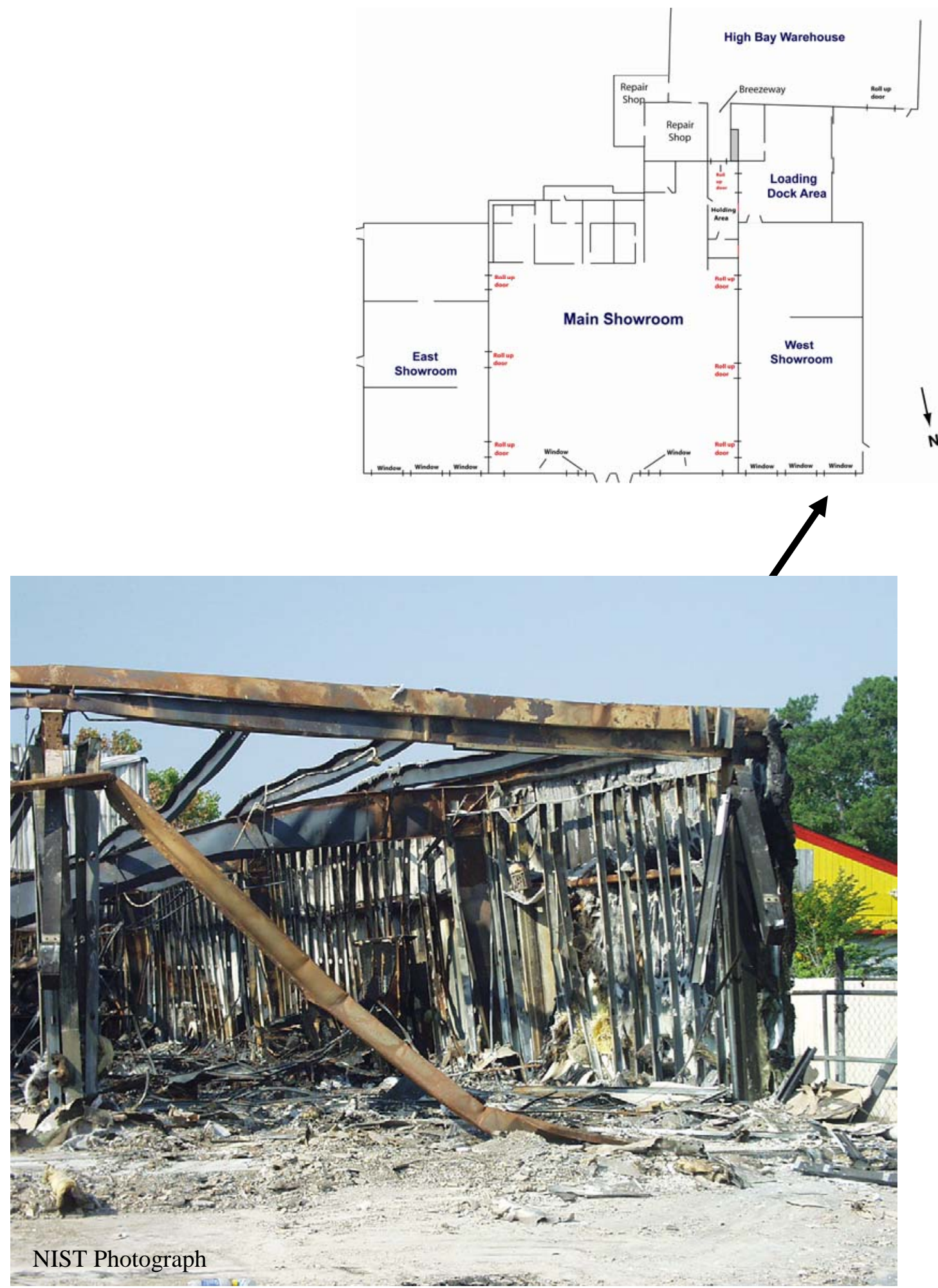

Figure E-14. Exterior view of west showroom looking into north-west corner of showroom. Vertical metal studs of interior wall visible in photo. Yellow automobile repair garage visible in right center background. 


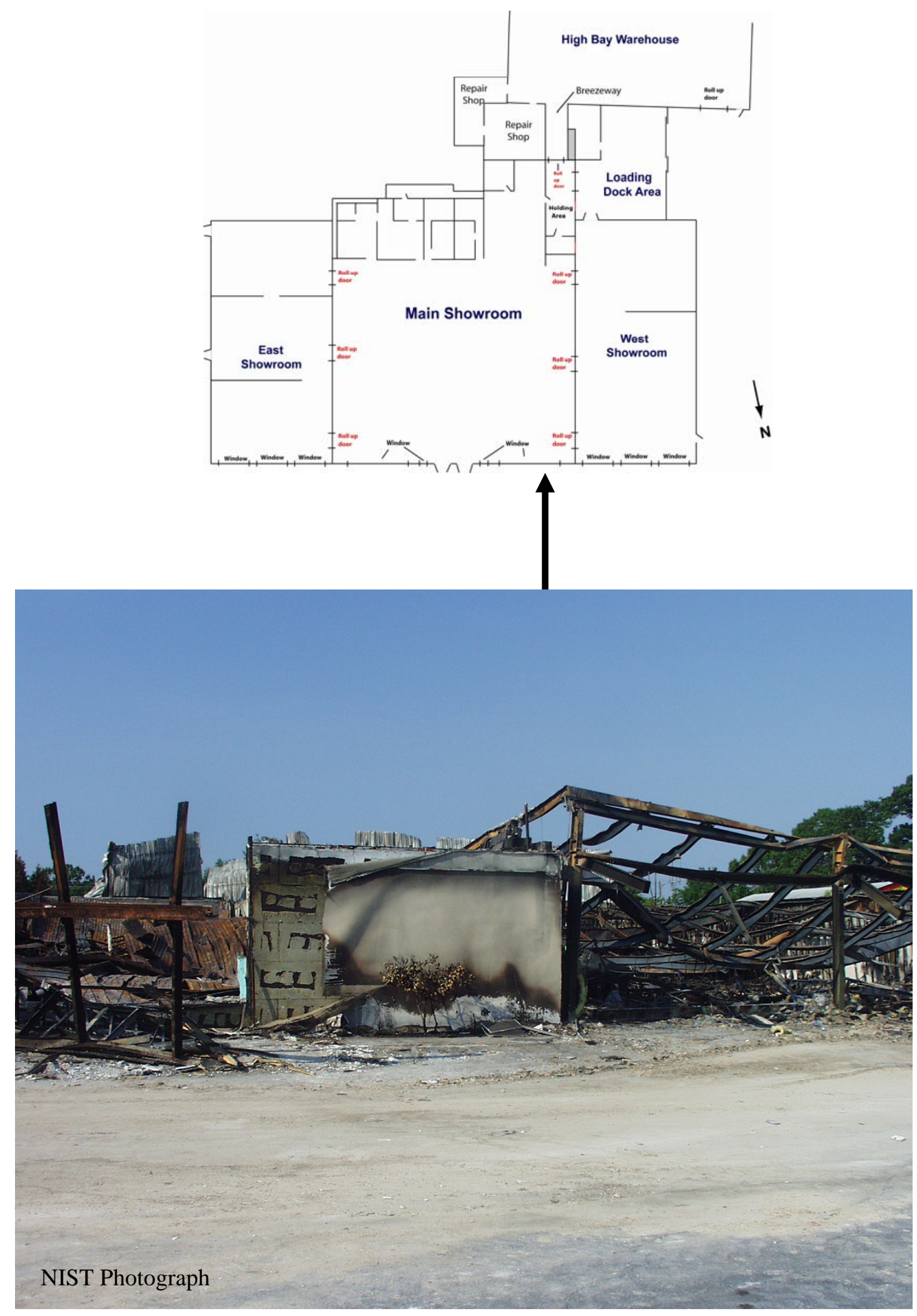

Figure E-15. Exterior view of front walls of main and west showrooms. Solid wall of main showroom is visible in center of photo. 


\section{E.2 WEST SHOWROOM INTERIOR PHOTOS}

A series of photos were taken after the fire of the inside of the west showroom. Portions of the parapet wall, façade, and roof had been removed as a safety measure to prevent debris falling on personnel.

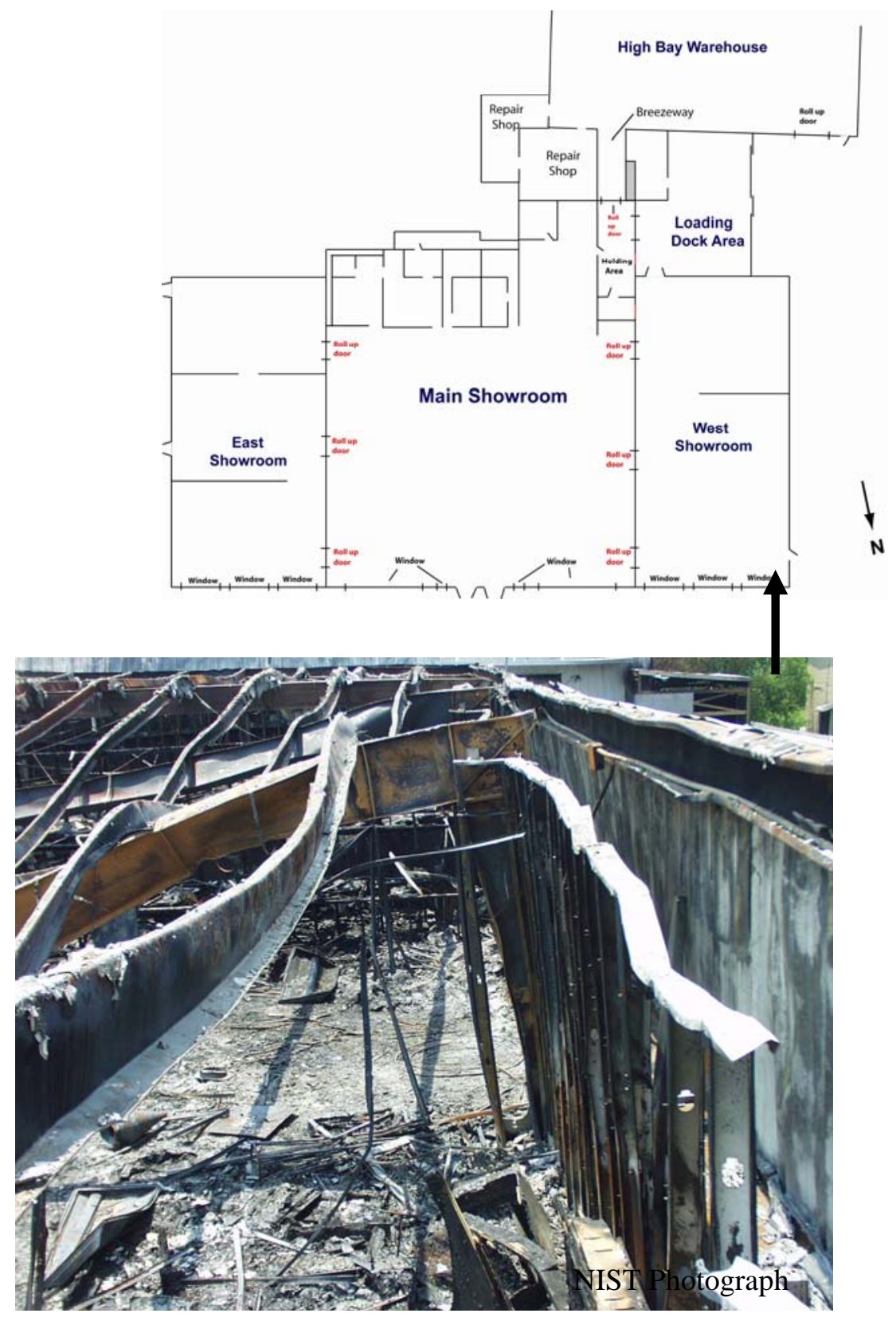

Figure E-16. Interior view of west showroom at top of exterior wall. View looking into showroom toward rear of showroom. 


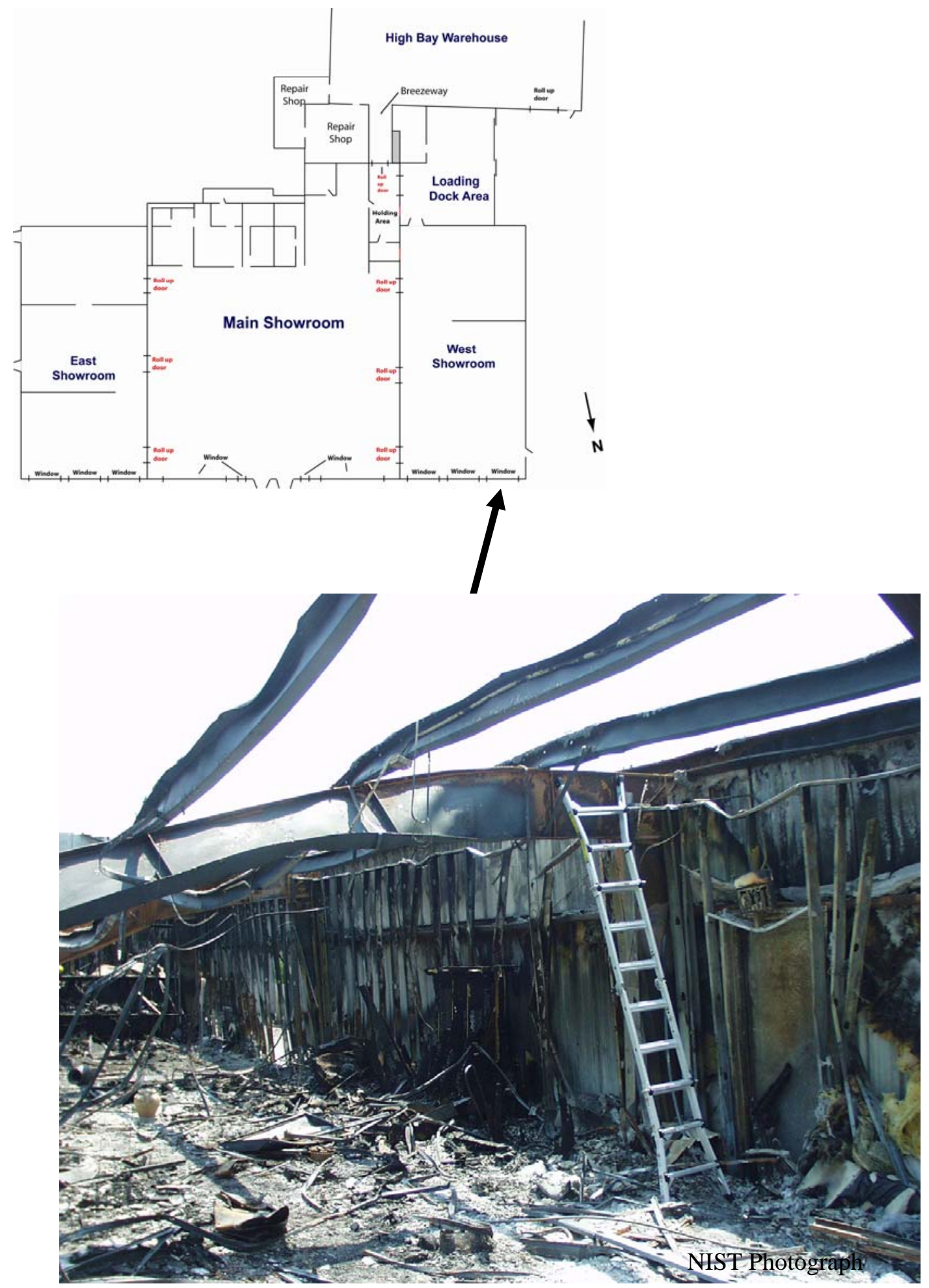

Figure E-17. Interior view of north-west corner of west showroom. Sagging Ibeam and roof Z-channel visible in photo. Exit door in west wall near front of showroom visible to the right of ladder. 

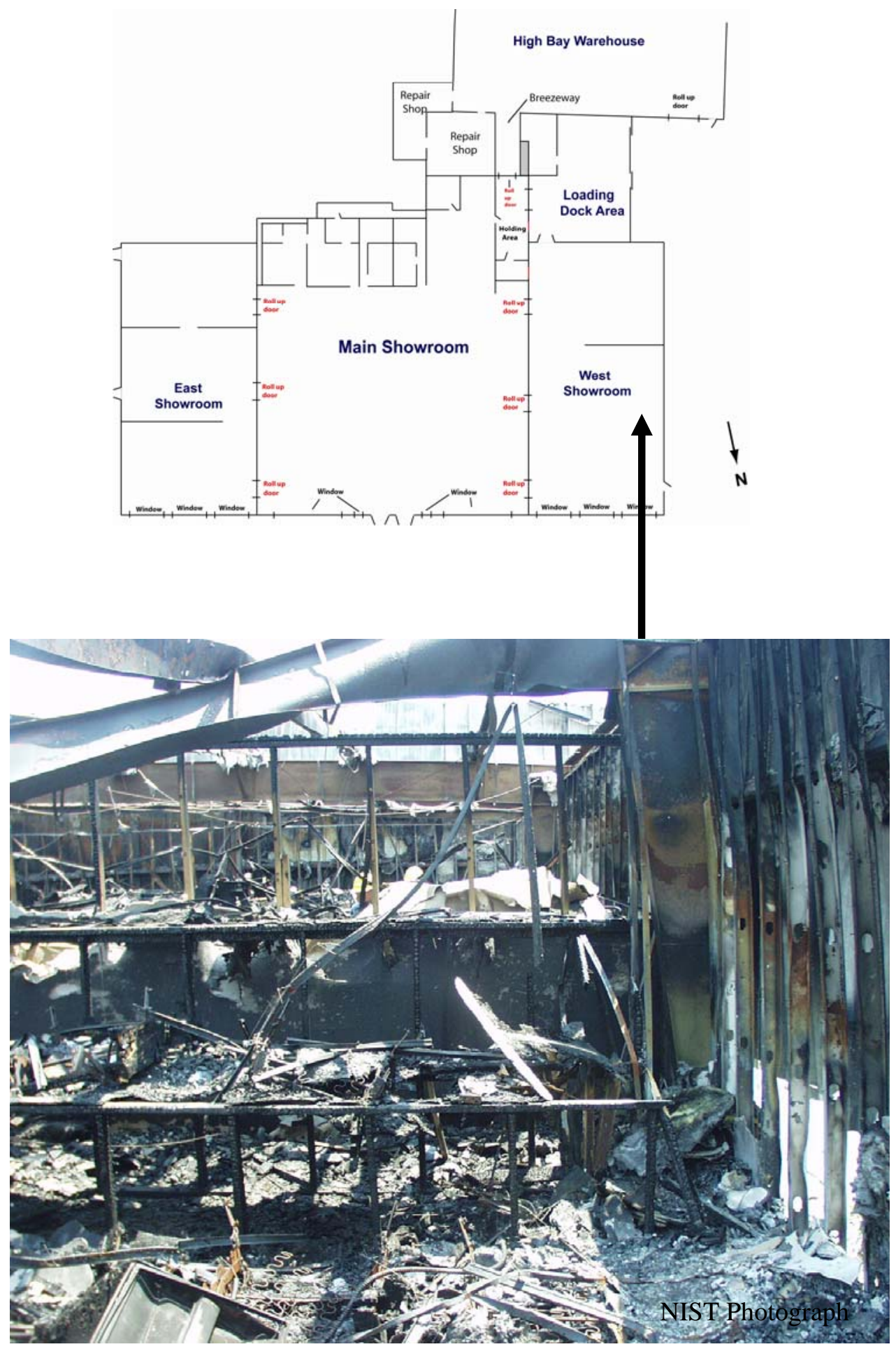

Figure E-18. Interior view along west wall of west showroom. Wood supports for two level display platforms visible in center of photo. Vertical wood studs of partition wall visible behind display platform remnants. Steel I-beam column and roof support also visible on right and top of photo. 


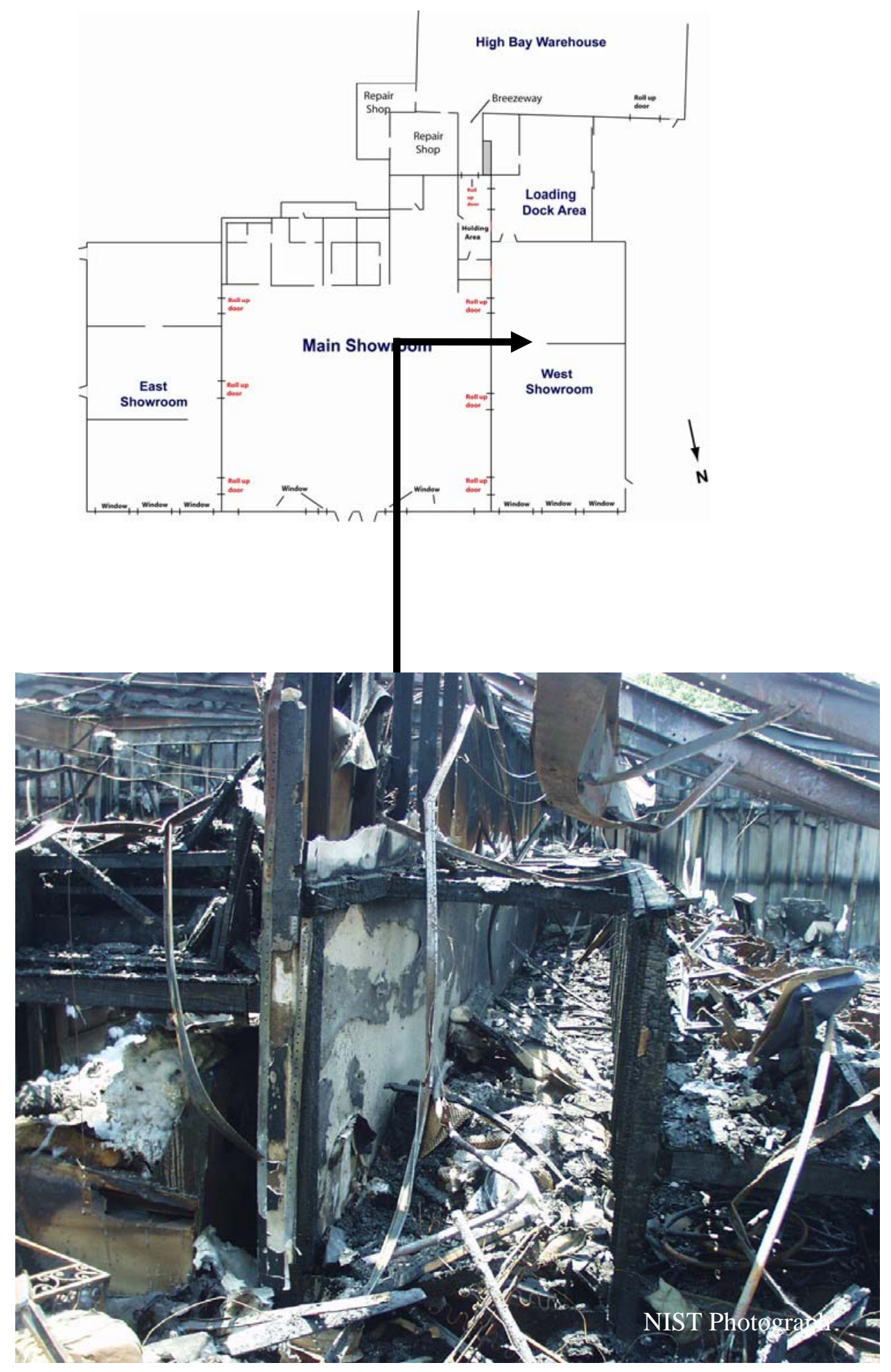

Figure E-19. Interior view of west showroom looking toward west wall. Three tiered recliner chair display shelves visible on right of partition wall. Two tiered sofa and futon display shelves visible on left of partition wall. 


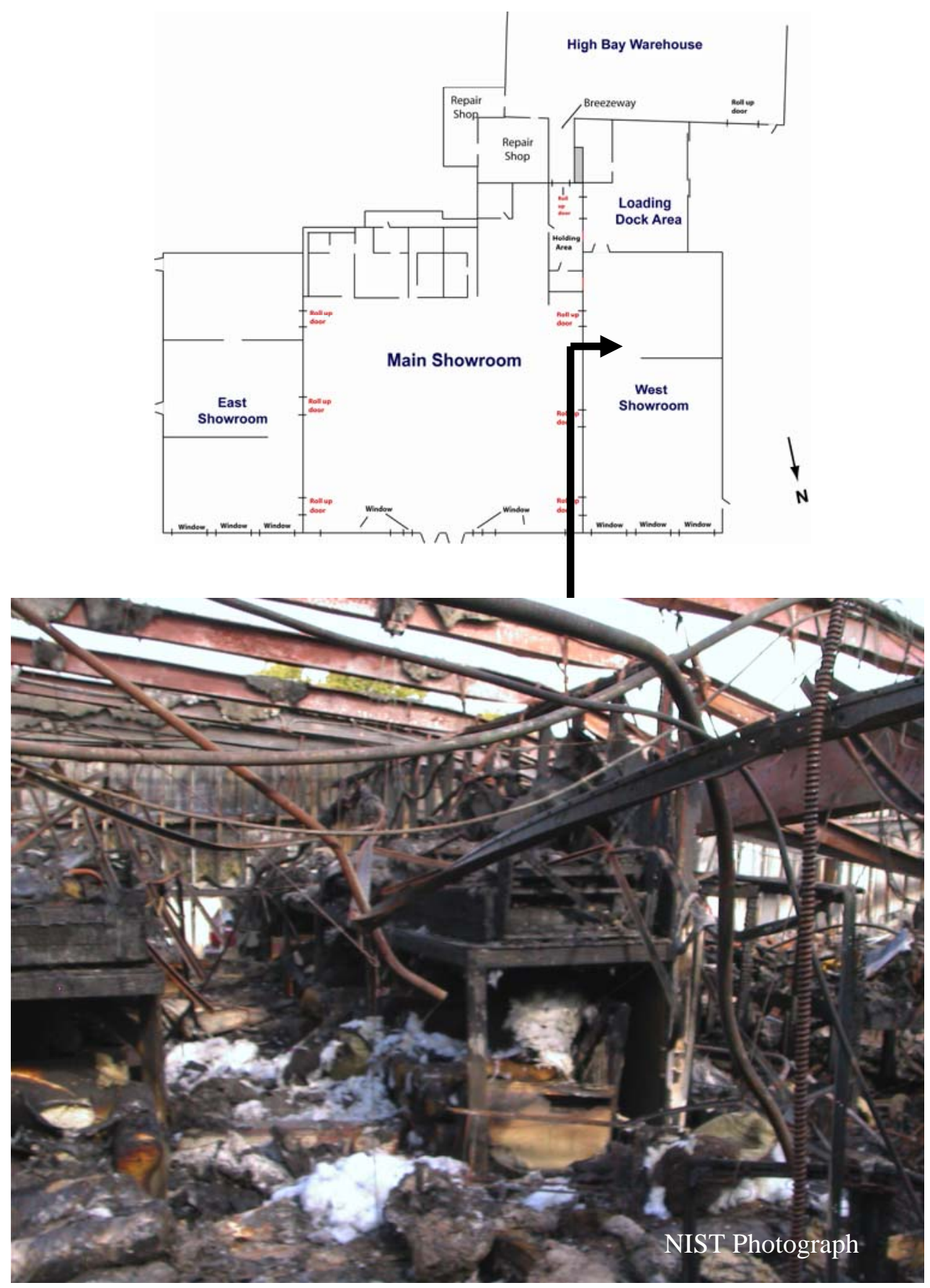

Figure E-20. Interior of rear portion of west showroom looking toward west wall. Two tiered sofa and futon display shelves visible. 


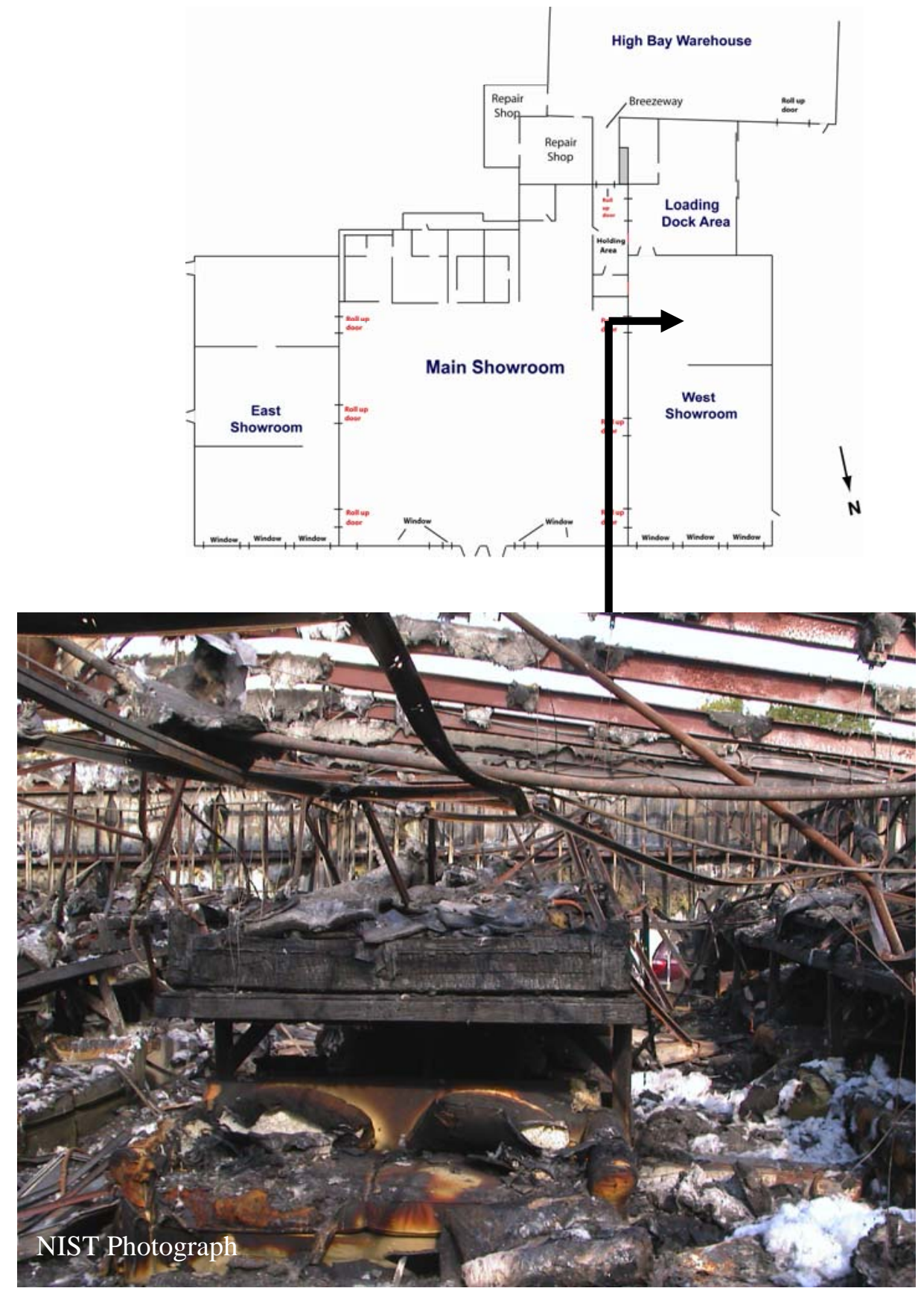

Figure E-21. Interior of rear portion of west showroom looking toward west wall. Two tiered sofa and futon display shelves visible. 


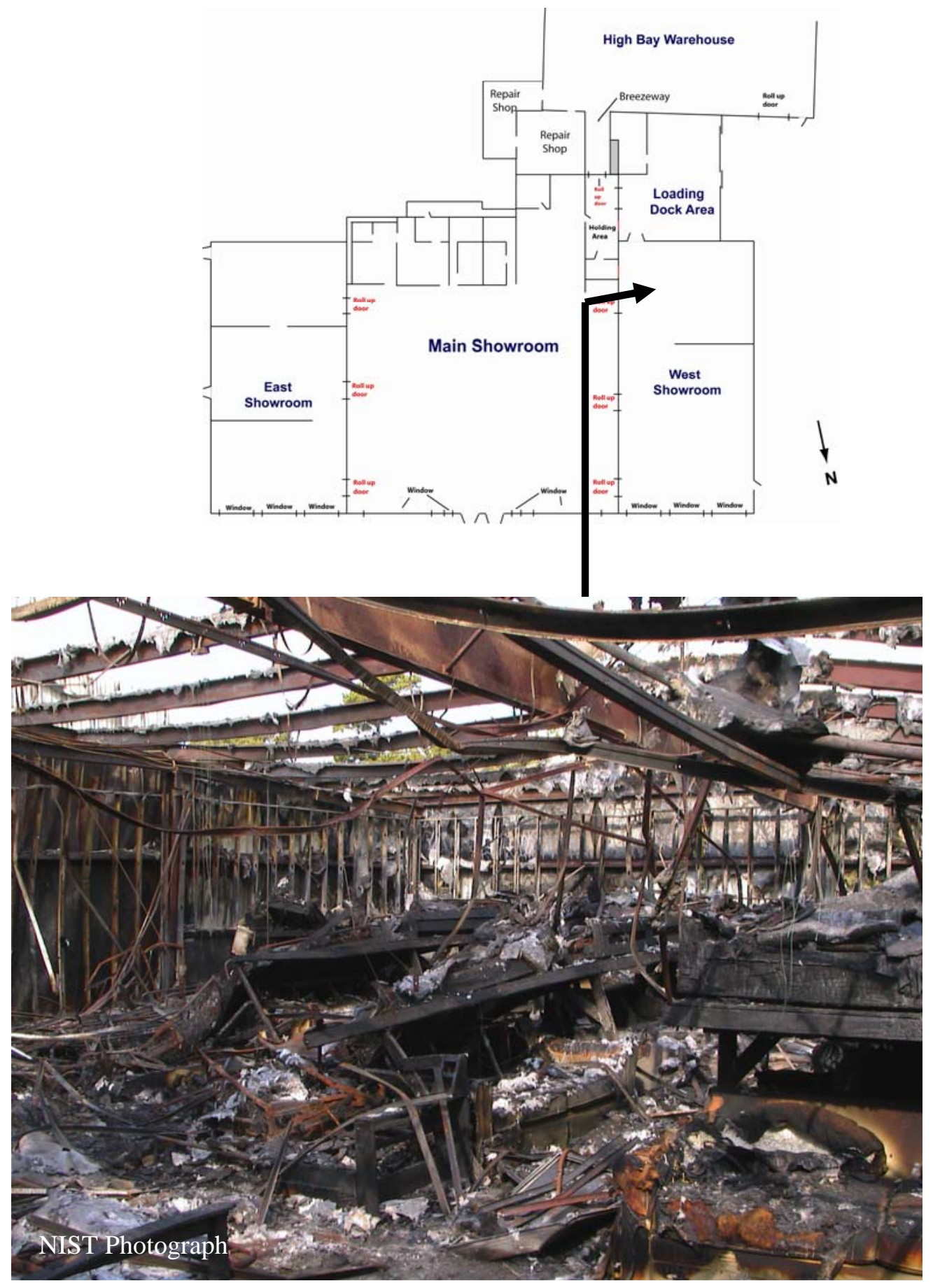

Figure E-22. Interior of rear portion of west showroom looking toward west rear corner. Two tiered sofa and futon display shelves visible. 


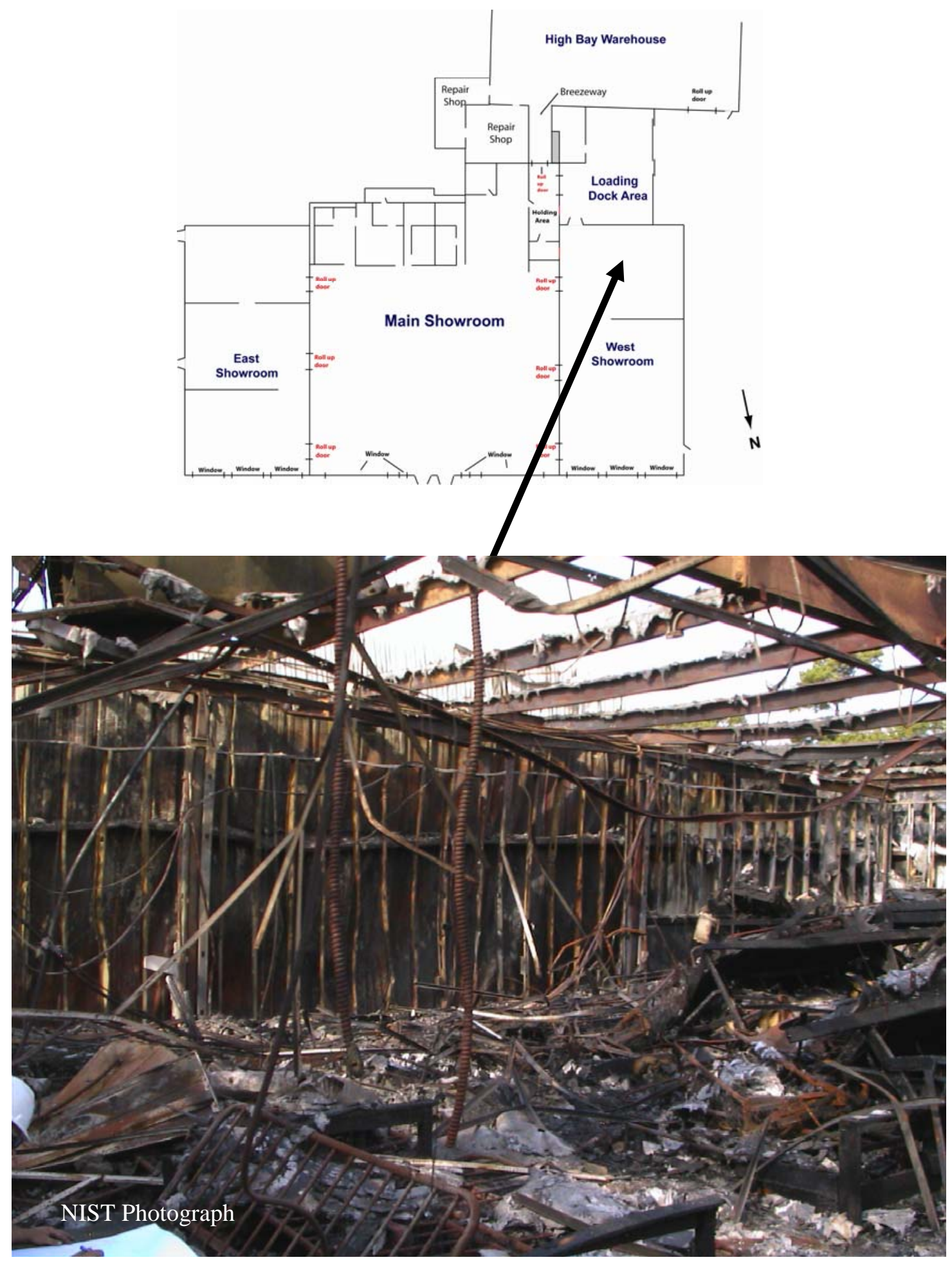

Figure E-23. Interior of rear portion of west showroom looking toward rear wall. Two tiered sofa and futon display shelves are visible on right side. 

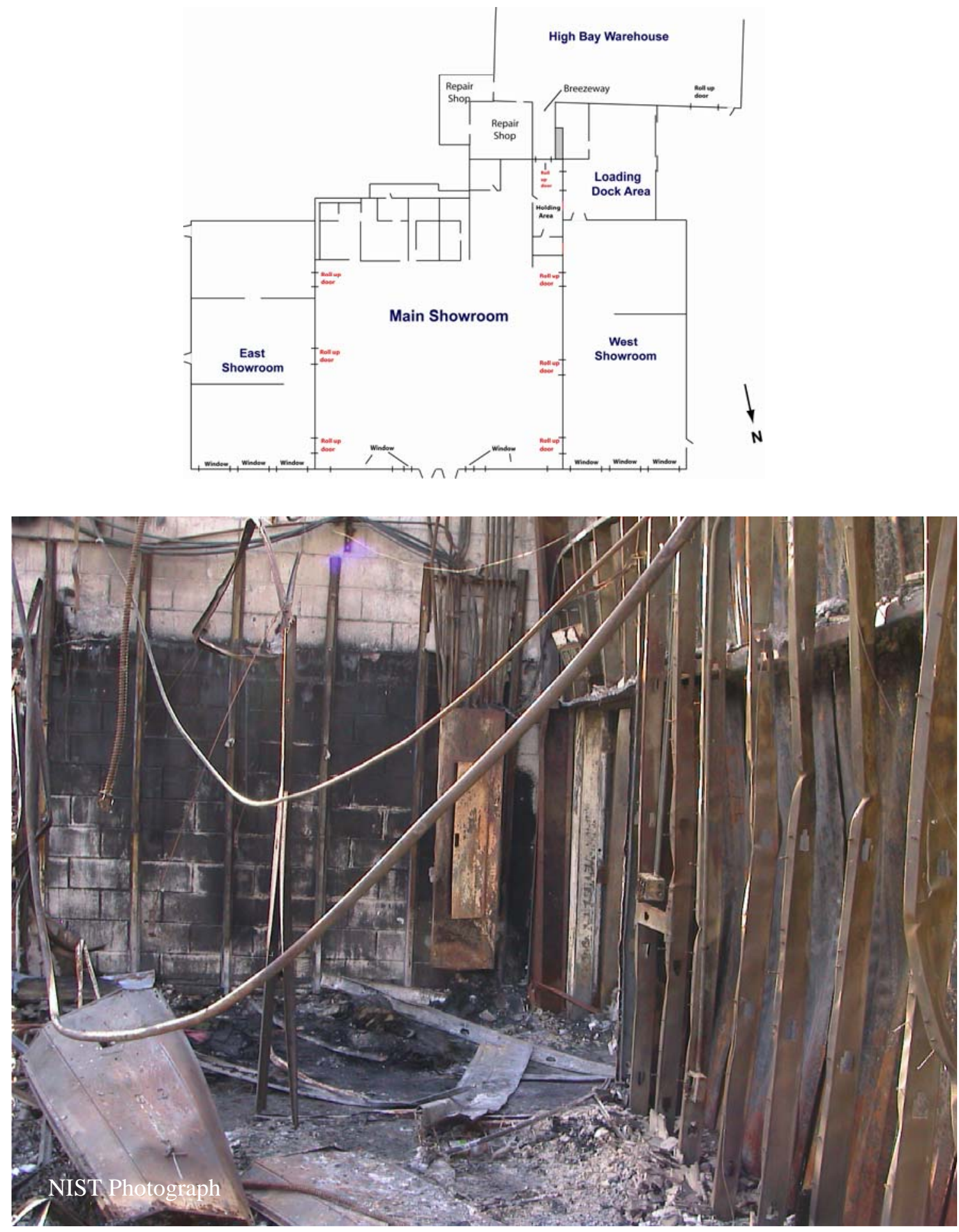

Figure E-24. Interior of west showroom looking along rear wall toward steel double doors. Doors opened into loading dock area, so not visible, but opening to loading dock in center of photo. 


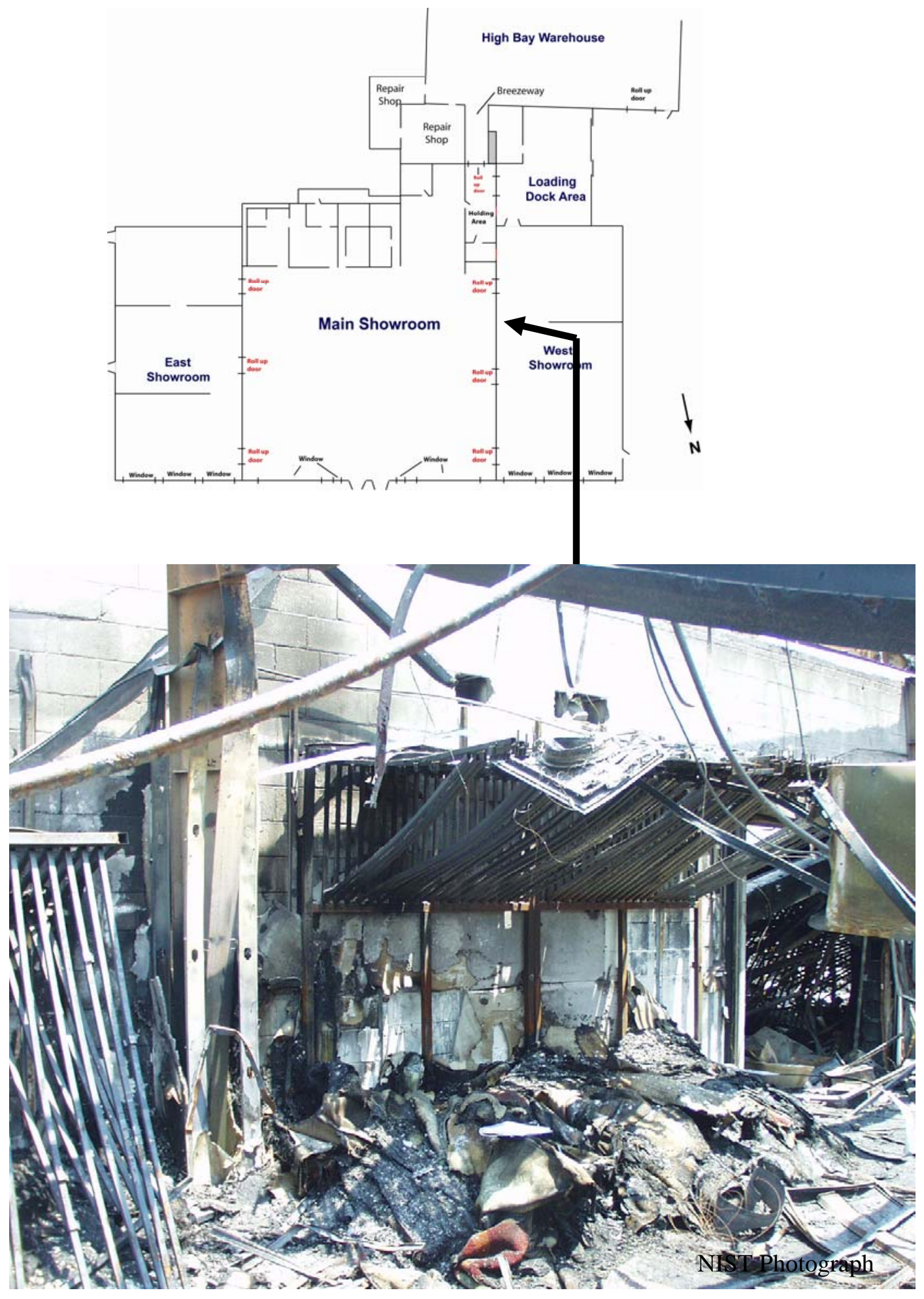

Figure E-25. Area rug support racks in west showroom. Open roll-down fire door visible on right of photo. 


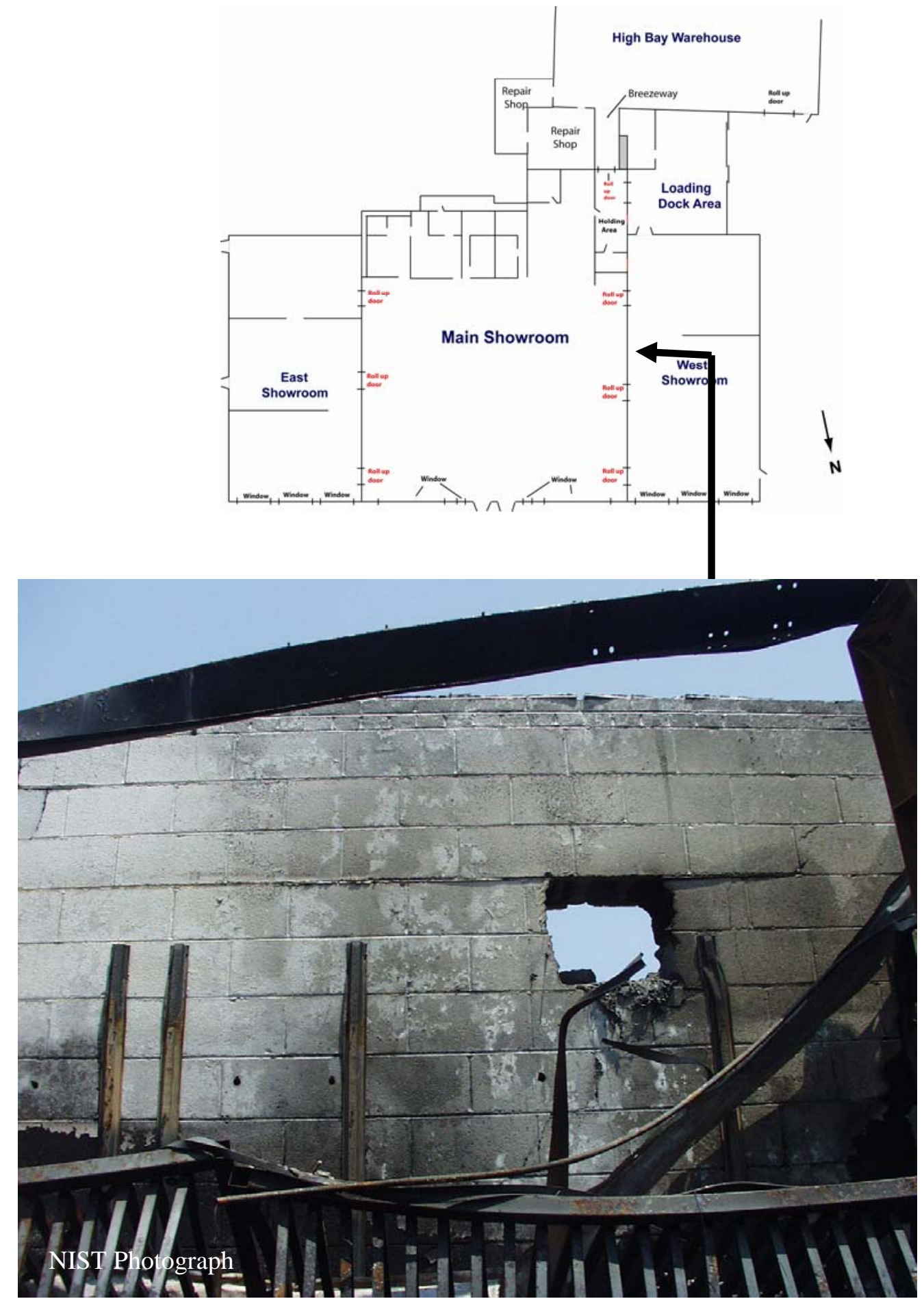

Figure E-26. Interior of west showroom above suspended ceiling looking toward main showroom. Open hole, approximately $0.5 \mathrm{~m}(1.5 \mathrm{ft}) \times 0.5 \mathrm{~m}(1.5 \mathrm{ft})$ opening through concrete block wall. This hole may have allowed smoke to move from interstitial space above suspended ceiling in main showroom to the interstitial space above the suspended ceiling in the west showroom. 

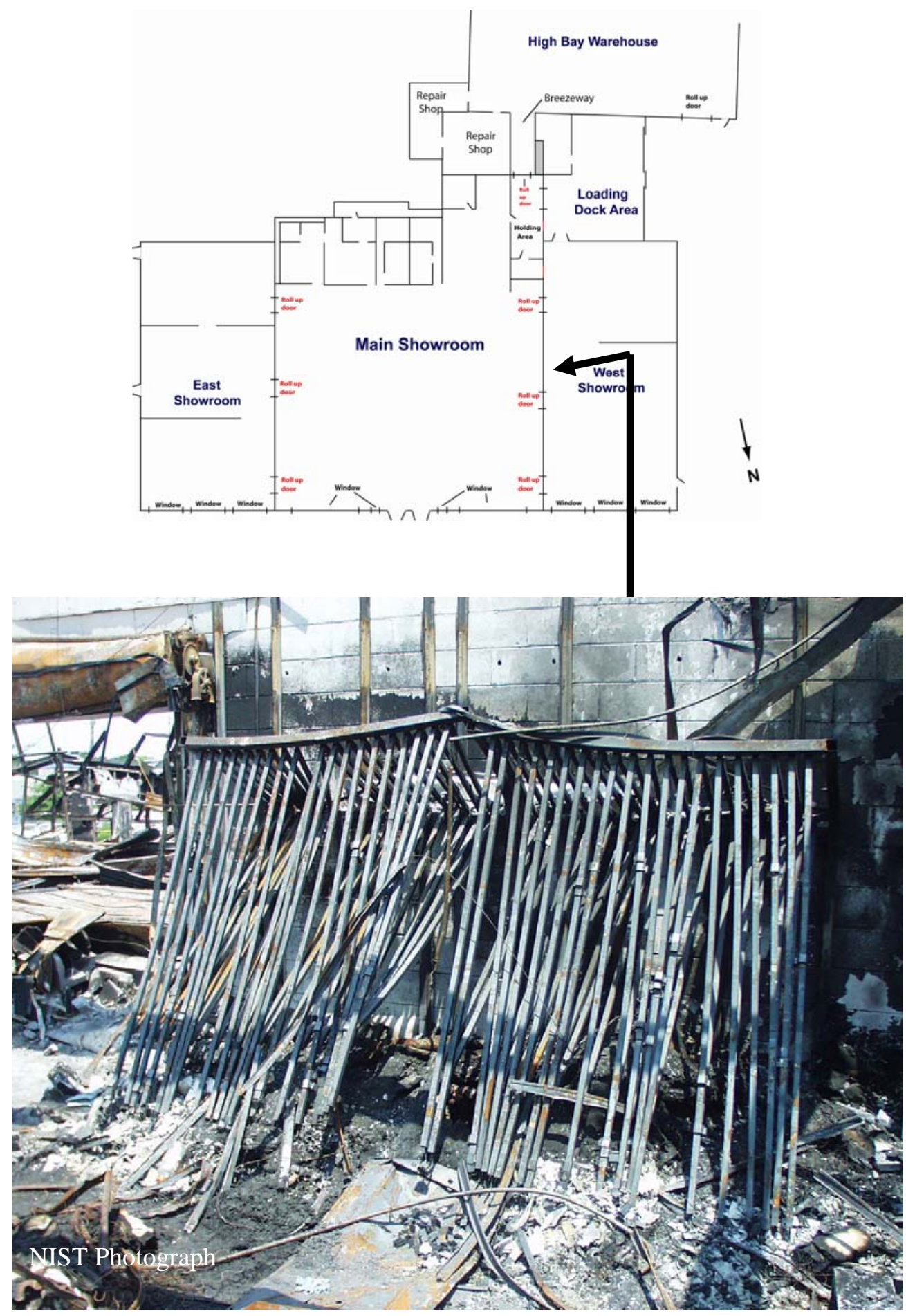

Figure E-27. Additional area rug support racks in west showroom. Open roll-down fire door visible on left of photo. 


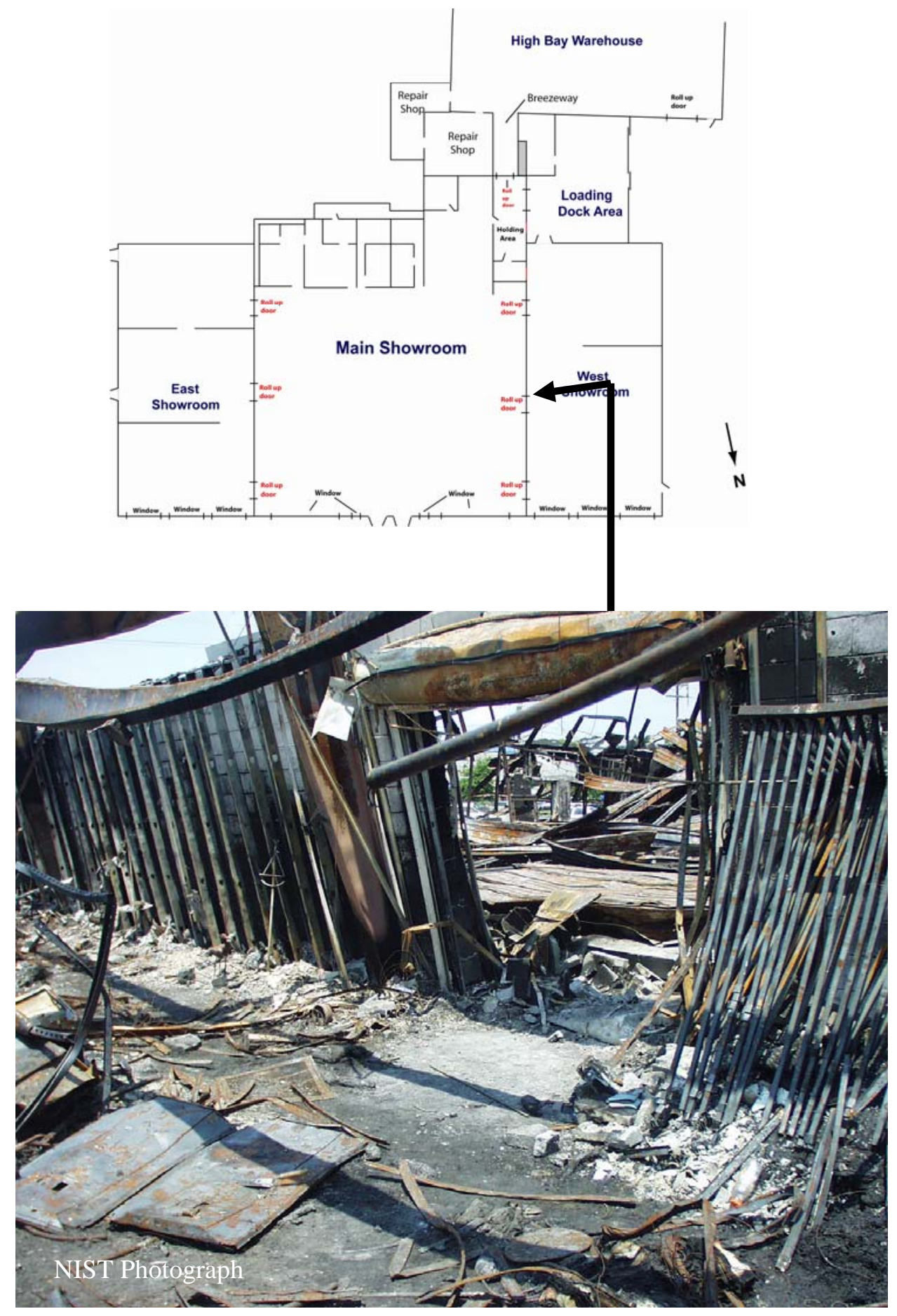

Figure E-28. Interior of west showroom looking through open roll-down fire door into main showroom. 


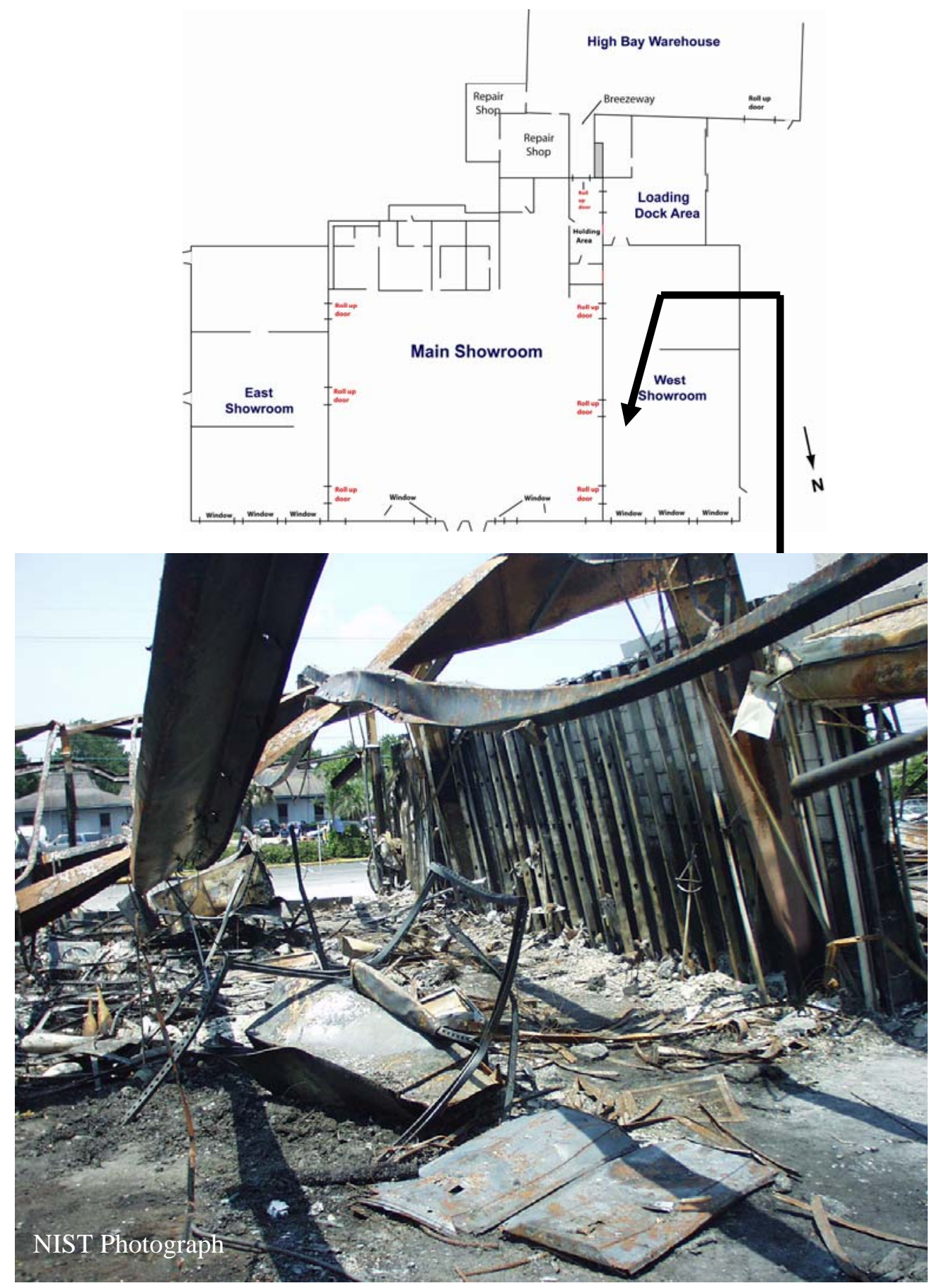

Figure E-29. Interior of west showroom along east wall toward front of store. Open roll-down fire door visible at right of photo. 

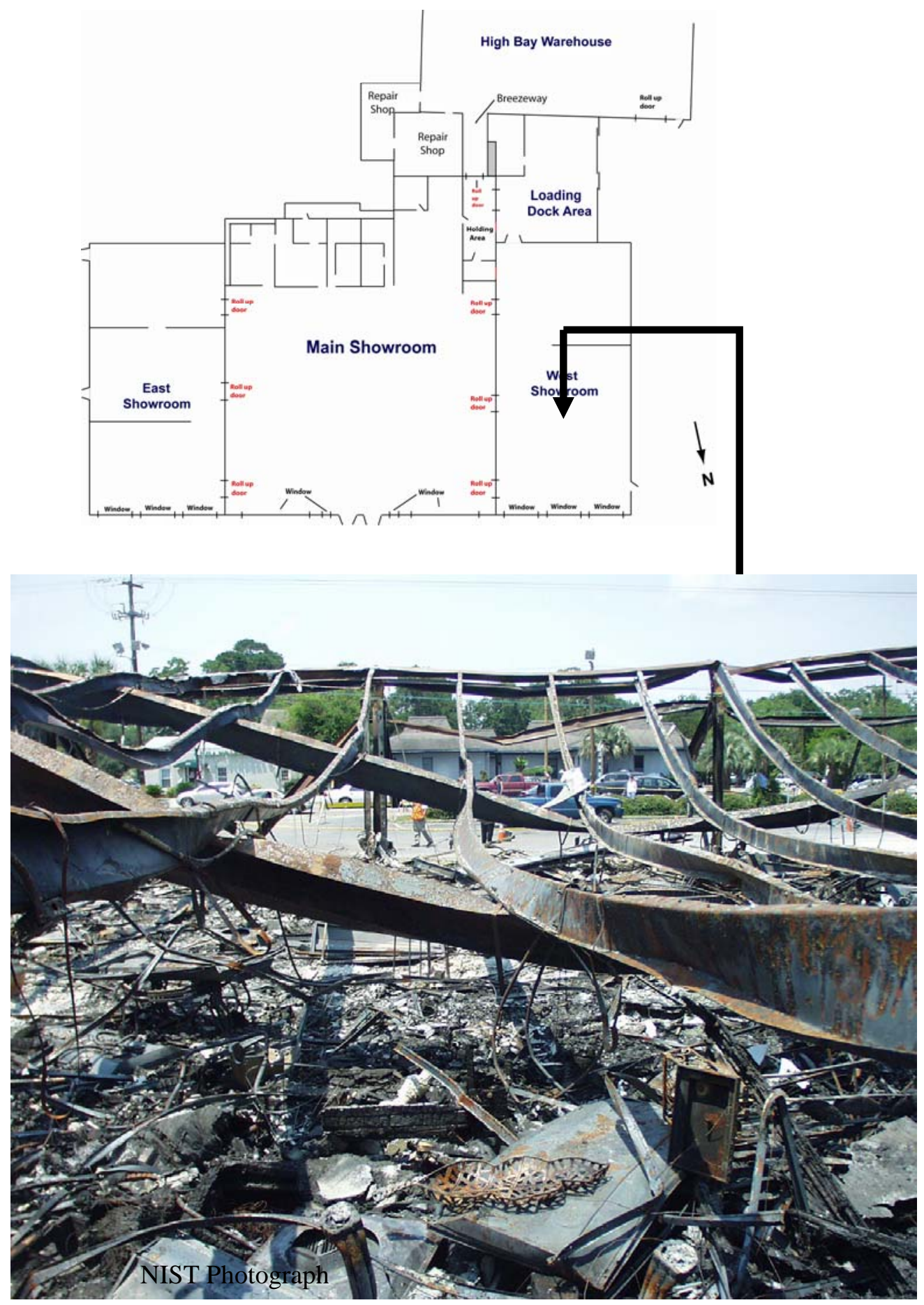

Figure E-30. Interior of west showroom looking toward front of showroom. Portions of two (left to right) I-beam steel supports and nine Z-channel roof supports are visible. Exposure to high temperatures caused I-beam and Zchannel to sag within $1 \mathrm{~m}$ (3.3 ft) of showroom floor. 


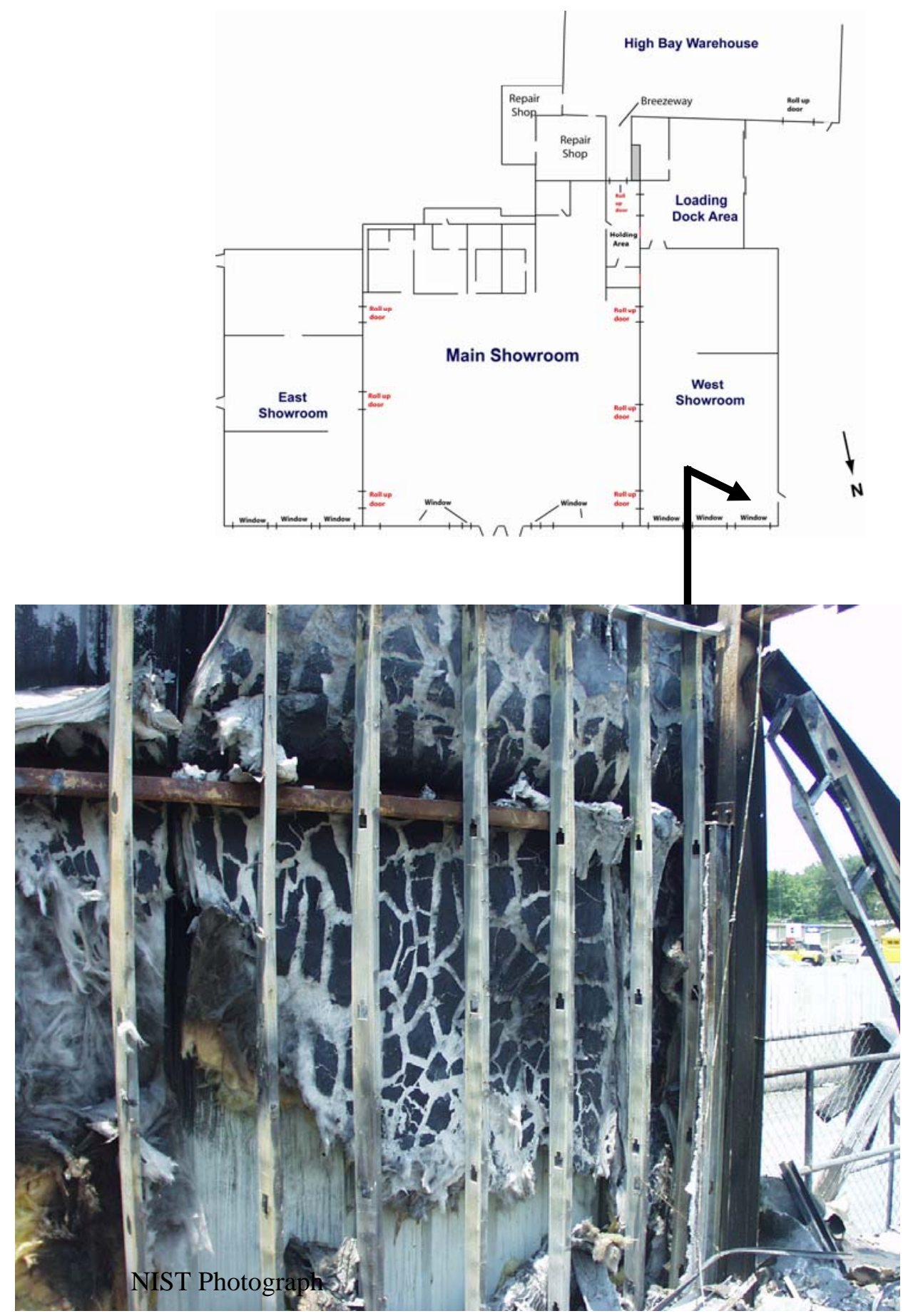

Figure E-31. Interior of west showroom looking at west wall near front of store. Exterior metal wall attached to exterior side of horizontal channel. Metal studs attached to inside face of horizontal channel. Gypsum wall board was installed over metal studs to form interior wall. Metal stud/gypsum wall appeared to extend approximately $0.3 \mathrm{~m} \mathrm{(1} \mathrm{ft)}$ above suspended ceiling along west wall. Fiberglass insulation is visible between metal studs and exterior metal siding. 
E-32 


\section{APPENDIX F. EAST SHOWROOM}

\section{F.1 EAST SHOWROOM EXTERIOR PHOTOS}

A series of photographs of the exterior of the east showroom were taken after the fire.

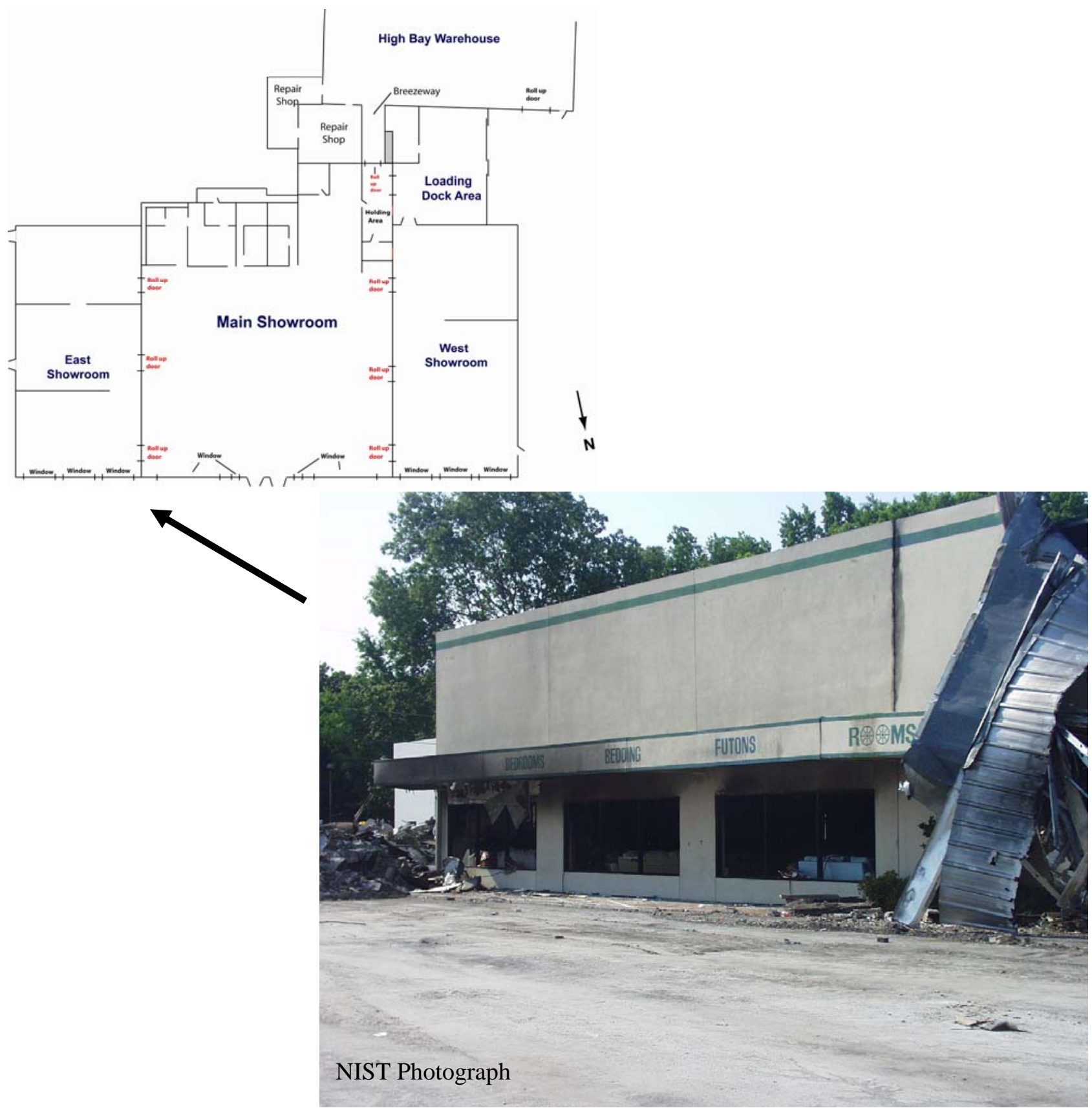

Figure F-1. Exterior view of east showroom from front parking lot. 

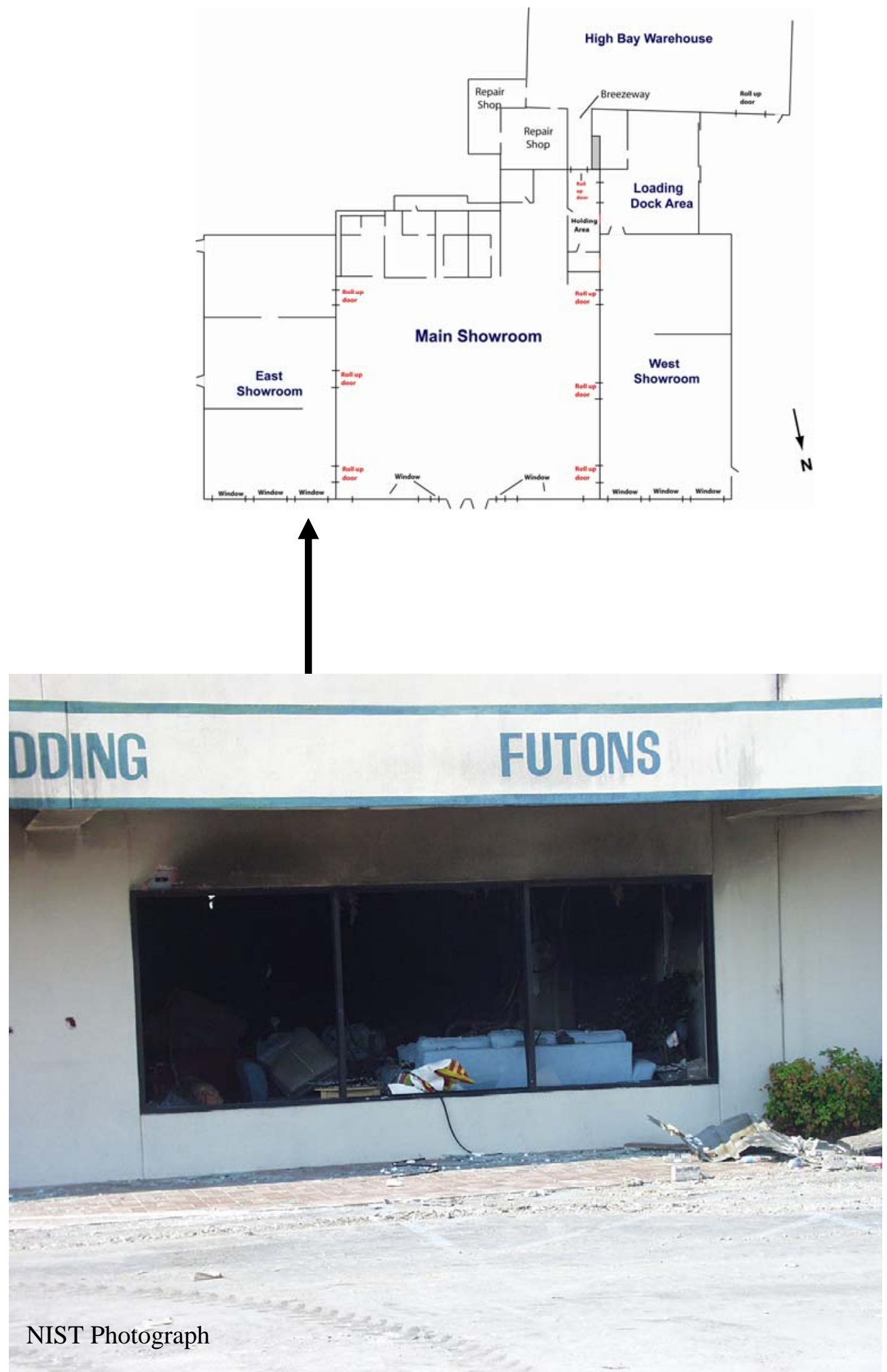

Figure F-2. Exterior view of east showroom looking into west-front window of the east showroom. 


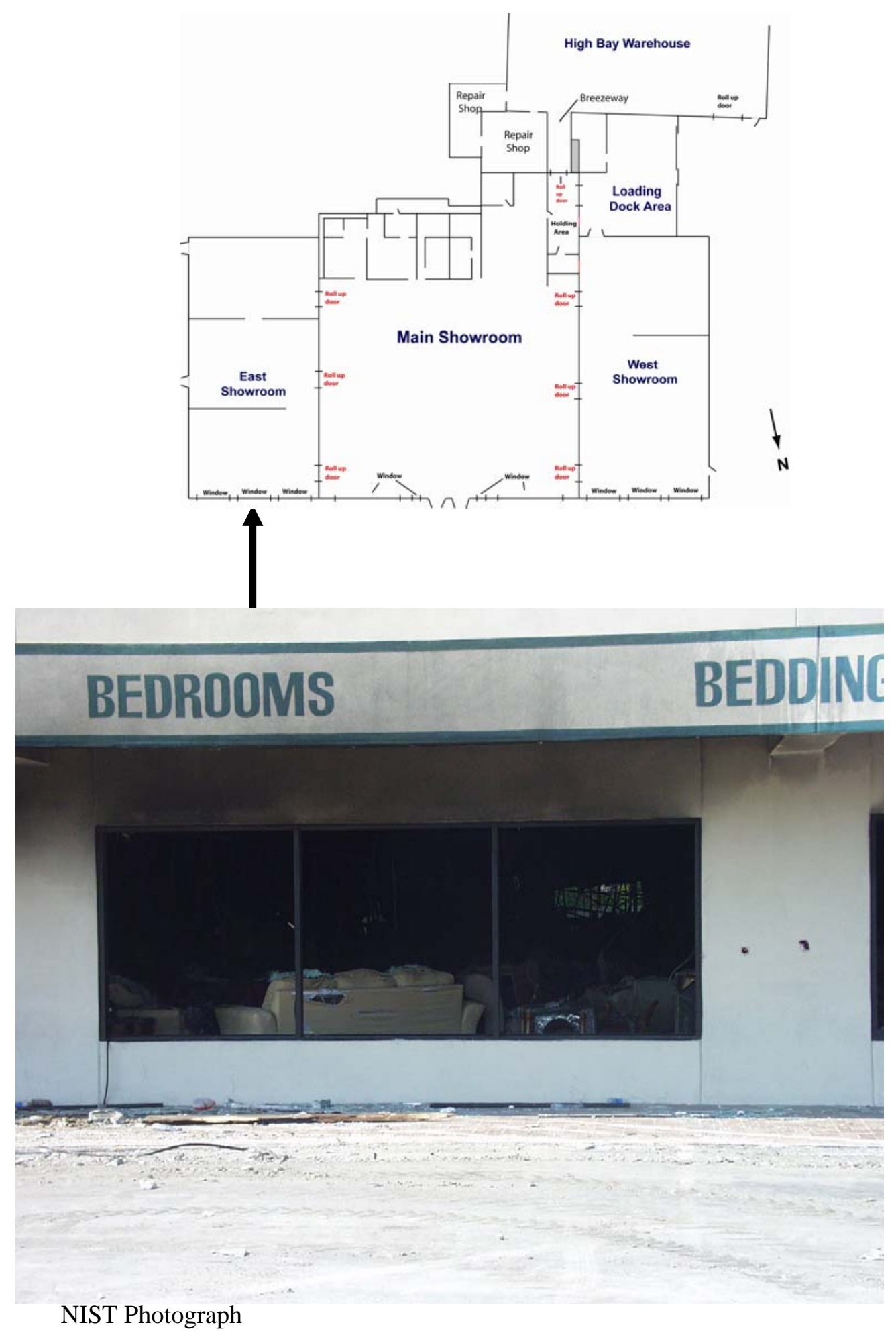

Figure F-3. Exterior view of east showroom looking into center-front window of the east showroom. 


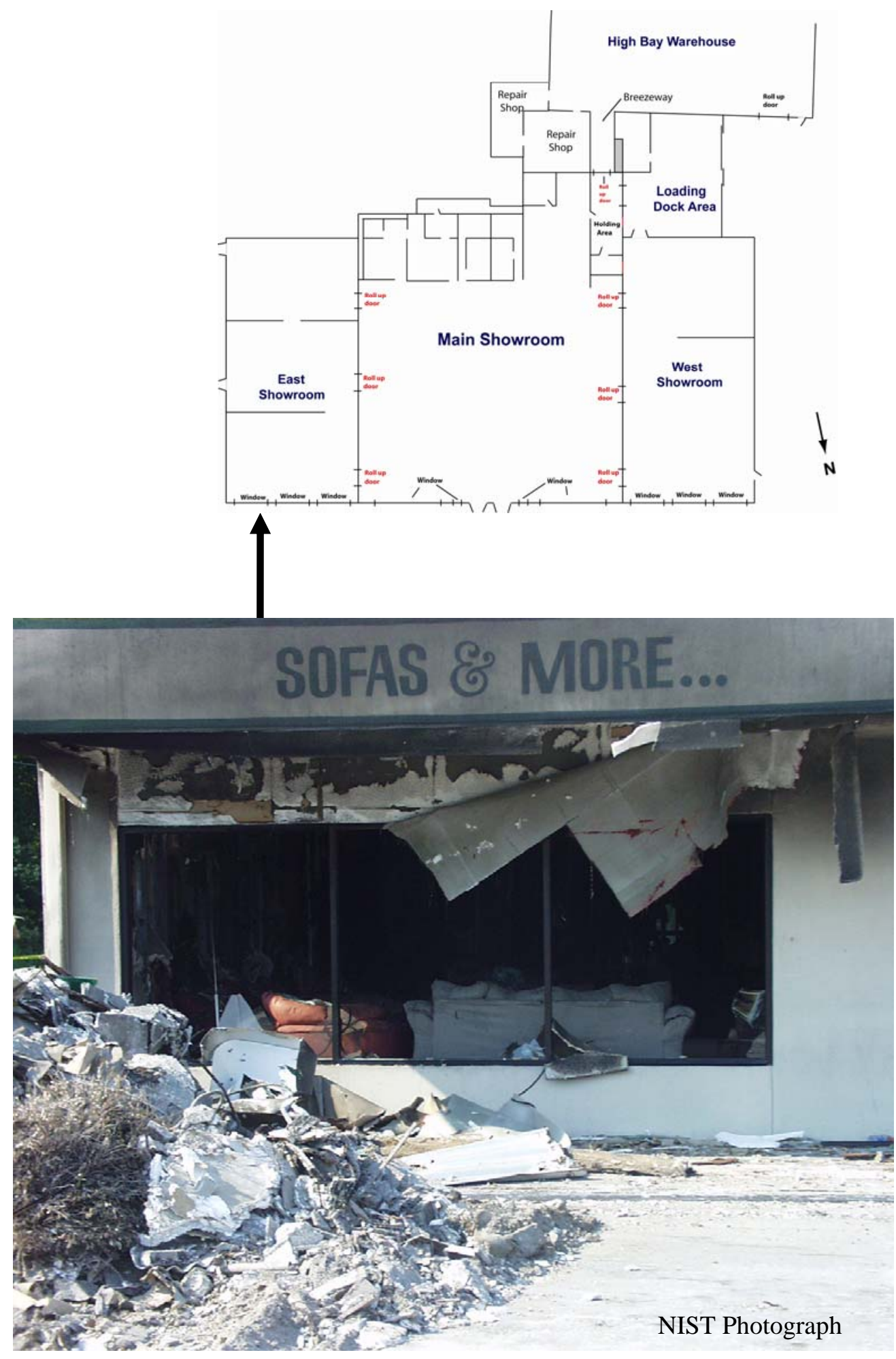

Figure F-4. Exterior view of east showroom looking into east-front window of the east showroom 


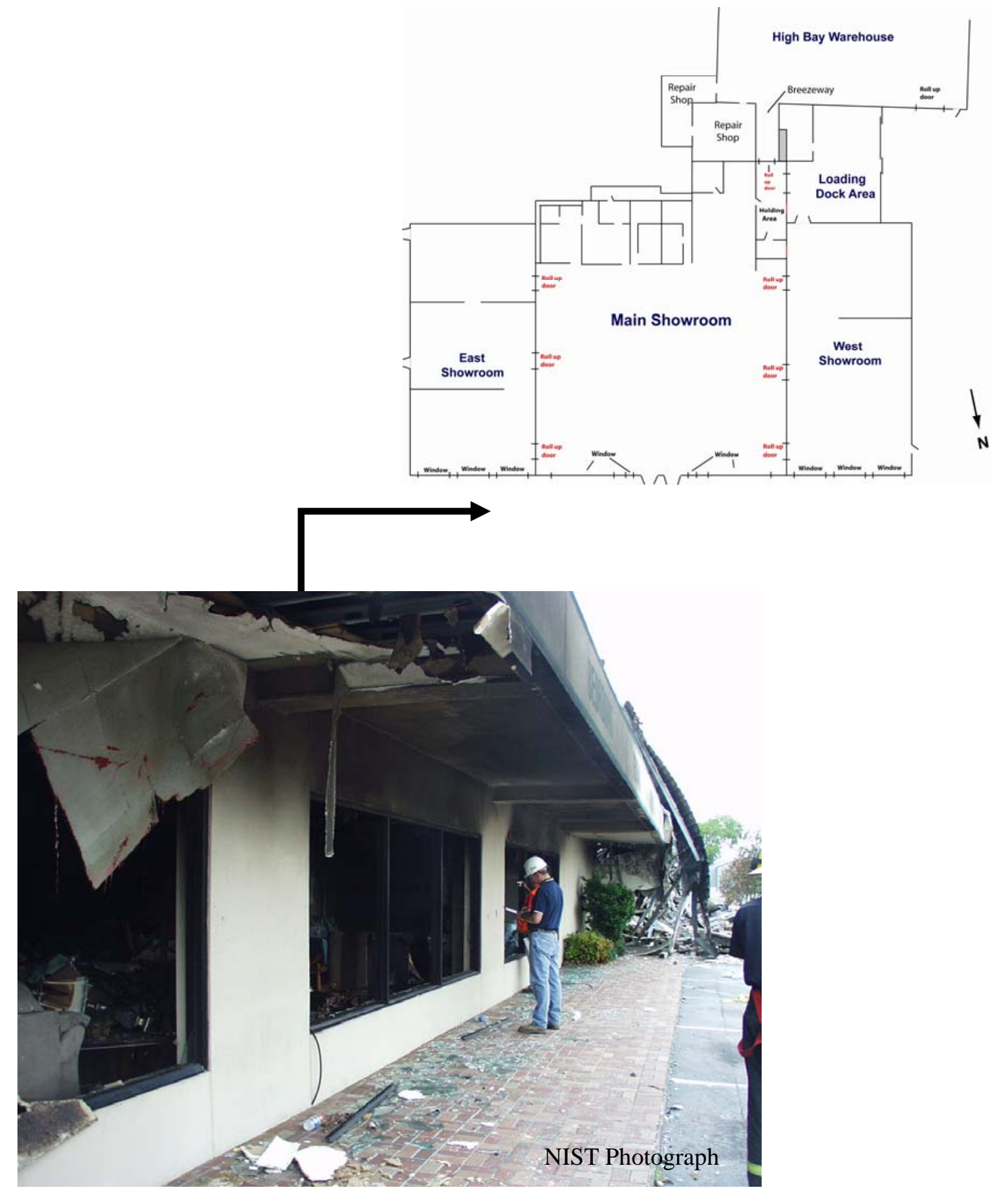

Figure F-5. Exterior view of front of east showroom looking toward main showroom. 


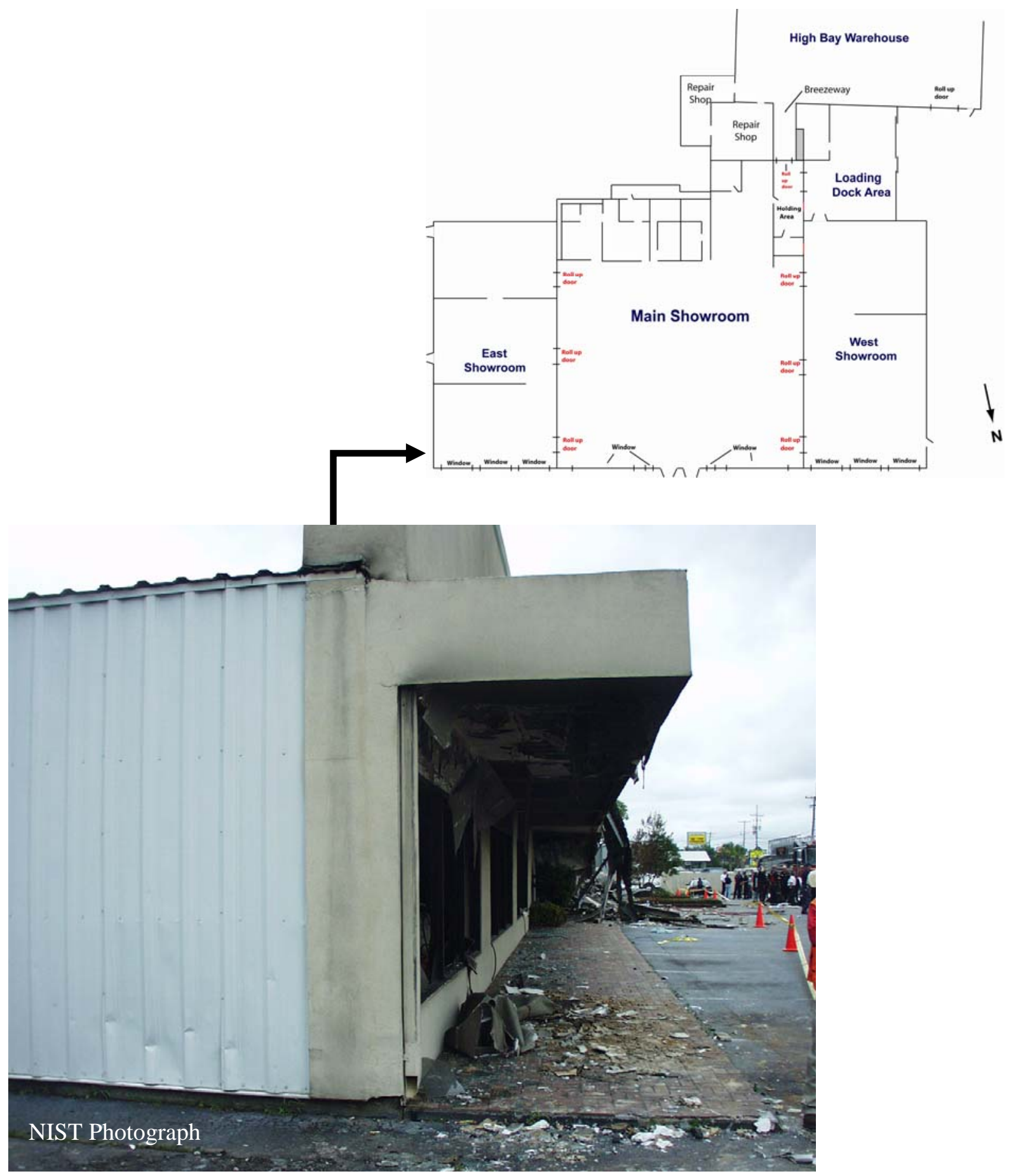

Figure F-6. Exterior view of front of east showroom looking toward main showroom from east parking lot. 


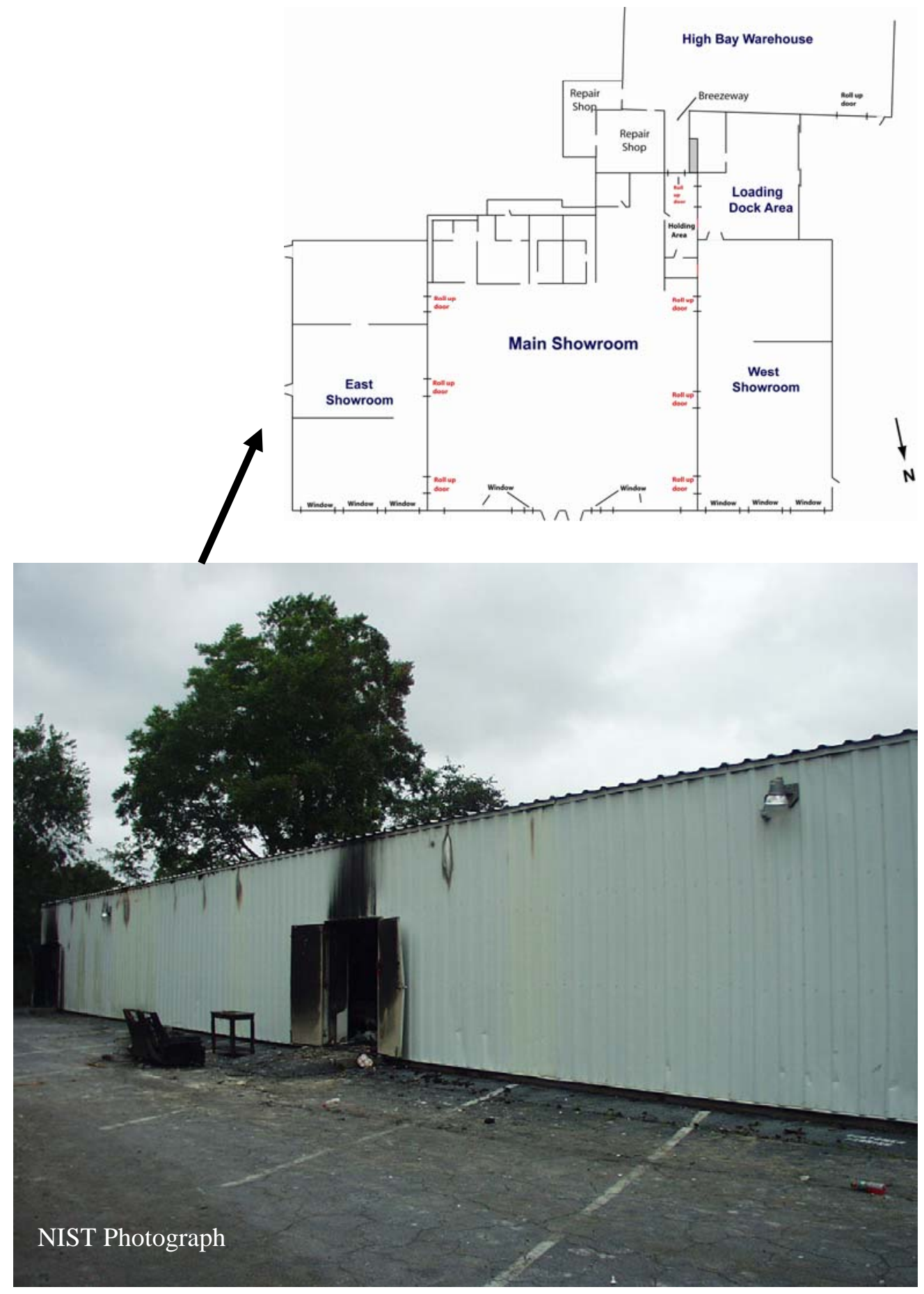

Figure F-7. Exterior view of east wall of east showroom. 


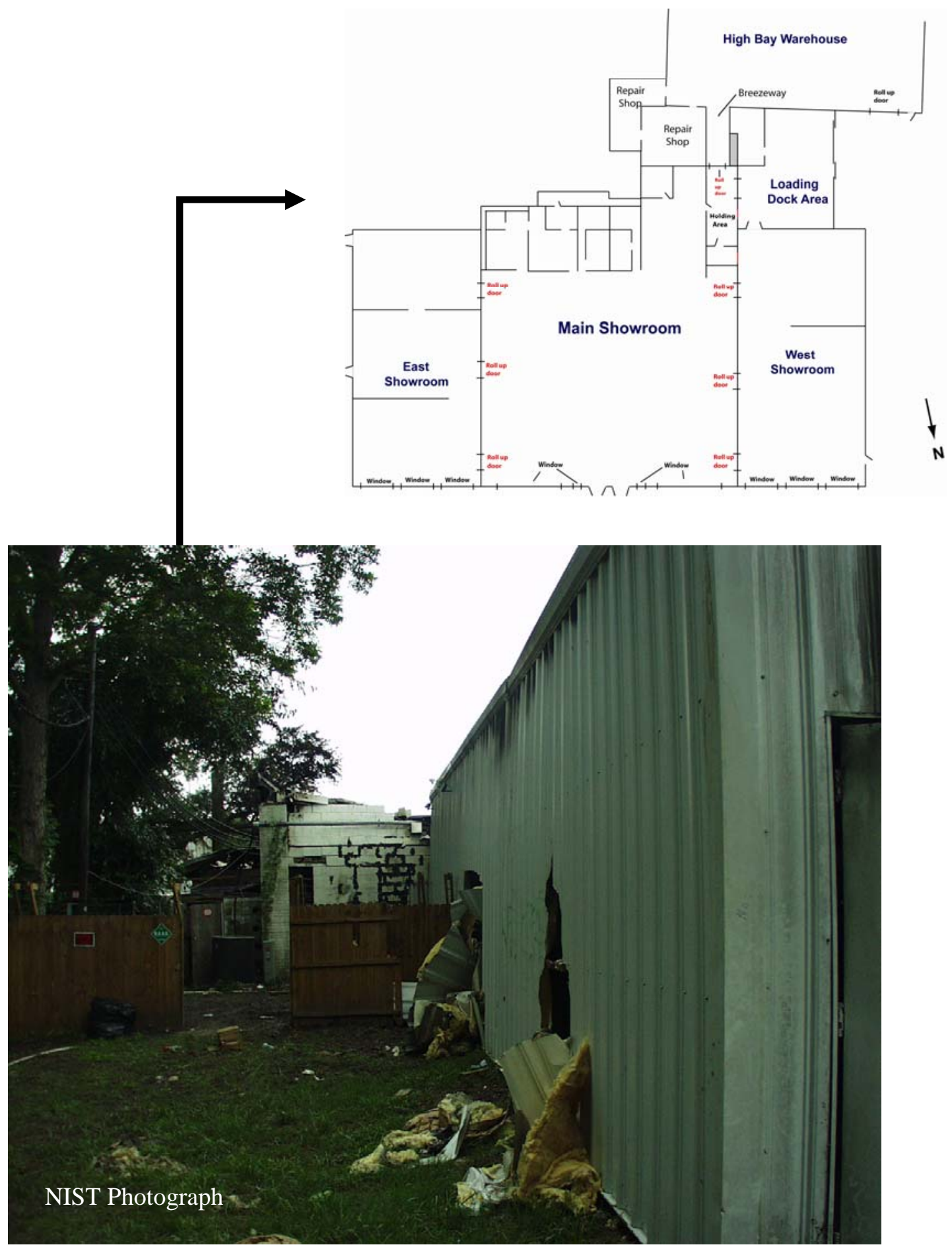

Figure F- 8. Rear wall of east showroom. 


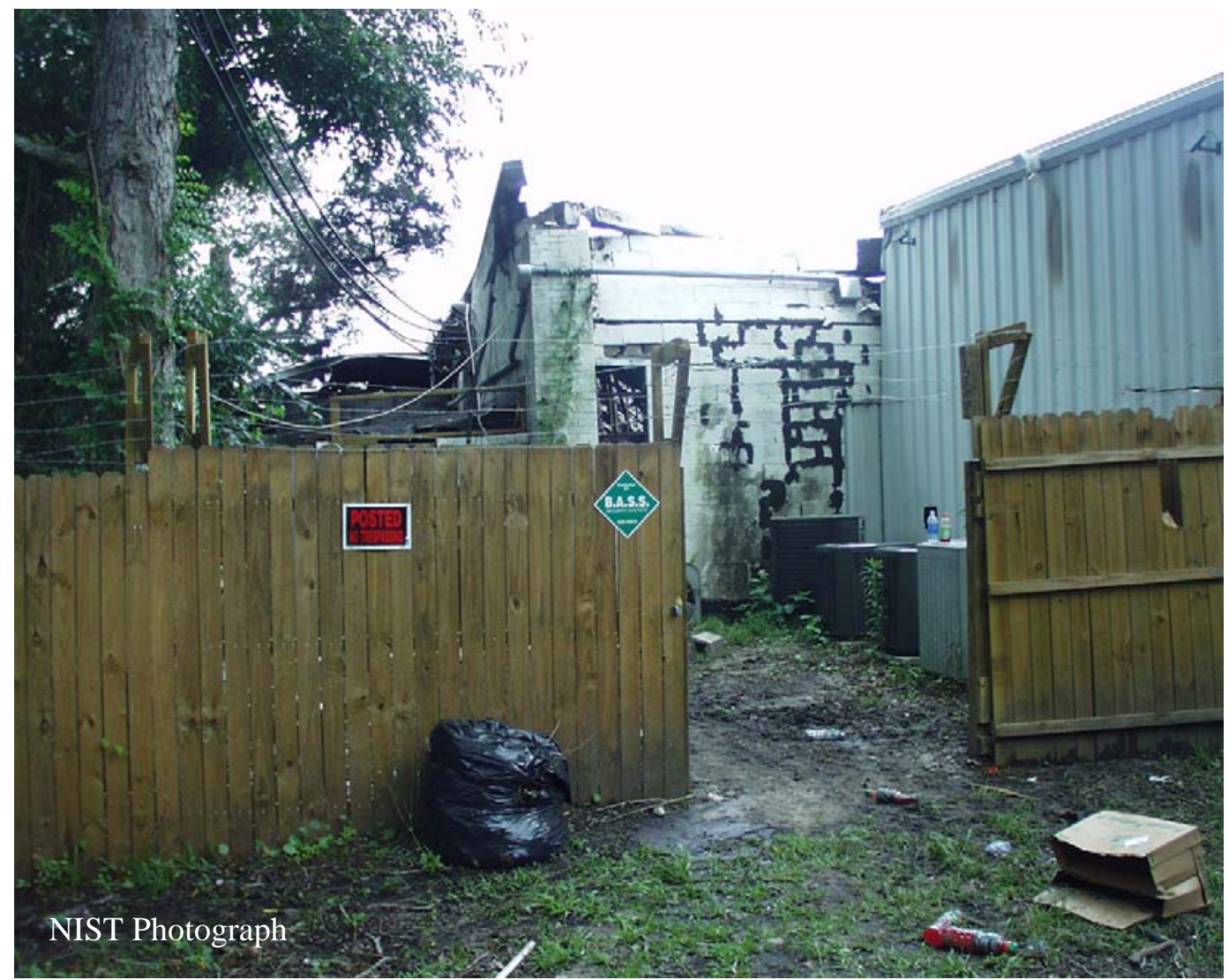

Figure F- 9. Wooden fence and gate at rear of east showroom.

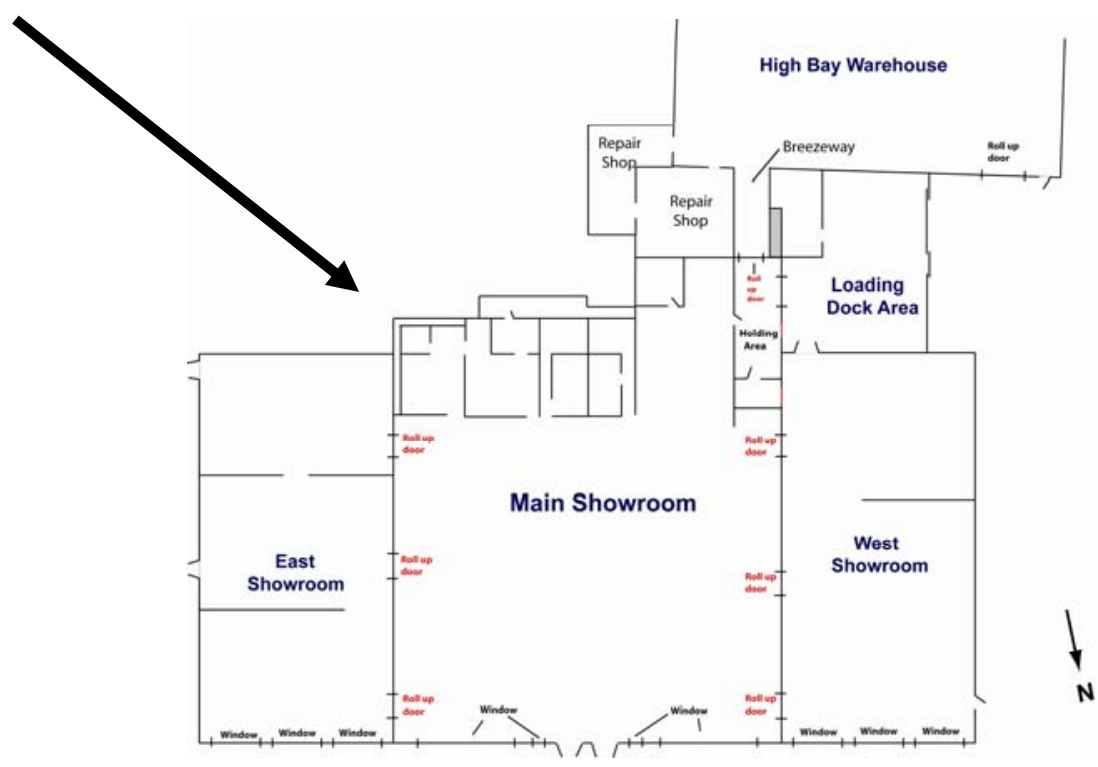




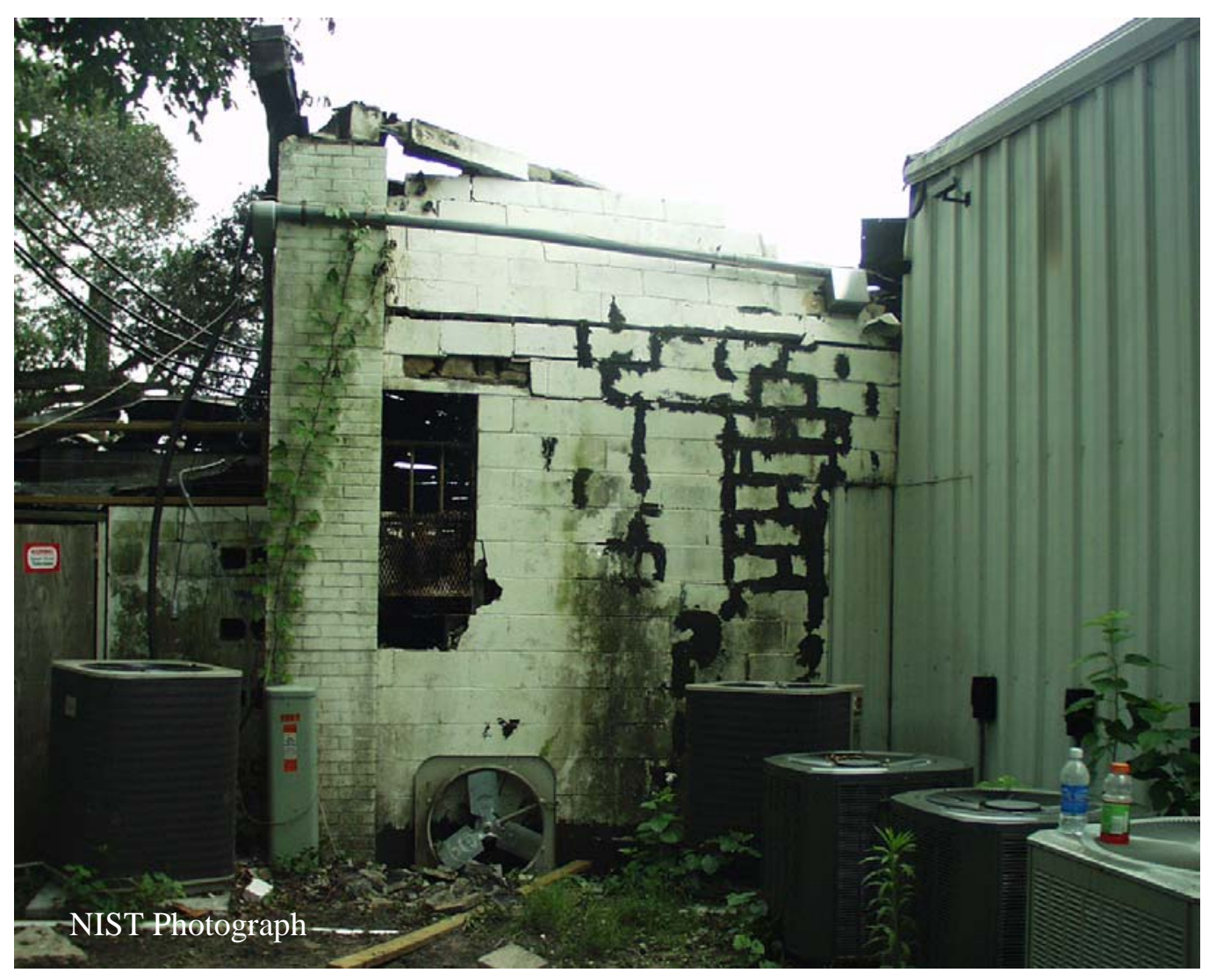

Figure F-10. Intersection of rear wall of east showroom with east wall of main showroom.

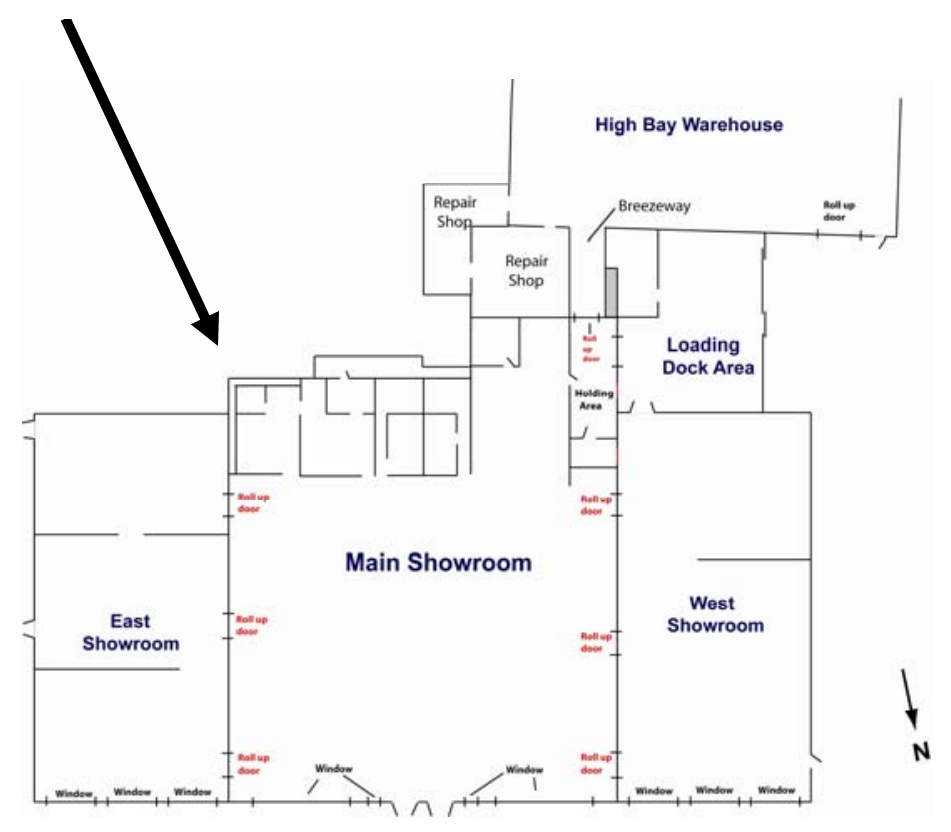




\section{F.2 EAST SHOWROOM INTERIOR PHOTOS}

A series of photographs of the interior of the east showroom were taken after the fire.

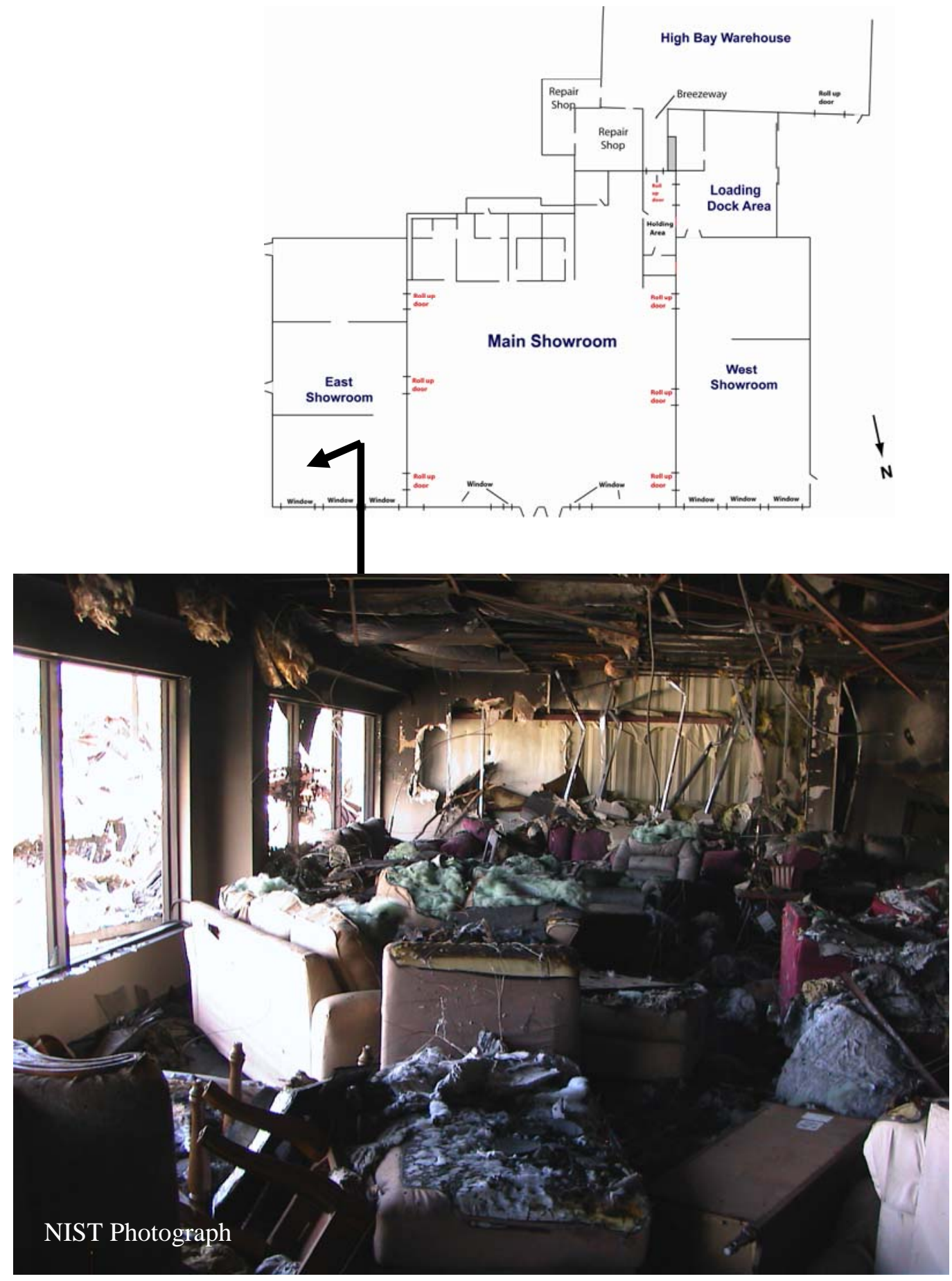

Figure F- 11. Interior view of east showroom looking toward front parking lot. 


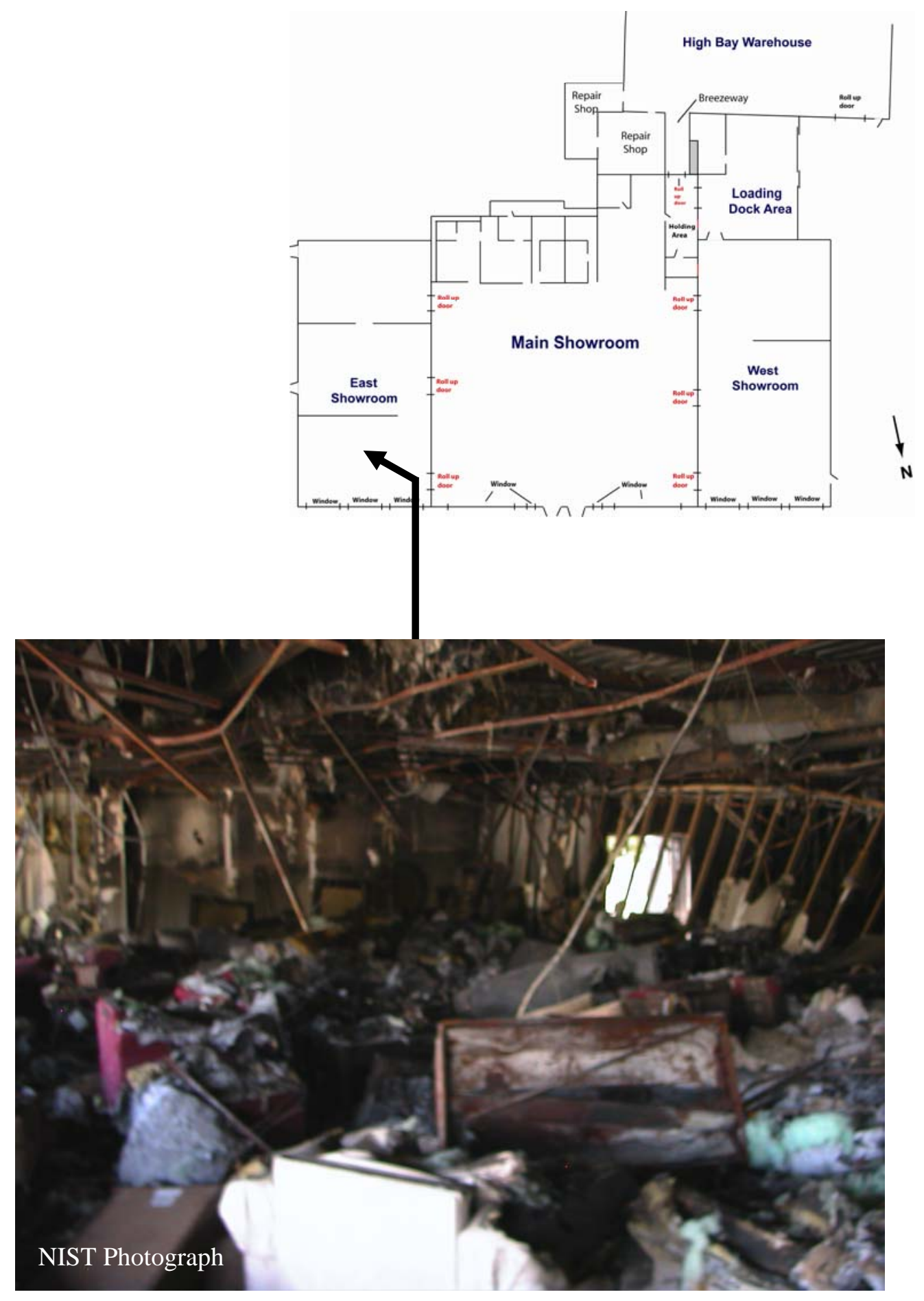

Figure F-12. Interior of east showroom. Wood stud partition wall visible in right background of photo. 


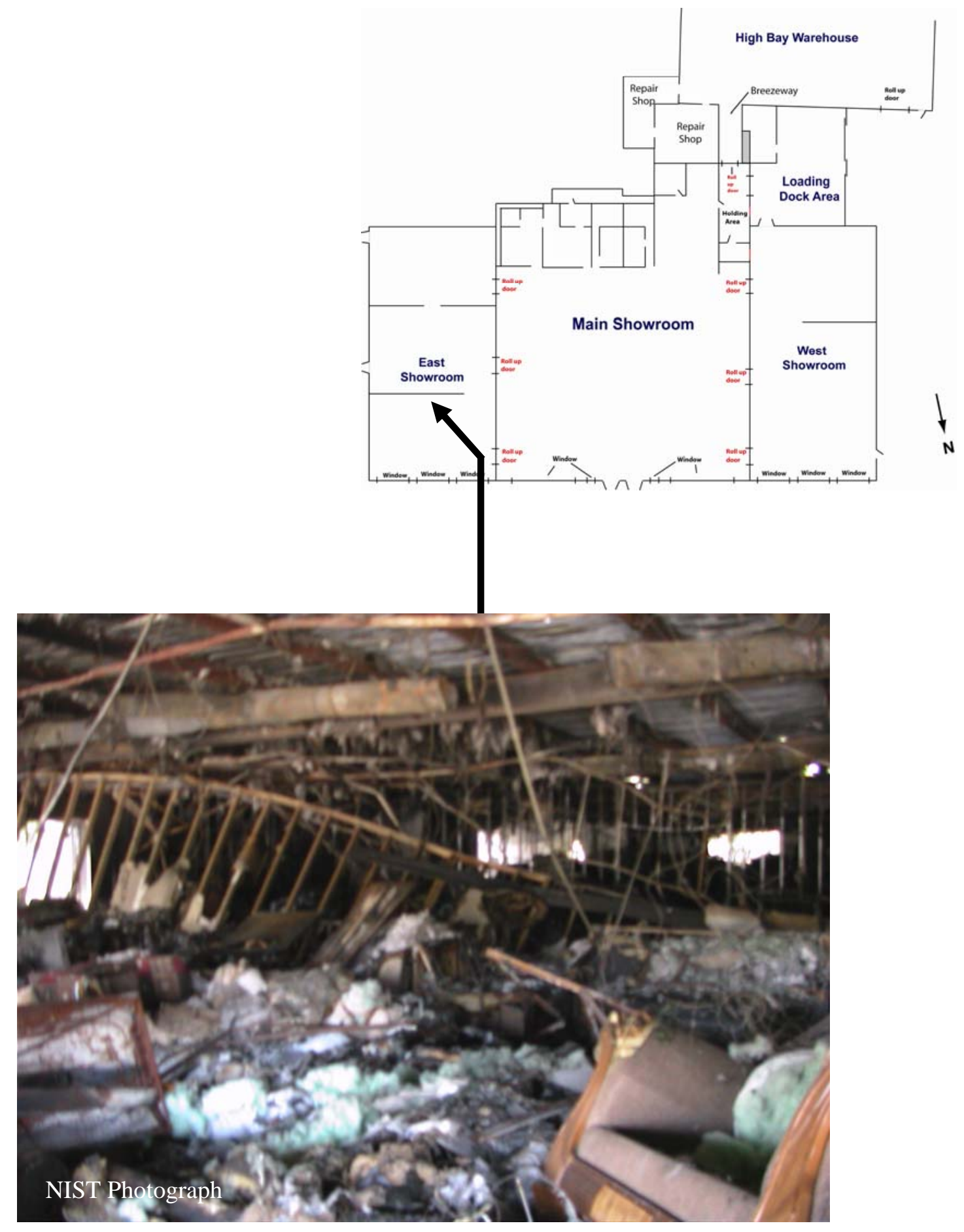

Figure F-13. Interior of east showroom looking toward rear corner. 


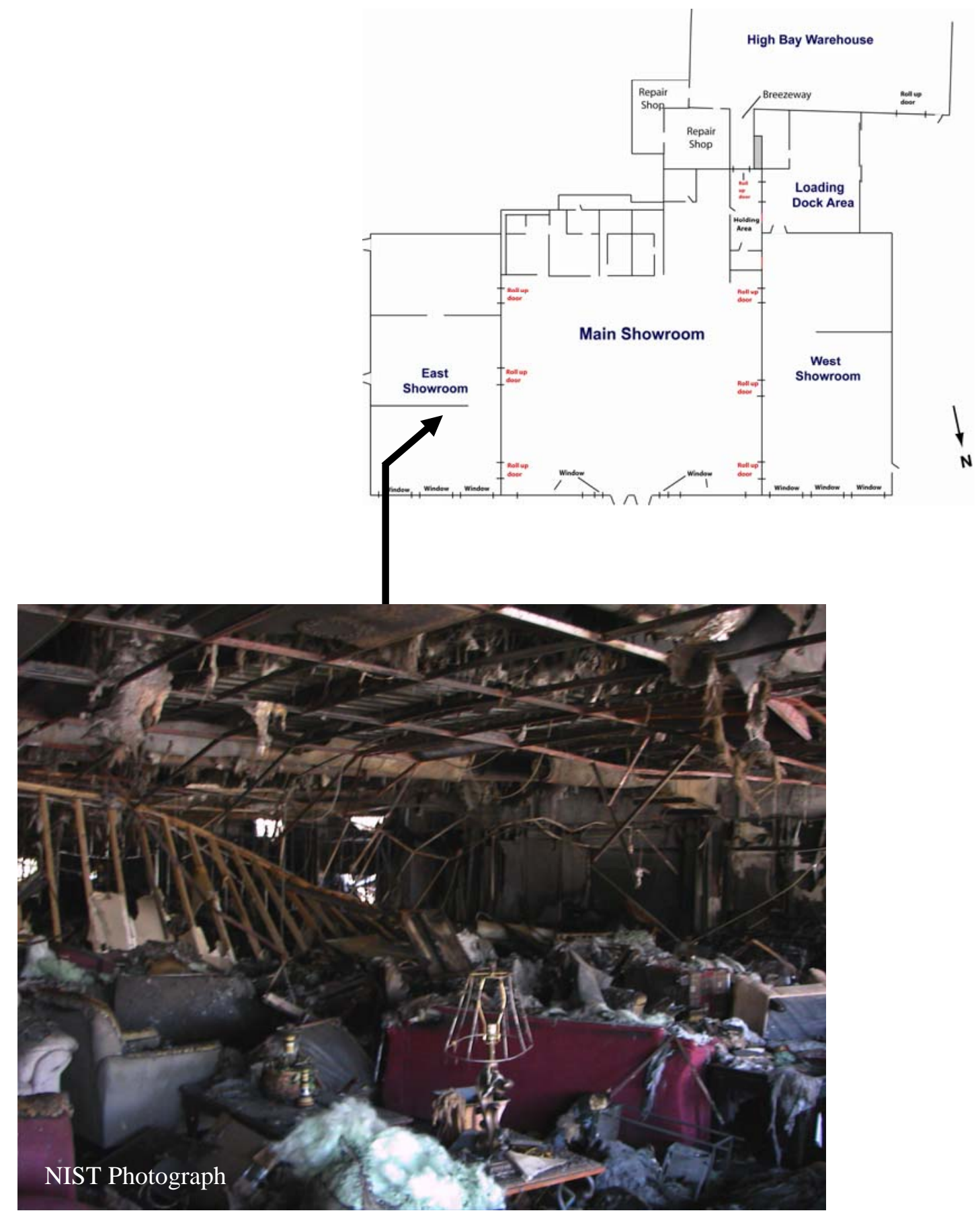

Figure F-14. Interior of east showroom looking toward main showroom. Closed roll-down fire door visible in center-right background. Partially closed roll-down fire door visible in center background. Photo displays less fire damage to furniture in front section of east showroom. 


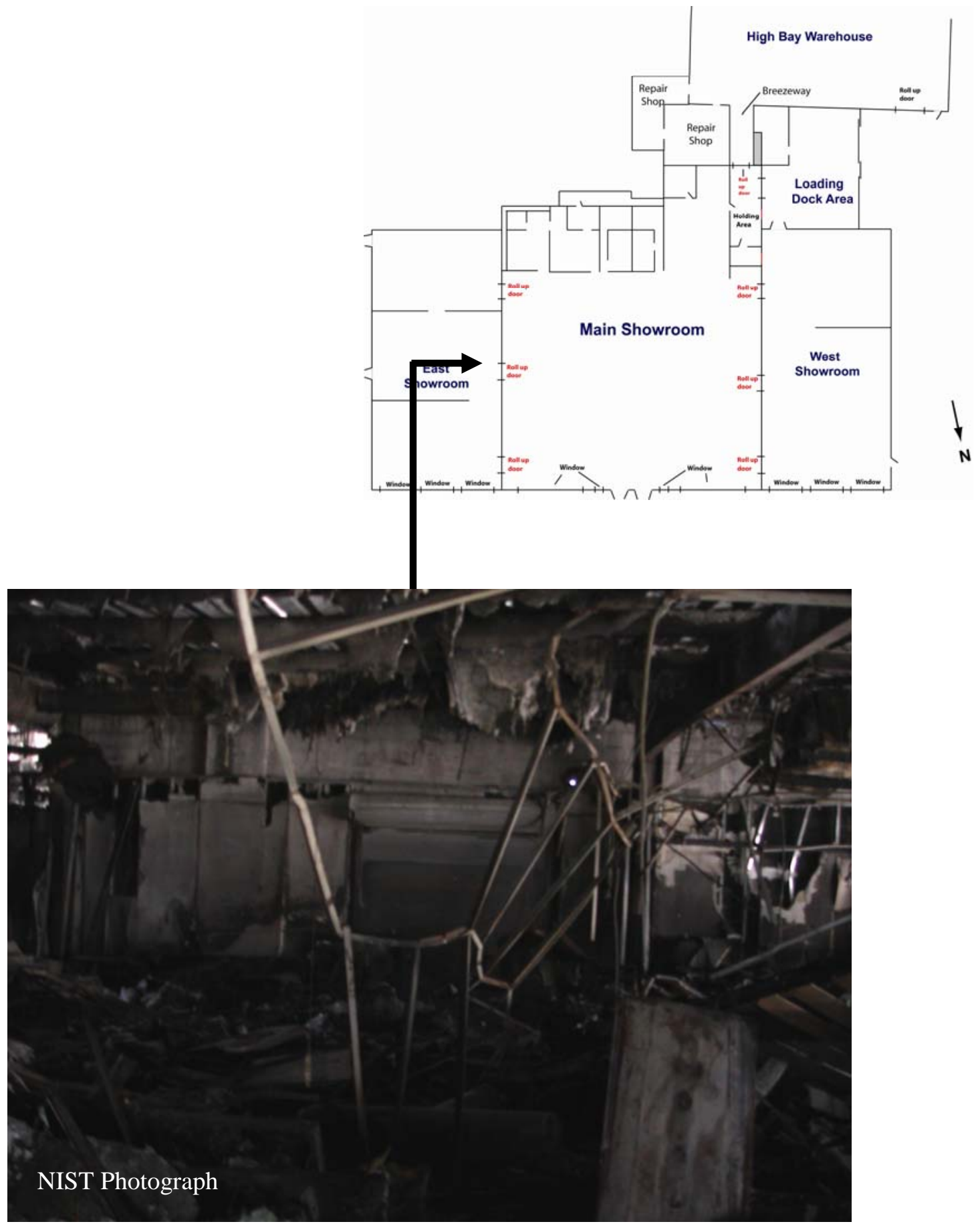

Figure F-15. Interior of east showroom looking toward main showroom. Closed roll-down fire door visible in center of photo. Photo displays more damage from fire in this center section of east showroom. 


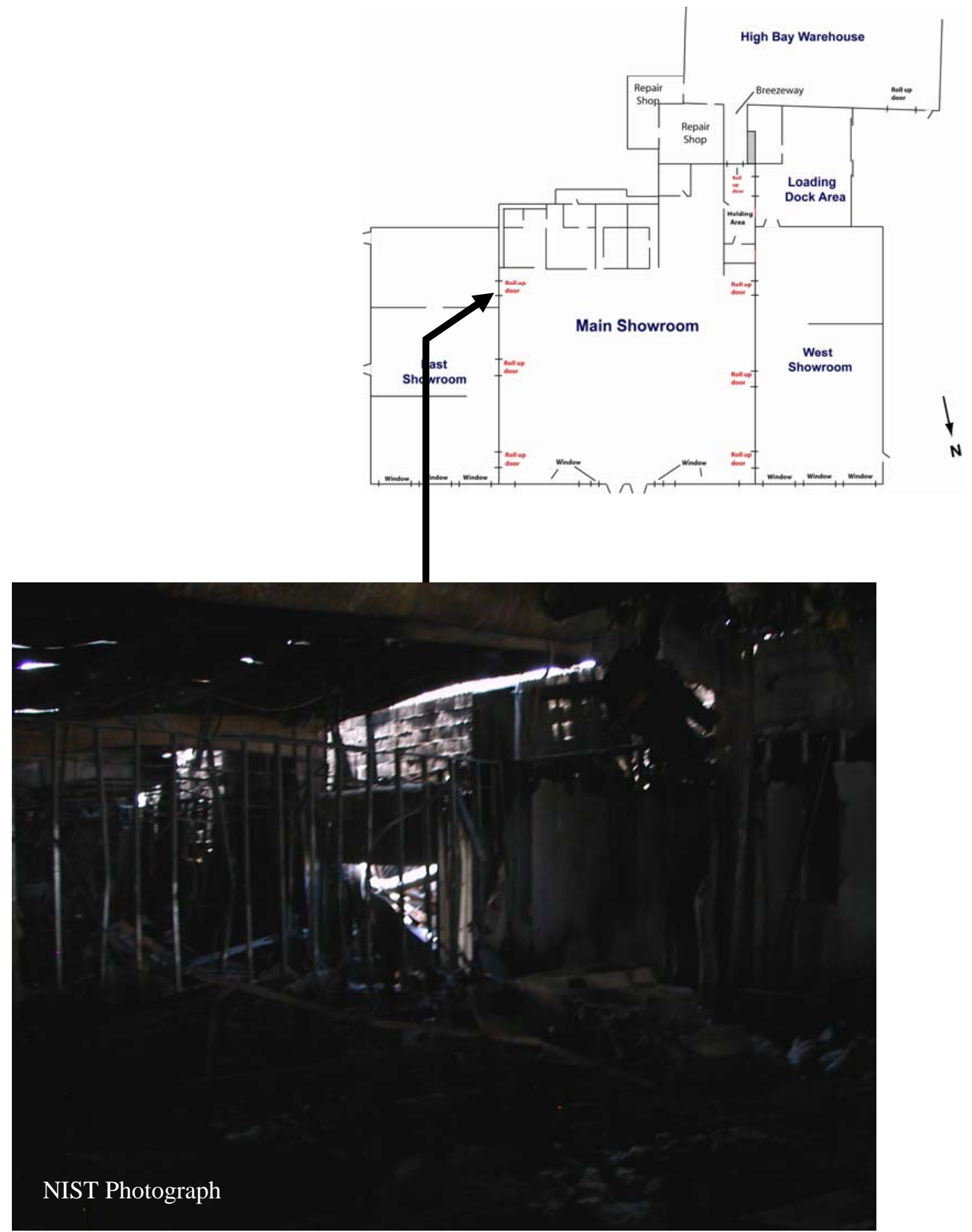

Figure F-16. Interior view of east showroom looking toward partially closed roll-down fire door. Metal studs of rear partition wall visible in left-center background of photo. 


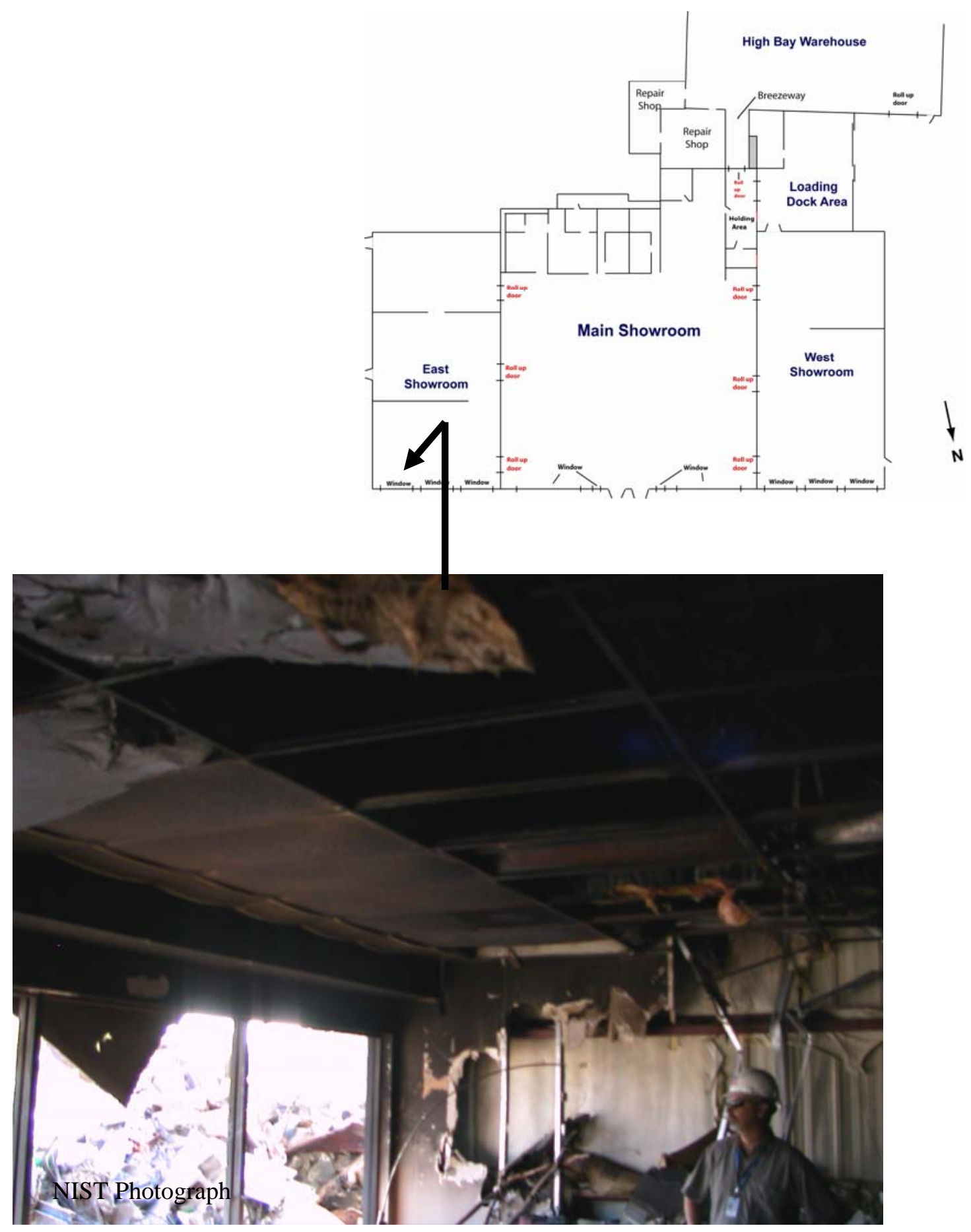

Figure F-17. Interior view of east showroom looking at drop ceiling. Exterior metal siding visible in center right of photo. Portions of gypsum wall board visible in center background of photo. 


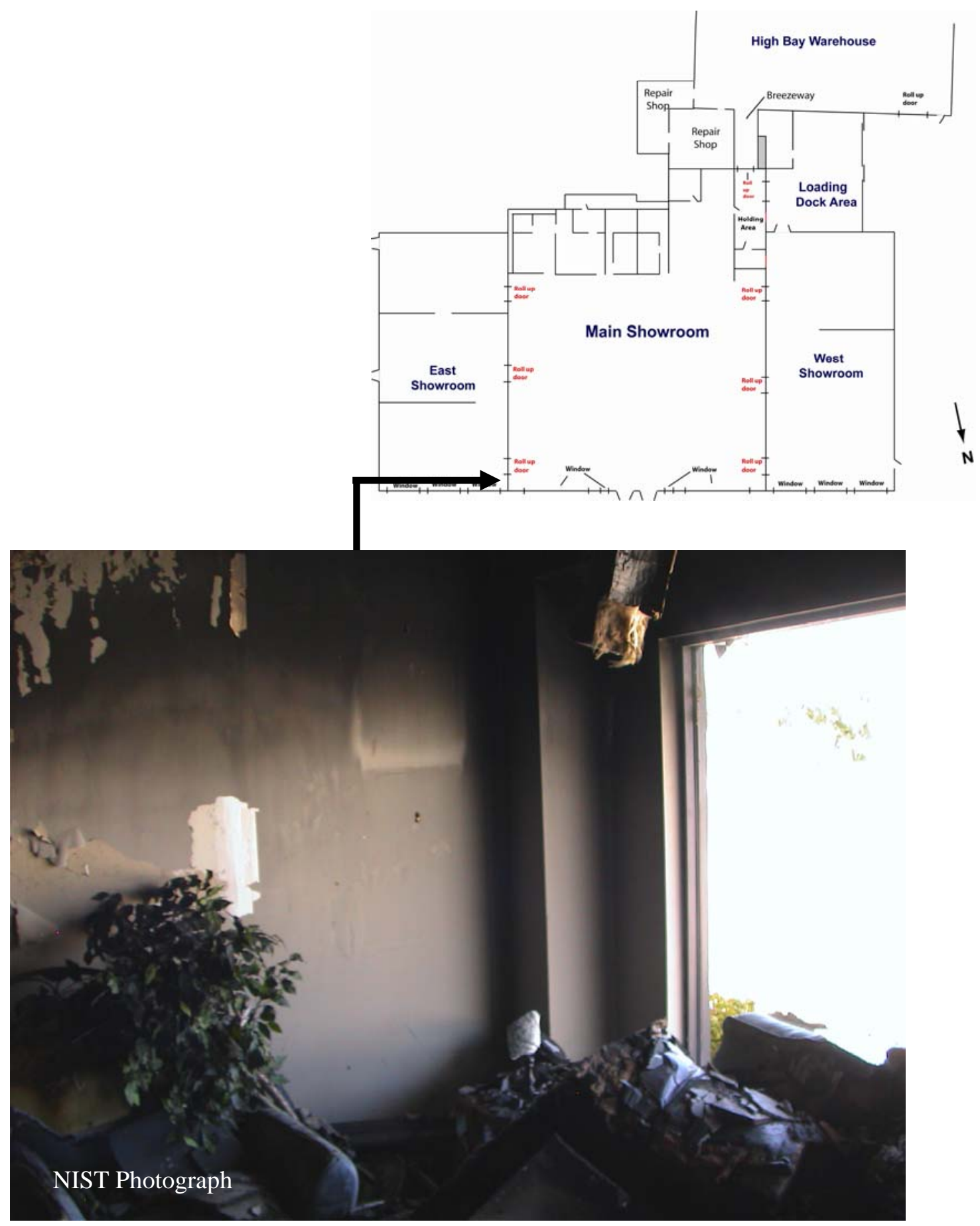

Figure F-18. Interior of east showroom near front window. 


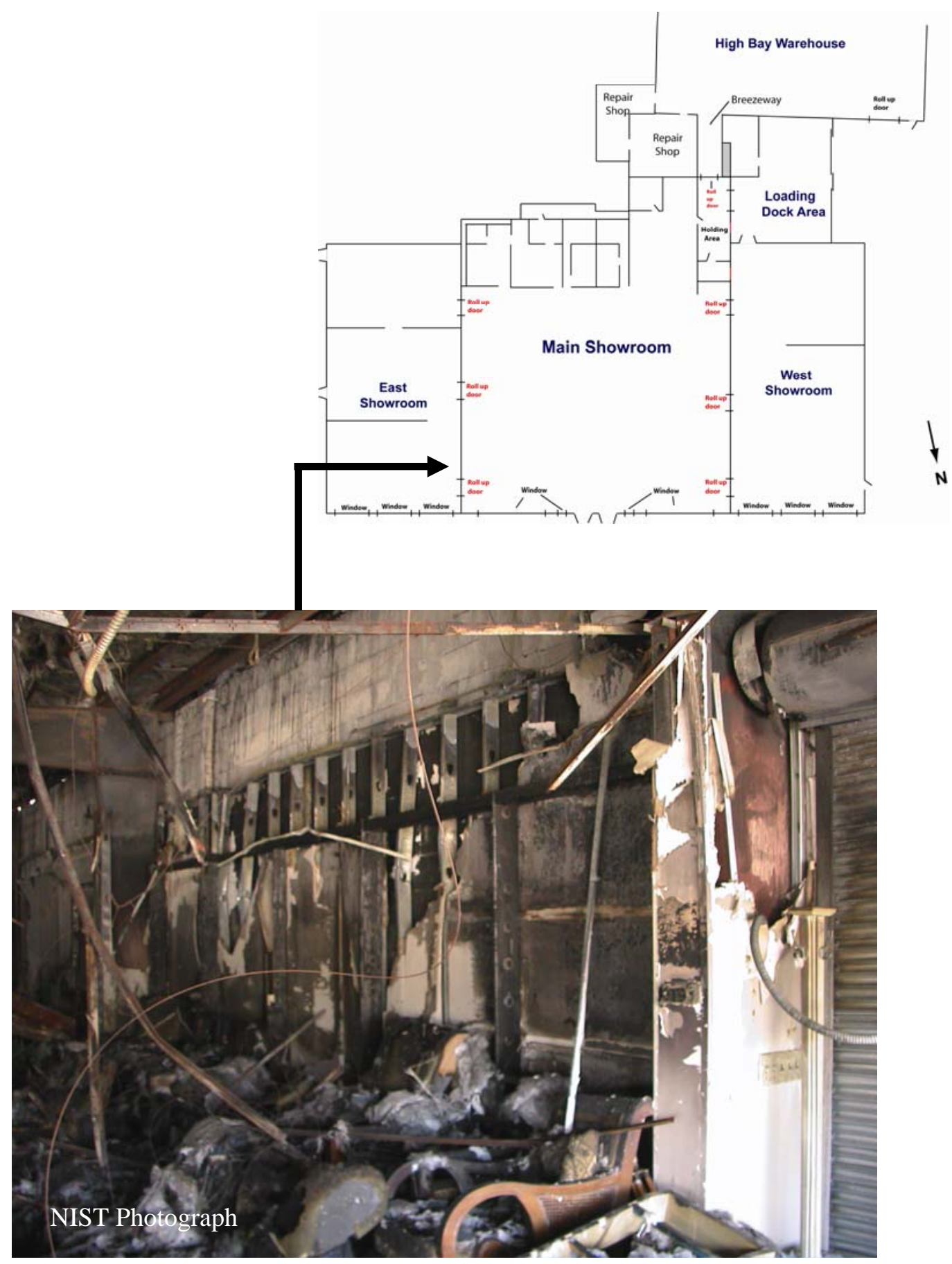

Figure F-19. Interior of east showroom. Closed roll-down fire door visible on right of image. 


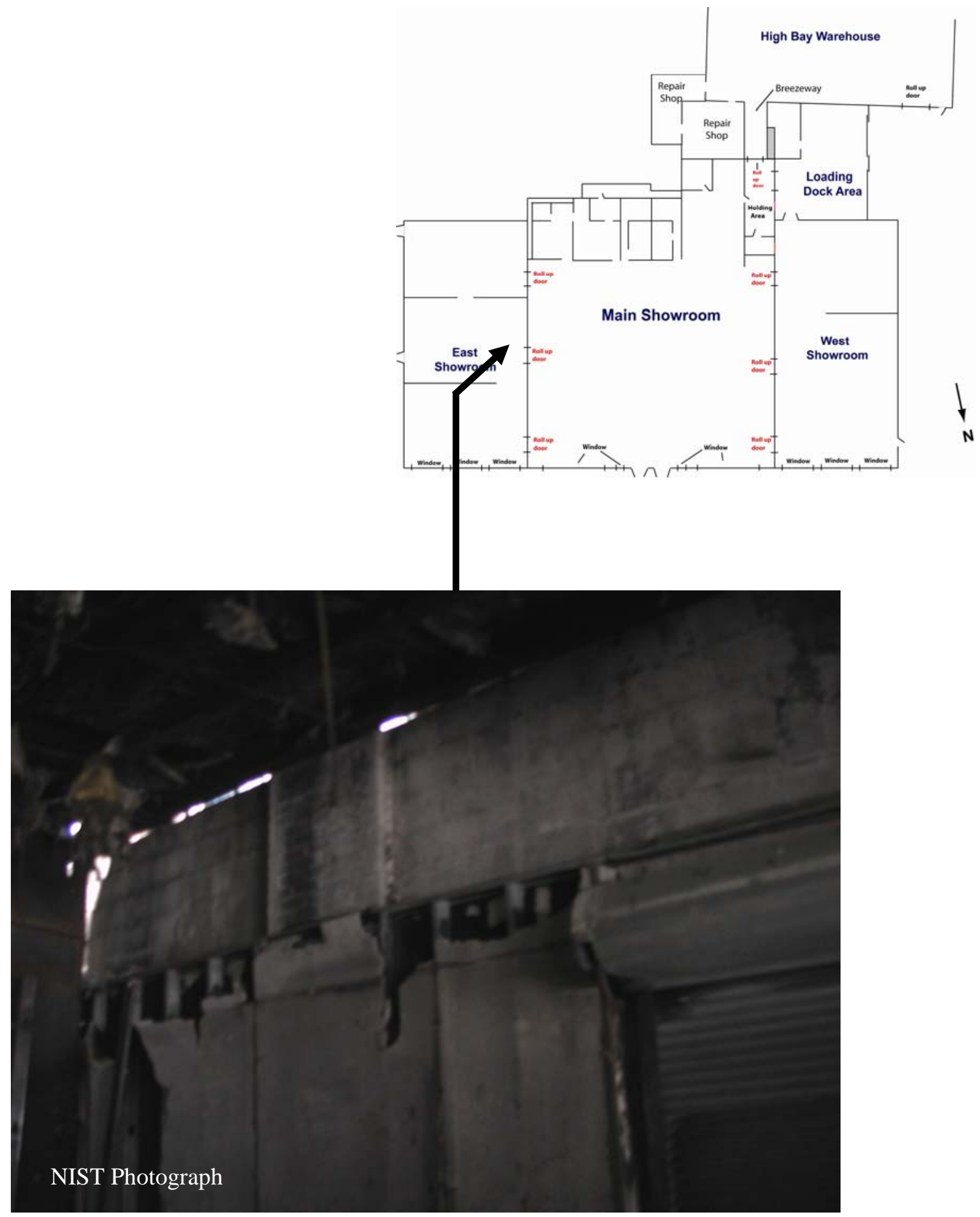

Figure F-20. Interior view of east showroom looking toward main showroom. Concrete block wall was originally exterior wall of grocery store. Gypsum wall board attached to metal stud which were attached to concrete block wall. Metal studs/gypsum wall board extended up to suspended ceiling, but not above suspended ceiling. 


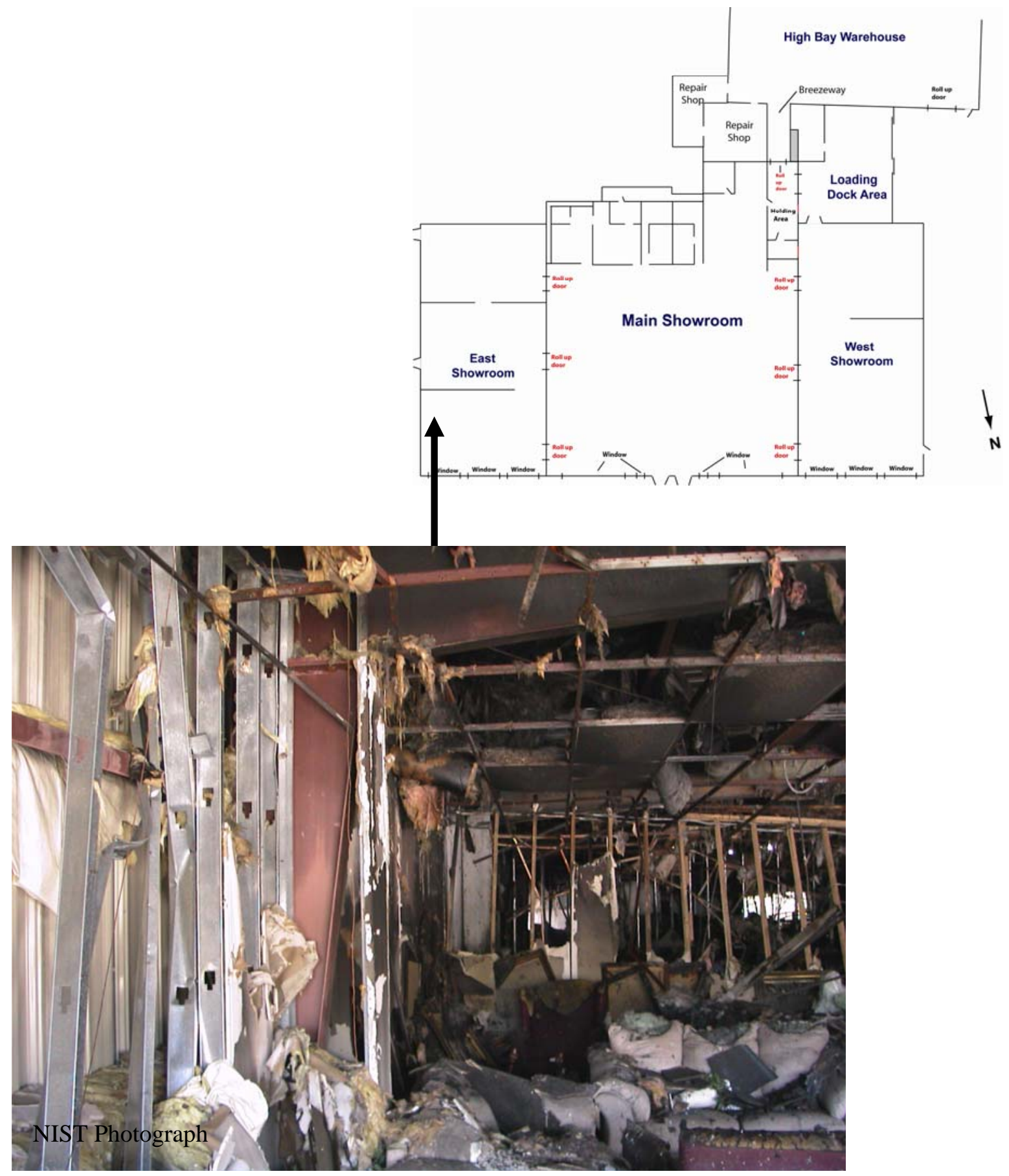

Figure F-21. Interior of east showroom looking along east wall toward rear of showroom. Exterior metal wall attached to exterior side of horizontal channel. Metal studs attached to inside face of horizontal channel. Gypsum wall board was installed over metal studs to form interior wall of showroom. Metal stud/gypsum wall appeared to extend approximately $0.3 \mathrm{~m}(1 \mathrm{ft}$ ) above drop ceiling along east wall. Reddish-brown steel I-beam column and roof beam also visible in photo. 


\section{ApPendix G. WARehouse PhOtographS}

\section{G.1 WAREHOUSE EXTERIOR PHOTOS}

Several days after the fire, photographs were taken of the exterior and to a more limited extent, the interior.

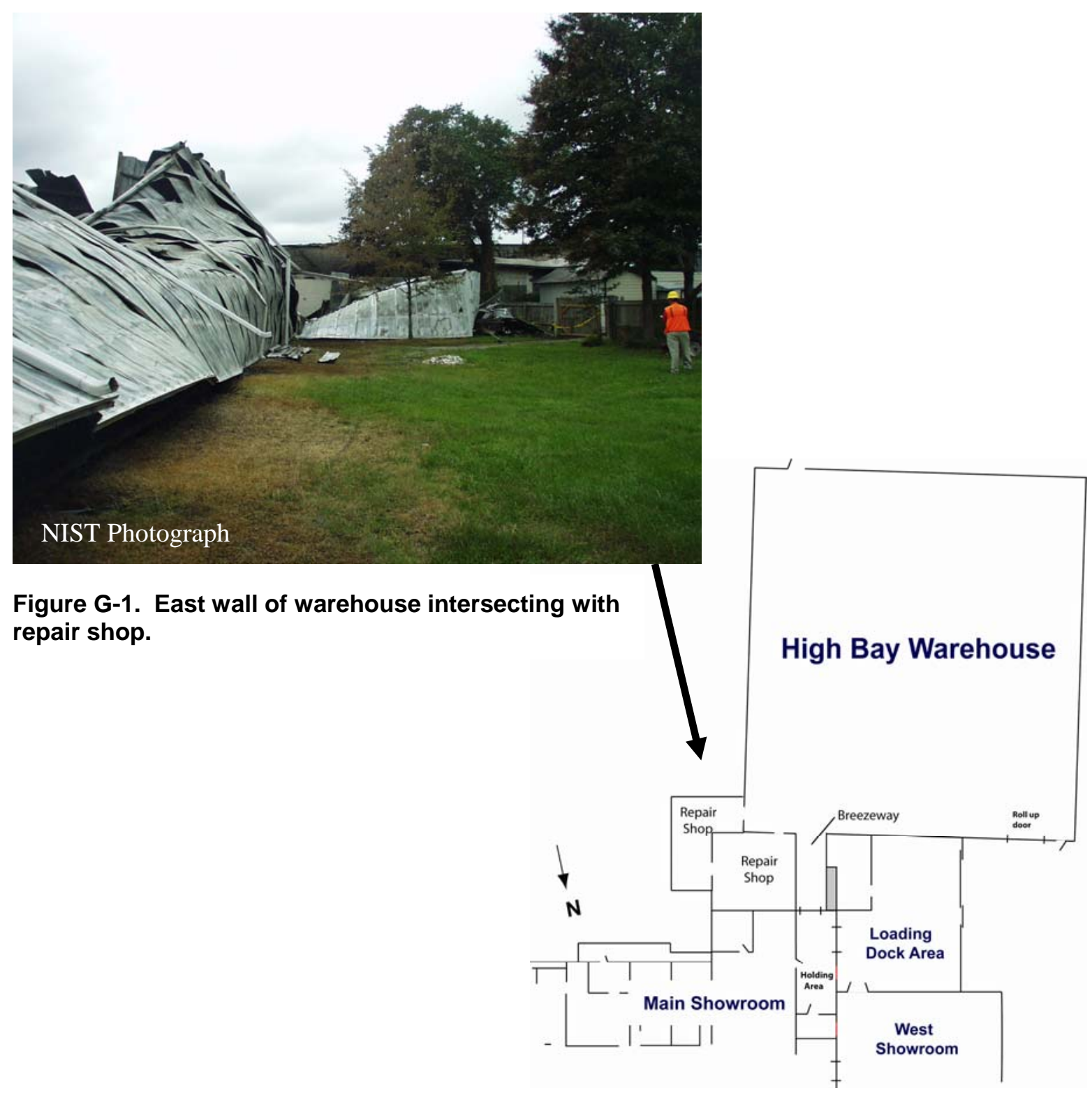




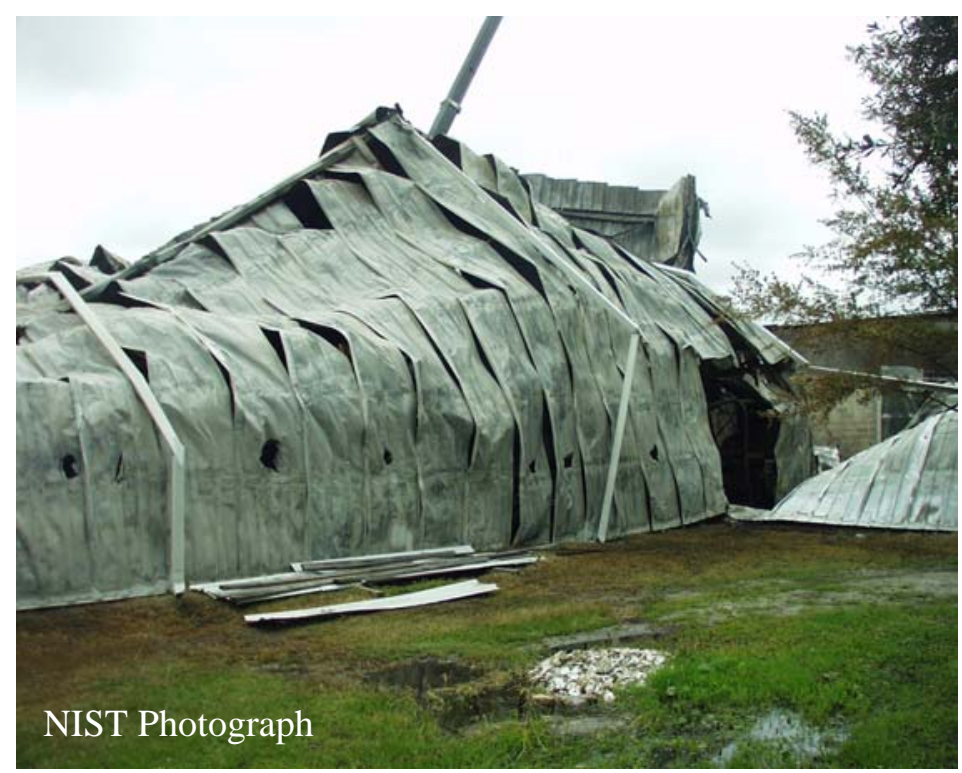

Figure G-2. East wall of warehouse intersecting repair shop. Open area at corner of warehouse wall was doorway from warehouse into repair shop.

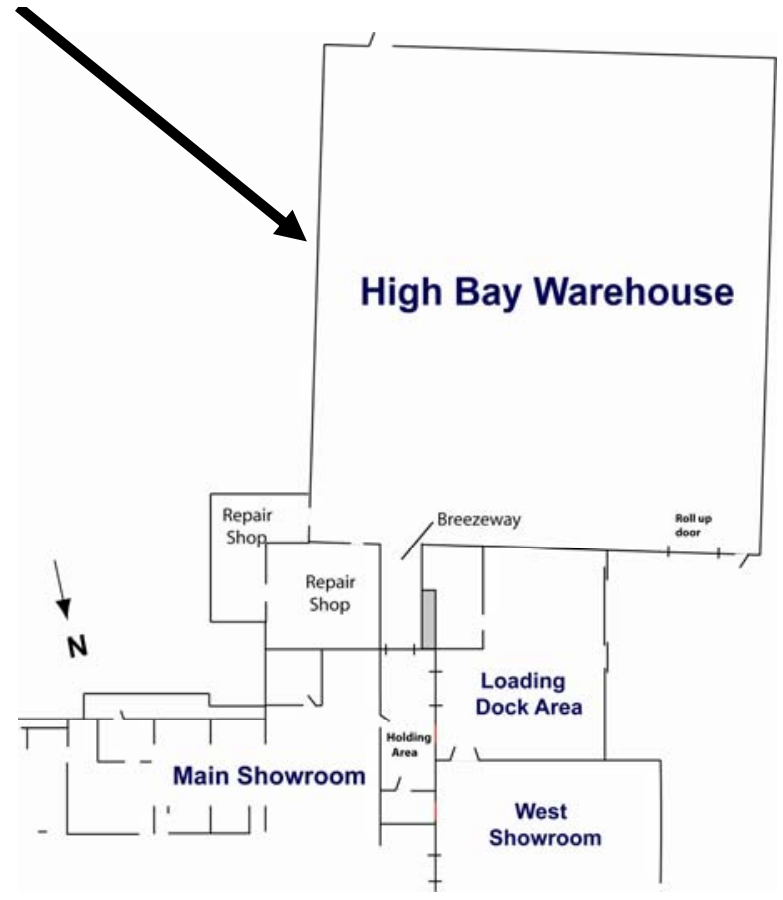



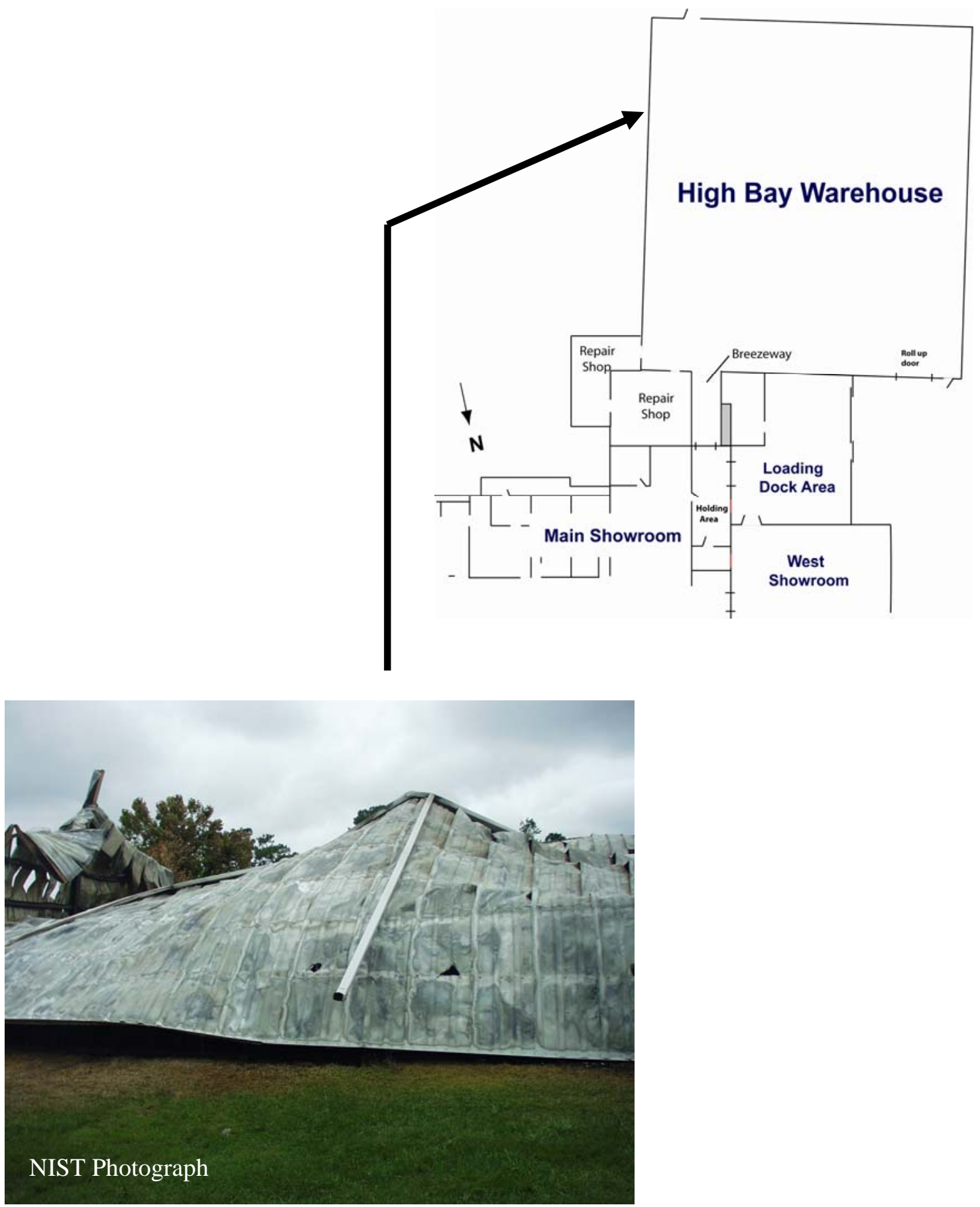

Figure G-3. East wall of warehouse. 


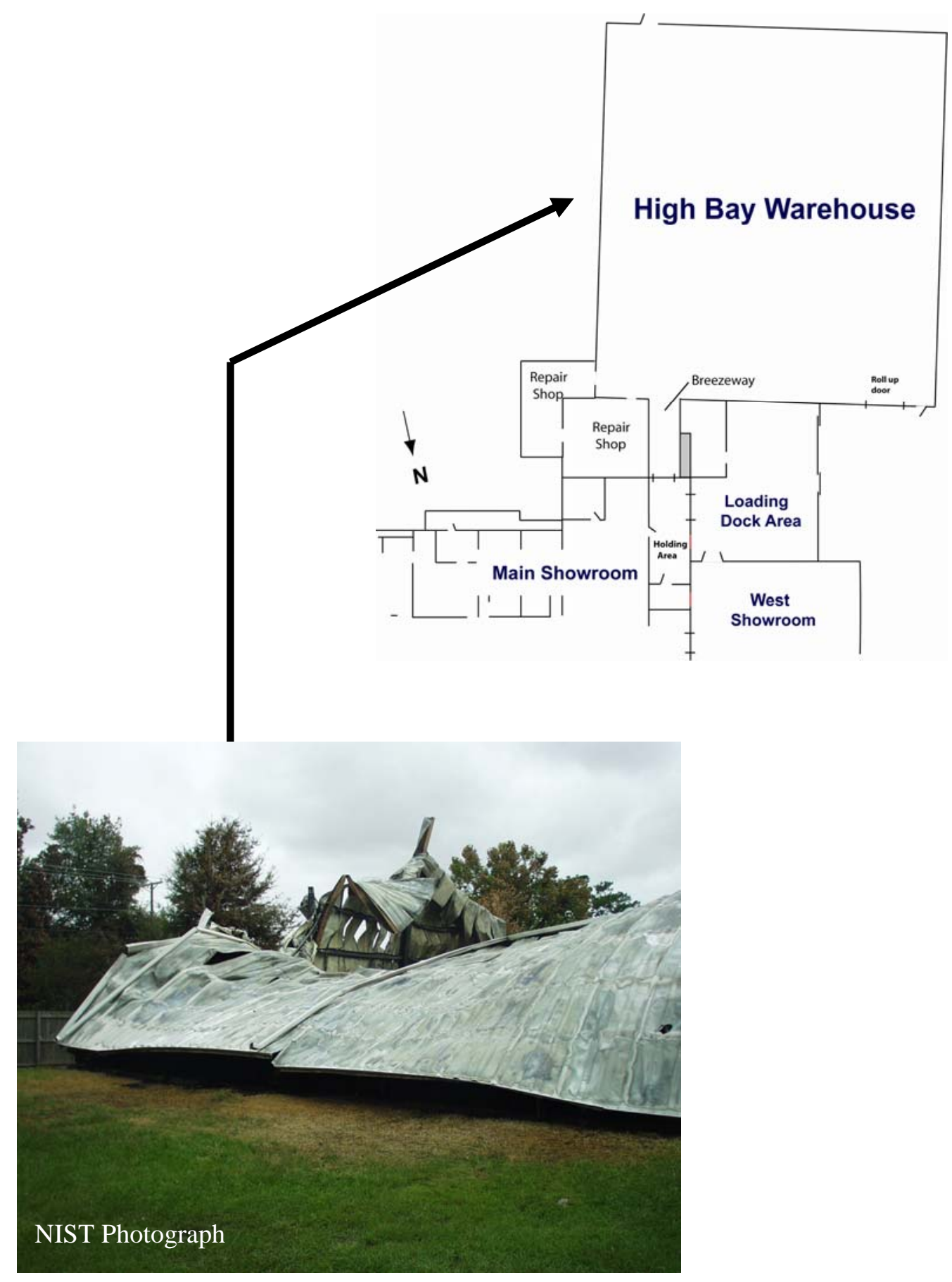

Figure G-4. East wall of warehouse intersecting with south wall. 


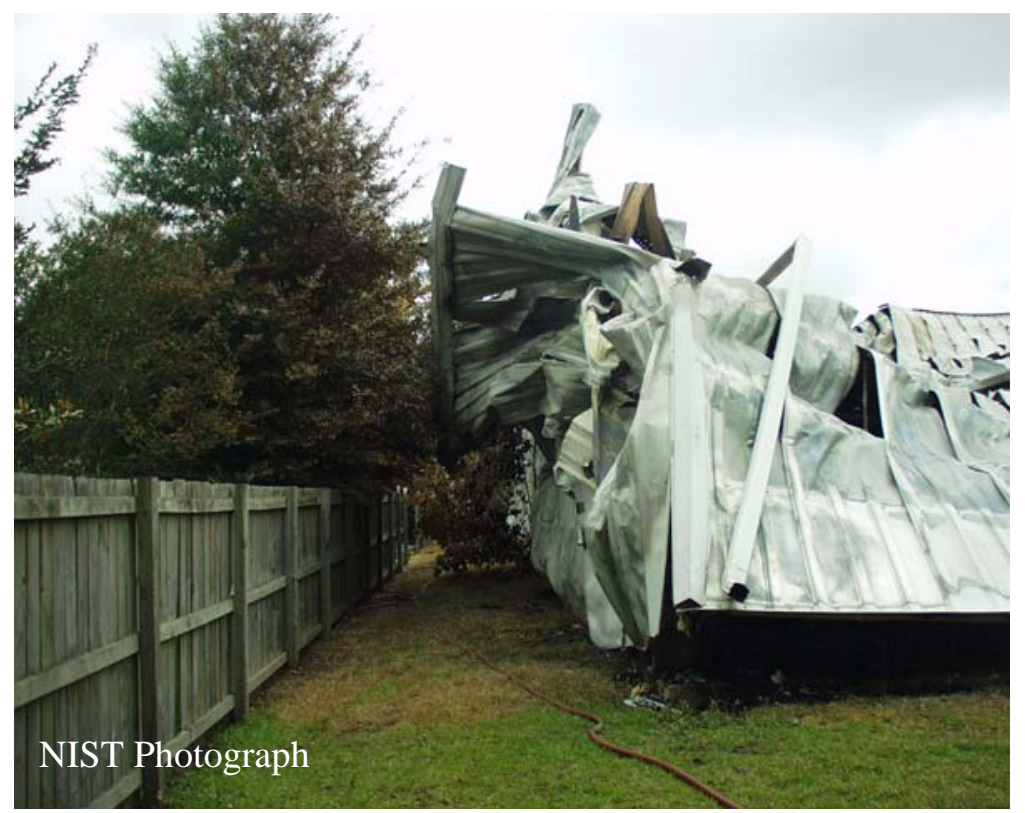

Figure G-5. Corner of south and east walls of warehouse.

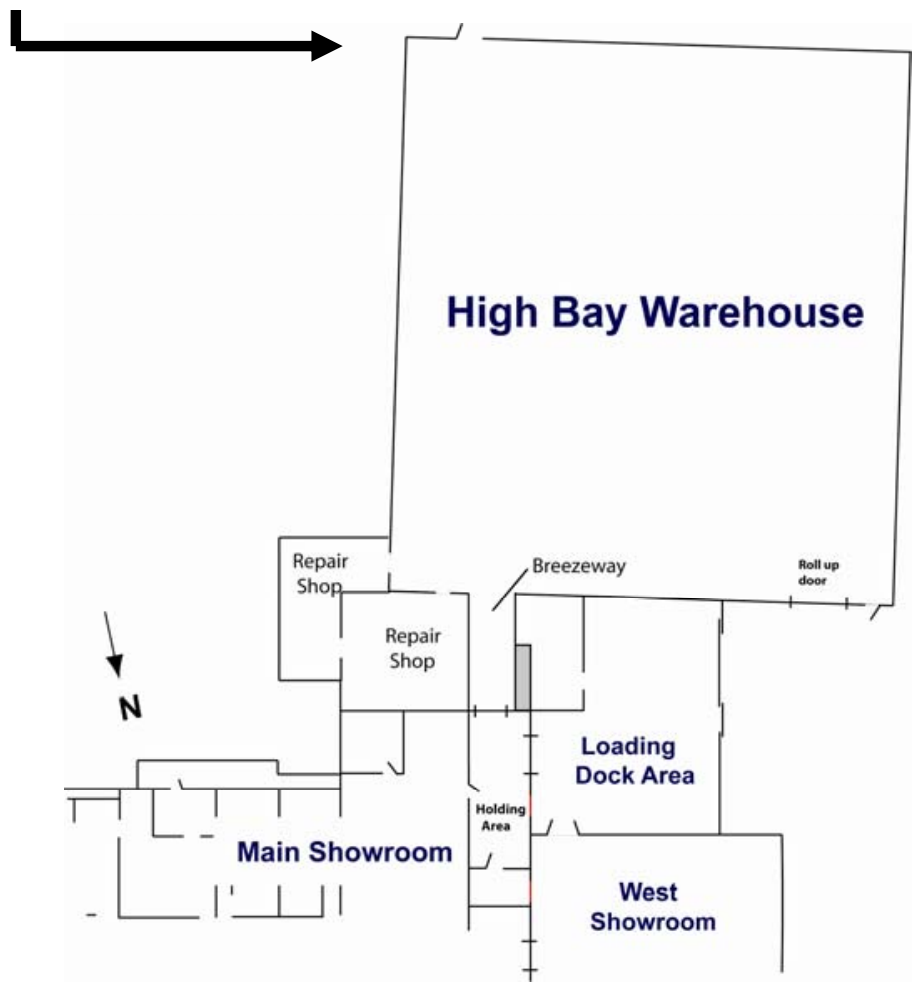




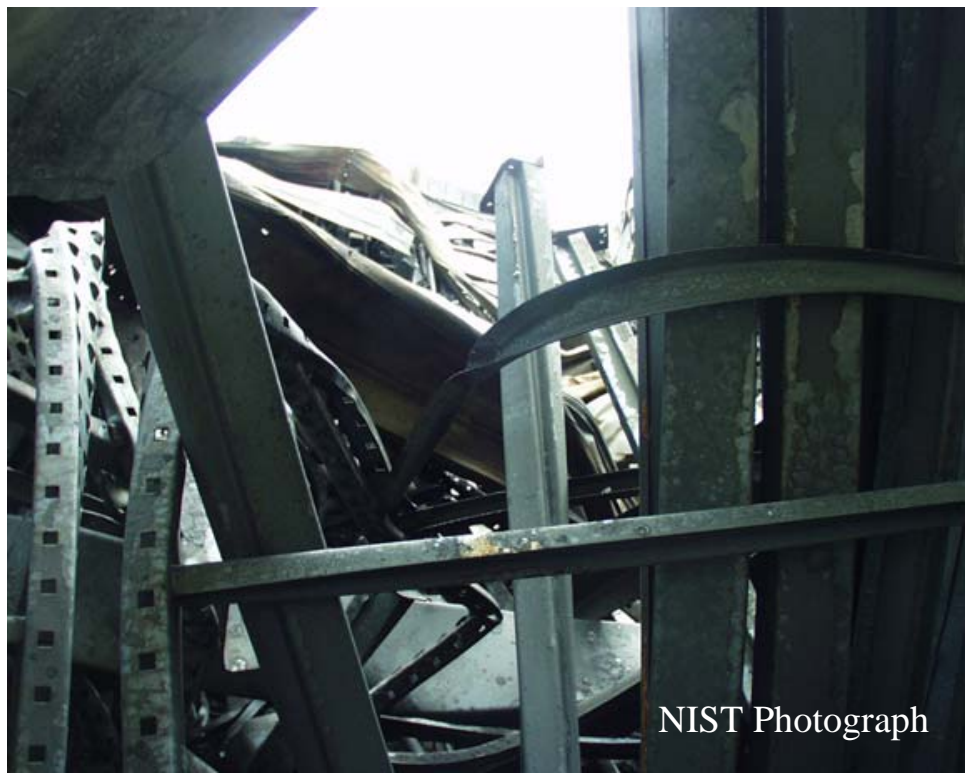

Figure G-6. View inside warehouse from outside south wall.

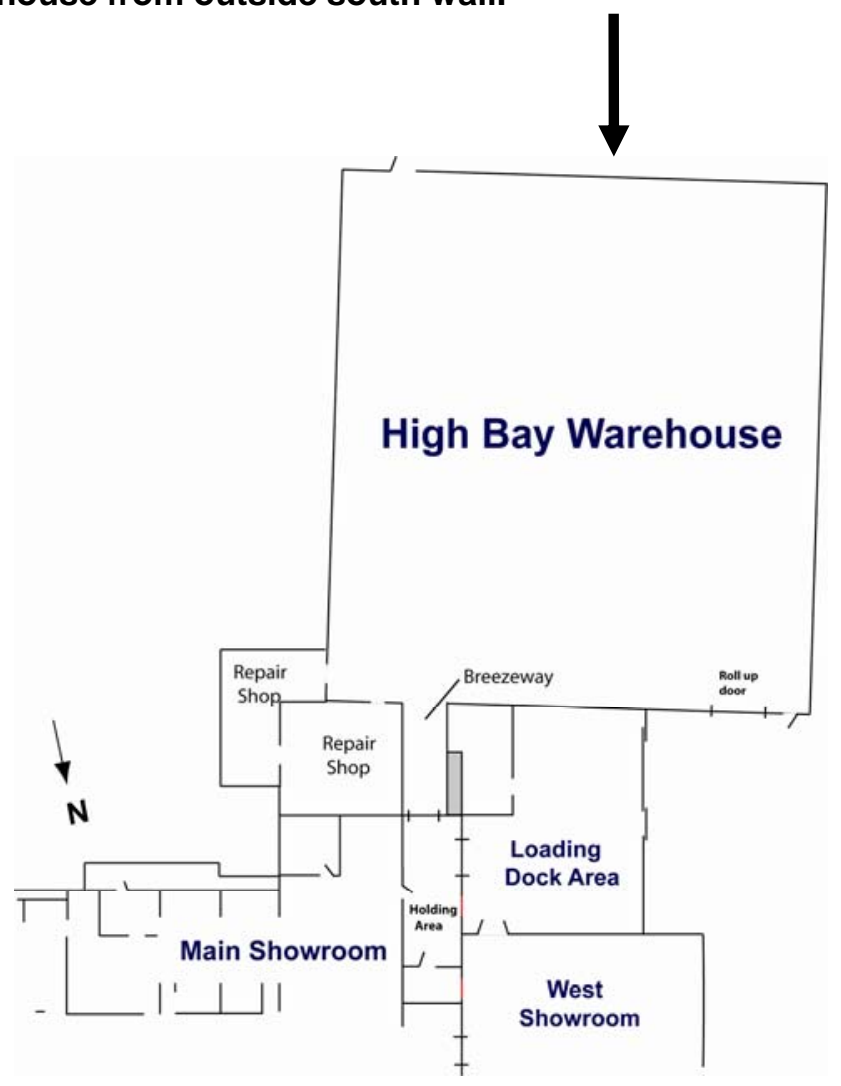




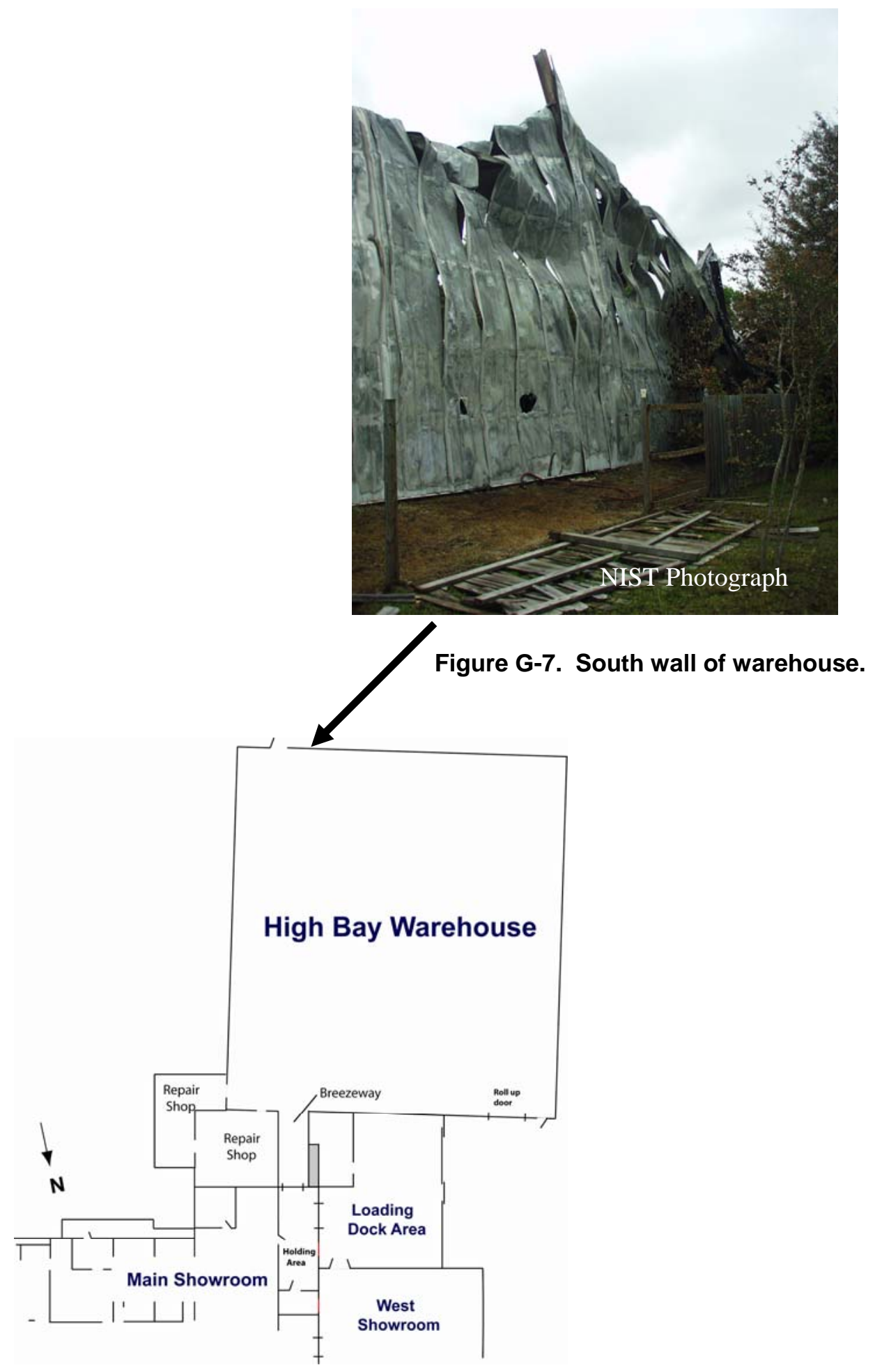




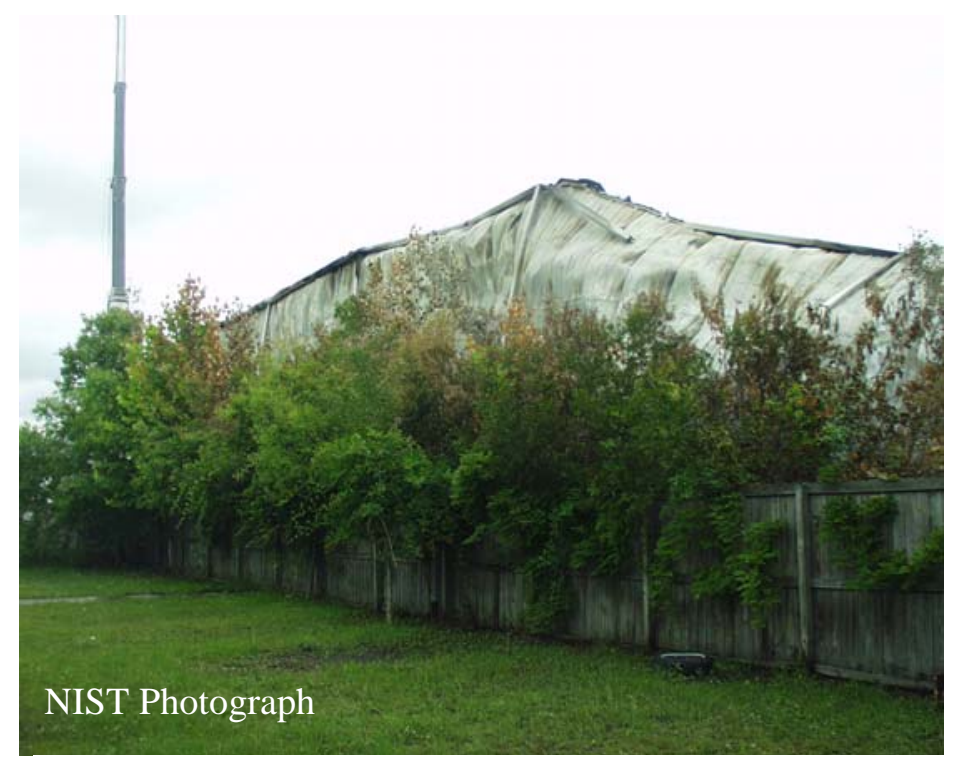

Figure G-8. West wall of warehouse behind fence and small trees.

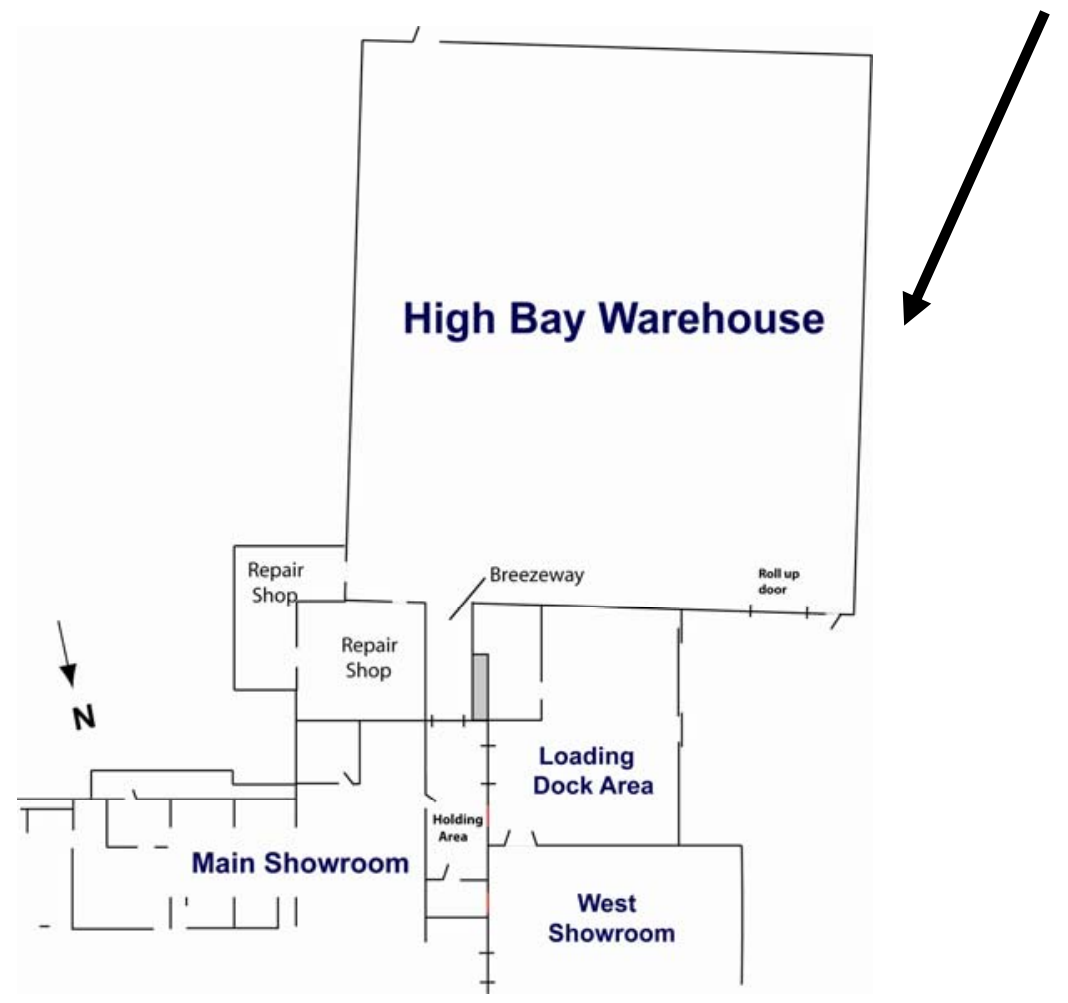



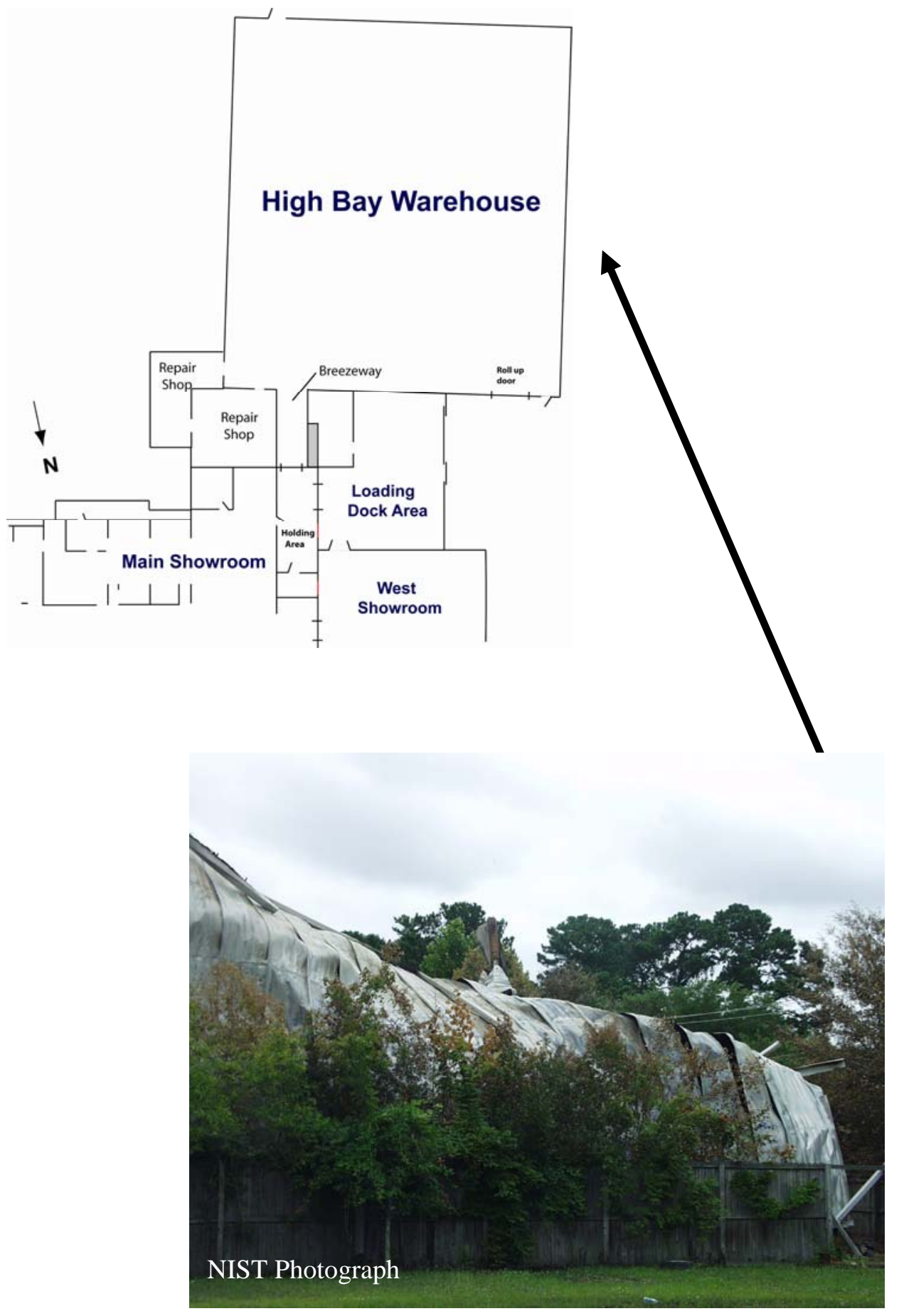

Figure G-9. West wall of warehouse. 


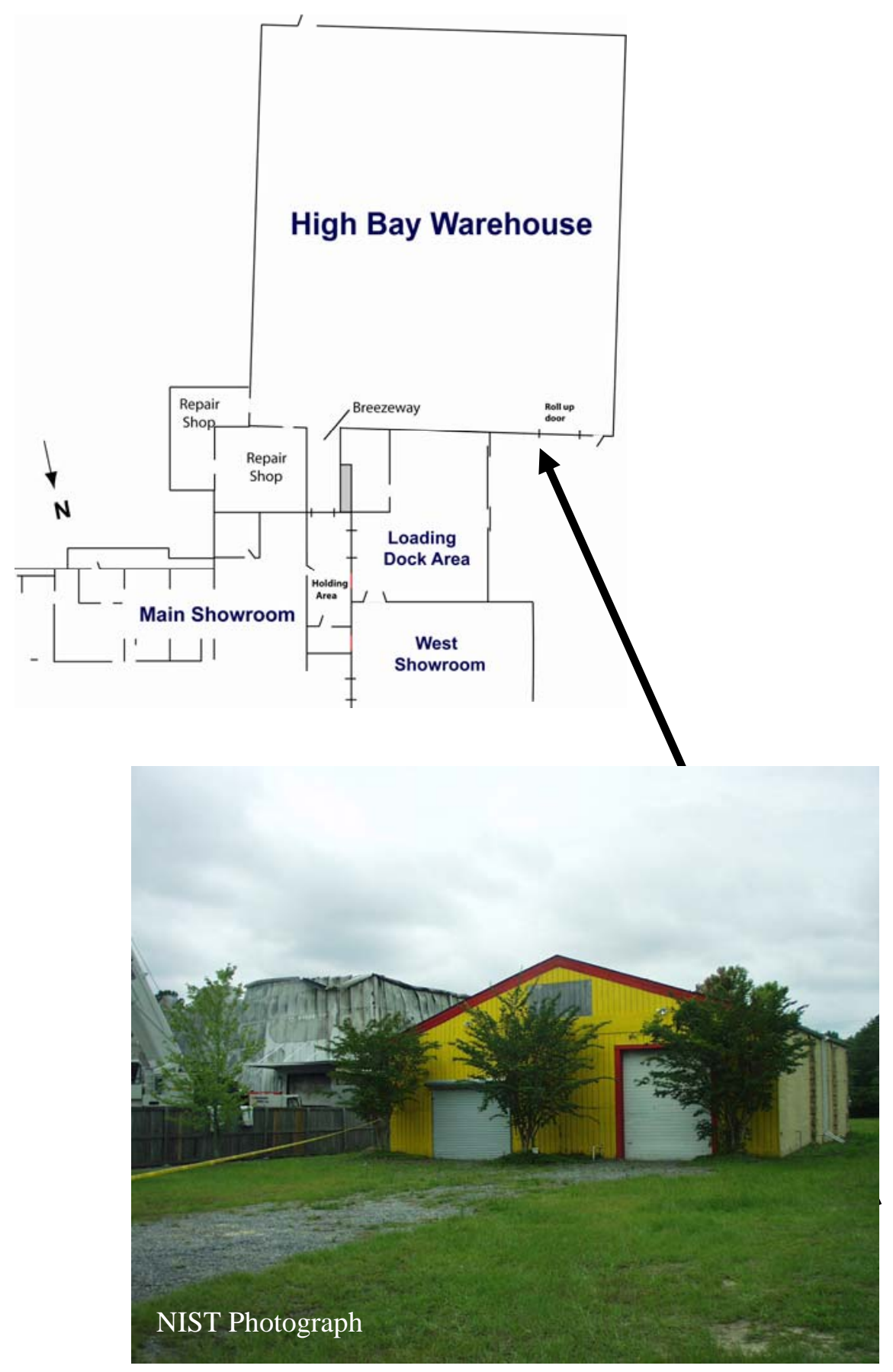

Figure G-10. Automobile repair garage, yellow structure, on lot adjacent to Sofa Super Store. Loading dock area of warehouse visible in background. 


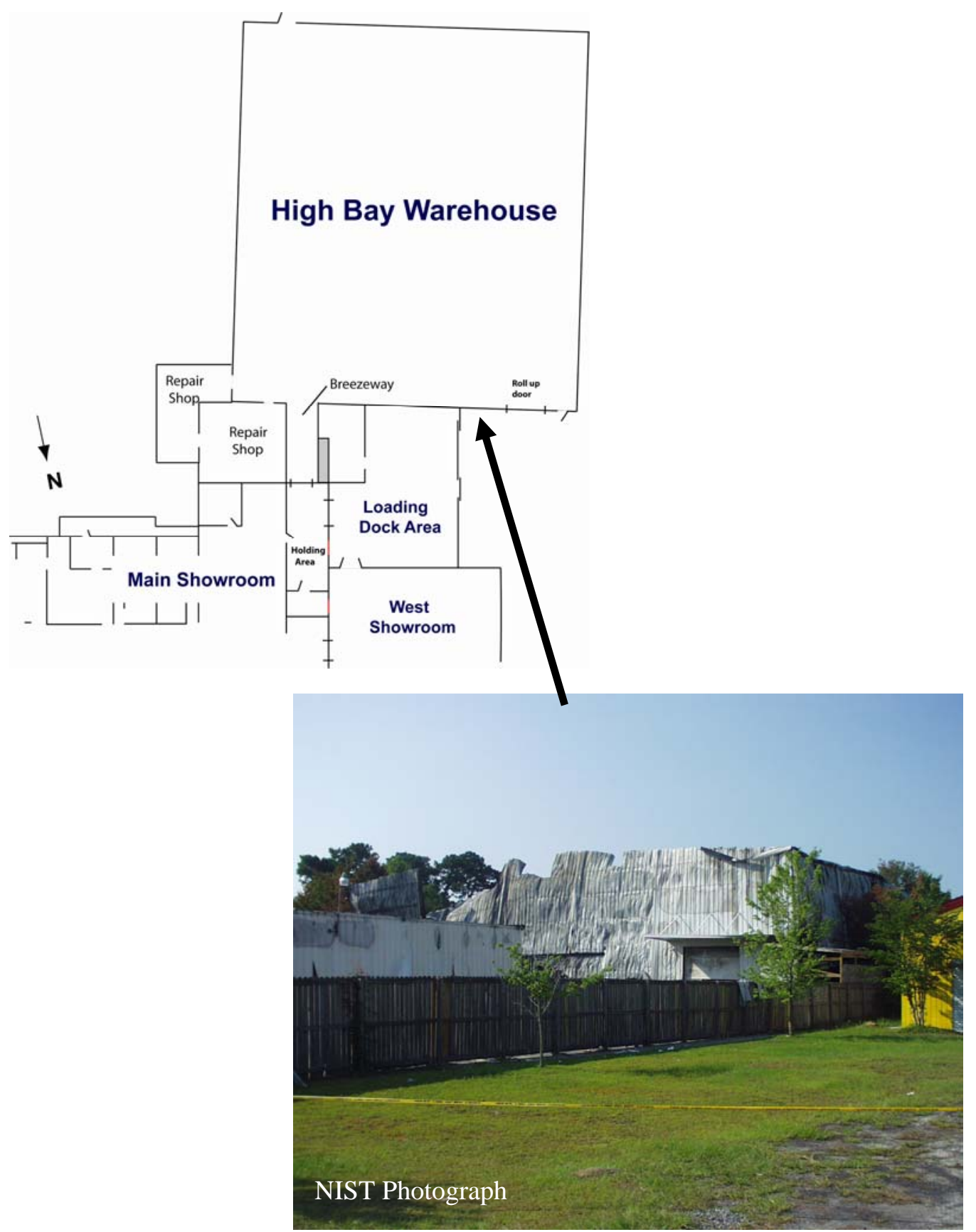

Figure G-11. West wall of west showroom and north wall of warehouse. 


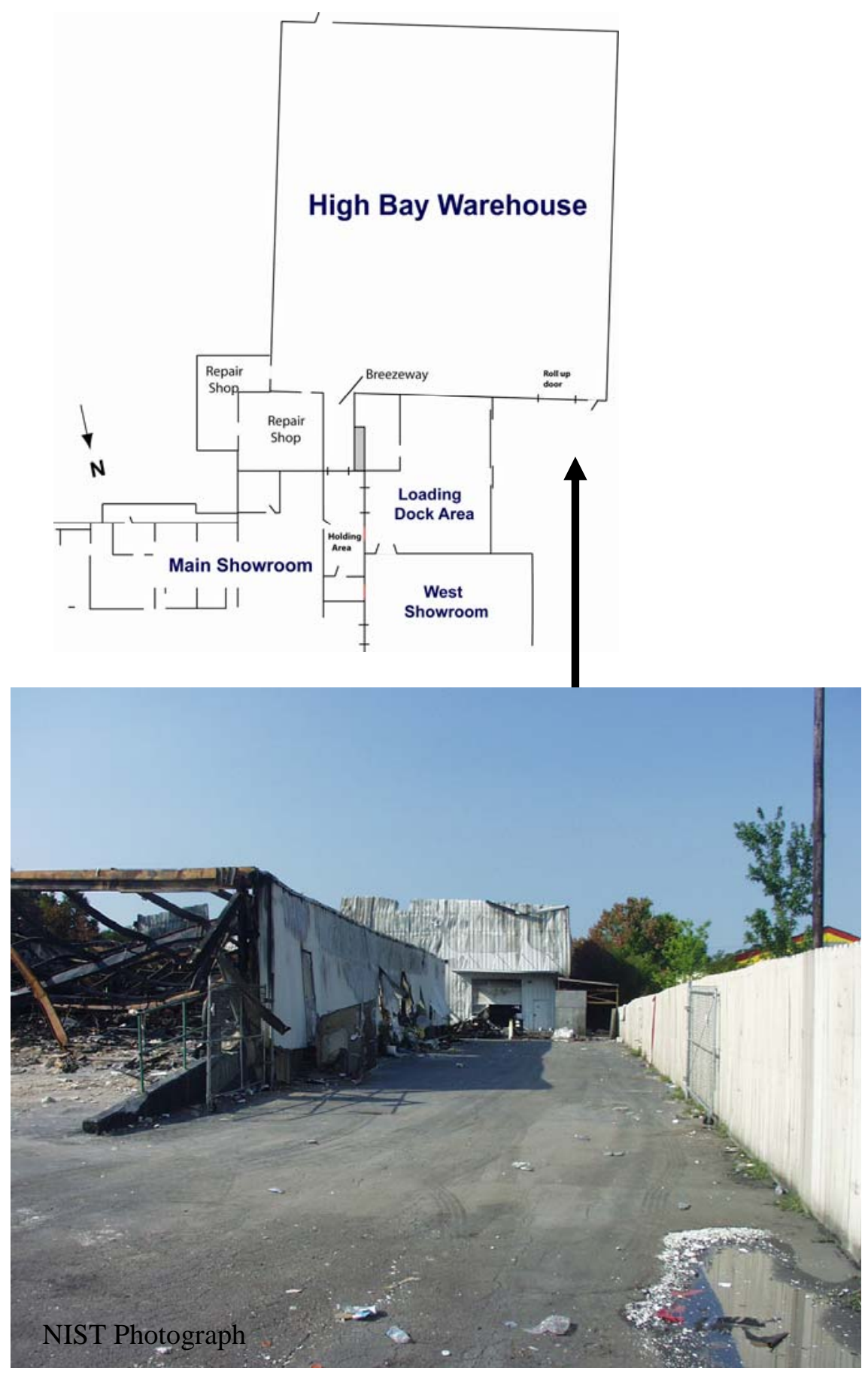

Figure G-12. Driveway leading along west showroom to warehouse and loading dock area. 


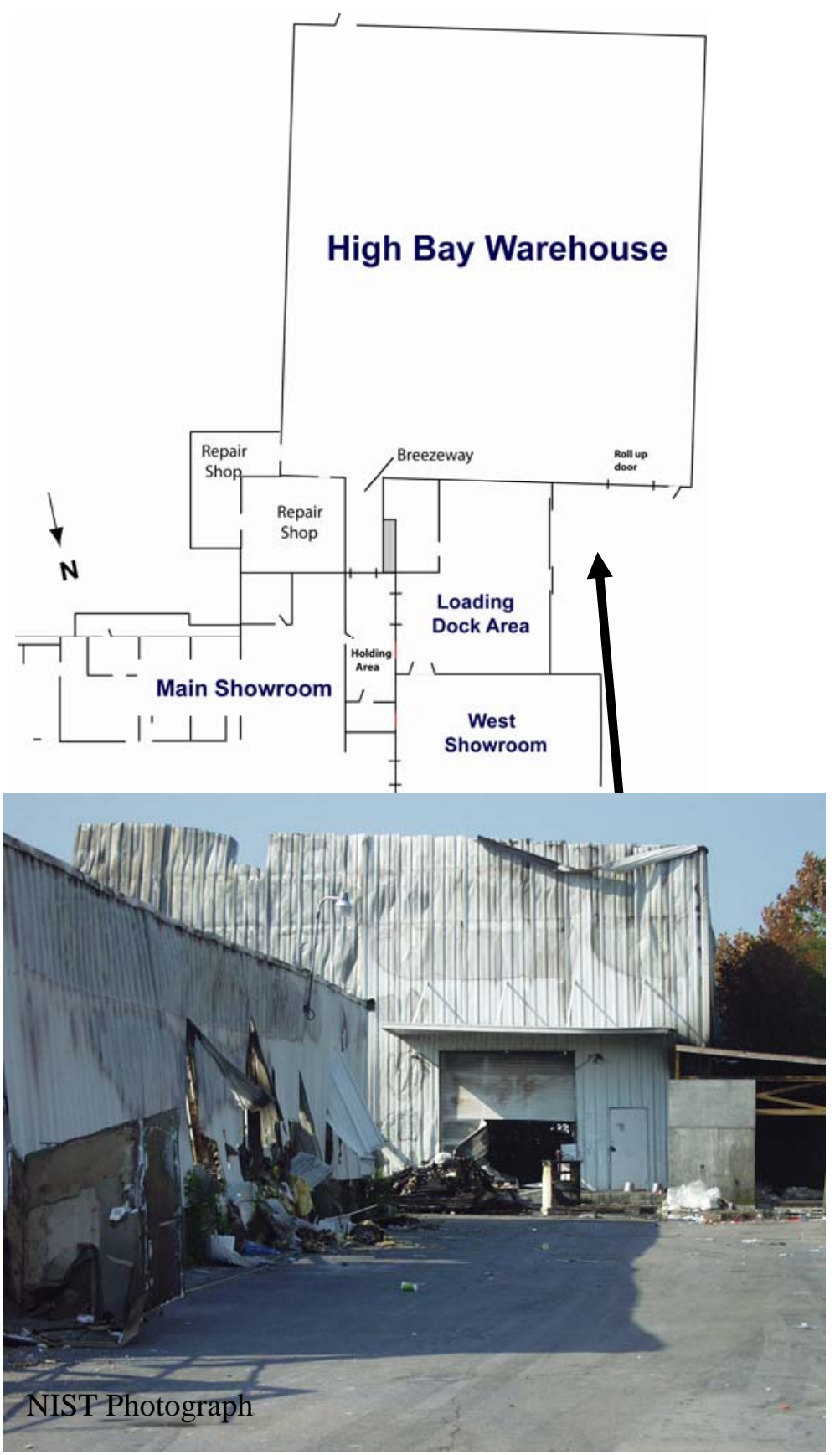

Figure G-13. West wall of west showroom and warehouse loading area. 


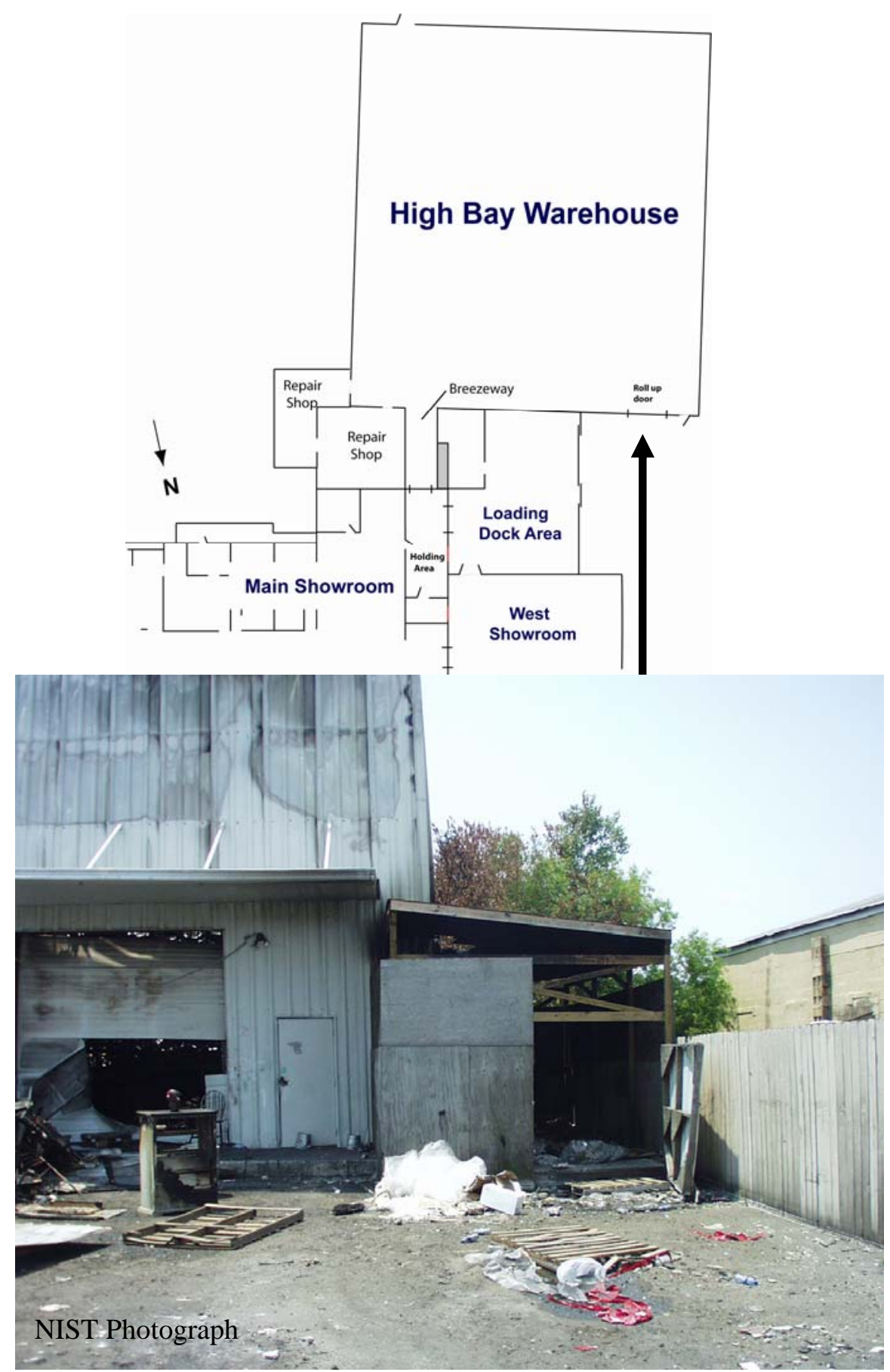

Figure G-14. Warehouse loading area visible on left and trash storage area visible on right. 


\section{G.2 WAREHOUSE INTERIOR PHOTOS}

Several days after the fire, photographs were taken of the interior of the warehouse. Due to safety concerns with structural stability of the warehouse, most interior photographs were taken from doorways or through openings rather than from inside the structure.

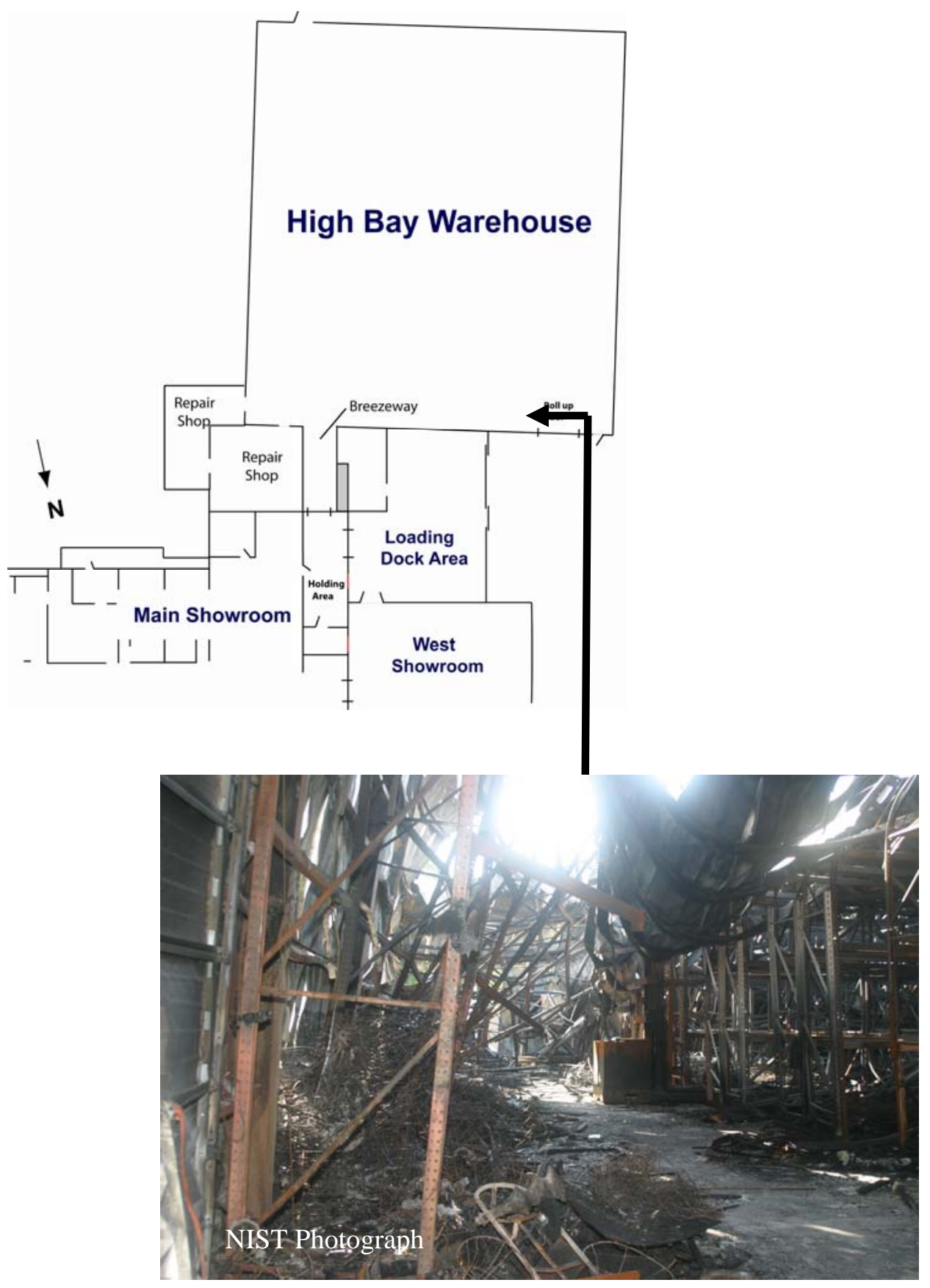

Figure G-15. Interior of warehouse looking from roll-down door toward repair shop doorway. 


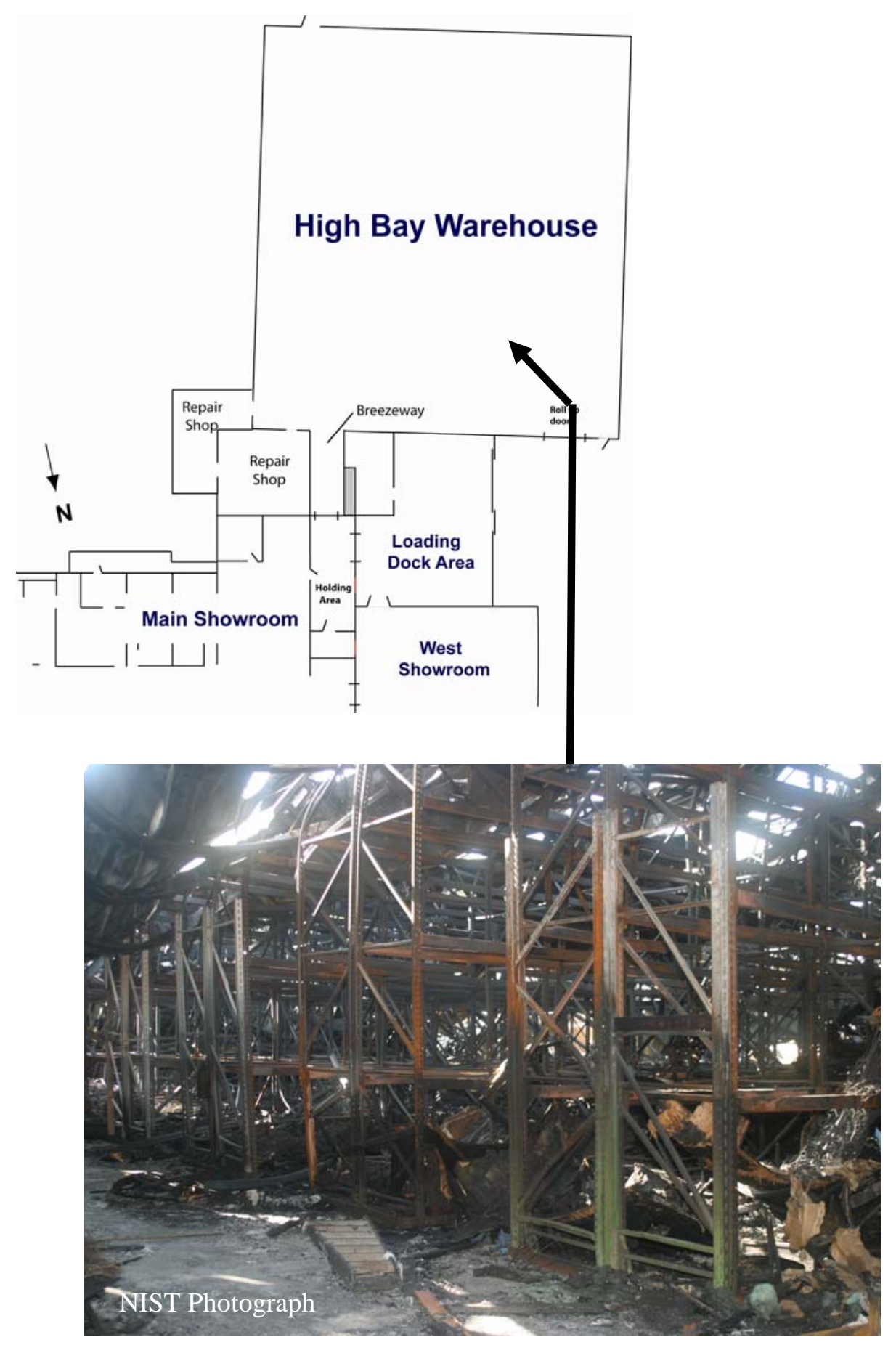

Figure G-16. High rack storage units inside warehouse. 


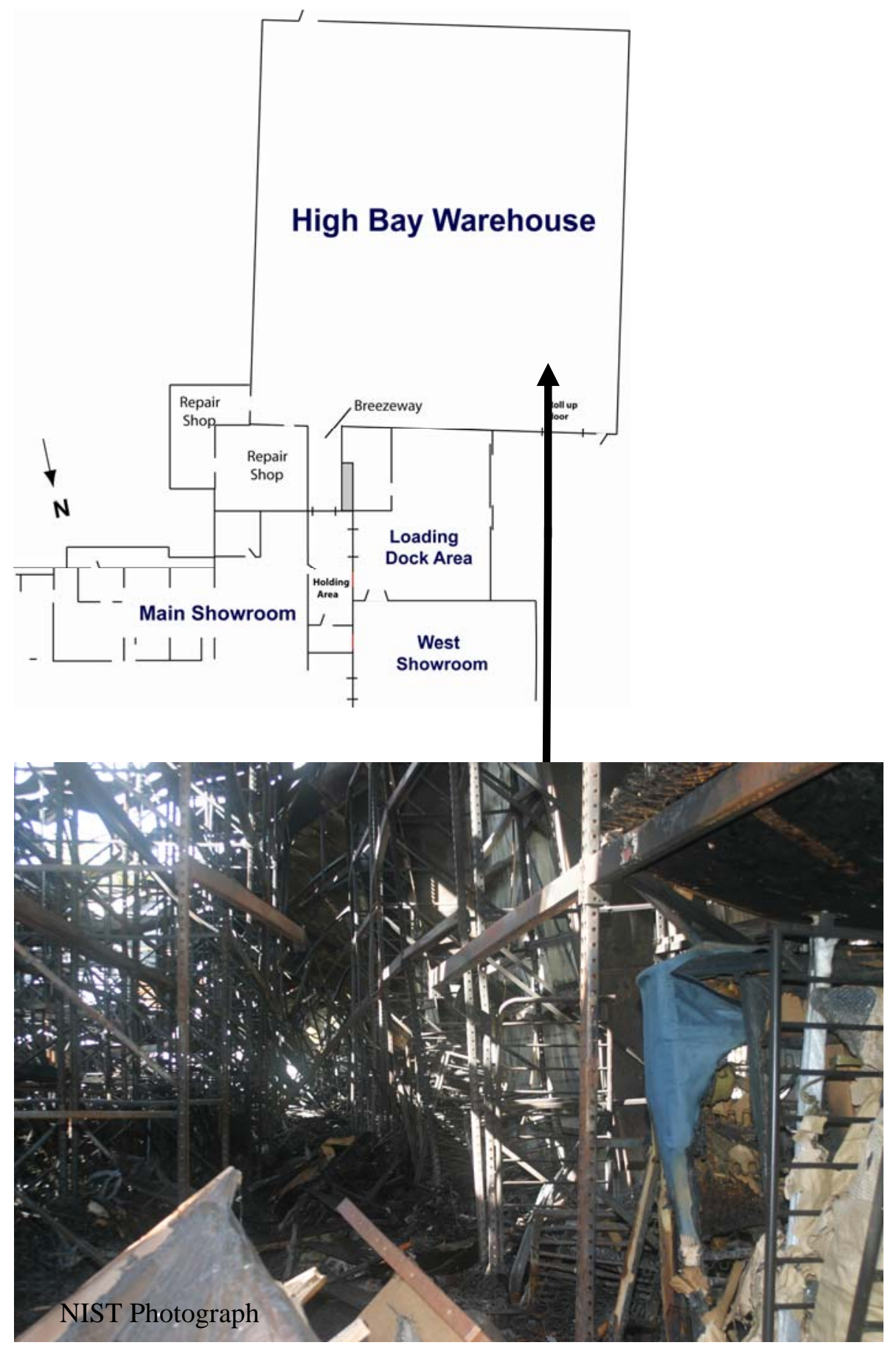

Figure G-17. High rack storage units inside warehouse. 

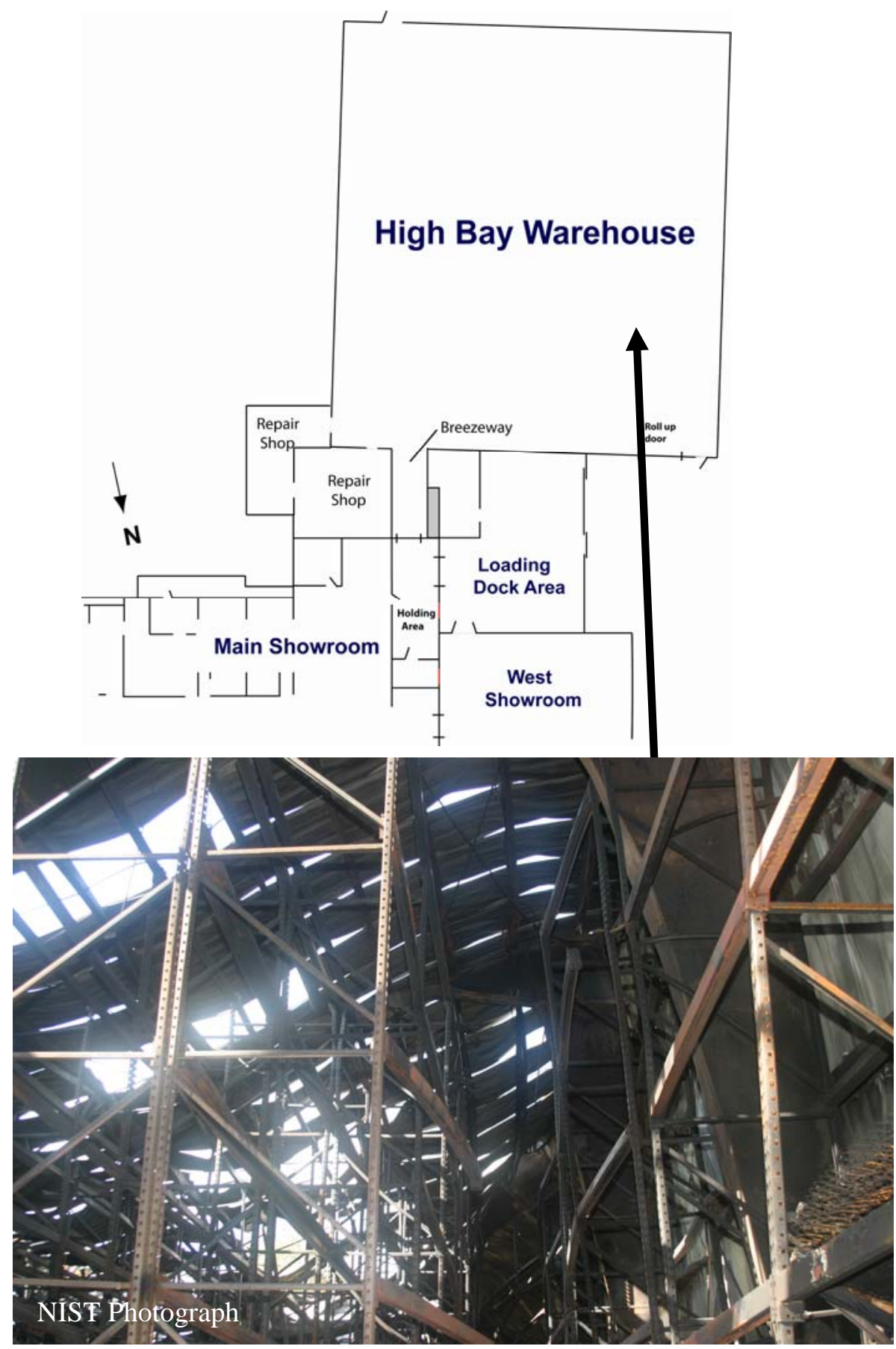

Figure G-18. Interior of warehouse looking toward roof. 


\section{APPENDIX H. REPAIR SHOPS}

\section{H.1 REPAIR SHOPS}

Two repair shops were added between the rear of the main showroom and the north wall of the warehouse. Both of these areas had metal siding over wood frame construction.

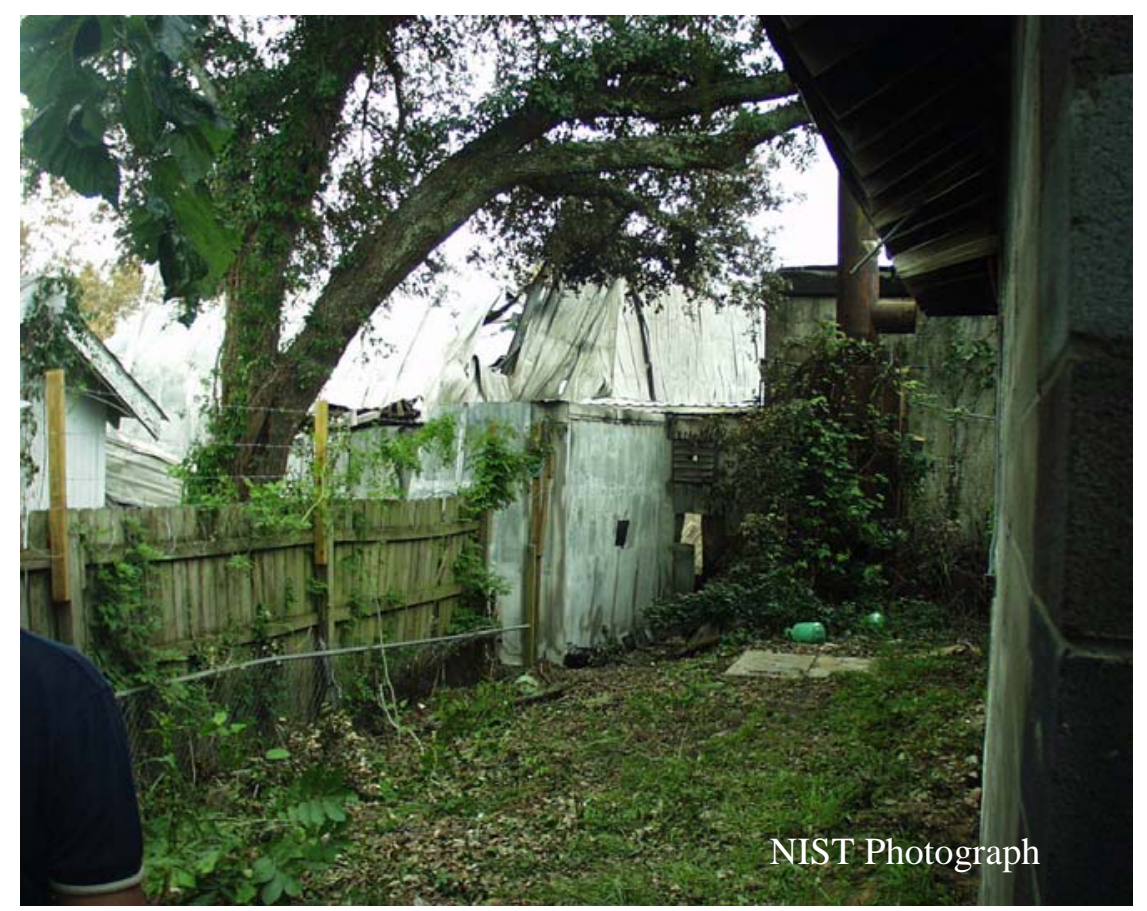

Figure H-1. North wall of repair shop looking toward warehouse from rear of main showroom. Small window-mount style air conditioner was installed in rectangular hole in center of wall.

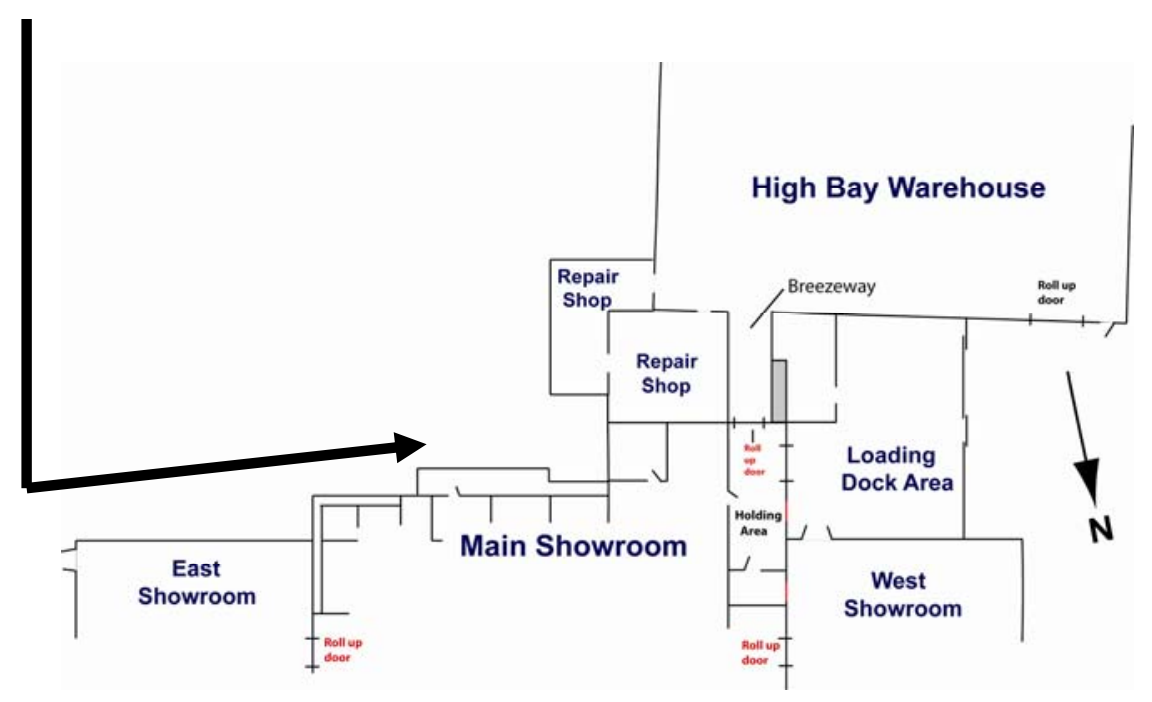




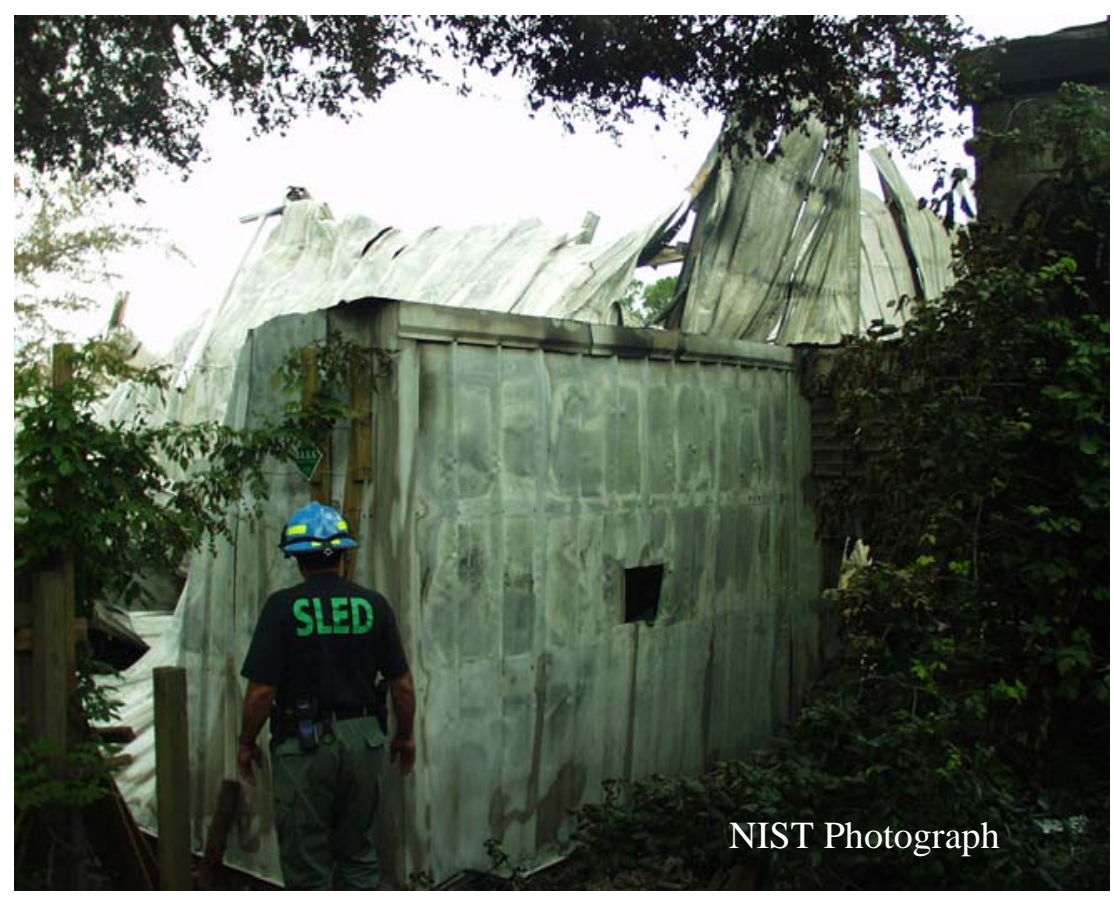

Figure H-2. North wall of repair shop looking toward warehouse, from rear of main showroom. Air conditioner was mounted in small rectangular hole in center of wall.

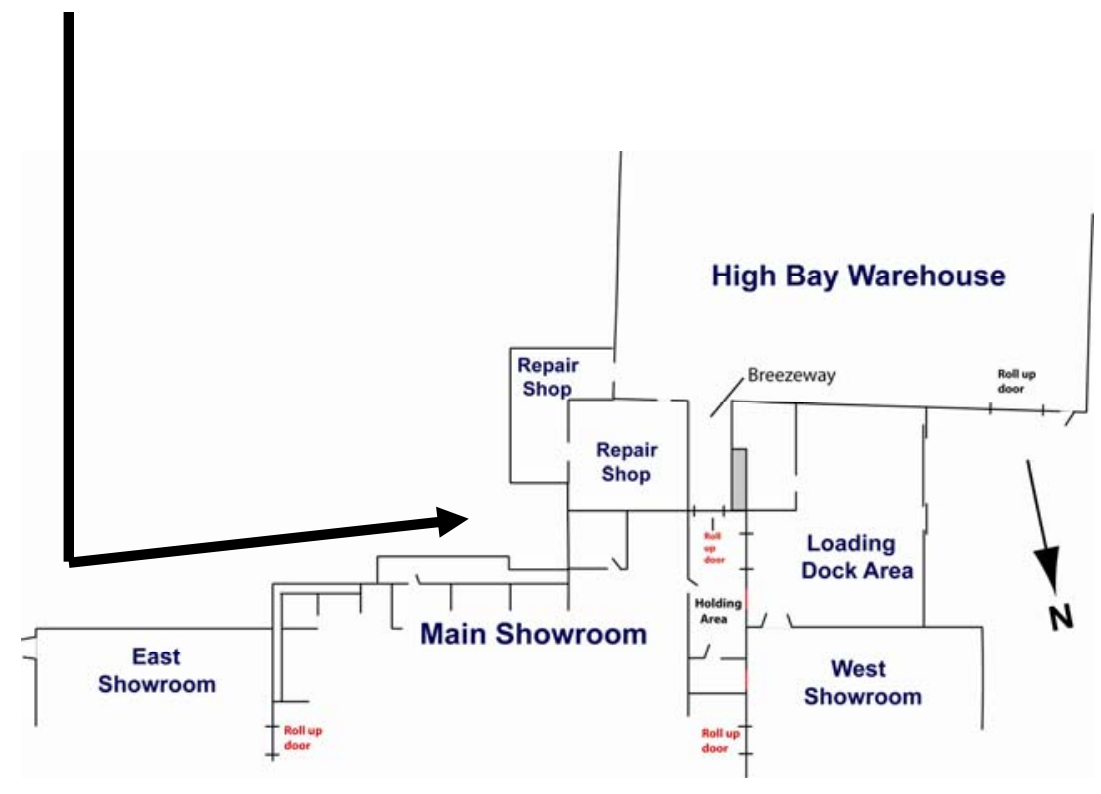




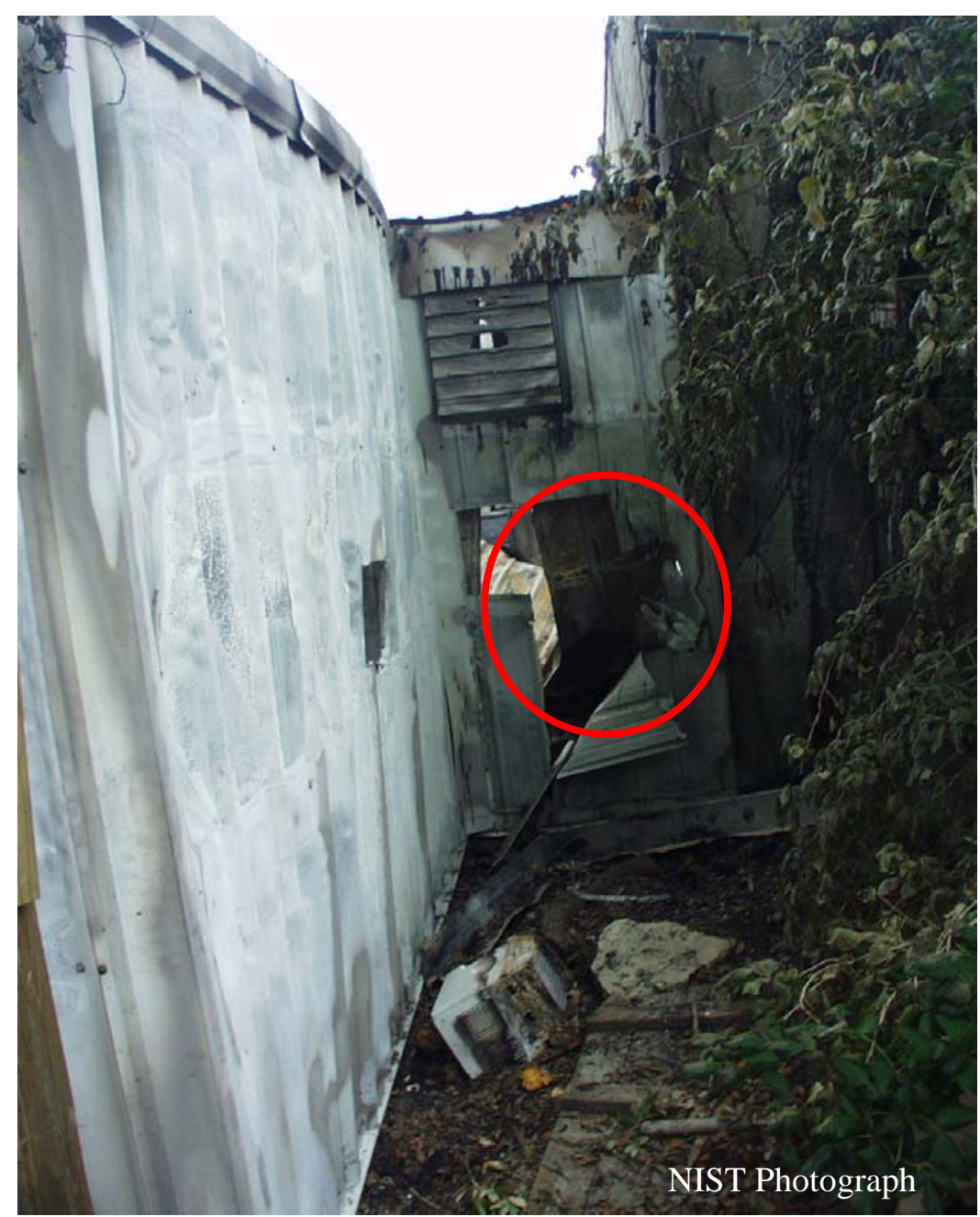

Figure H-3. Intersection of walls of two repair shops with rear wall of main showroom. Trapped employee was extracted from opening in metal siding visible in center of photo (circled area). Air conditioner which had been mounted in rectangular opening in wall fell out and was on ground below opening.

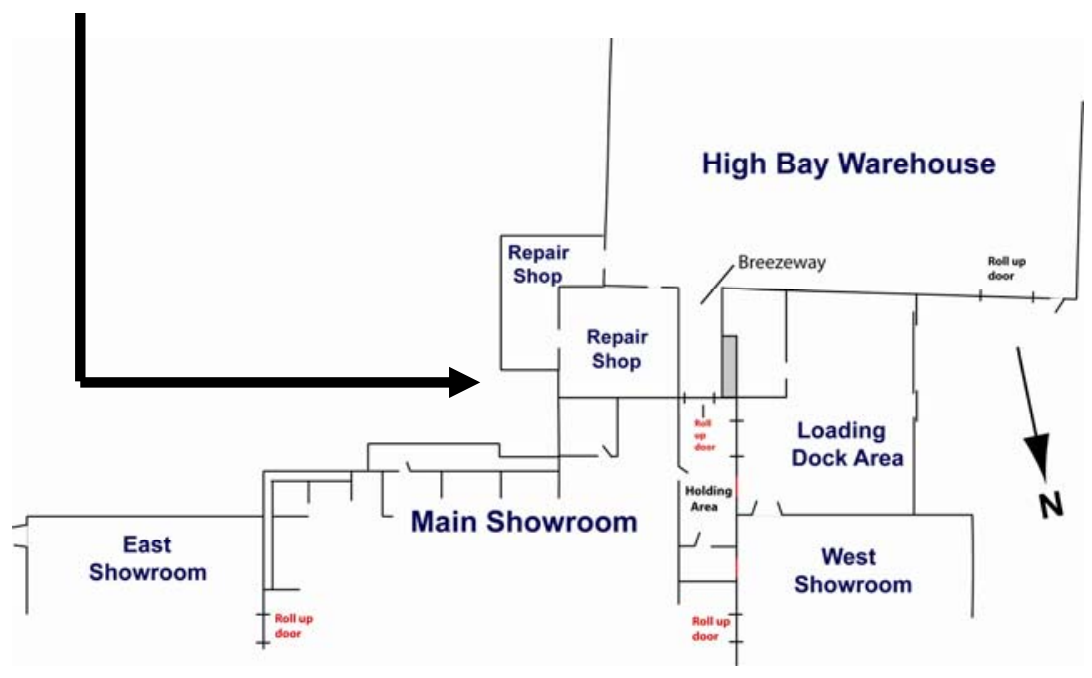




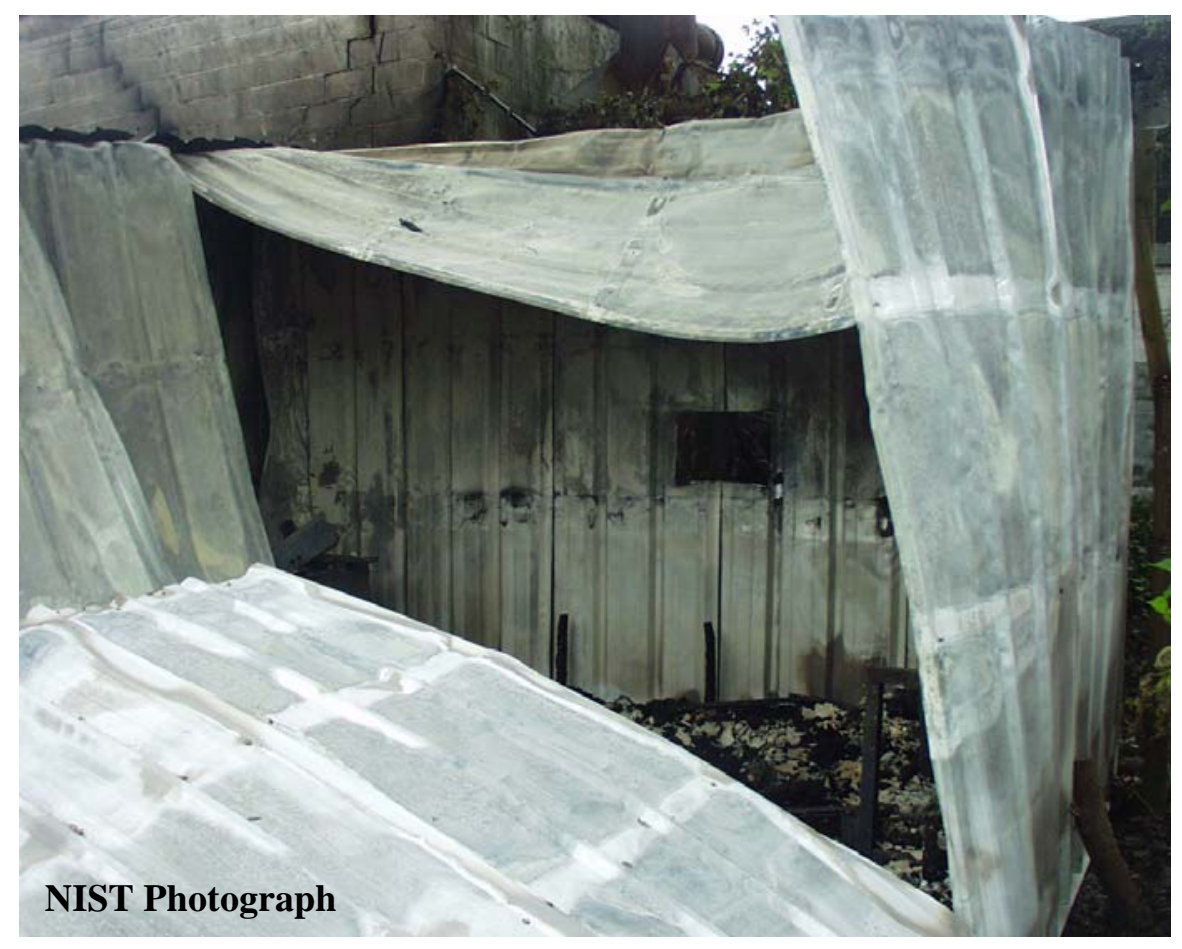

Figure H-4. View inside repair shop area.

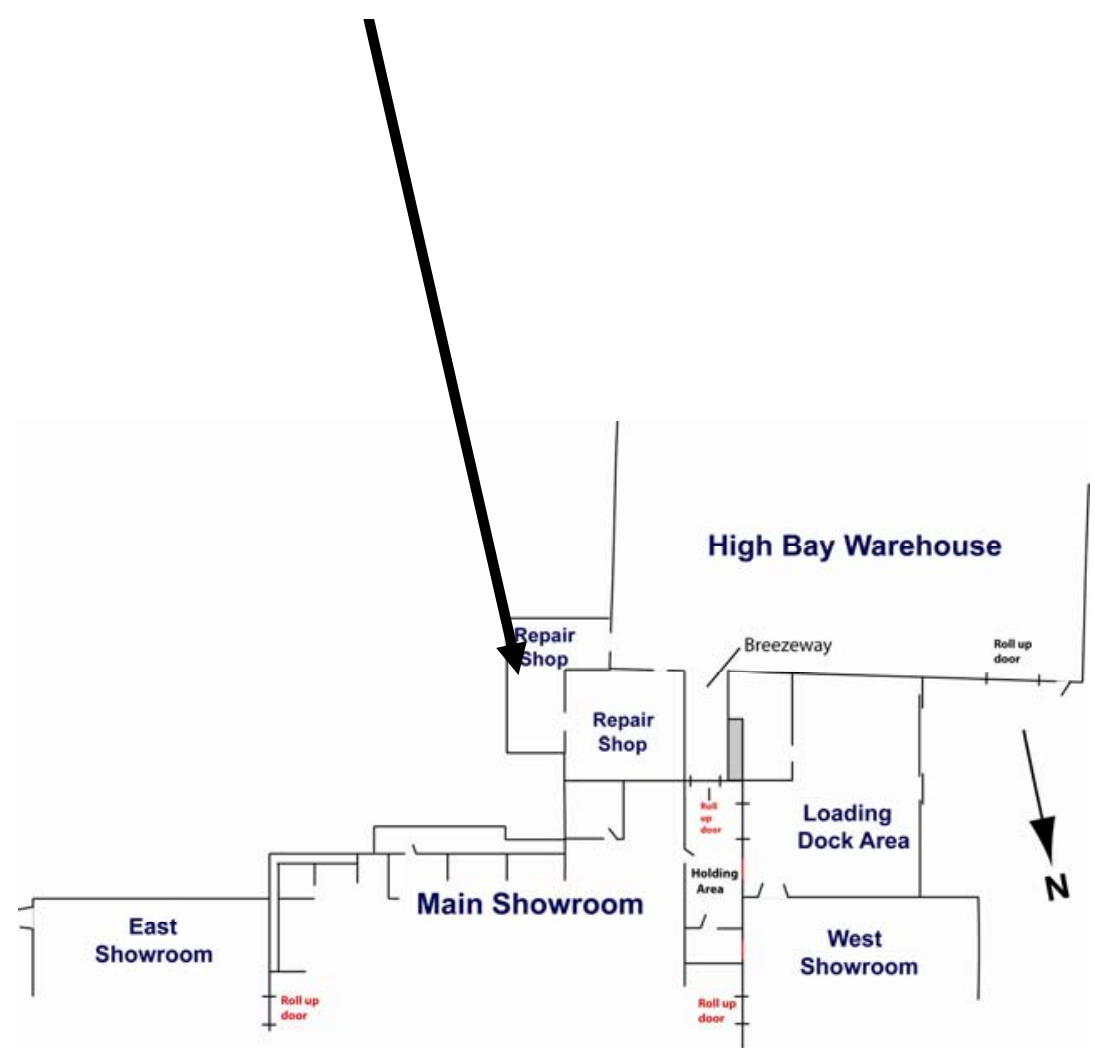




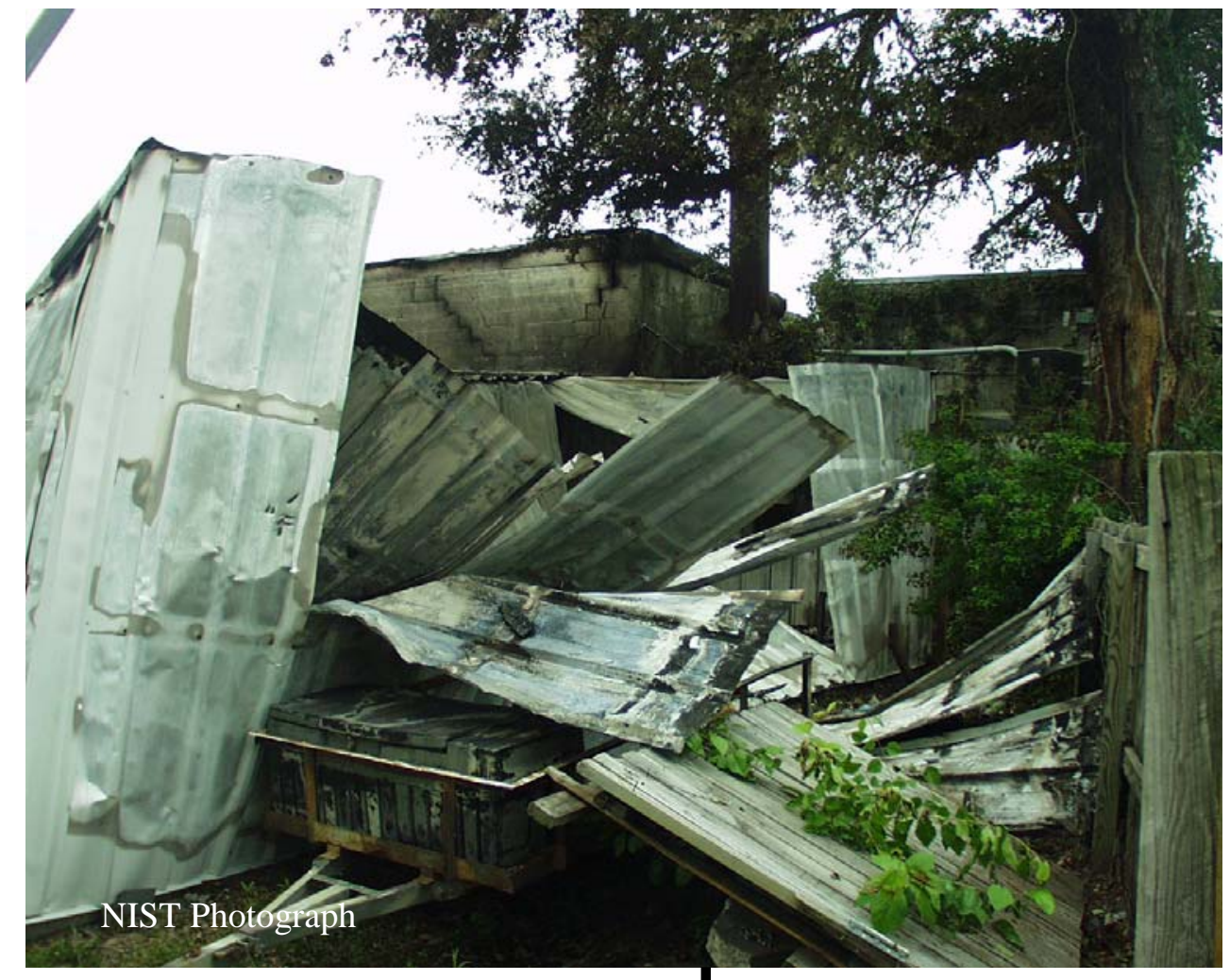

Figure H-5. East wall of repair shop.

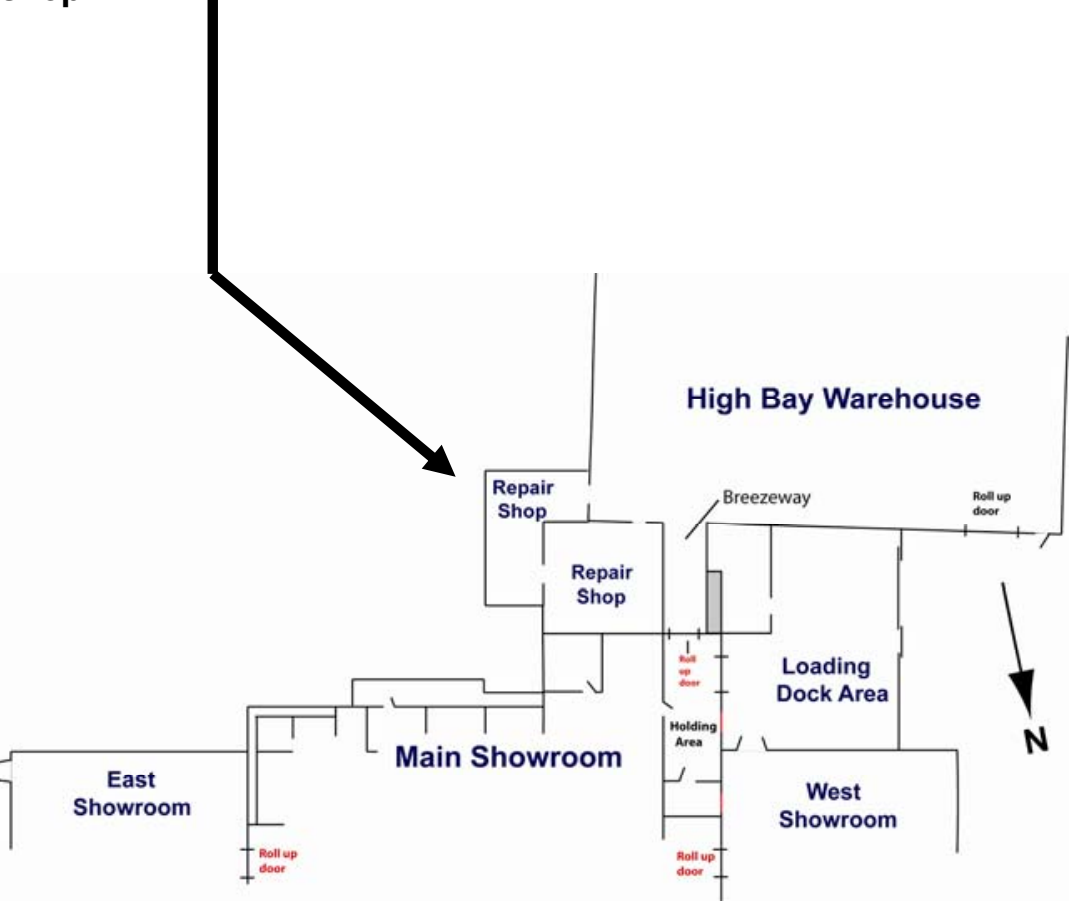




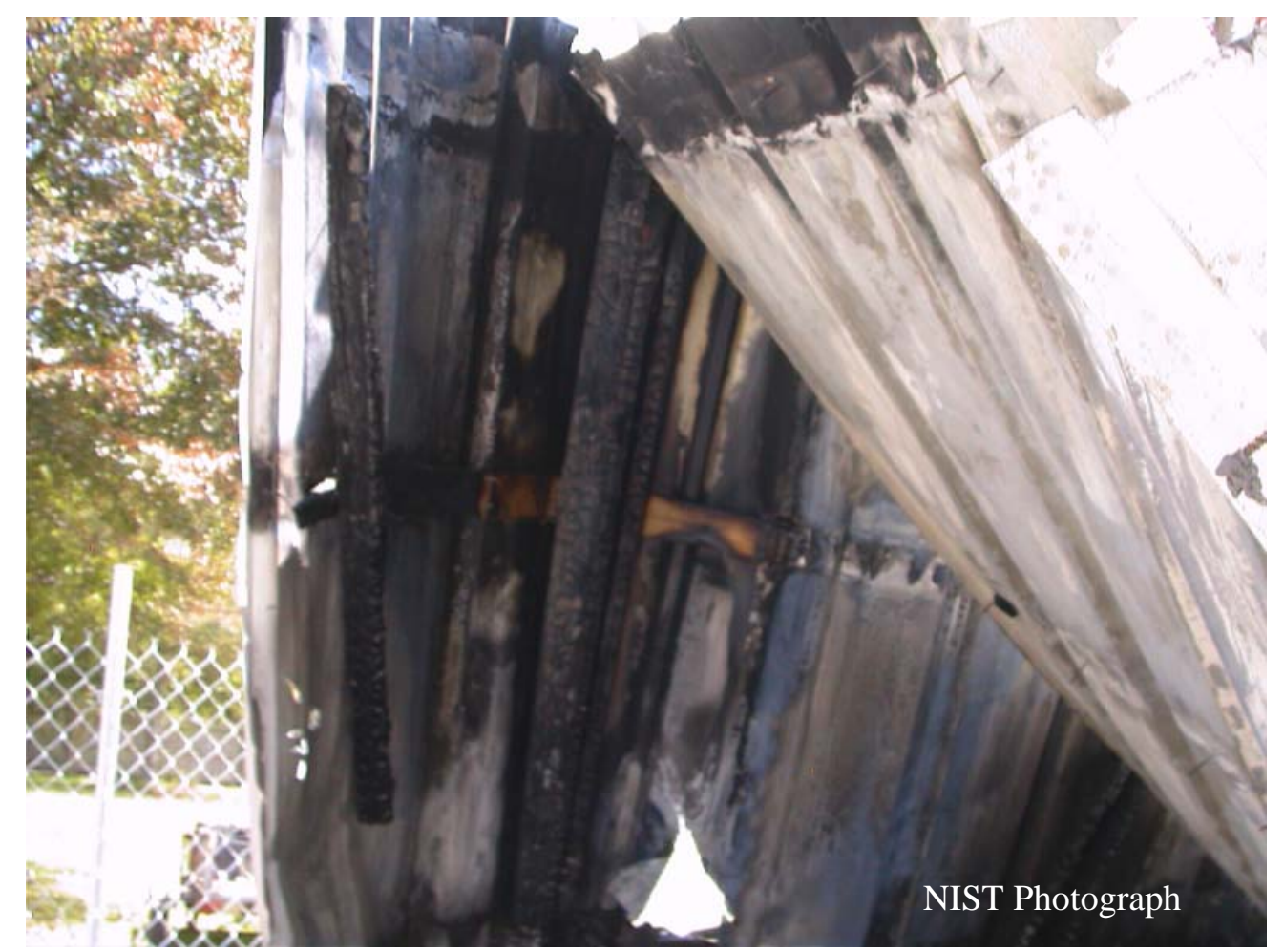

Figure H-6. Wood frame of repair shop.

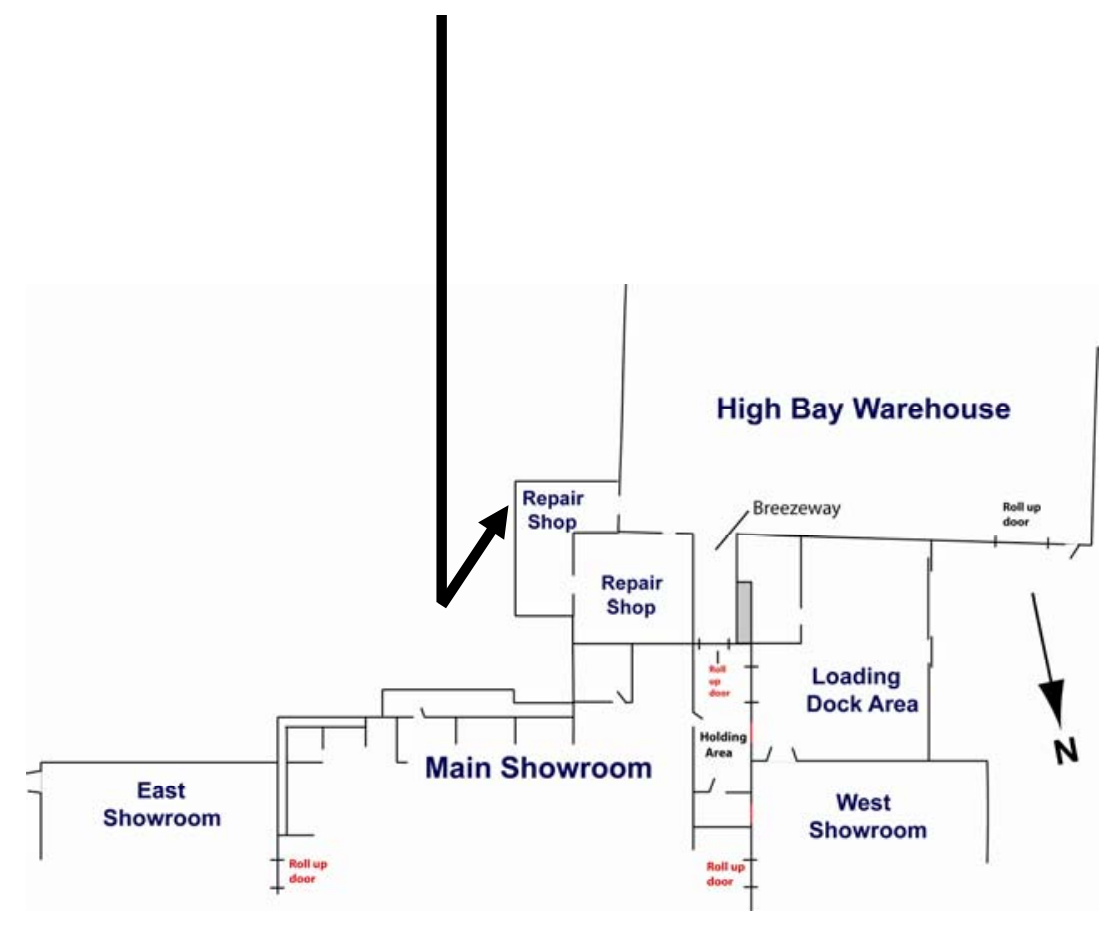




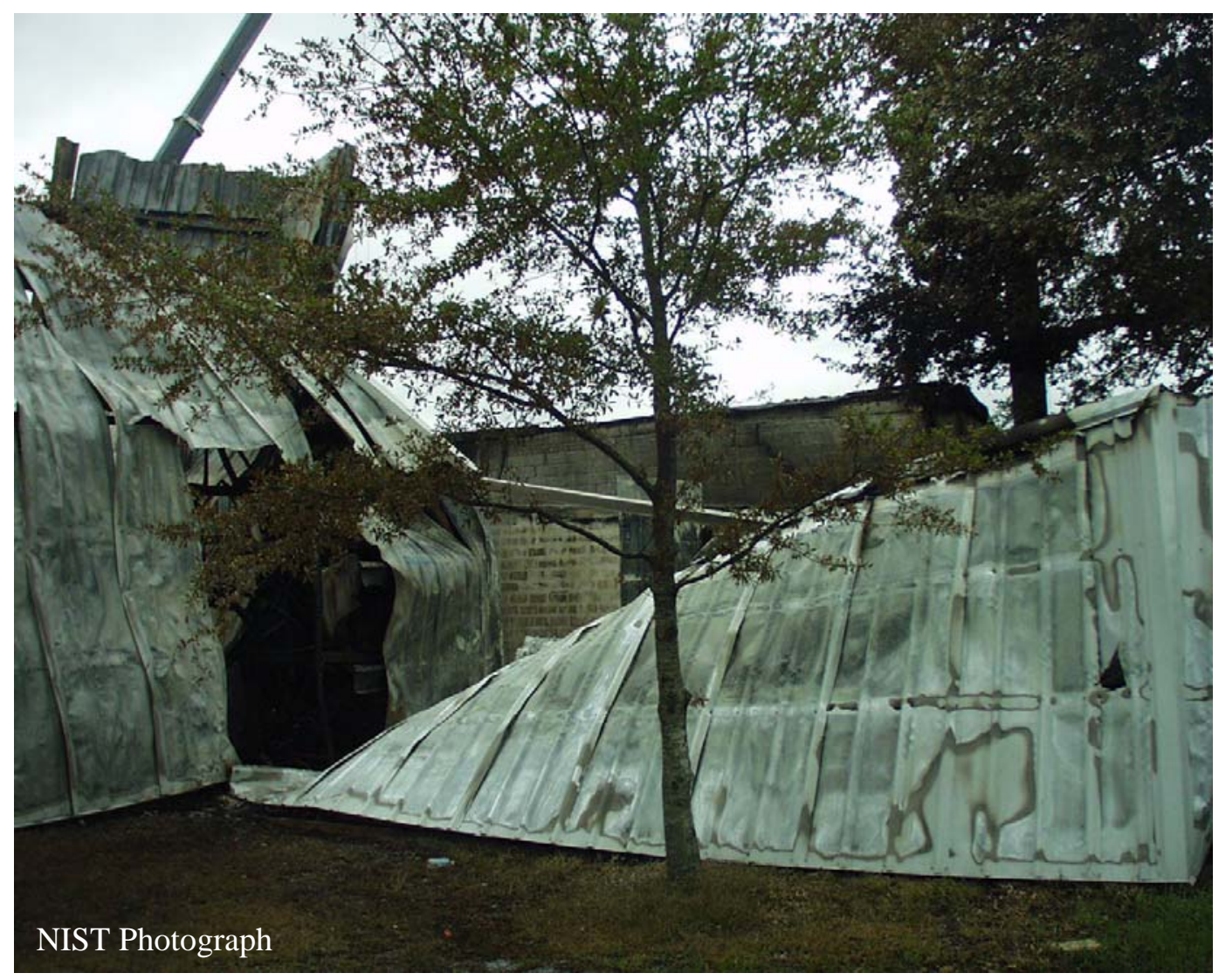

Figure H-7. South wall of repair shop. Rectangular opening was doorway from repair shop to warehouse.

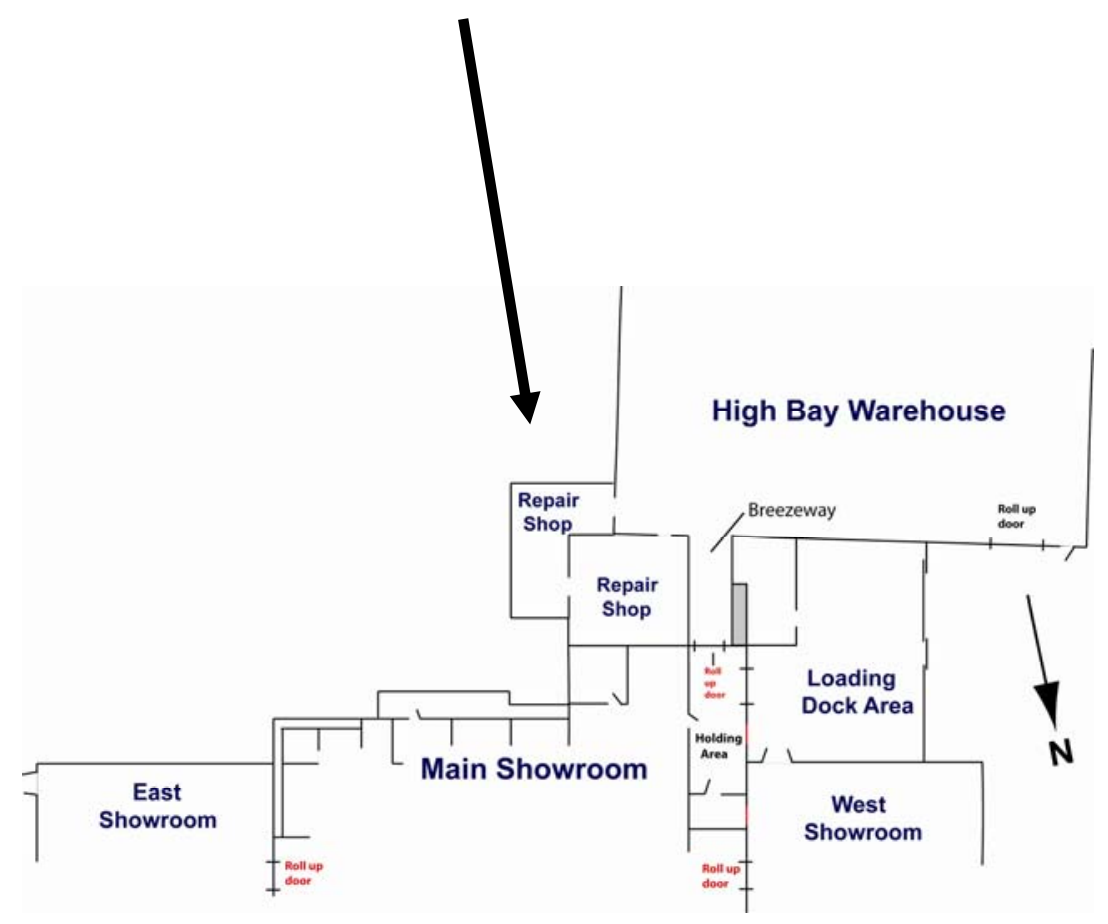




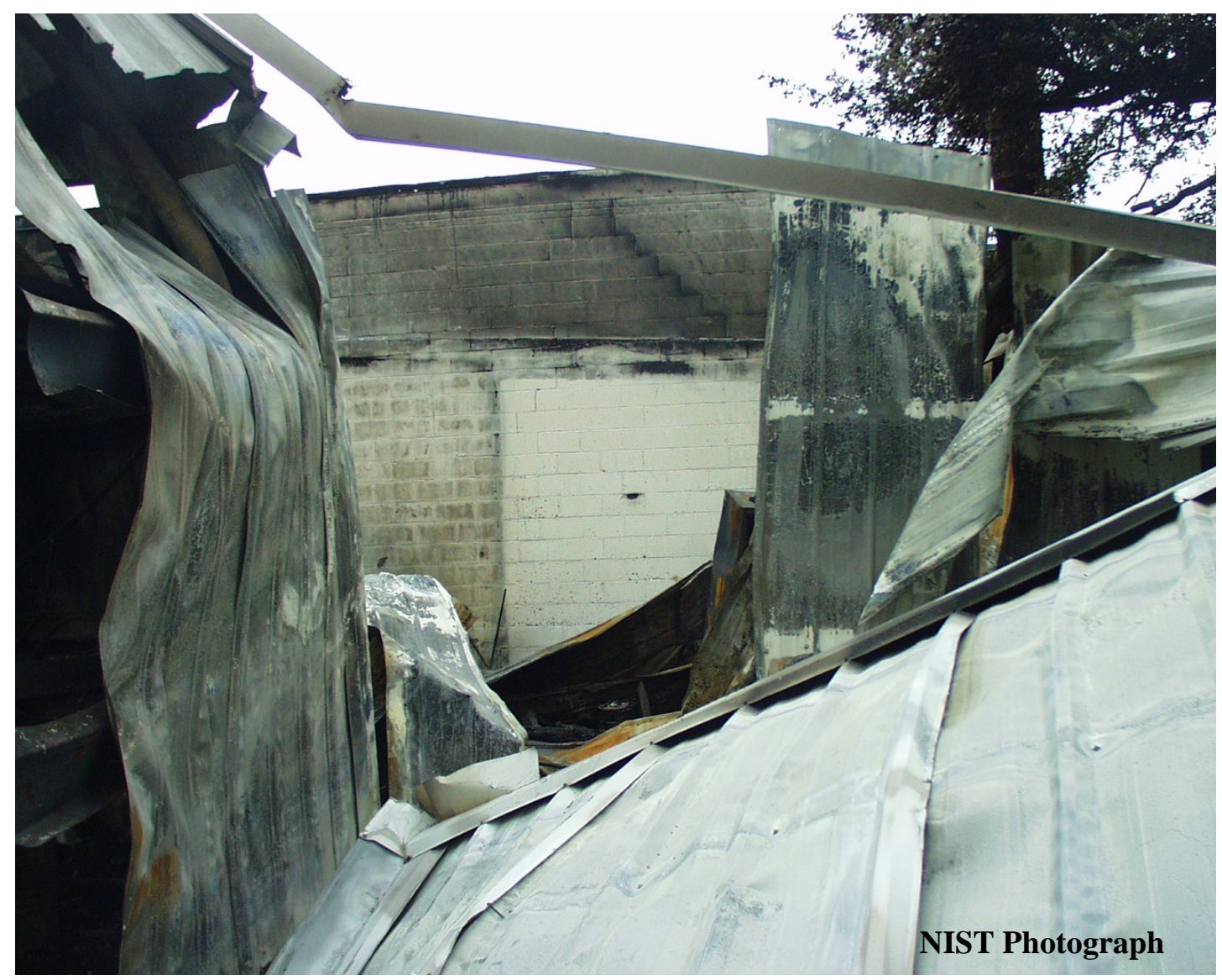

Figure H-8. Rear wall of main showroom visible through repair shop.

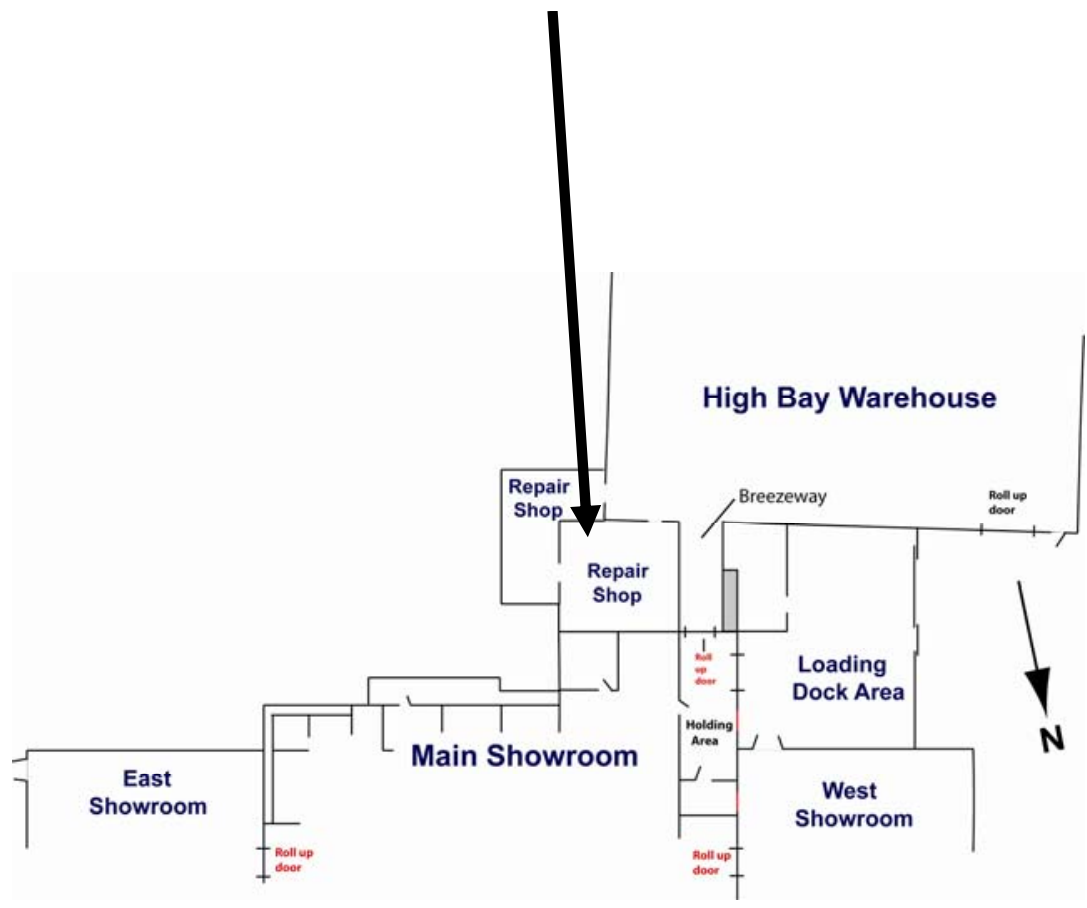




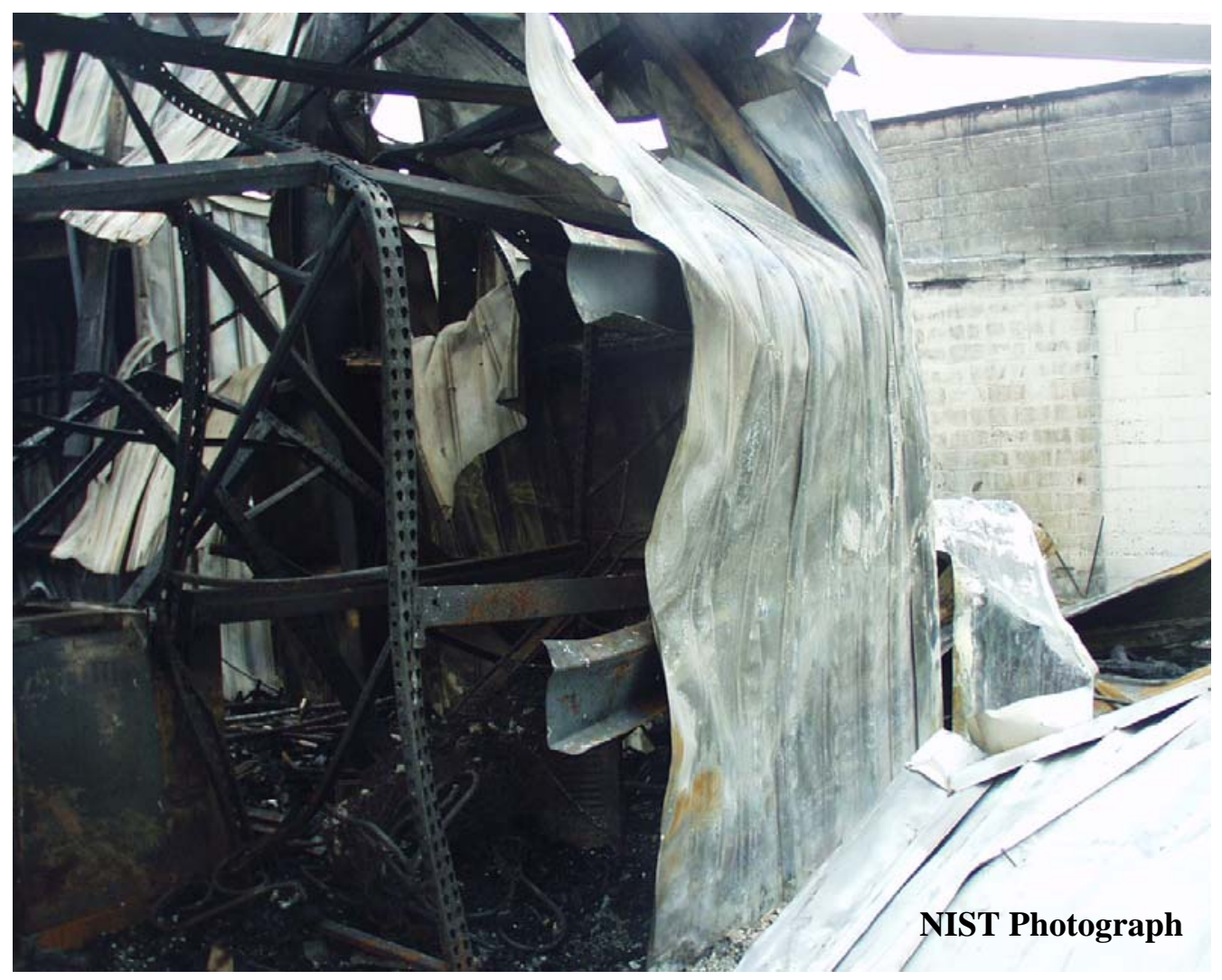

Figure H-9. Doorway from repair shop into warehouse.

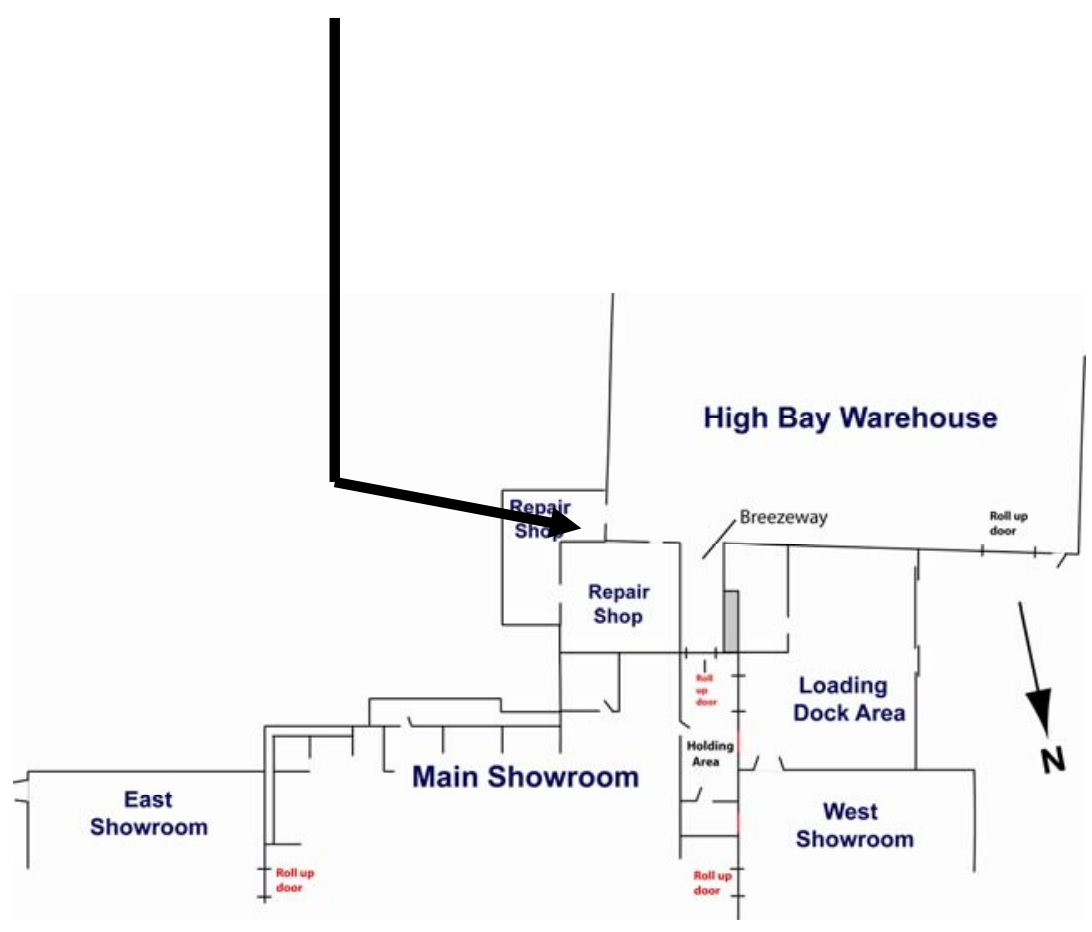




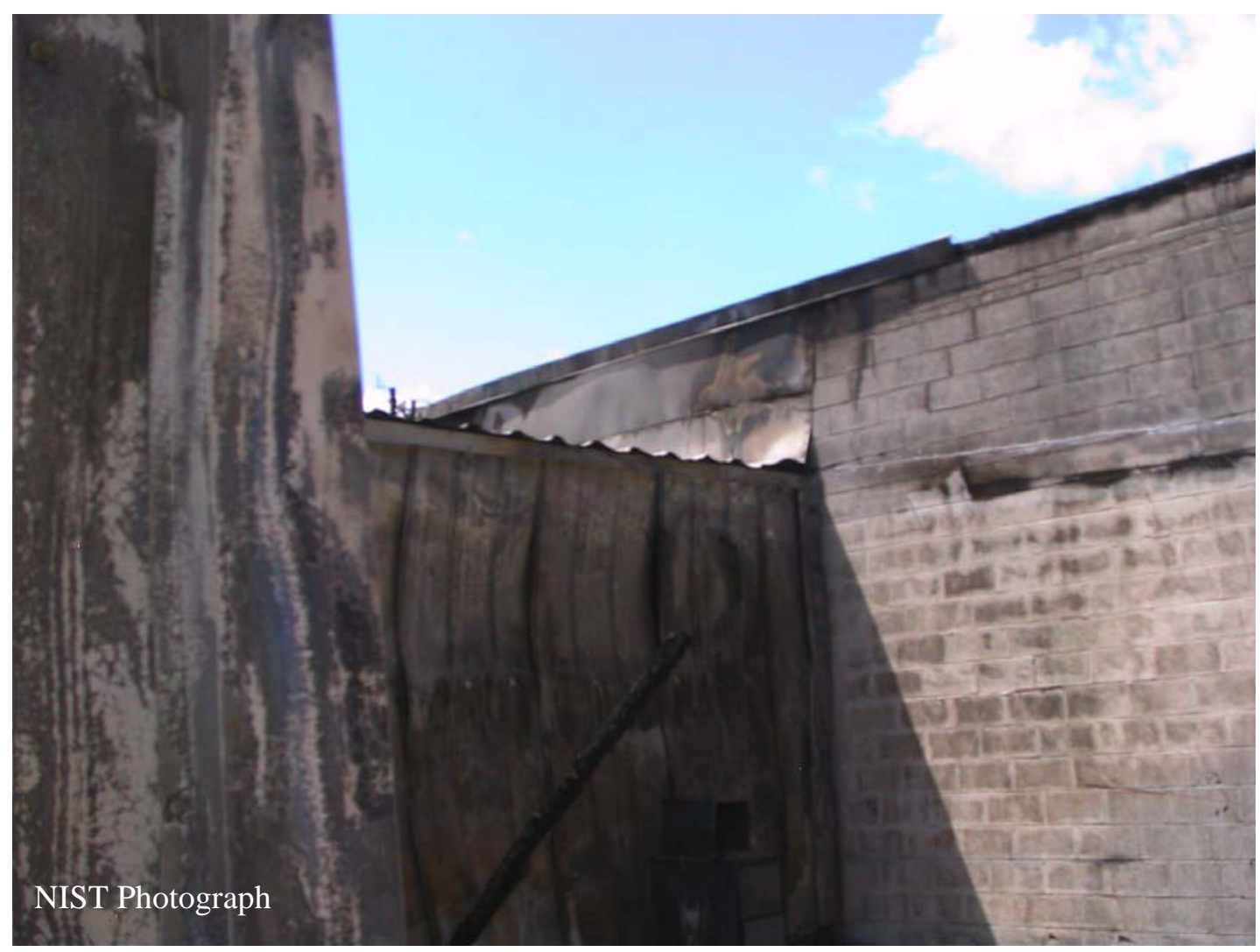

Figure H-10. Intersection of breezeway and rear wall of main showroom.

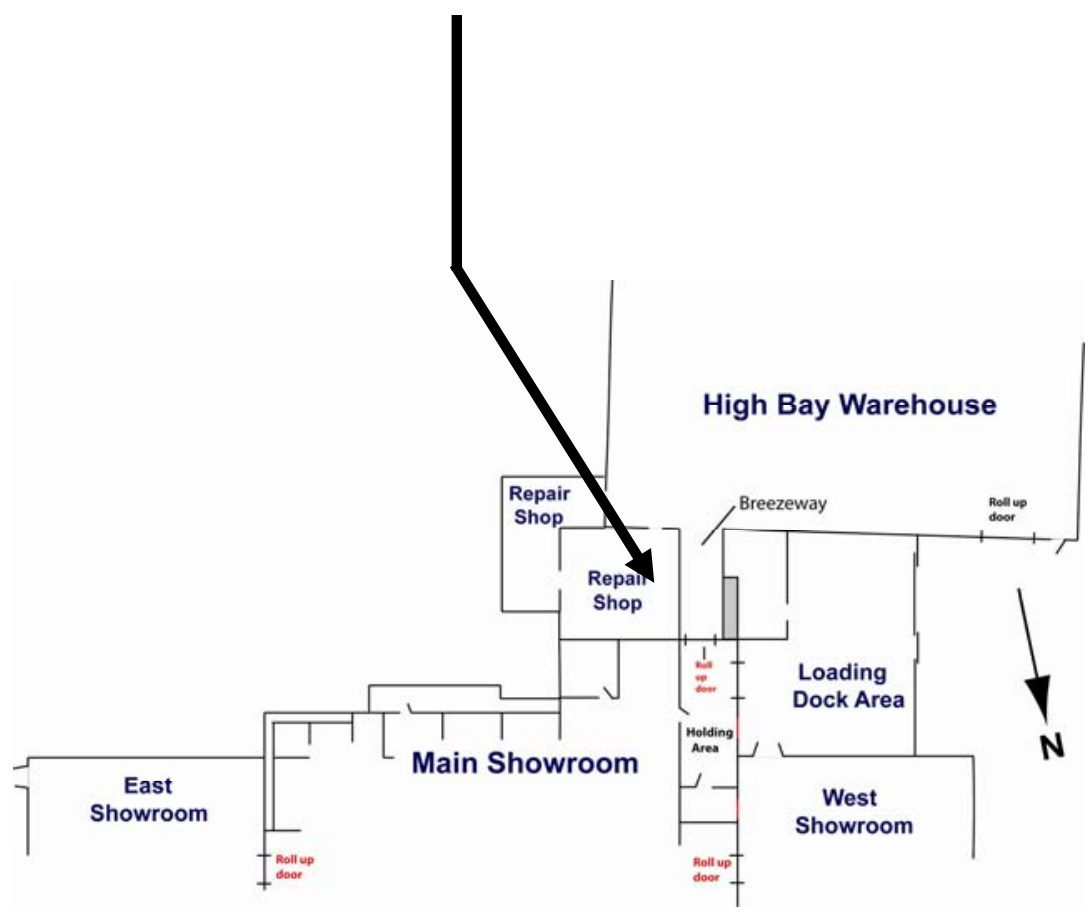




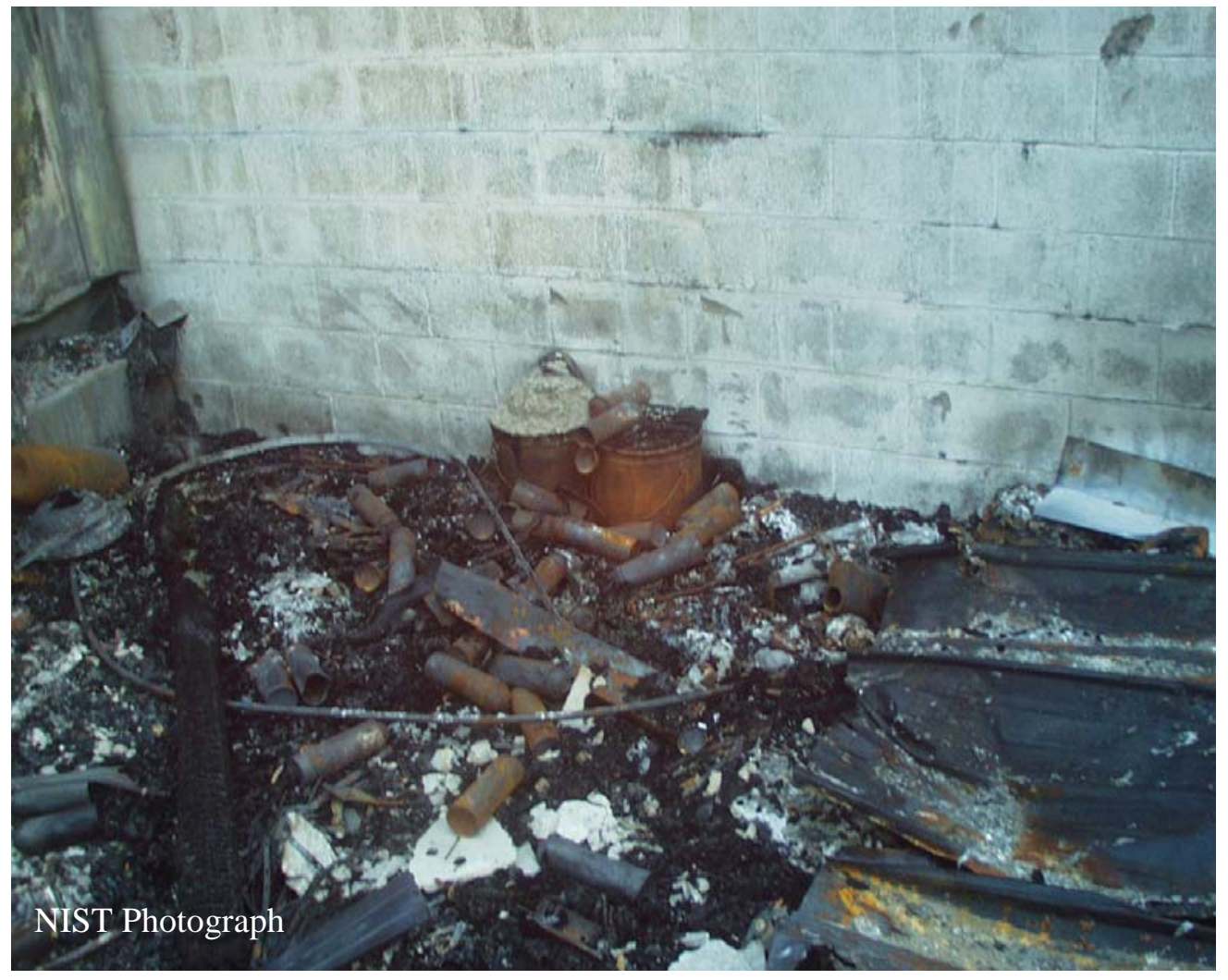

Figure H-11. Rear wall of main showroom formed north wall of repair shop.

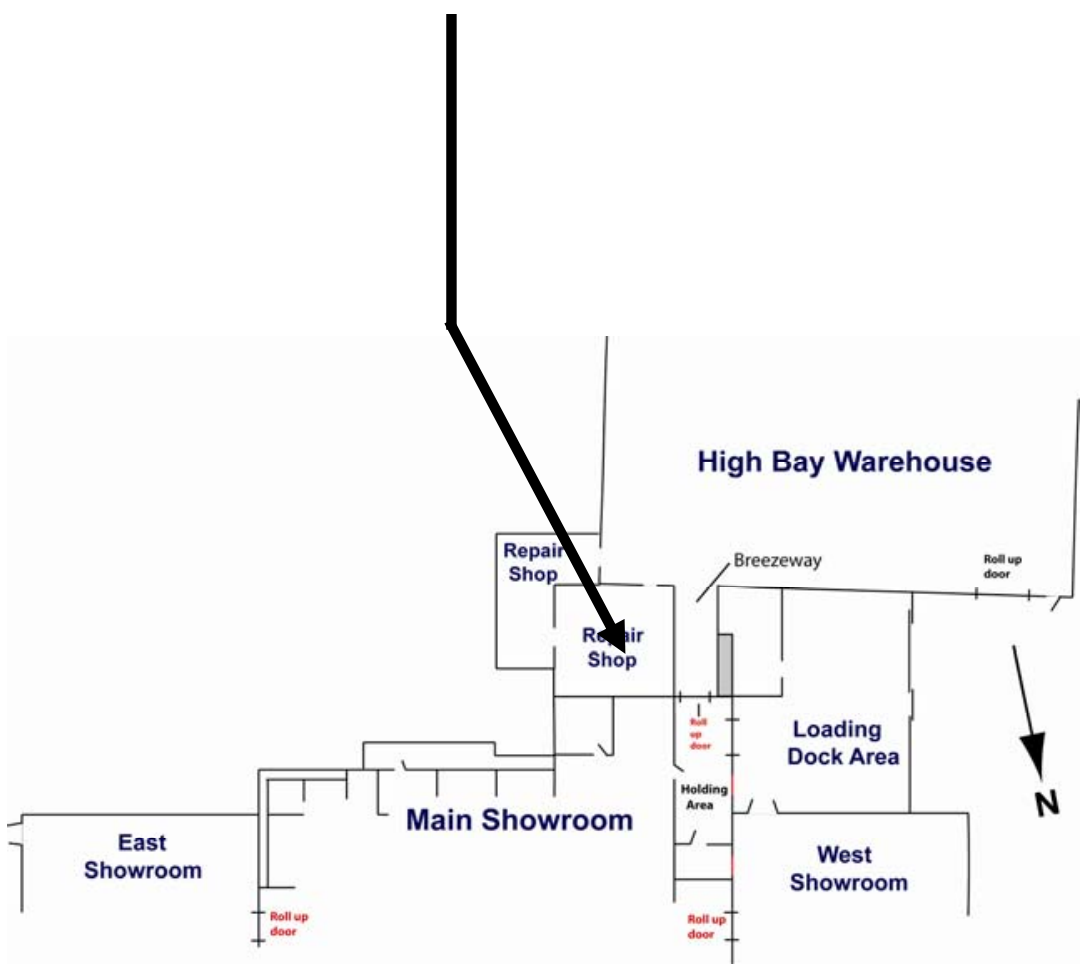




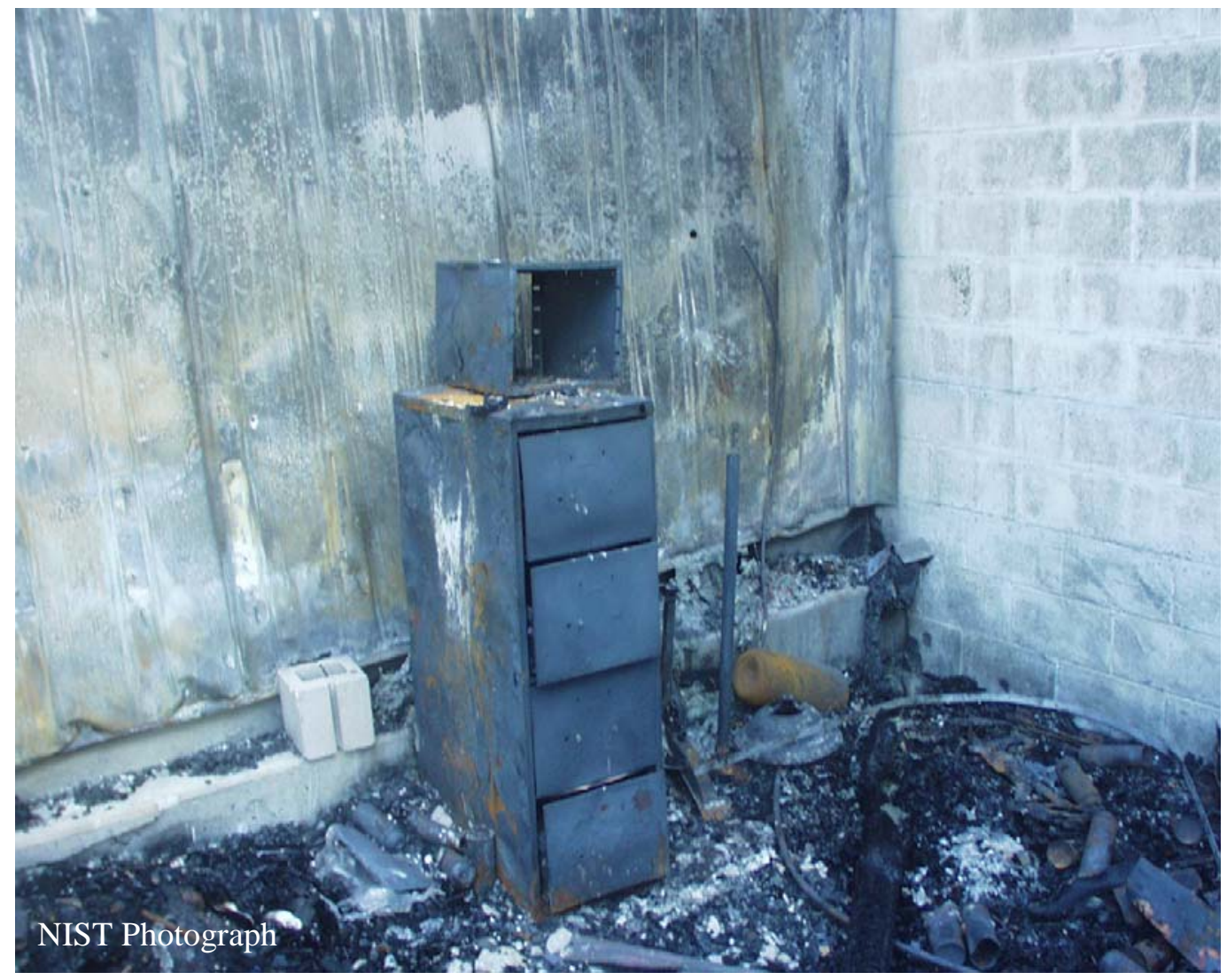

Figure H-12. Intersection of breezeway metal wall and rear wall of main showroom inside repair shop area.

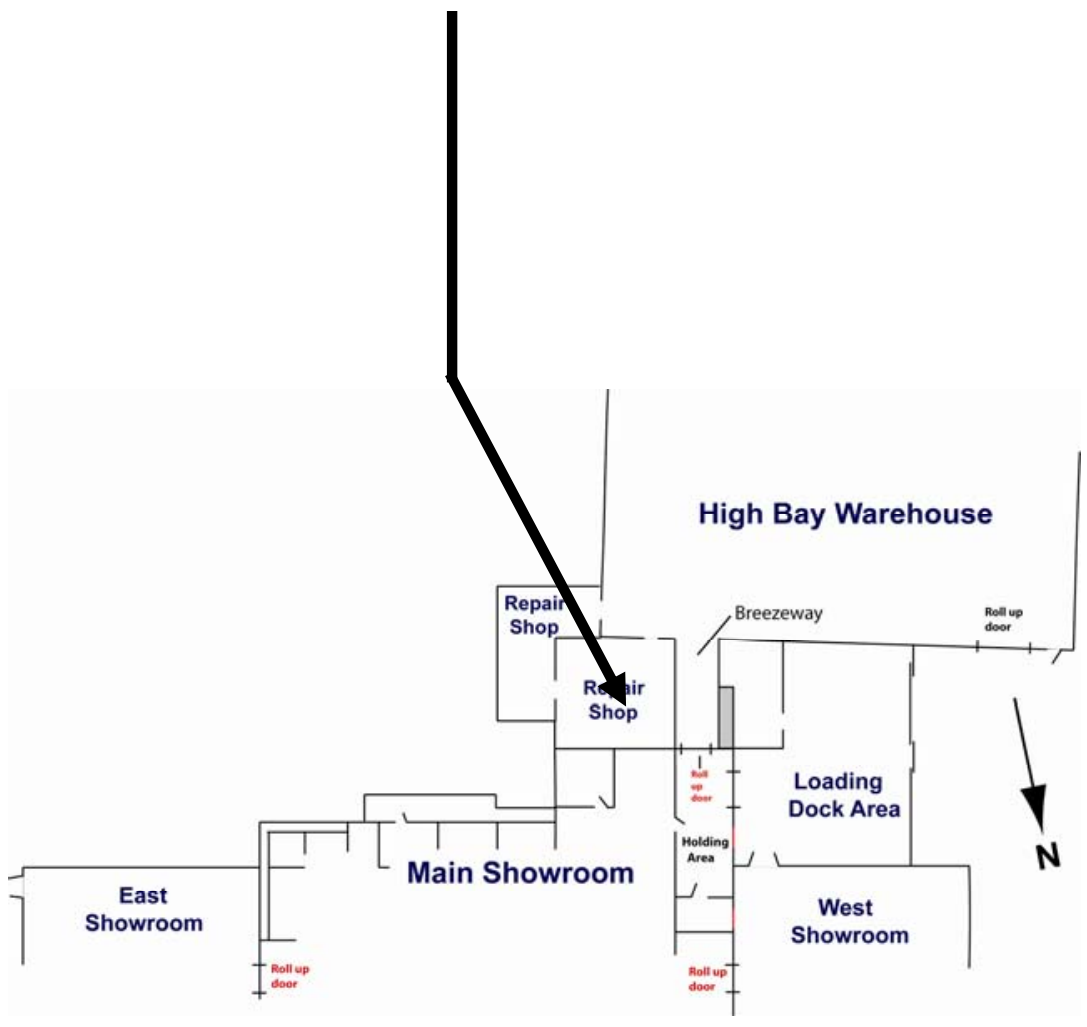




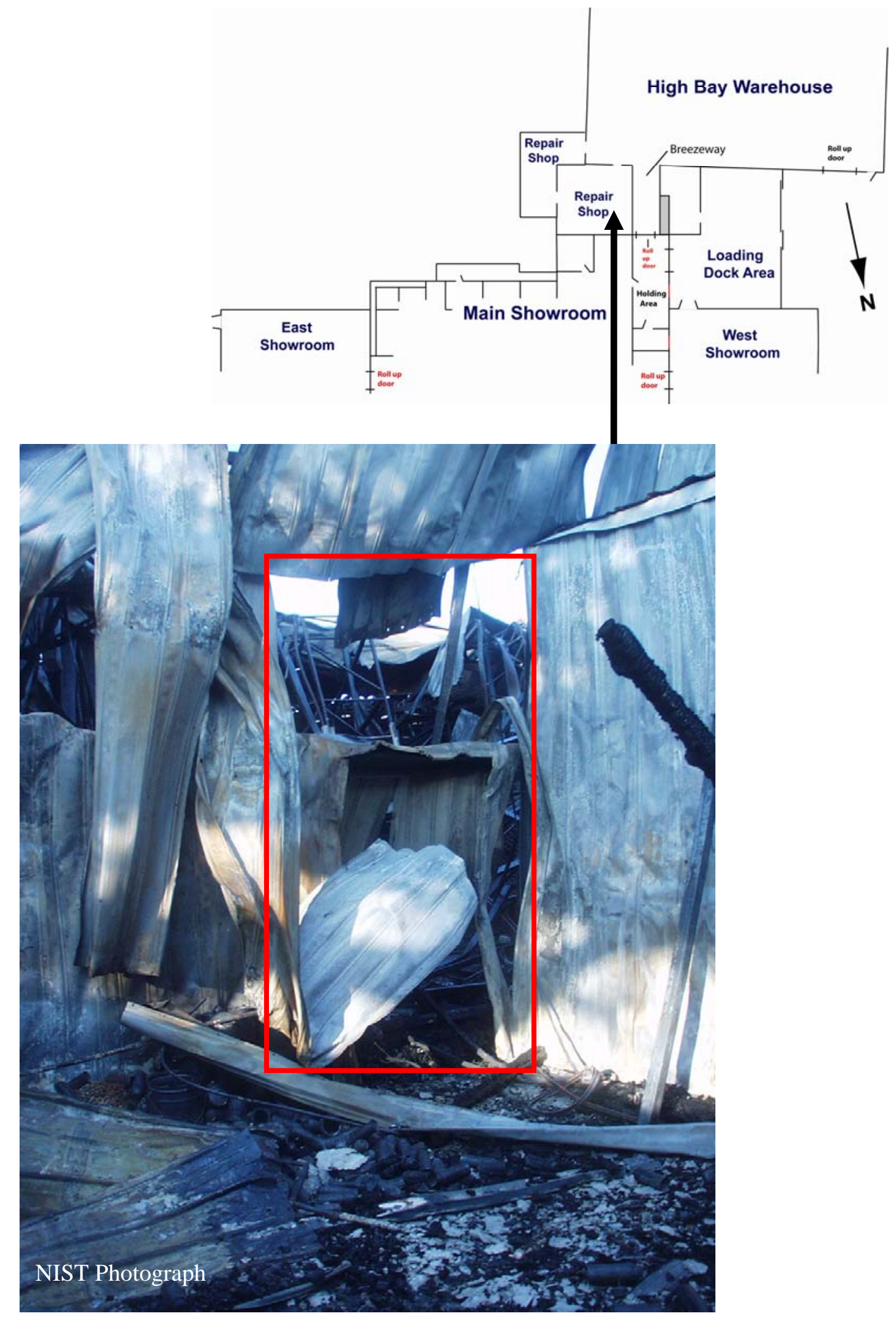

Figure H-13. Doorway opening from repair shop into warehouse. 

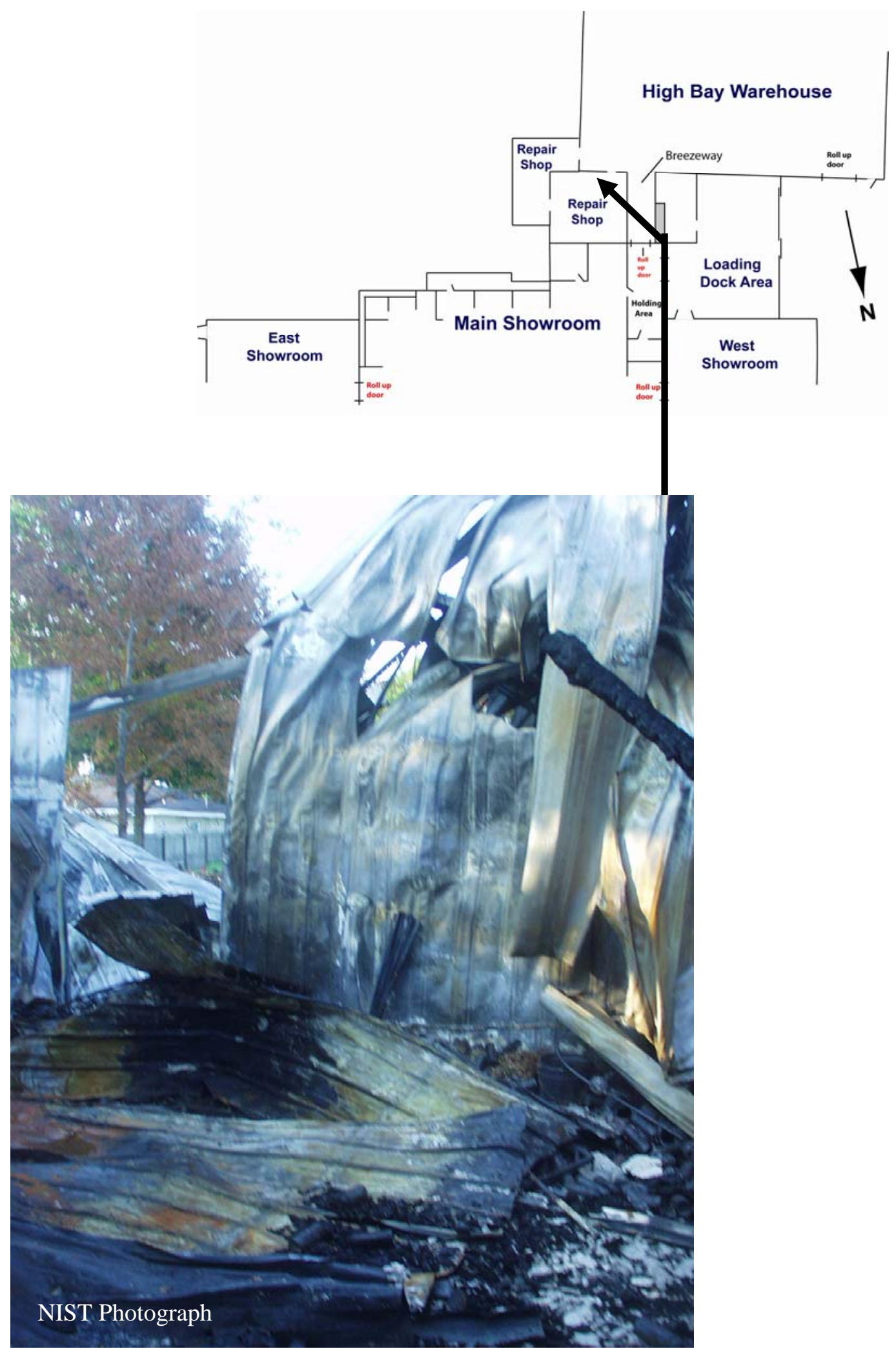

Figure H-14. Inside of repair shop looking out through other workshop. Northeast corner of warehouse visible in center of photo. 


\section{APPENDiX I. Rear WaLl of Main SHOWROOM}

\section{I.1 REAR WALL OF MAIN SHOWROOM}

In the original structure, there appeared to have been some areas or spaces that were external to the rear wall of the main showroom. These spaces may have housed the refrigeration equipment for the grocery store's freezers and refrigerated display cases. At some later time, the walls and doors leading to these areas were filled in with blocks.

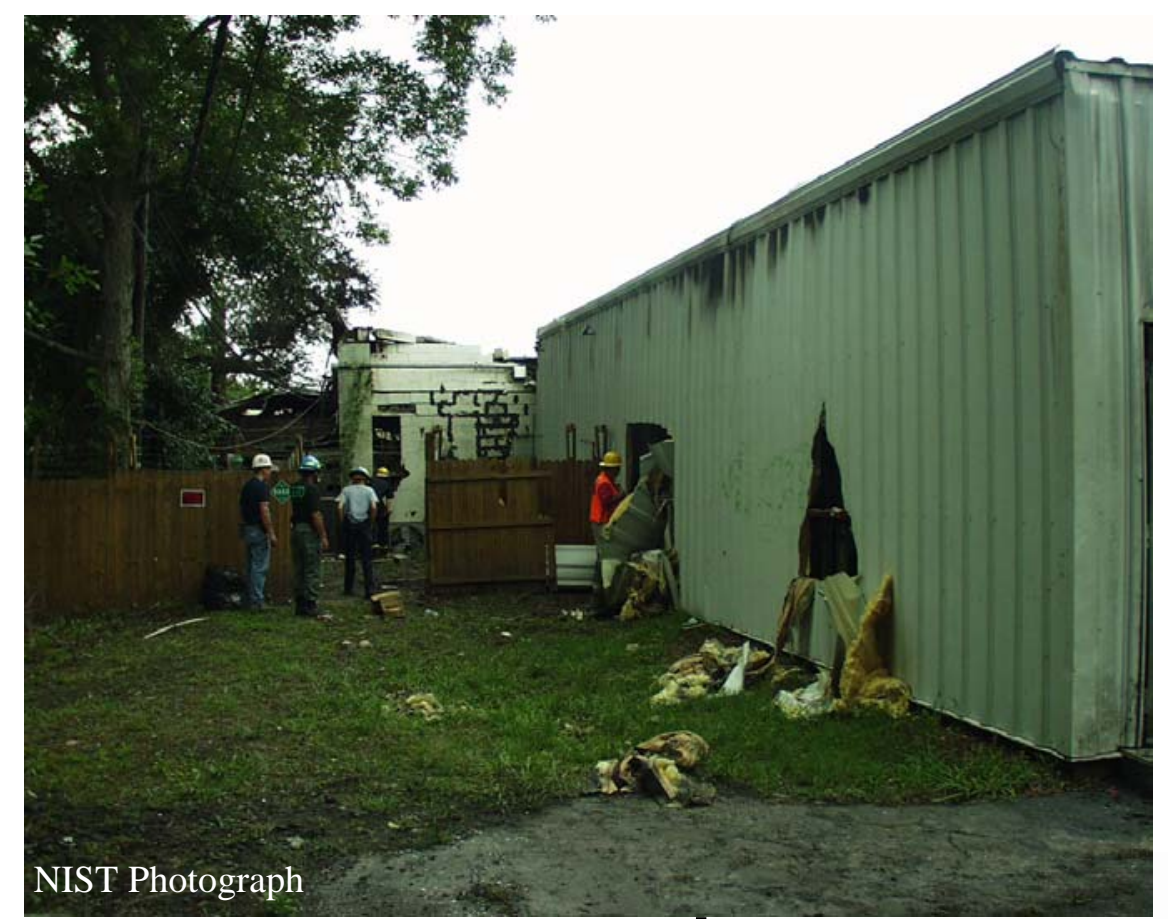

Figure I-1. Rear wall of store. Metal siding visible on the right is rear wall of east showroom. Rear wall of main showroom is visible beyond wood fence.

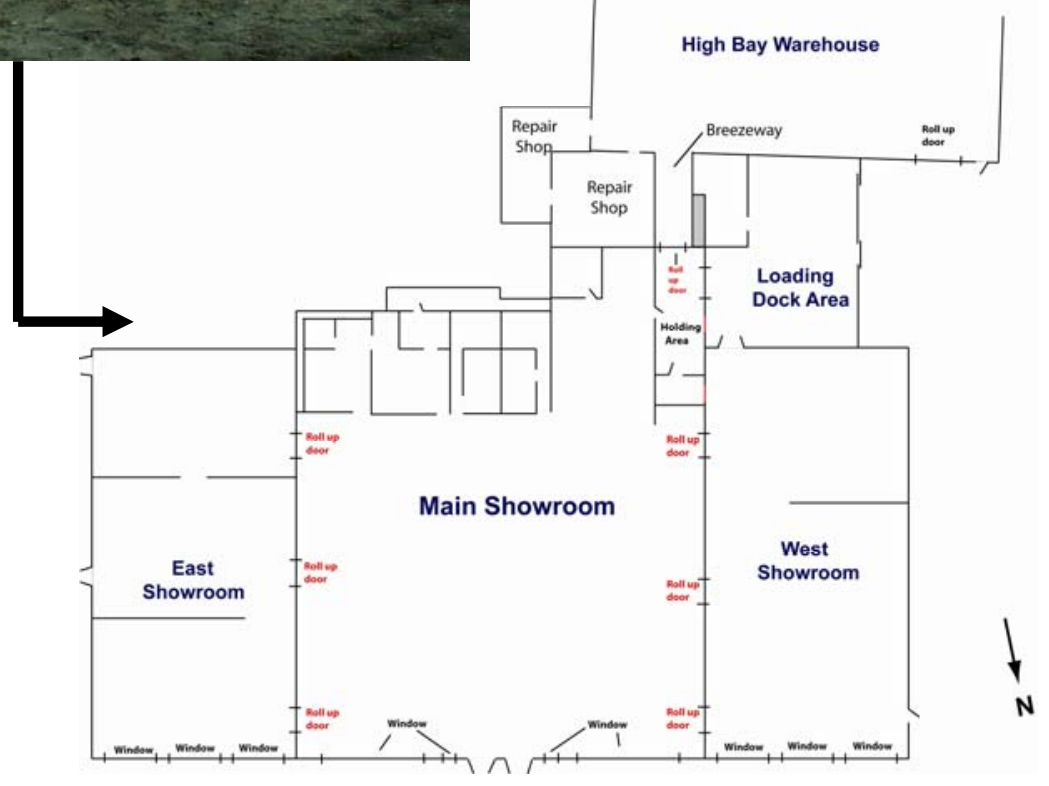




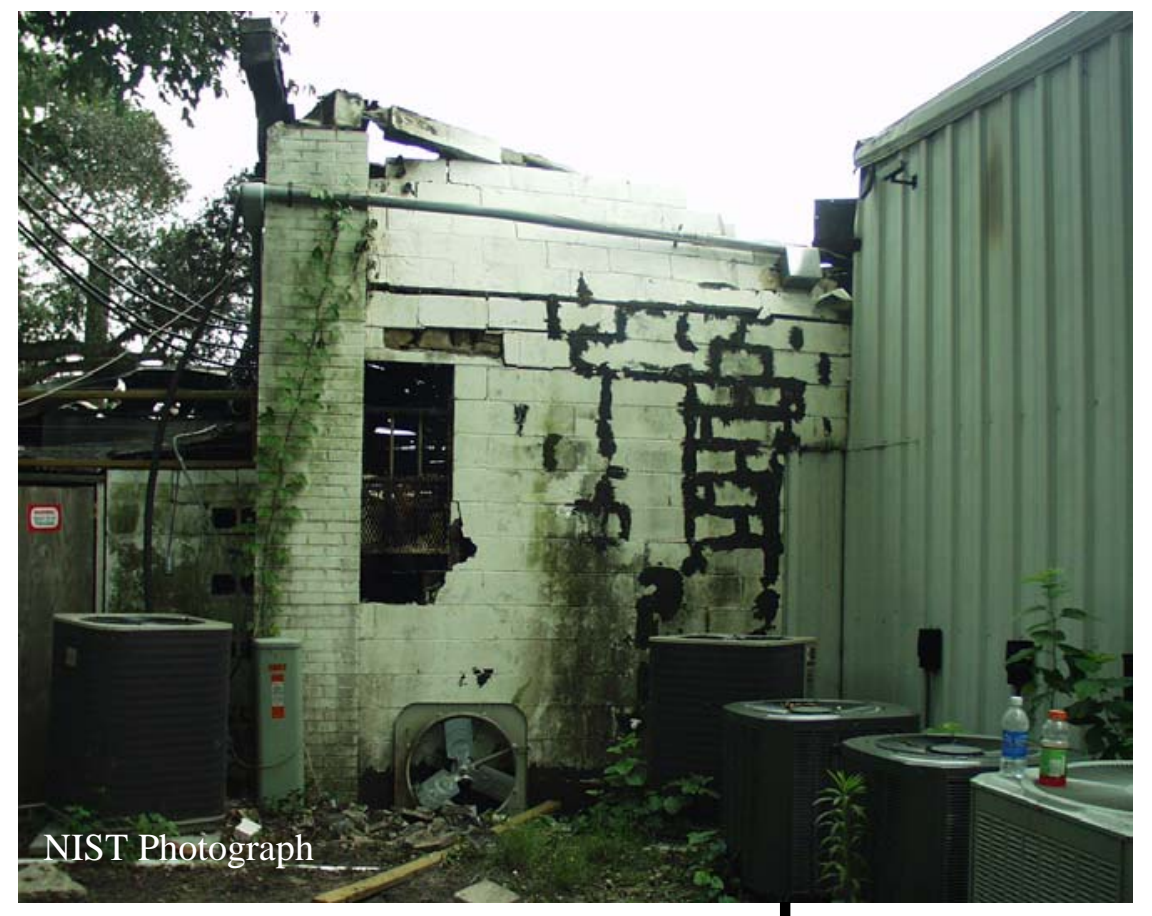

Figure I-2. Rear wall of store. Intersection of metal siding at rear of east showroom and concrete block wall at rear of main showroom.

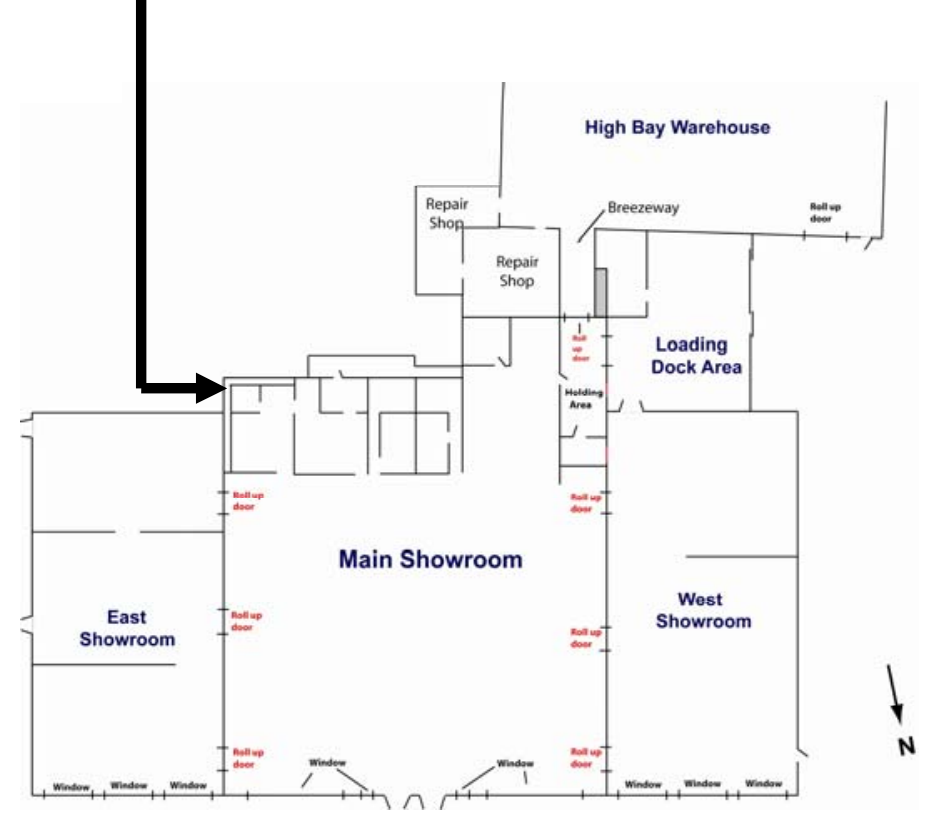




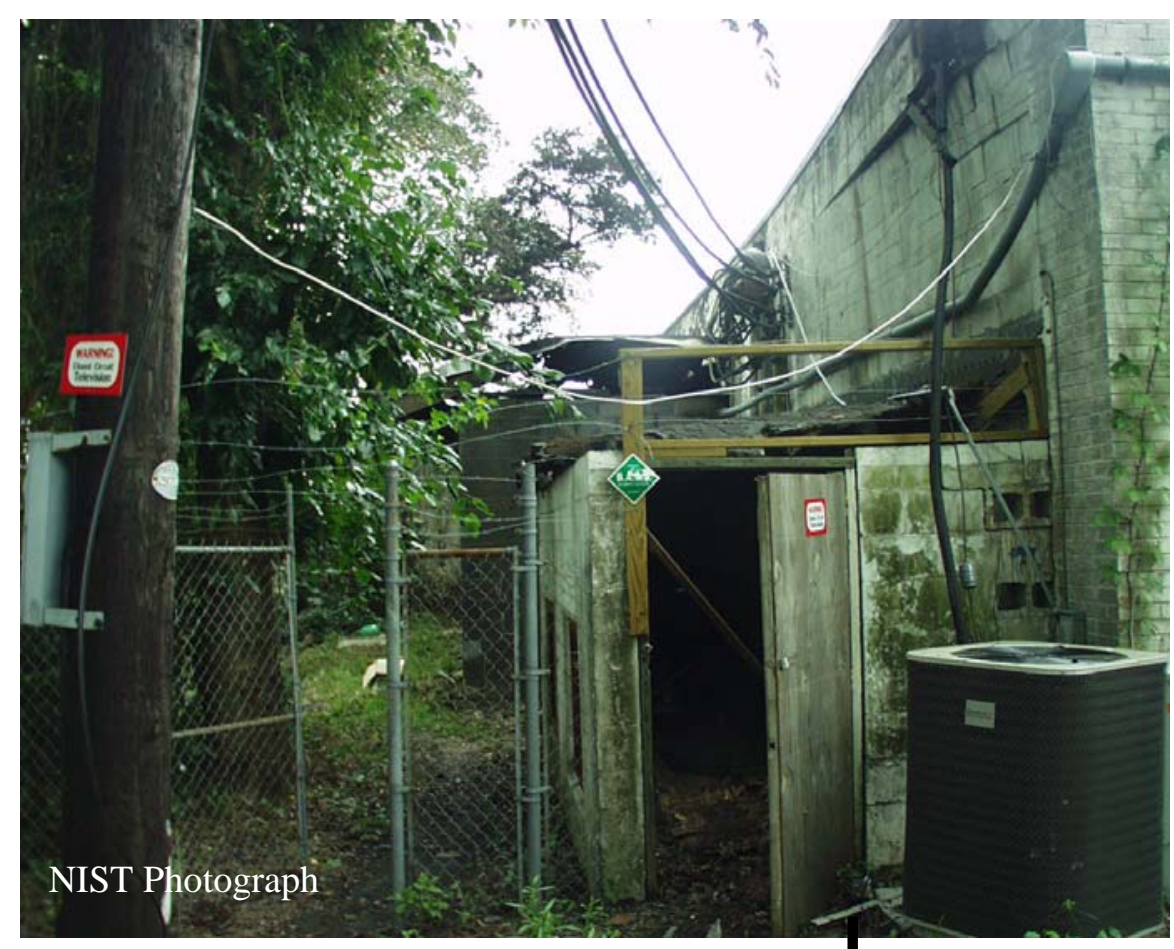

Figure I-3. Rear wall of main showroom.

When structure was used as a grocery store, refrigeration equipment was probably located in enclosed area attached to rear wall of main showroom. There did not appear to be a door opening between enclosed area and main showroom.

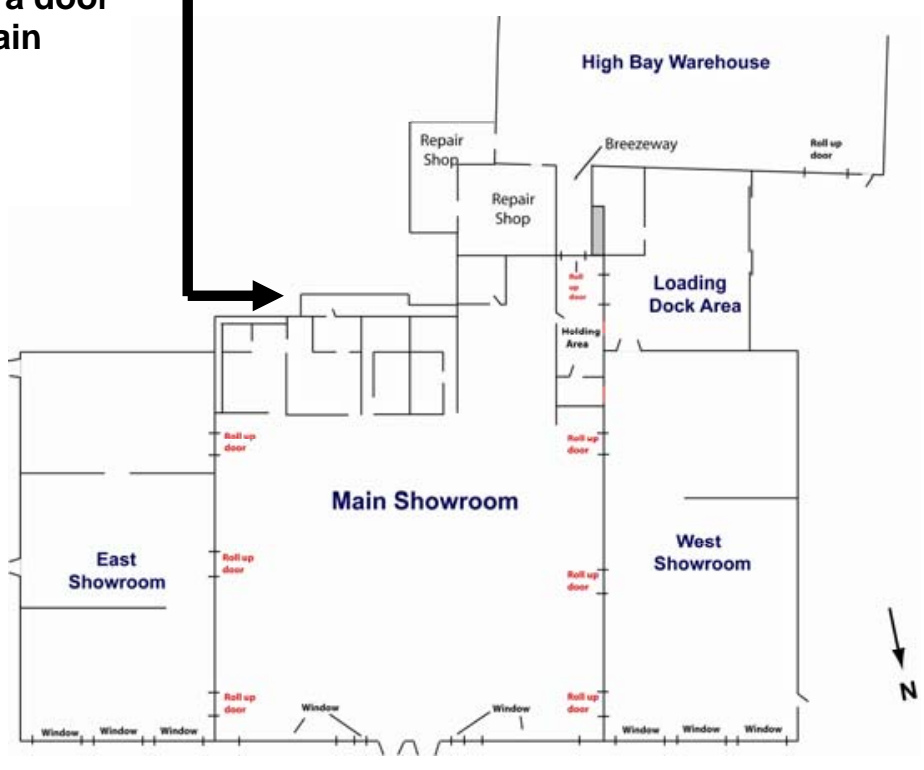




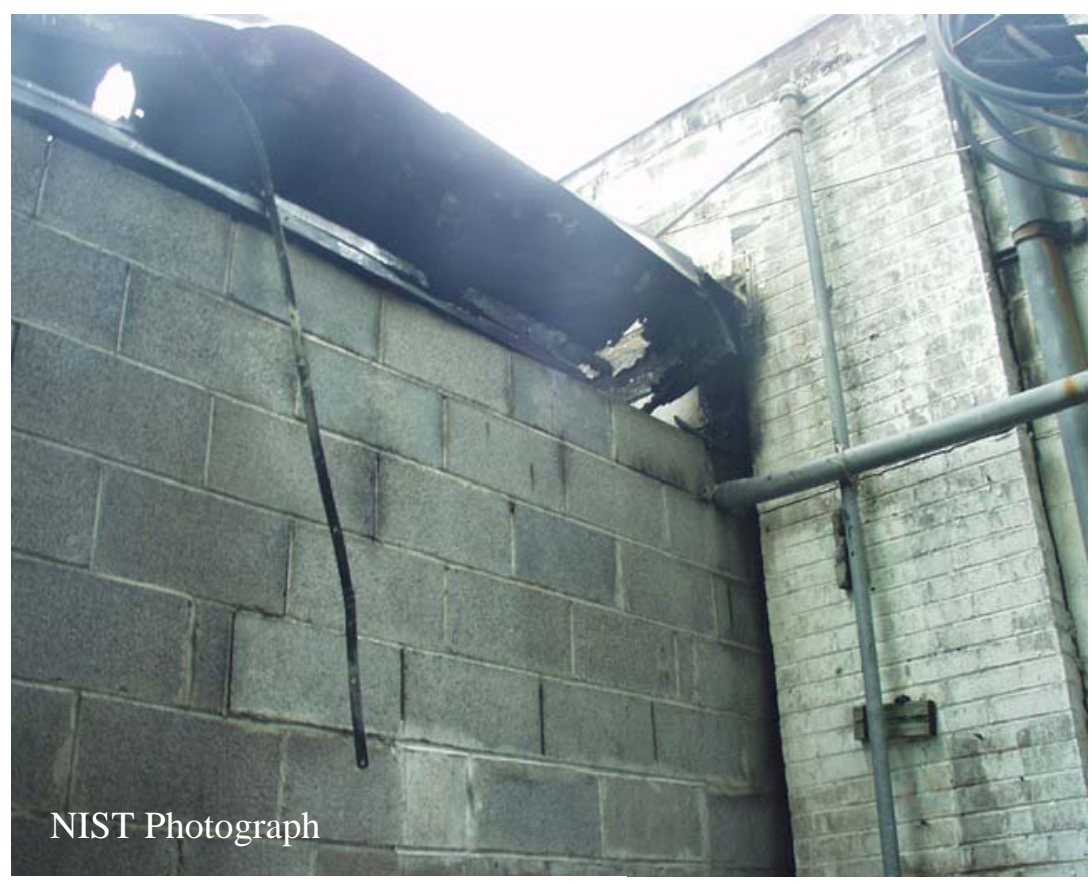

Figure I-4. Area on the rear wall of main showroom that was filled in with concrete blocks.

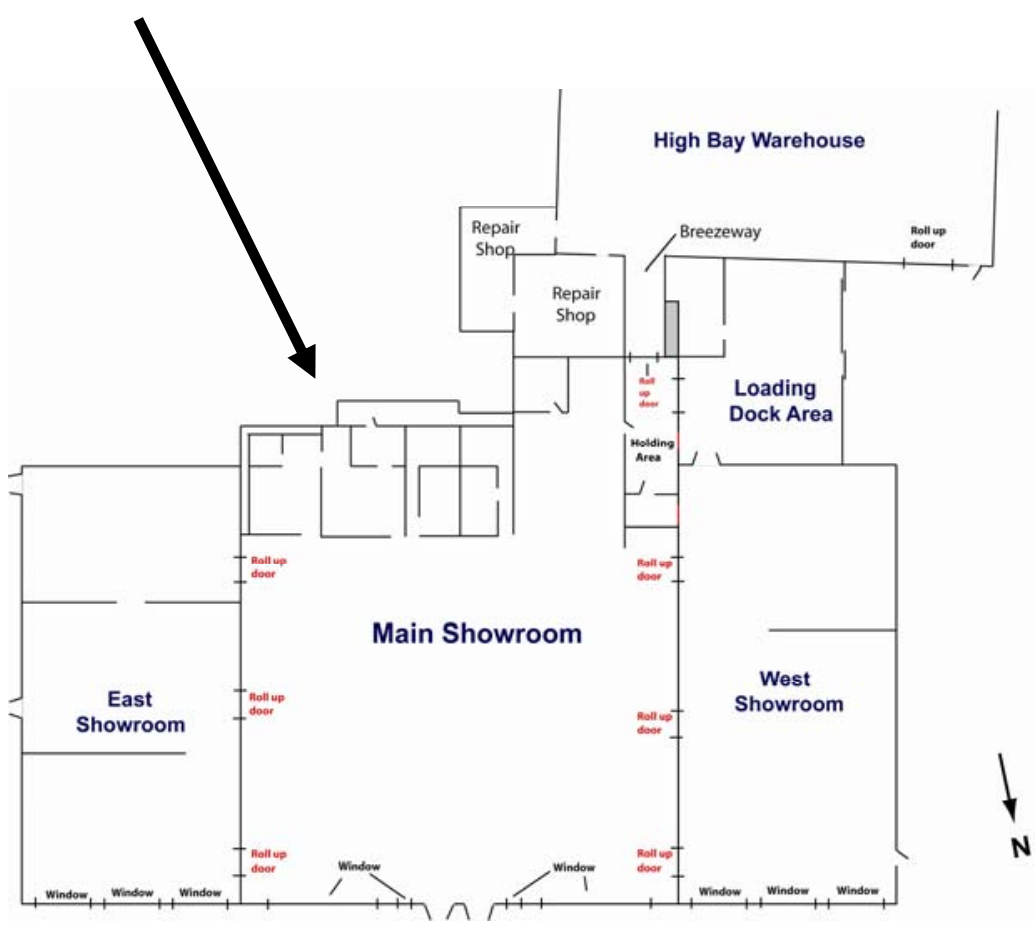




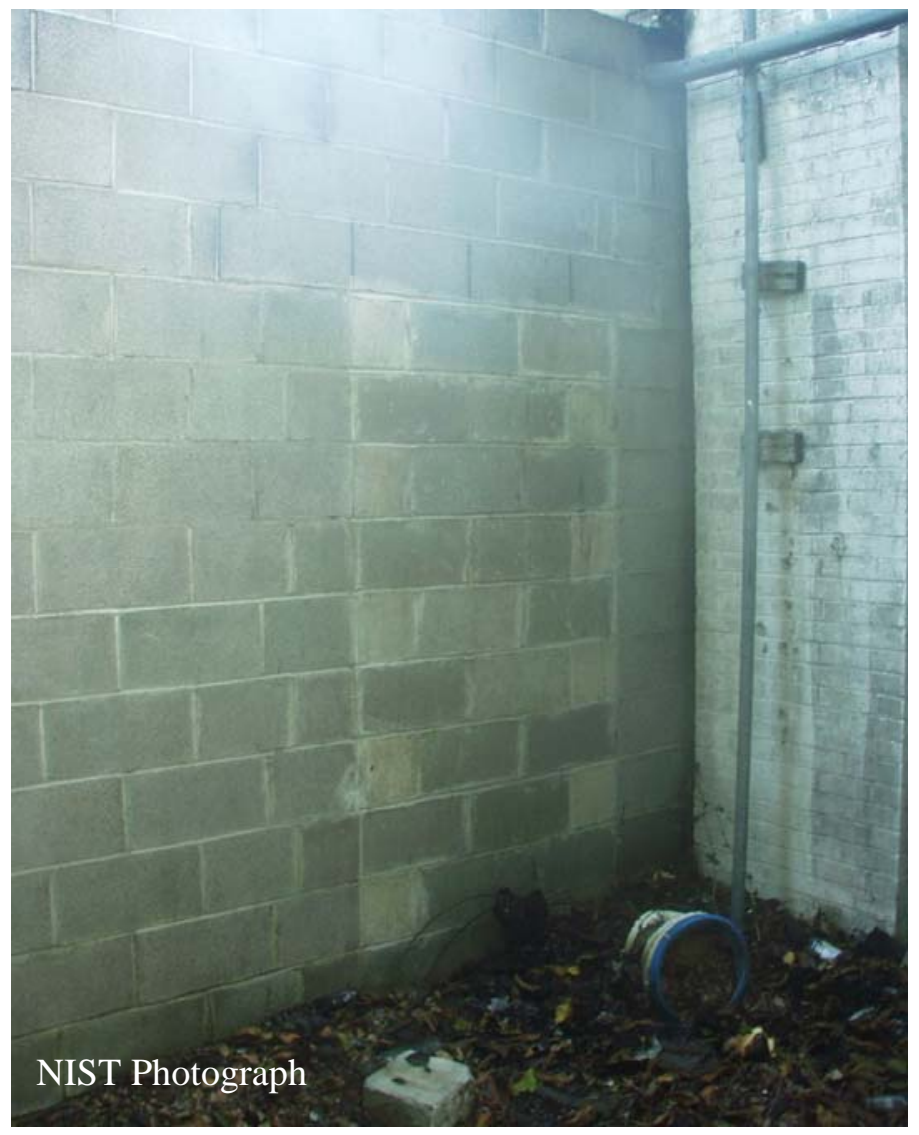

Figure 1-5. Doorway on the rear wall of main showroom that was filled in with concrete blocks.

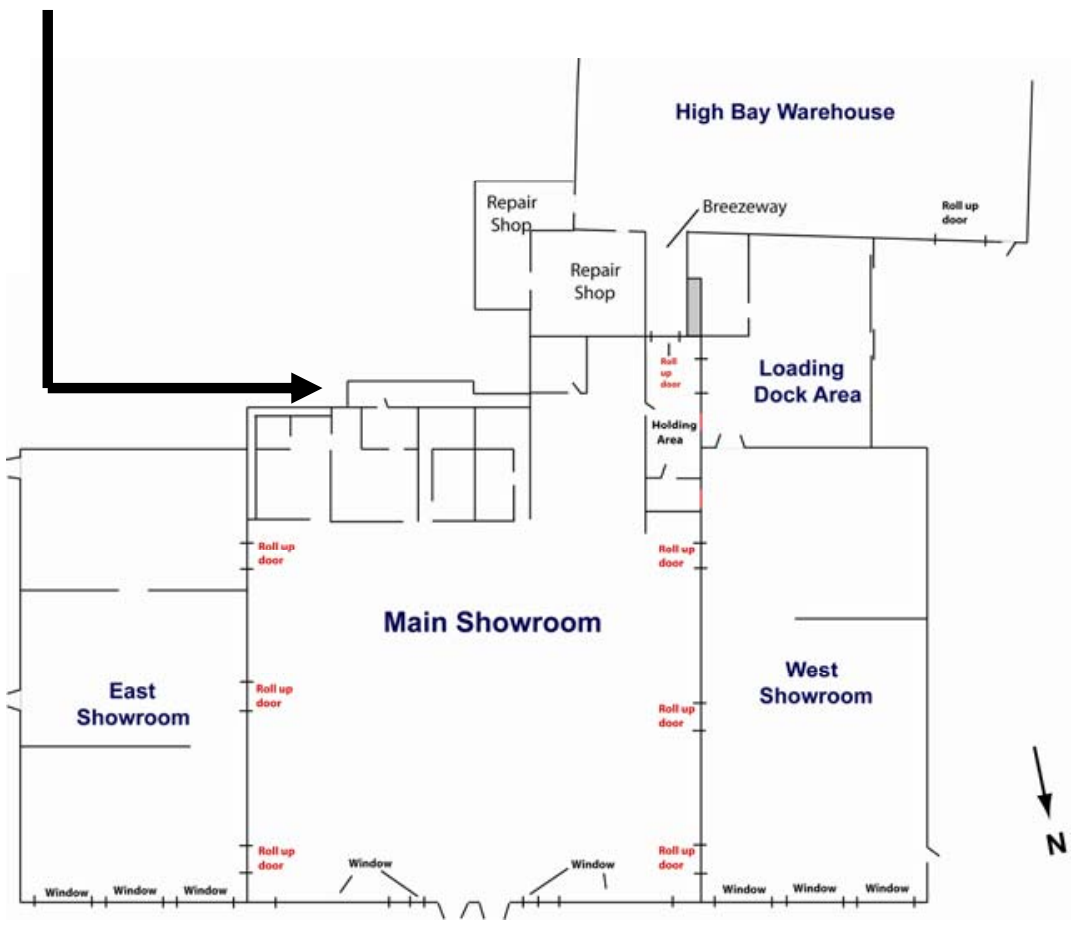




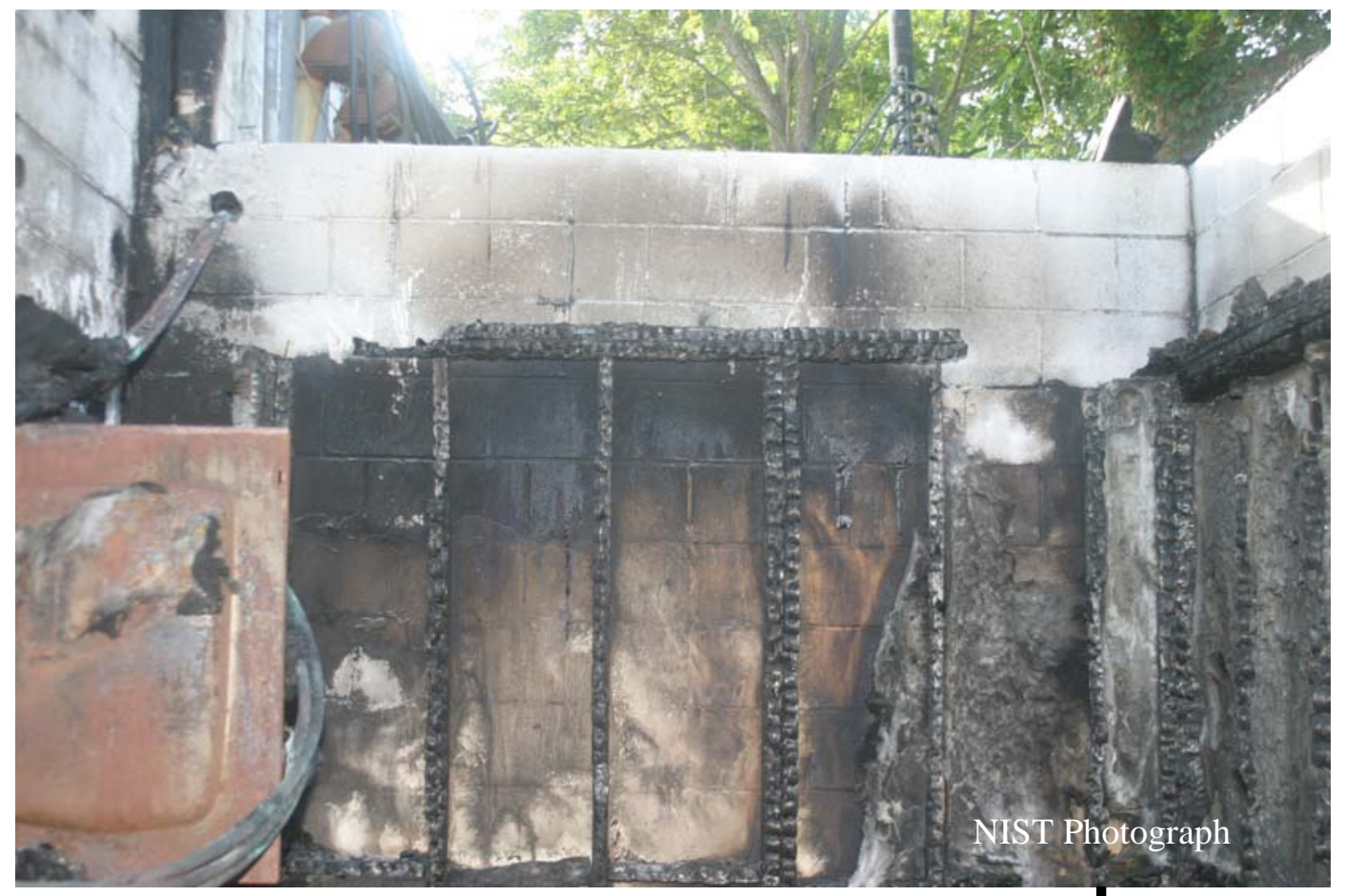

Figure I-6. Interior view of enclosed area at rear of main showroom. Doorway that was filled with concrete block is located beyond rusty metal electrical panel cover in left of photograph.

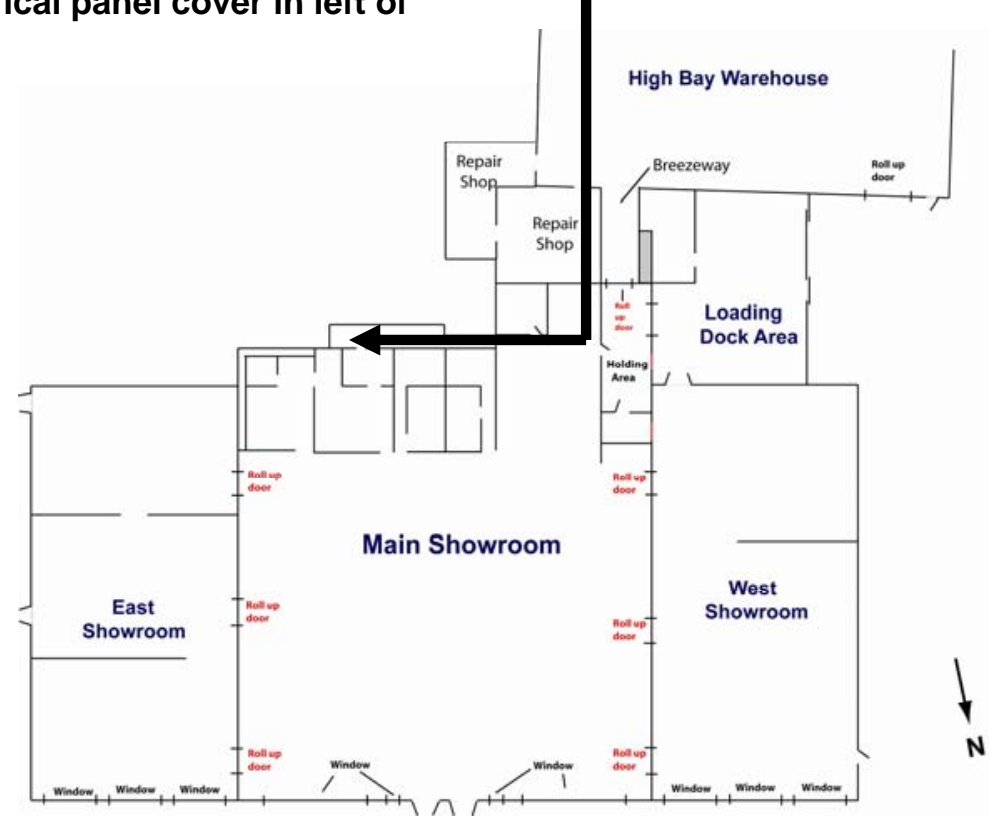




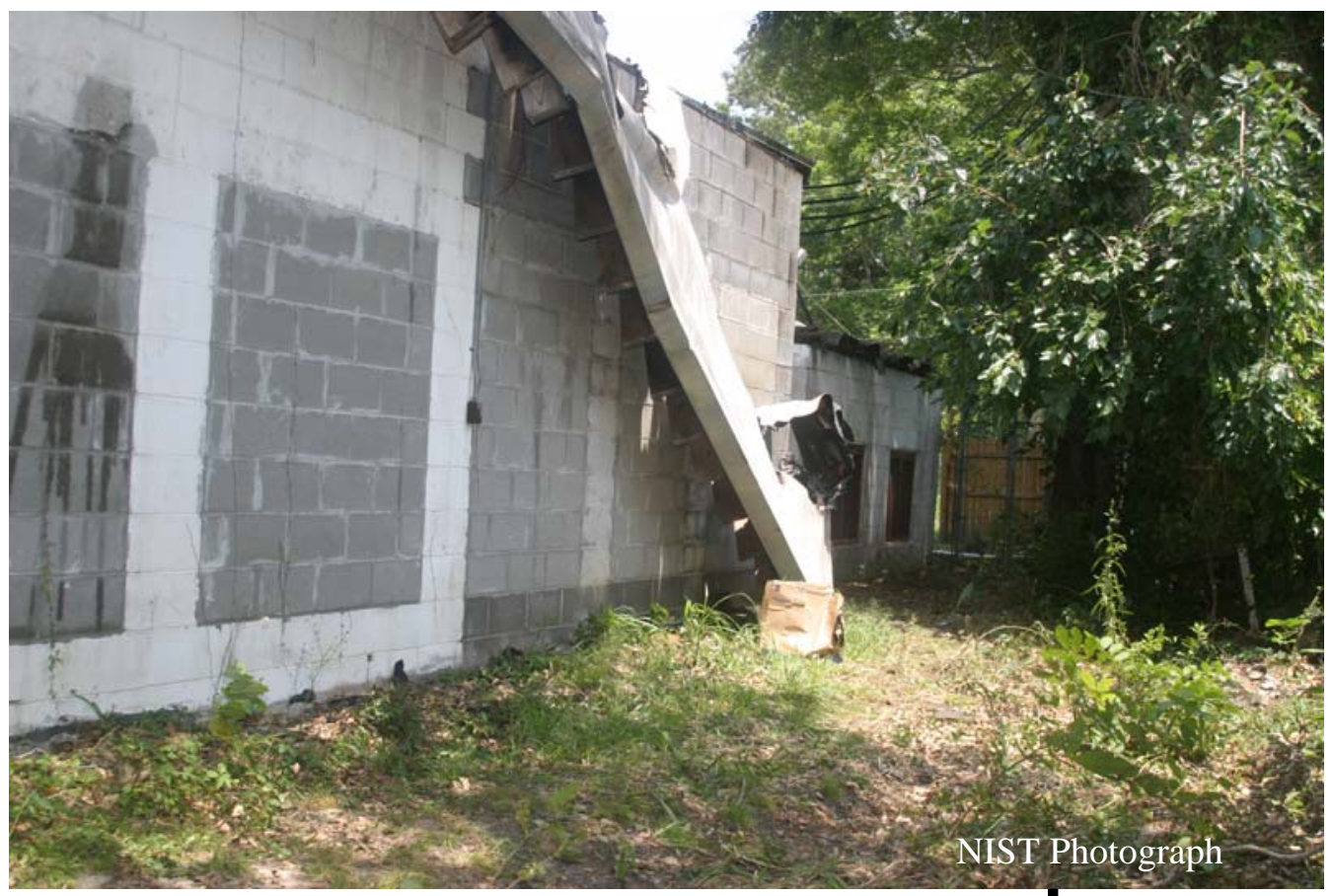

Figure 1-7. Rear wall of main showroom. Openings in wall have been filled with concrete block. Wood fence is visible in center right of photograph.

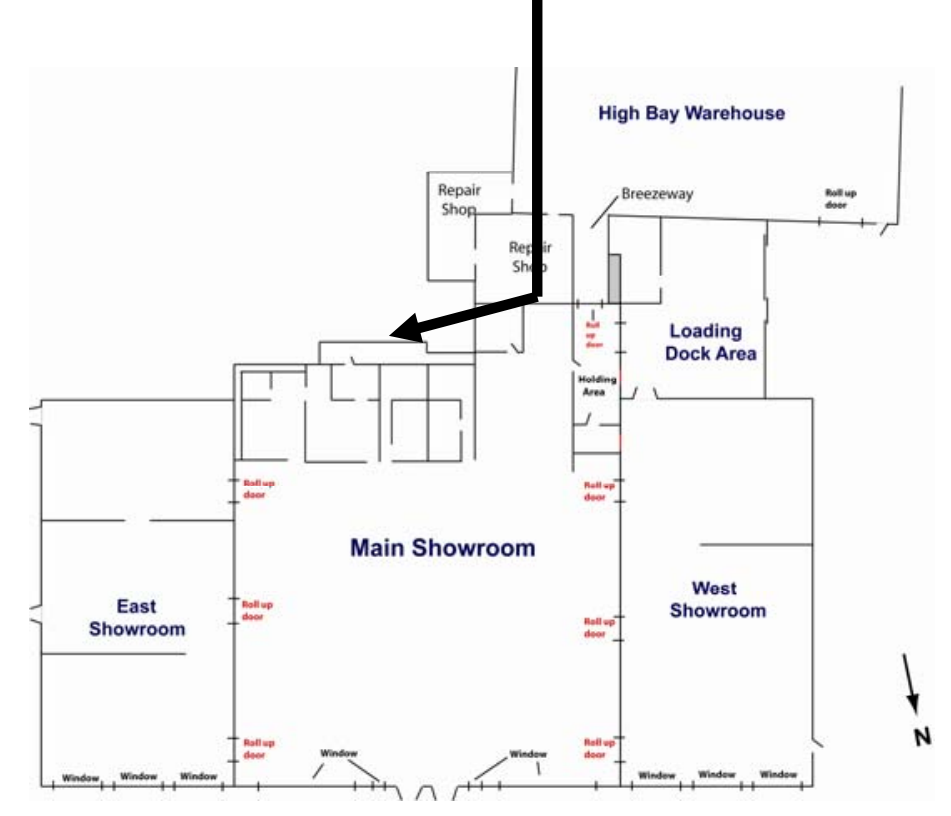




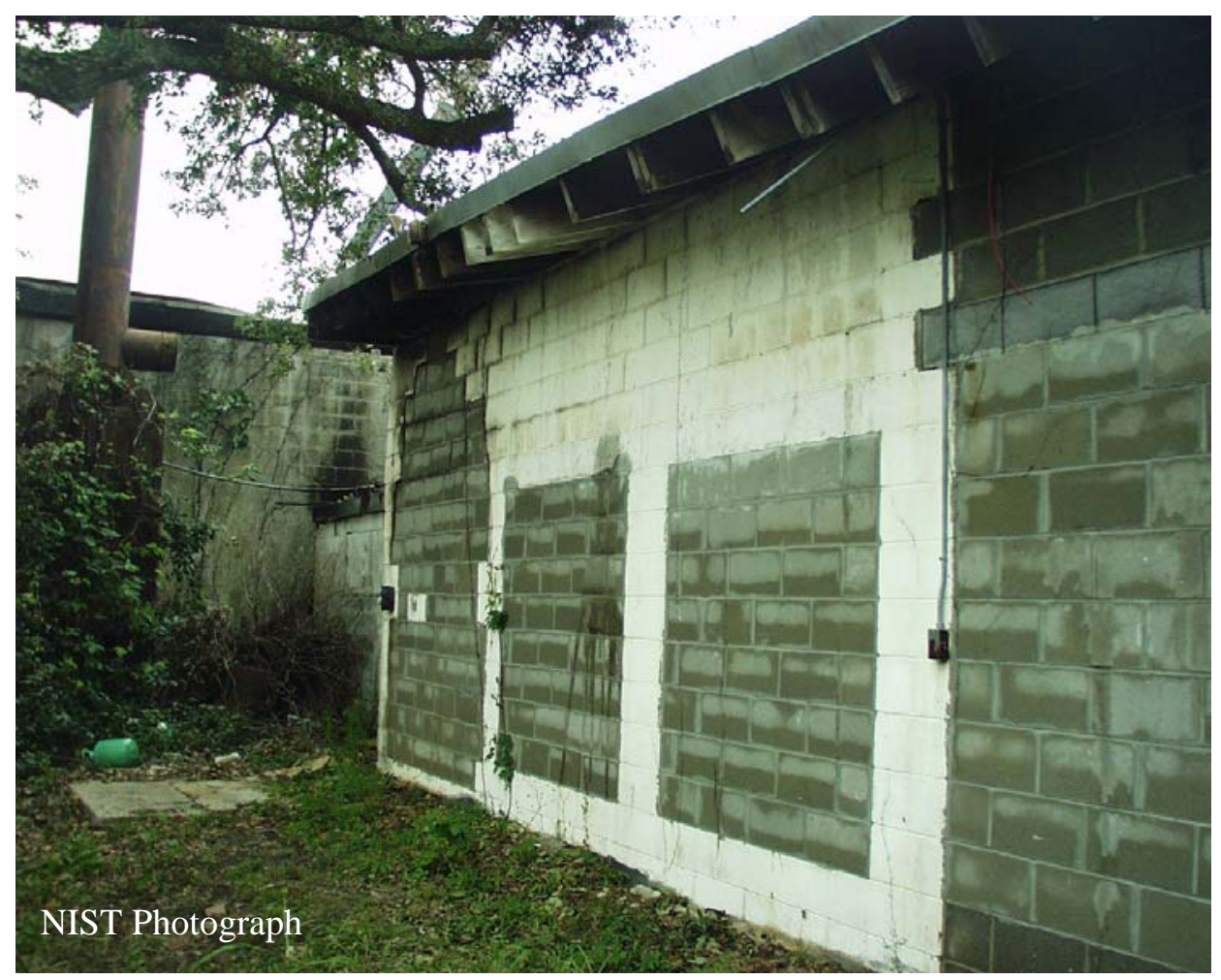

Figure I-8. Openings on the rear wall of main showroom that were filled in with concrete blocks.

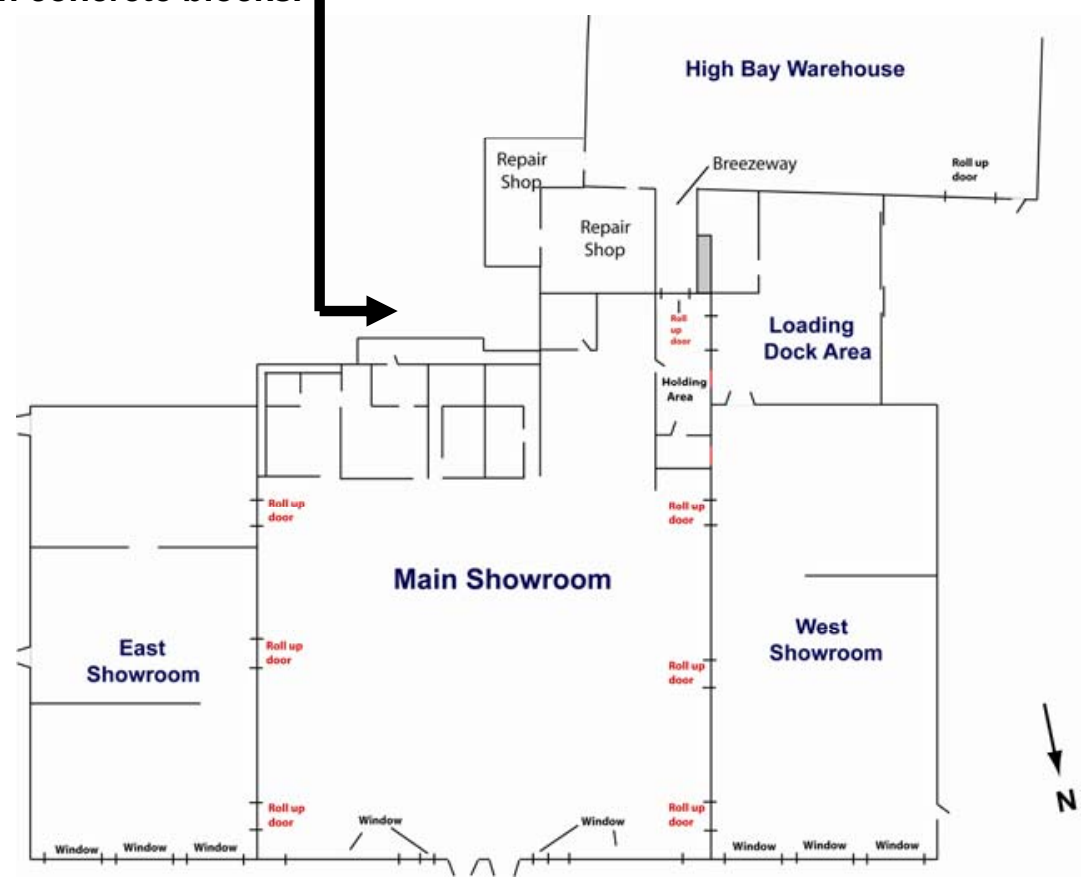




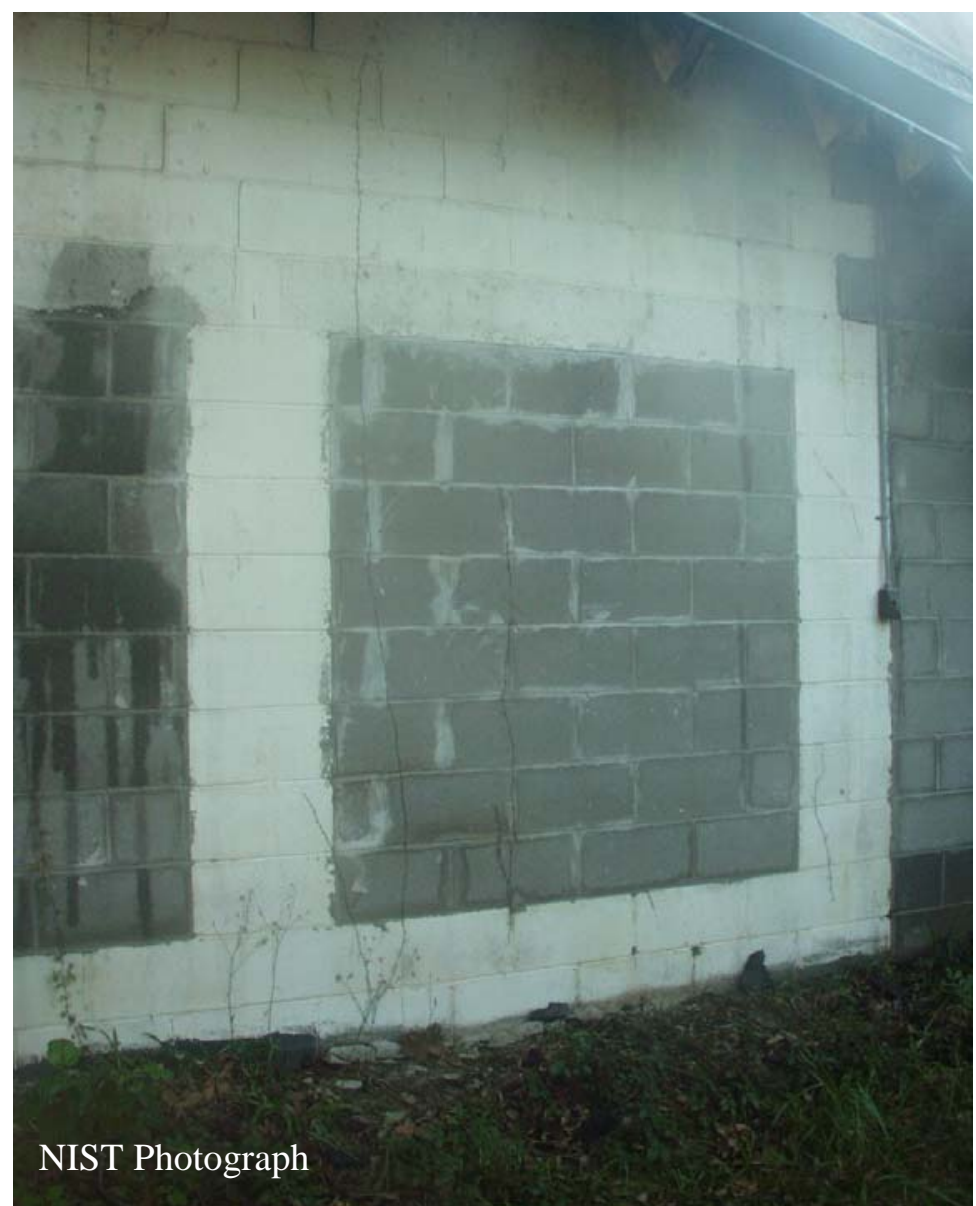

Figure 1-9. Openings on the rear wall of main showroom that were filled in with concrete blocks.

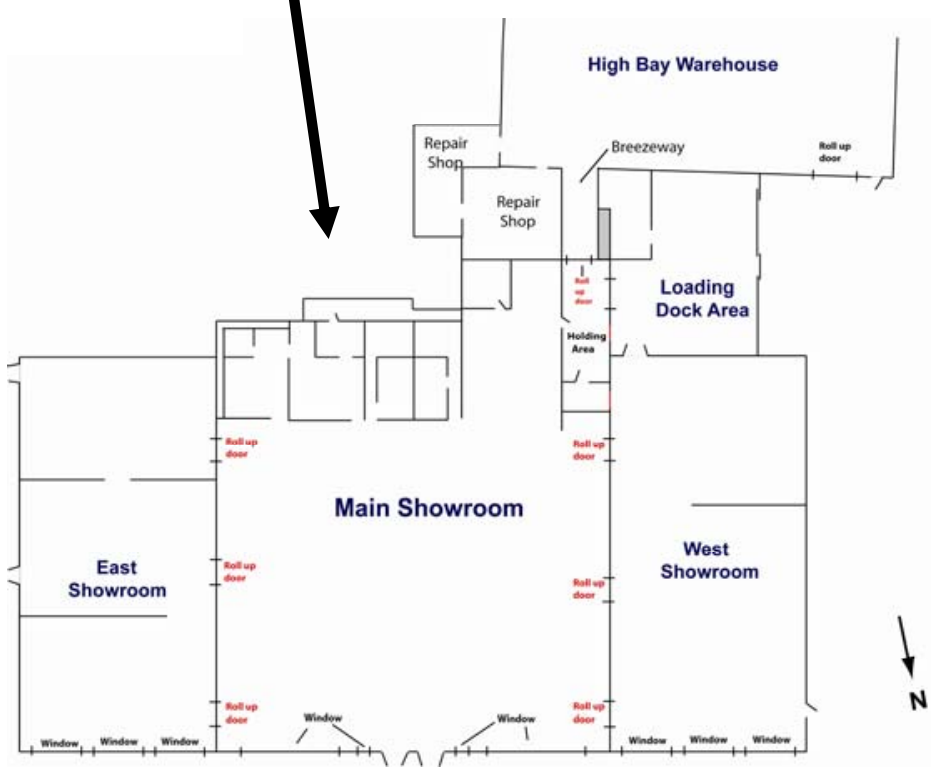




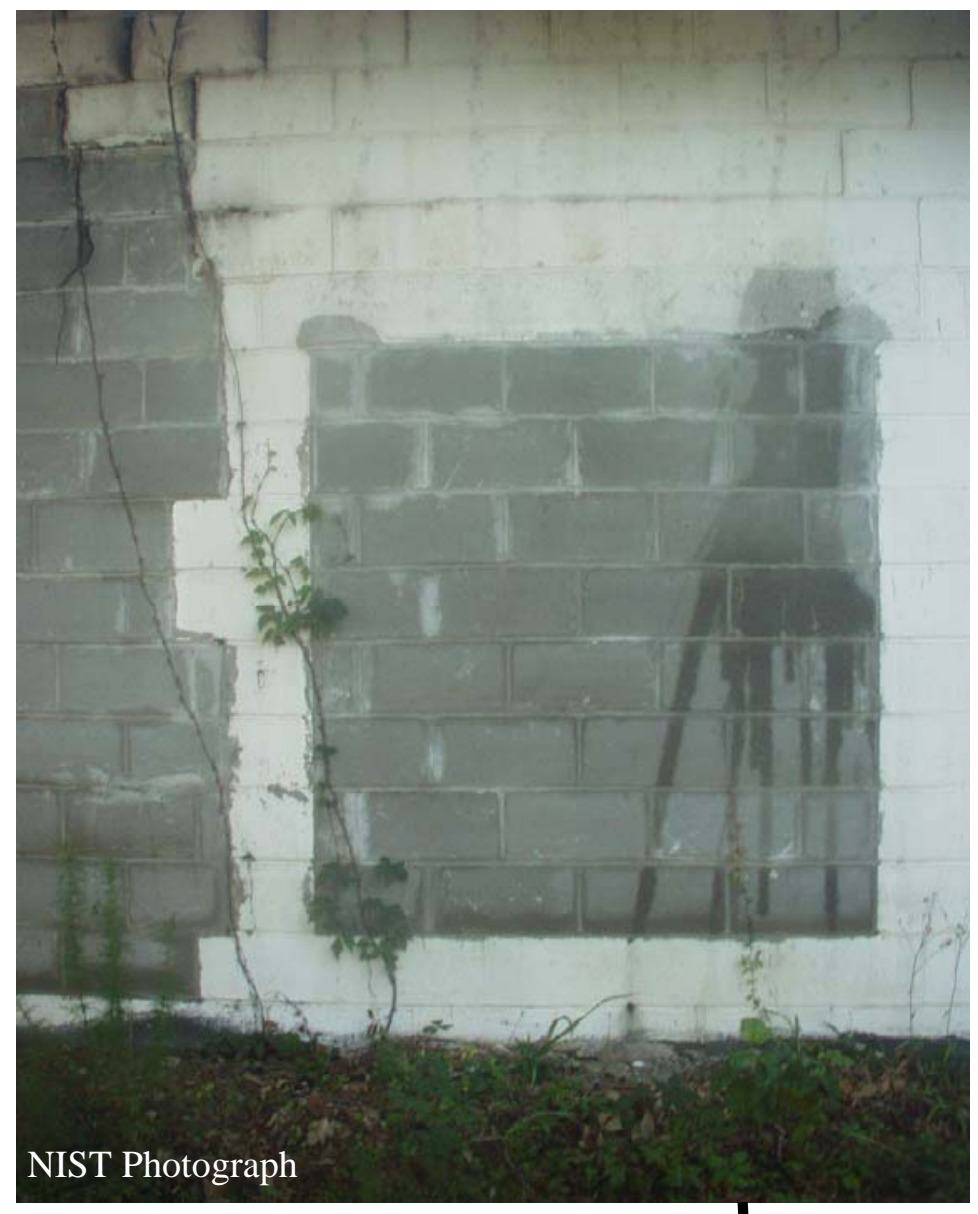

Figure I-10. Opening on the rear wall of main showroom that was filled in with concrete blocks.

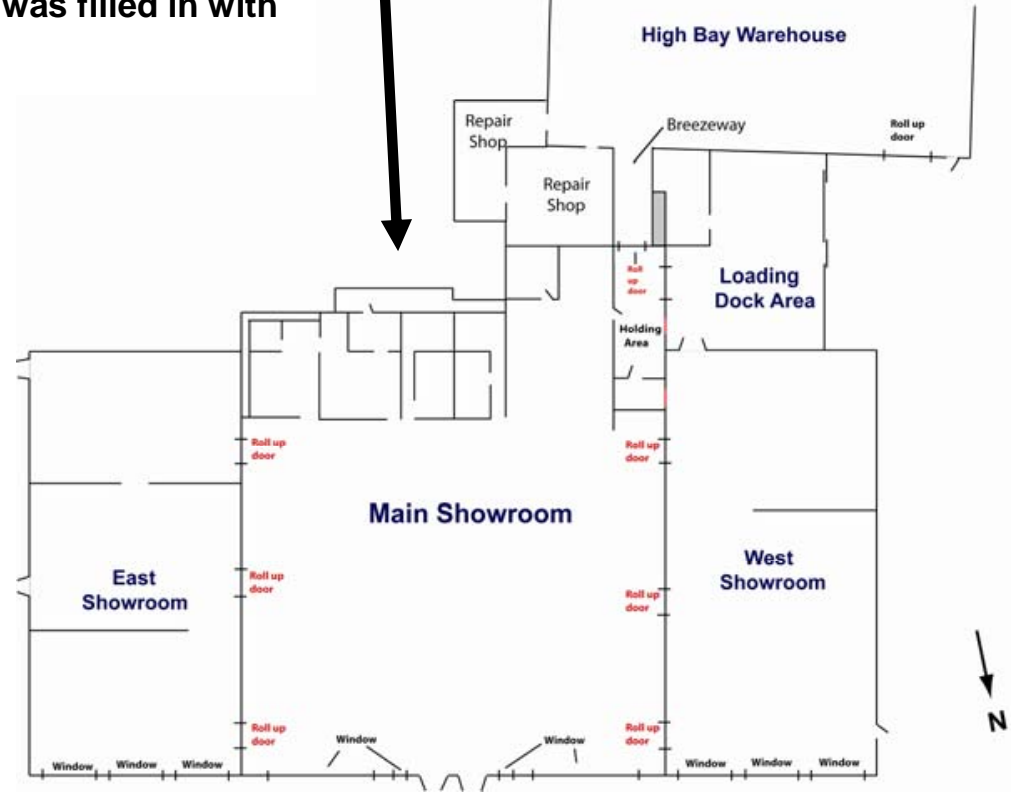




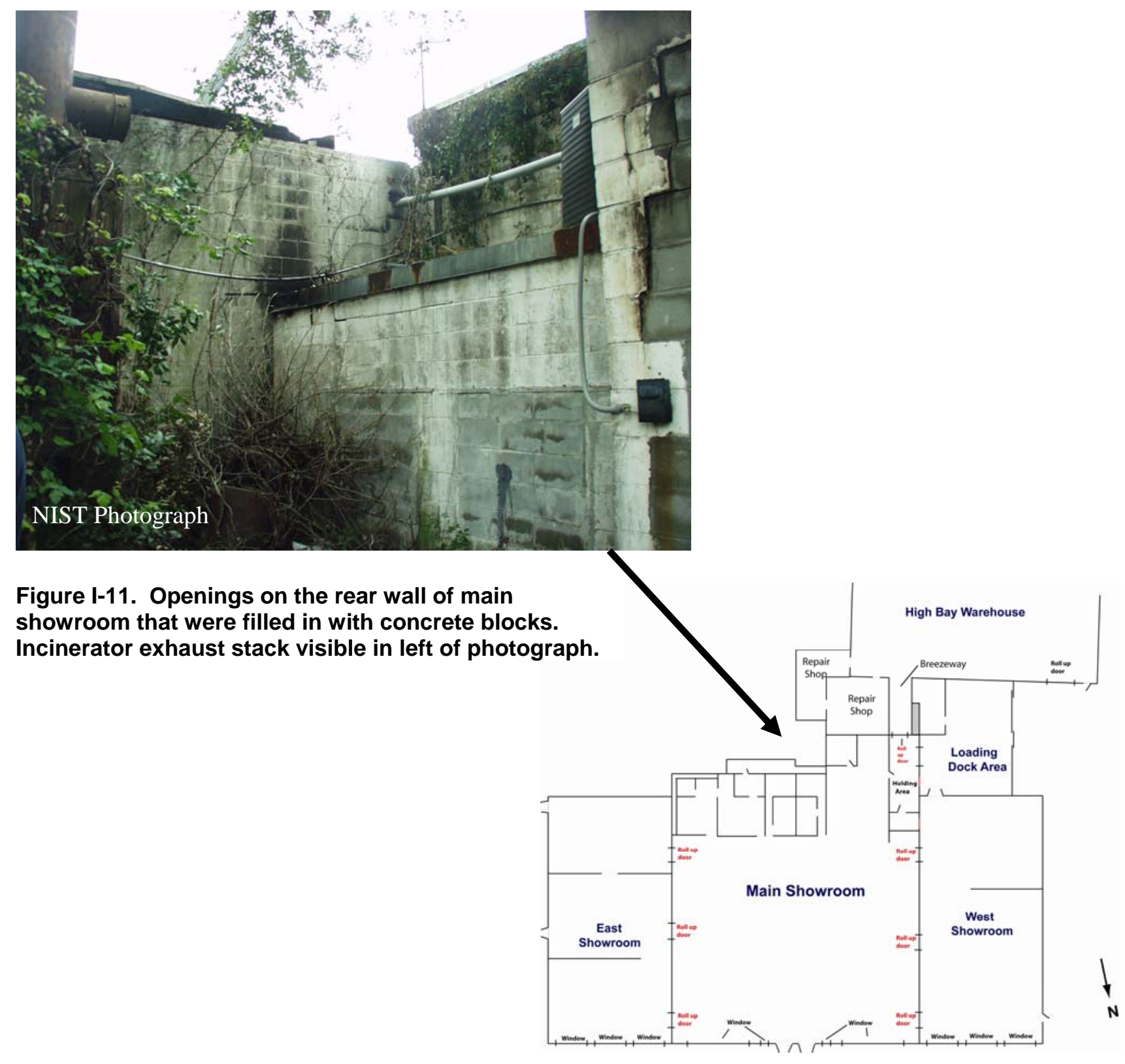




\section{I.2 REAR OF MAIN SHOWROOM AFTER REMOVAL OF WAREHOUSE}

After the demolition and removal of the warehouse, additional photographs were taken of the rear of the store.

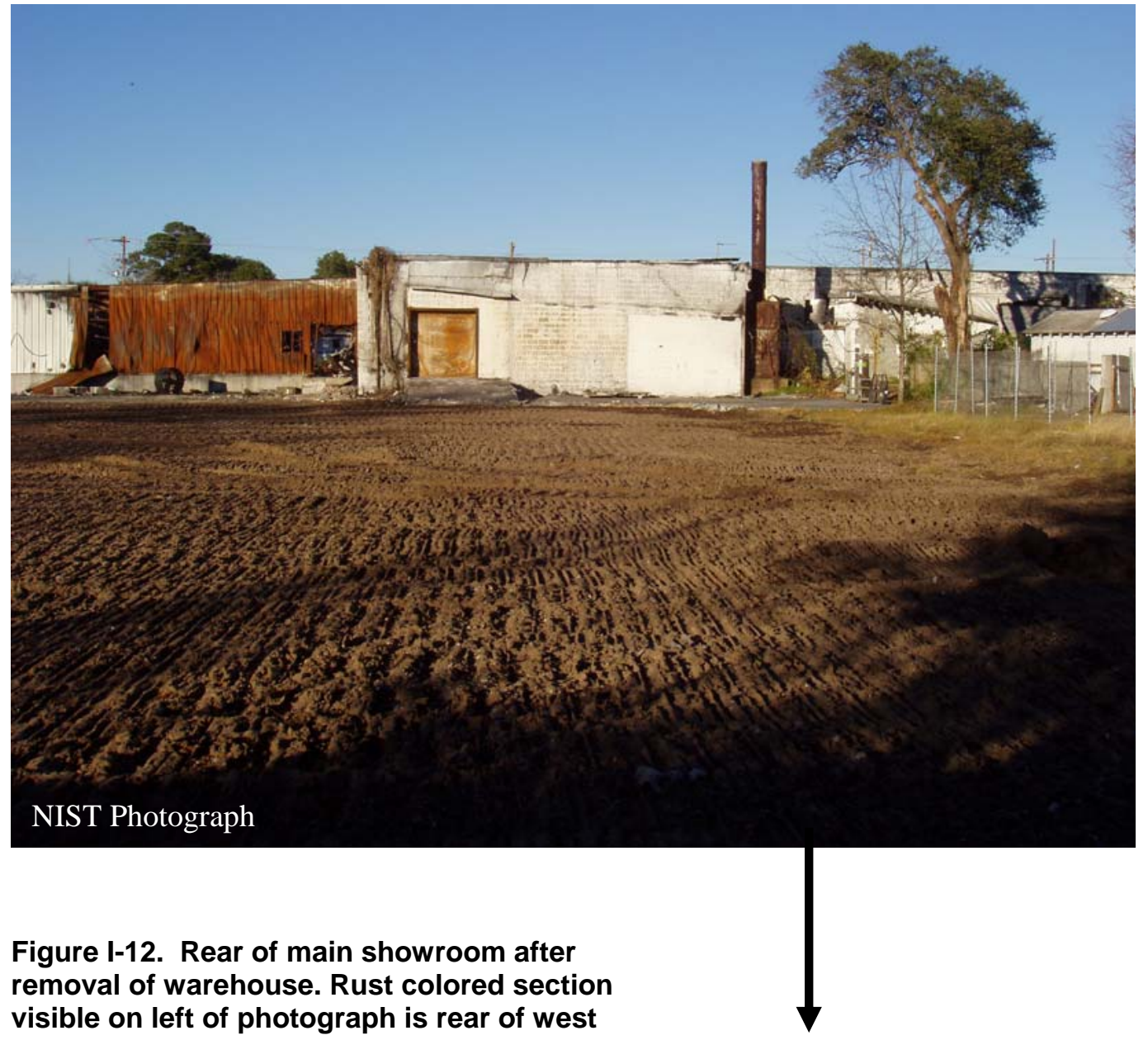
showroom.

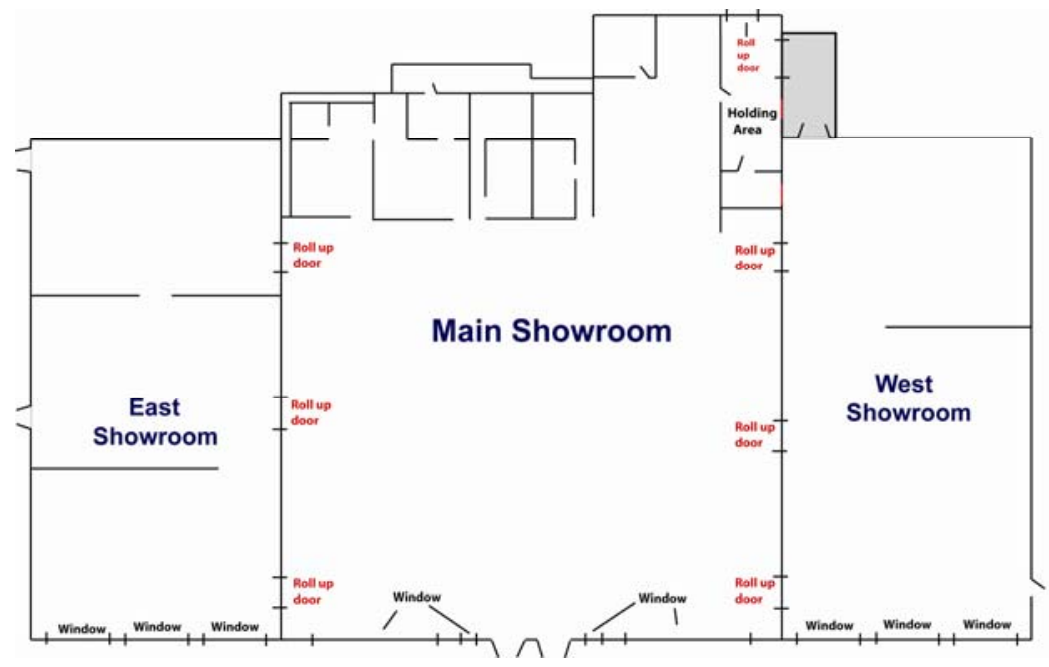




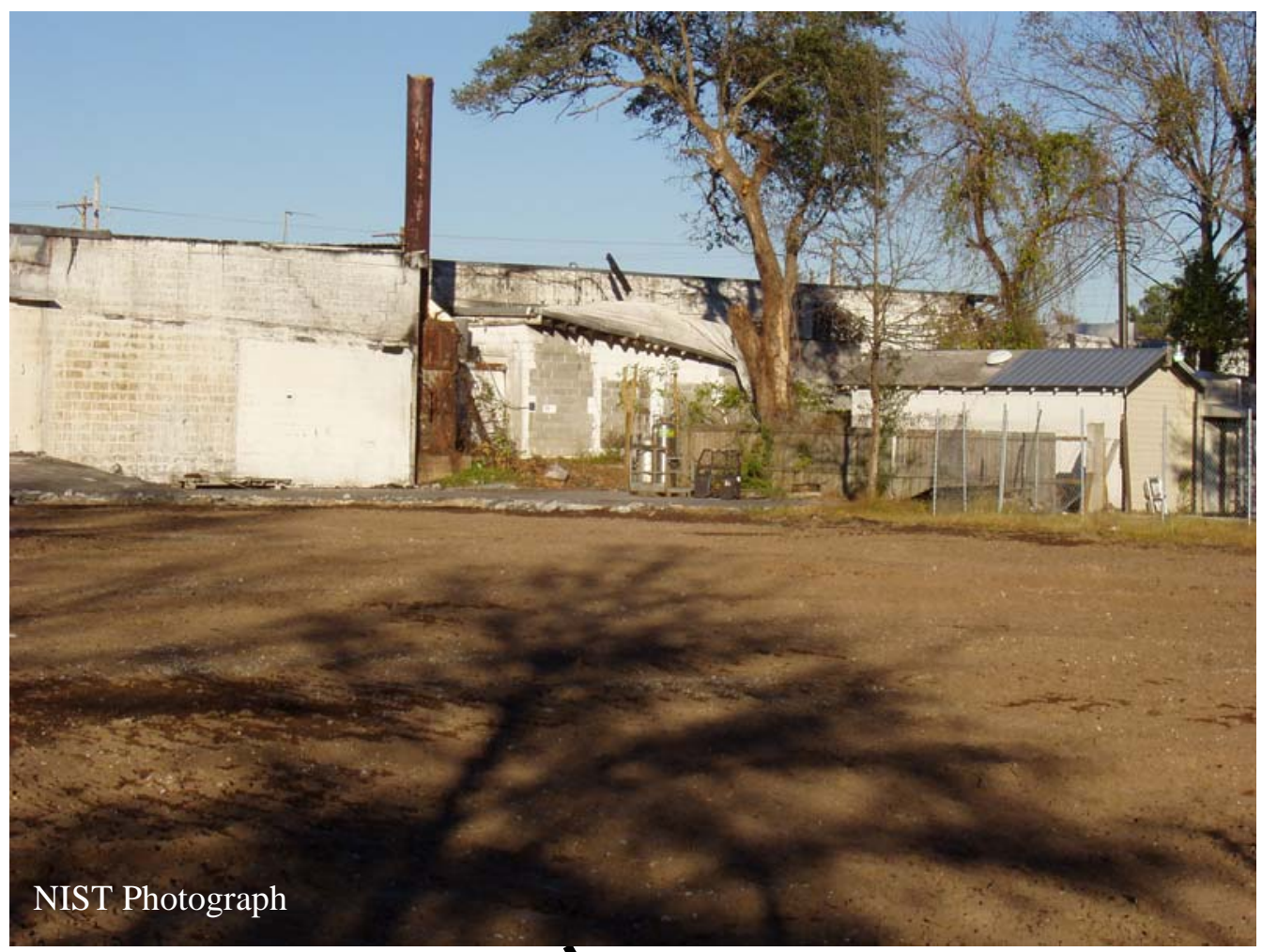

Figure l-13. Rear of main showroom after removal of warehouse.

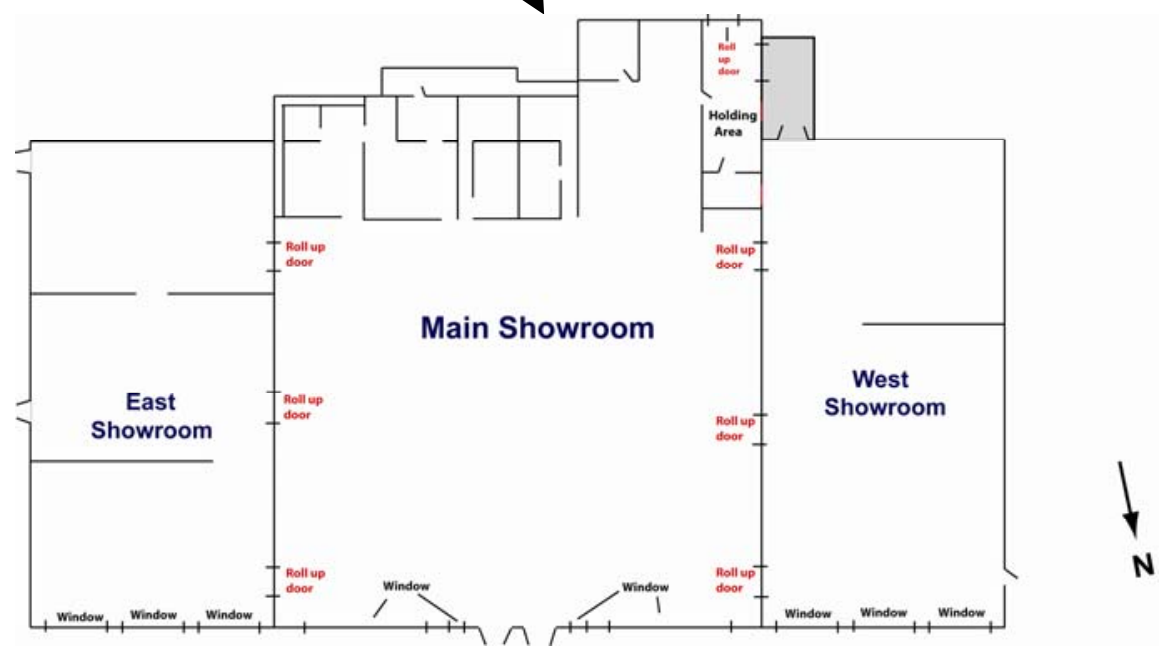




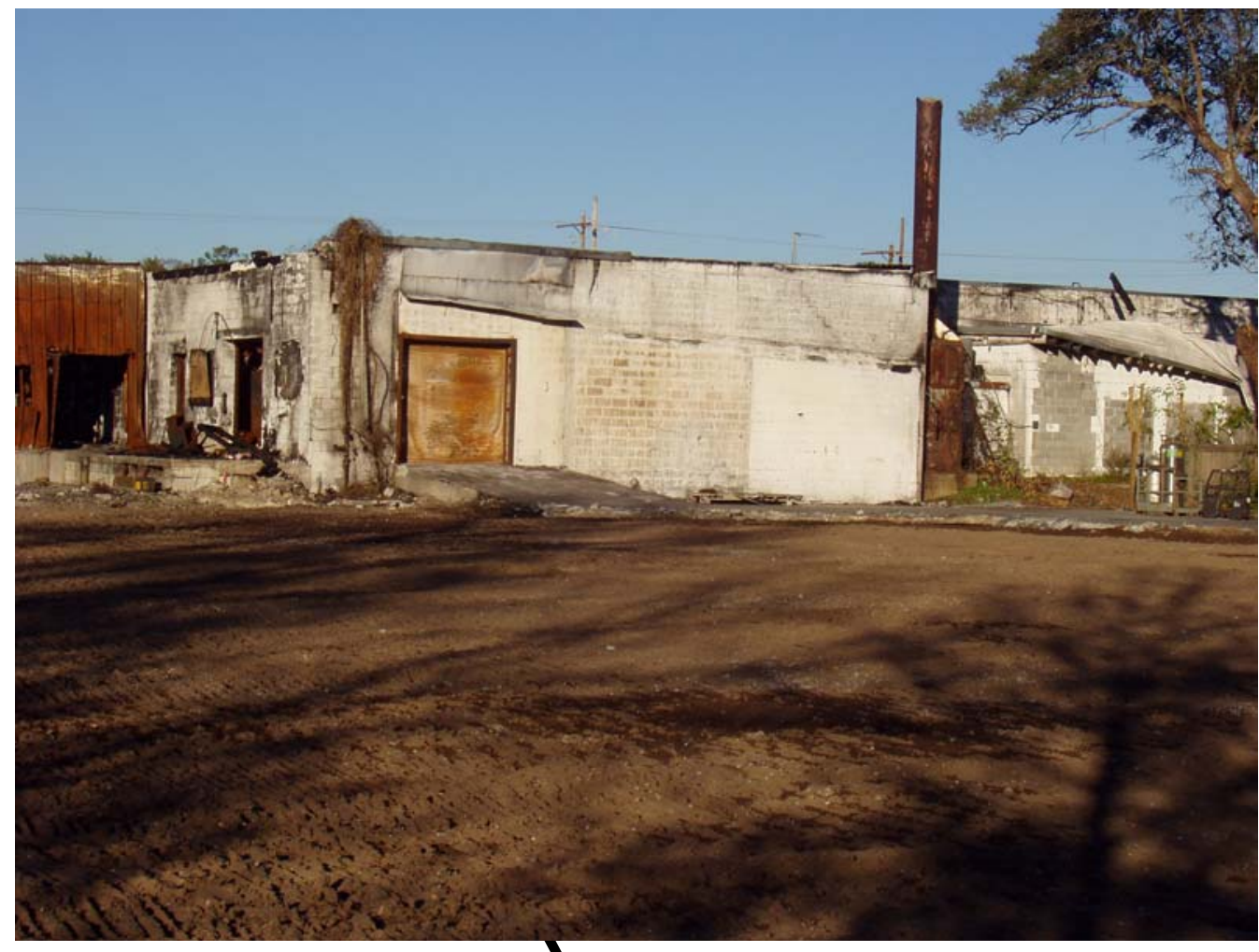

Figure I-14. Rear of main showroom after removal of warehouse.

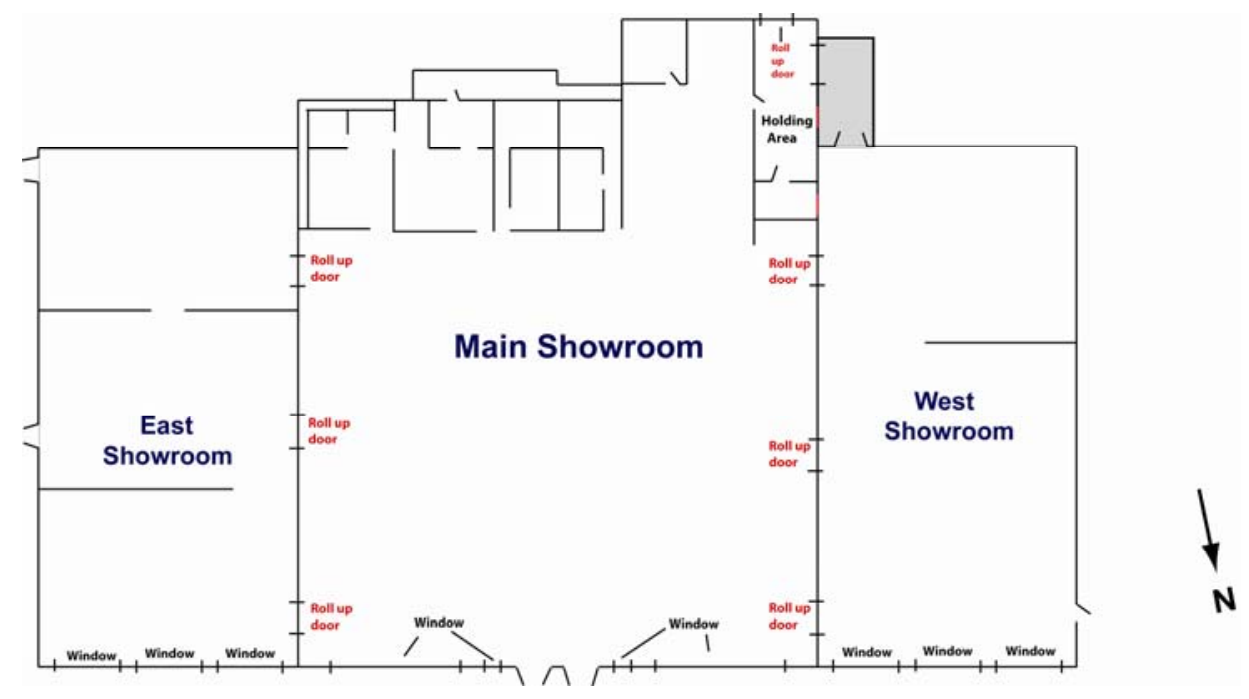




\section{Appendix J. Retall Merchandise Estimates}

\section{J.1 RETAIL MERCHANDISE}

Inventory and sales records of merchandise were stored at the Sofa Super Store. Although the inventory and sales records were not recovered from the fire scene, it is still useful to estimate how much merchandise might have been present.

Typical dimensions for different types of furniture are tabulated in Table J-1. Average values for mass and heat release rates for various types of furniture are also tabulated [1-5].

If one assumes a distribution of furniture items, and the fraction of floor space used to display the items, one can estimate the number of items that may have been displayed. The number of display items, as a function of various fractions of floor space used to display furniture, are tabulated in Tables J-2, J-3, and J-4. The loading dock area was used for staging furniture for delivery. Similar estimates for the loading dock are tabulated in Table J-5.

The warehouse used high rack storage to hold merchandise. Each of the 22 rows of racks appeared to be about 6 shelves high and $30.5 \mathrm{~m}$ (100 ft) long. Using the same dimensions for sofas, mattresses, and chairs, assuming each item required $150 \%$ of its width, and the fraction of rack space occupied, the number of items was estimated. These estimates are tabulated in Table J-6. 
Table J-1. Typical dimensions and average mass and energy content for range of furniture types.

\begin{tabular}{|c|c|c|c|c|c|c|c|c|c|c|c|}
\hline Item & in & $\mathrm{m}$ & in & $\mathrm{m}$ & in & $\mathrm{m}$ & $\mathrm{ft}^{2}$ & $\mathrm{~m}^{2}$ & lbs & $\mathrm{kg}$ & $\begin{array}{c}\text { Average Energy } \\
\text { Content } \\
\text { MJ }\end{array}$ \\
\hline Chair & 42 & 1.1 & 36 & 0.91 & 34 & 0.86 & 10.5 & 1.0 & 53 & 24 & 300 \\
\hline Mattress & 84 & 2.1 & 66 & 1.7 & 16 & 0.41 & 38.5 & 3.6 & 120 & 54 & 600 \\
\hline Table & 72 & 1.8 & 48 & 1.2 & 6 & 0.15 & 24 & 2.2 & 35 & 16 & 200 \\
\hline Rug & 96 & 2.4 & 96 & 2.4 & 0.5 & 0.012 & 64 & 6.0 & 35 & 16 & 200 \\
\hline
\end{tabular}


Table J-2. Number of items displayed in Main Showroom $\left(1585 \mathrm{~m}^{2}\right)$ for specific fraction of floor space used to display furniture.

\begin{tabular}{|c|c|c|c|c|c|c|c|c|c|c|}
\hline $\begin{array}{l}\text { Percent of } \\
\text { Available Floor } \\
\text { Space Used to } \\
\text { Display } \\
\text { Merchandise }\end{array}$ & Sofa & Chair & Mattress & Dresser & Table & Rug & $\begin{array}{c}\text { Estimated } \\
\text { Total } \\
\text { Items }\end{array}$ & $\begin{array}{c}\text { Total Mass } \\
\text { kg } \\
\text { (lbs) }\end{array}$ & $\begin{array}{l}\text { Estimated } \\
\text { Mass } \\
\text { Loading } \\
\mathrm{kg} / \mathrm{m}^{2} \\
\left(\mathrm{lbs} / \mathrm{ft}^{2}\right)\end{array}$ & $\begin{array}{c}\text { Estimated } \\
\text { Total } \\
\text { Energy } \\
\text { Content } \\
\text { GJ }\end{array}$ \\
\hline $40 \%$ & 45 & 90 & 23 & 60 & 39 & 38 & 293 & $\begin{array}{c}10,000 \\
(21,900)\end{array}$ & $\begin{array}{c}6.3 \\
(1.3)\end{array}$ & 120 \\
\hline $50 \%$ & 55 & 110 & 28 & 73 & 47 & 46 & 359 & $\begin{array}{c}12,200 \\
(26,800)\end{array}$ & $\begin{array}{c}7.7 \\
(1.6)\end{array}$ & 145 \\
\hline $60 \%$ & 68 & 136 & 34 & 90 & 58 & 57 & 444 & $\begin{array}{c}15,000 \\
(33,100)\end{array}$ & $\begin{array}{c}9.5 \\
(1.9)\end{array}$ & 180 \\
\hline $70 \%$ & 78 & 156 & 39 & 104 & 66 & 66 & 509 & $\begin{array}{c}17,300 \\
(38,000)\end{array}$ & $\begin{array}{l}10.9 \\
(2.2)\end{array}$ & 205 \\
\hline $80 \%$ & 90 & 180 & 45 & 120 & 77 & 77 & 589 & $\begin{array}{c}19,900 \\
(43,800)\end{array}$ & $\begin{array}{l}12.6 \\
(2.6)\end{array}$ & 240 \\
\hline
\end{tabular}


Table J-3. Number of items displayed in West Showroom $\left(652 \mathrm{~m}^{2}\right)$ for specific fraction of floor space used to display furniture.

\begin{tabular}{|c|c|c|c|c|c|c|c|c|c|c|}
\hline $\begin{array}{l}\text { Percent of } \\
\text { Available Floor } \\
\text { Space Used to } \\
\text { Display } \\
\text { Merchandise }\end{array}$ & Sofa & Chair & Mattress & Dresser & Table & Rug & $\begin{array}{c}\text { Estimated } \\
\text { Total } \\
\text { Items }\end{array}$ & $\begin{array}{c}\text { Total Mass } \\
\text { kg } \\
\text { (lbs) }\end{array}$ & $\begin{array}{c}\text { Estimated } \\
\text { Mass Loading } \\
\mathrm{kg} / \mathrm{m}^{2} \\
\left(\mathrm{lbs} / \mathrm{ft}^{2}\right)\end{array}$ & $\begin{array}{c}\text { Estimated } \\
\text { Total } \\
\text { Energy } \\
\text { Content } \\
\text { GJ }\end{array}$ \\
\hline $40 \%$ & 39 & 70 & 12 & 16 & 2 & 10 & 148 & $\begin{array}{c}5,400 \\
(11,900)\end{array}$ & $\begin{array}{c}8.3 \\
(1.7)\end{array}$ & 62 \\
\hline $50 \%$ & 48 & 86 & 14 & 19 & 2 & 12 & 182 & $\begin{array}{c}6,600 \\
(14,600)\end{array}$ & $\begin{array}{l}10.2 \\
(2.1)\end{array}$ & 80 \\
\hline $60 \%$ & 58 & 104 & 17 & 23 & 3 & 15 & 220 & $\begin{array}{c}8,000 \\
(17,700)\end{array}$ & $\begin{array}{l}12.3 \\
(2.5)\end{array}$ & 90 \\
\hline $70 \%$ & 66 & 119 & 20 & 26 & 3 & 17 & 251 & $\begin{array}{c}9,100 \\
(20,100)\end{array}$ & $\begin{array}{l}14.0 \\
(2.9)\end{array}$ & 105 \\
\hline $80 \%$ & 75 & 135 & 23 & 30 & 4 & 19 & 285 & $\begin{array}{c}10,400 \\
(23,000)\end{array}$ & $\begin{array}{l}15.9 \\
(3.3)\end{array}$ & 120 \\
\hline
\end{tabular}


Table J-4. Number of items displayed in East Showroom $\left(645 \mathrm{~m}^{2}\right)$ for specific fraction of floor space used to display furniture.

\begin{tabular}{|c|c|c|c|c|c|c|c|c|c|c|}
\hline $\begin{array}{l}\text { Percent of } \\
\text { Available Floor } \\
\text { Space Used to } \\
\text { Display } \\
\text { Merchandise }\end{array}$ & Sofa & Chair & Mattress & Dresser & Table & Rug & $\begin{array}{c}\text { Estimated } \\
\text { Total } \\
\text { Items }\end{array}$ & $\begin{array}{l}\text { Total } \\
\text { Mass } \\
\text { kg } \\
\text { (lbs) }\end{array}$ & $\begin{array}{c}\text { Estimated } \\
\text { Mass Loading } \\
\mathrm{kg} / \mathrm{m}^{2} \\
\left(\mathrm{lbs} / \mathrm{ft}^{2}\right)\end{array}$ & $\begin{array}{c}\text { Estimated } \\
\text { Total } \\
\text { Energy } \\
\text { Content } \\
\text { GJ }\end{array}$ \\
\hline $40 \%$ & 14 & 28 & 28 & 42 & 1 & 8 & 121 & $\begin{array}{c}5,100 \\
(11,300)\end{array}$ & $\begin{array}{c}8.0 \\
(1.6)\end{array}$ & 60 \\
\hline $50 \%$ & 18 & 36 & 36 & 54 & 1 & 11 & 156 & $\begin{array}{c}6,600 \\
(14,500)\end{array}$ & $\begin{array}{l}11.3 \\
(2.1)\end{array}$ & 80 \\
\hline $60 \%$ & 21 & 42 & 42 & 63 & 1 & 13 & 182 & $\begin{array}{c}7,700 \\
(17,000)\end{array}$ & $\begin{array}{l}12.0 \\
(2.4)\end{array}$ & 90 \\
\hline $70 \%$ & 25 & 50 & 50 & 84 & 1 & 15 & 216 & $\begin{array}{c}9,200 \\
(20,200)\end{array}$ & $\begin{array}{l}14.2 \\
(2.9)\end{array}$ & 110 \\
\hline $80 \%$ & 28 & 56 & 56 & 84 & 1 & 17 & 242 & $\begin{array}{c}10,300 \\
(22,600)\end{array}$ & $\begin{array}{l}15.9 \\
(3.3)\end{array}$ & 120 \\
\hline
\end{tabular}


Table J-5. Number of items staged in Loading Dock Area $\left(206 \mathrm{~m}^{2}\right)$ for specific fraction of floorspace used to display furniture.

\begin{tabular}{|c|c|c|c|c|c|c|c|c|c|c|}
\hline $\begin{array}{l}\text { Percent of } \\
\text { Available Floor } \\
\text { Space Used to } \\
\text { Display } \\
\text { Merchandise }\end{array}$ & Sofa & Chair & Mattress & Dresser & Table & Rug & $\begin{array}{c}\text { Estimated } \\
\text { Total } \\
\text { Items }\end{array}$ & $\begin{array}{l}\text { Total } \\
\text { Mass } \\
\text { kg } \\
\text { (lbs) }\end{array}$ & $\begin{array}{c}\text { Estimated } \\
\text { Mass Loading } \\
\mathrm{kg} / \mathrm{m}^{2} \\
\left(\mathrm{lbs} / \mathrm{ft}^{2}\right)\end{array}$ & $\begin{array}{c}\text { Estimated } \\
\text { Total } \\
\text { Energy } \\
\text { Content } \\
\text { GJ }\end{array}$ \\
\hline $30 \%$ & 9 & 9 & 9 & 9 & 1 & 5 & 42 & $\begin{array}{c}1,700 \\
(3,800)\end{array}$ & $\begin{array}{c}8.4 \\
(1.7)\end{array}$ & 20 \\
\hline $40 \%$ & 11 & 11 & 11 & 11 & 1 & 6 & 51 & $\begin{array}{c}2,100 \\
(4,600)\end{array}$ & $\begin{array}{l}10.2 \\
(2.1)\end{array}$ & 24 \\
\hline $50 \%$ & 15 & 15 & 15 & 15 & 2 & 8 & 70 & $\begin{array}{c}2,900 \\
(6,300)\end{array}$ & $\begin{array}{l}13.9 \\
(2.8)\end{array}$ & 33 \\
\hline $60 \%$ & 17 & 17 & 17 & 17 & 2 & 9 & 79 & $\begin{array}{c}3,300 \\
(7,200)\end{array}$ & $\begin{array}{l}15.8 \\
(3.2)\end{array}$ & 37 \\
\hline $70 \%$ & 20 & 20 & 20 & 20 & 2 & 10 & 92 & $\begin{array}{c}3,800 \\
(8,400)\end{array}$ & $\begin{array}{l}18.6 \\
(3.8)\end{array}$ & 44 \\
\hline $80 \%$ & 23 & 23 & 23 & 23 & 2 & 12 & 106 & $\begin{array}{c}4,400 \\
(9,700)\end{array}$ & $\begin{array}{l}21.4 \\
(4.4)\end{array}$ & 51 \\
\hline
\end{tabular}


Table J-6. Number of items stored in warehouse for specific fraction of rack space used to hold merchandise.

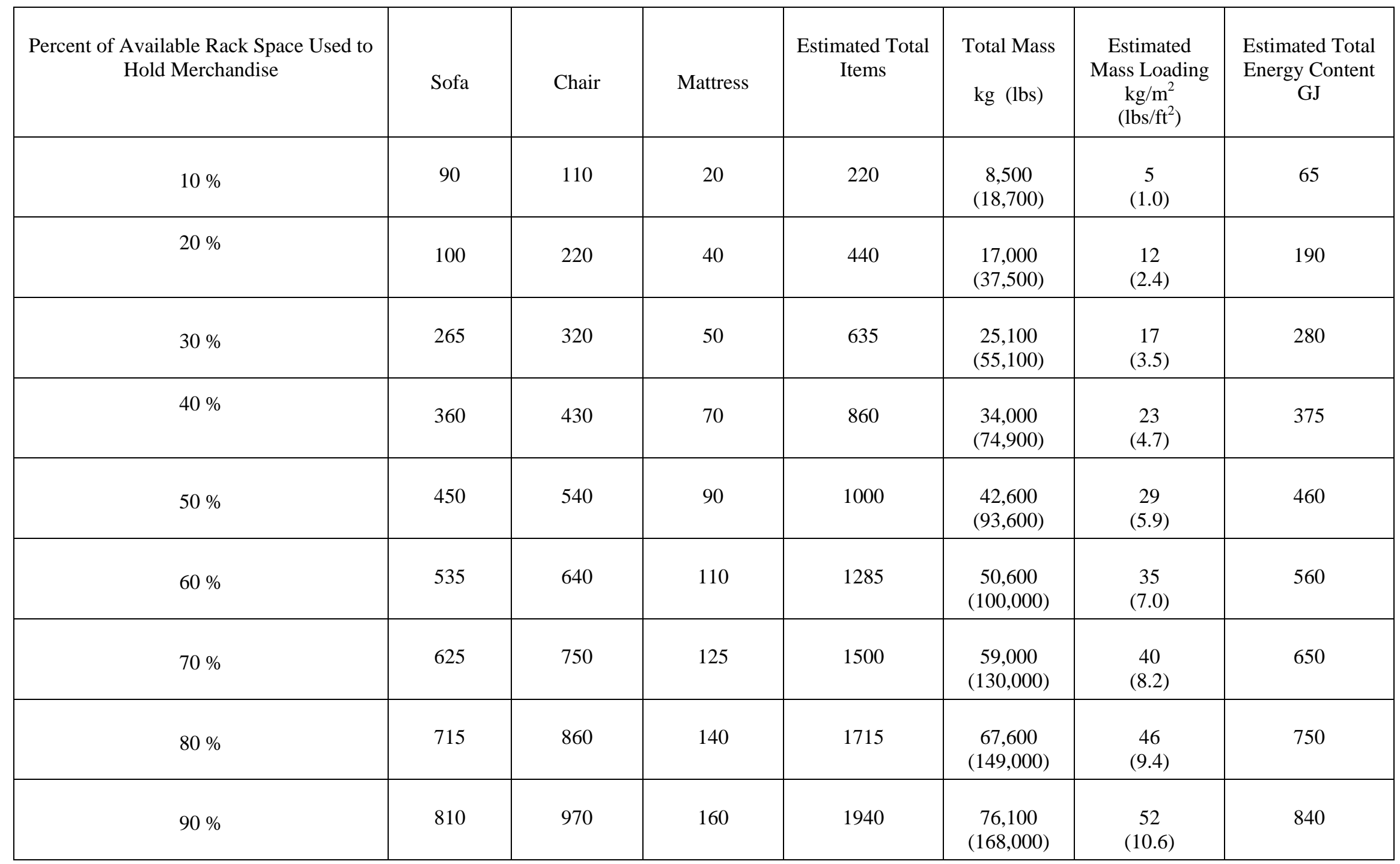




\section{J.2 REFERENCES FOR APPENDIX $\mathrm{J}$}

[1] Babrauskas, V., Lawson, J.R., Walton, W.D., and Twilley, W.H., ”Upholstered furniture Heat Release Rates Measured with a Furniture Calorimeter”, NBSIR 82-2604, National Institute of Standards and Technology, Gaithersburg, MD, 73 p., December 1982.

[2] Babrauskas, V., and Krasny, J., "Fire Behavior of Upholstered Furniture”, NBS Monograph 173, National Institute of Standards and Technology, Gaithersburg, MD, 101 p., Novermber 1985.

[3] Denize, Hamish, "The Combustion Behavior of Upholstered Furniture Materials in New Zealand”, Fire Engineering Research Report 00/04, University of Canterbury, Christchurch, New Zealand, March 2000.

[4] Stroup, D.W., Bryner, N., Lee, J.L., McElroy, J., Roadarmel, G., Twilley, W. H., "Structural Collapse Fire Tests: Single Story, Wood Frame Structures”, NISTIR 7094, National Institute of Standards and Technology, Gaithersburg, MD, March 2004.

[5] Firestone, James, “An Analysis of Furniture Heat Release Rates by Nordtest”, Fire Engineering Research Report 99/07, University of Canterbury, Christchurch, New Zealand, March 1999. 


\section{K. Computer Simulation OF Fire AND SMOKe SPREAD}

\section{K.1 INTRODUCTION}

\section{K.1.1 General}

Computer simulations, also known as numerical modeling, have been demonstrated to be useful, when properly applied, as a tool to help fill in details of the fire dynamics and to demonstrate the value of alternative building designs and fire safety measures [1]. When properly verified and validated, models can be used to obtain quantitative results. The Sofa Super Store simulation results are an approximation of the actual event, and should be considered considered as qualitative rather than quantitative. In other words, it is likely that the simulations do not return exactly the same results that might have been present in the real world situation, but they can provide a reasonable approximation of conditions. These simulated scenarios can then be used to further examine relative differences when simulations that include changes to the modeled environment are compared with each other.

This appendix provides a detailed description of the models and the inputs that were used to simulate the fire and smoke spread during the Sofa Super Store fire for five different scenarios. The computations included simulated fire and smoke spread, potential temperatures, and oxygen concentrations that may have existed in the actual incident. Each of the simulation results was analyzed and compared to published tenability criteria [2], excluding any protective clothing or use of self-contained breathing apparatus (SCBA). The input files for each of the simulation runs are included in Appendix L.

\section{K.1.2 Simulation of the Fire and Smoke Spread}

The value of the computer simulation of the Sofa Super Store fire is its ability to generate a clearer picture of the conditions that existed within the Sofa Super Store as the fire progressed. The simulation can be used to examine the change in the initial conditions and analyze possible interventions on the dynamics of the fire and the environment within the store. The store environment and fire dynamics are characterized in this study by the following dependent variables:

- heat release rate of the fire as a function of time;

- visibility as a function of location and time;

- temperature as a function of location and time;

- oxygen volume fraction as a function of location and time; and

- velocity of the air and fire plume as a function of location and time. 


\section{(i) Fire Growth and Spread Inside the Store}

The focus of this simulation was the examination of conditions that may have been present in the Sofa Super Store during the first 40 minutes (2400 s) after the fire department discovered the fire in the loading dock area. For these model simulations, the fire department was on scene at time $=0$, the fire was discovered at the rear of the west showroom at 2 minutes, E-12 began pumping water to E-10 (loading dock) at 10 minutes, broken radio calls began to indicate fire fighters in trouble at 16 minutes, front windows were vented at 24 minutes, fire was emerging from front windows at 26 minutes, last fire fighter successfully exited structure at 27 minutes, and the roof over the main showroom partially collapsed at 40 minutes. Each 40 minute simulation covered the time period from fire department's arrival at 7:11 p.m., to just after the partial collapse of the main showroom roof at 7:51 p.m.

\section{(ii) Fire-spread into Showrooms versus Warehouse}

The fire spread from the loading dock into both the warehouse and showrooms. The manner in which the fire spread to these two areas was significantly different. The spread of the fire into the showrooms was not reported by the fire fighters for at least 10 minutes after they entered the showrooms. The simulation was designed to provide insight into how the fire might have spread into the showrooms in a concealed or less visible manner. This simulation considered different scenarios for fire-spread into the showrooms, including 1) through the double doors at the rear of the west showroom, and 2) through the holding area.

The movement of the fire into the warehouse was observed and reported by the fire fighters (Section 3.3.6). The energy (heat) from the fire apparently moved through a metal wall and into the warehouse, igniting the fuel load stored in the warehouse. Because the movement of fire into the warehouse was straightforward, it was not the focus of this simulation.

\section{(iii) Impact of Sprinklers on the Loading Dock Fire}

Fire sprinklers are very effective in controlling the growth and spread of fires. Sprinklers were not installed in the showrooms, loading dock, or warehouse of the Sofa Super Store. The simulation considered the effect of sprinklers by including a scenario in which fire sprinklers were installed in the enclosed loading dock.

\section{(iv) Impact of Ventilation}

Ventilation can significantly impact how a fire grows and develops. The fire service often ventilates a structure by breaking windows or cutting holes in roofs in order to allow the smoke and hot gases to be exhausted from the structure. Less smoke and hot gases can improve visibility and make working conditions more tenable. Ventilation can also provide additional air to the fire and can result in a greater release of heat or energy.

The fire at the rear of the main showroom moved quickly toward the front of the store after the front windows were broken by the fire service. This simulation considered how different ventilation scenarios might have affected the fire-spread, including 1) breaking the front windows, and 2) leaving the front windows intact.

In addition to ventilating structures by breaking windows, a fire service fighting a fire might also cut holes in the roof. The simulation considered different scenarios involving holes in the roof, including: 1) a small opening above the holding area; and 2) a large opening above the rear of the main showroom. 


\section{K.1.3 Computer Model Simulations of the Fire Dynamics}

The simulation of the Sofa Super Store fire included the following five scenarios or cases:

1) Baseline Scenario - an approximation of what actually occurred in Sofa Super Store.

2) Sprinklered Scenario - fire sprinklers added to the loading dock.

3) Front Windows Intact Scenario - baseline case except front windows remained intact.

4) Small Vent Scenario - roof vented with a small hole, $1.7 \mathrm{~m}^{2}\left(18 \mathrm{ft}^{2}\right)$, above holding area front windows intact.

5) Large Vent Scenario - roof vented with a large hole, $5.9 \mathrm{~m}^{2}\left(64 \mathrm{ft}^{2}\right)$, above rear of main showroom, front windows intact.

The baseline scenario represents an approximation of what actually occurred in the store on June 18, 2007, based upon the evidence and time line described in the previous chapters.

\section{K.2 NIST FIRE DYNAMICS SIMULATOR OVERVIEW}

\section{K.2.1 General}

The NIST Fire Dynamics Simulator (FDS) is a computational fluid dynamics model of fire-driven fluid flow. It solves a form of the Navier-Stokes equations appropriate for low-speed, thermally driven flow with an emphasis on smoke and heat transport from fires [3]. A companion software program, Smokeview allows for the simulation results to be easily displayed. FDS Version 1 was publicly released in February 2000. The predictions performed here were made with version 5.1.0 and Smokeview, version 5.0.7. These applications were combined into a single file known as a release bundle (FDS_5.1.0SMV_5.1.0 - Released Jan. 8, 2008 - SVN Revision 1162) that was downloaded from the FDS-SMV development project site. ${ }^{1}$ Complete descriptions of the FDS model and Smokeview, as well as the technical references which support the model, are given in references [3-5].

Inputs required by FDS include the geometry of the structure, the computational cell size, the location of the fire source, the energy release rate of the fire source, the mass, geometry and thermal properties of walls, ceilings, floors, and furnishings, and the size, location, and timing of door and window openings to the outside of the structure. The selection of thermophysical properties and dimensions for the input parameters can have a significant impact on the outcome of the simulation, and because considerable uncertainty exists in the values of these parameters, a range of values is used.

For a specific set of inputs, FDS calculates the fire-spread and smoke movement within the Sofa Super Store. The results of the simulation including the spread of fire and smoke within the loading dock, holding area, and showrooms, are compared to the photographic and video record and the statements of witnesses to assess the agreement between the simulation and the actual fire. The input parameters are systematically adjusted and the simulation re-run (Table K-1). This process of refining the input parameters continues until the best possible agreement has been achieved.

\footnotetext{
${ }^{1}$ http://fds-smv.googlecode.com
} 
Table K-1. Range of Input Parameters and Simulation Values.

\begin{tabular}{|l|c|c|c|}
\hline \multicolumn{1}{|c|}{ Input Parameter } & Range & Simulation Value & Units \\
\hline Furniture Foam & & & \\
\hline Reference Temperature & $200-375$ & 350 & ${ }^{\circ} \mathrm{C}$ \\
\hline Density & $20-40$ & 20 & $\mathrm{~kg} / \mathrm{m}^{3}$ \\
\hline Specific Heat & $0.65-2.0$ & 1.4 & $\mathrm{~kJ} / \mathrm{kg}$ \\
\hline Conductivity & $0.034-1.60$ & 0.034 & $\mathrm{~W} /(\mathrm{m} \cdot \mathrm{K})$ \\
\hline Heat of Reaction & $800-2000$ & 1350 & $\mathrm{~kJ} / \mathrm{kg}$ \\
\hline Heat of Combustion & $20,000-50,000$ & 25,000 & $\mathrm{~kJ} / \mathrm{kg}$ \\
\hline Furniture Fabric/Covering & & & \\
\hline Reference Temperature & $200-375$ & 350 & \\
\hline Density & $0.1-100.0$ & 100 & $\mathrm{~kg} / \mathrm{m}^{3}$ \\
\hline Specific Heat & $1-100$ & 1 & $\mathrm{~kJ} / \mathrm{kg}$ \\
\hline Conductivity & $0.1-3.5$ & 0.1 & $\mathrm{~W} /(\mathrm{m} \cdot \mathrm{K})$ \\
\hline Heat of Reaction & $800-2000$ & 1500 & $\mathrm{~kJ} / \mathrm{kg}$ \\
\hline Heat of Combustion & $20,000-50,000$ & 15000 & $\mathrm{~kJ} / \mathrm{kg}$ \\
\hline Computational mesh cell dimension & $12.5-80.0$ & Multiple, see App. L & $\mathrm{cm}$ \\
\hline $\begin{array}{l}\text { Ventilation Points (doors, windows, } \\
\text { roof) }\end{array}$ & Based on observations & Multiple, see App. L & $\begin{array}{c}\mathrm{seconds} \\
{ }^{\circ} \mathrm{C}\end{array}$ \\
\hline
\end{tabular}

\section{K.2.2 Computational Mesh / Domain}

A computational fluid dynamics model requires that the room or building of interest be enclosed by a rectangular cuboid ${ }^{2}$ control volume that is divided into smaller rectangular cuboid computational cells. The CFD model computes the density, velocity, temperature, pressure and species concentration of the gases in each cell as it steps through time. Based on the laws of conservation of mass, momentum, species, and energy, the model tracks the generation and movement of fire gases. In FDS terminology a control volume of cells is called a mesh. More than one mesh is used to break up a large model into smaller blocks that can be distributed over different computers so they can work together in parallel to simulate a much larger volume that would otherwise exceed the capabilities of a single computer. This multiple mesh configuration of FDS (multi-mesh) was used to generate results for this study.

FDS predictions are sensitive to mesh size. Using a larger number of smaller cells will generally capture more features of the flow. However, the computation time increases more than linearly with the increase in the number of mesh cells. Computation times of one day on a fast computer are not uncommon but may increase to several months with a large number of mesh cells. Therefore, it is important to use the smallest number of mesh cells that will still capture the important features of the fire. One way to reduce the number of mesh cells is the use of the multi-mesh configuration described above. The multi-mesh techniques uses smaller mesh cells that capture more detail near fire sources and larger mesh cells with lower resolution farther away, where, in general, smoke and heat transport are the dominant phenomena of interest.

\footnotetext{
${ }^{2}$ Weisstein, Eric W. "Cuboid." From MathWorld--A Wolfram Web Resource. http://mathworld.wolfram.com/Cuboid.html
} 


\section{K.2.3 Geometry}

Building features such as walls, floors, windows, doors and furniture are described in FDS as "obstructions" (OBST in the input file, Appendix L). Since these obstructions are also cuboid, they must have sides that are either horizontal or vertical and no sloped or curved surfaces are allowed. The obstructions may be colored for identification and may be assigned material properties. The obstructions may be entered into the simulation with exact measurements from the building. However, FDS can only work with items that fall exactly on mesh cell boundaries. FDS takes the obstructions as they are defined in the input file and adjusts them to match the mesh cell boundaries. As a result, items may either grow or shrink to match the mesh. In most cases this does not have a major impact on the calculations, although it can result in walls with no thickness or walls with gaps at intersections. Usually these issues are resolved by adjusting the size of the object definition or slightly increasing mesh resolution to produce the desired model geometry.

\section{K.2.4 Heat Transfer and Materials}

Radiative heat transfer is included in the model and is shown as the solution of the radiation transport equation for a non-scattering gray gas. All solid surfaces are assigned thermal boundary conditions, plus information about their burning behavior. Heat and mass transfer to and from solid surfaces are based on empirical correlations. FDS utilizes material properties of the furnishings, walls, floors, and ceilings to compute fire growth and spread. When a surface material is defined for use in the FDS model, it is given a set of physical and thermal properties that are used by the model. Some of these properties such as thermal diffusivity, thermal conductivity, density and thickness, impact the heat transfer in the material. For materials that burn, additional parameters such as reference temperature, heat of combustion, heat of reaction and maximum burning rate, are specified. The properties for the materials, to the extent they were available, were taken from published fire data and references.

\section{K.2.5 Combustion}

The combustion process is handled by tracking where fuel and air mix at certain concentrations and react to produce heat. This is known as a mixture fraction combustion model [3], in which burning occurs in regions where the fuel and air are in specified stoichiometric proportion. This reaction processes generates heat along with products of combustion such as carbon monoxide (CO), carbon dioxide $\left(\mathrm{CO}_{2}\right)$ and water $\left(\mathrm{H}_{2} \mathrm{O}\right)$, that are tracked as they move throughout the meshes.

\section{K.2.6 Boundary Conditions}

Boundary conditions to planar rectangular regions on either a solid obstruction surface or on the boundary of the computational domain are defined in the FDS input file with the namelist group 'VENT' (Appendix L). These boundary conditions are generally referred to as vents in reference to the input parameter. One application for vents is to create openings from the model to ambient conditions outside the computational domain. In these simulations, vents were used to allow fresh air to enter the simulation domain and to allow smoke and heat to exit the domain. Vents may be either simple openings that allow natural flow to occur based on the buoyancy of the hot gases, or vents may use a specified or forced flow rate such as the flow from a fan. 
The VENT namelist group can also be used to introduce heat into the simulation and thus create the effect of a fire source in the model, by ejecting fuel according to a prescribed heat release rate ${ }^{3}$. Simulating the movement of the fire from the trash pile, through the wall, and into the loading dock, vents were used to initiate the fire on the loading dock. Later in the fire evolution, additional vents were used to simulate the spread of the fire from the holding area into the rear of the main showroom.

\section{K.2.7 Openings within a Mesh}

The placement of blocks (obstructions) within the mesh forms the structure of the building and its contents. The hydrodynamic calculations performed by FDS allow air, hot gases, smoke and flames to flow through the simulated building. Thermal radiation travels by line-of-sight and may be intercepted by obstacles within the meshes.

Normal buildings may appear tightly constructed, but there are many small openings or leaks within a building that allow for the flow of air or combustion products. Since objects can only exist at mesh boundaries, small leaks may be created by either using a very small mesh size, or by representing many small leaks by fewer large leaks.

During the course of a fire, some items within the building may be consumed by the fire or otherwise change position. FDS does not have the capability to calculate burn-through or collapse but the user can remove items during the course of the calculations. Items that are removed can represent objects that fall or are destroyed by fire, or objects that are changed by people such as doors or windows that are opened.

\section{K.2.8 Smokeview}

Smokeview is a scientific visualization program that was developed to display the results of an FDS model computation [4]. Smokeview allows the viewing of FDS results in three-dimensional images. Smokeview can display contours of temperature, velocity and gas concentration in planar slices. It can also display properties with iso-surfaces that are three-dimensional versions of a constant value of a property of interest. Iso-surfaces are most commonly used to provide a three-dimensional approximation of the flame surface where fuel and oxygen are mixing in a stoichiometric ratio, yielding a reaction and a flame surface.

\section{K.3 FDS INCIDENT SIMULATION OVERVIEW}

\section{K.3.1 Computational Domain and Mesh Size}

Collected on scene by the NIST team, data describing the geometry, material of constructions, and estimated number and type of retail merchandise were incorporated as input parameters for the FDS simulations. Figure K-1 shows a Smokeview rendering of the computational domain of the Sofa Super Store.

The computational domain for the incident simulation consisted of a total of 1,102,382 cells that encompassed a total volume of approximately $140,000 \mathrm{~m}^{3}\left(5\right.$ million $\left.\mathrm{ft}^{3}\right)$. Figure $\mathrm{K}-1$ shows an isometric view of the structure with cells to show relative scale. The black lines surrounding the structure indicate bounds of the volume within the computational domain. This outer perimeter reflects the computation of the fire and smoke within the building. It also includes the outdoor conditions that might have affected

\footnotetext{
${ }^{3}$ Heat Release Rate (HRR) is the rate at which a fire generates or releases energy or heat.
} 
the flow of air to the fire and the flow of smoke from the fire. Figures K-2 and K-3 show front and side views of the Sofa Super Store, respectively, as rendered by Smokeview. Two different cell sizes were used in the simulation. For volumes that contained parts of the structure, cells were $37.5 \mathrm{~cm}$ (14.8 in) on each side. Cells that were outside of the structure were used to track smoke movement, and were $75 \mathrm{~cm}$ (29.5 in) on each side. Table K-2 provides a summary of the meshes used.

Selecting the appropriate cell size required balancing the need to resolve critical dimensions and physical phenomena against the need to budget enough time to perform the hundreds of computer runs necessary to assess the importance of different variables on the outcome. The FDS input parameters were adjusted by comparing the simulation results with the available photos, videos, witness statements, and other documentation of the fire. Over 250 computer simulations were required to match the observed phenomena and time line. The simulation that best matched with observations and time lines is presented as the baseline case. Each of the other cases presented in this report used the baseline case as the starting point and was subsequently modified to incorporate fire sprinklers, maintain the front windows intact, or create openings in the roof.

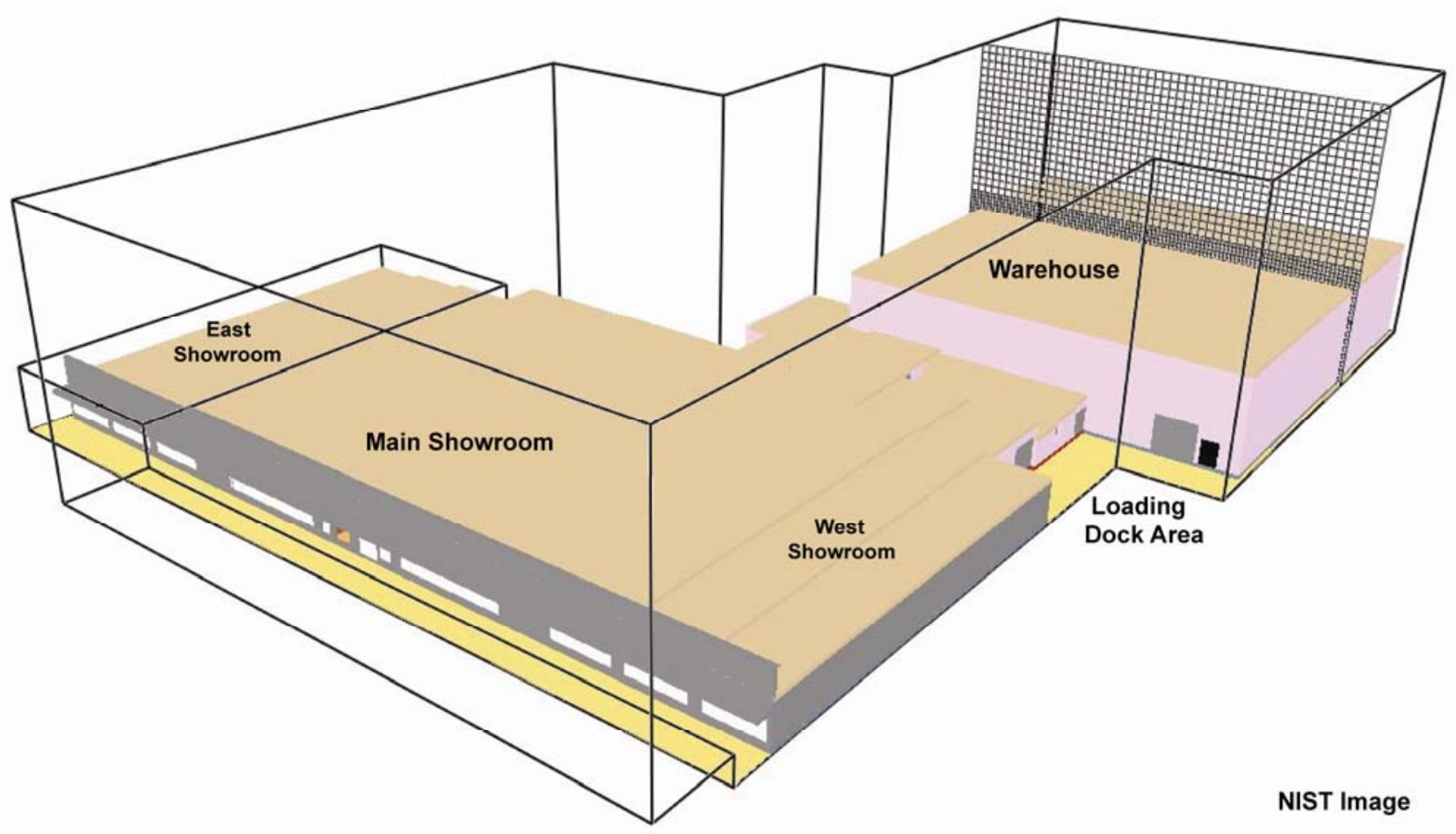

Figure K-1. Computational domain of the Sofa Super Store. Image rendered by Smokeview. 


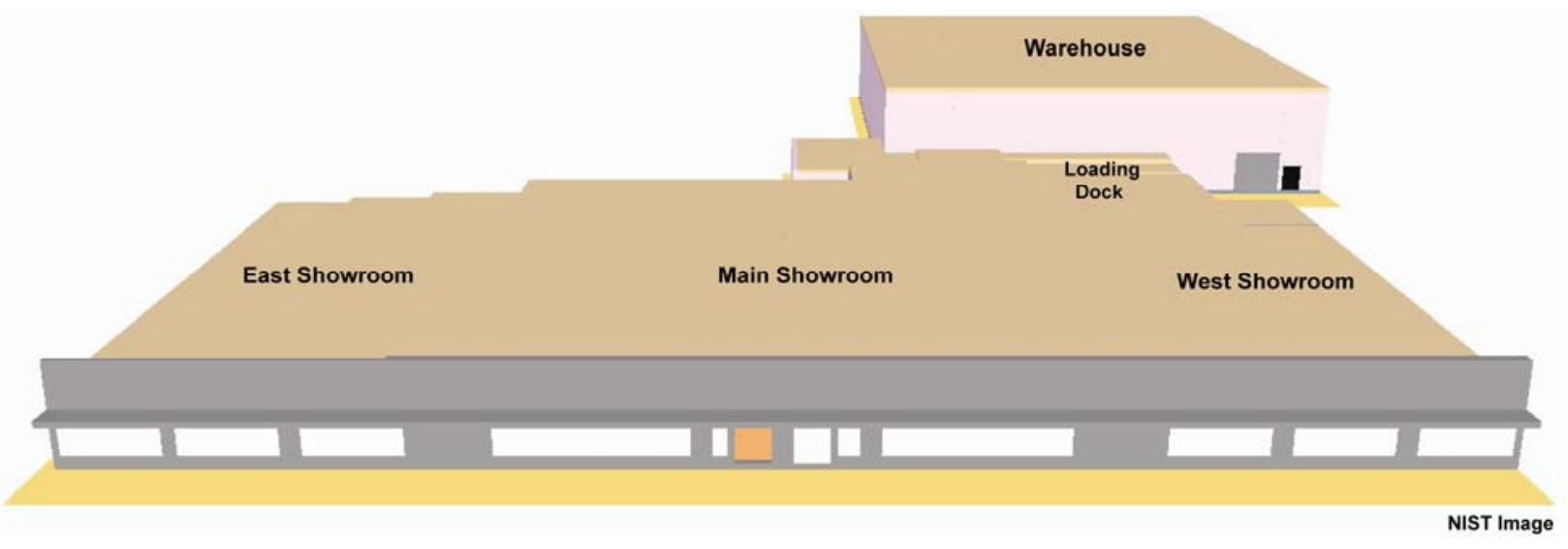

Figure K-2. Front view of the Sofa Super Store as rendered by Smokeview.

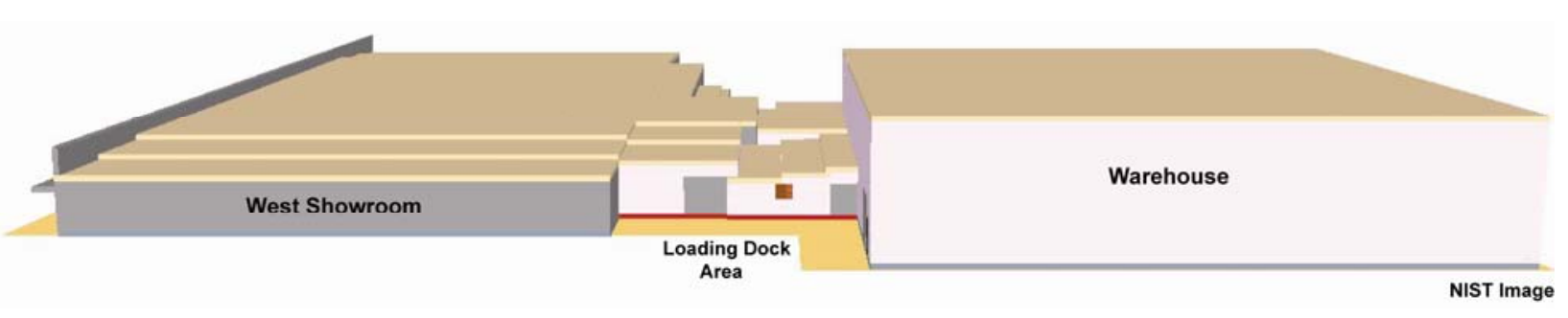

Figure K-3. Side view of the Sofa Super Store as rendered by Smokeview.

Table K-2. Summary of the computational meshes used in the Sofa Super Store simulations.

\begin{tabular}{|c|c|c|c|c|c|}
\hline Mesh & Width $(\mathrm{m})$ & Depth $(\mathrm{m})$ & Height $(\mathrm{m})$ & Cell Dimension $(\mathrm{cm})$ & Number of Cells \\
\hline 1 & 37.5 & 30.0 & 11.25 & 37.5 & 240,000 \\
\hline 2 & 37.5 & 33.75 & 6.0 & 37.5 & 144,000 \\
\hline 3 & 20.25 & 48.0 & 6.0 & 37.5 & 110,592 \\
\hline 4 & 18.0 & 40.5 & 6.0 & 37.5 & 82,944 \\
\hline 5 & 37.5 & 30.0 & 15.0 & 75.0 & 216,000 \\
\hline 6 & 30.0 & 33.75 & 11.25 & 37.5 & 36,000 \\
\hline 7 & 30.0 & 33.75 & 15.0 & 75.0 & 60,750 \\
\hline 8 & 37.5 & 33.75 & 20.25 & 75.0 & 46,656 \\
\hline 9 & 20.25 & 48.0 & 20.25 & 75.0 & 86,400 \\
\hline 10 & 9.0 & 45.0 & 11.25 & 37.5 & 14,400 \\
\hline 11 & 9.0 & 45.0 & 15.0 & 75.0 & 24,640 \\
\hline 12 & 57.75 & 7.5 & 24.0 & 75.0 & $\mathbf{1 , 1 0 2 , 3 8 2}$ \\
\hline \multicolumn{7}{|r|}{}
\end{tabular}

Note: Cells were $37.5 \mathrm{~cm}$ (14.8 in) on a side for meshes that contained parts of the structure. Cells were $75 \mathrm{~cm}$ (29.5 in) on each side for meshes that were outside the structure. 
Mesh size sensitivity has been examined in several studies as noted by McGrattan [3, 5; Vol. 2\&3]. The effectiveness of FDS in accurately modeling the motion of hot gases produced by a fire is based largely on the ratio of the heat release rate to the mesh cell dimension. The heat release rate can be expressed in terms of a characteristic diameter, $\mathrm{D}^{*}(\mathrm{~m})$, which is based on the HRR and physical properties.

$$
D^{*}=\left(\frac{\dot{Q}}{\rho_{\infty} c_{p} T_{\infty} \sqrt{g}}\right)^{2 / 5}
$$

In this expression, $\dot{Q}$ is the HRR of the fire $(\mathrm{kW}), \rho_{\infty}$ is the ambient density $\left(\mathrm{kg} / \mathrm{m}^{3}\right), c_{p}$ is the constant pressure specific heat $\left(\mathrm{kJ} \mathrm{kg}^{-1} \mathrm{~K}^{-1}\right), T_{\infty}$ is the ambient gas temperature $(\mathrm{K})$, and $g$ is the acceleration due to gravity $\left(\mathrm{m} / \mathrm{s}^{2}\right)$. Taking $\delta x(\mathrm{~m})$ as the mesh cell dimension, the ratio $D^{*} / \delta x$ provides a scale for the relative coarseness of the mesh. The accuracy of the simulation improves with increasing values of this ratio as the fire spans a larger number of mesh cells. It has been shown that ratios between 4 and 16 produce favorable results [6]. Using the peak HRR in the loading dock as the heat release rate ( $\bar{Q})$, the ratio $D^{* / \delta x}$ calculated for this simulation was approximately 14.8 .

The choice of computational mesh size influenced the selection of appropriate values for the initial conditions, boundary conditions and material properties, including the size and energy of the ignition source, heat transfer at the boundaries, and burning properties of the fuel. With the mesh size selected, the simulation computational domain required approximately 1.1 million mesh cells. Approximately four days were required to generate each 2400 second simulation. The FDS input parameters were adjusted by comparing the simulation results with the available photos, videos, witness statements and other documentation of the fire. This methodology has been used previously by NIST researchers in post-fire studies [7-15].

Figure K-4 is a plan view of the Sofa Super Store, as rendered by Smokeview, showing a layout of the furniture and walls. Furniture in the main showroom was consumed almost completely by the fire (see Figures B-15 to B-17, and Figures D-20 to D-25). There was some remaining furniture in the west showroom, primarily in the rear (south) end of the building (Figures E-17 to E-25). This area of the showroom stored sleep sofas in displays that were stacked two high on wood-framed racks. The damage in the east addition was much less severe because the roll-down doors closed when their fusible links released (Figures F-11 to F-16). The rear roll-down fire door between main and east showrooms was partially blocked by a coat rack and did not close completely. The warehouse contained multiple rows of high-rack storage. The fire consumed nearly all furniture in the warehouse (see Figures G-15 to G-17) and loading dock (see Figures B-4 to B-6). The consumption of fuel in the main and west showrooms was so extensive that the furniture layout used in the model was based largely on witness accounts. Witnesses described the showrooms as being "crowded" with furniture. The general description of the main showroom placed aisles down the center of the room, from the front door to the rear office area. Further, three aisles stretched across the showroom, east to west, aligned approximately with the rolldown doors. The remaining floor space was filled with upholstered furniture laid out tightly in typical rectilinear configurations. Because the exact layout was not known, furniture in the simulation was arranged in rows. Some of the rows were created by arranging furniture in "U" shaped configurations with a sofa and two chairs. In other places, rows consisted of single furniture items. The rear of the west addition was arranged in a manner that was similar to what could be discerned from the furniture that was in place during the post-fire scene examination.

Figure K-5 shows a rendered view from the interior of the main showroom. The light colored area in the upper right corner of this image is the rear roll-down door (roll-down fire door 2 in Figure 1-8) on the 


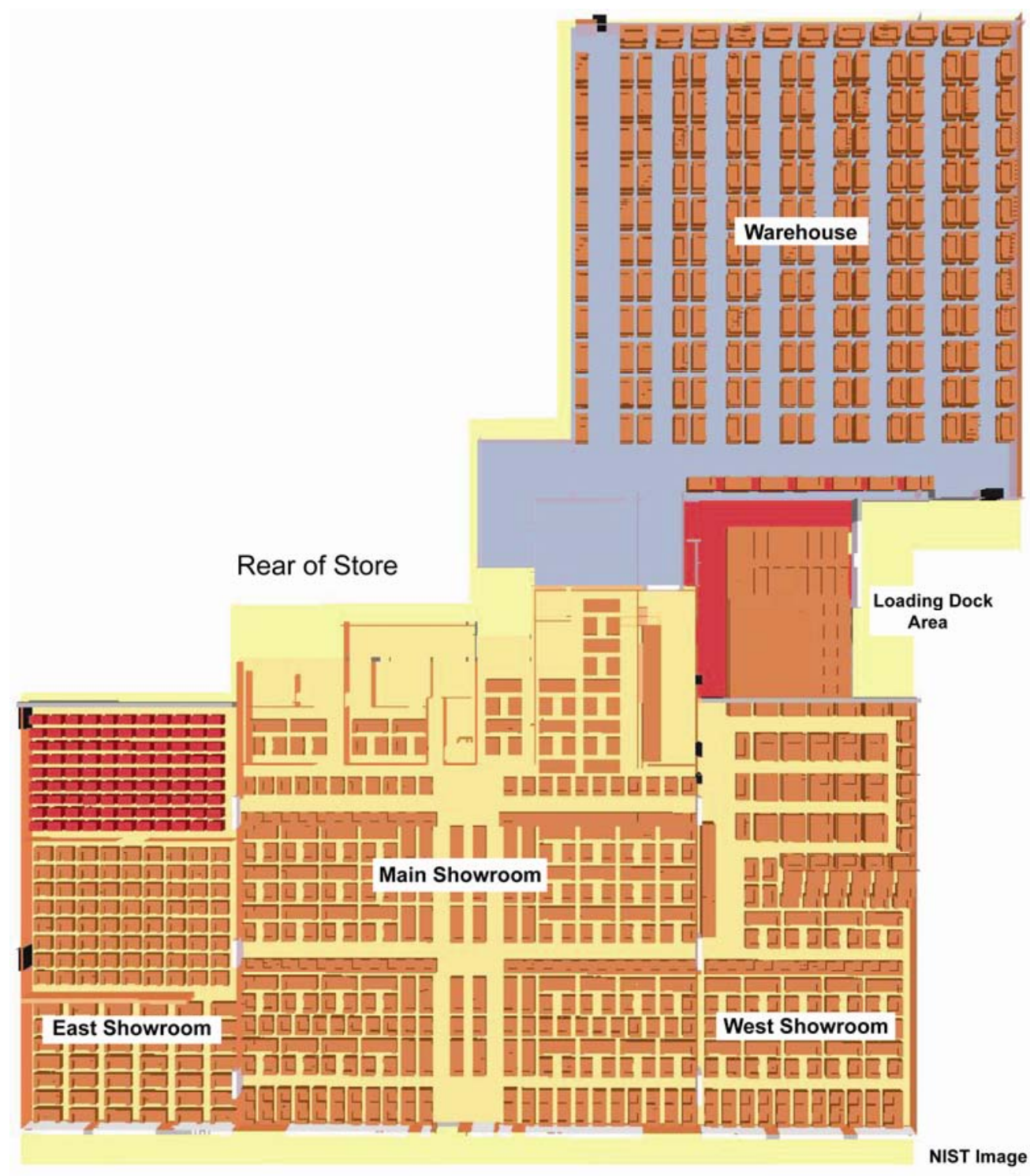

Front of Store

Figure K-4. Overhead view showing furniture layout used in the FDS simulations.

wall separating the main showroom from the west showroom. Figure K-6 shows a rendered image of the interior of the west showroom. To the left in this figure are two light colored areas. These represent the mid and rear roll-down doors (roll-down fire doors 2 and 3 in Figure 1-8) in the wall separating the west addition from the main showroom. The black area in the upper left of this figure is the location of the double steel doors that separated the west addition from the loading dock. Figure K-7 shows a close up view of the holding area, loading dock, and the rear areas of the main showroom and west addition. This figure shows the open non-fire roll-down door in the wall that separated the loading dock from the holding area. At the south end of the holding area, the light colored area represents the location of the roll-down door (fire door 1 in Figure 1-8) in the wall that separated the original structure from the breezeway that provided access to the warehouse. According to witness accounts this door closed early in the event and this would be consistent with the fusible links activating. 


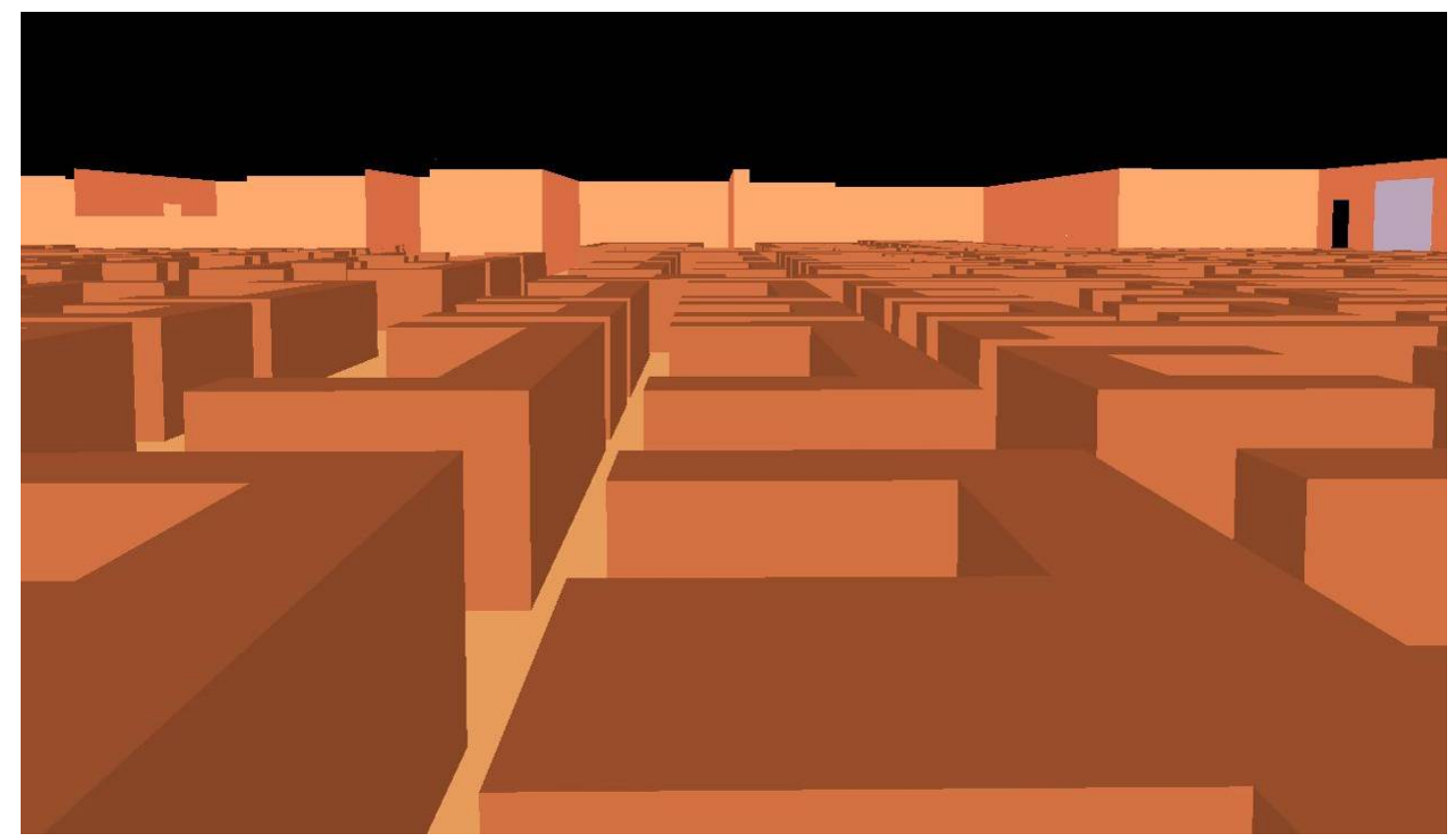

Figure K-5. Interior view of the main showroom. Viewpoint is the west side, looking towards the rear (south). The rear roll-down door for access to the west showroom is at the top right.

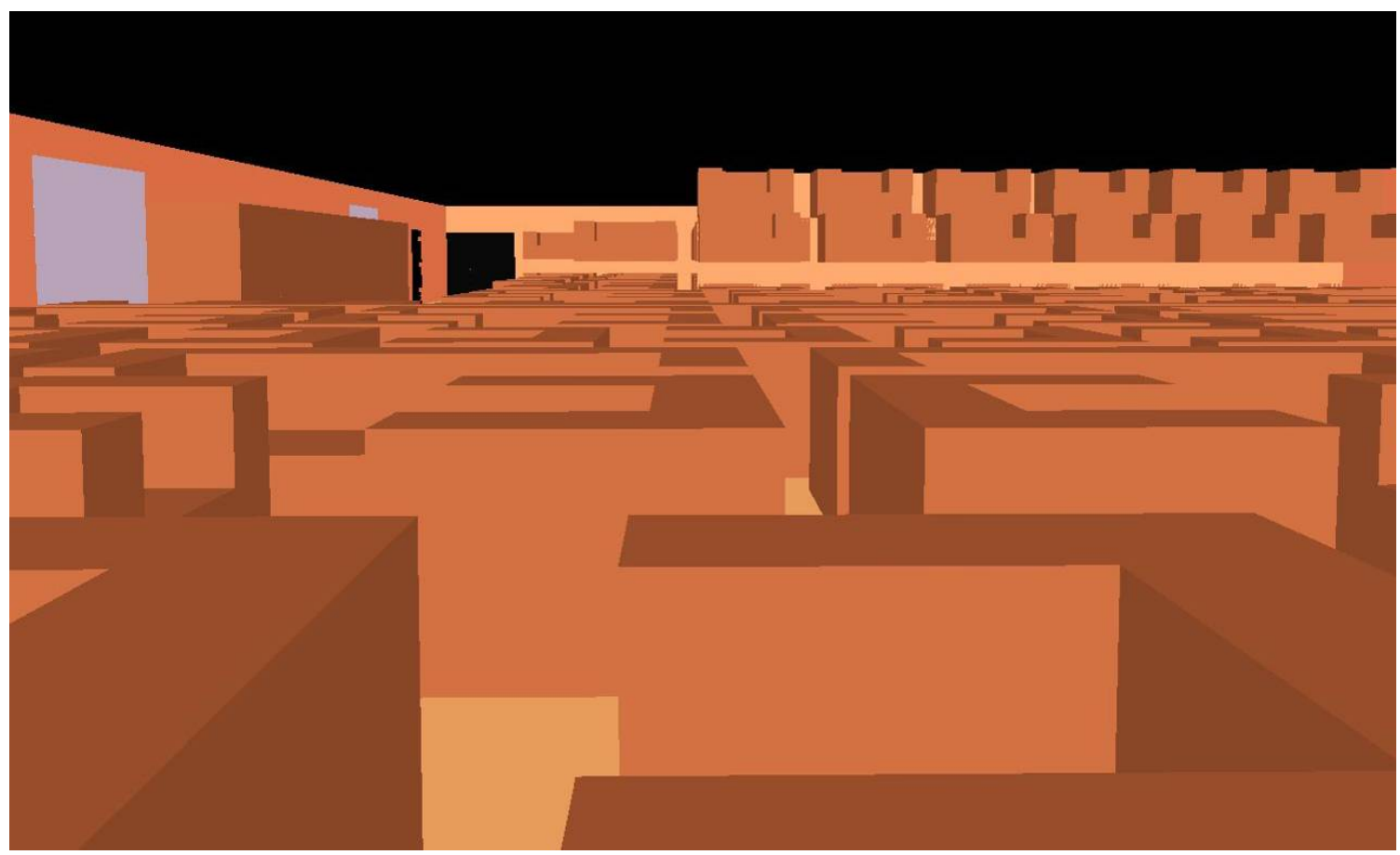

Figure K-6. Interior view of the west showroom. Viewpoint is the east side, looking towards the rear (south). The double door for access to the loading dock (black square) is at the top. 


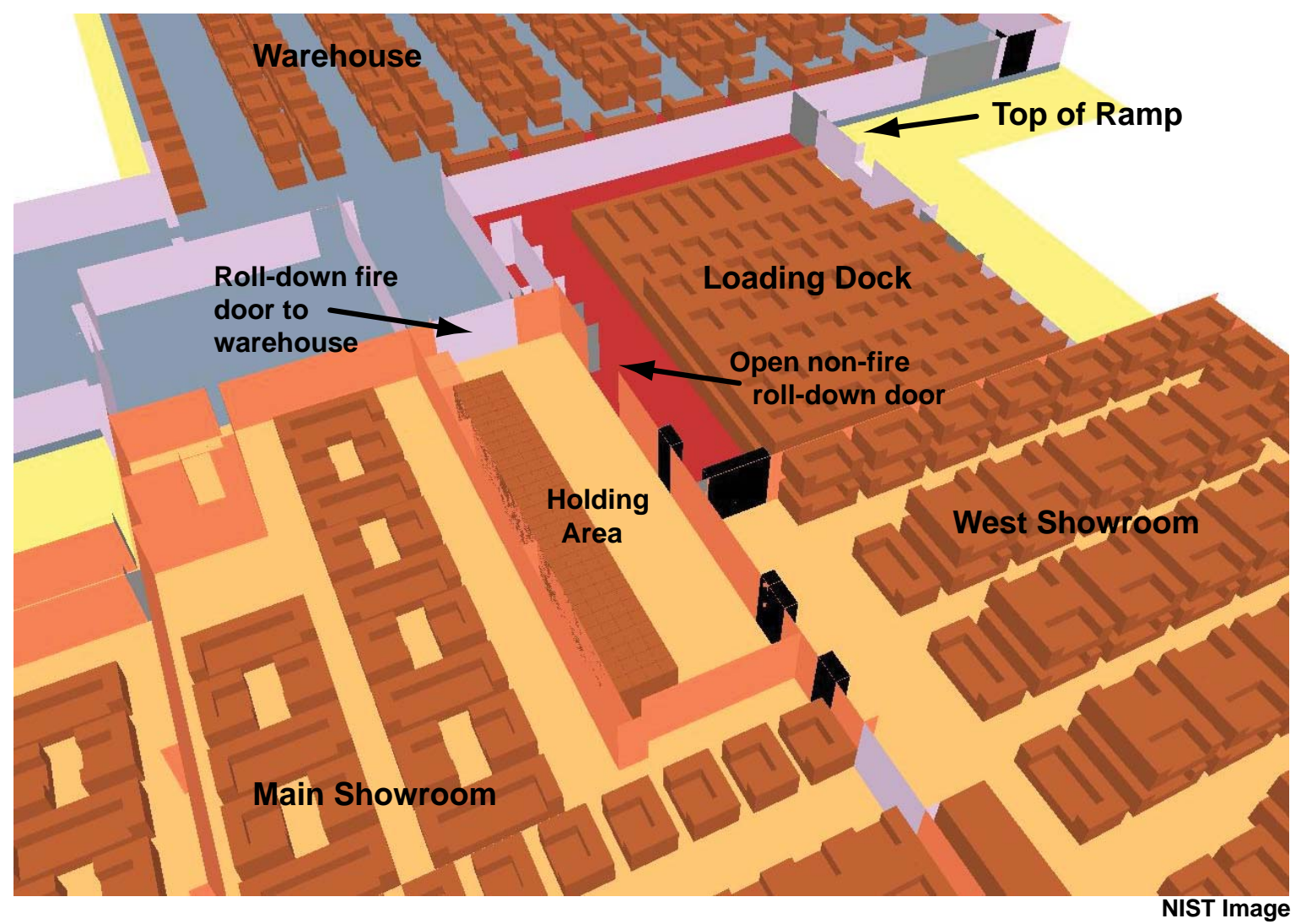

Figure K-7. Overhead view including sections of the main and west showrooms, the holding room, loading dock, breezeway, warehouse and work areas.

\section{K.3.2 Synchronizing the Model}

In order to develop an understanding of the fire progression and the conditions inside the structure, it was necessary to synchronize the simulated time line with the physical time line. The development of the incident time line was presented in Chapter 2. Since the earliest available photos and video were captured well into the event (approximately 13 minutes after the first dispatch), it was determined that the most appropriate way to synchronize the simulated time line with the physical time line was through a combination of radio transmission transcripts and witness statements.

The fire was first observed by a civilian driving along Savannah Highway in front of the store at 6:56 p.m. and was reported to store employees. A store manager attempted to put out the fire with a portable dry chemical fire extinguisher, but he was unsuccessful. He requested that other employees call 911. At 7:07 p.m., a report of a fire at the Sofa Super Store was received by the Charleston County 911 Emergency Center. After locating a second portable extinguisher, and heading back toward the loading dock, the store manager was unable to enter the loading dock because of the intense heat and smoke. From a position within the holding area, he discharged the second extinguisher into the loading dock. As he discharged the extinguisher, he heard one of the roll-down doors closing. The sound was presumably from the door (fire roll-down 1 in Figure K-7) closing at the end of the holding area. 
Battalion Chief 4 was the first city fire department unit to arrive on scene. At 7:10:46 p.m., BC-4 made a radio transmission to dispatch stating, "Bunch of trash and debris burning along side the building." That was followed 10 seconds later by, “...it may have got in the building. It’s right up against the wall.” Following these transmissions, BC-4 relocated to the front of the building to direct Engine 10 to back down the alley to the loading dock area. Engine 10 arrived on scene at 7:11:40 p.m. When BC-4 and E-10 captain returned to the loading dock area, flames were visible inside the loading dock through the opening in the wall that housed the exhaust fan. This sequence of events was used to synchronize the model time line to the physical time line, where time 0 in the simulation is equal to 7:10:53 p.m.

\section{K.3.3 Material Properties}

The assumption was made that all furnishings in the building were composites of foam and fabric, and that this material constituted the entire fuel load during the early stages of the fire. Other components of the fuel package, such as wood shelving or internal wood components, became involved later in the fire, but were not included in the modeling of the early stages of the fire. This material was labeled 'UPHOLSTERY' and was represented by a layer of foam covered by a thin layer of fabric. Table K-3 details the properties for each of these layers. Foam thickness was set equal to the mesh cell thickness. It was assumed that a single step reaction occurred for each material, and that all of the material was converted into gaseous fuel. The reaction was based on polyurethane foam, represented as $\mathrm{C}_{6.3} \mathrm{H}_{7.1} \mathrm{O}_{2.1} \mathrm{~N}$ [5, Appendix C, Table C.3]. The Reference Temperature is the temperature at which the mass fraction of specified material decreases at a specified reference rate $[3,5]$. The default reference rate of $0.1 \mathrm{~s}^{-1}$ was used. Other fuels such as flooring, wood display shelves, ceiling tile, paper documents, or wood framing, were not included as fuel for these simulations.

Interior finishes were categorized into the following list of materials: gypsum board, concrete block, concrete slab, sheet metal, steel, glass, carpet, ceiling tile, and wood. Table K-4 summarizes the properties assigned to materials in the simulation. Sheet metal and steel were assigned the same properties but were distinguished by thickness.

Table K-3. Material properties used to describe UPHOLSTERY.

\begin{tabular}{|c|c|c|c|c|c|c|c|}
\hline $\begin{array}{c}\text { Material } \\
\text { Name }\end{array}$ & $\begin{array}{c}\text { Thickness } \\
\text { (m) }\end{array}$ & $\begin{array}{c}\text { Thermal } \\
\text { Conductivity } \\
\left(\mathrm{W} \mathrm{m}^{-1}{ }^{\circ} \mathrm{C}^{-1}\right)\end{array}$ & $\begin{array}{c}\text { Specific } \\
\text { Heat } \\
\left(\mathbf{W ~ m}^{-1}\right. \\
\left.{ }^{\circ} \mathbf{C}^{-1}\right) \\
\end{array}$ & $\begin{array}{l}\text { Density } \\
\left(\mathrm{kg} / \mathrm{m}^{3}\right)\end{array}$ & $\begin{array}{c}\text { Reference } \\
\text { Temperature } \\
\left({ }^{\circ} \mathrm{C}\right)\end{array}$ & $\begin{array}{c}\text { Heat of } \\
\text { Reaction } \\
\text { (kJ/kg) }\end{array}$ & $\begin{array}{c}\text { Heat of } \\
\text { Combustion } \\
(\mathbf{k J} / \mathbf{k g})\end{array}$ \\
\hline Fabric & 0.002 & 0.100 & 1.0 & 100 & 350 & 1500 & 15000 \\
\hline Foam & 0.375 & 0.034 & 1.4 & 20 & 350 & 1350 & 25000 \\
\hline
\end{tabular}


Table K-4. Material properties assigned to obstructions that did not contribute to the overall fuel load.

\begin{tabular}{|c|c|c|c|c|}
\hline Material Name & $\begin{array}{c}\text { Thickness } \\
\text { (m) }\end{array}$ & 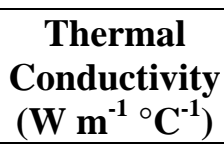 & $\begin{array}{c}\text { Specific } \\
\text { Heat } \\
\left(\mathrm{W} \mathrm{m}^{-1}{ }^{\circ} \mathrm{C}^{-1}\right)\end{array}$ & $\begin{array}{l}\text { Density } \\
\left(\mathrm{kg} / \mathrm{m}^{3}\right)\end{array}$ \\
\hline Gypsum Board [16] & 0.012 & 0.48 & 0.84 & 1440 \\
\hline Sheet Metal [16] & 0.0013 & 45.8 & 0.46 & 7850 \\
\hline Glass [16] & 0.005 & 0.76 & 0.84 & 2700 \\
\hline Carpet [5] & 0.006 & 0.16 & 9.0 & 750 \\
\hline Wood [16] & 0.04 & 0.14 & 2.85 & 640 \\
\hline Concrete [16] & 0.2 & 1.0 & 0.88 & 2100 \\
\hline Concrete Block $[5,17]$ & 0.2 & 0.7 & 0.84 & 1800 \\
\hline Steel [16] & 0.05 & 45.8 & 0.46 & 7850 \\
\hline Ceiling Tile [17] & 0.016 & 0.058 & 1.34 & 290 \\
\hline
\end{tabular}

The simulations did not include specific items as components of the fuel package because these items were considered to have negligible impact on the rapid fire spread through the showrooms during the early stages of the fire. These items included spray cans of solvents and paint at the rear of the main showroom and the foam insulation and membrane in the roof. Post-fire examination of an enclosed storage area outside the rear wall of the main showroom revealed dozens of spray cans that appeared to have contained paint or solvents. It was assumed that the fire spread across the main showroom to reach this storage area. Therefore, it was unlikely that the fuels in this storage area contributed significantly to the fire spread in the main showroom during the first 40 minutes of the fire.

The foam insulation and roof membrane were located above the metal roof decking. If flames from the fire had impinged on the underside of the metal decking and increased the temperature of the decking, the foam insulation could have softened, melted, and eventually pyrolyzed. The pyrolysis products would then have leaked into interstitial void space below the decking and above the suspended ceiling through cracks or holes in the metal decking. If the unburned fuel/pyrolysis products had combined with sufficient air, then it would have resulted in increased heat release rate.

The flames from the loading dock had direct access to the underside of the metal decking above the holding area because it lacked a suspended ceiling. However, a suspended ceiling had been installed above the rest of the main showroom. If the suspended ceiling tiles had remained intact, flames from fire on the showroom floor would not have been able to impinge on the underside of the metal decking and it would have been difficult for additional air to flow into the interstitial space.

Photographs taken during the fire demonstrate flames above the roof, but the flames appeared to be present only in close proximity to the holding area. During post-fire interviews, fire fighters did not report significant numbers of dislodged ceiling tiles or describe any flames above the ceiling except for around the holding area. This is consistent with the lack of flames on the roof, with the exception of the roof above the holding area. Since the direct impingement of flames appeared to be limited to the metal 
decking above the holding area, the energy content of the foam above the holding area was estimated to be less than $0.5 \%$ of the energy content of the fuels in the the enclosed loading dock. During the first 40 minutes of the fire, the quantity of smoke and unburned fuel from the loading dock that was channeled through the holding area into the void space was assumed to be much greater than the leakage of foam pyrolysis through the metal decking above the holding area.

\section{K.3.4 Boundary Conditions and Openings}

All sides of the computational domain were modeled as being open to the environment. This arrangement allowed air to be drawn into the domain and for combustion products to exit. An INERT ${ }^{4}$ surface was placed on the bottom of the domain to simulate the ground. This prevented the flow of air or other gases through the floor. The default initial conditions of $20^{\circ} \mathrm{C}\left(68^{\circ} \mathrm{F}\right)$ ambient temperature and 40 percent relative humidity with no wind were applied. Winds were out of the south at speeds in the $16 \mathrm{~km} / \mathrm{h}$ to $24 \mathrm{~km} / \mathrm{h}$ (10 mph to $15 \mathrm{mph}$ ) range on June 18, 2007 (Appendix A). It was assumed for modeling that the wind had negligible impact on fire conditions inside the showroom.

Doors and windows were opened during the simulation based on time estimates obtained from photos, video, radio transmissions and interview statements. Additionally, some venting was triggered by temperature events. Roofs on the loading dock and warehouse were equipped with skylights that failed at various points during the fire. Likewise, the roll-down fire doors on the warehouse breezeway and the east showroom were closed in the simulation when temperatures nearby reached the activation temperature of the fusible links, $73.9^{\circ} \mathrm{C}\left(165^{\circ} \mathrm{F}\right)$. Fusible links that were stamped with " $165^{\text {” " were }}$ observed by the NIST team in post-fire inspections. The performance or melting of the link at $73.9{ }^{\circ} \mathrm{C}$ $\left(165^{\circ} \mathrm{F}\right)$ was not tested by NIST.

The CFD used the front doors to the main showroom access the showroom to survey fire-spread, to pull hoses, and to conduct suppression. Throughout each simulation, the front doors were open. Time stamped photographs were used to determine the times at which the windows of the main showroom were vented by fire fighters at the scene. A series of photographs documented the removal of the front windows on the main showroom between 7:35:05 p.m. and 7:35:57 p.m. Photos were also used to estimate the times at which the windows on the west showroom failed. These windows failed between approximately 7:38 p.m. and 7:44 p.m. In the simulation, windows were removed at times corresponding to estimates based on photographs and not based on the interior conditions or material properties of the windows.

Radio transmission logs were used in conjunction with interview statements to estimate the time at which the door connecting the west showroom to the loading dock was opened. Upon arrival, the E-11 captain entered the main showroom through the front entrance and proceeded to the rear of the west showroom with the Assistant Chief (AC). When the AC opened the door, it was pulled out of his hand from the force of air rushing into the loading dock. They observed burning furniture and black smoke. The E-11 captain was able to close the door again with protected hands. The AC then ordered the E-11 captain to get an attack line and at 7:13:17 p.m., the E-11 captain requested an attack line. It is estimated that the door was opened 5 seconds before this transmission was made.

${ }^{4}$ INERT is the default SURF_ID in FDS. This allows for heat transfer but a surface with this property assigned will not react. K-15 
The same method was used to estimate the time that the exterior door to the loading dock was opened by the crew from E-10. The E-10 captain acknowledged arrival on scene at 7:11:40 p.m. The E-10 captain initially pulled the booster line and applied water to the burning debris outside the loading dock while fire fighters pulled the $3.8 \mathrm{~cm}$ (1.5 in) attack hose line. The E-10 engineer stretched a $6.4 \mathrm{~cm}$ (2.5 in) supply line up the driveway to Savannah Highway where he was met by the Fire Chief, who had arrived on scene at 7:16:32 p.m. The Fire Chief ordered the E-10 engineer back to his engine. When he arrived, the E-10 captain and fire fighter were exiting the loading dock to repair their hose. Based on the time of these events, it was estimated that the loading dock door was opened at approximately 7:14:30 p.m.

The timing associated with venting the loading dock roof, main showroom ceiling and roof and west showroom void space were all based on the conditions simulated by the model. NIST did not conduct full-scale tests to quantify the performance of roof, ceiling, or skylight assemblies. Photographs taken during the early stages of the fire show heavy smoke and flames exiting at the roof level. Figure K-8 shows a photograph taken at 7:28:15 p.m. This photo shows heavy smoke venting from the loading dock and rear of the main showroom. Flames are also visible and appear to be coming from the roof of the main showroom. Because it was not possible to determine the exact location or size of the holes in the ceiling and roof, several holes were used in the simulations to represent the ventilation that took place. Table K-5 summarizes the ventilation conditions used in the simulations.

\section{K.3.5 Fire Sources Used in the Simulation}

Although the cause of the fire was undetermined, the area of origin was identified [15]. The fire initiated on a pile of discarded furniture outside the loading dock. The discarded furniture was generally located in the corner formed by the loading dock exterior (west) wall and the ramp that was used to access the loading dock door. Figure K-9 shows a photograph of this area. The fire grew and spread into the loading dock through one or both of the following means: the void space beneath the wood floor, or through gaps in the construction. One witness, the employee who entered the loading dock from the interior of the store during the early stages of the fire said he "...observed flames entering from the exterior around the frame of the door at the top of the ramp.”

In the simulation, the fire was initiated ${ }^{5}$ inside the loading dock at floor level, between the door and the ventilation fan access hole. This is identified as "Loading Dock Source” in Figure K-10. This fire was $0.375 \mathrm{~m}$ x $1.875 \mathrm{~m}$ ( 1 x 5 mesh cells) and produced a peak HRR of $700 \mathrm{~kW}$. The fire source was activated at the start of the simulation, operated for $600 \mathrm{~s}$, and then shut off. No attempt was made to simulate the fire on the exterior side of the wall. The warehouse fire was initiated by a single mesh cell ( $0.375 \mathrm{~m} \times 0.375 \mathrm{~m})$ source that was positioned on the bottom row of furniture along the (north) wall separating the warehouse and the loading dock. The HRR was ramped up to a peak of $160 \mathrm{~kW}$ using a slow growth $\mathrm{t}^{2}$ curve [18], beginning at $410 \mathrm{~s}$ (7:17:43p.m.).

The route by which fire made its way through the holding area and into the main showroom is not precisely known. The most likely means for this to occur would have been for fire to breach the wall or door of the holding area into the rear of the main showroom, or for ignition to occur as a result of burning

\footnotetext{
${ }^{5}$ In FDS, the initiating fire is treated as an inflow of heat and combustion products through a vent. A vent was used within the simulation to introduce heat into the modeling domain and create the effect of a fire source in the model, by ejecting fuel according to a prescribed heat release rate.
} 
Table K-5. Opening locations and times used in the simulation.

\begin{tabular}{|c|c|c|c|c|}
\hline \multirow{2}{*}{ Location of Opening } & \multicolumn{2}{|c|}{ Condition } & \multirow{2}{*}{$\begin{array}{c}\text { Time of } \\
\text { Opening (s) }\end{array}$} & \multirow{2}{*}{ Clock Time } \\
\hline & Time & Temp & & \\
\hline Double doors connecting west showroom to loading dock & $\mathrm{X}$ & & 144 & $7: 13: 17$ \\
\hline Exterior loading dock door & $\mathrm{X}$ & & 219 & $7: 14: 32$ \\
\hline Holding area doors & $\mathrm{X}$ & & $\begin{array}{l}360 \\
600\end{array}$ & $\begin{array}{l}7: 16: 53 \\
7: 20: 53\end{array}$ \\
\hline Main showroom windows / doors & $\mathrm{X}$ & & $1457-1530$ & $\begin{array}{c}7: 35: 10- \\
7: 36: 23\end{array}$ \\
\hline West showroom windows & $\mathrm{X}$ & & $1637-1900$ & $\begin{array}{c}7: 38: 10- \\
7: 42: 31\end{array}$ \\
\hline Loading dock skylights & & $150^{\circ} \mathrm{C}$ & $214-909$ & $\begin{array}{c}7: 14: 27- \\
7: 26: 02 \\
\end{array}$ \\
\hline Warehouse skylights & & $150^{\circ} \mathrm{C}$ & $818-1409$ & $\begin{array}{c}7: 24: 31- \\
7: 34: 22 \\
\end{array}$ \\
\hline Main showroom roof & $\mathrm{X}$ & & $540 / 720 / 900$ & $\begin{array}{l}7: 19: 53 / \\
7: 22: 53 / \\
7: 25: 53\end{array}$ \\
\hline Loading dock roof & $\mathrm{X}$ & & 1000 & $7: 27: 33$ \\
\hline East roll up doors & & $73.9^{\circ} \mathrm{C}$ & $1057-1242$ & $\begin{array}{c}7: 28: 30- \\
7: 31: 35\end{array}$ \\
\hline Main showroom ceiling small & & $50^{\circ} \mathrm{C}$ & $289-897$ & $\begin{array}{c}7: 15: 42- \\
7: 25: 50\end{array}$ \\
\hline Main showroom ceiling & $\mathrm{X}$ & & 1000 & $7: 27: 33$ \\
\hline West showroom void space & $\mathrm{X}$ & & 270 & $7: 15: 23$ \\
\hline Warehouse roll up door & $\mathrm{X}$ & & 1200 & $7: 30: 53$ \\
\hline Warehouse man door & $\mathrm{X}$ & & 1500 & $7: 35: 53$ \\
\hline Loading dock large door & $\mathrm{X}$ & & 1717 & 7:39:30 \\
\hline
\end{tabular}


material dropping from above the ceiling. Because the route of spread was not known, fire spread into the main showroom was assumed to be initiated in the area near the holding area wall. This area is identified in Figure K-10 as "Main Showroom Source 1" and "Main Showroom Source 2." These initiating fires were ramped separately using parameters for ultrafast $\mathrm{t}^{2}$ fire curves [18]. Source 1 ( $0.75 \mathrm{~m} \times 3.4 \mathrm{~m}, 2 \mathrm{x} 9$ mesh cells) was initiated at 525 s (7:19:38 p.m.) and peaked at 4 MW. Source 2 (1.5 m x 2.6 m, 4 x 7 mesh cells) was initiated at $660 \mathrm{~s}$ (7:21:53 p.m.) and peaked at $6 \mathrm{MW}$. A range of fire development parameters and conditions was input into different model runs and the simulation results were compared to observations. The parameters and conditions described above provided simulation outputs that were most consistent with the observations.

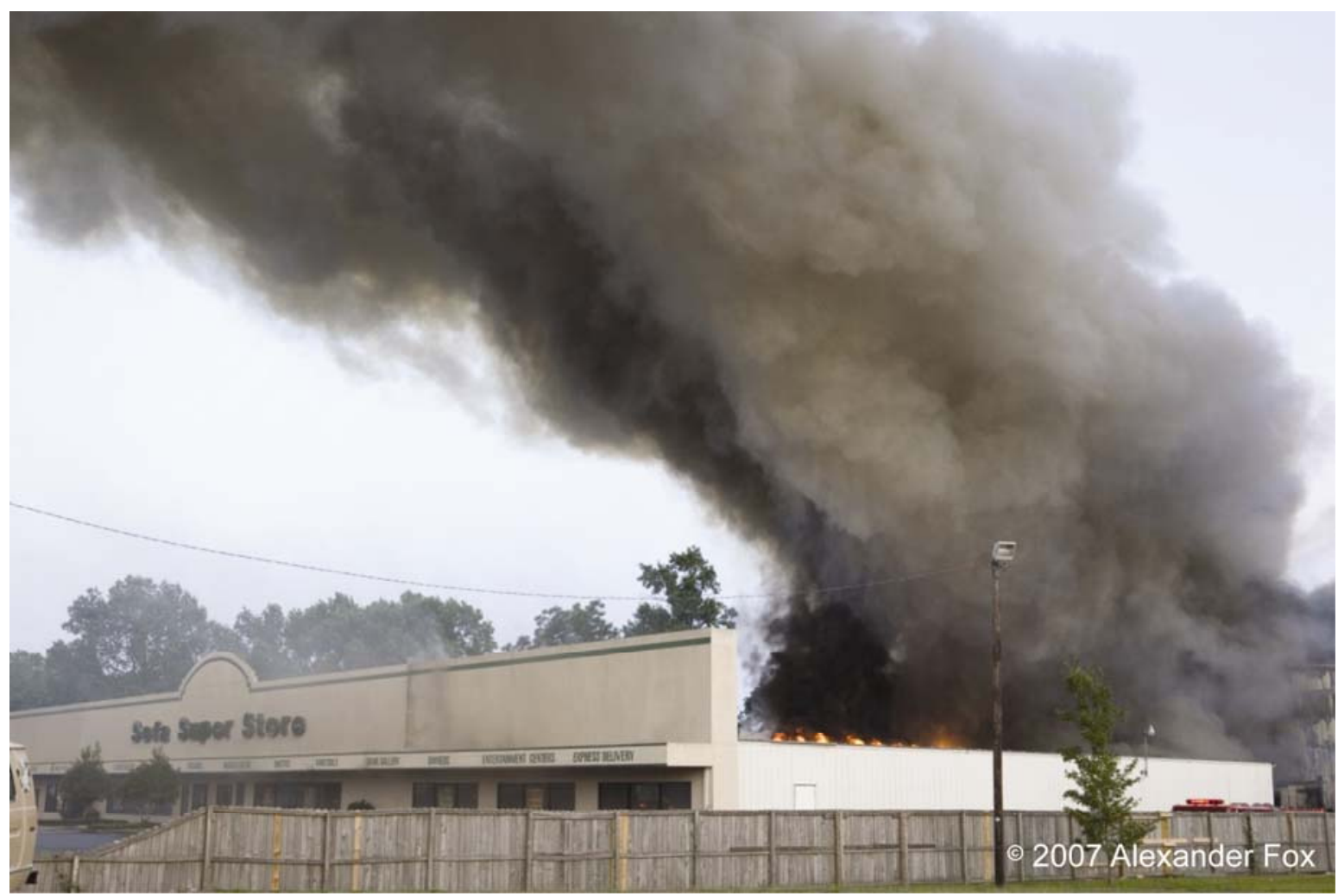

Figure K-8. Photograph showing the north and west sides of the showroom. Photo was taken at 7:28:15 p.m. 


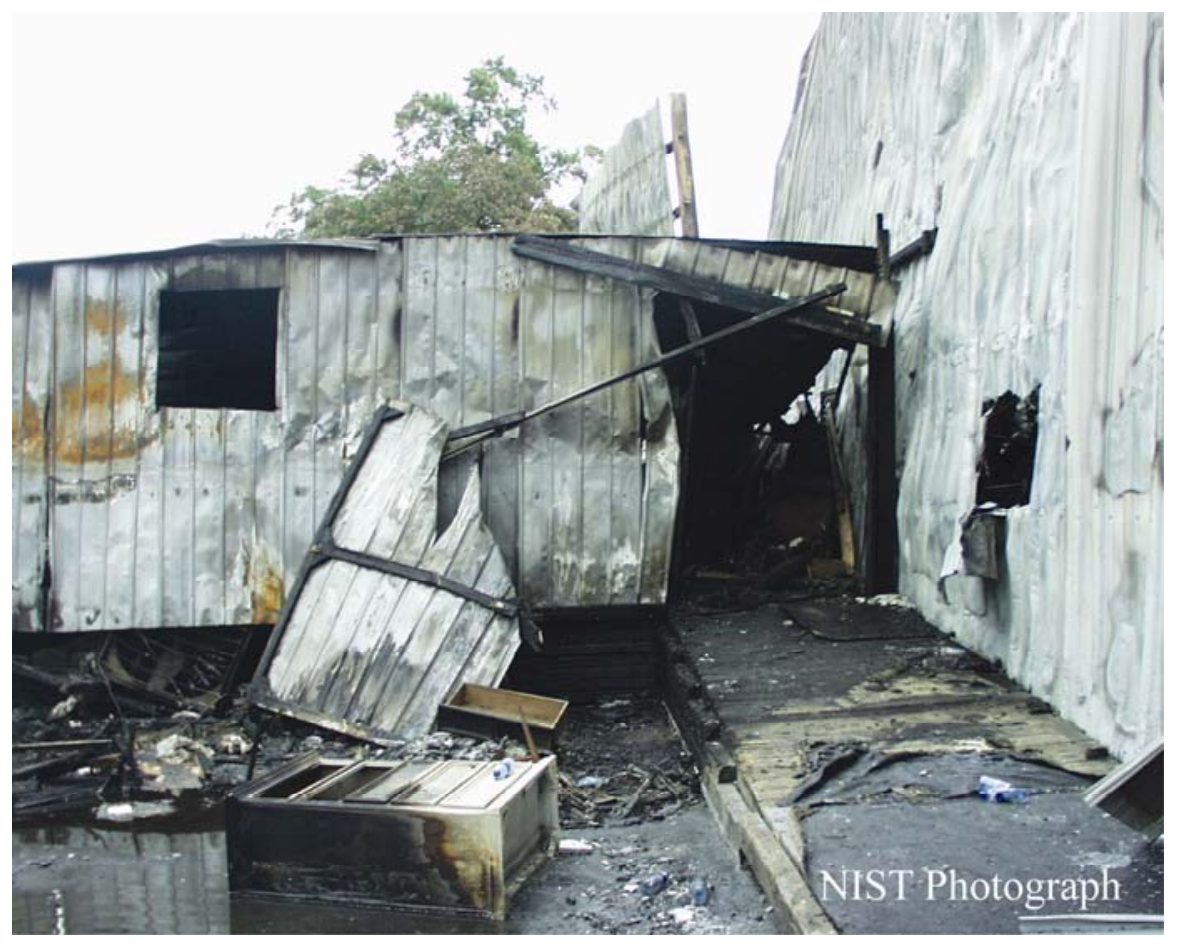

Figure K-9. Photograph showing the outside of the loading dock. This was identified as the area of fire origin.

\section{K.3.6 Fire Scenarios and Dependent Variables}

The value of a computer simulation is its ability to generate a clearer picture of the conditions that existed within the Sofa Super Store as the fire progressed. The simulation also allows researchers to examine the impact of changes in the initial environmental conditions and to evaluate possible interventions on the fire dynamics and the environment within the store. The store environment and fire dynamics are characterized in this study by the following dependent variables:

- heat release rate of the fire as a function of time

- visibility as a function of location and time

- temperature as a function of location and time

- oxygen volume fraction as a function of location and time

- velocity of the air and fire plume. 
The baseline scenario represents an approximation of what actually occurred in the store on June 18, 2007, based upon the evidence and time line described in the previous chapters. Four additional simulations were conducted that examined different intervention strategies: having installed fire sprinklers in the loading dock, maintaining the front window intact, venting the main showroom from the roof with a small opening (front window intact), and venting the main showroom from the roof with a large opening (front window intact).

The five scenarios are summarized in Table K-6, which includes a computer file name to which they may be referred.

Table K-6. Five computer simulation scenarios.

\begin{tabular}{|c|c|c|}
\hline $\begin{array}{c}\text { Scenario } \\
\text { Number }\end{array}$ & Description & Computer Filename \\
\hline 1 & Baseline & SSS_0254.fds \\
\hline 2 & Fire Sprinklers added inside loading dock & SSS_0255.fds \\
\hline 3 & Front windows intact & SSS_0256.fds \\
\hline 4 & $\begin{array}{c}\text { Front windows intact, } 1.7 \mathrm{~m}^{2}\left(18.2 \mathrm{ft}^{2}\right) \\
\text { ventilation in main showroom roof }\end{array}$ & SSS_0257.fds \\
\hline 5 & $\begin{array}{c}\text { Front windows intact, } 5.9 \mathrm{~m}^{2}\left(63.6 \mathrm{ft}^{2}\right) \\
\text { ventilation in main showroom roof }\end{array}$ & SSS_0258.fds \\
\hline
\end{tabular}

\section{K.4 BASELINE SIMULATION}

\section{K.4.1 Overall Accuracy}

The baseline simulation represents an estimate of what actually occurred in the Sofa Super Store based upon the evidence and time line described in the previous chapters. The resulting HRRs in the different portions of the store, visibility as estimated from the predicted smoke flows, and the temperatures and oxygen volume fractions at different elevations are presented in the following sections.

The following sections describe the simulation of properties that were not measured or observed on site. These simulated properties characterize the fire development (HRR, fire-spread rate, smoke movement, and temperature field) and are presented in the context of loss of tenability within the building due to lack of oxygen and heat.

\section{K.4.2 Heat Release Rate}

The total HRR for the fire, excluding the warehouse, is plotted as a function of time in Figure K-11. The HRR produced by the warehouse contents dominated the total HRR in the mid and late stages of the simulation, but since the breezeway door closed early in the fire time line, there was no flow or transfer of material between these areas of the structure. In addition, the fire department did not enter the warehouse to suppress the fire there. This analysis will focus on conditions in the loading dock and showrooms. 
The HRR grew quickly from the initiating $700 \mathrm{~kW}$ fire, reaching $90 \mathrm{MW}$ in $280 \mathrm{~s}$ as fire spread through the loading dock and into the holding area. Figure K-12 separates the HRR events in the loading dock, holding area and rear showroom, main showroom, and west showroom. During the first $500 \mathrm{~s}$, the loading dock fire dominated the HRR at about $85 \mathrm{MW}$. As the fire spread into the holding area and rear of the main showroom the HRR for the holding area and the rear of the main showroom began to increase to about $100 \mathrm{MW}$ at around $1200 \mathrm{~s}$. This increase in fire-spread to the holding area and main showroom was simulated by turning on Source 1 and 2 (Figure K-10) in the computer model. Main showroom Source 1 was activated at $525 \mathrm{~s}$ and Source 2 activated at $660 \mathrm{~s}$. After main showroom source 2 peaked at $900 \mathrm{~s}$, the HRR in the main showroom began to climb rapidly. Consistent with flame extension that was visible in fire photographs, at $1000 \mathrm{~s}$, a section of the loading dock roof was removed and the HRR increased in the loading dock and holding area. At 1200 s, fire spread through the holding area, driving the HRR above $90 \mathrm{MW}$ in the main showroom. After the fire consumed the readily available oxygen in the rear of the main showroom, the fire became underventilated and decreased in HRR. The total HRR continued to decrease until about $1450 \mathrm{~s}$. The windows at the front of the main showroom were broken out beginning at $1457 \mathrm{~s}$ (7:35:10 p.m.). The HRR increased after $1450 \mathrm{~s}$ and the increase in HRR would be consistent with the broken windows providing a fresh influx of oxygen to the fire in the rear of the main showroom. The HRR of the fire grew to over $100 \mathrm{MW}$ in the main showroom and $50 \mathrm{MW}$ in the west showroom. The energy release rate of the holding area/rear main showroom increased slightly and then leveled out at about $40 \mathrm{MW}$. The loading dock HRR exhibited a transient peak at about $50 \mathrm{MW}$ and then decreased to about $15 \mathrm{MW}$.

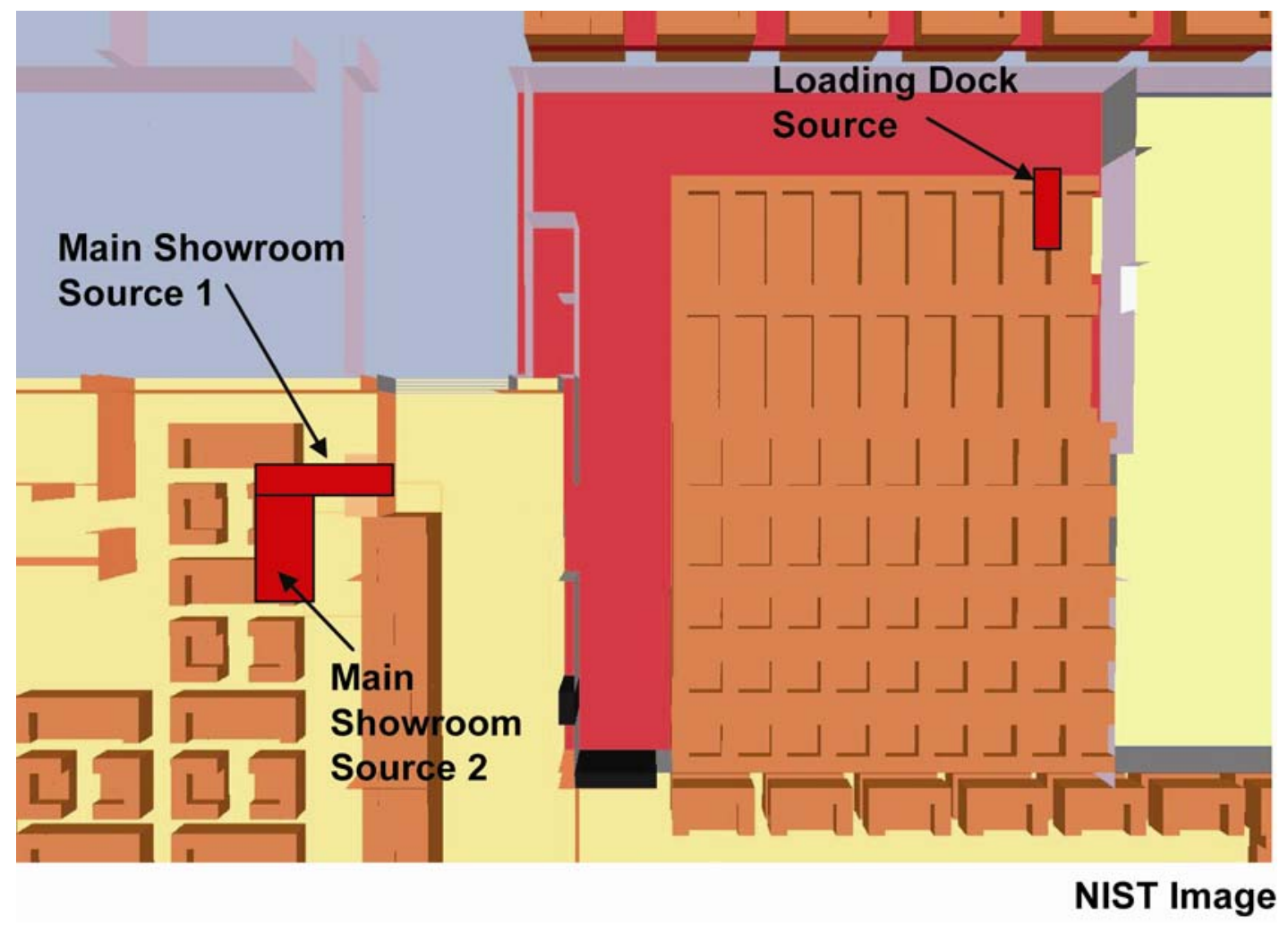

Figure K-10. Diagram showing locations of initiating fire sources in the loading dock and rear of main showroom. 


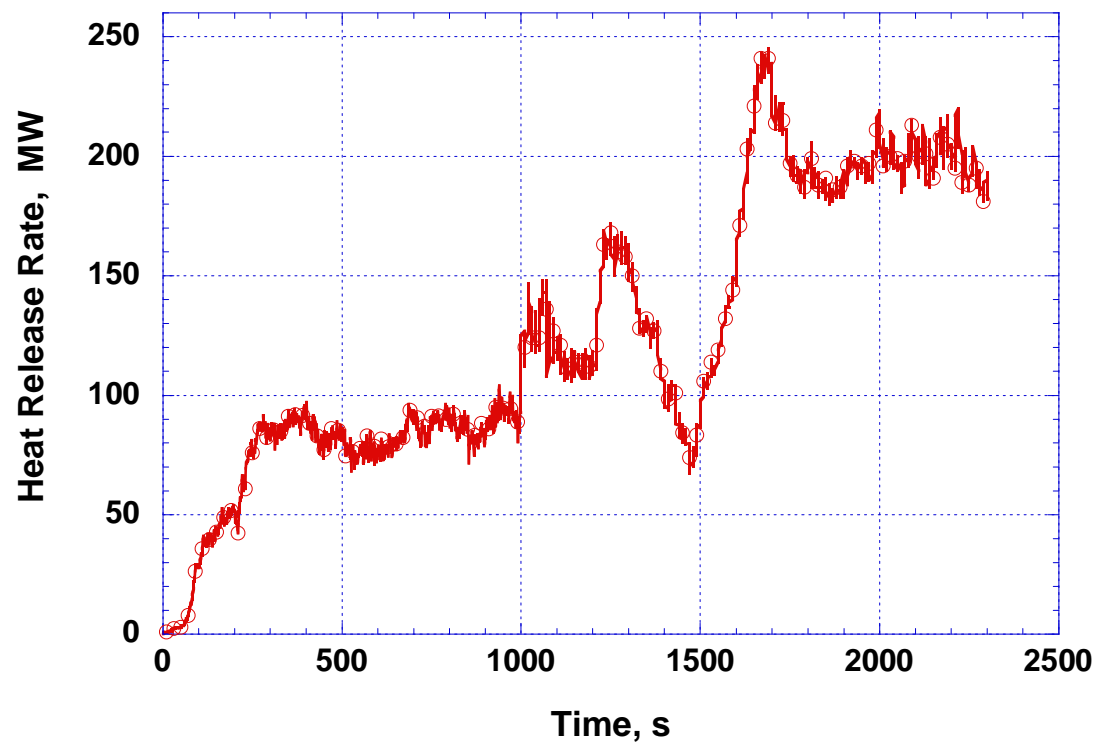

Figure K-11. Total heat release rate, excluding the warehouse.

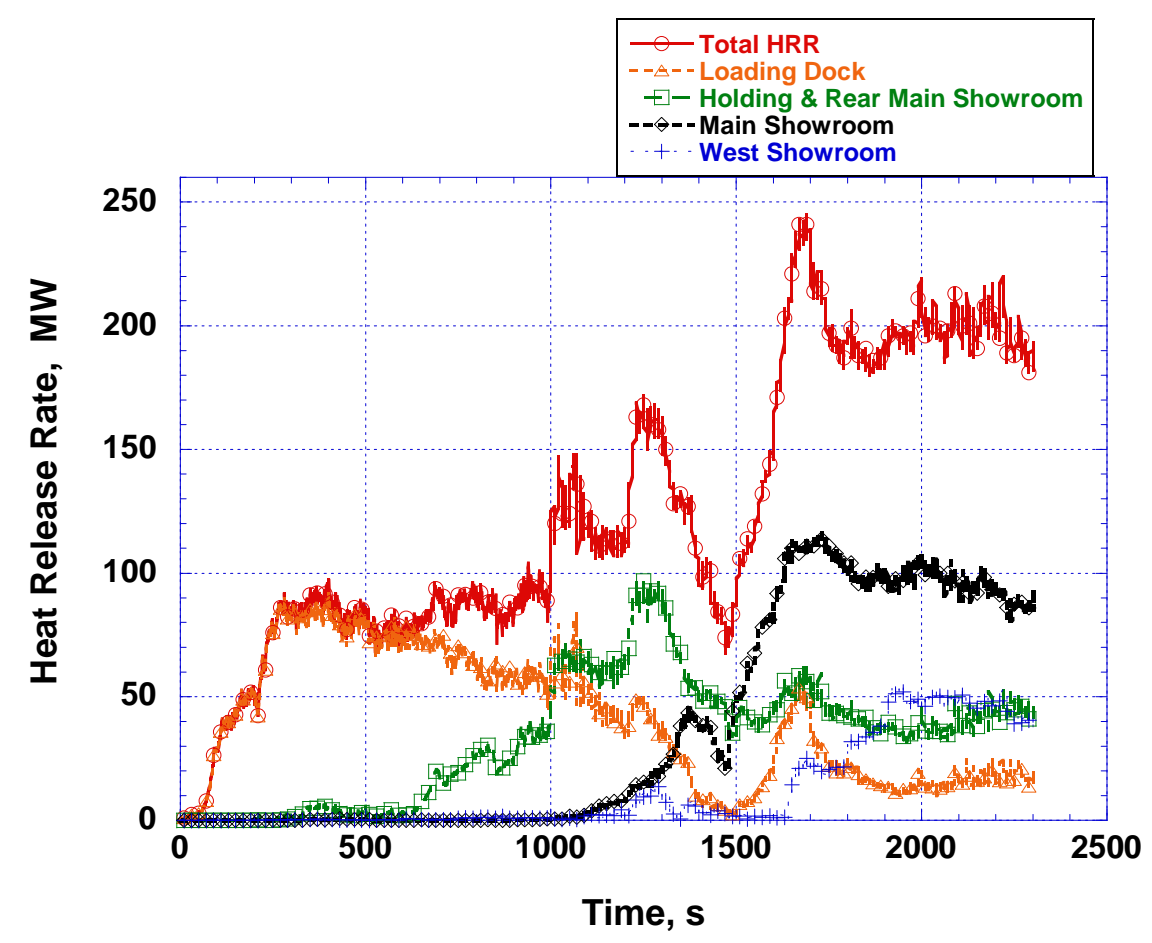

Figure K-12. Component heat release rates for the loading dock, holding area, main showroom, and west showroom. 


\section{K.4.3 Fire Growth and Smoke Spread}

The earliest available photos of the fire were taken at 7:22 p.m., approximately 13 minutes after the first dispatch. Therefore, knowledge of the early fire development is based on eyewitness accounts obtained during interviews of fire fighters. Some information was also gleaned from transcripts of the CFD radio transmissions. The fire initiated outside the loading dock in a pile of discarded furniture. This fire developed for an unknown period of time, and eventually spread into the loading dock area through the floor and gaps in the construction. The model fire was initiated inside the loading dock. The model time line was synchronized to the physical time line at 7:12:43 p.m., the time BC-4 made the statement to the AC, "Chief, I know it's inside that part of the building right there." This transmission was made after the BC-4 had guided E-10 down the alley to the loading dock area. The model time corresponding to this event was $110 \mathrm{~s}$. By this time, fire in the loading dock had progressed to the point that flames were venting from the exhaust fan in the west wall as well as through a gap between the roof sections of the loading dock. Figure K-13 is an image rendered $110 \mathrm{~s}$ into the simulation viewing the west side of the structure that shows fire and smoke venting from the loading dock wall and roof. It should be noted that the orange color in Smokeview tracked the location of stoichiometric fuel and air mixture. If the temperature were high, then the orange surface could be thought of as a flame; if the temperature were below a threshold value, then no flame was actually present, just a non-burning mixture of fuel and air.

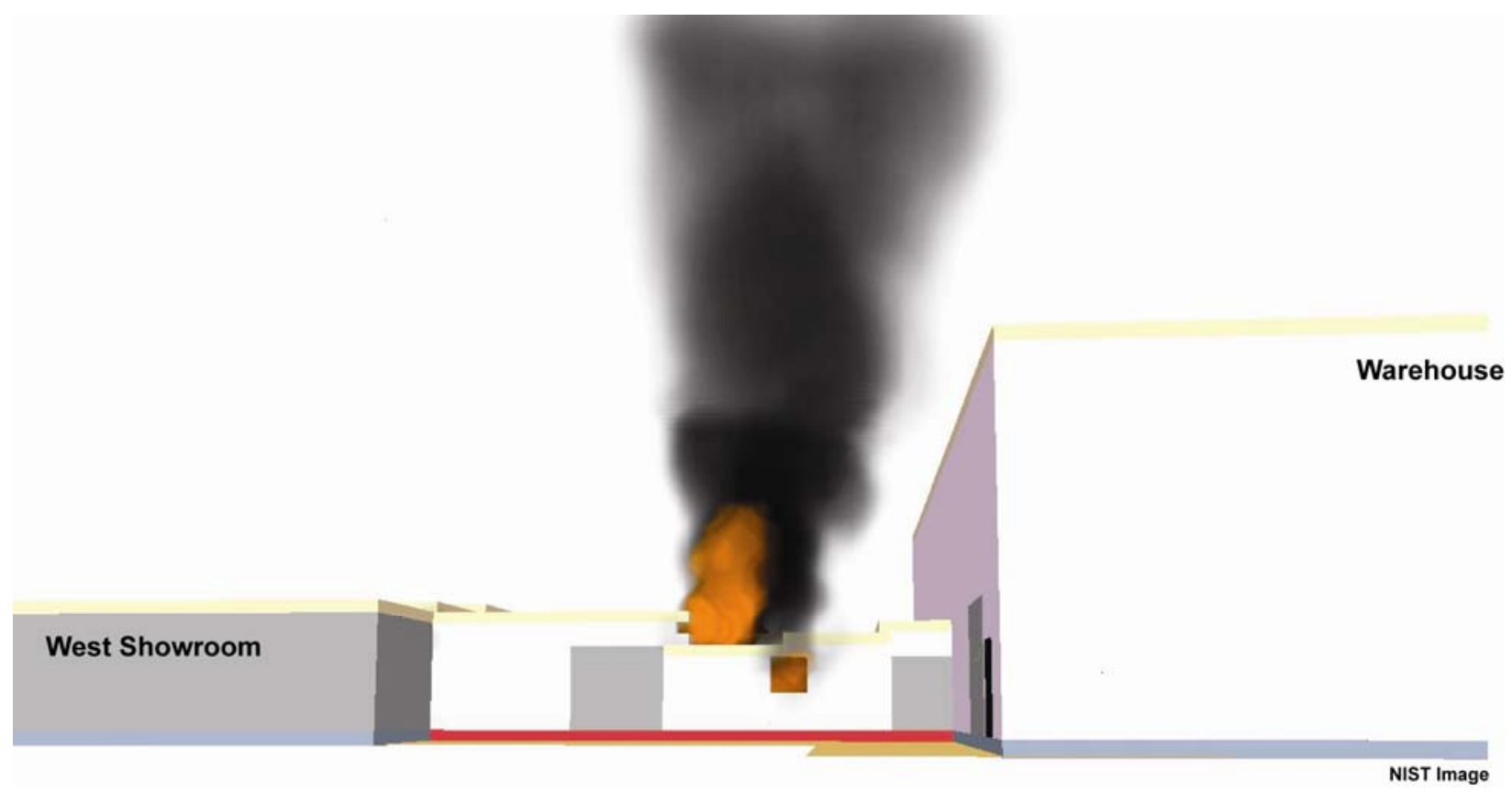

Figure K-13. Rendered image showing the west side of the loading dock 110 s into the simulation.

By this time (110 s), smoke had filled the loading dock and begun entering the void space above the main showroom ceiling through the holding area. The holding area was adjacent to the loading dock and was accessible through an open roll-down door. This room did not have a suspended ceiling and had shelves on which futon cushions were stored. Smoke and heated combustion gases produced by the fire passed 
into the void space above the main showroom. This development was concealed to fire fighters upon first entry into the building.

Figure K-14 shows a rendered image of the loading dock and showrooms at $110 \mathrm{~s}$. In this figure, obstructions below ceiling level have been removed and all remaining obstructions are transparent (indicated only by their outlines) to allow visible access to the fire and smoke. This figure shows that very early in the fire event smoke had entered the holding area through the open roll-down door and entered the void space above the main showroom ceiling.

The AC and E-10 captain entered the main showroom and initially observed clear conditions. Figure K-15 shows an image of the main showroom, from the perspective of a person standing at the front entrance and facing south west, towards the holding area. This image was rendered $80 \mathrm{~s}$ into the simulation (7:12:13 p.m.), approximately the time the AC entered the showroom and observed clear conditions.

At 7:13:17 p.m. (144 s) the E-11 captain called over his radio "I need an inch and a half inside this building." The AC opened the double doors connecting the west showroom to the loading dock and had the door pulled out of his hand by the force of air rushing into the loading dock. Figure K-16 shows a

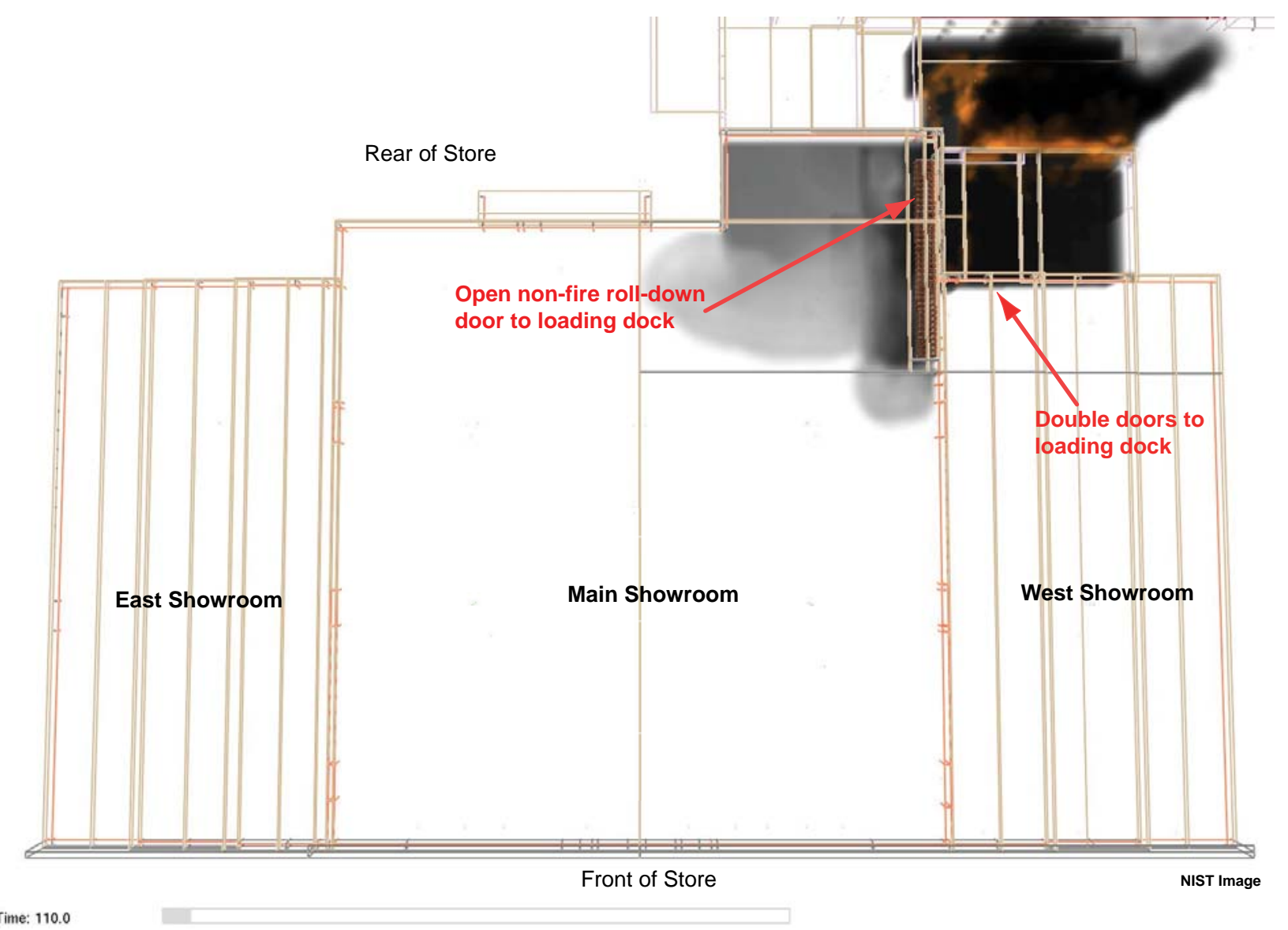

Figure K-14. Smoke filling in void space above main showroom. Image was rendered $110 \mathrm{~s}$ into the simulation. 


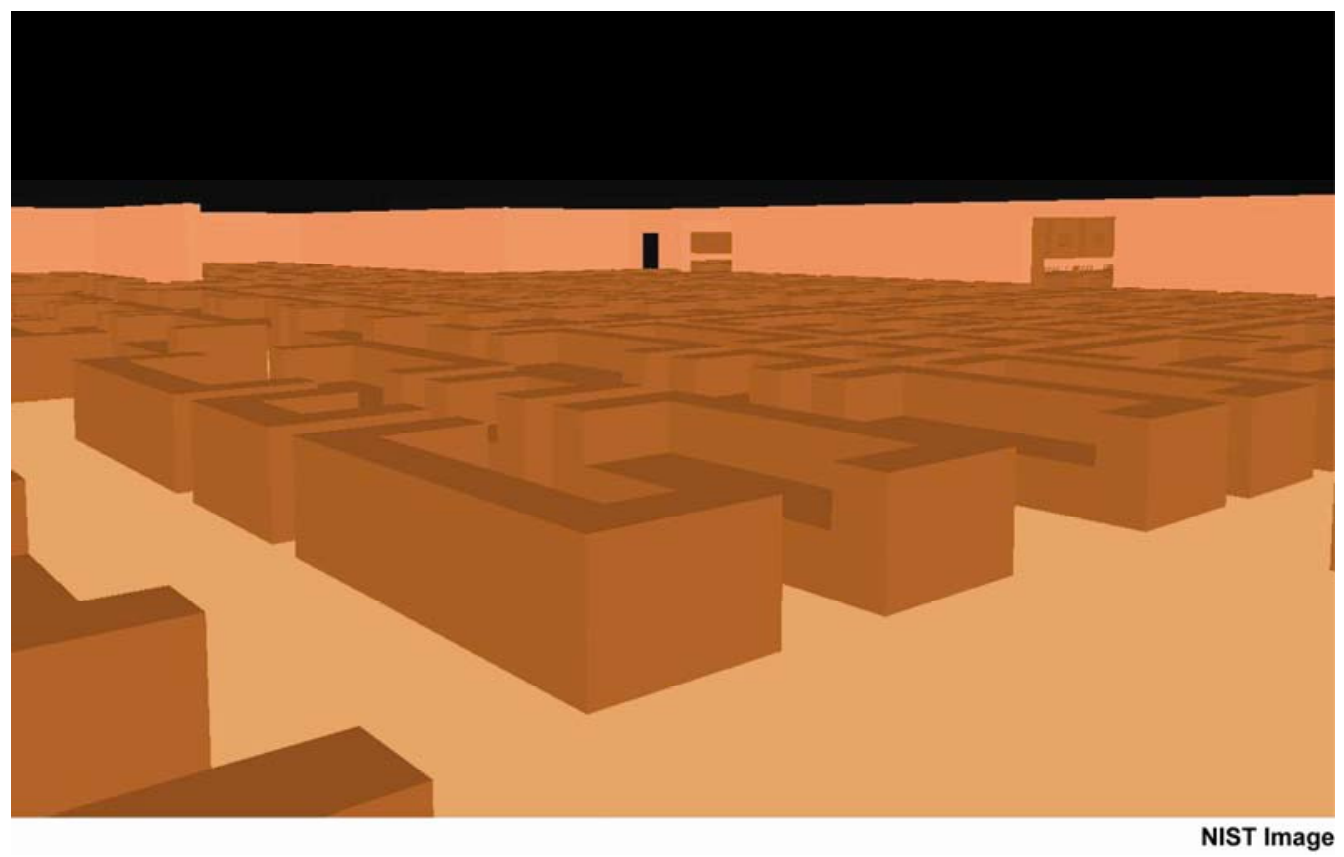

Figure K-15. Image rendered $80 \mathrm{~s}$ (7:12:13 p.m.) into the simulation from inside the main showroom, near the entrance. View is towards the south west corner of the main showroom.

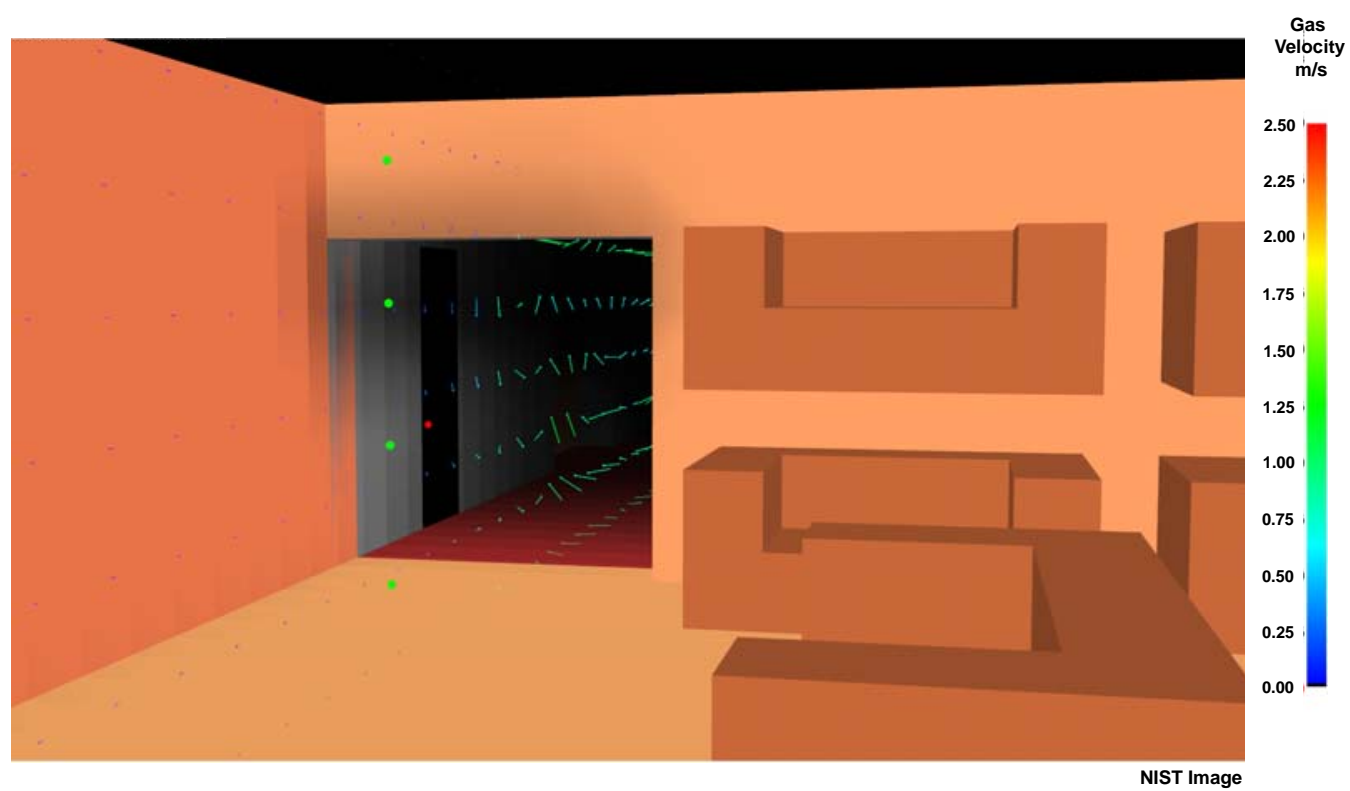

Figure K-16. Image rendered $145 \mathrm{~s}$ (7:13:18 p.m.) into the simulation from the west showroom, near the double doors that lead to the loading dock. Arrows indicate direction and relative speed of air moving from the showroom into the loading dock (The relative speed is proportional to the length of the arrow, i.e., longer arrows indicate higher relative speeds and color scale indicates velocity magnitude). 
rendered image of the double doors from inside the west showroom at $145 \mathrm{~s}, 1$ second after they were opened. The arrows are velocity vectors indicating direction and relative speed of the air passing through the doors.

Smoke penetration through the ceiling was modeled by creating openings in the rear of the main showroom when the adjacent temperature reached $50^{\circ} \mathrm{C}$. The openings measured $0.75 \mathrm{~m} \times 1.125 \mathrm{~m}(2 \times 3$ mesh cells) and were positioned as indicated in Figure K-17. Figure K-18 shows the progression of fire and smoke below the ceiling level $(2.6 \mathrm{~m})$ throughout the loading dock, holding area and showrooms between 300 s (7:15:53 p.m.) and 900 s (7:25:53 p.m.). The images in this figure have been clipped down to the level of the ceiling in the showrooms.

At $300 \mathrm{~s}$ (7:13:53 p.m.) there is light smoke in the rear of the main and west showrooms. Initially, smoke in the west showroom entered through the double doors while the smoke in the main showroom entered through the ceiling. The two ceiling holes positioned on the west side of the main showroom (identified as the dark black areas in the 300 second and 600 second images of Figure K-17) opened at $261 \mathrm{~s}$ (7:15:14 p.m.) and $291 \mathrm{~s} \mathrm{(7:15:44} \mathrm{p.m.),} \mathrm{respectively.} \mathrm{The} \mathrm{hole} \mathrm{on} \mathrm{the} \mathrm{centerline} \mathrm{of} \mathrm{the} \mathrm{main} \mathrm{showroom}$ (identified as the dark black area in the 420 second and 480 second images of Figure K-18) opened at 370 s (7:17:03p.m.), while the one placed on the east side opened at $976 \mathrm{~s}$ (7:27:09 p.m.). By $600 \mathrm{~s}$ (7:20:53 p.m.), the smoke layer had permeated the west and main showrooms, and there was evidence of a "tunneling" as fresh air was being pulled into the front door to supply oxygen. This is consistent with the account of Engineer 6, who entered the structure at approximately 7:24:00 p.m. (787 s) and reported a tunnel of clear space that extended several feet inside the showroom as fresh air was being pulled through the open door. Beyond this space he reported heavy smoke conditions. By 900 s (7:25:53 p.m.), the fire in the rear of the main showroom had grown to approximately $23 \mathrm{MW}$.

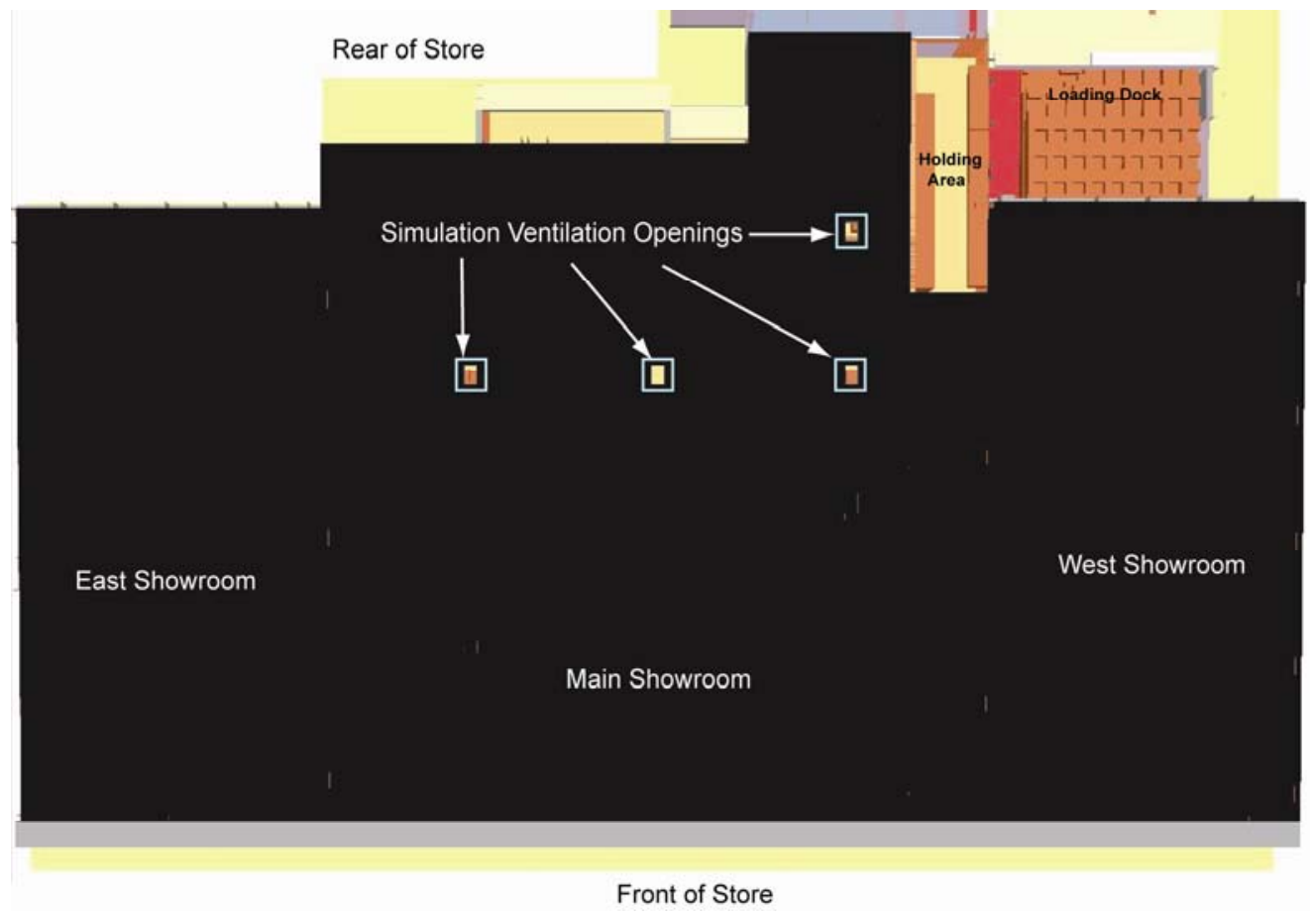

Figure K-17. Overhead image rendered with roof removed, showing locations of ceiling openings in main showroom to account for leakage. 


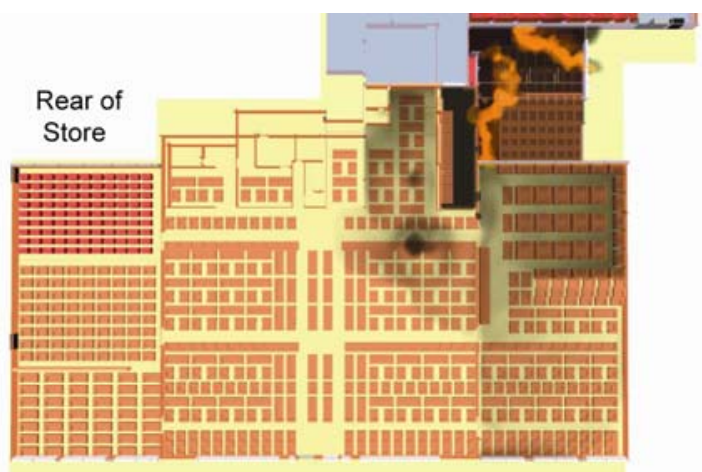

Front of Store

$$
300 \mathrm{~s}
$$

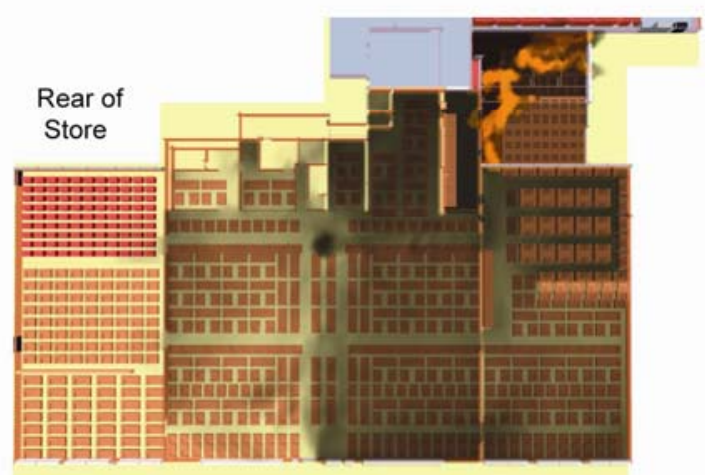

Front of Store

\section{$420 \mathrm{~s}$}

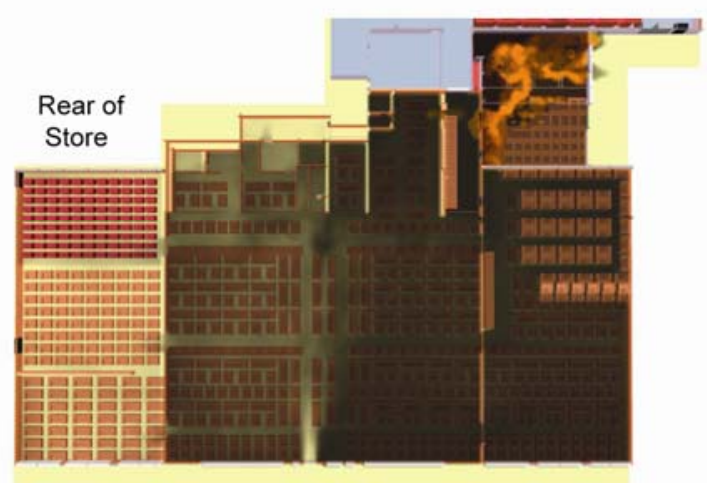

Front of Store

$$
600 \mathrm{~s}
$$

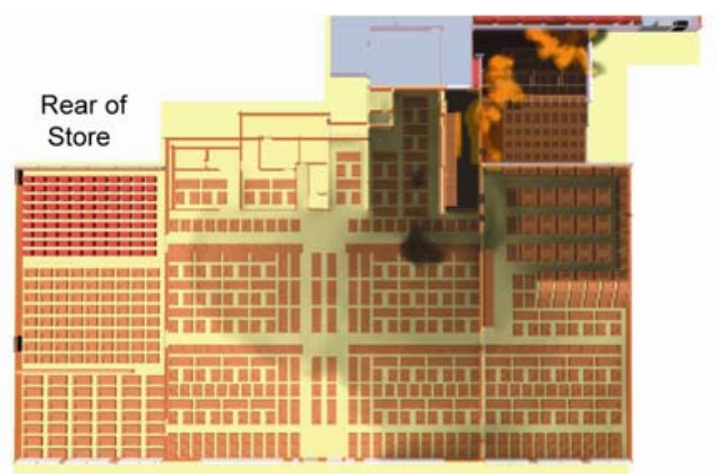

Front of Store

$360 \mathrm{~s}$

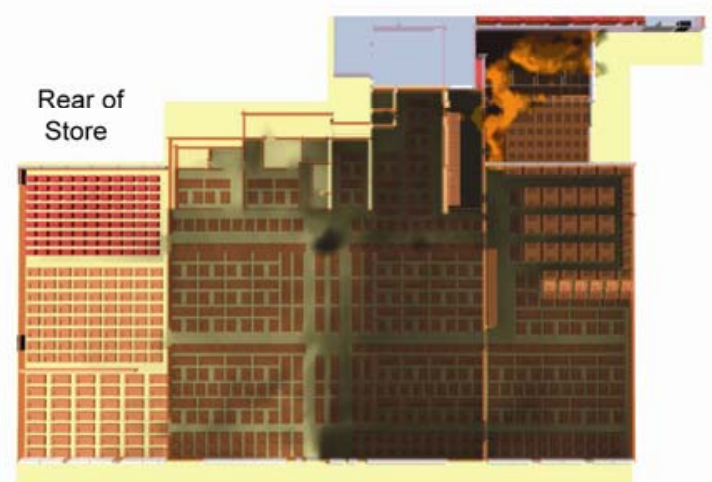

Front of Store

$480 \mathrm{~s}$

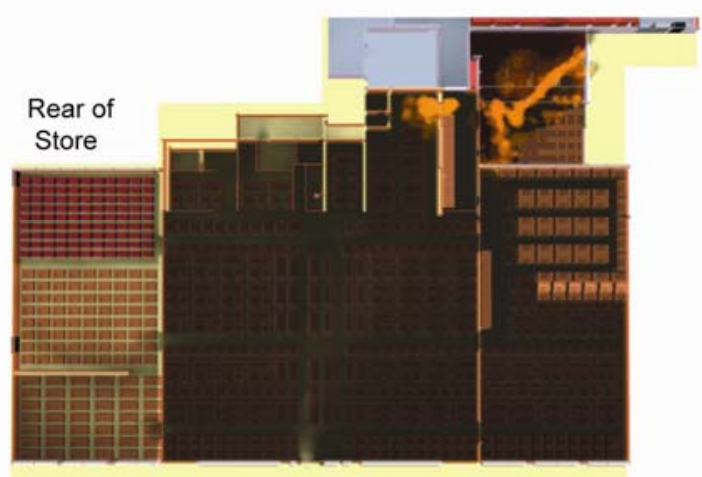

Front of Store

$900 \mathrm{~s}$

Figure K-18. View of fire and smoke progression from above the showrooms from $300 \mathrm{~s}$ to $900 \mathrm{~s}$ (7:15:53 p.m. to 7:25:53 p.m.). The images are clipped to view conditions below the ceiling. 
The captain and two firefighters from E-15 entered the main showroom at approximately 7:19:00 p.m. (487 s). According to reports, they encountered smoke and donned their self-contained breathing apparatus (SCBA) face pieces. Figure K-19 shows an image of the front of the main showroom, with the front wall removed, at $487 \mathrm{~s}$. This image shows that smoke had filled the void space above the ceiling and banked down to the showroom floor. Conditions on the east side, including the area near the entrance, are not as severe. However, as one progresses towards the rear and west, conditions degrade significantly.

E-19 reported on scene at 7:20:08 p.m. (555 s). The E-11 captain was making his way out of the structure to investigate the problem with the $6.4 \mathrm{~cm}$ (2.5 in) hand line at approximately the same time the crew from E-19 was entering the building. At this time, the E-11 captain noted heavy smoke conditions and increasing temperatures.

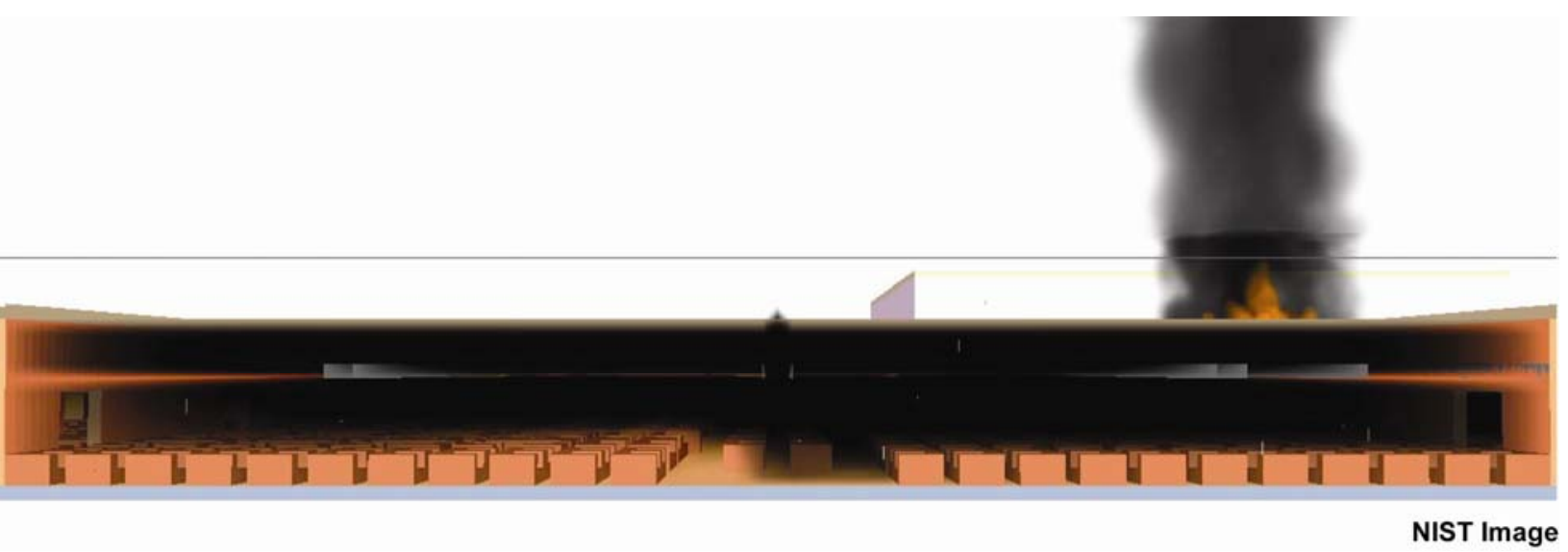

Figure K-19. Model rendering of view inside the front of the main showroom at $487 \mathrm{~s}$ (7:19:00 p.m.).

Figure K-20 and Figure K-21 show smoke filling in, respectively, the main and west showrooms from the perspective of someone at the front of either the main or west showroom. The sequence in Figure K-20 begins at $270 \mathrm{~s}$ (7:15:23p.m.), just after smoke began penetrating the ceiling. The sequence in Figure K21 begins at $240 \mathrm{~s}$ (7:14:53 p.m.). In both showrooms, conditions deteriorate considerably within a 150 second span.

At 1020 s (7:27:53 p.m.), the fire in the main showroom reached about $70 \mathrm{MW}$ and had begun moving forward towards the front of the showroom. Figure K-22 shows the progression of fire and smoke throughout the loading dock, holding area and showrooms between 1020 and $1620 \mathrm{~s}$. The front windows were vented during the period between 1457 to 1530 s (7:35:00 p.m. to 7:36:23 p.m.). Fire reached the front of the main showroom at approximately 1540 s (7:36:33 p.m.) and spread across the west side of the main showroom by $1590 \mathrm{~s}$ (7:37:23 p.m.).

Figures K-23 through K-27 show comparisons of the fire and smoke progression with available photos at various times during the critical time between 7:23 p.m. and 7:45 p.m. (740 s to $2040 \mathrm{~s}$ ). Figure K-23 shows one of the earliest photos of the fire, taken at 7:23:16 p.m. (743 s). At this time the fire was still mainly concentrated in the loading dock and holding area. Although the showrooms were charged with smoke, there was no smoke pushing out the open door at the front of the store. The fire had impinged on the underside of the roof over the holding area and flames were visible from the exterior at the roof level. At 1530s (7:36:23 p.m.), shown in Figure K-24, the main sections of the front windows had been 


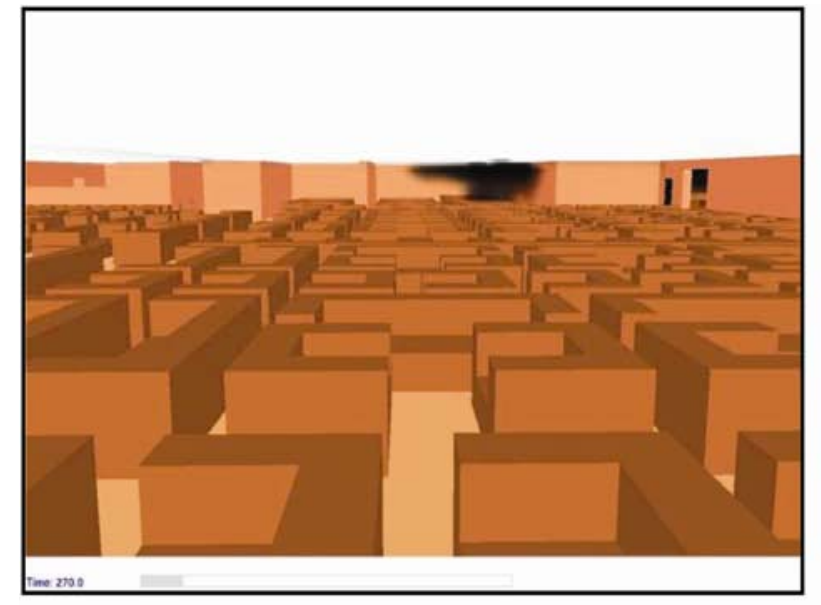

$270 \mathrm{~s}$

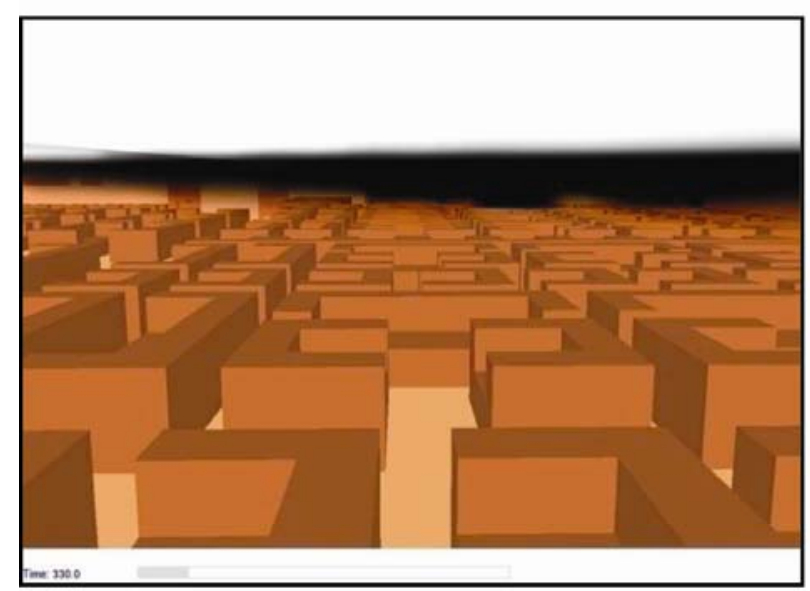

$330 \mathrm{~s}$

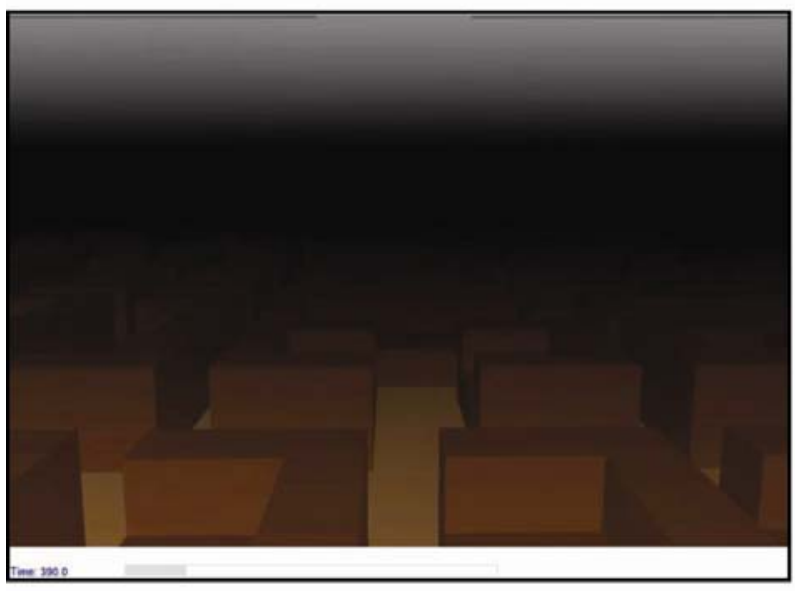

$390 \mathrm{~s}$

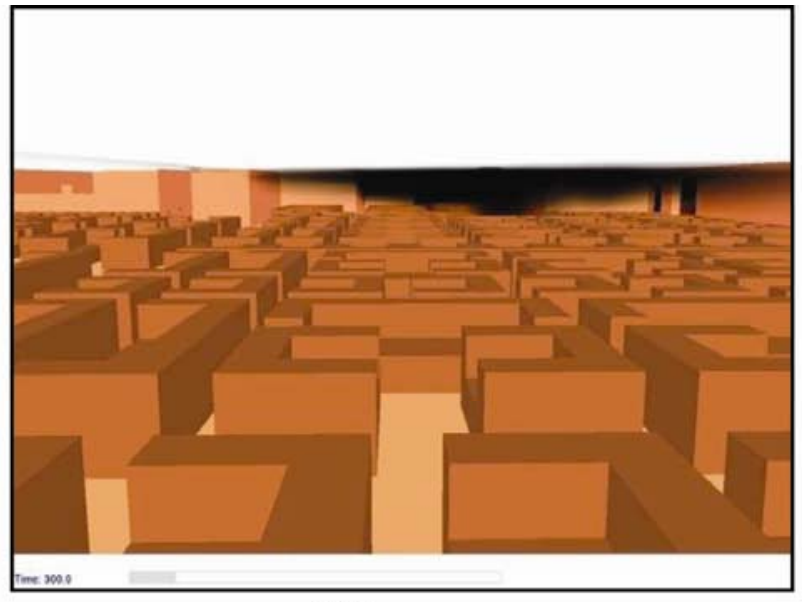

$300 \mathrm{~s}$

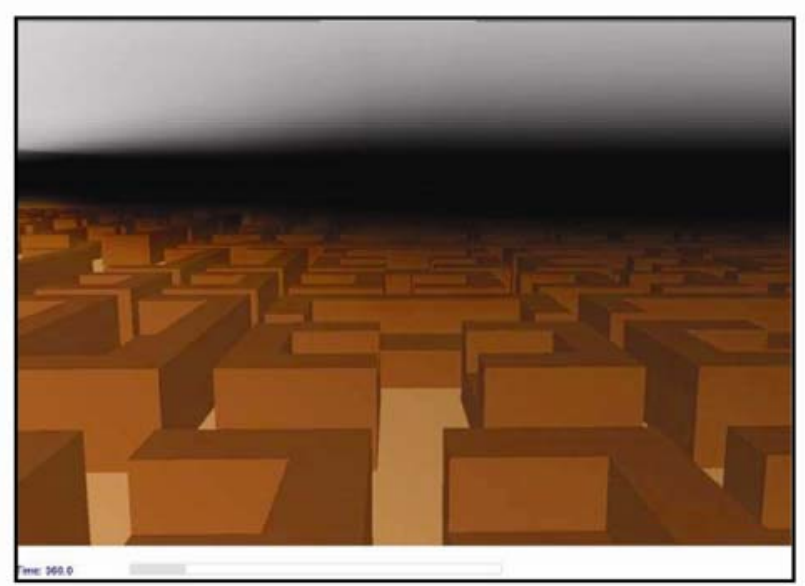

$360 \mathrm{~s}$

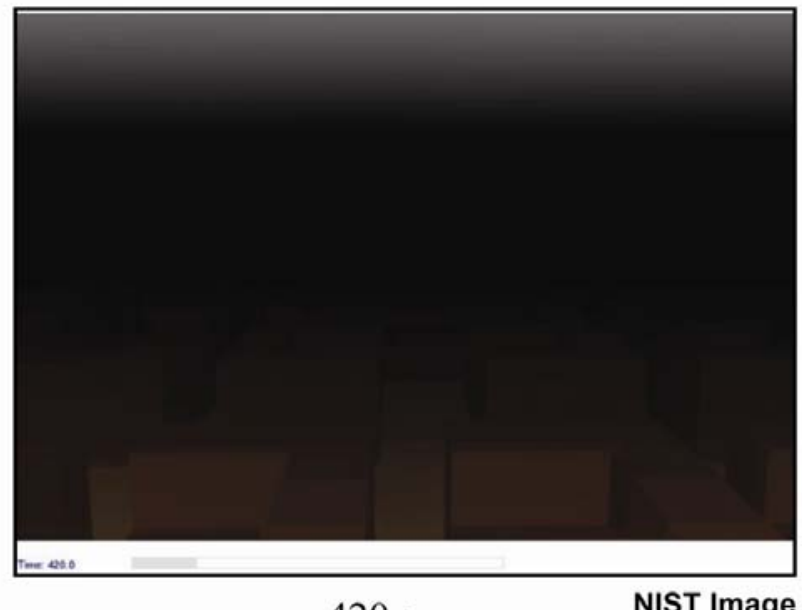

$420 \mathrm{~s}$

Figure K-20. Smoke progression inside the west side of the main showroom between $270 \mathrm{~s}$ to $420 \mathrm{~s}$ (7:15:23 p.m. to 7:17:53 p.m.). 


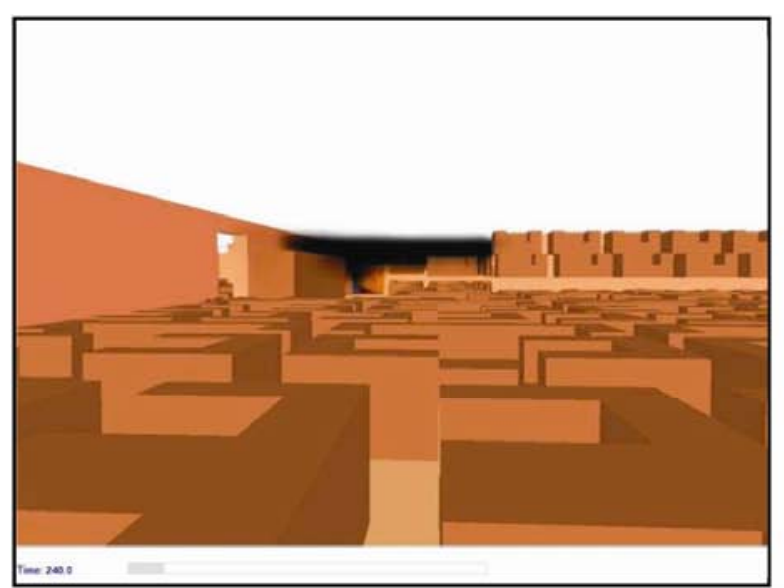

$240 \mathrm{~s}$

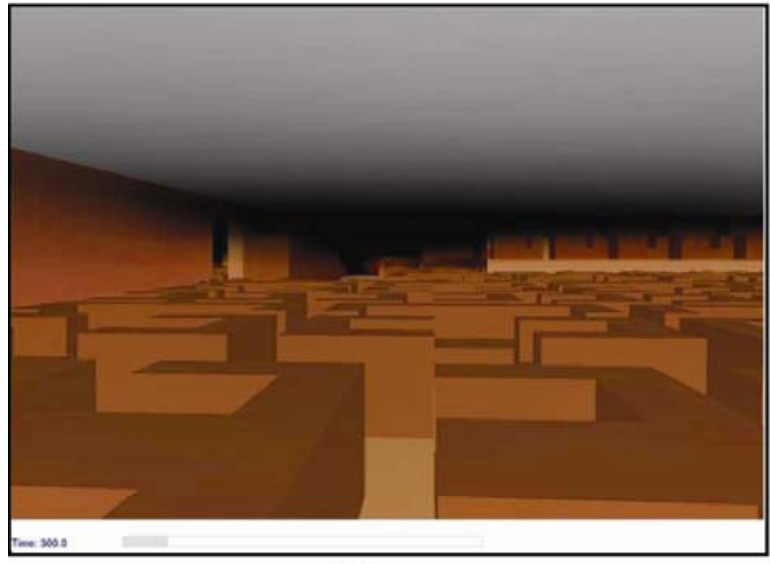

$300 \mathrm{~s}$

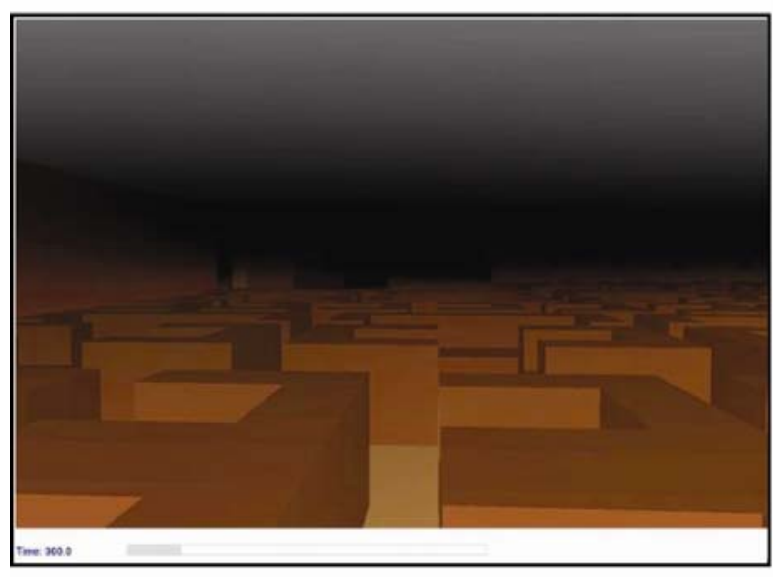

$360 \mathrm{~s}$

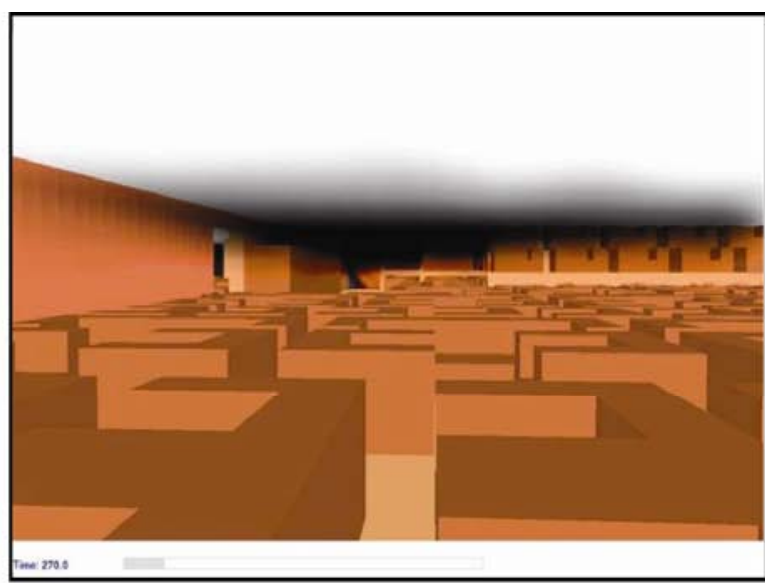

$270 \mathrm{~s}$

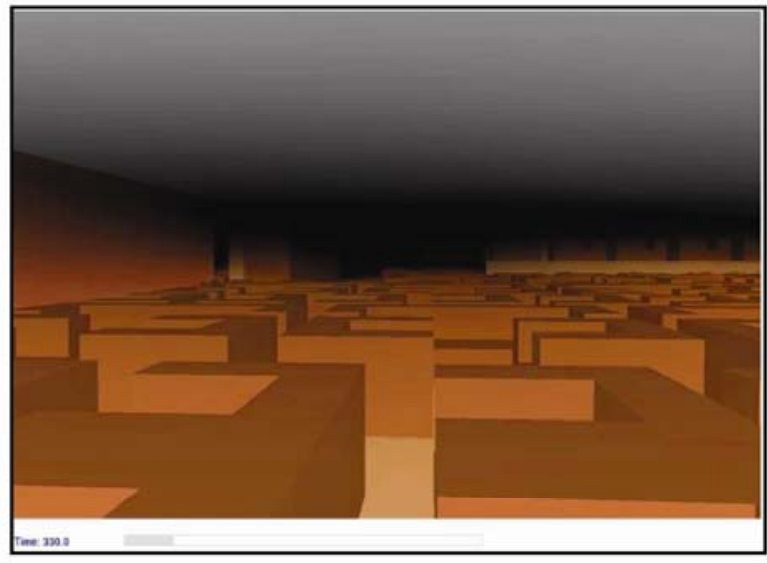

$330 \mathrm{~s}$

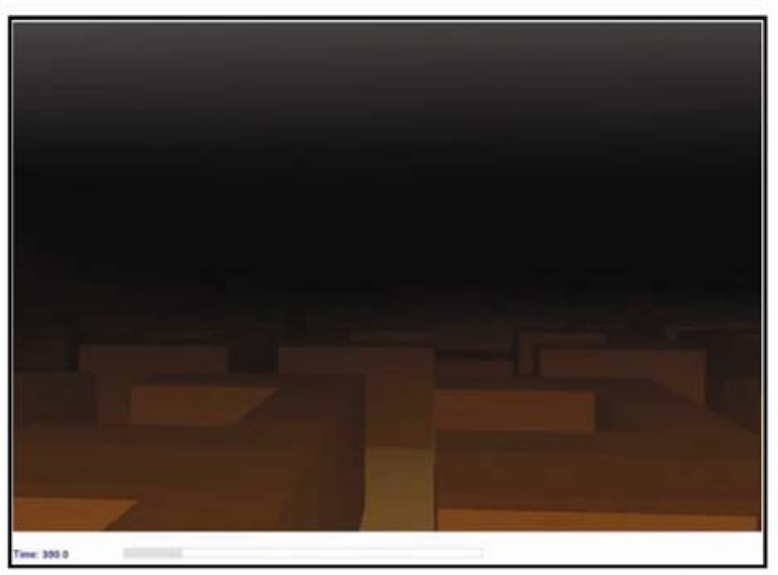

$390 \mathrm{~s}$ NIST Image

Figure K- 21. Smoke progression inside the west showroom between $240 \mathrm{~s}$ to $390 \mathrm{~s}$ (7:14:53p.m. to 7:17:23 p.m.). 


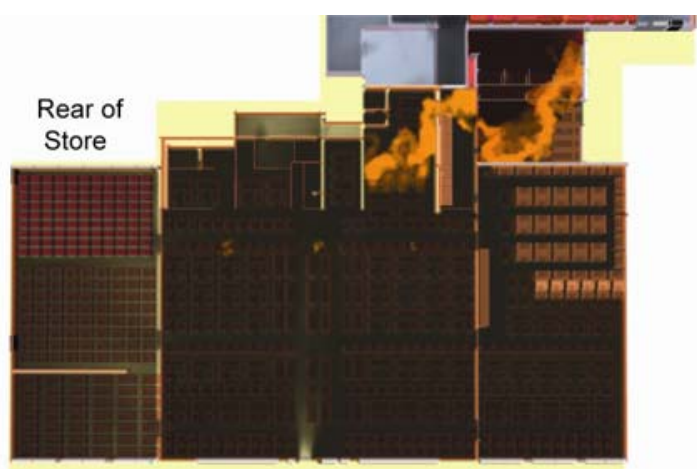

Front of Store

\section{$1020 \mathrm{~s}$}

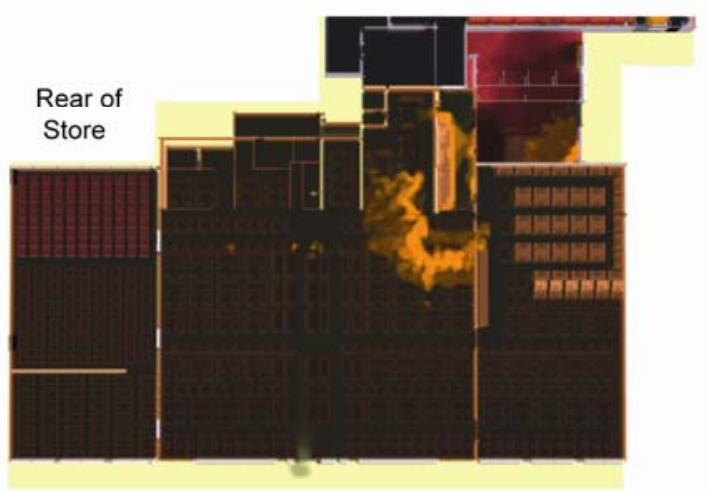

Front of Store

$1380 \mathrm{~s}$

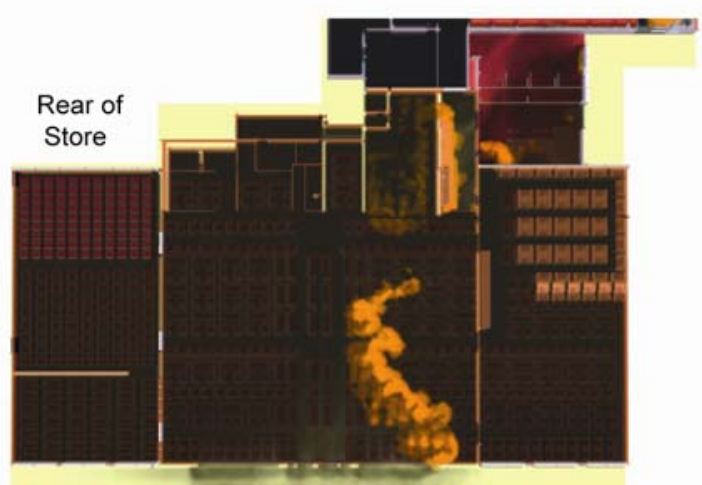

Front of Store

$1560 \mathrm{~s}$

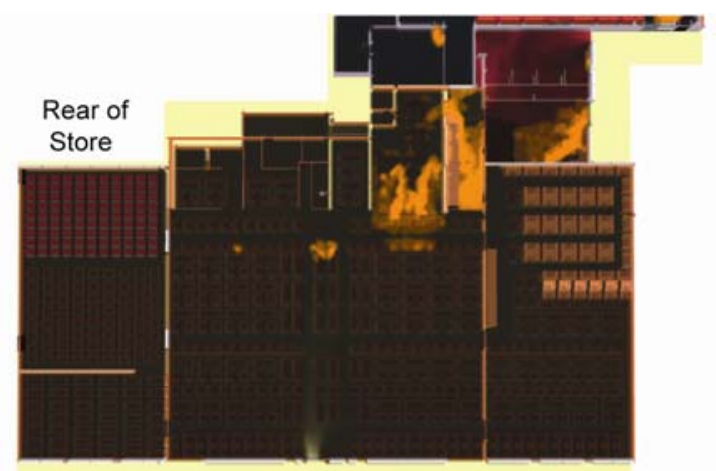

Front of Store

$1260 \mathrm{~s}$

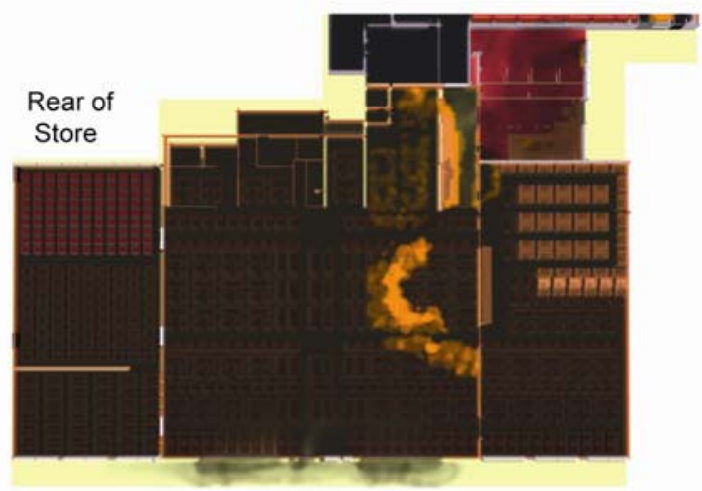

Front of Store

$1500 \mathrm{~s}$

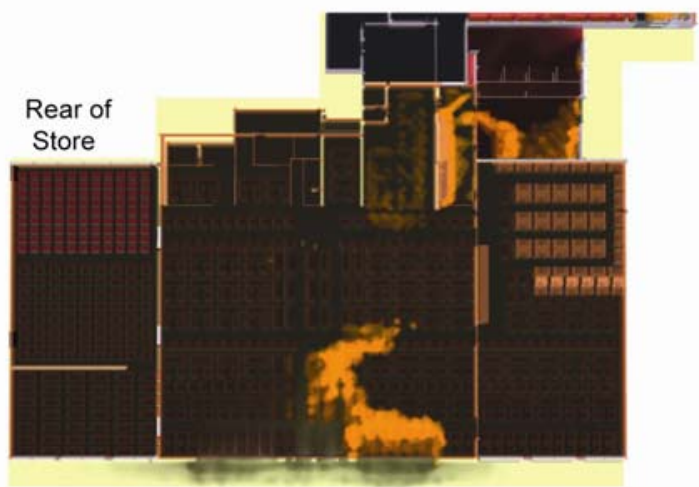

Front of Store

$1620 \mathrm{~s}$

Figure K-22. View of fire (orange color indicates stoichiometric boundary) and smoke progression from above the showrooms from $1020 \mathrm{~s}$ to $1620 \mathrm{~s}$ (7:27:53 p.m. to 7:37:53 p.m.). The images are clipped to view conditions below the ceiling. 

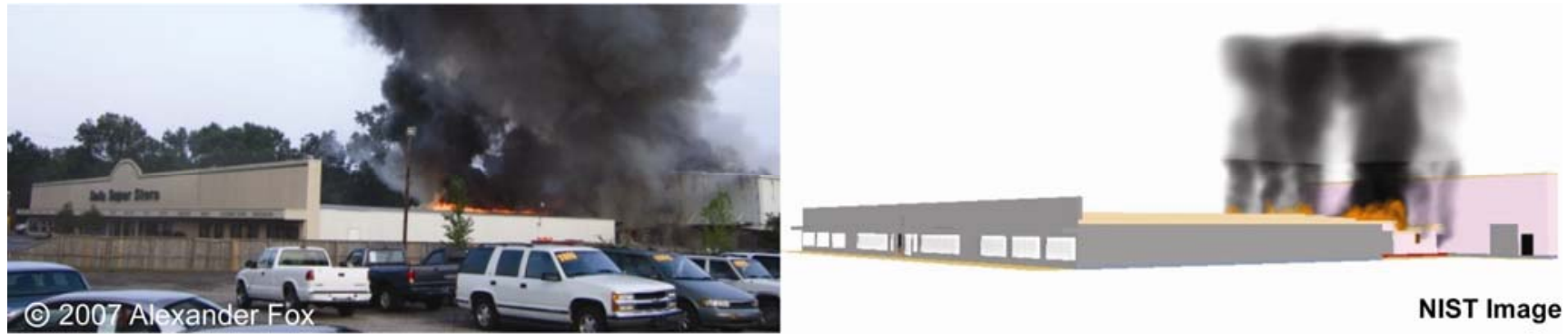

Figure K-23. Comparison between photo and rendered image from the simulation at 743 s (7:23:16 p.m.). The view is of the north west corner of the structure. Photo used with permission of Alexander Fox.

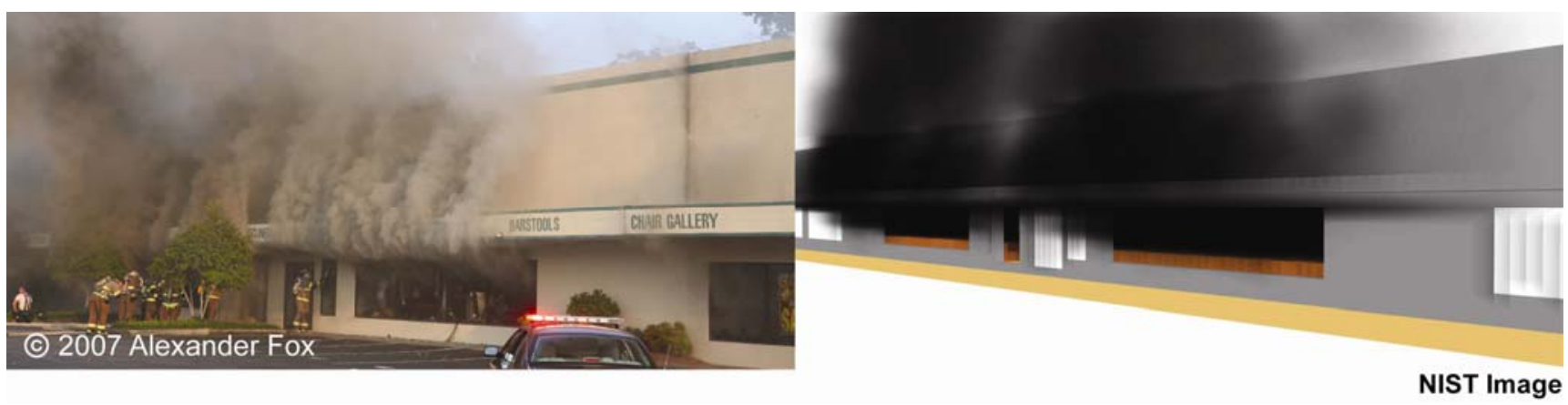

Figure K-24. Comparison between photo and rendered image from the simulation at 1530 s (7:36:23 p.m.). The view is of the front of the main showroom. Photo used with permission of Alexander Fox.
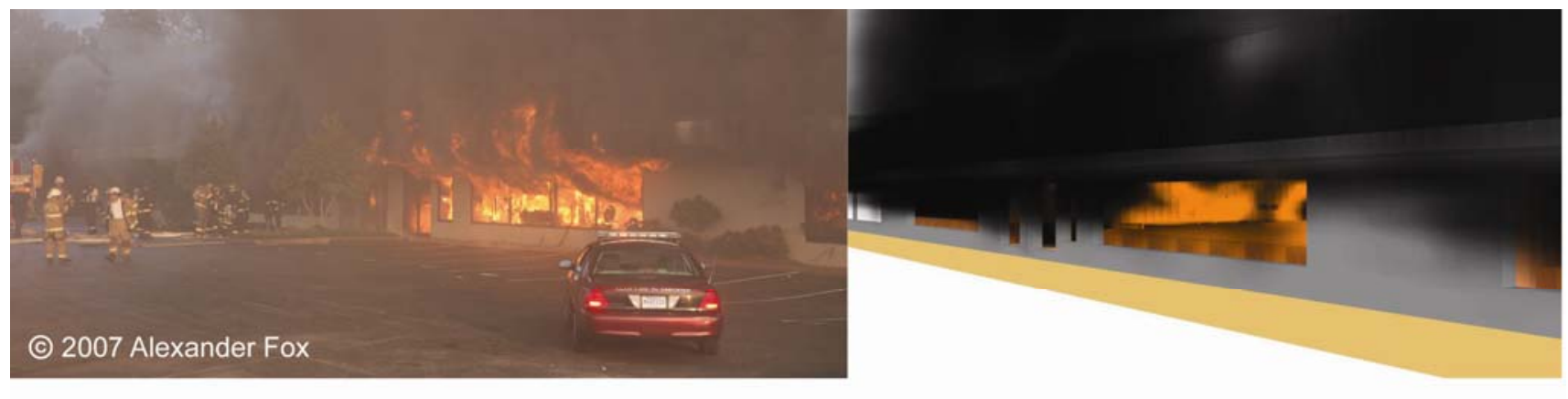

Figure K-25. Comparison between photo and rendered image from the simulation at 1655 s (7:38:28 p.m.). The view is of the front of the main showroom. Photo used with permission of Alexander Fox. 
broken out and smoke was pushing out the front of the main showroom. At this time, flames were not yet visible from the exterior through the front windows. By 1655 s (7:38:28 p.m.), shown in Figure K-25, fire had reached the front of the main showroom and had stretched across the west side of the main showroom. At 1686 s (7:38:59) flames were visible inside the west showroom, Figure K-26, and the first window in this side had failed from the heat exposure. By $1884 \mathrm{~s}$ (7:42:17), shown in Figure K-27, fire had stretched across the front of the west showroom and two of the three windows were broken out by fire.
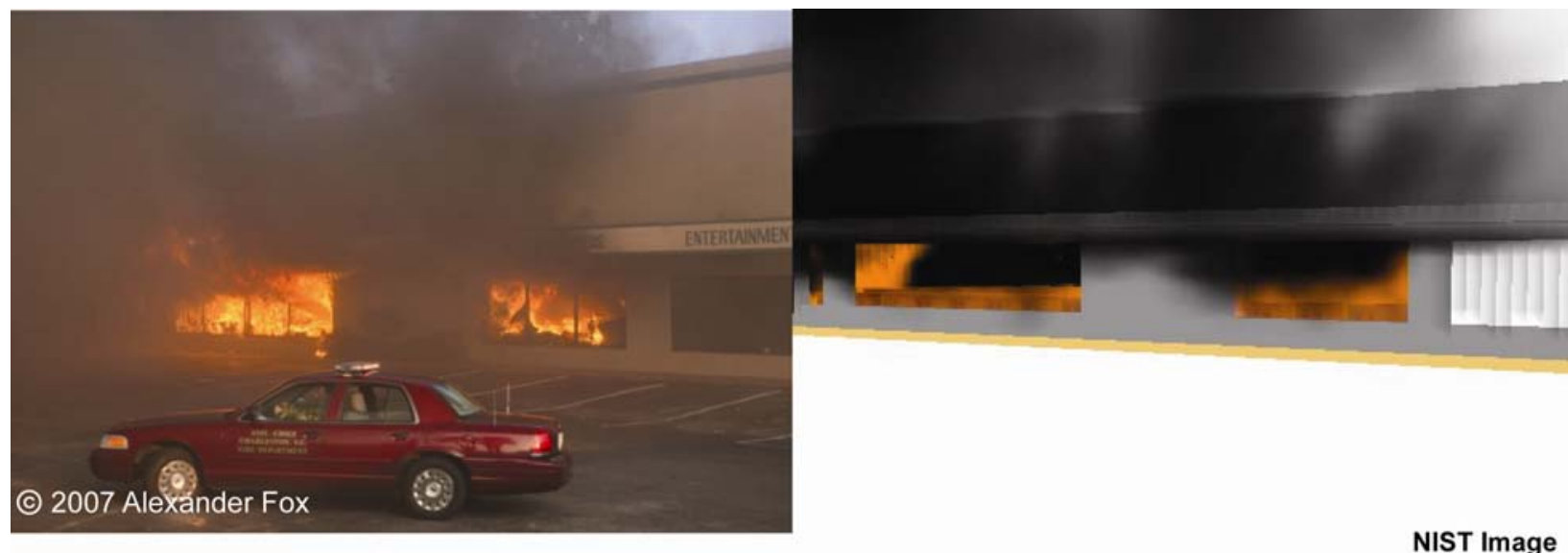

Figure K-26. Comparison between photo and rendered image from the simulation at $1686 \mathrm{~s}$ (7:38:59 p.m.). The view is of the front of the main showroom. Photo used with permission of Alexander Fox.

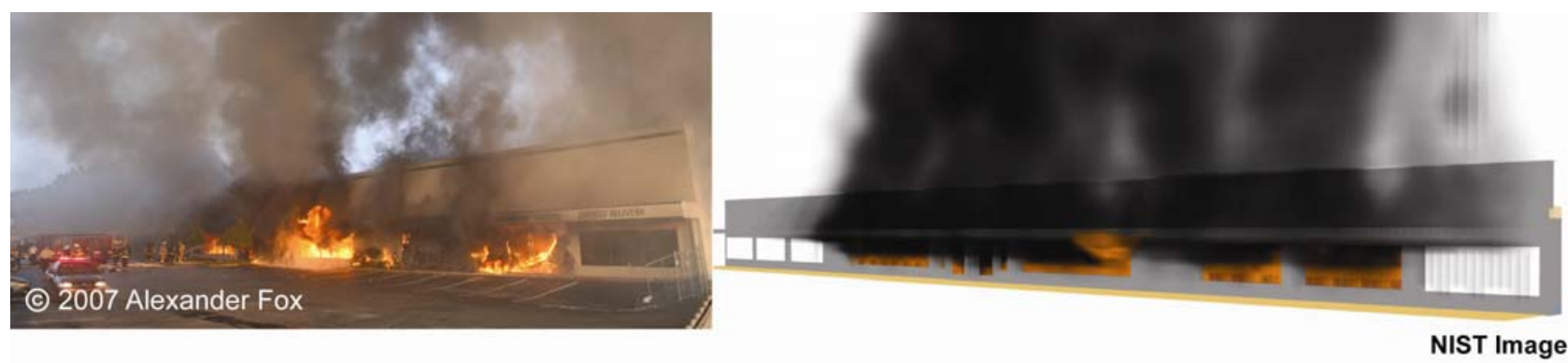

Figure K-27. Comparison between photo and rendered image from the simulation at 1884 s (7:42:17 p.m.). The view is of the front of the main and west showrooms. Photo used with permission of Alexander Fox. 


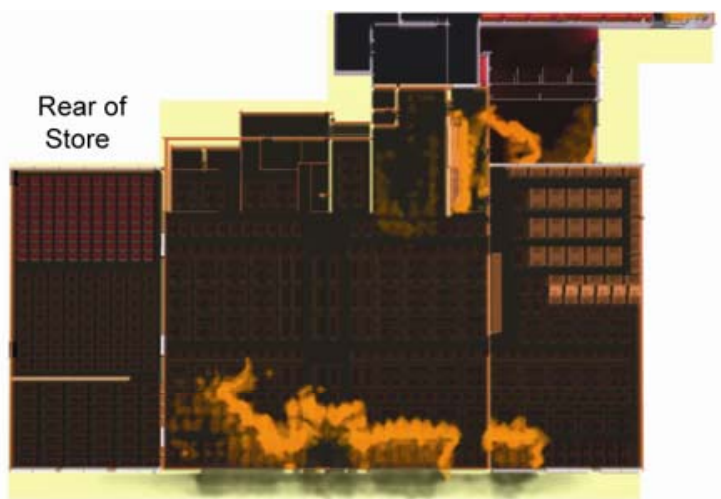

Front of Store

\section{0 seconds}

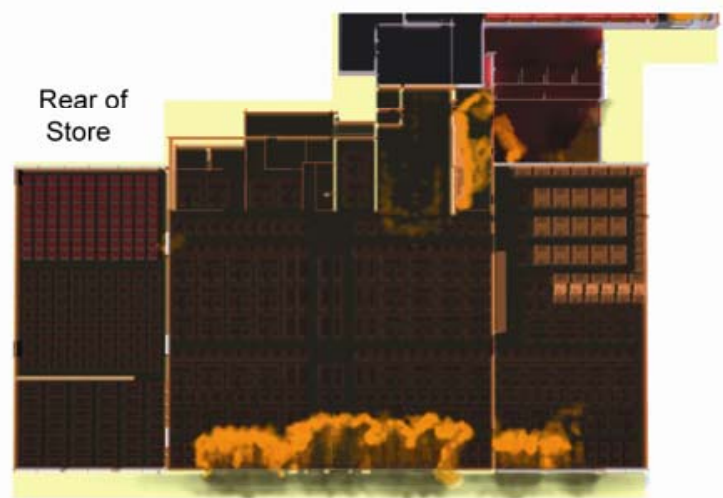

Front of Store

1800 seconds

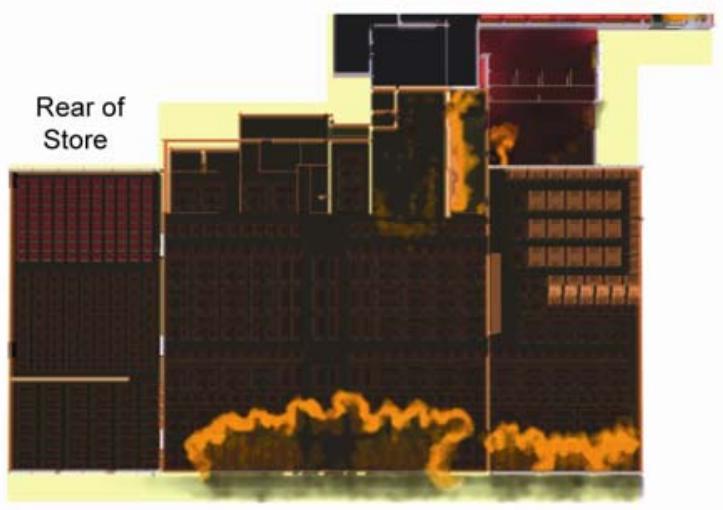

Front of Store

1920 seconds

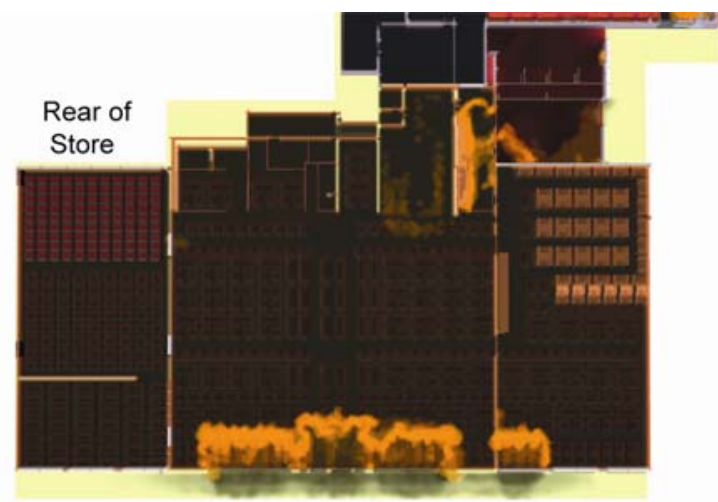

Front of Store

1740 seconds

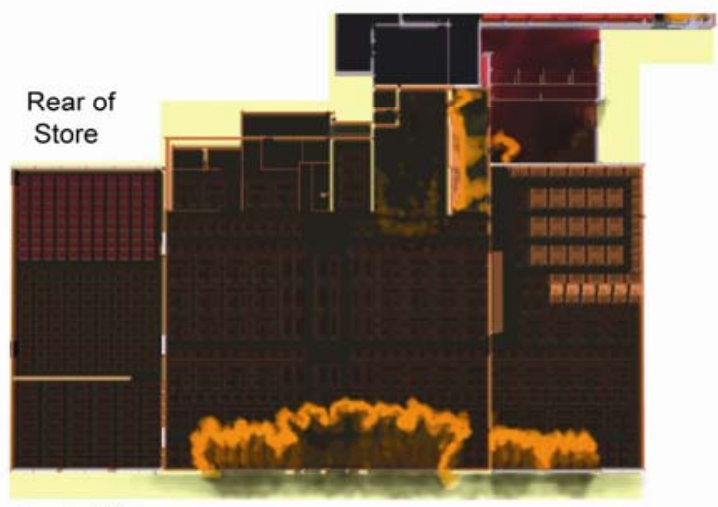

Front of Store

1860 seconds

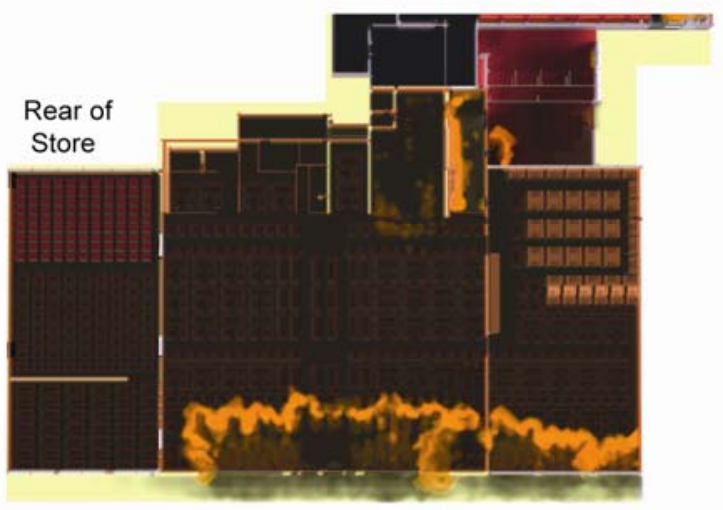

Front of Store

2040 seconds

Figure K-28. View of fire and smoke progression from above the showrooms from $1680 \mathrm{~s}$ to $2040 \mathrm{~s}$ (7:38:53 p.m. to 7:44:53 p.m.). The images are clipped to view conditions below the ceiling. 
Figure K-28 shows a series of images indicating the fire progression after it reached the front of the main showroom. By 1680 s (7:38:53 p.m.) the fire had moved into the front of the west showroom, causing the first window to fail. This is consistent with the image in Figure K-26, where the fire is visible in the west showroom through the window opening. Fire continued to spread across the front of the west showroom as seen in Figure K-28. The image in the sequence at 1860 s (7:41:53) is an overhead view of the image shown in Figure K-27.

\section{K.4.4 Tenability Criteria}

Purser [2] has published data that identify when conditions become untenable for humans. Purser provides an algorithm for estimating the time to lose consciousness due to low oxygen. At 0.12 volume fraction, the time is estimated at about five minutes. In a closed fire-engulfed environment, toxic gases (such as carbon monoxide) are likely to be present at incapacitating levels before the oxygen gets this low. Since the simulation tracks the oxygen volume fraction, it will be used as one indicator for tenability. A second indicator will be when the temperature exceeds $120^{\circ} \mathrm{C}\left(250^{\circ} \mathrm{F}\right)$ for about five minutes [2]. The effects of carbon monoxide, depleted oxygen, and temperature are dose-related, involving both magnitude and time of exposure [2]. Thus the time at which $120^{\circ} \mathrm{C}\left(250^{\circ} \mathrm{F}\right)$ is exceeded is before the time at which the person is incapacitated. For each of the simulations, the time for areas to become untenable ${ }^{6}$ due to elevated temperature ${ }^{7}$ or oxygen depletion ${ }^{8}$ will be tabulated. These incapacitation criteria are simplifications of complex studies and serve as a basis for appraising the relative effects of alternate fire scenarios.

The NIST study did not include analysis of the threat to protected fire fighters. If a fire fighter in turnout gear is utilizing a self-contained breathing apparatus (SCBA) and has an adequate supply of air, the fire fighter can temporarily survive higher temperatures and depleted external oxygen levels. This safety shell ends when the fire fighter runs out of tank air or remains within the hot fire environment too long.

\section{K.4.5 Temperature}

Temperatures were examined to assess tenability conditions that existed during the evolution of the fire.

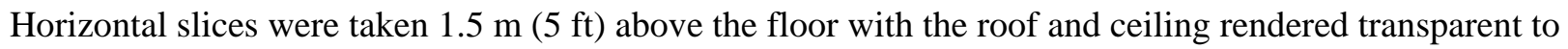
display the temperature distribution throughout the structure. Additionally, temperatures were tracked over time at different elevations at specific horizontal locations as shown in Figure K-29 with the filled red circles. These temperature monitoring locations were defined in the simulation in order to track temperatures at a specific location during each simulation. The labels in Figure K-29 and Table K-7 identify temperature locations (referred to as "sensor arrays") and are used throughout this report.

The three positions indicated to the left of the figure (front, mid, rear) were located, respectively, $3.38 \mathrm{~m}$ $(11.1 \mathrm{ft}), 14.62 \mathrm{~m}(48.0 \mathrm{ft})$, and $26.62 \mathrm{~m}(87.3 \mathrm{ft})$ from the front of the showrooms. Sensors in the west showroom were located near the center, $7.5 \mathrm{~m}$ (24.6 ft) from the west wall. In the west side of the main showroom, the front and mid sensors were positioned $7.9 \mathrm{~m}(25.9 \mathrm{ft})$ from the west wall. The rear and

\footnotetext{
${ }^{6}$ For this study, untenability was equated to incapacitation. A person is defined as incapacitated when he or she is unable to effect his or her own escape (ISO 13943)[16].

${ }^{7}$ For this study, temperature greater than $120^{\circ} \mathrm{C}\left(250{ }^{\circ} \mathrm{F}\right)$ for at least five minutes (300 seconds).

${ }^{8}$ For this study, oxygen volume fraction less than 0.12 for at least five minutes (300 seconds).
} 
corner sensors were located $9.8 \mathrm{~m}$ (32.2 ft) from the same wall; the corner position was $39 \mathrm{~m}$ (128.0 ft) from the front wall. The sensors positioned in the center of the main showroom were located $19.1 \mathrm{~m}$ $(62.7 \mathrm{ft})$ from the west wall of the main showroom. Sensors in the east side of the main showroom were positioned $30 \mathrm{~m}(98.4 \mathrm{ft})$ from the west wall of the main showroom.

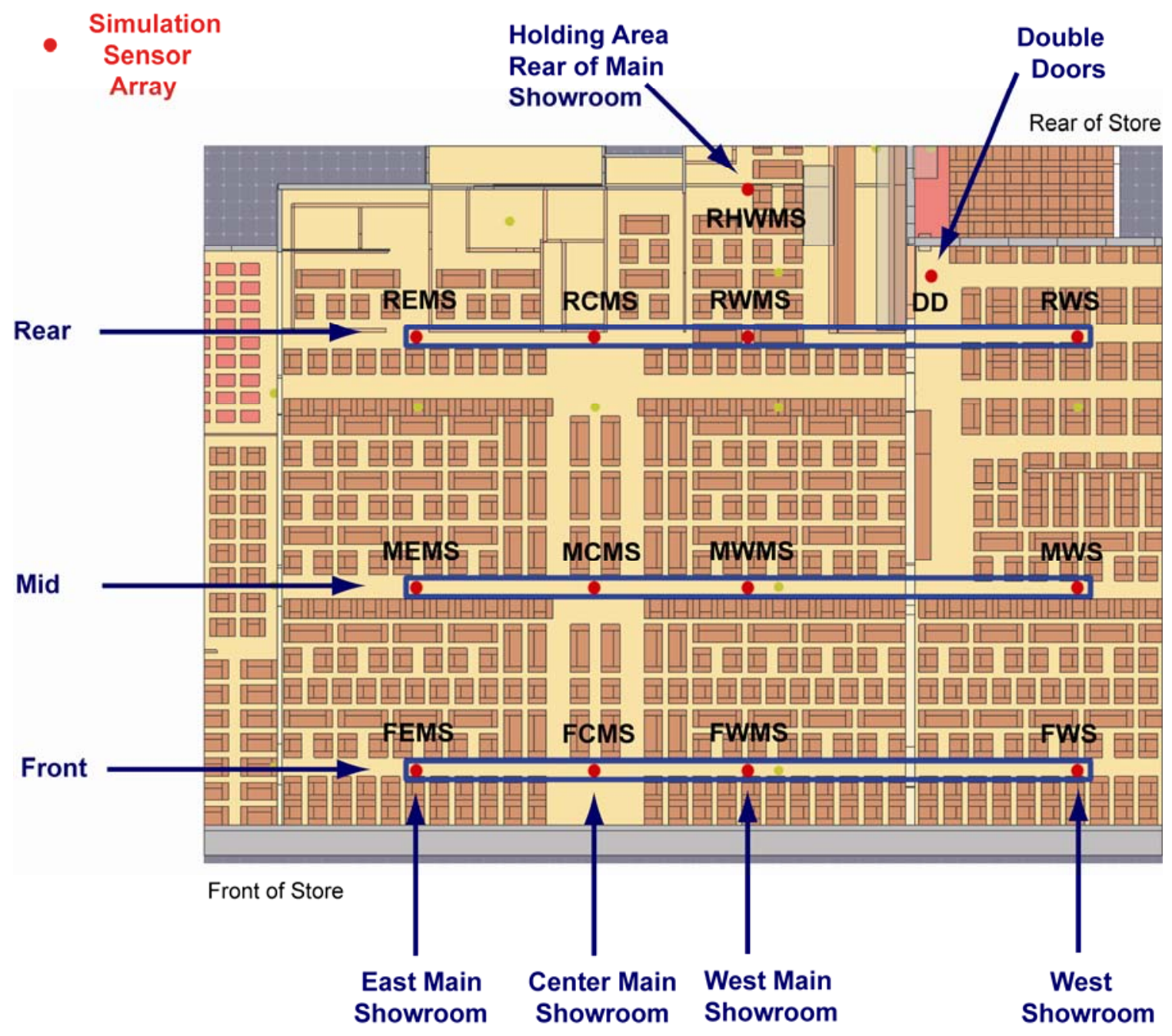

Figure K-29. Location of simulation sensor arrays in main and west showrooms. 
Table K-7. Position/Location Identifiers for simulation data.

\begin{tabular}{|l|c|}
\hline \multicolumn{1}{|c|}{ Location } & Identifier \\
\hline Front East Main Showroom & FEMS \\
\hline Mid East Main Showroom & MEMS \\
\hline Rear East Main Showroom & REMS \\
\hline Front Center Main Showroom & FCMS \\
\hline Mid Center Main Showroom & MCMS \\
\hline Rear Center Main Showroom & RCMS \\
\hline Front West Main Showroom & FWMS \\
\hline Mid West Main Showroom & MWMS \\
\hline Rear West Main Showroom & RWMS \\
\hline Rear Holding West Main Showroom & RHWMS \\
\hline Front West Showroom & FWS \\
\hline Mid West Showroom & MWS \\
\hline Rear West Showroom & RWS \\
\hline Double Doors & DD \\
\hline
\end{tabular}

Figure K-30 shows a series of images with a temperature slice $1.5 \mathrm{~m}(5 \mathrm{ft})$ from the floor rendered at a range of times between $900 \mathrm{~s}$ through $1620 \mathrm{~s}$ (7:25:53 p.m. through 7:37:53 p.m.). These images show the progression of the elevated temperatures from the loading dock, into the holding area, and eventually into the main showroom. Temperatures above $100{ }^{\circ} \mathrm{C}$ (approximately indicated by the border between dark blue and light blue) are first observed in the holding area approximately three minutes into the simulation. Note that the double doors separating the west showroom from the loading dock were opened at $144 \mathrm{~s}$, but the temperature in the west showroom remained low even as fire was spreading into the holding area.

Figure K-31 shows a chart of temperatures recorded in the west showroom, near the double doors. The

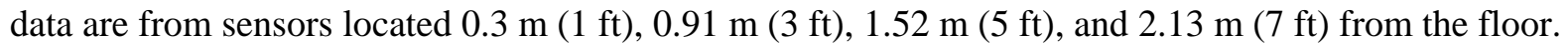
Figure K-31 shows temperatures remained below $100{ }^{\circ} \mathrm{C}$ through approximately $750 \mathrm{~s}$ (7:23:23 p.m.). The fire was positioned near the double doors to the loading dock, however, during the early stages much of the heat was venting through the loading dock roof and later through the main showroom roof. Air was being pulled through the doorway to feed oxygen to the fire in the loading dock and holding area. There was a surge in temperature in the upper layer lasting several minutes caused by fire progression into the showroom. At 900 s (7:25:53 p.m.), a section of the roof above the holding area was removed and this resulted in the subsequent temperature drop. Temperatures dropped below $100{ }^{\circ} \mathrm{C}$, but as the HRR increased in the main showroom the temperatures in both showrooms began to rise quickly. A second temperature surge, which began at approximately $1460 \mathrm{~s}$ (7:35:03 p.m.), was due to the fire entering the west showroom through the open roll-down door as it moved to the front of the 

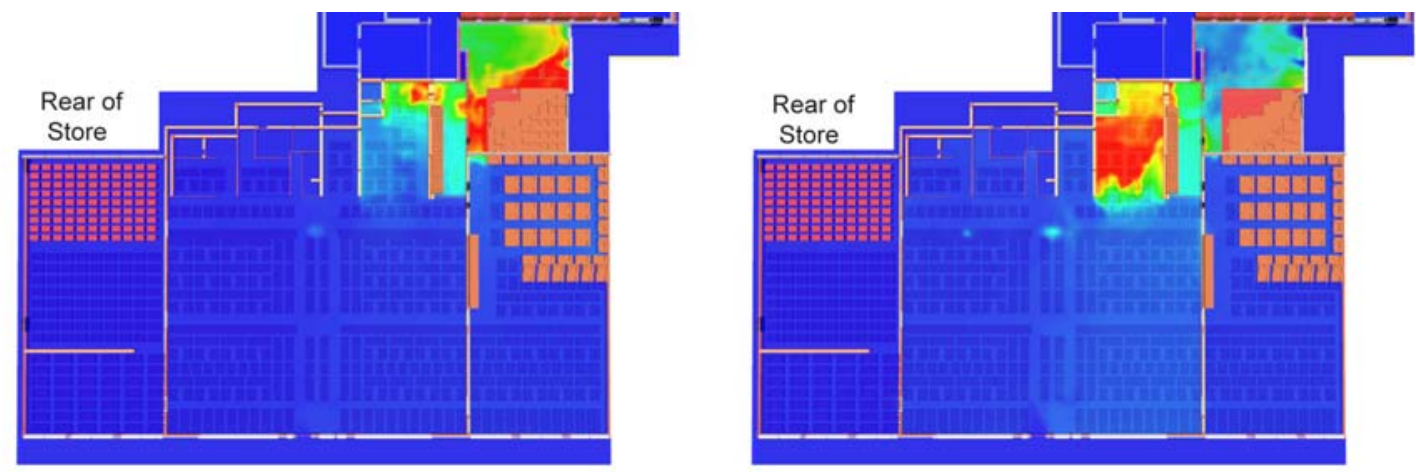

Front of Store

Front of Store

$$
1140 \mathrm{~s}
$$

Gas

$900 \mathrm{~s}$
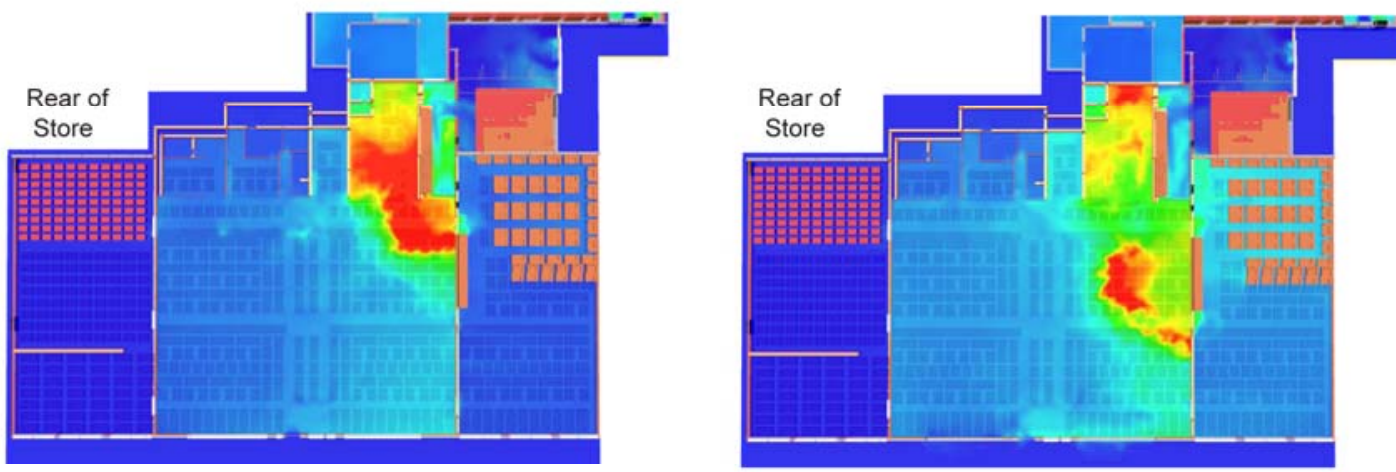

Front of Store

$1380 \mathrm{~s}$

Front of Store

$1500 \mathrm{~s}$

C

820

740

660

580

500

420

340

260

180

100
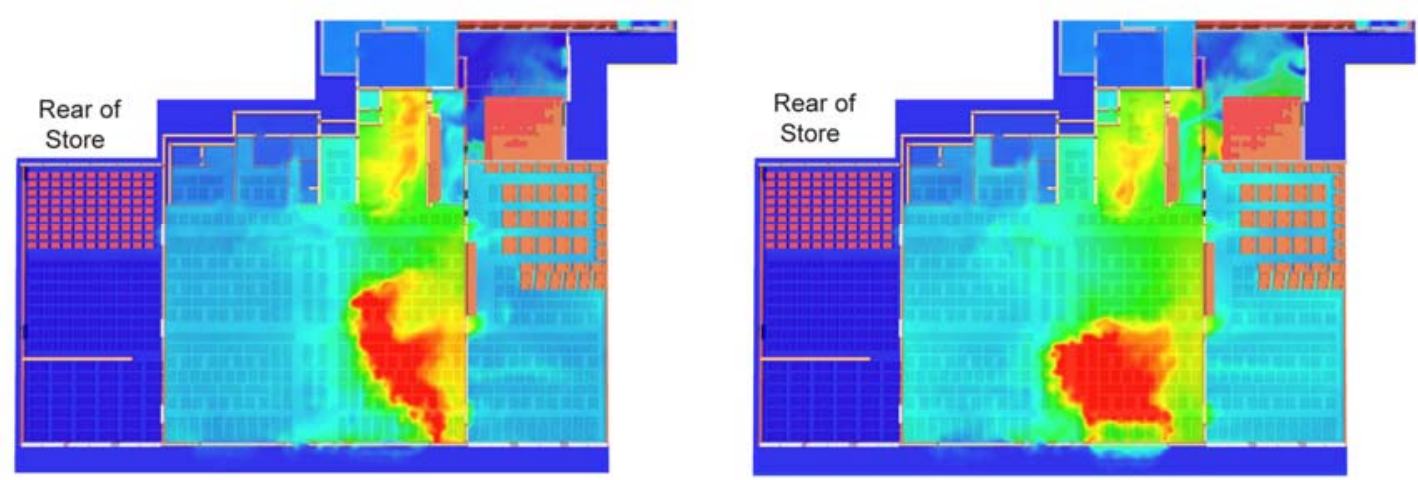

Front of Store

$$
1560 \mathrm{~s}
$$

Front of Store

NIST Image

$1620 \mathrm{~s}$

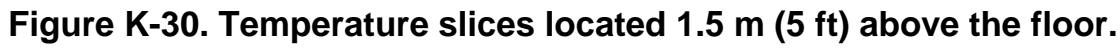




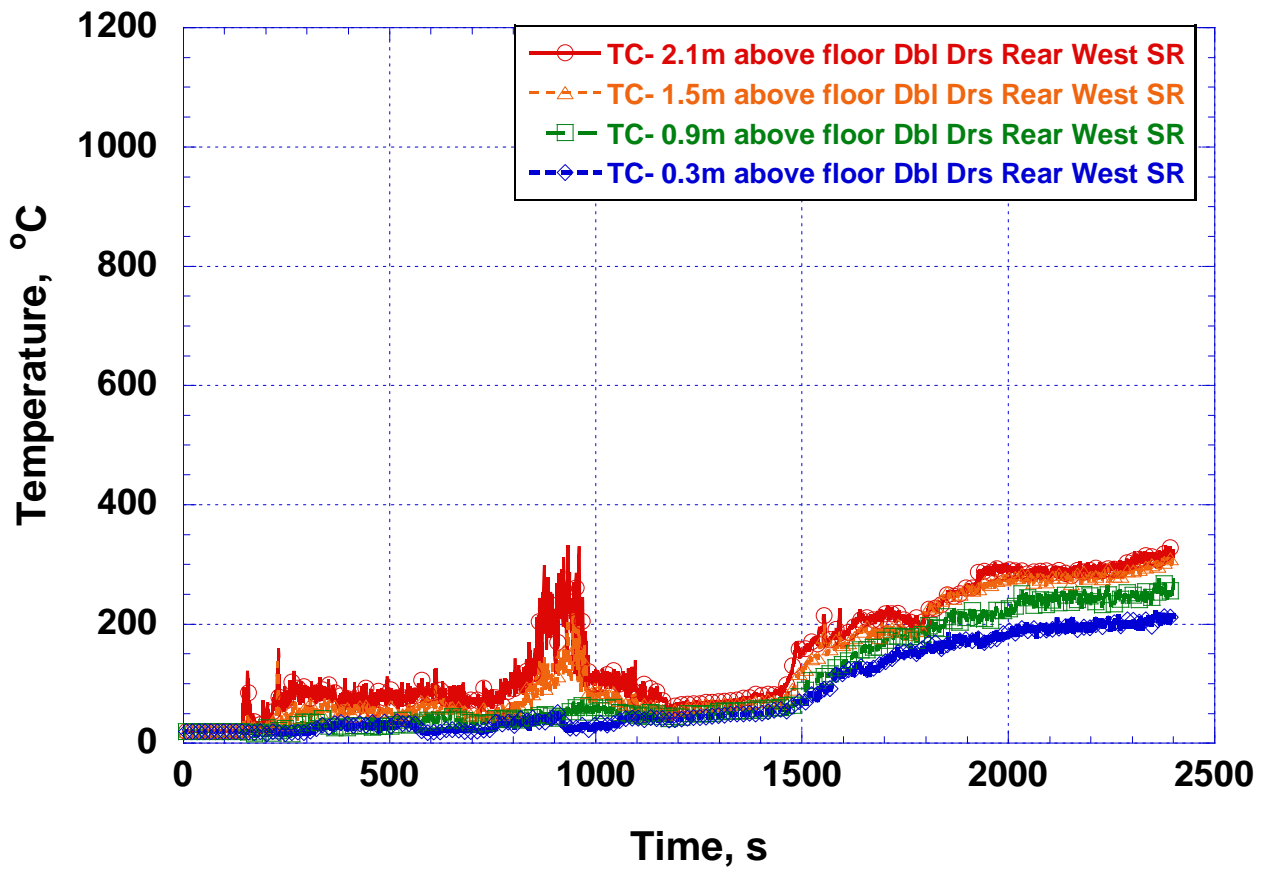

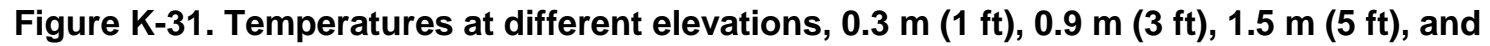
$2.1 \mathrm{~m} \mathrm{(7} \mathrm{ft)} \mathrm{in} \mathrm{rear} \mathrm{of} \mathrm{west} \mathrm{showroom} \mathrm{near} \mathrm{double} \mathrm{doors.}$

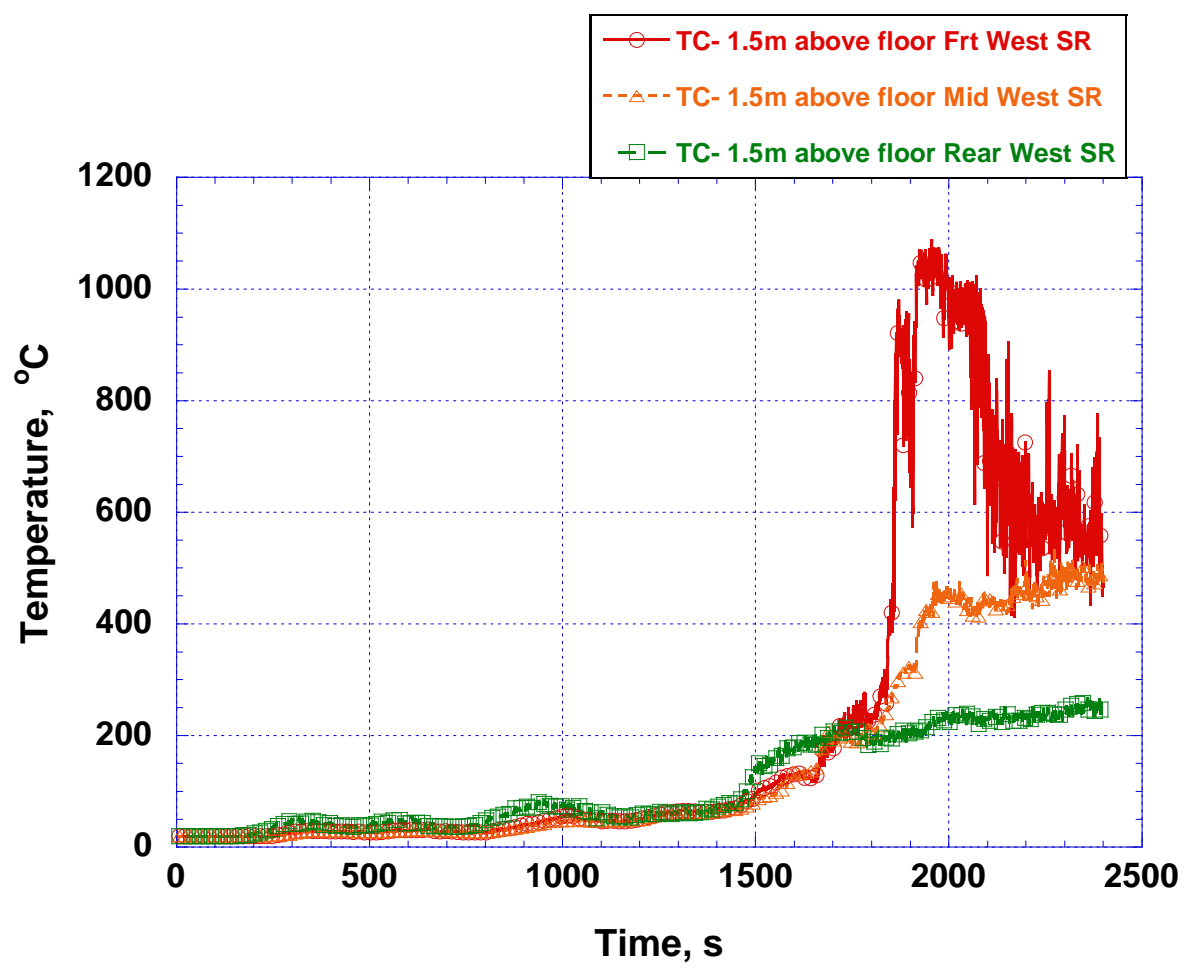

Figure K-32. West Showroom temperatures for $1.5 \mathrm{~m}(5 \mathrm{ft})$ above floor. 


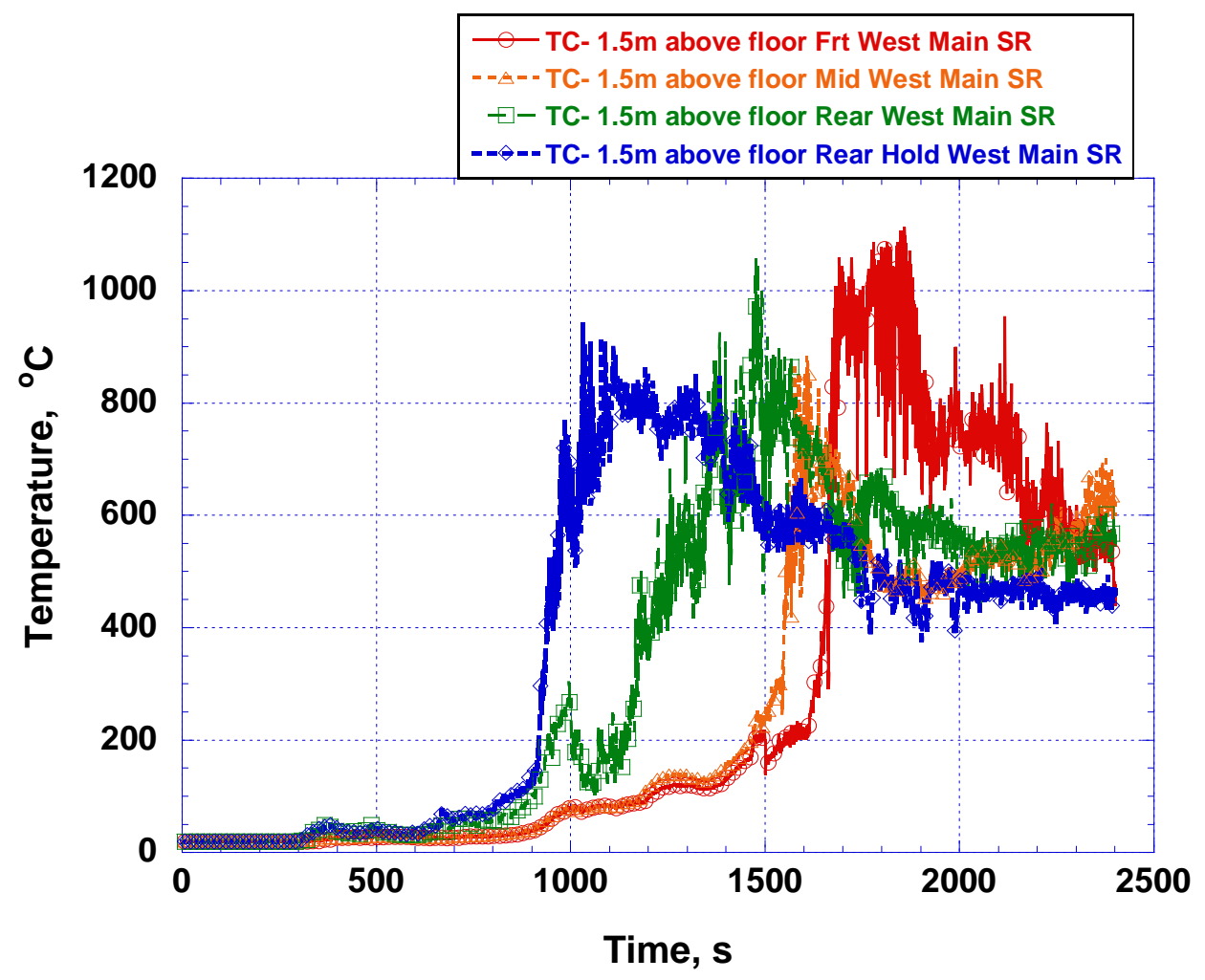

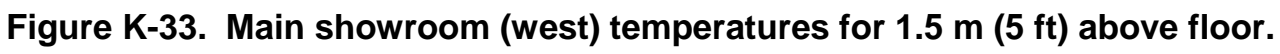

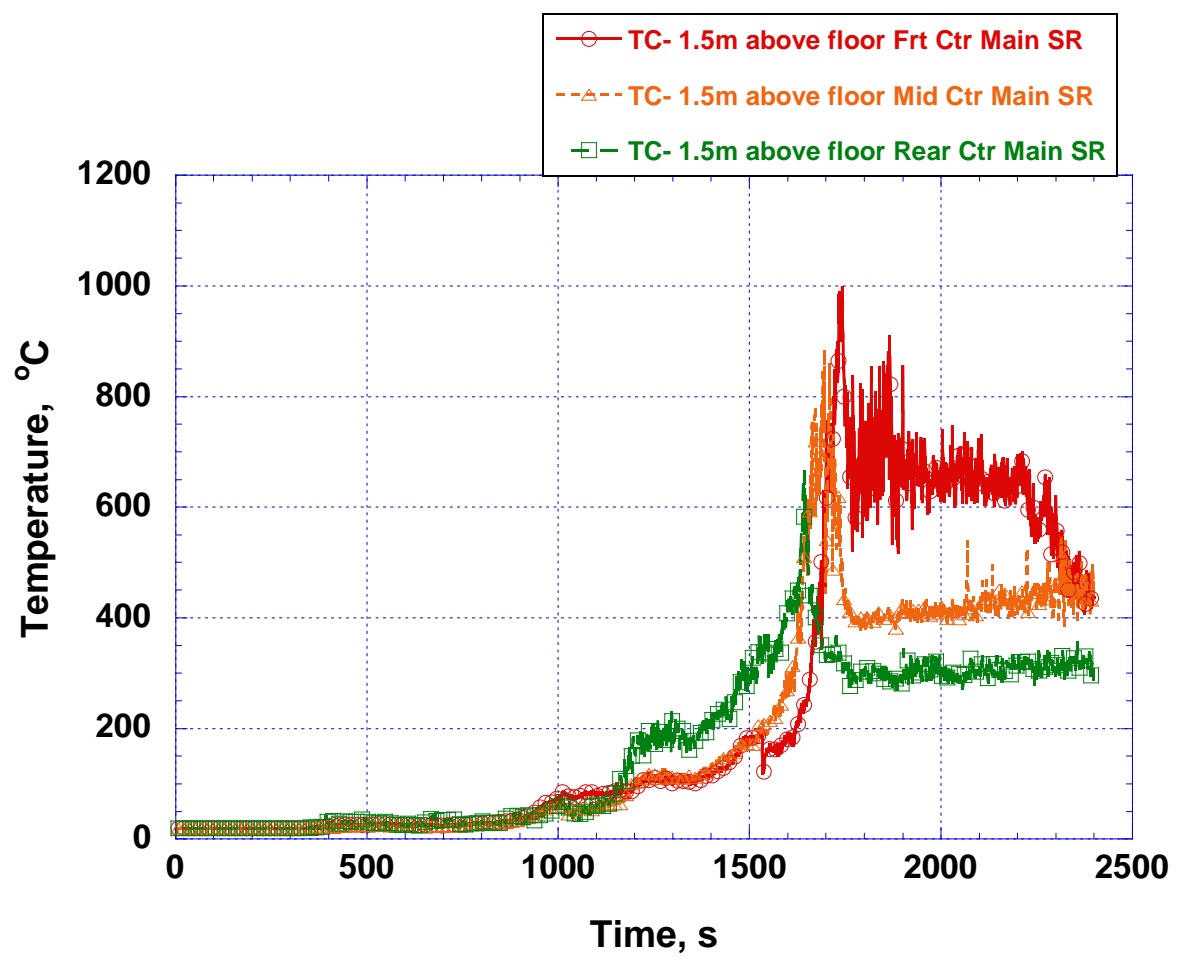

Figure K-34. Main showroom (center) temperatures for $1.5 \mathrm{~m} \mathrm{(5} \mathrm{ft)} \mathrm{from} \mathrm{floor.}$ 


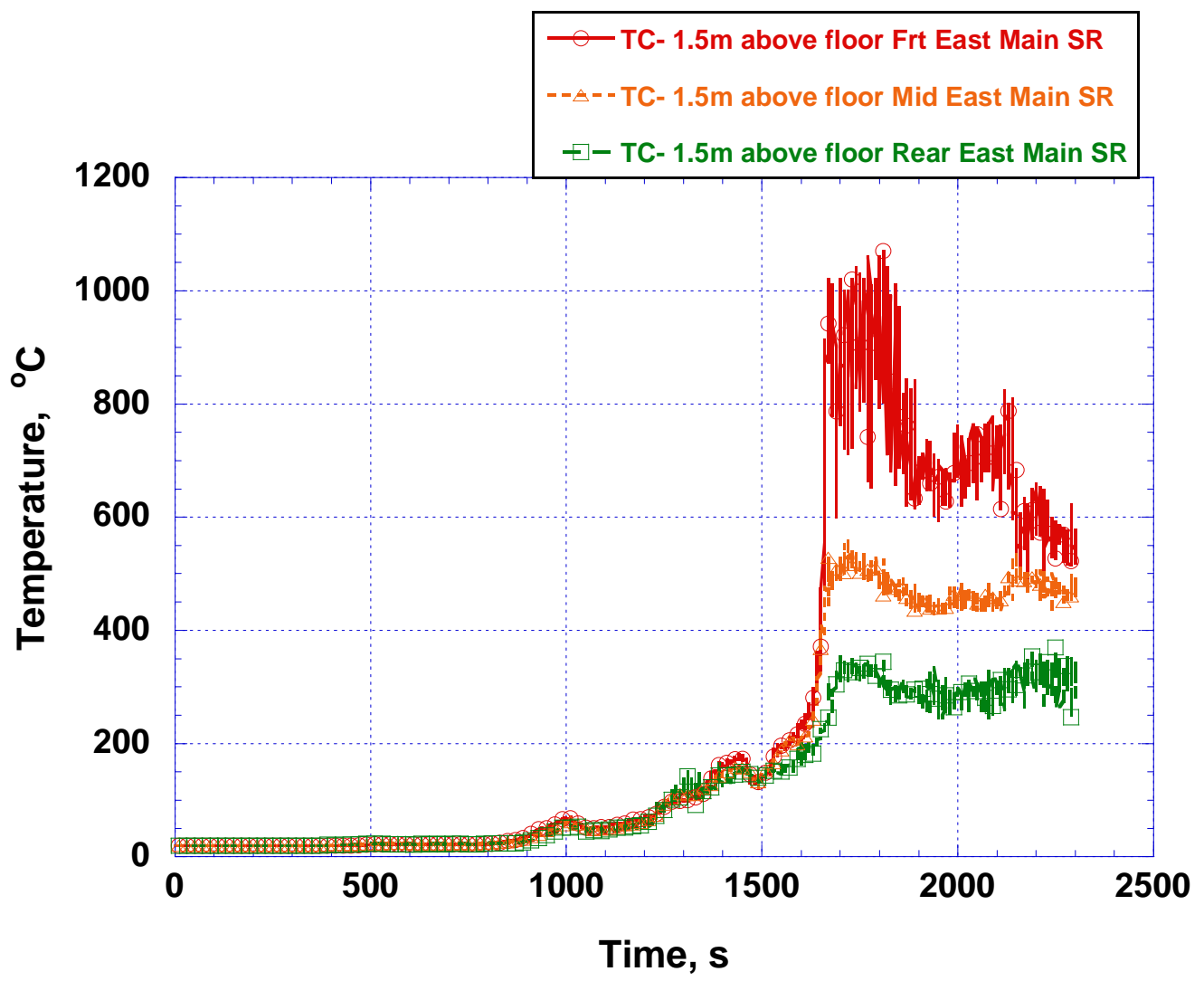

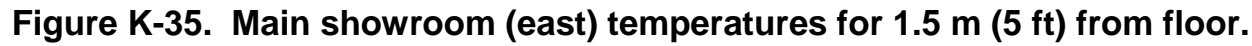

main showroom. It should be noted that the focus of suppression efforts was in this area of the showroom, and that the simulation does not take this into account.

Figures K-31 through K-35 show charts of temperatures recorded at the locations throughout the main

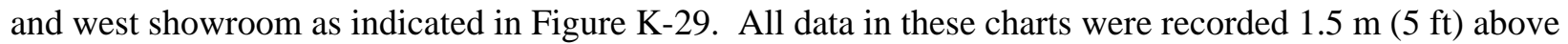
the floor. Figure K-31 shows temperature in the west showroom. Temperatures in the west showroom remained relatively low, below $100^{\circ} \mathrm{C}$, until the fire began moving toward the front of the main showroom. The temperature at the rear sensor surpassed $120^{\circ} \mathrm{C}$ at $1350 \mathrm{~s}$ (7:33:23 p.m.). As the fire in the main showroom continued to move forward, the temperature at the mid and front sensors reached $120^{\circ} \mathrm{C}$ at 1400 s (7:34:13 p.m.)

Figure K-33 shows a temperature chart that displays data from sensors located on the west side of the main showroom. This chart contains a fourth data location, labeled Rear Hold, which was identified in Figure K-29. Temperatures in the west side of the main showroom became elevated at an earlier point than in the other areas of the showroom because the fire moved across these sensors as it moved to the front of the showroom. The rear holding area location reached $120^{\circ} \mathrm{C}$ at $864 \mathrm{~s}$ (7:25:17 p.m.); the rear location was roughly $30 \mathrm{~s}$ behind, reaching $120^{\circ} \mathrm{C}$ at $900 \mathrm{~s}$ (7:25:53 p.m.). The mid and front locations reached $120^{\circ} \mathrm{C}$ simultaneously at $1250 \mathrm{~s}$ (7:31:43 p.m.). Temperatures in the center and east areas of the main showroom showed similar trends. 


\section{K.4.6 Oxygen Volume Fraction}

Figure K-36 shows a series of images with an oxygen slice $1.5 \mathrm{~m}(5 \mathrm{ft})$ from the floor rendered at a range of times between $360 \mathrm{~s}$ through $1440 \mathrm{~s}$ (7:16:53 p.m. through 7:34:53 p.m.).

Figures K-37 through K-40 show charts of oxygen volume fraction recorded at several locations throughout the main and west showroom. All data in these charts were recorded $1.5 \mathrm{~m}(5 \mathrm{ft})$ above the floor. The locations correspond to those used for the temperature results shown in Figures K-31 through K-35. The oxygen volume fraction at the sensor in the rear of west showroom fell below 0.12 at $1500 \mathrm{~s}$ (7:35:53 p.m.). As the fire in the main showroom continued to move forward, the oxygen at both the mid and front sensors reached 0.12 volume fraction at $1600 \mathrm{~s}$ (7:37:33 p.m.).

Figure K-38 shows a chart that displays oxygen data from sensors located on the west side of the main showroom. Oxygen in the west side of the main showroom became depleted at an earlier point than in the other areas of the showroom because this was the path of the fire as it moved to the front of the showroom. The holding area location reached 0.12 volume fraction at $950 \mathrm{~s}$ (7:26:43 p.m.); the rear

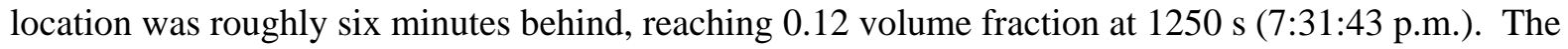
mid and front locations initially reached 0.12 volume fraction approximately the same time at $1400 \mathrm{~s}$ (7:34:13 p.m.). The mid and front locations then increased up to 0.15 volume fraction as the front windows were broken. Oxygen volume fractions in the center and east areas of the main showroom had similar trends.

\section{K.5 BASELINE SIMULATION WITH SPRINKLERS ADDED INSIDE THE LOADING DOCK}

\section{K.5.1 General}

A simulation was performed with code-compliant fire sprinklers added inside the enclosed loading dock to examine the effects that sprinklers might have had on the fire and the resulting environment inside the building. Otherwise, the simulation was identical to the baseline simulation. FDS has been shown to be able to predict the number of sprinklers activated and the approximate activation times, as well as trends, temperatures, heat fluxes and oxygen volume fractions in reasonable agreement with measured values [5; Vols. 2\&3]. However, the suppression physics in FDS is simplified and cannot capture all of the details of the suppression process.

The sprinkler system layout was designed in accordance with NFPA 13, Standard for the Installation of Sprinkler Systems [20]. The system was designed as an Ordinary Hazard Group 2 wet pipe system, assuming that the enclosed loading dock area was heated. An Ordinary Hazard Group 2 sprinkler system was utilized in order to provide a more conservative estimate for the design area/water density, as compared to an Extra Hazard design, for simulating the effects of sprinklers on fire conditions. The locations of the sprinklers within the enclosed loading dock are shown in Figure K-41. Note that given the dimensions of the cells in the simulation, the locations of the sprinklers in the simulation vary up to $2 \mathrm{~cm}$ (0.8 in) from the layout shown. The specific locations of the sprinklers are given in the FDS input file. The sprinkler characteristics and operating parameters are given in Table K-8. 


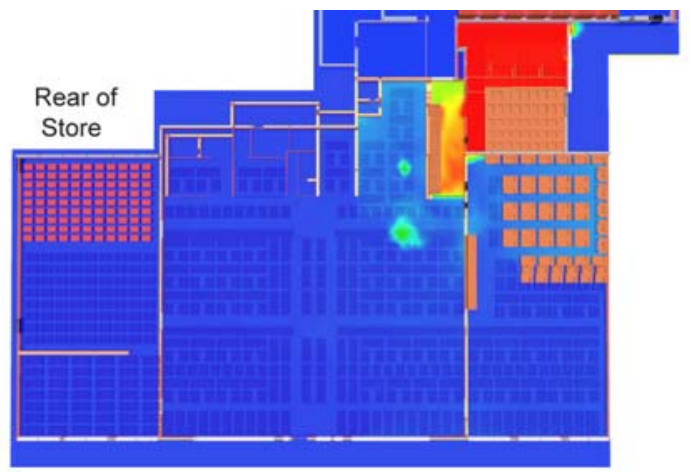

Front of Store

$360 \mathrm{~s}$

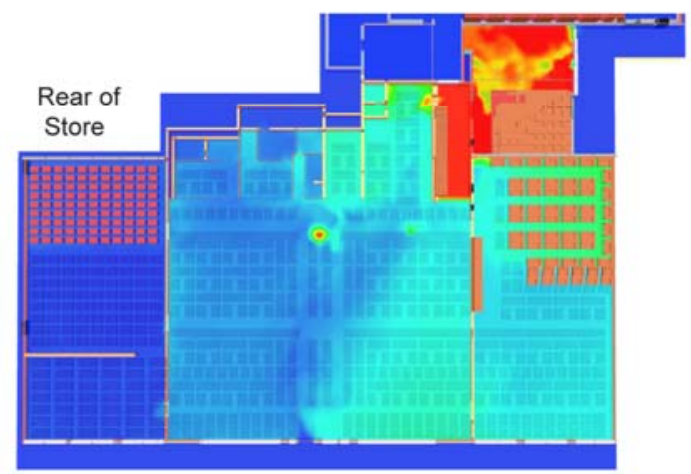

Front of Store

840 s

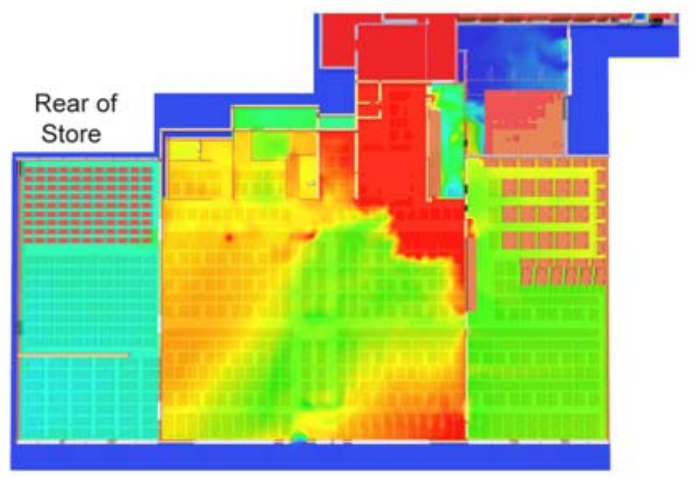

Front of Store

$1380 \mathrm{~s}$

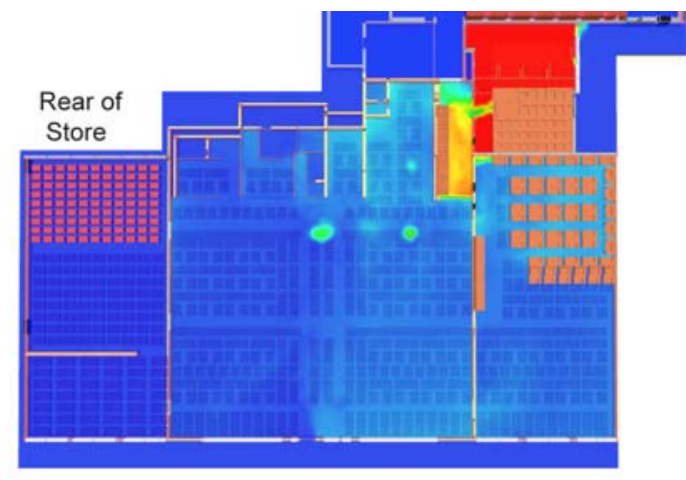

Oxygen

Volume

Front of Store

$480 \mathrm{~s}$

0.21

0.20

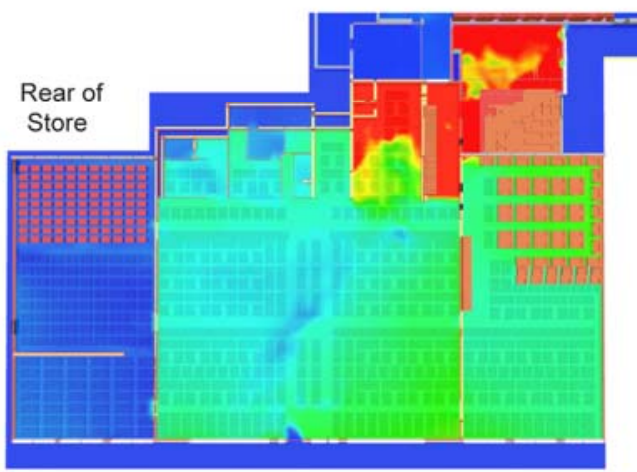

0.19

0.18

0.17

0.16

0.15

0.14

Front of Store

$960 \mathrm{~s}$

0.13

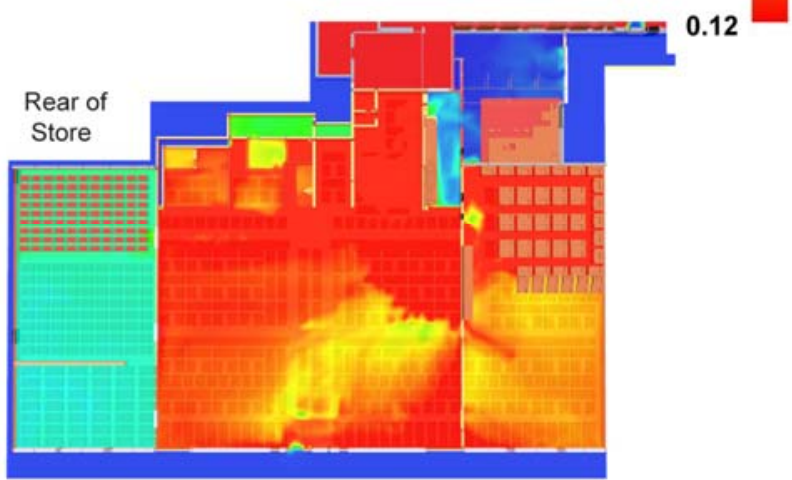

Front of Store

$1440 \mathrm{~s}$

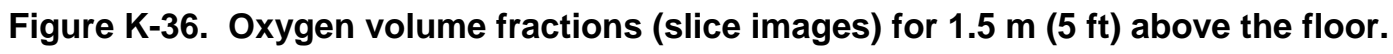




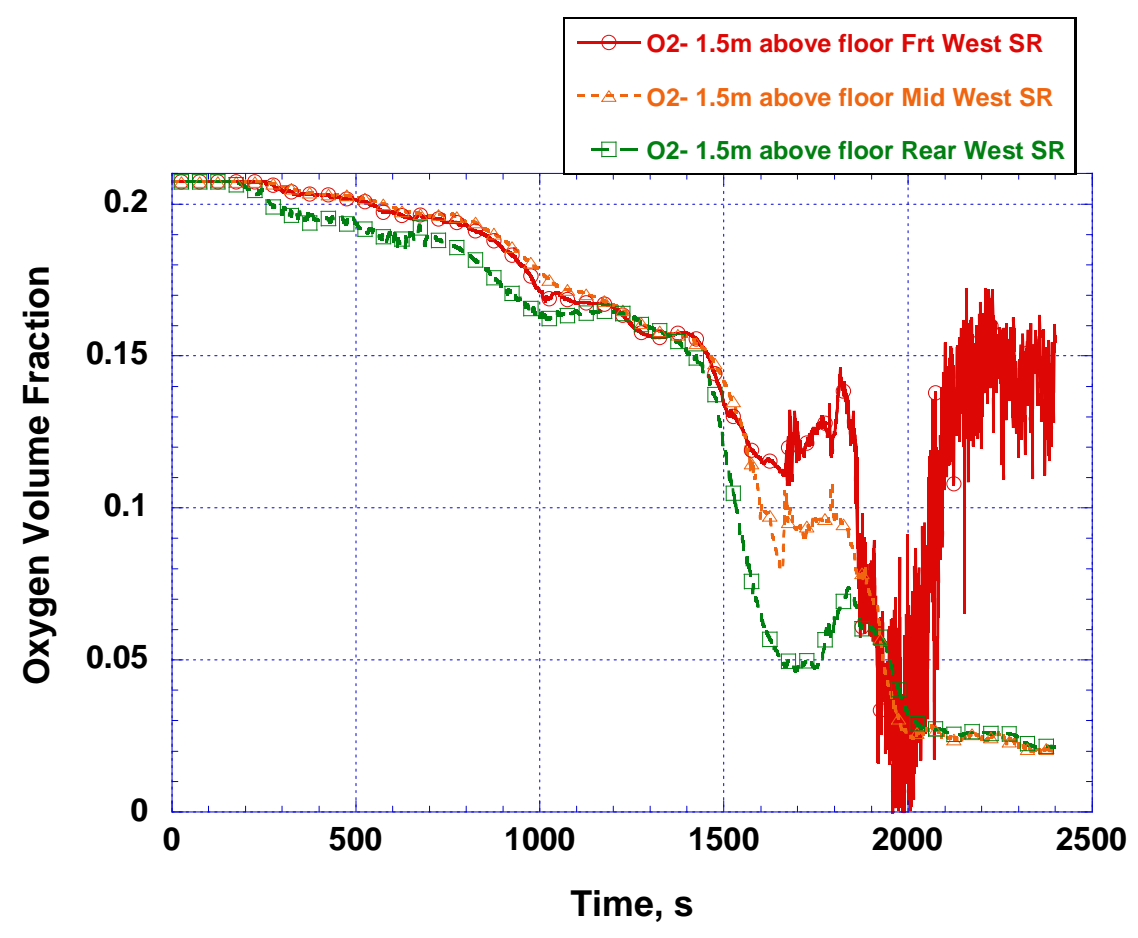

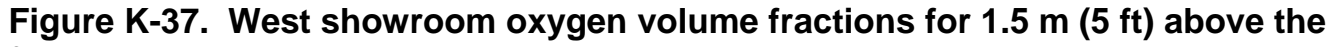
floor.

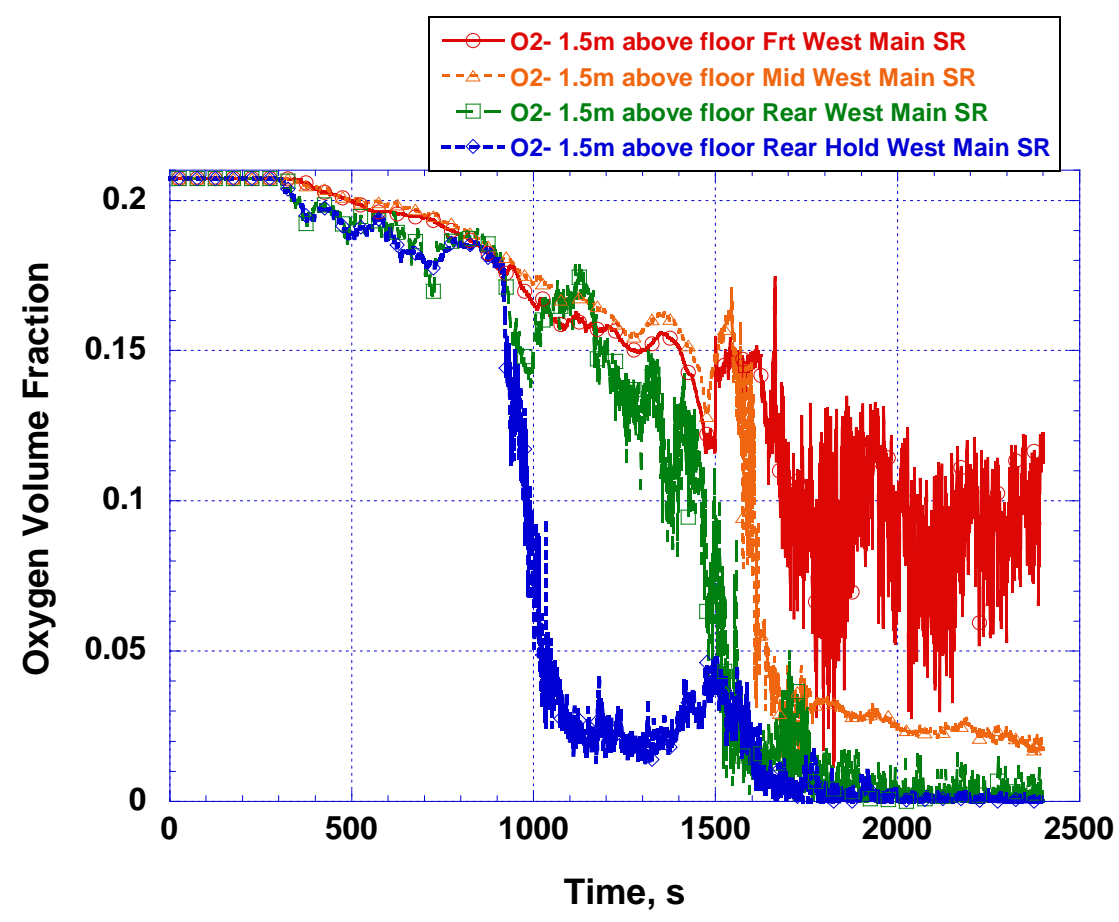

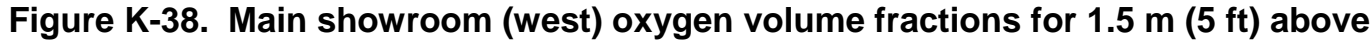
the floor. 


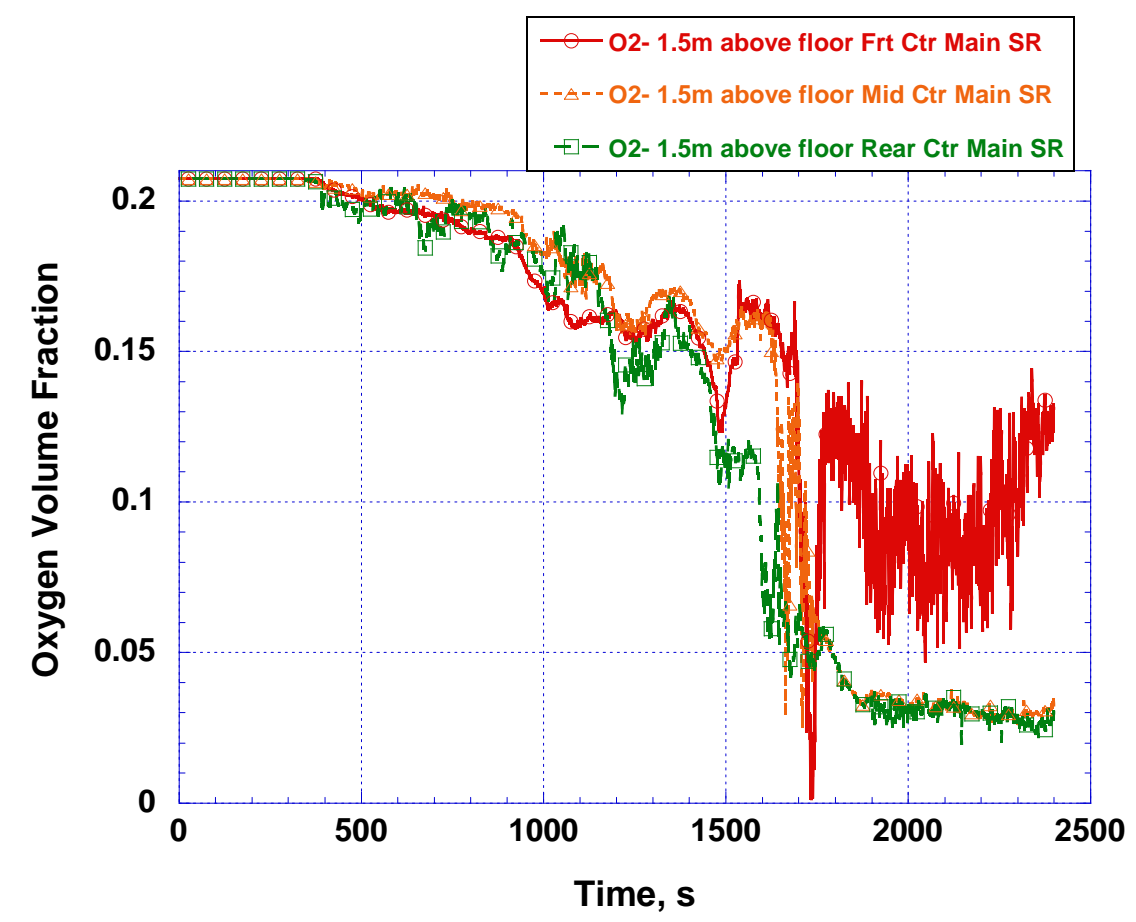

Figure K-39. Main showroom (center) oxygen volume fractions for $1.5 \mathrm{~m} \mathrm{(5} \mathrm{ft)}$ above the floor.

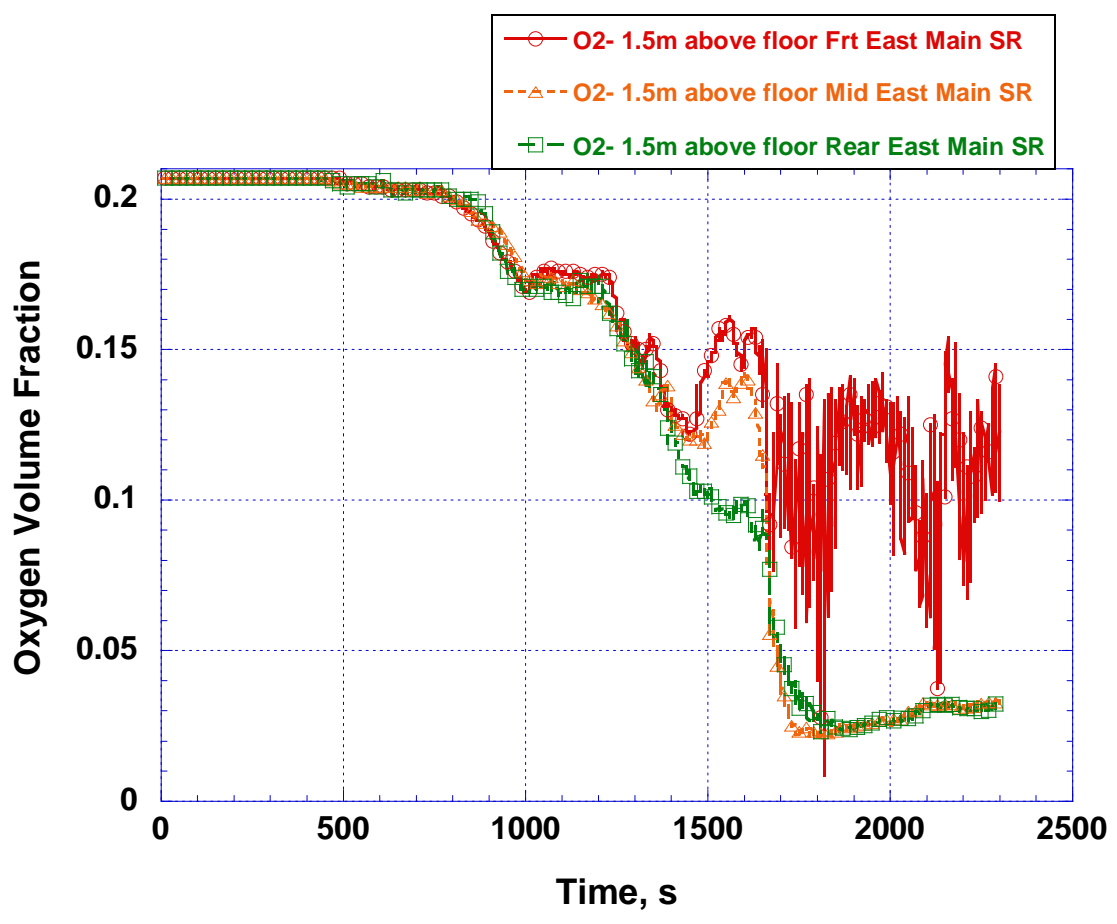

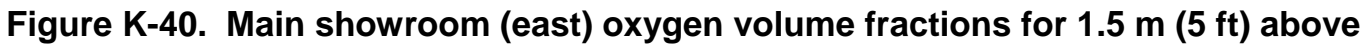
the floor. 


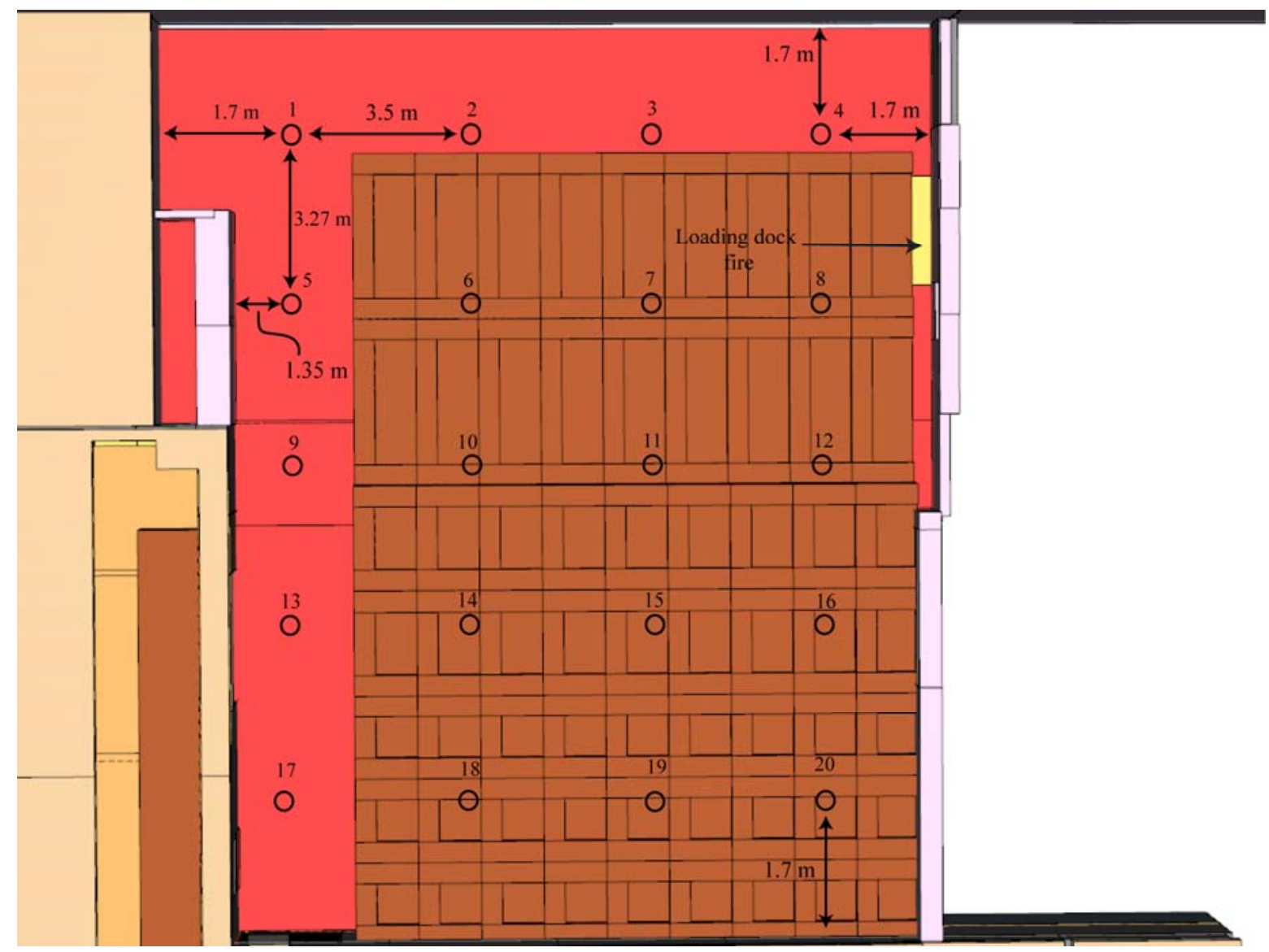

Figure K-41. Simulated sprinkler layout inside the enclosed loading dock.

Table K-8. Summary of sprinkler properties.

\begin{tabular}{|l|l|}
\hline \multicolumn{1}{|c|}{ Property } & \multicolumn{1}{c|}{ Value } \\
\hline Sprinkler Type & Pendant \\
\hline Operating Pressure & $1.7 \mathrm{bar}(25 \mathrm{psi})$ \\
\hline K-factor & $80.6 \mathrm{~L} \mathrm{~min}^{-1} \mathrm{bar}^{-1 / 2}\left(5.6 \mathrm{gpm} / \mathrm{psi}^{1 / 2}\right)$ \\
\hline Response Time Index & $40 . \mathrm{m}^{1 / 2} \mathrm{~s}^{1 / 2}\left(72 \mathrm{ft}^{1 / 2} \mathrm{~s}^{1 / 2}\right)$ \\
\hline C-Factor & 0 \\
\hline Activation Temperature & $74^{\circ} \mathrm{C}\left(165^{\circ} \mathrm{F}\right)$ \\
\hline Offset Distance & $0.10 \mathrm{~m}(3.9 \mathrm{in})$ \\
\hline Size Distribution & Global Average \\
\hline Median Volumetric Diameter & $675 \mu \mathrm{m}\left(2.66 \mathrm{x} 10^{-2} \mathrm{in}\right)$ \\
\hline Minimum Spray Angle & $75^{\circ}$ \\
\hline Maximum Spray Angle & $105^{\circ}$ \\
\hline Speed & $14 \mathrm{~m} / \mathrm{s}(46 \mathrm{ft} / \mathrm{s})$ \\
\hline
\end{tabular}


Twenty sprinklers were placed inside the loading dock, as shown by the black circles in Figure K-41. In this configuration, the maximum protection area of coverage per sprinkler was approximately $11.9 \mathrm{~m}^{2}$ $\left(128 \mathrm{ft}^{2}\right)$ with a water density of approximately $8.8 \mathrm{~L} \mathrm{~min}^{-1} \mathrm{~m}^{-2}\left(0.22 \mathrm{gpm} / \mathrm{ft}^{2}\right)$. Two sprinklers, labeled 4 and 8 in Figure K-41, activated in the simulation. Sprinkler 8 was the first to activate, at 50 s, while sprinkler 4 activated at $75 \mathrm{~s}$.

Figure K-42 shows a series of images indicating the progression of fire and smoke in the loading dock during the sprinklered simulation. These images show an overhead view, centered on the loading dock (the area shaded in red). The north end of the warehouse is shown at the top of the images and the south end of the west showroom is at the bottom. The southwest corner of the main showroom (including the holding area) is shown in the lower left corner. In these images, the roof and walls of the loading dock were made invisible in order to facilitate viewing the loading dock interior. Additionally, the roofs over the main and west showrooms were clipped so that the interior of the holding area was visible. The areas colored black are the showroom ceilings. The west showroom ceiling is only partially revealed because this addition had a sloped roof and only the upper section was clipped away.

The quantity of fire generated smoke increases through the first five images in this sequence, from 30 through $210 \mathrm{~s}$. Smoke spreads across the underside of the loading dock roof and begins to fill downward. At $210 \mathrm{~s}$, the smoke layer was low enough to begin spilling into the holding area where there is access to the space above the showroom ceiling. By $240 \mathrm{~s}$ however, the fire is no longer burning and the smoke is beginning to dissipate.

The same initiating fire was used in this scenario as for the baseline case, an ultrafast $\mathrm{t}^{2}$ growth curve [18] to a peak HRR of $750 \mathrm{~kW}$ and then held at this level for two minutes, after which time it was ramped linearly down over one minute. The intent of this fire was to ignite furniture in the loading dock. However, because the initiating fire was sufficient to activate two sprinklers (at $50 \mathrm{~s}$ and $75 \mathrm{~s}$ ), the fire did not spread to the nearby furniture on the loading dock.

\section{K.5.2 Temperature}

Figure K-43 shows the sprinkler temperature at each of the sprinkler locations. The graph indicates that the temperature at locations 4 and 8 reached $74^{\circ} \mathrm{C}$, at which point the sprinklers were activated in these locations. Temperatures at locations 1 and 2peaked in the $60^{\circ} \mathrm{C}$ to $70^{\circ} \mathrm{C}$ range but never reached the threshold for activation.

The predicted temperatures and oxygen volume fraction in the main and west showroom remained near ambient throughout since the fire in the loading dock was extinguished by the sprinklers. 


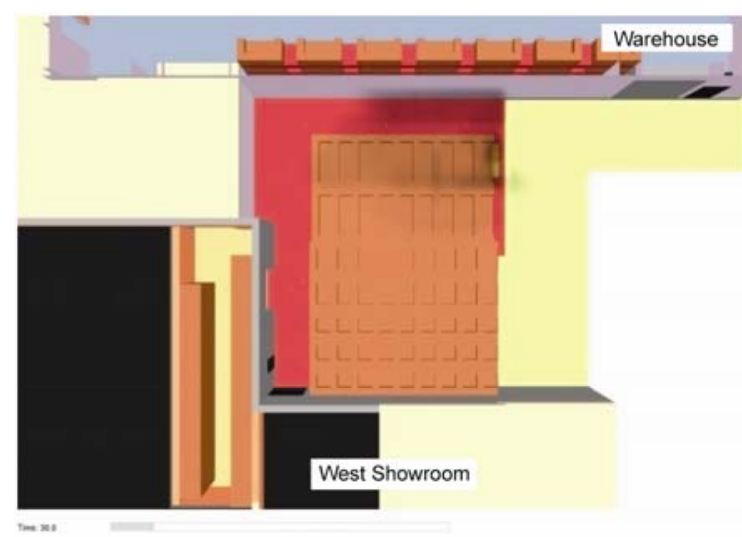

$30 \mathrm{~s}$

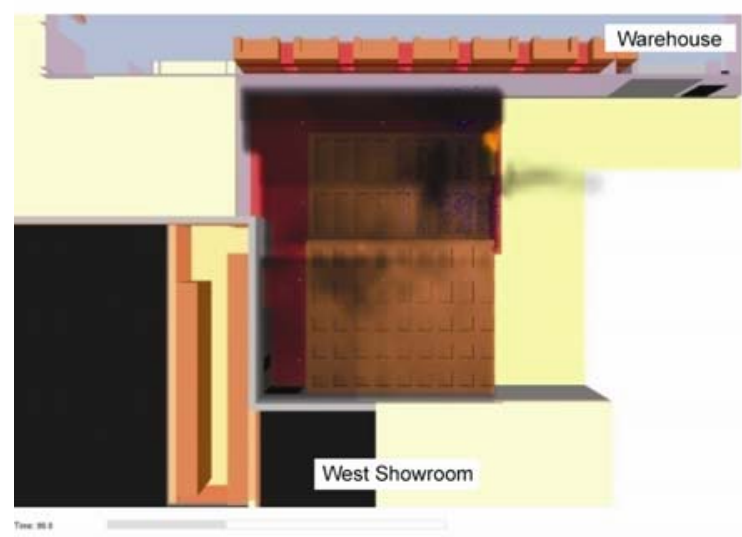

$90 \mathrm{~s}$

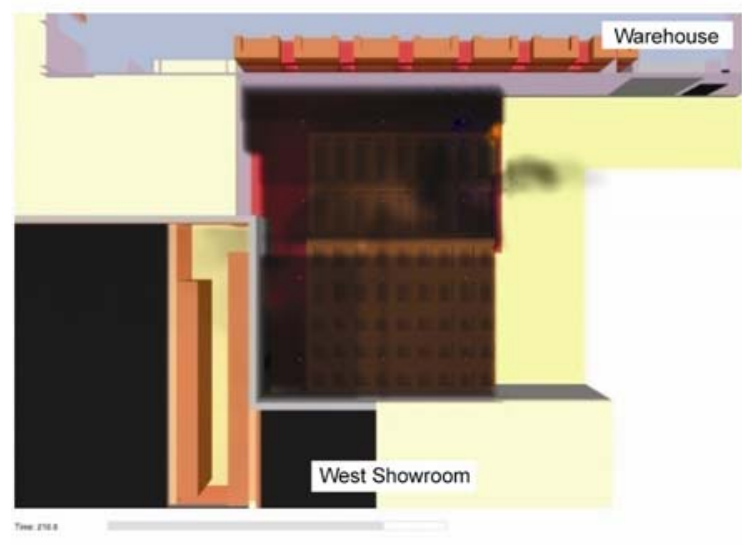

$210 \mathrm{~s}$

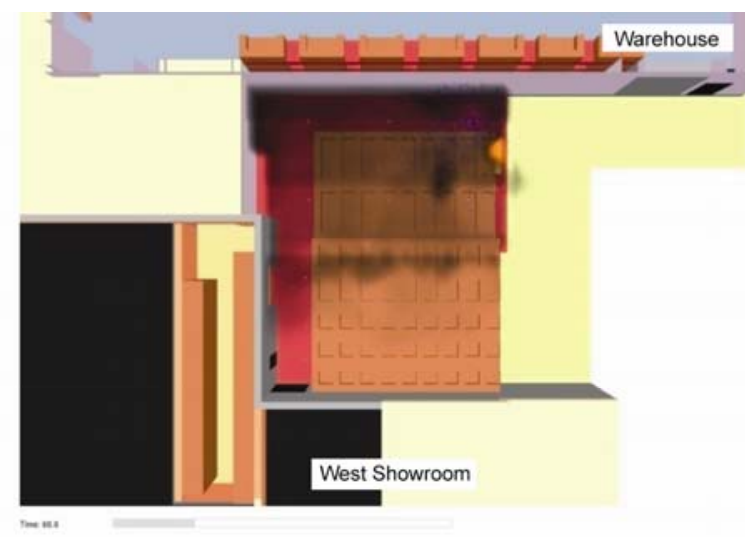

$60 \mathrm{~s}$

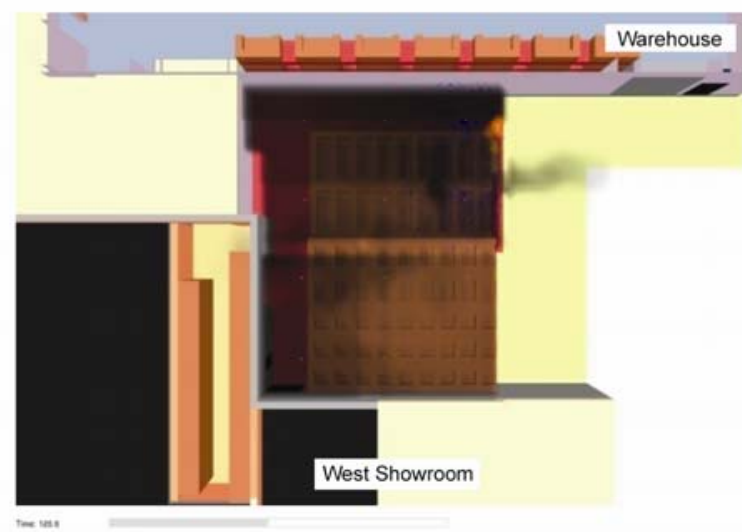

$120 \mathrm{~s}$

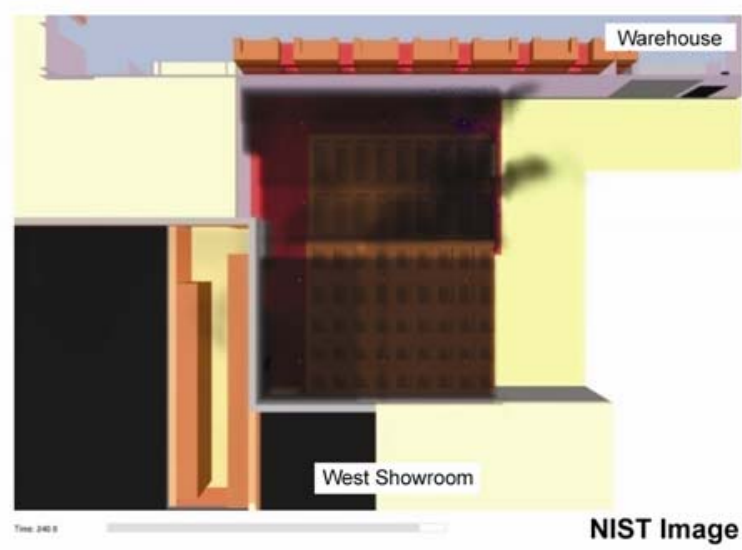

$240 \mathrm{~s}$

Figure K-42. Fire and smoke progression in the early stages of the sprinklered scenario. Sprinkler 8 was the first to activate, at $50 \mathrm{~s}$, while sprinkler 4 activated at $75 \mathrm{~s}$. 


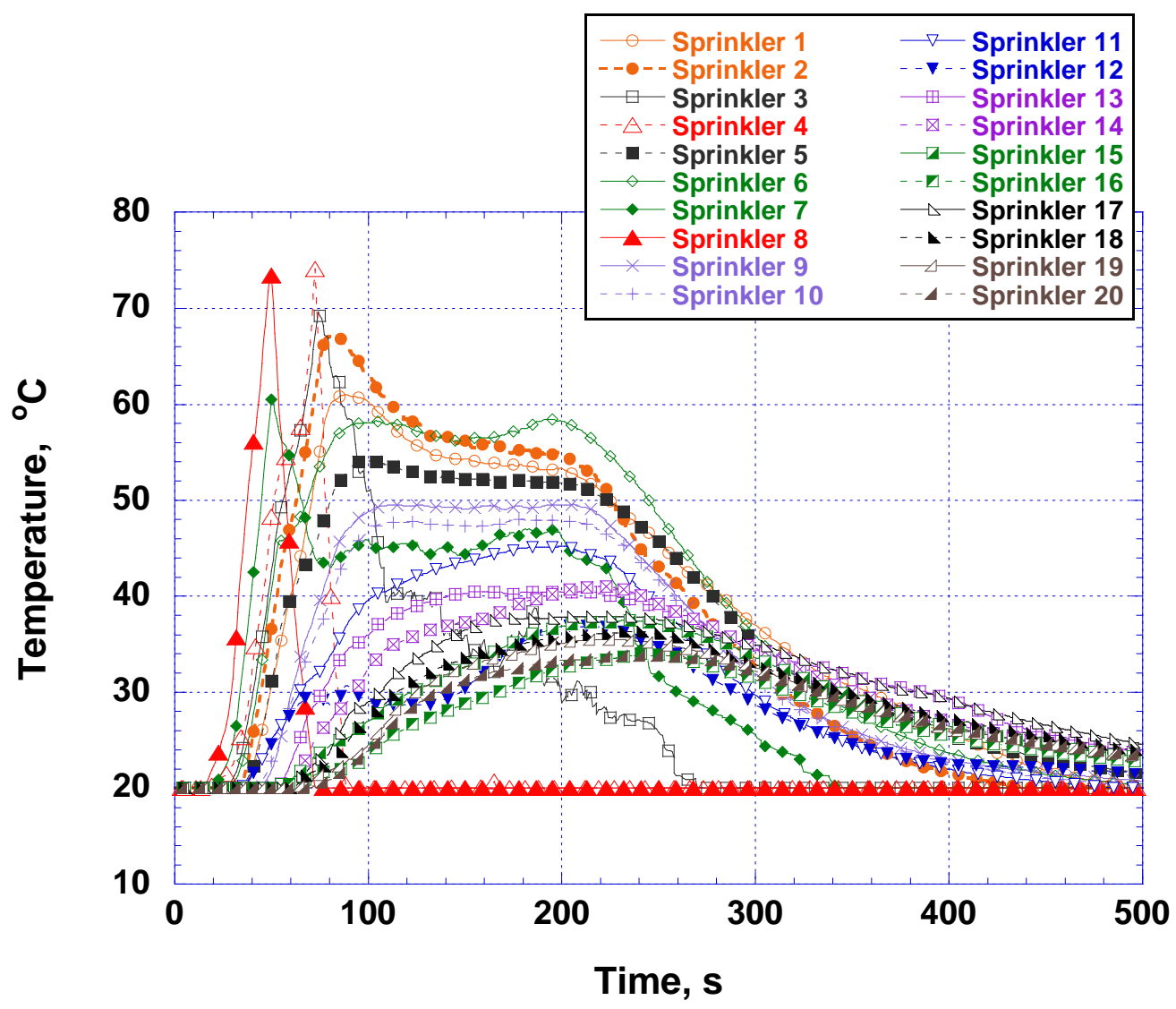

Figure K-43. Sprinkler temperatures inside the enclosed loading dock.

Figure K-44 shows a series of images with a temperature slice $1.5 \mathrm{~m}(5 \mathrm{ft})$ from the floor rendered at a range of times between $30 \mathrm{~s}$ through $300 \mathrm{~s}$. Note that the scale on these images is consistent with those in Figure K-30. These images show that the area of elevated temperatures is limited to the immediate vicinity of the fire inside the loading dock.

\section{K.5.3 Oxygen Volume Fraction}

A similar result is seen in the oxygen volume fraction data, shown in Figure K-45. This figure shows a series of images $1.5 \mathrm{~m}$ (5 ft) from the floor at times between $30 \mathrm{~s}$ through $240 \mathrm{~s}$. Depleted oxygen levels are observed in the vicinity of the holding area. The oxygen volume fraction at $1.5 \mathrm{~m}$ never drops below the tenability threshold of 0.12 (volume fraction). There is an increase in oxygen volume fraction between the final two images, as fresh air enters the holding area. 


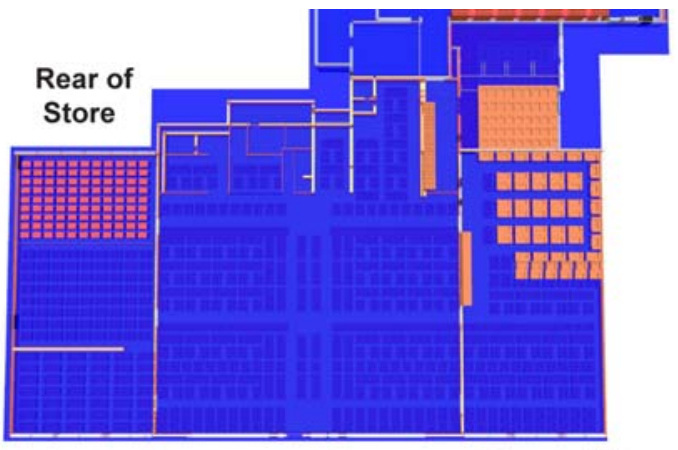

Front of Store

$30 \mathrm{~s}$

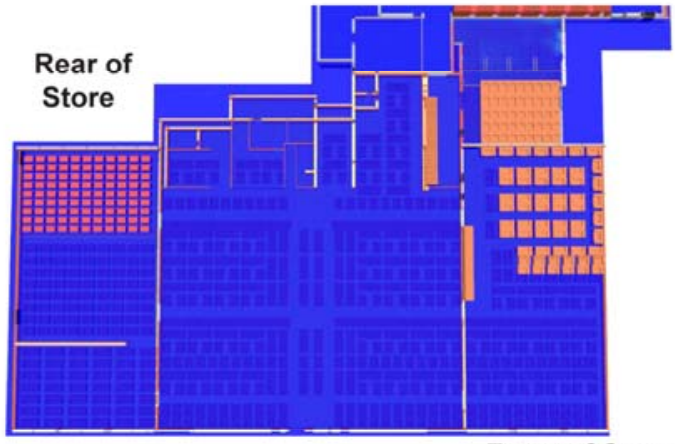

$120 \mathrm{~s}$

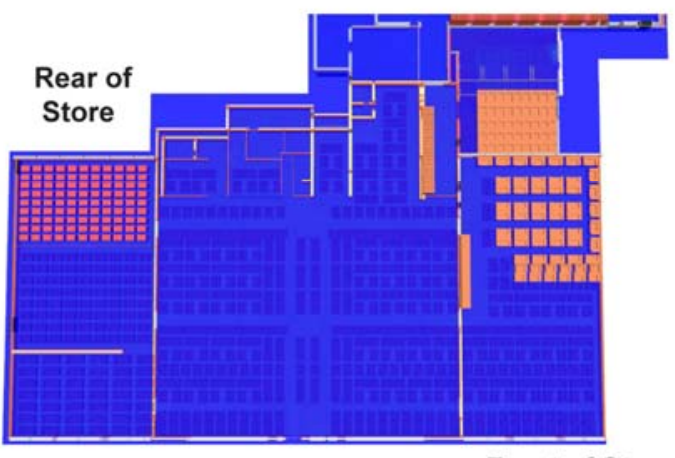

$240 \mathrm{~s}$

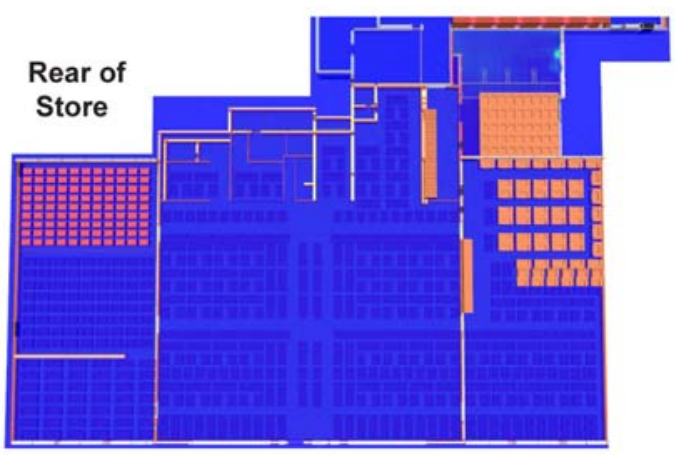

Gas

$60 \mathrm{~s}$

Front of Store

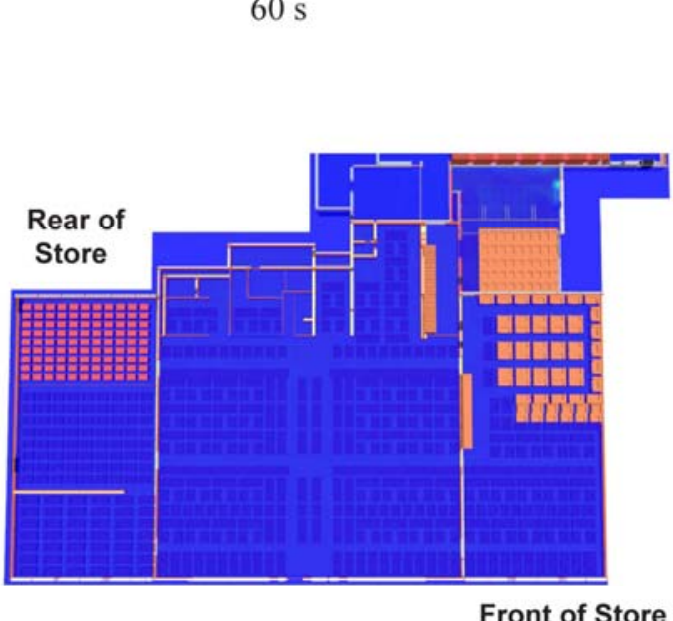

$180 \mathrm{~s}$

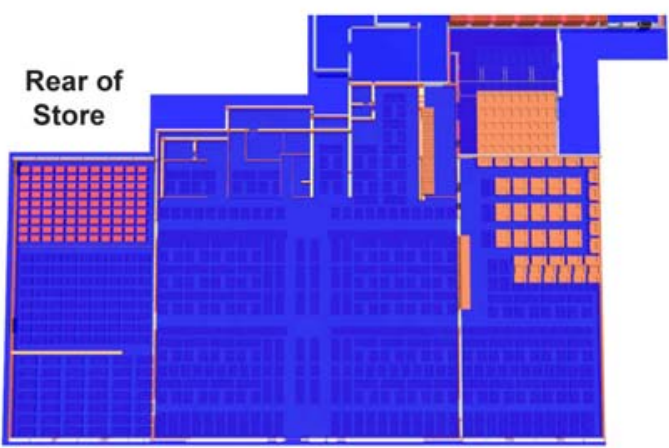

Front of Store

$300 \mathrm{~s}$

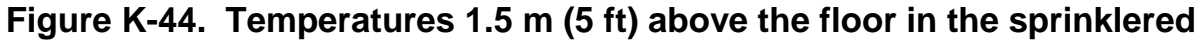
simulation. 


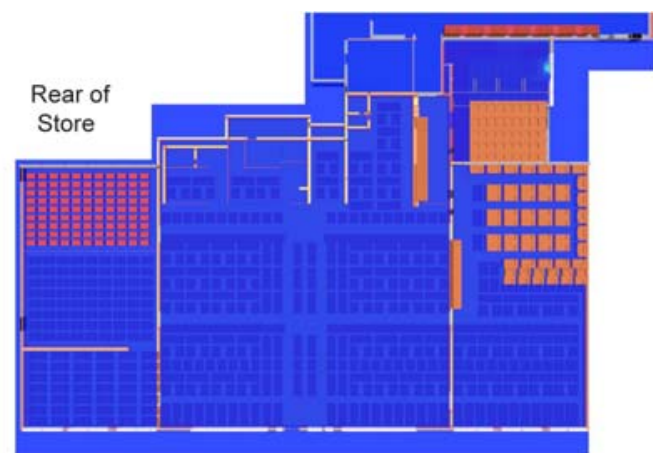

Front of Store

$30 \mathrm{~s}$

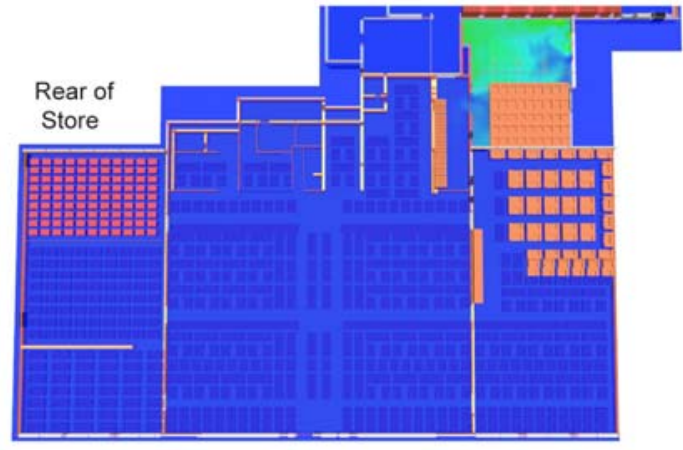

Front of Store

$120 \mathrm{~s}$

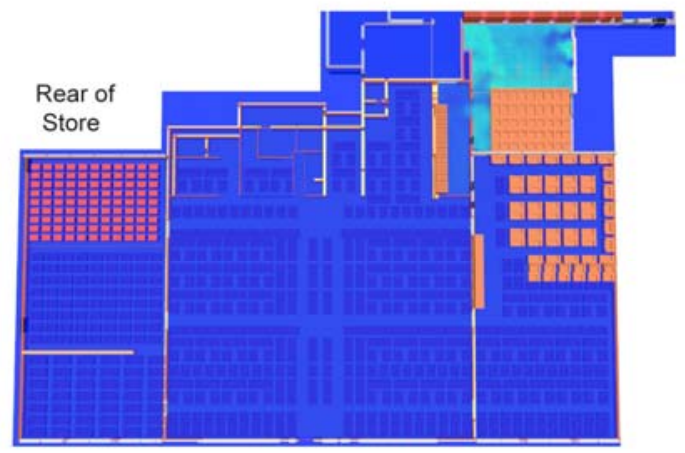

Front of Store

$240 \mathrm{~s}$

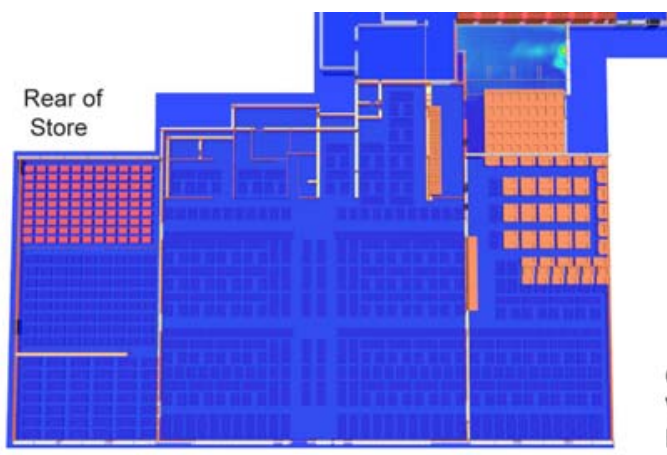

Volume

Fraction

Front of Store

$60 \mathrm{~s}$

0.20

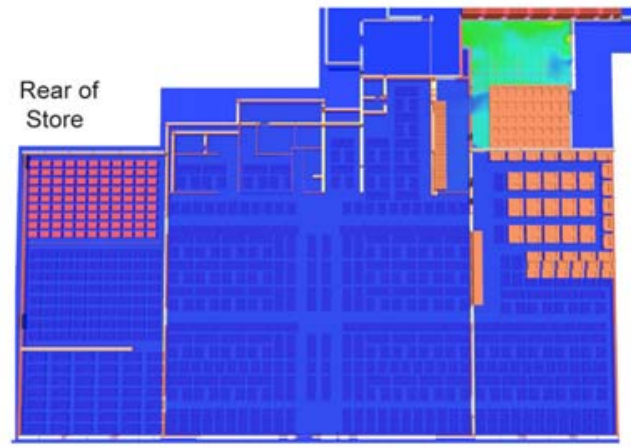

0.19

0.18

0.17

0.16

0.15

0.14

Front of Store

$180 \mathrm{~s}$

0.13

0.12

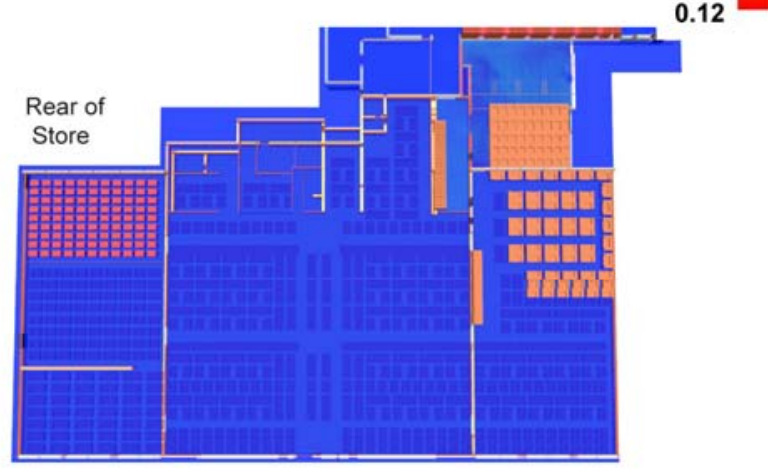

Front of Store

$300 \mathrm{~s}$

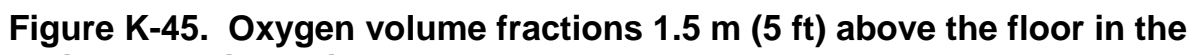
sprinklered simulation. 


\section{K.6 BASELINE WITH FRONT WINDOWS INTACT}

\section{K.6.1 General}

In this simulation, the windows at the north (front) end of the main and west showrooms were kept in place, while everything else remained fixed from the base scenario. The front entrance, through which fire department personnel entered and exited the main showroom, was kept open and all other ventilation parameters were unaltered. The results of this simulation are presented here.

\section{K.6.2 Fire Growth and Smoke Spread}

Figure K-46 shows the progression of fire and smoke throughout the loading dock, holding area and showrooms between $1020 \mathrm{~s}$ and $2040 \mathrm{~s}$ (7:27:53 p.m. through 7:44:53 p.m.). The first three images in this figure are indistinguishable from the corresponding images in Figure K-22 (the baseline simulation). The differences appear in the later images - $1500 \mathrm{~s}$ and beyond. Compared to Figure K-22, Figure K-46 shows far less burning in the northwest quadrant of the main showroom.

In the base simulation the fire HRR begins to diminish after $1380 \mathrm{~s}$, but when the front windows of the main showroom were taken out starting at $1457 \mathrm{~s}$, there was a rapid increase in the HRR. In this scenario, with the windows remaining intact, the fire HRR continues to decrease after $1380 \mathrm{~s}$. The fire moved toward the front of the showroom, where oxygen was still being supplied through the front door. With the front windows intact, the flames reached the front of the main showroom at $1800 \mathrm{~s}$ (7:40:53 p.m.), approximately 3 minutes longer than the base scenario. At 2040 s (7:44:53 p.m.) in the base case, the flames appear across the front of the main and west showrooms, while with the windows intact, the flames only appear across a small portion of the main showroom.

\section{K.6.3 Heat Release Rate}

Figure K-47 shows the change in HRR over time for this scenario and the baseline simulation. In both scenarios, HRR peaked at $120 \mathrm{MW}$ at around $1300 \mathrm{~s}$ after which the HRR decreased. This would be consistent with the fire becoming underventilated. The venting of the front windows in the main showroom at 1457 s corresponds with the subsequent rapid increase in HRR for the base case. In the simulation with the windows intact, the HRR did not increase at approximately $1500 \mathrm{~s}$, but continued to decrease until about $1600 \mathrm{~s}$. 


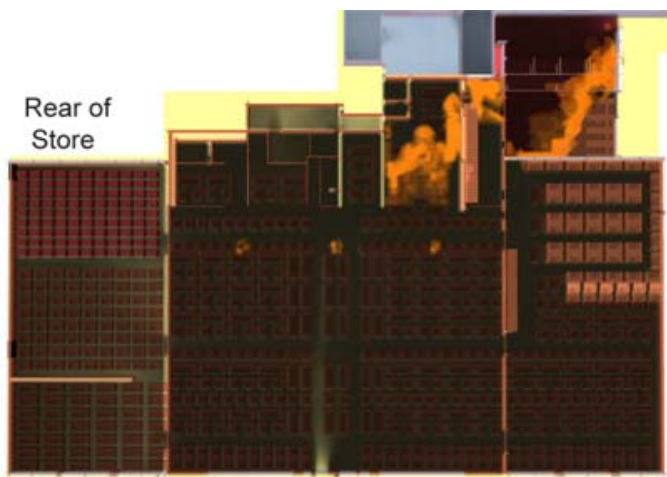

Front of Store

$1020 \mathrm{~s}$

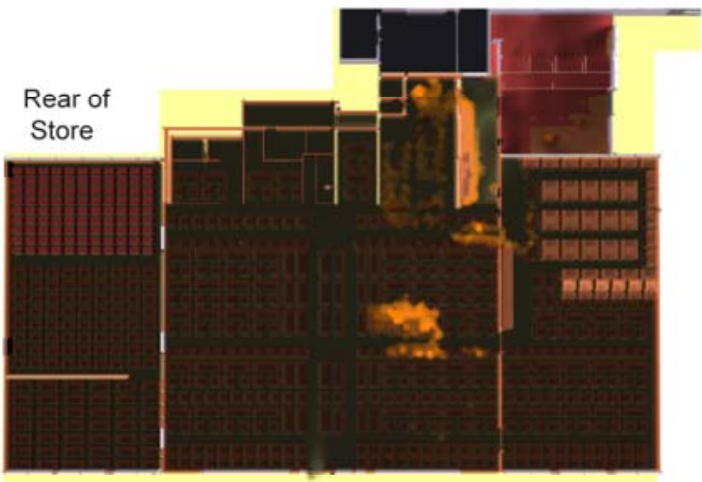

Front of Store

$1500 \mathrm{~s}$

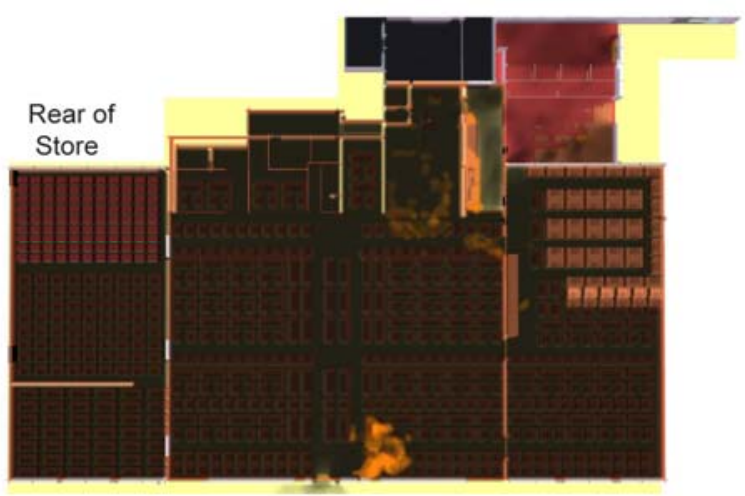

Front of Store

$1800 \mathrm{~s}$

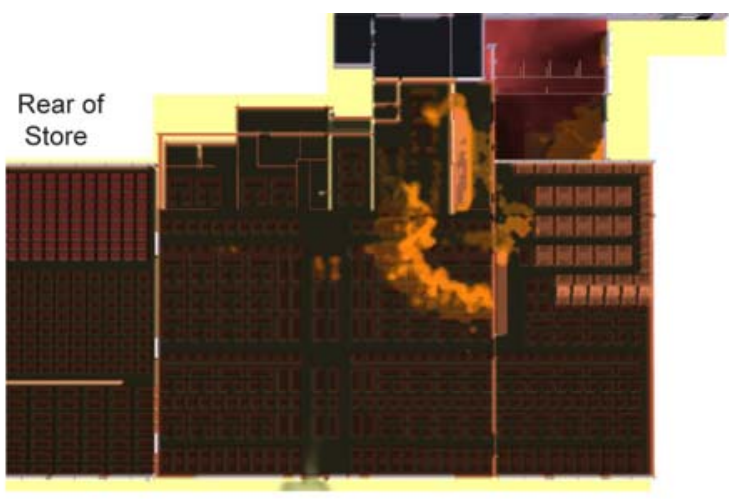

Front of Store

$1380 \mathrm{~s}$

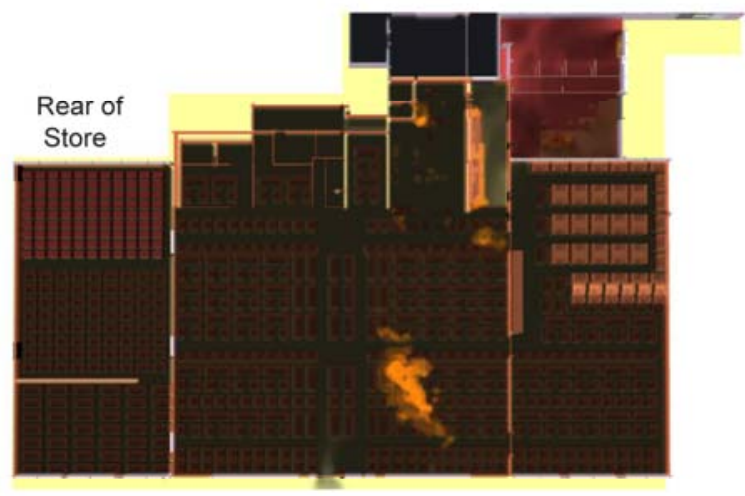

Front of Store

$1620 \mathrm{~s}$

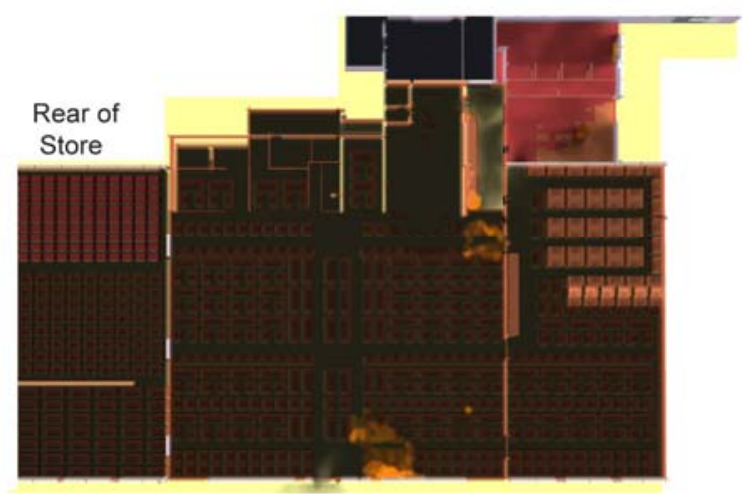

Front of Store

$2040 \mathrm{~s}$

Figure K-46. Fire and smoke progression in case with front windows intact. 


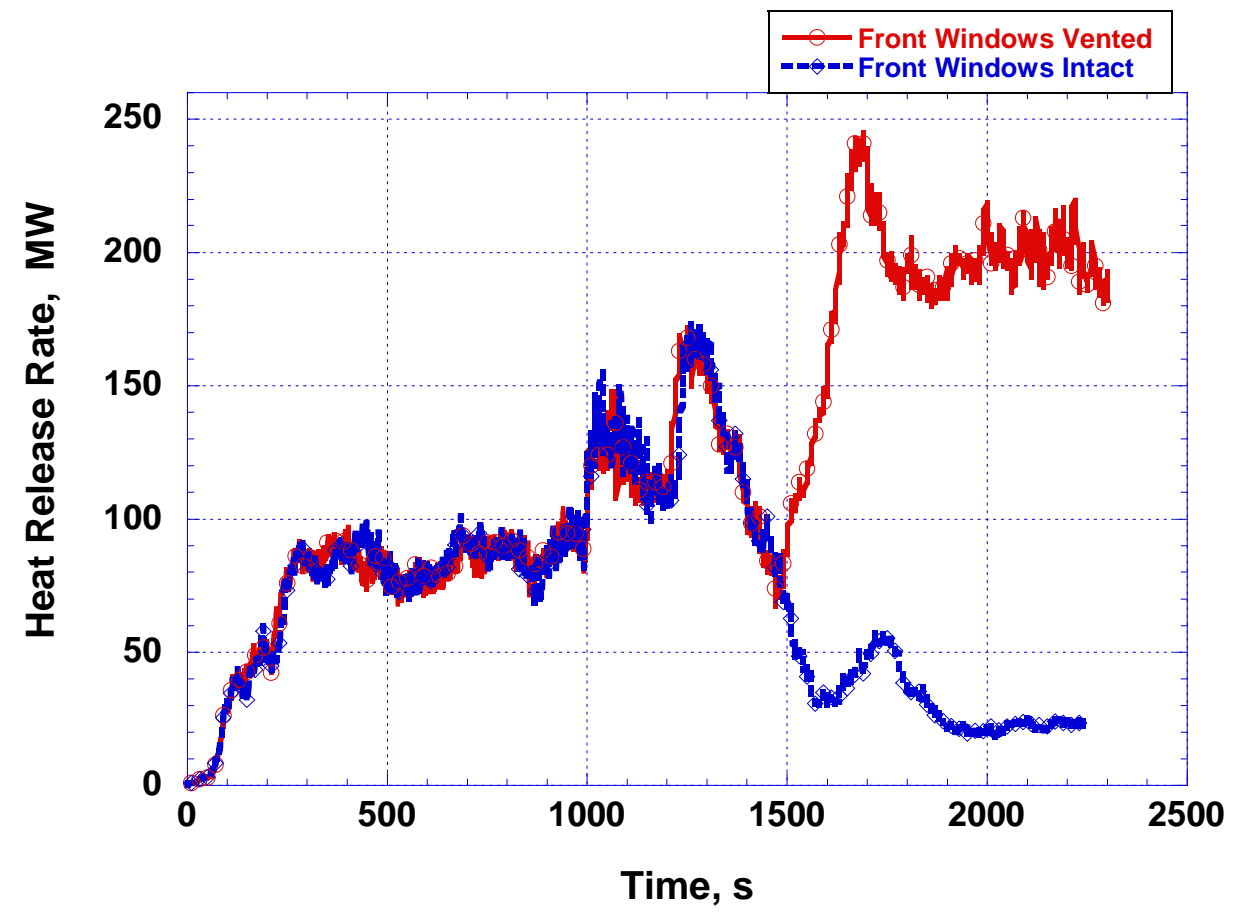

Figure K-47. Comparison of the heat release rate for simulations with front windows vented and front windows intact.

\section{K.6.4 Temperature}

Figure K-48 shows a series of images with a temperature slice $1.5 \mathrm{~m}(5 \mathrm{ft})$ from the floor rendered at a range of times between $900 \mathrm{~s}$ through $1620 \mathrm{~s}$ (7:25:53 p.m. through 7:37:53 p.m.). The trends in this figure are similar to those seen in Figure K-30 for the baseline simulation. For the windows intact scenario the high temperature areas, shown in red, diminishes between $1380 \mathrm{~s}$ and $1500 \mathrm{~s}$. After $1500 \mathrm{~s}$ the simulation displays less red areas in the windows intact case than for the same times in the baseline case Figure K-30.

Figures K-49 through K-53 show simulated temperature data at the same sensor locations used in Figure K-29 for the baseline simulation. For comparison purposes, the scale in these figures was kept the same as the corresponding scale in Figures K-31 through K-36. After 1500 s, the temperatures were lower in the scenario with the front windows intact as compared to the base case. The temperatures with the windows intact exceeded the $120^{\circ} \mathrm{C}$ tenability threshold at each sensor location before the time at which the windows would have been broken out.

\section{K.6.5 Oxygen Volume Fraction}

Figures K-54 through K-57 show oxygen volume fraction data for the front windows intact case that were recorded at the same sensor locations (Figure K-29) as the baseline case. For the first $1000 \mathrm{~s}$, the oxygen volume fraction in the west showroom Figures K-37 through K-40 drops to about 0.16 for both cases. 

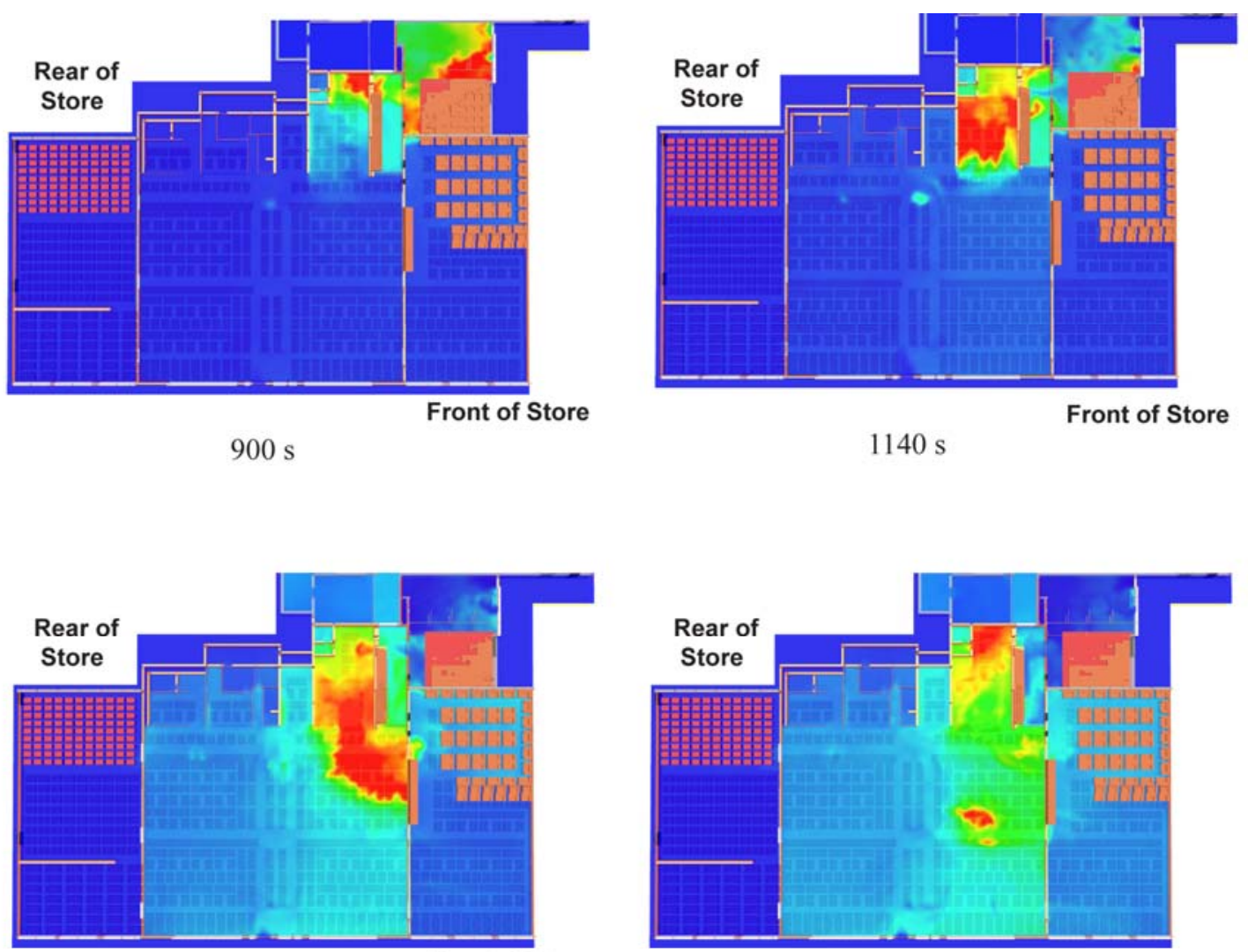

Front of Store

$1380 \mathrm{~s}$

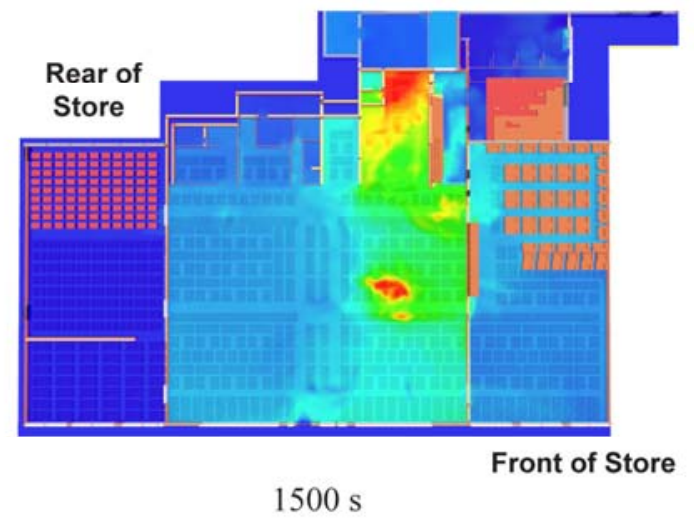

660

580

500

420

340

260

180

100

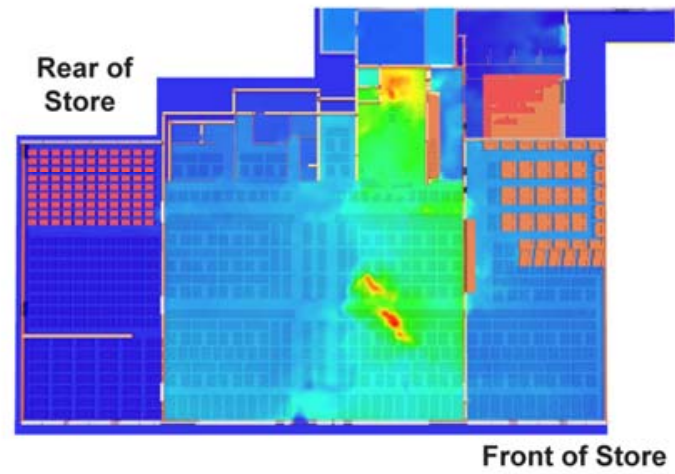

$1560 \mathrm{~s}$

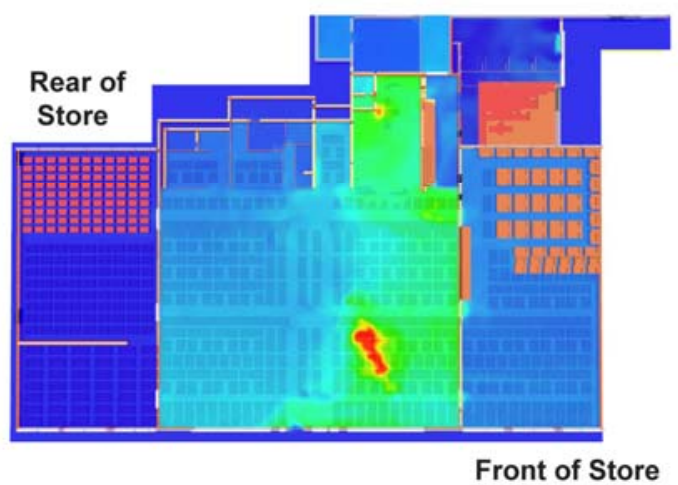

$1620 \mathrm{~s}$

Figure K-48. Temperature slices located $1.5 \mathrm{~m} \mathrm{(5} \mathrm{ft)} \mathrm{above} \mathrm{the} \mathrm{floor} \mathrm{in} \mathrm{simulation} \mathrm{with} \mathrm{front}$ windows intact. 


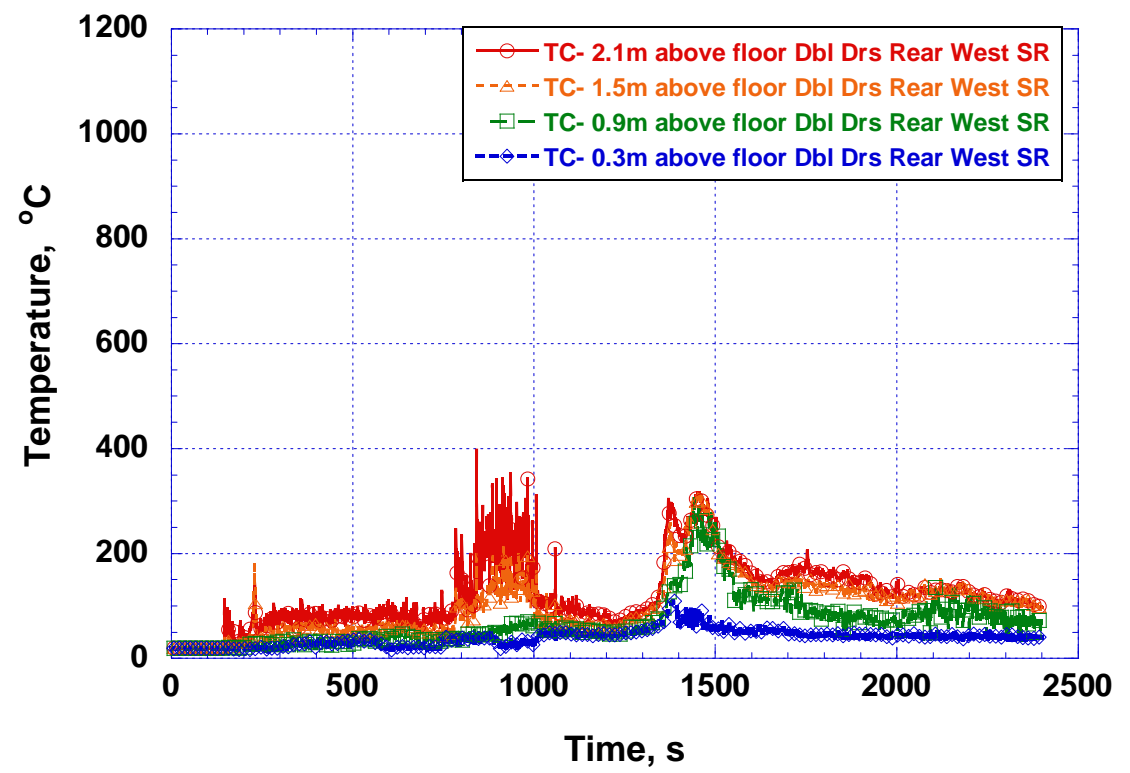

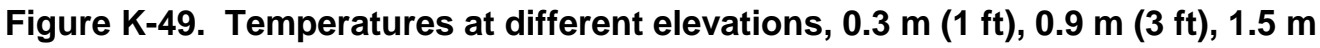
$(5 \mathrm{ft})$, and $2.1 \mathrm{~m}(7 \mathrm{ft})$ in rear of west showroom near double doors.

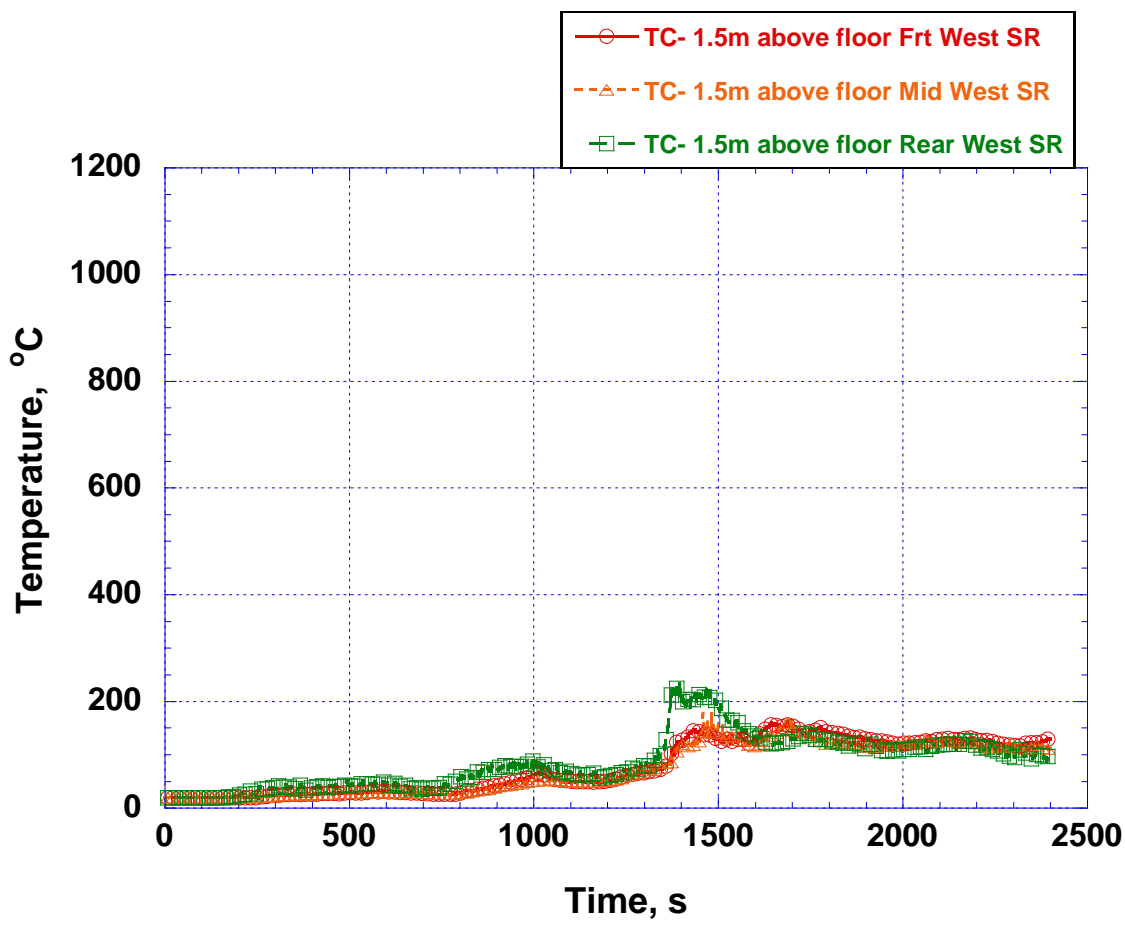

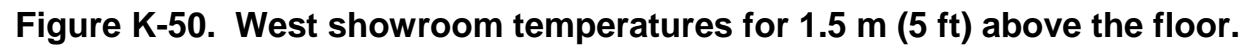




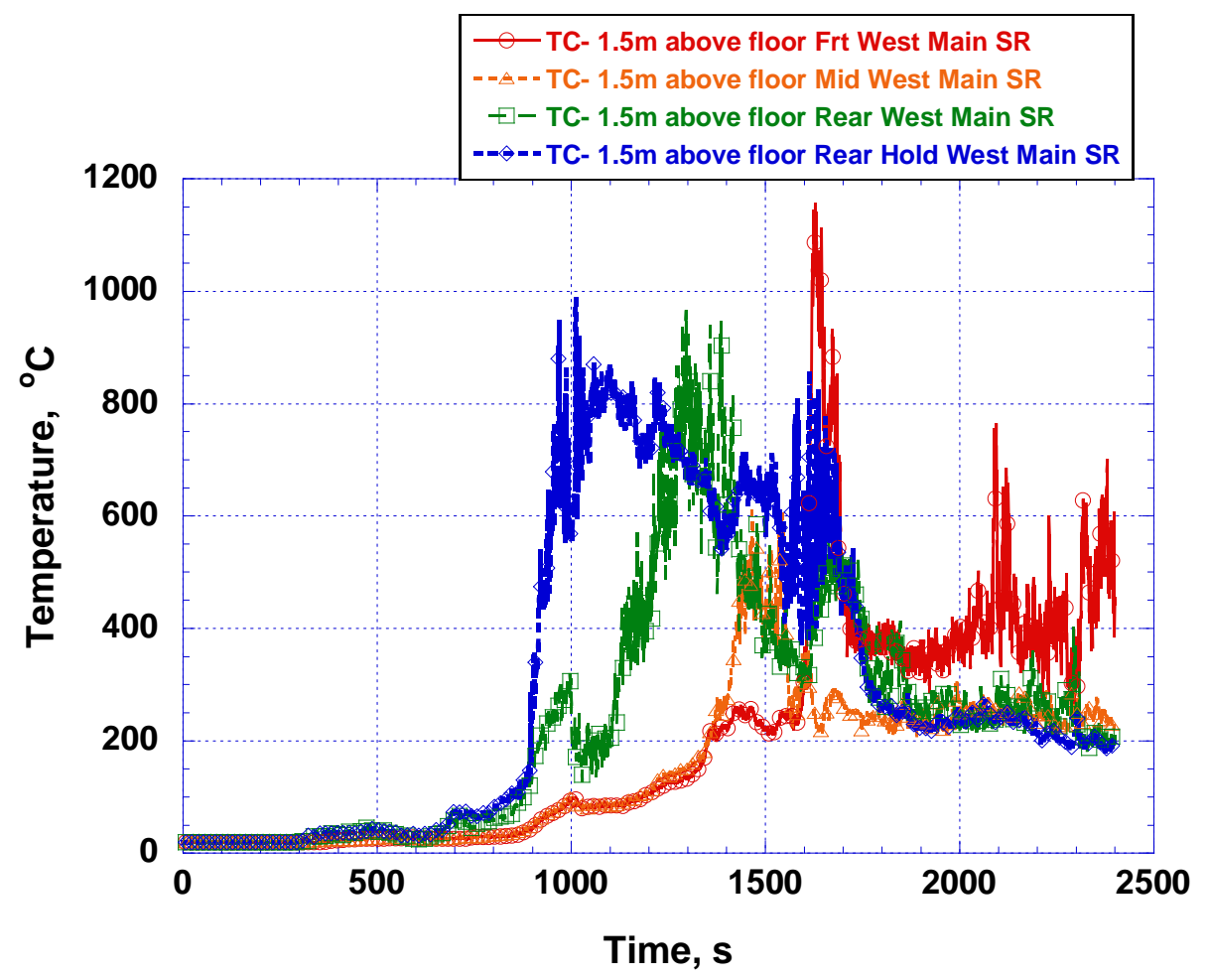

Figure K-51. Main showroom (west) temperatures for $1.5 \mathrm{~m} \mathrm{(5} \mathrm{ft)} \mathrm{above} \mathrm{the} \mathrm{floor.}$

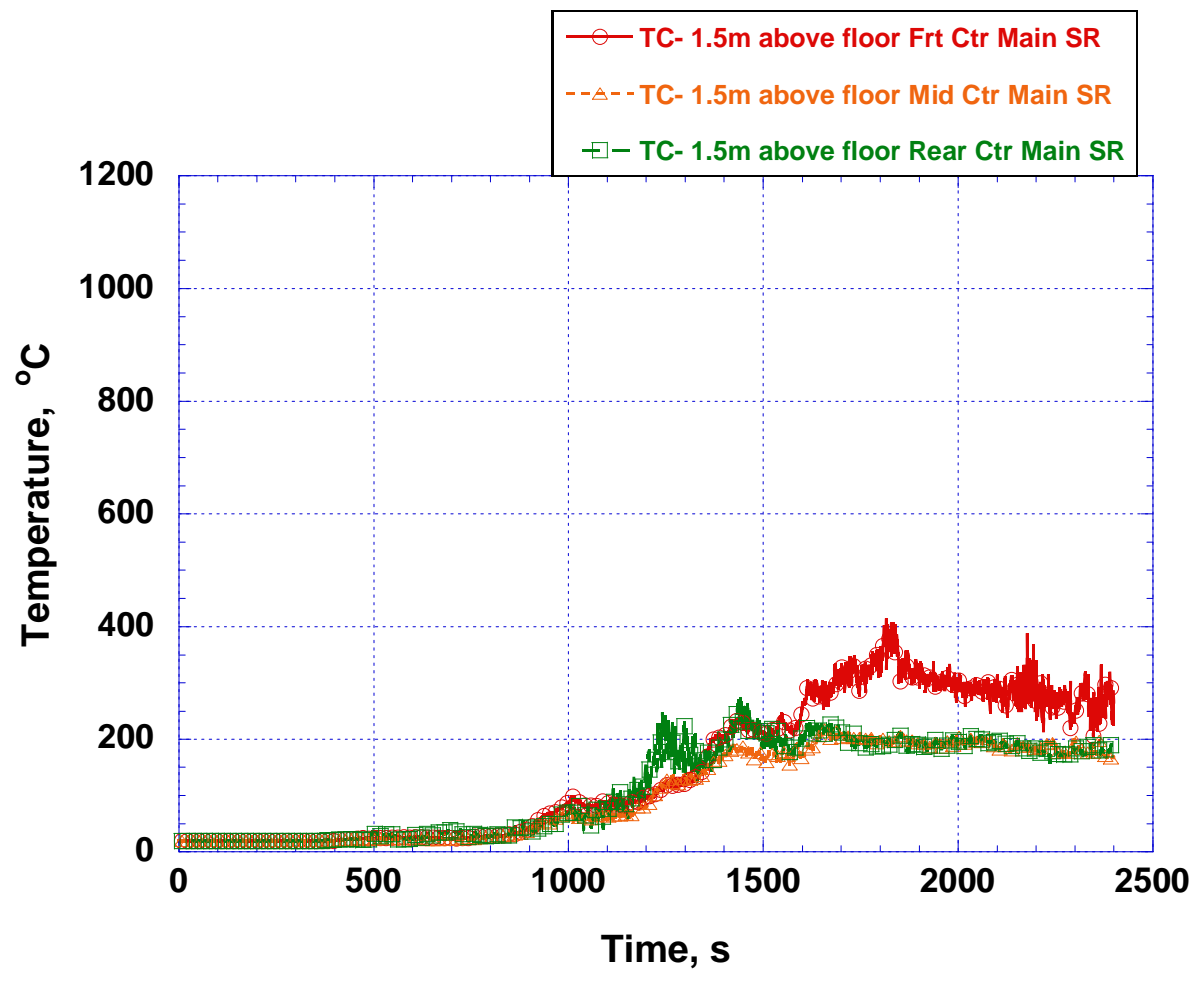

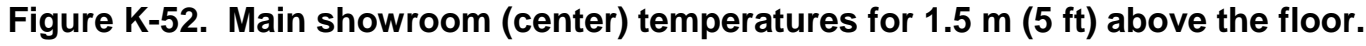




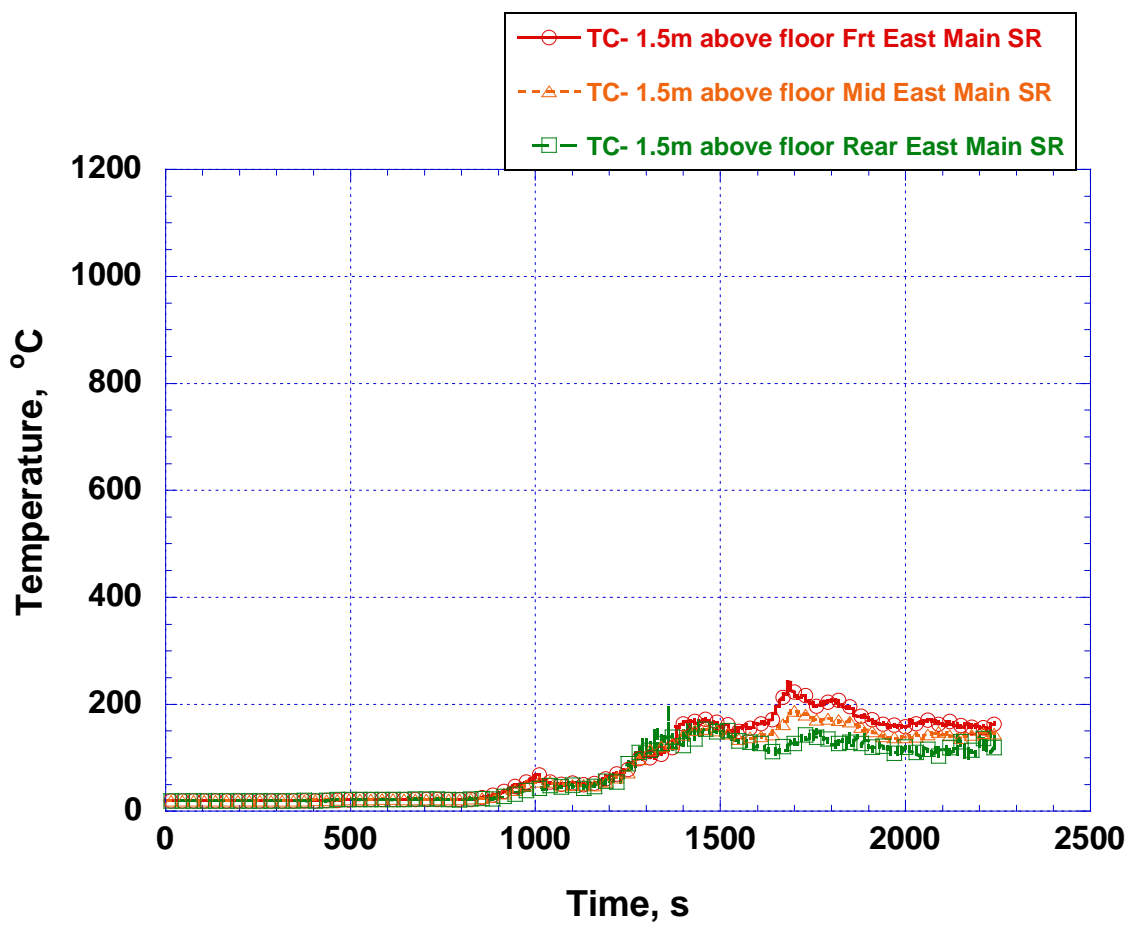

Figure K-53. Main showroom (east) temperatures for $1.5 \mathrm{~m} \mathrm{(5} \mathrm{ft)} \mathrm{above} \mathrm{the} \mathrm{floor.}$

For about the next $400 \mathrm{~s}$, the oxygen volume fraction decreases more slowly in both cases. By $1400 \mathrm{~s}$ the volume fraction drops below 0.1 for the windows intact case, but decreases to 0.15 for the base scenario. For the windows intact case, after the time when the windows would have been broken at $1457 \mathrm{~s}$, the oxygen volume fraction in the rear of the west showroom begins to increase and peak at about 0.12 at $1850 \mathrm{~s}$. The oxygen volume fractions in the mid and front of the west showroom decrease to 0.09 and 0.07 , respectively. The oxygen volume fractions in the rear of the main showroom (Figures K-55 and K-38) show similar trends during the first $1300 \mathrm{~s}$. For the windows intact case, the oxygen volume fraction became more steady between 0.07 and 0.09 . The oxygen volume fraction for the same location in the base scenario continues to drop to less than 0.02 . For the base case, after $1700 \mathrm{~s}$, the oxygen volume fraction is less than 0.03 for the holding area, rear, and mid showroom. For the windows intact scenario, at about the same time, $1700 \mathrm{~s}$, the oxygen volume fraction for the rear, mid, and front showroom locations are all above 0.07. Overall, the trends for both cases are similar during the early portion of the simulations, but after the time at which the windows were broken out in the base scenario, the oxygen volume fractions appear lower than in the front windows intact case for the rear holding, rear, and mid showroom locations.

Figure K-58 shows a series of images with an oxygen slice $1.5 \mathrm{~m}(5 \mathrm{ft})$ from the floor rendered at a range of times between $360 \mathrm{~s}$ and $1440 \mathrm{~s}$. As was the case with temperature, the conditions in the main and west showrooms become untenable because of oxygen depletion prior to $1440 \mathrm{~s}$ (7:34:53 p.m.). While minimum oxygen volume fractions were not as low in the case with the windows intact, they nonetheless dropped below 0.12 (indicated by the red shaded areas in Figure K-48) in the main and west showrooms. 


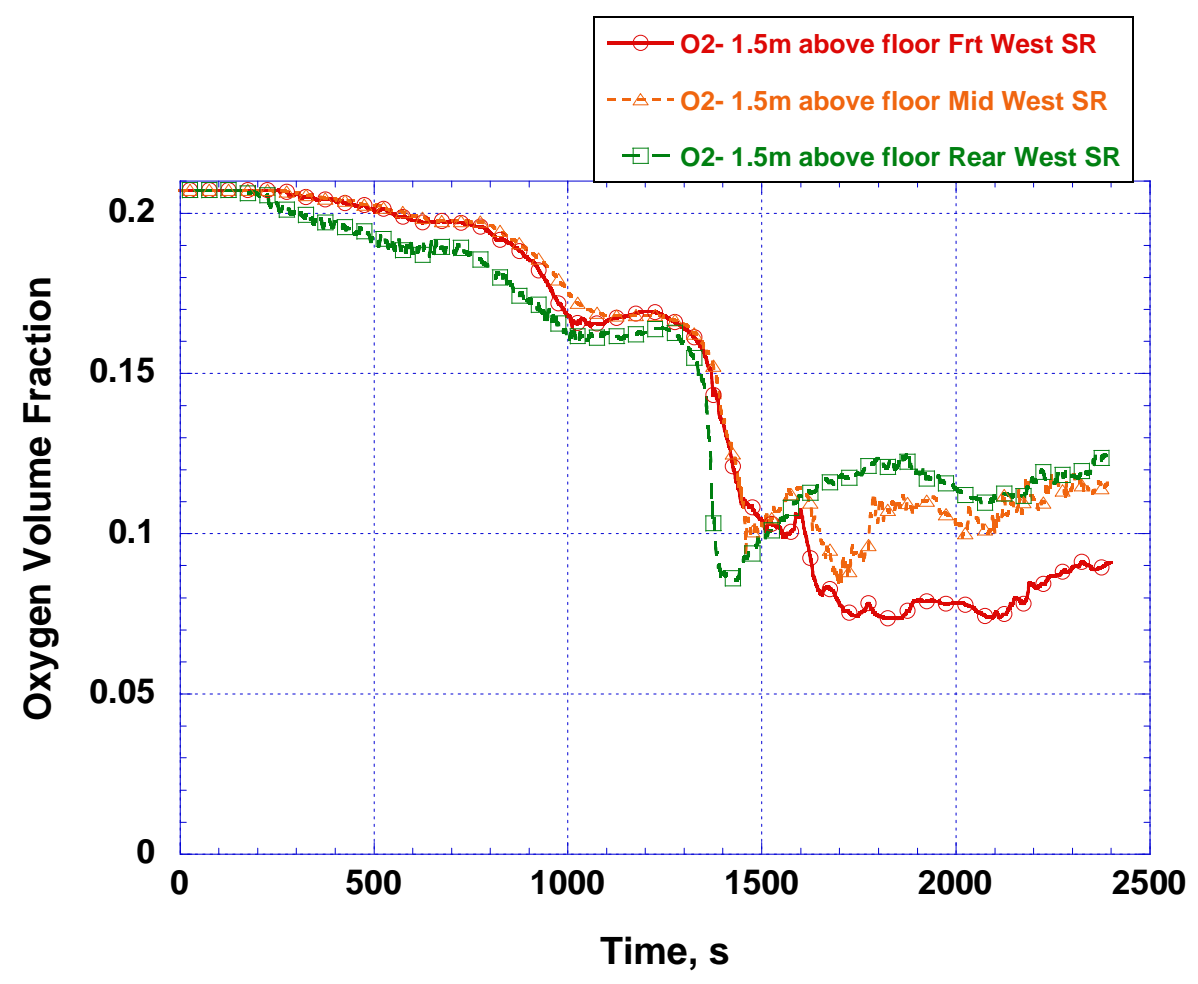

Figure K-54. West showroom oxygen volume fractions for $1.5 \mathrm{~m} \mathrm{(5} \mathrm{ft)} \mathrm{above} \mathrm{the} \mathrm{floor.}$

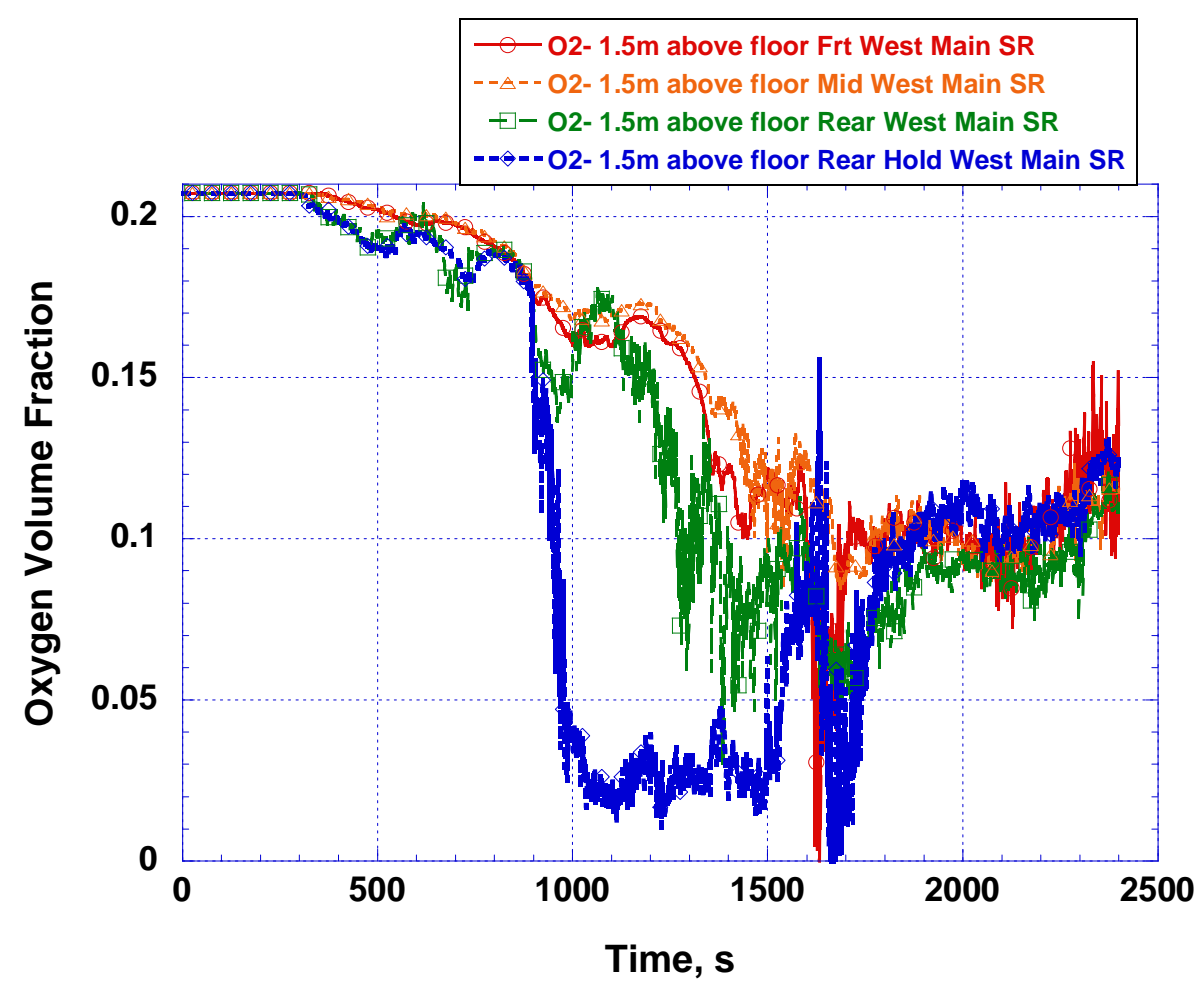

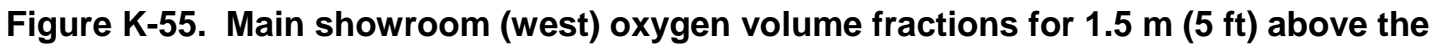
floor. 


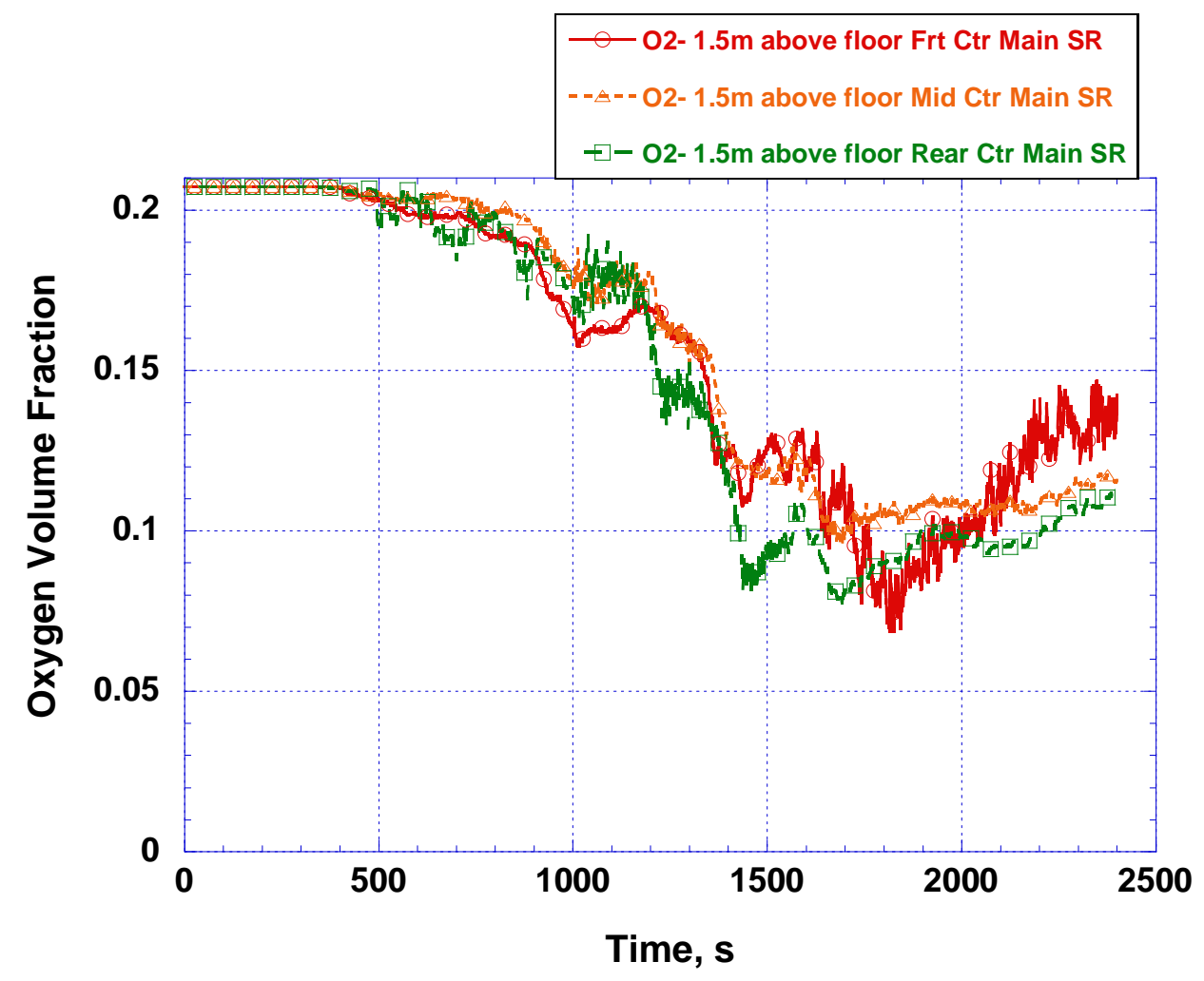

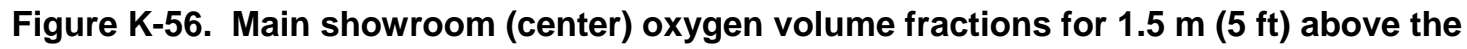
floor.

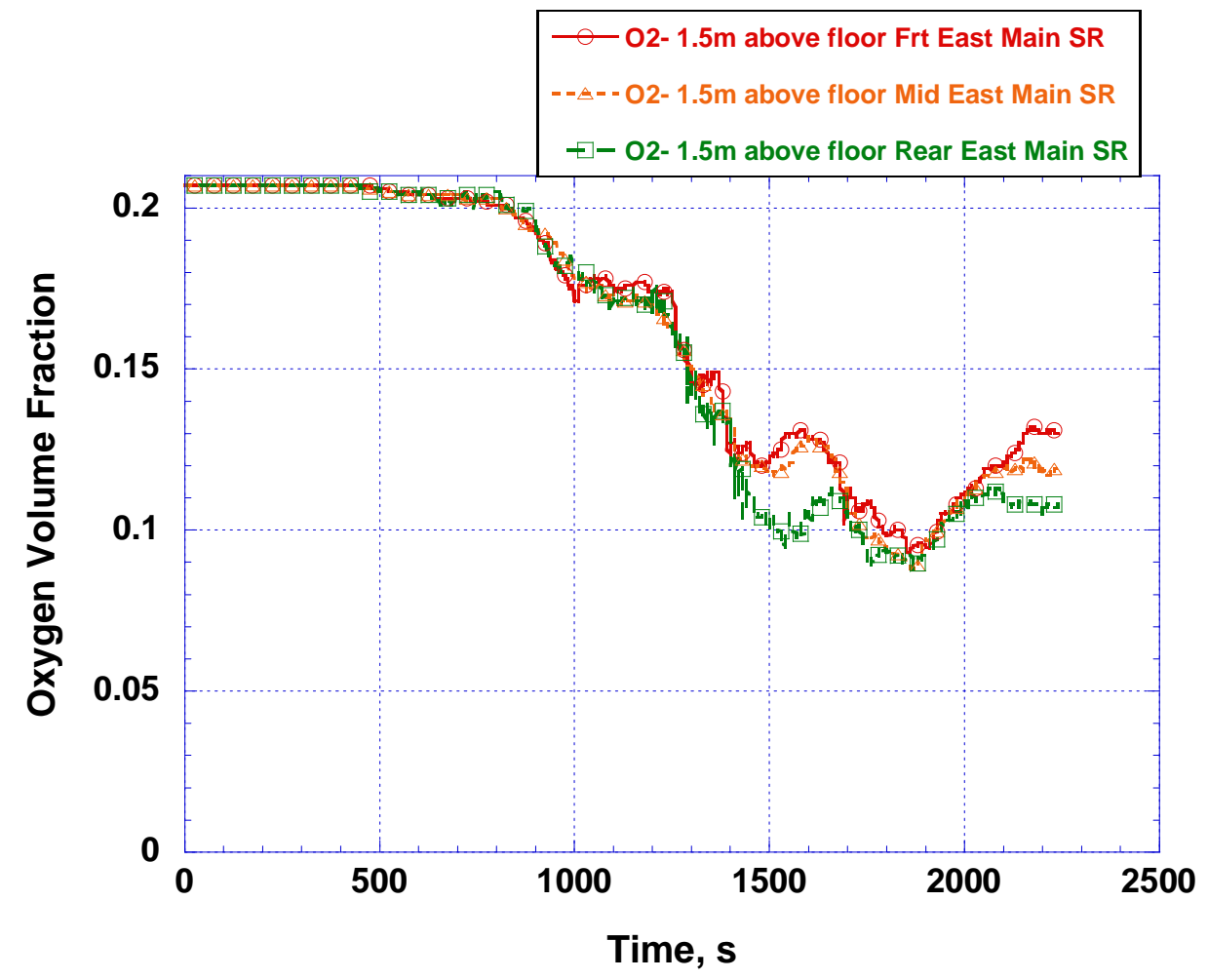

Figure K-57. Main showroom (east) oxygen volume fractions for $1.5 \mathrm{~m}(5 \mathrm{ft}$ ) above the floor. 


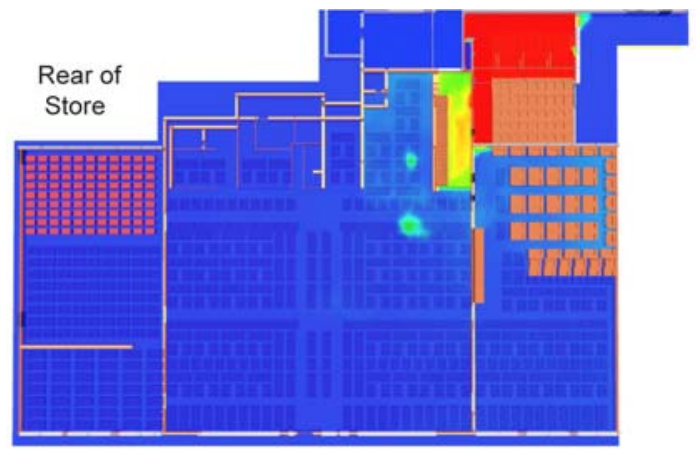

Front of Store

$360 \mathrm{~s}$

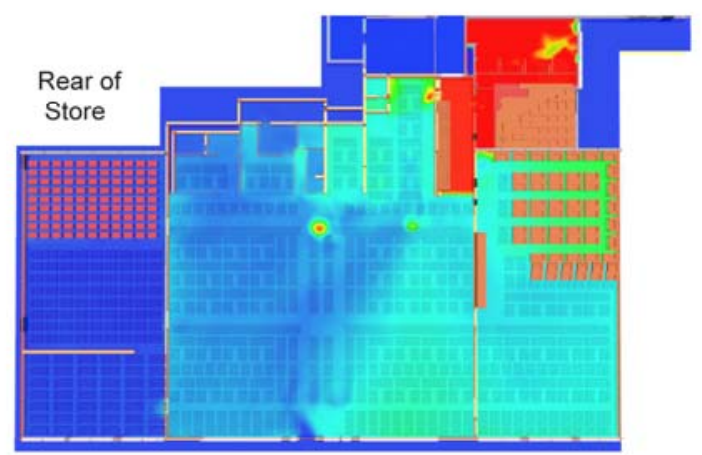

Front of Store

$$
840 \mathrm{~s}
$$

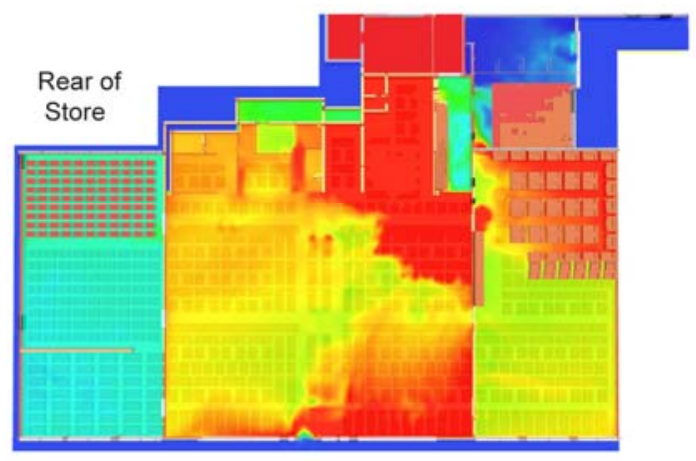

Front of Store

$$
1380 \mathrm{~s}
$$

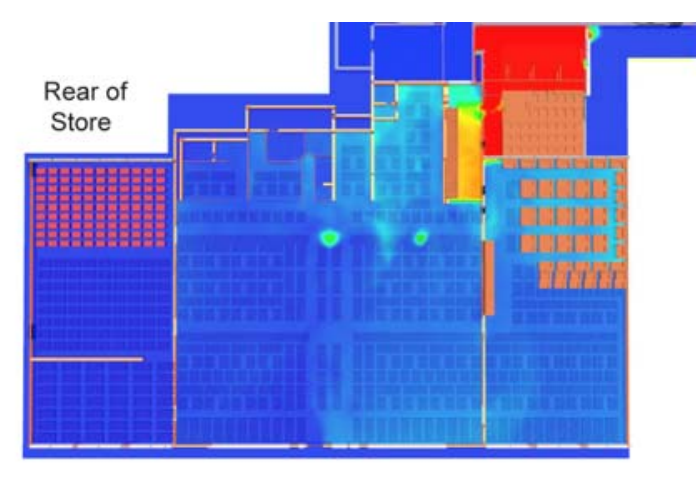

Front of Store

$480 \mathrm{~s}$

Volume

Fraction

0.21

0.20

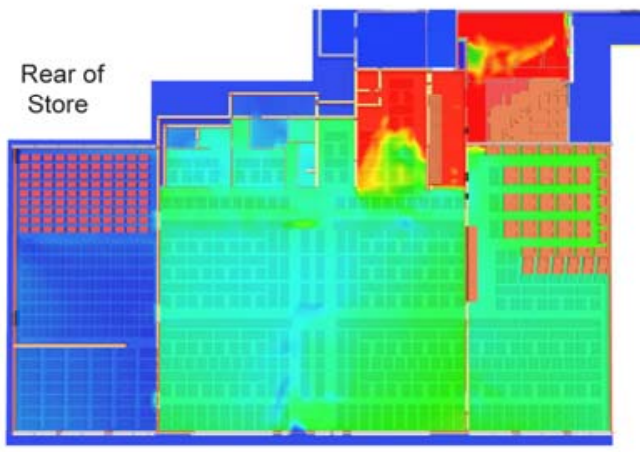

0.19

0.18

0.17

0.16

0.15

0.14

Front of Store

$960 \mathrm{~s}$

0.13

0.12

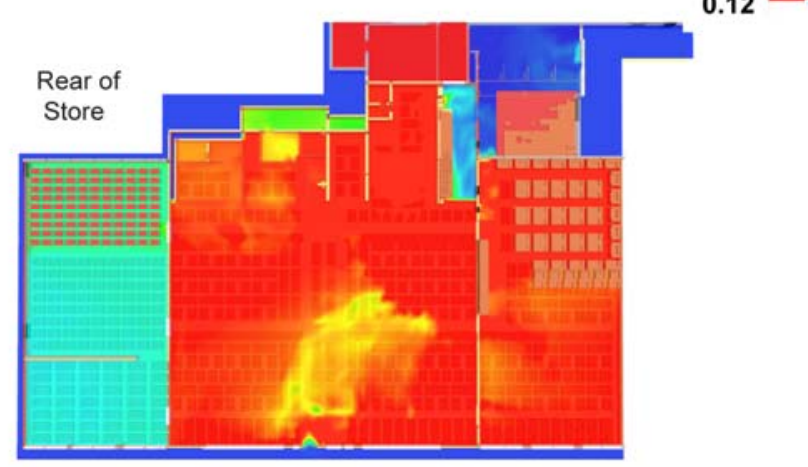

Front of Store

$1440 \mathrm{~s}$

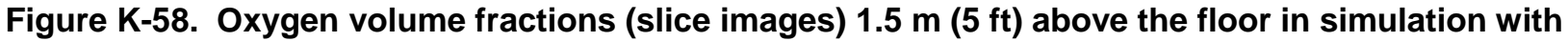
front windows intact. 


\section{K.7 VERTICAL VENTILATION - SMALL ROOF VENT}

\section{K.7.1 General}

In the this case a small vent or hole, $1.2 \mathrm{~m} \times 1.2 \mathrm{~m}(4 \mathrm{ft} \times 4 \mathrm{ft})$ was placed in the roof of the main showroom in the rear section of the store, near the holding area, providing an open area of $1.7 \mathrm{~m}^{2}$ $\left(18.2 \mathrm{ft}^{2}\right)$. This case was useful to examine because fire fighters will often vent a structure, either through cutting holes in the roof or breaking windows, to allow the hot gases to vent. As the hot smoke exhausts out the vent opening, visibility within the structure may improve and allow the fire fighters to locate and suppress the fire. For this case to approximate a more typical vent that might be created in a fire fighting

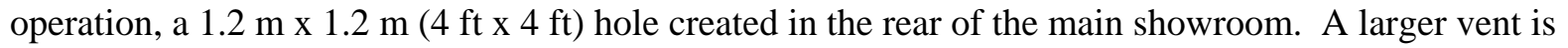
discussed in the next section of this appendix. Fire photographs also suggest that flames impinging on the underside of the roofing deck, may have caused an opening to be created above the holding area.

Creating a small vent opening at the rear of the main showroom allowed the impact of a small vent, either created by the fire fighters or by flame impingement, to be better characterized. The hole was opened one second after the start of the simulation and was positioned as shown in Figure K-59. As in the previous scenario the front windows were kept intact. No other changes were made to the ventilation conditions from the base scenario. The results of these simulations are presented here.

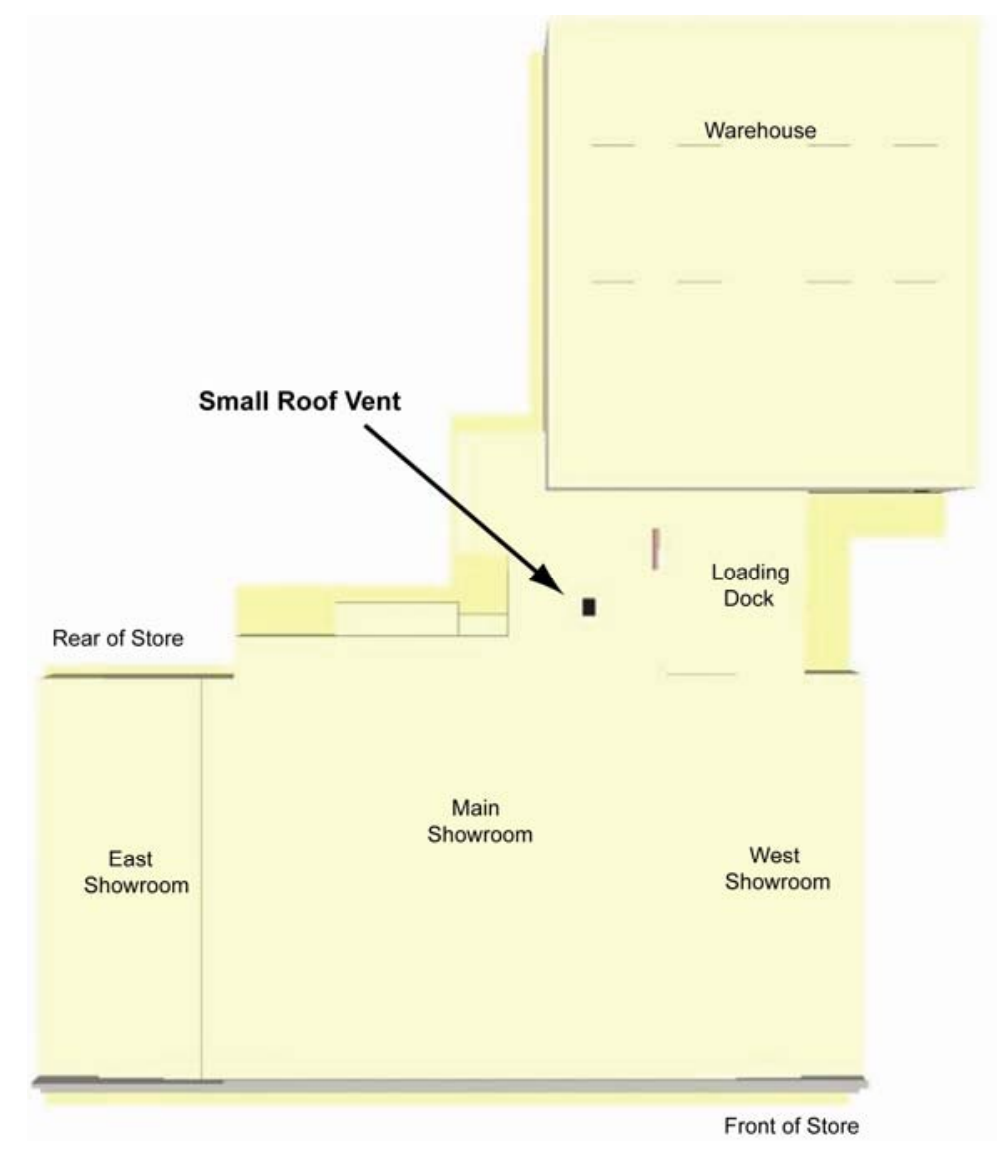

Figure K-59. Overhead view of the simulation domain with the small roof vent identified. 


\section{K.7.2 Fire Growth and Smoke Spread}

Figure K-60 shows the progression of fire and smoke throughout the loading dock, holding area and showrooms between 1020 and 2040 seconds. The images were taken at times identical to those shown in Figure K-46. Figures K-22 and K-28 shows fire progression in the base scenario, while Figure K-46 shows fire progression in the scenario with the front windows kept intact. The progression of fire in Figure K-60 appears very similar to that shown with the windows intact, Figure K-46. According to the simulation results, the addition of a small $\left(1.7 \mathrm{~m}^{2}\right)$ hole in the showroom roof did not appear to have asignificant impact on the spread of fire and smoke as compared to the windows intact case.

\section{K.7.3 Heat Release Rate}

Figure K-61 shows a plot that compares the HRR in this scenario with the HRR in the base scenario. The data sets are similar through approximately 1400 seconds. The peak in the data at 330 seconds is slightly higher than in the base scenario. This peak is attributed to the heated, fuel rich mixture reacting with air as it exits the roof vent. In both cases the HRR peaked at $170 \mathrm{MW}$, and then began to decrease in size as the oxygen in the showroom was consumed. In the base scenario the main showroom windows were vented at $1457 \mathrm{~s}$. It is at time that the data sets diverge, with the ventilated HRR rapidly increasing and the underventilated HRR continuing to decrease. Aside from small peaks at $320 \mathrm{~s}$ (small vent case) and at 1700 s (windows intact case), the HRR in this scenario is not significantly different from the scenario with just the front windows intact, shown in Figure K-47.

\section{K.7.4 Temperature}

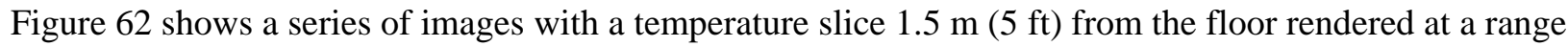
of times between $900 \mathrm{~s}$ to $1620 \mathrm{~s}$. The trends in this figure are similar to those seen in Figure K-48. The area of peak temperatures, shown in red, diminishes between 1380 and 1500 seconds. While there is an increase seen later in the simulation, shown by the image at 1620 seconds, overall the area of peak temperatures (area shaded in red) is smaller than that seen in the ventilated (base) case shown in Figure $\mathrm{K}-30$.

Figures K-63 through K-66 show temperature data recorded at various locations throughout the main and west showrooms. The sensor locations used here are identical to those shown in Figure K-29. For comparison purposes, the scale in these figures was kept the same as the corresponding scale in Figures K-31 through K-35 and in Figures K-49 through K-53. After 1500 s, the small vent case temperatures were significantly lower than the base case (Figures K-31 to K-35), but similar to the windows intact scenario (Figures K-49 to K53). The temperatures still exceeded the $120^{\circ} \mathrm{C}$ tenability threshold at each sensor location. According to the simulation results, the addition of a small $\left(1.7 \mathrm{~m}^{2}\right)$ hole in the showroom roof did not appear to have asignificant impact on the temperatures as compared to the windows intact case. 


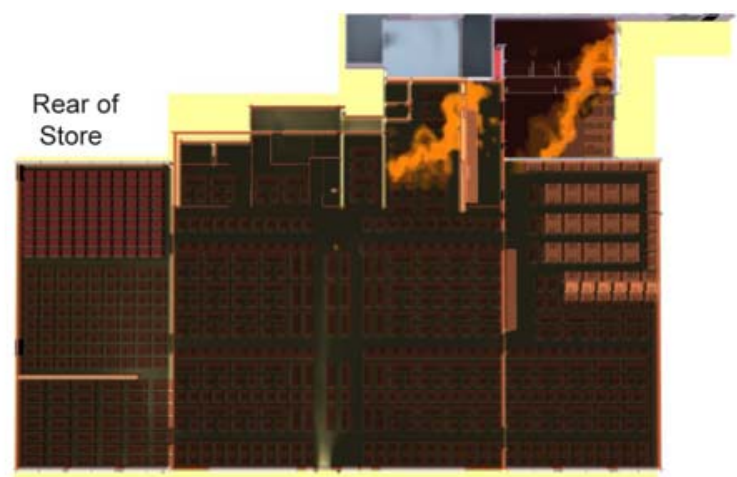

Front of Store

$1020 \mathrm{~s}$

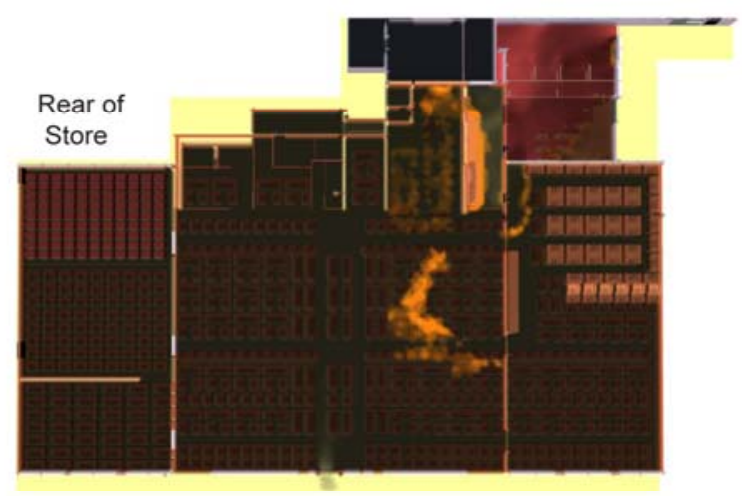

Front of Store

$1500 \mathrm{~s}$

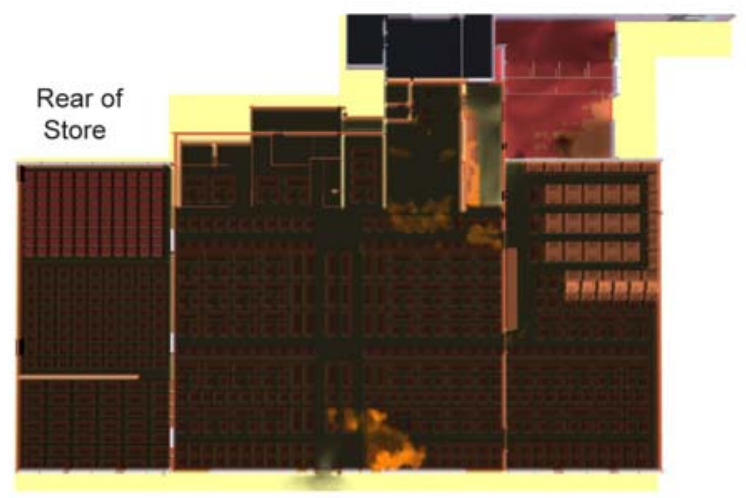

Front of Store

$1800 \mathrm{~s}$

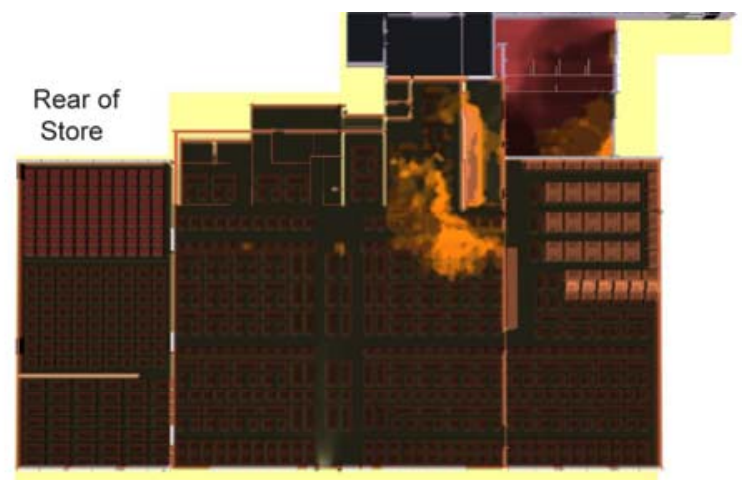

Front of Store

$1380 \mathrm{~s}$

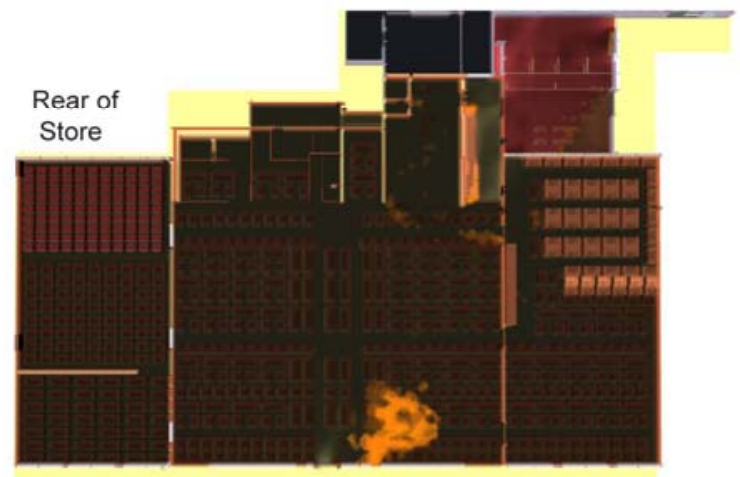

Front of Store

$1620 \mathrm{~s}$

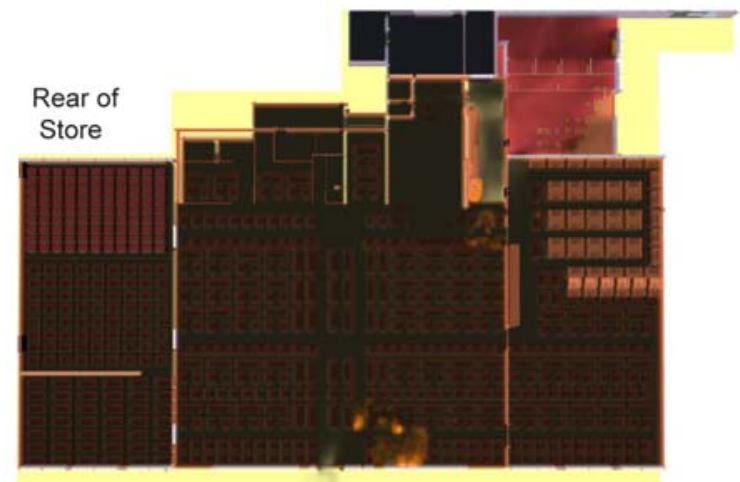

Front of Store

$2040 \mathrm{~s}$

Figure K-60. Fire and smoke progression in scenario with front windows intact and small $\left(1.69 \mathrm{~m}^{2}\right)$ roof vent in showroom as rendered by computer simulation. 


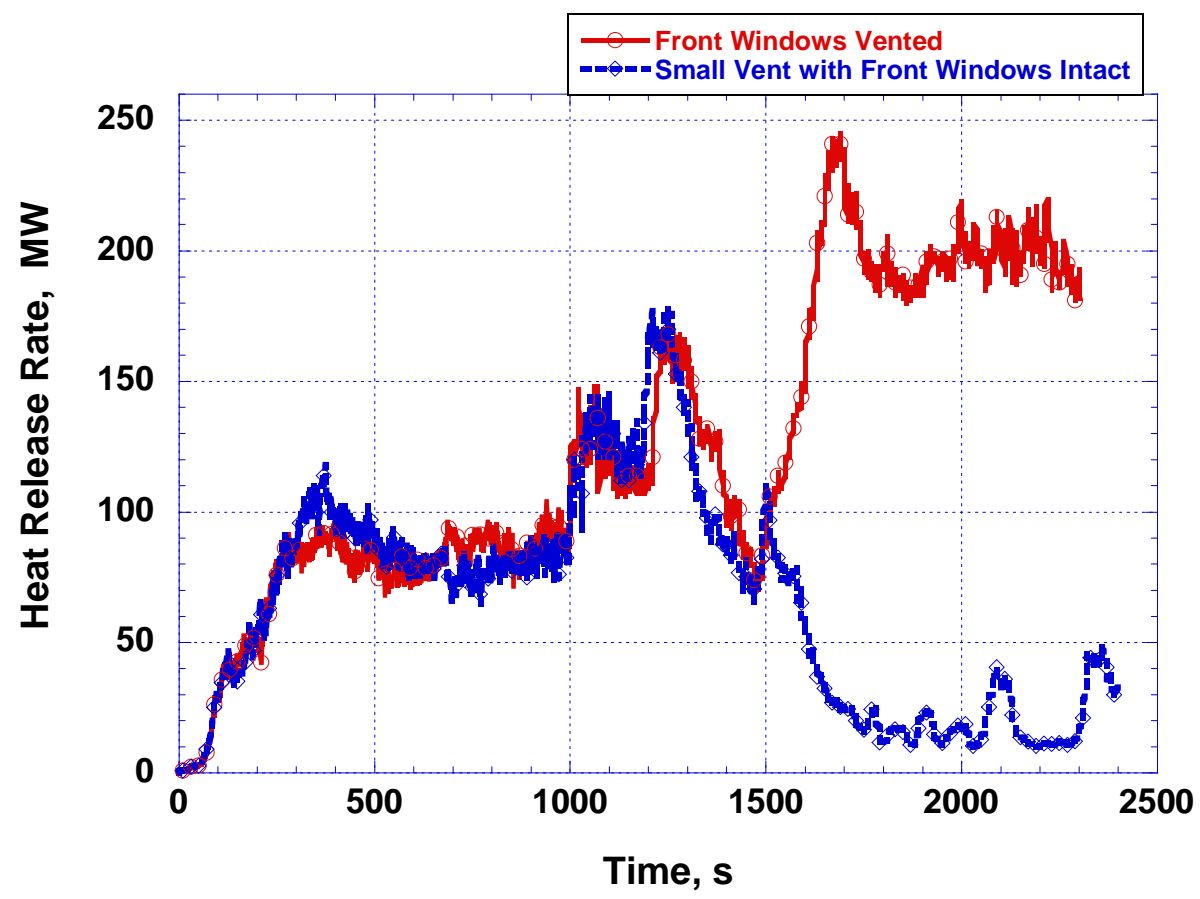

Figure K-61. Comparison of the heat release rate for the base scenario and the scenario with the front windows intact and a small $\left(1.69 \mathrm{~m}^{2}\right)$ vent in the main showroom roof.

\section{K.7.5 Oxygen}

Figure K-67 shows a series of images with an oxygen slice $1.5 \mathrm{~m}(5 \mathrm{ft})$ from the floor rendered at a range of times between $360 \mathrm{~s}$ to $1440 \mathrm{~s}$. As was the case with temperature, the conditions in most of the main and west showrooms become untenable because of oxygen depletion. Minimum oxygen volume fraction dropped below 0.12 (indicated by the red shaded areas in Figure K-67) in the rear of both the main and west showrooms. Minimum oxygen volume fraction dropped below 0.12 (indicated by the red shaded areas in Figure K-67) in the rear of both the main and west showrooms. Significant portions of the front of the main and west showrooms did not drop below an oxygen volume fraction of 0.12 by $1440 \mathrm{~s}$ (indicated by the yellow shaded areas in Figure K-67).

Figures K-68 to K-71 show oxygen data recorded at various locations throughout the main and west showrooms. The sensor locations used here are identical to those shown in Figure K-29. The data shown in these figures are similar to the data from the front window intact scenario (Figures K-54 to K-57) during the early stages of the simulation including the period in which the front windows were removed. Oxygen volume fractions fell below 0.12 at each sensor location before $1700 \mathrm{~s}$. 


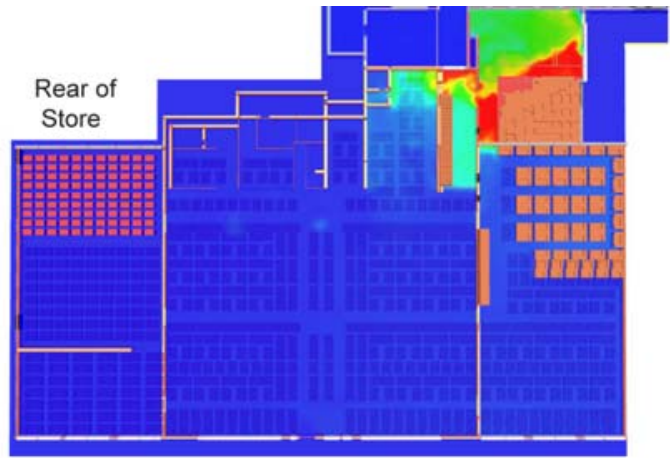

Front of Store

$900 \mathrm{~s}$

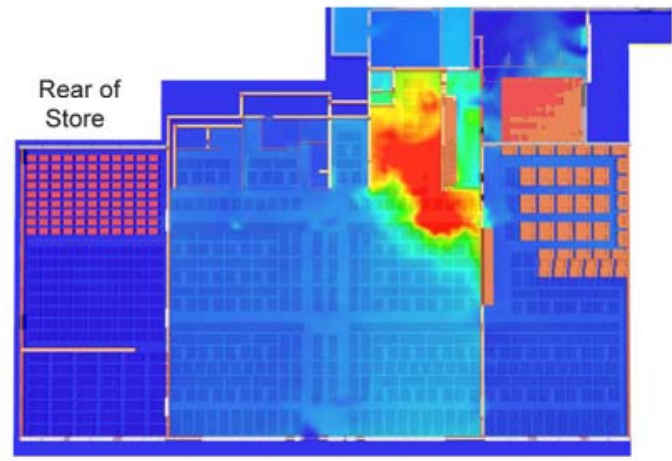

Front of Store

$1380 \mathrm{~s}$

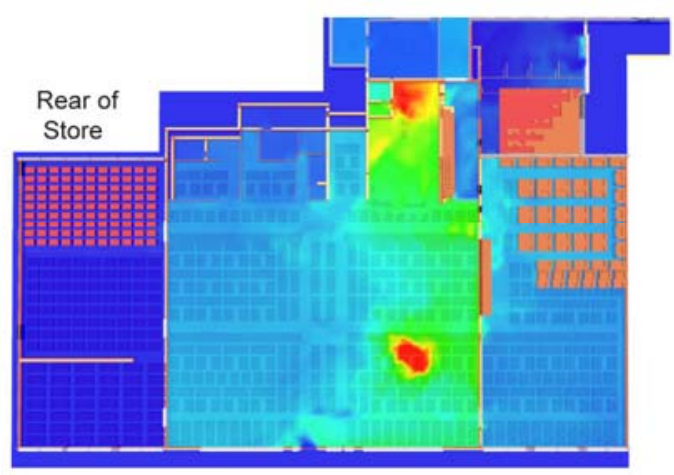

Front of Store

$1560 \mathrm{~s}$

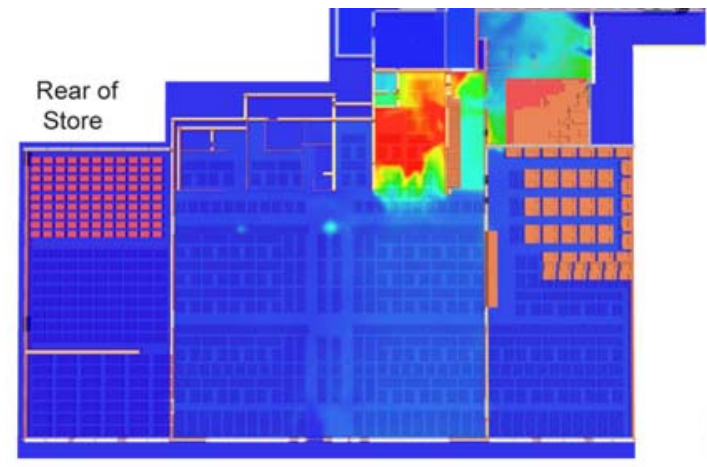

Gas

Front of Store

$1140 \mathrm{~s}$

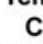

820

740

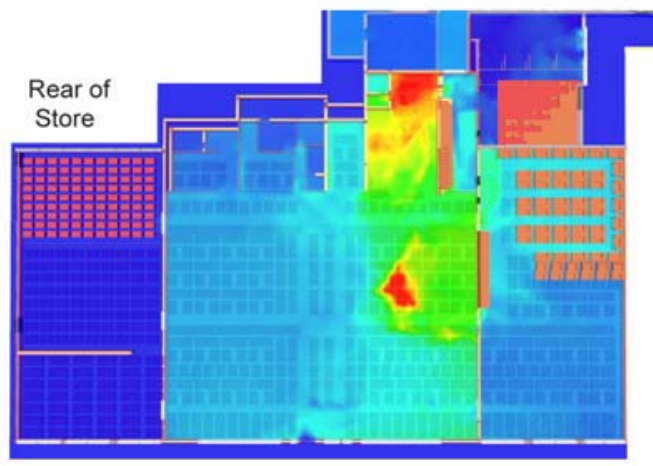

660

580

500

420

340

260

180

Front of Store

$1500 \mathrm{~s}$

100

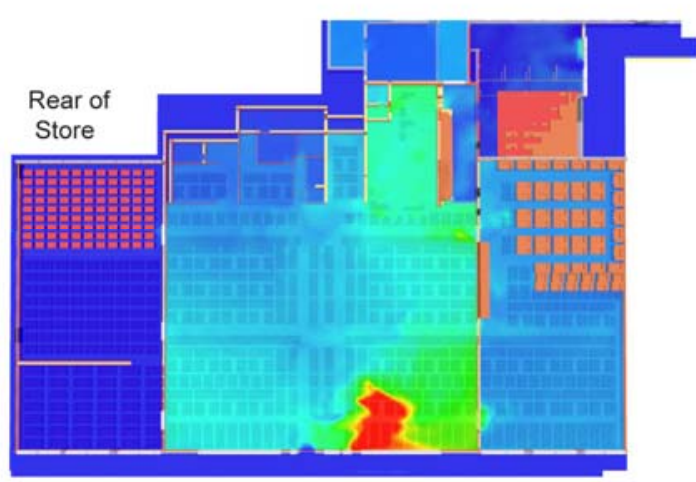

Front of Store

$1620 \mathrm{~s}$

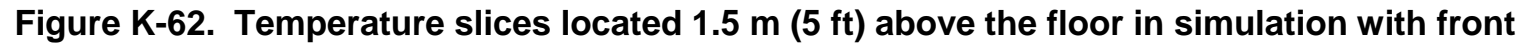
windows intact and small roof vent. 


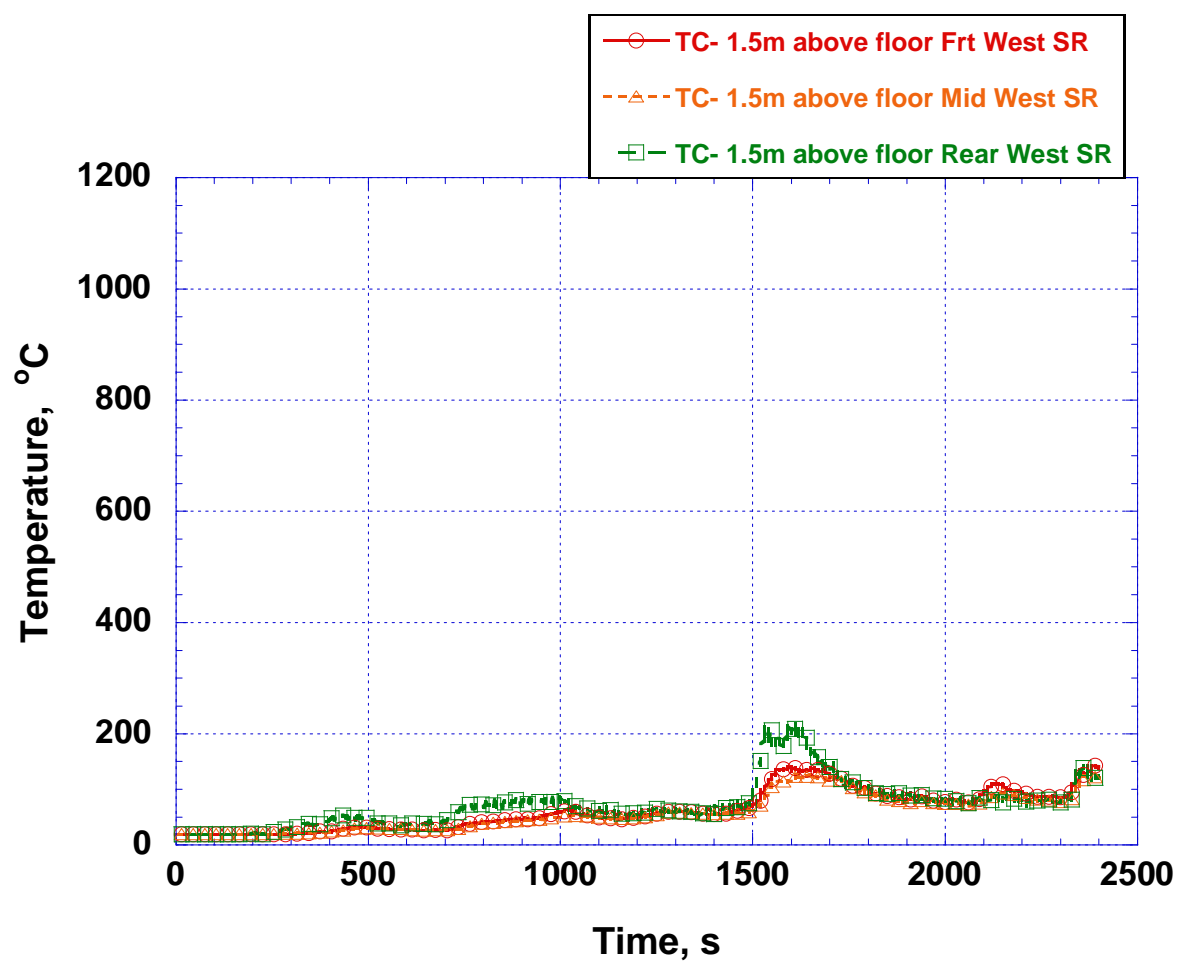

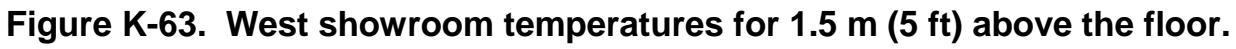

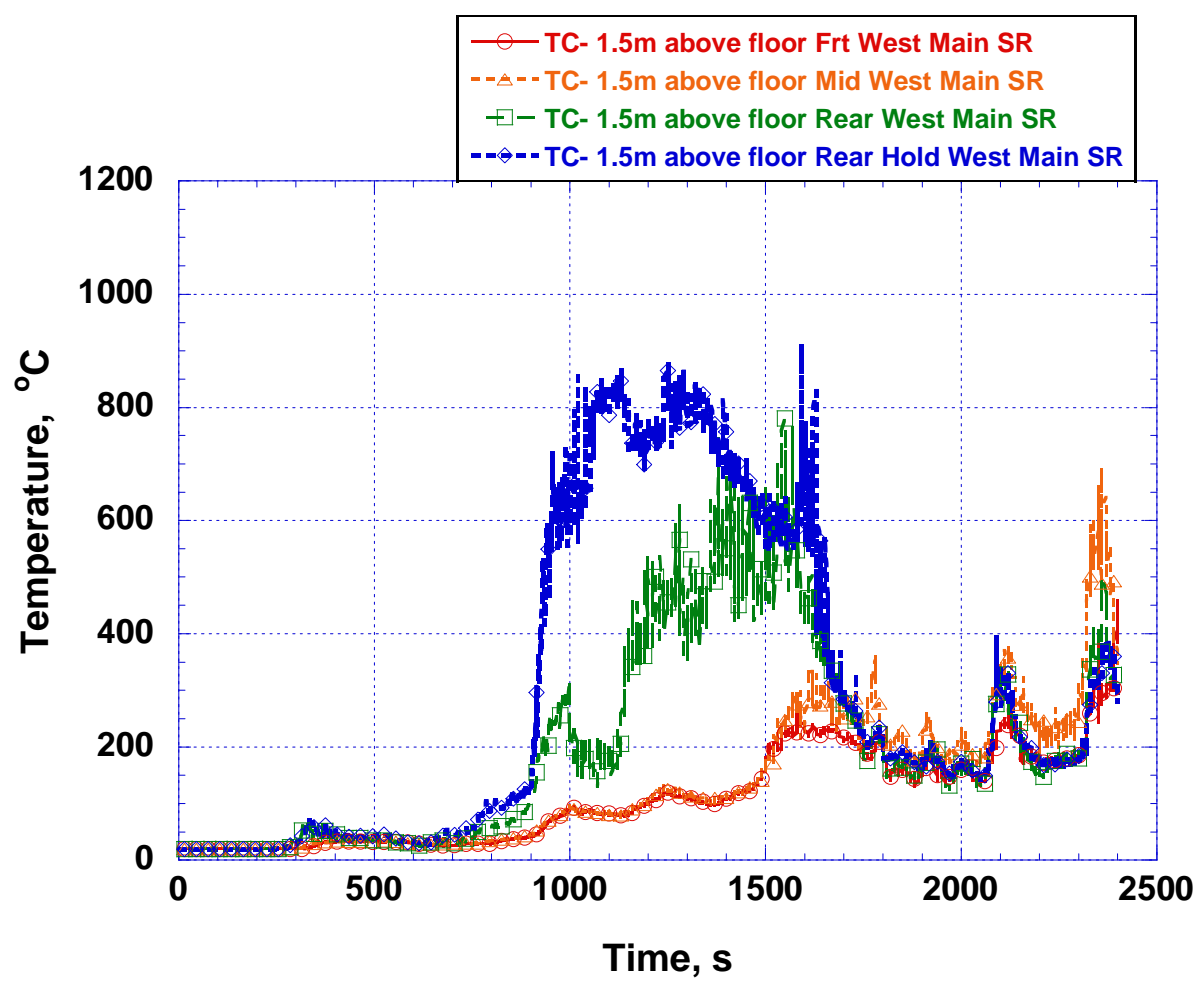

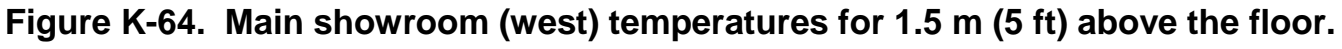




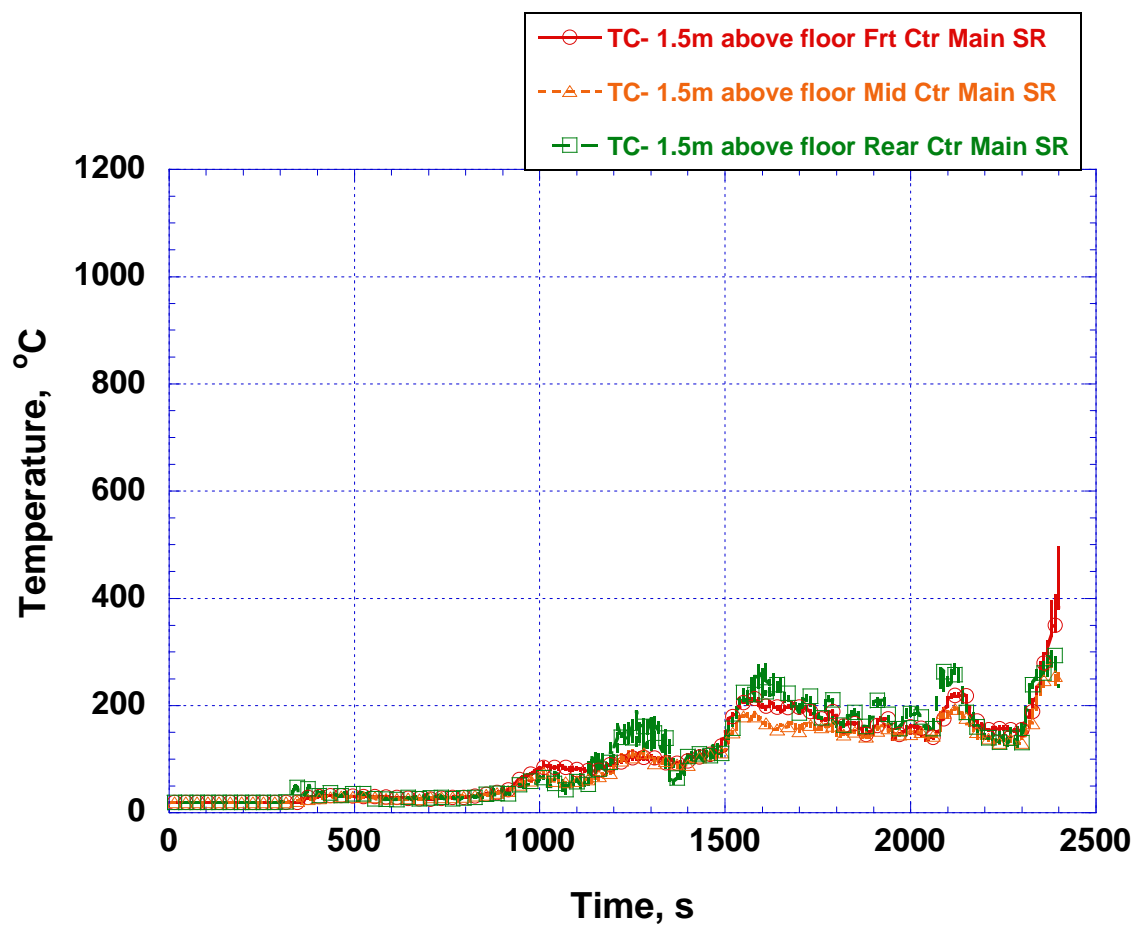

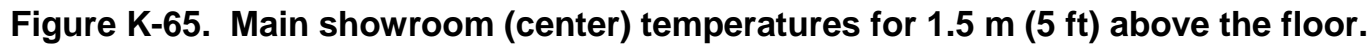

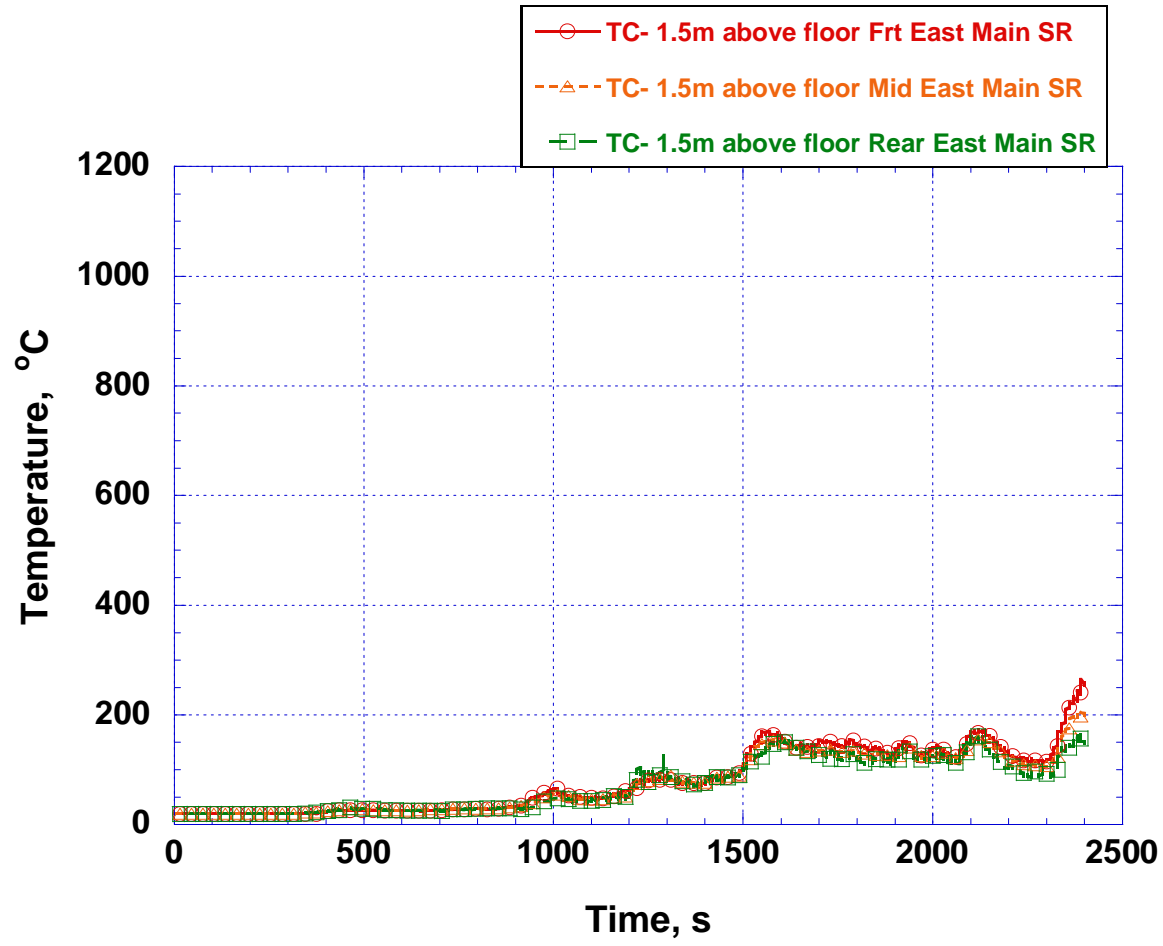

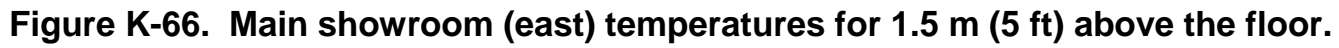




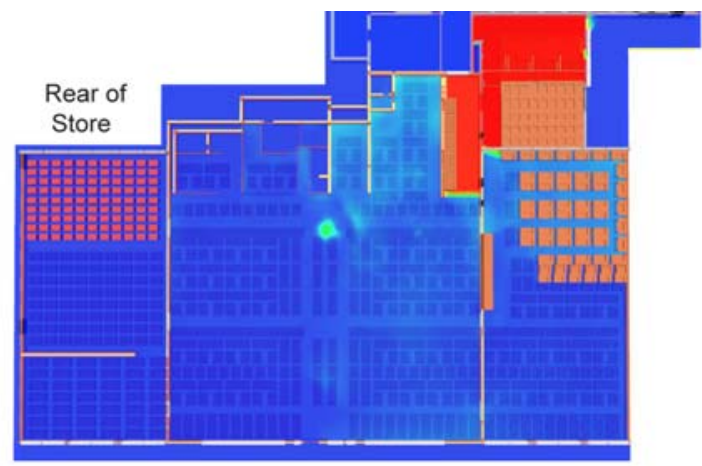

Front of Store

$360 \mathrm{~s}$

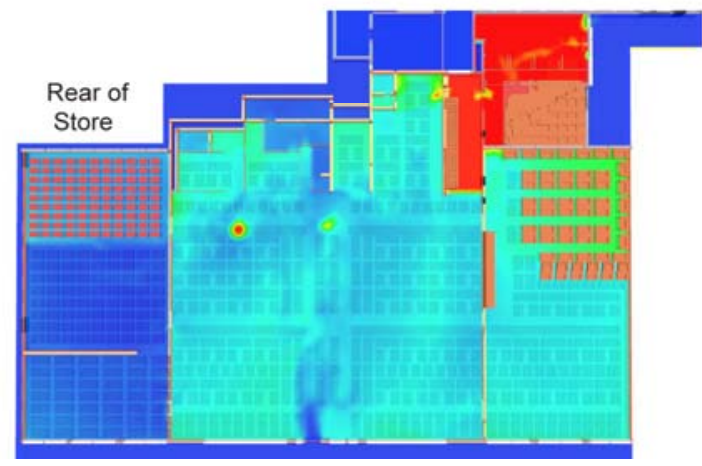

Front of Store

$840 \mathrm{~s}$

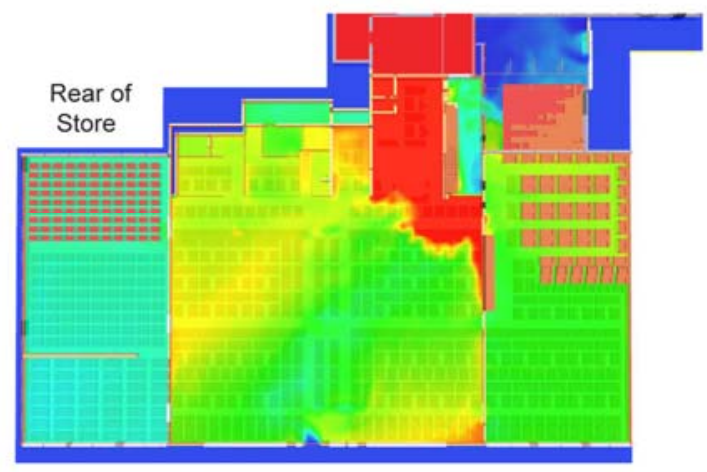

Front of Store

$1380 \mathrm{~s}$

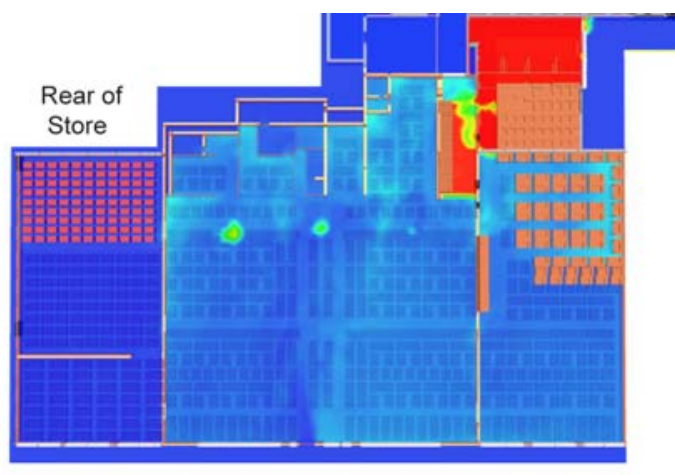

Front of Store

$480 \mathrm{~s}$

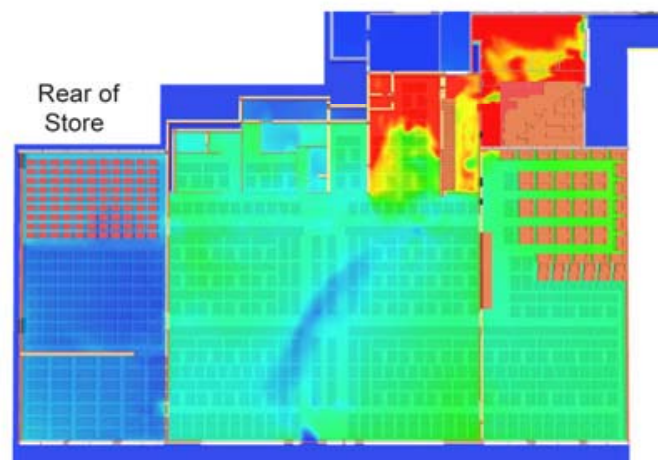

Front of Store

$960 \mathrm{~s}$

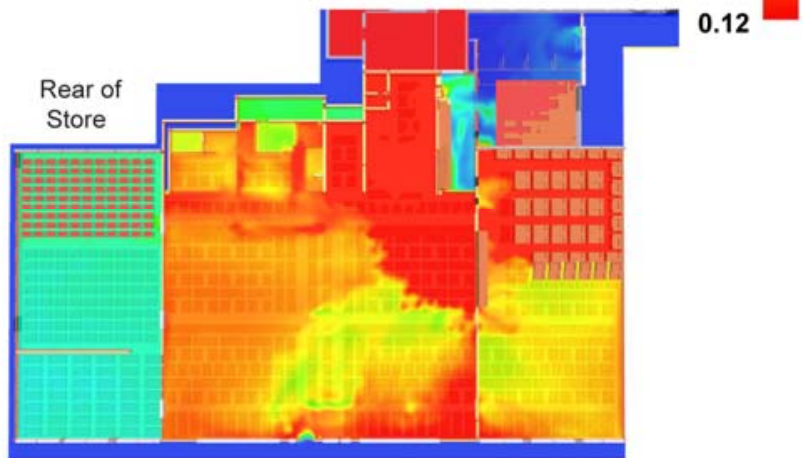

Front of Store

$1440 \mathrm{~s}$
0.14

0.13

Oxygen

Volume

Fraction

0.20

0.19

0.18

0.17

0.16

0.15

0.14

12

Figure K-67. Oxygen volume fractions (slice images) $1.5 \mathrm{~m} \mathrm{(5} \mathrm{ft)} \mathrm{above} \mathrm{the} \mathrm{floor} \mathrm{in} \mathrm{simulation} \mathrm{with}$ front windows intact and small roof vent. 


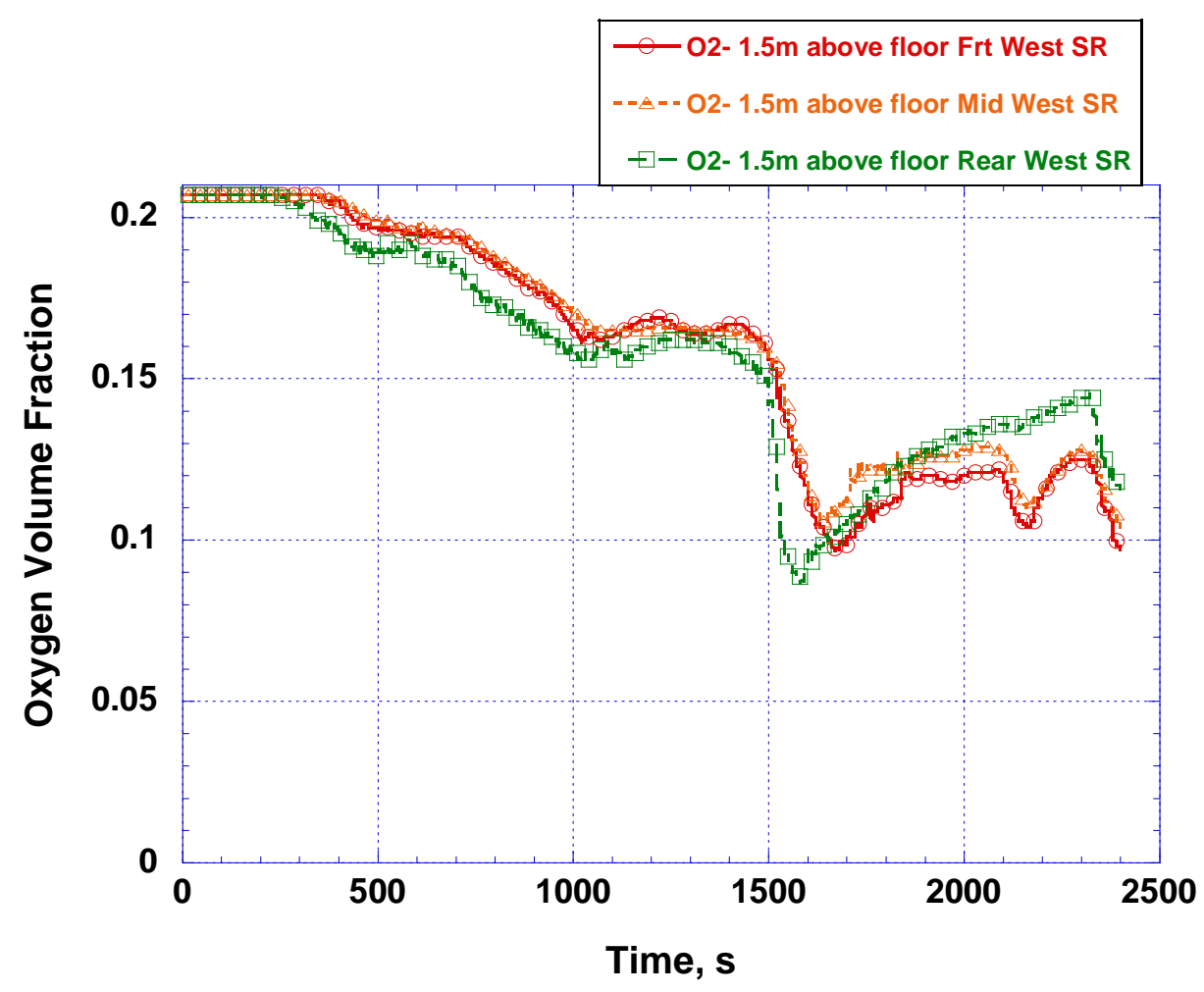

Figure K-68. West showroom oxygen volume fractions for $1.5 \mathrm{~m}(5 \mathrm{ft})$ above the floor.

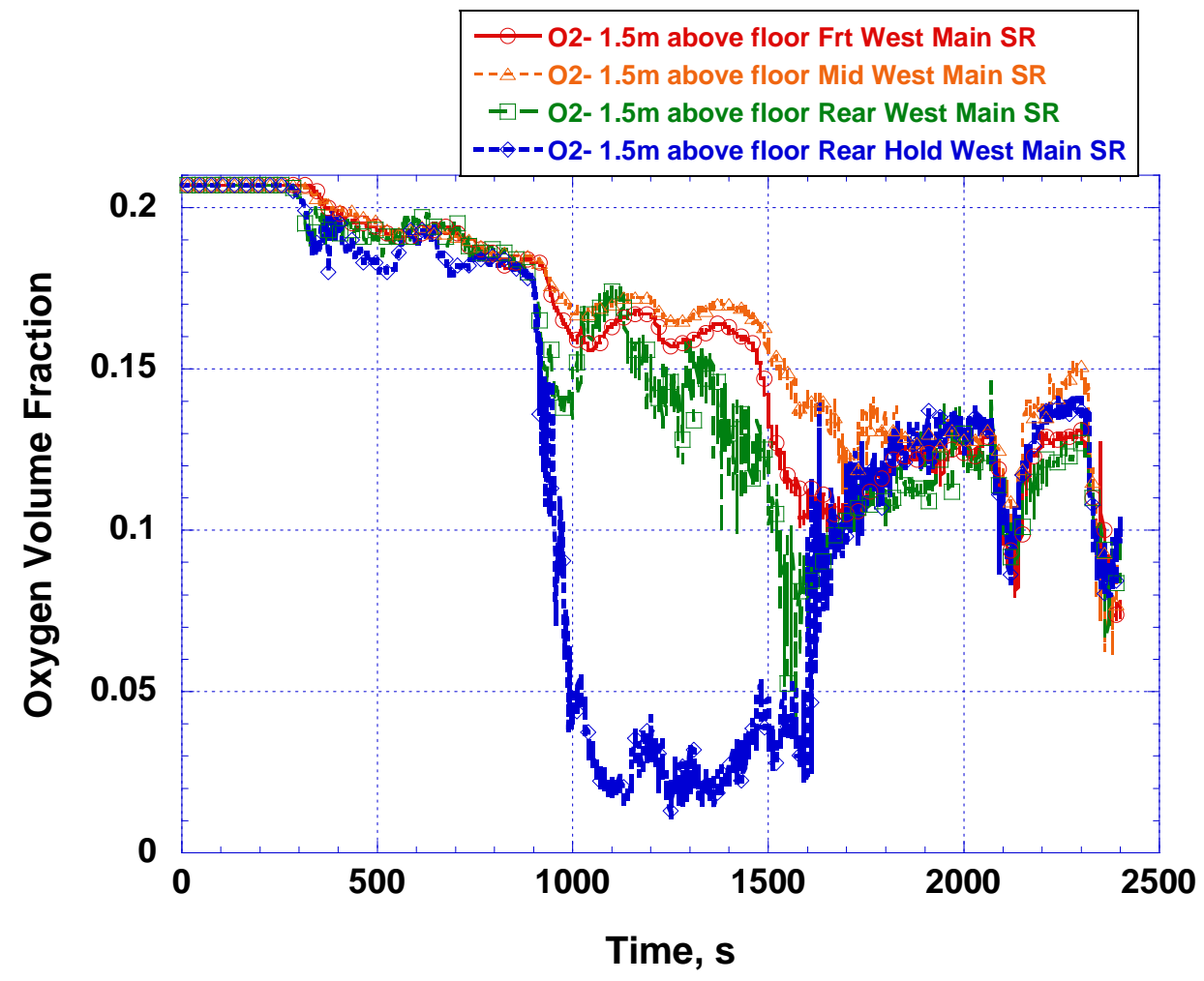

Figure K-69. Main showroom (west) oxygen volume fractions for $1.5 \mathrm{~m}(5 \mathrm{ft})$ above the floor. 


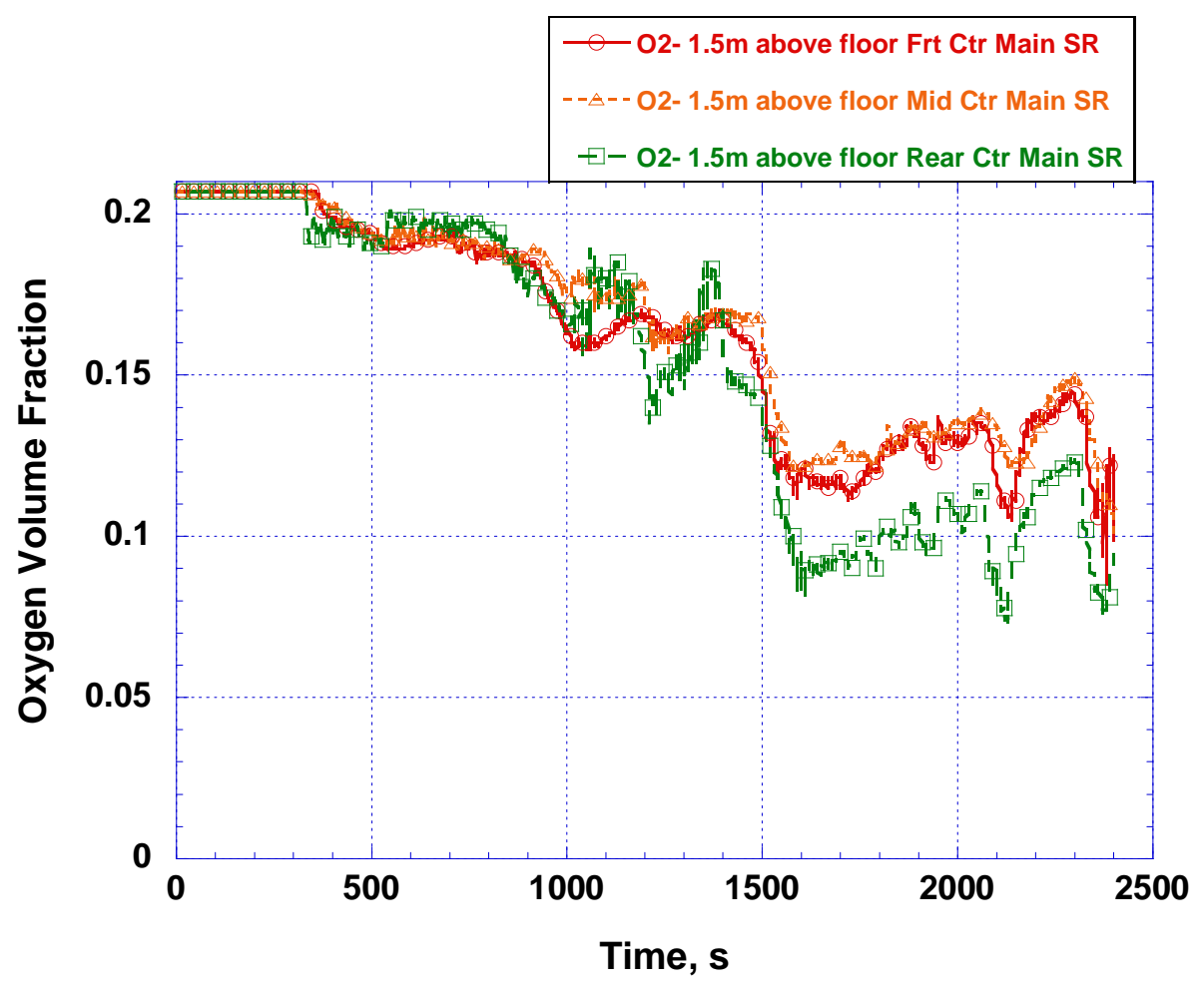

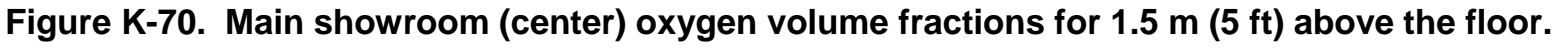

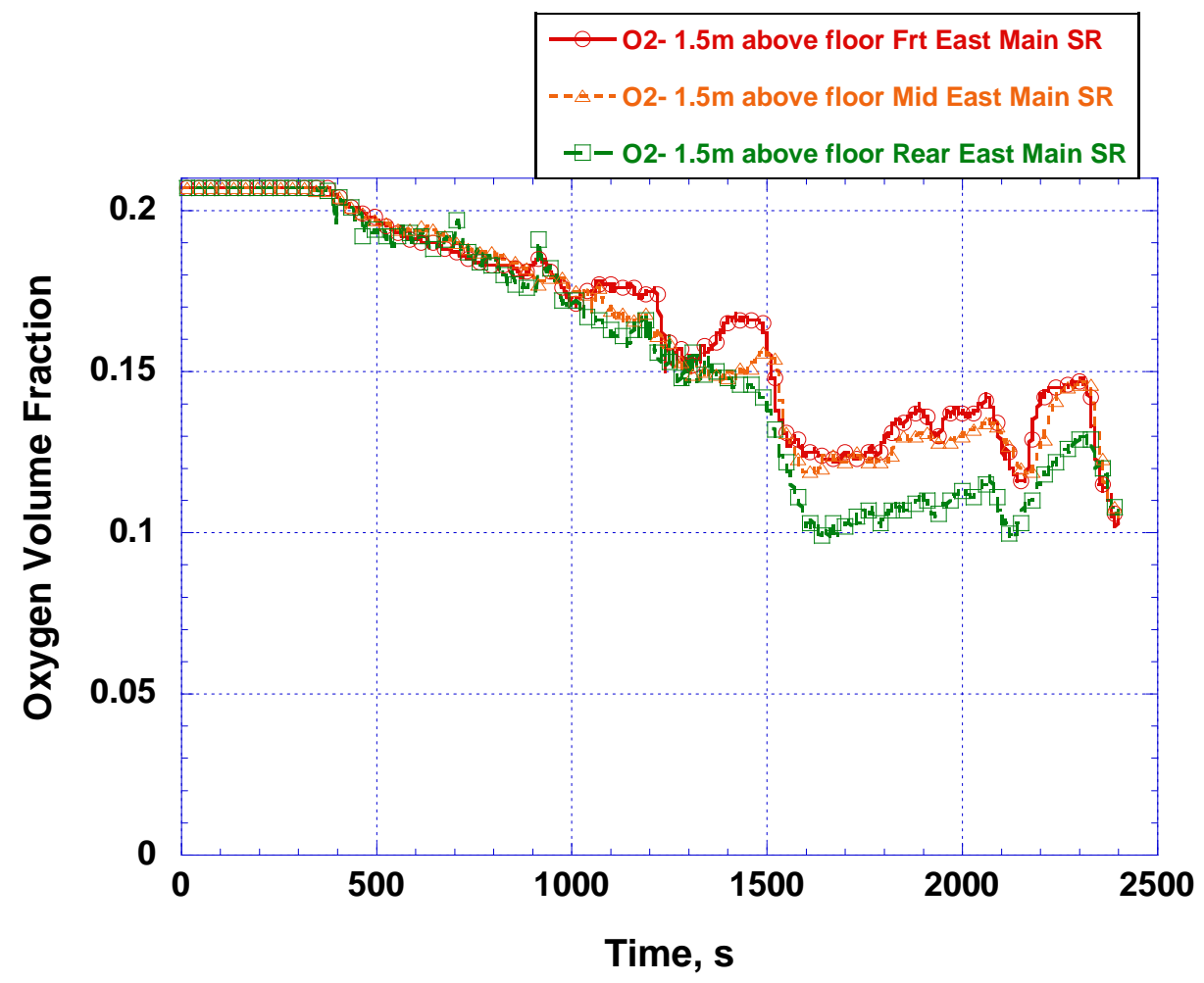

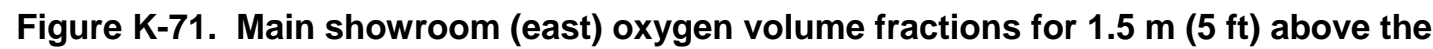
floor. 


\section{K.8 VERTICAL VENTILATION - LARGE ROOF VENT}

\section{K.8.1 General}

In the second vertical ventilation case a larger, $2.44 \mathrm{~m}$ x $2.44 \mathrm{~m}$ hole was used, providing an area of $5.9 \mathrm{~m}^{2}\left(63.6 \mathrm{ft}^{2}\right)$. This case was useful to examine because ventilation, either through cutting holes in the roof or breaking windows, is often used to allow the hot gases to vent. As the hot smoke exhausts out the vent opening, visibility within the structure may improve and allow quicker location and suppression of the fire.

The small vent scenario which was presented in the previous section did not appear to impact the fire growth significantly, so for this case, a much larger vent, $2.44 \mathrm{~m}$ x $2.44 \mathrm{~m}$ (8 ft x $8 \mathrm{ft}$ ), was created. The area of this large vent was not designed to simulate the actual fire, but to help understand how a large vertical vent might have impacted the fire growth and spread. The area of this large roof vent, $5.9 \mathrm{~m}^{2}$ $\left(63.6 \mathrm{ft}^{2}\right)$ was about 20 percent of the area of front windows, $34.1 \mathrm{~m}^{2}\left(367 \mathrm{ft}^{2}\right)$ which were broken out during the actual fire. The large roof hole was opened one second after the start of the simulation and was positioned as shown in Figure K-72. As in the previous scenario the front windows were kept intact. No other changes were made to the ventilation conditions from the base scenario. The results of these simulations are presented here.

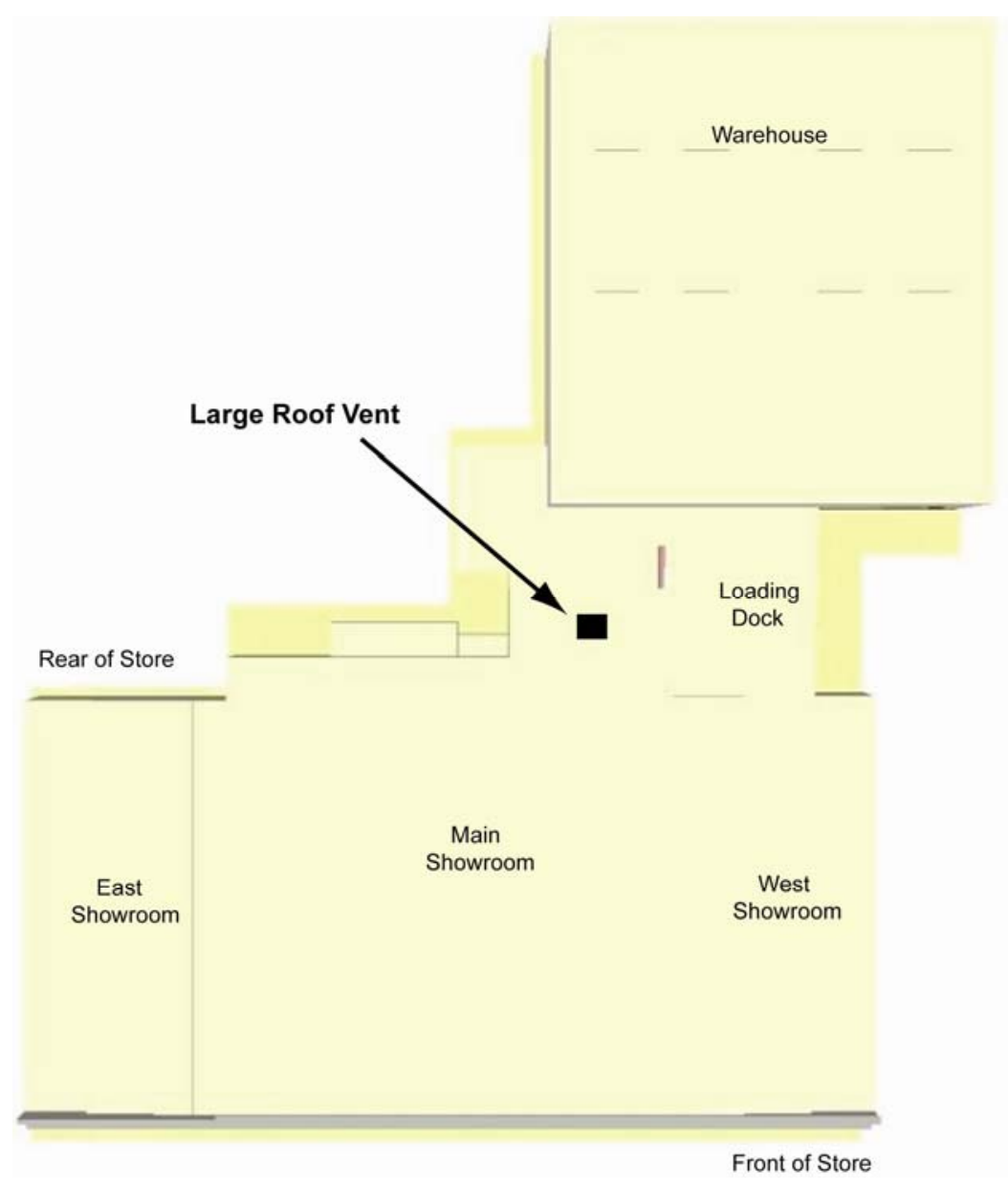

Figure K-72. Overhead view of the domain with the large roof vent identified. 


\section{K.8.2 Fire Growth and Smoke Spread}

Figure K-73 shows the progression of fire and smoke throughout the loading dock, holding area and showrooms between $1020 \mathrm{~s}$ and $2040 \mathrm{~s}$. The images were taken at times identical to those shown in Figures K-22, K-28, and Figure K-46. Figures K-22 and K-28 show fire progression in the base scenario, while Figure K-46 shows fire progression in the scenario with the front windows kept intact. The progression of fire in Figure K-73 appears very similar for the first 1500 s to that shown in Figures K-22 and K-46. However, for the large ventilation vent scenario, the flames do not appear to spread very quickly toward the front of the main showroom. The flames appear to reach the front windows by $1500 \mathrm{~s}$ in the base case and after $1620 \mathrm{~s}$ in the front windows intact case. For the large vent case, the flames required an additional $300 \mathrm{~s}$ before reaching the front windows at about $1800 \mathrm{~s}$. The large vertical vent $\left(5.9 \mathrm{~m}^{2}\right)$ slowed or delayed the movement of the fire from the rear of the main showroom toward the front of the main showroom as compared to the base and windows intact cases.

\section{K.8.3 Heat Release Rate}

Figure K-74 shows a plot that compares the HRR in this scenario with the HRR in the base scenario. Both the base and large vent cases are similar for the first $280 \mathrm{~s}$. While the base case HRR levels off at about $90 \mathrm{MW}$, the large vent case continues to increase to approximately $120 \mathrm{MW}$. By $500 \mathrm{~s}$, both cases have HRRs of between $80 \mathrm{MW}$ and $90 \mathrm{MW}$. At $950 \mathrm{~s}$, both HRR begin a steep increase that results in a $200 \mathrm{MW}$ peak for the large vent case and a lower $170 \mathrm{MW}$ peak for the base case. The additional heat release in the large vent case is consistent with the large roof hole allowing more unburned fuel to exhaust from the structure. This additional outflow is balanced by additional inflow which brings additional air to the fire and allows it to release more energy. In the base scenario the main showroom windows were vented between $1457 \mathrm{~s}$ to $1537 \mathrm{~s}$. It is during this interval that the data sets diverge, with the base case HRR rapidly increasing as the fire spreads toward the front of the main showroom. In the large roof vent case, the HRR continues to decrease until after $1600 \mathrm{~s}$ when the does begin to move toward the front of the main showroom. Initially, the large vent provides additional air to the fire allowing it to reach higher peaks (120 MW and $170 \mathrm{MW}$ ). Later in the fire, the large vent slows the movement of the fire and reduces it HRR.

\section{K.8.4 Temperature}

Figure K-75 shows a series of images with a temperature slice $1.5 \mathrm{~m}(5 \mathrm{ft})$ from the floor rendered at a range of times between $900 \mathrm{~s}$ to $1620 \mathrm{~s}$. The trends in this figure are different as compared to the windows intact case shown in Figure K-48. As was described in the flame spread and heat release discussion above, the temperatures are similar for the first $1200 \mathrm{~s}$. After $1380 \mathrm{~s}$, the temperatures in the main showroom increase for the windows intact case (Figure K-48), but the increase in the temperatures in the large vent scenario occur more slowly. The temperature data demonstrates that the movement of the fire toward the front of the main showroom is slowed in the large vent case and this is consistent with the flame spread and HRR data.

Figures K-76 through K-79 show temperature data recorded at various locations throughout the main and west showrooms. The sensor locations used here are identical to those shown in Figure K-29 and used 
previously in this report. For comparison purposes, the scale in these figures was kept the same as the corresponding scale in Figures K-31 through K-35 and in Figures K-49 through K-53. The large vent case displays similar overall peaks as with the base and front windows intact cases. For the large vent case, the temperatures in the mid- and front-locations of the main showroom are slower to increase. This is consistent with the fire not moving as quickly toward the front of the showroom. However, the slower moving fire produces higher temperatures in the center of the main showroom (Figure K-78). The large vertical vent impacted how quickly the temperatures peaked, but did not appear to produce significantly higher or lower temperatures than the base and windows intact cases.

\section{K.8.5 Oxygen}

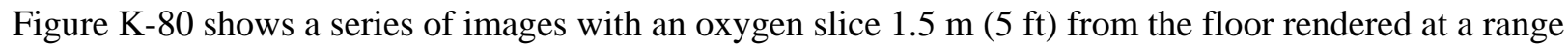
of times between $720 \mathrm{~s}$ to $1440 \mathrm{~s}$. During the first $1500 \mathrm{~s}$, the oxygen volume fraction is depleted to less than 0.12 in the rear of the main showroom, but remains above 0.15 in the rest of the main showroom as well as in the west showroom. It requires an additional $300 \mathrm{~s}$ until about $1800 \mathrm{~s}$ before the oxygen drops below 0.12 volume fraction. The large vertical vent caused the fire to move more slowly toward the front of the store as compared to the base and window intact cases.

Figures K-81 to K-84 show oxygen data recorded at various locations throughout the main and west showrooms. The sensor locations used here are identical to those shown in Figure K-29 and used previously in this report. For the first $1000 \mathrm{~s}$, the data shown in these figures are similar to the data from the front window intact scenario (Figures K-54 to K-57). After $1000 \mathrm{~s}$, the data from the holding area and west showroom remain similar to the front window intact case. For the large vent case, the oxygen is depleted more slowly in the rear of the main showroom than in the windows intact scenario. At about $1400 \mathrm{~s}$, the oxygen volume fraction in the rear of the main showroom drops from about 0.15 to 0.02 volume fraction in a very short time period. The oxygen volume fraction drops below the tenability criteria before $1500 \mathrm{~s}$ and remained low throughout the remainder of the simulation. 


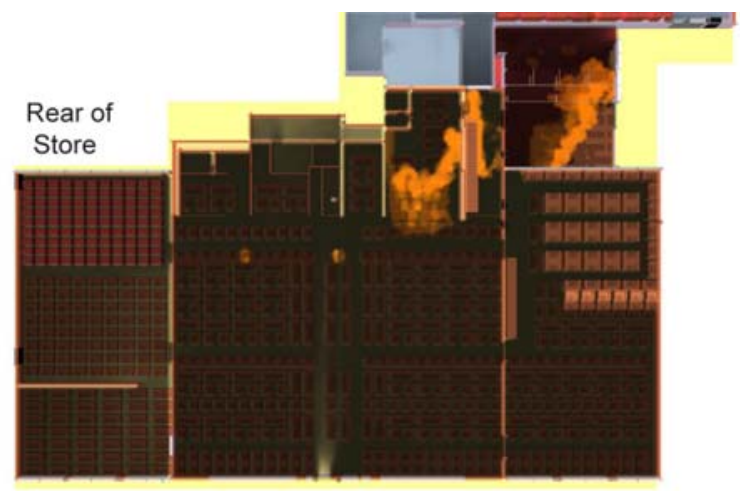

Front of Store

$1020 \mathrm{~s}$

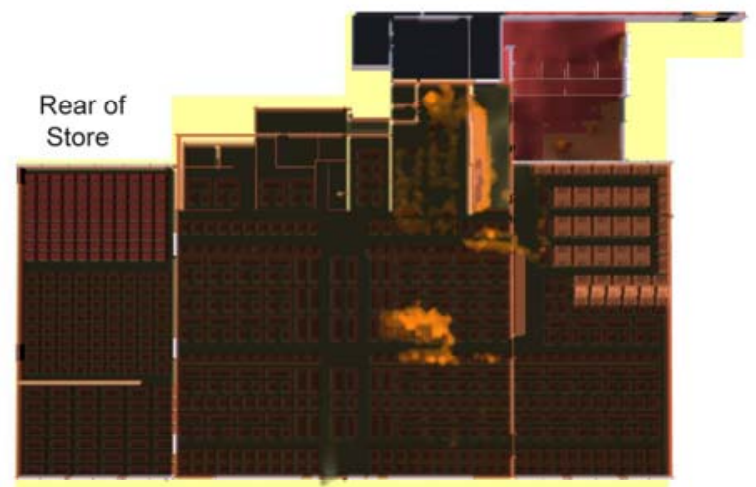

Front of Store

$1500 \mathrm{~s}$

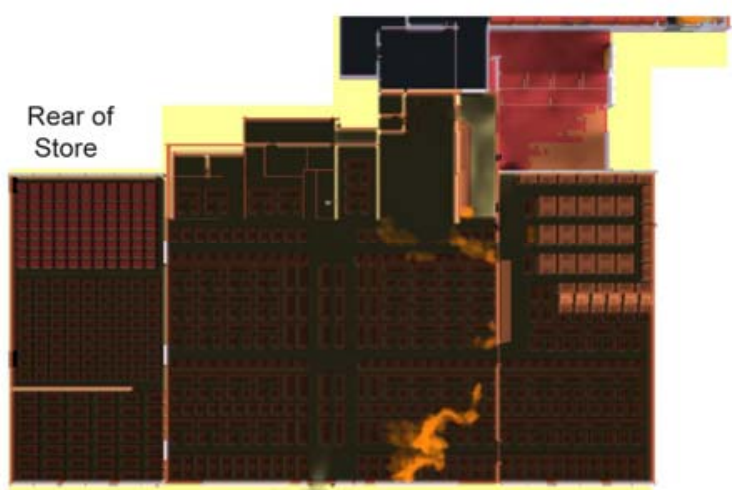

Front of Store

$1800 \mathrm{~s}$

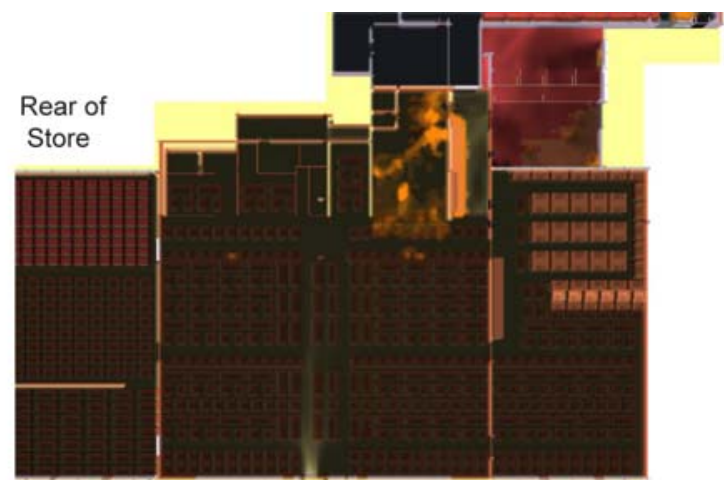

Front of Store

$1380 \mathrm{~s}$

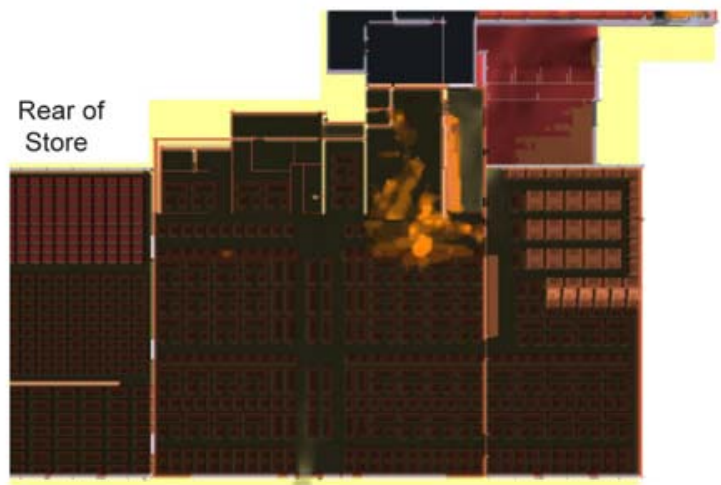

Front of Store

$1620 \mathrm{~s}$

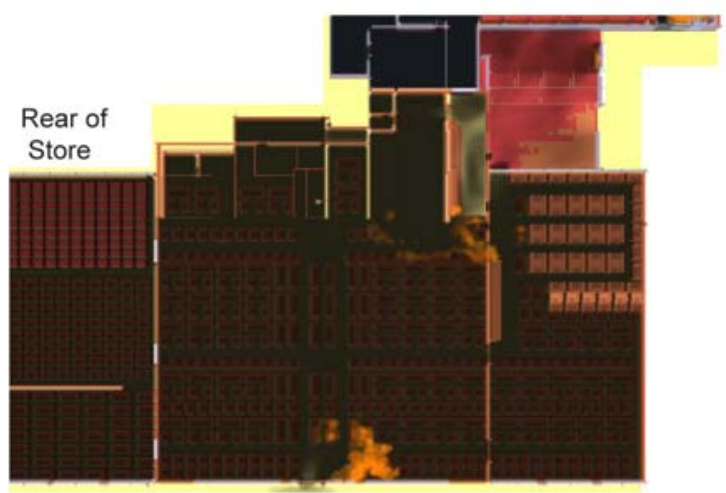

Front of Store

$2040 \mathrm{~s}$

Figure K-73. Fire and smoke progression in scenario with front windows intact and large $\left(5.9 \mathrm{~m}^{2}\right)$ roof vent in showroom as rendered by computer simulation. 


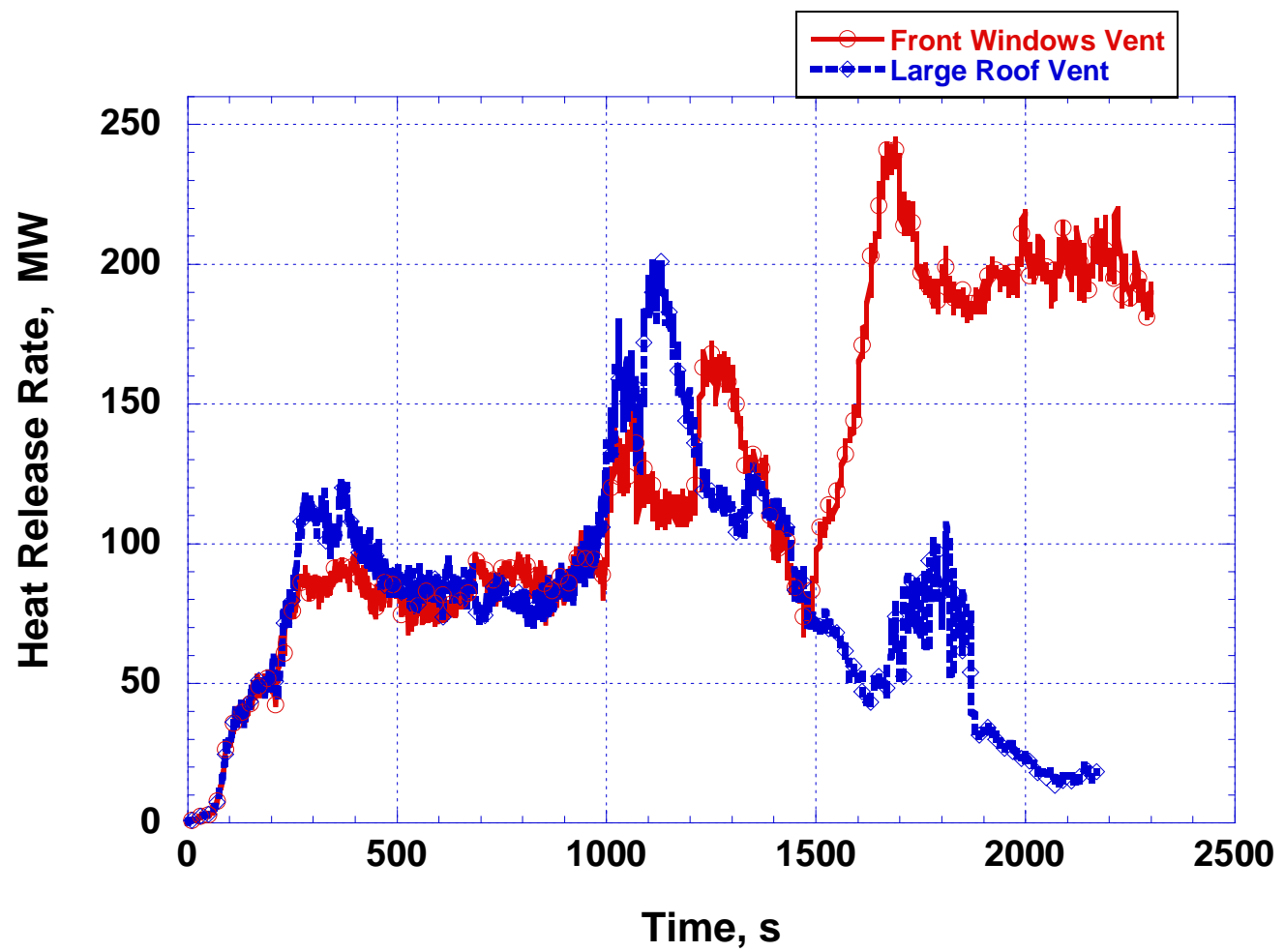

Figure K-74. Comparison of the heat release rate in simulations for the base scenario and the scenario with the front windows intact and a large $\left(5.91 \mathrm{~m}^{2}\right)$ vent in the main showroom roof. 


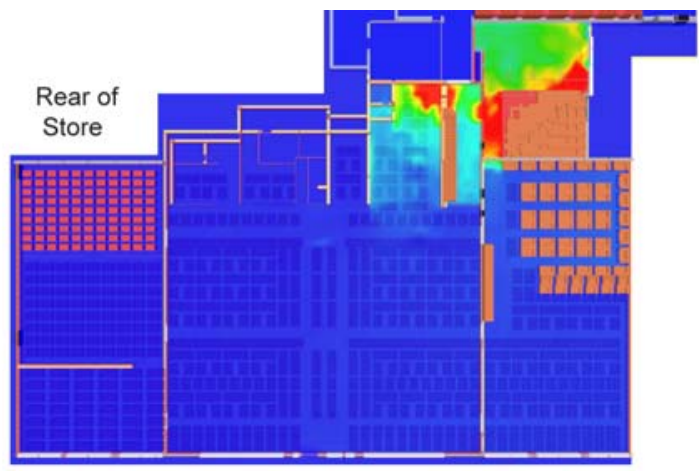

Front of Store

$900 \mathrm{~s}$

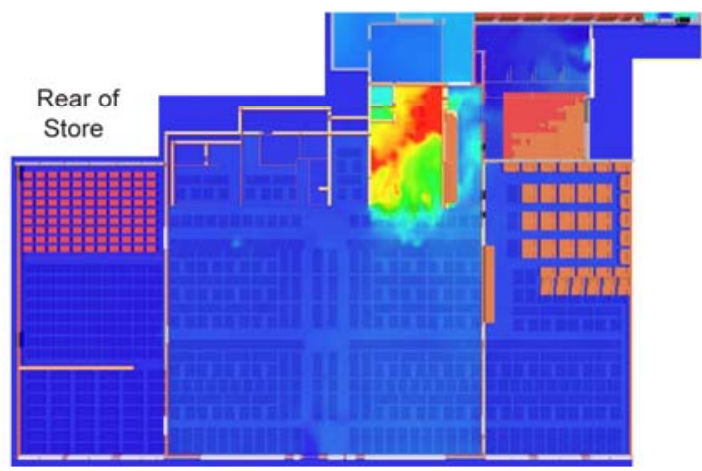

Front of Store

$1380 \mathrm{~s}$

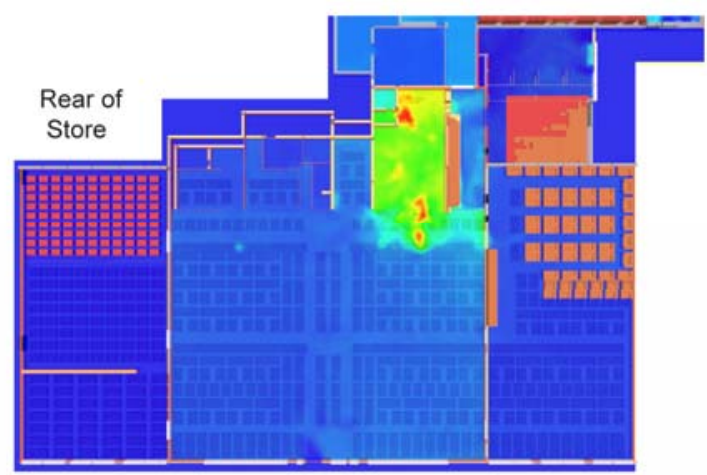

Front of Store

$1560 \mathrm{~s}$

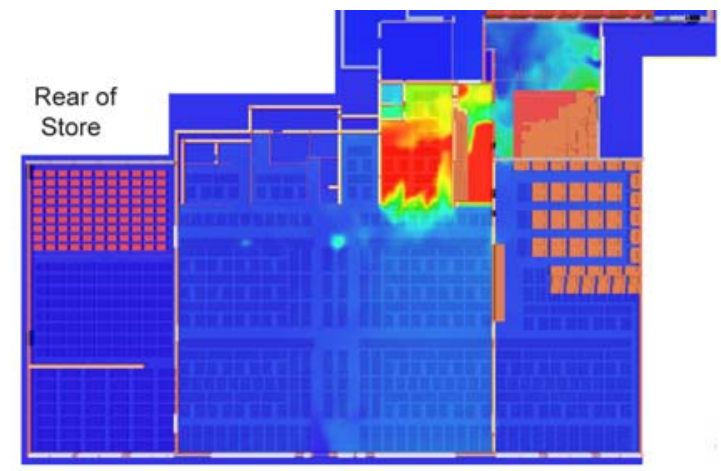

Front of Store

$1140 \mathrm{~s}$

Temp

C

820

740

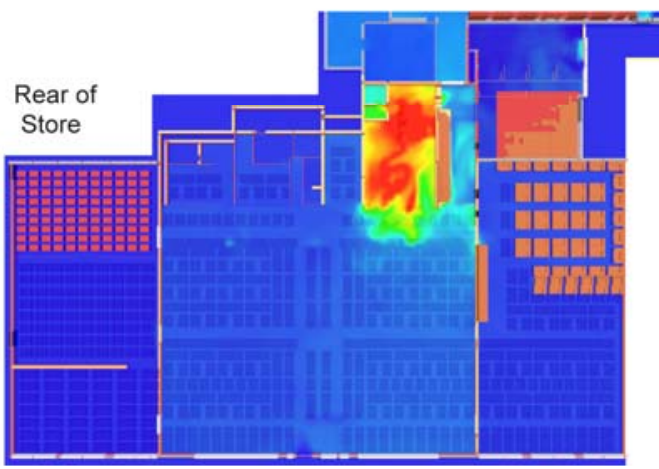

Front of Store

$1500 \mathrm{~s}$

100

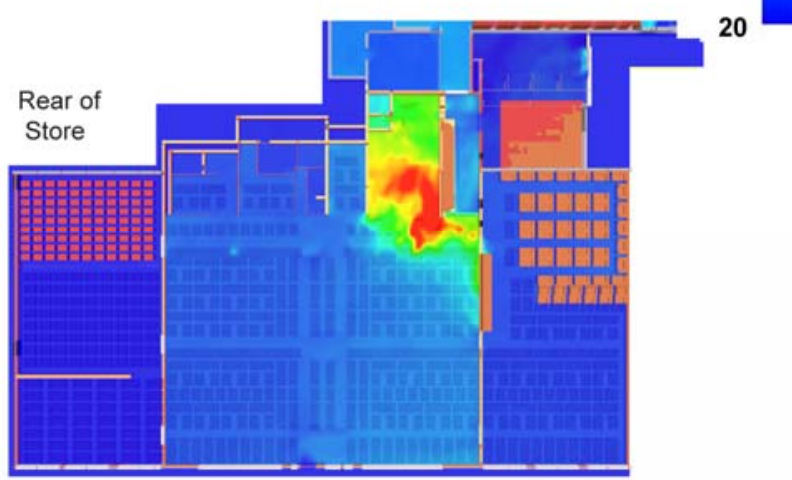

Front of Store

$1620 \mathrm{~s}$

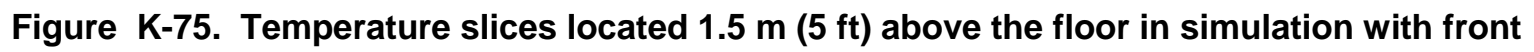
windows intact and large roof vent. 


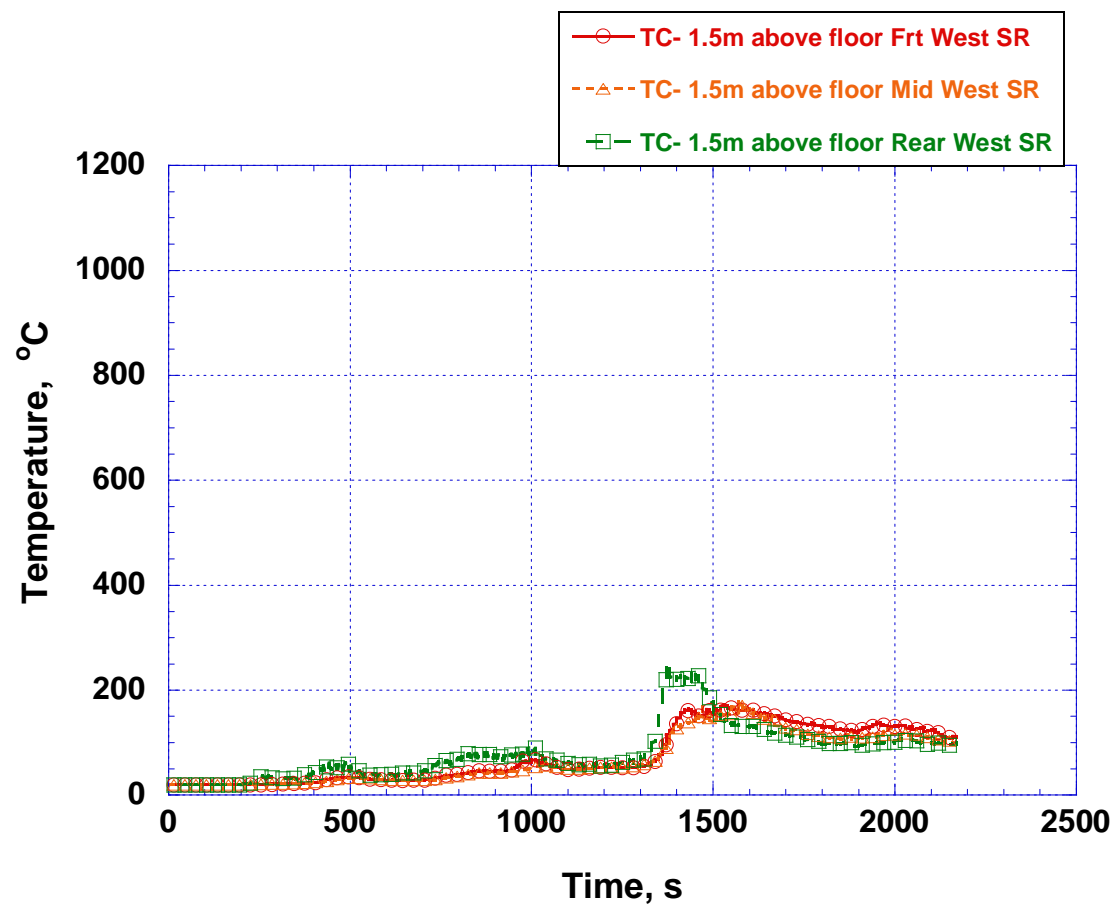

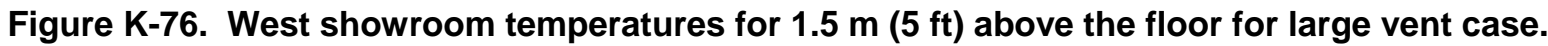

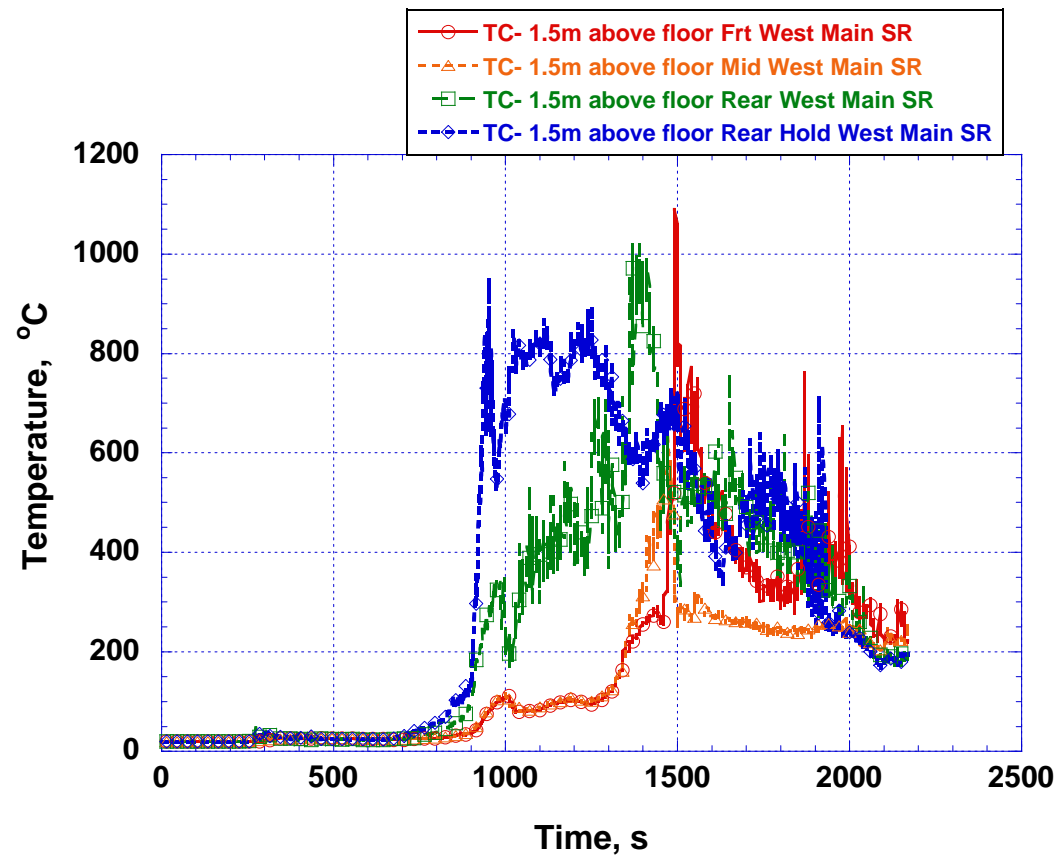

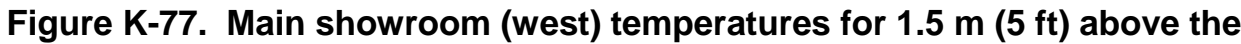
floor for large vent case. 


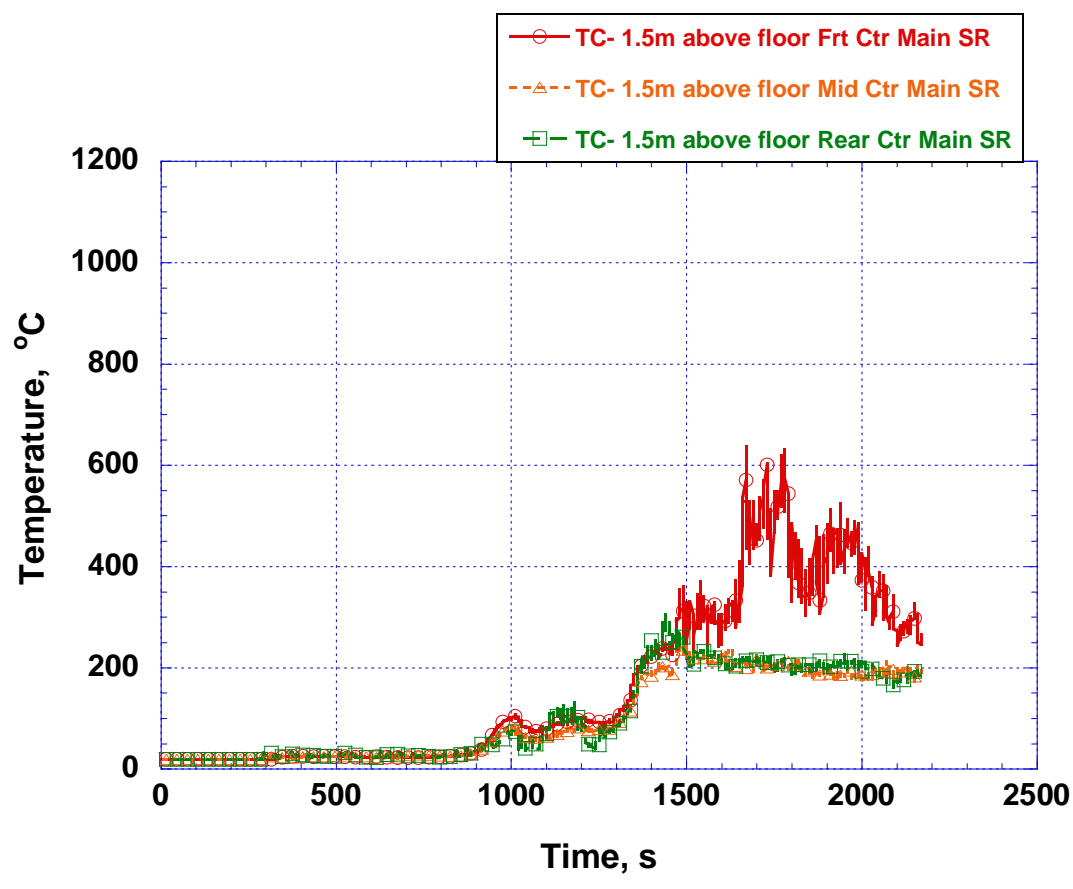

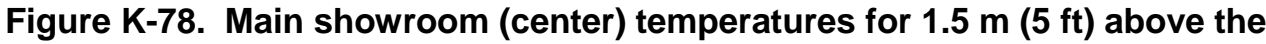
floor for large vent case.

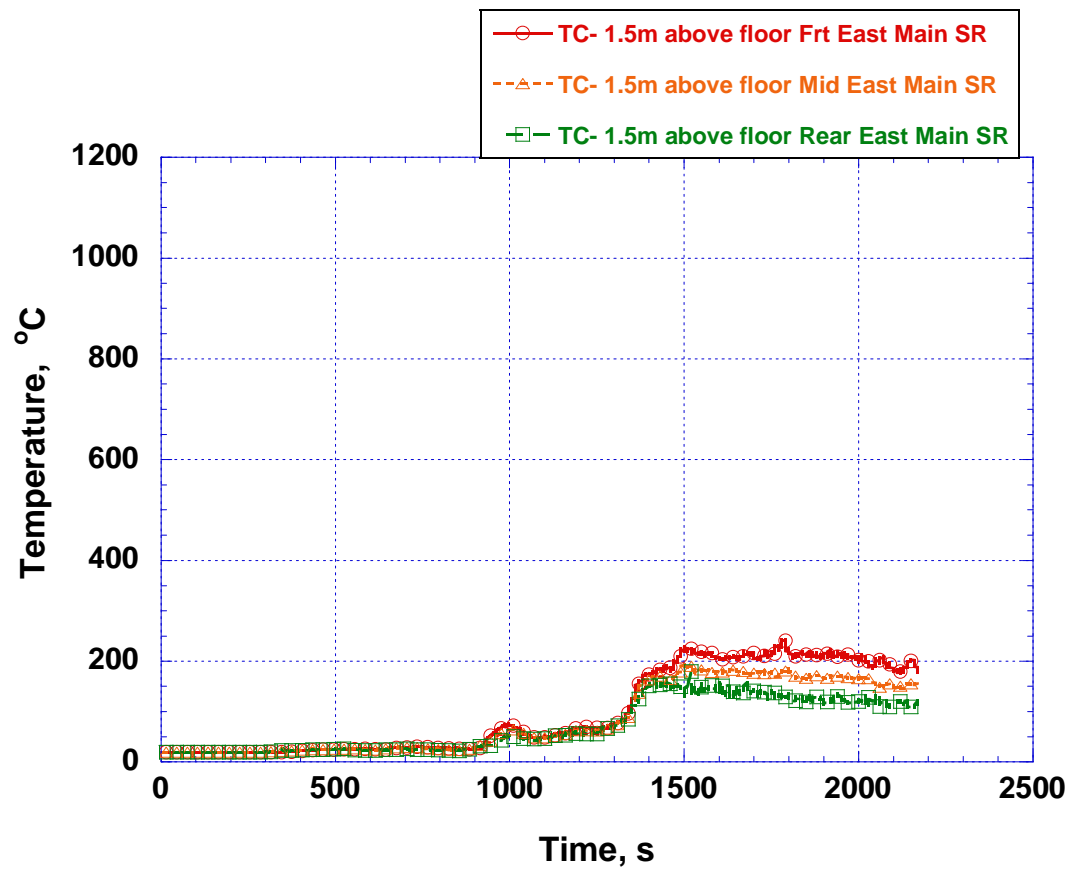

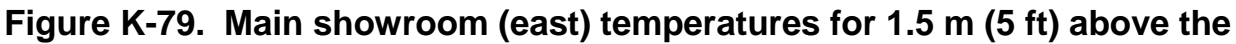
floor for large vent case. 


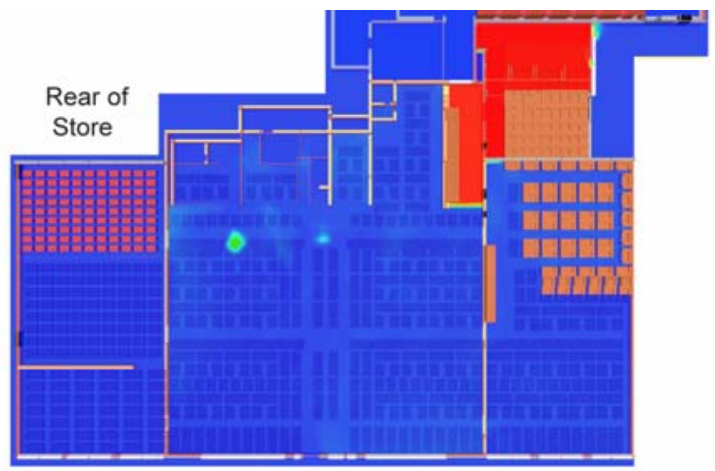

Front of Store

$360 \mathrm{~s}$

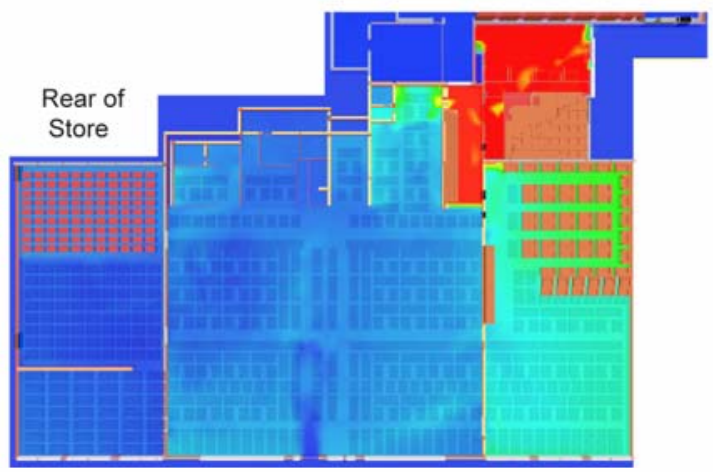

Front of Store

$840 \mathrm{~s}$

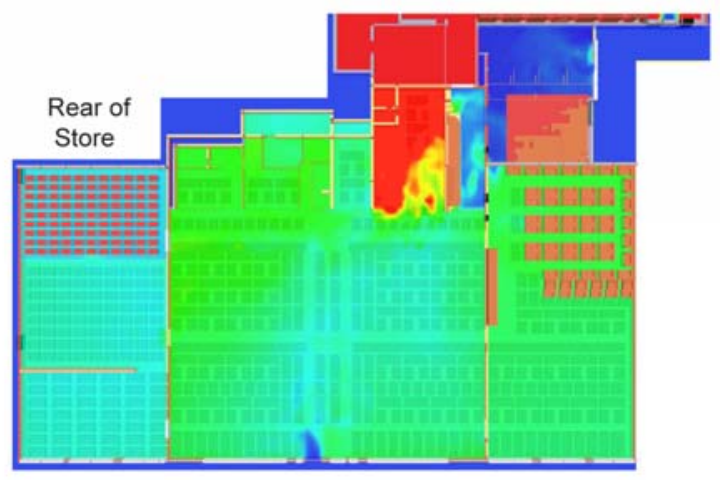

Front of Store

$1380 \mathrm{~s}$

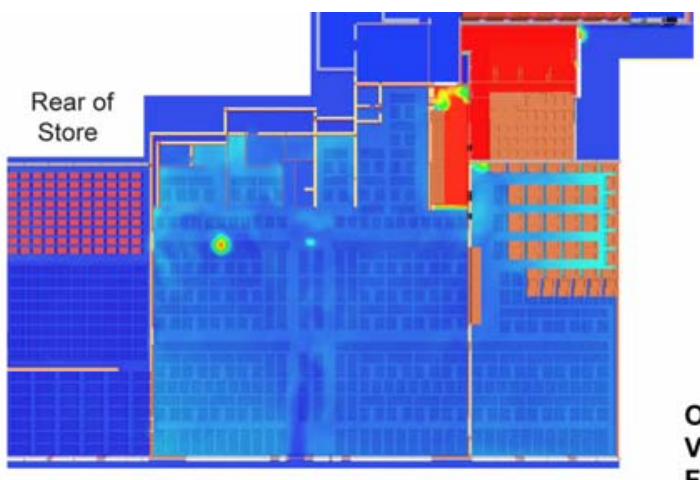

Front of Store

$480 \mathrm{~s}$

0.20

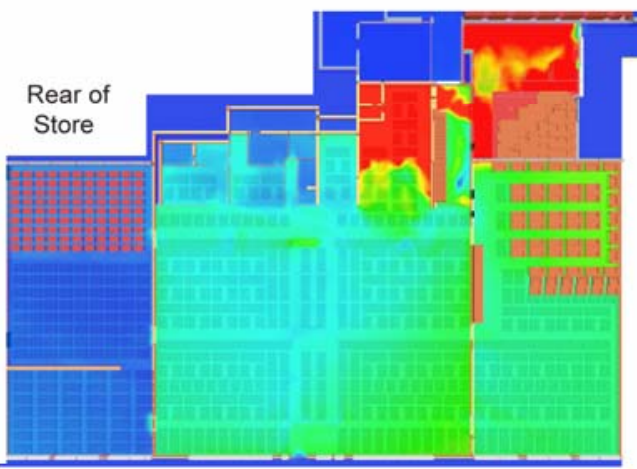

0.19

0.18

0.17

0.16

0.15

0.14

Front of Store

$960 \mathrm{~s}$

0.13

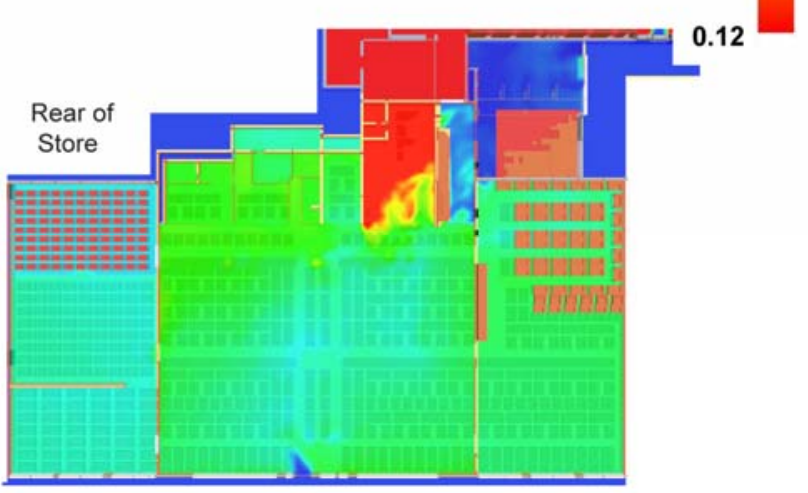

Front of Store

$1440 \mathrm{~s}$

Figure K-80. Oxygen volume fractions (slice images) $1.5 \mathrm{~m} \mathrm{(5} \mathrm{ft)} \mathrm{above} \mathrm{the} \mathrm{floor} \mathrm{in} \mathrm{simulation} \mathrm{with}$ front windows intact and large roof vent. 


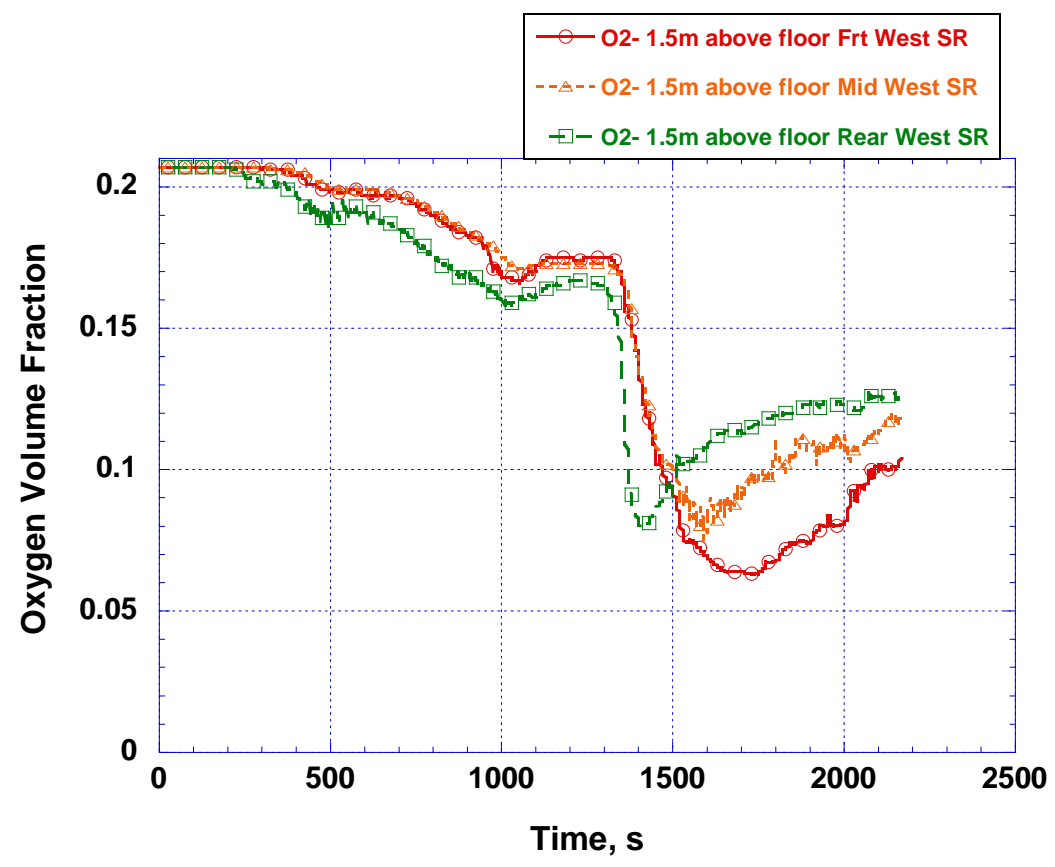

Figure K-81. West showroom oxygen volume fractions for $1.5 \mathrm{~m}(5 \mathrm{ft})$ above the floor for large vent case.

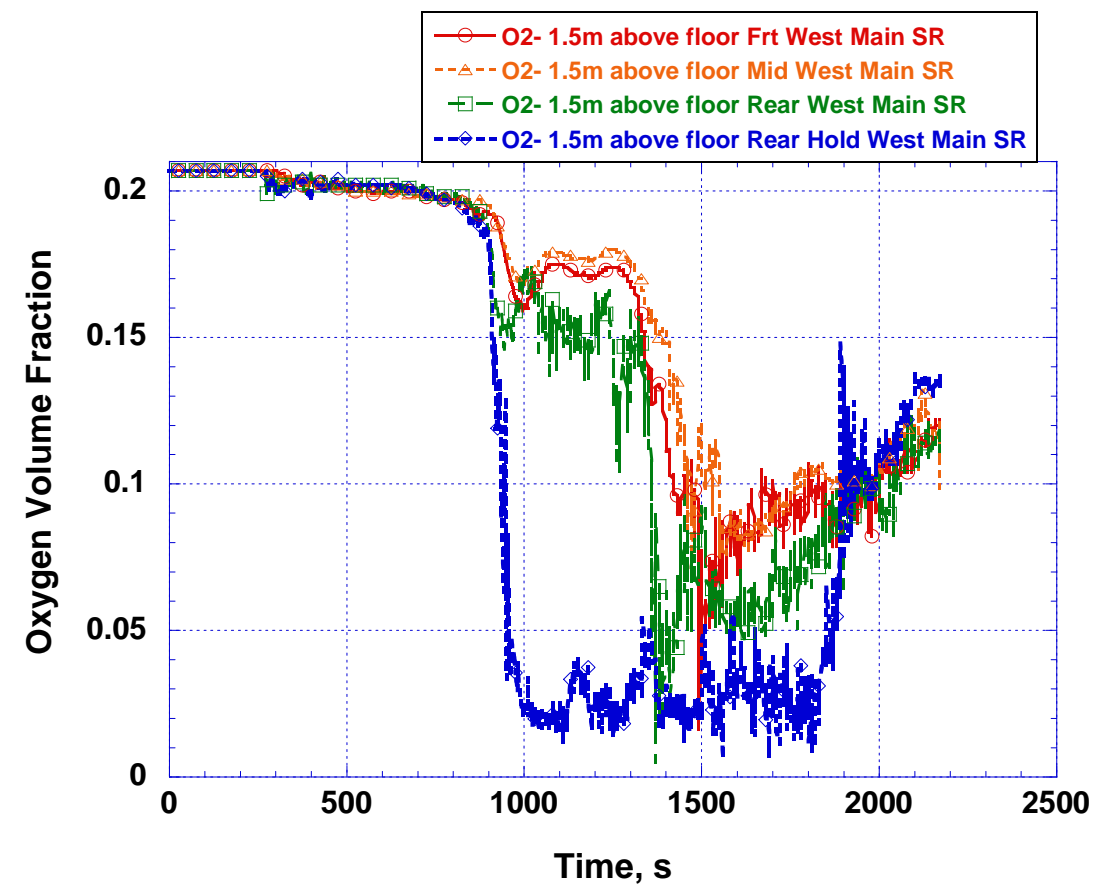

Figure K-82. Main showroom (west) oxygen volume fractions for $1.5 \mathrm{~m} \mathrm{(5} \mathrm{ft)}$ above the floor for large vent case. 


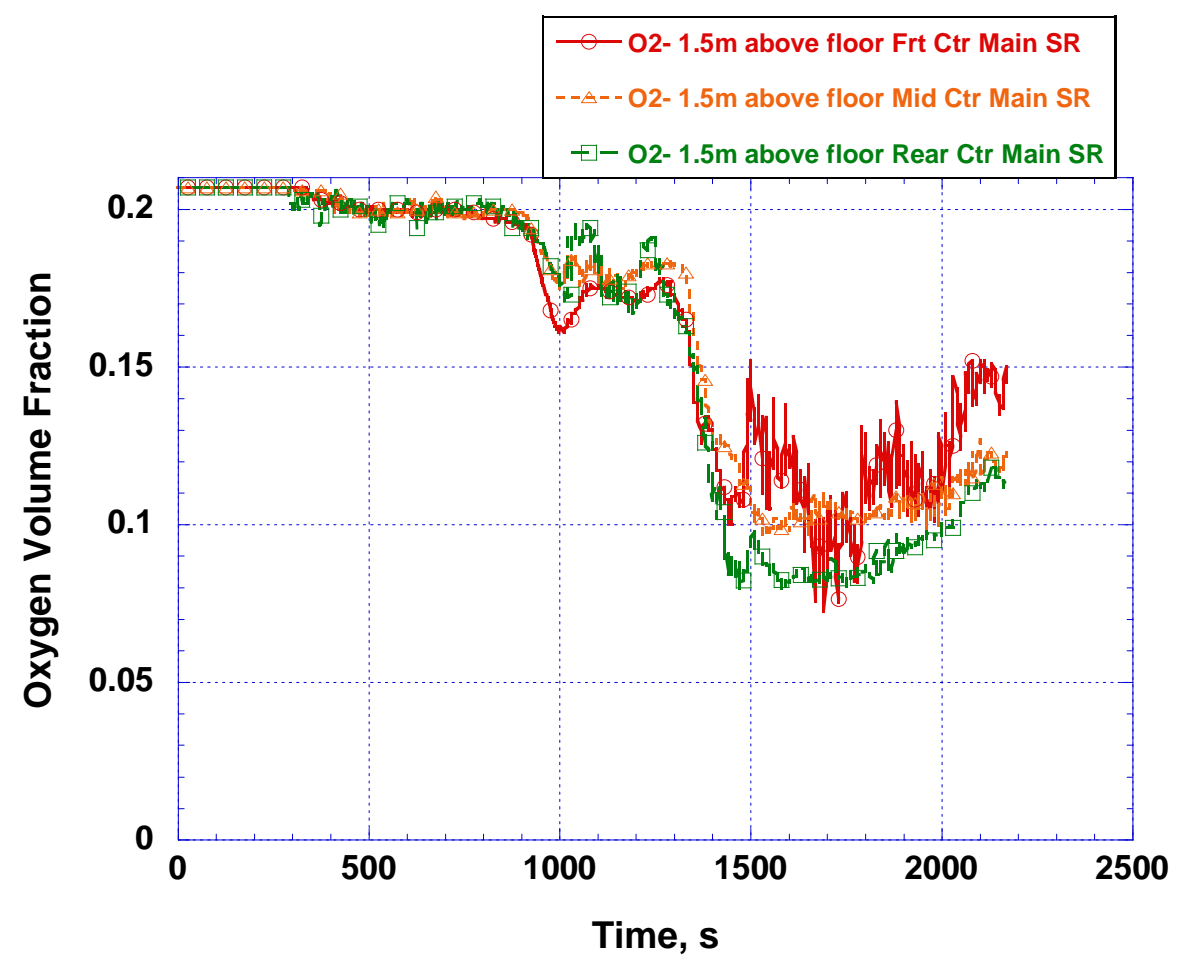

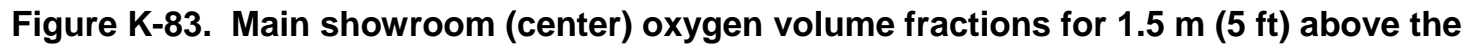
floor for large vent case.

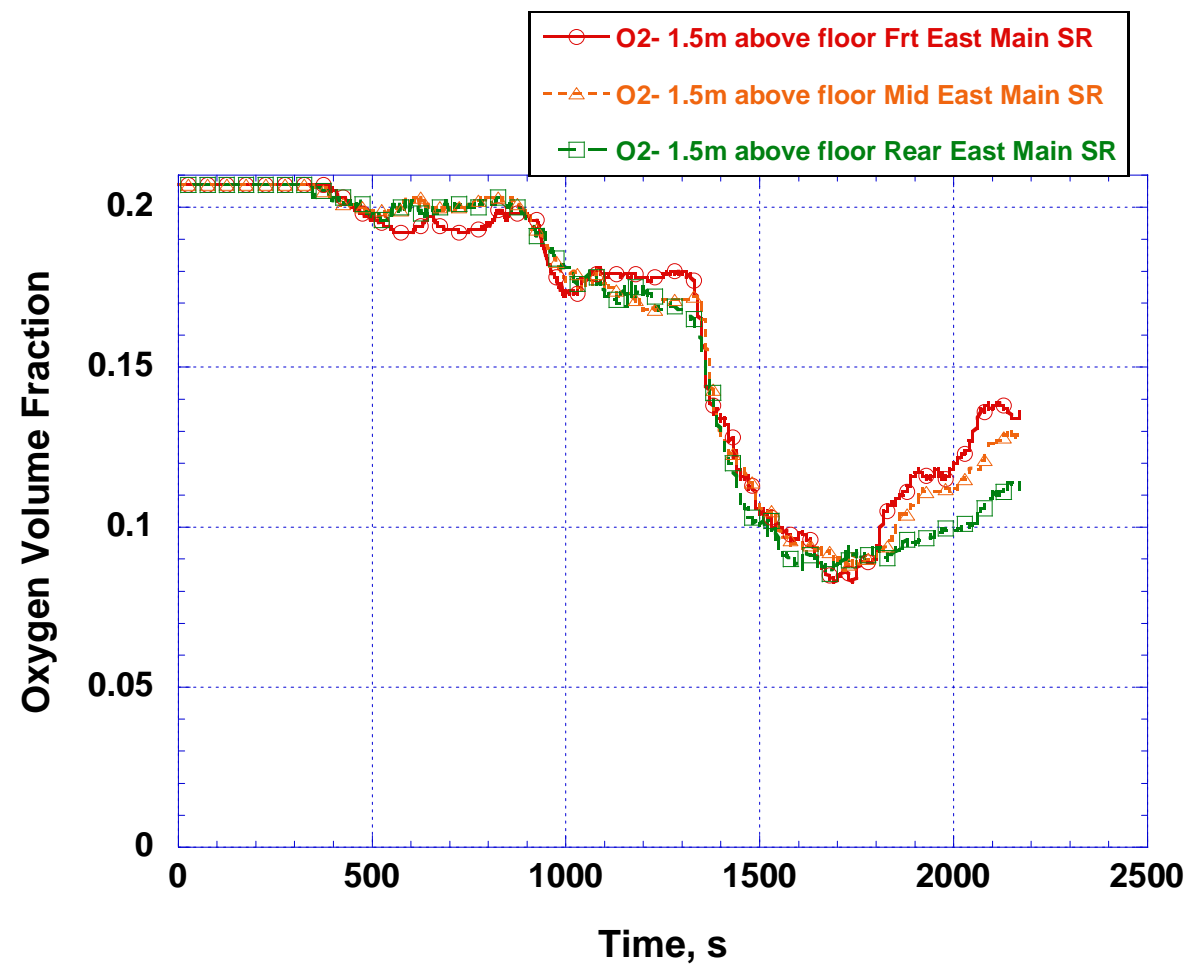

Figure K-84. Main showroom (east) oxygen volume fractions for $1.5 \mathrm{~m}(5 \mathrm{ft})$ above the floor for large vent case. 


\section{K.9 SUMMARY OF SIMULATION RESULTS}

\section{K.9.1 General}

Using FDS, NIST modeled the development of the fire in the Sofa Super Store during the fire that occurred on June 18, 2007, in which nine fire fighters lost their lives. This baseline fire scenario was constructed using measurements from the scene, witness interviews and discussions, time stamped photographs, and radio transmission logs. The simulation was run for $2400 \mathrm{~s}$, closely corresponding to the duration of the time that passes from the time that the fire started inside the loading dock to the time when the roof of the main showroom collapsed. Simulations were designed to provide insight into:

- fire and smoke spread inside the structure,

- fire-spread into the showrooms versus the warehouse,

- impact of sprinklers on the loading dock,and

- impact of ventilation, including breaking front windows and openings in the roof.

The insights provided by these simulations served as a basis for examining the potential effects of some intervention strategies. These were the installation of a fire sprinkler system, breaking the front windows of the building, and venting the rear of the showroom with two differently sized roof openings.

For each of the five simulations, the times for areas to become untenable due to elevated temperature or oxygen depletion are tabulated in Tables K-8 to K-13. These untenability criteria are simplifications of complex studies and serve as a basis for appraising the relative effects of alternate fire scenarios.

The NIST study did not include analysis of the threat to protected fire fighters. If a fire fighter in turnout gear is utilizing a self-contained breathing apparatus (SCBA) and has an adequate supply of air, the fire fighter can temporarily survive higher temperatures and depleted external oxygen levels. This safety shell ends when the fire fighter runs out of tank air or remains within the hot fire environment too long. 
Table K-9. Temperature and Oxygen Tenability Conditions for Rear of West Showroom.

\begin{tabular}{|c|c|c|c|c|c|c|}
\hline \multirow[b]{2}{*}{ Simulation } & \multicolumn{3}{|c|}{ Temperature } & \multicolumn{3}{|c|}{ Oxygen } \\
\hline & $\begin{array}{c}\text { Time to } \\
\text { Untenability } \\
\left(>120^{\circ} \mathrm{C}\right)^{1} \\
\text { S }\end{array}$ & $\begin{array}{l}\text { Peak } \\
\text { Temp } \\
{ }^{\circ} \mathrm{C}\end{array}$ & $\begin{array}{l}\text { Time to } \\
\text { Peak } \\
\text { Temp. } \\
\text { s }\end{array}$ & $\begin{array}{c}\text { Time to } \\
\text { Untenability } \\
(<0.12)^{2} \\
\mathrm{~s}\end{array}$ & $\begin{array}{c}\text { Minimum } \\
\text { Oxygen } \\
\text { Level } \\
\text { volume } \\
\text { fraction }\end{array}$ & $\begin{array}{l}\text { Time to } \\
\text { Minimum } \\
\text { Oxygen } \\
\text { Level } \\
\text { s }\end{array}$ \\
\hline Base & 1650 & 260 & 2300 & 1800 & 0.02 & 2300 \\
\hline Base with Sprinklers & \multicolumn{6}{|c|}{ Fire growth limited to loading dock. Conditions remained tenable. } \\
\hline $\begin{array}{l}\text { Front Windows } \\
\text { Intact }\end{array}$ & 1650 & 200 & 1400 & 1700 & 0.09 & 1450 \\
\hline Small Vent & 1800 & 210 & 1600 & 1800 & 0.09 & 1600 \\
\hline Large Vent & 1850 & 230 & 1400 & 1650 & 0.08 & 1400 \\
\hline
\end{tabular}

Table K-10. Temperature and Oxygen Tenability Conditions for Front of West Showroom.

\begin{tabular}{|c|c|c|c|c|c|c|}
\hline \multirow[b]{2}{*}{ Simulation } & \multicolumn{3}{|c|}{ Temperature } & \multicolumn{3}{|c|}{ Oxygen } \\
\hline & $\begin{array}{c}\text { Time to } \\
\text { Untenability } \\
\left(>120^{\circ} \mathrm{C}\right)^{1} \\
\mathrm{~s}\end{array}$ & $\begin{array}{c}\text { Peak } \\
\text { Temp } \\
\text { C }\end{array}$ & $\begin{array}{l}\text { Time to } \\
\text { Peak } \\
\text { Temp. } \\
\text { s }\end{array}$ & $\begin{array}{c}\text { Time to } \\
\text { Untenability } \\
(<0.12)^{2} \\
\mathrm{~s}\end{array}$ & $\begin{array}{c}\text { Minimum } \\
\text { Oxygen } \\
\text { Level } \\
\text { Volume } \\
\text { Fraction }\end{array}$ & $\begin{array}{c}\text { Time to } \\
\text { Minimum } \\
\text { Oxygen } \\
\text { Level } \\
\text { s }\end{array}$ \\
\hline Base & 1700 & 1100 & 1950 & 1900 & 0.2 & 1950 \\
\hline Base with Sprinklers & \multicolumn{6}{|c|}{ Fire growth limited to loading dock. Conditions remained tenable. } \\
\hline $\begin{array}{l}\text { Front Windows } \\
\text { Intact }\end{array}$ & 1700 & 140 & 1450 & 1750 & 0.07 & 1700 \\
\hline Small Vent & 1850 & 140 & 1600 & 1900 & 0.1 & 1700 \\
\hline Large Vent & 1700 & 170 & 1550 & 1700 & 0.06 & 1700 \\
\hline
\end{tabular}


Table K-11. Temperature and Oxygen Tenability Conditions for Rear of West Side of Main Showroom.

\begin{tabular}{|l|c|c|c|c|c|c|}
\hline \multirow{2}{*}{ Simulation } & \multicolumn{3}{|c|}{ Temperature } & \multicolumn{3}{c|}{ Oxygen } \\
\cline { 2 - 7 } & $\begin{array}{c}\text { Time to } \\
\text { Untenability } \\
\left(120^{\circ} \mathrm{C}\right)^{1} \\
\mathrm{~S}\end{array}$ & $\begin{array}{c}\text { Peak } \\
\text { Temp }\end{array}$ & $\begin{array}{c}\text { Time to } \\
\text { Peak } \\
\text { Temp. } \\
\mathrm{S}\end{array}$ & $\begin{array}{c}\text { Time to } \\
\text { Untenability } \\
(<0.12)^{2} \\
\mathrm{~s}\end{array}$ & $\begin{array}{c}\text { Minimum } \\
\text { Oxygen } \\
\text { Level } \\
\text { Volume } \\
\text { Fraction }\end{array}$ & $\begin{array}{c}\text { Time to } \\
\text { Minimum } \\
\text { Oxygen } \\
\text { Level } \\
\mathrm{s}\end{array}$ \\
\hline Base & 1200 & 850 & 1300 & 1550 & 0.01 & 1750 \\
\hline Base with Sprinklers & Fire growth limited to loading dock. Conditions remained tenable. \\
\hline $\begin{array}{l}\text { Front Windows } \\
\text { Intact }\end{array}$ & 1200 & 850 & 1300 & 1550 & 0.06 & 1400 \\
\hline Small Vent & 1200 & 600 & 1400 & 1800 & 0.07 & 1550 \\
\hline Large Vent & 1200 & 950 & 1400 & 1650 & 0.03 & 1400 \\
\hline $\begin{array}{l}\text { 1. Time for temperature to exceed } 1200^{\circ} \mathrm{C} \text { plus } 300 \mathrm{~s} . \\
\text { 2. Time for oxygen volume fraction less than } 0.12 \text { plus } 300 \mathrm{~s} .\end{array}$ \\
\hline
\end{tabular}

Table K-12. Temperature and Oxygen Tenability Condition for Front of West Side of Main Showroom.

\begin{tabular}{|c|c|c|c|c|c|c|}
\hline \multirow[b]{2}{*}{ Simulation } & \multicolumn{3}{|c|}{ Temperature } & \multicolumn{3}{|c|}{ Oxygen } \\
\hline & $\begin{array}{c}\text { Time to } \\
\text { Untenability } \\
\left(>120^{\circ} \mathrm{C}\right)^{1} \\
\mathrm{~S}\end{array}$ & $\begin{array}{c}\text { Peak } \\
\text { Temp } \\
\text { C }\end{array}$ & $\begin{array}{l}\text { Time to } \\
\text { Peak } \\
\text { Temp. } \\
\text { s }\end{array}$ & $\begin{array}{c}\text { Time to } \\
\text { Untenability } \\
(<0.12)^{2} \\
\mathrm{~s}\end{array}$ & $\begin{array}{c}\text { Minimum } \\
\text { Oxygen } \\
\text { Level } \\
\text { Volume } \\
\text { Fraction }\end{array}$ & $\begin{array}{c}\text { Time to } \\
\text { Minimum } \\
\text { Oxygen } \\
\text { Level } \\
\text { s }\end{array}$ \\
\hline Base & 1200 & 850 & 1300 & 1550 & 0.01 & 1750 \\
\hline Base with Sprinklers & \multicolumn{6}{|c|}{ Fire growth limited to loading dock. Conditions remained tenable. } \\
\hline $\begin{array}{l}\text { Front Windows } \\
\text { Intact }\end{array}$ & 1200 & 850 & 1300 & 1550 & 0.06 & 1400 \\
\hline Small Vent & 1200 & 600 & 1400 & 1800 & 0.07 & 1550 \\
\hline Large Vent & 1200 & 950 & 1400 & 1650 & 0.03 & 1400 \\
\hline
\end{tabular}


Table K-13. Temperature and Oxygen Tenability Conditions for Rear of East Side of Main Showroom.

\begin{tabular}{|l|c|c|c|c|c|c|}
\hline \multirow{2}{*}{ Simulation } & \multicolumn{3}{|c|}{ Temperature } & \multicolumn{3}{c|}{ Oxygen } \\
\cline { 2 - 7 } & $\begin{array}{c}\text { Time to } \\
\text { Untenability } \\
\left(>120^{\circ} \mathrm{C}\right)^{1} \\
\mathrm{~s}\end{array}$ & $\begin{array}{c}\text { Peak } \\
\text { Temp }\end{array}$ & $\begin{array}{c}\text { Time to } \\
\text { Peak } \\
\text { Temp. } \\
\mathrm{S}\end{array}$ & $\begin{array}{c}\text { Time to } \\
\text { Untenability } \\
(<0.12)^{2} \\
\mathrm{~s}\end{array}$ & $\begin{array}{c}\text { Minimum } \\
\text { Oxygen } \\
\text { Level } \\
\text { Volume } \\
\text { Fraction }\end{array}$ & $\begin{array}{c}\text { Time to } \\
\text { Minimum } \\
\text { Oxygen } \\
\text { Level } \\
\mathrm{s}\end{array}$ \\
\hline Base & 1550 & 1100 & 1550 & 1700 & 0.05 & 1900 \\
\hline Base with Sprinklers & Fire growth limited to loading dock. Conditions remained tenable. \\
\hline $\begin{array}{l}\text { Front Windows } \\
\text { Intact }\end{array}$ & 1550 & 800 & 1700 & 1700 & 0.06 & 1700 \\
\hline Small Vent & 1550 & 230 & 1550 & 1850 & 0.1 & 1600 \\
\hline Large Vent & 1600 & 1050 & 1500 & 1700 & 0.05 & 1500 \\
\hline $\begin{array}{l}\text { 1. Time for temperature to exceed } 120^{\circ} \mathrm{C} \text { plus } 300 \mathrm{~s} . \\
\text { 2. Time for oxygen volume fraction less than } 0.12 \text { plus } 300 \mathrm{~s} .\end{array}$ \\
\hline
\end{tabular}

Table K-14. Temperature and Oxygen Tenability Conditions for Front of East Side of Main Showroom.

\begin{tabular}{|c|c|c|c|c|c|c|}
\hline \multirow[b]{2}{*}{ Simulation } & \multicolumn{3}{|c|}{ Temperature } & \multicolumn{3}{|c|}{ Oxygen } \\
\hline & $\begin{array}{c}\text { Time to } \\
\text { Untenability } \\
\left(>120^{\circ} \mathrm{C}\right)^{1} \\
\text { S }\end{array}$ & $\begin{array}{c}\text { Peak } \\
\text { Temp } \\
\text { C }\end{array}$ & $\begin{array}{l}\text { Time to } \\
\text { Peak } \\
\text { Temp. } \\
\text { s }\end{array}$ & $\begin{array}{c}\text { Time to } \\
\text { Untenability } \\
(<0.12)^{2} \\
\mathrm{~s}\end{array}$ & $\begin{array}{c}\text { Minimum } \\
\text { Oxygen } \\
\text { Level } \\
\text { Volume } \\
\text { Fraction }\end{array}$ & $\begin{array}{l}\text { Time to } \\
\text { Minimum } \\
\text { Oxygen } \\
\text { Level } \\
\text { s }\end{array}$ \\
\hline Base & 1650 & 1000 & 1680 & 1400 & 0.08 & 1800 \\
\hline Base with Sprinklers & \multicolumn{6}{|c|}{ Fire growth limited to loading dock. Conditions remained tenable. } \\
\hline $\begin{array}{l}\text { Front Windows } \\
\text { Intact }\end{array}$ & 1650 & 230 & 1700 & 1750 & 0.09 & 1850 \\
\hline Small Vent & 1800 & 160 & 1550 & 2450 & 0.11 & 2150 \\
\hline Large Vent & 1650 & 220 & 1500 & 1750 & 0.08 & 1700 \\
\hline
\end{tabular}




\section{K.9.2 Baseline Simulation}

Results from the baseline simulation were tabulated with specific events from the fire ground in Table K-15. The simulation results are consistent with available photos, video, and witness accounts. The results indicate that fire spread from the loading dock into the holding area and void space above the main showroom. The fire also spread to the warehouse and to the rear of the west showroom. The simulation demonstrates that although the fire did spread into the rear of the west showroom, the spread through the double doors was limited. The fire moved into the main showroom through the holding area and then into the rear of the main showroom. The flow of the smoke into the holding area and up into the void space above the main showroom would have been hidden by the holding area partition wall and suspended ceiling of the main showroom.

The results indicate that as early as $270 \mathrm{~s}$ into the simulation, smoke may have begun to flow down through ventilation openings in the suspended ceiling and into the rear of the main showroom. By $300 \mathrm{~s}$, there is also a layer of smoke beginning to develop under the suspended ceiling in the rear of the west showroom. It cannot be concluded from the simulation whether an observer located in the main showroom would have noticed smoke in the rear main showroom at $300 \mathrm{~s}$, or would have been able to distinguish the source of that smoke. The smoke continued to flow down through ventilation openings and, after forming a substantial layer in the rear of the main showroom, began to spread throughout the main showroom. At about $400 \mathrm{~s}$, the simulation indicates that the smoke layer extended down to near the floor on the west side of the main showroom. As the fire spread from the holding area into the rear of the main showroom at around $500 \mathrm{~s}$, additional smoke was added to the smoke layer in the main showroom. As demonstrated by the rendering of smoke by the simulation, visibility became compromised in the showrooms within 8 minutes to 10 minutes. The simulation results are consistent with the E-11 captain reporting heavy smoke in the main showroom at 7:20 p.m., which would correspond to $555 \mathrm{~s}$ into the simulation.

At $900 \mathrm{~s}$, the baseline simulation results demonstrate dense black smoke would have been present from the floor to the ceiling in the main and west showrooms and less dense gray smoke in the east showroom. About a minute later at 7:27 p.m. there were fragmented radios transmissions suggesting fire fighters were "lost or trapped inside" and this would have been consistent with the dense black smoke in the showrooms. The model indicates that the fire continued to grow in the rear of the main showroom. By $1380 \mathrm{~s}$ in the simulation, the fire in the rear of the main showroom had exceeded $90 \mathrm{MW}$ and involved at least 15 percent of the main showroom. At about 7:35 p.m. (1450 s), the fire department vented the front windows and the fire spread extremely quickly to the front of the main showroom. Flames extended out the front windows on the west side of the main showroom at $1620 \mathrm{~s} \mathrm{(7:37:53} \mathrm{p.m.)} \mathrm{in} \mathrm{the} \mathrm{simulation.}$ This is in good agreement with fire ground photographs which show fire out the front window at 7:37 p.m.

During the base simulation, the loading dock, holding area, west showroom, and main showroom each exceeded the tenability thresholds (Tables K-8 to K-13). Although not specifically tabulated, but apparent from the temperature and oxygen plots (Figures K-33 and K-38), both the loading dock and the holding area became untenable early in the fire. The rear of the west side of the main showroom first became untenable due to high temperature at $1200 \mathrm{~s}$. The front of the west side of the main showroom exceeded the modeled temperature tenability threshold at $1350 \mathrm{~s}$. These times are consistent with the observed fire spreading from the rear to the front of the main showroom. The rear of the west showroom became untenable due to high temperature and oxygen depletion at $1650 \mathrm{~s}$ while the front of the west showroom exceeded both tenability thresholds at $1700 \mathrm{~s}$. The slightly longer time for the west showroom 
to exceed the tenability limits is consistent with the observed fire spreading through the main showroom first and then moving into the west showroom.

The simulation peak temperatures provide some understanding of the fire behavior within the building. The relatively low $210^{\circ} \mathrm{C}$ peak temperature in the rear of the west showroom is consistent with the understanding that the observed fire did not move directly through the rear of the west showroom to reach the main showroom. The higher simulation temperatures in the front of both showrooms, as compared to the rear of the showrooms, are consistent with additional oxygen being available in the front of the showrooms, relative to the less ventilated rear areas of the showroom.

The HRRs for the loading dock, main showroom, and west showroom are tabulated in Table K-16. The simulation indicates that the peak HRR for the loading dock was approximately $85 \mathrm{MW}$, the peak occurred at $280 \mathrm{~s}$, and the total energy released was 95 GJ. The peak heat release rate for main showroom was about one and a half times the loading dock at $135 \mathrm{MW}$ peak and $160 \mathrm{GJ}$ for total heat release. The west showroom values were a peak HRR of $50 \mathrm{MW}$ and total energy release of $30 \mathrm{GJ}$ which is consistent with the west showroom being about half the floor space of the main showroom and the rear third of the west showroom not being as involved in the fire. 
Table K-15. Observed Fire Ground and Computer Simulation Events.

\begin{tabular}{|c|c|c|c|}
\hline $\begin{array}{l}\text { Clock Time } \\
\text { hh:mm:ss }\end{array}$ & $\begin{array}{l}\text { Computer } \\
\text { Simulation } \\
\text { Time } \\
\text { s }\end{array}$ & Observed Fire Ground Event & Computer Simulation Event \\
\hline 6:56 p.m. & & Fire observed at rear of store & \\
\hline 7:07 p.m. & & Dispatch receives report of fire behind store & \\
\hline 7:10:46 p.m. & & BC-4 reports trash and debris fire outside loading dock & \\
\hline 7:10:53 p.m. & 0 & & $\begin{array}{l}\text { Computer simulation start time } \\
\text { Loading Dock Source Fire initiated (600 s duration and } \\
\text { ramped up to } 700 \mathrm{~kW} \text { ) }\end{array}$ \\
\hline 7:10:56 p.m. & 3 & BC 4 reports fire may have gotten into building & \\
\hline 7:11:40 p.m. & 47 & E-10 on scene & \\
\hline 7:12:13 p.m. & 80 & AC enters main showroom & Smoke not visible at rear of main showroom \\
\hline & 110 & & $\begin{array}{l}\text { Smoke filled loading dock } \\
\text { Smoke beginning to fill interstitial space above main } \\
\text { showroom }\end{array}$ \\
\hline 7:13:17 p.m. & 144 & Double doors at rear of west showroom opened & Double doors opened \\
\hline 7:13:18 p.m. & 145 & & $\begin{array}{l}\text { Fire pulling air from rear of west showroom into loading } \\
\text { dock through double doors. }\end{array}$ \\
\hline 7:14:23 p.m. & 270 & & $\begin{array}{l}\text { Smoke begins to flow through openings in suspended } \\
\text { ceiling in rear of main showroom }\end{array}$ \\
\hline 7:15:53p.m. & 300 & & $\begin{array}{l}\text { Fire-spread across entire loading dock } \\
\text { Smoke continuing to flow into interstitial space above } \\
\text { main showroom }\end{array}$ \\
\hline
\end{tabular}




\begin{tabular}{|c|c|c|c|}
\hline $\begin{array}{l}\text { Clock Time } \\
\text { hh:mm:ss }\end{array}$ & $\begin{array}{l}\text { Computer } \\
\text { Simulation } \\
\text { Time } \\
\text { s }\end{array}$ & Observed Fire Ground Event & Computer Simulation Event \\
\hline & & & $\begin{array}{l}\text { Light smoke flowing down through openings in } \\
\text { suspended ceiling into rear of main showroom } \\
\text { Light smoke below suspended ceiling in rear of west } \\
\text { showroom }\end{array}$ \\
\hline 7:16:32 p.m. & 339 & Fire Chief on scene & \\
\hline 7:16:53 p.m. & 360 & & $\begin{array}{l}\text { Loading dock fully involved in fire } \\
\text { Smoke continuing to flow into interstial space above } \\
\text { main showroom } \\
\text { Medium smoke flowing down through openings in } \\
\text { suspended ceiling into rear of main showroom } \\
\text { Medium smoke below suspended ceiling in rear of west } \\
\text { showroom } \\
\text { Light smoke below suspended ceiling in from of main } \\
\text { and west showrooms }\end{array}$ \\
\hline 7:17:23 p.m. & 390 & & $\begin{array}{l}\text { Conditions deteriorate in both main and west } \\
\text { showrooms }\end{array}$ \\
\hline \multirow[t]{2}{*}{ 7:17:33 p.m. } & 400 & & Warehouse Source Fire initiated \\
\hline & 420 & & $\begin{array}{l}\text { Smoke layer filling west side of main showroom } \\
\text { suspended ceiling to floor }\end{array}$ \\
\hline 7:18:53 p.m. & 480 & & $\begin{array}{l}\text { Holding area fully involved in fire } \\
\text { Smoke continuing to flow into interstial space above } \\
\text { main showroom } \\
\text { Medium smoke flowing down through openings in } \\
\text { suspended ceiling into rear of main showroom }\end{array}$ \\
\hline
\end{tabular}




\begin{tabular}{|c|c|c|c|}
\hline $\begin{array}{l}\text { Clock Time } \\
\text { hh:mm:ss }\end{array}$ & $\begin{array}{l}\text { Computer } \\
\text { Simulation } \\
\text { Time } \\
\text { s }\end{array}$ & Observed Fire Ground Event & Computer Simulation Event \\
\hline & & & $\begin{array}{l}\text { Medium smoke below suspended ceiling in rear of main } \\
\text { showroom } \\
\text { Medium smoke below suspended ceiling on west side of } \\
\text { main showroom } \\
\text { Light smoke below suspended ceiling on east side of } \\
\text { main showroom } \\
\text { Medium smoke below suspended ceiling of entire west } \\
\text { showroom }\end{array}$ \\
\hline 7:19:00 p.m. & 487 & $\begin{array}{l}\text { E-15 captain and two fire fighters enter main showroom } \\
\text { encountered smoke and donned SCBA facepieces }\end{array}$ & \\
\hline 7:19:38 p.m. & 525 & & $\begin{array}{l}\text { Main Showroom Fire Source } 1 \text { initiated (ramped up to } \\
4 \text { MW) }\end{array}$ \\
\hline 7:20:08 p.m. & 555 & $\begin{array}{l}\text { E-15 arrives on scene and as E- } 11 \text { captain exits structure } \\
\text { passes by E-15 crew entering structure. E- } 11 \text { captain } \\
\text { reports heavy smoke and increasing temperatures }\end{array}$ & \\
\hline 7:20:08 p.m. & & E-12 begins pumping water to E-10 at loading dock & \\
\hline 7:20:53 p.m. & 600 & & $\begin{array}{l}\text { Holding area fire has penetrated into rear of main } \\
\text { showroom } \\
\text { Fire burning in rear of main showroom and smoke from } \\
\text { fire flowing under suspended ceiling in main showroom } \\
\text { Heavy smoke in rear of main showroom } \\
\text { Medium to heavy smoke below suspended ceiling on } \\
\text { west side of main showroom }\end{array}$ \\
\hline
\end{tabular}




\begin{tabular}{|c|c|c|c|}
\hline $\begin{array}{l}\text { Clock Time } \\
\text { hh:mm:ss }\end{array}$ & $\begin{array}{l}\text { Computer } \\
\text { Simulation } \\
\text { Time } \\
\text { s }\end{array}$ & Observed Fire Ground Event & Computer Simulation Event \\
\hline & & & $\begin{array}{l}\text { Light to medium smoke below suspended ceiling on east } \\
\text { side of main showroom } \\
\text { Medium smoke below suspended ceiling of entire west } \\
\text { showroom }\end{array}$ \\
\hline 7:21:53 p.m. & 660 & & $\begin{array}{l}\text { Main Showroom Fire Source } 2 \text { initiated -(ramped up to } \\
6 \mathrm{MW} \text { ) }\end{array}$ \\
\hline 7:23:16 p.m. & 743 & $\begin{array}{l}\text { Early photograph shows flames on roof above rear of } \\
\text { main showroom and/or holding area (Figure 4-23) }\end{array}$ & \\
\hline 7:25:53 p.m. & 900 & & $\begin{array}{l}\text { Fire burning in rear of main showoom grown to about } \\
23 \text { MW. Smoke from fire flowing under suspended } \\
\text { ceiling in main showroom } \\
\text { Heavy smoke in rear of main showroom } \\
\text { Heavy smoke below suspended ceiling of entire main } \\
\text { showroom. } \\
\text { Heavy smoke below suspended ceiling of entire west } \\
\text { showroom } \\
\text { Light smoke below suspended ceiling of east showroom }\end{array}$ \\
\hline 7:27 p.m. & & $\begin{array}{l}\text { E-16 begins pumping water to E- } 11 \text { at front of store } \\
\text { Inaudible radio transmissions - "lost or trapped inside" }\end{array}$ & \\
\hline 7:27:53 p.m. & 1020 & & $\begin{array}{l}\text { Fire in main showroom continues to grow in the rear of } \\
\text { showroom. Fire has reached approximately } 90 \text { MW. }\end{array}$ \\
\hline 7:31:53 p.m. & 1260 & & $\begin{array}{l}\text { Fire continues to grow in southwest rear corner of main } \\
\text { showroom involving about } 10 \% \text { of main showroom }\end{array}$ \\
\hline 7:31 p.m. to & & Fire fighter call “Mayday” & \\
\hline
\end{tabular}




\begin{tabular}{|c|c|c|c|}
\hline $\begin{array}{l}\text { Clock Time } \\
\text { hh:mm:ss }\end{array}$ & $\begin{array}{l}\text { Computer } \\
\text { Simulation } \\
\text { Time } \\
\text { S }\end{array}$ & Observed Fire Ground Event & Computer Simulation Event \\
\hline 7:34 p.m. & & Fire Chief "we need to vacate building” & \\
\hline 7:33:53 p.m. & 1380 & & $\begin{array}{l}\text { Fire begins to spread out of southwest corner and } \\
\text { involves about } 15 \% \text { of main showroom. }\end{array}$ \\
\hline 7:35:10 p.m. & 1457 & Removal of front windows of main showroom & Main showroom windows opened \\
\hline 7:35:53 p.m. & 1500 & & $\begin{array}{l}\text { Fire spreading towards front of store on west side of } \\
\text { main showroom and involves about } 20 \% \text { of main } \\
\text { showroom }\end{array}$ \\
\hline 7:36 p.m. & & Thick black smoke rolls out front windows & \\
\hline 7:36:23 p.m. & 1530 & $\begin{array}{l}\text { Removal of additional front windows of main } \\
\text { showroom }\end{array}$ & Main showroom windows opened \\
\hline 7:36:53 p.m. & 1560 & & $\begin{array}{l}\text { Fire has spread to front of store on west side of main } \\
\text { showroom and involves about } 30 \% \text { of main showroom }\end{array}$ \\
\hline 7:37 p.m. & & Fire rolls out front windows of main showroom & \\
\hline 7:37:53 p.m. & 1620 & & $\begin{array}{l}\text { Fire spreading to east side of main showroom and front } \\
\text { of west showroom }\end{array}$ \\
\hline 7:38:00 p.m. & 1627 & Failure of front windows of west showroom & \\
\hline 7:44:00 p.m. & 1987 & Failure of additional front windows of west showroom & \\
\hline 7:50:53 p.m. & 2400 & & $\begin{array}{l}\text { End of simulation - } 40 \text { minutes after initial discovery of } \\
\text { fire by fire department }\end{array}$ \\
\hline 7:51 p.m. & & Portion of main showroom roof collapses & \\
\hline
\end{tabular}




\section{K.9.3 Fire Sprinklers Simulation}

In the simulation with fire sprinklers, the two sprinklers nearest the fire (in the southwest corner of the loading dock) activated early in the fire, at $50 \mathrm{~s}$ and $75 \mathrm{~s}$. The two sprinklers controlled the fire and prevented the fire from spreading into the showrooms or warehouse. As a result, the temperatures and oxygen volume fractions remained below untenability thresholds.

\section{K.9.4 Alternate Ventilation Scenarios}

Three simulations were performed in which the building ventilation conditions were altered from the baseline scenario.

In the first, the windows at the front of the main showroom were kept in place. While this did effectively reduce the peak heat release rate, conditions throughout the main and west showrooms became untenable due to excessive temperature and depleted oxygen. Untenability would have occurred prior to the time that the windows were removed in the base scenario.

In the second variation, a $1.7 \mathrm{~m}^{2}\left(18 \mathrm{ft}^{2}\right)$ vent was placed in the roof at the rear of the main showroom. The results of this simulation were not significantly different from the ventilation scenario with the front windows intact. With a vent or hole in the roof, the smoke and unburned fuel exited the structure and that if gases vented outside were ignited, the energy released would not have contributed to the energy inside the modeled structure.

In the final ventilation variation, the roof vent was expanded to $5.9 \mathrm{~m}^{2}\left(64 \mathrm{ft}^{2}\right)$. This enlargement did not change the peak HRR, temperature, or minimum oxygen volume fractions. However, the fire progression was delayed at the rear of the showroom by several minutes. This delay in the movement of the fire did not significantly change the time that conditions became untenable throughout the main and west showrooms. 
Table K-16. Fire Energetics for the Loading Dock, Main Showroom, and West Showroom.

\begin{tabular}{|c|c|c|c|c|c|c|c|c|c|}
\hline \multirow[b]{2}{*}{ Simulation } & \multicolumn{3}{|c|}{ Loading Dock } & \multicolumn{3}{|c|}{ Main Showroom } & \multicolumn{3}{|c|}{ West Showroom } \\
\hline & $\begin{array}{c}\text { Time to } \\
\text { Peak } \\
\text { HRR } \\
s\end{array}$ & $\begin{array}{l}\text { Peak } \\
\text { HRR } \\
\text { MW }\end{array}$ & $\begin{array}{c}\text { Total } \\
\text { Heat } \\
\text { Release } \\
\text { GJ }\end{array}$ & $\begin{array}{c}\text { Time to } \\
\text { Peak } \\
\text { HRR } \\
\text { s }\end{array}$ & $\begin{array}{c}\text { Peak } \\
\text { HRR } \\
\text { MW }\end{array}$ & $\begin{array}{c}\text { Total } \\
\text { Heat } \\
\text { Release } \\
\text { GJ }\end{array}$ & $\begin{array}{c}\text { Time to } \\
\text { Peak } \\
\text { HRR } \\
\text { s }\end{array}$ & $\begin{array}{c}\text { Peak } \\
\text { HRR } \\
\text { MW }\end{array}$ & $\begin{array}{c}\text { Total } \\
\text { Heat } \\
\text { Release } \\
\text { GJ }\end{array}$ \\
\hline Base & 280 & 85 & 95 & 1720 & 135 & 160 & 1940 & 50 & 30 \\
\hline Base with Sprinklers & 60 & 0.9 & 1.4 & & & & & & \\
\hline $\begin{array}{l}\text { Front Windows } \\
\text { Intact }\end{array}$ & & & & 1260 & 130 & 75 & & & \\
\hline
\end{tabular}




\section{K.10 REFERENCES FOR APPENDIX K}

[1] Nelson, H.E., "An Engineering Analysis of the Early Stages of Fire Development - The Fire at the Dupont Plaza Hotel and Casino- Dec. 31, 1986,” NBSIR 97-3560, National Institute of Standards and Technology (formerly the National Bureau of Standards), Gaithersburg, MD, April 1987.

[2] Purser, D.A., “Toxicity Assessment of Combustion Products," SFPE Handbook of Fire Protection Engineering, $3^{\text {rd }}$ ed. DiNenno, P.J., et al. (eds). National Fire Protection Association (NFPA) Quincy, MA, 2002.

[3] McGrattan, K.B., Hostikka, S., and Floyd, J.E., Fire Dynamics Simulator (Version 5), User’s Guide. NIST Special Publication 1019-5, National Institute of Standards and Technology, Gaithersburg, MD, October 2007.

[4] Forney, G.P., Smokeview (Version 5), A Tool for Visualizing Fire Dynamics Simulation Data, Volume I: User’s Guide. NIST Special Publication 1017-1, National Institute of Standards and Technology, Gaithersburg, MD, August 2007.

[5] National Institute of Standards and Technology, Gaithersburg, MD, USA, and VTT Technical Research Centre of Finland, Espoo, Finland. Fire Dynamics Simulator, Technical Reference Guide, 5th edition, October 2007. NIST Special Publication 1018-5 (four volume set).

[6] Hill, K., Dreisbach, J., Joglar, F., Najafi, B., McGrattan, K., Peacock, R., and Hamins, A., "Verification and Validation of Selected Fire Models for Nuclear Power Plant Applications." NUREG 1824, United States Nuclear Regulatory Commission, Washington, DC, 2007.

[7] Grosshandler, W.L., Bryner, N., Madrzykowski, D., and Kuntz, K., Report of the Technical Investigation of The Station Nightclub Fire. NIST NCSTAR 2, National Institute of Standards and Technology, Gaithersburg, MD, July 2005.

[8] McGrattan, K., Bouldin, C., and Forney, G., Federal Building and Fire Safety Investigation of the World Trade Center Disaster: Computer Simulation of the Fires in the WTC Towers. NIST NCSTAR 1-5F, National Institute of Standards and Technology, Gaithersburg, MD, September 2005.

[9] McGrattan, K.B., Hamins, A., and Evans, D.D., "Sprinklers, Vent and Draft Curtain Interaction Modeling and Experiment,” Fire Suppression and Detection Research Application Symposium, Research and Practice: Bridging the Gap, February 25-27, 1998, Orlando, FL Proceedings. National Fire Protection Research Foundation, Quincy, MA, 1998.

[10] Madrzykowski, D., and Vettori, R.L., "Simulation of the Dynamics of the Fire at 3146 Cherry Road NE, Washington D.C., May 30, 1999,” NISTIR 6510, National Institute of Standards and Technology, Gaithersburg, MD, April 2000.

[11] Madrzykowski, D., Forney, G.P., and Walton, W.D., "Simulation of the Dynamics of a Fire in a Two-Story Duplex-Iowa, Dec. 22, 1999,” NISTIR 6854, National Institute of Standards and Technology, Gaithersburg, MD, January 2002. 
[12] Sutula, J., “Applications of Fire Dynamics Simulator in Fire Protection Engineering Consulting,” Fire Protection Engineering, No. 14, Society of Fire Protection Engineers, Bethesda, MD, Spring 2002.

[13] Rehm, R.G., Pitts, W.M., Baum, H.R., Evans, D.D., Prasad, K., McGrattan, K.B., and Forney, G.P., “Initial Model for Fires in the World Trade Center Towers," NISTIR 6879, National Institute of Standards and Technology, Gaithersburg, MD, May, 2002.

[14] Sutula, J., "Practical Applications of Fire Dynamics Simulator in Fire Protection Engineering Consulting,” Fire Protection Engineering, No. 15, Society of Fire Protection Engineers, Bethesda, MD, Summer 2002.

[15] McGrattan, K.B., and Hamins, A., "Numerical Simulation of the Howard Street Tunnel Fire, Baltimore, MD, July 2001, NISTIR 6902, National Institute of Standards and Technology, Gaithersburg, MD, August 2002.

[16] Quintiere, J. G., Principles of Fire Behavior, Delmar Publishers, Albany, NY, 255 p., 1997.

[17] Incropera, F.P., and DeWitt, D.P., Fundamentals of Heat and Mass Transfer, Fifth Edition, John Wiley \& Sons, Hoboken, NJ, 969 p., 2002.

[18] Fire Dynamics, Chapter 2, The SFPE Handbook of Fire Protection Engineering, 4th edition, Society of Fire Protection Engineers, Quincy, MA page 2-29.

[19] ISO 13943, Fire Safety - Vocabulary, International Standards Organization, Geneva.

[20] NFPA 13, Standard for the Installation of Sprinkler Systems, National Fire Protection Association (NFPA) Quincy, MA 2007. 
K-98 


\section{APPENDIX L. COMPUTER SimULATION INPUT FILES}

\section{L.1 FIRE DYNAMIC SIMULATOR VERSION 5.1.0 (SVN 1162)}

(Note: Executable FDS 5.0.0 computer code included on accompanying DVD)

\section{L.1.1 Computer Simulation Runs}

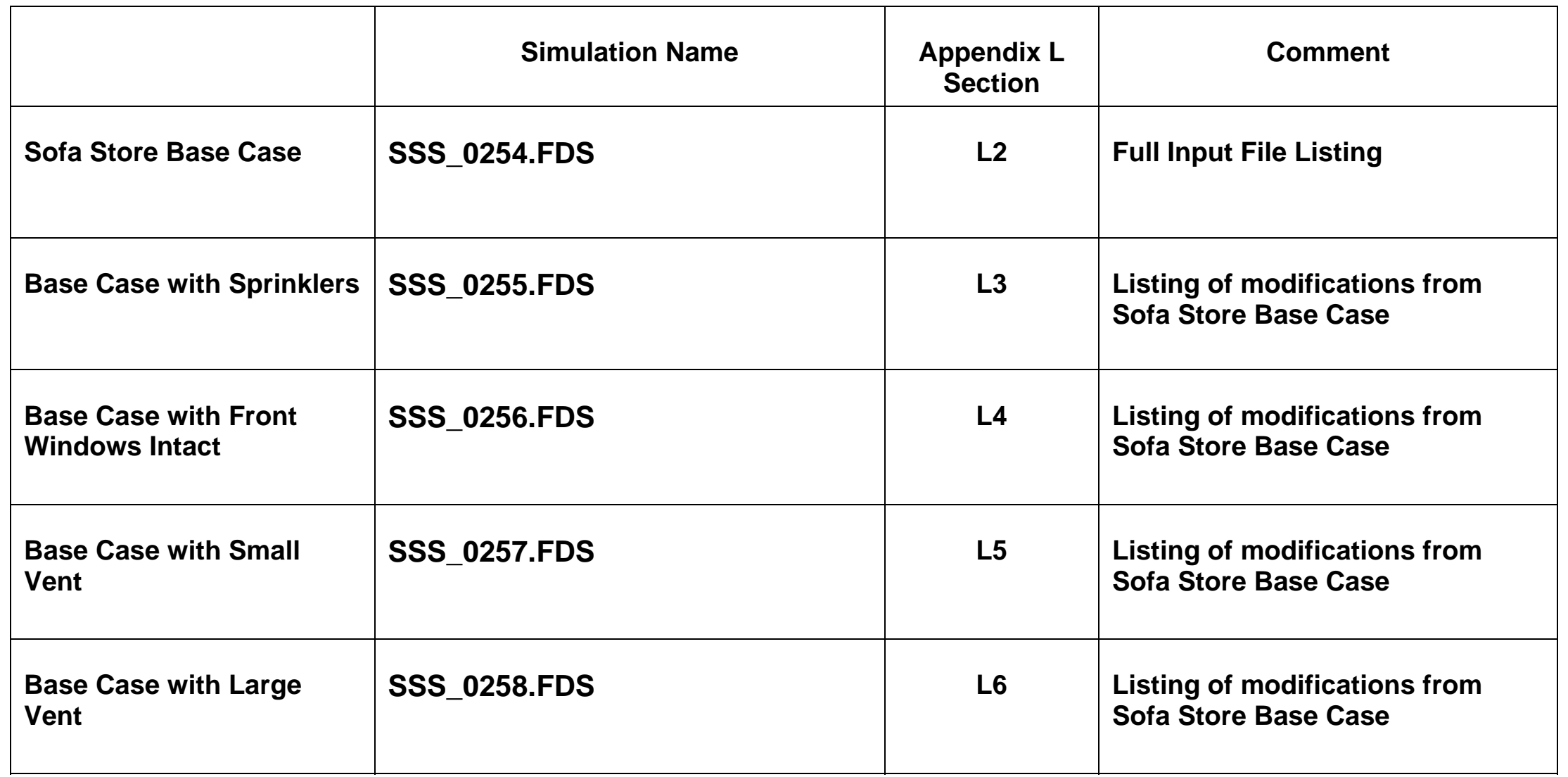




\section{L.2 Sofa Store Base Case}

SSS_0254.FDS

\&HEAD CHID= 'SSS_0254' TITLE='Sofa Super Store Reconstruction' /

\&MISC SURF-DEFAULT=' GB', BAROCLINIC=. FALSE. $/$
\&DUMP NFRAMES $=2400$, DT_RESTART $=120 . /$

\&REAC ID = 'POLYURETHANE'

SII Y_6.3 H-7.1 N 0_2.1, NFPA Handbook, Babrauskas'

SOOT_YIELD $=0.10$

$\begin{aligned} \mathrm{N} & =1.0 \\ \mathrm{C} & =6.3 \\ \mathrm{H} & =7.1\end{aligned}$

$H=7.1$
$\begin{aligned} & H \\ & 0\end{aligned}=2.1$

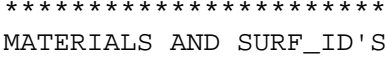

\&MATL ID = 'GYPSUM PLASTER'

FYI $=$ 'Quintiere, Fire Behavior'

CONDUCTIVITY $=0.48$

$\begin{aligned} \text { SPECIFIC_HEAT } & =0.84 \\ \text { DENSITY } & =1440 .,\end{aligned}$

$\begin{aligned} \text { \&SURF ID } & =\text { 'GB' } \\ \text { COLOR } & =\text { 'CORAL' }\end{aligned}$

MATL_ID= 'GYPSUM PLASTER

\&SURF ID='WINDOW'

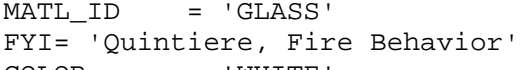

COLOR $=$ 'WHITT'

THICKNESS $=0.005$
BACKING='EXPOSED'

\&MATL ID=' 'GLASS'

$\begin{array}{ll}\text { CONDUCTIVITY } & =0.7 \\ \text { SPECIFIC_HEAT } & =0.84 \\ \text { DENSITY } & =2700.1\end{array}$

$\begin{aligned} \text { \&SURF ID } & =\text { 'SLAB } \\ \text { MATL_ID } & =\text { ' } C O N C R E T E '\end{aligned}$

FYI = 'Quintiere, Fire Behavior'

BACKING = 'INSULATED'

EMATL ID= 'CONCRETE'

$\begin{array}{ll}\text { CONDUCFIVITY } & =1.0 \\ \text { SPECIIIC_HEAT } & =0.88\end{array}$

$\begin{array}{ll}\text { SURF ID } & =\text { 'BLOCK WALL' } \\ \text { MATL_ID } & =\text { 'CONCRETE BLOCK' }\end{array}$

FYI $=$ 'Quintiere, Fire Behavior'

THICKNESS $\stackrel{=}{=} 0.2$

\&MATL ID = 'CONCRETE BLOCK'

$\begin{array}{ll}\text { CONDUCFIVITY } & =0.7 \\ \text { SPECIIIC_HEAT } & =0.84\end{array}$

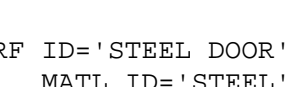

MATL ID =' STEEL'
COLOR='BLACK'
THICKNESS $=0.05 /$

\&MATL ID=' 'STEEL'

$\begin{array}{ll}\text { CONDUCTIVITY } & =45.8 \\ \text { SPECIFIC_HEAT } & =0.46 \\ \text { DENSITY }=7850.1 & \end{array}$

\&SURF ID $=$ 'SHEET_METAL' COLOR $=$ 'THISTL (13)

\&SURF ID = 'WOOD FLOR' $\begin{array}{ll}\text { MATL_ID } & =\text { 'WOOD' } \\ \text { CLOR } & =\text { 'BROWN }\end{array}$

\&SURF ID $=$ 'WOOD FURN $\begin{array}{ll}\text { MATLIID } & =\text { 'WOOD } \\ \text { COLOR } & =\text { 'BROWN } \\ \text { THICKNESS } & =0.013 /\end{array}$

\&MATL ID= 'WOOD' $\begin{array}{ll}\text { CONDUCTIVITY } & =0.14 \\ \text { SPECIFIC_HEAT } & =2.85 \\ \text { DENSITY }=640.1 & =2.85\end{array}$

ATL ID = 'CARPET MATERIAL' CONDUCTIVITY $=0.16$ DENSITY $=750$ $\begin{array}{ll}\text { N_REACTIONS } & =1 \\ \text { NU_FUEL } & =1\end{array}$ HEAT_OF_COMBUSTION $=1$. 22300 . $\begin{array}{ll}\text { HEAT_OF_COMBUSTION } & =22300 . \\ \text { HEA_OF_REATION } & =2000 . \\ \text { REFERENCE_TEMPERATURE } & =350 .\end{array}$

\&SURF ID $=$ 'CARPET' MATL_ID $=$ 'CARPET MATERIAL'
COLOR $=$ 'SANDY BROWN' THIKNESS 'SANDY BROWN' $=0.006$
THICR

\&SURF ID = 'ROOF_MAIN' MATLID $=$ 'STEEL'
COLOR $=$ 'TAN' $\begin{aligned} \text { THICKNESS } & =0.1 \\ \text { BACKING }= & \text { INSULATED } 1\end{aligned}$

\&SURF ID $=$ 'ROOF_LEFT' MATL ID $=$ 'STEEL'
COLOR $=$ 'TAN' $=0.1$
THICKNESS
BACKING = ' INSULATED' 1

\&SURF ID = 'ROOF_RIGHT' MATL_ID = 'STEEL' $\begin{aligned} & =0.1 \\ \text { THICKNESS } & 0.1 \\ \text { BACKING }= & \text { INSULATED } 1\end{aligned}$

SURF ID = 'ROOF_WORKSHOP' MATL_ID $=$ 'STEEL'
COLOR $=$ 'TAN' THICKNESS TAN $=0.1$

$\begin{array}{ll}\text { \&SURF ID } & =\text { 'ROOF_WAREHOUSE' } \\ \text { MATL_ID } & =\text { 'STEEL' }\end{array}$ MATLID $=$ 'STEEL'
COLOR $=$ 'TAN' THICKNESS
BACKING = ' INSULATED $=0.1$

$\begin{aligned} \text { \&SURF ID } & =\text { 'ROOF_LD' } \\ \text { MATL_ID } & =\text { 'STEEL' } \\ & =\text { 'TEN' }\end{aligned}$ 


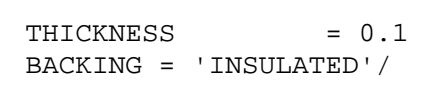

$\begin{aligned} \text { \&SURF ID } & =\text { 'ROOF_BREEZEWAY' } \\ \text { MATL_ID } & =\text { 'STEEL' }\end{aligned}$

MATL_ID = 'STEE'
COLOR $=$ 'TAN'

$\begin{aligned} & =0.1 \\ \text { THICKNESS } & \text { ' } \\ \text { BACKING }= & \text { INSULATED' } /\end{aligned}$

$\begin{aligned} \text { \&SURF ID } & =\text { 'ROOF_REAR' } \\ \text { MATL_ID } & =\text { 'STEEL' }\end{aligned}$

$\begin{aligned} &=0 \\ & \text { THICKNESS } 0 . \\ & \text { BACKING }=\text { 'INSULATED' }\end{aligned}$

\&SURF ID = 'ROOF_REAR_FLAT' MATLID $=$ 'STEEL'
COLOR $=$ 'TAN'

THICKNESS $=0$.

\&MATL ID $=$ 'FABRIC'

$\begin{aligned} \text { SPECIFIC_HEAT } & =1.0 \\ \text { CONDUCTIVITY } & =0.1\end{aligned}$

$\begin{aligned} \text { DENSITY } & =100.0\end{aligned}$

NU_FUEL $=1$.

REFERENCE TEMPERATURE $=350$.

$\begin{aligned} \text { HEAT_OF_REACTION } & =1500 . \\ \text { HEAT_OF_COMBUSTION } & =15000 .\end{aligned}$

\&MATL ID = 'FOAM'

CONDUCTIVITY $=0.034$

DENSITY $=20.0$

NGREACTIONS $=1$

REFERENCE_TEMPERATURE $=350$.

$\begin{aligned} \text { HEAT_OF_REACTION } & =1350 . \\ \text { HEAT_OF_COMBUSTION } & =25000 .\end{aligned}$

$\begin{aligned} \text { \&SURF ID } & =\text { 'UPHOLSTERY' } \\ \text { COLOR } & =\text { 'STELNA' }\end{aligned}$

$\begin{aligned} \operatorname{MATL} I D(1: 2,1) & ={ }^{\prime} \text { FABRIC ' , ' FOAM' } \\ \operatorname{THICKNESS}(1: 2) & =0.002,0.375 /\end{aligned}$

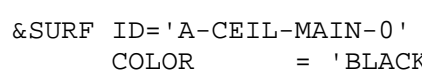

COLOR $=$ 'BLACK'
THICKNESS = 0.016
MATL_ID='ACOUSTIC_TILE'

MATL ID= 'ACOUSTIC_TILE'

$\begin{aligned} \text { CONDUCTVITY } & =0.058 \\ \text { DENSITY } & =290 /\end{aligned}$

$* * * * * * * * * x$
Meshes

EMESH IJK $=100,80,30, \quad \mathrm{XB}=28.8, \quad 66.3, \quad 23.3, \quad 53.3, \quad-0.24, \quad 11.01$ / Mesh 1240000

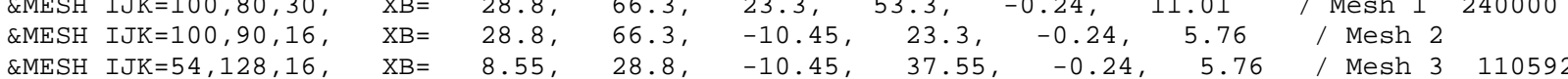

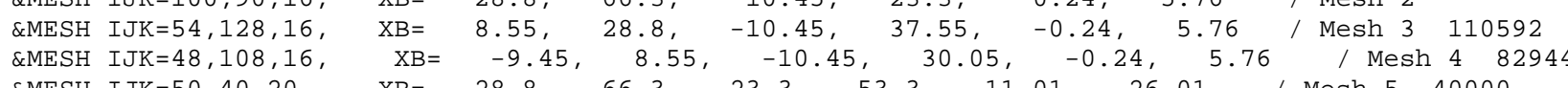
\&MESH IJK=40, 45, 20, $\mathrm{XB}=36.3,66.3, \quad 53.3, \quad 87.05, \quad 11.01,26.01 /$ Mesh 73600

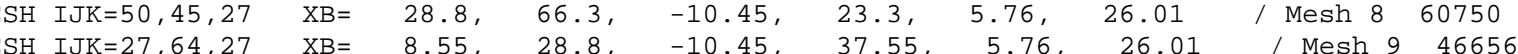

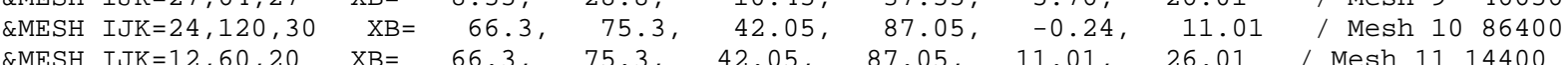

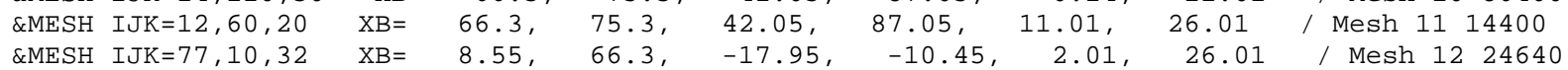

Domain Boundaries

QVENT MB='XMIN' SURF_ID='OPEN'/

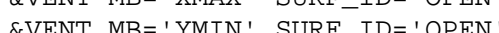

QVENT MB='YMAX' SURF-ID=' OPEN',
QVENT MBE' 'ZMTN' SURE-IDD' OPEN',

\&VENT MB' 'ZMAX' SURF-ID=' OPEN'/

\&VENT XB=-9.45,75.3, - 10.45, 87.05, - $0.24,-0.24$, SURF_ID=' INERT '/

Fires

QVENT $X B=60.66, \quad 61.05, \quad 41.5, \quad 43.5, \quad 0.15, \quad 0.15, \quad$ SURF_ID='FIRE_RAMP' $/$ Couch Fire

\&SURF ID='FIRE_RAMP', HRRPUA $=1000.0$ RAMP_Q $=$ 'RAMP_FIRE' $/$

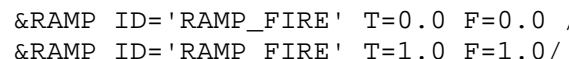

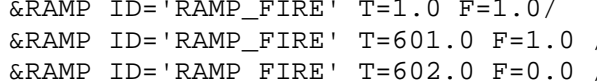

\&SURF ID=' BUPNER', HRPPUA=1000,

\&VENT XB $=56.55, \quad 56.925, \quad 47.3, \quad 47.675, \quad 0.51, \quad 0.51, \quad$ OUTLINE=.TRUE. SURF_ID= 'WH_FIRE_RAMP'

\&SURF ID='WH_FIRE RAMP' HRRPUA $=1000$. RAMP_Q $=$ 'WH_RAMP'/

\&RAMP ID='WH_RAMP' $T=0.0 \quad F=0.0 \%$

\&RAMP ID='WH_RAMP' T=440.0 $\quad F=0.02$,

$\begin{array}{ll} & \\ & \end{array}$

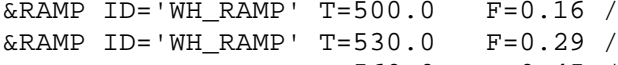

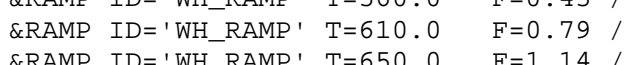

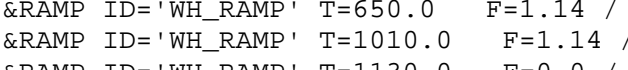

\&VENT XB= $41.55, \quad 44.92, \quad 35.67,36.41, \quad 0.14, \quad 0.14, \quad$ OUTLINE=
\&SURF TRUE,

TIRE'

SURF_ID=

'MAIN_SHOWROOM_FIRE' /

RRAMP ID='MAIN_SHOWROOM_RAMP', T=525.0 $\mathrm{F}=0.0 .0$,

\&RAMP ID='MAIN SHOWROOM_RAMP' T=585.0 $\quad F=0.169$

QRAMP ID='MAAN_SHOWROM_RAMP' T=615.0 $\quad \mathrm{F}=0.379$

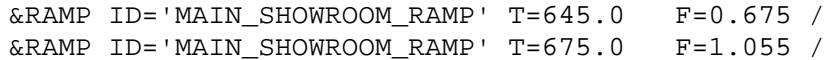

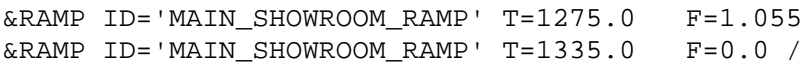

QVENT XB= $41.55,43.05, \quad 33.045, \quad 35.67,0.15, \quad 0.15, \quad$ OUTLINE=.TRUE. SURF_ID=

\&SURF ID='MAIN_SHOWROOM_FIRE2' HRRPUA=1500. RAMP_Q =' 'MAIN_SHOWROOM_RAMP2'

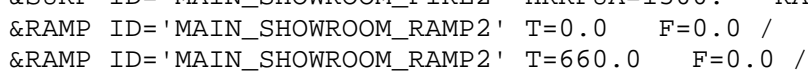

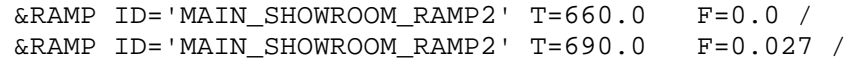

$\begin{array}{lll}\text { \&RAMP ID=' 'MAIN_SHOWROOM_RAMP2' } & T=720.0 & F=0.108\end{array}$

$\begin{array}{lll}\text { \&RAMP ID='MAIN_SHOWROOM_RAMP2' } & T=750.0 & \mathrm{~F}=0.244 / \\ \text { \&RAMP ID='MATNSHOWROOM_RAMP2' } & T=780.0 & \mathrm{~F}=0.433\end{array}$

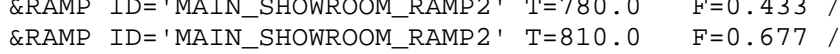

\&RAMP ID='MAIN_SHOWROOM_RAMP2' $\quad T=900.0 \quad \mathrm{~F}=1.029 /$

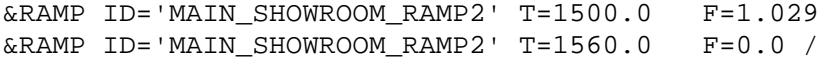

obstruction Surface Types

\&SURF ID='A-CEIL-MAIN- -9 '
COLOR='BLACK'

\&SURF ID='A-WALL-MAIN-WS'
COLOR ='SILVER' 
\&SURF ID='A-WALL-MAIN-SR'
COLOR='RED

COLOR= 'RED'
\&SURF ID IA-WALL-MAIN-FS
COLOE= 'CYAN'

\&SURF ID $=$ IA-WALL-MAIN - CB
COLOR $=$ 'GREN'/

$* \star * \star * * * \star * * *$
Windows

\&OBST XB=61. $01,66.1,-6.4,-6.55,0.54,2.26$ SURF_ID='WINDOW', DEVC_ID='RIGHT ADDITION WINDOWS 1'/ RIGHT ADDITION WINDOW
\&OBST $X B=54.5,59.93,-6.4,-6.55,0.54,2.26$ SURF_ID='WINDOW' , DEVC_ID='RIGHT ADDITION WINDOWS 2 '/ RIGHT ADDITION LOBST XB=48.45,53.4, -6.4, - 6.55, 0.54,2.26 SURF_ID='WINDOW', DEVC_ID='RIGHT ADDITION WINDOWS 3'/ RIGHT ADDITION
WINDOW

\&DEVC ID='RIGHT ADDITION WINDOWS $11, \quad X Y Z=63.5,-6.3,2.0$, QUANTITY=' 'TIME' SETPOINT $=1900.0$. INITIAL_STATE=. TRUE./

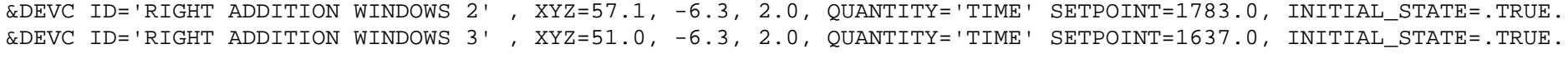
\&OBST XB=33.60,36.30,-6.4, -6.55, 0.54,2.26 SURF_ID='WINDOW'，DEVC_ID='MAIN STRUCTURE WINDOW R2'/ MAIN STRUCTURE WIIDOW
OOBST $X B=36.30,38.55,-6.4,-6.55,0.54,2.26$ SURF_ID='WINDOW' , DEVC_ID='MAIN STRUCTURE WINDOW R3'/ MAIN STRUCTURE
WTIDDT \&OBST $X B=38.55,40.80,-6.4,-6.55,0.54,2.26$ SURF_ID= 'WINDOW' , DEVC_ID='MAIN STRUCTURE WINDOW R4'/ MAIN STRUCTURE WOBST XB=40.80,43.35, - $6.4,-6.55,0.54,2.26$ SURF_ID='WINDOW' , DEVC_ID='MAIN STRUCTURE WINDOW R5'/ MAIN STRUCTURE

ROBST XB=31.53,32.54,-6.4,-6.55, 0.54,2.26 SURF_ID='WINDOW'，DEVC_ID='MAIN STRUCTURE WINDOW R1'/ MAIN STRUCTURE \&OBST XB=25.00, 25.77, -6.4, -6.55, 0.54,2.26 SURF_ID='WINDOW', DEVC_ID='MAIN STRUCTURE WINDOW L1'/ MAIN STRUCTURE WINDOW

\&OBST XB=13.95,16.42, -6.4, -6.55, 0.54,2.26 SURF_ID='WINDOW', DEVC_ID='MAIN STRUCTURE WINDOW L5' / MAIN STRUCTURE WINDOW
\&OBST $X B=16.42,19.05,-6.4,-6.55,0.54,2.26$ SURF_ID= 'WINDOW', DEVC_ID=' 'MAIN STRUCTURE WINDOW L4'/ MAIN STRUCTURE \&OBST XB=19.05, 21.67, - $6.4,-6.55,0.54,2.26$ SURF_ID='WINDOW', DEVC_ID=' 'MAIN STRUCTURE WINDOW L3'/ MAIN STRUCTURE WINDOW
ROBST XB=21.67, 23.85, - $6.4,-6.55,0.54,2.26$ SURF_ID='WINDOW', DEVC_ID=' MAIN STRUCTURE WINDOW L2'/ MAIN STRUCTURE EDEVC ID='MAIN STRUCTURE WINDDW L1', XYZ=25.4,-6.0,2.0, QUANTITY='TIME', SETPOINTT1497. 0 , INITIAL_STATE=. TRUE., \&DEVC ID='MAIN STRUCTURE WINDOW L4', $X Y Z=17.5,-6.0,2.0$, QUANTITY='TIME', SETPOINT=1467.0, INITIAL_STATE=.TRUE.,

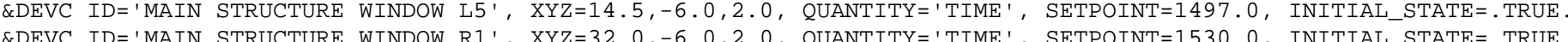
\&DEVC ID='MAIN STRUCTURE WINDOW R2', XYZ $=35.0,-6.0,2.0$, QUANTITY='TIME', SETPOINT=1465. $\theta$, INITIAL_STATE=.TRUE. \&DEVC ID='MAIN STRUCTURE WINDOW R3', XYZ=37.1,-6.0,2.0, QUANTITY='TIME', SETPOINT=1485.0, INITIAL_STATE=.TRUE.

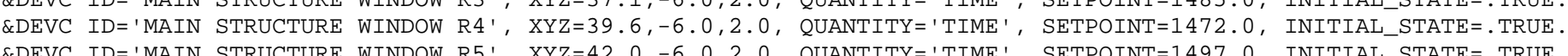

\&OBST XB=-3.16, $-8.2,-6.4,-6.55,0.58,2.26$ SURF_IDD='WINDOW' / LEFT ADDITION WINDOW QOBST XB=-2.17, $2.97,-6.4,-6.55,0.58,2.26$ SURF_ID' 'WINDOW' / LEFT ADDITION WINDW
\&OBST XB=4.16,9.3,-6.4, $-6.55,0.58,2.26$ SURF_ID 'WINDOW' / LEFT ADDITION WINDOW

\&OBST XB=67.5, 71.1, 46.25, 46.9, 0. 06, 3. 51 SURF_ID='SHEET_METAL' ', COLOR=' GRAY' / WAREHOUSE ROLL UP

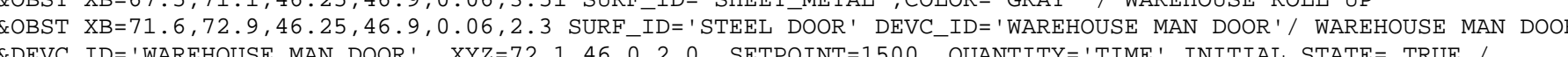
\&HOLE XB $=69.3,71.2,45.8,47.3,0.88,2.01$ DEVC_ID=' 'WH_ROLL' '

\&OBST XB=39.3, 40.58, 85.98, 86.2, ๑. 06, 2.39 SURF_ID='STEEL DOOR' / WAREHOUSE REAR DOO
\&HOLE XB=46, 86, 43.5, $45,9,46,8,0,06,3.5$ / BREEZEWAY ENTRANCE

\&HOLE XB=41.77, 42.97, 45.9, $46.8,0.06,2.05 /$ WORKSHOP ENTRANCE
$\&$ \&OLE XB $=37.582,38.882,48.7753,50.352,0.06,3.07 /$ L-SHAPED ADDITION ENTRANCE

\&HOLE XB $=34.0,34.5,42.8,44.3,0.06,2.05 /$ PASS THROUGH TO 10 G ROOM

\&OBST XB=29.25, 31. $05,-6.4,-6.55,0.07,2.26$, SURF_ID=' 'WINDOW', DEVC_ID=' MAIN STRUCTURE WINDOW R1'/ MAIN DOOR \&OBST XB $=-8.7,-8.2,27.01,28.9,0.07,2.18$, SURF_ID='STEEL DOOR' / LEFT ADDITION DOOR

EOBST XB=47.85, 48.4, 19. 044, 21.482, 2.48,4.29 SURF_ID6='GB' , 'GB' , 'GB', 'GB' , 'GB' , 'ROOF_MAIN' / SOFFET FOR RIGHT SID

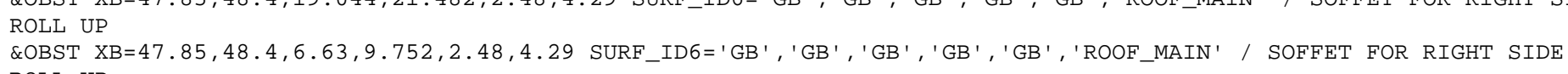

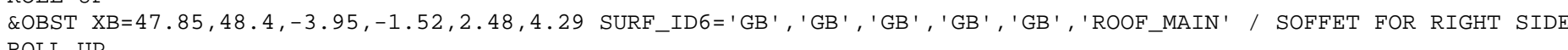

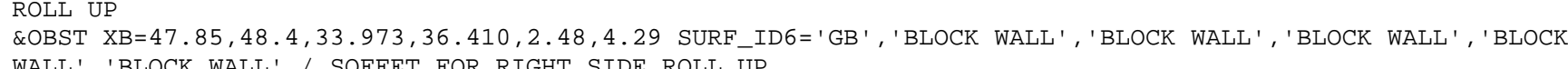

\&OBST XB=47.85, 48.4, 19. 044, 21.482, 0. 06, 2.48 SURF_ID='SHEET_METAL', DEVC_ID='RIGHT SIDE ROLL UP 1 ' / RIGHT SIDE \&OBST XB $=47.85,48.4,7.03,9.752,0.06,2.48$ SURF_ID=' SHEET_METAL', DEVC_ID='RIGHT SIDE ROLL UP 2'/ RIGHT SIDE ROLL \&OBST XB=47.85,48.4, $-3.95,-1.52,0.06,2.48$ SURF_ID='SHEET_METAL', DEVC_ID='RIGHT SIDE ROLL UP 3 '/ RIGHT SIDE ROLL \&DEVC ID='RIGHT SIDE ROLL UP 1 ', XYZ=48.2, 20.24, 2.48, QUANTITY=' TEMPERATURE ' $/$, SETPOINT=73.89,

\&DEVC ID='RIGHT SIDE ROLL UP 2 ', $X Y Z=48.2,8.5,2.48$, QUANTITY='TEMPERATURE' $/$, SETPOINT=73.89, INITIAL_STATE=.FALSE./ \&DEVC ID='RIGHT SIDE ROLL UP $3{ }^{\prime}$ ', XYZ=48.2, $2.76,2.48$, QUANTITY='TEMPERATURE' 1 , SETPOINT=73.89,

\&OBST XB=47.85, 48.4, 30.622,31.688, $0.06,2.03$ SURF_ID='STEEL DOOR' / RIGHT SIDE DOOR COBST XB $=47.85,48.4,24.68,25.747,0.06,2.03$ SURF_ID=' 'STEEL DOOR' / RIGHT SIDE DOOR
\&OBST XB $=47.85,48.4,22.396,23.310,0.06,2.03$ SURF_ID='STEEL DOOR'

\&OBST XB=9.3,9.6,-3.806,-1.368,0.06,2.48 SURF_ID='SHEET_METAL', DEVC_ID='LEFT SIDE ROLL UP 3 ' / LEFT SIDE ROLL UP

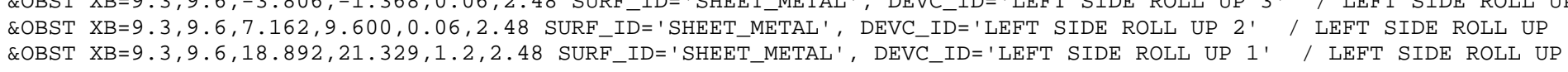
\&DEVC ID='LEFT SIDE ROLL UP 1 ', XYZ=9.1, 20.24,2.48, SETPOINT=73.89, QUANTITY=' TEMPERATURE', INITIAL_STATE=.FALSE.

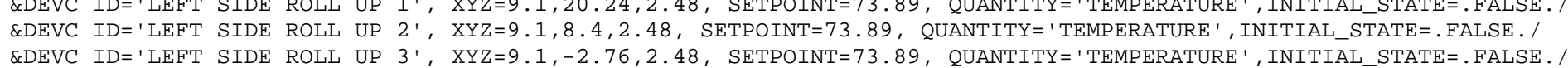

EOBST XB=9.3, 9.6, -3.806, -1.368, 2.48, 4.29 SURF_ID6='GB' , 'GB' , 'GB' , 'GB' , 'GB' , 'ROOF_MAIN' / SOFFET FOR LEFT SIDE ROL UP OBST XB=9.3, 9.6, 7.162, 9.600, 2.48,4.29 SURF_ID6='GB' , 'GB' , 'GB' , 'GB' , 'GB' , 'ROOF_MAIN' / SOFFET FOR LEFT SIDE ROLL

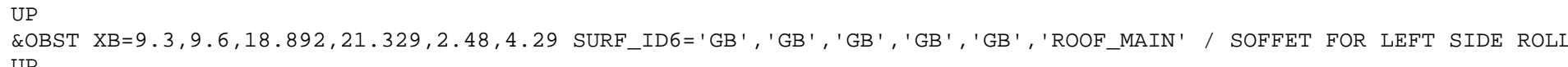

\&OBST XB=43.95, 46.35, 38.85, 39. . , 0. 96, 2. 48 SURF_ID='SHEET_METAL'， DEVC_ID=' BREEZEWAY ROLL UP' / BREEZEWAY ROLL UP \&DEVC ID='BREEZEWAY ROLL UP', XYZ=45.15,38.65, 2.48, SETPOINT=73.89, QUANTITY=' TEMPERATURE', INITIAL_STATE=. FALSE./

\&OBST XB=43.95, 46.35, 38.85, 39. $9,2.48,2.48$ SURF_IDG=' BLOCK WALL', 'BLOCK WALL', ' 'GB' ' 'BLOCK WALL' , 'BLOCK WALL' , 'BLOCK
WALL' SOFFET FOR WAREHOUSE ROLL UP' DOOR

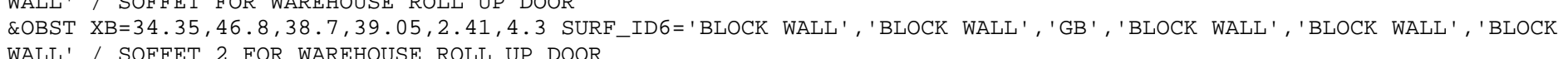

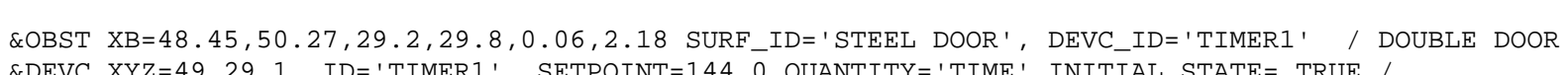

\&OBST XB=60.6, $61.0,34.33,37.13,0.07,2.73$ SURF_ID='SHEET_METAL' , COLOR=' GRAY' , DEVC_ID= ' TIMER_LD2' / LOADING DOCK BDEVC XYZ=61.8, 35.73, 2.4, ID=' TIMER_LD2', SETP0INT=1717. 0 , QUANTITY=' TIME', INITIAL_STATE=. TRUE./ 


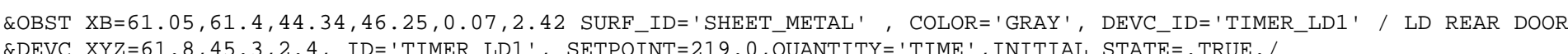
\&OBST XB=43.7, 47.8, 23.9, 24.1, $0.15,2.89$ / REAR HALL WALL

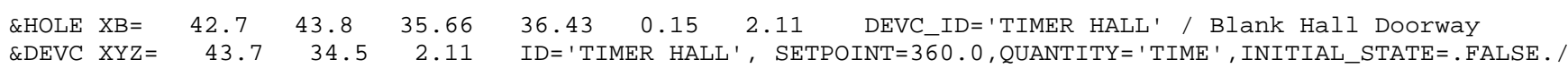

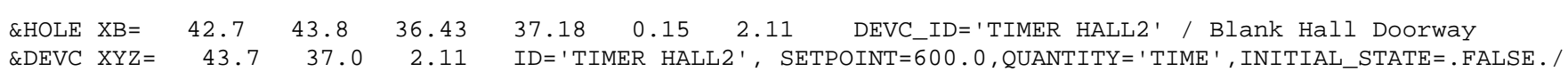
WAREHOUSE SKYLIGHTS

$\begin{array}{llllllllll}\text { \&HOLE XB }= & 63.75 & 60 & 48.33 & 49.24 & 8.1 & 9.5 & \text { DEVC_ID='SKYLIGHT } & 1{ }^{\prime} \prime & \text { WAREHOUSE SKYLIGHT } \\ \text { \&HOLE XB= } & 63.75 & 60 & 51.45 & 52.36 & 8.1 & 9.5 & \text { DEVC_ID='SKYLIGHT } & 11 & \text { WAREHOUSE SKYLIGHT }\end{array}$

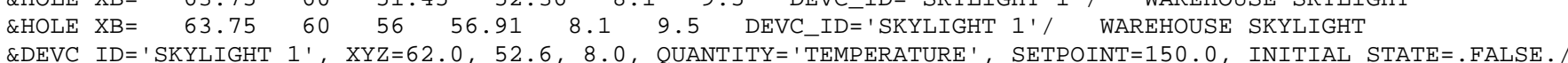

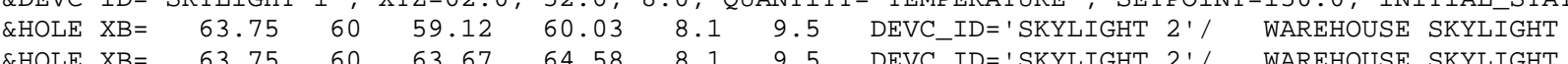

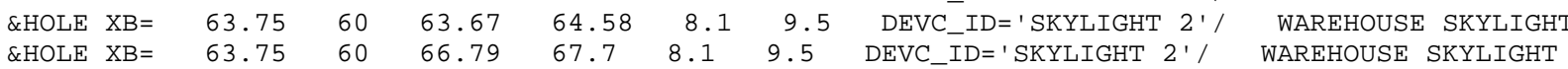

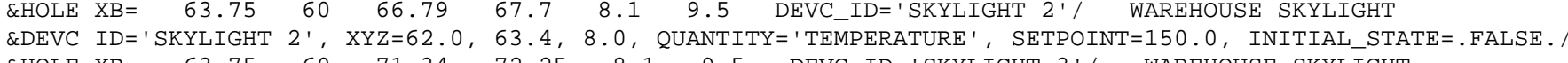

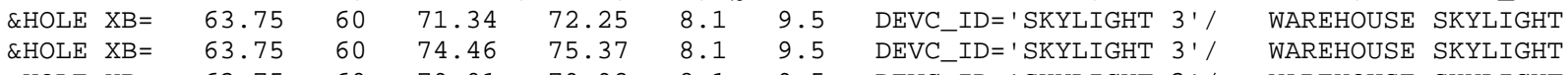

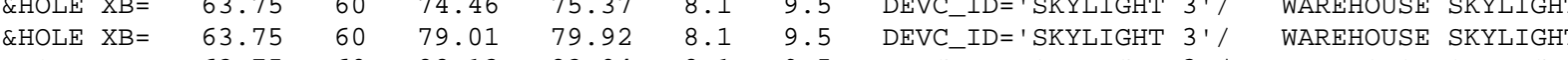

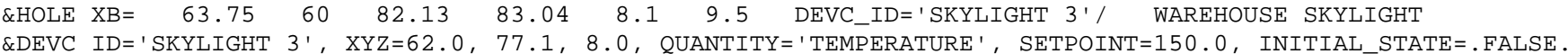

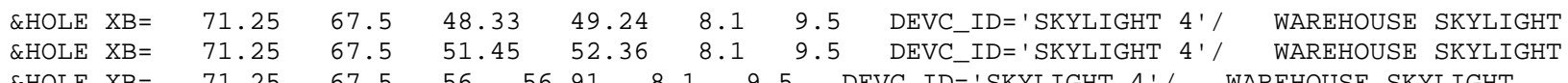
\&DEVC ID='SKYLIGHT 4 ', XYZ $=69.4,52.6,8.0$, QUANTITY='TEMPERATURE', SETPOINT=150. $\theta$, INITIAL STATE=.FALSE./

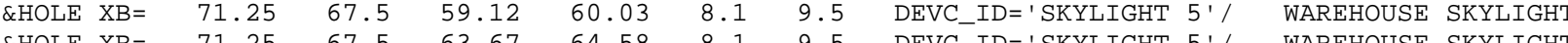

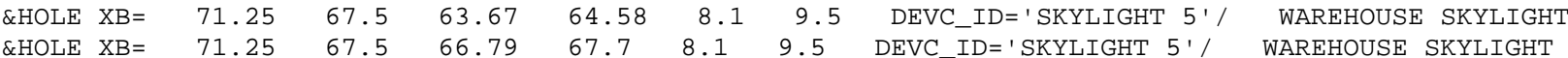
\&DEVC ID='SKYLIGHT 5 ', XYZ=69.4, 63.4, 8.0, QUANTITY='TEMPERATURE', SETPOINT=150.0, INITIAL_STATE=.FALSE.

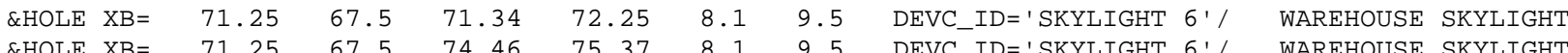

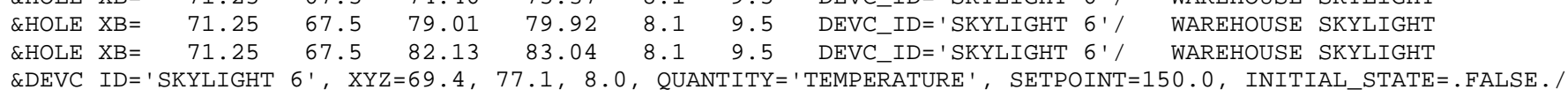

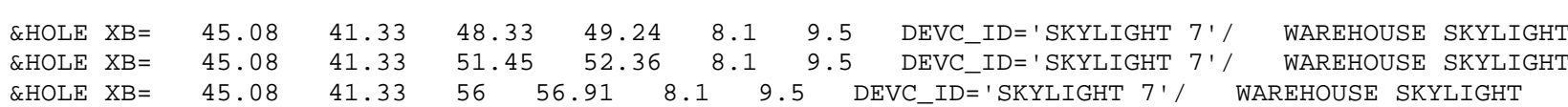
\&DEVC ID=' SKYLIGHT 71 , XYZ=43.2, 52.6, 8.0 , QUANTITY=' 'TEMPERATURE' SETPOINT=150.0, INITIAL_STATE=. FALSE.

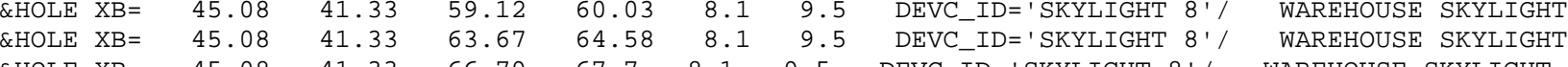

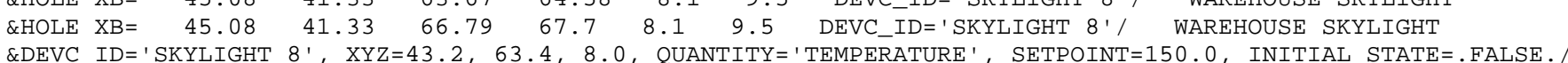

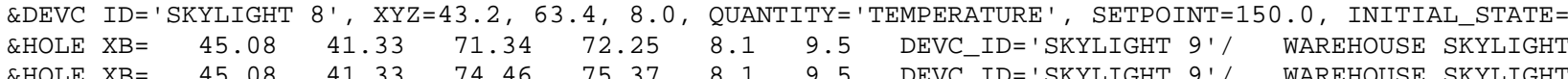

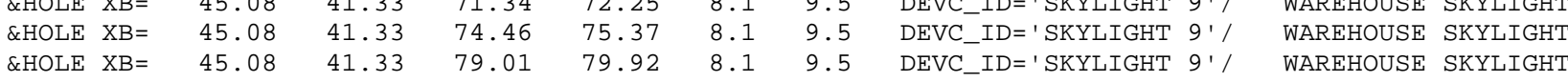

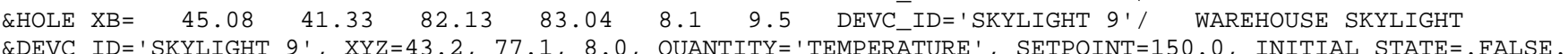

$\begin{array}{lllllllll}\text { \&HOLE XB= } & 48.83 & 52.58 & 48.33 & 49.24 & 8.1 & 9.5 & \text { DEVC_ID=' 'SKYLIGHT } 10 \text { ' } & \text { WAREHOUSE SKYLIGHT } \\ \text { \&HOLE XB= } & 48.83 & 52.58 & 51.45 & 52.36 & 8.1 & 9.5 & \text { DEVC_ID=' SKYLIGHT 10' } & \text { WAREHOUSE SKYLIGHT }\end{array}$

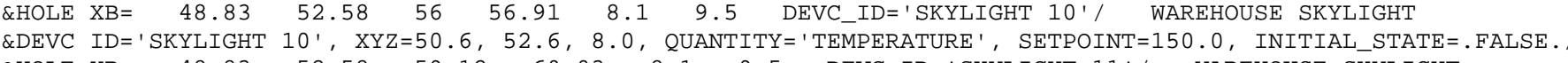

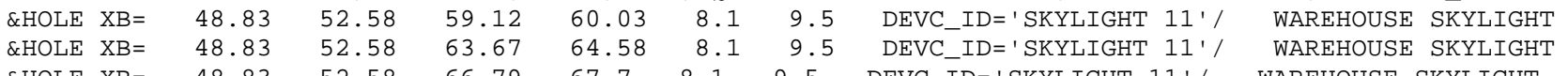
\&DEVC ID='SKYLIGHT 11 ', XYZ=50.6,63.4, 8.0, QUANTITY='TEMPERATURE', SETPOINT=150.0, INITIAL_STATE=. FALSE./

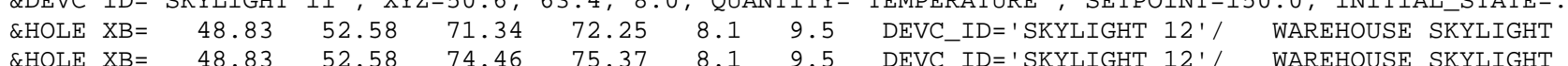

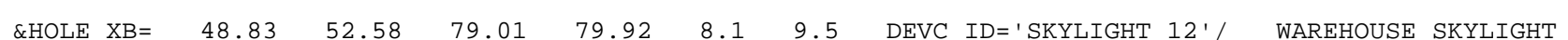

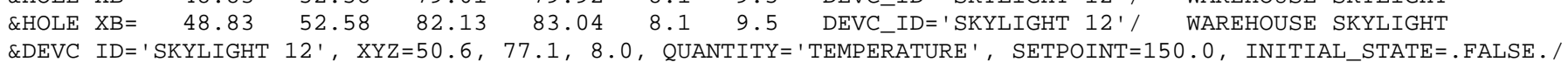

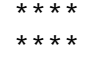

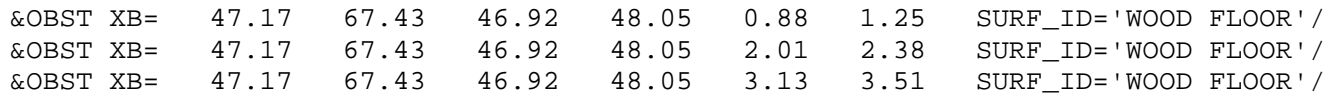

$\begin{array}{lllllllll}\text { \&OBST } X \mathrm{XB}= & 47.17 & 67.43 & 46.92 & 48.05 & 4.26 & 4.64 & \text { SURF-ID='WOOD FLOOR', } \\ \text { \&OBST } \mathrm{XB}= & 47.17 & 67.43 & 46.92 & 48.05 & 5.39 & 5.77 & \text { SURF_ID='WOOD FLOOR', }\end{array}$

$\begin{array}{llllllll}\text { \&OBST } X \mathrm{XB}= & 37.8 & 38.9 & 51.05 & 83.3 & 0.88 & 1.25 & \text { SURF_ID='WOOD FLOOR' } \\ \text { \&OBST } \mathrm{XB}= & 41.55 & 44.17 & 51.05 & 83.3 & 0.88 & 1.25 & \text { SURF_ID='WOOD FLOOR' }\end{array}$

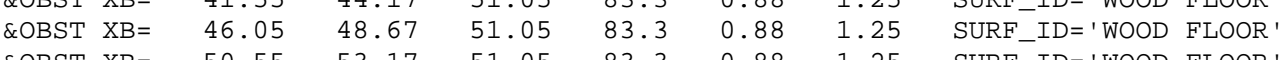

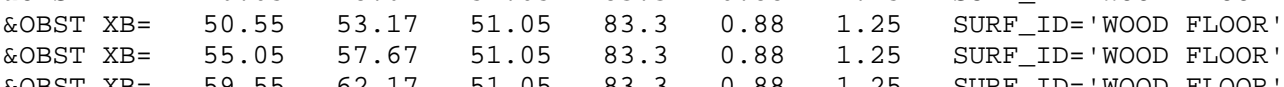

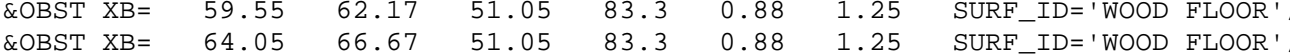

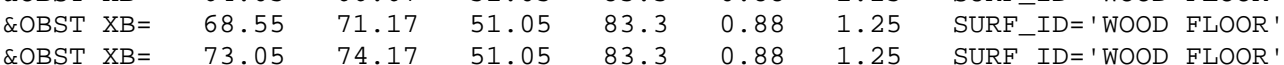

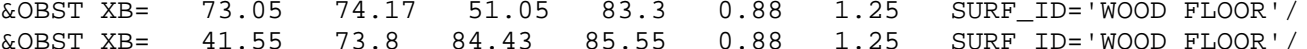

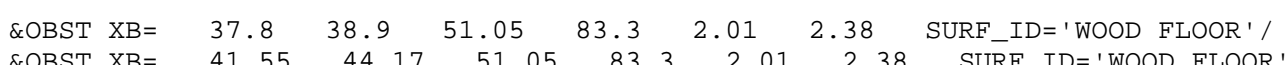

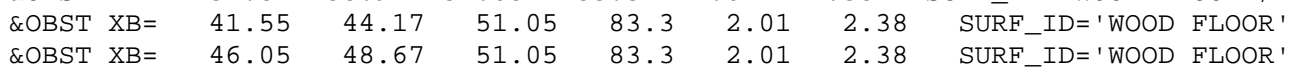

$\begin{array}{llllllll}\text { \&OBST } \mathrm{XB}= & 50.55 & 53.17 & 51.05 & 83.3 & 2.01 & 2.38 & \text { SURF_ID=' 'WOOD FLOOR'/ } \\ \text { \&OBST } \mathrm{XB}= & 55.05 & 57.67 & 51.05 & 83.3 & 2.01 & 2.38 & \text { SURF_ID ' 'WOOD FLOOR' }\end{array}$

$\begin{array}{llllllll}\text { \&OBST XB }= & 59.55 & 62.17 & 51.05 & 83.3 & 2.01 & 2.38 & \text { SURF-ID='WOOD FLOOR' } \\ \text { \&OBST XB= } & 64.05 & 66.67 & 51.05 & 83.3 & 2.01 & 2.38 & \text { SURF_IDE'WOOD FLOOR' }\end{array}$

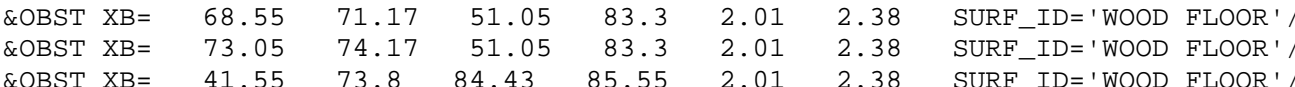

\&OBST $X B=\begin{array}{lllllll}37.8 & 38.9 & 51.05 & 83.3 & 3.13 & 3.51 & \text { SURF_ID='WOOD FLOOR' }\end{array}$

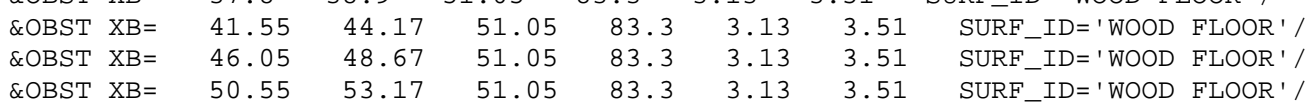

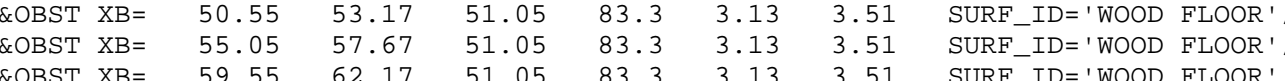

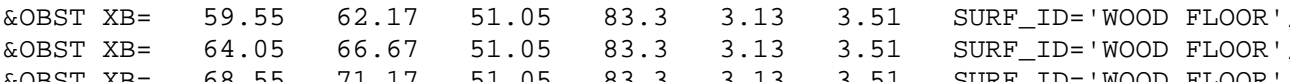

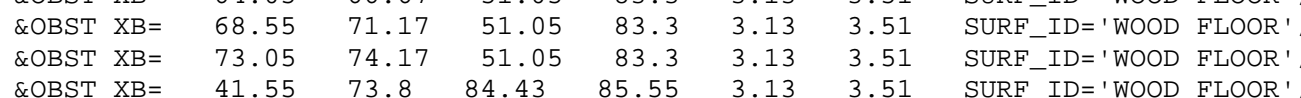

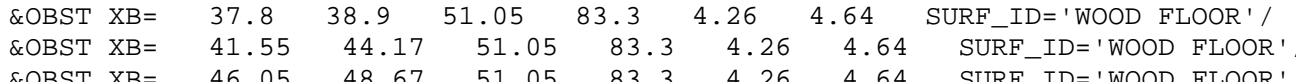

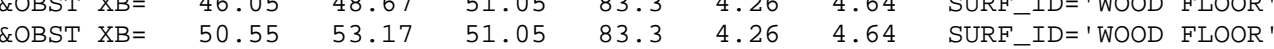

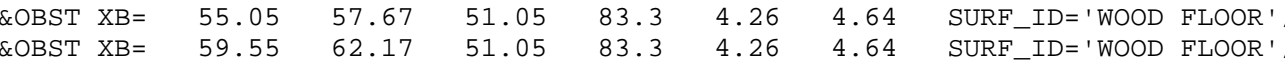

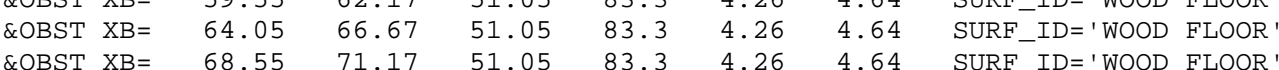

$\begin{array}{lllllllll}\text { COBST } \mathrm{XB}= & 73.05 & 74.17 & 51.05 & 83.3 & 4.26 & 4.64 & \text { SURF-ID='WOOD FLOOR' } \\ \text { \&OBST } \mathrm{XB}= & 41.55 & 73.8 & 84.43 & 85.55 & 4.26 & 4.64 & \text { SURF_ID='WOOD FLOR'' }\end{array}$

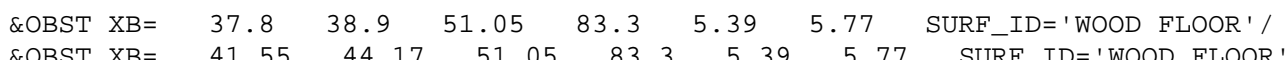

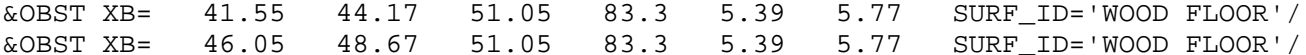

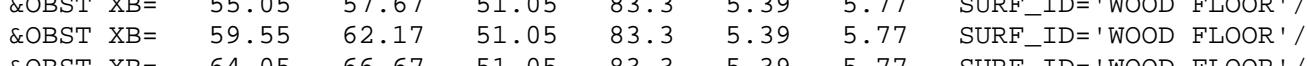

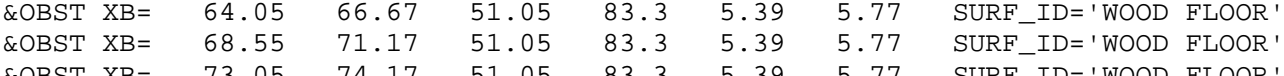

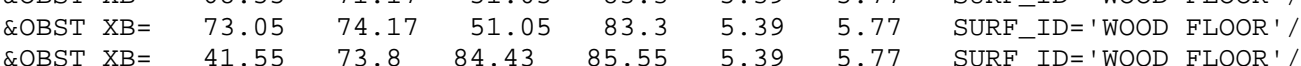

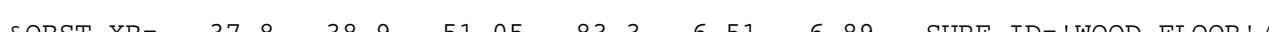




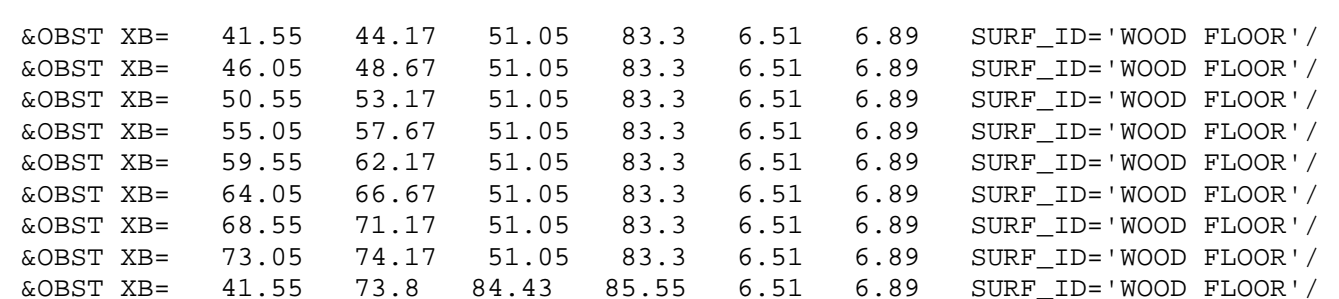

LD SKYLIGHTS

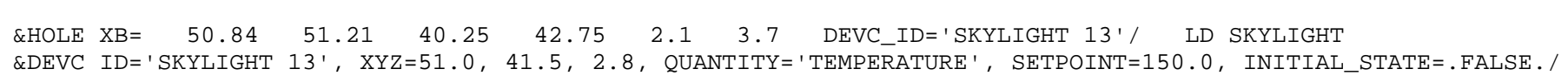

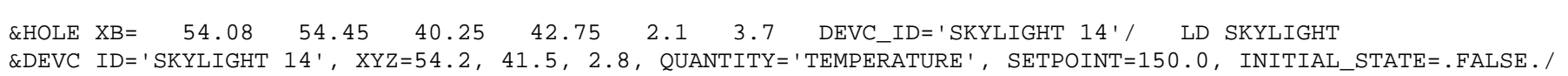

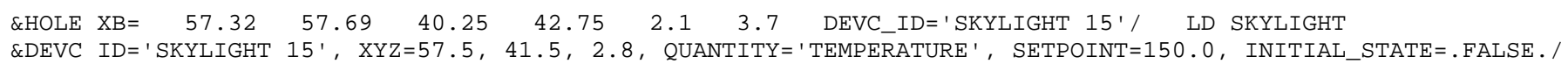
ROOF

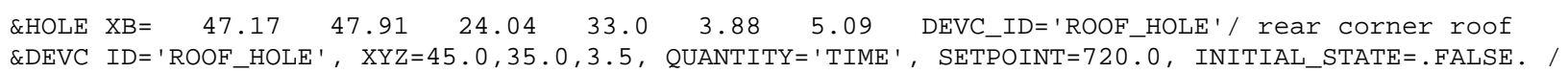

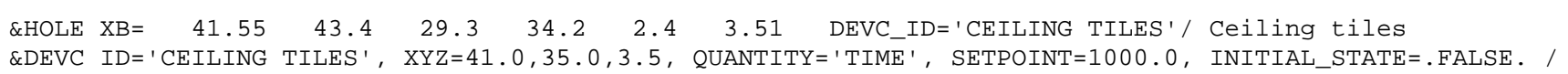

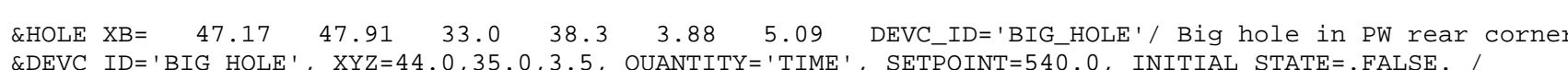

\&HOLE XB= $46.05 \quad 47.17 \quad 24.04$
\&DEVC ID 'BIG HOLE2

\&HOLE XB= $\quad \begin{array}{lllllll}17.55 & 18.3 & 18.8 & 19.92 & 2.4 & 3.51 & \text { DEVC_ID='CEILING_HOLE_T1' }\end{array}$

\&DEVC ID=' 'CEILING_HOLE_T1', XYZ=17.925,19.36,3.5, QUANTITY='TEMPERATURE'， SETPOINT=50.0， INITIAL_STATE=.FALSE. /

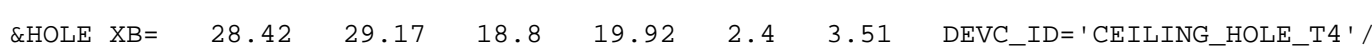

\&DEVC ID=' 'CEILING_HOLE_T4', XYZ=28.795, 19.36,3.5, QUANTITY='TEMPERATURE'， SETPOINT=50.0， INITIAL_STATE=.FALSE. /

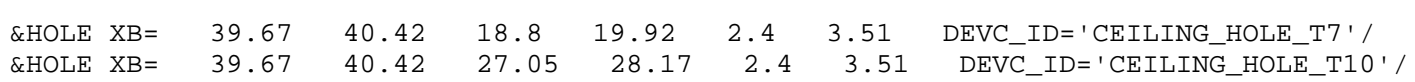

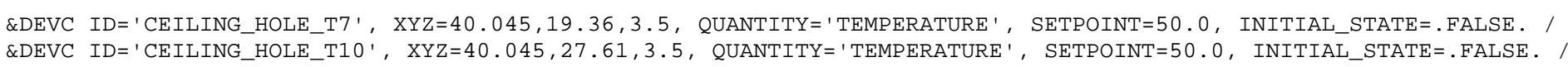
\&HOLE XB $=\begin{array}{lllllll}58.05 & 58.8 & 18.8 & 19.92 & 2.4 & 3.51 & \text { DEVC_ID='CEILING_HOLE_T11' }\end{array}$

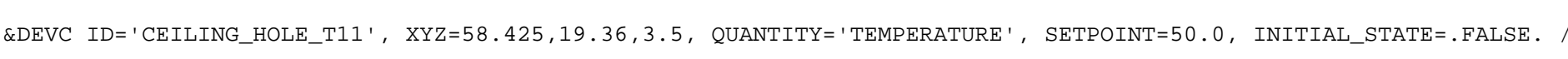
\&OBST XB=37.58,75.0, 46.25, 86.3, 8.7, 9.3 SURF_ID='ROOF_WAREHOUSE' / WAREHOUSE ROOF

\&OBST $X \mathrm{XB}=29.8,34.29,40.37,51.25,3.07,3.38$ SURF_ID= 'ROOF-WORKSHOP' / WORKSHOP ROOF
\&OBST XB $=34.2,37.55,46.25,51.25,3.07,3.38$ SURF IDE 'ROOF WORKSHOP' EOBST XB=34.2, 37.58, $39,46,25,3.07,3.38$ SURF_ID='ROOF_WORKSHOP' / WORKSHOP ROOF

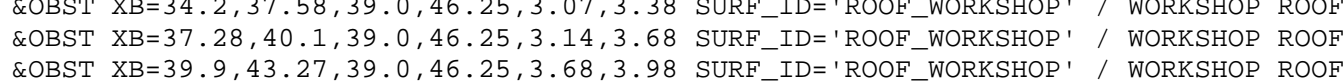
\&OBST XB=43.27, $47.1,39.0,46.85,3.68,3.98$ SURF_ID=' ROOF_BREEZEWAY' / BREEZEWAY ROOF
\&OBST XB=43.10,46.8, $45.8,46.9,3.51,3.88$ SURF_ID='ROOF_BREEZEWAY' / BREEZEWAY ROOF SUPP

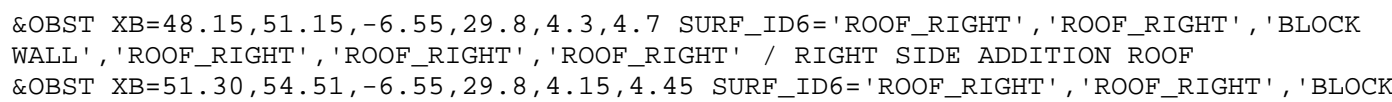

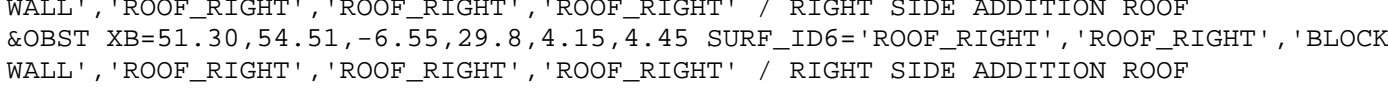

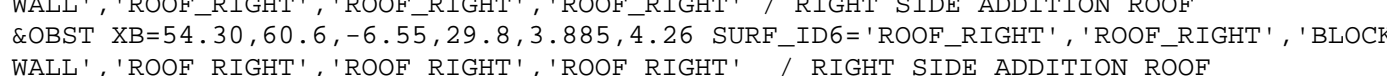

\&OBST XB=57. 00, 60.15, - $6.55,29.8,3.85,4.0$ SURF_ID6='ROOF_RIGHT', 'ROOF_RIGHT ', 'BLOCK
WALL', 'ROOF_RIGHT', 'ROOF_RIGHT', 'ROOF_RIGHT' / RIGHT SIDE ADDITION_ROOF \&OBST' XB=60.30,63.15,-6.55, 29.8, 3.51,4. 4 SURF_IDE=' ROOF_RIGHT', 'ROOF_RIGHT' ', 'BLOC

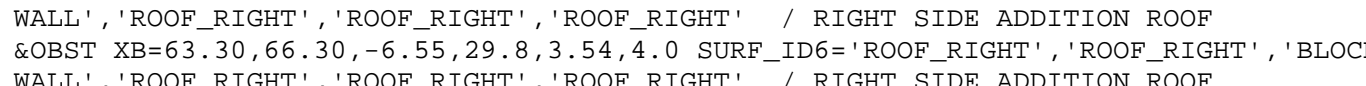
\&OBST XB=6.3,9.5, $-6.55,29.3,4.3,4.7$ SURF_IDG= 'ROOF_LEFT', 'ROOF_LEFT', 'BLOCK \&OBST $X \mathrm{BB}=2.97,6.45,-6.55,29.3,4.15,4.64$ SURF_ID6='ROOF_LEFT', 'ROOF_LEFT' ', 'BLOCK WALL', 'ROOF_LEFT', ROOF_LEFT', ROOF_LEFT'

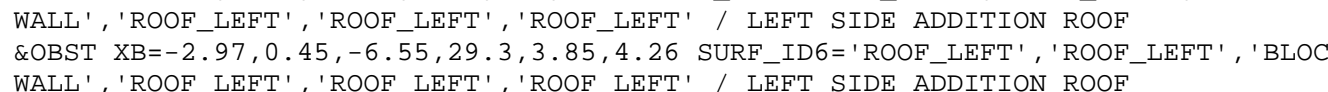
\&OBST' XB=-5.7, $-2.55,-6.55,29.3,3.5,3.85$ SURF_IDG=' 'ROOF_LEFT', 'ROOF_LEFT', 'BLOCK

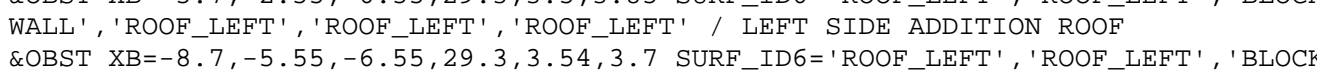

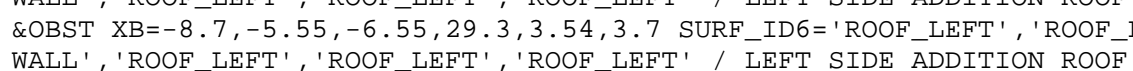

\&OBST XB $=9.3,48.41,-6.55,32.9,4.29,4.7$ SURF_ID6='ROOF_MAIN', 'ROOF_MAIN' ' 'BLOCK WALL', 'ROOF_MAIN' ' 'ROOF_MAIN', 'ROOF_MAIN' ' MAIN ROOF
COBST XB $=34.2,48.41,32.9,39.0,4.3,4.7$ SURF_ID='ROOF_MAIN' / MAIN ROOF REAR

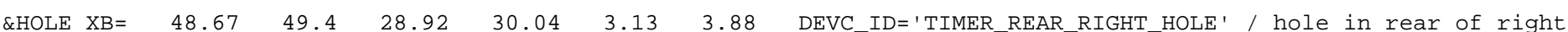

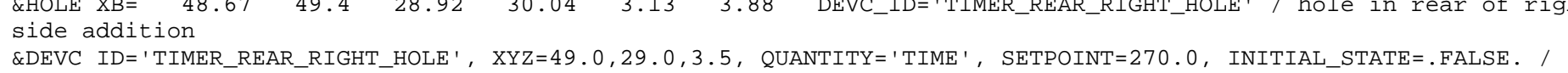
\&OBST XB=48.0, 51.15, 29.25, 29.8, 2.4, 4. 3 SURF_ID6='BLOCK WALL', 'BLOCK WALL', 'GB' , 'BLOCK WALL', 'BLOCK WALL' , 'BLOCK

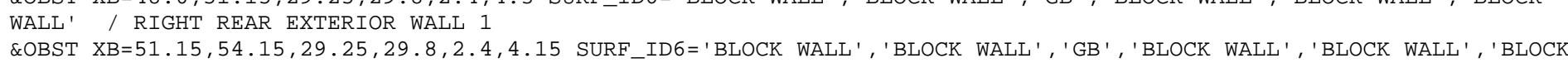
WALL' $\begin{aligned} & 1 \\ & \text { RIGHT' REAR EXTERTOR WALL } 2\end{aligned}$ \&OBST XB=54.15, $57.15,29.25,29.8,2.4,4.0$ SURF_IDG ROBST XB=57.15,60.15, 29.25, 29.8, 2.4, 3.85 SURF_ID6='BLOCK WALL', 'BLOCK WALL', ' 'GB' , 'BLOCK WALL', 'BLOCK WALL', 'BLOCK \&OBST XB=60.15, 63.15, 29.25, 29.8, 2.4, 3.69 SURF_ID6='BLOCK WALL' ' 'BLOCK WALL', ' 'GB' , 'BLOCK WALL', 'BLOCK WALL', 'BLOCK WOBST XB=63.15,66.15, 29.25, 29.8, 2.4, 3.54 SURF_ID6='BLOCK WALL', 'BLOCK WALL', ' 'GB' , 'BLOCK WALL', 'BLOCK WALL', 'BLOCK \&OBST XB=48.15, 51.15, - $6.54,-6.39,2.4,4.3$ SURF_IDG='BLOCK WALL' , 'BLOCK WALL', 'BLOCK WALL' , 'GB' , 'BLOCK WALL' , 'BLOCK \&OBST XB=51.15,54.15,-6.54,-6.39,2.4,4.15 SURF_ID6= 'BLOCK WALL', 'BLOCK WALL', 'BLOCK WALL', ' 'GB' , 'BLOCK WALL', 'BLOCK WALLT XBRIGHT FRONT EXTERIOR WALL 2 . 4 . SURF_ID6='BLOCK WALL', 'BLOCK WALL', 'BLOCK WALL', 'GB', 'BLOCK WALL', 'BLOCK

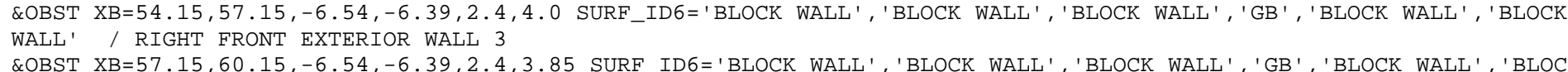

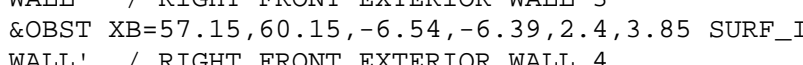

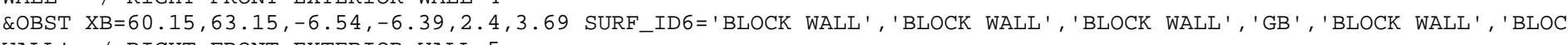

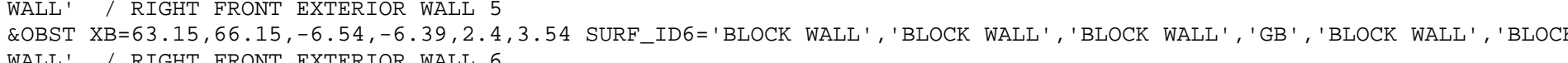

\&OBST XB=-8.7, - 5. 53, 28.95, 29.3, 2.41,3.5 SURF_ID6=' 'BLOCK WALL', 'BLOCK WALL', 'GB' ', 'BLOCK WALL' , 'BLOCK WALL' , 'BLOCK \&OBST XB=-5.53, -2.6, 28.95, 29.3, 2.41,3.65 SURF_ID6='BLOCK WALL', 'BLOCK WALL', 'GB' , 'BLOCK WALL', 'BLOCK WALL', 'BLOCK

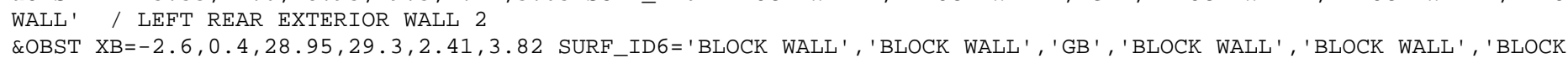
\&OBST XB=0.4,3.5, 28.95, 29.3, 2.41, 3.98 SURF_ID6= 'BLOCK WALL', 'BLOCK WALL', ' 'GB' , 'BLOCK WALL' , 'BLOCK WALL', 'BLOCK WALL' ' LEFT REAR EXTERTOR WALL 4 .
\&OBST XB $=3.5,6.5,28.95,29.3,2.41,4.13$ SURF_ID6= 'BLOCK WALL', 'BLOCK WALL', ' 'GB' ' 'BLOCK WALL', 'BLOCK WALL', 'BLOCK

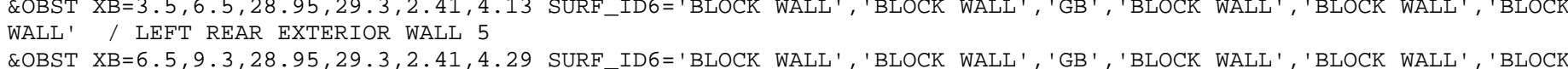
\&OBST XB $=6.5,9.3,28.95,29.3,2.41,4.29$
WALL' $/$ LEFT REAR EXTERIOR WALL' 6 \&OBST XB=-5.53,-2.6, - $6.55,-6.4,2.41,3.65$ SURF_ID6='BLOCK WALL', 'BLOCK WALL', 'BLOCK WALL', 'GB', 'BLOCK WALL', 'BLOCK

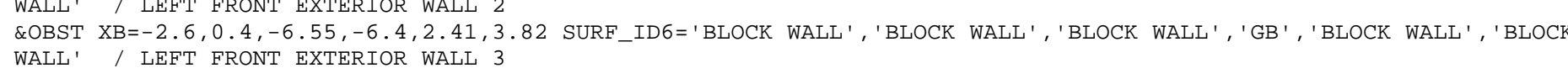


\&OBST XB=0.4,3.5, -6.55, -6.4, 2.41,3.98 SURF_ID6='BLOCK WALL', 'BLOCK WALL', 'BLOCK WALL' , 'GB' , 'BLOCK WALL' , 'BLOCK \&OBST XB=3.5,6.5, -6.55, - $6.4,2.41,4.13$ SURF_IDG='BLOCK WALL', 'BLOCK WALL', 'BLOCK WALL', ' 'GB' , 'BLOCK WALL', 'BLOCK

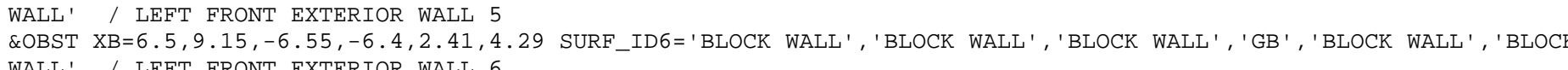

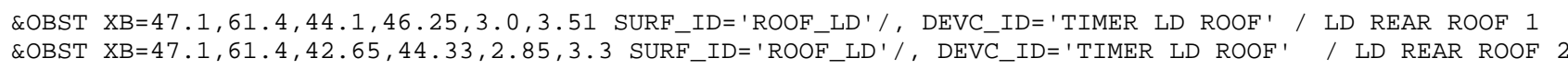

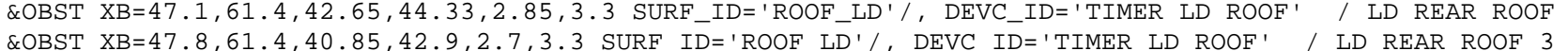

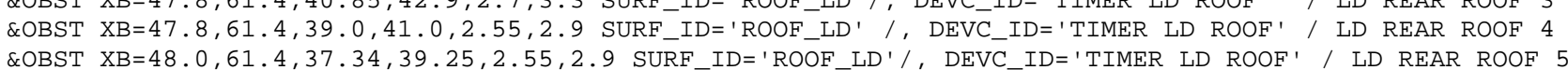

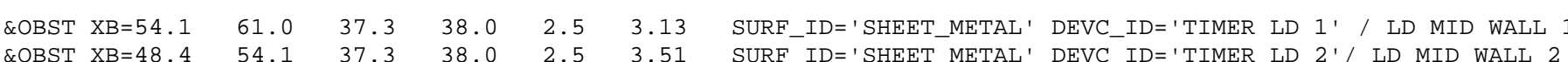

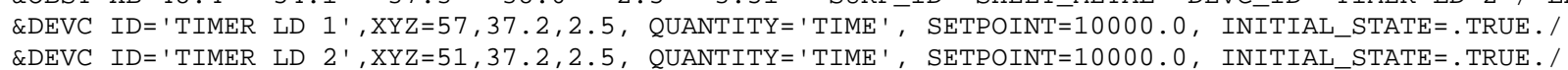

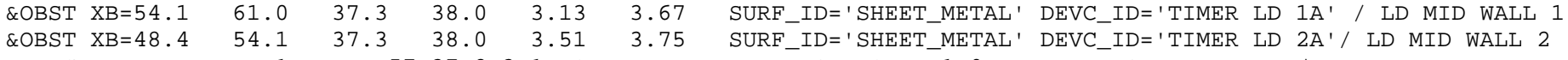
\&DEVC IDE' TIMER LD 2A', XYZ=51, 37.2,3.5, QUANTITY=' TIME', SETPOINT=1., , INITIAL STATE=. TRUE./

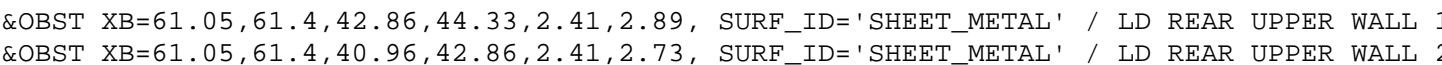

\&OBST XB=47.8,48.4,40.8,42.86, $0.07,2.89$, SURF_ID='SHEET_METAL' / LD REAR UPPER WALL 4

\&OBST XB $=47.1,48.15,42.72,42.86,0.07,3.043$, SURF_ID='SHEET_METAL' / LD REAR UPPER WALL

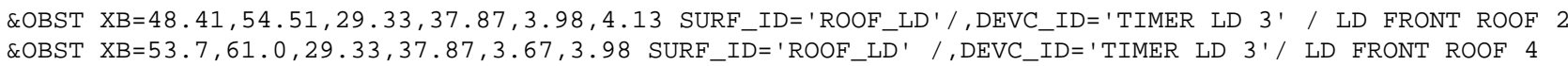

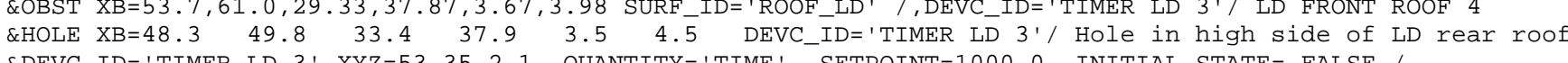

\&OBST XB $=18.45,29.4,32.87,33.87,3.14,3.65$ SURF_ID=' ROOF_REAR' / REAR ROOM ROOF

EOBST XB=18.45, 18.6, 32.87, 33.75, 3.2, 3.5 SURF_ID6=' 'BLOCK WALL', 'GB' , 'BLOCK WALL', 'BLOCK WALL', 'BLOCK WALL', 'BLOCK \&OBST XB=18.45,18.6, 33.75, 34.75, 3.2, 3.35 SURF_ID6='BLOCK WALL', 'GB' , 'BLOCK WALL', 'BLOCK WALL', 'BLOCK WALL', 'BLOCK

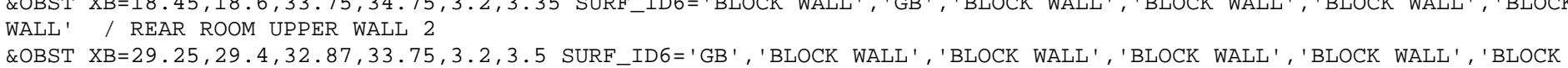

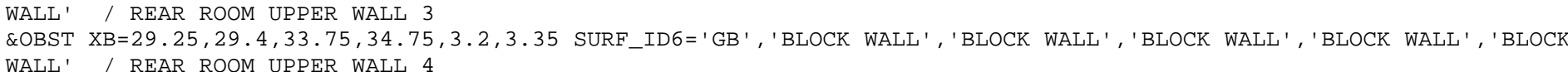
\&OBST XB=29.4, 34.2375, 32.9144, 35. 07, 2. 39, 2.85 SURF_ID='ROOF_REAR_FLAT'/ REAR ROOM FLAT ROOF $* * \star * * * * * * * \star *$
Store Front

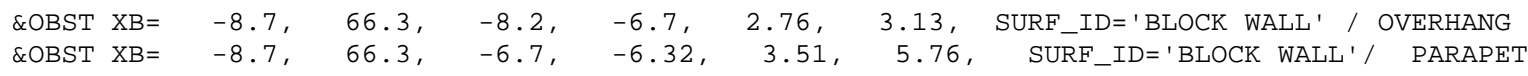
Output

\&SLCF PBX $=49.4$, QUANTITY='TEMPERATURE' $/$

\&SLCF PBX $=45.2$, QUANTTTY=' TEMPERATURE'

\&SLCF PBX 30.3 , QUANTITY='TEMPERATURE',
$\& S L C F$ PBX $=27.0$, QUANTITY=' TEMPERATURE',

\&SLCF PBY $=-2.8$, QUANTITY=' 'TEMPERATURE'
\&SLCF PBY $=8.4$, QUANTITY=' $T E M P E R A T U R E '$

\&SLCF PBY 30.55 , QUANTITY='TEMPERATURE'/
$\& S L C F$ PBY 35.3, QUANTITY='TEMPERATURE'

\&SLCF PBZ $=3.51$, QUANTITY=' 'TEMPERATURE'
\&LLCF PBZ $=1.63$,
QUANTITY $=$ 'TEMPRPATRRE'

\&SLCF PBX $=49.4$, QUANTITY='VELOCITY', VECTOR=. TRUE ./
\&SLCF PBX $=45.2$, QUANTITY= 'VELOCITY', VECTOR=.TRUE. $/$ \&SLCF PBX $=30.3$, QUANTITY='VELOCITY', VECTOR=.TRUE.
\&SLCF PBX $=27.0$, QUANTITY='VELOCITY', VECTOR $=$.TRUE. $/$

\&SLCF PBY=-2.8, QUANTITY='VELOCITY', VECTOR=.TRUE./ \&SLCF PBY $=8.4$, QUANTITY='VELOCITY', 'VECTOR=. TRUE./ \&SLCF PBY 30.55 , QUANTI TY= 'VELOCITY', VECTOR=. TRUE. \&SLCF PBZ $=3.51$, QUANTITY='VELOCITY', VECTOR=.TRUE./
\&SLCF PBZ=1.63, QUANTITY='VELOCITY', VECTOR=. TRUE./

\&SLCF PBX=49.4, QUANTITY=' oxygen' $/$

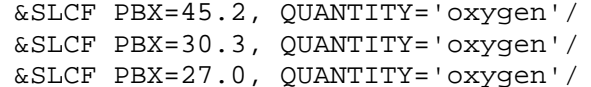

\&SLCF PBY=-2.8, QUANTITY=' oxygen'/ \&SLCF PBY $=30.50$, QUANTITY= 'Oxygen ',
\&SLCF PBY $=35.3$, QUANTITY=' oxygen'

\&SLCF PBZ $=3.51$, QUANTITY=' oxygen'
\&SLCF PBZ=1.63, QUANTITY=' oxygen'/

\&SLCF PBX $=40.4$, QUANTITY $=$ ' TEMPERATURE $1 /$

\&SLCF PBX $=40.4$, QUANTITY=' 'oxygen' 1 ',
\&SLCF PBX $=40.4$, QUANTITY='VELOCITY', VECTOR=. TRUE. I \&SLCF PBX $=42.3$, QUANTITY= 'TEMPERATURE' $/$

\&SLCF PBX $=42.3$, QUANTITY=' 'OXYGen',
\&SLCF PBX $=42.3$, QUANTITY' 'VELOCITY', VECTOR=. TRUE. I \&SLCF PBX $=38.17$, QUANTITY='TEMPERATURE' $/$ \&SLCF PBX=38.17, QUANTITY='OXYgen' '',
\&SLCF PBX=38.17, QUANTITY='VELOCITY', VECTOR=.TRUE. \&SLCF PBX $=19.05$, QUANTITY=' 'TEMPERATURE'/ \&SLCF PBX $=19.05$, QUANTITY=' OXYgen'
\&SLCF PBX $=19.05$, QUANTITY='VELOCITY', VECTOR=.TRUE. \&SLCF PBX $=0.3$, QUANTITY= 'TEMPERATURE'/ \&SLC PBX $=0.3$, QUANTITY=' OXYgen 1
\&SLCF PBX $=0.3$, QUANTITY='VELOCITY', VECTOR=. TRUE. \&SLCF PBX $=50.9$, QUANTITY $=$ 'TEMPERATURE '/ \&SLCF PBX $50.9,9$ QUANTTYY=' 'oxygen'
\&SLCF PBX $=50.9$, QUANTITY='VELOCITY', VECTOR=.TRUE. \&SLCF PBX $=57.3$, QUANTITY= 'TEMPERATURE' $/$

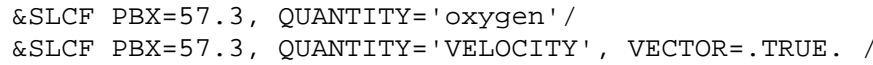
\&SLCF PBX $=63.7$, QUANTITY='TEMPERATURE' $/$ \&SLCF PBX $=63.7$, QUANTITY=' 'oXygen' 1
\&SLCF PBX $=63.7$, QUANTITY='VELOCITY', VECTOR=. TRUE. \&SLCF PBY $=20.3$, QUANTITY='TEMPERATURE'/ \&SLCF PBY $=20.3$, QUANTITY='VELOCITY', VECTOR=. TRUE. I \&SLCF PBY $=45.05$, OUANTITY=' TEMPERATURE' $/$ \&SLCF PBY 45.05 , Q QAANTITY=' OXYGEn $'$ '
\&SLCF PBY 45.05 , QUANTITY='VELOCITY', VECTOR=.TRUE. \&SLCF PBY=49.17, QUANTITY='TEMPERATURE'/

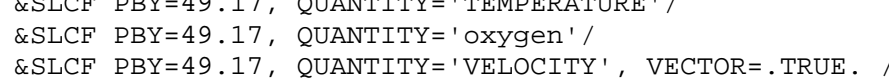
\&SLCF PBY $=39.8$, QUANTITY=' TEMPERATURE ' / \&SLCF PBY $=39.8$, QUANTITY=' 'OXYGen' 1 ,
\&SLCF PBY $=39.8$, QUANTITY='VELOCITY', VECTOR=. TRUE. I \&SLCF PBY $=67.18$, QUANTITY='TEMPERATURE'/

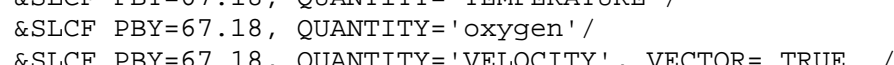


\&SLCF PBY $=84.05$, QUANTITY='TEMPERATURE' $/$

\&SLCF PBY=84. 05, QUANTITY=' 'OXYgen'1,
\&SLCF PBY $=84.05$, QUANTITY='VELOCITY', VECTOR=.TRUE.

\&SLCF PBZ $=0.50$, QUANTITY=' TEMPERATURE'

\&SLCF PBZ $=0.50$, QUANTITY=' 'OXYgen' $/$
\&SLCF PBZ $=0.50$, QUANTITY='VELOCITY', VECTOR=. TRUE.

\&SLCF PBZ $=2.38$, QUANTTTY=' TEMPERATURE' $/$

\&SLCF PBZ $=2.38$, QUANTITY=' $O$ OXgen'
\&SLCF PBZ $=2.38$, QUANTITY='VELOCITY', VECTOR=.TRUE. ,

\&SLCF PBZ $=3.13$, QUANTITY=' 'TEMPERATURE'/

\&SLCF PBZ=3.13, QUANTTYY=' oxygen'
\&SLCF PBZ $=3.13$, QUANTITY='VELOCITY', VECTOR=. TRUE. I

\&SLCF PBZ $=0.88$, QUANTITY=' TEMPERATURE' $/$

\&SLCF PBZ $=0.88$, QUANTITY=' OXYgen' $'$ ', VETOR=.TRUE. ,
\&SLCF PBZ $=0.88$, QUANTITY='VELOCITY', VECTOR

\&SLCF PBZ $=8.39$, QUANTITY= ' TEMPERATURE
\&SLCF

\&SLCF PBZ=8.39, QUANTITY=' OXYgen' $'$ ', VETOR=.TRUE. ,
\&SLCF PBZ $=8.39$, QUANTTYY='VELOCITY', VECOR

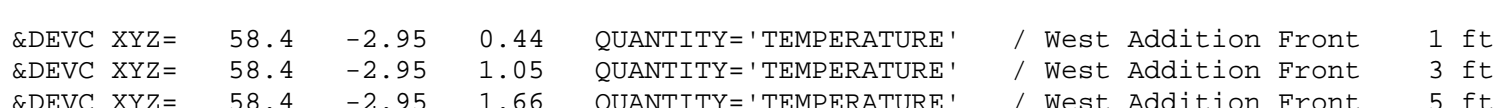

$\begin{array}{lllllll}\text { \&DEVC XYZ }= & 58.4 & -2.95 & 1.66 & \text { QUANTTYY=' 'TEMPERATURE' , West Addition Front } & 5 \mathrm{ft} \\ \text { \&DEVC XYZ= } & 58.4 & -2.95 & 2.27 & \text { QUANTITY='TEMPERATURE' / west Addition Front } & 7 \mathrm{ft}\end{array}$

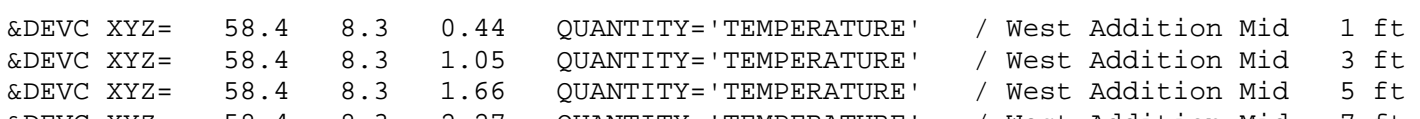

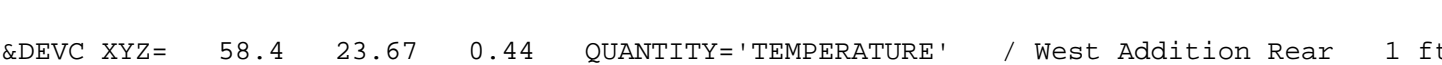

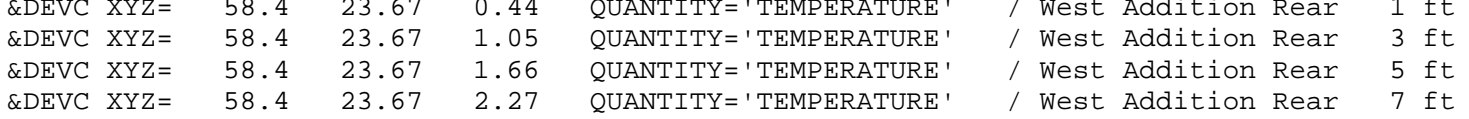

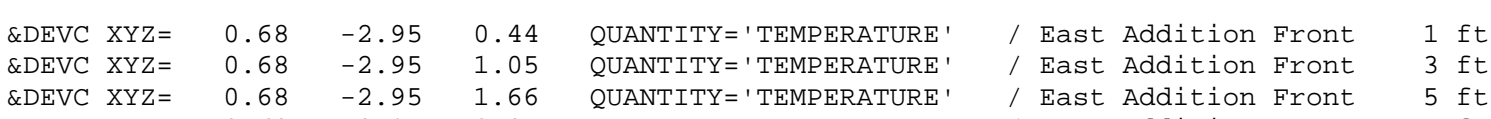

\&DEVC XYZ $=0.68$

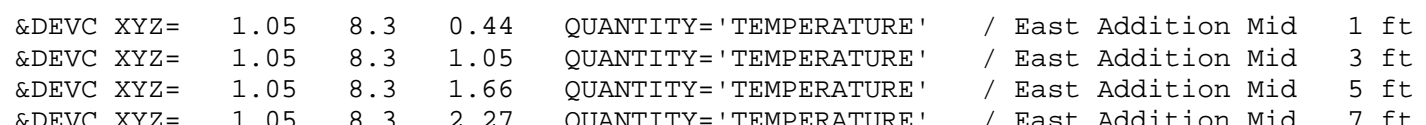

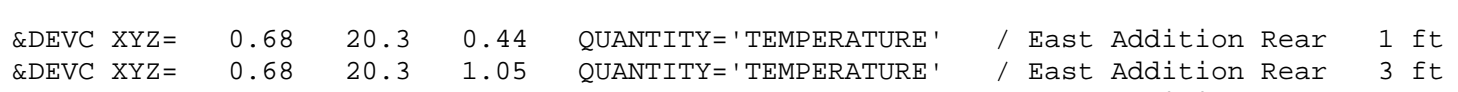

$\begin{array}{lllllll}\text { QDEVC XYZ }= & 0.68 & 20.3 & 1.66 & \text { QUANTITY='TEMPRRATURE', / East Addition Rear } & 5 \mathrm{ft} \\ \text { \&DEVC XYZ } & 0.68 & 20.3 & 2.27 & \text { QUANTITY='TEMPERATURE' } & \text {, East Addition Rear } & 7 \mathrm{ft}\end{array}$

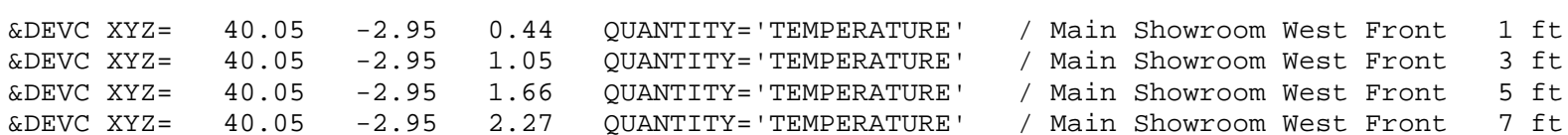

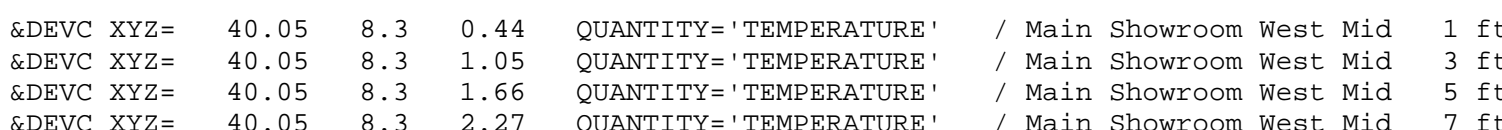

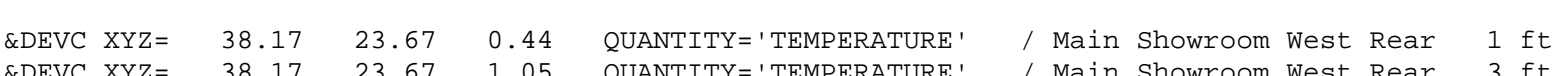

$\begin{array}{llllllll}\text { \&DEVC XYZ }= & 38.17 & 23.67 & 1.66 & \text { QUANTITY='TEMPERATURE' } & \text { / Main Showroom west Rear } & 5 \mathrm{ft} \\ \text { \&DEVC XYZ } & 38.17 & 23.67 & 2.27 & \text { QUANTITY='TEMPERATURE' } & \text { / Main Showroom west Rear } & 7 \mathrm{ft}\end{array}$

$\begin{array}{lllllllll}\text { EDEVC XYZ= } & 38.17 & 32.7 & 0.44 & \text { QUANTITY='TEMPERATURE' } & \text { Main Showroom west Rear } 2 & 1 \mathrm{ft} \\ \text { \&DEVC XYZ= } & 38.17 & 32.7 & 1.05 & \text { QUANTITY='TEMPERATURE', / Main Showroom west Rear } 2 & 3 \mathrm{ft}\end{array}$

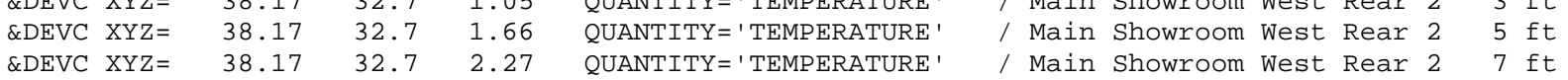

$\begin{array}{llllllll}\text { EDEVC XYZ }= & 28.8 & -2.95 & 0.44 & \text { QUANTITY='TEMPERATURE' , Main Showroom ctr Front } & 1 \mathrm{ft} \\ \text { \&DEVC XYZ } & 28.8 & -2.95 & 1.05 & \text { QUANTITY='TEMPERATURE' } & \text { / Main Showroom Ctr Front } & 3 \mathrm{ft}\end{array}$

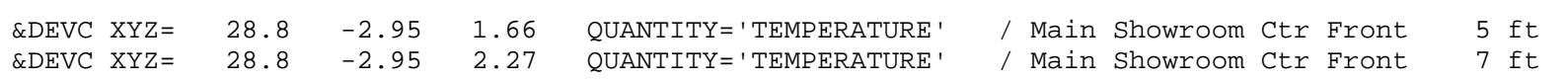

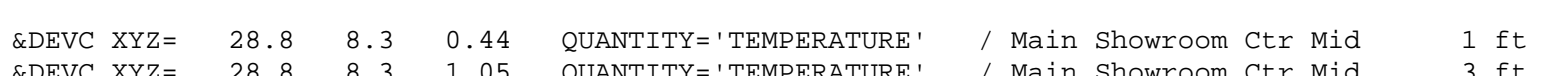

$\begin{array}{llllllll}\text { \&DEVC XYZ= } & 28.8 & 8.3 & 1.66 & \text { QUANTITY='TEMPRATURE', /Main Showroom Ctr Mid } & 5 \mathrm{ft} \\ \text { \&DEVC XYZ= } & 28.8 & 8.3 & 2.27 & \text { QUANTITY='TEMPERATURE' } & \text { / Main Showroom ctr Mid } & 7 \mathrm{ft}\end{array}$

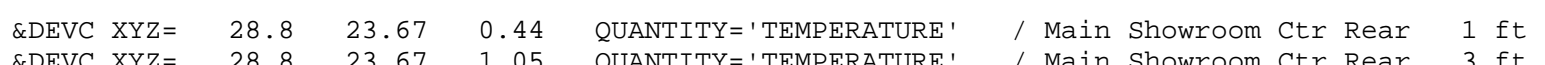

$\begin{array}{llllllll}\text { \&DEVC XYZ= } & 28.8 & 23.67 & 1.05 & \text { QUANTITY='TEMPERATURE', / Main Showroom ctr Rear } & 3 \mathrm{ft} \\ \text { \&DEVC XYZ= } & 28.8 & 23.67 & 1.66 & \text { QUANTITY='TEMPERATURE', } & \text { Main Showroom ctr Rear } & 5 \mathrm{ft} \\ \text { \&DEVC XYZ= } & 28.8 & 23.67 & 2.27 & \text { QUANTITY=' TEMPERATURE' } & \text { / Main Showroom Ctr Rear } & 7 \mathrm{ft}\end{array}$

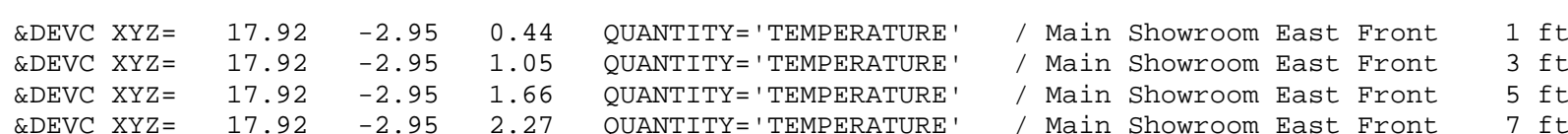

$\begin{array}{llllllll}\text { EDEVC XYZ }= & 17.92 & 8.3 & 0.44 & \text { QUANTITY='TEMPERATURE', / Main Showroom East Mid } & 1 \mathrm{ft} \\ \text { \&DEVC XYZ } & 17.92 & 8.3 & 1.05 & \text { QUANTITY='TEMPERATURE', } & \text { Main Showroom East Mid } & 3 \mathrm{ft}\end{array}$

$\begin{array}{lllllll}\text { \&DEVC XYZ }= & 17.92 & 8.3 & 1.66 & \text { QUANTITY='TEMPERATURE' } & \text { / Main Showroom East Mid } & 5 \mathrm{ft} \\ \text { \&DEVC XYZ } & 17.92 & 8.3 & 2.27 & \text { QUANTITY='TEMPERATURE' } & \text { / Main Showroom East Mid } & 7 \mathrm{ft}\end{array}$

$\begin{array}{lllllll}\text { \&DEVC XYZ= } & 17.92 & 23.67 & 0.44 & \text { QUANTITY='TEMPERATURE', / Main Showroom East Rear } & 1 \mathrm{ft} \\ \text { \&DEVC XYZ } & 17.92 & 23.67 & 1.05 & \text { QUANTITY='TEMPERATURE' } & \text { / Main Showroom East Rear } & 3 \mathrm{ft}\end{array}$

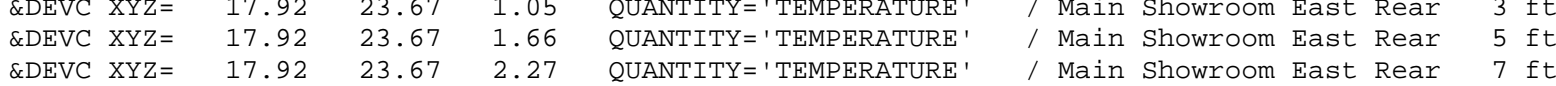

$\begin{array}{lllll}\text { EDEVC XYZ }= & 46.05 & 35.3 & 0.44 & \text { QUANTITY=' 'TEMPERATURE', / Holding Room } 1 \mathrm{ft} \\ \text { \&DVC XYZ } & 46.05 & 35.3 & 1.05 & \text { QUANTTY'T TEMERATURE' }\end{array}$

$\begin{array}{llllllll}\text { \&DEVC XYZ= } & 46.05 & 35.3 & 1.05 & \text { QUANTITY='TEMPERATURE', / Holding Room } & 3 \mathrm{ft} \\ \text { \&DEC XYZ= } & 46.05 & 35.3 & 1.66 & \text { QUANTIY=' 'EMPERATURE' } & \text { / Holding Room } & 5 \mathrm{ft} \\ \text { \&DEVC XYZ= } & 46.05 & 35.3 & 2.27 & \text { QUANTITY=' TEMPERATURE' } & \text { / Holding Room } & 7 \mathrm{ft}\end{array}$

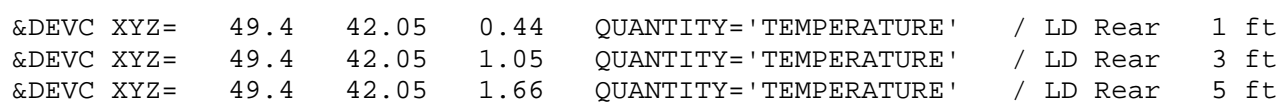

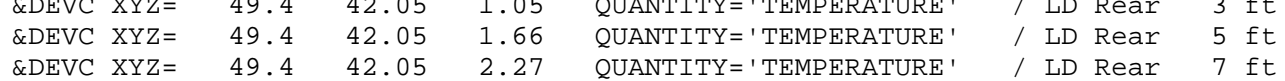

$\begin{array}{lllllllll}\text { \&DEVC XYZ= } & 49.4 & 35.3 & 0.44 & \text { QUANTITY='TEMPERATURE' } & \text { / LD Front } & 1 \mathrm{ft} \\ \text { \&DEVC XYZ }= & 49.4 & 35.3 & 1.05 & \text { QUANTIY='TEMPERATURE' } & \text {, LD Front } & 3 \mathrm{ft} \\ \text { Q }\end{array}$

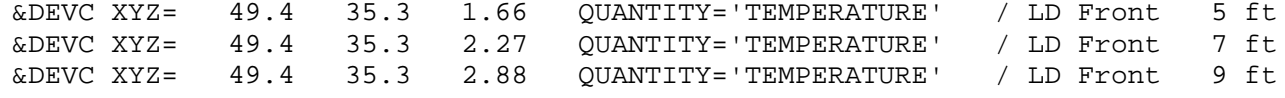

$\begin{array}{lllllllll}\text { \&DEVC XYZ= } & 32.55 & 48.4 & 0.44 & \text { QUANTITY='TEMPERATURE' } & \text { L Room } & 1 \mathrm{ft} \\ \text { \&DEVC XYZ= } & 32.55 & 48.4 & 1.05 & \text { QUANTITY='TEMPERATURE' } & \text {, L Room } & 3 \mathrm{ft} \\ \text { \&DEVC XYZ }= & 32.55 & 48.4 & 1.66 & \text { QUANTITY='TEMPERATURE' } & \text { L L Room } & 5 \mathrm{ft}\end{array}$

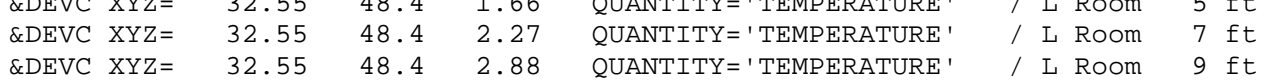

\begin{tabular}{llllllll} 
\&DEVC XYZ= & 38.9 & 42.4 & 0.44 & QUANTITY='TEMPERATURE', / Workshop & $1 \mathrm{ft}$ \\
\&DEVC XYZ= & 38.9 & 42.4 & 1.05 & QUANTITY='TEMPERATURE', / Workshop & $3 \mathrm{ft}$ \\
\hline
\end{tabular}

\&DEVC XYZ= 38.9 4. 42.4 1.66 QUANTITY='TEMPERATURE', / Workshop $5 \mathrm{ft}$

$\begin{array}{llllllll}\text { \&DEVC XYZ }= & 38.9 & 42.4 & 2.27 & \text { QUANTITY='TEMPERATURE', } & \text { / Workshop } & 7 \mathrm{ft} \\ \text { \&DEVC XYZ } & 38.9 & 42.4 & 2.88 & \text { QUANTITY='TEMPERATURE' } & \text { / workshop } & 9 \mathrm{ft}\end{array}$

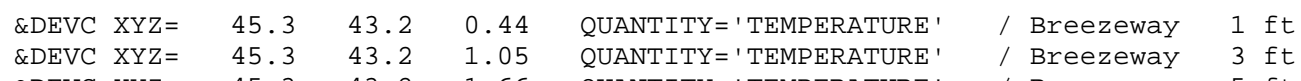

$\begin{array}{llllllll}\text { \&DEVC XYZ= } & 45.3 & 43.2 & 1.66 & \text { QUANTITY='TEMPERATURE', / Breezeway } & 5 \mathrm{ft} \\ \text { \&DEVC XYZ= } & 45.3 & 43.2 & 2.27 & \text { QUANTTY=' 'TEMPRRATURE' } & \text { / Breezeway } & 7 \mathrm{ft} \\ \text { \&DEVC XYZ= } & 45.3 & 43.2 & 2.88 & \text { QUANTITY=' TEMPERATURE' } & \text { / Breezeway } & 9 \mathrm{ft}\end{array}$

$\begin{array}{llllllll}\text { \&DEVC XYZ= } & 49.4 & 27.42 & 0.44 & \text { QUANTITY=' 'TEMPERATURE' } & \text { D Doors } & 1 \mathrm{ft} \\ \text { \&DEVC XYZ } & 49.4 & 27.42 & 1.05 & \text { OUANTTTY' TEPPERATURE' } & \text { / D Doors } & 3 \mathrm{ft}\end{array}$

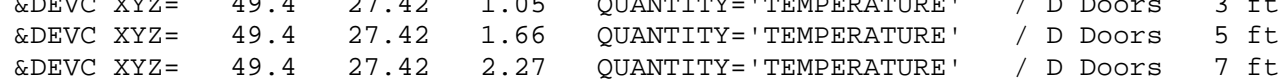

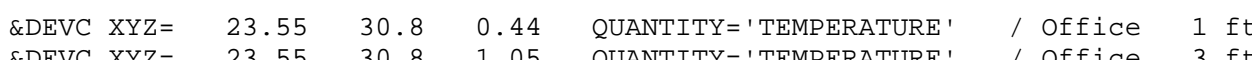

$\begin{array}{llllllll}\text { QDEVC XYZ } & 23.55 & 30.8 & 1.05 & \text { QUANTITY='TEMPERATURE' } & / \text { Office } & 3 \mathrm{ft} \\ \text { QDEVC XYZ } & 23.55 & 30.8 & 1.66 & \text { QUANTTY='TEMPERATURE' } & \text {, Office } & 5 \mathrm{ft} \\ \text { QDEVC XYZ } & 23.55 & 30.8 & 2.27 & \text { QUANTITY='TEMPERATURE' } & \text {, Office } & 7 \mathrm{ft}\end{array}$

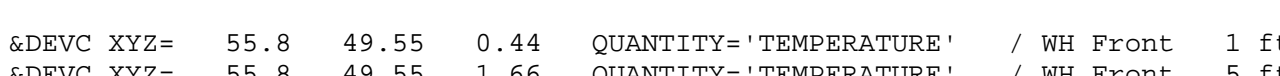

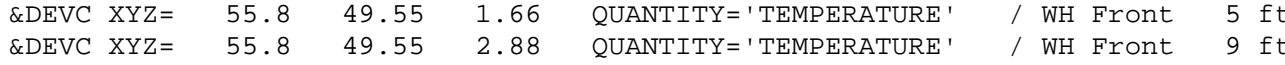

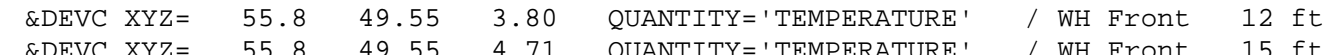




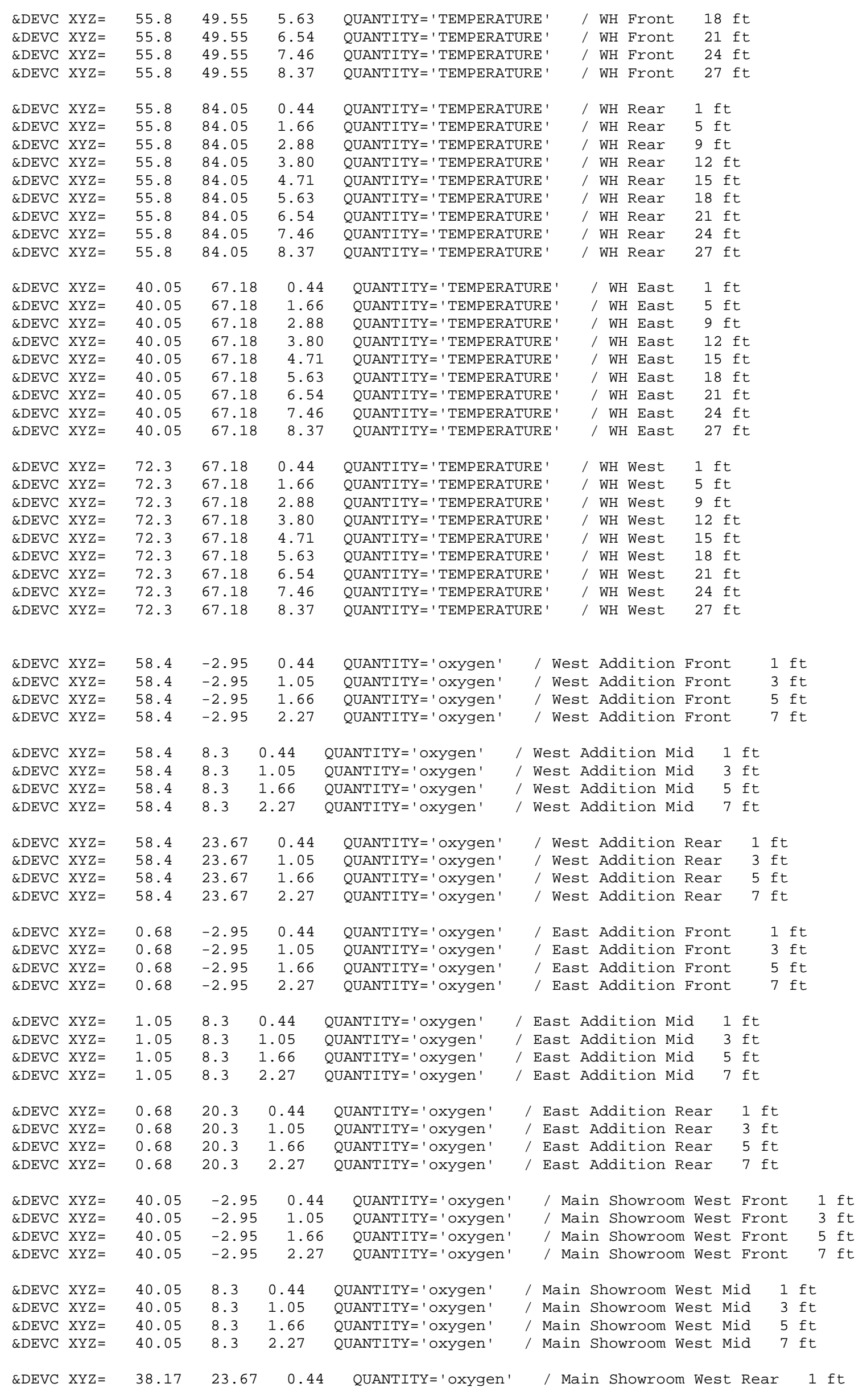

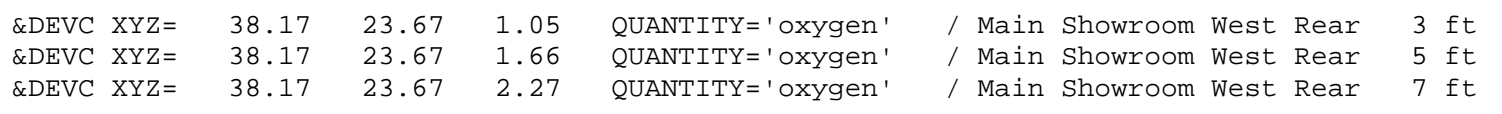

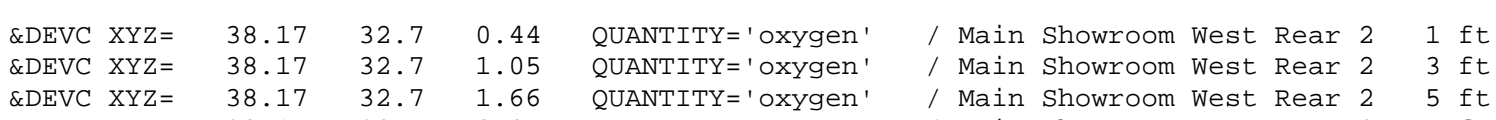

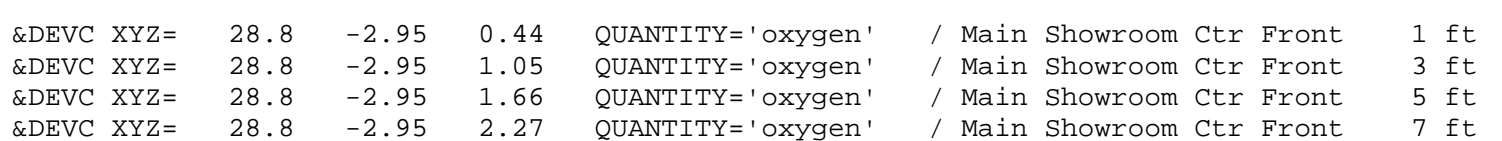

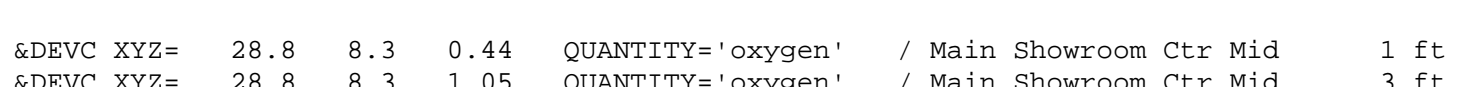

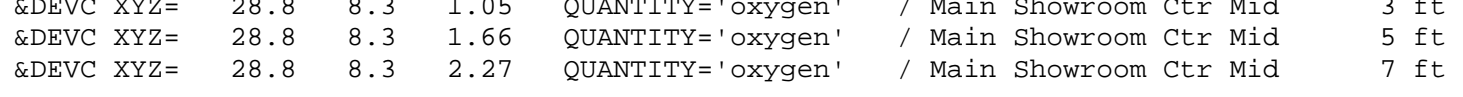
$\begin{array}{lllllll}\text { \&DEVC XYZ= } & 28.8 & 23.67 & 0.44 & \text { QUANTITY='oxygen' , Main Showroom Ctr Rear } & 1 \mathrm{ft} \\ \text { \&DEVC XYZ } & 28.8 & 23.67 & 1.05 & \text { QUANTITY=' oxygen' } & \text { Main Showroom Ctr Rear } & 3 \mathrm{ft}\end{array}$ $\begin{array}{llllllll}\text { \&DEV XYZ= } & 28.8 & 23.67 & 1.66 & \text { QUANTITY='oxygen' } & \text { / Main Showroom Ctr Rear } & 5 \mathrm{ft} \\ \text { \&DEVC XYZ= } & 28.8 & 23.67 & 2.27 & \text { QUANTITY='oxygen' } & \text { Main Showroom Ctr Rear } & 7 \mathrm{ft}\end{array}$ $\begin{array}{lllllll}\text { \&DEVC XYZ }= & 17.92 & -2.95 & 0.44 & \text { QUANTITY=' oxygen' } & \text { Main Showroom East Front } & 1 \mathrm{ft} \\ \text { \&DEVC XYZ } & 17.92 & -2.95 & 1.05 & \text { QUANTITY=' oxygen' } & \text {, Main Showroom East Front } & 3 \mathrm{ft}\end{array}$

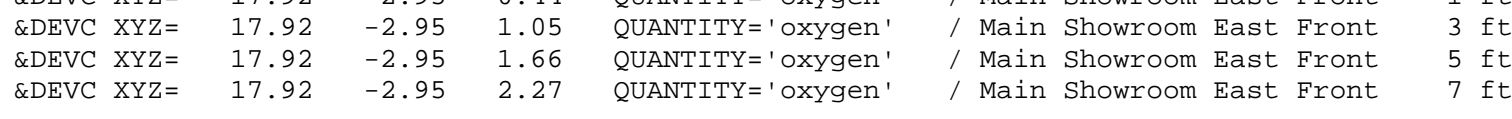

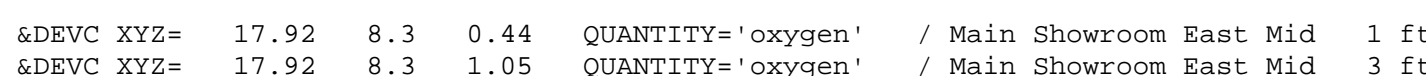
$\begin{array}{lllllll}\text { \&DEVC XYZ }= & 17.92 & 8.3 & 1.66 & \text { QUANTITY='oxygen' } & \text { / Main Showroom East Mid } & 5 \mathrm{ft} \\ \text { \&DEVC XYZ }= & 17.92 & 8.3 & 2.27 & \text { QUANTITY='oxygen' } & \text { Main Showroom East Mid } & 7 \mathrm{ft}\end{array}$

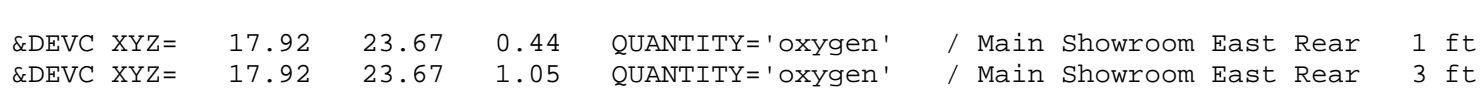

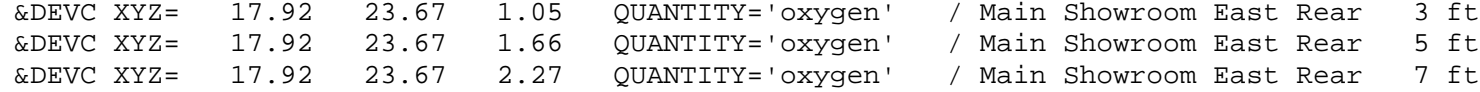
$\begin{array}{llllllll}\text { \&DEVC XYZ }= & 46.05 & 35.3 & 0.44 & \text { QUANTITY='oxygen' } & \text { / Holding Room } & 1 \mathrm{ft} \\ \text { \&DEVC XYZ } & 46.05 & 35.3 & 1.05 & \text { QUANTITY=' oxygen' } & \text { Holding Room } & 3 \mathrm{ft}\end{array}$ $\begin{array}{llllllll}\text { \&DEVC XYZ= } & 46.05 & 35.3 & 1.05 & \text { QUANTTYY=' 'oxygen', / Holding Room } & 3 \mathrm{ft} \\ \text { \&DVCV XYZ } & 46.05 & 35.3 & 1.66 & \text { QUANTTY= 'oxygen' } & \text {, Holding Room } & 5 \mathrm{ft} \\ \text { \&DEVC XYZ= } & 46.05 & 35.3 & 2.27 & \text { QUANTITY=' oxygen' } & \text { Holding Room } & 7 \mathrm{ft}\end{array}$

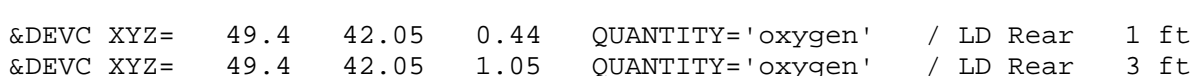

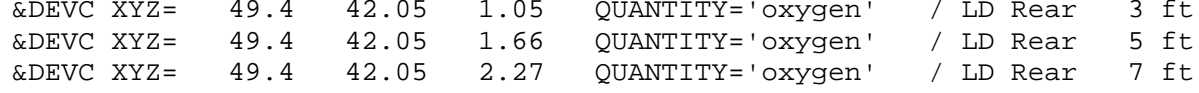

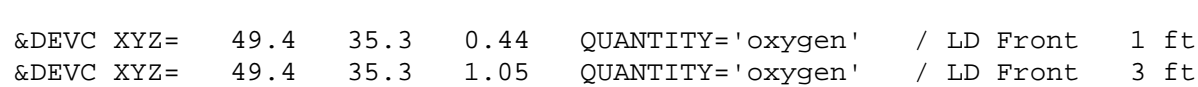

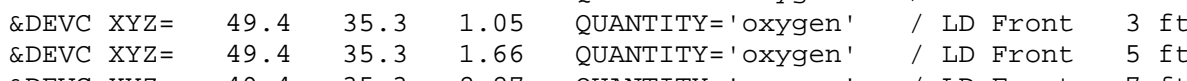
$\begin{array}{llllllll}\text { \&DEVC XYZ= } & 49.4 & 35.3 & 2.27 & \text { QUANTITY='oxygen', /, LD Front } & 7 \mathrm{ft} \\ \text { \&DEVC XYZ }= & 49.4 & 35.3 & 2.88 & \text { QUANTITY='oxygen' } & \text { LD Front } & 9 \mathrm{ft}\end{array}$ $\begin{array}{llllllll}\text { \&DEVC XYZ }= & 32.55 & 48.4 & 0.44 & \text { QUANTITY=' oxygen', / L Room } & 1 \mathrm{ft} \\ \text { \&DEVC XYZ } & 32.55 & 48.4 & 1.05 & \text { QUANTITY=' oxygen' } & \text {, L Room } & 3 \mathrm{ff}\end{array}$

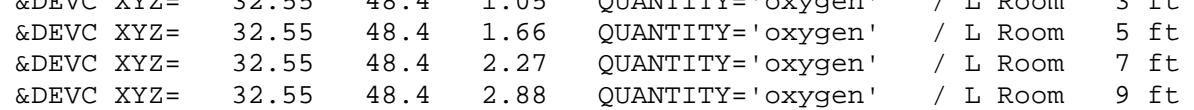
\&DEVC XYZ $=\begin{array}{lllllll}38.9 & 42.4 & 0.44 & \text { QUANTITY='oxygen' / Workshop } 1 \mathrm{ft}\end{array}$

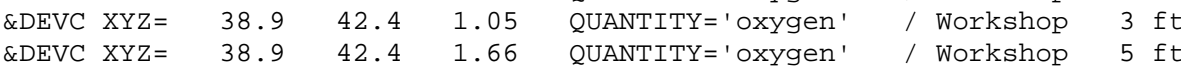
$\begin{array}{llllllll}\text { \&DEVC XYZ= } & 38.9 & 42.4 & 2.27 & \text { QUANTITY='oxygen' } & \text { / Workshop } & 7 \mathrm{ft} \\ \text { \&DEVC XYZ } & 38.9 & 42.4 & 2.88 & \text { QUANTITY='oxygen' } & \text {, workshop } & 9 \mathrm{ft}\end{array}$ $\begin{array}{llllllll}\text { \&DEVC XYZ= } & 45.3 & 43.2 & 0.44 & \text { QUANTITY=' 'oxygen' / Breezeway } & 1 \mathrm{ft} \\ \text { \&DEVC XYZ } & 45.3 & 43.2 & 1.05 & \text { QUANTITY='oxygen' } & \text { / Breezeway } & 3 \mathrm{ft}\end{array}$ $\begin{array}{llllllll}\text { \&DEVC XYZ= } & 45.3 & 43.2 & 1.66 & \text { QUANTITY='oxygen' } & \text { / Breezeway } & 5 \mathrm{ft} \\ \text { \&DEVC XYZ= } & 45.3 & 43.2 & 2.27 & \text { QUANTTY=' oxygen' } & \text { Breezeway } & 7 \mathrm{ft} \\ \text { \&DEVC XYZ= } & 45.3 & 43.2 & 2.88 & \text { QUANTITY=' oxygen' } & \text { Breezeway } & 9 \mathrm{ft}\end{array}$

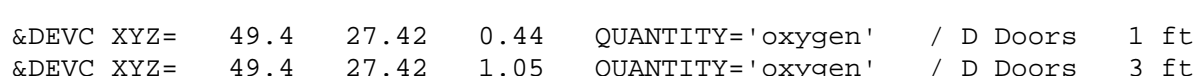
$\begin{array}{llllllllll}\text { \&DEVC XYZ }= & 49.4 & 27.42 & 1.66 & \text { QUANTITY='oxygen' } & \text { D Doors } & 5 \mathrm{ft} \\ \text { \&DEVC XYZ } & 49.4 & 27.42 & 2.27 & \text { QUANTITY='oxygen' } & \text { D Doors } & 7 \mathrm{ft}\end{array}$ 


\begin{tabular}{|c|c|c|c|c|c|}
\hline 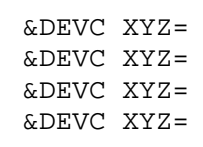 & $\begin{array}{l}23.55 \\
23.55 \\
23.55 \\
23.55\end{array}$ & $\begin{aligned} 30.8 \\
30.8 \\
30.8 \\
30.8\end{aligned}$ & $\begin{array}{l}0.44 \\
1.05 \\
1.66 \\
2.27\end{array}$ & 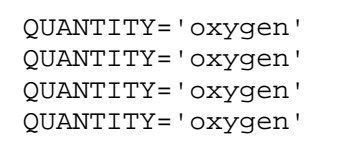 & $\begin{array}{l}1 \text { office } \\
\text { office } \\
\text { Office } \\
\text { office }\end{array}$ \\
\hline 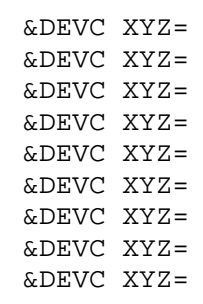 & 50.8 & 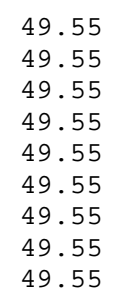 & $\begin{array}{l}0.44 \\
1.66 \\
2.88 \\
3.86 \\
4.71 \\
5.63 \\
6.54 \\
7.46 \\
8.37\end{array}$ & 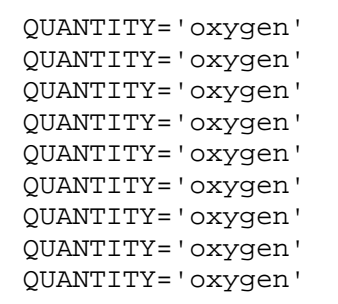 & 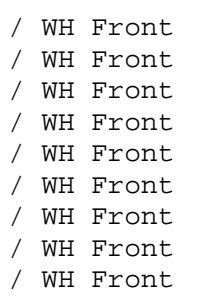 \\
\hline 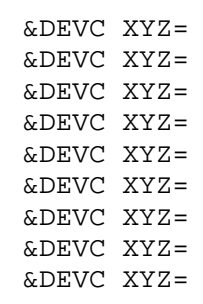 & 55.8 & $\begin{array}{l}84.05 \\
84.05 \\
84.05 \\
84.05 \\
84.05 \\
84.05 \\
84.05 \\
84.05 \\
84.05\end{array}$ & $\begin{array}{l}0.44 \\
1.66 \\
2.88 \\
3.80 \\
3.71 \\
5.63 \\
6.54 \\
7.46 \\
8.37\end{array}$ & 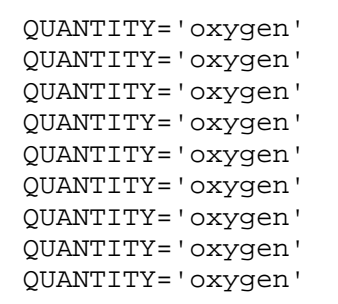 & 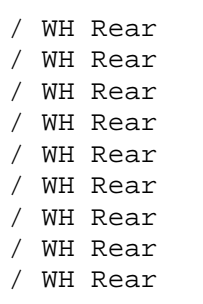 \\
\hline 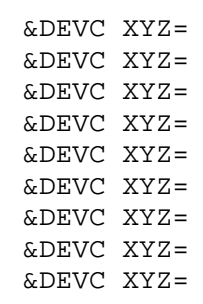 & $\begin{array}{l}40.05 \\
40.05 \\
40.05 \\
40.05\end{array}$ & $\begin{array}{l}67.18 \\
67.18 \\
67.18 \\
67.18 \\
67.18 \\
67.18 \\
67.18 \\
67.18 \\
67.18\end{array}$ & $\begin{array}{l}0.44 \\
1.66 \\
2.88 \\
3.88 \\
3.71 \\
5.73 \\
6.54 \\
7.44 \\
8.37\end{array}$ & 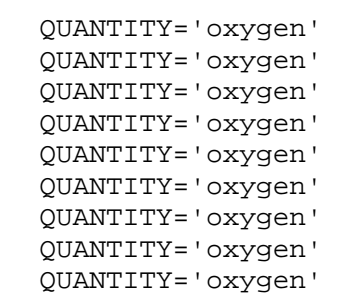 & 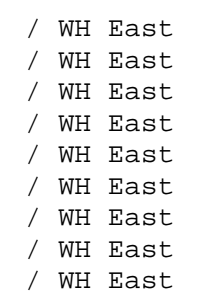 \\
\hline 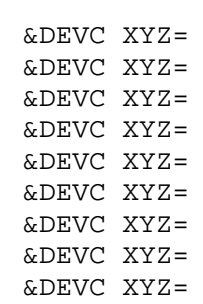 & & $\begin{array}{l}67.18 \\
67.18 \\
67.18 \\
67.18\end{array}$ & $\begin{array}{l}5.63 \\
6.54 \\
7.46 \\
8.37\end{array}$ & 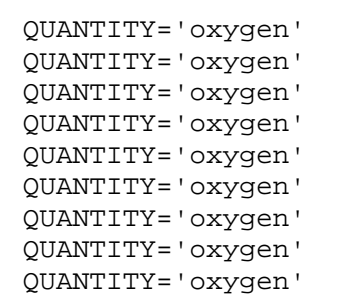 & 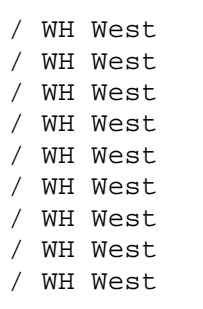 \\
\hline
\end{tabular}

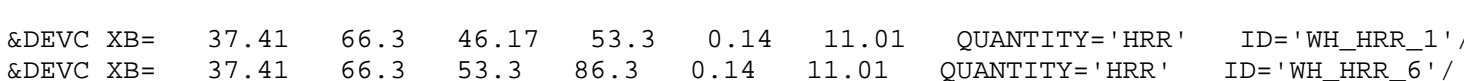

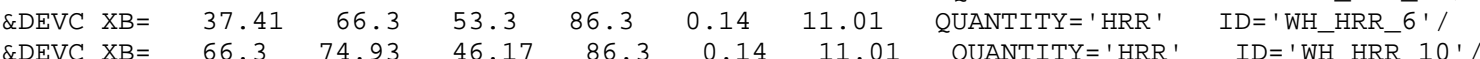

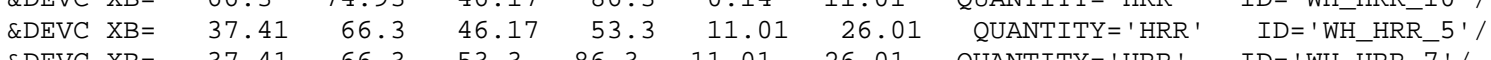

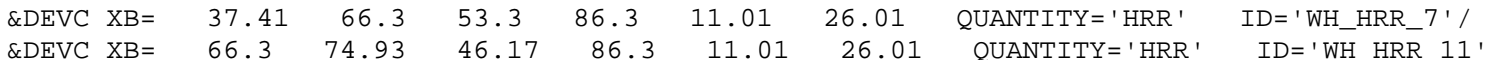

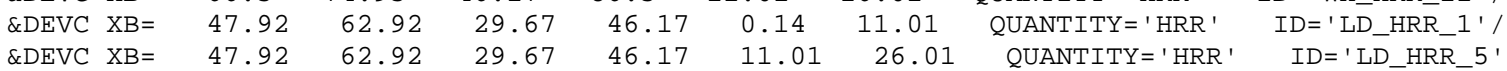

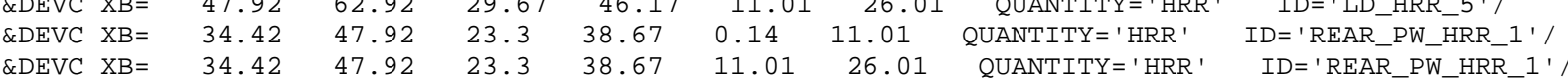

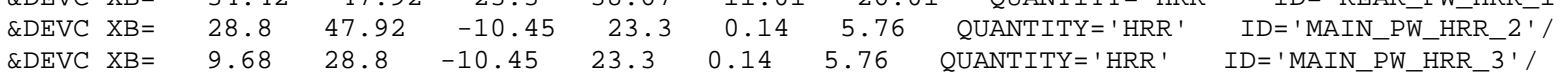

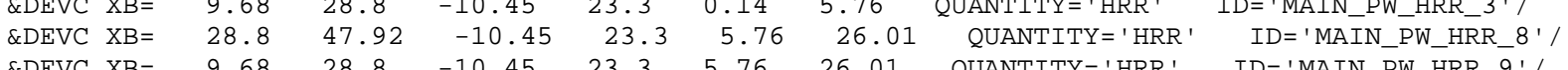

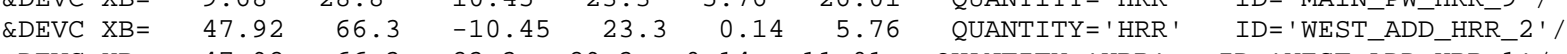

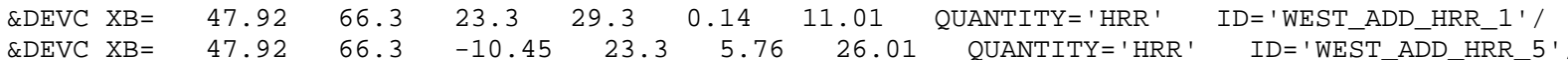
$\begin{array}{llllllll}\text { EDEVC XB }= & 47.92 & 66.3 & 23.3 & 29.3 & 11.01 & 26.01 & \text { QUANTITY='HRR' ID='WEST ADD HRR 8'/ }\end{array}$

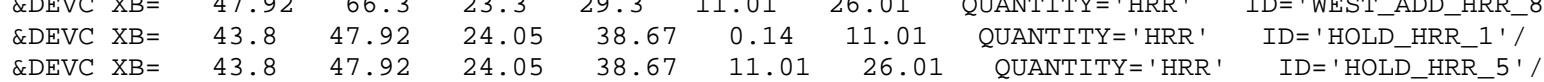
$* * * * * * * * * * * x$
Furniture

*** GROUP: LEVEL

$* \star *$ GROUP: ROW

*** GROUP: COUCH2 offset $=0.000000$, ๑. $.000000,0.000000$ rotate $=90.000000$

\&OBST XB=37.799999, 38.924999, 51.049999, 51.424999, 0.28,1.03, SURF_ID=' UPHOLSTERY' / Left Arm
\&OBST XB=37. $799999,38.924999,51.424999,52.924999,0.28,0.655$, SURF_ID= 'UPHOLSTERY' / Base

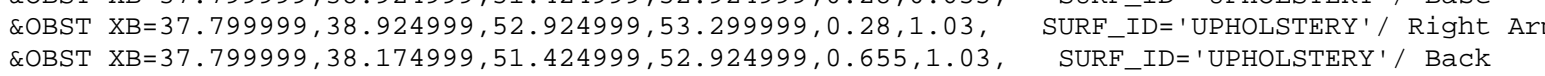
*** GROUP: COUCH2 offset $=0.000000,3.000000,0.000000$ rotate $=90.000000$

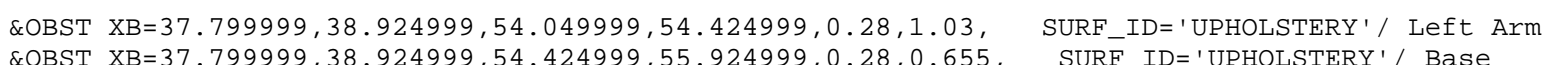

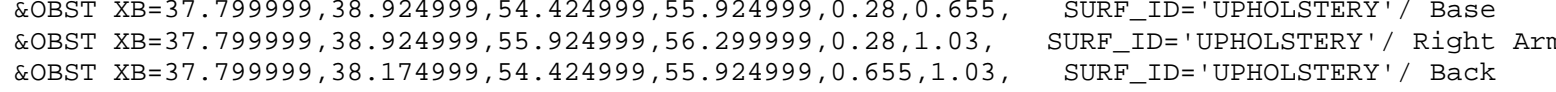

*** GROUP: COUCH2 offset $=0.000000,6.000000,0.000000$ rotate $=90.000000$

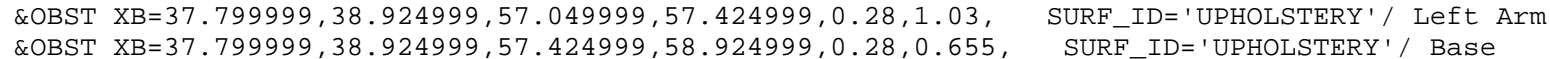

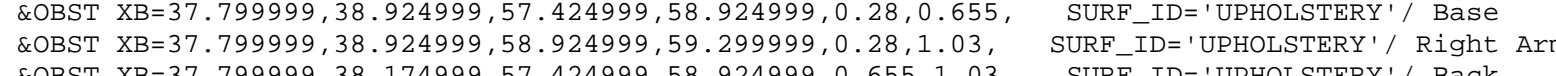

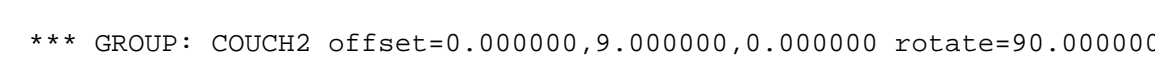

\&OBST XB=37.799999, 38. 924999, 60. $049999,60.424999,0.28,1.03, \quad$ SURF_ID= 'UPHOLSTERY' / Left Arm

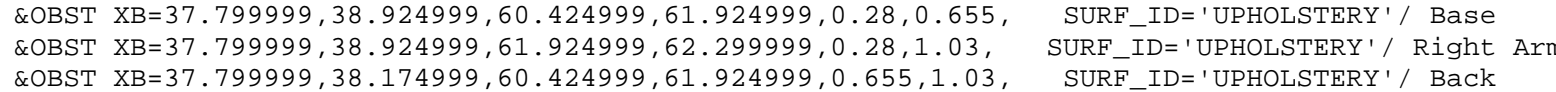

*** GROUP: COUCH2 offset $=0.000000,12.000000$, ๑. .000000 rotate $=90.000000$

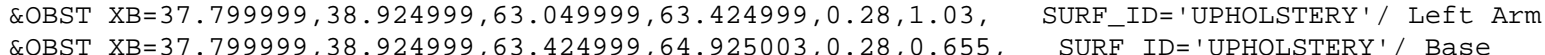

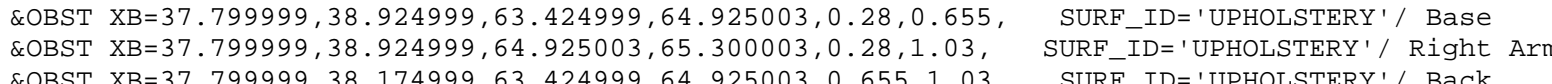

*** GROUP: COUCH2 offset $=0.000000,15.000000,0.000000$ rotate $=90.000000$

\&OBST XB=37.799999, 38. 924999, 66. 050003, 66. 425003, 0.28,1.03, SURFID=' 'UPHOLSTERY ' ' Left Arm

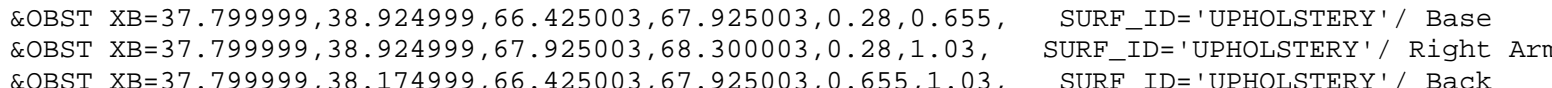

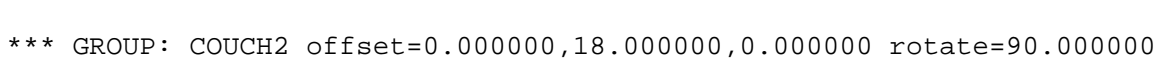

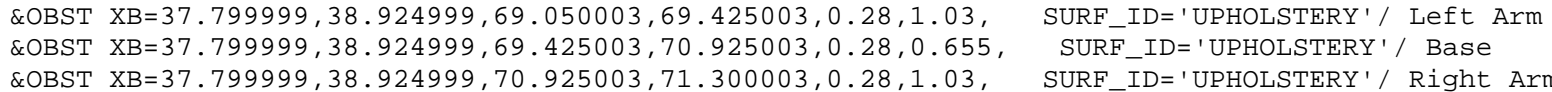

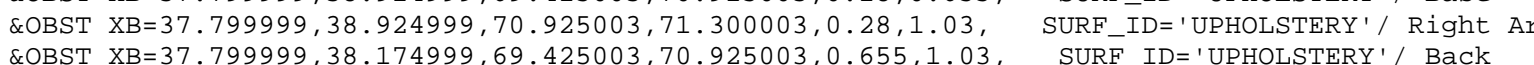

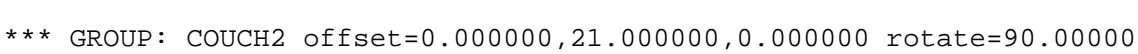

\&OBST XB=37.799999, 38.924999, 72. $.550003,72.425003,0.28,1.03$, SURF_ID=' 'UPHOLSTERY '/ Left Arm

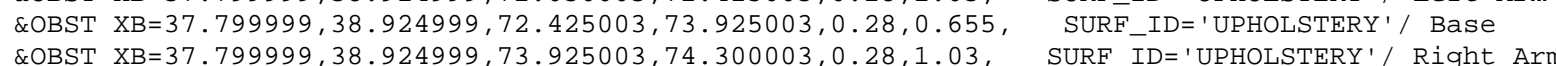
\&OBST XB=37.79999, 38.924999, $73.925003,74.300003,0.28,1.03, \quad$ SURF_ID=' 'UPHOLSTERY'/ Right Arm
\&OBST XB $\mathrm{X}=37.799999,38.174999,72.425003,73.925003,0.655,1.03, \quad$ SURF_ID=' UPHOLSTERY' / Back

*** GROUP: COUCH2 offset $=0.000000,24.000000,0.000000$ rotate $=90.000000$

\&OBST XB=37.799999, 38.924999, 75. $050003,75.425003,0.28,1.03$, SURF_ID=' 'UPHOLSTERY ' / Left Arm \&OBST XB=37.799999, 38.924999, 75.425003,76.925003, $0.28,0.655$, SURF_ID=' 'UPHOLSTERY'/ Base

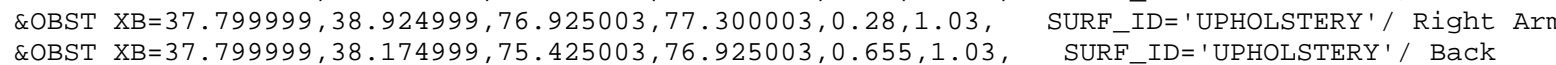

$* * *$ GROUP: COUCH2 offset $=0.000000,27.000000,0.000000$ rotate $=90.000000$

\&OBST XB=37.799999, 38. 924999, 78. $050003,78.425003,0.28,1.03$, SURF_ID=' 'UPHOLSTERY ' / Left Arm

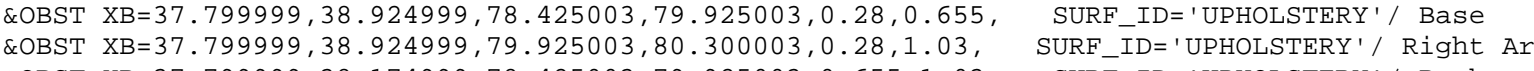

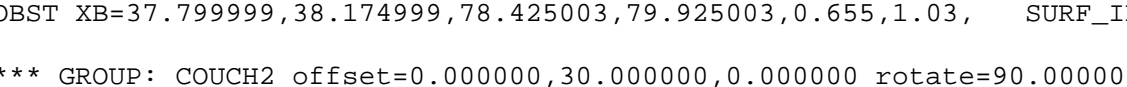

\&OBST XB=37.799999, 38.924999, 81. 050003, 81.425003, 0.28, 1. 03 , SURF_ID=' 'UPHOLSTERY ' / Left Arm

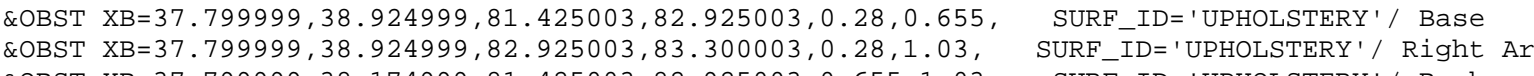

*** GROUP: BASE

*** GROUP: TWIN 
*** GROUP: ROW

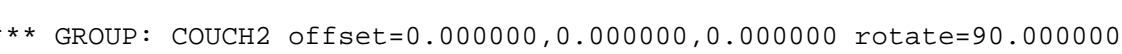

\&OBST $X \mathrm{XB}=43.049999,44.174999,51.049999,51.424999,0.28,1.03$, SURF_ID='UPHOLSTERY '/ Left Arm

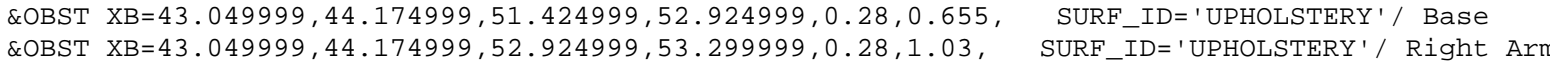
\&OBST XB $=43.04909$

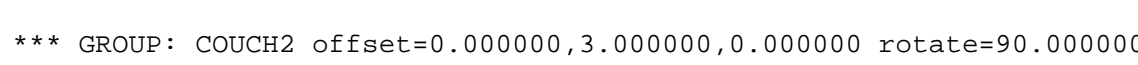

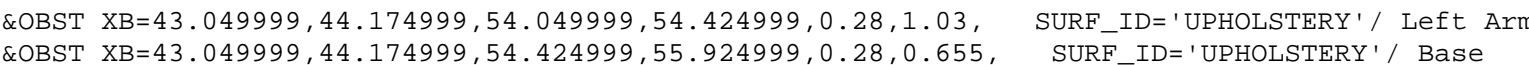
\&OBST XB=43. $449999,44.174999,55.924999,56.299999,0.28,1.03$, SURF_ID='UPHOLSTERY'/ Right Ar

*** GROUP: COUCH2 offset $=0.000000,6.000000,0.000000$ rotate $=90$. ๑ 00000

\&OBST XB=43. $049999,44.174999,57.049999,57.424999,0.28,1.03, \quad$ SURF_ID=' 'UPHOLSTERY' $/$ ' Left Arm
\&OBST XB=43.04999, 44.174999, $57.424999,58.924999,0.28,0.655$,
SURF_ID='UPHOLSTERY'/ Base \&OBST XB=43.049999, 44.174999, 58.924999, 59. 299999, $, .28,1.03$, SURF_ID='UPHOLSTERY'/ Right Ar

*** GROUP: COUCH2 offset $=0.000000,9.000000,0.000000$ rotate $=90.000000$

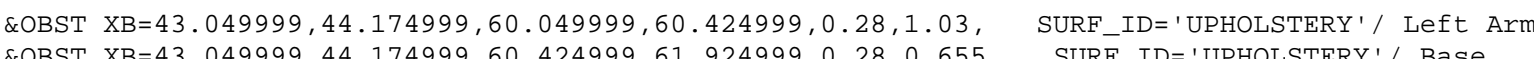

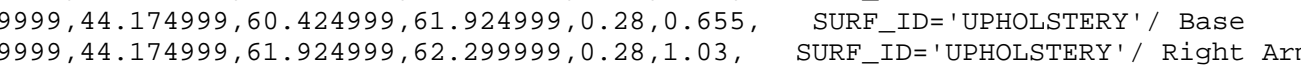

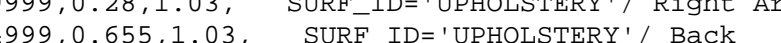

*** GROUP: COUCH2 offset $=0.000000,12.000000, \Theta .000000$ rotate $=90.000000$

\&OBST XB=43. 049999, 44.174999, 63. $049999,63.424999,0.28,1.03$, SURF_ID=' 'UPHOLSTERY'/ Left Arm \&OBST XB=43. $449999,44.174999,63.424999,64.925003,0.28,0.655$, SURF IDD 'UPHOLSTERY'/ Base \&OBST XB=43.049999, 43.424999, 63.424999,64.925003, $0.655,1.03$, SURF_ID =' 'UPHOLSTERY' / Back

*** GROUP: COUCH2 offset $=0.000000,15.000000,0.000000$ rotate $=90.000000$

\&OBST XB $=43.049999,44.174999,66.050003,66.425003,0.28,1.03$, SURF_ID=' 'UPHOLSTERY'/ Left Arm \&OBST XB $=43.049999,44.174999,66.425003,67.925003,0.28,0.655$, SURF_ID=' 'UPHOLSTERY '/ Base

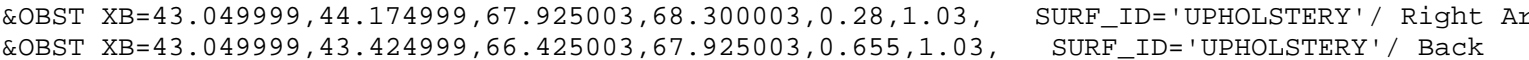

*** GROUP: COUCH2 offset $=0.000000,18.000000,0 . .000000$ rotate $=90.000000$

\&OBST XB $=43.049999,44.174999,69.050003,69.425003,0.28,1.03, \quad$ SURF ID = ' UPHOLSTERY ' / Left Arm

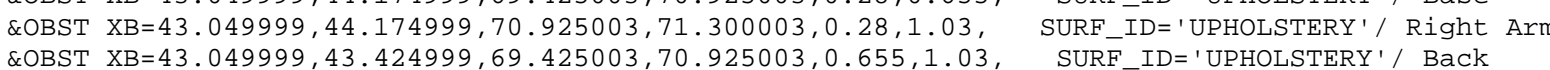

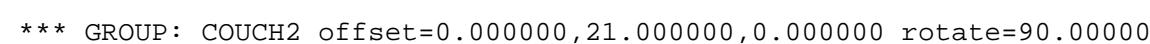

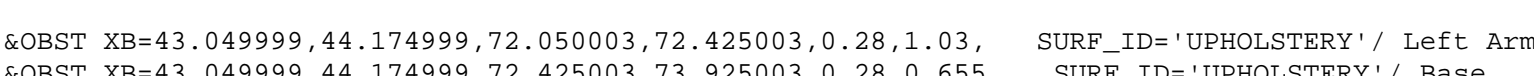

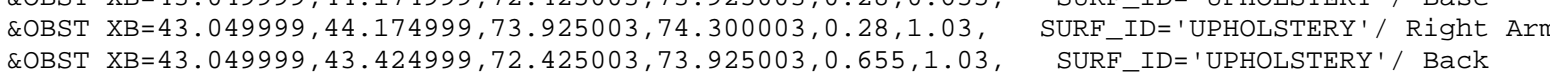

*** GROUP: COUCH2 offset $=0.000000,24.000000,0.000000$ rotate $=90,000000$

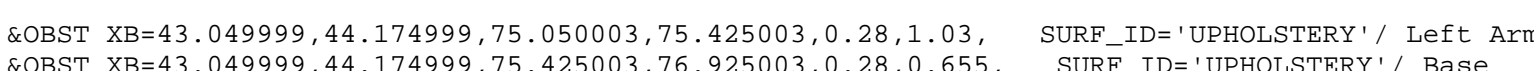

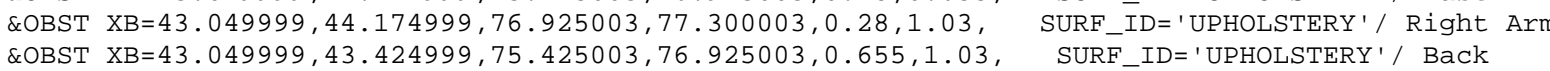

*** GROUP: COUCH2 offset $=0.000000,27.000000,0.000000$ rotate $=90.000000$

\&OBST XB=43. $049999,44.174999,78.050003,78.425003,0.28,1.03$, SURF_ID=' 'UPHOLSTERY' ' Left Arm

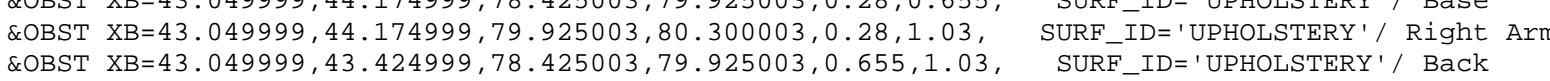
*** GROUP: COUCH2 offset $=0.000000,30.000000,0.000000$ rotate $=90.000000$

\&OBST XB=43. $049999,44.174999,81.050003,81.425003,0.28,1.03, \quad$ SURF_ID= 'UPHOLSTERY' $/$ 'Left Arm
\&OBST XB=43.04999, 44.174999, 81.425003, 82.925003, $0.28,0.655$,
SURF_ID='UPHOLSTERY' / Base

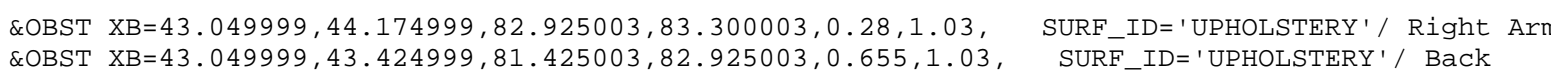
*** GROUP: ROW2

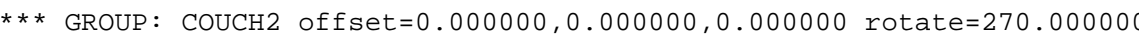

\&OBST XB=41.549999, 42.674999, 52.924999, 53.299999, $0.28,1.03$, SURF_ID=' 'UPHOLSTERY' ' Left Arm \&OBST XB=41.549999, $42.67499,51.049999,51.424999,0.82,1.03, \quad$ SURF_ID= 'UPHOLSTERY'/ Right Arm
\&OBST XB=42.29999, $42.674999,51.424999,52.924999,0.655,1.03$, SURF_ID='UPHOLSTERY'/ Back

*** GROUP: COUCH2 offset $=0.000000,3.000000,0.000000$ rotate $=270.000000$

\&OBST XB $=41.549999,42.674999,55.924999,56.299999,0.28,1.03, \quad$ SURF ID=' 'UPHOLSTERY ' $/$ Left Arm
\&OBST XB $=41.549999,42.674999,54.424999,55.924999,0.28,0.655, \quad$ SURF ID ' 'UPHOLSTERY' $/$ Base

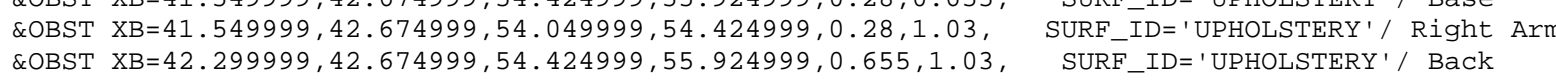

$* *$ GROUP: COUCH2 offset $=0.000000,6.000000,0.000000$ rotate $=270.000000$

EOBST $X \mathrm{X}=41.549999,42.674999,58.924999,59.299999,0.28,1.03, \quad$ SURF ID= 'UPHOLSTERY ' ' Left Arm

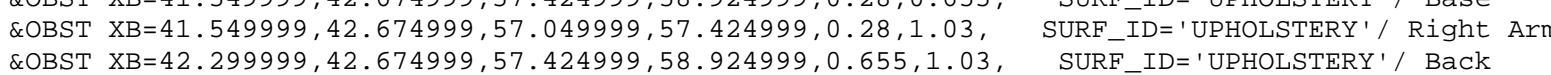

*** GROUP: COUCH2 offset $=0.000000,9.000000,0.000000$ rotate $=270.000000$

\&OBST XB=41.549999, 42.674999, 61.924999, 62. 299999, 0.28, 1. 03, SURF_ID= 'UPHOLSTERY' ' Left Arm \&OBST XB $=41.549999,42.674999,60.049999,60.424999,0.28,1.03$, SURF ID='UPHOLSTERY'/ \&OBST XB=42.299999, 42.674999, 60.424999, 61.924999, $\theta .655,1.03$, SURF_ID='UPHOLSTERY'/ Back

*** GROUP: COUCH2 offset $=0.000000,12.000000,0.000000$ rotate $=270.000000$

\&OBST XB=41.549999, 42.674999, 64.925003, 65. 300003, $, 0.28,1.03, \quad$ SURF_ID='UPHOLSTERY' ' Left Arm
\&OBST XB=41.549999, $42.674999,63.424999,64.925003,0.28,0.655$,
SURF_ID='UPHOLSTERY' $/$ Base \&OBST XB=41. 549999, 42.674999, 63. $049999,63.424999,0.28,1.03$, SURF ID='UPHOLSTERY'/ Rise \&OBST XB=42.299999, 42.674999, 63.424999, 64.925003, $0.655,1.03$, SURF_ID=' 'UPHOLSTERY' / Back

*** GROUP: COUCH2 offset $=0.000000,15.000000,0.000000$ rotate $=270.000000$

\&OBST XB=41.549999, 42.674999, 67. $925003,68.300003,0.28,1.03, \quad$ SURF_ID='UPHOLSTERY' ' Left Arm
\&OBST XB=41.549999, $42.674999,66.425003,67.925003,0.28,0.655$,
SURF_ID=' 'UPHOLSTERY'

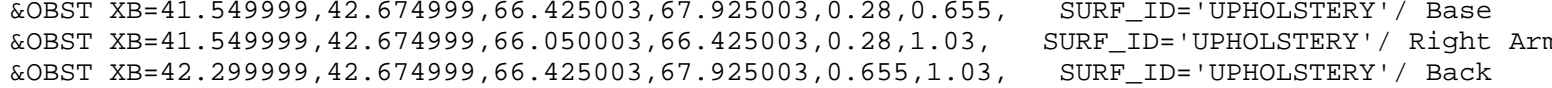

*** GROUP: COUCH2 offset $=0.000000,18.000000,0.000000$ rotate $=270.000000$

\&OBST XB $=41.549999,42.674999,70.925003,71.300003,0.28,1.03$,
\&OBST XB $\quad$ SURF_ID= 'UPHOLSTERY' $/$ Left Arm

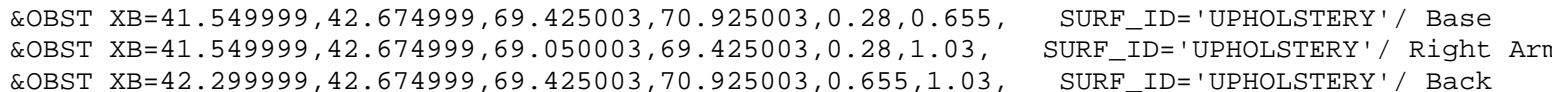

*** GROUP: COUCH2 offset $=0.000000,21.000000$, ,. .000000 rotate $=270.000000$

\&OBST XB $=41.549999,42.674999,73.925003,74.300003,0.28,1.03$, SURF_ID= 'UPHOLSTERY' / Left Arm

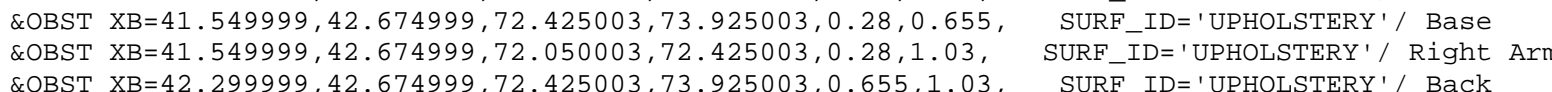

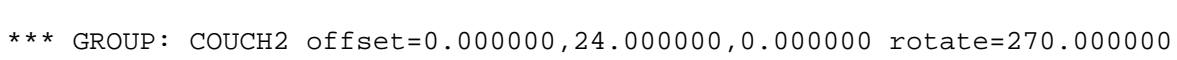

\&OBST XB=41.549999, 42.674999, 76.925003, 77.300003, 0.28,1.03, SURF_ID='UPHOLSTERY'/ Left Arm

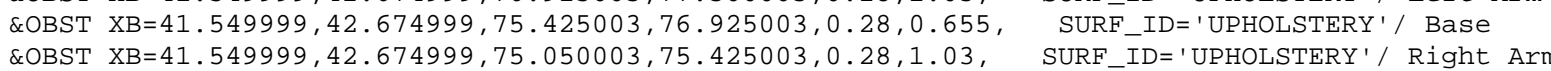
\&OBST XB $X 2.299999,42.674999,75,425003,76.925003,0.655,1.03$, SUPF_ID='UPHOLSTEPY'/ Back A

*** GROUP: COUCH2 offset $=0.000000,27.000000,0.000000$ rotate $=270.000000$

\&OBST XB=41.549999, 42.674999, 79.925003, 80.300003, 0.28,1.03, SURF_ID=' UPHOLSTERY'/ Left Arm \&OBST XB=41.549999, $42.674999,78.425003,79.925003,0.28,0.655$, SURF_ID='UPHOLSTERY' / Base

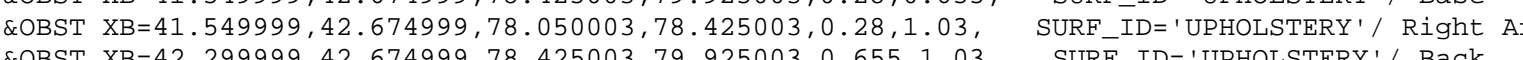

*** GROUP: COUCH2 offset $=0.000000,30.000000,0.000000$ rotate $=270.000000$ 
\&OBST XB=41.549999, 42.674999, 82. $925003,83.300003,0.28,1.03, \quad$ SURF_ID=' 'UPHOLSTERY ' 1 Left Arm
\&OBST XB=41.549999, $42.674999,81.425003,82.925003,0.28,0.655, \quad$ SURF_ID='UPHOLSTERY' / Base

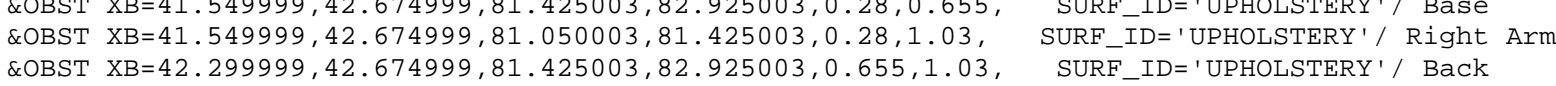
$* \star *$ GROUP: TWIN

$* * *$ GROUP: ROW

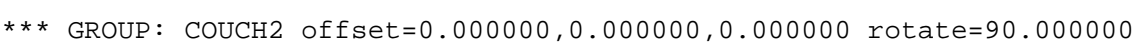

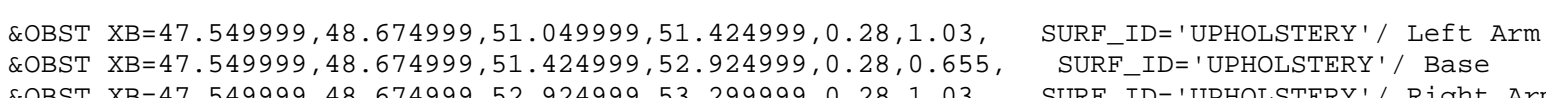

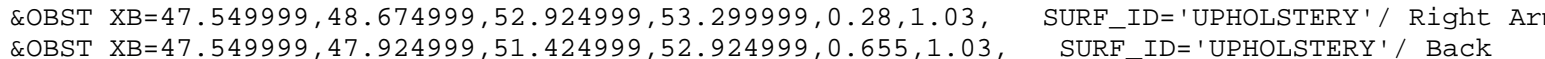

*** GROUP: COUCH2 offset $=0.000000,3.000000,0.000000$ rotate $=90.000000$

\&OBST XB=47.549999, 48.674999, 54. $449999,54.424999,0.28,1.03$, SURF_ID=' 'UPHOLSTERY' ' Left Arm

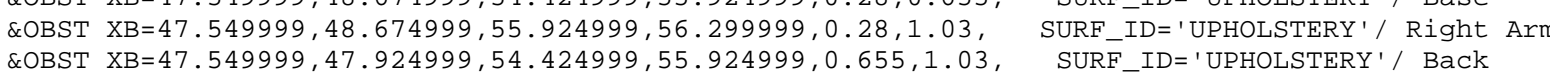
*** GROUP: COUCH2 offset $=0.000000,6.000000,0.000000$ rotate $=90.000000$

\&OBST XB=47.549999, 48.674999, 57. 049999, 57.424999, 0.28, 1. 03 , SURF_ID='UPHOLSTERY' / Left Arm \&OBST XB=47.549999, 48.674999, 58.924999, 59.299999, $0.28,1.03$, SURF_ID='UPHOLSTERY'/ Right Ar \&OBST XB=47.549999, 47. 924999, 57.424999, 58. $924999,0.655,1.03$, SURF_ID-"L

*** GROUP: COUCH2 offset $=0.000000,9.000000,0.000000$ rotate $=90.000000$

\&OBST XB=47.549999, 48.674999, 60. $049999,60.424999,0.28,1.03$, SURF_ID= 'UPHOLSTERY Y / Left Arm

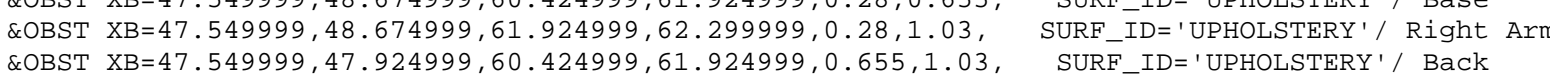

*** GROUP: COUCH2 offset $=0.000000,12.000000,0.000000$ rotate $=90.000000$

\&OBST XB=47.549999, 48.674999, 63. 049999, 63. 424999, $0.28,1.03$, SURF_ID= 'UPHOLSTERY' ', Left Arm

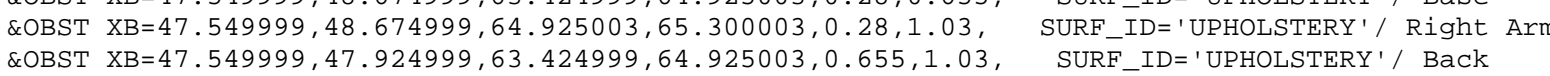

*** GROUP: COUCH2 offset $=0.000000,15.000000,0.000000$ rotate $=90.000000$

\&OBST XB=47.549999, 48.674999, 66. $050003,66.425003,0.28,1.03, \quad$ SURF_ID=' 'UPHOLSTERY' $/$ Left Arm
\&OBST XB=47.549999, $48.674999,66.425003,67.925003,0.28,0.655, \quad$ SURF_ID='UPHOLSTERY' Rase

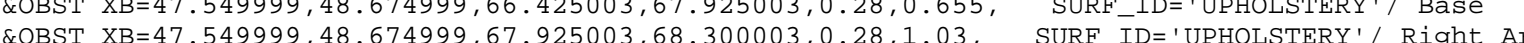
\&OBST XB $=47.549999,47.924999,66.425003,67.925003,0.655,1.03, \quad$ SURF_ID='UPHOLSTERY'/ Back

*** GROUP: COUCH2 offset $=0.000000,18.000000,0.000000$ rotate $=90$. . 000000

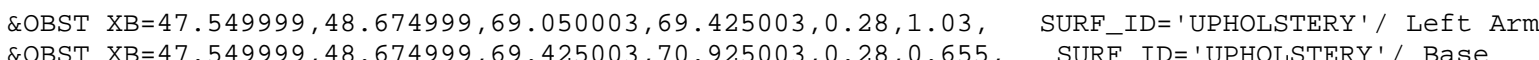

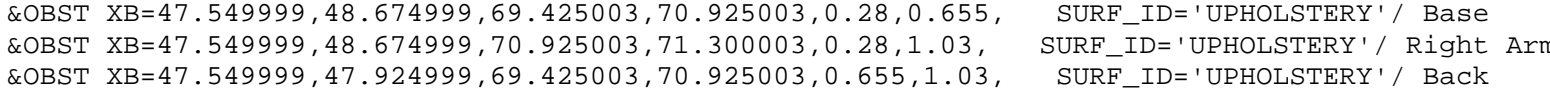

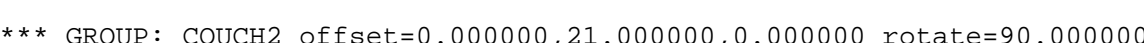

\&OBST XB $=47.549999,48.674999,72.050003,72.425003,0.28,1.03, \quad$ SURF-ID=' 'UPHOLSTERY'/ Left Arm

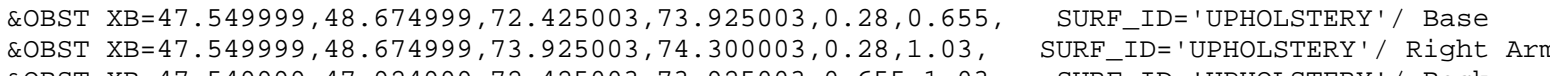
\&OBST $X B=47.549999,47.924999,72.425003,73.925003,0.655,1.03$, SURF_ID='UPPOLSTERY'/ Back

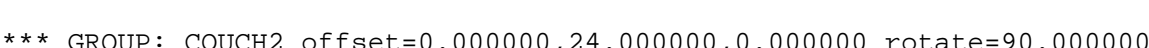
\&OBST XB $=47.549999,48.674999,75.050003,75.425003,0.28,1.03, \quad$ SURF_ID=' 'UPHOLSTERY ' $/$ Left Arm
\&OBST XB $47.549999,48.67499,775.425503,76.925003,0.28,0.655, \quad$ SURF_ID=' 'UPHOLSTERY' $/$ Base

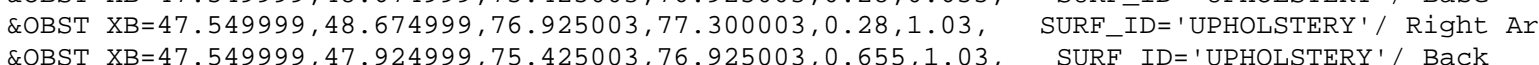

*** GROUP: COUCH2 offset $=0.000000,27.000000,0.000000$ rotate $=90 . .000000$

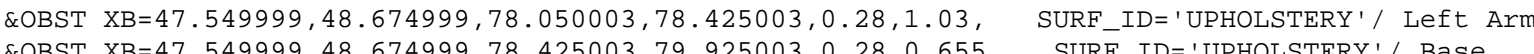

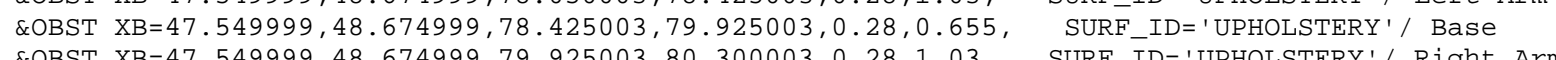

\&OBST XB $=47.549999,47,924999,78,425003,79,925003,0.655,1,03$, SURF_ID=' UPHOLSTERY' / Back *** GROUP: COUCH2 offset $=0.000000,30.000000,0.000000$ rotate $=90.000000$

\&OBST XB=47.549999, 48.674999, 81.050003, 81.425003, 0.28,1.03, SURF_ID='UPHOLSTERY'/ Left Arm \&OBST XB $=47.549999,48.674999,81.425003,82.925003,0.28,0.655$, SURF_ID='UPHOLSTERY'/ Base \&OBST XB=47.549999, 47.924999, 81.425003, 82.925003, $0.655,1.03$, SURF_ID=' 'UPHOLSTERY' / Back

*** GROUP: ROW2

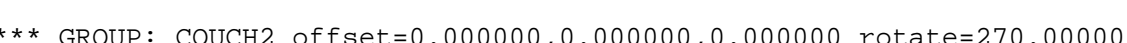

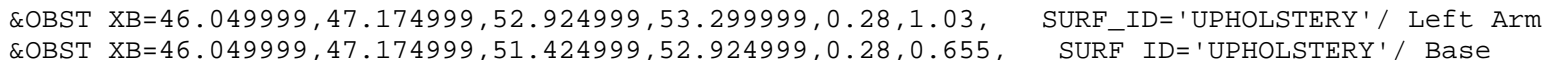

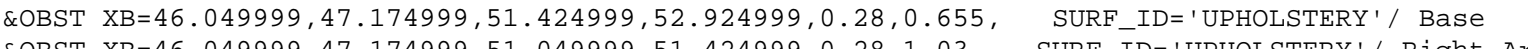
\&OBST XB=46. $849999,47.174999,51.049999,51.424999,0.28,1.03$,
\&OBST $X B=46.799999,47.174999,51.424999,52.924999,0.655,1.03$,
SURF_IDE'ID 'UPHOLSTERY' $/$ Back

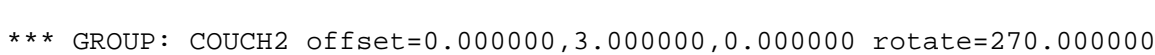

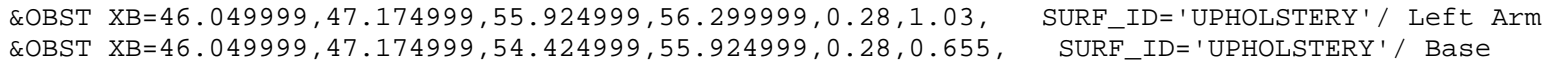
\&OBST XB=46.04999, 47.174999, 54.424999, 55. 224999, $0.28,0.655$, SURF ID='UPHOLSTERY' / Base \&OBST XB=46.799999, 47.174999, 54.424999, 55.924999, $0.655,1.03$, SURF_ID=' 'UPHOLSTERY' / Back

** GROUP: COUCH2 offset $=0.000000,6.000000$, ๑. .000000 rotate $=270.000000$

\&OBST XB $=46.049999,47.174999,58.924999,59.299999,0.28,1.03$,
SOBST

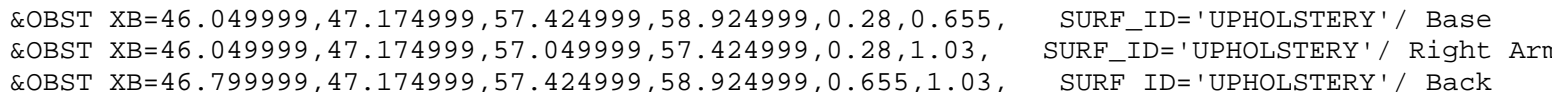

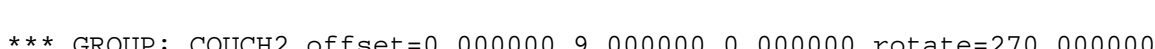

\&OBST XB=46.049999, 47.174999, 61.924999, 62.299999, 0.28,1.03, SURF_ID=' 'UPHOLSTERY ' / Left Arm QOBST XB $=46.04999,47.17499,00.424999,61.224999,0.28,0.055$, SURF_ID='UPHOLSTERY'/ Base EOBST XB=46. $049999,47.174999,60.049999,60.424999,0.28,1.03, \quad$ SURF_ID= 'UPHOLSTERY'/ Right Ar
EOBST XB $=46.799999,47.17499,60.424999,61.924999,0.655,1.03$, SURF_ID 'UPHOLSTERY' $/$ Back

*** GROUP: COUCH2 offset $=0.000000,12.000000,0.000000$ rotate $=270.000000$

\&OBST XB $=46.049999,47.174999,64.925003,65.300003,0.28,1.03$, SURF_ID= 'UPHOLSTERY'/ Left Arm \&OBST XB=46.049999, 47.174999,63.424999,64.925003, 0.28, 0.655, SURF_ID='UPHOLSTERY' / Base

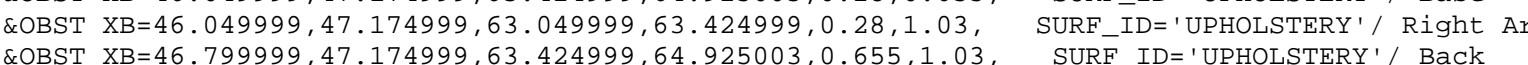

*** GROUP: COUCH2 offset $=0.000000,15.000000,0.000000$ rotate $=270.0000000$

\&OBST XB=46. 049999, 47.174999, 67.925003, 68. 300003, 0.28,1.03, SURF_ID=' UPHOLSTERY'/ Left Arm \&OBST XB=46.049999, 47.174999,66.425003, 67.925003, $0.28,0.655$, SURF_ID=' 'UPHOLSTERY' / Base \&OBST X $\mathrm{X}=46.049999,47.174999,66.050003,66.425003,0.28,1.03$, SURF_ID='UPHOLSTERY'/Right Ar

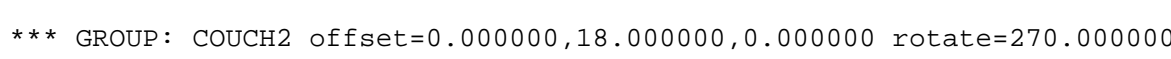

\&OBST XB=46. $049999,47.174999,70.925003,71.300003,0.28,1.03, \quad$ SURF ID= 'UPHOLSTERY ' $/$ Left Arm \&OBST XB $=46.049999,47.174999,69.425003,70.925003,0.28,0.655$, SURF_ID=' 'UPHOLSTERY'/ Base

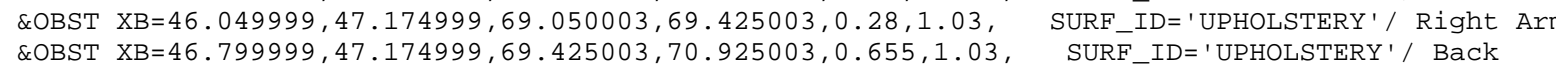

*** GROUP: COUCH2 offset $=0.000000,21.000000,0.000000$ rotate $=270.000000$

\&OBST XB=46. $049999,47.174999,73.925003,74.300003,0.28,1.03$, SURF_ID=' 'UPHOLSTERY ' / Left Arm \&OBST XB=46.049999, 47.174999, 72.425003,73.925003, $0.28,0.655$, SURF_ID='UPHOLSTERY'/ Base

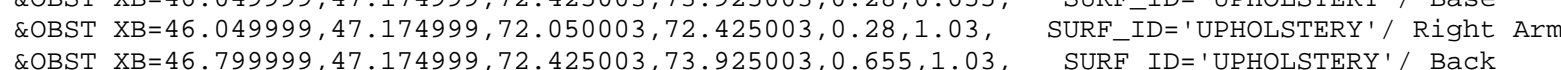

*** GROUP: COUCH2 offset $=0.000000,24.000000,0.000000$ rotate $=270.000000$

\&OBST XB=46. $049999,47.174999,76.925003,77.300003,0.28,1.03$, SURF_ID= 'UPHOLSTERY' ' Left Arm \&OBST XB $46.0499999,47.174999,75.050003,75.425003,0.28,1.03$, SURF_IDD'UPHOLSTERY'/ Right Ar

*** GROUP: COUCH2 offset $=0.000000,27.000000,0.000000$ rotate $=270.000000$

\&OBST XB=46. $049999,47.174999,79.925003,80.300003,0.28,1.03$, SURF_ID=' 'UPHOLSTERY'/ Left Arm 


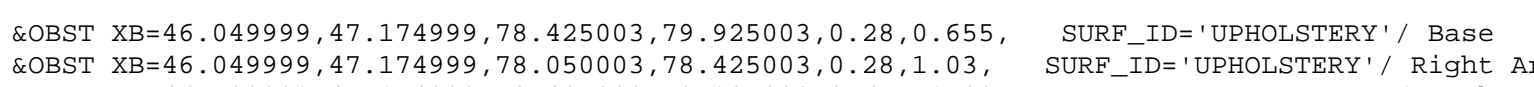

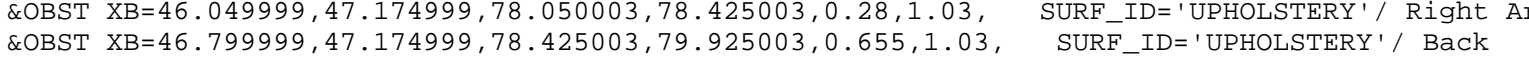

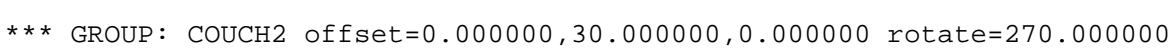

\&OBST XB=46. $049999,47.174999,82.925003,83.300003,0.28,1.03, \quad$ SURF_ID= 'UPHOLSTERY' 1 ' Left Arm
\&OBST XB=46.049999, 47.174999, $81.425003,82.925003,0.28,0.655$,
SURF_ID=' 'UPHOLSTERY'/ Base RoBST XB $=46.049999,47.174999,81.050003,81.425003,0.28,1.03$,
\&OBST XB $=46.799999,47.17499,81.425003,82.925003,0.655,1.03$,

\section{$* * *$ GROUP: TWIN}

$* * *$ GROUP: ROW

*** GROUP: COUCH2 offset $=0.000000,0.000000,0.000000$ rotate $=90.000000$

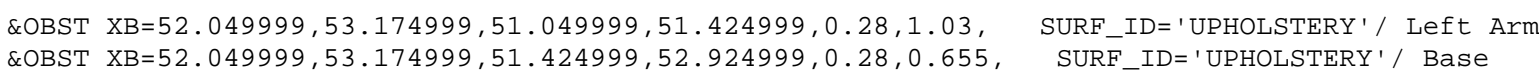

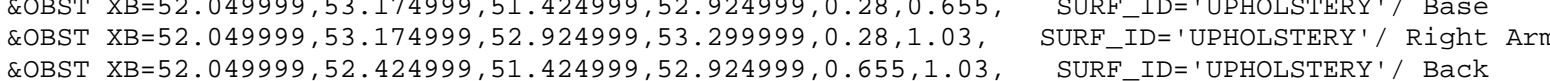
*** GROUP: COUCH2 offset $=0.000000,3.000000,0.000000$ rotate $=90.000000$

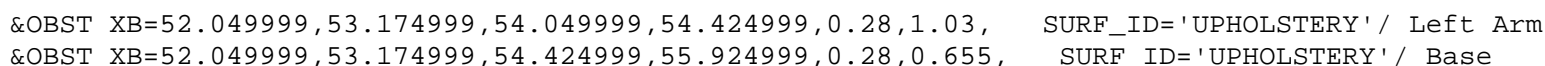

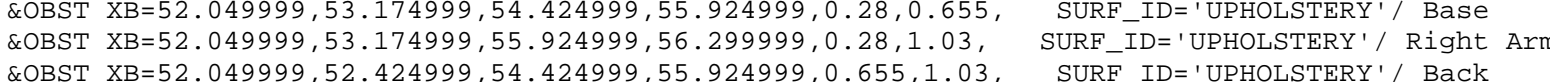

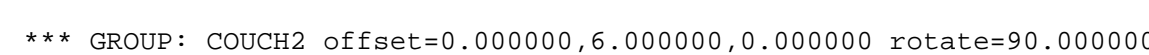

\&OBST XB=52. $049999,53.174999,57.049999,57.424999,0.28,1.03$, SURF_ID=' 'UPHOLSTERY' ' Left Arm

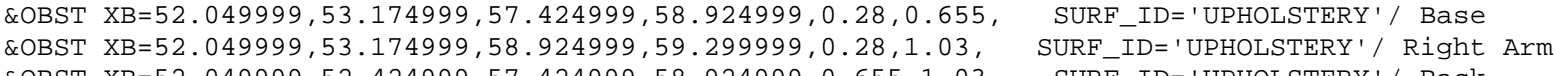

*** GROUP: COUCH2 offset $=0.000000,9.000000,0.0000000$ rotate $=90.000000$

\&OBST XB=52.049999, 53.174999, 60.049999, 60.424999, $0.28,1.03$, SURF_ID='UPHOLSTERY'/ Left Arm

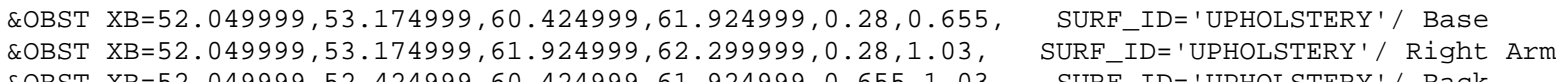

$* * *$ GROUP: COUCH2 offset $=0.000000,12.000000,0.000000$ rotate $=90.000000$

\&OBST XB=52. $049999,53.174999,63.049999,63.424999,0.28,1.03, \quad$ SURF_ID=' 'UPHOLSTERY ' 1 Left Arm
ROBST XB=52.049999, $53.174999,63.424999,64.925003,0.28,0.655, \quad$ SURF_ID=' 'UPHOLSTERY' / Base

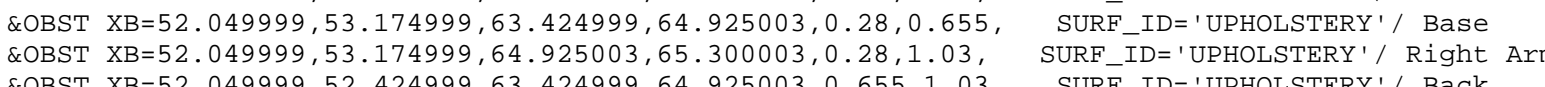

${ }_{* * *}$ GROUP: COUCH2 offset $=0.000000,15.000000,0.000000$ rotate $=90.000000$

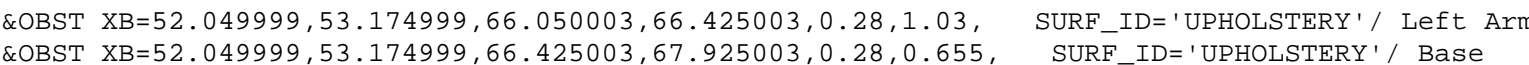
\&OBST XB =52.049999, 53.174999, 67.925003,68.300003, $0.28,1.03$, SURF-ID='UPHOLSTERY'/ Right Ar

*** GROUP: COUCH2 offset $=0.000000,18.000000,0.000000$ rotate $=90.000000$ \&OBST $X \mathrm{XB}=52.049999,53.174999,69.050003,69.425003,0.28,1.03, \quad$ SURF_ID=' 'UPHOLSTERY' $/$ Left Arm
\&OBST XB=52.049999, $53.174999,69.425003,70.925003,0.28,0.655, \quad$ SURF_ID=' 'UPHOLSTERY' / Base

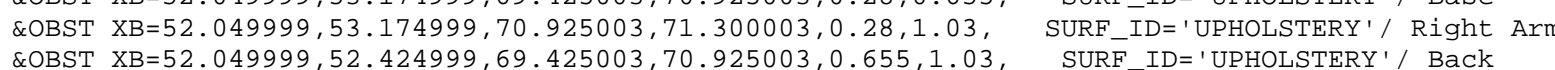

*** GROUP: COUCH2 offset $=0.000000,21.000000,0.000000$ rotate $=90.000000$

\&OBST XB=52. $449999,53.174999,72.050003,72.425003,0.28,1.03$, SURF_ID='UPHOLSTERY'/ Left Arm \&OBST XB=52. $049999,53.174999,72.425003,73.925003,0.28,0.655$, SURF_ID='UPHOLSTERY'/ Base \&OBST XB $52.049999,53.174999,72.925003,74.300003,0.28,1.03$, S SURF_ID='UPHOLSTERY'/ Right Ar *** GROUP: COUCH2 offset $=0.000000,24.000000,0.000000$ rotate $=90.000000$

\&OBST XB=52. $049999,53.174999,75.050003,75.425003,0.28,1.03$, SURF_ID='UPHOLSTERY'/ Left Arm

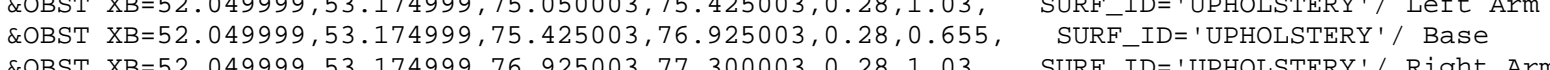

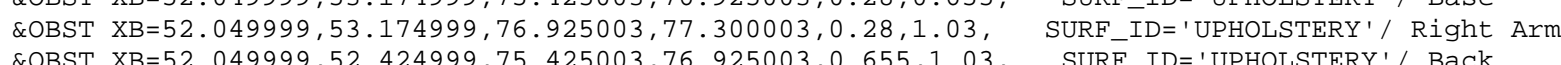

*** GROUP: COUCH2 offset $=0.000000,27.000000,0.000000$ rotate $=90.000000$

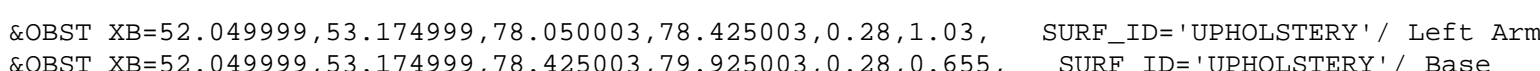

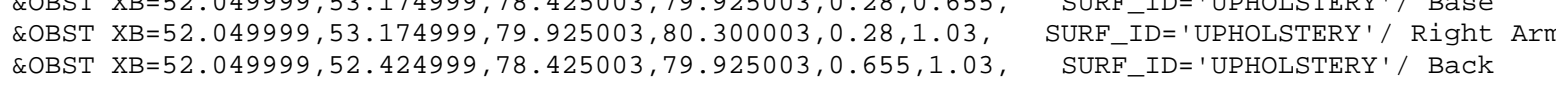
*** GROUP: COUCH2 offset $=0.000000,30.000000,0.000000$ rotate $=90.000000$

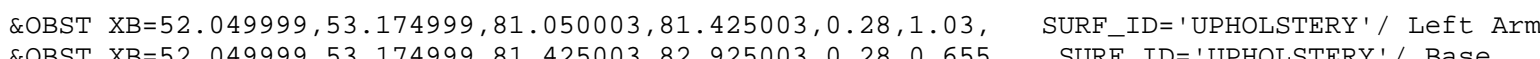

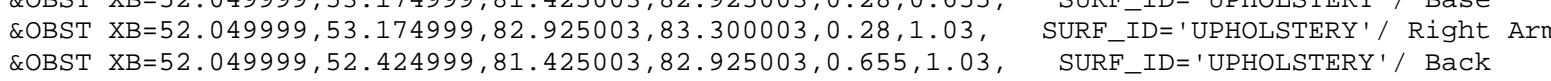

*** GROUP: ROW2

*** GROUP: COUCH2 offset $=0.000000,0.000000, \odot . .000000$ rotate $=270 . .000000$

\&OBST XB=50.549999, 51.674999, 52.924999, 53. 299999, $0.28,1.03, \quad$ SURF_ID= 'UPHOLSTERY'/ Left Arm
\&OBST XB=50.54999, $51.674999,51.424999,52.924999,0.28,0.655, \quad$ SURFID=' 'UPHOLSTERY'/ Base

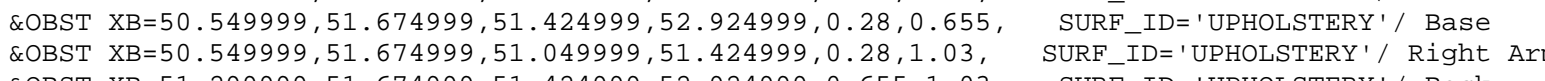

$* * *$ GROUP: COUCH2 offset $=0.000000,3.000000,0.000000$ rotate $=270.000000$

\&OBST XB=50.549999, 51.674999, 55. 924999, 56.299999, $0.28,1.03$, SURF_ID='UPHOLSTERY'/ Left Arm

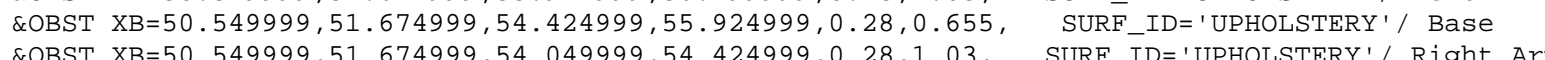
\&OBST XB=50.54999, $51.674999,54.049999,54.424999,0.28,1.03, \quad$ SURF_ID='UPHOLSTERY'/ Right Arn
\&OBST XB=51.299999, $51.674999,54.424999,55.924999,0.655,1.03, \quad$ SURF_ID='UPHOLSTERY'/ Back

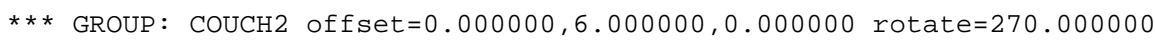

\&OBST XB=50.549999, 51.674999, 58.924999, 59.299999, 0.28,1.03, SURF_ID=' UPHOLSTERY'/ Left Arm \&OBST XB=50.549999, 51.674999, $57.424999,58.924999,0.28,0.655$, SURF_ID='UPHOLSTERY'/ Base

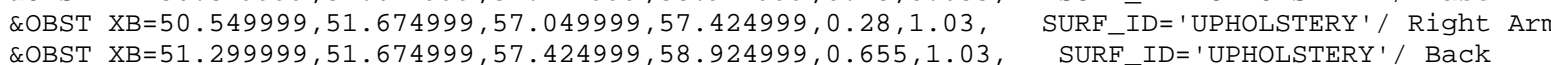

*** GROUP: Couch2 offset $=0.000000,9.000000,0.000000$ rotate $=270.000000$

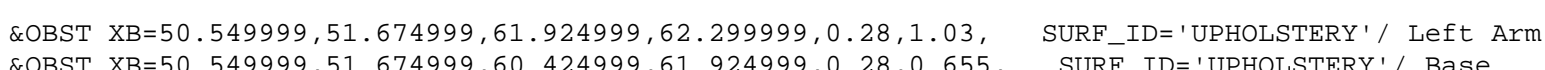

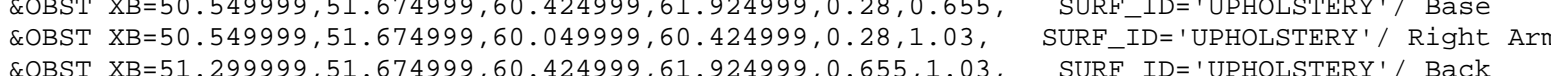

*** GROUP: COUCH2 offset $=0.000000,12.000000,0.000000$ rotate $=270.000000$

\&OBST XB=50.549999, 51.674999, 64.925003, 65.300003, $0.28,1.03$, SURF_ID=' 'UPHOLSTERY ' / Left Arm

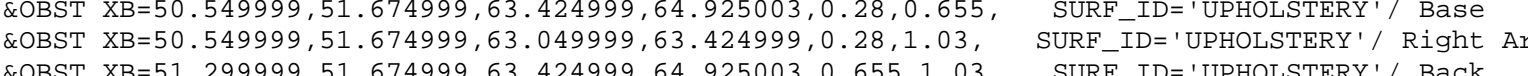

$* * *$ GROUP: COUCH2 offset $=0.000000,15.000000,0.000000$ rotate $=270.000000$

\&OBST XB=50.549999, 51.674999, 67.925003, 68.300003, $0.28,1.03$, SURF_ID=' 'UPHOLSTERY' / Left Arm \&OBST XB=50.549999, 51.674999, 66.050003, 66.425003,0.28,1.03, SURF_ID='UPHOLSTERY'/ Right Ar \&OBST XB $=51.299999,51.674999,66.425003,67.925003,0.655,1.03$, SURF_ID ='UPHOLSTERY'/ Back

*** GROUP: COUCH2 offset $=0.000000,18.000000,0.000000$ rotate $=270,000000$

\&OBST XB=50.549999, 51.674999, 70.925003, 71.300003, $0.28,1.03$, SURF_ID=' 'UPHOLSTERY' ' Left Arm

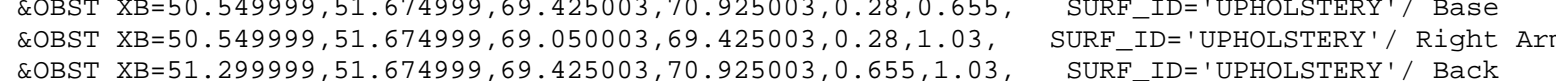

*** GROUP: COUCH2 offset $=0,000000,21,000000,0,000000$ rotate $=270,000000$

\&OBST XB=50.549999, 51.674999, 73.925003, 74.300003, 0.28, 1.03, SURF_ID=' 'UPHOLSTERY' / Left Arm \&OBST XB=50.54999, $51.674999,72.050003,72.425003,0.28,1.03, \quad$ SURF_ID=' 'UPHOLSTERY'/ Right Arm
\&OBST XB $\mathrm{X}=51.299999,51.674999,72.425003,73.925003,0.655,1.03, \quad$ SURF_ID=' 'UPHOLSTERY'/ Back *** GROUP: COUCH2 offset $=0.000000,24.000000,0.000000$ rotate $=270.000000$

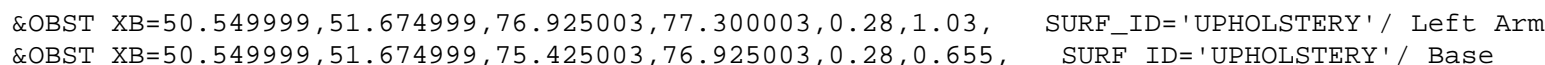


\&OBST XB=50.549999, 51.674999, 75. $050003,75.425003,0.28,1.03, \quad$ SURF_ID= 'UPHOLSTERY' $/$ Right Arm
\&OBST XB=51.29999, $51.674999,75.425003,76.925003,0.655,1.03, \quad$ SURF_ID='UPHOLSTERY'/ Back *** GROUP: COUCH2 off set $=0.0000000,27.000000,0.000000$ rotate $=270.000000$

\&OBST $X \mathrm{XB}=50.549999,51.674999,79.925003,80.300003,0.28,1.03, \quad$ SURF_ID='UPHOLSTERY '/ Left Arm \&OBST XB=50.54999, 51.674999, 78.425003, 79. $925003,0.28,0.655, \quad$ SURF_ID='UPHOLSTERY'/ Base
\&OBST XB=50.549999, $51.674999,78.050003,78.425003,0.28,1.03$,

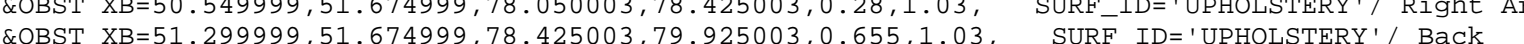

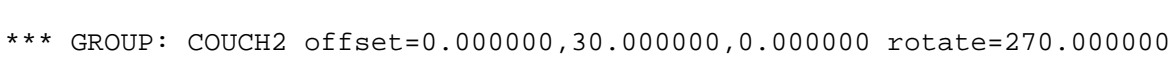

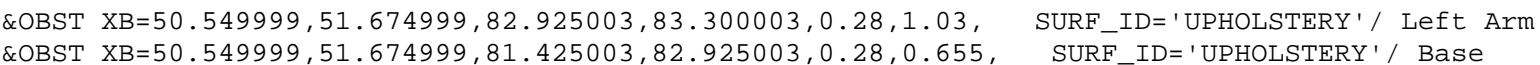
\&OBST XB=50.549999, 51.674999, 81. $050003,81.425003,0.28,1.03$, SURF_ID='UPHOLSTERY' '/ Right Aru $* * *$ GROUP: TWIN

*** GROUP: ROW

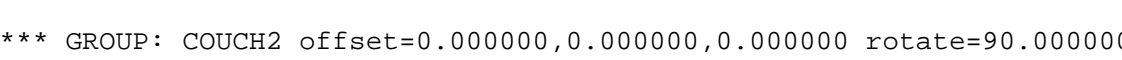

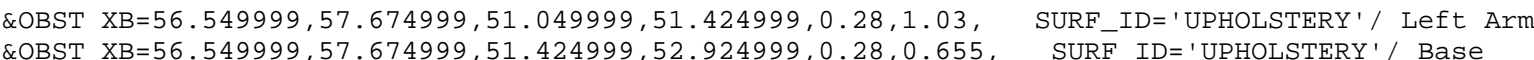
LOBST XB $=56.549999,57.674999,51.424999,52.924999,0.28,0.655, \quad$ SURF_ID='UPHOLSTERY'/ Base
\&OBST XB $=56.549999,57.674999,52.924999,53.299999,0.28,1.03$,
SURF_ID='UPHOLSTERY'/ Right Arm

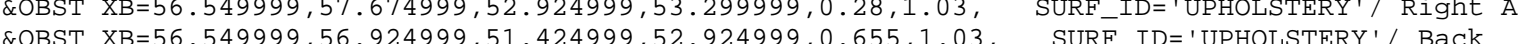
*** GROUP: COUCH2 offset $=0.000000,3.000000,0.0000000$ rotate $=90.000000$ \&OBST XB=56. $549999,57.674999,54.049999,54.424999,0.28,1.03, \quad$ SURF_ID=' 'UPHOLSTERY' 1 ' Left Arm
\&OBST XB=56.549999, $57.674999,54.424999,55.924999,0.28,0.655$,
SURF_ID='UPHOLSTERY'/ Base

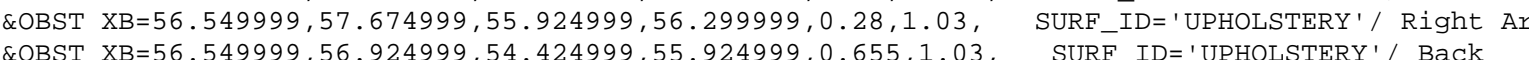

*** GROUP: COUCH2 offset $=0.000000,6.000000,0.000000$ rotate $=90.000000$

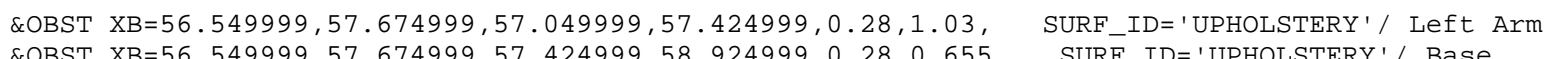
\&OBST XB=56.549999, $57.674999,58.924999,59.299999,0.28,1.03$, SURF_ID='UPHOLSTERY'/ Base SUF
IDE 'UPHOLSTERY' / Back

$* * *$ GROUP: COUCH2 offset $=0.000000,9.000000,0.000000$ rotate $=90.000000$

\&OBST XB=56.549999, 57.674999, 60. 049999, 60.424999, 0.28,1.03, SURF_ID='UPHOLSTERY '/ Left Arm

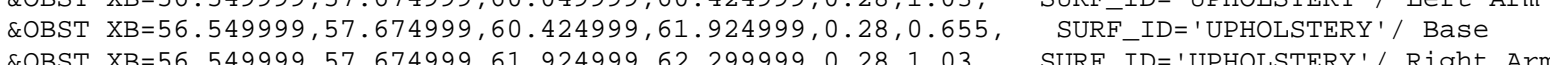

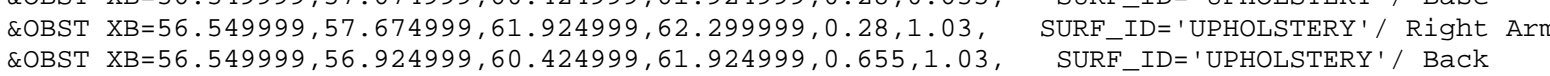

$* * *$ GROUP: COUCH2 offset $=0.000000,12.000000,0.000000$ rotate $=90.000000$

\&OBST XB=56.549999, 57.674999, 63. $049999,63.424999,0.28,1.03$, SURF_ID=' 'UPHOLSTERY'/ Left Arm \&OBST XB=56.549999, $57.674999,63.424999,64.925003,0.28,0.655, \quad$ SURF_ID='UPHOLSTERY'/ Base \&OBST XB=56.54999, 57.674999, 64.925003, 65.300003, $0.28,1.03$, SURF_ID='UPHOLSTERY'/ Right Ar
\&OBST XB=56.549999, $56.924999,63.424999,64.925003,0.655,1.03, \quad$ SURF ID=' 'UPHOLSTERY' / Back

*** GROUP: COUCH2 offset $=0.000000,15.000000,0.000000$ rotate $=90.000000$

\&OBST XB $=56.549999,57.674999,66.050003,66.425003,0.28,1.03, \quad$ SURF ID= 'UPHOLSTERY ' / Left Arm

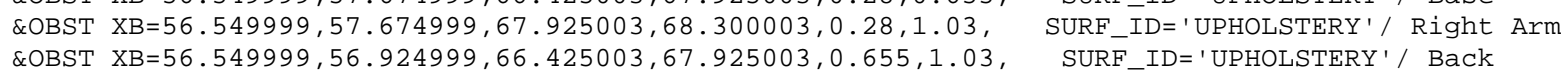
*** GROUP: COUCH2 offset $=0.000000,18.000000, \Theta .000000$ rotate $=90.000000$

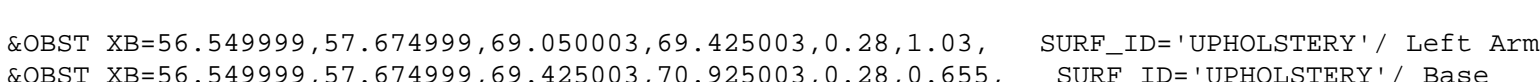
\&OBST XB $=56.549999,57.674999,70.925003,71.300003,0.28,1.03, \quad$ SURF_ID='UPHOLSTERY'/ Right Arm
\&OBST XB $=56.549999,56.924999,69.425003,70.925003,0.655,1.03, \quad$ SURF_ID='UPHOLSTERY'/ Back

*** GROUP: COUCH2 offset $=0.000000,21.000000,0.000000$ rotate $=90.000000$

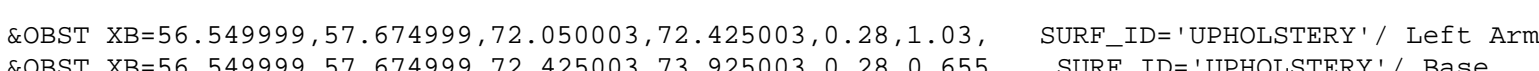

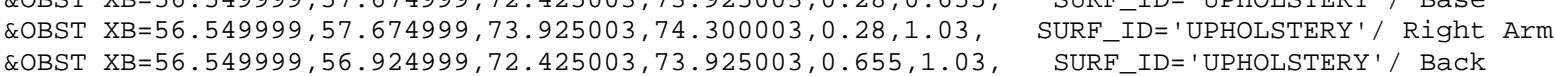

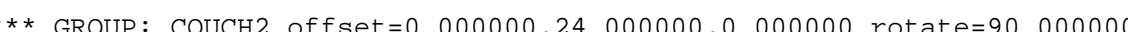

\&OBST XB=56.549999, 57.674999, 75. $050003,75.425003,0.28,1.03, \quad$ SURF_ID='UPHOLSTERY' ' Left Arm
\&OBST XB=56.549999, $57.674999,75.425003,76.925003,0.28,0.655$,
SURF_ID=' 'UPHOLSTERY' \&OBST XB $=56.549999,57.674999,76.925003,77.300003,0.28,1.03$, SURF-ID='UPHOLSTERY'/ Right Arm

*** GROUP: COUCH2 offset $=0.000000,27.000000,0.000000$ rotate $=90.000000$

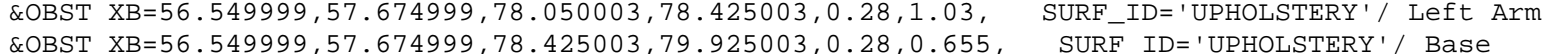

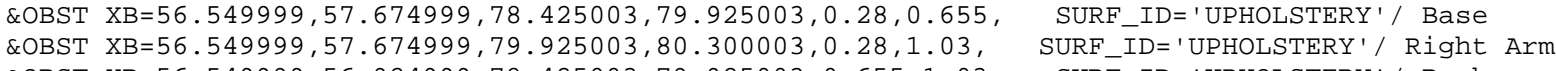
\&OBST XB=56.549999, 56.924999, 78.425003, 79.925003, 0.655, 1.03 , SURF_ID=' 'UPHOLSTERY'

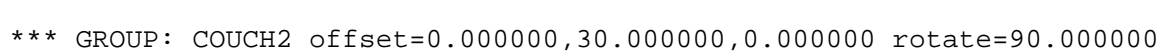

\&OBST XB=56.549999, $57.674999,81.050003,81.425003,0.28,1.03, \quad$ SURF_ID=' 'UPHOLSTERY'/' Left Arm
\&OBST X X=56.549999, $57.674999,81.425003,82.925003,0.28,0.655$,
SURF_ID='UPHOLSTERY'/ Base \&OBST XB $=56.549999,57.674999,82.925003,83.300003,0.28,1.03$, SURF ID ' 'UPHOLSTERY'/Right Ar

*** GROUP: ROW2

*** GROUP: COUCH2 offset $=0.000000,0.000000,0.000000$ rotate $=270.000000$

\&OBST XB $=55.049999,56.174999,52.924999,53.299999,0.28,1.03, \quad$ SURF ID=' 'UPHOLSTERY ' ' Left Arm

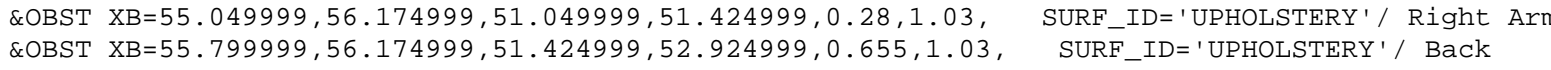

*** GROUP: COUCH2 offset $=0.000000,3.000000,0.000000$ rotate $=270.000000$

\&OBST XB=55. $049999,56.174999,55.924999,56.299999,0.28,1.03$, SURF_ID=' 'UPHOLSTERY' ' Left Arm

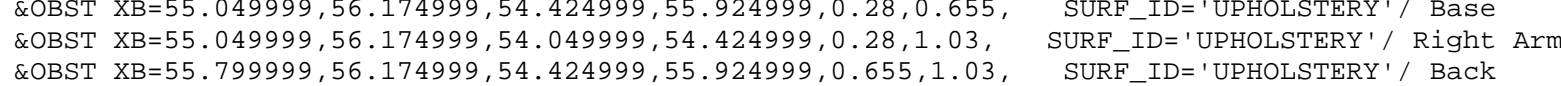
*** GROUP: COUCH2 offset $=0.000000,6.000000,0.000000$ rotate $=270.000000$

\&OBST XB=55. $049999,56.174999,58.924999,59.299999,0.28,1.03$, SURF_ID= 'UPHOLSTERY' / Left Arm \&OBST XB 5 . \&OBST XB=55.799999, 56.174999, 57.424999, 58.924999, $0.655,1.03$, SURF_ID='UPHOLSTERY'/ Back

*** GROUP: COUCH2 offset $=0.000000,9.000000,0.000000$ rotate $=270.000000$

\&OBST XB=55. 049999, 56. 174999, 61. 924999, 62. 299999, 0.28, 1. 03, SURFIDD=' UPHOLSTERY' ' Left Arm \&OBST $X \mathrm{~B}=55$. \&OBST XB=55.799999, 56.174999, 60.424999, 61.924999, $0.655,1.03$, SURF_ID='UPHOLSTERY'/ Back

*** GROUP: COUCH2 offset $=0.0000000,12.000000,0.000000$ rotate $=270.000000$

\&OBST XB=55. $049999,56.174999,64.925003,65.300003,0.28,1.03, \quad$ SURF_ID= 'UPHOLSTERY' $/$ Left Arm
\&OBST XB=55.04999, $56.174999,63.424999,64.925003,0.28,0.655, \quad$ SURF ID=' 'UPHOLSTERY' Rese

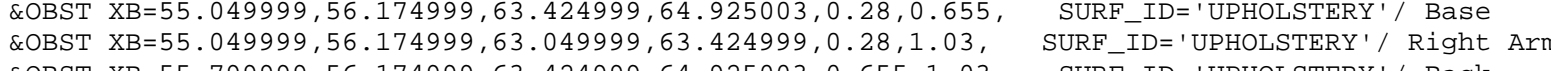

$* * *$ GROUP: COUCH2 offset $=0.000000,15.0000000,0.000000$ rotate $=270.000000$

\&OBST XB $=55.049999,56.174999,67.925003,68.300003,0.28,1.03, \quad$ SURF_ID=' UPHOLSTERY' ' Left Arm
\&OBST XB $=55.049999,56.174999,66.425003,67.025003,0.28,0.655, \quad$ SURF_ID=' 'UPHOLSTERY' $/$ Base

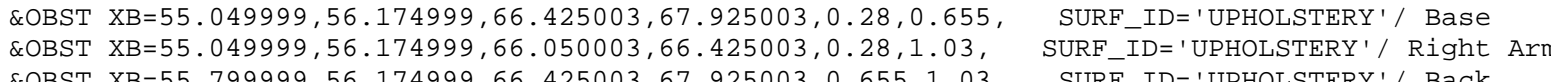

*** GROUP: COUCH2 offset $=0.000000,18.000000, \odot .000000$ rotate $=270.0000000$

\&OBST XB=55.049999, 56.174999, 70.925003,71.300003, 0.28,1.03, SURF_ID=' 'UPHOLSTERY' / Left Arm

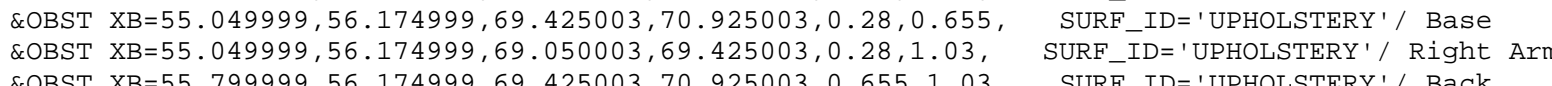

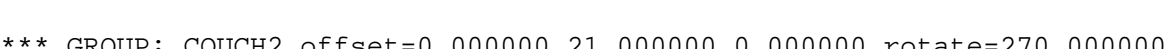
$-14$

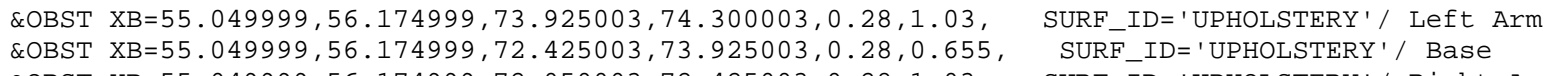


SOBST XB $=55,799999,56.174999,72,425003,73,925003,0.655,1.03$, SURF_ID=' UPHOLSTERY' / Back *** GROUP: COUCH2 offset $=0.000000,24.000000,0.000000$ rotate $=270$. ๑०००००

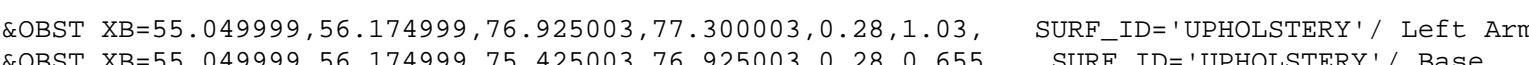

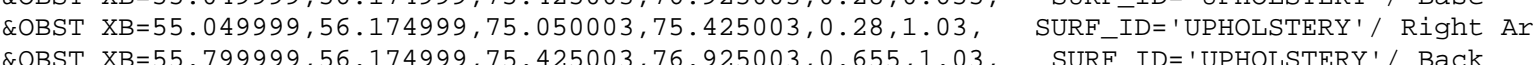

*** GROUP: COUCH2 offset $=0.000000,27.000000,0.000000$ rotate $=270.000000$

\&OBST XB=55. 049999, $56.174999,79.925003,80.300003,0.28,1.03$, SURF_ID='UPHOLSTERY'/ Left Arm \&OBST XB $=55.049999,56.174999,78.425003,79.925003,0.28,0.655$, SURF_ID=' 'UPHOLSTERY ' / Base \&OBST XB $=55.049999,56.174999,78.050003,78.425003,0.28,1.03$,
\&OBST XB $=55.799999,56.174999,78.425003,79.925003,0.655,1.03, \quad$ SURF_ID='UPHOLSTERY'/ Right Arm
SURF ID=' 'UPHOLSTERY'/ Back

*** GROUP: COUCH2 offset $=0.000000,30.000000,0.000000$ rotate $=270.000000$

\&OBST XB=55. $049999,56.174999,82.925003,83.300003,0.28,1.03$, SURF_ID=' UPHOLSTERY' ' Left Arm \&OBST XB=55.049999, 56.174999, 81.050003, 81.425003, 0.28, 1.03, SURF_ID='UPHOLSTERY'/ Right Ar

$* * *$ GROUP: TWIN

*** GROUP: ROW

*** GROUP: COUCH2 offset $=0.000000,0.000000,0.000000$ rotate $=90.000000$

\&OBST XB=61. 049999, 62.174999, 51. $049999,51.424999,0.28,1.03$, SURF_ID=' 'UPHOLSTERY'/ Left Arm \&OBST XB=61. $049999,62.174999,51.424999,52.924999,0.28,0.655$, SURF IID= 'UPHOLSTERY' / Base

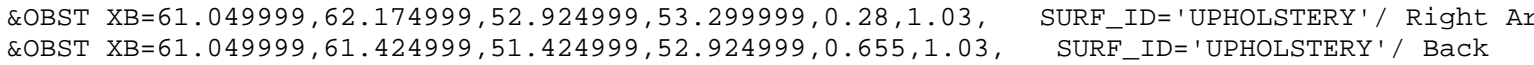

$* * *$ GROUP: COUCH2 offset $=0.000000,3$. .

\&OBST XB=61. $049999,62.174999,54.049999,54.424999,0.28,1.03$, SURF_ID=' 'UPHOLSTERY' / Left Arm

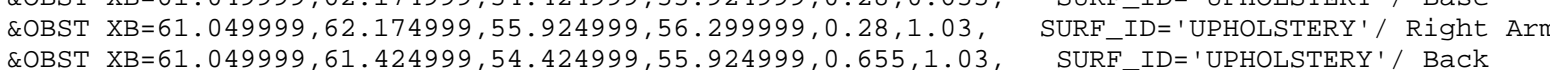

*** GROUP: COUCH2 offset $=0.000000,6.000000,0.000000$ rotate $=90.000000$

\&OBST XB=61. $049999,62.174999,57.049999,57.424999,0.28,1.03$, SURF-ID=' 'UPHOLSTERY' ' Left Arm

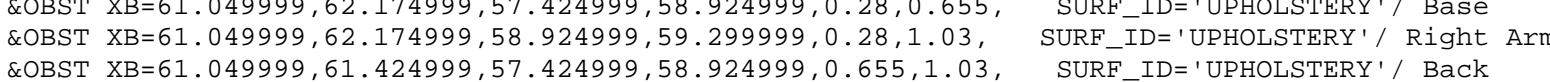

*** GROUP: COUCH2 offset $=0.000000,9.000000,0.000000$ rotate $=90.000000$

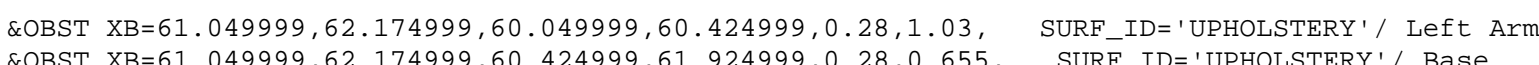

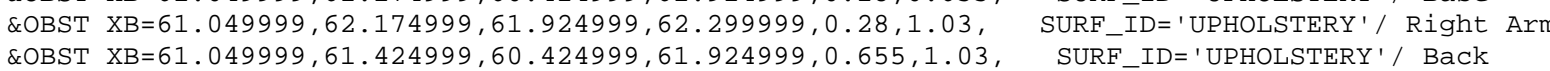

*** GROUP: COUCH2 offset $=0.000000,12.000000$, ๑. .000000 rotate $=90.0000000$

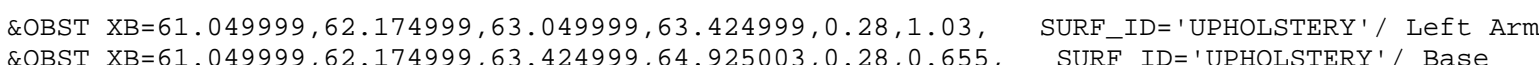

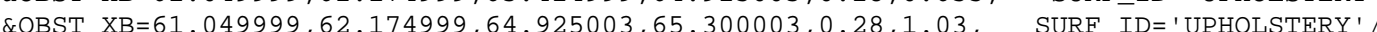
\&OBST XB=61. $049999,62.174999,64.925003,65.300003,0.28,1.03, \quad$ SURF_ID=' 'UPHOLSTERY'/ Right Ar
\&OBST XB=61.049999, 61.424999, 63.424999, 64. *** GROUP: COUCH2 off set $=0.000000,15.000000,0$. . 000000 rotate $=90.000000$

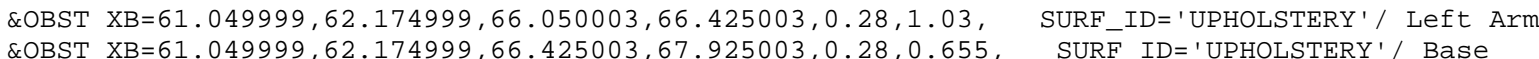
\&OBST XB $=61.049999,62.174999,67.02503,073003,0.22,1.03$, SURE ID='UPHOLSTERY'/ Rise \&OBST XB=61. $049999,62.174999,67.925003,68.300003,0.28,1.03$,
\&OBST $X \mathrm{~B}=61.049999,61.424999,66.425003,67.925003,0.655,1.03$,
SURF_ID= 'UPHOLSTERY'/ Right Ar

** GROUP: COUCH2 offset $=0.000000,18.000000,0.000000$ rotate $=90 . .000000$

\&OBST XB=61. $049999,62.174999,69.050003,69.425003,0.28,1.03, \quad$ SURF_ID=' 'UPHOLSTERY' $/$ Left Arm
\&OBST XB=61.04999, $62.174999,69.425003,70.925003,0.28,0.655, \quad$ SURF_ID='UPHOLSTERY' Rase QOBST XB $=61.049999,62.174999,70.925003,71.300003,0.28,1.03$, SURF-IDD='UPHOLSTERY $/$ ' Right Arm

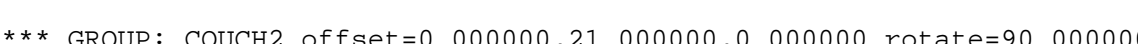

\&OBST XB=61. $049999,62.174999,72.050003,72.425003,0.28,1.03, \quad$ SURF_ID=' 'UPHOLSTERY' ' Left Arm
\&OBST XB=61.049999, 62.174999, $72.425003,73.925003,0.28,0.655, \quad$ SURF_ID='UPHOLSTERY'/ Base \&OBST XB $=61.049999,62.174999,73.925003,74.300003,0.28,1.03$, SURF-ID=' UPHOLSTERY'/ Right Ar

*** GROUP: COUCH2 offset $=0.000000,24.000000,0.000000$ rotate $=90$. . 00000

\&OBST XB=61. 049999, 62.174999, 75. 050003, 75. 425003, 0.28,1.03, SURF_ID= 'UPHOLSTERY' / Left Arm \&OBST XB=61.049999, 62.174999,75.425003,76.925003, $0.28,0.655$, SURF_ID='UPHOLSTERY' / Base

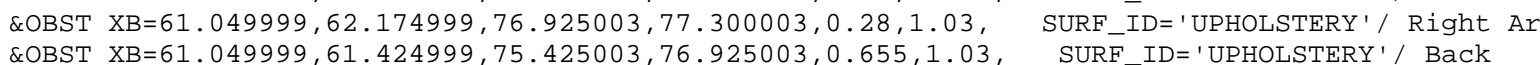

*** GROUP: COUCH2 offset $=0.000000,27.000000,0.000000$ rotate $=90 . .000000$

EOBST XB=61. $049999,62.174999,78.050003,78.425003,0.28,1.03$, SURF_ID='UPHOLSTERY'/ Left Arm \&OBST XB=61. $049999,62.174999,78.425003,79.925003,0.28,0.655$, SURF_ID=' UPHOLSTERY' / Base EOBST XB $=61.049999,61.424999,78.425003,79.925003,0.655,1.03$, SURF_ID='UPHOLSTERY'

*** GROUP: COUCH2 offset $=0.000000,30.000000,0.000000$ rotate $=90.000000$

\&OBST XB $=61.049999,62.174999,81.050003,81.425003,0.28,1.03$, SURF_ID=' 'UPHOLSTERY'/ Left Arm \&OBST XB $=61.049999,62.174999,81.425003,82.925003,0.28,0.655$, SURF_ID='UPHOLSTERY'/ Base

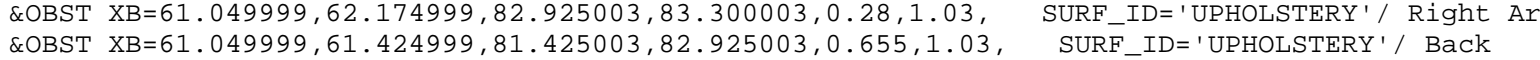

*** GROUP: ROW2

*** GROUP: COUCH2 offset $=0.000000$, ๑. ๑000000, ๑. .000000 rotate $=270.000000$

\&OBST XB=59.549999, 60.674999, 52. 924999, 53. $299999,0.28,1.03, \quad$ SURF_ID=' 'UPHOLSTERY'/' Left Arm
\&OBST XB=59.549999, 60.674999, $51.424999,52.924999,0.28,0.655, \quad$ SURF_ID='UPHOLSTERY'/ Base

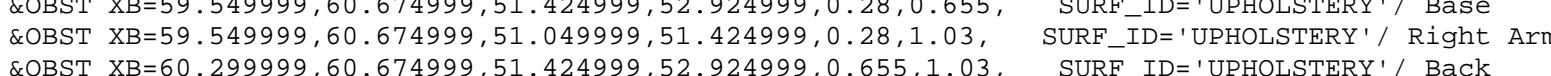

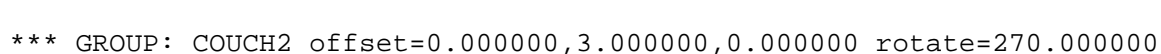

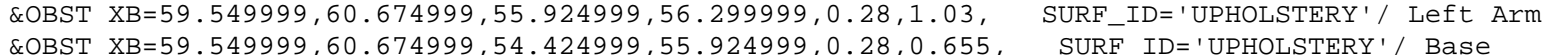

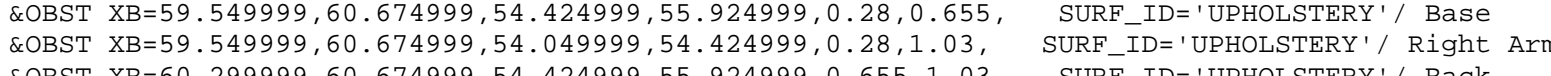

*** GROUP: COUCH2 offset $=0.000000,6.000000,0.000000$ rotate $=270.000000$

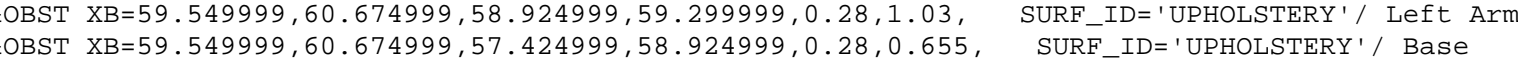

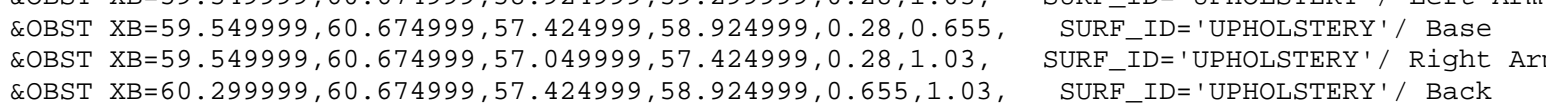

*** GROUP: COUCH2 offset $=0.000000,9.000000,0.000000$ rotate $=270.000000$

\&OBST XB=59.549999, 60.674999, 61.924999, 62. 299999, $0.28,1.03$, SURF_ID='UPHOLSTERY'/ Left Arm

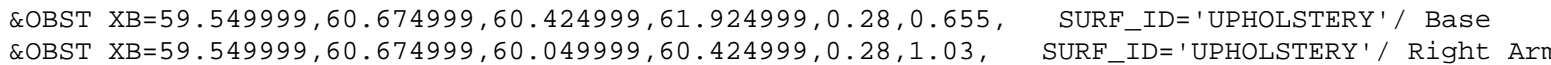

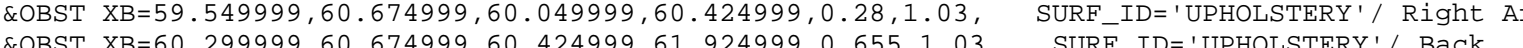

*** GROUP: COUCH2 offset $=0.000000,12.000000,0.000000$ rotate $=270.0000000$

\&OBST XB=59.549999, 60.674999, 64.925003,65.300003, 0.28,1.03, SURF_ID=' UPHOLSTERY'/ Left Arm

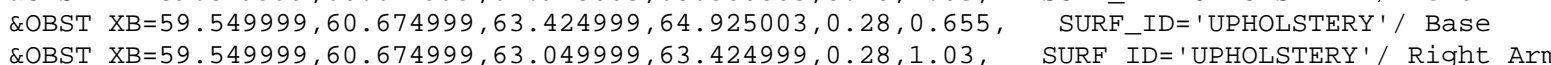
SOBST XB $=60.299999,60.674999,63.424999,64.925093,0.655,1.03$, SUPF_ID=' 'UPHOLSTERY'/ Bnck Ar

*** GROUP: COUCH2 offset $=0.000000,15.000000, \Theta . .000000$ rotate $=270.000000$

\&OBST XB=59.549999, 60.674999, 67. 925003, 68.300003, 0.28,1.03, SURFID=' 'UPHOLSTERY' ' Left Arm

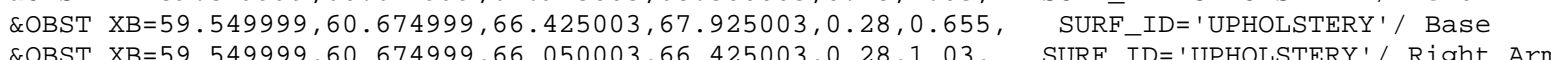

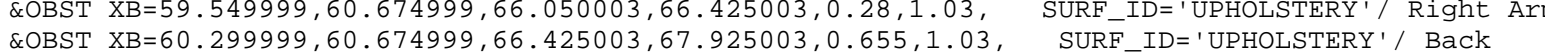

*** GROUP: COUCH2 offset $=0.000000,18.000000, \Theta . \bullet 00000$ rotate $=270.000000$

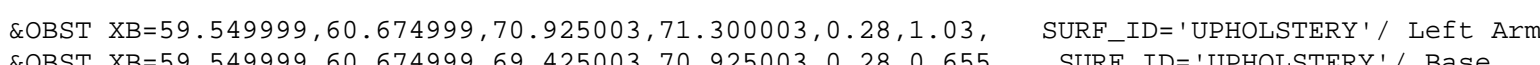
\&OBST XB=59.549999, 60.674999, 69.050003,69.425003, 0.28, 1.03, SURF_ID='UPHOLSTERY'/ Right Ar 
*** GROUP: COUCH2 offset $=0.000000,21.000000,0.000000$ rotate $=270.000000$

\&OBST XB=59.549999, 60.674999, 73.925003, 74.300003, $0.28,1.03$, SURF_ID= ' UPHOLSTERY ' / Left Arn

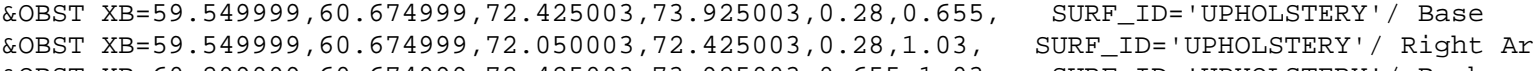
\&OBST XB=60.299999, 60.674999, 72.425003, 73.925003, $0.655,1.03$, SURF_ID='UPHOLSTERY'/ Back

*** GROUP: COUCH2 offset $=0.000000,24.000000,0.000000$ rotate $=270.000000$

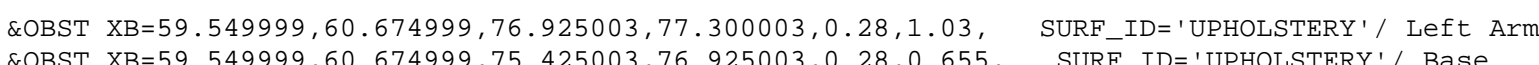

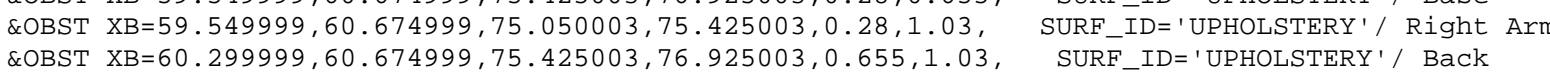

*** GROUP: COUCH2 offset $=0.000000,27.000000,0.000000$ rotate $=270.000000$

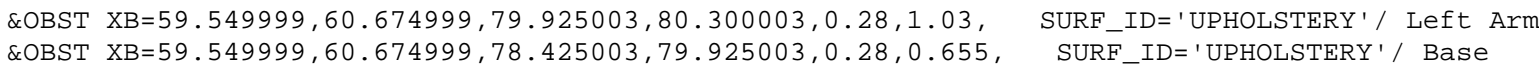

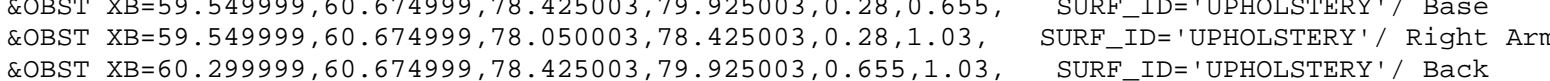
*** GROUP: COUCH2 offset $=0.000000,30.000000,0.000000$ rotate $=270.000000$

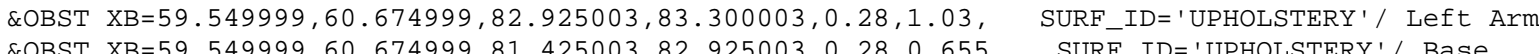

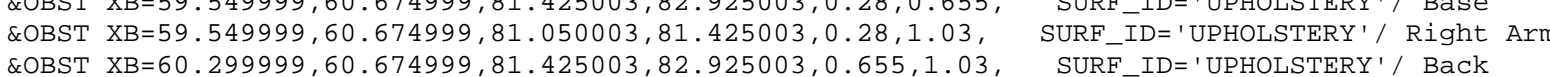

$* * *$ GROUP: TWIN

*** GROUP: ROW

*** GROUP: COUCH2 offset $=0.000000,0.000000,0.000000$ rotate $=90.000000$

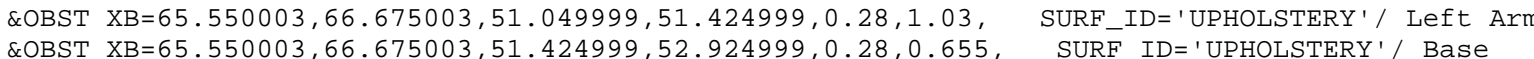
\&OBST XB $=65.550003,66.675003,52.924999,532.299999,0.28,1.03$ SURF ID='UPHOLSTERY'/ Base A \&OBST XB $65.550003,65.925003,51.424999,52.924999,0.655,1.03, \quad$ SURF_ID='UPHOLSTERY'/ Back

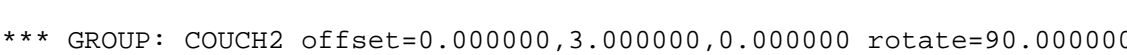

\&OBST XB=65.550003, 66.675003, 54. $949999,54.424999,0.28,1.03$, SURF_ID=' 'UPHOLSTERY ' / Left Arm

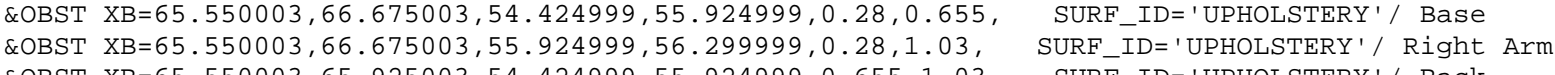

*** GROUP: COUCH2 offset $=0.000000,6.0000000,0.000000$ rotate $=90.0000000$

\&OBST XB $=65.5500003,66.675003,57.049999,57.424999,0.28,1.03, \quad$ SURF_ID='UPHOLSTERY'/ Left Arm

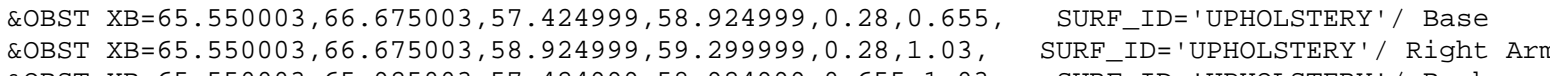

*** GROUP: COUCH2 offset $=0.0000000,9.000000,0.000000$ rotate $=90.000000$

OOBST XB $=65.550003,66.675003,60.049999,60.424999,0.28,1.03, \quad$ SURF_ID='UPHOLSTERY' ' Left Arm
OBST XB=65.550003,66. $675003,60.424999,61.924999,0.28,0.655, \quad$ SURF_ID=' 'UPHOLSTERY'/ Base \&OBST XB $=65.555003,66.675003,61.924999,62.299999, \theta .28,1.03$,
\&OBST XB

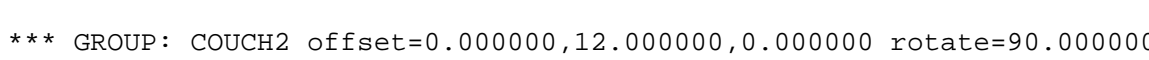

\&OBST XB $=65.550003,66.675003,63.049999,63.424999,0.28,1.03$, SURF-ID=' 'UPHOLSTERY ' / Left Arm \&OBST XB=65.550003, 66.675003,63.424999,64.925003, 0.28, 0.655, SURF_ID='UPHOLSTERY'/ Base

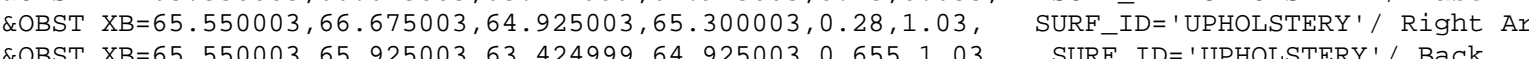

*** GROUP: COUCH2 offset $=0.000000,15.000000,0.000000$ rotate $=90.000000$

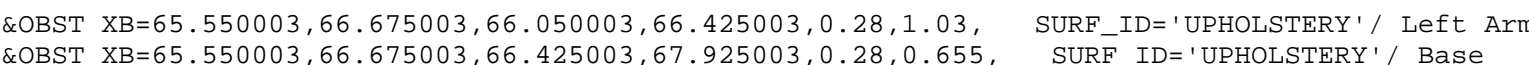
\&OBST XB=65.550003,66.675003, 67.925003,68.300003, $0.28,1.03$, SURF_ID=' 'UPHOLSTERY'/ Right Ar

*** GROUP: COUCH2 offset $=0.000000,18.000000,0.000000$ rotate $=90.000000$
\&OBST $X \mathrm{~B}=65.550003,66.675003,69.050003,69.425003,0.28,1.03, \quad$ SURF ID=' 'UPHOLSTERY' / Left Arm \&OBST XB $=65.550003,66.675003,69.425003,70.925003,0.28,0.655$, SURF_ID='UPHOLSTERY' / Base \&OBST $X \mathrm{~B}=65.550003,65.925003,69.425003,70.925003,0.655,1.03, \quad$ SURF_ID='UPHOLSTERY'/ Right $A$

*** GROUP: COUCH2 offset $=0.000000,21.0000000,0 . .000000$ rotate $=90$. . 000000

\&OBST XB=65.550003, 66.675003,72. $050003,72.425003,0.28,1.03$, SURF_ID=' 'UPHOLSTERY' ' Left Arm \&OBST XB=65.550003, $66.675003,73.925003,74.300003,0.28,1.03, \quad$ SURF_ID= 'UPHOLSTERY'/ Right Arm
\&OBST XB=65.550003, $65.925003,72.425003,73.925003,0.655,1.03$, SURF_ID='UPHOLSTERY'/ Back

*** GROUP: COUCH2 offset $=0.000000,24.000000,0.000000$ rotate $=90.000000$

\&OBST XB $=65.550003,66.675003,75.050003,75.425003,0.28,1.03, \quad$ SURF ID=' 'UPHOLSTERY ' $/$ Left Arm

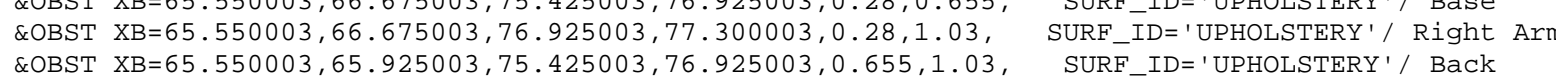

$* * *$ GROUP: COUCH2 offset $=0.000000,27.000000,0.000000$ rotate $=90.000000$

LOBST $X \mathrm{~B}=65.550003,66.675003,78.050003,78.425003,0.28,1.03, \quad$ SURF ID= ' ' UPHOLSTERY ' $/$ Left Arm

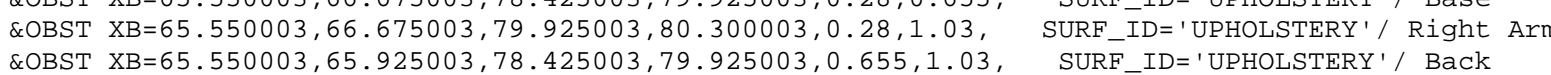

*** GROUP: COUCH2 offset $=0.000000,30.000000,0.000000$ rotate $=90.000000$

\&OBST XB $=65.550003,66.675003,81.050003,81.425003,0.28,1.03, \quad$ SURF ID = 'UPHOLSTERY ' $/$ Left Arm \&OBST $X \mathrm{XB}=65.550003,66.675003,82.925003,83,300003,0.28,1.03$, SURF ID=' UPHOLSTERY'/ Right A A In \&OBST XB $=65.550003,65.925003,81.425003,82.925003,0.655,1.03$, SURF_ID=' 'UPHOLSTERY'/ Back

$* * *$ GROUP: ROW2

*** GROUP: COUCH2 offset $=0.000000,0.000000,0.000000$ rotate $=270.000000$

\&OBST XB $=64.050003,65.175003,52.924999,53.299999,0.28,1.03, \quad$ SURF_ID='UPHOLSTERY '/ Left Arm \&OBST $X \mathrm{~B}=64.050003,65.175003,51.424999,52.924999,0.28,0.655$, SURF_ID=' 'UPHOLSTERY'/ Base \&OBST $X \mathrm{XB}=64.0500003,65.175003,51.049999,51.424999,0.28,1.03, \quad$ SURF $I D='$ 'UPHOLSTERY'/

*** GROUP: COUCH2 of fset $=0.000000,3.000000,0.000000$ rotate $=270.000000$

EOBST XB=64.050003, 65.175003, 55.924999, 56. 299999, 0. 28,1.03, SURF_ID='UPHOLSTERY'/ Left Arm \&OBST XB=64. $050003,65.175003,54.424999,55.924999,0.28,0.655, \quad$ SURF IDE='UPHOLSTERY' $/$ Base EOBST XB=64.800003, 65.175003, 54.424999, 55.924999, $0.655,1.03$, SURF_ID=' 'UPHOLSTERY'/ Back

$* * *$ GROUP: COUCH2 offset $=0.000000,6.000000,0.000000$ rotate $=270.000000$

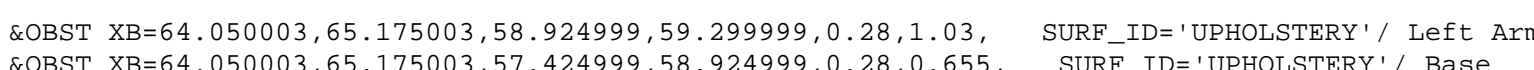
\&OBST $X \mathrm{XB}=64.050003,65.175003,57.049999,57.424999,0.28,1.03, \quad$ SURF_ID=' 'UPHOLSTERY'/ Right Arm
\&OBST XB $=64.800003,65.175003,57.424999,58.924999,0.655,1.03, \quad$ SURF_ID=' 'UPHOLSTERY'/ Back

*** GROUP: COUCH2 offset $=0.000000,9.000000,0.000000$ rotate $=270.000000$

\&OBST XB=64. $050003,65.175003,61.924999,62.299999,0.28,1.03$, SURF_ID=' 'UPHOLSTERY '/ Left Arm

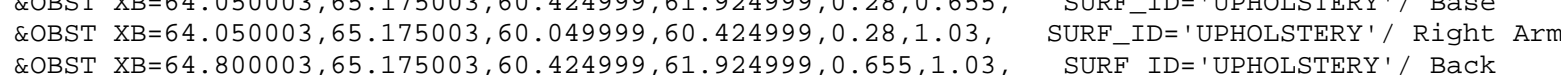

*** GROUP: COUCH2 offset $=0.000000,12.000000,0.0000000$ rotate $=270.000000$

\&OBST XB=64. $050003,65.175003,64.925003,65.300003,0.28,1.03$, SURF_ID=' 'UPHOLSTERY ' / Left Arm \&OBST XB=64.050003, 65.175003,63.049999, 63.424999, 0.28,1.03, SURF_ID='UPHOLSTERY'/ Right Ar \&OBST XB=64.800003,65.175003,63.424999,64.925003,0.655,1.03, SURF_ID='

*** GROUP: COUCH2 offset $=0.000000,15.000000,0.000000$ rotate $=270.000000$

\&OBST XB=64. $050003,65.175003,67.925003,68.300003,0.28,1.03$, SURF_ID=' 'UPHOLSTERY' ' Left Arm \&OBST $X \mathrm{~B}=64.050003,65.175003,66.050003,66.425003,0.28,1.03$, SURF ID=' 'UPHOLSTERY'/ Right Ar \&OBST $X \mathrm{~B}=64.800003,65.175003,66.425003,67.925003,0.655,1.03$, SURF_ID=' 'UPHOLSTERY'/ Back 


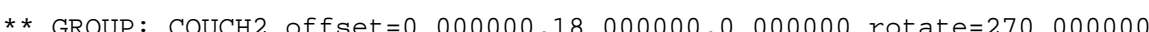

\&OBST XB=64. $950003,65.175003,70.925003,71.300003,0.28,1.03, \quad$ SURF_ID='UPHOLSTERY' ' Left Arm
\&OBST XB=64.050003, $65.175003,69.425003,70.925003,0.28,0.655$,
SURF_ID='UPHOLSTERY' \&OBST XB $=64.050003,65.175003,69.050003,69.425003,0.28,1.03$, SURF ID ' 'UPHOLSTERY'/ Right Arm

*** GROUP: COUCH2 offset $=0.000000,21.000000,0.000000$ rotate $=270$. .

\&OBST XB $=64.050003,65.175003,73.925003,74.300003,0.28,1.03, \quad$ SURF_ID='UPHOLSTERY '/ Left Arm

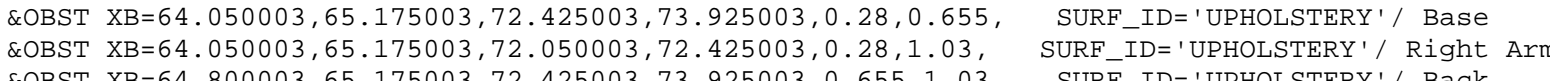

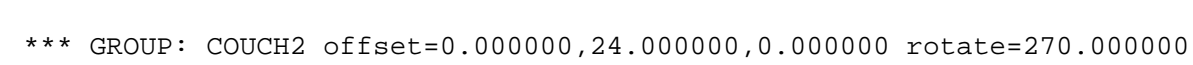

\&OBST XB=64.050003,65.175003, 76.925003,77.300003, 0.28,1.03, SURF_ID=' 'UPHOLSTERY' / Left Arm

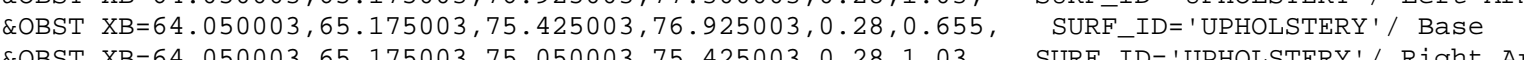

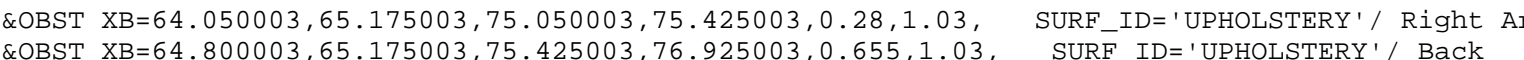

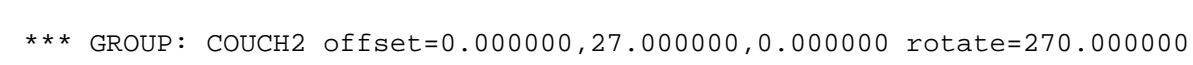

\&OBST XB $=64.0500003,65.175003,79.925003,80.300003,0.28,1.03, \quad$ SURF ID='UPHOLSTERY'/ Left Arm \&OBST $X \mathrm{~B}=64.050003,65.175003,78.425003,79.925003,0.28,0.655$, SURF_ID='UPHOLSTERY'/ Base \&OBST $X B=64.800003,65.175003,78,425003,79,925003,0.655,1,03$ S SUPF_ID=' 'UPHOLSTERY'/ *** GROUP: COUCH2 offset $=0.000000,30.000000,0.000000$ rotate $=270 . .000000$

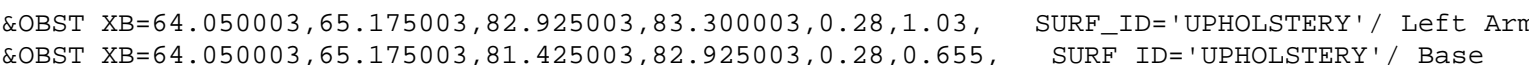
\&OBST XB $=64.0500003,65.175003,81.050003,81.425003,0.28,1.03$, S SURF ID ='UPHOLSTERY'/ Right Ar

*** GROUP: TWIN

*** GROUP: ROW

*** GROUP: COUCH2 offset $=0.0000000,0.000000,0.0 .000000$ rotate $=90.000000$

\&OBST XB $=70.050003,71.175003,51.049999,51.424999,0.28,1.03, \quad$ SURF_ID=' 'UPHOLSTERY ' 1 Left Arm
\&OBST XB=70.050003, 71.175003, $51.424999,52.924999,0.28,0.655, \quad$ SURF_ID='UPHOLSTERY' / Base \&OBST $X=70.050003,71.175003,55.924999,53.299999,0.28,1.03, \quad$ SURF_ID=' 'UPHOLSTERY'/Right A

*** GROUP: COUCH2 offset $=0.000000,3.000000,0.000000$ rotate $=90.000000$

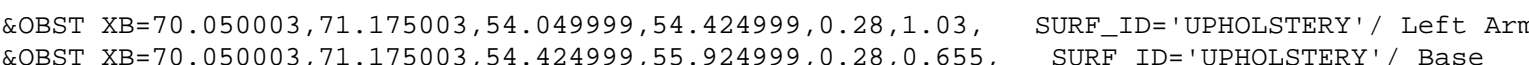

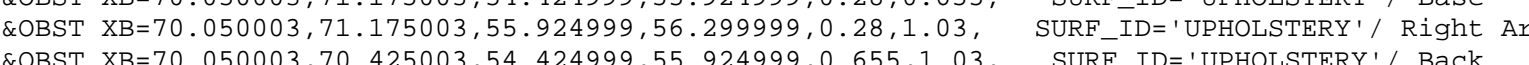

*** GROUP: COUCH2 offset $=0.000000,6.000000,0.000000$ rotate $=90.000000$

\&OBST XB=70. 050003, 71.175003, 57. 049999, 57. 424999, 0.28,1.03, SURF_ID='UPHOLSTERY'/ Left Arm \&OBST XB=70.050003, 71.175003, 57. 424999, 58. 224999, $0.28,0.655$, SURF ID='UPHOLSTERY '/ Base

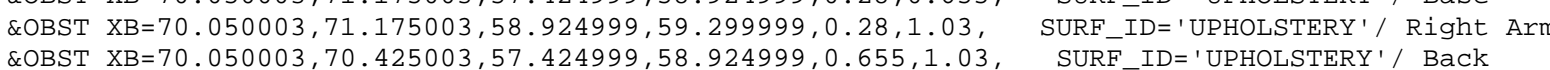

*** GROUP: COUCH2 offset $=0.000000,9.000000,0.000000$ rotate $=90$. . $\bullet 00000$

\&OBST XB $=70.050003,71.175003,60.049999,60.424999,0.28,1.03$, SURF_ID='UPHOLSTERY'/ Left Arm \&OBST XB=70.050003, 71.175003,60.424999,61.924999, $0.28,0.655$, SURF_ID='UPHOLSTERY'/ Base

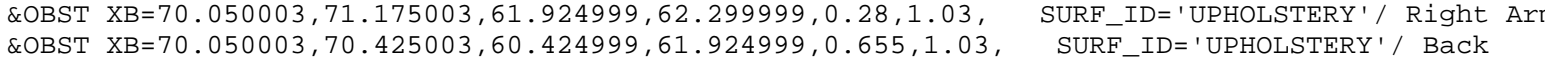

*** GROUP: CouCH2 offset $=0.000000,12.000000,0.000000$ rotate $=90.000000$

\&OBST XB $=70.050003,71.175003,63.049999,63.424999,0.28,1.03$, SURF_ID=' 'UPHOLSTERY'/ Left Arm \&OBST XB=70.050003, 71.175003, 63.424999,64.925003, $0.28,0.655$, SURF_ID='UPHOLSTERY'/ Base

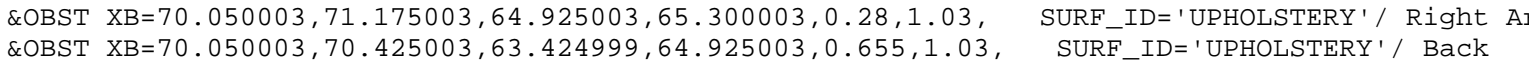

*** GROUP: COUCH2 offset $=0.000000,15.000000,0.000000$ rotate $=90.000000$

\&OBST $X B=70.050003,71.175003,66.050003,66.425003,0.28,1.03$, SURF_ID='UPHOLSTERY ' / Left Arm
\&OBST XB $=70.050003,71.175003,66.425003,67.925003,0.28,0.655$, SURF_ID=' UPHOLSTERY '/ Base

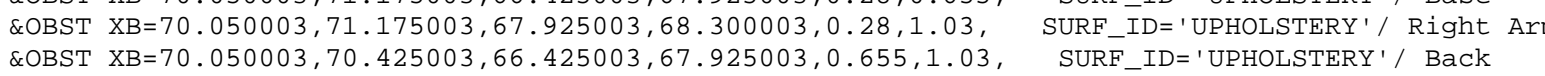
*** GROUP: COUCH2 offset $=0.000000,18.000000,0.000000$ rotate $=90.000000$

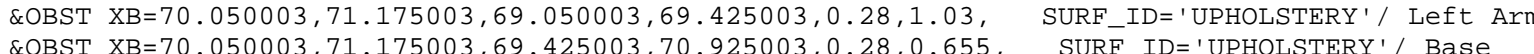
\&OBST XB $=70.050003,71.175003,70.925003,71,300003,0.28,1,03, \quad$ SURF ID='UPHOLSTERY'/ Right Ar \&OBST $X \mathrm{~B}=70.050003,70.425003,69.425003,70.925003,0.655,1.03$, SURF_ID=' 'UPHOLSTERY' / Back

*** GROUP: COUCH2 offset $=0.000000,21.000000,0.000000$ rotate $=90.000000$

\&OBST XB $=70.050003,71.175003,72.050003,72.425003,0.28,1.03, \quad$ SURF_ID=' 'UPHOLSTERY' $/$ Left Arm
\&OBST XB $=70.050003,71.175003,72.425003,73.925003,0.28,0.655$,
SURF ID='UPHOLSTERY' / Base \&OBST XB=70.050003, 71.175003, 73.02503,74.300003, $0.28,1.03$, SUPF ID='UPHOLSTERY'/ Base

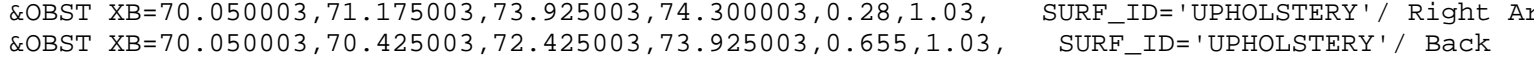

*** GROUP: COUCH2 offset $=0.000000,24.000000$, ๑. .000000 rotate $=90.0000000$

\&OBST XB=70.050003, 71.175003, 75. $050003,75.425003,0.28,1.03, \quad$ SURF_ID='UPHOLSTERY ' / Left Arm \&OBST $\mathrm{XB}=70.050003,17.170003,75.425003,76.925003,0.28,0.655$, SURF-ID='UPHOLSTERY' / Base COBST $X B=70.050003,70.425003,75.425003,76.925003,0.655,1.03$, SURF_ID=' 'UPHOLSTERY' / Back

*** GROUP: COUCH2 offset $=0.000000,27.000000$, ๑. .000000 rotate $=90 . .000000$

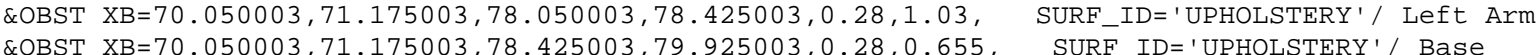
\&OBST $X \mathrm{XB}=7.0050003,71.175003,78.425003,79.925003,0.28,0.055, \quad$ SURF_ID='UPHOLSTERY'/ Base

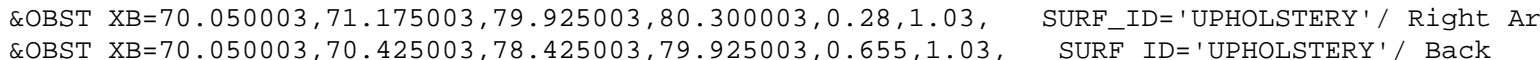

*** GROUP: COUCH2 offset $=0.000000,30.000000$, ๑. .000000 rotate $=90.000000$

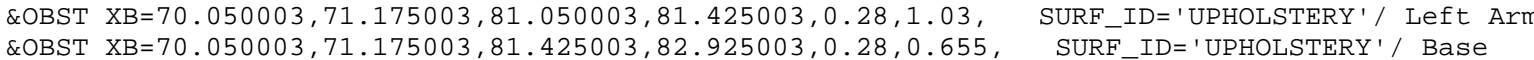

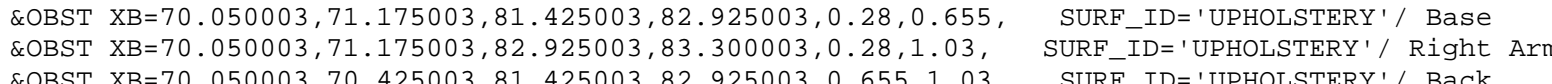

*** GROUP: ROW2

*** GROUP: COUCH2 offset $=0.000000, \Theta . .000000,0.000000$ rotate $=270.000000$

\&OBST XB=68.550003, 69.675003, 52.924999, 53.299999, $0.28,1.03$, SURF_ID=' 'UPHOLSTERY' ' Left Arm

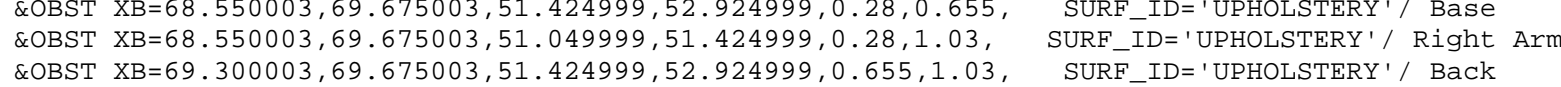

*** GROUP: COUCH2 offset $=0.000000,3.000000,0.0000000$ rotate $=270.000000$

\&OBST XB=68. $5500003,69.675003,55.924999,56.299999,0.28,1.03, \quad$ SURF_ID=' UPHOLSTERY ' $/$ Left Arm \&OBST XB=69.300003, 69.675003, $54.424999,55.924999,0.655,1.03$, SURF_ID='UPHOLSTERY'/ Back

*** GROUP: COUCH2 offset $=0.000000,6.000000,0.000000$ rotate $=270.000000$

\&OBST XB $=68.550003,69.675003,58.924999,59.299999,0.28,1.03, \quad$ SURF_ID= 'UPHOLSTERY ' / Left Arm \&OBST XB $69.300003,69.675003,57.424999,58.924999,0.655,1.03, \quad$ SURF_ID=' 'UPHOLSTERY'/ Right Ar
SURF_ID=' UPHOLSTERY'/ Back

** GROUP: COUCH2 offset $=0.000000,9.000000,0.000000$ rotate $=270.000000$

\&OBST XB=68. $550003,69.675003,61.924999,62.299999,0.28,1.03, \quad$ SURF_ID=' 'UPHOLSTERY' $/$ Left Arm
\&OBST XB $=68.550003,69.675003,60.424999,61.924999,0.28,0.655, \quad$ SURF ID=' 'UPHOLSTERY' Rase

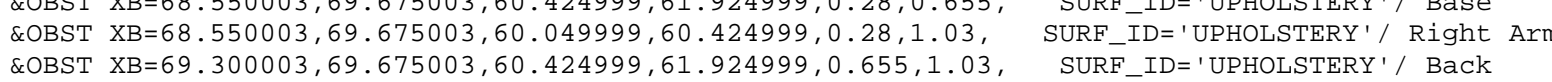

*** GROUP: COUCH2 offset $=0.0000000,12.000000,0.000000$ rotate $=270.000000$

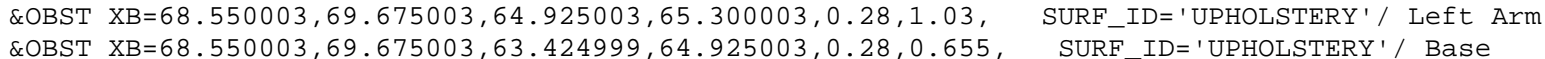

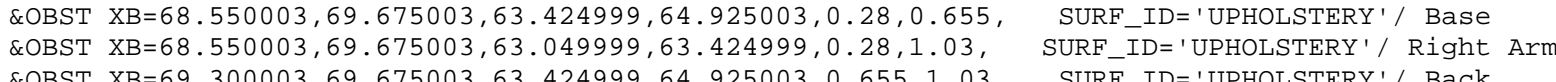

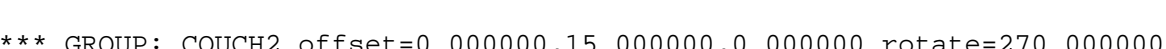




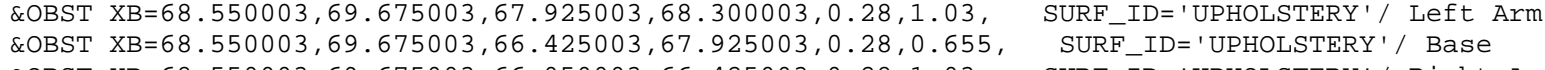
\&OBST XB $=68.550003,69.675003,66.050003,66.425003,0.28,1.03$, SURF *** GROUP: coucH2 offset $=0.000000,18.000000,0.000000$ rotate $=270.000000$ \&OBST XB $=68.550003,69.675003,70.925003,71.300003,0.28,1.03, \quad$ SURF_ID= 'UPHOLSTERY' ' Left Arm

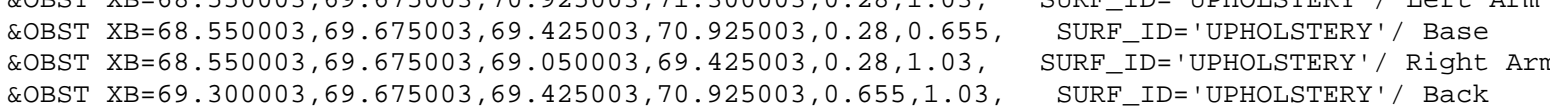

${ }^{*}{ }^{* *}$ GROUP: COUCH2 offset $=0.000000,21.000000,0.000000$ rotate $=270.000000$

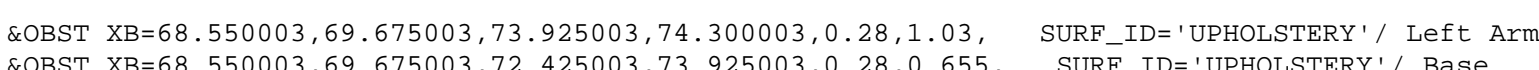
\&OBST XB $=68.550003,69.675003,72.050003,72.425003,0.28,1.03$, SURF ID ='UPHOLSTERY' $/$ Right Ar

*** GROUP: COUCH2 offset $=0.000000,24.000000,0.000000$ rotate $=270.000000$

\&OBST XB $=68.550003,69.675003,76.925003,77.300003,0.28,1.03$, SURF_ID='UPHOLSTERY'/ Left Arm \&OBST XB=68.550003, 69.675003, 75.425003,76.925003, 0.28, 0.655, SURF_ID='UPHOLSTERY'/ Base \&OBST XB $=68.550003,69.675003,75.050003,75.425003,0.28,1.03, \quad$ SURF_ID='UPHOLSTERY'/ Right Arm
\&OBST XB $=69.300003,69.675003,75.425003,76.925003,0.655,1.03$,
SURF_ID='UPHOLSTERY' $/$ Back

*** GROUP: COUCH2 offset $=0.000000,27.000000,0.000000$ rotate $=270.000000$

\&OBST $X B=68.550003,69.675003,79.925003,80.300003,0.28,1.03$, SURF_ID='UPHOLSTERY Y' Left Arm

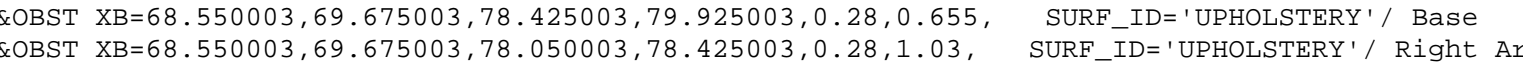
BOBST XB=69. $300003,69.675003,78.425003,79.925003,0.655,1.03$, SURF ID='UPHOLSTEPY'/ Back

*** GROUP: COUCH2 offset $=0.000000,30.000000,0.000000$ rotate $=270.000000$

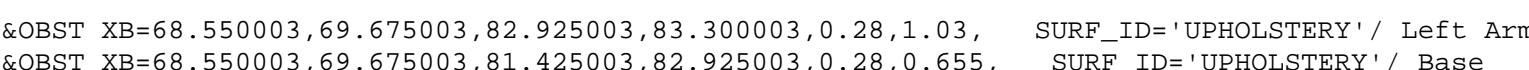

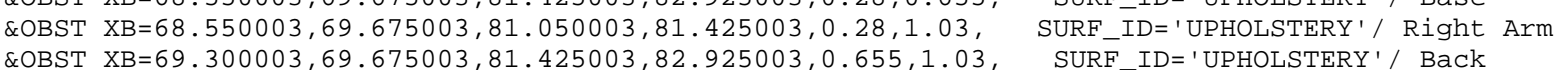

\section{*** GROUP: ROW2}

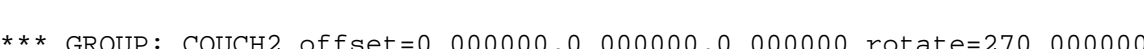

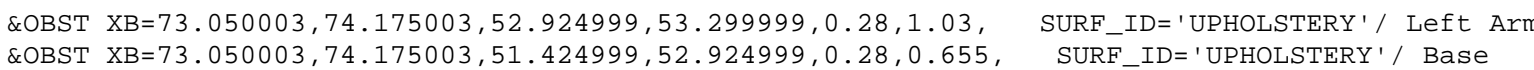

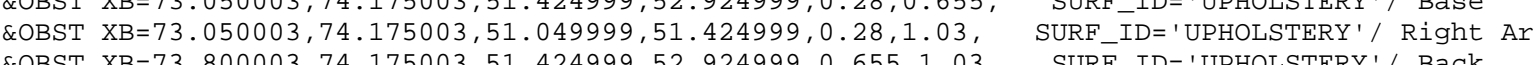

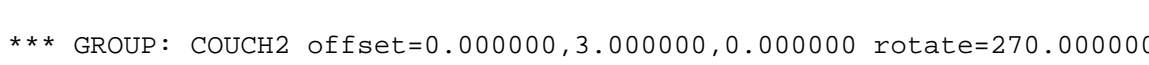

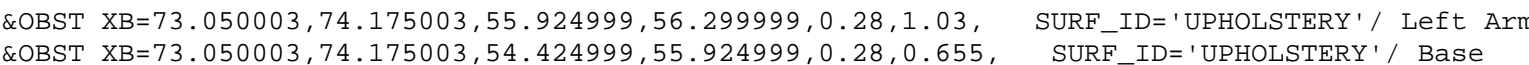
\&OBST XB $=73.050003,74.175003,54.049999,54.424999,0.28,1.03$, SURF_ID='UPHOLSTERY'/ Right Ar

*** GROUP: COUCH2 offset $=0.000000,6.000000,0.000000$ rotate $=270.0000000$

\&OBST XB $=73.050003,74.175003,58.924999,59.299999,0.28,1.03, \quad$ SURF_ID= 'UPHOLSTERY' / Left Arm \&OBST XB=73. $050003,74.175003,57.424999,58.924999,0.28,0.655$, SURF_ID='UPHOLSTERY'/ Base \&OBST $X \mathrm{XB}=73.050003,74.175003,57.049999,57.424999,0.28,1.03, \quad$ SURF_ID='UPHOLSTERY'/' Right Ar

*** GROUP: COUCH2 offset $=0.000000,9 . \bullet 00000,0.000000$ rotate $=270.000000$

\&OBST XB $=73.050003,74.175003,61.924999,62.299999,0.28,1.03$, SURF_ID='UPHOLSTERY'/ Left Arm \&OBST XB=73. $050003,74.175003,60.424999,61.924999,0.28,0.655$, SURF_ID='UPHOLSTERY' / Base \&OBST $\mathrm{BB}=73.050003,74.175003,60.449999,60.424999,0.28,1.03$, SURF_ID='UPHOLSTERY'/ RIght Ar $\star \star \star$ GROUP: COUCH2 offset $=0.000000,12.000000,0.000000$ rotate $=270.000000$

\&OBST XB=73. $050003,74.175003,64.925003,65.300003,0.28,1.03$, SURF_ID= 'UPHOLSTERY' ' Left Arm \&OBST XB=73.050003, 74.175003, 63.424999, 64.925003, 0.28, 0.655, SURF-ID='UPHOLSTERY'/ Base

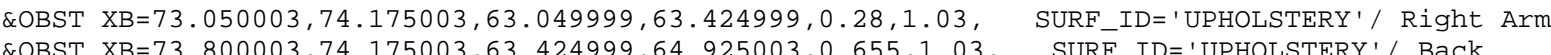

*** GROUP: COUCH2 offset $=0.000000,15.000000, \Theta .000000$ rotate $=270.000000$

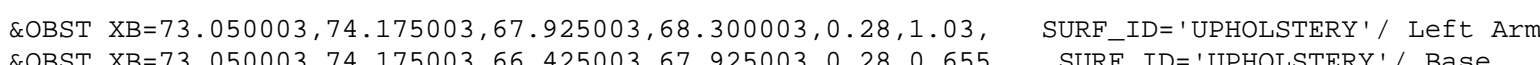

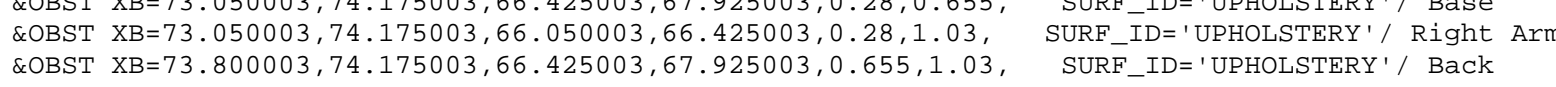
*** GROUP: COUCH2 offset $=0.000000,18.000000,0.000000$ rotate $=270, .000000$

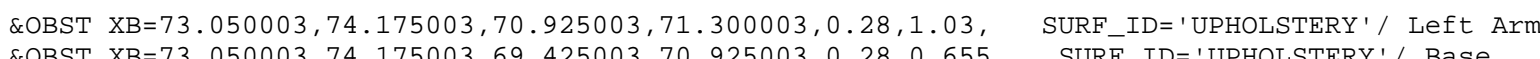

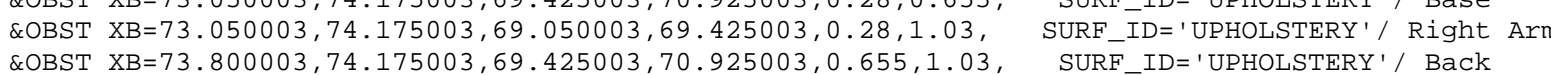

*** GROUP: COUCH2 offset $=0.000000,21.000000,0.000000$ rotate $=270.000000$

\&OBST $X B=73.050003,74.175003,73.925003,74.300003,0.28,1.03$, SURF_ID=' UPHOLSTERY' ' Left Arm

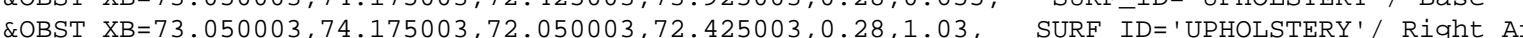
\&OBST $X B=73.800003,74.175003,72.425003,73.925003,0.655,1.03$, SURF_ID='UPHOLSTERY' / Back

*** GROUP: COUCH2 offset $=0.000000,24.000000,0.000000$ rotate $=270.000000$

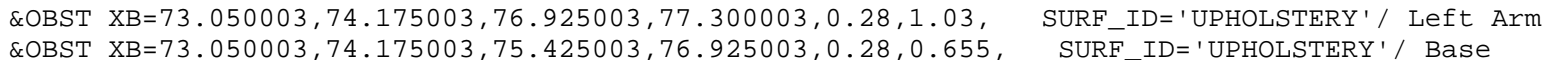

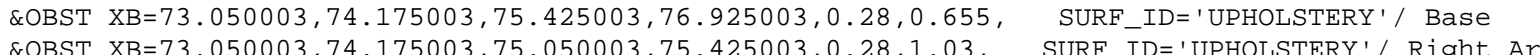
QOBST $X B=73.800003,74.175003,75.425003,76.925003,0.655,1.03$, SURF_ID ' 'UPHOLSTERY'/ Back

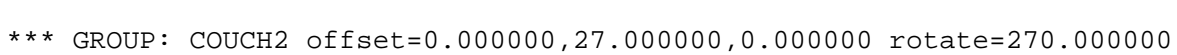

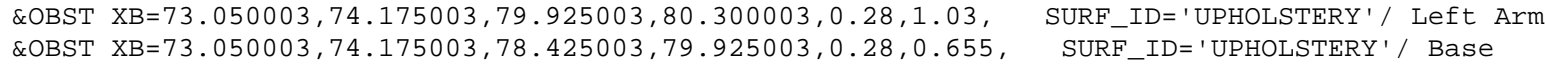

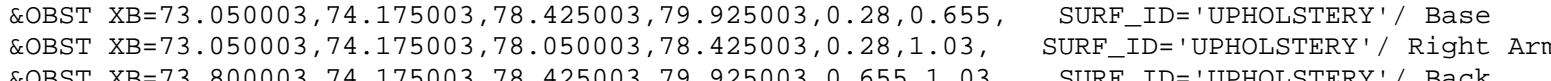

*** GROUP: COUCH2 offset $=0.000000,30.000000,0.000000$ rotate $=270.000000$

\&OBST XB $=73.050003,74.175003,82.925003,83.300003,0.28,1.03, \quad$ SURF_ID= 'UPHOLSTERY ' / Left Arm

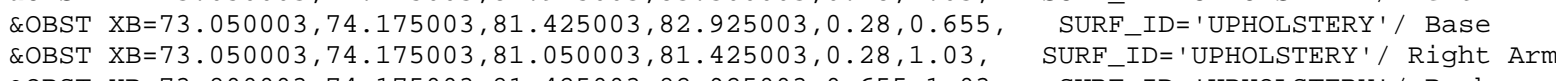

*** GROUP: SHORT_ROW

${ }_{*}^{* *}$ GROUP: COUCH2 offset $=0.000000,0.000000,0.000000$ rotate $=90.000000$

\&OBST XB $=67.044998,67.419998,46.91,48.035,0.28,1.03, \quad$ SURF_ID= 'UPHOLSTERY' ' Left Arm \&OBST XB=65.169998, 65.544998, 46.91, 48. $035,0.28,1.03$,
\&OBST $X \mathrm{~B}=65.544998,67.044998,46.91,47.285,0.655,1.03$,

*** GROUP: COUCH2 offset $=0.000000,3.000000$, ๑. .00000 rotate $=90.000000$

\&OBST XB=64. 044998, 64.419998, 46.91, 48. 035, 0.28, 1. 03, SURF_ID='UPHOLSTERY'/ Left Arm \&OBST XB $=62.169998,62.544998,46.91,48.035,0.28,1.03$, SURF_ID='UPHOLSTERY'/ Right Arm \&OBST XB=62.544998,64.044998, 46.91, 47.285, 0.655, 1. 13 , SURF_ID='UPHOLSTERY' / Back

*** GROUP: COUCH2 offset $=0.000000,6.000000,0.000000$ rotate $=90.000000$

\&OBST XB=61.044998, 61.419998, 46.91, 48.035, $0.28,1.03$, SURF_ID=' 'UPHOLSTERY' ' Left Arm

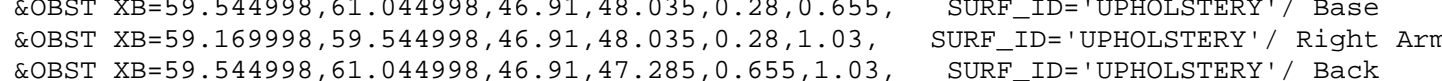

*** GROUP: COUCH2 offset $=0.000000,9.000000,0.000000$ rotate $=90 . .000000$

EOBST XB=58. 044998, 58.419998, 46.91, 48. 035, 0.28, 1. 03 , SURFIDD ' UPHOLSTERY ' ' Left Arm

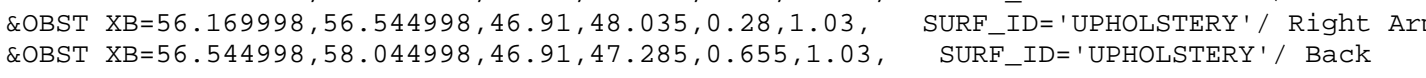
*** GROUP: COUCH2 offset $=0.000000,12.000000,0.000000$ rotate $=90.000000$ \&OBST XB=55. $044998,55.419998,46.91,48.035,0.28,1.03, \quad$ SURF_ID=' 'UPHOLSTERY' ' Left Arm
\&OBST XB=53.544998, $55.044998,46.91,48.035,0.28,0.655$,
SURF_ID=' UPHOLSTERY' $/$ Base 
\&OBST XB=53.169998,53.544998, 46.91, 48. $035,0.28,1.03, \quad$ SURF_ID= 'UPHOLSTERY '/ Right Arm
\&OBST XB=53.544998,55.044998, $46.91,47.285,0.655,1.03, \quad$ SURF_ID=' UPHOLSTERY' / Back *** GROUP: COUCH2 offset $=0.000000,15.000000,0.000000$ rotate $=90$. . 000000

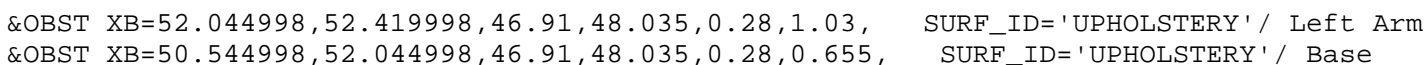
\&OBST XB $=50.544998,52.044998,46.91,48.035,0.28,0.655$,
\&OBST XB $=50.169998,50.544998,46.91,48.035,0.28,1.03$,

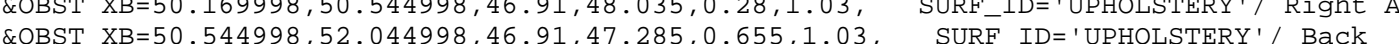

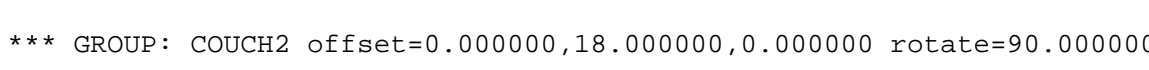
\&OBST XB $=49.044998,49.419998,46.91,48.035,0.28,1.03$,
\&OBST XB $=47.544998,49.044998,46.91,48.035,0.28,0.655$,

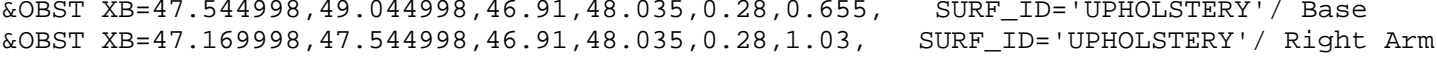
*** GROUP: ROW

*** GROUP: COUCH2 offset $=0.000000,0.000000,0.000000$ rotate $=90.000000$

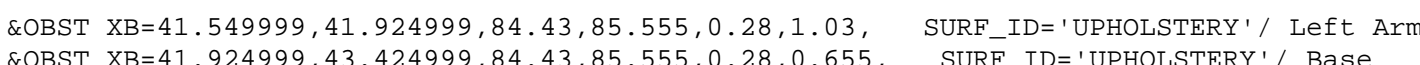

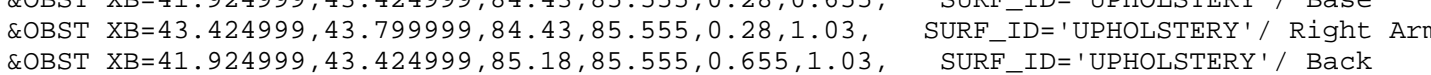

*** GROUP: COUCH2 offset $=0.000000,3.000000,0.000000$ rotate $=90.000000$

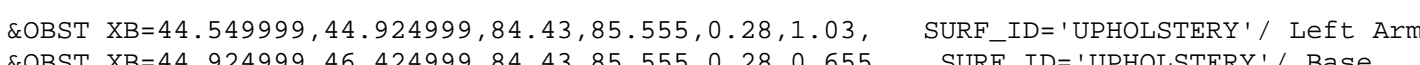

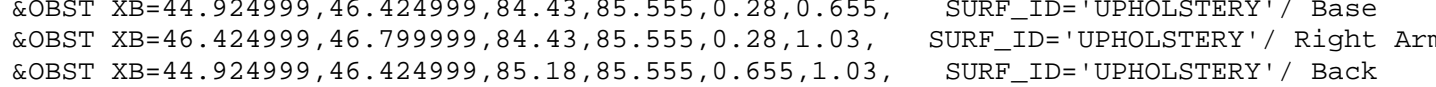
$* * *$ GROUP: COUCH2 offset $=0.000000,6.000000,0.000000$ rotate $=90.000000$

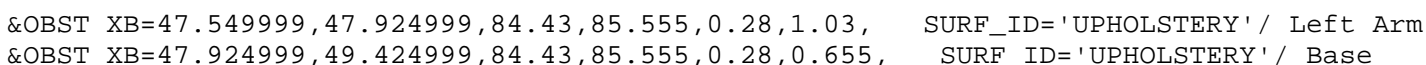

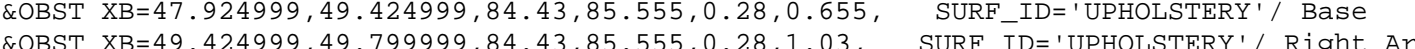

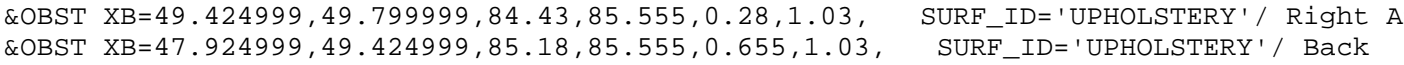

*** GROUP: COUCH2 offset $=0.000000,9.000000,0.000000$ rotate $=90.000000$

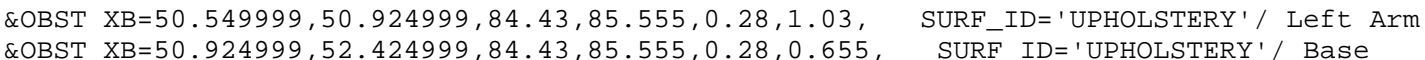

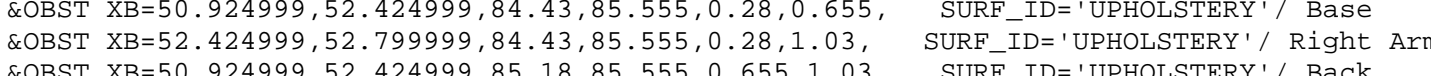

*** GROUP: COUCH2 offset $=0.000000,12.000000,0.0000000$ rotate $=90.000000$

\&OBST XB=53.549999, 53.924999, 84. 43, 85.555, 0.28,1.03, SURF_ID='UPHOLSTERY'/ Left Arm

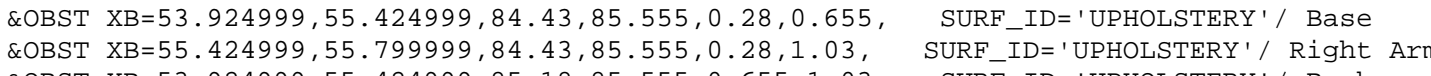
*** GROUP: COUCH2 offset $=0.000000,15.000000,0.000000$ rotate $=90.000000$

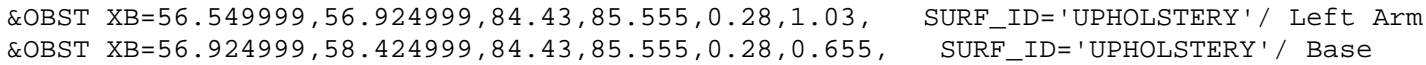

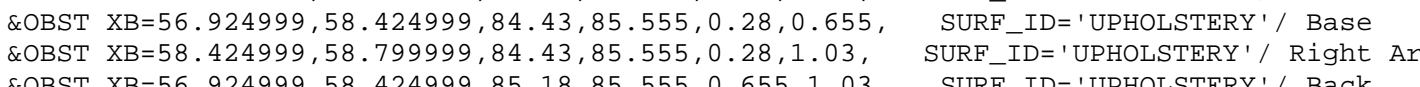
*** GROUP: COUCH2 offset $=0.000000,18.000000,0.000000$ rotate $=90.000000$

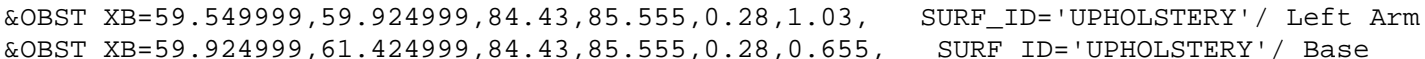

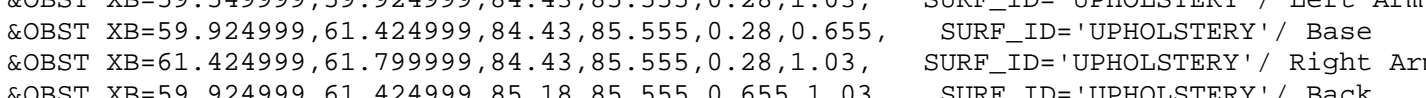

*** GROUP: COUCH2 offset $=0.000000,21.000000,0.000000$ rotate $=90.000000$

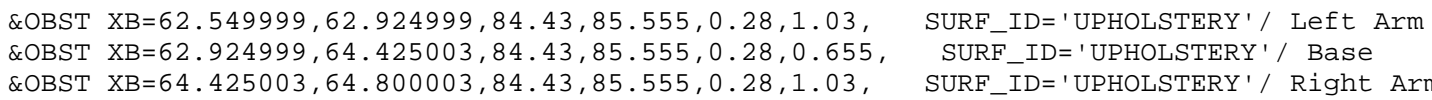
\&OBST XB $=64.425003,64.800003,84.43,85.555,0.28,1.03$ ' SURF_ID= 'UPHOLSTERY'/ Right Ar
\&OBST XB $=62.924999,64.425003,85.18,85.555,0.655,1.03, \quad$ SURF_ID 'UPHOLSTERY' / Back

*** GROUP: COUCH2 offset $=0.000000,24.000000,0.000000$ rotate $=90.000000$
EOBST XB=65.550003, 65.925003, 84.43, 85.555, 0.28,1.03, SURF_ID='UPHOLSTERY'/ Left Arm \&OBST XB $=65.925003,67.425003,84.43,85.555,0.28,0.655$, SURF_ID='UPHOLSTERY'/ Base

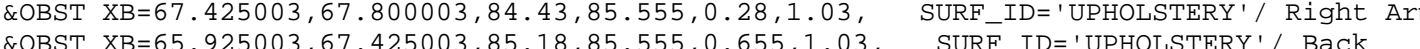

*** GROUP: COUCH2 offset $=0.000000,27.0000000,0.000000$ rotate $=90$. .

\&OBST XB $=68.550003,68.925003,84.43,85.555,0.28,1.03, \quad$ SURF_ID = 'UPHOLSTERY ' $/$ Left Arm \&OBST XB=7. $425003,70.800003,84.43,85.555,0.28,1.03$,
\&OBST $X B=68.925003,70.425003,85.18,85.555,0.655,1.03$,

*** GROUP: COUCH2 offset $=0.000000,30.000000,0.000000$ rotate $=90.000000$

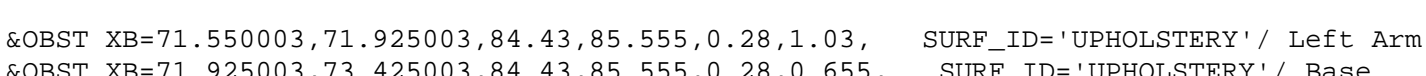
$\begin{aligned} & \text { \&OBST } X B=73.425003,73.800003,84.43,85.555,0.28,1.03, \\ & \text { \&OBST } X B=71.925003,73.425003,85.18,85.555,0.655,1.03,\end{aligned}$ SURF_ID='UPHOLSTERY'/ Right Arm
SURF_ID='UPHOLSTERY'/ Back

*** GROUP: LEVEL

$* * *$ GROUP: ROW

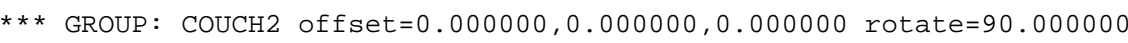

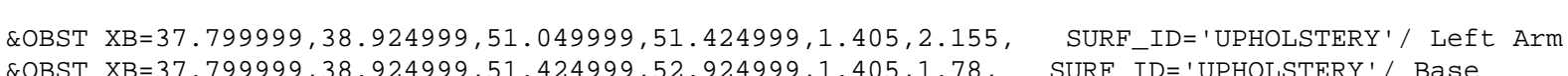

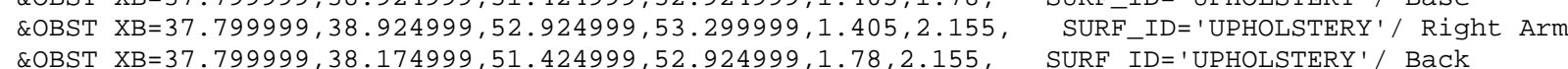

${ }^{*} *$ GROUP: COUCH2 offset $=0.000000,3.000000,0.000000$ rotate $=90.000000$

\&OBST XB=37.799999, 38.924999, 54. 049999, 54.424999, 1.405, 2.155, SURF_ID=' 'UPHOLSTERY '/ Left Arm

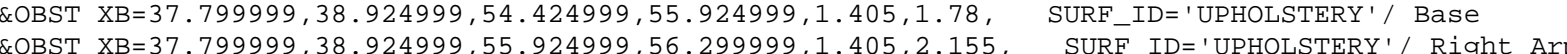
\&OBST XB=37.799999, 38.174999, $54.424999,55.924999,1.78,2.155, \quad$ SURF_ID='UPHOLSTERY'/ Back

*** GROUP: COUCH2 offset $=0.000000,6.000000,0.000000$ rotate $=90.000000$

\&OBST XB=37.799999, 38.924999, 57. 049999, 57.424999, 1.405, 2.155, SURF_ID= 'UPHOLSTERY' / Left Arm

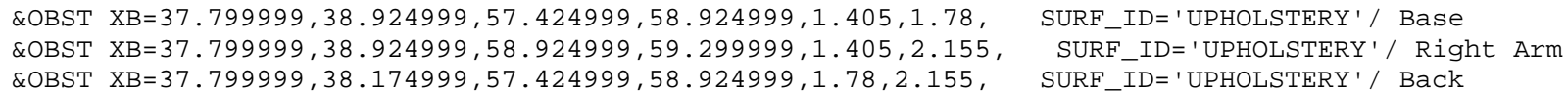

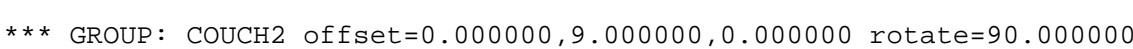

\&OBST XB $=37.799999,38.924999,60.049999,60.424999,1.405,2.155$, SURF_ID=' UPHOLSTERY ' / Left Arm

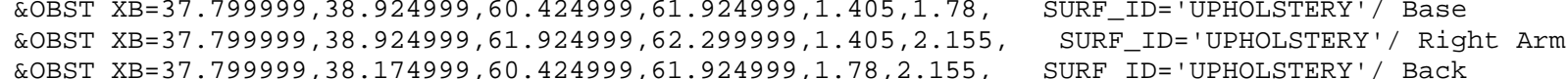

*** GROUP: COUCH2 offset $=0.000000,12.000000,0.000000$ rotate $=90.0000000$

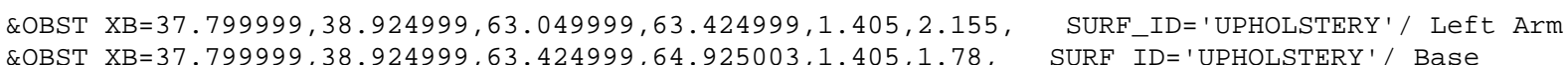

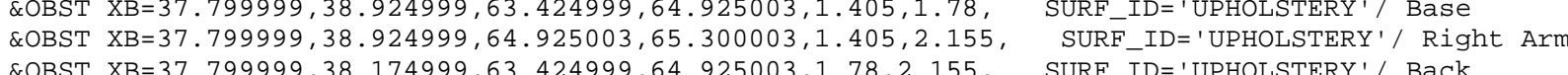

*** GROUP: COUCH2 offset $=0.000000,15.000000,0.000000$ rotate $=90.0000000$

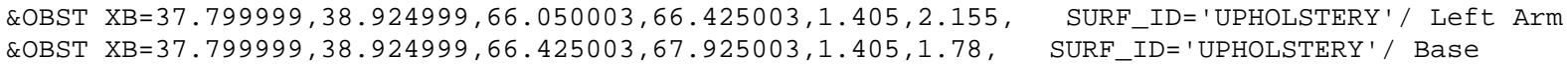
\&OBST XB=37.799999, 38. 924999, 67.925003, 68.300003,1.405, 2.155, SUURF IDD='UPHOLSTERY'/ Right Arm

*** GROUP: COUCH2 offset $=0.000000,18.000000,0.000000$ rotate $=90.000000$

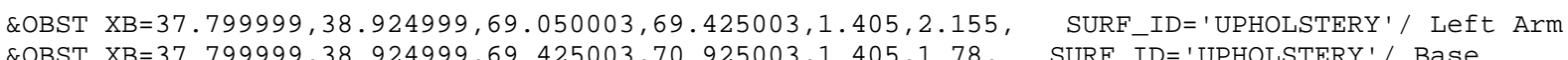

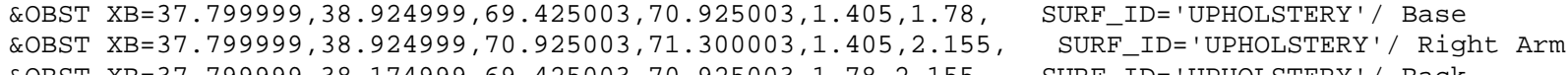

*** GROUP: COUCH2 offset $=0.000000,21.000000,0.000000$ rotate $=90 . \bullet 000000$

\&OBST XB=37.799999, 38.924999, 72.050003, 72.425003, 1.405, 2.155, SURF_ID=' 'UPHOLSTERY'/ Left Arm

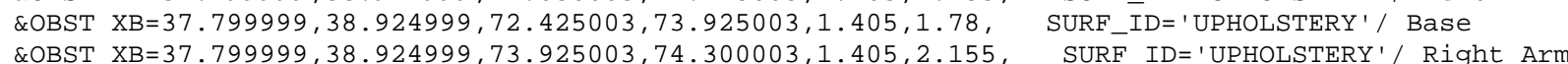


SOBST XB=37. 799999, $38.174999,72,425003,73,925003,1.78,2.155$, SURF_ID=' UPHOLSTERY' / Back

*** GROUP: COUCH2 offset $=0.000000,24.000000,0.000000$ rotate $=90.000000$

\&OBST XB $=37.799999,38.924999,75.050003,75.425003,1.405,2.155$, SURF_ID=' UPHOLSTERY '/ Left Arm \&OBST XB $=37.799999,38.924999,75.425003,76.925003,1.405,1.78$, $\quad$ SURF_ID='UPHOLSTERY'/ Base \&OBST XB $=37.799999,38.174999,75.425003,76.92503,1.78,2.155$, SURF $I D=$ 'UPHOLSTERY' Right

*** GROUP: COUCH2 offset $=0.000000,27.000000, \Theta . .000000$ rotate $=90 . .000000$

\&OBST XB $=37.799999,38.924999,78.050003,78.425003,1.405,2.155$, SURF_ID=' 'UPHOLSTERY'/ Left Arm \&OBST XB=37.799999, 38.924999, 78.425003, 79.925003, 1.405,1.78, SURF_ID='UPHOLSTERY'/ Base QOBST $X \mathrm{~B}=37.799999,38.924999,79.925003,80.300003,1.405,2.155$, SURF_ID='UPHOLSTERY/Right Ar

*** GROUP: COUCH2 offset $=0.000000,30.000000,0.000000$ rotate $=90.000000$

\&OBST XB=37.799999, 38.924999, 81. $050003,81.425003,1.405,2.155$, SURF_ID=' 'UPHOLSTERY '/ Left Arm

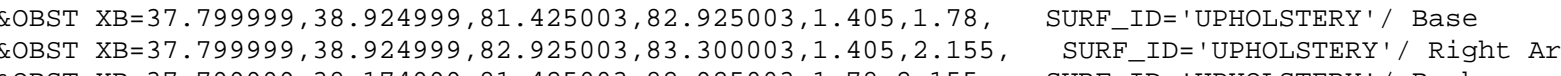

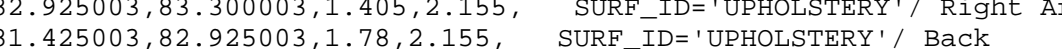

$* * *$ GROUP: BASE

*** GROUP: TWIN

*** GROUP: ROW

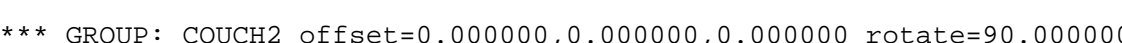

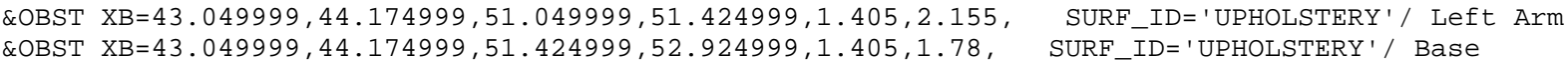

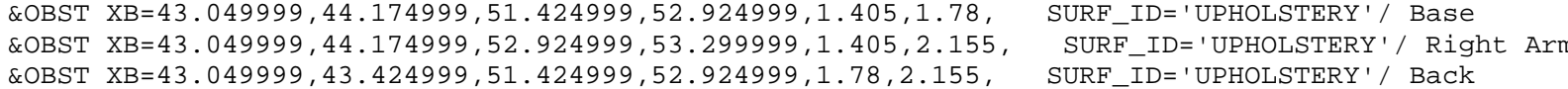

*** GROUP: COUCH2 offset $=0.000000,3.000000,0.000000$ rotate $=90.000000$

\&OBST XB $=43.049999,44.174999,54.049999,54.424999,1.405,2.155$, SURF_ID='UPHOLSTERY' / Left Arm

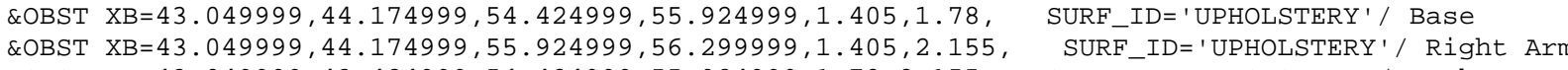

** GROUP: COUCH2 offset $=0,00000,6,000000,0,000000$, rotate $=90,000000$

EOBST XB $=43.049999,44.174999,57.049999,57.424999,1.405,2.155$, SURF_ID=' 'UPHOLSTERY '/ Left Arm QOBST XB=43.04999, 44.174999, 57.424999, 58.924999, 1.405, 1.78, SURF_ID='UPHOLSTERY'/ Base

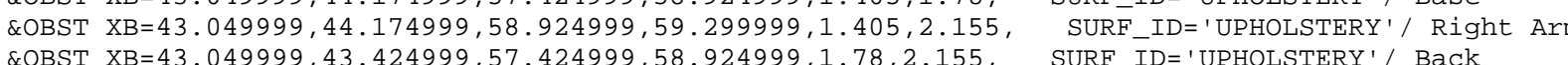

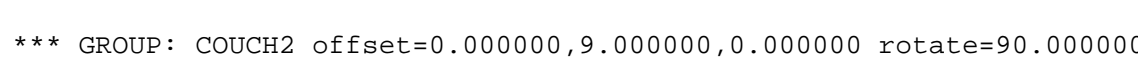

\&OBST XB=43.049999, 44.174999,60.049999,60.424999, 1.405, 2.155, SURF_ID=' 'UPHOLSTERY'/ Left Arm \&OBST XB=43.049999, 44.174999, 60.424999, 61.924999, 1.405, 1.78, SURF_ID='UPHOLSTERY'/Base Arm

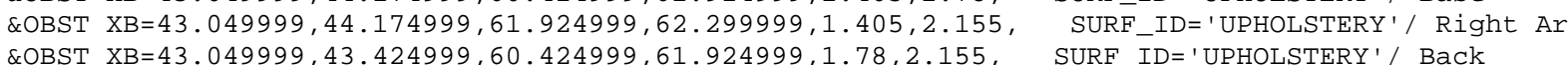

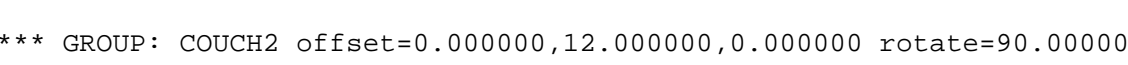

\&OBST XB=43.049999, 44.174999, 63.049999,63.424999, 1.405, 2.155, SURF_ID=' 'UPHOLSTERY'/ Left Arm LOBST XB=43.049999, 44.174999, 63.424999, 64.925003,1.405, 1.78, SURF_IDD='UPHOLSTERY'/ Base

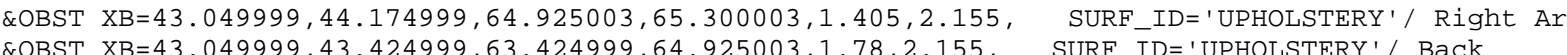

*** GROUP: COUCH2 offset $=0.000000,15.000000,0.000000$ rotate $=90.000000$

8OBST XB=43.049999, 44.174999, 66.050003, 66.425003, 1.405, 2.155, SURF_ID=' 'UPHOLSTERY'/ Left Arm \&OBST XB=43. $049999,44.174999,66.425003,67.925003,1.405,1.78$, SURF_ID='UPHOLSTERY'/ Base \&OBST XB=43.04999, $44.174999,67.925003,68.300003,1.405,2.155$, SURF_ID='UPHOLSTERY'/ Right Ar

* ** GROUP: COUCH2 offset $=0.000000,18 . .000000,0.000000$ rotate $=90.000000$

EOBST XB $=43.049999,44.174999,69.050003,69.425003,1.405,2.155$, SURF_ID= 'UPHOLSTERY' / Left Arm COBST XB $=43.049999,44.174999,69.425003,70.925003,1.405,1.78$, SURF IDE' 'UPHOLLTERY'/ Base

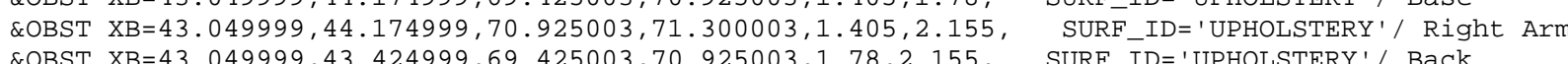

** GROUP: COUCH2 offset $=0,000000,21.000000,0.000000$ rotate $=90, .000000$

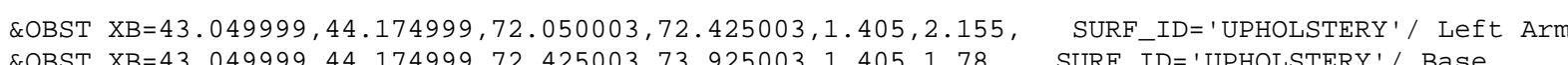

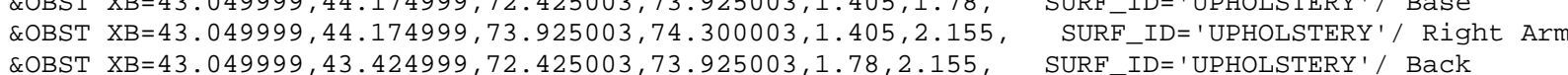

*** GROUP: COUCH2 offset $=0.000000,24 . \odot 00000,0.000000$ rotate $=90.000000$

\&OBST XB $=43.049999,44.174999,75.050003,75.425003,1.405,2.155, \quad$ SURF_ID=' UPHOLSTERY '/ Left Arm
\&OBST XB $=43.049999,44.174999,75.425003,76.925003,1.405,1.78, \quad$ SURF_ID='UPHOLSTERY'/Base Arm

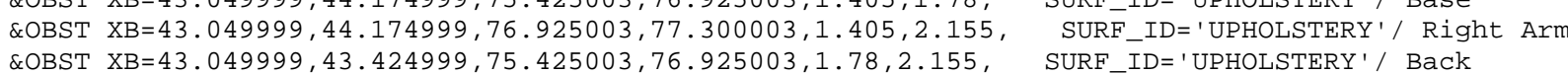

*** GROUP: COUCH2 offset $=0.000000,27.000000,0.000000$ rotate $=90.000000$

\&OBST XB=43. $049999,44.174999,78.050003,78.425003,1.405,2.155$, SURF_ID=' 'UPHOLSTERY ' / Left Arm

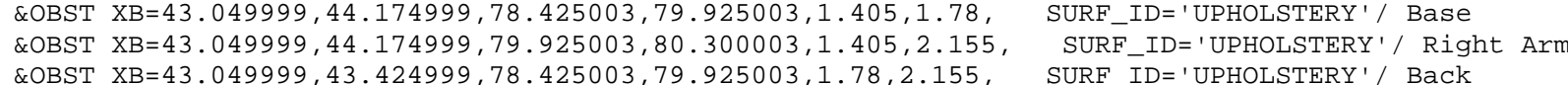

*** GROUP: COUCH2 offset $=0.000000,30.000000,0.000000$ rotate $=90.000000$

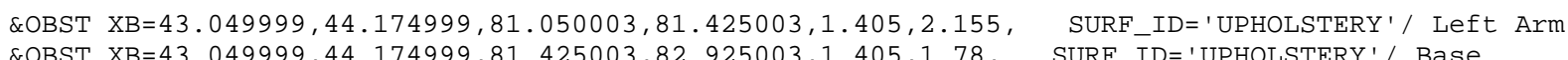
\&OBST XB $=43.049999,44.174999,82.925003,83.300003,1.405,2.155, \quad$ SURF ID IDHO'UPOLSTERY'/ Right Ar \&OBST XB=43. $049999,43.424999,81.425003,82.925003,1.78,2.155$, SURF_ID= 'UPHOLSTERY' / Back

*** GROUP: ROW2

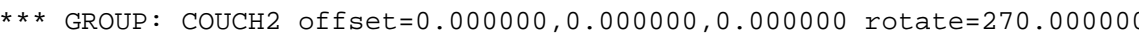

\&OBST XB=41.549999, 42.674999, 52.924999, 53.299999, 1.405, 2.155, SURF_ID=' 'UPHOLSTERY'/ Left Arm \&OBST XB=41.549999, 42.674999, 51.424999, 52. $224999,1.405,1.78$, SURF ID =' 'UPHOLSTERY'/ Base

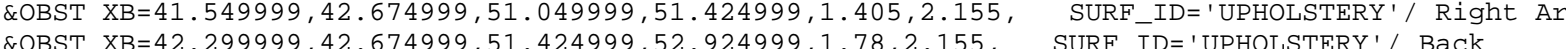

$* * *$ GROUP: COUCH2 offset $=0.000000,3.000000,0.000000$ rotate $=270.000000$

\&OBST XB=41.549999, 42.674999, 55.924999, 56. 299999, 1.405, 2.155, SURF_ID=' 'UPHOLSTERY' / Left Arm \&OBST XB=41.549999, 42.674999, $54.049999,54.424999,1.405,2.155$, SURF_ID=' UPHOLSTERY'/ Right Ar

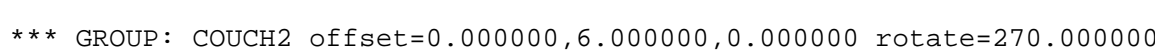

\&OBST XB=41.549999, 42.674999, 58.924999, 59.299999, 1.405, 2.155, SURF_ID='UPHOLSTERY' / Left Arm \&OBST XB=41.549999, 42.674999, 57.049999, 57.424999, 1.405, 2.155, SURF_ID='UPHOLSTERY'/ Right Ar

*** GROUP: COUCH2 off set $=0.000000,9.000000,0.000000$ rotate $=270.000000$

\&OBST XB=41.549999, 42.674999, 61.924999, 62.299999, 1.405, 2.155, SURF_ID=' UPHOLSTERY' / Left Arm \&OBST XB=41.549999, 42.674999, 60.049999, 60.424999, 1.405, 2.155, SURF_ID='UPHOLSTERY'/Right Ar \&OBST XB=42.299999, 42.674999, 60.42499, 61.924999, 1.78, 2.155, SURF_ID='UPHOLSTERY'/ Back

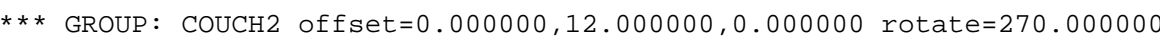

\&OBST XB=41.549999, 42.674999, 64.925003,65.300003, 1.405, 2.155, SURF_ID= 'UPHOLSTERY' / Left Arm \&OBST XB=41.549999,42.674999,63.049999,63.424999,1.405,2.155, SURF_ID='UPHOLSTERY'/ Right Ar \&OBST XB=42.299999, 42.674999, 63.424999, 64.925003, 1.78, 2.155, SURF_ID='UPHOLSTERY'/ Back

*** GROUP: COUCH2 offset $=0.000000,15,000000,0.000000$ rotate $=270,000000$

\&OBST XB=41.549999, 42.674999, 67.925003, 68.300003, 1.405, 2.155, SURF_ID=' 'UPHOLSTERY '/ Left Arm \&OBST XB=41.549999, 42.674999,66.050003,66.425003,1.405, 2.155, SURF ID=' 'UPHOLSTERY'/ Right Ar \&OBST XB=42.299999, 42.674999, 66.425003,67.925003,1.78,2.155, SURF_ID='UPHOLSTERY'/ Back *** GROUP: COUCH2 offset $=0.000000,18.000000,0.000000$ rotate $=270.000000$

\&OBST XB $=41.549999,42.674999,70.925003,71.300003,1.405,2.155, \quad$ SURF_ID= 'UPHOLSTERY' $/$ Left Arm
ROBST XB $=41.549999,42.674999,69.425003,70.925003,1.405,1.78$ SURF_ID='UPHOLSTERY' 
\&OBST XB=41.549999, 42.674999, 69. $050003,69.425003,1.405,2.155, \quad \begin{gathered}\text { SURF_ID=' UPHOLSTERY '/ Right Arm } \\ \text { \&OBST XB }=42.299999,42.674999,69.425003,70.925003,1.78,2.155,\end{gathered}$ SURF_ID='UPHOLSTERY'/ Back *** GROUP: COUCH2 off set $=0.0000000,21.000000,0.000000$ rotate $=270.000000$

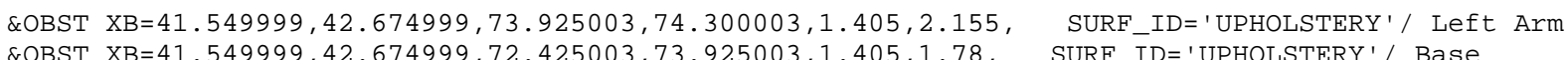

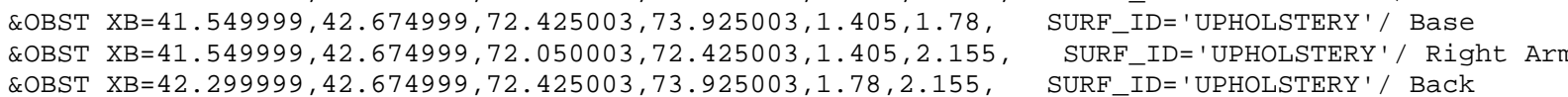
*** GROUP: COUCH2 offset $=0.000000,24.000000,0.000000$ rotate $=270.000000$

\&OBST XB $=41.549999,42.674999,76.925003,77.300003,1.405,2.155$, SURF_ID='UPHOLSTERY'/ Left Arm \&OBST XB=41.549999, 42.674999, 75.425003, 76.925003,1.405, 1.78, SURF_ID='UPHOLSTERY'/ Base Arm \&OBST XB $=42.299999,42.674999,75.425003,76,925003,1.78,2.155$, SURF ID ='UPHOLSTERY'/ Rick Ar

*** GROUP: COUCH2 offset $=0.000000,27.000000,0.000000$ rotate $=270.000000$

\&OBST XB=41.549999, 42.674999, 79.925003, 80.300003,1.405, 2.155, SURF_ID=' 'UPHOLSTERY ' / Left Arm

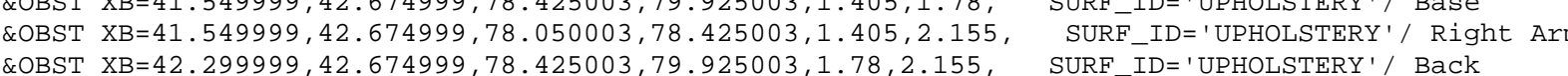

*** GROUP: COUCH2 off set $=0.000000,30.000000,0.000000$ rotate $=270.000000$

LOBST XB $=41.549999,42.674999,82.925003,83.300003,1.405,2.155$, SURF_ID=' 'UPHOLSTERY ' / Left Arm \&OBST XB=41.549999, 42.674999, 81. $050003,81.425003,1.405,2.155$, SURF_ID='UPHOLSTERY'/ Right Ar

*** GROUP: TWIN

$* * *$ GROUP: ROW

*** GROUP: COUCH2 offset $=0.000000, \odot . .000000,0.000000$ rotate $=90.000000$

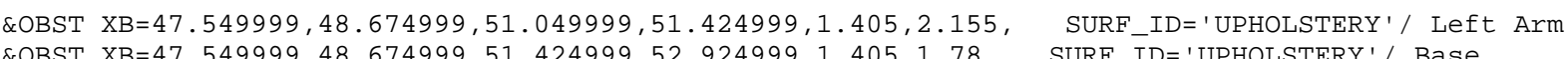
\&OBST XB=47.549999, 48.674999, 52.924999, 53.299999, 1.405, 2.155, SURF_ID=' 'UPHOLSTERY'/ Right Ar *** GROUP: COUCH2 offset $=0.000000,3.000000,0.000000$ rotate $=90.000000$

\&OBST XB=47.549999, 48.674999, 54.049999, 54.424999, 1.405, 2.155, SURF_ID=' 'UPHOLSTERY'/ Left Arm COBST XB $=47.549999,48.674999,54.424999,55.924999,1.405,1.78, \quad$ SURF-IDE' 'UPHOLSTERY'/ Base

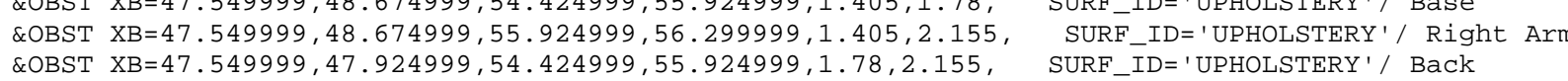
*** GROUP: COUCH2 offset $=0.000000,6.000000,0.000000$ rotate $=90.000000$

\&OBST XB=47.549999, 48.674999, 57. 049999, 57.424999, 1.405, 2.155, SURF_ID='UPHOLSTERY'/ Left Arm

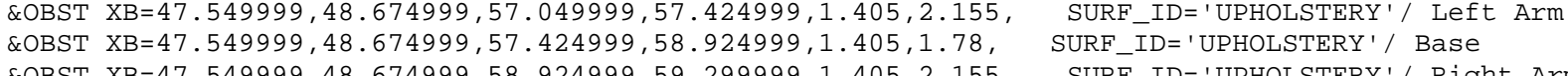

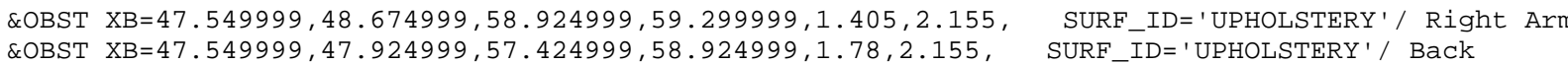

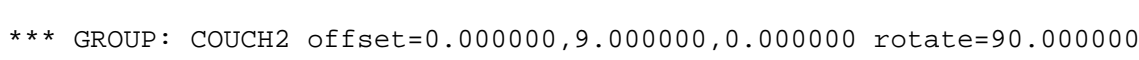

\&OBST XB=47.549999, 48.674999, 60. 449999, 60.424999, 1.405, 2.155, SURF_ID=' 'UPHOLSTERY ' / Left Arm

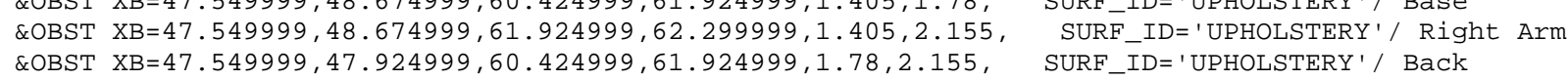
$* * *$ GROUP: COUCH2 offset $=0.000000,12.0000000,0.000000$ rotate $=90.000000$

\&OBST XB $=47.549999,48.674999,63.049999,63.424999,1.405,2.155, \quad$ SURF_ID= ' 'UPHOLSTERY ' / Left Arm
\&OBST XB

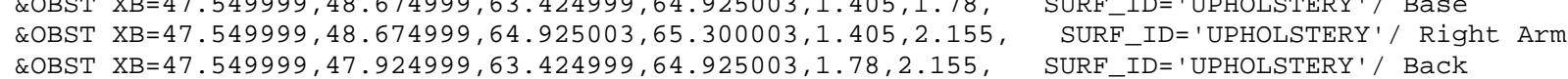

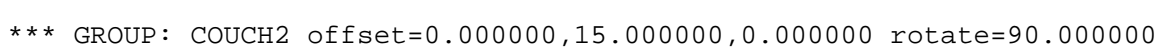

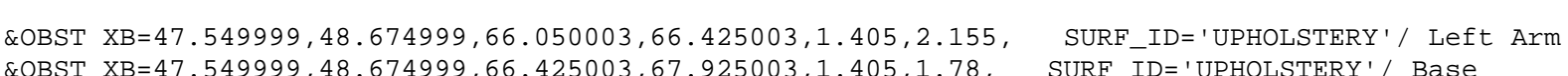

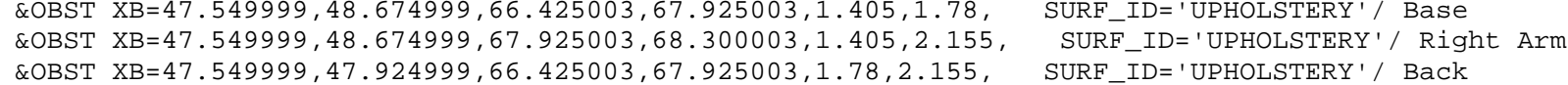

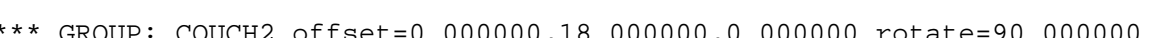

\&OBST XB $=47.549999,48.674999,69.050003,69.425003,1.405,2.155, \quad$ SURF_ID= 'UPHOLSTERY ' $/$ Left Arm
\&OBST XB=47.549999, $48.674999,69.425003,70.925003,1.405,1.78, \quad$ SURF_ID='UPHOLSTERY'

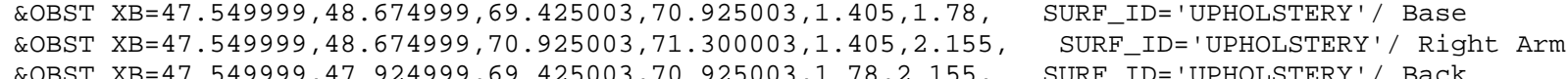

$* * *$ GROUP: COUCH2 offset $=0.000000,21.000000,0.000000$ rotate $=90.000000$

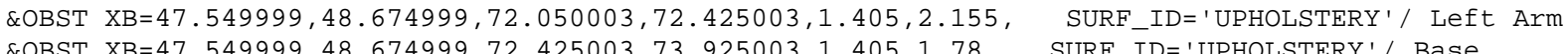

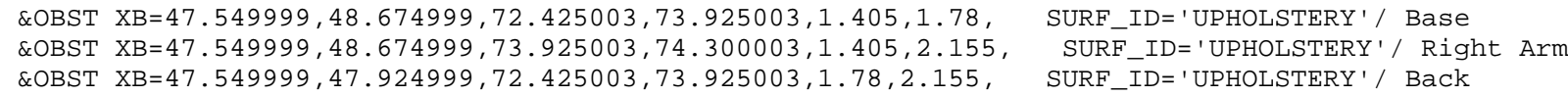

*** GROUP: COUCH2 offset $=0.000000,24 . .000000,0.000000$ rotate $=90.000000$

\&OBST XB $=47.549999,48.674999,75.050003,75.425003,1.405,2.155$,
\&OBST XB $=47.549999,48.674999,75.425003,766.925003,1.405,1.78, \quad$ SURF_ID=' 'UPHOLSTERY '/ Left Arm
SURF_ID=' 'UPHOLSTERY'/ Base

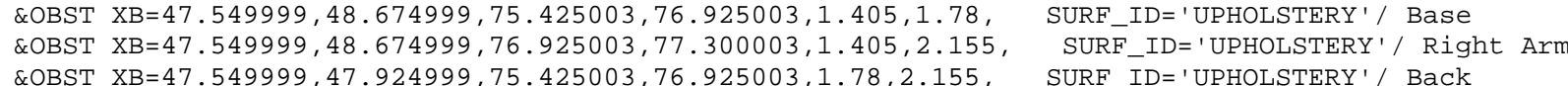
*** GROUP: COUCH2 offset $=0.000000,27.000000,0.000000$ rotate $=90.000000$

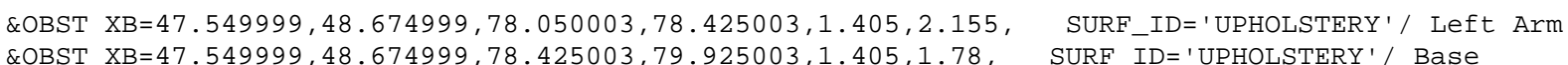

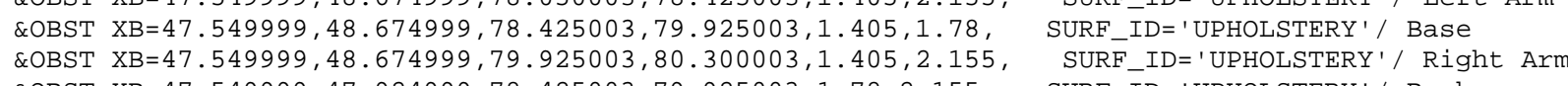

*** GROUP: COUCH2 offset $=0.000000,30.000000,0.000000$ rotate $=90.000000$

\&OBST XB=47.549999, 48.674999, 81.050003, 81.425003,1.405, 2.155, SURF_ID=' 'UPHOLSTERY '/ Left Arm

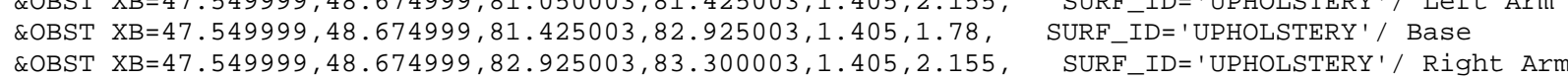
\&OBST XB $=47.549999,47.924999,81.425003,82.925003,1.78,2.155$, SURF_ID='UPHOLSTERY' '/ Back

$* * *$ GROUP: ROW2

*** GROUP: COUCH2 offset $=0.000000,0.000000,0.000000$ rotate $=270.000000$

\&OBST XB $=46.049999,47.174999,52.924999,53.299999,1.405,2.155$, SURF_ID=' 'UPHOLSTERY ' / Left Arm

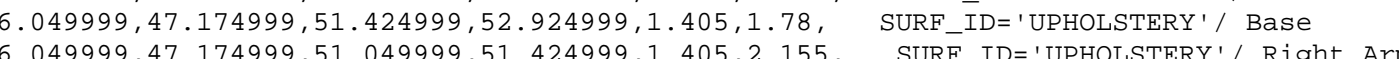
\&OBST XB=46. $049999,47.174999,51.049999,51.424999,1.405,2.155$,
\&OBST XB $\quad$ S $=46.799999,47.174999,51.424999,52.924999,1.78,2.155$,
SURF_ID=' 'UPHOLSTERY' $1 /$ Back

*** GROUP: COUCH2 offset $=0.000000,3.000000,0.000000$ rotate $=270.000000$

\&OBST XB $=46.049999,47.174999,55.924999,56.299999,1.405,2.155$, SURF_ID= ' UPHOLSTERY ' / Left Arm \&OBST X LOBST XB=46. $049999,47.174999,54.049999,54.424999,1.405,2.155$,
\&OBST XB=46.79999, $47.174999,54.424999,55.924999,1.78,2.155$,
SURF_IDE='UPHOLSTERY'/ Back

*** GROUP: COUCH2 offset $=0.000000,6.000000,0.0000000$ rotate $=270.000000$

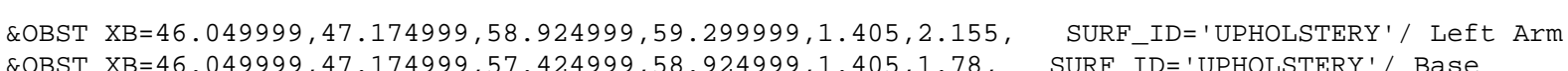
QOBST XB $=46.049999,47.174999,57.049999,57.424999,1.405,2.155$,
QOBST

*** GROUP: COUCH2 offset $=0.000000,9.000000,0.0000000$ rotate $=270.000000$

\&OBST XB $=46.049999,47.174999,61.924999,62.299999,1.405,2.155, \quad$ SURF_ID='UPHOLSTERY ' / Left Arm
\&OBST XB $=46.049999,47.174999,60.424999,61.924999,1.405,1.78, \quad$ SURF_ID='UPHOLSTERY'

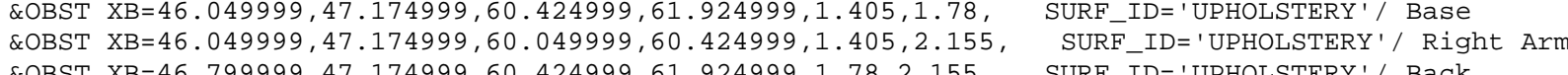
$* * *$ GROUP: COUCH2 offset $=0.000000,12.000000,0.000000$ rotate $=270$. .000000

\&OBST XB $=46.049999,47.174999,64.925003,65.300003,1.405,2.155$, SURF_ID=' 'UPHOLSTERY ' / Left Arm

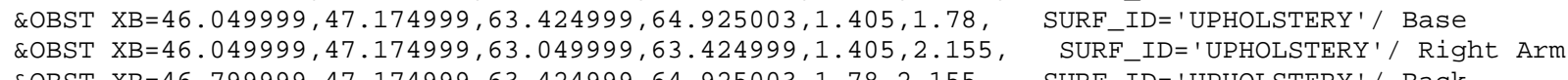
*** GROUP: COUCH2 offset $=0.000000,15.000000,0.000000$ rotate $=270.000000$

\&OBST XB=46.049999, 47.174999, 67.925003, 68.300003, 1.405, 2.155, SURF_ID=' 'UPHOLSTERY'/ Left Arm \&OBST XB=46. $049999,47.174999,66.425003,67.925003,1.405,1.78$,
\&OBST XB=46. 
\&OBST XB=46.799999, $47.174999,66.425003,67.925003,1.78,2.155$, SURE_ID=' UPHOLSTERY' / Back

*** GROUP: COUCH2 offset $=0.000000,18.000000,0.000000$ rotate $=270$. ๑०००००

\&OBST XB=46.049999, 47.174999, 70.925003, 71.300003, 1.405, 2.155, SURF_ID=' 'UPHOLSTERY ' / Left Arm \&OBST XB=46.049999, 47.174999, 69.050003, 69.425003,1.405, 2.155, SURF_ID=' 'UPHOLSTERY'/ Right Arm

*** GROUP: COUCH2 offset $=0.000000,21.000000,0.000000$ rotate $=270.000000$

\&OBST XB=46. 049999, 47.174999, 73.925003, 74. 300003, 1.405, 2.155, SURF_ID=' 'UPHOLSTERY'/ Left Arm \&OBST XB=46.049999, 47.174999, $72.425003,73.925003,1.405,1.78$, SURF_ID='UPHOLSTERY'/ Base QOBST $X \mathrm{~B}=46.79999,47.174999,72.050003,72.425003,1.405,2.155$, SURF_ID='UPHOLSTERY//Right Ar

*** GROUP: COUCH2 offset $=0.000000,24.000000,0.000000$ rotate $=270.000000$

\&OBST XB=46. $049999,47.174999,76.925003,77.300003,1.405,2.155$, SURF_ID=' 'UPHOLSTERY'/ Left Arm \&OBST XB=46.049999, 47.174999, 75.425003, 76.925003,1.405, 1.78, SURF_ID='UPHOLSTERY'/ Base COBST XB=46. $049999,47.174999,75.050003,75.425003,1.405,2.155$,
COBST XB $=46.799999,47.174999,75.425003,76.925003,1.78,2.155$,
SURF_IDE='UPHOLSTERY '/ Right Arm
SURF 'UPHOLSTERY'/ Back $* * *$ GROUP: COUCH2 offset $=0.000000,27.000000,0.000000$ rotate $=270.000000$

\&OBST XB=46. $049999,47.174999,79.925003,80.300003,1.405,2.155$, SURF_ID=' 'UPHOLSTERY' / Left Arm

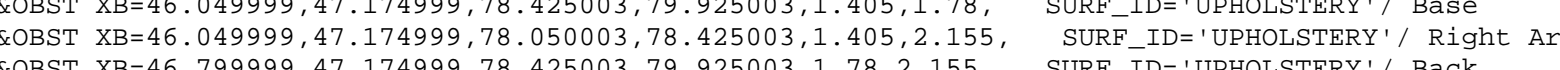

*** GROUP: COUCH2 offset $=0.000000,30.000000,0.000000$ rotate $=270.000000$

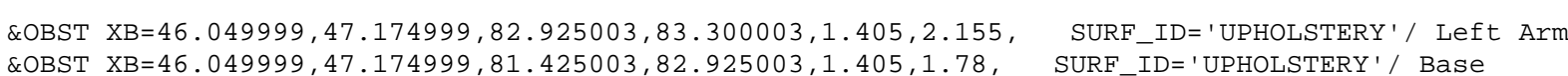

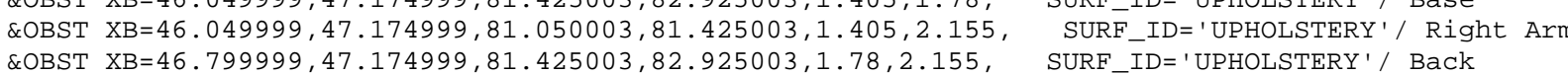

\section{*** GROUP: TWIN}

$* * *$ GROUP: ROW

*** GROUP: COUCH2 offset $=0.000000,0.000000,0.000000$ rotate $=90.000000$

\&OBST XB $=52.049999,53.174999,51.049999,51.424999,1.405,2.155, \quad$ SURF_ID=' 'UPHOLSTERY '/ Left Arm

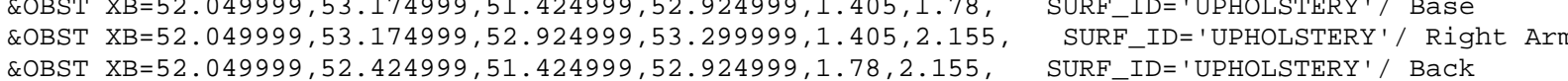
*** GROUP: COUCH2 offset $=0.000000,3.000000,0.000000$ rotate $=90.000000$

\&OBST XB $=52.049999,53.174999,54.049999,54.424999,1.405,2.155, \quad$ SURF_ID=' 'UPHOLSTERY ' / Left Arm

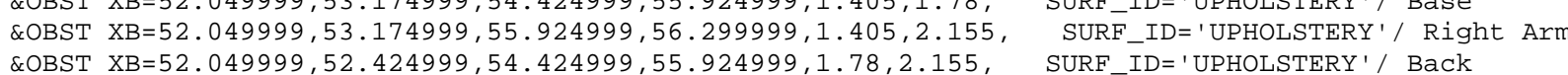

*** GROUP: COUCH2 offset $=0.000000,6.000000,0.000000$ rotate $=90.000000$

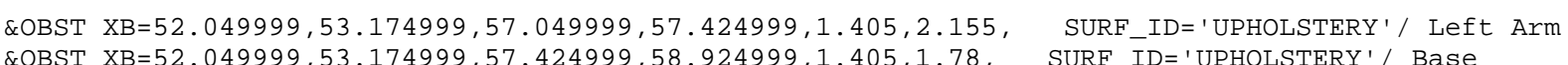

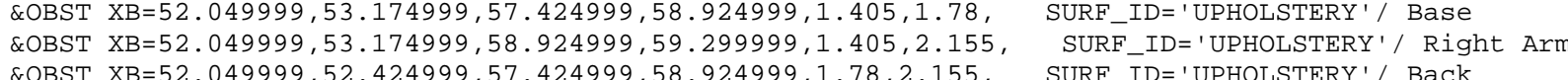
*** GROUP: COUCH2 offset $=0.000000,9.000000,0.000000$ rotate $=90.000000$

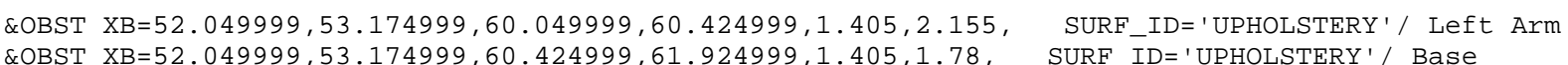

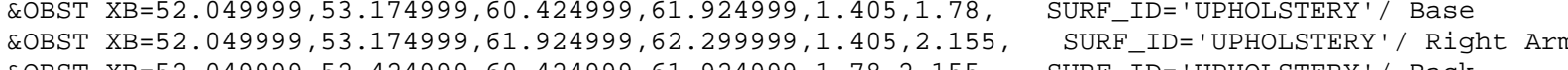

$* * *$ GROUP: COUCH2 offset $=0.000000,12.000000,0.000000$ rotate $=90 . .000000$

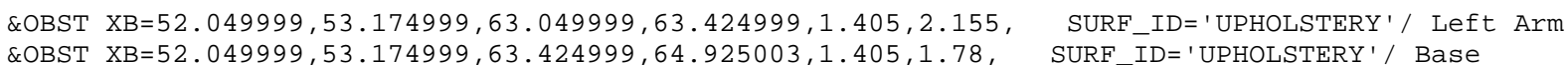

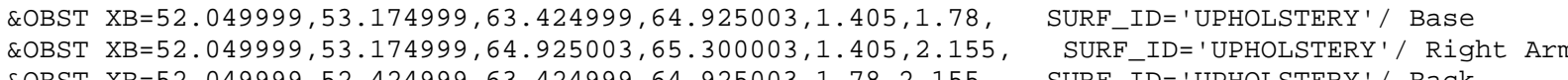

*** GROUP: COUCH2 offset $=0.000000,15.0000000,0.000000$ rotate $=90 . .000000$

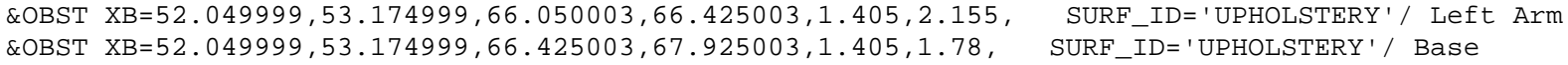
\&OBST XB=52.049999, 53.174999, 67.925003,68.300003,1.405, 2.155, SURF_ID=' 'UPHOLSTERY'/ Right Ar

*** GROUP: COUCH2 offset $=0.000000,18.000000,0.000000$ rotate $=90 . .000000$

\&OBST XB=52.049999, 53.174999, 69.050003, 69.425003,1.405, 2.155, SURF_ID=' 'UPHOLSTERY '/ Left Arm \&OBST XB=52.049999, 53.174999, 69.425003, 70.925003, 1.405, 1.78, SURF_ID='UPHOLSTERY'/ Base

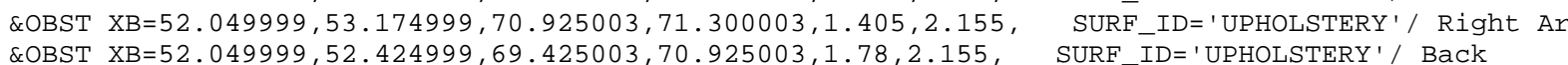

*** GROUP: COUCH2 offset $=0.000000,21.000000,0.000000$ rotate $=90 . .000000$

\&OBST XB=52.049999, 53.174999, 72.050003, 72.425003, 1.405, 2.155, SURF_ID='UPHOLSTERY'/ Left Arm

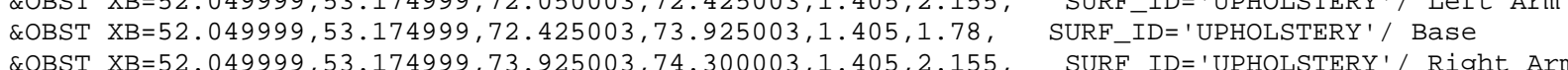

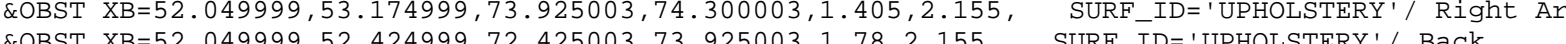

*** GROUP: COUCH2 offset $=0.000000,24.000000,, 0.000000$ rotate $=90.000000$

\&OBST XB $=52.049999,53.174999,75.050003,75.425003,1.405,2.155$, SURF_ID=' 'UPHOLSTERY'/ Left Arm

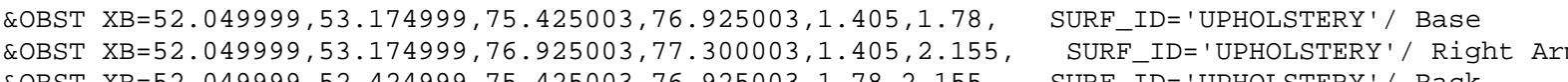

*** GROUP: COUCH2 offset $=0.000000,27.000000,0.000000$ rotate $=90.000000$

\&OBST XB=52. $049999,53.174999,78.050003,78.425003,1.405,2.155$, SURF_ID= 'UPHOLSTERY ' / Left Arm

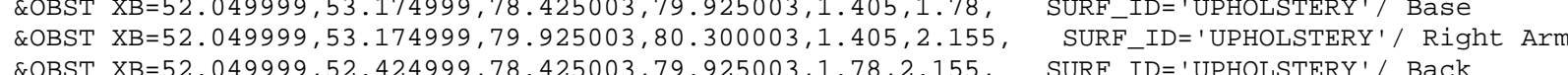

*** GROUP: COUCH2 offset $=0.000000,30.000000,0.000000$ rotate $=90.000000$

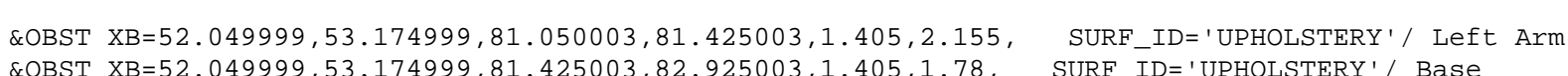

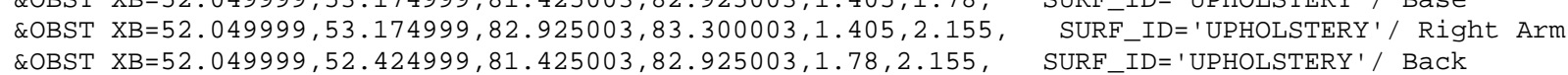

\section{*** GROUP: ROW2}

** GROUP: COUCH2 offset=0.

\&OBST XB $=50.549999,51.674999,52.924999,53.299999,1.405,2.155, \quad$ SURF_ID='UPHOLSTERY '/ Left Arm
\&OBST XB $=50.549999,51.674999,51.424999,52.924999,1.405,1.78$,
SURF_ID='UPHOLSTERY' $/$ Base \&OBST XB $50.549999,51.674999,51.049999,51.424999,1.405,2.155$, SURF IDD='UPHOLSTERY'/Right Arm

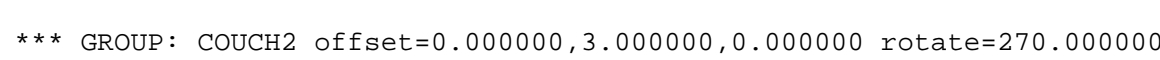

\&OBST XB=50.549999, 51.674999, 55.924999, 56. 299999, 1.405, 2.155, SURF_ID=' 'UPHOLSTERY'/ Left Arm \&OBST XB=50.549999, $51.674999,54.424999,55.924999,1.405,1.78, \quad$ SURF_ID='UPHOLSTERY'/ Base \&OBST XB=50.54999,

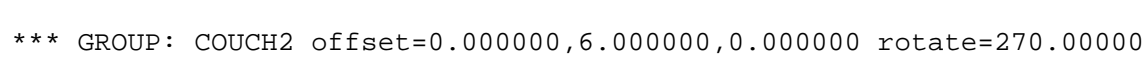

\&OBST XB=50.549999, 51.674999, 58.924999, 59.299999, 1.405, 2.155, SURF_ID='UPHOLSTERY'/ Left Arm

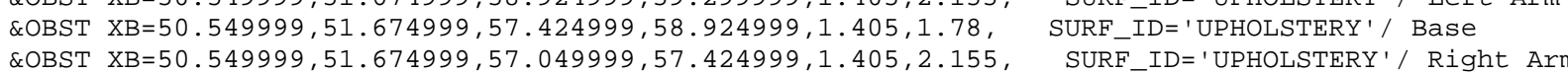
\&OBST XB $51.299999,51.674999,57.424999,58.924999,1.78,2.155$, SURF_ID='UPHOLSTERY'/ Rick Ar

*** GROUP: COUCH2 offset $=0.000000,9.000000,0.000000$ rotate $=270.000000$

\&OBST XB=50.549999, 51.674999, 61.924999, 62. 299999, 1.405, 2.155, SURF_ID=' 'UPHOLSTERY'/ Left Arm

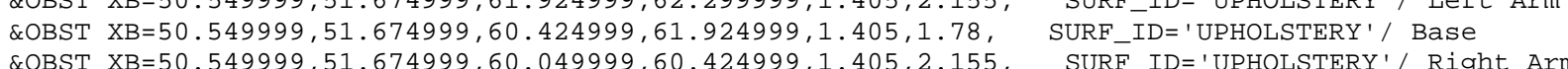
\&OBST XB=51.299999, 51.674999, 60.424999, 61.924999, 1.78, 2.155, SURF_ID=' 'UPHOLSTERY'/ Right Ar

$* * *$ GROUP: COUCH2 offset $=0.000000,12.000000,0.000000$ rotate $=270.000000$

\&OBST XB=50.549999, 51.674999, 64.925003, 65.300003,1.405, 2.155, SURF_ID='UPHOLSTERY' / Left Arm \&OBST XB=50.549999, 51.674999, 63.049999, 63.424999, 1.405, 2.155, SURF-ID='UPHOLSTERY'/ Right Ar 
*** GROUP: COUCH2 offset $=0.000000,15.000000,0.000000$ rotate $=270.000000$

\&OBST XB=50.549999, 51.674999, 67.925003, 68.300003, 1.405, 2.155, SURF_ID=' 'UPHOLSTERY '/ Left Arm

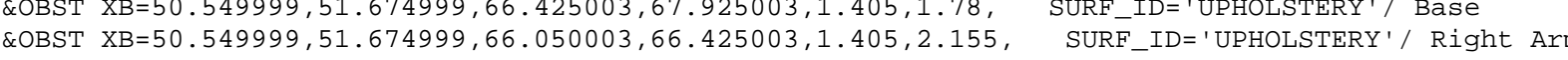
\&OBST XB=51.299999, 51.674999, 66.425003, 67.925003,1.78, 2.155, SURF_ID=' UPHOLSTERY'/ Back

*** GROUP: COUCH2 offset $=0.000000,18,000000,0.000000$ rotate $=270.000000$

\&OBST XB=50.549999, 51.674999, 70.925003, 71.300003,1.405, 2.155, SURF_ID= 'UPHOLSTERY '/ Left Arm
\&OBST XB=50.549999, $51.674999,69.425003,70.925003,1.405,1.78, \quad$ SURF_ID='UPHOLSTERY' $/$ Base

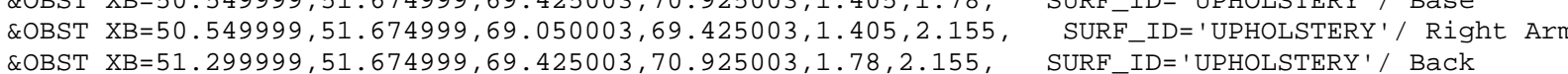

*** GROUP: COUCH2 offset $=0.000000,21.000000,0.000000$ rotate $=270.000000$

\&OBST XB=50.549999, 51.674999, 73.925003, 74.300003,1.405, 2.155, SURF_ID='UPHOLSTERY '/ Left Arm
\&OBST XB=50.549999, $51.674999,72.425003,73.925003,1.405,1.78, \quad$ SURF_ID='UPHOLSTERY'/ Base

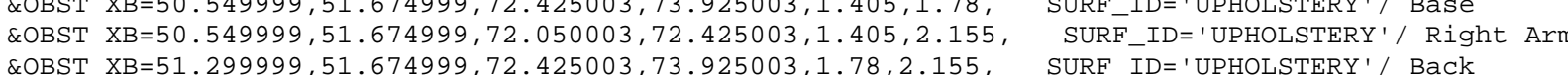
*** GROUP: COUCH2 offset $=0.000000,24.000000,0.000000$ rotate $=270.000000$

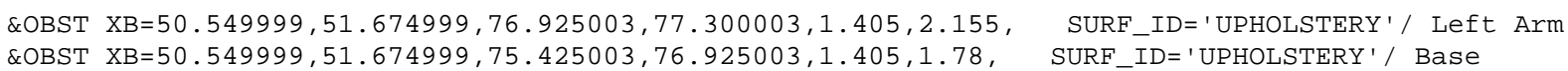
COBST XB $=50.549999,51.674999,75.050003,75.425003,1.405,2.155$,
SOBST

*** GROUP: COUCH2 offset $=0.000000,27.000000,0.0000000$ rotate $=270.000000$

\&OBST XB=50.549999, 51.674999, 79.925003, 80.300003, 1.405, 2.155, SURF_ID=' 'UPHOLSTERY' ' Left Arm
\&OBST XB=50.549999, $51.674999,78.425003,79.925003,1.405,1.78, \quad$ SURF_ID='UPHOLSTERY'

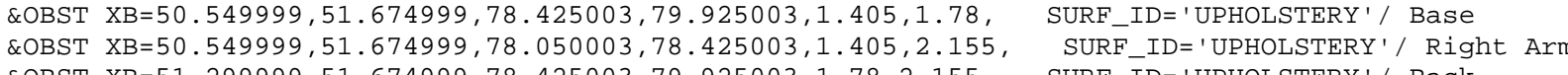
(1) SURF_ID $=$ 'L

OOBST XB=50.549999, 51.674999, 82.925003, 83.300003,1.405, 2.155, SURF_ID='UPHOLSTERY' / Left Arm SOBST XB $50.54999,51.674999,81.425003,82.925003,1.405,1.78$, SURF_ID='UPHOLSTERY'/ Base ATh \&OBST $X B=51.299999,51.674999,81.425003,82.925003,1.78,2.155$, SURF_ID='UPHOLSTERY'/ Back Ar

*** GROUP: TWIN

*** GROUP: ROW

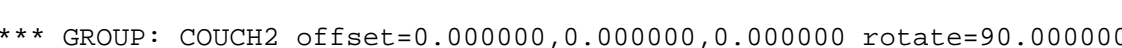

\&OBST XB $=56.549999,57.674999,51.049999,51.424999,1.405,2.155$, SURF_ID=' 'UPHOLSTERY '/ Left Arm

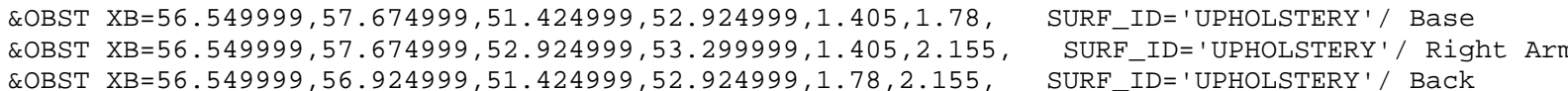

*** GROUP: COUCH2 offset $=0.0000000,3.000000,0.000000$ rotate $=90.000000$

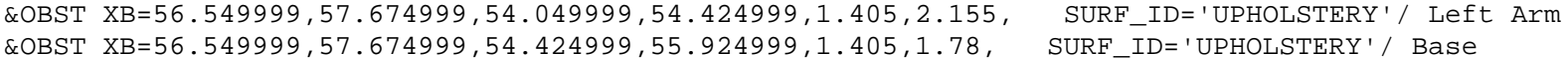

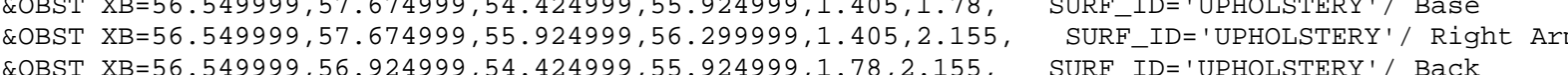

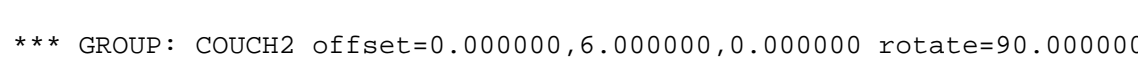

\&OBST XB $=56.549999,57.674999,57.049999,57.424999,1.405,2.155$, SURF_ID='UPHOLSTERY'/ Left Arm \&OBST XB=56.549999, $57.674999,57.424999,58.924999,1.405,1.78$, SURF_ID='UPHOLSTERY'/ Base

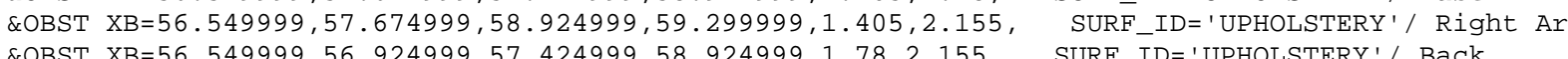
*** GROUP: COUCH2 offset $=0.000000,9.000000,0.0000000$ rotate $=90.000000$

\&OBST XB=56.549999, 57.674999,60.049999, 60.424999, 1.405, 2.155, SURF_ID=' 'UPHOLSTERY'/ Left Arm \&OBST XB=56.549999, 57.674999, 60.424999, 61.924999, 1.405, 1.78, SURF_ID='UPHOLSTERY'/ Base Arm \&OBST XB $=56.549999,57.674999,61.92499,62.299999,1.45,2.155$, SURF IDE=' 'UPHOLSTERY'/ Right Ar

*** GROUP: COUCH2 offset $=0.000000,12.000000,0.000000$ rotate $=90.000000$
\&OBST XB=56.549999, 57.674999, 63. 449999, 63.424999, 1.405, 2.155, SURF_ID=' 'UPHOLSTERY'/ Left Arm \&OBST XB=56.549999, $57.674999,63.424999,64.925003,1.405,1.78$, SURF-IDE'UPHOLSTERY'/ Base عOBST XE *** GROUP: COUCH2 offset $=0.000000,15.000000,0.000000$ rotate $=90.000000$

\&OBST XB=56.549999, 57.674999, 66. $050003,66.425003,1.405,2.155$, SURF_ID=' 'UPHOLSTERY' / Left Arm

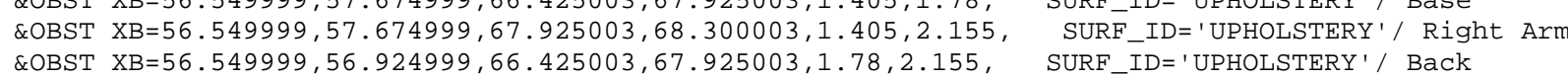
*** GROUP: COUCH2 offset $=0.000000,18.000000,0.000000$ rotate $=90.000000$

\&OBST XB=56.549999, 57.674999, 69. 050003, 69.425003, 1.405, 2.155, SURF_ID= 'UPHOLSTERY' / Left Arm

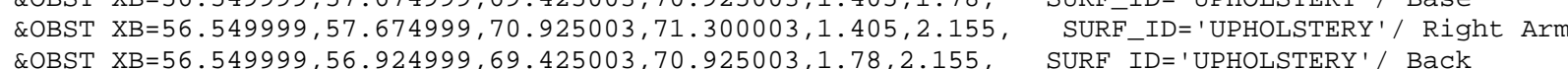

*** GROUP: COUCH2 offset $=0.000000,21.000000,0.000000$ rotate $=90.000000$

\&OBST XB $=56.549999,57.674999,72.050003,72.425003,1.405,2.155, \quad$ SURF_ID=' 'UPHOLSTERY ' / Left Arm

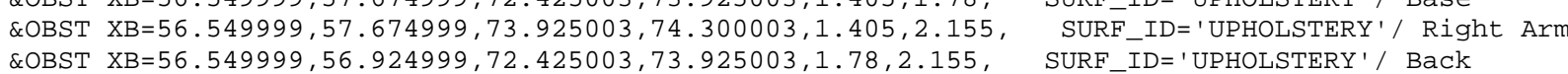

*** GROUP: COUCH2 offset $=0 ., 000000,24 ., 000000,0 ., 000000$ rotate $=90 ., 000000$

\&OBST XB=56.549999, $57.674999,75.050003,75.425003,1.405,2.155$, SURF_ID=' 'UPHOLSTERY'/ Left Arm \&OBST XB $56.549999,57.674999,76.925003,77.300003,1.405,2.155$, SURF ID='UPHOLSTERY'/ Rase Ar Ar \&OBST XB=56.549999, 56.924999, 75.425003,76.925003,1.78,2.155, SURF_ID='UPHOLSTERY'/ Back

*** GROUP: COUCH2 offset $=0.000000,27.000000,0.000000$ rotate $=90.000000$

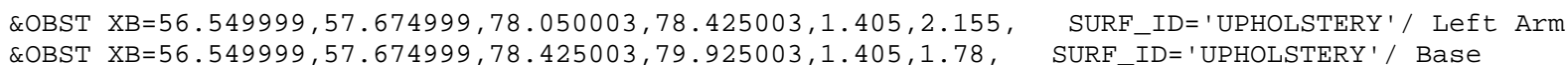
COBST XB $=56.549999,57.674999,79.925003,80.300003,1.405,2.155$, SURE_ID='UPHOLSTERY'/ Right Ar \&OBST X $\mathrm{B}=56.549999,56.924999,78.425003,79.925003,1.78,2.155$, SURF_ID=' 'UPHOLSTERY'/ Back

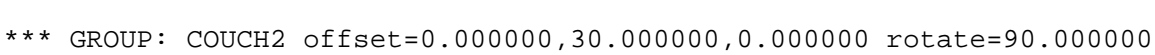

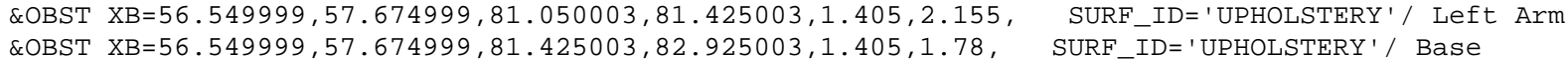
\&OBST XB $=56.549999,57.679999,82.925003,83.300003,1.405,2.155$, SURF ID ' 'UPHOLSTCRY'/ Right Ar COBST XB $\mathrm{X}=56.549999,56.924999,81.425003,82.925003,1.78,2.155$, SURF_ID='UPHOLSTERY'/ Back

*** GROUP: ROW2

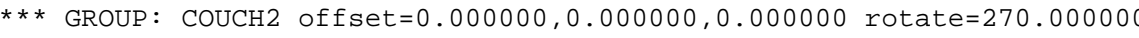

\&OBST XB=55. $449999,56.174999,52.924999,53.299999,1.405,2.155$, SURF_ID=' 'UPHOLSTERY'/ Left Arm

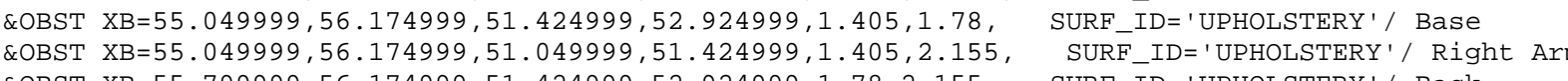

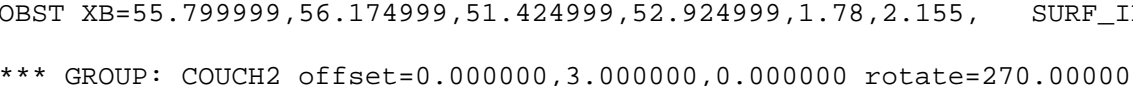

\&OBST XB $=55.049999,56.174999,55.924999,56.299999,1.405,2.155$, SURF_ID=' 'UPHOLSTERY'/ Left Arm QOBST XB $=5.049999,56.174999,54.049999,54.424999,1.405,2.155$,
QOBST

*** GROUP: COUCH2 offset $=0.000000,6.000000,0.000000$ rotate $=270.000000$

\&OBST XB $=55.049999,56.174999,58.924999,59.299999,1.405,2.155$, SURF_ID=' 'UPHOLSTERY'/ Left Arm \&OBST XB=55.049999, 56.174999, $57.049999,57.424999,1.405,2.155$, SURF_ID='UPHOLSTERY'/ Right Arm \&OBST XB=55.79999, $56.174999,57.424999,58.924999,1.78,2.155$, SURF_ID='UPHOLSTERY'/ Back

*** GROUP: COUCH2 offset $=0.000000,9.000000,0.000000$ rotate $=270.000000$

EOBST XB=55. 449999, 56.174999, 61.924999, 62.299999, 1.405, 2.155, SURF_ID=' UPHOLSTERY'/ Left Arm

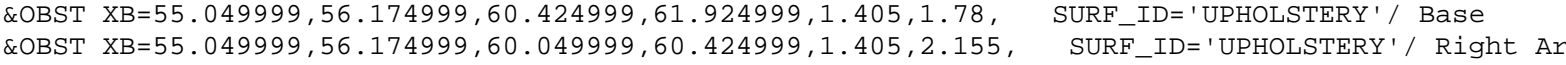
Q
\&OBST XB $X=55.799999,56.174999,60.424999,61.924999,1.78,2.155$, 


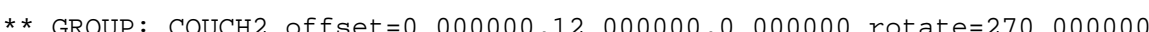

\&OBST XB=55. $049999,56.174999,64.925003,65.300003,1.405,2.155, \quad$ SURF_ID='UPHOLSTERY'/ Left Arm
\&OBST XB=55.049999, $56.174999,63.424999,64.925003,1.405,1.78, \quad$ SURF_ID='UPHOLSTERY'/ Base

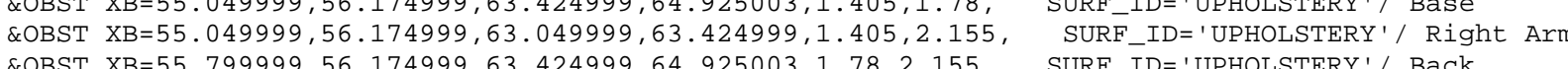

*** GROUP: COUCH2 offset $=0.000000,15.000000,0.000000$ rotate $=270.000000$

\&OBST XB $=55.049999,56.174999,67.925003,68.300003,1.405,2.155, \quad$ SURF_ID=' 'UPHOLSTERY '/ Left Arm
COBST XB

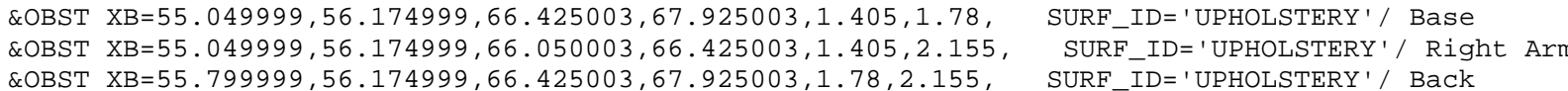
*** GROUP: COUCH2 offset $=0.000000,18.000000,0.000000$ rotate $=270.000000$

\&OBST XB $=55.049999,56.174999,70.925003,71.300003,1.405,2.155$, SURF_ID=' 'UPHOLSTERY '/ Left Arm \&OBST XB $55.049999,56.174999,69.425003,70.925003,1.456,1.78, \quad$ SURF_ID='UPHOLSTERY'/ Base COBST XB $=55.049999,56.174999,69.050003,69.425003,1.405,2.155, \quad$ SURF_ID='UPHOLSTERY'/Right Ar
\&OBST XB=55.799999, $56.174999,69.425003,70.925003,1.78,2.155$, SURF_ID='UPHOLSTERY'/Back

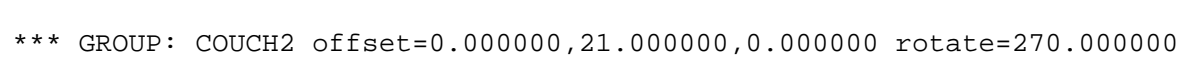

\&OBST XB=55. 049999, 56. 174999, 73.925003, 74.300003,1.405, 2.155, SURF_ID=' 'UPHOLSTERY'/ Left Arm \&OBST XB=55. $449999,56.174999,72.425003,73.925003,1.405,1.78$, SURF_ID='UPHOLSTERY'// Base \&OBST XB=55.04999, *** GROUP: COUCH2 offset $=0.000000,24.000000,0.000000$ rotate $=270.000000$

\&OBST XB $=55.049999,56.174999,76.925003,77.300003,1.405,2.155$, SURF_ID=' 'UPHOLSTERY'/ Left Arm

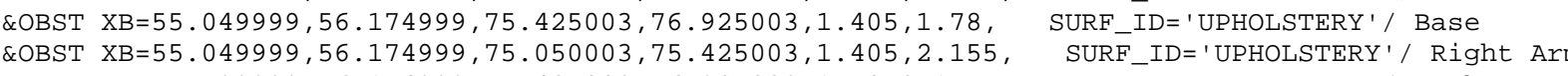

*** GROUP: COUCH2 offset $=0.000000,27.000000,0.000000$ rotate $=270.000000$

EOBST XB=55. $049999,56.174999,79.925003,80.300003,1.405,2.155$, SURF_ID=' 'UPHOLSTERY'/ Left Arm QOBST XB=55.049999, 56.174999, 78.425003, 79. 925003,1.405, 1.78, SURF_ID='UPHOLSTERY'/ Base Arm \&OBST XB $=55.799999,56.174999,78.425003,79.925003,1.78,2.155$, SURF ID='UPHOLSTERY'/ Right Ar

*** GROUP: COUCH2 offset $=0.000000,30.000000,0.000000$ rotate $=270.000000$

\&OBST XB $=55.049999,56.174999,82.925003,83.300003,1.405,2.155$, SURF_ID=' 'UPHOLSTERY'/ Left Arm \&OBST XB=55.049999, 56.174999, 81.425003, 82.925003, 1.405, 1.78, SURF ID='UPHOLSTERY'/ Base \&OBST XB=55. $449999,56.174999,81.050003,81.425003,1.405,2.155$, SURF_ID=' 'UPHOLSTERY'/ Right Ar

*** GROUP: TWIN

*** GROUP: ROW

*** GROUP: COUCH2 offset $=0.000000,0.000000,0.000000$ rotate $=90.000000$

\&OBST XB=61. $449999,62.174999,51.049999,51.424999,1.405,2.155$, SURF_ID=' 'UPHOLSTERY'/ Left Arm

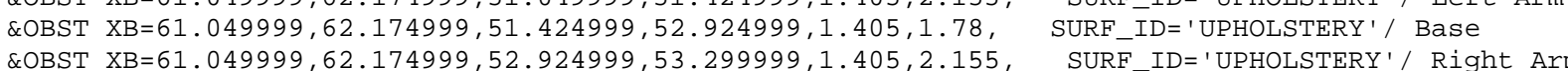
COBST $X \mathrm{~B}=61.049999,62.174999,52.924999,53.299999,1.405,2.155$, SURF_ID='UPHOLSTERY//Right Ar

$* * *$ GROUP: COUCH2 offset $=0.000000,3.000000,0.000000$ rotate $=90.000000$

\&OBST XB=61. 049999, 62.174999, 54. 049999, 54.424999, 1.405, 2.155, SURF_ID=' 'UPHOLSTERY'/ Left Arm \&OBST XB=61.049999, 62.174999, $54.424999,55.924999,1.405,1.78$, SURF-ID='UPHOLSTERY'/ Base COBST XB=61.049999, 62.174999, 55.924999,56.299999, 1.405,2.155, SURF_ID='UPHOLSTERY'/Right Ar

$* * *$ GROUP: COUCH2 offset $=0.000000,6.000000,0.000000$ rotate $=90.000000$

\&OBST XB=61. 049999, 62.174999, 57. 049999, 57.424999, 1.405, 2.155, SURF_ID='UPHOLSTERY'/ Left Arm \&OBST XB=61.049999, 62.174999, 57.424999, 58.924999, 1.405, 1.78, SURF IDD='UPHOLSTERY'/ Base \&OBST XB=61. $849999,62.174999,58.924999,59.299999,1.405,2.155$,
\&OBST XB=61.04999, $61.424999,57.424999,58.924999,1.78,2.155$,

*** GROUP: COUCH2 offset $=0.000000,9.000000,0.000000$ rotate $=90.000000$

\&OBST XB=61. 049999, 62.174999, 60. 049999, 60.424999, 1.405, 2.155, SURF_ID=' 'UPHOLSTERY '/ Left Arm
ROBST XB=61. 049999, 62.174999, 60.424999, 61.924999, 1.405, 1.78, SURF_ID=' UPHOLSTERY'/ Base

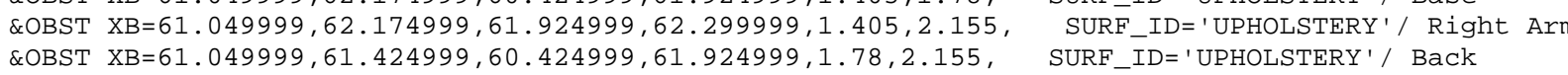
*** GROUP: COUCH2 offset $=0.000000,12.000000,0.000000$ rotate $=90.000000$

\&OBST XB $=61.049999,62.174999,63.049999,63.424999,1.405,2.155, \quad$ SURF_ID=' 'UPHOLSTERY '/ Left Arm

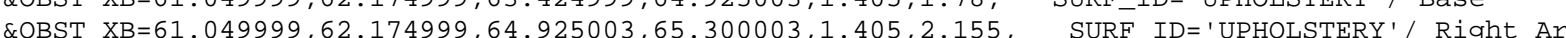
\&OBST XB=61. $049999,61.424999,63.424999,64.925003,1.78,2.155$, SURF_ID='UPHOLSTERY' / Back

*** GROUP: COUCH2 offset $=0.000000,15.000000,0.000000$ rotate $=90.000000$

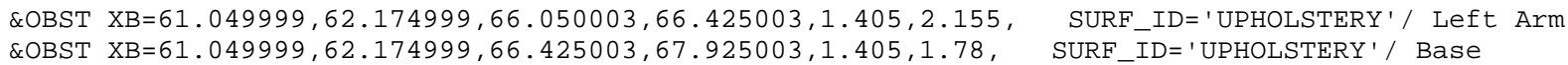

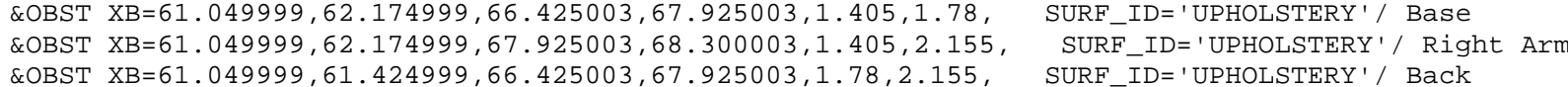

*** GROUP: COUCH2 offset $=0.000000,18.000000$, ๑. .00000 rotate $=90.000000$

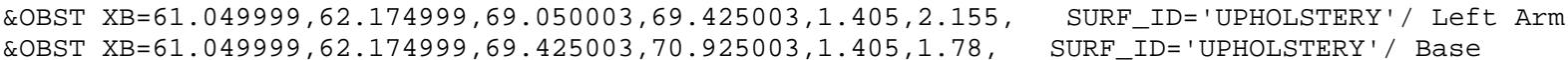

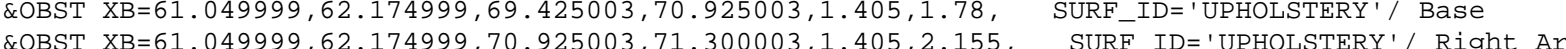
\&OBST XB=61.049999, 61.424999, $69.425003,70.925003,1.78,2.155$, SURF_ID='UPHOLSTERY'/ Back

*** GROUP: COUCH2 offset $=0.000000,21.000000,0.000000$ rotate $=90.000000$

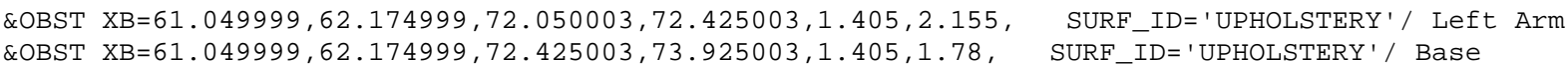
LOBST XB $61.049999,02.174999,72.425003,73.925003,1.405,1.78, \quad$ SURF_ID='UPHOLSTERY'/ Lase

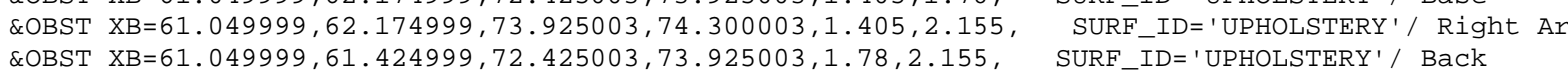

*** GROUP: COUCH2 offset $=0.000000,24.000000$, ๑. .000000 rotate $=90.000000$

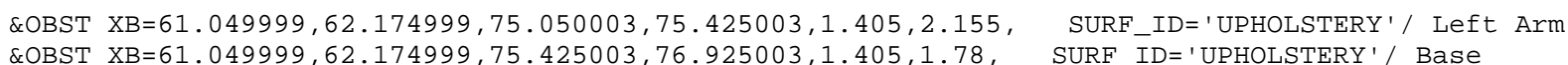

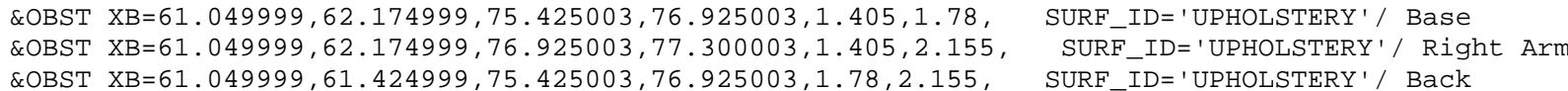

*** GROUP: COUCH2 offset $=0.000000,27.000000,0.0000000$ rotate $=90.0000000$

\&OBST XB=61.049999, 62.174999, 78.050003, 78.425003,1.405, 2.155, SURF_ID=' 'UPHOLSTERY'/ Left Arm \&OBST $X \mathrm{XB}=61.049999,62.174999,78.425003,79.925003,1.405,1.78$,
\&OBST XB $\mathrm{X}=61.049999,62.174999,79.925003,80.300003,1.405,2.155$,

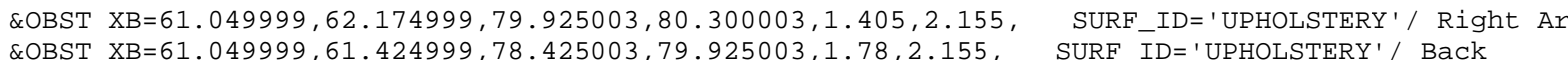

*** GROUP: COUCH2 offset $=0.000000,30.000000,0.000000$ rotate $=90 . .000000$

\&OBST XB=61.049999, 62.174999, 81.050003, 81.425003,1.405, 2.155, SURF_ID=' 'UPHOLSTERY'/ Left Arm \&OBST XB=61.049999, 62.174999, $81.425003,82.925003,1.405,1.78$, SURF_ID='UPHOLSTERY'/ Base

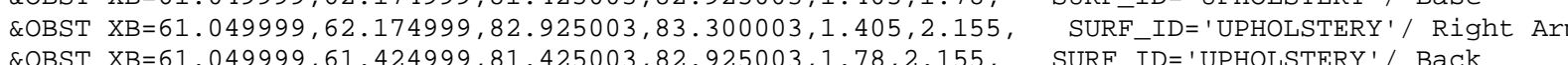

*** GROUP: ROW2

*** GROUP: COUCH2 offset $=0,000000,0,000000,0.000000$ rotate $=270,00000$

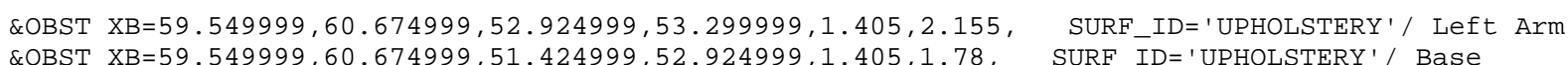
\&OBST XB $=59.549999,60.674999,51.049999,51.424999,1.405,2.155$, SURE ID='UPHOLSTERY'/ Right Ar \&OBST XB $=60.299999,60.674999,51.424999,52.924999,1.78,2.155$, SURF_ID='UPHOLSTERY'/ Back

*** GROUP: COUCH2 offset $=0.000000,3.000000,0.000000$ rotate $=270.000000$

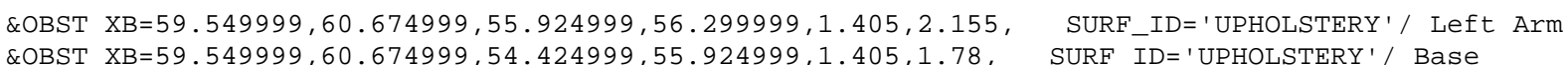

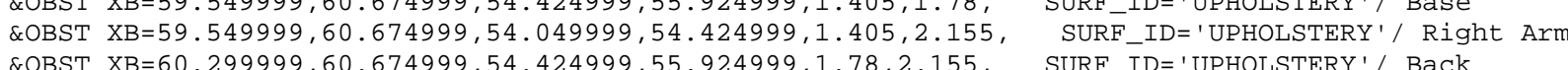

$* * *$ GROUP: COUCH2 offset $=0.000000,6.000000,0.000000$ rotate $=270.000000$

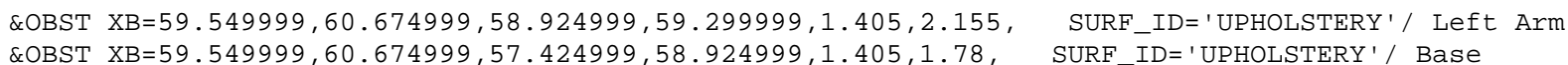

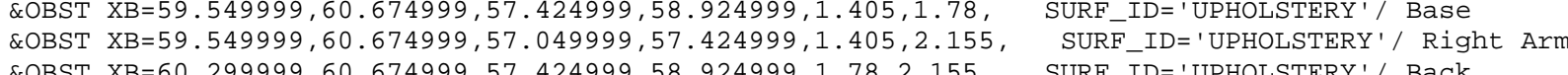

*** GROUP: COUCH2 offset $=0.000000,9.000000,0.000000$ rotate $=270.000000$ 
ROBST XB $=59.549999,60.674999,61.924999,62.299999,1.405,2.155, \quad$ SURF_ID=' 'UPHOLSTERY '/ Left Arm
\&OBST XB $=59.549999,60.674999,60.424999,61.924999,1.405,1.78$,
SURF_ID='UPHOLSTERY' $/$ Base \&OBST XB $59.549999,60.674999,60.049999,60.424999,1.475,2.155$, SURF IDD='UPHOLSTERY'/ Right Ar *** GROUP: COUCH2 offset=0. $000000,12.000000,0.000000$ rotate $=270.000000$

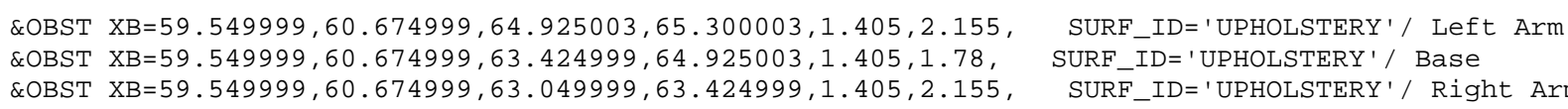

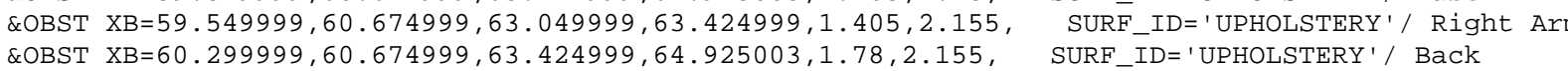
*** GROUP: COUCH2 offset $=0.000000,15.000000,0.000000$ rotate $=270.000000$

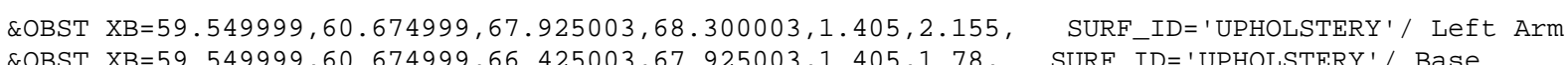
\&OBST XB=59.549999,60.674999, 66. $050003,66.425003,1.405,2.155$, SURF $\quad$ SIDE='UPHOLSTERY'/ Right Ar *** GROUP: COUCH2 offset $=0.000000,18.000000,0.000000$ rotate $=270.000000$

\&OBST XB=59.549999, 60.674999, 70.925003, 71.300003,1.405, 2.155, SURF_ID=' 'UPHOLSTERY'/ Left Arm QOBST XB $=59.549999,60.674999,69.425003,70.925003,1.405,1.78$, SURF IDD='UPHOLSTERY'/ Base Arm

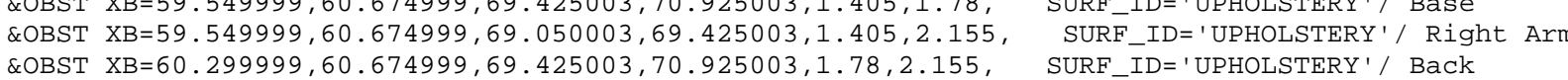

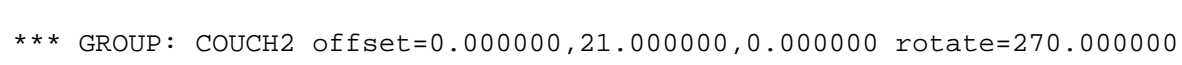

\&OBST XB=59.549999, 60.674999, 73.925003, 74.300003,1.405, 2.155, SURF_ID=' 'UPHOLSTERY' / Left Arm \&OBST XB=59.549999, 60.674999, 72.425003, 73.925003,1.405, 1.78, SURF_ID='UPHOLSTERY'/ Base Ar \&OBST XB=59.54999, 60.674999, 72.050003, 72.425003,1.405, 2.155, SURF_ID='UPHOLSTERY' $/$ Right Ar
\&OBST XB=60.299999, $60.674999,72.425003,73.925003,1.78,2.155, \quad$ SURF_ID='UPHOLSTERY'/ Back *** GROUP: COUCH2 offset $=0.000000,24.000000,0.000000$ rotate $=270$. .

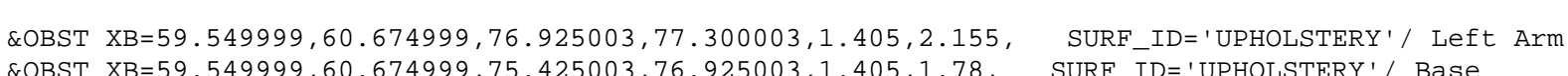
\&OBST XB=59.549999,60.674999, 75.050003,75.425003,1.405,2.155, SURF_ID=' 'UPHOLSTERY'/ Right Ar 1.78, 2.155, SURF_ID='UNOLSTERY'/ Back *** GROUP: COUCH2 offset $=0.000000,27.000000,0.000000$ rotate $=270.000000$

\&OBST XB=59.549999, 60.674999, 79.925003, 80.300603, 1.405, 2.155, SURF_ID=' 'UPHOLSTERY'/ Left Arm

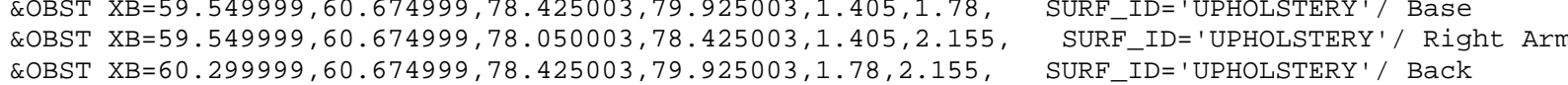
*** GROUP: COUCH2 offset $=0.000000,30.000000,0.000000$ rotate $=270.000000$

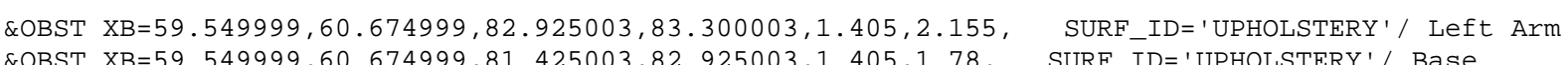

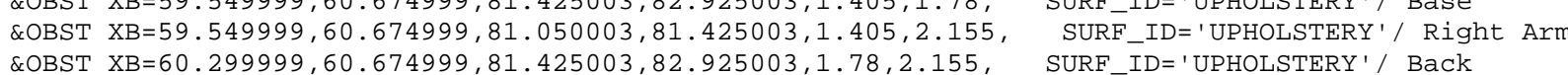

\section{*** GROUP: TWIN}

*** GROUP: ROW

*** GROUP: COUCH2 offset $=0.000000,0.000000,0.000000$ rotate $=90.000000$

\&OBST XB $=65.5500003,66.675003,51.049999,51.424999,1.405,2.155, \quad$ SURF_ID= ' UPHOLSTERY' '/ Left Arm
\&OBST XB $=65.550003,66.675003,51.424999,52.924999,1.405,1.78, \quad$ SURF_IDD='UPHOLSTERY'/Base \&OBST XB=65.550003,66. $675003,52.924999,53.299999,1.405,2.155$,
\&OBST XB=65.550003, SURF_ID=' 'UPHOLSTERY '/ Right Arm
SURF_ID='UPHOLSTERY'/ Back

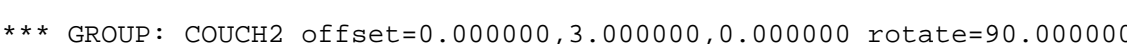

\&OBST XB $=65.550003,66.675003,54.049999,54.424999,1.405,2.155, \quad$ SURF_ID=' 'UPHOLSTERY ' / Left Arm

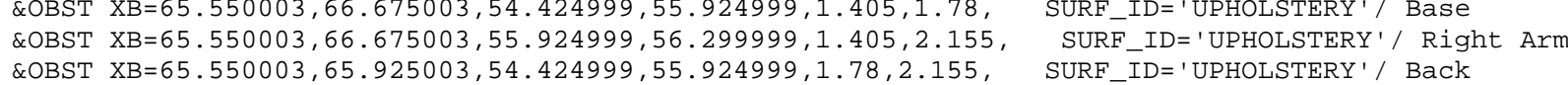
*** GROUP: COUCH2 offset $=0.0000000,6.000000,0.000000$ rotate $=90$. . 0000000

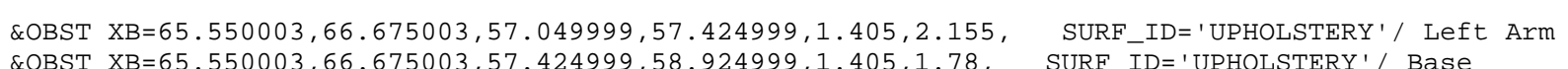

\&OBST XB $=65.550003,66.675003,58.924999,59.299999,1.405,2.155$,
\&OBST XB=65.55003, SURF_ID=' 'UPHOLSTERY '/ Right Arm
SURF_ID='UPHOLSTERY' $/$ Back

*** GROUP: COUCH2 offset $=0.000000,9.000000,0.0000000$ rotate $=90.000000$

\&OBST XB $=65.550003,66.675003,60.049999,60.424999,1.405,2.155, \quad$ SURF_ID='UPHOLSTERY '/ Left Arm
\&OBST XB $=65.550003,66.675003,60.424999,61.924999,1.405,1.78$,
SURF_ID='UPHOLSTERY' $/$ Base

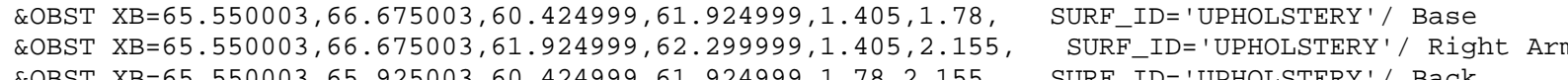

*** GROUP: COUCH2 offset $=0.0000000,12.0000000,0.000000$ rotate $=90.0000000$

\&OBST XB=65. $550003,66.675003,63.049999,63.424999,1.405,2.155$,
\&OBST XB=65.550003,66.675003,63.424999,64.925093,1.405,1.78, SURF_ID=' 'UPHOLSTERY' $/$ Left Arm
SURF_ID='UPHOLSTERY'/ Base

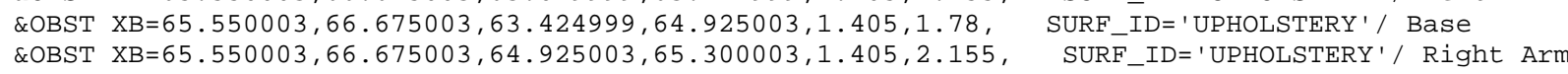
\&OBST XB $=65.550003,66.675003,64.925003,65.300003,1.405,2.155, \quad$ SURF_ID='UPHOLSTERY' Right A
\&OBST XB $=65.550003,65.925003,63.424999,64.925003,1.78,2.155$,

*** GROUP: COUCH2 offset $=0.000000,15.000000,0.000000$ rotate $=90.000000$

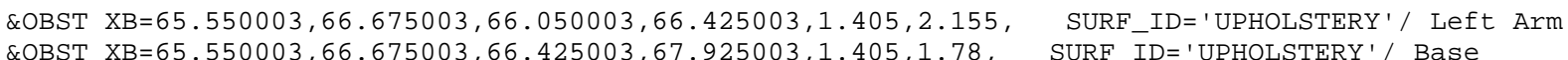

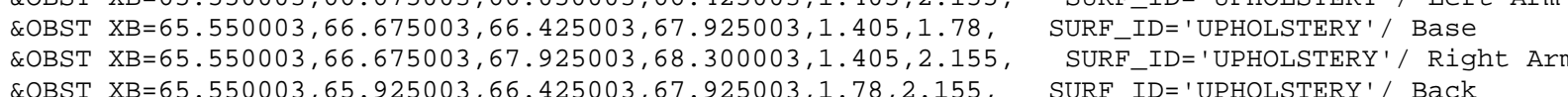

*** GROUP: COUCH2 offset $=0.000000,18.000000,0.000000$ rotate $=90.000000$

\&OBST XB=65.550003, 66.675003, 69.050003,69.425003, 1.405, 2.155, SURF_ID=' 'UPHOLSTERY'/ Left Arm

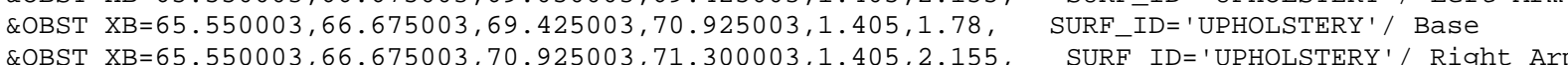
\&OBST XB $=65.550003,65.925093,69.425003,70.925003,1.78,2.155$, SURF_ID='UPHOLSTERY'/ Right Ar

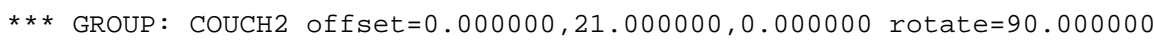

\&OBST XB=65.550003, 66.675003, $72.050003,72.425003,1.405,2.155$, SURF_ID=' 'UPHOLSTERY'/ Left Arm \&OBST XB=65.550003, 66.675003, 72.425003,73.925003,1.405, 1.78, SURF_ID='UPHOLSTERY'/ Base

$\star \star \star$ GROUP: COUCH2 offset $=0.000000,24.000000,0.000000$ rotate $=90.000000$

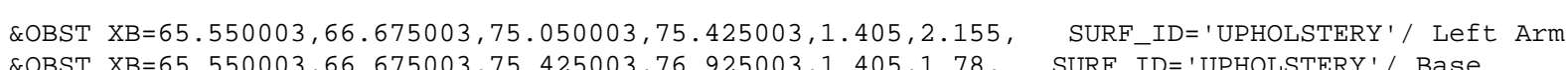

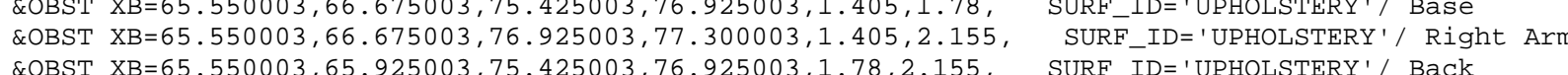
$* \star \star$ GROUP: COUCH2 offset $=0.000000,27.000000,0.000000$ rotate $=90.000000$

\&OBST XB $=65.550003,66.675003,78.050003,78.425003,1.405,2.155$, SURF_ID= 'UPHOLSTERY' / Left Arm

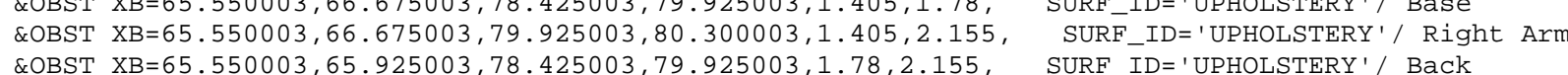

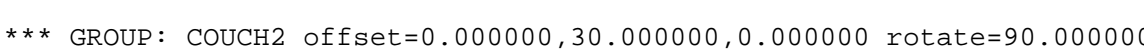

\&OBST XB=65.550003, 66.675003, 81. 0500003, 81.425003, 1.405, 2.155, SURF_ID=' UPHOLSTERY'/ Left Arm \&OBST XB=65.550003,66.675003, $82.925003,83.300003,1.405,2.155, \quad$ SURF_ID='UPHOLSTERY'/ Right Ar \&OBST XB=65.550003, 65.925003, 81.425003, 82.925003,1.78, 2.155, SURF_ID='UPHOLSTERY'/ Back

\section{$* *$ GROUP: ROW2}

** GROUP: COUCH2 offset $=0.000000, \diamond .0000000,0.000000$ rotate $=270.000000$

\&OBST XB $=64.050003,65.175003,52.924999,53.299999,1.405,2.155$, SURF_ID=' 'UPHOLSTERY'/ Left Arm \&OBST XB $=64.050003,65.175003,51.424999,52.924999,1.405,1.78, \quad$ SURF_ID='UPHOLSTERY'/ Base \&OBST $X \mathrm{~B}=64.800003,65.175003,51.424999,52.924999,1.78,2.155$, SURF_ID=' UPHOLSTEPY'/ Right Ar

*** GROUP: COUCH2 offset $=0.000000,3.000000,0.000000$ rotate $=270.0000000$

\&OBST XB $=64.050003,65.175003,55.924999,56.299999,1.405,2.155$, SURF_ID='UPHOLSTERY'/ Left Arm \&OBST XB=64.050003, 65.175003, $54.424999,55.924999,1.405,1.78$, SURF_ID='UPHOLSTERY'/ Base

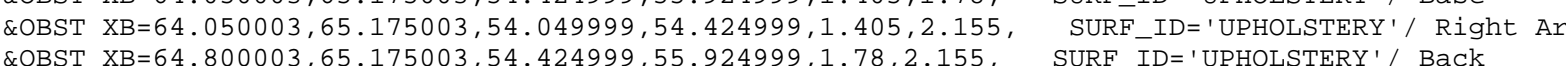

*** GROUP: COUCH2 offset $=0.000000,6.000000,0.000000$ rotate $=270.000000$ 
\&OBST XB $=64.050003,65.175003,58.924999,59.299999,1.405,2.155, \quad$ SURF_ID= 'UPHOLSTERY ' $/$ Left Arm
\&OBST XB $=64.050003,65.175003,57.424999,58.924999,1.405,1.78, \quad$ SURF_ID='UPHOLSTERY' $/$ Base

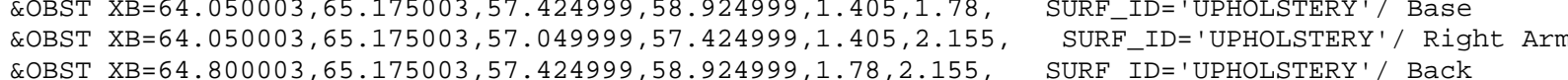
*** GROUP: COUCH2 offset $=0.000000,9.000000,0.0000000$ rotate $=270.000000$

\&OBST XB $64.050003,65.175003,61.924999,62.299999,1.405,2.155$, SURF_ID=' 'UPHOLSTERY '/ Left Arm

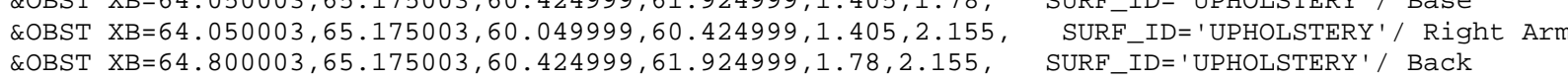
*** GROUP: COUCH2 offset $=0.000000,12.000000,0.000000$ rotate $=270.000000$

\&OBST XB $=64.050003,65.175003,64.925003,65.300003,1.405,2.155$, SURF_ID=' 'UPHOLSTERY '/ Left Arm

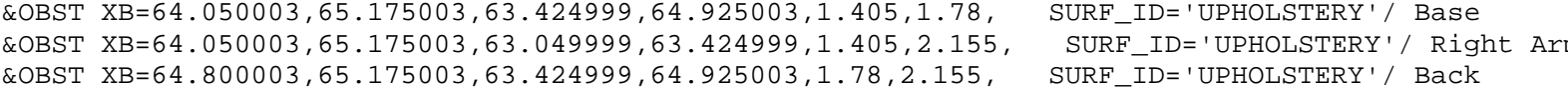

*** GROUP: COUCH2 offset $=0.000000,15.000000,0.000000$ rotate $=270.000000$

\&OBST XB $=64.050003,65.175003,67.925003,68.300003,1.405,2.155, \quad$ SURF_ID='UPHOLSTERY '/ Left Arm
\&OBST XB $=64.050003,65.175003,66.425003,67.925003,1.405,1.78, \quad$ SURF_ID ='UPHOLSTERY'
Base \&OBST XB $=64.050003,65.175003,66.050003,66.425003,1.405,2.155$,
\&OBST XB $=64.800003,65.175003,66.425003,67.925003,1.78,2.155$,
SURF_IDE='UPHOLSTERY '/ Right Arm
SURF 'UPHOLSTERY'/ Back *** GROUP: COUCH2 offset $=0.000000,18.000000,0.000000$ rotate $=270.000000$

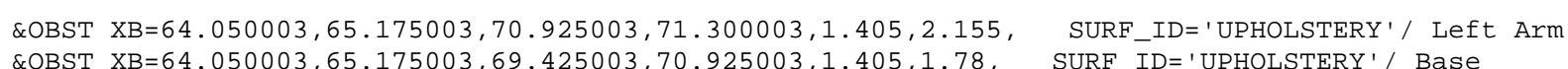

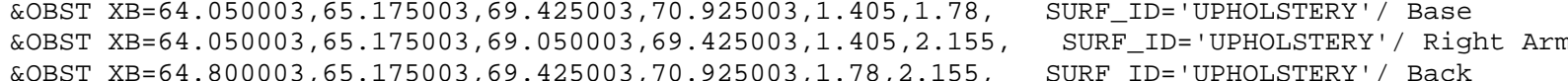
*** GROUP: COUCH2 offset $=0.000000,21.000000,0.000000$ rotate $=270.000000$

\&OBST XB=64. $050003,65.175003,73.925003,74.300003,1.405,2.155$,
\&OBST XB=64.050003, SURF-ID=' UPHOLSTERY '/ Left Arm
SURF ID='UPHOLSTERY'/ Base

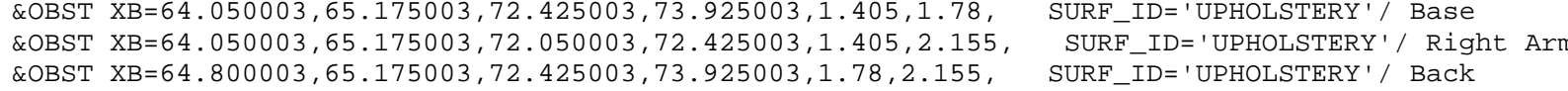
*** GROUP: COUCH2 offset $=0.000000,24.000000,0.000000$ rotate $=270.000000$

\&OBST XB $=64.050003,65.175003,76.925003,77.300003,1.405,2.155$,
\&OBST XB $=64.050003,65.175003,75.425003,76.925003,1.405,1.78$,
SURF IDEID=' 'UPHOLSTERY '/ Left Arm

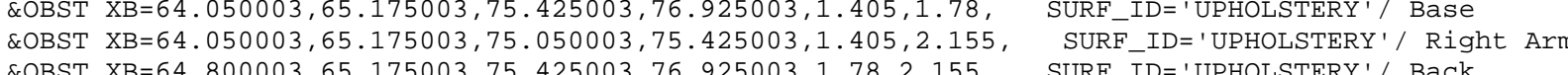

*** GROUP: COUCH2 offset $=0.000000,27.000000,0.000000$ rotate $=270.000000$

\&OBST XB $64.050003,65.175003,79.925003,80.300003,1.405,2.155$, SURF_ID='UPHOLSTERY '/ Left Arm

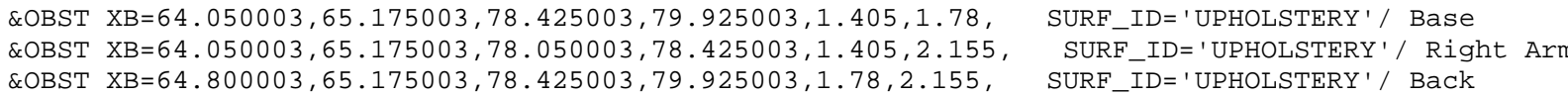
*** GROUP: COUCH2 offset $=0.000000,30.000000,0.000000$ rotate $=270.000000$

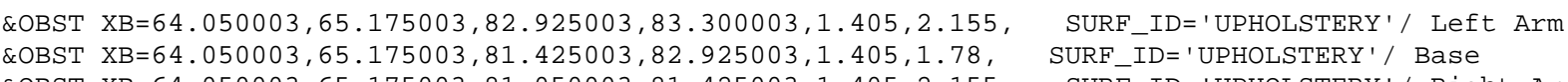
\&OBST XB $=64.050003,65.175003,81.050003,81.425003,1.455,2.155$,
\&OBST XB $=64.800003,65.175003,81.425003,82.925003,1.78,2.155$,
SURF_IDE='UPHOLSTERY '/ Right Arm

$* * *$ GROUP: TWIN

*** GROUP: ROW

*** GROUP: COUCH2 offset $=0.000000$, ๑. ๑००९००,,. .000000 rotate $=90$. . 000000

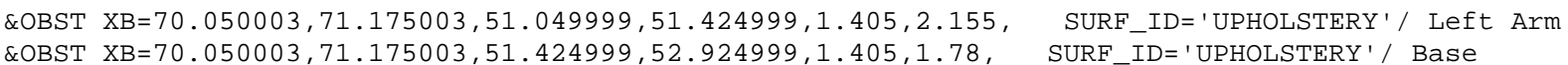
\&OBST XB $70.050003,71.175003,52.924999,53.299999,1.405,2.155$,
\&OBST XB $70.050003,70.425003,51.424999,52.924999,1.78,2.155$, *** GROUP: COUCH2 offset $=0.000000,3.000000,0.0000000$ rotate $=90.000000$

\&OBST XB $=70.050003,71.175003,54.049999,54.424999,1.405,2.155$, SURF_ID=' 'UPHOLSTERY '/ Left Arm

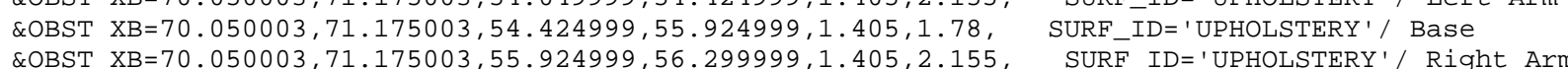

\&OBST XB $=70.050003,70.425003,54.424999,55.924999,1.78,2.155$, SURF_ID=' 'UPHOLSTERY' / Back

*** GROUP: COUCH2 offset $=0.000000,6.000000,0.000000$ rotate $=90 . .000000$

\&OBST XB=70. 050003, 71.175003, 57. 049999, 57.424999, 1.405, 2.155, SURF_ID='UPHOLSTERY'/ Left Arm

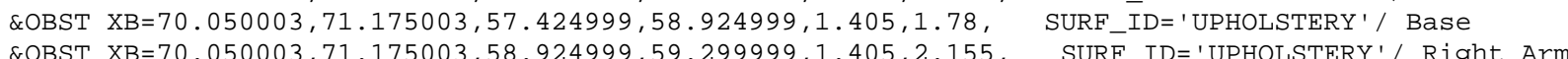
COBST $X \mathrm{~B}=70.050003,70.425003,57.424999,58.299999,1.405,2.155$, SURF-ID=' 'UPHOLSTERY/ Right

*** GROUP: COUCH2 offset $=0.000000,9.000000,0.000000$ rotate $=90.000000$

\&OBST XB $=70.050003,71.175003,60.049999,60.424999,1.405,2.155$, SURF_ID=' 'UPHOLSTERY'/ Left Arm \&OBST XB=70.050003, 71.175003, 60.424999, 61. 924999, 1.405, 1.78, SURF_ID='UPHOLSTERY'/ Base \&OBST XB=70.050003, 71.175003, 61.924999, 62.299999, 1.405, 2.155, SURF_ID='UPHOLSTERY'/ Right
\&OBST XB=70.050003, $70.425003,60.424999,61.924999,1.78,2.155$,
SURF_ID='UPHOLSTERY'/ Back

*** GROUP: COUCH2 offset $=0.000000,12.000000,0.000000$ rotate $=90.000000$

\&OBST XB=70. 050003, 71.175003,63. 049999, 63.424999, 1.405, 2.155, SURF_ID=' 'UPHOLSTERY'/ Left Arm

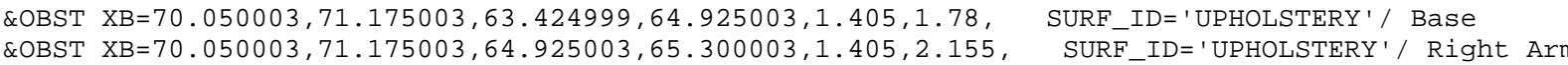


*** GROUP: COUCH2 offset $=0.0000000,15 . \bullet 000000,0.000000$ rotate $=90.000000$

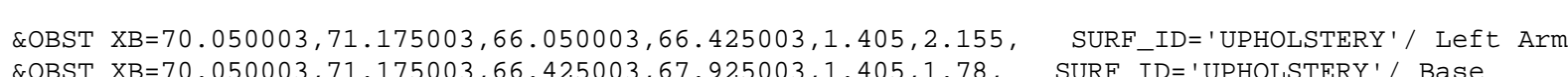

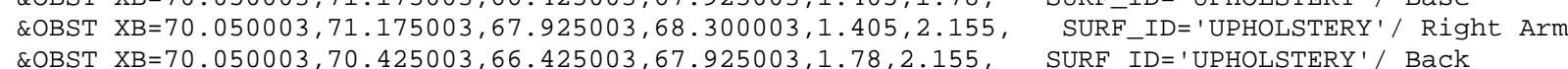

*** GROUP: COUCH2 offset $=0.000000,18.000000,0.000000$ rotate $=90.000000$

\&OBST XB=70. 050003, 71.175003, 69. 050003, 69.425003,1.405, 2.155, SURF_ID=' UPHOLSTERY ' / Left Arm

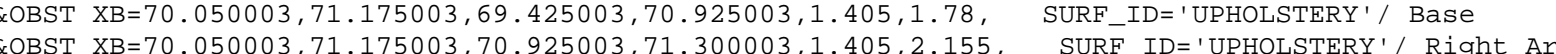
\&OBST XB $=70.050003,70.425003,69.425003,70.925003,1.78,2.155, \quad$ SURF_ID='UPHOLSTERY'/ Back

*** GROUP: COUCH2 offset $=0.000000,21.000000,0.000000$ rotate $=90.000000$

\&OBST XB=70.050003, 71.175003, 72.050003,72.425003,1.405, 2.155, SURF_ID='UPHOLSTERY ' / Left Arm \&OBST XB $70.050003,71.175003,73.925003,74.300003,1.405,2.155$, SURF_ID=' 'UPHOLSTERY' / Right Ar QOBST XB $=70.050003,70.425003,72.425003,73.925003,1.78,2.155$, SURF_ID='UPHOLSTERY'/ Back

*** GROUP: COUCH2 offset $=0.000000,24.000000,0.000000$ rotate $=90.000000$

\&OBST XB $=70.0500003,71.175003,75.050003,75.425003,1.405,2.155$, SURF_ID=' 'UPHOLSTERY' ' Left Arm \&OBST $X B=70.050003,71.175003,76,925003,77,300003,1.405,2,155$, SURF ID='UPHOLSTERY'/Right Arm QOBST $X \mathrm{~B}=70.050003,70.425003,75.425003,76.925003,1.78,2.155$, SURF_ID='UPHOLSTERY'/ Back

*** GROUP: COUCH2 offset $=0.000000,27.000000$, ๑. .000000 rotate $=90 . .000000$

\&OBST XB $=70.050003,71.175003,78.050003,78.425003,1.405,2.155, \quad$ SURF_ID=' 'UPHOLSTERY '/ Left Arm
\&OBST XB=70.050003, $71.175003,78.425003,79.925003,1.405,1.78$,
SURF_ID='UPHOLSTERY' $/$ Base

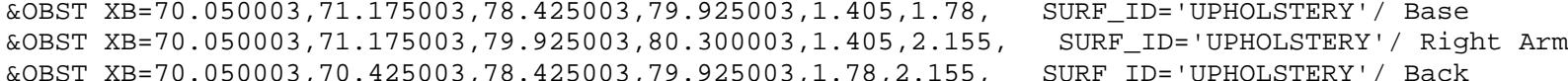

*** GROUP: COUCH2 offset $=0.000000,30.000000$, ๑. 000000 rotate $=90.000000$

\&OBST XB $=70.050003,71.175003,81.050003,81.425003,1.405,2.155$,
\&OBST XB $=70.050003,71.175003,81.425003,82.925003,1.405,1.78$,
SURF-IDE='UPHOLSTERY' $/$ ' Base

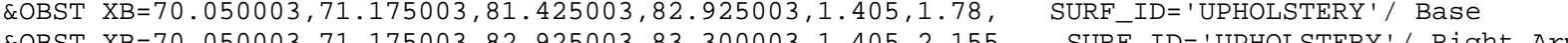
\&OBST $X \mathrm{XB}=70.050003,71.175003,82.925003,83.300003,1.405,2.155, \quad$ SURF_ID='UPHOLSTERY'/Right Ar
\&OBST XB=70.050003, 70.425003,81.425003, 82.925003,1.78,2.155, SURF_ID='UPHOLSTERY'/ Back

*** GROUP: ROW2

*** GROUP: COUCH2 offset $=0.000000, \Theta . .000000,0.000000$ rotate $=270.000000$

\&OBST XB=68.550003, 69.675003, 52.924999, 53.299999, 1.405, 2.155, SURF_ID='UPHOLSTERY' / Left Arm \&OBST XB=68.550003, 69.675003, 51.424999, 52.924999, 1.405, 1.78, SURF_ID='UPHOLSTERY'/ Base

*** GROUP: COUCH2 offset $=0.000000,3.000000,0.000000$ rotate $=270.000000$

\&OBST XB $=68.550003,69.675003,55.924999,56.299999,1.405,2.155$, SURE_ID=' 'UPHOLSTERY' / Left Arm 


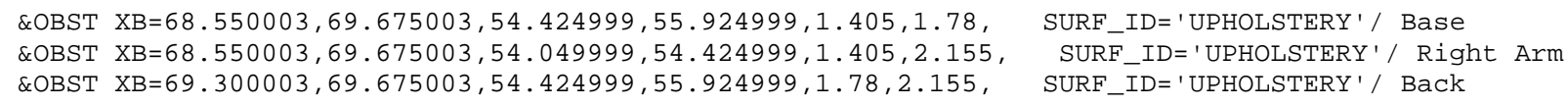
*** GROUP: COUCH2 offset $=0.000000,6.000000,0.000000$ rotate $=270.000000$

COBST XB $=68.550003,69.675003,58.924999,59.299999,1.405,2.155, \quad$ SURF_ID= 'UPHOLSTERY '/ Left Arm
\&OBST XB=68.550003,69.675003, $57.424999,58.924999,1.405,1.78, \quad$ SURF_ID='UPHOLSTERY' $/$ Base

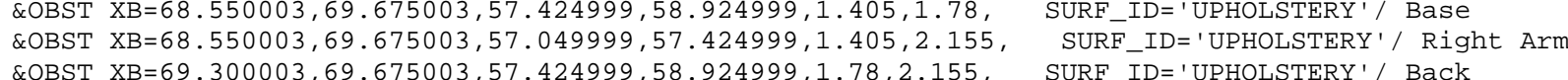

*** GROUP: COUCH2 offset $=0.000000,9.0000000,0.000000$ rotate $=270.000000$

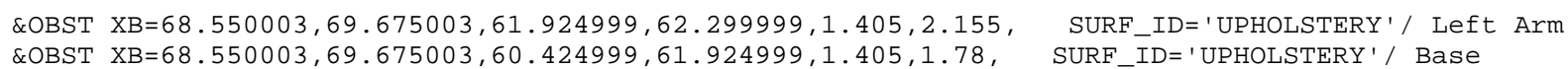

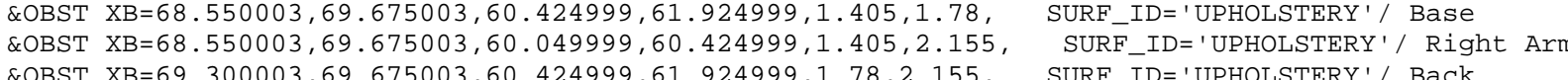

*** GROUP: COUCH2 offset $=0.000000,12.000000,0.000000$ rotate $=270.000000$

\&OBST XB $=68.550003,69.675003,64.925003,65.300003,1.405,2.155$, SURF_ID=' 'UPHOLSTERY '/ Left Arm

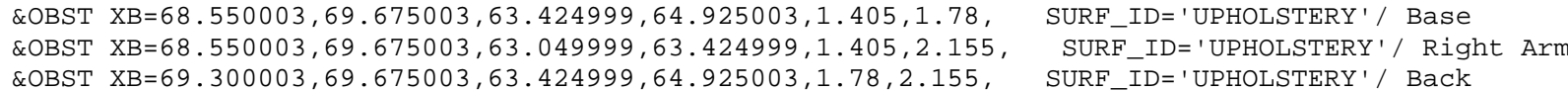

*** GROUP: COUCH2 offset $=0.000000,15.000000,0.000000$ rotate $=270$. . ๑००००००

\&OBST XB=68.550003, 69.675003, 67.925003, 68.300003,1.405, 2.155, SURF_ID=' 'UPHOLSTERY '/ Left Arm

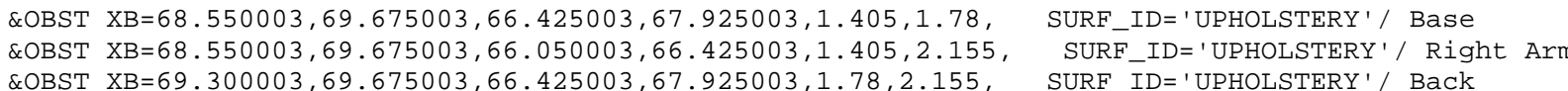

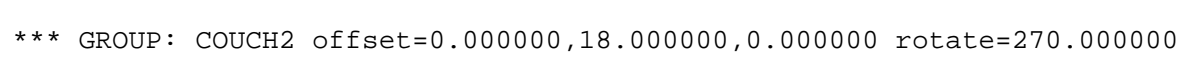

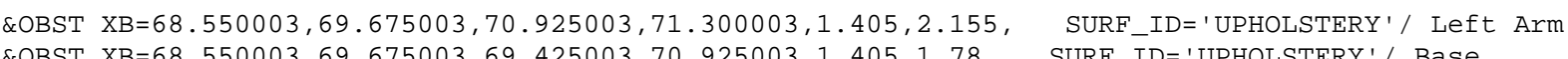
\&OBST XB=68.550003,69.675003,69.425003,70.925003,1.405,1.78, SURF_ID='UPHOLSTERY'/ Base Arm

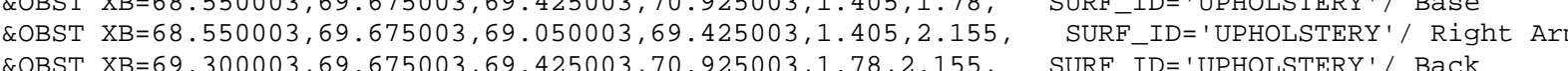
*** GROUP: COUCH2 offset $=0.000000,21.000000,0.0000000$ rotate $=270.000000$

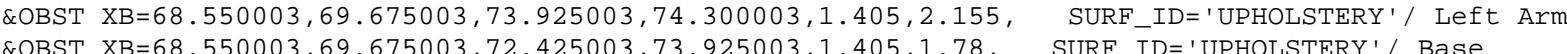

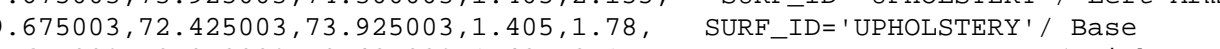
\&OBST XB=68.550003,69.675003,

*** GROUP: COUCH2 offset $=0.000000,24.000000,0.000000$ rotate $=270.000000$

EOBST XB $=68.550003,69.675003,76.925003,77.300003,1.405,2.155$, SURF_ID=' 'UPHOLSTERY ' / Left Arm \&OBST XB=68.550003, 69.675003, 75.425003, 76.925003,1.405,1.78, SURF_ID='UPHOLSTERY'/ Base

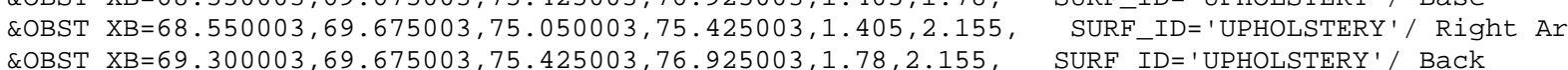

*** GROUP: COUCH2 offset $=0.000000,27.000000, \odot .000000$ rotate $=270.000000$

\&OBST XB=68.550003, 69.675003, 79.925003, 80.300003,1.405, 2.155, SURF_ID=' 'UPHOLSTERY'/ Left Arm

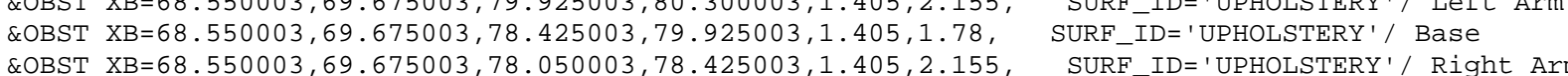
COBST $\mathrm{XB}=69.350003,69.675003,78.050003,78.425003,1.405,2.155$, SURF-ID='UPHOLSTERY/Right Ar

$* * *$ GROUP: COUCH2 offset $=0.000000,30.000000,0.000000$ rotate $=270.000000$

\&OBST XB=68.550003, 69.675003, 82.925003, 83.300003,1.405, 2.155, SURF_ID=' 'UPHOLSTERY'/ Left Arm

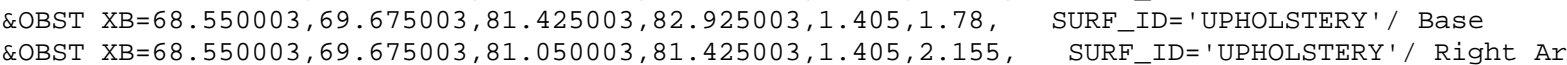
LOBST $X \mathrm{BB}=68.550003,69.675003,81.050003,81.425003,1.405,2.155$, SURF_ID='UPHOLSTERY'/ Right Ar

*** GROUP: ROW2

*** GROUP: COUCH2 offset $=0.000000, \diamond ., 000000, \diamond ., 000000$ rotate $=270.000000$

COBST XB $=73.050003,74.175003,52.924999,53.299999,1.405,2.155, \quad$ SURF_ID=' 'UPHOLSTERY '/ Left Arm
COBST XB $=73.050003,74.175003,51.424999,52.924999,1.405,1.78$,
SURF_ID='UPHOLSTERY' Base

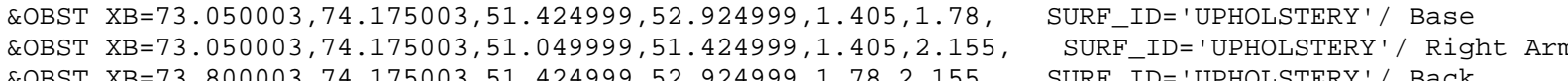

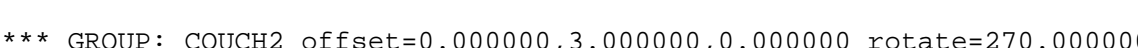

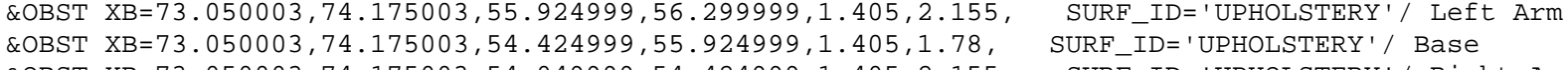
\&OBST XB=73.050003, 74.175003, 54.049999,54.424999, 1.405, 2.155, SURF_ID='UPHOLSTERY'/ Right Ar

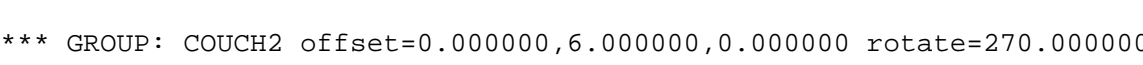

\&OBST XB=73.050003, 74.175003,58.924999, 59.299999, 1.405, 2.155, SURF_ID=' 'UPHOLSTERY'/ Left Arm \&OBST XB=73.050003, 74.175003, $57.424999,58.924999,1.405,1.78$, SURF_ID='UPHOLSTERY'/ Base \&OBST XB=73.050003,
\&OBST XB $73.800003,74.175003,57.424999,58.924999,1.78,2.155$,

*** GROUP: COUCH2 offset $=0.000000,9.000000,0.000000$ rotate $=270.000000$

\&OBST XB=73.050003, 74.175003, 61.924999, 62.299999, 1.405, 2.155, SURF_ID='UPHOLSTERY '/ Left Arm

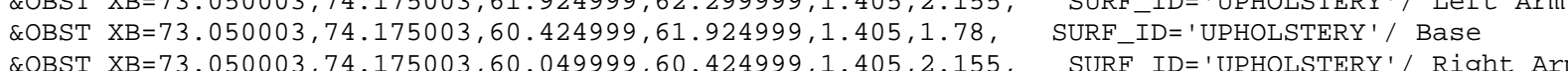
EOBST XB $=73.800003,74.175003,60.424999,61.924999,1.78,2.155$, SURF IID='UPHOLSTERY/ Right Ar

$* * *$ GROUP: COUCH2 offset $=0.000000,12.000000,0.000000$ rotate $=270.000000$

\&OBST $X \mathrm{~B}=73.050003,74.175003,64.925003,65.300003,1.405,2.155$, SURF_ID=' 'UPHOLSTERY'/ Left Arm

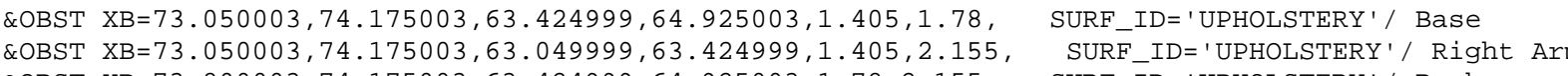

*** GROUP: COUCH2 offset $=0.000000,15.000000,0.000000$ rotate $=270.000000$

\&OBST XB=73. $050003,74.175003,67.925003,68.300003,1.405,2.155$, SURF_ID= 'UPHOLSTERY ' / Left Arm

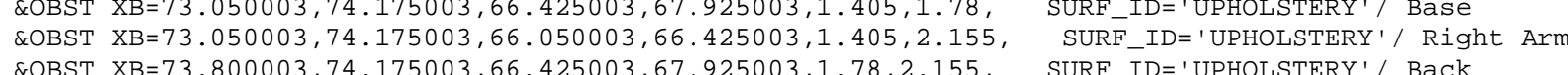

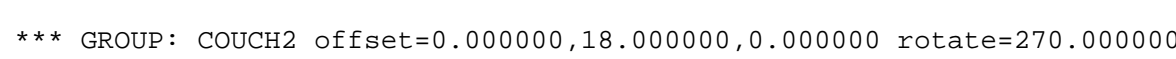

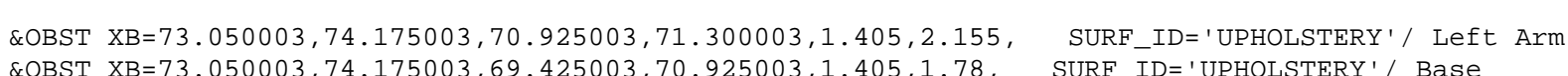

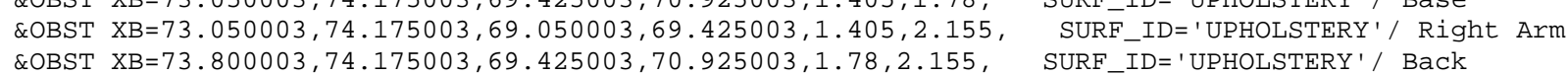

*** GROUP: COUCH2 offset $=0.000000,21.000000,, 0.0000000$ rotate $=270.000000$

\&OBST $X \mathrm{XB}=73.050003,74.175003,73.925003,74.300003,1.405,2.155, \quad$ SURF_ID=' 'UPHOLSTERY ' / Left Arm

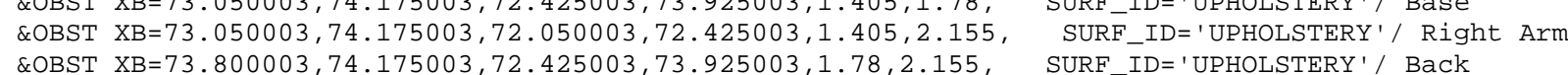

*** GROUP: COUCH2 offset $=0.000000,24.000000,0.000000$ rotate $=270.000000$

\&OBST XB=73. $0500003,74.175003,76.925003,77.300003,1.405,2.155$, SURF_ID='UPHOLSTERY' / Left Arm

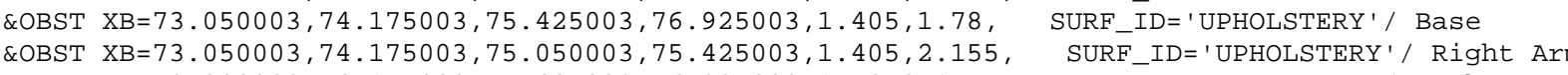
\&OBST XB=73.800003, 74.175003, 75.425003,76.925003, 1.78, 2.155, SURF_ID='UPHOLSTERY'/ Back

*** GROUP: COUCH2 offset $=0,000000,27,000000,0,000000$ rotate $=270,000000$

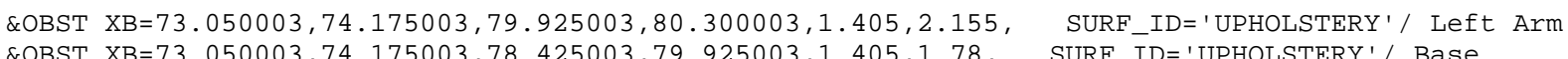

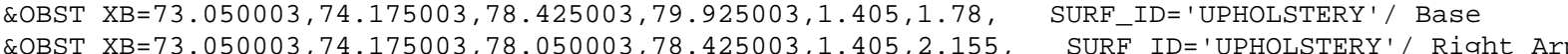
\&OBST $X B=73.800003,74.175003,78.425003,79.925003,1.78,2.155, \quad$ SURF_ID='UPHOLSTERY'/ Back

*** GROUP: COUCH2 offset $=0.000000,30.000000,0.000000$ rotate $=270.000000$

\&OBST $X \mathrm{X}=73.050003,74.175003,82.925003,83.300003,1.405,2.155, \quad$ SURF_ID= 'UPHOLSTERY '/ Left Arm
\&OBST XB $\mathrm{XB}=73.050003,74.175003,81.425003,82.925003,1.405,1.78, \quad$ SURF_ID 'UPHOLSTERY' \&OBST XB $=73.050003,74.175003,81.050003,81.425003,1.405,2.155$, , SURF_ID='UPHOLSTERY' $/$ Right Ar
\&OBST XB $73.800003,74.175003,81.425003,82.925003,1.78,2.155$,
SURF_ID=' 'UPHOLSTERY'/ Back

*** GROUP: SHORT_ROW

*** GROUP: COUCH2 offset $=0.000000,0.000000,0.000000$ rotate $=90.000000$

\&OBST XB $=67.044998,67.419998,46.91,48.035,1.405,2.155, \quad$ SURF_ID=' 'UPHOLSTERY' / Left Arm
\&OBST XB \&OBST $X \mathrm{~B}=65.169998,65.544998,46.91,48.035,1.405,2.155$, SURF_ID='UPHOLSTERY'/ Right Ar 
*** GROUP: COUCH2 offset $=0.000000,3.000000,0.000000$ rotate $=90.000000$

\&OBST $X \mathrm{X}=64.044998,64.419998,46.91,48.035,1.405,2.155, \quad$ SURF_ID=' 'UPHOLSTERY ' / Left Arm \&OBST XB=62.169998, 62. $444998,46.91,48.035,1.405,2.155, \quad$ SURF_ID='UPHOLSTERY'/ Right Ar \&OBST XB=62.544998, 64. $044998,46.91,47.285,1.78,2.155$, SURF_ID=' UPHOLSTERY'/ Back

*** GROUP: COUCH2 offset $=0.000000,6.000000,0.000000$ rotate $=90.000000$

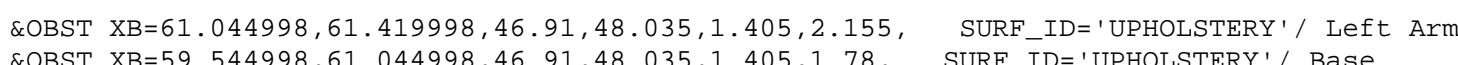

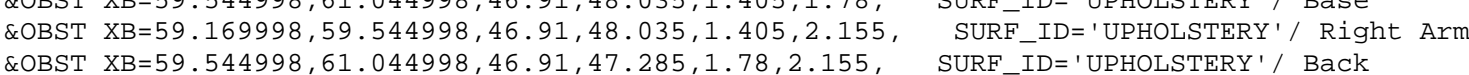

*** GROUP: COUCH2 offset $=0.000000,9.000000,0.000000$ rotate $=90.000000$

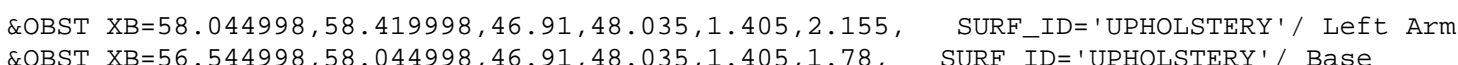
Q
COBST XB X $=5.169998,56.544998,46.91,48.035,1.405,2.155$,

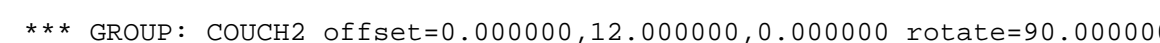

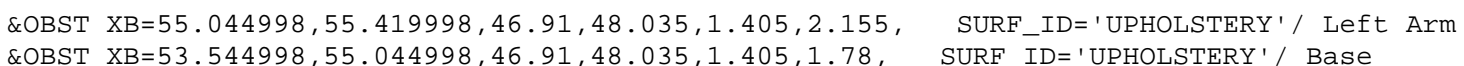
\&OBST XB
\&OBST XB $=53.169998,53.544998,46.91,48.035,1.405,2.155$,

*** GROUP: COUCH2 offset $=0.000000,15.000000,0.000000$ rotate $=90.000000$

\&OBST XB=52. $444998,52.419998,46.91,48.035,1.405,2.155$, SURF_ID= 'UPHOLSTERY' / Left Arm

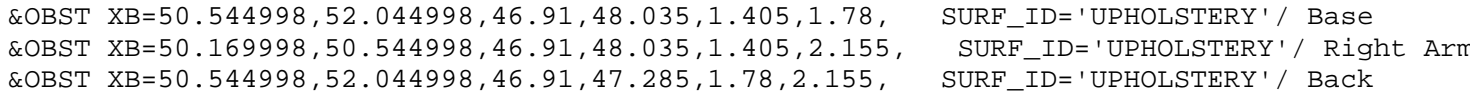

*** GROUP: COUCH2 offset $=0.000000,18.0000000,0.000000$ rotate $=90.000000$

\&OBST XB=49. 044998, 49.419998, 46.91, 48.035, 1.405, 2.155, SURF_ID=' 'UPHOLSTERY'/ Left Arm

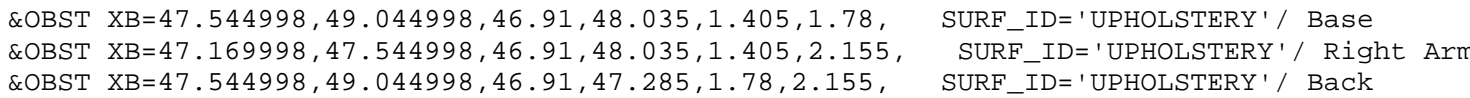

*** GROUP: ROW

*** GROUP: COUCH2 offset $=0.000000,0.000000,0.000000$ rotate $=90 . .000000$

\&OBST $X B=41.549999,41.924999,84.43,85.555,1.405,2.155, \quad$ SURF_ID=' 'UPHOLSTERY' / Left Arm \&OBST XB=43.424999, 43.799999, 84.43,85.555, 1.405, 2.155, SURF_ID='UPHOLSTERY'/ Right Ar \&OBST XB=41.924999, 43.424999, 85.18, 85.555, 1.78, 2.155, SURF_ID='UPHOLSTERY' / Back

*** GROUP: COUCH2 offset $=0.000000,3.000000,0.000000$ rotate $=90.000000$

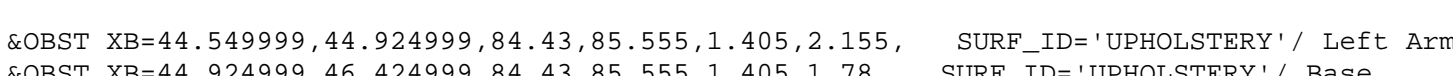

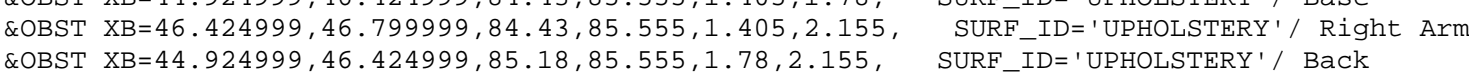

*** GROUP: COUCH2 offset $=0.000000,6.000000,0.000000$ rotate $=90.000000$

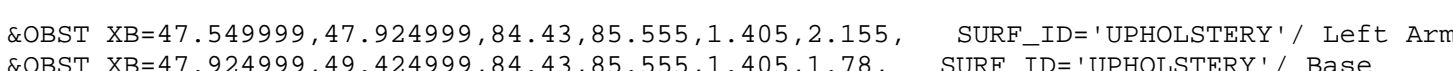

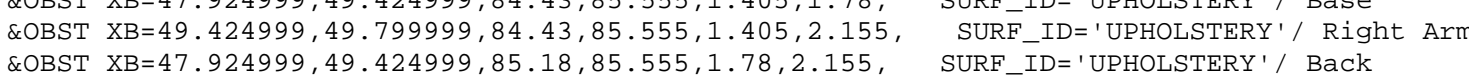
*** GROUP: COUCH2 offset $=0.000000,9$. . ०००००००,,. .000000 rotate $=90 . .000000$

\&OBST XB=50.549999, 50.924999, 84.43, 85.555, 1.405, 2.155, SURF_ID='UPHOLSTERY'/ Left Arm

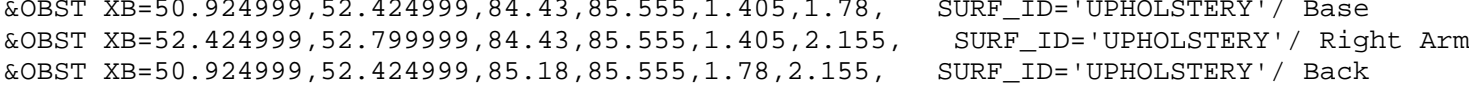
*** GROUP: COUCH2 offset $=0.000000,12.000000,0.000000$ rotate $=90 . .000000$

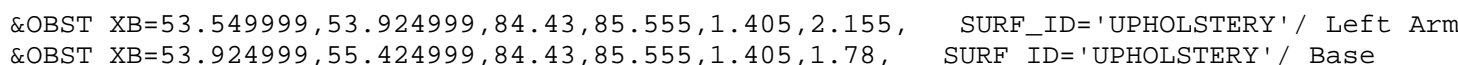

\&OBST XB=55.424999, 55.799999, 84.43, 85.555, 1.405, 2.155, SURF_ID='UPHOLSTERY'
\&OBST XBight Arm

*** GROUP: COUCH2 offset $=0.000000,15.000000,0.0000000$ rotate $=90.0000000$

LOBST XB=56.549999, 56. $924999,84.43,85.555,1.405,2.155, \quad$ SURF_ID=' 'UPHOLSTERY'/ Left Arm
\&OBST XB $\mathrm{X}=56.924999,58.424999,84.43,85.555,1.405,1.78$,

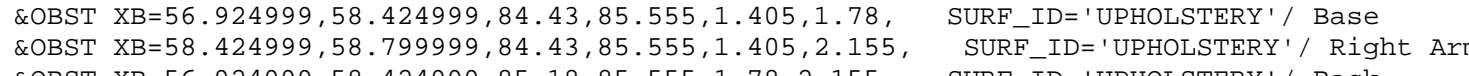

*** GROUP: COUCH2 offset $=0.000000,18.000000,0.000000$ rotate $=90.000000$

OBST XB=59.549999, 59. 924999, 84.43,85.555, 1.405, 2.155, SURF_ID='UPHOLSTERY'/ Left Arm \&OBST XB=59.924999, 61.424999, 84.43,85.555, 1.405, 1.78, SURF_ID='UPHOLSTERY'/ Base

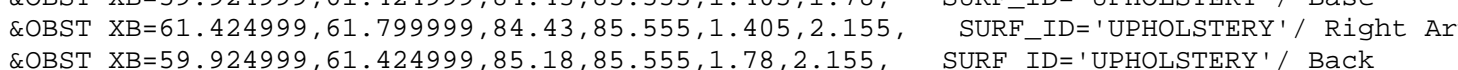

*** GROUP: COUCH2 offset $=0.000000,21.000000,0.000000$ rotate $=90.000000$

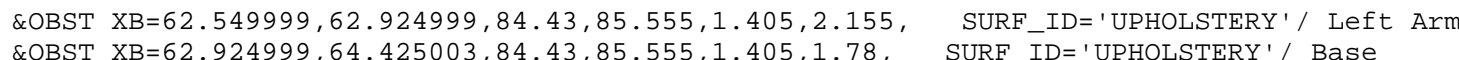

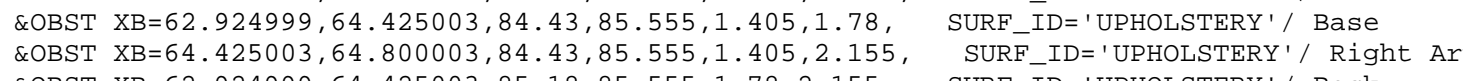

*** GROUP: COUCH2 offset $=0.000000,24.000000,0.000000$ rotate $=90.000000$

\&OBST XB $=65.550003,65.925003,84.43,85.555,1.405,2.155$, SURF_ID=' 'UPHOLSTERY' / Left Arm \&OBST XB $=65.925003,67.425003,84.43,85.555,1.405,1.78$, SURF_ID='UPHOLSTERY'/ Base

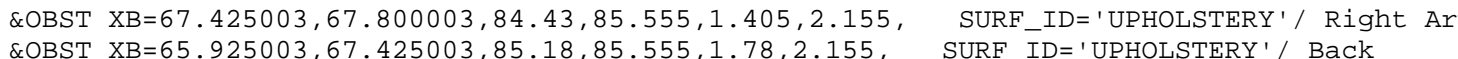

$* * *$ GROUP: COUCH2 of fset $=0.000000,27.000000,0.000000$ rotate $=90.000000$

\&OBST XB $=68.550003,68.925003,84.43,85.555,1.405,2.155$, SURF_ID='UPHOLSTERY'/ Left Arm \&OBST XB $=68.925003,70.425003,84.43,85.555,1.405,1.78$, SURF IDE='UPHOLSTERY'/ Base \&OBST XB $=70.425003,70.800003,84.43,85.555,1.405,2.155$,
\&OBST XB $=68.925003,70.425003,85.18,85.555,1.78,2.155$,
SURF_ID=' 'UPHOLSTERY'/ Back

$\star \star *$ GROUP: COUCH2 offset $=0.000000,30.000000,0.000000$ rotate $=90.000000$

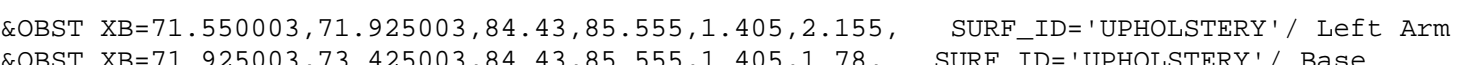
\&OBST XB $=73.425003,73.800003,84.43,85.555,1.405,2.155$, SURF_ID=' 'UPHOLSTERY'/Right Ar

$* * *$ GROUP: LEVEL

*** GROUP: ROW

*** GROUP: COUCH2 offset $=0.000000,0.000000,0.000000$ rotate $=90.000000$

\&OBST XB=37.799999, 38.924999, 51. 449999, 51.424999, 2.53,3.28, SURF_ID=' 'UPHOLSTERY' ' Left Arm COBST $X \mathrm{XB}=37.799999,38.924999,52.924999,53.299999,2.53,3.28$,
\&OBST $X \mathrm{~B}=37.799999,38.174999,51.424999,52.924999,2.905,3.28, \quad$ SURF_IDE 'UPHOLSTERY'/ Right Arm
SURF_ID='UPHOLSTERY' $/$ Back

*** GROUP: COUCH2 offset $=0.000000,3.000000,0.000000$ rotate $=90.000000$

\&OBST XB=37.799999, 38.924999, 54. $449999,54.424999,2.53,3.28$, SURF_ID=' 'UPHOLSTERY' ' Left Arm

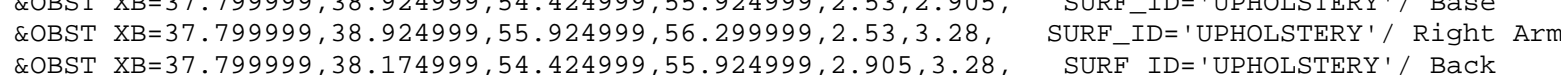

*** GROUP: COUCH2 offset $=0.000000,6.000000,0.000000$ rotate $=90.000000$

\&OBST XB=37.799999, 38.924999, 57. 049999, 57. 424999, 2.53, 3.28, SURF_ID=' 'UPHOLSTERY ' / Left Arm \&OBST XB=37.799999, 38.924999, 58.924999, 59.299999, 2.53,3.28, SURF_ID='UPHOLSTERY'/Right Ar 'UPHOLSTERY' / Back

*** GROUP: CoUCH2 offset $=0.000000,9.000000,0.000000$ rotate $=90.000000$

\&OBST XB $=37.799999,38.924999,60.049999,60.424999,2.53,3.28, \quad$ SURF_ID= ' UPHOLSTERY ' / Left Arm \&OBST XB $=37.799999,38.924999,61.924999,62.299999,2.53,3.28, \quad$ SURF_IDE' UPHOLSTERY' $/$ ' Right Ar
\&OBST XB $=37.799999,38.174999,60.424999,61.924999,2.905,3.28, \quad$ SURF_IDE 'UPHOLSTERY' / Back 


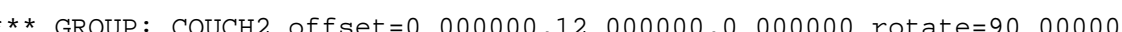

\&OBST XB=37. $799999,38.924999,63.049999,63.424999,2.53,3.28, \quad$ SURF_ID=' 'UPHOLSTERY' ', Left Arm
\&OBST XB=37.799999, 38.924999, $63.424999,64.925003,2.53,2.905$,
SURF_IDE' 'UPHOLSTERY'/ Base

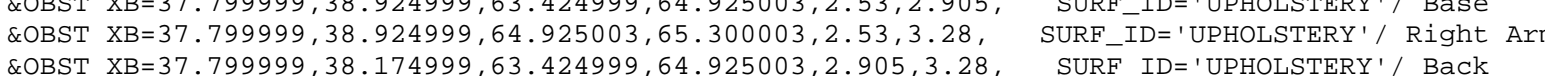

*** GROUP: COUCH2 offset $=0.000000,15.000000,0.000000$ rotate $=90$. . 000000

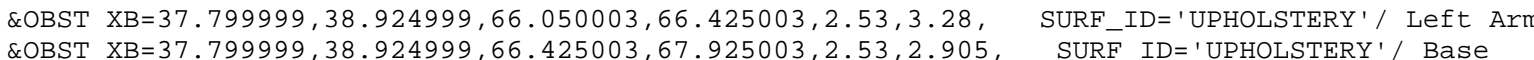

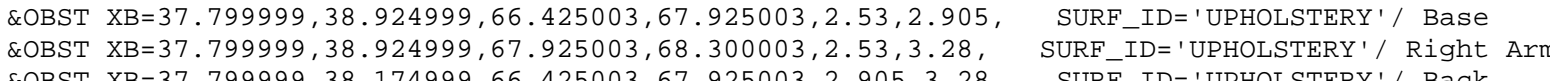

*** GROUP: COUCH2 off set $=0.000000,18.000000,0.000000$ rotate $=90.000000$

\&OBST XB $=37.799999,38.924999,69.050003,69.425003,2.53,3.28$, SURF_ID='UPHOLSTERY ' / Left Arm \&OBST XE=37.799999, 38.924999,69.425003, 70.925503, 2.53,2.905, SURF_ID='UPHOLSTERY'/ Base

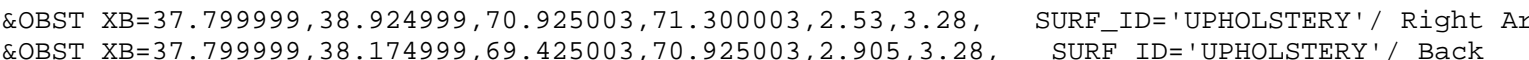

*** GROUP: COUCH2 offset $=0.000000,21.0000000,0 . .000000$ rotate $=90 . .000000$

\&OBST XB $=37.799999,38.924999,72.050003,72.425003,2.53,3.28$, SURFID=' 'UPHOLSTERY'/ Left Arm \&OBST XB=37.799999, 38.924999, 72.425003,73.925003, 2.53,2.905, SURF_ID='UPHOLSTERY'/ Base \&OBST $X \mathrm{~B}=37.799999,38.174999,72,425003,73,925003,2.905,3,28, \quad$ SUPE_ID='UPHOLSTEPY'/ RIght Ar

*** GROUP: COUCH2 offset $=0.000000,24.000000,0.000000$ rotate $=90.000000$

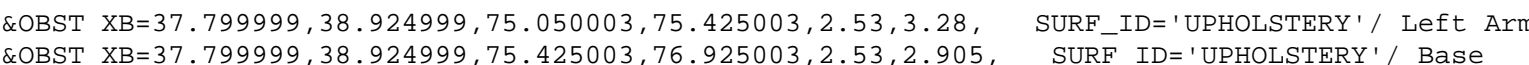
\&OBST XB=37.799999, 38.924999, 76.925003, 77.300003, 2.53,3.28, SURF_ID='UPHOLSTERY'/ Right Ar

*** GROUP: COUCH2 offset $=0.000000,27.000000,0.000000$ rotate $=90.0000000$

\&OBST XB=37.799999, 38. 924999, 78. 050003, 78.425003, 2.53,3.28, SURF_ID=' 'UPHOLSTERY'/ Left Arm

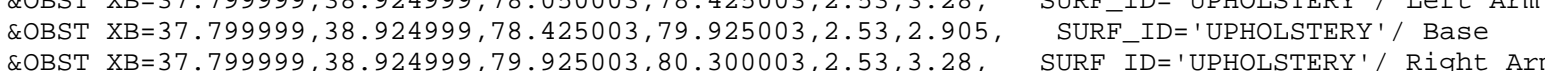
COBST $X \mathrm{~B}=37.799999,38.924999,79.925003,80.300003,2.53,3.28, \quad$ SURF_ID=' 'UPHOLSTERY'/ Right Arm
\&OBST $X \mathrm{~B}=37.799999,38.174999,78.425003,79.925003,2.905,3.28, \quad$ SURF_ID=' 'UPHOLSTERY'/ Back

*** GROUP: COUCH2 offset $=0.000000,30.000000, \Theta .000000$ rotate $=90.000000$

\&OBST XB=37.799999, 38.924999, 81. 050003, 81.425003, 2.53,3.28, SURF_ID=' 'UPHOLSTERY'/ Left Ar

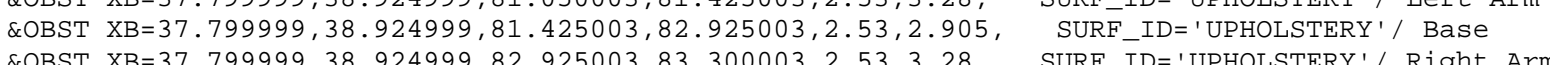

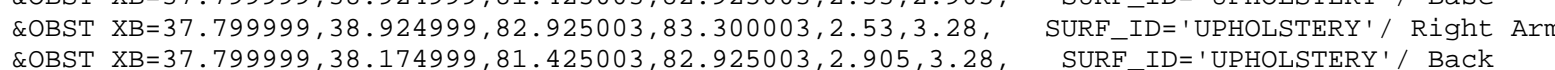

*** GROUP: BASE

*** GROUP: TWIN

$* * *$ GROUP: ROW

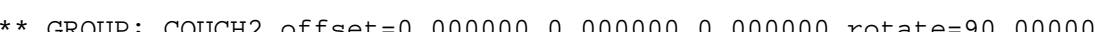

\&OBST XB=43. $049999,44.174999,51.049999,51.424999,2.53,3.28, \quad$ SURF_ID= 'UPHOLSTERY ' / Left Arm
\&OBST XB=43.04999, 44.174999, $51.424999,52.924999,2.53,2.905$, SURF ID=' 'UPHOLSTERY' Base SURF ID='UPHOLSTERY'/ \&OBST XB=43.049999, $43.424999,51.424999,52.924999,2.905,3.28$, SURF_ID='UPHOLSTERY'/ Back *** GROUP: COUCH2 offset $=0.000000,3.000000,0.0000000$ rotate $=90.000000$

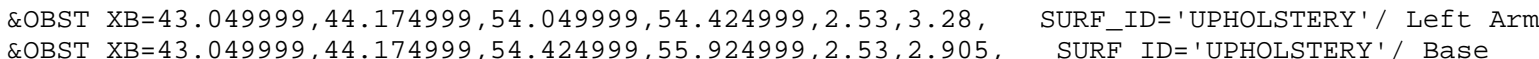
\&OBST XB $=3.049999,44.17499,55.024999,56.299999,2.533 .28$, SURE ID=' UPHOLSTERY'/ Rise \&OBST $X \mathrm{XB}=43.049999,44.174999,55.924999,56.299999,2.53,3.28, \quad$ SURF_ID=' 'UPHOLSTERY'/ Right Ar

** GROUP: COUCH2 offset $=0.0000000,6.000000,0.0 .000000$ rotate $=90.0000000$

\&OBST XB=43. $049999,44.174999,57.049999,57.424999,2.53,3.28, \quad$ SURF_ID=' 'UPHOLSTERY' $/$ Left Arm
SURF

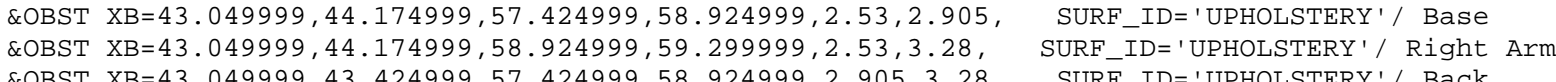

** GROUP: COUCH2 offset $=0.000000,9.000000,0.000000$ rotate $=90 ., 000000$

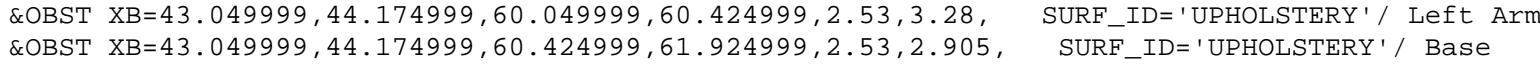
\&OBST XB=43.049999, 44.174999, 61.924999, 62.299999, 2.53,3.28, SURF-ID=' UPHOLSTERY'/ Right Ar

*** GROUP: COUCH2 offset $=0.000000,12.000000,0.000000$ rotate $=90.000000$

\&OBST XB=43. 049999, 44.174999, 63. 049999, 63. 424999, 2.53,3.28, SURFID=' 'UPHOLSTERY ' / Left Arm \&OBST XB=43.049999, 44.174999, 63.424999,64.925003, 2.53, 2.905, SURF_ID=' 'UPHOLSTERY'/ Base

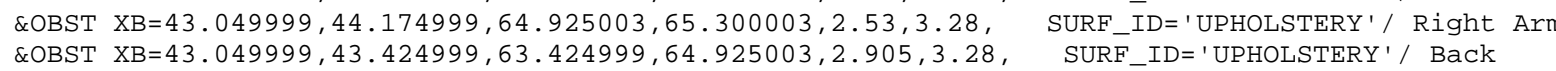

*** GROUP: COUCH2 offset $=0.000000,15.000000,0.000000$ rotate $=90 . .000000$

\&OBST XB=43. $049999,44.174999,66.050003,66.425003,2.53,3.28$, SURF_ID='UPHOLSTERY'/ Left Arm \&OBST XB=43. 049999, 44.174999, 66. $425003,67.925003,2.53,2.905, \quad$ SURF_IDE 'UPHOLSTERY' / Base LOBST XB $=43.049999,43.424999,66.425003,67.925003,2.905,3.28$, SURF_ID=' SUPHOLSTERY'/ Back

*** GROUP: COUCH2 off set $=0.000000,18.000000,0.000000$ rotate $=90.000000$

\&OBST XB=43. 049999, 44.174999, 69. 050003, 69.425003, 2.53,3.28, SURF_ID=' UPHOLSTERY'/ Left Arm \&OBST XB=43.049999, 44.174999, 69.425003,70.925003, 2.53,2.905, SURF_ID='UPHOLSTERY' / Base

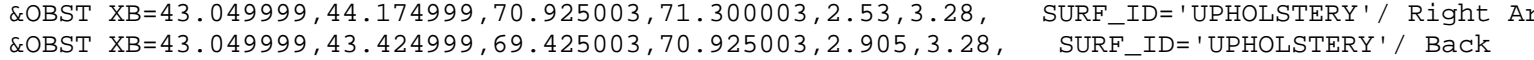

*** GROUP: COUCH2 offset $=0.000000,21.000000,0.000000$ rotate $=90.000000$

\&OBST XB=43. $049999,44.174999,72.050003,72.425003,2.53,3.28, \quad$ SURF_ID=' 'UPHOLSTERY' ' Left Arm

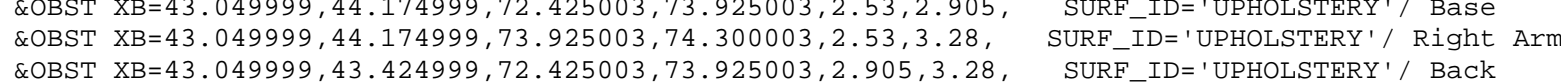

*** GROUP: COUCH2 offset $=0.000000,24.000000,0.000000$ rotate $=90.000000$

\&OBST XB=43. $049999,44.174999,75.050003,75.425003,2.53,3.28, \quad$ SURF-ID=' 'UPHOLSTERY' $/$ Left Arm
LOBST XB $=43.04999,44.174999,75.425003,76.925003,2.53,2.905, \quad$ SURF ID=' 'UPHOLSTERY' $/$ Base

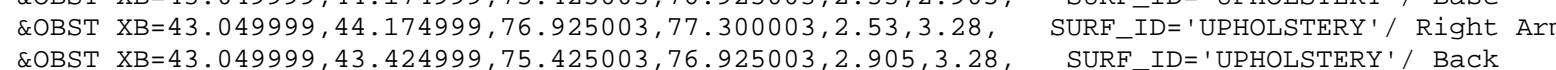

*** GROUP: COUCH2 offset $=0.000000,27.000000,0.000000$ rotate $=90.000000$

\&OBST XB $=43.049999,44.174999,78.050003,78.425003,2.53,3.28, \quad$ SURF ID= ' UPHOLSTERY ' / Left Arm

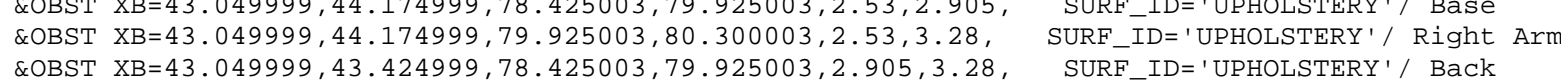

*** GROUP: COUCH2 offset $=0.000000,30.000000,0.000000$ rotate $=90.000000$

\&OBST XB=43. $049999,44.174999,81.050003,81.425003,2.53,3.28$, SURFIDD ' UPHOLSTERY ' ' Left Arm

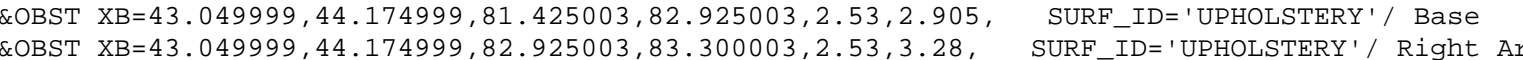
\&OBST XB=43. $049999,43.424999,81.425003,82.925003,2.905,3.28$, SURF_ID='UPHOLSTERY'/ Back

*** GROUP: ROW2

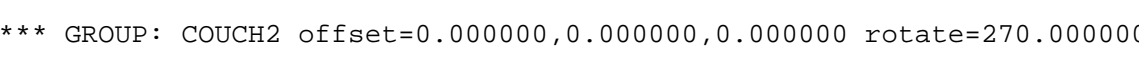

\&OBST XB=41.549999, 42.674999, 52.924999,53.299999, 2.53,3.28, SURF_ID='UPHOLSTERY'/ Left Arm

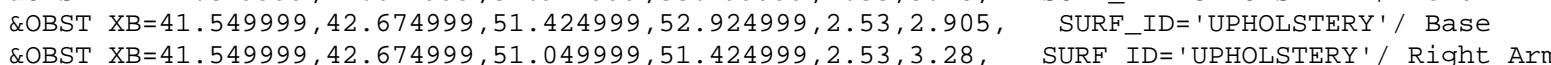
\&OBST XB $=42.299999,42.674999,51.424999,52.924999,2.905,3.28$, SURF ID ='UPHOLSTERY'/ Back

*** GROUP: COUCH2 offset $=0.000000,3.000000,0.000000$ rotate $=270.000000$

\&OBST XB=41.549999, 42.674999, 55.924999, 56. 299999, 2.53,3.28, SURF_ID=' 'UPHOLSTERY'/ Left Arm

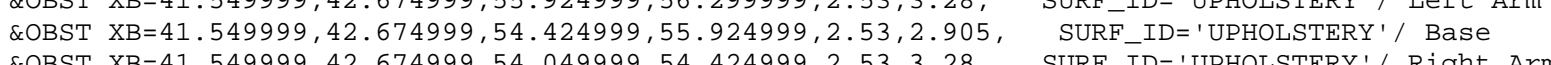

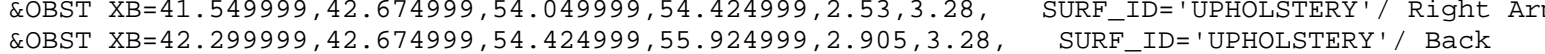

*** GROUP: COUCH2 offset $=0.000000,6.000000,0.000000$ rotate $=270.000000$

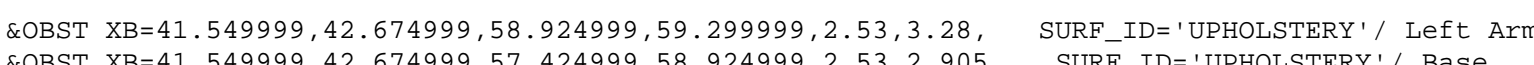
\&OBST XB=41.549999, 42.674999, $57.049999,57.424999,2.53,3.28$, SURF_ID='UPHOLSTERY'/ Right Ar 
*** GROUP: COUCH2 offset $=0.000000,9.000000,0.000000$ rotate $=270.000000$ \&OBST XB=41.549999, 42.674999, 61.924999, 62. 299999, 2.53,3.28, SURF_ID=' 'UPHOLSTERY ' / Left Arm \&OBST XB =41.549999, 42.674999,60.049999,66.424999, $2.53,2.28$, SURF_ID='UPHOLSTERY'/ Right Ar \&OBST XB=42.299999, 42.674999, 60.424999, 61.924999, 2.905, 3.28, SURF_ID='UPHOLSTERY'/ Back *** GROUP: COUCH2 offset $=0.000000,12.000000,0.000000$ rotate $=270.000000$

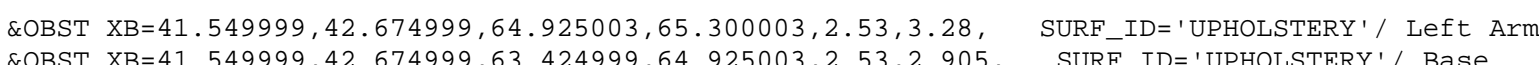

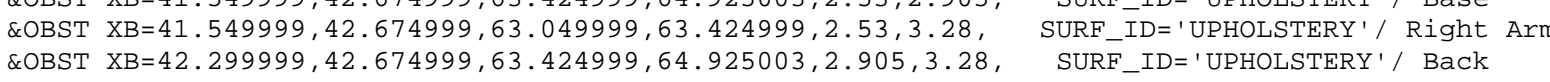

*** GROUP: COUCH2 offset $=0.000000,15.000000,0.000000$ rotate $=270.000000$

\&OBST XB=41.549999, 42.674999, 67.925003, 68.300003, 2.53,3.28, $\quad$ SURF_ID=' 'UPHOLSTERY' ' Left Arm
\&OBST XB=41.549999, 42.674999,66.425003,67.925003,2.53,2.905,
SURF_ID='UPHOLSTERY'/ Base

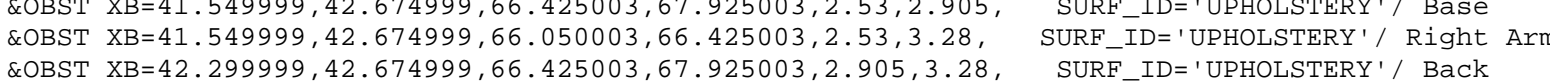
*** GROUP: COUCH2 offset $=0.000000,18.000000,0.000000$ rotate $=270.000000$

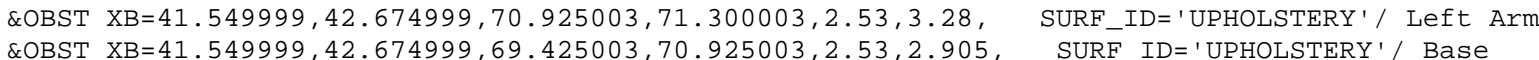

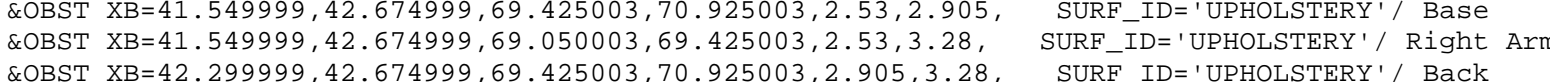

*** GROUP: COUCH2 offset $=0.000000,21.000000,0.000000$ rotate $=270.000000$

\&OBST $X \mathrm{XB}=41.549999,42.674999,73.925003,74.300003,2.53,3.28, \quad$ SURF_ID='UPHOLSTERY ' 1 Left Arm
\&OBST XB $\mathrm{X}=41.549999,42.674999,72.425003,73.925003,2.53,2.905$,
SURF ID='UPHOLSTERY' / Base

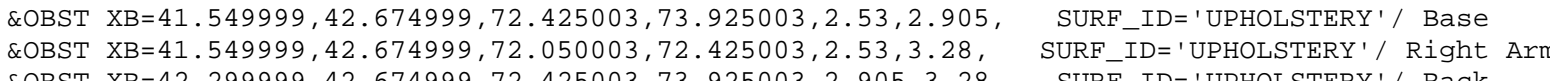

*** GROUP: COUCH2 offset $=0.000000,24.000000,0.000000$ rotate $=270.000000$

\&OBST XB $=41.549999,42.674999,76.925003,77.300003,2.53,3.28$, SURF_ID='UPHOLSTERY'/ Left Arm

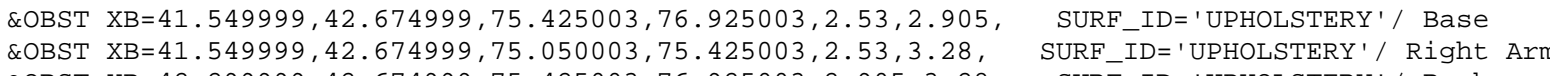
\&OBST $X B=42.299999,42.674999,75.425003,76.925003,2.905,3.28$, SURF_ID= 'UPHOLSTERY' / Back

*** GROUP: COUCH2 offset $=0.000000,27.000000,0.000000$ rotate $=270.000000$

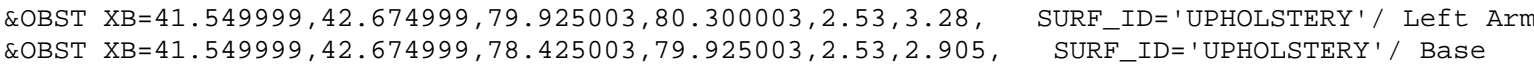
\&OBST XB=41.549999, 42.674999, 78.050003, 78.425003,2.53,3.28, SURF-ID='UPHOLSTERY'/ Right Ar

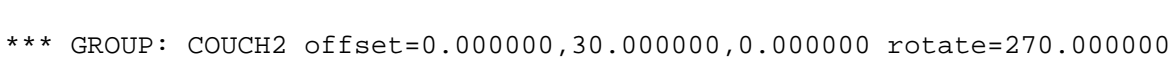

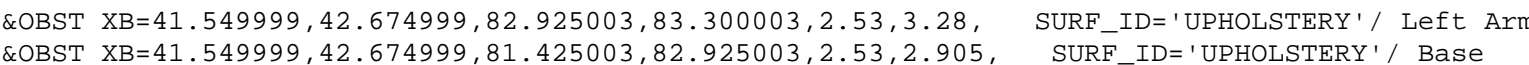
\&OBST XB=41.549999, 42.674999, 81.050003, 81.425003,2.53,3.28, SURF_ID='UPHOLSTERY' '/ Right Ar

$* * *$ GROUP: TWIN

*** GROUP: ROW

*** GROUP: COUCH2 offset $=0.000000,0.000000,0.000000$ rotate $=90$. . 000000

\&OBST $X \mathrm{XB}=47.549999,48.674999,51.049999,51.424999,2.53,3.28, \quad$ SURF_ID= 'UPHOLSTERY' $/$ Left Arm
\&OBST XB $=47.549999,48.674999,51.424999,52.924999,2.53,2.905, \quad$ SURF_ID=' 'UPHOLSTERY' $/$ Base \&OBST XB $=47.549999,48.674999,52.924999,53.299999,2.53,3.28$, SURF_ID=' 'UPHOLSTERY' ' Right Ar

$* * *$ GROUP: COUCH2 offset $=0.000000,3.000000,0.000000$ rotate $=90.000000$

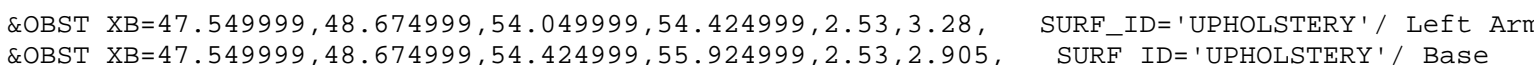
\&OBST XB=47.549999, 48.674999, 55.924999, 56.299999, 2.53,3.28, SURF_ID=' 'UPHOLSTERY'/ Right Ar

*** GROUP: COUCH2 offset $=0.000000,6.000000,0.000000$ rotate $=90.000000$
\&OBST XB $=47.549999,48.674999,57.049999,57.424999,2.53,3.28$, SURF_ID='UPHOLSTERY '/ Left Arm \&OBST XB=47.549999, 48.674999, 57.424999, 58.924999, 2.53,2.905, SURF_ID='UPHOLSTERY'/ Base \&OBST XB $=47.549999,47.974999,58.924999,59.299999,2.53,3.28$, SURF_IDE' 'UPHOLSTERY'/ Right Ar

*** GROUP: COUCH2 offset $=0.000000,9.000000,0.000000$ rotate $=90.000000$

\&OBST XB=47.549999, 48.674999, 60. 049999, 60.424999, 2.53,3.28, SURF_ID='UPHOLSTERY'/ Left Arm

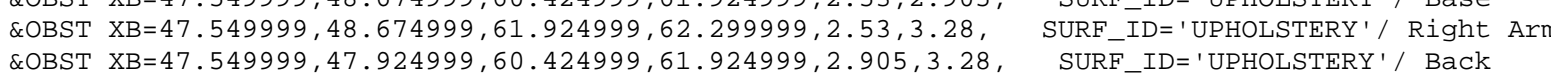

*** GROUP: COUCH2 offset $=0.000000,12.000000,0.000000$ rotate $=90.000000$

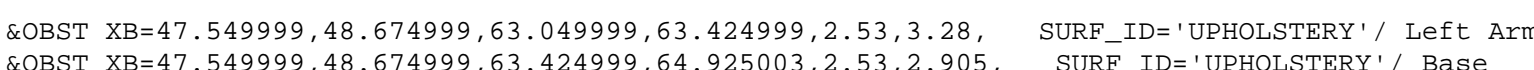

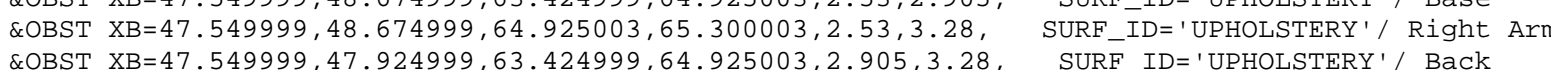

$* * *$ GROUP: COUCH2 offset $=0.000000,15.000000,0.000000$ rotate $=90.000000$

\&OBST XB=47.549999, 48.674999, 66. 050003, 66. 425003, 2.53,3.28, SURF_IDD ' UPHOLSTERY' / Left Arm

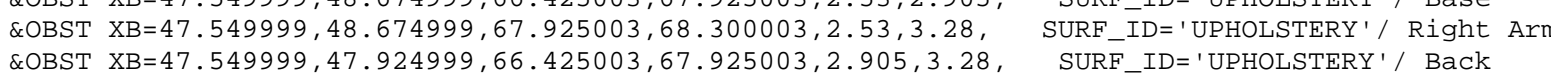

*** GROUP: COUCH2 offset $=0.000000,18.000000,0.000000$ rotate $=90.000000$

\&OBST XB $=47.549999,48.674999,69.050003,69.425003,2.53,3.28$, SURF ID= 'UPHOLSTERY ' $/$ Left Arm \&OBST XB $=47.549999,48.674999,70.925003,71.300003,2.53,3.28$,
\&OBST XB $=47.549999,47.924999,69.425003,70.925003,2.905,3.28, \quad$ SURF_ID=' 'UPHOLSTERY'/ Right Ar
SURF_ID='UPHOLSTERY'/ Back

*** GROUP: COUCH2 offset $=0.000000,21.000000,0.000000$ rotate $=90.000000$

\&OBST XB=47. $549999,48.674999,72.050003,72.425003,2.53,3.28, \quad$ SURF_ID=' 'UPHOLSTERY' ' Left Arm
\&OBST XB=47.549999, $48.674999,72.425003,73.925003,2.53,2.905$,
SURF_ID='UPHOLSTERY' $/$ Base \&OBST XB $=47.549999,48.674999,73.925003,74.300003,2.53,3.28$, SURF ID='UPHOLSTERY'/ Rise A \&OBST XB=47.549999, 47.924999, 72.425003, 73.925003, 2.905, 3.28, SURF_ID=' 'UPHOLSTERY' / Back

** GROUP: COUCH2 offset $=0.000000,24.000000,0.000000$ rotate $=90.000000$

\&OBST XB=47.549999, 48.674999, 75. $050003,75.425003,2.53,3.28, \quad$ SURF_ID='UPHOLSTERY' ' Left Arm
\&OBST XB=47.549999, $48.674999,75.425003,76.925003,2.53,2.905$,
SURF_ID=' 'UPHOLSTERY'

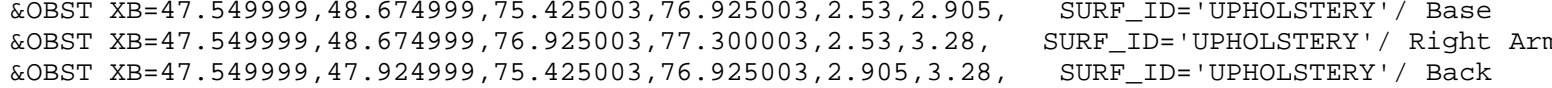

*** GROUP: COUCH2 offset $=0.000000,27.000000$, ๑. .000000 rotate $=90.0000000$

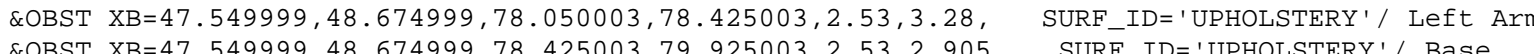

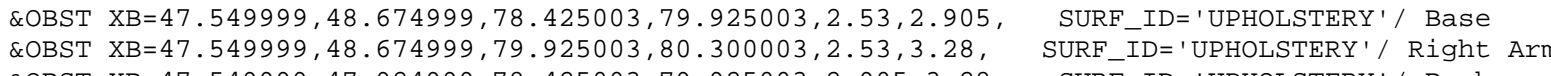

*** GROUP: COUCH2 offset $=0.0000000,30.000000,0.000000$ rotate $=90.000000$

\&OBST XB $=47.549999,48.674999,81.050003,81.425003,2.53,3.28$, SURF_ID='UPHOLSTERY'/ Left Arm

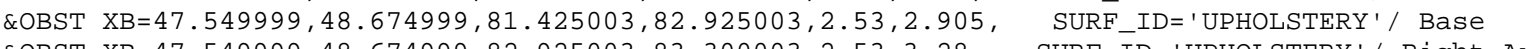
\&OBST XB $=47.549999,47.924999,81.425003,82.925003,2.905,3.28$, SURF ${ }^{\prime}$ ID=' 'UPHOLSTERY'/ Back

*** GROUP: ROW2

*** GROUP: COUCH2 of fset $=0.000000,0.000000,0.000000$ rotate $=270.000000$

\&OBST XB=46. $049999,47.174999,52.924999,53.299999,2.53,3.28$, SURF_ID=' 'UPHOLSTERY ' / Left Arm \&OBST XB=46.049999, 47.174999, 51.049999, 51.424999, 2.53,3.28, SURF_ID='UPHOLSTERY'/ Right Ar \&OBST XB=46.799999, 47.174999, 51.424999, 52.924999, 2.905, 3.28, SURF_ID= 'UPHOLSTERY' / Back

$\star * *$ GROUP: COUCH2 offset $=0.000000,3.000000,0.000000$ rotate $=270.000000$

\&OBST XB=46. $049999,47.174999,55.924999,56.299999,2.53,3.28$, SURF_ID='UPHOLSTERY' ' Left Arm \&OBST XB=46. $049999,47.174999,54.049999,54.424999,2.53,3.28$, SURE ID=' 'UPHOLSTERY'/R \&OBST XB=46.799999, $47.174999,54.424999,55.924999,2.905,3.28, \quad$ SURF_ID ' 'UPHOLSTERY'/ Back 


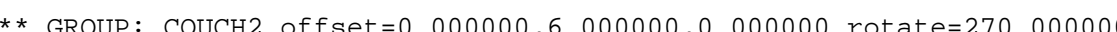

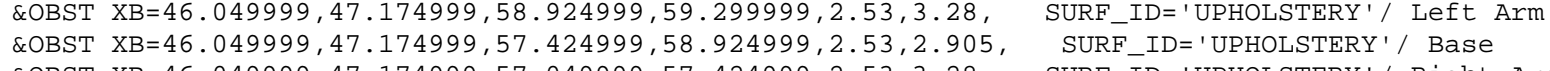

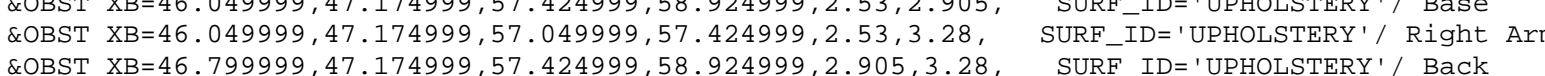

*** GROUP: COUCH2 offset $=0.000000,9$. ๑०००००,,. .00000 rotate $=270.000000$

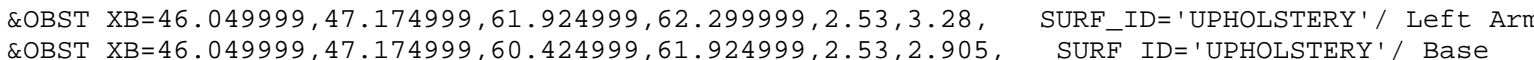

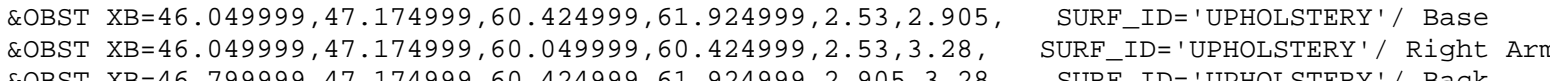

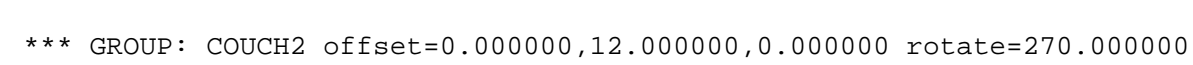

\&OBST XB=46.049999, $47.174999,64.925003,65.300003,2.53,3.28$, SURF_ID='UPHOLSTERY'/ Left Arm \&OBST XB=46. $949999,47.174999,63.424999,64.925003,2.53,2.905$, SURF_ID='UPHOLSTERY'/ Base \&OBST $X \mathrm{XB}=46.049999,47.174999,63.049999,63.424999,2.53,3.28, \quad$ SURF_ID='UPHOLSTERY'/ Right Ar
\&OBST $X \mathrm{~B}=46.799999,47.174999,63.42499,64.925003,2.905,3.28$, SURFID='UPHOLSTERY' Back

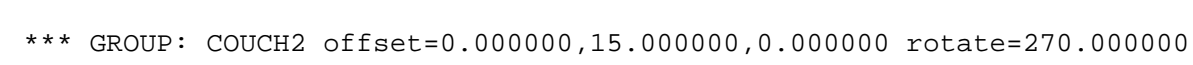

\&OBST XB $=46.0499999,47.174999,67.925003,68.300003,2.53,3.28$, SURF ID='UPHOLSTERY'/ Left Arm \&OBST XB=46. $949999,47.174999,66.425003,67.925003,2.53,2.905$, SURF_ID='UPHOLSTERY'/ Base \&OBST XB=46.04999, $47.174999,66.050003,66.425003,2.53,3.28, \quad$ SURF_ID='UPHOLSTERY'/ RIght A

*** GROUP: COUCH2 offset $=0.000000,18.000000,0.000000$ rotate $=270.000000$

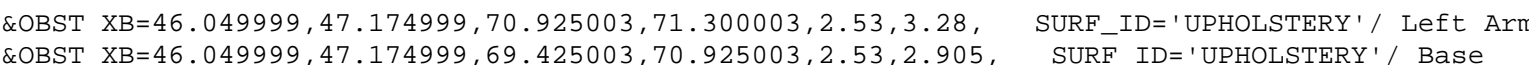
\&OBST XB=46. $049999,47.174999,69.050003,69.425003,2.53,3.28$, SURF_ID=' 'UPHOLSTERY'/ Right Ar

$* * *$ GROUP: COUCH2 offset $=0.000000,21.000000,0.000000$ rotate $=270.000000$ \&OBST XB $=46.049999,47.174999,73.925003,74.300003,2.53,3.28$,
\&OBST XB $=46.049999,47.174999,72.425003,73.925003,2.53,2.905, \quad$ SURF_ID=' 'UPHOLSTERY' $/$ Left Arm
SURF_ID=' 'UPHOLSTERY' / Base

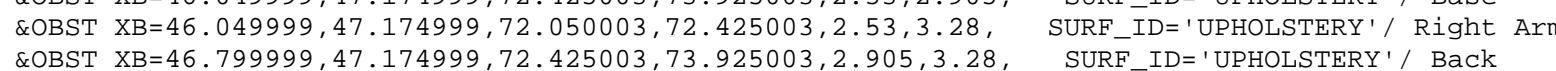

*** GROUP: COUCH2 offset $=0.000000,24.000000,0.000000$ rotate $=270.000000$

\&OBST XB=46.049999, 47.174999, 76.925003, 77.300003, 2.53,3.28, SURF_ID='UPHOLSTERY'/ Left Arm

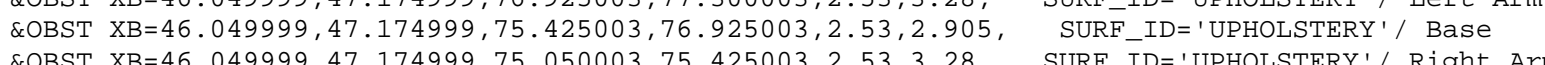

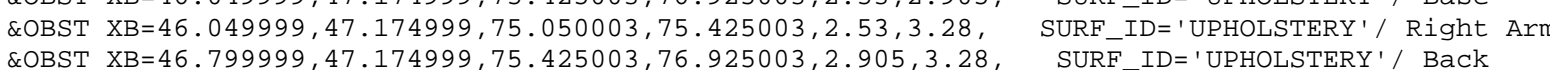

*** GROUP: COUCH2 offset $=0.000000,27.000000,0.000000$ rotate $=270.000000$

\&OBST XB=46. $049999,47.174999,79.925003,80.300003,2.53,3.28$, SURF_ID=' 'UPHOLSTERY'/ Left Arm QOBST XB $=46.049999,47.174999,78.425003,79.925003,2.53,2.905$, SURF ${ }_{\text {IDD }}$ 'UPHOLSTERY'/ Base \&OBST XB=46. $949999,47.174999,78.050003,78.425003,2.53,3.28, \quad$ SURF_ID='UPHOLSTERY'/ Right Ar
\&OBST XB $=46.799999,47.174999,78.425003,79.925003,2.905,3.28$,
SURF_ID=' 'UPHOLSTERY'/ Back

*** GROUP: COUCH2 offset $=0.000000,30.000000,0.000000$ rotate $=270.000000$

\&OBST XB=46. $049999,47.174999,82.925003,83.300003,2.53,3.28$, SURF_ID=' 'UPHOLSTERY'/ Left Arm

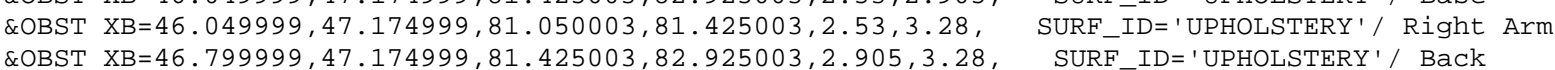

\section{$* * *$ GROUP: TWIN}

*** GROUP: ROW

*** GROUP: CoucH2 offset $=0.000000,0.000000,0.000000$ rotate $=90.000000$

\&OBST $X \mathrm{X}=52.049999,53.174999,51.049999,51.424999,2.53,3.28$, SURF_ID='UPHOLSTERY '/ Left Arm \&OBST XB $=52.049999,53.174999,51.424999,52.924999,2.53,2.905, \quad$ SURF IDD IUPHOLSTERY' $^{\prime}$, Base

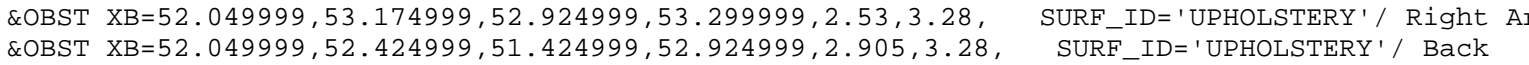

*** GROUP: COUCH2 offset $=0.000000,3$. .

\&OBST XB=52.049999, 53.174999, 54.049999, 54.424999, 2.53,3.28, SURF_ID='UPHOLSTERY'/ Left Arm
\&OBST XB=52. $049999,53.174999,54.424999,55.924999,2.53,2.905$, SURF_ID='UPHOLSTERY'/ Base

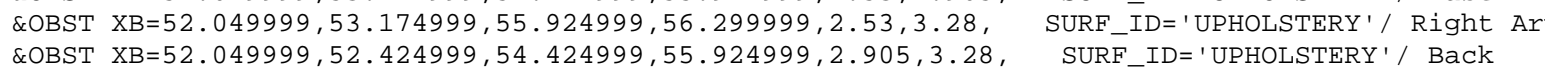
*** GROUP: COUCH2 offset $=0.000000,6.000000,0.000000$ rotate $=90.000000$

\&OBST $X \mathrm{XB}=52.049999,53.174999,57.049999,57.424999,2.53,3.28, \quad$ SURF_ID=' 'UPHOLSTERY'/ Left Arm
\&OBST XB $\mathrm{B}=52.049999,53.174999,57.424999,58.924999,2.53,2.905$,
SURF_ID=' 'UPHOLSTERY'/ Base \&OBST XB LOBST XB=52.049999, 52.424999, 57.424999, 58. $924999,2.905,3.28$, SURF_ID ' 'UPHOLSTERY' / Back

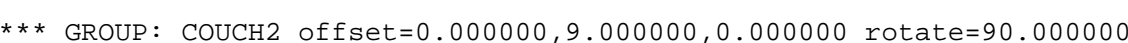

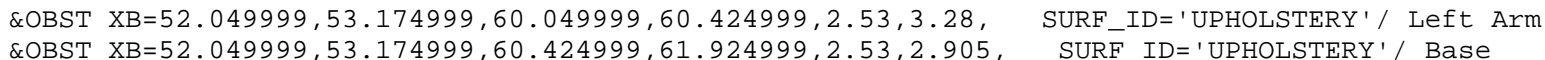

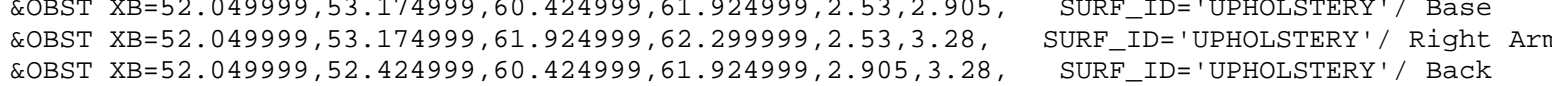

*** GROUP: COUCH2 offset $=0.000000,12.000000$, ๑. .000000 rotate $=90.0000000$

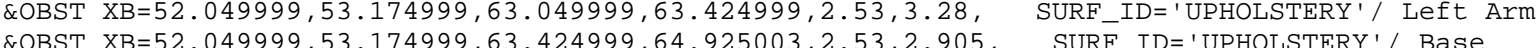

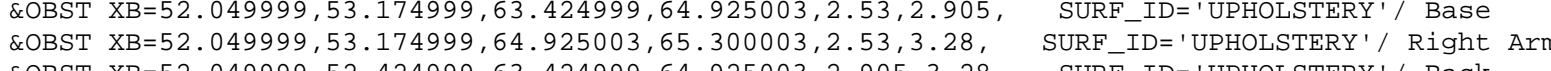

*** GROUP: COUCH2 off set $=0.000000,15.000000,0.000000$ rotate $=90.000000$

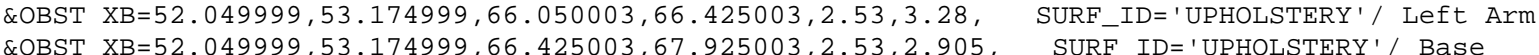
\&OBST $X \mathrm{~B}=52.04999,53.17499,66.425003,67.925003,2.53,2.905$, SURF_ID='UPHOLSTERY'/ Base

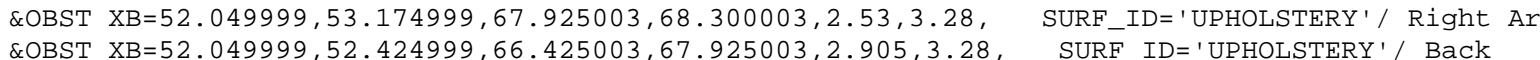

*** GROUP: COUCH2 offset $=0.000000,18.000000$, ๑. 000000 rotate $=90.000000$

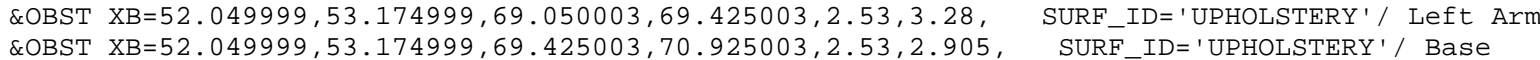

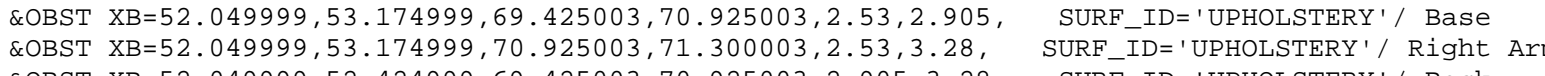

*** GROUP: COUCH2 offset $=0.000000,21.000000,0.000000$ rotate $=90.000000$

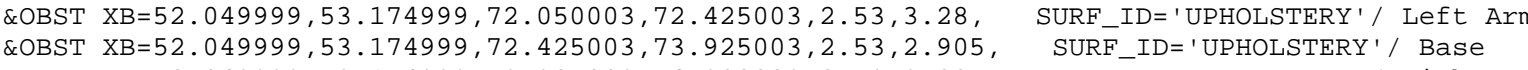

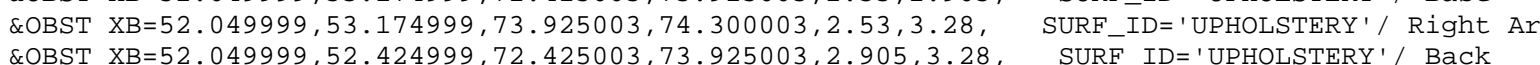

*** GROUP: COUCH2 offset $=0.000000,24.000000,0.000000$ rotate $=90 . .000000$

\&OBST XB=52.049999, 53.174999, 75.050003,75.425003, 2.53,3.28, SURF_ID='UPHOLSTERY'/ Left Arm \&OBST XB=52. $049999,53.174999,75.425003,76.925003,2.53,2.905$, SURF_ID=' 'UPHOLSTERY' / Base

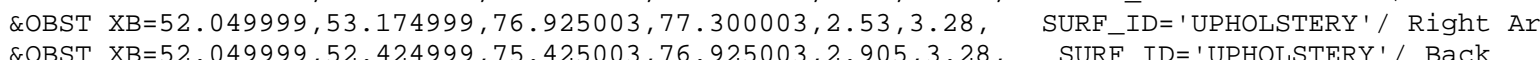

*** GROUP: COUCH2 offset $=0.000000,27.0000000,0 . .000000$ rotate $=90.0000000$

\&OBST XB=52. $049999,53.174999,78.050003,78.425003,2.53,3.28$, SURF ID=' 'UPHOLSTERY ' / Left Arm \&OBST XB $=52.049999,53.174999,78.425003,79.925003,2.53,2.905$, SURF_ID=' UPHOLSTERY'/ Base

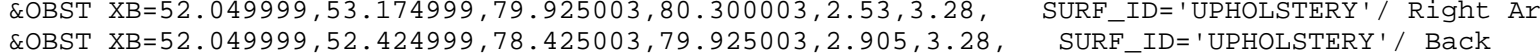

*** GROUP: COUCH2 offset $=0.000000,30.000000,0.000000$ rotate $=90.000000$

\&OBST XB=52.049999, 53.174999, 81.050003, 81.425003, 2.53,3.28, SURF_ID=' 'UPHOLSTERY ' ' Left Arm

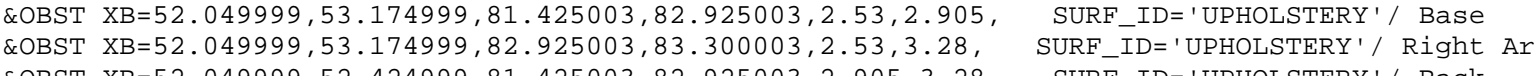

*** GROUP: ROW2

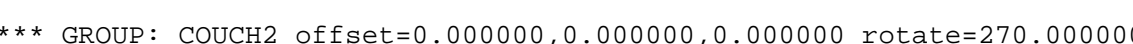

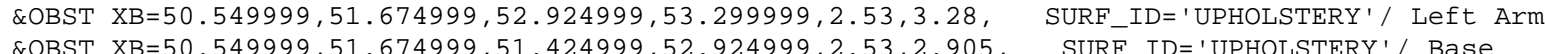

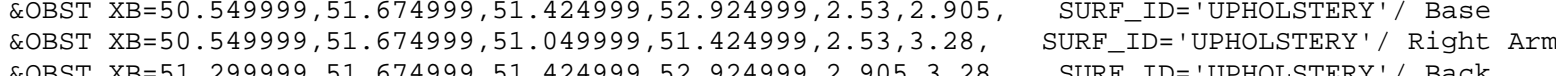

*** GROUP: COUCH2 offset $=0.000000,3.000000,0.000000$ rotate $=270.000000$ 
\&OBST XB $=50.549999,51.674999,55.924999,56.299999,2.53,3.28, \quad$ SURF_ID=' 'UPHOLSTERY '/ Left Arm
\&OBST XB $=50.549999,51.674999,54.424999,55.924999,2.53,2.905, \quad$ SURF_IDD' 'UPHOLSTERY' $/$ Base \&OBST XB=50.549999, 51.674999, 54. $049999,54.424999,2.53,3.28$, SURE ID =' UPHOLSTERY'/ Right Ar A SURF_ID='UPHOLSTERY' / Back

*** GROUP: COUCH2 offset $=0.000000,6.000000,0.000000$ rotate $=270.000000$

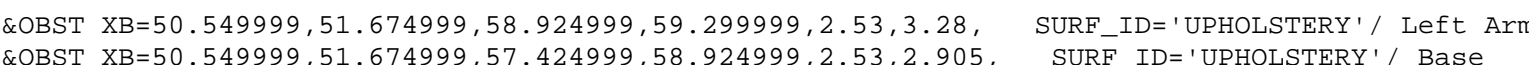
\&OBST XB=50.549999, 51.674999, 57.049999, 57.424999, 2.53,3.28, SURF_ID='UPHOLSTERY'// Right Ar

*** GROUP: COUCH2 offset $=0.000000,9.000000,0.000000$ rotate $=270.000000$

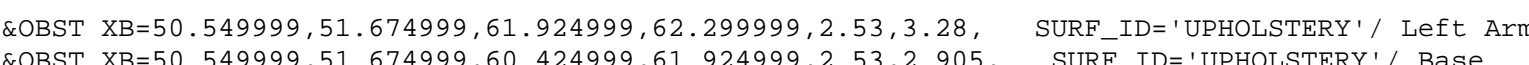
\&OBST XB $=50.549999,51.674999,60.049999,66.424999,2.53,3.28, \quad$ SURF ID= 'UPHOLSTERY'/ Right Ar $* * *$ GROUP: COUCH2 offset $=0.000000,12.000000,0.000000$ rotate $=270.000000$

\&OBST XB=50.549999, 51.674999, 64.925003, 65.300003, 2.53,3.28, SURF_ID=' UPHOLSTERY'/ Left Arm

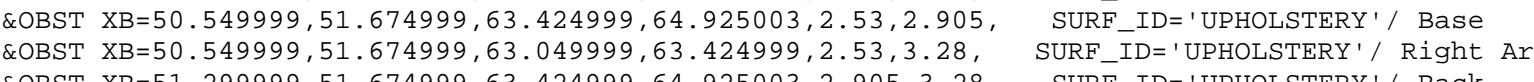

*** GROUP: CoUCH2 offset $=0.000000,15.000000,0.000000$ rotate $=270.000000$

\&OBST XB $=50.549999,51.674999,67.925003,68.300003,2.53,3.28$, SURF_ID=' 'UPHOLSTERY'/ Left Arm \&OBST XB=50.549999, $51.674999,66.425003,67.925003,2.53,2.905, \quad$ SURF_ID='UPHOLSTERY'/ Base \&OBST XB=50.549999, $51.674999,66.050003,66.425003,2.53,3.28$,
\&OBST XB=51.29999, $51.674999,66.425003,67.925003,2.905,3.28$,
SURF_IDE_ID='UPHOLSTERY' / Back

$* * *$ GROUP: COUCH2 offset $=0.000000,18.000000,0.000000$ rotate $=270.000000$

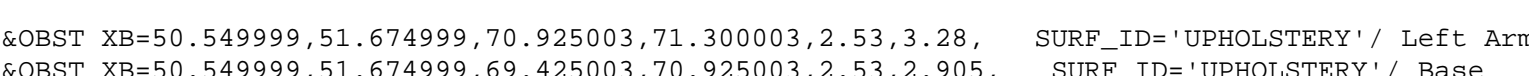

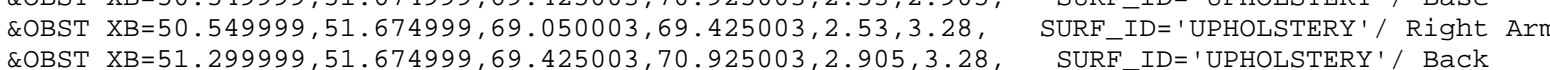

*** GROUP: COUCH2 offset $=0.000000,21.000000,0.000000$ rotate $=270.000000$

\&OBST XB $=50.549999,51.674999,73.925003,74.300003,2.53,3.28$, SURF-ID=' 'UPHOLSTERY'/ Left Arm

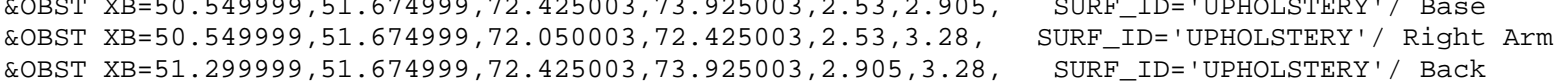
*** GROUP: COUCH2 offset $=0,000000,24.000000, \Theta ., 000000$ rotate $=270,000000$

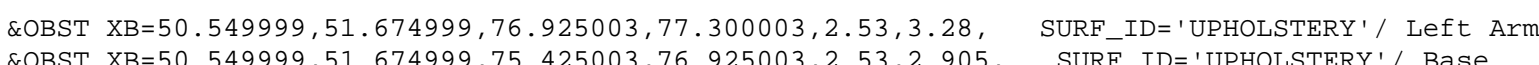
COBST $X \mathrm{XB}=50.549999,51.674999,75.050003,75.425003,2.53,3.28$,
\&OBST XB=51.299999, $51.674999,75.425003,76.925003,2.905,3.28, \quad$ SURF_ID='UPHOLSTERY'/ Right Arm
SURF_ID=' 'UPHOLSTERY' $/$ Back

*** GROUP: COUCH2 offset $=0.000000,27.000000,0.000000$ rotate $=270.000000$

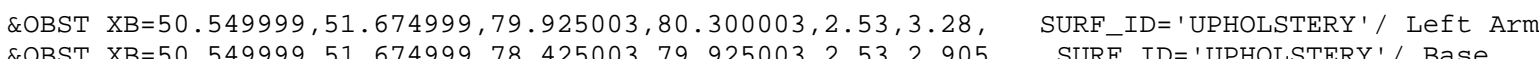
\&OBST XB $=50.54999,51.074999,78.050003,78,425003,2,53,3.28$, SUPF_ID='UPHOLSTERY'/ Right Arm \&OBST XB=51.299999, $51.674999,78.425003,79.925003,2.905,3.28$, SURF_ID=' UPHOLSTERY'/ Back

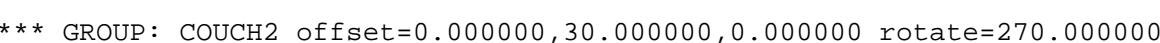

\&OBST XB $=50.549999,51.674999,82.925003,83.300003,2.53,3.28, \quad$ SURF_ID= 'UPHOLSTERY'/ Left Arm \&OBST XB $50.549999,51.674999,81.050003,81.425003,2.53 .328$, SUPF ID='UPHOLSTERY'/ Rise \&OBST XB=51.299999, $51.674999,81.425003,82.925003,2.905,3.28$, SURF_ID=' 'UPHOLSTERY'/ Back

$* * *$ GROUP: TWIN

*** GROUP: ROW

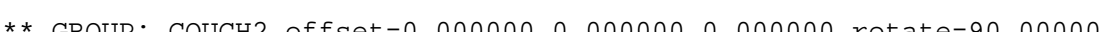

\&OBST XB=56.549999, 57.674999, 51. $049999,51.424999,2.53,3.28, \quad$ SURF_ID='UPHOLSTERY' ' Left Arm
\&OBST XB=56.549999, $57.674999,51.424999,52.924999,2.53,2.905, \quad$ SURF_ID=' 'UPHOLSTERY' $/$ Base
\&OBST XB=56.549999, $57.674999,52.924999,53.299999,2.53,3.28, \quad$ SURF_ID= 'UPHOLSTERY' 1 Right Arm
\&OBST XB=56.549999, $56.924999,51.424999,52.924999,2.905,3.28, \quad$ SURF_ID=' 'UPHOLSTERY' / Back

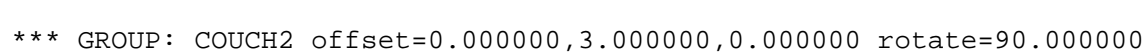

\&OBST XB=56. $549999,57.674999,54.049999,54.424999,2.53,3.28, \quad$ SURF_ID=' 'UPHOLSTERY'/' Left Arm
\&OBST XB=56.549999, $57.674999,54.424999,55.924999,2.53,2.905$,
SURF_ID='UPHOLSTERY'/ Base

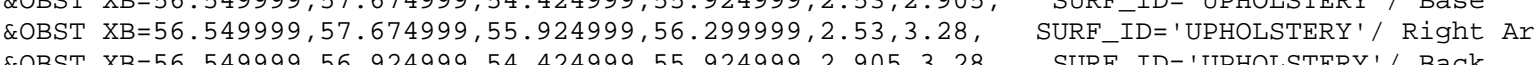

*** GROUP: COUCH2 offset $=0.0000000,6.000000,, 0.000000$ rotate $=90.000000$

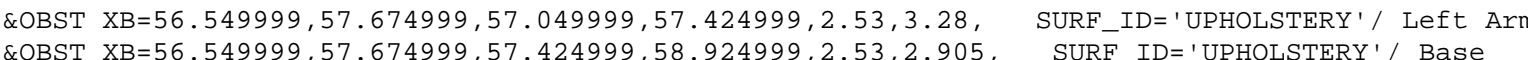
\&OBST XB=56.549999, $57.674999,55.424999,58.924999,2.53,2.905$, SURF_ID='UPHOLSTERY'/ Base

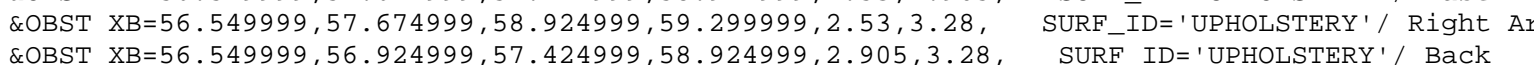

*** GROUP: COUCH2 offset $=0.000000,9.000000,0.000000$ rotate $=90.000000$

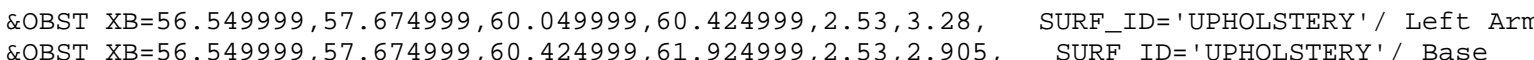

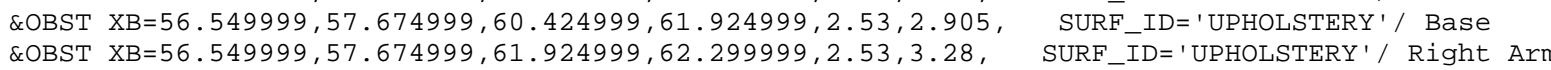

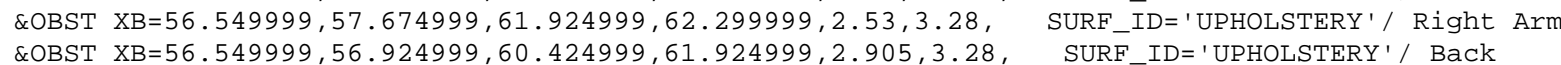

*** GROUP: COUCH2 offset $=0.000000,12.000000,0.000000$ rotate $=90.000000$

\&OBST XB=56.549999, 57.674999, 63.049999, 63.424999, 2.53,3.28, SURF_ID=' 'UPHOLSTERY'/ Left Arm

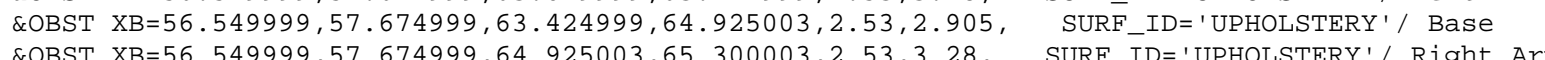
KOBST XB=56.549999, 56.924999, 63.424999,64.925003, 2.905, 3.28, SURF_ID='UPHOLSTERY'/ Back

*** GROUP: COUCH2 offset $=0.000000,15.000000,0.000000$ rotate $=90.000000$

\&OBST XB $=56.549999,57.674999,66.050003,66.425003,2.53,3.28$, SURF_ID='UPHOLSTERY'/ Left Arm \&OBST XB $=56.549999,57.674999,66.425003,67.925003,2.53,2.905, \quad$ SURF $I$ ID $=$ 'UPHOLSTERY' '/ Base

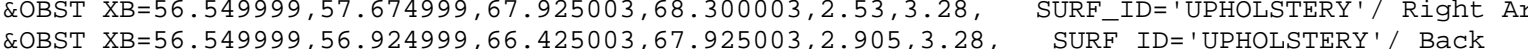

*** GROUP: CouCH2 offset $=0.000000,18.000000,0.000000$ rotate $=90.000000$

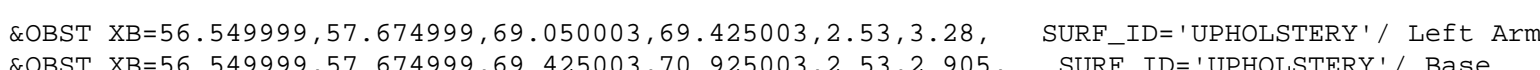

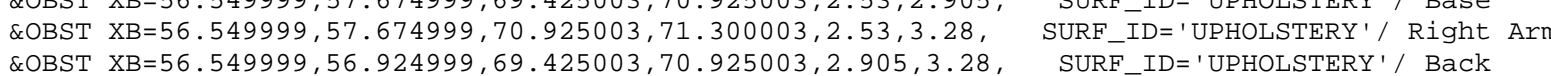

*** GROUP: COUCH2 offset $=0.000000,21.000000,0.000000$ rotate $=90.000000$

\&OBST XB=56.549999, $57.674999,72.050003,72.425003,2.53,3.28$, SURF_ID=' 'UPHOLSTERY' ' Left Arm

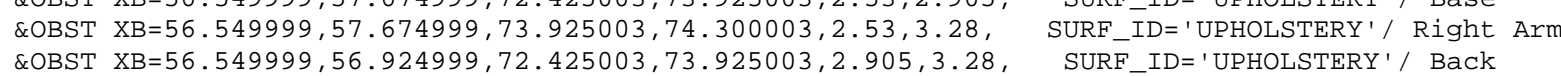

*** GROUP: COUCH2 offset $=0.000000,24.000000,0.000000$ rotate $=90.000000$

\&OBST XB=56.549999, 57.674999, 75. 050003, 75.425003, 2.53,3.28, SURF_ID=' UPHOLSTERY'/ Left Arm \&OBST XB=56.549999, 57.674999, 76.925003, 77.300003,2.53,3.28, SURF_ID='UPHOLSTERY'/ Right Ar \&OBST XB=56.549999, 56. 924999, 75.425003, 76. 925003, 2.905, 3.28, SURF_ID='UPHOLSTERY'/ Back

*** GROUP: COUCH2 offset $=0.000000,27.000000,0.000000$ rotate $=90.000000$

\&OBST XB=56.549999, $57.674999,78.050003,78.425003,2.53,3.28$, SURF_ID=' 'UPHOLSTERY' ' Left Arm 源 \&OBST XB=56.549999, 56. 924999, 78.425003, 79.925003, 2.905, 3.28, SURF_ID='UPHOLSTERY'/ Back

*** GROUP: COUCH2 offset $=0.000000,30.000000,0.000000$ rotate $=90.000000$

\&OBST XB=56.549999, 57.674999, 81. $0500003,81.425003,2.53,3.28$, SURF ID=' 'UPHOLSTERY ' / Left Arm \&OBST XB $=56,549999,57.674999,82,925003,83,300003,2,53,3.28, \quad$ SUPF-ID='UPHOLSTERY'/ \&OBST XB=56.549999, 56. $924999,81.425003,82.925003,2.905,3.28$, SURF_ID='UPHOLSTERY' / Back

*** GROUP: ROW2

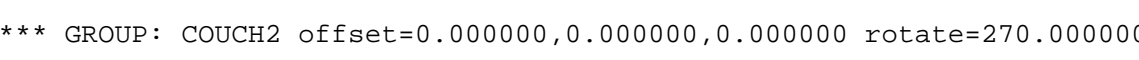


\&OBST XB=55. $049999,56.174999,52.924999,53.299999,2.53,3.28, \quad$ SURF_ID= 'UPHOLSTERY ' /' Left Arm
\&OBST XB=55.049999, $56.174999,51.424999,52.924999,2.53,2.905, \quad$ SURF_ID=' 'UPHOLSTERY' / Base

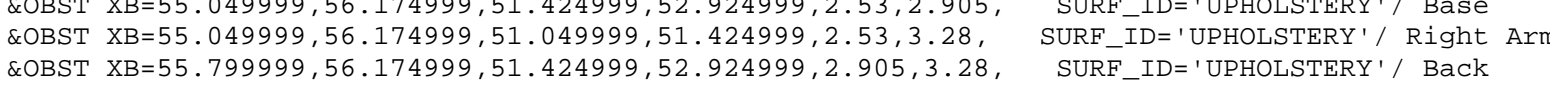
*** GROUP: COUCH2 offset $=0.000000,3.000000,0.000000$ rotate $=270.0000000$

\&OBST XB=55. 049999, 56.174999, 55. 924999, 56. 299999, 2.53,3.28, SURF_ID=' 'UPHOLSTERY '/ Left Arm

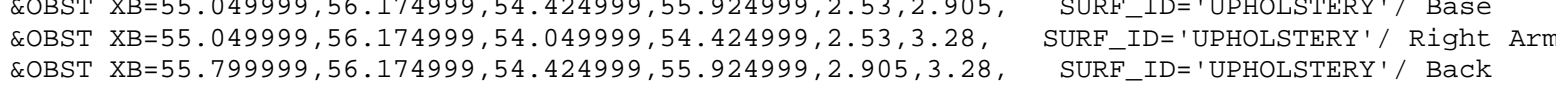
*** GROUP: COUCH2 offset $=0.000000,6.000000,0.000000$ rotate $=270.000000$

\&OBST $X \mathrm{XB}=55.049999,56.174999,58.924999,59.299999,2.53,3.28, \quad$ SURF_ID=' 'UPHOLSTERY' $/$ Left Arm

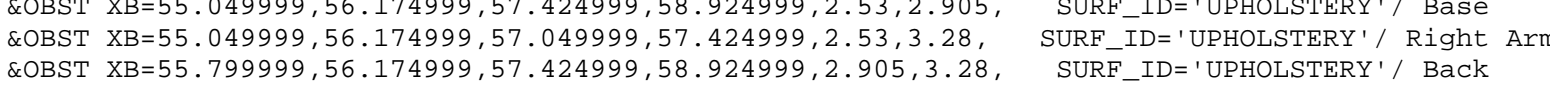
*** GROUP: COUCH2 offset $=0.000000,9.000000,0.000000$ rotate $=270.000000$

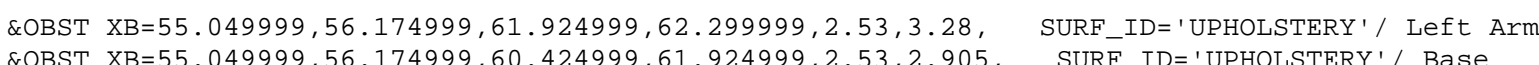

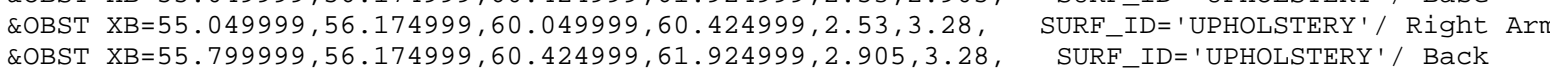

*** GROUP: COUCH2 offset $=0.000000,12.000000,0.000000$ rotate $=270.000000$

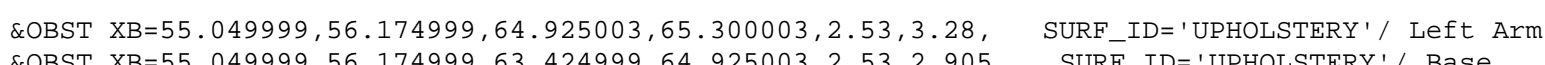

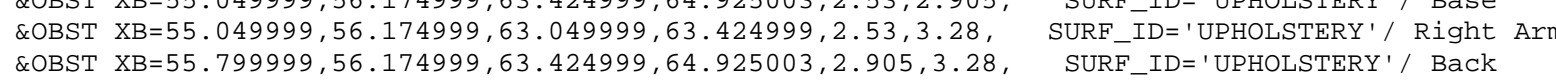
*** GROUP: COUCH2 offset $=0.000000,15.000000,0.000000$ rotate $=270.000000$

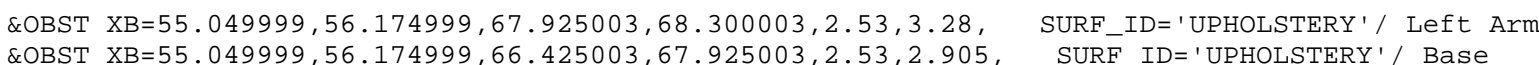
\&OBST XB=55. $049999,56.174999,66.050003,66.425003,2.53,3.28$, SURE TD='UPHOLSTERY'/ Right Ar \&OBST XB $=55.799999,56.174999,66.425003,67.925003,2.905,3.28, \quad$ SURF_ID=' 'UPHOLSTERY'/ Back

*** GROUP: COUCH2 offset $=0.000000,18.000000,0.000000$ rotate $=270.000000$

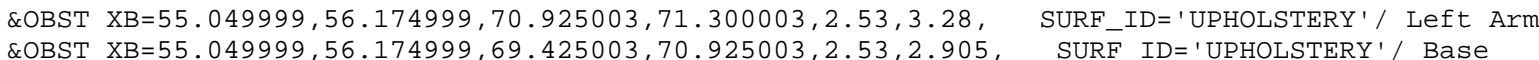
\&OBST XB $55.049999,56.174999,69.050003,69.42503,2.53,3.28, \quad$ SURF_IDD='UPHOLSTERY'/ Right Arm

** GROUP: COUCH2 offset $=0.000000,21.000000,0.000000$ rotate $=270.000000$

\&OBST XB $=55.049999,56.174999,73.925003,74.300003,2.53,3.28$, SURF_ID=' UPHOLSTERY' ' Left Arm

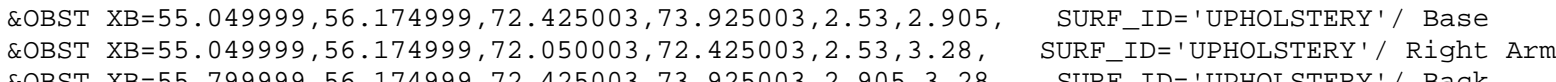

$* \star *$ GROUP: COUCH2 offset $=0.000000,24.000000$, ๑. .000000 rotate $=270$. ๑

\&OBST XB $=55.049999,56.174999,76.925003,77.300003,2.53,3.28, \quad$ SURF_ID=' UPHOLSTERY '/' Left Arm
\&OBST XB $=55.049999,56.174999,75.425003,76.925003,2.53,2.905, \quad$ SURF_ID='UPHOLSTERY' / Base

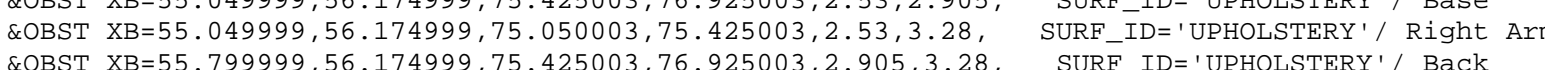
*** GROUP: COUCH2 offset $=0.000000,27.000000,0.000000$ rotate $=270$. . 000000

\&OBST XB $=55.049999,56.174999,79.925003,80.300003,2.53,3.28$, SURF_ID='UPHOLSTERY'/ Left Arm \&OBST XB $=55.049999,56.174999,78.425003,79.925003,2.53,2.905$, SURF_ID= 'UPHOLSTERY' / Base

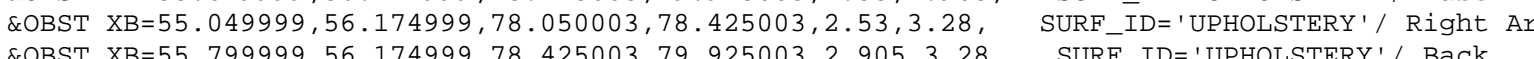
*** GROUP: COUCH2 offset $=0.000000,30.000000,0.000000$ rotate $=270.000000$

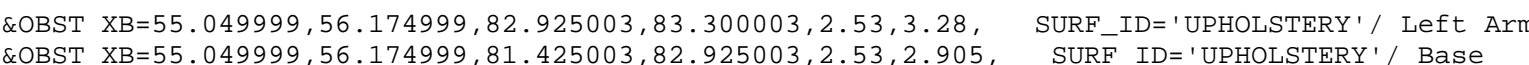
\&OBST XBB $55.049999,56.174999,81.055003,81.425503,2.53,3.28$, SURF_ID=' 'UPHOLSTERY'/ Right Ar *** GROUP: TWIN
*** GROUP: ROW

*** GROUP: COUCH2 offset $=0.000000,0.000000,0.000000$ rotate $=90 . .00000$

\&OBST XB=61. 049999, 62.174999, 51.049999, 51.424999, 2.53,3.28, SURF_ID=' 'UPHOLSTERY'/ Left Arm LOBST XB $=61.049999,62.174999,51.424999,52.924999,2.53,2.905$, SURF_ID=' 'UPHOLSTERY '/ Base LOBST XB=61.049999, 61.424999, 51.424999, 52.924999, 2.905, 3.28, SURF_ID='UPHOLSTERY'/ Right Ar

*** GROUP: COUCH2 offset $=0.000000,3.000000,0.000000$ rotate $=90.000000$

\&OBST $X \mathrm{XB}=61.049999,62.174999,54.049999,54.424999,2.53,3.28$, SURF_ID=' 'UPHOLSTERY'/ Left Arm \&OBST XB=61. $049999,62.174999,54.424999,55.924999,2.53,2.905$, SURF_ID='UPHOLSTERY' / Base

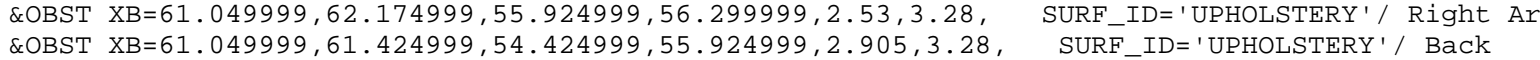

*** GROUP: COUCH2 offset $=0.000000,6.000000,0.000000$ rotate $=90.000000$

\&OBST XB=61. $049999,62.174999,57.049999,57.424999,2.53,3.28$, SURF_ID='UPHOLSTERY'// Left Arm

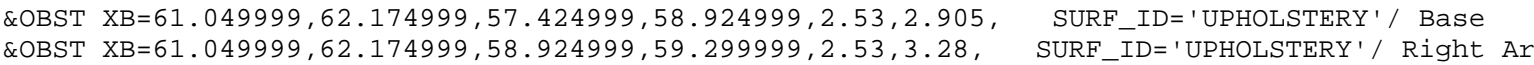
عOBST XB $=61.049999,61.424999,57.424999,58.924999,2.905,3.28, \quad$ SURF_IDE 'UPHOLSTERY'/ Back

*** GROUP: COUCH2 offset $=0.000000,9.0000000,0.000000$ rotate $=90.000000$

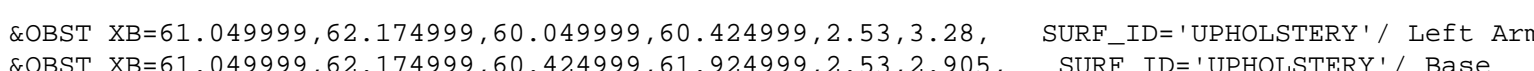
\&OBST XB=61. $049999,62.174999,61.924999,62.299999,2.53,3.28$,
\&OBST XB=61.04999, $61.424999,60.424999,61.924999,2.905,3.28, \quad$ SURF_ID= 'UPHOLSTERY'/ Right Arm
SURF_ID='UPHOLSTERY'/ Back

*** GROUP: COUCH2 offset $=0.000000,12.000000,0.000000$ rotate $=90.000000$

\&OBST XB=61. $049999,62.174999,63.049999,63.424999,2.53,3.28$, SURF_ID=' 'UPHOLSTERY' ' Left Arm

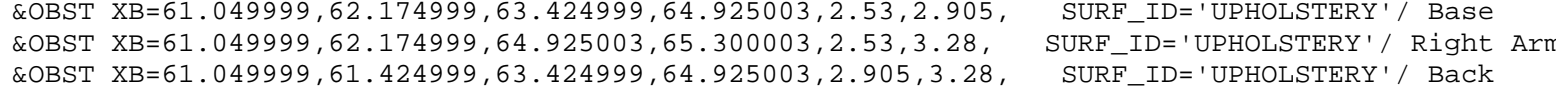

*** GROUP: COUCH2 offset $=0.000000,15.000000,0.000000$ rotate $=90.000000$

\&OBST XB $=61.049999,62.174999,66.050003,66.425003,2.53,3.28$, SURF.ID= 'UPHOLSTERY ' $/$ Left Arm \&OBST XB $61.049999,62.174999,67.925003,68.300003,2.53,3.28$, SURF IDD='UPHOLSTERY'/ Pight Ar \&OBST XB=61.049999, 61.424999, 66.425003, 67.925003, 2.905, 3.28, SURF_ID=' 'UPHOLSTERY' / Back

*** GROUP: COUCH2 offset $=0.000000,18.000000,0.000000$ rotate $=90.000000$

\&OBST XB=61. 049999, 62.174999, 69.050003, 69.425003, 2.53,3.28, SURF_ID=' 'UPHOLSTERY'/ Left Arm

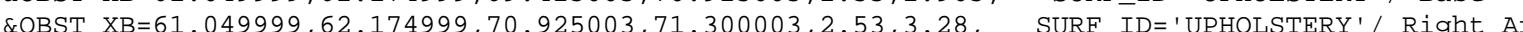
\&OBST XB=61. $049999,61.424999,69.425003,70.925003,2.905,3.28$, SURF_ID=' 'UPHOLSTERY' / Back

*** GROUP: COUCH2 offset $=0.000000,21.000000,0.000000$ rotate $=90.000000$

\&OBST XB=61.049999, 62.174999, 72.050003,72.425003, 2.53,3.28, SURF_ID=' 'UPHOLSTERY' 1 Left Arm QOBST XB=61. $049999,62.174999,73.925003,74.300003,2.53,3.28, \quad$ SURF IDD 'UPHOLSTERY'/ Right Arm

*** GROUP: COUCH2 offset $=0.000000,24.000000,0.000000$ rotate $=90.0000000$

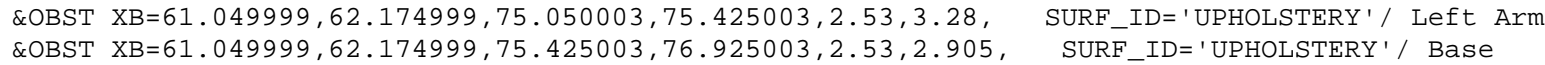

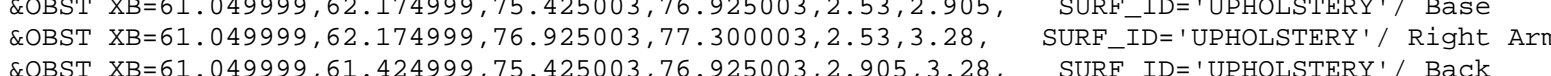

*** GROUP: COUCH2 offset $=0.000000,27.000000$, ๑. .000000 rotate $=90.000000$

\&OBST XB $=61.049999,62.174999,78.050003,78.425003,2.53,3.28, \quad$ SURF_ID='UPHOLSTERY' 1 ' Left Arm

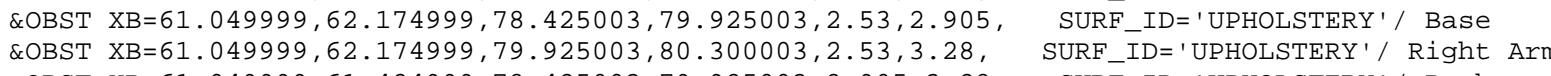
\&OBST XB=61.049999, 61.424999, 78. 425003, 79.925

** GROUP: COUCH2 offset $=0.000000,30.000000,0.000000$ rotate $=90.000000$

\&OBST XB=61.049999, 62.174999, 81.050003, 81.425003, 2.53,3.28, SURF_ID='UPHOLSTERY '/ Left Arm 
\&OBST XB=61. 049999, 61. $424999,81.425003,82.925003,2.905,3.28$, SURE ID=' UPHOLSTERY' / Back

*** GROUP: ROW2

*** GROUP: COUCH2 offset $=0.000000,0.000000,0.000000$ rotate $=270$. . 000000

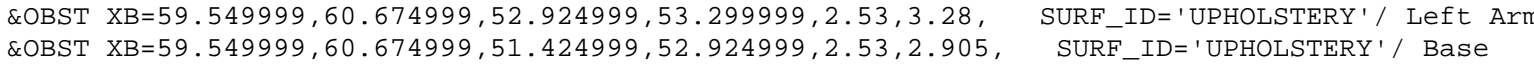

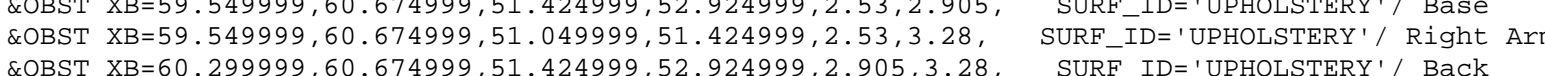
$\star * \star$ GROUP: COUCH2 offset $=0.000000,3.000000,0.000000$ rotate $=270.000000$

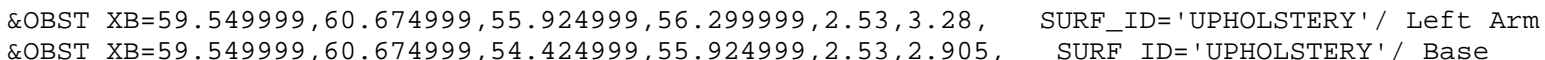

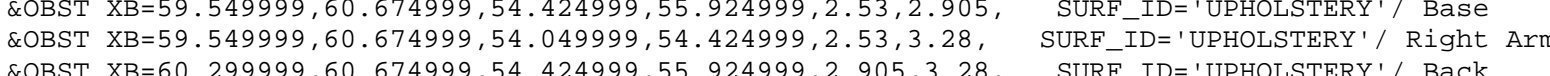

*** GROUP: COUCH2 offset $=0.000000,6.000000,0.000000$ rotate $=270.000000$

\&OBST XB=59.549999, 60.674999, 58.924999, 59.299999, 2.53,3.28, SURF_ID='UPHOLSTERY' ' Left Arm

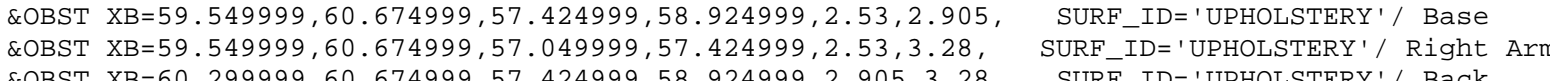

*** GROUP: COUCH2 offset $=0.000000,9.000000,0.0000000$ rotate $=270.000000$

\&OBST XB=59.549999, 60.674999, 61.924999, 62.299999, 2.53,3.28, SURF_ID='UPHOLSTERY ' / Left Arm

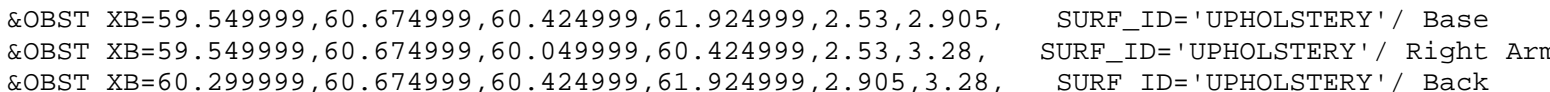

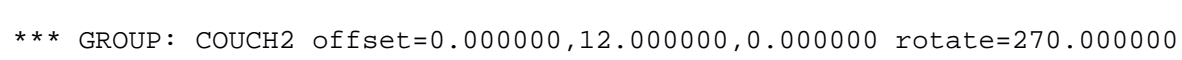

\&OBST XB=59. $549999,60.674999,64.925003,65.300003,2.53,3.28, \quad$ SURF_ID= ' UPHOLSTERY' 1 ' Left Arm
\&OBST XB=59.549999, 60.674999, 63.424999,64.925003, 2.53,2.905, SURF_ID=' 'UPHOLSTERY'/ Base \&OBST XB $=59.549999,60.674999,63.049999,63.424999,2.53,3.28$, SURF_ID=' UPHOLSTERY'/ Right Ar

*** GROUP: COUCH2 offset $=0.000000,15.000000$, ๑. .000000 rotate $=270.000000$

\&OBST XB=59. $549999,60.674999,67.925003,68.300003,2.53,3.28, \quad$ SURF_ID= 'UPHOLSTERY' 1 ' Left Arm
\&OBST XB=59.549999, $60.674999,66.425003,67.925003,2.53,2.905$,
SURF_ID=' UPHOLSTERY'/ Base \&OBST XB=59.549999, 60.674999, 66.050003,66.425003, 2.53,3.28, SURF_ID=' UPHOLSTERY' '/Right Ar

*** GROUP: COUCH2 offset $=0.000000,18.000000,0.000000$ rotate $=270.000000$

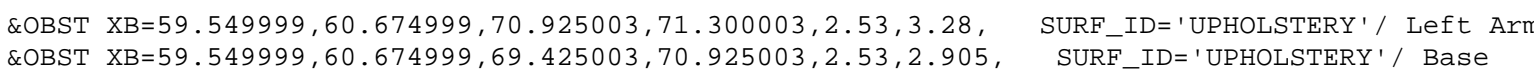
\&OBST XB=59.549999, $60.674999,69.050003,69.425003,2.53,3.28$,
\&OBST XB=60.29999, $60.674999,69.425003,70.925003,2.905,3.28, \quad$ SURF_IDD 'UPHOLSTERY'/ Right Arm
SURF_ID='UPHOLSTERY'/ Back

*** GROUP: COUCH2 offset $=0.000000,21.000000,0.000000$ rotate $=270.000000$

\&OBST XB=59.549999, 60.674999, 73.925003, 74.300003, 2.53,3.28, SURF_ID='UPHOLSTERY'/ Left Arm

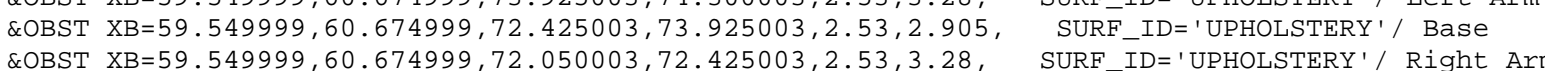
\&OBST XB $=60.299999,60.674999,72.425003,73.925003,2.905,3.28, \quad$ SURF_ID=' 'UPHOLSTERY'/ Right Ar

*** GROUP: COUCH2 offset $=0.000000,24.000000,0.000000$ rotate $=270.000000$

\&OBST XB=59.549999, 60.674999, 76.925003, 77. 300003, 2.53,3.28, SURF_ID=' 'UPHOLSTERY'/ Left Arm \&OBST XB $=59.549999,60.674999,75.425003,76.925003,2.53,2.905$, SURF_ID='UPHOLSTERY' / Base

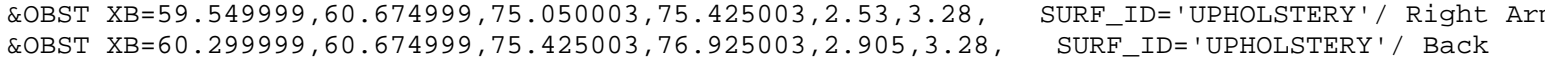

$\star \star \star *$ GROUP: COUCH2 offset $=0.000000,27.000000,0.000000$ rotate $=270 . .000000$

\&OBST XB=59.549999, 60.674999, 79. $925003,80.300003,2.53,3.28$, SURF_ID=' UPHOLSTERY '/ Left Arm \&OBST XB $=59.549999,60.674999,78.425003,79.925003,2.53,2.905, \quad$ SURF_ID= 'UPHOLSTERY' '/ Base 8OBST XB=60.299999, 60.674999, 78.425003, 79.925003,2.905, 3.28, SURF ID='UPHOLSTEPY' / Back

*** GROUP: COUCH2 offset $=0.000000,30.000000,0.000000$ rotate $=270.000000$

\&OBST XB=59.549999, 60.674999, 82.925003, 83.300003, 2.53, 3.28, SURF_ID=' 'UPHOLSTERY' / Left Arm
\&OBST XB=59.549999, 60.674999, 81. 425003, 82. 925003, 2.53, 2. 905, SURF_ID= 'UPHOLSTERY'/ Base

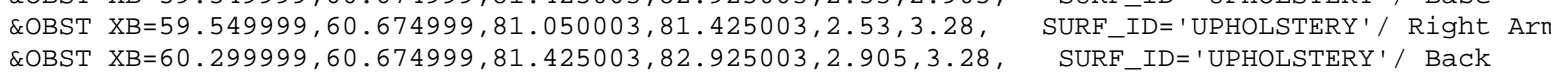

*** GROUP: TWIN

*** GROUP: ROW

*** GROUP: COUCH2 offset $=0.000000,0.000000,0.000000$ rotate $=90.000000$

\&OBST XB $=65.550003,66.675003,51.049999,51.424999,2.53,3.28, \quad$ SURF IDE= 'UPHOLSTERY' ' Left Arm
\&OBST XB $=65.550003,66.675003,51.424999,52.924999,253,2.905$,
SURF IDE' 'UPHOLSTERY' / Base

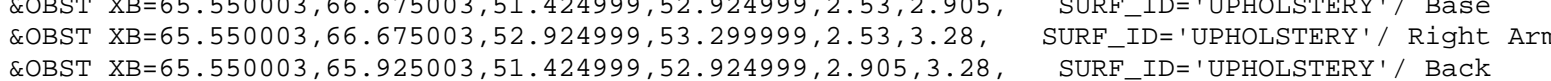

*** GROUP: COUCH2 offset $=0.000000,3.000000,0.000000$ rotate $=90 . .000000$

\&OBST XB $=65.550003,66.675003,54.049999,54.424999,2.53,3.28$, SURF_ID=' 'UPHOLSTERY'/ Left Arm \&OBST XB=65.550003, 65.925003,54.424999, 55.924999, $2.905,3.28$, SURF_ID='UPHOLSTERY'/ Back

*** GROUP: COUCH2 offset $=0.000000,6.000000,0.000000$ rotate $=90.000000$

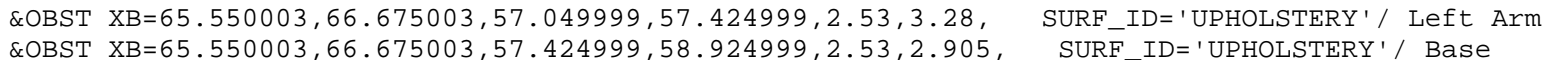
\&OBST XB $=65.550003,66.675003,58.924999,59.299999,2.53,3.28$, SURF ID='UPHOLSTERY'/ Rise A \&OBST XB=65.550003,65.925003,57.424999,58.924999, 2.905,3.28, SURF_ID=' 'UPHOLSTERY'/ Back

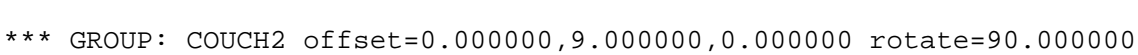

\&OBST XB $=65.550003,66.675003,60.049999,60.424999,2.53,3.28, \quad$ SURF_ID=' UPHOLSTERY' ' Left Arm
\&OBST XB $=65.550003,66.675003,60.424999,61.924999,2.53,2.905, \quad$ SURF_IDD='UPHOLSTERY' $/$ Base

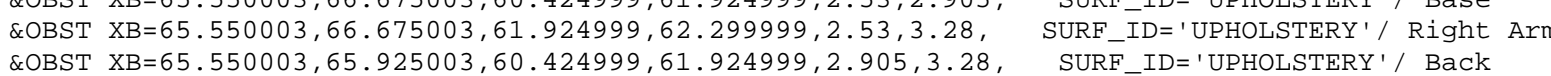

*** GROUP: COUCH2 offset $=0.000000,12.000000,0.000000$ rotate $=90.000000$

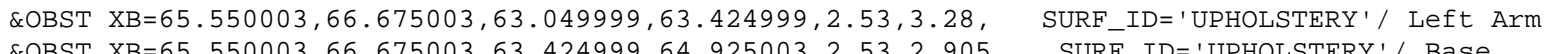

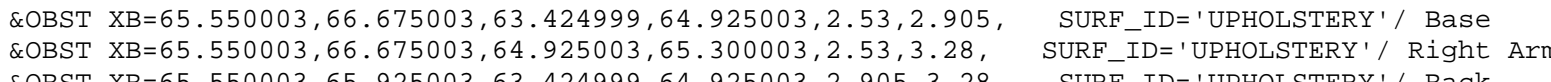

*** GROUP: COUCH2 offset $=0.000000,15.000000,0.000000$ rotate $=90.0000000$

\&OBST XB $=65.550003,66.675003,66.050003,66.425003,2.53,3.28, \quad$ SURF_ID=' 'UPHOLSTERY' ' Left Arm
\&OBST XB $=65.550003,66.675003,66.425003,67.925003,2.53,2.905, \quad$ SURF_ID=' UPHOLSTERY' / Base

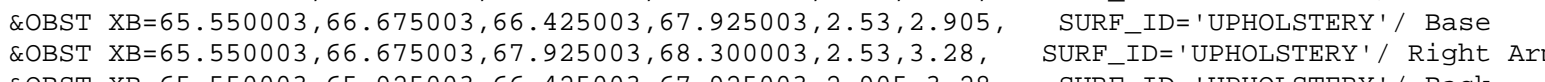

$* * *$ GROUP: COUCH2 offset $=0.000000,18.000000,0.000000$ rotate $=90.000000$

OBST XB $=65.550003,66.675003,69.050003,69.425003,2.53,3.28$, SURF_ID='UPHOLSTERY '/ Left Arm \&OBST XB=65.550003,66.675003, 69.425003,70.925003, 2.53,2.905, SURF_ID='UPHOLSTERY'/ Base

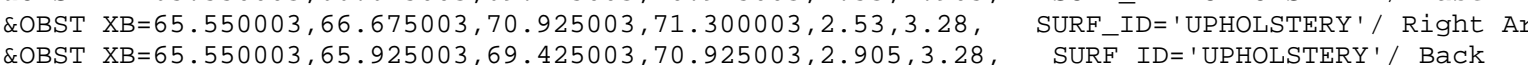

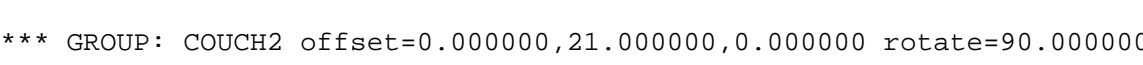

\&OBST XB $=65.550003,66.675003,72.050003,72.425003,2.53,3.28$, SURF_ID=' 'UPHOLSTERY'/ Left Arm

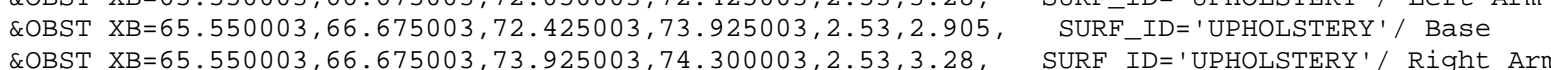
\&OBST XB $=65.550003,65.925003,72.425003,73.925003,2.905,3.28$, SUPF_ID=' 'UPHOLSTERY'/ Bnck Ar

*** GROUP: COUCH2 offset $=0.000000,24.000000,0.000000$ rotate $=90.000000$

\&OBST XB $=65.550003,66.675003,75.050003,75.425003,2.53,3.28$, SURFID = 'UPHOLSTERY ' ' Left Arm \&OBST XB $=65.555003,66.675003,75.425003,76.925003,2.53,2.905$, SUURF IDD' 'UPHOLSTERY'/ Base

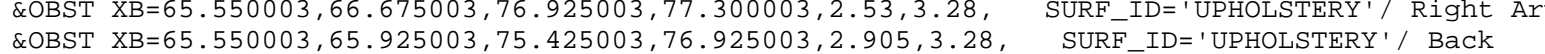

*** GROUP: COUCH2 offset $=0.000000,27.000000,0.000000$ rotate $=90.0000000$

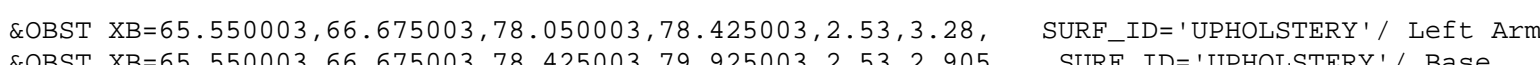
\&OBST XB=65.550003,66.675003,79.925003,80.300003,2.53,3.28, SURF-ID='UPHOLSTERY'/Right Ar 
*** GROUP: COUCH2 offset $=0.000000,30.000000,0.000000$ rotate $=90.000000$ \&OBST $X \mathrm{XB}=65.550003,66.675003,81.050003,81.425003,2.53,3.28$, SURF_ID=' ' UPHOLSTERY ' / Left Arm

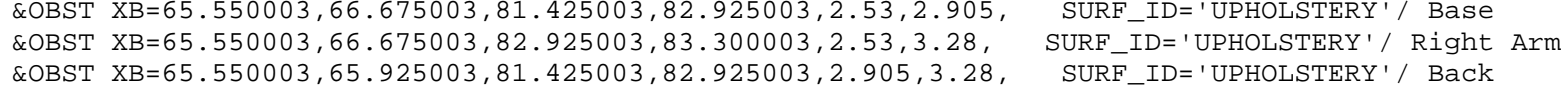
*** GROUP: ROW2

*** GROUP: COUCH2 offset $=0.000000, \odot . \bullet 000000, \Theta . \bullet 000000$ rotate $=270.000000$

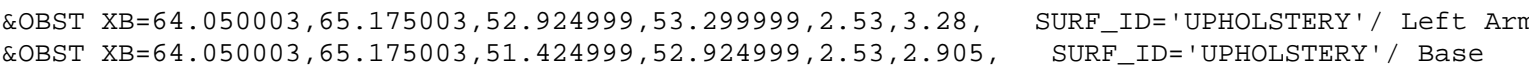
\&OBST XB $=64.050003,65.175003,51.049999,51.424999,2.53,3.28$, SURF_ID='UPHOLSTERY'/ Right Ar

$* * *$ GROUP: COUCH2 offset $=0.000000,3.000000,0.000000$ rotate $=270.000000$

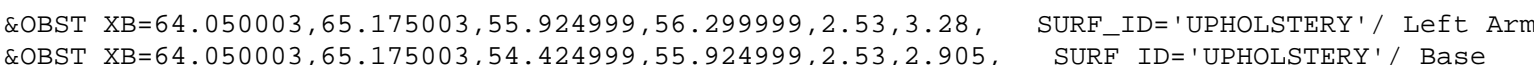

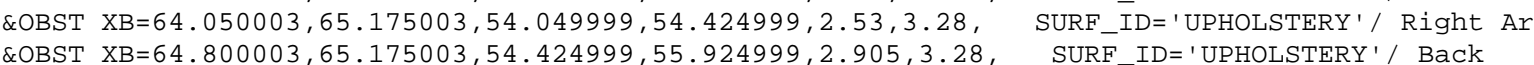

$* * *$ GROUP: COUCH2 offset $=0.000000,6.000000,0.000000$ rotate $=270.0000000$

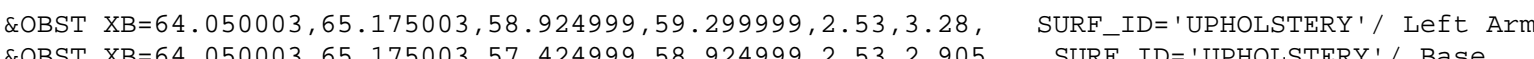
\&OBST XB=64. $050003,65.175003,57.049999,57.424999,2.53,3.28$, SURF_ID='UPHOLSTERY'/Right Ar

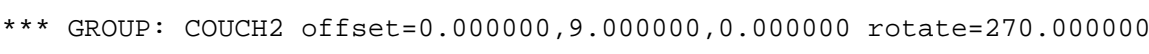

\&OBST XB=64.050003, 65.175003, 61.924999, 62. 299999, 2.53,3.28, SURF_ID= 'UPHOLSTERY' / Left Arm \&OBST XB $=64.050003,65.175003,60.424999,61.924999,2.53,2.905$, SURF_ID=' 'UPHOLSTERY' / Base \&OBST XB $=64.050003,65.175003,60.049999,60.424999,2.53,3.28$,
\&OBST XB $=64.800003,65.175003,60.424999,61.924999,2.905,3.28$,
SURF_IDE_ID=' 'UPHOLSTERY' / Back

*** GROUP: COUCH2 offset $=0.000000,12.000000,0.000000$ rotate $=270.000000$

\&OBST XB $=64.050003,65.175003,64.925003,65.300003,2.53,3.28$, SURF_ID=' 'UPHOLSTERY'/ Left Arm \&OBST $X \mathrm{XB}=64.050003,65.175003,63.424999,64.925003,2.53,2.905$, SURF_ID= 'UPHOLSTERY '/ Base

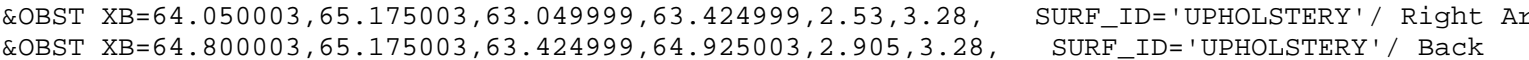

*** GROUP: COUCH2 offset $=0.000000,15.000000,0.000000$ rotate $=270.000000$

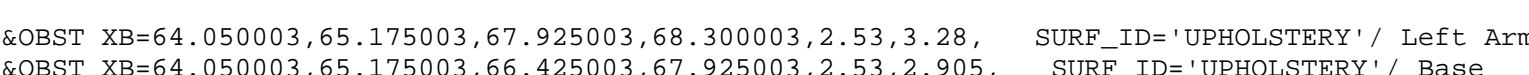

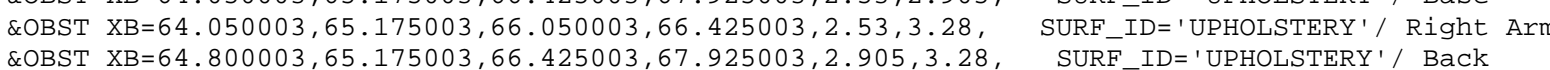
$* * *$ GROUP: COUCH2 offset $=0.000000,18.000000,0.0000000$ rotate $=270.000000$

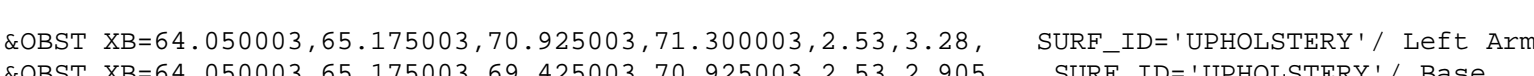

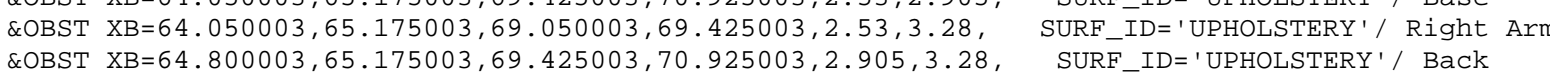

** GROUP: COUCH2 offset $=0.000000,21.000000,0.000000$ rotate $=270,000000$

\&OBST XB $=64.050003,65.175003,73.925003,74.300003,2.53,3.28, \quad$ SURF.IDE= 'UPHOLSTERY' 1 ' Left Arm
ROBST XB $=64.050003,65.175003,72.425003,73.925003,2.53,2.905$,
SURF. IDE' 'UPHOLSTERY'/ Base

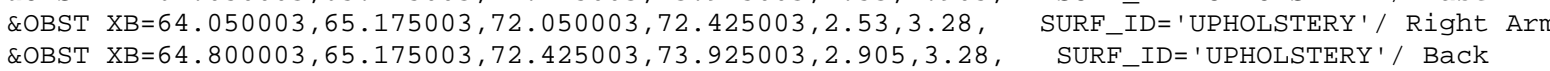

*** GROUP: COUCH2 offset $=0.000000,24.000000,0.000000$ rotate $=270.000000$

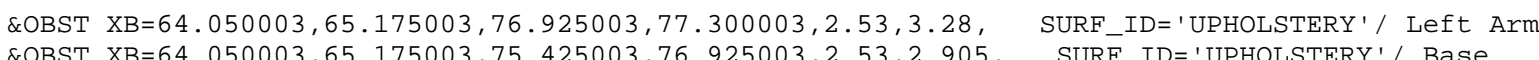

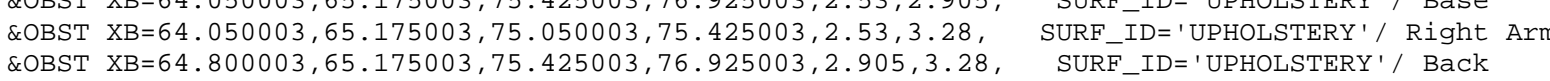
*** GROUP: COUCH2 offset $=0.000000,27.000000,0.000000$ rotate $=270.000000$

\&OBST XB=64. $050003,65.175003,79.925003,80.300003,2.53,3.28, \quad$ SURF_ID='UPHOLSTERY' ' Left Arm
\&OBST XB=64.050003,65.175003, $78.425003,79.925003,2.53,2.905, \quad$ SURF_ID=' 'UPHOLSTERY' $/$ Base
\&OBST $X \mathrm{XB}=64.050003,65.175003,78.050003,78.425003,2.53,3.28, \quad$ SURF_ID= 'UPHOLSTERY'
\&OBST $X \mathrm{~B}=64.800003,65.175003,78.425003,79.925003,2.905,3.28, \quad$ SURF_ID=' 'UPHOLSTERY' $/$ Back *** GROUP: COUCH2 offset $=0.000000,30.000000,0.000000$ rotate $=270.000000$

\&OBST XB=64. $050003,65.175003,82.925003,83.300003,2.53,3.28, \quad$ SURF_ID=' 'UPHOLSTERY' '/ Left Arm
\&OBST XB=64.050003, $65.175003,81.425003,82.925003,2.53,2.905$,
SURF_ID='UPHOLSTERY'/ Base

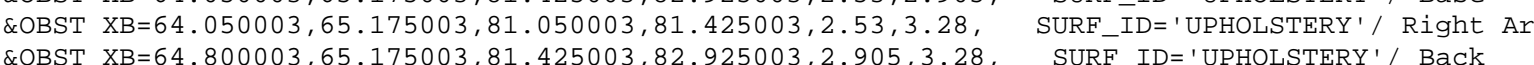

$\star \star \star *$ GROUP: TWIN

*** GROUP: ROW

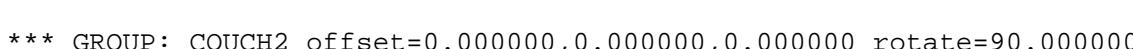

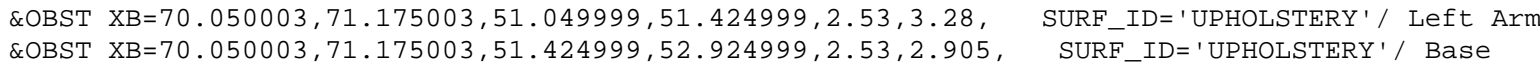

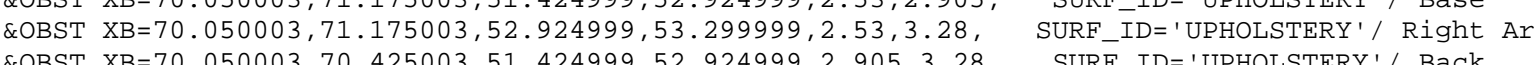

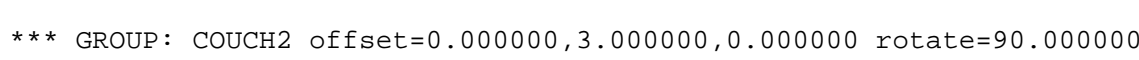

\&OBST XB=70.050003, 71.175003, 54. $049999,54.424999,2.53,3.28, \quad$ SURF_ID=' 'UPHOLSTERY' ' Left Arm

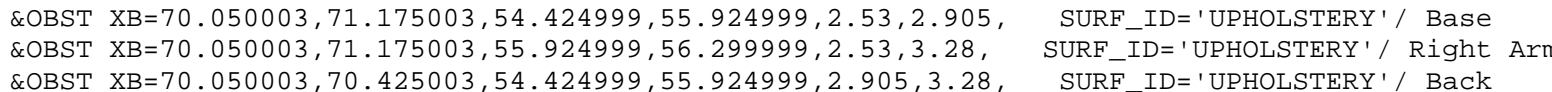

*** GROUP: COUCH2 offset $=0.000000,6.000000,0.000000$ rotate $=90 . .000 \odot 00$

\&OBST XB=70.050003, 71.175003, 57.049999, 57.424999, 2.53,3.28, SURF_ID='UPHOLSTERY '/ Left Arm

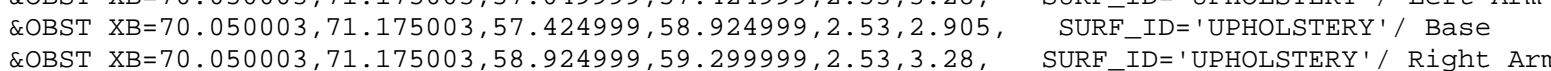
\&OBST XB=70.050003,

*** GROUP: COUCH2 offset $=0.000000,9.000000,0.000000$ rotate $=90.000000$

\&OBST XB=70.050003, 71.175003, 60.049999, 60.424999, 2.53, 3.28, SURF_ID='UPHOLSTERY'/ Left Arm \&OBST $X B=70.050003,71.175003,60.424999,61.924999,2.53,2.905$, SURF_ID=' 'UPHOLSTERY'/ Base \&OBST XB=70.050003,71.175003, 61.924999, 62.299999, $2.53,3.28$, SURF_ID='UPHOLSTERY'/ Right Ar

*** GROUP: COUCH2 offset $=0.000000,12.000000,0.000000$ rotate $=90 . .000000$

\&OBST XB=70.050003, 71.175003, 63. 049999, 63.424999, 2.53, 3.28, SURF_ID='UPHOLSTERY'/ Left Ar \&OBST XB $=70.050003,71.175003,63.424999,64.925003,2.53,2.905, \quad$ SURF IDE='UPHOLSTERY' '/ Base
\&OBST XB EOBST XB=70. $050003,70.425003,63.424999,64.925003,2.905,3.28$, SURF_ID='UPHOLSTERY'/ Right Ar

$* * *$ GROUP: COUCH2 offset $=0.000000,15.000000,0.000000$ rotate $=90.000000$

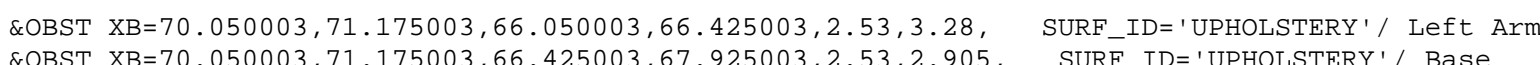

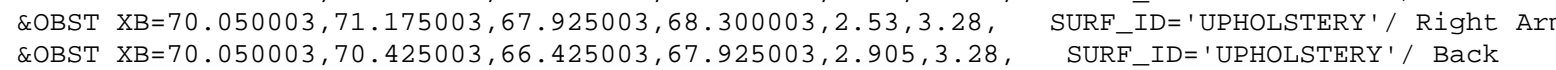

*** GROUP: COUCH2 offset $=0.000000,18.000000,0.000000$ rotate $=90.000000$

\&OBST XB=70.050003, 71.175003, 69.050003,69.425003, 2.53,3.28, SURF_ID=' 'UPHOLSTERY' ' Left Arm

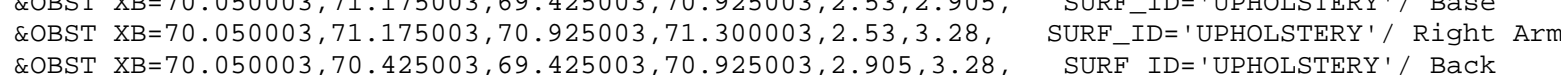

*** GROUP: COUCH2 offset $=0.000000,21.000000,0.000000$ rotate $=90.000000$

\&OBST XB=70. $050003,71.175003,72.050003,72.425003,2.53,3.28$, SURF_ID=' 'UPHOLSTERY ' / Left Arm \&OBST XB $=70.050003,71.175003,73.925003,74.300003,2.53,3.28$, SURF_ID='UPHOLSTERY'/Right Ar \&OBST XB=70.050003, 70.425003, 72.425003, 73.925003, 2.905,3.28, SURF_ID='UPHOLSTERY'/ Back

*** GROUP: COUCH2 offset $=0.000000,24.000000,0.000000$ rotate $=90.000000$

\&OBST $X \mathrm{XB}=70.050003,71.175003,75.050003,75.425003,2.53,3.28, \quad$ SURF_ID=' 'UPHOLSTERY'/ Left Arm \&OBST $X \mathrm{~B}=70.0500003,71.175003,76.925003,77.300003,2.53,3.28$, SURE IDD 'UPHOLSTERY'/ Right Ar \&OBST XB=70.050003, 70.425003, 75.425003, 76.925003, 2.905, 3.28, SURF_ID='UPHOLSTERY'/ Back 
** GROUP: COUCH2 offset $=0,000000,27.000000,0,000000$ rotate $=90,000000$

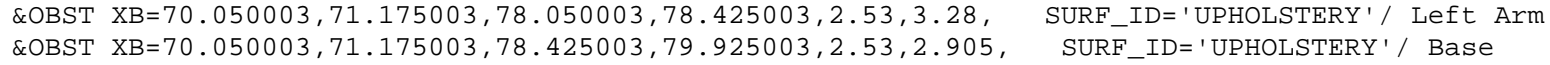

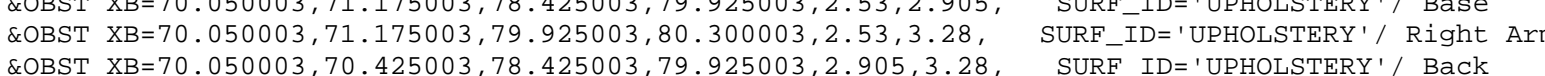

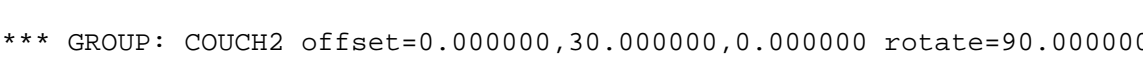

\&OBST $X B=70.050003,71.175003,81.050003,81.425003,2.53,3.28$, SURF_ID=' 'UPHOLSTERY'/ Left Arm

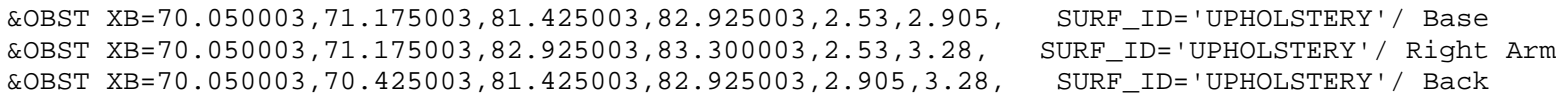

*** GROUP: ROW2

*** GROUP: COUCH2 offset $=0.000000,0.000000,0.000000$ rotate $=270.000000$

\&OBST XB=68.550003, 69.675003, 52.924999, 53.299999, 2.53,3.28, SURF_ID=' UPHOLSTERY' ' Left Arm

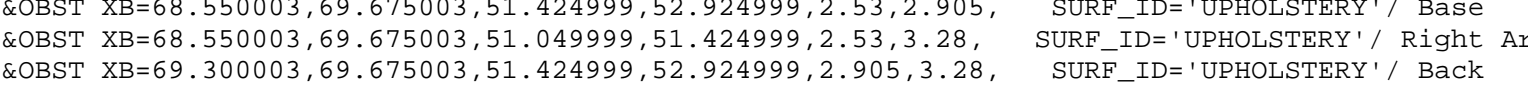
*** GROUP: COUCH2 offset $=0.000000,3.000000,0.000000$ rotate $=270.000000$

\&OBST XB=68.550003, 69.675003, 55.924999, 56.299999, 2.53,3.28, SURF_ID='UPHOLSTERY' / Left Arm \&OBST XB $=68.550003,69.675003,54.049999,54.424999,2.53,3.28$, SURF_ID='UPHOLSTERY'/ Right Ar \&OBST XB=69. $300003,69.675003,54.424999,55.924999,2.905,3.28$, SURF_ID= 'UPHOLSTERY' / Back

*** GROUP: COUCH2 offset $=0.000000,6.000000,0.000000$ rotate $=270.000000$

\&OBST XB=68.550003, 69.675003,58.924999, 59. 299999, 2.53,3.28, SURF_ID=' 'UPHOLSTERY' ' Left Arm

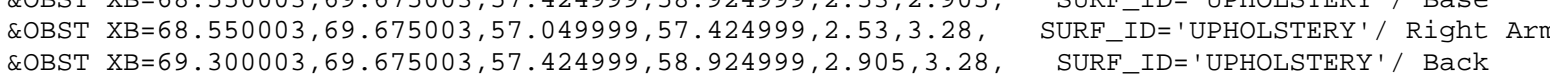

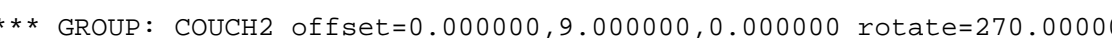

OOBST XB $=68.550003,69.675003,61.924999,62.299999,2.53,3.28$, SURF ID=' 'UPHOLSTERY ' ' Left Arm

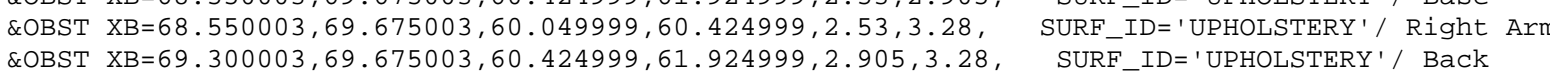

*** GROUP: COUCH2 offset $=0.000000,12.000000,0.000000$ rotate $=270.000000$

\&OBST $X \mathrm{XB}=68.550003,69.675003,64.925003,65.300003,2.53,3.28, \quad$ SURF_ID='UPHOLSTERY ' 1 Left Arm
\&OBST $X \mathrm{~B}=68.550003,69.675003,63.424999,64.925003,253,2905, \quad$ SURF ID='UPHOLSTERY' / Base \&OBST XB $=68.550003,69.675003,63.049999,63.42499,2,53,23,28$, QOBST XB $=69.300003,69.675003,63.424999,64.925003,2.905,3.28$, SURF_ID 'UPHOLSTERY' / Back

*** GROUP: COUCH2 offset $=0.000000,15.000000,0.000000$ rotate $=270$. .

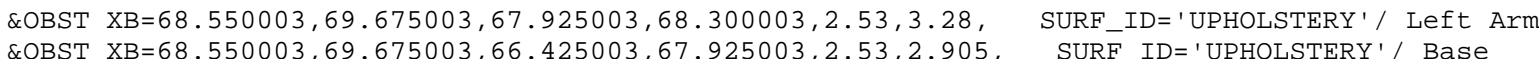

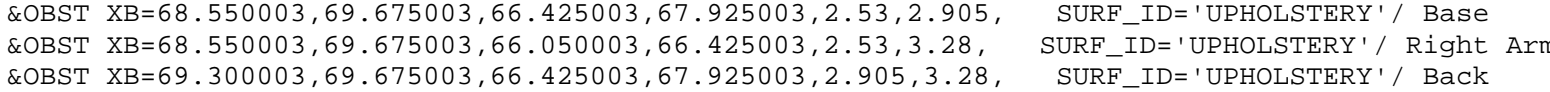

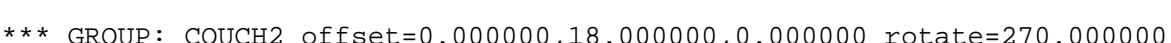

\&OBST XB $=68.5500003,69.675003,70.925003,71.300003,2.53,3.28$, SURF-ID=' 'UPHOLSTERY'/ Left Arm

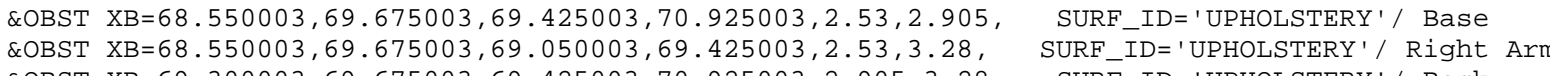
\&OBST $\mathrm{XB}=69.300003,69.675003,69.425003,70.925003,2.905,3.28$, SURF_ID='UPPOLSTERY'/ Back

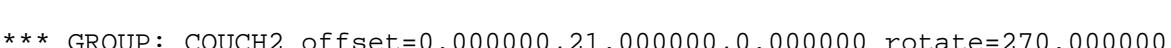

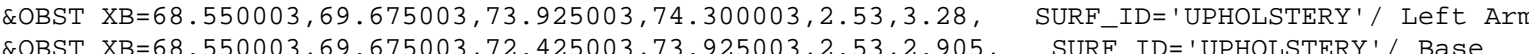

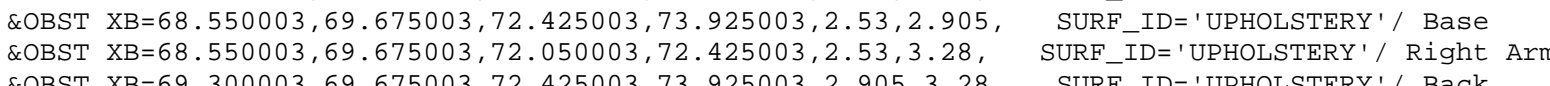

*** GROUP: COUCH2 offset $=0.000000,24.000000,0.0000000$ rotate $=270.000000$

\&OBST XB $=68.5500003,69.675003,76.925003,77.300003,2.53,3.28, \quad$ SURF_ID='UPHOLSTERY ' ' Left Arm

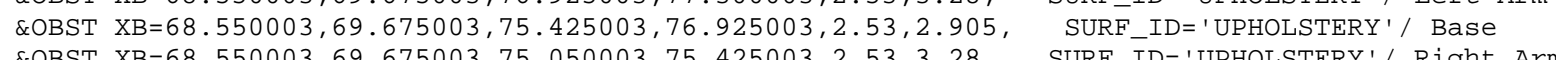

\&OBST XB=69. 300003, 69.675003, 75, 425003, 76, 925003, 2.905, 3. 28, SURF_ID=' UPHOLSTERY'/ Back *** GROUP: COUCH2 offset $=0.000000,27.000000,0.000000$ rotate $=270.000000$

\&OBST XB=68.550003, 69.675003, 79.925003, 80.300003, 2.53,3.28, SURF_ID='UPHOLSTERY'/ Left Arm \&OBST XB $=68.550003,69.675003,78.425003,79.925003,2.53,2.905$, SURF_ID=' 'UPHOLSTERY' / Base KOBST $X \mathrm{~B}=69.300003,69.675003,78.425003,79.925003,2.905,3.28, \quad$ SURF_ID=' 'UPHOLSTERY'/ Back

*** GROUP: COUCH2 offset $=0.000000,30.000000,0.000000$ rotate $=270.000000$

\&OBST $X \mathrm{XB}=68.550003,69.675003,82.925003,83.300003,2.53,3.28$, SURF_ID=' 'UPHOLSTERY'/ Left Arm \&OBST XB $=68.550003,69.675003,81.425003,82.925003,2.53,2.905$, SURF_ID='UPHOLSTERY'/ Base

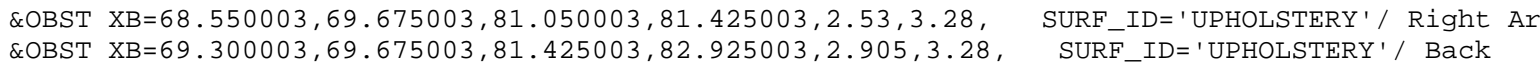

$* \star *$ GROUP: ROW2

*** GROUP: COUCH2 offset $=0.000000, \odot ., 000000,0.000000$ rotate $=270 ., 000000$

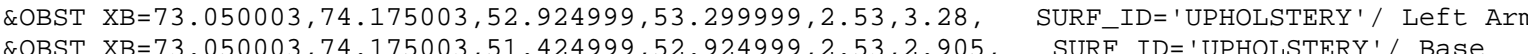
\&OBST $\mathrm{XB}=73.050003,74.175003,51.424999,52.924999,2.53,2.905, \quad$ SURF IDE' UPHOLSTERY' \&OBST XB=73.800003, 74.175003, 51.424999, 52.924999, 2.905, 3.28, SURF_ID='UPHOLSTERY'/ Back

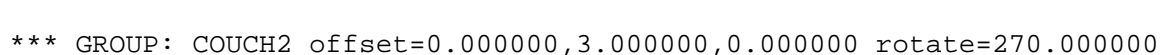

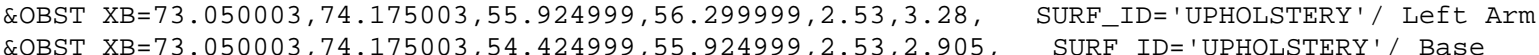

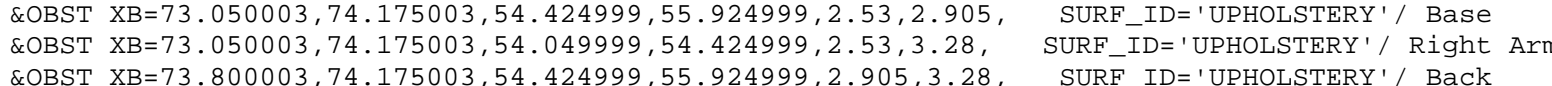

*** GROUP: COUCH2 offset $=0.000000,6.000000,0.0000000$ rotate $=270.000000$

\&OBST XB $=73.050003,74.175003,58.924999,59.299999,2.53,3.28, \quad$ SURF_ID=' 'UPHOLSTERY ' / Left Arm \&OBST XB $=73.050003,74.175003,57.424999,58.924999,2.53,2.905$, SURF_ID='UPHOLSTERY'/ Base \&OBST XB=73.050003, 74. 175003, 57. $049999,57.424999,2.53,3.28$,
\&OBST XURF_IDE' UPHOLSTERY'/ Right Ar

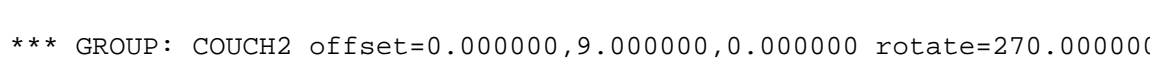

\&OBST XB=73.050003, 74.175003, 61.924999, 62. 299999, 2.53,3.28, SURF_ID=' 'UPHOLSTERY' / Left Arm \&OBST XB=73.050003,74.175003,60.424999,61.924999, $2.53,2.905$, SURF_ID=' 'UPHOLSTERY'/ Base

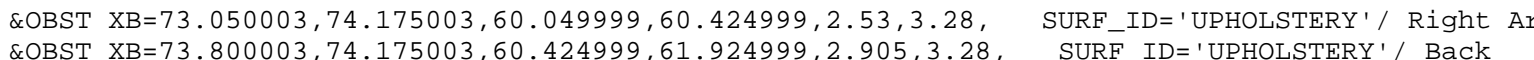

*** GROUP: COUCH2 offset $=0.000000,12.000000,0.000000$ rotate $=270.0000000$

\&OBST XB=73.050003, 74.175003,64.925003,65.300003, 2.53,3.28, SURF_ID='UPHOLSTERY'/ Left Arm \&OBST XB=73.050003,74.175003,63.424999,64.925003, 2.53, 2.905, SURF_ID='UPHOLSTERY' / Base

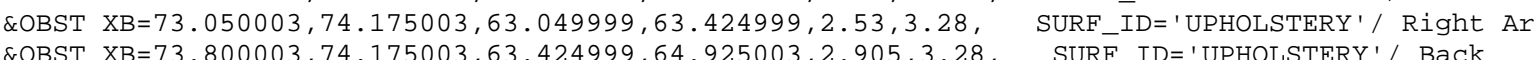

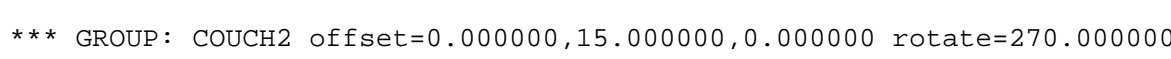

\&OBST XB=73. $050003,74.175003,67.925003,68.300003,2.53,3.28, \quad$ SURF_ID= ' 'UPHOLSTERY' ' Left Arm SURF, ID= UPHOLSTERY'/ Base

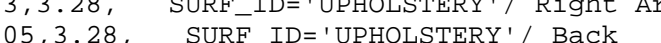

*** GROUP: COUCH2 offset $=0.000000,18.000000, \Theta . \bullet 00000$ rotate $=270.000000$

\&OBST XB=73.050003, 74.175003, 70.925003, 71.300003, 2.53,3.28, SURF_ID=' 'UPHOLSTERY ' / Left Arm \&OBST XB=73.050003,74.175003, 69.425003,

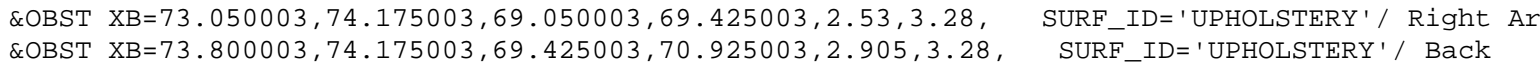

*** GROUP: couCH2 offset $=0.000000,21.000000,0.000000$ rotate $=270.000000$

\&OBST $X B=73.050003,74.175003,73.925003,74.300003,2.53,3.28$, SURF_ID= 'UPHOLSTERY' ' Left Arm

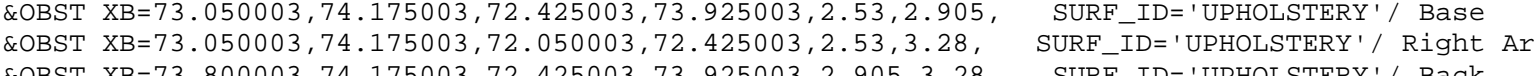

*** GROUP: COUCH2 offset $=0.000000,24.000000,0.000000$ rotate $=270.000000$

\&OBST XB=73.050003, 74.175003, 76.925003, 77. 300003, 2.53,3.28, SURF_ID='UPHOLSTERY'/ Left Arm 
\&OBST XB $=73.050003,74.175003,75.425003,76.925003,2.53,2.905, \quad$ SURF_ID=' 'UPHOLSTERY' $/$ Base
\&OBST XB=73.050003,74.175003, 75.050003, 75.425003,2.53,3.28,
SURF_ID='UPHOLSTERY'

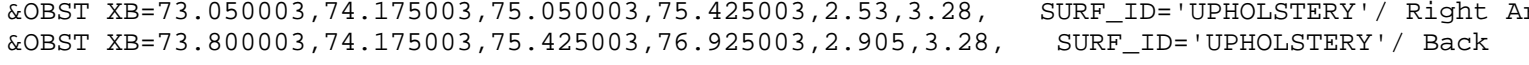
*** GROUP: COUCH2 offset $=0.000000,27.000000,0.000000$ rotate $=270$. . 000000

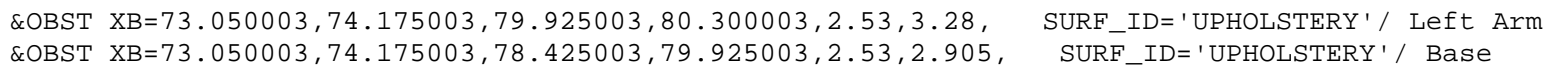

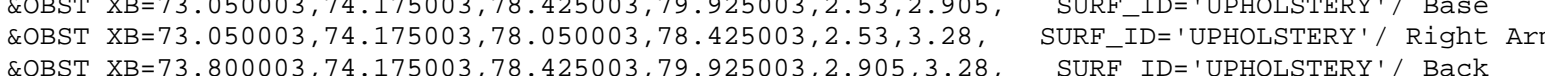
*** GROUP: COUCH2 offset $=0.000000,30.000000,0.000000$ rotate $=270.000000$

\&OBST XB=73.050003, 74.175003, 82.925003, 83.300003, 2.53,3.28, SURF_ID ' 'UPHOLSTERY' ' Left Arm

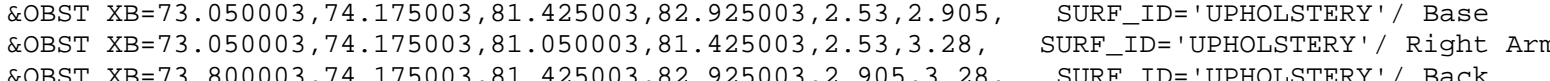

$* * *$ GROUP: SHORT_ROW

$* * *$ GROUP: COUCH2 offset $=0.000000,0.000000,0.000000$ rotate $=90.000000$

\&OBST XB=67. $044998,67.419998,46.91,48.035,2.53,3.28$, SURF_ID='UPHOLSTERY'/ Left Arm \&OBST XB=65.544998, 67. $044998,46.91,48.035,2.53,2.905$, SURF -ID='UPHOLSTERY' $/$ Base

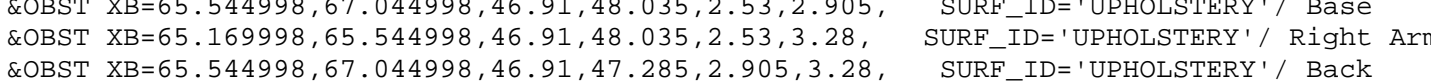

*** GROUP: COUCH2 offset $=0.0000000,3.000000,0.000000$ rotate $=90.000000$

EOBST XB=64. $044998,64.419998,46.91,48.035,2.53,3.28$, SURF_ID='UPHOLSTERY '/ Left Arm

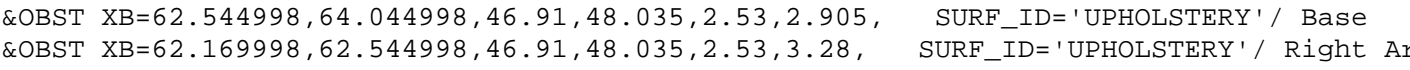
\&OBST XB=62.544998,64.044998, 46.91,47.285,2.905,3.28, SURF_ID='UPHOLSTERY' / Back

$* * *$ GROUP: COUCH2 offset $=0.000000,6.000000,0.000000$ rotate $=90$. . 000000

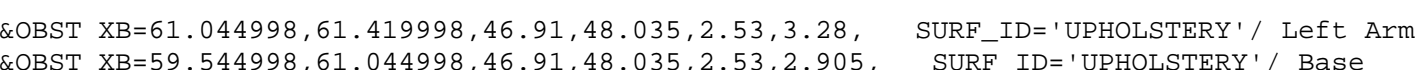

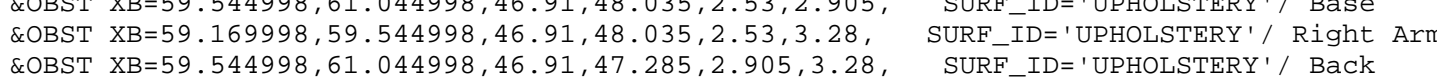

*** GROUP: COUCH2 offset $=0.000000,9.000000,0.000000$ rotate $=90.000000$

\&OBST XB $=58.044998,58.419998,46.91,48.035,2.53,3.28$, SURF_ID='UPHOLSTERY' ' Left Arm

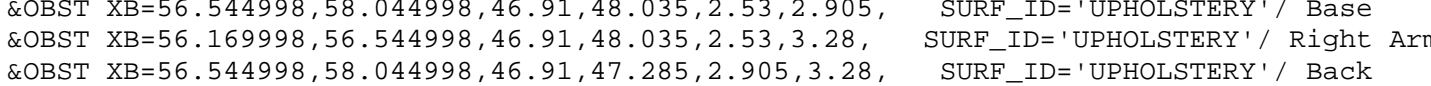

*** GROUP: COUCH2 offset $=0.000000,12.000000,0.000000$ rotate $=90.000000$

\&OBST XB=55. $044998,55.419998,46.91,48.035,2.53,3.28$, SURF IDD ' UPHOLSTERY ' ' Left Arm

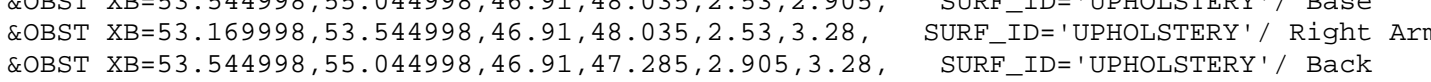

*** GROUP: COUCH2 offset $=0 ., 000000,15$. .

\&OBST XB $=52.044998,52.419998,46.91,48.035,2.53,3.28$, SURF_ID=' 'UPHOLSTERY ' $/$ Left Ar

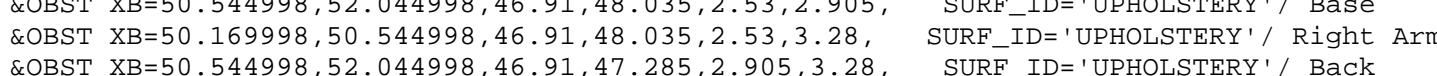

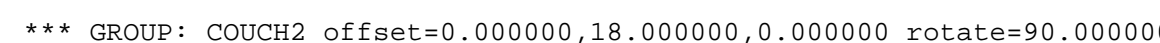

\&OBST XB=49. $044998,49.419998,46.91,48.035,2.53,3.28, \quad$ SURF_ID= 'UPHOLSTERY' ' Left Arm \&OBST XB-47.169998, 47.54999, 46.91, EOBST XB=47.169998, $47.544998,46.91,48.035,2.53,3.28$,
\&OBST XB $47.544998,49.044998,46.91,47.285,2.905,3.28$,
SURF_IDE='UPHOLSTERY'/ Back

*** GROUP: ROW

*** GROUP: COUCH2 offset $=0.000000,0.0000000,0.000000$ rotate $=90.000000$

\&OBST XB $=41.549999,41.924999,84.43,85.555,2.53,3.28$, SURF_ID='UPHOLSTERY'/ Left Arm

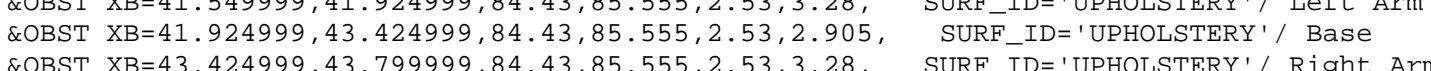

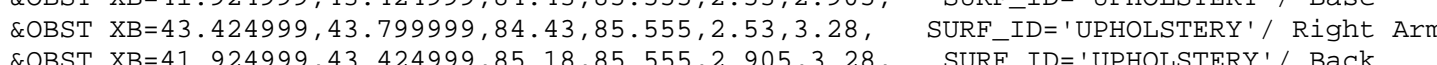

** GROUP: COUCH2 offset $=0.000000,3.000000,0.000000$ rotate $=90.000000$

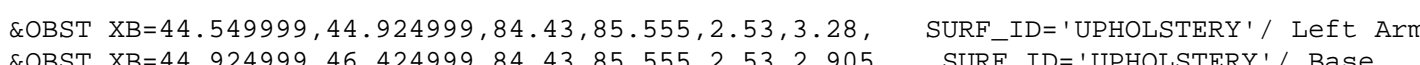

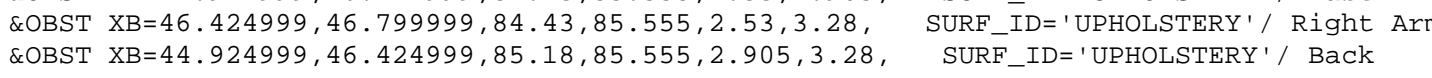
*** GROUP: COUCH2 offset $=0.000000,6.000000,0.000000$ rotate $=90.000000$

\&OBST XB $=47.549999,47.924999,84.43,85.555,2.53,3.28, \quad$ SURF_ID= 'UPHOLSTERY' ' Left Arm
\&OBST XB $=47.924999,49.424999,84.43,85.555,2.53,2.905$,
SURF ID ='UPHOLSTERY' $/$ Base

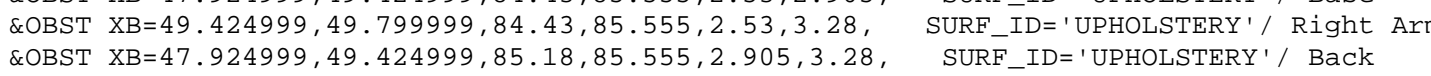

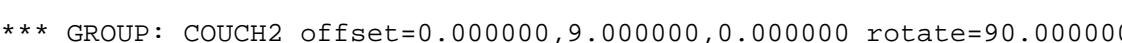

\&OBST XB=50.549999, 50.924999, 84.43, 85.555, 2.53,3.28, SURFIDD='UPHOLSTERY' / Left Arm

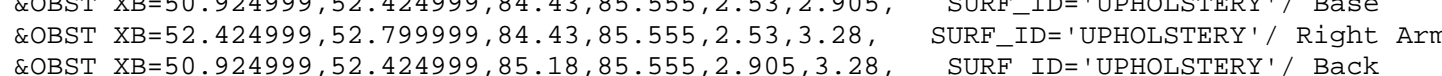

*** GROUP: COUCH2 offset $=0.000000,12.000000,0.000000$ rotate $=90.000000$

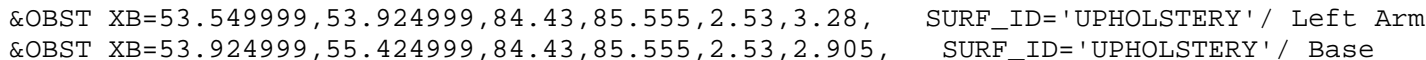
\&OBST XB $=55.424999,55.799999,84.43,85.555,2.53,3.28$, SURE TD ='UPHOLSTERY' Right \&OBST XB $=53.924999,55.424999,85.18,85.555,2.905,3.28$, SURF_ID='UPHOLSTERY'/ Back

*** GROUP: COUCH2 offset $=0.000000,15.000000,0.000000$ rotate $=90 . .000000$

ROBST XB $=56.549999,56.924999,84.43,85.555,2.53,3.28, \quad$ SURF_IDE 'UPHOLSTERY' ' Left Arm
\&OBST XB $=56.924999,58.424999,84.43,85.555,2.53,2.905$,
SURF_ID='UPHOLSTERY' / Base

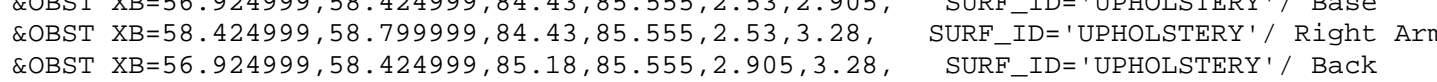

*** GROUP: COUCH2 offset $=0.000000,18.000000$, ๑. .000000 rotate $=90.000000$

\&OBST XB=59.549999, 59.924999, 84.43,85.555, 2.53,3.28, SURF_ID='UPHOLSTERY' ' Left Arm

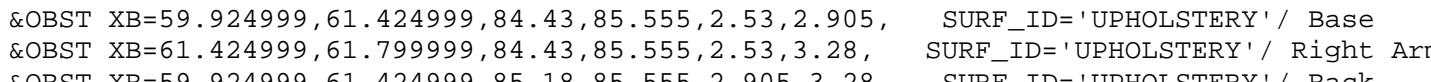

*** GROUP: COUCH2 offset $=0.000000,21.000000,0.000000$ rotate $=90.0000000$

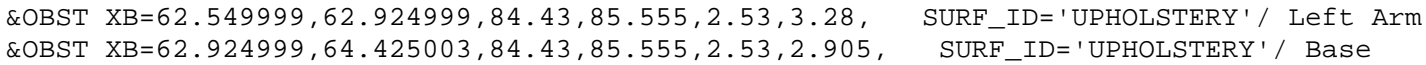

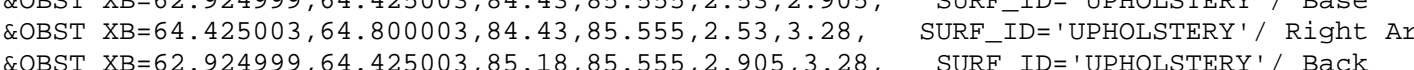

*** GROUP: COUCH2 offset $=0.000000,24.000000,0.000000$ rotate $=90.000000$

\&OBST XB=65.550003,65.925003, 84.43,85.555, 2.53,3.28, SURF_ID='UPHOLSTERY'/ Left Arm EOBST XB $=65.925003,67.425003,84.43,85.555,2.53,2.905, \quad$ SURF_ID=' 'UPHOLSTERY'/ Base COBST $X \mathrm{~B}=65.925003,67.425003,85,18,85,555,2.905,3.28$, SURF_ID='UPHOLSTERY'/ Right A

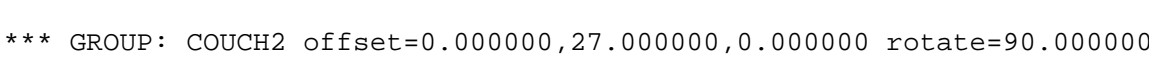
\&OBST XB $68.550003,68.925003,84.43,85.555,2.53,3.28$, SURF_ID='UPHOLSTERY' ' Left Arm \&OBST XB $=68.925003,70.425003,84.43,85.555,2.53,2.905$, SURF_ID='UPHOLSTERY' / Base

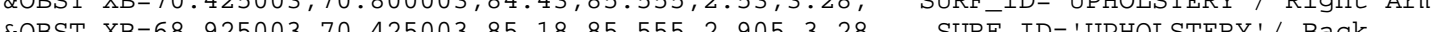

*** GROUP: COUCH2 offset $=0.000000,30.000000,0.000000$ rotate $=90 . .000000$

EOBST XB=71.550003,71.925003, 84. 43, 85. 555, 2.53,3.28, SURF_ID=' 'UPHOLSTERY ' / Left Arm

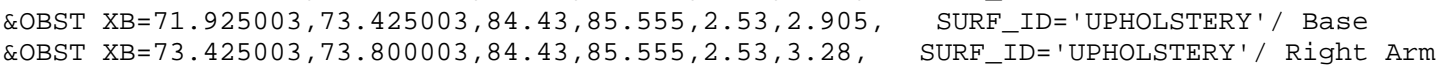

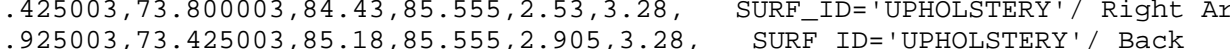

*** GROUP: LEVEL

*** GROUP: ROW

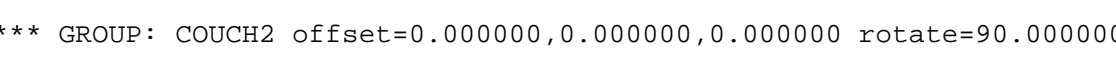


\&OBST XB=37.799999, 38. $244999,51.049999,51.424999,3.655,4.405, \quad$ SURF_ID= 'UPHOLSTERY '/ Left Arm
\&OBST XB=37.799999, 38.924999, $51.424999,52.924999,3.655,4.03, \quad$ SURF_ID='UPHOLSTERY' $/$ Base

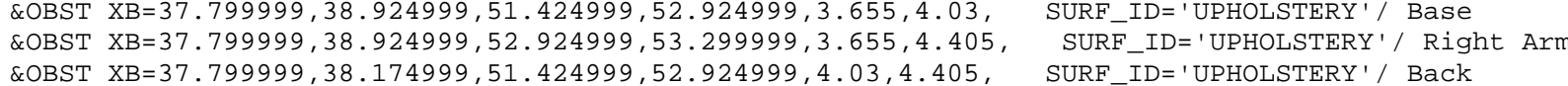

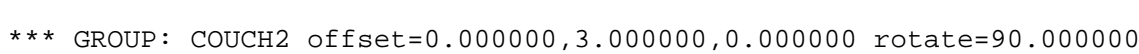

\&OBST XB=37.799999, 38.924999, 54. 449999, 54.424999, 3.655, 4.405, SURF_ID=' 'UPHOLSTERY ' / Left Arm

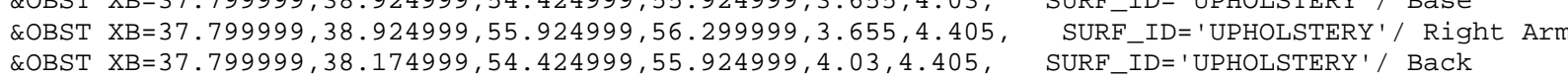

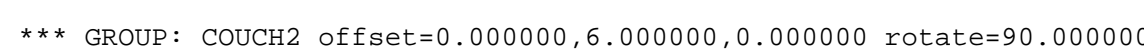

\&OBST XB=37.799999, 38. 924999, 57. 449999, 57.424999, 3.655, 4.405, SURF_ID=' 'UPHOLSTERY'/ Left Arm

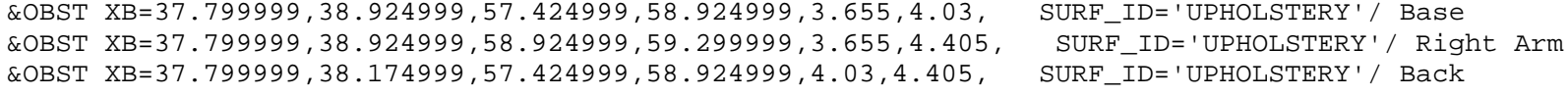

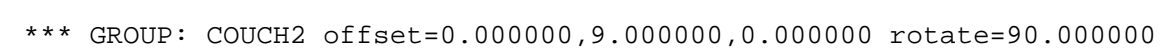

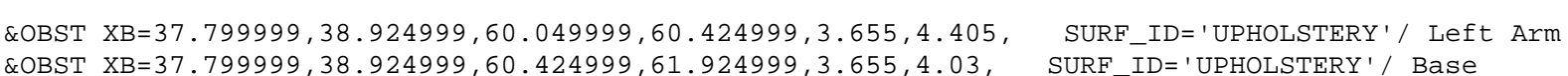

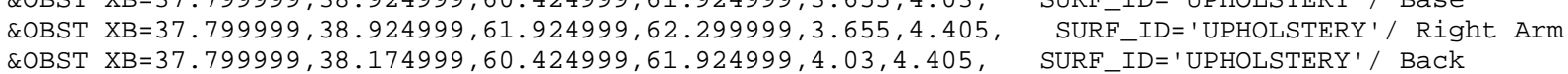

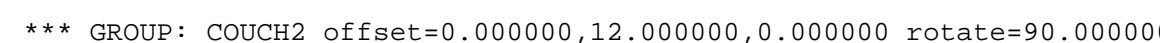

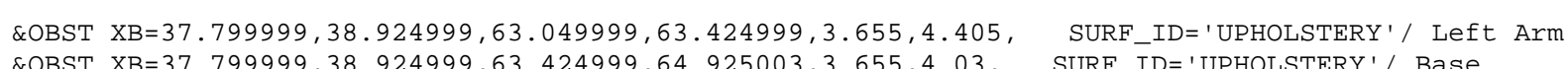

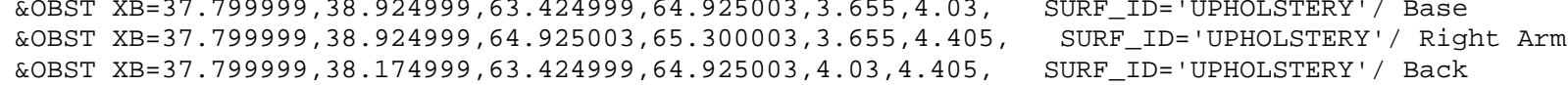
*** GROUP: COUCH2 offset $=0.000000,15.000000,0.000000$ rotate $=90.000000$

\&OBST XB $=37.799999,38.924999,66.050003,66.425003,3.655,4.405, \quad$ SURF_ID= 'UPHOLSTERY '/ Left Arm
\&OBST XB $=37.799999,38.924999,66.425003,67.925003,3.655,4.03$,
SURF_ID='UPHOLSTERY' 1 Base

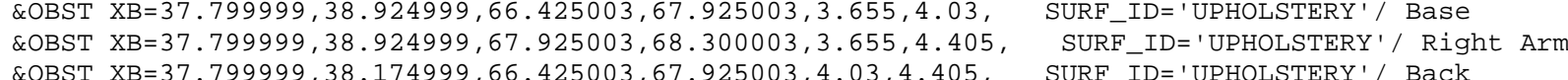
*** GROUP: COUCH2 offset $=0.000000,18.000000,0.000000$ rotate $=90.000000$

\&OBST XB=37. $799999,38.924999,69.050003,69.425003,3.655,4.405$,
\&OBST XB=37.79999, SURF_ID=' 'UPHOLSTERY' / Left Arm
SURF IDE'UPHOLSTERY'/ Base

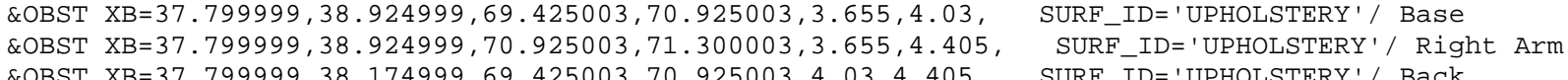

*** GROUP: COUCH2 offset $=0.000000,21.000000,0.0000000$ rotate $=90.000000$

\&OBST XB $=37.799999,38.924999,72.050003,72.425003,3.655,4.405$, SURF_ID=' 'UPHOLSTERY '/ Left Arm

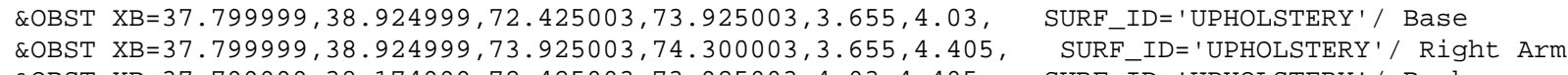
*** GROUP: COUCH2 offset $=0.000000,24.000000,0.000000$ rotate $=90.000000$

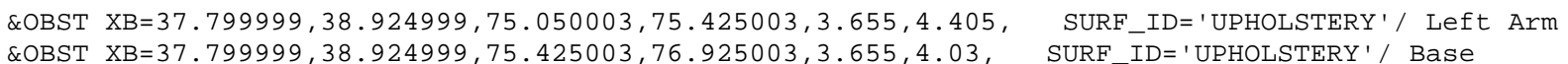

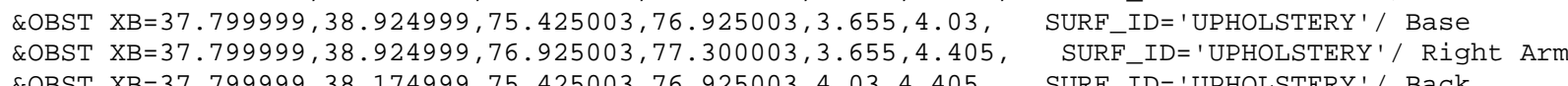
*** GROUP: COUCH2 offset $=0.000000,27.000000,0.000000$ rotate $=90.000000$

\&OBST XB $=37.799999,38.924999,78.050003,78.425003,3.655,4.405$, SURF_ID=' 'UPHOLSTERY'/ Left Arm \&OBST XB=37.799999, 38.924999, $78.425003,79.925003,3.655,4.03$, SURF_ID='UPHOLSTERY'/Base Arm

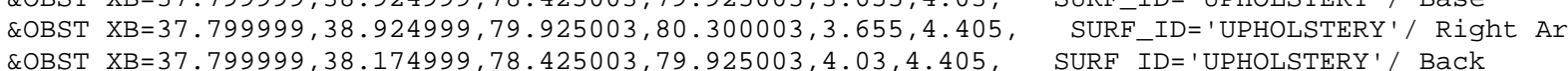
*** GROUP: COUCH2 offset $=0.000000,30.000000,0.000000$ rotate $=90.000000$

\&OBST XB $=37.799999,38.924999,81.050003,81.425003,3.655,4.405$, SURF_ID='UPHOLSTERY'/ Left Arm COBST XB=37.799999, 38.924999, 82. $925003,83.300003,3.655,4.405$, SURF_IDE' 'UPHOLSTERY'/ Base *** GROUP: BASE
*** GROUP: TWIN

*** GROUP: ROW

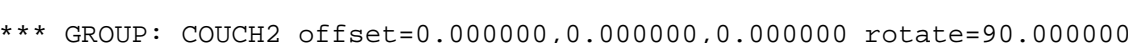

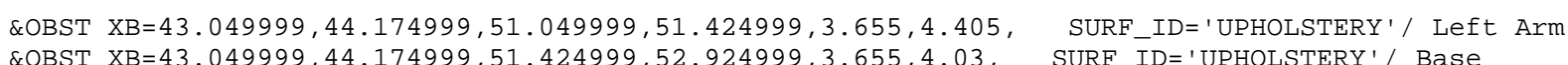

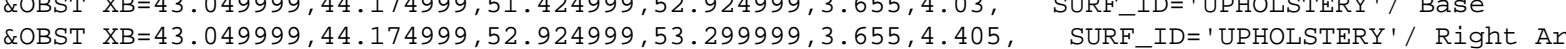
\&OBST XB $=43.049999,43.424999,51.424999,52.924999,4.03,4.405$, SURF_ID=' 'UPHOLSTERY'/ Back

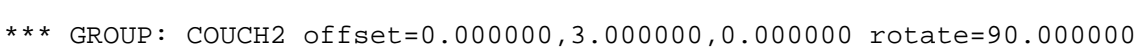

\&OBST XB $=43.049999,44.174999,54.049999,54.424999,3.655,4.405, \quad$ SURF_ID= 'UPHOLSTERY ' / Left Arm
\&OBST XB $=43.049999,44.174999,54.424999,55.924999,3.655,4.03, \quad$ SURF_ID='UPHOLSTERY'

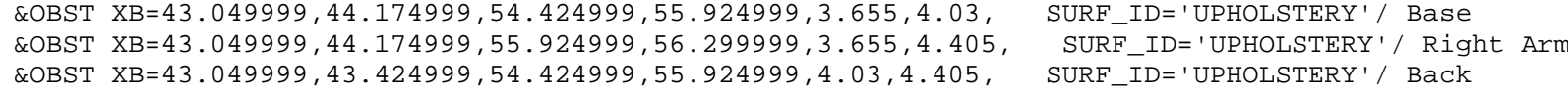

*** GROUP: COUCH2 offset $=0.000000,6.000000,0.000000$ rotate $=90.000000$

\&OBST XB $=43.049999,44.174999,57.049999,57.424999,3.655,4.405, \quad$ SURF_ID='UPHOLSTERY '/ Left Arm
\&OBST XB=43.04999, 44.174999, $57.424999,58.924999,3.655,4.03$ SURF ID='UPHOLSTERY'/ Base

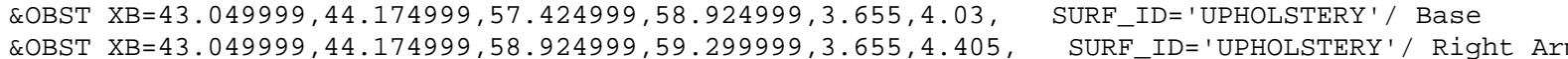
\&OBST XB=43.049999, 43.424999, 57.424999, 58.924999, 4.03,4.405, SURF_ID='UPHOLSTERY'/ Back

*** GROUP: COUCH2 offset $=0.0000000,9.000000,, 0.000000$ rotate $=90.000000$

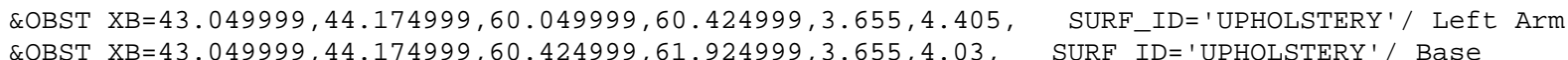
ROBST XB $=43.049999,44.174999,60.424999,61.924999,3.655,4.03, \quad$ SURF_ID=' 'UPHOLSTERY'/ Base
\&OBST XB $=43.049999,44.174999,61.92499,62.299999,3.655,4.405$,
SURF_ID 'UPHOLSTERY'/ Right Arm

COBST XB=43. 049999, 44.174999, 63. 049999, 63.424999, 3.655,4.405, SURF_ID='UPHOLSTERY'/ Left Arm

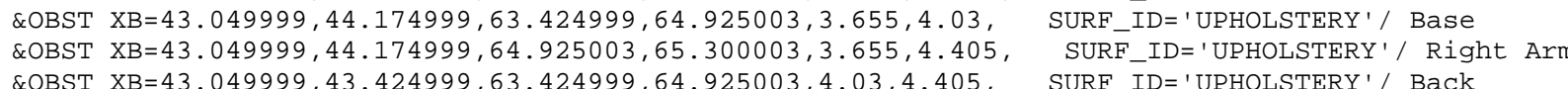

*** GROUP: COUCH2 offset $=0.000000,15.000000,0.000000$ rotate $=90.0000000$

\&OBST XB=43.049999, 44.174999, 66.050003, 66. 425003, 3.655, 4.405, SURF_ID=' 'UPHOLSTERY'/ Left Arm \&OBST XB=43.049999, 44.174999, 66.425003, 67. $925003,3.655,4.03$, SURF_ID='UPHOLSTERY'/ Base

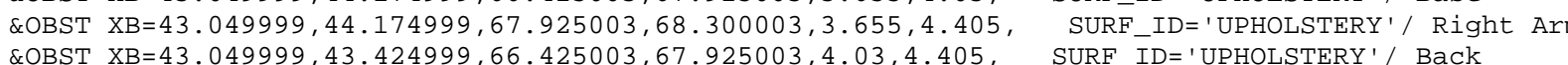

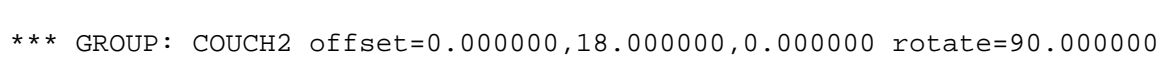

\&OBST XB=43.049999, 44.174999, 69.050003, 69.425003, 3.655, 4.405, SURF_ID=' 'UPHOLSTERY '/ Left Arm

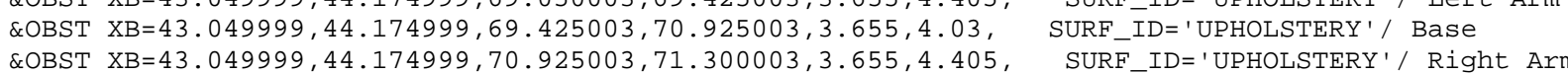

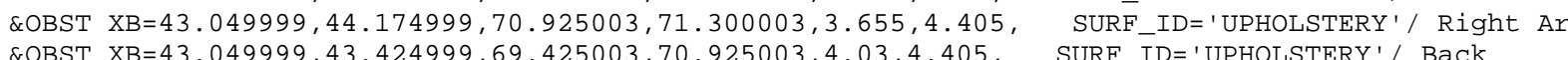

*** GROUP: COUCH2 offset $=0.000000,21.000000,0.000000$ rotate $=90.0000000$

\&OBST XB $=43.049999,44.174999,72.050003,72.425003,3.655,4.405$, SURF_ID=' 'UPHOLSTERY ' / Left Arm

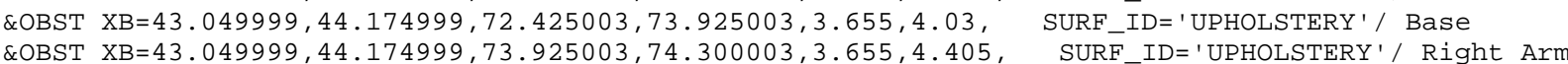
\&OBST XB $\times$ - $43.049999,43.424999,72.425003,73.925003,4.03,4.405$, SURF_ID='UPHOLSTERY'/ Right Ar

*** GROUP: COUCH2 offset $=0.000000,24.000000,0.000000$ rotate $=90.000000$

\&OBST XB=43. 049999, 44.174999, 75. 050003, 75.425003, 3.655, 4.405, SURF_ID=' 'UPHOLSTERY'/ Left Arm

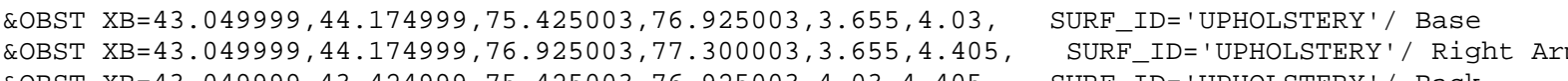
\&OBST XB=43. $049999,43.424999,75.425003,76.925003,4.03,4.405$, SURF_ID $='$ 'UP

\&OBST XB $=43.049999,44.174999,78.050003,78.425003,3.655,4.405$, SURF_ID=' 'UPHOLSTERY ' / Left Arm

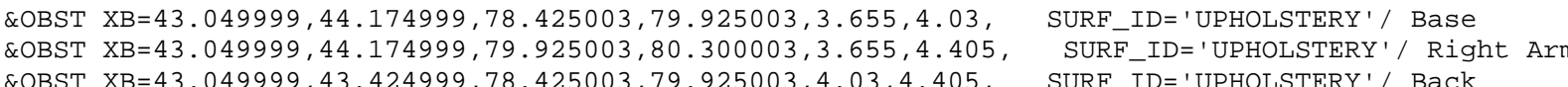

*** GROUP: COUCH2 offset $=0.000000,30.000000,0.000000$ rotate $=90.000000$

EOBST XB=43. $049999,44.174999,81.050003,81.425003,3.655,4.405$, SURF_ID=' 'UPHOLSTERY'/ Left Arm 


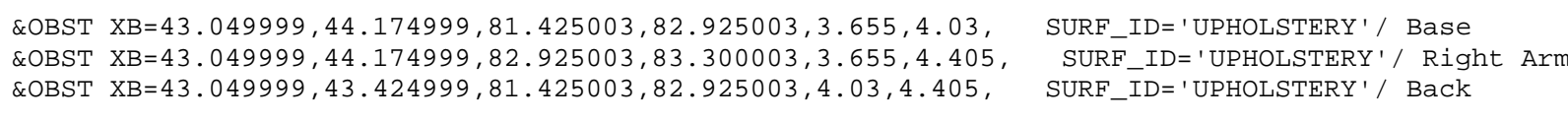

*** GROUP: ROW2

$* * *$ GROUP: COUCH2 offset $=0.000000,0.000000,0.000000$ rotate $=270.000000$

\&OBST XB=41.549999, 42.674999, 52.924999, 53. 299999, 3.655, 4.405, SURF_ID=' 'UPHOLSTERY ' / Left Arm

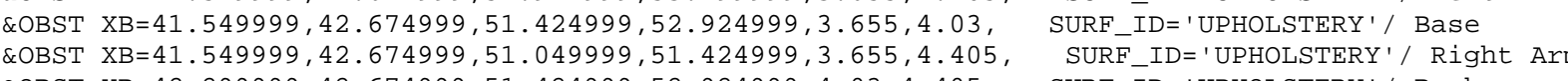

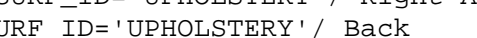

*** GROUP: COUCH2 offset $=0.000000,3.000000,0.000000$ rotate $=270.000000$

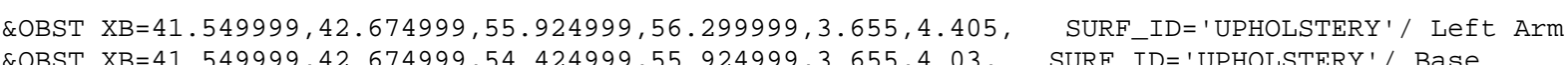

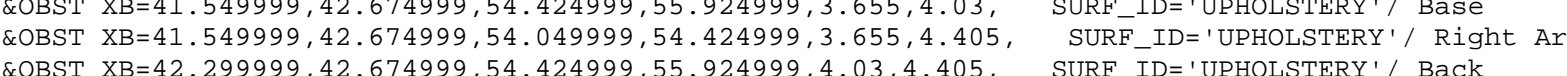

${ }_{* \star *}$ GROUP: COUCH2 offset $=0.000000,6.000000,0.000000$ rotate $=270.000000$

\&OBST XB=41.549999, 42.674999, 58.924999, 59. 299999, 3.655, 4.405, SURF_ID=' 'UPHOLSTERY'/ Left Arm \&OBST XB $=41.549999,42.674999,57.424999,58.924999,3.655,4.03$, SURF IDD='UPHOLSTERY'/ Base Arm

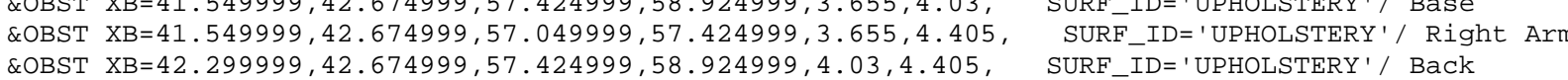

*** GROUP: COUCH2 offset $=0.000000,9.000000,, . .000000$ rotate $=270.000000$

\&OBST XB=41.549999, 42.674999, 61.924999, 62. 299999, 3.655, 4.405, SURF_ID=' 'UPHOLSTERY '/ Left Arm \&OBST XB=41.549999, 42.674999,60.424999, 61.924999, 3.655, 4.03, SURF_ID='UPHOLSTERY'/ Base Ar COBST XB $=41.549999,42.674999,60.049999,60.424999,3.655,4.405$, SURF_ID=' 'UPHOLSTERY '/ Right Arm
COBST XB $=42.299999,42.674999,60.424999,61.924999,4.03,4.405, \quad$ SURF_ID=' 'UPHOLSTERY' $/$ Back *** GROUP: COUCH2 offset $=0.000000,12.000000,0.000000$ rotate $=270$. .

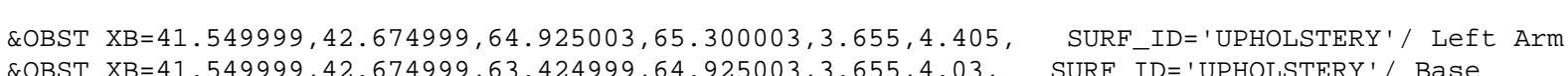
\&OBST XB=41.549999, 42.674999,63.049999,63.424999,3.655,4.405, SURF_ID=' 'UPHOLSTERY'/ Right Ar

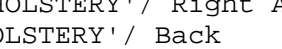
*** GROUP: COUCH2 offset $=0.000000,15.000000,0.000000$ rotate $=270.000000$

\&OBST XB $=41.549999,42.674999,67.925003,68.300003,3.655,4.405, \quad$ SURF_ID=' 'UPHOLSTERY ' / Left Arm

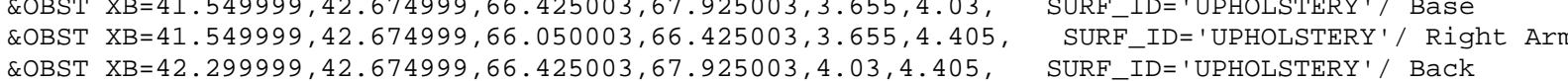
*** GROUP: COUCH2 offset $=0.000000,18.000000,0.000000$ rotate $=270.000000$

\&OBST XB $=41.549999,42.674999,70.925003,71.300003,3.655,4.405, \quad$ SURF_ID= 'UPHOLSTERY '/ Left Arm
\&OBST XB $=41.549999,42.674999,69.425003,70.925003,3.655,4.03$, SURF IDD='UPHOLSTERY'/ Base Arm

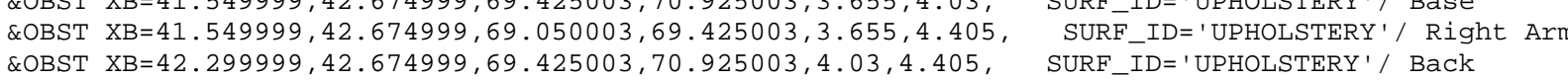

*** GROUP: COUCH2 offset $=0.000000,21.000000,0.000000$ rotate $=270.000000$

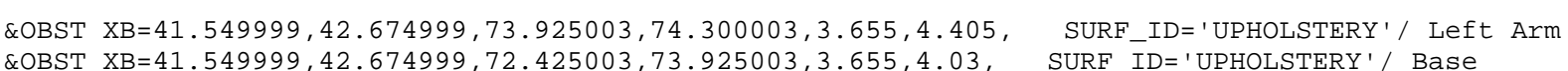

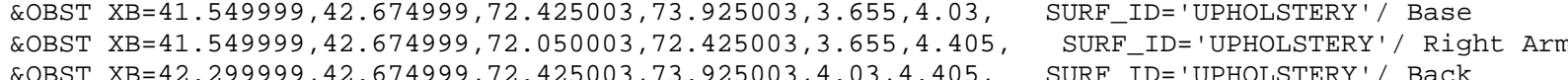
*** GROUP: COUCH2 offset $=0.000000,24.000000,0.000000$ rotate $=270.000000$

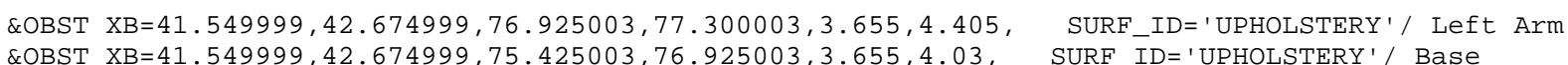

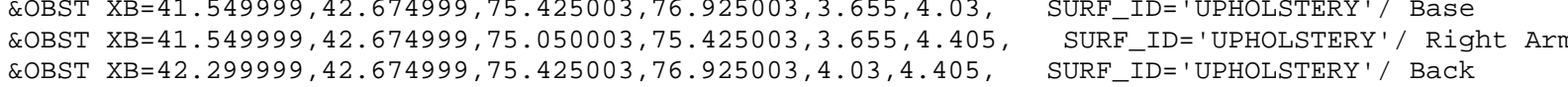

*** GROUP: COUCH2 offset $=0.000000,27.000000,0.000000$ rotate $=270.000000$

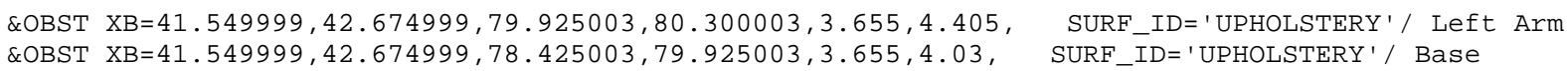

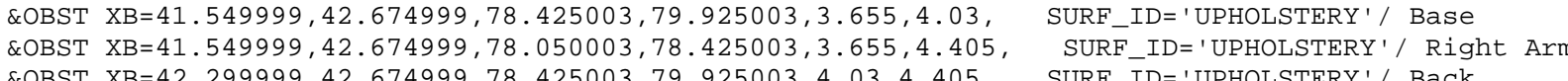

*** GROUP: COUCH2 offset $=0.000000,30.000000,0.000000$ rotate $=270.0000000$
\&OBST XB=41. $549999,42.674999,82.925003,83.300003,3.655,4.405, \quad$ SURF_ID=' 'UPHOLSTERY' $/$ Left Arm
\&OBST X $\mathrm{B}=41.549999,42.674999,81.425003,82.925003,3.655,4.03, \quad$ SURF_ID='UPHOLSTERY'/ Base

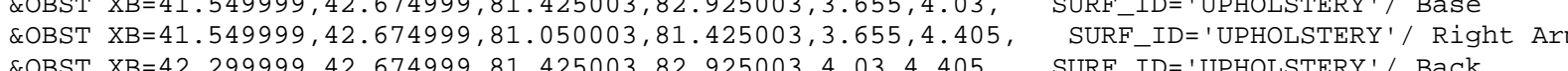

*** GROUP: TWIN

*** GROUP: ROW

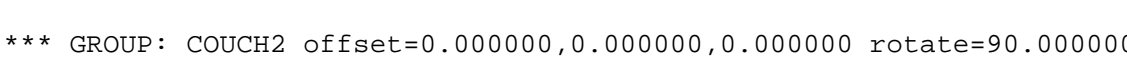

COBST XB $=47.549999,48.674999,51.049999,51.424999,3.655,4.405, \quad$ SURF_ID=' 'UPHOLSTERY'/ Left Arm
\&OBST XB $47.549999,48.674999,51.424999,52.924999,3.655,4.03$, SURF_ID='UPHOLSTERY' $/$ Base

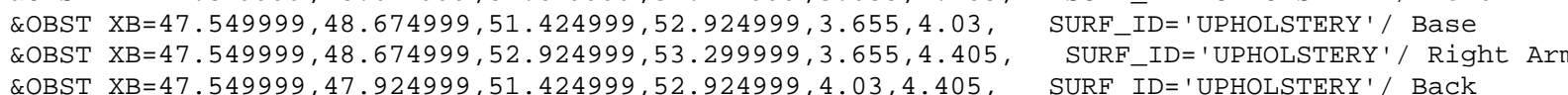

*** GROUP: COUCH2 offset $=0.000000,3.000000,0.000000$ rotate $=90.000000$

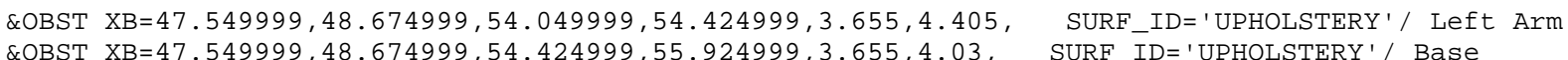

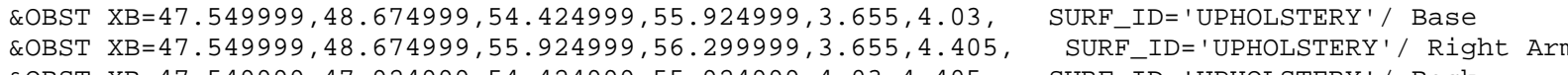

$* * *$ GROUP: COUCH2 offset $=0.000000,6.0000000,0.000000$ rotate $=90.000000$

\&OBST XB=47.549999, 48.674999, 57. 049999, 57.424999, 3.655, 4.405, SURF_ID=' 'UPHOLSTERY'/ Left Arm

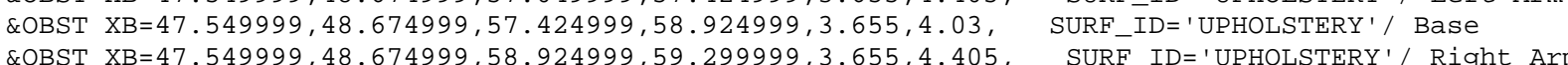

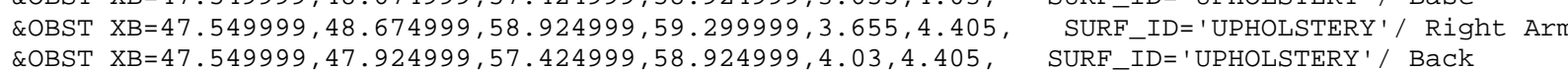

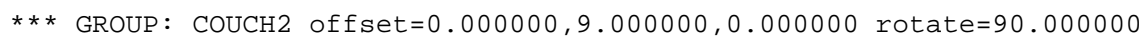

\&OBST XB=47.549999, 48.674999, 60. 049999, 60.424999, 3.655, 4.405, SURF_ID=' 'UPHOLSTERY '/ Left Arm \&OBST XB $=47.549999,48.674999,60.424999,61.924999,3.655,4.03$, SURF-ID='UPHOLSTERY'/ Base \&OBST XB $=47.549999,47.924999,60.424999,61.924999,4.03,4.405$, SURF IID='UPHOLSTERY'/ Right Ar

*** GROUP: CouCH2 offset $=0.000000,12.000000,0.000000$ rotate $=90.000000$

\&OBST XB $=47.549999,48.674999,63.049999,63.424999,3.655,4.405$, SURF_ID=' 'UPHOLSTERY ' / Left Arm

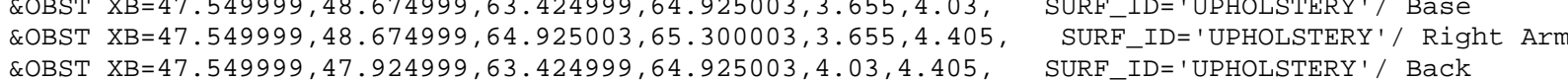

$* \star *$ GROUP: COUCH2 offset $=0.000000,15.000000,0.000000$ rotate $=90.000000$

\&OBST XB=47. $549999,48.674999,66.050003,66.425003,3.655,4.405$, SURF_ID= 'UPHOLSTERY' / Left Arm

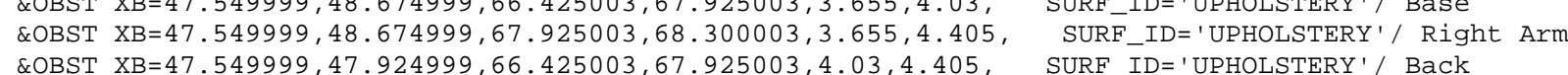

*** GROUP: COUCH2 offset $=0.000000,18.000000,0.000000$ rotate $=90.000000$

\&OBST XB=47. $549999,48.674999,69.050003,69.425003,3.655,4.405$, SURF_ID=' 'UPHOLSTERY' / Left Arm \&OBST XB=47.549999, $48.674999,70.925003,71.302003,3.655,4.405, \quad$ SURF_ID='UPHOLSTERY'/Right Arm \&OBST XB=47.549999, 47.924999, 69.425003, 70.925003, 4.03,4.405, SURF_ID='UPHOLSTERY'/ Back

*** GROUP: COUCH2 offset $=0.000000,21.000000,0.000000$ rotate $=90.000000$

\&OBST XB=47.549999, 48.674999, 72. $050003,72.425003,3.655,4.405$, SURF_ID= 'UPHOLSTERY' / Left Arm \&OBST XB $=47.549999,48.674999,73.925003,74.300003,3.655,4.405$, SURF_ID='UPHOLSTERY'/ Right Ar \&OBST XB=47.549999, 47.924999, 72.425003, 73.925003, 4.03, 4.405, SURF_ID='UPHOLSTERY'/ Back

*** GROUP: COUCH2 offset $=0.000000,24.000000,0.000000$ rotate $=90.000000$

\&OBST XB $=47.549999,48.674999,75.050003,75.425003,3.655,4.405$, SURF_ID=' 'UPHOLSTERY '/ Left Arm \&OBST XB=47.549999, 48.674999, 76.925003,77.300003, 3.655,4.405, SURF ID ='UPHOLSTERY'/ Rase \&OBST XB=47.549999, 47.924999, 75.425003, 76.925003, 4.03,4.405, SURF_ID='UPHOLSTERY'/ Back

*** GROUP: COUCH2 offset $=0.000000,27.000000,0.000000$ rotate $=90.000000$

\&OBST XB $=47.549999,48.674999,78.050003,78.425003,3.655,4.405$, SURF_ID=' 'UPHOLSTERY'/ Left Arm 
\&OBST XB=47.549999, 48.674999, $79.925003,80.300003,3.655,4.405, \quad \begin{aligned} & \text { SURF_ID= 'UPHOLSTERY '/ Right Arm } \\ & \text { \&OBST XB } \mathrm{X}=47.549999,47.924999,78.425003,79.925003,4.03,4.405,\end{aligned}$ SURF_ID='UPHOLSTERY'/ Back *** GROUP: COUCH2 offset $=0.000000,30.000000, \odot . .000000$ rotate $=90.000000$

ROBST XB $=47.549999,48.674999,81.050003,81.425003,3.655,4.405$, SURF_ID=' 'UPHOLSTERY' $/$ Left Arm

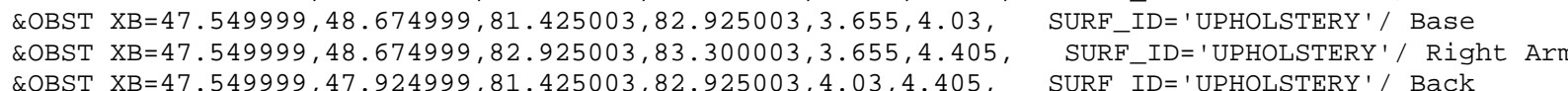
$* * *$ GROUP: ROW2

*** GROUP: COUCH2 of fset $=0.000000, \Theta .000000,0.000000$ rotate $=270.000000$

\&OBST XB=46. $049999,47.174999,52.924999,53.299999,3.655,4.405$, SURF_ID='UPHOLSTERY ' / Left Arm \&OBST XB=46.049999, 47.174999, 51.049999, 51.424999,3.655,4.405, SURF_ID='UPHOLSTERY'/Right Ar \&OBST XB=46.799999, 47.174999, 51.424999, 52.924999, 4.03,4.405, SURF_ID='UPHOLSTERY'/ Back

*** GROUP: COUCH2 offset $=0.000000,3.000000,0.000000$ rotate $=270.000000$

\&OBST XB $=46.049999,47.174999,55.924999,56.299999,3.655,4.405, \quad$ SURF_ID='UPHOLSTERY '/ Left Arm
\&OBST XB $=46.049999,47.174999,54.424999,55.924999,3.655,4.03, \quad$ SURF_ID ='UPHOLSTERY'
Base

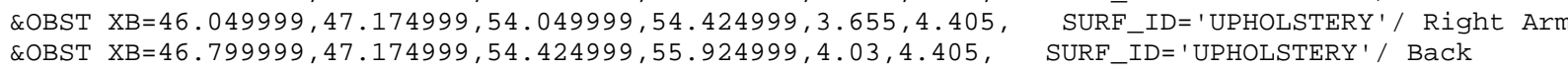
*** GROUP: COUCH2 offset $=0.000000,6.000000,0.000000$ rotate $=270.000000$

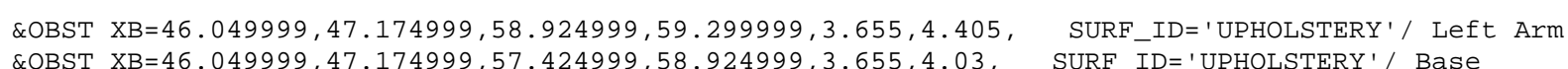

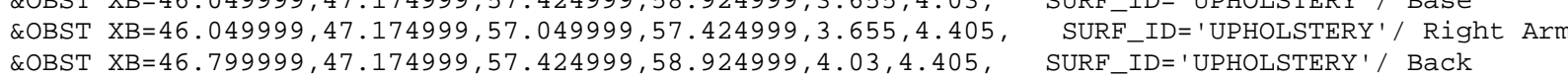
$* * *$ GROUP: COUCH2 offset $=0.000000,9.000000,0.000000$ rotate $=270.000000$

\&OBST XB=46. $849999,47.174999,61.924999,62.299999,3.655,4.405$,
\&OBST XB=46.04999, $47.174999,60.424999,61.924999,3.655,4.03$,
SURF_ID= 'UPHOLSTERY' 1 Left Arm
SURF'UPHOLSTERY'

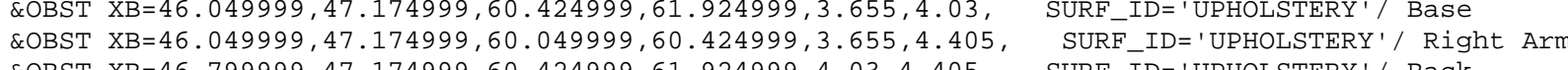

*** GROUP: COUCH2 offset $=0.000000,12.000000,0.000000$ rotate $=270.000000$

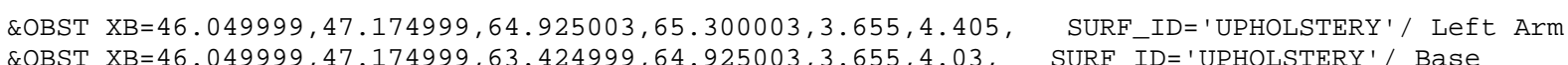

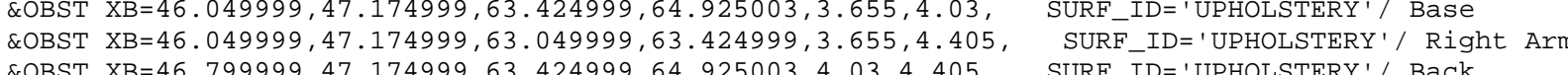

*** GROUP: COUCH2 offset $=0.000000,15.000000,0.0000000$ rotate $=270.000000$

\&OBST XB=46. $049999,47.174999,67.925003,68.300003,3.655,4.405$, SURF_ID=' 'UPHOLSTERY '/ Left Arm

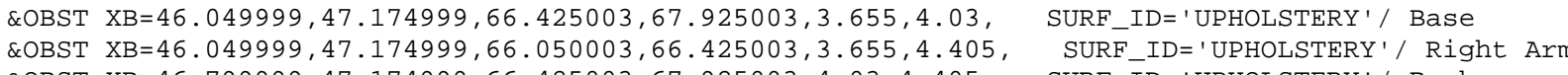
\&OBST XB=46.799999, 47.174999, 66.425003, 67.925003,4.03,4.405, SURF_ID='UPHOLSTERY'/ Back

*** GROUP: COUCH2 offset $=0.000000,18.000000,0.000000$ rotate $=270.000000$

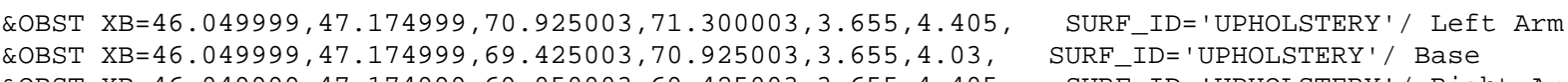

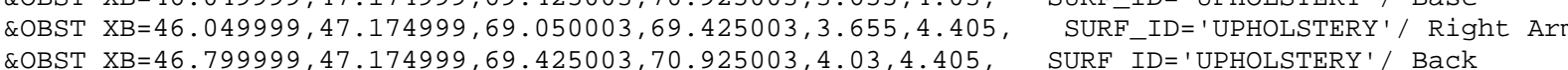
*** GROUP: COUCH2 offset $=0.000000,21.000000,0.0000000$ rotate $=270$. . 000000

\&OBST XB $=46.049999,47.174999,73.925003,74.300003,3.655,4.405$, SURF_ID=' 'UPHOLSTERY'/ Left Arm \&OBST XB=46.049999, 47.174999, $72.425003,73.925003,3.655,4.03$, SURF_ID='UPHOLSTERY'/Base Arm

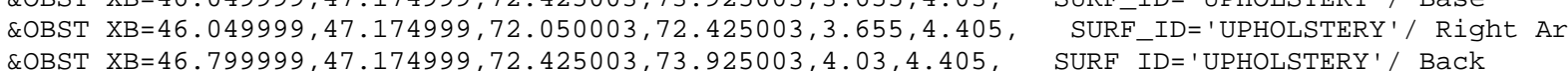
*** GROUP: COUCH2 offset $=0.000000,24.000000,0.000000$ rotate $=270.000000$

\&OBST XB=46.049999, 47.174999, 76.925003, 77. 300003, 3.655, 4.405, SURF_ID='UPHOLSTERY'/ Left Arm QOBST $\mathrm{XB}=46.049999,47.174999,75.425003,76.925003,3.655,4.03$, SURF_ID='UPHOLSTERY'/ Base ATm

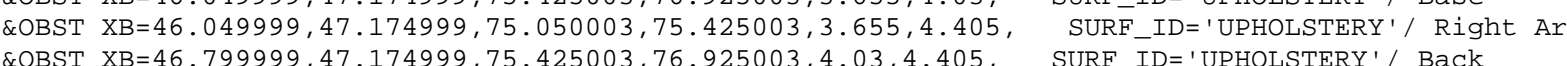

*** GROUP: COUCH2 offset $=0.000000,27.000000,0.000000$ rotate $=270.000000$
\&OBST XB=46. 049999, 47.174999, 79. $925003,80.300003,3.655,4.405$, SURF_ID=' 'UPHOLSTERY' / Left Arm \&OBST XB=46.049999, 47.174999, 78.425003,79.925003,3.655,4.03, SURF-ID='UPHOLSTERY'/ Base

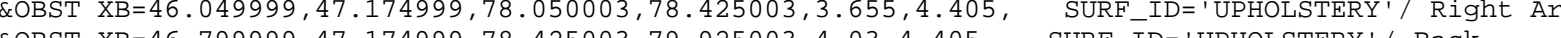

*** GROUP: COUCH2 offset $=0.0000000,30 . .000000,0.000000$ rotate $=270.000000$

\&OBST XB=46. $049999,47.174999,82.925003,83.300003,3.655,4.405$, SURF_ID=' 'UPHOLSTERY' / Left Arm

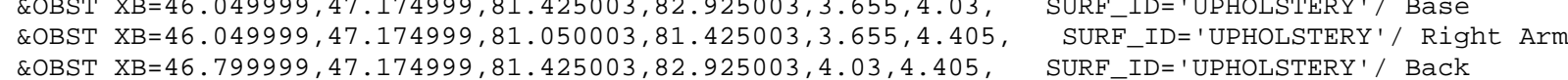

*** GROUP: TWIN

*** GROUP: ROW

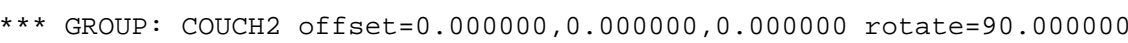

\&OBST XB=52. 049999, 53.174999, 51. 049999, 51.424999, 3.655, 4.405, SURF_ID=' 'UPHOLSTERY'/ Left Arm

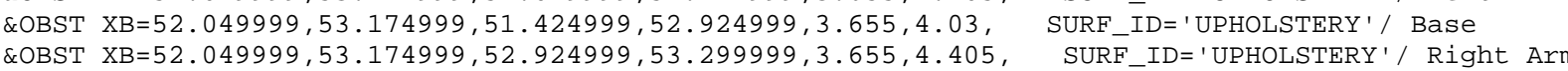
\&OBST XB=52.049999, 52.424999, 51.424999, 52.924999, $4.03,4.405$, SURF-ID=' 'UPHOLSTEPY'/ Back

*** GROUP: COUCH2 offset $=0.000000,3.000000,0.000000$ rotate $=90.000000$

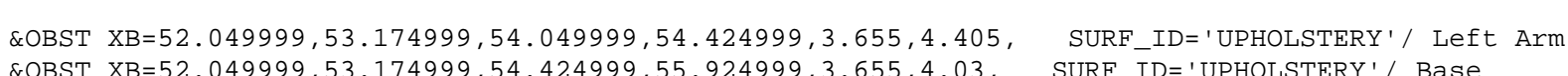

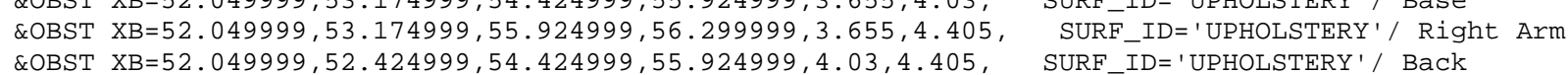

$* * *$ GROUP: COUCH2 offset $=0.000000,6.000000,0.000000$ rotate $=90$. .

\&OBST XB=52. 049999, 53.174999, 57. 049999, 57.424999, 3.655, 4.405, SURF_ID='UPHOLSTERY ' / Left Arm

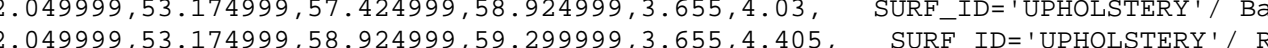
\&OBST XB=52.049999, $52.424999,57.424999,58.924999,4.03,4.405$, SURF_ID='UPHOLSTERY'/ Back

*** GROUP: COUCH2 offset $=0.0000000,9.000000,0.000000$ rotate $=90.000000$

\&OBST XB=52.049999, 53.174999, 60.049999, 60.424999, 3.655, 4.405, SURF_ID=' 'UPHOLSTERY '/ Left Arm

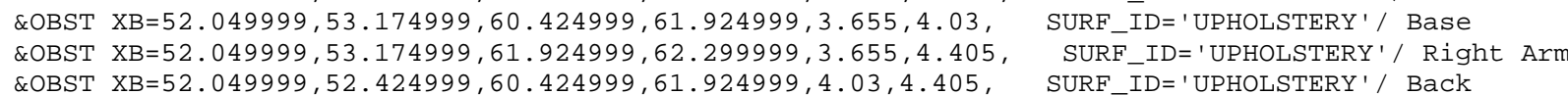
*** GROUP: COUCH2 offset $=0.000000,12.000000,0.000000$ rotate $=90.000000$

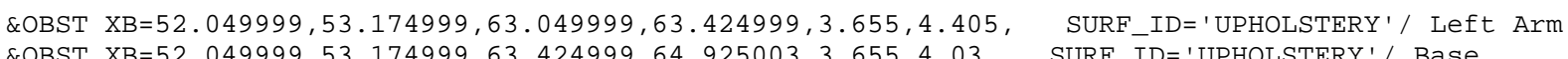
\&OBST XB $=52.049999,53.174999,64.925093,65.300003,3.655,4.405$, SURF ID='UPHOLSTERY'/ Right Arm \&OBST XB=52.049999, 52.424999,63.424999,64.925003,4.03,4.405, SURF_ID='UPHOLSTERY'/ Back

*** GROUP: COUCH2 offset $=0.000000,15.000000$, ๑. .000000 rotate $=90 . .000000$

\&OBST XB=52. $049999,53.174999,66.050003,66.425003,3.655,4.405, \quad$ SURF_ID= 'UPHOLSTERY '/ Left Arm
8OBST XB=52.04999, $53.174999,66.425003,67.925003,3.655,4.03$ SURF ID='UPHOLSTERY'/ Base

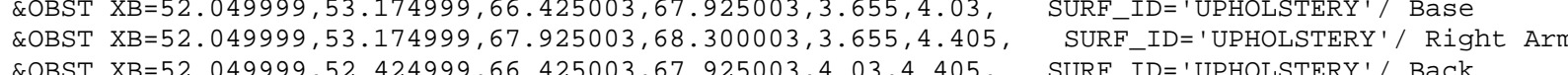

*** GROUP: COUCH2 offset $=0.000000,18.000000$, ๑. 000000 rotate $=90.000000$

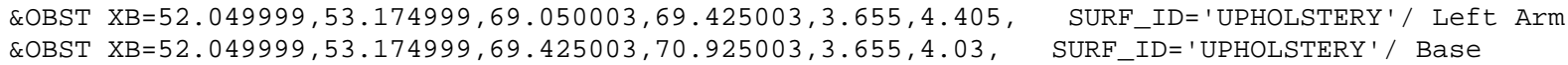

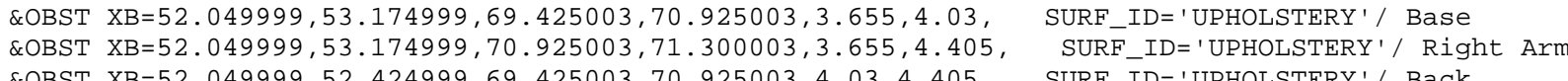

*** GROUP: COUCH2 offset $=0.000000,21.000000,0.000000$ rotate $=90.000000$

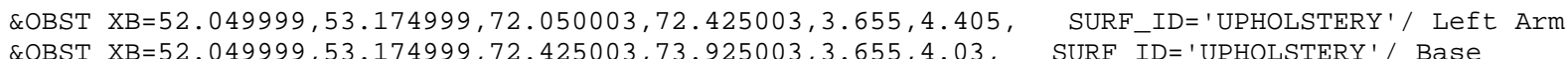

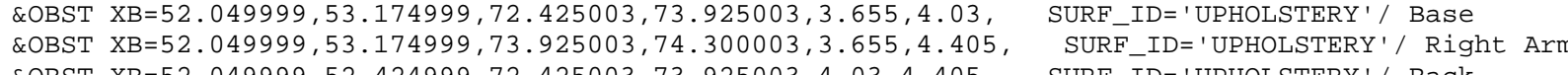

*** GROUP: COUCH2 off set $=0.0000000,24.000000,0$. . .000000 rotate $=9 \odot . .000000$

\&OBST XB $=52.049999,53.174999,75.050003,75.425003,3.655,4.405$, SURF_ID='UPHOLSTERY'/ Left Arm

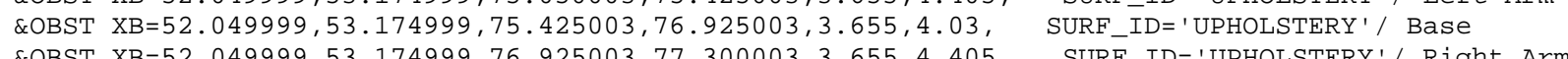


\&OBST XB $=52,049999,52,424999,75,425003,76,925003,4,03,4,405$, SURE_ID=' UPHOLSTERY' / Back

** GROUP: COUCH2 offset $=0.000000,27.000000,0.000000$ rotate $=90.000000$

\&OBST XB $=52.049999,53.174999,78.050003,78.425003,3.655,4.405$, SURF_ID=' 'UPHOLSTERY '/ Left Arm \&OBST XB=52.049999, 53.174999, 79.925003, 80.300003,3.655,4.405, SURF_ID=' 'UPHOLSTERY'// Base

*** GROUP: COUCH2 offset $=0.000000,30.000000,0.000000$ rotate $=90.000000$

\&OBST XB $=52.049999,53.174999,81.050003,81.425003,3.655,4.405$, SURF_ID=' 'UPHOLSTERY'/ Left Arm \&OBST XB $=52.049999,53.174999,81.425003,82.925003,3.655,4.03, \quad$ SURF_ID='UPHOLSTERY'/ Base 20BST XB=52.04999, $53.174999,82.925003,83.300003,3.655,4.405$, SURF_ID='UPHOLSTYRY'/Right Ar

*** GROUP: ROW2

** GROUP: COUCH2 offset=0, 000000, ๑. 000000, ๑.

\&OBST XB $=50.549999,51.674999,52.924999,53.299999,3.655,4.405$, SURF_ID=' 'UPHOLSTERY '/ Left Arm

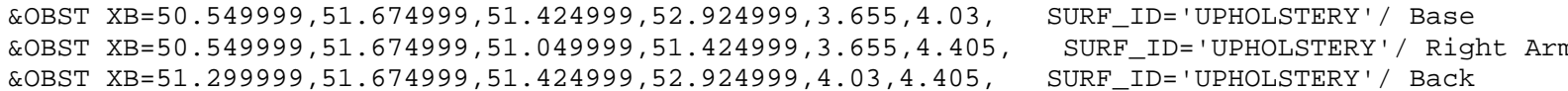

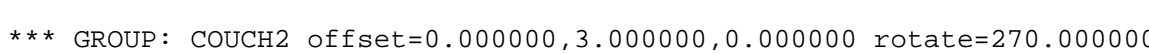

\&OBST XB $=50.549999,51.674999,55.924999,56.299999,3.655,4.405$, SURF_ID=' 'UPHOLSTERY '/ Left Arm

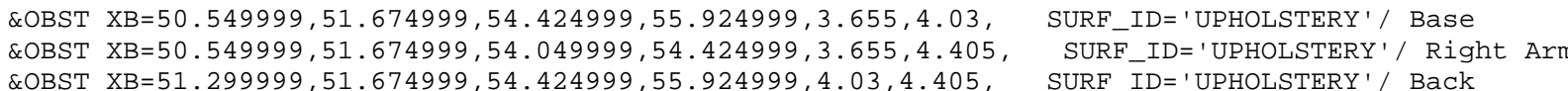
*** GROUP: COUCH2 offset $=0.000000,6.000000,0.000000$ rotate $=270.000000$

\&OBST XB $=50.549999,51.674999,58.924999,59.299999,3.655,4.405$, SURF_ID='UPHOLSTERY'/ Left Arm \&OBST XB=50.54999, $51.674999,57.424999,58.924999,3.655,4.03$, SURF_ID='UPHOLSTERY'/ Base Arm

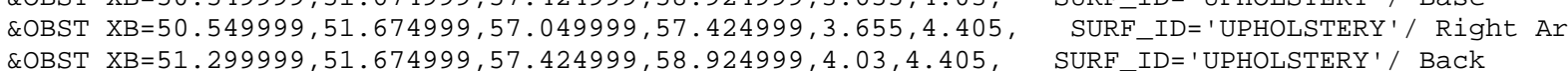
*** GROUP: COUCH2 offset $=0.000000,9.000000,0.000000$ rotate $=270.0000000$

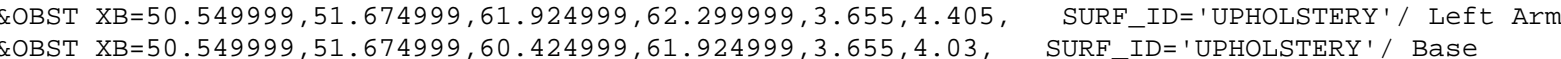
$\begin{array}{ll} & \\ & \end{array}$ \&OBST XB $=51.299999,51.674999,60.424999,61.924999,4.03,4.405$, SURF_ID='UPHOLSTERY'/ Rack Ar

*** GROUP: COUCH2 offset $=0.000000,12.000000,0.000000$ rotate $=270$. .

\&OBST XB=50.549999, 51.674999,64.925003, 65.300003, 3.655, 4.405, SURF_ID=' 'UPHOLSTERY'/ Left Arm QOBST XB=50.549999, 51.674999, 63. 424999, 64.925003, 3.655, 4.03, SURF_ID='UPHOLSTERY'/ Base Arm

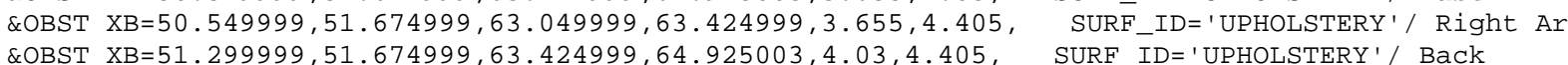

*** GROUP: COUCH2 offset $=0.000000,15.000000,0.000000$ rotate $=270.000000$

\&OBST XB=50.549999, 51.674999, 67. $925003,68.300003,3.655,4.405$, SURF_ID=' 'UPHOLSTERY'/ Left Arm

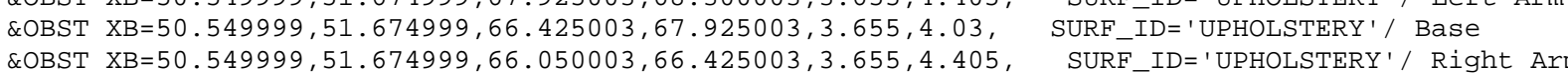
\&OBST XB=51.299999, $51.674999,66.425003,67.925003,4.03,4.405$, SURF_ID='UPHOLSTERY'/ Back

*** GROUP: COUCH2 offset $=0.000000,18.000000,0.000000$ rotate $=270.000000$

\&OBST XB $=50.549999,51.674999,70.925003,71.300003,3.655,4.405$, SURF_ID=' 'UPHOLSTERY'/ Left Arm $\begin{array}{lll}\text { \&OBST XB }=50.549999,51.674999,69.425003,70.925003,3.655,4.03, & \text { SURF_ID='UPHOLSTERY'/ Base } \\ \text { SOBST } & \text { SURFID='UPHOLSTERY'// Right Ar }\end{array}$ COBST XB $=50.549999,51.674999,69.050003,69.425003,3.655,4.405, \quad$ SURF_ID=' 'UPHOLSTERY'/ Right Arm
\&OBST XB $=51.299999,51.674999,69.425003,70.925003,4.03,4.405$,
SURF-ID=' 'UPHOLSTERY'/ Back

*** GROUP: CoUCH2 offset $=0.000000,21.000000,0.000000$ rotate $=270.000000$

\&OBST XB=50.549999, 51.674999, 73.925003, 74.300003, 3.655, 4.405, SURF_ID=' 'UPHOLSTERY'/ Left Arm

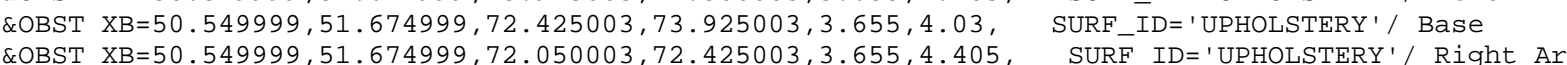

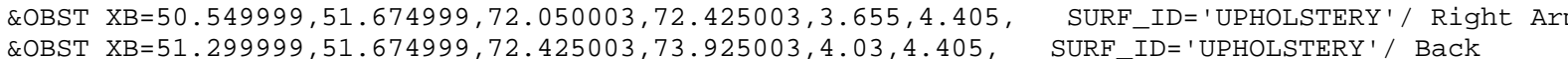
*** GROUP: COUCH2 offset $=0.000000,24.000000,0.000000$ rotate $=270.000000$

\&OBST XB=50.549999, 51.674999, 76.925003, 77.300003, 3.655, 4.405, SURF_ID=' 'UPHOLSTERY' / Left Arm
\&OBST XB $=50.549999,51.674999,75,425003,76.925003,3.655,4.03$ SURF ID=' UPHOLSTERY'/ Base

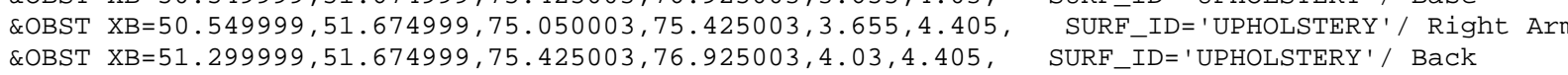
*** GROUP: COUCH2 offset $=0.000000,27.000000, \Theta .000000$ rotate $=270.000000$

\&OBST XB $=50.549999,51.674999,79.925003,80.300003,3.655,4.405, \quad$ SURF_ID='UPHOLSTERY'/ Left Arm \&OBST XB $=50.549999,51.674999,78.050003,78.425003,3.655,4.405$, SURF ID='UPHOLSTERY'/ Right Ar \&OBST XB $=51.299999,51.674999,78.425003,79.925003,4.03,4.405$, SURF_ID=' 'UPHOLSTERY'/ Back

*** GROUP: COUCH2 offset $=0.000000,30.000000,0.000000$ rotate $=270.000000$

\&OBST XB $=50.549999,51.674999,82.925003,83.300003,3.655,4.405, \quad$ SURF_ID='UPHOLSTERY'/ Left Arm \&OBST XB $50.549999,51.674999,81.050003,81.425003,3.655,4.405$, SURF $I D=$ 'UPHOLSTEPY' $/$ Right Ar \&OBST XB=51.299999, 51.674999, 81.425003, 82.925003,4.03,4.405, SURF_ID='UPHOLSTERY'/ Back Ar

*** GROUP: TWIN

*** GROUP: ROW

*** GROUP: COUCH2 offset $=0.000000,0.000000,0.000000$ rotate $=90.000000$

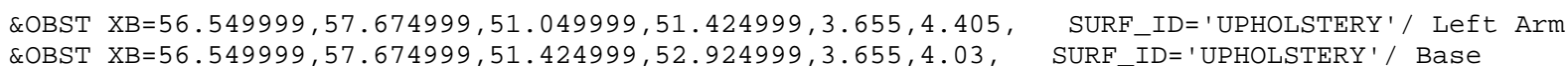
\&OBST XB $=5.549999,57.674999,52.924999,53.299999,3.655,4.405$, SURF ID=' 'UPHOLSTERY'/ Right AP

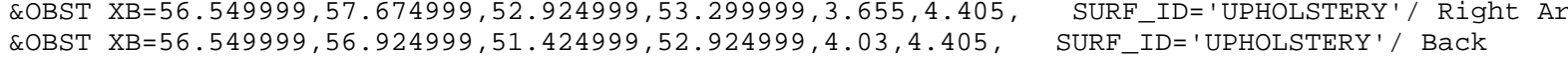

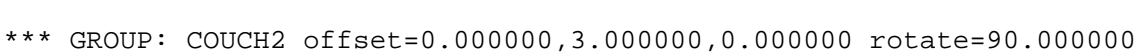

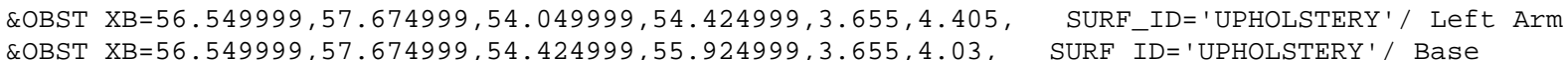

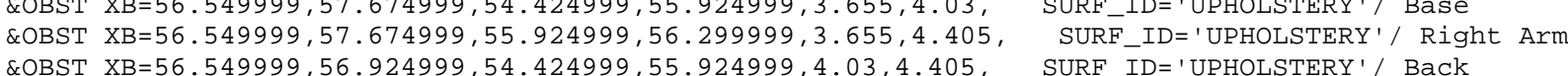

*** GROUP: COUCH2 offset $=0$. . $000000,6.0000000,0.000000$ rotate $=90$. .

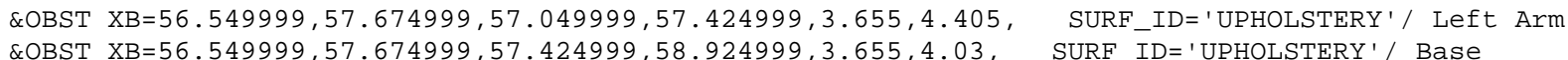

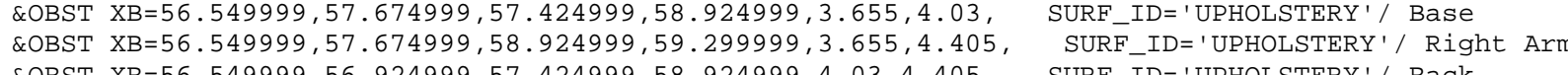

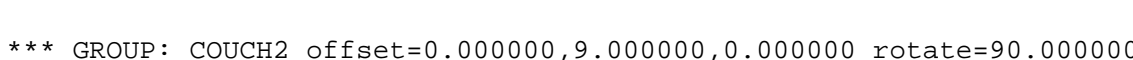

COBST XB=56.549999, 57.674999, 60. $049999,60.424999,3.655,4.405, \quad$ SURF_ID=' 'UPHOLSTERY '/ Left Arm
SURF_ID='UPHOLSTERY' $/$ Base

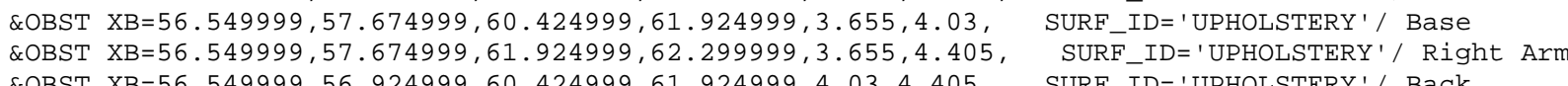

*** GROUP: COUCH2 offset $=0.000000,12.000000,0.0000000$ rotate $=90.000000$

\&OBST XB=56.549999, 57.674999, 63.049999, 63.424999, 3.655, 4.405, SURF_ID=' 'UPHOLSTERY'/ Left Arm \&OBST XB $=56.549999,57.674999,63.424999,64.925003,3.655,4.03$, SURF_ID='UPHOLSTERY' '/ Base

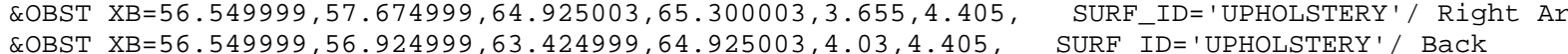

*** GROUP: COUCH2 offset $=0.000000,15.0000000,0 . .000000$ rotate $=90 . .000000$

\&OBST XB=56.549999, 57.674999, 66.050003,66.425003, 3.655, 4.405, SURF_ID=' 'UPHOLSTERY'/ Left Arm

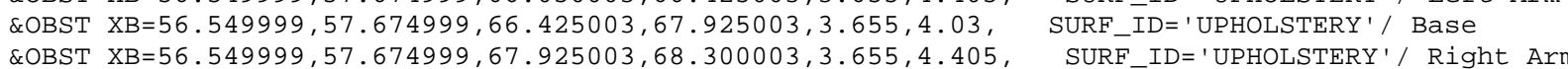
ROBST XB $56.549999,56.924999,66.425003,67.925003,4.03,4.405$, SURF_ID='UPHOLSTERY'/ Rigkt Arm

*** GROUP: COUCH2 offset $=0.000000,18.000000,0.000000$ rotate $=90.000000$

\&OBST XB=56.549999, 57.674999, 69.050003, 69.425003, 3.655, 4.405, SURF_ID=' 'UPHOLSTERY'/ Left Arm

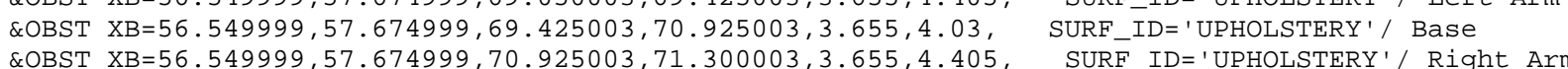
\&OBST XB $=56.549999,56.924999,69.425003,70.925003,4.03,4.405$, SURF IDD='UPHOLSTERY'/ Rigk Ar

*** GROUP: COUCH2 offset $=0.000000,21.000000,0.000000$ rotate $=90.000000$

\&OBST XB $=56.549999,57.674999,72.050003,72.425003,3.655,4.405$, SURF_ID=' 'UPHOLSTERY'/ Left Arm \&OBST XB=56.549999, 57.674999, 73.925003,74. $3500003,3.655,4.405$, SURF-ID='UPHOLSTERY'/ Right Arn 
*** GROUP: COUCH2 offset $=0.000000,24.000000,0.000000$ rotate $=90.000000$

\&OBST XB $=56.549999,57.674999,75.050003,75.425003,3.655,4.405, \quad$ SURF_ID=' 'UPHOLSTERY '/ Left Arm \&OBST XB=56.549999, $57.674999,76.925003,77.300003,3.655,4.405$, SURF_ID='UPHOLSTERY'/ Right Ar \&OBST XB $=56.549999,56.924999,75.425003,76.925003,4.03,4.405$, SURF_ID='UPHOLSTERY'/ Back *** GROUP: COUCH2 offset $=0.000000,27.000000,0.000000$ rotate $=90.000000$

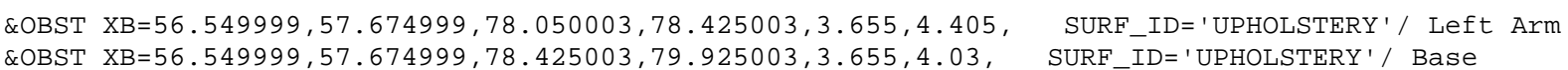

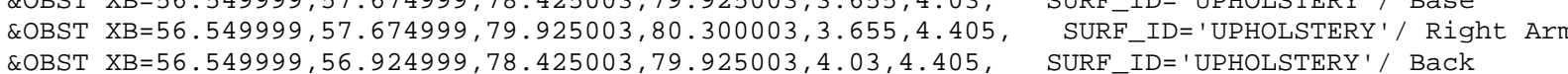

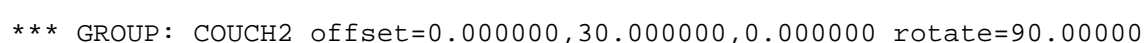

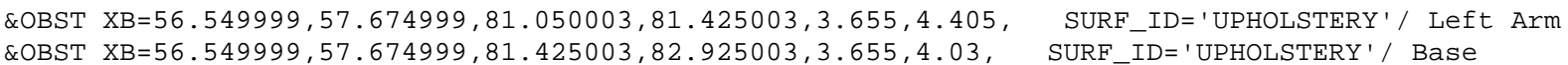

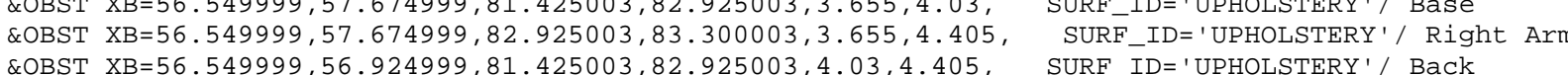
*** GROUP: ROW2

$* * *$ GROUP: COUCH2 offset $=0.000000,0.000000,0.000000$ rotate $=270.000000$

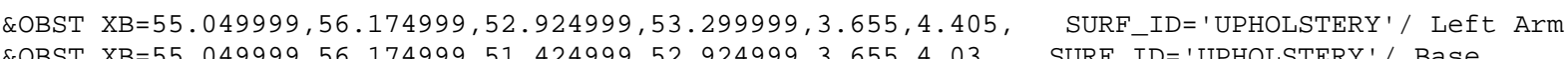

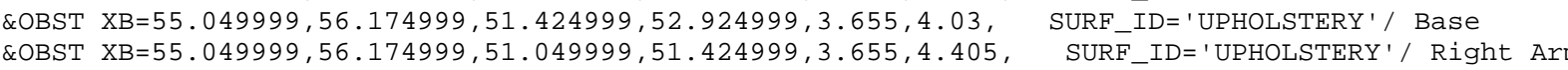

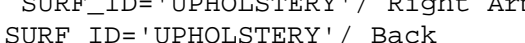

*** GROUP: COUCH2 offset $=0.000000,3.000000, \Theta .000000$ rotate $=270.000000$

\&OBST XB $=55.049999,56.174999,55.924999,56.299999,3.655,4.405$, SURF_ID=' 'UPHOLSTERY'/ Left Arm QOBST XB $55.049999,56.174999,54.424999,55.924999,3.655,4.03$, SURF IDE' 'UPHOLSTERY'/ Base

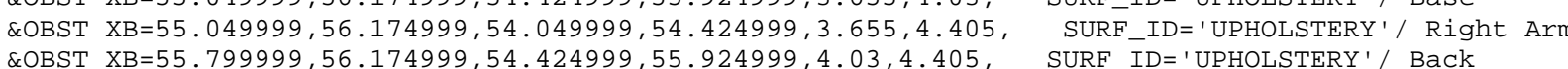

*** GROUP: COUCH2 offset $=0.000000,6.000000,0.000000$ rotate $=270.000000$

\&OBST XB=55. 049999, 56.174999, 58.924999, 59. 299999, 3.655, 4.405, SURF_ID=' 'UPHOLSTERY' / Left Arm \&OBST XB=55. $049999,56.174999,57.424999,58.924999,3.655,4.03$, SURF_ID='UPHOLSTERY'/ Base

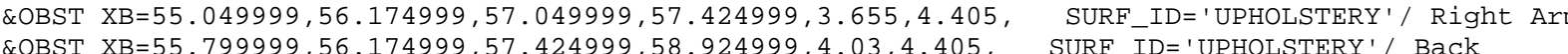
*** GROUP: COUCH2 offset $=0.000000,9.000000,0.000000$ rotate $=270.000000$

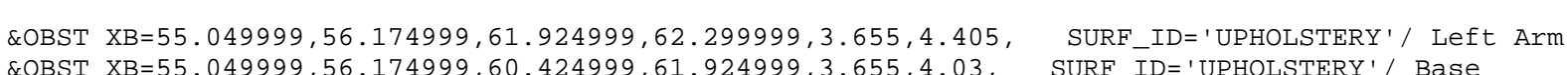
\&OBST XB=55.04999, 56.174999, 60.04999, 60.424999, $3.655,4.405$, SURF_ID=' 'UPHOLSTERY'/R Right Ar
\&OBST XB=55.799999, $56.174999,60.424999,61.924999,4.03,4.405$, SURF_ID='UPHOLSTERY'/ Back *** GROUP: COUCH2 offset $=0.000000,12.000000,0.000000$ rotate $=270.000000$

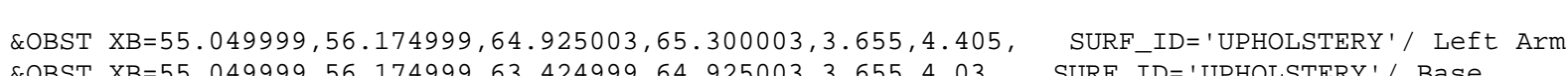

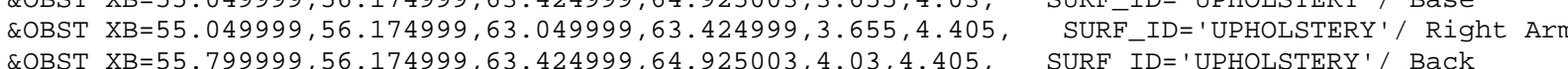
*** GROUP: COUCH2 offset $=0.0000000,15.000000,0.000000$ rotate $=270.0000000$

ROBST XB=55. $449999,56.174999,67.925003,68.300003,3.655,4.405, \quad$ SURF_ID= 'UPHOLSTERY ' / Left Arm
\&OBST XB=55.049999, $56.174999,66.425003,67.925003,3.655,4.03, \quad$ SURF_ID='UPHOLSTERY' Base \&OBST XB=55. $049999,56.174999,66.050003,66.425003,3.655,4.405$,
\&OBST XB=55.799999, S66.174999, $66.425003,67.925003,4.03,4.405$, SURF_IDE' 'UPHOLSTERY '/ Right Arm
SURHOLSTERY'/ Back *** GROUP: COUCH2 offset $=0.000000,18.000000,0.000000$ rotate $=270.000000$

LOBST XB=55. $049999,56.174999,70.925003,71.300003,3.655,4.405$, SURF_ID='UPHOLSTERY' / Left Arm

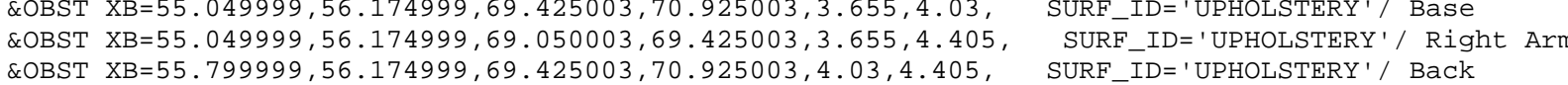
*** GROUP: COUCH2 offset $=0.000000,21.000000,0.000000$ rotate $=270.000000$

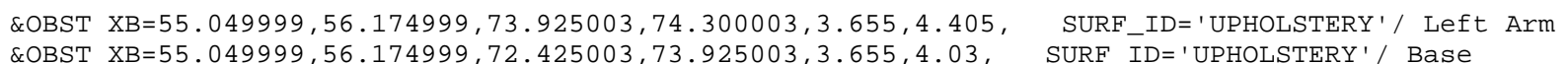

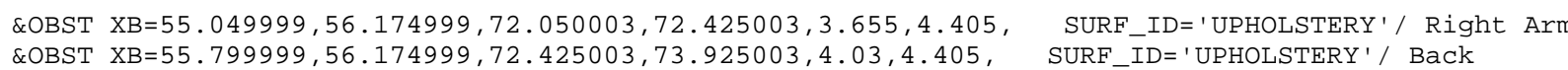
*** GROUP: COUCH2 offset $=0.0000000,24.0000000,0.000000$ rotate $=270.0000000$

COBST XB=55. $049999,56.174999,76.925003,77.300003,3.655,4.405, \quad$ SURF_ID='UPHOLSTERY' $/$ Left Arm
\&OBST XBE55.049999, $56.174999,75.425003,76.925003,3.655,4.03, \quad$ SURF_ID='UPHOLSTERY'/ Base

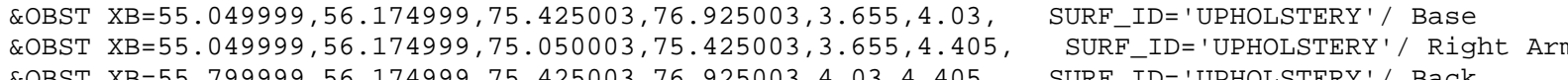

*** GROUP: COUCH2 offset $=0.000000,27.000000,0.0000000$ rotate $=270.0000000$

\&OBST XB $=55.049999,56.174999,79.925003,80.300003,3.655,4.405$, SURF_ID='UPHOLSTERY'/ Left Arm \&OBST XB=55.049999, $56.174999,78.425003,79.925003,3.655,4.03$, SURF_ID='UPHOLSTERY'/ Base

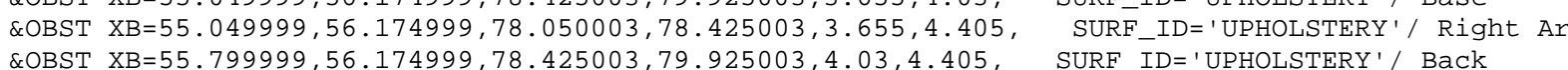

*** GROUP: COUCH2 offset $=0.000000,30.000000,0.000000$ rotate $=270.000000$

\&OBST XB=55. 049999, 56.174999, 82.925003, 83.300003, 3.655, 4.405, SURF_ID=' 'UPHOLSTERY'/ Left Arm \&OBST XB $=55.049999,56.174999,81.425003,82.925003,3.655,4.03$, SURF_ID='UPHOLSTERY'/ Base

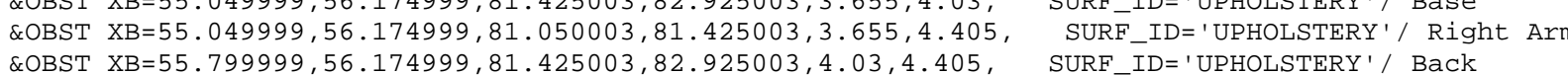

$* * *$ GROUP: TWIN

*** GROUP: ROW

*** GROUP: COUCH2 offset $=0.000000, \odot . .000000, \odot . .000000$ rotate $=90 . .000000$

\&OBST XB=61.049999, 62.174999, 51.049999, 51.424999, 3.655, 4.405, SURF_ID=' 'UPHOLSTERY'/ Left Arm

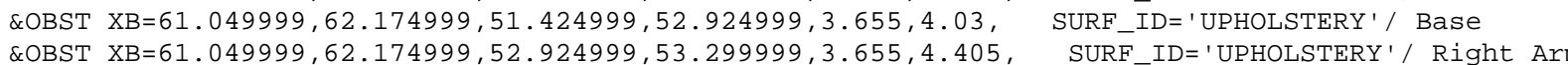

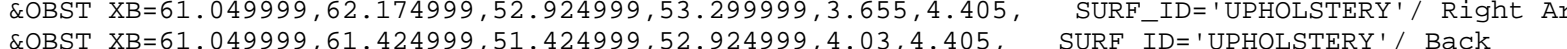

*** GROUP: COUCH2 offset $=0.000000,3.000000,0.000000$ rotate $=90.000000$

\&OBST XB=61.049999, 62.174999, 54.049999, 54.424999, 3.655, 4.405, SURF_ID='UPHOLSTERY'/ Left Arm \&OBST XB $=61.049999,62.174999,54.424999,55.924999,3.655,4.03$,
\&OBST XB $\mathrm{X}=61.049999,62.174999,55.924999,56.299999,3.655,4.405, \quad$ SURF_ID='UPHOLSTERY'/ Base
SURF_ID='UPHOLSTERY'/ Right Ar

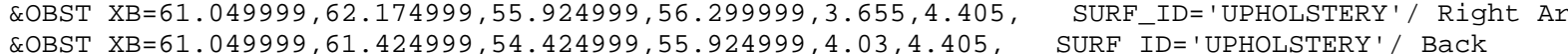

*** GROUP: COUCH2 offset $=0.000000,6.000000,0.000000$ rotate $=90.000000$

\&OBST XB=61. 049999, 62.174999, 57. 049999, 57.424999, 3.655, 4.405, SURF_ID='UPHOLSTERY'/ Left Arm \&OBST XB $61.049999,62.174999,57.42499,58.924999,3.655,4.03$, SURF-ID=' 'UPHOLSTERY'/ Base 2OBST XB=61.04999, $2.174999,58.924999,59.299999,3.655,4.405$, SURF_ID='UPHOLSTERY//Right Ar

*** GROUP: COUCH2 offset $=0.000000,9.000000,0.000000$ rotate $=90.000000$

\&OBST XB=61. 049999, 62.174999, 60. 049999, 60.424999, 3.655, 4.405, SURF_ID=' 'UPHOLSTERY ' / Left Arm \&OBST XB=61.049999, 62.174999, 61.924999, 62.299999, 3.655, 4.405, SURF_ID=' 'UPHOLSTERY'/ Right Ar

*** GROUP: COUCH2 offset $=0.000000,12.000000,0.000000$ rotate $=90.000000$

\&OBST XB=61. $049999,62.174999,63.049999,63.424999,3.655,4.405$, SURF_ID= 'UPHOLSTERY' / Left Arm

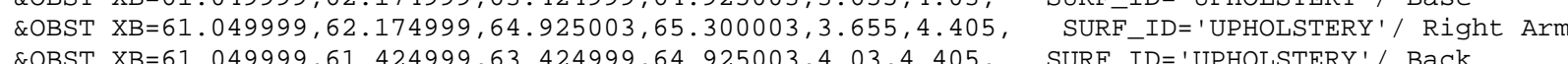

*** GROUP: COUCH2 offset $=0.000000,15.000000,0.000000$ rotate $=90.000000$

\&OBST XB=61. $049999,62.174999,66.050003,66.425003,3.655,4.405$, SURF_ID=' 'UPHOLSTERY' / Left Arm \&OBST XB=61.049999, 62.174999, 67.925003, 68.300003, 3.655,4.405, SURF_ID='UPHOLSTERY'/ Right Arm

*** GROUP: COUCH2 offset $=0.000000,18.000000,0.000000$ rotate $=90 ., 000000$

\&OBST XB=61. $449999,62.174999,69.050003,69.425003,3.655,4.405$, SURF_ID=' 'UPHOLSTERY'/ Left Arm \&OBST XB $61.049999,62.174999,70.925003,71.300003,3.655,4.405$, SURF_ID 'UPHOLSTERY' $/$ Right Ar \&OBST XB=61.049999,61.424999, 69.425003, 70.925003,4.03,4.405, SURF_ID=' UPHOLSTERY'/ Back 
** GROUP: COUCH2 offset=0, 000000, 21.000000,0.000000 rotate=90,

\&OBST XB $=61.049999,62.174999,72.050003,72.425003,3.655,4.405, \quad$ SURF_ID=' 'UPHOLSTERY'/ Left Arm
\&OBST XB $=61.049999,62.174999,72.425003,73.925003,3.655,4.03, \quad$ SURF_ID='UPHOLSTERY' $/$ Base

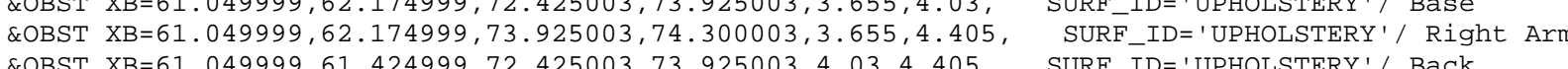

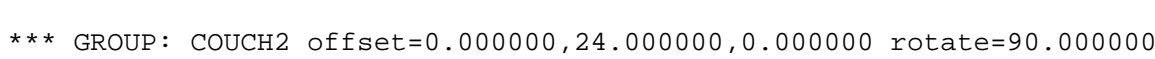

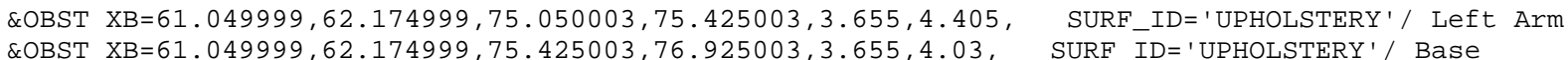

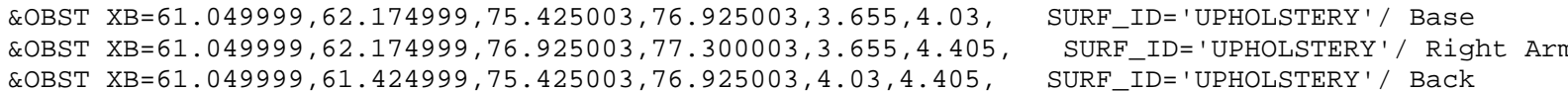

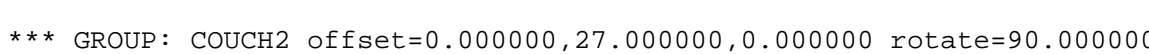

\&OBST XB=61.049999, 62.174999, 78.050003, 78.425003,3.655, 4.405, SURF_ID='UPHOLSTERY' / Left Arm

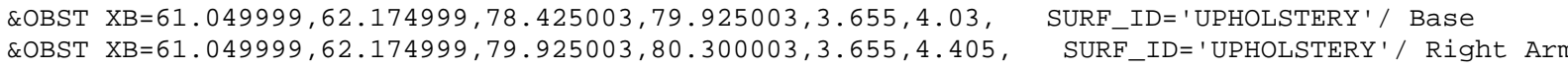
\&OBST XB $61.049999,61.424999,78.425003,79.925003,4.03,4.405$, SURF_ID='UPHOLSTERY'/ Back Ar

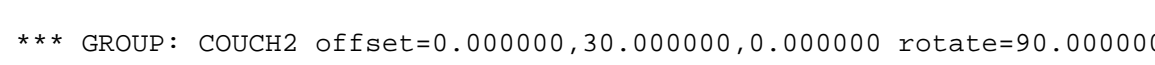

\&OBST XB=61.049999, 62.174999, 81.050003, 81.425003, 3.655, 4.405, SURF_ID=' 'UPHOLSTERY'/ Left Arm \&OBST XB=61.049999, 62.174999, 81.425003, 82.925003, 3.655,4.03, SURF_ID='UPHOLSTERY'/ Base \&OBST XB=61.049999, 61. 424999, 81.425003,82.925093,4.03,4.405, SURE-ID='UPHOLSTERY'/ Rack Ar

*** GROUP: ROW2

** GROUP: COUCH2 offset $=0.000000,0.000000,0.000000$ rotate $=270.000000$

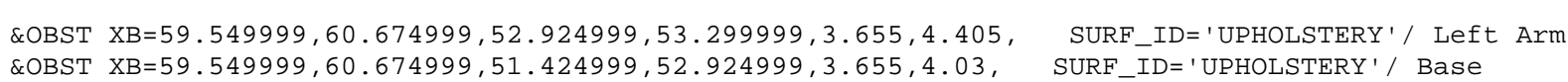

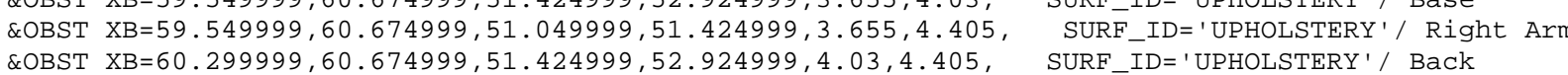

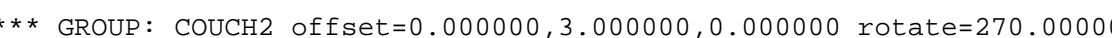

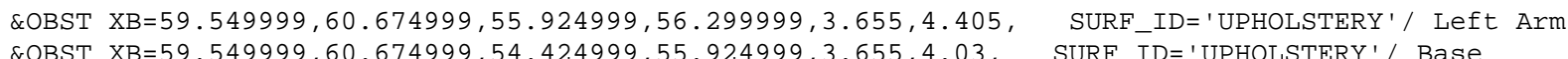

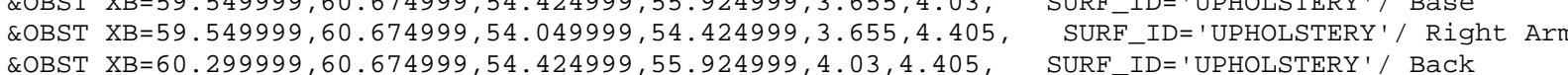

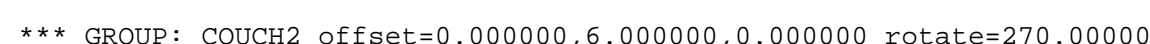

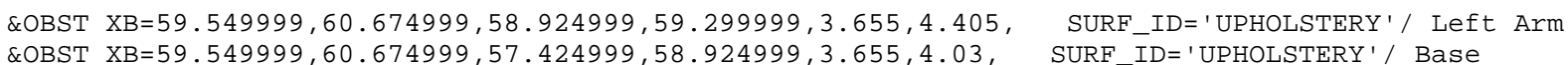

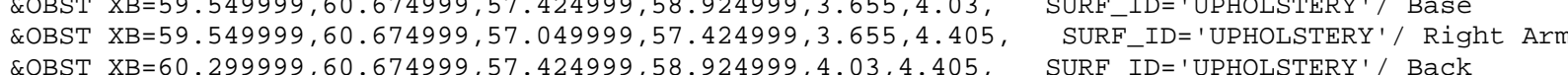
*** GROUP: COUCH2 offset $=0.000000,9.000000,0.000000$ rotate $=270.000000$

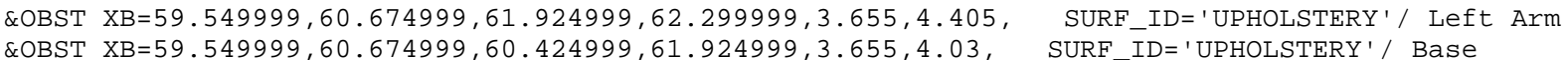

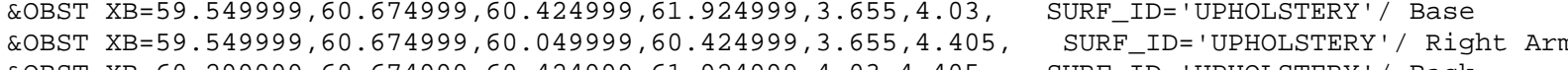

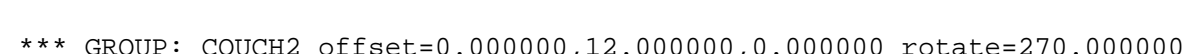

\&OBST XB=59.549999, 60.674999, 64.925003, 65.300003,3.655, 4.405, SURF_ID=' 'UPHOLSTERY '/ Left Arm

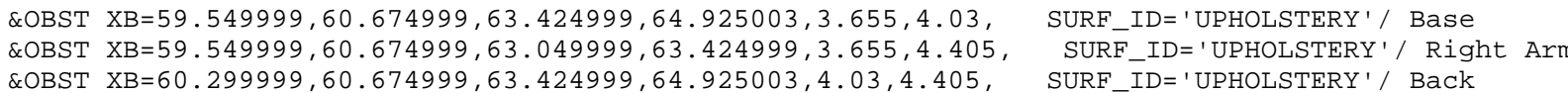
*** GROUP: COUCH2 offset $=0.000000,15.000000,0.000000$ rotate $=270$. ๑००००००

\&OBST XB=59.549999, 60.674999, 67.925003, 68.300003,3.655, 4.405, SURF_ID=' 'UPHOLSTERY '/ Left Arm

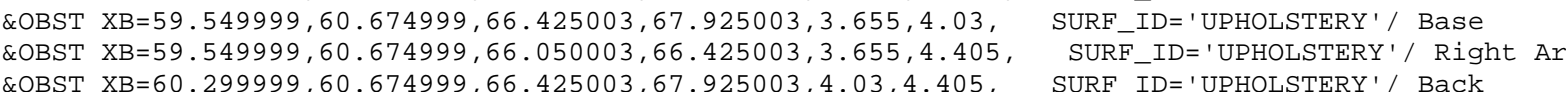

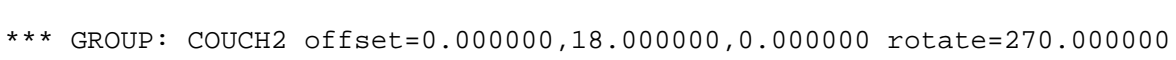

\&OBST XB=59.549999, 60.674999, 70.925003, 71.300003,3.655, 4.405, SURF_ID=' 'UPHOLSTERY '/ Left Arm

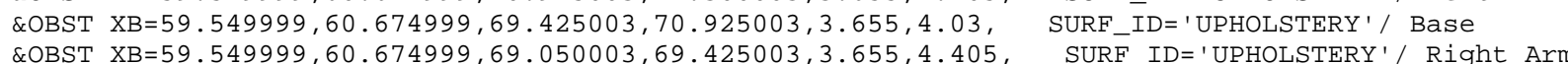

\&OBST XB=60, 299999, 60. 674999, 69, 425003, 70, 925003, 4. 03, 4. 405, SURF_ID=' UPHOLSTERY'/ Back

*** GROUP: COUCH2 offset $=0.000000,21.000000,0.000000$ rotate $=270.000000$

\&OBST XB $=59.549999,60.674999,73.925003,74.300003,3.655,4.405$, SURF_ID='UPHOLSTERY' / Left Arm \&OBST XB $59.549999,60.674999,72.425003,73.925003,3.655,4.03$, SURF_ID=' 'UPHOLSTERY'/ Base COBST XB

*** GROUP: COUCH2 offset $=0.000000,24.000000,0.000000$ rotate $=270.000000$

\&OBST XB=59.549999, 60.674999, 76.925003, 77. 300003, 3.655, 4.405, SURF_ID=' 'UPHOLSTERY'/ Left Arm \&OBST XB $=59.549999,60.674999,75.425003,76.925003,3.655,4.03$, SURF_ID='UPHOLSTERY'/ Base LOBST XB $=59.549999,60.674999,75.050003,75.425003,3.655,4.405, \quad$ SURF_IDE' 'UPHOLSTERY'/ Right
\&OBST

*** GROUP: COUCH2 offset $=0.000000,27.000000,0.000000$ rotate $=270.000000$

\&OBST XB $=59.549999,60.674999,79.925003,80.300003,3.655,4.405$, SURF_ID=' UPHOLSTERY'/ Left Arm \&OBST XB=59.54999, 60.674999, $78.425003,79.925003,3.655,4.03, \quad$ SURF_ID='UPHOLSTERY'/ $/$ Base

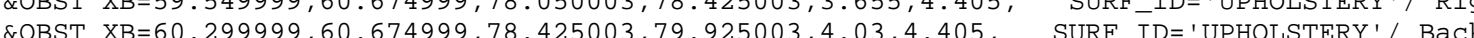

*** GROUP: COUCH2 offset $=0.000000,30.000000,0.0000000$ rotate $=270.000000$

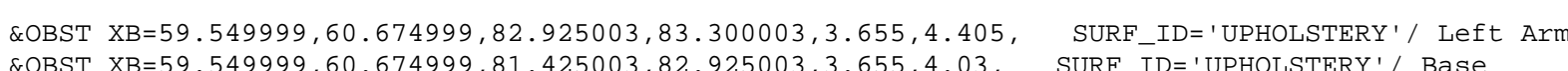

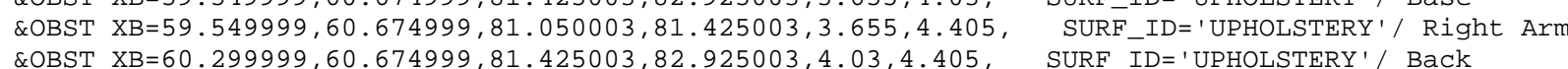

*** GROUP: TWIN

*** GROUP: RoW

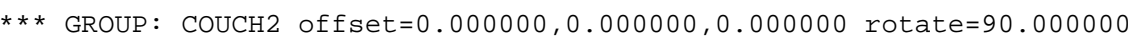

\&OBST XB $=65.550003,66.675003,51.049999,51.424999,3.655,4.405$, SURF_ID=' 'UPHOLSTERY '/ Left Arm

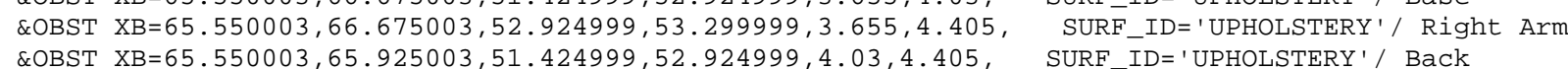

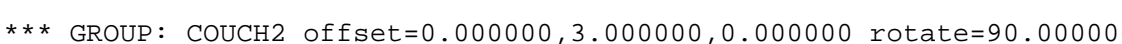

\&OBST XB=65. $550003,66.675003,54.049999,54.424999,3.655,4.405$, SURF_ID= 'UPHOLSTERY' / Left Arm

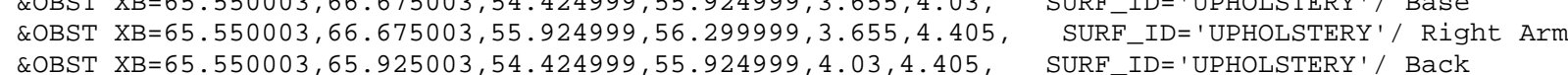

*** GROUP: COUCH2 offset $=0.000000,6.000000,0.000000$ rotate $=90$. .

\&OBST XB=65.550003, 66.675003, 57. 049999, 57.424999, 3.655, 4.405, SURF_ID='UPHOLSTERY' / Left Arm \&OBST XB $=65.550003,66.675003,58.924999,59.299999,3.655,4.405$, SURF SURF_IDD='UPHOLSTERY' $/$ Right Ar \&OBST XB=65.550003, 65.925003, 57.424999, 58.924999, 4.03,4.405, SURF_ID=' 'UPHOLSTERY'/ Back

*** GROUP: COUCH2 offset $=0.000000,9.000000,0.000000$ rotate $=90.000000$

\&OBST XB=65.550003, 66.675003, 60. 0499999, 60.424999, 3.655, 4.405, SURFIDD='UPHOLSTERY ' Left Arm
EOBST XB=65.550003,66.67503, \&OBST XB-65.550003,66.675003, 61.92499,62.29999, 3.655,4.405, SUPF ID='UPHOLSTEPY'/ \&OBST XB $=65.550003,65.925003,60.424999,61.924999,4.03,4.405$, SURF_ID=' 'UPHOLSTERY'/ Back

*** GROUP: COUCH2 offset $=0.000000,12.000000,0.000000$ rotate $=90.000000$

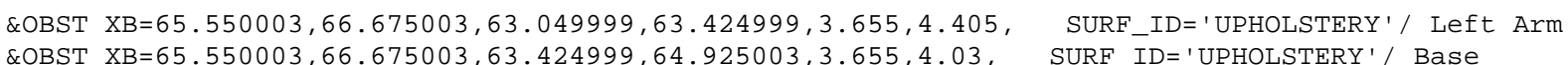

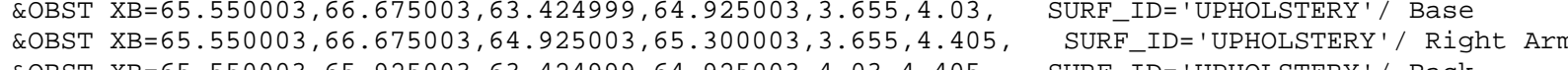

*** GROUP: COUCH2 offset $=0.000000,15.000000,0.000000$ rotate $=90.000000$

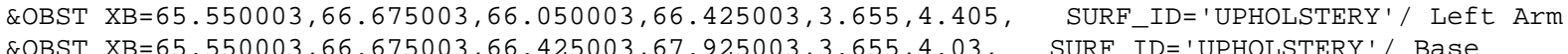
\&OBST XB $=65.550003,66.675003,67.925003,68.300003,3.655,4.405, \quad$ SURF_ID=' 'UPHOLSTERY '/ Right Arm
\&OBST XB=65.550003,65.925003,66.425003,67.925003,4.03,4.405, SURF_ID='UPHOLSTERY'/ Back

*** GROUP: COUCH2 offset $=0.000000,18.000000,0.000000$ rotate $=90.000000$ 
\&OBST XB $65.550003,66.675003,69.050003,69.425003,3.655,4.405, \quad$ SURF_ID=' 'UPHOLSTERY '/ Left Arm
\&OBST XB $=65.550003,66.675003,69.425003,70.925003,3.655,4.03$,
SURF_ID='UPHOLSTERY' $/$ Base \&OBST XB $65.550003,66.675003,70.925003,71.300003,3.655,4.405$, SURF_ID='UPHOLSTERY'/ Right Ar

*** GROUP: COUCH2 offset $=0.000000,21.000000,0.000000$ rotate $=90.000000$

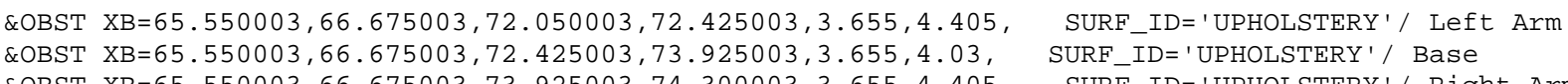

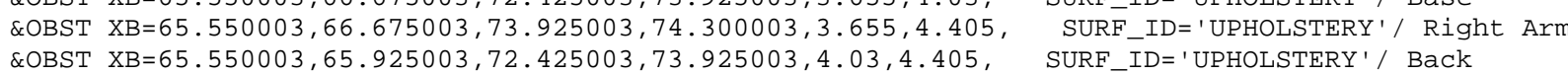

${ }^{* * *}$ GROUP: COUCH2 offset $=0.000000,24.000000,0.000000$ rotate $=90.000000$

\&OBST XB $=65.550003,66.675003,75.050003,75.425003,3.655,4.405, \quad$ SURF_ID= ' 'UPHOLSTERY ' / Left Arm \&OBST XB=65.550003,66.675003,76.925003,77.300003,3.655,4.405, SULRF_ID='UPHOLSTERY'/ Right ArI

*** GROUP: COUCH2 off set $=0.000000,27.000000,0.000000$ rotate $=90.000000$

\&OBST $X \mathrm{~B}=65.550003,66.675003,78.050003,78.425003,3.655,4.405$, SURF_ID=' UPHOLSTERY'/ Left Arm \&OBST XB $=65.550003,66.675003,78.425003,79.925003,3.655,4.03$, SURF IDD='UPHOLSTERY'/ Base Arm

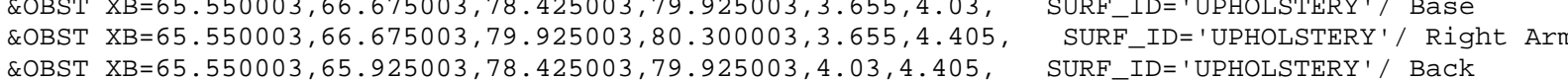

*** GROUP: COUCH2 offset $=0.000000,30.0000000,0.0000000$ rotate $=90.0000000$

\&OBST XB $=65.550003,66.675003,81.050003,81.425003,3.655,4.405$, SURF_ID='UPHOLSTERY' / Left Arm

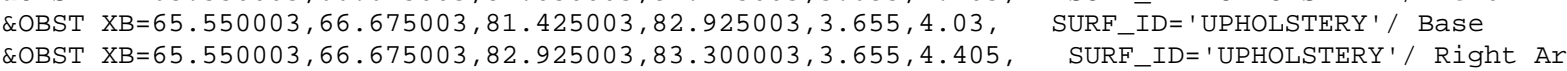
dOBST XB $65.550003,65.025003,82.425003,82.9250303,4.03,4.405$, SUPF - ID=' UPHOLSTERY / Right ATM

*** GROUP: ROW2

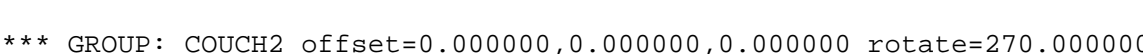

\&OBST XB $=64.050003,65.175003,52.924999,53.299999,3.655,4.405$, SURF_ID=' 'UPHOLSTERY '/ Left Arm

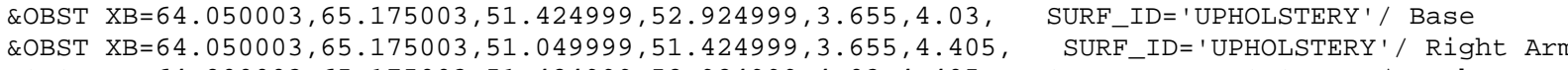
*** GROUP: COUCH2 offset $=0.000000,3.000000, \Theta .000000$ rotate $=270.000000$

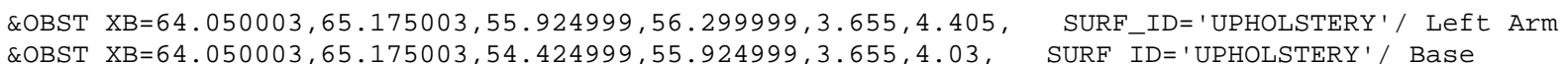

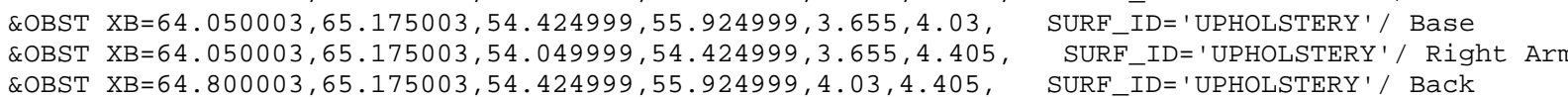

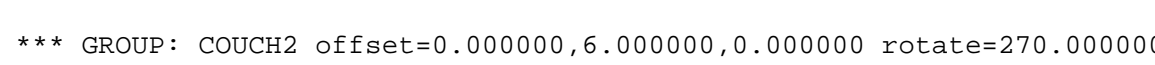
QOBST XB $=64.050003,65.175003,58.924999,59.299999,3.655,4.405, \quad$ SURF_ID=' 'UPHOLSTERY '/ Left Arm
\&OBST XBE $64.050003,65.175003,57.42499,58.924999,3.655,4.03$, SURF_ID='UPHOLSTER'

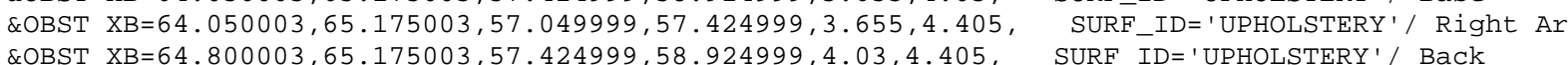

*** GROUP: COUCH2 offset $=0$. .

\&OBST XB=64.050003, 65.175003, 61.924999, 62. 299999, 3.655, 4.405, SURF_ID='UPHOLSTERY '/ Left Arm \&OBST XB=64.050003, 65.175003,60.424999, 61.924999, 3.655, 4.03, SURF_ID='UPHOLSTERY'/ Base \&OBST XB=64. $850003,65.175003,60.049999,60.424999,3.655,4.405$,
\&OBST XB=64.800003, $65.175003,60.424999,61.924999,4.03,4.405$,

*** GROUP: COUCH2 offset $=0.000000,12.000000,0.000000$ rotate $=270.000000$

\&OBST XB $=64.050003,65.175003,64.925003,65.300003,3.655,4.405$, SURF_ID=' 'UPHOLSTERY'/ Left Arm \&OBST XB=64.050003,65.175003,63.424999,64.925003,3.655,4.03, SURF_ID='UPHOLSTERY'/ Base Arm \&OBST XB=64. $050003,65.175003,63.049999,63.424999,3.655,4.405$, SURF-IDE' UPHOLSTERY'/ Right Ar $\star \star \star$ GROUP: COUCH2 offset $=0.000000,15.000000,0.000000$ rotate $=270.000000$

\&OBST XB $=64.050003,65.175003,67.925003,68.300003,3.655,4.405$, SURF_ID=' 'UPHOLSTERY'/ Left Arm

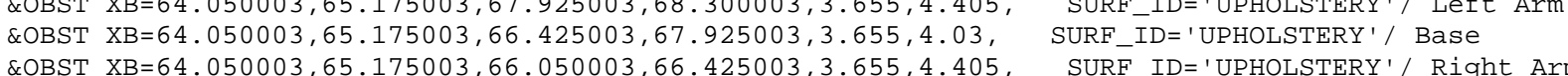
\&OBST XB=64. $050003,65.175003,66.050003,66.425003,3.655,4.405$,
\&OSST XB
*** GROUP: COUCH2 offset $=0.000000,18.000000,0.000000$ rotate $=270.000000$

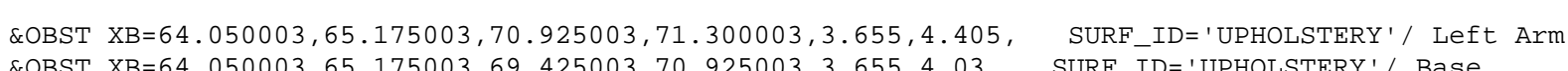

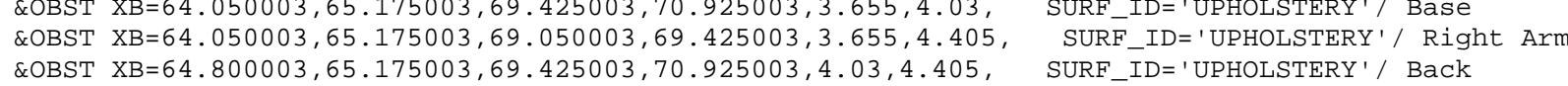

*** GROUP: COUCH2 offset $=0.0000000,21.000000,0.000000$ rotate $=270.000000$

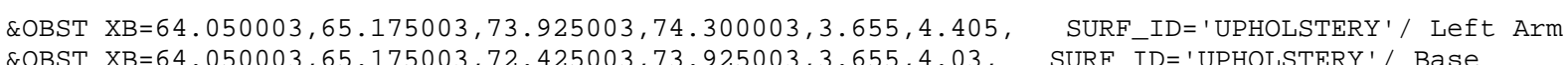

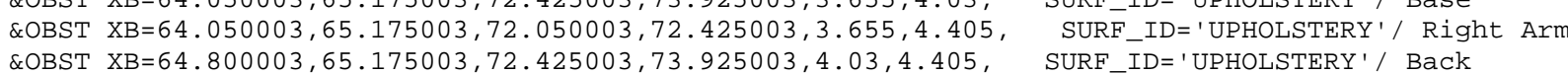

*** GROUP: COUCH2 offset $=0.000000,24.000000,0.000000$ rotate $=270.000000$

\&OBST XB=64. $050003,65.175003,76.925003,77.300003,3.655,4.405$, SURF_ID='UPHOLSTERY ' / Left Arm

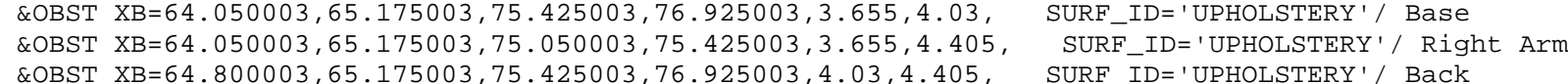

*** GROUP: COUCH2 offset $=0.000000,27.000000,0.000000$ rotate $=270.000000$

\&OBST XB $=64.050003,65.175003,79.925003,80.300003,3.655,4.405, \quad$ SURF_ID= 'UPHOLSTERY ' / Left Arm
\&OBST XB $=64.050003,65.175003,78.425003,79.925003,3.655,4.03$,
SURF_ID ='UPHOLSTERY'

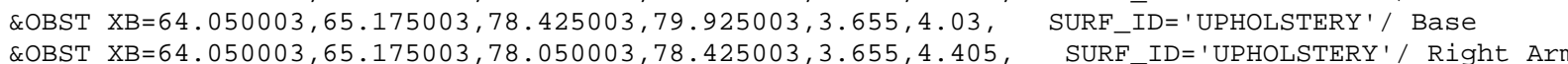
\&OBST XB $=64.800003,65.175003,78.425003,79.925003,4.03,4.405$, SURF_ID='UPHOLSTERY' / Back

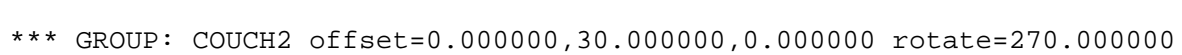

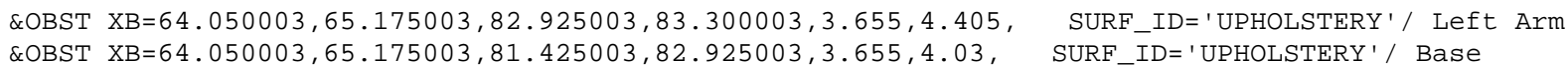

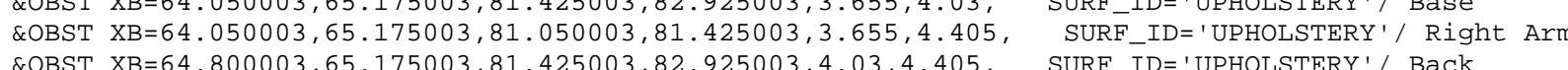

*** GROUP: TWIN

$* * *$ GROUP: ROW

** GROUP: COUCH2 offset $=0.000000$, ๑. ๑00000, ๑. ๑००००00 rotate $=90.000000$

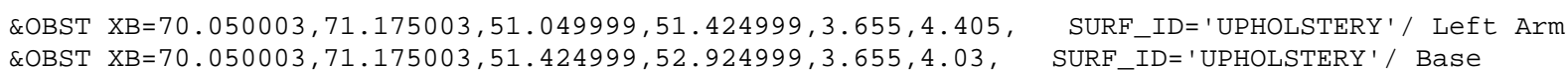

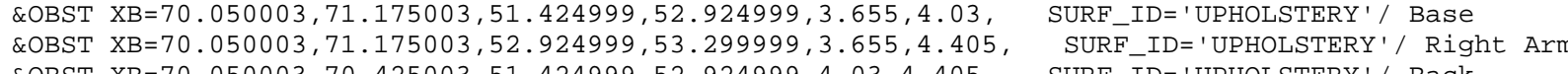

*** GROUP: COUCH2 offset $=0.000000$, 3. .000000, ๑. .000000 rotate $=90.000000$

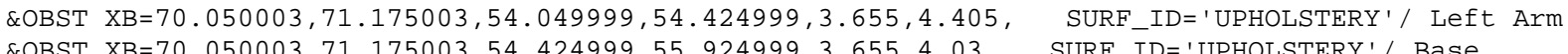

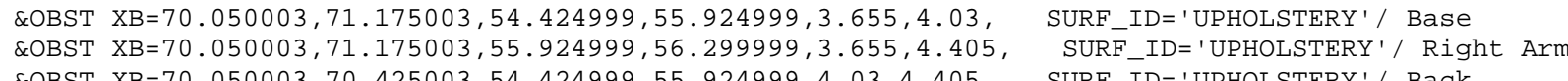

*** GROUP: COUCH2 offset $=0.000000,6.000000,0.000000$ rotate $=90.000000$

\&OBST XB $=70.050003,71.175003,57.049999,57.424999,3.655,4.405$, SURF_ID=' 'UPHOLSTERY'/ Left Arm \&OBST XB=70.050003,71.175003,57.424999,58.924999, $3.655,4.03$, SURF_IDE'UPHOLSTERY'/ Base

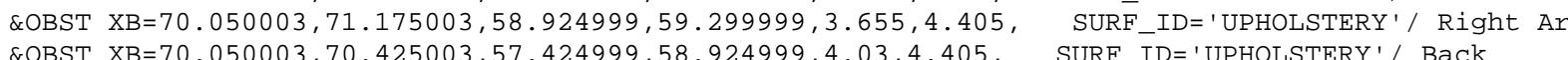

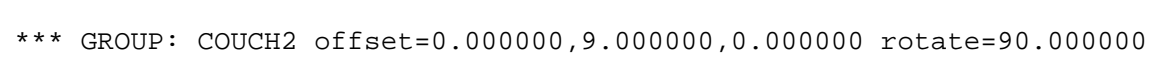

EOBST XB $=70.050003,71.175003,60.049999,60.424999,3.655,4.405$, SURF_ID=' 'UPHOLSTERY'/ Left Arm \&OBST XB $=70.050003,71.175003,60.424999,61.924999,3.655,4.03, \quad$ SURF_ID='UPHOLSTERY'/ Base

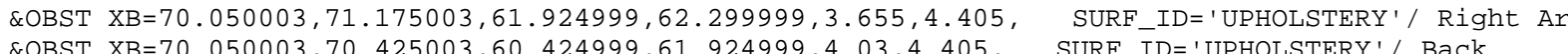

*** GROUP: COUCH2 offset $=0.0000000,12.000000,0.000000$ rotate $=90.000000$

\&OBST XB=70.050003, 71.175003,63.049999, 63.424999, 3.655, 4.405, SURF_ID='UPHOLSTERY'/ Left Arm \&OBST XB=70.050003, 71.175003, 63.424999,64.925003, 3.655,4.03, SURF_ID='UPHOLSTERY'/ Base \&OBST $X$ B.70.7050

*** GROUP: COUCH2 offset $=0.000000,15.000000,0.000000$ rotate $=90.000000$ 
\&OBST $X B=70.050003,71.175003,66.050003,66.425003,3.655,4.405, \quad$ SURF_ID= 'UPHOLSTERY ' $/$ Left Arm
\&OBST XB $\mathrm{X}=70.050003,71.175003,66.425003,67.925003,3.655,4.03, \quad$ SURF_ID='UPHOLSTERY' $/$ Base

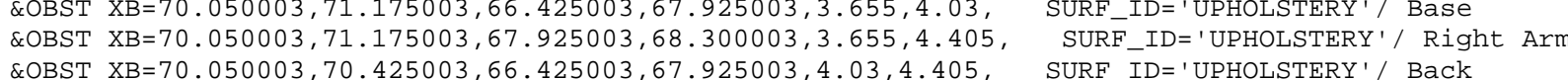
*** GROUP: COUCH2 offset $=0.000000,18.000000,0.000000$ rotate $=90.0000000$

\&OBST XB=70. $050003,71.175003,69.050003,69.425003,3.655,4.405, \quad$ SURF_ID=' 'UPHOLSTERY ' / Left Arm

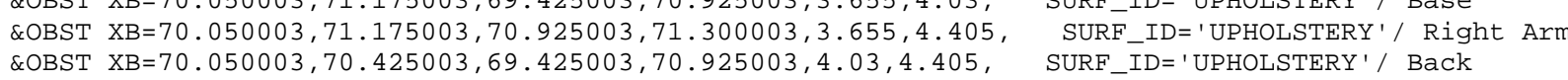
*** GROUP: COUCH2 offset $=0.000000,21.000000,0.000000$ rotate $=90.000000$

\&OBST XB=70. $050003,71.175003,72.050003,72.425003,3.655,4.405, \quad$ SURF_ID=' 'UPHOLSTERY'/ Left Arm

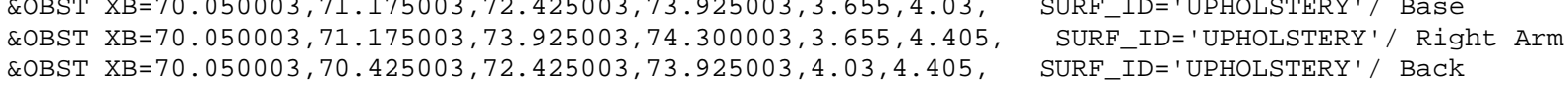
*** GROUP: COUCH2 offset $=0.000000,24.000000,0.000000$ rotate $=90.000000$

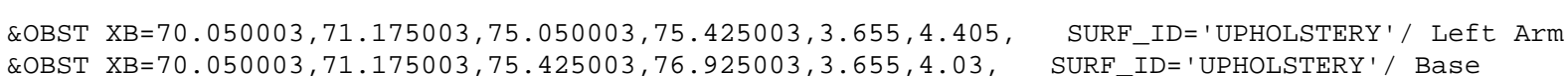

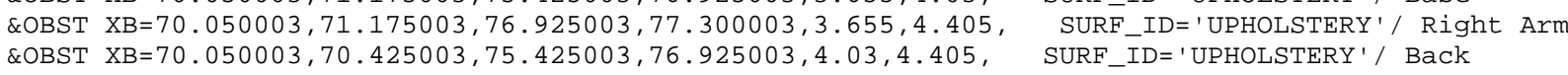

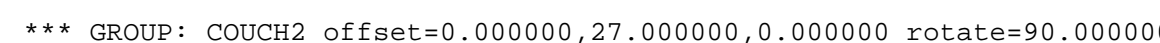

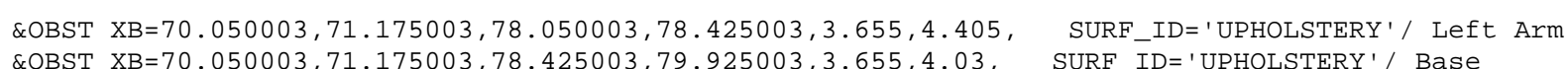

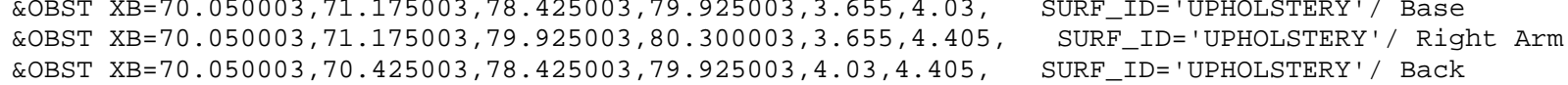
*** GROUP: COUCH2 offset $=0.000000,30.000000,0.000000$ rotate $=90.000000$

\&OBST XB $=70.050003,71.175003,81.050003,81.425003,3.655,4.405$, SURF_ID='UPHOLSTERY' '/ Left Arm

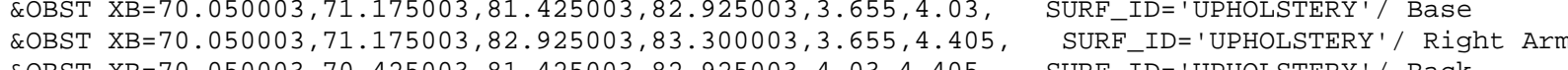

\section{$* * *$ GROUP: ROW2}

$* * *$ GROUP: COUCH2 offset $=0.000000,0.000000,0.000000$ rotate $=270.000000$

\&OBST XB=68.550003, 69.675003, 52.924999, 53.299999, 3.655, 4.405, SURF_ID=' 'UPHOLSTERY'/ Left Arm \&OBST XB $=8.5500003,69.675003,51.424999,52.924999,3.655,4.03, \quad$ SURF-IDE' 'UPHOLSTERY'/ Base

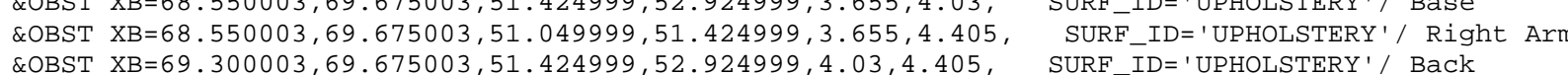

*** GROUP: COUCH2 offset $=0.000000,3.000000,0.000000$ rotate $=270.000000$

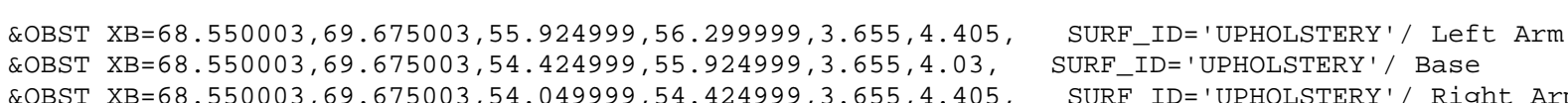

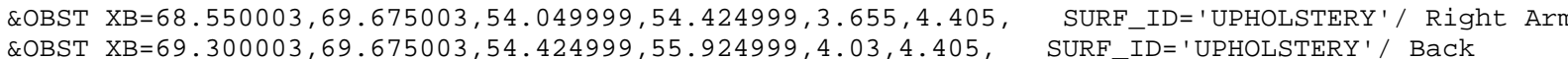

*** GROUP: COUCH2 offset $=0.000000,6.000000,0.000000$ rotate $=270.000000$

\&OBST XB=68.550003, 69.675003, 58.924999, 59.299999, 3.655, 4.405, SURF_ID=' 'UPHOLSTERY ' / Left Arm

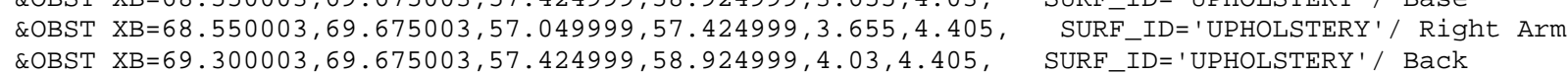

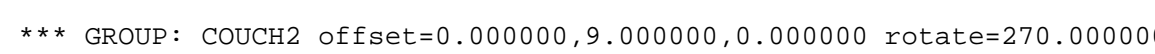

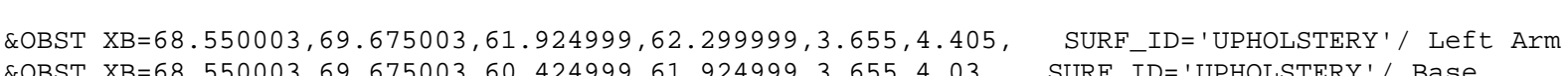
\&OBST XB $=68.550003,69.675003,60.049999,66.424999,3.655,4.405, \quad$ SURF_ID='UPHOLSTERY'/ Right Arm
\&OBST XB $=69.30003,69.675003,60.424999,61.924999,4.03,4.405$,
SURF_ID='UPHOLSTERY'/ Back *** GROUP: COUCH2 offset $=0.000000,12.000000,0.000000$ rotate $=270.000000$

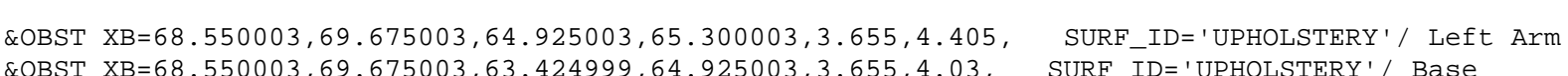

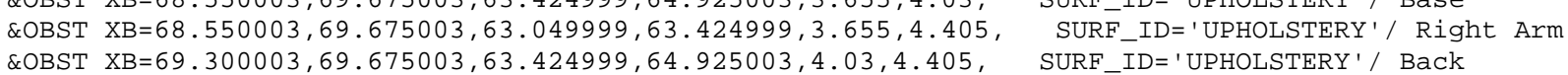

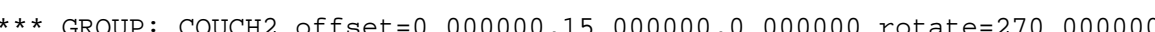

\&OBST XB=68.550003, $69.675003,67.925003,68.300003,3.655,4.405, \quad$ SURF_ID='UPHOLSTERY ' / Left Arm
\&OBST XB=68.550003,69.675003,66.425003,67.925003,3.655,4.03, SURF_ID='UPHOLSTERY'

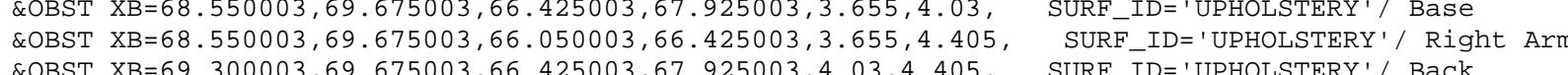

*** GROUP: COUCH2 offset $=0.000000,18.000000,0.000000$ rotate $=270.000000$

\&OBST XB $=68.5500003,69.675003,70.925003,71.300003,3.655,4.405$, SURF_ID= 'UPHOLSTERY' ' Left Arm \&OBST XB=68.550003,69.675003,69.425003,70.925003,3.655,4.03, SURF_ID='UPHOLSTERY'/ Base \&OBST XB $=69.300003,69.675003,69.425003,70.925003,4.03,4.405$, SURF_ID='UPHOLSTERY'/ Back Ar

*** GROUP: COUCH2 offset $=0.000000,21.0000000,0.000000$ rotate $=270.000000$

\&OBST XB $=68.550003,69.675003,73.925003,74.300003,3.655,4.405, \quad$ SURF_ID=' 'UPHOLSTERY '/ Left Arm
\&OBST XB $=68.550003,69.675003,72.425003,73.925003,3.655,4.03$, SURF_ID='UPHOLSTERY'/ Base

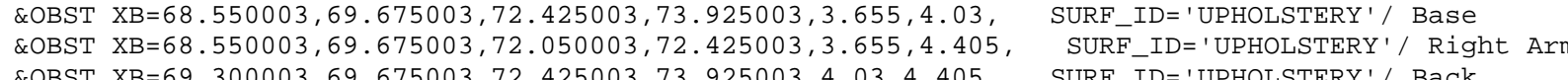
*** GROUP: COUCH2 offset $=0.000000,24.000000,0.000000$ rotate $=270.0000000$

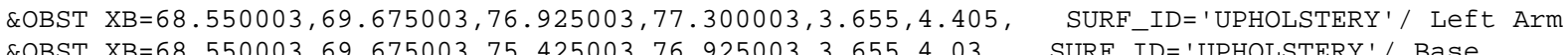
\&OBST XB $=68.550003,69.675003,75.425003,76.925003,3.655,4.03, \quad$ SURF_ID='UPHOLSTERY'/ Base

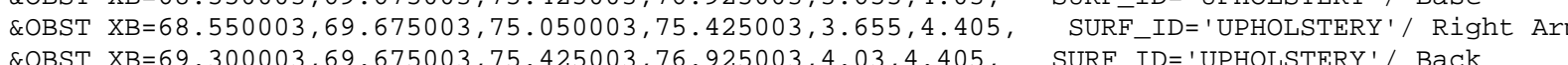

*** GROUP: COUCH2 offset $=0.000000,27.000000,0.000000$ rotate $=270.000000$

\&OBST XB $=68.550003,69.675003,79.925003,80.300003,3.655,4.405$, SURF_ID=' 'UPHOLSTERY'/ Left Arm

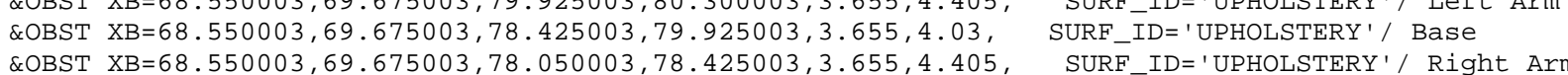

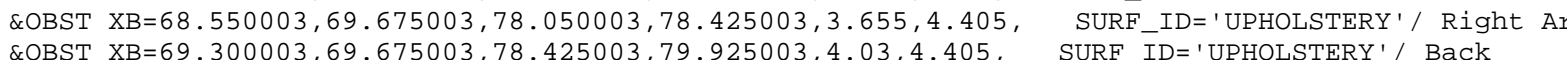

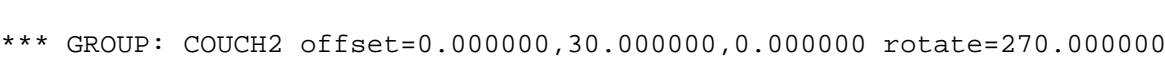

\&OBST XB $=68.550003,69.675003,82.925003,83.300003,3.655,4.405$, SURF_ID=' 'UPHOLSTERY '/ Left Arm \&OBST XB $=68.550003,69.675003,81.425003,82.925003,3.655,4.03$, SURF_ID='UPHOLSTERY' / Base

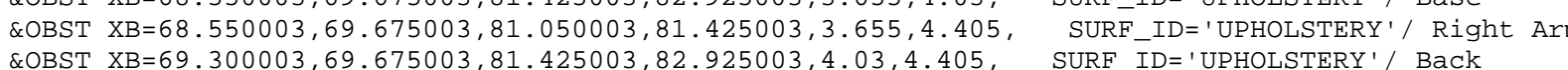

*** GROUP: ROW2

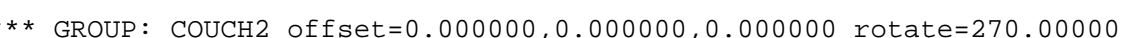

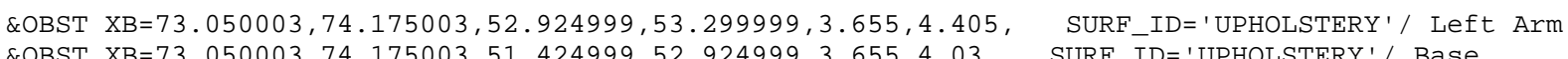

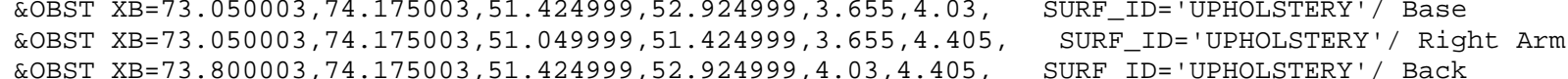

*** GROUP: COUCH2 offset $=0.000000,3.000000,0.000000$ rotate $=270.000000$

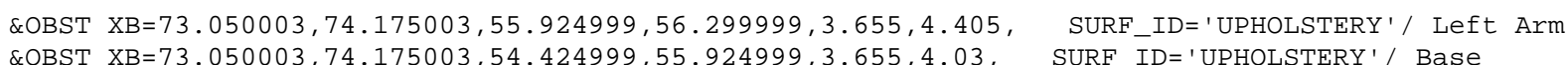

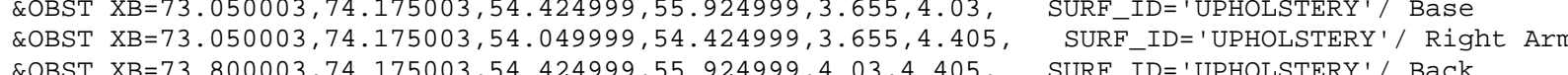

*** GROUP: COUCH2 offset $=0.000000,6.0000000,0.000000$ rotate $=270.000000$

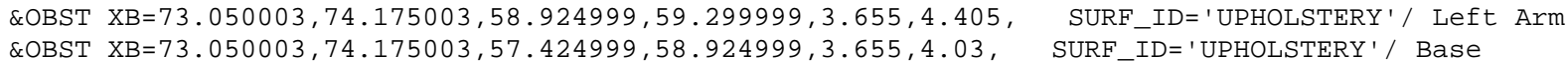
COBST XB XB
QOBS

*** GROUP: COUCH2 offset $=0.000000,9.000000,0.000000$ rotate $=270.000000$

\&OBST XB=73.050003, 74.175003, 61. 924999, 62.299999, 3.655, 4.405, SURF_ID='UPHOLSTERY'/ Left Arm

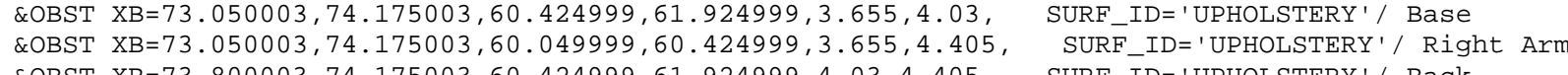

*** GROUP: COUCH2 offset $=0.000000,12.000000,0.000000$ rotate $=270.000000$

\&OBST XB $=73.050003,74.175003,64.925003,65.300003,3.655,4.405$, SURF_ID=' 'UPHOLSTERY '/ Left Arm

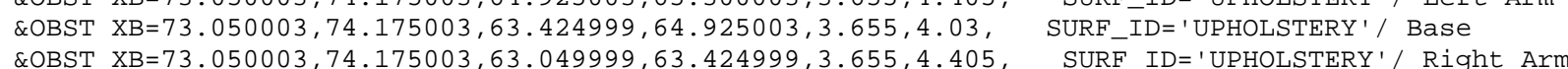


\&OBST XB $=73,800000,74.175003,63,424999,64,925003,4,03,4,405$, SURE_ID=' UPHOLSTERY' / Back *** GROUP: COUCH2 offset $=0.000000,15$. $.00000,0.000000$ rotate $=270.000000$

\&OBST XB $=73.050003,74.175003,67.925003,68.300003,3.655,4.405$, SURF_ID='UPHOLSTERY ' / Left Arm \&OBST XB=73.050003, 74.175003, 66. $050003,66.425003,3.655,4.405$, SURF_ID=' 'UPHOLSTERY'/ Right Arm

*** GROUP: COUCH2 offset $=0.000000,18.000000,0.000000$ rotate $=270.000000$

\&OBST XB=73.050003, 74.175003, 70.925003, 71.300003,3.655, 4.405, SURF_ID=' 'UPHOLSTERY'/ Left Arm \&OBST XB=73.050003, 74.175003, 69.425003,70.925003,3.655,4.03, SURF_IDD='UPHOLSTERY'/ Base QOBST $X \mathrm{~B}=73.050003,74.175003,69.050003,69.425003,3.655,4.405$, SURF-ID='UPHOLSTYRY/Right Ar

*** GROUP: CoUCH2 offset $=0.000000,21.000000,0.000000$ rotate $=270.000000$

\&OBST XB $=73.050003,74.175003,73.925003,74.300003,3.655,4.405$, SURF_ID=' 'UPHOLSTERY'/ Left Arm \&OBST XB=73.050003, 74.175003, 72.425003,73.925003,3.655,4.03, SURF_ID='UPHOLSTERY'/ Base

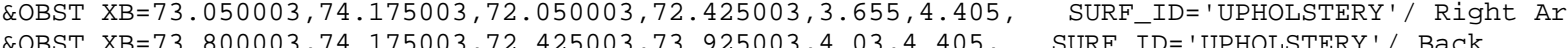
$* * *$ GROUP: COUCH2 offset $=0.000000,24.000000,0.000000$ rotate $=270.000000$

\&OBST XB $=73.050003,74.175003,76.925003,77.300003,3.655,4.405$, SURF_ID=' 'UPHOLSTERY' / Left Arm \&OBST XB $=73.050003,74.175003,75.050003,75.425003,3.655,4.405$, SURF_ID=' 'UPHOLSTERY'/ Right Ar

*** GROUP: COUCH2 offset $=0.000000,27.000000,0.000000$ rotate $=270.000000$

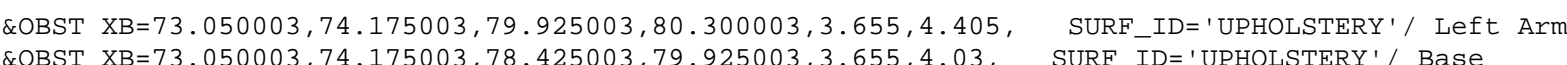

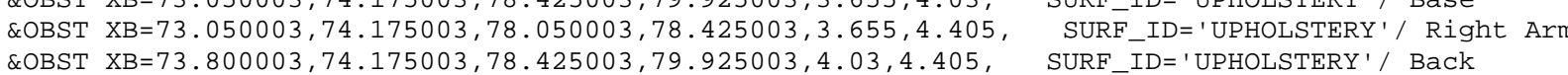
*** GROUP: COUCH2 offset $=0,000000,30 ., 000000,0,000000$ rotate $=270,000000$

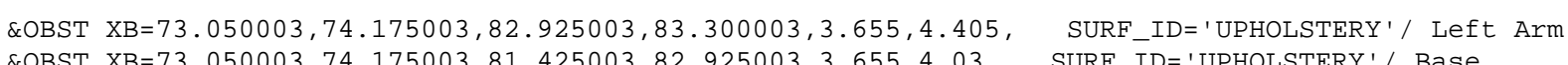

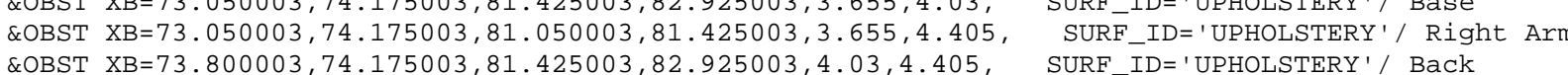

\section{** GROUP: SHORT_ROW}

*** GROUP: COUCH2 offset $=0.000000,0.000000,0.000000$ rotate $=90.000000$

\&OBST XB $=67.044998,67.419998,46.91,48.035,3.655,4.405$, SURF_ID=' 'UPHOLSTERY' / Left Arm

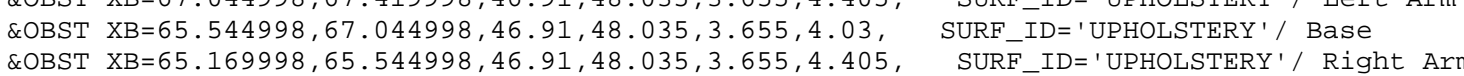
COBST $X \mathrm{~B}=65.169998,65.544998,46.91,48.035,3.655,4.405, \quad$ SURF_ID='UPHOLSTERY '/ Right Ar
COBST XB=65.544998,67.044998, 46.91,47.285,4.03,4.405, SURF_ID='UPHOLSTERY'/ Back

*** GROUP: COUCH2 offset $=0.000000,3.000000,0.000000$ rotate $=90.000000$

\&OBST XB $=64.044998,64.419998,46.91,48.035,3.655,4.405$, SURF_ID=' 'UPHOLSTERY'/ Left Ar OOBST XB $=62.544998,64.044998,46.91,48.035,3.655,4.03$,
SOBST \&OBST XB $=62.169998,62.544998,46.91,48.035,3.655,4.405, \quad$ SURF_ID='UPHOLSTERY'
\&OBST XB Right
B $=62.544998,64.044998,46.91,47.285,4.03,4.405$,

$* \star *$ GROUP: COUCH2 offset $=0.000000,6.000000,0.000000$ rotate $=90.000000$

\&OBST XB $61.044998,61.419998,46.91,48.035,3.655,4.405$, SURF_ID='UPHOLSTERY'/ Left Arm \&OBST XB $59.544998,61.044998,46.91,48.035,3.655,4.03$, SURF ID = 'UPHOLSTERY'/ Base \&OBST XB $=59.169998,59.544998,46.91,48.035,3.655,4.405$,
\&OBST XB $=59.544998,61.044998,46.91,47.285,4.03,4.405$,

*** GROUP: COUCH2 offset $=0.000000,9.000000,0.000000$ rotate $=90.000000$

\&OBST XB=58. $044998,58.419998,46.91,48.035,3.655,4.405, \quad$ SURF_ID=' UPHOLSTERY' / Left Arm

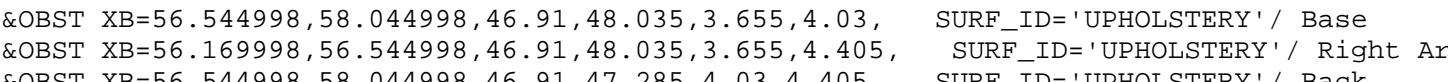

*** GROUP: COUCH2 offset $=0.000000,12.000000,0 . .000000$ rotate $=90.000000$

\&OBST XB=55.044998, 55.419998, 46.91, 48.035, 3.655,4.405, SURF_ID='UPHOLSTERY' / Left Arm
ROBST XB $=53.544998,55,044998,46.91,48,035,3.655,4.03$, SURF ID=' 'UPHOLSTERY'/, Base COBST $X \mathrm{~B}=53.169998,53.544998,46.91,48.035,3.655,4.405$,
\&OBST $X \mathrm{~B}=53.544998,55.044998,46.91,47.285,4.03,4.405$,

$* * *$ GROUP: COUCH2 offset $=0.000000,15.000000,0.000000$ rotate $=90.000000$

\&OBST XB=52. 044998, 52.419998, 46.91, 48. 035, 3.655, 4.405, SURF_ID=' 'UPHOLSTERY' / Left Arm \&OBST XB $=50.56998,50.544998,46,91,48.035,3.055,4,405$, \&OBST XB $\mathrm{B}=50.544998,52.044998,46.91,47.285,4.03,4.405, \quad$ SURF_ID='UPHOLSTERY'/ Back
\&

*** GROUP: COUCH2 offset $=0.000000,18.000000,0.000000$ rotate $=90.000000$

\&OBST XB $=49.044998,49.419998,46.91,48.035,3.655,4.405, \quad$ SURF_ID='UPHOLSTERY' / Left Arm
\&OBST XB $=47.544998,49.044998,46.91,48.035,3.655,4.03$,

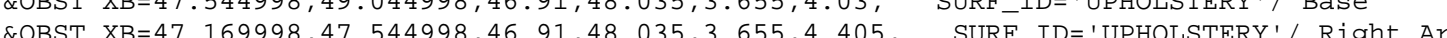
\&OBST XB $=47.544998,49.044998,46.91,47.285,4.03,4.405$, SURF_ID=' 'UPHOLSTERY'/ Back

*** GROUP: ROW

$* * *$ GROUP: COUCH2 offset $=0.000000,0.000000,0.000000$ rotate $=90.000000$

\&OBST XB=41.549999, $41.924999,84.43,85.555,3.655,4.405$, SURF_ID='UPHOLSTERY'/ Left Arm \&OBST XB $=41.924999,43.424999,84.43,85.555,3.655,4.03$, SURF-ID='UPHOLSTERY'/ Base LOBST XB $43.424999,43.799999,84.43,85.555,3.655,4.405$, SURF_ID 'UPHOLSTERY'/ Right Ar

*** GROUP: COUCH2 offset $=0.000000,3.000000,0.000000$ rotate $=90.000000$

\&OBST XB=44.549999, 44.924999, 84.43, 85.555, 3.655, 4.405, SURF_ID=' 'UPHOLSTERY' / Left Arm \&OBST $X \mathrm{XB}=46.424999,46.799999,84.43,85.555,3.655,4.405, \quad$ SURF_ID='UPHOLSTERY'/ Right Arm
\&OBST XB=44.924999, $46.424999,85.18,85.555,4.03,4.405$,

*** GROUP: COUCH2 offset $=0.000000,6.000000,0.000000$ rotate $=90.000000$

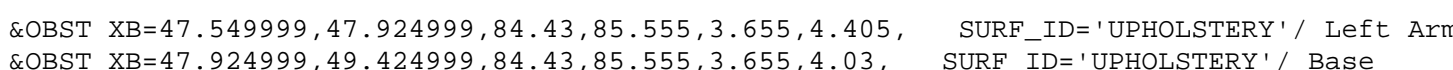
COBST XB=49.424999, 49. $799999,84.43,85.555,3.655,4.405, \quad$ SURF_ID='UPHOLSTERY'/ Right Arm
\&OBST XB $=47.924999,49.424999,85.18,85.555,4.03,4.405, \quad$ SURF_ID='UPHOLSTERY'/ Back *** GROUP: COUCH2 offset $=0.000000,9$. . ००००००, $\odot . .000000$ rotate $=90.000000$

\&OBST $X \mathrm{XB}=50.549999,50.924999,84.43,85.555,3.655,4.405, \quad$ SURF_ID= ' ' UPHOLSTERY ' / Left Arm

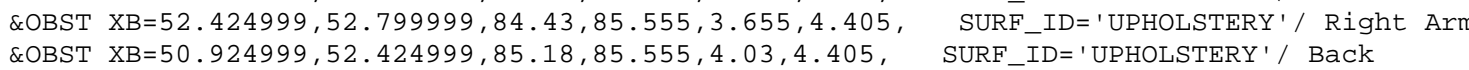

*** GROUP: COUCH2 offset $=0.000000,12.000000,0.000000$ rotate $=90.000000$

\&OBST XB $=53.549999,53.924999,84.43,85.555,3.655,4.405$, SURF_ID=' 'UPHOLSTERY' / Left Ar

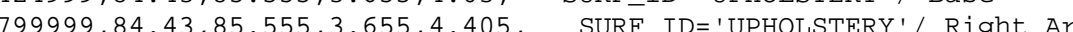
QOBST XB $=53.924999,55.424999,85.18,85.555,4.03,4.405$, SURF_ID='UPHOLSTERY'/ Back

*** GROUP: COUCH2 offset $=0.000000,15.000000,0.000000$ rotate $=90.000000$

\&OBST XB=56.549999, 56. 924999, 84.43, 85.555, 3.655, 4.405, SURF-ID=' 'UPHOLSTERY' / Left Ar \&OBST XB=58.424999, 58.799999, 84.43, 85.555, 3.655,4.405, SURF_ID='UPHOLSTERY'/ Right Arm
\&OBST XB=56.924999, $58.424999,85.18,85.555,4.03,4.405, \quad$ SURF_ID='UPHOLSTERY' 1 Back

*** GROUP: COUCH2 offset $=0.000000,18.000000,0.000000$ rotate $=90.000000$

\&OBST XB=59. 549999, 59.924999, 84. $43,85.555,3.655,4.405, \quad$ SURF_ID='UPHOLSTERY' '/ Left Arm
\&OBST XB=59.924999, $61.424999,84.43,85.555,3.655,4.03$,

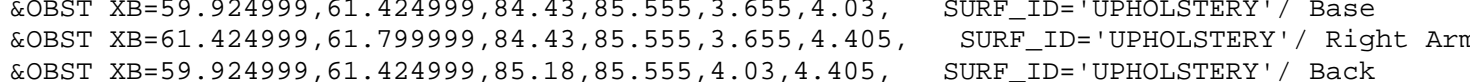

*** GROUP: COUCH2 offset $=0.000000,21.000000,0.000000$ rotate $=90.000000$

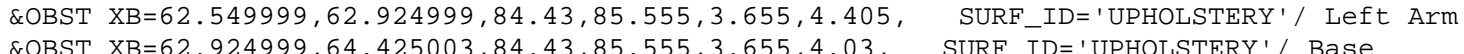

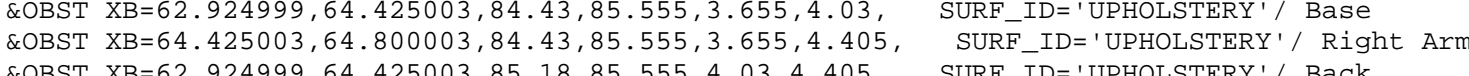

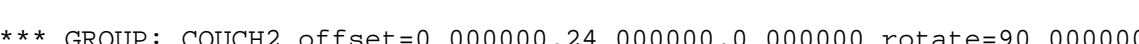




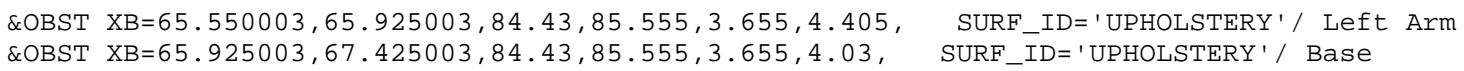

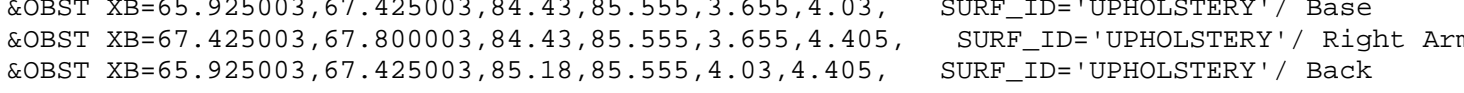

*** GROUP: COUCH2 offset $=0.000000,27.000000,0.000000$ rotate $=90.000000$

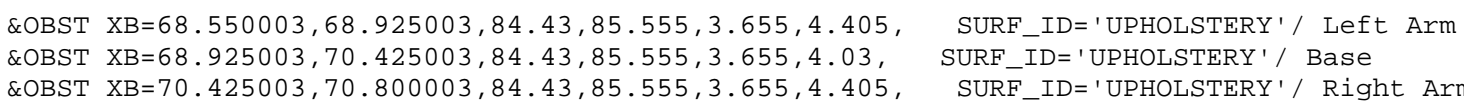
COBST $X \mathrm{XB}=70.425003,70.800003,84.43,85.555,3.655,4.405, \quad$ SURF_ID=' 'UPHOLSTERY'/ Right
\&OBST $\mathrm{XB}=68.925003,70.425003,85.18,85.555,4.03,4.405, \quad$ SURF_ID='UPHOLSTERY'/ Back

*** GROUP: COUCH2 offset $=0.000000,30.000000,0.000000$ rotate $=90.000000$

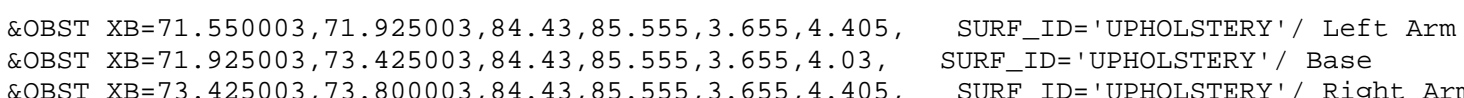

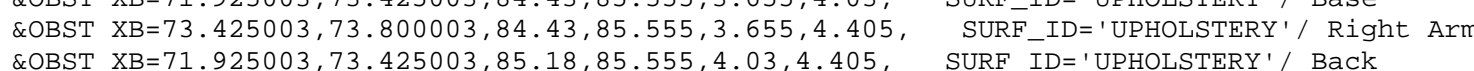

*** GROUP: LEVEL

$* * *$ GROUP: ROW

*** GROUP: COUCH2 offset $=0.000000,0.000000,0.000000$ rotate $=90.000000$

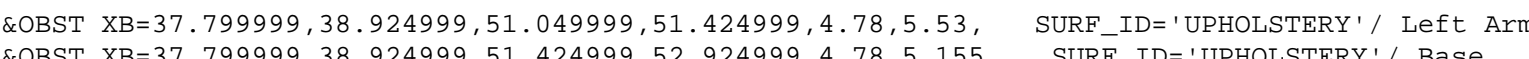

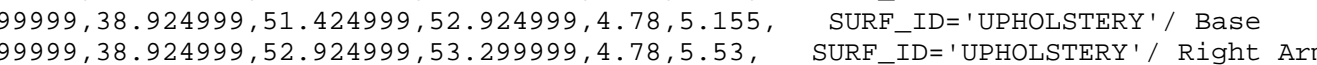
ROBST XB =37.799999, 38.174999, 51.424999, 52.924999, 5.155, 5.53, SURF_ID=' 'UPHOLSTERY' / Back

** GROUP: COUCH2 offset $=0.000000,3.000000,0.0000000$ rotate $=90 . .000000$

\&OBST XB=37.799999, 38. 924999, 54.049999, 54.424999, 4.78,5.53, SURF_ID='UPHOLSTERY'/ Left Arm \&OBST XB $=37.799999,38.924999,54.424999,55.924999,4.78,5.155$, SURF-ID=' UPHOLSTERY' '/ Base

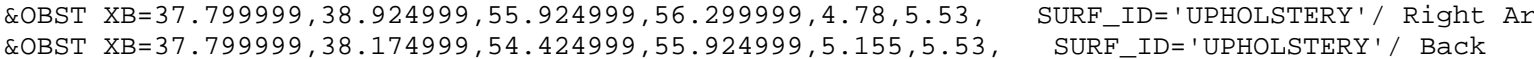

*** GROUP: COUCH2 offset $=0.000000,6.000000,0.000000$ rotate $=90.000000$

\&OBST XB=37.799999, 38. 924999, 57. .49999, 57.424999, 4.78, 5.53, SURF_ID=' 'UPHOLSTERY'/ Left Arm \&OBST XB=37.799999, 38.924999, 57.424999, 58. 924999, 4.78, 5.155, SURF_ID=' 'UPHOLSTERY' / Base \&OBST XB=37.79999, 38. 924999, 58.92499, 59.299999, 4.78,5.53, $\quad$ SURF_ID='UPHOLSTERY'/ Right Ar
\&OBST XB $=37.799999,38.174999,57.424999,58.924999,5.155,5.53, \quad$ SURF_ID=' 'UPHOLSTERY' $/$ Back

*** GROUP: COUCH2 offset $=0.000000,9.000000,0.000000$ rotate $=90.000000$

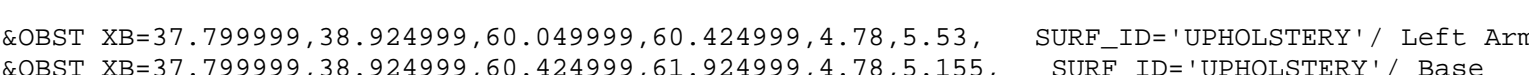
\&OBST $X \mathrm{XB}=37.799999,38.924999,61.924999,62.299999,4.78,5.53$,
\&OBST $X \mathrm{XB}=37.799999,38.174999,60.424999,61.924999,5.155,5.53, \quad$ SURF_ID='UPHOLSTERY'/ Right Arm
SURF_ID=' 'UPHOLSTERY' / Back

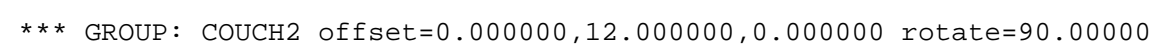

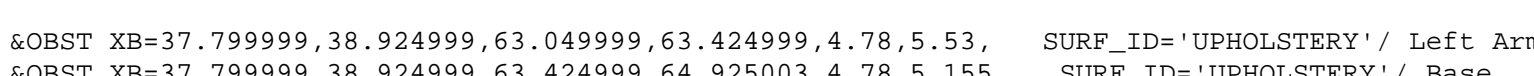

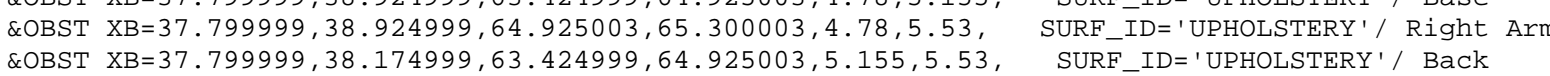

*** GROUP: COUCH2 offset $=0.000000,15.000000,0.000000$ rotate $=90, .000000$

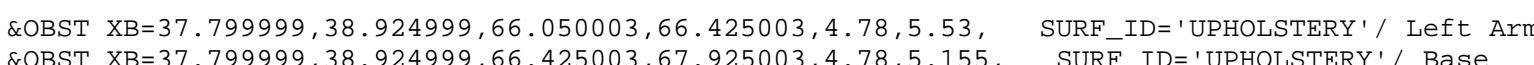
COBST $X \mathrm{XB}=37.799999,38.924999,67.925003,68.300003,4.78,5.53$,
\&OBST $X \mathrm{XB}=37.799999,38.174999,66.425003,67.925003,5.155,5.53, \quad$ SURF_ID='UPHOLSTERY'/ Right Arm
SURF_ID=' 'UPHOLSTERY'/ Back

*** GROUP: COUCH2 offset $=0.000000,18.000000,0.000000$ rotate $=90.000000$

\&OBST XB=37.799999, 38.924999, 69. 050003, 69.425003, 4.78, 5.53, SURF_ID=' 'PPHOLSTERY' ' Left Arm

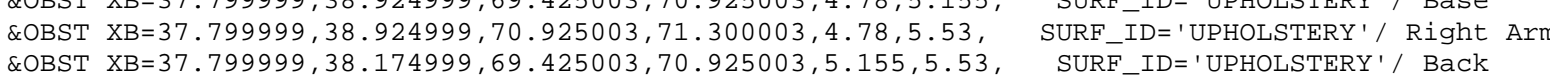
*** GROUP: COUCH2 offset $=0.000000,21.000000,0.000000$ rotate $=90.000000$

\&OBST XB=37. $799999,38.924999,72.050003,72.425003,4.78,5.53, \quad$ SURF_ID= 'UPHOLSTERY' $/$ ' Left Arm
\&OBST XB=37.79999, $38.924999,72.425003,73.925003,4.78,5.155$,
SURF_ID=' 'UPHOLSTERY' / Base

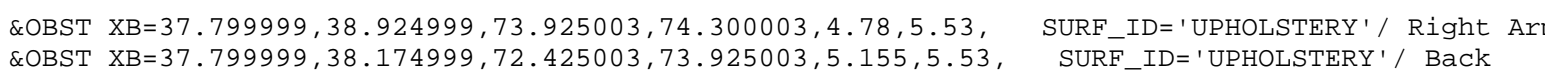

*** GROUP: COUCH2 offset $=0.000000,24.000000,0.000000$ rotate $=90.000000$

\&OBST XB=37. $799999,38.924999,75.050003,75.425003,4.78,5.53, \quad$ SURF_ID=' 'UPHOLSTERY'/' Left Arm
\&OBST XB=37.799999, 38.924999, $75.425003,76.925003,4.78,5.155$,
SURF_ID='UPHOLSTERY'/ Base \&OBST XB $=37.799999,38.924999,76.925003,77.300003,4.78,5.53$, SURF IDE='UPHOLSTERY' '/ Right Ar

*** GROUP: COUCH2 offset $=0.000000,27.000000,0.000000$ rotate $=90 . .000000$ \&OBST XB $=37.799999,38.924999,78.050003,78.425003,4.78,5.53, \quad$ SURF_ID=' 'UPHOLSTERY ' / Left Arm
\&OBST XB $=37.799999,38.92499,78.425003,79.925003,4.78,5.155, \quad$ SURF
IID ' UPHOLSTRRY' $/$ Base

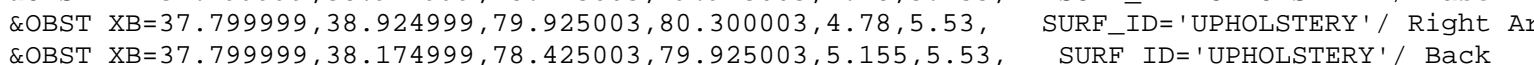

*** GROUP: COUCH2 offset $=0.000000,30.000000,0.000000$ rotate $=90 . .000000$

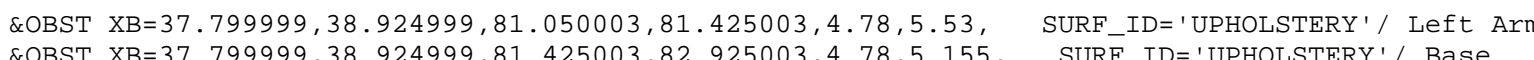

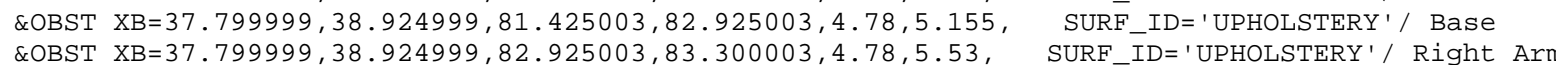

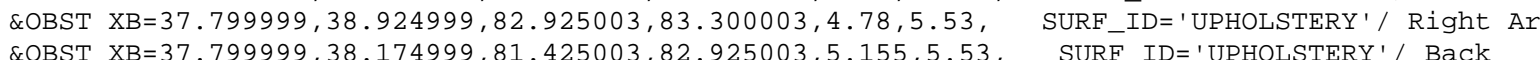

*** GROUP: BASE

$* \star \star$ GROUP: TWIN

*** GROUP: ROW

*** GROUP: COUCH2 offset $=0.000000,0.000000,0.000000$ rotate $=90.000000$

\&OBST XB=43. $049999,44.174999,51.049999,51.424999,4.78,5.53$, SURF_ID=' 'UPHOLSTERY' ' Left Arm

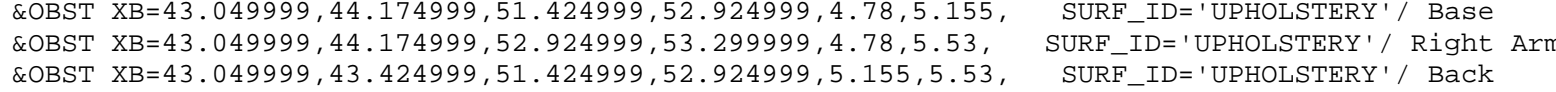

$* * *$ GROUP: COUCH2 offset $=0.0000000,3.000000,0.000000$ rotate $=90.000000$

\&OBST XB=43. 049999, 44.174999, 54. 049999, 54.424999, 4.78, 5.53, SURF_ID=' 'UPHOLSTERY' / Left Arm \&OBST XB=43. \&OBST XB=43. $049999,43.424999,54.424999,55.924999,5.155,5.53$, SURF_ID=' 'UPHOLSTERY' / Back

*** GROUP: COUCH2 offset $=0.000000,6.000000,0.000000$ rotate $=90.000000$

\&OBST XB=43. $049999,44.174999,57.049999,57.424999,4.78,5.53, \quad$ SURF_ID=' 'UPHOLSTERY' ' Left Arm
\&OBST $\mathrm{XB}=43.04999,44.174999,57.424999,58.924999,4.78,5.155$, SURE ID 'UPHOLSTEPY' \&OBST XB $=43.049999,44.174999,58,924999,59,299999,4.78,5.53$, SURF-ID='UPHOLSTERY'/ Right Arm LOBST XB=43. 049999, 43.424999, 57.424999, 58.924999, 5.155, 5.53, SURF_ID=' 'UPHOLSTERY' / Back

*** GROUP: COUCH2 offset $=0.000000,9.000000,0.000000$ rotate $=90.000000$

\&OBST XB=43. $049999,44.174999,60.049999,60.424999,4.78,5.53, \quad$ SURF_ID= 'UPHOLSTERY' ' Left Arm
\&OBST XB=43. $049999,44.174999,60.424999,61.924999,4.78,5.155$, SURF ID=' 'UPHOLSTERY' / Base \&OBST XB $=43.049999,44.174999,61.924999,62.299999,4.78,5.53$, SURF-ID='UPHOLSTERY' 1 Right Arm

*** GROUP: COUCH2 offset $=0.000000,12.000000,0.000000$ rotate $=90.0000000$

\&OBST XB $=43.049999,44.174999,63.049999,63.424999,4.78,5.53, \quad$ SURF_ID=' UPHOLSTERY' ' Left Arm
\&OBST XB $=43.049999,44.174999,63.424999,64.925003,4.78,5.155, \quad$ SURF_ID=' 'UPHOLSTERY' $/$ Base

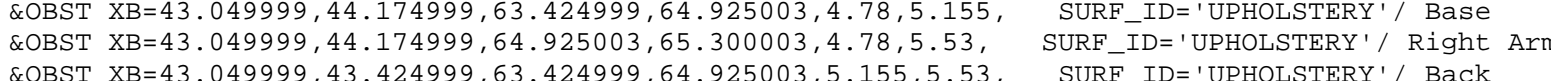

*** GROUP: COUCH2 offset $=0.000000,15.000000,0.000000$ rotate $=90.000000$

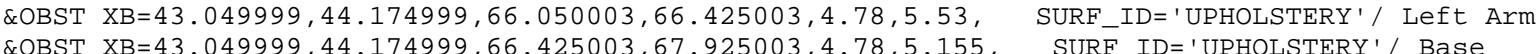

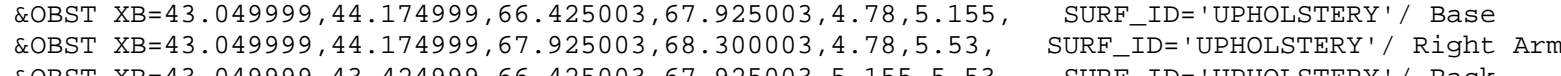

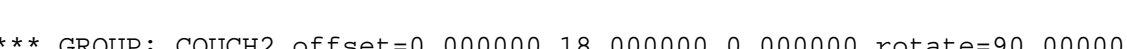

\&OBST XB=43. 049999, 44.174999, 69. $050003,69.425003,4.78,5.53$, SURF_ID= 'UPHOLSTERY ' / Left Arm $-47$ 
\&OBST XB=43, $049999,43.424999,69,425003,70,925003,5,155,5.53$, SURE_ID=' UPHOLSTERY' / Back ** GROUP: COUCH2 offset $=0.000000,21.000000,0.000000$ rotate $=90.000000$

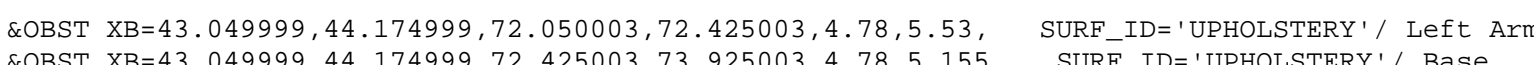
\&OBST XB=43. $049999,44.174999,73.925003,74.300003,4.78,5.53$, SURF_ID='UPHOLSTERY'/ Right Ar

*** GROUP: COUCH2 offset $=0.000000,24.000000,0.000000$ rotate $=90.000000$

\&OBST XB $=43.049999,44.174999,75.050003,75.425003,4.78,5.53$, SURF_ID='UPHOLSTERY'/ Left Arm \&OBST XB $=43.049999,44.174999,75.425003,76.925003,4.78,5.155$, SURF_ID='UPHOLSTERY' '/ Base

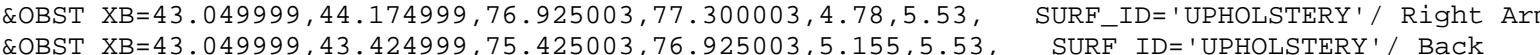

*** GROUP: COUCH2 offset $=0.000000,27.000000,0.000000$ rotate $=90.000000$

\&OBST XB=43. $849999,44.174999,78.050003,78.425003,4.78,5.53$, SURF_ID=' UPHOLSTERY' ' Left Arm \&OBST XB $=43.049999,43.424999,78.425003,79.925003,5.155,5.53$ S SUPF_ID= ' UPPOLSTERY' / Back *** GROUP: COUCH2 offset $=0.000000,30.000000,0.000000$ rotate $=90.000000$

\&OBST XB=43. 049999, 44.174999, 81. 050003, 81.425003, 4.78,5.53, SURF_ID=' 'UPHOLSTERY' / Left Arm \&OBST XB=43.049999, 44.174999, 82.925003, 83.300003, 4.78,5.53, SURF_ID='UPHOLSTERY'/Right Ar \&OBST $X B=43.049999,43.424999,81.425003,82.925003,5.155,5.53$, SURF_ID='UPHOLSTERY' / Back

*** GROUP: ROW2

*** GROUP: COUCH2 offset $=0.000000,0.000000,0.000000$ rotate $=270.000000$

\&OBST XB $=41.549999,42.674999,52.924999,53.299999,4.78,5.53$, SURF_ID=' 'UPHOLSTERY' / Left Arm \&OBST XB=41.549999, $42.674999,51.424999,52.924999,4.78,5.155$, SURF_ID='UPHOLSTERY'/ Base

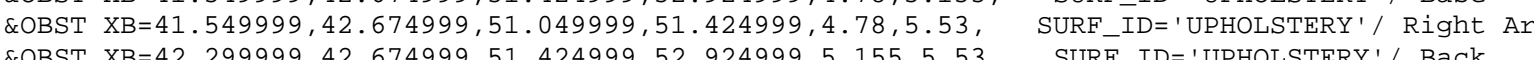
*** GROUP: COUCH2 offset $=0.000000,3.000000,0.000000$ rotate $=270.0000000$ \&OBST XB=41.549999, 42.674999, 55.924999, 56. 299999, 4.78, 5.53, $\quad$ SURF_ID=' 'UPHOLSTERY' 1 ' Left Arm
\&OBST XB=41.549999, 42.674999, $54.424999,55.924999,4.78,5.155$,
SURF_ID=' UPHOLSTERY'/ Base \&OBST XB=41.549999, 42.674999, 54. 4499999, 54.4244999,4.78,5.53, SURF-ID=' 'UPHOLSTERY'/ Right Ar

*** GROUP: COUCH2 offset $=0.000000,6.000000,0.000000$ rotate $=270.0000$

\&OBST XB=41.549999, 42.674999, 58.924999, 59. 299999, 4.78,5.53, SURF_ID=' 'UPHOLSTERY' / Left Arm \&OBST XB=41.549999, 42.674999, 57.424999, 58.924999, 4.78,5.155, SURF_ID='UPHOLSTERY'/ Base

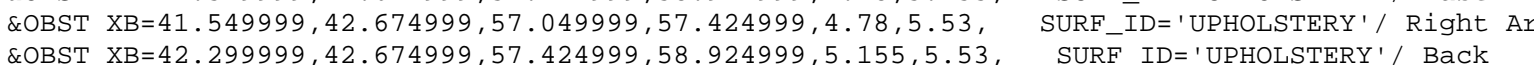

*** GROUP: COUCH2 offset $=0.000000,9.000000,0.000000$ rotate $=270.000000$

EOBST XB=41.549999, 42.674999, 61.924999, 62. 299999, 4.78, 5.53, SURF_ID=' 'UPHOLSTERY'/ Left Arm \&OBST XB=41.549999, $42.674999,60.424999,61.924999,4.78,5.155$, SURF_ID='UPHOLSTERY'/Base\&OBST XX=41.549999, $42.674999,60.049999,60.424999,4.78,5.53$, SURF_IDE' UPHOLSTERY' $/$ Right Ar

*** GROUP: COUCH2 offset $=0.000000,12.000000,0.000000$ rotate $=270.000000$

\&OBST XB $41.549999,42.674999,64.925003,65.300003,4.78,5.53$, SURF_ID=' 'UPHOLSTERY'/ Left Arm \&OBST XB $=41.549999,42.674999,63.424999,64.925003,4.78,5.155$, SURF_ID='UPHOLSTERY'/ Base

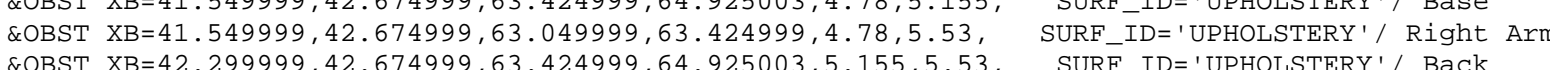

*** GROUP: COUCH2 offset $=0.000000,15.000000,0.000000$ rotate $=270.000000$

\&OBST $X B=41.549999,42.674999,67.925003,68.300003,4.78,5.53$, SURF_ID='UPHOLSTERY '/ Left Arm \&OBST XB=41.549999, 42.674999, 66.425003, 67.925003, 4.78, 5.155, SURF_ID=' 'UPHOLSTERY' '/ Base

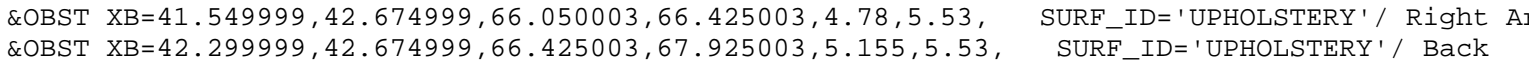

*** GROUP: COUCH2 offset $=0.000000,18.000000,0.000000$ rotate $=270.000000$

\&OBST XB=41.549999, $42.674999,70.925003,71.300003,4.78,5.53$, SURF_ID=' 'UPHOLSTERY' / Left Arm
\&OBST $X \mathrm{X}=41.549999,42.674999,69.425003,70.925003,4.78,5.155, \quad$ SURF_ID= 'UPHOLSTERY' / Base

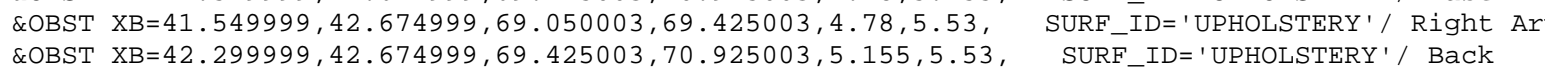
*** GROUP: COUCH2 offset $=0.000000,21.000000,0.000000$ rotate $=270.000000$

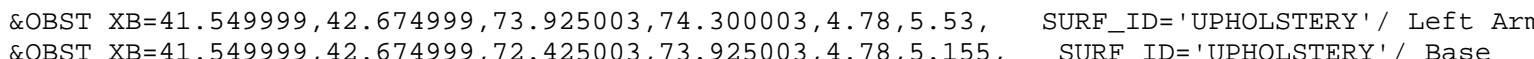
\&OBST XB $=41.549909,42.674999,72.05003,73,425003,4,78,5.55$, SUPF_TD='UPHOLSTERY'/ Right A COBST XB=42.299999, 42.674999, 72.425003, 73.925003,5.155, 5.53, SURF_ID=' 'UPHOLSTERY'/ Back

*** GROUP: COUCH2 offset $=0.000000,24.000000,0.000000$ rotate $=270.000000$

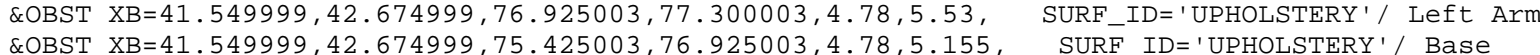

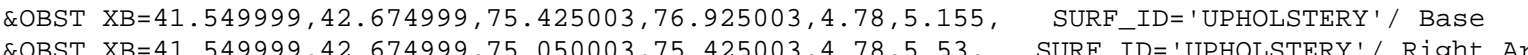
\&OBST XB $=41.549999,42.674999,75.050003,75.425003,4.78,5.53$,
\&OBST $X B=42.299999,42.674999,75.425003,76.925003,5.155,5.53$,
SURF_ID=' 'UPHOLSTERY'/ Right Ar

*** GROUP: COUCH2 offset $=0.000000,27.000000,0.000000$ rotate $=270.000000$

\&OBST XB $=41.549999,42.674999,79.925003,80.300003,4.78,5.53, \quad$ SURF_ID=' UPHOLSTERY' 1 Left Arm

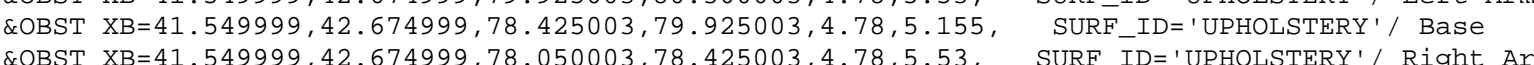
COBST $X B=42.299999,42.674999,78.425003,79.925003,5.155,5.53$, SURF ID=' 'UPHOLSTERY' / Back

*** GROUP: COUCH2 offset $=0.000000,30.0000000,0.000000$ rotate $=270.000000$

\&OBST XB=41.549999, 42.674999, 82.925003, 83.300003,4.78,5.53, SURF_ID='UPHOLSTERY'/ Left Arm COBST $X \mathrm{~B}=41.55999,42.67499,81.425003,82.925003,4.78,5.155$, SURF ID=' UPHOLSTERY'/ Base \&OBST $X \mathrm{~B}=41.549999,42.674999,81.050003,81.425003,4.78,5.53$,
\&OBST $X \mathrm{~B}=42.299999,42.674999,81.425003,82.925003,5.155,5.53$,
SURF_IDE'ID='UPHOLSTERY'/ Back

*** GROUP: TWIN

*** GROUP: ROW

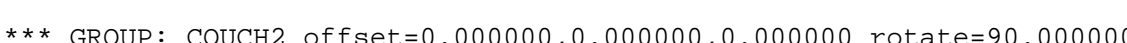

\&OBST XB $=47.549999,48.674999,51.049999,51.424999,4.78,5.53$, SURF_ID= ' UPHOLSTERY' / Left Arm

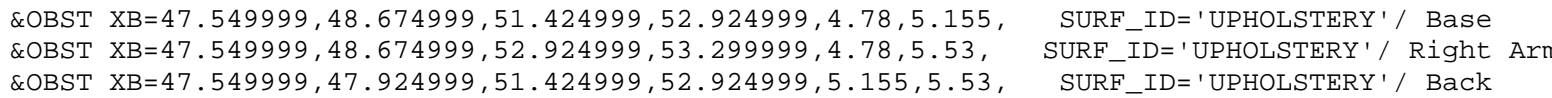

*** GROUP: COUCH2 offset $=0.000000,3.000000,0.000000$ rotate $=90.000000$

\&OBST XB $=47.549999,48.674999,54.049999,54.424999,4.78,5.53, \quad$ SURF_ID=' 'UPHOLSTERY' ' Left Arm
\&OBST XB $=47.549999,48.674999,54.424999,55.924999,4.78,5.155$,
SURF_ID='UPHOLSTERY'

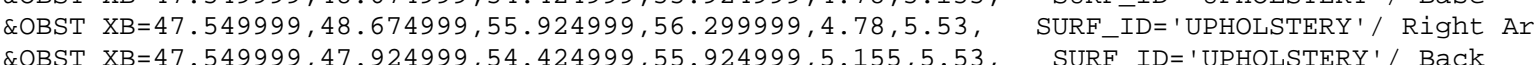

*** GROUP: COUCH2 offset $=0.000000,6.000000,0.000000$ rotate $=90.000000$

\&OBST XB $=47.549999,48.674999,57.049999,57.424999,4.78,5.53$, SURF_ID=' 'UPHOLSTERY' '/ Left Arm \&OBST XB=47.549999, 48.674999, 57.424999, 58.924999, 4.78, 5.155, SURF_ID='UPHOLSTERY'/ Base \&OBST XB $=47.549999,47.924999,57.424999,58.924999,5.155,5.53$ S SURF ID ='UPHOLSTERY' $/$ Back

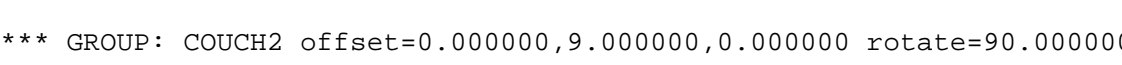

\&OBST XB=47.549999, 48.674999, 60. 049999, 60.424999, 4.78,5.53, SURF_ID='UPHOLSTERY'/ Left Arm

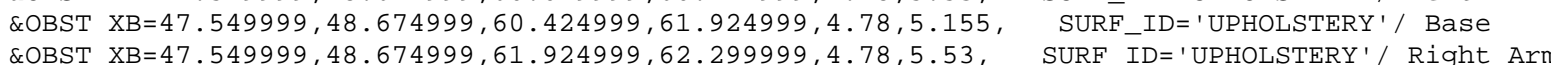
\&OBST XB=47.549999, 47.924999, 60.424999, 61.924999, 5.155, 5.53, SURF ID=' 'UPHOLSTERY'/ Back

*** GROUP: COUCH2 offset $=0.000000,12.000000,0.000000$ rotate $=90.000000$

\&OBST XB $=47.549999,48.674999,63.049999,63.424999,4.78,5.53$, SURF_ID=' UPHOLSTERY ' / Left Arm \&OBST XB $=47.549999,48.674999,63.424999,64.925003,4.78,5.155$, SURF ${ }_{\text {IDD ' 'UPHOLSTERY' }}$ Base ROBST XB=47.549999, $48.674999,64.925003,65.300003,4.78,5.53$,
\&OBST XB

$* * *$ GROUP: COUCH2 offset $=0.000000,15.000000,0.000000$ rotate $=90.000000$

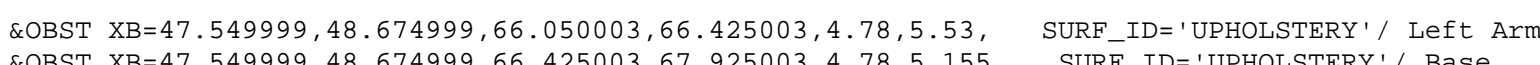
\&OBST XB=47.549999, 48.674999,67.925003,68.300003,4.78,5.53, SURF_ID='UPHOLSTERY'/Right Ar 
*** GROUP: COUCH2 offset $=0.000000,18.000000,0.000000$ rotate $=90.000000$ \&OBST XB=47.549999, 48.674999, 69. $050003,69.425003,4.78,5.53$, SURF_ID=' ' UPHOLSTERY ' / Left Arm \&OBST XB $=47.549999,48.674999,70.925003,71.300003,4.78,5.53$, SURF_ID='UPHOLSTERY'/ Right Ar \&OBST XB=47.549999, 47.924999, 69.425003, 70.925003, 5.155, 5.53, SURF_ID='UPHOLSTERY'/ Back *** GROUP: COUCH2 offset $=0.000000,21.000000,0.000000$ rotate $=90.000000$

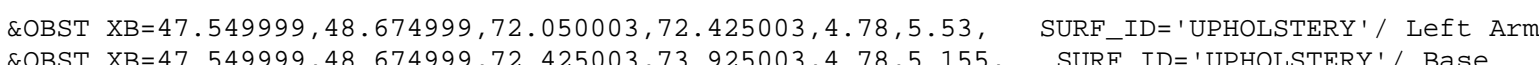

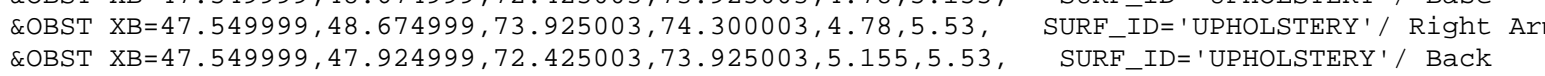

*** GROUP: COUCH2 offset $=0.000000,24.000000,0.000000$ rotate $=90.000000$

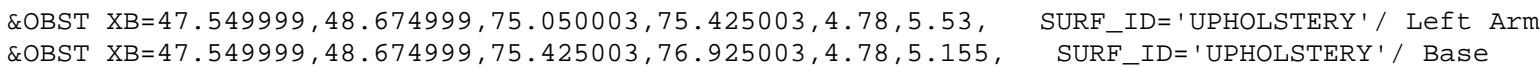

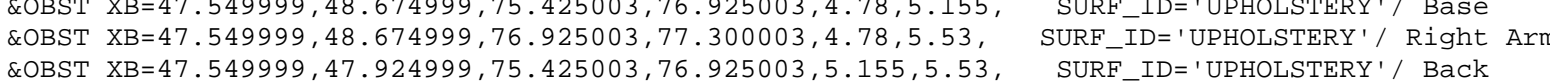
*** GROUP: COUCH2 offset $=0.000000,27.000000,0.000000$ rotate $=90.000000$

\&OBST XB=47.549999, $48.674999,78.050003,78.425003,4.78,5.53, \quad$ SURF_ID=' 'UPHOLSTERY ' ' Left Arm
\&OBST XB $=47.549999,48.674999,78.425003,79.925003,4.78,5.155, \quad$ SURF ID ' 'UPHOLSTERY' Q
\&OBST XB X $47.549999,48.674999,79.925003,80.300003,4.78,5.53$,

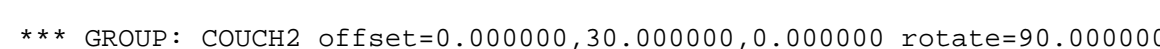

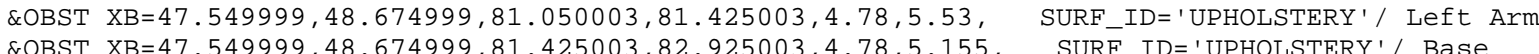

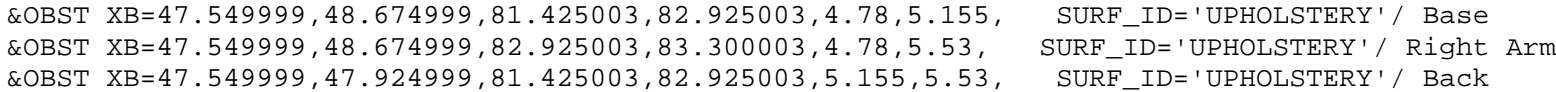

$* * *$ GROUP: ROW2

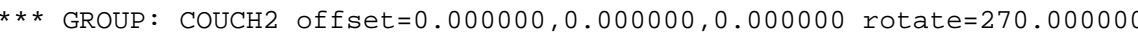

\&OBST XB $=46.049999,47.174999,52.924999,53.299999,4.78,5.53$, SURF_ID=' 'UPHOLSTERY '/ Left Arm \&OBST XB $=46.049999,47.174999,51.424999,52.924999,4.78,5.155, \quad$ SURF IDD='UPHOLSTERY'/ Base
SURE IDD='UPHOLSTERY'/Right Ar \&OBST XB=46. $049999,47.174999,51.049999,51.424999,4.78,5.53$,
\&OBST XB $=46.799999,47.174999,51.424999,52.924999,5.155,5.53$,
SURF_IDE='UPHOLSTERY'/ Right Ar

*** GROUP: COUCH2 offset $=0.000000,3.000000,0.000000$ rotate $=270.000000$

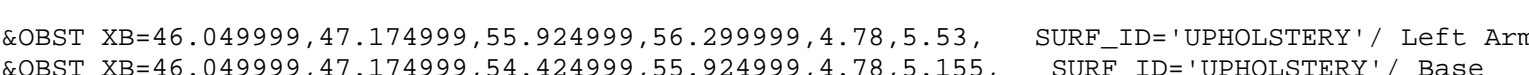

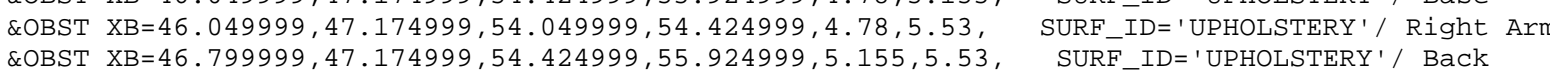

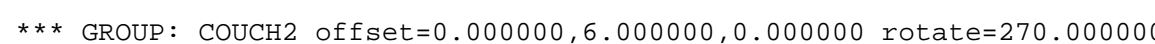

\&OBST $X \mathrm{XB}=46.049999,47.174999,58.924999,59.299999,4.78,5.53$, SURF-ID=' 'UPHOLSTERY' $/$ Left Arm

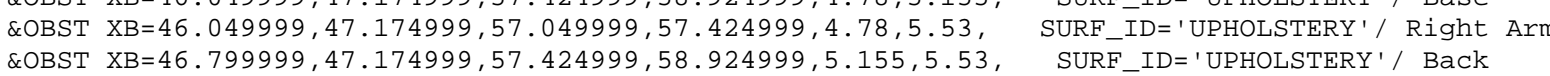

*** GROUP: COUCH2 offset $=0.000000,9.000000,0.000000$ rotate $=270.000000$

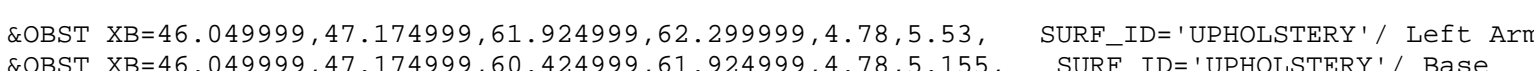

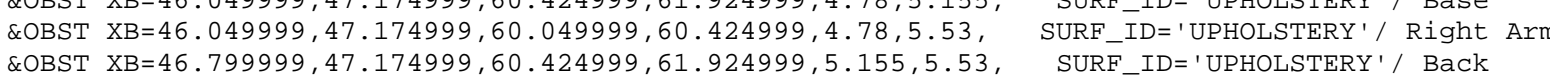

*** GROUP: COUCH2 of fset $=0.000000,12.000000,0.000000$ rotate $=270.000000$

\&OBST XB=46. $049999,47.174999,64.925003,65.300003,4.78,5.53$, SURF_ID=' 'UPHOLSTERY' ' Left Arm

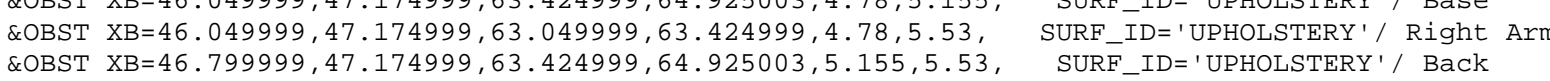
*** GROUP: COUCH2 offset $=0.000000,15.000000,0.000000$ rotate $=270.000000$

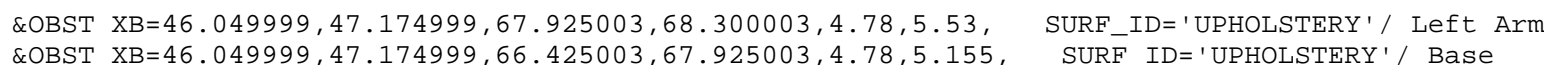

\&OBST XB=46. $049999,47.174999,66.050003,66.425003,4.78,5.53, \quad$ SURF_ID= 'UPHOLSTERY '/ Right Arm
\&OBST XB=46.799999, $47.174999,66.425003,67.925003,5.155,5.53, \quad$ SURF_ID=' 'UPHOLSTERY' / Back *** GROUP: COUCH2 offset $=0.000000,18.000000,0.000000$ rotate $=270.000000$

\&OBST XB=46. $049999,47.174999,70.925003,71.300003,4.78,5.53, \quad$ SURF_ID=' 'UPHOLSTERY'/' Left Arm
\&OBST XB=46.049999, 47.174999,69.425003,70.925003,4.78,5.155, $\quad$ SURF_ID='UPHOLSTERY'/ Base

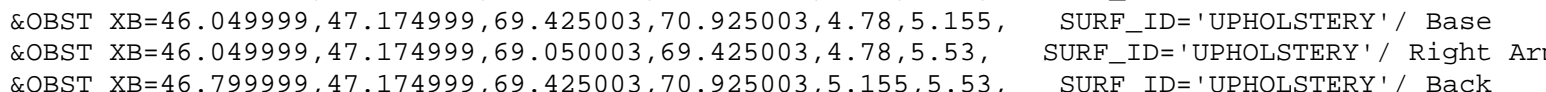

*** GROUP: COUCH2 offset $=0.000000,21.000000,0.000000$ rotate $=270.000000$

\&OBST XB=46.049999, 47.174999, 73.925003, 74.300003,4.78,5.53, SURF_ID=' 'UPHOLSTERY'/ Left Arm \&OBST XB=46. $049999,47.174999,72.425003,73.925003,4.78,5.155$, SURF_ID=' UPHOLSTERY'/ Base

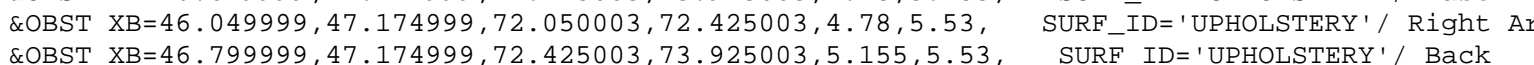

*** GROUP: COUCH2 offset $=0.000000,24.000000,0.000000$ rotate $=270.000000$

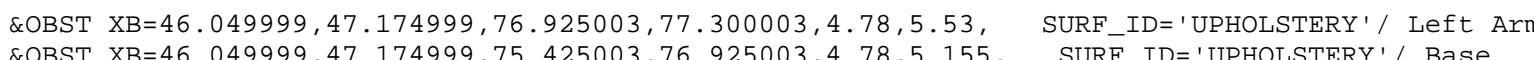

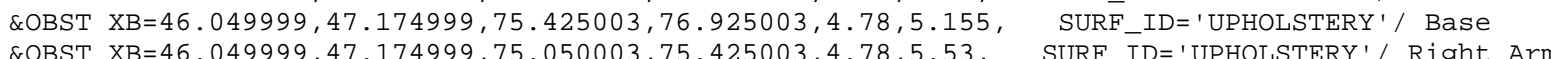

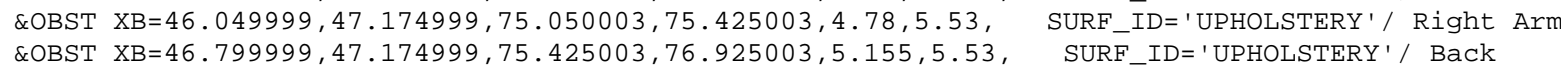

*** GROUP: COUCH2 offset $=0.000000,27.000000, \Theta .000000$ rotate $=270.000000$

OBST XB=46. $049999,47.174999,79.925003,80.300003,4.78,5.53$, SURF_ID='UPHOLSTERY'/ Left Arm \&OBST XB=46. $\odot 49999,47.174999,78.425003,79.925003,4.78,5.155$, SURF_ID='UPHOLLTERY' / Base

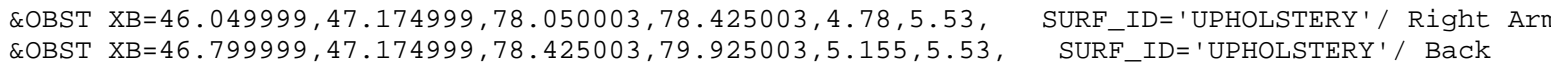

*** GROUP: COUCH2 offset $=0.000000,30.000000,0.000000$ rotate $=270.000000$

\&OBST XB=46. 049999, 47.174999, 82.925003, 83.300003, 4.78, 5.53, SURF_ID=' 'UPHOLSTERY'/ Left Arm \&OBST XB $=46.049999,47.174999,81.425003,82.925003,4.78,5.155$, SURF $=$ ID=' 'UPHOLSTERY'/ Base

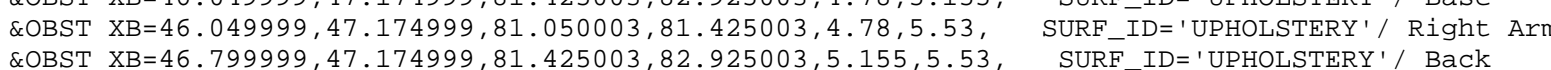

*** GROUP: TWIN

*** GROUP: ROW

*** GROUP: COUCH2 offset $=0.000000,0.000000,0.000000$ rotate $=90 . .000000$

\&OBST XB $=52.049999,53.174999,51.049999,51.424999,4.78,5.53$, SURF_ID=' 'UPHOLSTERY '/ Left Arm \&OBST XB $=52.049999,53.174999,51.424999,52.224999,4.78,5.155, \quad$ SURF IDE='UPHOLSTERY' ' Base

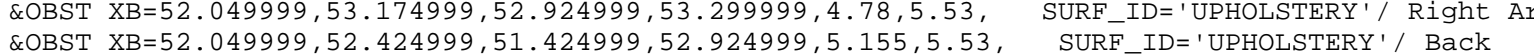

*** GROUP: COUCH2 offset $=0.000000,3.000000,0.000000$ rotate $=90.000000$

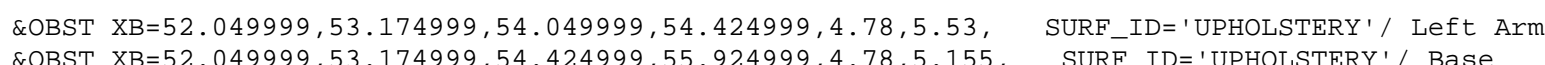

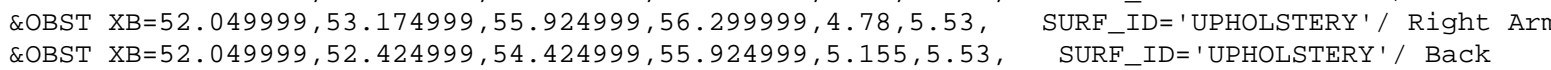

*** GROUP: COUCH2 offset $=0.000000,6.000000,0.000000$ rotate $=90.000000$

\&OBST XB=52. $049999,53.174999,57.049999,57.424999,4.78,5.53$, SURF_ID=' 'UPHOLSTERY' '/ Left Arm

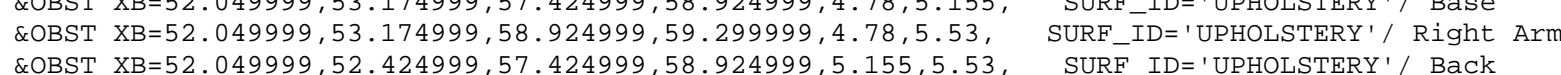

*** GROUP: COUCH2 offset $=0.000000,9.0000000,0.000000$ rotate $=90.000000$

\&OBST XB=52. $049999,53.174999,60.049999,60.424999,4.78,5.53$, SURF_ID=' 'UPHOLSTERY ' / Left Arm \&OBST XB=52.049999, 53.174999, 61.924999, 62.299999, 4.78, 5.53, SURF_ID='UPHOLSTERY'/Right Ar 61.924999, 5.155, 5.53, SURF_ID='UPHOLSTERY' / Back

*** GROUP: COUCH2 offset $=0.000000,12.000000,0.000000$ rotate $=90.000000$

\&OBST XB=52. 049999, 53.174999, 63. 049999, 63.424999, 4.78,5.53, SURF_ID='UPHOLSTERY '/ Left Arm \&OBST XB=52.049999, 53.174999,64.925003, 65.300003, 4.78,5.53, SURE ID='UPHOLSTERY'/ \&OBST XB $X 52.049999,52.424999,63.424999,64.925003,5.155,5.53$, SURF_ID=' 'UPHOLSTERY'/ Back 
** GROUP: COUCH2 offset $=0,000000,15,000000,0,000000$ rotate $=90,000000$

\&OBST XB=52. $049999,53.174999,66.050003,66.425003,4.78,5.53, \quad$ SURF_ID=' 'UPHOLSTERY' ', Left Arm
\&OBST XB=52.049999, $53.174999,66.425003,67.925003,4.78,5.155$,
SURF_IDE' UPHOLSTERY'/ Base

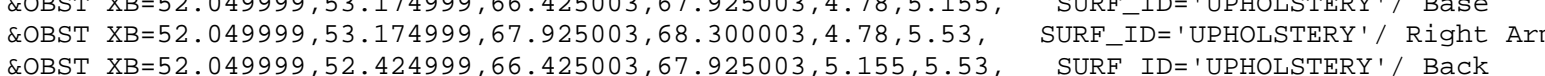

*** GROUP: COUCH2 offset $=0.000000,18.000000$, ๑. .0000000 rotate $=90.000000$

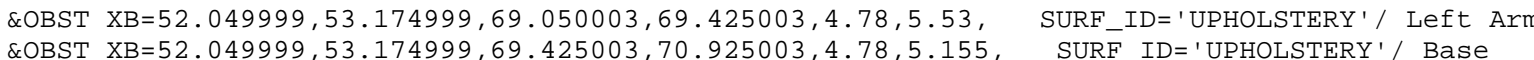

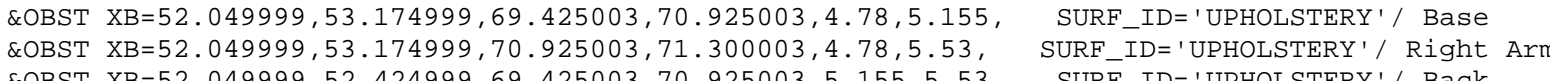

*** GROUP: COUCH2 off set $=0.000000,21.000000,0.000000$ rotate $=90.000000$

\&OBST XB=52. $449999,53.174999,72.050003,72.425003,4.78,5.53$, SURF_ID=' 'UPHOLSTERY' $/$ Left Arm \&OBST XBE $52.049999,53.174999,72.425003,73.925003,4.78,5.155$, SURF_ID='UPHOLSTERY'/ Base \&OBST XB=52.
\&OBST XB $X$ B

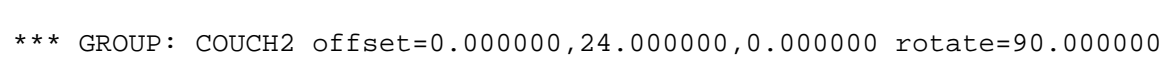

\&OBST XB=52. $049999,53.174999,75.050003,75.425003,4.78,5.53$, SURF_ID=' 'UPHOLSTERY'/ Left Arm \&OBST XB=52. $849999,53.174999,75.425003,76.925003,4.78,5.155$, SURF_ID='UPHOLSTERY'/ Base \&OBST $X \mathrm{~B}=52.049999,52.424999,75,425003,76,925093,5.155,5,53, \quad$ SURF_ID='UPHOLSTEY'/ Right Ar

*** GROUP: COUCH2 offset $=0.000000,27.000000,0.000000$ rotate $=90 . .000000$

\&OBST XB=52. $049999,53.174999,78.050003,78.425003,4.78,5.53, \quad$ SURF_ID=' 'UPHOLSTERY' 1 , Left Arm
\&OBST XB=52.049999, $53.174999,78.425003,79.925003,4.78,5.155$,
SURF_ID='UPHOLSTERY'/ Base \&OBST XB=52.049999, 53.174999, $79.925003,80.300003,4.78,5.53$, SURF_ID=' 'UPHOLSTERY'//Right Ar

*** GROUP: CoucH2 offset $=0.000000,30.000000, \odot .000000$ rotate $=90.000000$

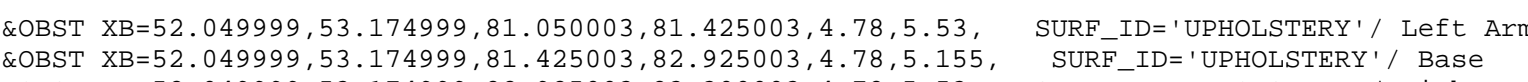

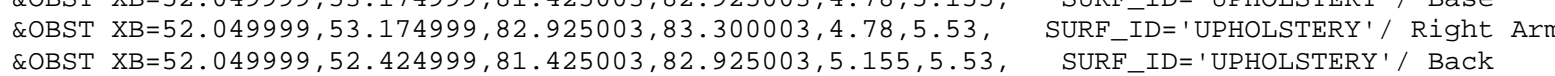

*** GROUP: ROW2

*** GROUP: COUCH2 offset $=0.000000,0.000000,0.000000$ rotate $=270.000000$

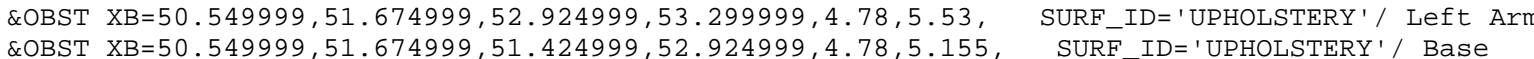
\&OBST XB $=50.549999,51.674999,51.049999,51.429999,4.78,5.53$, SURF - ID='UPHOLSTERY'/ Rige A A OOBST XB=51.299999, 51.674999, 51.424999, 52.924999, $5.155,5.53$, SURF_ID 'UPHOLSTERY'/ Back

*** GROUP: COUCH2 offset $=0.000000,3.000000,0.000000$ rotate $=270.000000$

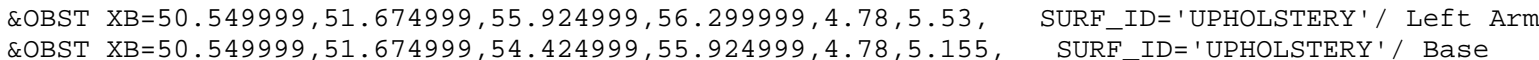
\&OBST XB $50.549999,51.674999,54.049999,54.424999,4.78,5.53$, SURF_ID ' SUPHOLSTERY'/ Right Arm

*** GROUP: COUCH2 offset $=0.000000,6.000000,0.000000$ rotate $=270.000000$

\&OBST XB $=50.549999,51.674999,58.924999,59.299999,4.78,5.53$, SURF_ID='UPHOLSTERY ' / Left Arm

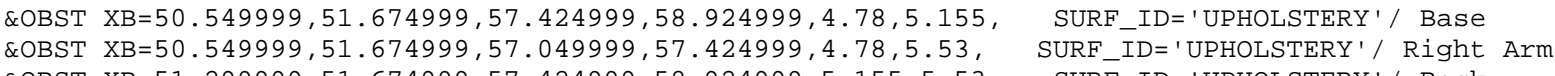
\&OBST XB=51.299999, $51.674999,57.424999,58.924999,5.155,5.53$, SURF_ID='UPHOLSTERY'/ Back

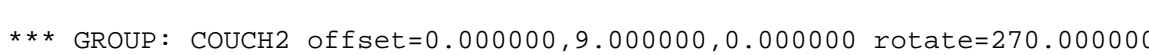

\&OBST XB $=50.549999,51.674999,61.924999,62.299999,4.78,5.53$,
\&OBST XB $X=50.549999,51.674999,60.424999,61.924999,4.78,5.155$, \&OBST XB=50.549999, $51.674999,60.424999,61.924999,4.78,5.155, \quad$ SURF_ID=' 'UPHOLSTERY'/ Base

*** GROUP: COUCH2 offset $=0.000000,12.000000,0.0000000$ rotate $=270.000000$

\&OBST XB=50.549999, 51.674999, 64.925003, 65.300003, 4.78,5.53, SURF_ID='UPHOLSTERY' ' Left Arm \&OBST XB
\&OBST XB=51. 299999, 51. 674999, 63, 424999, 64, 925003, 5.155, 5.53, SURF_ID=' UPHOLSTERY'/ Back *** GROUP: COUCH2 offset $=0.000000,15.000000,0.000000$ rotate $=270.000000$

\&OBST XB $=50.549999,51.674999,67.925003,68.300003,4.78,5.53$, SURF_ID=' 'UPHOLSTERY'/ Left Arm \&OBST XB=50.549999, 51.674999, 66.425003, 67. $925003,4.78,5.155$, SURF_ID='UPHOLSTERY' / Base LOBST XB=51.299999, $51.674999,66.425003,67.925003,5.155,5.53$, SURF_ID='UPHOLSTERY'/ Back

*** GROUP: COUCH2 offset $=0.000000,18.000000,0.000000$ rotate $=270.000000$

\&OBST XB $=50.549999,51.674999,70.925003,71.300003,4.78,5.53$, SURF_ID=' 'UPHOLSTERY'/ Left Arm \&OBST XB $=50.549999,51.674999,69.425003,70.925003,4.78,5.155$, SURF_ID='UPHOLSTERY'/ Base

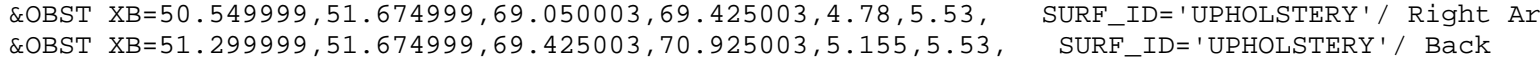

*** GROUP: COUCH2 offset $=0.000000,21.000000,0.000000$ rotate $=270.000000$

\&OBST XB=50.549999, 51.674999, 73.925003, 74.300003, 4.78,5.53, SURF_ID='UPHOLSTERY'/ Left Arm

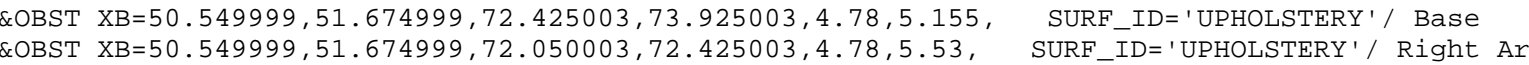
\&OBST XB=51.299999, $51.674999,72.425003,73.925003,5.155,5.53$, SURF ID='UPHOLSTEPY' / Back

*** GROUP: COUCH2 offset $=0.000000,24 . .000000,0.000000$ rotate $=270 . .000000$

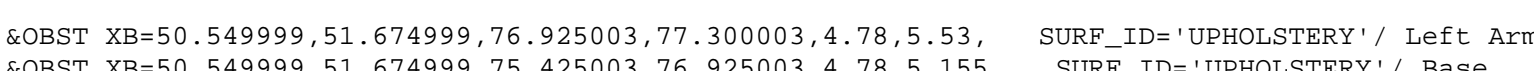

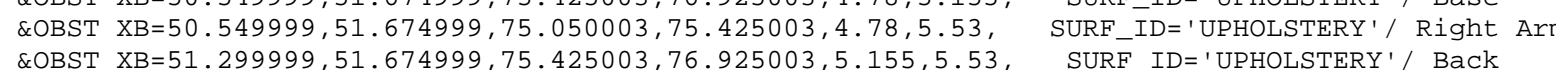

*** GROUP: COUCH2 offset $=0.000000,27.000000,0.000000$ rotate $=270.000000$

\&OBST XB=50.549999, $51.674999,79.925003,80.300003,4.78,5.53$, SURF_ID=' 'UPHOLSTERY' ' Left Arm \&OBST XB=50.549999, 51.674999, 78.050003, 78.425003,4.78,5.53, SURF_ID='UPHOLSTERY' $/$ Right Ar \&OBST XB=51.299999, 51.674999, 78.425003, 79.925003, $5.155,5.53$, SURF_ID='UPHOLSTERY'/ Back

*** GROUP: COUCH2 offset $=0.000000,30.000000,0.000000$ rotate $=270.000000$

\&OBST XB $=50.549999,51.674999,82.925003,83.300003,4.78,5.53, \quad$ SURF ID= 'UPHOLSTERY' $/$ Left Arm \&OBST XB=50.549999, 51.674999, 81, $050003,81.425003,4.78,5.53$, SURF - ID $=$ ' UPHOLSTERY'/ Bight \&OBST XB=51.299999, 51.674999, 81.425003, 82.925003, 5.155, 5.53, SURF_ID='UPHOLSTERY' / Back

*** GROUP: TWIN

*** GROUP: ROW

*** GROUP: COUCH2 offset $=0.000000,0.000000,0.000000$ rotate $=90 . .000000$

\&OBST XB=56.549999, 57.674999, 51.049999, 51.424999, 4.78,5.53, SURF_ID= 'UPHOLSTERY' / Left Arm 源 \&OBST XB=56.549999, 56. $924999,51.424999,52.924999,5.155,5.53$, SURF_ID='UPHOLSTERY'/ Back

*** GROUP: COUCH2 offset $=0.000000,3.000000,0.000000$ rotate $=90.000000$

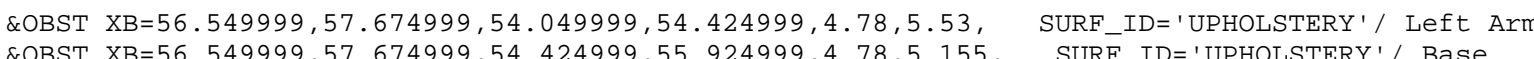
\&OBST XB=56.549999, 56.924999, 54.424999, 55.924999, 5.155,5.53, SURF_ID='UPHOLSTERY'/ Back

*** GROUP: COUCH2 offset $=0.000000,6.000000,0.0000000$ rotate $=90.000000$

\&OBST XB=56.549999, 57.674999, 57. 4049999, 57.424999, $4.78,5.53, \quad$ SURF_ID='UPHOLSTERY' ' Left Arm
\&OBST XB $=56.549999,57.674999,57.424999,58.924999,4.78,5.155$,
SURF_ID='UPHOLSTERY' / Base

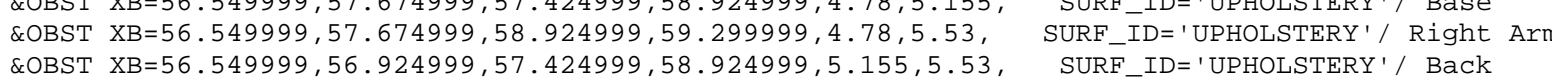

*** GROUP: COUCH2 offset $=0.000000,9.000000,0.0000000$ rotate $=90.000000$

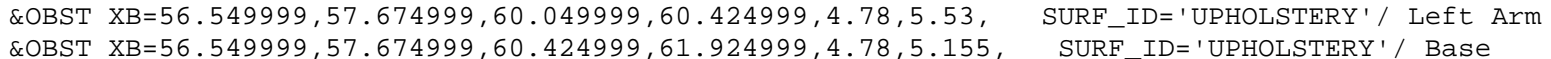

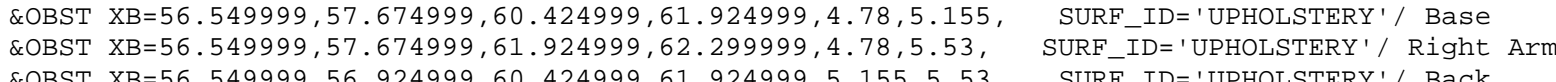

*** GROUP: COUCH2 offset $=0.000000,12.000000,0.000000$ rotate $=90.000000$ 


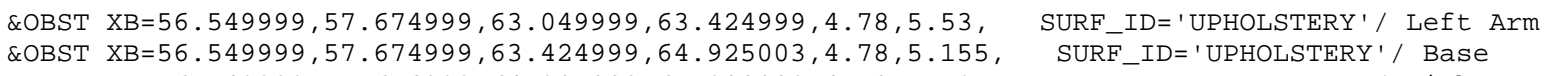

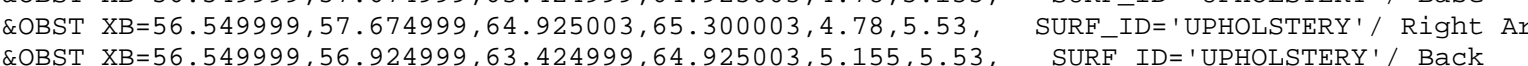

*** GROUP: COUCH2 offset $=0.000000,15.000000,0.000000$ rotate $=90.000000$

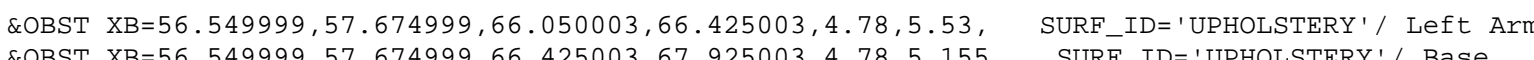
\&OBST XB=56.54999, $57.674999,67.925003,68.300003,4.78,5.53$, SURF_ID='UPHOLSTERY'/R Right Ar

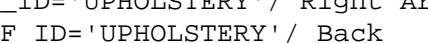

*** GROUP: COUCH2 offset $=0.000000,18.000000,0.000000$ rotate $=90.000000$

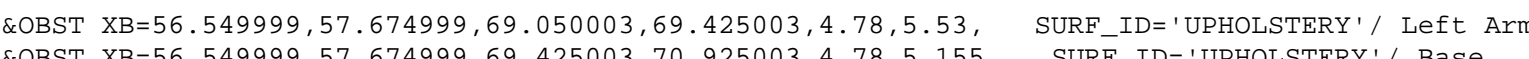

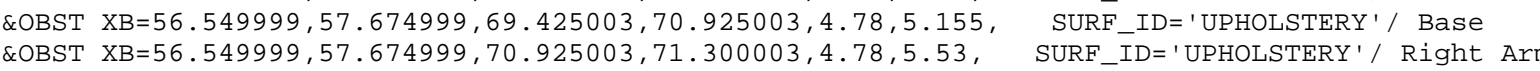

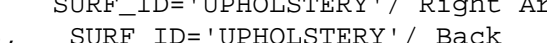

*** GROUP: COUCH2 offset $=0.000000,21.000000, \Theta . .000000$ rotate $=90.000000$

\&OBST XB=56.549999, $57.674999,72.050003,72.425003,4.78,5.53$, SURF_ID='UPHOLSTERY'/ Left Arm \&OBST XB $56.549999,57.674999,72.425003,73.925003,4.78,5.155$, SURF_ID= 'UPHOLSTERY' / Base \&OBST XB $=56.549999,57.674999,73.925003,74.300003,4.78,5.53, \quad$ SURF_ID='UPHOLSTERY'/ Right Arm
\&OBST XB $=56.549999,56.924999,72.425003,73.925003,5.155,5.53$,
SURF_ID='UPHOLSTERY' / Back

*** GROUP: CouCH2 offset $=0.000000,24.000000,0.000000$ rotate $=90.000000$

\&OBST XB $=56.549999,57.674999,75.050003,75.425003,4.78,5.53$, SURF_ID=' 'UPHOLSTERY'/ Left Arm \&OBST XB=56.549999, $57.674999,75.425003,76.925003,4.78,5.155$, SURF_ID=' 'UPHOLSTERY' / Base \&OBST XB=56.549999, 57. $674999,76.925003,77.300003,4.78,5.53, \quad$ SURF_ID=' 'UPHOLSTERY'/ Right Ar
\&OBST XB=56.549999, $56.924999,75.425003,76.925003,5.155,5.53, \quad$ SURF_ID='UPHOLSTERY'/ Back

$* * *$ GROUP: COUCH2 offset $=0.000000,27.000000,0.000000$ rotate $=90.000000$

\&OBST XB $=56.549999,57.674999,78.050003,78.425003,4.78,5.53, \quad$ SURF ID= ' 'PPHOLSTERY' ' Left Arm

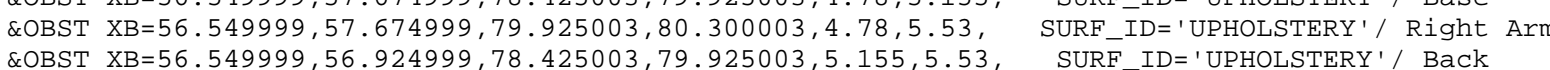

*** GROUP: COUCH2 offset $=0.000000,30.000000,0.000000$ rotate $=90.000000$

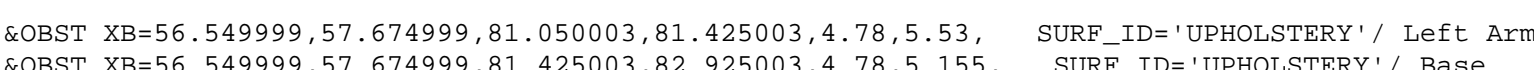

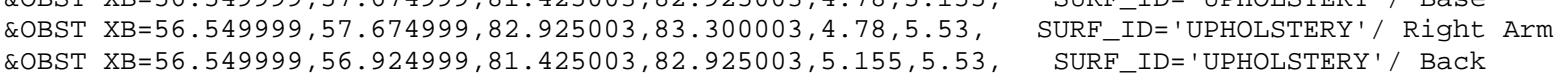

*** GROUP: ROW2

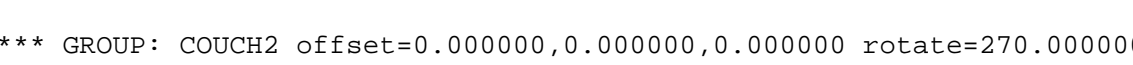

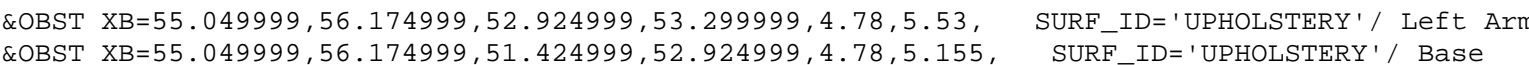
\&OBST XB $=55.049999,56.174999,51.049999,51.424999,4.78,5.53, \quad$ SURF-ID=' UPHOLSTERY'/ Right Ar

*** GROUP: COUCH2 offset $=0.000000,3.000000,0.000000$ rotate $=270.000000$

\&OBST XB=55. 049999, 56. 174999, 55. 924999, 56. 299999, 4.78, 5.53, SURFID=' UPHOLSTERY ' / Left Arm \&OBST XB $=55.049999,56.174999,54.424999,55.924999,4.78,5.155, \quad$ SURF_ID='UPHOLSTERY'/ Base
COBST XB $=55.049999,56.174999,54.049999,54.424999,4.78,5.53$,
SURF_ID='UPHOLSTERY'/Right Ar

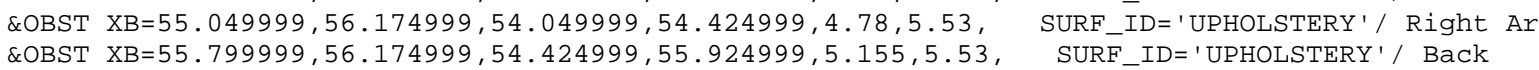

*** GROUP: COUCH2 offset $=0.000000,6.000000,0.000000$ rotate $=270.000000$

\&OBST XB=55. 049999, 56.174999, 58.924999, 59.299999, 4.78,5.53, SURF_ID='UPHOLSTERY'/ Left Arm \&OBST XB=55. $849999,56.174999,57.424999,58.924999,4.78,5.155$, SURF_ID='UPHOLSTERY'/ Base \&OBST XB=55. $449999,56.174999,57.049999,57.424999,4.78,5.53$, SURF_ID='UPHOLSTERY'/ Right Ar *** GROUP: COUCH2 offset $=0.000000,9.000000,0.000000$ rotate $=270.000000$

\&OBST XB=55.049999, 56.174999, 61.924999, 62.299999, 4.78,5.53, SURF_ID='UPHOLSTERY'/ Left Arm

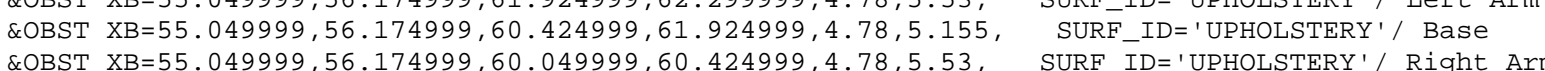

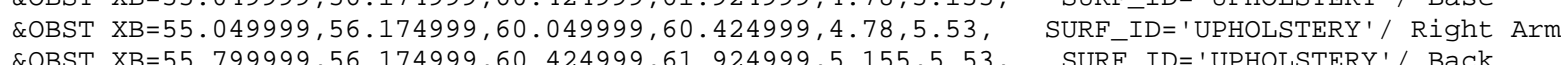

*** GROUP: COUCH2 offset $=0.000000,12.000000, \odot .000000$ rotate $=270.000000$

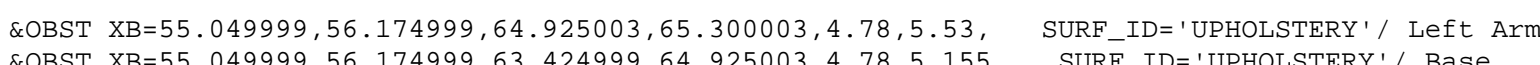

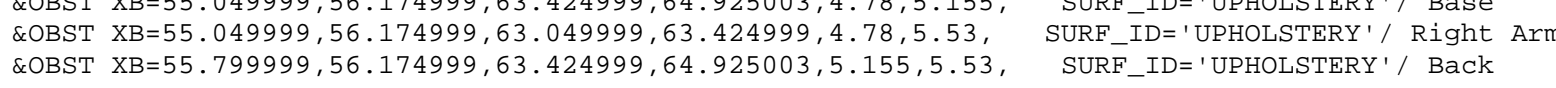
*** GROUP: COUCH2 offset $=0.0000000,15.000000,0.000000$ rotate $=270.000000$

\&OBST XB=55.049999, 56.174999, 67.925003, 68.300003,4.78,5.53, $\quad$ SURF_ID=' 'UPHOLSTERY'/ Left Arm
\&OBST XB=55. $049999,56.174999,66.425003,67.925003,4.78,5.155$,
SURFID='UPHOLSTERY'/ Base

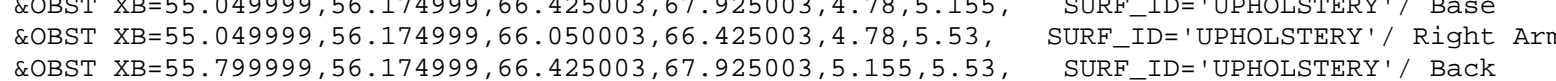

*** GROUP: COUCH2 offset $=0.000000,18.000000,0.000000$ rotate $=270.000000$

\&OBST XB=55. $049999,56.174999,70.925003,71.300003,4.78,5.53$, SURF_ID='UPHOLSTERY' ' Left Arm

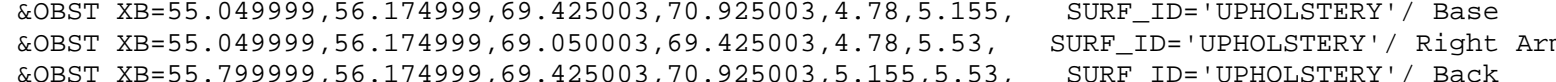

*** GROUP: COUCH2 offset $=0.000000,21.000000,0.000000$ rotate $=270.000000$

\&OBST XB=55. $049999,56.174999,73.925003,74.300003,4.78,5.53$,
\&OBST XB=55. $049999,56.174999,72.425003,73.925003,4.78,5.155$,
SURF_ID='UPHOLSTERY ' ' Left Arm

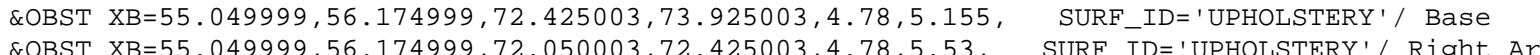
EOBST $X \mathrm{~B}=55.049999,56.174999,72.050003,72.425003,4.78,5.53, \quad$ SURF_ID=' 'UPHOLSTERY'/ Right Ar
EOBST XB $=55.799999,56.174999,72.425003,73.925003,5.155,5.53$, SURF_ID='UPHOLSTERY'/ Back

*** GROUP: COUCH2 offset $=0.000000,24.000000,0.000000$ rotate $=270.000000$

\&OBST XB $=55.049999,56.174999,76.925003,77.300003,4.78,5.53, \quad$ SURF_ID='UPHOLSTERY' ' Left Arm
\&OBST XB $=55.049999,56.174999,75.425003,76.925003,4.78,5.155, \quad$ SURF_IDD='UPHOLSTERY' $/$ Base

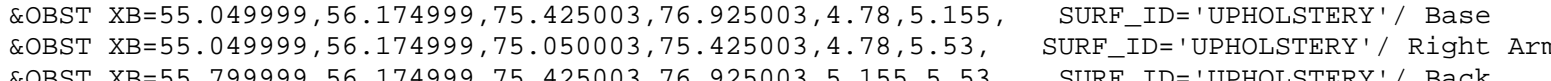

*** GROUP: COUCH2 offset $=0.000000,27.000000,0.000000$ rotate $=270.000000$

\&OBST XB=55. 049999, 56.174999, 79.925003, 80. 300003, 4.78,5.53, SURF_ID=' 'UPHOLSTERY' / Left Arm \&OBST XB $=55.049999,56.174999,78.425003,79.925003,4.78,5.155, \quad$ SURF_ID=' 'UPHOLSTERY'/ Base
\&OBST XB $=55.049999,56.17499,78.050003,78.425003,4.78,5.53$

*** GROUP: COUCH2 offset $=0.000000,30.000000,0.000000$ rotate $=270.000000$

\&OBST XB=55. 049999, 56. $174999,82.925003,83.300003,4.78,5.53, \quad$ SURF_ID=' 'UPHOLSTERY'/ Left Arm
\&OBST XB X $55.049999,56.174999,81.425003,82.925003,4.78,5.155, \quad$ SURF_ID=' UPHOLSTERY' / Base

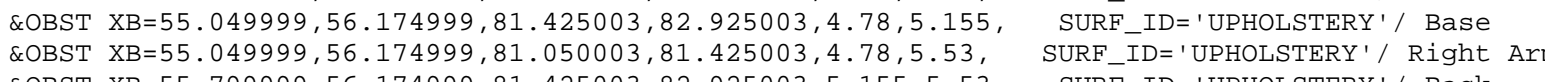

\section{*** GROUP: TWIN}

*** GROUP: ROW

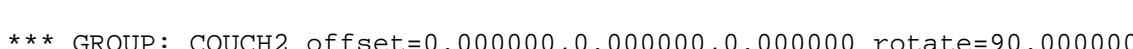

\&OBST XB $=61.049999,62.174999,51.049999,51.424999,4.78,5.53$, SURF_ID='UPHOLSTERY '/ Left Arm

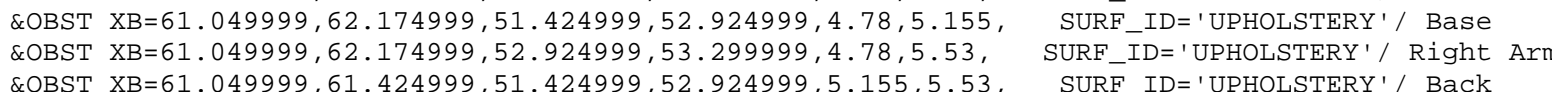

*** GROUP: COUCH2 offset $=0.000000,3.000000,0.000000$ rotate $=90.000000$

\&OBST XB $=61.049999,62.174999,54.049999,54.424999,4.78,5.53$, SURF ID=' 'UPHOLSTERY'/ Left Arm

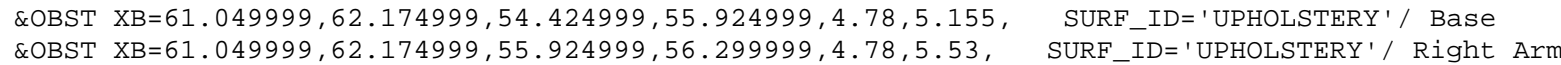
\&OBST XB $61.049999,61.424999,54.424999,55,924999,5.155,5,53$, SUPF_ID='UPHOLSTEPY'/ Righ A

*** GROUP: COUCH2 offset $=0.0000000,6.000000,0.000000$ rotate $=90.000000$

EOBST XB=61.049999, 62.174999, 57. 049999, 57.424999, 4.78,5.53, SURF_ID='UPHOLSTERY '/ Left Arm EOBST XB=61.049999, 62.174999,57.424999, 58.924999, $4.78,5.155$, SURF_ID='UPHOLSTERY' / Base

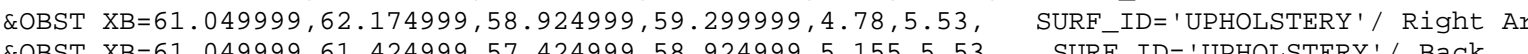

*** GROUP: COUCH2 offset $=0.000000,9.000000,0.000000$ rotate $=90.000000$ 
\&OBST XB=61. $049999,62.174999,60.049999,60.424999,4.78,5.53, \quad$ SURF_ID= 'UPHOLSTERY ' /' Left Arm
\&OBST XB=61.049999, 62.174999, 60.424999,61.924999, $4.78,5.155, \quad$ SURF_ID=' 'UPHOLSTERY' / Base

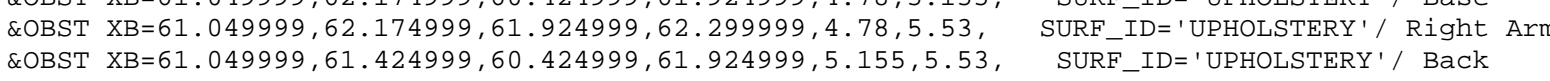
*** GROUP: COUCH2 offset $=0.000000,12.000000,0.0000000$ rotate $=90.000000$

\&OBST XB=61. $049999,62.174999,63.049999,63.424999,4.78,5.53$, SURF_ID=' 'UPHOLSTERY ' $/$ Left Arm

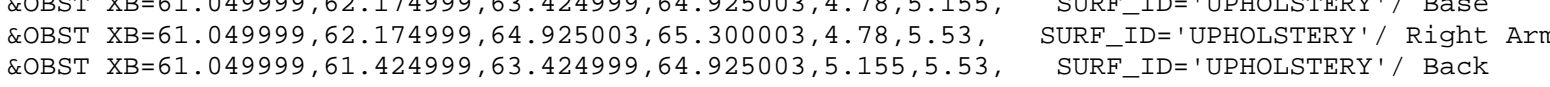

*** GROUP: COUCH2 offset $=0.000000,15.000000,0.000000$ rotate $=90.000000$

\&OBST $X \mathrm{XB}=61.049999,62.174999,66.050003,66.425003,4.78,5.53$, SURF_ID=' 'UPHOLSTERY' $/$ Left Arm \&OBST XB=61.049999, 62.174999, 67.925003,68.300003, 4.78,5.53, SURF_ID='UPHOLSTERY'/Right Ar \&OBST XB=61.049999, 61.424999, 66.425003,67.925003, 5.155,5.53, SURF_ID='UPHOLSTERY'/ Back

*** GROUP: COUCH2 offset $=0.000000,18.000000,0.000000$ rotate $=90.000000$

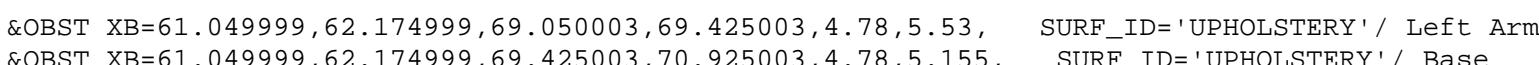
COBST XB=61. $049999,62.174999,70.925003,71.300003,4.78,5.53$,
\&OBST XB=61.049999, $61.424999,69.425003,70.925003,5.155,5.53, \quad$ SURF_ID='UPHOLSTERY'/ Right Arm
SURF_ID=' 'UPHOLSTERY' $/$ Back

*** GROUP: COUCH2 offset $=0.000000,21.000000,0.000000$ rotate $=90.000000$

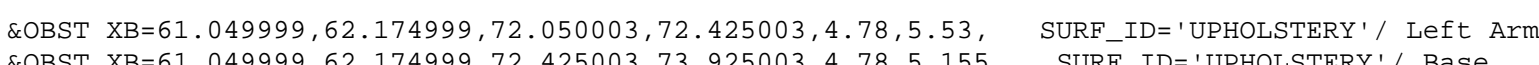

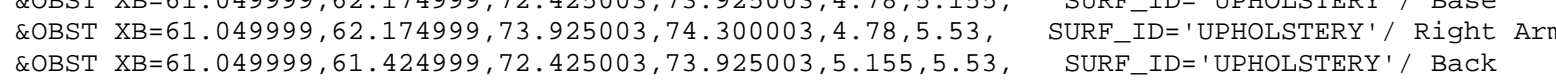
*** GROUP: COUCH2 offset $=0.000000,24.000000,0.000000$ rotate $=90.000000$

\&OBST XB=61. $049999,62.174999,75.050003,75.425003,4.78,5.53$,
\&OBST XB=61.049999, $62.174999,75.425003,76.925003,4.78,5.155$,
SURF_ID='UPHOLSTERY' '/ Left Arm

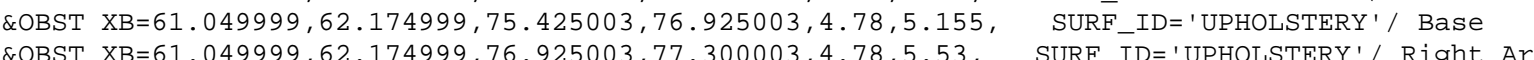
\&OBST XB=61. $049999,62.174999,76.925003,77.300003,4.78,5.53$,
\&OBST XB=61.049999, $61.424999,75.425003,76.925003,5.155,5.53$,
SURF_IDD='UPHOLSTERY'/ Back

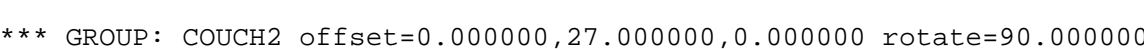

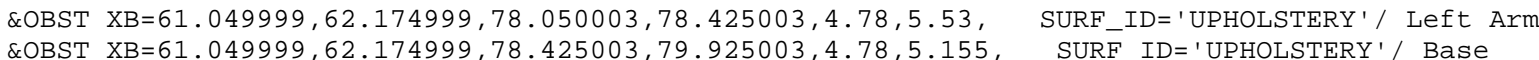

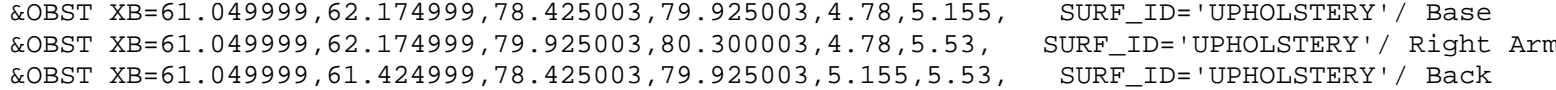

*** GROUP: COUCH2 offset $=0.000000,30.000000,0.000000$ rotate $=90.000000$

\&OBST XB $=61.049999,62.174999,81.050003,81.425003,4.78,5.53$, SURF_ID='UPHOLSTERY'/ Left Arm

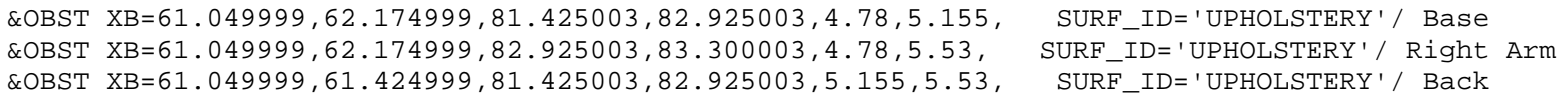

*** GROUP: ROW2

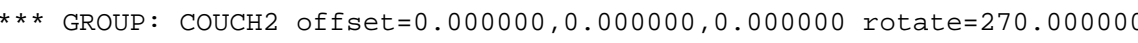

\&OBST XB=59.549999, 60.674999, 52.924999, 53.299999, 4.78, 5.53, SURF_ID=' 'UPHOLSTERY' ' Left Arm

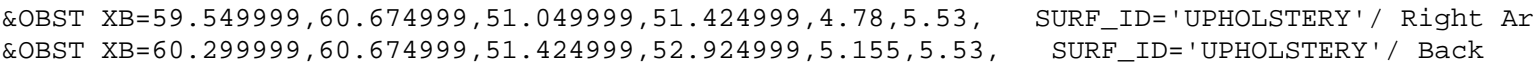
*** GROUP: COUCH2 offset $=0.000000,3.000000,0.000000$ rotate $=270.000000$

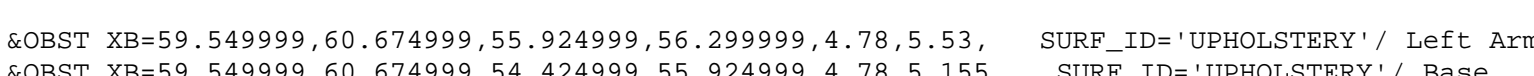

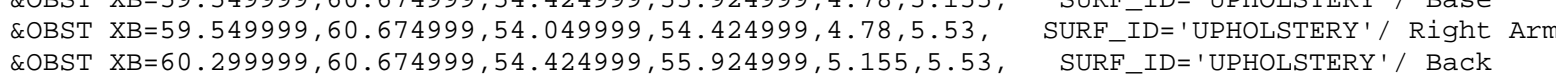
*** GROUP: COUCH2 offset $=0.000000,6.000000,0.000000$ rotate $=270.000000$

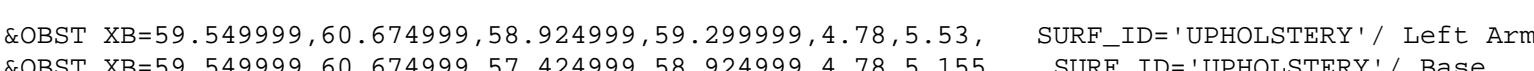

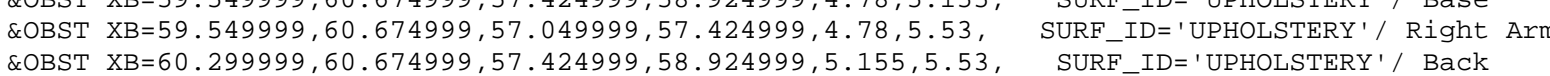

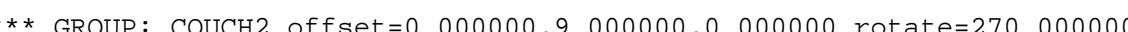

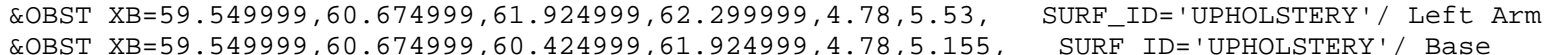

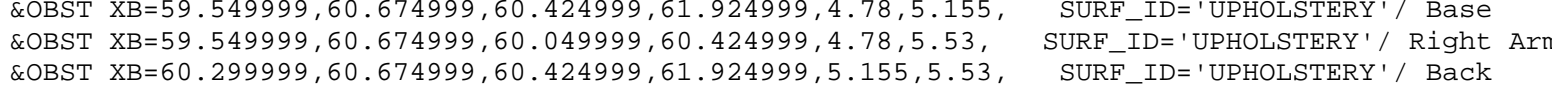

*** GROUP: COUCH2 offset $=0.000000,12.000000,0.000000$ rotate $=270.000000$

\&OBST XB $=59.549999,60.674999,64.925003,65.300003,4.78,5.53, \quad$ SURF_ID=' 'UPHOLSTERY' ' Left Arm

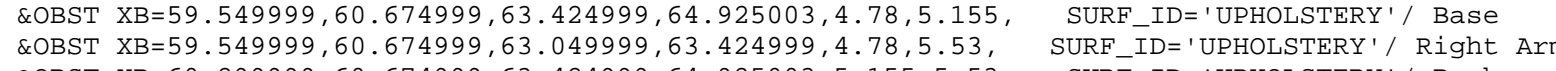
\&OBST XB=60. 299999, 60.674999, 63.424999, 64.925003, 5. 155, 5.53, SURF ID=' UPHOLSTERY' / Back

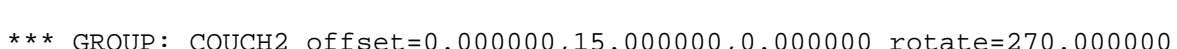

\&OBST XB=59. $549999,60.674999,67.925003,68.300003,4.78,5.53, \quad$ SURF_ID=' 'UPHOLSTERY'/' Left Arm
\&OBST XB=59.549999, 60.674999,66.425003,67.925003,4.78,5.155,
SURF_ID='UPHOLSTERY'/ Base

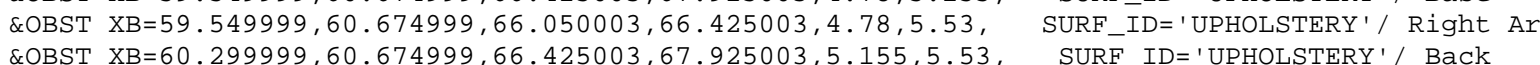

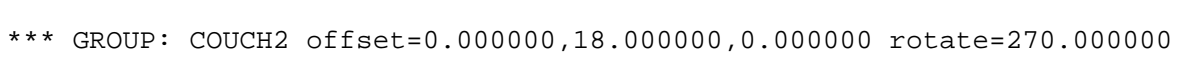

\&OBST XB=59.549999, 60.674999, 70.925003,71.300003, 4.78,5.53, SURF_ID=' 'UPHOLSTERY ' / Left Arm \&OBST XB=59.549999,60.674999,69.425003,70.925003,4.78,5.155, SURF_ID='UPHOLSTERY'/ Base

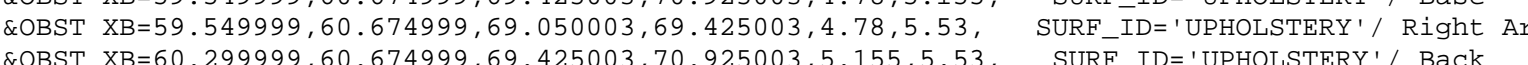

*** GROUP: COUCH2 offset $=0.000000,21.000000,0.000000$ rotate $=270.000000$

\&OBST XB=59.549999, 60.674999, 73.925003, 74.300003, 4.78,5.53, SURF_ID=' 'UPHOLSTERY'/ Left Arm

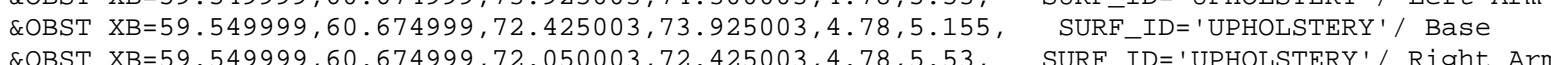

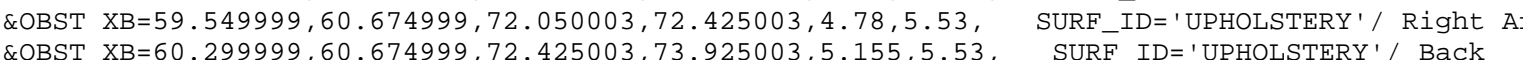

*** GROUP: COUCH2 offset $=0.000000,24.000000,0.000000$ rotate $=270.000000$

\&OBST XB=59.549999, 60.674999, 76.925003, 77. 300003, 4.78, 5.53, SURF_ID='UPHOLSTERY'/ Left Arm \&OBST XB=59.549999, 60.674999, 75.425003, 76.925003, 4.78, 5.155, SURF_ID='UPHOLSTERY' / Base \&OBST XB=59.549999,66.6747999, 75.

*** GROUP: COUCH2 offset $=0.000000,27.000000,0.000000$ rotate $=270.000000$

EOBST XB=59.549999, 60.674999, 79.925003, 80.300003, 4.78, 5.53, SURF_ID=' UPHOLSTERY'/ Left Arm \&OBST XB=59. $549999,60.674999,78.425003,79.925003,4.78,5.155$, SURF_ID='UPHOLSTERY' / Base \&OBST XB $=59.549999,60.674999,78.050003,78.425003,4.78,5.53$,
\&OBST XB $\quad$ SURF_ID=' 'UPHOLSTERY'/ Right Ar

*** GROUP: COUCH2 offset $=0.000000,30.000000,0.000000$ rotate $=270.000000$

\&OBST XB=59. $549999,60.674999,82.925003,83.300003,4.78,5.53, \quad$ SURF_ID= ' 'UPHOLSTERY' ' Left Arm
\&OBST XB=59.54999, 60.674999, $81.425003,82.925003,4.78,5.155$,
SURF IDE' 'UPHOLSTERY' / Base

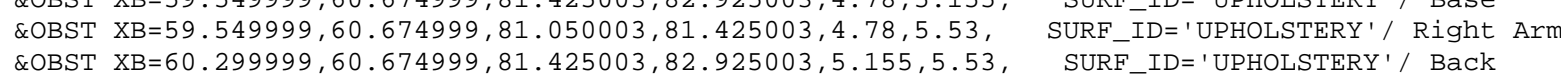

*** GROUP: TWIN

*** GROUP: ROW

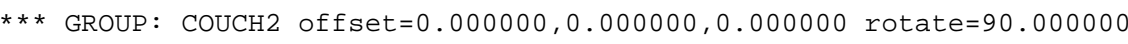

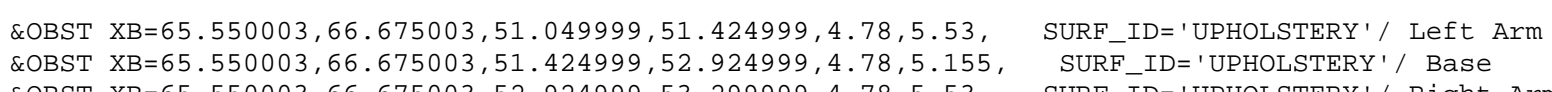

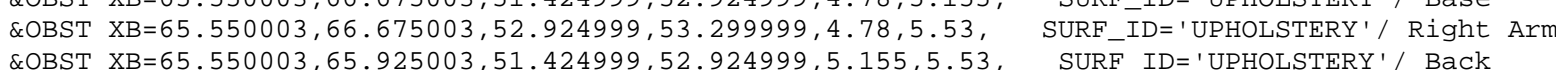

*** GROUP: COUCH2 offset $=0.000000,3.000000,0.000000$ rotate $=90.000000$

\&OBST XB $=65.550003,66.675003,54.049999,54.424999,4.78,5.53, \quad$ SURF-ID=' 'UPHOLSTERY '/ Left Arm

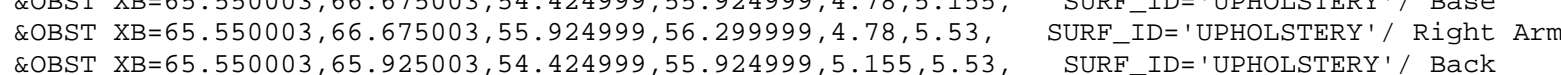

*** GROUP: COUCH2 offset $=0.000000,6.000000,0.000000$ rotate $=90.000000$

\&OBST XB=65.550003,66.675003, 57, $049999,57.424999,4.78,5.53$, SURF_ID='UPHOLSTERY' / Left Arm 
\&OBST XB $=65.550003,66.675003,57.424999,58.924999,4.78,5.155, \quad$ SURF_ID='UPHOLSTERY'/ Base
\&OBST XB=65.550003,66.675003, $58.924999,59.299999,4.78,5.53, \quad$ SURF_ID='UPHOLSTERY'/ Right A \&OBST $X \mathrm{X}=65.550003,66.675003,58.924999,59.299999,4.78,5.53, \quad$ SURF_ID=' 'UPHOLSTERY'
\&OBST $\mathrm{XB}=65.550003,65.925003,57.424999,58.924999,5.155,5.53, \quad$ SURF_ID ' 'UPHOLSTERY' $/$ Back *** GROUP: COUCH2 offset $=0.000000,9.000000,0.000000$ rotate $=90.000000$

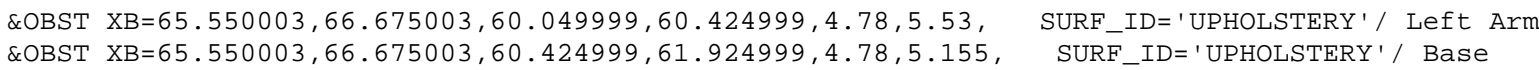

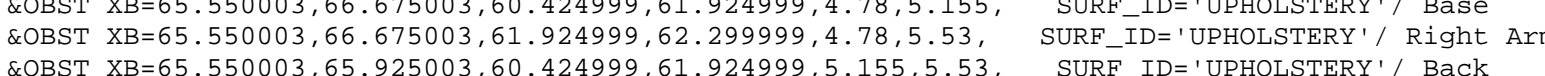
$* * *$ GROUP: COUCH2 offset $=0.000000,12.000000,0.000000$ rotate $=90.000000$

\&OBST XB $=65.550003,66.675003,63.049999,63.424999,4.78,5.53, \quad$ SURF_ID=' UPHOLSTERY ' $/$ ' Left Arm
\&OBST XB $=65.550003,66.675003,63.424999,64.925003,4.78,5.155$,
SURF_ID=' 'UPHOLSTERY' $/$ Base COBST XB $65.550003,66.675003,64.925003,65.300003,4.78,5.53$,
COBST

*** GROUP: COUCH2 offset $=0.000000,15.000000,0.000000$ rotate $=90.000000$

\&OBST XB $65.5500003,66.675003,66.050003,66.425003,4.78,5.53$, SURF_ID= 'UPHOLSTERY' / Left Arm

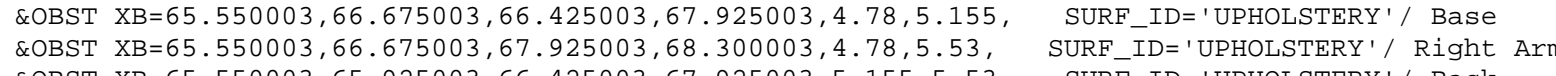

${ }_{* \star *}$ GROUP: COUCH2 off set $=0.000000,18.000000,0.000000$ rotate $=90.000000$

\&OBST XB $=65.550003,66.675003,69.050003,69.425003,4.78,5.53$, SURF_ID='UPHOLSTERY ' / Left Arm

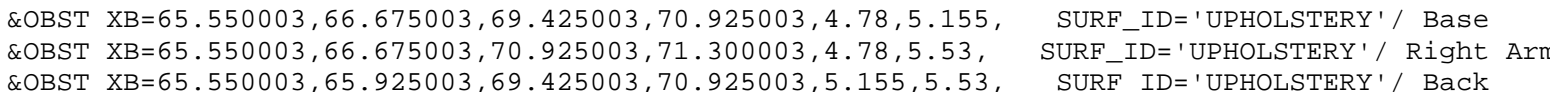

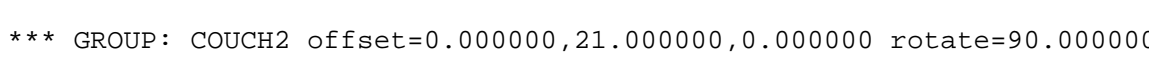

\&OBST XB=65. $550003,66.675003,72.050003,72.425003,4.78,5.53, \quad$ SURF_ID=' 'UPHOLSTERY' 1 ' Left Arm
\&OBST XB=65.550003, $66.675003,72.425003,73.925003,4.78,5.155$,
SURF_ID='UPHOLSTERY'/ Base \&OBST XB $=65.550003,66.675003,72.425003,73.925003,4.78,5.155$,
\&OBST XB $=65.550003,66.675003,73.925003,74.30003,4.78,5.53$

*** GROUP: COUCH2 offset $=0.000000,24.000000,0.000000$ rotate $=90.000000$

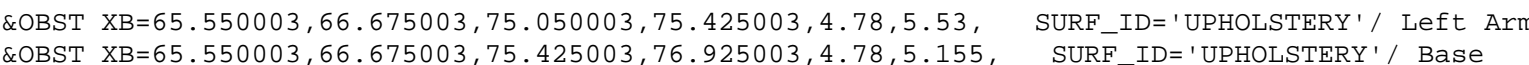
\&OBST XB=65.550003, 66.675003, 76.925003,77.300003,4.78,5.53, SURF_ID='UPHOLSTERY'/ Right A

$* *$ GROUP: COUCH2 offset $=0.000000,27.000000,0.000000$ rotate $=90.000000$

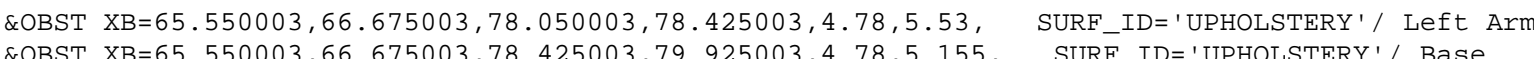
\&OBST XB=65.550003, 66.675003, 78.425003, 79.925003, 4.78, 5.155, SURF_ID='UPHOLSTERY'/ Base

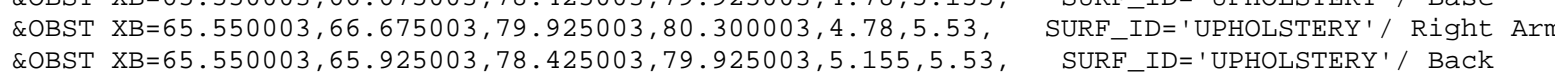

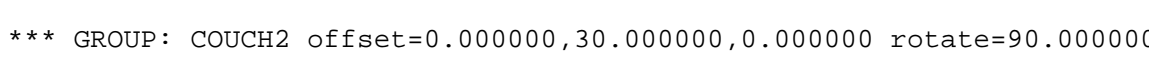

\&OBST XB=65.550003, 66.675003, 81.050003, 81.425003, 4.78,5.53, SURF_ID='UPHOLSTERY'/ Left Arm

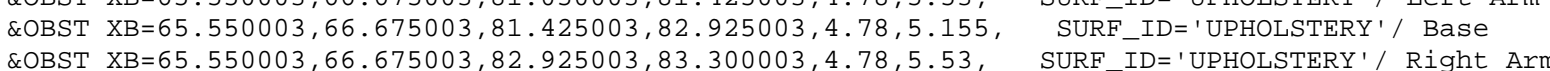

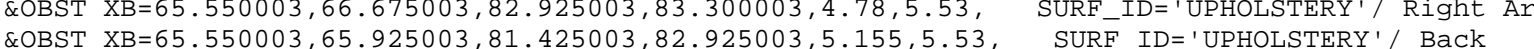

*** GROUP: ROW2

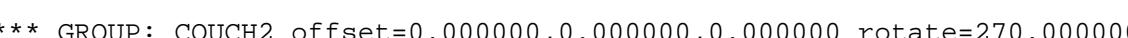

\&OBST $X \mathrm{XB}=64.050003,65.175003,52.924999,53.299999,4.78,5.53, \quad$ SURF_ID=' 'UPHOLSTERY' ' Left Arm \&OBST XB $=04.0503,65.175003,51.04999,51.424999,4.78,5.53$, SURF ID=' 'UPHOLSTERY'/ Rige \&OBST XB $=64.050003,65.175003,51.049999,51.424999,4.78,5.53$,
\&OBST $X \mathrm{~B}=64.800003,65.175003,51.424999,52.924999,5.155,5.53$,
SURF_ID=' 'UPHOLSTERY'/ Right Ar

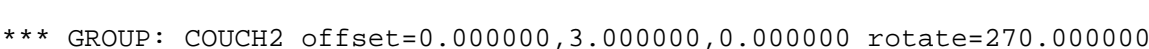

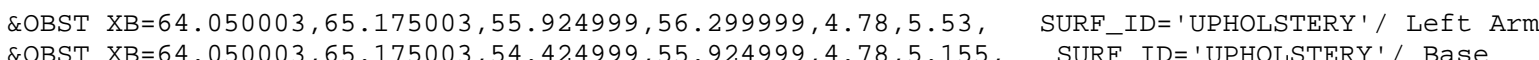

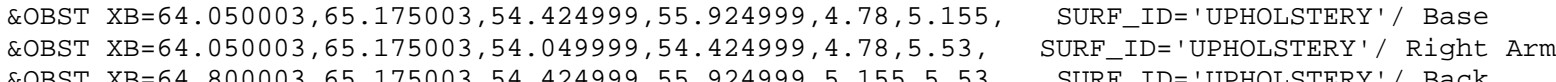

** GROUP: COUCH2 offset $=0.000000,6.000000,0.0000000$ rotate $=270.000000$

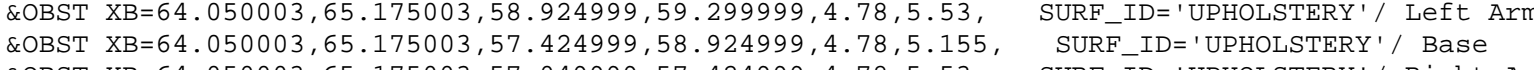
\&OBST XB $=64.050003,65.175003,57.049999,57.424999,4.78,5.53$, SURF_ID='UPHOLSTERY'/ Right Ar

*** GROUP: COUCH2 offset $=0.000000,9.000000,0.000000$ rotate $=270.000000$

\&OBST XB $64.050003,65.175003,61.924999,62.299999,4.78,5.53$, SURF_ID='UPHOLSTERY' ' Left Arm

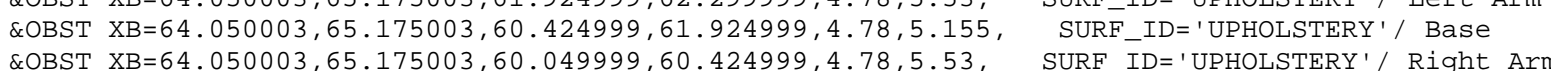

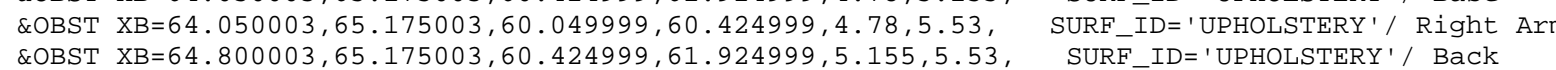

*** GROUP: COUCH2 offset $=0.000000,12.000000,0.000000$ rotate $=270.000000$

\&OBST $X \mathrm{~B}=64.050003,65.175003,64.925003,65.300003,4.78,5.53$, SURF_ID=' 'UPHOLSTERY'/ Left Arm

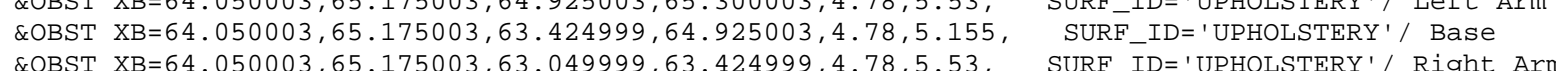

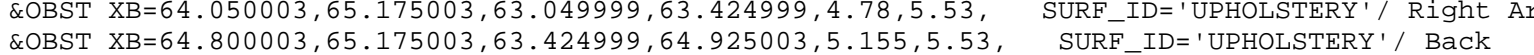

*** GROUP: CoUCH2 offset $=0.000000,15.000000,0.000000$ rotate $=270.000000$

\&OBST $X \mathrm{~B}=64.050003,65.175003,67.925003,68.300003,4.78,5.53$, SURF_ID=' UPHOLSTERY '/ Left Arm \&OBST XB=64.050003,65.175003,66.425003,67.925003, 4.78,5.155, SURF_ID='UPHOLSTERY' / Base

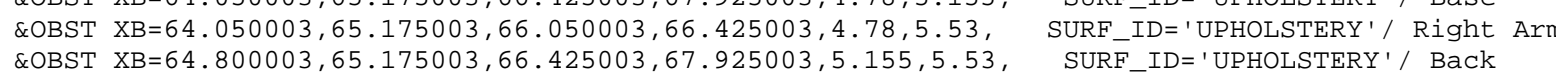

*** GROUP: COUCH2 offset $=0.000000,18.000000,0.000000$ rotate $=270.000000$

\&OBST XB=64. $050003,65.175003,70.925003,71.300003,4.78,5.53$, SURF_ID=' 'UPHOLSTERY ' Left Arm

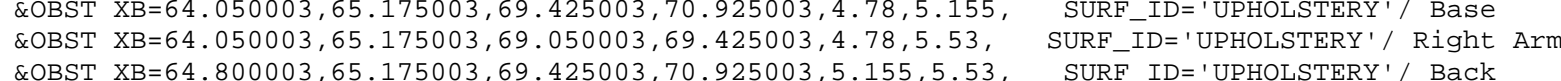

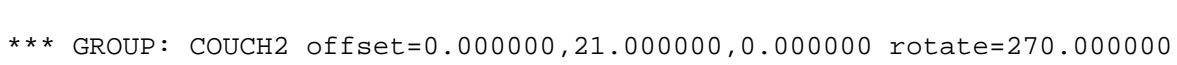

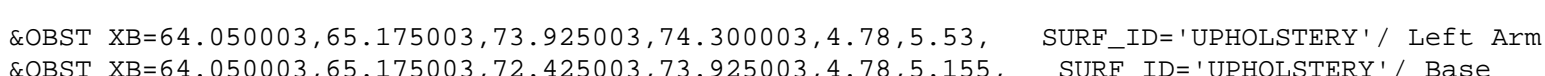

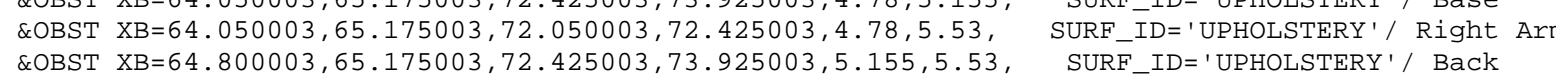

*** GROUP: COUCH2 offset $=0.000000,24.000000,, 0.0000000$ rotate $=270.000000$

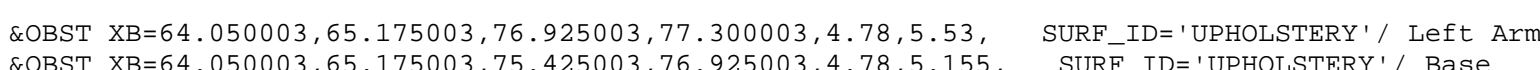

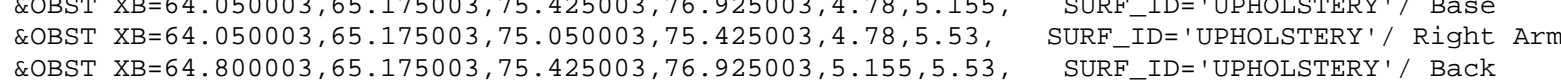

*** GROUP: COUCH2 offset $=0.000000,27.000000,0.000000$ rotate $=270.000000$

\&OBST XB=64. $950003,65.175003,79.925003,80.300003,4.78,5.53$, SURF_IDD ' UPHOLSTERY' / Left Arm

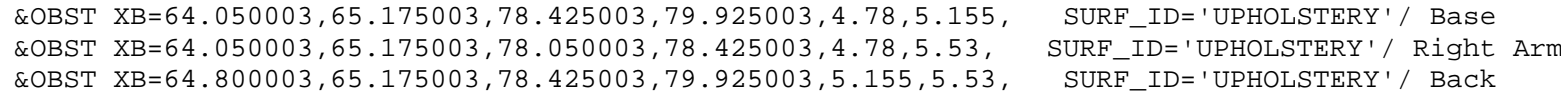

*** GROUP: COUCH2 offset $=0.000000,30.000000,0.000000$ rotate $=270.000000$

\&OBST XB=64. $950003,65.175003,82.925003,83.300003,4.78,5.53, \quad$ SURF_ID=' 'UPHOLSTERY' ' Left Arm \&OBST XB $\mathrm{B}=64.050003,65.17503,81,05$ \&OBST XB=64.800003, 65.175003,81.425003, 82.925003, $5.155,5.53$, SURF_ID=' UPHOLSTERY'/ Back

*** GROUP: TWIN

*** GROUP: ROW

*** GROUP: COUCH2 offset $=0.000000,0.000000,0.000000$ rotate $=90.000000$

\&OBST $X \mathrm{X}=70.050003,71.175003,51.049999,51.424999,4.78,5.53, \quad$ SURF-ID=' 'UPHOLSTERY ' / Left Arm \&OBST $X \mathrm{XB}=70.050003,71.175003,52.924999,53.299999,4.78,5.53, \quad$ SURF_ID= 'UPHOLSTERY' $/$ Right Arm
\&OBST XB $=70.050003,70.425003,51.424999,52.924999,5.155,5.53, \quad$ SURF_ID=' 'UPHOLSTERY' $/$ Back *** GROUP: COUCH2 offset $=0.000000,3.000000,0.000000$ rotate $=90.000000$

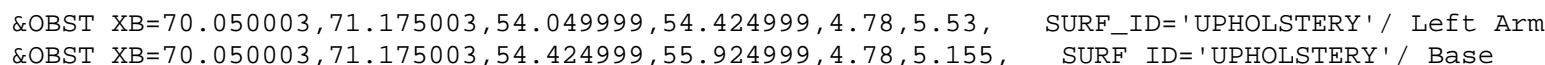


\&OBST XB=70. $050003,71.175003,55.924999,56.299999,4.78,5.53, \quad$ SURF_ID= 'UPHOLSTERY' $/$ Right Arm
\&OBST XB=70.050003, $70.425003,54.424999,55.924999,5.155,5.53, \quad$ SURF_ID='UPHOLSTERY'/ Back *** GROUP: COUCH2 offset $=0.000000,6.000000,0.000000$ rotate $=90$. . 000000

\&OBST XB $=70.050003,71.175003,57.049999,57.424999,4.78,5.53, \quad$ SURF_ID=' 'UPHOLSTERY'/ Left Arm

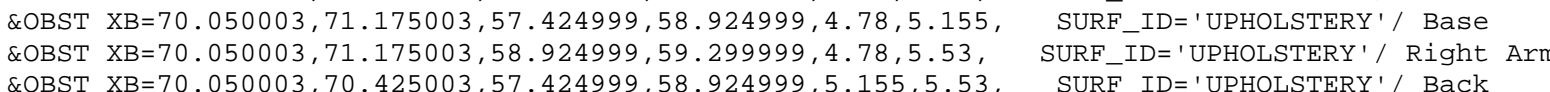

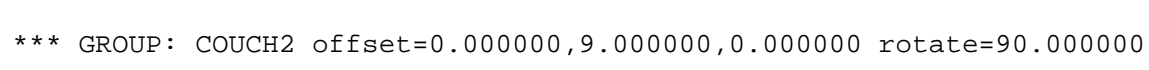

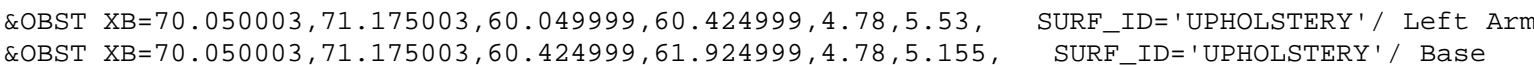
\&OBST XB $=70.050003,71.175003,61.924999,62.299999,4.78,5.53$, SURF_ID='UPHOLSTERY'/ Right Ar

*** GROUP: COUCH2 offset $=0.000000,12.000000,0.000000$ rotate $=90.000000$

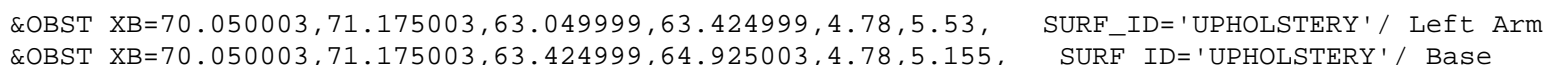

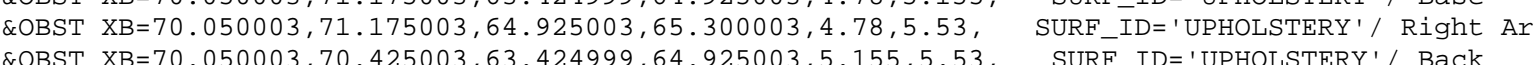

${ }_{* * *}$ GROUP: COUCH2 offset $=0.000000,15.000000,0.000000$ rotate $=90.0000000$

\&OBST XB $=70.050003,71.175003,66.050003,66.425003,4.78,5.53, \quad$ SURF-ID=' 'UPHOLSTERY ' $/$ Left Arm ROBST XB $\mathrm{B}=70.050003,71.175003,67.925003,68.300003,4.78,5.53$, SURF_ID='UPHOLSTERY' / Base \&OBST XB $=70.050003,70.425003,66.425003,67.925003,5.155,5.53$, SURF_ID= 'UPHOLSTERY' / Back

$* * *$ GROUP: COUCH2 offset $=0.000000,18.000000,0.000000$ rotate $=90.000000$

\&OBST $X \mathrm{XB}=70.050003,71.175003,69.050003,69.425003,4.78,5.53$, SURF_ID='UPHOLSTERY '/ Left Arm \&OBST XB $=70.0500003,71.175003,69.425003,70.925003,4.78,5.155$, SURF IDE='UPHOLSTERY '/ Base

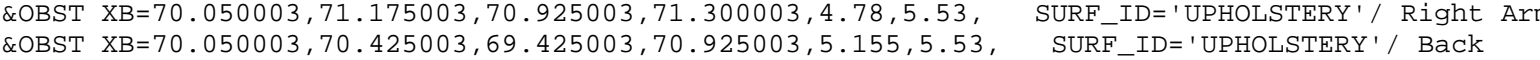

*** GROUP: COUCH2 offset $=0.000000,21.000000,0.000000$ rotate $=90.000000$

\&OBST XB $=70.050003,71.175003,72.050003,72.425003,4.78,5.53$, SURF_ID='UPHOLSTERY'/ Left Arm \&OBST XB $=70.050003,71.175003,72.425003,73.925003,4.78,5.155$, SURF_ID='UPHOLSTERY' / Base

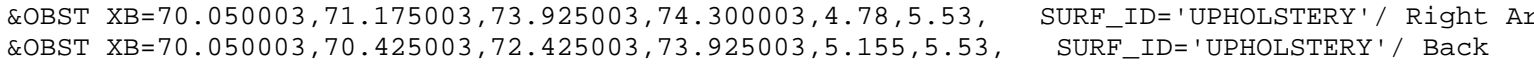

*** GROUP: COUCH2 offset $=0.000000,24.000000,0 . .000000$ rotate $=90.000000$

\&OBST XB $=70.050003,71.175003,75.050003,75.425003,4.78,5.53, \quad$ SURF-ID=' 'UPHOLSTERY' ' Left Arm

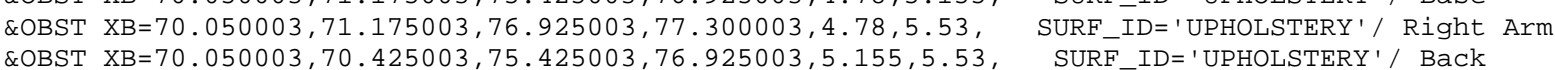

*** GROUP: COUCH2 offset $=0.000000,27.000000,0.000000$ rotate $=90.000000$

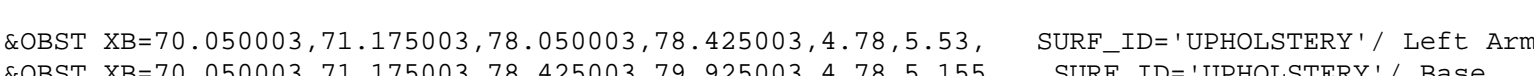

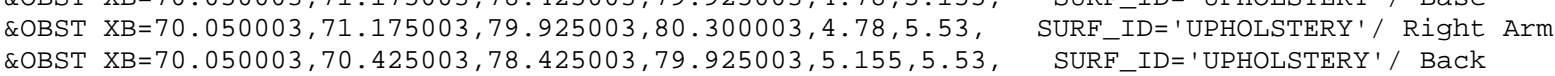

*** GROUP: COUCH2 offset $=0.000000,30.000000,0.000000$ rotate $=90,000000$

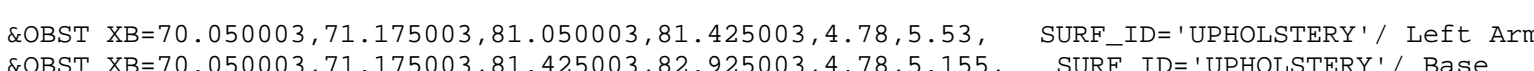

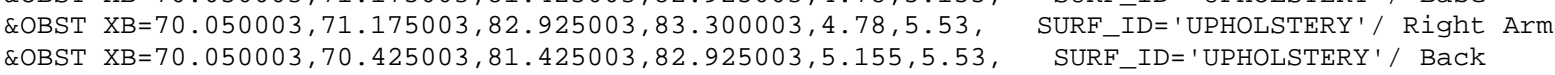

*** GROUP: ROW2

** GROUP: COUCH2 offset $=0.000000,0.000000,0.000000$ rotate $=270.000000$

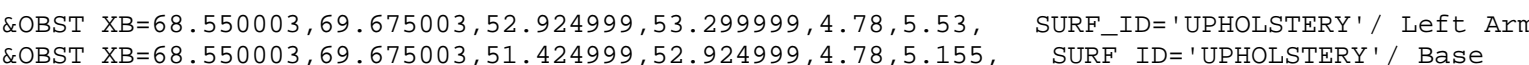
\&OBST XB=68.550003, 69.675003, 51. $449999,51.424999,4.78,5.53$, SURF_ID=' 'UPHOLSTERY'/ Right Ar

*** GROUP: COUCH2 offset $=0.000000,3.000000,0.000000$ rotate $=270.000000$
\&OBST XB $=68.550003,69.675003,55.924999,56.299999,4.78,5.53$, SURF ID=' 'UPHOLSTERY '/ Left Arm \&OBST XB $=68.550003,69.675003,54.424999,55.924999,4.78,5.155$, SURF_ID='UPHOLSTERY' / Base \&OBST XB $=69.3000003,69.675003,54.049999,54.424999,4.78,5.53$, SURF_ID=' UPHOLSTERY'/ Right Ar

*** GROUP: COUCH2 of fset $=0.000000,6.000000,0 . .000000$ rotate $=270.000000$

\&OBST XB=68.550003, 69.675003, 58.924999, 59. 299999, 4.78, 5.53, SURF_ID=' 'UPHOLSTERY' ' Left Arm

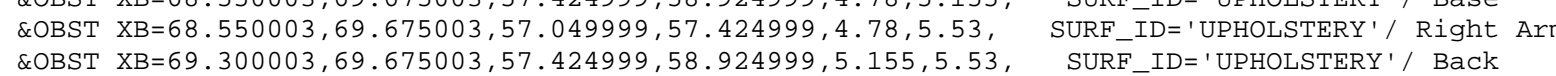

*** GROUP: COUCH2 offset $=0.000000,9.000000,0.000000$ rotate $=270.000000$

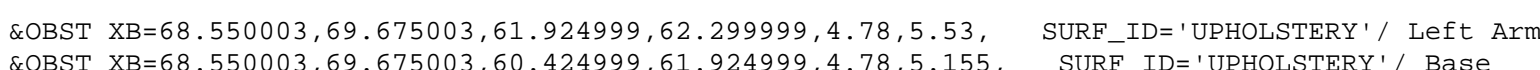

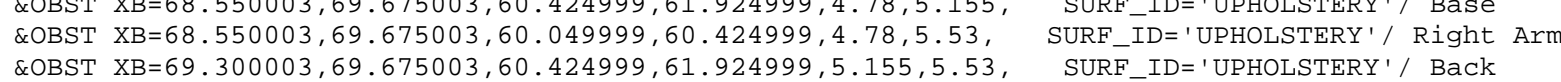

*** GROUP: COUCH2 offset $=0.000000,12.000000,0.000000$ rotate $=270.000000$

2OBST XB $68.550003,69.675003,64.925003,65.300003,4.78,5.53, \quad$ SURF_ID= 'UPHOLSTERY' / Left Arm

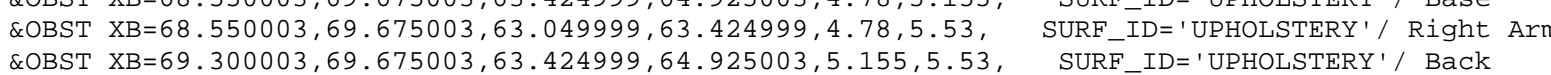

*** GROUP: COUCH2 offset $=0.000000,15.000000,0.000000$ rotate $=270.000000$

\&OBST $X B=68.550003,69.675003,67.925003,68.300003,4.78,5.53$, SURF_ID=' UPHOLSTERY' ' Left Arm عOBST XB=68.550003, \&OBST XB $69.300003,69.675003,66.425003,67.925003,5.155,5.53$, SURF_ID='UPHOLSTERY'/ Back

*** GROUP: COUCH2 offset $=0.000000,18.000000,0.000000$ rotate $=270.000000$

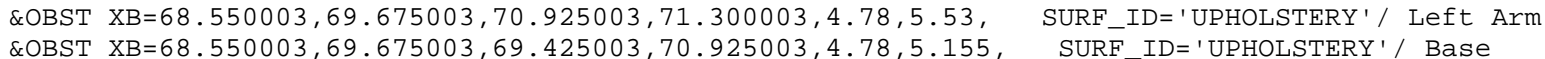

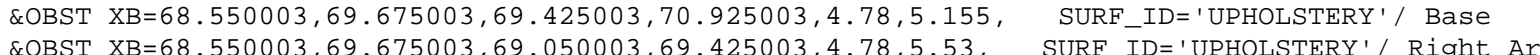
\&OBST XB=69.300003,69.675003, 69.425003, 70.925003, 5.155, 5.53, SURF_ID=' 'UPHOLSTERY' / Back

*** GROUP: COUCH2 offset $=0.000000,21.000000,0.000000$ rotate $=270.000000$

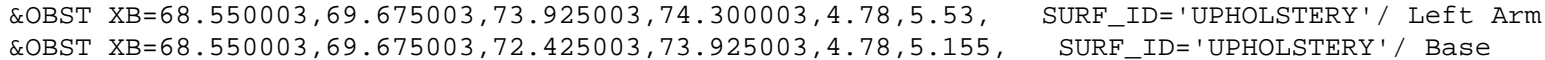

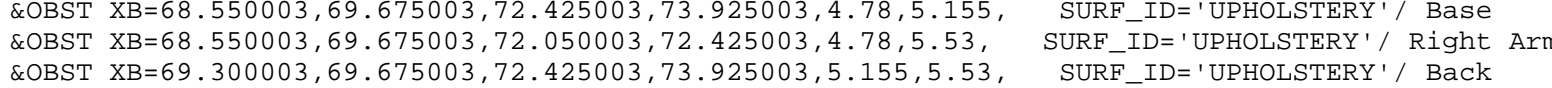

*** GROUP: COUCH2 offset $=0.000000,24.000000,0.000000$ rotate $=270.000000$

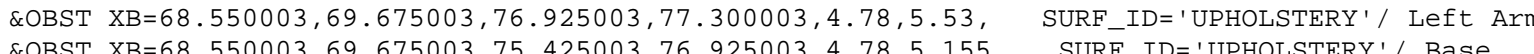

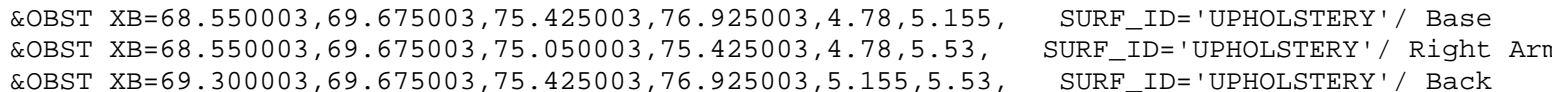

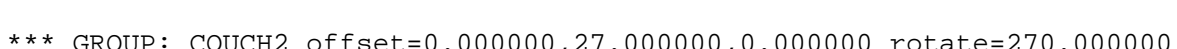

\&OBST XB $=68.550003,69.675003,79.925003,80.300003,4.78,5.53$, SURF_ID='UPHOLSTERY' / Left Arm

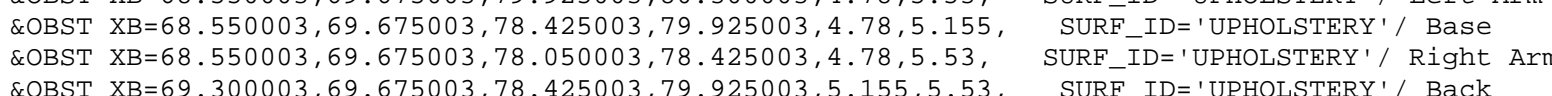

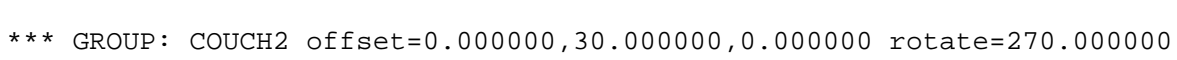

\&OBST XB $=68.550003,69.675003,82.925003,83.300003,4.78,5.53$, SURF_ID='UPHOLSTERY'/ Left Arm \&OBST XB $=68.5550003,69.675003,81.425003,82.925003,4.78,5.155$, SURF_ID='UPHOLSTERY' $/$ Base QOBST XB $=69.300003,69.675003,81.425003,82.925003,5.155,5.53$, SUPF_ID='UPHOLSTERY'/ Right Ar

*** GROUP: ROW2

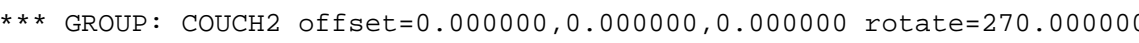

\&OBST XB $=73.050003,74.175003,52.924999,53.299999,4.78,5.53$, SURF_ID='UPHOLSTERY'/ Left Arm \&OBST XB=73.050003, 74.175003, 51.049999, 51.424999, 4.78, 5.53, SURE ID='UPHOLSTERY'/ \&OBST XB=73.800003, 74.175003, 51.424999, 52.924999, 5.155, 5.53, SURF_ID='UPHOLSTERY'/ Back 


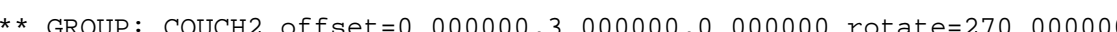

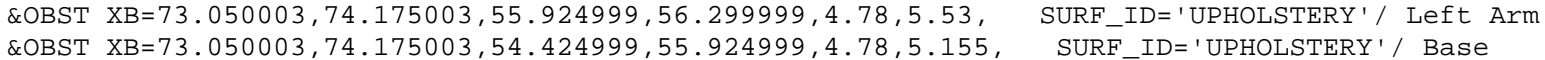
\&OBST XB $=73.050003,74.175003,54.049999,54.424999,4.78,5.53, \quad$ SURF IDD 'UPHOLSTERY'/ Right Arm

*** GROUP: COUCH2 offset $=0.000000,6.0000000,0.000000$ rotate $=270.0000000$

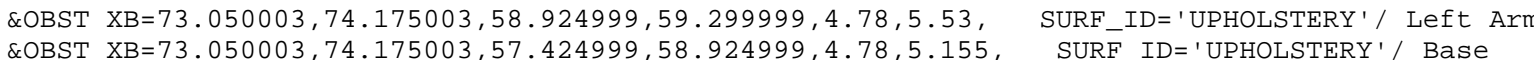

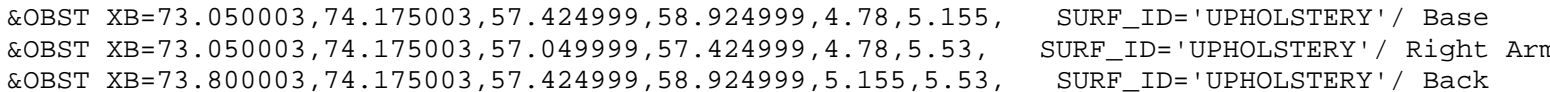

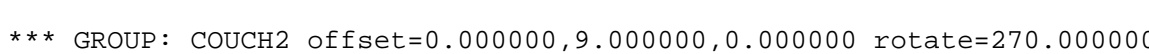

\&OBST XB $=73.050003,74.175003,61.924999,62.299999,4.78,5.53$, SURF_ID=' 'UPHOLSTERY'/ Left Arm \&OBST XB $=73.050003,74.175003,60.424999,61.924999,4.78,5.155, \quad$ SURF_ID='UPHOLSTERY'/ Base
\&OBST XB $=73.050003,74.175003,60.049999,60.424999,4.78,5.53$,
SURF_ID='UPHOLSTERY'/Right Ar \&OBST $X B=73.0500003,74.175003,60.049999,60.424999,4.78,5.53$, SURF_ID=' 'UPHOLSTERY'/ Right Ar

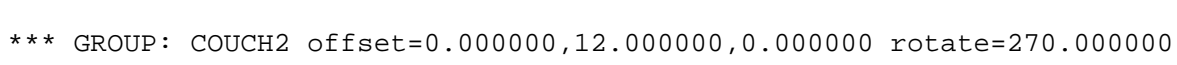

\&OBST XB $=73.050003,74.175003,64.925003,65.300003,4.78,5.53$, SURFID=' 'UPHOLSTERY'/ Left Arm

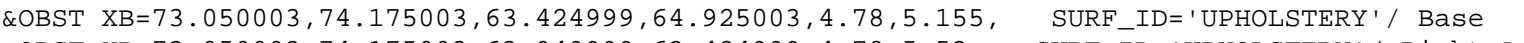

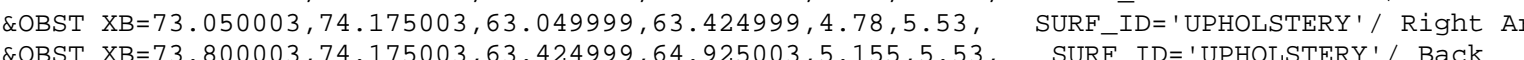
$* * *$ GROUP: COUCH2 offset $=0.000000,15.000000,0.000000$ rotate $=270.000000$

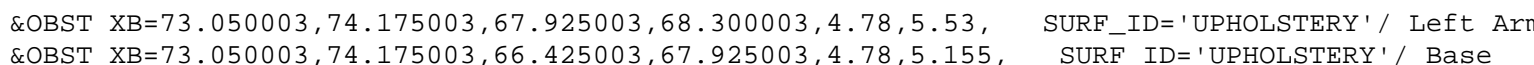
\&OBST XE=73. $050003,74.175003,66.050003,66.425503,4.78,5.53$, SURF_ID='UPHOLSTERY'/ Right Ar

*** GROUP: COUCH2 offset $=0.000000,18.000000,0.000000$ rotate $=270.000000$

\&OBST XB=73.050003, 74.175003, 70.925003, 71.300003,4.78,5.53, SURF_ID='UPHOLSTERY'/ Left Arm \&OBST XB=73. $050003,74.175003,69.425003,70.925003,4.78,5.155$, SURF_ID='UPHOLSTERY'/ Base

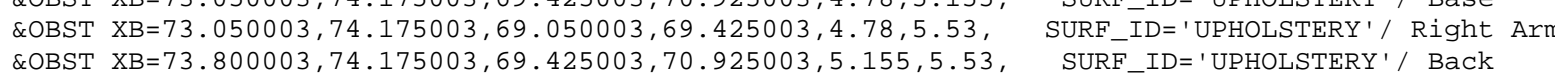

*** GROUP: COUCH2 offset $=0.000000,21.000000,0.000000$ rotate $=270.000000$

\&OBST XB=73. $050003,74.175003,73.925003,74.300003,4.78,5.53$, SURF_ID=' 'UPHOLSTERY'/ Left Arm

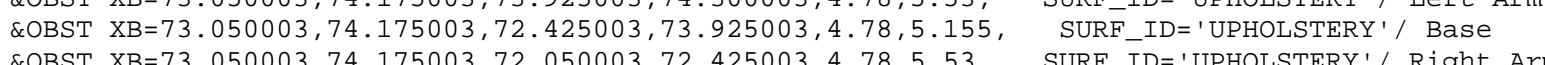

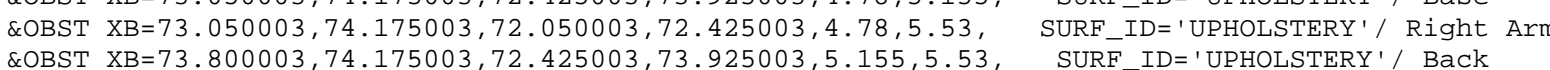

*** GROUP: COUCH2 offset $=0.000000,24.000000,0.000000$ rotate $=270.000000$

\&OBST XB $=73.050003,74.175003,76.925003,77.300003,4.78,5.53$, SURF_ID=' 'UPHOLSTERY'/ Left Arm \&OBST XB $=73.0500003,74.175003,75.425003,76.925003,4.78,5.155$, SURF_ID='UPHOLSTERY' / Base \&OBST $X B=73.050003,74.175003,75.050003,75.425003,4.78,5.53, \quad$ SURF_ID='UPHOLSTERY'/ Right Ar
\&OBST $X \mathrm{X}=73.800003,74.175003,75.425003,76.925003,5.155,5.53$,
SURF_ID=' 'UPHOLSTERY'/ Back

*** GROUP: COUCH2 offset $=0.000000,27.000000,0.000000$ rotate $=270.000000$

\&OBST XB=73. $050003,74.175003,79.925003,80.300003,4.78,5.53$, SURF_ID=' 'UPHOLSTERY' ' Left Arm

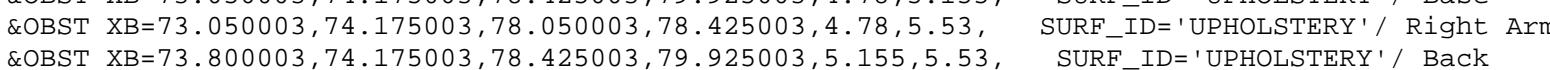
*** GROUP: COUCH2 offset $=0.000000,30.000000,0.000000$ rotate $=270.000000$

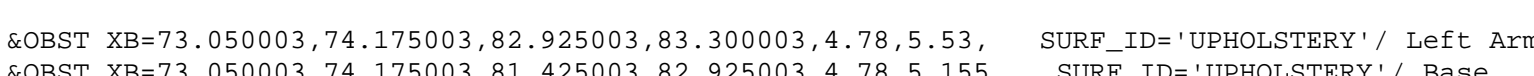

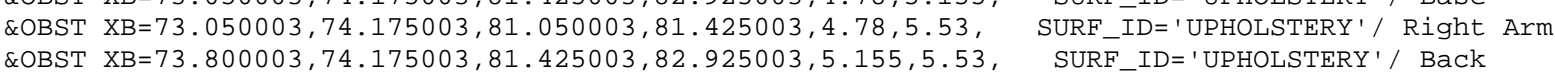

\section{*** GROUP: SHORT_ROW}

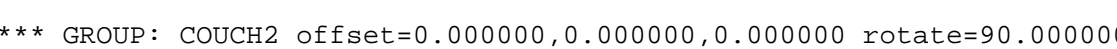

\&OBST XB=67.044998, 67.419998, 46.91, 48. $035,4.78,5.53$, SURF_ID= 'UPHOLSTERY ' ' Left Arm

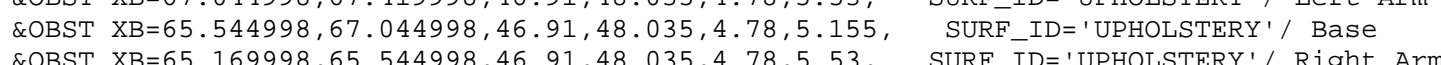

\&OBST XB=65, $544998,67,044998,46,91,47,285,5,155,5,53$, SURF_ID=' UPHOLSTERY' $/$ Back

*** GROUP: COUCH2 offset $=0.000000,3.000000,0.000000$ rotate $=90.000000$

\&OBST XB=64.044998, 64.419998, 46.91, 48.035, 4.78, 5.53, SURF_ID='UPHOLSTERY'/ Left Arm \&OBST $X \mathrm{XB}=62.544998,64.044998,46.91,48.035,4.78,5.155, \quad$ SURF_ID=' 'UPHOLSTERY '/ Base (NOBST

*** GROUP: COUCH2 offset $=0.000000,6.000000,0.000000$ rotate $=90.000000$

\&OBST XB=61. $444998,61.419998,46.91,48.035,4.78,5.53$, SURF_ID='UPHOLSTERY'/ Left Arm \&OBST XB=59. $544998,61.044998,46.91,48.035,4.78,5.155$, SURF_ID=' 'UPHOLSTERY'/ Base

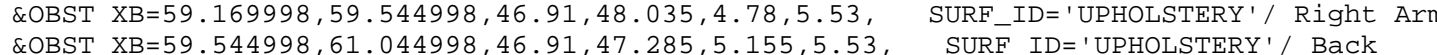

*** GROUP: COUCH2 offset $=0.000000,9.000000,0.000000$ rotate $=90.000000$

\&OBST XB=58. 044998, 58.419998, 46.91, 48. 035, 4.78, 5.53, SURF_ID='UPHOLSTERY'/ Left Arm

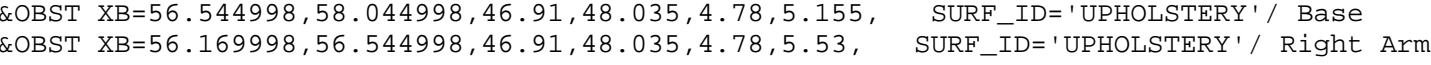

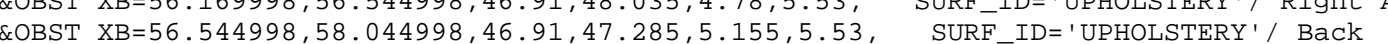

$* \star *$ GROUP: COUCH2 offset $=0.000000,12.000000,0.000000$ rotate $=90.000000$

\&OBST XB=55. 044998, 55.419998, 46.91, 48. 035, 4.78, 5.53, SURF ID= 'UPHOLSTERY ' ' Left Arm

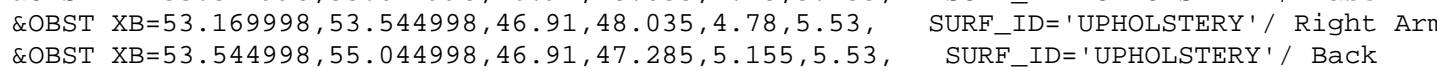

$\star * \star$ GROUP: COUCH2 offset $=0.000000,15.000000,0.000000$ rotate $=90.000000$

\&OBST XB $=52.044998,52.419998,46.91,48.035,4.78,5.53, \quad$ SURF_ID='UPHOLSTERY'/ Left Arm \&OBST XB=50.169998, 50.544998, 46.91, $48.035,4.78,5.53$, SURF_ID='UPHOLSTERY'/ Right Arm \&OBST XB=50.544998, 52.044998, 46.91, 47.285, $5.155,5.53$, SURF_ID=' 'UPHOLSTERY'/'Back

*** GROUP: COUCH2 offset $=0.000000,18.000000,0.000000$ rotate $=90.000000$

\&OBST XB=49. $944998,49.419998,46.91,48.035,4.78,5.53$, SURF_ID= 'UPHOLSTERY ' ' Left Arm \&OBST XB=47.169998, 47.544998, 46.91,48.035,4.78,5.53, SURF ID=' 'UPHOLSTERY'/ Right A \&OBST XB $=47.544998,49.044998,46.91,47.285,5.155,5.53$, SURF_ID='UPHOLSTERY'/ Back

*** GROUP: ROW

*** GROUP: COUCH2 offset $=0.000000,0.000000,0.000000$ rotate $=90.000000$

EOBST XB=41.549999, 41.924999, 84.43,85.555, 4.78,5.53, SURF_ID= 'UPHOLSTERY ' / Left Arm \&OBST XB=41.924999, 43.424999, 84.43, 85.555, 4.78,5.155, SURF_ID='UPHOLSTERY'/ Base

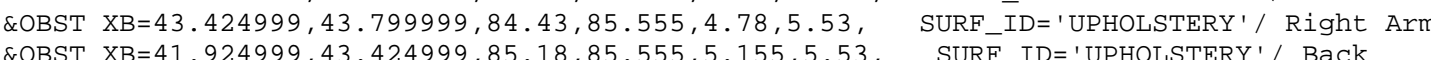

*** GROUP: COUCH2 offset $=0.000000,3.000000,0.000000$ rotate $=90.000000$

\&OBST XB=44.549999, 44.924999, 84.43, 85.555, 4.78,5.53, SURF_ID='UPHOLSTERY'/ Left Arm \&OBST XB=46.424999, 46.799999, 84.43, 85.555, 4.78,5.53, SURF_ID='UPHOLSTERY'/ Right Arm

*** GROUP: COUCH2 offset $=0.000000,6.000000,0.000000$ rotate $=90.000000$

\&OBST XB $=47.549999,47.924999,84.43,85.555,4.78,5.53, \quad$ SURF_ID='UPHOLSTERY ' ' Left Arm \&OBST XB=49.424999, 49.799999, 84.43, 85. 555, 4.78,5.53, SURF - ID = 'UPHOLSTERY' '/ Right Arm

*** GROUP: COUCH2 offset $=0.000000,9.000000,0.000000$ rotate $=90.000000$

\&OBST XB=50.549999, 50.924999, 84.43, 85.555,4.78,5.53, SURF_ID='UPHOLSTERY'/ Left Arm \&OBST XB=52.424999, $52.799999,84.43,85.555,4.78,5.53, \quad$ SURF_ID=' 'UPHOLSTERY'/ Right Arm
\&OBST XB=50.924999, $52.424999,85.18,85.555,5.155,5.53, \quad$ SURF_ID='UPHOLSTERY'/ Back

*** GROUP: COUCH2 offset $=0.000000,12.000000,0.000000$ rotate $=90.000000$

COBST XB=53.549999, 53.924999, 84.43,85.555,4.78,5.53, SURE_ID= 'UPHOLSTERY' / Left Arm 


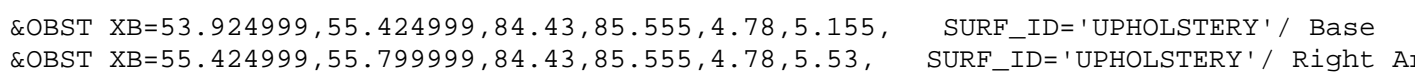
COBST XB $=55.424999,55.799999,84.43,85.555,4.78,5.53$,
\&OBST XB $=53.92499,55.424999,85.18,85.555,5.155,5.53$,
SURF_IDE='IUPHOLSTERY'/ Back $* * *$ GROUP: COUCH2 offset $=0.000000,15.000000,0.000000$ rotate $=90.000000$

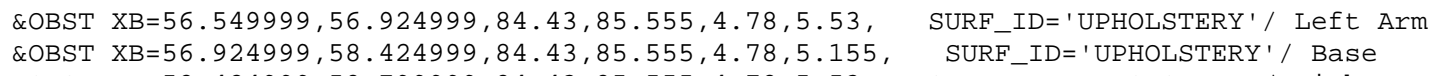

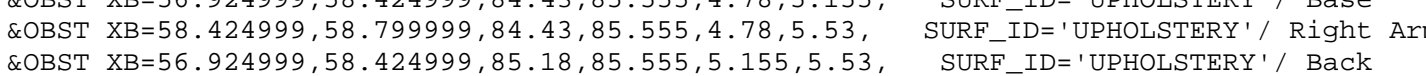
*** GROUP: COUCH2 offset $=0.000000,18.000000,0 . .000000$ rotate $=90.000000$

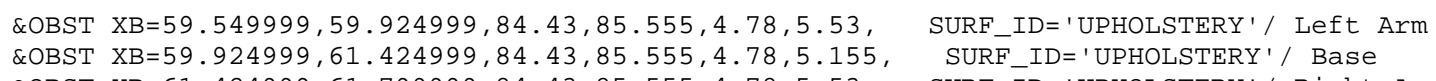

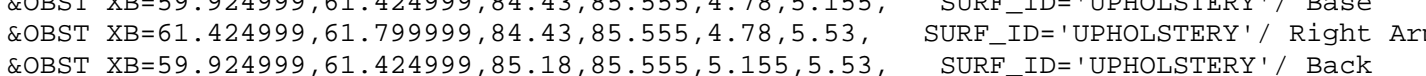

$\star \star \star *$ GROUP: COUCH2 offset $=0.000000,21.000000,0.000000$ rotate $=90.0000000$

\&OBST XB $=62.549999,62.924999,84.43,85.555,4.78,5.53$,
\&OBST XB $=62.924999,64.425003,84.43,85.555,4.78,5.155$,
SURF_ID=' 'UPHOLSTERY' ' Left Arm
SURFID=' 'UPHOLSTERY' / Base

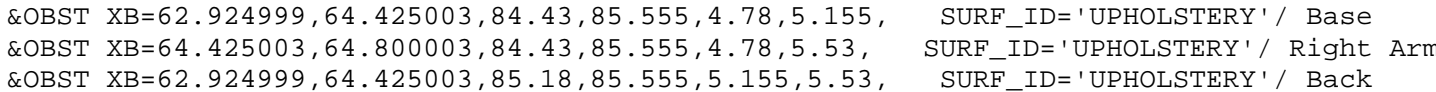

*** GROUP: COUCH2 offset $=0.000000,24.0000000,0$. . 000000 rotate $=90$. . 0000000

\&OBST XB=65.550003, 65.925003, 84. 43, 85. 555, 4.78,5.53, SURF_ID='UPHOLSTERY' ' Left Arm

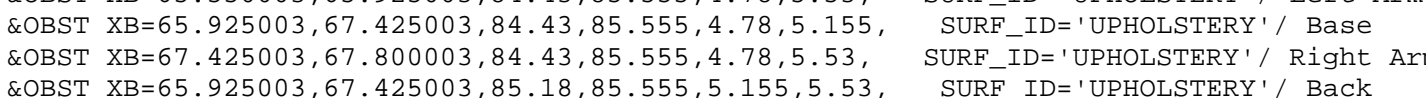

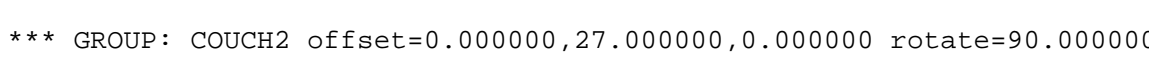
COBST XB=68.550003, 68.925003, 84. 43, $85.555,4.78,5.53, \quad$ SURF_ID='UPHOLSTERY' ' Left Arm
\&OBST XB $68.925003,70.425003,84.43,85.555,4.78,5.155$,
SURF_ID='UPHOLSTERY' / Base

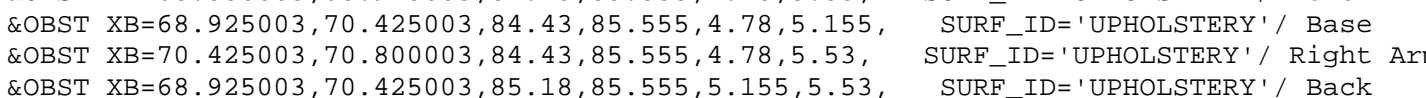

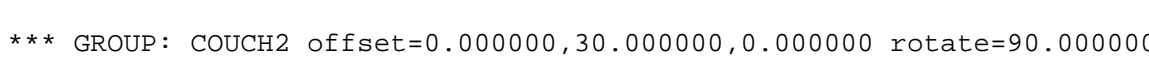

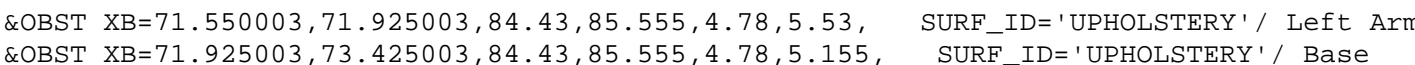
\&OBST XB=73.425003, 73.800003, 84.43, 85.555, 4.78,5.53, SURF-ID='UPHOLSTERY'/Right Arm

\section{*** GROUP: LEVEL}

*** GROUP: ROW

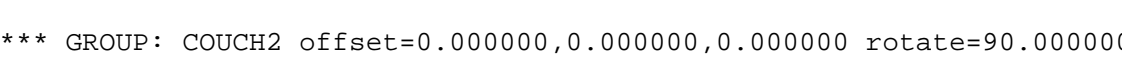

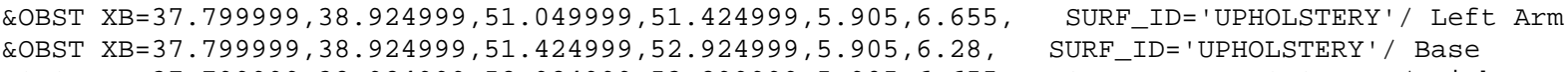
\&OBST XB=37.799999, 38.924999, 52.924999, 52.29999, $5.905,6.655$, SURF ID='UPHOLSTYRY/ Right Ar

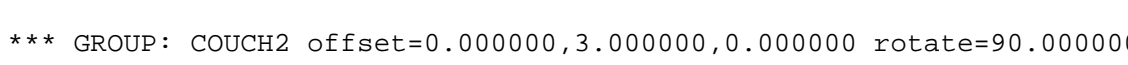

\&OBST XB=37.799999, 38.924999, 54. 049999, 54.424999, 5.905, 6.655, SURF_ID=' 'UPHOLSTERY'/ Left Arm \&OBST XB=37.799999, 38.924999, 54.424999, 55.924999, 5.905, 6.28, SURF_ID='UPHOLSTERY'/ Base

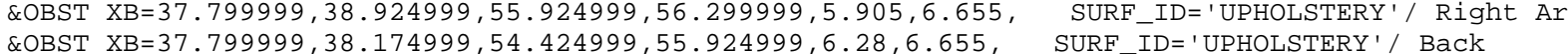

*** GROUP: COUCH2 offset $=0.000000,6.000000,0.000000$ rotate $=90 . .00000$

\&OBST XB=37.799999, 38.924999, 57. 049999, 57.424999, 5.905, 6.655, SURF_ID=' 'UPHOLSTERY'/ Left Arm \&OBST XB=37.799999, 38.924999, 57.424999, 58.924999, 5.905, 6.28, SURF_ID='UPHOLSTERY'/ Base \&OBST XB=37.79999, 38.924999, 58.924999, 59. 299999, 5.905,6.655, SURF_ID='UPHOLSTERY'/ Right Ar *** GROUP: COUCH2 offset $=0.000000,9.000000,0.000000$ rotate $=90.000000$

\&OBST XB=37.799999, 38.924999, 60. $449999,60.424999,5.905,6.655$, SURF_ID=' 'UPHOLSTERY'/ Left Arm

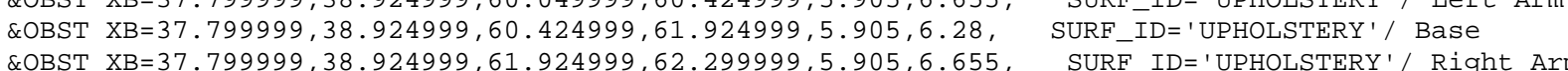

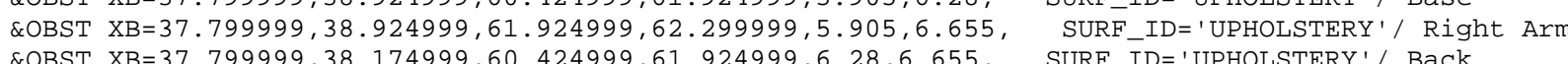

** GROUP: COUCH2 offset $=0.000000,12.000000,0.000000$ rotate $=90.000000$

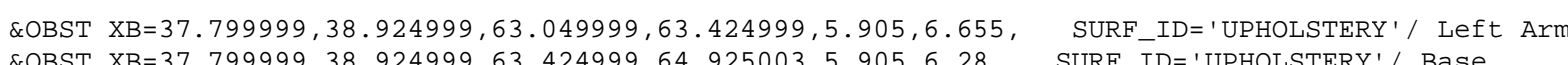

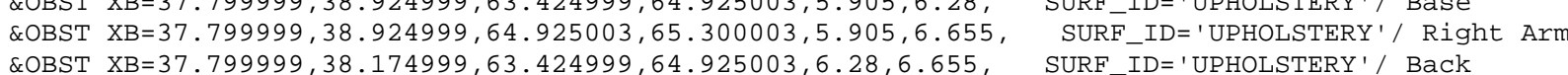

*** GROUP: COUCH2 offset $=0.000000,15.000000,0.000000$ rotate $=90.000000$

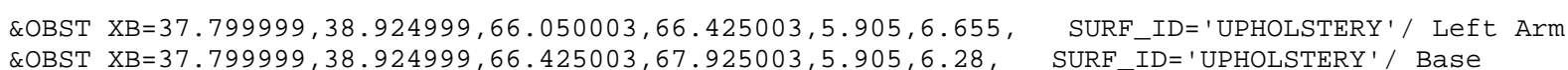

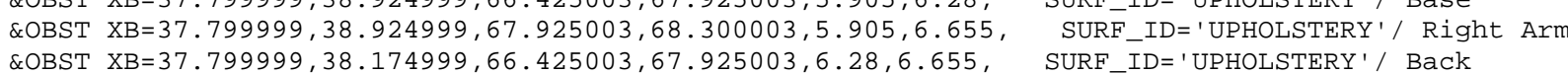
*** GROUP: COUCH2 offset $=0.000000,18.000000,0.000000$ rotate $=90.000000$

\&OBST XB $=37.799999,38.924999,69.050003,69.425003,5.905,6.655$, SURF_ID=' 'UPHOLSTERY ' / Left Arm

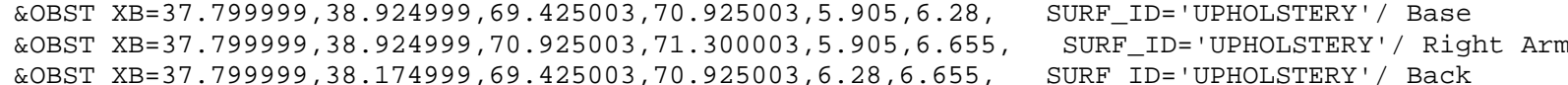
*** GROUP: COUCH2 offset $=0.000000,21.000000,0.000000$ rotate $=90.000000$

\&OBST XB $=37.799999,38.924999,72.050003,72.425003,5.905,6.655, \quad$ SURF_ID= 'UPHOLSTERY' $/$ Left Arm
\&OBST XB $=37.799999,38.924999,72.425003,73.925003,5.905,6.28$,
SURF $I D=$ 'UPHOLSTERY'

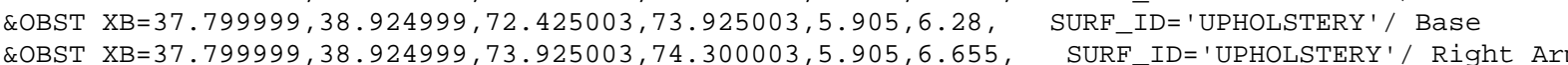
\&OBST X $X=37.799999,38.174999,72.425003,73.925003,6.28,6.655$, SURF_IDD='UPHOLSTERY' / Back

*** GROUP: COUCH2 offset $=0.000000,24.000000,0.000000$ rotate $=90.000000$

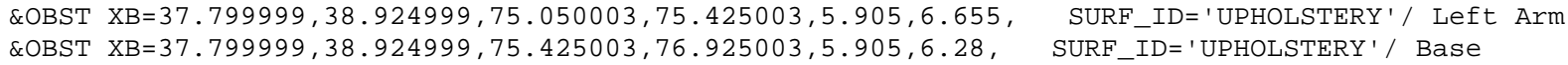

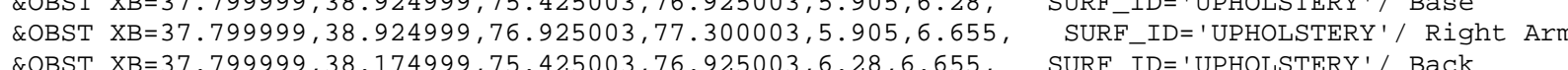

*** GROUP: COUCH2 offset $=0.000000,27.000000,0.000000$ rotate $=90.000000$

QOBST XB $=37.799999,38.924999,78.050003,78.425003,5.905,6.655$,
SURF_ID='UPHOLSTERY' / Left Arm

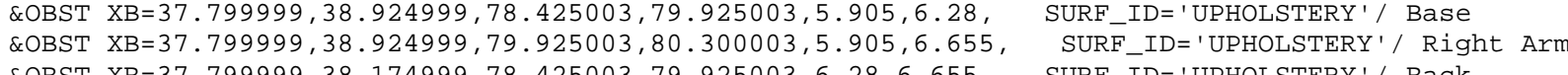

*** GROUP: COUCH2 offset $=0.000000,30.000000,0.000000$ rotate $=90.000000$

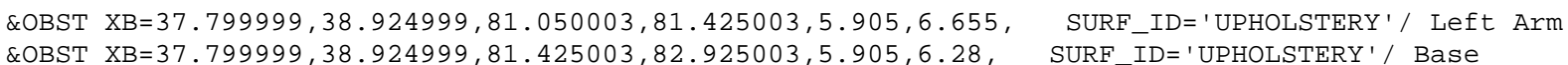

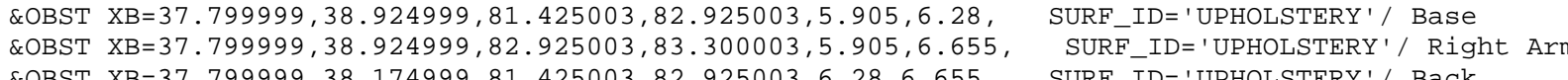

\section{*** GROUP: BASE}

$* * *$ GROUP: TWIN

*** GROUP: ROW

${ }^{* * *}$ GROUP: COUCH2 offset $=0.000000,0.000000,0.000000$ rotate $=90.000000$

\&OBST XB $=43.049999,44.174999,51.049999,51.424999,5.905,6.655$, SURF_ID=' 'UPHOLSTERY'/ Left Arm \&OBST XB=43.049999, 44.174999, 52.924999, 53.299999, 5.905,6.655, SURF_ID='UPHOLSTERY'/ Right Ar *** GROUP: COUCH2 offset $=0.000000,3.000000,0.000000$ rotate $=90.000000$

\&OBST XB=43. $049999,44.174999,54.049999,54.424999,5.905,6.655$, SURF_ID='UPHOLSTERY' / Left Arm

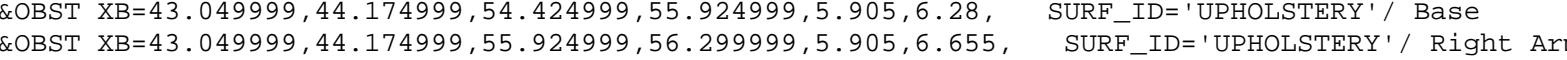

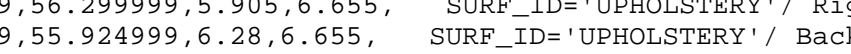

*** GROUP: COUCH2 offset $=0.000000,6.000000,0.000000$ rotate $=90.000000$

\&OBST XB=43. $849999,44.174999,57.049999,57.424999,5.905,6.655$, SURF_ID= 'UPHOLSTERY' / Left Arm

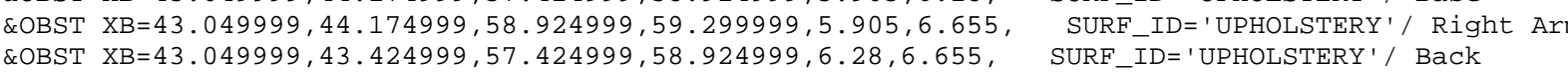


** GROUP: COUCH2 offset $=0.000000,9,000000,0,000000$ rotate $=90,000000$

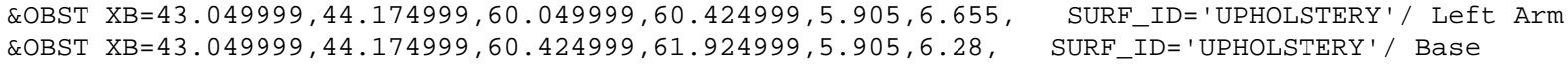
\&OBST XB=43.049999, 44.174999, 61.924999, 62. 299999, $5.905,6.655$, SURF_ID='UPHOLSTERY' $/$ Right Arm

*** GROUP: COUCH2 offset $=0.000000,12.0000000,0.000000$ rotate $=90$. . 000000

\&OBST XB $=43.049999,44.174999,63.049999,63.424999,5.905,6.655$,
SURF_ID=' 'UPHOLSTERY ' / Left Arm

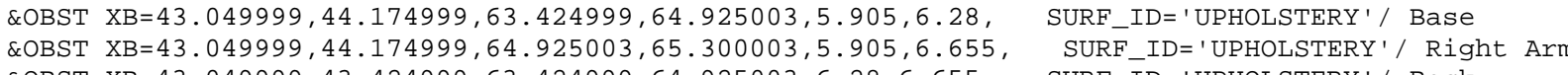
*** GROUP: COUCH2 offset $=0.0000000,15.000000,0.000000$ rotate $=90.000000$

\&OBST XB=43.049999, 44.174999, 66. 050003, 66. 425003, 5.905, 6.655, SURF_ID=' 'UPHOLSTERY '/ Left Arm \&OBST $X \mathrm{X}=43.049999,44.174999,66.425003,67.925003,5.905,6.28, \quad$ SURF-ID=' 'UPHOLSTERY'/ Base COBST XB=43.04999, $44.174999,67.925003,68.300003,5.905,6.655$, SURF_ID='UPHOLSTERY'/Right Ar
\&OBST XB $=43.049999,43.424999,66.425003,67.925003,6.28,6.655$,
SURF_ID='UPHOLSTERY'/ Back

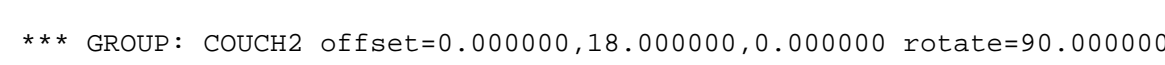

\&OBST XB=43.049999, 44.174999, 69.050003, 69.425003, 5.905, 6.655, SURF_ID=' 'UPHOLSTERY'/ Left Arm \&OBST XB=43. $449999,44.174999,69.425003,70.925003,5.905,6.28$, SURF_ID=' 'UPHOLSTERY'/ Base

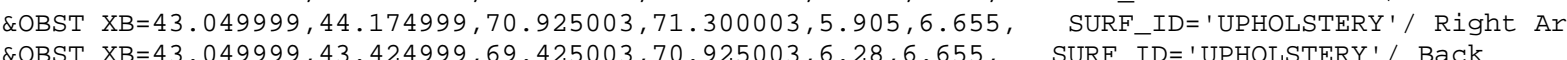
*** GROUP: COUCH2 offset $=0.000000,21.000000,0.000000$ rotate $=90.000000$ \&OBST X X=43.049999, $44.174999,73.925003,74.300003,5.905,6.655$, SURF_ID='UPHOLSTERY'/ Base

*** GROUP: COUCH2 offset $=0.000000,24.000000,0.000000$ rotate $=90.000000$

\&OBST XB=43.049999, 44.174999, 75.050003, 75.425003, 5.905, 6.655, SURF_ID=' 'UPHOLSTERY'/ Left Arm QOBST XB=43.049999, 44.174999, 75.425003, 76. 925003, $5.905,6.28$, SURF_ID='UPHOLSTERY'/ Base Ar عOBST XB $=43.049999,43.424999,75.425003,76.925003,6.28,6.655$, SURF ID

*** GROUP: Couch2 offset $=0.000000,27.000000,0.000000$ rotate $=90.000000$

\&OBST XB $=43.049999,44.174999,78.050003,78.425003,5.905,6.655$, SURF_ID=' 'UPHOLSTERY'/ Left Arm

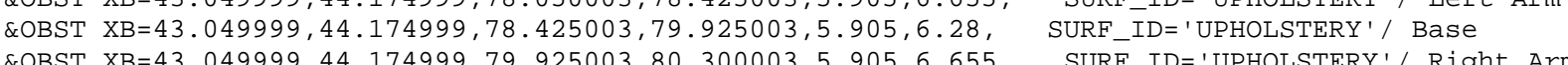

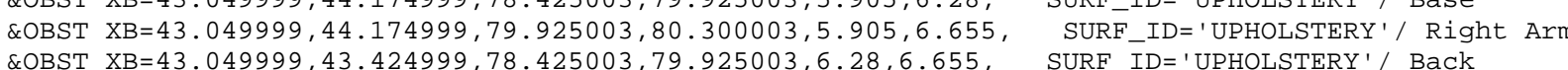

*** GROUP: COUCH2 offset $=0.000000,30.000000,0.000000$ rotate $=90.000000$

\&OBST XB=43. 049999, 44.174999, 81. 050003, 81.425003, 5.905, 6.655, SURF_ID=' 'UPHOLSTERY'/ Left Arm \&OBST XB=43.049999, 44.174999, 81.425003, 82.925003, 5.905, 6.28, SURF_ID=' 'UPHOLSTERY'/ Base

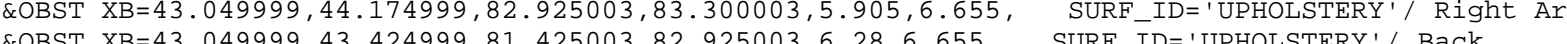

$* * *$ GROUP: ROW2

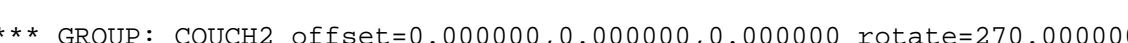

COBST XB $=41.549999,42.674999,52.924999,53.299999,5.905,6.655$, SURF ID=' 'UPHOLSTERY '/ Left Arm

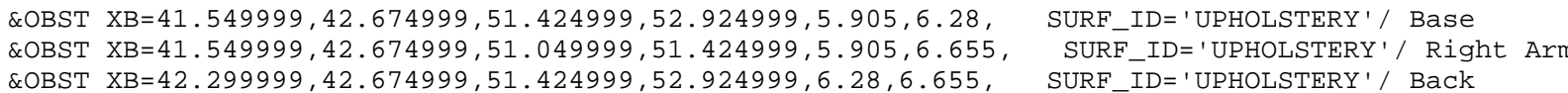

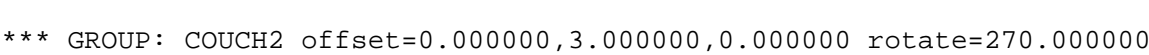

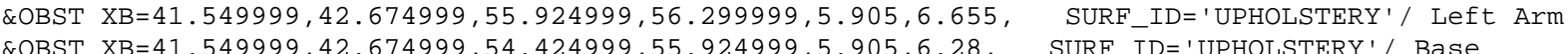

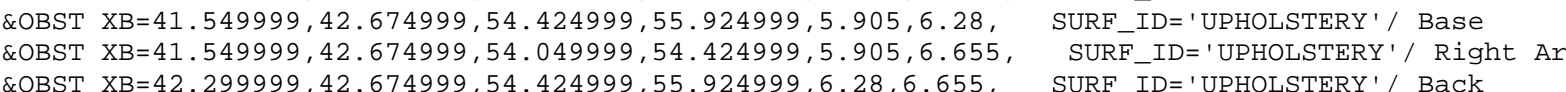

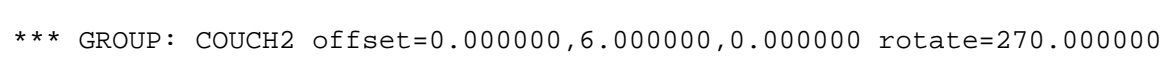

\&OBST XB=41.549999, 42.674999, 58.924999, 59. 299999, 5.905, 6.655, SURF_ID= 'UPHOLSTERY' / Left Arm

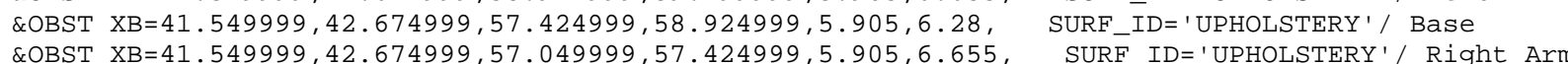

\&OBST XB=42. 299999, 42. 674999, 57, 424999, 58, 924999, 6. 28,6.655, SURF_ID=' UPHOLSTERY'/ Back

*** GROUP: COUCH2 offset $=0.000000,9.000000,0.000000$ rotate $=270.000000$

\&OBST XB=41.549999, 42.674999, 61.924999, 62.299999, 5.905, 6.655, SURF_ID='UPHOLSTERY'/ Left Arm

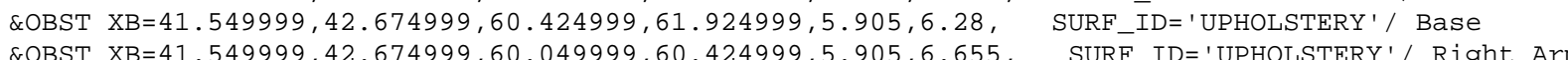
OOBST XB

*** GROUP: COUCH2 offset $=0.000000,12.000000,0.000000$ rotate $=270.000000$

\&OBST XB=41.549999, 42.674999,64.925003, 65.300003, 5.905, 6.655, SURF_ID=' 'UPHOLSTERY'/ Left Arm \&OBST XB $41.549999,42.674999,63.424999,64.925003,5.905,6.28$, SURF-ID='UPHOLSTERY'/ Base \&OBST XB=41.54999, 42.674999, 63. $449999,63.424999,5.905,6.655$, SURF_ID='UPHOLSTERY'/ Right
\&OBST XB=42.29999, $42.674999,63.424999,64.925003,6.28,6.655$,
SURF_ID='UPHOLSTERY'/ Back

*** GROUP: COUCH2 offset $=0.000000,15.000000,0.000000$ rotate $=270.000000$

\&OBST XB $41.549999,42.674999,67.925003,68.300003,5.905,6.655$, SURF_ID=' 'UPHOLSTERY' / Left Arm \&OBST XB=41.549999, 42.674999,66.425003, 67.925003,5.905,6.28, SURF-ID='UPHOLSTERY'/ Base \&OBST XB=41.549999, 42.674999,66.050003,66.425003,5.905,6.655, SURF_ID='UPHOLSTERY'/ Right Ar *** GROUP: COUCH2 offset $=0.0000000,18.000000,0.000000$ rotate $=270.000000$

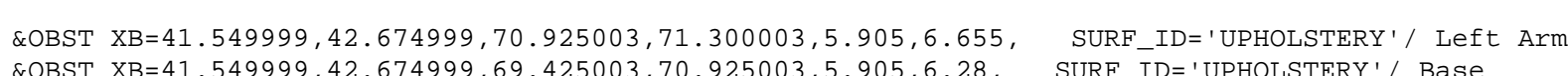

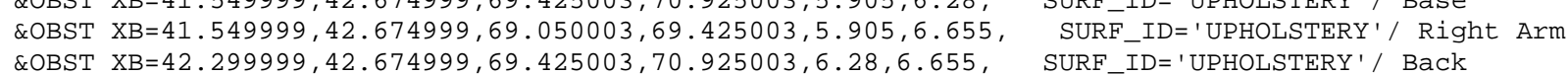

*** GROUP: COUCH2 offset $=0.000000,21.000000,0.000000$ rotate $=270.000000$

\&OBST XB $=41.549999,42.674999,73.925003,74.300003,5.905,6.655$, SURF_ID=' UPHOLSTERY ' / Left Arm

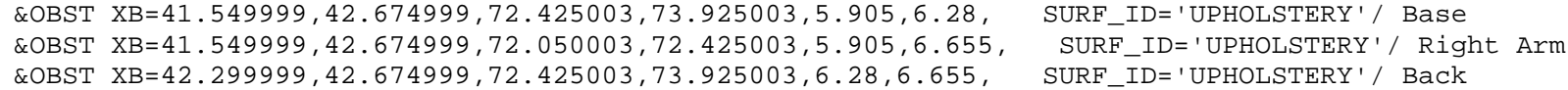

*** GROUP: COUCH2 offset $=0.000000,24.000000,0.000000$ rotate $=270.000000$

\&OBST XB=41.549999, 42.674999, 76.925003, 77.300003, 5.905, 6.655, SURF_ID=' 'UPHOLSTERY'/ Left Arm

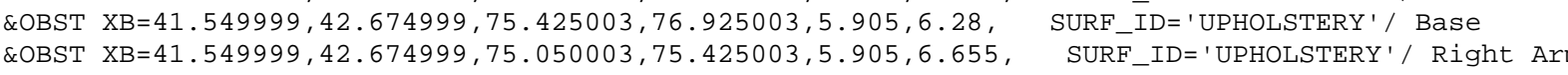
\&OBST XB=42.299999, 42.674999, 75.425003, 76.925003,6.28,6.655, SURF_ID='UPHOLSTERY'/ Back

*** GROUP: COUCH2 offset $=0.000000,27.000000, \Theta .000000$ rotate $=270.000000$

\&OBST XB=41.549999, 42.674999, 79.925003, 80.300003, 5.905, 6.655, SURF_ID=' 'UPHOLSTERY ' / Left Arm \&OBST XB=41.549999, 42.674999, 78. 2050093, 78.425003, $5.905,6.655$, SURE ID=''UPHOLSTERY'/ Right Ar \&OBST XB=42.299999, 42.674999, 78.425003, 79.925003,6.28,6.655, SURF_ID='UPHOLSTERY'/ Back

*** GROUP: COUCH2 offset $=0.000000,30.000000,0.000000$ rotate $=270.000000$

\&OBST XB=41.549999, 42.674999, 82.925003, 83.300003, 5.905, 6.655, SURF_ID=' 'UPHOLSTERY '/ Left Arm

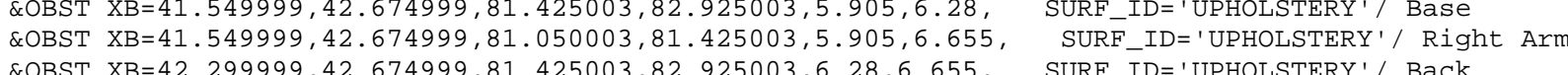

*** GROUP: TWIN

*** GROUP: ROW

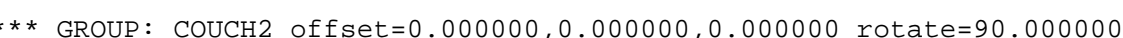

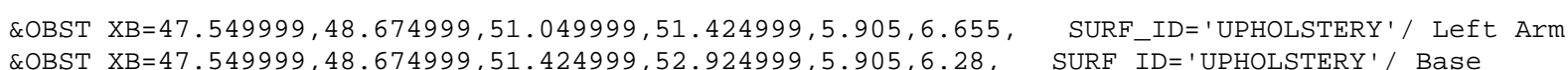
\&OBST XB $=47.549999,48.674999,52.924999,53.299999,5.905,6.655$, SURF SID='UPHOLSTERY'/ Right Arm

$* * *$ GROUP: COUCH2 offset $=0.000000,3.000000,0.000000$ rotate $=90.000000$

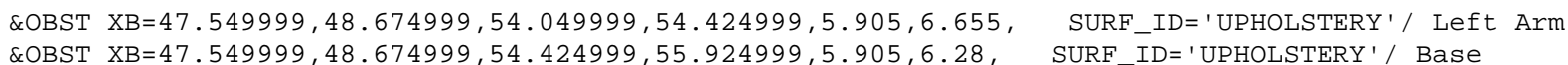
\&OBST XB=47.54999, $48.674999,55.924999,56.299999,5.905,6.655$, SURF_ID=' 'UPHOLSTERY'/R Right Arm
\&OBST XB=47.549999, $47.924999,54.424999,55.924999,6.28,6.655$,
SURF_ID='UPHOLSTERY'/ Back

*** GROUP: COUCH2 offset $=0.000000,, 6.000000,, 0.000000$ rotate $=90.0000000$ 
\&OBST XB $=47.549999,48.674999,57.049999,57.424999,5.905,6.655, \quad$ SURF_ID= 'UPHOLSTERY '/ Left Arm

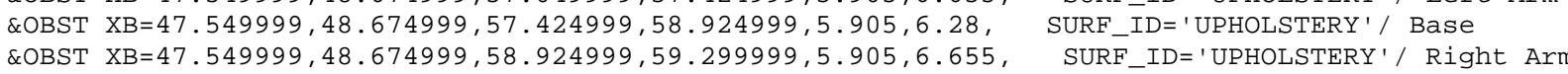
\&OBST XB=47.549999, 47.924999, 57.424999, 58. 924999, 6.28,6.655, SURF_ID='UPHOLSTERY'/ Back

*** GROUP: COUCH2 offset $=0.000000,9.000000,0.000000$ rotate $=90.000000$

\&OBST XB $=47.549999,48.674999,60.049999,60.424999,5.905,6.655$, SURF_ID=' 'UPHOLSTERY'/ Left Arm QOBST XB=47.549999, 48.674999, 60.424999, 61.924999, 5.905, 6.28, SURF_ID='UPHOLSTERY'/ Base Ar \&OBST XB $=47.549999,47.924999,60.424999,61.924999,6.28,6.655$, SURF IDD' 'UPHOLSTERY'/ Rack Ar

*** GROUP: COUCH2 offset $=0.0000000,12.000000,0.0000000$ rotate $=90 . .000000$

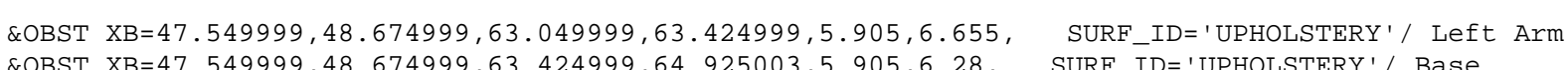
\&OBST XB=47.549999, 48.674999, 64.925003, 65.300003,5.905,6.655, SUURF_ID=' 'UPHOLSTERY'// Right ArI

*** GROUP: COUCH2 offset $=0.000000,15.000000,0.000000$ rotate $=90.000000$

\&OBST XB=47.549999, 48.674999, 66. 050003, 66.425003, 5.905, 6.655, SURF_ID=' 'UPHOLSTERY'/ Left Arm

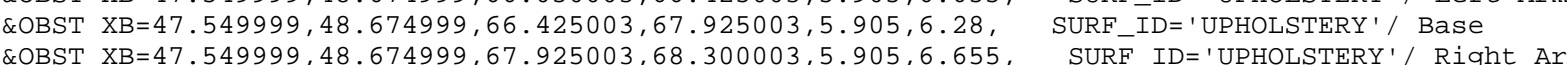
LOBST XB=47.54999, $48.674999,67.925003,68.300003,5.905,6.655$, SURF-ID=' 'UPHOLSTERY'/ Right Arm
\&OBST XB $=47.549999,47.924999,66.425003,67.925003,6.28,6.655$, SURF-ID=' 'UPHOLSTERY'/ Back

*** GROUP: CouCH2 offset $=0.000000,18.000000,0.000000$ rotate $=90.000000$

\&OBST XB=47.549999, 48.674999, 69. 050003, 69.425003, 5.905, 6.655, SURF_ID=' 'UPHOLSTERY' ' Left Arm \&OBST XB=47.549999, 48.674999, 69.425003, 70.925003, 5.905, 6.28, SURF_ID='UPHOLSTERY'/ Base Ar

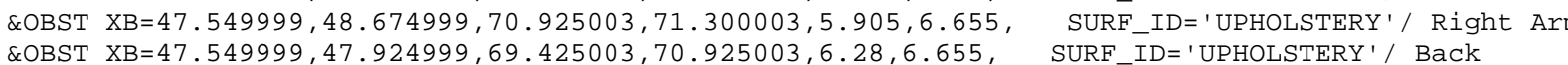

$* * *$ GROUP: COUCH2 offset $=0.000000,21.000000,0.000000$ rotate $=90.000000$

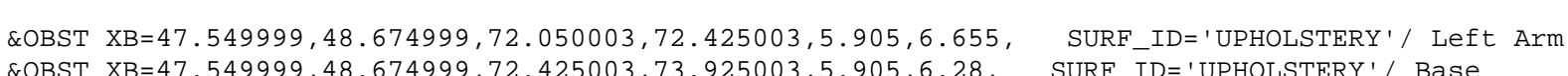
\&OBST XB=47.549999, 48.674999, 73.925003,74.300003,5.905,6.655, SURF_ID=' 'UPHOLSTERY'/ Right Ar

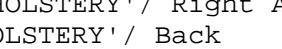

*** GROUP: COUCH2 offset $=0.000000,24.000000,0.0000000$ rotate $=90.000000$

\&OBST XB $=47.549999,48.674999,75.050003,75.425003,5.905,6.655$, SURF_ID=' 'UPHOLSTERY '/ Left Arm

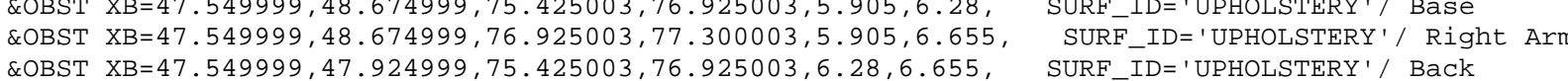
*** GROUP: COUCH2 offset $=0.000000,27,000000,0.000000$ rotate $=90,000000$

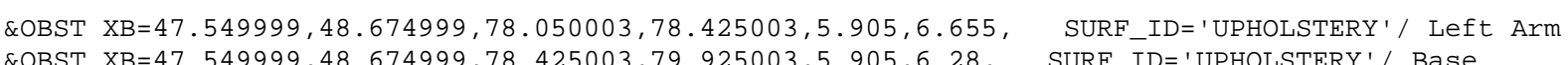

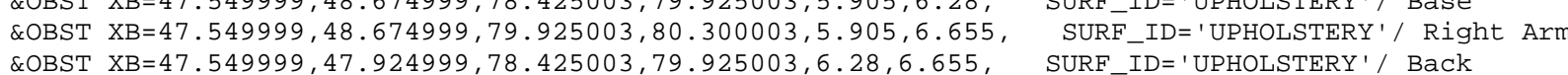

*** GROUP: COUCH2 offset $=0.000000,30.000000,0.000000$ rotate $=90.000000$

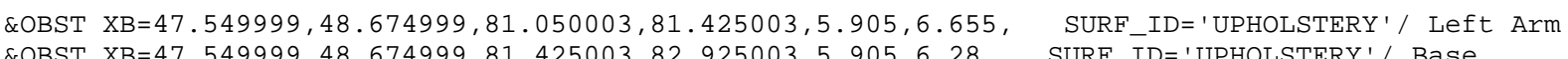
\&OBST XB $=47.549999,48.674999,82.925003,83.300003,5.905,6.655$, SURF_ID=' 'UPHOLSTERY' $/$ Right Arm
\&OBST XB $=47.549999,47.924999,81.425003,82.925003,6.28,6.655$, SURF_ID='UPHOLSTERY' 1 Back

*** GROUP: ROW2

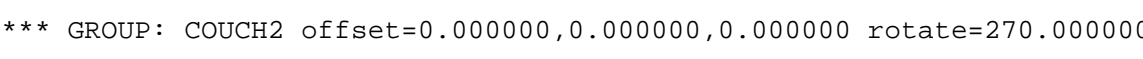

\&OBST XB=46.049999, 47.174999, 52.924999, 53.299999, 5.905, 6.655, SURF_ID=' 'UPHOLSTERY'/ Left Arm \&OBST XB=46. 049999, 47.174999, 51.424999, 52.924999, 5.905, 6.28, SURF_ID='UPHOLSTERY'/ Base \&OBST XB=46.04999, $47.174999,51.049999,51.424999,5.905,6.655$, SURF_ID='UPHOLSTERY'/Right Ar

*** GROUP: COUCH2 offset $=0.000000,3.000000,0.0000000$ rotate $=270.000000$

\&OBST XB=46. 049999, 47.174999, 55.924999, 56. 299999, 5.905, 6.655, SURF_ID=' 'UPHOLSTERY'/ Left Arm COBST XB $=46.049999,47.174999,54.424999,55.924999,5.905,6.28, \quad$ SURF IDE' 'UPHOLLTERY'/ Base

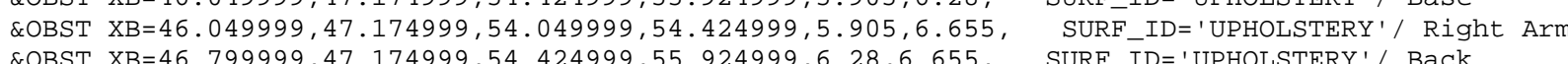

*** GROUP: COUCH2 offset $=0,000000,6,000000,0,000000$ rotate $=270, .000000$

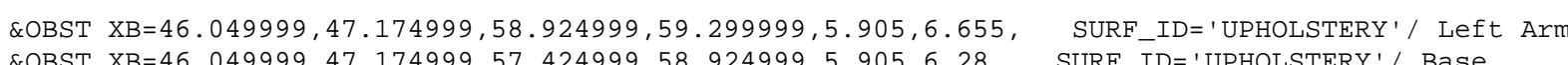

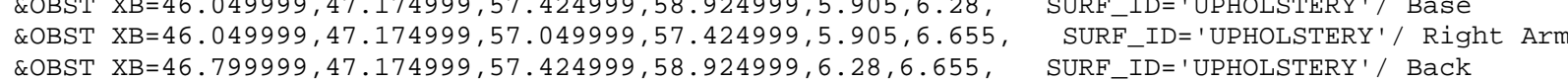

*** GROUP: COUCH2 offset $=0.000000,9.000000,0.000000$ rotate $=270.000000$

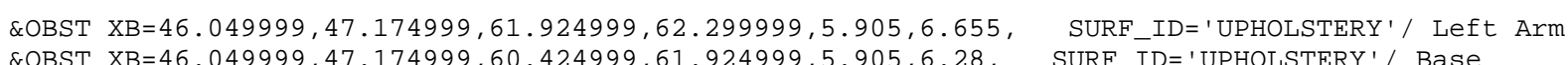

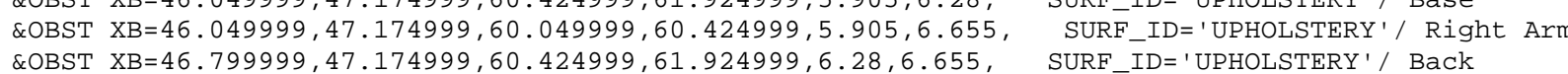

*** GROUP: COUCH2 offset $=0.000000,12.000000,0.000000$ rotate $=270.000000$

\&OBST XB=46. $449999,47.174999,64.925003,65.300003,5.905,6.655$, SURF_ID=' 'UPHOLSTERY '/ Left Arm

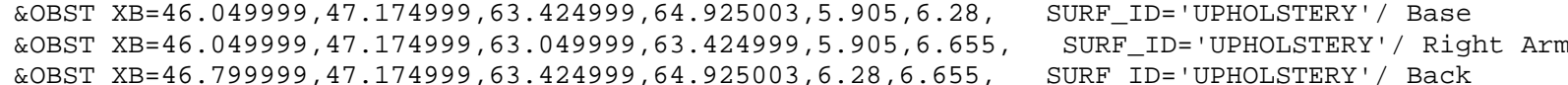

*** GROUP: COUCH2 offset $=0.000000,15.000000,0.000000$ rotate $=270.000000$

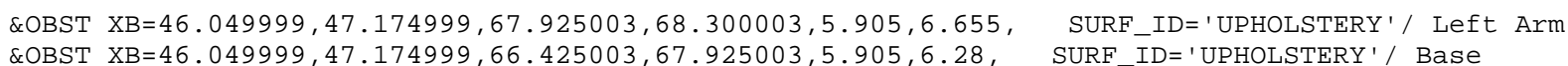
\&OBST
\&OBST $X B=46.049999,47.174999,66.050903,66.425003,5.905,6.655$,

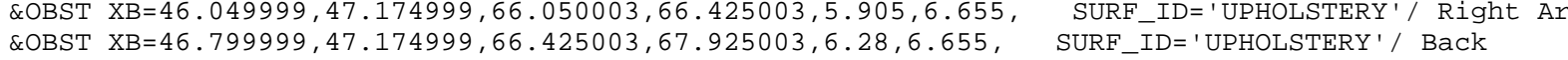

*** GROUP: COUCH2 offset $=0.000000,18.000000,0.000000$ rotate $=270$. . ๑०००००

\&OBST XB $=46.049999,47.174999,70.925003,71.300003,5.905,6.655$,
\&OBST XB $=46.049999,47.174999,69.425003,70.925003,5.905,6.28$,
SURF IDE='UPHOLSTERY' '/ Base

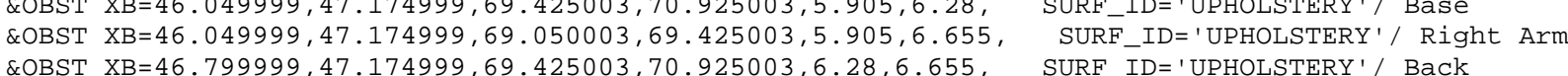

*** GROUP: COUCH2 offset $=0.000000,21.000000,0.000000$ rotate $=270.000000$

\&OBST XB $=46.049999,47.174999,73.925003,74.300003,5.905,6.655$,
SURF_ID='UPHOLSTERY' $/$ Left Arm

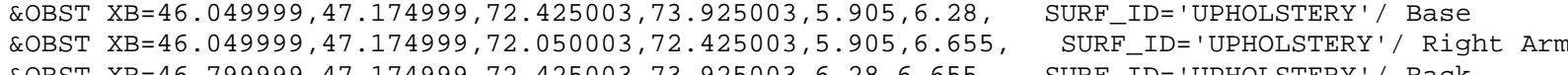

*** GROUP: COUCH2 offset $=0.000000,24$. $.000000,0.000000$ rotate $=270.000000$

COBST XB $=46.049999,47.174999,76.925003,77.300003,5.905,6.655, \quad$ SURF_ID='UPHOLSTERY'/ Left Arm
OBBST XB $=46.049999,47.174999,75.425003,76.925003,5.905,6.28$,

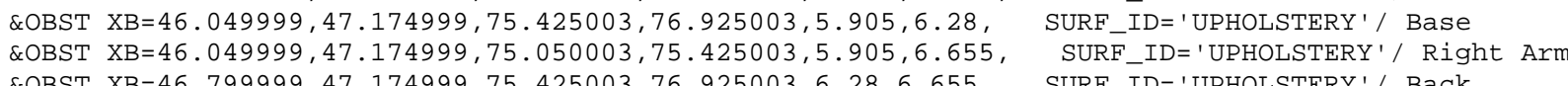

*** GROUP: COUCH2 offset $=0.000000,27.000000,0.0000000$ rotate $=270.0000000$

EOBST XB $=46.049999,47.174999,79.925003,80.300003,5.905,6.655$, SURF_ID=' 'UPHOLSTERY'/ Left Arm \&OBST XB=46.04999, $47.174999,78.425003,79.925003,5.905,6.28, \quad$ SURF_ID='UPHOLSTERY'/ Base \&OBST XB=46.049.

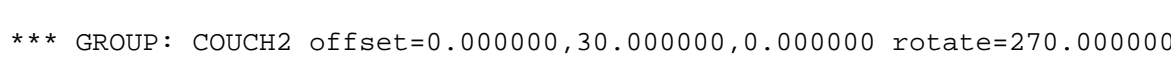

\&OBST XB=46.049999, 47.174999, 82.925003, 83.300003,5.905,6.655, SURF_ID='UPHOLSTERY'/ Left Arm \&OBST XB=46.049999, $47.174999,81.425003,82.925003,5.905,6.28$, SURF_ID='UPHOLSTERY' / Base

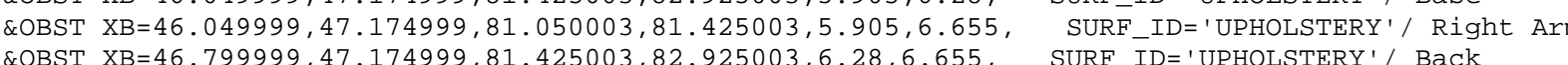

*** GROUP: TWIN

$* * *$ GROUP: ROW

*** GROUP: COUCH2 offset $=0.000000,0.000000,0.000000$ rotate $=90 . .000000$

\&OBST XB=52.049999, 53.174999, 51.049999, 51.424999, 5.905, 6.655, SURF_ID='UPHOLSTERY'/ Left Arm \&OBST XB=52.049999, 53.174999, 51.424999, 52. 924999, 5.905, 6.28, SURF_ID='UPHOLSTERY'/ Base \&OBST XB.52.049 X

*** GROUP: COUCH2 offset $=0.000000,3.000000,0.000000$ rotate $=90.000000$ 
\&OBST XB=52. $049999,53.174999,54.049999,54.424999,5.905,6.655, \quad$ SURF_ID=' 'UPHOLSTERY '/ Left Arm
\&OBST XB=52.049999, $53.174999,54.424999,55.924999,5.905,6.28, \quad$ SURF_ID='UPHOLSTERY'/ Base

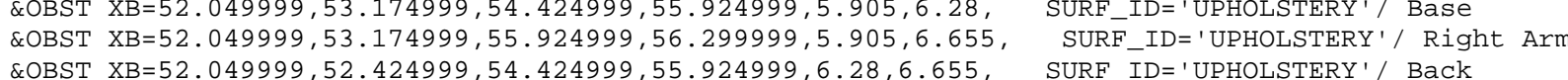
*** GROUP: COUCH2 offset $=0.000000,6.000000,0.000000$ rotate $=90.000000$

\&OBST XB=52. 049999, 53.174999, 57. 4949999, 57.424999, 5. 905, 6.655, SURF_ID='UPHOLSTERY ' / Left Arm

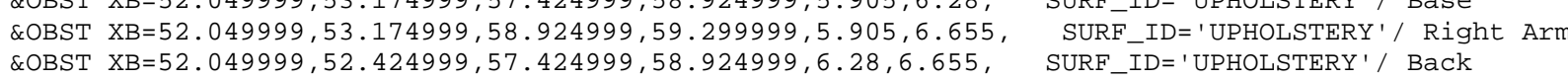

*** GROUP: COUCH2 offset $=0.000000,9.000000,0.000000$ rotate $=90.000000$

\&OBST XB $=52.049999,53.174999,60.049999,60.424999,5.905,6.655, \quad$ SURF_ID=' 'UPHOLSTERY '/ Left Arm

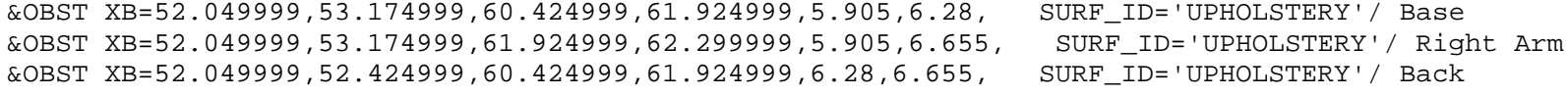
$* * *$ GROUP: COUCH2 offset $=0.000000,12.000000,0.000000$ rotate $=90.000000$

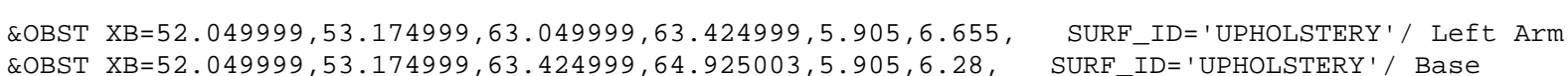

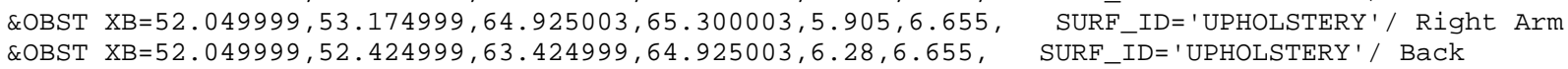
*** GROUP: COUCH2 offset $=0.000000,15.000000,0.000000$ rotate $=90.000000$

\&OBST XB=52. 049999, 53.174999, 66. 0550003, 66. 425003, 5.905, 6.655, SURF_ID=' 'UPHOLSTERY'/ Left Arm

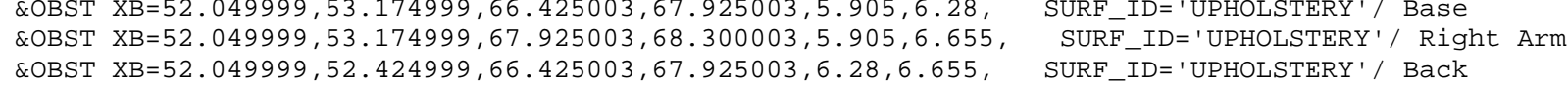
*** GROUP: COUCH2 offset $=0.000000,18.000000,0.000000$ rotate $=90.000000$

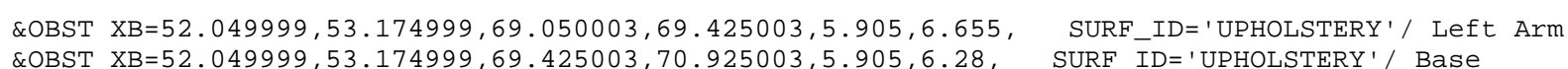

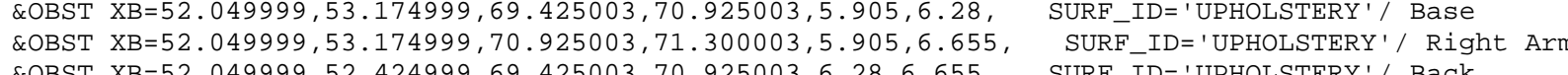
*** GROUP: COUCH2 offset $=0.000000,21.000000,0.000000$ rotate $=90.000000$

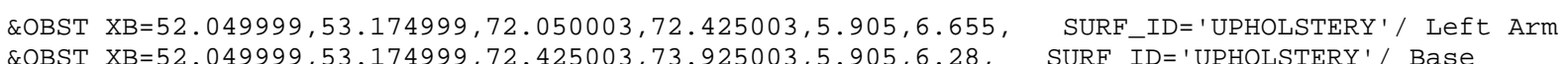

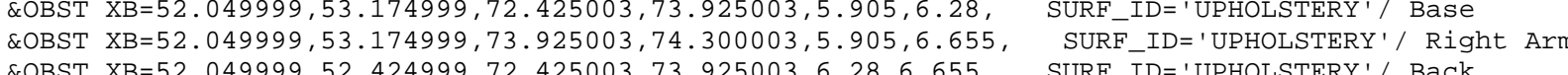

$* \star *$ GROUP: COUCH2 offset $=0.000000,24.000000,0.0000000$ rotate $=90.000000$

\&OBST XB=52. $049999,53.174999,75.050003,75.425003,5.905,6.655$, SURF_ID=' 'UPHOLSTERY '/ Left Arm

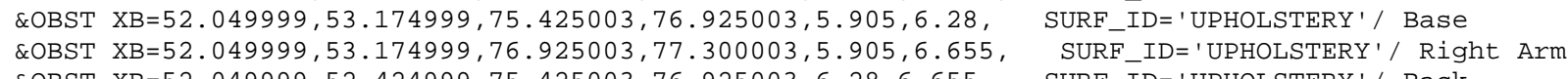
*** GROUP: COUCH2 offset $=0.000000,27.000000,0.000000$ rotate $=90.000000$

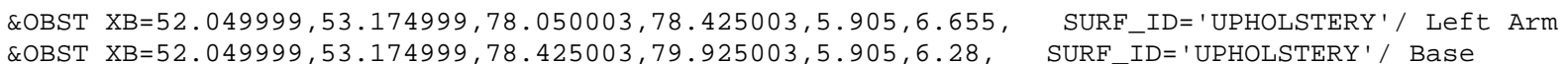

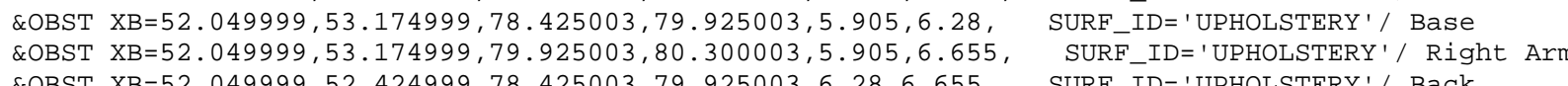
*** GROUP: COUCH2 offset $=0.000000,30.000000,0.000000$ rotate $=90.000000$

\&OBST XB=52.049999, 53.174999, 81.050003, 81.425003, 5.905, 6.655, SURF_ID=' 'UPHOLSTERY'/ Left Arm \&OBST XB $=52.049999,53.174999,81.425003,82.925003,5.905,6.28$, SURF_ID ='UPHOLSTERY'/ Base

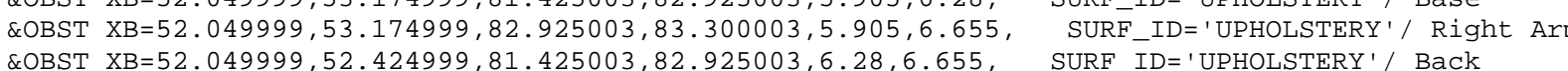
*** GROUP: ROW2

*** GROUP: COUCH2 offset $=0.000000,0.000000,0.000000$ rotate $=270.000000$

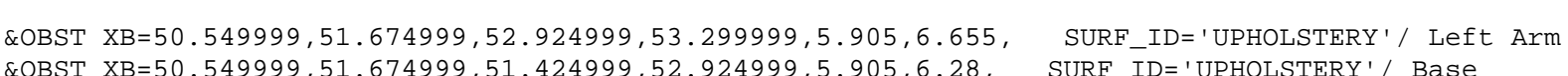

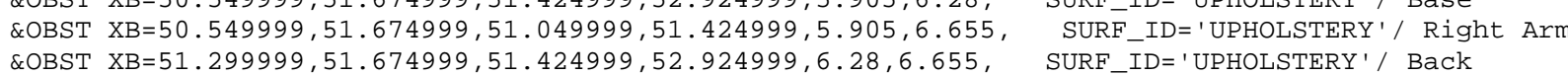

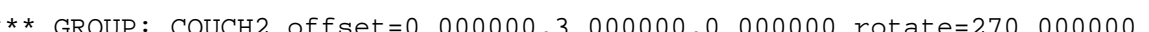

COBST XB=50.54999, $51.674999,55.924999,56.299999,5.905,6.655, \quad$ SURF_ID='UPHOLSTERY ' / Left Arm
\&OBST XB=50.549999, $51.674999,54.424999,55.924999,5.905,6.28$,
SURF_ID='UPHOLSTERY'

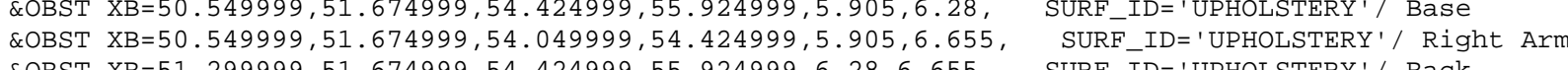
*** GROUP: COUCH2 offset $=0.000000,6.000000,0.000000$ rotate $=270.000000$

\&OBST XB=50.549999, 51.674999, 58.924999, 59.299999, 5.905, 6.655, SURF_ID='UPHOLSTERY'/ Left Arm

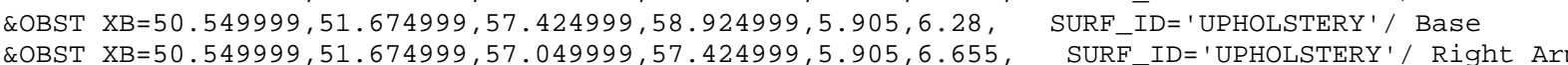
\&OBST XB $=51.299999,51.674999,57.424999,58.924999,6.28,6.655$, SURF ID='UPHOLSTERY'/ Back Ar

*** GROUP: COUCH2 offset $=0.0000000,9.000000,0.000000$ rotate $=270.0000000$

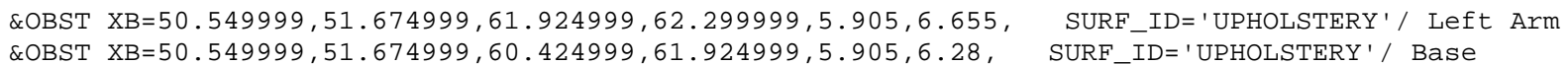

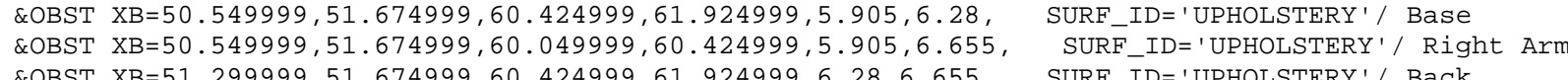
*** GROUP: COUCH2 of fset $=0.000000,12.000000,0.000000$ rotate $=270.0000000$

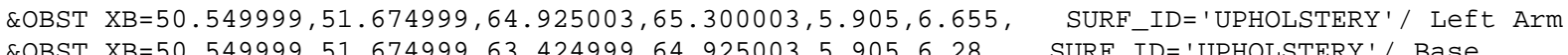
\&OBST XB=50.549999, $51.674999,63.424999,64.925003,5.905,6.28, \quad$ SURF_ID='UPHOLSTERY'/ Base

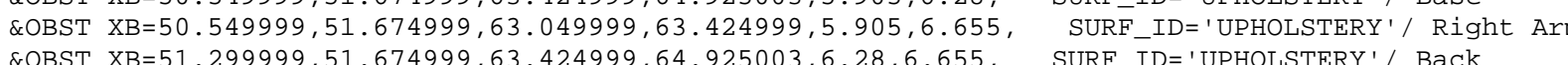

*** GROUP: COUCH2 offset $=0.000000,15.000000,0.000000$ rotate $=270.000000$

\&OBST XB=50.549999, 51.674999, 67.925003, 68.300003,5.905,6.655, SURF_ID='UPHOLSTERY'/ Left Arm

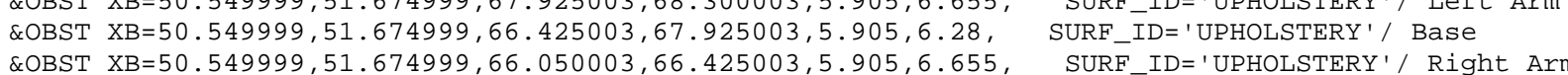
\&OBST XB

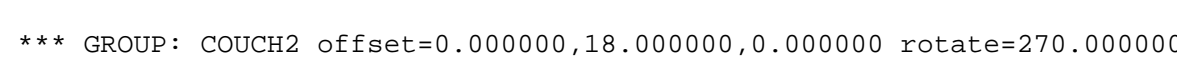

\&OBST XB=50.549999, 51.674999, 70.925003, 71.300003,5.905,6.655, SURF_ID=' 'UPHOLSTERY'/ Left Arm

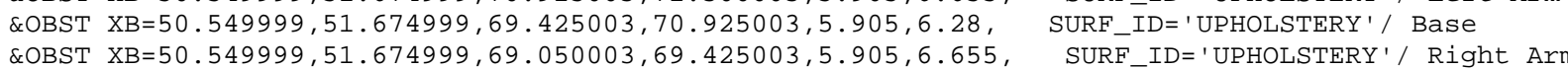

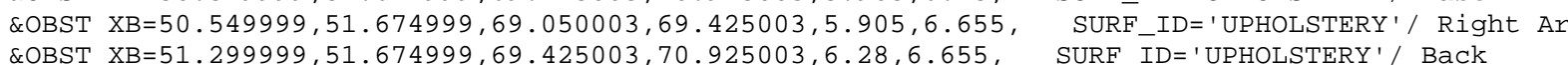

*** GROUP: COUCH2 offset $=0.000000,21.000000,0.000000$ rotate $=270.000000$

\&OBST XB=50.549999, 51.674999, 73.925003, 74.300003, 5.905,6.655, SURF_ID=' 'UPHOLSTERY'/ Left Arm

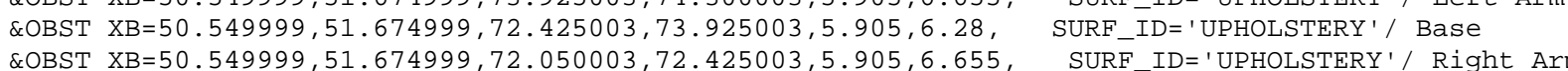
SOBST XB $=51.299999,51.674999,72.425003,73.425003,6.905,6.655$, SURF_ID=' 'UPHOLSTERY'/ Right Ar

*** GROUP: COUCH2 offset $=0.000000,24.000000,0.000000$ rotate $=270.000000$

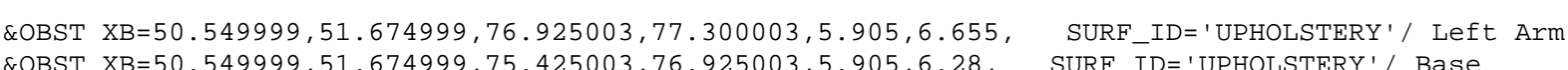

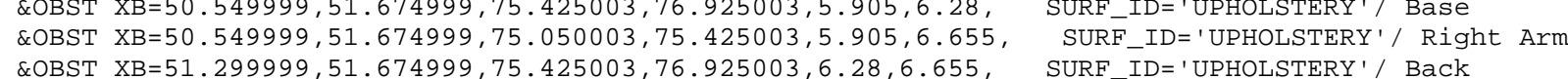

*** GROUP: COUCH2 offset $=0.000000,27.0000000,0.0000000$ rotate $=270.000000$

\&OBST XB $=50.549999,51.674999,79.925003,80.300003,5.905,6.655$, SURF_ID=' 'UPHOLSTERY '/ Left Arm

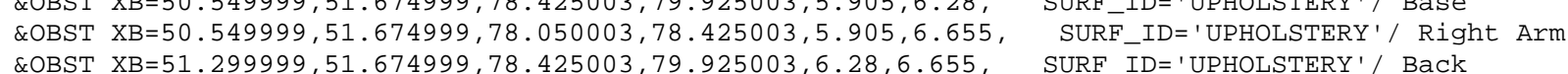

*** GROUP: COUCH2 offset $=0.000000,30.000000,0.000000$ rotate $=270.000000$

\&OBST XB=50.549999, 51.674999, 82.925003, 83.300003, 5.905, 6.655, SURF_ID='UPHOLSTERY' / Left Arm \&OBST XB=50.549999, 51.674999, 81.050003, 81.425003, 5.905, 6.655, SURF_ID='UPHOLSTERY'/ Right Arm

\section{*** GROUP: TWIN}

*** GROUP: ROW

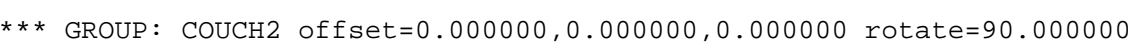

\&OBST XB $=56.549999,57,674999,51.049999,51.424999,5.905,6.655$, SURE_ID=' 'UPHOLSTERY' / Left Arm 


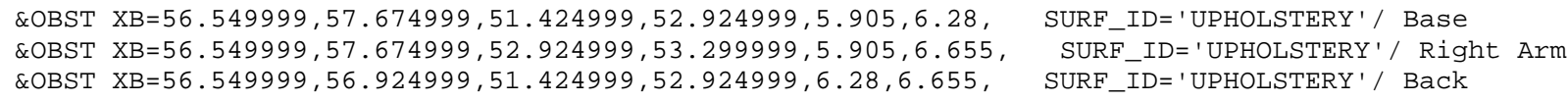
$* * *$ GROUP: COUCH2 offset $=0.000000,3.000000,0.000000$ rotate $=90.000000$

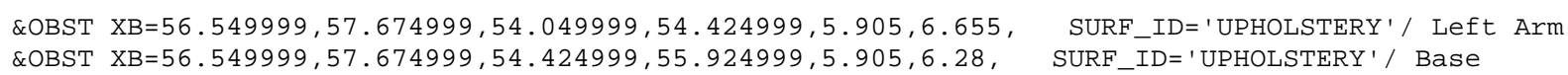

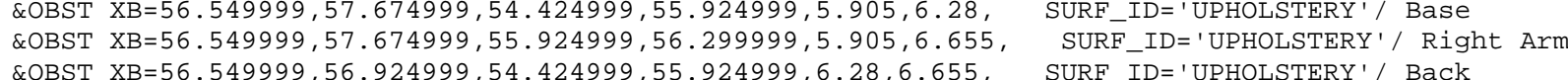

*** GROUP: COUCH2 offset $=0.000000,6.000000,0.000000$ rotate $=90.000000$

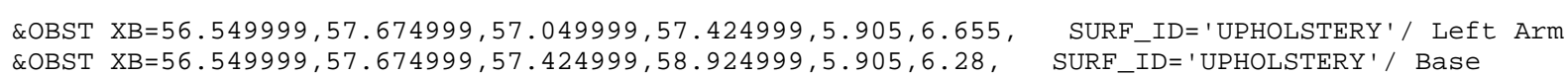

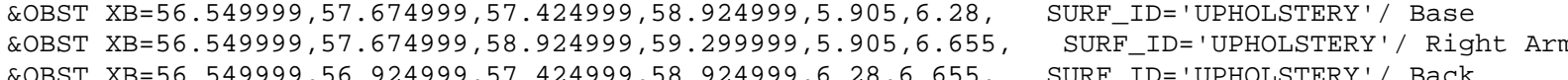

$* * *$ GROUP: COUCH2 offset $=0.000000,9$. ๑०००००,, .000000 rotate $=90.000000$

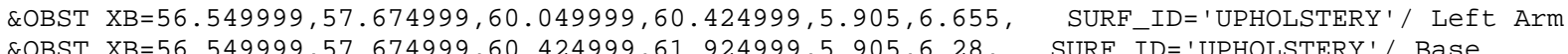

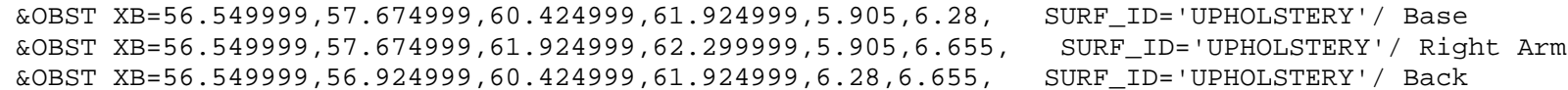

*** GROUP: COUCH2 offset $=0.000000,12.000000$, ๑. .0000000 rotate $=90.000000$

\&OBST XB $=56.549999,57.674999,63.049999,63.424999,5.905,6.655$, SURF_ID=' 'UPHOLSTERY ' / Left Arm

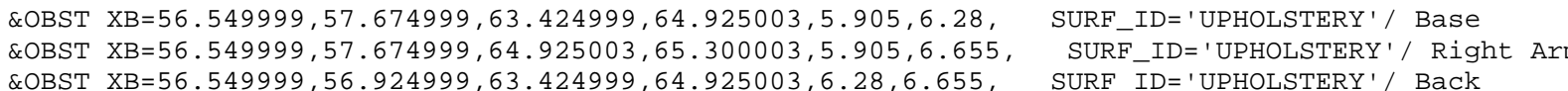
*** GROUP: COUCH2 offset $=0.000000,15.000000,0.000000$ rotate $=90$. . 000000

\&OBST XB $=56.549999,57.674999,66.050003,66.425003,5.905,6.655$, SURF_ID=' 'UPHOLSTERY ' / Left Arm
ROBST XB $=56.549999,57.674999,66.425003,67.925003,5.905,6.28$ \&OBST XB=56.549999, $57.674999,66.425003,67.925003,5.905,6.28$, SURF_ID='UPHOLSTERY'/ Base ATm

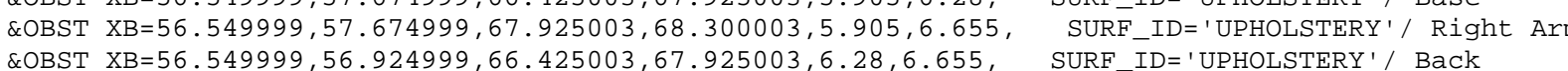
*** GROUP: COUCH2 offset $=0.000000,18.000000,0.000000$ rotate $=90.000000$

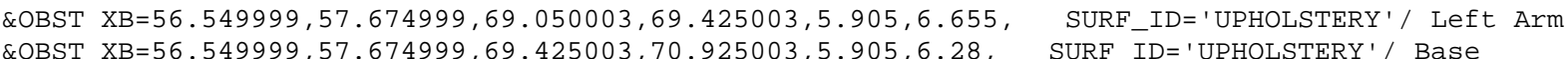
\&OBST XB=56.549999, 57.674999, 70.925003, 71.300003,5.905,6.655, SURF_IDD='UPHOLSTERY'/ Right Ar

*** GROUP: COUCH2 offset $=0.000000,21.000000,0.000000$ rotate $=90.000000$

\&OBST XB=56.549999, 57.674999, 72.050003, 72.425003,5.905, 6.655, SURF_ID=' 'UPHOLSTERY ' / Left Arm \&OBST XB $=56.549999,57.674999,72.425003,73.925003,5.905,6.28$, SURF_ID='UPHOLSTERY'/ Base

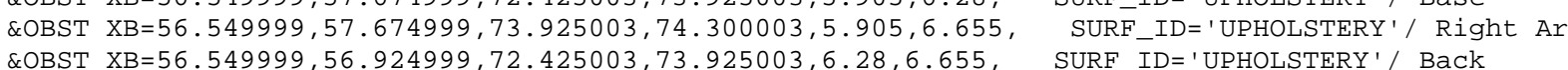

*** GROUP: COUCH2 offset $=0.000000,24.000000,0.000000$ rotate $=90.000000$

\&OBST XB $=56.549999,57.674999,75.050003,75.425003,5.905,6.655$, SURF_ID=' 'UPHOLSTERY'/ Left Arm

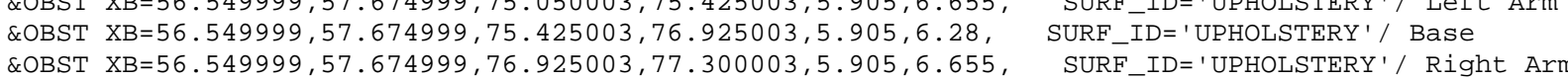
\&OBST XB $=56.549999,56.924999,75.425003,76.925003,6.28,6.655$, SURF ID ' 'UPHOLSTERY'/ Rack Ar

*** GROUP: COUCH2 offset $=0.000000,27.000000,0.000000$ rotate $=90.000000$

\&OBST XB $=56.549999,57.674999,78.050003,78.425003,5.905,6.655$, SURF_ID=' 'UPHOLSTERY' / Left Arm

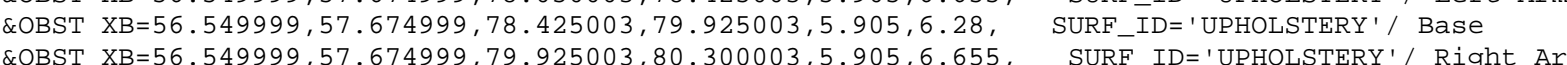

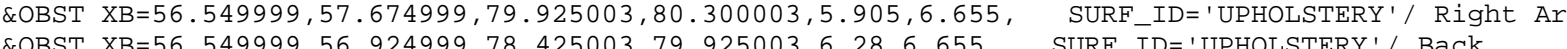

*** GROUP: COUCH2 offset $=0.000000,30.000000,0.000000$ rotate $=90.000000$

\&OBST XB=56.549999, 57.674999, 81. 050003, 81.425003, 5.905, 6.655, SURF_ID='UPHOLSTERY'/ Left Arm

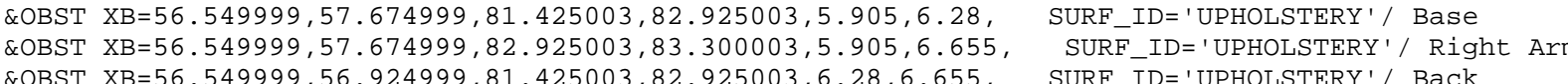

*** GROUP: ROW2

** GROUP: COUCH2 offset $=0.000000$, ๑. ๑000000, ๑. .000000 rotate $=270.000000$
\&OBST XB $=55.049999,56.174999,52.924999,53.299999,5.905,6.655, \quad$ SURF_ID=' 'UPHOLSTERY '/ Left Arm
\&OBST XB \&OBST XB=55.049999, 56.174999, $51.049999,51.424999,5.905,6.655$, SURF_ID=' 'UPHOLSTERY'/ Right Ar

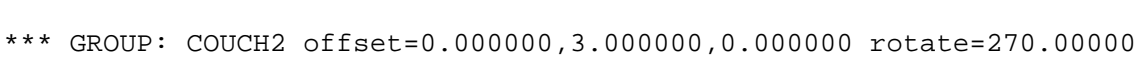

\&OBST XB $=55.049999,56.174999,55.924999,56.299999,5.905,6.655$, SURF_ID=' 'UPHOLSTERY '/ Left Arm \&OBST XB=55.049999, 56.174999, 54.424999, 55.924999, 5.905, 6.28, SURF_ID='UPHOLSTERY'/ Base

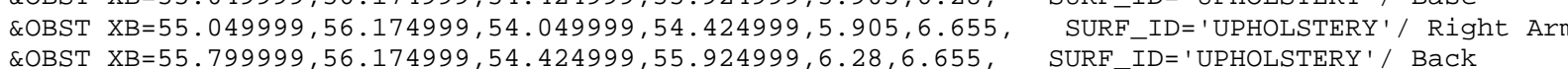

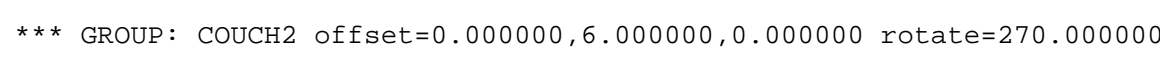

\&OBST XB=55. 049999, 56.174999, 58.924999, 59. 299999, 5.905, 6.655, SURF_ID='UPHOLSTERY'/ Left Arm

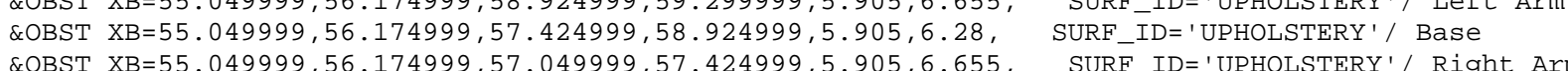
QOBST XB $55.79999,56.174999,57.424999,58.924999,6.28,6.655$, SURF IDD='UPHOLSTERY/Right Ar

*** GROUP: COUCH2 off set $=0.000000,9.000000,0.000000$ rotate $=270.000000$

\&OBST XB=55. 049999, 56.174999, 61. 924999, 62. 299999, 5.905, 6.655, SURF_ID=' 'UPHOLSTERY'/ Left Arm \&OBST XB=55. $049999,56.174999,60.424999,61.924999,5.905,6.28$, SURF-ID=' 'UPHOLSTERY' ' Base \&OBST XB $\times 55.799999,56.174999,60.049999,60.424999,5.905,6.655$, SURF_ID='UPHOLSTERY'/Right Ar

*** GROUP: COUCH2 offset $=0.000000,12.000000,0.000000$ rotate $=270.000000$

\&OBST XB=55. $049999,56.174999,64.925003,65.300003,5.905,6.655$, SURF_ID= 'UPHOLSTERY ' / Left Arm

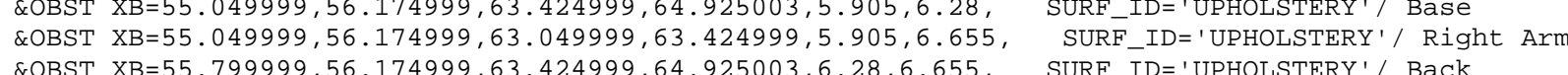

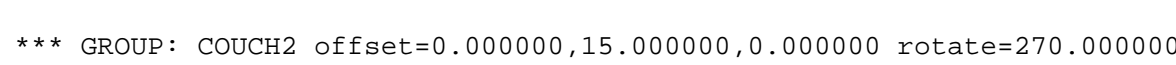

\&OBST XB=55. 049999, 56.174999, 67.925003, 68.300003, 5.905, 6.655, SURF_ID=' 'UPHOLSTERY '/ Left Arm

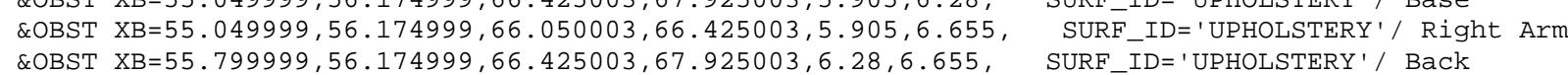

*** GROUP: COUCH2 offset $=0.0000000,18.0000000,0.0000000$ rotate $=270.000000$

\&OBST XB=55. $049999,56.174999,70.925003,71.300003,5.905,6.655$, SURF_ID=' 'UPHOLSTERY'/ Left Arm

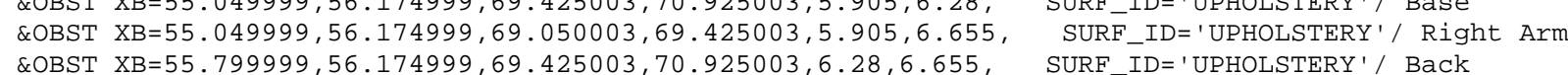

*** GROUP: COUCH2 offset $=0.000000,21.000000,0.000000$ rotate $=270.000000$

\&OBST XB=55. $049999,56.174999,73.925003,74.300003,5.905,6.655$, SURF_ID='UPHOLSTERY' / Left Arm \&OBST XB=55. $049999,56.174999,72.050003,72.425003,5.905,6.655, \quad$ SURF_ID =' 'UPHOLSTERY' $/$ Right Ar \&OBST XB=55.799999, 56.174999, 72.425003,73.925003,6.28,6.655, SURF_ID='UPHOLSTERY'/ Back

*** GROUP: COUCH2 offset $=0.000000,24.000000,0.000000$ rotate $=270.000000$

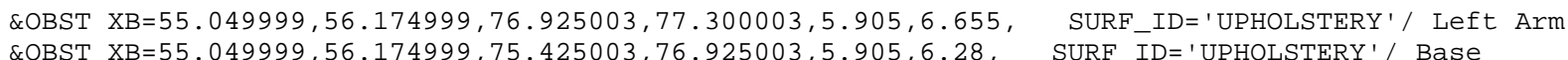
\&OBST XB $=55.049999,56.174999,75.050003,75,425003,5.905,6.655$, SURE IDE' 'UPHOLSTERY'/ Right Ar \&OBST XB $=55.799999,56.174999,75.425003,76.925003,6.28,6.655$, SURF_ID='UPHOLSTERY'/ Back

*** GROUP: COUCH2 offset $=0.000000,27.000000,0.000000$ rotate $=270.000000$

\&OBST XB=55. $449999,56.174999,79.925003,80.300003,5.905,6.655, \quad$ SURF ID=' 'UPHOLSTERY'/ Left Arm
\&OBST XB=55.04999, $56.174999,78.425003,79.925003,5.905,6.28$,
SURF IDE'UPHOLSTERY'/ Base

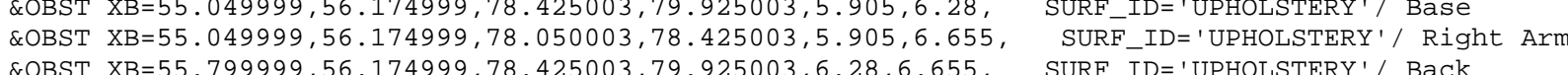

*** GROUP: COUCH2 offset $=0.000000,30.000000,0.000000$ rotate $=270.000000$

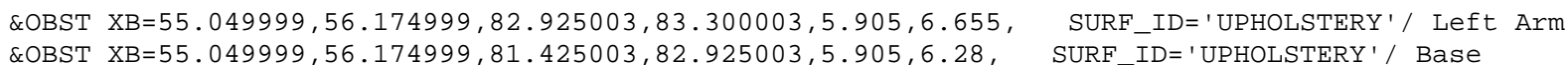

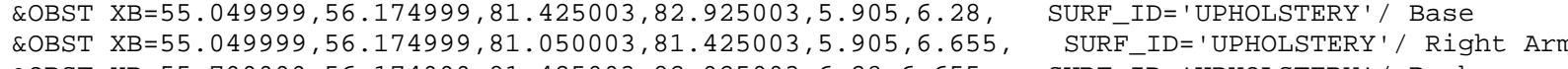
*** GROUP: TWIN 
*** GROUP: ROW

** GROUP: COUCH2 offset $=0.000000,0.000000,0.000000$ rotate $=90 . .000000$

\&OBST XB=61.049999, 62.174999, 51. 049999, 51.424999, 5.905, 6.655, SURF_ID=' 'UPHOLSTERY'/ Left Arm

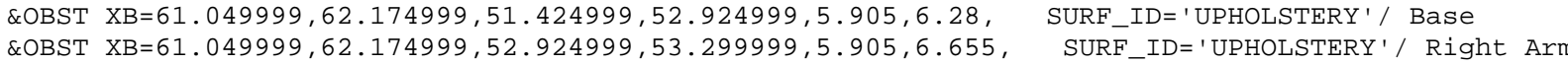
\&OBST XB $61.049999,61.424999,51.424999,52.924999,6.28,6.655$, SURF IDD='UPHOLSTERY'/ Rigk Ar *** GROUP: COUCH2 offset $=0.000000,3.000000,0.000000$ rotate $=90.000000$

\&OBST XB=61.049999, 62.174999, 54.049999, 54.424999, 5.905, 6.655, SURF_ID='UPHOLSTERY'/ Left Arm

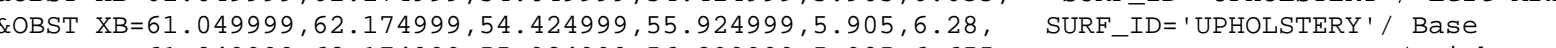
\&OBST XB=61.049999, $62.174999,55.924999,56.299999,5.905,6.655$, SURF_ID='UPHOLSTERY'/ Right Ar
\&OBST XB $61.049999,61.424999,54.424999,55.924999,6.28,6.655$

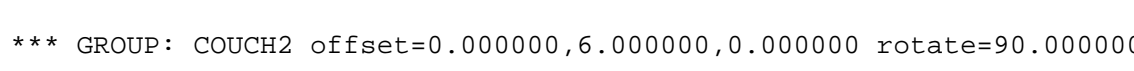

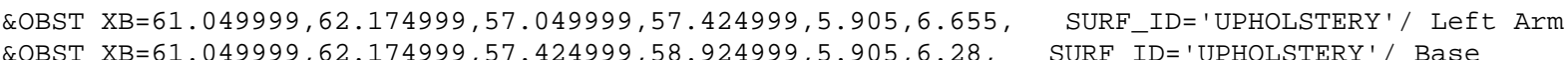

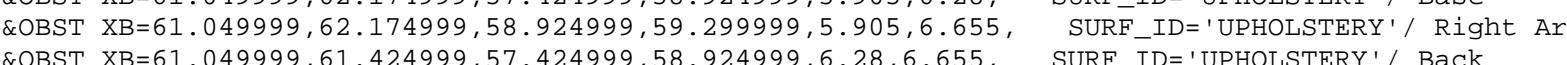

*** GROUP: COUCH2 offset $=0.000000,9.000000,0.000000$ rotate $=90$. .

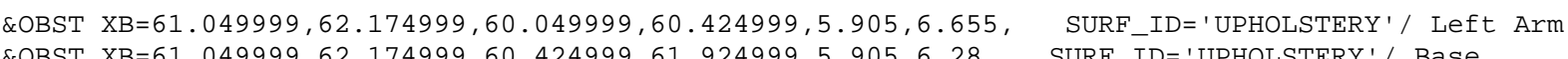
\&OBST XB=61.049999, 62.174999, 61.924999, 62.299999, 5.905,6.655, SURF_ID='UPHOLSTERY'/ Right Ar

*** GROUP: COUCH2 offset $=0.000000,12 . .000000,0.000000$ rotate $=90 . .000000$

\&OBST XB=61. $449999,62.174999,63.049999,63.424999,5.905,6.655$, SURF_ID=' 'UPHOLSTERY'/ Left Arm QOBST XB $=61.049999,62.174999,63.424999,64.925003,5.905,6.28, \quad$ SURF IDE 'UPHOLSTERY'/ Base

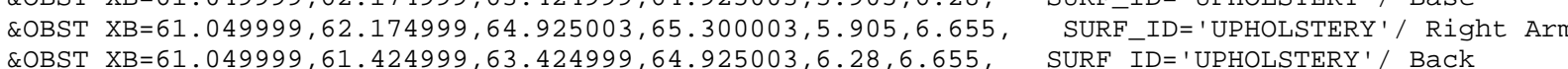

*** GROUP: COUCH2 offset $=0.000000,15.000000,0.000000$ rotate $=90.000000$

\&OBST XB=61. 049999, 62.174999, 66. 050003, 66. 425003, 5.905, 6.655, SURF_ID=' 'UPHOLSTERY'/ Left Arm \&OBST XB=61.049999, 62.174999, 66.425003, 67. 925003, 5.995,6.28, SURF_ID='UPHOLSTERY'/ Base

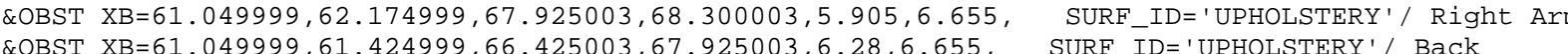

*** GROUP: COUCH2 offset $=0.000000,18.000000,0 . .000000$ rotate $=90.000000$

\&OBST XB $=61.049999,62.174999,69.050003,69.425003,5.905,6.655, \quad$ SURF_ID=' 'UPHOLSTERY '/ Left Arm \&OBST XB=61. $049999,62.174999,70.925003,71.300003,5.905,6.655$, SURF_ID='UPHOLSTERY'/Right Ar
\&OBST XB=61.04999, $61.424999,69.425003,70.925003,6.28,6.655$, SURF_ID='UPHOLSTERY'/ Back

*** GROUP: COUCH2 offset $=0.000000,21.000000,0.000000$ rotate $=90.000000$

\&OBST XB=61. $049999,62.174999,72.050003,72.425003,5.905,6.655$, SURF_ID=' 'UPHOLSTERY '/ Left Arm

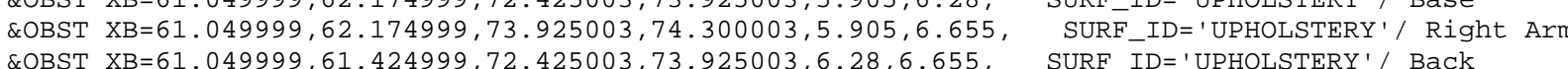

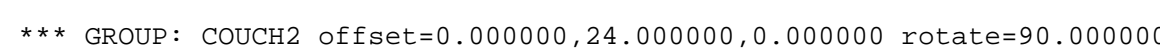

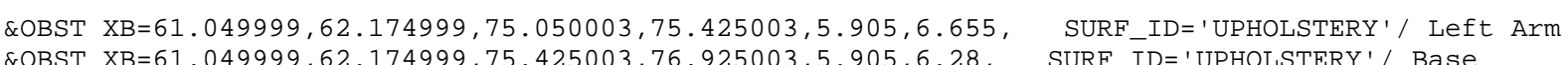

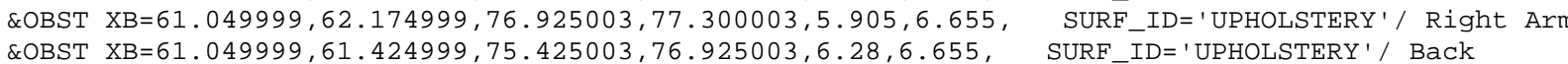
*** GROUP: COUCH2 offset $=0.000000,27.000000,0.000000$ rotate $=90.000000$

\&OBST XB=61. $049999,62.174999,78.050003,78.425003,5.905,6.655$, SURF_IDD 'UPHOLSTERY ' / Left Arm

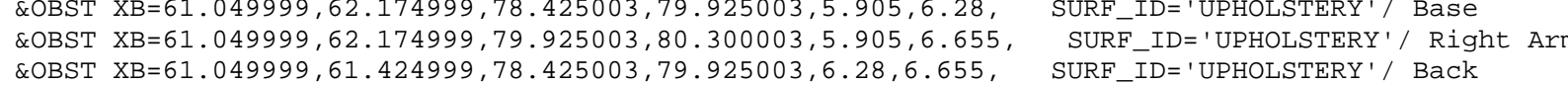
*** GROUP: COUCH2 offset $=0.000000,30.000000,0.000000$ rotate $=90.000000$ \&OBST XB $=61.049999,62.174999,81.050003,81.425003,5.905,6.655$, SURF_ID='UPHOLSTERY ' / Left Arm
\&OBST XB=61. $049999,62.174999,82.925003,83.300003,5.905,6.655, \quad \begin{aligned} & \text { SURF_ID=' 'UPHOLSTERY '/ Right Arm } \\ & \text { \&OBST XB=61.049999, } 61.424999,81.425003,82.925003,6.28,6.655, \\ & \text { SURF_ID='UPHOLSTERY' } / \text { Back }\end{aligned}$ *** GROUP: ROW2

*** GROUP: COUCH2 offset $=0.000000,0.000000,0.000000$ rotate $=270.000000$

\&OBST XB=59.549999, 60.674999, 52.924999, 53.299999, 5.905, 6.655, SURF_ID=' 'UPHOLSTERY'/ Left Arm \&OBST XB=59.549999, 60.674999, 51.049999, 51.424999, 5.905,6.655, SURF_ID=' 'UPHOLSTERY'/ Right Ar

$* * *$ GROUP: COUCH2 offset $=0.000000,3.000000,0.000000$ rotate $=270.000000$

\&OBST XB=59.549999, 60.674999, 55.924999, 56.299999, 5.905, 6.655, SURF_ID=' 'UPHOLSTERY '/ Left Arm

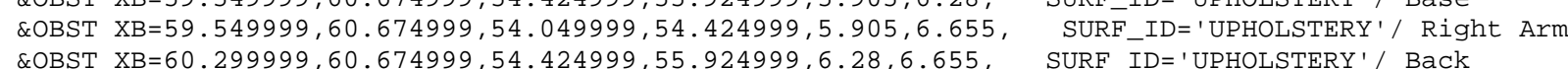

*** GROUP: COUCH2 offset $=0.000000,6.000000,0.000000$ rotate $=270.000000$

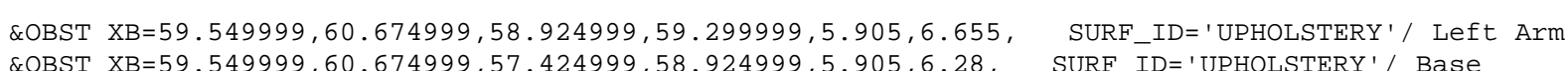

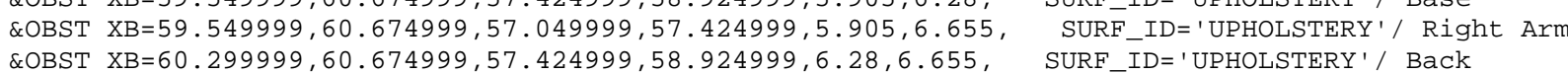

*** GROUP: COUCH2 offset $=0$, .

\&OBST XB $=59.549999,60.674999,61.924999,62.299999,5.905,6.655$, SURF_ID=' 'UPHOLSTERY ' / Left Arm \&OBST XB $=59.549999,60.674999,60.049999,60.424999,5,905,6.655$, SURF ID='UPHOLSTERY'/ Right Ar \&OBST XB=60.299999, 60.674999, 60.424999, 61.924999,6.28,6.655, SURF_ID='UPHOLSTERY'/ Back

$* * *$ GROUP: COUCH2 offset $=0.000000,12.000000,0.000000$ rotate $=270.000000$

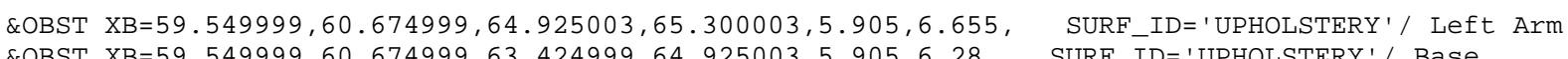
\&OBST XB $=59.549999,60.674999,63.049999,63.424999,5.905,6.655$, SURF ID=' 'UPHOLSTERY'/ Right Ar \&OBST XB=60.299999, 60.674999, 63.424999, 64.925003,6.28,6.655, SURF_ID='UPHOLSTERY'/ Back

*** GROUP: COUCH2 offset $=0.000000,15.000000,0.000000$ rotate $=270.000000$

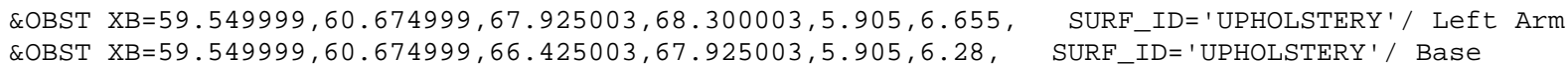

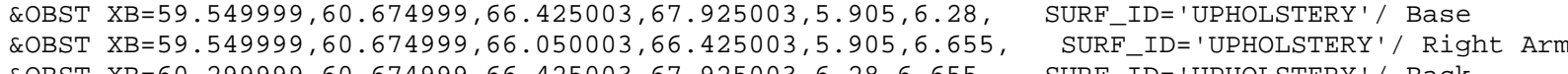

*** GROUP: COUCH2 offset $=0.000000,18.0000000,0.000000$ rotate $=270.000000$

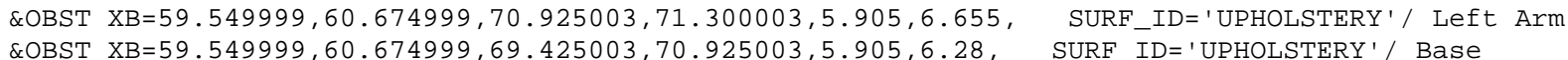

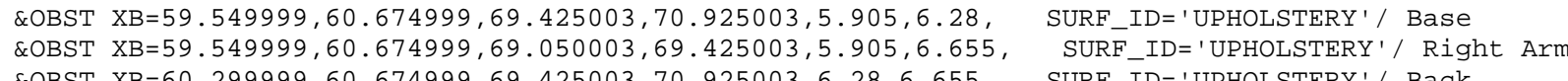

*** GROUP: COUCH2 offset $=0.0000000,21.000000,0.0000000$ rotate $=270.000000$

\&OBST XB=59.549999, 60.674999, 73.925003, 74.300003,5.905, 6.655, SURF_ID=' 'UPHOLSTERY'/ Left Arm \&OBST XB=59.54999, 60.674999, $72.425003,73.925003,5.905,6.28$, SURF_ID='UPHOLSTERY'/ Base

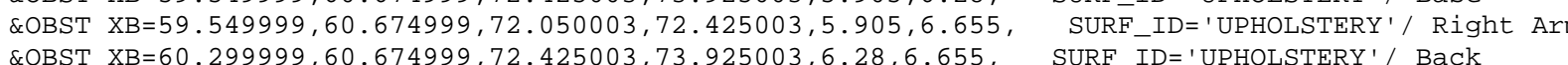

*** GROUP: COUCH2 offset $=0.000000,24.000000,0.000000$ rotate $=270.000000$

\&OBST XB=59.549999, 60.674999, 76.925003, 77.300003,5.905,6.655, SURF_ID='UPHOLSTERY'/ Left Arm \&OBST XB $=59.549999,60.674999,75.425003,76.925003,5.905,6.28, \quad$ SURF_ID='UPHOLSTERY'/ Base

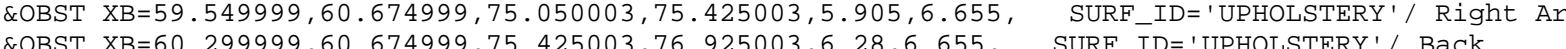

*** GROUP: COUCH2 offset $=0.000000,27.000000,0.000000$ rotate $=270.000000$

\&OBST XB=59.549999, 60.674999, 79.925003, 80.300003,5.905,6.655, SURF_ID=' 'UPHOLSTERY'/ Left Arm \&OBST XB=59.549999, 60.674999, 78.425003, 79.925003, 5.905, 6.28, SURF_ID='UPHOLSTERY'/ Base \&OBST XB $X$ B

*** GROUP: COUCH2 offset $=0.000000,30.000000,0.000000$ rotate $=270.000000$ 


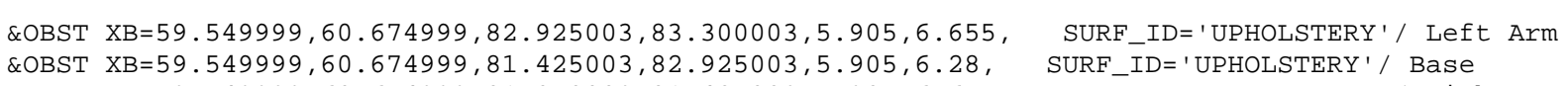

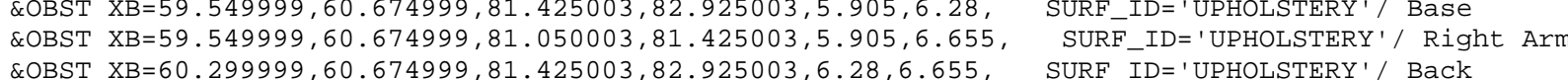
$* \star *$ GROUP: TWIN

*** GROUP: ROW

*** GROUP: COUCH2 offset $=0.000000, \Theta . \bullet 00000,0.000000$ rotate $=90 . .000000$

\&OBST XB=65.550003, 66. 675003, 51. $049999,51.424999,5.905,6.655$, SURF_ID=' 'UPHOLSTERY' / Left Arm \&OBST XB $=65.550003,66.675003,51.424999,52.924999,5.905,6.28, \quad$ SURF_ID='UPHOLSTERY'/ Base QOBST $X \mathrm{BB}=65.550003,66.675003,52.924999,53.299999,5.905,6.655$, SURF_ID='UPHOLSTERY'/Right Ar

*** GROUP: COUCH2 offset $=0.000000,3.000000,0.000000$ rotate $=90.000000$

\&OBST XB $=65.550003,66.675003,54.049999,54.424999,5.905,6.655$, SURF_ID=' 'UPHOLSTERY ' / Left Arm

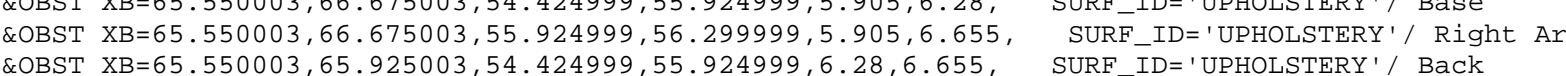
$* * *$ GROUP: COUCH2 offset $=0.000000,6.000000,0.000000$ rotate $=90.000000$

\&OBST XB $=65.550003,66.675003,57.049999,57.424999,5.905,6.655$, SURF_ID=' 'UPHOLSTERY' / Left Arm \&OBST XB=65.550003,66.675003, 58.924999, 59.299999, 5.905,6.655, SURF_ID=' 'UPHOLSTERY'/ Right Ar *** GROUP: COUCH2 offset $=0.000000,9.000000,0.000000$ rotate $=90.000000$

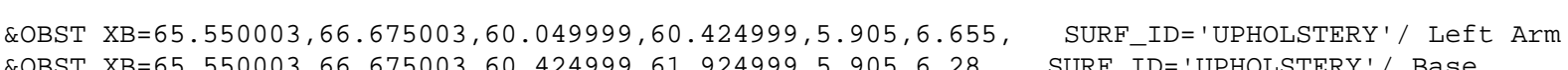

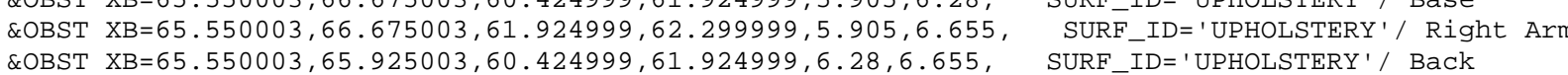

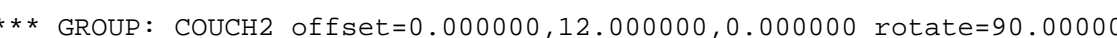

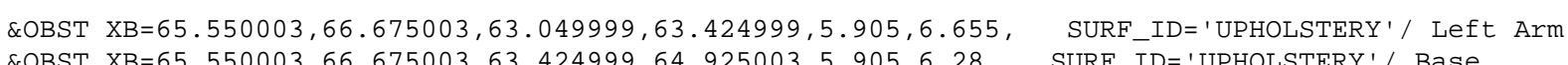

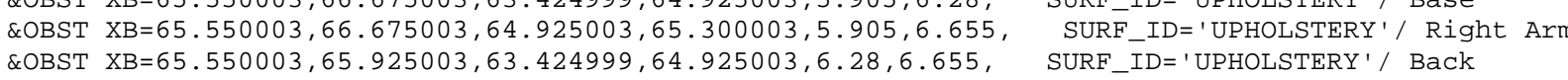
*** GROUP: COUCH2 offset $=0.000000,15.000000,0.000000$ rotate $=90.000000$

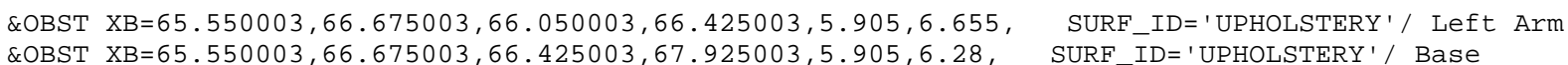

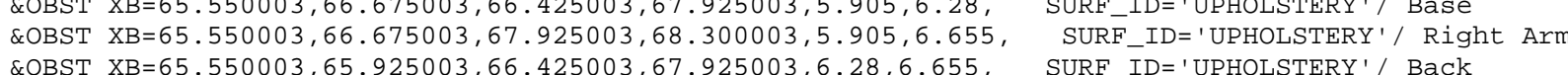
*** GROUP: COUCH2 offset $=0.000000,18.000000,, 0.000000$ rotate $=90 . .000000$

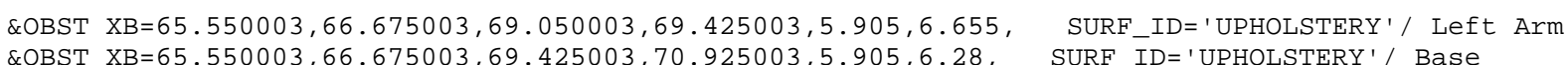

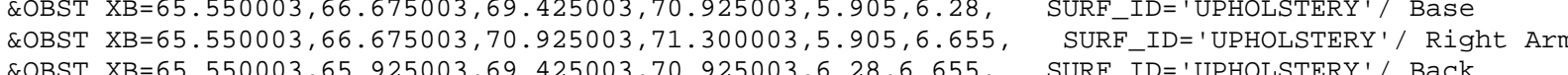

*** GROUP: COUCH2 offset $=0.000000,21.000000,0.000000$ rotate $=90.000000$

\&OBST XB $=65.550003,66.675003,72.050003,72.425003,5.905,6.655$, SURF_ID=' 'UPHOLSTERY '/ Left Arm

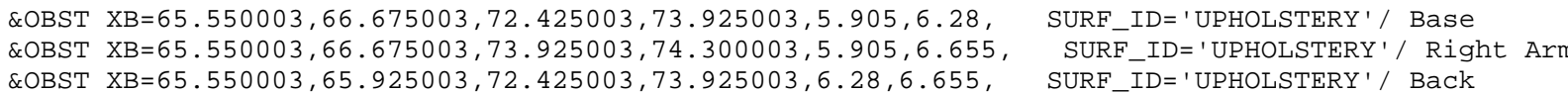

*** GROUP: COUCH2 offset $=0.000000,24.000000,0.000000$ rotate $=90$. . 000000

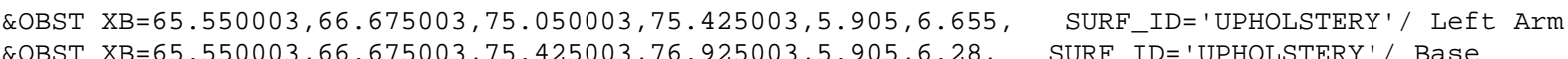

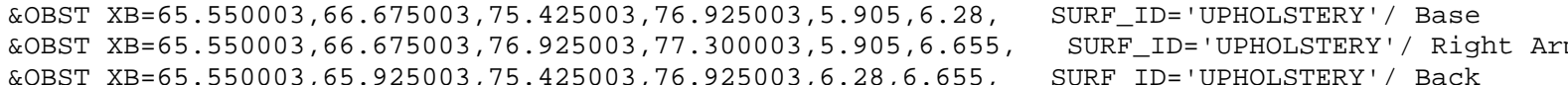
*** GROUP: COUCH2 offset $=0.000000,27.000000,0.000000$ rotate $=90.000000$

\&OBST XB=65.550003, 66.675003, 78. $050003,78.425003,5.905,6.655$, SURF_ID= 'UPHOLSTERY ' / Left Arm

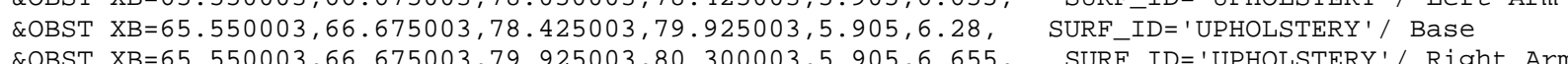

\&OBST XB $=65.550003,65.925003,78.425003,79.925003,6.28,6.655$, SURF ID=' 'UPHOLSTERY' / Back

*** GROUP: COUCH2 offset $=0.000000,30.000000,0.000000$ rotate $=90.000000$

\&OBST XB $=65.550003,66.675003,81.050003,81.425003,5.905,6.655$, SURF_ID='UPHOLSTERY'/ Left Arm \&OBST XB $=65.550003,66.675003,81.425003,82.925003,5.905,6.28$, SURF_ID=' 'UPHOLSTERY'/ Base OOBST $X \mathrm{~B}=65.50003,65.07503,82.925003,83.300003,5.905,6.655$, SURF_ID=' 'UPHOLSTERY/ Right

*** GROUP: ROW2

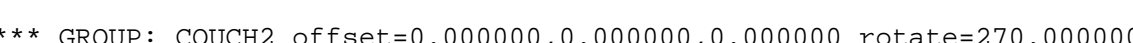

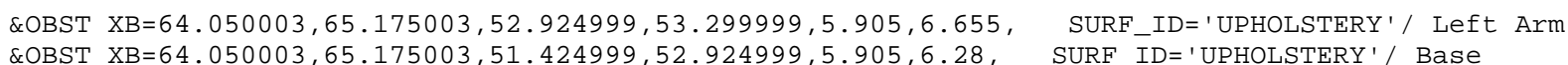

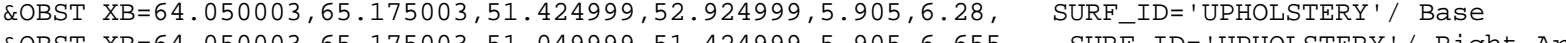
\&OBST XB $64.800003,65.175003,51.424999,52.924999,6.28,6.655$, SURF_ID=' 'UPHOLSTERY'/ Back

*** GROUP: COUCH2 offset $=0.000000,3.000000,0.000000$ rotate $=270.0000000$

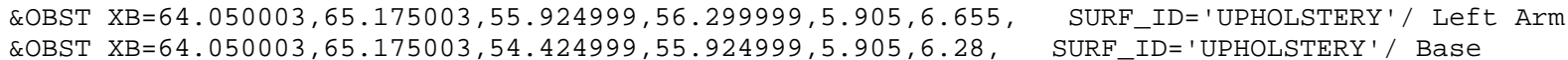

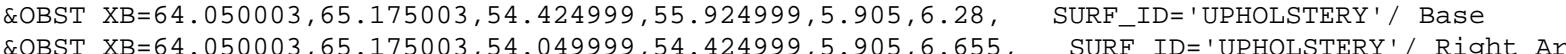
\&OBST XB=64.800003, 65.175003, 54.424999, 55.924999, 6. 28,6.655, SURF_ID='UPHOLSTERY'/ Back Ar

*** GROUP: COUCH2 offset $=0.000000,6.0000000,0.000000$ rotate $=270.000000$

\&OBST XB $=64.050003,65.175003,58.924999,59.299999,5.905,6.655$,
\&OBST XB SURF_ID= 'UPHOLSTERY ' / Left Arm
SURF ID='UPHOLSTERY' \&OBST XB $64.050003,65.175003,57.424999,587.924499,5.905,6.28, \quad$ SURF_ID='UPHOLSTERY'/ Lase

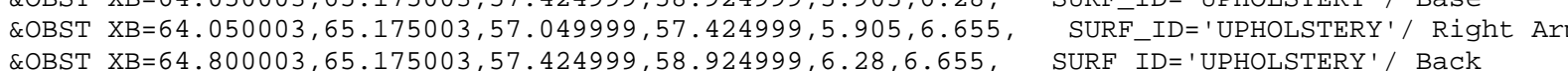

*** GROUP: COUCH2 offset $=0.000000,9.000000,0.000000$ rotate $=270.0000000$

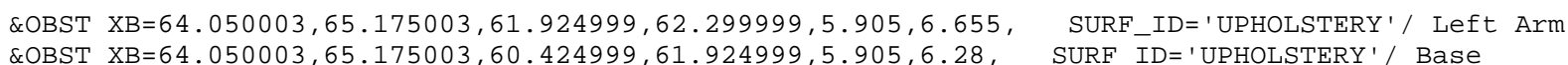

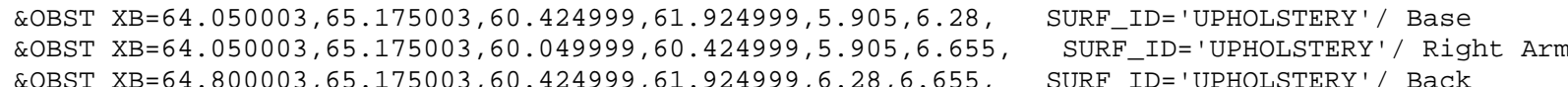

*** GROUP: COUCH2 offset $=0.000000,12.000000,0.000000$ rotate $=270.000000$

\&OBST XB=64.050003, 65.175003,64.925003,65.300003, 5.905, 6.655, SURF_ID=' 'UPHOLSTERY'/ Left Arm \&OBST XB=64.050003, 65.175003,63.424999, 64.925003,5.905, 6.28, SURF_ID='UPHOLSTERY'/ Base

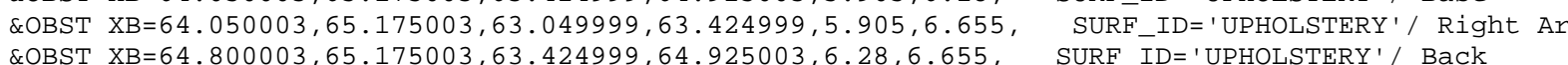

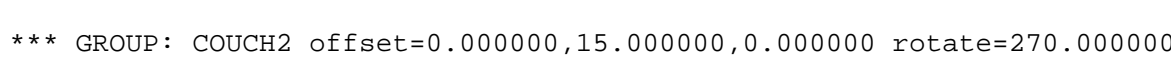

\&OBST XB $=64.050003,65.175003,67.925003,68.300003,5.905,6.655$, SURF_ID=' 'UPHOLSTERY'/ Left Arm \&OBST XB=64.050003,65.175003,66.425003, 67.925003, 5.905, 6.28, SURF_ID='UPHOLSTERY'/ Base

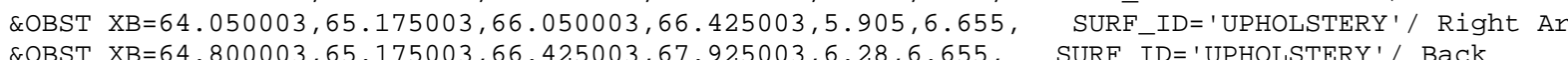

*** GROUP: COUCH2 offset $=0.000000,18.000000,0.000000$ rotate $=270.000000$ \&OBST XB $=64.050003,65.175003,70.925003,71.300003,5.905,6.655, \quad$ SURF_ID=' 'UPHOLSTERY '/ Left Arm
\&OBST XB $64.050003,65.175003,69.425003,70.925003,5.905,6.28$,
SURF_ID=' 'UPHOLSTEY' $/$ Base

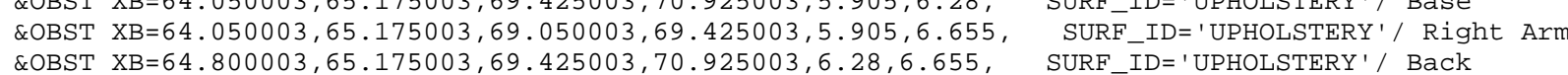

*** GROUP: COUCH2 offset $=0.000000,21.000000,0.000000$ rotate $=270.000000$

\&OBST XB=64. 050003, 65.175003, 73.925003, 74.300003, 5.905,6.655, SURF_ID=' 'UPHOLSTERY'/ Left Arm \&OBST XB $=64.050003,65.175003,72.425003,73.925003,5.905,6.28$, SURF IDE 'UPHOLSTERY'/ Base \&OBST $X \mathrm{BB}=64.050003,65.175003,72.050003,72.425003,5.905,6.655$, SURF_ID=' 'UPHOLSTERY'/ Right Ar

*** GROUP: couCH2 offset $=0.000000,24.000000,0.000000$ rotate $=270.000000$

\&OBST XB $=64.050003,65.175003,76.925003,77.300003,5.905,6.655$, SURF_ID=' 'UPHOLSTERY ' / Left Arm \&OBST XB $=64.050003,65.175003,75.050003,75.425003,5.905,6.655$,
\&OST

*** GROUP: COUCH2 offset $=0.000000,27.000000,0.000000$ rotate $=270.000000$

\&OBST XB $=64.050003,65.175003,79.925003,80.300003,5.905,6.655$, SURF_ID=' 'UPHOLSTERY' / Left Arm 


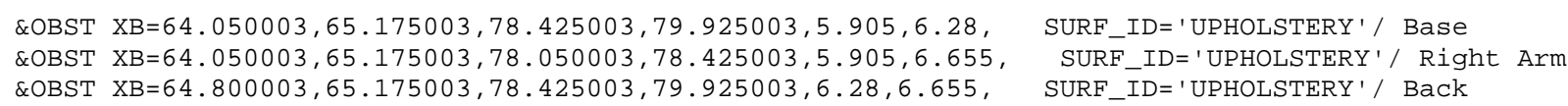
*** GROUP: COUCH2 offset $=0.000000,30.000000,0.000000$ rotate $=270.000000$

\&OBST XB $=64.050003,65.175003,82.925003,83.300003,5.905,6.655, \quad$ SURF_ID= 'UPHOLSTERY '/ Left Arm
\&OBST XB=64.050003,65.175003, $81.425003,82.925003,5.905,6.28, \quad$ SURF_ID='UPHOLSTERY' $/$ Base

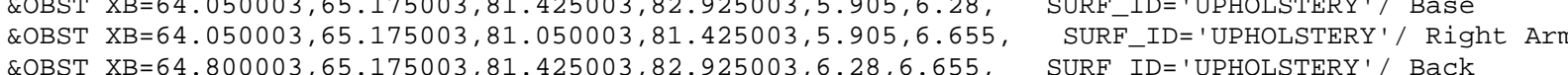

\section{$* * *$ GROUP: TWIN}

$* * *$ GROUP: ROW

*** GROUP: COUCH2 offset $=0.000000,0.000000,0.000000$ rotate $=90.000000$

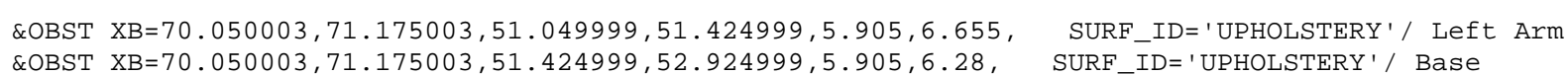

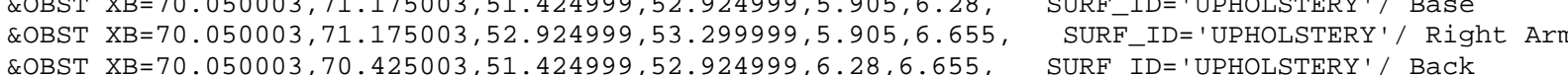
*** GROUP: COUCH2 offset $=0.000000,3.000000,0.000000$ rotate $=90 . .000000$

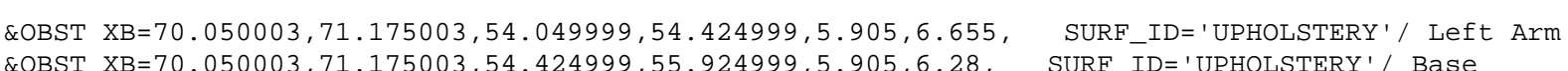

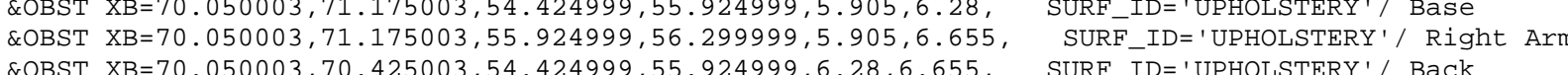

$* * *$ GROUP: COUCH2 offset $=0.000000,6.000000,0.000000$ rotate $=90.000000$

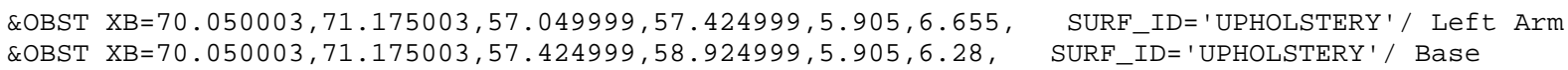

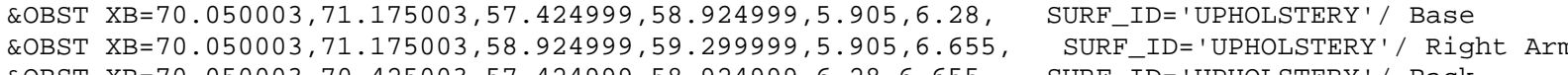

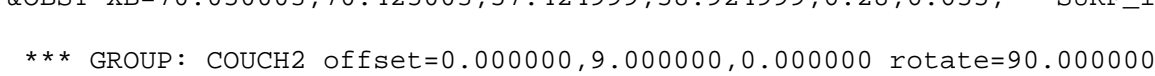

\&OBST XB $=70.050003,71.175003,60.049999,60.424999,5.905,6.655$, SURF_ID=' 'UPHOLSTERY'/ Left Arm

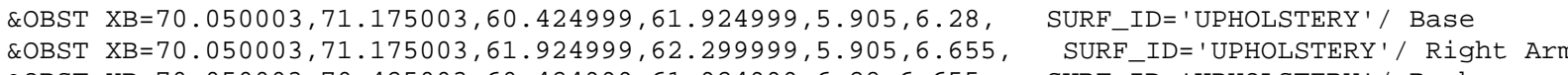
*** GROUP: COUCH2 offset $=0.000000,12.000000,0.000000$ rotate $=90.000000$

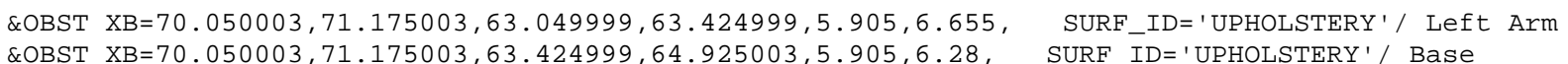

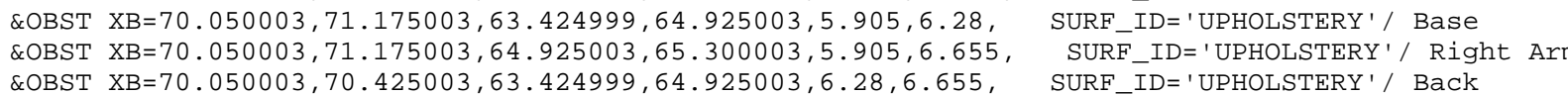

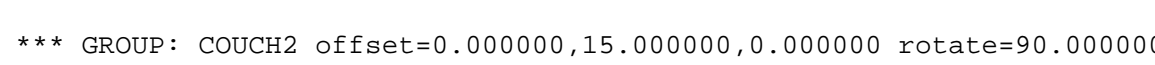

\&OBST XB=70.050003, 71.175003, 66. $050003,66.425003,5.905,6.655$, SURF_ID=' 'UPHOLSTERY'/ Left Arm \&OBST XB $=70.050003,71.175003,66.425003,67.925003,5.905,6.28$, SURF_ID='UPHOLSTERY'/Base

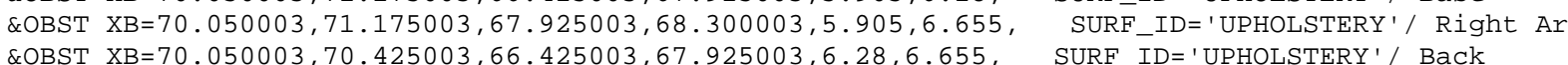

*** GROUP: COUCH2 offset $=0.000000,18.000000,0.000000$ rotate $=90.000000$

\&OBST XB $=70.050003,71.175003,69.050003,69.425003,5.905,6.655$, SURF_ID=' 'UPHOLSTERY ' / Left Arm \&OBST XB=70.050003, 71.175003, 69.425003, 70.925003, 5.905, 6.28, SURF_ID='UPHOLSTERY'// Base

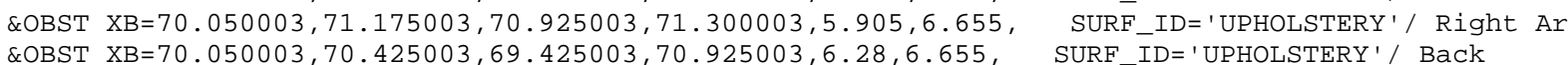

*** GROUP: COUCH2 offset $=0.000000,21.000000,0 . .000000$ rotate $=90.000000$

\&OBST XB $=70.050003,71.175003,72.050003,72.425003,5.905,6.655$, SURF_ID=' 'UPHOLSTERY '/ Left Arm \&OBST XB=70.050003, 71.175003,72.425003,73.925003,5.905, 6.28, SURF_ID='UPHOLSTERY'/ Base Arm \&OBST XB=70.050003, *** GROUP: COUCH2 offset $=0.000000,24.000000,0.000000$ rotate $=90.000000$

\&OBST $X B=70.050003,71.175003,75.050003,75.425003,5.905,6.655$, SURF_ID=' 'UPHOLSTERY'/ Left Arm

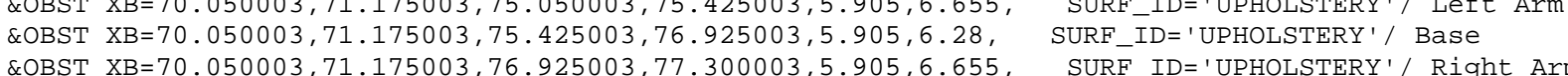

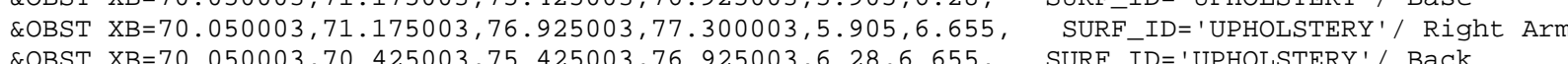

*** GROUP: COUCH2 offset $=0.000000,27.000000,0.000000$ rotate $=90.000000$

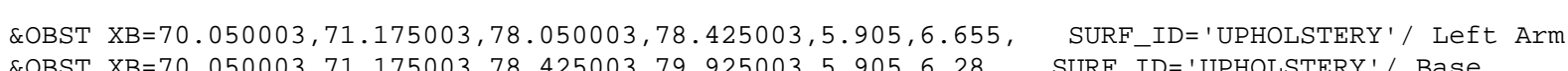

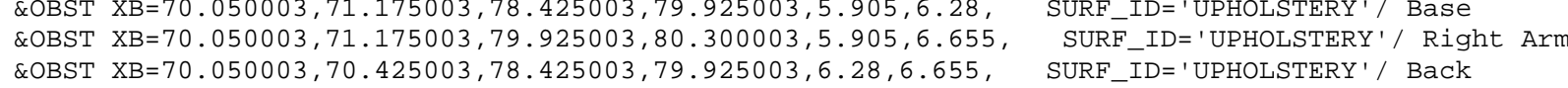
*** GROUP: COUCH2 offset $=0.000000$, , 30. $.000000,0.000000$ rotate $=90.000000$

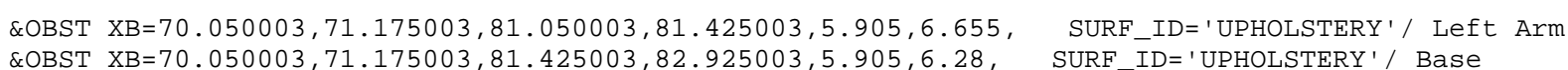

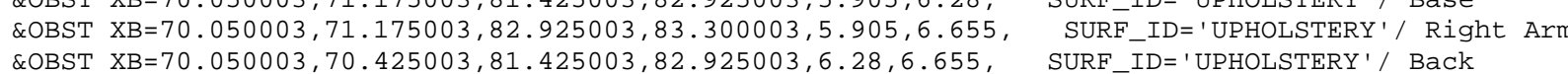

*** GROUP: ROW2

*** GROUP: COUCH2 offset $=0.000000,0.0000000,0 . .000000$ rotate $=270 . .000000$

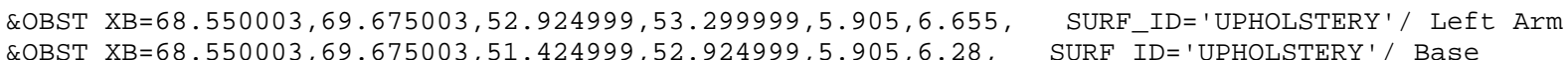

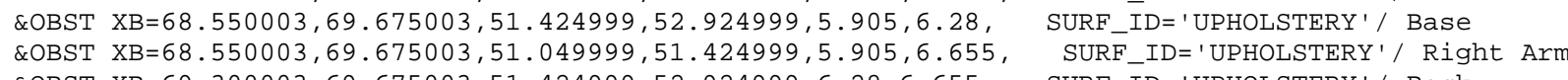

$* * *$ GROUP: COUCH2 offset $=0.000000,3.000000,0.000000$ rotate $=270.000000$

\&OBST XB $=68.550003,69.675003,55.924999,56.299999,5.905,6.655$, SURF_ID=' 'UPHOLSTERY'/ Left Arm

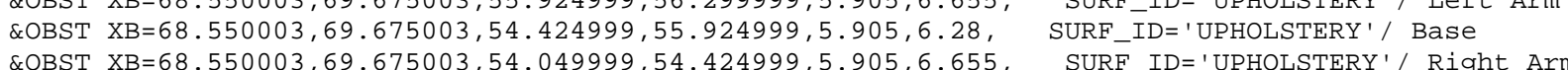

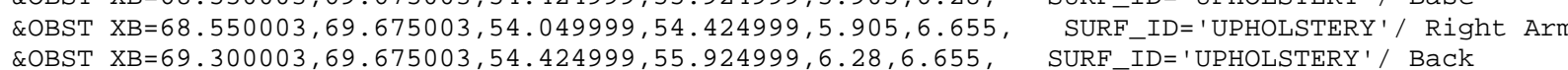

*** GROUP: COUCH2 off set $=0.000000,6.000000,0.000000$ rotate $=270.000000$

\&OBST XB=68.550003, 69.675003, 58.924999, 59.299999, 5.905, 6.655, SURF_ID=' 'UPHOLSTERY'/ Left Arm

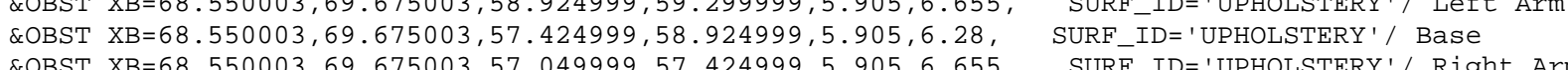

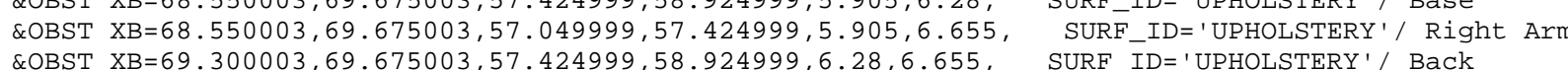

*** GROUP: Couch2 offset $=0.000000,9.000000,0 . .000000$ rotate $=270.000000$

\&OBST XB $=68.550003,69.675003,61.924999,62.299999,5.905,6.655$, SURF_ID=' 'UPHOLSTERY ' / Left Arm

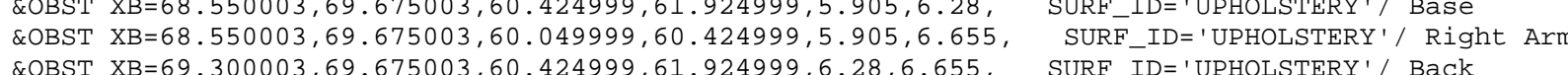
*** GROUP: COUCH2 offset $=0.000000,12.000000,0.000000$ rotate $=270.000000$

\&OBST XB $=68.550003,69.675003,64.925003,65.300003,5.905,6.655$, SURF_ID=' 'UPHOLSTERY '/ Left Arm

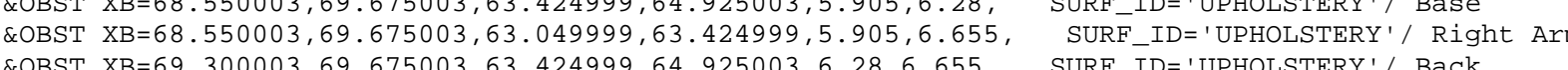

*** GROUP: COUCH2 offset $=0.000000,15.000000,0.000000$ rotate $=270.000000$

\&OBST XB=68.550003, 69.675003,67.925003,68.300003,5.905, 6.655, SURF_ID=' UPHOLSTERY'/ Left Arm

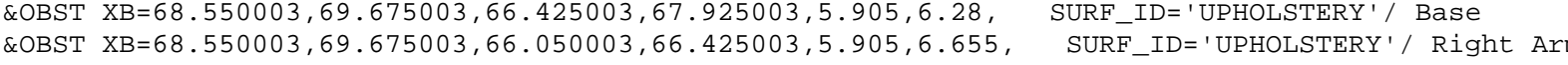
\&OBST XB=68.550003, $69.675003,66.050003,66.425003,5.905,6.655$, SURF_ID=' 'UPHOLSTERY'/ Rigk
\&OBST XB $69.300003,69.675003,66.425003,67.925003,6.28,6.655$,

*** GROUP: COUCH2 offset $=0.000000,18.000000,0.000000$ rotate $=270.000000$

\&OBST XB $=68.550003,69.675003,70.925003,71.300003,5.905,6.655$, SURF_ID=' 'UPHOLSTERY '/ Left Arm \&OBST XB $=68.550003,69.675003,69.050003,69.425003,5.905,6.655$, SURF_ID=' UPHOLSTERY'/ Right Ar \&OBST XB=69.300003, 69.675003, 69.425003, 70.925003, 6.28,6.655, SURF_ID='UPHOLSTERY'/ Back

** GROUP: COUCH2 offset $=0.000000,21,000000,0,000000$ rotate $=270,000000$

\&OBST XB $=68.550003,69.675003,73.925003,74.300003,5.905,6.655$, SURF_ID=' 'UPHOLSTERY '/ Left Arm \&OBST XB $=68.550003,69.675003,72.050003,72.425003,5.905,6.655$, SURF ID=' 'UPHOLSTERY' $/$ Richt Ar \&OBST XB=69. $300003,69.675003,72.425003,73.925003,6.28,6.655$, SURF_ID='UPHOLSTERY'/ Back *** GROUP: COUCH2 offset $=0.000000,24.000000,0.000000$ rotate $=270.000000$ \&OBST XB $68.550003,69.675003,76.925003,77.300003,5.905,6.655$, SURF_ID='UPHOLSTERY'/ Left Arm
\&OBST XB $6.6855003,69.67503,75.42500,76.925003,5.955,6.28$ 


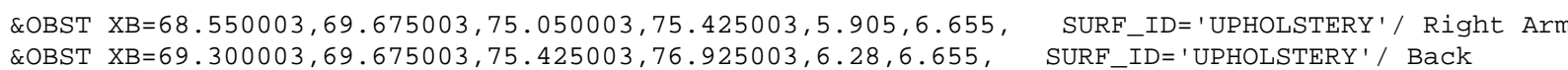
*** GROUP: COUCH2 offset $=0.000000,27.000000,0.000000$ rotate $=270.000000$

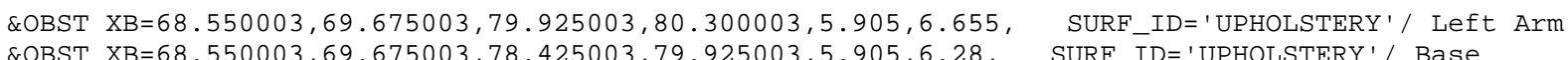

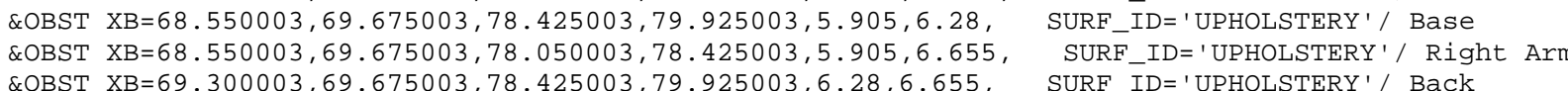
*** GROUP: COUCH2 offset $=0.000000,30.000000,0.000000$ rotate $=270.000000$

\&OBST XB $=68.550003,69.675003,82.925003,83.300003,5.905,6.655$, SURF_ID=' 'UPHOLSTERY'/ Left Arm \&OBST XB=68.550003, 69.675003, 81.425003, 82.925003, 5.905, 6.28, SURF_ID='UPHOLSTERY'/ Base Arm

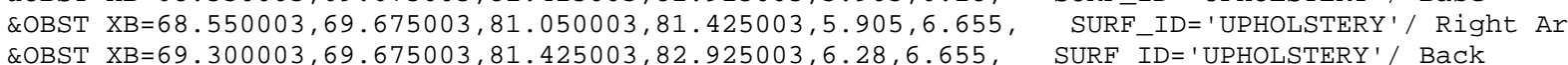

*** GROUP: ROW2

*** GROUP: COUCH2 offset $=0.000000,0.000000,0.000000$ rotate $=270.000000$

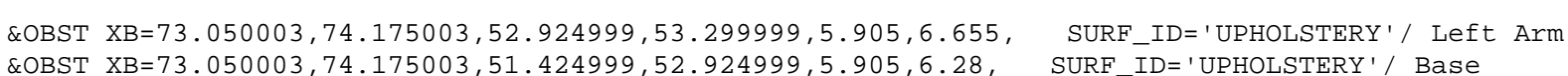
\&OBST XB $=73.050003,74.175003,51.049999,51.424999,5.905,6.655$,
\&OBST XB $=73.800003,74.175003,51.424999,52.924999,6.28,6.655$,
SURF_IDE='UPHOLSTERY '/ Right Arm
SURF 'UPHOLSTERY'/ Back

*** GROUP: COUCH2 offset $=0.000000,3.000000,0.000000$ rotate $=270.000000$

\&OBST XB $=73.050003,74.175003,55.924999,56.299999,5.905,6.655$, SURF ID=' 'UPHOLSTERY' / Left Arm

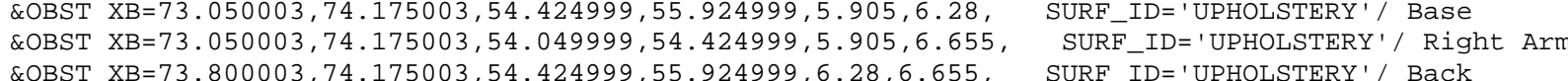
*** GROUP: COUCH2 offset $=0 ., 000000,6$. . $000000,0.000000$ rotate $=270.000000$

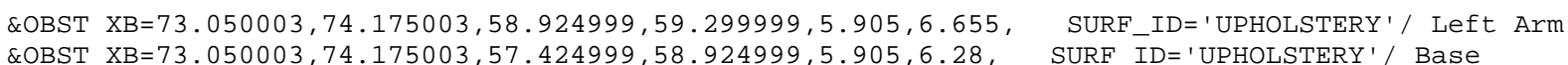

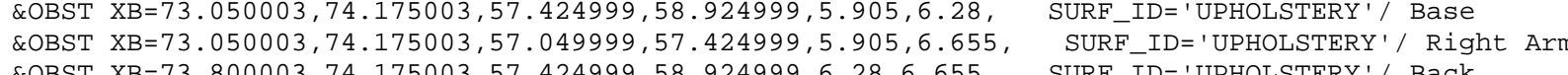

$* * *$ GROUP: COUCH2 offset $=0.000000,9$. . 000000, ०. .000000 rotate $=270.000000$

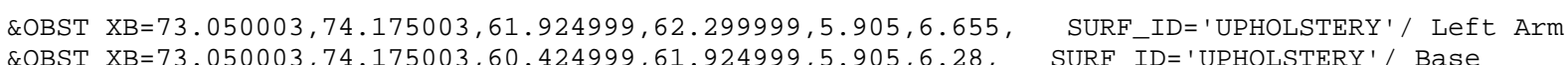

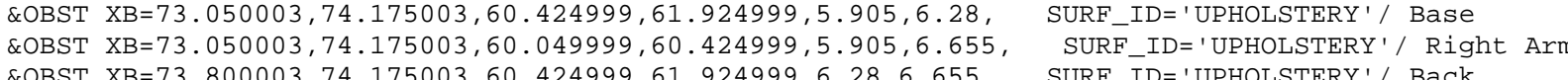

*** GROUP: COUCH2 offset $=0.000000,12.000000,0.0000000$ rotate $=270.000000$

\&OBST XB $=73.050003,74.175003,64.925003,65.300003,5.905,6.655$, SURF_ID=' 'UPHOLSTERY '/ Left Arm

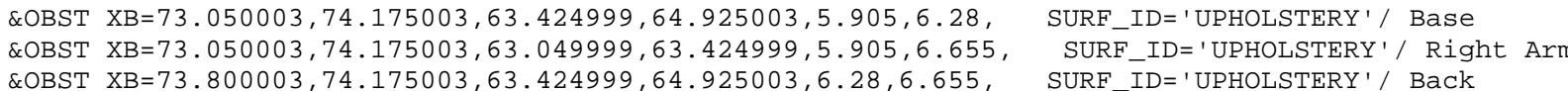

*** GROUP: COUCH2 offset $=0.0000000,15.000000,0.000000$ rotate $=270.000000$

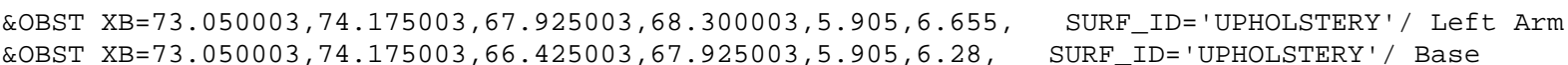
\&OBST $X \mathrm{~B}=73.050003,74.175003,66.425003,67.925003,5.905,6.28$, SURF_ID='UPHOLSTERY'/ Base Arm

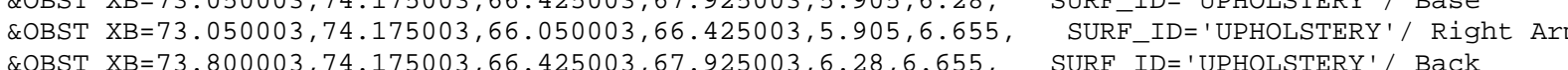
*** GROUP: COUCH2 offset $=0.000000,18.000000,0.000000$ rotate $=270$. .

EOBST XB $=73.050003,74.175003,70.925003,71.300003,5.905,6.655$, SURF_ID=' 'UPHOLSTERY'/ Left Arm \&OBST XB=73.050003, 74.175003,69.425003, 70.925003, 5.905,6.28, SURF_ID='UPHOLSTERY'/ Base

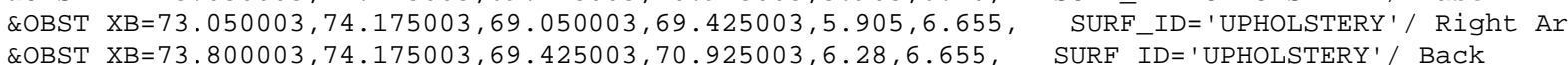
*** GROUP: COUCH2 offset $=0.000000,21.000000,0.000000$ rotate $=270.000000$

\&OBST XB $=73.050003,74.175003,73.925003,74.300003,5.905,6.655$, SURF_ID=' 'UPHOLSTERY'/ Left Arm

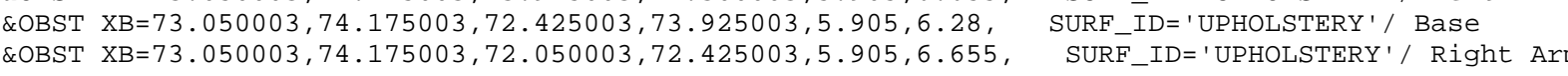
ID= 'UPHOLSTERY' / Back

*** GROUP: COUCH2 offset $=0.000000,24.000000,0.000000$ rotate $=270.000000$
\&OBST XB $=73.050003,74.175003,76.925003,77.300003,5.905,6.655$, SURF_ID=' 'UPHOLSTERY'/ Left Arm \&OBST XB=73.050003,74.175003,75.425003,76.925003,5.905,6.28, SURF-ID='UPHOLSTERY'/ Base (2OBST XB

$* * *$ GROUP: COUCH2 offset $=0.0000000,27.000000,0.000000$ rotate $=270.000000$

\&OBST XB=73. $050003,74.175003,79.925003,80.300003,5.905,6.655$, SURF_ID=' 'UPHOLSTERY '/ Left Arm

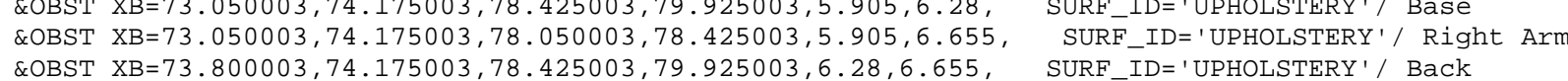

*** GROUP: COUCH2 offset $=0.000000,30.000000,0.000000$ rotate $=270.0000000$

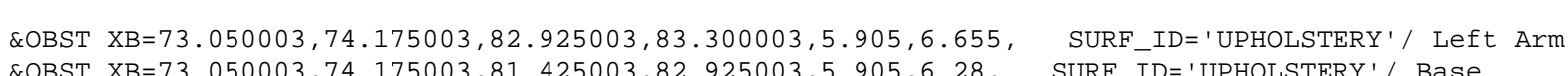

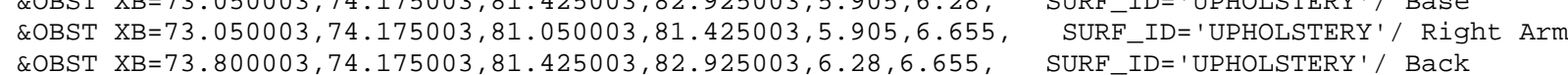

*** GROUP: SHORT_ROW

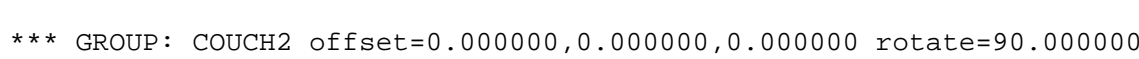

\&OBST XB $=67.044998,67.419998,46.91,48.035,5.905,6.655, \quad$ SURF_ID='UPHOLSTERY' $/$ Left Arm
\&OBST XB $=65.544998,67.044998,46.91,48.035,5.905,6.28$

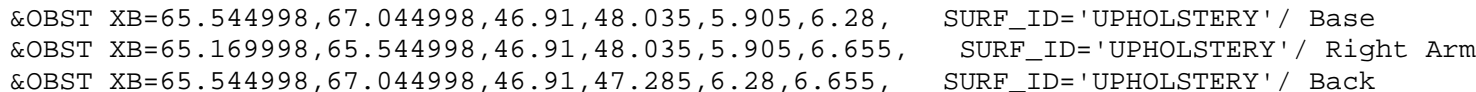

*** GROUP: COUCH2 offset $=0.000000,3.000000,0.000000$ rotate $=90.000000$ \&OBST XB $=64.044998,64.419998,46.91,48.035,5.905,6.655$, SURF_ID=' 'UPHOLSTERY' / Left Arm

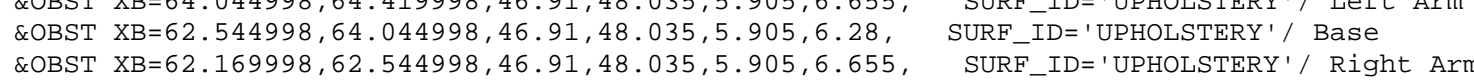
\&OBST XB $=62.544998,64.044998,46.91,47,285,6.28,6.655$, SURF_ID=' UPHOLSTERY' $/$ Back

*** GROUP: COUCH2 offset $=0.000000,6.000000,0.000000$ rotate $=90.000000$

\&OBST XB=61.044998, 61.419998, 46.91, 48.035, 5.905, 6.655, SURF_ID=' 'UPHOLSTERY'/ Left Arm \&OBST XB=59.544998, 61.044998, 46.91, 48.035, 5.905, 6.28, SURF_ID='UPHOLSTERY'/ Base \&OBST XB=59.169998, 59.544998, 46.91,48.035, $5.905,6.655$, SURF_ID='UPHOLSTERY'/ Right Ar
\&OBST XB=59.544998, $61.044998,46.91,47.285,6.28,6.655$,
SURE_ID='UPHOLSTERY'/ Back

*** GROUP: COUCH2 offset $=0.0000000,9.000000,0.000000$ rotate $=90 . .000000$

\&OBST XB=58.044998, 58.419998, 46.91, 48.035, 5.905,6.655, SURF_ID=' UPHOLSTERY'/ Left Arn

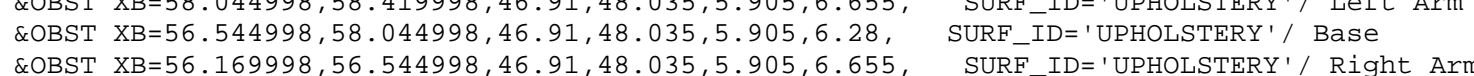
COBST XB=56.544998, $58.044998,46.91,47.285,6.28,6.655$, SURF_ID=' UPHOLSTERY'/ Back

*** GROUP: COUCH2 offset $=0.000000,12.000000,0.000000$ rotate $=90.000000$

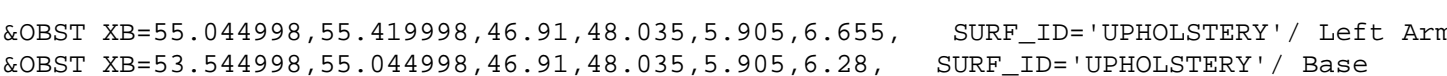
\&OBST XB=53.169998, 53.544998, 46.91,48.035, 5.905, 6.655, SURF_ID='UPHOLSTERY'/ Right Arm
\&OBST XB=53.544998, $55.044998,46.91,47.285,6.28,6.655$,
SURF_ID='UPHOLSTERY' $/$ Back

*** GROUP: COUCH2 offset $=0.000000,15.000000,0.000000$ rotate $=90.000000$

\&OBST XB=52. $044998,52.419998,46.91,48.935,5.905,6.655$, SURF_ID='UPHOLSTERY'/ Left Arm

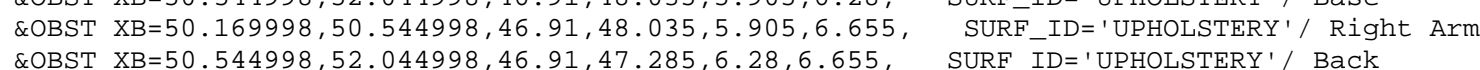

$\star \star \star *$ GROUP: COUCH2 offset $=0.000000,18.000000,0.000000$ rotate $=90.000000$

\&OBST XB $=49.044998,49.419998,46.91,48.035,5.905,6.655, \quad$ SURF_ID=' UPHOLSTERY' / Left Arm \&OBST XB $=47.169998,47.544998,46.91,48.035,5.905,6.655$, SURF_ID='UPHOLSTERY'/ Right Arm \&OBST XB=47.544998,

*** GROUP: ROW

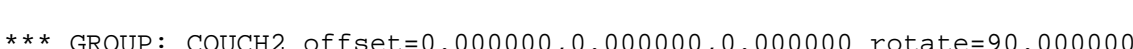

\&OBST XB=41.549999, 41.924999, 84.43, 85.555, 5.995, 6.655, SURF_ID=' 'UPHOLSTERY '/ Left Arm

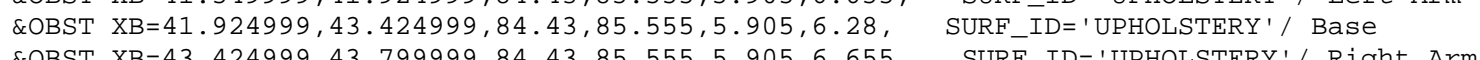


\&OBST XB $=41.924999,43,424999,85,18,85,555,6,28,6,655$, SURF ID=' UPHOLSTERY' / Back *** GROUP: COUCH2 offset $=0.000000,3.000000,0.000000$ rotate $=90.000000$ \&OBST XB $=44.549999,44.924999,84.43,85.555,5.905,6.655$, SURF_ID= 'UPHOLSTERY Y Left Arm

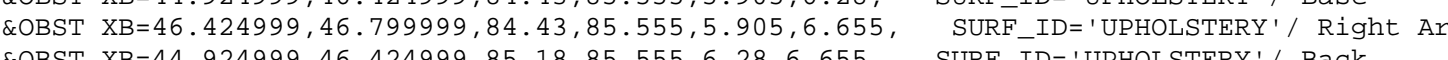

$* * *$ GROUP: COUCH2 offset $=0.000000,6.000000,0.000000$ rotate $=90.000000$

\&OBST XB $=47.549999,47.924999,84.43,85.555,5.905,6.655$, SURF_ID=' UPHOLSTERY' / Left Arm

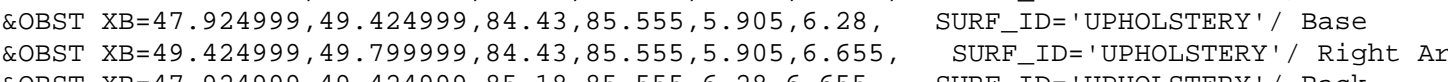

*** GROUP: COUCH2 offset $=0.000000,9.000000,0.000000$ rotate $=90.000000$

\&OBST XB=50.549999, 50.924999, 84.43,85.555, 5.905,6.655, SURF_ID='UPHOLSTERY' / Left Arm \&OBST $\mathrm{XB}=50.924999,52.424999,84.43,85.555,5.905,6.28$, SURF_ID ='UPHOLSTERY'/ Base A QOBST XB=52.424999, $52.799999,84.43,85.555,5.905,6.655$

** GROUP. COUCH2 off

\&OBST XB=53.549999, 53.924999, 84.43, 85.555, 5.905, 6.655,

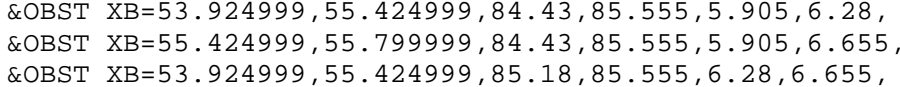

*** GROUP: COUCH2 offset $=0,00000,15,000000,0,000000$ ro

SURF_ID=' 'UPHOLSTERY'/ Right Arm
SURF_ID='UPHOLSTERY'/ Back

rotate $=90.000000$

SURF_ID='UPHOLSTERY'/ Left Arm SURF_ID='UPHOLSTERY'/ Right Ar SURF_ID=' 'UPHOLSTERY' / BaCK

\&OBST XB $=56.549999,56.924999,84.43,85.555,5.995,6.655, \quad$ SURF_ID=' 'UPHOLSTERY ' / Left Arm \&OBST XB $=58.424999,58.799999,84.43,85.555,5.905,6.655, \quad$ SURF_ID='UPHOLSTERY'/ Right Arm
\&OBST XB $=56.924999,58.424999,85.18,85.555,6.28,6.655$, *** GROUP: COUCH2 offset $=0.000000,18.000000,0.000000$ rotate $=90.000000$

\&OBST XB=59.549999, 59.924999, 84.43, 85.555, 5.905, 6.655, SURF_ID=' UPHOLSTERY' ' Left Arm

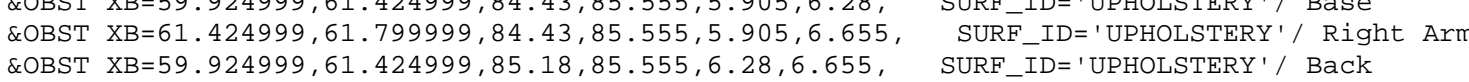

*** GROUP: COUCH2 offset $=0.000000,21.000000,0.000000$ rotate $=90.000000$

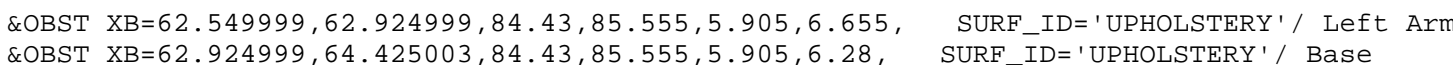

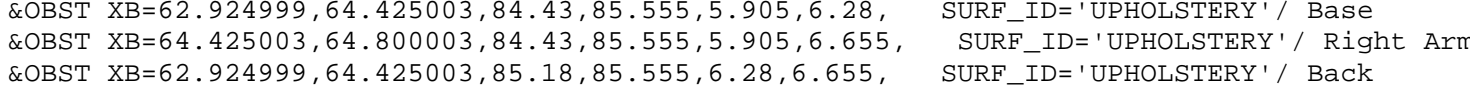

*** GROUP: COUCH2 offset $=0.000000,24.000000,0.000000$ rotate $=90.000000$

\&OBST XB $=65.550003,65.925003,84.43,85.555,5.905,6.655$, SURF_ID=' 'UPHOLSTERY'/ Left Arm

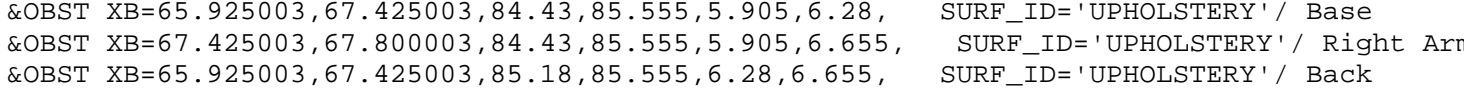

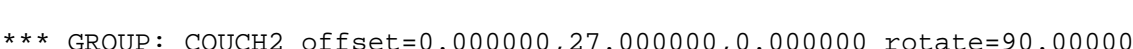

\&OBST XB $=68.550003,68.925003,84.43,85.555,5.905,6.655$, SURF_ID='UPHOLSTERY'/ Left Arm

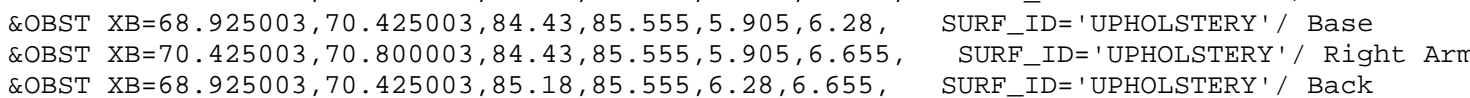

** GROUP: COUCH2 offset $=0.000000,30.000000,0.000000$ rotate $=90.000000$

\&OBST XB $71.550003,71.925003,84.43,85.555,5.905,6.655$, SURF_ID='UPHOLSTERY'/ Left Arm QOBST $X \mathrm{~B}=71.925003,73.425003,84.43,85.555,5.905,6.28$, SURF_ID='UPHOLSTERY'/ Base

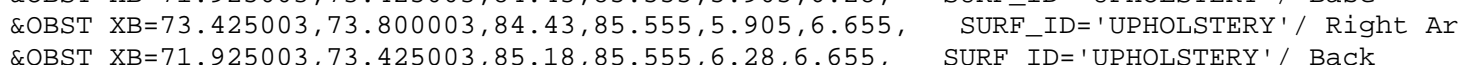

*** GROUP: LEVEL

*** GROUP: ROW

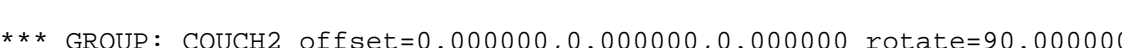

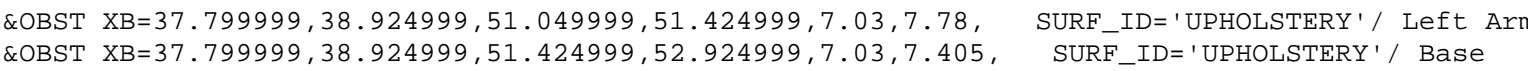

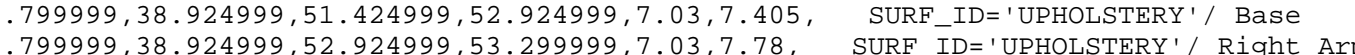

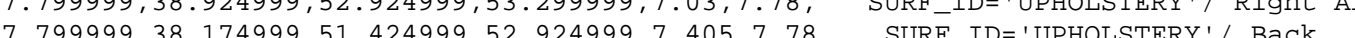

*** GROUP: COUCH2 offset $=0.000000,3.000000,0.000000$ rotate $=90.000000$

\&OBST XB $=37.799999,38.924999,54.049999,54.424999,7.03,7.78$, SURF ID= ' 'UPHOLSTERY ' / Left Arm \&OBST XB=37.799999, 38.924999, 54.424999, 55.924999, 7.03, 7.405, SURF_ID='UPHOLSTERY'/ Base

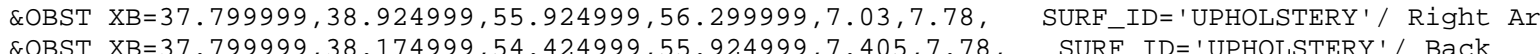

*** GROUP: COUCH2 offset $=0.000000,6.000000,0.000000$ rotate $=90.000000$

\&OBST XB=37.799999, 38.924999, 57.049999, 57.424999, 7.03, 7.78, SURF_ID='UPHOLSTERY'/ Left Arm \&OBST XB=37.79999, 38.924999, 57.424999, 58. 924999, 7.03, 7.405, SURF_ID='UPHOLSTERY'/ Base EOBST XB $=37.799999,38.174999,57.424999,58.924999,7.405,7.78$, SURF_ID='UPHOLSTERY SURF ID 'UPHOLSTERY'/ Back

*** GROUP: COUCH2 offset $=0.000000,9.000000,0.000000$ rotate $=90.000000$

\&OBST XB=37.799999, 38.924999, 60. $449999,60.424999,7.03,7.78$, SURF_ID=' 'UPHOLSTERY '/ Left Arm \&OBST XB=37.799999, 38.924999, 60.424999,61.924999, 7.03, 7.405, SURF_ID='UPHOLSTERY'/ Base

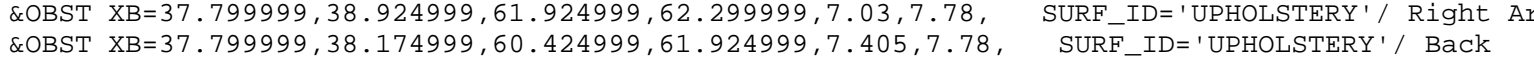

*** GROUP: COUCH2 offset $=0.000000,12.000000,0.000000$ rotate $=90.000000$

\&OBST XB=37.799999, 38.924999, 63. 049999, 63.424999, 7.03, 7.78, SURF_ID=' 'UPHOLSTERY' ' Left Arm

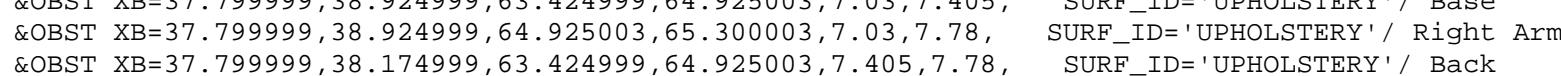

*** GROUP: COUCH2 offset $=0.000000,15.000000,0.000000$ rotate $=90.000000$

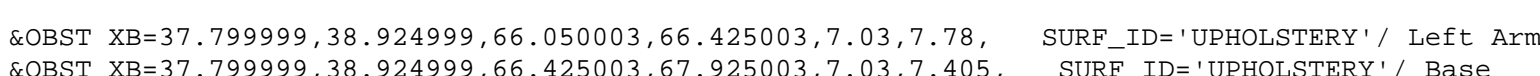
\&OBST XB=37.799999, 38. 924999, 67. $925003,68.300003,7.03,7.78, \quad$ SURF_ID=' 'UPHOLSTERY'/ Right Arm
\&OBST $X \mathrm{~B}=37.799999,38.174999,66.425003,67.925003,7.405,7.78, \quad$ SURF_ID=' 'UPHOLSTERY'/ Back

*** GROUP: COUCH2 offset $=0.000000,18.000000,0.000000$ rotate $=90.000000$

\&OBST XB=37.799999, 38.924999, 69. 050003, 69.425003, 7.03, 7.78, SURF_ID=' 'UPHOLSTERY' ' Left Arm

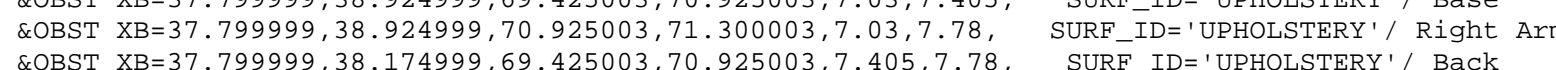

*** GROUP: COUCH2 offset $=0.000000,21.000000,0.000000$ rotate $=90.000000$

\&OBST XB=37.799999, 38. 924999, 72. $050003,72.425003,7.03,7.78$, SURFID ='UPHOLSTERY' ' Left Arm

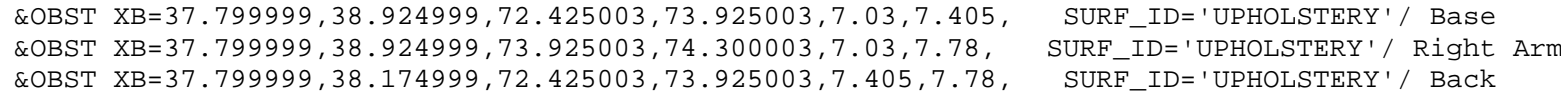

*** GROUP: COUCH2 offset $=0.000000,24.000000,0.000000$ rotate $=90.000000$

\&OBST XB $=37.799999,38.924999,75.050003,75.425003,7.03,7.78, \quad$ SURF ID=' 'UPHOLSTERY'/ Left Arm \&OBST XB $=37.79999,38.924999,76,925003,77,300003,7,03,7,78$, \&OBST XB $=37.799999,38.174999,75.425003,76.925003,7.405,7.78$, SURF_ID='UPHOLSTERY'/ Back

*** GROUP: COUCH2 offset $=0.000000,27.000000,0.000000$ rotate $=90.000000$

\&OBST XB=37.799999, 38. 924999, 78. 050003, 78.425003, 7. 03, 7.78, $\quad$ SURF_ID=' 'UPHOLSTERY'/ Left Arm
\&OBST XB=37.79999, 38.924999, 78.425003,79.925003,7.03,7.405, SURFID='UPHOLSTERY' Rase

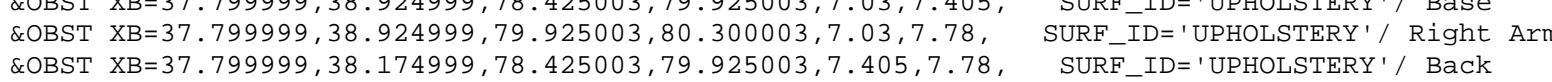

*** GROUP: COUCH2 offset $=0.000000,30.000000$, ๑. .000000 rotate $=90.0000000$

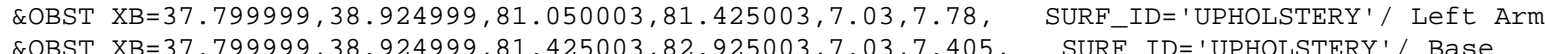

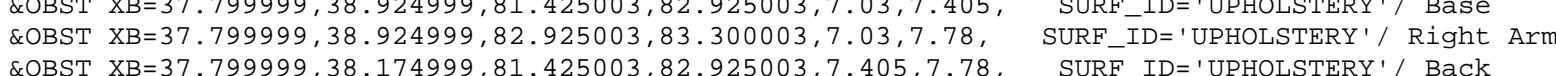
*** GROUP: BASE 
*** GROUP: TWIN

*** GROUP: ROW

** GROUP: COUCH2 offset $=0.000000,0.000000,0.000000$ rotate $=90 . .000000$

\&OBST XB=43. 049999, 44.174999, 51. $049999,51.424999,7.03,7.78, \quad$ SURF_ID=' UPHOLSTERY '/ Left Arm

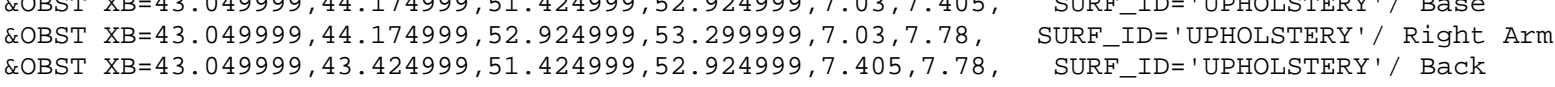

*** GROUP: COUCH2 offset $=0.000000,3.000000,0.000000$ rotate $=90.000000$

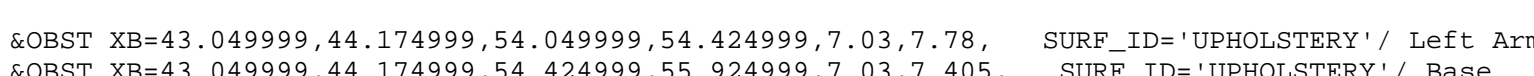

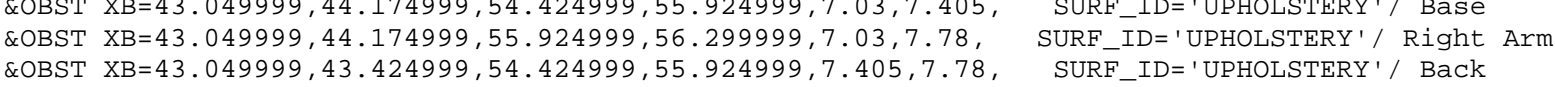

*** GROUP: COUCH2 offset $=0.000000,6.000000,0.000000$ rotate $=90.000000$

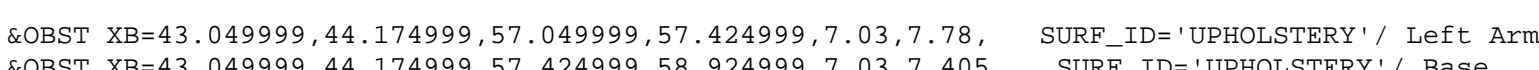
COBST XB $=43.049999,44.174999,58.924999,59.299999,7.03,7.78$,
COBST XB $=43.049999,43.424999,57.424999,58.924999,7.405,7.78, \quad$ SURF_ID='UPHOLSTERY'/ Right Arm
SURF_ID=' 'UPHOLSTERY' $/$ Back

$* * *$ GROUP: COUCH2 offset $=0.000000,9.000000,0.000000$ rotate $=90.000000$

\&OBST XB=43. 049999, 44. 174999, 60. 049999, 60.424999, 7. $03,7.78$, SURF.ID=' 'UPHOLSTERY' ' Left Arm

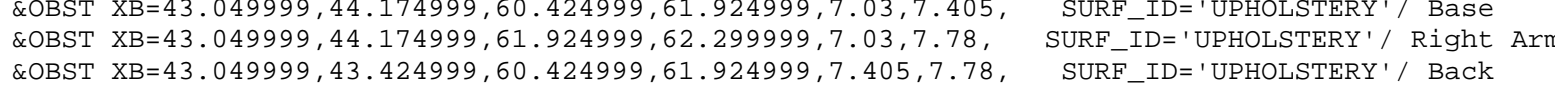

*** GROUP: COUCH2 offset $=0.000000,12.000000,0.000000$ rotate $=90.000000$

\&OBST XB=43. $949999,44.174999,63.049999,63.424999,7.03,7.78$, SURF_ID=' UPHOLSTERY' ' Left Arm

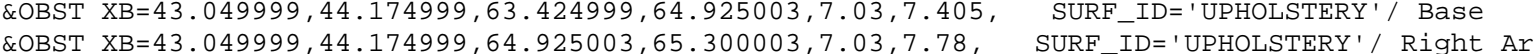

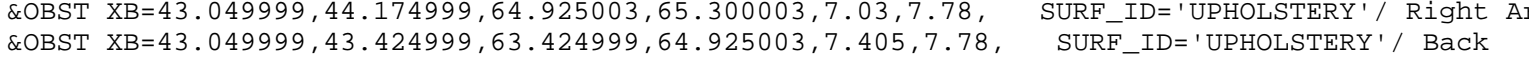

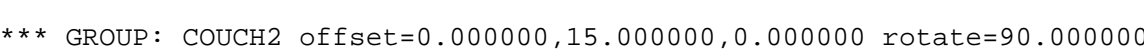

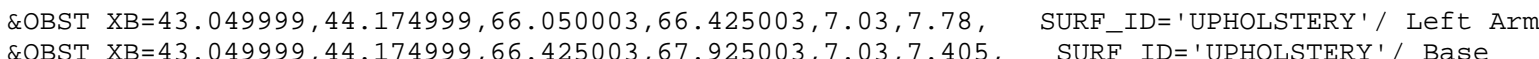

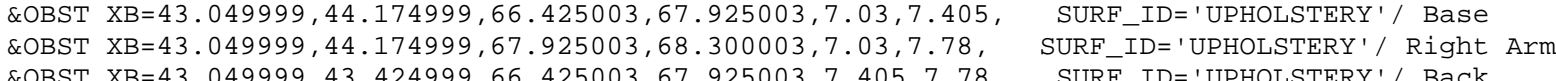

$* * *$ GROUP: COUCH2 offset $=0.000000,18.000000,0.0000000$ rotate $=90.000000$

\&OBST XB=43. 049999, 44.174999, 69.050003, 69.425003, 7. 03, 7.78, SURF_ID='UPHOLSTERY' '/ Left Arm

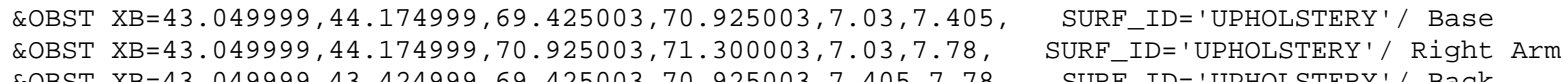

*** GROUP: COUCH2 offset $=0.000000,21.000000,0.000000$ rotate $=90.000000$

\&OBST XB $=43.049999,44.174999,72.050003,72.425003,7.03,7.78, \quad$ SURF_ID='UPHOLSTERY' ' Left Arm
\&OBST XB $=43.049999,44.174999,72.425003,73.925003,7.03,7.405$,
SURF_ID=' 'UPHOLSTERY' $/$ Base

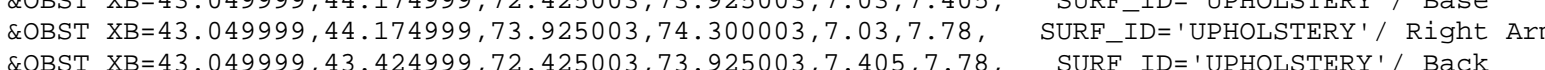

*** GROUP: COUCH2 offset $=0.000000,24.000000,0.000000$ rotate $=90$. . 000000

\&OBST XB $=43.049999,44.174999,75.050003,75.425003,7.03,7.78$, SURF_ID='UPHOLSTERY' $/$ Left Arm \&OBST XB $=43.049999,44.174999,75.425003,76.925003,7.03,7.405$, SURF_ID=' 'UPHOLSTERY' / Base

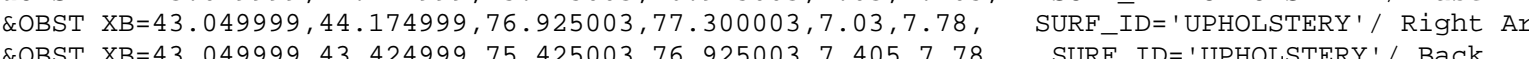

*** GROUP: COUCH2 offset $=0.000000,27.000000,0.000000$ rotate $=90.000000$

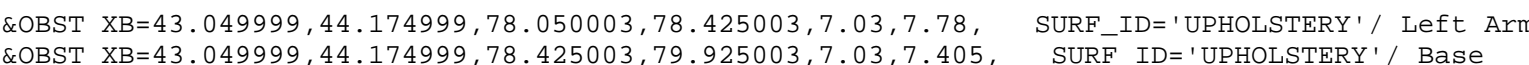
\&OBST XB=43.049999, 44.174999, $79.925003,80.300003,7.03,7.78, \quad$ SURF ID=' 'UPHOLSTERY' '/ Right Ar

*** GROUP: COUCH2 offset $=0.000000,30.000000, \Theta .000000$ rotate $=90.000000$
\&OBST XB=43. 049999, 44.174999, 81. 050003, 81.425003, 7.03, 7.78, SURF_ID='UPHOLSTERY'/ Left Arm \&OBST XB=43. $049999,44.174999,81.425003,82.925003,7.03,7.405$, SURF_ID='UPHOLSTERY'/ Base

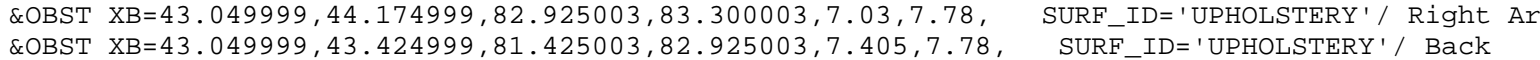

$* * *$ GROUP: ROW2

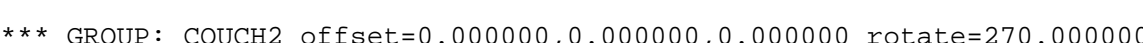

\&OBST XB $=41.549999,42.674999,52.924999,53.299999,7.03,7.78$, SURF_ID='UPHOLSTERY '/ Left Arm

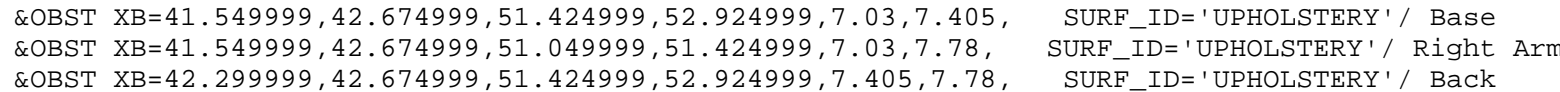

*** GROUP: COUCH2 offset $=0.000000,3.000000,0.000000$ rotate $=270.000000$

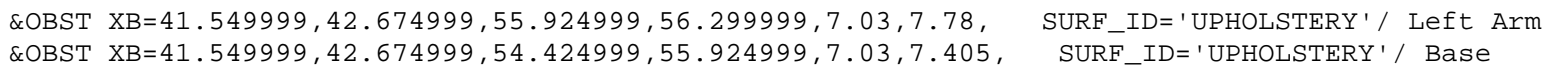
\&OBST XB=41.549999, 42.674999, 54.049999, 54. 424999, 7.03, 7.78, SURF_ID=' 'UPHOLSTERY'/ Right Ar
EOBST XB $=42.299999,42.674999,54.424999,55.924999,7.405,7.78, \quad$ SURF_ID='UPHOLSTERY' $/$ Back

*** GROUP: COUCH2 offset $=0.000000,6.000000,0.000000$ rotate $=270$. .

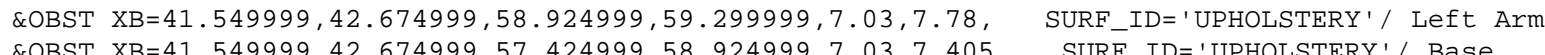

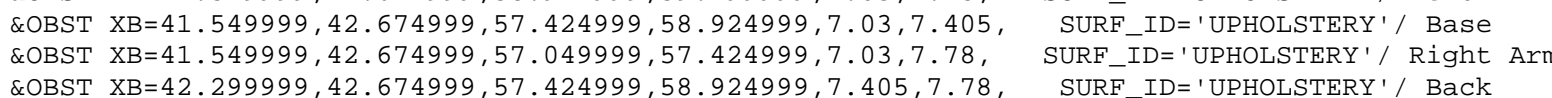

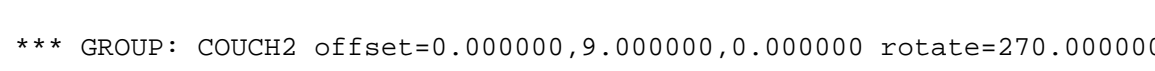

\&OBST XB $=41.549999,42.674999,61.924999,62.299999,7.03,7.78$, SURF_ID='UPHOLSTERY '/ Left Arm

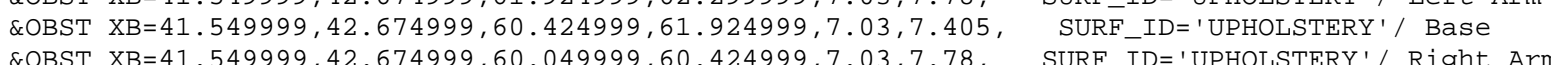
\&OBST XB-41.54999,

*** GROUP: COUCH2 offset $=0.000000,12.000000,0.000000$ rotate $=270.000000$

\&OBST XB=41.549999, 42.674999, 64.925003, 65.300003, 7.03, 7.78, SURF_ID='UPHOLSTERY'/ Left Arm \&OBST XB=41.549999, 42.674999, 63.424999, 64.925003, 7.03, 7.405, SURF_ID=' 'UPHOLSTERY'/ Base \&OBST XB $=41.549999,42.674999,63.049999,63.424999,7.03,7.78, \quad$ SURF_ID= 'UPHOLSTERY' '/ Right Arm
\&OBST XB $=42.299999,42.674999,63.424999,64.925003,7.405,7.78, \quad$ SURF_ID=' 'UPHOLSTERY' / Back

*** GROUP: COUCH2 offset $=0.000000,15.000000,0.000000$ rotate $=270.000000$

\&OBST XB $=41.549999,42.674999,67.925003,68.300003,7.03,7.78$, SURF_ID=' 'UPHOLSTERY '/ Left Arm EOBST XB $=41.549999,42.674999,66.425003,67.925003,7.03,7.405, \quad$ SURF IID='UPHOLSTERY'/ Base
SURST EOBST XB $=42.299999,42.674999,66.425003,67.925003,7.405,7.78$, SURF_ID='UPHOLSTERY'/ Right A

*** GROUP: COUCH2 offset $=0.000000,18.000000,0.000000$ rotate $=270.000000$

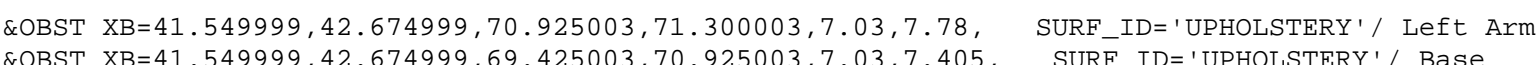

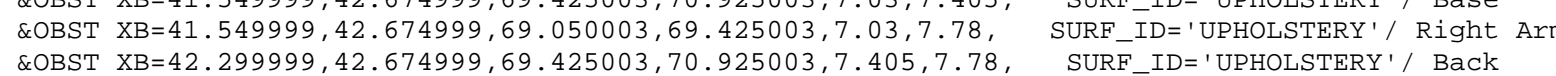

*** GROUP: COUCH2 offset $=0.0000000,21.000000,0.000000$ rotate $=270.000000$

\&OBST XB=41.549999, 42.674999, 73.925003, 74.300003, 7. $03,7.78$, SURF_ID=' 'UPHOLSTERY' ' Left Arm

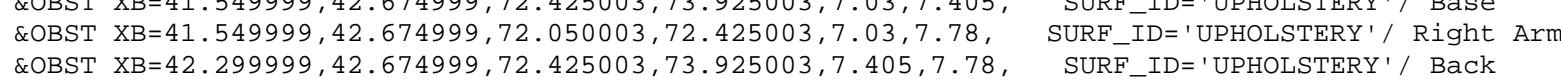

*** GROUP: COUCH2 offset $=0.000000,24.000000,0.000000$ rotate $=270.000000$

\&OBST XB=41.549999, 42.674999, 76.925003, 77. 300003, 7. 03, 7.78, SURF_ID=' 'UPHOLSTERY' / Left Arm \&OBST XB=41.549999, 42.674999, 75. 050003, 75.425003, 7.03, 7.78, SURF_ID='UPHOLSTERY'/Right Ar \&OBST XB=42.299999, 42.674999, 75.425003, 76. 925003, 7.405, 7.78, SURF_ID='UPHOLSTERY' / Back

*** GROUP: COUCH2 offset $=0.000000,27.000000,0.000000$ rotate $=270.000000$

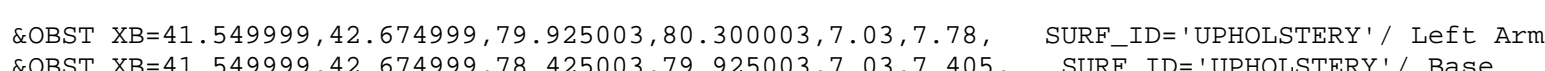

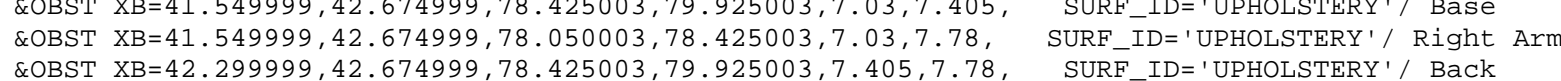




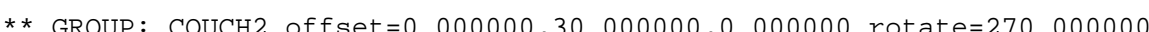

LOBST XB=41.549999, 42.674999, 82. $925003,83.300003,7.03,7.78, \quad$ SURF_ID='UPHOLSTERY' ' Left Arm
\&OBST XB $=41.549999,42.674999,81.425003,82.925003,7.03,7.405, \quad$ SURF_ID='UPHOLSTERY' $/$ Base

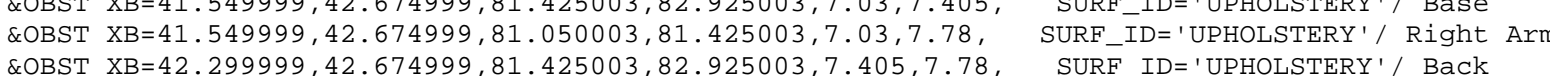

$* * *$ GROUP: TWIN

$* * *$ GROUP: ROW

*** GROUP: COUCH2 offset $=0.0000000,0.000000,0.000000$ rotate $=90.000000$

\&OBST XB=47.549999, 48.674999, 51.049999, 51.424999, 7.03, 7.78, $\quad$ SURF_ID='UPHOLSTERY' ' Left Arm
\&OBST XB=47.549999, $48.674999,51.424999,52.924999,7.03,7.405, \quad$ SURF_ID='UPHOLSTERY'/ Base \&OBST XB $=47.549999,48.674999,52.924999,53.299999,7.03,7.78$, SURF ID ' UPHOLSTERY'/ Right Arm

$* * *$ GROUP: COUCH2 offset $=0.000000,3.000000,0.000000$ rotate $=90.000000$

\&OBST XB=47.549999, 48.674999, 54. $049999,54.424999,7.03,7.78$, SURF_ID=' 'UPHOLSTERY'/ Left Arm

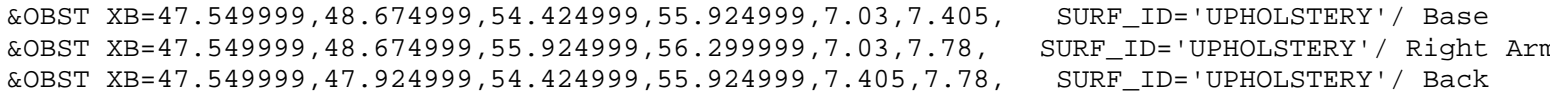

*** GROUP: COUCH2 offset $=0.0000000,6.000000,0.000000$ rotate $=90.000000$

\&OBST XB $=47.549999,48.674999,57.049999,57.424999,7.03,7.78$, SURF_ID='UPHOLSTERY'/ Left Arm

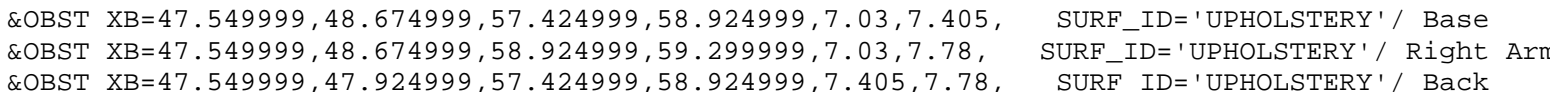

*** GROUP: COUCH2 offset $=0.0000000,9.000000,0.000000$ rotate $=90 . .000000$

\&OBST XB=47.549999, 48.674999, 60. 049999, 60.424999, 7. 03, 7.78, $\quad$ SURF_ID=' 'UPHOLSTERY' '/ Left Arm
\&OBST XB=47.549999, 48.674999,60.424999,61.924999,7.03,7.405, $\quad$ SURF_ID='UPHOLSTERY'/ Base \&OBST XB $=47.549999,48.674999,61.924999,62.299999,7.03,7.78$, SURF ID='UPHOLSTERY' 1 Right Ar

*** GROUP: COUCH2 offset $=0.000000,12.000000,, \odot .000000$ rotate $=90.0000000$

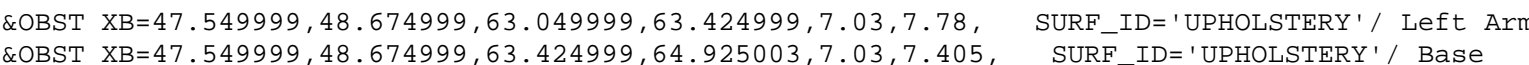
\&OBST XB=47.549999, 48.674999,64.925003,65.300003,7.03,7.78, SURF_ID=' 'UPHOLSTERY'/ Right Ar

*** GROUP: COUCH2 offset $=0.000000,15.000000,0.000000$ rotate $=90.0000000$

\&OBST XB $=47.549999,48.674999,66.050003,66.425003,7.03,7.78$, SURF ID= 'UPHOLSTERY ' / Left Arm \&OBST XB=47.549999, 48.674999, 66.425003, 67.925003, 7.03, 7.405, SURF_ID='UPHOLSTERY'/ Base COBST $X \mathrm{~B}=47.549999,48.674999,67.925003,68.300003,7.03,7.78, \quad$ SURF_ID=' 'UPHOLSTERY'/ Right Arm
\&OBST XB=47.549999, $47.924999,66.425003,67.925003,7.405,7.78, \quad$ SURF_ID=' 'UPHOLSTERY'/ Back

*** GROUP: COUCH2 offset $=0.000000,18.000000,0 . .000000$ rotate $=90$. . 000000

EOBST XB=47. $549999,48.674999,69.050003,69.425003,7.03,7.78$, SURF_ID='UPHOLSTERY'/ Left Arm \&OBST XB=47.549999, 48.674999,69.425003,70.9250003,7.03,7.405, SURF ID='UPHOLSTERY'/ Base \&OBST XB $=47.549999,47.924999,69.425003,70.925003,7.405,7.78, \quad$ SURF_ID=' 'UPHOLSTERY'/ Right Ar

*** GROUP: COUCH2 offset $=0.000000,21.000000,0.000000$ rotate $=90.000000$

\&OBST XB $=47.549999,48.674999,72.050003,72.425003,7.03,7.78$, SURF_ID='UPHOLSTERY'/ Left Arm

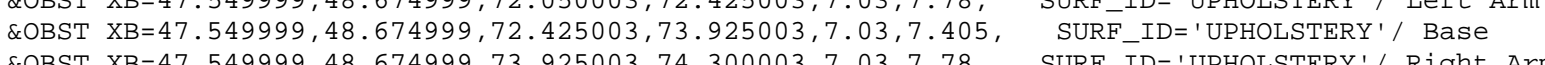

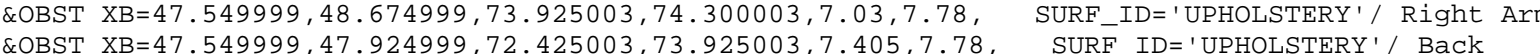

*** GROUP: COUCH2 offset $=0.000000,24.000000,0.000000$ rotate $=90.000000$

\&OBST XB $=47.549999,48.674999,75.050003,75.425003,7.03,7.78$, SURF_ID=' 'UPHOLSTERY'/ Left Arm \&OBST XB=47.549999, 48.674999, 75. 425003, 76.925003, 7.03, 7.405, SURF_ID=' 'UPHOLSTERY' / Base

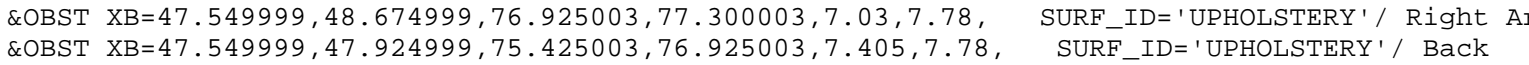

*** GROUP: COUCH2 offset $=0.000000,27.000000,0.000000$ rotate $=90.000000$

\&OBST XB=47.549999, 48.674999, 78.050003, 78.425003, 7.03,7.78, SURF_ID=' UPHOLSTERY' / Left Arm
\&OBST XB $=47.549999,48.674999,78.425003,79.925003,7.03,7.405$, SURF_ID=' UPHOLSTERY' / Base

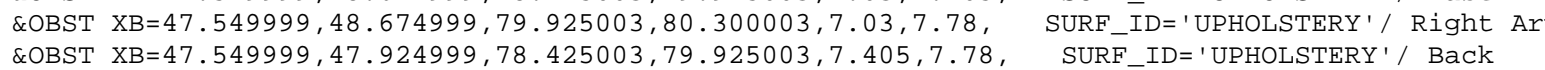

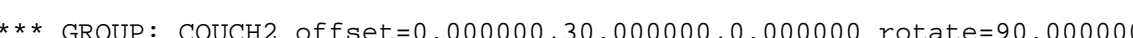

\&OBST XB=47.549999, 48.674999, 81.050003, 81.425003,7.03, 7.78, SURF_ID=' 'UPHOLSTERY'/ Left Arm \&OBST XB=47.549999, 47.924999, 81.425003, 82.925003, 7.405, 7.78, SURF_ID=' 'UPHOLSTERY' / Back

*** GROUP: ROW2

*** GROUP: COUCH2 offset $=0.000000,0.000000, \Theta .000000$ rotate $=270.000000$

\&OBST XB=46.049999, 47.174999, 52.924999, 53.299999, 7.03, 7.78, SURF_ID='UPHOLSTERY'/ Left Arm

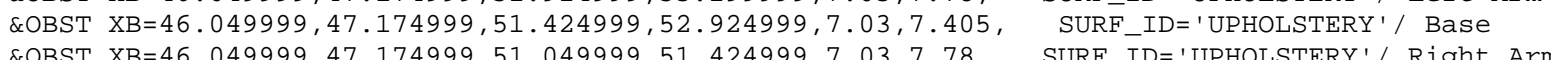
\&OBST $X \mathrm{~B}=46.799999,47.174999,51.424999,52.924999,7.405,7.78$, SURF_ID= 'UPHOLSTERY' $/$ ' Back

*** GROUP: COUCH2 offset $=0.000000,3.000000,0.000000$ rotate $=270.000000$

\&OBST XB $=46.049999,47.174999,55.924999,56.299999,7.03,7.78$, SURF_ID=' 'UPHOLSTERY'/ Left Arm \&OBST XB=46.049999, 47.174999, 54.424999, 55.924999, 7.03, 7.405, SURF_ID='UPHOLSTERY'/ Base

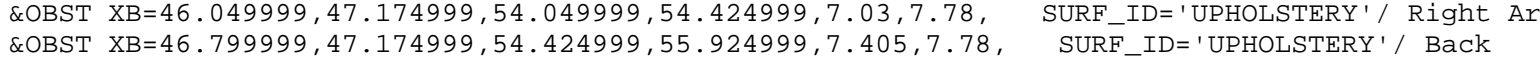

*** GROUP: COUCH2 offset $=0.000000,6.000000,0.000000$ rotate $=270.000000$

\&OBST XB=46. $049999,47.174999,58.924999,59.299999,7.03,7.78$, SURF_ID=' 'UPHOLSTERY' ' Left Arm

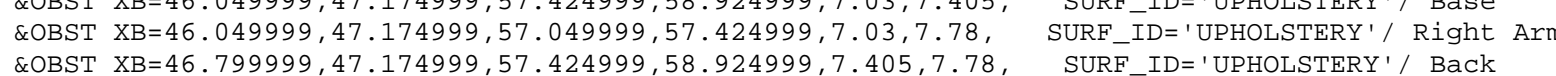

*** GROUP: COUCH2 offset $=0.000000,9.000000,0.000000$ rotate $=270.000000$

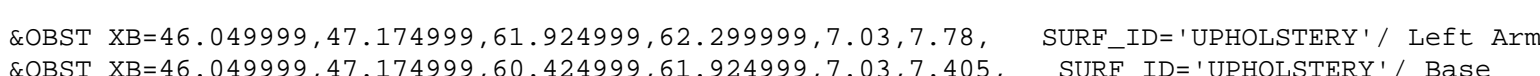
\&OBST XB=46. $049999,47.174999,60.049999,60.424999,7.03,7.78, \quad$ SURF_ID=' 'UPHOLSTERY'/ Right Arm
\&OBST XB=46.799999, $47.174999,60.424999,61.924999,7.405,7.78, \quad$ SURF_ID=' 'UPHOLSTERY'/ Back

*** GROUP: COUCH2 offset $=0.000000,12.000000,, 0.000000$ rotate $=270.000000$

\&OBST XB=46. $049999,47.174999,64.925003,65.300003,7.03,7.78, \quad$ SURF_ID=' 'UPHOLSTERY' ' Left Arm

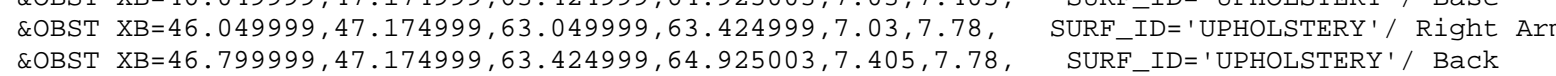

*** GROUP: COUCH2 offset $=0.000000,15.000000,0.000000$ rotate $=270.000000$

\&OBST XB=46. $049999,47.174999,67.925003,68.300003,7.03,7.78$, SURFIDD' 'UPHOLSTERY' ' Left Arm

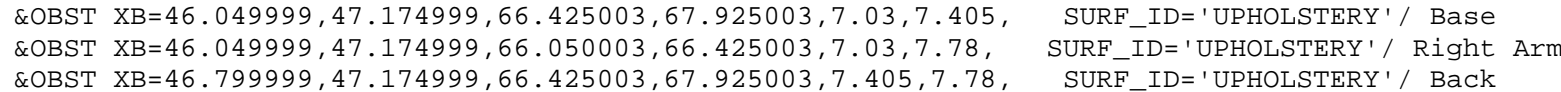

*** GROUP: COUCH2 offset $=0.000000,18.000000,0.000000$ rotate $=270.000000$

\&OBST XB=46. 049999, 47.174999, 70.925003, 71.300003,7.03,7.78, SURF_ID=' 'UPHOLSTERY'/ Left Arm \&OBST $X \mathrm{~B}=46.049999,47.174999,69.050003,69.425003,7.03,7.78, \quad$ SURF_ID=' 'UPHOLSTERY'/ Right Ar
\&OBST *** GROUP: COUCH2 offset $=0.000000,21.000000,0.000000$ rotate $=270.000000$

\&OBST XB=46. $049999,47.174999,73.925003,74.300003,7.03,7.78, \quad$ SURF_ID=' 'UPHOLSTERY'/ Left Arm
\&OBST XB $=46.049999,47.174999,72.425003,73.925003,7.03,7.405, \quad$ SURF ID=' 'UPHOLSTERY' Rase

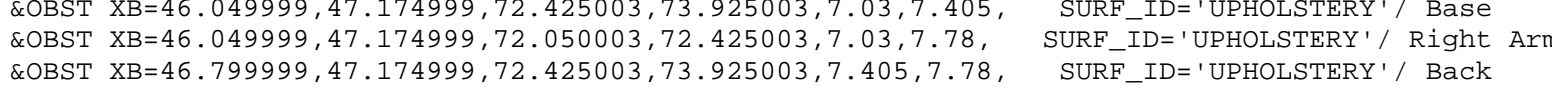

*** GROUP: COUCH2 offset $=0.000000,24.000000,0.000000$ rotate $=270.000000$

\&OBST XB=46. $049999,47.174999,76.925003,77.300003,7.03,7.78, \quad$ SURF_ID='UPHOLSTERY' 1 Left Arm
\&OBST XB $=46.049999,47.174999,75.425003,76.925003,7.03,7.405, \quad$ SURF ID='UPHOLSTERY' / Base

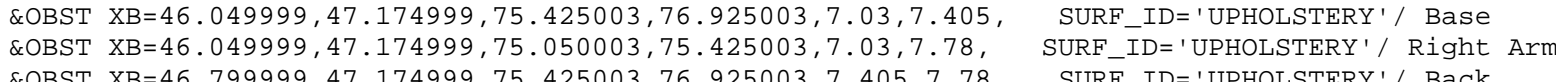

*** GROUP: COUCH2 offset $=0.000000,27.0000000,0.0000000$ rotate $=270.0000000$ 
\&OBST XB $=46.049999,47.174999,79.925003,80.300003,7.03,7.78, \quad$ SURF_ID=' 'UPHOLSTERY' $/$ Left Arm
\&OBST XB $=46.049999,47.174999,78.425003,79.925003,7.03,7.405, \quad$ SURF_ID='UPHOLSTERY' $/$ Base , SURE_ID='UPHOLSTERY' / Back

*** GROUP: COUCH2 offset $=0.000000,30.000000,0.000000$ rotate $=270.000000$

\&OBST XB $=46.049999,47.174999,82.925003,83.300003,7.03,7.78$, SURF_ID='UPHOLSTERY'/ Left Arm \&OBST XB=46. $049999,47.174999,81.425003,82.925003,7.03,7.405$, SURF_ID='UPHOLSTERY' / Base \&OBST XB=46. $049999,47.174999,81.050003,81.425003,7.33,7.78, \quad$ SURF_ID=' 'UPHOLSTERY'/ Right Arm
\&OBST XB=46.79999, $47.174999,81.425003,82.925003,7.405,7.78, \quad$ SURF_ID='UPHOLSTERY'/ Back

*** GROUP: TWIN

*** GROUP: ROW

*** GROUP: COUCH2 offset $=0.000000,0.000000,0.000000$ rotate $=90.000000$

\&OBST XB=52. $049999,53.174999,51.049999,51.424999,7.03,7.78, \quad$ SURF_ID=' 'UPHOLSTERY' $/$ Left Arm
\&OBST XB=52.04999, $53.174999,51.424999,52.924999,7.03,7.405$,
SURF_ID='UPHOLSTERY'/ Base \&OBST XB=52. 049999, 53.174999, 52.924999, 53.299999, 7. 03, 7.78, SURF ID= 'UPHOLSTERY'/ Right Ar

$* * *$ GROUP: COUCH2 offset $=0.000000,3.000000,0.000000$ rotate $=90.0000000$

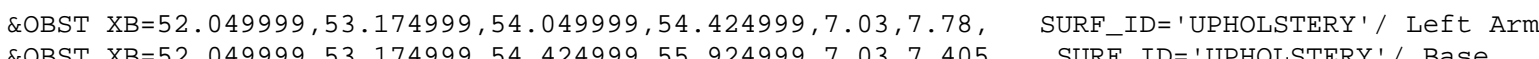
\&OBST XB=52. $049999,53.174999,55.924999,56.299999,7.03,7.78$, SURF_ID='UPHOLSTERY'/Right Ar

*** GROUP: COUCH2 offset $=0.000000,6.000000,0.000000$ rotate $=90.000000$

\&OBST XB=52.049999, 53.174999, 57.049999, 57.424999, 7. 03, 7.78, SURF_ID='UPHOLSTERY'/ Left Arm \&OBST XB=52. $049999,53.174999,57.424999,58.924999,7.03,7.405$, SURF_ID='UPHOLSTERY '/ Base \&OBST XB=52. $049999,53.174999,58.924999,59.299999,7.03,7.78$,
\&OBST XB=52.049999, 52.424999, $57.424999,58.924999,7.405,7.78$,
SURF_IDE'ID='UPHOLSTERY' / Back

$* * *$ GROUP: COUCH2 offset $=0.000000,9.000000,0.000000$ rotate $=90.000000$

\&OBST XB=52. $049999,53.174999,60.049999,60.424999,7.03,7.78$, SURF_ID=' 'UPHOLSTERY'/ Left Arm COBST $X \mathrm{XB}=52.049999,53.174999,60.424999,61.924999,7.03,7.405, \quad$ SURF_ID=' 'UPHOLSTERY'/ Base
\&OBST XB $\mathrm{X}=52.049999,53.174999,61.924999,62.299999,7.03,7.78$,
SURF_ID='UPHOLSTERY'/ Right Ar

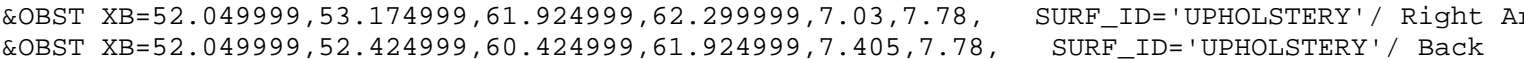

*** GROUP: COUCH2 offset $=0.000000,12.000000,0 . .000000$ rotate $=90.000000$

\&OBST XB=52. $049999,53.174999,63.049999,63.424999,7.03,7.78, \quad$ SURF-ID=' 'UPHOLSTERY' ' Left Arm

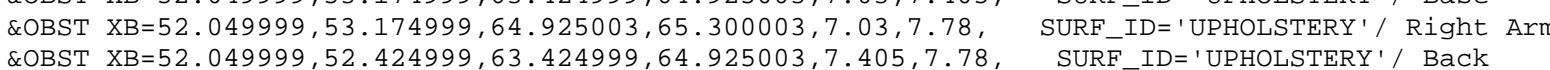

*** GROUP: COUCH2 offset $=0.000000,15.000000,0.000000$ rotate $=90.000000$

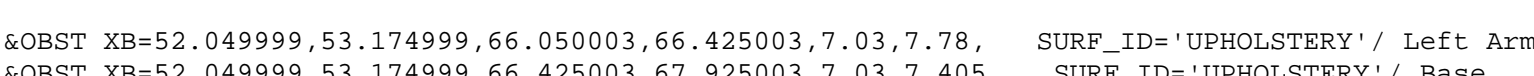

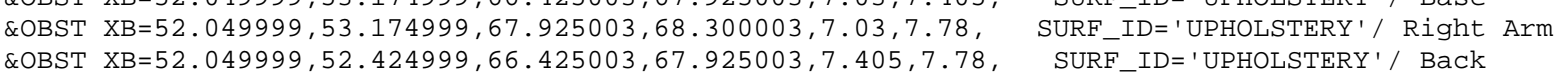

*** GROUP: COUCH2 offset $=0,000000,18,000000,0.000000$ rotate $=90, .000000$

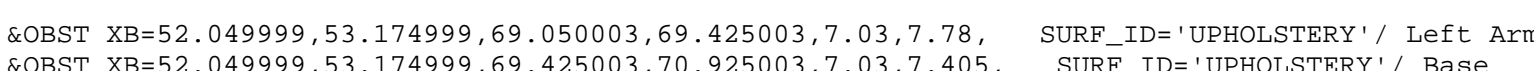

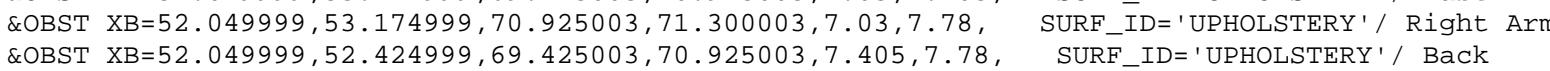

*** GROUP: COUCH2 offset $=0.000000,21$. .

\&OBST XB=52. $049999,53.174999,72.050003,72.425003,7.03,7.78$, SURF_ID=' 'UPHOLSTERY' ' Left Arm

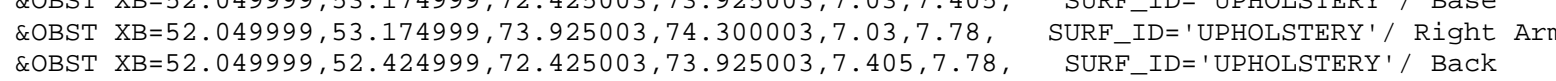
*** GROUP: COUCH2 offset $=0.000000,24.000000,0.000000$ rotate $=90.000000$

\&OBST XB=52. $049999,53.174999,75.050003,75.425003,7.03,7.78, \quad$ SURF_ID=' 'UPHOLSTERY' $/$ 'Left Arm
\&OBST XB=52.04999, $53.174999,75.425003,76.925003,7.03,7.405$,
SURF_ID=' 'UPHOLSTERY' / Base
\&OBST XB=52. $049999,53.174999,76.925003,77.300003,7.03,7.78, \quad$ SURF_ID= 'UPHOLSTERY'
\&OBST XB Right Arm
SB $52.049999,52.424999,75.425003,76.925003,7.405,7.78, \quad$ SURF_ID=' 'UPHOLSTERY' / Back

*** GROUP: COUCH2 offset $=0.000000,27.000000,0.000000$ rotate $=90.000000$

\&OBST XB=52. $049999,53.174999,78.050003,78.425003,7.03,7.78, \quad$ SURF_ID=' 'UPHOLSTERY'/' Left Arm
\&OBST XB=52.049999, 53.174999, $78.425003,79.925003,7.03,7.405, \quad$ SURF_ID='UPHOLSTERY'/ Base

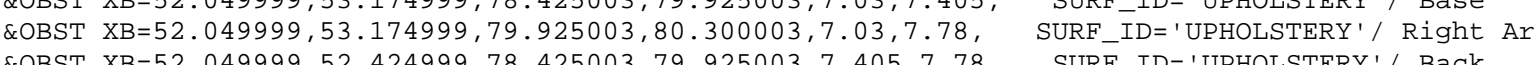

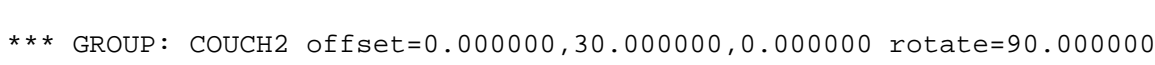

\&OBST XB=52. 049999, 53.174999, 81.050003, 81.425003,7.03,7.78, SURF_ID=' 'UPHOLSTERY'/ Left Arm \&OBST XB=52. $849999,53.174999,81.425003,82.925003,7.03,7.405$, SURF_ID=' 'UPHOLSTERY' / Base

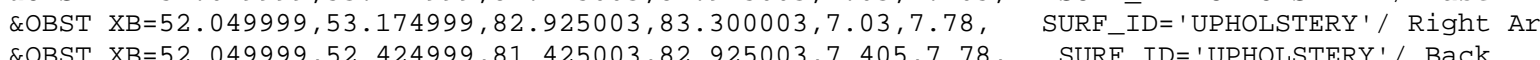

*** GROUP: ROW2

*** GROUP: COUCH2 offset $=0.000000,0.000000,0.000000$ rotate $=270.000000$

\&OBST $X \mathrm{XB}=50.549999,51.674999,52.924999,53.299999,7.03,7.78, \quad$ SURF ID = ' 'PPHOLSTERY ' / Left Arm

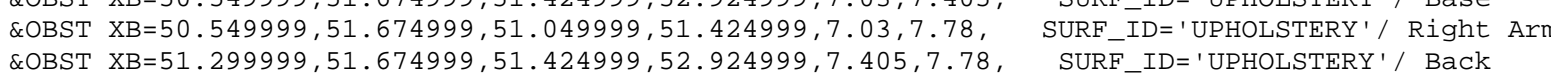

*** GROUP: COUCH2 offset $=0.000000,3.000000,0.000000$ rotate $=270.000000$

\&OBST XB=50.549999, 51.674999, 55.924999, 56. 299999, 7. 03,7.78, SURF_ID=' 'UPHOLSTERY' ' Left Arm \&OBST XB-50.549999, $51.674999,54,049999,54.424999,7.03,7,78$, \&OBST XB=51.299999, 51.674999, $54.424999,55.924999,7.405,7.78$, SURF_ID='UPHOLSTERY'/ Back

*** GROUP: COUCH2 offset $=0.000000,6.000000,0.000000$ rotate $=270.000000$

\&OBST $X \mathrm{XB}=50.549999,51.674999,58.924999,59.299999,7.03,7.78, \quad$ SURF_ID=' 'UPHOLSTERY'/ Left Arm
\&OBST XB $X 50.549999,51.674999,57.424999,58.924999,7.03,7.405, \quad$ SURF ID ='UPHOLSTERY'/ Base \&OBST XB $=5.549999,51.674999,57.049999,57.424999,7.03,7.78$, SURF ID='UPHOLSTERY'/ Rise A ZOBST XB=51.299999, 51.674999, 57.424999, 58.924999, 7.405, 7.78, SURF_ID=' 'UPHOLSTERY'/ Back

** GROUP: COUCH2 offset $=0.000000,9$. . $000000,0.000000$ rotate $=270.000000$

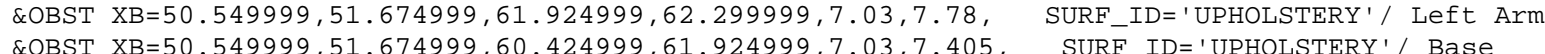

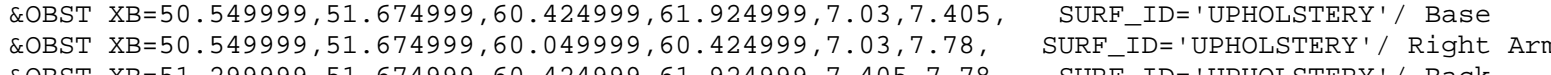

*** GROUP: COUCH2 offset $=0.000000,12.000000,0.000000$ rotate $=270.0000000$

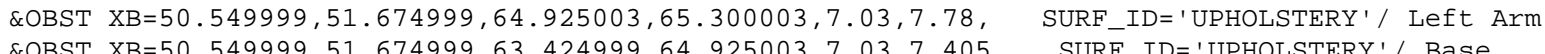

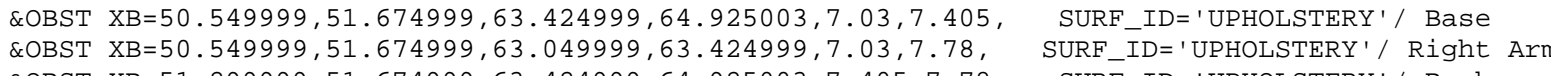

*** GROUP: COUCH2 offset $=0.000000,15.000000,0.000000$ rotate $=270.000000$

\&OBST XB=50.549999, $51.674999,67.925003,68.300003,7.03,7.78$, SURF_ID='UPHOLSTERY'/ Left Arm

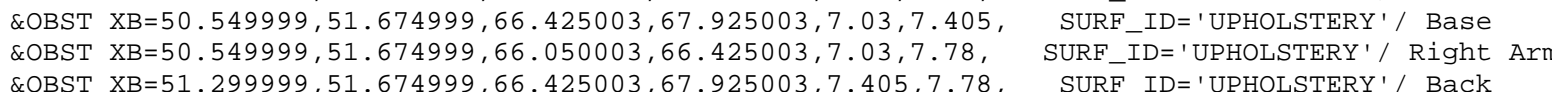

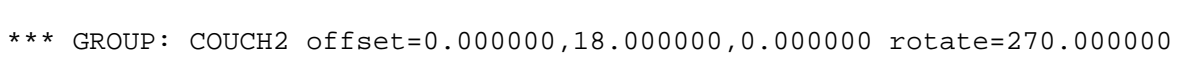

\&OBST XB=50.549999, 51.674999,70.925003, 71.300003, 7.03, 7.78, SURF_ID='UPHOLSTERY'/ Left Arm \&OBST XB=50.54999, $51.674999,69.425003,70.925003,7.03,7.405, \quad$ SURF_ID='UPHOLSTERY'/ Base
\&OBST XB $\mathrm{X}=50.549999,51.674999,69.050003,69.425003,7.03,7.78$,
SURF_ID='UPHOLSTERY'/Right Arm \&OBST XB $=51$. 299999, $51.674999,69.425003,70.925003,7.405,7.78$, SURF ID=' UPHOLSTEPY' $/$ Rack Ar

*** GROUP: COUCH2 offset $=0.0000000,21.000000,0.000000$ rotate $=270.0000000$ \&OBST XB $=50.549999,51.674999,73.925003,74.300003,7.03,7.78, \quad$ SURF_ID=' 'UPHOLSTERY' ' Left Arm
\&OBST XB $=50.549999,51.674999,72.425003,73.925003,7.03,7.405, \quad$ SURF_IDD' 'UPHOLSTERY' $/$ Base

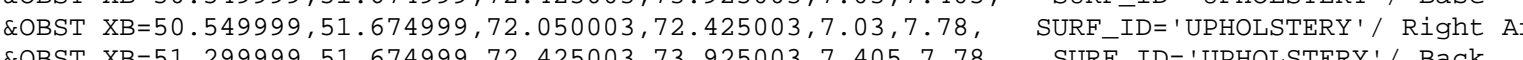

*** GROUP: COUCH2 offset $=0.000000,24.000000,0.000000$ rotate $=270.000000$ 


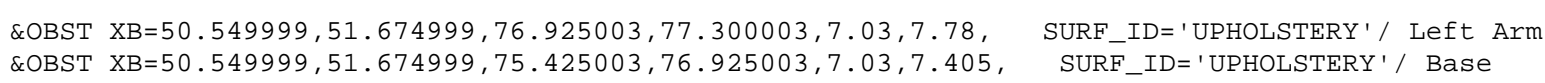

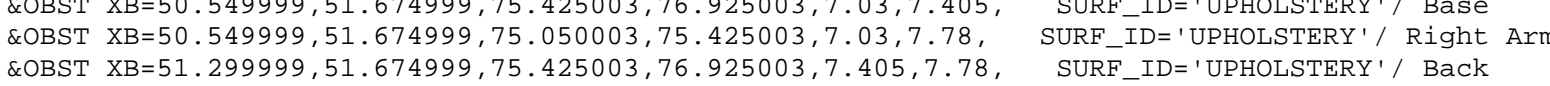
*** GROUP: COUCH2 offset $=0.000000,27.0000000,0.0000000$ rotate $=270.000000$

\&OBST XB $=50.549999,51.674999,79.925003,80.300003,7.03,7.78, \quad$ SURF_ID='UPHOLSTERY'/ Left Arm

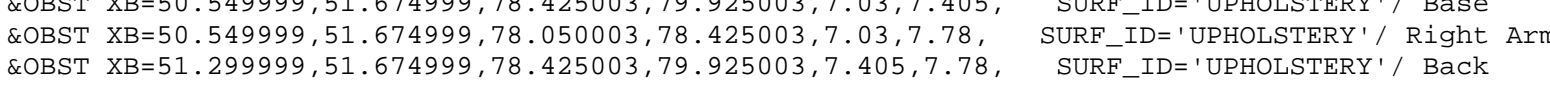
*** GROUP: COUCH2 offset $=0.000000,30.000000,0.000000$ rotate $=270.000000$

\&OBST XB=50.549999, 51.674999, 82. $925003,83.300003,7.03,7.78, \quad$ SURF_ID='UPHOLSTERY'/ Left Arm

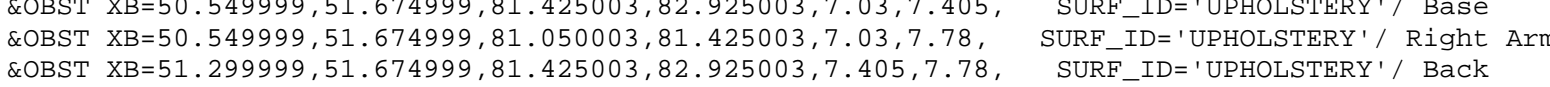

*** GROUP: TWIN

*** GROUP: ROW

*** GROUP: COUCH2 offset $=0.000000,0.000000,0.000000$ rotate $=90.000000$

\&OBST XB $=56.549999,57.674999,51.049999,51.424999,7.03,7.78, \quad$ SURF_ID='UPHOLSTERY'/ Left Arm

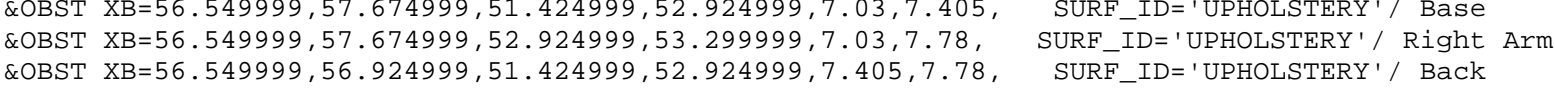
$* *$ GROUP: COUCH2 offset $=0.000000,3.000000,0.000000$ rotate $=90.000000$

\&OBST XB=56.549999, 57.674999, 54. 449999, 54.424999, 7.03, 7.78, SURF_ID=' 'UPHOLSTERY' ' Left Arm

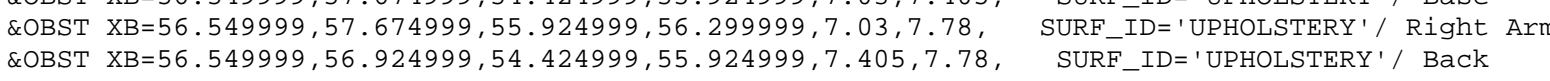
*** GROUP: COUCH2 offset $=0.000000,6.000000,0.000000$ rotate $=90.000000$

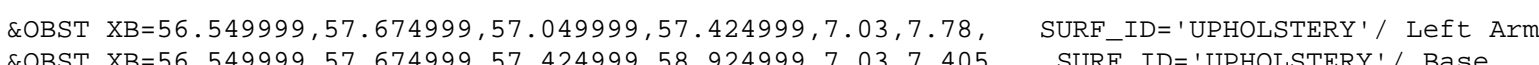

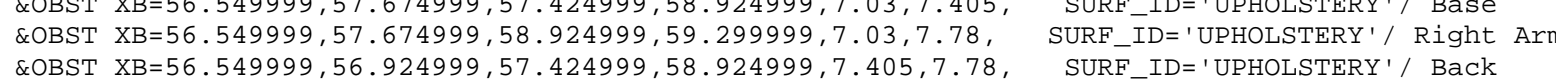

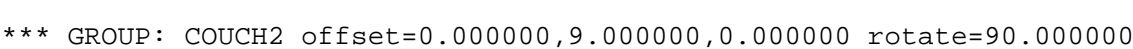

\&OBST XB=56.549999, 57.674999, 60. $049999,60.424999,7.03,7.78, \quad$ SURF_ID='UPHOLSTERY' ' Left Arm
\&OBST XB=56.549999, $57.674999,60.424999,61.924999,7.03,7.405, \quad$ SURF_ID=' 'UPHOLSTERY'/ Base

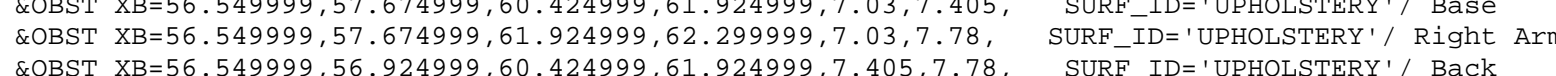
*** GROUP: COUCH2 offset $=0.000000,12.000000,0.000000$ rotate $=90 . \bullet 00000$

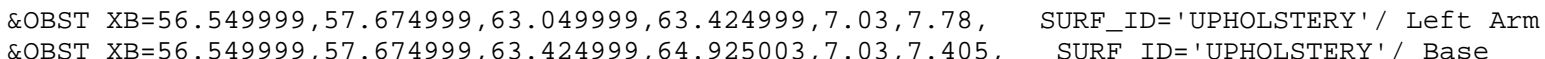

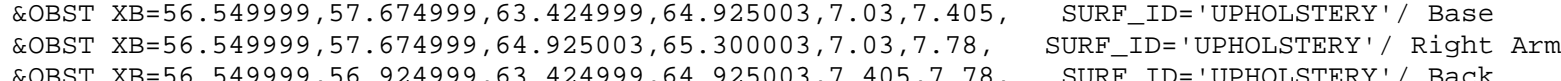

*** GROUP: COUCH2 offset $=0.000000,15.000000,0.000000$ rotate $=90.000000$

\&OBST XB $56.549999,57.674999,66.050003,66.425003,7.03,7.78$, SURF_ID='UPHOLSTERY '/ Left Arm

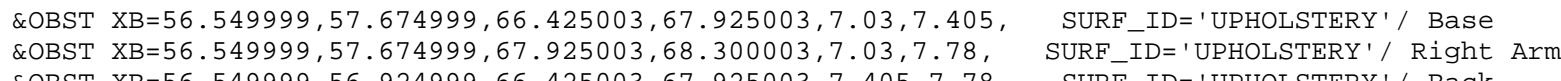

*** GROUP: COUCH2 offset $=0.000000,18.000000,, 0.000000$ rotate $=90.000000$

\&OBST XB $=56.549999,57.674999,69.050003,69.425003,7.03,7.78, \quad$ SURF_ID=' UPHOLSTERY'/' Left Arm
\&OBST XB $=56.549999,57.674999,69.425003,70.925003,7.03,7.405, \quad$ SURF_ID='UPHOLSTERY' $/$ Base

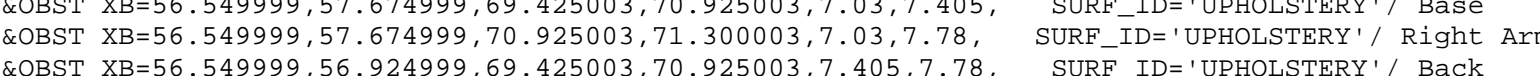
*** GROUP: COUCH2 offset $=0.000000,21.0000000,0.0000000$ rotate $=90.0000000$

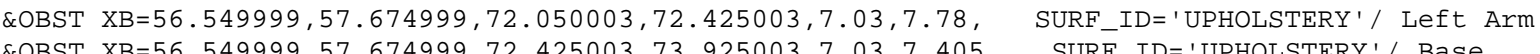

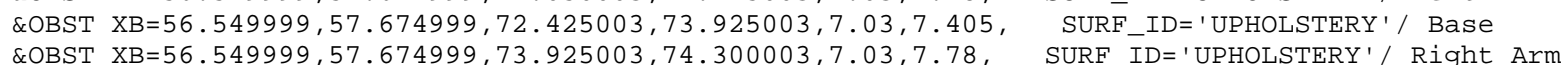

\&OBST XB $=56,549999,56,924999,72,425003,73,925003,7,405,7,78$, SURF_ID=' UPHOLSTERY'/ Back *** GROUP: COUCH2 offset $=0.000000,24.000000,0.000000$ rotate $=90.000000$

\&OBST XB=56.549999, 57.674999, 75.050003,75.425003, 7.03, 7.78, SURF_ID='UPHOLSTERY'/ Left Arm \&OBST XB $=56.549999,57.674999,75.425003,76.925003,7.03,7.405$, SURF_ID='UPHOLSTERY'/ Base LOBST XB=56.549999, 56.924999, 75.425003, 76.925003,7.405,7.78, SURF_ID='UPHOLSTERY'/ Back

*** GROUP: COUCH2 offset $=0.000000,27.000000,0.000000$ rotate $=90.000000$

\&OBST $X \mathrm{XB}=56.549999,57.674999,78.050003,78.425003,7.03,7.78$, SURF_ID=' 'UPHOLSTERY'/ Left Arm \&OBST XB=56.549999, $57.674999,78.425003,79.925003,7.03,7.405, \quad$ SURF_ID='UPHOLSTERY '/ Base

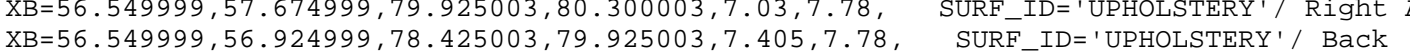

*** GROUP: COUCH2 offset $=0.000000,30.000000,0.000000$ rotate $=90.000000$

\&OBST XB=56.549999, 57.674999, 81.050003, 81.425003, 7.03, 7.78, SURF_ID='UPHOLSTERY'/ Left Arm

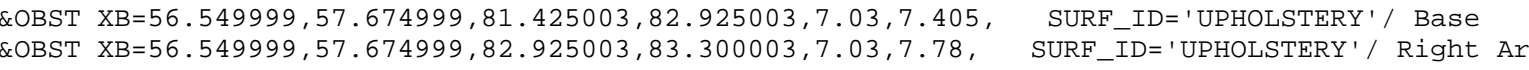
عOBST XB=56.549999, 56.924999, 81.425003, 82.925003, $7.405,7.78$, SURF IDE' 'UPPOLSTEPY'/ Back

*** GROUP: ROW2

*** GROUP: COUCH2 offset $=0.000000$, ๑. ๑000000,,. .000000 rotate $=270.0000000$

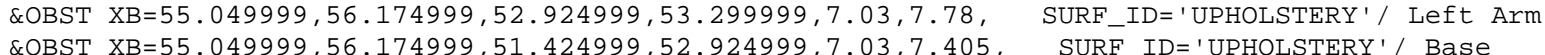

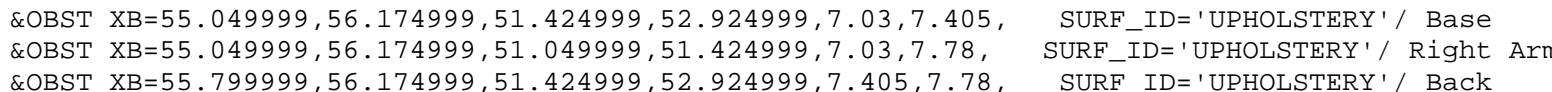

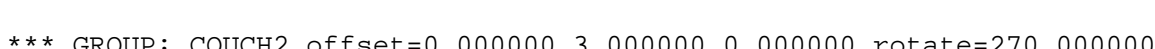

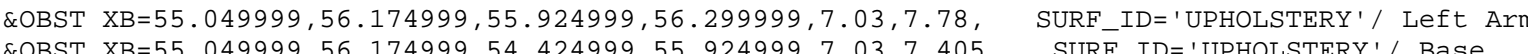
\&OBST XB $=55.049999,56.174999,54.424999,55.924999,7.03,7.405$, SURF_ID='UPHOLSTERY'/ Base

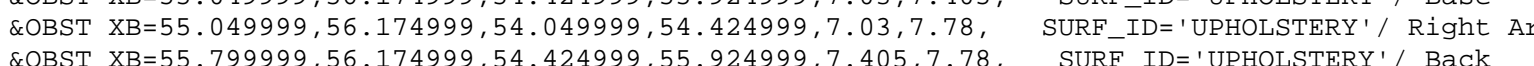

*** GROUP: COUCH2 offset $=0.000000,6.000000,0.000000$ rotate $=270.000000$

\&OBST XB=55.049999, 56.174999, 58.924999, 59. 299999, 7. 03, 7.78, SURF_ID=' 'UPHOLSTERY' / Left Arm

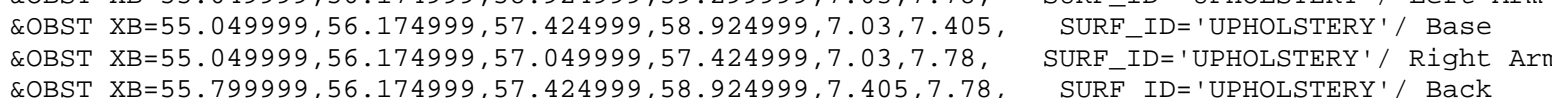

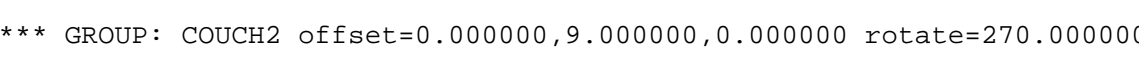

\&OBST XB=55. 049999, 56.174999, 61.924999, 62.299999, 7. 03, 7.78, SURF_ID='UPHOLSTERY'/ Left Arm \&OBST XB=55. $049999,56.174999,60.424999,61.924999,7.03,7.405$, SURF_ID='UPHOLSTERY' / Base \&OBST XB=55.
\&OBST X X $=55.799999,56.174999,60.424999,61.924999,7.405,7.78, \quad$ SURF_ID='UPHOLSTERY'/ Right Ar

*** GROUP: COUCH2 offset $=0.000000,12.000000,0.000000$ rotate $=270.000000$

\&OBST XB=55. $049999,56.174999,64.925003,65.300003,7.03,7.78$, SURFID= 'UPHOLSTERY ' / Left Arm \&OBST XB $=55.049999,56.174999,63.424999,64.925003,7.03,7.405$, SURF_ID='UPHOLSTERY'/ Base \&OBST XB $=55.049999,56.174999,63.049999,63.424999,7.03,7.78$,
\&OBST XB $X 55.799999,56.174999,63.424999,64.925003,7.405,7.78$,
SURF_IDD=' 'UPHOLSTERY' / Back

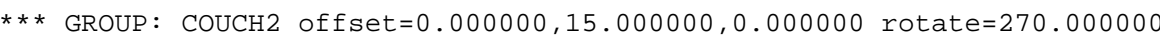

\&OBST XB=55.049999, 56.174999, 67. 925003, 68.300003, 7.03, 7.78, SURF_ID=' 'UPHOLSTERY ' / Left Arm

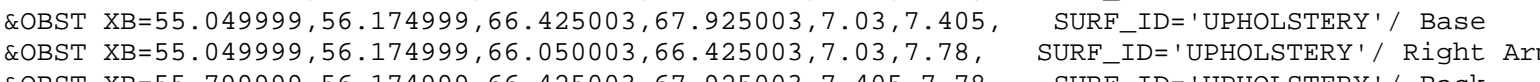

*** GROUP: coucH2 offset $=0.000000,18.000000,0.000000$ rotate $=270.000000$

\&OBST XB=55. $049999,56.174999,70.925003,71.300003,7.03,7.78, \quad$ SURF-ID=' 'UPHOLSTERY'/ Left Arm \&OBST XB=55. 049999, 56.174999, 69.050003, 69.425003, 7.03, 7.78, SURF_ID='UPHOLSTERY'/ Right Ar

*** GROUP: COUCH2 offset $=0.000000,21.000000,0.000000$ rotate $=270.000000$

\&OBST XB=55. $049999,56.174999,73.925003,74.300003,7.03,7.78$, SURF_ID='UPHOLSTERY'/ Left Arm 
\&OBST XB=55. $049999,56.174999,72.425003,73.925003,7.03,7.405, \quad$ SURF_ID=' 'UPHOLSTERY' $/$ Base
\&OBST XB=55.049999, $56.174999,72.050003,72.425003,7.03,7.78, \quad$ SURF_ID='UPHOLSTERY'
Right A

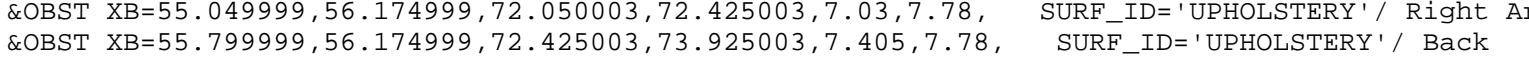
*** GROUP: COUCH2 offset $=0.000000,24.000000,0.000000$ rotate $=270.000000$

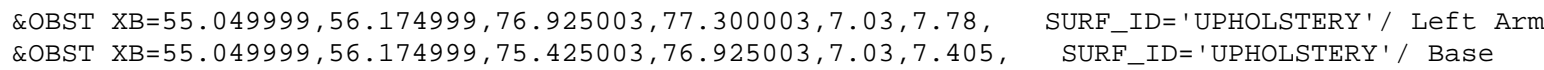

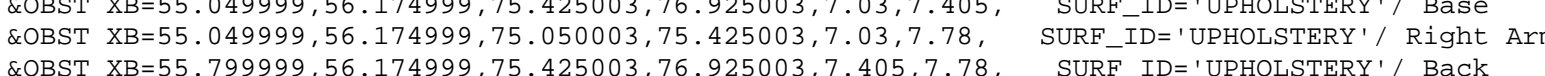
$* * *$ GROUP: COUCH2 offset $=0.000000,27.000000,0.000000$ rotate $=270.000000$

\&OBST XB=55. $049999,56.174999,79.925003,80.300003,7.03,7.78, \quad$ SURF_ID='UPHOLSTERY' '/' Left Arm
\&OBST XB $=55.049999,56.174999,78.425003,79.925003,7.03,7.405, \quad$ SURF_ID=' 'UPHOLSTERY' / Base

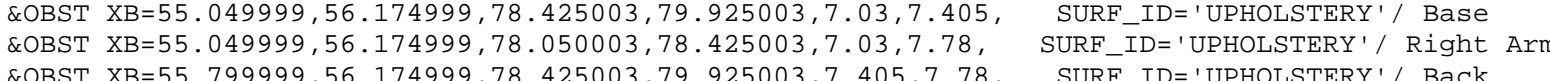

*** GROUP: COUCH2 offset $=0.000000,30.000000,0.000000$ rotate $=270.000000$

\&OBST XB=55. 0499999, 56.174999, 82.925003, 83. 300003, 7. 03, 7.78, SURF_ID=' 'UPHOLSTERY'/ Left Arm

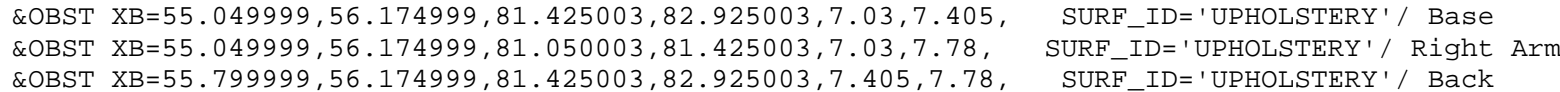

*** GROUP: TWIN

*** GROUP: ROW

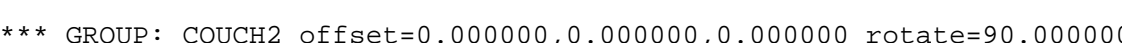

\&OBST XB $=61.049999,62.174999,51.049999,51.424999,7.03,7.78$, SURF_ID='UPHOLSTERY'/ Left Arm

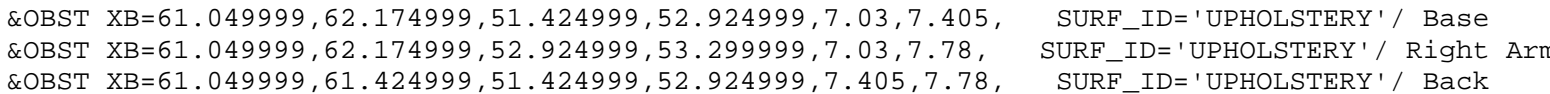

*** GROUP: COUCH2 offset $=0.000000,3$. . .000000, ๑. ๑. 0000000 rotate $=90.0000000$

\&OBST XB $=61.049999,62.174999,54.049999,54.424999,7.03,7.78$, SURF_ID=' 'UPHOLSTERY'/ Left Arm

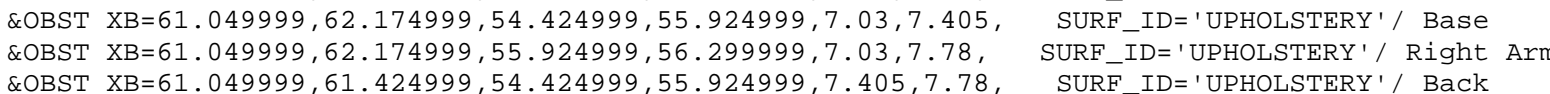

*** GROUP: COUCH2 offset $=0.0000000,6.000000,0.000000$ rotate $=90.000000$

\&OBST XB $=61.049999,62.174999,57.049999,57.424999,7.03,7.78, \quad$ SURF-ID=' 'UPHOLSTERY'/ Left Arm \&OBST XB=61.049999, 62.174999,57.424999,58.924999, 7.03, 7.405, SURF_ID='UPHOLSTERY'/ Base

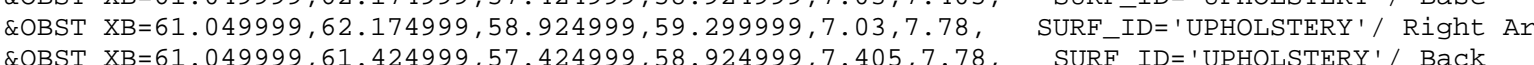

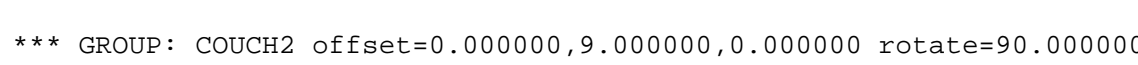

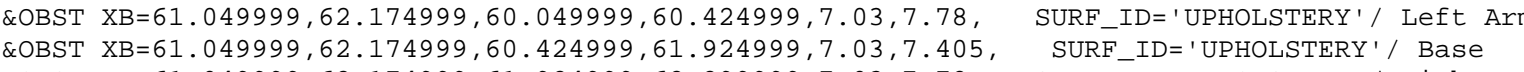

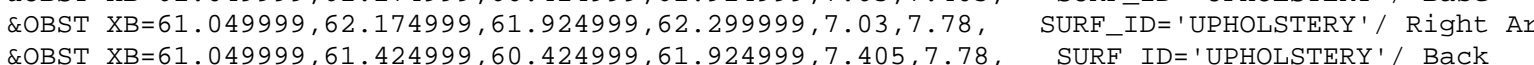

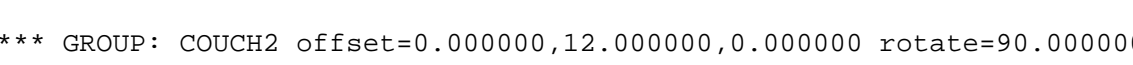

\&OBST XB $=61.049999,62.174999,63.049999,63.424999,7.03,7.78$, SURF ID= 'UPHOLSTERY ' / Left Arm \&OBST XB=61. $049999,62.174999,63.424999,64.925003,7.03,7.405$, SURF_ID='UPHOLSTERY'/ Base

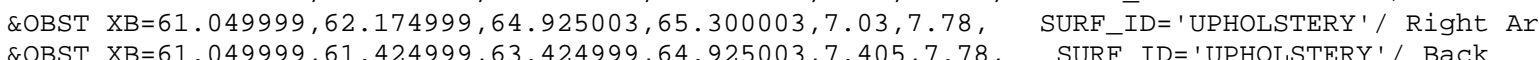

*** GROUP: COUCH2 offset $=0.000000,15.000000,0 . .000000$ rotate $=90 . .000000$

8OBST XB=61.049999, 62.174999, 66.050003, 66.425003, 7.03, 7.78, SURF_ID='UPHOLSTERY' L Left Ar ZOBST XB=61.049999, 62.174999,66.425003, 67.925003, 7.03, 7.405, SURF_ID='UPHOLSTERY'/ Base QOBST $X \mathrm{~B}=61.0499,62.47499,67.925003,68.300003,7.03,7.78$, SURF-ID=' 'UPHOLSTERY'/ Right Arm

*** GROUP: COUCH2 offset $=0.000000,18.000000,0.000000$ rotate $=90 . .000000$

\&OBST XB=61. 049999, 62.174999, 69. 050003, 69.425003, 7.03, 7.78, SURF_ID='UPHOLSTERY'/ Left Arm

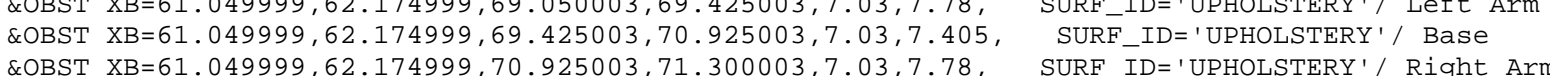

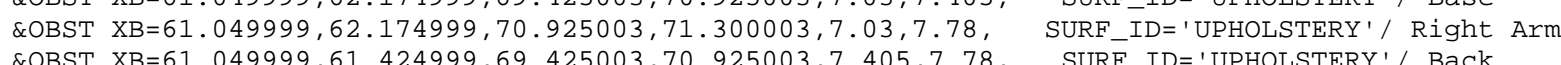

*** GROUP: COUCH2 offset $=0,000000,21.000000,0,000000$ rotate $=90, .000000$

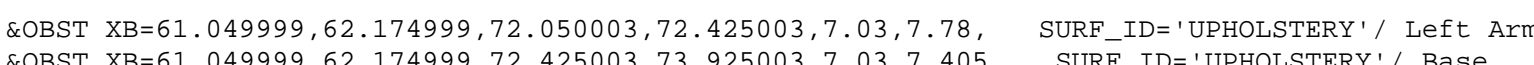

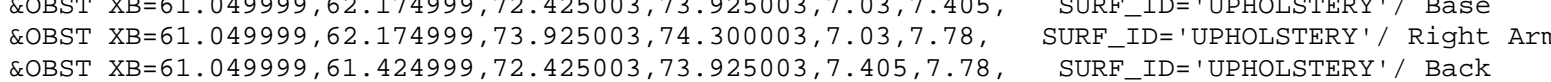

*** GROUP: COUCH2 offset $=0.000000,24.000000,0.000000$ rotate $=90.000000$

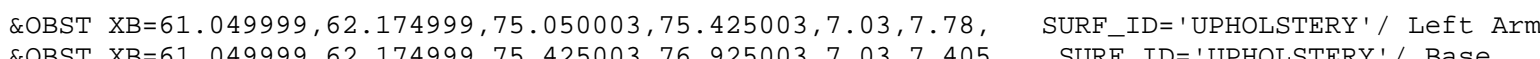

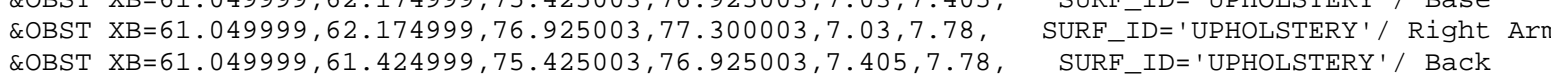

*** GROUP: COUCH2 offset $=0.000000,27.000000,0.000000$ rotate $=90.000000$

\&OBST XB $=61.049999,62.174999,78.050003,78.425003,7.03,7.78$, SURF ID=' 'UPHOLSTERY ' / Left Arm

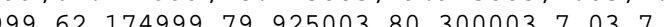
\&OBST $X B=61.049999,61.424999,78.425003,79.925003,7.405,7.78, \quad$ SURF_ID='UPHOLSTERY'/ Back

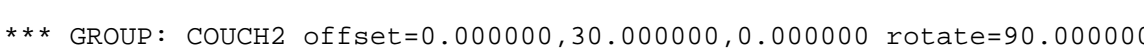

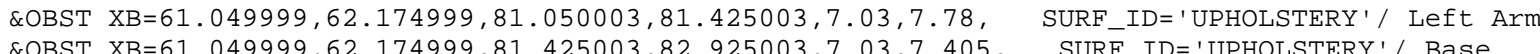
\&OBST XB=61. $049999,62.174999,82.925003,83.300003,7.03,7.78$, SURF-ID='UPHOLSTERY'/ Rise \&OBST XB=61. $049999,61.424999,81.425003,82.925003,7.405,7.78$, SURF_ID=' 'UPHOLSTERY' / Back

*** GROUP: ROW2

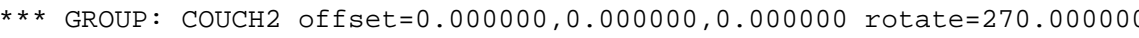

\&OBST XB=59.549999, 60.674999, 52.924999, 53. 299999, 7.03, 7.78, SURF_ID=' 'UPHOLSTERY'/ Left Arm \&OBST XB $=59.549999,60.674999,51.424999,52.924999,7.03,7.405$, SURF_ID='UPHOLSTERY'/ Base \&OBST XB $=59.549999,60.674999,51.049999,51.424999,7.03,7.78$,
\&OBST XB $=60.299999,60.674999,51.424999,52.924999,7.405,7.78$,
SURF_IDE'ID=' UPHOLSTERY' / Back

$\star \star \star$ GROUP: COUCH2 offset $=0.000000,3.000000,0.000000$ rotate $=270.000000$

\&OBST XB $=59.549999,60.674999,55.924999,56.299999,7.03,7.78, \quad$ SURF ID=' 'UPHOLSTERY ' ' Left Arm

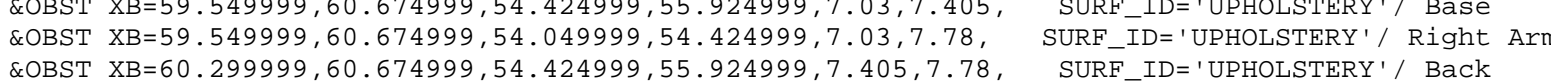

*** GROUP: COUCH2 offset $=0.000000,6.000000,0.000000$ rotate $=270.000000$

\&OBST XB=59.549999, 60.674999, 58.924999, 59. 299999, 7. 03, 7.78, SURF_ID=' 'UPHOLSTERY ' / Left Arm \&OBST XB=59.549999, $60.67499,577.04999,57.424999,7.33,7.78, \quad$ SURF_ID='UPHOLSTERY'/ Right Arm
\&OBST XB=60.29999, $60.674999,57.424999,58.924999,7.405,7.78, \quad$ SURF_ID='UPHOLSTERY'/ Back

*** GROUP: COUCH2 offset $=0.000000,9.000000,0.0000000$ rotate $=270.000000$

\&OBST XB=59.549999, 60.674999, 61.924999, 62. 299999, 7. 03, 7.78, SURF_ID=' 'UPHOLSTERY' / Left Arm \&OBST XB=59.549999, 60.674999, 60.049999, 60.424999, 7.03, 7.78, SURF_ID='UPHOLSTERY'/ Right Ar \&OBST XB=60.299999, 60.674999, 60.424999, 61.924999, 7.405, 7.78, SURF_ID='UPHOLSTERY' / Back

*** GROUP: COUCH2 offset $=0.000000,12.000000,0.000000$ rotate $=270,000000$

\&OBST XB=59.549999, 60.674999, 64.925003, 65.300003, 7. 03, 7.78, SURF_ID=' 'UPHOLSTERY' ' Left Arm

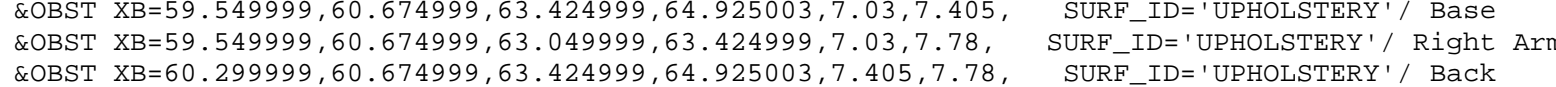

*** GROUP: COUCH2 offset $=0,000000,15,000000,0,000000$ rotate $=270,000000$

\&OBST XB=59.549999, 60.674999, 67.925003, 68.300003, 7.03, 7.78, SURF_ID=' 'UPHOLSTERY'/ Left Arm

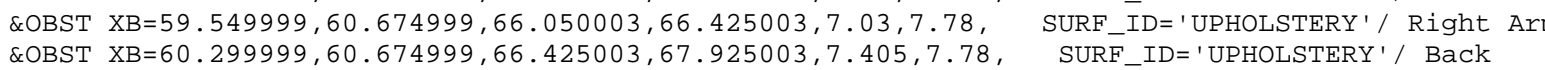
*** GROUP: COUCH2 offset $=0.000000,18.000000,0.000000$ rotate $=270.000000$

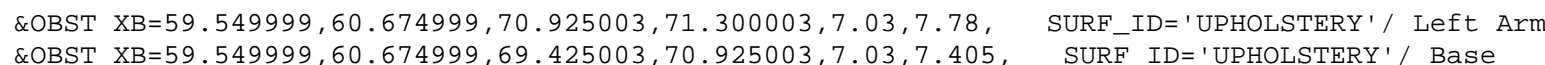


\&OBST XB=59. $549999,60.674999,69.050003,69.425003,7.03,7.78, \quad$ SURF_ID= 'UPHOLSTERY' $/$ Right Arm
\&OBST XB=60.29999, $60.674999,69.425003,70.925003,7.405,7.78, \quad$ SURF_ID='UPHOLSTERY'/ Back *** GROUP: COUCH2 offset $=0.000000,21.000000,0.000000$ rotate $=270.000000$

\&OBST XB=59.549999, 60.674999, 73.925003, 74.300003,7.03,7.78, SURF_ID='UPHOLSTERY '/ Left Arm

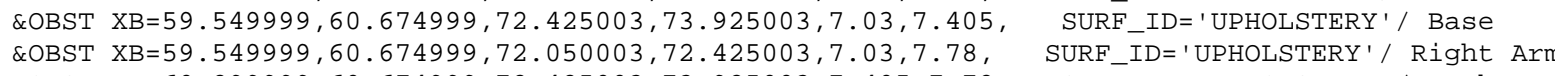

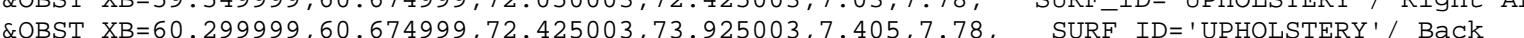
*** GROUP: COUCH2 offset $=0.000000,24.000000,0.000000$ rotate $=270.000000$

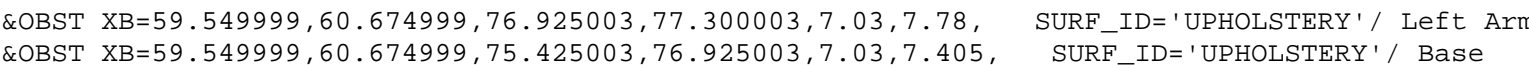
\&OBST XB=59.549999, 60.674999, 75. $050003,75.425003,7.03,7.78$, SURF_ID='UPHOLSTERY'//Right Ar

*** GROUP: COUCH2 offset $=0.000000,27.000000,0.000000$ rotate $=270.000000$ \&OBST XB=59. $549999,60.674999,79.925003,80.300003,7.03,7.78, \quad$ SURF_ID= 'UPHOLSTERY' 1 ' Left Arm
\&OBST XB=59.549999, 60.674999, $78.425003,79.925003,7.03,7.405$,
SURF_ID=' 'UPHOLSTERY'/ Base

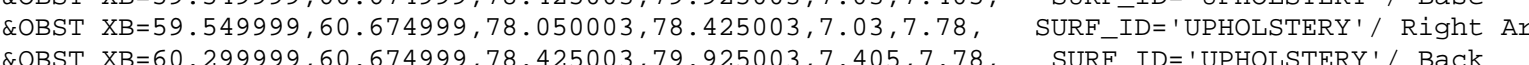
${ }_{*} * *$ GROUP: COUCH2 offset $=0.000000,30.000000,0.000000$ rotate $=270.000000$

\&OBST XB $=59.549999,60.674999,82.925003,83.300003,7.03,7.78$, SURF-ID=' 'UPHOLSTERY ' / Left Arm \&OBST XB=59.549999,60.674999, $81.05003,81.425003,7.03,7.78$, SURF_ID='UPHOLSTERY' / Base

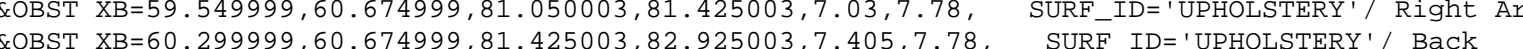

*** GROUP: TWIN

*** GROUP: ROW

*** GROUP: COUCH2 offset $=0.000000,0.000000,0.000000$ rotate $=90.000000$

\&OBST XB $=65.550003,66.675003,51.049999,51.424999,7.03,7.78$, SURF ID=' 'UPHOLSTERY ' / Left Arm

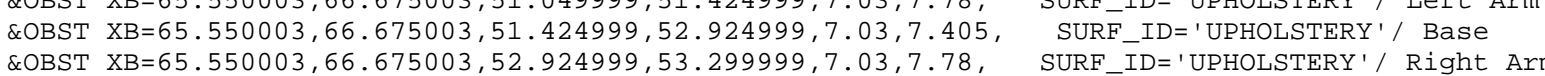
COBST $X \mathrm{~B}=65.550003,66.675003,52.924999,53.299999,7.03,7.78, \quad$ SURF_ID=' 'UPHOLSTERY'/ Right Arm
\&OBST $X \mathrm{~B}=65.550003,65.925003,51.424999,52.924999,7.405,7.78, \quad$ SURF_ID=' 'UPHOLSTERY'/ Back

*** GROUP: COUCH2 offset $=0.000000,3.000000,0.000000$ rotate $=90.000000$

\&OBST $X \mathrm{XB}=65.550003,66.675003,54.049999,54.424999,7.03,7.78$, SURF_ID=' UPHOLSTERY '/ Left Arm

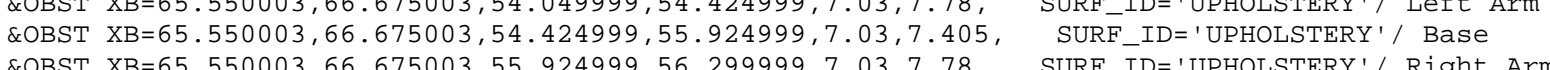

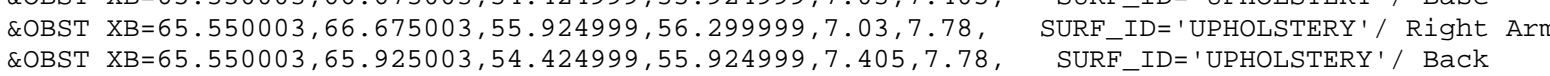

*** GROUP: COUCH2 offset $=0.000000,6.000000,0.000000$ rotate $=90.000000$

\&OBST XB=65.550003, 66.675003, 57.049999, 57.424999, 7. 03, 7.78, SURF_ID='UPHOLSTERY'/ Left Arm \&OBST XB $=65.550003,66.675003,57.424999,58.924999,7.03,7.405$, SURF_ID='UPHOLSTERY' / Base

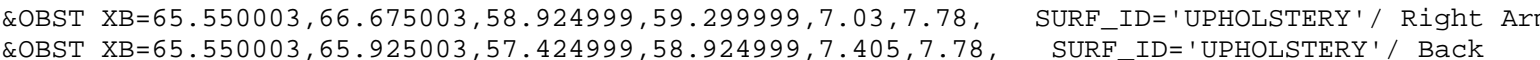

$* * *$ GROUP: COUCH2 offset $=0.000000,9 . .000000,0.000000$ rotate $=90.0000000$

\&OBST $X \mathrm{X}=65.550003,66.675003,60.049999,60.424999,7.03,7.78, \quad$ SURF-ID=' 'UPHOLSTERY ' / Left Arm

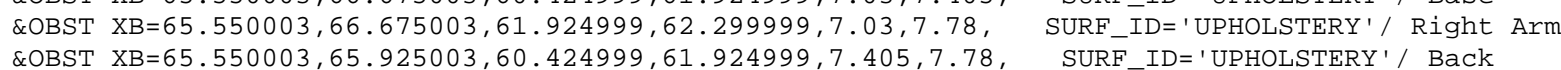
*** GROUP: COUCH2 offset $=0.000000,12.000000,0.000000$ rotate $=90.000000$

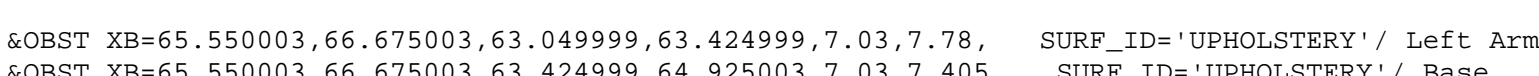

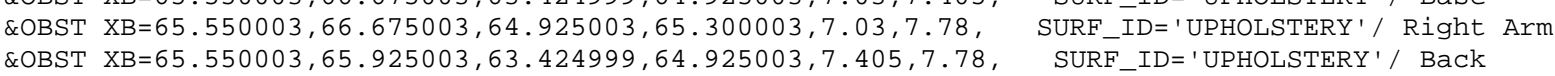

*** GROUP: COUCH2 offset $=0.000000,15.000000,0.000000$ rotate $=90.000000$

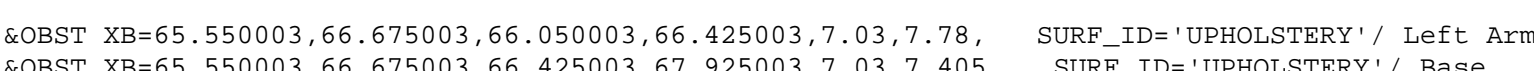

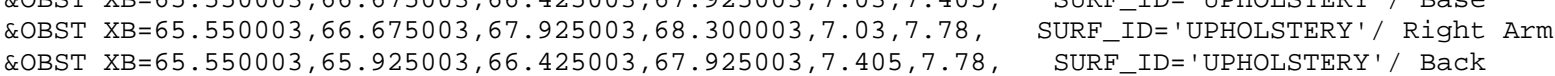

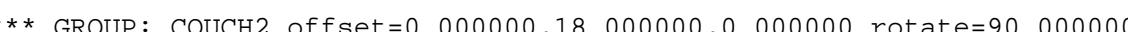

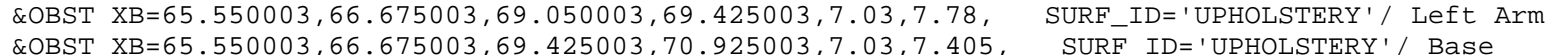
\&OBST XB $=65.550003,66.675003,70.925003,71.300003,7.03,7.78, \quad$ SURF_IDE'UPHOLSTERY'/ Right Arm

*** GROUP: COUCH2 offset $=0.000000,21.000000,0.000000$ rotate $=90.000000$

\&OBST XB=65.550003, 66.675003, 72.050003, 72.425003,7.03,7.78, SURF_ID='UPHOLSTERY' ' Left Arm

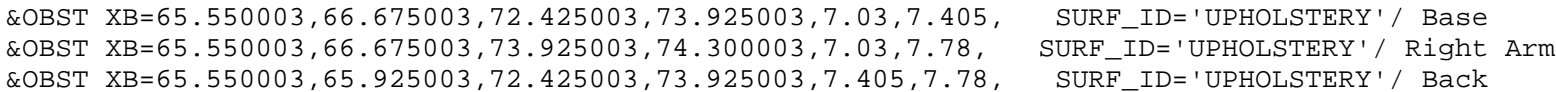

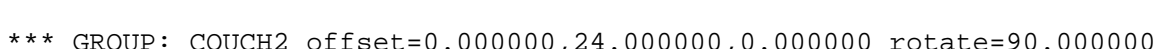

\&OBST XB $=65.550003,66.675003,75.050003,75.425003,7.03,7.78, \quad$ SURF_ID=' 'UPHOLSTERY' ' Left Arm
\&OBST XB $=65.550003,66.675003,75.425003,76.925003,7.03,7.405, \quad$ SURF_ID='UPHOLSTERY' $/$ Base

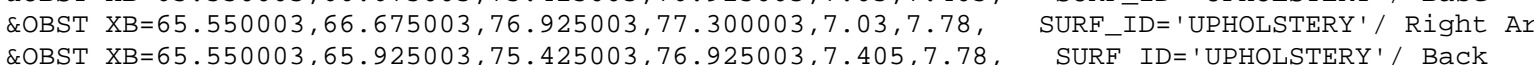

*** GROUP: COUCH2 offset $=0.000000,27.000000,0.000000$ rotate $=90.000000$

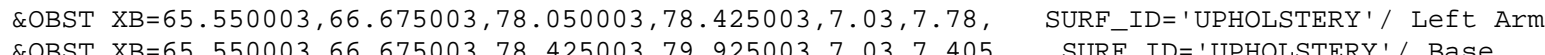

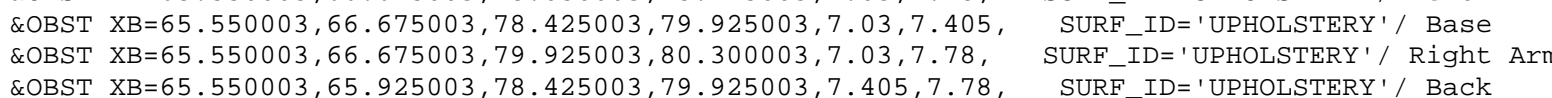

*** GROUP: COUCH2 offset $=0.000000$, 3०. $.000000,0.000000$ rotate $=90 . .000000$

\&OBST XB $=65.550003,66.675003,81.050003,81.425003,7.03,7.78$, SURF_ID='UPHOLSTERY'/ Left Arm

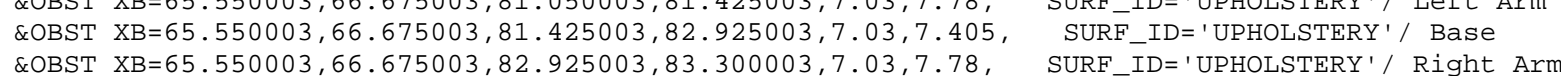

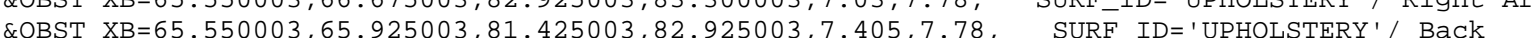

*** GROUP: ROW2

** GROUP: COUCH2 offset $=0.000000, \Theta ., 000000,0.000000$ rotate $=270.000000$

\&OBST XB=64. $050003,65.175003,52.924999,53.299999,7.03,7.78$, SURF_IDD ' UPHOLSTERY' / Left Arm

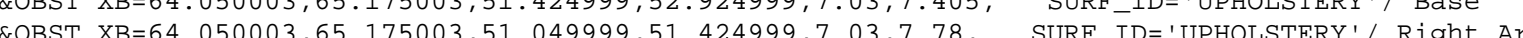
\&OBST XB=64.800003, 65.175003, 51.424999, 52.924999, 7.405, 7.78, SURF_ID=' 'UPHOLSTERY' / Back

*** GROUP: COUCH2 offset $=0.000000,3.000000,0.000000$ rotate $=270.000000$

\&OBST XB $64.050003,65.175003,55.924999,56.299999,7.03,7.78$, SURFIDD='UPHOLSTERY' ' Left Arm \&OBST XB $64.800003,65.175003,54.424999,55.924999,7.405,7.78$, SURF_ID='UPHOLSTERY'/ Back

*** GROUP: COUCH2 offset $=0.000000,6.000000,0.000000$ rotate $=270.000000$

\&OBST XB=64. $050003,65.175003,58.924999,59.299999,7.03,7.78, \quad$ SURF_ID='UPHOLSTERY' 1 ' Left Arm

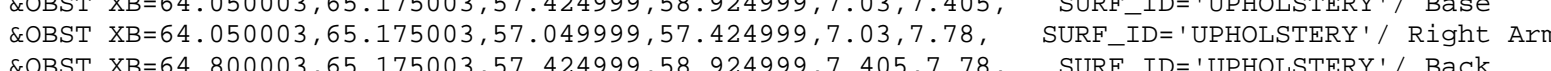

$* * *$ GROUP: COUCH2 offset $=0.000000,9.000000,0.000000$ rotate $=270.000000$

\&OBST XB=64. $050003,65.175003,61.924999,62.299999,7.03,7.78, \quad$ SURF_ID=' 'UPHOLSTERY' '/ Left Arm
\&OBST XB=64.050003, 65.175003,60.424999,61.924999,7.03,7.405, SURF_ID='UPHOLSTERY'/ Base

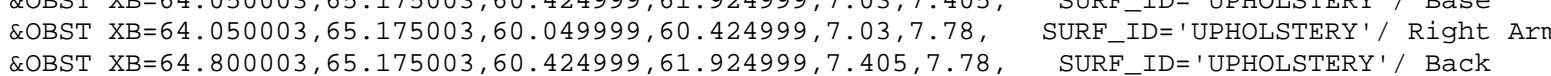

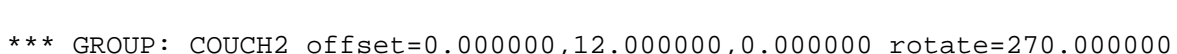

\&OBST XB $=64.0500003,65.175003,64.925003,65.300003,7.03,7.78, \quad$ SURF_ID='UPHOLSTERY' 1 ' Left Arm

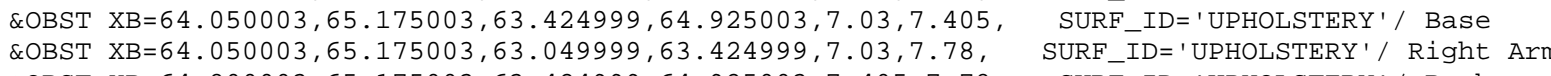

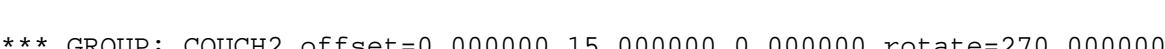

\&OBST XB $=64.050003,65.175003,67.925003,68.300003,7.03,7.78$, SURF_ID='UPHOLSTERY ' / Left Arm 
\&OBST XB $=64.8000003,65.175003,66,425003,67,925003,7,405,7,78$, SURF_ID=' UPHOLSTERY' / Back *** GROUP: COUCH2 offset $=0.000000,18.000000,0.000000$ rotate $=270$. ๑

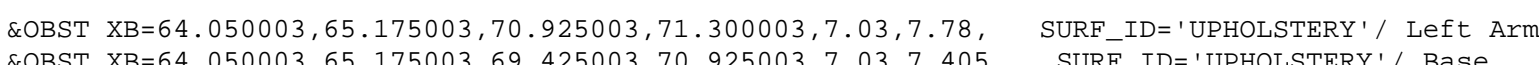

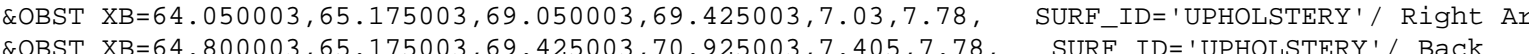

*** GROUP: COUCH2 offset $=0.000000,21.000000,0.000000$ rotate $=270.000000$

\&OBST XB $=64.050003,65.175003,73.925003,74.300003,7.03,7.78$, SURF_ID='UPHOLSTERY'/ Left Arm \&OBST XB $=64.0500003,65.175003,72.425003,73.925003,7.03,7.405$, SURF_IDD' 'UPHOLSTERY' $/$ Base

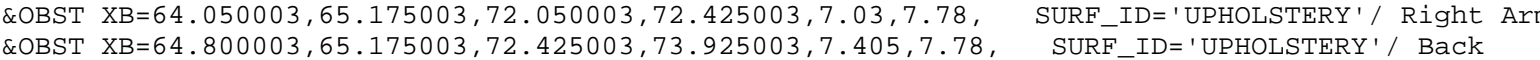

*** GROUP: CoUCH2 offset $=0.000000,24.000000,0.000000$ rotate $=270.000000$

\&OBST XB=64. $050003,65.175003,76.925003,77.300003,7.03,7.78$, SURF_ID=' UPHOLSTERY' ' Left Arm QOBST XB $=64.050003,65.175003,75.050003,75.425003,7.03,7.78$, SURF_ID='UPHOLSTERY'/ Right Ar

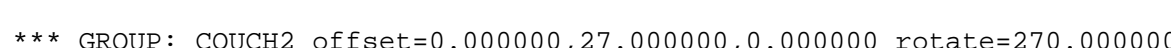

\&OBST XB=64.050003, 65.175003,79.925003, 80.300003, 7. 03, 7.78, SURF_ID='UPHOLSTERY' / Left Arm \&OBST XB=64.050003,65.175003, 78.050003, 78.425003, 7.03, 7.78, SURF_ID='UPHOLSTERY'/ Right Ar \&OBST XB=64.800003,65.175003, 78.425003,79.925003,7.405,7.78, SURF_ID='UP

*** GROUP: COUCH2 offset $=0.000000,30.000000,0.000000$ rotate $=270,000000$

\&OBST XB=64. $050003,65.175003,82.925003,83.300003,7.03,7.78$, SURF_ID=' 'UPHOLSTERY' ' Left Arm

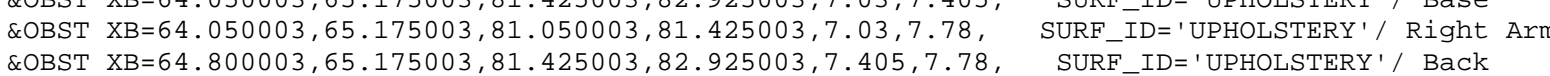

\section{*** GROUP: TWIN}

*** GROUP: ROW

*** GROUP: COUCH2 offset $=0.000000,0.000000,0.000000$ rotate $=90.000000$

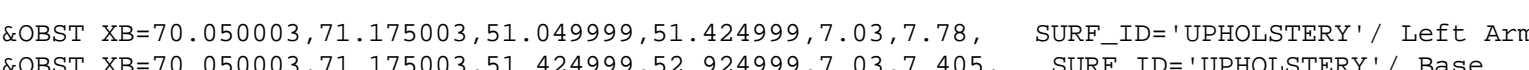

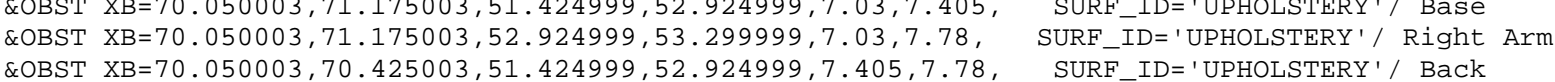

*** GROUP: COUCH2 offset $=0.000000,3.000000,0.000000$ rotate $=90.000000$

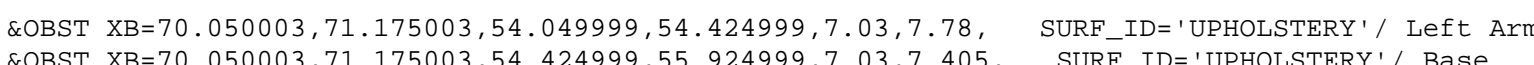
COBST $X \mathrm{XB}=70.050003,71.175003,55.924999,56.299999,7.03,7.78$,
\&OBST $X \mathrm{~B}=70.050003,70.425003,54.424999,55.924999,7.405,7.78, \quad$ SURF_ID='UPHOLSTERY'/ Right Arm
SURF_ID=' 'UPHOLSTERY' $/$ Back

*** GROUP: COUCH2 offset $=0.000000,6.000000,0.000000$ rotate $=90 . .000000$

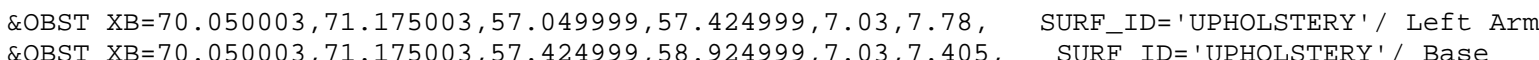
\&OBST XB $=70.050003,71.17503,58,024999,59,299999,7,03,7,78$, SURE ID='UPHOLSTEPY'/Right Arm \&OBST XB=70. $050003,71.175003,58.924999,59.299999,7.03,7.78, \quad$ SURF_ID=' 'UPHOLSTERY'/ Right Ar
\&OBST XB=70.050003, $70.425003,57.424999,58.924999,7.405,7.78, \quad$ SURF_ID='UPHOLSTERY'/ Back

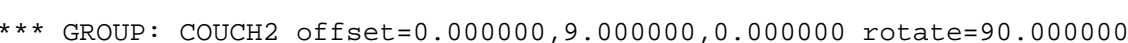

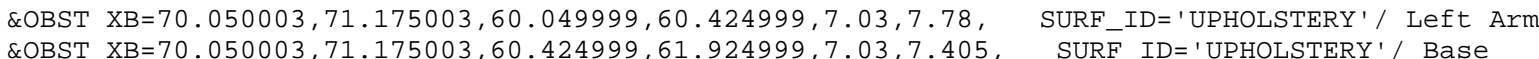

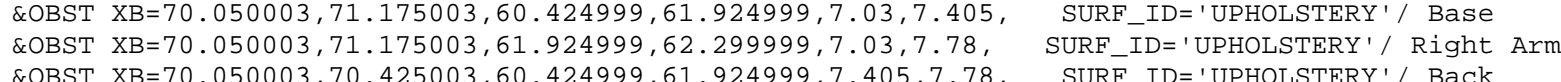

*** GROUP: COUCH2 offset $=0.000000,12.000000,0.000000$ rotate $=90.000000$

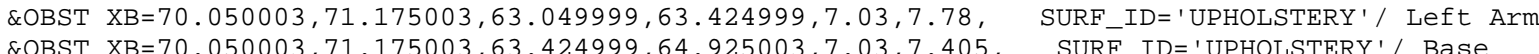

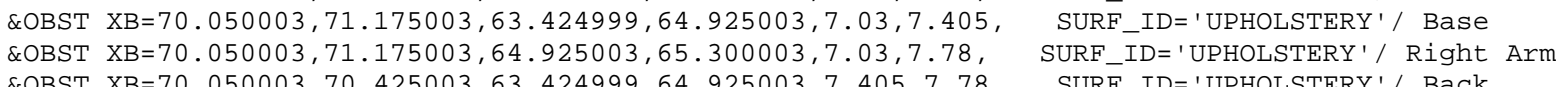

** GROUP: COUCH2 offset $=0.000000,15.0000000,0.000000$ rotate $=90.000000$
\&OBST XB=70. $050003,71.175003,66.050003,66.425003,7.03,7.78, \quad$ SURF_ID=' 'UPHOLSTERY' ' Left Arm
\&OBST XB=70.050003, 71.175003, $66.425003,67.925003,7.03,7.405, \quad$ SURF_ID='UPHOLSTERY'/ Base \&OBST XB=70.050003, 71.175003, 67.925003, 68.300003, 7.03, 7.78, SURF-ID='UPHOLSTERY'/ Right Ar

*** GROUP: COUCH2 offset $=0.000000,18.000000,0.000000$ rotate $=90 . .000000$ LOBST XB $=70.050003,71.175003,69.050003,69.425003,7.03,7.78, \quad$ SURF_ID=' 'UPHOLSTERY'/ Left Arm
\&OBST XB $=70.050003,71.175003,69.425003,70.925003,7.03,7.405, \quad$ SURF_ID='UPHOLSTERY'/ Base \&OBST XB=70. $050003,71.175003,70.925003,71.300003,7.03,7.78, \quad$ SURF_ID=' 'UPHOLSTERY'/ Right Arm
\&OBST XB=70.050003,70.425003,69.425003,70.925003,7.405,7.78, SURF_ID=' 'UPHOLSTERY'/ Back

*** GROUP: COUCH2 offset $=0.000000,21.000000,0.000000$ rotate $=90 . .000000$

\&OBST XB=70. $050003,71.175003,72.050003,72.425003,7.03,7.78$, SURF_ID='UPHOLSTERY'/ Left Arm

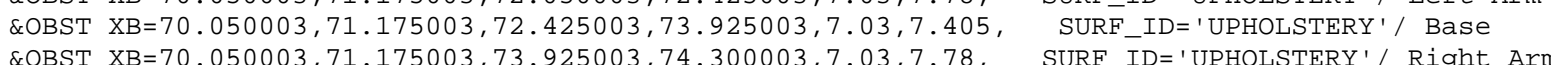
\&OBST XB=70.050003, 70.425003, 72.425003, 73.925003,7.405, 7.78 , SURF_ID='UPHOLSTERY'/ Back

*** GROUP: COUCH2 offset $=0.000000,24.000000,0.000000$ rotate $=90.000000$

\&OBST $X B=70.050003,71.175003,75.050003,75.425003,7.03,7.78$, SURF_ID='UPHOLSTERY'/ Left Arm \&OBST XB=70.050003, 71.175003,75.425003,76.925003, 7.03,7.405, SURF_ID='UPHOLSTERY'/ Base

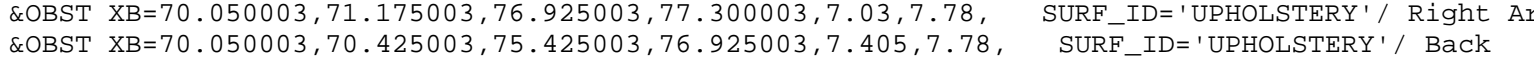

*** GROUP: COUCH2 offset $=0.000000,27.000000,0.000000$ rotate $=90.000000$

\&OBST XB=70.050003, 71.175003, 78. 050003, 78.425003, 7. $03,7.78$, SURF_ID=' 'UPHOLSTERY' ' Left Arm

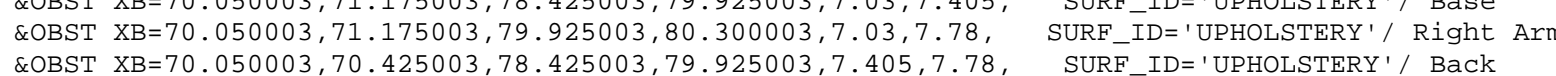

*** GROUP: COUCH2 offset $=0.000000,30.000000,0.000000$ rotate $=90.000000$

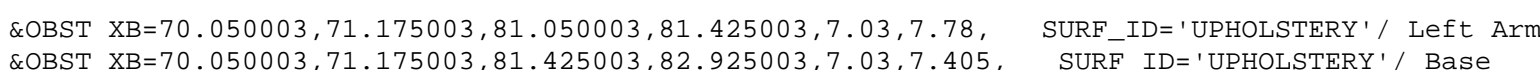

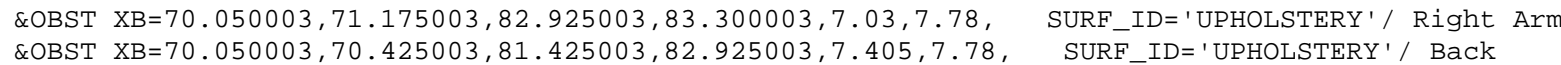

*** GROUP: ROW2

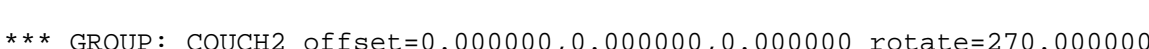

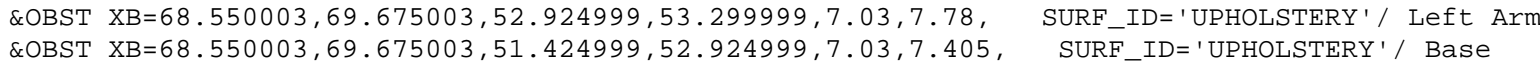

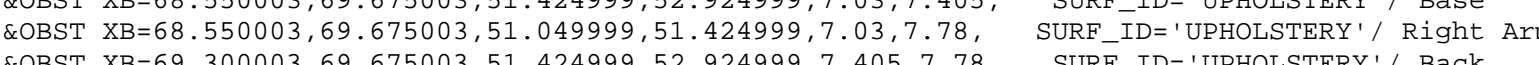

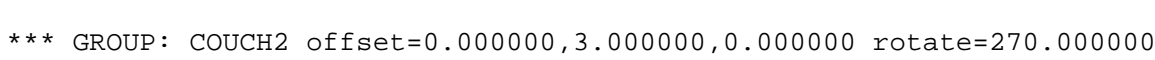

\&OBST XB $=68.550003,69.675003,55.924999,56.299999,7.03,7.78$, SURF_ID=' 'UPHOLSTERY'/ Left Arm \&OBST XB $=68.550003,69.675003,54.424999,55.924999,7.03,7.405, \quad$ SURF_ID='UPHOLSTERY'/ Base
\&OBST XB $=68.550003,69.675003,54.049999,54.424999,7.03,7.78, \quad$ SURF_IDE'UPHOLSTERY'/ Right Ar

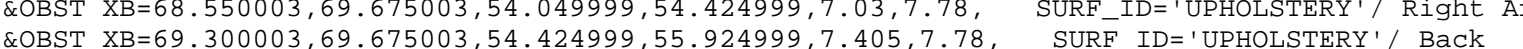

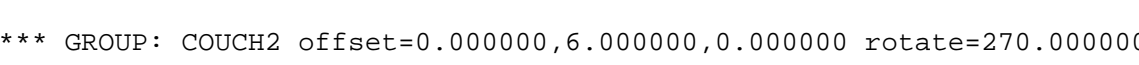

\&OBST XB $=68.550003,69.675003,58.924999,59.299999,7.03,7.78$, SURF_ID=' 'UPHOLSTERY'/ Left Arm LOBST XB $=68.550003,69.675003,57.424999,58.924999,7.03,7.405, \quad$ SURF IDE='UPHOLSTERY'/ Base
LOBST XB $=68.550003,69.675003,57.04999,57.424999,7.03,7.78$,
SURF ID 'UPHOLSTERY'/ Right Ar SOBST XB $=69.300003,69.67503,57.424999,58.924999,7.405,7.78$, SUPF_ID=' 'UPHOLSTERY'/ Bnck Ar

*** GROUP: COUCH2 offset $=0.000000,9.000000,0.000000$ rotate $=270.000000$

\&OBST XB $=68.550003,69.675003,61.924999,62.299999,7.03,7.78$, SURF_ID=' 'UPHOLSTERY ' / Left Arm

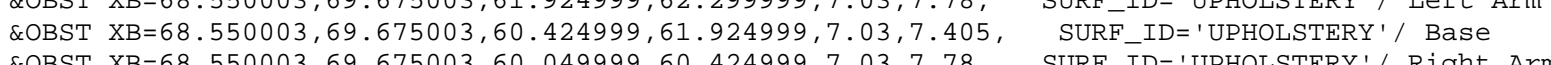
KOBST XB=69.300003,69.675003, 60.424999,61.924999, $7.405,7.78$, SURF_ID='UPHOLSTERY'/ Back

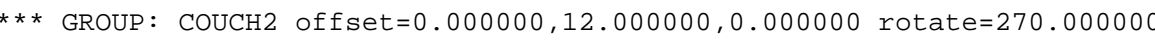

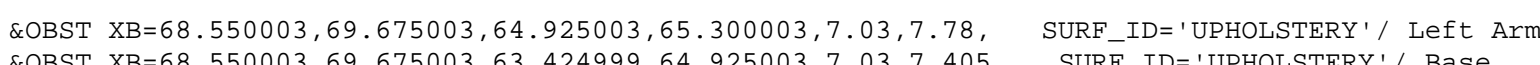
\&OBST XB $=68.550003,69.675003,63.049999,63.424999,7.03,7.78$, SURF_ID='UPHOLSTERY'/ Right Ar 
*** GROUP: COUCH2 offset $=0.000000,15.000000,0.000000$ rotate $=270.000000$

\&OBST $X \mathrm{XB}=68.550003,69.675003,67.925003,68.300003,7.03,7.78, \quad$ SURF_ID= ' UPHOLSTERY ' / Left Arn \&OBST XB $=68.550003,69.675003,66.050003,66.425003,7.03,7.78$, SURF_ID='UPHOLSTERY'/ Right Ar \&OBST XB=69.300003, 69.675003,66.425003, 67.925003, 7.405, 7.78, SURF_ID='UPHOLSTERY'/ Back

*** GROUP: COUCH2 offset $=0,000000,18,000000,0.000000$ rotate $=270 ., 000000$

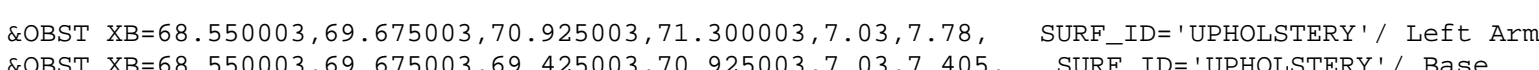

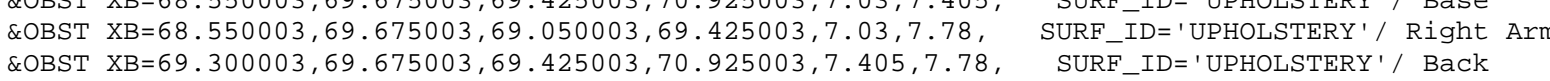

*** GROUP: COUCH2 offset $=0.000000,21.000000, \odot .000000$ rotate $=270.000000$

\&OBST XB $=68.550003,69.675003,73.925003,74.300003,7.03,7.78$,
\&OBST XB $=68.550003,69.675003,72.425003,73.925003,7.03,7.405$,
SURF_ID=' 'UPHOLSTERY ' 1 'Left Arm

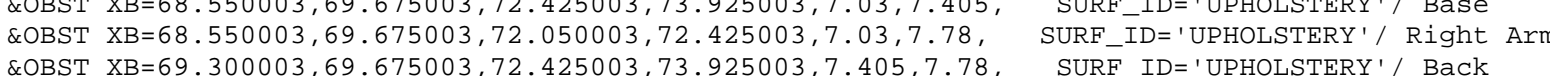
*** GROUP: COUCH2 offset $=0.000000,24.000000,0.000000$ rotate $=270.000000$

\&OBST XB $=68.550003,69.675003,76.925003,77.300003,7.03,7.78, \quad$ SURF_ID=' 'UPHOLSTERY ' ' Left Arm
\&OBST XB $=68.550003,69.675003,75.425003,76.925003,7.03,7.405, \quad$ SURF ID ' 'UPHOLSTERY'

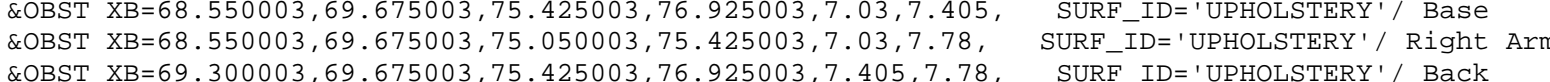

*** GROUP: COUCH2 offset $=0.000000,27.000000,0.000000$ rotate $=270.000000$

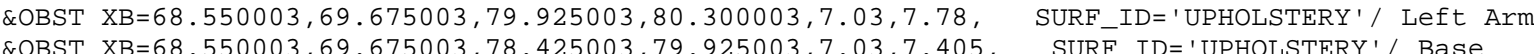

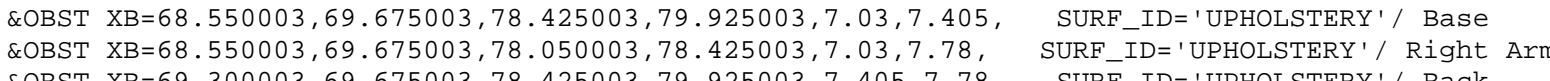

*** GROUP: COUCH2 offset $=0.000000$, 30. $000000,0.000000$ rotate $=270.000000$

\&OBST XB $=68.550003,69.675003,82.925003,83.300003,7.03,7.78$, SURF_ID='UPHOLSTERY'/ Left Arm

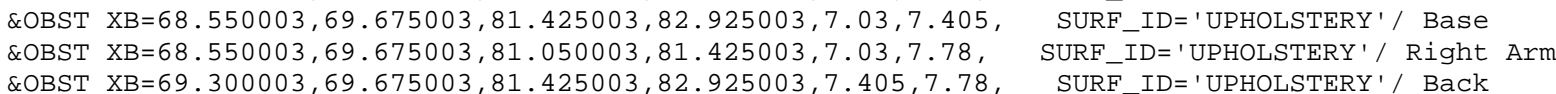

*** GROUP: ROW2

*** GROUP: COUCH2 offset $=0.000000,0.000000,0.000000$ rotate $=270.000000$

\&OBST $X \mathrm{XB}=73.050003,74.175003,52.924999,53.299999,7.03,7.78, \quad$ SURF-ID='UPHOLSTERY'/ Left Arm

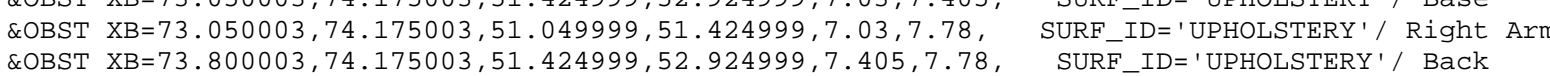

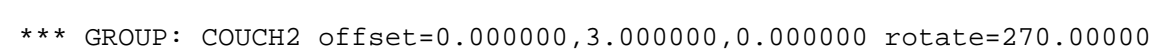

\&OBST $X B=73.050003,74.175003,55.924999,56.299999,7.03,7.78, \quad$ SURF_ID=' 'UPHOLSTERY' $/$ Left Arm

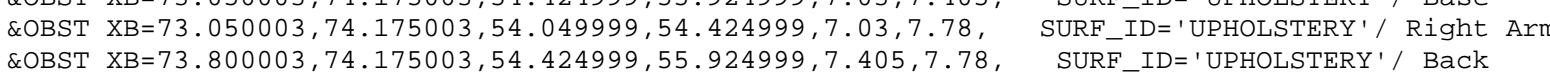

*** GROUP: COUCH2 offset $=0.000000,6.000000,0.000000$ rotate $=270.000000$

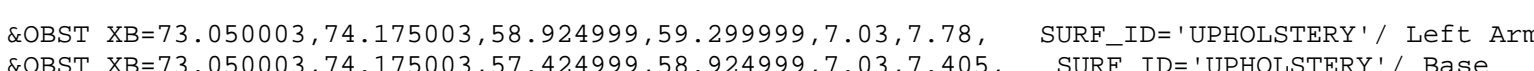

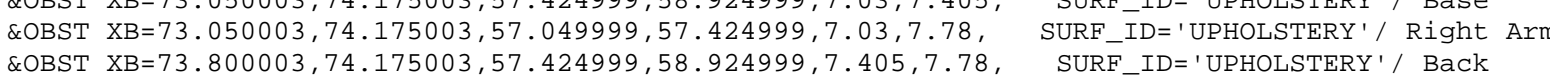

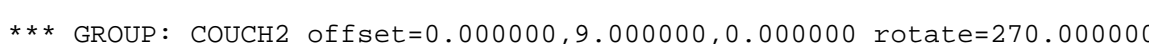

\&OBST XB $=73.0500003,74.175003,61.924999,62.299999,7.03,7.78$, SURF_ID=' 'UPHOLSTERY ' / Left Arm COBST XB $=73.050003,74.175003,60.049999,60.424999,7.03,7.78, \quad$ SURF_ID='UPHOLSTERY' $/$ Right Arm
\&OBST XB $=73.800003,74.175003,60.424999,61.924999,7.405,7.78, \quad$ SURF_ID=' 'UPHOLSTERY' $/$ Back *** GROUP: COUCH2 offset $=0.0000000,12.000000,0.000000$ rotate $=270.000000$ \&OBST XB=73.050003, 74.175003, 64.925003, 65. 300003, 7. 03, 7.78, $\quad$ SURF_ID='UPHOLSTERY' ' Left Arm
\&OBST XB=73.050003, $74.175003,63.424999,64.925003,7.03,7.405, \quad$ SURF_ID=' 'UPHOLSTERY' $/$ Base
\&OBST XB=73. $050003,74.175003,63.049999,63.424999,7.03,7.78, \quad$ SURF_ID= 'UPHOLSTERY '/ Right Arm
\&OBST XB $73.800003,74.175003,63.424999,64.925003,7.405,7.78, \quad$ SURF_ID=' 'UPHOLSTERY' / Back *** GROUP: COUCH2 offset $=0.000000,15.000000,0.000000$ rotate $=270.000000$

\&OBST XB=73. $050003,74.175003,67.925003,68.300003,7.03,7.78, \quad$ SURF_ID=' 'UPHOLSTERY'/' Left Arm
\&OBST XB=73.050003, 74.175003,66.425003,67.925003,7.03,7.405,
SURF_ID='UPHOLSTERY'/ Base

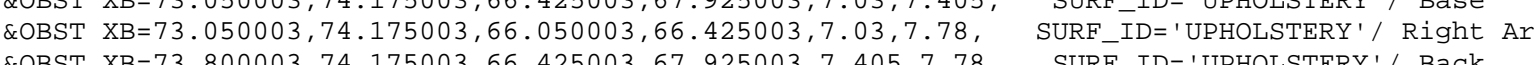

*** GROUP: COUCH2 offset $=0.000000,18.000000,0.000000$ rotate $=270.000000$

\&OBST $X B=73.050003,74.175003,70.925003,71.300003,7.03,7.78$,
SURF_ID= 'UPHOLSTERY'/ Left Arm
SURF ID=' 'UPHOLSTERY' / Base \&OBST $X \mathrm{XB}=73.050003,74.175003,69.425003,70.925003,7.03,7.405, \quad$ SURF_ID='UPHOLSTERY'/ Base
\&OBST XB $=73.050003,74.175003,69.050003,69.425003,7.03,7.78, \quad$ SURF IDD='UPHOLSTERY'/ Right Ar

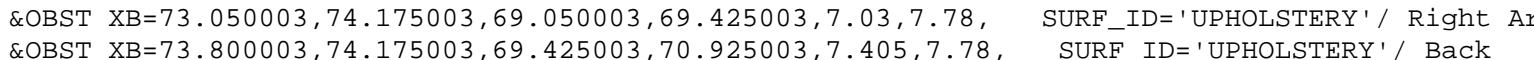

*** GROUP: COUCH2 offset $=0.000000,21.000000,0.000000$ rotate $=270.000000$

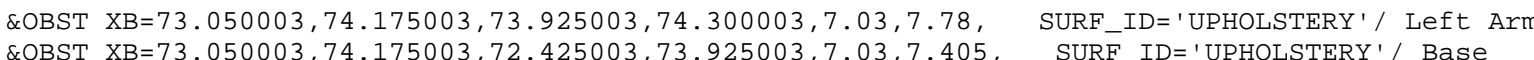
\&OBST XB $=73.050003,74.175003,72.050003,72.425003,7.03,7.78$, SURF_ID='UPHOLSTERY'/ Right Ar

*** GROUP: CoucH2 offset $=0.0000000,24.000000,0.000000$ rotate $=270.000000$

\&OBST $X \mathrm{~B}=73.050003,74.175003,76.925003,77.300003,7.03,7.78$, SURF_ID=' 'UPHOLSTERY'/ Left Arm

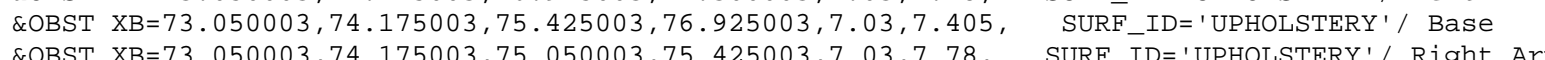
\&OBST XB=73. $050003,74.175003,75.050003,75.425003,7.03,7.78, \quad$ SURF_ID='UPHOLSTERY'/ Right Arn
\&OBST XB=73.800003, 74.175003, $75.425003,76.925003,7.405,7.78, \quad$ SURF_ID='UPHOLSTERY'/ Back

*** GROUP: COUCH2 offset $=0.000000,27.000000,0.000000$ rotate $=270.000000$

\&OBST XB $=73.050003,74.175003,79.925003,80.300003,7.03,7.78$, SURF_ID='UPHOLSTERY'/ Left Arm \&OBST XB=73.050003, 74.175003,78.425003,79.925003, 7.03,7.405, SURF_ID='UPHOLSTERY'/ Base \&OBST XB $=73.050003,74.175003,78.050003,78.425003,7.03,7.78, \quad$ SURF_ID=' 'UPHOLSTERY'/ Right Ar
\&OBST $X B=73.800003,74.175003,78.425003,79.925003,7.405,7.78$,
SURF_ID=' 'UPHOLSTERY' / Back

*** GROUP: COUCH2 offset $=0.000000,30.000000,0.000000$ rotate $=270.000000$

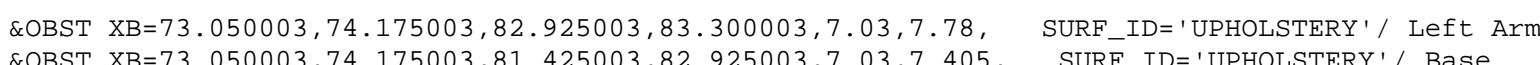

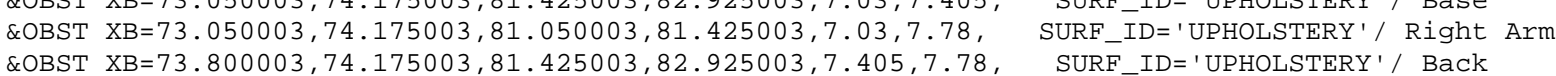

$* * *$ GROUP: SHORT_ROW

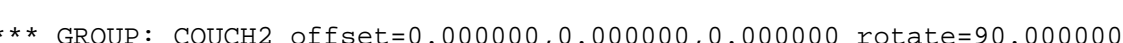

\&OBST XB $=67.044998,67.419998,46.91,48.035,7.03,7.78$, SURF_ID='UPHOLSTERY' ' Left Arm

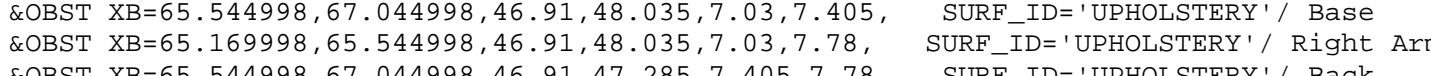

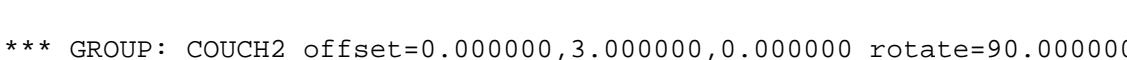

\&OBST XB=64.044998, 64.419998, 46.91, 48.035, 7.03, 7.78, SURF_ID='UPHOLSTERY' / Left Arm \&OBST XB-62.544998, 64. $444998,46.91,48.035,7.03,7.405$, SURF_ID='UPHOLSTERY'/ Base \&OBST XB=62.544998,64. $944998,46.91,47.285,7.405,7.78, \quad$ SURF_ID=' 'UPHOLSTERY' $/$ Back

*** GROUP: COUCH2 offset $=0.0000000,6.000000,0.000000$ rotate $=90$. . 000000

OBST XB=61. $044998,61.419998,46.91,48.035,7.03,7.78$, SURF_ID=' 'UPHOLSTERY'/ Left Arm COBST XB=59.544998, 61. $044998,46.91,48.035,7.03,7.405$, SURF_ID='UPHOLSTERY'/ Base \&OBST XB $=59.544998,61.044998,46.91,47.285,7.405,7.78$, SURF ID='UPHOLSTERY'/ Rack *** GROUP: COUCH2 offset $=0.0000000,9.000000,0.000000$ rotate $=90 . .000000$ \&OBST XB=58. 044998, 58.419998, 46.91, 48. $035,7.03,7.78$, SURF_ID='UPHOLSTERY' ' Left Arm

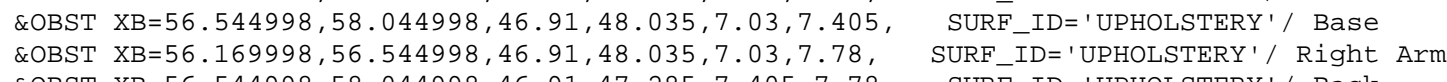

*** GROUP: COUCH2 offset $=0.0000000,12.000000,0.0000000$ rotate $=90.000000$ 
\&OBST XB=55. $044998,55.419998,46.91,48.035,7.03,7.78, \quad$ SURF_ID=' 'UPHOLSTERY ' $/$ Left Arm
\&OBST XB=53.544998, $55.044998,46.91,48.035,7.03,7.405, \quad$ SURF_ID=' 'UPHOLSTERY' $/$ Base

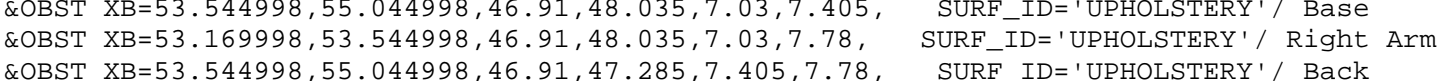
*** GROUP: COUCH2 offset $=0.000000,15.000000,0.0000000$ rotate $=90.000000$

\&OBST XB $=52.044998,52.419998,46.91,48.035,7.03,7.78$, SURF_ID='UPHOLSTERY' ' Left Arm QOBST XB $=50.169998,50.544998,46.91,48.035,7.03,7.78, \quad$ SURF_ID='UPHOLSTERY'/Right A \&OBST XB $=50.544998,52.044998,46.91,47.285,7.405,7.78$, SURF_ID 'UPHOLSTERY'/ Back

*** GROUP: COUCH2 offset $=0.000000,18.000000,0.000000$ rotate $=90.000000$

\&OBST XB=49. $044998,49.419998,46.91,48.035,7.03,7.78, \quad$ SURF_ID= 'UPHOLSTERY ' / Left Arm \&OBST XB=47.169998, 47.544998, 46.91, 48.035, 7. $03,7.78, \quad$ SURF_ID='UPHOLSTERY' 1 'Right A \&OBST XB=47.544998, 49.044998, 46.91, 47.285, 7.405, 7.78, SURF_ID=' 'UPHOLSTERY'/ Back

*** GROUP: ROW

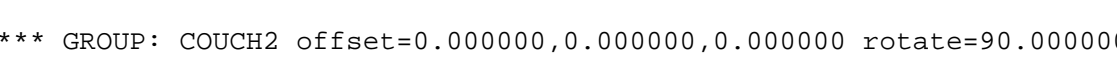

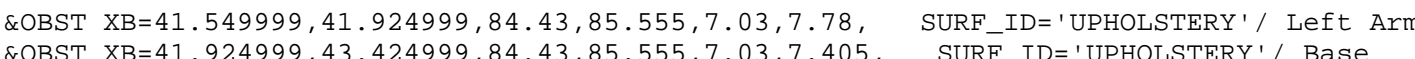
\&OBST XB $=41.924999,43.424999,84.43,85.555,7.03,7.405, \quad$ SURF-ID=' 'UPHOLSTERY '/ Base
\&OBST XB $=43.424999,43.799999,84.43,85.555,7.03,7.78$, \&OBST XB $=41.924999,43.424999,85,18,85.555,7.405,7.78$, SURF_ID='UPHOLSTEPY' Righ

*** GROUP: COUCH2 offset $=0.000000,3.000000,0.000000$ rotate $=90.000000$

\&OBST XB=44.549999, 44.924999, 84.43, 85.555, 7.03, 7.78, SURF_ID=' 'UPHOLSTERY ' ' Left Arm \&OBST XB $=44.924999,46.424999,84.43,85.555,7.03,7.405, \quad$ SURF IDE= 'UPHOLSTERY '/ Base
\&OBST XB \&OBST XB $=46.424999,46.799999,84.43,85.555,7.03,7.78$,
\&OBST XB $44.924999,46.424999,85.18,85.555,7.405,7.78$,
SURF_IDE_ID 'UPHOLSTERY' 'UPHOLSTERY' $/$ Right Back

*** GROUP: COUCH2 offset $=0.000000,6 . \bullet 000000, \bullet . .000000$ rotate $=90 . .00000$

EOBST XB=47.549999, 47.924999, 84.43,85.555, 7.03, 7.78, SURF_ID= 'UPHOLSTERY ' / Left Arm \&OBST XB $47.92499,49.42499,84.43,85.555,7.03,7.405$,
SORST

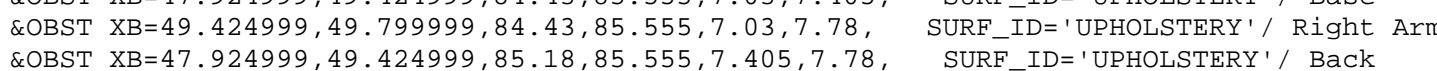

*** GROUP: COUCH2 offset $=0.000000,9.000000,0.000000$ rotate $=90.000000$

\&OBST XB=50.549999, 50.924999, 84.43, 85.555, 7.03, 7.78, SURF_ID='UPHOLSTERY'/ Left Arm

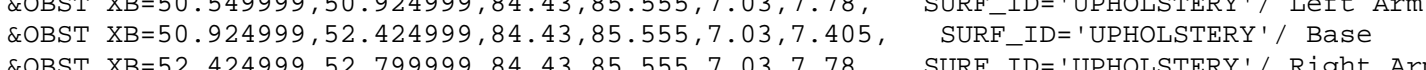
\&OBST XB=52.424999, 52.799999, $84.43,85.555,7.03,7.78, \quad$ SURF_ID='UPHOLSTERY'/ Right Ar
\&OBST XB=50.924999, $52.424999,85.18,85.555,7.405,7.78, \quad$ SURF_ID='UPHOLSTERY'/ Back

*** GROUP: COUCH2 offset $=0.000000,12.000000,0.000000$ rotate $=90.000000$

\&OBST XB=53.549999, 53.924999, 84.43,85.555, 7.03, 7.78, SURF_ID='UPHOLSTERY'/ Left Arm

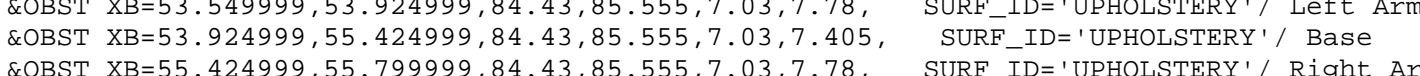

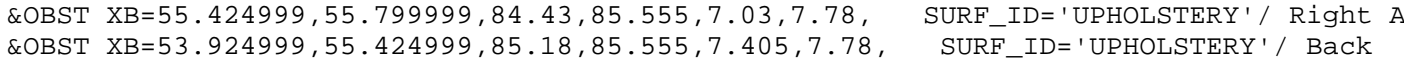

*** GROUP: COUCH2 offset $=0.000000,15.000000,0.000000$ rotate $=90.000000$

\&OBST XB $=56.549999,56.924999,84.43,85.555,7.03,7.78$, SURF_ID='UPHOLSTERY'/ Left Arm

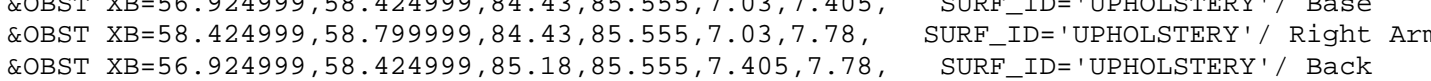

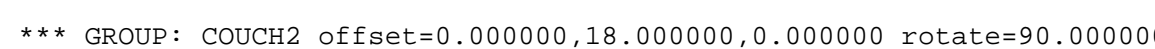

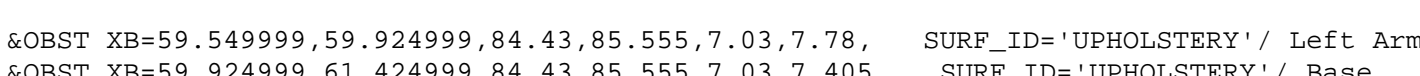

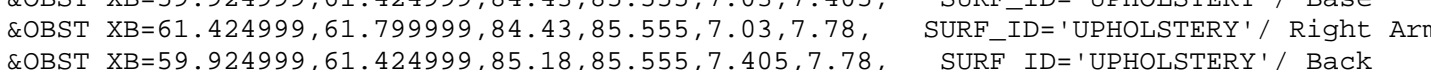

*** GROUP: COUCH2 offset $=0.000000,21.000000,0.000000$ rotate $=90.000000$

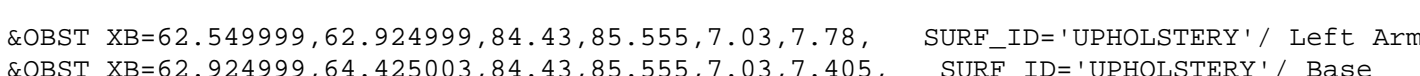

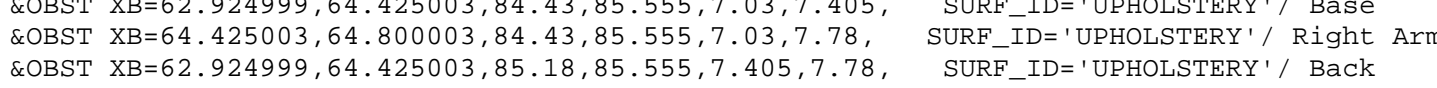

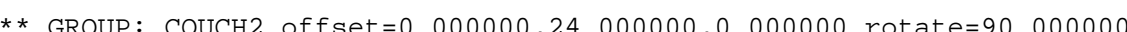

\&OBST XB $=65.550003,65.925003,84.43,85.555,7.03,7.78$,
COBST XB $=65.925003,67.425003,84.43,85.555,7.03,7.405$,

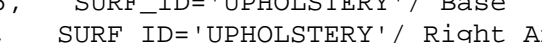
\&OBST XB $=65.925003,67.425003,85.18,85.555,7.405,7.78$, SURF_ID=' 'UPHOLSTERY'/ Back

*** GROUP: COUCH2 offset $=0.000000,27.000000,0.000000$ rotate $=90.0000000$

\&OBST XB=68.550003, 68.925003, 84. 43, 85.555, 7.03,7.78, SURF_ID='UPHOLSTERY' ' Left Arm \&OBST XB=70.425003,70.800003,84.43,85.555,7.03.7.78, SURF IDD='UPHOLSTTERY'/ Base \&OBST XB $68.925003,70.425003,85.18,85.555,7.405,7.78$, SURF_ID=' 'UPHOLSTERY' / Back

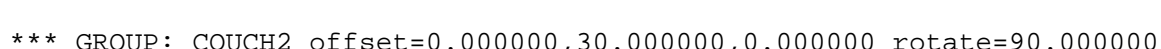

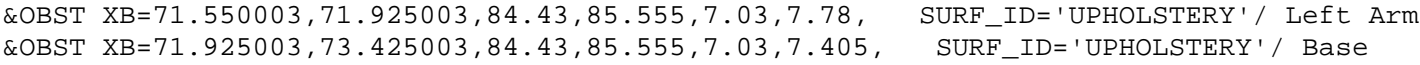
\&OBST XB=73.425003,73.800003, 84.43, 85.555, 7. $03,7.78$, SURF_ID ='UPHOLSTERY'/ Right Ar

$* * *$ GROUP: CHAIR_STACK

*** GROUP: CHAIR_ROW

*** GROUP: CHATR offset $=0.000000,0.000000,0.140000$ rotate $=0.000000$

\&OBST XB=55. $049999,55.424999,12.05,13.175,0.28,1.03, \quad$ SURF_ID=' 'UPHOLSTERY ' ' Left Arm

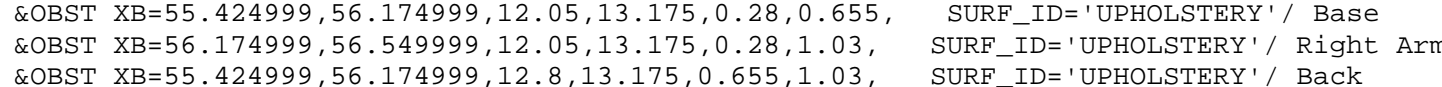
*** GROUP: CHAIR offset $=1.875000,0.000000,0.140000$ rotate $=0.000000$

\&OBST XB $=56.924999,57.299999,12.05,13.175,0.28,1.03, \quad$ SURF_ID='UPHOLSTERY' $/$ Left Arm
\&OBST XB $=57.299999,58.049999,12.05,13.175,0.28,0.655$, SURF ID='UPHOLSTERY' $/$ Base COBST XB=58. $049999,58.424999,12.05,13.175,0.28,1.03$, S SURF IDE 'UPHOLSTERY' Right \&OBST XB=57. 299999, 58. 049999, 12.8, 13.175, $0.655,1.03$, SURF_ID='UPHOLSTERY'/ Back

*** GROUP: CHAIR offset $=3.750000,0.000000,0.140000$ rotate $=0.000000$

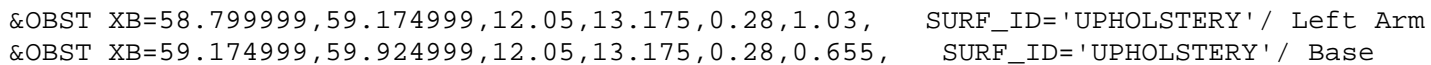
\&OBST XB $59.924999,60.299999,12.05,13.175,0.28,1.03$, SURF IDE'UPHOLSTERY'/ Base \&OBST XB $=59.174999,59.924999,12.8,13.175,0.655,1.03$, SURF_ID=' 'UPHOLSTERY'/ Rack

*** GROUP: CHAIR offset $=5.625000,0.000000,0.140000$ rotate $=0.000000$

\&OBST XB=60.674999, 61. 0499999, 12. $05,13.175,0.28,1.03$, SURF_ID='UPHOLSTERY'/' Left Arm

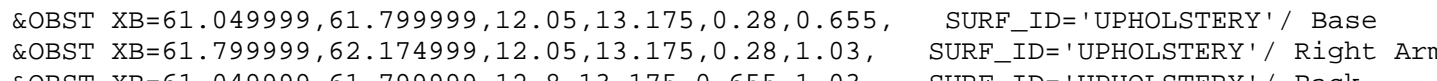

$* * *$ GROUP: CHAIR offset $=7.500000,0.000000,0.140000$ rotate $=0.000000$

\&OBST XB=62.549999, 62.924999, 12.05, 13.175, 0.28,1.03, SURF_ID='UPHOLSTERY'/ Left Arm \&OBST XB $62.024999,63.674999,12.05,13.175,0.28,0.655$, SURF_IDE' UPHOLSTERY'/ Base

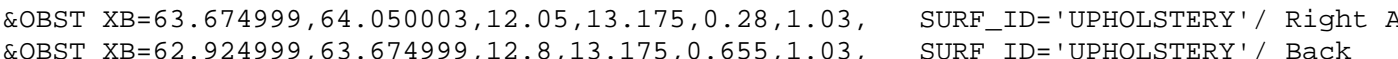

*** GROUP: CHAIR offset $=9.375000,0.000000,0.140000$ rotate $=0.000000$

\&OBST XB $=64.425003,64.800003,12.05,13.175,0.28,1.03, \quad$ SURF_ID='UPHOLSTERY'/ Left Arm \&OBST XB=64. $800003,65.550003,12.05,13.175,0.28,0.655$, SURF_ID=' 'UPHOLSTERY'/ Base \&OBST XB $64.800003,65.550003,12.8,13.175,0.655,1.03$ ' SUPF-ID=' 'UPHOLSTERY'/ Rack

*** GROUP: CHAIR_ROW

*** GROUP: CHAIR offset $=0.000000,0.000000,0.140000$ rotate $=0.000000$

\&OBST XB $=55.049999,55.424999,13.18,14.305,1.155,1.905$, SURF_ID='UPHOLSTERY ' / Left Arm \&OBST XB=56.174999, 56.549999,13.18, 14.305,1.155,1.905, SURF_ID='UPHOLSTERY'/ Right Ar \&OBST XB=55.424999,56.174999,13.93,14.305, 1.53,1.905, SURF_ID='UPHOLSTERY'/ Back 
$* *$ GROUP: CHATR offset $=1.875000,0.000000,0.140000$ rotate $=0.000000$

\&OBST XB=56.924999, 57.299999, 13.18, 14.305, 1.155, 1.905, SURF_ID=' 'UPHOLSTERY' ' Left Arm
\&OBST XB=57.299999, $58.049999,13.18,14.305,1.155,1.53, \quad$ SURF_ID='UPHOLSTERY'

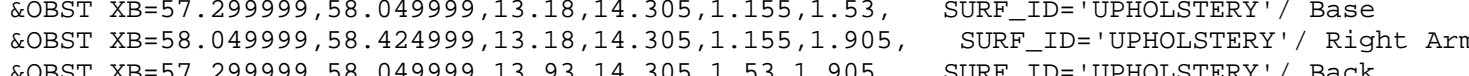

*** GROUP: CHAIR offset $=3.750000,0.000000,0.140000$ rotate $=0.000000$

\&OBST XB=58.799999, 59.174999, 13.18,14.305, 1.155, 1.905, SURF_ID=' 'UPHOLSTERY'/ Left Arm

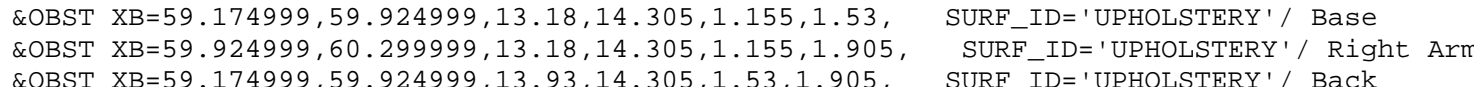
$* * *$ GROUP: CHAIR offset $=5.625000,0.000000,0.140000$ rotate $=0.000000$

\&OBST XB=60.674999, 61. 049999, 13.18,14.305, 1.155, 1.905, SURF_ID=' 'UPHOLSTERY'/ Left Arm
SURF_ID='UPHOLSTERY'/ Base

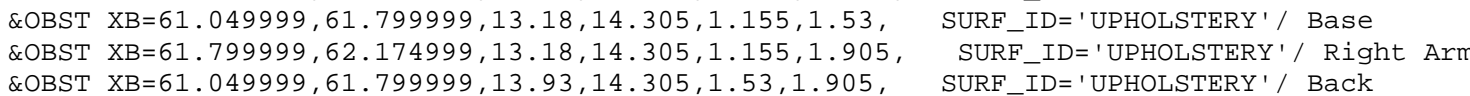

*** GROUP: CHAIR offset $=7.500000,0.000000,0.140000$ rotate $=0.000000$

\&OBST XB=62.549999, 62.924999, 13.18, 14.305, 1.155, 1.905, SURF_ID=' 'UPHOLSTERY'/ Left Arm

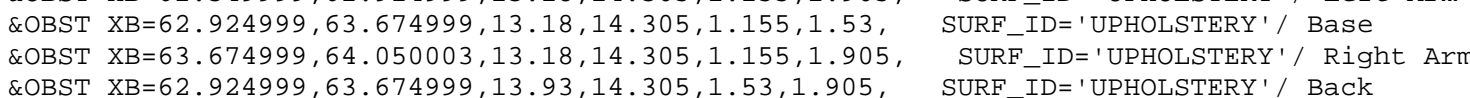
*** GROUP: CHAIR offset $=9.375000,0.000000,0.140000$ rotate $=0.000000$ \&OBST XB $=64.425003,64.800003,13.18,14.305,1.155,1.905$,
SURF_ID=' UPHOLSTERY' $/$ Left Arm
SURF_ID='UPHOLSTERY'

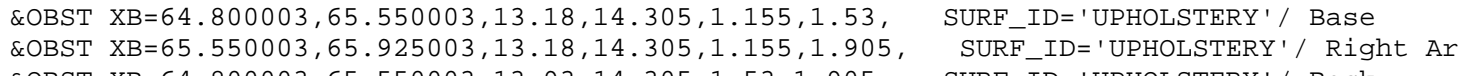

\section{$* * *$ GROUP: CHAIR_ROW}

** GROUP: CHAIR offset $=0.000000,0.000000,0.140000$ rotate $=0.000000$

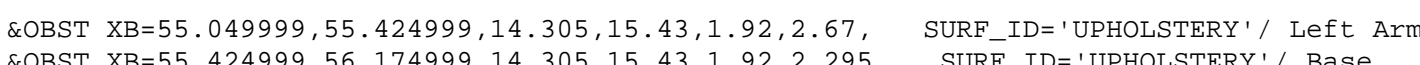

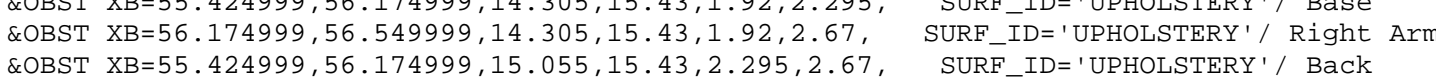

$* * *$ GROUP: CHAIR offset $=1.875000,0.000000,0.140000$ rotate $=0.000000$

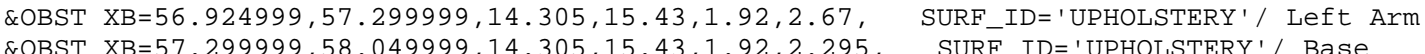

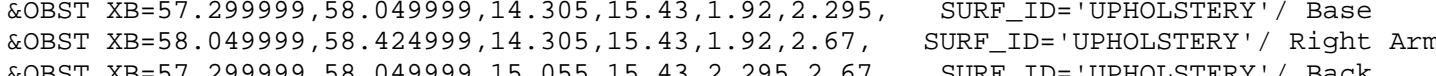
*** GROUP: CHAIR offset $=3.750000,0.000000,0.140000$ rotate $=0.000000$

\&OBST XB $=58.799999,59.174999,14.305,15.43,1.92,2.67, \quad$ SURF_ID='UPHOLSTERY' $/$ Left Arm
\&OBST XB $=59.174999,59.924999,14.305,15.43,1.92,2.295$,
SURFID='UPHOLSTERY'

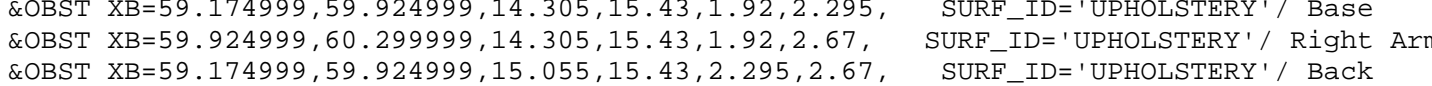

*** GROUP: CHAIR offset $=5.625000,0.000000,0.140000$ rotate $=0.000000$

\&OBST XB=60.674999, 61. 049999, 14.305, 15.43,1.92, 2.67, SURF_ID='UPHOLSTERY' ' Left Arm

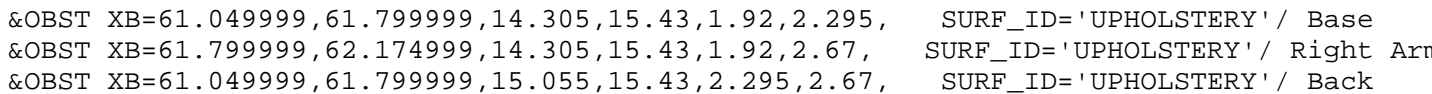

*** GROUP: CHAIR offset $=7.500000,0.000000,0.140000$ rotate $=0.000000$

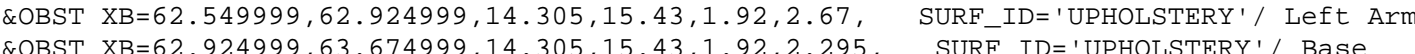

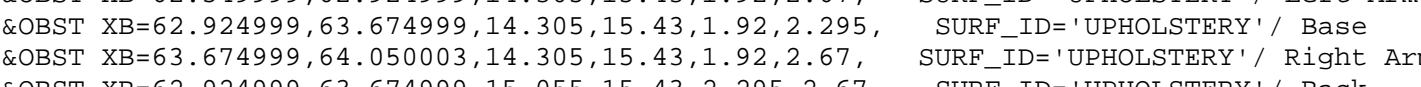

*** GROUP: CHAIR offset $=9.375000,0.000000,0.140000$ rotate $=0.000000$

\&OBST XB=64.425003, 64.800003,14.305, 15.43,1.92, 2.67, SURF_ID='UPHOLSTERY' ' Left Arm

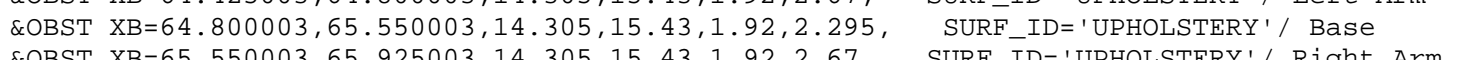

\&OBST XB=64. $8000003,65.550003,15,055,15,43,2.295,2.67$, SURF ID=' 'UPHOLSTERY' / Back

*** GROUP: LOVESEAT_STACK

$* * *$ GROUP: LOVESEAT_ROW

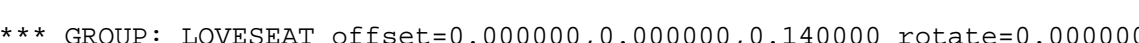

\&OBST XB=50.549999, 50.924999, 28.174999, 29. 299999, $0.28,1.03$, SURF_ID='UPHOLSTERY '/ Left Arm

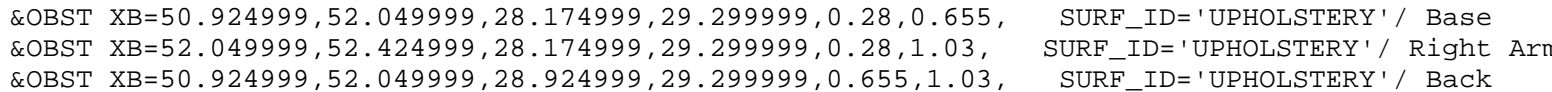

*** GROUP: LOVESEAT offset $=2.250000,0.000000,0.140000$ rotate $=0.000000$

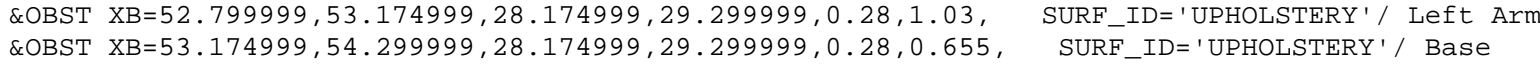

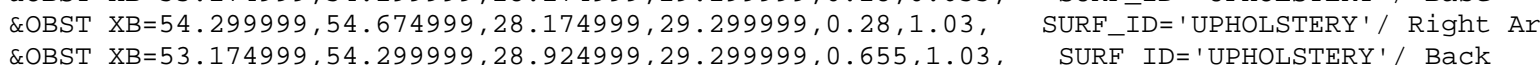

*** GROUP: LOVESEAT off set $=4.500000,0.000000,0.140000$ rotate $=0.000000$

\&OBST XB=55. 049999, 55.424999, 28.174999, 29. 299999, $0.28,1.03$, SURF_ID=' 'UPHOLSTERY ' / Left Arm \&OBST XB=55.424999, $56.549999,28.174999,29.299999,0.28,0.655$, SURF_ID='UPHOLSTERY' / Base

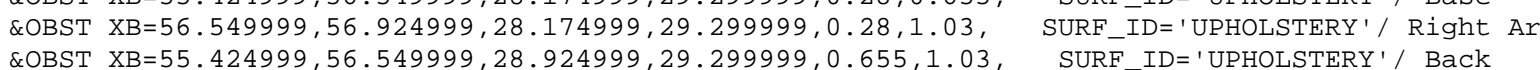

*** GROUP: LOVESEAT offset $=6.750000, \Theta .000000,0.140000$ rotate $=0.000000$

\&OBST XB=57.299999, 57.674999, 28.174999, 29. 299999, 0.28,1.03, SURF_ID=' 'UPHOLSTERY ' Left Arm LOBST XB=57.674999, $58.799999,28.174999,29.299999,0.28,0.655$, SURF_ID='UPHOLSTERY'/ Base \&OBST XB=57.674999, $58.799999,28.924999,29.299999,0.655,1.03$ S SURF ID='UPHOLSTERY'/ Back A

$* \star *$ GROUP: LOVESEAT of fset $=9.000000,0.000000,0.140000$ rotate $=0.000000$

\&OBST XB=59.549999, 59.924999, 28.174999, 29. 299999, 0.28,1.03, SURF_ID=' 'UPHOLSTERY'/ Left Arm

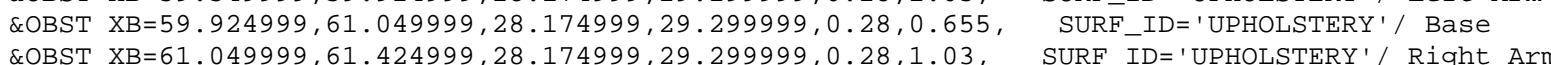
\&OBST XB=61. $049999,61.424999,28.174999,29.299999,0.28,1.03$,
\&OBST XB=59.924999, $61.049999,28.924999,29.299999,0.655,1.03, \quad$ SURF_ID=' 'UPHOLSTERY'/ Right Arm
SURF_ID=' 'UPHOLSTERY'/ Back

*** GROUP: LOVESEAT offset $=11.250000,0.000000,0.140000$ rotate $=0.000000$

\&OBST XB=61.799999, 62.174999, 28.174999, 29. 299999, 0. 28,1.03, SURF_ID=' UPHOLSTERY'/ Left Ar

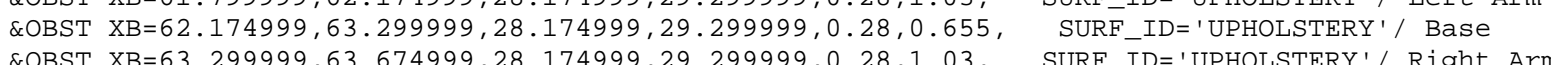
EOBST XB=62.174999, 63. 299999, 28.924999, 29.299999, $0.655,1.03$, SURF_ID=' 'UPHOLSTERY'/ Right Ar

$* * *$ GROUP: LOVESEAT offset $=13.500000,0.000000,0.140000$ rotate $=0.000000$

\&OBST XB=64. 050003, 64.425003, 28.174999, 29. 299999, $0.28,1.03$, SURF ID=' 'UPHOLSTERY ' / Left Arm

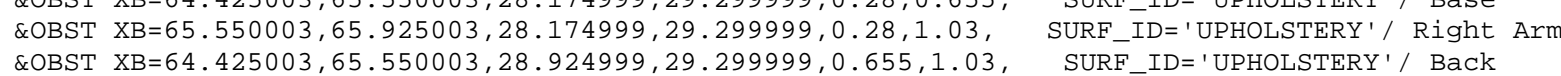

$* * *$ GROUP: LOVESEAT_ROW

*** GROUP: LOVESEAT offset $=0.000000, \odot . .000000,0.140000$ rotate $=0$. ๑ 000000

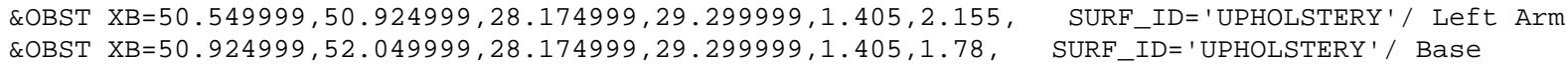
\&OBST XB $=52.049999,52.424999,28.174999,29.299999,1.405,2.155$, SURF $=$ ID='UPHOLSTERY'/ Right Arm *** GROUP: LOVESEAT offset $=2.250000,0.000000,0.140000$ rotate $=0.000000$

\&OBST XB=52.799999, 53.174999, 28.174999, 29.299999, 1.405, 2.155, SURF_ID='UPHOLSTERY'/ Left Arm

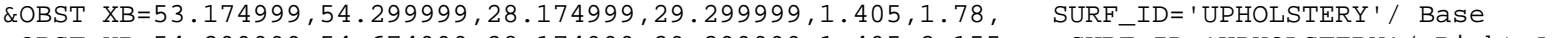
\&OBST XB $54.299999,54.674999,28.174999,29.299999,1.405,2.155, \quad$ SURF ID='UPHOLSTERY' $/$ Right Ar

*** GROUP: LOVESEAT off set $=4.500000,0.000000,0.140000$ rotate $=0.000000$

\&OBST XB=55. 049999, 55.424999, 28.174999, 29.299999, 1.405, 2.155, SURF_ID=' 'UPHOLSTERY'/ Left Arm 
\&OBST XB $=55.424999,56.549999,28,924999,29,299999,1.78,2.155$, SURE_ID=' UPHOLSTERY' / Back ** GROUP: LOVESEAT off set $=6.750000,0.000000,0.140000$ rotate $=0.000000$

\&OBST XB $=57.299999,57.674999,28.174999,29.299999,1.405,2.155$, SURF_ID=' 'UPHOLSTERY ' / Left Arm COBST XB $=58.799999,59.174999,28.174999,29.299999,1.405,2.155$, SURF_ID=' 'UPHOLSTERY'/ Right Arm

*** GROUP: LOVESEAT of $\mathrm{s}$ set $=9.000000,0.000000,0.140000$ rotate $=0.000000$

\&OBST XB $=59.549999,59.924999,28.174999,29.299999,1.405,2.155$, SURF_ID=' 'UPHOLSTERY' / Left Arm \&OBST XB=59.924999, 61. $049999,28.174999,29.299999,1.405,1.78$, SURF_ID='UPHOLSTERY' / Base QOBST XB=61.04999, 61.424999, 28.174999, 29.299999, 1.405, 2.155, SURF_ID='UPHOLSTYRY/Right Ar

$* * *$ GROUP: LOVESEAT of $\mathrm{fset}=11.250000,0.000000,0.140000$ rotate $=0.000000$

\&OBST XB=61.799999, 62.174999, 28.174999, 29. 299999, 1.405, 2.155, SURF_ID=' UPHOLSTERY' / Left Arm OBST XB=63.299999,63.674999, 28.174999, 29.299999,1.405, 1.78, SURF_ID=' 'UPHOLSTERY'/ Base SURF_ID=' 'UPHOLSTERY'/ Right Arm
SURF_ID='UPHOLSTERY'/ Back $* * *$ GROUP: LOVESEAT offset $=13.500000,0.0000000,0.140000$ rotate $=0.000000$

\&OBST XB=64. $050003,64.425003,28.174999,29.299999,1.405,2.155$, SURF_ID=' 'UPHOLSTERY' / Left Arm \&OBST XB=65.550003,65.925003, 28.174999, 29.299999, 1.405, 2.155, SURF_ID=' 'UPHOLSTERY'/ Right Ar

\section{*** GROUP: LOVESEAT_STACK2}

** GROUP: LOVESEAT_SHORT_ROW

*** GROUP: LOVESEAT of fset $=0.000000,0.000000,0.140000$ rotate $=0.000000$

\&OBST XB=63.299999, 63.674999, 25.549999, 26. 674999, 0.28,1.03, SURF_ID=' 'UPHOLSTERY' / Left Arm

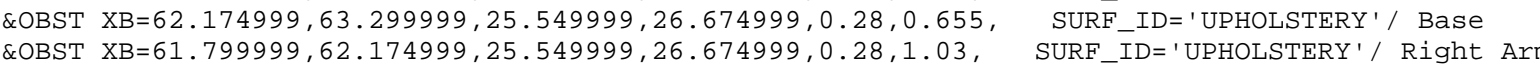
\&OBST XB $=62.174999,63.299999,25.549999,25.924999,0.655,1.03$, SURF_ID=' 'UPHOLSTERY' / Back

$* * *$ GROUP: LOVESEAT off set $=2.250000,0.000000,0.140000$ rotate $=0.000000$

عOBST XB=61. $049999,61.424999,25.549999,26.674999,0.28,1.03$, SURF_ID=' 'UPHOLSTERY '/ Left Arm

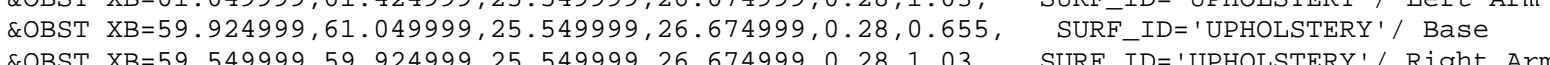

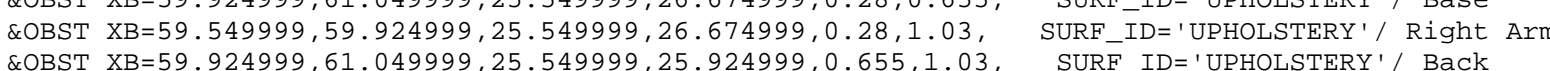

$* * *$ GROUP: LOVESEAT offset $=4.500000,0.000000,0.140000$ rotate $=0.000000$

\&OBST XB=58.799999, 59.174999, 25.549999, 26.674999, 0.28,1.03, SURF_ID='UPHOLSTERY' / Left Arm

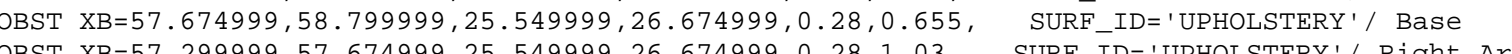

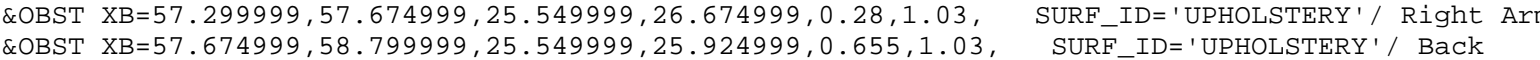

*** GROUP: LOVESEAT of $\mathrm{set}=6.750000,0.000000,0.140000$ rotate $=0.000000$

\&OBST XB=56.549999, 56.924999, 25.549999, 26.674999, $0.28,1.03$, SURF_ID=' 'UPHOLSTERY ' ' Left Arm

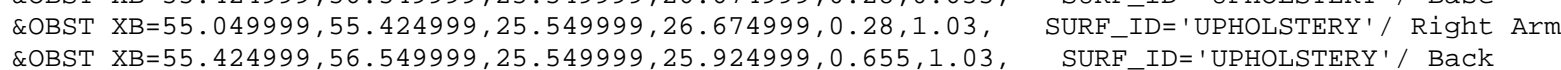

*** GROUP: LOVESEAT of fset $=9.000000,0.000000,0.140000$ rotate $=0 . .000000$

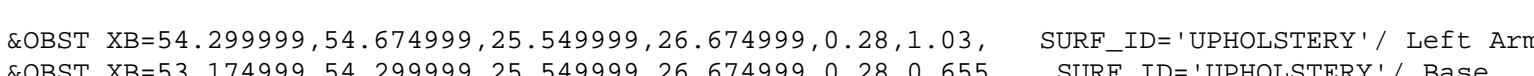
\&OBST XB=52.79999, $53.174999,25.549999,26.674999,0.28,1.03$,
\&OBST XB $\quad$ SURF_ID = 'UPHOLSTERY'/ Right Arm
SURF_ID ='UPHOLSTERY'/ Back

*** GROUP: LOVESEAT_SHORT_ROW

*** GROUP: LOVESEAT offset $=0.0000000,0.000000,0.140000$ rotate $=0.000000$

\&OBST XB $=63.299999,63.674999,25.549999,26.674999,1.405,2.155$, SURF_ID=' 'UPHOLSTERY ' / Left Arm \&OBST XB $=62.174999,63.299999,25.549999,26.674999,1.405,1.78, \quad$ SURF - ID $=$ 'UPHOLSTERY' 1 ' Base
\&OBST XB=62.174999, 63. 299999, 25.549999, 25, 924999, 1.78, 2.155, SURF_ID=' UPHOLSTERY'/ Back ** GROUP: LOVESEAT of $f$ set $=2.250000,0.000000,0.140000$ rotate $=0.000000$

\&OBST XB=61. 449999, 61. 424999, 25.549999, 26.674999, 1.405, 2.155, SURF_ID='UPHOLSTERY'/ Left Arm

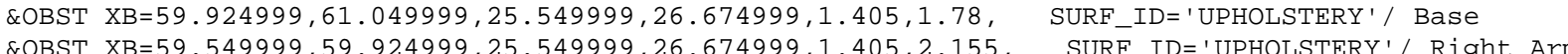
2OBST XB-59.54999,

*** GROUP: LOVESEAT of f set $=4.500000,0.000000,0.140000$ rotate $=0.000000$

\&OBST XB=58.799999, 59.174999, 25.549999, 26.674999, 1.405, 2.155, SURF_ID=' 'UPHOLSTERY'/ Left Arm \&OBST XB $57.674999,58.799999,25.549999,26.674999,1.405,1.78, \quad$ SURF_ID='UPHOLSTERY'/ Base \&OBST XB=57.29999, 57.674999, 25.54999, 26.674999, $1.405,2.155$, SURF_ID='UPHOLSTERY'/ Right
\&OBST XB=57.674999, $58.799999,25.549999,25.924999,1.78,2.155$,

$* * *$ GROUP: LOVESEAT off set $=6.750000,0.000000,0.140000$ rotate $=0.000000$

\&OBST XB=56.549999, 56.924999, 25.549999, 26.674999, 1.405, 2.155, SURF_ID=' UPHOLSTERY'/ Left Arm

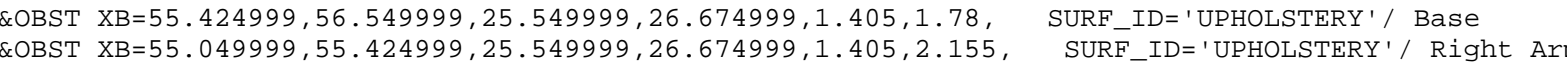
\&OBST XB $=55.424999,56.549999,25.549999,25.924999,1.78,2.155$, SUPF-ID='UPHOLSTERY $/$ Back

$\star \star *$ GROUP: LOVESEAT of fset $=9.000000,0.000000,0.140000$ rotate $=0.000000$

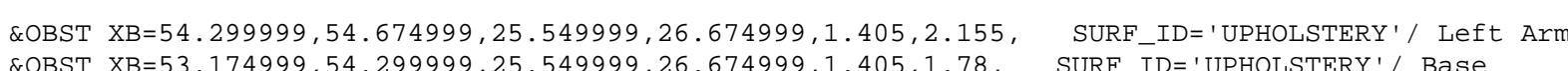

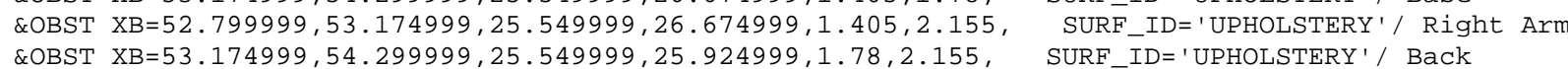

*** GROUP: LOVESEAT_STACK2 FLIP

*** GROUP: LOVESEAT_SHORT_ROW

*** GROUP: LOVESEAT off $\mathrm{set}=0.000000,0.000000,0.140000$ rotate $=0.000000$

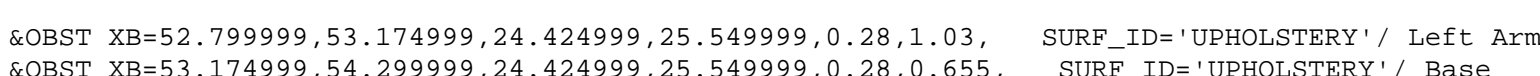
\&OBST XB=54.299999, 54.674999, 24.424999, 25.549999, $0.28,1.03, \quad$ SURF_ID=' 'UPHOLSTERY'/ Right Arm
\&OBST XB=53.174999, $54.299999,25.174999,25.549999,0.655,1.03, \quad$ SURF ID=' 'UPHOLSTERY'/ Back

$* * *$ GROUP: LOVESEAT offset $=2.250000,0.000000,0.140000$ rotate $=0.000000$

\&OBST XB=55. $049999,55.424999,24.424999,25.549999,0.28,1.03$, SURF_ID=' 'UPHOLSTERY' ' Left Arm

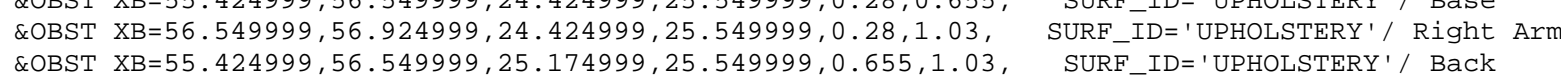

*** GROUP: LOVESEAT off set $=4.500000,0.000000,0.140000$ rotate $=0.000000$

\&OBST XB=57. 299999, 57.674999, 24.424999, 25.549999, 0.28,1.03, SURFID=' 'UPHOLSTERY' ' Left Arm

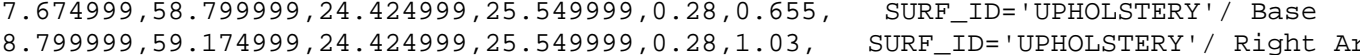
\&OBST XB=57.674999, 58.799999, 25.174999, 25.549999, 0.655, 1. 03, SURF_ID=' 'UPHOLSTERY'/ Back

*** GROUP: LOVESEAT off $\mathrm{set}=6.750000,0.000000,0.140000$ rotate $=0.000000$

\&OBST XB=59.549999, 59. 924999, 24.424999, 25.549999, $0.28,1.03$, SURF_ID= 'UPHOLSTERY ' / Left Arm \&OBST XB=61. $049999,61.424999,24.424999,25.5$ \&OBST XB=59.924999, 61.049999, 25.174999, 25.549999, $0.655,1.03$, SURF_ID='UPHOLSTERY'/ Back

*** GROUP: LOVESEAT offset $=9.000000,0.000000,0.140000$ rotate $=0.000000$

\&OBST XB=61.799999, 62.174999, 24.424999, 25.549999, $0.28,1.03, \quad$ SURF_ID=' 'UPHOLSTERY' $/$ Left Arm
\&OBST XB=62.174999, 63.299999, 24.424999, $25.549999,0.28,0.655, \quad$ SURF ID=' 'UPHOLSTERY' Rase

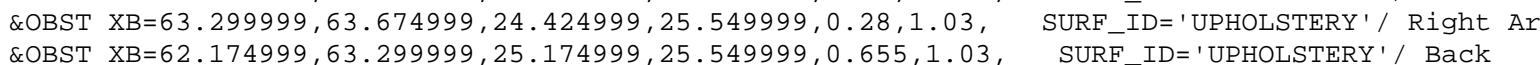

*** GROUP: LOVESEAT_SHORT_ROW

*** GROUP: LOVESEAT offset $=0.000000,0.000000,0.140000$ rotate $=0.000000$

\&OBST XB=52.799999, 53.174999, 24.424999, 25.549999, 1.405, 2.155, SURF_ID=' 'UPHOLSTERY ' / Left Arm

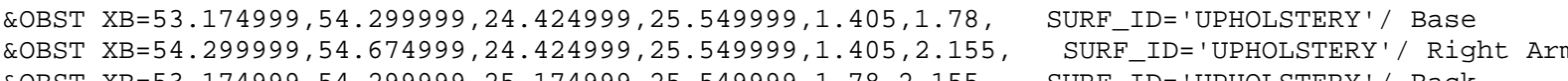


*** GROUP: LOVESEAT offset $=2.250000,0.000000,0.140000$ rotate $=0.000000$

\&OBST $X B=55.049999,55.424999,24.424999,25.549999,1.405,2.155, \quad$ SURF_ID=' 'UPHOLSTERY ' / Left Arm \&OBST XB=56.549999, 56. $524999,24.424999,25.549999,1.405,2.155$, SURF_ID=' UPHOLSTERY'/ Right Ar \&OBST XB=55.424999, 56.549999, 25.174999, 25.549999, 1.78, 2.155, SURF_ID='UPHOLSTERY'/ Back $\star \star *$ GROUP: LOVESEAT off set $=4.500000,0.000000,0.140000$ rotate $=0.000000$

\&OBST XB=57.299999, 57.674999, 24.424999, 25.549999, 1.405, 2.155, SURF_ID= 'UPHOLSTERY '/ Left Arm
\&OBST XB=57.674999, $58.799999,24.424999,25.549999,1.405,1.78, \quad$ SURF_ID='UPHOLSTERY' $/$ Base

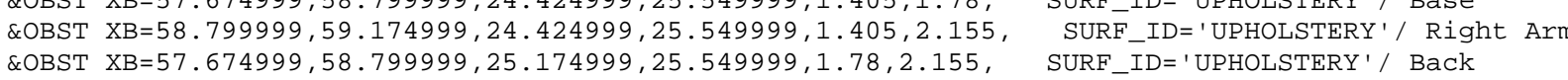

$* * *$ GROUP: LOVESEAT of fset $=6.750000,0.000000,0.140000$ rotate $=0.000000$

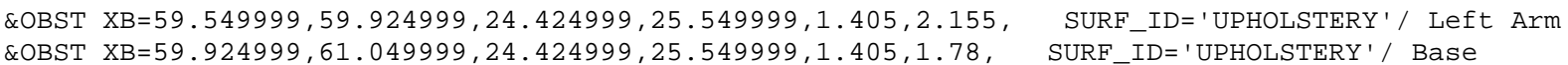

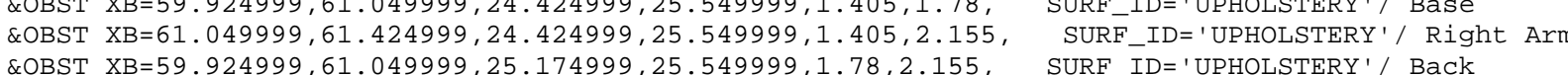
*** GROUP: LOVESEAT off $\mathrm{set}=9.000000, \Theta . \bullet 00000,0.140000$ rotate $=0.000000$

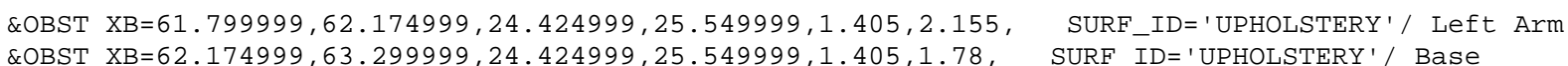

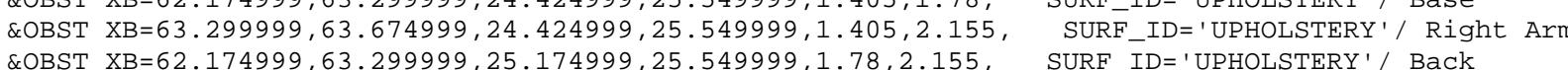

*** GROUP: LOVESEAT_STACK2

*** GROUP: LOVESEAT_SHORT_ROW

** GROUP: LOVESEAT of fset $=0.000000,0.000000,0.140000$ rotate $=0.000000$

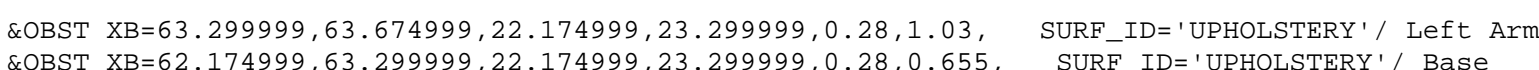
\&OBST XB $61.799999,62.174999,22.174999,23.299999,0.28,1.03$, SURF_ID=' 'UPHOLSTERY'/ Right Arm
\&OBST XB=62.174999, 63. 299999, 22.17499, 22.549999, $0.655,1.03$, SURF_ID 'UPHOLSTERY' $/$ Back

*** GROUP: LOVESEAT of fset $=2.250000,0.000000,0.140000$ rotate $=0.000000$

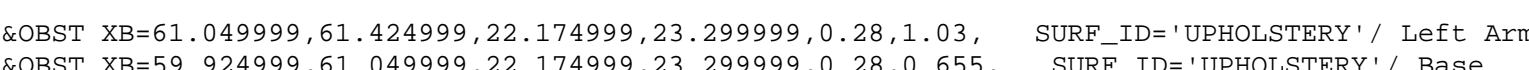

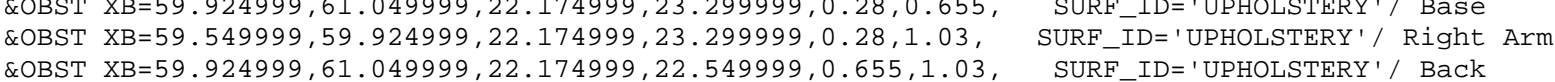

*** GROUP: LOVESEAT offset $=4.500000,0.000000,0.140000$ rotate $=0.000000$

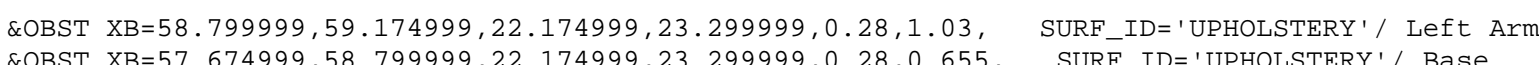

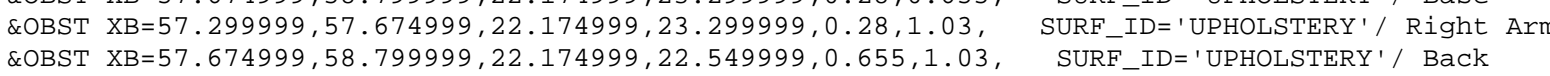

*** GROUP: LOVESEAT off set $=6.750000,0.000000,0.140000$ rotate $=0.000000$

\&OBST XB $=56.549999,56.924999,22.174999,23.299999,0.28,1.03, \quad$ SURF_ID= 'UPHOLSTERY' 1 ' Left Arm
ROBST XB

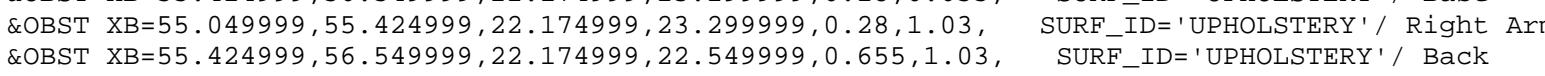

*** GROUP: LOVESEAT offset $=9.000000,0.000000,0.140000$ rotate $=0.000000$

\&OBST XB=54.299999, 54. 674999, 22.174999, 23. 299999, $0.28,1.03, \quad$ SURF_ID='UPHOLSTERY ' ' Left Arm
\&OBST XB=53.174999, $54.299999,22.174999,23.299999,0.28,0.655, \quad$ SURF_ID=' 'UPHOLSTERY' / Base

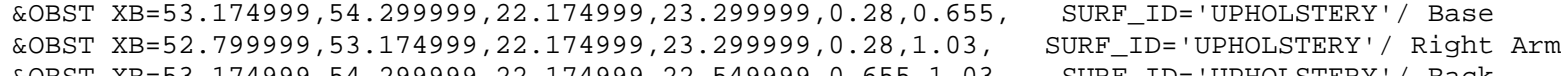

*** GROUP: LOVESEAT_SHORT_ROW

*** GROUP: LOVESEAT of fset $=0.000000,0.000000,0.140000$ rotate $=0.000000$

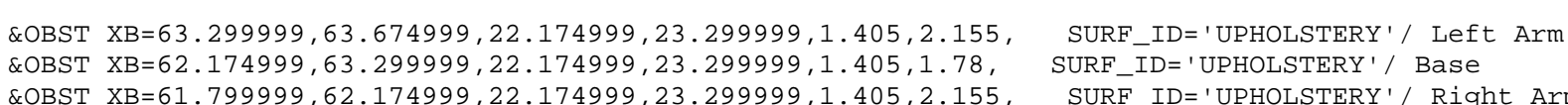

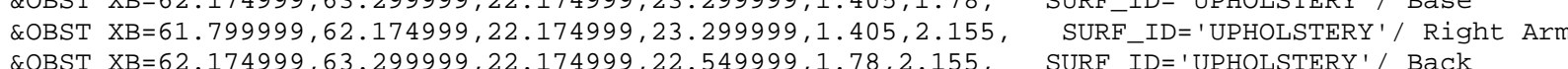

*** GROUP: LOVESEAT offset $=2.250000,0,000000, \Theta .140000$ rotate $=0,000000$

\&OBST XB=61. 0499999, 61.424999, 22.174999, 23.299999, 1.405, 2.155, SURF_ID=' 'UPHOLSTERY ' / Left Arm

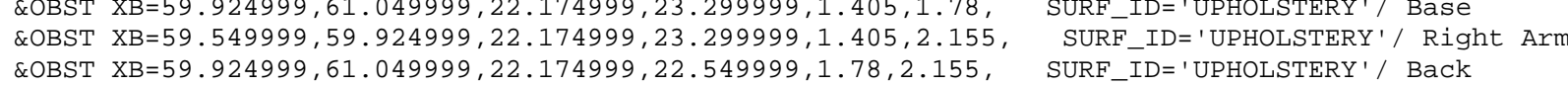

$* * *$ GROUP: LOVESEAT offset $=4.500000,0.000000,0.140000$ rotate $=0 . .000000$

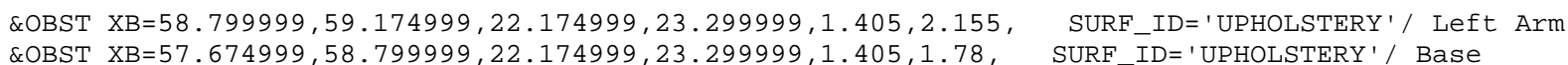

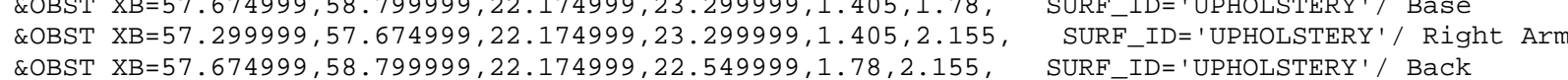

*** GROUP: LOVESEAT offset $=6.750000,0.000000,0.140000$ rotate $=0.000000$

\&OBST XB=56.549999, 56.924999, 22.174999, 23.299999, 1.405, 2.155, SURF_ID=' 'UPHOLSTERY '/ Left Arm

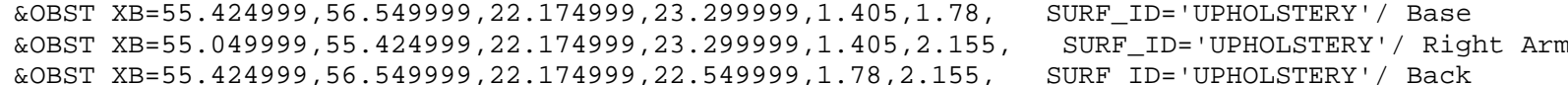

*** GROUP: LOVESEAT offset $=9.000000,0.000000,0.140000$ rotate $=0.000000$

\&OBST XB $=54.299999,54.674999,22.174999,23.299999,1.405,2.155, \quad$ SURF_ID= 'UPHOLSTERY ' / Left Arm
\&OBST XB $\mathrm{X}=53.174999,54.29999,22.174999,23.29999,1.405,1.78$,
SURF $\mathrm{ID}=$ 'UPHOLSTERY'

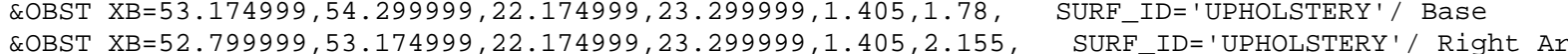

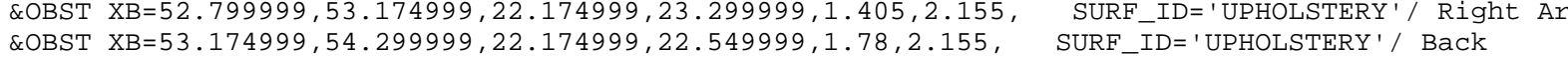

*** GROUP: LOVESEAT_STACK2_FLIP

*** GROUP: LOVESEAT_SHORT_ROW

$* * *$ GROUP: LOVESEAT offset $=0.000000,0.000000,0.140000$ rotate $=0 ., 000000$

\&OBST $X \mathrm{XB}=52.799999,53.174999,21.049999,22.174999,0.28,1.03, \quad$ SURF_ID=' 'UPHOLSTERY'/ Left Arm
\&OBST XB $X 53.174999,54.299999,21.049999,22.174999,0.28,0.655, \quad$ SURF ID=' 'UPHOLSTERY'/ Base \&OBST XB=54.299999, $54.674999,21.049999,22.174999,0.28,1.03$, SURE IDD='UPHOLSTERY' \&OBST XB=53.174999, 54.299999, 21.799999, 22.174999, $0.655,1.03$, SURF_ID=' 'UPHOLSTERY' / Back

** GROUP: LOVESEAT off set $=2.250000,0.000000,0.140000$ rotate $=0.000000$

\&OBST XB=55. $049999,55.424999,21.049999,22.174999,0.28,1.03, \quad$ SURF_ID='UPHOLSTERY' ' Left Arm
\&OBST XB=55.424999, $56.549999,21.04999,22.174999,0.28,0.655, \quad$ SURF_ID=' 'UPHOLSTERY'

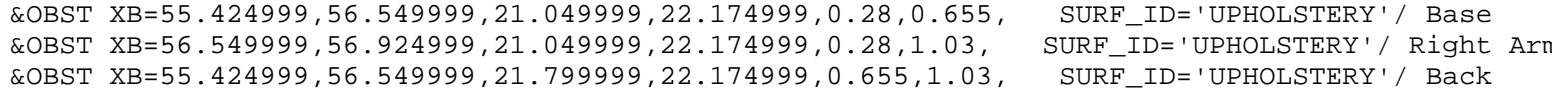

$* * *$ GROUP: LOVESEAT offset $=4.500000,0.000000,0.140000$ rotate $=0.0000000$

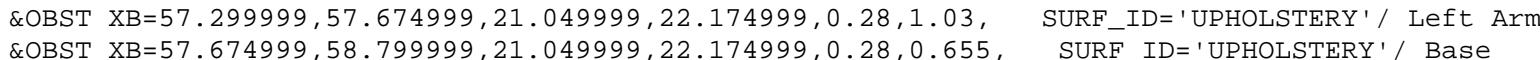

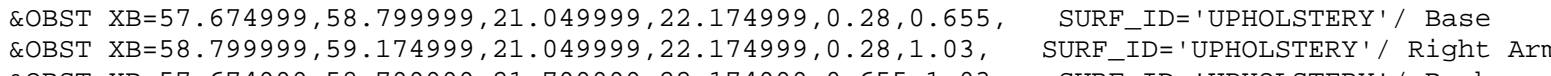

*** GROUP: LOVESEAT offset $=6.750000,0.000000,0.140000$ rotate $=0.000000$

\&OBST XB=59.549999, 59. 924999, 21. 049999, 22.174999, 0.28, 1.03, SURF_ID='UPHOLSTERY' / Left Arm

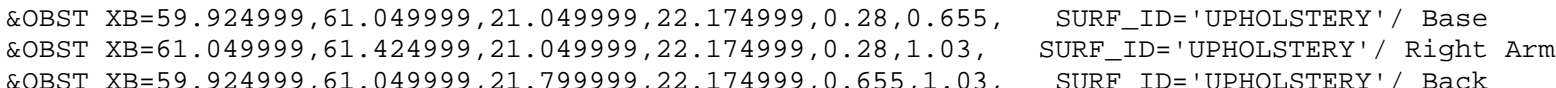

*** GROUP: LOVESEAT of fset $=9.000000, \Theta .000000,0.140000$ rotate $=0.0000000$

\&OBST XB=61.799999, 62.174999, 21.049999, 22.174999, 0.28,1.03, SURF_ID=' 'UPHOLSTERY'/ Left Arm \&OBST XB $=62.174999,63.299999,21.049999,22.174999,0.28,0.655$, SURF_ID='UPHOLSTERY' $/$ Base \&OBST XB $=62.174999,63.299999,21.799999,22.174999,0.2655,1.03$, SUPF_ID='UPHOISTEPY'/

*** GROUP: LOVESEAT_SHORT_ROW

*** GROUP: LOVESEAT offset $=0.000000,0.000000,0.140000$ rotate $=0.000000$

\&OBST XB=52.799999, 53.174999, 21. 049999, 22.174999, 1.405, 2.155, SURF_ID=' 'UPHOLSTERY '/ Left Arm \&OBST XB $=54.299999,54.674999,21.049999,22.174999,1.405,2.155$, SURF IDE='UPHOLSTERY'/Right Ar \&OBST XB=53.174999, 54.299999, 21.799999, 22.174999, 1.78, 2.155, SURF_ID='UPHOLSTERY'/ Back 
** GROUP: LOVESEAT Offset=2.250000, 0.000000, 0.140000 rotate $=0.000000$

\&OBST XB $=55.049999,55.424999,21.049999,22.174999,1.405,2.155$,
\&OBST XB=55.424999, $56.549999,21.049999,22.174999,1.405,1.78$,
SURF_IDE=' 'UPHOLSTERTY'/ Base \&OBST XB $=56.549999,56.924999,21.049999,22.174999,1.405,2.155$, SURF_ID=' UPHOLSTERY'/ Right Arm

*** GROUP: LOVESEAT offset $=4.500000,0.000000,0.140000$ rotate $=0.000000$

\&OBST XB $=57.299999,57.674999,21.049999,22.174999,1.405,2.155, \quad$ SURF_ID=' 'UPHOLSTERY '/ Left Arm
SORST XB

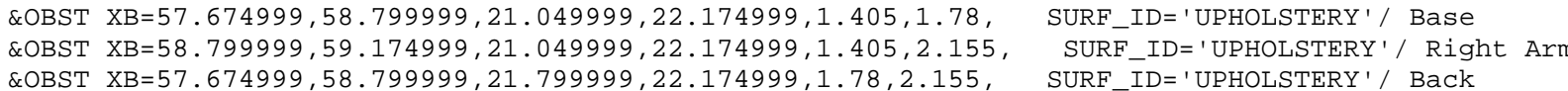

$* * *$ GROUP: LOVESEAT off set $=6.750000,0.000000,0.140000$ rotate $=0.000000$

\&OBST XB $=59.549999,59.924999,21.049999,22.174999,1.405,2.155$, SURF_ID='UPHOLSTERY'/ Left Arm \&OBST XB $59.924999,61.042999,21.049999,22.174999,1.405,1.78$, SURF_ID='UPHOLSTERY'/ Base \&OBST XB $=61.04999,61.42499,21.049999,22.174999,1.405,2.155, \quad$ SURF_ID='UPHOLSTERY'/Right Ar
\&OBST XB $=59.924999,61.049999,21.799999,22.174999,1.78,2.155$, SURF_ID='UPHOLSTERY'/ Back $* * *$ GROUP: LOVESEAT offset $=9.000000,0.000000,0.140000$ rotate $=0.000000$

\&OBST XB=61.799999,62.174999, 21.049999, 22.174999, 1.405, 2.155, SURF_ID=' 'UPHOLSTERY'/ Left Arm \&OBST XB=62.174999, 63. 299999, 21. 449999, 22.174999, 1.405, 1.78, SURF_IDD='UPHOLSTERY'/ Base \&OBST $X$ B

*** GROUP: LOVESEAT_STACK2

$* * *$ GROUP: LOVESEAT_SHORT_ROW

** GROUP: LOVESEAT offset $=0.000000,0.000000,0.140000$ rotate $=0.000000$

\&OBST XB=63.299999, 63.674999, 18.799999, 19.924999, $0.28,1.03$, SURF_ID=' 'UPHOLSTERY'/ Left Arm \&OBST XB $=61.799999,62.174999,18.799999,19.929999,0.28,1.03$, SURF ID='UPHOLSTERY'/ Rige A \&OBST XB $=62.174999,63.299999,18.799999,19.174999,0.655,1.03$, SURF_ID='UPHOLSTERY' / Back

*** GROUP: LOVESEAT offset $=2.250000,0.000000,0.140000$ rotate $=0.000000$

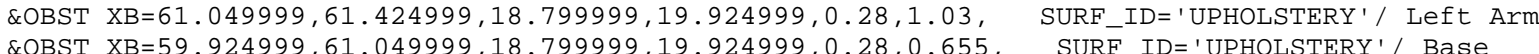
COBST XB
QOB $59.549999,59.924999,18.799999,19.924999,0.28,1.03$,

*** GROUP: LOVESEAT off set $=4.500000,0.000000,0.140000$ rotate $=0.000000$

\&OBST XB=58.799999, 59.174999, 18.799999, 19.924999, $0.28,1.03$, SURF_ID=' 'UPHOLSTERY'/ Left Arm

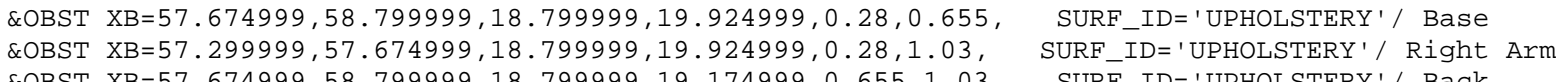

*** GROUP: LOVESEAT of $f$ set $=6.750000,0.000000,0.140000$ rotate $=0$. .

\&OBST XB=56.549999, 56. 924999, 18.799999, 19.924999, $0.28,1.03, \quad$ SURF_ID='UPHOLSTERY '/' Left Arm
\&OBST XB $\mathrm{XB}=55.424999,56.549999,18.799999,19.924999,0.28,0.655, \quad$ SURF_ID=' 'UPHOLSTERY' / Base

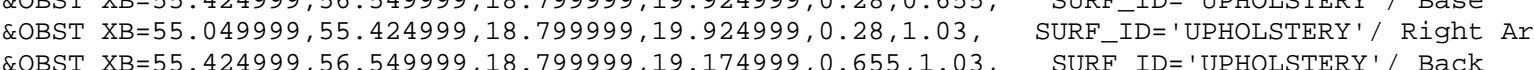

*** GROUP: LOVESEAT off set $=9.000000$, $. .000000,0.140000$ rotate $=0.000000$

\&OBST XB=54.299999, 54.674999, 18.799999, 19.924999, $0.28,1.03, \quad$ SURF_ID='UPHOLSTERY'/ Left Arm
\&OBST XB=53.174999, $54.299999,18.799999,19.924999,0.28,0.655, \quad$ SURF_ID='UPHOLSTERY'/ Base \&OBST XB $=53.174999,54.299999,18.799999,19.924999,0.28,0.655, \quad$ SURF_ID='UPHOLSTERY'/ Base LOBST XB $=52.799999,53.174999,18.799999,19.924999,0.28,1.03$,
\&OBST XB $=53.174999,54.299999,18.799999,19.174999,0.655,1.03$,

*** GROUP: LOVESEAT_SHORT_ROW

*** GROUP: LOVESEAT of $f$ set $=0.000000,0.000000,0.140000$ rotate $=0.000000$

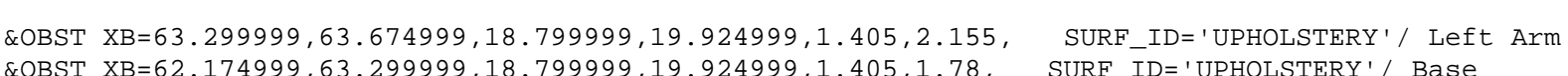

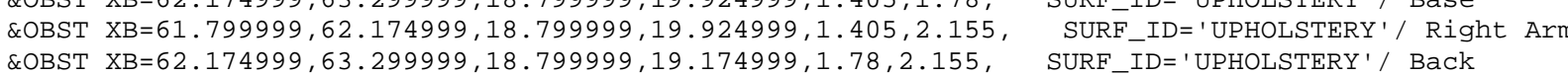

** GROUP: LOVESEAT offset $=2.250000,0.000000,0.140000$ rotate $=0$. .

\&OBST XB $=61.049999,61.424999,18.799999,19.924999,1.405,2.155, \quad$ SURF_ID=' 'UPHOLSTERY' / Left Arm
\&OBST XB $=59.924999,61.049999,18.799999,19.924999,1.405,1.78$,
SURF_ID='UPHOLSTERY'

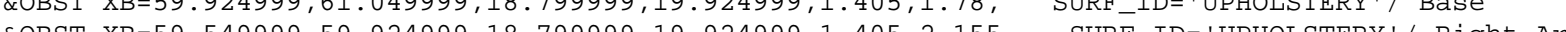
\&OBST XB=59.924999, 61.049999, 18.799999, 19.174999, 1.78, 2.155, SURF_ID='UPHOLSTERY'/ Back Ar

$* *$ GROUP: LOVESEAT off set $=4.500000,0.000000,0.140000$ rotate $=0.000000$

\&OBST XB $=58.799999,59.174999,18.799999,19.924999,1.405,2.155, \quad$ SURF_ID='UPHOLSTERY'/ Left Arm \&OBST XB $=57.674999,58.799999,18.799999,19.924999,1.405,1.78, \quad$ SURF_ID=' 'UPHOLSTERY'/ Base
\&OBST XB $=57.299999,57.674999,18.79999,19.924999,1.405,2.155, \quad$ SURF_IDD' 'UPHOLSTERY'/ Right Arm \&OBST XB=57.674999, 58.799999, 18.799999, 19.174999, 1.78, 2.155, SURF_ID='UPHOLSTERY'/ Back

*** GROUP: LOVESEAT offset $=6.750000,0.000000,0.140000$ rotate $=0.000000$

\&OBST XB $=56.549999,56.924999,18.799999,19.924999,1.405,2.155, \quad$ SURF_ID=' 'UPHOLSTERY'/ Left Arm
\&OBST XB $=55.424999,56.549999,18.799999,19.924999,1.405,1.78, \quad$ SURF_ID=' 'UPHOLSTERY' Base \&OBST XB=55. $449999,55.424999,18.799999,19.924999,1.405,2.155, \quad$ SURF_ID='UPHOLSTERY'/Right Ar
\&OBST XB=55.424999, $56.549999,18.799999,19.174999,1.78,2.155, \quad$ SURF_ID='UPHOLSTERY'/Back

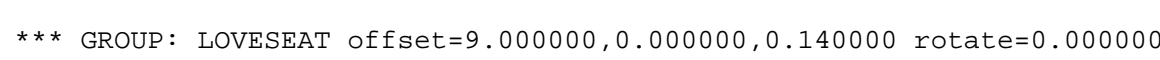

\&OBST XB=54.299999, 54.674999, 18.799999, 19.924999, 1.405, 2.155, SURF_ID=' UPHOLSTERY ' / Left Arm \&OBST XB=53.174999, $54.299999,18.799999,19.924999,1.405,1.78$, SURF_ID='UPHOLSTERY'/ Base

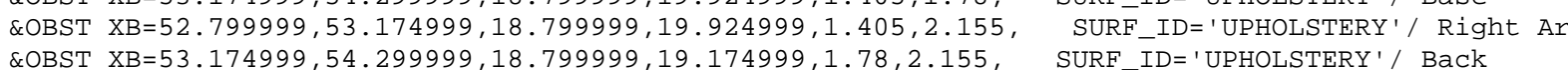

*** GROUP: LOVESEAT_STACK2_FLIP

$* * *$ GROUP: LOVESEAT_SHORT_ROW

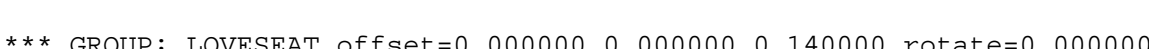

\&OBST XB=52.799999, 53.174999, 17.674999, 18.799999, $, 0.28,1.03, \quad$ SURF_ID='UPHOLSTERY' ' Left Arm
\&OBST XB $=53.174999,54.299999,17.674999,18.799999,0.28,0.655, \quad$ SURF_ID='UPHOLSTERY' $/$ Base

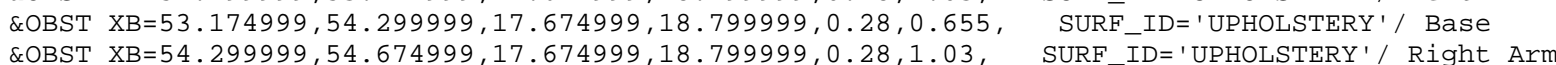

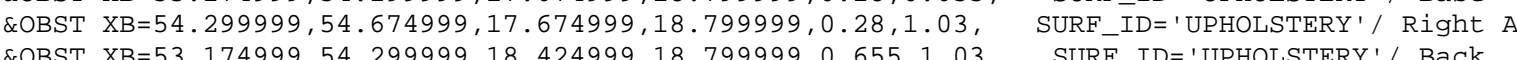

*** GROUP: LOVESEAT offset $=2.250000,0.000000,0.140000$ rotate $=0.000000$

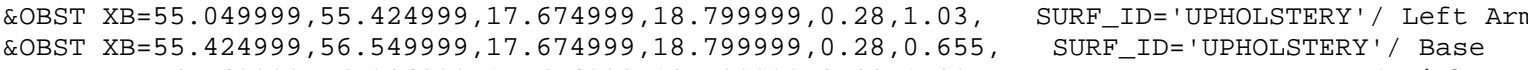

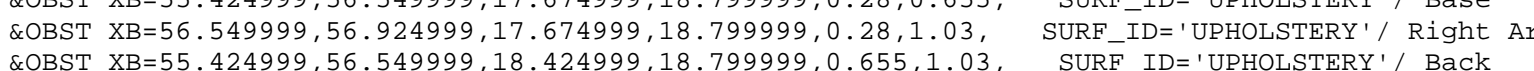

*** GROUP: LOVESEAT of fset $=4.500000,0.000000,0.140000$ rotate $=0.000000$

\&OBST XB=57.299999, 57.674999, 17.674999, 18.799999, 0.28,1.03, SURF_ID='UPHOLSTERY'/ Left Arm \&OBST XB=57.674999, 58.799999, 17.674999, 18.799999, 0.28, 0.655, SURF_ID='UPHOLSTERY'/ Base

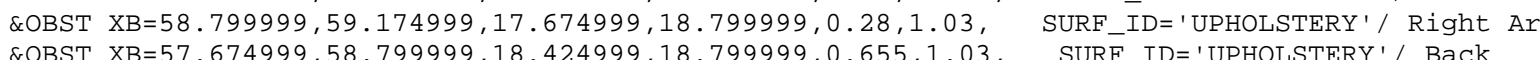

$* * *$ GROUP: LOVESEAT off $\mathrm{set}=6.750000,0.000000,0.140000$ rotate $=0.000000$

\&OBST XB=59.549999, 59. 924999, 17.674999, 18.799999, $0.28,1.03$, SURF ID=' 'UPHOLSTERY ' / Left Arm \&OBST XB $59.924999,61.049999,17.674999,18.799999,0.28,0.655$, SURF_ID=' 'UPHOLSTERY' / Base

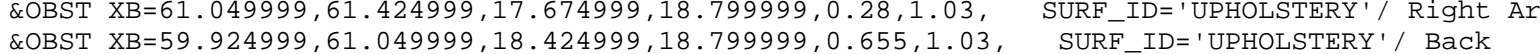

*** GROUP: LOVESEAT off set $=9.000000,0.000000,0.140000$ rotate $=0.000000$

\&OBST XB=61.799999, 62.174999, 17.674999, 18.799999, $0.28,1.03$, SURF_ID= 'UPHOLSTERY' ' Left Arm \&OBST XB $=62.174999,63.299999,17.674999,18.799999,0.28,0.655$, SURF_ID=' 'UPHOLSTERY' / Base \&OBST XB=63.29999, 63.674999, 17.674999, 18.799999, $0.28,1.03, \quad$ SURF_ID='UPHOLSTERY'/ Right Arm
\&OBST XB=62.174999, 63.299999, 18.424999, 18.799999, $0.655,1.03$,
SURF_ID='UPHOLSTERY'/ Back

*** GROUP: LOVESEAT_SHORT_ROW

*** GROUP: LOVESEAT off set $=0.000000,0.000000,0.140000$ rotate $=0.000000$

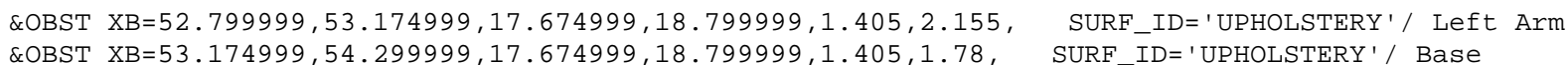

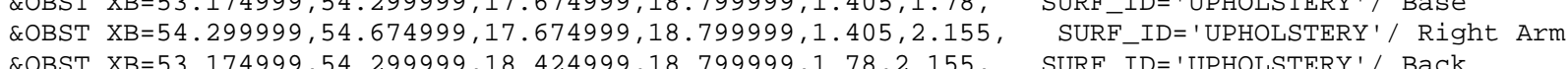

*** GROUP: LOVESEAT offset $=2.250000, \odot .000000,0.140000$ rotate $=0 ., 000000$ 
\&OBST XB $=55.049999,55.424999,17.674999,18.799999,1.405,2.155, \quad$ SURF_ID=' 'UPHOLSTERY '/ Left Arm
\&OBST XB $=55.424999,56.549999,17.674999,18.799999,1.405,1.78, \quad$ SURF_ID='UPHOLSTERY'
Base \&OBST XBE $56.549999,56.924999,17.674999,18.799999,1.405,2.155$, SURF_ID=' UPHOLSTERY '/ Right Ar $* * *$ GROUP: LOVESEAT offset $=4.500000,0.000000,0.140000$ rotate $=0.000000$

OOBST XB $=57.299999,57.674999,17.674999,18.799999,1.405,2.155$, SURF_ID='UPHOLSTERY '/ Left Arm

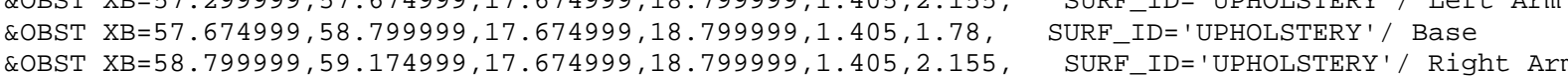
\&OBST XB=57.674999, $58,79999,17.64499,11877$

$* * \star$ GROUP: LOVESEAT offset $=6.750000,0.000000,0.140000$ rotate $=0.000000$

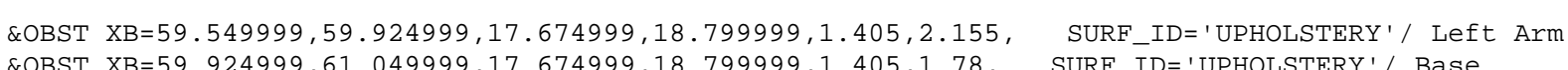
\&OBST XB=61.049999, 61.424999, 17.674999, 18.799999, 1.405, 2.155, SURF_ID='UPHOLSTERY'/ Right Ar *** GROUP: LOVESEAT offset $=9.000000,0.000000,0.140000$ rotate $=0.000000$

\&OBST XB=61.799999, 62.174999, 17.674999, 18.799999, 1.405, 2.155, SURF_ID='UPHOLSTERY'/ Left Arm \&OBST XB $=62.174999,63.299999,17.674999,18.799999,1.405,1.78$, SURF IDD='UPHOLSTERY' $/$ Base Arm

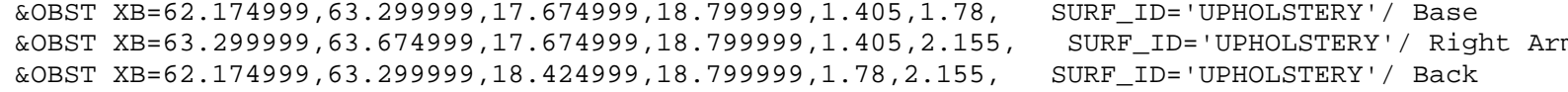

$* * *$ GROUP: LOVESEAT_STACK2_90

*** GROUP: LOVESEAT_SHORT_ROW

*** GROUP: LOVESEAT off $\mathrm{set}=0.000000,0.000000,0.140000$ rotate $=0.000000$

\&OBST XB $=64.800003,65.925003,27.419998,27.794998,0.28,1.03, \quad$ SURF_ID='UPHOLSTERY ' $/$ ' Left Arm
\&OBST XB $=64.800003,65.925003,26.294998,27.419998,0.28,0.655, \quad$ SURF_ID=' 'UPHOLSTERY' $/$ Base \&OBST XB $=64.800003,65.925003,25.919998,26.294998,0.28,1.03$, SURF-ID='UPHOLSTERY'/ Right Ar

$* * *$ GROUP: LOVESEAT offset $=2.250000,0.000000,0.140000$ rotate $=0.000000$

\&OBST $X \mathrm{XB}=64.800003,65.925003,25.169998,25.544998,0.28,1.03, \quad$ SURF_ID='UPHOLSTERY ' $/$ Left Arm
\&OBST $X \mathrm{~B}=64.800003,65.925003,24.044998,25.169998,0.28,0.655, \quad$ SURF_ID='UPHOLSTERY' / Base

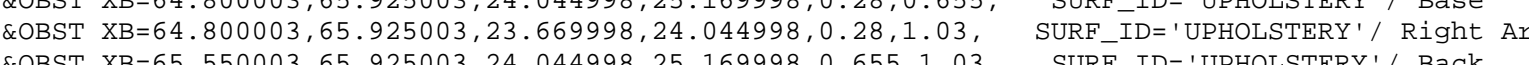

$* * *$ GROUP: LOVESEAT offset $=4.500000,0.000000,0.140000$ rotate $=0.000000$

\&OBST XB=64. 800003, 65. $925003,22.919998,23.294998,0.28,1.03, \quad$ SURF_ID= 'UPHOLSTERY' 1 ' Left Arm
\&OBST XB=64.800003, 65. $925003,21.794998,22.919998,0.28,0.655$,
SURF_ID=' 'UPHOLSTERY'/ Base \&OBST XB $=64.800003,65.925003,21.419998,21.794998,0.28,1.03$, SURF-ID=' 'UPHOLSTERY'/ Right Ar

${ }_{* * *}$ GROUP: LOVESEAT off $\mathrm{set}=6.750000,0.000000,0.140000$ rotate $=0.000000$

\&OBST $X B=64.800003,65.925003,20.669998,21.044998,0.28,1.03$, SURF_ID='UPHOLSTERY'/ Left Arm

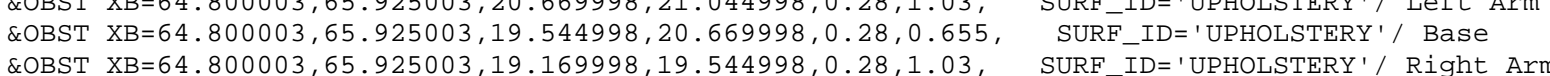

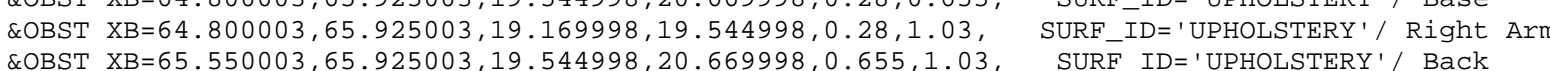

*** GROUP: LOVESEAT offset $=9.000000,0.000000,0.140000$ rotate $=0.000000$

\&OBST $X B=64.800003,65.925003,18.419998,18.794998,0.28,1.03$, SURF_ID=' 'UPHOLSTERY'/ Left Arm COBST $\mathrm{XB}=64.800003,65.925003,17.294998,18.419998,0.28,0.655, \quad$ SURF_ID=' 'UPHOLSTERY' / Base
COBST XB $\mathrm{X}=64.800003,65$

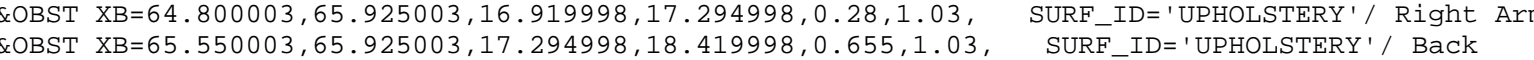

$* * *$ GROUP: LOVESEAT_SHORT_ROW

** GROUP: LOVESEAT of fset $=0.000000,0.0000000,0.140000$ rotate $=0.000000$

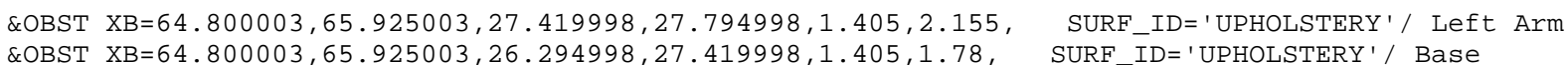

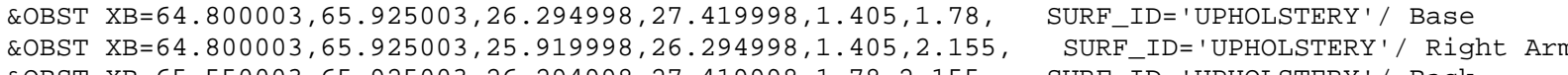

*** GROUP: LOVESEAT of $\mathrm{fset}=2.250000,0.000000,0.140000$ rotate $=0.000000$

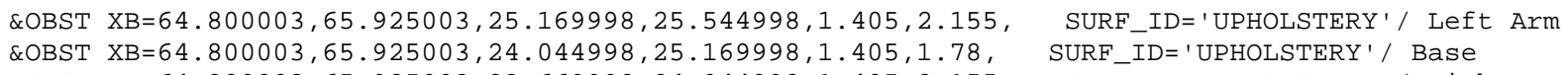
\&OBST XB=64.800003, 65.925003, 23.669998, 24.044998, 1.405,2.155, SURF_ID='UPHOLSTERY'/Right Ar

$* \star *$ GROUP: LOVESEAT offset $=4.500000,0.000000,0.140000$ rotate $=0.000000$

\&OBST XB $=64.800003,65.925003,22.919998,23.294998,1.405,2.155$, SURF_ID= 'UPHOLSTERY ' / Left Arm \&OBST XB=64.800003, 65. 925003, 21.794998, 22.919998, 1.405, 1.78, SURF_ID='UPHOLSTERY'/ Base

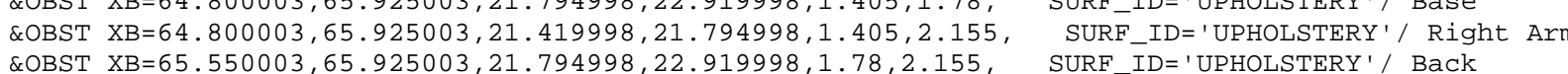

$* * *$ GROUP: LOVESEAT offset $=6.750000,0.000000,0.140000$ rotate $=0.000000$

\&OBST XB $=64.800003,65.925003,20.669998,21.044998,1.405,2.155$, SURF_ID=' 'UPHOLSTERY'/ Left Arm

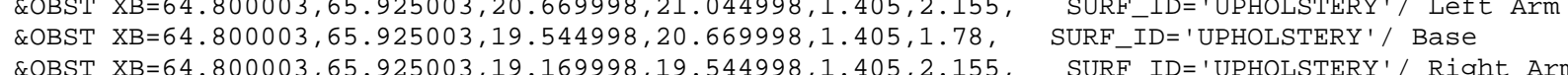
\&OBST XB $=65.550003,65.925093,19.544998,20.669998,1.78,2.155$, SURF_ID='UPHOLSTERY'/ Right Ar

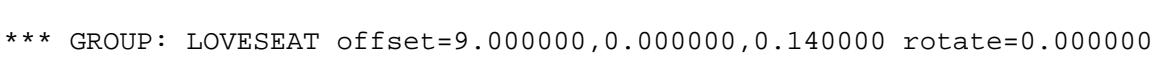

\&OBST XB=64.800003, 65.925003, 18.419998, 18.794998, $1.405,2.155$, SURF_ID=' 'UPHOLSTERY'/ Left Arm

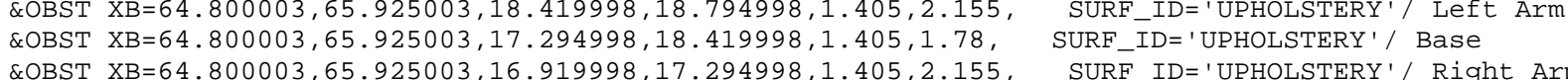

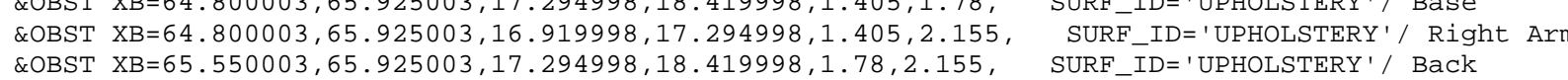

*** GROUP: LOVESEAT_STACK2

*** GROUP: LOVESEAT_SHORT_ROW

$* * *$ GROUP: LOVESEAT off $\mathrm{set}=0.000000,0.000000,0.140000$ rotate $=0.0000000$

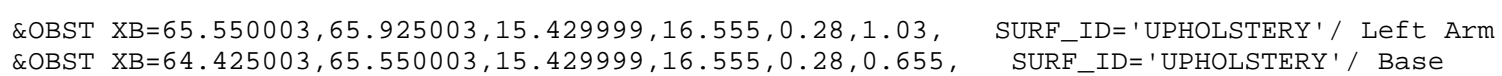

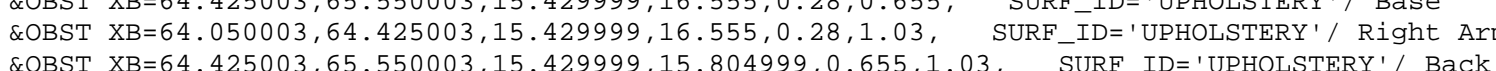

*** GROUP: LOVESEAT off $\mathrm{set}=2.250000,0.000000,0.140000$ rotate $=0.000000$ \&OBST XB $=63.299999,63.674999,15.429999,16.555,0.28,1.03, \quad$ SURF_ID= 'UPHOLSTERY' $/$ Left Arm
\&OBST XB $=62.174999,63.29999,15.429999,16.555,0.28,0.655, \quad$ SURF_ID='UPHOLSTERY' $/$ Base \&OBST XB=61.799999, 62.174999, 15.429999, 16.555, 0.28,1.03, SURF_ID=' 'UPHOLSTERY'/ Right Arm

*** GROUP: LOVESEAT off $\mathrm{set}=4.500000,0.000000,0.140000$ rotate $=0.000000$ \&OBST XB=61. $049999,61.424999,15.429999,16.555,0.28,1.03$, SURF.ID=' 'UPHOLSTERY' $/$ Left Arm \&OBST XB=59.924999, 61. 049999, 15.429999, 16. 555, 0.28, 0.655, SURF_ID=' 'UPHOLSTERY'/ Base

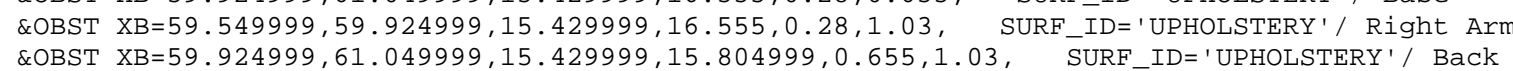

*** GROUP: LOVESEAT offset $=6.750000,0.000000,0.140000$ rotate $=0.000000$

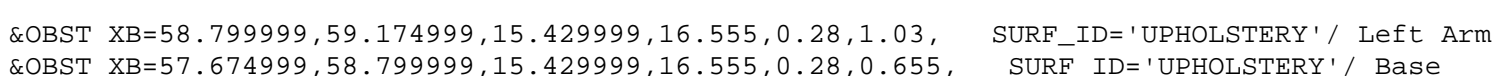
\&OBST XB=57.299999, $57.674999,15.429999,16.555,0.28,1.03$, SURF_ID='UPHOLSTERY'/Right Ar

*** GROUP: LOVESEAT off $\mathrm{set}=9.000000,0.000000,0.140000$ rotate $=0.000000$

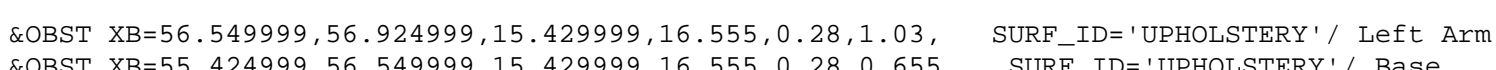

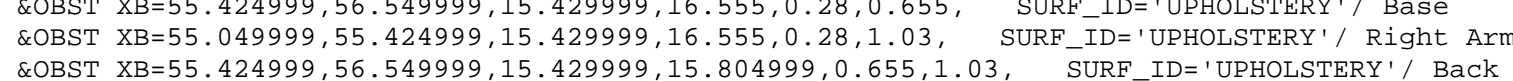

*** GROUP: LOVESEAT_SHORT_ROW

*** GROUP: LOVESEAT of $\mathrm{fset}=0.000000,0.0000000,0.140000$ rotate $=0.000000$

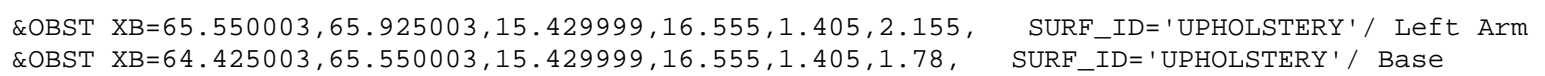

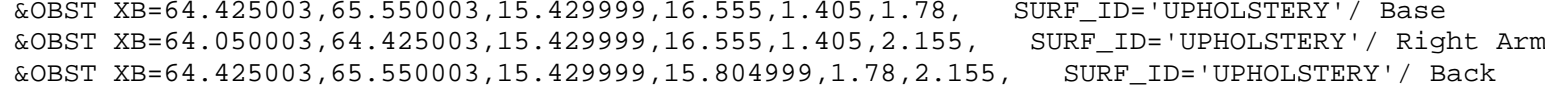

*** GROUP: LOVESEAT off $\mathrm{set}=2.250000,0.000000,0.140000$ rotate $=0.0000000$ 
\&OBST XB=63.299999, 63.674999, 15.429999, 16.555,1.405, 2.155, SURF_ID='UPHOLSTERY '/ Left Arm \&OBST $X \mathrm{X}=62.174999,63.299999,15.429999,16.555,1.405,1.78, \quad$ SURF_ID='UPHOLSTERY'/ Base
\&OBST XB $\mathrm{X}=61.799999,62.174999,15.429999,16.555,1.405,2.155$,
SURF ID='UPHOLSTERY'/ Right Ar \&OBST XB=61.79999, $62.174999,15.429999,16.555,1.405,2.155, \quad$ SURF_ID=' 'UPHOLSTERY'/ Right A
\&OBST XB $62.174999,63.299999,15.429999,15.804999,1.78,2.155, \quad$ SURF_ID='UPHOLSTERY' / Back

*** GROUP: LOVESEAT of $\mathrm{fset}=4.500000,0.000000,0.140000$ rotate $=0.000000$

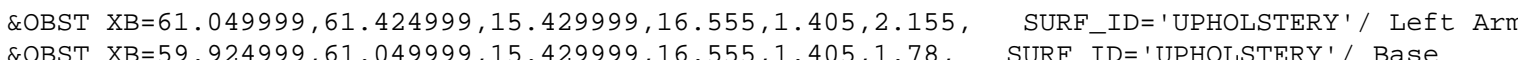
\&OBST XB=59.549999, 59.924999, 15. 429999, 16.555, 1.405, 2.155, SURF_ID='UPHOLSTERY'/Right Ar

*** GROUP: LOVESEAT offset $=6.750000,0.000000,0.140000$ rotate $=0.000000$

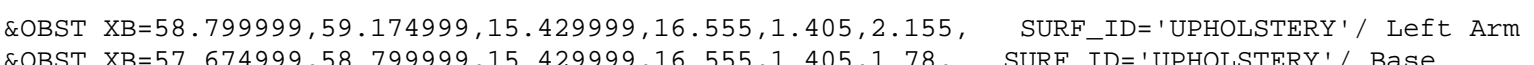
\&OBST XB=57. $299999,57.674999,15.429999,16.555,1.405,2.155, \quad$ SURF_ID=' 'UPHOLSTERY ' / Right Arm
\&OBST XB=57.674999, $58.799999,15.429999,15.804999,1.78,2.155$,
SURF_ID=' UPHOLSTERY' $/$ Back

$* * *$ GROUP: LOVESEAT of fset $=9.000000,0.000000,0.140000$ rotate $=0.000000$

EOBST XB=56.549999, 56. 924999, 15.429999, 16.555, 1.405, 2.155, SURF_ID='UPHOLSTERY'/ Left Arm

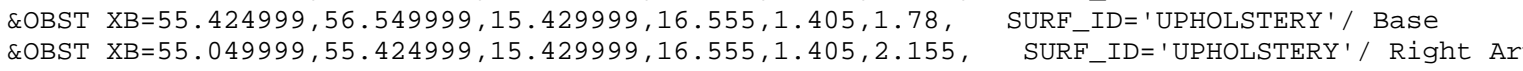

\section{*** GROUP: LHS_FRONT}

*** GROUP: SHORT_ROW2

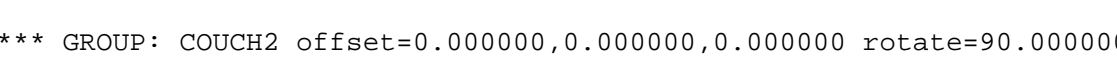

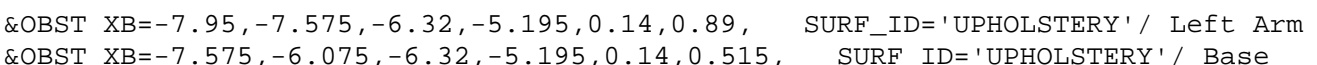

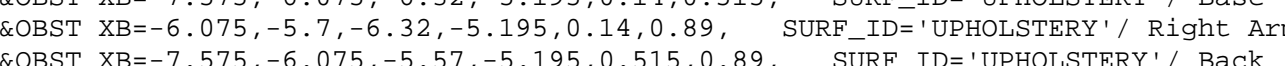

*** GROUP: COUCH2 offset $=0.000000,3.000000,0.000000$ rotate $=90.000000$ ROBST XB=-4.95, $-4.575,-6.32,-5.195,0.14,0.89, \quad$ SURF_ID='UPHOLSTERY ' / Left Arm
\&OBST $X B=-4.575,-3.075,-6.32,-5.195,0.14,0.515$,
SURF_ID='UPHOLSTERY' $/$ Base \&OBST XB $=-3.075,-2.7,-6.32,-5.195,0.14,0.89, \quad$ SURF ID =' 'UPHOLSTERY' $/$ Right Ar

*** GROUP: COUCH2 offset $=0.000000,6.000000,0.000000$ rotate $=90.000000$

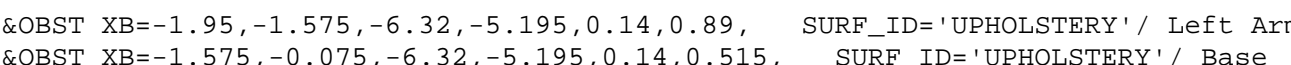

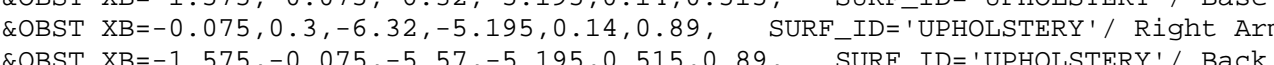
*** GROUP: COUCH2 offset $=0.000000,9.000000,0.000000$ rotate $=90.000000$ \&OBST XB=1.05, $1.425,-6.32,-5.195,0.14,0.89$, SURF_ID= 'UPHOLSTERY ' / Left Arm \&OBST $\mathrm{XB}=1.425,2.925,-6.32,-5.195,0.14,0.515$, SURF_ID='UPHOLSTERY'/ Base

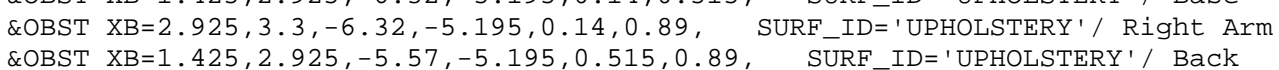

*** GROUP: COUCH2 offset $=0.000000,12.000000,0.000000$ rotate $=90.000000$ \&OBST XB=4.05, 4. 425, -6.32, -5.195, 0.14, 0.89, SURF_ID='UPHOLSTERY' / Left Arm

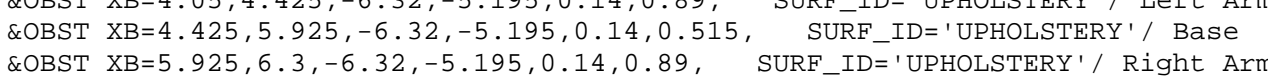

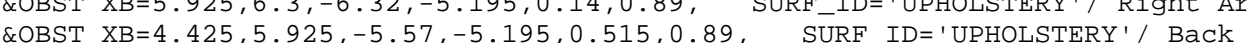

*** GROUP: COUCH2 offset $=0.000000,15.000000,0.000000$ rotate $=90.000000$ \&OBST XB=7.05,7.425, -6.32, $-5.195,0.14,0.89$, SURF_ID='UPHOLSTERY' / Left Arm

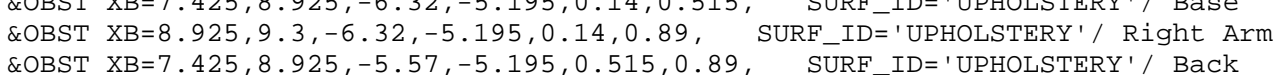

*** GROUP: SHORT_ROW2

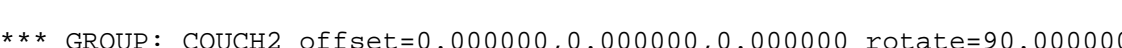

ROBST XB=-7.95, $-7.575,-4.82,-3.695,0.14,0.89, \quad$ SURF_ID='UPHOLSTERY '/ Left Arm
\&OBST XB=-7.575, -6.075,-4.82,-3.695, $0.14,0.515, \quad$ SURF_ID='UPHOLSTERY' $/$ Base \&OBST XB $=-6.075,-5.7,-4.82,-3.695,0.14,0.89$, SURF_ID=' 'UPHOLSTERY'/ Right Ar

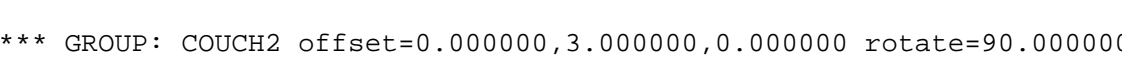

\&OBST XB=-4.95, $-4.575,-4.82,-3.695,0.14,0.89$, SURF_ID=' 'UPHOLSTERY' / Left Arm
SURF ID='UPHOLSTERY' $/$ Base

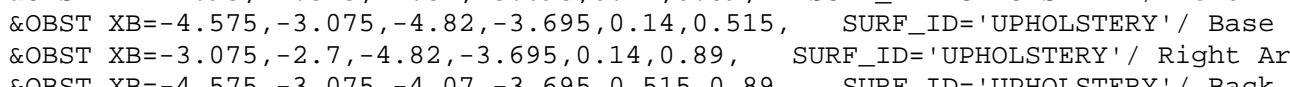

*** GROUP: COUCH2 offset $=0.000000,6.000000, \Theta .000000$ rotate $=90.0000000$

\&OBST XB $=-1.95,-1.575,-4.82,-3.695,0.14,0.89$, SURF_ID= ' UPHOLSTERY' / Left Ar \&OBST XB $=-1.575,-0.075,-4.82,-3.695,0.14,0.515$, SURF_ID='UPHOLSTERY' / Base

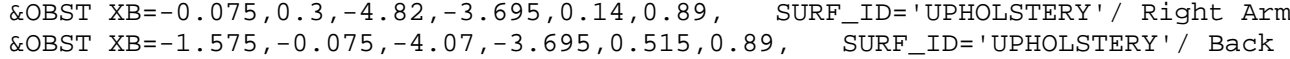

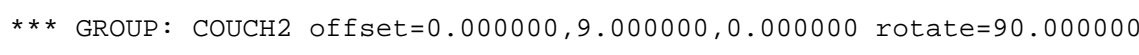

\&OBST $X \mathrm{X}=1.05,1.425,-4.82,-3.695,0.14,0.89$, SURF_ID=' UPHOLSTERY ' / Left Arm QOBST XB $=1.425,2.925,-4.82,-3.695, \theta .14,0.515, \quad$ SURF-ID='UPHOLSTERY'/ Base \&OBST XB=2.925, $3.3,-4.82,-3.695,0.14,0.89, \quad$ SURF_ID=' 'UPHOLSTERY' / Right Arm
\&OBST XB=1.425, $2.925,-4.07,-3.695,0.515,0.89, \quad$ SURF_ID=' 'UPHOLSTERY' / Back

*** GROUP: COUCH2 offset $=0.000000,12.000000,0.000000$ rotate $=90.000000$

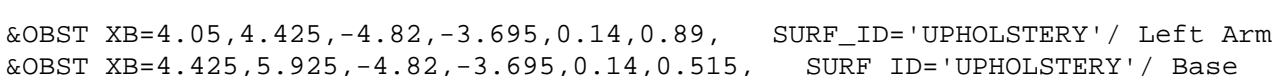

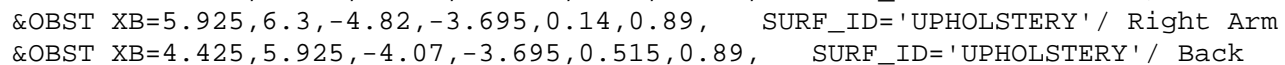

$* * *$ GROUP: COUCH2 offset $=0.000000,15.000000,0.000000$ rotate $=90.000000$

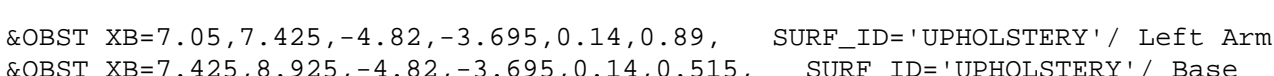

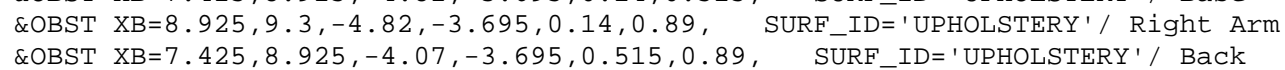

\section{*** GROUP: SHORT_ROW2}

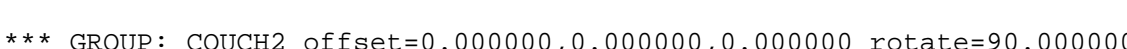

\&OBST XB $=-7.95,-7.575,-3.32,-2.195,0.14,0.89, \quad$ SURF_IDE' UPHOLSTERY' ' Left Arm
\&OBST XB $=-7.575,-6.075,-3.32,-2.195,0.14,0.515, \quad$ SURF_ID 'UPHOLSTERY' $/$ ' Base

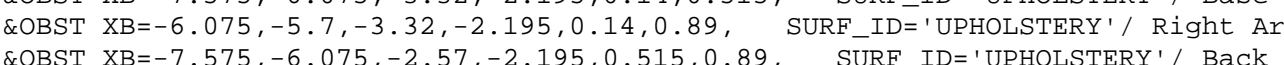

*** GROUP: COUCH2 offset $=0.000000,3.000000,0.000000$ rotate $=90.000000$

\&OBST XB $=4.95,-4.575,-3.32,-2.195,0.14,0.89$, SURF_ID= 'UPHOLSTERY' / Left Arm

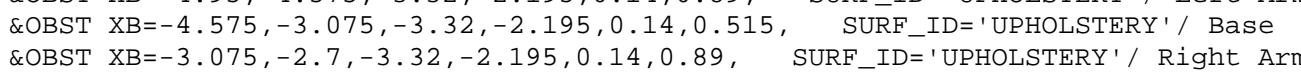
\&OBST XB $=-4.575,-3.075,-2.57,-2.195,0.515,0.89$, SURF_ID=' 'UPHOLSTERY' $/$ Back

*** GROUP: COUCH2 offset $=0.000000,6.000000,0.000000$ rotate $=90.000000$ \&OBST XB $=-1.95,-1.575,-3.32,-2.195,0.14,0.89$, SURF_ID=' UPHOLSTERY' / Left Arm

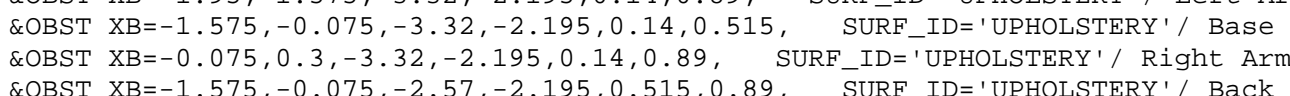

*** GROUP: COUCH2 offset $=0.000000,9.000000,0.000000$ rotate $=90 . .00000$

\&OBST $X \mathrm{XB}=1.05,1.425,-3.32,-2.195,0.14,0.89$, SURF_ID= 'UPHOLSTERY ' / Left Arm

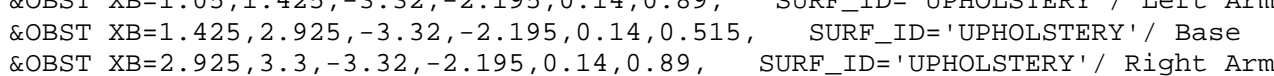
\&OBST $X \mathrm{~B}=2.925,3.3,-3.32,-2.195,0.14, \theta .89$,
\&OBST $\mathrm{XB}=1.425,2.925,-2.57,-2.195,0.515,0.89$, SURF_ID='UPHOLSTERY' / Right Arm
SURF_ID=' UPHOLSTERY'/ Back

*** GROUP: COUCH2 offset $=0.000000,12.000000,0.000000$ rotate $=90.000000$

\&OBST $X \mathrm{XB}=4.05,4.425,-3.32,-2.195,0.14,0.89, \quad$ SURF ID = 'UPHOLSTERY ' $/$ Left Arm \&OBST X X B =5.925,6.3,-3.32,-2.195, $1.14,0.89$, SURF_IDE' 'UPHOLSTERY'/ Right Arm 
*** GROUP: COUCH2 offset $=0.000000,15.000000,0.000000$ rotate $=90.000000$

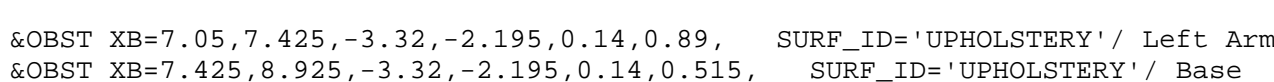
\&OBST XB $=8.925,9.3,-3.32,-2.195,0.14,0.89$, SURF_IDF' 'UPHOLSTERY' $/$ Right Arm \&OBST XB $=7.425,8.925,-2.57,-2.195,0.515,0.89$, SURF_ID='UPHOLSTERY' $/$ Back $* * *$ GROUP: SHORT_ROW2

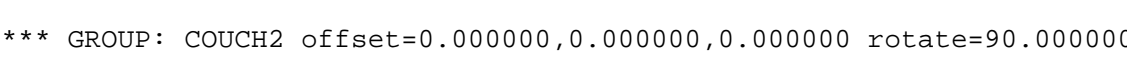
\&OBST XB=-7.95,-7.575, $-1.82,-0.695,0.14,0.89, \quad$ SURF_ID='UPHOLSTERY' $/$ Left Arm
\&OBST XB=-7.575,-6.075,-1.82,-0.695, 0.14,0.515, SURF_ID='UPHOLSTERY' $/$ Base

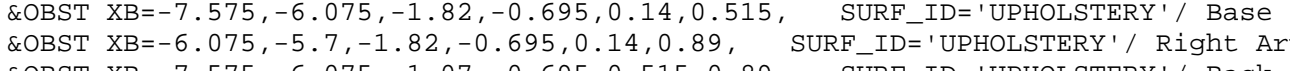

*** GROUP: COUCH2 offset $=0.000000,3.000000,0.000000$ rotate $=90.000000$

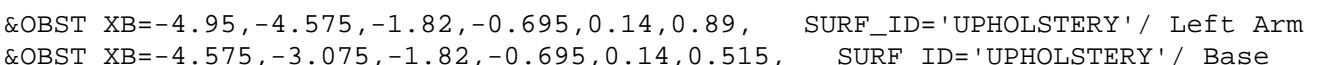

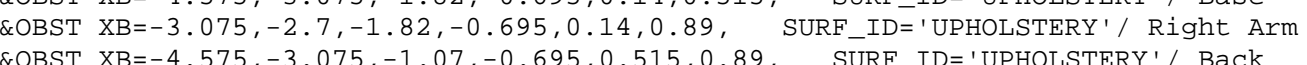
*** GROUP: COUCH2 offset $=0.000000,6.000000,0.000000$ rotate $=90.000000$ \&OBST XB $=-1.95,-1.575,-1.82,-0.695,0.14,0.89, \quad$ SURF_IDE 'UPHOLSTERY ' / Left Arm
\&OBST XB=-1.575, $-0.075,-1.82,-0.695,0.14,0.515$, SURF_ID='UPHOLSTERY ' $/$ Base \&OBST XB=- $-0.075,0.3,-1.82,-0.695,0.14,0.89$, SURF_ID='UPHOLSTERY' $/$ Right Ar *** GROUP: COUCH2 offset $=0.000000,9.000000,0.000000$ rotate $=90.000000$ \&OBST XB $=1.05,1.425,-1.82,-0.695,0.14,0.89$, SURF_ID= ' UPHOLSTERY ' ' Left Arm \&OBST XB $=2.925,3.3,-1.82,-0.695,0.14,0.89$, , SURF_ID ' 'UPHOLSTERY'/Right Arm

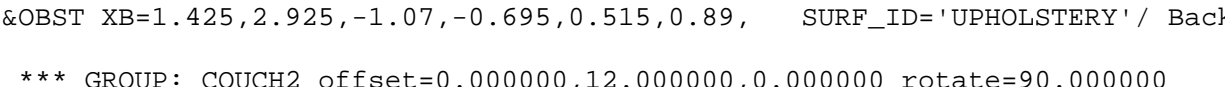
\&OBST $\mathrm{XB}=4.05,4.425,-1.82,-0.695,0.14,0.89$, SURF_ID=' 'UPHOLSTERY ' $/$ Left Arm

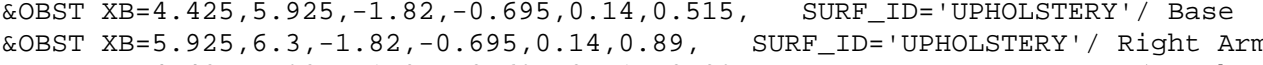
*** GROUP: COUCH2 offset $=0.000000,15.000000,0 . .000000$ rotate $=90.000000$

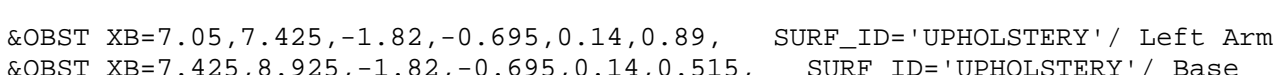

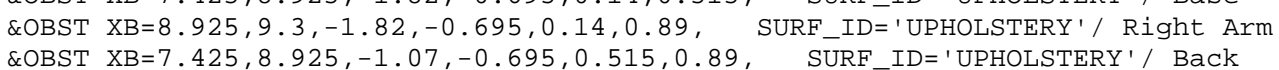
*** GROUP: SHORT_ROW2

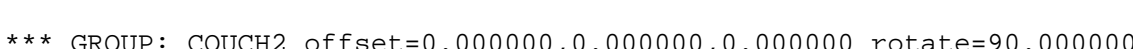

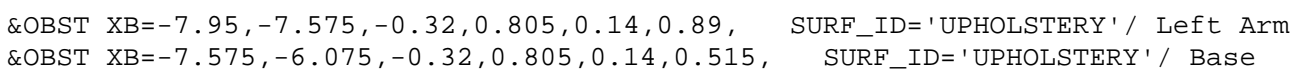

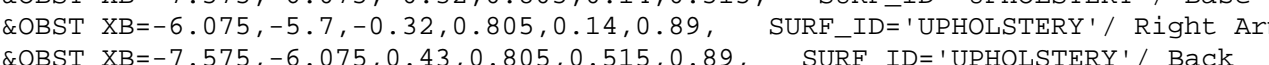
*** GROUP: COUCH2 offset $=0.000000,3.000000,0.000000$ rotate $=90.000000$ \&OBST $X B=-4.95,-4.575,-0.32,0.805,0.14,0.89, \quad$ SURF_ID='UPHOLSTERY ' / Left Arm
\&OBST $X B=-4.575,-3.075,-0.32,0.805,0.14,0.515, \quad$ SURF_ID='UPHOLSTERY' $/$ Base \&OBST XB $=-4.575,-3.075,-0.32,0.805,0.14,0.515, \quad$ SURF_ID='UPHOLSTERY' $/$ Base *** GROUP: COUCH2 offset $=0.000000,6.000000,0.000000$ rotate $=90.000000$ \&OBST XB=-1.95, $-1.575,-0.32,0.805,0.14,0.89, \quad$ SURF_ID= 'UPHOLSTERY ' / Left Arm
\&OBST XB $=-1.575,-0.075,-0.32,0.805,0.14,0.515$, SURF ID='UPHOLSTERY' $/$ Base QOBST XB $=-0.075,0.3,-0.32,0.805,0.14,0.89$, SURF-ID='UPHOLSTERY'/ Right Arm

*** GROUP: COUCH2 offset $=0.000000,9.000000,0.000000$ rotate $=90.000000$
\&OBST $X B=1.05,1.425,-0.32,0.805,0.14,0.89, \quad$ SURF_ID='UPHOLSTERY'/ Left Arm
\&OBST XB=1.425,2.925,-0.32,0.805, $0.14,0.515, \quad$ SURF_ID=' 'UPHOLSTERY' $/$ Base \&OBST XB=2.925,3.3, $-0.32,0.805,0.14,0.89$, SURF_ID =' 'UPHOLSTERY' $/$ Right Ar \&OBST XB=1.425, 2.925, $0.43,0.805,0.515,0.89$, SURF_ID='UPHOLSTERY '/ BaCK \&OBST XB $=4.05,4.425,-0.32,0.805,0.14,0.89, \quad$ SURF_ID=' 'UPHOLSTERY' / Left Arm
\&OBST XB $=4.425,5.925,-0.32,0.805,0.14,0.515, \quad$ SURF_IDF' UPHOLSTERY' Base

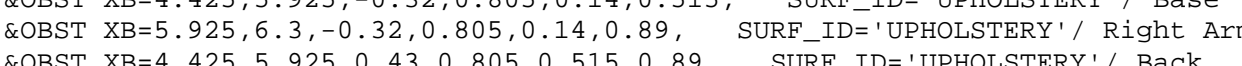

*** GROUP: COUCH2 offset $=0.000000,15.0000000,0 . .000000$ rotate $=90$. .

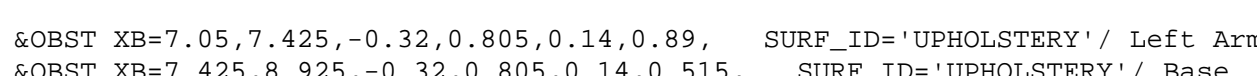
\&OBST XB=8.925, $9.3,-0.32,0.805,0.14,0.89, \quad$ SURF_IDE ' UPHOLSTERY ' / Right Arm
\&OBST XB=7.425,8.925, $0.43,0.805,0.515,0.89, \quad$ SURF_ID=' 'UPHOLSTERY'/ Back

\section{$* * *$ GROUP: SHORT_ROW2}

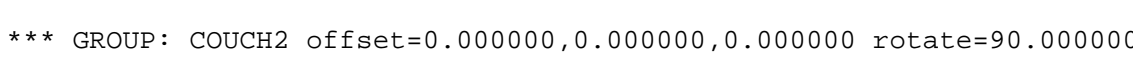

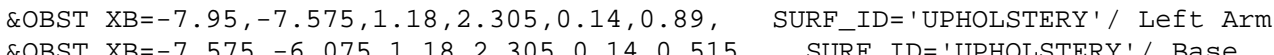
EOBST XB $=-7.575,-6.075,1.18,2.305,0.14,0.515$, SURF_ID='UPHOLSTERY' $/$ Base \&OBST XB=-6. $075,-5.7,1.18,2.305,0.14, \theta .89$,
\&OBST $X B=-7.575,-6.075,1.93,2.305,0.515,0.89$,

*** GROUP: COUCH2 offset $=0.000000,3.000000,0.000000$ rotate $=90.000000$ \&OBST XB=-4.95, $-4.575,1.18,2.305,0.14,0.89, \quad$ SURF_ID='UPHOLSTERY $1 /$ Left Arm
\&OBST $X \mathrm{~B}=-4.575,-3.075,1.18,2.305,0.14,0.515$,
SURF_ID='UPHOLSTERY' $/$ Base COBST XB $=-4.575,-3.075,1.18,2.305,0.14,0.515, \quad$ SURF_ID='UPHOLSTERY' $/$ Base
\&OBST XB $=-3.075,-2.7 .1 .18,2.305,0.14,0.89$, SURF IDD 'UPHOLSTERY' $/$ Right Arm \&OBST XB=-3.075,
\&OBST $X B=-4.575,-3.075,1.93,2.305,0.515,0.89, \quad$ SURF_ID=' 'UPHOLSTERY' / Back

${ }^{* * *}$ GROUP: COUCH2 offset $=0.000000,6.000000,0.000000$ rotate $=90.000000$

\&OBST XB $=-1.95,-1.575,1.18,2.305,0.14,0.89$, SURF_ID='UPHOLSTERY ' / Left Arm \&OBST $X B=-1.575,-0.075,1.18,2.305,0.14,0.515$, SURF_ID= 'UPHOLSTERY' / Base \&OBST $X B=-0.075,0.3,1.18,2.305,0.14,0.89, \quad$ SURF_IDE= 'UPHOLSTERY ' / Right Arm
\&OBST $X B=-1.575,-0.075,1.93,2.305,0.515,0.89$, SUR_IDE' 'UPHOLSTERY'/ Back

*** GROUP: COUCH2 offset $=0.000000,9.000000,0.000000$ rotate $=90.000000$

\&OBST XB=1.05, 1.425, 1.18, 2.305, 0.14, 0.89 , SURF_ID='UPHOLSTERY ' / Left Arm

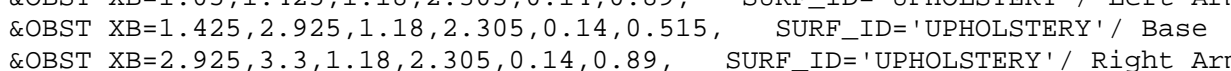

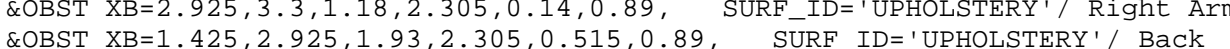

*** GROUP: COUCH2 offset $=0.000000,12.000000,0.000000$ rotate $=90.000000$

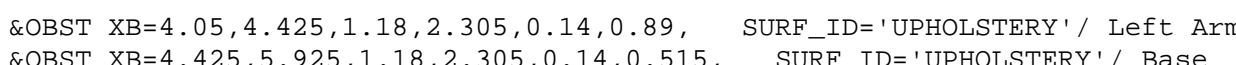

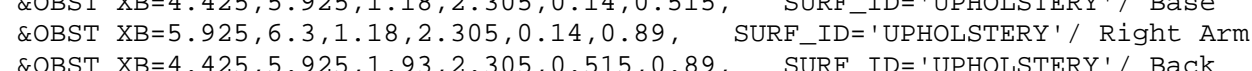

*** GROUP: COUCH2 offset $=0.000000,15.000000,0.000000$ rotate $=90.000000$ \&OBST XB $=7.05,7.425,1.18,2.305,0.14,0.89$, SURF ID= 'UPHOLSTERY' / Left Arm

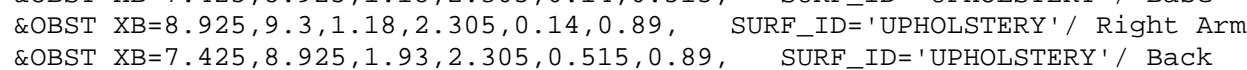

$* * *$ GROUP: SHORT_ROW2

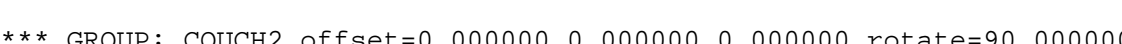

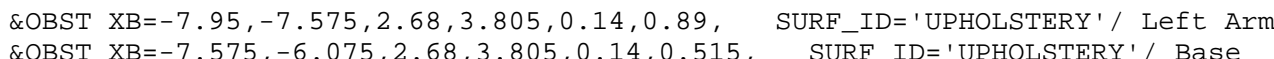

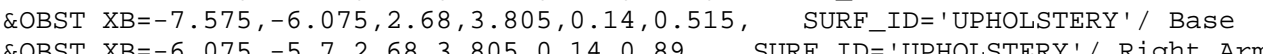

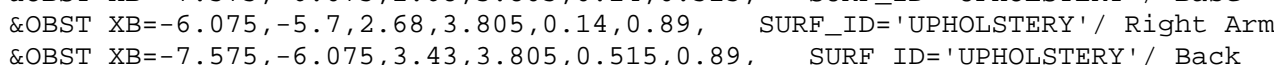

*** GROUP: COUCH2 offset $=0.000000,3.000000,0.000000$ rotate $=90.000000$

\&OBST XB=-4.95, $-4.575,2.68,3.805,0.14,0.89, \quad$ SURF_ID=' 'UPHOLSTERY ' / Left Arm
\&OBST XB $=-4.575,-3.075,2.68,3.805,0.14,0.515, \quad$ SURF_ID=' UPHOLSTERY' $/$ Base L-81 
\&OBST XB $=-4.575,-3.075,3.43,3.805,0.515,0.89, \quad$ SURE_ID= ' UPHOLSTERY' / Back

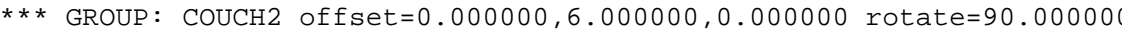
EOBST XB $=-1.95,-1.575,2.68,3.805,0.14,0.89$, SURF_ID = 'UPHOLSTERY' / Left Arm

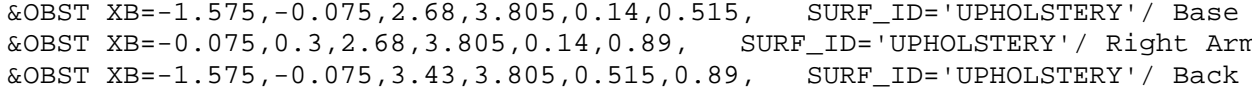
*** GROUP: COUCH2 offset $=0.000000,9.000000,0.000000$ rotate $=90.000000$ \&OBST $X \mathrm{~B}=1.05,1.425,2.68,3.805,0.14,0.89$, SURF_ID='UPHOLSTERY' ' Left Ar \&OBST XB $=1.425,2.925,2.68,3.805,0.14,0.515$, SURF IDD = 'UPHOLSTERY' $/$ Base

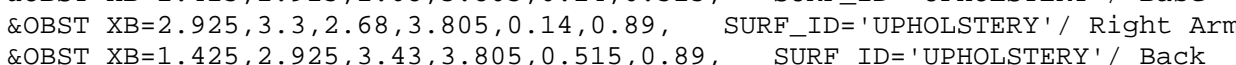

*** GROUP: COUCH2 offset $=0.000000,12.000000,0.000000$ rotate $=90.000000$ \&OBST XB=4. $05,4.425,2.68,3.805,0.14,0.89$, SURF_ID=' UPHOLSTERY' ' Left Arm *** GROUP: COUCH2 offset $=0.000000,15.000000,0.000000$ rotate $=90.000000$ \&OBST XB=7.05,7.425, 2.68,3.805, $0.14,0.89$, SURF_ID=' UPHOLSTERY' ' Left Arm \&OBST XB=8.925,9.3, 2.68, 3.805, $0.14,0.89$, SURF_ID='UPHOLSTERY'/ Right Ar

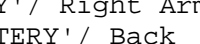

\section{*** GROUP: LHS_MID}

*** GROUP: CHAIR_ROW2

$* *$ GROUP: CHAIR offset $=0.000000,0.000000,0.140000$ rotate $=0.000000$

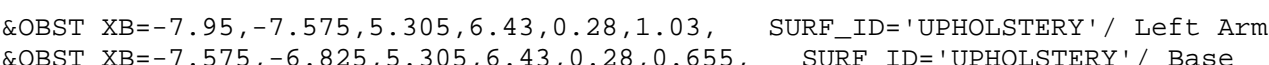
\&OBST XB $=-6.825,-6.45,5.305,6.43,0.28,1.03$, SURF_ID ='UPHOLSTERY'/ Right Ar

$* * *$ GROUP: CHAIR offset $=1.875000,0.000000,0.140000$ rotate $=0.000000$

\&OBST XB $=-6.075,-5.7,5.305,6.43,0.28,1.03$, SURF_ID='UPHOLSTERY' / Left Arm \&OBST XB=-5.7, $-4.95,5.305,6.43,0.28,0.655, \quad$ SURF_ID='UPHOLSTERY'/ Base QOBST $X$ B $=4.5,7,-4.575,5.305,6.43,0.28,1.03$, SURF_ID='UPHOLSTERY/ Right Ar

$* * *$ GROUP: CHAIR offset $=3.750000,0.000000,0.140000$ rotate $=0.000000$

EOBST XB=-4.2, $-3.825,5.305,6.43,0.28,1.03$, SURF_ID='UPHOLSTERY' / Left Arm

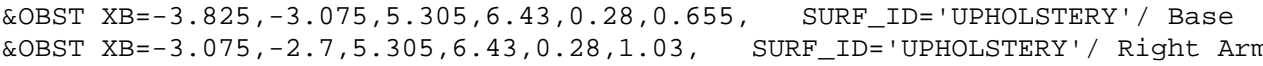
作

$\star * \star$ GROUP: CHAIR offset $=5.625000,0.000000,0.140000$ rotate $=0.000000$ \&OBST $X \mathrm{XB}=-2.325,-1.95,5.305,6.43,0.28,1.03$, SURF_ID=' 'UPHOLSTERY ' / Left Arm

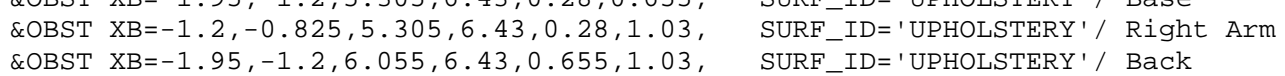
*** GROUP: CHAIR offset $=7.500000,0.000000,0.140000$ rotate $=0.000000$

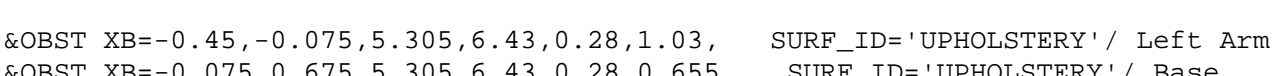
\&OBST XB=0.675,1.05,5.305, $6.43,0.28,1.03$, SURF_ID='UPHOLSTERY'/ Right Arm
\&OBST $X \mathrm{~B}=-0.075,0.675,6.055,6.43,0.655,1.03$, SURF_ID='UPHOLSTERY' $/$ Back

*** GROUP: CHAIR offset $=9.375000,0.000000,0.140000$ rotate $=0.000000$

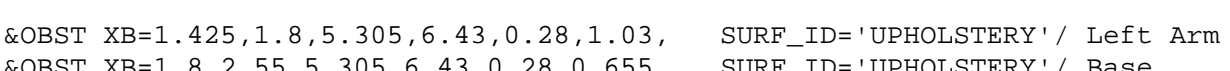

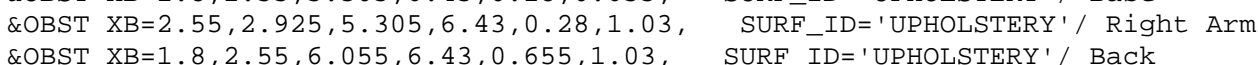

** GROUP: CHAIR offset $=11.250000,0.000000,0.140000$ rotate $=0.000000$

\&OBST XB $=3.3,3.675,5.305,6.43,0.28,1.03$, SURF_ID='UPHOLSTERY'/ Left Arm
COBST XB $=3.675,4.425,5.305,6.43,0.28,0.655$, SURF_ID='UPHOLSTERY'

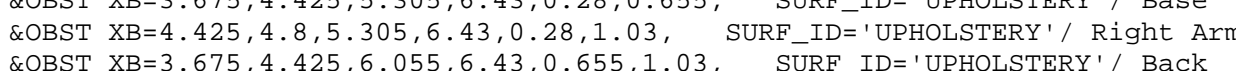

*** GROUP: CHAIR offset $=13.125000,0.000000,0.140000$ rotate $=0.000000$

\&OBST XB $5.175,5.55,5.305,6.43,0.28,1.03, \quad$ SURF_ID=' 'UPHOLSTERY' / Left Arm \&OBST $X \mathrm{~B}=5.55,6.3,5.305,6.43,0.28,0.655, \quad$ SURF_ID= 'UPHOLSTERY'/ Base
COBST XB $=6.3,6.675,5.305,6.43,0.28,1.03$, \&OBST XB $=5.55,6.3,6.055,6.43,0.655,1.03$, SURF ID=' 'UPHOLSTERY' / Back

*** GROUP: CHAIR off set $=15.0000000,0.000000,0.140000$ rotate $=0.000000$

\&OBST XB $=7.05,7.425,5.305,6.43,0.28,1.03, \quad$ SURF_IDE' 'UPHOLSTERY'/ Left Arm
\&OBST XB $=7.425,8.175,5.305,6.43,0.28,0.655, \quad$ SURF_ID 'UPHOLSTERY' $/$ Base

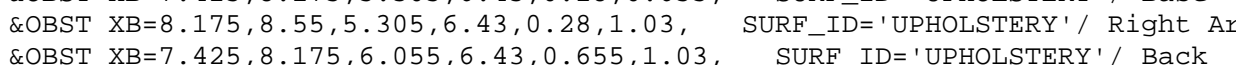

*** GROUP: CHAIR_ROW2

*** GROUP: CHAIR offset $=0.000000,0.000000,0.140000$ rotate $=0.000000$

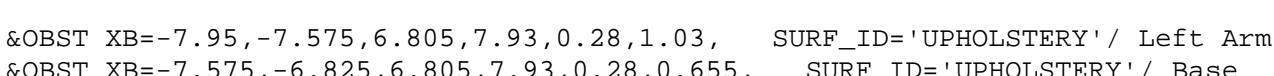

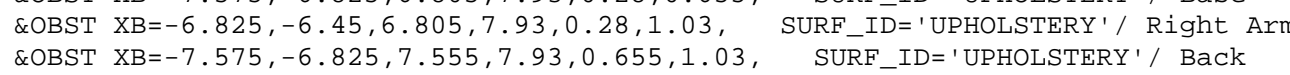

*** GROUP: CHAIR offset $=1.875000,0.000000,0.140000$ rotate $=0.000000$

\&OBST XB=-6. $075,-5.7,6.805,7.93,0.28,1.03, \quad$ SURF_ID= 'UPHOLSTERY ' ' Left Arm

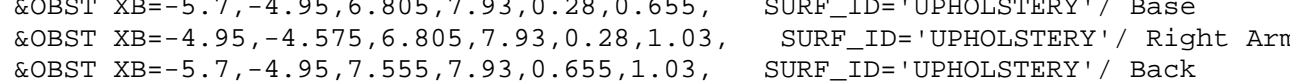

*** GROUP: CHAIR offset $=3.750000,0.000000,0.140000$ rotate $=0.000000$

\&OBST XB=-4.2, - 3.825, 6. 805, 7.93, 0.28, 1. 03, SURFIDD='UPHOLSTERY'/ Left Arm \&OBST $X \mathrm{~B}=-3,075,-2.7,6.805,7,93,0.28,1.03$, SURF_ID='UPHOLSTERY' / Right Arm \&OBST XB=-3.825, -3. 075, 7.555, 7.93, 0.655, 1.03, SURF_ID='UPHOLSTERY'// Back

*** GROUP: CHAIR offset $=5.625000,0.000000,0.140000$ rotate $=0.000000$

\&OBST XB $=-2.325,-1.95,6.805,7.93,0.28,1.03, \quad$ SURF_ID= 'UPHOLSTERY' / Left Arm
\&OBST XB $=-1.95,-1.26 .805,93,0.28,0655$ SURE_ID=' UPHOLSTERY' / Base \&OBST XB $=-1.2,-0.825,6.805,7,93,0.28,1,03, \quad$ SURE ID=' 'UPHOLSTERY'/ Right Arm QOBST XB=-1.2, $-0.825,6.805,7.93,0.28,1.03, \quad$ SURF_ID='UPHOLSTERY'/ Right Ar
\&OBST XB $=-1.95,-1.2,7.555,7.93,0.655,1.03, \quad$ SURF_ID='UPHOLSTERY'// Back

*** GROUP: CHAIR offset $=7.500000,0.000000,0.140000$ rotate $=0.000000$

\&OBST XB=-0.45, $-0.075,6.805,7.93,0.28,1.03, \quad$ SURF_ID='UPHOLSTERY ' ' Left Arm
\&OBST $X \mathrm{~B}=-0.075,0.675,6.805,7.93,0.28,0.655, \quad$ SURF_ID='UPHOLSTERY'

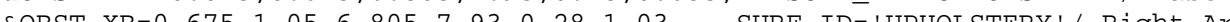
COBST XB $=0.675,1.05,6.805,7.93,0.28,1.03$, SURF_ID='UPHOLSTERY' / Right Arm
\&OBST XB=-0.075, $0.675,7.555,7.93,0.655,1.03$, SURF_ID='UPHOLSTERY'/ Back

*** GROUP: CHAIR offset $=9.375000,0.000000,0.140000$ rotate $=0.000000$

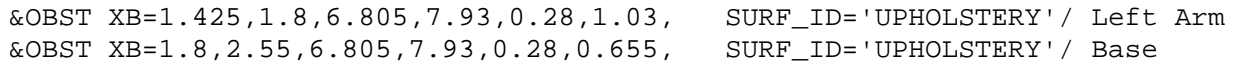

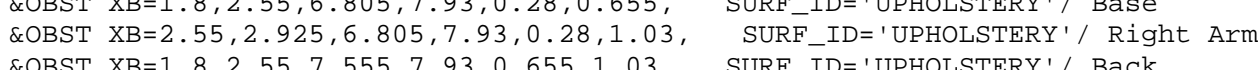

*** GROUP: CHAIR offset $=11.250000,0.000000,0.140000$ rotate $=0.000000$

\&OBST XB=3.3,3.675,6.805,7.93,0.28,1.03, SURF_ID=' 'UPHOLSTERY ' / Left Arm \&OBST XB $=3.675,4.425,6.805,7.93,0.28,0.655$, SURF \&OBST XB $=4.425,4.8,6.805,7.93,0.28,1.03$, SURF_ID='UPHOLSTERY'/ Right Ar
COBST XB $=3.675,4.425,7.555,7.93,0.655,1.03$, SURF_ID='UPHOLSTERY' $/$ Back

*** GROUP: CHAIR offset $=13.125000,0.000000,0.140000$ rotate $=0.000000$

\&OBST XB=5.175, 5. 55, 6.805, 7.93, 0.28,1.03, SURF_ID='UPHOLSTERY'/ Left Arm \&OBST XB $=5.55,6.3,6.805,7.93,0.28,0.655$, SURF_ID='UPHOLSTERY'/ Base 
\&OBST XB $=5,55,6,3,7.555,7,93,0,655,1,03$, SURE_ID=' 'UPHOLSTERY' / Back ** GROUP: CHAIR offset $=15.000000,0.000000,0.140000$ rotate $=0.000000$

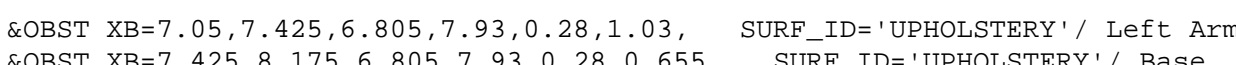

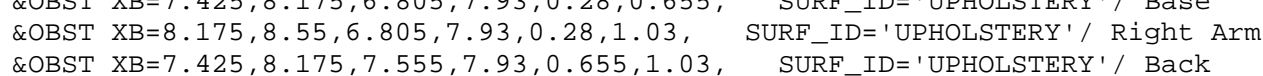

*** GROUP: CHAIR_ROW2

*** GROUP: CHAIR offset $=0.000000,0.000000,0.140000$ rotate $=0.000000$

\&OBST $X \mathrm{XB}=-7.95,-7.575,8.305,9.43,0.28,1.03, \quad$ SURF_ID='UPHOLSTERY '/ Left Arm
\&OBST XB $=-7.575,-6.825,8.305,9.43,0.28,0.655, \quad$ SURF_ID=' 'UPHOLSTERY' $/$ Base

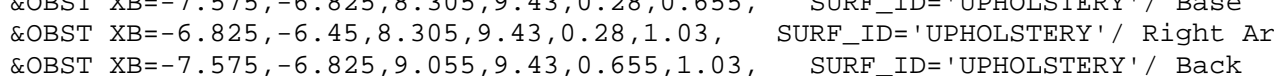

*** GROUP: CHAIR offset $=1.875000,0.000000,0.140000$ rotate $=0.000000$ \&OBST $X \mathrm{XB}=-6.075,-5.7,8.305,9.43,0.28,1.03, \quad$ SURF_ID='UPHOLSTERY' $/$ Left Arm

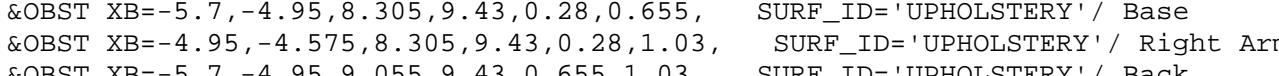
\&OBST X $\mathrm{B}=-5.7,-4.95,9.055,9.43,0.655,1.03$, SURF_ID='UPHOLSTERY'/ Back

*** GROUP: CHAIR offset $=3.750000,0.0000000,0.140000$ rotate $=0.000000$ \&OBST XB=-4.2, $-3.825,8.305,9.43,0.28,1.03$, SURF_ID='UPHOLSTERY' / Left Arm

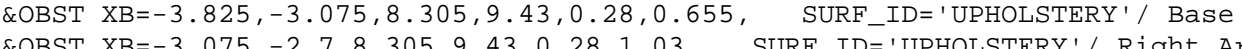
\&OBST $X \mathrm{XB}=-3.075,-2.7,8.305,9.43,0.28,1.03$, SURF_ID='UPHOLSTERY'/Right Arm
\&OBST $X \mathrm{~B}=-3.825,-3.075,9.055,9.43,0.655,1.03$, SURF_ID='UPHOLSTERY'/ Back *** GROUP: CHAIR offset $=5.625000,0.000000,0.140000$ rotate $=0.000000$ \&OBST XB=-2.325, -1.95, 8.305, 9.43, 0.28,1.03, SURF_ID='UPHOLSTERY'/ Left Arm
\&OBST XB=-1.95,-1.2, $8.305,9.43,0.28,0.655, \quad$ SURF_IDE'UPHOLSTERY'/ Base

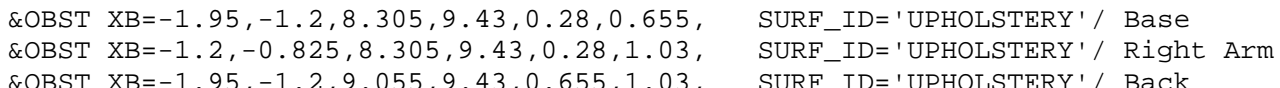
*** GROUP: CHAIR offset $=7.500000,0.0000000,0.140000$ rotate $=0.000000$

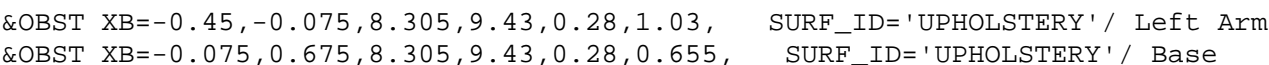
\&OBST XB $=0.675,1.05,8.305,9.43,0.28,1.03$, SURF ID ' UPHOLSTERY'/ Right Ar

$* *$ GROUP: CHAIR offset $=9.375000,0.000000,0.140000$ rotate $=0.000000$ \&OBST XB $=1.425,1.8,8.305,9.43,0.28,1.03$, SURF_ID=' 'UPHOLSTERY' '/ Left Arm
\&OBST XB $=1.8,2.55,8.305,9.43,0.28,0.655$

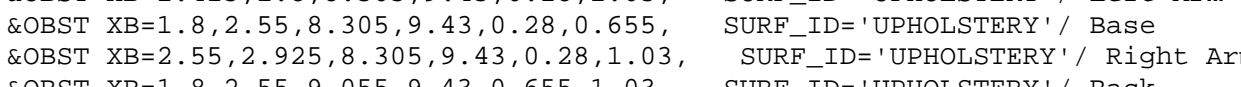
*** GROUP: CHAIR offset $=11.250000,0.000000,0.140000$ rotate $=0.000000$ \&OBST XB=3.3, 3.675, 8.305, 9.43, 0.28,1.03, SURF_ID=' 'UPHOLSTERY ' / Left Arm \&OBST XB=3.675,4.425,8.305,9.43,0.28,0.655, SURF_ID='UPHOLSTERY'/ Base

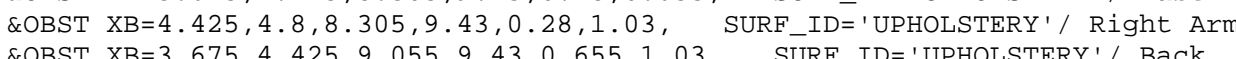
$* * *$ GROUP: CHAIR offset $=13.125000,0.000000,0.140000$ rotate $=0.000000$ \&OBST XB=5.175, 5. 55, 8.305, 9.43, $0.28,1.03$, SURF_ID='UPHOLSTERY' / Left Arm \&OBST XB $=5.55,6.3,8.305,9.43,0.28,0.655$, SURF-ID=' 'UPHOLSTERY'/ Base EOBST XB $=6.3,6.675,8.305,9.43,0.28,1.03$, SURF-IDD' 'UPHOLSTERY'/ Right Arm

*** GROUP: CHAIR offset $=15.000000,0.000000,0.140000$ rotate $=0.000000$ \&OBST XB=7.05, 7.425,8.305, 9.43, $0.28,1.03$, SURF_ID=' 'UPHOLSTERY'/ Left Arm

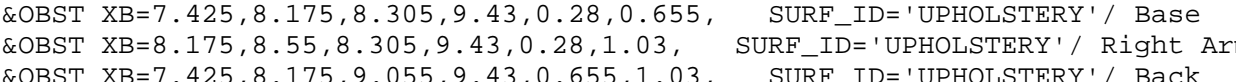

*** GROUP: CHAIR_ROW2

*** GROUP: CHAIR offset $=0.0000000, \Theta .0000000,0.1400000$ rotate $=0 . .000000$

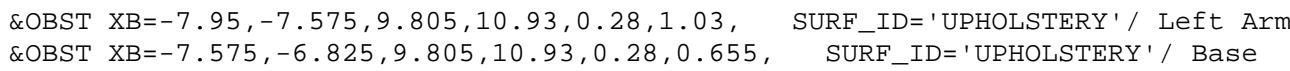
COBST XB $=-6.825,-6.45,9.805,10.93,0.28,1.03$, SURF_ID='UPHOLSTERY' $/$ Right Ar

*** GROUP: CHAIR offset $=1.875000,0.000000,0.140000$ rotate $=0.000000$ \&OBST XB=-6. $075,-5.7,9.805,10.93,0.28,1.03$, SURF_ID= 'UPHOLSTERY' / Left Arm \&OBST XB $=4.45,-4.575,9.805,10.93,0.28,1.03$, SUFF_IIDD 'UPHOLSTERY' / Right Ar

*** GROUP: CHAIR offset $=3.750000,0.000000,0.140000$ rotate $=0.000000$ \&OBST XB $=-4.2,-3.825,9.805,10.93,0.28,1.03, \quad$ SURF_ID= 'UPHOLSTERY ' / Left Arm
SOBST \&OBST XB $=-3.825,-3.075,9.805,10.93,0.28,0.655$, SURF_IDE'UPHOLSTERY'
ROBST \&OBST $X B=-3.825,-3.075,10,555,10,93,0,655,1,03, \quad$ SD

*** GROUP: CHAIR offset $=5.625000,0.000000,0.140000$ rotate $=0.000000$

\&OBST XB=-2.325, -1.95, 9.805,10.93, 0.28,1.03, SURF_ID=' 'UPHOLSTERY '/ Left Ar

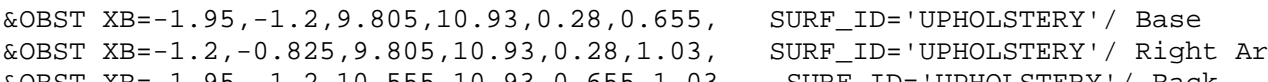

$* * *$ GROUP: CHAIR offset $=7.500000,0.000000,0.140000$ rotate $=0.000000$

\&OBST XB $=-0.45,-0.075,9.805,10.93,0.28,1.03$, SURF_ID='UPHOLSTERY Y' Left Arm

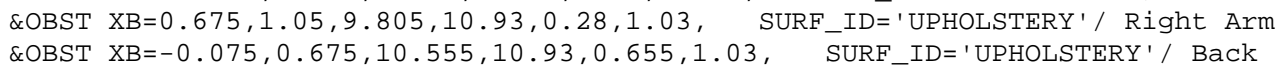

*** GROUP: CHAIR offset $=9.375000,0.000000,0.140000$ rotate $=0.000000$

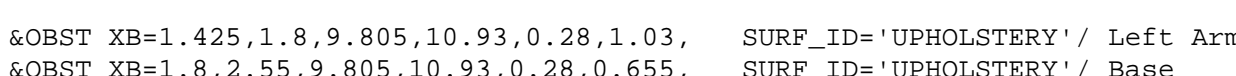
COBST $X \mathrm{XB}=2.55,2.925,9.805,10.93,0.28,1.03$,
\&OBST $\mathrm{XB}=1.8,2.55,10.555,10.93,0.655,1.03$,
SURF_ID='ID='UPHOLLSTERRY'/ Rack

*** GROUP: CHAIR offset $=11.250000,0.000000,0.140000$ rotate $=0.000000$ \&OBST XB=3.3,3.675,9.805, 10.93, $0.28,1.03$, SURF ID ' 'UPHOLSTERY' / Left Arm

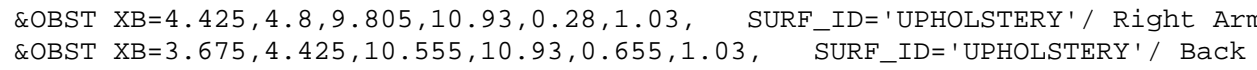

$* * *$ GROUP: CHAIR offset $=13.125000,0.000000,0.140000$ rotate $=0.000000$ 2OBST XB=5.175, 5.55,9.805,10.93, $0.28,1.03$, SURF_ID='UPHOLSTERY' / Left Arm \&OBST XB=6.3,6.675,9.805,10.93,0.28,1.03, SURF-ID='UPHOLSTERY'/ Pight Ar \&OBST XB $=5.55,6.3,10.555,10.93,0.655,1.03$, SURF_ID=' UPHOLSTERY'/ Back

*** GROUP: CHAIR offset $=15.000000,0.000000,0.140000$ rotate $=0.000000$

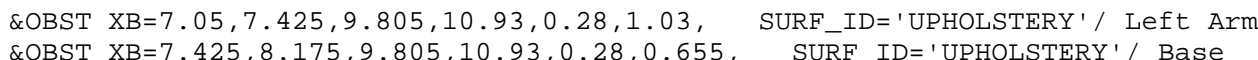
EOBST $X \mathrm{X}=8.175,8.55,9.805,10.93,0.28,1.03, \quad$ SURF_ID=' 'UPHOLSTERY' $/$ Right Arm
EOBST $\mathrm{XB}=7.425,8.175,10.555,10.93,0.655,1.03$, SURF_ID='UPHOLSTERY'/ Back

*** GROUP: CHAIR_ROW2

$* * *$ GROUP: CHAIR offset $=0.000000,0.000000,0.140000$ rotate $=0.000000$

\&OBST XB $=-7.95,-7.575,11.305,12.43,0.28,1.03$, SURF_ID=' 'UPHOLSTERY' / Left Arm \&OBST XB $=-7.575,-6.825,11.305,12.43,0.28,0.655$, SURF-ID=' 'UPHOLSTERY' ' Base \&OBST XB=-7.575, $-6.825,12.055,12.43,0.655,1.03$, SURF_ID=' 'UPHOLSTERY' / Back

$* * *$ GROUP: CHAIR offset $=1.875000,0.000000,0.140000$ rotate $=0.000000$

\&OBST XB $=-6.075,-5.7,11.305,12.43,0.28,1.03$,
\&OBST XB $=-5.75$ SURFIDD ' UPHOLSTERY' 1 ' Left Arm \&OBST XB $=-4.95,-4.575,11.305,12.43,0.28,1.03$, SURF_ID=' 'UPHOLSTERY'/ Right Ar 
*** GROUP: CHAIR offset $=3.750000,0.000000,0.140000$ rotate $=0.000000$

\&OBST $X \mathrm{X}=-4.2,-3.825,11.305,12.43,0.28,1.03$, SURF ID = 'UPHOLSTERY ' / Left Arm

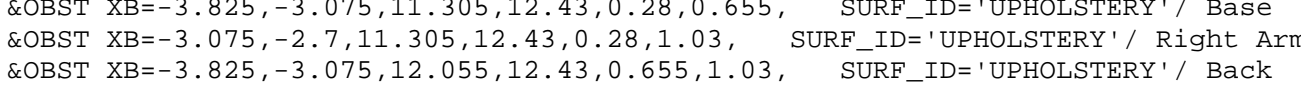
*** GROUP: CHAIR offset $=5.625000,0.000000,0.140000$ rotate $=0.000000$

ROBST XB $=-2.325,-1.95,11.305,12.43,0.28,1.03, \quad$ SURF_ID=' 'UPHOLSTERY' / Left Arm
\&OBST XB $=-1.95,-1.2,11.305,12.43,0.28,0.655, \quad$ SURF_ID='UPHOLSTERYY' Base

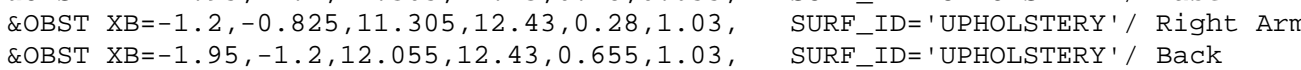

$* * *$ GROUP: CHAIR offset $=7.500000,0.000000,0.140000$ rotate $=0.000000$

\&OBST XB=-0.45, $-0.075,11.305,12.43,0.28,1.03, \quad$ SURF_ID= 'UPHOLSTERY' ' Left Ar
\&OBST XB=-0.075, $0.675,11.305,12.43,0.28,0.655, \quad$ SURF_ID=' UPHOLSTERY' 1 Base \&OBST $X \mathrm{XB}=0.675,1.05,11.305,12.43,0.28,1.03, \quad$ SURF_ID='UPHOLSTERY' $/$ Right Arm
\&OBST $\mathrm{XB}=-0.075,0.675,12.055,12.43,0.655,1.03, \quad$ SURF_ID='UPHOLSTERY'/ Back $* * *$ GROUP: CHAIR offset $=9.375000,0.000000,0.140000$ rotate $=0.000000$

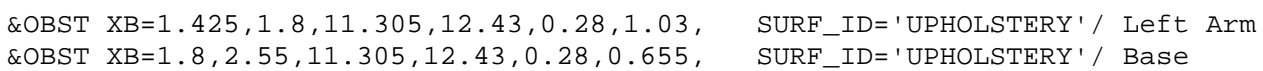

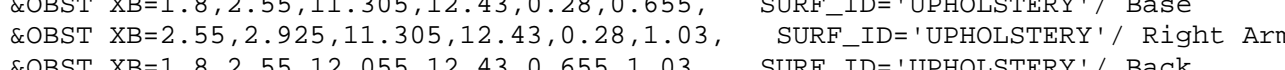

*** GROUP: CHAIR offset $=11.250000,0.000000,0.140000$ rotate $=0.000000$

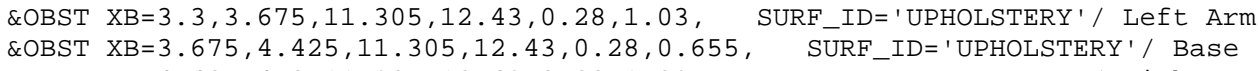
\&OBST $X B=4.425,4.8,11.305,12.43,0.28,1.03$, SURF_ID='UPHOLSTERY'/ Right Arm

*** GROUP: CHAIR offset $=13.125000,0.0000000,0.1400000$ rotate $=0.000000$

\&OBST XB $5.175,5.55,11.305,12.43,0.28,1.03$, SURF_ID=' UPHOLSTERY ' / Left Arm

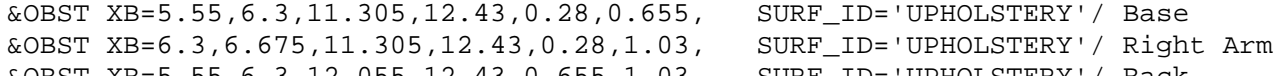

*** GROUP: CHAIR offset $=15.0000000,0.000000,0.140000$ rotate $=0.000000$

\&OBST $X \mathrm{X}=7.05,7.425,11.305,12.43,0.28,1.03, \quad$ SURF_ID= 'UPHOLSTERY ' / Left Arm
COBST $X \mathrm{~B}=7.425,8.175,11.305,12.43,0.28,0.655, \quad$ SURF_ID=' 'UPHOLSTERY' $/$ Base

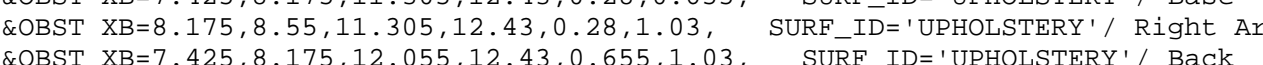

*** GROUP: CHAIR_ROW2

*** GROUP: CHAIR offset $=0.000000,0.000000,0.140000$ rotate $=0.000000$

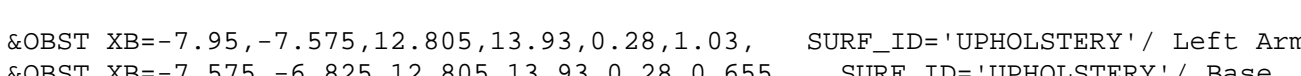

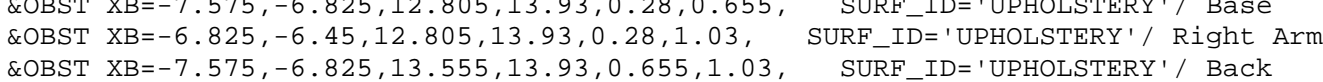

*** GROUP: CHAIR offset $=1.875000,0.000000,0.140000$ rotate $=0.000000$

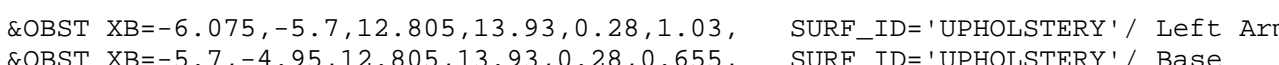
\&OBST XB- $-4.95,-4.575,12.805,13.93,0.28,1.03$,
\&OBST XB =-5.7,

*** GROUP: CHATR offset $=3.750000,0.000000,0.140000$ rotate $=0.000000$

\&OBST XB $=-4.2,-3.825,12.805,13.93,0.28,1.03$, SURF ID = 'UPHOLSTERY Y / Left Arm \&OBST XB $=-3.075,-2.7,12.805,13.93,0.28,1,03$, SURF ID =' 'UPHOLSTERY'

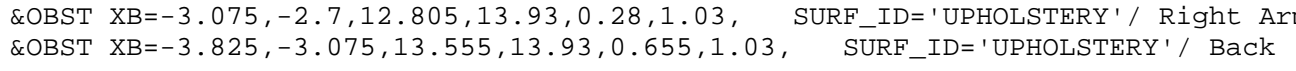
*** GROUP: CHAIR offset $=5.625000,0.000000,0.140000$ rotate $=0.000000$ \&OBST $X B=-2.325,-1.95,12.805,13.93,0.28,1.03, \quad$ SURF_ID='UPHOLSTERY '/ Left Arm
\&OBST $X B=-1.95,-1.2,12.805,13.93,0.28,0.655$,
SURF_ID='UPHOLSTERY'/ Base

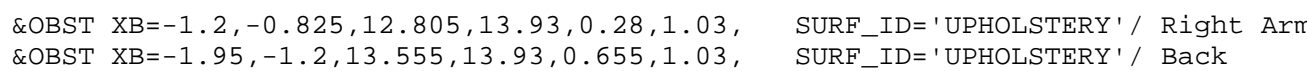
$* * *$ GROUP: CHAIR offset $=7.500000,0.000000,0.140000$ rotate $=0.000000$

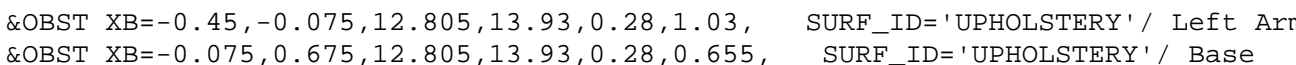

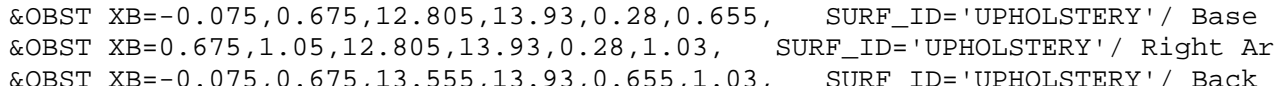

*** GROUP: CHAIR offset $=9.375000,0.000000,0.140000$ rotate $=0.000000$ $\begin{array}{ll}\text { \&OBST } X B=1.425,1.8,12.805,13.93,0.28,1.03, & \text { SURF_ID=' 'UPHOLSTERY' / Left Arm } \\ \text { SURF_ID ' UPHOLSTEPY', eft }\end{array}$ \&OBST XB=1.8, 2.55, 12.805, 13.93, $0.28,0.655$, SURF-ID='UPHOLSTERY'/ Base

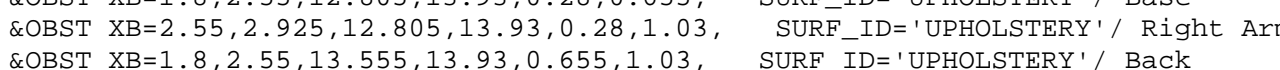
*** GROUP: CHAIR offset $=11.250000,0.000000,0.140000$ rotate $=0.000000$ \&OBST XB=3.3,3.675,12.805,13.93, $0.28,1.03$, SURF_ID=' 'UPHOLSTERY' ' Left Arm
\&OBST XB=3.675,4.425,12.805,13.93,0.28,0.655, SURF_ID='UPHOLSTERY' $/$ Base COBST $X \mathrm{~B}=4.425,4.8,12.805,13.93,0.28,1.03$, SURF ID =' 'UPHOLSTERY'/ Right Arm

$* * *$ GROUP: CHAIR offset $=13.125000,0.000000,0.140000$ rotate $=0.000000$ \&OBST XB=5.175, 5.55, 12.805, 13.93, 0.28,1.03, SURF_ID='UPHOLSTERY' / Left Arm \&OBST $X B=5.55,6.3,12.805,13.93,0.28,0.655$, SURF_ID='UPHOLSTERY' / Base

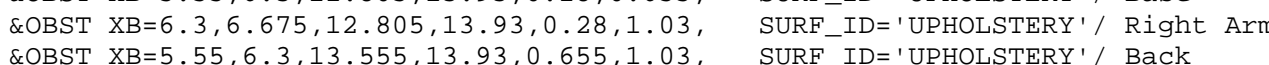

*** GROUP: CHAIR offset $=15.000000,0.000000,0.140000$ rotate $=0.000000$

\&OBST $X B=7.05,7.425,12.805,13.93,0.28,1.03$, SURF_ID = 'UPHOLSTERY' / Left Arm \&OBST XB $=7.425,8.175,12.805,13.93,0.28,0.655$, SURF-ID=' UPHOLSTERY'/ Base

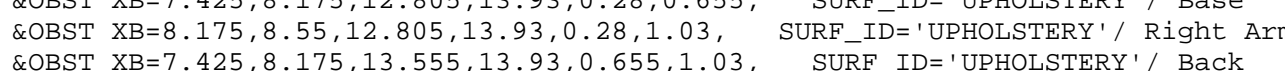

$* * *$ GROUP: CHAIR_ROW2

$* * *$ GROUP: CHAIR offset $=0.000000,0.000000,0.140000$ rotate $=0.000000$

\&OBST XB $=-7.95,-7.575,14.305,15.43,0.28,1.03, \quad$ SURF_ID=' 'UPHOLSTERY'/ Left Arm
COBST XB $=-7.575,-6.825,14.305,15.43,0.28,0.655, \quad$ SURF_ID='UPHOLSTERY' \&OBST XB=-6.825, -6.45,14.305, 15.43, $0.28,1.03$, SURF IDD='UPHOLSTERY' ' Right Arm

*** GROUP: CHAIR offset $=1.875000,0.000000,0.140000$ rotate $=0.000000$

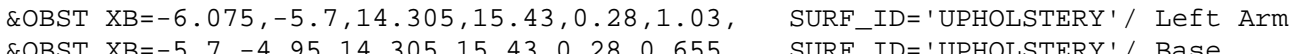

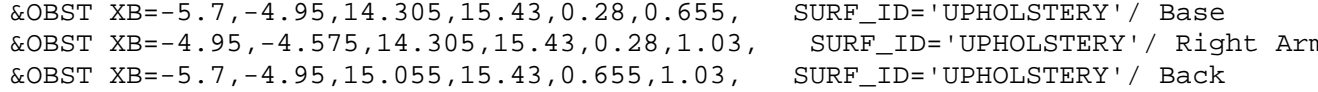

*** GROUP: CHAIR offset $=3.750000,0.000000,0.140000$ rotate $=0.000000$

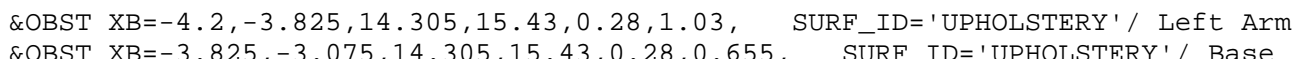
\&OBST XB $=-3.225,-3.075,14.305,45.43,0.28,0.655$, SURF_ID='UPHOLSTERY' / Base \&OBST XB=-3.825, $-3.075,15.055,15.43,0.655,1.03$, SURF ID=' 'UPHOLSTERY' $/$ Back

*** GROUP: CHAIR offset $=5.625000,0.000000,0.140000$ rotate $=0.000000$

\&OBST XB $=-2.325,-1.95,14.305,15.43,0.28,1.03$, SURF_ID='UPHOLSTERY'/ Left Arm \&OBST XB $=-1.95,-1.2,14.305,15.43,0.28,0.655$, SURF_ID='UPHOLSTERY'/ Base \&OBST $X \mathrm{~B}=1.2,-1.225,15.305,15.43,0.28,1.03$, SURF-ID='UPHOLSTERY'/ Right A

$* \star *$ GROUP: CHAIR offset $=7.500000,0.000000,0.140000$ rotate $=0.000000$ \&OBST XB $=-0.45,-0.075,14.305,15.43,0.28,1.03$, SURF_ID ='UPHOLSTERY'/ Left Ar \&OBST XB $=-0.075,0.675,14.305,15.43,0.28,0.655$, SURF_ID='UPHOLSTERY'/ Base

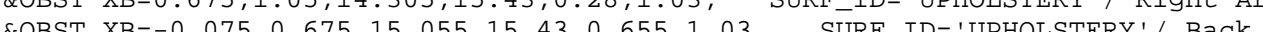

$* * *$ GROUP: CHAIR offset $=9.375000,0.000000,0.140000$ rotate $=0.000000$ 


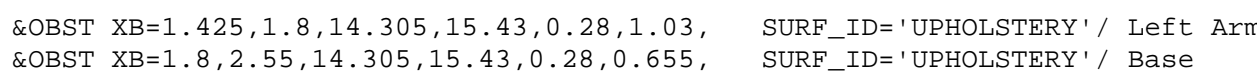

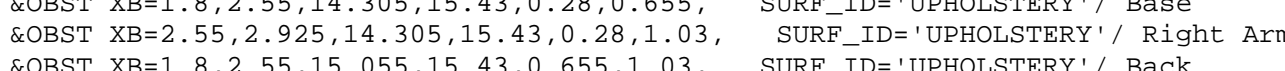

*** GROUP: CHAIR offset $=11.250000,0.000000,0.140000$ rotate $=0.000000$ \&OBST $X \mathrm{XB}=3.3,3.675,14.305,15.43,0.28,1.03, \quad$ SURF_ID= 'UPHOLSTERY ' / Left Arm \&OBST $X B=4.425,4.8,14.305,15.43,0.28,1.03$, SURF_ID='UPHOLSTERY' / Right Arm *** GROUP: CHAIR offset $=13.125000,0.000000,0.140000$ rotate $=0.000000$ \&OBST XB=5.175, 5.55, 14.305, 15.43, 0.28,1.03, SURF_ID= 'UPHOLSTERY ' / Left Arm \&OBST $\mathrm{XB}=6.3,6.675,14.305,15.43,0.28,1.03$, SURF_ID=' 'UPHOLSTERY'/ Right Arm \&OBST XB=5.55, 6.3, 15.055, 15.43, $0.655,1.03$, SURF_ID='UPHOLSTERY'/ BaCK

$* * *$ GROUP: CHAIR offset $=15.000000,0.000000,0.140000$ rotate $=0.000000$

\&OBST $X \mathrm{X}=7.05,7.425,14.305,15.43,0.28,1.03, \quad$ SURF-ID=' 'UPHOLSTERY'/ Left Arm
\&OBST $X \mathrm{~B}=7.425,8.175,14.305,15.43,0.28,0.655$, SURF ID='UPHOLSTERY'
Sase COBST $X B=8.175,8.55,14.305,15.43,0.28,1.03$,
\&OBST $X B=7.425,8.175,15.055,15.43,0.655,1.03$, SURF_IDE 'UPHOLSTERY'/ Right Arm
SURF_ID=' 'UPHOLSTERY'/ Back

*** GROUP: CHAIR_ROW2

** GROUP: CHAIR offset $=0.000000,0.000000,0.140000$ rotate $=0.000000$

\&OBST XB=-7.95,-7.575, 15.805, 16.93, $0.28,1.03, \quad$ SURF_ID='UPHOLSTERY'/ Left Arm
\&OBST XB=-7.575, $-6.825,15.805,16.93,0.28,0.655$, SURF_ID='UPHOLSTERY'/ Base \&OBST $X \mathrm{~B}=-6.825,-6.45,15.805,16.93,0.28,1.03$, SURF-ID='UPHOLSTERY'/ Right Ar

$* * *$ GROUP: CHAIR offset $=1.875000,0.000000,0.140000$ rotate $=0.000000$ \&OBST XB $=-6.075,-5.7,15.805,16.93,0.28,1.03, \quad$ SURF_ID=' 'UPHOLSTERY' '/ Left Arm
\&OBST XB =-5.7.

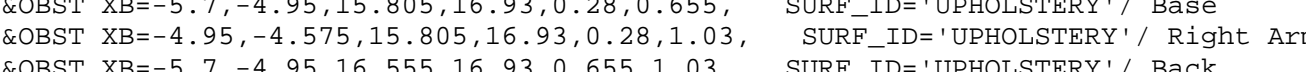

$* * *$ GROUP: CHAIR offset $=3.750000,0.000000,0.140000$ rotate $=0.000000$

\&OBST XB $=-4.2,-3.825,15.805,16.93,0.28,1.03$, SURF ID = 'UPHOLSTERY ' / Left Arm

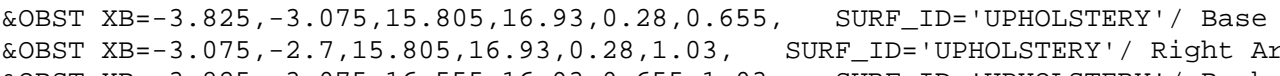

$* \star *$ GROUP: CHAIR offset $=5.625000,0.000000,0.140000$ rotate $=0.000000$

\&OBST XB $=-2.325,-1.95,15.805,16.93,0.28,1.03$, SURF_ID='UPHOLSTERY'/ Left Ar \&OBST XB=-1.95, -1.2, 15.805, 16.93, 0.28, , .655, SURF-ID='UPHOLSTERY'/ Base

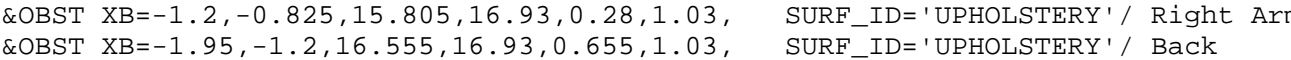

*** GROUP: CHAIR offset $=7.500000,0.000000,0.140000$ rotate $=0.000000$

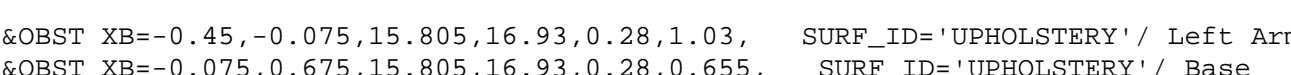
\&OBST $X \mathrm{~B}=0.675,1.05,15.805,16.93,0.28,1.03$, SURF_ID='UPHOLSTERY' '/ Right Ar

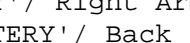

$* * *$ GROUP: CHAIR offset $=9.375000,0.000000,0.140000$ rotate $=0.000000$

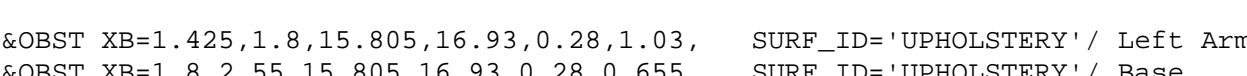

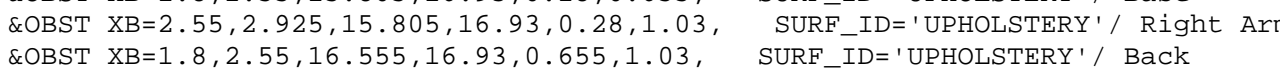

** GROUP: CHAIR offset $=11.250000,0.000000,0.140000$ rotate $=0.000000$

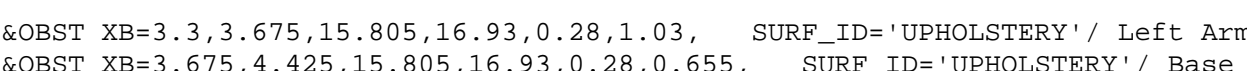
\&OBST $X \mathrm{XB}=4.425,4.8,15.805,16.93,0.28,1.03$, SURF_ID='UPHOLSTERY'/Right Arm
\&OBST $\mathrm{XB}=3.675,4.425,16.555,16.93,0.655,1.03, \quad$ SURF_ID=' 'UPHOLSTERY' $/$ Back
** GROUP: CHAIR offset $=13.125000,0.000000,0.140000$ rotate $=0.000000$

\&OBST $X \mathrm{XB}=5.175,5.55,15.805,16.93,0.28,1.03, \quad$ SURF_ID=' 'UPHOLSTERY '/ Left Arm
\&OBST $X \mathrm{~B}=5.55,6.3,15.805,16.93,0.28,0.655$,
SURF_IDD' UPHOLSTERY'/ Base \&OBST XB=6.3,6.675,15.805,16.93,0.28,1.03, SURF-ID=' UPHOLSTERY'/ Right Arm \&OBST XB $=5.55,6.3,16.555,16.93,0.655,1.03$, SURF_ID=' 'UPHOLSTERY'/ Back

*** GROUP: CHAIR offset $=15.000000$, ๑. ๑००००००,. .140000 rotate $=0.000000$

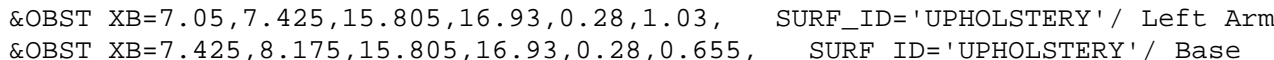
EOBST $X \mathrm{X}=7.425,8.175,15.805,16.93,0.28,0.655, \quad$ SURF-ID='UPHOLSTERY'
EOBST $X \mathrm{~B}=8.175,8.55,15.805,16.93,0.28,1.03$, \&OBST $X \mathrm{~B}=7.425,8.175,16.555,16.93,0.655,1.03$, SURF_ID=' UPHOLSTERY' $/$ Back

*** GROUP: LHS_REAR

** GROUP: WOOD ROW

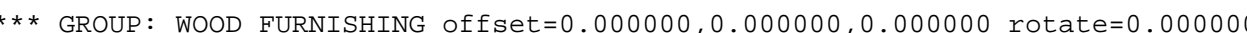
\&OBST XB=-7.95, - $6.825,18.42,19.17,0.14,1.64$, SURF_ID='WOOD FLOOR'/

*** GROUP: WOOD FURNISHING offset $=1.500000,0.000000,0.000000$ rotate $=0.000000$ \&OBST XB $=-6.45,-5.325,18.42,19.17,0.14,1.64$, SURF_ID='WOOD FLOOR' /

*** GROUP: WOOD FURNISHING offset $=3.000000,0.000000,0.000000$ rotate $=0.000000$ \&OBST XB=-4. $95,-3.825,18.42,19.17,0.14,1.64$, SURF_ID= 'WOOD FLOOR' /

** GROUP: WOOD FURNISHING offset $=4.500000,0.000000,0.000000$ rotate $=0.000000$ \&OBST XB $=-3.45,-2.325,18.42,19.17,0.14,1.64$, SURF_ID='WOOD FLOOR'/

*** GROUP: WOOD FURNISHING offset $=6.000000,0.000000,0.000000$ rotate $=0.000000$ \&OBST XB $=-1.95,-0.825,18.42,19.17,0.14,1.64$, SURF_ID='WOOD FLOOR'/

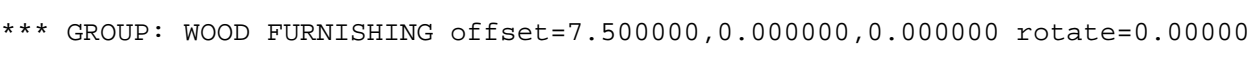
\&OBST XB=- $0.45,0.675,18.42,19.17,0.14,1.64, \quad$ SURF_ID= 'WOOD FLOOR'/

*** GROUP: WOOD FURNISHING offset $=9.000000,0.000000,0.000000$ rotate $=0.000000$ \&OBST XB=1.05, 2.175, 18.42, 19.17, 0.14,1.64, SURF_ID='WOOD FLOOR'

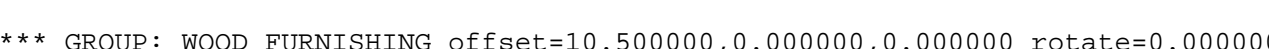
\&OBST XB=2.55, 3.675, 18.42, 19.17, $0.14,1.64$, SURF_ID='WOOD FLOOR'

*** GROUP: WOOD FURNISHING offset $=12.000000,0.000000,0.0000000$ rotate $=0.000000$ \&OBST XB=4.05, 5.175, 18.42, 19.17, 0.14,1.64, SURF_ID= 'WOOD FLOOR'/

*** GROUP: WOOD FURNISHING offset $=13.5000$. \&OBST XB=5.55, 6.675, 18.42, 19.17, 0.14,1.64, SURF_ID='WOOD FLOOR'/

$* * *$ GROUP: WOOD FURNISHING offset $=15.000000,0.000000,0.000000$ rotate $=0.000000$ \&OBST XB=7.05, 8.175, 18.42, 19.17, 0.14,1.64, SURF_ID='WOOD FLOOR' '

*** GROUP: WOOD ROW

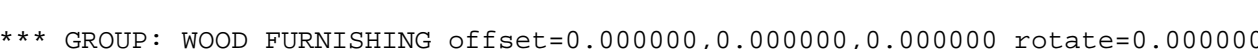
\&OBST XB=-7.95, $-6.825,19.545,20.295,0.14,1.64$, SURF_ID='WOOD FLOOR'

** GROUP: WOOD FURNISHING offset $=1.500000,0.000000,0.000000$ rotate $=0.000000$ \&OBST XB=-6.45, $-5.325,19.545,20.295,0.14,1.64, \quad$ SURF_ID='WOOD FLOOR'

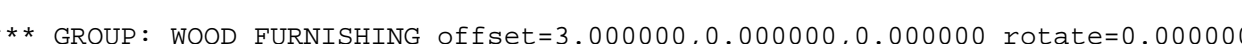
\&OBST XB=-4.95, $-3.825,19.545,20.295,0.14,1.64$, SURF_ID='WOOD FLOOR'/ 


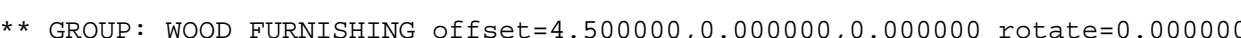
\&OBST XB $=-3.45,-2.325,19.545,20.295,0.14,1.64, \quad$ SURF_ID= 'WOOD FLOOR'

*** GROUP: WOOD FURNISHING offset $=6.000000,0.000000,0.000000$ rotate $=0.000000$ \&OBST XB $=-1.95,-0.825,19.545,20.295,0.14,1.64, \quad$ SURF_ID='WOOD FLOOR'

*** GROUP: WOOD FURNISHING offset $=7.500000,0.000000,0.000000$ rotate $=0.000000$ \&OBST XB $=-0.45,0.675,19.545,20.295,0.14,1.64$, SURF_ID='WOOD FLOOR' '

*** GROUP: WOOD FURNISHING offset $=9.000000,0.000000,0.000000$ rotate $=0.000000$ \&OBST XB=1. 05, 2.175, 19.545, 20.295, 0.14,1.64, SURF_ID='WOOD FLOOR'/

*** GROUP: WOOD FURNISHING offset $=10.500000,0.000000,0.000000$ rotate $=0.000000$ \&OBST XB=2.55, 3. 675, 19.545, 20.295, 0.14,1.64, SURF_ID='WOOD FLOOR' /

$* * *$ GROUP: WOOD FURNISHING of fset $=12.000000,0.000000,0.000000$ rotate $=0.000000$ \&OBST XB=4. $05,5.175,19.545,20.295,0.14,1.64$, SURF_ID='WOOD FLOOR' /

*** GROUP: WOOD FURNISHING offset $=13.500000,0.000000,0.000000$ rotate $=0.000000$ \&OBST XB=5.55, 6.675, 19.545, 20.295, $0.14,1.64$, SURF_ID='WOOD FLOOR' /

*** GROUP: WOOD FURNISHING offset $=15.000000,0.000000,0.000000$ rotate $=0.000000$ \&OBST XB=7. 05, 8.175, 19.545, 20.295, 0.14,1.64, SURF_ID='WOOD FLOOR' /

*** GROUP: WOOD ROW

$* * *$ GROUP: WOOD FURNISHING of fset $=0.000000,0.000000,0.000000$ rotate $=0.000000$ \&OBST XB=-7.95, - $6.825,20.67,21.42,0.14,1.64$, SURF_ID='WOOD FLOOR'/

*** GROUP: WOOD FURNISHING offset $=1.500000,0.000000,0.000000$ rotate $=0.000000$ \&OBST XB=-6.45, $-5.325,20.67,21.42,0.14,1.64$, SURF_ID='WOOD FLOOR' /

$* * *$ GROUP: WOOD FURNISHING offset $=3.000000,0.000000,0.000000$ rotate $=0.000000$ EOBST XB=-4.95, $-3.825,20.67,21.42,0.14,1.64$, SURF_ID='WOOD FLOOR'/

*** GROUP: WOOD FURNISHING offset $=4.500000,0.000000,0.000000$ rotate $=0.000000$ \&OBST XB=-3.45, $-2.325,20.67,21.42,0.14,1.64$, SURF_ID='WOOD FLOOR' /

*** GROUP: WOOD FURNISHING offset $=6.000000,0.000000,0.000000$ rotate $=0.000000$ \&OBST XB=-1.95, - $0.825,20.67,21.42,0.14,1.64$, SURF_ID='WOOD FLOOR' /

*** GROUP: WOOD FURNISHING offset $=7.500000,0.000000,0.000000$ rotate $=0.000000$ \&OBST XB=- $0.45,0.675,20.67,21.42,0.14,1.64$, SURF_ID=' 'WOOD FLOOR'

*** GROUP: WOOD FURNISHING offset $=9.000000,0.000000,0.000000$ rotate $=0.000000$ \&OBST XB=1.05, 2.175, 20.67, 21.42, 0.14,1.64, SURF_ID='WOOD FLOOR'/

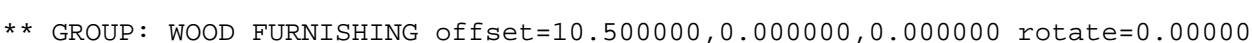
\&OBST XB=2.55, 3.675, 20.67, 21.42, $0.14,1.64, \quad$ SURF_ID='WOOD FLOOR'

** GROUP: WOOD FURNISHING offset $=12.000000,0.000000,0.000000$ rotate $=0.000000$ \&OBST XB=4. 05, 5.175, 20.67, 21.42, $0.14,1.64$, SURF_ID='WOOD FLOOR'/

*** GROUP: WOOD FURNISHING offset $=13.500000,0.000000,0.000000$ rotate $=0.000000$ \&OBST XB=5.55, 6. 675, 20.67, 21.42, $0.14,1.64$, SURF_ID='WOOD FLOOR' '

*** GROUP: WOOD FURNISHING offset $=15.000000,0.000000,0.000000$ rotate $=0.000000$ \&OBST XB=7. $05,8.175,20.67,21.42,0.14,1.64, \quad$ SURF_ID= 'WOOD FLOOR'/
** GROUP: WOOD ROW

*** GROUP: WOOD FURNISHING offset $=0.000000,0.000000,0.000000$ rotate $=0.000000$ \&OBST XB=-7.95, -6.825, 21.795, 22.545, 0.14,1.64, SURF_ID= 'WOOD FLOOR'/

*** GROUP: WOOD FURNISHING offset $=1.500000,0.000000,0.000000$ rotate $=0.000000$ \&OBST XB=-6.45,-5.325, 21.795, 22.545, 0.14,1.64, SURF_ID='WOOD FLOOR'

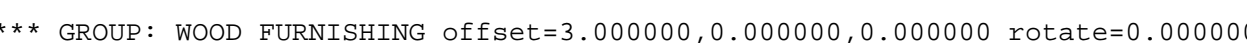
\&OBST XB=-4.95, $-3.825,21.795,22.545,0.14,1.64$, SURF_ID='WOOD FLOOR'

** GROUP: WOOD FURNISHING offset $=4.500000,0.000000,0.000000$ rotate $=0.000000$ \&OBST XB=-3.45, $-2.325,21.795,22.545,0.14,1.64$, SURF_ID='WOOD FLOOR'

*** GROUP: WOOD FURNISHING offset $=6.000000,0.000000,0.000000$ rotate $=0.000000$ \&OBST XB=-1.95, $-0.825,21.795,22.545,0.14,1.64$, SURF_ID= 'WOOD FLOOR'/

*** GROUP: WOOD FURNISHING offset $=7.500000,0.000000,0.000000$ rotate $=0.000000$ \&OBST XB=- $-4.45,0.675,21.795,22.545,0.14,1.64$, SURF_ID=' 'WOOD FLOOR'/

*** GROUP: WOOD FURNISHING offset $=9.000000,0.000000,0.000000$ rotate $=0.000000$ \&OBST XB=1. 05, 2.175, 21.795, 22.545, 0.14, 1.64, SURF_ID='WOOD FLOOR'/

** GROUP: WOOD FURNISHING offset $=10.500000,0.000000,0.000000$ rotate $=0.000000$ \&OBST XB=2.55, 3. 675, 21.795, 22.545, 0.14,1.64, SURF_ID='WOOD FLOOR' /

*** GROUP: WOOD FURNISHING offset $=12.000000,0.000000,0.000000$ rotate $=0.000000$ \&OBST XB=4.05, 5.175, 21.795, 22.545, 0.14,1.64, SURF_ID='WOOD FLOOR' /

*** GROUP: WOOD FURNISHING offset $=13.500000,0.000000,0.000000$ rotate $=0.000000$ \&OBST XB=5.55, 6.675, 21.795, 22.545, 0.14,1.64, SURF_ID='WOOD FLOOR' /

*** GROUP: WOOD FURNISHING offset $=15.000000,0.000000$,, .000000 rotate $=0.000000$ \&OBST XB=7.05, 8.175, 21.795, 22.545, 0.14,1.64, SURF_ID='WOOD FLOOR'/

*** GROUP: WOOD ROW

*** GROUP: WOOD FURNISHING offset $=0.000000,0.000000,0.000000$ rotate $=0.000000$ \&OBST XB=-7.95, $-6.825,22.92,23.67,0.14,1.64$, SURF_ID='WOOD FLOOR'/

*** GROUP: WOOD FURNISHING offset $=1.500000,0.000000,0.000000$ rotate $=0.000000$ \&OBST XB=-6.45, $-5.325,22.92,23.67,0.14,1.64$, SURF_ID='WOOD FLOOR' /

*** GROUP: WOOD FURNISHING of $f$ set $=3.000000,0.000000,0.000000$ rotate $=0.000000$ \&OBST XB=-4.95, $-3.825,22.92,23.67,0.14,1.64$, SURF_ID='WOOD FLOOR' /

*** GROUP: WOOD FURNISHING offset $=4.5000000,0.0000000,0.000000$ rotate $=0.000000$ \&OBST XB=-3.45, $-2.325,22.92,23.67,0.14,1.64$, SURF_ID='WOOD FLOOR'/

** GROUP: WOOD FURNISHING offset $=6.000000, \bullet . ๑ 00000, \odot . .000000$ rotate $=0.000000$ \&OBST XB=-1.95, $-0.825,22.92,23.67,0.14,1.64$, SURF_ID='WOOD FLOOR'/

*** GROUP: WOOD FURNISHING offset $=7.500000,0.000000,0.000000$ rotate $=0.000000$ \&OBST XB=- $0.45,0.675,22.92,23.67,0.14,1.64$, SURF_ID=' 'WOOD FLOOR'

*** GROUP: WOOD FURNISHING offset $=9.000000,0.0000000,0.000000$ rotate $=0.000000$ \&OBST XB=1.05, 2.175, 22.92, 23.67, 0.14, 1.64, SURF_ID='WOOD FLOOR' ' 
*** GROUP: WOOD FURNISHING of fset $=10.500000,0.000000,0.000000$ rotate $=0.000000$ \&OBST XB=2.55, 3.675, 22.92, 23.67, 0.14,1.64, SURF_ID='WOOD FLOOR'/

*** GROUP: WOOD FURNISHING offset $=12.000000,0.000000,0.000000$ rotate $=0.000000$ \&OBST XB=4.05, 5.175, 22.92, 23.67, $0.14,1.64$, SURF_ID='WOOD FLOOR'

$* * *$ GROUP: WOOD FURNISHING offset $=13.500000,0.000000,0.000000$ rotate $=0.000000$ \&OBST XB=5.55, 6.675, 22.92, 23.67, 0.14,1.64, SURF_ID='WOOD FLOOR'/

*** GROUP: WOOD FURNISHING offset $=15.000000,0.000000,0.000000$ rotate $=0.000000$ EOBST $X B=7.05,8.175,22.92,23.67,0.14,1.64, \quad$ SURF_ID='WOOD FLOOR'/

$* * *$ GROUP: WOOD ROW

*** GROUP: WOOD FURNISHING offset $=0.000000,0.000000$, ๑. .000000 rotate $=0.000000$ \&OBST XB=-7.95, -6.825, 24. 045, 24.795, 0.14,1.64, SURF_ID=' 'WOOD FLOOR'/

*** GROUP: WOOD FURNISHING offset $=1.500000,0.000000,0.000000$ rotate $=0.000000$ \&OBST XB=-6.45, -5.325, 24. 045, 24.795, 0.14,1.64, SURF_ID='WOOD FLOOR'

** GROUP: WOOD FURNISHING offset $=3,000000,0,000000,0.000000$ rotate $=0.000000$ \&OBST XB=-4.95, $-3.825,24.045,24.795,0.14,1.64$, SURF_ID='WOOD FLOOR'

*** GROUP: WOOD FURNISHING offset $=4.500000,0.000000,0.000000$ rotate $=0.000000$ \&OBST XB=-3.45, - 2.325, 24. 045, 24.795, 0.14,1.64, SURF_ID= 'WOOD FLOOR'/

*** GROUP: WOOD FURNISHING offset $=6.000000,0.000000,0.000000$ rotate $=0.000000$ EOBST $X B=-1.95,-0.825,24.045,24.795,0.14,1.64$, SURF_ID= 'WOOD FLOOR',

*** GROUP: WOOD FURNISHING offset $=7.500000,0.000000,0.000000$ rotate $=0.000000$ \&OBST XB=- $0.45,0.675,24.045,24.795,0.14,1.64, \quad$ SURF_ID=' WOOD FLOOR'

$* * *$ GROUP: WOOD FURNISHING offset $=9.000000,0.000000,0.000000$ rotate $=0.000000$ EOBST XB=1.05, 2.175, 24. 045, 24.795, 0.14,1.64, SURF_ID='WOOD FLOOR'/

*** GROUP: WOOD FURNISHING offset $=10.500000,0.000000,0.000000$ rotate $=0.000000$ \&OBST XB=2.55, 3.675, 24. 045, 24.795, 0.14,1.64, SURF_ID='WOOD FLOOR' /

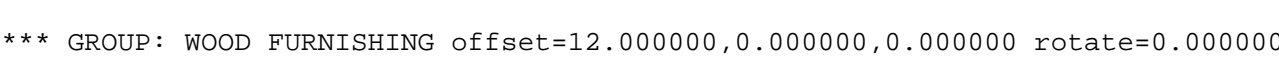
\&OBST XB=4. 05, 5.175, 24. 045, 24.795, 0.14,1.64, SURF_ID='WOOD FLOOR' /

*** GROUP: WOOD FURNISHING offset $=13.500000,0.000000,0.000000$ rotate $=0.000000$ \&OBST XB=5.55, 6.675, 24. 045, 24.795, 0.14,1.64, SURF_ID='WOOD FLOOR'/

*** GROUP: WOOD FURNISHING offset $=15.0000000, \Theta . .000000,0.000000$ rotate $=0.000000$ \&OBST XB=7. 05, 8.175, 24.045, 24.795, 0.14,1.64, SURF_ID= 'WOOD FLOOR' /

*** GROUP: WOOD ROW

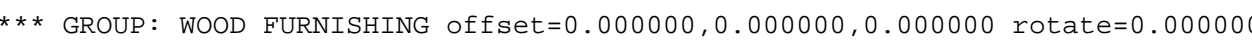
\&OBST XB=-7.95, - $6.825,25.17,25.92,0.14,1.64$, SURF_ID='WOOD FLOOR' /

*** GROUP: WOOD FURNISHING offset $=1.500000,0.000000,0.000000$ rotate $=0.000000$ \&OBST XB=-6.45, $-5.325,25.17,25.92,0.14,1.64$, SURF_ID= 'WOOD FLOOR' /

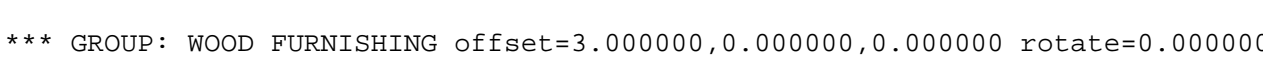
\&OBST XB=-4.95, $-3.825,25.17,25.92,0.14,1.64$, SURF_ID='WOOD FLOOR' $/$

*** GROUP: WOOD FURNISHING offset $=4.500000,0.000000,, 0.000000$ rotate $=0.000000$
\&OBST XB=-3.45, $-2.325,25.17,25.92,0.14,1.64$, SURF_ID='WOOD FLOOR'/ ** GROUP: WOOD FURNISHING offset $=6.000000,0.000000,0.000000$ rotate $=0.000000$ \&OBST XB=-1.95, $-0.825,25.17,25.92,0.14,1.64$, SURF_ID='WOOD FLOOR'/

*** GROUP: WOOD FURNISHING offset $=7.500000,0.000000,0.000000$ rotate $=0.000000$ \&OBST XB=- $0.45,0.675,25.17,25.92,0.14,1.64$, SURF_ID='WOOD FLOOR'

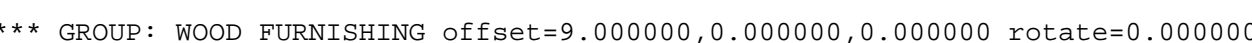
\&OBST XB=1.05, 2.175, 25.17, 25.92, 0.14,1.64, SURF_ID='WOOD FLOOR'/

** GROUP: WOOD FURNISHING offset $=10.500000,0.000000,0.000000$ rotate $=0.000000$ \&OBST XB=2.55, 3.675, 25.17, 25.92, 0.14,1.64, SURF_ID='WOOD FLOOR'/

*** GROUP: WOOD FURNISHING off fet $=12.000000,0.000000,0.000000$ rotate $=0.000000$ \&OBST XB=4.05, 5.175, 25.17, 25.92, 0.14,1.64, SURF_ID='WOOD FLOOR'/

*** GROUP: WOOD FURNISHING offset $=13.500000,0.000000,0.000000$ rotate $=0.000000$ \&OBST XB=5.55, $6.675,25.17,25.92,0.14,1.64, \quad$ SURF_ID='WOOD FLOOR'/

*** GROUP: WOOD FURNISHING of fset $=15.000000,0.000000,0.000000$ rotate $=0.000000$ EOBST $X B=7.05,8.175,25.17,25.92,0.14,1.64$, SURF_ID='WOOD FLOOR'

*** GROUP: WOOD ROW

*** GROUP: WOOD FURNISHING offset $=0.000000,0.000000,0.000000$ rotate $=0.000000$ \&OBST XB=-7.95, -6.825, 26.295, 27.045, 0.14,1.64, SURF_ID='WOOD FLOOR'/

*** GROUP: WOOD FURNISHING offset $=1.500000,0.000000,0.000000$ rotate $=0.000000$ \&OBST XB=-6.45, $-5.325,26.295,27.045,0.14,1.64$, SURF_ID=' 'WOOD FLOOR', *** GROUP: WOOD FURNISHING offset $=3.000000,0.000000,0.000000$ rotate $=0.000000$ \&OBST XB=-4.95, - $3.825,26.295,27.045,0.14,1.64$, SURF_ID='WOOD FLOOR'/

*** GROUP: WOOD FURNISHING offset $=4.500000,0.000000,0.000000$ rotate $=0.000000$ \&OBST XB $=-3.45,-2.325,26,295,27,045,0.14,1.64$, SURF_ID= 'WOOD FLOOR',

*** GROUP: WOOD FURNISHING offset $=6.000000,0.000000,0.000000$ rotate $=0.000000$ \&OBST $X B=-1.95,-0.825,26.295,27.045,0.14,1.64, \quad$ SURF_ID= 'WOOD FLOOR'/

*** GROUP: WOOD FURNISHING offset $=7.500000,0.000000,0.000000$ rotate $=0.000000$ \&OBST XB $=-0.45,0.675,26.295,27.045,0.14,1.64$, SURF_ID='WOOD FLOOR' /

*** GROUP: WOOD FURNISHING of $f$ set $=9.000000,0.000000,0.000000$ rotate $=0.000000$ \&OBST XB=1.05, 2.175, 26.295, 27.045, 0.14,1.64, SURF_ID='WOOD FLOOR'/

*** GROUP: WOOD FURNISHING offset $=10.500000,0.000000,, 0.000000$ rotate $=0.000000$ \&OBST XB=2.55, 3.675, 26.295, 27.045, 0.14,1.64, SURF_ID='WOOD FLOOR'/

*** GROUP: WOOD FURNISHING offset $=12.0000000,0.000000,, 0.0000000$ rotate $=0.0000000$ \&OBST XB=4.05, 5.175, 26.295, 27.045, 0.14,1.64, SURF_ID='WOOD FLOOR' /

*** GROUP: WOOD FURNISHING offset $=13.500000,0.000000,0.000000$ rotate $=0.000000$ \&OBST XB=5.55, 6.675, 26.295, 27. 045, 0.14,1.64, SURF_ID='WOOD FLOOR' /

*** GROUP: WOOD FURNISHING offset $=15.000000,0.000000,0.000000$ rotate $=0.000000$ \&OBST $X B=7.05,8.175,26.295,27.045,0.14,1.64$, SURF_ID= 'WOOD FLOOR' I 
** GROUP: WOOD ROW

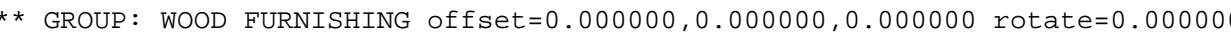
\&OBST XB=-7.95, $-6.825,27.42,28.17,0.14,1.64$, SURF_ID='WOOD FLOOR'/

$\star * \star$ GROUP: WOOD FURNISHING offset $=1.500000,0.000000,0.000000$ rotate $=0.000000$ \&OBST XB=-6.45, $-5.325,27.42,28.17,0.14,1.64$, SURF_ID='WOOD FLOOR' /

*** GROUP: WOOD FURNISHING offset $=3.000000,0.000000,0.000000$ rotate $=0.000000$ \&OBST XB=-4.95, $-3.825,27.42,28.17,0.14,1.64$, SURF_ID='WOOD FLOOR' /

*** GROUP: WOOD FURNISHING of $f$ set $=4.500000,0.000000,0 . .000000$ rotate $=0.000000$ \&OBST XB=-3.45, $-2.325,27.42,28.17,0.14,1.64$, SURF_ID='WOOD FLOOR' $/$

$* * *$ GROUP: WOOD FURNISHING offset $=6.000000,0 . \bullet 00000,0 . .000000$ rotate $=0 . .000000$ \&OBST XB=-1.95, - $0.825,27.42,28.17,0.14,1.64$, SURF_ID= 'WOOD FLOOR' $/$

*** GROUP: WOOD FURNISHING offset $=7.500000,0.000000,0.000000$ rotate $=0.000000$ \&OBST XB=-0.45, $0.675,27.42,28.17,0.14,1.64$, SURF_ID=' 'WOOD FLOOR'

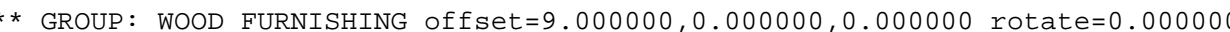
\&OBST XB=1.05, 2.175, 27.42, 28.17, 0.14,1.64, SURF_ID= 'WOOD FLOOR'/

*** GROUP: WOOD FURNISHING offset $=10.500000,0.000000, \Theta .000000$ rotate $=0.000000$ \&OBST XB=2.55, 3.675, 27.42, 28.17, $0.14,1.64$, SURF_ID= 'WOOD FLOOR'/

*** GROUP: WOOD FURNISHING offset $=12.000000,0.000000,0.000000$ rotate $=0.000000$ \&OBST $X \mathrm{XB}=4.05,5.175,27.42,28.17,0.14,1.64, \quad$ SURF_ID= 'WOOD FLOOR'

$* \star *$ GROUP: WOOD FURNISHING offset $=13.500000, \Theta . \odot 00000, \Theta . \diamond 00000$ rotate $=0.000000$ \&OBST XB=5.55, 6.675, 27.42, 28.17, $0.14,1.64$, SURF_ID='WOOD FLOOR'

*** GROUP: WOOD FURNISHING offset $=15.0000000,0.0000000,0.000000$ rotate $=0.000000$ \&OBST $X \mathrm{~B}=7.05,8.175,27.42,28.17,0.14,1.64, \quad$ SURF_ID='WOOD FLOOR'/

*** GROUP: LR_ROW

*** GROUP: LR

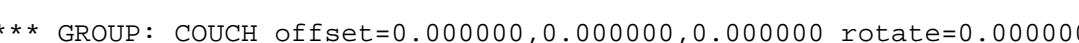

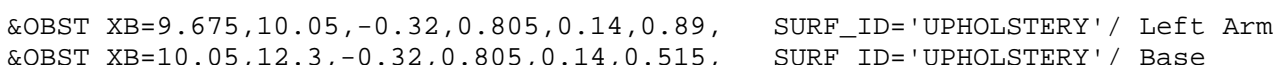

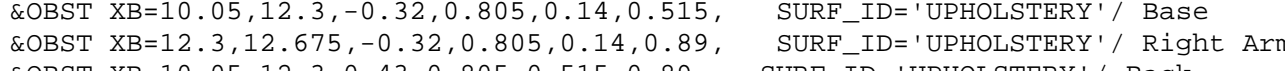

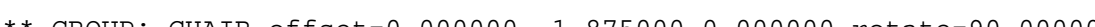

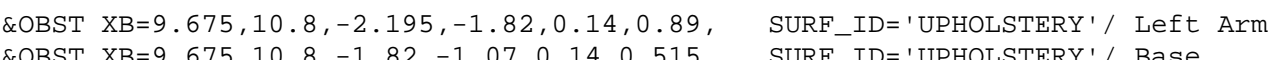

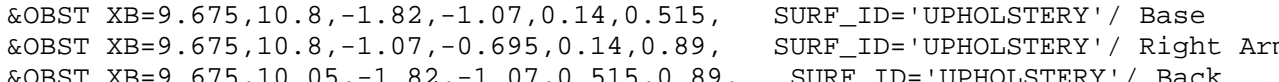

$* * *$ GROUP: CHAIR offset $=1.875000,-1.875000,0.000000$ rotate $=270.000000$

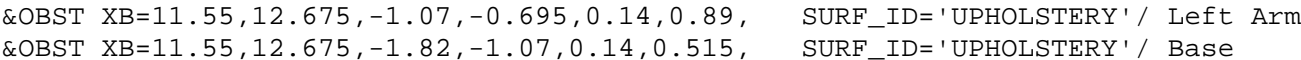

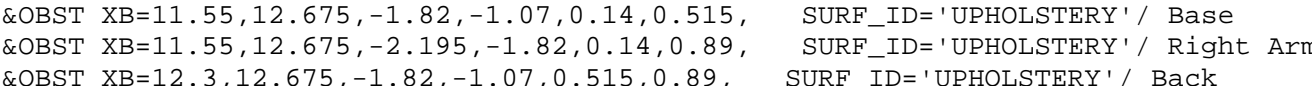

*** GROUP: LR

$* * *$ GROUP: COUCH offset $=0.000000, \odot . \bullet .00000,0.000000$ rotate $=0 . \bullet 000000$
\&OBST XB $=13.05,13.425,-0.32,0.805,0.14,0.89$, SURF_ID= 'UPHOLSTERY' / Left Ar

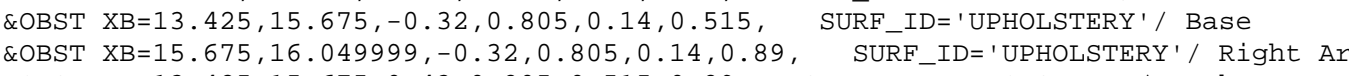

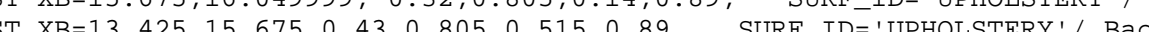

*** GROUP: CHAIR offset $=0.000000,-1.875000,0.000000$ rotate $=90.000000$

\&OBST XB=13.05, 14.175, $-2.195,-1.82,0.14,0.89$, SURF_ID=' 'UPHOLSTERY' ' Left Arm \&OBST XB=13.05,14.175,-1.07,-0.695,0.14,0.89, SURF_ID=' 'UPHOLSTERY'// Right Arm

$* * *$ GROUP: CHAIR offset $=1.875000,-1.875000,0.000000$ rotate $=270.000000$

\&OBST XB=14.925, 16. $049999,-1.07,-0.695,0.14,0.89, \quad$ SURF_ID= ' 'UPHOLSTERY ' / Left Arm

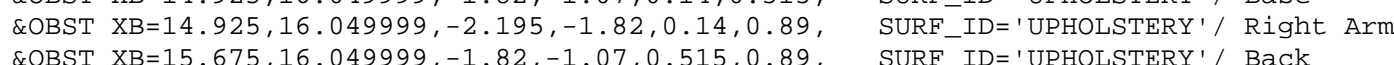

*** GROUP: LR

*** GROUP: COUCH offset $=0.000000,0.000000,0.000000$ rotate $=0.000000$

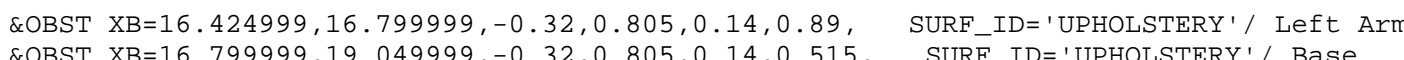
\&OBST XB=19.049999, $19.424999,-0.32,0.805,0.14,0.815$, SURF_ID='UPHOLSTERY'/ Righ \&OBST XB $=16.799999,19.049999,0.43,0.805,0.515,0.89$, SURF_ID='UPHOLSTERY' / Back

** GROUP: CHAIR offset $=0.000000,-1.875000,0.000000$ rotate $=90.000000$

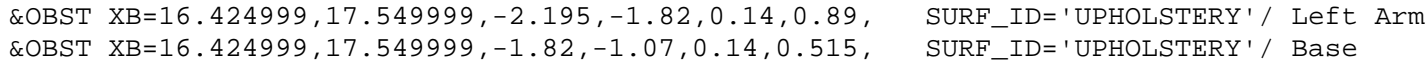

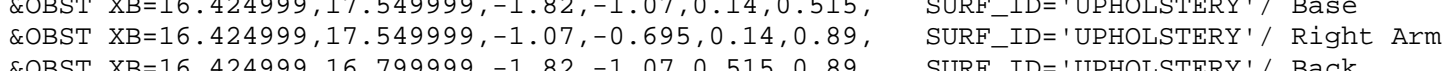

** GROUP: CHATR offset $=1.875000,-1.875000,0.000000$ rotate $=270.0000000$

\&OBST XB=18.299999, 19.424999, $-1.07,-0.695,0.14,0.89, \quad$ SURF_ID='UPHOLSTERY' ' Left Arm

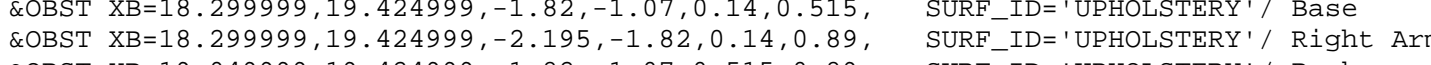

\section{$* * *$ GROUP: LR}

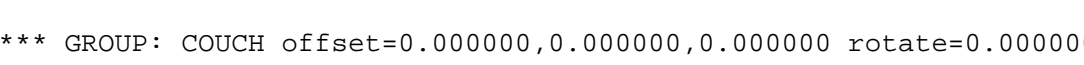

\&OBST XB $=19.799999,20.174999,-0.32,0.805,0.14,0.89$, SURF_ID='UPHOLSTERY'/ Left Arm \&OBST XB=20.174999, 22.424999, - $0.32,0.805,0.14,0.515$, SURF_ID='UPHOLSTERY'/ Base \&OBST XB $=22.424999,22.799999,-0.32,0.805,0.14,0.89$,
\&OBST XB SURF-ID= 'UPHOLSTERY'/ Right Ar

$* * *$ GROUP: CHAIR offset $=0.000000,-1.875000,0.000000$ rotate $=90.000000$

\&OBST XB $=19.799999,20.924999,-2.195,-1.82,0.14,0.89, \quad$ SURF_ID=' 'UPHOLSTERY'/ Left Arm \&OBST XB=19.799999, $20.924999,-1.07,-0.695,0.14,0.89$, SURF-ID='UPHOLSTERY'/' Base S
SURE_ID ='UPHOLSTERY'/ Back

$* * *$ GROUP: CHAIR offset $=1.875000,-1.875000,0.000000$ rotate $=270.000000$

\&OBST XB=21.674999, 22.799999, $-1.07,-0.695,0.14,0.89$, SURF_ID='UPHOLSTERY'/ Left Arm \&OBST $X \mathrm{~B}=21.674999,22.799999,-2.195,-1.82,0.14,0.89, \quad$ SURF_ID= 'UPHOLSTERY' $/$ Base

*** GROUP: COUCH offset $=12.750000,-1.875000,0.000000$ rotate $=90.000000$

\&OBST XB $=23.174999,24.299999,-2.195,-1.82,0.14,0.89$, SURF_ID='UPHOLSTERY' / Left Ar \&OBST XB=23.174999, 24. 299999, -1.82, $0.43,0.14,0.515$, SURF_ID='UPHOLSTERY'/ Base \&OBST XB $X$ B $=23.174999,23.549999,-1.82,0.43,0.515,0.89$, SURF

*** GROUP: COUCH offset $=14.250000,-1.875000,0.0000000$ rotate $=90.000000$ 
\&OBST XB=24.674999, 25.799999, $-2.195,-1.82,0.14,0.89, \quad$ SURF_ID=' 'UPHOLSTERY '/ Left Arm
\&OBST XB=24.674999, 25.799999, $-1.82,0.43,0.14,0.515, \quad$ SURF_ID='UPHOLSTERY'/ Base

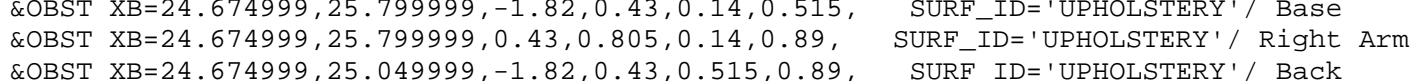

*** GROUP: LR

*** GROUP: COUCH offset $=0.000000,0.000000,0.000000$ rotate $=0.000000$

\&OBST XB=44.915001, 45.290001, - $0.32,0.805,0.14,0.89$, SURF_ID='UPHOLSTERY' / Left Arm

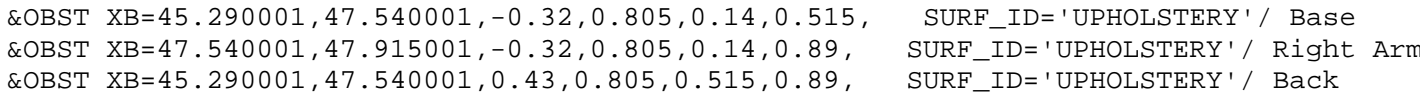

*** GROUP: CHAIR offset $=0.000000,-1.875000,0.000000$ rotate $=90 . .000000$

\&OBST XB=44.915001, 46. $040001,-2.195,-1.82,0.14,0.89$, SURF_ID= 'UPHOLSTERY' / Left Arm \&OBST XB=44.915001, 46. $040001,-1.82,-1.07,0.14,0.515$, SURF_ID='UPHOLSTERY'// Base عOBST XB=44.91501, $45.290001,-1.82,-1,07,0.515,8.89$, SURF-ID= 'UPHOLSTERY'/ Right A *** GROUP: CHAIR offset $=1.875000,-1.875000,0.000000$ rotate $=270.000000$ \&OBST XB=46.790001, 47.915001, $-1.07,-0.695,0.14,0.89$, SURF_ID='UPHOLSTERY'/ Left Arm \&OBST XB=46.790001, 47.915001, -1.82,-1.07, 0.14, 0.515 , SURF_ID='UPHOLSTERY'/ Base \&OBST XB=46.79001, 47.915001, $-2.195,-1.82,0.14,0.89$, SURF_IDD' 'UPHOLSTERY'/ Right A

$* * *$ GROUP: LR

** GROUP: COUCH offset $=0.000000,0.000000,0.000000$ rotate $=0.000000$

\&OBST XB=41.540001, 41.915001, - $0.32,0.805,0.14,0.89$, SURF_ID='UPHOLSTERY'/ Left Arm \&OBST XB=41.915001, 44.164997, - $0.32,0.805,0.14,0.515$, SURF_ID=' 'UPHOLSTERY'/ Base

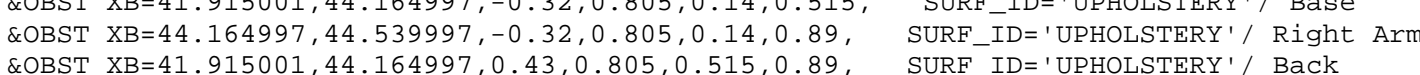

*** GROUP: CHAIR offset $=0.000000,-1.875000,0.000000$ rotate $=90.000000$

\&OBST XB=41.540001, 42.664997, $-2.195,-1.82,0.14,0.89$, \&OBST $X B=41.540001,42.664997,-1.82,-1.07,0.14,0.515$, \&OBST XB=41.540001, 42.664997,-1.07,-0.695, $14.14,0.89$,

SURE ID= 'UPHOLSTERY' / Left Arm

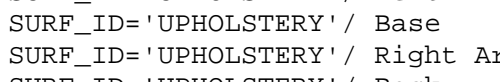
SURF_ID ='UPHOLSTER

*** GROUP: CHAIR offset $=1.875000,-1.875000,0.000000$ rotate $=270.000000$

LOBST XB=43.414997, 44.539997, $-1.07,-0.695,0.14,0.89, \quad$ SURF_ID= 'UPHOLSTERY Y' Left Arm \&OBST XB=43.414997, 44.539997, $-2.195,-1.82,0.14,0.89$, SURF_ID='UPHOLSTERY'/ Right A

*** GROUP: LR

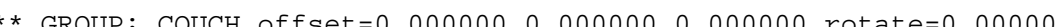

OOBST XB $=38.165001,38.540001,-0.32,0.805,0.14,0.89$, SURF_ID='UPHOLSTERY' ' Left Arm

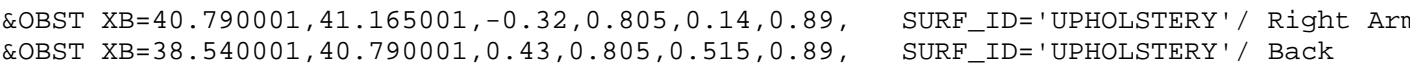

*** GROUP: CHAIR offset $=0.000000,-1.875000,0.000000$ rotate $=90 . .00000$

\&OBST XB=38.165001, 39. 290001, - 2.195,-1.82, $0.14,0.89$, SURF_ID='UPHOLSTERY' ' Left Arm

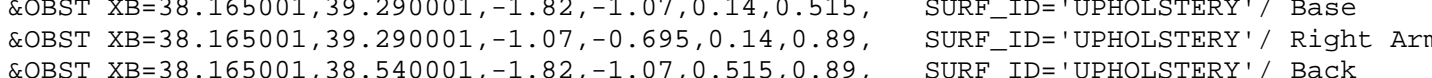

$* * *$ GROUP: CHAIR offset $=1.875000,-1.875000,0.000000$ rotate $=270.0000000$

\&OBST XB=40.040001,41.165001, $-1.07,-0.695,0.14,0.89, \quad$ SURF_ID='UPHOLSTERY' '/ Left Arm

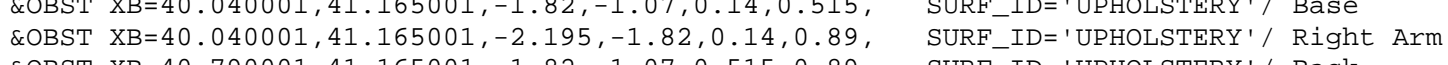

*** GROUP: LR

*** GROUP: COUCH offset $=0.000000,0.0000000,0.000000$ rotate $=0.000000$

\&OBST XB $=34.790001,35.165001,-0.32,0.805,0.14,0.89$, SURF_ID='UPHOLSTERY'/ Left Arm

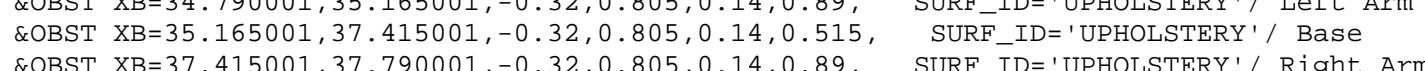
\&OBST XB=37.415001, $37.790001,-0.32,0.805,0.14,0.89$, SURF_ID ='UPHOLSTERY'/Right A

*** GROUP: CHATR offset $=0.0000000,-1.875000,0.000000$ rotate $=90.000000$

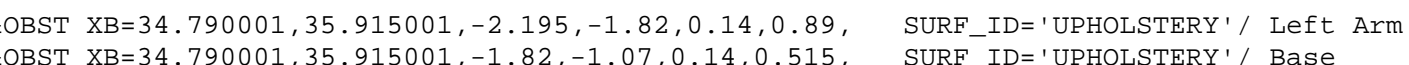

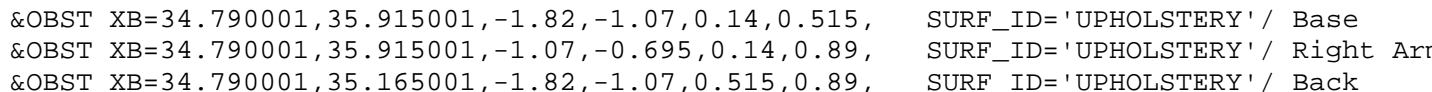

$* * *$ GROUP: CHAIR offset $=1.875000,-1.875000,0.000000$ rotate $=270.000000$

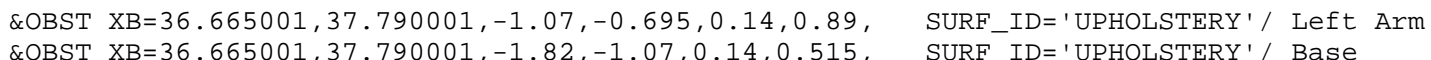
\&OBST XB=36.665001,37.790001,-2.195,-1.82,0.14,0.89, SURF-ID=' 'UPHOLSTERY'/, Right A

*** GROUP: CouCH offset $=22.865000,-1.875000,0.000000$ rotate $=270.0000000$

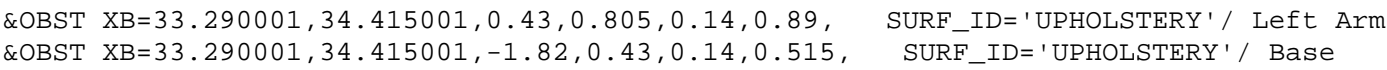

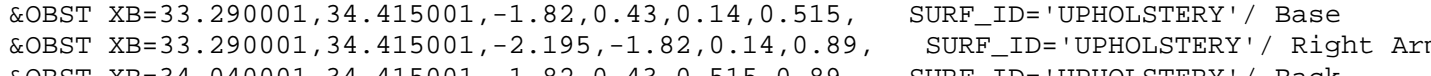

*** GROUP: COUCH offset $=21.365000,-1.875000,0.000000$ rotate $=270.000000$

OBST XB=31.790001, 32.915001, $0.43,0.805,0.14,0.89$, SURF ID='UPHOLSTERY' ' Left Arm ZOBST XB=31.

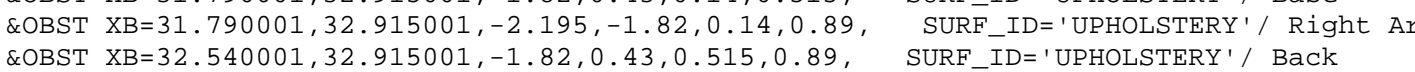

\section{*** GROUP: LR_ROW}

$* * *$ GROUP: LR

*** GROUP: COUCH offset $=0.000000,0.000000,0.000000$ rotate $=0.000000$

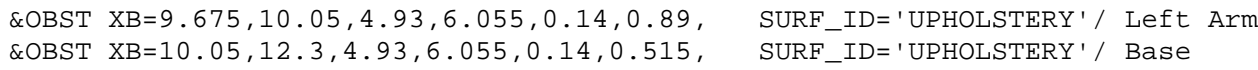

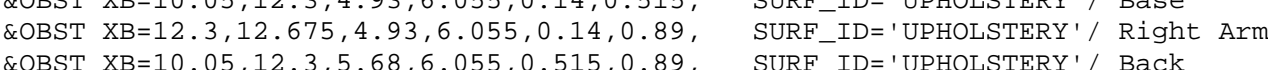

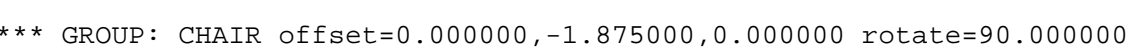

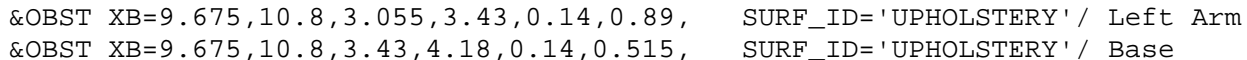

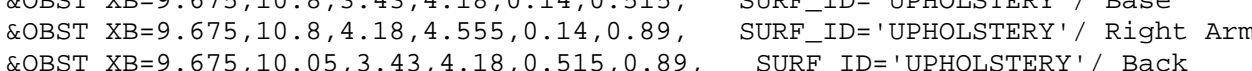

$* * *$ GROUP: CHAIR offset $=1.875000,-1.875000,0.000000$ rotate $=270.0000000$

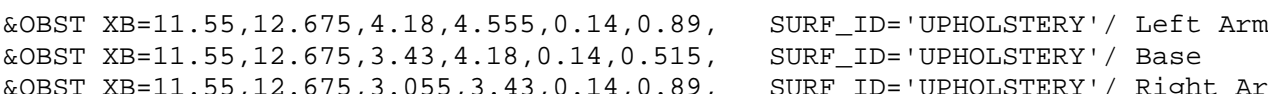

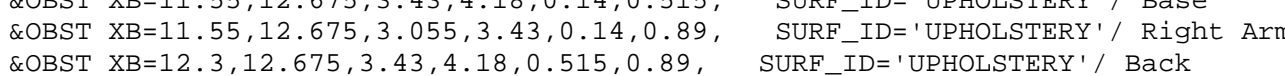

$* * *$ GROUP: LR

*** GROUP: COUCH offset $=0.000000,0.000000,0.000000$ rotate $=0.000000$

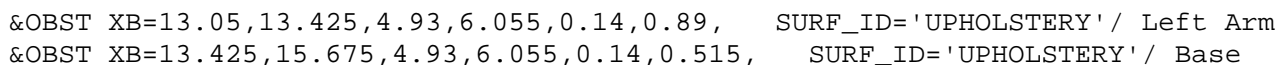

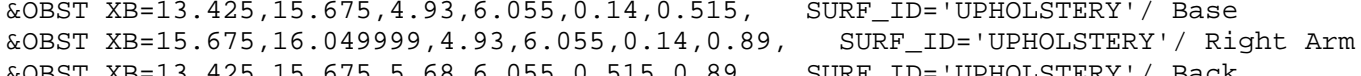




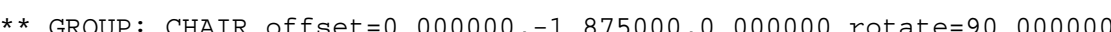

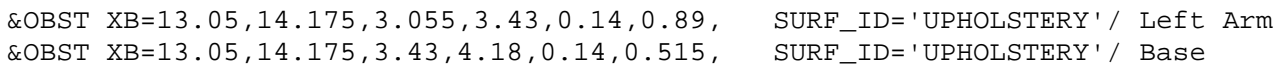

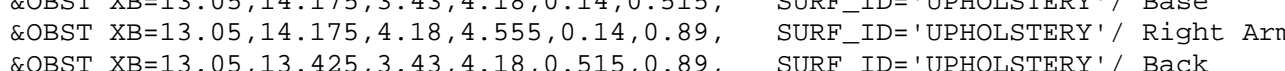

*** GROUP: CHAIR offset $=1.875000,-1.875000,0.000000$ rotate $=270.000000$ \&OBST XB=14.925, 16. $049999,4.18,4.555,0.14,0.89$, SURF_ID='UPHOLSTERY' ' Left Arm

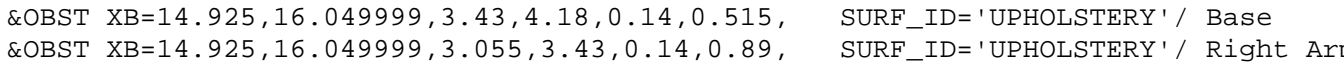

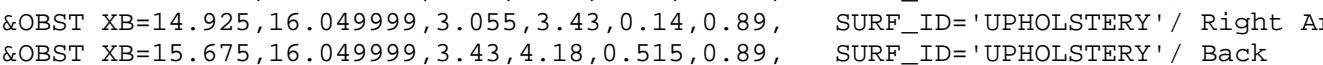

*** GROUP: LR

*** GROUP: COUCH offset $=0.000000,0.000000,0.000000$ rotate $=0.0000000$

\&OBST XB=16.424999, 16.799999, 4.93,6.055, 0.14, 0.89, SURF_ID='UPHOLSTERY' ' Left Arm

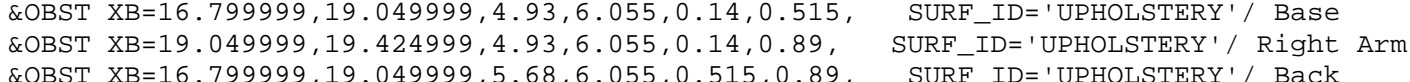

*** GROUP: CHAIR offset $=0.000000,-1.875000,0.000000$ rotate $=90.000000$

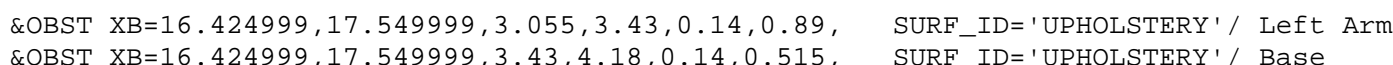

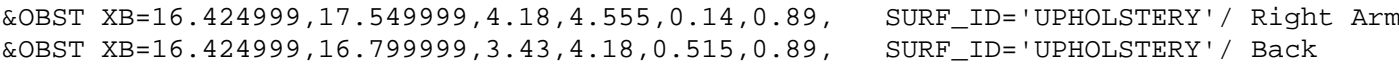
$* * *$ GROUP: CHAIR offset $=1.875000,-1.875000,0.000000$ rotate $=270.000000$

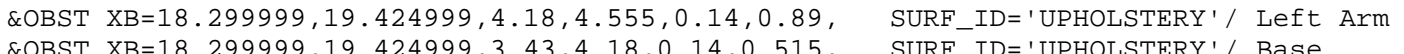
\&OBST XB=18.299999, 19.42499, 3.055, 3.43, $0.14,0.89$,
\&OBST XB $=19.049999,19.424999,3.43,4.18,0.515,0.89$,

*** GROUP: LR

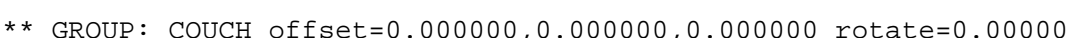

\&OBST XB $=19.799999,20.174999,4.93,6.055,0.14,0.89$, SURF_ID=' 'UPHOLSTERY' $/$ Left Arm

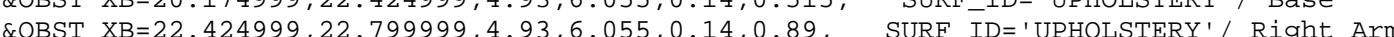

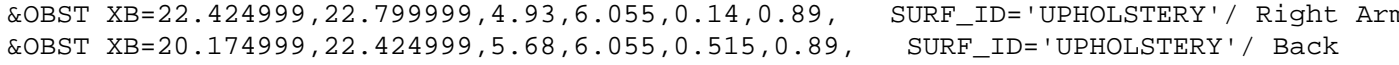

*** GROUP: CHAIR offset $=0.000000,-1.875000,0.000000$ rotate $=90.000000$

\&OBST XB=19.799999, 20.924999, 3. 055, 3.43, 0.14, 0.89 , SURF_ID= 'UPHOLSTERY '/ Left Arm

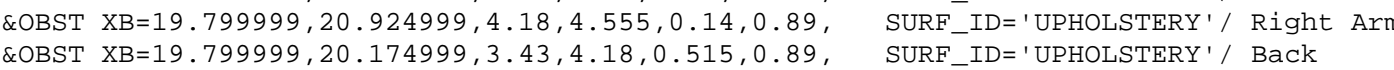

*** GROUP: CHAIR offset $=1.875000,-1.875000,0.000000$ rotate $=270.000000$

\&OBST XB=21.674999, 22.799999, 4.18,4.555, 0.14, 0.89 , SURF_ID=' 'UPHOLSTERY ' / Left Arm

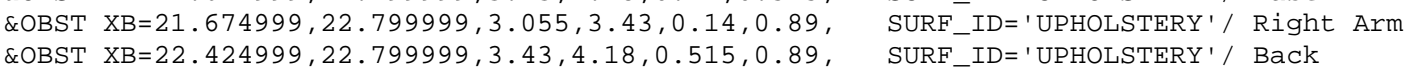

*** GROUP: COUCH offset $=12.750000,-1.875000,0.000000$ rotate $=90.000000$

\&OBST XB=23.174999, 24. 299999, 3. 055, 3.43, 0.14, 0.89, SURF_ID=' 'UPHOLSTERY' '/ Left Arm

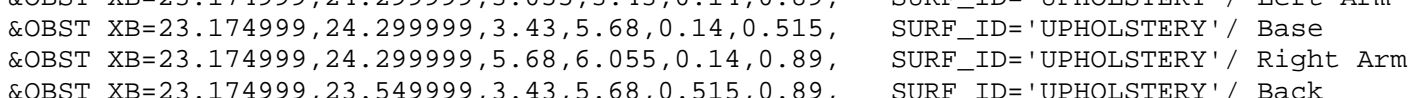

*** GROUP: COUCH offset $=14.250000,-1.875000,0.000000$ rotate $=90.000000$
\&OBST XB=24.674999, 25.799999, 3. $055,3.43,0.14,0.89$, SURF_ID='UPHOLSTERY' / Left Arm \&OBST XB $24.674999,25.799999,3.43,5.68,0.14,0.515$, SURF-ID=' UPHOLSTERY' '/ Base Q

$* * *$ GROUP: LR

** GROUP: COUCH offset $=0.000000$, ๑. .000000, ๑. .0000000 rotate $=0.0000000$

\&OBST XB=44.915001, 45.290001, 4.93,6.055, 0.14, 0.89, SURF_ID='UPHOLSTERY' ' Left Arm \&OBST XB \&OBST XB=45. 290001, 47.540001, 5.68, 6. 055, 0.515, 0.89, SURF_ID='UPHOLSTERY'/ Back

*** GROUP: CHAIR offset $=0,000000,-1.875000,0,000000$ rotate $=90,000000$

\&OBST XB=44.915001, 46. $040001,3.055,3.43,0.14,0.89$, SURF_ID= 'UPHOLSTERY ' / Left Arm

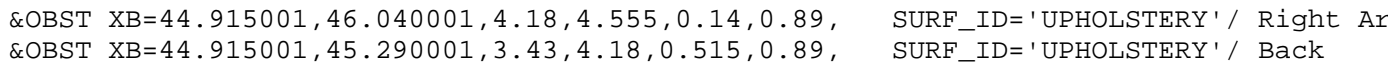

$* * *$ GROUP: CHAIR offset $=1.875000,-1.875000,0.000000$ rotate $=270.000000$

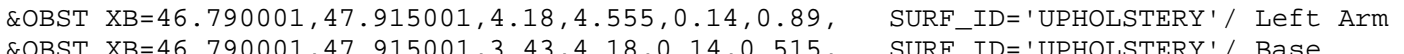
\&OBST XB $=46.790001,47.915001,3.055,3.43,0.14,0.89, \quad$ SURE-ID=' 'UPHOLSTERY' Right Ar \&OBST XB=47.540001, 47.915001,3.43,4.18,0.515, 0.89 , SURF_ID='UPHOLSTERY'/ Back

*** GROUP: LR

*** GROUP: COUCH offset $=0.000000,0.000000,0.000000$ rotate $=0.000000$

\&OBST $X \mathrm{XB}=41.540001,41.915001,4.93,6.055,0.14,0.89$, SURF_ID='UPHOLSTERY' $/$ Left Ar

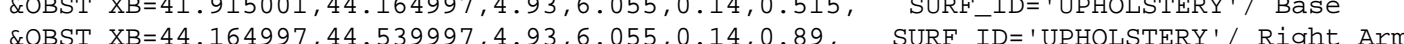
\&OBST XB=41.915001, 44.164997, 5.68,6.055, 0.515, 0.89, SURF_ID='UPHOLSTERY' / Back

*** GROUP: CHAIR offset $=0.000000,-1.875000,0.000000$ rotate $=90.000000$

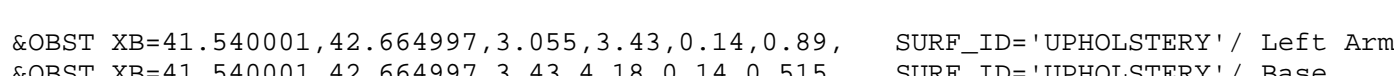

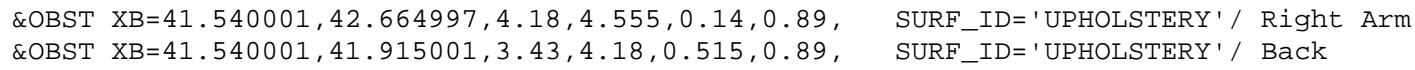

*** GROUP: CHAIR offset $=1.875000,-1.875000,0.000000$ rotate $=270.000000$

\&OBST XB=43.414997, 44.539997, 4.18,4.555, 0.14, ब. 89 , SURF-ID=' 'UPHOLSTERY' / Left Ar \&OBST XB=43.414997, 44.539997, 3. $055,3.43,0.14,0.89$, SURE-ID=' 'UPHOLSTERY'/ Right Ar \&OBST XB=44.164997, 44.539997, 3.43,4.18,0.515, 0.89 , SURF_ID='UPHOLSTERY'/ Back

*** GROUP: LR

** GROUP: COUCH offset $=0.000000,0.000000,0.000000$ rotate $=0.000000$

\&OBST $X \mathrm{XB}=38.165001,38.540001,4.93,6.055,0.14,0.89, \quad$ SURF_ID= 'UPHOLSTERY ' $/$ Left Ar \&OBST XB=40.790001, 41.165001, 4.93,6.055, 0.14, 0.89, SURF_ID='UPHOLSTERY'/Right Arm \&OBST XB=38.540001, 40.790001, $5.68,6.055,0.515,0.89$, SURF_ID=' 'UPHOLSTERY' / Back

*** GROUP: CHAIR offset $=0.000000,-1.875000,0.000000$ rotate $=90.000000$

\&OBST XB=38.165001, 39.290001, 3. $055,3.43,0.14,0.89$, SURF_ID=' UPHOLSTERY' / Left Arm \&OBST XB=38.165001, 39. 290001,4.18,4.555, $0.14,0.89$, SURF_IDD' 'UPHOLSTERY'/ Right Arn *** GROUP: CHAIR offset $=1.875000,-1.875000,0.000000$ rotate $=270.000000$

\&OBST XB=40. $9400001,41.165001,4.18,4.555,0.14,0.89$, SURF ID=' UPHOLSTERY' / Left Arm 


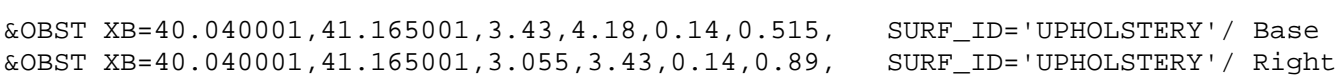
\&OBST XB $=40.040001,41.165001,3.055,3.43,0.14,0.89$,
\&OBST XB $=40.790001,41.165001,3.43,4.18,0.515,0.89$,

*** GROUP: LR

*** GROUP: COUCH offset $=0.000000,0.000000,0.000000$ rotate $=0.000000$

\&OBST $X \mathrm{XB}=34.790001,35.165001,4.93,6.055,0.14,0.89$, SURF_ID='UPHOLSTERY I' Left Ar

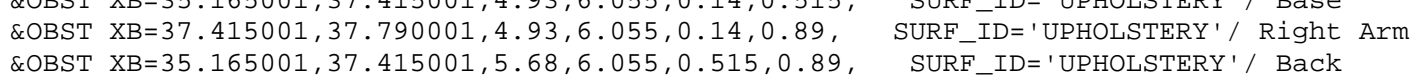

*** GROUP: CHAIR offset $=0.000000,-1.875000,0.000000$ rotate $=90.000000$

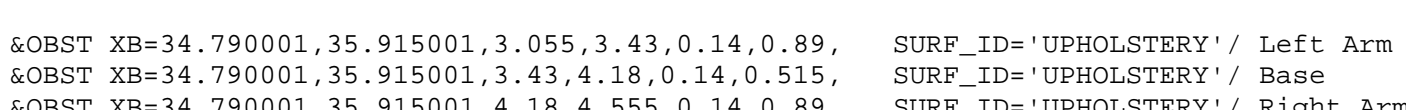

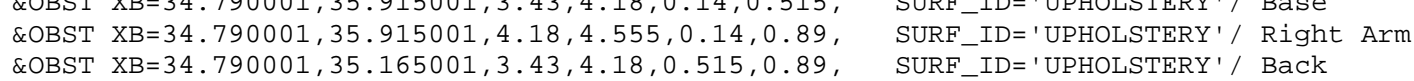

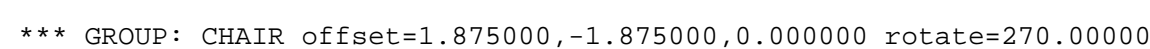

\&OBST XB=36.665001, 37. $790001,4.18,4.555,0.14,0.89$, SURF_ID=' 'UPHOLSTERY '/ Left Arm \&OBST $X \mathrm{~B}=36.665001,37.790001,3.055,3.43,0.14,0.89$, SURF_ID='UPHOLSTERY', Pase $\mathrm{XB}=37.41501,37.79001,3.055,3.43,0.14,0.89, \quad$ SURF-ID=' 'UPHOLSTERY', Right

*** GROUP: COUCH offset $=22.865000,-1.875000,0.000000$ rotate $=270.000000$

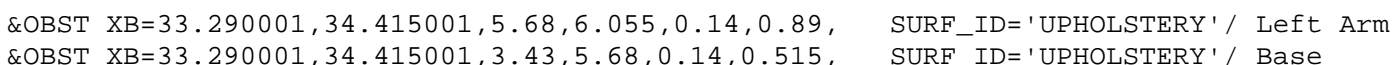

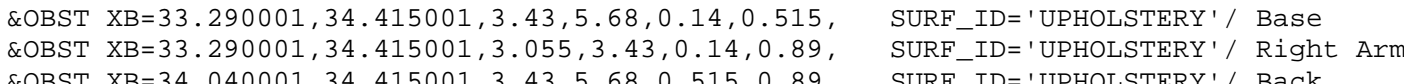
*** GROUP: couch offset $=21.365000,-1.875000,0.000000$ rotate $=270.000000$

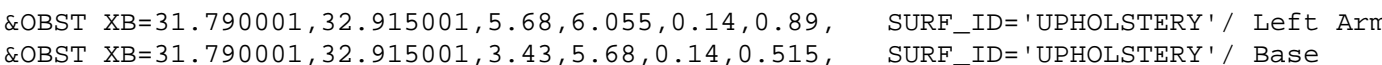
\&OBST XB=31.790001, 32.915001,3.055, 3.43, $0.14,0.89$, SURF_ID='UPHOLSTERY'/ Right Arm

\section{$* * *$ GROUP: LR_RO}

** GROUP: LR

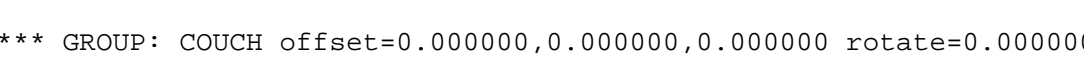

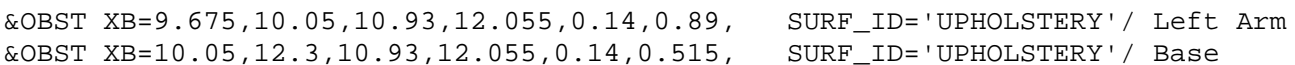

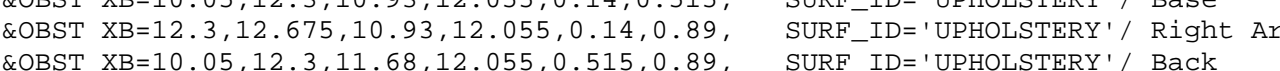

*** GROUP: CHAIR offset $=0.0000000,-1.875000,0.000000$ rotate $=90.000000$

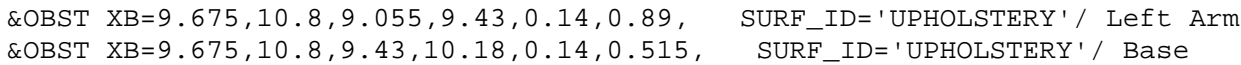

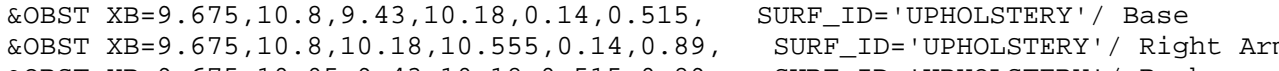

*** GROUP: CHAIR Of

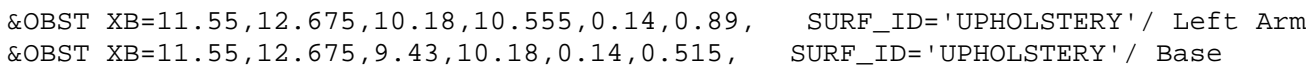

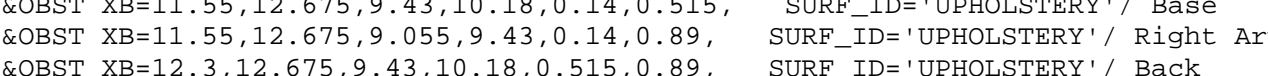

*** GROUP: LR

*** GROUP: COUCH offset $=0.0000000,0.000000,0.000000$ rotate $=0.0000000$
ROBST XB $=13.05,13.425,10.93,12.055,0.14,0.89, \quad$ SURF_ID=' 'UPHOLSTERY' $/$ Left Arm
\&OBST XB $=13.425,15.675,10.93,12.055,0.14,0.515, \quad$ SURF_ID='UPHOLSTERY' $/$ Base

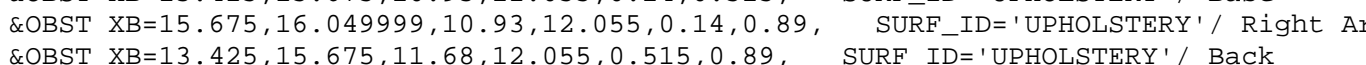

*** GROUP: CHAIR offset $=0.000000,-1.875000,0.000000$ rotate $=90 . .000000$

\&OBST XB $=13.05,14.175,9.055,9.43,0.14,0.89$, SURF_ID=' 'UPHOLSTERY' $/$ Left Arm

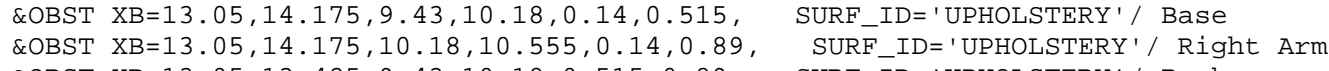
\&OBST XB=13.05,13.425, $9.43,10.18,0.515,0.89$, SURF ID='UPHOLSTERY'/ Back

*** GROUP: CHAIR offset $=1.875000,-1.875000,0.000000$ rotate $=270.000000$

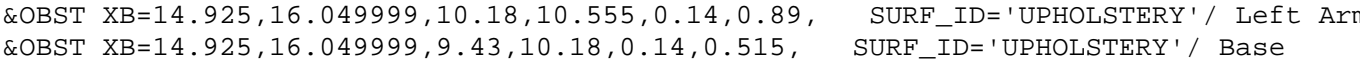
\&OBST XB-14.925, $16.049090,0.43,10.18,0.14,0.515$, SURF_IDD='UPHOLSTERY'/ Base

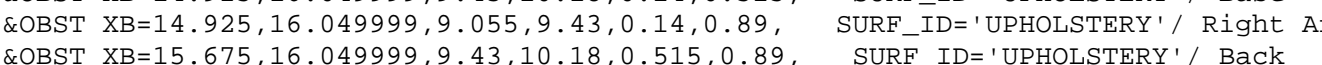

$* * *$ GROUP: LR

** GROUP: COUCH offset $=0.000000,0.000000,0.000000$ rotate $=0.000000$

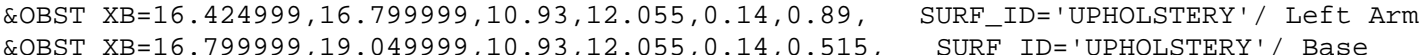

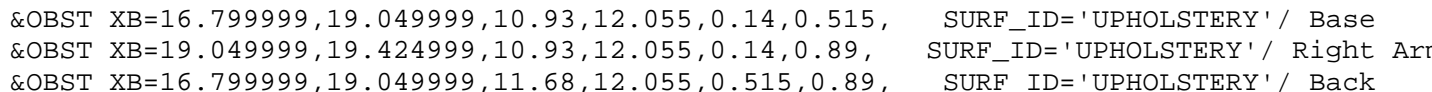

*** GROUP: CHAIR offset $=0.000000,-1.875000,0.000000$ rotate $=90.000000$

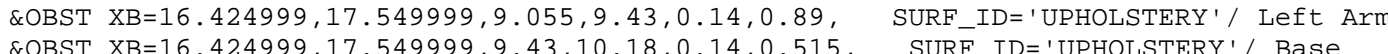
QOBST $X B=162499,17.5499,0.43,10.18,0.14,0.515$, SURF_ID='UHOLSTERY' / Base \&OBST XB=16.424999, 16.799999, 9.43,10.18, 0.515, 0.89, SURF_ID='UPHOLSTERY'/ Back

$* *$ GROUP: CHAIR offset $=1.875000,-1.875000,0.000000$ rotate $=270.000000$

\&OBST XB=18.299999, 19.424999, 10.18,10.555, 0.14, 0.89 , SURFIID='UPHOLSTERY' / Left Arm \&OBST XB $18.299999,19.424999,9.055,9.43,0.14,0.89$, SURF IDD' UPHOLSTERY', Right Arm \&OBST $X B=19.049999,19.424999,9.43,10.18, \theta .515, \theta .89, \quad$ SURF_ID='UPHOLSTERY'/ Back

*** GROUP: LR

*** GROUP: COUCH offset $=0.000000,0.000000,0.000000$ rotate $=0.0000000$

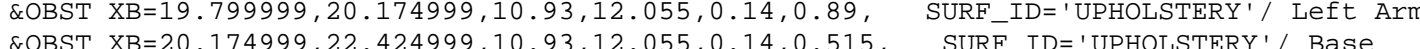

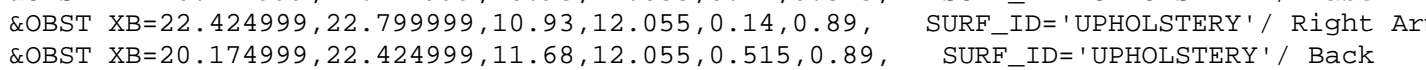

*** GROUP: CHAIR offset $=0.000000,-1.875000,0.000000$ rotate $=90.000000$

\&OBST XB=19.799999, 20.924999, 9. 055, 9.43, 0.14, Ө.89, SURF_ID=' 'UPHOLSTERY ' / Left Ar \&OBST XB=19.799999, 20.924999, 10.18, 10.555, 0.14, 0.89 , SURF_ID='UPHOLSTERY'/ Right Ar \&OBST XB=19.799999, 20.174999, 9.43,10.18, $0.515,0.89$, SURF_ID='UPHOLSTERY'/ Back

*** GROUP: CHATR offset $=1.875000,-1.875000,0.000000$ rotate $=270.000000$

\&OBST XB=21.674999, 22.799999, 10.18,10.555, 0.14, $\Theta .89$, SURF_ID='UPHOLSTERY'/ Left Ar \&OBST XB $=21.674999,22.799999,9.055,9.43,0.14,0.89$,
COBST XB $=22.424999,22.799999,9.43,10.18,0.515,0.89$,
SURF_IDE='UPHOLSTERY' 'UPHOLSTERY' $/$ Right

** GROUP: COUCH offset $=12.750000,-1.875000,0.000000$ rotate $=90.000000$ 
\&OBST XB=23.174999, 24.299999, 9. 055, 9.43, 0.14, $0.89, \quad$ SURF_ID= 'UPHOLSTERY' $/$ Left Arm
\&OBST XB=23.174999, 24.29999, $9.43,11.68,0.14,0.515, \quad$ SURF_ID='UPHOLSTERY' 1 Base

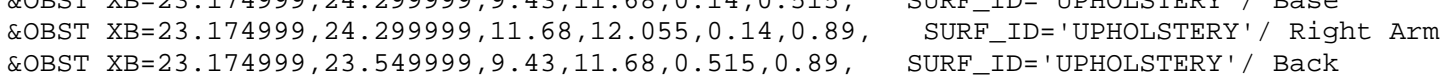
$* * *$ GROUP: COUCH offset $=14.250000,-1.875000,0.000000$ rotate $=90$. . \&OBST XB=24.674999, 25.799999, 9. 055, 9.43, 0.14, 0.89 , SURF_ID=' UPHOLSTERY ' / Left Arm SURF_ID='UPHOLSTERY'/ Right Ar \&OBST XB=24.67

\section{*** GROUP: LR}

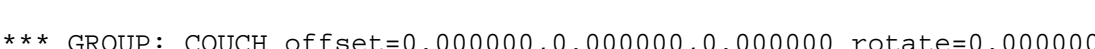

\&OBST XB=44.915001, 45.290001, 10.93,12.055, 0.14, 0.89, SURF_ID='UPHOLSTERY'/ Left Arm \&OBST XB=45.290001, 47.540001, 10.93, 12.055, $0.14,0.515$, SURF_ID='UPHOLSTERY'/ Base \&OBST $X \mathrm{~B}=47.54001,47.915001,10.93,12.055,0.14,0.89$, SURF_ID='UPHOLSTERY'/ Right A

*** GROUP: CHAIR offset $=0.0000000,-1.875000,0.000000$ rotate $=90.000000$

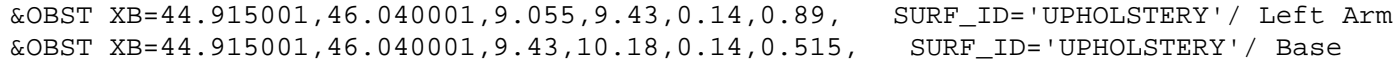
COBST XB=44.91501, 46. $040001,10.18,10.555,0.14, \theta .89$, SURF-ID='UPHOLSTERY'/ Right Arm

*** GROUP: CHAIR offset $=1.875000,-1.875000,0.000000$ rotate $=270.000000$

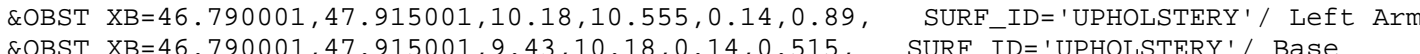

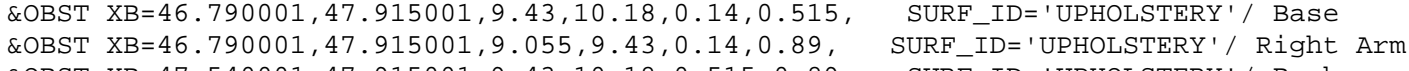

*** GROUP: LR

*** GROUP: COUCH offset $=0.000000,0.000000,0.000000$ rotate $=0.000000$

\&OBST XB=41.540001, 41.915001, 10.93,12.055, 0.14,, .89 , SURF_ID='UPHOLSTERY ' / Left Arm

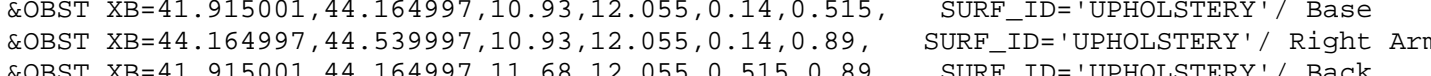

*** GROUP: CHAIR offset $=0.000000,-1.875000,0.000000$ rotate $=90.000000$

\&OBST XB $=41.540001,42.664997,9.055,9.43,0.14,0.89$, SURF_ID=' 'UPHOLSTERY ' 1 Left Ar

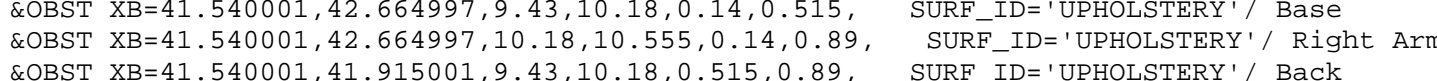
*** GROUP: CHAIR offset $=1.875000,-1.875000,0.000000$ rotate $=270.000000$

\&OBST $X \mathrm{XB}=43.414997,44.539997,10.18,10.555,0.14,0.89$, SURF_ID=' 'UPHOLSTERY ' / Left Arm

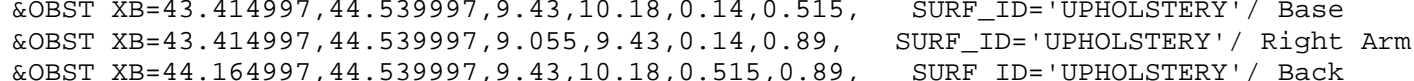

*** GROUP: LR

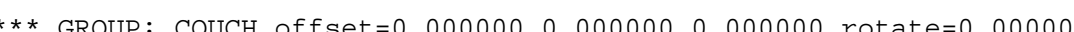

\&OBST $X \mathrm{XB}=38.165001,38.540001,10.93,12.055,0.14,0.89$, SURF_ID=' UPHOLSTERY' ' Left Arm

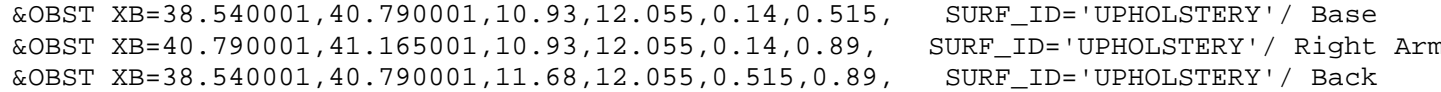

$* * *$ GROUP: CHAIR offset $=0.000000,-1.875000,0.000000$ rotate $=90.000000$

\&OBST XB=38.165001, 39.290001, 9.055, 9.43, 0.14, Ө.89, SURF_ID='UPHOLSTERY' / Left Arm
\&OBST XB=38.165001, 39. 290001, 9.43,10.18, 0.14, 0.515, SURF_ID=' UPHOLSTERY'/ Base

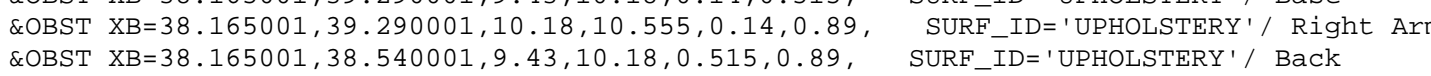
*** GROUP: CHAIR offset $=1.875000,-1.875000,0.000000$ rotate $=270.000000$

\&OBST XB $=40.040001,41.165001,10.18,10.555,0.14,0.89$, SURF _ID= 'UPHOLSTERY' $/$ Left Ar \&OBST XB $=40.040001,41.165001,9.055,9.43,0.14,0.89$, SURE_ID='UPHOLSTERY'/ Right Ar \&OBST $X B=40.790001,41.165001,9.43,10.18,0.515,0.89$,
SURF_ID ='UPHOLSTERY' / Back

*** GROUP: LR

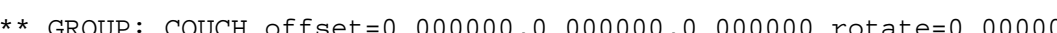

\&OBST $X \mathrm{XB}=34.790001,35.165001,10.93,12.055,0.14,0.89$, SURF_ID= 'UPHOLSTERY ' ' Left Arm

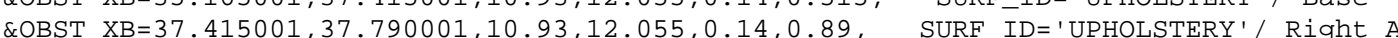

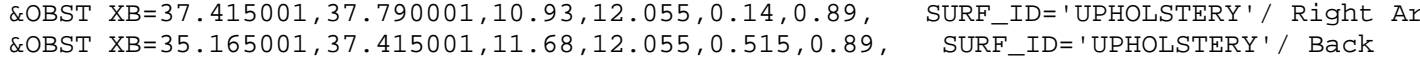

${ }^{*} \star \star$ GROUP: CHAIR offset $=0.000000,-1.875000,0.000000$ rotate $=90.000000$

\&OBST XB $=34.790001,35.915001,9.055,9.43,0.14,0.89$, SURF_ID=' UPHOLSTERY ' / Left Arm

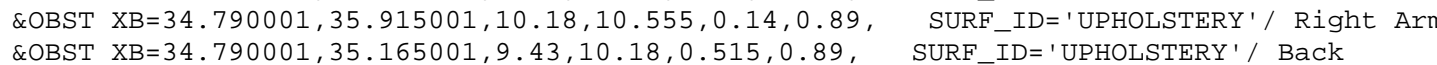
$* * *$ GROUP: CHAIR offset $=1.875000,-1.875000,0.000000$ rotate $=270.000000$

\&OBST XB=36.665001, 37. $790001,10.18,10.555,0.14,0.89, \quad$ SURF_ID='UPHOLSTERY' $/$ Left Ar

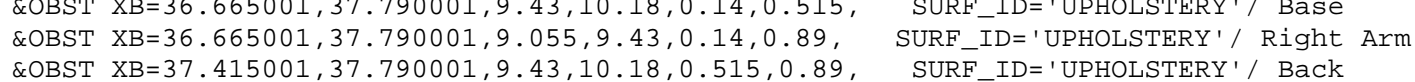

*** GROUP: COUCH offset $=22.865000,-1.875000, \Theta . .000000$ rotate $=270.000000$

\&OBST XB=33.290001,34.415001, 11.68, 12.055, 0.14, 0.89, SURF_ID='UPHOLSTERY'/ Left Arm \&OBST $X \mathrm{XB}=33.290001,34.415001,9.43,11.68,0.14,0.515, \quad$ SURF_ID='UPHOLSTERY'/

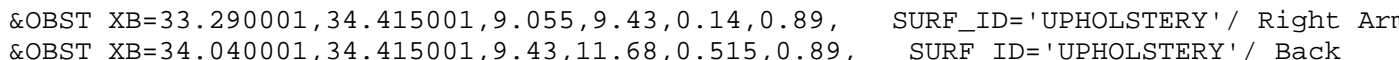

*** GROUP: COUCH offset $=21.365000,-1.875000,0.000000$ rotate $=270.0000000$

\&OBST XB $=31.790001,32.915001,11.68,12.055,0.14,0.89$, SURF_ID='UPHOLSTERY' $/$ Left Ar \&OBST XB=31.790001, 32.915001, $9.43,11.68,0.14,0.515$, SURF_ID='UPHOLSTERY'/ Base \&OBST $X \mathrm{XB}=31.790001,32.915001,9.055,9.43,0.14,0.89, \quad$ SURF-ID='UPHOLSTERY'/ Right Ar
\&OBST $\mathrm{XB}=32.540001,32.915001,9.43,11.68,0.515,0.89$,

*** GROUP: LR_ROW

*** GROUP: LR

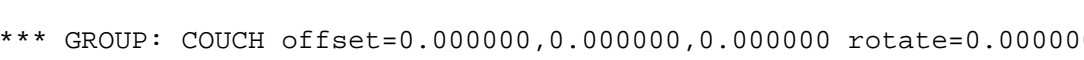
\&OBST XB=9.675, 10.05, 14.3,15.425, 0.14, 0.89, SURF_ID= 'UPHOLSTERY '/ Left Arm

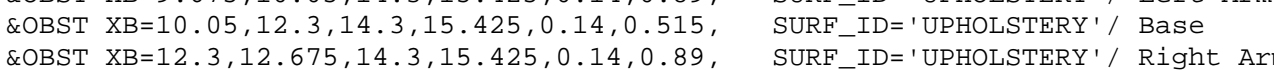
\&OBST XB-12.3, $12.675,14.3,15.425,0.14,0.89$, SURF-ID=' 'HOLSTERYY Right Ar

*** GROUP: CHAIR offset $=0.000000,-1.875000,0.000000$ rotate $=90.000000$

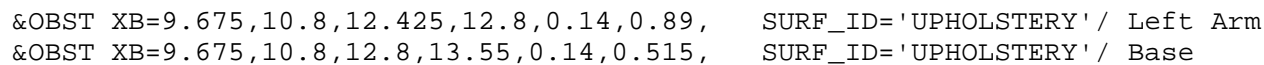

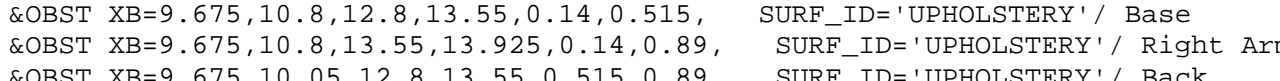

$* * *$ GROUP: CHAIR offset $=1.875000,-1.875000,0.000000$ rotate $=270.000000$ \&OBST XB $=11.55,12.675,13.55,13.925,0.14,0.89$, SURF_ID='UPHOLSTERY' / Left Arm L-92 
*** GROUP: LR

*** GROUP: COUCH offset $=0.000000,0.000000,0.000000$ rotate $=0.000000$

\&OBST XB $=13.05,13.425,14.3,15.425,0.14,0.89$, SURF ID = ' UPHOLSTERY ' / Left Arm

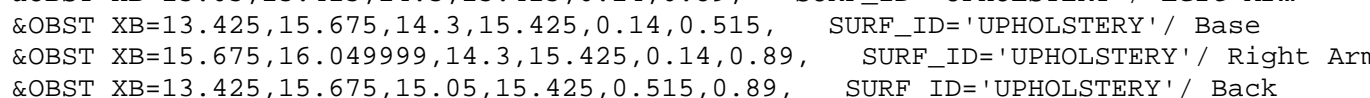

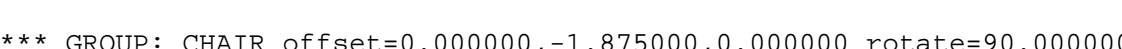
$\begin{array}{ll}\text { \&OBST XB }=13.05,14.175,12.425,12.8,0.14,0.89, & \text { SURF_ID='UPHOLSTERY '/ Left Arm } \\ \text { \&OBST XB }=13.05,14.175,12.8,13.55,0.14,0.515, & \text { SURF_ID='UPHOLSTERY' }\end{array}$

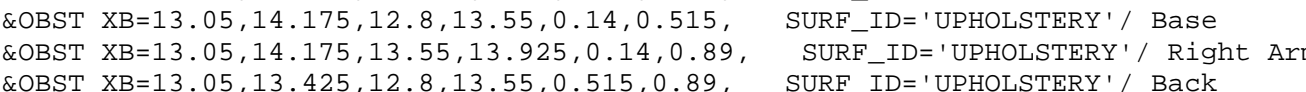
*** GROUP: CHAIR offset $=1.875000,-1.875000,0.000000$ rotate $=270.000000$ \&OBST XB=14.925, 16. 0499999, 13.55,13.925, 0.14, 0.89, SURF_ID='UPHOLSTERY'/ Left Arm \&OBST XB=14.925, 16.049999,12.8,13.55, 0.14, 0.515, SURF_ID='UPHOLSTERY'/ Base \&OBST XB=14.925, $16.449999,12.425,12.8,0.14,0.89$, SURF-ID='UPHOLSTERY'/ Right Ar

*** GROUP: LR

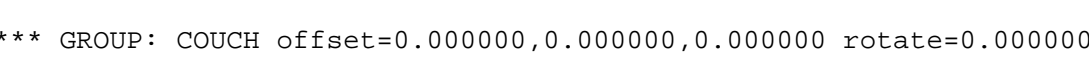

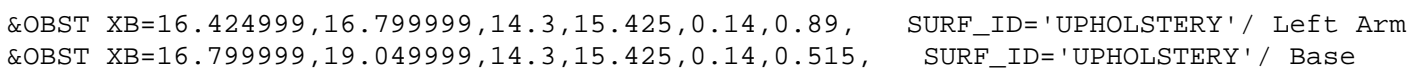

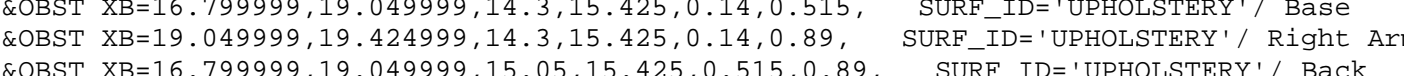

*** GROUP: CHAIR offset $=0.0000000,-1.875000,0.000000$ rotate $=90$. ๑०००००

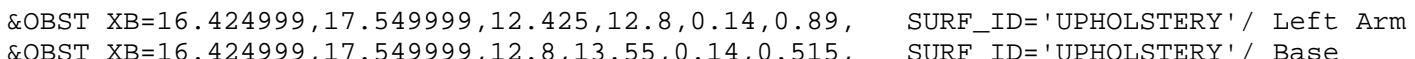

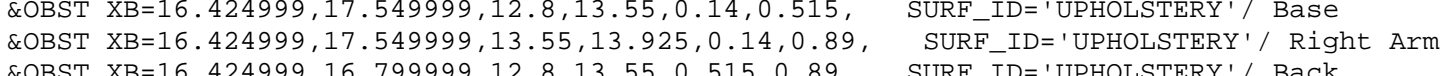
*** GROUP: CHAIR offset $=1.875000,-1.875000,0.000000$ rotate $=270.000000$

\&OBST XB=18.299999, 19.424999, 13.55,13.925, 0.14, 0.89, SURF_ID='UPHOLSTERY'/ Left Arm

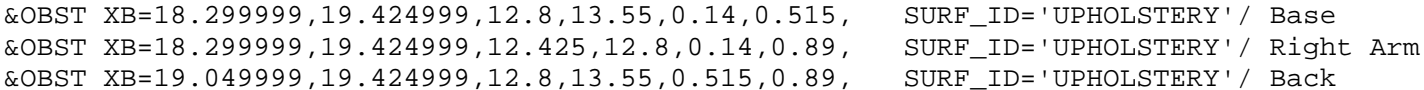

*** GROUP: LR

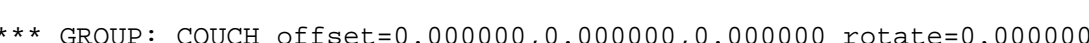

\&OBST XB $=19.799999,20.174999,14.3,15.425,0.14,0.89$, SURF_ID='UPHOLSTERY' / Left Arm

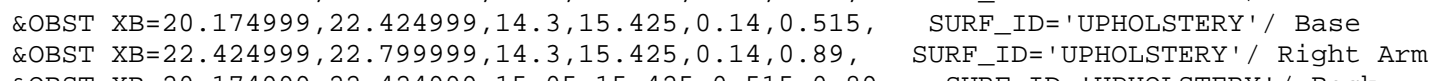

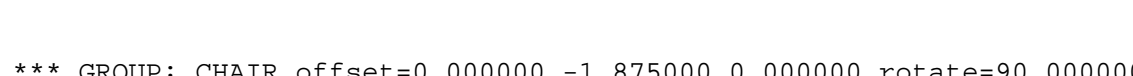

\&OBST XB=19.799999, 20.924999, 12.425, 12.8, $0.14,0.89$, SURF_ID='UPHOLSTERY' ' Left Arm \&OBST XBE=19.799999, $20.924999,13.55,13.925,0.14,0.89, \quad$ SURF_ID='UPHOLSTERY' / Right Arm
\&OBST XB=19.79999, $20.17499,12.8,13.55,0.515,0.89, \quad$ SURF_ID='UPHOLSTERY'/ Back *** GROUP: CHAIR offset $=1.875000,-1.875000,0.000000$ rotate $=270.000000$

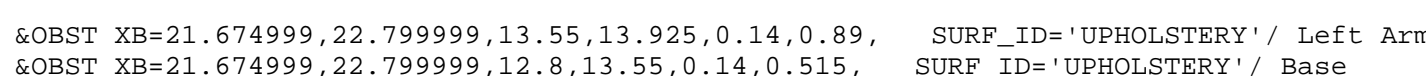

*** GROUP: COUCH offset $=12.750000,-1.875000,0.000000$ rotate $=90.000000$ \&OBST XB $=23.174999,24.299999,12.425,12.8,0.14, \Theta .89, \quad$ SURF_ID=' 'UPHOLSTERY' '/ Left Arm

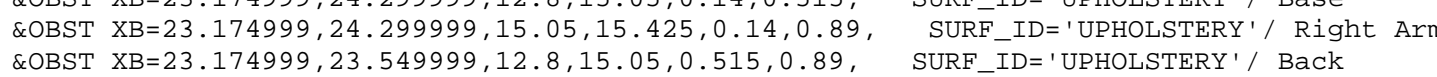
*** GROUP: COUCH offset $=14.250000,-1.875000,0.000000$ rotate $=90.0000000$

\&OBST XB=24.674999, 25.799999, 12.425, 12.8, $0.14,0.89, \quad$ SURF_ID=' 'UPHOLSTERY ' / Left Arm

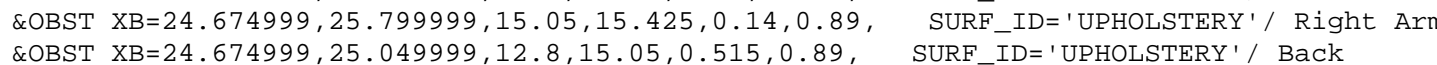

$* \star *$ GROUP: LR

*** GROUP: COUCH offset $=0.000000$, ๑. .000000,,$~ \odot . ~ 000000$ rotate $=0.000000$

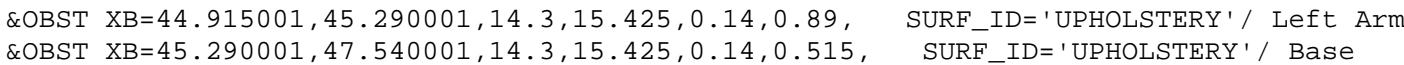

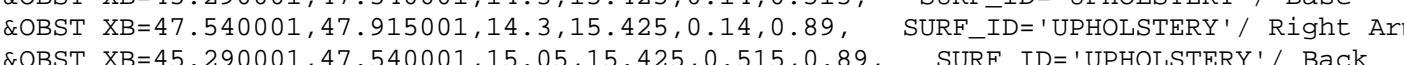

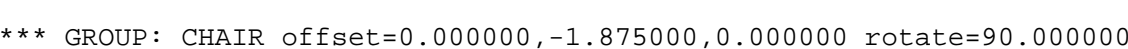

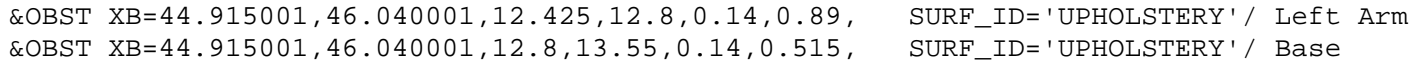

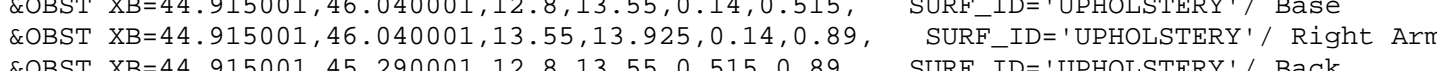
*** GROUP: CHAIR offset $=1.875000,-1.875000,0.000000$ rotate $=270.0000000$

\&OBST XB=46.790001, 47.915001, 13.55, 13.925, 0.14, 0.89 , SURF_ID='UPHOLSTERY'/ Left Arm

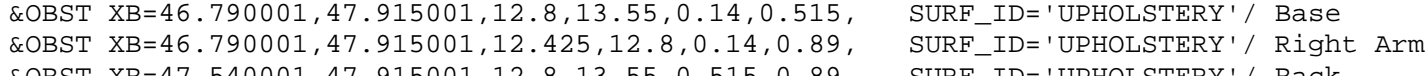

$* * *$ GROUP: LR

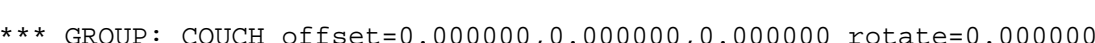

\&OBST XB=41.540001, 41.915001, 14.3,15.425, 0.14, 0.89, SURF_ID='UPHOLSTERY' / Left Arm

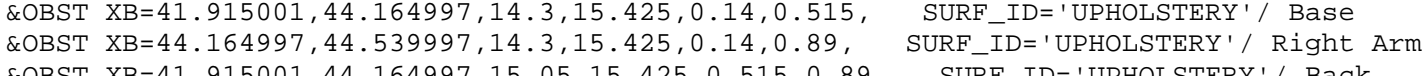

*** GROUP: CHAIR offset $=0.000000,-1.875000,0.000000$ rotate $=90.000000$

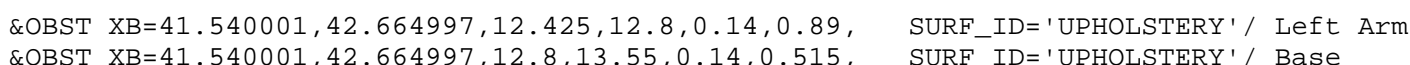

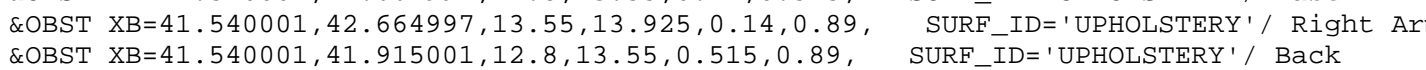
*** GROUP: CHAIR offset $=1.875000,-1.875000,0.000000$ rotate $=270.000000$

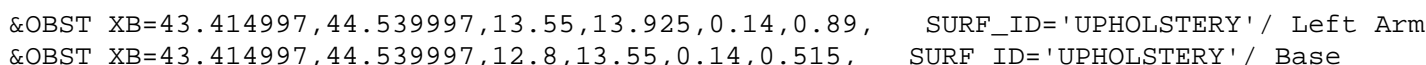

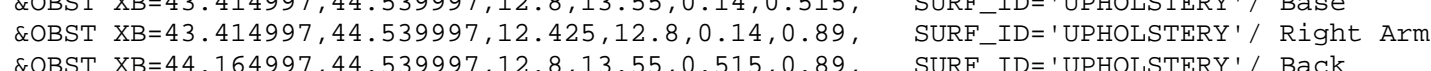

*** GROUP: LR

*** GROUP: COUCH offset $=0.000000,0.000000,0.000000$ rotate $=0.0000000$

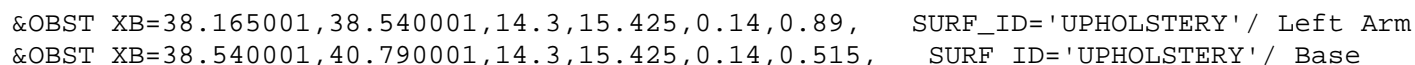




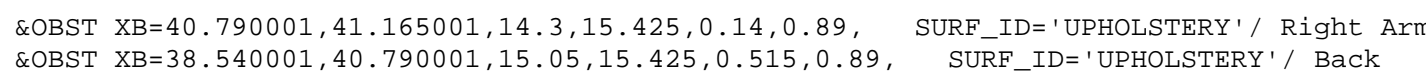

*** GROUP: CHAIR offset $=0.0000000,-1.875000,0.000000$ rotate $=90.000000$

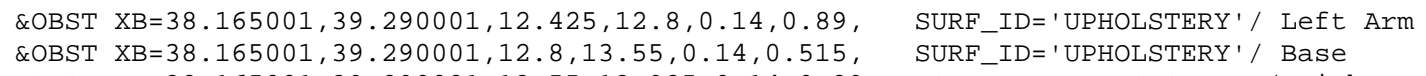

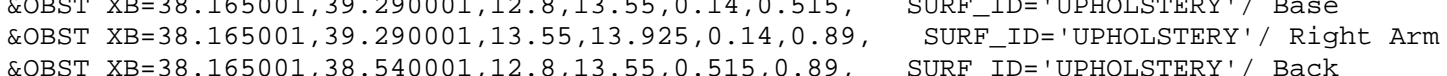
$* * *$ GROUP: CHAIR offset $=1.875000,-1.875000,0.000000$ rotate $=270.000000$

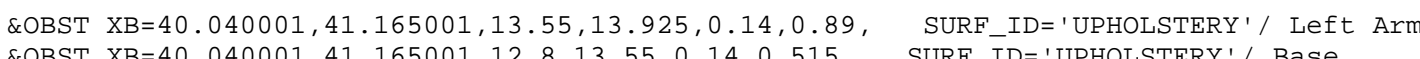

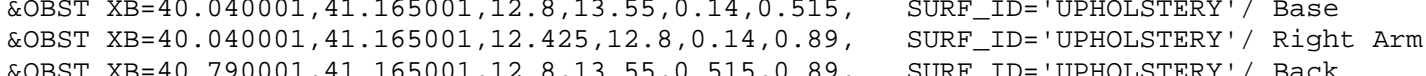

*** GROUP: LR

*** GROUP: COUCH offset $=0.000000,0.000000,0.000000$ rotate $=0.000000$

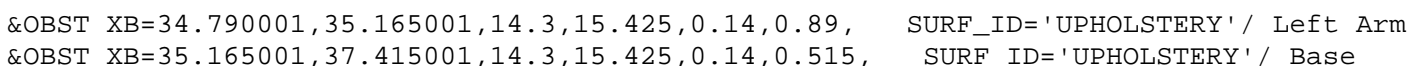

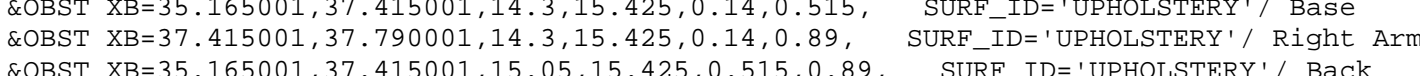

*** GROUP: CHAIR offset $=0.000000,-1.875000,0.000000$ rotate $=90.000000$

\&OBST XB=34.790001,35.915001, 12.425, 12.8, $0.14,0.89$, SURF_ID= 'UPHOLSTERY ' ' Left Arm

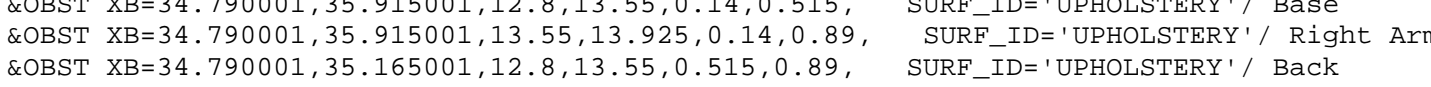
** GROUP: CHAIR offset $=1.875000,-1.875000,0.000000$ rotate $=270.000000$

\&OBST XB=36.665001, 37.790001,13.55,13.925, 0.14, 0.89 , SURF_ID='UPHOLSTERY' / Left Ar

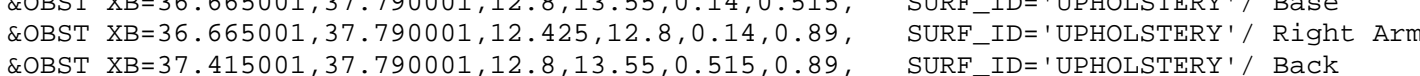

*** GROUP: CouCH offset $=22.865000,-1.875000, \Theta .000000$ rotate $=270.000000$

\&OBST XB=33.290001, 34. 415001, 15. 05, 15.425, 0.14, 0.89, SURF_ID= 'UPHOLSTERY ' / Left Arm

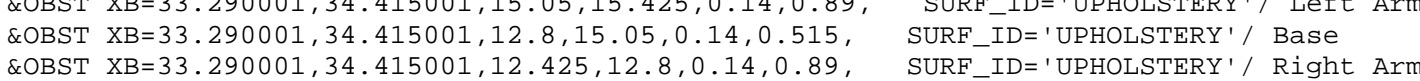

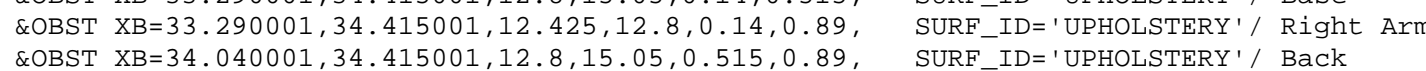
*** GROUP: COUCH offset $=21.365000,-1.875000, \Theta .000000$ rotate $=270.000000$

\&OBST XB=31.790001, 32.915001, 15. 05, 15.425, 0.14, 0.89, SURF_ID='UPHOLSTERY' / Left Arn

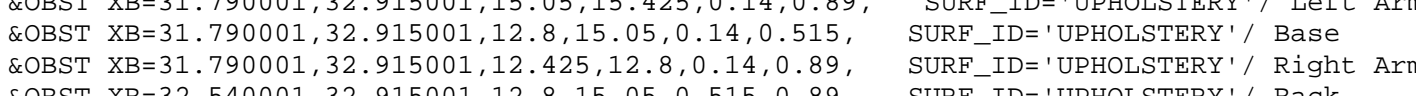

*** GROUP: LR_ROW

$* * *$ GROUP: LR

*** GROUP: COUCH offset $=0.000000,0.000000,0.000000$ rotate $=0.000000$

\&OBST XB=9.675, 10. $05,17.67,18.795,0.14,0.89$, SURF_ID= 'UPHOLSTERY' / Left Arm

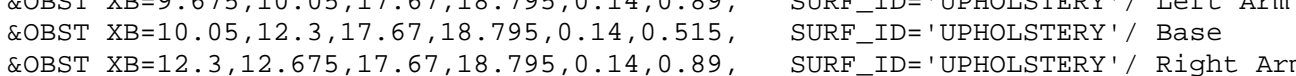
\&OBST XB=12.3,12.675,17.67,18.795, $14.14,0.89$, SURF-ID=' 'UPHOLSTERY'/ Right Ar

*** GROUP: CHAIR offset $=0.000000,-1.875000,0.000000$ rotate $=90.000000$

\&OBST XB=9.675,10.8, 15.795, 16.17, $0.14,0.89$, SURF_ID=' 'UPHOLSTERY Y / Left Arm

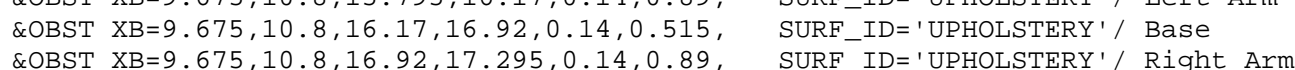

\&OBST XB $=9.675,10,05,16,17,16,92,0.515,0.89$, SURF_ID=' 'UPHOLSTERY ' / Back

*** GROUP: CHAIR offset $=1.875000,-1.875000,0.000000$ rotate $=270.000000$

\&OBST XB=11.55, 12.675, 16.92, 17.295, 0.14, 0.89 , SURF_ID='UPHOLSTERY'/ Left Arm

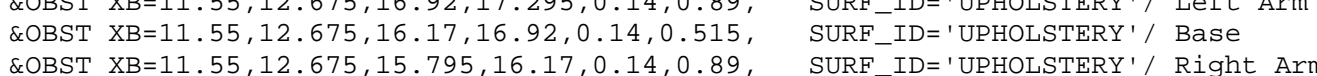
QOBST $X \mathrm{~B}=12.35,12.675,16.17,16.92,0.515,0.89$, SURF_ID='UPHOLSTERY// Right Arm

*** GROUP: LR

*** GROUP: COUCH offset $=0.0000000,0.000000,0.0000000$ rotate $=0.000000$

\&OBST XB=13.05, 13.425, 17.67, 18.795, 0.14, 0.89, SURF_ID='UPHOLSTERY' / Left Ar \&OBST XB=13.425, 15.675, 17.67, 18.795, 0.14, 0.515, SURF_ID='UPHOLSTERY'/ Base \&OBST $X \mathrm{XB}=15.655,16.049999,17.67,18.795,0.14,0.89$, SURF_IDD='UPHOLSTERY'/ Right Ar

*** GROUP: CHAIR offset $=0.0000000,-1.875000,0.0000000$ rotate $=90.000000$

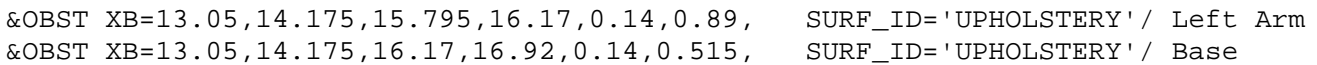

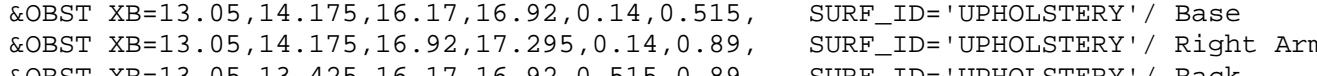

** GROUP: CHAIR offset $=1.875000,-1.875000,0.000000$ rotate $=270.000000$ \&OBST XB=14.925, 16. 049999, 16.92, 17.295, 0.14, 0.89, SURF_ID='UPHOLSTERY'/ Left Arm

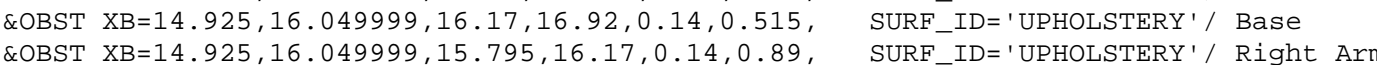
\&OBST $X \mathrm{XB}=14.925,16.049999,15.795,16.17,0.14,0.89$ ' SURF-ID='UPHOLSTERY' RIght

*** GROUP: LR

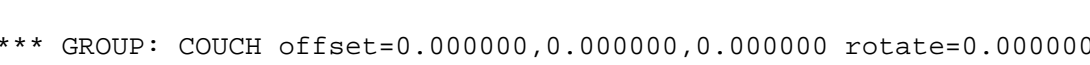

\&OBST XB=16.424999, 16.799999, 17.67,18.795, 0.14,, .89 , SURF_ID='UPHOLSTERY'/ Left Arm

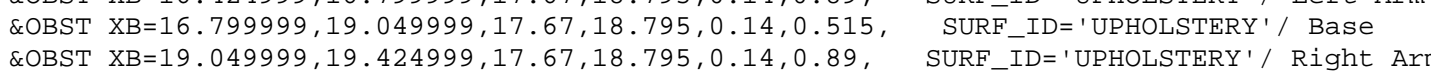

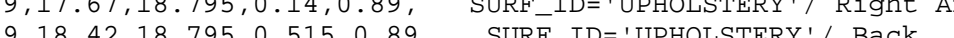

*** GROUP: CHAIR offset $=0.000000,-1.875000,0.000000$ rotate $=90.000000$

$\begin{array}{lll}\text { ROBST XB } 16.424999,17.549999,15.795,16.17,0.14,0.89, & \text { SURF_ID='UPHOLSTERY'/' Left Arm } \\ \text { \&OBST XB }=16.424999,17.549999,16.17,16.92,0.14,0.515, & \text { SURF-ID='UPHOLSTERY'/ Base }\end{array}$

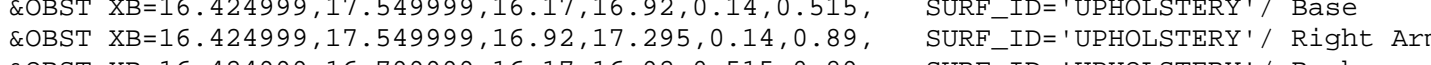

** GROUP: CHAIR offset $=1.875000,-1.875000,0.000000$ rotate $=270.000000$

\&OBST XB=18.299999, 19.424999, 16. 92, 17.295, 0.14, 0.89 , SURF_ID='UPHOLSTERY' / Left Arm

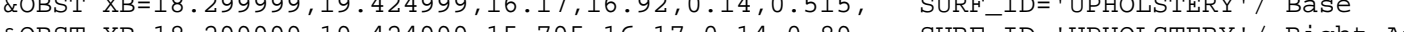

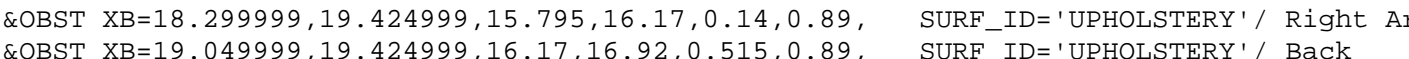

$* * *$ GROUP: LR

** GROUP: COUCH offset $=0.000000$, ๑. ๑00000,,. .000000 rotate $=0.000000$

\&OBST XB=19.799999, 20.174999, 17.67, 18.795, 0.14,, 8.89 , SURF_ID='UPHOLSTERY' ' Left Arm

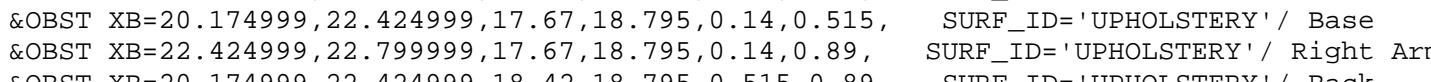

*** GROUP: CHAIR offset $=0.0000000,-1.875000,0.000000$ rotate $=90.000000$

\&OBST XB=19.799999, 20.924999, 15.795, 16.17, $9.14,0.89, \quad$ SURF_ID=' 'UPHOLSTERY '/ Left Arm
\&OBST XB $=19.799999,20.924999,16.17,16.92,0.14,0.515$, 
\&OBST XB=19.799999, 20.924999, 16.92,17.295, $0.14,0.89, \quad$ SURF_ID='UPHOLSTERY' Right Arm
\&OBST XB=19.799999, 20.174999,16.17,16.92, $0.515,0.89, \quad$ SURF_ID='UPHOLSTERY'/ Back $* * *$ GROUP: CHAIR offset $=1.875000,-1.875000,0.000000$ rotate $=270.000000$ \&OBST XB=21.674999, 22.799999, 16.92,17.295, 0.14, 0.89 , SURF_ID='UPHOLSTERY'/ Left Arm

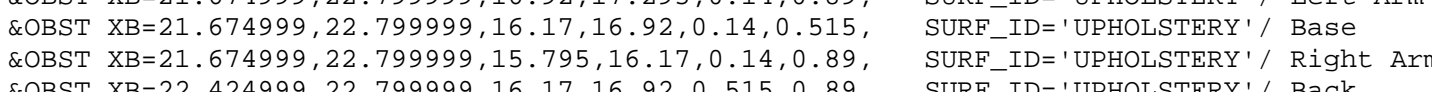

*** GROUP: COUCH offset $=12.750000,-1.875000,0.000000$ rotate $=90.000000$

\&OBST XB=23.174999, 24.299999, 15.795,16.17, 0.14, 0.89, SURF_ID='UPHOLSTERY' / Left Arm \&OBST XB=23.174999, 24.299999, 18.42, 18.795, 0.14,0.89, SURF ID=' 'UPHOLSTERY' QOBST XB=23.174999, 23.549999, 16.17, 18.42, $0.515,0.89$, SURF_ID='UPHOLSTERY'/ Back

*** GROUP: COUCH offset $=14.250000,-1.875000,0.000000$ rotate $=90.000000$

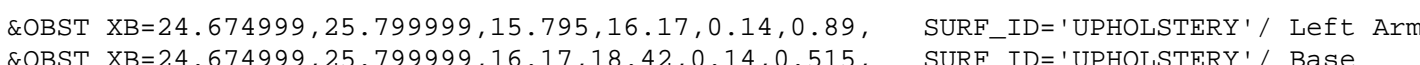

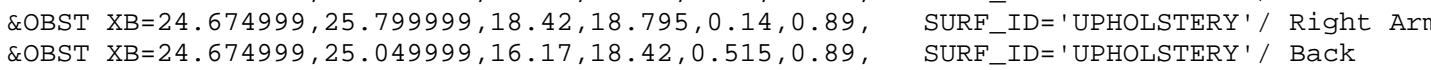

*** GROUP: LR

*** GROUP: COUCH offset $=0.000000,0.000000,0.000000$ rotate $=0.000000$

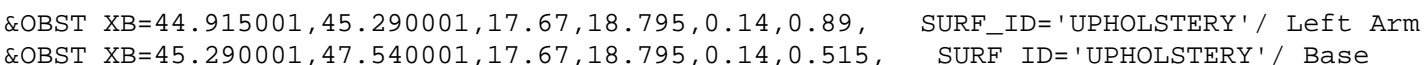
\&OBST XB=47.540001, 47.915001,17.67, 18.795, $0.14,0.89$, SURF_ID='UPHOLSTERY'/ Right Arm

*** GROUP: CHAIR offset $=0.000000,-1.875000,0.000000$ rotate $=90.000000$

EOBST $X \mathrm{XB}=44.915001,46.040001,15.795,16.17,0.14,0.89, \quad$ SURF_ID='UPHOLSTERY' / Left Arm

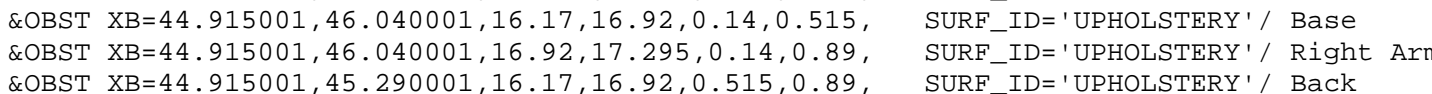

$* * *$ GROUP: CHAIR offset $=1.875000,-1.875000,0.000000$ rotate $=270.0000000$

$\begin{array}{ll}\text { \&OBST XB }=46.790001,47.915001,16.92,17.295,0.14,0.89, & \text { SURF_ID='UPHOLSTERY'/' Left Arm } \\ \text { \&OBST XB }=46.790001,47.915001,16.17,16.92,0.14,0.515, & \text { SURF_ID='UPHOLSTERY'/ Base }\end{array}$

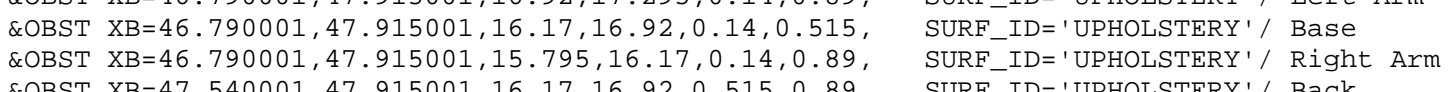

*** GROUP: LR

*** GROUP: COUCH offset $=0.000000,0.000000,0.000000$ rotate $=0.000000$

\&OBST XB $=41.540001,41.915001,17.67,18.795,0.14,0.89, \quad$ SURF_ID='UPHOLSTERY' $/$ ' Left Arm
\&OBST XB $=41.915001,44.164997,17.67,18.795,0.14,0.515$,
SURF_ID ='UPHOLSTERY' / Base

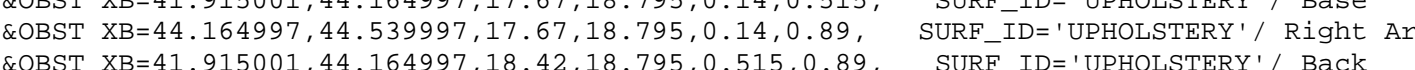

*** GROUP: CHAIR offset $=0.0000000,-1.875000,0.000000$ rotate $=90$. . 000000

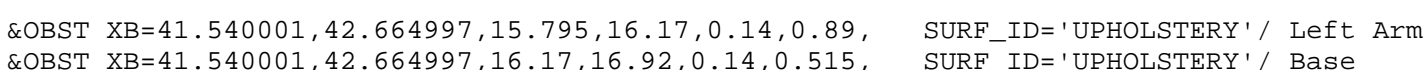

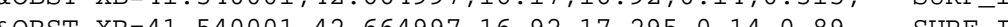

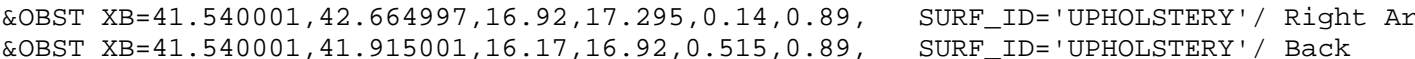

*** GROUP: CHAIR offset $=1.875000,-1.875000,0.000000$ rotate $=270.0000000$

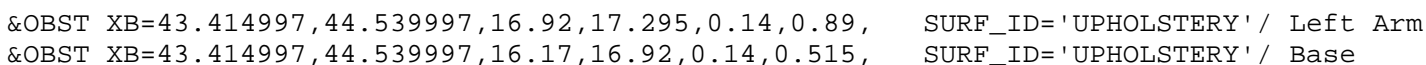

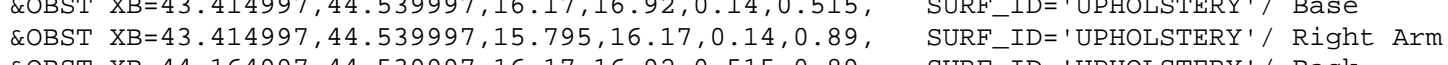

*** GROUP: LR

*** GROUP: COUCH offset $=0$, .

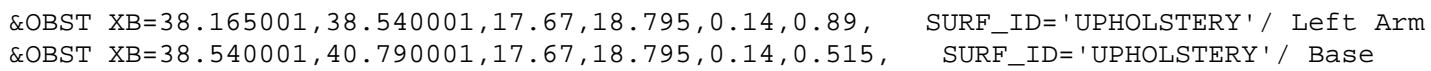
\&OBST XB=40.790001,41.165001, 17.67, 18.795, $0.14,0.89$, SURF IDE' UPHOLSTERY'/' Right Ar

*** GROUP: CHAIR offset $=0.000000,-1.875000,0.000000$ rotate $=90 . .000000$

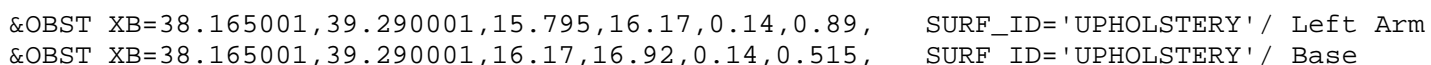

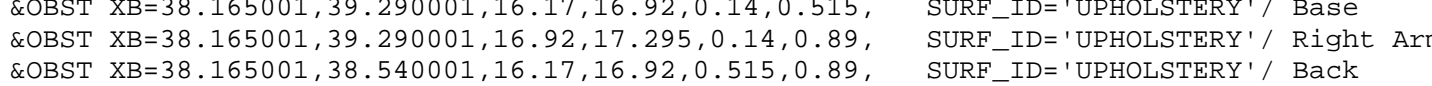

*** GROUP: CHAIR offset $=1.875000,-1.875000,0.000000$ rotate $=270$. . 0000000

\&OBST XB=40.040001, 41.165001, 16.92,17.295, 0.14, $0.89, \quad$ SURF_ID='UPHOLSTERY'/ Left Arm

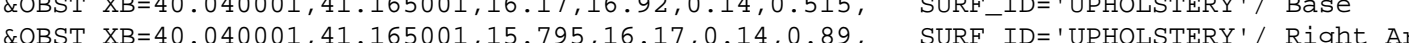
\&OBST $X B=40.790001,41.165001,16.17,16.92,0.515,0.89, \quad$ SURF_ID=' 'UPHOLSTERY' ' Right A

*** GROUP: LR

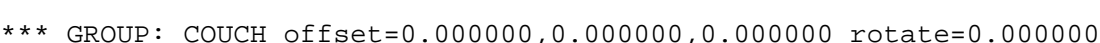

\&OBST XB $=34.790001,35.165001,17.67,18.795,0.14,0.89$,
COBST XB $=35.165001,37.415001,17.67,18.795,0.14,0.515$,

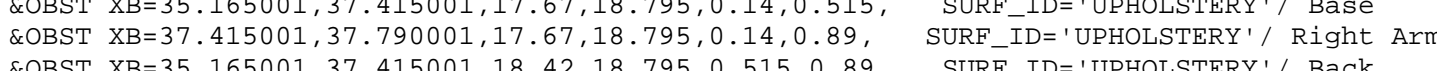

*** GROUP: CHAIR offset $=0.000000,-1.875000,0.000000$ rotate $=90.000000$

\&OBST XB=34.790001, 35. 915001, 15.795, 16.17, $0.14,0.89$, SURF_ID='UPHOLSTERY ' / Left Arm \&OBST XB=34.790001,35.915001, 16.92,17.295, 0.14, 0.89 , SURF_ID='UPHOLSTERY'/ Right Arm \&OBST XB $=34.790001,35.165001,16.17,16.92,0.515,0.89$, SURF_ID='UPHOLSTERY'/ Back

$* * *$ GROUP: CHAIR offset $=1.875000,-1.875000,0.000000$ rotate $=270.000000$

\&OBST XB=36.665001, 37. 790001, 16.92, 17.295, 0.14, 0.89 , SURFIID='UPHOLSTERY' / Left Arm

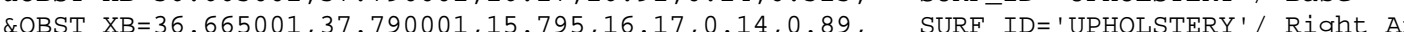
\&OBST XB=37.415001, 37.790001,16.17,16.92, $0.515,0.89$, SURF_ID=' UPHOLSTERY'/ Back

*** GROUP: COUCH offset $=22.865000,-1.875000,0.000000$ rotate $=270.000000$

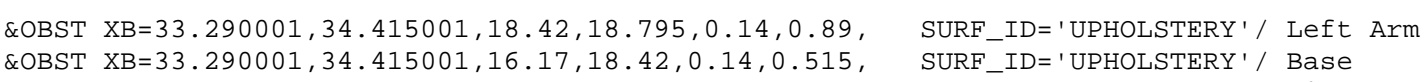
\&OBST $X=33.290001,34.415001,15.795,16.17,0.14,0.89$, SURF_IDD' 'UPHOLSTERY'

*** GROUP: COUCH offset $=21.365000,-1.875000,0.000000$ rotate $=270.000000$

\&OBST XB=31.790001, 32.915001, 18.42, 18.795, $0.14,0.89$, SURF_ID='UPHOLSTERY' / Left Arm

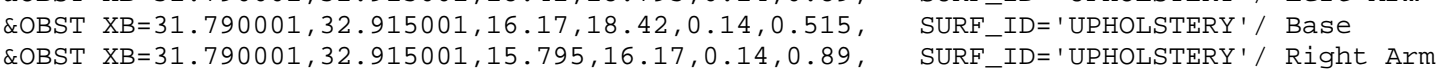

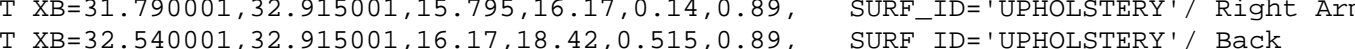

*** GROUP: CHAIR_ROW3

*** GROUP: CHAIR offset $=0.000000,0.000000,0.140000$ rotate $=90.000000$

\&OBST XB=9.68, 10.805, 1.17, $1.545,0.28,1.03, \quad$ SURF_ID='UPHOLSTERY'/ Left Arm
\&OBST XB=9.68,10.805, $1.545,2.295,0.28,0.655, \quad$ SURF_ID='UPHOLSTERY' $/$ Base

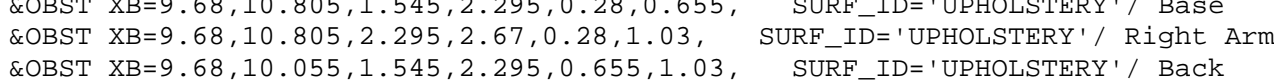

*** GROUP: CHAIR offset $=1.500000,0.000000,0.140000$ rotate $=90.000000$ 
\&OBST $X \mathrm{XB}=11.18,12.305,1.17,1.545,0.28,1.03, \quad$ SURF_ID= 'UPHOLSTERY ' $/$ Left Arm
\&OBST $X \mathrm{~B}=11.18,12.305,1.545,2.295,0.28,0.655, \quad$ SURF_ID=' 'UPHOLSTERY' $/$ Base \&OBST XB=11.18, $12.055,205,2.07,0.28,0.055$, SURF ID 'UPHOLSTERY'/ Right Ar \&OBST XB=11.18, 11. 555, 1. $545,2.295,0.655,1.03$, SURF_ID=' 'UPHOLSTERY'/ Back

${ }^{* * *}$ GROUP: CHAIR offset $=3.000000,0.000000,0.140000$ rotate $=90.000000$

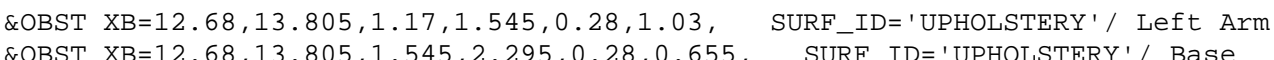
COBST XB=12.68, 13.805, 2.295, 2.67, $0.28,1.03$, SURF ID='UPHOLSTERY'/ Right Ar

$* * *$ GROUP: CHAIR offset $=4.500000,0.000000,0.140000$ rotate $=90.000000$

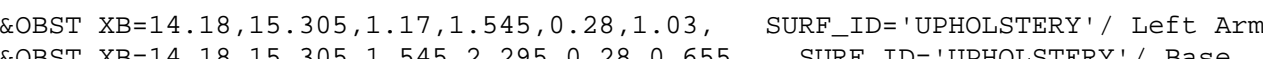
\&OBST $\mathrm{XB}=14.18,15.305,2.295,2.67,0.28,1.03$, SURF-ID=' 'UPHOLSTERY' '/ Right Ar

*** GROUP: CHAIR offset $=6.000000,0.000000,0.140000$ rotate $=90.000000$

\&OBST XB $=15.68,16.805,1.17,1.545,0.28,1.03$, SURF_ID= 'UPHOLSTERY ' / Left Arm \&OBST XB=15.68,16.805, 1.545, 2.295, 0.28, 0.655, SURF_ID='UPHOLSTERY' / Base

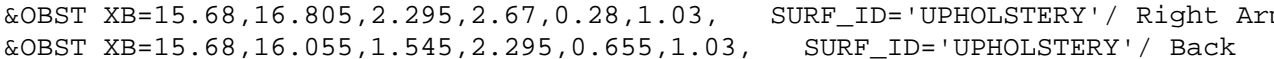

$* * *$ GROUP: CHAIR offset $=7.500000,0.000000,0.140000$ rotate $=90.000000$

\&OBST XB=17.18,18.305, 1.17, 1.545, 0.28,1.03, SURF_ID='UPHOLSTERY' / Left Arm

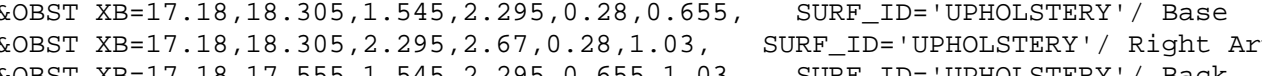
*** GROUP: CHAIR offset $=9.000000,0.000000,0.140000$ rotate $=90.000000$ \&OBST XB $=18.68,19.805,1.17,1.545,0.28,1.03, \quad$ SURF_IDD ' UPHOLSTERY ' $/$ Left Arm
\&OBST XB $=18.68,19.805,1.545,2.295,2.28,0.655$, SURF ID ' UPHOLSTERY' $/$ Base \&OBST $X \mathrm{XB}=18.68,19.805,2.295,2.67,0.28,1.03$, SURF_ID='UPHOLSTERY'/ Right Arm
\&OBST $\mathrm{XB}=18.68,19.055,1.545,2.295,0.655,1.03, \quad$ SURF_ID='UPHOLSTERY'// Back *** GROUP: CHAIR offset $=10.500000,0.000000,0.140000$ rotate $=90.000000$ \&OBST $X B=20.18,21.305,1.17,1.545,0.28,1.03$, SURF ID=' 'UPHOLSTERY ' / Left Arm

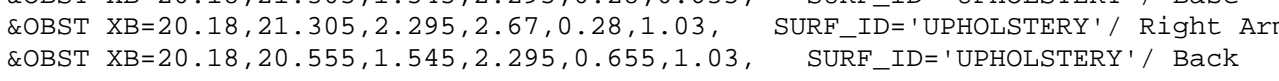
$* * *$ GROUP: CHAIR offset $=12.000000,0.000000,0.140000$ rotate $=90.000000$

\&OBST $X B=21.68,22.805,1.17,1.545,0.28,1.03, \quad$ SURF_ID='UPHOLSTERY $/$ Left Arm
\&OBST $X \mathrm{~B}=21.68,22.805,1.545,2.295,0.28,0.655$,
SURF_ID='UPHOLSTERY' $/$ Base

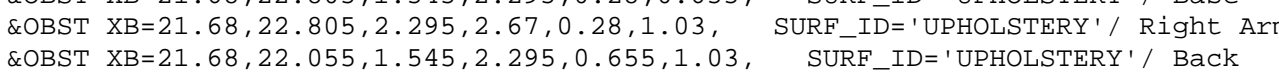

$* * *$ GROUP: CHAIR offset $=13.500000,0.000000,0.140000$ rotate $=90.000000$

\&OBST XB=23.18, 24.305, 1.17, 1.545, 0.28, 1. 03, SURF_IDD ' UPHOLSTERY ' / Left Arm \&OBST XB $=23.18,24.305,2.295,2.67,0.28,1.03$, SURE ID $=$ 'UPHOLSTERY' $/$ R/ght \&OBST XB=23.18, 23.555, 1.545, 2.295, 0.655,1.03, SURF_ID='UPHOLSTERY'/ Back

*** GROUP: CHAIR offset $=15.000000,0.000000,0.140000$ rotate $=90.000000$

\&OBST XB=24.68,25.805, 1.17, $1.545,0.28,1.03, \quad$ SURF_ID='UPHOLSTERY' / Left Arm
\&OBST XB=24.68,25.805,1.545,2.295,0.28,0.655, SURF_ID='UPHOLSTERY' / Base

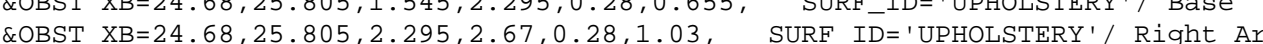
\&OBST $\mathrm{XB}=24.68,25.805,2.295,2.67,0.28,1.03, \quad$ SURF_ID=' 'UPHOLSTERY'/ Right Ar
\&OBST XB $=24.68,25.055,1.545,2.295,0.655,1.03, \quad$ SURF_ID='UPHOLSTERY'/ Back

*** GROUP: CHAIR_ROW4

*** GROUP: CHAIR_ROW3

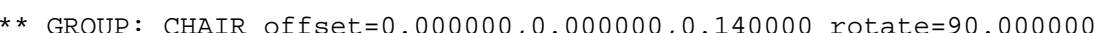

\&OBST XB=46.799999, $47.924999,2.295,2.67,0.28,1.03, \quad$ SURF_ID='UPHOLSTERY ' ' Left Arm
\&OBST XB $=46.799999,47.924999,1.545,2.295,0.28,0.655$,
SURF_ID='UPHOLSTERY' $/$ Base
ROBST XB=46.799999, 47. 924999, $1.17,1.545,0.28,1.03, \quad$ SURF_ID= 'UPHOLSTERY ' $/$ Right Arm
\&OBST XB $=47.549999,47.924999,1.545,2.295,0.655,1.03, \quad$ SURF_ID='UPHOLSTERY '/ Back *** GROUP: CHAIR offset $=1.500000,0.0000000,0.140000$ rotate $=90.000000$

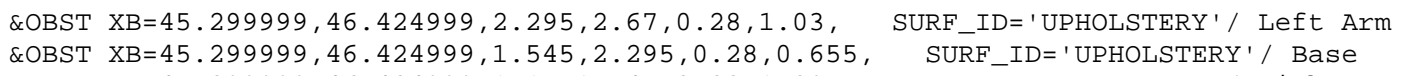

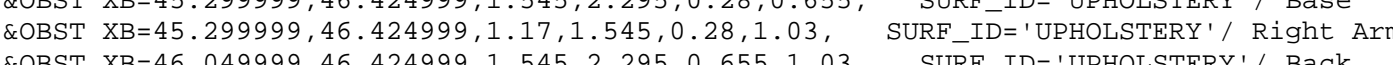

*** GROUP: CHAIR offset $=3.000000,0.000000,0.140000$ rotate $=90.000000$

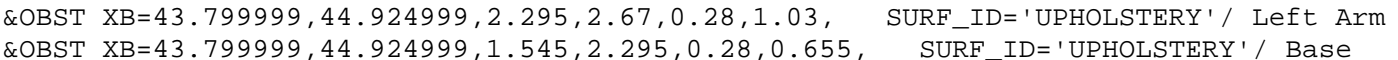

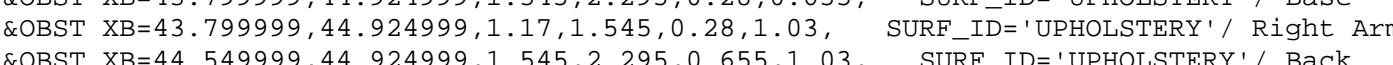

*** GROUP: CHAIR offset $=4.500000,0.000000,0.140000$ rotate $=90.000000$ \&OBST $X \mathrm{XB}=42.299999,43.424999,2.295,2.67,0.28,1.03, \quad$ SURF_ID='UPHOLSTERY ' / Left Arm
\&OBST XB=42.299999, $43.424999,1.545,2.295,0.28,0.655$,
SURF_ID=' UPHOLSTERY' $/$ Base

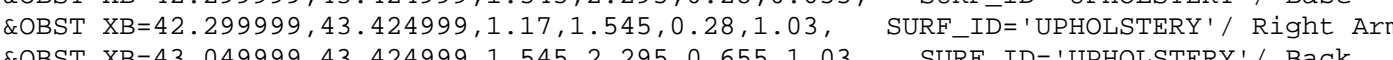

*** GROUP: CHAIR offset $=6.0000000,0.000000,0.140000$ rotate $=90.0000000$

\&OBST XB $=40.799999,41.924999,2.295,2.67,0.28,1.03$, SURF_ID=' 'UPHOLSTERY ' / Left Arm \&OBST XB=40.799999, 41.924999, 1.545, 2.295, 0. 28, 0.655 , SURF_ID='UPHOLSTERY' / Base COBST $X \mathrm{XB}=40.799999,41.924999,1.17,1.545,0.28,1.03, \quad$ SURF_ID=' 'UPHOLSTERY' ' Right Arm
\&OBST XB=41.549999, $41.924999,1.545,2.295,0.655,1.03, \quad$ SURF_ID='UPHOLSTERY' $/$ Back

$* * *$ GROUP: CHAIR offset $=7.500000,0.000000,0.140000$ rotate $=90.000000$

\&OBST XB=39.299999, 40.424999, 2.295, 2.67, 0.28,1.03, SURF_ID=' 'UPHOLSTERY'/ Left Arm \&OBST XB $=39.299999,40.424999,1.545,2.295,0.28,0.655$, SURF IDE= 'UPHOLSTERY' / Base \&OBST XB=39.29999, $40.424999,1.17,1.545, \theta .28,1.03, \quad$ SURF_ID='UPHOLSTERY' Right Arm
\&OBST XB $=40.049999,40.424999,1.545,2.295,0.655,1.03$,
SURF_ID=' 'UPHOLSTERY'/ Back

$* * *$ GROUP: CHAIR offset $=9.000000,0.000000,0.140000$ rotate $=90.000000$

\&OBST XB=37.799999, 38.924999, 2.295, 2.67, 0.28,1.03, SURF_ID=' 'UPHOLSTERY ' / Left Arm

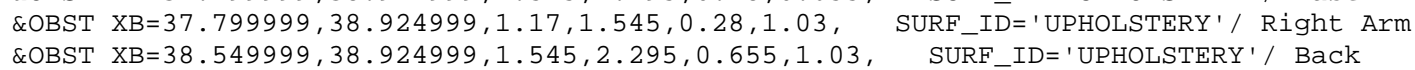

*** GROUP: CHAIR offset $=10.500000,0.000000,0.140000$ rotate $=90.000000$

\&OBST $X \mathrm{XB}=36.299999,37.424999,2.295,2.67,0.28,1.03, \quad$ SURF_ID= 'UPHOLSTERY ' / Left Arm

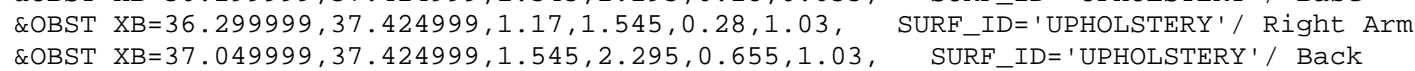

$* * *$ GROUP: CHAIR offset $=12.000000,0.000000,0.140000$ rotate $=90.000000$

\&OBST XB $=34.799999,35.924999,2.295,2.67,0.28,1.03$, SURF_ID=' 'UPHOLSTERY' '/ Left Arm \&OBST XB=34.799999, 35.924999, 1.17, 1.545, 0. 28, 1. 03, SURF_ID='UPHOLSTERY'/ Right Ar \&OBST XB $=35.549999,35.924999,1.545,2.295,0.655,1.03$, SURF_ID='UPHOLSTERY'/ Back

*** GROUP: CHAIR offset $=13.500000,0.000000,0.140000$ rotate $=90.000000$

\&OBST XB=33.299999, 34.424999, 2.295, 2.67,0.28,1.03, SURF-ID=' 'UPHOLSTERY' / Left Arm \&OBST XB=33.299999, 34. 424999, 1.17, 1.545, 0.28, 1.03, SURF ID='UPHOLSTERY'/ Right Ar \&OBST XB=34. 049999, 34. 424999, 1. 545, 2.295, 0.655, 1. 03, SURF_ID=' UPHOLSTERY' / Back

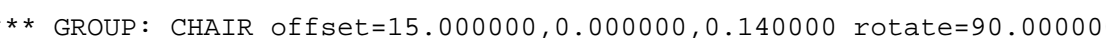

\&OBST XB $=31.799999,32.924999,2.295,2.67,0.28,1.03$, SURF_ID=' 'UPHOLSTERY ' / Left Arm \&OBST XB=31.799999, 32.924999, 1.17, 1.545, $28,1.03$, SURE ID='UPHOLSTERY'/ Right Ar \&OBST XB $=32.549999,32.924999,1.545,2.295,0.655,1.03, \quad$ SURF_ID ='UPHOLSTERY'/ Back

*** GROUP: CHAIR_ROW3

$* * *$ GROUP: CHAIR offset $=0.000000,0.000000,0.140000$ rotate $=90.000000$ 


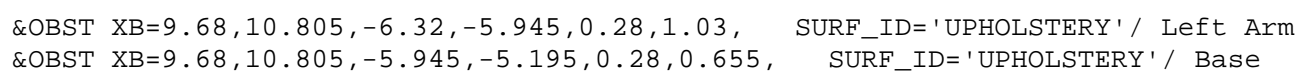
COBST XB=9.68, 10.805, $-5.195,-4.82,0.28,1.03$, S SURF_IDE'UPHOLSTERY'/ Right Arm
\&OBST XB $=9.68,10.055,-5.945,-5.195,0.655,1.03, \quad$ SURF_ID='UPHOLSTERY'/ Back *** GROUP: CHAIR offset $=1.500000,0.0000000,0.140000$ rotate $=90.0000000$ \&OBST $X B=11.18,12.305,-6.32,-5.945,0.28,1.03$, SURF_ID='UPHOLSTERY ' / Left Arm

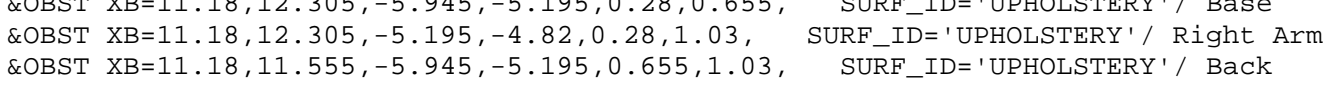
*** GROUP: CHAIR offset $=3.000000,0.000000,0.140000$ rotate $=90.000000$ \&OBST XB=12.68, 13.805, - $6.32,-5.945,0.28,1.03$, SURF_ID='UPHOLSTERY' / Left Arm \&OBST XB=12.68,13.805, - $5.195,-4.82,0.28,1.03$, SURF_ID='UPHOLSTERY' '/ Right Ar \&OBST XB $=12.68,13.055,-5.945,-5.195,0.655,1.03$, SURF_ID='UPHOLSTERY'/ Back *** GROUP: CHAIR offset $=4.500000,0.000000,0.140000$ rotate $=90.000000$ \&OBST XB $=14.18,15.305,-6.32,-5.945,0.28,1.03$, SURF_ID='UPHOLSTERY' / Left Arm
\&OBST XB $=14.18,15.305,-5.945,-5.195,0.28,0.655$,
SURF_ID='UPHOLSTERY' / Base COBST $X \mathrm{X}=14.18,15.305,-5.195,-4.82,0.28,1.03$,
\&OBST $X \mathrm{XB}=14.18,14.555,-5.945,-5.195,0.655,1.03$,

$* * *$ GROUP: CHAIR offset $=6.000000,0.0000000,0.140000$ rotate $=90.000000$

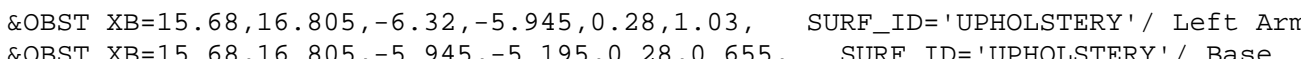
\&OBST XB $=15.68,16.805,-5.195,-4.82,0.28,1.03, \quad$ SURFID='UPHOLSTERY '/ Right Arm
\&OBST XB $=15.68,16.055,-5.945,-5.195,0.655,1.03, \quad$ SURF_ID=' 'UPHOLSTERY' $/$ Back *** GROUP: CHAIR offset $=7.500000,0.0000000,0.140000$ rotate $=90.000000$

\&OBST XB $=17.18,18.305,-6.32,-5.945,0.28,1.03, \quad$ SURF_IDE='UPHOLSTERY' $/$ Left Arm
\&OBST XB $17.18,18.305,-5.945,-5.195,0.28,0.655, \quad$ SURF_ID=' 'UPHOLSTERY'/B Base \&OBST XB $17.18,18.305,-5,195,-4.82,0.28,1.03$, SURF ID $=$ 'UPHOLSTERY' \&OBST XB $=17.18,18.305,-5.195,-4.82,0.28,1.03$, SURF_ID ='UPHOLSTERY' Right Arm
\&OBST XB $17.18,17.555,-5.945,-5.195,0.655,1.03$,
SURF_ID=' 'UPHOLSTERY'/ Back

*** GROUP: CHAIR offset $=9.0000000,0.000000,0.140000$ rotate $=90 . .000000$

ROBST XB $18.68,19.805,-6.32,-5.945,0.28,1.03, \quad$ SURF_ID= 'UPHOLSTERY ' Left Arm
\&OBST XB $=18.68,19.805,-5.945,-5.195,0.28,0.655, \quad$ SURF_ID='UPHOLSTERY ' $/$ Base

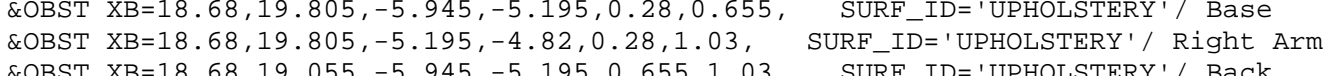

$* * *$ GROUP: CHAIR offset $=10.500000,0.0000000,0.140000$ rotate $=90.0000000$

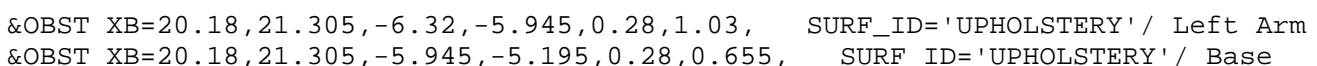

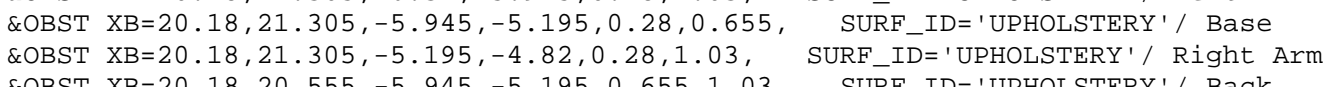

$* * *$ GROUP: CHAIR offset $=12.000000,0.000000,0.140000$ rotate $=90.000000$

ROBST XB $=21.68,22.805,-6.32,-5.945,0.28,1.03, \quad$ SURF_ID='UPHOLSTERY' ' Left Arm
\&OBST XB $=21.68,22.805,-5.945,-5.195,0.28,0.655, \quad$ SURF_ID=' 'UPHOLSTERY'/ Base

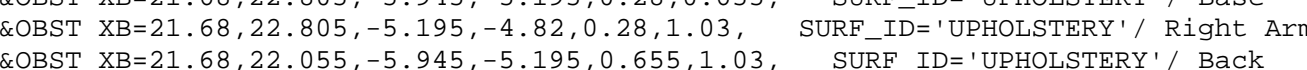
$* * *$ GROUP: CHAIR offset $=13.500000,0.000000,0.140000$ rotate $=90.0000000$

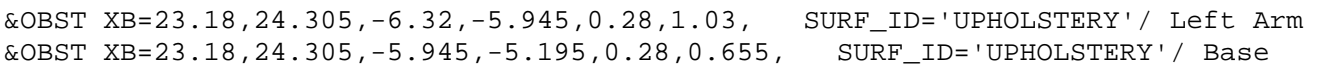

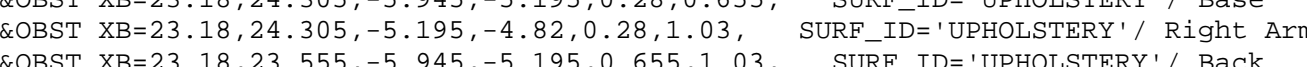
*** GROUP: CHAIR offset $=15.000000,0.000000,0.140000$ rotate $=90.000000$

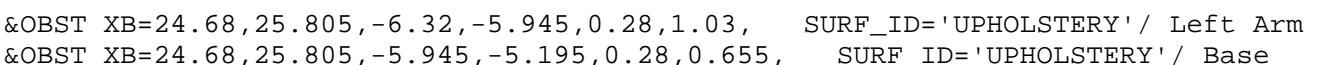
\&OBST XB $24.68,25.805,-5.195,-4.82,0.28,1.03$, SURF ID='UPHOLSTERY' '/ Right Arm
** GROUP: CHAIR_ROW3

*** GROUP: CHAIR offset $=0.000000,0.000000,0.140000$ rotate $=90.000000$

\&OBST XB=46.799999, 47.924999, -5.195, - 4.82, 0.28, 1. 03, SURF_ID= 'UPHOLSTERY'/ Left Arm \&OBST XB=46.799999, 47.924999, $-5.945,-5.195,0.28,0.655$, SURF IDD= 'UPHOLSTERY' $/$ Base

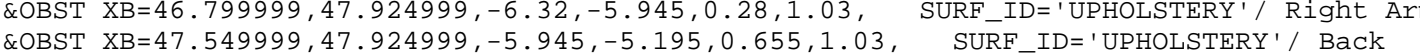

$* * *$ GROUP: CHAIR offset $=1.500000,0.000000,0.140000$ rotate $=90.000000$

\&OBST XB=45.299999, 46.424999, $-5.195,-4.82,0.28,1.03$, SURF_ID='UPHOLSTERY' / Left Arm \&OBST XB=45.299999, 46.424999, $-5.945,-5.195,0.28,0.655$, SURF ID = ' 'UPHOLSTERY' '/ Base

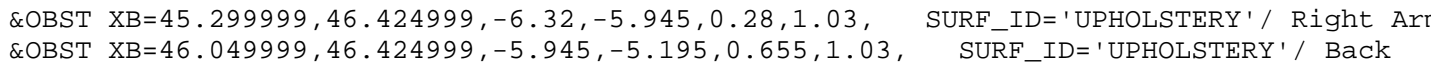

$* * *$ GROUP: CHAIR offset $=3.000000,0.000000,0.140000$ rotate $=90.000000$

\&OBST XB $=43.799999,44.924999,-5.195,-4.82,0.28,1.03$, SURF_ID='UPHOLSTERY' ' Left Arm

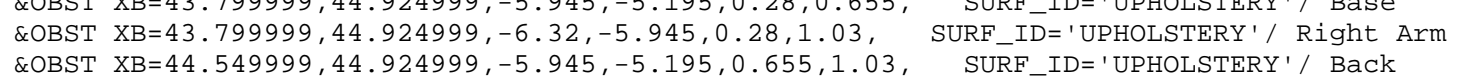

*** GROUP: CHAIR offset $=4.500000,0.000000,0.140000$ rotate $=90$. . 000000

\&OBST XB $42.299999,43.424999,-5.195,-4.82,0.28,1.03, \quad$ SURF_ID=' 'UPHOLSTERY ' $/$ Left Arm
\&OBST XB $=42.29999,43.424999,-5.945,-5.195,0.28,0.655, \quad$ SURF ID='UPHOLSTERY' $/$ Base COBST XB=42.299999, 43.424999, $-6.32,-5.945,0.28,1.03$, SURF_ID='UPHOLSTERY' 1 Right Arm
\&OBST XB=43.049999, $43.424999,-5.945,-5.195,0.655,1.03$, SURF_ID=' 'UPHOLSTERY'/ Back

$* * *$ GROUP: CHAIR offset $=6.000000,0.000000,0.140000$ rotate $=90.000000$

\&OBST XB=40.799999, 41.924999, $-5.195,-4.82,0.28,1.03$, SURF_ID= 'UPHOLSTERY' / Left Arm

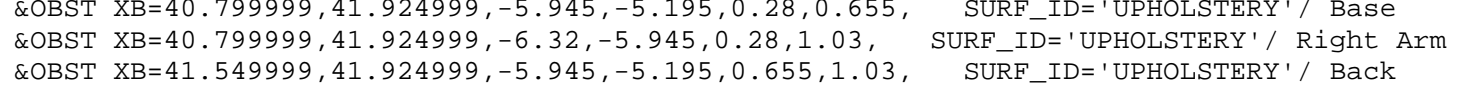

$* * *$ GROUP: CHAIR offset $=7.500000,0.000000,0.140000$ rotate $=90.000000$

\&OBST $\mathrm{XB}=39.299999,40.424999,-5.195,-4.82,0.28,1.03$, SURF_ID=' 'UPHOLSTERY ' $/$ Left Arm \&OBST XB $=39.299999,40.424999,-6.32,-5.945,0.28,1.03$, SURF ID ='UPHOLSTERY' 'Right \&OBST XB $=40.049999,40.424999,-5.945,-5.195,0.655,1.03$, SURF_ID='UPHOLSTERY'/ Back

$* * *$ GROUP: CHAIR offset $=9.000000,0.000000,0.140000$ rotate $=90.000000$

\&OBST XB=37.799999, 38.924999, $-5.195,-4.82,0.28,1.03$, SURF_ID=' 'UPHOLSTERY' ' Left Arm \&OBST XB $=37.799999,38.924999,-6.32,-5.945,0.28,1.03$, SURF $I \bar{D}=$ 'UPHOLSTERY' \&OBST XB=38.549999, 38.924999, -5.945, - $5.195,0.655,1.03$, SURF_ID='UPHOLSTERY'/ Back

*** GROUP: CHAIR offset $=10.500000,0.000000,0.140000$ rotate $=90.000000$

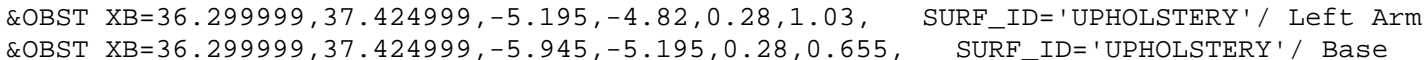

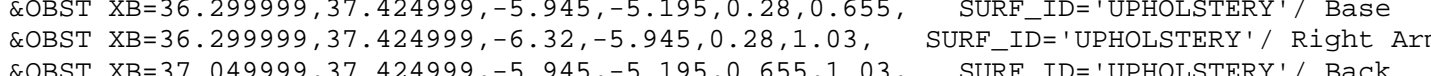

*** GROUP: CHAIR offset $=12.000000,0.000000,0.140000$ rotate $=90.000000$

\&OBST XB=34.799999, 35. 924999, $-5.195,-4.82,0.28,1.03, \quad$ SURF_ID='UPHOLSTERY' $/$ Left Arm
\&OBST XB $=34.799999,35.924999,-5.945,-5.195,0.28,0.655, \quad$ SURF_ID ='UPHOLSTERY' $/$ Base

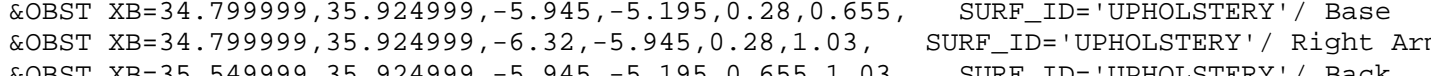

*** GROUP: CHAIR offset $=13.500000,0.000000,0.140000$ rotate $=90 . .000000$

\&OBST XB=33.299999, 34.424999, $-5.195,-4.82,0.28,1.03, \quad$ SURF_ID='UPHOLSTERY' $/$ Left Arm

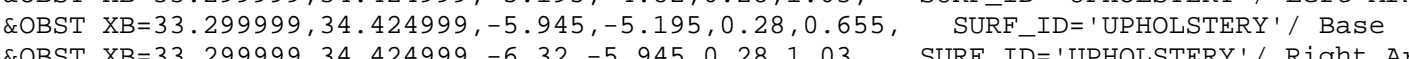

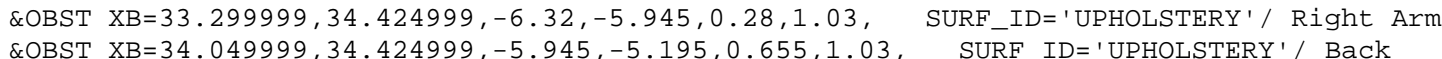

*** GROUP: CHATR offset $=15$,

\&OBST XB=31.799999, 32.924999, $-5.195,-4.82,0.28,1.03$, SURFIID='UPHOLSTERY' / Left Arm $-97$ 
\&OBST XB $=32.549999,32.924999,-5,945,-5,195,0,655,1,03$, SURE ID=' 'UPHOLSTERY' / Back

*** GROUP: CHAIR_ROW4

**** GROUP: CHAIR_ROW3

** GROUP: CHAIR offset $=0.000000,0.000000,0.140000$ rotate $=90.000000$

\&OBST XB=46.799999, 47. 924999, -3.695, -3.32, 0.28,1.03, SURF_ID='UPHOLSTERY' ' Left Arm OBST XB=46.799999, 47.924999, $-4.82,-4.445,0.28,1.03$, S SURF_ID=' 'UPHOLSTERY'/ Right Ar

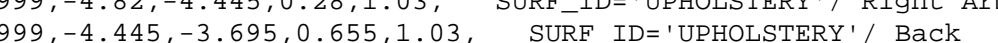

*** GROUP: CHAIR offset $=1.500000,0.000000,0.140000$ rotate $=90.000000$

\&OBST XB=45.299999, 46. 424999, -3.695, -3.32, 0. 28,1.03, SURF_ID=' 'UPHOLSTERY' ' Left Arm \&OBST XB $=45.299999,46.424999,-4.82,-4.445,0.28,1.03$, SURF-IID='UPHOLLTERY' / Right A

*** GROUP: CHAIR offset $=3.000000,0.000000,0.140000$ rotate $=90.000000$

EOBST XB=43.799999, 44.924999, -3.695,-3.32, 0.28,1.03, SURF_ID='UPHOLSTERY'/ Left Arm \&OBST XB=43.799999, 44.924999, $-4.445,-3.695,0.28,0.655, \quad$ SURF IDD= 'UPHOLSTERY' / Base عOBST XB=44.549999, $44.924999,-4.445,-3.695,0.655,1.03, \quad$ SURF_ID =' UPHOLSTERY' $/$ ' Back

*** GROUP: CHAIR offset $=4.500000,0.000000,0.140000$ rotate $=90.000000$

\&OBST XB=42.299999, 43.424999, - - $.695,-3.32,0.28,1.03$, SURF_ID='UPHOLSTERY'/ Left Arm \&OBST XB=42.299999, 43.424999, $-4.445,-3.695,0.28,0.655$, SURF_ID='UPHOLSTERY' ' Base EOBST XB $=42.299999,43.424999,-4.82,-4.445,0.28,1.03, \quad$ SURF_ID= 'UPHOLSTERY' $/$ Right Ar
EOBST XB $=43.049999,43.424999,-4.445,-3.695,0.655,1.03, \quad$ SURF_ID ' 'UPHOLSTERY ' / Back $* * *$ GROUP: CHAIR offset $=6.000000,0.000000,0.140000$ rotate $=90.000000$

\&OBST XB=40.799999, 41.924999, $-3.695,-3.32,0.28,1.03, \quad$ SURF-ID= 'UPHOLSTERY ' / Left Arm \&OBST XB-40.799999, $41.924999,-4.82,-4.445,0.28,1.03$,
\&OBST XB $41.549999,41.924999,-4.445,-3.695,0.655,1.03$,

*** GROUP: CHAIR offset $=7.500000,0.000000,0.140000$ rotate $=90.000000$

\&OBST XB=39.299999, 40.424999, -3.695, -3.32, $0.28,1.03$, SURF ID = 'UPHOLSTERY ' / Left Arn

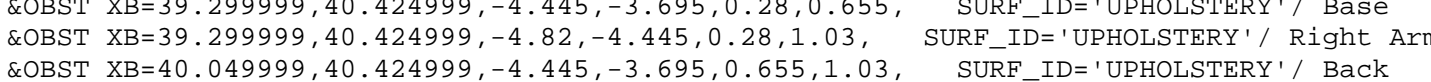
$* * *$ GROUP: CHAIR offset $=9.000000,0.000000,0.140000$ rotate $=90.000000$

\&OBST XB=37.799999, 38. 924999, $-3.695,-3.32,0.28,1.03$, SURF_ID = 'UPHOLSTERY ' / Left Arm COBST XB $=37.799999,38.924999,-4.82,-4.445,0.28,1.03$,
\&OBST XB $38.549999,38.924999,-4.445,-3.695,0.655,1.03$, SURF-IDE='UPHOLSTERY'/ Right Arm
SURF_ID=' UPHOLSTERY'/ Back

*** GROUP: CHAIR offset $=10.500000,0.000000,0.140000$ rotate $=90.000000$

\&OBST XB=36.299999, 37. 424999, -3.695, -3.32, 0.28,1.03, SURFIDD='UPHOLSTERY' / Left Ar \&OBST XB=36.299999, 37. $424999,-4.82,-4.445,0.28,1.03$, SURF ID ='UPHOLSTERY'/ Right A \&OBST XB $=37.049999,37.424999,-4.445,-3.695,0.655,1.03$, SURF_ID=' UPHOLSTERY'/ Back *** GROUP: CHAIR offset $=12.000000,0.000000,0.140000$ rotate $=90.000000$

ROBST XB $=34.799999,35.924999,-3.695,-3.32,0.28,1.03, \quad$ SURF_ID= 'UPHOLSTERY ' ' Left Arm
\&OBST XB $34.799999,35.924999,-4.445,-3.695,0.28,0.655$,
SURF_ID='UPHOLSTERY' $/$ Base

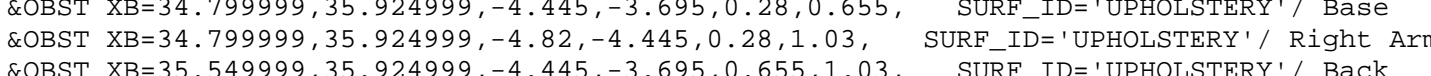

$* * *$ GROUP: CHAIR offset $=13.500000,0.0000000,0.140000$ rotate $=90.000000$

\&OBST XB $=33.299999,34.424999,-3.695,-3.32,0.28,1.03, \quad$ SURF_ID= 'UPHOLSTERY ' ' Left Arm
\&OBST XB $33.29999,34.424999,-4.445,-3.695,0.28,0.655, \quad$ SURF_ID=' UPHOLSTERY'

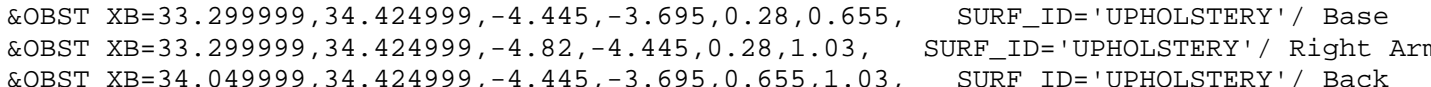

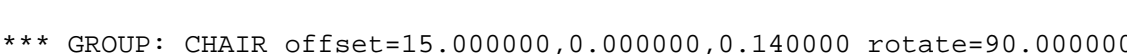

ROBST XB=31.799999, 32. 924999, $-3.695,-3.32,0.28,1.03, \quad$ SURF_ID='UPHOLSTERY' $/$ Left Arm
\&OBST XB $=31.799999,32.924999,-4.445,-3.695,0.28,0.655, \quad$ SURF_ID='UPHOLSTERY' $/$ Base

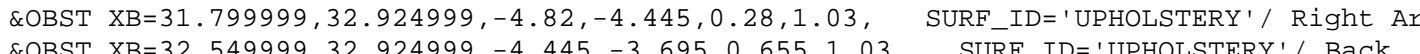

*** GROUP: CHAIR_ROW3

$* * *$ GROUP: CHAIR offset $=0.000000,0.000000,0.140000$ rotate $=90.000000$

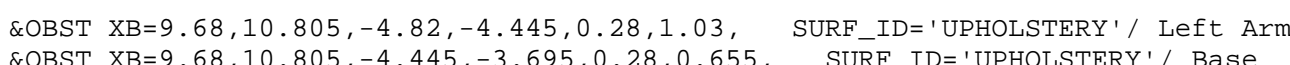

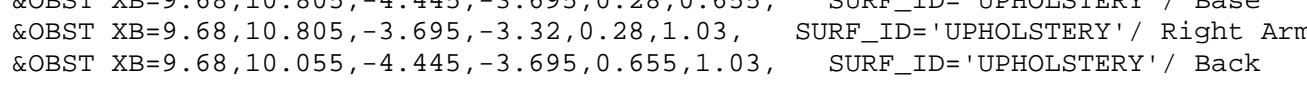

** GROUP: CHAIR offset $=1.500000,0.000000,0.140000$ rotate $=90.000000$

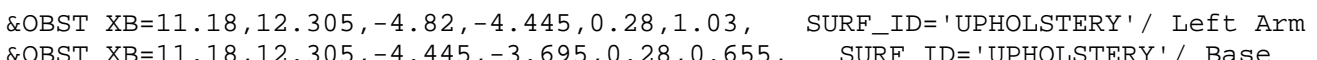

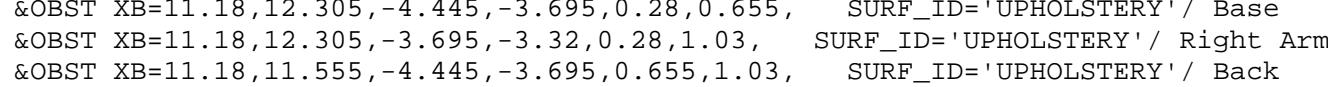

$* * *$ GROUP: CHAIR offset $=3.0000000,0.000000,0.140000$ rotate $=90.000000$

\&OBST XB $12.68,13.805,-4.82,-4.445,0.28,1.03, \quad$ SURF_ID='UPHOLSTERY ' / Left Arm
\&OBST XB $=12.68,13.805,-4.445,-3.695,0.28,0.655$, SURF ID='UPHOLSTERY'/ Base

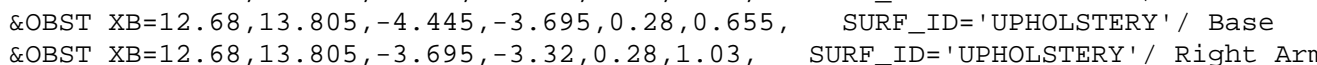
\&OBST $X B=12.68,13.805,-3.695,-3.32, \theta .28,1.03$,
\&OBST $X B=12.68,13.055,-4.445,-3.695,0.655,1.03$,

*** GROUP: CHAIR offset $=4.500000,0.000000,0.140000$ rotate $=90$. .

\&OBST XB $=14.18,15.305,-4.82,-4.445,0.28,1.03, \quad$ SURF_ID='UPHOLSTERY' Left Arm
\&OBST XB $=14.18,15.305,-4.445,-3.695,0.28,0.655, \quad$ SURF_ID=' 'UPHOLSTERY'/ Base

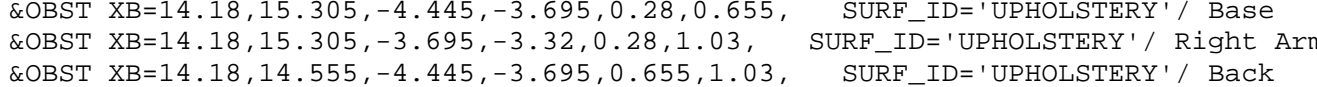

*** GROUP: CHAIR offset $=6.000000,0.000000,0.140000$ rotate $=90.000000$

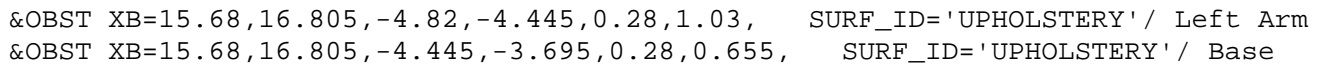

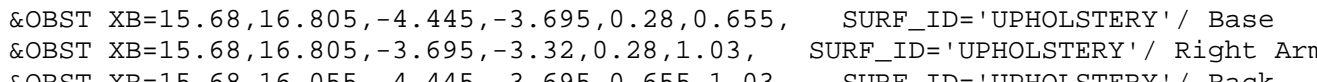

$* * *$ GROUP: CHAIR offset $=7.500000$, $, .000000,0.140000$ rotate $=90.000000$

\&OBST XB $=17.18,18.305,-4.82,-4.445,0.28,1.03, \quad$ SURF_ID=' 'UPHOLSTERY'/ Left Arm
COBST XB $=17.18,18.305,-4.445,-3.695,0.28,0.655, \quad$ SURF_ID='UPHOLSTERY'
Base

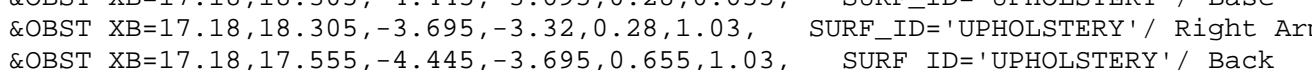

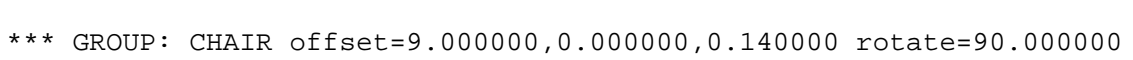

\&OBST XB $18.68,19.805,-4.82,-4.445,0.28,1.03$, SURF-ID=' 'UPHOLSTERY' / Left Arm

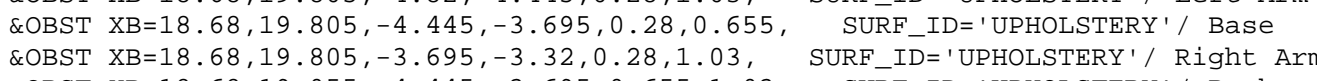
SURE ID=' 'UPHOLSTERY'/ Back

*** GROUP: CHAIR offset $=10.500000,0.000000,0.140000$ rotate $=90.000000$ \&OBST XB $=20.18,21.305,-4.82,-4.445,0.28,1.03$, SURFIID=' 'UPHOLSTERY' / Left Arm

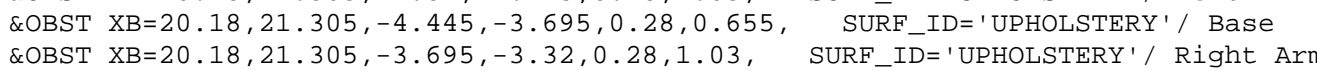
\&OBST XB $=20.18,20,555,-4,445,-3,65,0.655,1,03$, SURF_ID=' UPHOLSTERY'/ Back

$\star * \star$ GROUP: CHAIR offset $=12.000000,0.0000000,0.140000$ rotate $=90.000000$

\&OBST XB $=21.68,22.805,-4.82,-4.445,0.28,1.03$, SURF IDD ' UPHOLSTERY' ' Left Arm \&OBST XB=21.68, 22.805, - $3.695,-3.32,0.28,1.03$, , SURF_ID='UPHOLSTERY'/ Right Ar

*** GROUP: CHAIR offset $=13.500000,0.000000,0.140000$ rotate $=90.000000$

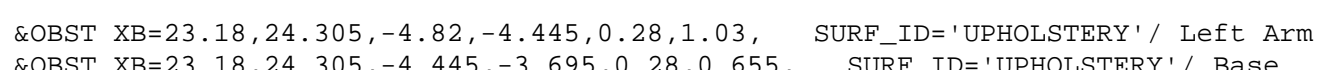
\&OBST $X \mathrm{X}=23.18,24.305,-3.695,-3.32,0.28,1.03$, SURF_ID='UPHOLSTERY'/ Right Arm 
*** GROUP: CHAIR of $f$ set $=15.000000,0.000000,0.140000$ rotate $=90.000000$

ROBST XB $=24.68,25.805,-4.82,-4.445,0.28,1.03$, SURF ID = ' 'PHOLSTERY ' $/$ Left Arm

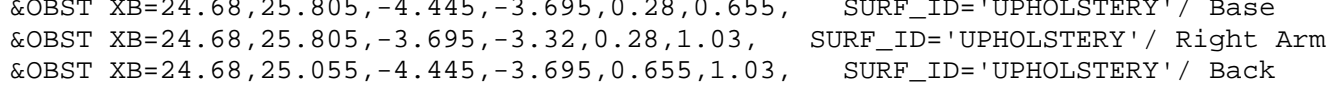

\section{*** GROUP: LR}

*** GROUP: COUCH offset $=0.000000,0.000000,0.000000$ rotate $=0.000000$

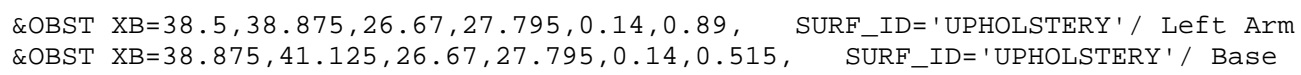

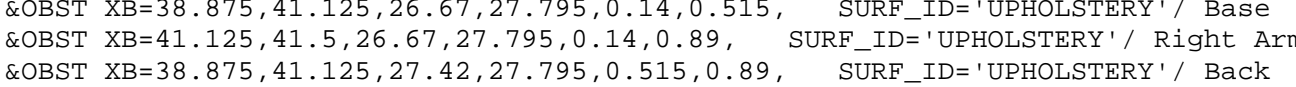

*** GROUP: CHAIR offset $=0.000000,-1.875000,0.000000$ rotate $=90.000000$

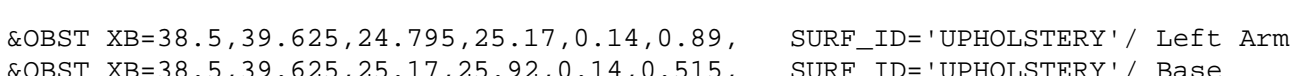

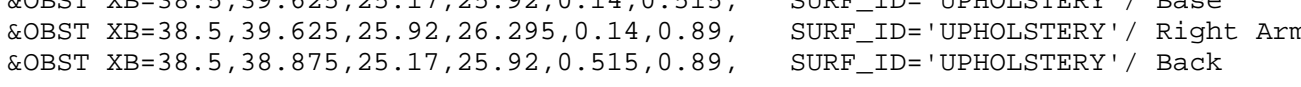

*** GROUP: CHAIR offset $=1.875000,-1.875000,0.000000$ rotate $=270.000000$

\&OBST $\mathrm{XB}=40.375,41.5,25.92,26.295,0.14,0.89, \quad$ SURF_ID=' 'UPHOLSTERY ' / Left Arm

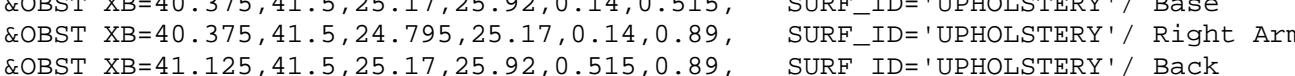

\section{*** GROUP: LR}

** GROUP: COUCH offset $=0.000000,0.000000,0.000000$ rotate $=0.000000$

\&OBST XB=38.5,38.875,30.049999, 31. 174999, $0.14, \Theta .89, \quad$ SURF_ID=' 'UPHOLSTERY' ' Left Arm \&OBST XB $=41.125,41.5,30.049999,31.174999,0.14, \theta .89$,
\&OBST XB $38.875,41.125,30.799999,31.174999,0.515,0.89$,
SURF_ID=' 'UPHOLSTERY '/ Right Arn
SURF_ID=' 'UPHOLSTERY'/ Back

*** GROUP: CHAIR offset $=0.000000,-1.875000,0.000000$ rotate $=90.000000$ \&OBST XB=38.5, 39.625, 28.174999, 28. 549999, 0.14, 0.89, SURF_ID= 'UPHOLSTERY ' / Left Arm \&OBST XB=38.5, 39.625, 29.299999, 29.674999, $0.14,0.89$, SURF_ID='UPHOLSTERY'/ Right Ar \&OBST XB $=38.5,38.875,28.549999,29.299999,0.515,0.89$, SURF_ID *** GROUP: CHAIR offset $=1.875000,-1.875000,0.000000$ rotate $=270.000000$ \&OBST XB=40.375, 41.5, 29.299999, 29.674999, $0.14,0.89$, SURF_ID=' 'UPHOLSTERY ' / Left Arm

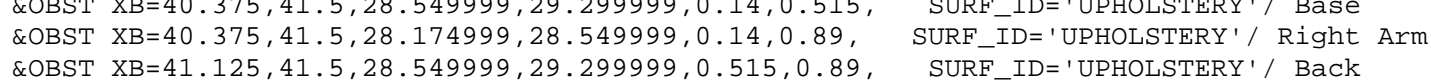

*** GROUP: LR

** GROUP: COUCH offset $=0.000000,0.000000,0.000000$ rotate $=0.000000$

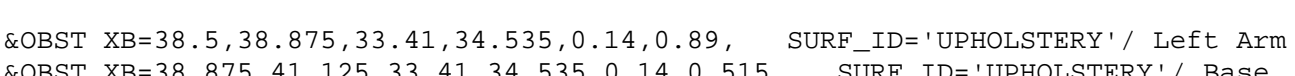
COBST $X B=41.125,41.5,33.41,34.535,0.14,0.89$,
\&OBST $X B=38.875,41.125,34.16,34.535,0.515,0.89$,
SURF_ID='UPHOLSTERY'/ Right Arm
SURF_ID='UPHOLSTERY' / Back

*** GROUP: CHAIR offset $=0.000000,-1.875000,0.000000$ rotate $=90.000000$ \&OBST XB=38.5, 39.625, 31.535, 31.91, $0.14,0.89$, SURF_ID= 'UPHOLSTERY ' / Left Arm

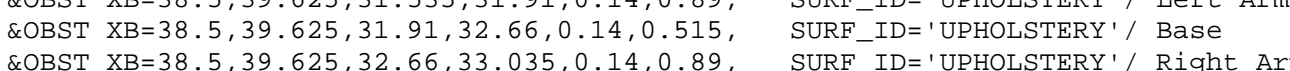

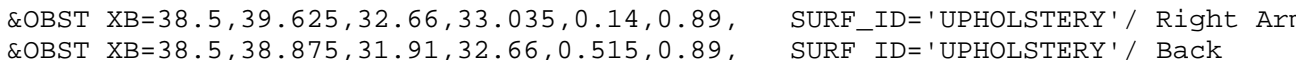

*** GROUP: CHATR offset $=1.875000,-1.875000,0,000000$ rotate $=270,000000$

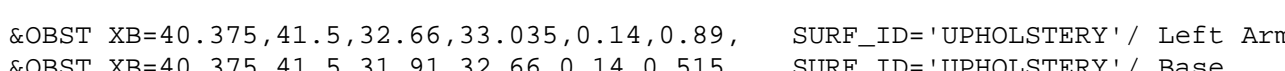

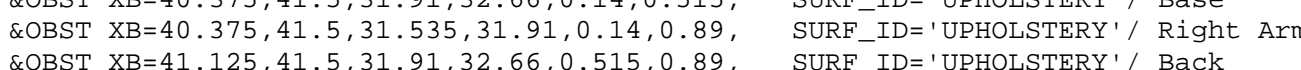

*** GROUP: LR

*** GROUP: CouCH offset $=0.000000,0.000000,0.000000$ rotate $=0.000000$

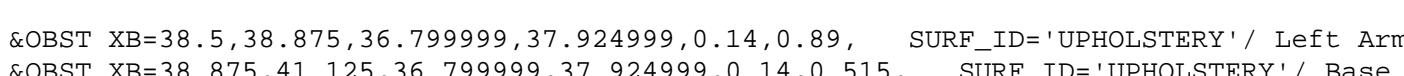

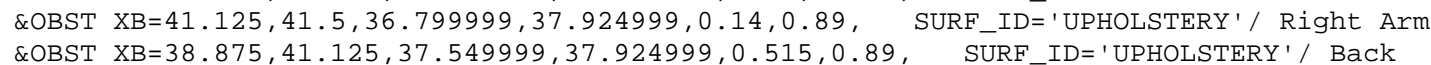

*** GROUP: CHAIR offset $=0.000000,-1.875000,0.000000$ rotate $=90 . .000000$

\&OBST XB=38.5, 39.625, 34. 924999, 35. 299999, 0.14, 0.89, SURF_ID='UPHOLSTERY'/ Left Arm \&OBST XB $=38.5,39.625,35.299999,36.049999,0.14,0.515$, SURE ID='UPHOLSTERY'/ Base-

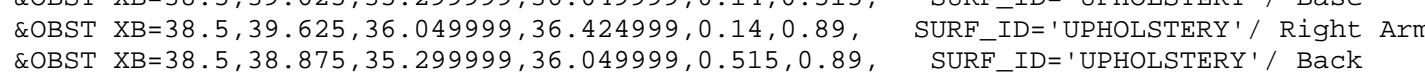

*** GROUP: CHAIR offset $=1.875000,-1.875000,0.000000$ rotate $=270.000000$

\&OBST XB=40.375, 41.5, 36. 049999, 36.424999, $0.14,0.89$, SURF_ID=' 'UPHOLSTERY ' / Left Arm

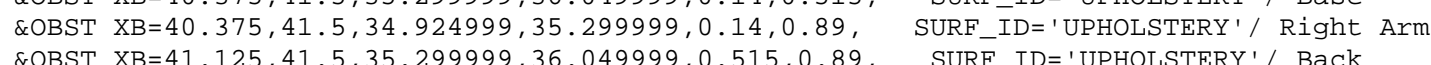

$* * *$ GROUP: COUCH_TWIN

*** GROUP: COUCH offset $=0.000000,0.000000,0.000000$ rotate $=90.000000$

\&OBST XB=27.299999, 28.424999, $-2.2,-1.825,0.14,0.89$, SURF_ID='UPHOLSTERY' / Left Arm \&OBST XB=27.299999, 28.424999, $-1.825,0.425,0.14,0.515$, SURF_ID=' 'UPHOLSTERY'/ Base \&OBST XB $=27.299999,27.674999,-1.825,0.425,0.515,0.89$, SURF IDE' 'UPHOLSTERY'/ Back

*** GROUP: COUCH offset $=1.875000,0.000000,0.000000$ rotate $=270.000000$ \&OBST XB=29.174999, 30.299999, 0.425, ब.8, $0.14,0.89$, SURF_ID=' 'UPHOLSTERY ' / Left Arm

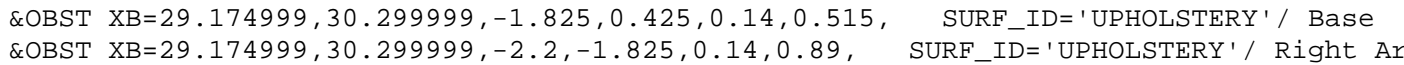

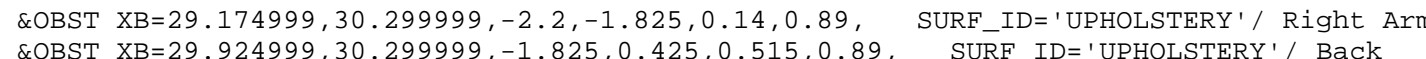

*** GROUP: COUCH_TWIN

*** GROUP: COUCH offset $=0.000000,0.0000000,0.000000$ rotate $=90.000000$

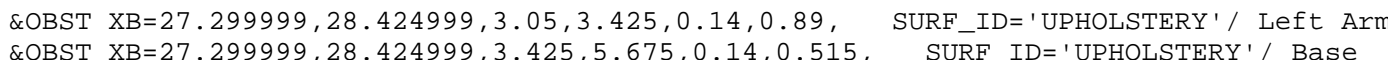
\&OBST XB=27.299999, 28.424999, 5.675,6.05, $14,0.89$, SURE ID ='UPHOLSTERY'/ Right Ar \&OBST XB $=27.299999,27.674999,3.425,5.675,0.515,0.89$, SURF_ID='UPHOLSTERY'/ Back

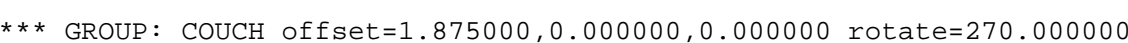

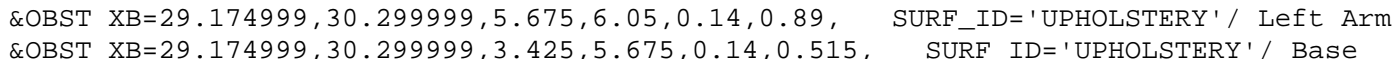

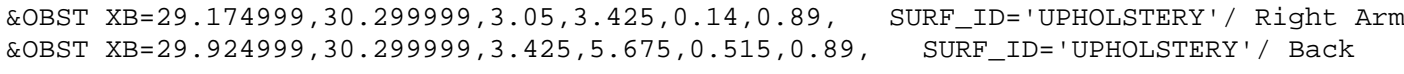

*** GROUP: COUCH_TWIN

*** GROUP: COUCH offset $=0.0000000,0.000000,0.000000$ rotate $=90.000000$

\&OBST XB=27.299999, 28.424999, 9. $95,9.425,0.14,0.89$, SURF-ID='UPHOLSTERY' / Left Arm \&OBST XB=27.299999, 28.424999, 11.675, 12.05, 0.14, 14.89 , SURF_ID='UPHOLSTERY'/ Right Ar 
** GROUP: COUCH offset $=1.875000,0.000000,0.000000$ rotate $=270.000000$

\&OBST XB=29.174999, 30. 299999, 11.675, 12.05, $0.14,0.89, \quad$ SURF_ID=' 'UPHOLSTERY' ' Left Ar \&OBST XB=29.174999, 30. 299999, 9. $05,9.425,0.14,0.89$, SURF IDE' 'UPHOLSTERY' 1 'Right Ar \&OBST XB=29.924999, 30.299999, $9.425,11.675,0.515,0.89$, SURF_ID='UPHOLSTERY' $/$ Back

\section{*** GROUP: COUCH_TWIN}

*** GROUP: COUCH offset $=0.000000,0 . .000000, \Theta . .000000$ rotate $=90 . .000000$

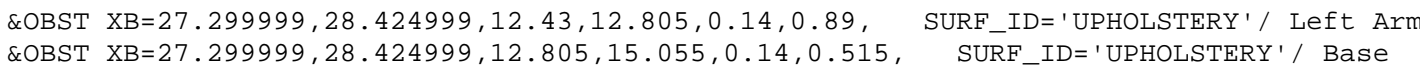
\&OBST XB=27. 299999, 28.424999, 15. $055,15.43,0.14,0.89$, SURF ID='UPHOLSTERY' ' Right Ar

*** GROUP: COUCH offset $=1.875000,0.000000,0.000000$ rotate $=270.000000$

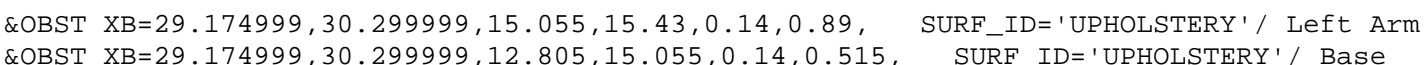
\&OBST XB $=29.174999,30.299999,12.43,12.805,0.14,0.89$,

*** GROUP: COUCH_TWIN

*** GROUP: COUCH offset $=0.000000,0.000000,0.000000$ rotate $=90.000000$

\&OBST XB $=27.299999,28.424999,15.8,16.174999,0.14,0.89, \quad$ SURF_ID= 'UPHOLSTERY ' Left Arm
\&OBST XB $=27.29999,28.424999,16.174999,18.424999,0.14,0.515, \quad$ SURF_ID 'UPHOLSTERY' A B \&OBST XB $=27.299999,28.424999,18.424999,18.799999,0.14,0.89$, SURF_ID='UPHOLSTERY' 1 Right Arm
\&OBST XB $=27.299999,27.674999,16.174999,18.424999,0.515,0.89$,
SURF_ID=' 'UPHOLSTERY' $/$ Back $* * *$ GROUP: COUCH offset $=1.875000,0.000000,0.000000$ rotate $=270.000000$

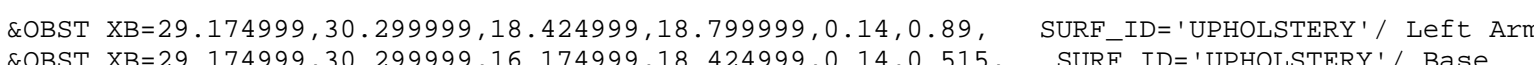

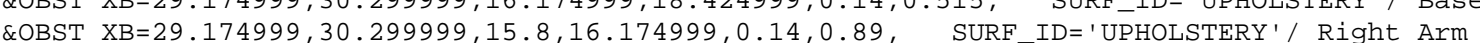
\&OBST XB=29.174999, 30.299999,15.8, 16.174999, $0.14,0.89, \quad$ SURF_ID='UPHOLSTERY'/ Right A Arm
\&OBST XB=29.924999, 30.29999, $16.174999,18.424999,0.515,0.89, \quad$ SURF_IDE'UPHOLSTERY'/ Back

*** GROUP: CHAIR_TWIN

*** GROUP: CHAIR of fset $=0.000000,0.000000,0.000000$ rotate $=90.000000$

\&OBST XB $=27.299999,28.424999,1.17,1.545,0.14,0.89$, SURF_ID= 'UPHOLSTERY' / Left Arm \&OBST XB $=27.299999,28.424999,1.545,2.295,0.14,0.515, \quad$ SURF-ID= 'UPHOLSTERY'/ Base

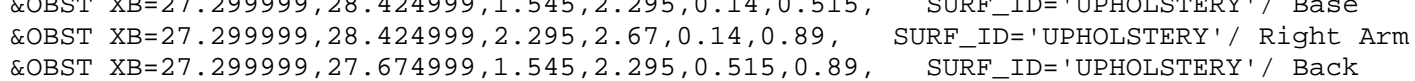
$* * *$ GROUP: CHAIR offset $=1.875000,0.000000,0.000000$ rotate $=270.000000$

\&OBST XB=29.174999, 30.299999, 2.295, 2.67, 0.14, 0.89 , SURF_ID='UPHOLSTERY' / Left Arm \&OBST $X \mathrm{XB}=29.174999,30.29999,1.545,2.295,0.14,0.515$,
\&OBST $X \mathrm{~B}=29.174999,30.299999,1.17,1.545,0.14,0.89$,
SURF ID ID = 'UPHOLSTERY' 'Pight Arm

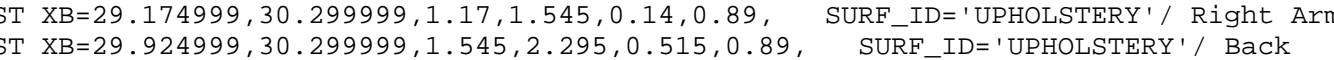

\section{*** GROUP: RHS_ROW}

$* * *$ GROUP: LR

*** GROUP: COUCH offset $=0.000000,0.000000,0.0000000$ rotate $=0.000000$

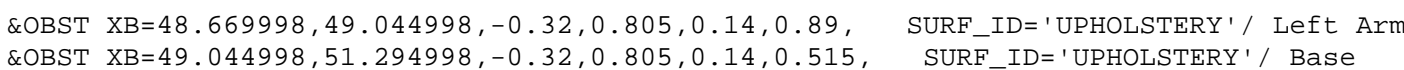
\&OBST XB=51.294998, 51.669998,-0.32, $0.805,0.14,0.89$, SURF_IDE' 'UPHOLSTERY'/ Right Ar

$* * *$ GROUP: CHAIR offset $=0.0000000,-1.875000,0.0000000$ rotate $=90.000000$

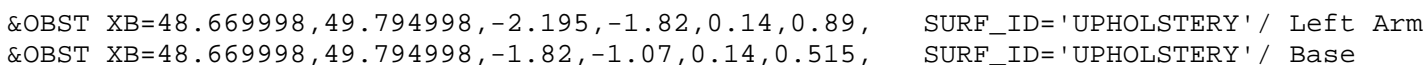

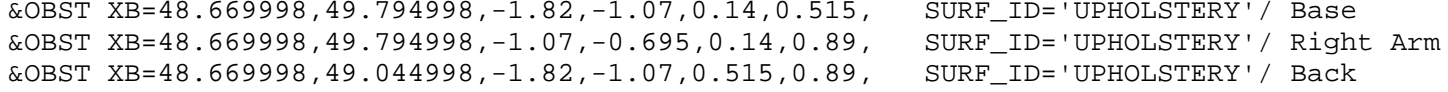

*** GROUP: CHAIR offset $=1.875000,-1.875000,0.0000000$ rotate $=270.000000$
\&OBST XB=50.544998, 51.669998, $-1.07,-0.695,0.14,0.89, \quad$ SURF_ID=' 'UPHOLSTERY ' / Left Arm
\&OBST XB=50.544998, $51.669998,-1.82,-1.07,0.14,0.515, \quad$ SURF_ID=' 'UPHOLSTERY'

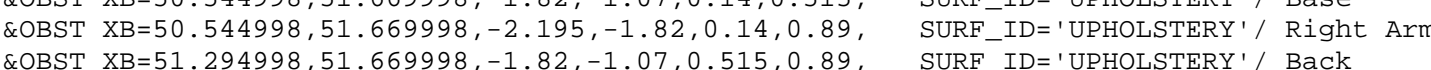

*** GROUP: LR

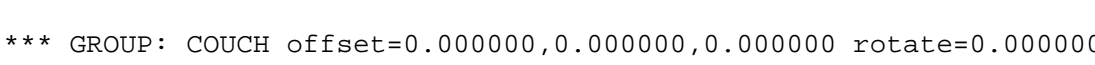

\&OBST XB=52. $044998,52.419998,-0.32,0.805,0.14,0.89$, SURF_ID=' 'UPHOLSTERY'/ Left Arm \&OBST XB $=52.419998,54.669998,-0.32,0.805,0.14,0.515$, SURF_ID='UPHOLSTERY'/ Base

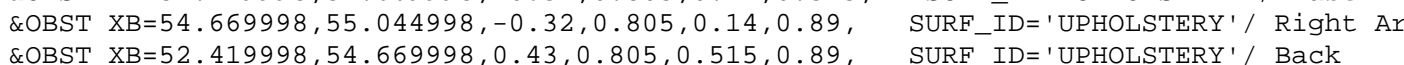

*** GROUP: CHAIR offset $=0.000000,-1.875000,0.000000$ rotate $=90$. . 000000

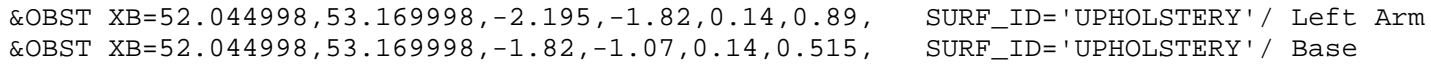
\&OBST XB $=52.044998,53.199998,-1.07,-0.695,0.14,0.89, \quad$, SURF ID=' 'UPHOLSTTRY' Base \&OBST XB=52. $044998,52.419998,-1.82,-1.07,0.515,0.89$, SURF_ID=' UPHOLSTERY'/ Back

*** GROUP: CHAIR offset $=1.875000,-1.875000,0.000000$ rotate $=270.000000$

\&OBST XB=53.919998, 55. 044998, -1.07, - $0.695,0.14,0.89, \quad$ SURF_ID= 'UPHOLSTERY ' / Left Arm

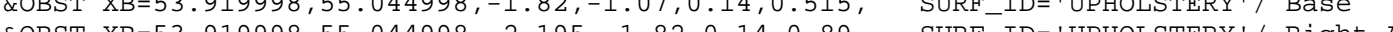

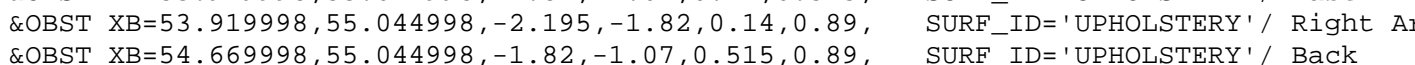

*** GROUP: LR

** GROUP: COUCH offset $=0.000000,0.000000,0.000000$ rotate $=0.000000$

\&OBST XB=55.419998, 55.794998, - $0.32,0.805,0.14,0.89, \quad$ SURF_ID='UPHOLSTERY' / Left Arm COBST XB $=55.794998,58.044998,-0.32,0.805,0.14,0.515$,
\&OBST XB

*** GROUP: CHAIR offset $=0.000000,-1.875000,0.0000000$ rotate $=90.000000$

\&OBST XB=55.419998, 56.544998, $-2.195,-1.82,0.14,0.89, \quad$ SURF_ID=' 'UPHOLSTERY ' / Left Arm \&OBST XB $=55.419998,55.794998,-1.82,-1.07,0.515,0.89$,
SURF ID=' 'UPHOLSTERY'/ Back $* * *$ GROUP: CHAIR offset $=1.875000,-1.875000,0.000000$ rotate $=270.000000$

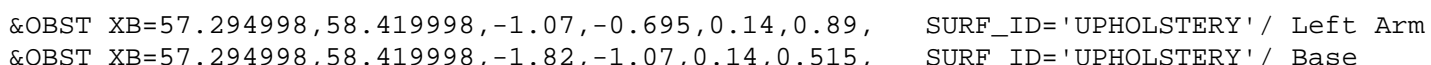

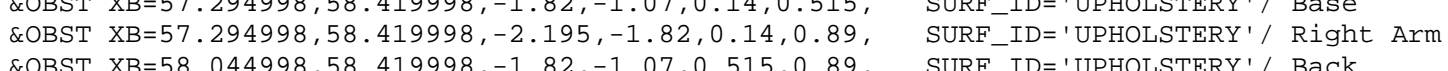

*** GROUP: LR

*** GROUP: COUCH offset $=0.000000,0.000000,0.000000$ rotate $=0.000000$

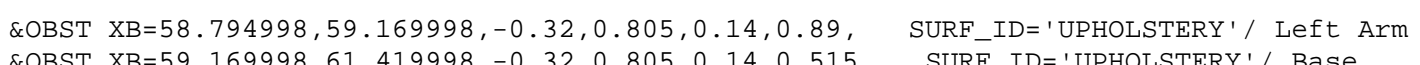

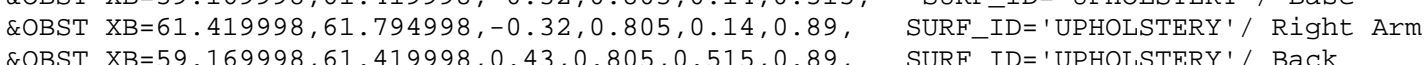

$\star * *$ GROUP: CHAIR offset $=0.000000,-1.875000,0.000000$ rotate $=90.000000$

\&OBST XB=58.794998, 59.919998, $-2.195,-1.82,0.14,0.89$, SURF_ID='UPHOLSTERY'/ Left Arm

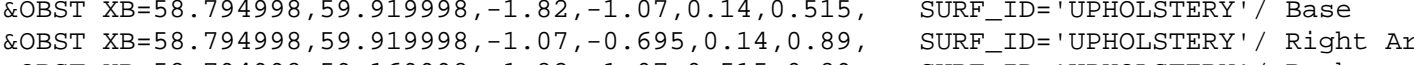

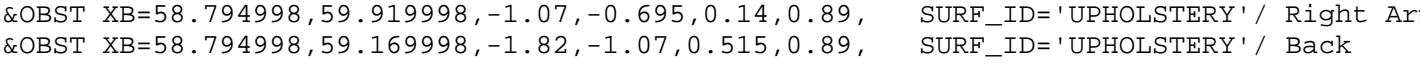


** GROUP: CHATR offset $=1.875000,-1.875000,0.000000$ rotate $=270.000000$

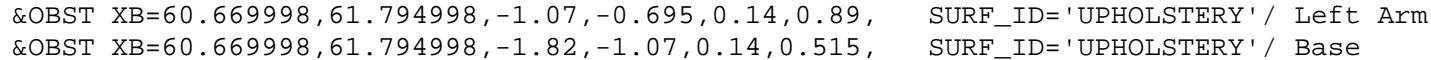
\&OBST XB=60.669998, 61.794998, -2.195,-1.82,0.14,0.89, SURF-IDE'UPHOLSTERY', Right Arm

*** GROUP: LR

*** GROUP: COUCH offset $=0.000000,0.000000,0.000000$ rotate $=0.000000$

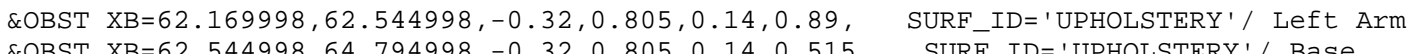
\&OBST XB $=64.794998,65.169998,-0.32,0.805,0.14,0.89, \quad$ SURF_IDE='UPHOLSTERY'

*** GROUP: CHAIR offset $=0.000000,-1.875000,0.000000$ rotate $=90.000000$

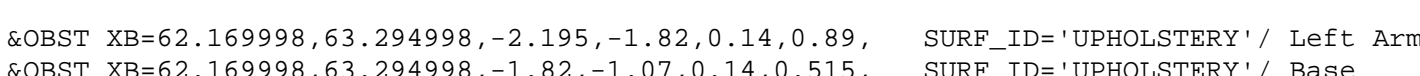

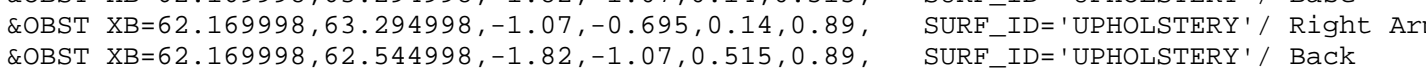

*** GROUP: CHAIR offset $=1.875000,-1.875000,0.000000$ rotate $=270.000000$

\&OBST XB $=64.044998,65.169998,-1.07,-0.695,0.14,0.89, \quad$ SURF_ID= 'UPHOLSTERY ' ' Left Arm

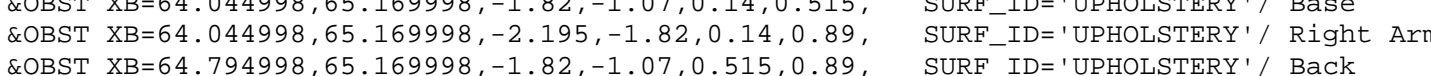

\section{*** GROUP: RHS_ROW}

$* * *$ GROUP: LR

*** GROUP: COUCH offset $=0.000000,0.000000,0.000000$ rotate $=0.000000$

\&OBST XB $=48.669998,49.044998,4.93,6.055,0.14,0.89$, SURF_ID= ' UPHOLSTERY' $/$ Left Arm
\&OBST XB $=49.044998,51.294998,4.93,6.055,0.14,0.515, \quad$ SURF_ID 'UPHOLSTERY' $/$ Base

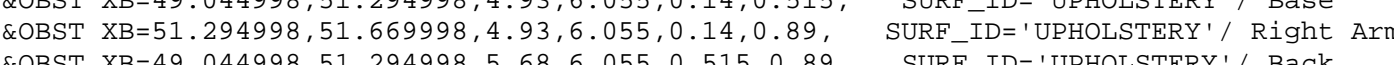

(a)

\&OBST XB=48.669998, 49.794998, 3. 055, 3.43, $0.14,0.89$, SURF_ID='UPHOLSTERY'/ Left Arm \&OBST X X=48.669998, 49.794998, 3.43, 4.18, $0.14,0.515$, SURF_IDD' UPHOLSTERY' \&OBST XB $48.669998,49.794998,4.18,4.555,0.14,0.89$, SURF_ID='UPHOLSTERY'/ Right Arm
\&OBST XB $48.669998,49.944998,3.43,48,0.515,0.89$, *** GROUP: CHAIR offset $=1.875000,-1.875000,0.000000$ rotate $=270.000000$

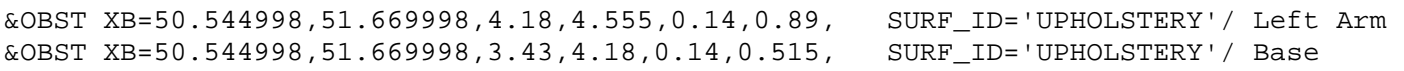

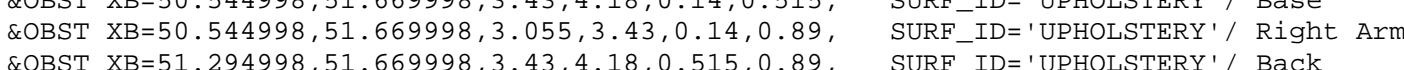

*** GROUP: LR

*** GROUP: COUCH offset $=0.000000$, ๑. .000000, ๑. .000000 rotate $=0.000000$

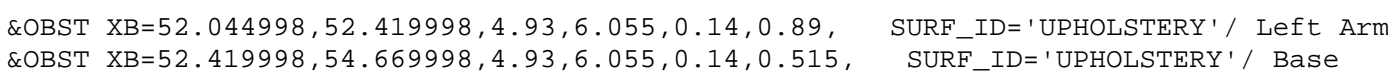

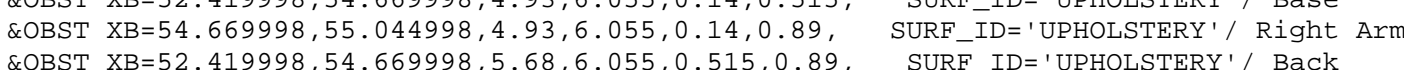

*** GROUP: CHAIR offset $=0.0000000,-1.875000,0.000000$ rotate $=90.0000000$

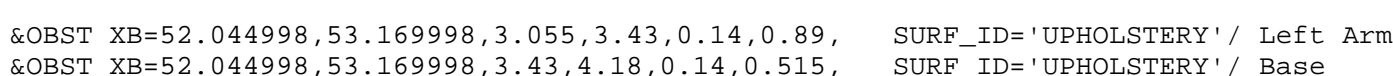

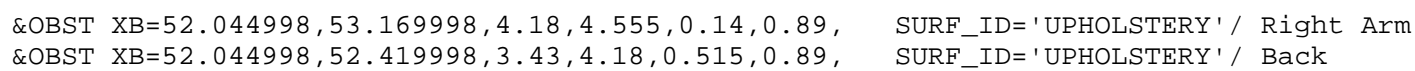
*** GROUP: CHAIR offset $=1.875000,-1.875000,0.000000$ rotate $=270.000000$

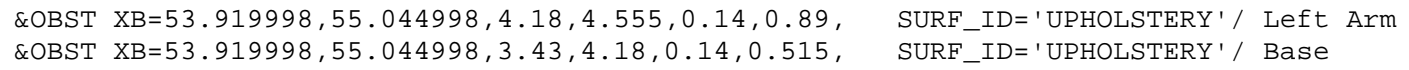

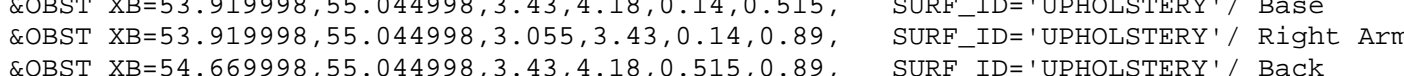

*** GROUP: LR

*** GROUP: Couch offset $=0.000000$, $, .000000,0.0 .000000$ rotate $=0.000000$

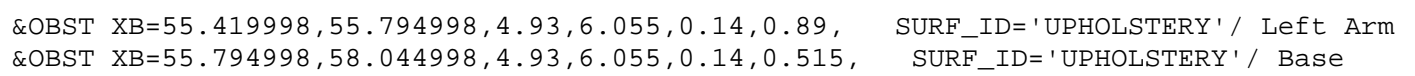

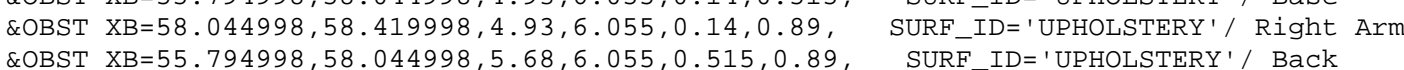

*** GROUP: CHAIR offset $=0.000000,-1.875000,0.0000000$ rotate $=90.000000$

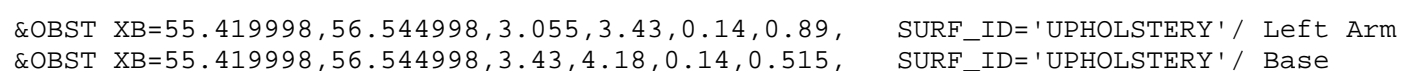
\&OBST XB $=55.419998,56.544998,4.18,4.555,0.14,0.89, \quad$ SURE IDE' UPHOLSTERY' ' Rase \&OBST XB=55.419998, 55.794998, 3.43,4.18,0.515, 1.89 , SURF_ID='UPHOLSTERY'/ Back

*** GROUP: CHAIR offset $=1.875000,-1.875000,0.000000$ rotate $=270.000000$

$\begin{array}{ll}\text { \&OBST XB=57. } 294998,58.419998,4.18,4.555,0.14,0.89, & \text { SURF_ID='UPHOLSTERY' } / \text { Left Arm } \\ \text { \&OBST XB=57.294998, } 58.419998,3.43,4.18,0.14,0.515, & \text { SURF_ID='UPHOLSTERY' }\end{array}$

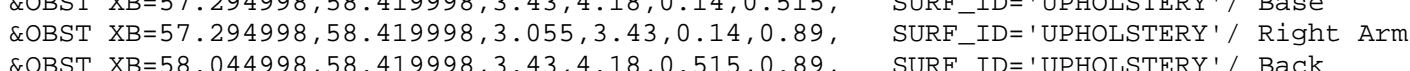

*** GROUP: LR

*** GROUP: COUCH offset $=0.000000$, ๑. .000000, ๑. 0000000 rotate $=0.000000$

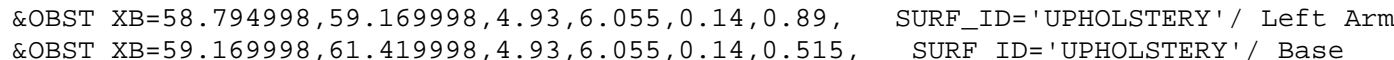

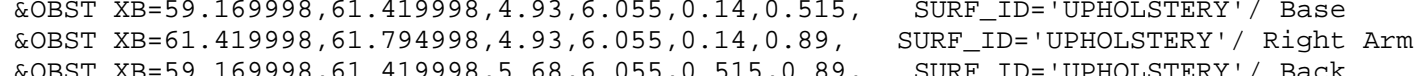

$* * *$ GROUP: CHAIR offset $=0.000000,-1.875000,0.000000$ rotate $=90 . .000000$

\&OBST $X \mathrm{XB}=58.794998,59.919998,3.055,3.43,0.14,0.89, \quad$ SURF_ID=' 'UPHOLSTERY ' / Left Arm

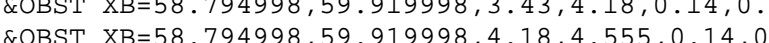
\&OBST XB=58.794998, 59.169998, $3.43,4.18,0.515,0.89$, SURF_ID='UPHOLSTERY'/ Back

*** GROUP: CHAIR offset $=1.875000,-1.875000,0.0000000$ rotate $=270.000000$

\&OBST XB=60.669998, 61.794998, 4.18,4.555, $0.14,0.89$, SURF_ID='UPHOLSTERY ' / Left Arm \&OBST XB=60.669998, 61.794998, $3.055,3.43,0.14,0.89$, SURF-ID='UPHOLSTERY'/ Right Ar

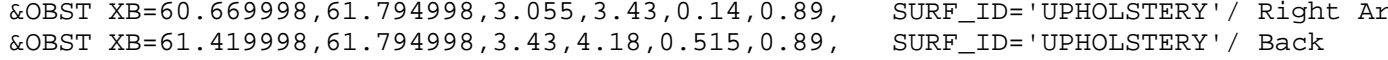

*** GROUP: LR

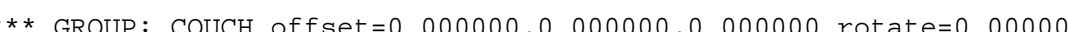

\&OBST $X \mathrm{XB}=62.169998,62.544998,4.93,6.055,0.14,0.89$, SURF_ID='UPHOLSTERY' ' Left Arm

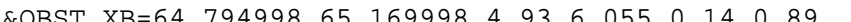
\&OBST XB=62.544998,64.794998,5.68,6.055, 0.515, 0.89, SURF_ID='UPHOLSTERY' / Back

*** GROUP: CHAIR offset $=0.000000,-1.875000,0.000000$ rotate $=90.000000$

\&OBST XB=62.169998, 63.294998, 3. 055, 3.43, $0.14,0.89, \quad$ SURF_ID= 'UPHOLSTERY' / Left Arm 


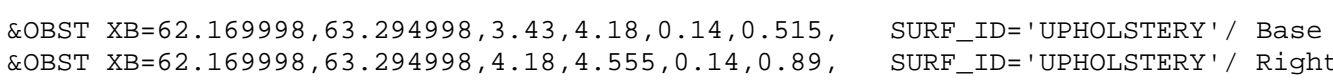

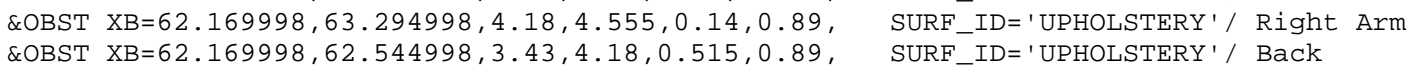
*** GROUP: CHAIR offset $=1.875000,-1.875000,0.000000$ rotate $=270.000000$

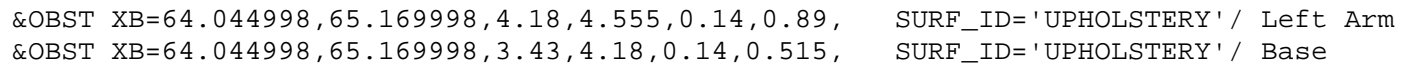

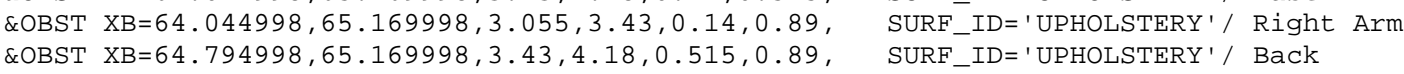

*** GROUP: LR

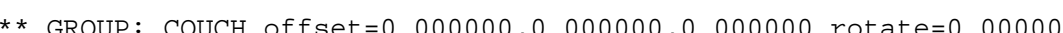

\&OBST XB=52.049999, 52.424999, 10.55, 11.675, 0.14, 0.89 , SURF_ID= 'UPHOLSTERY ' ' Left Arm

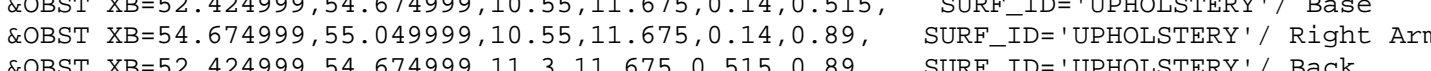

*** GROUP: CHAIR offset $=0.000000,-1.875000,0.000000$ rotate $=90.000000$

\&OBST XB=52. $449999,53.174999,8.675,9.05,0.14,0.89$, SURF_ID=' 'UPHOLSTERY'/ Left Arm

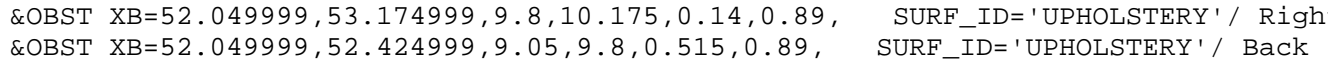

$* * *$ GROUP: CHAIR offset $=1.875000,-1.875000,0.000000$ rotate $=270.000000$

\&OBST XB=53.924999, 55. 049999, 9.8, 10.175, 0.14, 0.89 , SURF_ID=' 'UPHOLSTERY ' $/$ Left Ar

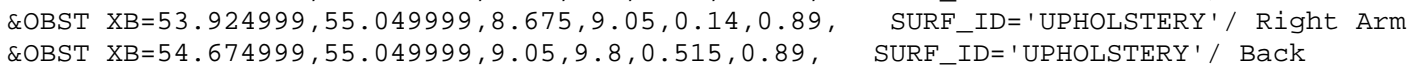

*** GROUP: LR

*** GROUP: COUCH of fset $=0.000000,0.000000,0.000000$ rotate $=0.000000$

\&OBST XB $=55.419998,55.794998,10.55,11.675,0.14,0.89$, SURF ID= 'UPHOLSTERY' ' Left Arm \&OBST XB=58.044998, 58.419998, 10.55, 11.675, 0.14, 0.89 , SURF_ID='UPHOLSTERY'/ COBST XB=55.794998, 58.044998, 11.3,11.675, 0.515, 0.89 , SURF_ID='UPHOLSTERY'/ Back

*** GROUP: CHAIR offset $=0.000000,-1.875000,0.000000$ rotate $=90.000000$

EOBST XB=55.419998, 56.544998, 8.675, 9. 05, 0.14, 0.89 , SURF_ID='UPHOLSTERY'/ Left Arm

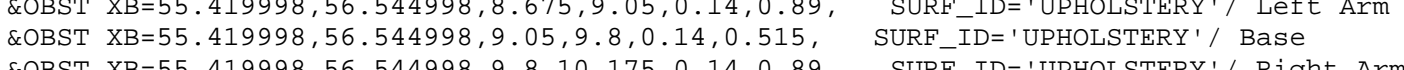
\&OBST XB=55.419998,56.544998,9.8,10.175, $0.14,0.89$, SURF_ID='UPHOLSTERY'/ Right AP

$* * *$ GROUP: CHAIR offset $=1.875000,-1.875000,0.000000$ rotate $=270.000000$

EOBST XB=57.294998, 58.419998, 9.8,10.175, 0.14, 0.89 , SURF_ID='UPHOLSTERY'/ Left Arm OBSST XB $=57.294998,58.419998,9.05,9.8,0.14,0.515$, SURF_ID='UPHOLSTERY'/ Base \&OBST XB $=57.294998,58.419998,8.675,9.05,0.14,0.89$,
SURF
SURF_IDE='UPHOLSTERY '/ Right Arm

*** GROUP: LR

** GROUP: COUCH offset $=0.000000,0.000000,0.000000$ rotate $=0.000000$

EOBST XB=58.799999, 59.174999, 10.55, 11.675, 0.14, 0.89, SURF_ID='UPHOLSTERY'/ Left Arm

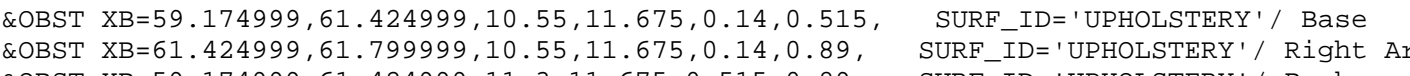

** GROUP: CHAIR offset $=0.000000,-1.875000,0.000000$ rotate $=90 . .000000$
ROBST XB $=58.799999,59.924999,8.675,9.05,0.14,0.89, \quad$ SURF IDE' 'UPHOLSTERY' / Left Arm \&OBST XB=58.799999, 59.924999, 9. 05, 9.8, $0.14,0.515$, SURF_ID=' 'UPHOLSTERY'/ Base \&OBST XB $=58.799999,59.174999,9.05,9.8,0.515,0.89, \quad$ SURF_ID='UPHOLSTERY'/ Right

*** GROUP: CHAIR offset $=1.875000,-1.875000,0.000000$ rotate $=270.000000$

\&OBST XB $=60.674999,61.799999,9.8,10.175,0.14,0.89$, SURF_ID='UPHOLSTERY' ' Left Arm \&OBST XB=60.674999, 61.799999, 8.675, 9. 05, $4.14,0.89$, SURF_ID='UPHOLSTERY'// Right Arm

$* * *$ GROUP: LR

*** GROUP: COUCH offset $=0.000000,0.000000,0.000000$ rotate $=0.000000$

\&OBST XB=62.169998, $62.544998,10.55,11.675,0.14,0.89$, SURF_ID='UPHOLSTERY'/ Left Arm

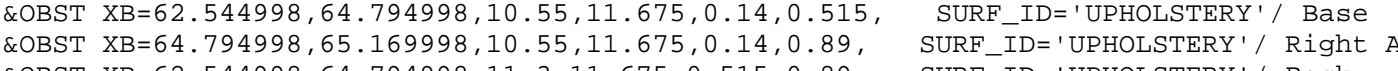

, $-1.875000,0.000000$ rotate $=90.000000$

\&OBST XB=62.169998, 63. 294998, 8. 675, 9. 05, 0.14, 0.89, SURF_ID='UPHOLSTERY' / Left Arm

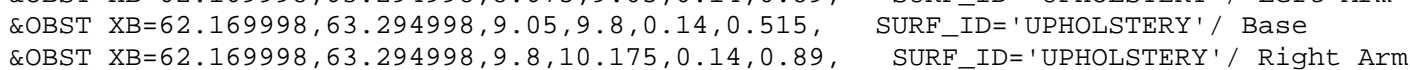
\&OBST $X \mathrm{~B}=62.169998,62.544998,9.05,9.8,0.515,0.89$, SURF_ID=' 'UPHOLSTERY'/ Right

*** GROUP: CHAIR offset $=1.875000,-1.875000,0.000000$ rotate $=270$. ๑०९०००

\&OBST XB=64. 044998, 65.169998, 9.8,10.175, 0.14, 0.89, SURF_ID='UPHOLSTERY'/ Left Arm

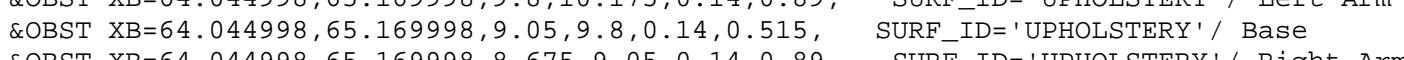
\&OBST XB $=44.044998,65.169998,8.675,9.05,0.14,0.89$, SURF_ID='UPHOLSTERY'/ Right Ar

\section{*** GROUP: CHAIR_ROW4}

*** GROUP: CHAIR_ROW3

$* * *$ GROUP: CHATR offset $=0 ., 000000,0 ., 000000,0.140000$ rotate $=90.000000$

\&OBST XB $=63.669998,64.794998,2.295,2.67,0.28,1.03$, SURF_ID=' 'UPHOLSTERY ' / Left Arm \&OBST XB=63.669998,64.794998, 1.17, 1.545,0.28, 1.03, SURE IDD='UPHOLSTERY' Right Ar \&OBST XB $=64.419998,64.794998,1.545,2.295,0.655,1.03$, SURF_ID='UPHOLSTERY'/ Back

*** GROUP: CHAIR offset $=1.500000,0.000000,0.140000$ rotate $=90$. .

\&OBST XB=62.169998, 63.294998, 2.295, 2. 67, $0.28,1.03$, SURF_ID= 'UPHOLSTERY' $/$ Left Arm
\&OBST XB=62.169998,63.294998,1.545,2.295, $0.28,0.655$, SURF_ID='UPHOLSTERY'

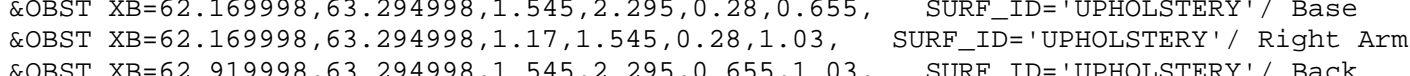

*** GROUP: CHAIR offset $=3.000000,0.000000,0.140000$ rotate $=90.000000$

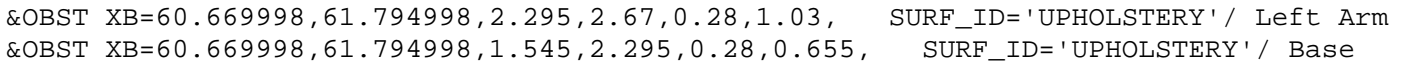

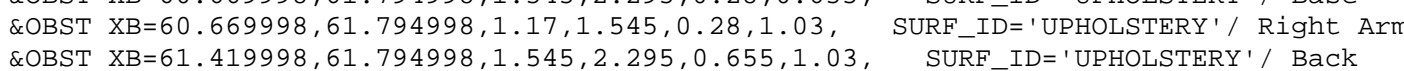

$* * *$ GROUP: CHAIR offset $=4.500000,0.0000000,0.140000$ rotate $=90.000000$

\&OBST XB $=59.169998,60.294998,2.295,2.67,0.28,1.03, \quad$ SURF_ID= 'UPHOLSTERY' $/$ Left Arm
\&ORST

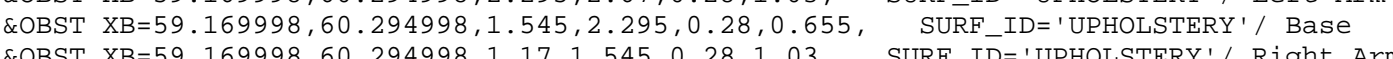

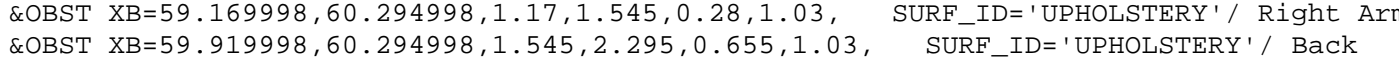

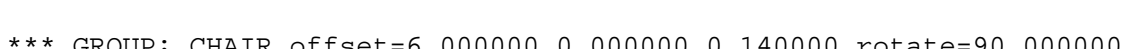

\&OBST XB=57.669998, 58.794998, 2.295, 2.67, 0.28,1.03, SURF_ID='UPHOLSTERY'/ Left Arm

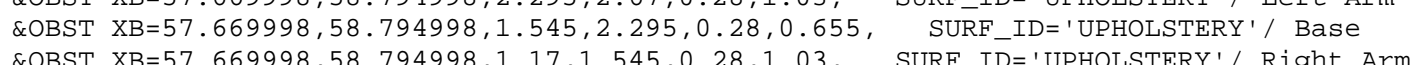


\&OBST XB $=58.419998,58,794998,1.545,2,295,0.655,1.03$, SURE ID = ' UPHOLSTERY ' / Back *** GROUP: CHAIR offset $=7.500000,0.000000,0.140000$ rotate $=90.000000$

\&OBST XB=56.169998, 57. 294998, 2.295, 2.67, 0.28, 1.03, SURF ID=' 'UPHOLSTERY' / Left Arm

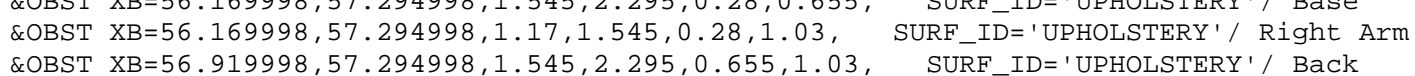

*** GROUP: CHAIR offset $=9.000000,0.000000,0.140000$ rotate $=90.000000$

\&OBST XB=54.669998, 55.794998, 2.295, 2.67, 0. 28, 1. 03 , SURF_ID='UPHOLSTERY' / Left Arm \&OBST XB $54.669998,55.794998,1.545,2.295,0.28,0.655$, SURF_ID=' 'UPHOLSTERY' ' / Base \&OBST XB $=54.669998,55.794998,1.17,1.545,0.28,1.03$,
\&OBST XB $=55.419998,55.794998,1.545,2.295,0.655,1.03$,

*** GROUP: CHAIR offset $=10.500000,0.000000,0.140000$ rotate $=90.000000$

\&OBST XB=53.169998, 54.294998, 2.295, 2.67, 0.28,1.03, SURF_ID='UPHOLSTERY'/ Left Arm \&OBST XB $53.169998,54.294998,1.545,2.295,0.28,0.655$, SURF_ID='UPHOLSTERY ' / Base

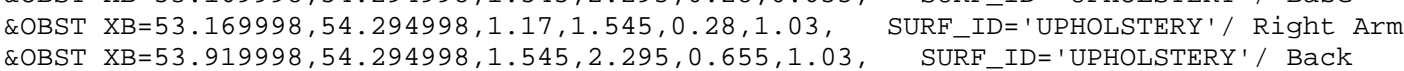

$* * *$ GROUP: CHAIR offset $=12.000000,0.000000,0.140000$ rotate $=90.000000$

\&OBST XB $=51.669998,52.794998,2.295,2.67,0.28,1.03, \quad$ SURF_ID='UPHOLSTERY ' / Left Arm \&OBST XB=51.669998, 52.794998, 1.17, 1.545, 0. 28,1.03, SURF_ID='UPHOLSTERY'/ Right Ar \&OBST XB $=52.419998,52.794998,1.545,2.295,0.655,1.03$, SURF_IDE-ULSTEYRY'/ Back

*** GROUP: CHAIR offset $=13.500000,0.000000,0.140000$ rotate $=90.000000$

\&OBST XB=50.169998, 51.294998, 2.295, 2.67, 0.28,1.03, SURF_ID= ' UPHOLSTERY' / Left Arm

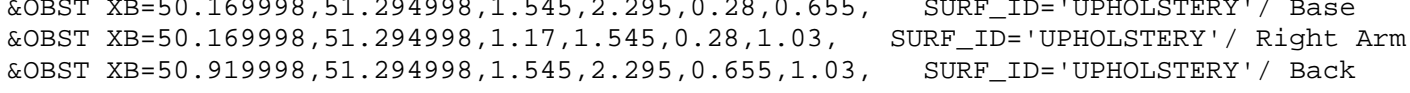

*** GROUP: CHAIR offset $=15.000000,0.000000,0.140000$ rotate $=90.000000$

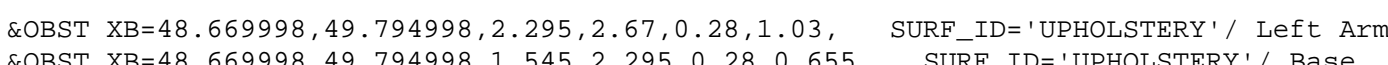

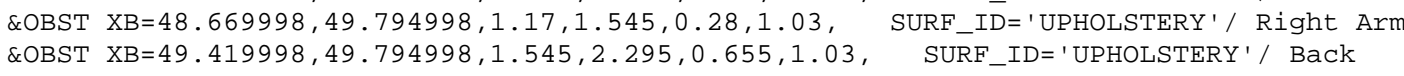

*** GROUP: CHAIR270

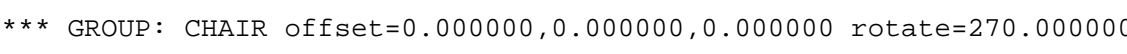

\&OBST XB $=64.050003,65.175003,2.295,2.67,0.14,0.89$, SURF_ID=' 'UPHOLSTERY Y / Left Arm \&OBST XB $64.050003,65.175003,1.17,1.545,0.14,0.89$, SURF_ID='UPHOLSTERY' ' Right Arm

*** GROUP: CHAIR_ROW4

*** GROUP: CHAIR_ROW3

$* * *$ GROUP: CHAIR offset $=0.000000,0.000000,0.140000$ rotate $=90.000000$

\&OBST XB $63.669998,64.794998,-5.195,-4.82,0.28,1.03$, SURF IDD ' UPHOLSTERY Y / Left Arm \&OBST XBE $63.669998,64.794998,-6.32,-5.945,0.28,1.03$, SURF ID 'UPOLLSTERY' $'$ Right A

${ }^{* * *}$ GROUP: CHAIR offset $=1.500000,0.000000,0.140000$ rotate $=90.000000$

\&OBST XB=62.169998, 63. 294998, -5.195, -4.82, 0.28,1.03, SURF_ID='UPHOLSTERY Y / Left Arm \&OBST XB=62.169998, 63. 294998, -5.945, -5.195, 0.28, 0.655, SURF_ID='UPHOLSTERY'/ Base ROBST XB $=62.169998,63.294998,-6.32,-5.945,0.28,1.03, \quad$ SURF_ID='UPHOLSTERY'/ Right Arm
\&OBST XB $=62.919998,63.294998,-5.945,-5.195,0.655,1.03, \quad$ SURF_ID=' UPHOLSTERY'/ Back

$* * *$ GROUP: CHAIR offset $=3.000000,0.000000,0.140000$ rotate $=90.000000$

\&OBST XB=60.669998, 61.794998, $-5.195,-4.82,0.28,1.03$, SURF_ID='UPHOLSTERY'/ Left Arm

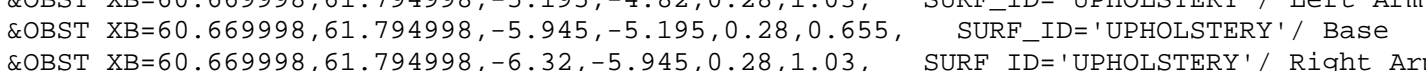

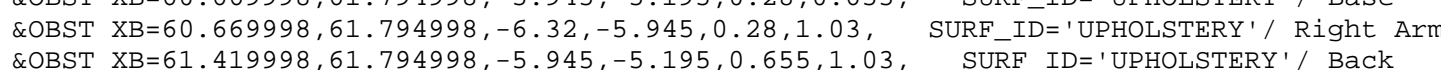

*** GROUP: CHAIR offset $=4.500000,0.000000,0.140000$ rotate $=90.000000$

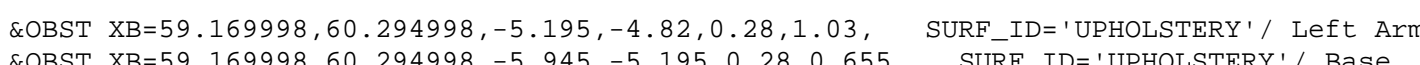
\&OBST XB=59.169998, 60.294998, $-6.32,-5.945,0.28,1.03, \quad$ SURF_ID='UPHOLSTERY' ' Right Arm
\&OBST XB=59.919998, 60.294998, $-5.945,-5.195,0.655,1.03, \quad$ SURF_ID=' UPHOLSTERY'/ Back

*** GROUP: CHAIR offset $=6.000000,0.000000,0.140000$ rotate $=90.000000$

\&OBST XB $=57.669998,58.794998,-5.195,-4.82,0.28,1.03, \quad$ SURF_ID= 'UPHOLSTERY' ' Left Arm
\&OBST XB $=57.669998,58.794998,-5.945,-5.195,0.28,0.655$,
SURF ID='UPHOLSTERY'/ Base COBST XB $=57.669998,58.794998,-6.32,-5.945,0.28,1.03, \quad$ SURF_ID='UPHOLSTERY'/ Right Arm
\&OBST XB $=58.419998,58.794998,-5.945,-5.195,0.655,1.03, \quad$ SURF_ID ' 'UPHOLSTERY'/ Back

*** GROUP: CHAIR offset $=7.500000,0.000000,0.140000$ rotate $=90.000000$

\&OBST XB=56.169998, 57. 294998, -5.195, -4.82, 0.28,1.03, SURF ID='UPHOLSTERY' / Left Arm \&OBST XB $=56.169998,57,294998,-6,32,-5,945,0.28,1,03$, SURE TD ='UPHOLSTERY' $/$ Right A \&OBST XB $=56.919998,57.294998,-5.945,-5.195, \theta .655,1.03$, SURF_ID='UPHOLSTERY' / Back

$* * *$ GROUP: CHAIR offset $=9.000000,0.0000000,0.140000$ rotate $=90.000000$

\&OBST XB $=54.669998,55.794998,-5.195,-4.82,0.28,1.03$,
\&OBST XB $=54.669998,55.794998,-5.945,-5.195,0.28,0.655$,
SURF_ID='UPHOLSTERY' $/$ Left Arm
SURFID='UPHOLSTERY' / Base

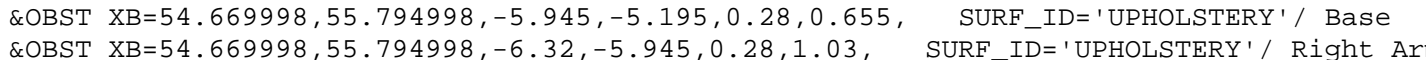
\&OBST XB $=55.419998,55.794998,-5.945,-5.195,0.655,1.03$, SURF_ID='UPPOLSTERY'/ Back

*** GROUP: CHAIR offset $=10.500000,0.000000,0.140000$ rotate $=90.000000$

\&OBST XB $=53.169998,54.294998,-5.195,-4.82,0.28,1.03$,
\&OBST XB $=53.169998,54.294998,-5.945,-5.195,0.28,0.655$,
SURF_ID='UPHOLSTERY'/ Left Arm
SURF_ID='UPHOLSTERY'/ Base LOBST XB $=53.169998,54.294998,-6.32,-5.945,0.28,1.03$,
COBST XB $=53.919998,54.294998,-5.945,-5.195,0.655,1.03$,

*** GROUP: CHAIR offset $=12.000000,0.000000,0.140000$ rotate $=90.000000$

\&OBST XB=51.669998, 52.794998, -5.195, - 4.82, $0.28,1.03$, SURF_ID=' UPHOLSTERY' ' Left Arm

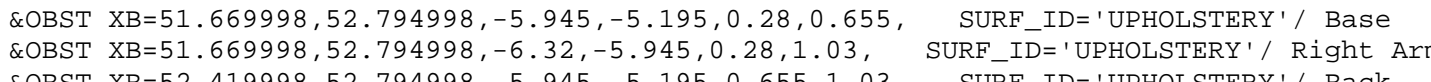

*** GROUP: CHAIR offset $=13.500000,0.000000,0.140000$ rotate $=90.000000$

ROBST XB $=50.169998,51.294998,-5.195,-4.82, \theta .28,1.03$, SURF_IDE' 'UPHOLSTERY' $/$ Left Arm
\&OBST XB $=50.169998,51.294998,-5.945,-5.195,0.28,0.655$,
SURF_ID='UPHOLSTERY'/ Base

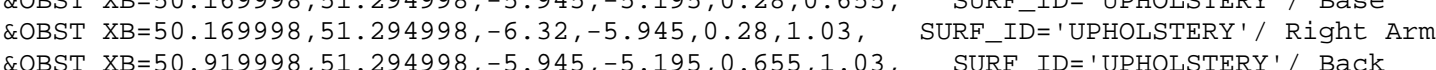

$* * *$ GROUP: CHAIR offset $=15.000000,0.000000,0.140000$ rotate $=90.000000$

\&OBST XB $48.669998,49.794998,-5.195,-4.82,0.28,1.03, \quad$ SURF_ID= 'UPHOLSTERY' $/$ Left Arm
SURF ID='UPHOLSTERY' $/$ Base \&OBST XB $=48.669998,49.794998,-5.945,-5.195,0.28,0.655$, SURF_ID $=$ 'UPHOLSTERY' $/$ Base

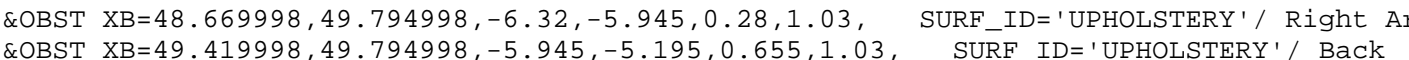

*** GROUP: CHAIR270

*** GROUP: CHAIR offset $=0.000000,0.000000,0.000000$ rotate $=270.000000$

\&OBST XB $=64.050003,65.175003,-5.195,-4.82,0.14,0.89$, SURF_ID= 'UPHOLSTERY' ' Left Arm \&OBST XB $=64.050003,65.175003,-6.32,-5.945,0.14,0.89$, SURF_ID ='UPHOLSTERY' / Right Ar \&OBST XB=64.800003, 65.175003, $-5.945,-5.195,0.515,0.89$, SURF_ID=' 'UPHOLSTERY' / Back

*** GROUP: CHAIR_ROW4

*** GROUP: CHAIR_ROW3

$* * *$ GROUP: CHAIR offset $=0.000000,0.000000,0.140000$ rotate $=90.000000$

\&OBST XB $=63.669998,64.794998,-3.695,-3.32,0.28,1.03$, SURF_ID=' 'UPHOLSTERY ' / Left Arm

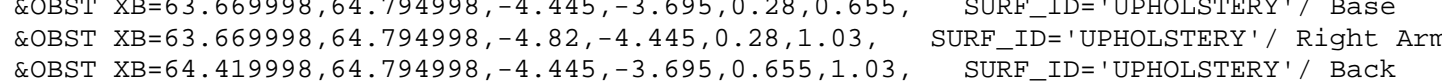


$* *$ GROUP: CHATR offset $=1.500000,0.000000,0.140000$ rotate $=90.000000$

LOBST XB $=62.169998,63.294998,-3.695,-3.32,0.28,1.03, \quad$ SURF_ID= 'UPHOLSTERY ' / Left Arm
\&OBST XB $=62.169998,63.294998,-4.445,-3.695,0.28,0.655$,
SURF_ID='UPHOLSTERY' $/$ Base \&OBST XBE62. $169998,63.294998,-4.82,-4.445,0.28,1.03$,
\&OBST XB $62.919998,63.29498,-4.445,-3.695,0.655,1.03$,

*** GROUP: CHAIR offset $=3.000000,0.000000,0.140000$ rotate $=90.000000$

\&OBST XB=60.669998, 61.794998, -3.695, -3.32, $0.28,1.03$, SURF_ID=' 'UPHOLSTERY ' ' Left Arm

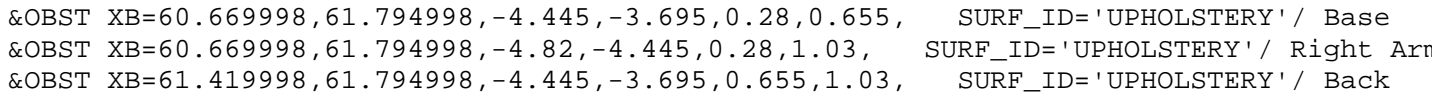

*** GROUP: CHAIR offset $=4.500000,0.0000000,0.140000$ rotate $=90$. .

\&OBST XB=59.169998, 60.294998, - 3.695, -3.32, 0.28,1.03, SURF_ID=' 'UPHOLSTERY ' ' Left Arm \&OBST XBE $59.169998,60.294998,-4.445,-3.695,0.28,0.655$, SURF-ID=' 'UPHOLSTERY' '/ Base \&OBST XB=59.919998, 60.294998, -4.445,-3.695,0.655,1.03, SURF ID ='UPHOLSTERY'

*** GROUP: CHAIR offset $=6.000000,0.000000,0.140000$ rotate $=90.000000$

\&OBST XB $=57.669998,58.794998,-3.695,-3.32,0.28,1.03$,
SOBST XB $57.669998,58.794998,-4.445,-3.695,0.28,0.655$,
SURF_IDE ' UPHOLSTERY' ' Left Arm \&OBST XB=57.669998, 58.794998, -4.445, -3.695, 0.28, 0.655 , SURF_ID='UPHOLSTERY' / Base

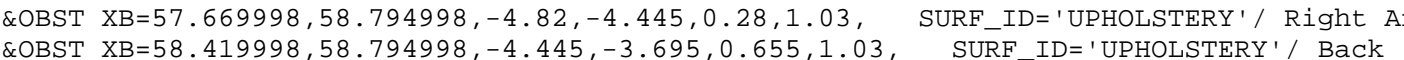

$* * *$ GROUP: CHAIR offset $=7.500000,0.000000,0.140000$ rotate $=90.000000$

\&OBST XB=56.169998, 57.294998, $-3.695,-3.32,0.28,1.03, \quad$ SURF_ID='UPHOLSTERY' $/$ Left Arm
\&OBST XB $=56.169998,57.294998,-4.445,-3.695,0.28,0.655$,
SURF ID ='UPHOLSTERY' $/$ Base COBST $X \mathrm{XB}=56.169998,57.294998,-4.82,-4.445,0.28,1.03$, SURF-ID='UPPOLSTERY'/ Right A

*** GROUP: CHAIR offset $=9.000000,0.000000,0.140000$ rotate $=90.000000$

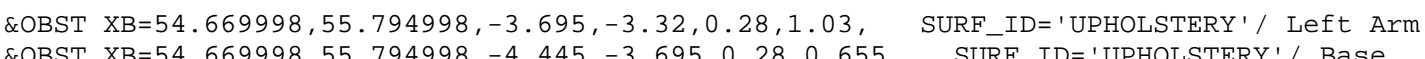
\&OBST XB=54.669998, 55.794998, $-4.82,-4.445,0.28,1.03$, SURF_ID='UPHOLSTERY'/Right A

*** GROUP: CHAIR offset $=10.500000,0.000000,0.140000$ rotate $=90.000000$

\&OBST XB=53.169998, 54.294998, -3.695,-3.32, 0.28, 1. 03, SURF_ID='UPHOLSTERY'/ Left Arm \&OBST XB $=53.169998,54.294998,-4.445,-3.695,0.28,0.655$, SURF ID = 'UPHOLSTERY' ' Base \&OBST XB=53.169998,54.294998, $-4.82,-4.445,0.28,1.03$, S SURF_IDE'UPHOLSTERY' $/$ Right Ar
\&OBST XB=53.919998, $54.294998,-4.445,-3.695,0.655,1.03$, SURF_ID='UPHOLSTERY'/ Back

*** GROUP: CHAIR offset $=12.000000,0.000000,0.140000$ rotate $=90.000000$

EOBST XB=51.669998, 52.794998, $-3.695,-3.32,0.28,1.03$, SURF_ID='UPHOLSTERY' / Left Arm QOBST XB=51.669998,52.794998, $-4.445,-3.695,0.28,0.655$, SURF_ID=' 'UPHOLSTRRY' Base

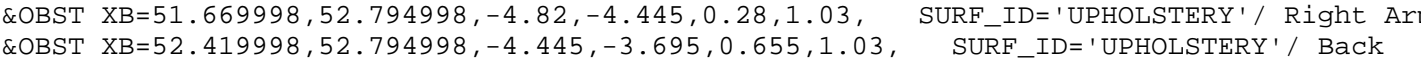

$* * *$ GROUP: CHAIR offset $=13.500000,0.000000,0.140000$ rotate $=90.000000$

\&OBST XB $=50.169998,51.294998,-3.695,-3.32,0.28,1.03, \quad$ SURF ID= 'UPHOLSTERY ' ' Left Arm

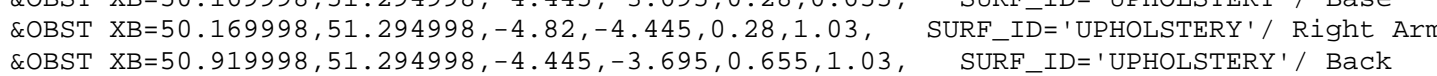

$* * *$ GROUP: CHAIR offset $=15.000000,0.0000000,0.140000$ rotate $=90.000000$

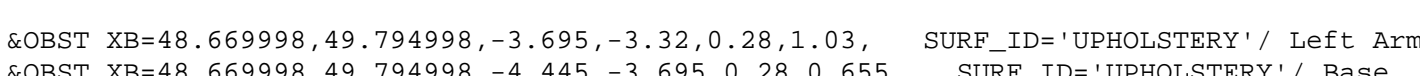
\&OBST XB=48.669998, 49.794998, $-4.82,-4.445,0.28,1.03$,
\&OBST XB $=49.419998,49.794998,-4.445,-3.695,0.655,1.03$,
SURF_ID = 'UPHOLSTERY' / Right Arm

*** GROUP: CHAIR270

** GROUP: CHAIR offset $=0.000000,0.000000,0.000000$ rotate $=270.000000$

\&OBST XB $=64.050003,65.175003,-3.695,-3.32,0.14,0.89, \quad$ SURF_ID= 'UPHOLSTERY ' ' Left Arm
\&OBST XB $64.050003,65.175003,-4.445,-3.695,0.14,0.515$, SURF ID=' UPHOLSTERY' \&OBST XB $=64.0550003,65.175003,-4.445,-3.695,0.14,0.515$, SULPF_ID=' 'UPHOLSTERY' $/$ Base
SOBST XB $=64.800003,65.175003,-4.445,-3.695,0.515,0.89$, SURE_ID=' 'UPHOLSTERY' / Back

*** GROUP: LD CHAIR ROW

$* * *$ GROUP: CHAIR offset $=0.000000,0.000000,0.000000$ rotate $=90.000000$

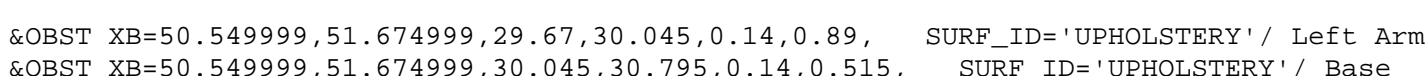
\&OBST XB=50.54999, 51.674999, 30.795, 31.17, $1.14,0.89, \quad$ SURF_ID='UPHOLSTERY'/ Right Arm
\&OBST XB=50.549999, $50.924999,30.045,30.795,0.515,0.89, \quad$ SURF_ID='UPHOLSTERY'/ Back

$* * *$ GROUP: CHAIR offset $=1.125000,0.000000,0.000000$ rotate $=90.000000$

\&OBST XB $=51.674999,52.799999,29.67,30.045,0.14,0.89, \quad$ SURF ID ' 'UPHOLSTERY' ' Left Arm
\&OBST XB

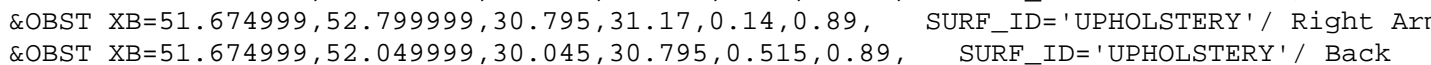

$* * *$ GROUP: CHAIR offset $=2.250000,0.000000,0.000000$ rotate $=90.000000$

\&OBST XB=52.799999, 53. 924999, 29.67, 30. 045, 0.14, 0.89 , SURF ID = 'UPHOLSTERY' ' Left Arm

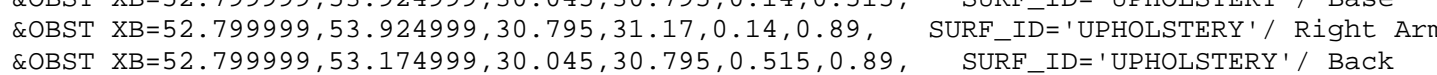

$* * *$ GROUP: CHATR offset $=3.375000,0.000000,0.000000$ rotate $=90,000000$

\&OBST XB $=53.924999,55.049999,29.67,30.045,0.14,0.89$, SURF-ID=' 'UPHOLSTERY' 1 ' Left Ar \&OBST XB $=53.924999,55.049999,30.795,31.17,0.14,0.89$, SURE ID $=$ 'UPHOLSTTRY' ' Right \&OBST XB=53.924999, $54.299999,30.045,30.795, \theta .515,0.89$, SURF_ID=' UPHOLSTERY' / Back

$\star \star *$ GROUP: CHAIR offset $=4.500000,0.0000000,0.000000$ rotate $=90.000000$

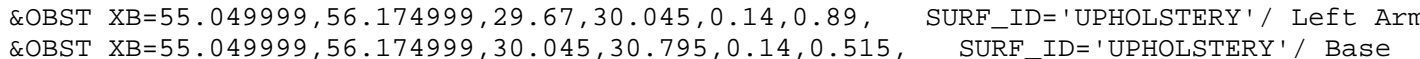
\&OBST XB X $55.049999,56.174999,30.795,31.17,0.14,0.89$, SURF ID $=$ ' 'UPHOLSTERY' \&OBST XB=55. $449999,55.424999,30.045,30.795,0.515,0.89$, SURF_ID=' 'UPHOLSTERY' / Back

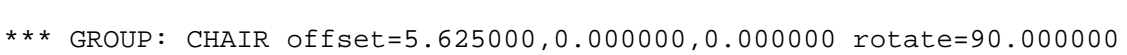

\&OBST XB $=56.174999,57.299999,29.67,30.045,0.14,0.89, \quad$ SURF_ID='UPHOLSTERY'/ Left Arm
\&OBST XB $=56.174999,57.299999,30.045,30.795,0.14,0.515$,
SURF ID='UPHOLSTERY' $/$ Base

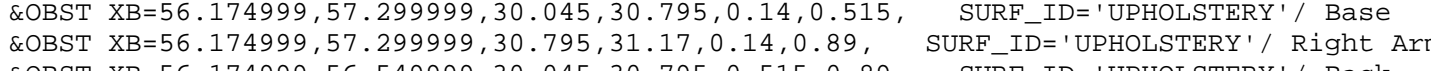

*** GROUP: CHAIR offset $=6.750000,0.000000$, ๑. .000000 rotate $=90$. . 000000

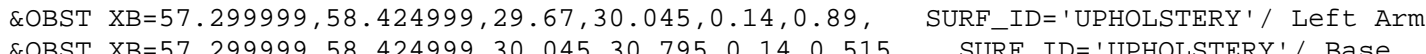

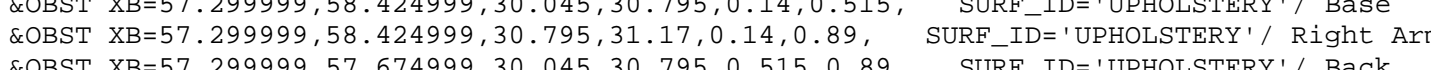

*** GROUP: CHAIR offset $=7.875000,0$. ๑ 000000, ๑. .000000 rotate $=90$. .

\&OBST XB=58.424999,59.549999, 29.67, 30.045, 0.14, 0.89 , SURF_ID='UPHOLSTERY' / Left Arm \&OBST XB $58.424999,59.549999,30.045,30.795,0.14,0.515$, SURFF_DID=' 'OPHOLSTERY' B Base \&OBST XB=58.424999, 58.799999, 30. 045, 30.795, $0.515,0.89$, SURF ID=' 'UPHOLSTERY' ' Back

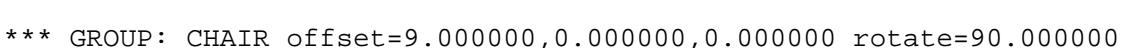

OBST XB=59.549999, 60.674999, 29.67, 30.045, 0.14, 0.89 , SURF_ID='UPHOLSTERY' / Left Arm \&OBST XB=59.549999, $60.674999,30.045,30.795,0.14,0.515$, SURF ID= 'UPHOLSTERY' $/$ Base \&OBST XB=59.549999, $59.924999,30.045,30.795,0.515,0.89$, SURF ID='UPHOLSTEPY' ' Back

*** GROUP: LD CHAIR ROW

*** GROUP: CHAIR offset $=0.000000,0.000000,0.000000$ rotate $=90.000000$

\&OBST XB=50.549999, 51.674999, 31.17,31.545, $0.14,0.89, \quad$ SURF_ID= 'UPHOLLTERY ' / Left Arm

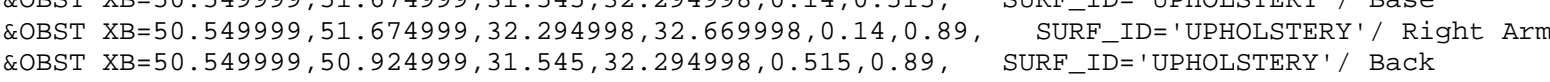




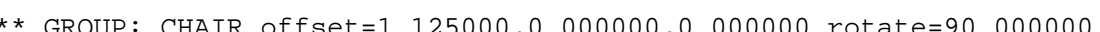

\&OBST XB $=51.674999,52.799999,31.17,31.545,0.14,0.89, \quad$ SURF_ID= 'UPHOLSTERY ' / Left Arm
\&OBST XB $=51.674999,52.799999,31.545,32.294998,0.14,0.515, \quad$ SURF_ID=' 'UPHOLSTERY'/ Bas

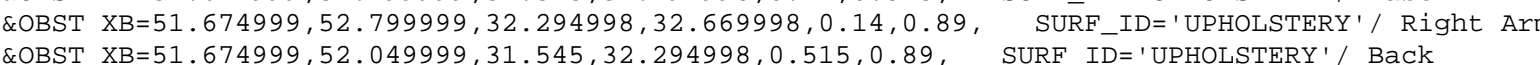

$* *$ GROUP: CHAIR offset $=2.250000,0.000000,0.000000$ rotate $=90.000000$

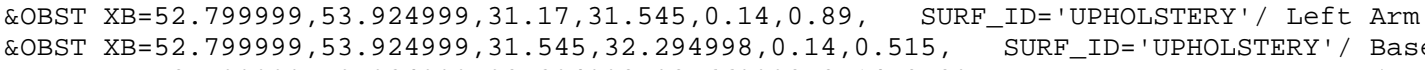

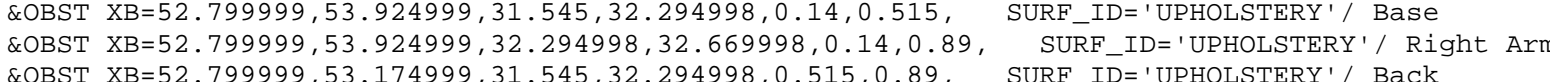
*** GROUP: CHAIR offset $=3.375000,0.000000,0.000000$ rotate $=90.000000$ COBST XB $=53.924999,55.049999,31.17,31.545,0.14,0.89, \quad$ SURF_ID= 'UPHOLSTERY' / Left Arm
OBST XB $=53.924999,55.049999,31.545,32.294998,0.14,0.515, \quad$ SURF_ID ' 'UPHOLSTERY' $/$ Base

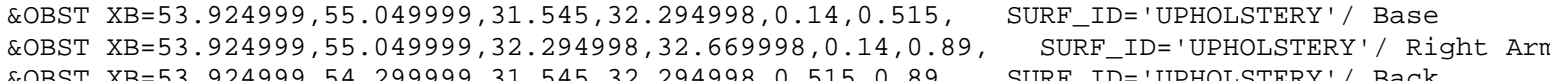
*** GROUP: CHAIR offset $=4.500000,0.000000,0.000000$ rotate $=90.000000$

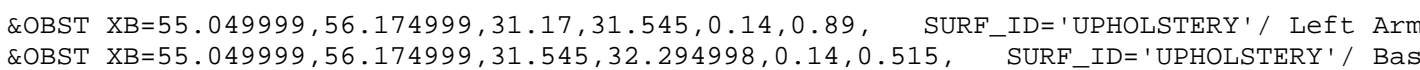

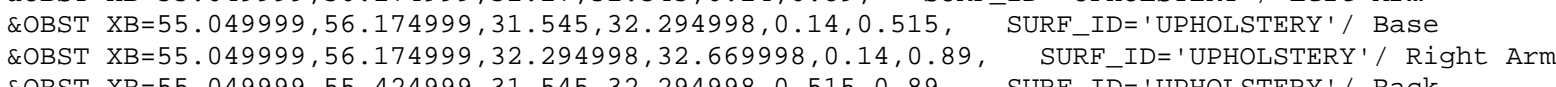
$* * *$ GROUP: CHAIR offset $=5.625000,0.000000,0.000000$ rotate $=90.000000$

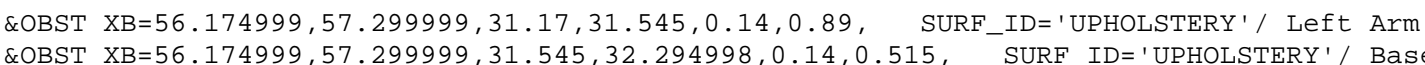
\&OBST XB $=56.174999,57.299999,32.294998,32.669998,0.14,0.89$, SURF-ID='UPHOLSTERY'/ Right Ar ${ }^{* \star *}$ GROUP: CHAIR offset $=6.750000,0.000000,0.0000000$ rotate $=90.0000000$

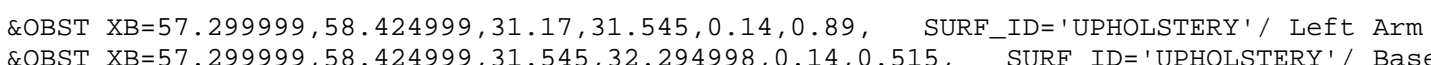

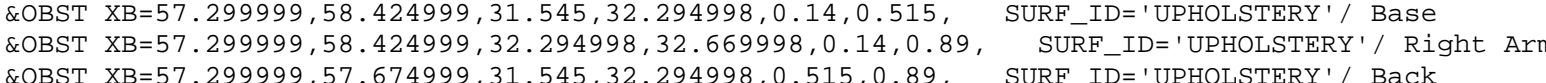
*** GROUP: CHAIR offset $=7.875000,0.000000,0.000000$ rotate $=90.000000$

\&OBST XB=58.424999, 59.549999, 31.17, 31.545, $0.14,0.89$, SURF_ID='UPHOLSTERY ' / Left Arm

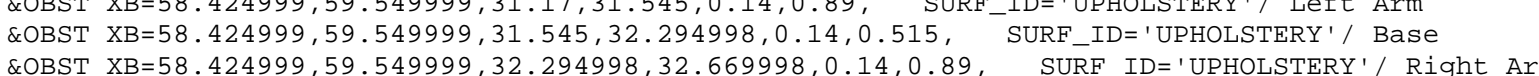

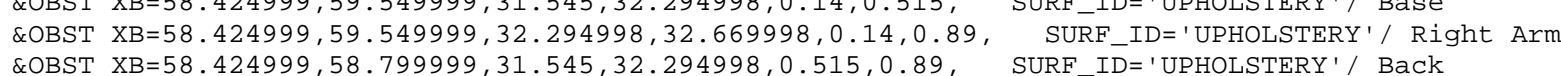
*** GROUP: CHAIR offset $=9.000000,0.000000,0.000000$ rotate $=90.000000$

\&OBST XB=59.549999, 60.674999, 31.17, 31.545, $0.14,0.89$, SURF_ID='UPHOLSTERY' / Left Arm

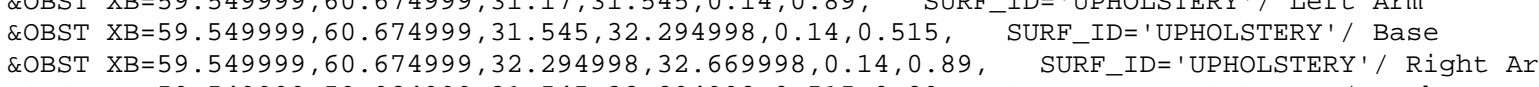

*** GROUP: LD LS ROW

*** GROUP: LOVESEAT Offset=0,

\&OBST XB $=50.549999,51.674999,34.169998,34.544998,0.14,0.89$, SURF_ID=' 'UPHOLSTERY'/ Left Arm

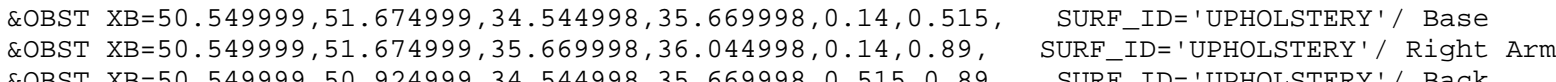
\&OBST XB=50.549999, 50.924999, 34.544998, 35.669998, $0.515,0.89$, SURF_ID=' UPHOLSTERY' / Back

*** GROUP: LOVESEAT offset $=1.125000,0.000000,0.000000$ rotate $=90$. ๑००००००

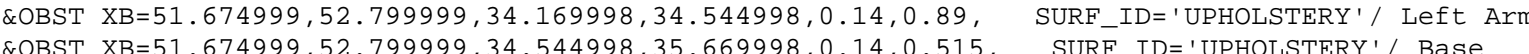

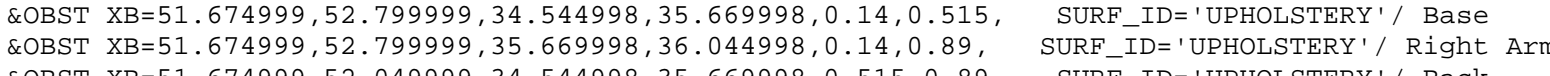

*** GROUP: LOVESEAT offset $=2.250000,0.000000,, 0.000000$ rotate $=90.000000$

\&OBST XB=52.799999, 53.924999, 34.169998, 34.544998, 0.14, 0.89, SURF_ID='UPHOLSTERY' ' Left Arm

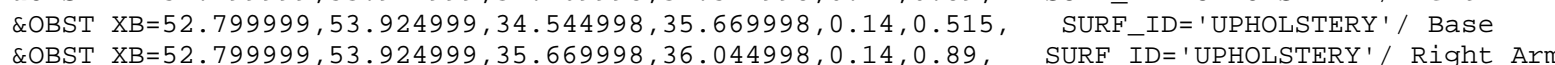

\&OBST XB=52. 799999, 53. 174999, 34. 544998, 35. 669998, 0.515, ๑. 89, SURF_ID=' UPHOLSTERY'/ Back *** GROUP: LOVESEAT of fset $=3.375000,0.000000,0.000000$ rotate $=90.000000$

\&OBST XB $=53.924999,55.049999,34.169998,34.544998,0.14,0.89$, SURF_ID=' 'UPHOLSTERY'/ Left Arm \&OBST XB=53. 924999, 55. 049999, 34.544998, 35. 669998, $0.14,0.515$, SURF_ID='UPHOLSTERY / Base LOBST XB=53.924999, 54.299999, 34.544998, 35.669998, $0.515,0.89$, SURF_ID='UPHOLSTERY'/ Right Ar

*** GROUP: LOVESEAT offset $=4.500000,0.000000,0.000000$ rotate $=90.000000$

\&OBST XB=55. 049999, 56.174999, 34.169998, 34.544998, 0.14, 0.89, SURF_ID=' 'UPHOLSTERY '/ Left Arm \&OBST XB=55. 049999, 56.174999, 34.544998, 35.669998, 0.14, 0.515 , SURF_ID='UPHOLSTERY'/ Base

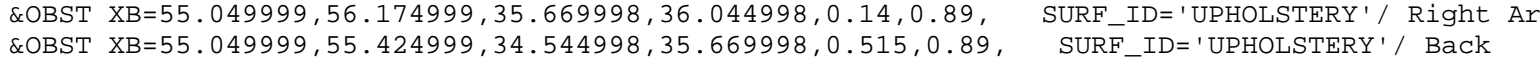

$* * *$ GROUP: LOVESEAT offset $=5.625000,0.000000,0.000000$ rotate $=90.000000$

\&OBST XB=56.174999, 57.299999, 34.169998, 34.544998, 0.14, 0.89, SURF_ID='UPHOLSTERY'/ Left Arm \&OBST XB=56.174999, 57. 299999, 34.544998, 35.669998, 0.14, 0.515, SURF_ID='UPHOLSTERY'/ Base \&OBST $X \mathrm{~B}=56.174999,57.299999,35.669998,36.044998,0.14,0.89$, SURF_ID='UPHOLSTERY'/ Right Ar $* \star *$ GROUP: LOVESEAT of $\mathrm{set}=6.750000,0.000000,0.000000$ rotate $=90.000000$

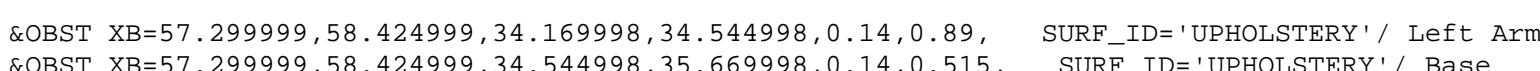

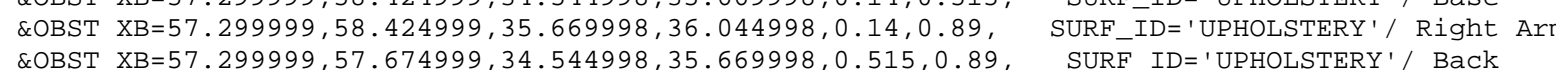

*** GROUP: LOVESEAT of fset $=7.875000,0.000000,0.000000$ rotate $=90.000000$

\&OBST XB=58.424999, 59.549999, 34.169998, 34.544998, $0.14,0.89$, SURF_ID=' 'UPHOLSTERY' ' Left Arm \&OBST XB=58.424999, 59.549999, 35.669998, 36.044998, $0.14,0.89$, SURF_ID=' 'UPHOLSTERY'/ Right Ar \&OBST XB=58.424999, 58.799999, 34.544998, 35.669998, 0.515, 0.89, SURF_ID='UPHOLSTERY'/ Back

*** GROUP: LOVESEAT of fset $=9.000000,0.000000,0.000000$ rotate $=90 . .000000$

\&OBST XB=59.549999, 60.674999, 34.169998, 34.544998, $0.14,0.89$, SURF_IDD ' UPHOLSTERY' / Left Arm \&OBST XB $=59.549999,60.674999,35,669998,36.044998,0.14,0.89$, SURF ID='UPHOLSTERY'/ Righe \&OBST XB=59.549999, 59. 924999, 34.544998, 35.669998, $0.515,0.89$, SURF_ID='UPHOLSTERY'/ Back

*** GROUP: LD LS ROW

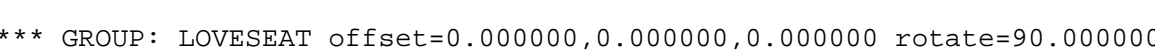

\&OBST XB=50.549999, 51.674999, 36.049999, 36.424999, 0.14, 0.89, SURF_ID='UPHOLSTERY '/ Left Arm \&OBST XB=50.549999, $51.674999,36.424999,37.549999,0.14,0.515$, SURF_ID='UPHOLSTERY'/ Base \&OBST XB=50.549999, $5.674999,37.549999,37.924999,0.14,0.89$, SURF_IDE= UPHOLSTERY' Right Ar

*** GROUP: LOVESEAT offset $=1.125000,0.000000,0.000000$ rotate $=90.000000$

\&OBST XB=51.674999, 52.799999, 36. $049999,36.424999,0.14,0.89$, SURF ID=' 'UPHOLSTERY ' / Left Arm \&OBST XB $51.674999,52.799999,36.424999,37.549999,0.14,0.515$, SURF_ID=' 'UPHOLSTERY' / Base

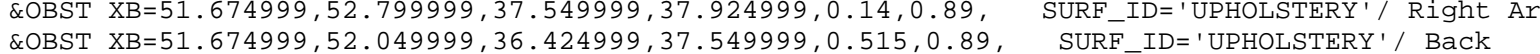

*** GROUP: LOVESEAT of $f$ set $=2.250000,0.000000,0.000000$ rotate $=90.000000$

\&OBST XB=52.799999, 53.924999, 36. 049999, 36.424999, $0.14,0.89$, SURF_ID=' 'UPHOLSTERY ' / Left Arm \&OBST XB=52.799999, 53.924999, 36. 424999, 37.549999, $0.14,0.515$, SURF_ID='UPHOLSTERY'/ Base

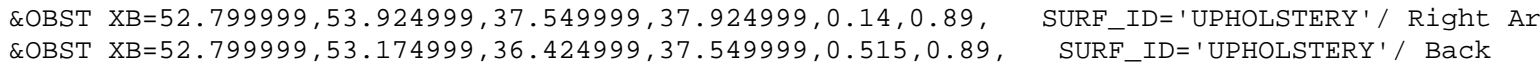

*** GROUP: LOVESEAT of fset $=3.375000,0.000000,0.000000$ rotate $=90.000000$

\&OBST XB=53.924999, 55. 049999, 36. $449999,36.424999,0.14,0.89$, SURF_ID= 'UPHOLSTERY' ' Left Arm \&OBST XB $=53.924999,55.049999,37.549999,37.924999,0.14,0.89$, SURF ID $=$ 'UPHOLSTERY'/Right Ar

*** GROUP: LOVESEAT of fset $=4.500000,0.000000,0.000000$ rotate $=90.000000$

\&OBST XB=55. $049999,56.174999,36.049999,36.424999,0.14,0.89$, SURF_ID=' 'UPHOLSTERY'/ Left Arm 
\&OBST XB=55. $049999,56.174999,36.424999,37.549999,0.14,0.515, \quad$ SURF_ID= 'UPHOLSTERY' $/$ Base
\&OBST XB=55.04999, $56.174999,37.549999,37.924999,0.14,0.89, \quad$ SURF_ID='UPHOLSTERY'
Right A

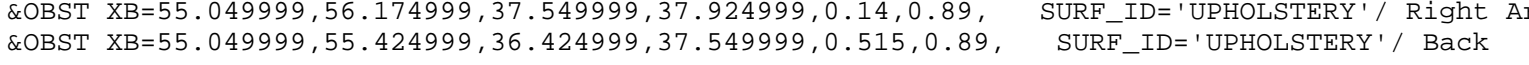
$* * *$ GROUP: LOVESEAT offset $=5.625000, \odot .000000, \odot . \odot 00000$ rotate $=90 . .000000$

LOBST XB=56.174999, 57.299999, 36. $449999,36.424999,0.14,0.89, \quad$ SURF_ID=' 'UPHOLSTERY '/ Left Arm
\&OBST XB=56.174999, $57.299999,36.424999,37.549999,0.14,0.515, \quad$ SURF_ID='UPHOLSTERY' $/$ Base

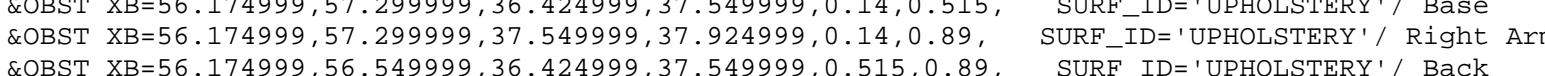
$* * *$ GROUP: LOVESEAT offset $=6.750000,0.000000,0.000000$ rotate $=90 . .000000$

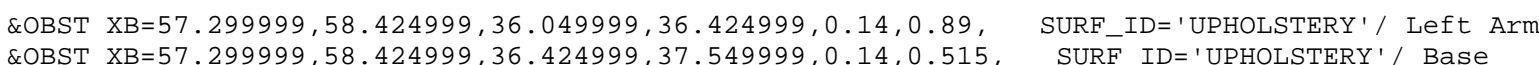

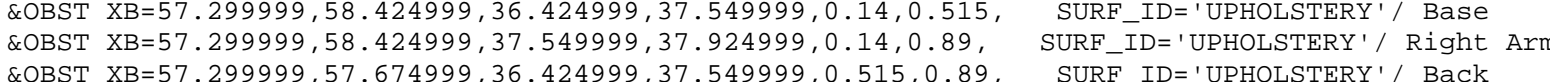

$* * *$ GROUP: LOVESEAT of $f$ set $=7.875000,0.000000,0.000000$ rotate $=90.000000$

\&OBST XB $58.424999,59.549999,36.049999,36.424999,0.14,0.89$, SURF_ID='UPHOLSTERY ' / Left Arm

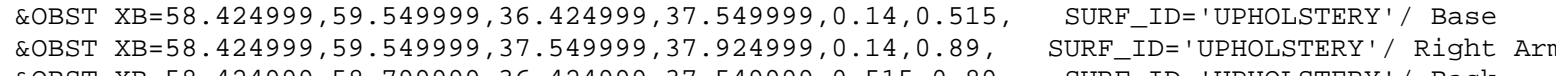
\&OBST XB=58.424999, $58.799999,36.424999,37.549999,0.515,0.89$, SURF_ID= 'UPHOLSTERY'/ Back

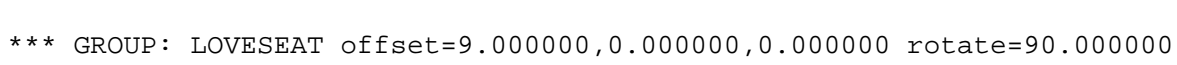

\&OBST XB=59.549999, 60.674999, 36. $049999,36.424999,0.14,0.89$, SURF_ID=' 'UPHOLSTERY'/ Left Arm

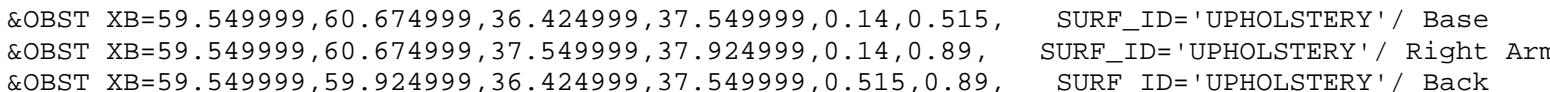

$* \star *$ GROUP: LD COUCH ROW

*** GROUP: COUCH offset $=0.000000,0.000000,0.000000$ rotate $=90.000000$

\&OBST XB $=50.549999,51.674999,37.919998,38.294998,0.14,0.89, \quad$ SURF-ID='UPHOLSTERY '/' Left Arm

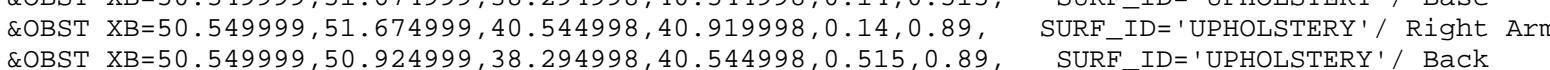

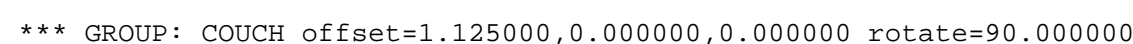

\&OBST XB=51.674999, 52.799999, 37.919998, 38. 294998, $0.14,0.89$, SURF_ID=' 'UPHOLSTERY ' ' Left Arm

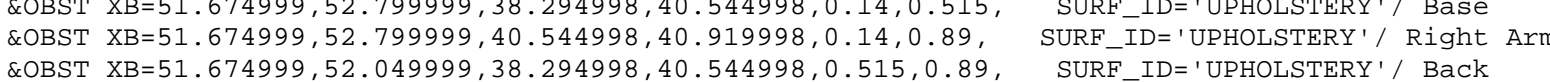
*** GROUP: COUCH offset $=2.250000,0.000000,0.000000$ rotate $=90.000000$

\&OBST XB=52.799999, 53. 924999, 37. 919998, 38. 294998, 0.14, 0.89, SURF_ID=' 'UPHOLSTERY' ' Left Arm

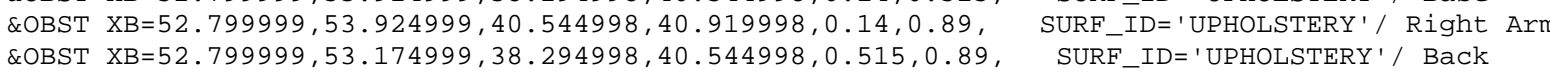

*** GROUP: COUCH offset $=3.375000,0.000000$,,. .000000 rotate $=90.000000$

\&OBST XB=53.924999, 55. 049999, 37. 919998, 38. 294998, 0.14, 0.89, SURF_ID= 'UPHOLSTERY ' / Left Arm \&OBST XB-53.92499, $55.04999,38.29$

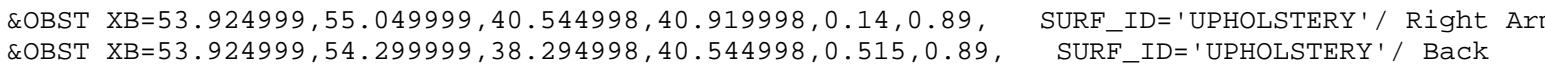
*** GROUP: COUCH offset $=4.500000,0.000000,0.000000$ rotate $=90 . .000000$

\&OBST XB=55. $049999,56.174999,37.919998,38.294998,0.14,0.89, \quad$ SURF_ID='UPHOLSTERY ' ' Left Arm
\&OBST XB=55.04999, $56.174999,38.294998,40.544998,0.14,0.515$,
SURF_ID=' 'UPHOLSTERY' / Base \&OBST XB $=55.049999,56.174999,40.544998,40.919998,0.14,0.89$, SURF ID='UPHOLSTERY'/ Rise

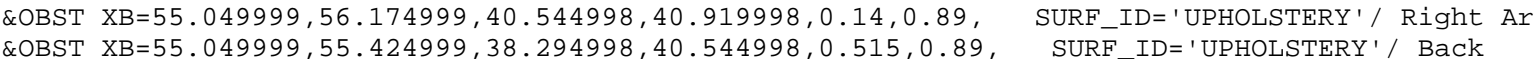

*** GROUP: COUCH offset $=5.625000,0.000000$,, .000000 rotate $=90.000000$

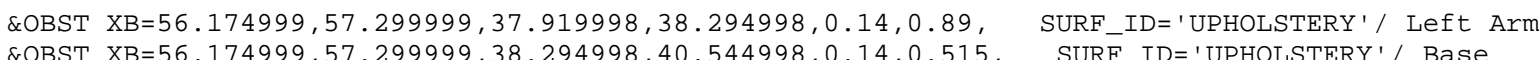

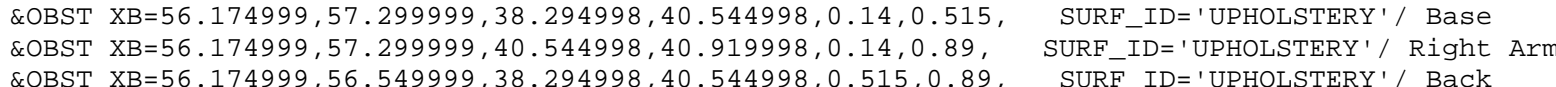

** GROUP: COUCH offset $=6.750000,0.0000000,0.000000$ rotate $=90.0000000$
\&OBST XB $=57.299999,58.424999,37.919998,38.294998,0.14,0.89, \quad$ SURF_ID=' ' UPHOLSTERY ' / Left Arm
\&OBST XB $=57.299999,58.424999,38.294998,40.544998,0.14,0.515, \quad$ SURF_IDD 'UPHOLSTERY' $/$ Base \&OBST XB=57.299999, $58.424999,40.544998,40.919998,0.14,0.89$, SURF_ID='UPHOLSTERY'/ Right Ar

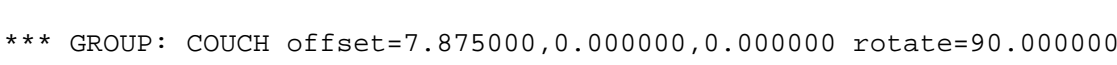

\&OBST XB=58.424999, 59. 549999, 37. 919998, 38. 294998, 0.14, 0.89 , SURF_ID='UPHOLSTERY' ' Left Arm \&OBST XB=58.424999, 59.549999, 38.294998, 40.544998, $0.14,0.515$, SURF_ID=' 'UPHOLSTERY' / Base \&OBST XB=58.42499, $59.549999,40.544998,40.919998,0.14,0.89$,
\&OBST XB=58.424999, $58.799999,38.294998,40,544998,0.515,0.89$,
SURF_ID='UPHOLSTERY'/ Right Ar

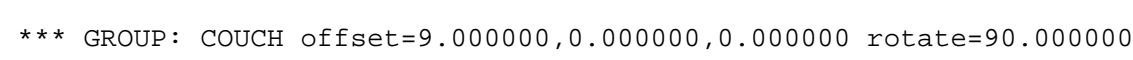

EOBST XB=59.549999, 60.674999, 37. 919998, 38. 294998, 0.14, 0.89, SURF_ID='UPHOLSTERY'/ Left Arm

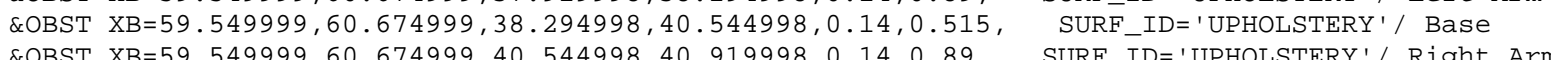

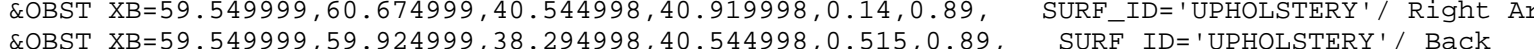

*** GROUP: LD COUCH ROW

*** GROUP: COUCH offset $=0.000000,, 0.000000,0.000000$ rotate $=90.000000$

\&OBST XB $=50.549999,51.674999,40.91,41.285,0.14,0.89, \quad$ SURF_ID='UPHOLSTERY ' / Left Arm
\&OBST XB $=50.549999,51.674999,41.285,43.535,0.14,0.515, \quad$ SURF_ID='UPHOLSTERY' $/$ Base \&OBST XB $50.549999,51.674999,43.535,43.91,0.14,0.89$, SURF ID ='UPHOLSTERY' COBST XB=50.549999, $50.924999,41.285,43.535,0.515,0.89$, SURF_ID =' UPHOLSTERY' / Back

*** GROUP: COUCH offset $=1.125000,0.000000,, 0.000000$ rotate $=90$. .

ROBST XB=51.674999, 52.799999, 40.91, $41.285,0.14,0.89, \quad$ SURF_ID='UPHOLSTERY'/ Left Arm
\&OBST XB=51.674999, $52.799999,41.285,43.535,0.14,0.515, \quad$ SURF_ID='UPHOLSTERY'/ Base

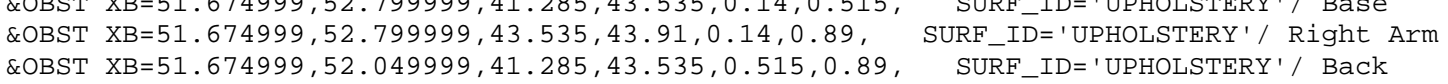

*** GROUP: COUCH offset $=2.250000,0.000000$, ๑. .000000 rotate $=90.000000$

\&OBST XB $=52.799999,53.924999,40.91,41.285,0.14,0.89, \quad$ SURF_ID='UPHOLSTERY'/ Left Arm
EOBST XB $=52.799999,53.924999,41.285,43.535,0.14,0.515, \quad$ SURF ID ' 'UPHOLSTEPY'

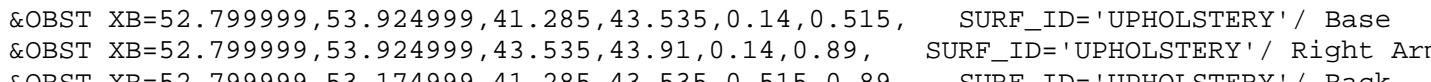

*** GROUP: COUCH offset $=3.375000,0.000000,0.000000$ rotate $=90 . .000000$

\&OBST XB $=53.924999,55.049999,40.91,41.285,0.14,0.89, \quad$ SURF_IDE' 'UPHOLSTERY' / Left Arm
\&OBST XB $=53.924999,55.049999,41.285,43.535,0.14,0.515, \quad$ SURF_ID='UPHOLSTERY'/ Base

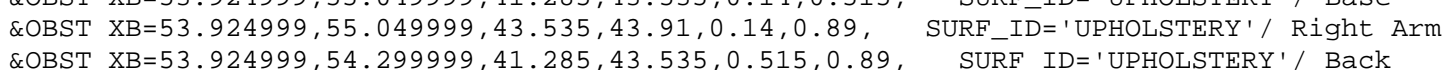

*** GROUP: Couch offset $=4.500000,0.000000,0.000000$ rotate $=90 . .000000$

\&OBST XB=55. 049999, 56.174999, 40.91, 41.285, 0.14, 0.89 , SURF_ID='UPHOLSTERY' / Left Arm EOBST XB $=55.049999,56.174999,41.285,43.535,0.14,0.515$, SURF ID $=$ ' 'OPHOLSTERY' ' Base

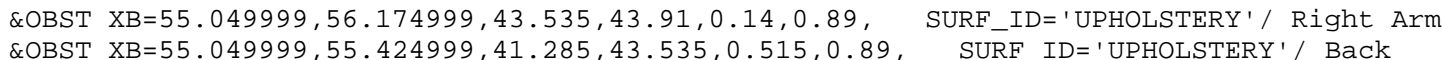

*** GROUP: COUCH offset $=5.625000, \Theta . .000000,0.000000$ rotate $=90 . .000000$ \&OBST XB=56.174999, 57. 299999, 40.91, 41.285, 0.14, 0.89 , SURF_ID='UPHOLSTERY'/ Left Arm

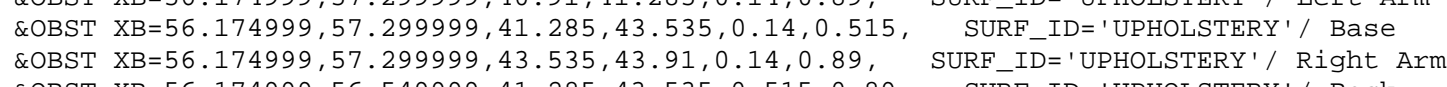

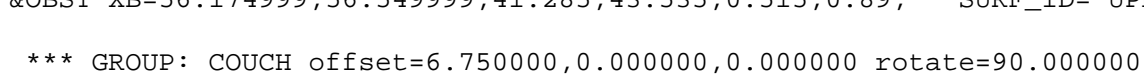

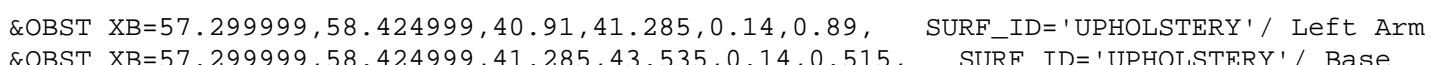
EOBST XB=57. $299999,58.424999,41.285,43.535,0.14,0.515, \quad$ SURF-ID='UPHOLSTERY'

*** GROUP: CoUCH offset $=7.875000,0.000000,0.000000$ rotate $=90.000000$

\&OBST XB=58.424999, 59.549999, 40.91,41.285, 0.14, 0.89 , SURF_ID=' 'UPHOLSTERY'/ Left Arm EOBST XB=58.424999, $59.549999,43.535,43.91,0.14,0.89$, SURF ID='UPHOLSTERY'/ Right Arm 
*** GROUP: COUCH offset $=9.000000,0.000000,0.000000$ rotate $=90.000000$

\&OBST XB=59.549999, 60.674999, 40.91, 41.285, 0.14, 0.89 , SURF IDD ' 'UPHOLSTERY ' / Left Arm \&OBST XB=59.549999, 60.674999, 43.535, 43.91,0.14,0.89, SURF ID ' 'UPHOLSTERY' ' Right \&OBST XB=59.549999, 59.924999, 41.285, 43.535, 0.515, 0.89, SURF_ID='UPHOLSTERY'/ Back

*** GROUP: LD CHAIR ROW

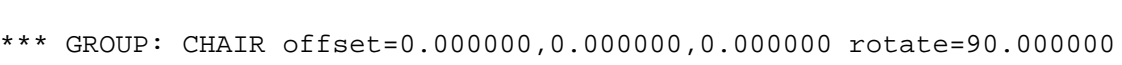

\&OBST XB=50.549999, 51.674999, 32.669998, 33. $044998,0.14,0.89, \quad$ SURF_ID=' 'UPHOLSTERY ' ' Left Arm
\&OBST XB=50.549999, $51.674999,33.044998,33.794998,0.14,0.515, \quad$ SURF ID=' 'UPHOLSTERY'/ Base \&OBST XB $=50.549999,51.674999,33.794998,34.169998,0.14,0.89$, SURF_ID='UPHOLSTERY'// Right Ar

$* * *$ GROUP: CHAIR offset $=1.125000,0.000000,0.000000$ rotate $=90.000000$

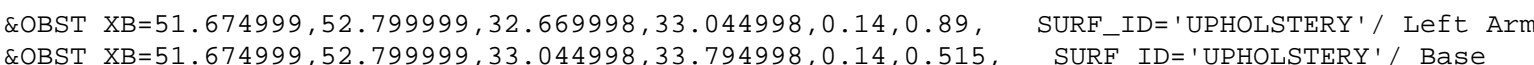

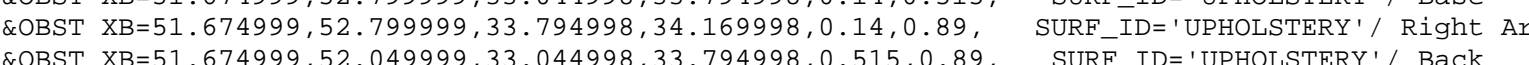

$* * *$ GROUP: CHAIR offset $=2.250000,0.000000,0.000000$ rotate $=90.000000$

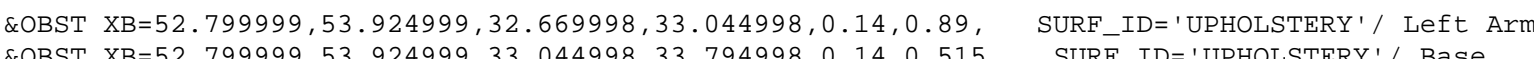
\&OBST XB=52.799999, 53.924999, 33.794998, 34.169998, $0.14,0.89$, SURF_ID='UPHOLSTERY'/Right A

** GROUP: CHAIR offset $=3.375000,0.0000000,0.000000$ rotate $=90.000000$

EOBST XB=53.924999, 55. 049999, 32.669998, 33.044998, 0.14, 0.89, SURF_ID=' 'UPHOLSTERY'/ Left Ar \&OBST XB $=53.924999,55.049999,33.044998,33.794998,0.14,0.515$, SURF_ID=' 'UPHOLSTERY' $/$ Base \&OBST XB=53.924999, 54.299999, 33.044998, 33.794998, $0.515,0.89$, SURF_ID='UPHOLSTERY'/Right Arm

*** GROUP: CHAIR offset $=4.500000,0.000000,0.000000$ rotate $=90.000000$

\&OBST XB $=55.049999,56.174999,32.669998,33.044998,0.14,0.89$, SURF_ID=' 'UPHOLSTERY '/ Left Arm \&OBST XB $=55.049999,56.174999,33.044998,33.794998,0.14,0.515$, SURF_ID='UPHOLSTERY '/ Base

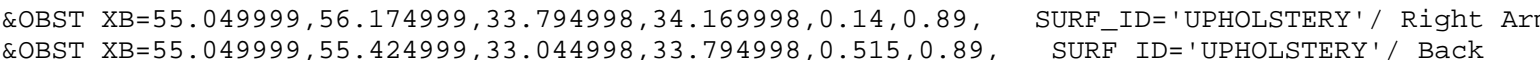

$* * *$ GROUP: CHAIR offset $=5.625000,0.000000,0.000000$ rotate $=90.000000$

\&OBST XB $=56.174999,57.299999,32.669998,33.044998,0.14,0.89, \quad$ SURF ID= ' UPHOLSTERY '/ Left Arm

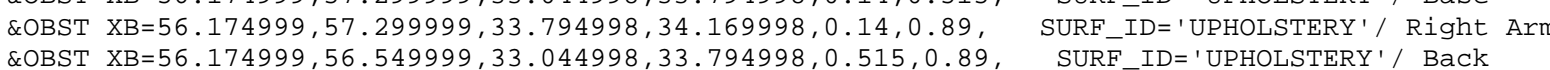
$* * *$ GROUP: CHAIR offset $=6.750000,0.000000, \Theta . .000000$ rotate $=90 ., 000000$

\&OBST $X B=57.299999,58.424999,32.669998,33.044998,0.14,0.89, \quad$ SURF-ID=' 'UPHOLSTERY' ' Left Arm

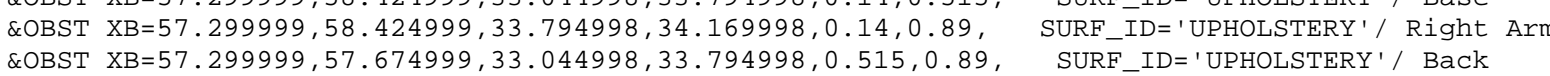

$* \star *$ GROUP: CHAIR offset $=7.875000,0.000000,0.000000$ rotate $=90.000000$

\&OBST XB=58.424999, 59.549999, 32.669998, 33. 044998, 0.14, 0.89 , SURF_ID= 'UPHOLSTERY' ' Left Arm

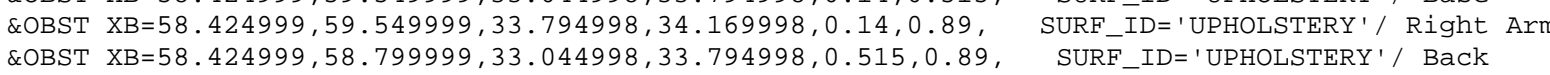

*** GROUP: CHAIR offset $=9.000000,0.000000,0.000000$ rotate $=90.000000$

\&OBST XB=59.549999, 60.674999, 32.669998, 33. 044998, $0.14,0.89$, SURF_ID=' 'UPHOLSTERY' ' Left Arm

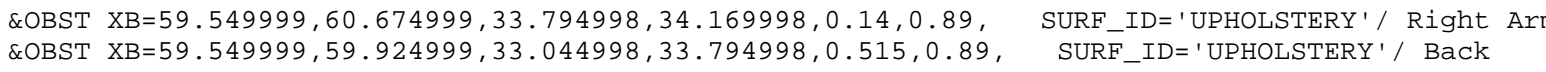

*** GROUP: LD CHAIR ROW

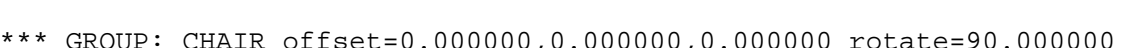

ROBST XB=50.549999, 51.674999, 29.67, 30. $045,1.4,2.15, \quad$ SURF_ID='UPHOLSTERY ' / Left Arm
\&OBST XB=50.549999, $51.674999,30.045,30.795,1.4,1.775, \quad$ SURF_ID='UPHOLSTERY' $/$ Base \&OBST XB=50.549999, 51.674999, 30.795, 31.17, 1.4, 2.15, SURF-ID='UPHOLSTERY'/ Right Ar

*** GROUP: CHAIR offset $=1.125000,0.000000,0.000000$ rotate $=90.000000$

\&OBST XB $=51.674999,52.799999,29.67,30.045,1.4,2.15, \quad$ SURF_ID= 'UPHOLSTERY' ' Left Arm
SUPST

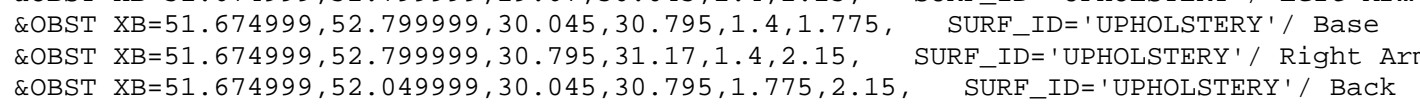

*** GROUP: CHAIR offset $=2.250000,0.000000,0.000000$ rotate $=90.000000$

\&OBST XB=52.799999, 53.924999, 29.67, 30.045, 1.4, 2.15, SURF_ID= 'UPHOLSTERY ' / Left Arm

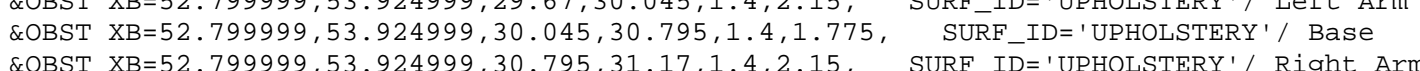
\&OBST XB $=52.799999,53.174999,30.045,30.795,1.775,2.15$, SURF_ID=' 'UPHOLSTERY'/ Back

*** GROUP: CHAIR offset $=3.375000,0.000000,0.000000$ rotate $=90.000000$

\&OBST XB=53.924999, 55. $049999,29.67,30.045,1.4,2.15$, SURF_ID=' UPHOLSTERY' / Left Arm \&OBST XB $=53.924999,55.049999,30.045,30.795,1.4,1.775$, SURF_ID=' 'UPHOLSTERY' $/$ Base

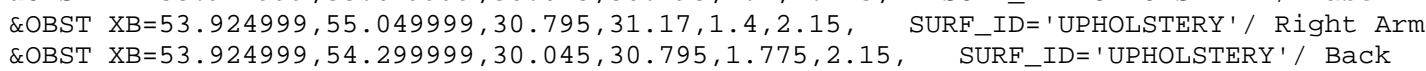

*** GROUP: CHAIR of fset $=4.500000,0.000000,0.000000$ rotate $=90.000000$

\&OBST XB $=55.049999,56.174999,29.67,30.045,1.4,2.15, \quad$ SURF_ID= 'UPHOLSTERY ' / Left Arm

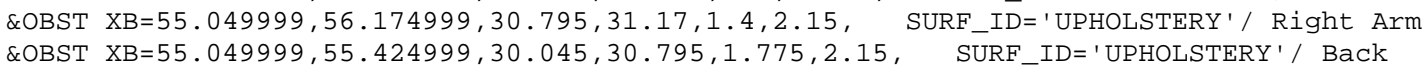

$* * *$ GROUP: CHAIR offset $=5.625000,0.000000,0.000000$ rotate $=90.000000$

\&OBST XB=56.174999, 57. 299999, 29.67, 30.045,1.4,2.15, SURF_ID=' UPHOLSTERY ' / Left Arm

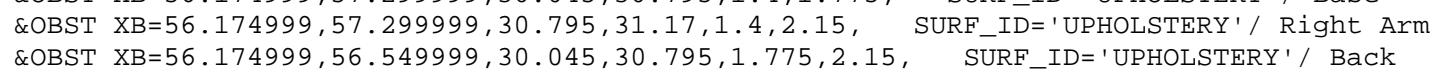

*** GROUP: CHAIR offset $=6.750000,0.000000,0.000000$ rotate $=90.000000$

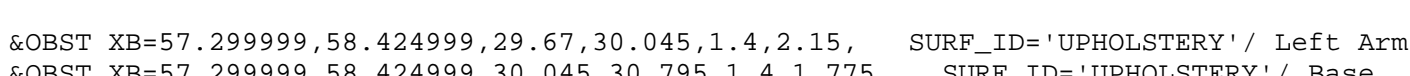
\&OBST XB $=57.299999,58.424999,30.795,31.17,1.4,2.15$, SURF_ID='UPHOLSTERY'/ Right Arn
\&OBST XB $=57.299999,57.674999,30.045,30.795,1.775,2.15, \quad$ SURF_ID='UPHOLSTERY'/ Back

*** GROUP: CHAIR offset $=7.875000,0.000000,0.000000$ rotate $=90.000000$

\&OBST XB=58.424999, 59.549999, 29.67, 30.045,1.4, 2.15, SURF_ID='UPHOLSTERY ' / Left Arm \&OBST XB $-58.424999,59.549999,30.795,31.17,1.4,2.15, \quad$ SURF_ID=' 'UPHOLSTERY' \&OBST XB=58.424999, 58.799999, 30.045, 30.795, $1.775,2.15$, SURF_ID=' UPHOLSTERY'/ Back

*** GROUP: CHAIR offset $=9.000000,0.000000,0.000000$ rotate $=90.000000$

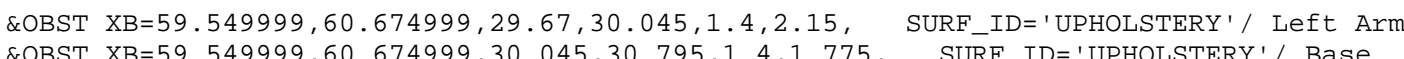
\&OBST XB=59.549999,60.674999,30.795,31.17,1.4,2,15, SURE ID ='UPHOLSTERY'/ Right Ar \&OBST XB $=59.549999,59.924999,30.045,30.795,1.775,2.15$, SURF_ID=' 'UPHOLSTERY'/Back

*** GROUP: LD CHAIR ROW

$* * *$ GROUP: CHAIR offset $=0.000000,0.000000,0.000000$ rotate $=90.000000$

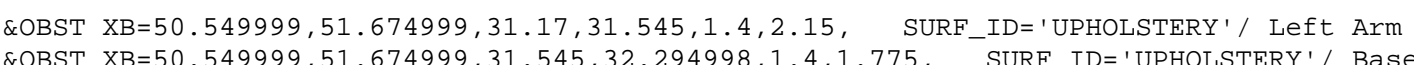
\&OBST XB=50.549999, $51.674999,32.294998,32.669998,1.4,2.15$, SURF_ID='UPHOLSTERY' $/$ Right ArI

*** GROUP: CHAIR offset $=1.125000,0.000000,0.000000$ rotate $=90.000000$

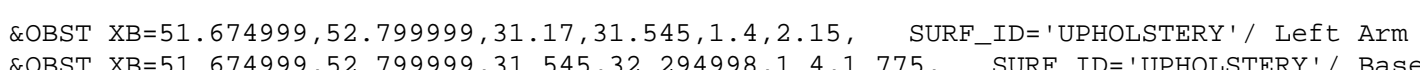
\&OBST XB=51.674999, $52.799999,32.294998,32.669998,1.4,2.15$, SURF_ID='UPHOLSTERY'/ Right Arm 
*** GROUP: CHAIR offset $=2.250000,0.000000,0.000000$ rotate $=90.000000$

ROBST XB=52.799999, 53.924999, 31.17, 31.545,1.4,2.15, SURF_ID=' 'UPHOLSTERY ' / Left Arm

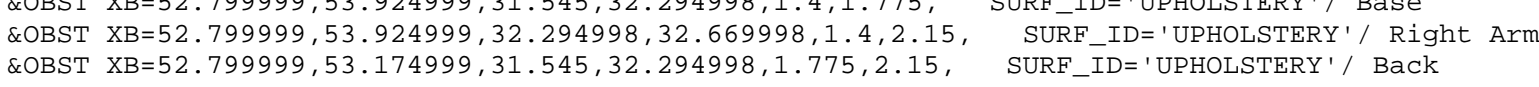
*** GROUP: CHAIR offset $=3.375000,0.000000,0.000000$ rotate $=90.000000$

\&OBST XB=53.924999, 55. 049999, 31.17, 31.545, 1.4, 2.15, SURF_ID=' 'UPHOLSTERY ' / Left Arm
\&OBST XB=53.924999, $55.049999,31.545,32.294998,1.4,1.775$, SURF_ID='UPHOLSTERY'/ Base

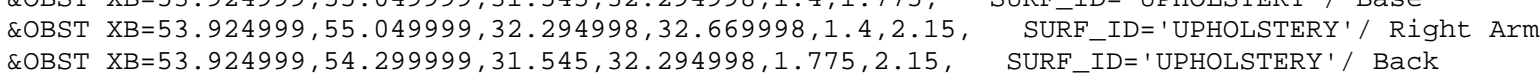
$* * *$ GROUP: CHAIR offset $=4.500000,0.000000,0.000000$ rotate $=90 . .000000$

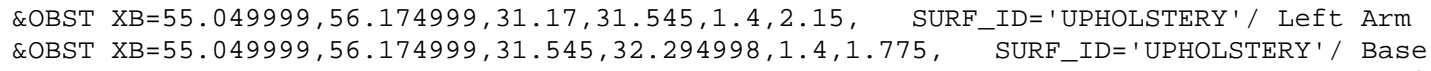

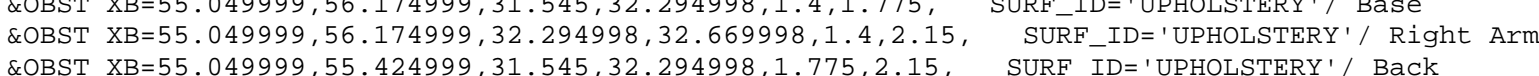
*** GROUP: CHAIR offset $=5.625000,0.000000,0.000000$ rotate $=90.000000$

ROBST XB $=56.174999,57.299999,31.17,31.545,1.4,2.15, \quad$ SURF_ID=' 'UPHOLSTERY ' Left Arm
\&OBST XB $=56.174999,57.299999,31.545,32.294998,1.4,1.775$, SURF_ID='UPHOLSTERY' $/$ Base

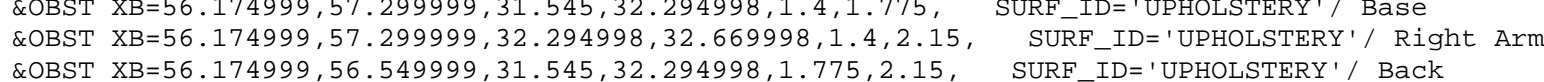

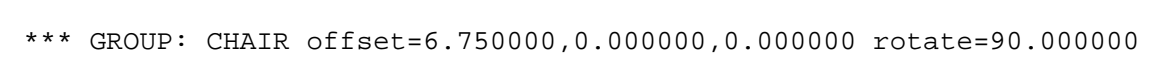

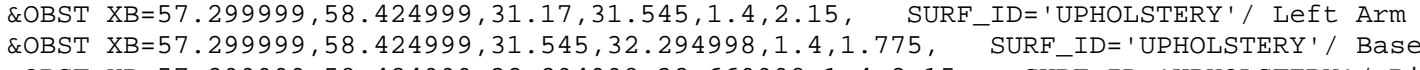

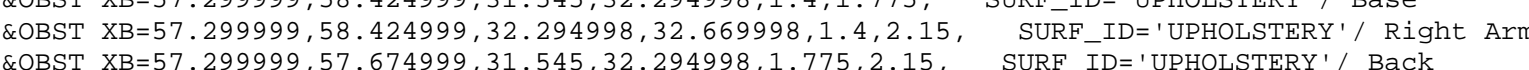

*** GROUP: CHAIR offset $=7.875000,0.000000,0.000000$ rotate $=90.000000$

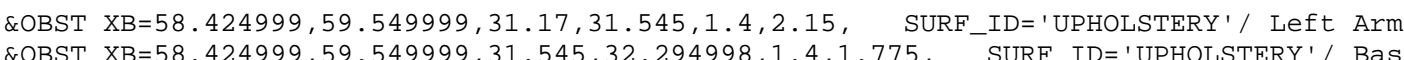

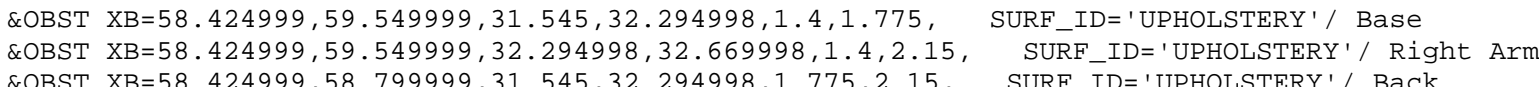

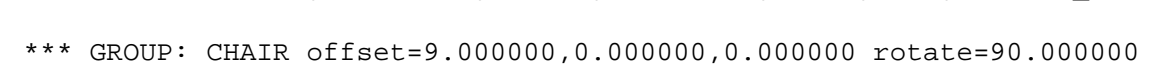

\&OBST XB=59.549999, 60.674999, 31.17, 31.545, 1.4, 2.15, SURF_ID=' 'UPHOLSTERY' / Left Arm
\&OBST XB $=59.549999,60.674999,31.545,32.294998,1.4,1.775$, SURF_ID='UPHOLSTERY'/ BaS

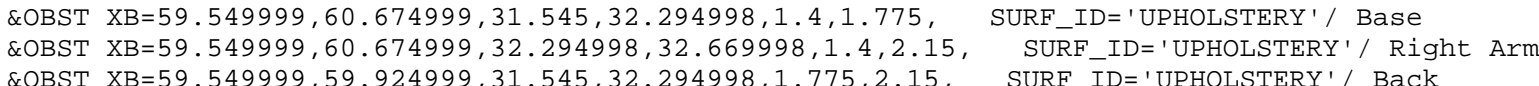
$* * *$ GROUP: LD LS ROW

*** GROUP: LOVESEAT offset $=0.000000, \odot . \bullet 00000,0.000000$ rotate $=90.000000$

\&OBST $X B=50.549999,51.674999,34.169998,34.544998,1.4,2.15, \quad$ SURF_ID= 'UPHOLSTERY ' ' Left Arm \&OBST XB $=50.549999,51.674999,35.669998,36.044998,1.4,2.15, \quad$ SURF-ID=' 'UPHOLSTERY ' $/$ Right Arm
\&OBST XB $=50.549999,50.924999,34.544998,35.669998,1.775,2.15, \quad$ SURF_ID=' UPHOLSTERY' $/$ Back *** GROUP: LOVESEAT off $\mathrm{set}=1.125000,0.000000,0.000000$ rotate $=90.000000$

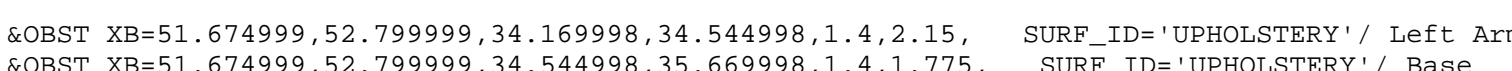

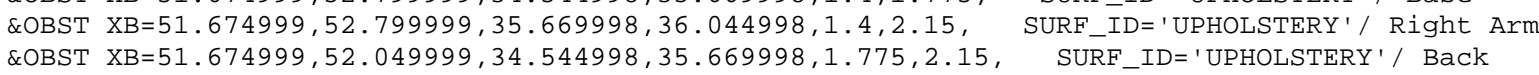
*** GROUP: LOVESEAT of fset $=2.250000,0.000000,0.000000$ rotate $=90.000000$

\&OBST XB $=52.799999,53.924999,34.169998,34.544998,1.4,2.15$, SURF-ID=' 'UPHOLSTERY' $/$ Left Ar

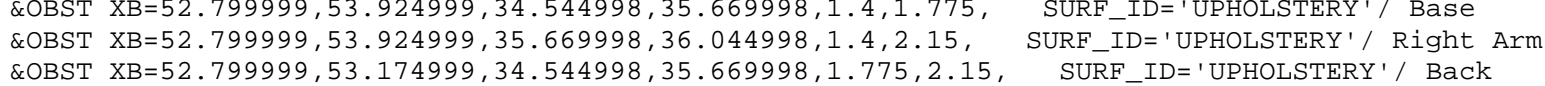
*** GROUP: LOVESEAT offset $=3.375000,0.000000,0.000000$ rotate $=90.000000$

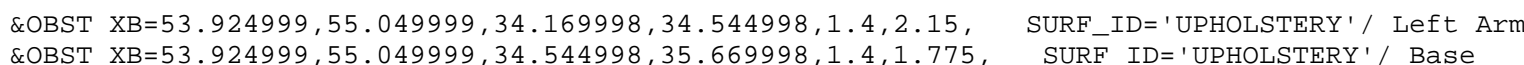

\&OBST XB=53.924999, 55. 049999, 35.669998, 36. 044998, 1.4, 2.15, $\quad$ SURF_ID= 'UPHOLSTERY ' / Right Arm
\&OBST XB=53.924999, $54.299999,34.544998,35.669998,1.775,2.15, \quad$ SURF_ID=' UPHOLSTERY' $/$ Back

$* * *$ GROUP: LOVESEAT offset $=4.500000,0.000000,0.000000$ rotate $=90.000000$

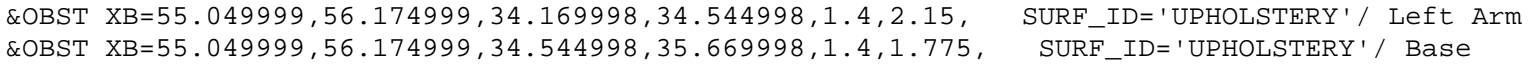

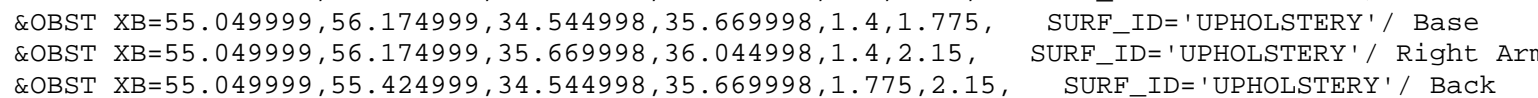

*** GROUP: LOVESEAT of fset $=5.625000,0.000000,0.000000$ rotate $=90.000000$

\&OBST XB=56.174999, 57. 299999, 34.169998, 34.544998, 1.4, 2.15, $\quad$ SURF_ID='UPHOLSTERY' '/' Left Arm
\&OBST XB=56.174999, 57.299999, 34.544998, 35.669998, 1.4, $1.775, \quad$ SURF_ID=' UPHOLSTERY'/ Base EOBST XB=56.174999,57.299999, 34.544998, 35.669998, 1.4,1.775, SURF_ID=''UPHOLSTERY'/ Base

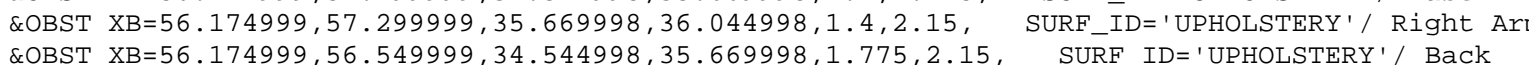

*** GROUP: LOVESEAT offset $=6.750000,0.000000,0.000000$ rotate $=90.000000$

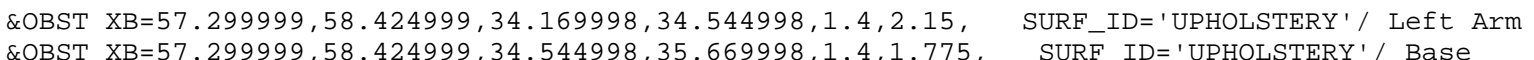
\&OBST XB=57.299999, 58.424999, 35.669998, 36. $044998,1.4,2.15$, SURF_IDE'UPHOLSTERY'/R Right Ar

*** GROUP: LOVESEAT of $f$ set $=7.875000,0.000000,0.000000$ rotate $=90 . .000000$

\&OBST XB=58.424999, 59.549999, 34.169998, 34.544998, 1.4, 2.15, SURF_ID= 'UPHOLSTERY' / Left Arm \&OBST XB=58.424999, 59.549999, 34.544998, 35. 669998, 1.4, 1.775 , SURF IDD=' 'UPHOLSTERY'/ Base

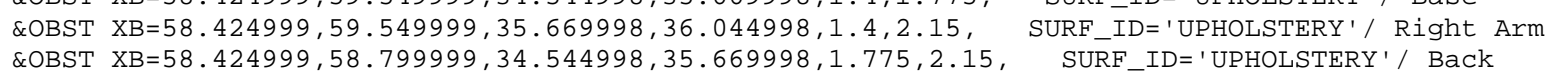

*** GROUP: LOVESEAT offset $=9.000000,0.000000, \odot .000000$ rotate $=90.000000$

\&OBST XB=59.549999, 60.674999, 34.169998, 34.544998, 1.4, 2.15, SURF_ID= 'UPHOLSTERY' / Left Arm

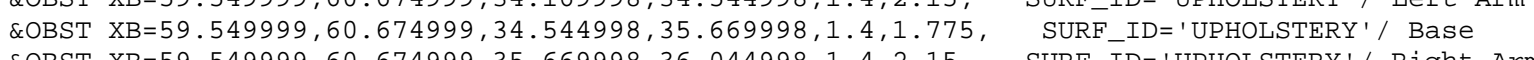

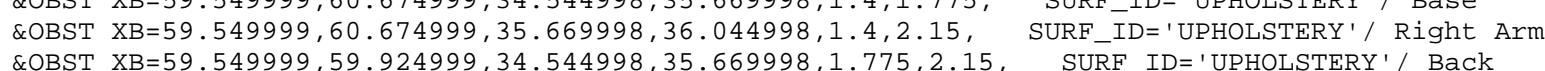

*** GROUP: LD LS ROW

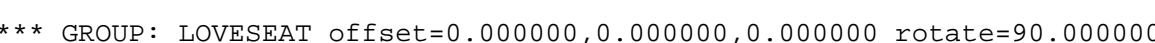

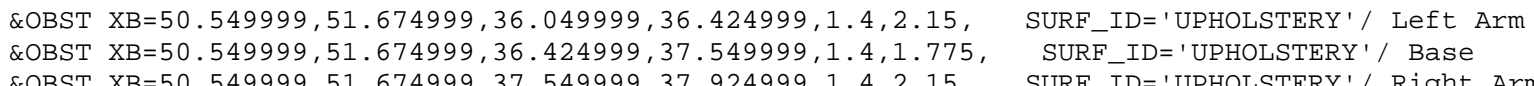
COBST XB=50.54999, $51.674999,37.549999,37.924999,1.4,2.15, \quad$ SURF_ID=' UPHOLSTERY'/ Right Ar
\&OBST XB=50.549999, $50.924999,36.424999,37.549999,1.775,2.15, \quad$ SURF_ID=' 'UPHOLSTERY' $/$ Back

*** GROUP: LOVESEAT offset $=1.125000,0.000000,0.000000$ rotate $=90.000000$

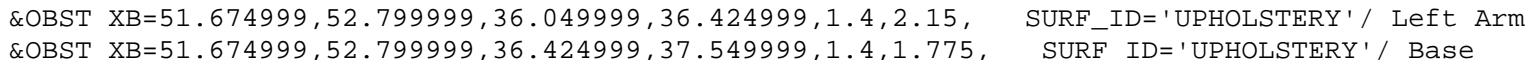

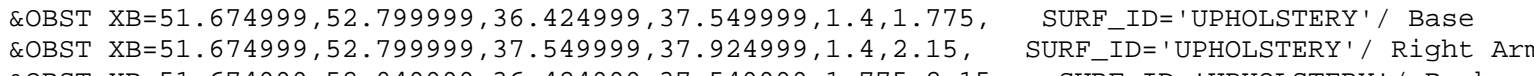

*** GROUP: LOVESEAT offset $=2.250000,0.000000,0.000000$ rotate $=90 . .000000$

\&OBST XB $=52.799999,53.924999,36.049999,36.424999,1.4,2.15$, SURF_ID='UPHOLSTERY ' / Left Arm

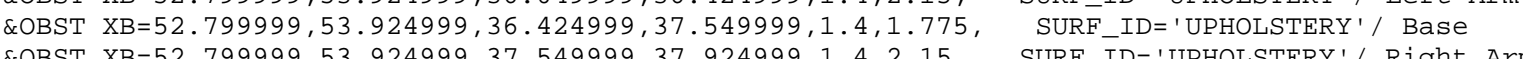

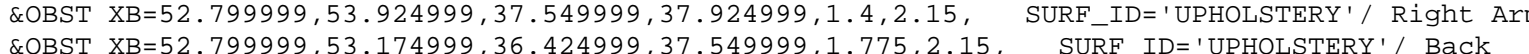

$* * *$ GROUP: LOVESEAT off $\mathrm{set}=3.375000,0.000000,0.0000000$ rotate $=90.000000$

\&OBST XB=53.924999, 55. 049999, 36. 049999, 36.424999, 1.4, 2.15, SURF_ID='UPHOLSTERY'/ Left Arm \&OBST XB=53.924999, 55. $449999,36.424999,37.549999,1.4,1.775$, SURF_ID='UPHOLSTERY'/ Base \&OBST XB=53.924999, 54.29999, 36.424999, 37.549999, $1.775,2.15$, SURF_ID ' 'UPHOLSTEPY'/ Back

$\star \star \star$ GROUP: LOVESEAT offset $=4.500000,0.000000,0.0000000$ rotate $=90.000000$

EOBST XB=55. 049999, 56.174999, 36. 049999, 36.424999, 1.4, 2.15, SURF_ID='UPHOLSTERY'/ Left Arm EOBST XB=55. $049999,56.174999,36.424999,37.549999,1.4,1.775$, SURF_ID='UPHOLSTERY'/ Base

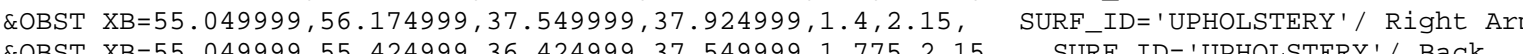

*** GROUP: LOVESEAT offset $=5.625000, \Theta .000000,0.000000$ rotate $=90.000000$ 
\&OBST XB=56.174999, 57.299999, 36. 049999, 36. 424999, 1.4, 2.15, $\quad$ SURF_ID=' 'UPHOLSTERY ' ' Left Arm
\&OBST XB=56.174999, $57.299999,36.424999,37.549999,1.4,1.775, \quad$ SURF_ID='UPHOLSTERY' / Base

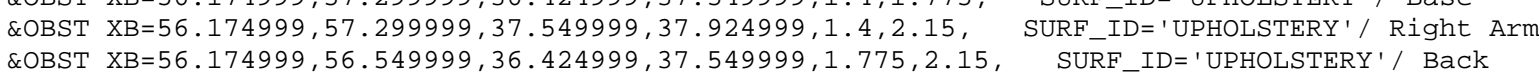
$* * *$ GROUP: LOVESEAT offset $=6.750000,0.000000,0.000000$ rotate $=90.000000$

\&OBST XB=57.299999, 58.424999, 36. $049999,36.424999,1.4,2.15$, SURF_ID=' 'UPHOLSTERY ' / Left Arm \&OBST XB $=57.299999,58.424999,37.549999,37.924999,1.4,2.15, \quad$ SURF-ID=' 'UPHOLSTERY' $/$ Right Arm
\&OBST XB $=57.299999,57.674999,36.424999,37.549999,1.775,2.15, \quad$ SURF_ID=' UPHOLSTERY' $/$ Back

*** GROUP: LOVESEAT of fset $=7.875000,0.000000,0.000000$ rotate $=90.000000$

\&OBST $X \mathrm{XB}=58.424999,59.549999,36.049999,36.424999,1.4,2.15$, SURF_ID='UPHOLSTERY' ' Left Arm

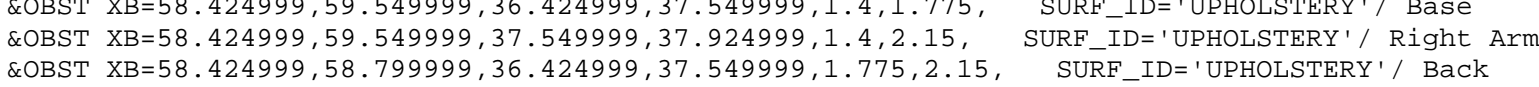
*** GROUP: LOVESEAT offset $=9.000000,0.000000,0.000000$ rotate $=90.000000$

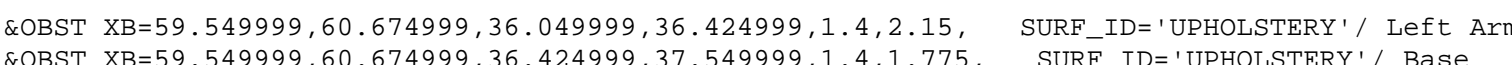

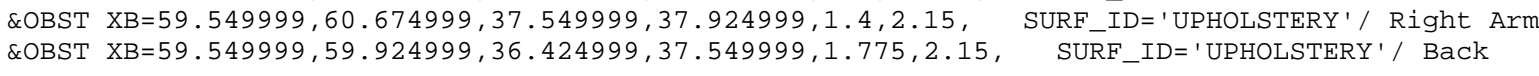

*** GROUP: LD COUCH ROW

*** GROUP: COUCH offset $=0.000000,0.000000, \odot .000000$ rotate $=90 . .000000$

OBST XB=50.549999, 51.674999, 37. $919998,38.294998,1.4,2.15, \quad$ SURF_ID=' 'UPHOLSTERY' ' Left Arm
OBST XB=50.549999, $51.674999,38.294998,40.544998,1.4,1.775, \quad$ SURF_ID='UPHOLSTERY' / Base OBST XB=50.549999, 51.674999, 40.544998, 40.919998, 1.4,2.15, SURF ID='UPHOLSTERY'/ Right Arm

*** GROUP: Couch offset $=1.125000,0.000000,0.000000$ rotate $=90.000000$

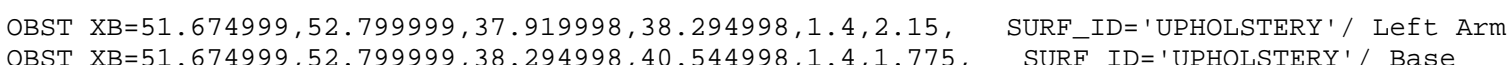
OBST XB $=51.674999,52.799999,38.294998,40.544998,1.4,1.775, \quad$ SURF_IDE' 'UPHOLSTERY'/ Base

*** GROUP: Couch of fset $=2.250000,0.000000,0.000000$ rotate $=90.000000$

OBST XB=52.799999, 53.924999, 37. 919998, 38.294998, 1.4, 2.15, SURF_ID='UPHOLSTERY '/ Left Arm OBST XB=52.799999, 53.924999, 38.294998, 40.544998, 1.4, $1.775, \quad$ SURF IDD='UPHOLSTERY '/ Base
OBST XB OBST XB $=52.799999,53.924999,40.544998,40.919998,1.4,2.15, \quad$ SURF_ID=' 'UPHOLSTERY'/ Right Arm
OBST XB $=52.799999,53.174999,38.294998,40.544998,1.775,2.15$,
SURF_ID=' UPHOLSTERY' $/$ Back

*** GROUP: COUCH offset $=3.375000,0.000000,0.000000$ rotate $=90.000000$

OBST XB=53.924999, 55. 049999, 37. $919998,38.294998,1.4,2.15$, SURF_ID=' UPHOLSTERY'/ Left Arm OBST XB $=53.924999,55.049999,38.294998,40.544998,1.4,1.775, \quad$ SURF_ID= 'UPHOLSTERY' / Base OBST XB $=53.924999,55.049999,40.544998,40.919998,1.4,2.15, \quad$ SURF_ID=' 'UPHOLSTERY'/ Right Ar A
OBST XB $=53.924999,54.299999,38.294998,40.544998,1.775,2.15, \quad$ SURF_ID='UPHOLSTERY'/ Back

*** GROUP: COUCH offset $=4.500000,0.000000,0.000000$ rotate $=90.000000$

OBST $X B=55.049999,56.174999,37.919998,38.294998,1.4,2.15$, SURF_ID=' 'UPHOLSTERY' ' Left Arm OBST XB=55.049999, 56.174999, 40.544998, 40.919998, 1.4, 2.15, SURF_ID='UPHOLSTERY'/ Right Arm
OBST XB $=55.049999,55.424999,38.294998,40.544998,1.775,2.15, \quad$ SURF_ID='UPHOLSTERY'/ Back $* * *$ GROUP: COUCH offset $=5.625000,0.000000,0.000000$ rotate $=90.000000$

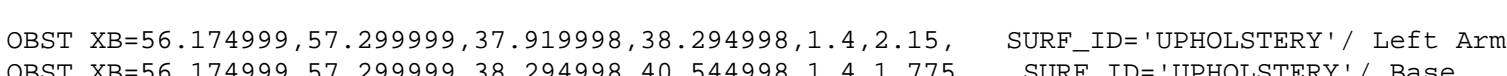

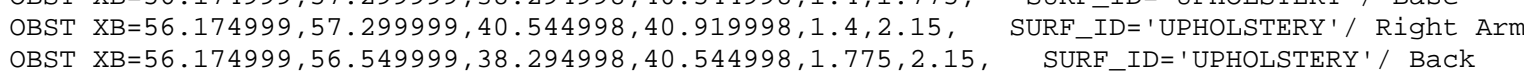
*** GROUP: COUCH offset $=6.750000,0.000000,0.000000$ rotate $=90.000000$

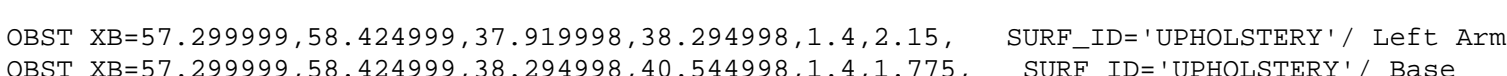

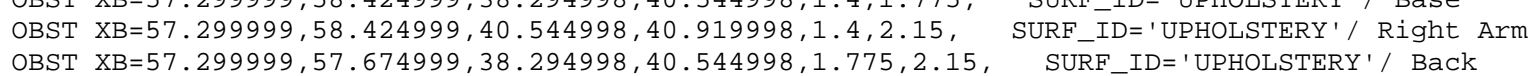

** GROUP: COUCH offset $=7.875000,0.000000,0.000000$ rotate $=90$. .

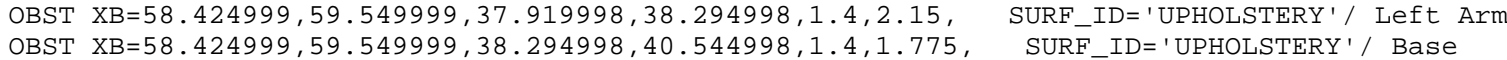
OBST XB $58.424999,59.549999,40.544998,40.919998,1.4,2.15$,
OBST

*** GROUP: COUCH offset $=9.000000,0.000000$, ๑. 000000 rotate $=90.000000$

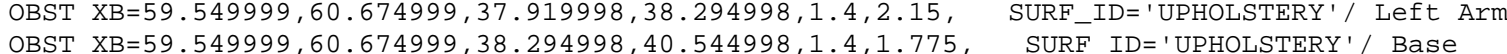
OBST XB $=59.54999,60.674999,38.294998,40.54498,1.4,4.15$, SURF

OBST XB=59.549999, 59.924999, 38. 294998, 40.544998, 1.775, 2.15, SURF_ID='UPHOLSTERY' ' Back

*** GROUP: LD COUCH ROW

*** GROUP: COUCH offset $=0.000000,0.000000,0.000000$ rotate $=90.000000$

OBST $X B=50.549999,51.674999,40.91,41.285,1.4,2.15$, SURF ID= ' UPHOLSTERY' ' Left Arm OBST XB=50.549999, 51.674999, $43.535,, 43.91,1.4,2.75$, S SURF_IDE' 'UPHOLSTERY' $/$ Right Ar

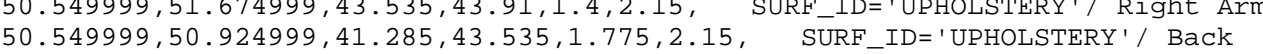

*** GROUP: COUCH offset $=1.125000,0.000000,, 0.000000$ rotate $=90.000000$

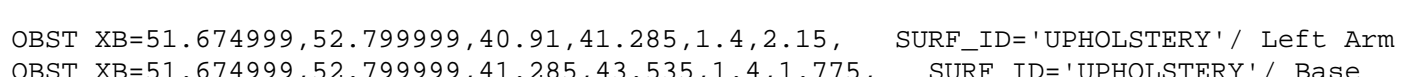
OBST $X B=51.674999,52.799999,43.535,43.91,1.4,2.15, \quad$ SURF_ID ='UPHOLSTERY' $/$ Right Arm
OBST XB=51.674999, $52.049999,41.285,43.535,1.775,2.15, \quad$ SURF_ID=' 'UPHOLSTERY'/ Back

*** GROUP: COUCH offset $=2.250000,0.000000,0.000000$ rotate $=90.000000$

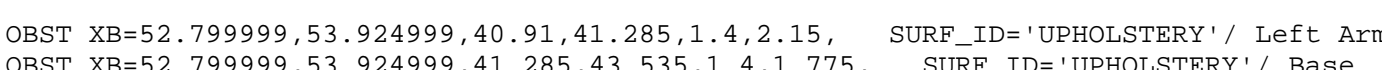

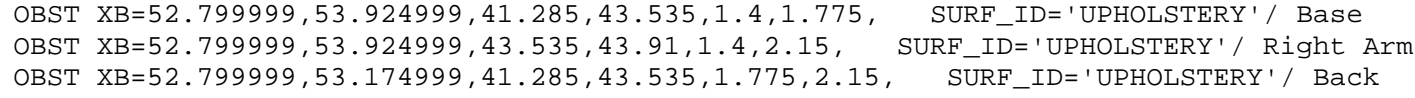

*** GROUP: COUCH offset $=3.375000,0.000000,0.000000$ rotate $=90.000000$

OBST XB=53.924999, 55. 049999, 40.91, 41.285, 1.4, 2.15, SURF_ID=' 'UPHOLSTERY ' / Left Arm OBST XB $=53.924999,55.049999,43.535,43.91,1.4,2.15, \quad$ SURF IDE 'UPHOLSTERY' 1 'Right OBST XB=53.924999, 54.299999, 41.285, 43.535, 1.775, 2.15, SURF_ID=' 'UPHOLSTERY'/' Back

$* * *$ GROUP: COUCH offset $=4.500000,0.000000,0.000000$ rotate $=90.000000$

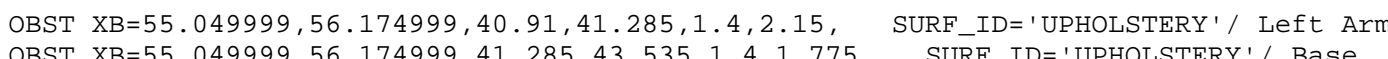
OBST XB $=55040$ OBST $X B=55.049999,55.424999,41.285,43.535,1.775,2.15$, SURF_IDE'UPHOLSTERY' SURF_ID 'UPHOLSTERY'/ Back

*** GROUP: COUCH offset $=5.625000,0.0000000,0.000000$ rotate $=90$. .

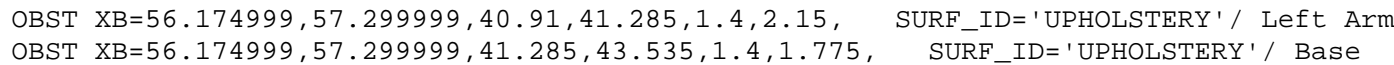
OBST XB $=56.174999,57.299999,43.535,43.91,1.4,2.15$,
OBST XB $=56.174999,56.54999,41.285,43.535,1.775,2.15$

*** GROUP: COUCH offset $=6.750000,0.000000,0.0000000$ rotate $=90.000000$

OBST XB $=57.299999,58.424999,40.91,41.285,1.4,2.15, \quad$ SURF_ID=' 'UPHOLSTERY ' $/$ Left Arm
OBST XB $=57.299999,58.424999,41.285,43.535,1.4,1.755, \quad$ SURF_DD='UPHOLSTERY' OBST $X B=57.299999,58.424999,43.535,43.91,1.4,2.15$, SURF ID=' UPHOLSTERY' ' Base

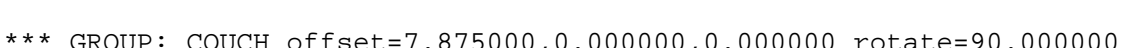

OBST XB=58.424999, 59.549999, 40.91, 41.285, 1.4, 2.15, SURF_ID='UPHOLSTERY'/ Left Arm
OBST XB=58.424999, $59.549999,41.285,43.535,1.4,1.775, \quad$ SURF ID='UPHOLSTERY'

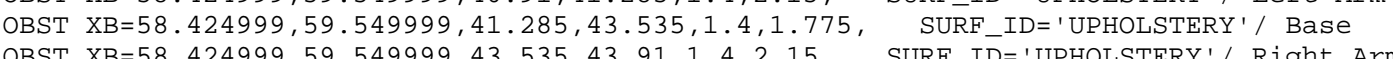
OBST XB=58.42499, $59.549999,43.535,43.91,1.4,2.15$, SURF_ID='UPHOLSTERY' / Right Arm
OBST XB $=58.424999,58.799999,41.285,43.535,1.775,2.15$, SURF_ID=' UPHOLSTERY'

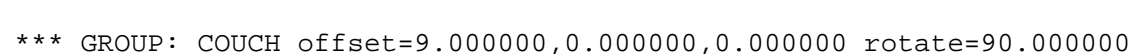

OBST XB=59.549999, 60.674999, 40.91, 41.285,1.4, 2.15, SURF_ID='UPHOLSTERY'/ Left Arm

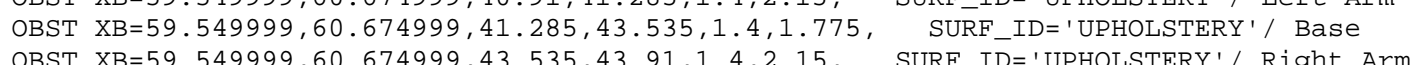


OBST XB=59.549999, 59, $924999,41.285,43.535,1.775,2.15$, SURF_ID=' UPHOLSTERY' / Back

*** GROUP: LD CHAIR ROW

*** GROUP: CHAIR offset $=0.000000,0.0000000,0.000000$ rotate $=90.000000$

\&OBST XB=50.549999, 51.674999, 32.669998, 33. $044998,1.4,2.15, \quad$ SURF_ID='UPHOLSTERY'/' Left Arm
\&OBST XB=50.549999, 51.674999, 33.044998,33.794998,1.4,1.775, SURF_ID='UPHOLSTERY'/ Base \&OBST XB=50.549999, $51.674999,33.794998,34.169998,1.4,2.15, \quad$ SURF IDE 'UPHOLSTERY'/ Right Arm

*** GROUP: CHAIR offset $=1.125000,0.000000,0.000000$ rotate $=90.000000$

LOBST XB=51.674999, 52.799999, 32.669998, 33. $044998,1.4,2.15, \quad$ SURF_ID=' 'UPHOLSTERY ' / Left Arm
\&OBST XB=51.674999, $52.799999,33.044998,33.794998,1.4,1.775, \quad$ SURF_ID='UPHOLSTERY'/ Base

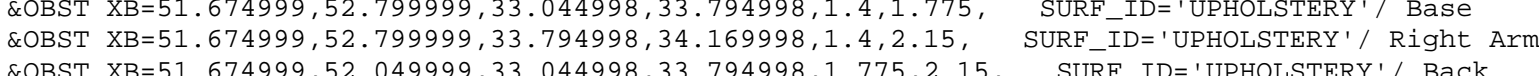

$* * *$ GROUP: CHAIR offset $=2.250000,0.000000,0.000000$ rotate $=90$. ๑०००००

\&OBST XB=52.799999, 53.924999, 32.669998, 33.044998, 1.4, 2.15, SURF_ID='UPHOLSTERY ' I Left Arm \&OBST XB $=52.799999,53.924999,33.044998,33.794998,1.4,1.775$,
\&OBST XB $=52.799999,53.92499,33.794988,34.169998,1.4,2.15$

*** GROUP: CHAIR offset $=3.375000, \Theta .000000,0.000000$ rotate $=90.000000$

\&OBST XB=53. 924999, 55. 049999, 32.669998, 33. 044998, 1.4, 2.15, SURF_ID=' 'UPHOLSTERY ' / Left Arm COBST XB-53.924999, $55.049999,33.044998,33.794998,1.4,1.775$, SURF_ID=' 'UPHOLSTERY'/ Base

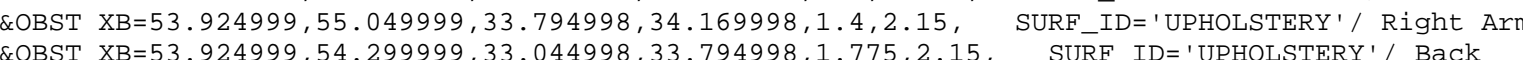
*** GROUP: CHAIR offset $=4.500000,0.0000000,0.000000$ rotate $=90 . .000000$

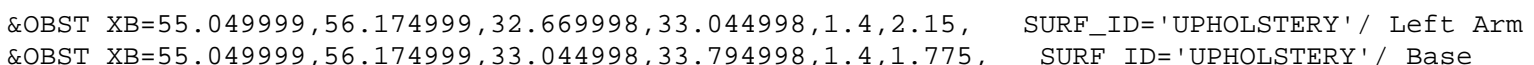

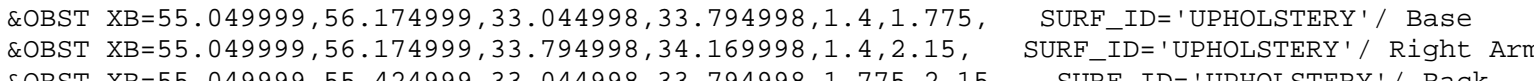

$* * *$ GROUP: CHAIR offset $=5.625000,0.000000,0.000000$ rotate $=90.000000$

\&OBST XB=56.174999, 57.299999, 32.669998, 33. $044998,1.4,2.15, \quad$ SURF_ID=' 'UPHOLSTERY' ' Left Arm
\&OBST XB=56.174999, $57.299999,33.044998,33.794998,1.4,1.775, \quad$ SURF_ID='UPHOLSTERY'/ Base \&OBST XBE $56.174999,57.299999,33.794998,34.169998,1.4,2.15$, SURF_IDD' 'UPHOLSTERY'/ Right Ar

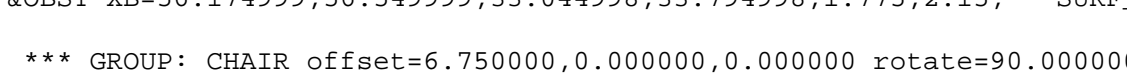
\&OBST XB $=57.299999,58.424999,32.669998,33.044998,1.4,2.15, \quad$ SURF_ID=' 'UPHOLSTERY ' / Left Arm
\&OBST XB $=57.299999,58.424999,33.044998,33.794998,1.4,1.775, \quad$ SURF_ID='UPHOLSTERY'/ Base \&OBST XB=57. $299999,58.424999,33.794998,34.169998,1.4,2.15, \quad$ SURF_ID='UPHOLSTERY'/ Right Ar
\&OBST XB=57. 29999, $57.674999,33.944998,33.794998,1.775,2.15, \quad$ SURE_ID='UPHOLSTEPY'/ Back

*** GROUP: CHAIR offset $=7.875000,0.000000,0.000000$ rotate $=90.000000$

\&OBST XB=58.424999, 59.549999, 32.669998, 33.044998, 1.4, 2.15, SURF_ID='UPHOLSTERY' / Left Arm

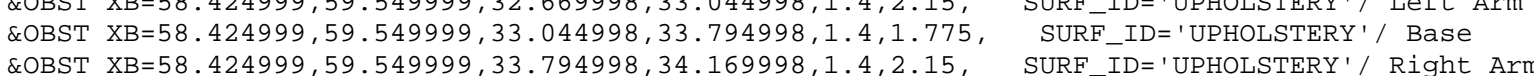

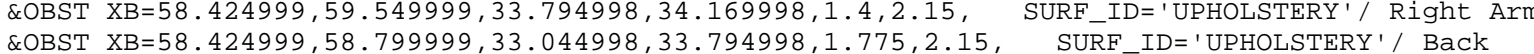

*** GROUP: CHAIR offset $=9.000000,0.000000,0.000000$ rotate $=90.000000$

\&OBST XB=59.549999, 60.674999, 32.669998, 33.044998, 1.4, 2.15, SURF_ID= 'UPHOLSTERY' / Left Arm

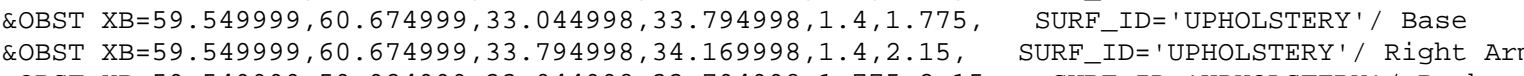

\section{$* * *$ GROUP: CHAIR_ROW5}

*** GROUP: CHAIR offset $=0.000000,0.000000,0.140000$ rotate $=180.000000$

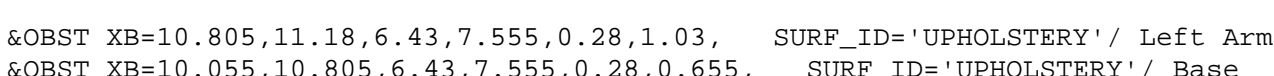

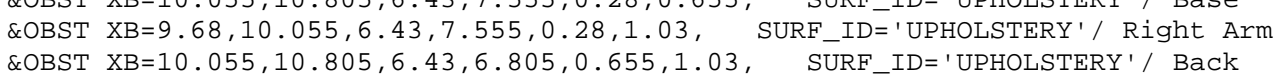

** GROUP: CHAIR offset $=1.500000,0.000000,0.140000$ rotate $=180 ., 000000$

\&OBST $X \mathrm{X}=12.305,12.68,6.43,7.555,0.28,1.03, \quad$ SURF_ID='UPHOLSTERY '/ Left Arm
\&OBST XB=11.555, 12.305, $6.43,7.555,0.28,0.655, \quad$ SURF_ID='UPHOLSTERY' / Base \&OST $X \mathrm{X}=11.18,11.555,6.43,7.555,0.28,1.03$,
\&OBST $X \mathrm{~B}=11.555,12.305,6.43,6.805,0.655,1.03$,

*** GROUP: CHAIR offset $=3.000000,0.000000,0.140000$ rotate $=180.000000$

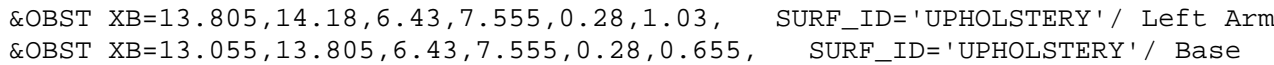
QOBST $X \mathrm{~B}=13.055,13.805,6.43,7.555,0.28,0.655, \quad$ SURF_ID='UPHOLSTERY'
COBST $X \mathrm{~B}=12.68,13.055,6.43,7.555,0.28,1.03, \quad$ SURF_ID= 'UPHOLSTERY' \&OBST XB=13.055, 13.805, 6.43, 6.805, 0.655,1.03, SURF_ID=' 'UPHOLSTERY'/ Back

*** GROUP: CHAIR offset $=4.500000,0.000000,0.140000$ rotate $=180.000000$

\&OBST $X B=15.305,15.68,6.43,7.555,0.28,1.03, \quad$ SURF_ID= 'UPHOLSTERY ' Left Arm
\&OBST XB=14.555, 15.305, $6.43,7.555,0.28,0.655, \quad$ SURF_ID=' 'UPHOLSTERY' $/$ Base

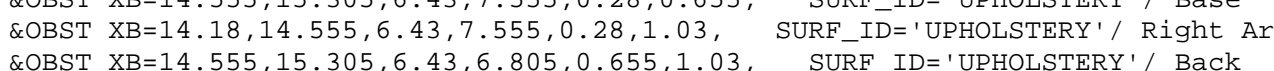

*** GROUP: CHAIR offset $=6.000000,0.000000,0.140000$ rotate $=180.000000$

\&OBST $X \mathrm{X}=16.805,17.18,6.43,7.555,0.28,1.03, \quad$ SURF_ID=' UPHOLSTERY ' Left Arm
\&OBST $X \mathrm{~B}=16.055,16.805,6.43,7.555,0.28,0.655, \quad$ SURF_ID=' 'UPHOLSTERY' $/$ Base

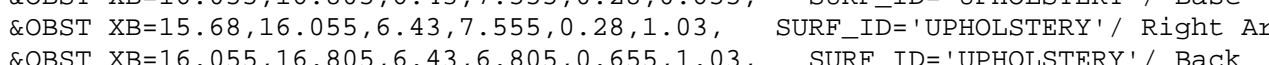

*** GROUP: CHAIR offset $=7.500000,0.000000,0.140000$ rotate $=180.0000000$

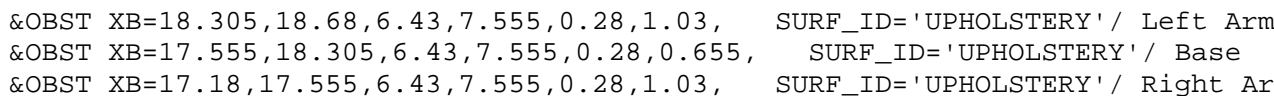
\&OBST XB=17.18, 17.555, $6.43,5.55,0.28,1.03, \quad$ SURF_ID='UPHOLSTERY'/ Right Ar
\&OBST XB=17.555,18.305, $6.43,6.805,0.655,1.03$, SURF_ID=' UPHOLSTERY'/ Back

$* * *$ GROUP: CHAIR offset $=9.000000,0.000000,0.140000$ rotate $=180.000000$

\&OBST XB $=19.805,20.18,6.43,7.555,0.28,1.03$, SURF_ID= 'UPHOLSTERY' / Left Arm \&OBST $X B=19.055,19.805,6.43,7.555,0.28,0.655$, SURF_ID='UPHOLSTERY' / Base

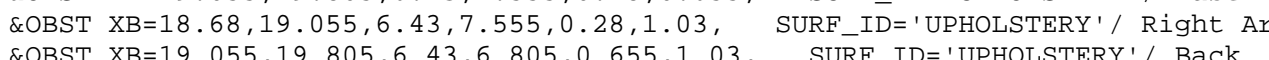

*** GROUP: CHAIR offset $=10.500000,0.000000,0.140000$ rotate $=180.000000$

\&OBST XB=21.305, 21.68,6.43,7.555, 0.28,1.03, SURF_ID= ' UPHOLSTERY' ' Left Arm \&OBST XB $=20.555,21.305,6.43,7.555,0.28,0.655$, SURF_ID='UPHOLSTERY' / Base \&OBST $X \mathrm{XB}=20.18,20.555,6.43,7.555,0.28,1.03$, SURF_ID= 'UPHOLSTERY' ' Right Ar

$* * *$ GROUP: CHAIR offset $=12.0000000,0.000000,0.140000$ rotate $=180.000000$

LOBST $X B=22.805,23.18,6.43,7.555,0.28,1.03, \quad$ SURF_ID= ' UPHOLSTERY ' / Left Arm
LOBST XB $=22.055,22.805,6.43,7.555,0.28,0.655$, SURF_ID=' 'UPHOLSTERY' $/$ Base

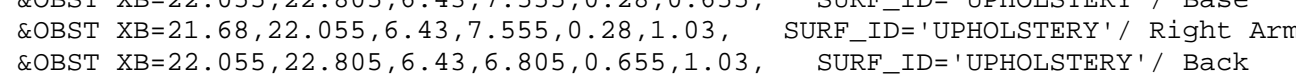

$* * *$ GROUP: CHAIR offset $=13.500000,0.000000,0.140000$ rotate $=180.000000$

\&OBST $\mathrm{XB}=24.305,24.68,6.43,7.555,0.28,1.03$, SURF_ID= 'UPHOLSTERY' ' Left Arm

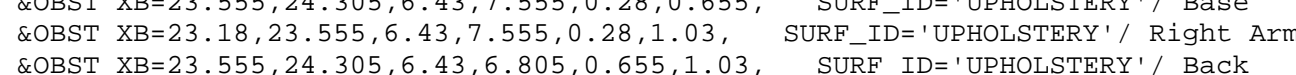

$* \star *$ GROUP: CHAIR offset $=15.000000,0.000000,0.140000$ rotate $=180.000000$

\&OBST $X \mathrm{XB}=25.805,26.18,6.43,7.555,0.28,1.03, \quad$ SURF_ID= 'UPHOLSTERY ' / Left Arm \&OBST XB $=24.68,25.055,6.43,7.555,0.28,1.03$, SURF_ID= 'UPHOLSTERY'/ Right Ar

*** GROUP: CHAIR_ROW5

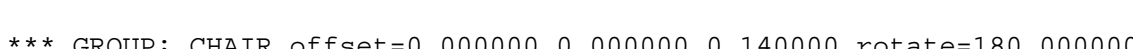

\&OBST XB=32.924999, 33.299999, 6.43,7.555, 0.28,1.03, SURF_ID='UPHOLSTERY' '/' Left Arm $-110$ 
\&OBST XB=32.174999, 32.924999, 6.43, 6. 805, 0. 655, 1. 03, SURE_ID=' 'UPHOLSTERY' / Back *** GROUP: CHAIR offset $=1.500000,0.000000,0.140000$ rotate $=180.000000$

LOBST XB=34.424999, 34.799999, 6.43,7.555, 0.28, 1.03 , SURF IDD ' UPHOLSTERY ' ' Left Arm \&OBST XB $33.299999,33.674999,6.43,7.555,0.28,1.03$, SURF IDE' UPHOLSTERY' '/ Right Arm

$* * *$ GROUP: CHAIR offset $=3.000000,0.000000,0.140000$ rotate $=180.000000$

\&OBST XB=35.924999, 36. 299999, 6.43, 7. 555, 0.28,1.03, SURF_ID='UPHOLSTERY'/ Left Arm \&OBST XB=35. 174999, 35. $924999,6.43,7.555,0.28,0.655, \quad$ SURF ID = 'UPHOLSTERY '/ Base

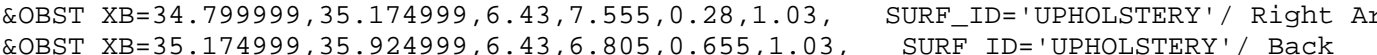

$* * *$ GROUP: CHAIR offset $=4.500000,0.0000000,0.140000$ rotate $=180.000000$

\&OBST XB $=37.424999,37.799999,6.43,7.555,0.28,1.03$, SURF_ID='UPHOLSTERY'/ Left Arm \&OBST XB $=36.674999,37.424999,6.43,7.555,0.28,0.655$, SURF_ID='UPHOLSTERY ' / Base

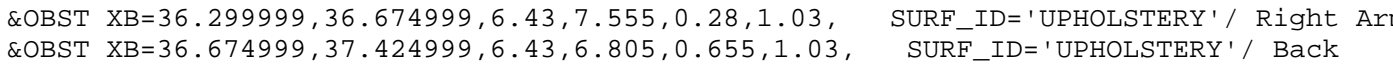

$* * *$ GROUP: CHAIR offset $=6.0000000,0.000000,0.140000$ rotate $=180.000000$

\&OBST XB=38.924999, 39.299999, $6.43,7.555,0.28,1.03$, SURF_ID=' UPHOLSTERY'/ Left Arm \&OBST XB=37.799999, 38.174999, 6.43, 7.555, 0.28,1.03, SURF_ID='UPHOLSTERY'/ Right Arm \&OBST XB=38.174999, 38.924999, 6.43,6.805, 0.655, 1.03 , SURF_ID=' 'UPHOLSTERY' / Back

$* *$ GROUP: CHAIR offset $=7.500000,0.000000,0.140000$ rotate $=180.000000$

\&OBST XB $=40.424999,40.799999,6.43,7.555,0.28,1.03$, SURF_ID=' 'UPHOLSTERY ' / Left Arm \&OBST XB $39.299999,39.679999,6.43,7.555,0.28,1.03$,
\&OBST XB $39.674999,40.424999,6.43,6.805,0.655,1.03$,
SURF_IDE 'UPHOLSTERY'/ Right Arm

*** GROUP: CHAIR offset $=9.000000,0.000000,0.140000$ rotate $=180.000000$

\&OBST XB=41.924999, 42.299999,6.43,7.555, 0.28,1.03, SURF_ID='UPHOLSTERY' ' Left Arm

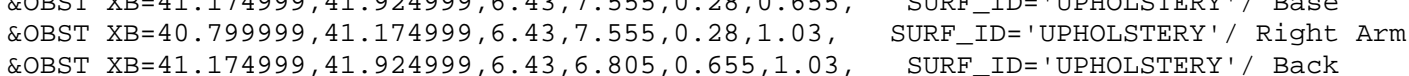

$* * *$ GROUP: CHAIR offset $=10.500000,0.000000,0.140000$ rotate $=180.000000$

\&OBST XB $=43.424999,43.799999,6.43,7.555,0.28,1.03$, SURF_ID='UPHOLSTERY ' ' Left Arm

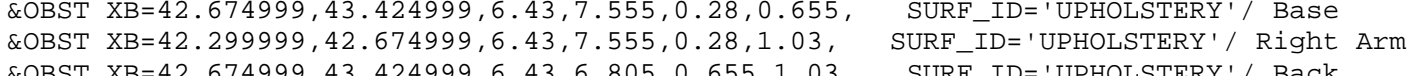

*** GROUP: CHAIR offset $=12.000000,0.000000,0.140000$ rotate $=180 . .000000$

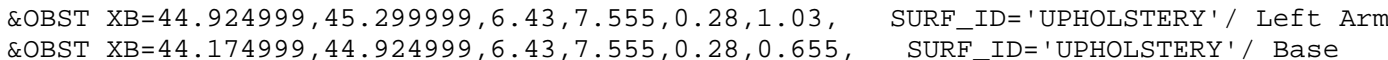

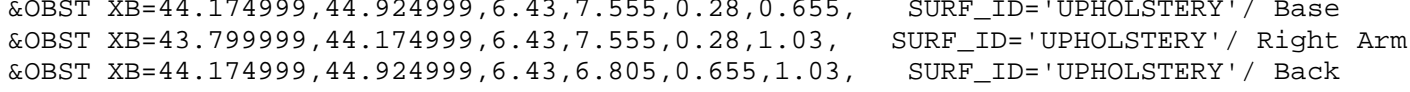

*** GROUP: CHAIR offset $=13.500000,0.0000000,0.140000$ rotate $=180.0000000$

\&OBST XB $=46.424999,46.799999,6.43,7.555,0.28,1.03$, SURF_ID=' 'UPHOLSTERY' ' Left Arm

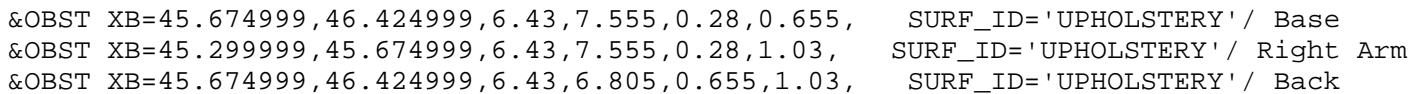

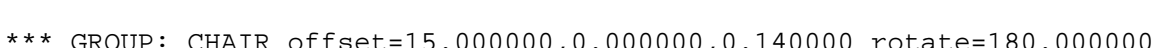

\&OBST XB $=47.924999,48.299999,6.43,7.555,0.28,1.03, \quad$ SURF_ID='UPHOLSTERY' $/$ Left Arm
\&OBST XB $=47.174999,47.924999,6.43,7.555,0.28,0.655$,
SURF ID ='UPHOLSTERY' $/$ Base \&OBST XB=47.174999, $47.924999,6.43,7.555,0.28,0.655$, SURF_ID='UPHOLSTERY'/ Base

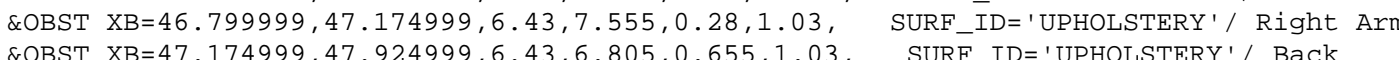
*** GROUP: CHAIR_ROW5

$\star \star *$ GROUP: CHAIR offset $=0.0000000,0.000000,0.140000$ rotate $=180.0000000$

\&OBST XB=49.794998, 50.169998,6.43,7.555, $0.28,1.03$, SURF_ID='UPHOLSTERY' / Left Ar
\&OBST XB $=49.044998,49.794998,6.43,7.555,0.28,0.655$, SURF_ID=' 'UPHOLSTERY'/ Base COBST XB $=48.669998,49.044998,6.43,7.555,0.28,1.03$,
\&OBST XB $=49.044998,49.794998,6.43,6.805,0.655,1.03$, *** GROUP: CHAIR offset $=1.500000,0.0000000,0.140000$ rotate $=180.000000$

\&OBST XB=51.294998, 51.669998, 6.43, 7.555, 0.28, 1.03, SURF_ID='UPHOLSTERY ' / Left Arm \&OBST XB $=50.169998,50.544998,6.43,7.555,0.28,1.03$, SURF ID=' 'UPHOLSTERY'/ Right Arm \&OBST XB $=50.544998,51.294998,6.43,6.805,0.655,1.03$, SURF_ID='UPHOLSTERY' / Back

*** GROUP: CHAIR offset $=3.000000,0.000000,0.140000$ rotate $=180.000000$

ROBST XB $=52.794998,53.169998,6.43,7.555,0.28,1.03, \quad$ SURF_ID='UPHOLSTERY' ' Left Arm
\&OBST XB $=52.044998,52.794998,6.43,7.555,0.28,0.655, \quad$ SURF_ID='UPHOLSTERY' $/$ Base

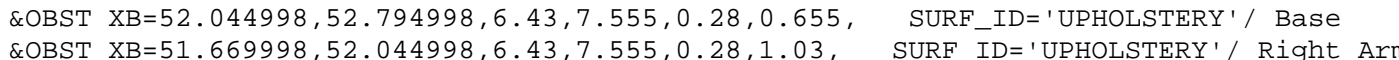

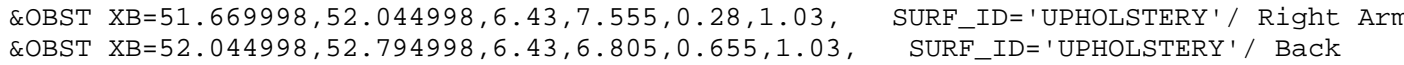

$* * *$ GROUP: CHAIR offset $=4.500000,0.000000,0.140000$ rotate $=180.000000$

\&OBST XB $=54.294998,54.669998,6.43,7.555,0.28,1.03, \quad$ SURF_ID=' 'UPHOLSTERY' ' Left Arm

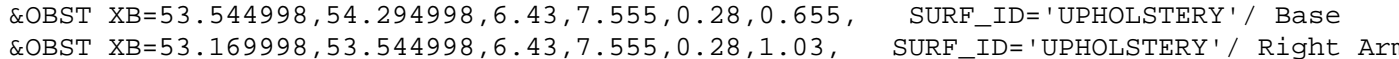
\&OBST XB $=53.544998,54.294998,6.43,6.805,0.655,1.03$, SURF_ID='UPHOLSTERY'/ Back

*** GROUP: CHAIR offset $=6.000000,0.000000,0.140000$ rotate $=180 . .000000$

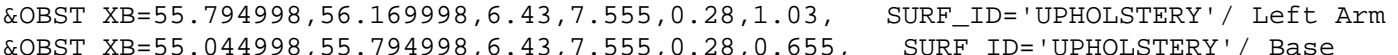

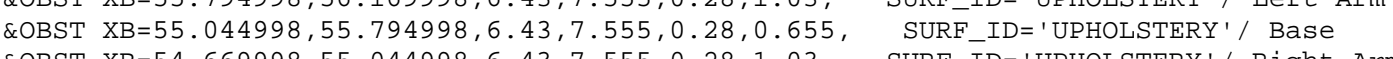

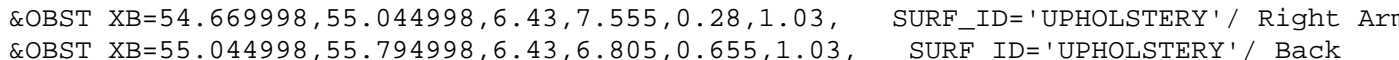

*** GROUP: CHAIR offset $=7.500000,0.000000,0.140000$ rotate $=180.000000$

\&OBST XB=57.294998, 57.669998,6.43,7.555, 0.28,1.03, SURF_ID=' 'UPHOLSTERY' 1 ' Left Arm \&OBST XB $=56.544998,57.294998,6.43,7.555,0.28,0.655$, SURF_ID='UPHOLSTERY'/ Base \&OBST XB=56.169998, 56.544998,6.43,7.555, 0.28,1.03, SURF_ID=' 'UPHOLSTERY'/ Right Arm
\&OBST XB=56.544998,57.294998,6.43,6.805, 0.655,1.03, SURF_ID='UPHOLSTERY'/ Back

*** GROUP: CHAIR offset $=9.000000,0.000000,0.140000$ rotate $=180.000000$

\&OBST XB=58.794998, 59.169998,6.43,7.555, 0.28,1.03, SURF_ID='UPHOLSTERY'/ Left Arm \&OBST XB $=58.044998,58.794998,6.43,7.555,0.28,0.655$, SURF_ID=' 'UPHOLSTERY'/ Base

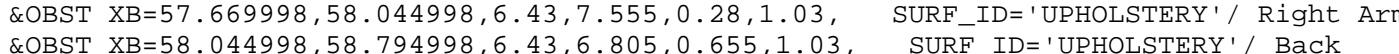

*** GROUP: CHAIR offset $=10.500000$, ๑. ๑๑0000, 0.140000 rotate $=180.000000$

\&OBST XB $=60.294998,60.669998,6.43,7.555,0.28,1.03$, SURF_ID='UPHOLSTERY' / Left Arm \&OBST XB $=59.544998,60.294998,6.43,7.555,0.28,0.655$, SURF_ID='UPHOLSTERY' / Base

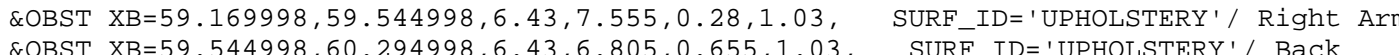

$* * *$ GROUP: CHAIR offset $=12.000000,0.000000,0.140000$ rotate $=180.000000$

\&OBST XB=61.794998, 62.169998,6.43,7.555, 0.28,1.03, SURF_ID='UPHOLSTERY'/ Left Arm \&OBST XB=60.669998, 61. $044998,6.43,7.555,0.28,1.03$, SURF_ID='UPHOLSTERY'/ Right Arm

*** GROUP: CHAIR offset $=13.500000,0.000000,0.140000$ rotate $=180.000000$

\&OBST $\mathrm{XB}=63.294998,63.669998,6.43,7.555,0.28,1.03$, SURF_ID=' 'UPHOLSTERY'/ Left Arm

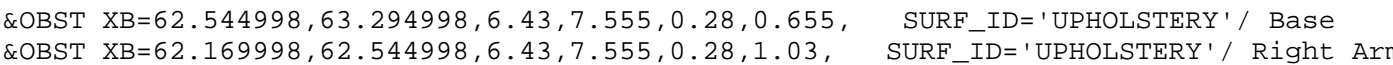
COBST XB=62.169998, 62.544998,6.43,7.555, $0.28,1.03$, SURF-ID='UPHOLSTERY'/ Right Arm

*** GROUP: CHAIR offset $=15.000000,0.000000,0.140000$ rotate $=180.000000$

\&OBST $X \mathrm{XB}=64.794998,65.169998,6.43,7.555,0.28,1.03$, SURF_ID=' 'UPHOLSTERY' $/$ Left Arm \&OBST XB=63.669998, 64.044998,6.43,7.555, 0.28,1.03, SURF_ID='UPHOLSTERY'/Right Arm

$* * *$ GROUP: CHAIR_ROW5

*** GROUP: CHAIR offset $=0.000000,0.000000,0.140000$ rotate $=180.000000$ 


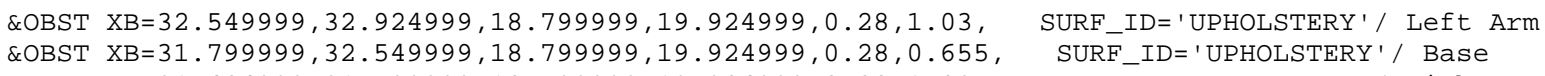
\&OBST XB=31.424999, 31.799999, 18.799999, 19.924999, $0.28,1.03$, SURF_LD='UPHOLSTER''/Right Arn SURF_ID='UPHOLSTERY'/ Back

$* * *$ GROUP: CHAIR offset $=1.500000,0.000000,0.140000$ rotate $=180.000000$

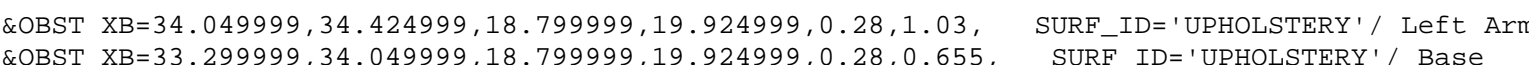
COBST XB $=32.924999,33.299999,18.799999,19.924999,0.28,1.03$, SURF-ID=' 'UPHOLSTERY'/ Right Ar

$* * *$ GROUP: CHAIR offset $=3.000000,0.000000,0.140000$ rotate $=180.000000$

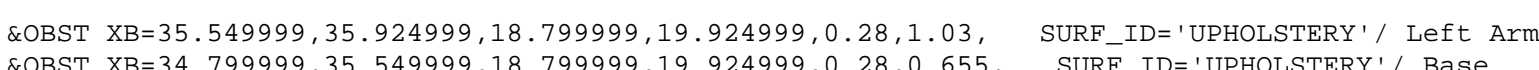
\&OBST XB=34.424999, 34.799999, 18.799999, 19.924999, $0.28,1.03$, SURF-ID='UPHOLSTERY'/ Right Ar *** GROUP: CHAIR offset $=4.500000,0.000000,0.140000$ rotate $=180.000000$

\&OBST XB=37. $049999,37.424999,18.799999,19.924999,0.28,1.03$, SURF_ID='UPHOLSTERY ' / Left Arm \&OBST XB=36.299999, 37. $049999,18.799999,19.924999,0.28,0.655$, SURF $=$ ID ='UPHOLSTERY'/ Base

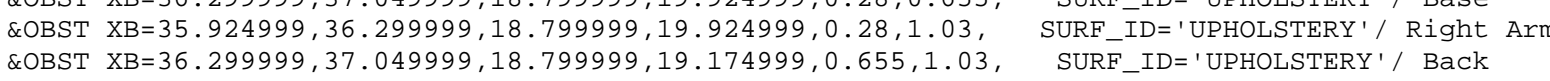

*** GROUP: CHAIR offset $=6.000000,0.000000,0.140000$ rotate $=180.000000$

\&OBST XB=38.549999, 38.924999, 18.799999, 19.924999, 0.28, 1. 03 , SURF_ID='UPHOLSTERY'/ Left Arm

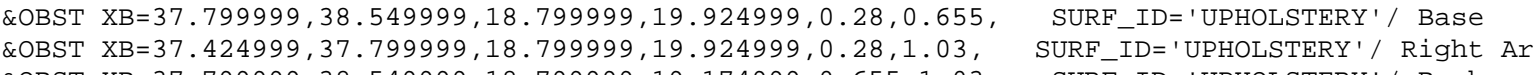
BOBST XB=37.799999, 38.549999, 18.799999, 19.174999, $0.655,1.03$, SURF_ID='UPHOLSTERY'/ Back

$* * *$ GROUP: CHAIR offset $=7.500000,0.000000,0.140000$ rotate $=180.000000$

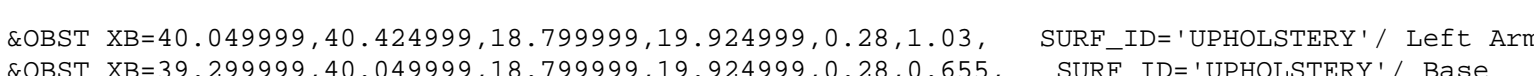

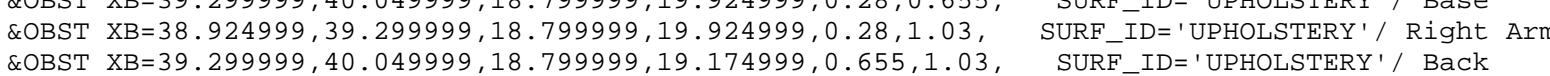

$\star \star *$ GROUP: CHAIR offset $=9.000000,0.000000,0.140000$ rotate $=180.000000$

\&OBST XB=41.549999, 41.924999, 18.799999, 19.924999, $0.28,1.03$, SURF_ID=' 'UPHOLSTERY' ' Left Arm

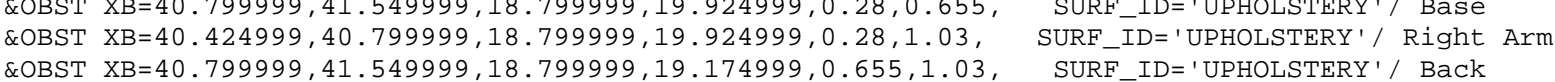

$\star \star *$ GROUP: CHAIR offset $=10.500000,0.000000, \Theta .140000$ rotate $=180.000000$

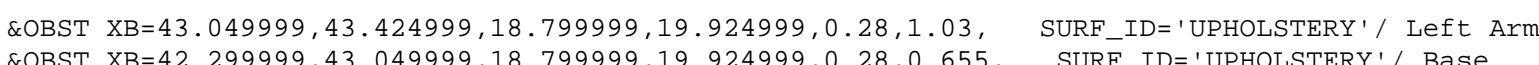

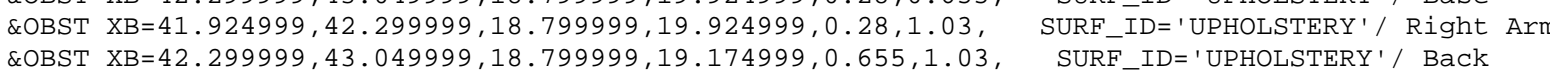

*** GROUP: CHAIR offset $=12.000000,0.0000000,0.140000$ rotate $=180.0000000$

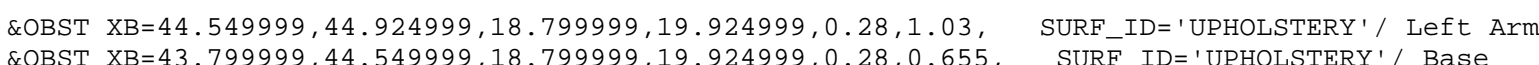
\&OBST XB=43.424999, 43.799999, 18.799999, 19.924999, $0.28,1.03$, SURF_ID='UPHOLSTERY'/ Right Ar
\&OBST XB=43.79999, $44.549999,18.799999,19.174999,0.655,1.03$, SURF_ID='UPHOLSTERY'/ Back *** GROUP: CHAIR offset $=13.500000,0.0000000,0.140000$ rotate $=180.000000$

\&OBST XB=46. $049999,46.424999,18.799999,19.924999,0.28,1.03, \quad$ SURF_ID= 'UPHOLSTERY' $/$ Left Arm
COBST XB=45.29999, $46.049999,18.799999,19.924999,0.28,0.655, \quad$ SURF ID ='UPHOLSTERY' Rase

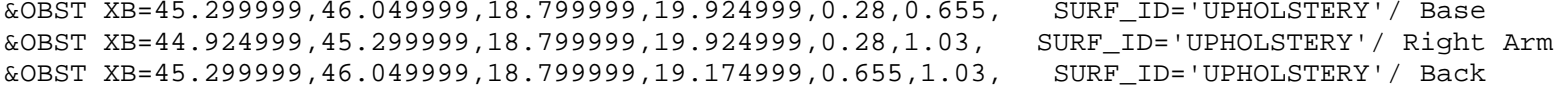

** GROUP: CHAIR offset $=15.000000,0.0000000,0.140000$ rotate $=180$. .00000

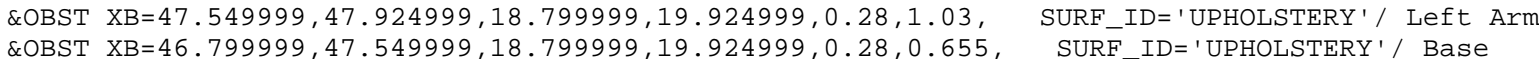

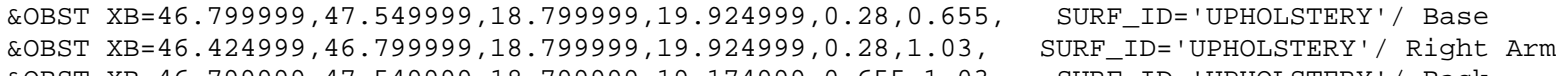
*** GROUP: CHATR_ROW5
*** GROUP: CHAIR offset $=0.000000,0.000000,0.140000$ rotate $=180.000000$

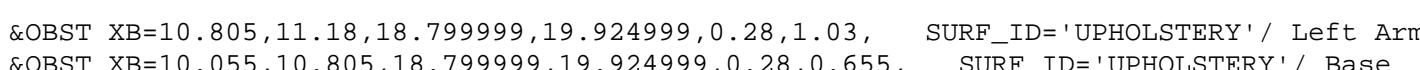

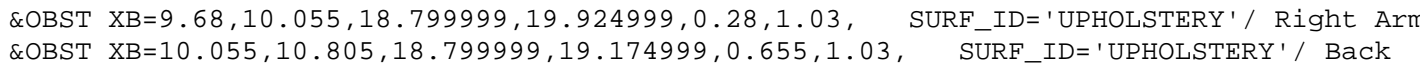
*** GROUP: CHAIR offset $=1.500000,0.000000,0.140000$ rotate $=180.000000$

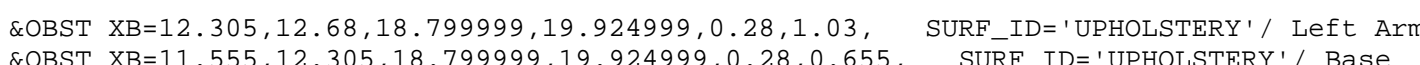

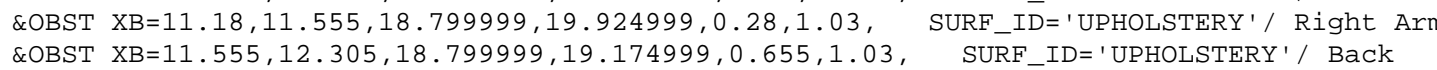

** GROUP: CHAIR offset $=3.0000000,0.000000,0.140000$ rotate $=180 . .000000$

\&OBST XB $13.805,14.18,18.799999,19.924999,0.28,1.03$, SURF_ID='UPHOLSTERY' ' Left Arm \&OBST XB=12.68, 13.055, 18.799999, 19.924999, $0.28,1.03$ SURE ID='UPHOLSTERY'/ Right A

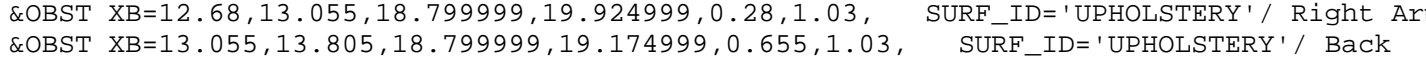

$* * *$ GROUP: CHAIR offset $=4.500000,0.000000,0.140000$ rotate $=180.000000$

\&OBST XB $=15.305,15.68,18.799999,19.924999,0.28,1.03, \quad$ SURF_ID='UPHOLSTERY ' / Left Arm
COBST XB $=14.555,15.305,18.799999,19.924999,0.28,0.655$,
SURF_ID='UPHOLSTERY'

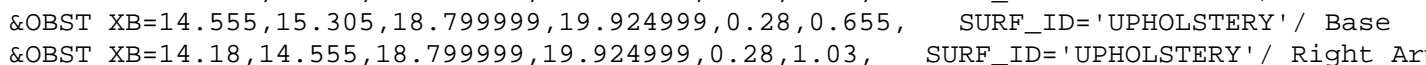
\&OBST XB $14.555,15.305,18.799999,19.174999, \theta .655,1.03$, SURF_ID='UPHOLSTERY'/ Back

*** GROUP: CHAIR offset $=6.000000,0.000000,0.140000$ rotate $=180$. .

ROBST XB $=16.805,17.18,18.799999,19.924999,0.28,1.03, \quad$ SURF_ID='UPHOLSTERY' $/$ Left Arm
\&OBST XB $16.055,16.805,18.799999,19.924999,0.28,0.655, \quad$ SURF_ID 'UPHOLSTERY' $/$ Base

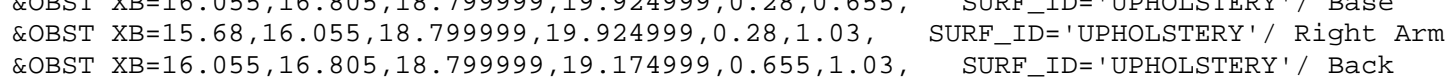

*** GROUP: CHAIR offset $=7.500000,0.000000,0.140000$ rotate $=180.000000$

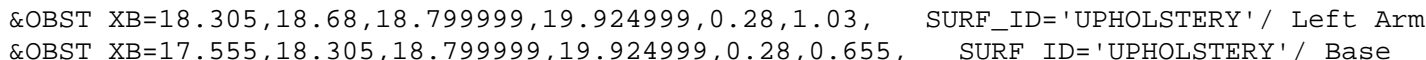
COBST XB $X 17.18,17.555,18.79999,19.924999,0.28,0.055$, SURF EOBST $X B=17.18,17.555,18.799999,19.924999,0.28,1.03, \quad$ SURF_ID='UPHOLSTERY'/ Right Arm
LOBST XB=17.555, 18.305, 18.79999, 19.174999, $0.655,1.03, \quad$ SURF_ID=' UPHOLSTERY'/ Back

*** GROUP: CHAIR offset $=9.000000,0.0000000,0.140000$ rotate $=180.000000$

ROBST XB $=19.805,20.18,18.799999,19.924999,0.28,1.03, \quad$ SURF_IDE' 'UPHOLSTERY' $/$ Left Arm
\&OBST XB $19.055,19.805,18.799999,19.924999,0.28,0.655, \quad$ SURF_ID 'UPHOLSTERY' $/$ Base

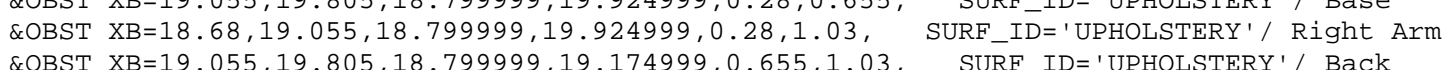

$* * *$ GROUP: CHAIR offset $=10.500000,0.000000,0.140000$ rotate $=180.000000$

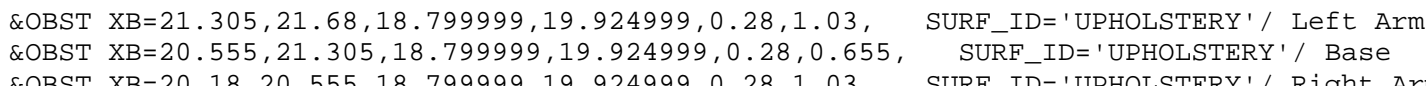

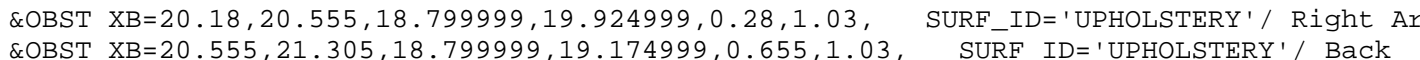

*** GROUP: CHAIR offset $=12.000000,0.000000,, 0.140000$ rotate $=180 . .000000$

\&OBST XB=22.805, 23.18, 18.799999, 19.924999, $0.28,1.03$, SURF_ID= 'UPHOLSTERY' ' Left Arm COBST XB $=22.055,22.805,18.799999,19.924999,0.28,0.655, \quad$ SURF_ID='UPHOLSTERY' $/$ Base
COBST XB $=21.68,22.055,18.799999,19.924999,0.28,1.03$,

*** GROUP: CHAIR offset $=13.500000,0.000000,0.140000$ rotate $=180.000000$

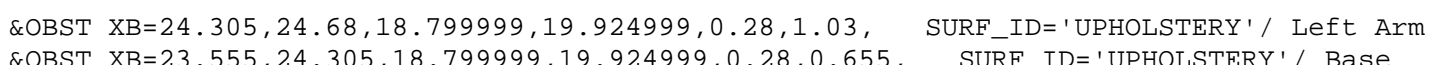
\&OBST $X B=23.18,23.555,18.799999,19.924999,0.28,1.03$, SURF_ID='UPHOLSTERY'/ Right Arm

*** GROUP: CHAIR offset $=15.000000, \Theta . \bullet 00000,0.140000$ rotate $=180.000000$

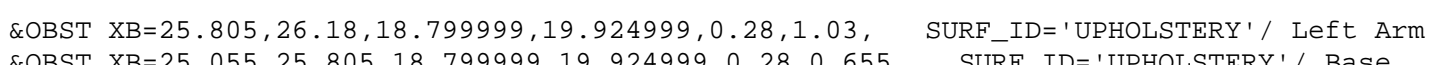
\&OBST XB $=24.68,25.055,18.799999,19.924999,0.28,1.03$, ' 


\section{** GROUP: CHAIR_ROW4}

$* * *$ GROUP: CHAIR_ROW3

$* * *$ GROUP: CHAIR offset $=0.000000,0.000000,0.140000$ rotate $=90.000000$

\&OBST XB=46.799999, 47.924999, 22.545, 22.92, 0.28,1.03, SURF_ID= 'UPHOLSTERY'/ Left Arm

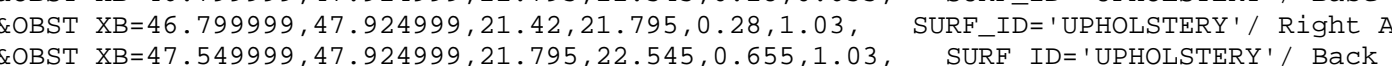

*** GROUP: CHAIR offset $=1.500000,0.000000,0.140000$ rotate $=90.000000$

\&OBST XB=45.299999, 46.424999, 22.545, 22.92, $9.28,1.03$, SURF_ID='UPHOLSTERY ' / Left Arm \&OBST XB=45. 299999, 46.424999, 21.42, 21.795, 0.28,1.03, SURF_ID ='UPHOLSTERY'/ Right A \&OBST XB=46.049999, 46.424999, 21.795, 22.545, 0.655, 1. 03, SURF_ID=' 'UPHOLSTERY' $/$ Back

$\star \star \star *$ GROUP: CHAIR offset $=3.000000,0.000000,0.140000$ rotate $=90.000000$

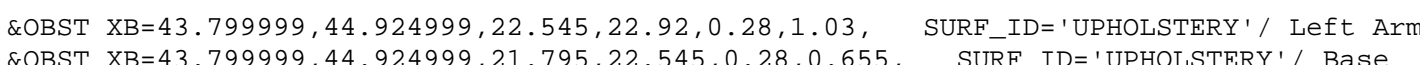
\&OBST XB $=43.799999,44.924999,21.42,21.795,0.28,1.03$,
\&OBST XB $44.549999,44.924999,21.795,22.545,0.655,1.03, \quad$ SURFIID='UPHOLSTERY'/ Right Arm
SURF_ID=' UPHOLSTERY'/ Back

$* * *$ GROUP: CHAIR offset $=4.500000,0.000000,0.140000$ rotate $=90.000000$

\&OBST XB=42.299999, 43. 424999, 22.545, 22.92, 0.28,1. 03, SURF_IDD ' UPHOLSTERY ' / Left Arm

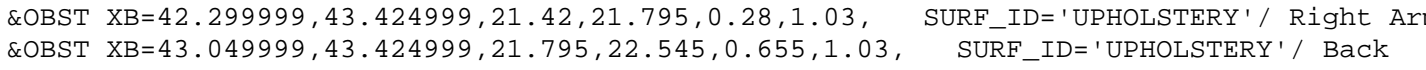
*** GROUP: CHAIR offset $=6.0000000,0.000000,0.140000$ rotate $=90.000000$

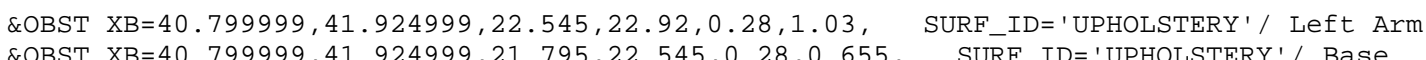
\&OBST XB-40.799999, $41.24999,21.42,21.795,0.28,1.053$, SURR ID $=$ ' UPHOLSTEPY' \&OBST XB=41.549999, 41.924999, 21.795, 22.545, $0.655,1.03$, SURF_ID='UPHOLSTERY'/ Back

*** GROUP: CHAIR offset $=7.5000000,0.000000,0.140000$ rotate $=90.000000$

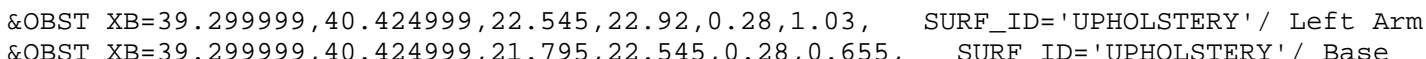

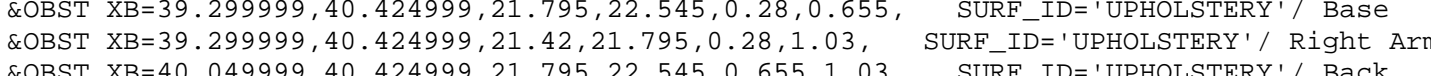

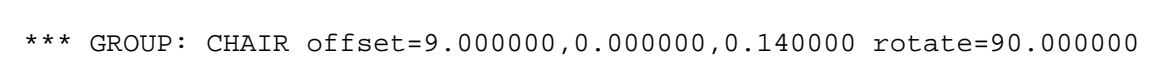

\&OBST XB=37.799999, 38. 924999, 22.545, 22.92, 0.28,1.03, SURF_ID= 'UPHOLSTERY ' / Left Arm

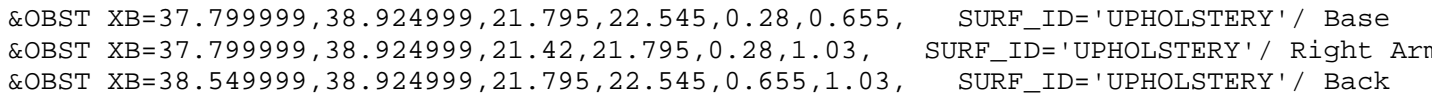

$* * *$ GROUP: CHAIR offset $=10.500000,0.000000,0.140000$ rotate $=90.000000$

ROBST XB $=36.299999,37.424999,22.545,22.92,0.28,1.03, \quad$ SURF_IDE'UPHOLSTERY' / Left Arm
\&OBST XB $=36.299999,37.424999,21.795,22.545,0.28,0.655$,
SURF_ID=' UPHOLSTERY' $/$ Base

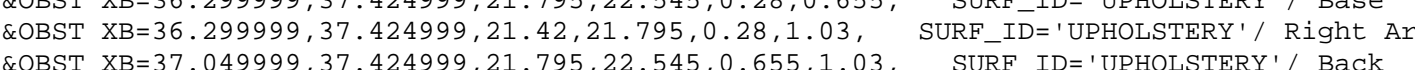

*** GROUP: CHAIR offset $=12.000000,0.000000,0.140000$ rotate $=90.000000$

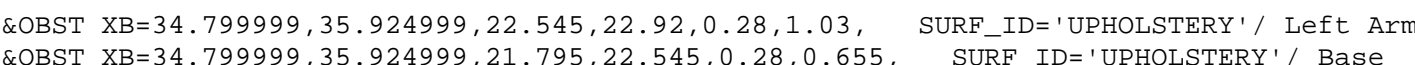

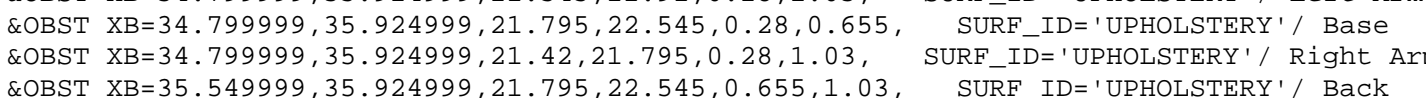

*** GROUP: CHAIR offset $=13.500000,0.000000,0.140000$ rotate $=90.000000$

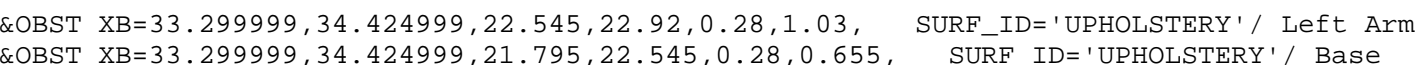
COBST XB=33.299999, 34. 424999, 21.42, 21.795, 0.28, 1. 03, SURF-ID='UPHOLSTERY'/Right Ar

$* * *$ GROUP: CHAIR offset $=15.000000,0.000000,0.140000$ rotate $=90.000000$
\&OBST XB=31.799999, 32.924999, 22.545, 22.92, 0.28,1.03, SURF_ID=' 'UPHOLSTERY' / Left Arm \&OBST XB $=31.799999,32.924999,21.795,22.545,0.28,0.655$, SURF_ID=' 'UPHOLSTERY' '/ Base \&OBST $X \mathrm{X}=32.799999,32.924999,21.42,21.795,0.28,1.03, \quad$ SURF_ID='UPHOLSTERY' $/$ Right Ar

*** GROUP: CHAIR_ROW3

*** GROUP: CHAIR offset $=0.000000, \Theta ., 000000,0.140000$ rotate $=90.0000000$

\&OBST $\mathrm{XB}=9.68,10.805,21.42,21.795,0.28,1.03$, SURF_ID= 'UPHOLSTERY' $/$ Left Arm

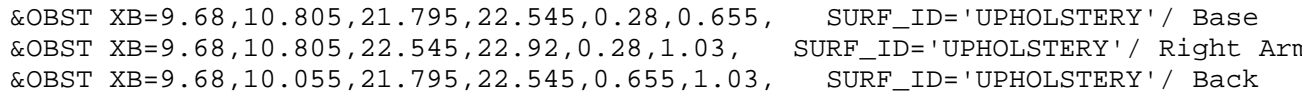

*** GROUP: CHAIR offset $=1.500000,0.000000,0.140000$ rotate $=90.000000$

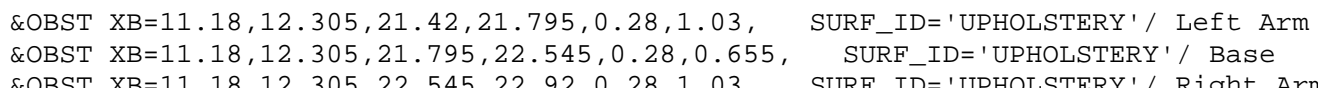

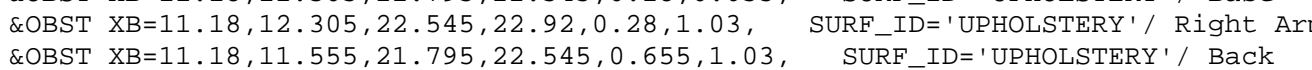

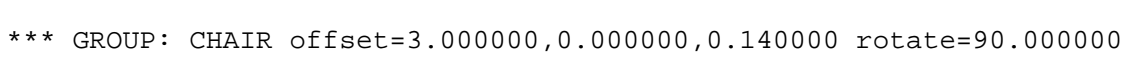

\&OBST XB $=12.68,13.805,21.42,21.795,0.28,1.03, \quad$ SURF_ID= ' UPHOLSTERY ' ' Left Arm
8OBST XB $=12.68,13.805,21.795,22.545,0.28,0.655$, SURF ID='UPHOLSTERY' \&OBST XB=12.68, 13.805, 21.795, 22.545, $0.28,0.65$, SURF_ID='UPHOLSTERY' / Base

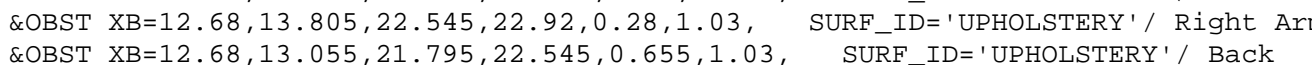

*** GROUP: CHAIR offset $=4.500000,0.000000,0.140000$ rotate $=90.000000$

\&OBST XB=14.18, 15.305, 21.42, 21.795, 0.28, 1.03, SURF_ID='UPHOLSTERY'/ Left Arm

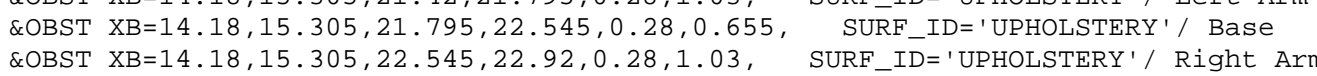
\&OBST XB=14.18,14.555, 21.795, 22.545, $0.655,1.03$, SURF ID ='UPHOLSTERY'

$* * *$ GROUP: CHAIR of $f$ set $=6.000000,0.000000,0.140000$ rotate $=90.000000$

\&OBST XB $=15.68,16.805,21.42,21.795,0.28,1.03$, SURF-ID='UPHOLSTERY' / Left Arm \&OBST XB $=15.68,16.805,21.795,22.545,0.28,0.655$, SURF_ID='UPHOLSTERY'/ Base \&OBST XB=15.68,16.805, 22. 545, 22.92, $0.28,1.03$, SURF_ID='UPHOLSTERY' ' Right Arm

*** GROUP: CHAIR offset $=7.500000,0.000000,0.140000$ rotate $=90.000000$

\&OBST XB=17.18, 18.305, 21.42, 21.795, 0.28,1.03, SURF_ID=' 'UPHOLSTERY'/ Left Arm \&OBST XB $=17.18,18.305,21.795,22.545,0.28,0.655$, SURF_ID='UPHOLSTERY' / Base

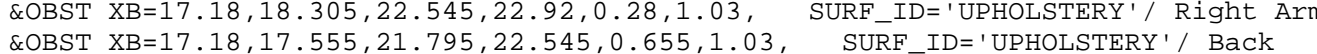

$* * *$ GROUP: CHAIR offset $=9.000000,0.000000,0.140000$ rotate $=90.000000$

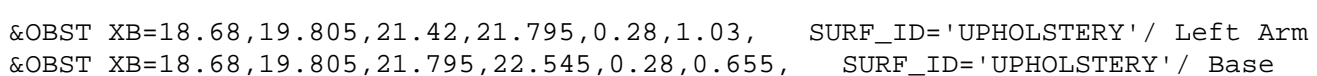

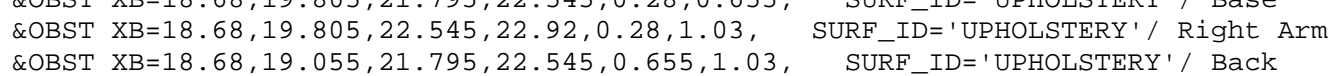

*** GROUP: CHAIR offset $=10.500000,0.000000,0.140000$ rotate $=90.000000$

\&OBST XB $=20.18,21.305,21.42,21.795,0.28,1.03$, SURF ID = 'UPHOLSTERY ' ' Left Arm

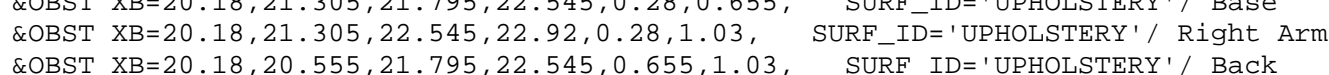

$* * *$ GROUP: CHAIR offset $=12.000000,0.000000,0.140000$ rotate $=90.000000$

\&OBST XB=21.68, 22.805, 21.42, 21.795, $0.28,1.03$, SURF_ID='UPHOLSTERY ' Left Arm \&OBST XB=21.68, 22.805, 22. .545, 22.92, $0.28,1.03$, SURF_ID='UPHOLSTERY' '/ Right Arm \&OBST XB=21.68, 22. $055,21.795,22.545,0.655,1.03$, SURF_ID='LPLLSTERY'/ Back

*** GROUP: CHAIR offset $=13.500000,0.000000,0.140000$ rotate $=90.000000$

\&OBST XB $=23.18,24.305,21.42,21.795,0.28,1.03$, SURF ID ' 'UPHOLSTERY ' / Left Arm \&OBST XB $=23.18,24.305,22.545,22.92,0.28,1.03$, S SURF_ID $=$ 'UPHOLSTERY'/ Right Arm \&OBST XB $=23.18,23.555,21.795,22.545,0.655,1.03$, SURF_ID='UPHOLSTERY'/ Back 
$* *$ GROUP: CHATR offset $=15.000000,0.000000,0.140000$ rotate $=90.000000$

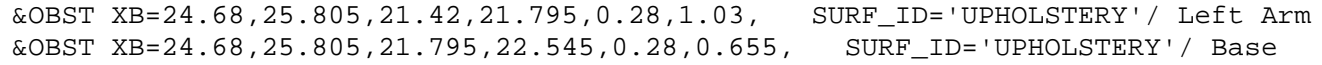

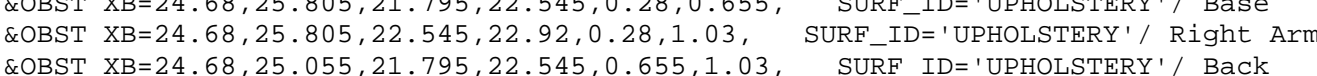

*** GROUP: LR

*** GROUP: COUCH offset $=0.000000,0.000000,0.000000$ rotate $=0.000000$

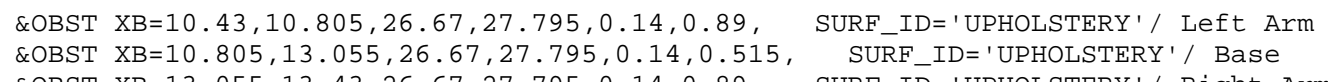

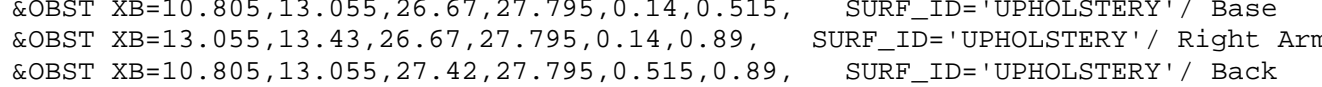

*** GROUP: CHAIR offset $=0.000000,-1.875000,0.000000$ rotate $=90.000000$ COBST XB $=10.43,11.555,24.795,25.17,0.14,0.89$,
SOBST XURF-ID=' 'UPHOLSTERY' 1 ' Left Arm

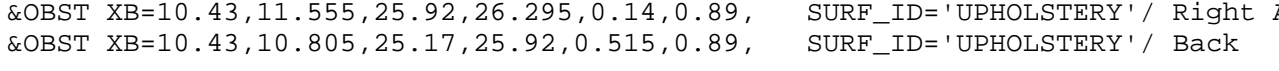

*** GROUP: CHAIR offset $=1.875000,-1.875000,0.000000$ rotate $=270.000000$

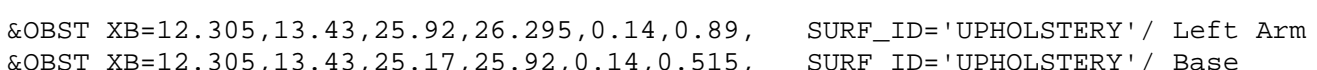

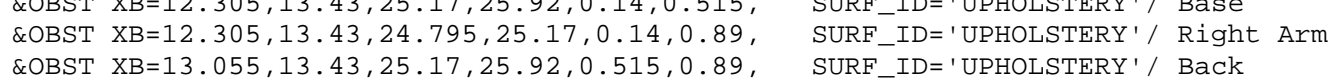

*** GROUP: LR

** GROUP: COUCH offset $=0.000000,0.000000,0.000000$ rotate $=0.000000$

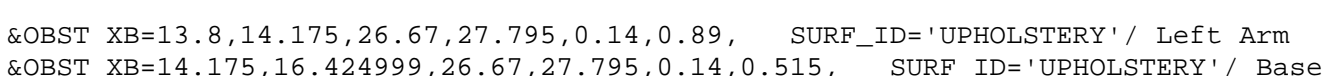

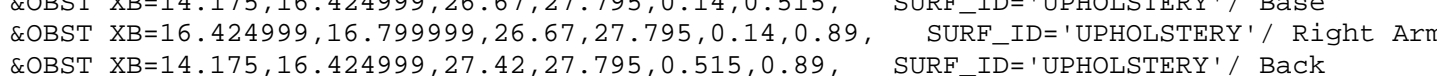

$* * *$ GROUP: CHAIR offset $=0.000000,-1.875000,0.000000$ rotate $=90.000000$ \&OBST XB $=13.8,14.925,24.795,25.17,0.14,0.89$, SURF_ID= 'UPHOLSTERY ' / Left Arm

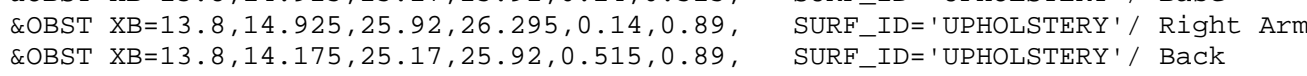

*** GROUP: CHAIR offset $=1.875000,-1.875000,0.000000$ rotate $=270.000000$

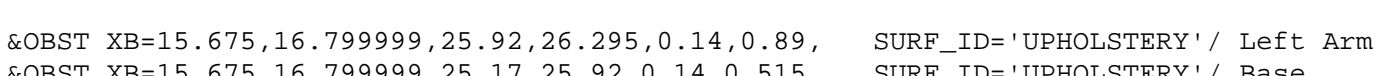

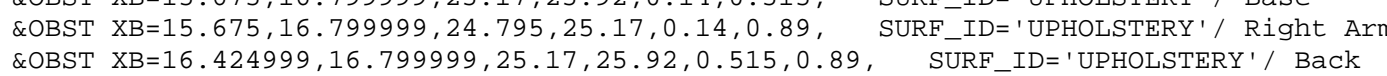

*** GROUP: LR

*** GROUP: COUCH offset $=0.000000,0.000000,0.000000$ rotate $=0.000000$

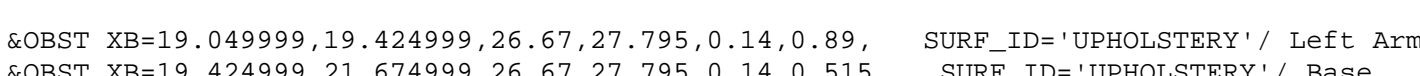
\&OBST XB=21.674999, 22. $049999,26.67,27.795,, 0.14,0.89$
\&OBST XB

*** GROUP: CHAIR offset $=0.000000,-1.875000,0.000000$ rotate $=90.000000$

EOBST XB=19. $049999,20.174999,24.795,25.17,0.14,0.89$, SURF_ID= 'UPHOLSTERY '/ Left Arm \&OBST XB=19.049999, 20.174999, 25.17, 25.92, $0.14,0.515, \quad$ SURF-ID='UPHOLSTERY', Base An

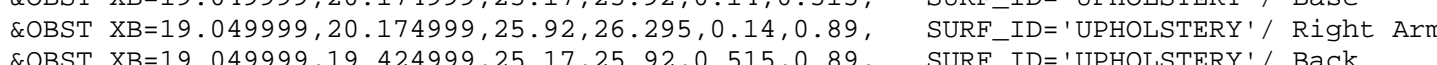

*** GROUP: CHAIR offset $=1.875000,-1.875000,0.000000$ rotate $=270,000000$

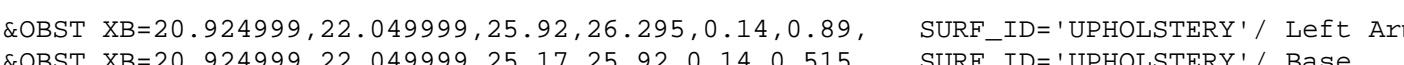

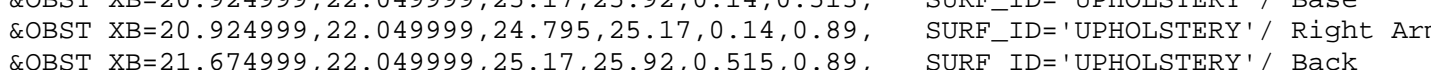

*** GROUP: LR

*** GROUP: COUCH offset $=0.000000,0.000000,0.000000$ rotate $=0.000000$

\&OBST XB $=22.42,22.795,26.67,27.795,0.14,0.89, \quad$ SURF ID = 'UPHOLSTERY ' $/$ Left Arm

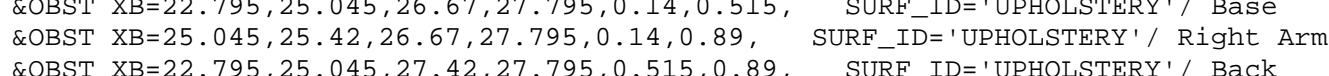

*** GROUP: CHAIR offset $=0.000000,-1.875000,0.000000$ rotate $=90 . .00000$

\&OBST XB=22.42, 23.545, 24.795, 25.17, $.14,0.89$, SURF_ID='UPHOLSTERY'/ Left Arm \&OBST $X \mathrm{XB}=22.42,23.545,25.17,25.92,0.14,0.515, \quad$ SURF-ID= 'UPHOLSTERY' / ' Base SOBST $X \mathrm{BB}=22.42,22.725,25.17,25.92,0.515,0.89, \quad$ SURF-ID=' 'UPHOLSTERY'/ Right Arm

$* * *$ GROUP: CHAIR offset $=1.875000,-1.875000,0.000000$ rotate $=270.000000$

\&OBST XB=24.295, 25.42, 25.92, 26. 295, 0.14, 0.89 , SURF_ID='UPHOLSTERY' ' Left Arm \&OBST XB=24.295, 25.42, 24.795, 25.17, 0.14, 9.89 , SURF_ID=' UPHOLSTERY' / Right Arm

*** GROUP: LR

*** GROUP: COUCH offset $=0.000000,0.000000,0.000000$ rotate $=0.000000$

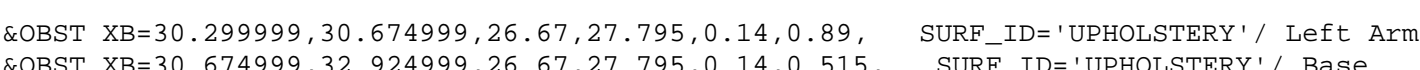
\&OBST XB $=32.924999,33.299999,26.67,27.795,0.14,0.89$, SURF_ID='UPHOLSTERY'/ Right A

*** GROUP: CHAIR offset $=0.000000,-1.875000,0.000000$ rotate $=90.000000$

\&OBST XB=30.299999, 31.424999, 24.795, 25.17, 0.14, 0.89 , SURF_ID='UPHOLSTERY' / Left Arm \&OBST XB=30.299999, 31.424999, 25.17, 25.92, 0.14, 0.515 , SURF_ID='UPHOLSTERY'/ Base

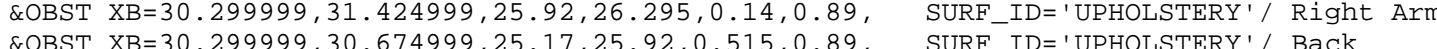

$* * *$ GROUP: CHAIR offset $=1.875000,-1.875000,0.000000$ rotate $=270.000000$

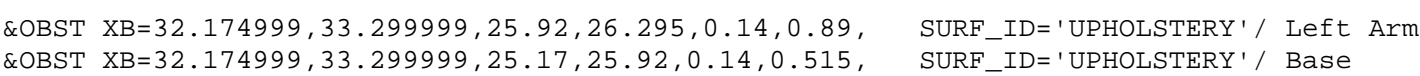
\&OBST XB=32.174999, 33.299999, 24.795, 25.17, $1.14,0.89$, SURF-ID='UPHOLSTERY' ' Right Arm

*** GROUP: LR

** GROUP: COUCH of fset $=0.000000,0.000000,0.000000$ rotate $=0.000000$

\&OBST XB=30.299999, 30.674999, 30. 049999, 31.174999, 0.14, 0.89, SURF_ID='UPHOLSTERY '/ Left Ar \&OBST XB $=30.674999,32.924999,30.049999,31.174999,0.14,0.515, \quad$ SURF $=$ ID= 'UPHOLSTERY'/ Base

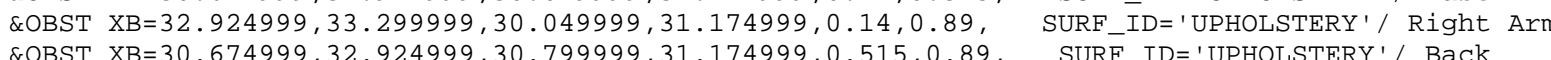

*** GROUP: CHAIR offset $=0.0000000,-1.875000,0.000000$ rotate $=90 ., 000000$

\&OBST XB $=30.299999,31.424999,28.174999,28.549999,0.14,0.89$, SURF_ID=' 'UPHOLSTERY ' / Left Arm

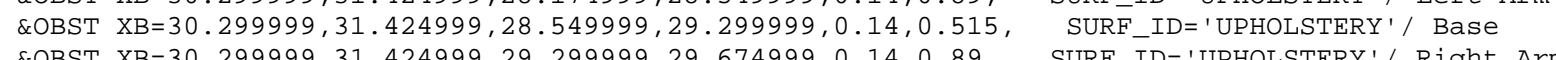


\&OBST XB=30, 299999, 30.674999, 28.549999, 29, 299999, 0.515, 0.89, SURE_ID=' UPHOLSTERY' / Back $* * *$ GROUP: CHAIR of fset $=1.875000,-1.875000,0.000000$ rotate $=270.000000$

\&OBST XB=32.174999, 33. 299999, 29. 299999, 29.674999, $0.14,0.89$, SURF_IDD ' UPHOLSTERY ' / Left Arm \&OBST XB $=32.174999,33.299999,28.174999,28.549999,0.14,0.89$, SURF_ID='UPHOLSTERY'/ Right Ar

*** GROUP: LR

** GROUP: COUCH offset $=0.000000,0.000000,0.000000$ rotate $=0.000000$

\&OBST XB=34.799999, 35.174999, 30. 049999, 31.174999, 0.14, 0.89, SURF_ID='UPHOLSTERY'/ Left Arm \&OBST XB=35.174999, 37.424999, 30. $449999,31.174999,0.14,0.515$, SURF_ID='UPHOLSTERY'/ Base \&OBST XB $=37.424999,37.799999,30.049999,31.174999,0.14,0.89$, SURF_ID='UPHOLSTERY'/ Right Ar

*** GROUP: CHAIR offset $=0.000000,-1.875000,0.000000$ rotate $=90.000000$

\&OBST XB=34.799999, 35. 924999, 28.174999, 28.549999, 0.14,0.89, SURF_ID=' 'UPHOLSTERY' ' Left Ar \&OBST XB=34.799999, 35.924999, 28.549999, 29.299999, $0.14,0.515$, SURF_ID='UPHOLSTERY'/ Base

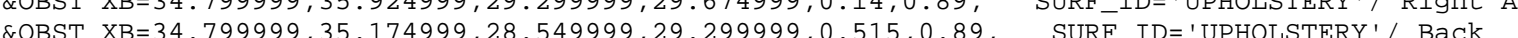
$* * *$ GROUP: CHAIR offset $=1.875000,-1.875000,0.000000$ rotate $=270.000000$

\&OBST XB $=36.674999,37.799999,29.299999,29.674999,0.14,0.89, \quad$ SURF ID=' 'UPHOLSTERY ' $/$ Left Arm \&OBST XB=36.674999, 37.799999, 28.549999, 29.299999, 0.14, 0.515, SURF_ID='UPHOLSTERY'/ Base \&OBST XB=36.674999, $37.799999,28.174999,28.549999,0.14,0.89, \quad$, SURF $=$ ID $=$ 'UPHOLSTERY'/ RIght Ar

*** GROUP: LR

*** GROUP: COUCH offset $=0.000000,0.000000,0.000000$ rotate $=0.000000$

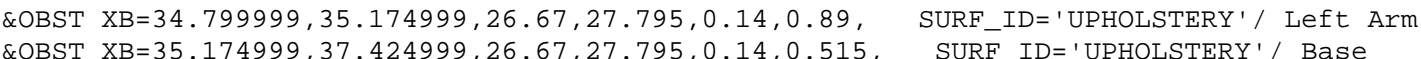
\&OBST XB=37.424999, 37.799999, 26.67, 27.795, 0.14, 0.89 , SURF_ID= 'UPHOLSTERY'/ Right A

*** GROUP: CHAIR offset $=0.0000000,-1.875000,0.000000$ rotate $=90.0000000$

$\begin{array}{ll}\text { \&OBST XB }=34.799999,35.924999,24.795,25.17,0.14,0.89, & \text { SURF_ID='UPHOLSTERY' } / \text { Left Arm } \\ \text { \&OBST XB }=34.799999,35.924999,25.17,25.92,0.14,0.515, & \text { SURF-ID='UPHOLSTERY' }\end{array}$

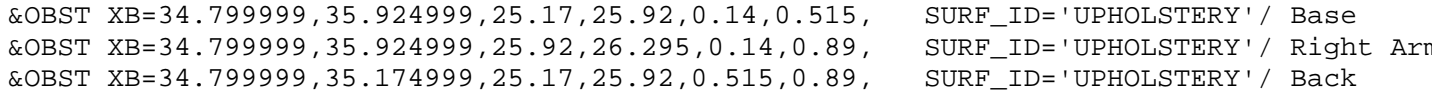

*** GROUP: CHAIR offset $=1.875000,-1.875000,0.000000$ rotate $=270.000000$

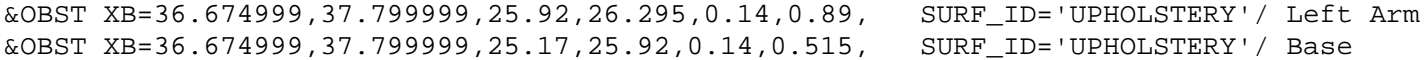
COBST XB=36.674999,37.799999, 24.795, 25.17, $0.14, \theta .89$, SURF-ID='IPHOLSTERY'/, Right Ar

\section{*** GROUP: COUCH2270}

*** GROUP: COUCH2 offset $=0.000000,0,000000,0.000000$ rotate $=270.000000$

\&OBST XB=51.299999, 52.424999, 19.545, 19.92, $0.14,0.89$, SURF_ID=' 'UPHOLSTERY' ' Left Ar

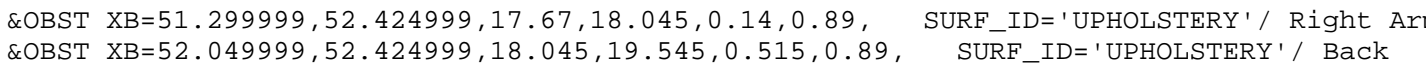

*** GROUP: COUCH2270

*** GROUP: COUCH2 offset $=0.000000,0.000000,0.000000$ rotate $=270.000000$
ROBST XB $=51.299999,52.424999,22.924999,23.299999,0.14,0.89$, SURF ID ='UPHOLSTERY '/ Left Arm \&OBST XB $=51.299999,52.424999,21.424999,22.924999,0.14,0.515$, SURF_ID='UPHOLSTERY'/ Base \&OBST $X B=51.299999,52.424999,21.04999$

*** GROUP: COUCH2270

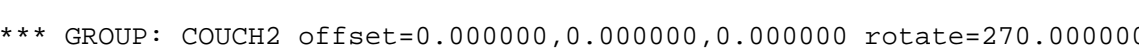

\&OBST XB=51.299999, 52.424999, 26.295, 26.67, 0.14, 0.89 , SURF_ID=' 'UPHOLSTERY' ' Left Arm

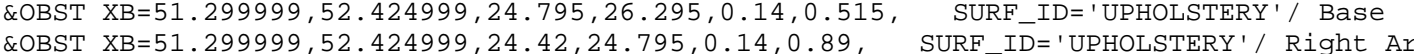
\&OBST XB=52.049999, 52.424999, 24.795, 26.295, 0.515, 0.89, SURF_ID=' UPHOLSTERY'/ Back

$* * *$ GROUP: LOVESEAT270

** GROUP: LOVESEAT off $\mathrm{set}=0.000000,0 . .000000,0.000000$ rotate $=270 . .000000$

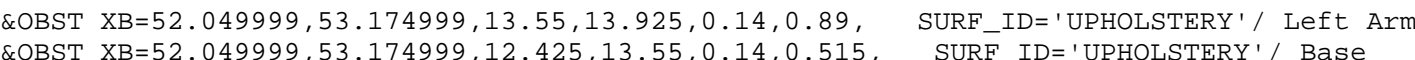

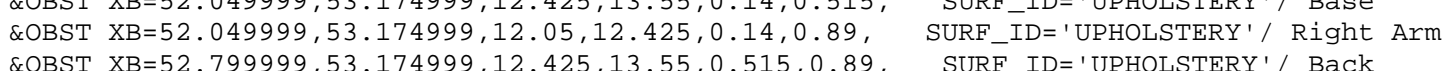

$* * *$ GROUP: LOVESEAT270

** GROUP: LOVESEAT offset $=0, .000000, \Theta ., 000000,0.000000$ rotate $=270,000000$

\&OBST XB=53.540001, 54.665001, 13.55, 13.925, 0.14, 0.89 , SURF_ID=' 'UPHOLSTERY ' / Left Ar \&OBST XB $=53.540001,54.665001,12.05,12.425,0.14,0.89$, SURF ID=' 'UPHOLSTERY'/ Right

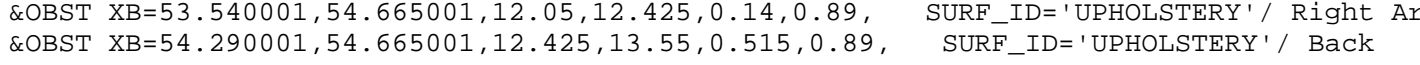

*** GROUP: LOVESEAT270

*** GROUP: LOVESEAT of fset $=0.000000,0.000000,0.000000$ rotate $=270.000000$

\&OBST XB=52.049999, 53.174999, 15.8, 16.174999, 0.14, 0.89 , SURF_ID='UPHOLSTERY'/ Left Ar \&OBST XB=52.049999, $53.174999,14.675,15.8,0.14,0.515$, SURF_ID=' 'UPHOLSTERY'/ Base\&OBST XB $=52.049999,53.174999,14.3,14.675,0.14,0.89$, SURF_ID='UPHOLSTERY'/ Right Ar

$* * *$ GROUP: LOVESEAT270

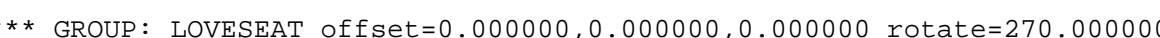

\&OBST XB=53.549999, 54.674999, 15.8,16.174999, $0.14,0.89$, SURF_ID=' 'UPHOLSTERY' / Left Arm \&OBST XB $=53.549999,54.674999,14.3,14,675,0.14,0.89$, SURF ID=' UPHOLSTERY'/ Right Arm \&OBST XB=54.299999, 54.674999, 14.675, 15.8, 0.515, 0.89, SURF_ID=' UPHOLSTERY'/ Back

*** GROUP: COUCH offset $=38.549999,23.299999,0.000000$ rotate $=0.000000$

\&OBST XB=38.549999, 38.924999, 23.299999, 24.424999, $0.14,0.89$, SURF_ID=' 'UPHOLSTERY ' / Left Arm \&OBST XB=41.174999, 41.549999, 23. 299999, 24.424999, $0.14,0.89$, SURF_ID=' 'UPHOLSTERY'/ Right Ar \&OBST XB=38.924999, 41.174999, 24.049999, 24.424999, $0.515,0.89$, SURF_ID=' UPHOLSTERY' / Back

*** GROUP: COUCH offset $=34.799999,23.299999,0.000000$ rotate $=0.000000$

\&OBST XB=34.799999, 35.174999, 23.299999, 24.424999, $0.14,0.89$, SURF_ID=' 'UPHOLSTERY ' / Left Arm \&OBST XB $=37.424999,37.729999,23.299999,24,424999,0.14,0.89$, SURF ID='UPHOLSTERY'/Right Ar \&OBST XB=35.174999, 37.424999, 24. 049999, 24.424999, $0.515,0.89$, SURF_ID= 'UPHOLSTERY' / Back

\section{Hallway}

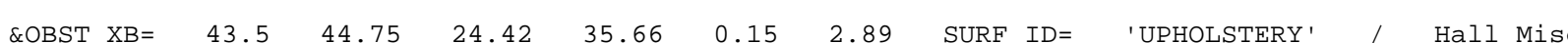

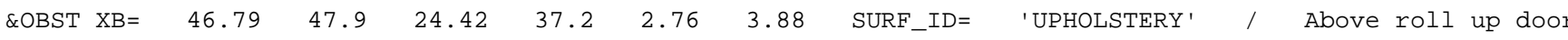


Wall RHS Addition

\&OBST $\quad \mathrm{XB}=48.4,49.42,9.98,16.54,0,2.0, \quad$ SURF_ID $=$ UPHOLSTERY , \&OBST $\mathrm{XB}=48.4,49.42,16.54,19.17,0,2.0, \quad$ SURF_ID $=$ UPHOLSTERY ,

$++++$

obstructions

\&OBST XB=-8.35661671323343, 66. $240157480315,-6.51511303022606,28.9560579121158,-0.304800609601219,-$
0.304800609601219 SURF_ID='A-CETL-MAIN-0, $/ 1$

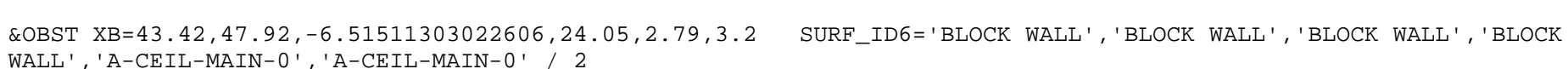

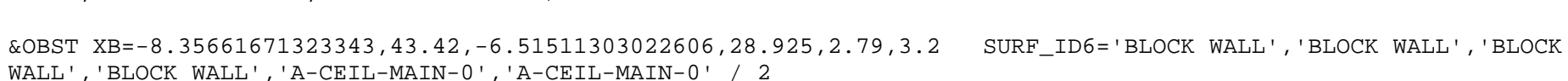

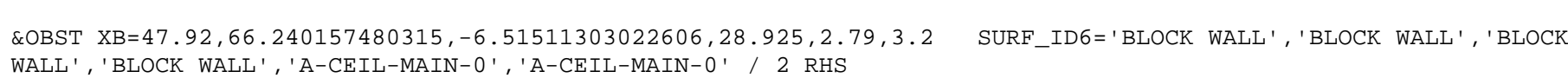

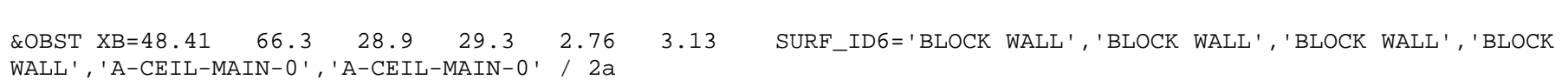

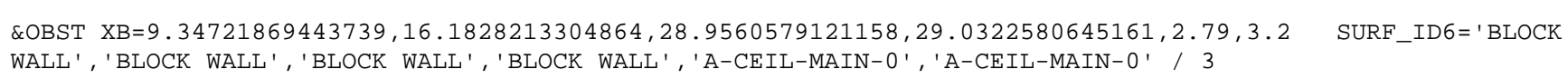

\&OBST XB=9. $34721869443739,48.0822961645923,28.9560579121158,32.9438658877318,-0.304800609601219,-0.304800609601219$
SURF_ID6=' BLOCK WALL', 'BLOCK WALL', 'BLOCK WALL', 'BLOCK WALL', 'A-CEIL-MAIN-O', 'A-CEIL-MAIN-0' / 4

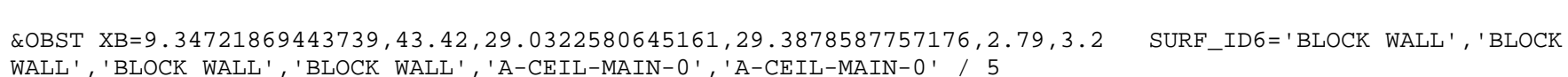

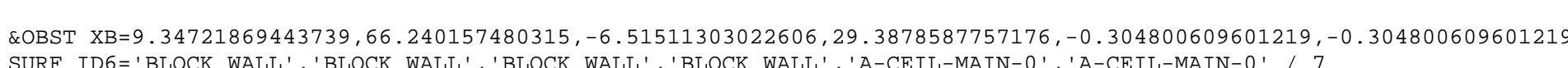

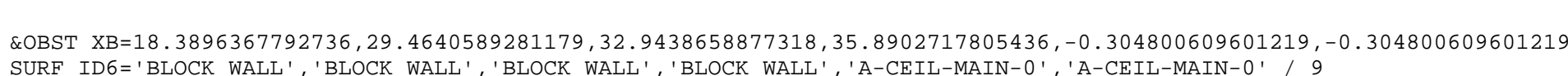

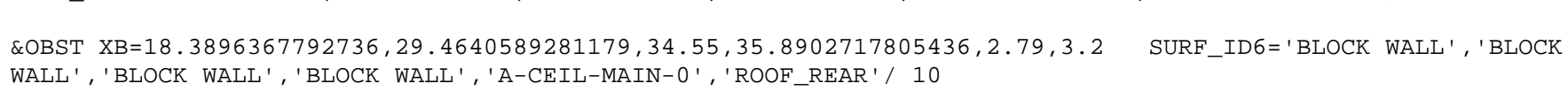

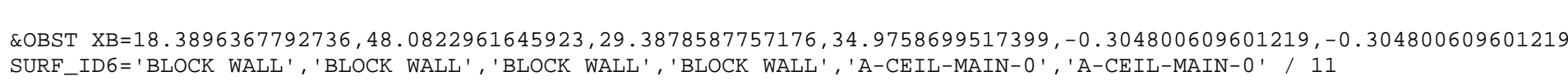

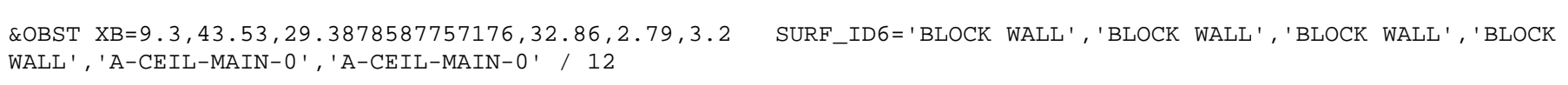

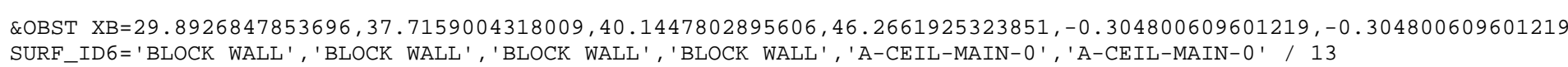

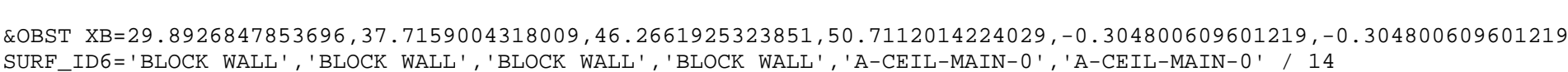

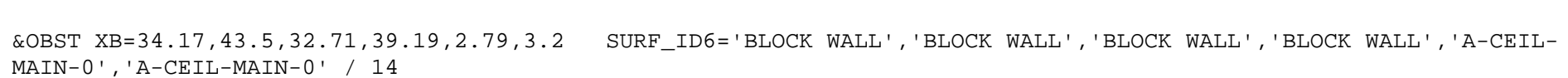

\&OBST XB=34. $1471932943866,48.0822961645923,29.3878587757176,38.9509779019558,-0.304800609601219,-0.304800609601219$
SURF ID= 'A-CEIL-MATN-0' $/ 17$

\&OBST XB=34. 2360934721869, 46.8091186182372, 38.9509779019558, 46.2661925323851, - $0.304800609601219,-0.304800609601219$

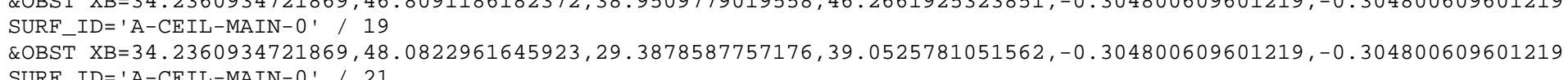

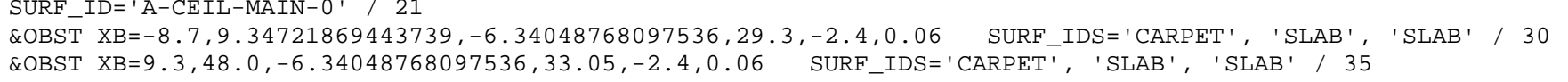

\&OBST XB=48. $0822961645923,66.3,-6.4,29.8,-2.4,0.06$ SURF_IDS=' CARPET' ', 'SLAB', 'SLAB' / 36
\&OBST XB=47.8790957581915,47.9806959613919,-3.97034544069088, $-1.53194056388113,-2.4,0.06$ SURF_IDS=' CARPET',

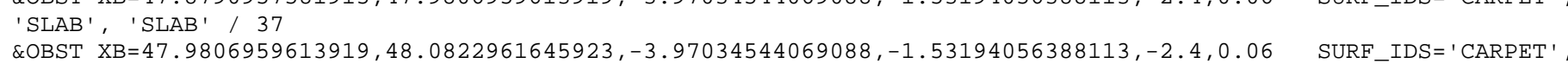

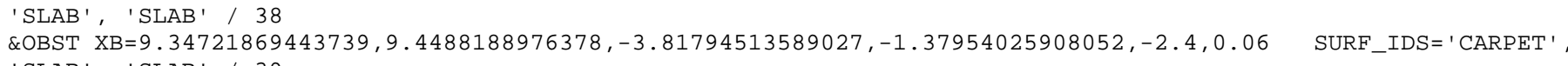

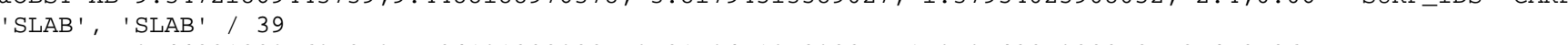

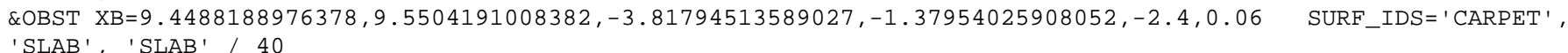

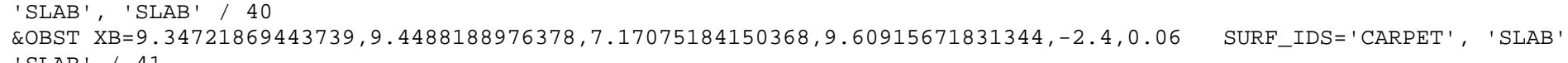
'SLAB' $/ 41$
\&OBST XB $=9.4488188976378,9.5504191008382,7.17075184150368,9.60915671831344,-2.4,0.06 \quad$ SURF_IDS=' 'CARPET ', ' 'SLAB',

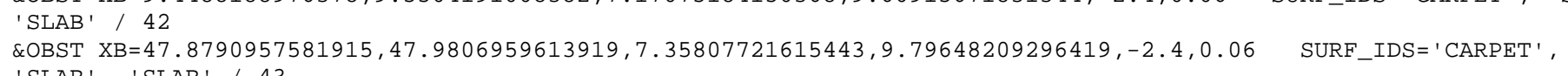

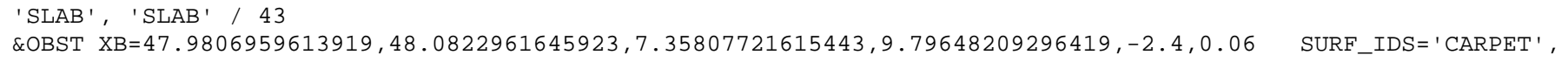
'SLAB', 'SLAB' 144
\&OBST XB=-0.592138684277369, $1.84626619253239,17.5514351028702,17.738760477521,-2.4,0.06 \quad$ SURF_IDS=' 'CARPET',

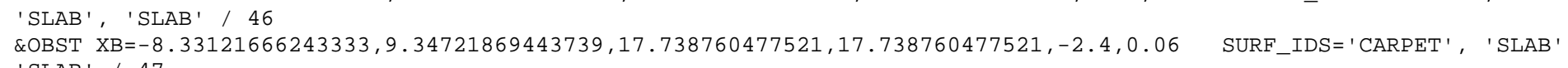
'SLAB' $/ 47$
\&OBST $X \mathrm{~B}=9.34721869443739,9.4488188976378,18.9484378968758,21.3868427736855,-2.4,0.06 \quad$ SURF_IDS=' 'CARPET' , 'SLAB', EOBST XB=9.4488188976378, 9.5504191008382, 18.9484378968758, 21.3868427736855, $-2.4,0.06 \quad$ SURF_IDS=' 'CARPET ', ' 'SLAB',

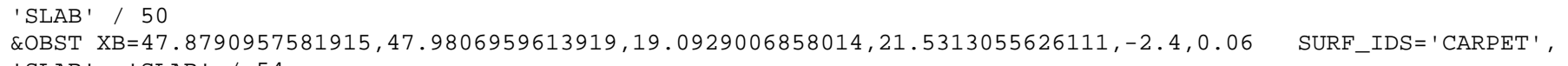
'SLAB' ' 'SLAB' 54
\&OBST XB $47.9806959613919,48.0822961645923,19.0929006858014,21.5313055626111,-2.4,0.06 \quad$ SURF_IDS=' 'CARPET' ',

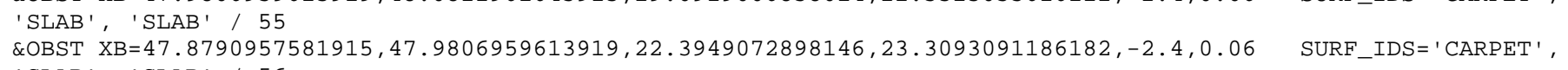

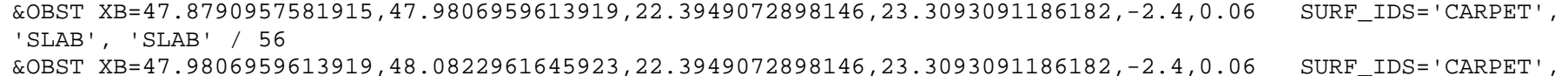
'SLAB', 'SLAB' / 59 9 \&OBST 'XB=26.9558039116078, 29.3211836423673, 24.1046482092964, 24. 4681864363729, $-2.4,0.06 \quad$ SURF_IDS=' 'CARPET ',

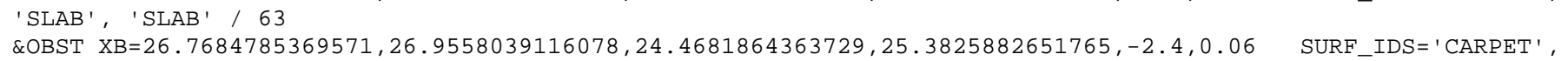
'SLAB' ' 'SLAB' / 67
\&OBST XB $\mathrm{X}=47.8790957581915,47.9806959613919,24.7825120650241,25.6969138938278,-2.4,0.06 \quad$ SURF_IDS=' 'CARPET',

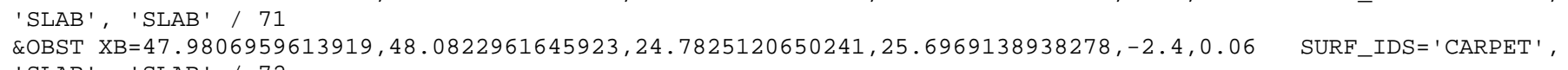

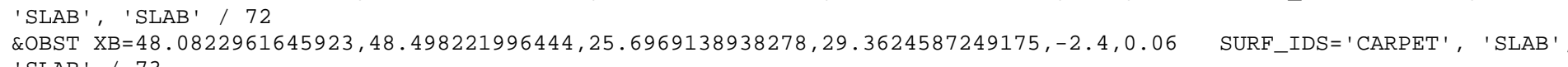
\&OBST XB=23. 9538354076708, 25. $0206375412751,28.8957327914656,29.0830581661163,-2.4,0.06 \quad$ SURF_IDS=' 'CARPET ' , 'SLAB' ' 'SLAB' $/ 78$
CLBST $X B=18.5896621793244,18.7769875539751,29.0830581661163,30.1498602997206,-2.4,0.06 \quad$ SURF_IDS=' 'CARPET ',

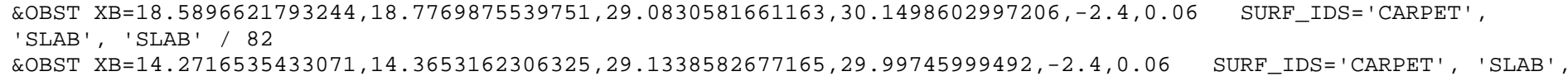

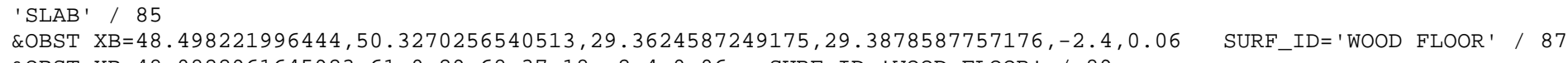

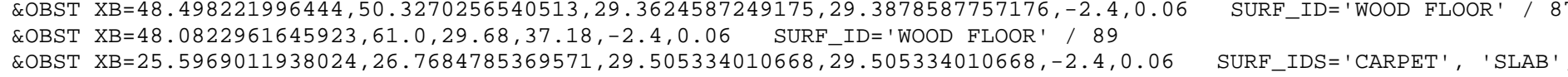

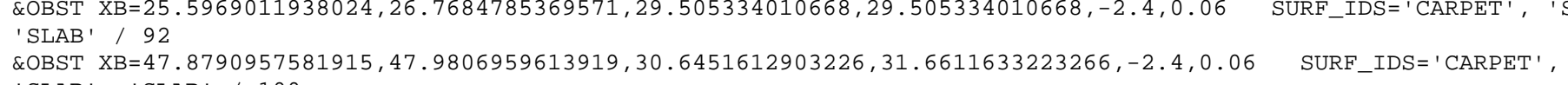

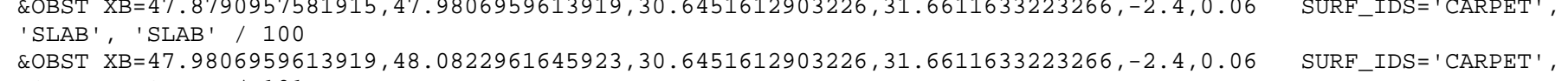

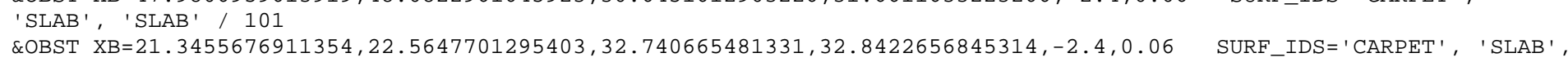

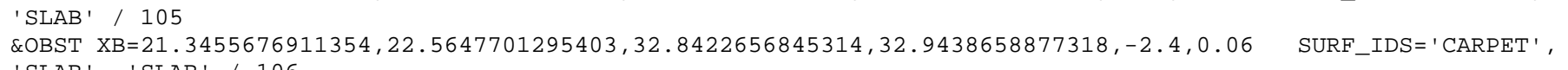

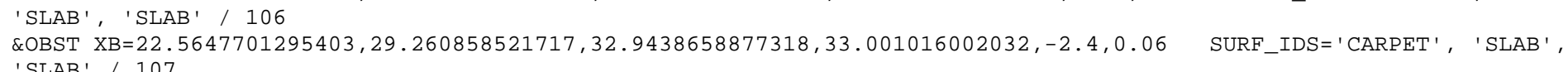

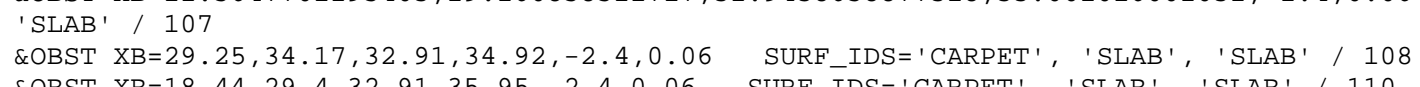

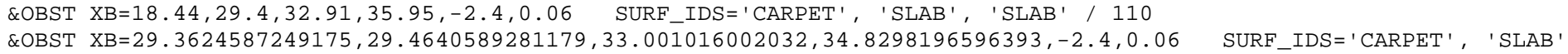
'SLAB' 1113
\&OBST XB=47.8790957581915, $47.9806959613919,33.9471678943358,36.3855727711455,-2.4,0.06$ SURF_IDS=' 'CARPET' ,

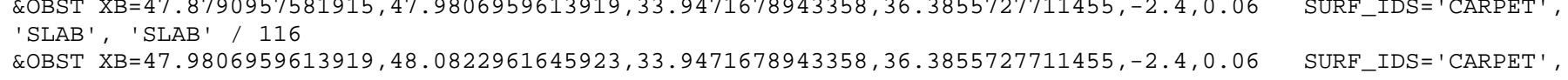

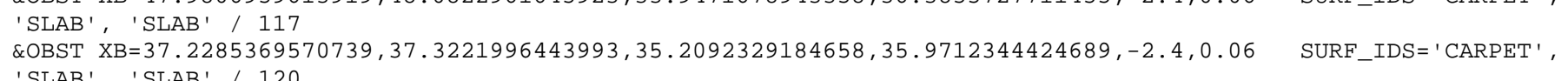

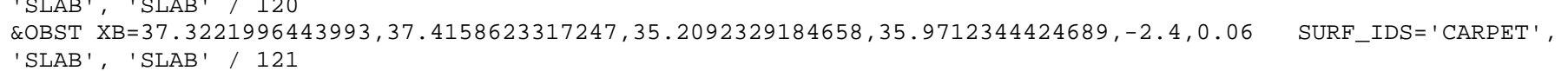


\&OBST XB=34.3503937007874, 37.2285369570739, 36.1823723647447, 36.1823723647447, -2.4, ๑.06 SURF_IDS=' CARPET' ,

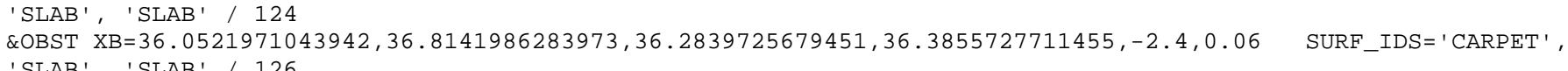
COBST 'XB=37. $4237998475997,47.8790957581915,36.2839725679451,36.3855727711455,-2.4,0.06 \quad$ SURF_IDS= 'CARPET ',
'SLAB', 'SLAB' $/ 127$

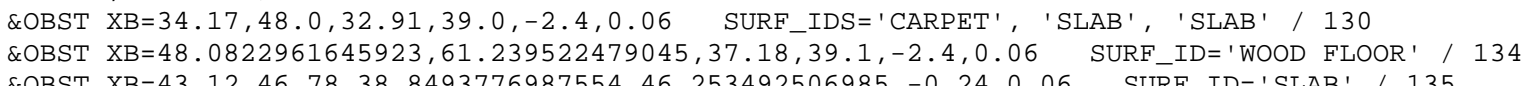

\&OBST XB=46.8091186182372, 61.239522479045, 39.0525781051562, 46.1772923545847, -24, 0.06 SURF_ID='WOOD FLOOR' / 137

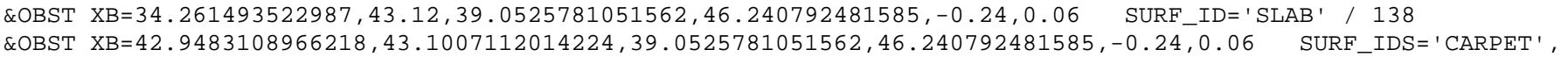

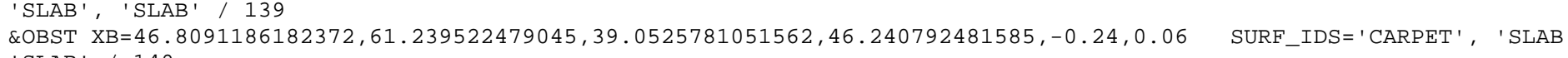

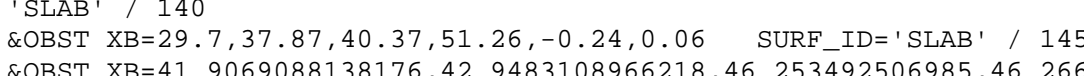

$\begin{array}{ll} & \\ & \end{array}$

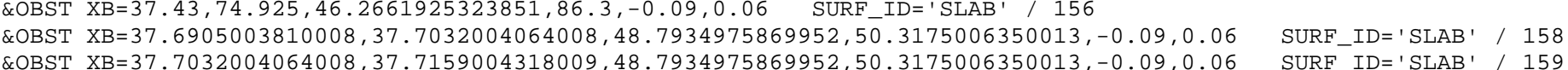

\&OBST XB $=-8.33121666243333,-8.22009144018288,-6.34048768097536,-6.34048768097536,0,2.46697993395987 \quad$ /SURF_ID='A-
WALL-MATN-WS' $/ 163$

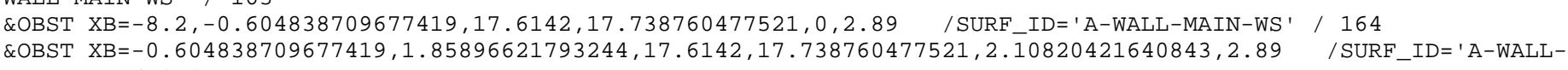

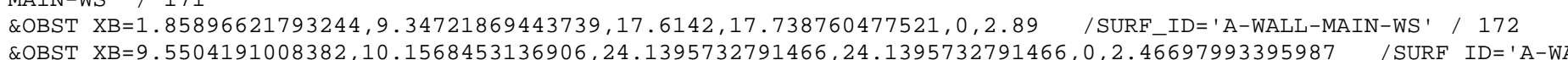

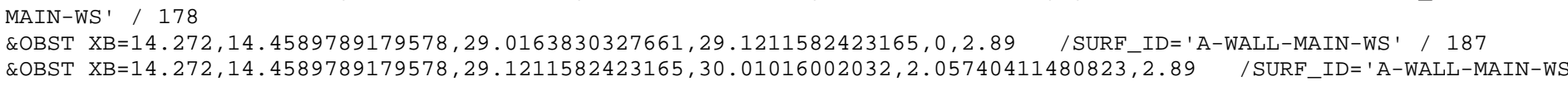

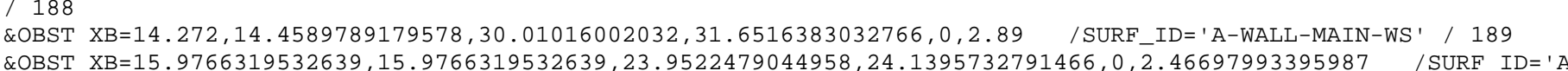
WALL -MAIN-WS' $/ 192$
\&OBST XB=16. $0528321056642,16.0528321056642,28.8290576581153,29.0163830327661,0,2.46697993395987$ ISURF_ID= 'A-
WALL

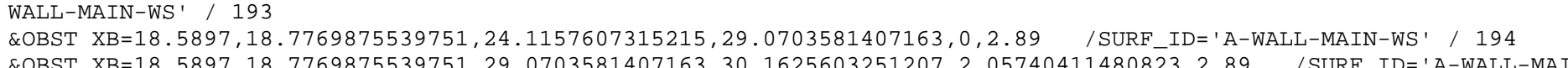

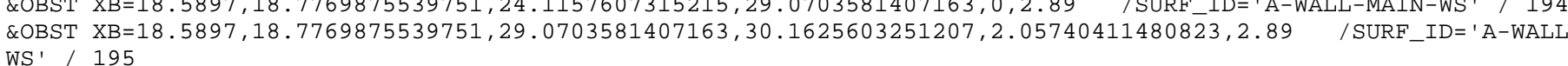

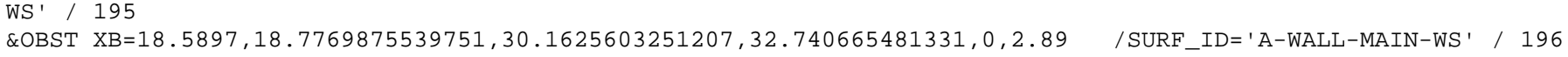

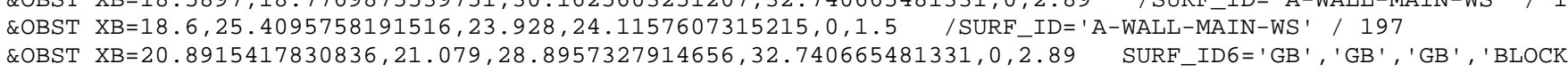

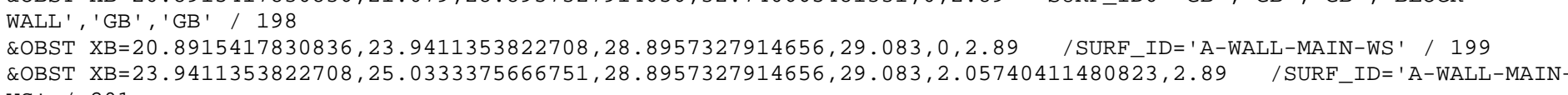

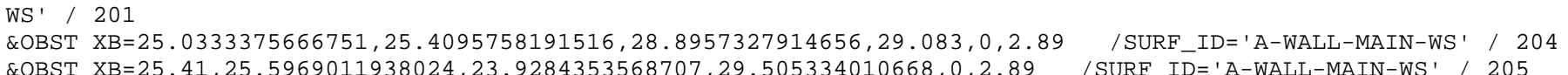

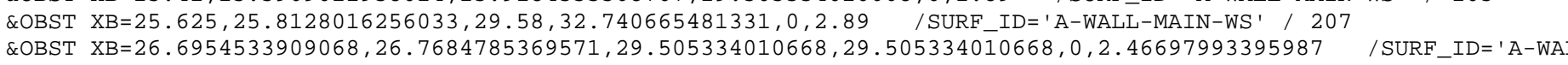
\&OBST XB=28. $0924561849124,28.0924561849124,26.0890271780544,26.2763525527051,0,2.46697993395987$ /SURF_ID= 'AWALL-MAIN-WS' / 216
EOBST XB $=28.0924561849124,29.3211836423673,26.0890271780544,26.276,0,2.89 \quad$ /SURF_ID= 'A-WALL-MAIN-WS' / 217

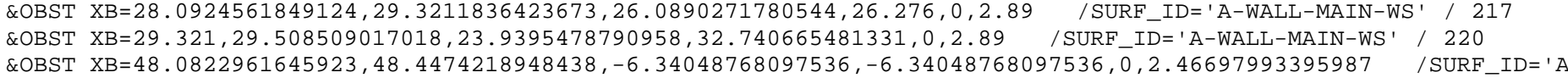
WALL-MAIN-WS' $/ 237$
COBST XB=55.1943103886208, 55.1943103886208, 15.3956057912116, 15.5829311658623, $0,2.46697993395987$ /SURF_ID='A

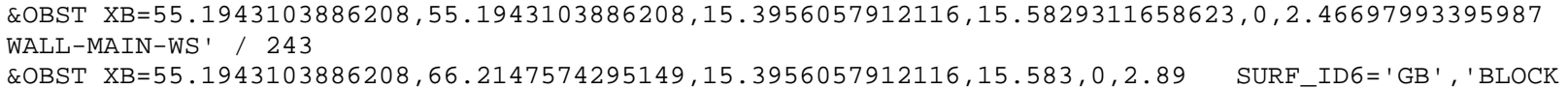

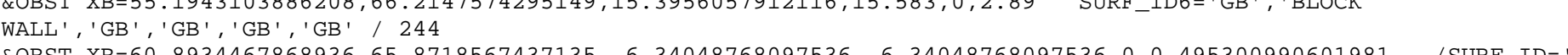
WALL-MAIN-WS' $/ 246$
COBST XB=-8.35661671323343, $-8.22009144018288,-6.55,-6.4,-0.304800609601219,3.5$ SURF_ID6='BLOCK WALL', 'BLOCK WALL', 'BLOCK WALL', 'GB', 'BLOCK WALL', 'BLOCK WALL', 249 , 24.3040

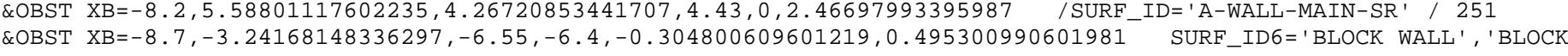
WALL', 'BLOCK WALL', 'GB' ', 'BLCCK WALL', 'BLOCK WALL' ' 252
ROBST' XB=-8.22009144018288, $-3.24168148336297,-6.55,-6.4,2.20980441960884,2.46697993395987$ SURF_ID6='BLOCK

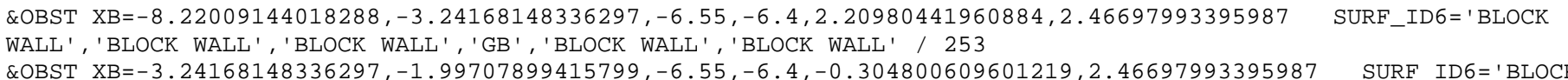
WALL', 'BLOCK WALL', 'BLOCK WALL', 'GB', 'BLOCK WALL', 'BLOCK WALL', 254

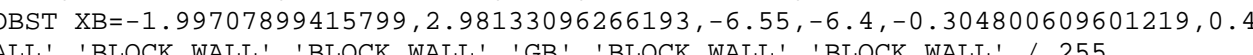
\&OBST' XB=-1.99707899415799, 2.98133096266193, $,-6.55{ }^{\prime},-1.4,2.20980441960884,2.46697993395987$ SURF_ID6=' BLOCK
EOBST XB=2.98133096266193, 4.24022098044196, $-6.55,-6,4,-0.304800609601219,2.46697993395987$ SURF ID6=' BLOCK

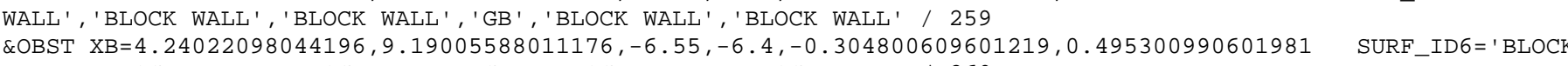
WALL', 'BLOCK WALL', 'BLOCK WALL', 'GB', 'BLOCK WALL', 'BLOCK WALL' $/ 260$

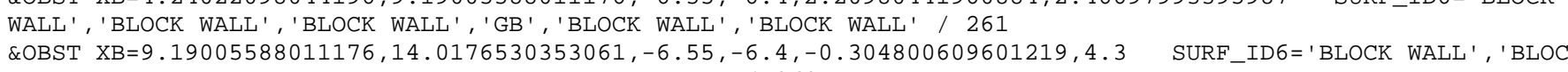
WALL', 'BLOCK WALL', 'GB', 'BLOCK WALL', 'BLOCK WALL' 1 262

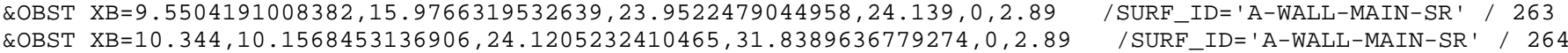

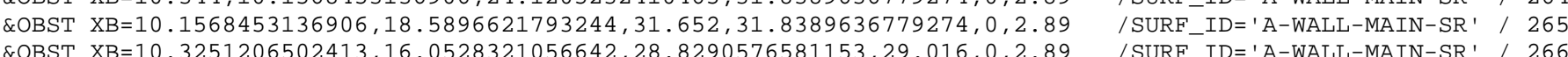

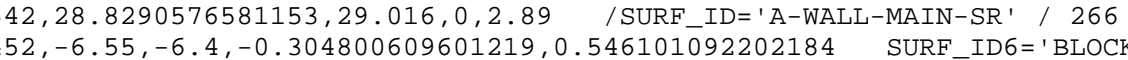
WALL', 'BLOCK WALL', 'BLOCK WALL', 'GB' ', 'BLOCK WALL', 'BLOCK WALL' 1267
\&OBST' XB $=14.0176530353061,23.7966725933452,-6.55,-6.4,2.20980441960884,4.3$
SURF_ID6= 'BLOCK WALL', ' 'BLOCK \&OBST XB=23.7966725933452, 24.9396748793498, -6.55, $-6.4,-0.304800609601219,4.3$ SURF_ID6=' BLOCK WALL', 'BLOCK WALL', 'BLOCK WALL', 'GB', 'BLOCK WALL', 'BLOCK WALL' 279 WAUL' \&OBST XB=24.9396748793498, 25.8286766573533, $-6.55,-26.4,2.20980441960884,4.3$ SURF_ID6= 'BLOCK WALL' ' 'BLOCK

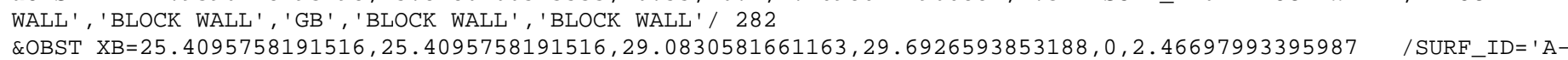
WALL-MAIN-SR' $\quad 285$
OOBST XB=25.4095758191516, 25.6254762509525, 29.505, 29.6926593853188, $0,2.89 \quad$ /SURF_ID='A-WALL-MAIN-SR' / 286 \&OBST XB=25.7937515875032, 25.8572517145034, 29.6926593853188, 29,6926593853188, $0,2.46697993305987$ /SURF ID ='A-

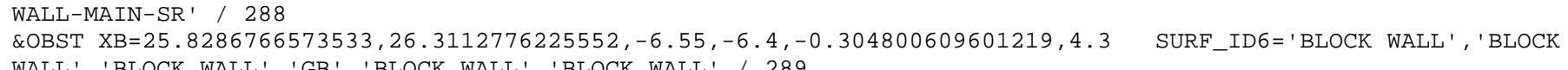

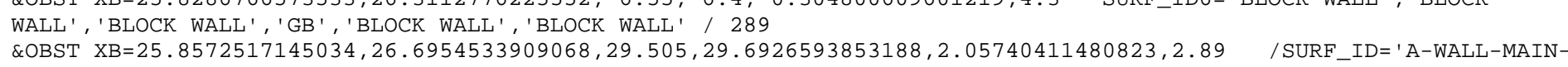
SR' ' 290
\&OBST XB=26.3112776225552, $28.1654813309627,-6.55,-6.4,-0.304800609601219,0.15$ SURF_ID6='BLOCK WALL' , 'BLOCK WALL', 'BLOCK WALL', 'GB', 'BLOCK WALL', 'BLOCK WALL' 1291 . 20.4

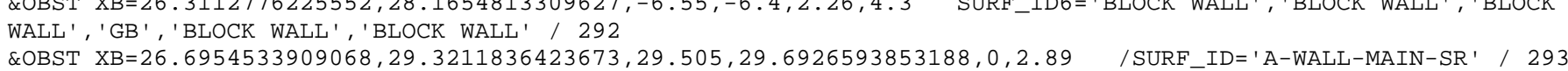

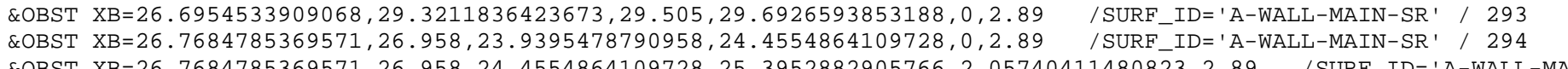
SR' $/ 2$ ' 295
\&OBST XB=26.7684785369571, 26. $958,25.3952882905766,29.5243840487681,0,2.89 \quad$ /SURF_ID= 'A-WALL-MAIN-SR' / 296

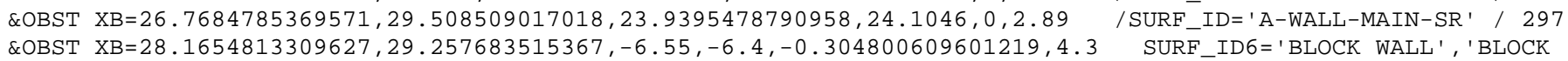
WALL' ', 'BLOCK WALL', 'GB', 'BLOCK WALL', 'BLOCK WALL' ' 1300
\&OBST XBE29.25768351536', 31. $1118872237744,-6.55,-6.4,-0.304800609601219,0.15$ SURF_ID6= 'BLOCK WALL', 'BLOCK \&OBST' XB=29.257683515367, 31. 1118872237744, -6.55, -6.4,2.26, 4.3 SURF_IDG=' BLOCK WALL', 'BLOCK WALL', ' BLOCK \&OBST XB=31.1118872237744,31.5944881889764,-6.55,-6.4,-0.3048006

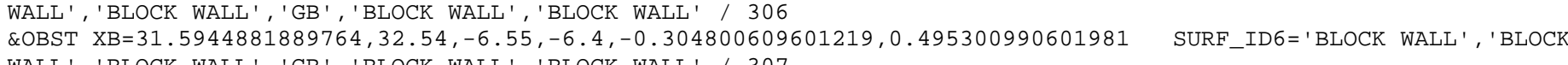

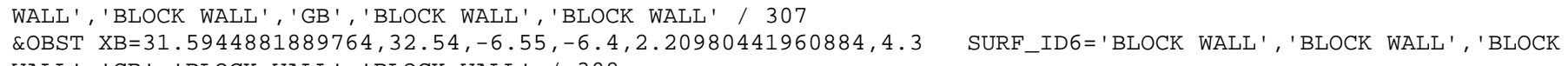

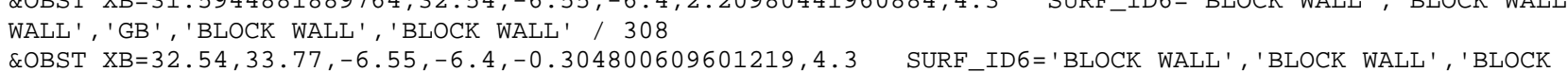

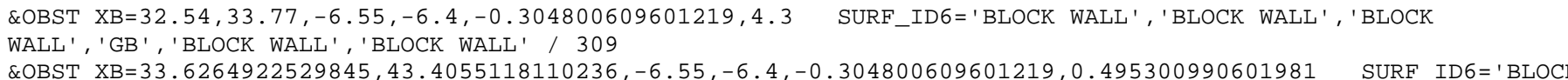
\&OBST' XB=33.36, 43.4055118110236, $-6.55,-6.4,2.20980441960884,4.3$ SURF_ID6=' 'BLOCK WALL', 'BLOCK WALL', 'BLOCK \&OBST $X \mathrm{XB}=34,3503937007874,37,4158623317247,34,3281686563373,34,515,0,2.89$ /SURE ID='A-WALL-MATN-SR'

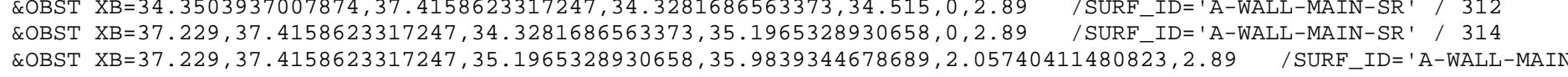

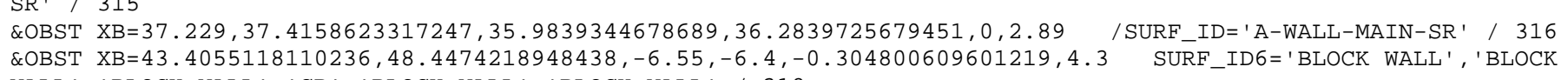
\&OBST' $X \mathrm{X}=43.12,43.65,23.8506477012954,38.8493776987554,0,2.89 \quad$ /SURF ID= 'A-WALL-MAIN-SR' / 319 QOBST XB=48.4474218948438, 53.4258318516637,-6.55,-6.4,-0.304800609601219, 0.495300990601981 SURF_ID6='BLOCK

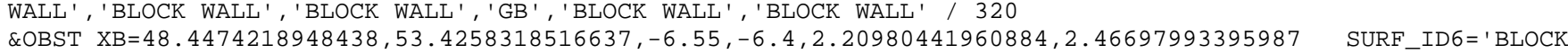

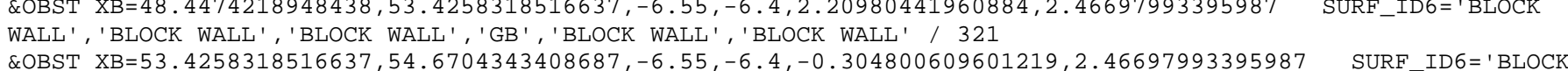

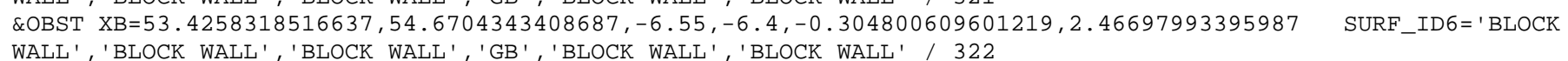

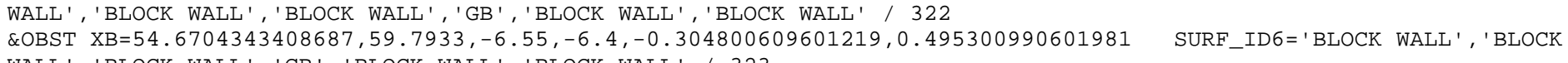
ROBST' XB=54.6704343408687, 59.7933, -6. 55, -6.4, 2. 20980441960884, 2.46697993395987 SURF_ID6='BLOCK WALL', 'BLOCK 


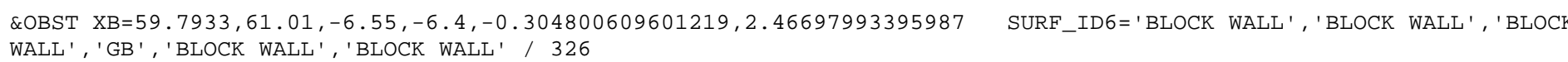

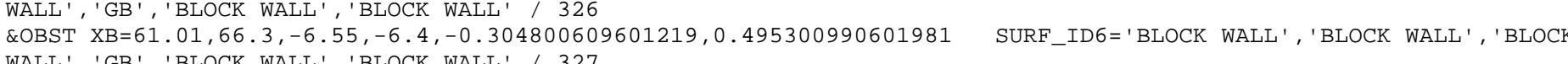

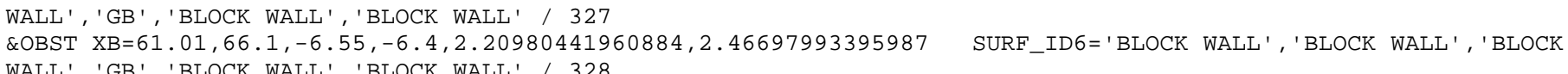
\&OBST' XB=-8.7, $-8.2,-6.7,6.83737617475235,-0.0063500127000254,3.512$ SURF_ID6=' 'BLOCK WALL', 'GB' , 'BLOCK WALL', 'BLOCK WALL', 'BLOCK WALL', 'BLOCK WALL' 1330
\&OBST XB=-8.7, $-8.2,6.83737617475235,8.69157988315977,2.15990431800864,3.512$ SURF_ID6='BLOCK WALL', ' 'GB' , 'BLOCK

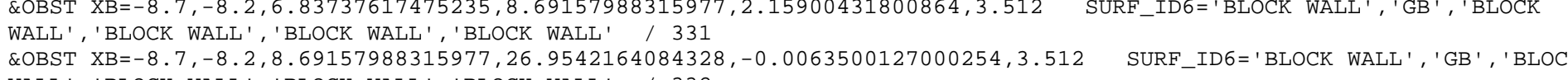
WALL', 'BLOCK WALL', 'BLOCK WALL', 'BLOCK WALL' 1332
ROBST' XB=-8.7 $-8.2,26.9542164084328,28.8084201168402,2.15900431800864,3.512$ SURF_ID6= 'BLOCK WALL' , 'GB' ' 'BLOCK

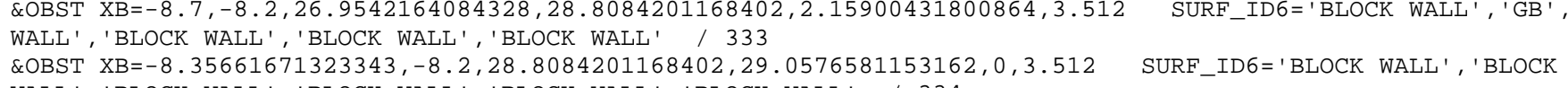

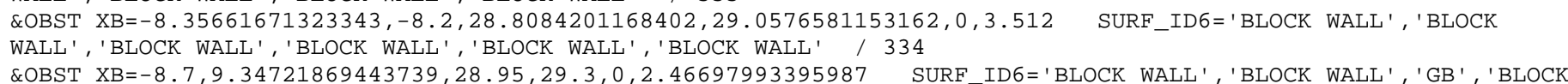

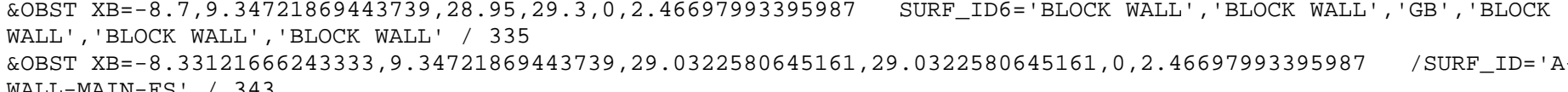

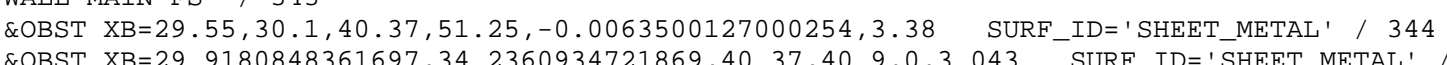

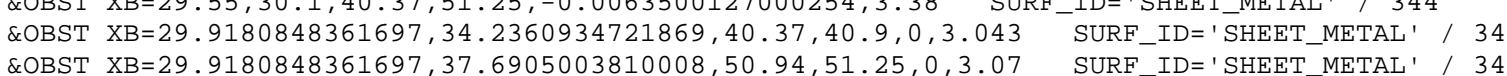
\&OBST XB $=34.2360934721869,34.5,40.1701803403607,46.2661925323851,0,3.38$ SURF_ID='SHEET_METAL'/ 351

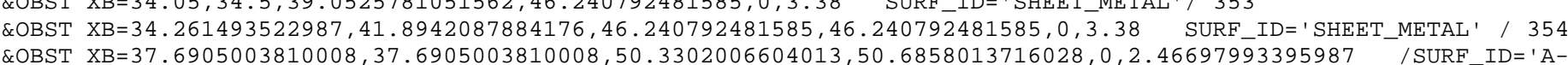

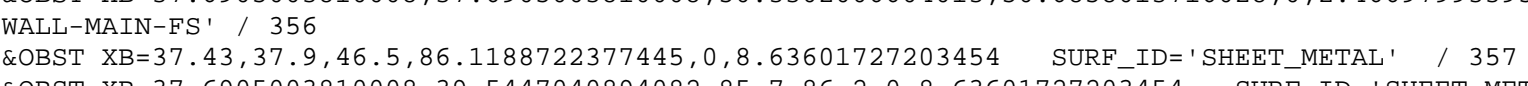

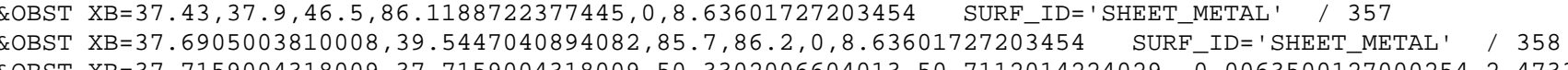
\&OBST $X B=37.7159004318009,39.5447040894082,86.0934721869444,86.0934721869444,0,8.63601727203454$ /SURF_ID= 'AWALL-MAIN-FS' 362
\&OBST XB=39.5447040894082, 40.4845059690119, 85.7, 86. 2, 2.05740411480823, 8.63601727203454 SURF_ID=' SHEET_METAL' / 364
RoBST XB $=39.5447040894082,40.4845059690119,86.1188722377445,86.1188722377445,2.05740411480823,8.63601727203454$

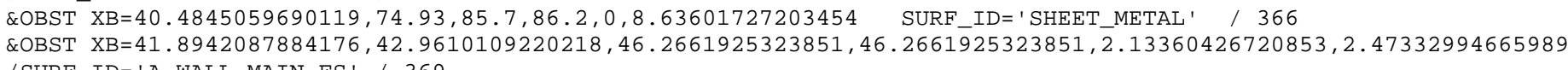
${ }^{2}$ COBST ${ }^{-} X \mathrm{XB}=42.9610109220218,43.1007112014224,46.240792481585,46.240792481585,0,2.46697993395987$ /SURF_ID= 'A-WALLMAIN-FS' $/ 370$
\&OBST XB $42.9610109220218,43.1261112522225,46.2661925323851,46.2661925323851,-0.0063500127000254,2.47332994665989$ \&OBST XB=43.1007112014224, 43.1007112014224, 39.0525781051562, 46.240792481585, $0,3.68$ SURF_ID=' SHEET_METAL' / 372
\&OBST XB $33.1261112522225,43.1261112522225,39.0525781051562,46.2661925323851,0,3.68$
/SURF_ID='A-WALL-MAIN-FS'

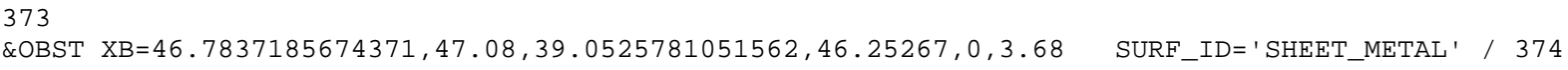

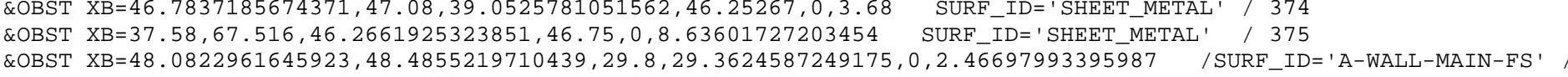
\&OBST XB=48. $0822961645923,48.4855219710439,29.3878587757176,29.3878587757176,-0.0063500127000254,2.47332994665989$

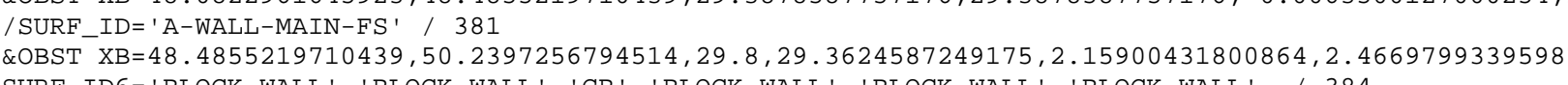

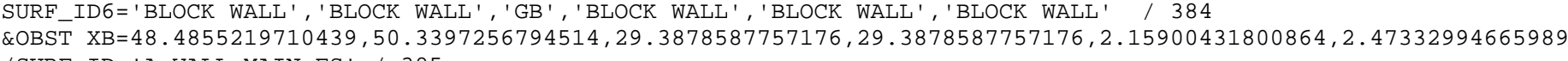
ISURF_ID='A-WALL-MAIN-FS' $/ 385$
COBST $X \mathrm{~B}=50.27,66.3,29.2,29.8,0,2.46697993395987$
SURF_ID6='BLOCK WALL', 'BLOCK WALL', 'GB' ', 'BLOCK WALL', 'BLOCK

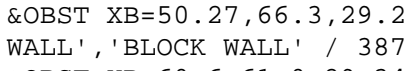

\&OBST XB=60.6, 61. $0,29.34,34.33,0.15,3.67$ SURF_ID=' 'SHEET_METAL' / 392

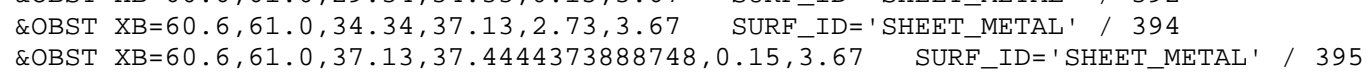

\&OBST XB $=61.05,61.239522479045,37.34,40.51,0.15,2.417$ SURF_ID= 'SHEET_METAL' / 398
\&OBST XB $=61.05,61.239522479045,40.51,41.54,0.15,1.32$, SURF_ID = 'SHEET_METAL'

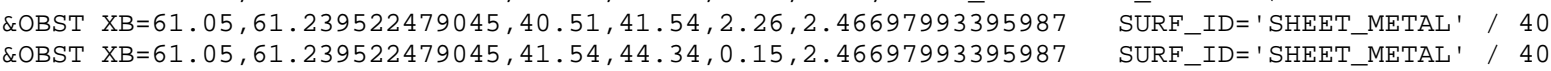

$\begin{array}{ll}\text { \&OBST XB=61. } 05,61.239522479045,41.54,44.34,0.15,2.46697993395987 & \text { SURF_ID=' 'SHEET_METAL' / } 401 \\ \text { \&OBST XB=61.05, } 61.239522479045,44.34,46.1899923799848,2.41,3.04 & \text { SURF_ID=' SHEET_METAL' / } 402\end{array}$

\&OBST XB=61.239522479045, 61.239522479045, 46.1899923799848, 46.240792481585, 0, 2.46697993395987 /SURF_ID='A-WALL-

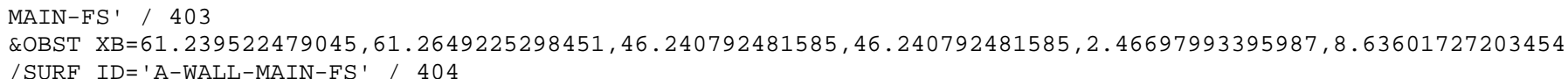
\&OBST XB=61.2649225298451, 61.2649225298451, 46.1899923799848, 46.240792481585, 0, 2.46697993395987 ISURF_ID='A-WALL-
ROBST XB=65.9, 66. 240157480315, - $6.52781305562611,29.3878587757176,-0.0063500127000254,3.512$ SURF_IDG=' GB' ', 'BLOCK

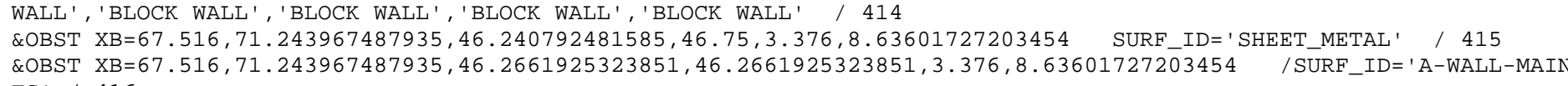
\&OBST XB $=71.243967487935,71.7,46.240792481585,46.75,0,8.63601727203454$ SURF_ID=' SHEET_METAL' / 417 COBST XB $71.7,72.9,46.240792481585,46.75,2.20980441960884,8.63601727203454$ SURR_ID= 'SHEET_METAL' / 419 ROBST XB=73. $4473710947422,74.5967741935484,46.240792481585,46.240792481585,0,8.63601727203454$ /SURF_ID='A-WALL-

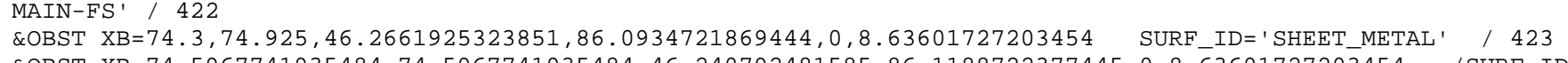

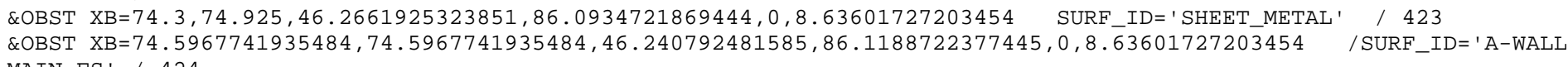
MAIN-FS' $/ 424$
\&OBST XB $=9.34721869443739,9.34721869443739,-3.83064516129032,-1.36684023368047,2.46380492760986,2.46697993395987$ \&OBST XB=9. 34721869443739, 9. 34721869443739, 7.15805181610363, 9. 62185674371349, 2. 46380492760986, 2. 46697993395987 ISURF-ID='A-WALL-MAIN-CB', 428
\&OBST $X \mathrm{~B}=9.34721869443739,9.34721869443739,18.9357378714757,21.3995427990856,2.46380492760986,2.46697993395987$ ISURF_ID ='A-WALL-MAIN-CB' 1431
/SOBST

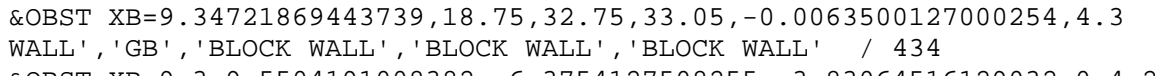

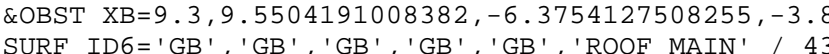

QOBST XB=9. 5504191008382, 9.5504191008382, $-3.83064516129032,-1.36684023368047,2.46380492760986,2.46697993395987$ ISURF-ID='A-WALL-MAIN-CB' / 436

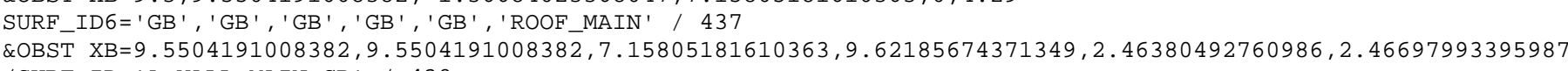

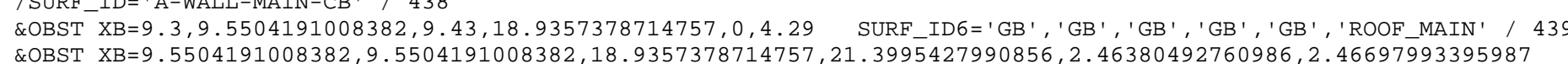
/SURF_ID='A-WALL-MAIN-CB' $/ 440$

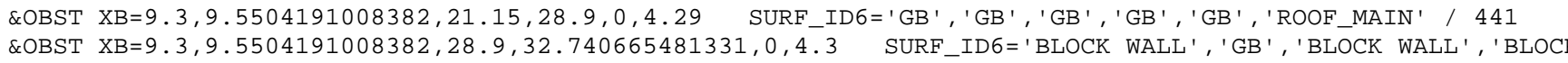
WALL', 'BLOCK WALL', 'BLOCK WALL',

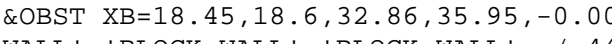
\&OBST' XB=18.45, 29.4, 35.65, 35.95, - $0.0063500127000254,3.2$ SURF_ID6='BLOCK WALL', 'BLOCK WALL', ' 'GB' , 'BLOCK WALL', 'BLOCK WALLL', 'BLOCK WALL' 1445
OOBST' XB $=18.742062484125,21.1,32.740665481331,32.91,0,4.3 \quad$ SURF_ID6=' 'BLOCK WALL', 'BLOCK WALL' , ' 'GB' , 'BLOCK WALL', 'BLOCK WALL', 'BLOCK WALL' $/ 449$

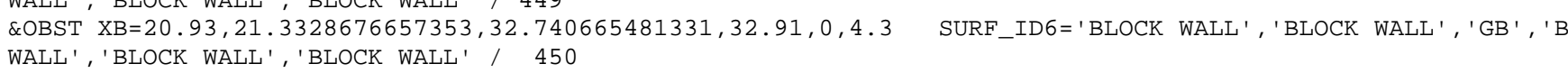
\&OBST $X B=21.3328676657353,22.5774701549403,32.740665481331,32.91,2.05740411480823,4.3$ SURF ID6=' 'BLOCK

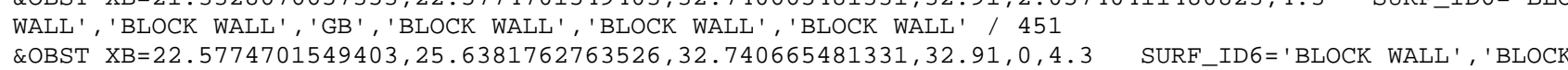

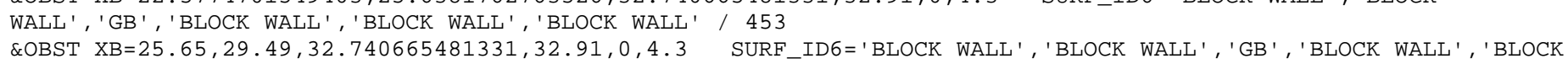
WALL', 'BLOCK WALL', 455
\&OBST' XB=29.25, 29.4, 32.72, 34.7726695453391, $1.79,3.2$ SURF_ID6='GB' , 'BLOCK WALL' , 'BLOCK WALL' , 'BLOCK WALL', 'BLOCK ROBST' XB $=29.25,29.4,34.77,35.95,-0.0063500127000254,3.2$ SURF_ID6=' GB', 'BLOCK WALL', 'BLOCK WALL', 'BLOCK

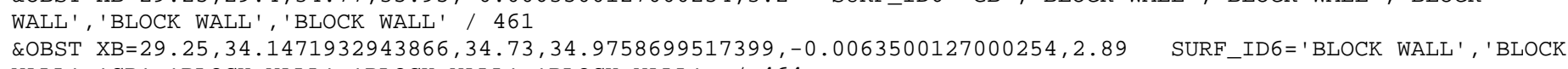

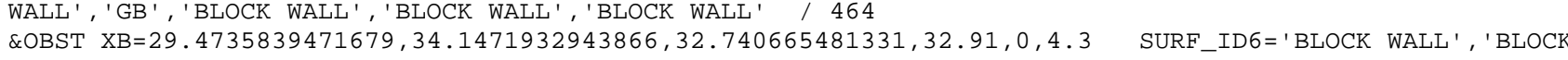

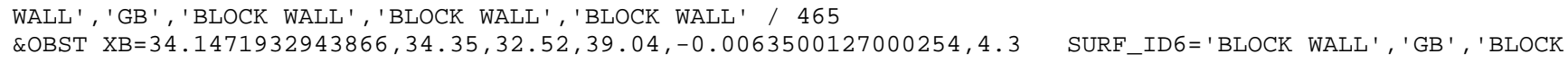

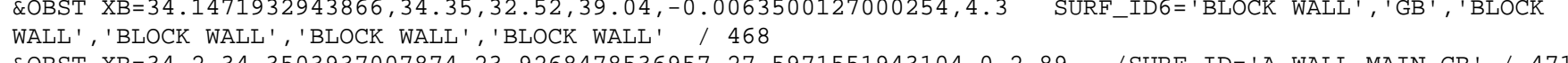
\&OBST XB=34.2, 34.3503937007874, 23.9268478536957, 27.5971551943104, 0, 2.89 /SURF_ID='A-WALL-MAIN-CB' / 471

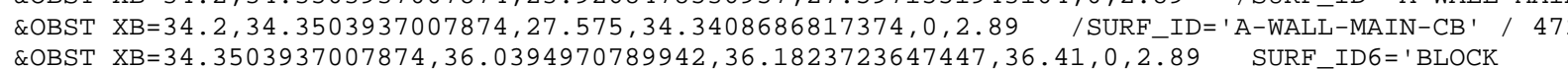
WALL', 'GB', 'GB', 'GB' 'GB' ' 'GB' ROBST' XB=34. $3503937007874,37.2205994411989,38.8493776987554,39.05,0,2.46697993395987$ /SURF_ID= 'A-WALL-MAIN-CB' OOBST XB=36.0394970789942, 36.8268986537973, 36.1823723647447, 36.41, 2.05740411480823, $2.89 \quad$ /SURF_ID= 'A-WALL-MAIN-

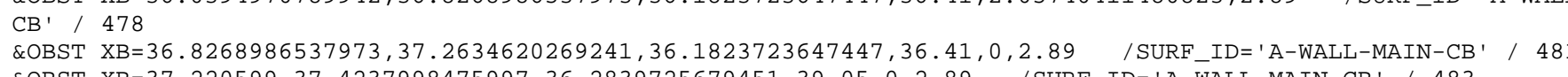

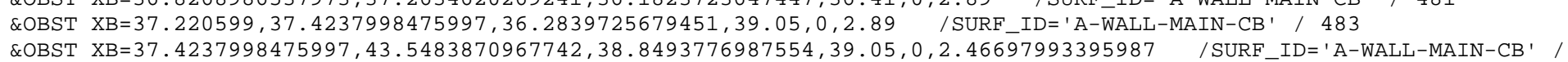
484
\&OBST XB=43.1261112522225, 43.9468503937008, 38.85, 39.0525781051562, 0, 2.46697993395987 SURF_ID6=' BLOCK

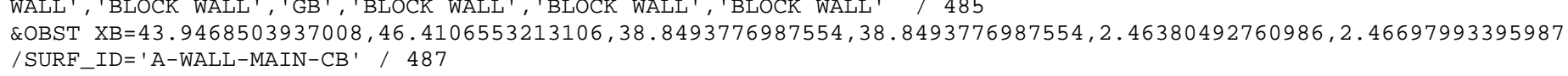


\&OBST XB=43. $9468503937008,46.4106553213106,39.0525781051562,39.0525781051562,2.46380492760986,2.46697993395987$ COBST XB=46. 31, 46.7837185674371, 38. 85, 39.0525781051562, 0, 2.46697993395987 SURF_ID6='BLOCK WALL', 'BLOCK

عOBST' $X B=46,72,48,0822961645023,38,7,39.0525781051562,-0,0063500127000254,4,3$ SURE_ID6='BLOCK WALL' , 'BLOCK \&OBST XB=47. 8790957581915, $47.8790957581915,-3.98304546609093,-1.51924053848108,2.46380492760986,2.46697993395987$ \&OBST XB $=47.8790957581915,47.8790957581915,7.34537719075438,9.80918211836424,2.46380492760986,2.46697993395987$

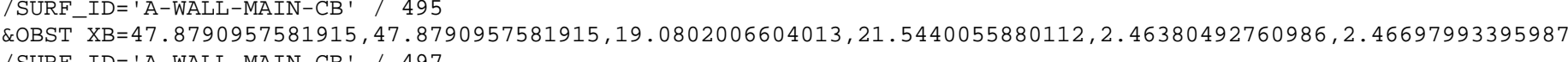
\&OBST XB=47. .8790957581915, 47. .8790957581915, 33.9344678689357, 36. 3982727965456, 2.46380492760986, 2. 46697993395987

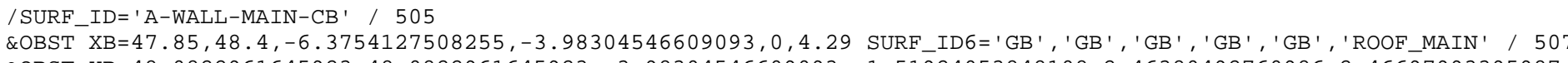

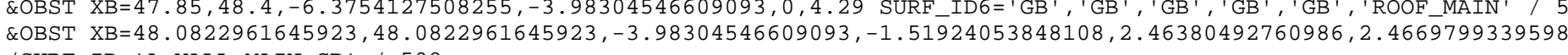

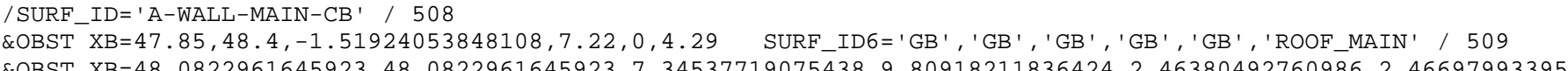

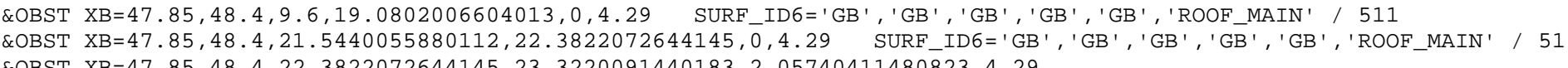

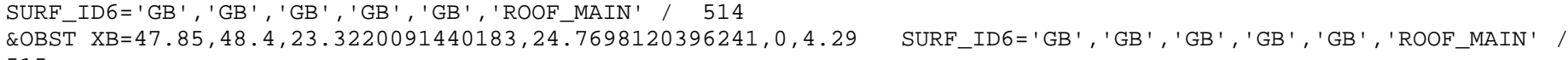

\&OBST XB=47.85, 48.4, 24.7698120396241, 25.65, 2.05740411480823, 4.29 /SURF_ID='A-WALL-MAIN-CB' / 516

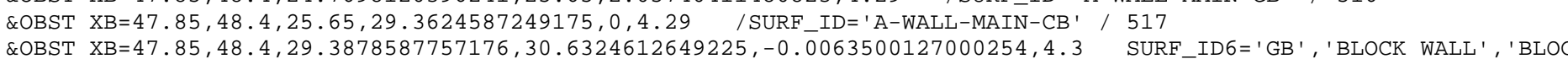
\&OBST XB=47.85, 48.4, 29.3878587757176, 30.6324612649225, -0.0063500127000254,4.3 SURF_IDO='GB', 'BLOCK WALL', 'BLOCK \&OBST' XB $=47.85,48.4,30.6324612649225,31.6738633477267,2.05740411480823,4.3$ SURF_ID6= 'GB' , 'BLOCK WALL', ' 'BLOCK WALL', 'BLOCK WALL', 'BLOCK WALL', 'BLOCK WALL' / 519 , $201000254,4.3$ SURF_ID6=' GB', 'BLOCK WALL', 'BLOCK WALL', 'BLOCK
COBST' XB $=47.85,48.4,31.6738633477267,34.04,-0.0063500127000254,3$

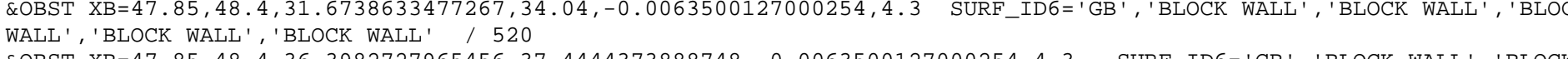
\&OBST XB=47.85, 48.4, 36.3982727965456, 37.4444373888748, - $0.0063500127000254,4.3$ SURF_ID6=' GB', ' 'BLOCK WALL', 'BLOCK

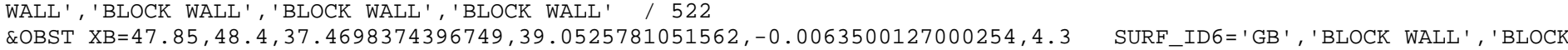
\&OBST XB=47.85, $48.4,37.4698374396749,39.0525781051562$,
WALL', 'BLOCK WALL', 'BLOCK WALL', 'BLOCK WALL' $/ 523$ 


\section{L.3 SSS 0255.FDS BASE CASE WITH SPRINKLERS}

1: (Modified CHID value.)

\&HEAD CHID='sss_0255' TITLE='Sofa Super Store Reconstruction'/

245-286 (Relative to SSS_0254.FDS): (Removed the following Warehouse and Main Showroom Ramps.)

VENT XB=56.55 56.925 47.3 47.675 0.51 0.51 OUTLINE=.TRUE. SURF_ID= 'WH_FIRE_RAMP' / Warehouse Fire

SURF ID='WH_FIRE_RAMP' HRRPUA=1000. RAMP_Q='WH_RAMP'/

RAMP ID='WH_RAMP' T=0.0 F=0.0 /

RAMP ID='WH RAMP' T=410.0 F=0.0 /

RAMP ID='WH_RAMP' T=440.0 F=0.02/

RAMP ID='WH RAMP' T=470.0 F=0.07/

RAMP ID='WH_RAMP' T=500.0 F=0.16 /

RAMP ID='WH RAMP' T=530.0 F=0.29/

RAMP ID='WH_RAMP' T=560.0 F=0.45 /

RAMP ID='WH_RAMP' T=610.0 F=0.79 /

RAMP ID='WH_RAMP' T=650.0 F=1.14 /

RAMP ID='WH_RAMP' T=1010.0 F=1.14 /

RAMP ID='WH_RAMP' T=1130.0 F=0.0 /

VENT XB= $41.5544 .92 \quad 35.67 \quad 36.41 \quad 0.14 \quad 0.14 \quad$ OUTLINE=.TRUE. SURF_ID= 'MAIN_SHOWROOM_FIRE' / SURF ID='MAIN_SHOWROOM_FIRE' HRRPUA=1500. RAMP_Q='MAIN_SHOWROOM_RAMP'/

RAMP ID='MAIN_SHOWROOM_RAMP' T=0.0 F=0.0 /

RAMP ID='MAIN_SHOWROOM_RAMP' T=525.0 F=0.0/

RAMP ID='MAIN_SHOWROOM_RAMP' T=555.0 F=0.042

RAMP ID='MAIN_SHOWROOM_RAMP' T=585.0 F=0.169/

RAMP ID='MAIN_SHOWROOM_RAMP' T=615.0 F=0.379 /

RAMP ID='MAIN_SHOWROOM_RAMP' T=645.0 F=0.675 /

RAMP ID='MAIN SHOWROOM RAMP' T=675.0 F=1.055 /

RAMP ID='MAIN_SHOWROOM_RAMP' T=1275.0 F=1.055 /

RAMP ID='MAIN_SHOWROOM_RAMP' T=1335.0 F=0.0 /

VENT $\quad \mathrm{XB}=41.5543 .0533 .04535 .67 \quad 0.15 \quad 0.15 \quad$ OUTLINE=.TRUE. SURF ID= 'MAIN_SHOWROOM_FIRE2' SURF ID='MAIN_SHOWROOM_FIRE2' HRRPUA=1500. RAMP_Q='MAIN_SHOWROOM_RAMP2'/

RAMP ID='MAIN_SHOWROOM_RAMP2' T=0.0 F $=0.0$ /

RAMP ID='MAIN SHOWROOM RAMP2' T=660.0 F=0.0

RAMP ID='MAIN_SHOWROOM_RAMP2' T=690.0 F=0.027 /

RAMP ID='MAIN SHOWROOM RAMP2' T=720.0 F=0.108 /

RAMP ID='MAIN_SHOWROOM_RAMP2' T=750.0 F=0.244 /

RAMP ID='MAIN_SHOWROOM_RAMP2' T=780.0 F=0.433 /

RAMP ID='MAIN_SHOWROOM_RAMP2' T=810.0 F=0.677 /

RAMP ID='MAIN_SHOWROOM_RAMP2' T=900.0 F=1.029 /

RAMP ID='MAIN_SHOWROOM_RAMP2' T=1500.0 F=1.029 /

RAMP ID='MAIN_SHOWROOM_RAMP2' T=1560.0 F=0.0 /

1181-1221 (Relative to SSS_0255.FDS): (Inserted Sprinkler definition and Sprinkler Devices in the Loading Dock Area.) Sprinklers

\&PART ID='water drops',

WATER=.TRUE.

AGE $=10.00$,

SAMPLING_FACTOR $=10$,

QUANTITIES='DROPLET_DIAMETER','DROPLET_TEMPERATURE','DROPLET_AGE',

DIAMETER $=675$,

GAMMA_D=2.43/

\&PROP ID='Sprk'

QUANTITY='SPRINKLER LINK TEMPERATURE',

DROPLETS_PER_SECOND $=1000$,

$\mathrm{RTI}=40.00$,

C FACTOR $=0.0$,

ACTIVATION_TEMPERATURE=74.,

PART_ID='water drops',

OFFSET $=0.1000$,

K_FACTOR=80.6,

OPERATING_PRESSURE $=1.7$,

DROPLET_VELOCITY $=14.00$,

SPRAY_ANGLE=75.00,105.00/

\&DEVC ID='Sprinkler 1', PROP_ID='Sprk', XYZ=48.8, 44.6, 2.8/ \&DEVC ID='Sprinkler 2', PROP_ID='Sprk', XYZ=52.4, 44.6, 2.8/ \&DEVC ID='Sprinkler 3', PROP_ID='Sprk', XYZ=56.0, 44.6, 2.8/ \&DEVC ID='Sprinkler 4', PROP_ID='Sprk', XYZ=59.4, 44.6, 2.8/ \&DEVC ID='Sprinkler 5', PROP_ID='Sprk', XYZ=48.8, 41.2, 2.6/ \&DEVC ID='Sprinkler 6', PROP ID='Sprk', XYZ=52.4, 41.2, 2.6/ \&DEVC ID='Sprinkler 7', PROP_ID='Sprk', XYZ=56.0, 41.2, 2.6/ \&DEVC ID='Sprinkler 8', PROP ID='Sprk', XYZ=59.4, 41.2, 2.6/ \&DEVC ID='Sprinkler 9', PROP_ID='Sprk', XYZ=48.8, 38.0, 2.2/ 
\&DEVC ID='Sprinkler 10', PROP_ID='Sprk', XYZ=52.4, 38.0, 2.2/ \&DEVC ID='Sprinkler 11', PROP_ID='Sprk', XYZ=56.0, 38.0, 2.2/ \&DEVC ID='Sprinkler 12', PROP_ID='Sprk', XYZ=59.4, 38.0, 2.2/ \&DEVC ID='Sprinkler 13', PROP_ID='Sprk', XYZ=48.8, 34.8, 3.4/ \&DEVC ID='Sprinkler 14', PROP_ID='Sprk', XYZ=52.4, 34.8, 3.4/ \&DEVC ID='Sprinkler 15', PROP ID='Sprk', XYZ=56.0, 34.8, 3.4/ \&DEVC ID='Sprinkler 16', PROP_ID='Sprk', XYZ=59.4, 34.8, 3.4/ \&DEVC ID='Sprinkler 17', PROP_ID='Sprk', XYZ=48.8, 31.4, 3.4/ \&DEVC ID='Sprinkler 18', PROP_ID='Sprk', XYZ=52.4, 31.4, 3.4/ \&DEVC ID='Sprinkler 19', PROP_ID='Sprk', XYZ=56.0, 31.4, 3.4/ \&DEVC ID='Sprinkler 20', PROP_ID='Sprk', XYZ=59.4, 31.4, 3.4/ ****************************************** 


\section{L.4 SSS 0256.FDS BASE CASE WITH WINDOWS INTACT}

1: (Modified CHID value.)

\&HEAD CHID='sss_0256' TITLE='Sofa Super Store Reconstruction'/

313-315 (Relative to SSS_0256.FDS): (Disabled Front Windows with Time SETPOINT at 10000.0)

\&DEVC ID='RIGHT ADDITION WINDOWS 1' , XYZ=63.5, -6.3, 2.0, QUANTITY='TIME' SETPOINT=10000.0, INITIAL_STATE=.TRUE./

\&DEVC ID='RIGHT ADDITION WINDOWS 2' , XYZ=57.1, -6.3, 2.0, QUANTITY='TIME' SETPOINT=10000.0, INITIAL_STATE=.TRUE./

\&DEVC ID='RIGHT ADDITION WINDOWS 3' , XYZ=51.0, -6.3, 2.0, QUANTITY='TIME' SETPOINT=10000.0, INITIAL_STATE=.TRUE./

331-340 (Relative to SSS_0256.FDS): (Disabled Front Windows with Time SETPOINT at 10000.0)

\&DEVC ID='MAIN STRUCTURE WINDOW L1', XYZ=25.4,-6.0,2.0, QUANTITY='TIME', SETPOINT=10000.0, INITIAL_STATE=.TRUE./

\&DEVC ID='MAIN STRUCTURE WINDOW L2', XYZ=23.2,-6.0,2.0, QUANTITY='TIME', SETPOINT=10000.0, INITIAL_STATE=.TRUE./

\&DEVC ID='MAIN STRUCTURE WINDOW L3', XYZ=20.0,-6.0,2.0, QUANTITY='TIME', SETPOINT=10000.0, INITIAL_STATE=.TRUE./

\&DEVC ID='MAIN STRUCTURE WINDOW L4', XYZ=17.5,-6.0,2.0, QUANTITY='TIME', SETPOINT=10000.0, INITIAL_STATE=.TRUE./

\&DEVC ID='MAIN STRUCTURE WINDOW L5', XYZ=14.5,-6.0,2.0, QUANTITY='TIME', SETPOINT=10000.0, INITIAL_STATE=.TRUE./

\&DEVC ID='MAIN STRUCTURE WINDOW R1', XYZ=32.0,-6.0,2.0, QUANTITY='TIME', SETPOINT=10000.0, INITIAL_STATE=.TRUE./

\&DEVC ID='MAIN STRUCTURE WINDOW R2', XYZ=35.0,-6.0,2.0, QUANTITY='TIME', SETPOINT=10000.0, INITIAL_STATE=.TRUE./

\&DEVC ID='MAIN STRUCTURE WINDOW R3', XYZ=37.1,-6.0,2.0, QUANTITY='TIME', SETPOINT=10000.0, INITIAL_STATE=.TRUE./

\&DEVC ID='MAIN STRUCTURE WINDOW R4', XYZ=39.6,-6.0,2.0, QUANTITY='TIME', SETPOINT=10000.0, INITIAL_STATE=.TRUE./

\&DEVC ID='MAIN STRUCTURE WINDOW R5', XYZ=42.0,-6.0,2.0, QUANTITY='TIME', SETPOINT=10000.0, INITIAL_STATE=.TRUE./ 
1: (Modified CHID value.)

\&HEAD CHID='sss_257' TITLE='Sofa Super Store Reconstruction'/

310-312 (Relative to SSS_0257.FDS): (Disabled Front Windows with Time SETPOINT at 10000.0)

\&DEVC ID='RIGHT ADDITION WINDOWS 1' , XYZ=63.5, -6.3, 2.0, QUANTITY='TIME' SETPOINT=10000.0, INITIAL_STATE=.TRUE./

\&DEVC ID='RIGHT ADDITION WINDOWS 2' , XYZ=57.1, -6.3, 2.0, QUANTITY='TIME' SETPOINT=10000.0, INITIAL_STATE=.TRUE./

\&DEVC ID='RIGHT ADDITION WINDOWS 3' , XYZ=51.0, -6.3, 2.0, QUANTITY='TIME' SETPOINT=10000.0, INITIAL_STATE=.TRUE./

328-337 (Relative to SSS_0257.FDS): (Disabled Front Windows with Time SETPOINT at 10000.0)

\&DEVC ID='MAIN STRUCTURE WINDOW L1', XYZ=25.4,-6.0,2.0, QUANTITY='TIME', SETPOINT=10000.0, INITIAL_STATE=.TRUE./

\&DEVC ID='MAIN STRUCTURE WINDOW L2', XYZ=23.2,-6.0,2.0, QUANTITY='TIME', SETPOINT=10000.0, INITIAL_STATE=.TRUE./

\&DEVC ID='MAIN STRUCTURE WINDOW L3', XYZ=20.0,-6.0,2.0, QUANTITY='TIME', SETPOINT=10000.0, INITIAL_STATE=.TRUE./

\&DEVC ID='MAIN STRUCTURE WINDOW L4', XYZ=17.5,-6.0,2.0, QUANTITY='TIME', SETPOINT=10000.0, INITIAL_STATE=.TRUE./

\&DEVC ID='MAIN STRUCTURE WINDOW L5', XYZ=14.5,-6.0,2.0, QUANTITY='TIME', SETPOINT=10000.0, INITIAL_STATE=.TRUE./

\&DEVC ID='MAIN STRUCTURE WINDOW R1', XYZ=32.0,-6.0,2.0, QUANTITY='TIME', SETPOINT=10000.0, INITIAL_STATE=.TRUE./

\&DEVC ID='MAIN STRUCTURE WINDOW R2', XYZ=35.0,-6.0,2.0, QUANTITY='TIME', SETPOINT=10000.0, INITIAL_STATE=.TRUE./

\&DEVC ID='MAIN STRUCTURE WINDOW R3', XYZ=37.1,-6.0,2.0, QUANTITY='TIME', SETPOINT=10000.0,

INITIAL_STATE=.TRUE./

\&DEVC ID='MAIN STRUCTURE WINDOW R4', XYZ=39.6,-6.0,2.0, QUANTITY='TIME', SETPOINT=10000.0, INITIAL_STATE=.TRUE./

\&DEVC ID='MAIN STRUCTURE WINDOW R5', XYZ=42.0,-6.0,2.0, QUANTITY='TIME', SETPOINT=10000.0, INITIAL_STATE=.TRUE./

616 (Relative to SSS_0257.FDS): (Inserted typical sized vertical vent in roof, open from time zero for maximum ventilation.) \&HOLE XB= $\begin{array}{llllll}40.8 & 41.9 & 34.9 & 36.4 & 3.88 & 5.09\end{array} /$ rear corner roof 


\section{L.6 SSS_0258.FDS WINDOWS INTACT CASE WITH LARGE VENT}

1: (Modified CHID value.)

\&HEAD CHID='sss_0258' TITLE='Sofa Super Store Reconstruction'/

326-328 (Relative to SSS_0258.FDS): (Disabled Front Windows with Time SETPOINT at 10000.0)

\&DEVC ID='RIGHT ADDITION WINDOWS 1' , XYZ=63.5, -6.3, 2.0, QUANTITY='TIME' SETPOINT=10000.0, INITIAL_STATE=.TRUE./

\&DEVC ID='RIGHT ADDITION WINDOWS 2' , XYZ=57.1, -6.3, 2.0, QUANTITY='TIME' SETPOINT=10000.0, INITIAL_STATE=.TRUE./

\&DEVC ID='RIGHT ADDITION WINDOWS 3' , XYZ=51.0, -6.3, 2.0, QUANTITY='TIME' SETPOINT=10000.0, INITIAL_STATE=.TRUE./

344-353 (Relative to SSS_0258.FDS): (Disabled Front Windows with Time SETPOINT at 10000.0)

\&DEVC ID='MAIN STRUCTURE WINDOW L1', XYZ=25.4,-6.0,2.0, QUANTITY='TIME', SETPOINT=10000.0, INITIAL_STATE=.TRUE./

\&DEVC ID='MAIN STRUCTURE WINDOW L2', XYZ=23.2,-6.0,2.0, QUANTITY='TIME', SETPOINT=10000.0,

INITIAL_STATE=.TRUE./

\&DEVC ID='MAIN STRUCTURE WINDOW L3', XYZ=20.0,-6.0,2.0, QUANTITY='TIME', SETPOINT=10000.0, INITIAL_STATE=.TRUE./

\&DEVC ID='MAIN STRUCTURE WINDOW L4', XYZ=17.5,-6.0,2.0, QUANTITY='TIME', SETPOINT=10000.0, INITIAL_STATE=.TRUE./

\&DEVC ID='MAIN STRUCTURE WINDOW L5', XYZ=14.5,-6.0,2.0, QUANTITY='TIME', SETPOINT=10000.0, INITIAL_STATE=.TRUE./

\&DEVC ID='MAIN STRUCTURE WINDOW R1', XYZ=32.0,-6.0,2.0, QUANTITY='TIME', SETPOINT=10000.0, INITIAL_STATE=.TRUE./

\&DEVC ID='MAIN STRUCTURE WINDOW R2', XYZ=35.0,-6.0,2.0, QUANTITY='TIME', SETPOINT=10000.0, INITIAL STATE=.TRUE./

\&DEVC ID='MAIN STRUCTURE WINDOW R3', XYZ=37.1,-6.0,2.0, QUANTITY='TIME', SETPOINT=10000.0, INITIAL_STATE=.TRUE./

\&DEVC ID='MAIN STRUCTURE WINDOW R4', XYZ=39.6,-6.0,2.0, QUANTITY='TIME', SETPOINT=10000.0, INITIAL_STATE=.TRUE./

\&DEVC ID='MAIN STRUCTURE WINDOW R5', XYZ=42.0,-6.0,2.0, QUANTITY='TIME', SETPOINT=10000.0, INITIAL_STATE=.TRUE./

619-620 (Relative to SSS_0258.FDS): (Inserted Large Sized Vertical Vent in Roof, Opened at 1 Second into the simulation for maximum ventilation.) \&HOLE XB $=40.05 \quad 42.67 \quad 34.55 \quad 36.8 \quad 3.88 \quad 5.09 \quad$ DEVC_ID='ROOF_HOLE'/ rear corner roof \&DEVC ID='ROOF_HOLE', XYZ=44.0,35.0,3.5, QUANTITY='TIME', SETPOINT=1.0, INITIAL_STATE=.FALSE. / 


\section{APPENDIX M. FIRE INSPECTION AND BUILDING PRE-PLAN}

\section{M.1 CITY OF CHARLESTON FIRE INSPECTION}

The City of Charleston Fire Department visited the Sofa Super Store to perform a fire inspection. The inspection order completed by the fire department is shown in Figures M-1 and M-2. For inclusion in this appendix, the form was divided into top and bottom portions.

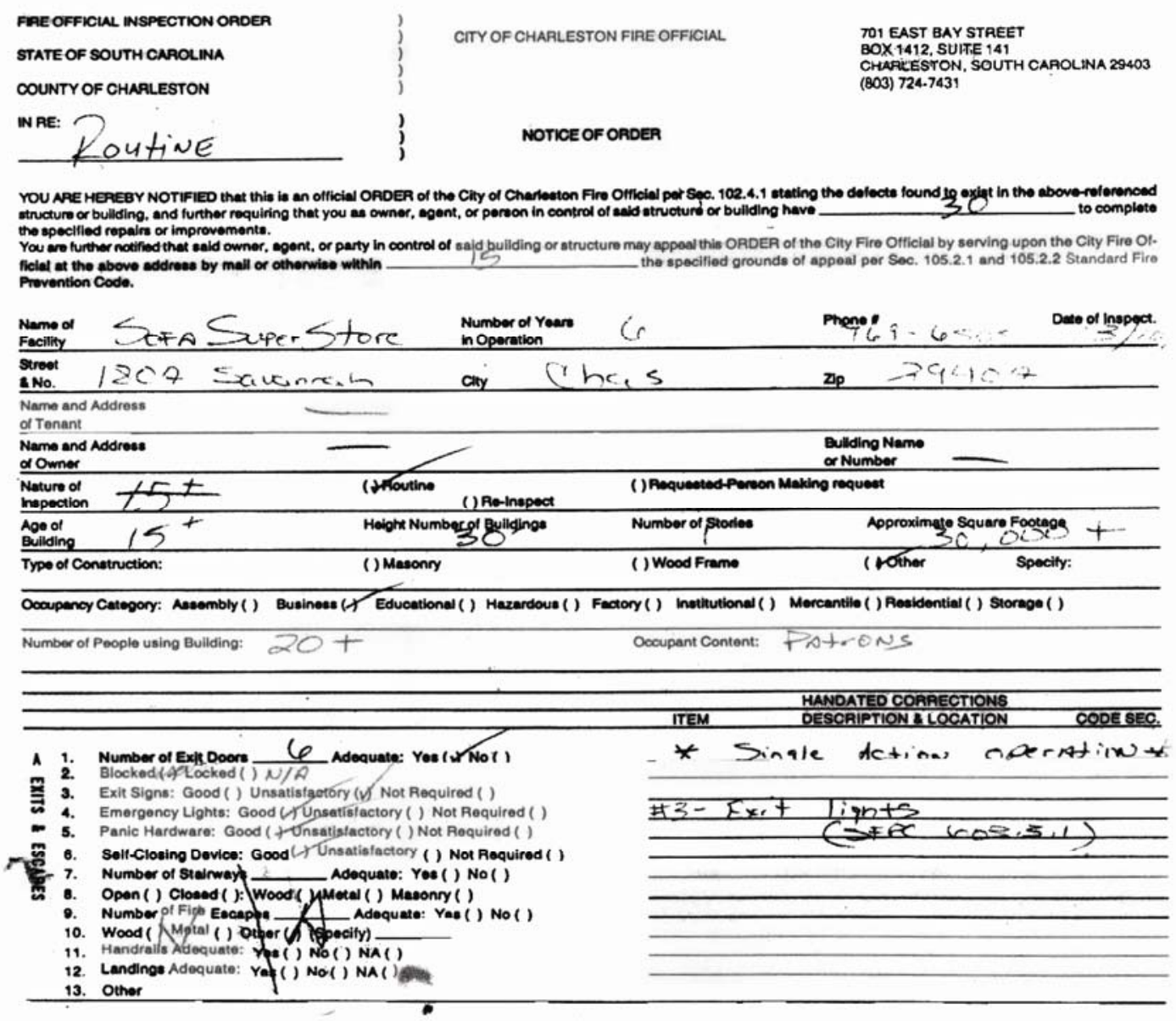

Figure M-1. Top portion of Fire Official Inspection Order for Sofa Super Store 
B 1. Firo Alarm: Yes () No (HAdequate: Yes () No ()

2. Smoke Detoctors: Yos () No (t Adequate: Yos () No ()

疍 3. Sprinkler System: Yes ( ) No ( ) Adequate: Yes ( ) No (G)

4. Standplpe Syatom: Yoe () No (LAdequate: Yoe () No ()

5. Number of Fire Extinguighers $5 T$ Adequate: Yes M No( )

B. Doto lewe oherged $97-\operatorname{Good}(T$ Unsatisfactory ()

7. Fixed Hood Extinguishe f Syotem; Yes ( ) No () Not Required ()

8. Dato Last Sonvicoof S A Aloquato: Yee ( ) No ()

9. Other

c 1. Fire Ratod Corridore-Walle: Yes (h Nor () Adequate: Yes () No ()

2. Fire Rated Cellings: Yoe ( ) No ( T Adequato: Yes () No ()

3. Fleme Spread Rating Adequate: Yes Y ( No ()

(4. Fire \& Drat Stopping Adequate: Yes (D) No()
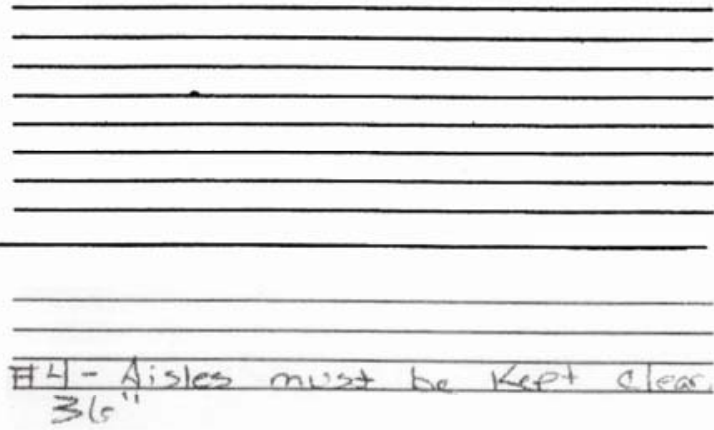

5. Other

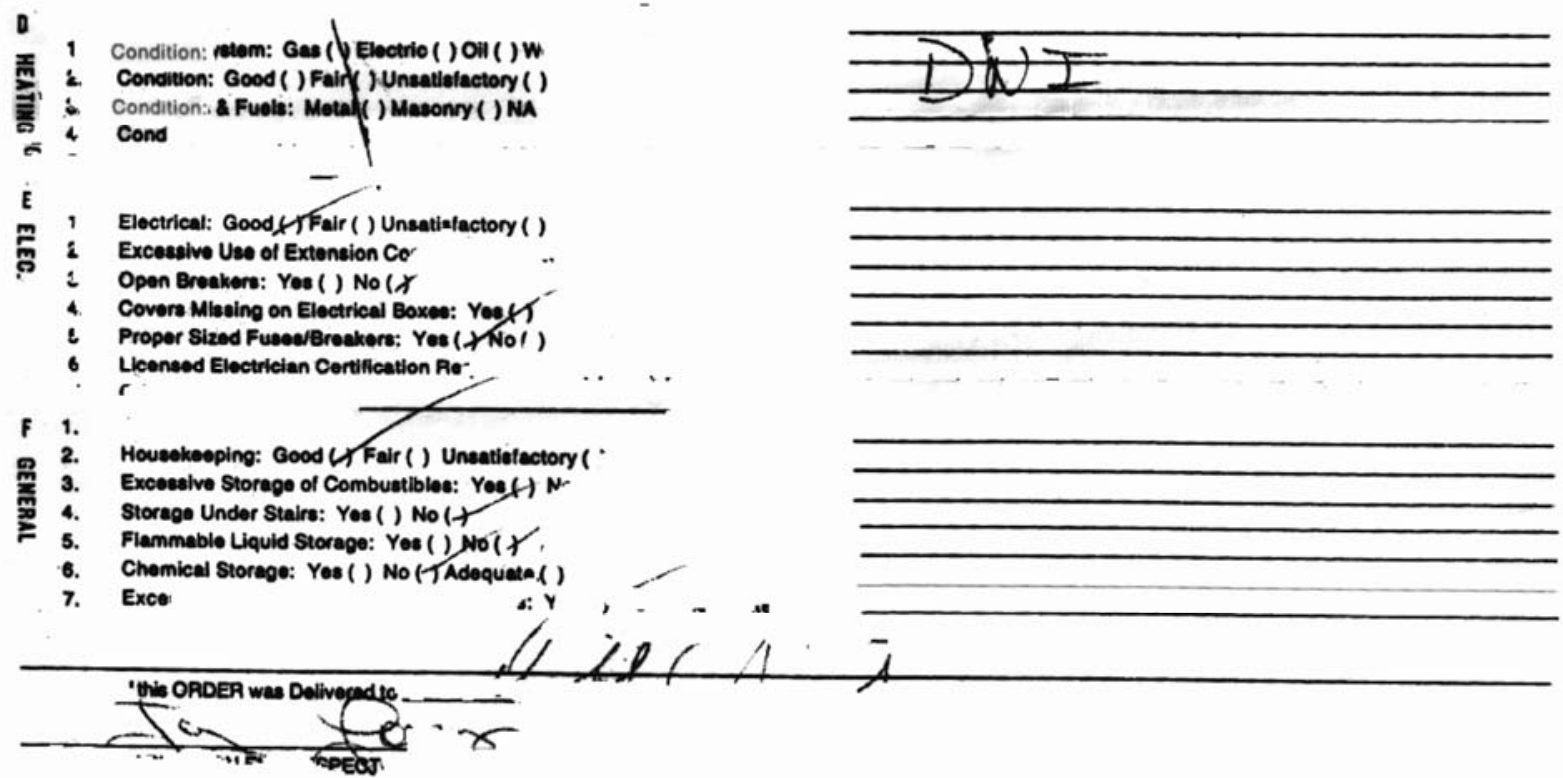

Figure M-2. Bottom portion of Fire Official Inspection Order for Sofa Super Store. 


\section{M.2 CITY OF CHARLESTON FIRE DEPARTMENT PRE-PLANNING}

First due companies, Engines 11, and Engine 10, and Ladder 5 inspected the Sofa Super Store on 4/26/06 for the purpose of pre-planning their response. The inspection noted a hydrant location to the rear of the warehouse on Pebble Road at the intersection with Sarah Road. The fire department also made note of the locations of the main electrical switch and gas shut-off valve.

\section{City of Charleston Fire Department PRE-PLANNING BULLDING INSPECTIONS Capt. G. GArdner.}

Company Eng $11, E n g, 10$ o L5 Date o4 fole ob Officer acct Copt. L, GAroin. Address 1807 Savannah Hwy. uccupant Sofa Super Store.

Owner Herb Goldstein.

Emergency \# (Key Holder) Je rry alford. $559-9951$ westeruluk lsox. 663

Lives Involved: Day $15-18$. Night

Type of Building Metal, Block No. Floors Type of Roof Comp. Construction Metal Bloch

F.D. Connections $N / 4 \quad$ Standpipes iv

Auto Sprinkler $N \mid A$ Location Main Valve $N / A$

Fire Alarm Indicator ij|

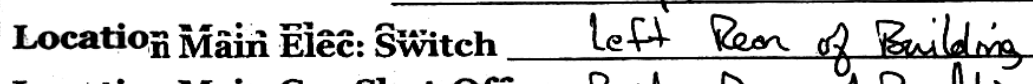

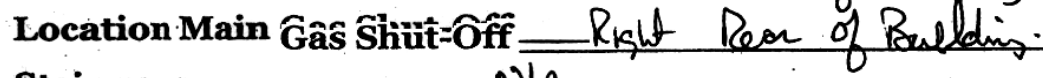

Stairways N N/A

Elevators N N $\quad$ A Fire Escapes

Vertical Openings Scuttle Holes

Fire Doors _ : H. Hallways N/A Exits 8

Best Way To Enter Building: Day Front

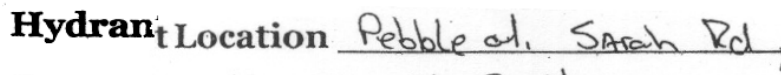
Contents. House hoold fumiture ot iffice Equipoment

Exposures to be covered Texaco gas Station + Car deabrohip Date of Last Extinguisher Recharged $\mathrm{CO} 2$ Dry Chemical

Additional Comments and/or Concerns Mag 2006 Night frond.

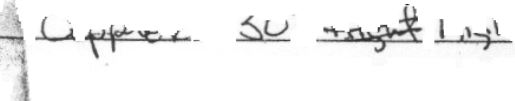

Figure M-3. Pre-Plannning Building Inspection for Sofa Super Store. 


\section{M.3 CITY OF CHARLESTON FIRE DEPARTMENT PRE-PLANNING SKETCH}

The hand drawn sketch of the store floorplan did not appear to note the enclosed loading dock area. The sketch did, however, include the locations of roll-down fire doors and exit doors. The floorplan sketch was not drawn to scale.

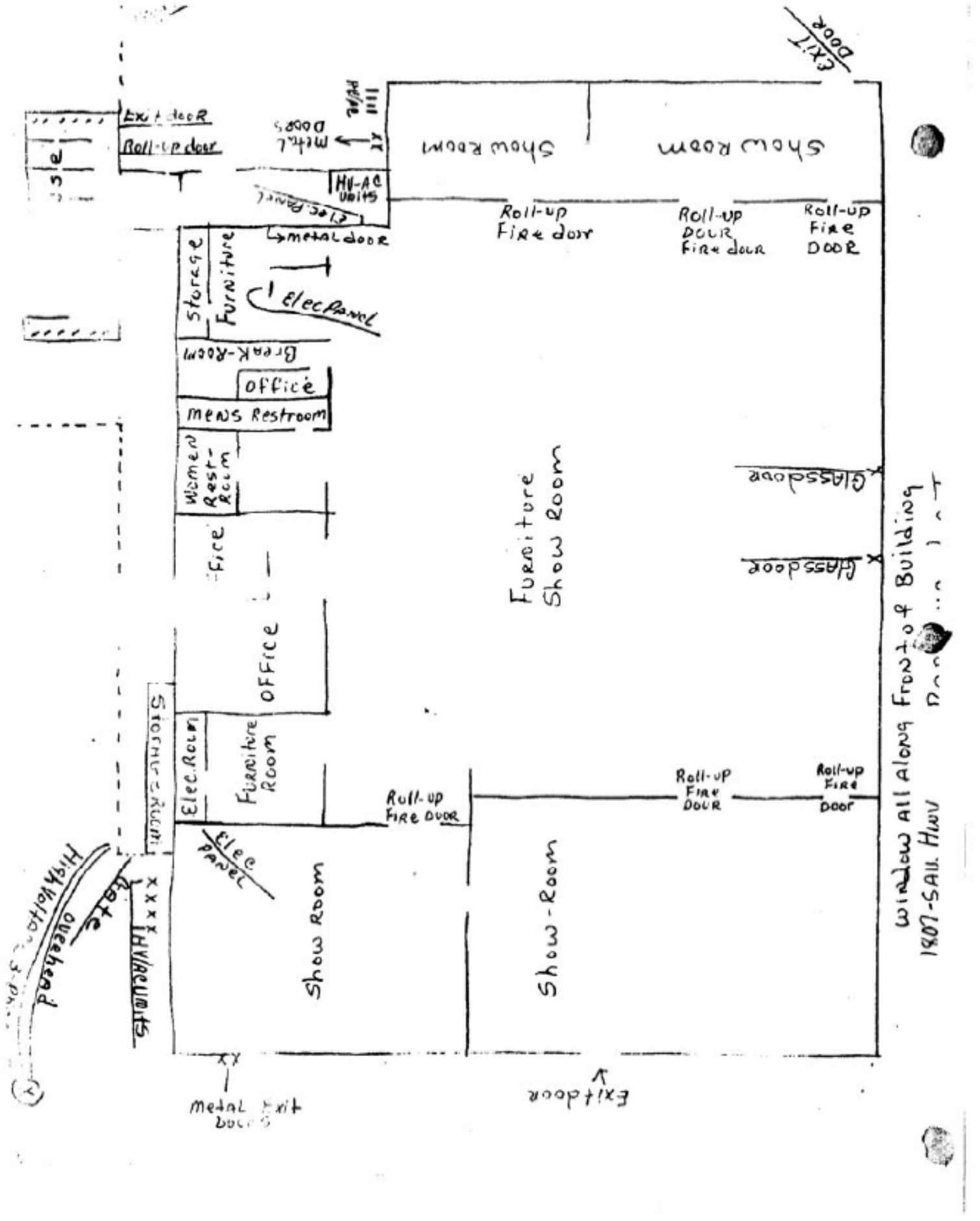

Figure M-4. Sketch of floor plan for Sofa Super Store. 


\section{Appendix N. Additional Aerial Images}

\section{N.1 AERIAL IMAGES}

Aircraft routinely record digital images for mapping, construction, utilities, remote sensing, and land surveying. Aerial photographs can be taken when an aircraft is directly overhead or at an angle. NIST obtained aerial images from DigitalGlobe ${ }^{1}$ of the West Ashley area of the city of Charleston from 1989,

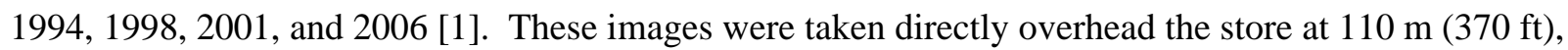
$230 \mathrm{~m}$ (740 ft), $340 \mathrm{~m}(1100 \mathrm{ft}), 450 \mathrm{~m}(1480 \mathrm{ft})$, and $1120 \mathrm{~m}(3700 \mathrm{ft})$ resolutions ${ }^{2}$. Angle or oblique views of the Sofa Super Store were obtained from Pictometry ${ }^{1}$ from north, south, east, and west views at 40 degree angles [2].

\section{N.2 TOP DOWN OR OVERHEAD AERIAL IMAGES}

Top images were recorded for a range of years and at resolutions as summarized in Table $\mathrm{N}-1$.

Table N-1. Top View Aerial Images for Sofa Super Store.

\begin{tabular}{|c|c|c|c|c|c|}
\hline Date & \multicolumn{5}{|c|}{ Image Resolution } \\
\hline DigitalGlobe[1] & $110 \mathrm{~m}(370 \mathrm{ft})$ & $230 \mathrm{~m}(740 \mathrm{ft})$ & $340 \mathrm{~m}(1100 \mathrm{ft})$ & $450 \mathrm{~m}$ (1480 ft) & $1130 \mathrm{~m}(3690 \mathrm{ft})$ \\
\hline 2/12/1989 & & & Figure N-1 & & \\
\hline 2/14/1994 & & & Figure N-2 & & \\
\hline 3/01/1998 & Figure N-3 & Figure N-4 & Figure N-5 & & \\
\hline $4 / 01 / 2001$ & Figure N-6 & Figure N-7 & Figure N-8 & & \\
\hline $12 / 1 / 2006$ & & Figure N-9 & & Figure N-10 & Figure N-11 \\
\hline Pictometry[2] & \multicolumn{2}{|c|}{ Narrow View } & \multicolumn{3}{|c|}{ Wide View } \\
\hline 3/11/2007 & \multicolumn{2}{|c|}{ Figure N-12 } & \multicolumn{3}{|c|}{ Figure N-13 } \\
\hline
\end{tabular}

\footnotetext{
${ }^{1}$ Certain commercial entities, equipment, products, or materials are identified in this document in order to describe a procedure or concept adequately or to trace the history of the procedures and practices used. Such identification is not intended to imply recommendation, endorsement, or implication that the entities, products, materials, or equipment are necessarily the best available for the purpose.

${ }^{2}$ Image resolution refers to meters or feet per pixel in an image. A $110 \mathrm{~m}$ resolution image had 2500 pixels with each pixel representing $0.045 \mathrm{~m}$. This translated into an image displaying an area approximately $110 \mathrm{~m}$ x $110 \mathrm{~m}$. Similarly, a $1120 \mathrm{~m}$ resolution displayed an area $1120 \mathrm{~m}$ x $1120 \mathrm{~m}$.
} 


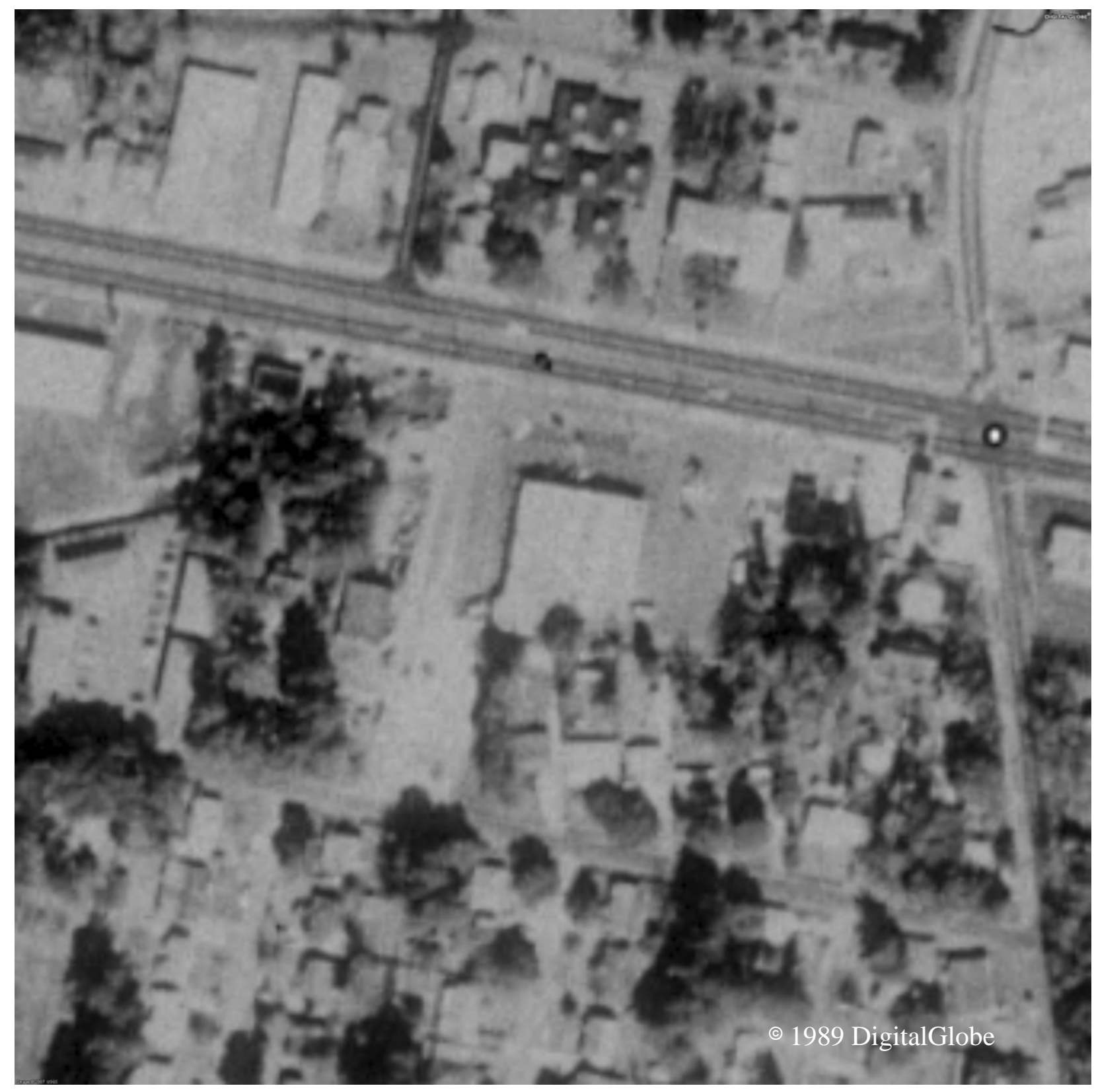

Figure N-1. Top view image recorded 2/12/1989 at 340 m (1100 ft) resolution. 


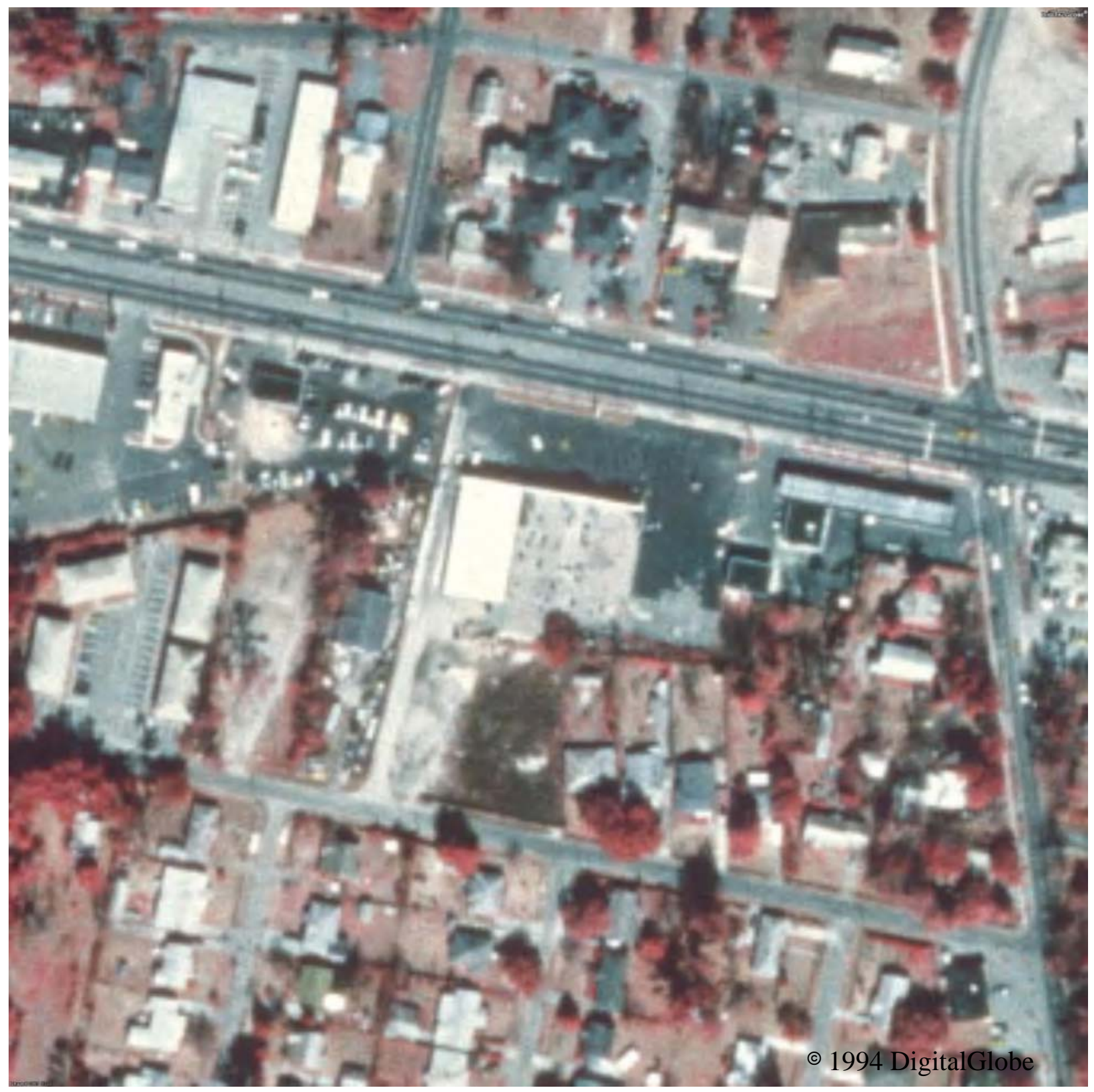

Figure N-2. Top view image recorded 2/14/1994 at 340 m (1100 ft) resolution. 


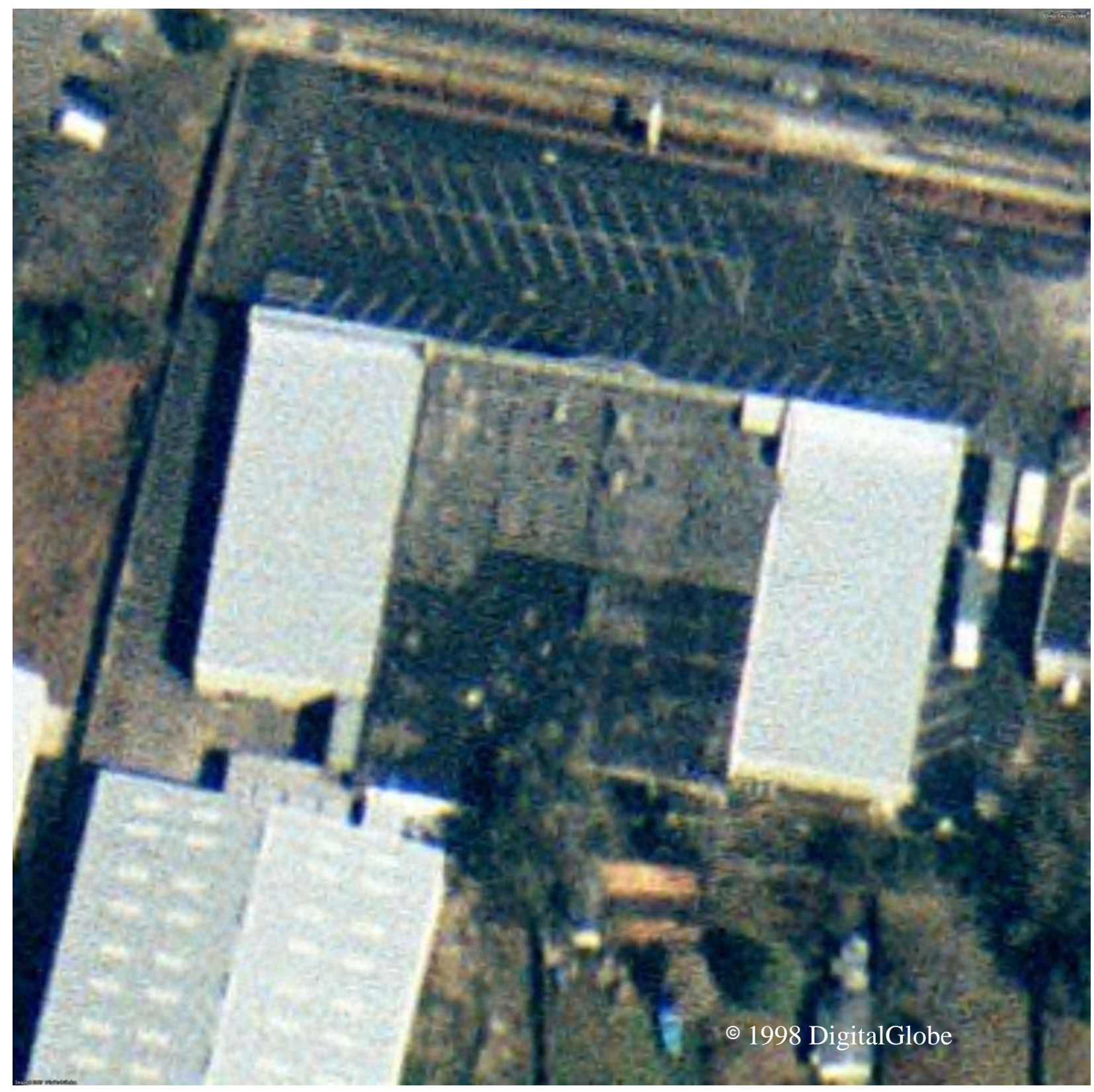

Figure N-3. Top view image recorded 3/1/1998 at $110 \mathrm{~m}$ (370 ft) resolution. 


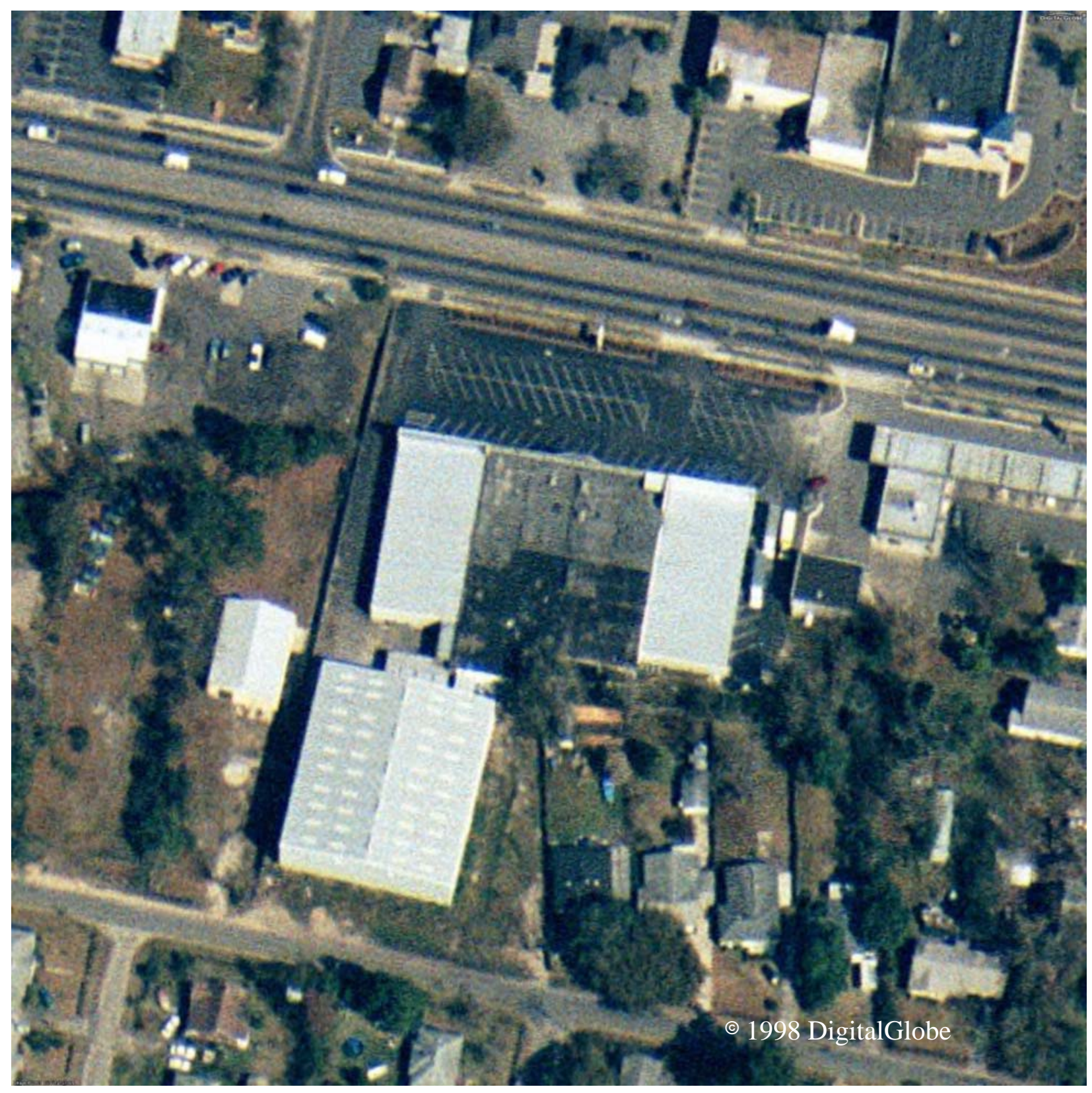

Figure N-4. Top view image recorded 3/1/1998 at $230 \mathrm{~m}$ (740 ft) resolution. 


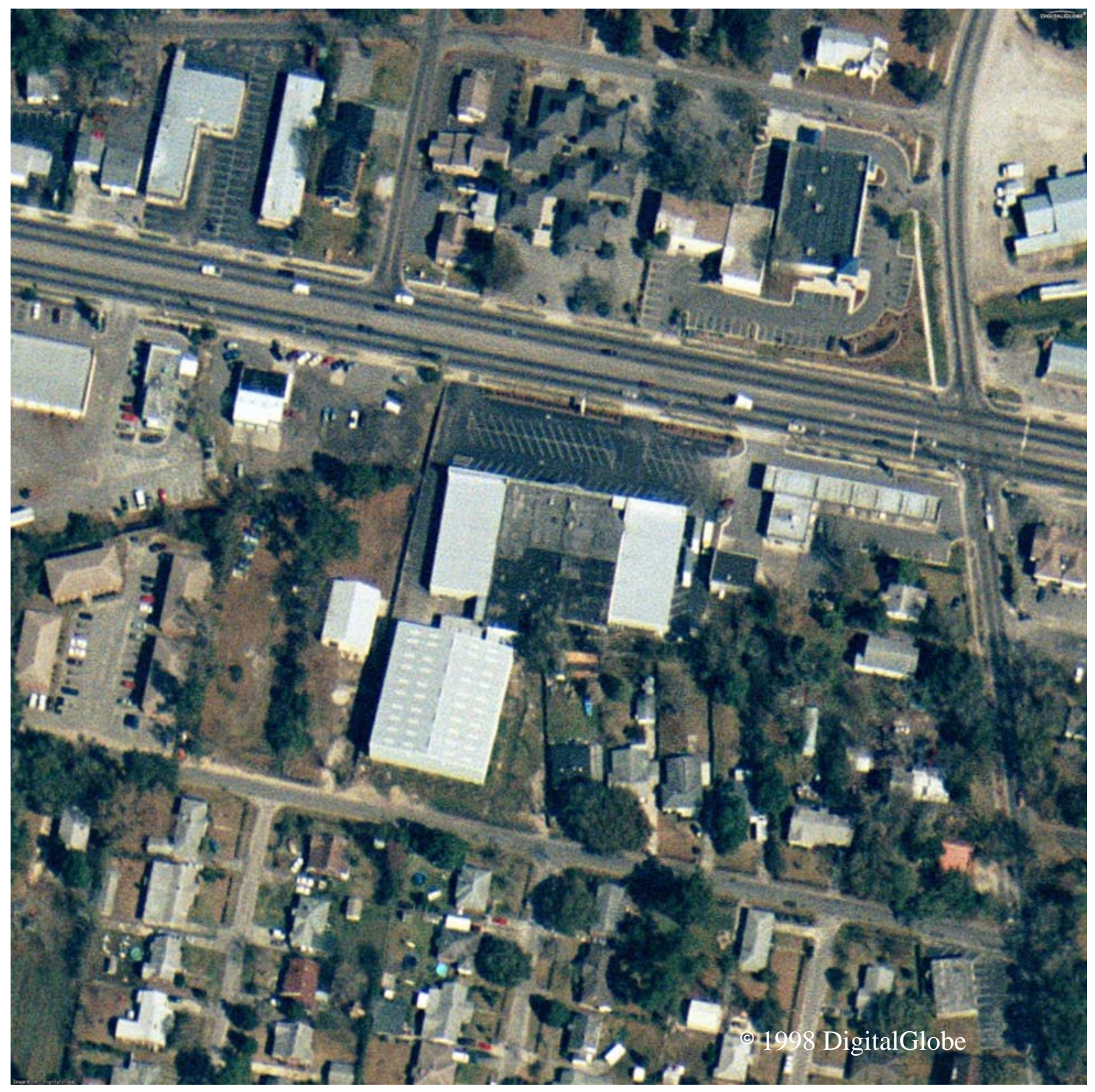

Figure N-5. Top view image recorded 3/1/1998 at 340 m (1100 ft) resolution. 


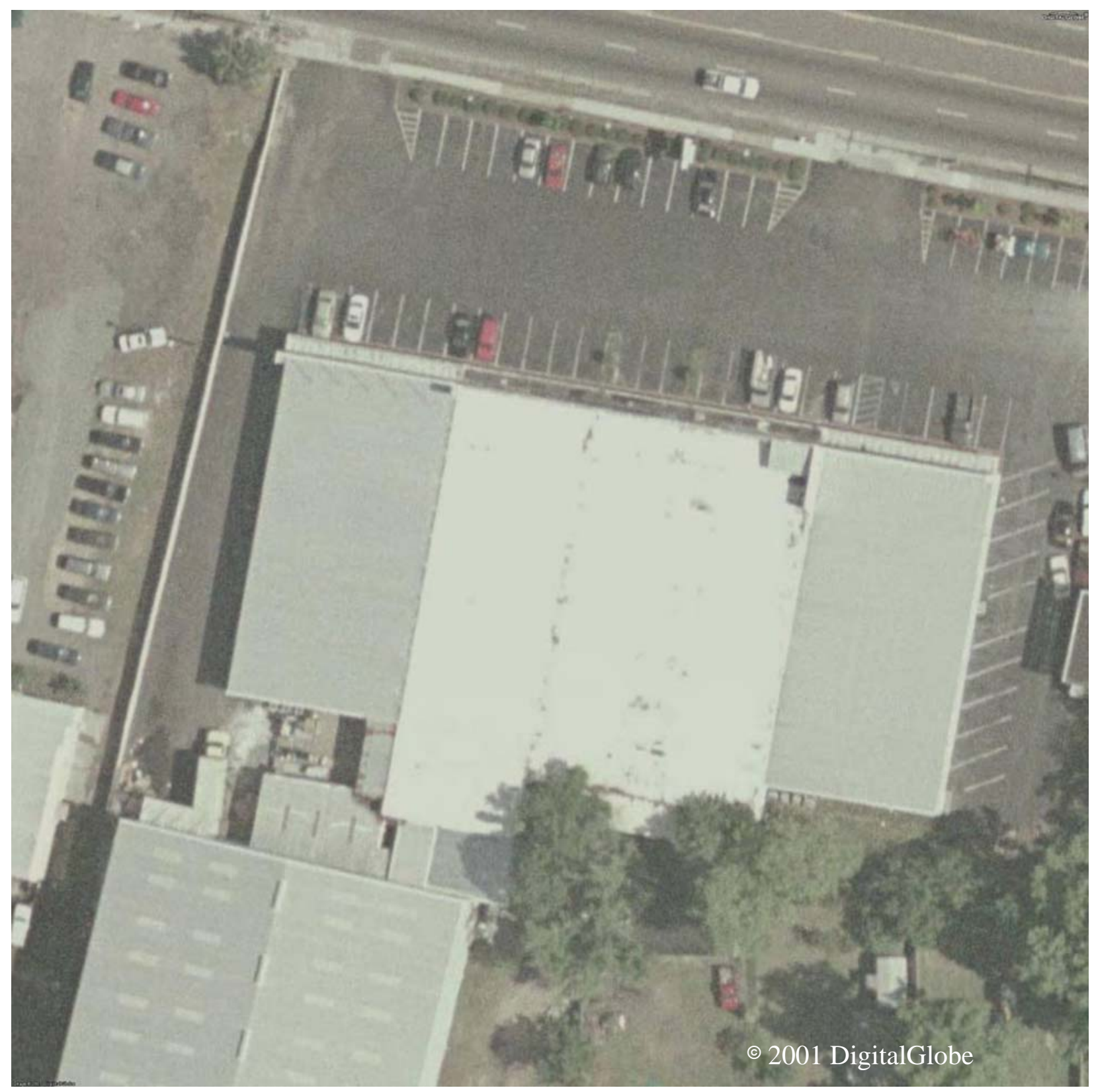

Figure N-6. Top view image recorded 4/1/2001 at $110 \mathrm{~m}(370 \mathrm{ft})$ resolution. 


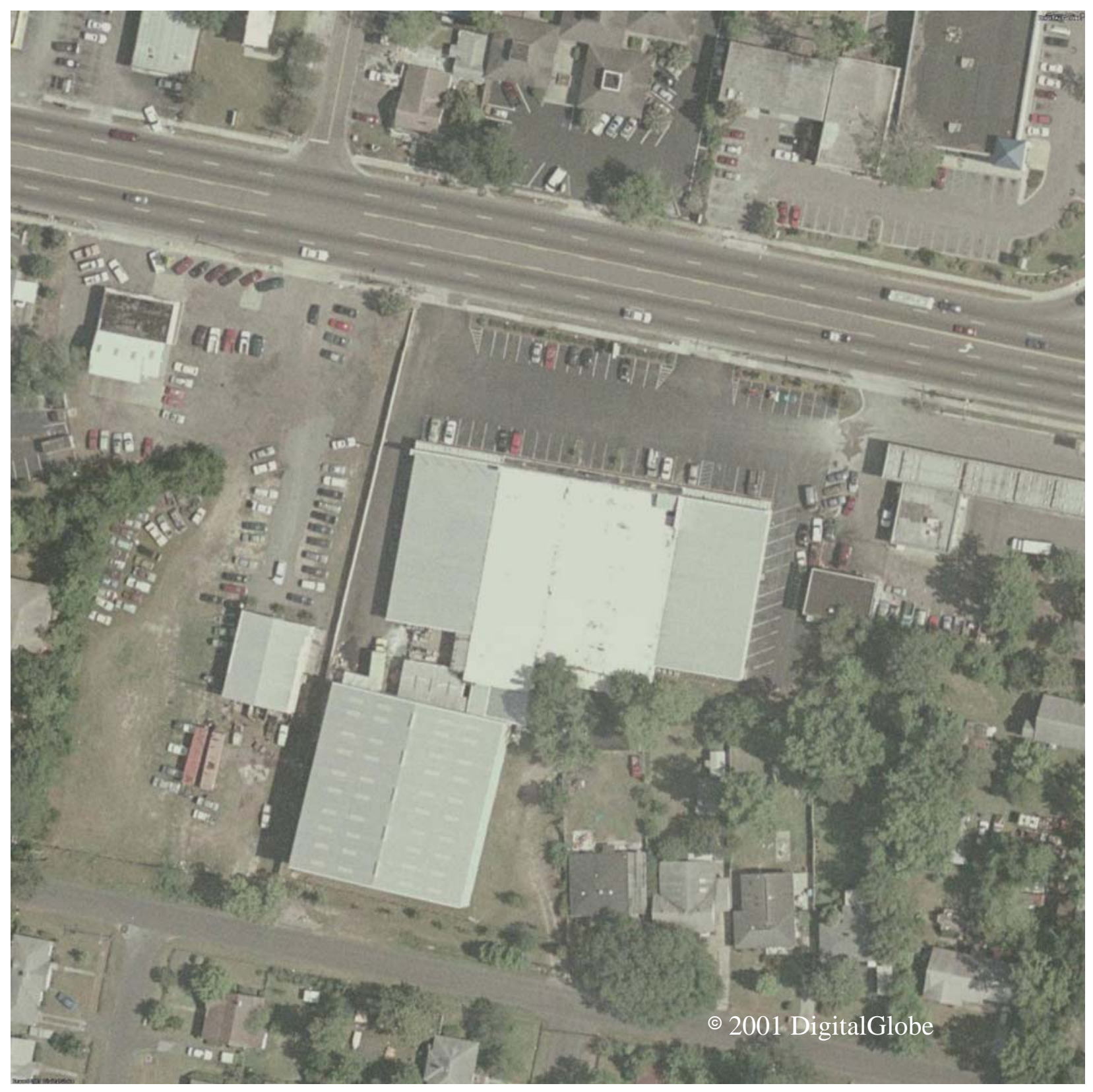

Figure N-7. Top view image recorded 4/1/2001 at $230 \mathrm{~m}$ (740 ft) resolution. 


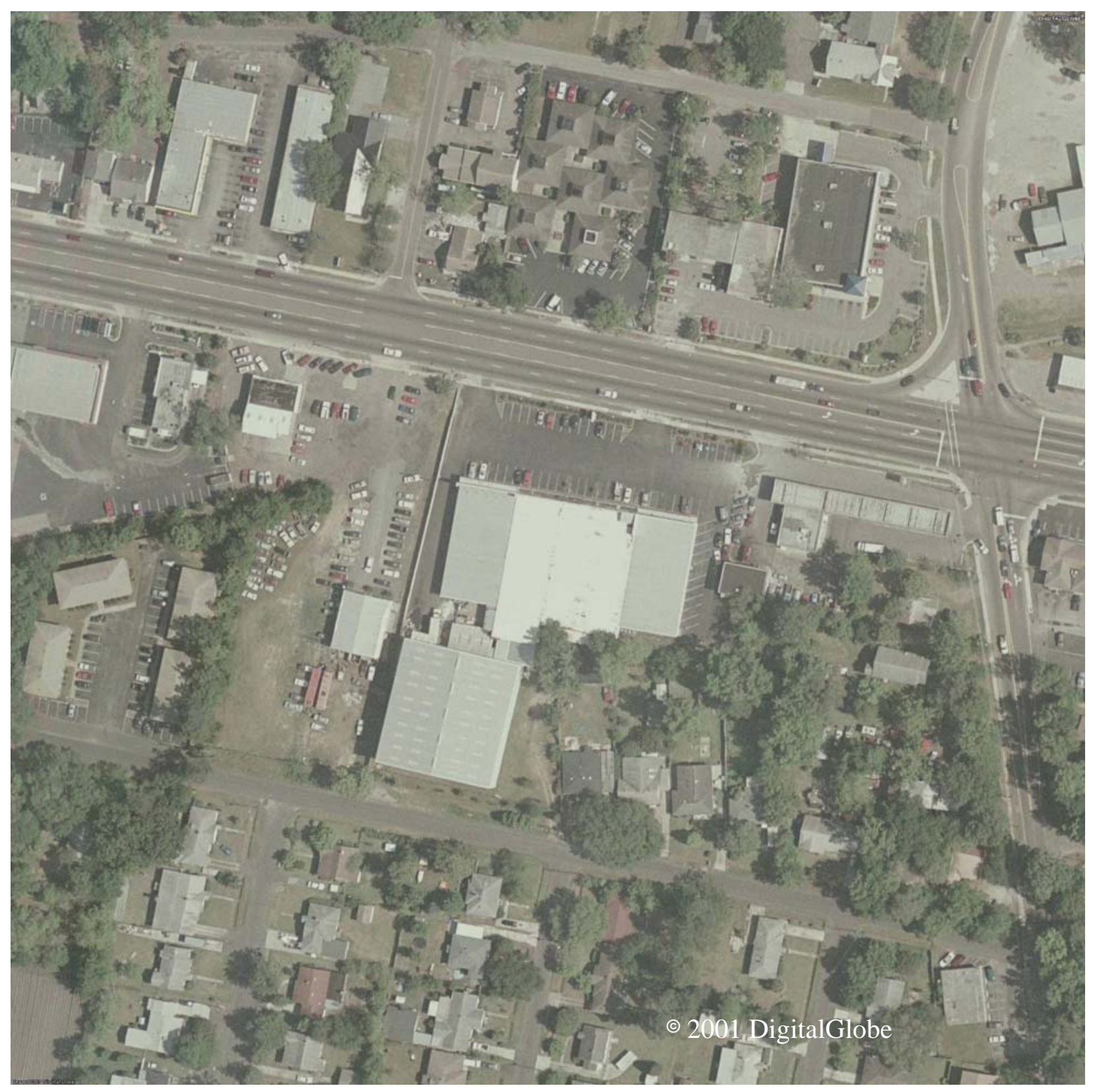

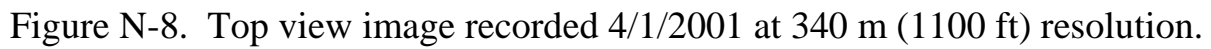




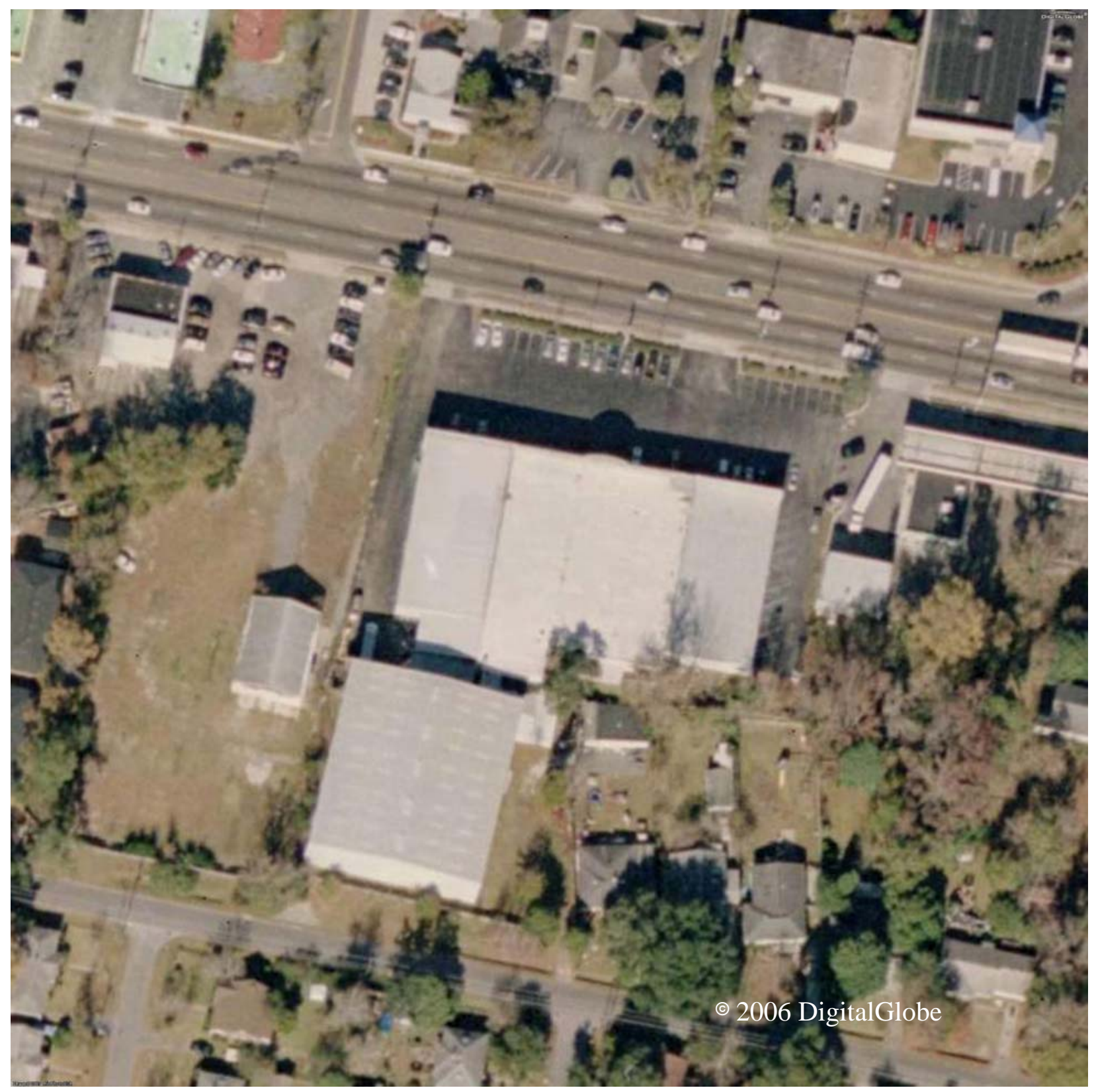

Figure N-9. Top view image recorded 12/1/2006 at 230 m (740 ft) resolution. 


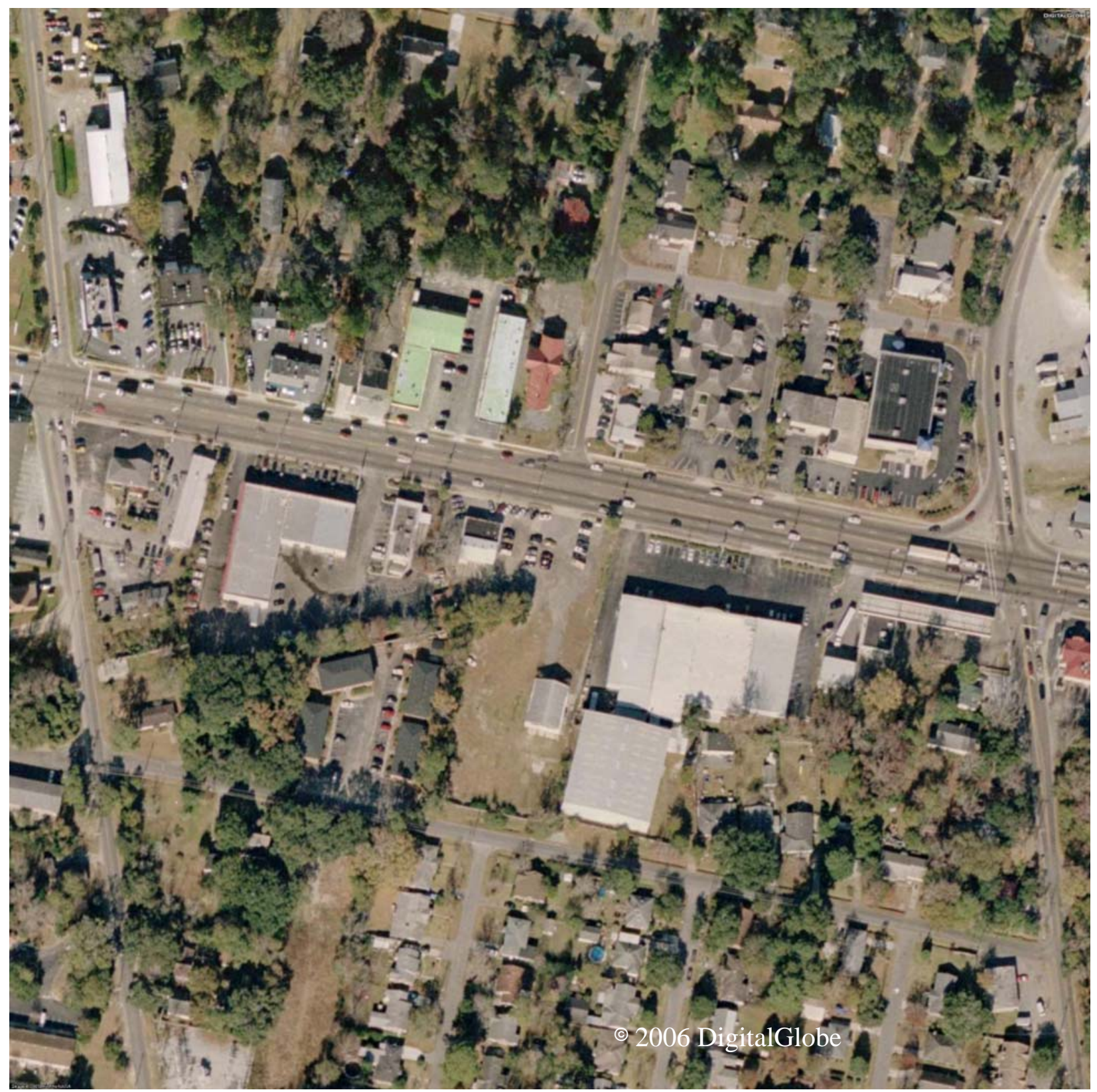

Figure N-10. Top view image recorded 12/1/2006 at $450 \mathrm{~m}(1480 \mathrm{ft})$ resolution. 


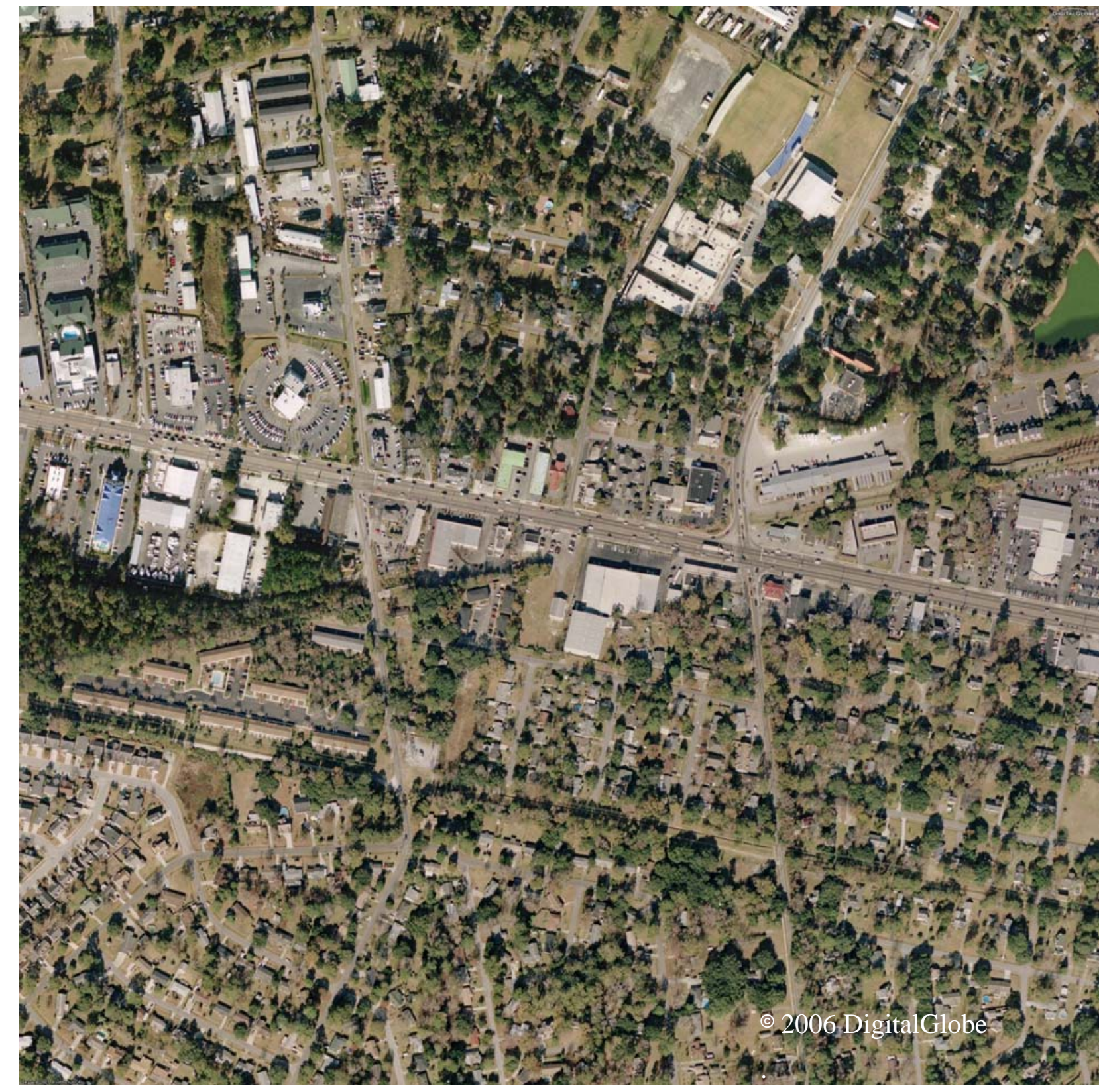

Figure N-11. Top view image recorded 12/1/2006 at 1130 m (3690 ft) resolution. 


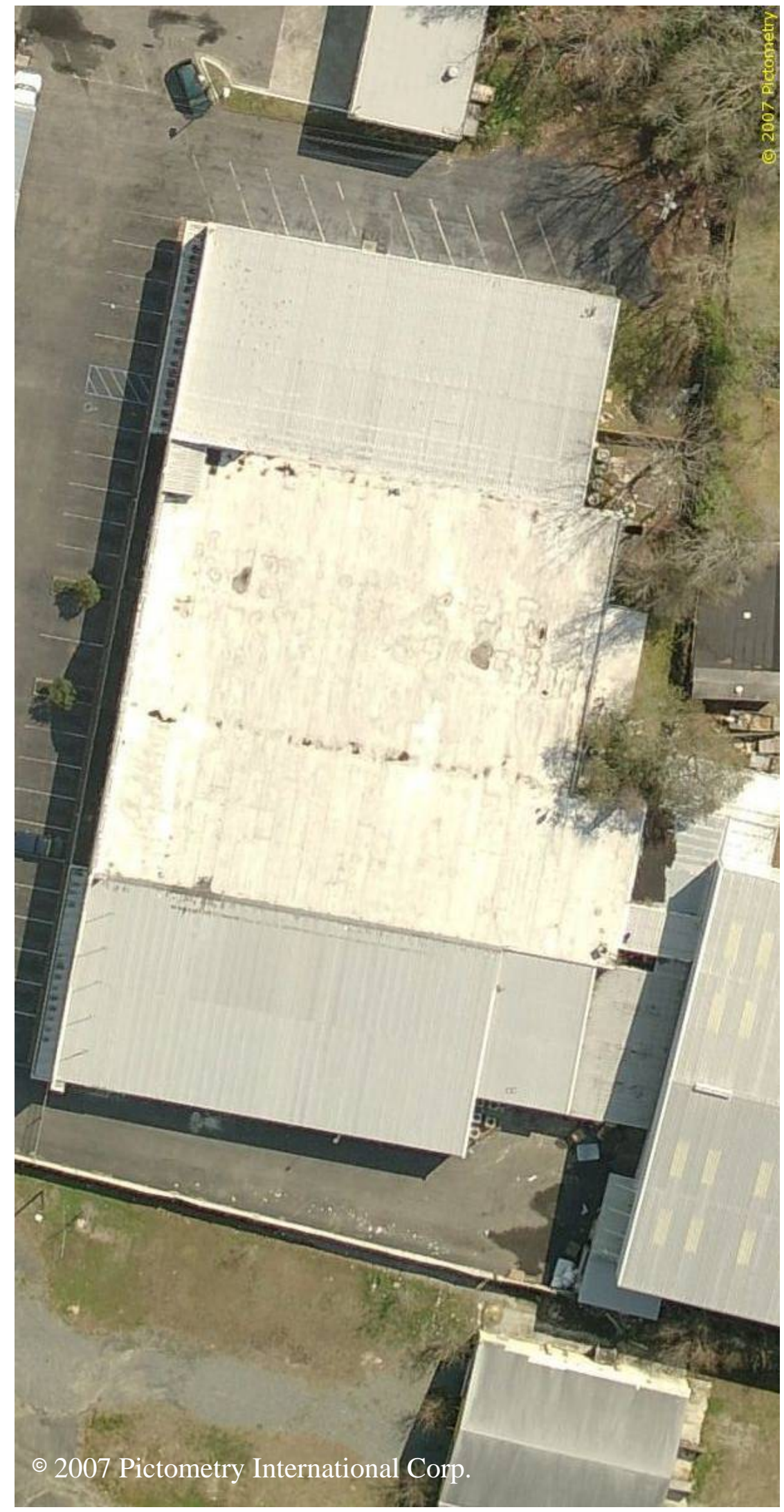

Figure N-12. Top view image recorded 3/11/2007 with narrow field of view. 


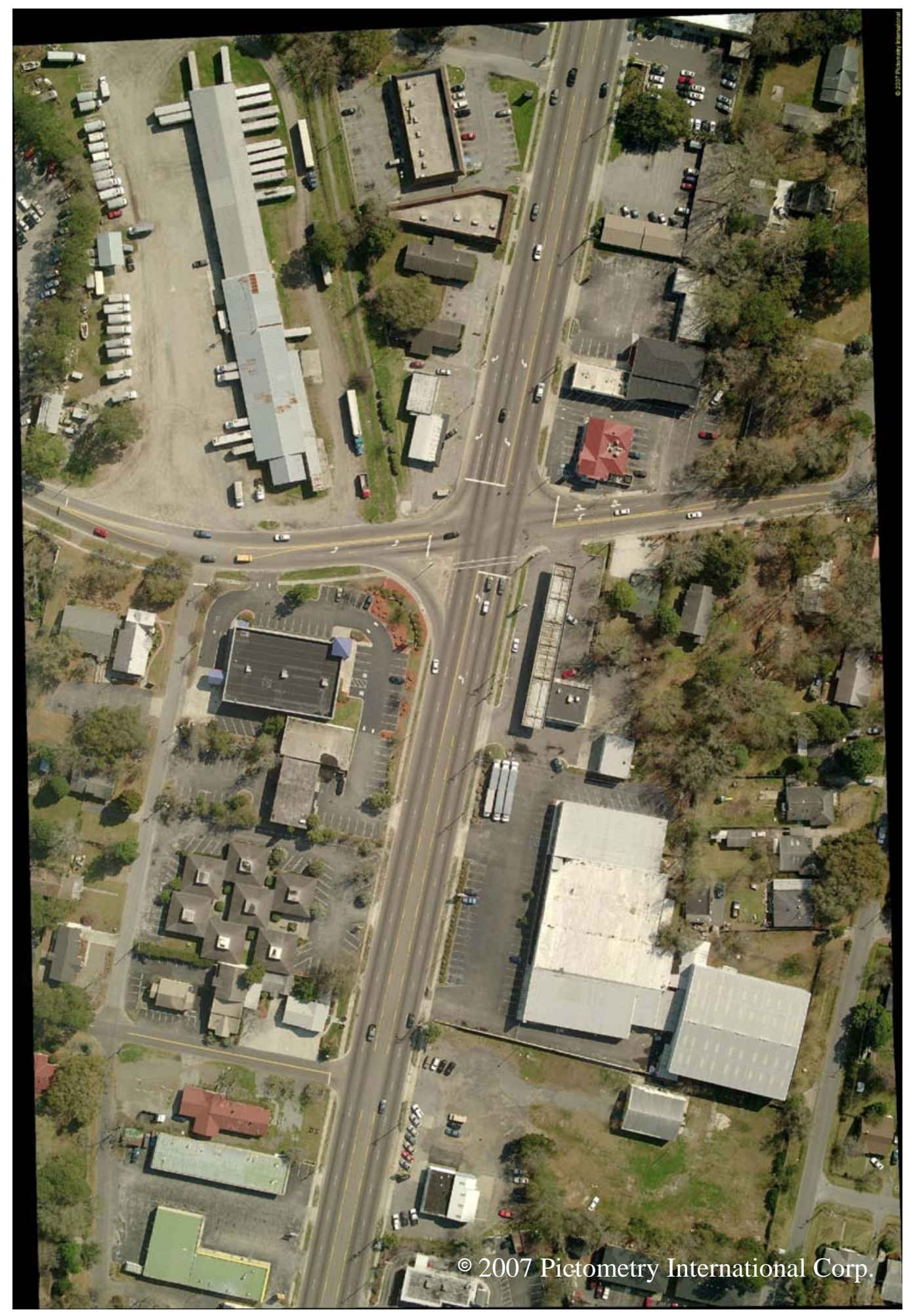

Figure N-13. Top view image recorded 3/11/2007 with wide field of view. 


\section{N.3 ANGLED OR OBLIQUE AERIAL IMAGES}

Oblique images were recorded on 3/11/2007 from north, east, south, and west perspectives and are summarized in Table N-2.

Table N-2. Oblique Aerial Images for Sofa Super Store.

\begin{tabular}{|l|c|c|c|c|}
\hline \multicolumn{1}{|c|}{ Date } & \multicolumn{3}{|c|}{ Image Perspective } \\
\hline \multirow{3}{*}{ 3/11/2007 } & North & East & South & West \\
Narrow View & Figures N-14 & Figure N-16 & Figure N-18 & Figure N-20 \\
\hline 3/11/2007 & & & & \\
Wide View & Figures N-15 & Figure N-17 & Figure N-19 & Figure N-21 \\
\hline
\end{tabular}




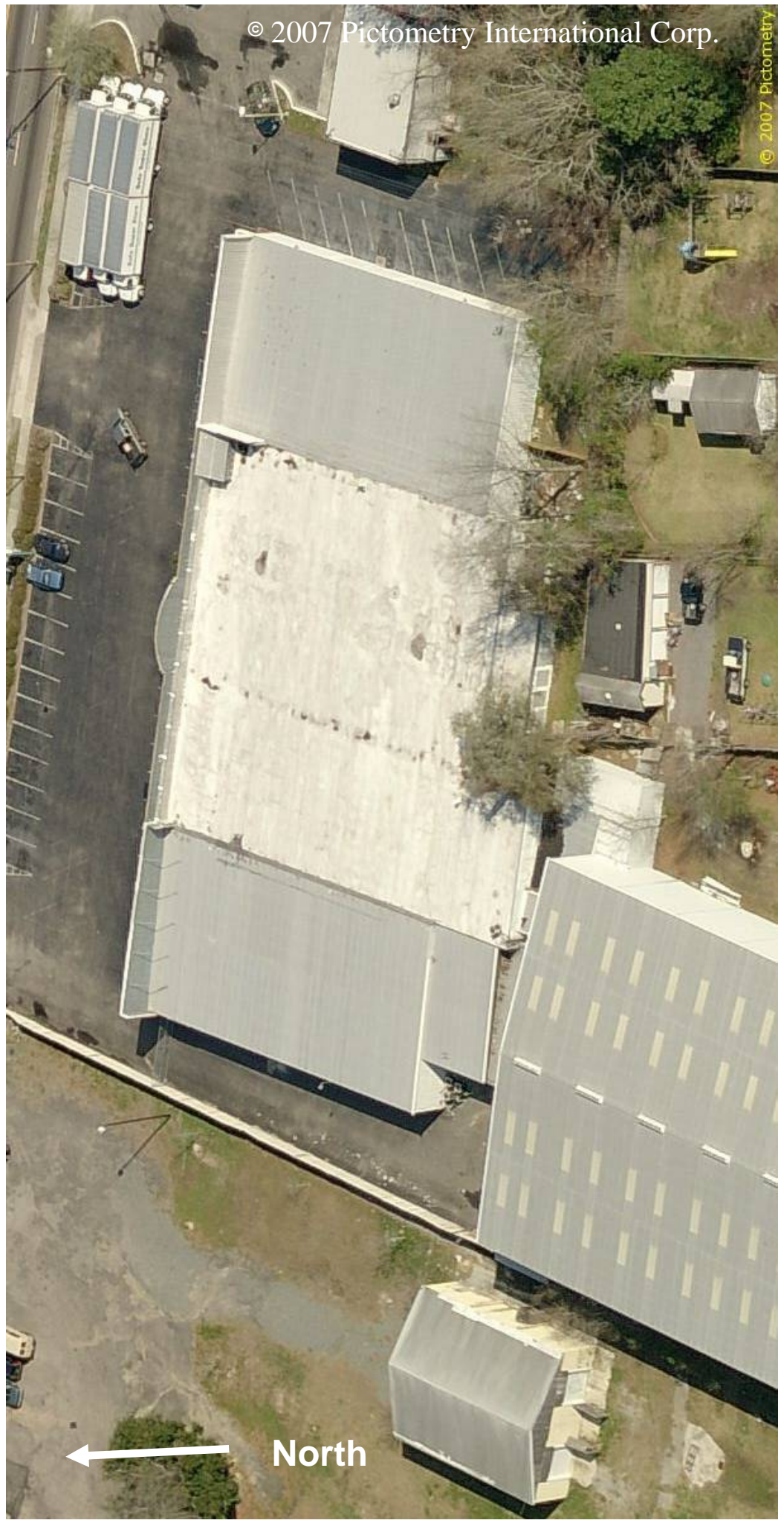

Figure N-14. Oblique view image recorded 3/11/2007 looking north with narrow field of view. 


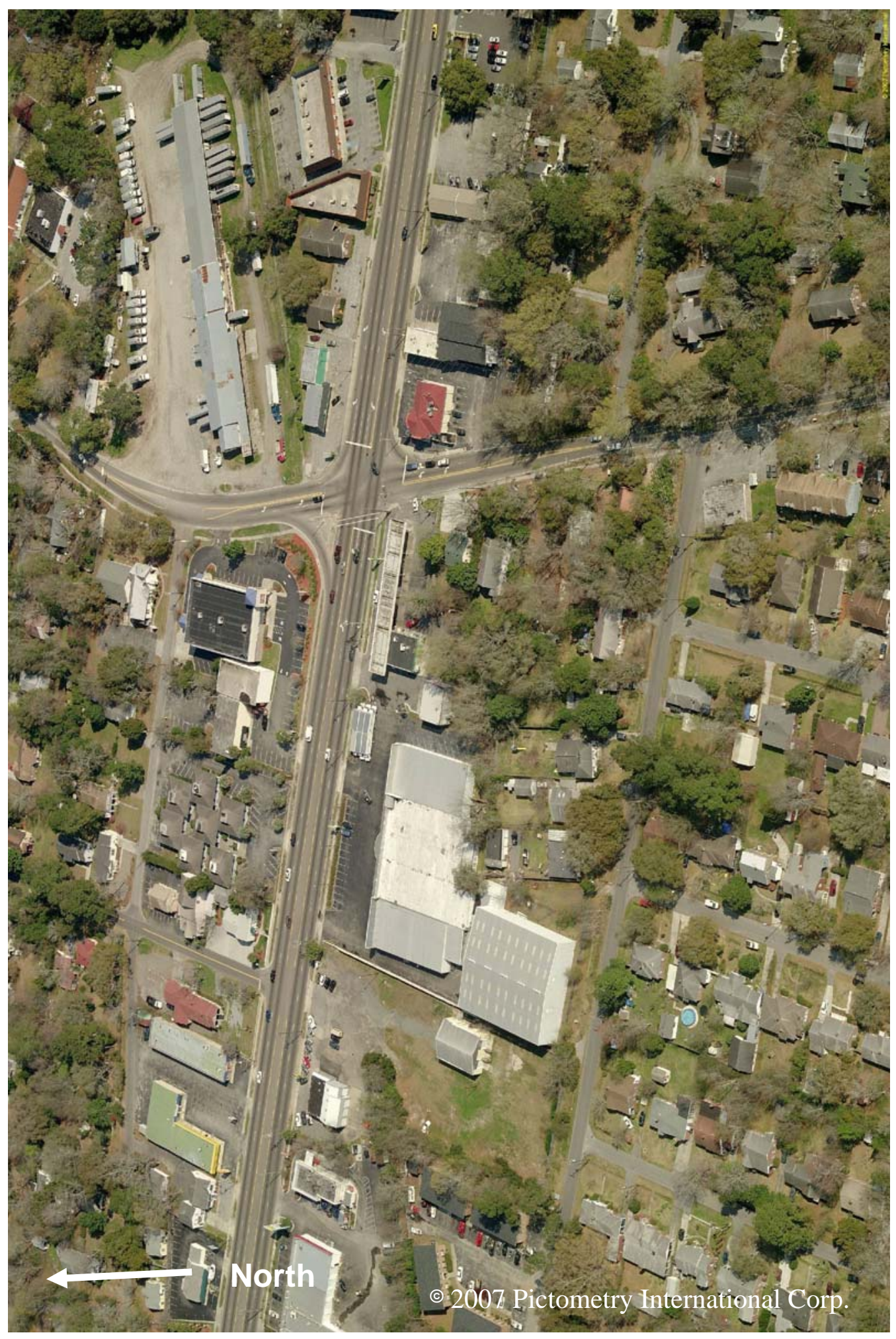

Figure N-15. Oblique view image recorded 3/11/2007 looking north with wide field of view. 


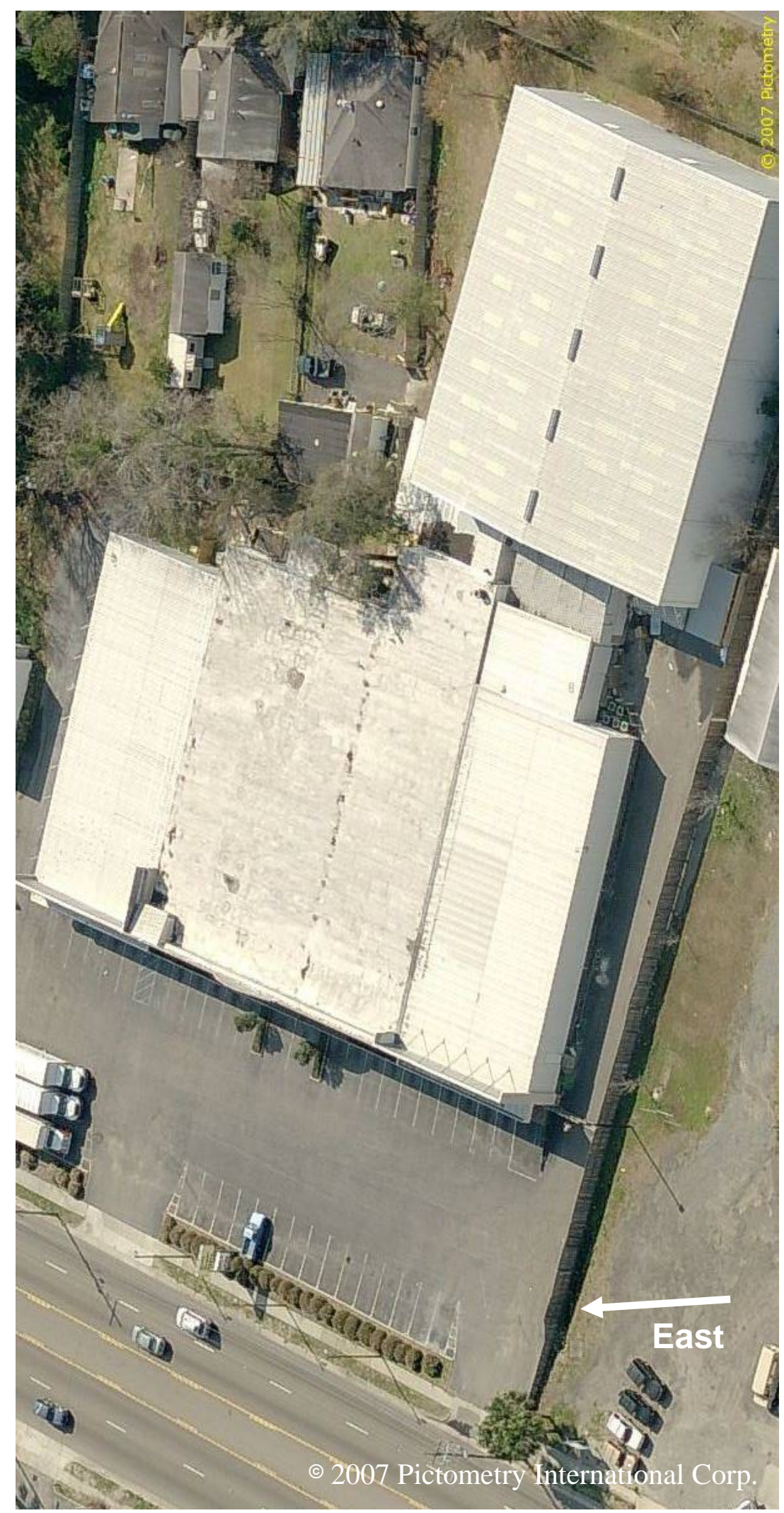

Figure N-16. Oblique view image recorded 3/11/2007 looking east with narrow field of view. 


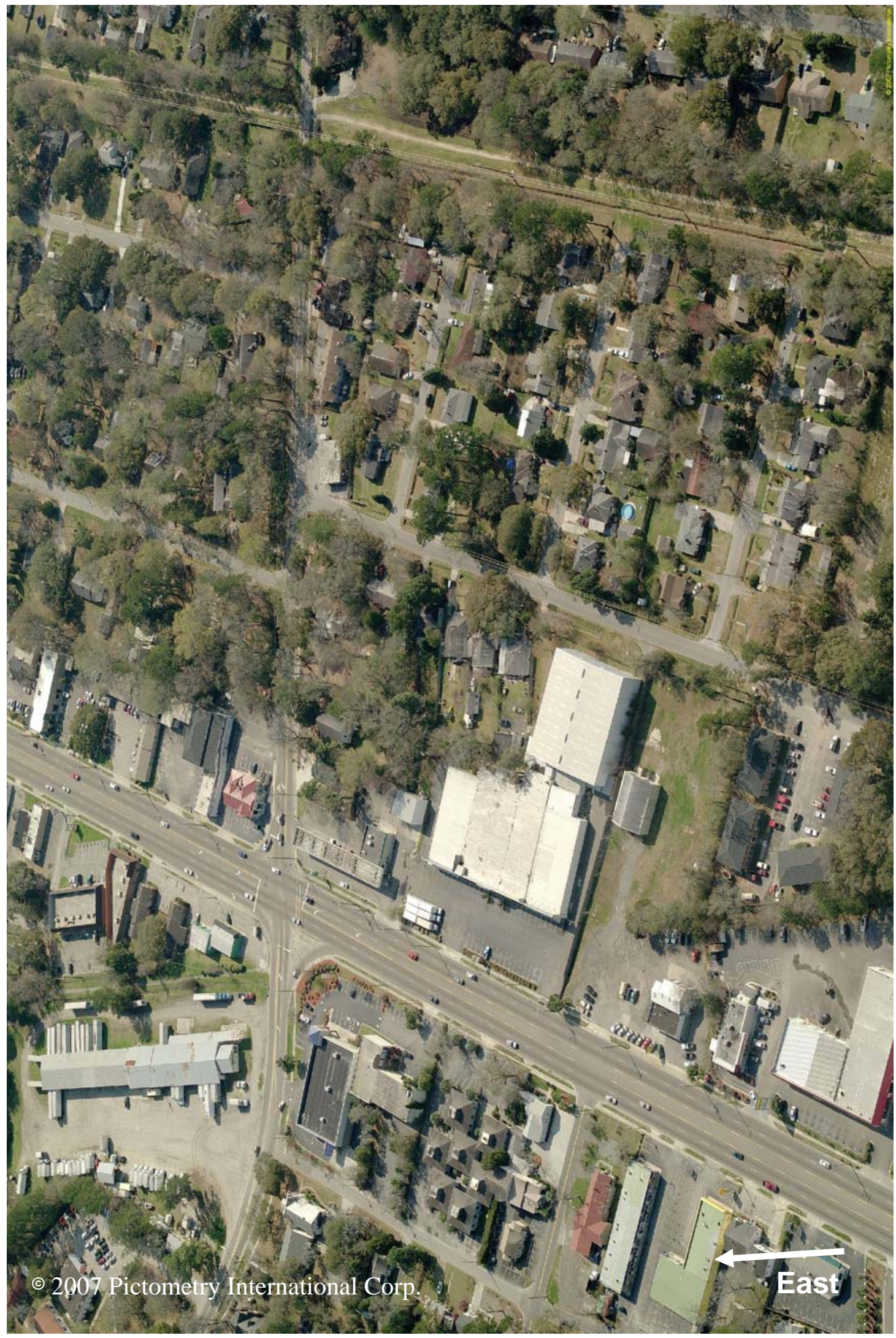

Figure N-17. Oblique view image recorded 3/11/2007 looking east with wide field of view. 


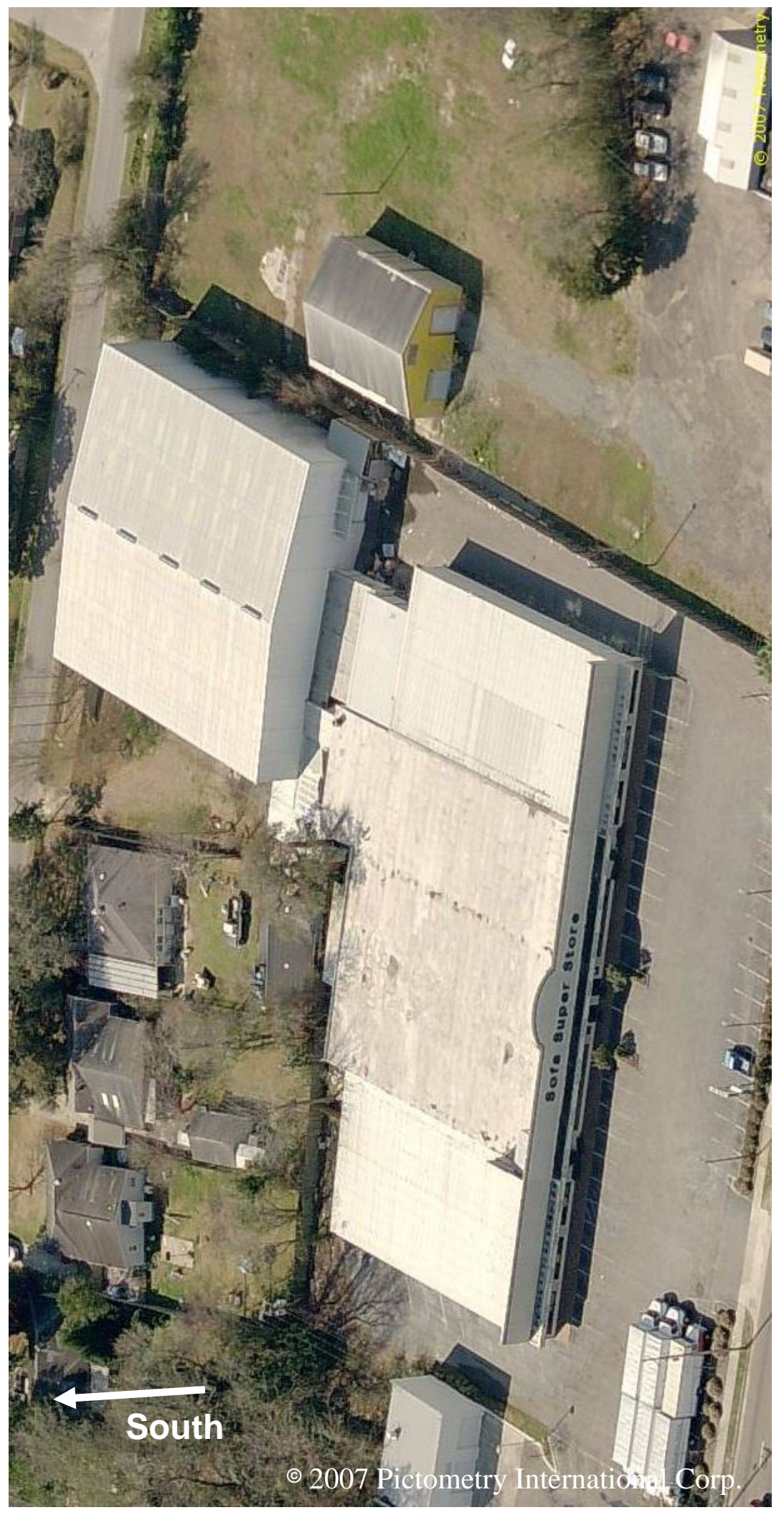

Figure N-18. Oblique view image recorded 3/11/2007 looking south with narrow field of view. 


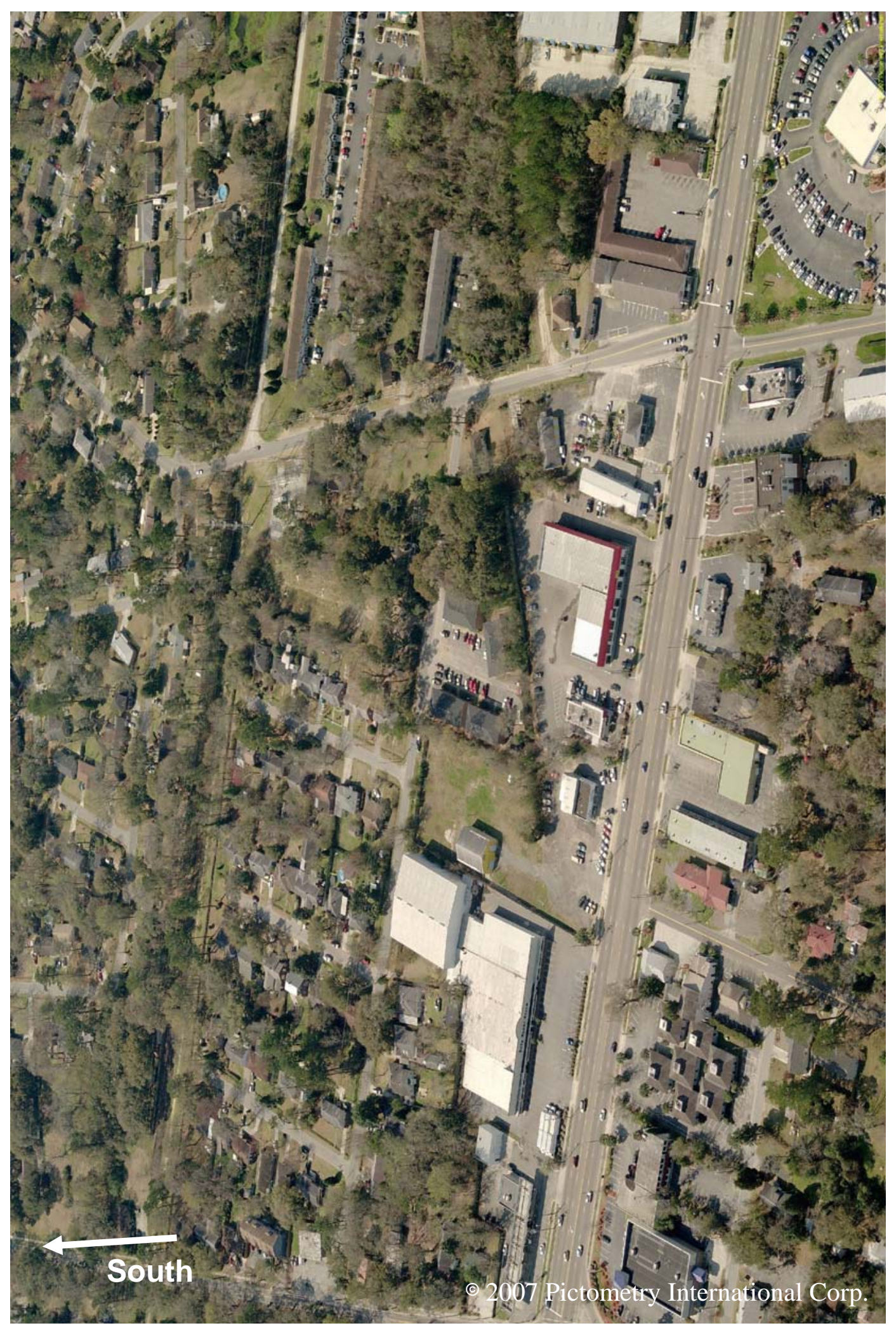

Figure N-19. Oblique view image recorded 3/11/2007 looking south with wide field of view. 


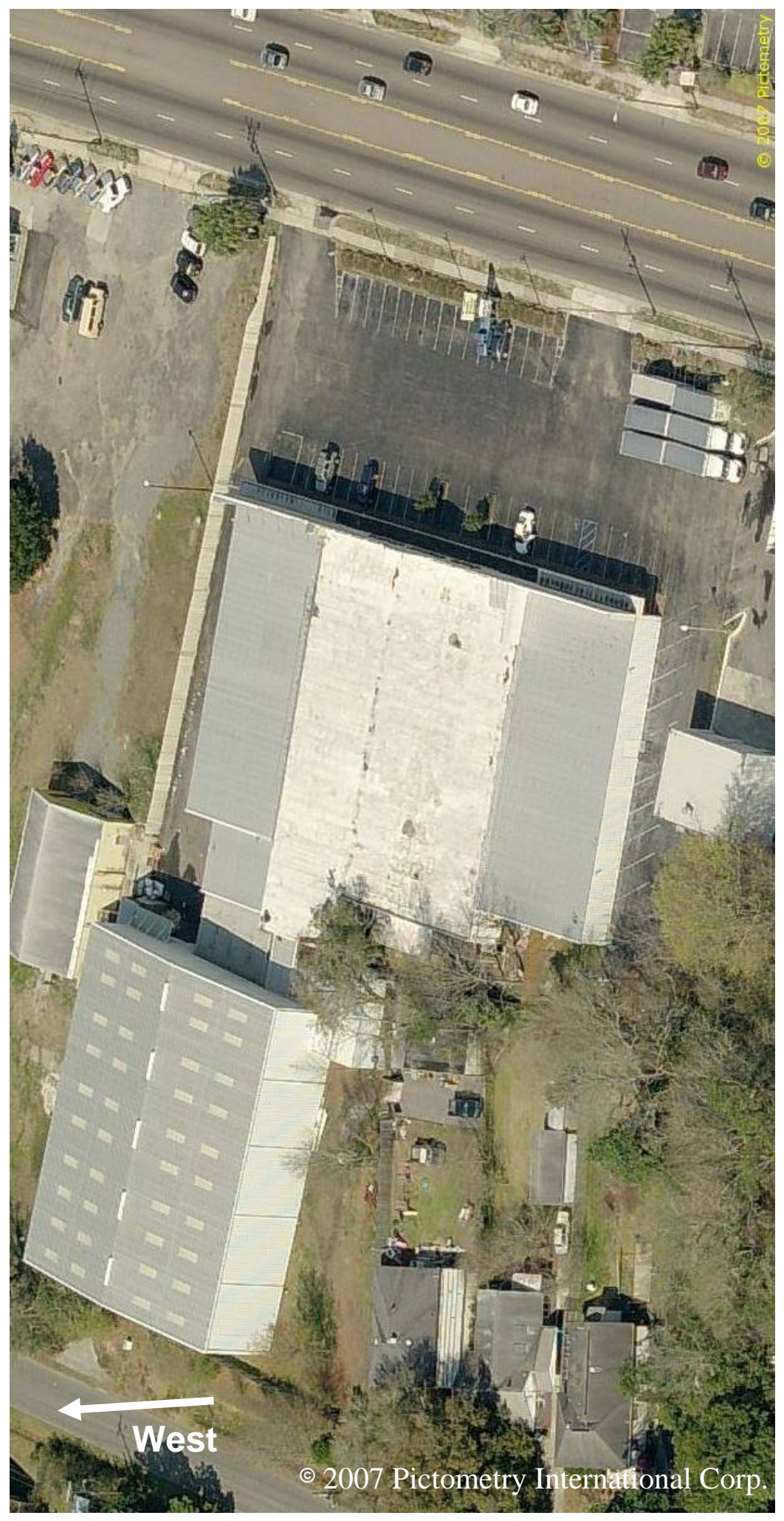

Figure N-20. Oblique view image recorded 3/11/2007 looking west with narrow field of view. 


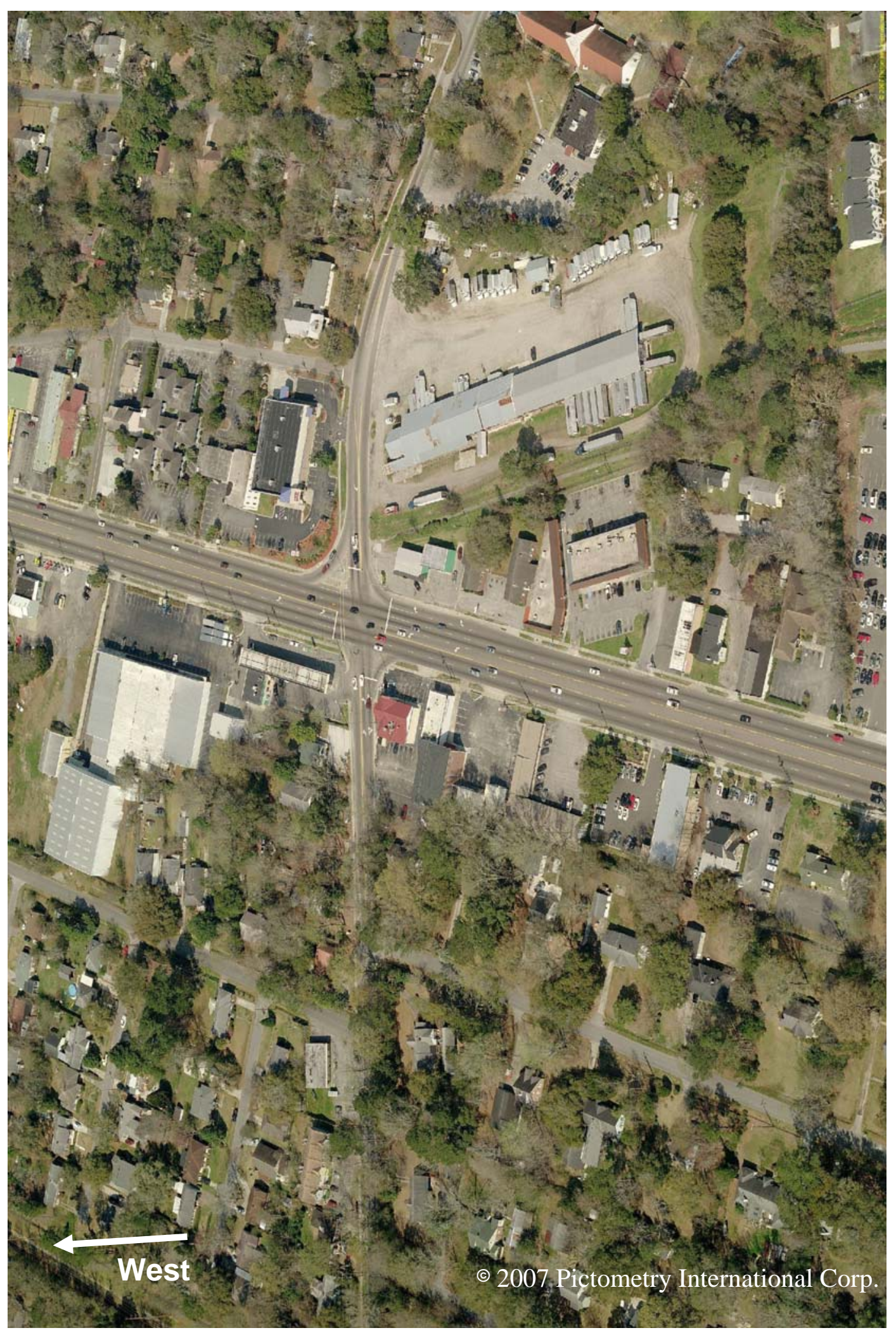

Figure N-21. Oblique view image recorded 3/11/2007 looking west with wide field of view. 


\section{N.4 REFERENCES FOR APPENDIX N}

[1] http://www.digitalglobe.com/ - DigitalGlobe, Longmont, CO 80503, 303-684-4000.

[2] http://www.pictometry.com/ - Pictometry, Rochester, NY 14623, 888-771-9714. 


\section{APPENDIX O. ROLL-DOWN DOORS}

\section{O.1 ROLL-DOWN DOORS}

The original configuration of this structure as a grocery store did not include any roll-down fire doors. The structure did have a non-fire roll-down door that allowed access to the south-west corner of the structure. When the west showroom was added in 1994, three roll-down fire doors were added to the west wall of the main showroom. In 1995, when the east showroom was built, three additional roll-down fire doors were installed in the east wall of the main showroom. As the warehouse was constructed in 1996, another roll-down fire door was placed at the entrance to the breezeway that led to the warehouse. 

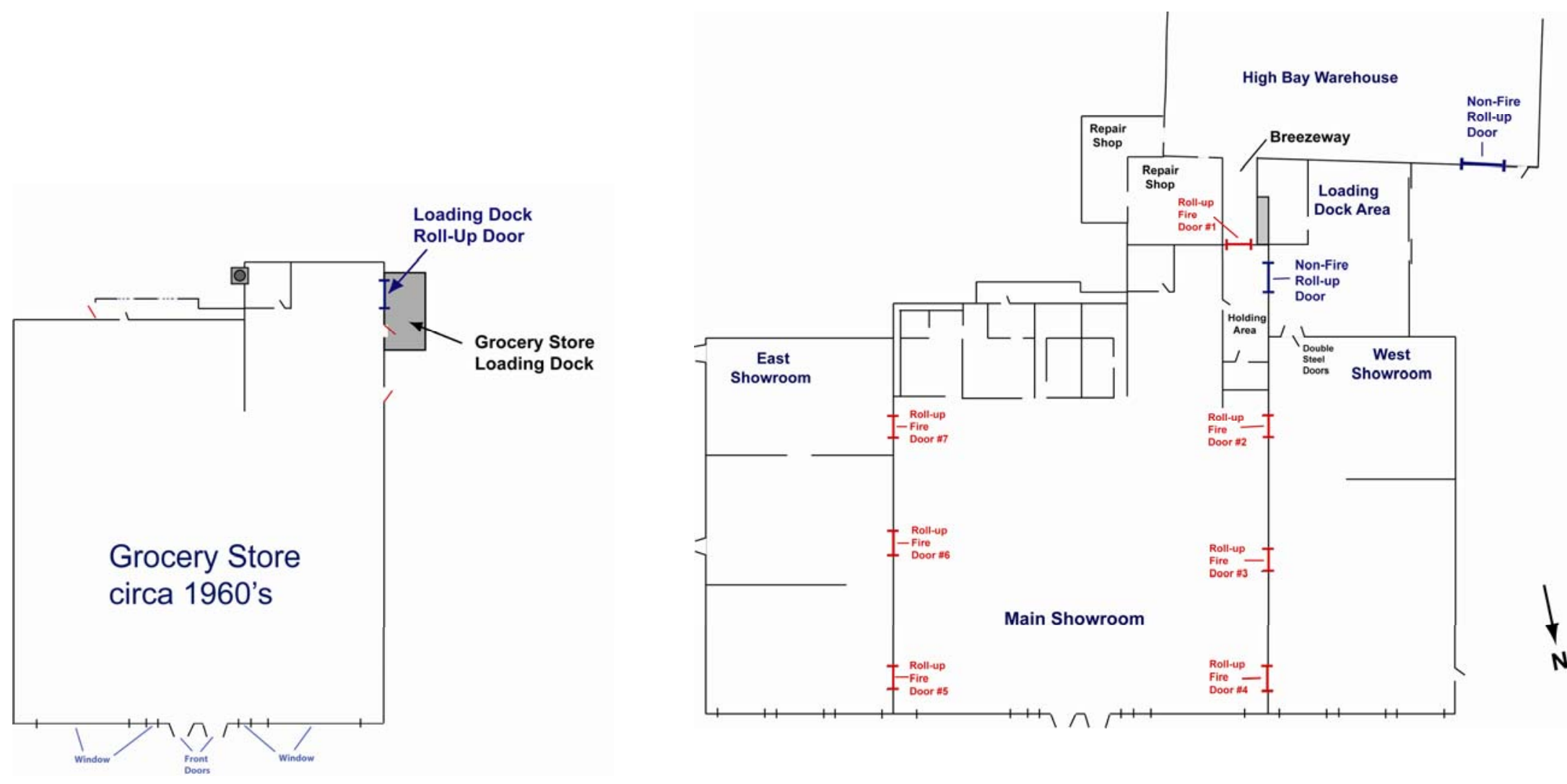

Figure 0-1. Before 1994, the structure included a single roll-down door at the loading dock (left side of figure). This door was not a fire door. By 1996, eight additional roll-down doors had been added (right side of figure). Six of these doors were installed

between the main showroom and east and west additions and were fire doors. An additional fire door was installed between the holding area and the warehouse. Each of these fire doors was designed to separate two interior spaces. An additional non-fire rolldown door was also installed in the warehouse in the loading area. 
O.2 WAREHOUSE ROLL-DOWN DOOR

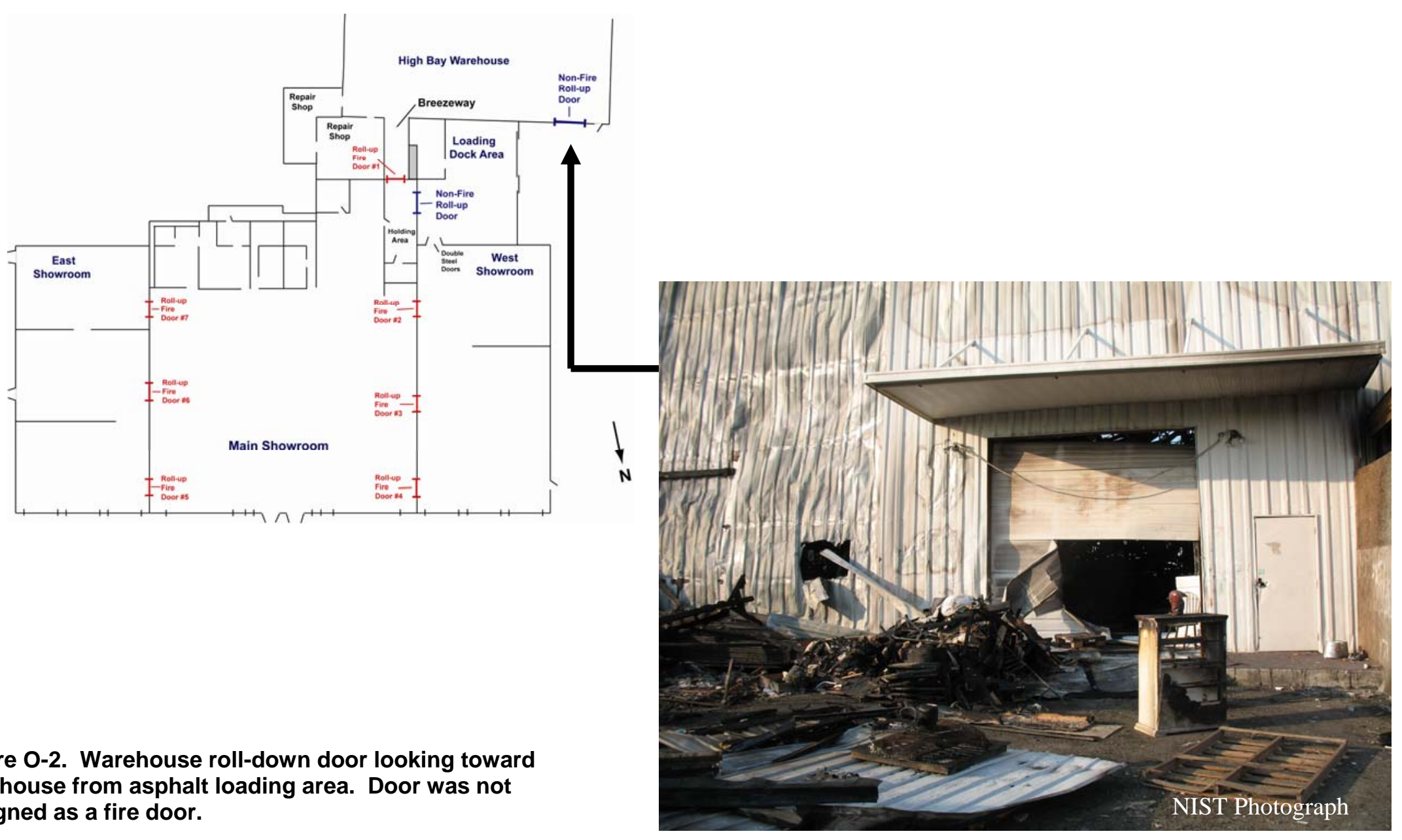




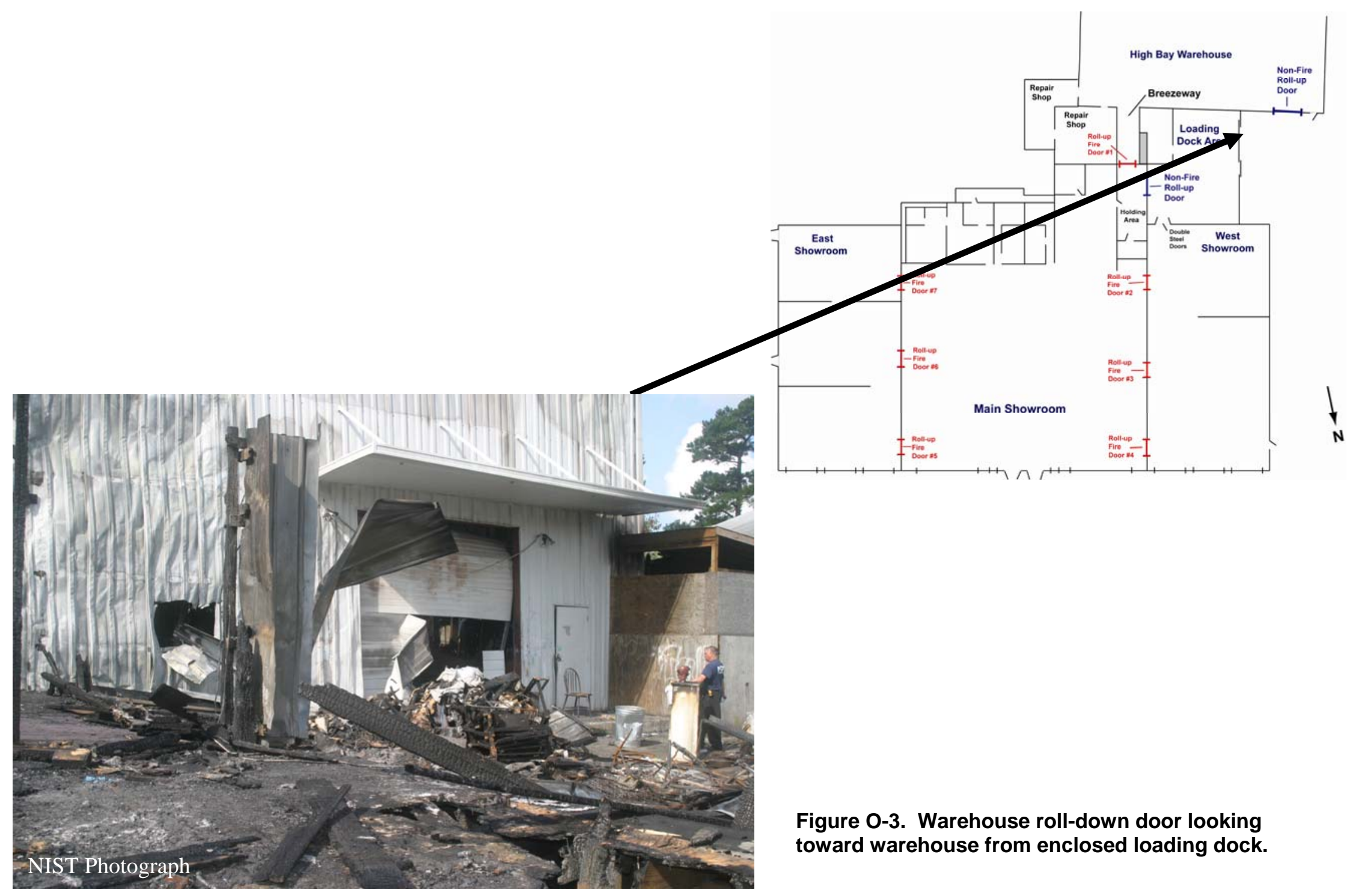




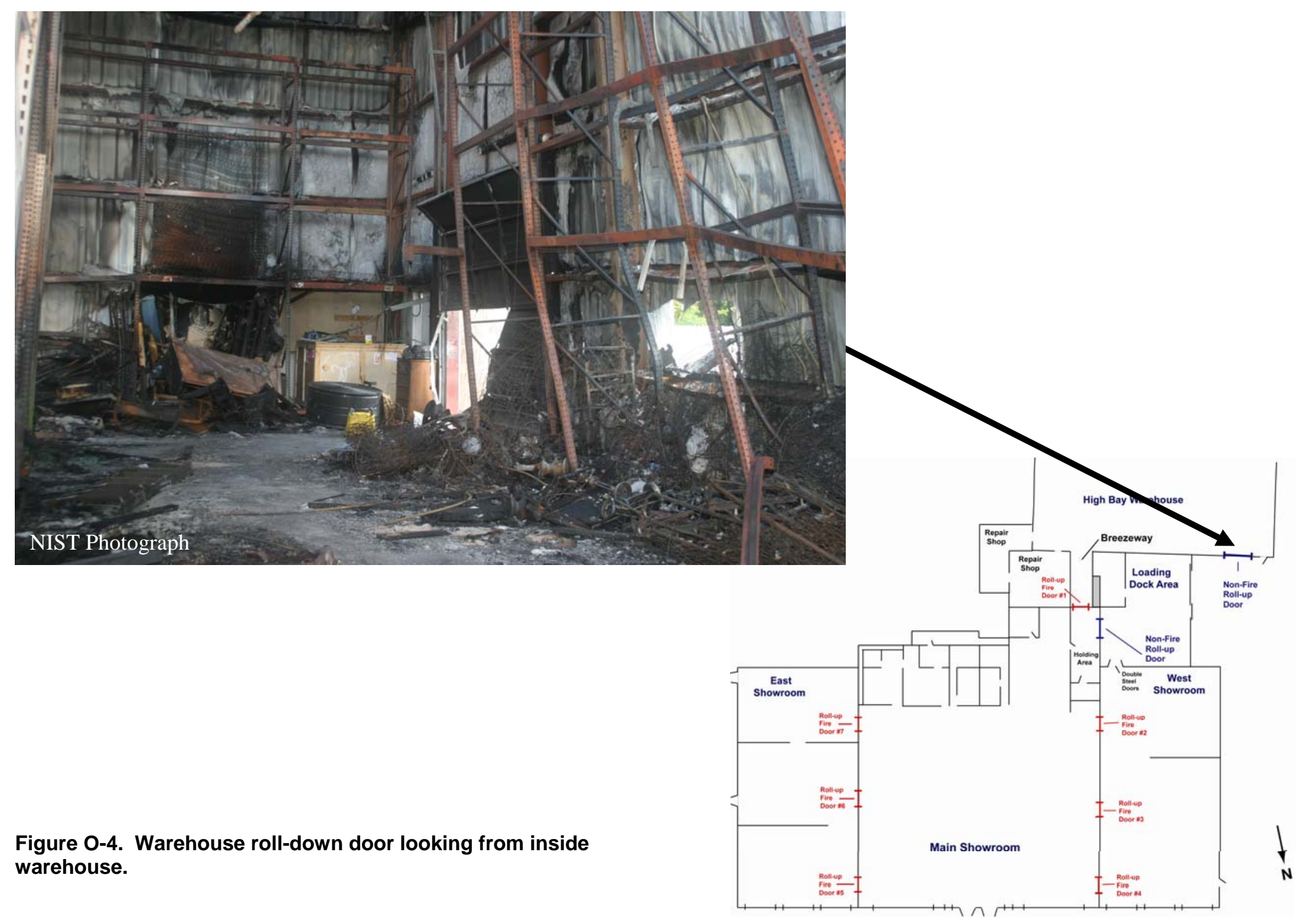




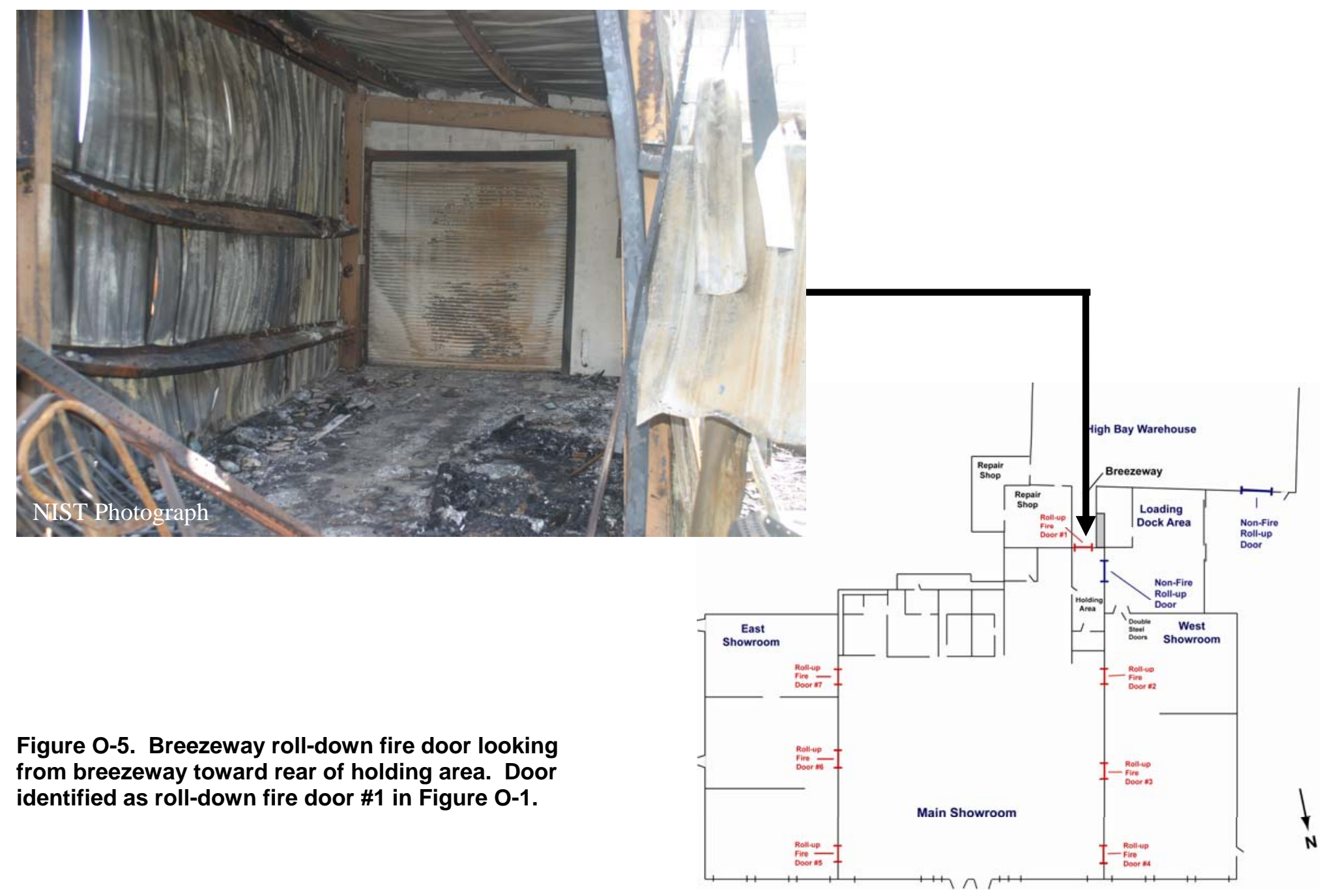




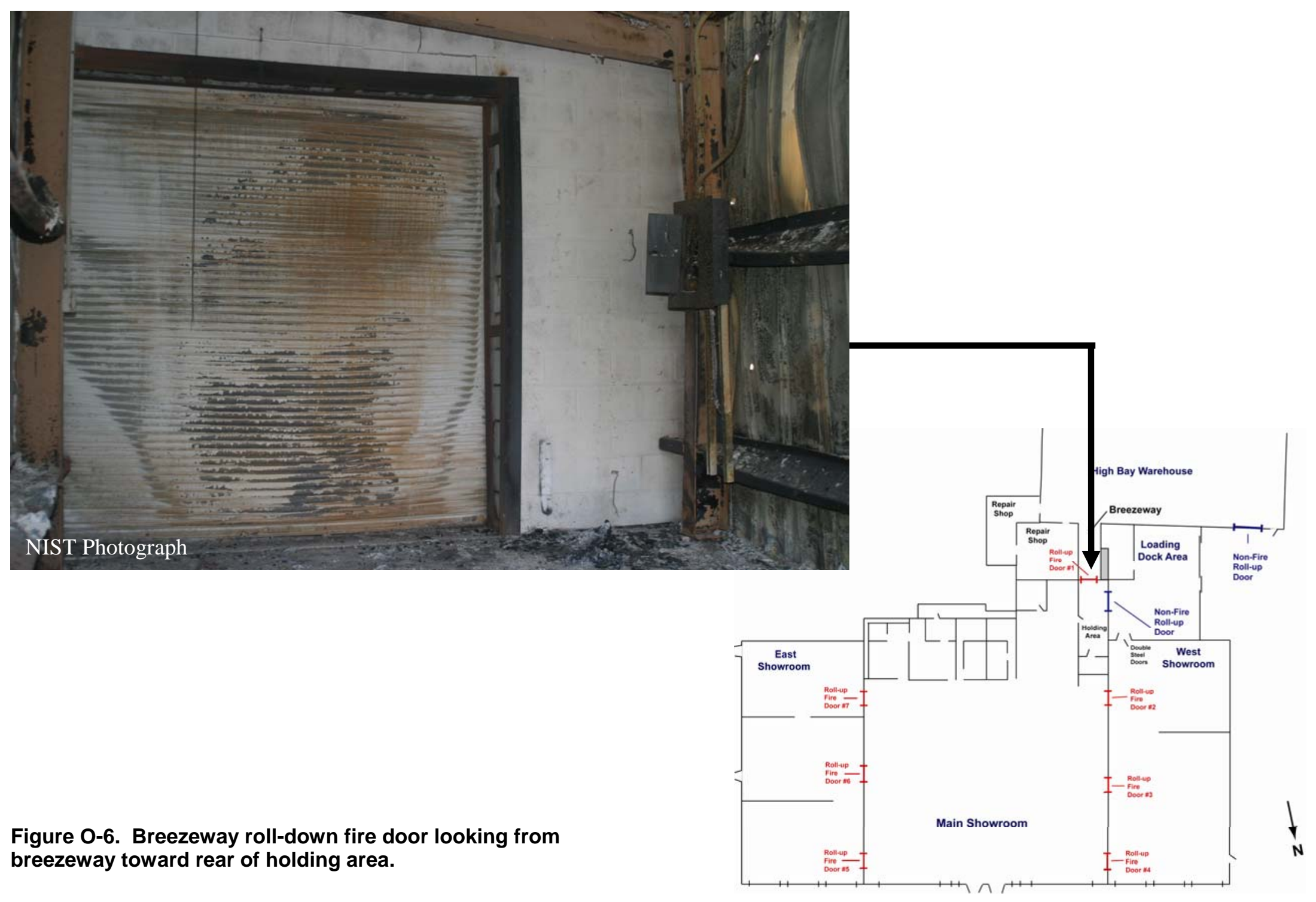




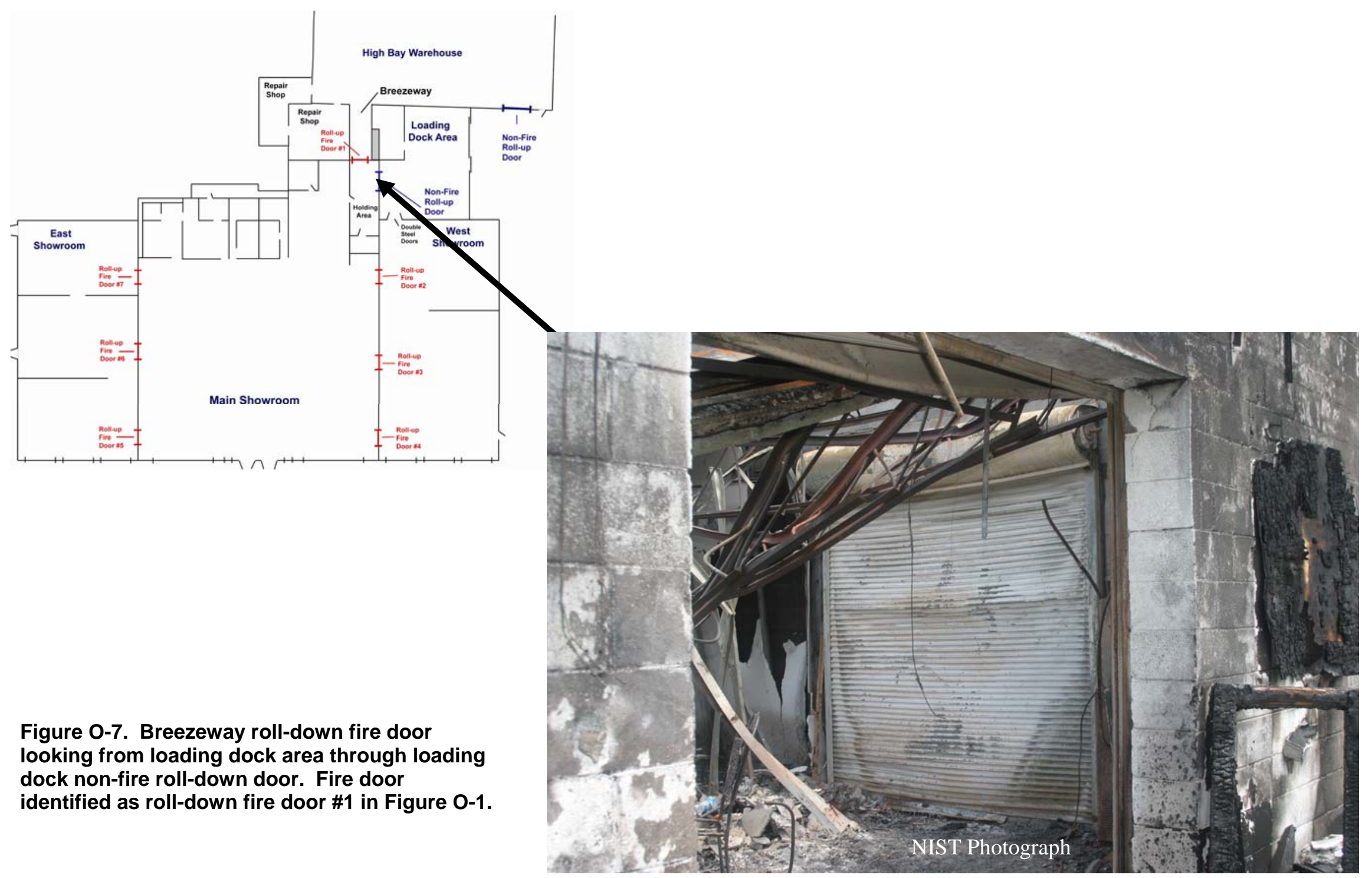




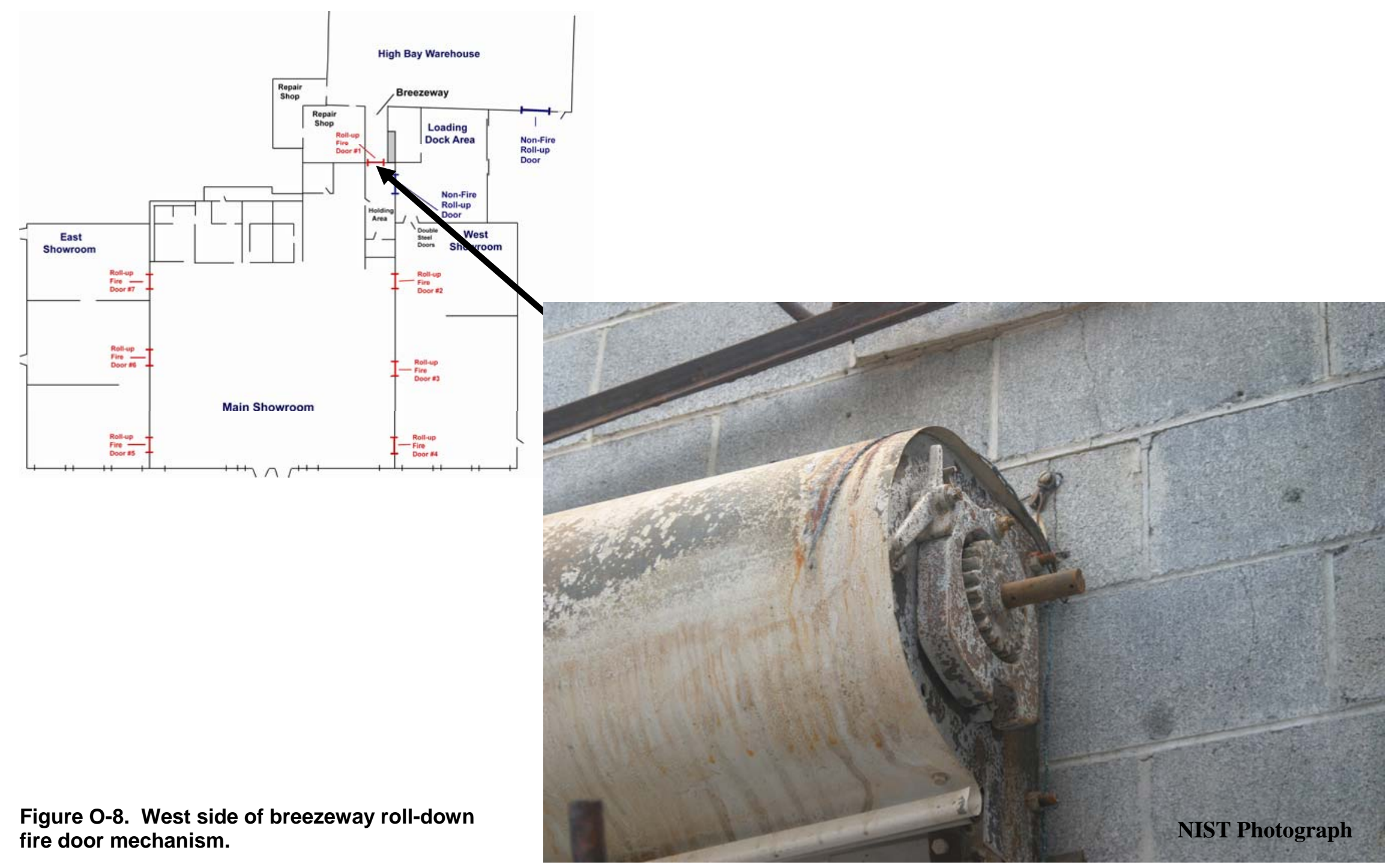




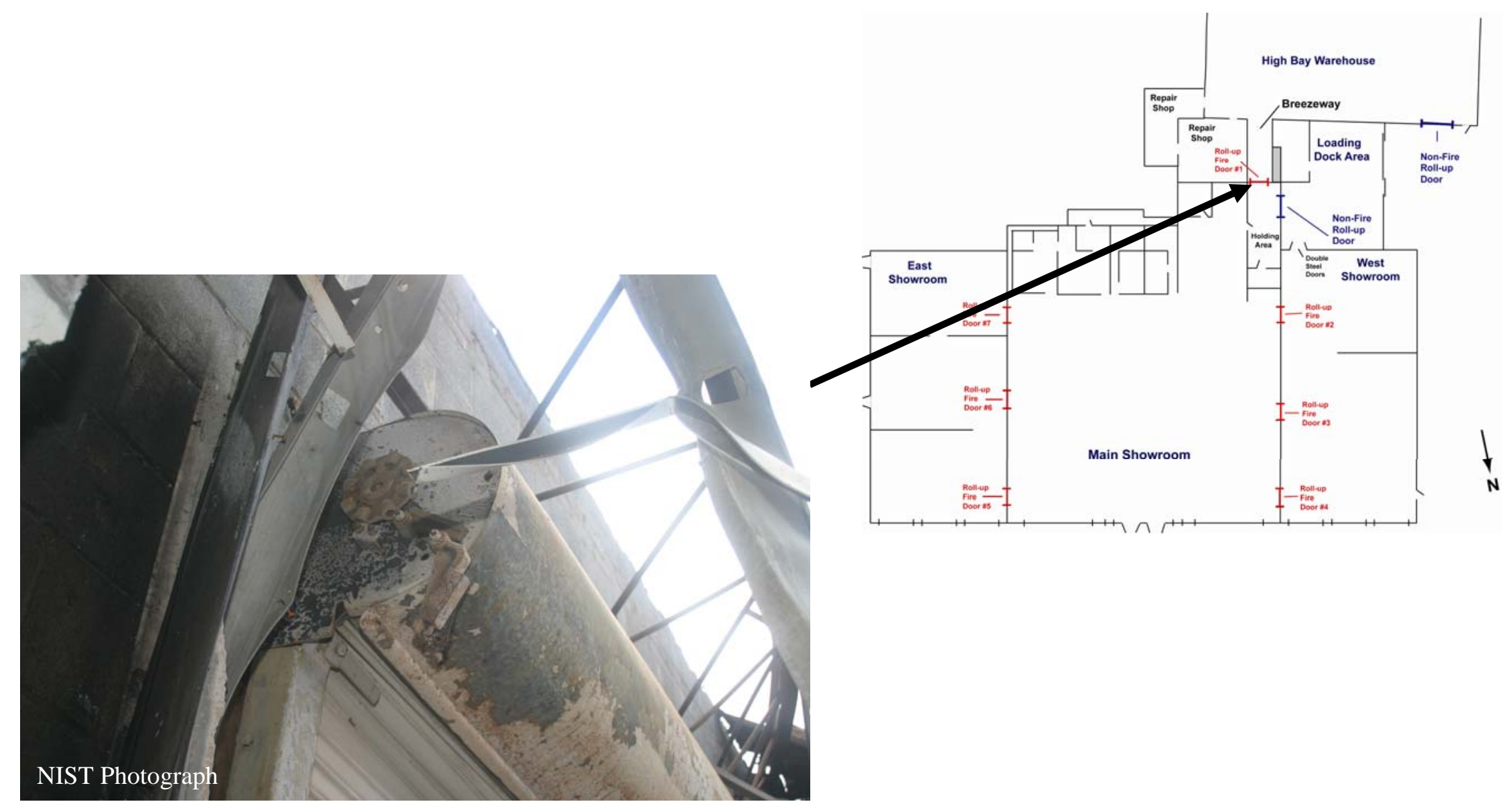

Figure 0-9. East side of breezeway roll-down fire door mechanism. 


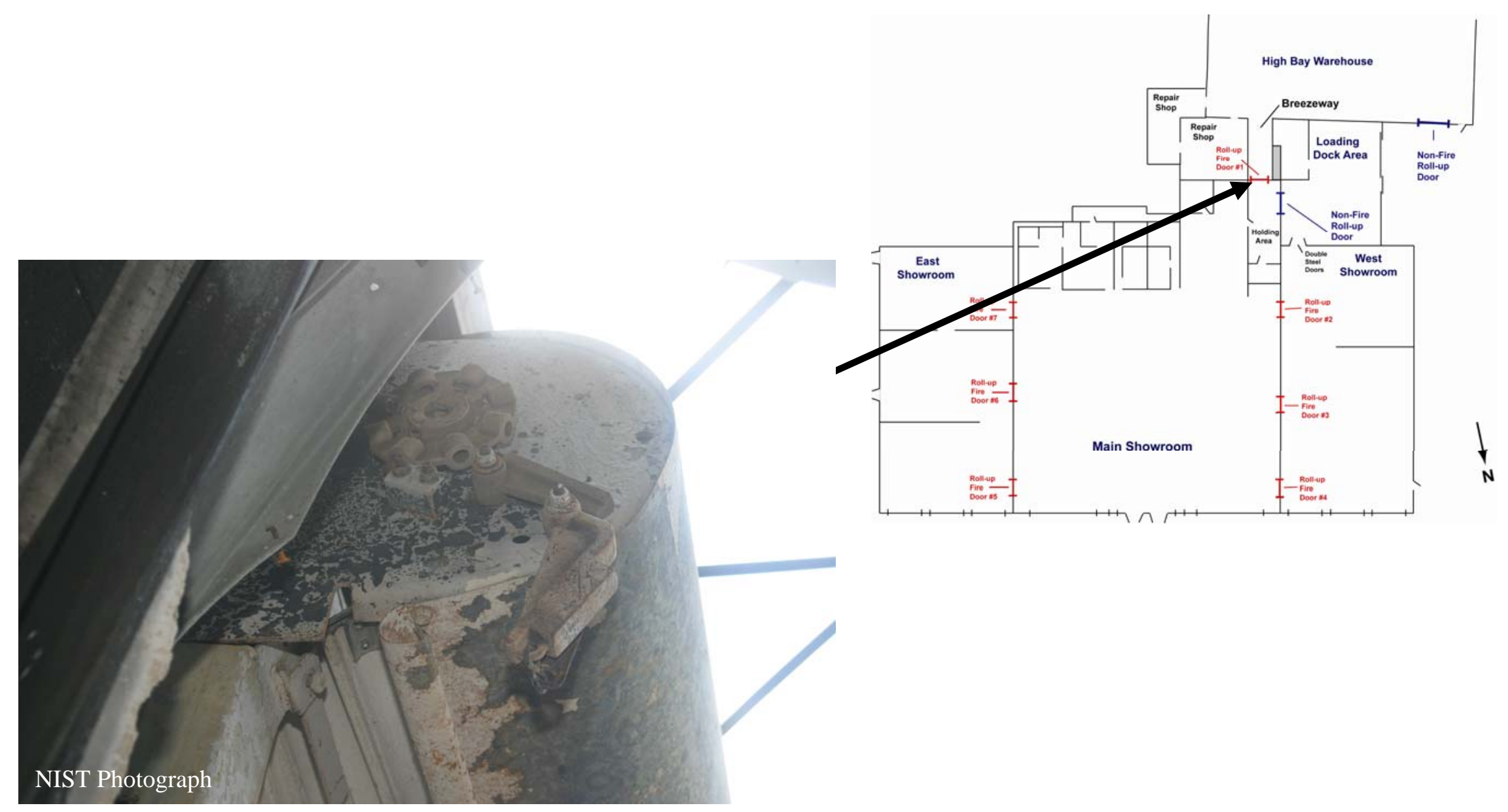

Figure 0-10. East side of breezeway roll-down fire door mechanism. 


\section{O.4 HOLDING AREA/LOADING DOCK ROLL-DOWN DOOR}

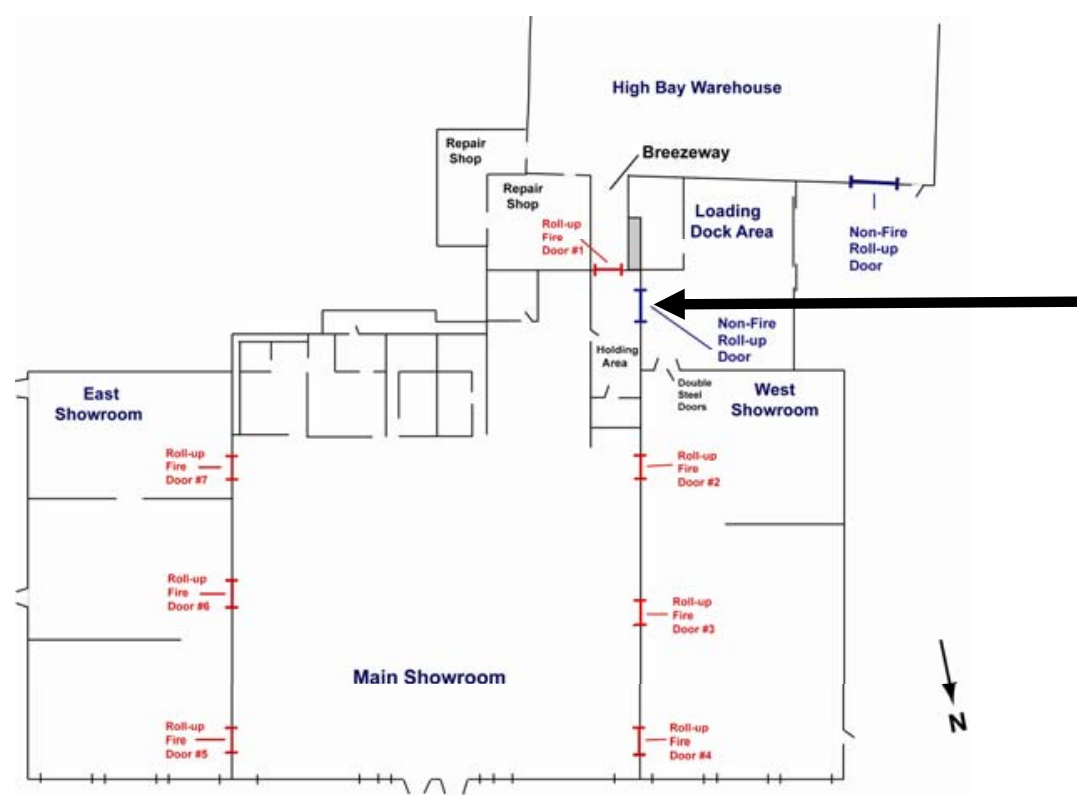

Figure 0-11. Non-fire roll-down loading dock door looking toward rear of main showroom. Door was not designed as a fire door.

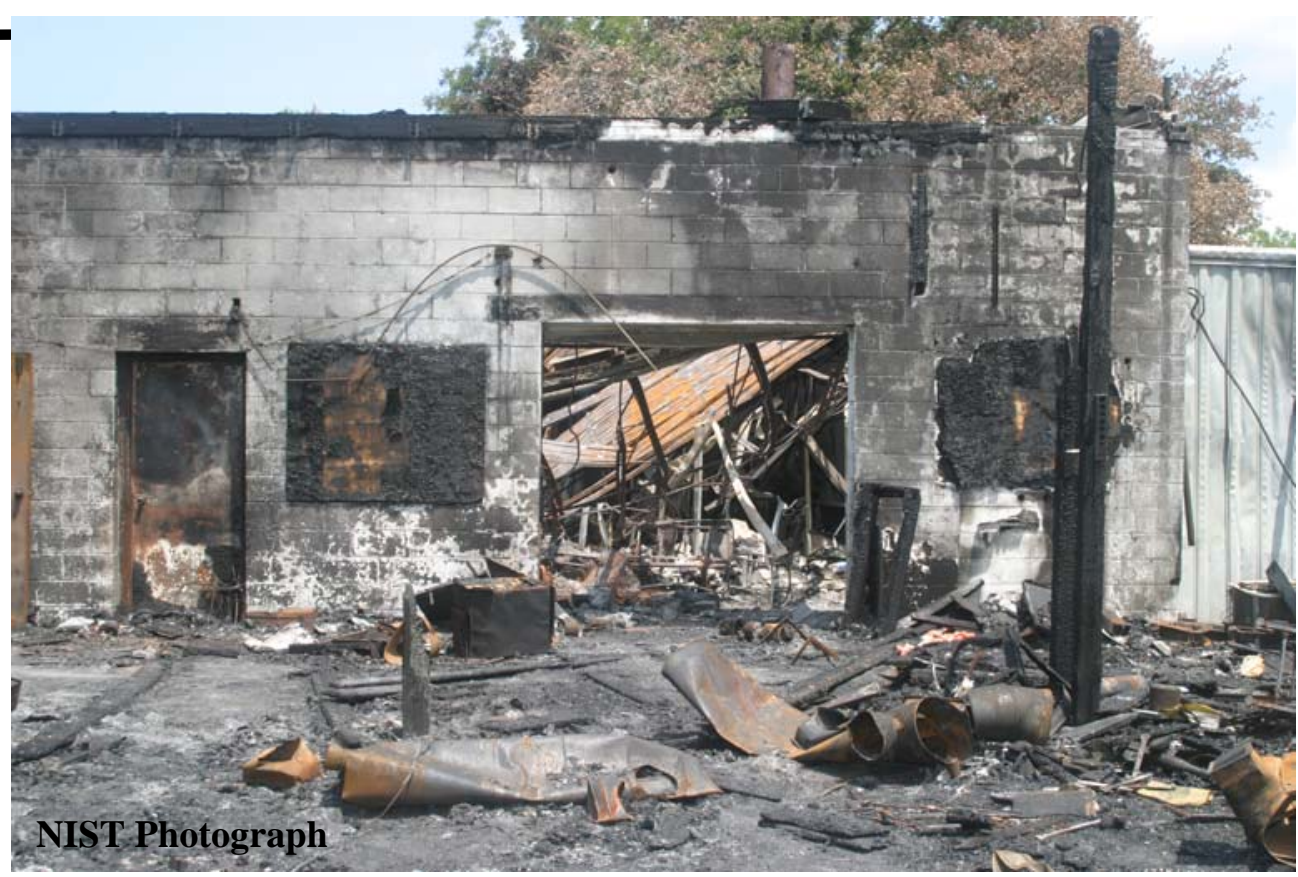




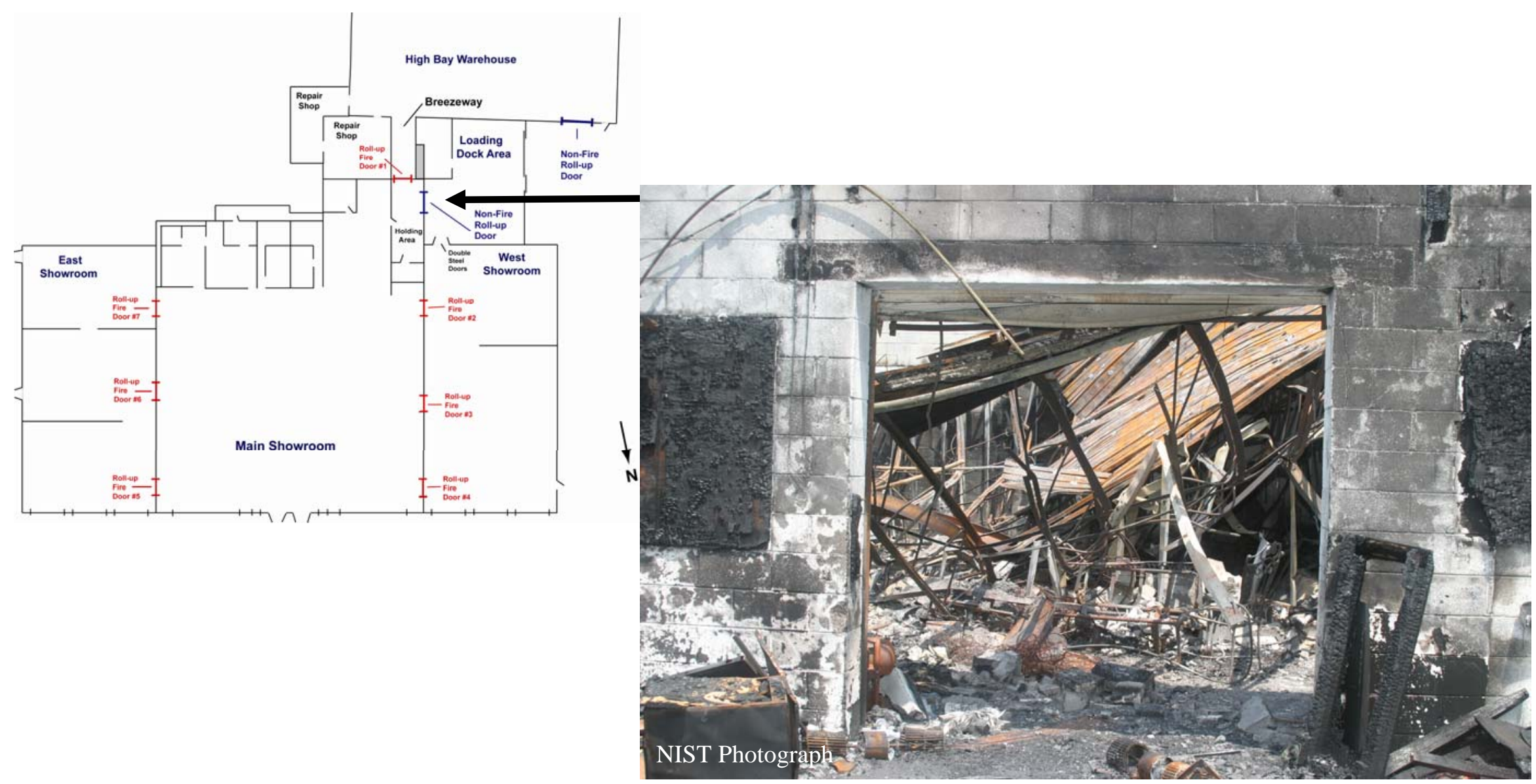

Figure 0-12. Non-fire roll-down loading dock door looking toward rear of main showroom. 


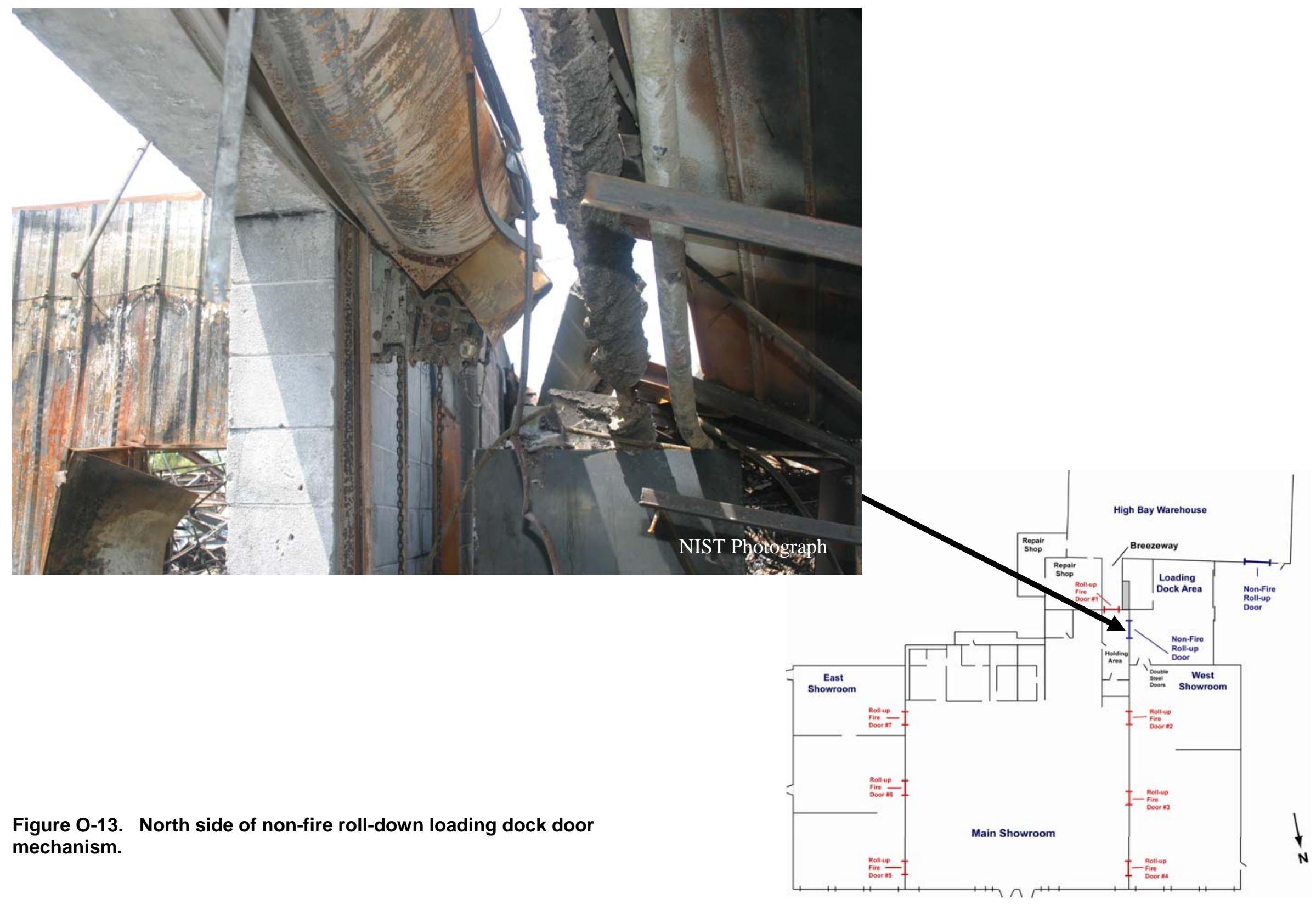




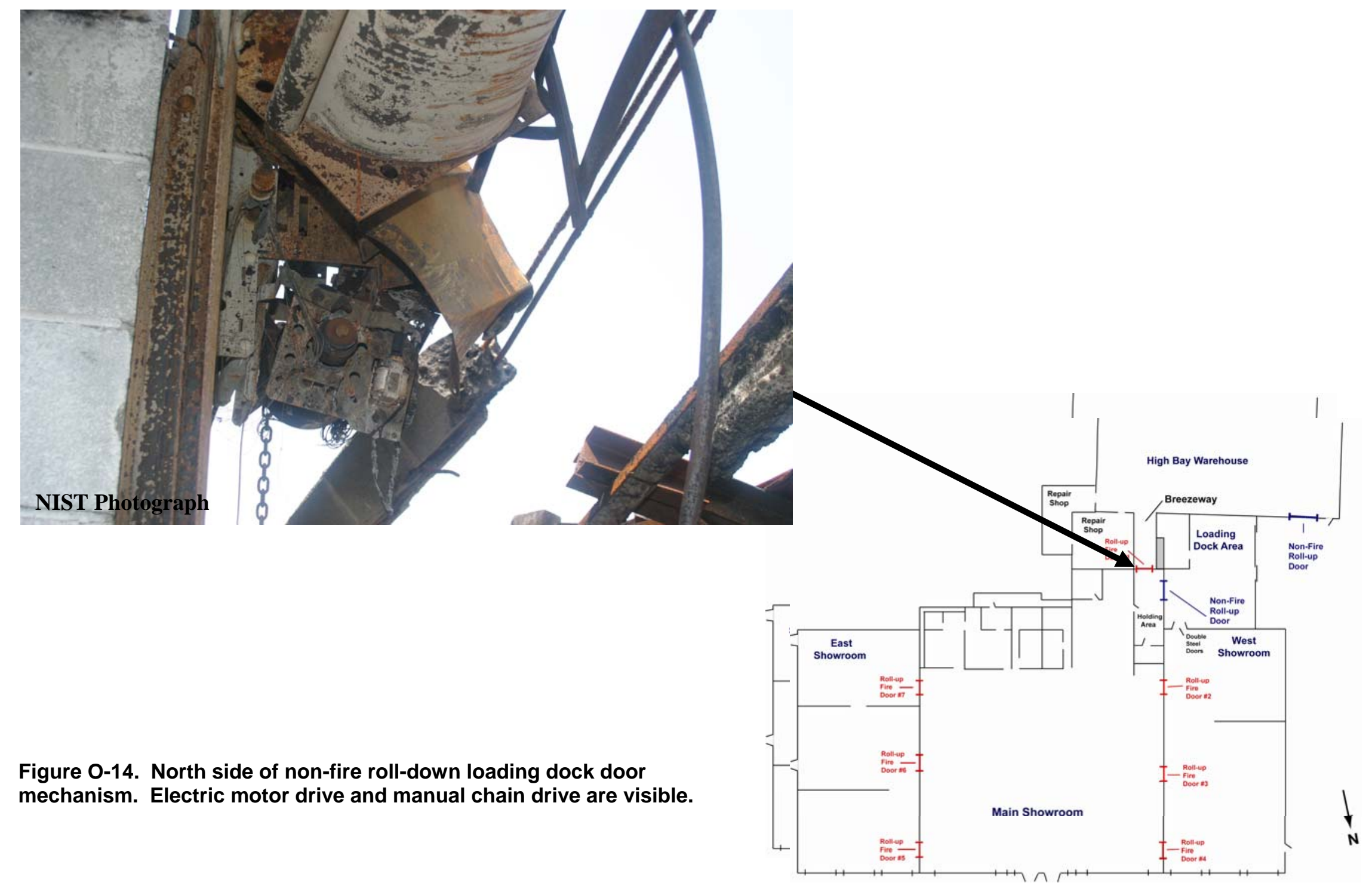




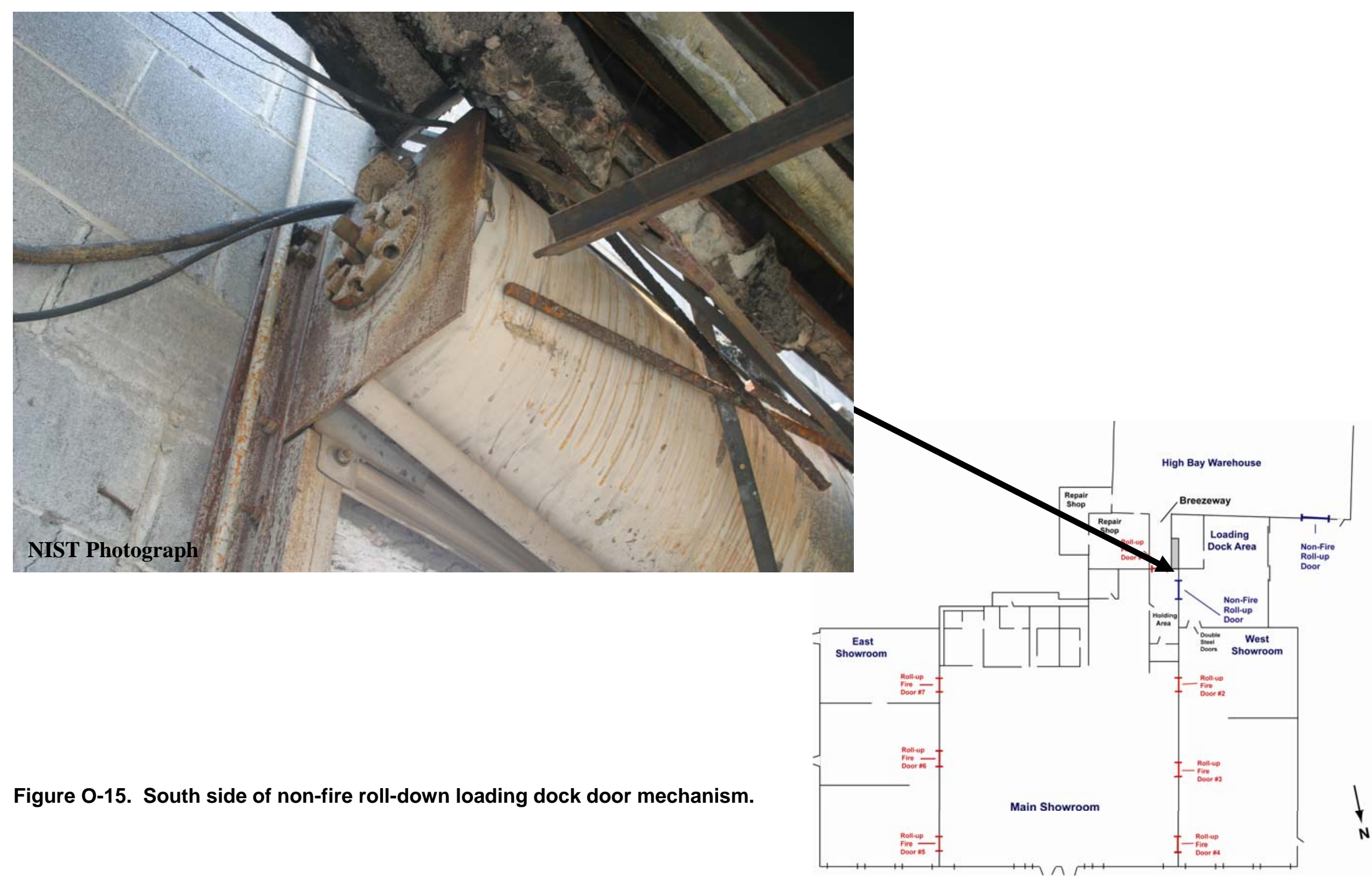


O.5 MAIN SHOWROOM I WEST SHOWROOM ROLL-DOWN DOORS

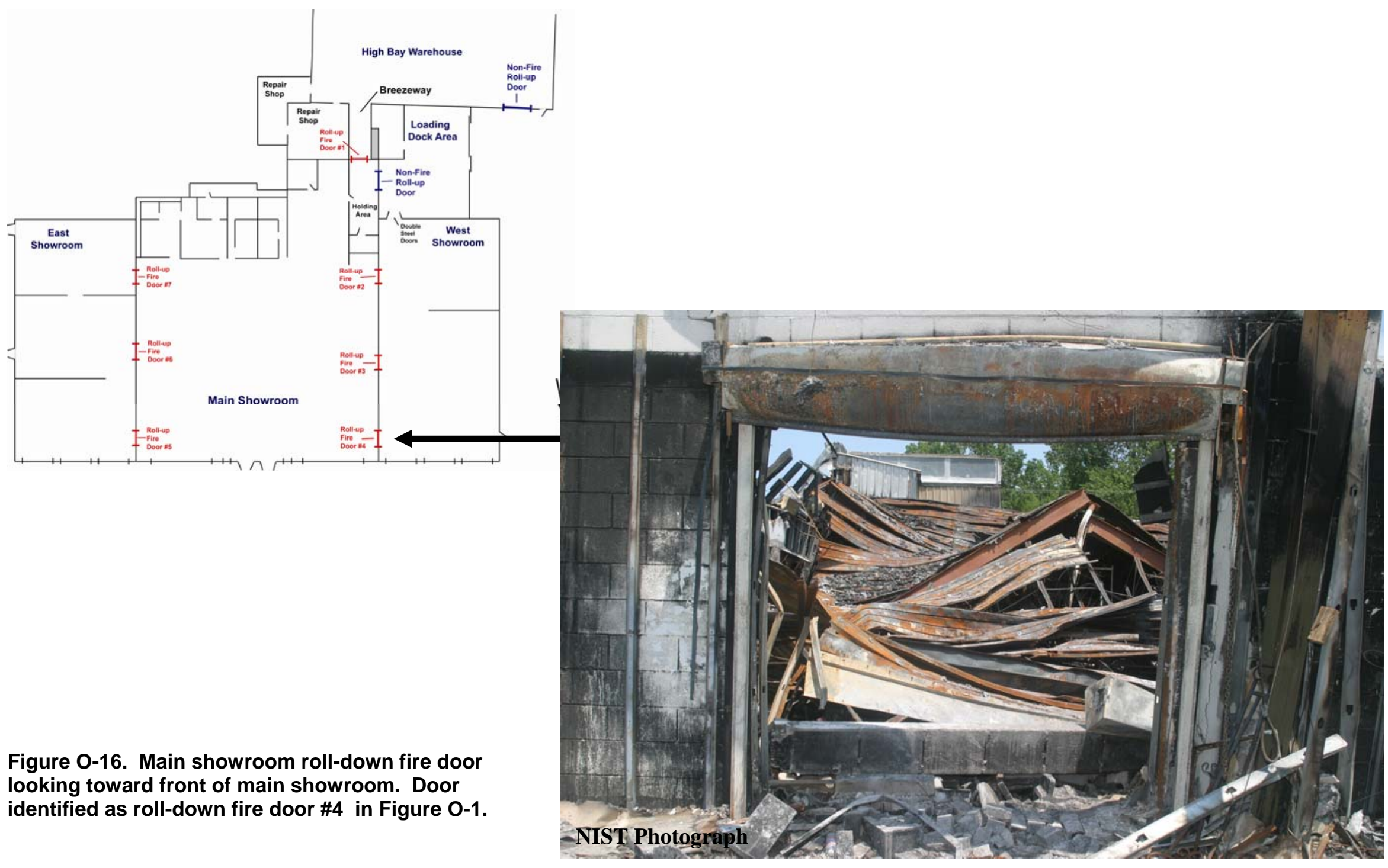




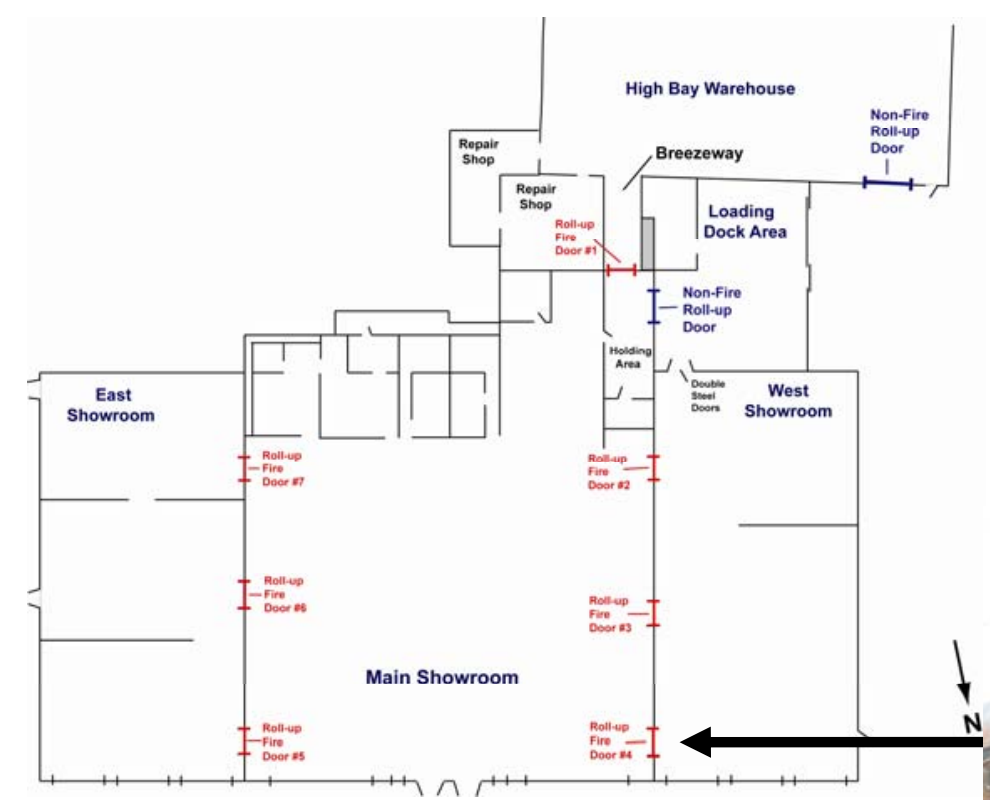

Figure 0-17. South side of operating mechanism for roll-down fire door \#4. 


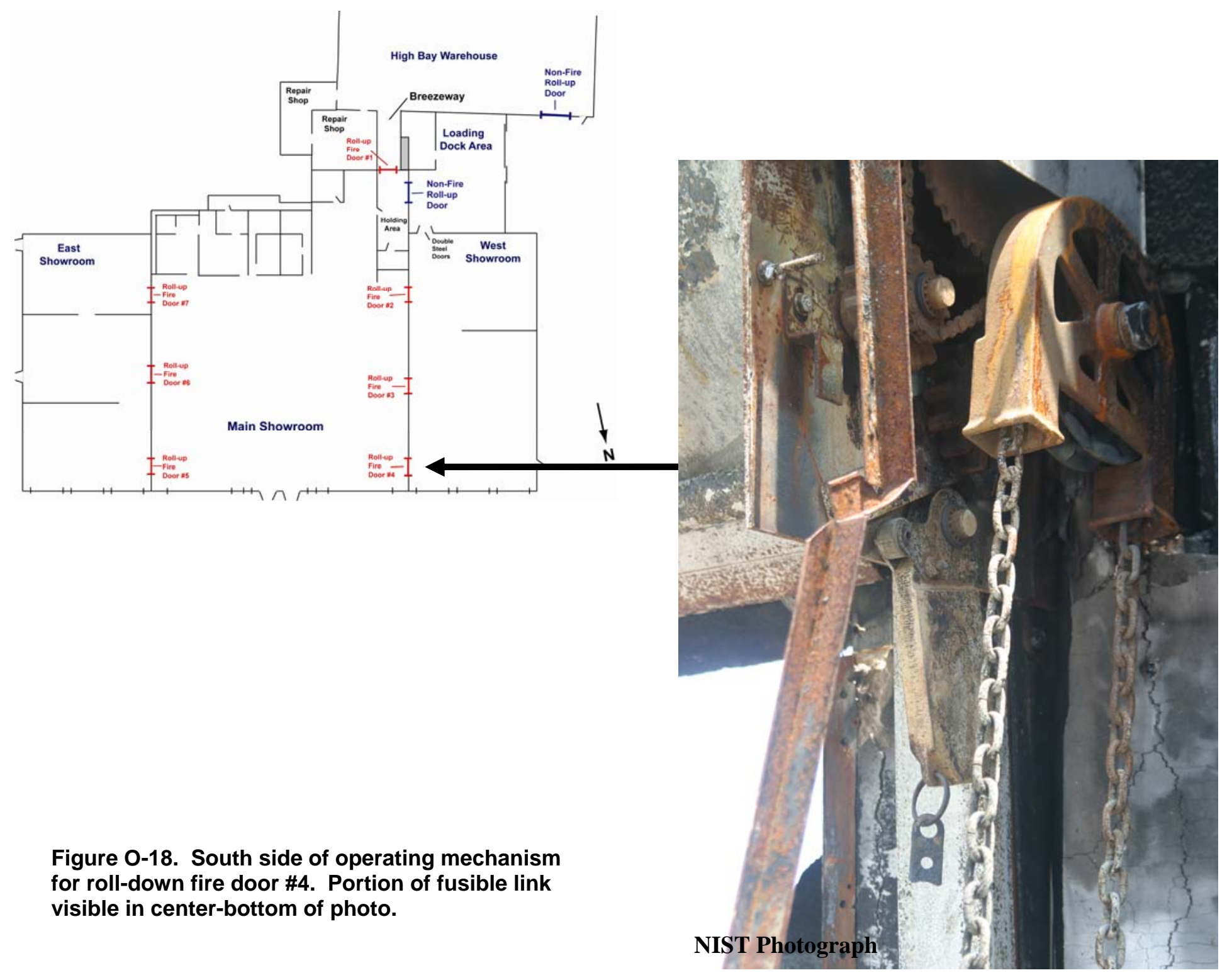




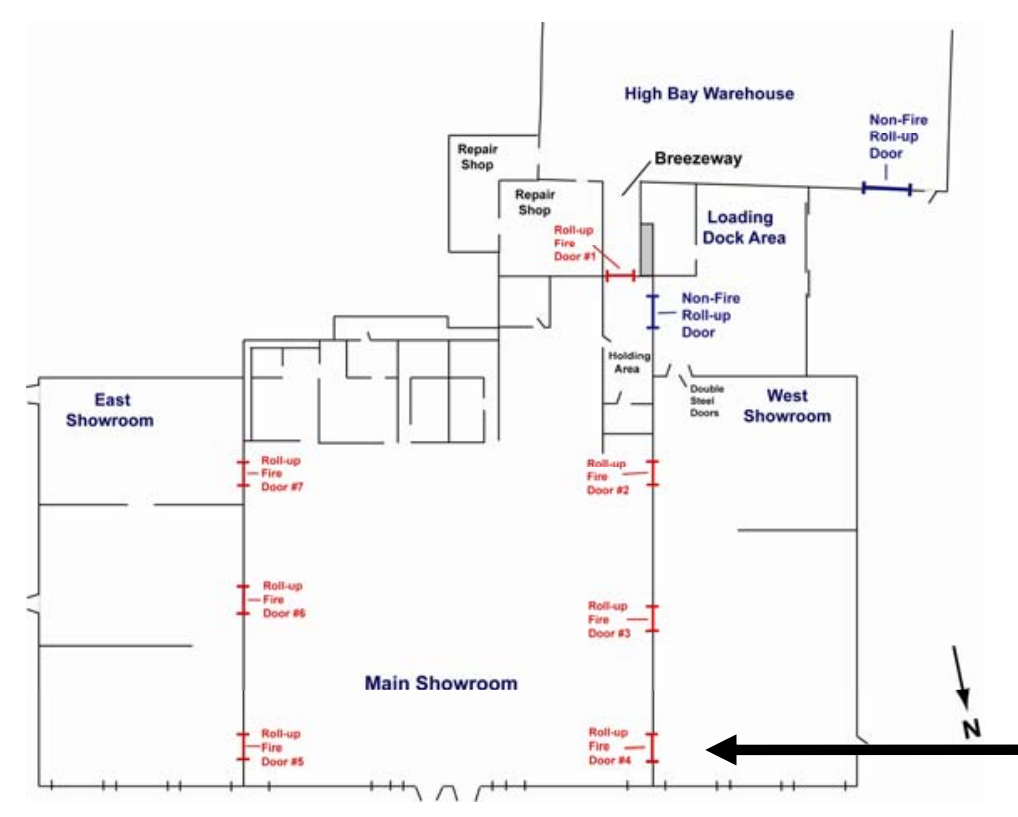

Figure 0-19. North side of operating mechanism for rolldown fire door \#4. Portion of fusible link visible in centerbottom of photo.

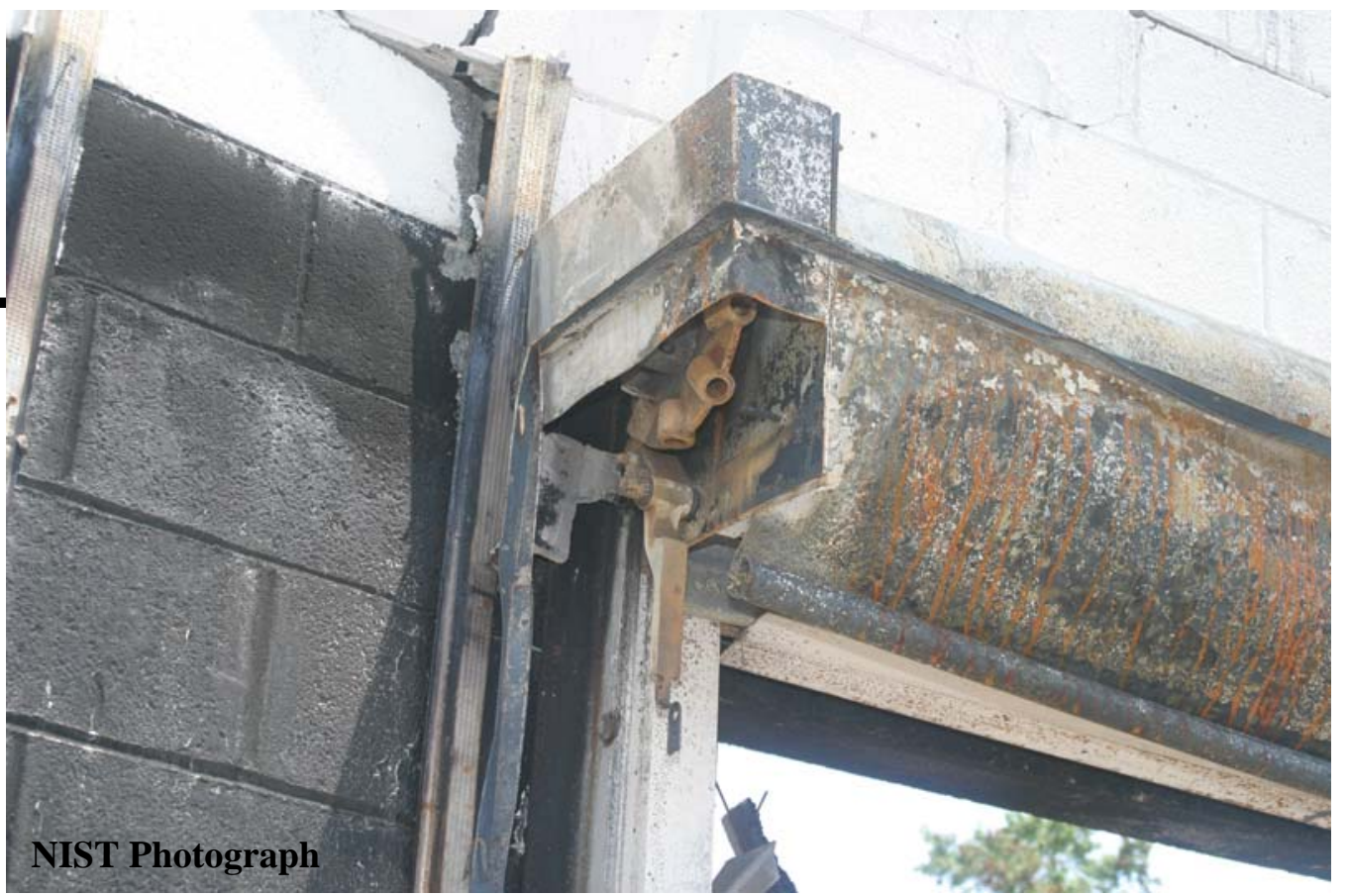




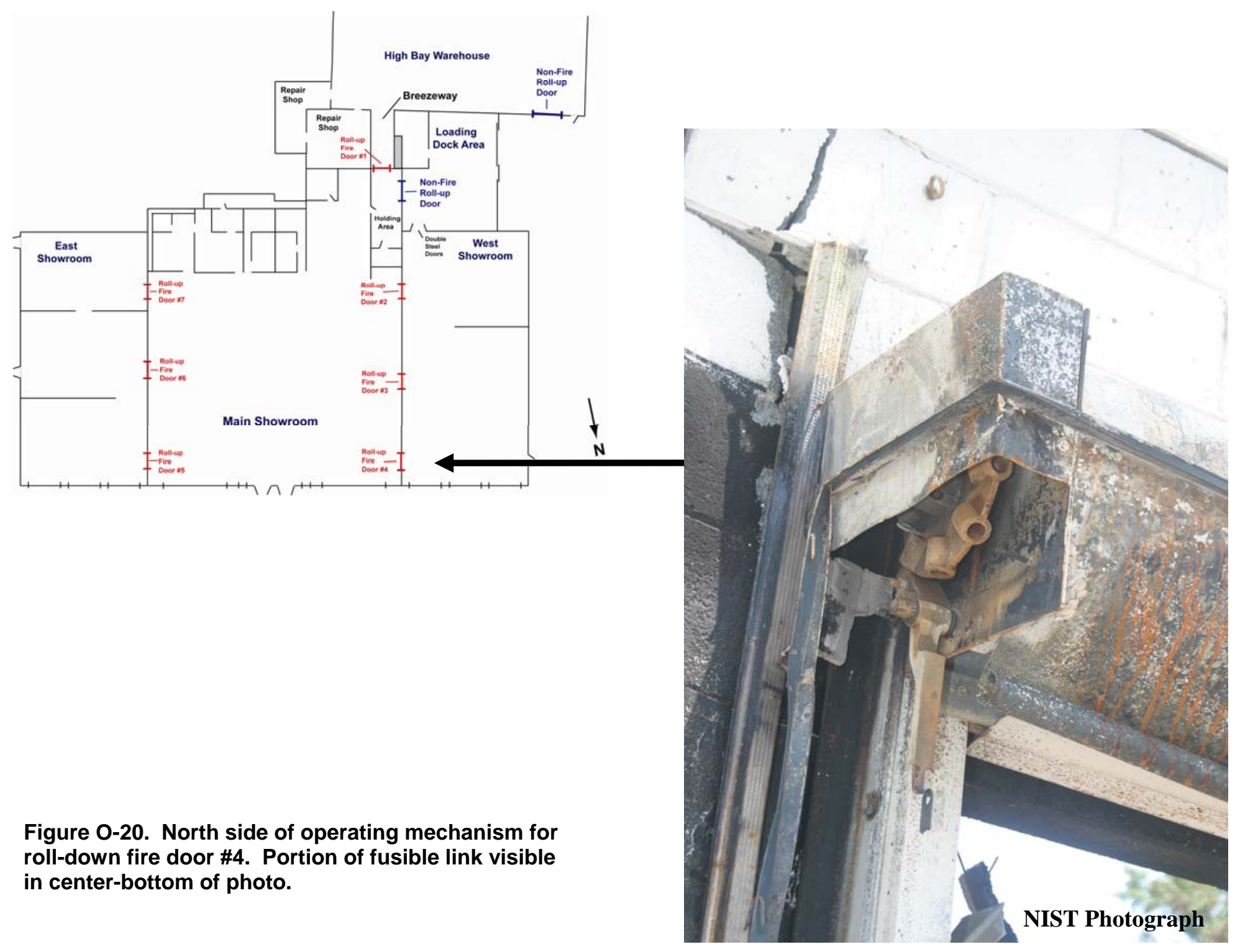




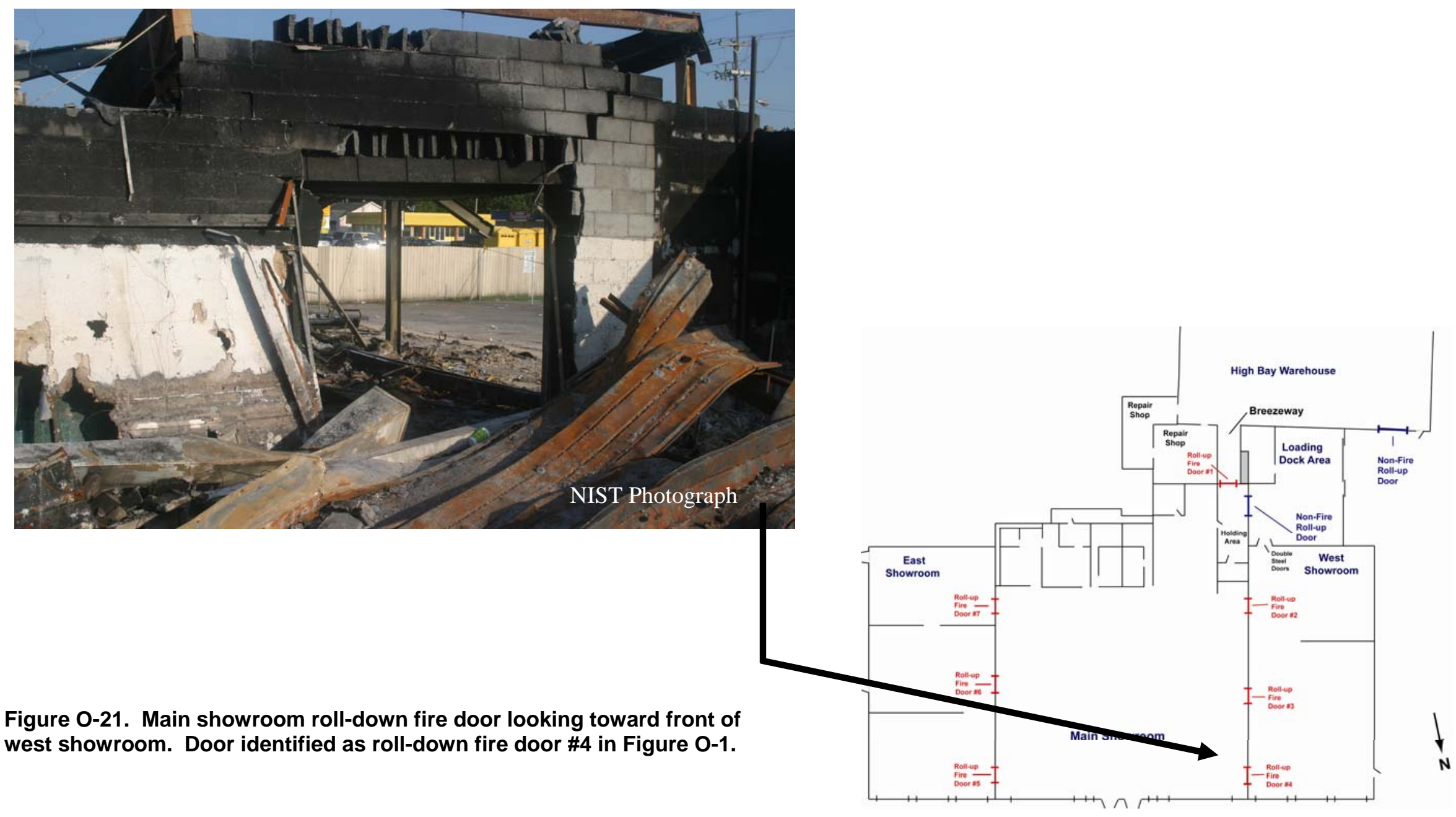




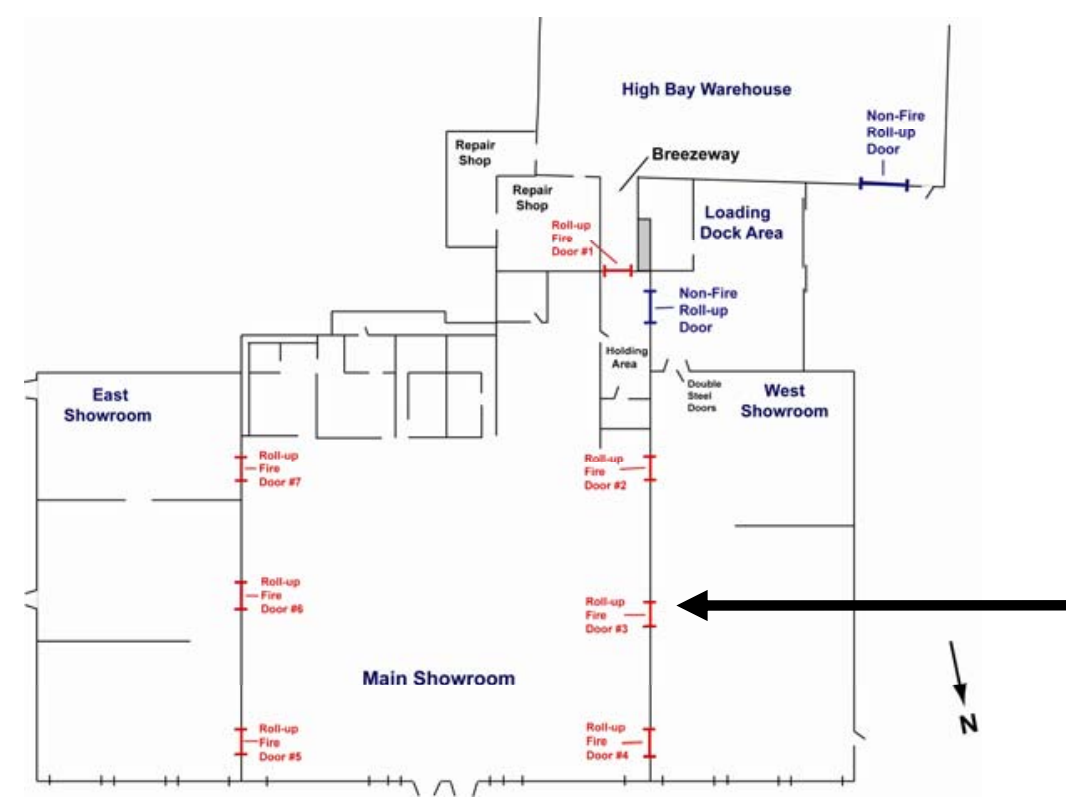

Figure 0-22. Main showroom roll-down fire door looking toward middle of main showroom. Door identified as rolldown fire door \#3 in Figure 0-1.

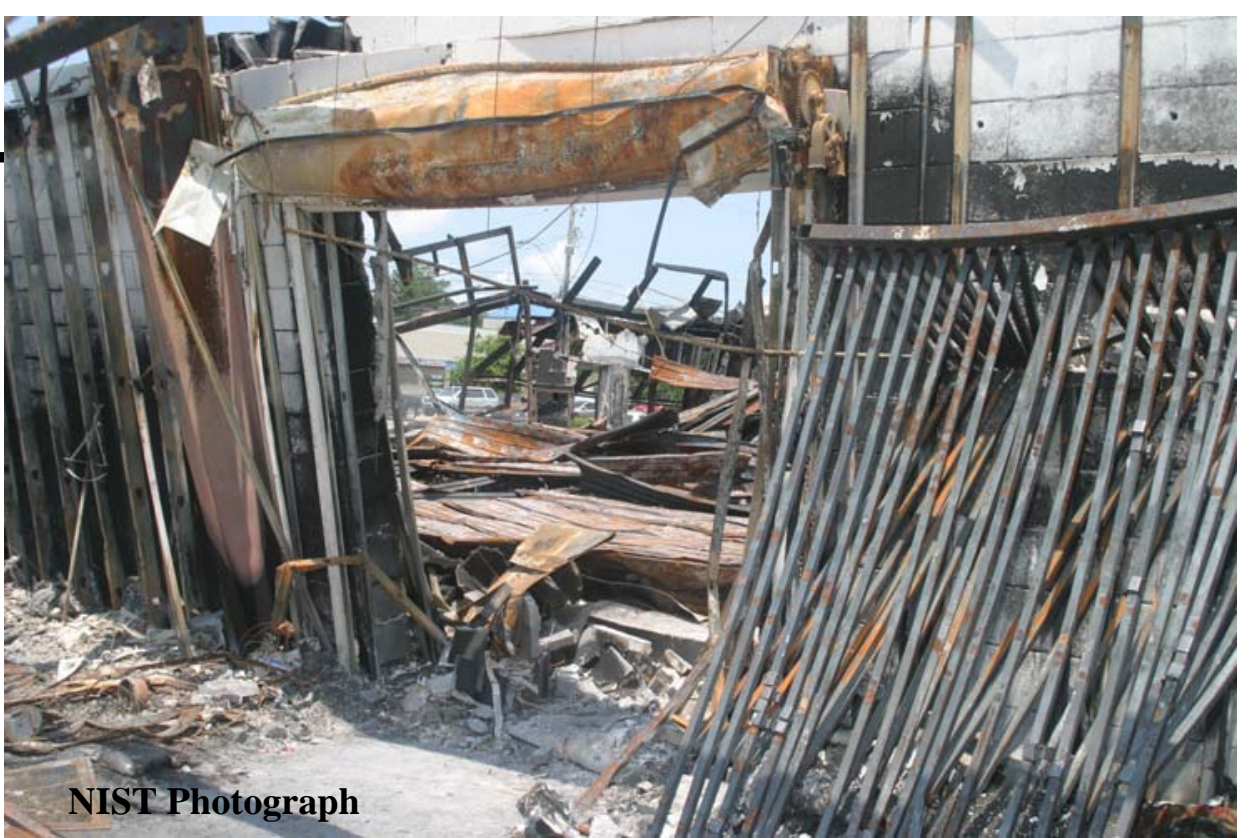




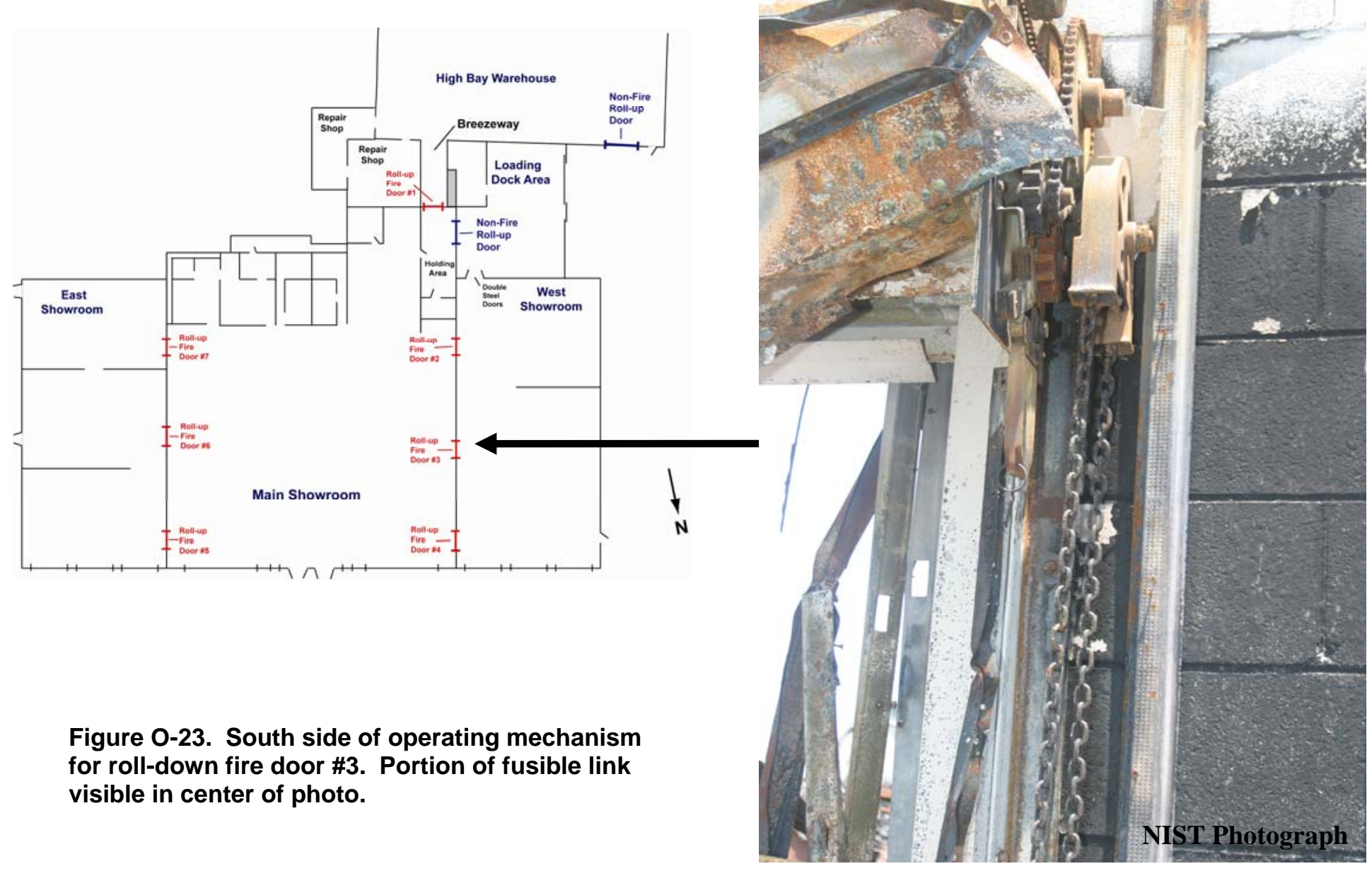




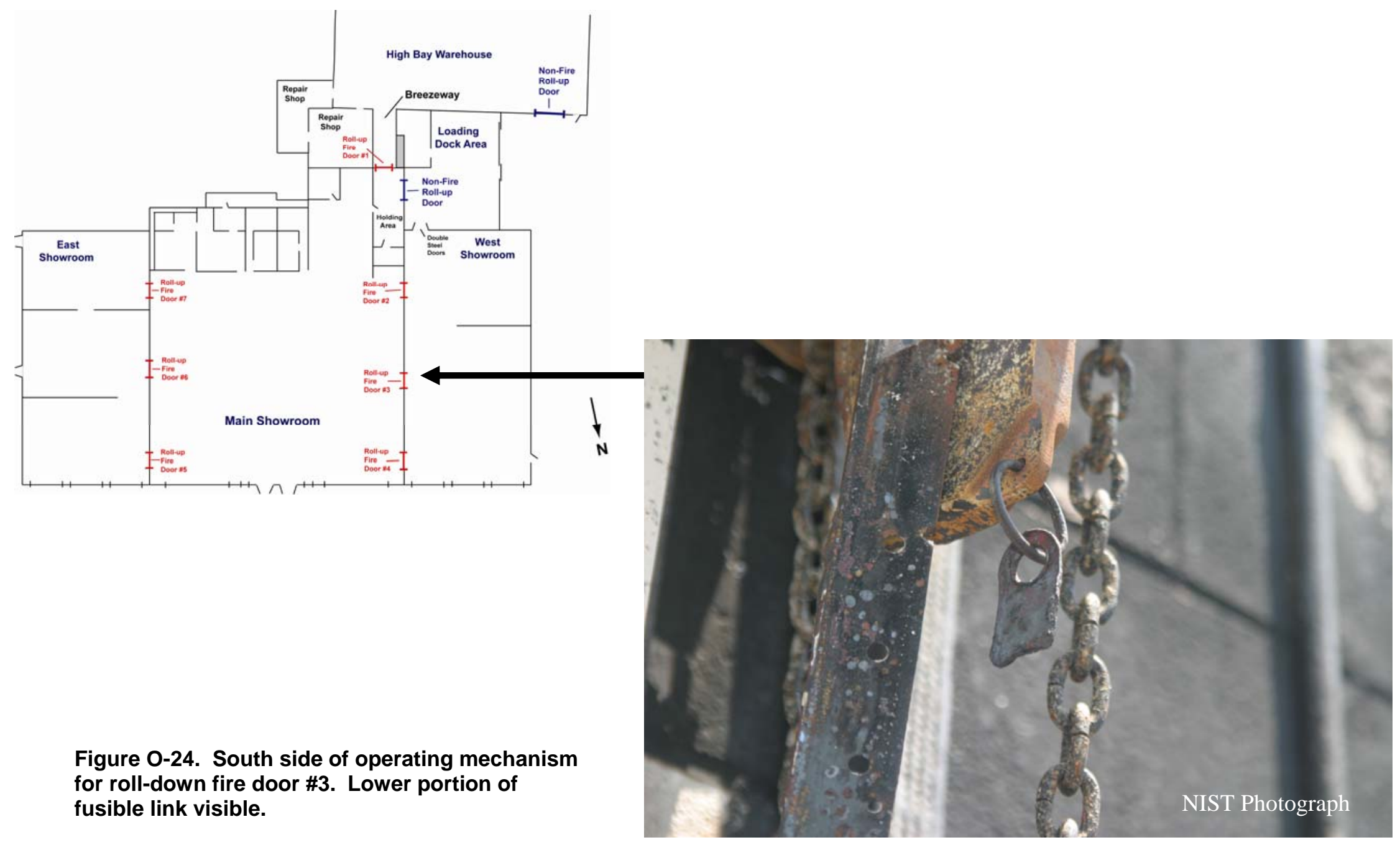




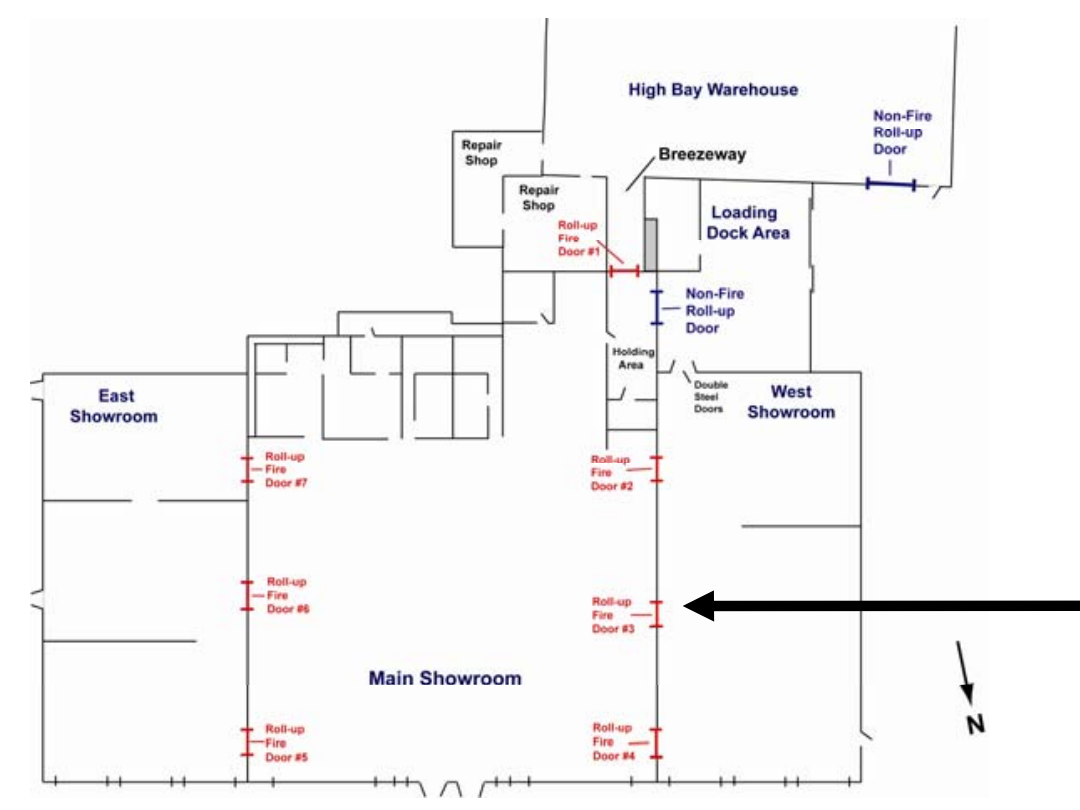

Figure 0-25. South side of operating mechanism for roll-down fire door \#3. Upper portion of fusible link visible in center of photo.

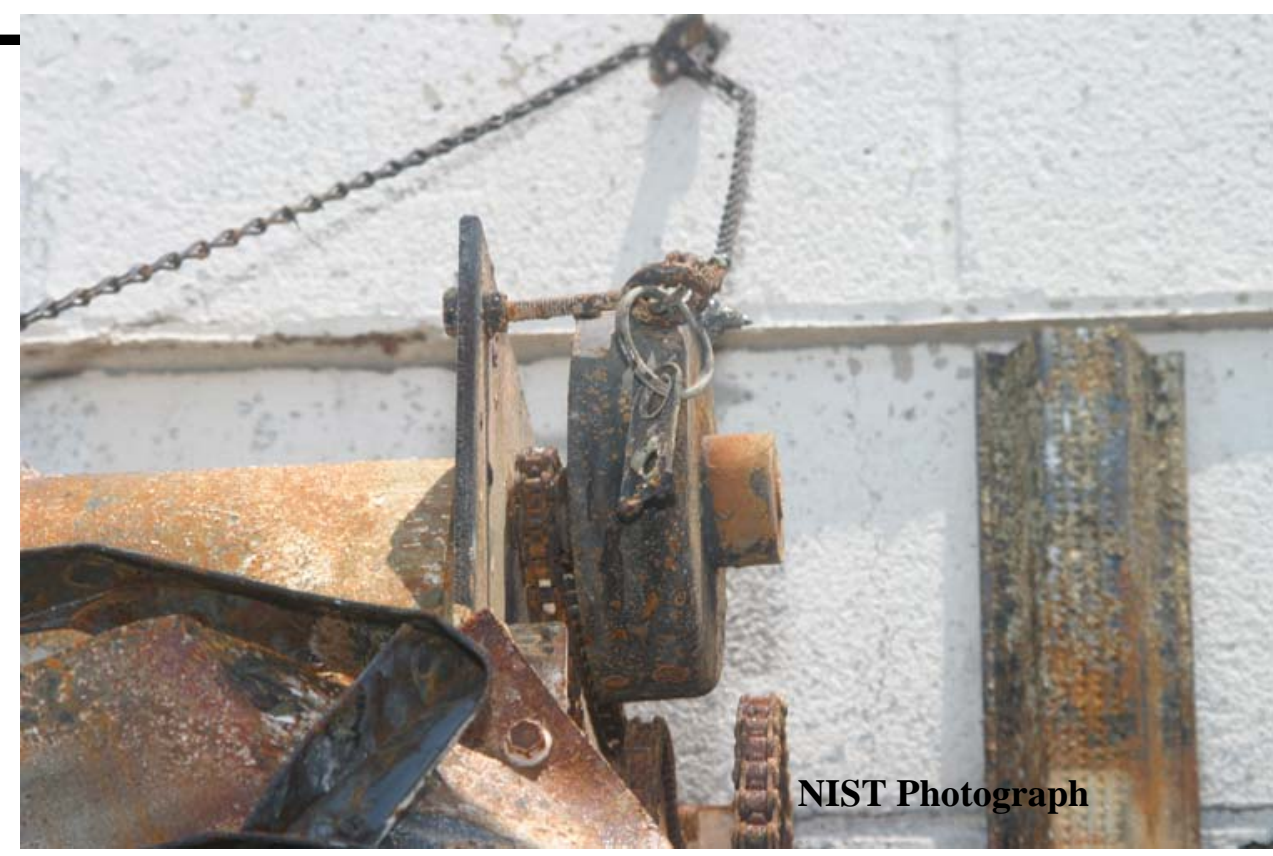




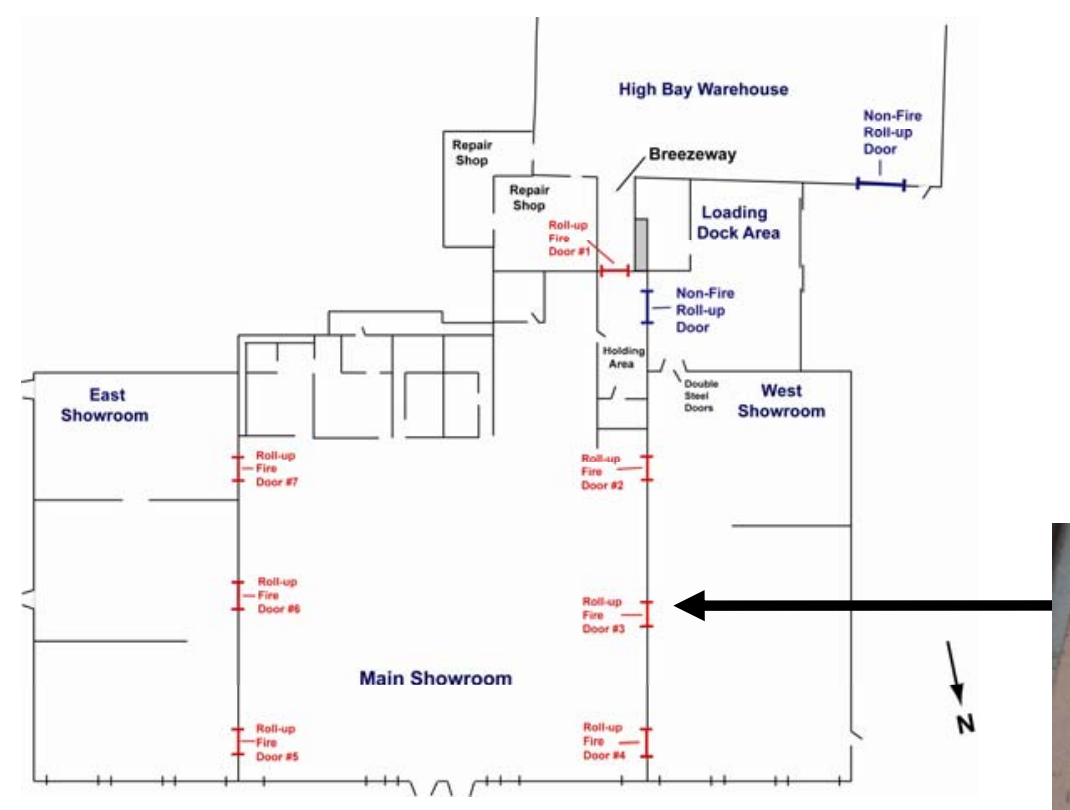

Figure 0-26. North side of operating mechanism for roll-down fire door \#3. Portion of fusible link visible in center of photo.

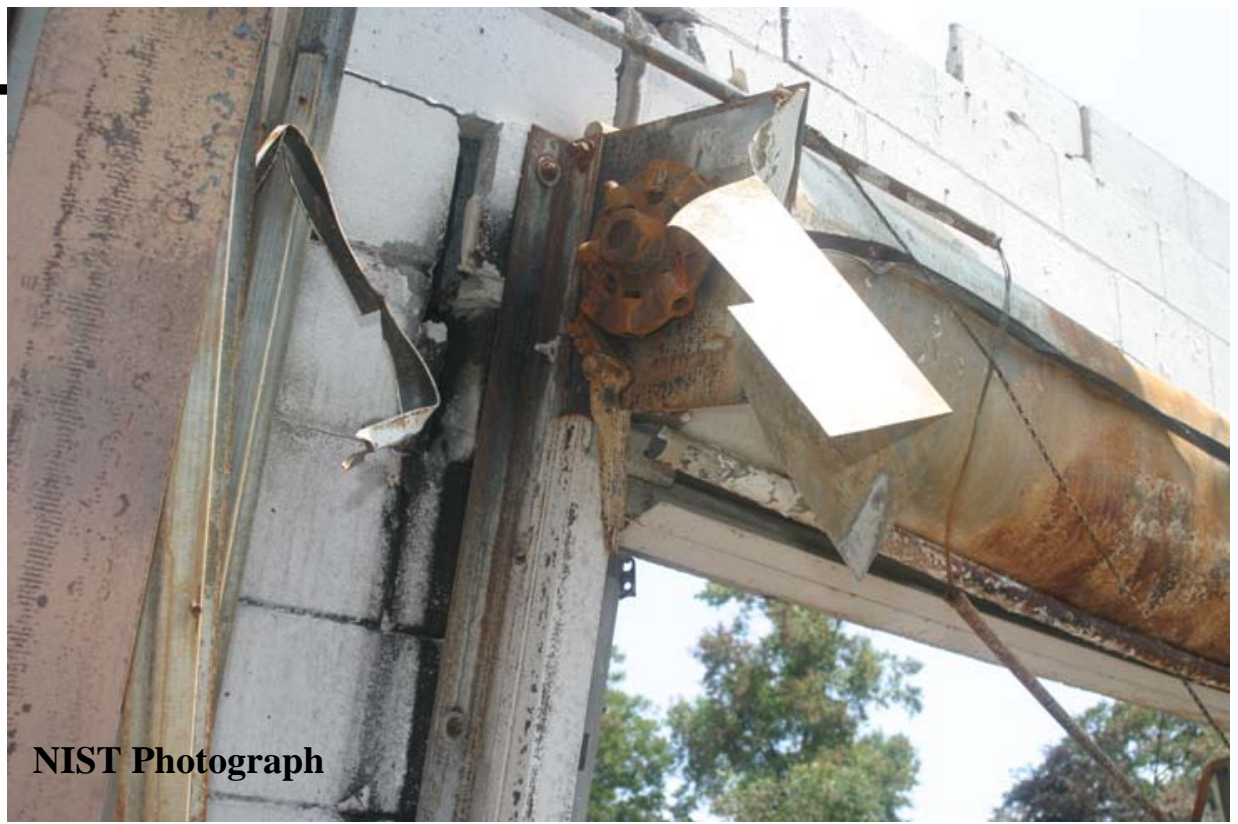




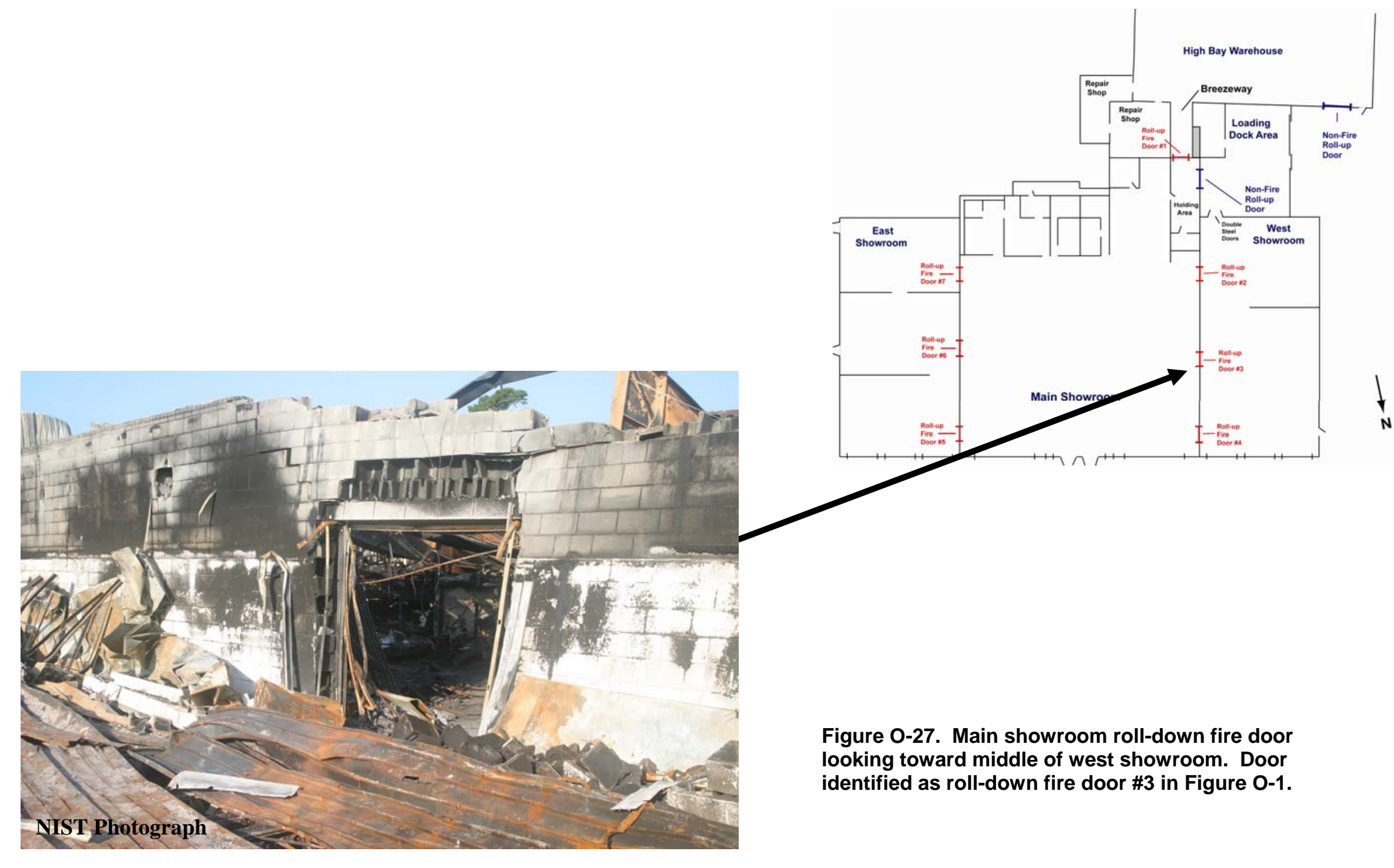




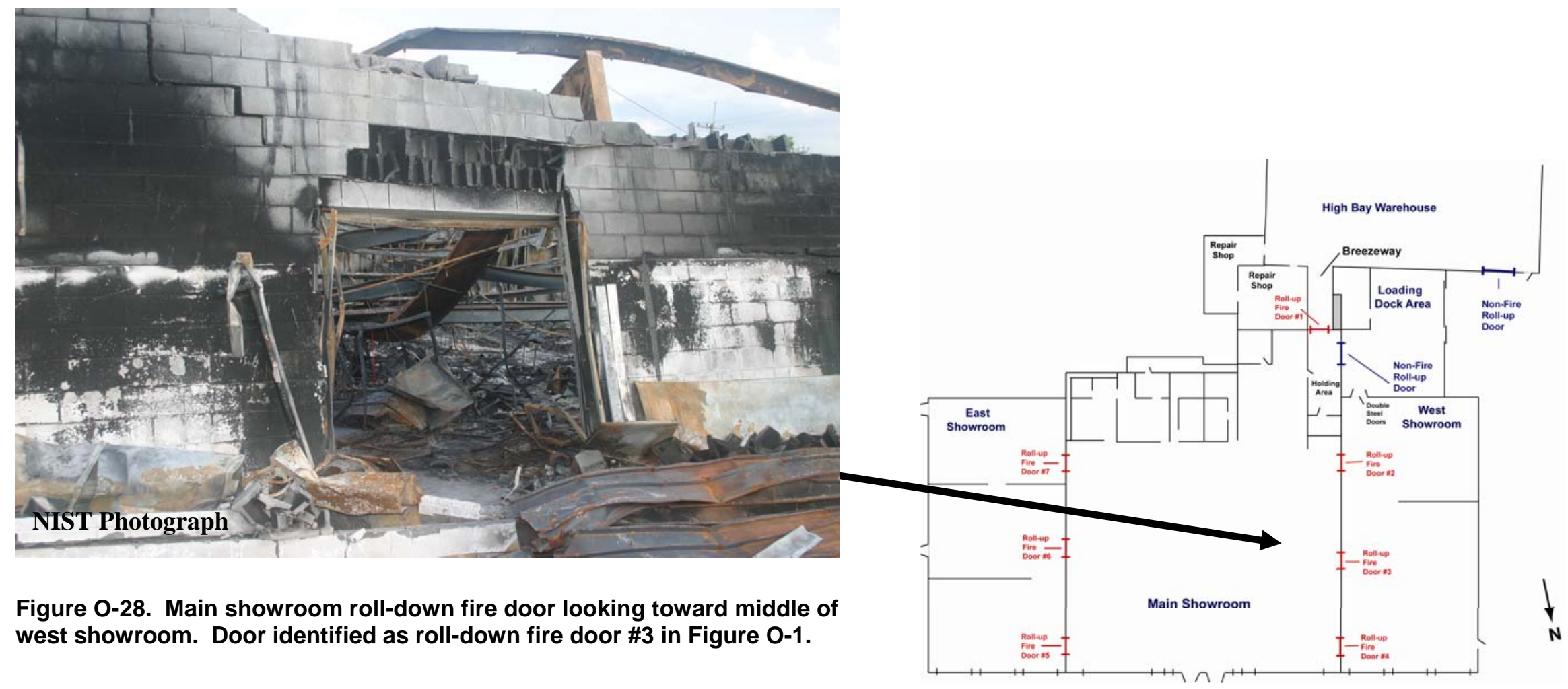




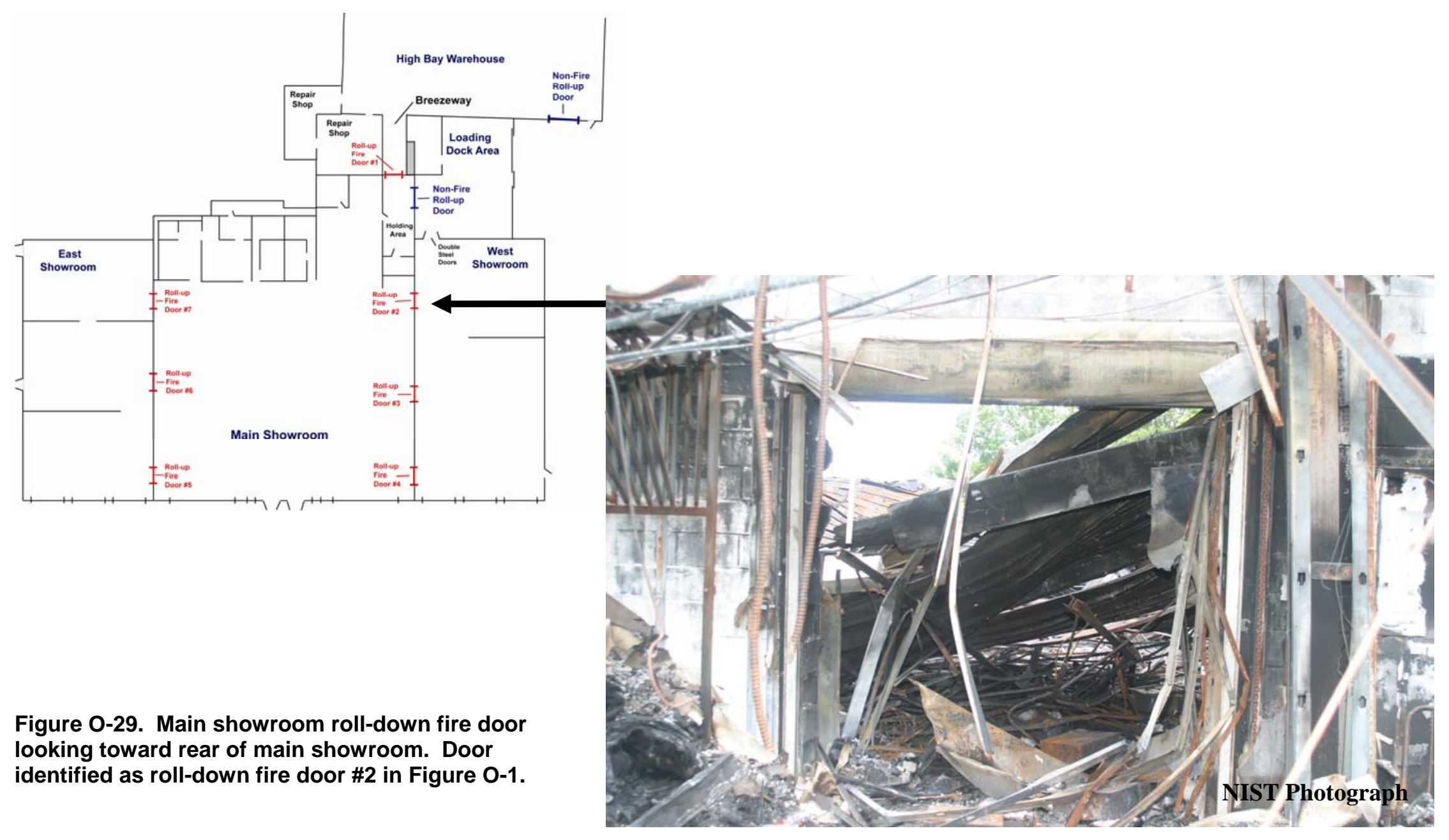



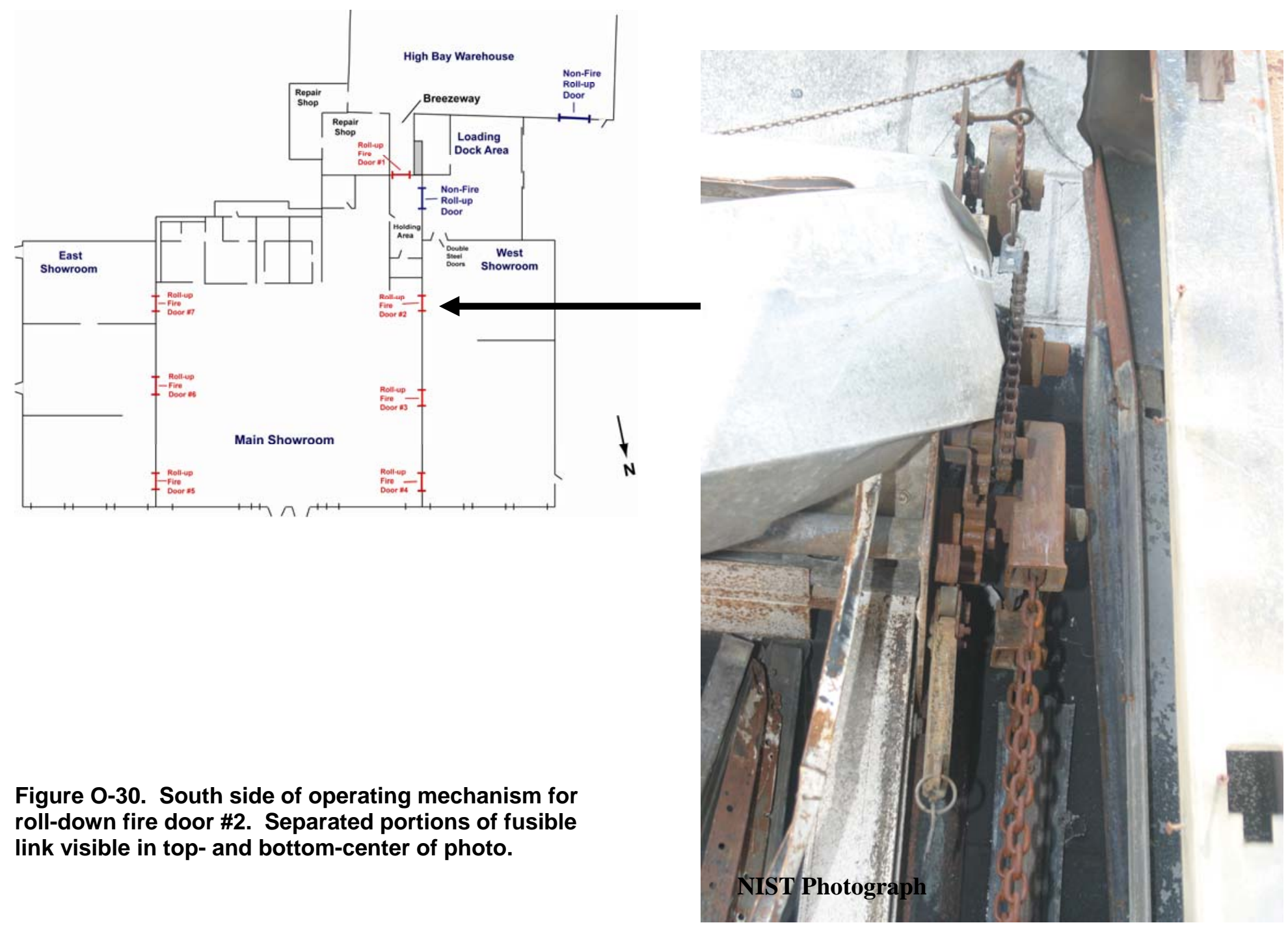

Figure 0-30. South side of operating mechanism for roll-down fire door \#2. Separated portions of fusible link visible in top- and bottom-center of photo. 

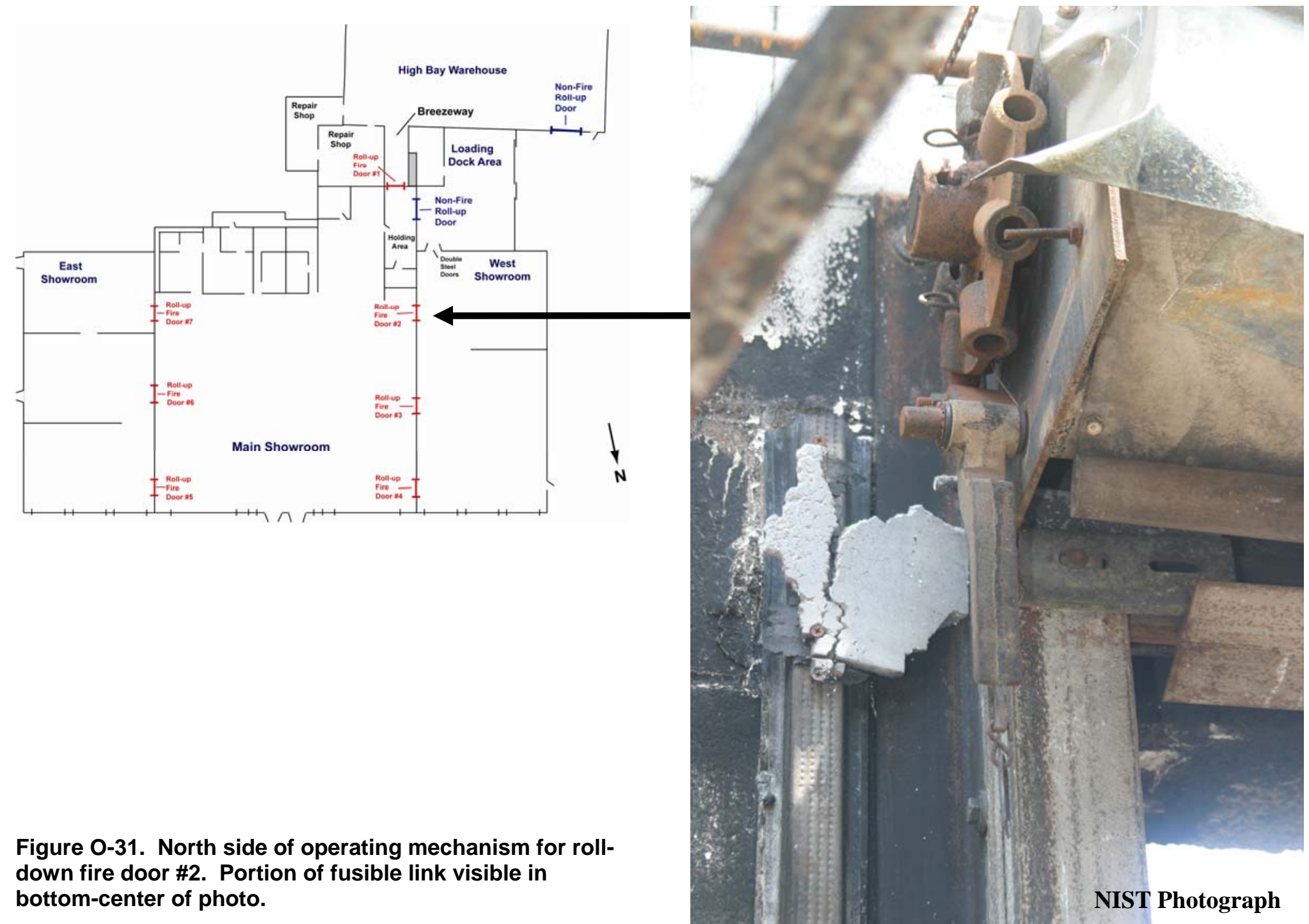

Figure 0-31. North side of operating mechanism for rolldown fire door \#2. Portion of fusible link visible in bottom-center of photo. 


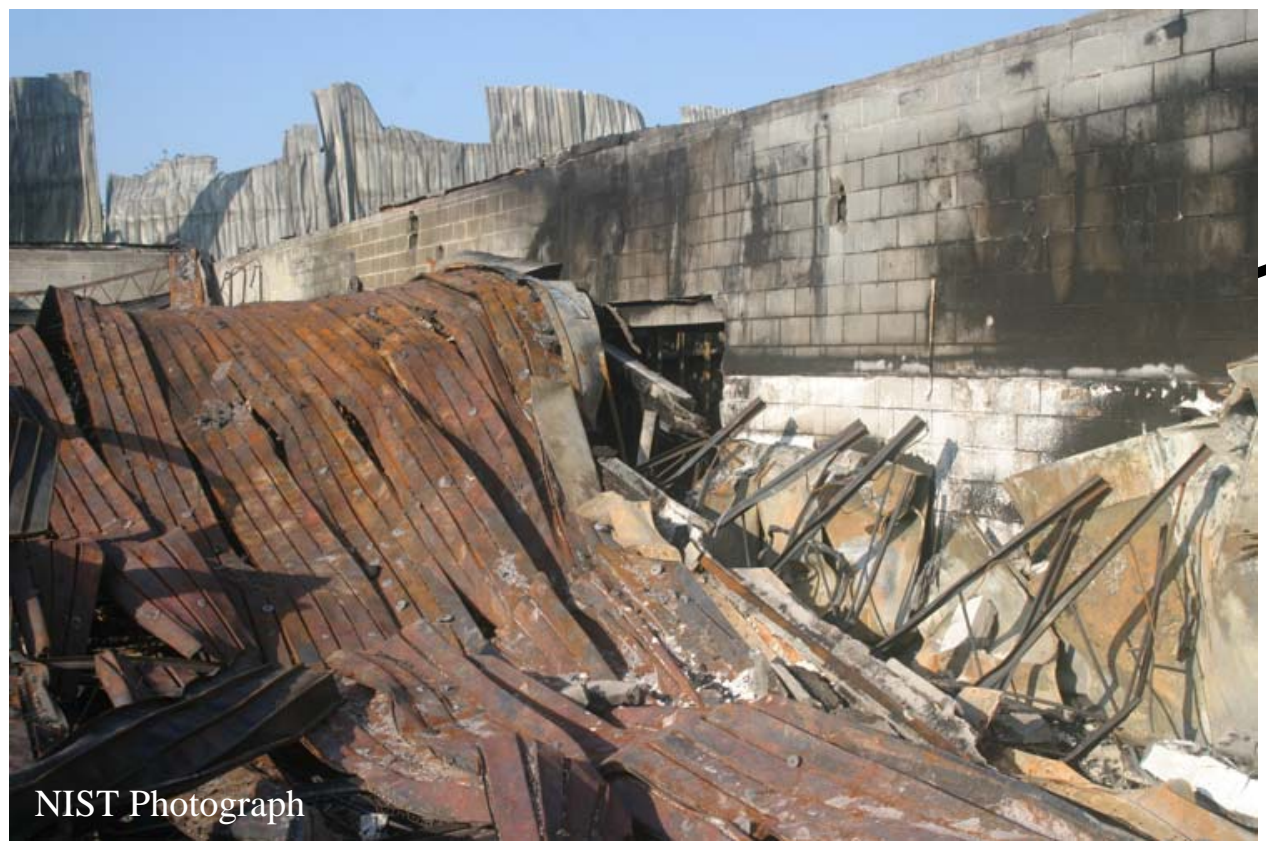

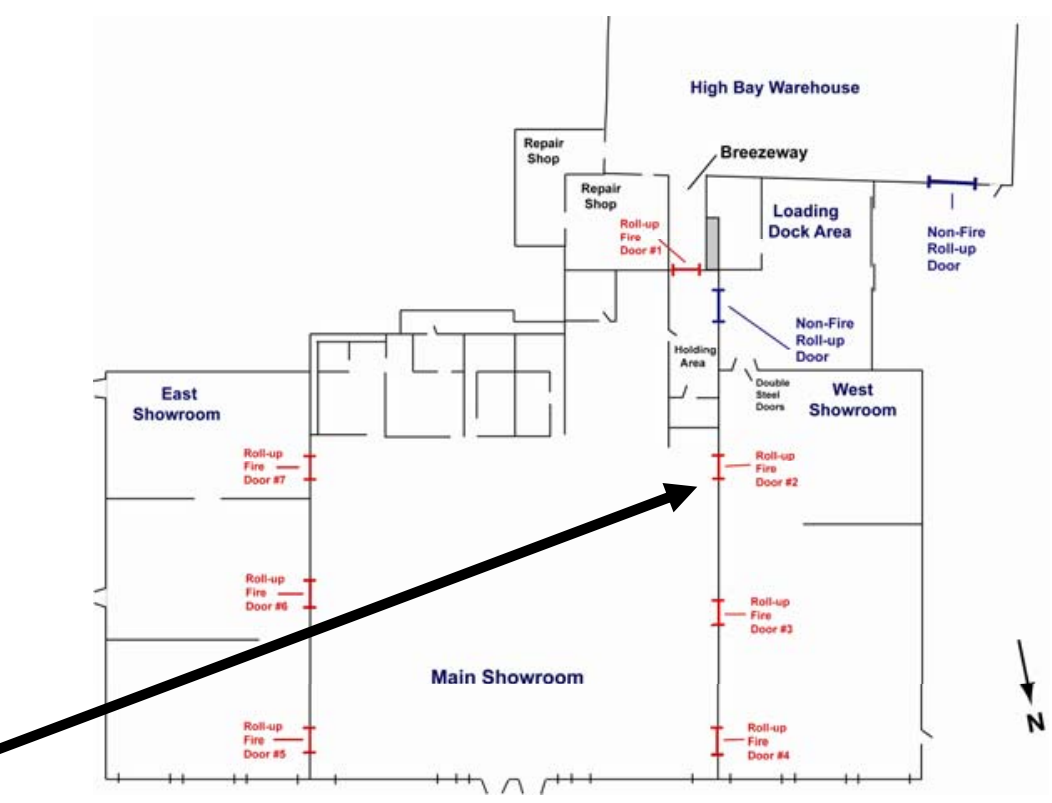

Figure 0-32. Main showroom roll-down fire door looking toward rear of west showroom. Door identified as roll-down fire door \#2 in Figure 0-1. 


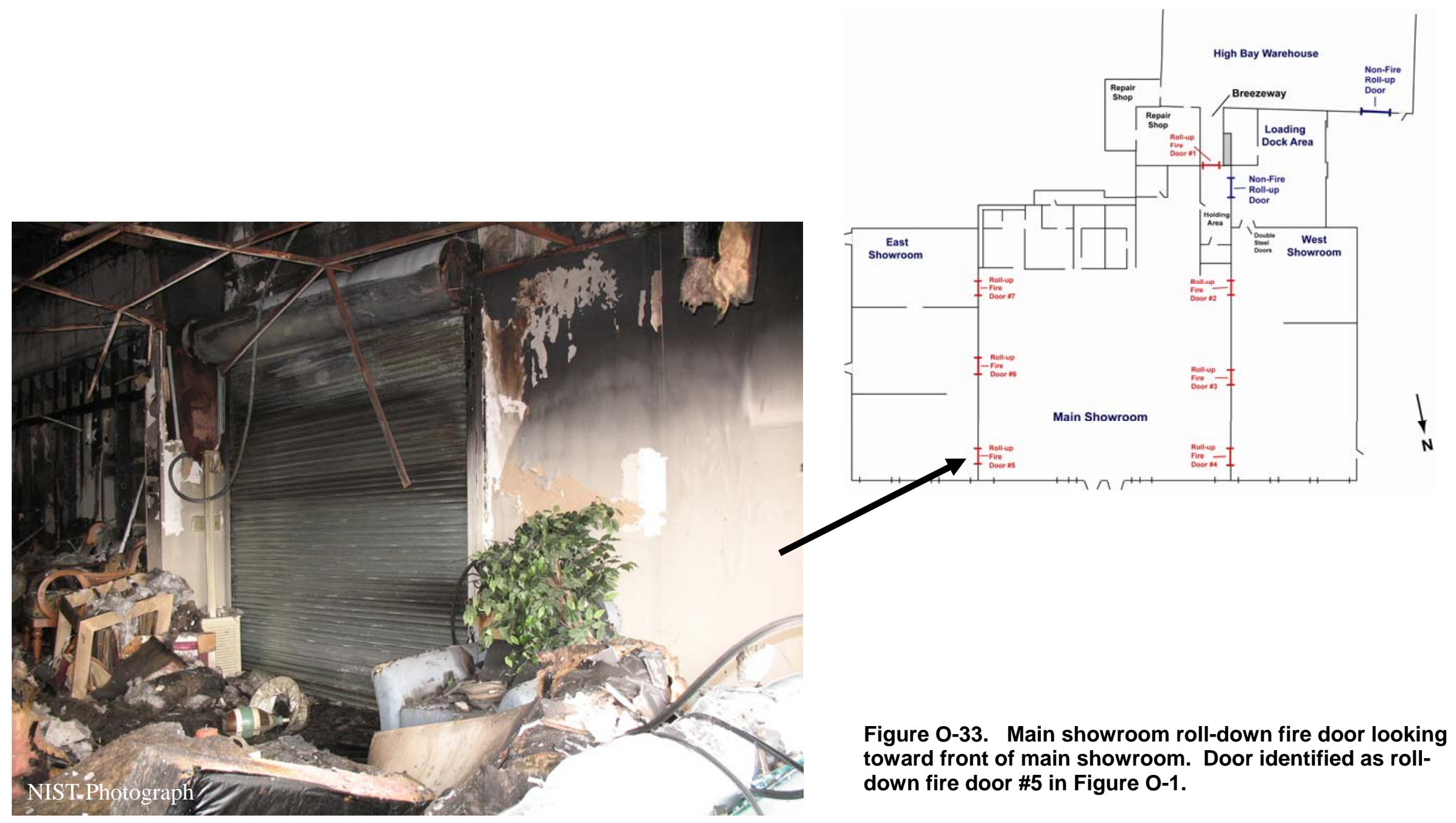




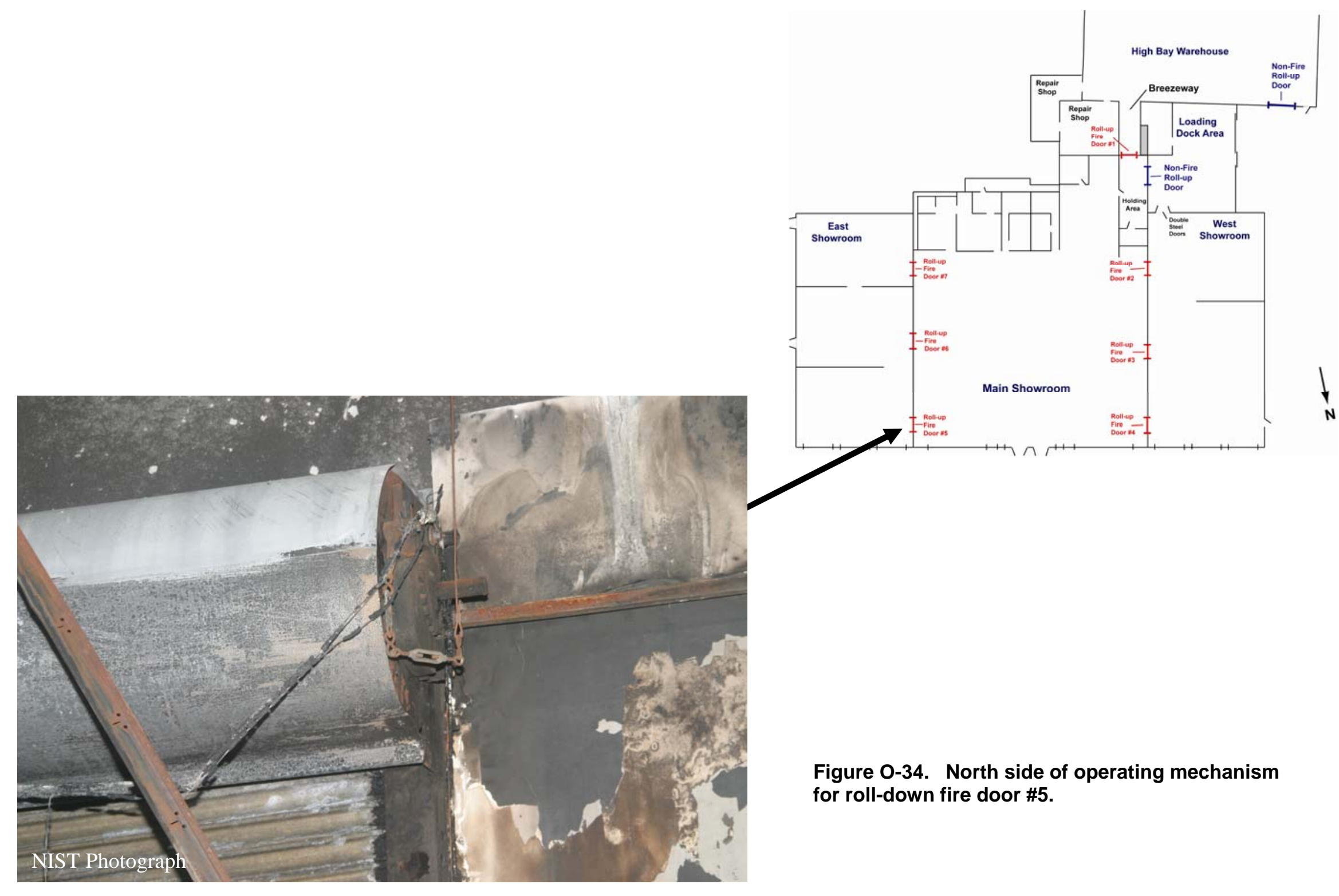




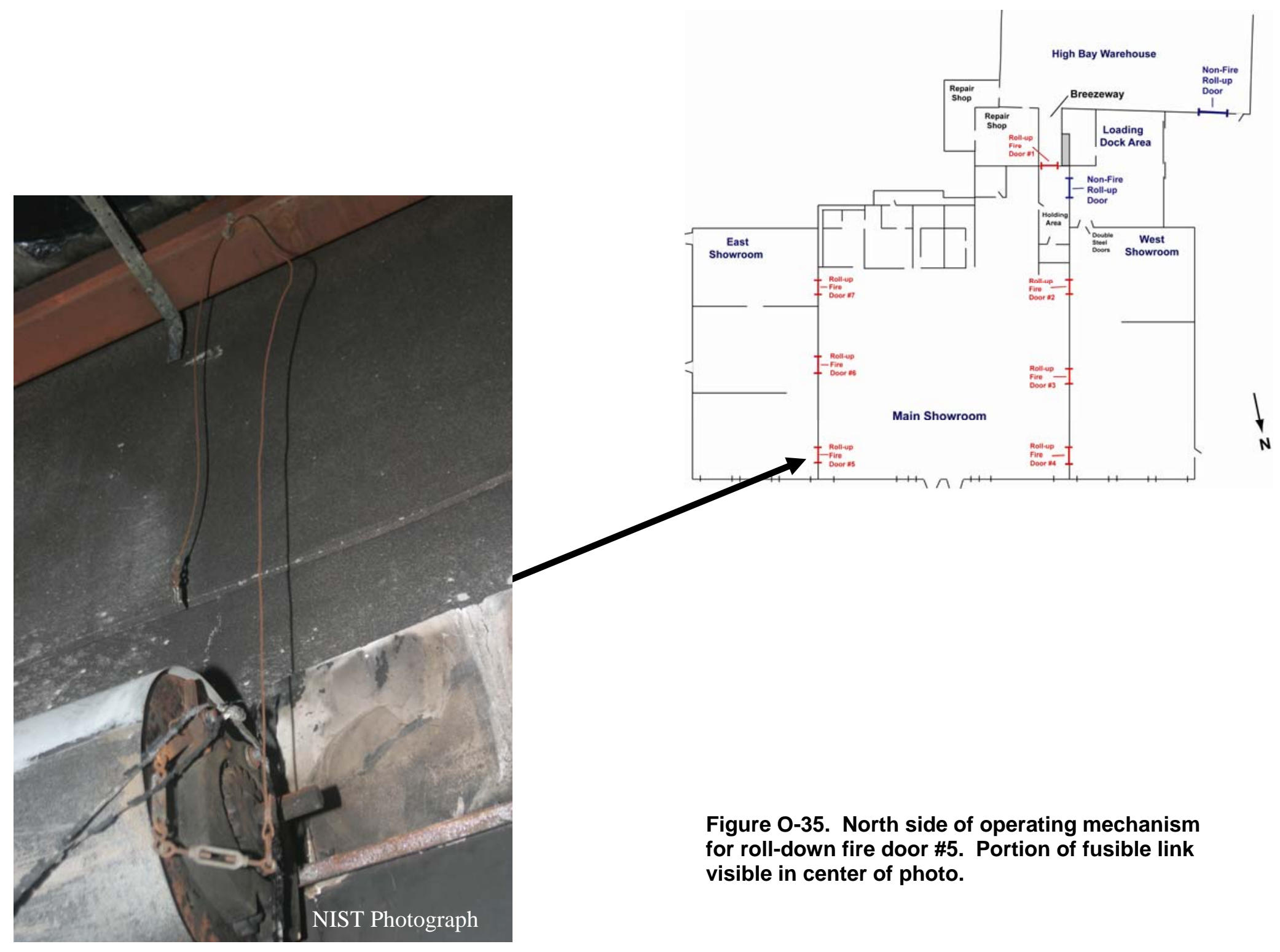




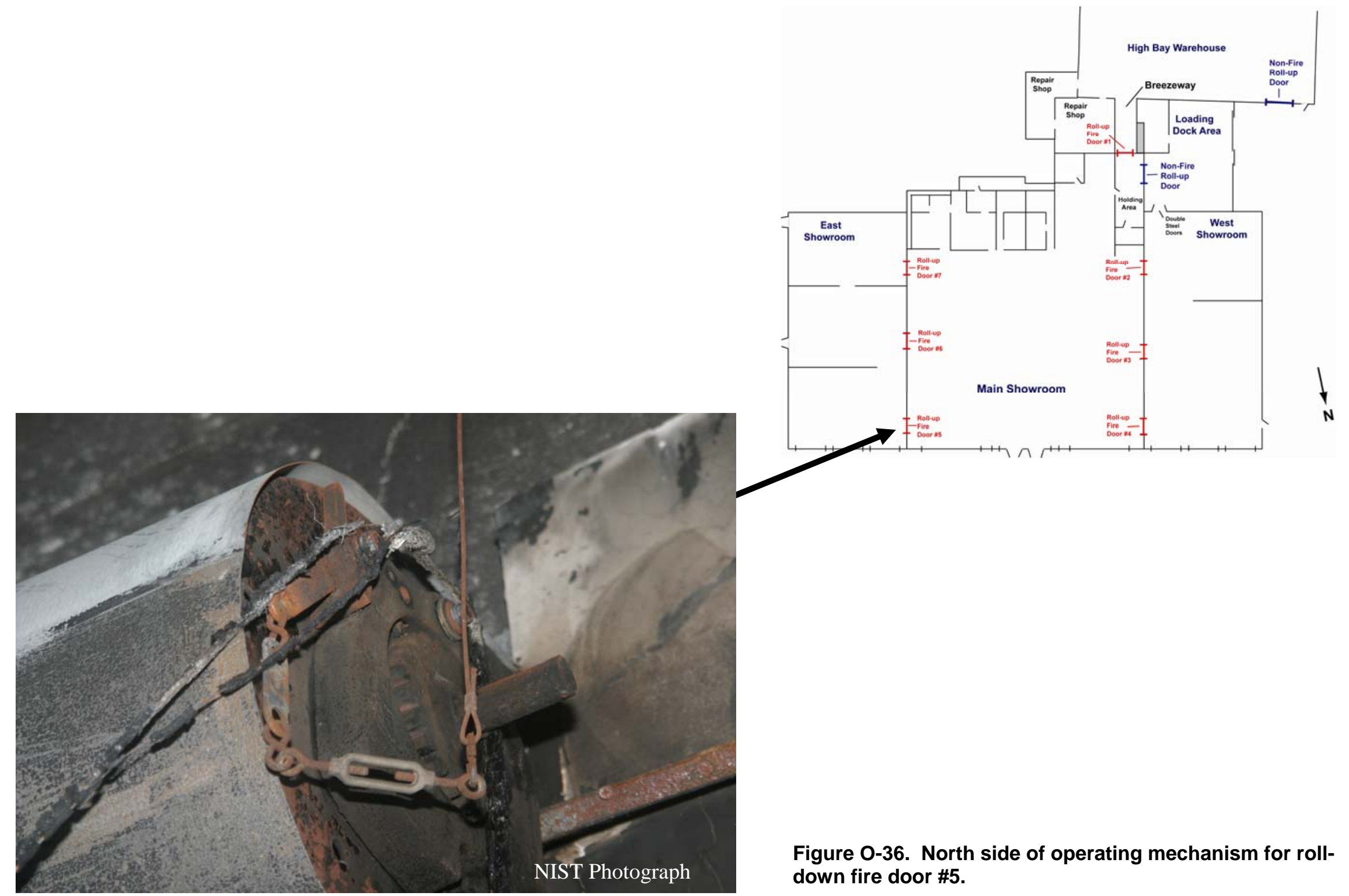




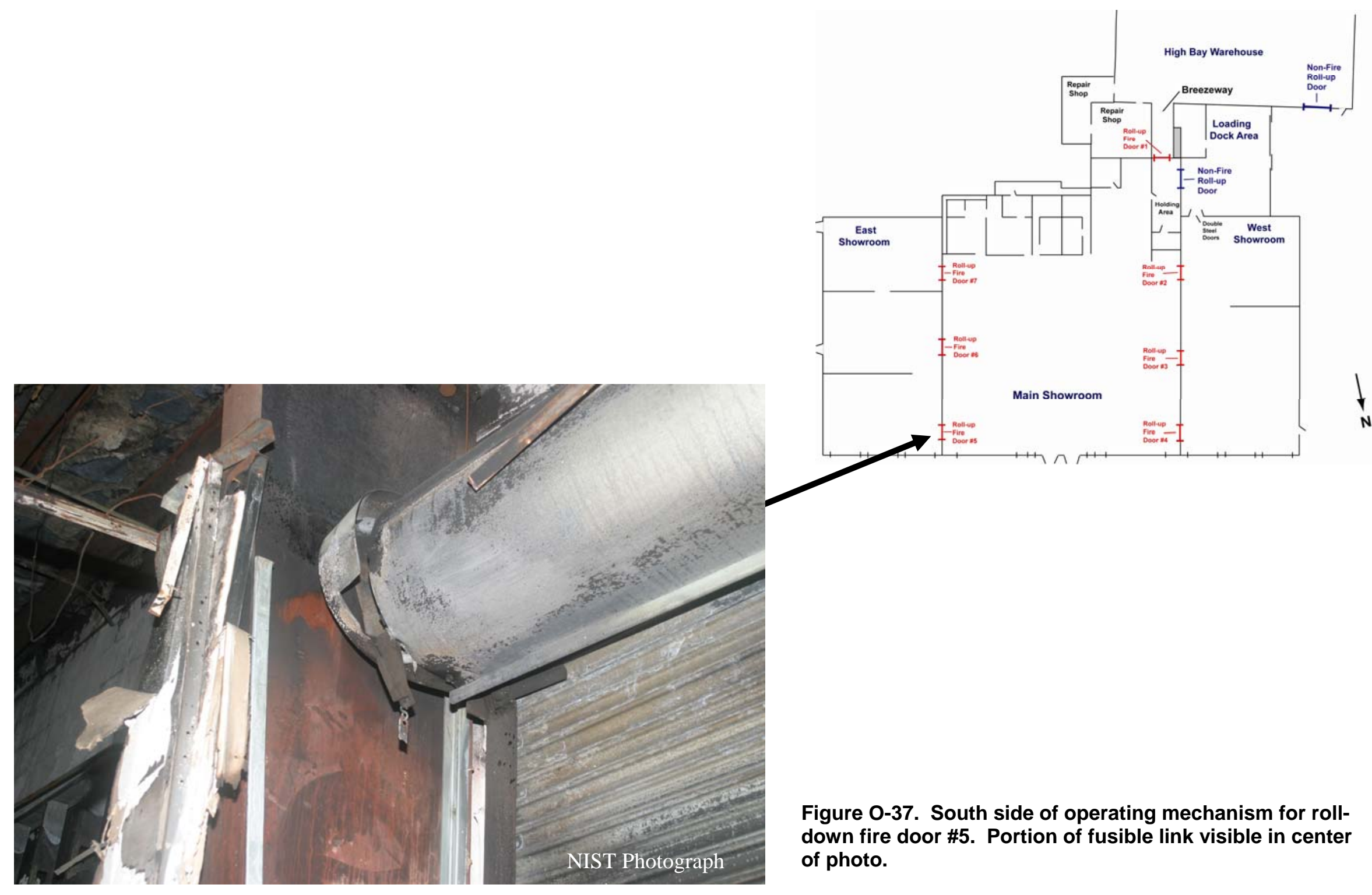




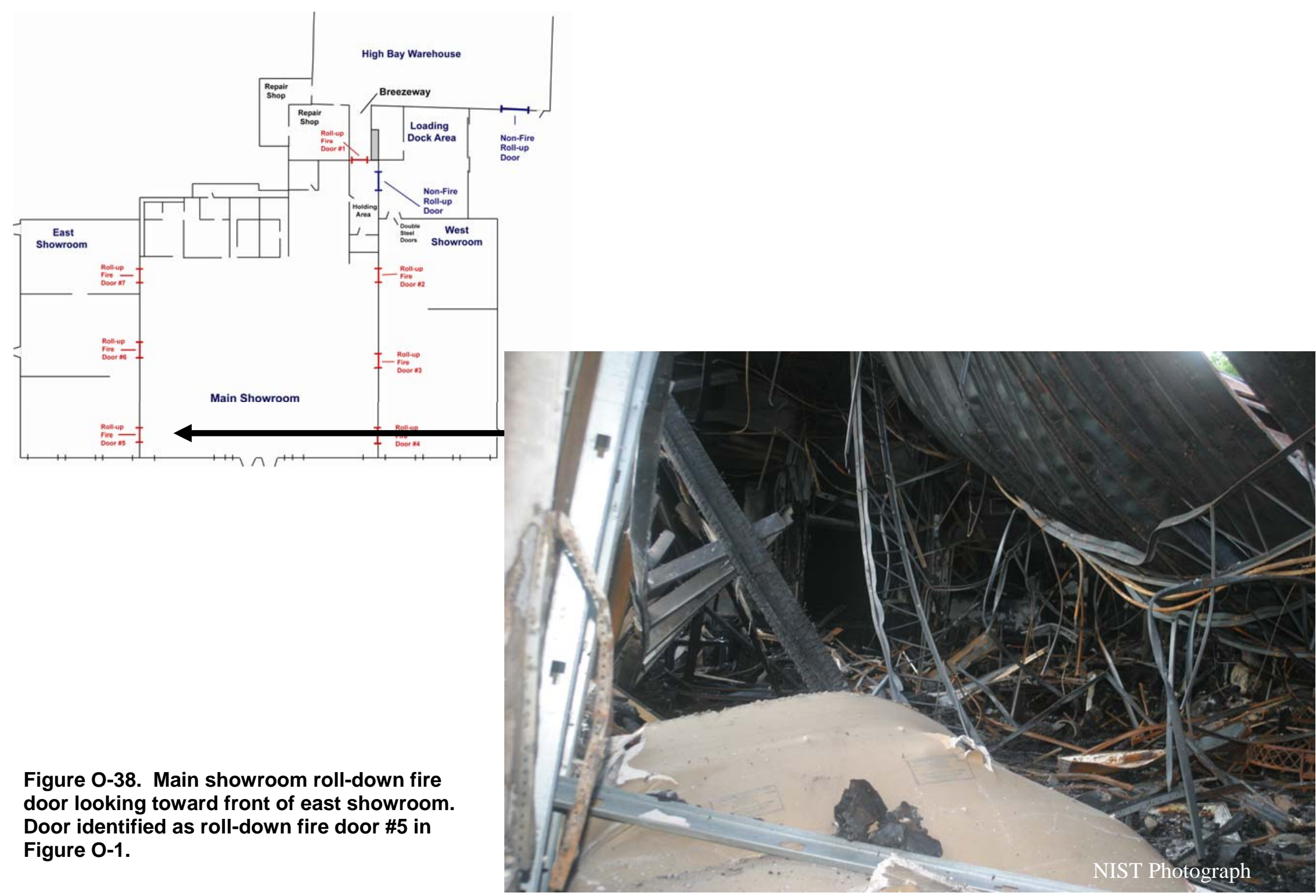

O-39 


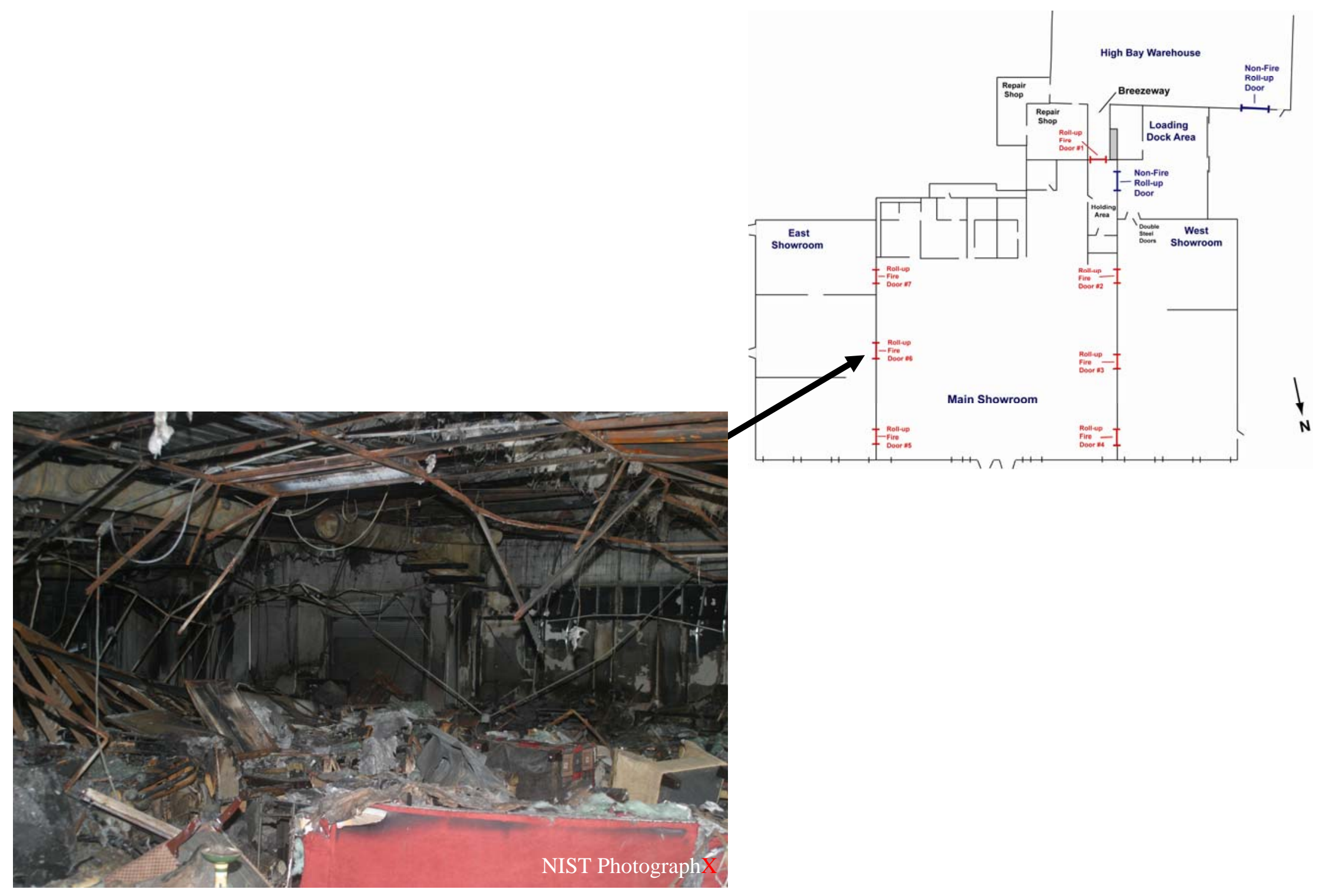

Figure 0-39. Main showroom roll-down fire door looking toward middle of main showroom. Door identified as roll-down fire door \#6 in Figure 01. 


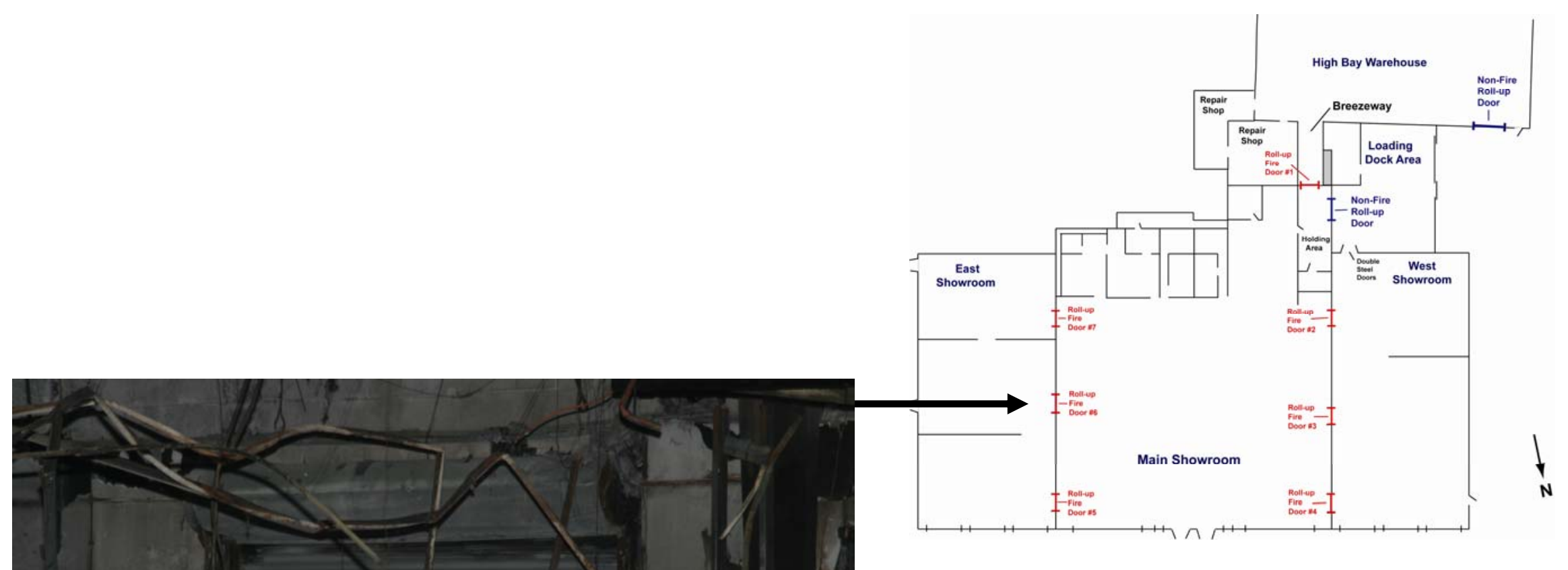

Figure 0-40. Main showroom roll-down fire door looking toward middle of main showroom. Door identified as roll-down fire door \#6 in Figure 0-1. 


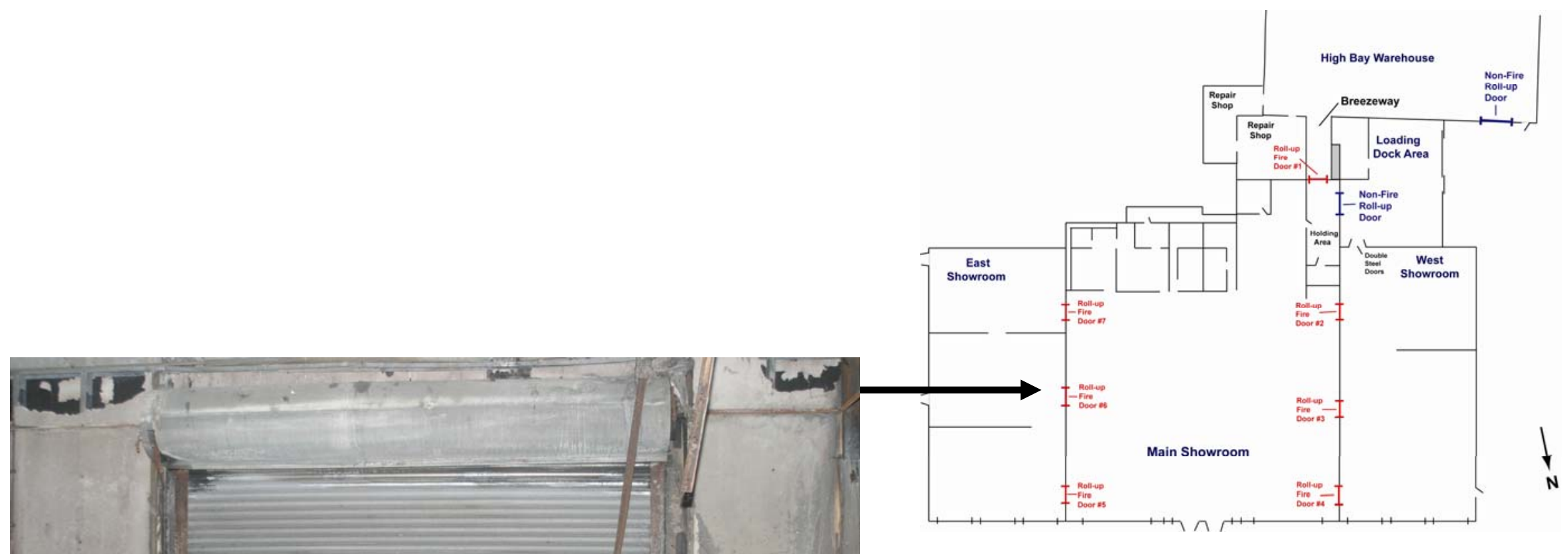

Figure 0-41. Main showroom roll-down fire door looking toward middle of main showroom. 


$$
E A
$$




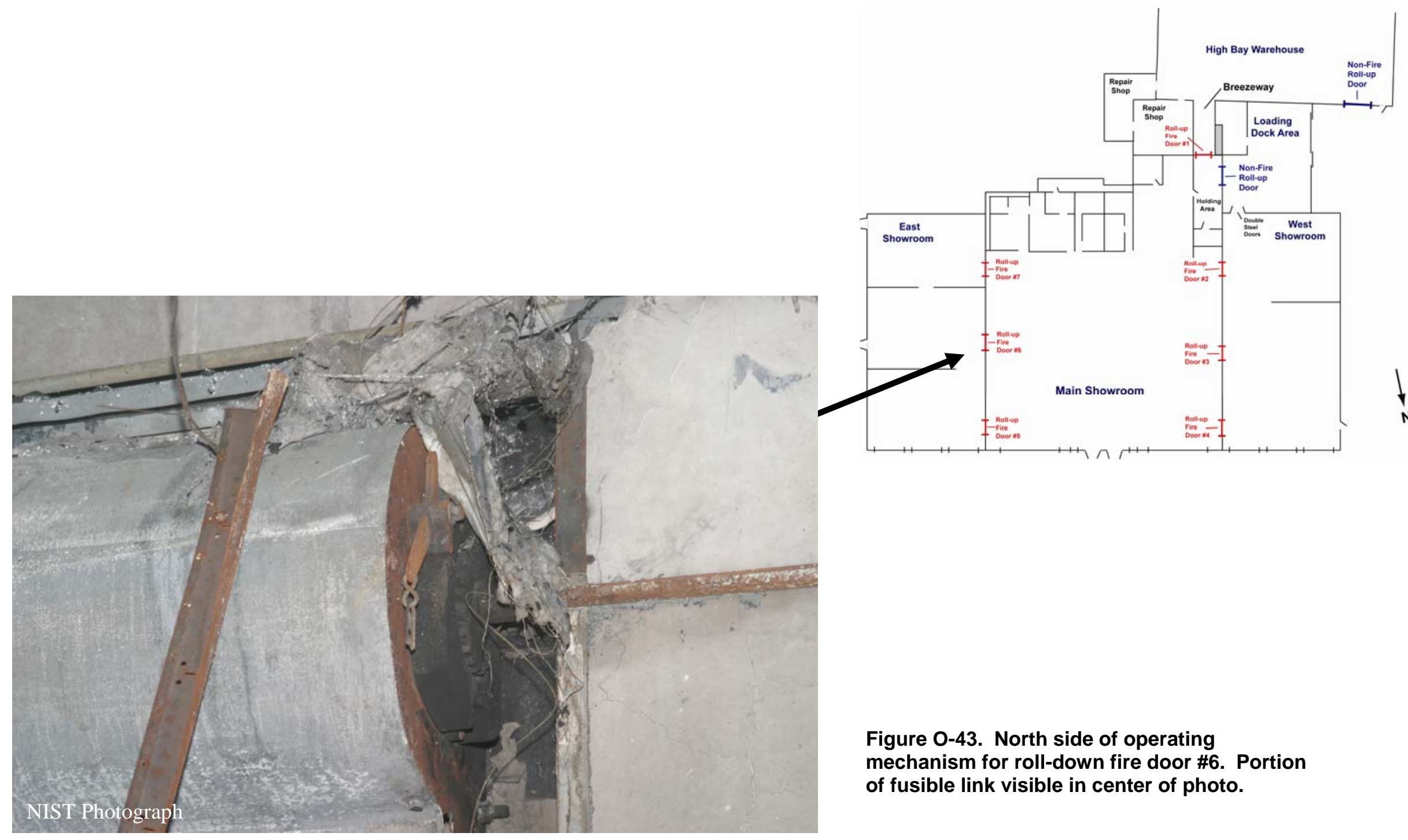



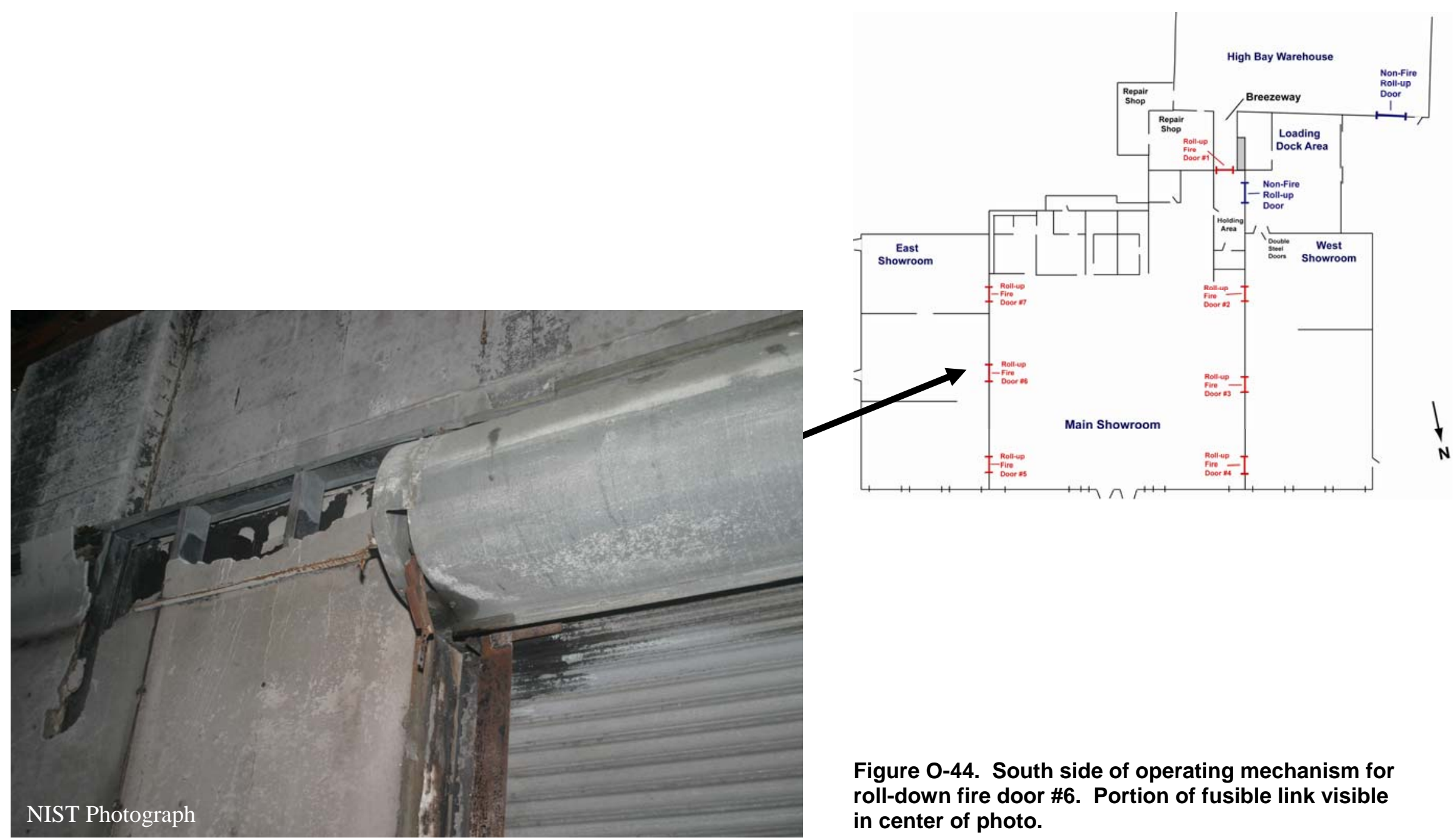

Figure 0-44. South side of operating mechanism for roll-down fire door \#6. Portion of fusible link visible in center of photo. 

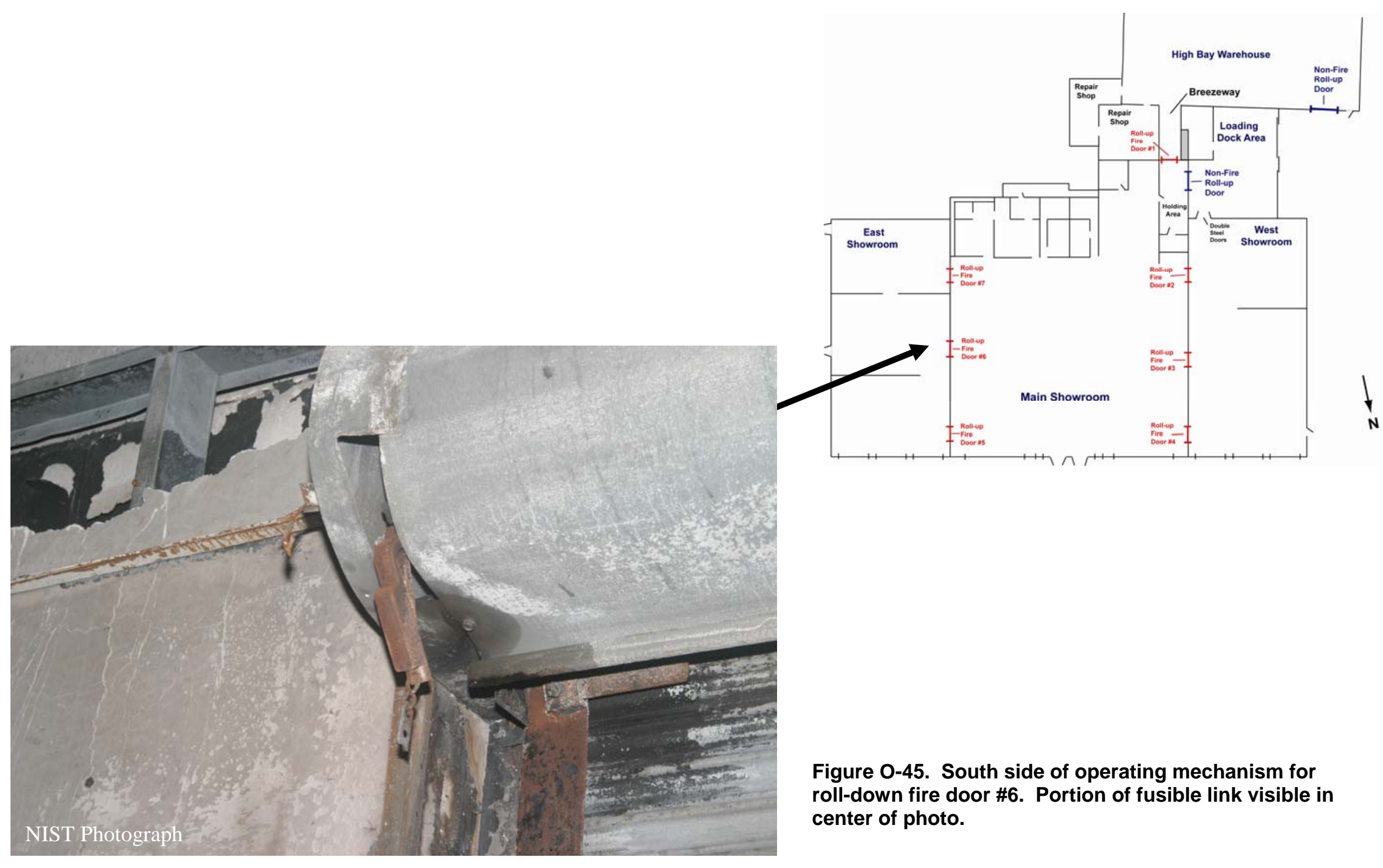

Figure 0-45. South side of operating mechanism for roll-down fire door \#6. Portion of fusible link visible in center of photo. 


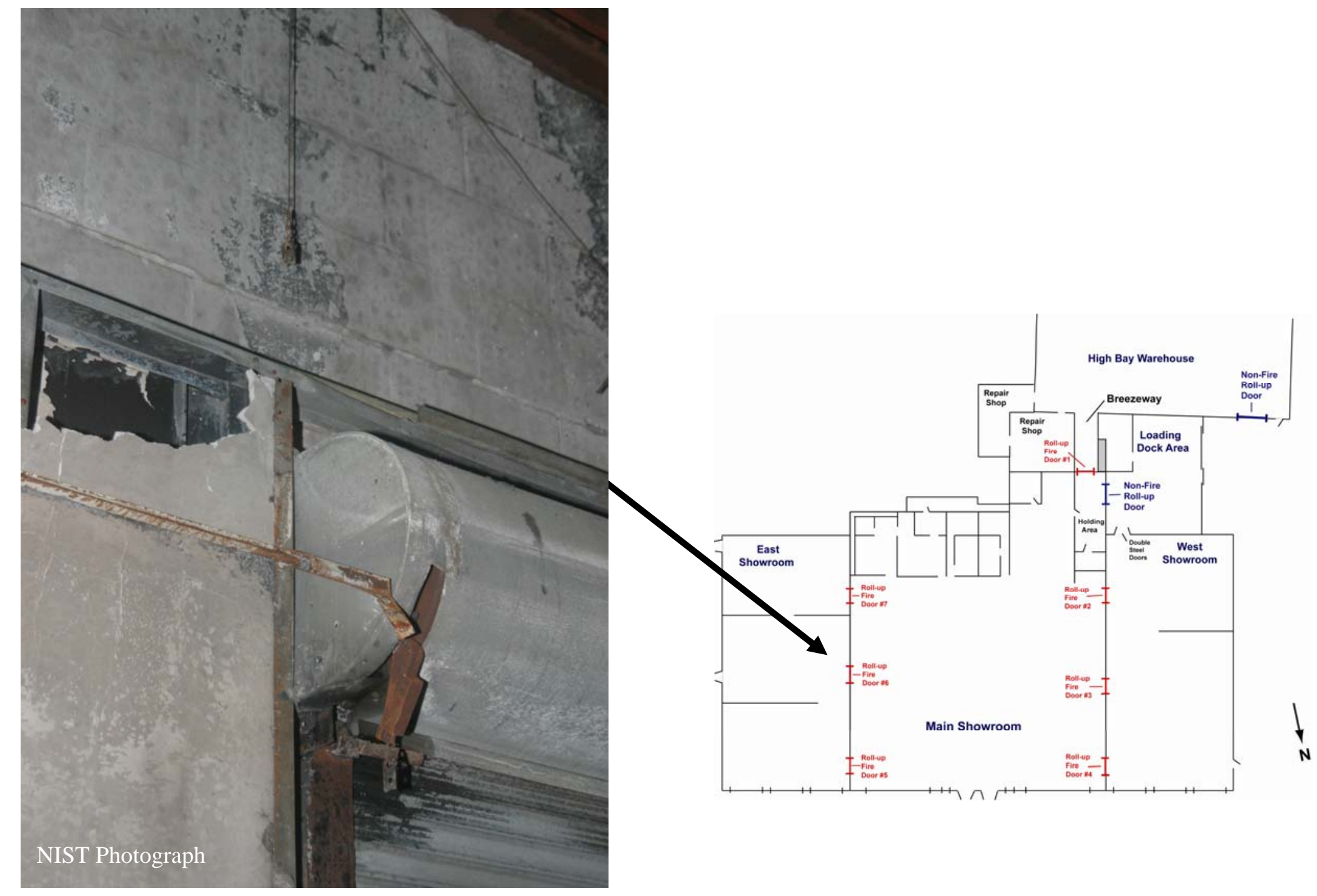

Figure 0-46. South side of operating mechanism for roll-down fire door \#6. Portion of fusible link visible in center of photo. 


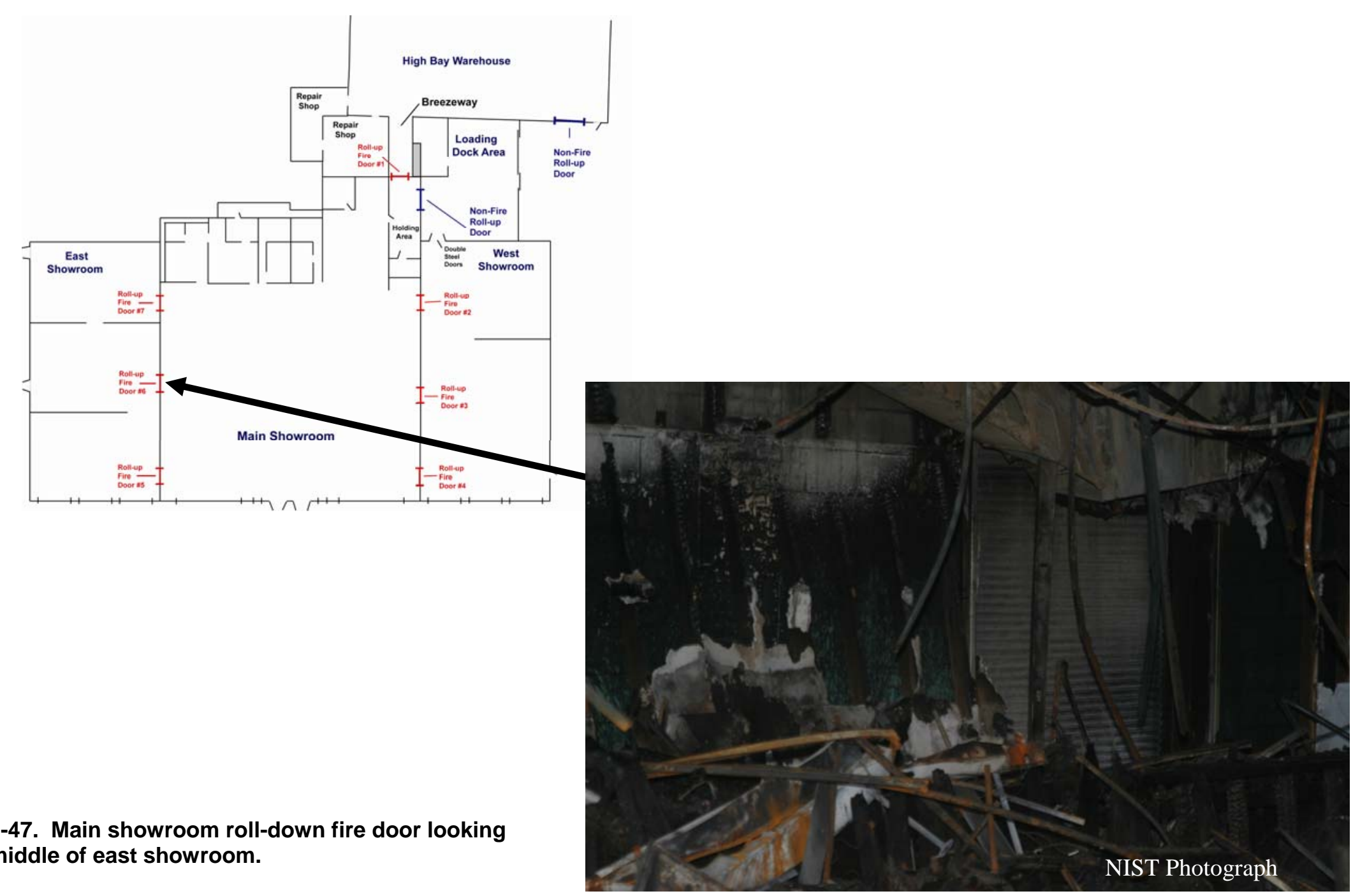

Figure 0-47. Main showroom roll-down fire door looking toward middle of east showroom. 


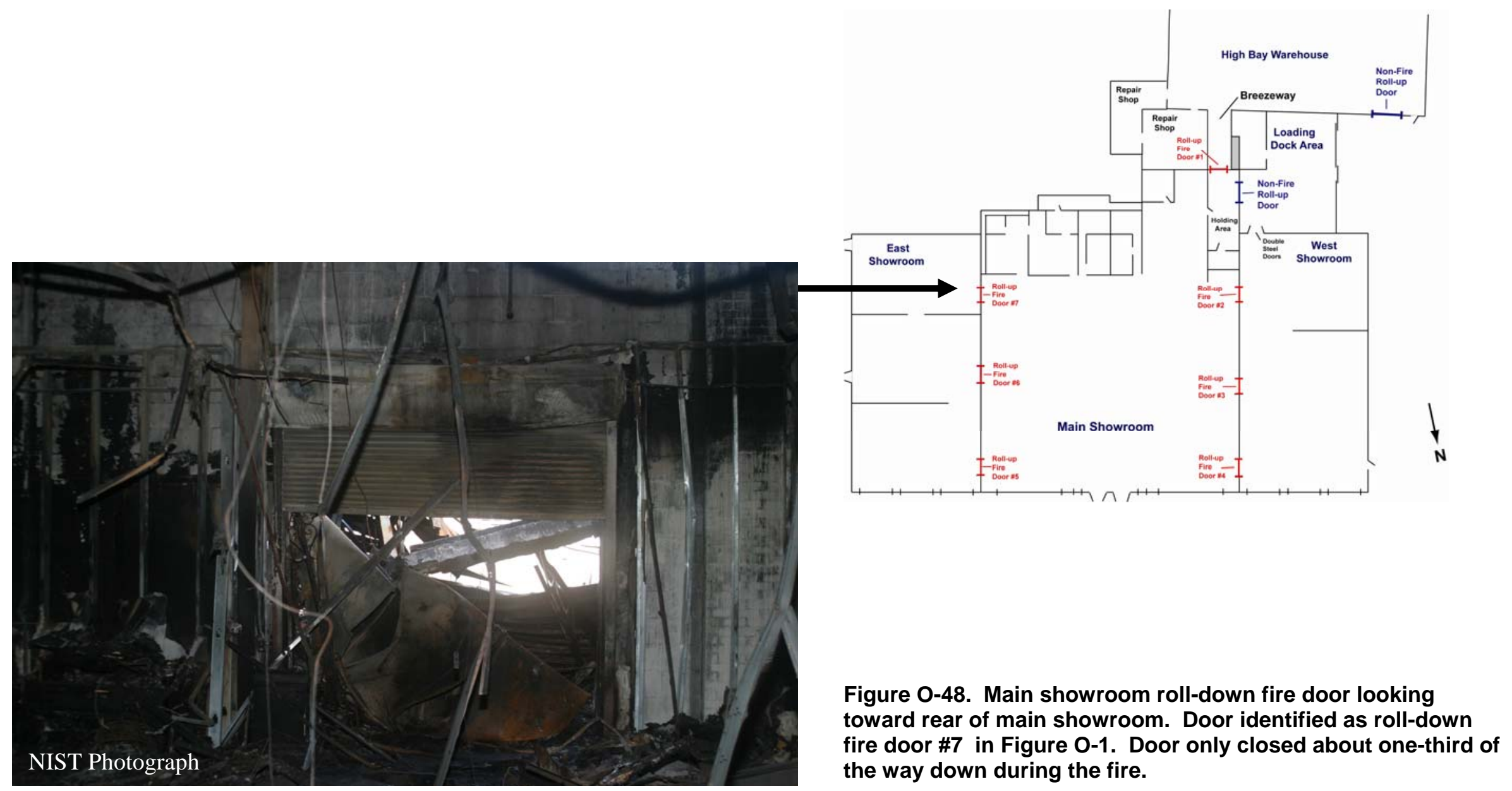




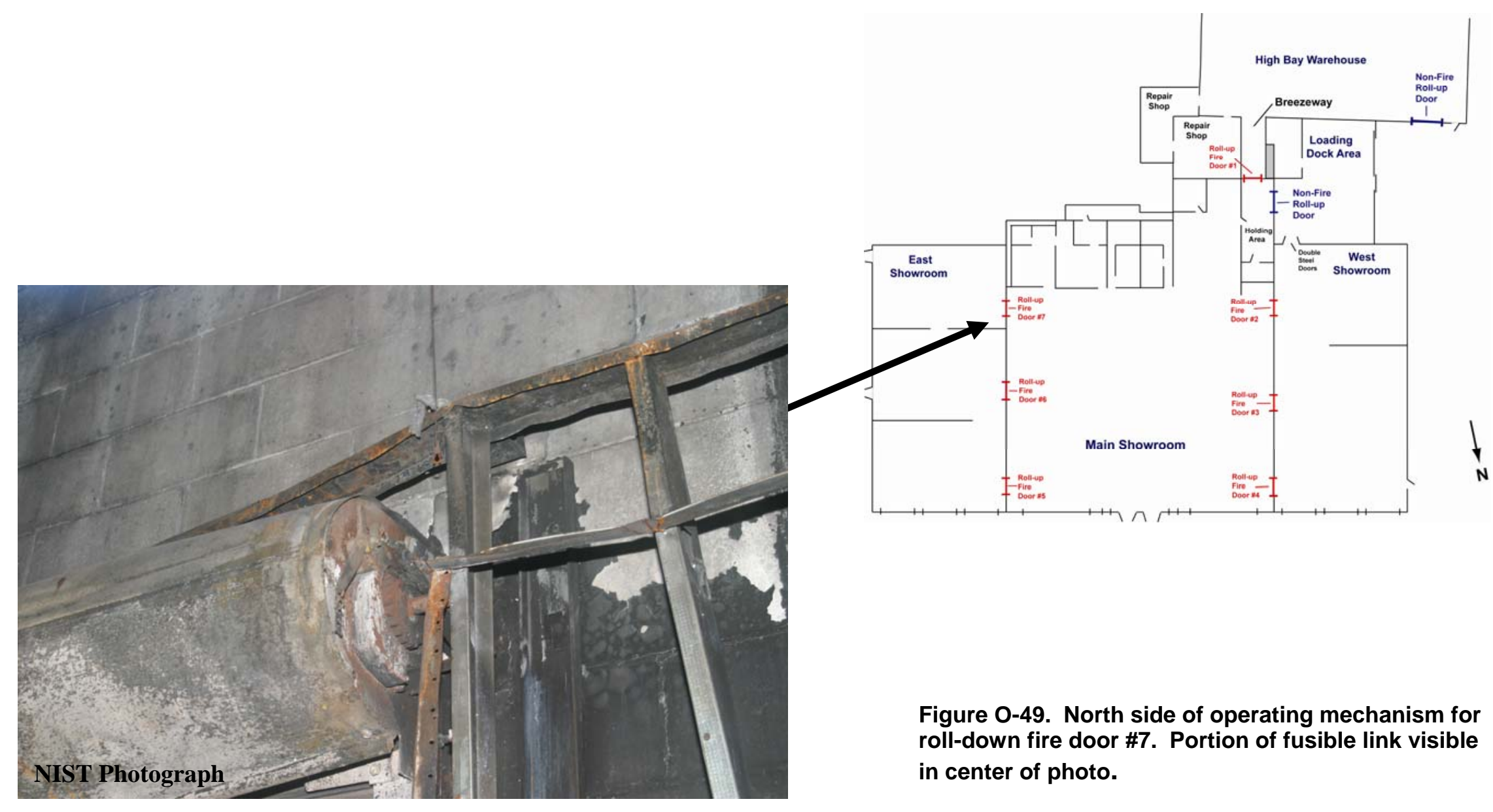




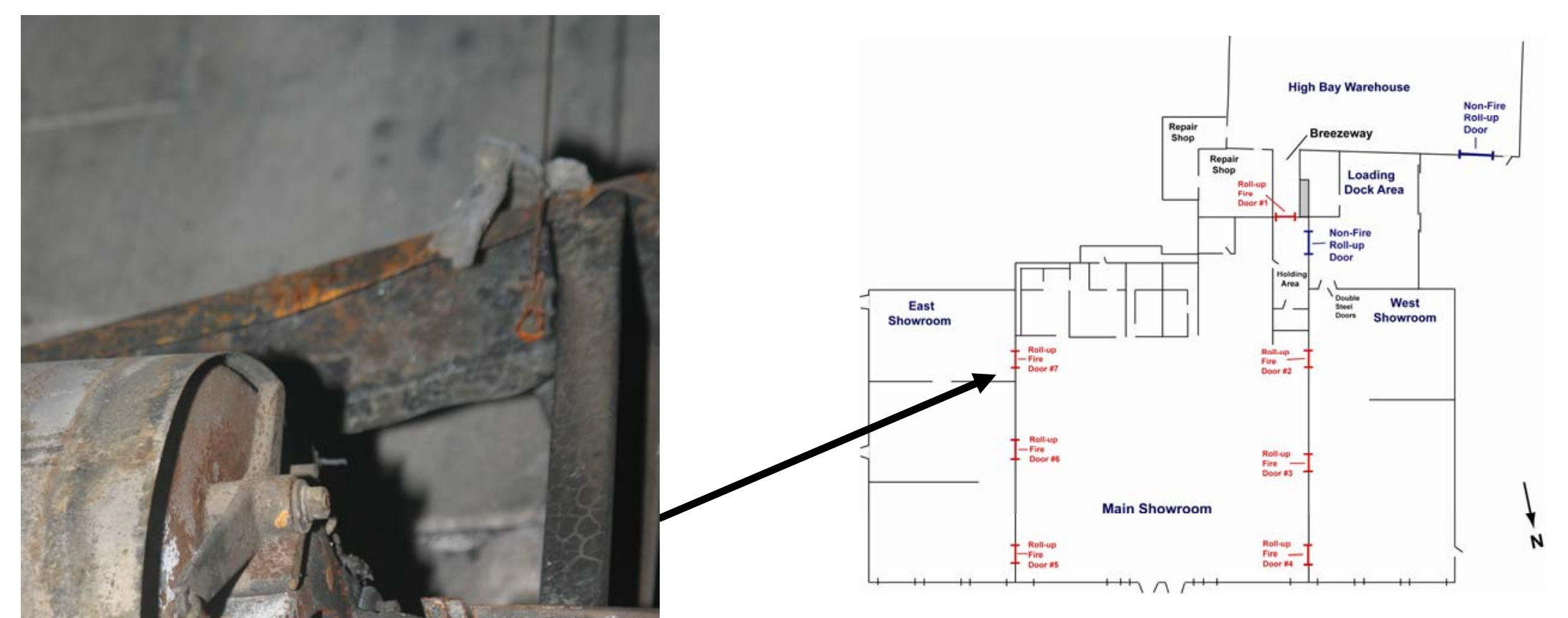
roll-down fire door \#7. Portion of fusible link visible in center of photo. 


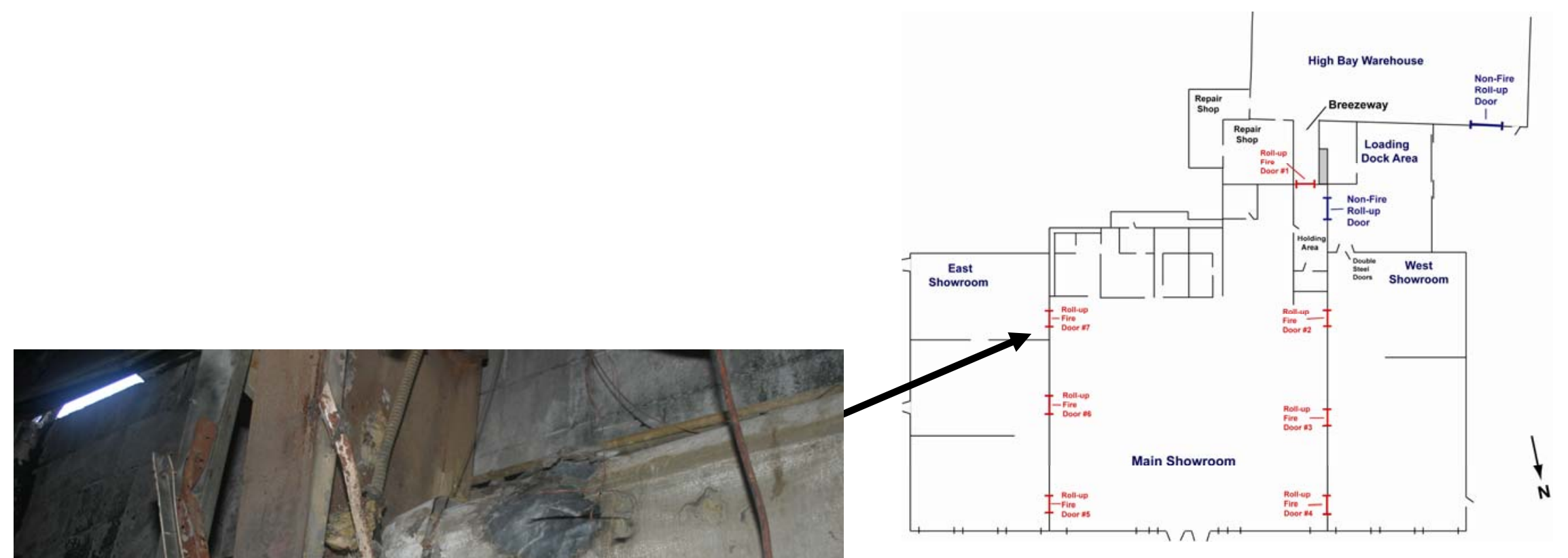

Figure 0-51. South side of operating mechanism for rolldown fire door \#7. Portion of fusible link visible in center 


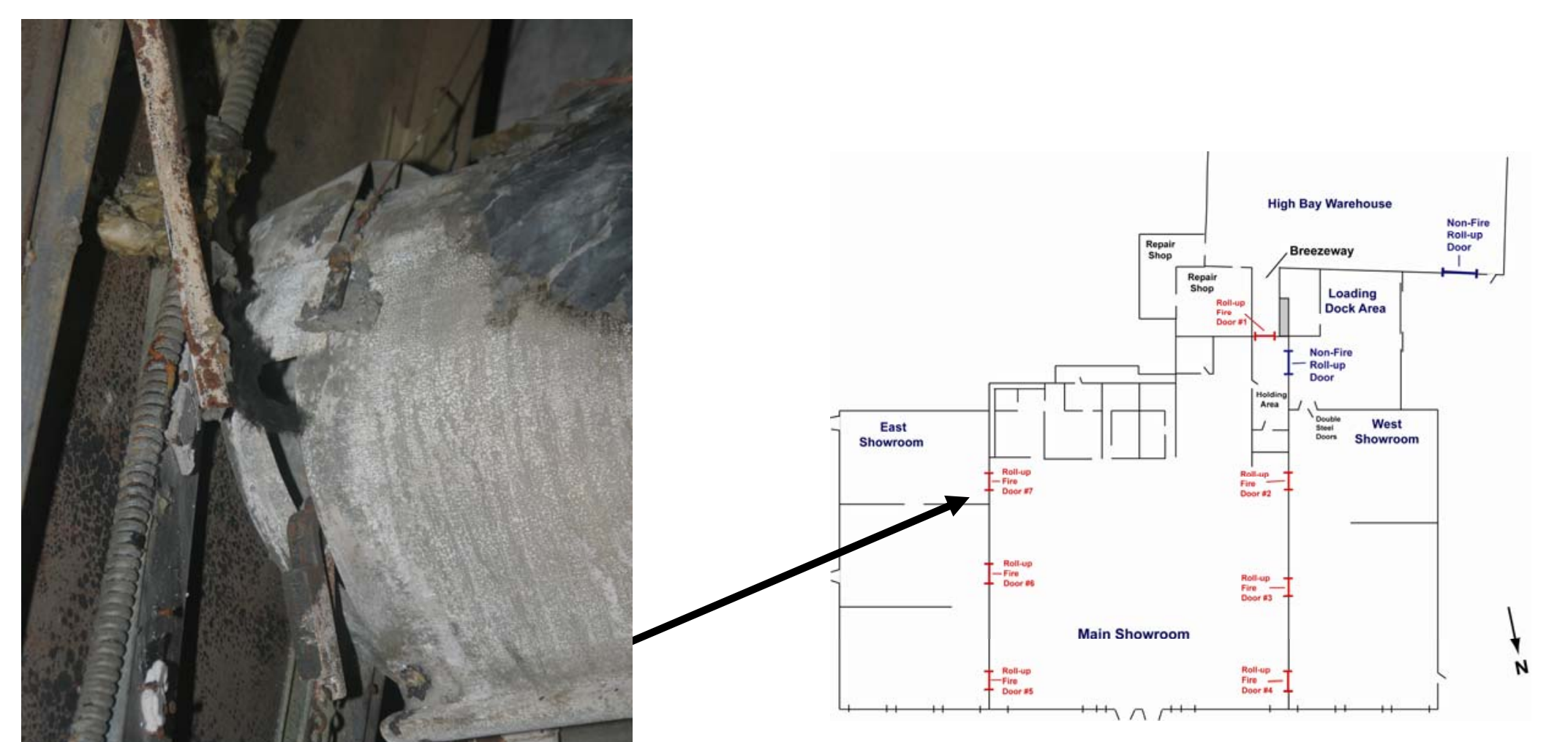

Figure 0-52. South side of operating mechanism for roll-down fire door \#7. Portion of fusible link visible in center of photo. 


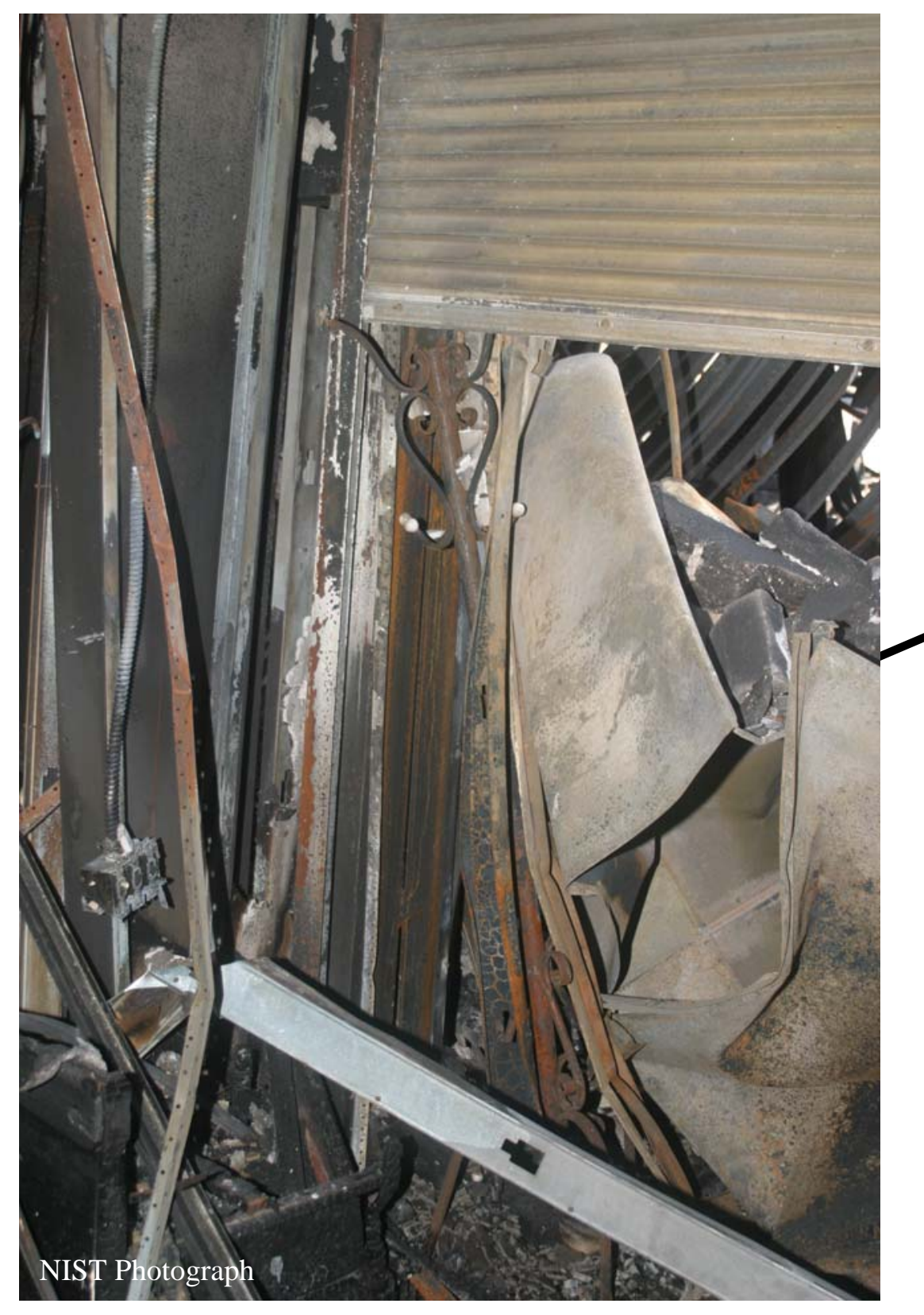

Figure 0-53. Main showroom roll-down fire door looking toward rear of main showroom. Door identified as roll-down fire door \#7 in Figure 0-1. Movement of door impeded by coat rack. Door only closed about one-third of the way. 


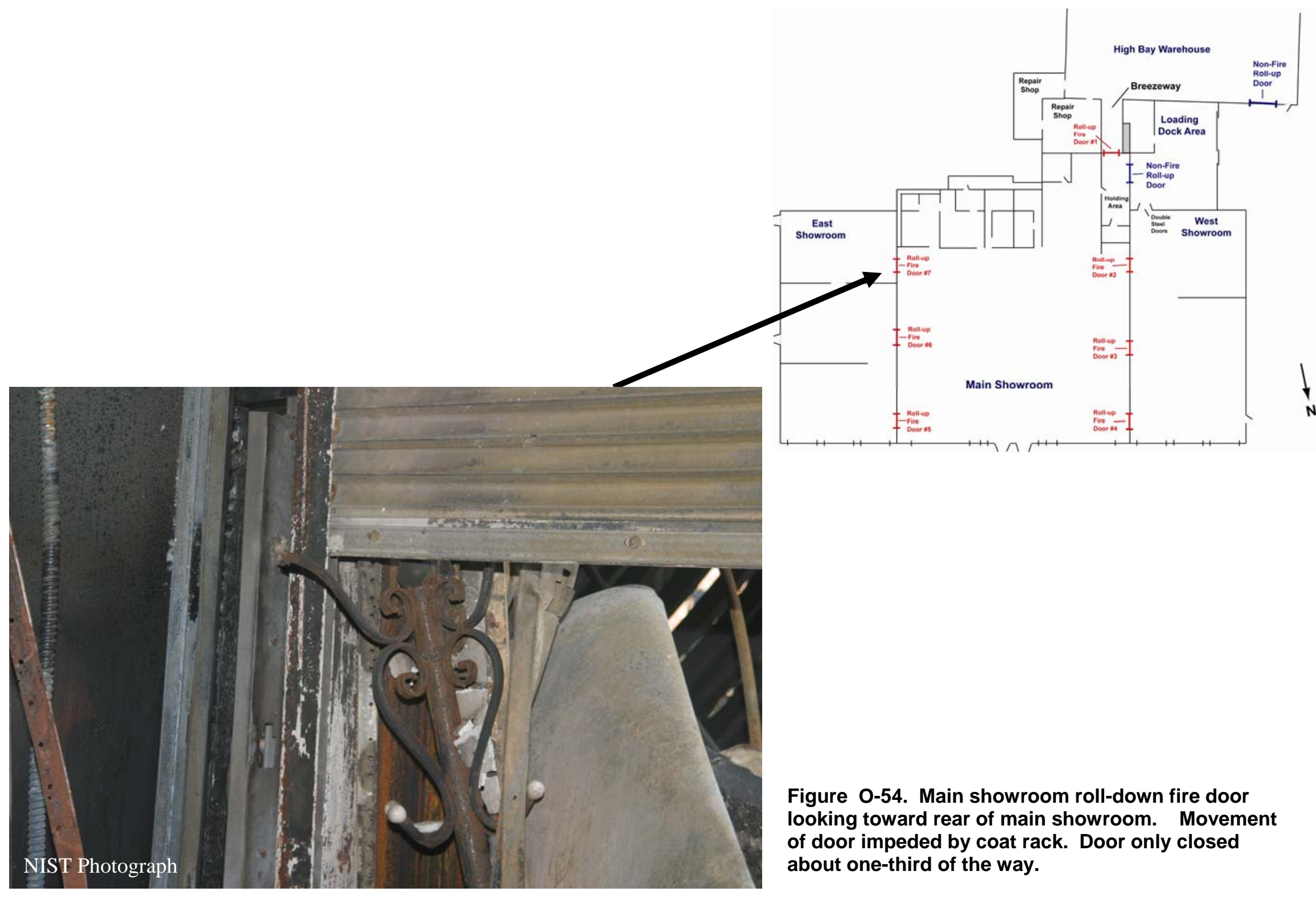




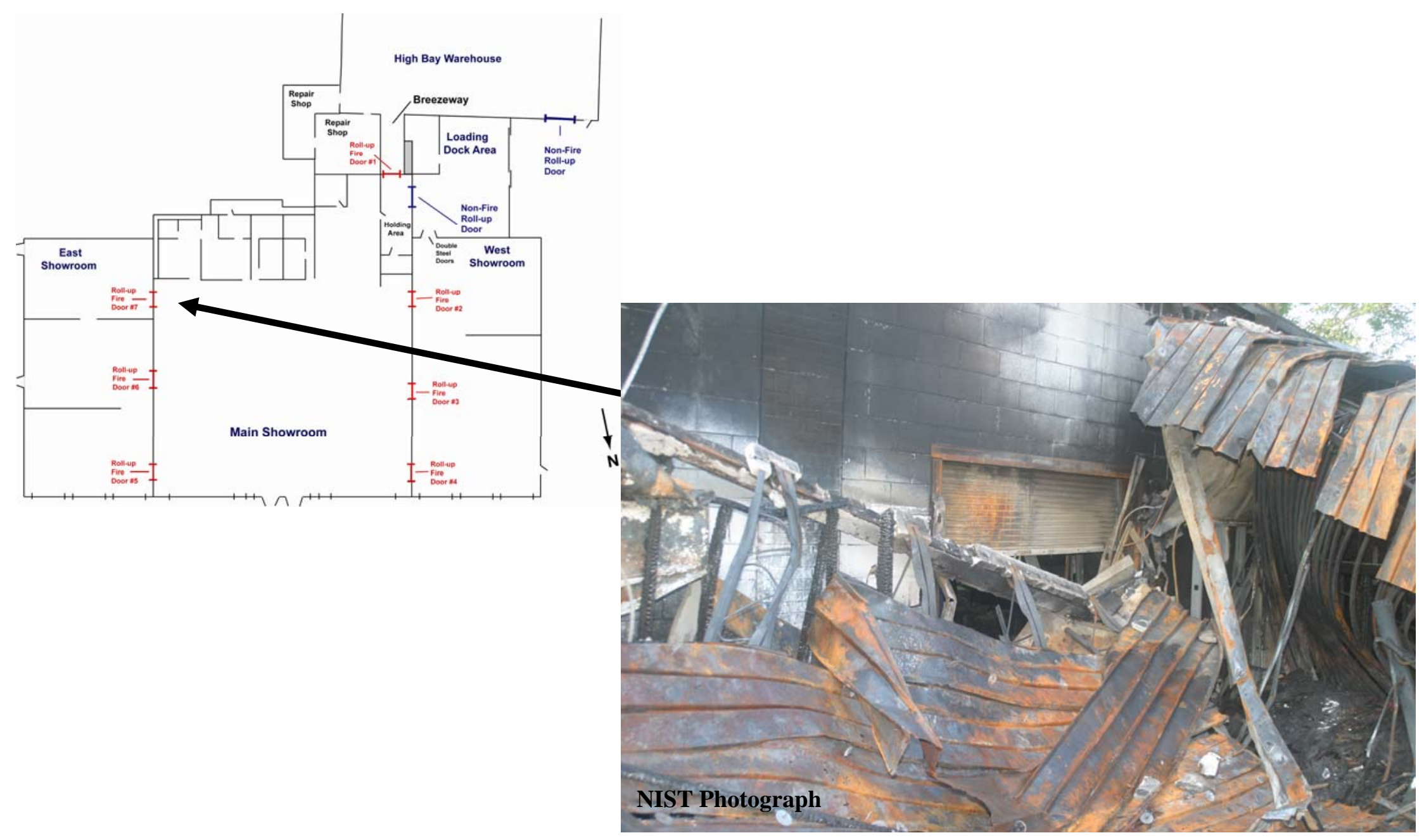

Figure 0-55. Main showroom roll-down fire door looking toward rear of east showroom. Door identified as roll-down fire door \#7 in Figure 0-1. Door only closed about one-third of the way down during the fire. 


\section{APPENDIX P. LOADING DOCK FUELS}

\section{P.1 LOADING DOCK FUELS}

A range of fuels were located within the loading dock. The component of the fuel load due to retail merchandise was estimated in Appendix J. The hydrocarbon solvents, wood framing, wood decking, and carpeting were also estimated. The solvents, framing, decking, and carpeting were estimated to have energy contents of 2.9 GJ, 29.3 GJ, 63.6 GJ, and 7.3 GJ, respectively, for a total of about $100 \mathrm{GJ}$.

\section{P.2 HYDROCARBON SOLVENTS}

Post fire inspection demonstrated at least 28 gallon (3.785 l) containers of various solvents including acetone, naphtha, and lacquer thinner. The intense heat of the fire on the loading dock had caused some of the container bottoms and screw-on caps to be separated from the container. Most of the containers appeared to be naphtha although there were several acetone and laquer thinner containers as well. The relative distribution of the different solvents, 19 naphtha, 3 acetone, and 6 lacquer thinner, was combined with density and heat of combustion data to estimate the energy content of the solvents. These values for the solvents are tabulated in Table P-1. The estimated total energy content of the solvents was approximately 2.9 GJ.

Table P-1. Estimated Energy Content of Solvents on Loading Dock

\begin{tabular}{|c|c|c|c|c|}
\hline Solvent & $\begin{array}{l}\text { Volume } \\
\text { Liter } \\
\text { (gallon) }\end{array}$ & $\begin{array}{c}\text { Density } \\
\text { kg/m3 }\end{array}$ & $\begin{array}{c}\text { Heat of } \\
\text { Combustion } \\
\text { MJ/kg } \\
\text { (MJ/gallon) }\end{array}$ & $\begin{array}{c}\text { Estimated } \\
\text { Energy Content } \\
\text { MJ }\end{array}$ \\
\hline Acetone & $\begin{array}{l}11.4 \\
(3)\end{array}$ & 785 & $\begin{array}{c}30 \\
(90)\end{array}$ & 270 \\
\hline Naphtha & $\begin{array}{l}71.9 \\
(19)\end{array}$ & 665 & $\begin{array}{c}43 \\
(108)\end{array}$ & 2060 \\
\hline Lacquer Thinner & $\begin{array}{l}22.7 \\
(6)\end{array}$ & 820 & $\begin{array}{c}29 \\
(90)\end{array}$ & 540 \\
\hline \multicolumn{4}{|c|}{ Estimated Total Energy Content } & 2870 \\
\hline
\end{tabular}




\section{P.3 WOOD FRAMING}

The loading dock construction also included significant amounts of wood in the framing and decking. Wood studs were incorporated in the west walls and along the warehouse wall. Post- fire inspection also demonstrated several partition walls inside the loading dock that also used wood studs. Wood columns were also used to support the wood roof joists. The estimated number of studs, columns, rafters, and joists was combined with density and heat of combustion data to estimate the energy content of the framing. These values for the framing are tabulated in Table P-2. The estimated energy content of the framing was about $29.3 \mathrm{GJ}$.

Table P-2. Estimated Energy Content of Wood Framing in Loading Dock

\begin{tabular}{|c|c|c|c|c|c|c|c|}
\hline $\begin{array}{c}\text { Wood } \\
\text { Component }\end{array}$ & Number & $\begin{array}{c}\text { Cross } \\
\text { Section } \\
\mathrm{cm}^{2} \\
\left(\mathrm{in}^{2}\right)\end{array}$ & $\begin{array}{c}\text { Height } \\
\text { m } \\
\text { (ft) }\end{array}$ & $\begin{array}{c}\text { Total } \\
\text { Volume } \\
\mathrm{m}^{3} \\
\left(\mathrm{ft}^{3}\right)\end{array}$ & $\begin{array}{c}\text { Total } \\
\text { Mass } \\
\text { kg }\end{array}$ & $\begin{array}{c}\begin{array}{c}\text { Heat of } \\
\text { Combustion }\end{array} \\
\mathrm{MJ} / \mathrm{kg}\end{array}$ & $\begin{array}{c}\text { Estimated } \\
\text { Energy } \\
\text { Content } \\
\text { MJ }\end{array}$ \\
\hline $\begin{array}{l}\text { Exterior Walls, } \\
\text { studs }\end{array}$ & 62 & $\begin{array}{c}37 \\
(5.7)\end{array}$ & $\begin{array}{l}2.44 \\
(8)\end{array}$ & $\begin{array}{c}0.59 \\
(20.7)\end{array}$ & 320 & 15 & 4,800 \\
\hline $\begin{array}{l}\text { Interior Partition } \\
\text { Walls, studs }\end{array}$ & 26 & $\begin{array}{c}37 \\
(5.7)\end{array}$ & $\begin{array}{r}2.44 \\
(8)\end{array}$ & $\begin{array}{l}0.23 \\
(8.2)\end{array}$ & 130 & 15 & 1,900 \\
\hline $\begin{array}{l}\text { Support Columns } \\
9 \mathrm{ft} \text { High Section }\end{array}$ & 5 & $\begin{array}{c}79 \\
(12.3)\end{array}$ & $\begin{array}{l}2.74 \\
(9)\end{array}$ & $\begin{array}{l}0.11 \\
(3.8)\end{array}$ & 60 & 15 & 890 \\
\hline $\begin{array}{l}\text { Support Columns } \\
12 \mathrm{ft} \mathrm{High} \\
\text { Section }\end{array}$ & 5 & $\begin{array}{r}186 \\
(28.9)\end{array}$ & $\begin{array}{l}3.66 \\
(12)\end{array}$ & $\begin{array}{c}0.34 \\
(12.0)\end{array}$ & 190 & 15 & 2,900 \\
\hline $\begin{array}{l}\text { Roof Supports } \\
50 \mathrm{ft} \text { long rafters } \\
\& \text { joists }\end{array}$ & 35 & $\begin{array}{r}56 \\
(8.7)\end{array}$ & $\begin{array}{r}15 \\
(50)\end{array}$ & $\begin{array}{l}2.9 \\
(82)\end{array}$ & 1250 & 15 & 19,000 \\
\hline $\begin{array}{l}\text { Estimated } \\
\text { Totals }\end{array}$ & & & & $\begin{array}{c}3.5 \\
(130)\end{array}$ & 1950 & & 29,500 \\
\hline
\end{tabular}




\section{P.4 WOOD DECKING}

The wood decking appeared to have been constructed in several different sections using different wood materials. The lower layer of the decking or floor appeared to be pressure treated planking approximately $2.54 \mathrm{~cm}$ (1 in) thick. A second layer of $1.27 \mathrm{~cm}(0.5 \mathrm{in})$ thick oriented strand board, particle board, or plywood had been installed on top of the pressure treated planking. The estimated floor area and layer thicknesses were combined with density and heat of combustion data to estimate the energy content of the decking. These values for the decking are tabulated in Table P-3. The estimated energy content of the decking was about $63.6 \mathrm{GJ}$.

Table P-3. Estimated Energy Content of Wood Decking in Loading Dock

\begin{tabular}{|c|c|c|c|c|c|c|}
\hline Component & $\begin{array}{l}\text { Area } \\
\mathrm{m}^{2} \\
\left(\mathrm{ft}^{2}\right)\end{array}$ & $\begin{array}{c}\text { Thickness } \\
\text { cm } \\
\text { (in) }\end{array}$ & $\begin{array}{c}\text { Total } \\
\text { Volume } \\
\mathrm{m}^{3} \\
\left(\mathrm{ft}^{3}\right)\end{array}$ & $\begin{array}{c}\text { Total } \\
\text { Mass } \\
\text { kg }\end{array}$ & $\begin{array}{c}\text { Heat of } \\
\text { Combustion } \\
\text { MJ/kg }\end{array}$ & $\begin{array}{c}\text { Estimated } \\
\text { Energy } \\
\text { Content } \\
\text { MJ }\end{array}$ \\
\hline Planking & $\begin{array}{c}206 \\
(2200)\end{array}$ & $\begin{array}{l}2.54 \\
(1)\end{array}$ & $\begin{array}{c}5.2 \\
(180)\end{array}$ & 2800 & 15 & 42,400 \\
\hline $\begin{array}{l}\text { OSB/Particle } \\
\text { Board/Plywood }\end{array}$ & $\begin{array}{c}206 \\
(2200)\end{array}$ & $\begin{array}{l}1.27 \\
(0.5)\end{array}$ & $\begin{array}{l}2.6 \\
(90)\end{array}$ & 1400 & 15 & 21,200 \\
\hline $\begin{array}{l}\text { Estimated } \\
\text { Totals }\end{array}$ & & & $\begin{array}{c}7.8 \\
(270)\end{array}$ & 4200 & & 63,600 \\
\hline
\end{tabular}

\section{P.5 CARPETING}

Carpeting appeared have be laid over portions of the wood flooring. Assuming that the carpeting had been installed over $50 \%$ of the loading dock floor, and that the total energy content of the carpeting was approximately $72 \mathrm{MJ} / \mathrm{m}^{2}$ [2], the total energy content of the carpeting was estimated to be $7.3 \mathrm{GJ}$. 


\section{P.6 REFERENCES FOR APPENDIX $P$}

[1] SFPE Handbook of Fire Protection Engineering, Fourth Edition, DiNenno, P.J. editor-in-chief, National Fire Protection Association, Quincy, MA, 2008.

[2] Grosshandler, W. L.; Bryner, N. P.; Madrzykowski, D.; Kuntz, K., "Report of the Technical Investigation of The Station Nightclub Fire: Appendices. Volume 2", NIST NCSTAR 2: Volume 2; National Institute of Standards and Technology, Gaithersburg, MD, 414 p. June 2005.

[3] Bryner, N. P.; Kerber, S., "Simulation of the Dynamics of a Fire in the Basement of a Hardware Store - New York, June 17, 2001”, NISTIR 7137; National Institute of Standards and Technology, Gaithersburg, MD, 57 p. May 2004.

[4] Denize, Hamish, "The Combustion Behavior of Upholstered Furniture Materials in New Zealand”, Fire Engineering Research Report 00/04, University of Canterbury, Christchurch, New Zealand, March 2000.

[5] Firestone, James, “An Analysis of Furniture Heat Release Rates by Nordtest”, Fire Engineering Research Report 99/07, University of Canterbury, Christchurch, New Zealand, March 1999. 


\section{Appendix Q. Technical Reports, Media Articles, Web Pages, AND OTHER RESOURCES}

\section{Q.1 TECHNICAL REPORTS}

[1] Routley, G. J., Chiaramonte, M., Crawford, B., Piringer, P., Roche, K., and Sendelbach, T., "Firefighter Fatality Investigative Report, Sofa Super Store, 1807 Savannah Highway, Charleston, South Carolina, June 18, 2007”, City of Charleston Post Incident Assessment and Review Team Phase I Report,” City of Charleston. October 16, 2007.

[2] Routley, G. J., Chiaramonte, M., Crawford, B., Piringer, P., Roche, K., and Sendelbach, T., "Firefighter Fatality Investigative Report, Sofa Super Store, 1807 Savannah Highway, Charleston, South Carolina, June 18, 2007” City of Charleston Post Incident Assessment and Review Team Phase II Report, City of Charleston May 15, 2008.

[3] "Bureau of Alcohol, Tobacco, Firearms, and Explosives (ATF) National Response Team Origin and Cause Report: Sofa Super Store Fire”; U.S. Department of Justice; Investigation Number 763010-07-0172; Report Number 32, June 22, 2007.

[4] “Investigative Summary,” S. C. Department of Labor, Licensing, and Regulation Office of Occupational Safety and Health; Summary Number 20374270, undated report, file recorded 9/21/2007.

[5] "Nine Career Fire Fighters Die in Rapid Fire Progression at Commercial Furniture Showroom South Carolina,” NIOSH. Fatality Assessment and Control Evaluation Investigation Report \# F2007-18. Feb. 11, 2009. http://www.cdc.gov/niosh/fire/reports/face200718.html.

[6] "Report of S.C. OSHA Findings in June 18, 2007 Charleston Sofa Super Store Fire,” South Carolina Department of Labor, Licensing and Regulation, Office of Occupational Safety and Health; undated report, file recorded 9/21/2007.

\section{Q.2 MEDIA ARTICLES}

[1] Parks, N., "West Ashley Sofa Super Store ablaze, roof collapses, smoke visible for miles,” The Post and Courier. Jun. 18, 2007. http://www.postandcourier.com/news/2007/jun/18/sofa _super_store_ablaze/.

[2] Bartelme, T., Haglund, N., Parks, N., and Smith, G., "Names released of nine firefighters killed in Sofa Super Store in West Ashley,” The Post and Courier. Jun. 19, 2007. http://www.postand courier.com/news/2007/jun/19/firefighters_lost/.

[3] Associated Press, “9 firefighters die in S. C. blaze,” msnbc.com. Jun. 20, 2007. http://www.msn bc.msn.com/id/19301684.

[4] Smith, B., “Charleston Blaze Kills 9 Firefighters,” washingtonpost.com. Jun. 20, 2007. http://www.washingtonpost.com/wp-dyn/content/article/2007/06/19/AR2007061900336. html?hpid=topnews.

[5] "Cause of death of firefighters was smoke inhalation and burns,” The Post and Courier. Jun. 20, 2007. http://www.postandcourier.com/news/2007/jun/20/cause_death_firefighters_was_smoke _inhalation_and_/. 
[6] Bartelme, T., Behre, R., Birmingham, N., Fennell, E., Findlay, P., Haglund, N., Johnson, J., Menchaca, R., and Petersen, B., "Fallen Firefighters," The Post and Courier. Jun. 20, 2007. http://www.postandcourier.com/news/2007/jun/20/nine_fallen_firefighters/.

[7] Stech, K., “Rescued from the flames,” The Post and Courier. Jun. 20, 2007. http://www.post andcourier.com/news/2007/jun/20/rescued_from_flames/.

[8] Stech, K., “2 St. Andrews firefighters saved store employee,” The Post and Courier. Jun. 21, 2007. http://www.postandcourier.com/news/2007/jun/21/st_andrews_firefighters_saved _store_employee/.

[9] Menchaca, R. “911 transcripts from Monday's fire released,” The Post and Courier. Jun. 21, 2007. http://www.postandcourier.com/news/2007/jun/21/911_transcripts_mondays_fire _released/.

[10] Behre, R., "Lack of sofa store sprinkler system prompts debate over requirements,” The Post and Courier. Jun. 21, 2007. http://www.postandcourier.com/news/2007/jun/21/lack_sofa_store _sprinkler_system_prompts_debate_/.

[11] Menchaca, R. and Smith, G., "Loss prompts grief, provokes questions," The Post and Courier. Jun. 21, 2007. http://www.postandcourier.com/news/2007/jun/21/grief_questions/.

[12] “Sofa Super Store showed no signs of danger for firefighters,” The Post and Courier. Jun. 21, 2007. http://www.postandcourier.com/news/2007/jun/21/sofa_super_store_showed_no _signs_danger_firefighte/.

[13] Parks, N., “A solemn day,” The Post and Courier. Jun. 22, 2007. http://www.postandcourier.com/ news/2007/jun/22/a_solemn_day/.

[14] Smith, G., "Faces of those lost haunt survivors,” The Post and Courier. Jun. 22, 2007. http://www.postandcourier.com/news/2007/jun/22/faces_those_lost_haunt_survivors/.

[15] Findlay, P., "Husband saw store as posing great risk,” The Post and Courier. Jun. 22, 2007. http://www.postandcourier.com/news/2007/jun/22/firefighter_saw_store_as_posing_great_risk/.

[16] Bartelme, T., and Menchaca, R., "Steel trusses like those in the Sofa Super Store can mask growing fires,” The Post and Courier. Jun. 22, 2007. http://www.postandcourier.com/news /2007/jun/22/steel_trusses_those_sofa_super_store_can_mask_grow/.

[17] “ATF National Response Team Origin and Cause Report: Sofa Super Store Fire”; Investigation Number 763010-07-0172; Report Number 32, June 22, 2007. The Post and Courier.

[18] Bartelme, T. and Menchaca, R., "Danger can hide in roof trusses,” The Post and Courier. Jun. 23, 2007. http://www.postandcourier.com/news/2007/jun/23/danger_can_hide_roof_trusses/.

[19] “Fire started in loading dock, ATF confirms,” The Post and Courier. Jun. 23, 2007. http://www.postandcourier.com/news/2007/jun/23/fire_started_loading_dock_atf_confirms/.

[20] Stech, K., “Super Sofa Store building had long history,” The Post and Courier. Jun. 23, 2007. http://www.postandcourier.com/news/2007/jun/23/super_sofa_store_building_had_long_history/.

[21] Mitchell, O., “Deadly Fire Investigation,” WCBD-TV2. Jun. 24, 2007.

[22] Kropf, S. and Stech, K., “Fire began at store's loading dock,” The Post and Courier. Jun. 24, 2007. http://www.postandcourier.com/news/2007/jun/24/fire_began_at_stores_loading_dock/.

[23] Menchaca, R. and Pardue, D., "Local, federal guidelines in conflict,” The Post and Courier. Jun. 24, 2007. http://www.postandcourier.com/news/2007/jun/24/local_federal_guidelines_conflict/. 
[24] Stech, K., "Blaze started in illegally built area,” The Post and Courier. Jun. 26, 2007. http://www.postandcourier.com/news/2007/jun/26/blaze_started_illegally_built_area/.

[25] Findlay, P., "If firefighters see furniture, they see fuel,” The Post and Courier. Jun. 27, 2007. http://www.postandcourier.com/news/2007/jun/27/if_firefighters_see_furniture_they_see_fuel/.

[26] Menchaca, R. and Pardue, D., “Fire union wants changes,” The Post and Courier. Jun. 28, 2007. http://www.postandcourier.com/news/2007/jun/28/fire_union_wants_changes/.

[27] Bartelme, T., “Sofa store lacked smoke detectors,” The Post and Courier. Jun. 29, 2007. http://www.postandcourier.com/news/2007/jun/29/sofa_store_lacked_smoke_detectors/.

[28] Parks, N., “Reporting on tragedy hits home,” The Post and Courier. Jun. 30, 2007. http://www.postandcourier.com/news/2007/jun/30/reporting_on_tragedy_hits_home/.

[29] Menchaca, R., "Smokers always were careful, store employee says,” The Post and Courier. Jun. 30, 2007. http://www.postandcourier.com/news/2007/jun/30/smokers_always_were_careful_store _employee_says/.

[30] Menchaca, R. and Smith, G., “Are City of Charleston's firefighting techniques too bold?” The Post and Courier. Jul. 1, 2007. http://www.postandcourier.com/news/2007/jul/01/tradition_risk/.

[31] Parks, N., "Firefighters to be interviewed by NIOSH investigators," The Post and Courier. Jul. 6, 2007. http://www.postandcourier.com/news/2007/jul/06/firefighters_be_interviewed_by_niosh _investigato/.

[32] Phillips, S., "Report that second-guessed firefighters falls short,” The Post and Courier. Jul. 6, 2007. http://www.postandcourier.com/news/2007/jul/06/report_second_guessed/.

[33] Findlay, P., "Department's insurance rating could be re-evaluated,” The Post and Courier. Jul. 15, 2007. http://www.postandcourier.com/news/2007/jul/15/departments_insurance_rating_could _be_re10621/.

[34] Smith, G., “Charleston launches own investigation into tragedy,” The Post and Courier. Jul. 26, 2007. http://www.postandcourier.com/news/2007/jul/26/charleston_launches_own _investigation_in11460/.

[35] Menchaca, R. and Smith, G., "Lapse in protection concerns some experts," The Post and Courier. Jul. 26, 2007. http://www.postandcourier.com/news/2007/jul/26/lapse_protection_concerns _some_experts11434/.

[36] Kropf, S., “Injunction preserves wreckage of sofa store inferno,” The Post and Courier. Jul. 26, 2007. http://www.postandcourier.com/news/2007/jul/26/injunction_preserves_wreckage _sofa_store11455/.

[37] Menchaca, R. and Smith, G., "Heat sensors weren’t used in fatal fire,” The Post and Courier. Jul. 29, 2007. http://www.postandcourier.com/news/2007/jul/29/heat_sensors_werent _used_fatal_fire11687/.

[38] Menchaca, R., "Panel assembled to review fatal blaze,” The Post and Courier. Aug. 3, 2007. http://www.postandcourier.com/news/2007/aug/03/panel_assembled_review_fatal_blaze12080/.

[39] Menchaca, R. and Smith, G., "Agency to study sofa store fire,” The Post and Courier. Aug. 4, 2007. http://www.postandcourier.com/news/2007/aug/04/agency_study_sofa_store_fire12144/.

[40] Menchaca, R. and Smith, G., “Anguish, courage,” The Post and Courier. Aug. 11, 2007. http://www.postandcourier.com/news/2007/aug/11/anguish_courage/. 
[41] Beam, A., "Pleas and prayers amid the flames," The Charlotte Observer. Aug. 11, 2007. http://www.charlotte.com/112/story/232235.html.

[42] Menchaca, R. "Fire experts: Charleston Fire Dept. must change,” The Post and Courier. Aug. 18, 2007. http://www.postandcourier.com/news/2007/aug/18/fire_panel_cfd_must_change13370/.

[43] Menchaca, R. and Smith, G. “Trapped: The story of nine Charleston firefighters' deaths,” The Post and Courier. Aug. 19, 2007. http://www.charleston.net/news/2007/aug/19/trapped _story_nine_charleston_firefighters_deaths/.

[44] Kropf, S. and Smith, G., "Investigators seize more fire evidence,” The Post and Courier. Aug. 22, 2007. http://www.postandcourier.com/news/2007/aug/22/investigators_seize_more_fire _evidenceit13652/.

[45] Bartelme, T., “After 11 months, origin of blaze not known,” The Post and Courier. May 16, 2008. http://archives.postandcourier.com/archive/arch08/0508/arc05166264227.shtml.

[46] Menchaca, R. and Smith, G., "Report: Fire Department's culture, store’s code violations created time bomb,” The Post and Courier. May 16, 2008. http://archives.postandcourier.com/archive/ arch08/0508/arc05166264245.shtml.

[47] “What’s next?” The Post and Courier. May 16, 2008. http://www.postandcourier.com/ news/2008/may/16/whats_next/?print.

[48] Pardue, D., and Smith, G., “Hidden Killer,” The Post and Courier. Jun. 7, 2009. http://archives. postandcourier.com/archive/arch09/0609/arc06078086223.shtml.

[49] Mccue, D. "Updated: Firefighters sue Sofa Store, others (includes links to complaints)," Charleston City Paper. Jan. 26, 2010. http://www.charlestoncitypaper.com/charleston/fourfirefighters-sue-over-ill-effects-of-sofa-store-fire/Content?oid=1710626.

\section{Q.3 WEB PAGES}

[1] Harrison, S., "Site of Fatal Charleston Fire Lacked Permit,” Engineering News Record. Jun. 23, 2007. http://www.enr.com.

[2] “Large Store Fire Tactics,” Firetactics.Com. 3D Firefighting. SOG5050/2007/1. Jun. 25, 2007. http://www.firetactics.com.

[3] “Update: Charleston Fire Department Claims Boosters Were Lifelines,” Firefighter Hourly. Jul. 16, 2007. http://www.firefighterhourly.com/firefighter_hourly/2007/07/update-charlest.html.

[4] “Charleston Fire: Flashover Is Nothing New,” Firefighter Hourly. Jul. 29, 2007. http://www.firefighterhourly.com/firefighter_hourly/2007/07/charleston-f-12.html.

[5] “Charleston Fire: Thermal Imagers Not In Use,” Firefighter Hourly. Jul. 29, 2007. http://www.firefighterhourly.com/firefighter_hourly/2007/07/charleston-f-13.html.

[6] “Charleston Fire: Early Signs of Fire above Ceiling?” Firefighter Hourly. Jul. 30, 2007. http://www.firefighterhourly.com/firefighter_hourly/2007/07/charleston-f-15.html.

[7] “Charleston Fire: Road to Recovery,” Firefighter Hourly. Jul. 30, 2007. http://www.firefighterhourly.com/firefighter_hourly/2007/07/charleston-f-14.html. 
[8] "Lawsuit filed over the death of Charleston firefighter Melvin Champaign,” Oct. 1, 2007. Motley Rice Attorneys at Law. http://www.motleyrice.com/news/view/lawsuit-filed-over-the-death-ofcharleston-sc-firefighter-melvin-champaign.

[9] “Last Chance to Evacuate?” Firetactics.Com; SOG5050/2007/1; June 25, 2007; http://www.firetactics.com.

[10] Sofa Super Store. http://sofasuperstore.com/default.asp.

\section{Q.4 OTHER RESOURCES}

[1] "Wrongful Death and Survival Action,” Carl Champaign, Sr. vs. Sofa Super Store, Inc., Golden Family Limited Partnership, RLB, Inc., and etc., State of South Carolina County of Charleston In the Court of Common Pleas Ninth Judicial Circuit, Case No. 07-CP-10-3186. Oct. 1, 2007.

[2] Baker, F. J., "Lessons Learned Charleston Sofa Super Store Fire: Part 1,” The American Society of Safety Engineers. Perspectives. Vol. 8, No. 2. 2009.

[3] Baker, F. J., “Lessons Learned Charleston Sofa Super Store Fire: Part 2,” The American Society of Safety Engineers. FireLine. Vol. 1, No. 1, pp. 4-8. 2009. 
